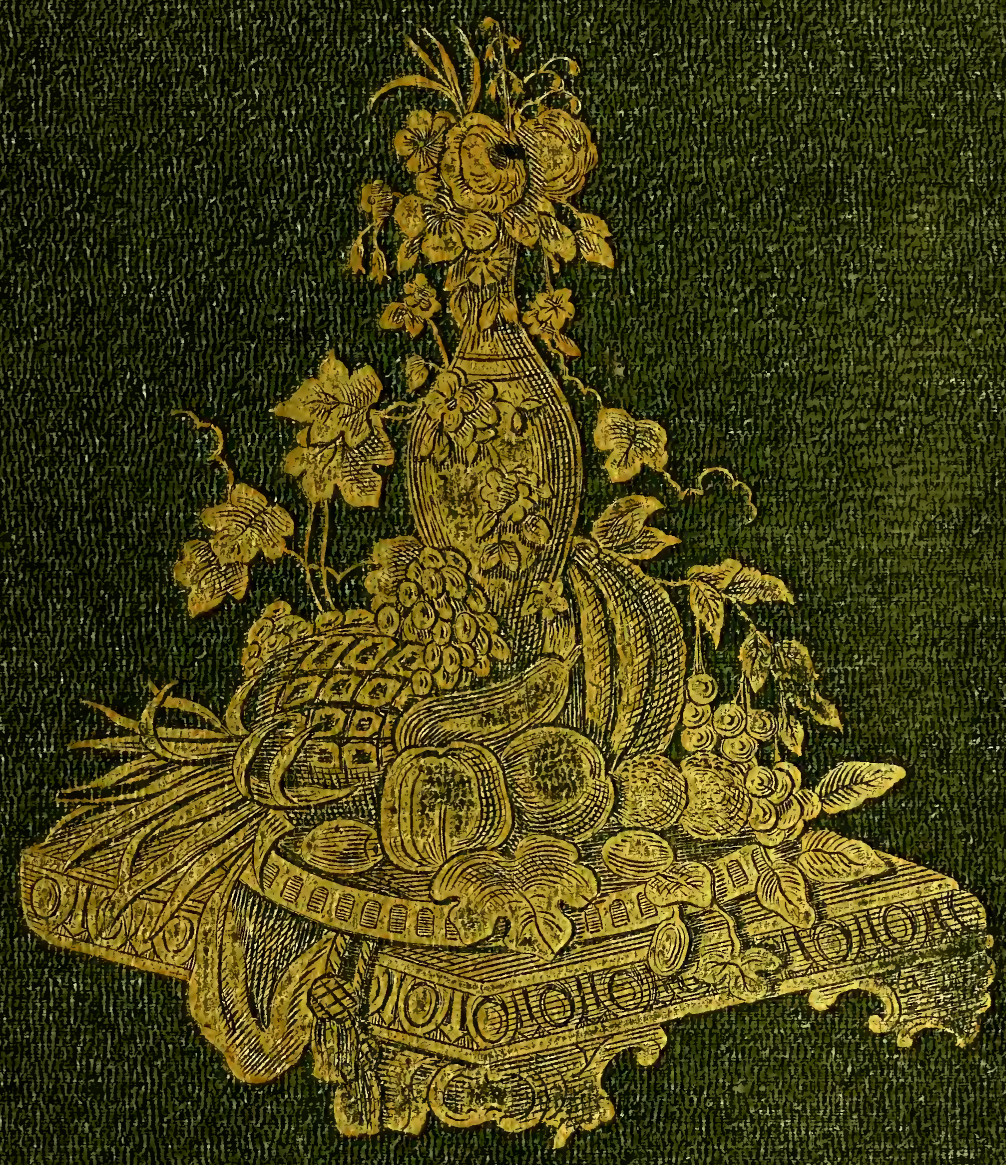





T H E

\section{BOOK OF THE GARDEN}


PRINTED BY WILLIAM BLACKWOOD AND SONS, EDINBURGH. 


\title{
BOOK OF THE GARDEN,
}

\author{
BY \\ CH A R L E M M ¿ I N T O H \\ F. R. P.S. F.R.S.S.A. \\ CORRESPONDING MEMBER OF THE IONDON HORTICUITURAI, SOCIETY, THE MASSACHUSETTS \\ HORTICULTURAL SOCIETY, AND THB ROYAI, CALEDONIAN HORTICUITURAL SOCIETY, FTC, \\ ATE CURATOR OF THE ROYAL GARDENS OF HIS MAJFSTY THE KING OF THE \\ GAACE THR DUKK OF BUCCHEUCH, AT DAIKFITH PAIACR.
}

IN TWO VOLUMES

V O L. I I.-C U L T U R A L

WITH 279 ILLUSTRATIONS

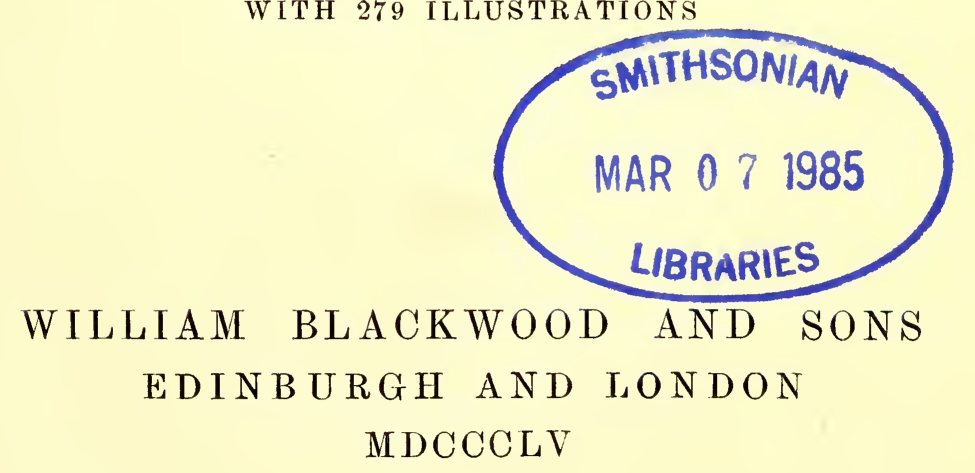





\section{0 N T E N T S.}

THE CULINARY OR KITCHEN GARDEN.

PAGE

INTRODUCTORY REMARKS,

§1. Sketch of the History of Culinary Vegetables, . . 3

2. Disposition of the Crops in the Kitchen Garden, . . 7

3. The Quantities of Seeds and Roots necessary to Crop a Garden, 8

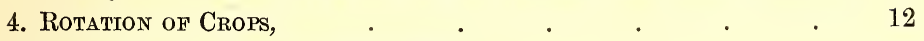

5. Transplanting Culinary Esculents, . . . . . $\quad 24$

6. Proposed Arrangement, ..$\quad$. . . . 26

Chapter I.-Alliaceous Plants,

\$1. The Shallot, . . . . . . . . 27

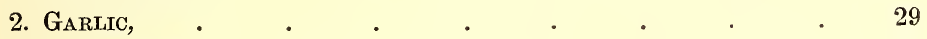

3. Rocambole,.$\quad$. $\quad$. $\quad$.

4. THe ONION, . . . . . . . . . $i b$.

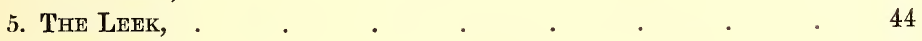

6. The Chive, .

Chapter II.-Leguminous Plants, or the Pulse Tribe,

§1. The PeA, . . . . . . . . . . . . . 48

2. The Bean, . . . . . . . . . . 62

3. The Kidney or Frence Bean, . . . . . . . . 71

4. The SCarlet Runner, . . . . . . . . . 78

Chapter III.-Brassicaceous Plants, or the Cabbage Tribe,

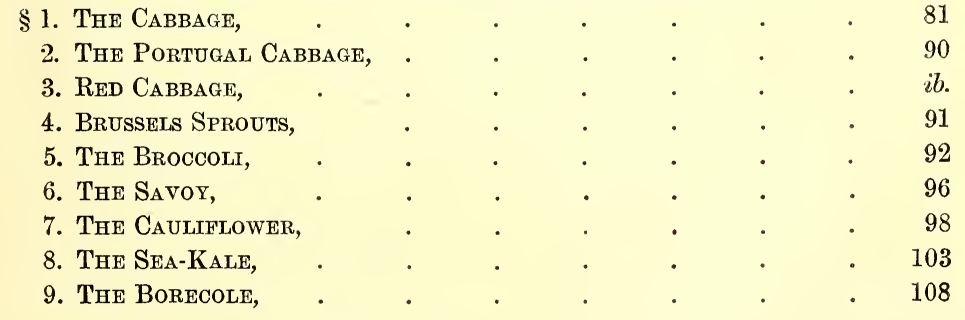

CHAPter IV.-Asparaginous Plants, . . . . . . 117

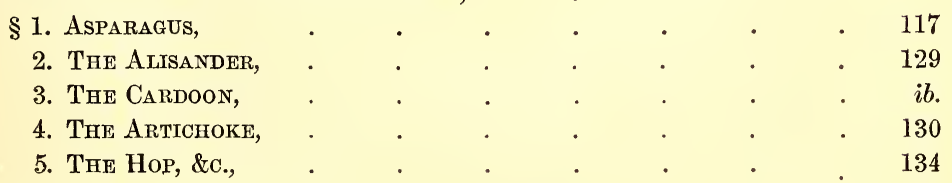


CHAPTER V.-Spinaceous Plants,

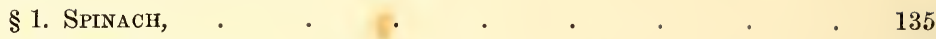

2. Wild Spinach, . . . . . . . . 137

3. French or Mountain Spinach, . . . . . . 138

4. New Zealand Spinach, . . $\quad$ : . . . $\quad i b$.

5. Garden Patience, \&c., . . . . . . . . $i b$.

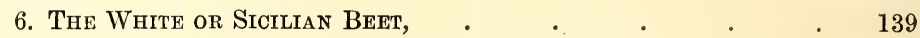

Chapter Vi.-Acetariaceous or Salad Plant,

§1. Celert, . . . . . . . . . . . .

2. Celertac, or Turnip-rooted Celert, . $\quad$. $\quad . \quad$. 150

3. The lettuce, . . . . . . . . . . 151

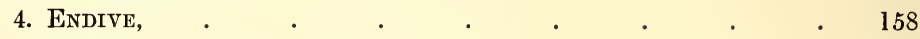

5. SUCCORY, . . . . . . . . . 164

6. Dandelion, Tarragon, \&., $\quad$. . . . . . $\quad$. 166

7. White and Black Mestard, . . . . . . . 167

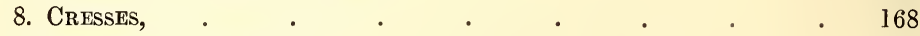

9. Purslane, Chervil, \&c. . . . . . . . 171

10. RAPE, CoRn-SALAD, \&C., . . . . . . . . . $\quad$. $i b$.

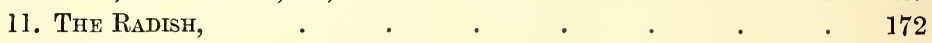

CHAPTER VII.-Esculent-rooted Plants, . . . . . . 176

§1. THE Carrot, . . . . . . . . . 176

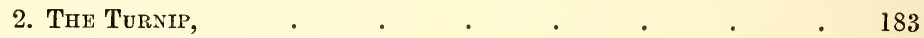

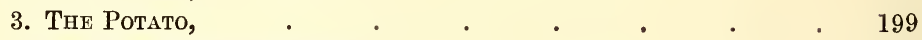

4. The Jerusalem Artichoke, . . . . . . . 223

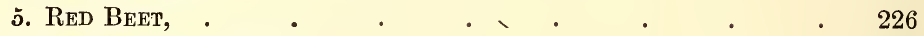

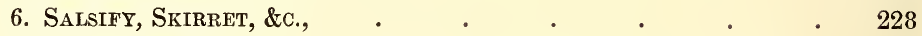

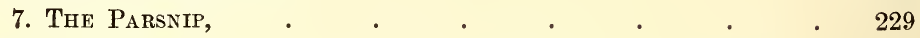

176

9
6
8
9

CHapter ViII.-Oleraceous Plants, or Pot-Herbs, comprising such as are used for Garnishing and Seasoning,

$\S 1$. Parsley,

2. Dill, Borage, \&c.,

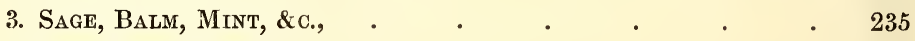

4. BASIL, SAVORY, \&C., . . . . . . $\quad$. 237

CHapter IX.-Medicinal Plants,

CHAPTER X.-Edible Fungi,

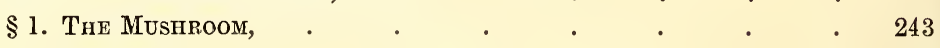

2. THE MOREL, . . . . . . . 252

3. The Truffle, . $\quad$. $\quad$. $\quad$. $\quad$. 253

CHAPTER XI.-Miscellaneous Vegetables,

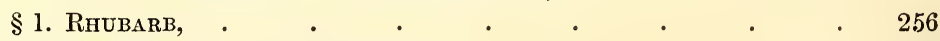

2. The Tomato and EgG-Plant, $\quad$. $\quad$. $\quad$. $\quad$. 258

3. The Gourd and Pumpkin, . . . . . 259

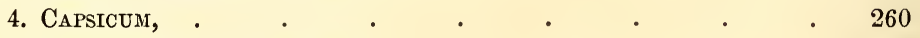

5. Rosemart and Lavender, $\quad . \quad$. $\quad . \quad$. $\quad$. $\quad i b$.

6. The Horse-Radish, $\quad$. $\quad$. $\quad$. $\quad$. $\quad$. $\quad 261$

CHAPTER XII.-SOILS, . . . . . . . . 263

§ 1. Their Origin and Nomenclature, $\quad$. $\quad$. $\quad$. $\quad$. 263

2. Determination of their Nature, \&C., . . . . . . 270

3. Their Improvement, . . . . . . . 277

4. WATERING, . $\quad$. $\quad$. $\quad$. 
CHAPTER XIII.-MaNuRES,

$\S$ l. KINDS OF THEM, \&c.,

2. Organic ManURES, . . . . . . . 290

3. InORganic ManURES, • : • • . • • 296

THE HARDY FRUIT GARDEN.

INTRODUCTION,

\$ 1. Selection of Kinds, \&C., . . . . . . . . . 299

2. The Origin of New Fruits, . . . . . . 303

3. On the duration, in a healthy state, of Fruit Trees, • 306

CHAPTER I.-Propagation,

$\S 1$. Propagation by SeEd, . . . . . . . 30 . 3

2. Crossing or Hybridising, . . . . . . . 313

3. Propagation by Grafting, . . . . . . 322

4. Propagation by Budding, . . . . . . 338

5. Propagation by Cuttings, . . . . . . 346

6. Propagation by Layers, . . . . . . . . 351

CHAPTER II.-Planting, . . . . . . . . 356

CHAPTER III.-Pruning and Training, . . . . . . . 389

CHAPTER IV.-The Apple, . . . . . . . . . . . 411

CHAPTER V.-The Pear, . . . . . . . 447

CHapter VI.-The Peach and Nectarine, . . . . 485

$\S 2$. Forcing the Peach and Nectarine, . . . . 501

CHAPTER VII.-The APRicot, . . . . . . . 517

CHAPTER VIII.—The Plum, . . . . . . . 524

CHAPTER IX.-The Cherry, . . . . . . . . 537

Chapter X.-Forcing the Apricot, Plum, and Cherry, . 546

CHAPTER XI.-The Fig, . . . . . . . 551

CHAPTER XII.-The Almond, Quince, Medlar, \&c., . . $\quad 559$

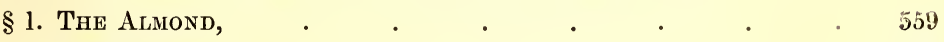

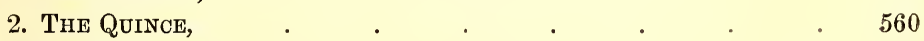

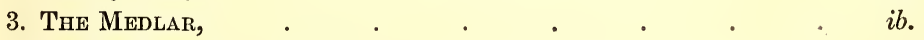

4. The Walnut, . $\quad . \quad$. $\quad . \quad 561$

5. The Chestnut, or Spanish or Sweet Chestnut, . $\quad 562$

6. The Fllbert, . . . . . . . . . 563

CHapter XIII. - The Currant, Raspberry, Gooseberry, StrawBERRY, \&C.

§ 1. The Red, White, and Black Currant, . $\quad$. $\quad$. $\quad . \quad 566$

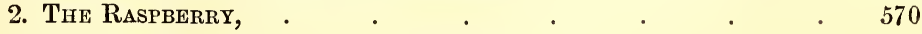

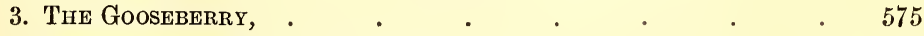

4. The Strawberry, . $\quad . \quad$. $\quad . \quad$. $\quad . \quad . \quad 581$

5. The Mulberry, . . . . . . . . 592

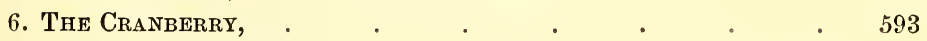


CHAP'TER XIV.-The Grape Vine,

CHAP'TER XV.-The Pine Apple,

CHAPTER XVI.-The Cucumber and Melon,

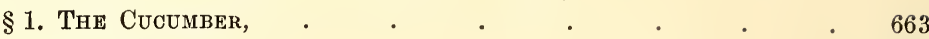

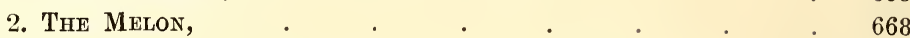

CHAPTER XVII.-Management of the Fruit-Room,

THE FLOWER GARDEN.

CHAPTER I.-Plant-Houses,

§ 1. Camellia-House, . . . . . . . . . . . 678

2. ORCHID-House, .

3. Bulb-House, .

4. Orangert, . . . . . . . . . . 700

5. Heath-House, . . . . . . . . . 703

6. Aquarium, $\quad . \quad$. $\quad . \quad$. . . . 710

7. The Stove or Tropical Plant House, . . . . 712

8. Succulent-House, . . . . . . . . . 719

9. The Conservatory, . . . . . . . . . . 721

10. The Greenhouse, . . . . . . . . . 724

11. Flower-Forcing House or Pits, . . . . . . 729

12. Cold or Conservative Pits, . . . . . . . 733

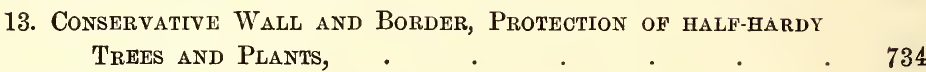

14. Miscellaneous Observations,

CHAPTER II.-The Open Flower-Garden, . . . . . 742

§ l. American Garden,

2. Rosary, . . . . . . . . . 750

3. The Lawn, . $\quad . \quad$. $\quad . \quad$. $\quad . \quad$. $\quad .772$

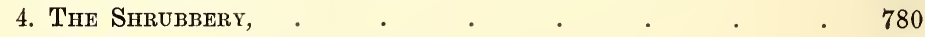

5. The Florists' Flower Garden, . . . . . . 796

6. The Alpine Garden and Rockery, . . . . 814

7. The Perennial-Plant Flower Garden, . . . 815

8. The Parterre Garden, . . . . . . . . 816

9. The Reserve Garden, . . . . . . . 822

10. Insects injurious in the Plant-Houses and Flower-Garden, 823

GLOSSARY of some of the most important Technical Terms employed IN THIS WORK, .

indeX to Select Lists of Vegetables and Fruits, . . 838

INDEX to Select Lists of Plants, . . . . . . $\quad$. 850

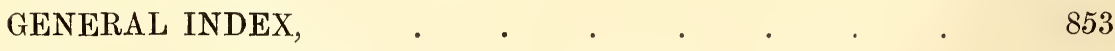




\section{A D V E R T I S E M E T.}

IN presenting to the public the first part of the Practical or Cultural volume of The Book of the Garden, we have only a few remarks to premise as to the general plan of arrangement we have employed, and the reasons which have induced us to adopt an arrangement which is, to a great extent, a departure from that hitherto followed in similar books.

Works on Practical Gardening have, for the most part, been arranged in the calender form, no doubt with a view to render them, in the estimation of their authors, more convenient for reference. There are, however, objections to this mode of arrangement, which we think may be avoided by adopting the sectional or separate garden division, as the operations in any of these departments may be carried on irrespective of the others-a mode of culture which is, in fact, practised in our largest and best-managed establishments, in all of which the subdivision of labour is found to be admirably adapted for facilitating the multifarious operations of the whole. Besides, some people have a predilection for one of these departments more than for another, and many are content with one of them only.

We had hoped that a seasonal arrangement might have been adopted, and that it would have combined all the advantages of the calender form, and have avoided its principal defects. The attempt to carry out this scheme has, however, shown us that it inevitably involved a want of connection and a degree of confusion, which could not be otherwise than most embarrassing to the reader, while it necessitated an amount of repetition which would have made it impossible to comprise the cultural department of the garden in a single volume, without sacrificing that minuteness of detail which is essential to the highest value of such a work.

By the mode of arrangement we have finally resolved on, the reader will more readily find the information he seeks; each subject assumes a more connected form when treated on as a whole, than if it were referred 
to in different places; and the necessity for frequent reference and much repetition is wholly done away. On these grounds we think the advantage will be sufficiently apparent of treating on the operations of the KITCHEN or Culinary Garden, the Hardy Fruit Garden, the Forcing Garden, and the Flower Garden, including Plant-Houses and Pleasure Grounds, \&c., as distinct in themselves.

In discussing the various subjects which collectively constitute any of these general divisions, we have adopted a mode of arrangement which we believe to be as complete as is attainable; - our great object being to systematise the whole, by bringing together, in our accounts of their culture, such productions as have a natural affinity to each other.

As regards the descriptive lists of the most approved Fruits, Vegetables, Flowering Plants, and Ornamental Trees, Shrubs, \&c., we avail ourselves of the present as a fit opportunity for correcting former lists, and adding those of recent introduction or origin, when of sufficient merit, to the lists of a similar nature which have appeared in practical works such as "THE BooK of the GARDEN" professes to be. This is the more important, because, with the exception of Mr Hogg's excellent work, "British Pomology," which treats on the apple exclusively, there has been no book of a similar description to the present published in Britain since our "Practical Gardener" and "The Orchard," both of which, in this respect, are now very far behind the requirements of the present age. The excellent descriptive "Fruit Catalogue of the London Horticultural Society," and the no less valuable "Orchard," by the late Mr G. Lindley, and "The Fruit Cultivator," by the late Mr Rogers, stand in a similar position to the works already named. The only channels through which the new and improved varieties of Fruits, Vegetables, and Plants of general interest have reached the public, (since the "Practical Gardener" was last revised by us, more than twelve years ago,) have been the horticultural periodicals, and the nurserymen's and seedsmen's trade catalogues; although, during this period, more important additions have been made to all of these classes than during any former period of the same extent. These lists, valuable as they certainly have been in making us acquainted with every novelty as it appeared, have, from their nature, scattered the information sought for over a wide extent of volumes and tracts, which renders the task of referring to them expensive and often exceedingly difficult. To these additions we may add the many fine fruits of American origin, and the vast number of new ornamental plants which, at the date of the works referred to, were wholly unknown in this country. 
From them, selected lists of such varieties as are suitable to our climate, \&c., will be made.

The arrangement of these lists will be as follows :-

The most approved and recently introduced Esculent Seeds and Roots will accompany the articles to which they respectively belong in the Culinary Garden.

The most approved and recently obtained Hardy Fruits will in like manner be found in the Hardy Fruit Garden.

The Tropical Fruits in the Forcing Garden. And

The more rare, choice, and interesting Trees of Ornament, \&c., Flowering Plants, \&c., will accompany the Flower Garden.

We have given some brief details of the practices of the London marketgardeners, who, it must be admitted, are the best culinary gardeners in the world. This is a subject scarcely hinted at by authors on gardening since the days of Abercrombie, the merits of whose excellent works (we mean the original editions) are mainly owing to the copious details he gave of the market-gardening of his day. As nearly a century has now elapsed since he wrote his first work, and as during that period a corresponding improvement has taken place in that department, as well as in that of private gardening, a work of this kind would be incomplete without a notice of these excellent modes of culture.

Little or nothing has been published concerning the London practice since that time, and private gardeners, in general, know little how things are there managed. There has been a reserve on the part of the former in affording information, and an unwillingness on the side of the latter to undergo the hard work to which they would be subjected, were they to spend a year in a market-garden, rather than two or three loitering about a nursery-too often a tax upon the proprietor, and losing much of their own valuable time. We here allude to young gardeners only, who would acquire a much greater amount of useful information in the general routine of their profession were they to spend a year in a first-rate London market-garden, than they could do in a dozen years, toiling nearly as hard, in very inferior places in the country. We are far from insinuating that a nursery is a bad school for a young gardener; on the contrary, no man can have much pretension to a thorough knowledge of his business, unless he has spent a part of his career in a firstrate establishment of that kind. In it he learns what he could not do in a private garden; he learns the most approved methods of propagation, has many 
opportunities of studying the nomenclature of Fruits, of attaining a general knowledge of Plants, and the best modes of taking up and packing Trees, and of becoming acquainted with the new and rarer ornamental and useful Trees and Shrubs, upon which decorative gardening is destined in future so much to depend. In many of these establishments he may obtain some practice in laying out grounds, as nurserymen of high repute are often employed as landscape-gardeners. In fact, every gardener wishing to excel in his profession, should spend a couple of years in such establishments, and one at least in a London market-garden.

To one department of great importance, both to the practical gardener and the amateur, and which has hitherto hardly received the attention it merits in practical works - that of the diseases, insects, and other enemies, to the attacks of which the various products of the garden are liable-especial attention has been paid. Full descriptions have been given of these from the best authorities, supplemented by actual observation, and also full details of the most approved methods of prevention and cure; while the insects in particular have been much more extensively figured than has, it is believed, ever before been done in any single work, and this on a scale and with a minuteness which will make the identification of them easy even to a comparatively superficial observer.

We have thought it expedient to give the European names of culinary vegetables and fruits, more especially the former, on account of our increasing intercourse with the Continent, and the quantities of seeds which are yearly brought or sent into this country, and often put into the hands of those unacquainted with the language in which the names are given. Readers of such popular Continental works as the "Bon Jardinier," the "Utrechtsche Hovinier," "Garten Zietung," "Verstandige Gartner," and similar books on gardening, may be assisted by a reference to the paragraph General Remarks at the end of each section.

Throughout the whole of this department of our subject, it will be our special aim to enter into all necessary minuteness of detail; to avoid all technicalities of term, or, wherever we are compelled to employ these, to append full explanations of them; and, in short, to make the "Book of THE GARDEN," as to its cultural department, so precise and complete in all its directions, that it may suffice to the tyro as his guide, from the most rudimental operations of gardening onward, and render the amateur in a great degree independent of other assistance; while, to the experienced gardener, we trust it will be found the best and most practical work of reference extant. 
T H E

\title{
BOOK OF THE GARDEN
}

\author{
C U L T U R A L
}



T H E

\section{ULINARY OR KITCHEN GARDEN.}

\section{INTRODUCTORY REMARKS.}

\section{§ 1.-SKETCH OF THE HISTORX OF CULINARY VEGETABLES.}

THE culinary or kitchen garden, (jardin potager of the French,) with all its varied productions, if not the most ornamental, will be admitted to be the most useful of all the departments of gardening. Many of the crops cultivated in those of the present day were raised, and we have no great right to suppose otherwise than that they were so to a very creditable extent, although upon much less scientific principles, soon after the Deluge, if not prior to that epoch. They are early mentioned in the histories of the nations of the greatest antiquity. The onion, the leek, the cucumber, and the garlic, were in extensive cultivation in Egypt long before the exodus of the children of Israel ; and herbs for seasoning cooked dishes were also well known at an equally early period. Of the means employed in their production we have no records left earlier than the date of the foundation of Rome. Soon after that period we find the environs of that city in a state of cultivation much like what exists around large towns and cities of our own time-namely, as market-gardens, in which were cultivated many of the culinary vegetables such gardens at present produce. One important difference, however, may be remarkedthe culinary gardens around ancient Rome were cultivated by the chief men, who were also the proprietors, and they themselves wrought the ground with their own hands; and hence several of the most celebrated families-the Pisones, the Cicerones, the Fabii, the Lentuli, \&c.derived their patronymics from ancestors who had distinguished themselves in the cultivation of culinary vegetables. Thus, Pisum, a Pea ; Cicer, a Chick-pea ; Faba, a Bean ; Lentulus, a Lentil, \&c.

Nor did some of their greatest historians consider it beneath their literary dignity to record many interesting hints regarding the modes of culture employed. Cato, Varro, Columella, Palladius, Pliny, Virgil, Martial, \&c., have left us quite enough, in those portions of their writings which have been handed down to us, to satisfy us that the culture of culinary vegetables was well understood and fully appreciated in their days. The former of these has left us in detail the Roman mode of cultivating asparagus, which was the last vegetable written upon by him, and may serve as a pretty good example of ancient Roman gardening. "You must work a spot that is moist, or which has richness and depth of soil. Make the beds so that you may be able to clean and weed them on each side; let there be a distance of half a foot between the plants. Set in the seed, two or three in a place, in a straight line, cover with mould, then scatter some compost over the beds. At the vernal equinox, when the plants come up, weed often, and take care that the asparagus is not plucked up with the weeds. The year you plant them, cover them with straw during the winter, that 
they may not be killed. In the beginning of the spring after, dress and weed them. The third year after you have sown them, burn the haulm in the beginning of the spring. Do not weed them before the plants come up, that you may not hurt the stools. The third or fourth year you may pluck them close by the root; if you break them off, they yield side-shoots, and some will die. You may take them until they run to seed. The seed is ripe in autumn. When you have gathered the seed, burn the haulm, and, when the plants begin to shoot, weed and manure. After eight or nine years, when the beds are old, lay out a spot, work and manure it well, then make drills where you may plant some roots ; set them well apart that you may dig between them. Take care that they are not injured. Carry as much sheep's dung as you can on the beds: it is best for this purpose; other manures produce weeds."

The globe artichoke is said by Pliny, book xix. chap. viii., to have been more esteemed, and to have obtained a higher price, than any other garden herb. $\mathrm{He}$ also informs us that the commoners of Rome were prohibited by an arbitrary law from eating this vegetable. $\mathrm{He}$, at the same time, censures his countrymen for their vanity and prodigality as regards the serving up such things to their tables as the very asses and other beasts refuse to eat, for fear of pricking their lips. The same writer, book xix. chap. iv., tells us that asparagus, which formerly grew wild, was, in his time, carefully cultivated in gardens, particularly at Ravenna, where the heads were so large that three of them would weigh a pound.

Basil, which stands now so high in the gastronomic art, that a new-made alderman would spurn a basin of turtle if not seasoned with it, was condemned by Chrysippus, more than two hundred years before Christ, as an enemy to the sight and a robber of the wits. Diodorus and Hollerus entertained equally superstitious notions regarding it. Philistis, Plistonicus, and others, extolled its vitrues, and recommended it as strongly as it had been formerly condemned. Pliny says, the Romans sowed the seeds of this plant with maledictions and ill words, believing that the more it was cursed the better it would prosper; and when they wished for a crop, they trod it down with their feet, and prayed to the gods that it might not vegetate!

The bean was cultivated both by the earlier Greeks and by the Athenians, who offered them as oblations to the gods-a practice, according to Pliny, afterwards followed by the Romans; and Lemprière states that bacon was added to the beans in the offerings to Carnanot, as he says, so much to gratify the palate of the goddess, as to represent the simplicity of their ancestors. The beet was highly prized by the Greeks, who used to offer it on silver to Apollo at Delphos. They used also to eat the leaves in preference to lettuce, and, by laying a small weight on the plant, they blanched it, much as gardeners of the present day lay a tile over endive plants for a like purpose. Pliny says, beets are, of all garden herbs, the lightest roots ; that they are eaten, as well as the leaves, with lentils and beans; and that the best way to eat them is with mustard, to give a -taste to their dull flatness. So highly was the cabbage esteemed by the ancients, that two of their leading physicians each wrote a book on the properties of the plant. Phillips tells us that the ancient Romans, having banished physicians out of their territories, preserved their health for six hundred years, and soothed their infirmities, by the use of this vegetable alone. Pliny goes to great length on the use and culture of the cabbage, which, he says, may be cut as coleworts at all times of the year; so may they be sown and set all the year through; but he adds that the most appropriate season is after the autumnal equinox; and also remarks that, after the first cutting, they yield abundance of excellent tops. Powdered nitre, sea-weed, and asses' dung, were used as a fitting manure for them. "There are," Pliny remarks, "many kinds of coleworts at Rome," and amongst them, one received in his time "from the vale of Aricia, with an exceedingly great head and an infinite number of leaves, which gather round and close together"probably the first type of our hearting cabbage, for those previously described by him appear to have been open-hearted or true coleworts. He afterwards, however, says, "there are some coles which 
stretch out into a round shape, others extend in breadth, and are very full of fleshy brawns, and some possess heads twelve inches thick." He also remarks, that all the varieties eat sweeter from being touched with the frost, an opinion prevalent at the present day. In the Roman culture we find that the sprouts were planted as well as the young plantsa discovery made a few years since, and recorded in one of our most costly horticultural works, and described nearly as Columella did many hundred years ago.

Fennel was cultivated largely by the Romans as a garden herb, and so much used in the kitchen that there were few meats seasoned or vinegar sauces served up without it. "A good housewife," says Pliny, "will go into her herb-garden instead of a spice-shop for her seasonings, and thus preserve the health of her family by saving her purse."

From an anecdote related by Herodotus, in connection with the murder of Smerdis by his brother Cambyses, it is quite evident that lettuces were served at the royal tables of the Persian kings five hundred and fifty years before Christ. The ancient Romans, however, appear to have known only one sort, which Pliny describes as a black variety. Suspicion prevailed of their having a deleterious effect; but, after Antonius Musa cured the Emperor Augustus Cæsar by means of this plant, that suspicion vanished, and, as Pliny says, men began to devise means of growing them at all seasons of the year. The variety of lettuce cultivated by the Greeks is stated to have grown "high and large." Those employed by the Romans in the days of Pliny were the purple sort, with a large root, the Egyptian, Cilician, Cappadocian, \&c. Great pains, he says, were taken to make them cabbage, and they were earthed up with sea-sand to blanch them and give them heart. The white lettuce was noticed as being the least hardy even in that mild climate.

Salads were much esteemed amongst the ancients, and even poets sang their praises. Ovid, in his "Philemon and Baucis," says-

"A garden-salad was the third supply, Of endive, radishes, and succory."

VOL. II.
And Columella also thus notices the endive-

"And endives, which the blunted palate please."

The endive is said by modern botanists to be a native of the East Indies. This would lead us to believe them little acquainted with European historical authors; for, besides Ovid and Columella, Pliny also mentions endive in the eighth chapter of his twentieth book. Horace alludes to the plant under the name of cicorea, in lib. i. ode 31 ; Virgil makes special mention of the marigold in the second eclogue of his "Bucolics;" and Catullus thus notices the marjoram, in the "Epithalamium of Julia and Manlius"-

"Bind your brows with the sweet-smelling marjoram."

Mint is equally honoured by Ovid's notice of it, from which we learn that the humbler classes used to perfume their tables by rubbing the plant upon them before serving their supper; and só highly was mint thought of at Rome, that Pliny says, "you will not see a husbandman's board in the country, but all the meats, from one end to the other, are seasoned with mint." The humble creeping Penny Royal formed the subject of a consultation of physicians held in Pliny's chamber, the result of which was, that they agreed that a chaplet of this plant was, without comparison, far better for giddiness and swimming of the head than one of roses. We have met with no account of the cultivation of the mushroom in the authors of antiquity, but that they were in extensive use is quite evident, had we no better authority than the circumstance of one of them, the Agaricus Cæsareus having been made the vehicle of poison by Agrippina for her husband, Tiberius Claudius. That they were as highly prized amongst the epicures of ancient Rome as they are by those of modern London, is evident from what Pliny says of them, in his 6 th book, chap. 8 , "the last device of our epicures to sharpen their appetites, and tempt them to eat inordinately, is the cooking of mushrooms ;" and, in the $23 \mathrm{~d}$ chap. of his $22 \mathrm{~d}$ book, he adds, "there are some dainty wantons of such fine taste, and who study their appetite to such excess, that they dress mushrooms with their own hands, that they may feed on the 
odour during the time they are handling and preparing their food."

Mustard must have been cultivated in Syria while our Saviour was upon earth, as it is mentioned in one of His beautiful parables as being the least seed that was sown in the field. The mustard here referred to does not appear, from the circumstance of the size of its seed, to be the mustard of our times. This has led to various conjectures, none of which have much plausibility. The mustard of the present time was cultivated in Rome, in Pliny's time, to the extent of three varieties.

The origin or native country of the well-known onion is unknown. Pliny, in book 20, chap. 5, enumerates all the countries from whence the Greeks as well as the Romans procured different varieties of this root, but declares he could never discover where they ever grew wild. Palladius, a Greek physician, recommends the onion to be sown with savory, in which curious opinion Pliny agrees, observing that onions prosper better when savory is sown with them. It was a current opinion, we should state, in those days, that certain plants had an antipathy to, or a sympathy with, each other. However absurd such an idea may appear to us, we should take into consideration that the opinions of the ancients may not have been faithfully handed down to us, or that their works may admit of a somewhat different construction. "We find," Phillips observes, "that all the plants which they recommend to be sown or planted together are of very opposite natures ; and there may be more reason in the system pursued by the ancients than is generally allowed; for plants drawing the same juice from the earth must naturally weaken each other; whereas those requiring different nutriment may, in some degree, assist each other, each feeding on juices that are prejudicial to plants of the other species." In this there is great truth. The garlic was in use at as early a time as the onion; the want of both was lamented by the Israelites in the wilderness. The Egyptians worshipped it, and are said to wish that they may enjoy it in paradise. The Greeks held it in such abhorrence, that those who ate it were regarded as profane. The Romans gave it to their labourers to strengthen them, to their soldiers to excite courage, and fed their game-cocks on it previous to fighting them. The eschalot (or shallot, as it is often written) is a species of onion, and was well known to both the Greeks and Romans. Pliny states, book 19, chap. 6, that the best leeks were brought from Egypt, and names Aricia, now called Riccia, in Italy, as celebrated for them in his time, and says, "it is not long since leeks were brought into great notice and esteem by the Emperor Nero, who used to eat them for several days in every month to clear his voice, eating them with oil only, and abstaining from bread on those leek-eating days," which absurdity led his people to give him the cognomen of Porrophagus. Pliny, book 20 , chap. 11, informs us that parsley was in great repute in his time, all classes partaking of it largely in their pottage, and that there was not a salad or sauce presented at the table without it. The Emperor Tiberius held parsnips in high repute, and had them annually brought to Rome from Germany, from the neighbourhood of Gelduba on the Rhine, where they were said to have been grown in great perfection. The Greeks sowed their pease in November; the Romans did not plant theirs till the spring.

So highly did the ancient Greeks esteem the radish, that, in offering their oblations to Apollo, they presented turnips in lead, and beet in silver, whereas radishes were presented in beaten gold. The Greeks appear to have been acquainted with three varieties of this plant; and Moschian, one of their chief physicians, wrote a whole book on the radish alone, so highly did he think of it. Pliny observes, that radishes grow best in saline soils, or when they are watered with salt-water; and hence, he says, the radishes of Egypt are better than any in the world, on account of their being there supplied with nitre. He gives some account of the kinds grown at Rome in his day, one of which he describes as being so clear and transparent that one may see through them. The transparent variety of our day has not this property to the same extent. The size also to which the radishes of those days are said to have attained far exceeded those of the present, nor do we think such would be appreciated by our modern radish-eaters. Tragus mentions radishes that weighed $40 \mathrm{lb}$; 
and Amatus states, he has seen some of 60 lb. ; while Matthiole declares having met with them $100 \mathrm{lb}$. each. Somewhat in accordance to views recently promulgated in regard to increasing the size of roots, these monster radishes are said to have been produced by carefully taking off the leaves. The ancients used them boiled; the Roman physicians, however, directed them to be eaten raw with salt, as in our modern practice, and that they should be taken in the morning before any other food.

Savory and lavender are recommended by Virgil as fitting plants to be set near bee-hives-

"The verdant lavender must there abound,

There savory shed its pleasant sweets around."

They were used by the Romans to mix with cool salads, such as lettuce and radishes. Vinegar flavoured with savory and other aromatic herbs was much used by the ancients to dip their animal food in previous to eating, or as we now do mint-sauce, with lamb. Sorrel was cultivated in Pliny's time, as he makes mention of "garden sorrel, or sour dock." Thyme was imported to Rome from Attica, and cultivated for the sake of bees. Watercress was eaten by the ancients along with lettuce, to counteract the cold nature of that vegetable.

We have above given a cursory view of the ancient history of culinary vegetables. That we have improved in culture, and in the production of superior varieties, is undeniable ; but that we have not added very greatly to the number of species is equally apparent. The periods when indigenous vegetables became reclaimed, or brought into a cultivated state, as well as the dates of the introduction of exotic ones, will be found under their respective heads.

\section{$\S$ 2.-DISPOSITION OF THE CROPS IN THE KITCHEN-GARDEN.}

$A$ systematic mode of arranging the contents of the kitchen-garden, although no one can doubt the propriety of such a course, has, in the majority of cases (not even excepting some of our very best gardens), been next to disregarded. The plants cultivated rank as either peren- nials of several: years' standing, or as annuals, and some few biennials occupying the same ground only one, or at most two years. We see, therefore, no good reason why two distinct classes of arrangement should not be formed; but as it occurs in practice, as will be hereafter shown, that the annuals and perennials comprising our olitory or sweet herbs are more conveniently cultivated when set apart by themselves, we shall exclude them from our present arrangement, adding, however, the strawberry, gooseberry, currant, and raspberry, which belong properly to the fruit-garden, but are in general cultivated in the quarters of the kitchen-garden. By such an arrangement, a better rotation of crops may be followed out, and the garden made to assume a more methodical appearance. Where, however, sufficient slips of ground surround the main garden, these perennial crops may be therein planted, and, having stood their allotted time, successional plantations of them may be made to occupy one or more of the quarters within the main garden, in extent according to the quantity required to meet the demand. When these are thus removed (which removal, in the case of the gooseberry, currant, and raspberry, should take place at periods of from seven to twelve years), the ground they occupied will be in a very fit state to afford a change of soil to some of the annual crops cultivated during that time on the ground now to be occupied by the others. The peremnial crops to which we refer (exclusive of the fruits above) are, asparagus, sea-kale, rhubarb, and globe artichokes. Where the three former are forced upor the taking-up plan, an annual change of soil is attained; and as artichokes and strawberries should be replanted yearly, or in most cases once in two years, this increases the opportunity of carrying out rotation. No doubt the three first, in good soils, and where they are allowed to come into use at their natural seasons, will last for from seven to twelve years, or even much longer, but we see no advantage in continuing them beyond the last-named period. For these reasons, we would group the gooseberry, currant, and raspberry in a plot by themselves, and not dispose them in the scattered manner 
they usually are met with, occupying more room, less conveniently protected, exposed to having their roots mutilated by digging about them, and, last of all, affording by their removal no useful rotation. The other perennial crops may be planted next to them, and, if allowed to remain, either to be forced on the ground, (as is usually done in the case of the seakale and rhubarb in particular, and in that of the asparagus occasionally), or to be allowed to come in at their natural season, then the whole quarter may be destroyed at once (presuming a succession is established in that adjoining), and the ground they occupied cropped with annual crops, for which the manurial applications hitherto given will render it exceedingly fit, particularly for carrots, onions, \&c., which seldom succeed on newly-manured ground. That, however, occupied with the gooseberries, currants, and raspberries, being less enriched, must now have manure applied to it. The strawberry crop will now, by about the seventh year, have travelled, by yearly changes, to the further side of the adjoining quarter, followed up by the successional crops of sea-kale, asparagus, and rhubarb, and, last of all, by currants, gooseberries, and raspberries. In this way, these crops may be made to circulate round the quarters or divisions of the main garden ; and if the changes appear to be too rapid, an occasional shift to the slips may be had recourse to.

No doubt an excellent rotation is afforded, when a portion of these crops is taken up annually for forcing, the ground vacated being next occupied with annual crops ; but this rotation, although equally good in principle, is less systematic in appearance.

The extent of ground occupied by any one species of esculent must ever depend on the quantity of the particular sort required to meet the demand. The following is about the proportions: Pease occupy, in general, the largest breadth in most gardens; always so, when the family are resident in the country; somewhat less, when they only arrive about the 1st of August (a very usual period), as crops before that are not required. The cabbage tribe comes next in order, and would occupy the larger space, were it not that the crops for the latter end of winter and early spring are planted on the ground from which the first crops of pease have been gathered. Turnips would stand next, were it not that asparagus, sea-kale, and rhubarb, to be forced during winter on the taking-up principle, require to be extensively grown, on account of the roots being destroyed when the crop is gathered; and this is more especially the case when a family requires them throughout the winter and in spring from the open ground also. Potatoes would rank next, were it expedient to grow them in gardens (beyond the early crops), which it is not; for, from some hitherto unexplained cause, they never are of so good a quality in richly-manured gardens, as in the less highly manured fields. Carrots, leeks, and onions follow-the former, however, being a precarious crop. Celery and spinach would occupy as large a space as the last, were it not that the latter, particularly for winter crops, which are the principal, is not sown till the latter end of July or beginning of Angust, on ground from which probably the early crop of pease has been removed. Garden and kidney beans, and scarlet runners, follow-the two latter having a preference, in most gardens, to the former. Parsnips, globe artichoke, and beet are the next in order, followed by parsley, endive, salsify, skirret, scorzonera, and Jerusalem artichokes; garlic, shallot, rocambole, succory, chervil, and all manner of pot and sweet herbs, find a place in the olitory or herbgarden ; and lastly, lettuce, of which there is a perpetual sowing and planting, being usually, unless grown upon a very large scale, accommodated with room amongst other crops, as its duration in summer is short-and during winter it is placed in sheltered places, by the bottoms of walls, \&c.

\section{§ 3.-THE QUANTITIES OF SEEDS AND ROOTS NECESSARY TO CROP A GAR- DEN.}

The young gardener will find himself, upon taking charge of a garden for the first time, in a much greater dilemma, when about to make up his seed order for the ensuing year, than in any other part of his charge. To order without some certain knowledge of the quantities 
required, entails not only an unnecessary expense on the owner, but if the quantities be not properly proportioned to each other, and each in as near a proportion as possible to the quantity of produce required, he will be in a second dilemma, when he finds he has more of one sort than he requires, and too little of some others.

Various estimates have been formed, and scales of quantities drawn out, ever since the days of Gordon of Fountain Bridge, who published his "Pocket Dictionary" in 1774. That great difficulty attends even an approximation to the truth, so as to be of universal application, will be sufficiently evident, when we take into consideration that some families use a greater quantity of vegetables than others; some requiring them all the year over, while others are content to have them at such times as they are produced naturally in the open air. Some prefer more of one sort than of others, and there are many esculents which some reject altogether. Much also depends on whether an English or French cook steers the helm of affairs in the kitchen: the former, we have invariably found, uses the greater quantity; while the latter, while he uses them in less bulk, is far more particular in having them young, and in proper season.

Again, there is the nature of the soil and the situation to be taken into consideration, as well as whether the garden is protected from game, or open to their attacks. With these data all to be taken into account, it is quite clear that only an approximation to the exact quantities can be arrived at, even by the most conversant in those matters; but that approximation is better than none at all. Before, however, stating what we conceive to be the necessary quantities under these circumstances, we must premise a few words of warning to those gentlemen who make up their own seed-lists, and who are always on the look-out for cheap houses, and advertised seed sales by auction-in too many cases got up by a set of swindlers, whose chief personal characteristics are a pair of huge mustaches, and an affectation of being totally ignorant of the English language. To such we say, Take the responsibility upon yourselves; and when you find no crop forth- coming, blame neither your gardener nor your soil. The more respectable the firm you purchase from is, the more certain you may be of avoiding disappointment. Besides, you have a remedy against the one; none whatever against the other. The law will afford you protection, and award you full and fair compensation for loss in the one case, but you may whistle for it in the other. Besides, firms of respectability have a character to maintain, of far more importance to them than any gain that could arise out of any fraudulent transaction.

We greatly object to most of the former seed estimates published, because they are in general below the mark; and much as we deprecate thick sowing, still, seeds are now so cheap that it were false economy to have such a deficient supply at first, as to be compelled to re-sow a crop cut off by insects, frost, or other accidents, to which all such crops are liable; or to be without a supply at hand, in the event of the crop being entirely destroyed.

We give below the seed estimate of Gordon, of 1774 , to show the quantities he allows, but more particularly to show that many of the vegetables still popular with us were so nearly a century ago.

"A seed bill for a private family, calculated to crop a garden consisting of an acre of ground. -4 lb. early Charlton pease ; 4 lb. Nicol's early Hotspur do.; 4 lb. golden early do.; 4 lb. common Hasting, or Essex Reading do.; 4 lb. Turkey Hotspur, or long marrow-fat do.; 4 lb. dwarf marrow-fat do.; 4 lb. Leadman's long dwarf do.; 1 lb. early Mazagan beans ; $1 \mathrm{lb}$. early Lisbon do.; 2 lb. long-podded do.; 4 lb. Tokar do.; 4 lb. Windsor or Turkey do.; $\frac{1}{2}$ lb. Barbary kidney do.; $\frac{1}{2}$ lb. Battersea do.; $\frac{1}{2}$ lb. Strasburg onion ; 3 oz. red Spanish do.; 1 oz. silverskinned do.; 4 oz. London leek ; 4 oz. orange carrot; 1 oz. early horn do.; 2 oz. Dutch parsnip ; $\frac{1}{2}$ lb. early Dutch turnip ; 2 oz. yellow do.; 2 oz. red-topped do.; $\frac{1}{2}$ lb. shallot; $\frac{1}{2}$ lb. garlic ; 4 oz. early London short-topped radish ; 2 oz. salmon do.; 1 oz. turnip-rooted do.; 1 oz. black Spanish do.; 6 dr. cabbage lettuce ; 4 dr. ice or white Cos do.; 2 dr. Silesia do.; 2 dr. tennis-ball do.; 2 dr. brown Dutch do.; 4 oz. curled cress ; 2 oz. broad-leaved do.; 4 oz. common do.; 
$\frac{1}{2}$ oz. Indian do.; 2 oz. curled parsley; 2 oz. common do.; $\frac{1}{2}$ lb. white mustard; $\frac{1}{2}$ oz. curled endive ; 4 dr. Italian celery ; 4 dr. long green cucumber ; 4 dr. short prickly do.; $2 \mathrm{dr}$. best melons; $1 \mathrm{lb}$. round spinach ; $\frac{1}{2}$ lb. prickly do.; 1 oz. French or Mountain do.; 1 oz. white beet; 1 oz. green do. ; 2 oz. red beet, or beet-rave; $\frac{1}{2}$ oz. cauliflower ; $\frac{1}{2}$ oz. white broccoli ; $\frac{1}{2}$ oz. purple do.; 1 oz. early Yorkshire cabbage ; 1 oz. sugar-loaf early do. ; $\frac{1}{2}$ oz. red Dutch do. ; 4 oz. late Alnwick do. ; $\frac{1}{2}$ lb. green savoy ; 2 oz. yellow do. ; 2 oz. Kilmaurs kale ; 4 oz. scarlet beans."

Our own estimate, in a similar case, is this; but as the varieties of cultivated vegetables are now so numerous, we shall omit specifying them, referring the reader to our select lists, which will be found appended to each subject, as well as leaving unnoticed some few kinds not generally expected to be found in a garden of the limited extent of one acre: Pease, 36 qts. ; garden beans, 10 qts.; French or kidney beans, 4 qts.-provided none are forced. Scarlet runners, 2 qts. ; cabbage of early sorts, 8 oz. ; savoys, 4 oz. ; Brussels sprouts, 3 oz. ; cauliflower, 4 oz.; broccoli of sorts, $8 \mathrm{oz}$; ; borecoles, $4 \mathrm{oz}$. ; red cabbage, 2 oz.; late or drumhead do., 2 oz. ; kohl-rabi, 2 oz. ; onions, 12 oz. ; carrots, $8 \mathrm{oz}$. -if none are forced. Turnip, white sorts, 16 oz.; yellow do., 6 oz.; celery, 2 oz. ; spinach, 8 qts. ; red beet, 4 oz.; yellow do., 2 oz.; leeks, 4 oz.; parsnips, 4 oz. ; salsify, 2 oz. ; skirret, 2 oz. ; scorzonera, 2 oz.; endive, 4 oz. ; lettuce, 4 oz. ; radish, 3 pts.-if none are forced. Mustard, $1 \frac{1}{2}$ qts., and cress the same-if neither are forced. Parsley, 4 oz. - 2 oz. curled, and 2 oz. plain. Of roots, early potatoes, $1 \frac{1}{2}$ bush. ; of late do., 3 bush.; Jerusalem artichokes, 1 pck.; garlic, $\frac{1}{2} \mathrm{lb}$. ; shallots, $2 \mathrm{lb}$.

It does not, however, exactly follow that a garden of 20 acres extent requires twenty times the quantities shown above; this, however paradoxical it may appear to the uninitiated, is well known to the practical cultivator. An example may be given in the case of a seed-bed of cabbage, cauliflower, leeks, \&c. A first planting is taken from the most forward plants, which, in general, are those arising from seed of the preceding year, or of those of that year most fully matured, compared with less perfectly ripened seeds, even of the same year's growth. They also arise from seed of the previous year's production being stronger than seeds of one or two year's standing, thus producing a very necessary succession in the hands of those who know how to manage matters rightly ; and, consequently, the experienced gardener, availing himself of the knowledge of which he has long been possessed, will and does take such plants in succession, as he well knows, under favourable circumstances, the stronger plants will come first to maturity, or else they will run to seed, and be useless. This misfortune is, however, made up for in the second planting, and this is succeeded by the third. In the meanwhile, let us endeavour to set aside the too prevalent opinion that seed of the previous year's growth alone should be sown, with some few exceptions. Than this a more erroneous notion could not, we think, have been driven into the craniums of those of our wiseacres, "the advocates for new seed." The honest seedsman does an act of great kindness, and confers an important benefit on his customers, when he mixes seeds of 1851 , 1852, and 1853 together; for, provided they have been well kept, the advantage in the case of many seeds (take the whole of the Brassicæ as an example) is very mich in favour of the purchaser. For ourselves, we would not thank any man for seeds of cauliflower, broccoli, turnip, \&c., of last year's production; and to avoid the chance of disappointment from this, we invariably keep by us in stock seeds of this natural order, of from two to five or six years. Our older gardeners, while they have handed down to us volumes of rules, have been very sparing in paying us the same compliment in regard to reasons. We have hardly had one assigned for the invariable rule of their carrying their cucumber and melon seeds in their pockets for months before they committed them to the soil. An ancient sage says, "there is reason in the boiling of an egg ;" so is there in carrying the seeds referred to in the warm dry atmosphere of a pocket. It is in consequence of the genial heat bringing about a state of maturity in the seed, which our cold climate, compared with Persia, the country of the melon, is incapable of doing; and hence the practice of 
keeping such seeds for years, until they, as it were, arrive at maturity in consequence of age, or are brought artificially to that state by the means (or other means similar) above stated, which is correct in principle. In the case of the natural order above referred to (the Brassicæ), seeds of three ages have been found of vast advantage - a circumstance, if we recollect rightly, brought before the public some years ago by $\mathrm{Mr}$ Archibald Gorrie, who, in reference to the turnip (and all the Brassicæ may be taken in the same category), says the plants from seeds of the previous year, being stronger, vegetate first, and afford food for the fly, while those from the seed of the year previous to that follow in succession; and if vegetation be going on rapidly, these may escape wholly the attacks of insects ; if not, the older seed of all, which vegetates last, is certain to escape, because the fly has had wherewithal to satiate its voracious appetite; and by the time the third in succession comes into leaf, the insects have undergone their transformation. This is also a reason for thicksowing under peculiar circumstances. Nor is the preservation from the fly all that arises from fully matured seed. If we only take a crop of early Dutch turnip as an example, it will be found that the plants raised from seed of the previous year will be exceedingly prone to run to seed without bulbing; and should they even do so, the bulbs will be neither so firm nor so well shaped as those from seed of two, three, or more years' saving. The tops, in the former case, will be large, and consequently monopolise for themselves much of the material which ought naturally to have gone to the bulb; in the latter case the tops will be small, the bulbs large and well formed ;-indeed, the proper preponderance will be, in this case, maintained between bulb and leaves. Cauliflower coming prematurely into flower-or what is technically called buttoning, because the abortive flower produced is not much larger than a good-sized button, and seldom worth half as much-may be offered as another example. Many other cases might be given; these, however, may suffice.

Returning, however, to how an expert gardener manages with less seed, in proportion to his wants, than one of less experience, and in a more humble way of business : the former has a gradual succession to maintain, which scarcely admits of separate sowings to supply the niceness of his calculations ; he therefore trusts to the three gradations, in which his seed-bed seldom disappoints him. He acts accordingly, and instead of taking the largest and strongest plants only, he takes part of all the three, and thus fills a much greater space of ground than he who calculates only on one crop, and contents himself with the first and strongest plants his bed produces, and, regardless of the others, digs them down, and trusts to another sowing, perhaps a month or more hence; which sowing, by the way, may suit his looser way of doing business. The gardener who has the superintendence of first-rate gardens is supposed to have a much greater knowledge of the principles of his profession-at least he should have so-than one whose field of operations is limited to a quarter of an acre, and who is often from circumstances ignorant of the rudimentary principles upon which he should act. These and amateurs are the class to which the sin of extravagance and too thick sowing is chargeable, and they in general consume far more seed, from misapplication, than is necessary. Fearful at sowing, either from want of confidence in the seed itself, or from being ignorant of the mode of proving its quality previous to sowing, they console themselves in either case with the certainty of being safe, and therefore sow unreasonably thick. There are, no doubt, on the other hand, some who, from penuriousness, err in sowing too thin, and not taking into calculation the losses by insects, frosts, and the doubtful quality of the seed, which such a class is of all the most likely to experience, having purchased cheap; and, in consequence, they find that their crop is a total failure. There is, however, no doubt that thin-sowing is preferable to thick-sowing, so far as the plant is concerned; and if seeds are to be depended upon, and were they of sufficient value to make it a measure of economy to plant them singly, instead of sowing them in the usual manner, there is no doubt, barring accidents, that the former mode would be preferable to the latter, and therefore one quarter of the seed usually ordered would suffice. 


\section{$\S$ 4.-ROTATION OF CROPS.}

The necessity of a rotation of cropsthat is, not sowing or planting the same ground with the same species of seed or plants from which it has been cleared, but introducing a succeeding crop of as dissimilar a kind as possible-is founded upon two facts, namely, the excrementitions of the plants, and the exhausting of the soil by them. Brugmanns supposed he had discovered that some plants exude an acid fluid from their spongioles, which may be regarded as a peculiar kind of excretion, which becomes obnoxious to roots of the same kind, but not so to those of another species; and Macaireasserts that this property is almost general throughout the vegetable kingdom. De Candolle was of this opinion, and conjectured that the soil was not only rendered unfit for the growth of the same species in consequence of these excretions, but believed that, acting as a manure, they improved the soil for other species. This, coupled with the supposition that plants cannot digest their own excretions, seemingly explains to us why the soil becomes deteriorated by one kind of plant having long grown in it, and its unfitness to support a crop of the same kind until the fecal matter in it shall have become decomposed. Daubeny and Gyde deny this, and say that these excretions are not injurious.

Gyde's opinion was, that though plants have no power of selection, "but take into their texture any solution offered to their roots, they have little or no power of again excreting it; that any excretions are only of the true sap; and that plants watered with excretions receive no injury by it." This opinion has occupied, to some extent, the attention of physiologists within these two or three years, but, as it appears, as yet without any conclusive result having been arrived at.

In connection with this, Dr Lindley remarks ("Theory of Horticulture," p. 21), "In addition to their feeding properties, roots are the organs by which plants rid themselves of the secreted matter, which is either superfluous or deleterious to them. If you place a plant of succory in water, it will be found that the roots will, by degrees, render the water bitter, as if opium had been mixed with it; a spurge (Euphorbia) will render it acrid, and a leguminous plant mucilaginous; and if you poison one half of the roots of any plant, the other half will throw the poison off again from the system. Hence it follows, that if roots are so circumstanced that they cannot constantly advance into fresh soil, they will, by degrees, be surrounded by their own excrementitious secretions. It would also seem to follow that, under the circumstances just named, they would be poisoned, because they have little power of refusing to take up whatever matter is presented to them in a fitting state. But it is by no means certain that the excrementitious matter of all plants is poisonous either to themselves or to others; and therefore the consequences of roots growing in soil from which they cannot advance are uncertain, and only to be judged of by actual inquiry into the nature of the secretions." On the power of selection of food, the same high authority observes (p. 18), "Powerful as the absorbing action of roots is found to be, those organs have little or no power of selecting their food; but they appear in most cases to take up whatever is presented to them in a sufficiently attenuated form. Their feeding properties depend upon the mere hygrometrical force of their tissue, sei in action in a peculiar manner by the vital principle. This force must be supposed to depend upon the action of the capillary tubes, of which every part of a vegetable membrane must of necessity consist, although they are in all cases invisible to the eye, even when aided by the most powerful microscopes. Whatever matter is presented to such a set of tubes will, we must suppose, be attracted through them, provided its molecules are sufficiently minute; and as we have no reason to believe that there is, in general, any difference in the size of the molecules of either gaseous matter, or fluids consisting principally of water, it will follow that one form of such matters will be absorbed by the roots of plants as readily as another. For this reason plants are peculiarly liable to injury from the presence of deleterious matter in the earth; and it is probable that, if in many cases they reject it, it is because it does not acquire a sufficient state of tenuity, as in the case of certain coloured infusions. 
This, although apparently a general rule, is not without its exceptions. If a pea and a grain of wheat are planted in the same soil, and placed in the same circumstances, it will be found that the latter will absorb silex from the soil, the former none; and this shows pretty clearly the power of selection. Dr Daubeny has shown that certain plants will not absorb strontian; and Saussure that the spiral-rooted polygonum, while it took up common salt (muriate of soda) freely, refused to absorb a solution of acetate of lime. "It is a curious fact," Dr Lindley remarks, "that the poisonous substances which are fatal to man are equally so to plants, and in nearly the same way; so that by presenting opium or arsenic, or any metallic or alkaline poison, to its roots, a tree may be destroyed as readily as a human being."

Cropping the same ground year after year with the same species of plant, or replanting a forest with the same species of trees, or indeed planting a young fruit-tree in the same situation and soil from which another had been removed, is attended with failure, for, in addition to the cause assigned above, the soil becomes exhausted of those parts it originally contained and has given out for the support of the previous crop, though it may still retain material of a different character, sufficient to enter into the constitution of a crop of a dissimilar kind. "As the inorganic materials which enter into the composition of plants vary much in their nature and relative proportions, it is evident that a soil may contain those necessary for the growth of certain species, while it may be deficient in those required by others. It is on this principle," says Professor Balfour, "that the rotation of crops proceeds-those plants succeeding each other in rotation which require different inorganic compounds for their growth. In ordinary cases, except in the case of very fertile virgin soil, a crop, by being constantly grown in successive years in the same field, will deteriorate in a marked degree. Dr Daubeny has put this to the test of experiment, by causing plants to grow on the same and on different plots in successive years, and noting the results :VOL. II.
A verage of 5 years.

Potatoes, $\{$ in the same plot, $72.9 \mathrm{lb}$. tubers.

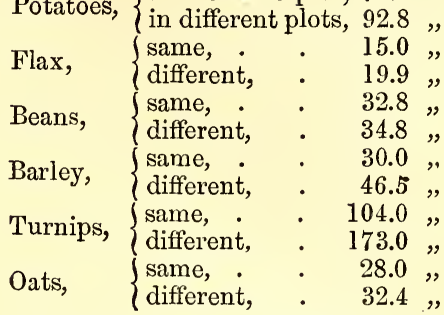

"This shows a manifest advantage in shifting crops, varying from 1 to 75 per cent, the deficiency of inorganic matter being the chief cause of difference." Manual of Botany.

"The prevailing opinion," Loudon remarks, "has long been that plants exhaust the soil generally of vegetable food, particularly of that kind of food which is peculiar to the crops growing on it for the time being. For example, both potatoes and onions exhaust the soil generally; while the potato deprives it of something that is necessary to insure the reproduction of a good crop of potatoes, and the onion of something which is necessary for the reproduction of a large crop of onions. According to the theory of De Candolle, both crops exhaust the soil generally, and both render it unfit for the particular kind of crop ; but this injury, according to his hypothesis, is not effected by depriving the soil of the particular kind of nutriment necessary for the particular kind of species, but by excreting into it substances peculiar to the species with which it has been cropped; which substances render it unfit for having these crops repeated." Sub. Hort., p. 436 . Both these theories have been disputed, and this by practical reasoners, who naturally enough ask, How do they apply to plants long confined to the same soil-an orange tree, for examplewhich has luxuriated, without being either poisoned or starved, within the limits of a three-feet square box for a score of years? and how do peremnial plants exist in the same soil for as great a length of time? The advocates of the one theory say the annual dropping and decay of the foliage supply at once general and particular nourishment. This does not, we suspect, apply to the orange tree we have taken as an example, because the leaves, if they even fell on the surface of the soil in the 
box, would be daily removed. The others say the same droppings of the leaves, by the general nourishment which they supply, neutralise the particular excretions. Liebig advocated the exhausting principle, and showed, chemically, that the roots of trees and plants in time exhaust those principles contained in the soil which are most conducive to their respective wants. This appears to be both a conclusive and simple way of settling the question. He afterwards modified these views, and remarks, p. 33, edit. 1843, "Transformations of existing compounds are constantly taking place during the whole life of a plant, in consequence of which, and as the results of these transformations, there are produced gaseous matters which are excreted by the leaves and blossoms, solid excrements deposited in the bark, and fluid soluble substances which are eliminated by the roots. Such excretions are most abundant immediately before the formation and during the continuance of the blossoms; they diminish after the development of the fruit. Substances containing a large proportion of carbon are excreted by the roots and absorbed by the soil. Through the expulsion of these matters, unfitted for nutrition, the soil receives again, with usury, the carbon which it had at first yielded to the young plants as food, in the form of carbonic acid. The soluble matter thus acquired by the soil is still capable of decay and putrefaction, and, by undergoing these processes, furnishes renewed sources of nutrition to another generation of plants, and becomes humus."

We have many instances in practice, where the same crop has been grown on the same soil for many successive years, vide article ONION; and many others of a like kind could be adduced. $\mathrm{Mr}$ Stephens, in his excellent "Book of the Farm," vol. ii. p. 455, reasons on this subject practically, and we think judiciously. He says, "Experience has demonstrated that one crop after another of the same kind greatly reduces the fertility of all classes of soils. This conclusion might be drawn from reason as well as experience, since it is reasonable to suppose that crops of the same kind take the same sort of food out of the same kind of soil. Experience has also demonstrated that one crop after another, of a different kind, does not materially reduce the condition of soils. This deduction, then, seems fair, that the condition of the soil is best maintained by taking different crops after one another; and as every crop, though of different kind, and deriving support from the soil, assists in exhausting it, a limit must be put to the number of crops that should follow one another. Though all crops derive food from the soil, one kind appropriates food in a different degree from another; and even the same crop takes food in different quantities, according to the state its product is allowed to proceed." Plants which ripen their seed, as cabbages, turnips, \&c., when the ostensible object is to produce seed, draw more strongly on the soil than those which are grown for their leaves and bulbs only, as spinach and beet, \&c. Hence the practice, in gardens, of uselessly allowing plants to shoot up into flower, much less seed, in spring, cannot be too severely reprobated.

The excrementitious theory is ingenious, if not even correct, and has occupied the attention of the chemist for many years. At the request of De Candolle, M. Macaire of Geneva instituted a series of experiments, which led him to conclude that, in the formation of the seed, or other nutritious parts of plants, the sap is digested; that it takes up certain elements, and deposits others, which are the residue of the process; and these, being no longer necessary for the formation of the seed, are rejected by the vital action of the plant, and exude by the roots. "Our ignorance of the functions of vegetable life prevents us from seeing the effects produced on the sap by the expansion of the blossoms, or the ripening of the seed; but experience leads us to perceive that certain plants thrive best after certain others, and that in this case they are always of distinct and different natures, and of different natural botanical families. Macaire and other scientific men observed the change that took place in the water in which wheat had been made to grow. They found a deposit in the water of the nature of bitter extract, and this they concluded to be excrementitious. Beans grew well in this water; and, on the other hand, wheat throve in the water in which beans had grown."-Donaldson on Soils and Manures, p. 30. The effects of fal- 
lowing land, or deep-digging, ridging, and trenching, are said to be the sweetening the soil, because the excrementitious matter becomes washed out by the rains, decomposed by the action of light and air, or buried beyond the reach of the roots, and may remain so until decomposed or completely changed by some unseen and as yet imperfectly understood cause.

The alternation of crops becomes also necessary, as a safeguard against the attacks of insect enemies. Thus, some of the insects which are most injurious to the Brassica tribe, for example, by depositing their eggs in the soil, when the period of their own brief existence terminates, secure by this means a numerous progeny to commit their baneful depredations on the succeeding crop; whereas, if a different kind of plant were substituted, it is, in many cases, certain that they would die of starvation, rather than feed on food of a character different from that destined for them by nature. Take, for example, a plant of their own natural order, the black mustard, (Sinapis nigra,) which has been recommended to be sown on ground infected with the larvæ of insects feeding on the cabbage tribe : the roots of the mustard being too acrid for them, they have actually died of starvation.

As a restorative or compensation to the soil for a continued cropping with the same species of crops, certain materials, forming in themselves the inorganic constituents of plants, have been recommended. Indeed, chemically speaking, one piece of ground may possibly be made to produce the same species of crop ad infinitum. To carry out, however, these ideas, it will be necessary to ascertain the matter abstracted from the soil by such crops, and then to add to them, at each sowing or planting, an equivalent, and something more, of the ingredients of the same nature as that of which the ground has been robbed by the preceding crop. This is, however, only meeting the subject midway, if even so much.

To ascertain correctly what is the food of plants, we must first ascertain what they themselves are composed of; for whatever elements constitute their structure, these elements are their true food: therefore it is the plant more than the soil whose component parts should be determined. Chemists have laboured for years in determining the qualities of soils and manures, while they have ly far too much neglected the analysis of the plants themselves, which is the first and most important consideration.

It is a pretty generally received opinion amongst many cultivators that each species of plant requires a distinct species of food to be presented to it from the soil ; but vegetable physiologists have shown that the organs of one plant derive their food from substances which concur in the true nutrition of plants generally; or, that is to say, plants of the most opposite characters and properties, as articles of food or vehicles of poison, will not only exist, but flourish in the same flower-pot of earth or of manure-a circumstance opposed to the theory that each species requires a different element of food.

M. Boussingault favoured the opinion that there was no absolute necessity for a rotation of crops "when dung and labour can be readily procured. Nevertheless," he says, "there are certain plants which cannot be reproduced upon the same soil advantageously, except at intervals more or less remote. The cause of this exigence on the part of certain plants is still obscure, and the hypotheses propounded for clearing it up are far from satisfactory. One of the marked advantages of alternate culture is the periodic cultivation of plants which improve the soil. In this way a sort of compensation is made for exhaustion. The main thing to be secured, in the rotation of crops, is such a system as shall enable the husbandman to attain the greatest amount of vegetable production with the least manure, and in the shortest possible time. This system can alone be realised by employing, in the course of rotation, those plants which draw largely from the atmosphere. The best plan of rotation in theory is that in which the quantity of organic matter obtained most exceeds the quantity of organic matter introduced into the soil in the shape of manure. This does not hold in practice. It is less the surplus amount of organic matter over that contained in the manure, than the value of the same matter, which concerns the cultivator. The excess required, and the form in which it should 
be produced, must vary widely according to locality, commercial demand, and the habits of the people, considered wholly apart from theoretical provisions. One point in theory which should agree with practice is this, that in no case is it possible to expect more organic matter, and particularly more azotised organic matter, than the excess of the same matter contained in the manure which is consumed in course of the rotation. By acting upon another presumption, the productiveness of the soil would be infallibly lessened. Hence it may be inferred how closely the study of rotations is connected with that of the exhaustion of the soil."”

Such is a brief epitome of the two leading theories-hypotheses, perhaps, more properly they should be called. The practical deductions to be drawn from them differ little, and add as little to what observing cultivators have long been taught by experience-namely, that crops, the plants of which have a close affinity to each other, belong to the same natural order, or resemble each other in structure or habit, should not follow each other in cultivation. Cruciferous plants-that is, the cabbage, kale, or turnip tribe-should be followed by those of the leguminous order, or the pea and bean tribe, and vice versa. Deep-rooting plants, like the beet, carrot, parsnip, \&c., should be followed by such as spinach, lettuce, \&c., and vice versa. Again, plants which have been grown for their fruit, such as seedcabbage, and even pease, beans, scarlet runners, although the three latter do not remain to ripen their seeds, take more from the soil than cabbage, celery, or spinach, which are grown for their leaves alone. Perennial plants, such as sea-kale, asparagus, globe artichoke, \&c., should never succeed each other, but should be succeeded by onions, leeks, or other annual crops of short duration, of essentially different characters. Indeed, no two exhausting crops should succeed each other, but rather the least exhausting, such as lettuce, endive, \&c., should be succeeded by cabbage or the like.

The following excellent article on rotation cropping appeared in "The Gardeners' Chronicle," and deserves the special attention of every cultivator, whether the field of his operations be large or small. "Rotation crops consist of those most required for culinary purposes, such as pease, beans, French beans, broccoli, cabbage, turnip, carrots, parsnip, beet, onions, savoys, spinach (winter), and winter greens, celery, cauliflower, leeks. Pease and beans should be sown from February to June." In Scotland we sow on till the beginning of August, as our moist mild climate is more favourable for producing crops during September, October, November, and we have had them occasionally up to nearly Christmas, seasons when pease have hitherto been almost unknown in the neighbourhood of London, where the drought and heat prevent their natural development. The London Horticultural Society have this season taken up this matter, and we believe that, by counteracting the effects of heat and drought, both of which are pretty much under the control of the cultivator, green pease will soon be seen in Covent Garden market on Lord Mayor's Day, as they are now on the 1st of June. "The first crop of pease will be clear for early broccoli in the end of June, and for the other seasons until September, for later broccoli, savoys, borecole, Brussels sprouts, collards or coleworts, and spring cabbage. This crop should have a slight crop of manure. Broccoli ground will be cleared of early sorts by winter"-in Scotland, say the end of November- " and should be ridged up all winter for a crop of carrots, which should be sown as early as possible. The later broccoli, colewort, sprouts, \&c., will make way by April or the beginning of May for beet, parsnip, scorzonera, and salsify. Carrots, beet, and parsnips will be clear in the beginning of November, when the ground must be again ridged up for winter, and have a good coat of dung ready for cauliflowers, onions, garlic, and shallots, the two latter being planted in November, and also the principal crop of turnips sown in the end of March and April. Cauliflower, onions, and turnips will be clear from July to September ; the cauliflower, shallots, \&c., in July, for autumn spinach and endive; the onions for winter spinach, and the turnips for spring onions, winter lettuce, and other secondary crops. Spinach, endive, and spring onions will be clear by the end of May for savoys, winter 
greens, red cabbage, cauliflower, and leeks, all of which require a moderate coat of manure. Savoys, winter greens, red cabbage, \&c., will be ready for early potatoes in April and May. Potatoes will make way in July and August for turnips, spring cabbage, late broccoli, and such crops, if wanted. Turnips, cabbage, and broccoli may be cleared in May for celery and cardoon trenches, if all the ground is wanted; but if not, the cabbage may be allowed to remain for sprouts during all the summer. The intermediate spaces between the trenches may be planted with lettuce or any other secondary crops : dung must be given for celery, of course. Celery and similar crops will, in part, make way in autumn, when the ground should be ridged up for the winter, and the remainder as soon as the entire crop is clear; the ground will then be ready for French beans, scarlet runners, cauliflower, cucumber, and tomatos, in the end of April or beginning of May. French beans will be clear by November, when the ground should be again ridged up all winter, to be ready for pease and beans as at first begun. This will make eight or ten years between the return of the principal crops to the same place, and, by judicious management of the secondary division" (such as salads and short-lived crops) "among the rotation crops, every space of ground between one crop and the other may be occupied to advantage during the intervals of cropping."

The following shows the order of rotation :-1, pease and beans; 2 , broccoli, savoys, winter greens, collards - i. e., spring cabbage ; 3, carrots, parsnips, beet, scorzonera, salsify, "skirrets, Hamburg parsley;" 4, onions, cauliflower, turnips; 5 , spinach, spring onions, and other secondary crops ; 6 , savoy, broccoli, winter greens, red cabbage, leeks; 7 , potatoes; 8, turnip, cabbage, broccoli ; 9, celery, cardoons; 10, French beans, \&c.

"Secondary crops are those of the shortest duration, such as lettuces, radishes, small salads, annual herbs, and very early pease and beans, (sown in November,) very early cauliflowers, very early turnips, and early potatoes, all of which will require a warm south border."

This is a specimen of the rotation practised by the best cultivators around Lon- don, and, taking the difference of latitude into consideration, it is calculated for a great part of Scotland and Ireland. It will be remarked that this system of rotation comprises only the annual or biennial crops, and therefore we propose it as the precursor course to the systematic arrangement of the contents of the kitchen-garden sketched out, (vide p. 7,) and we know of no better rotation that can be followed. This rotation embraces eight years, but, by following ours as an addition course, the change may be extended to the eleventh or twelfth year. The author of the above rotation is one of the best cultivators around London, and calculates, of course, that each crop is to be removed as soon after it is fit for immediate use as the consumption calls for, and hence the rapidity of his movements. The private gardener can do the same thing by keeping up a very close succession (as elsewhere noticed); but, unfortunately, in many private gardens, from causes the cultivator has no control over, crops are allowed to come to too full a state of maturity before they are commenced upon, and kept lingering on the ground long after they should be consigned to the rot heap, or, much better, trenched into the ground, restoring to it much of those elements they had abstracted from it.

According to $\mathrm{Mr}$ Prideaux, in "The Gardeners' Chronicle," 1848, quoted by Mr Stephens in "The Book of the Farm," vol. ii. p. 453, the following quantities of mineral ingredients are removed from an acre of soil by a single crop of beans. "Beans, of a crop of 25 bushels of grain and $2800 \mathrm{lb} .=1$ ton $5 \mathrm{cwt}$. of straw, carry off from an acre of soil these quantities :-

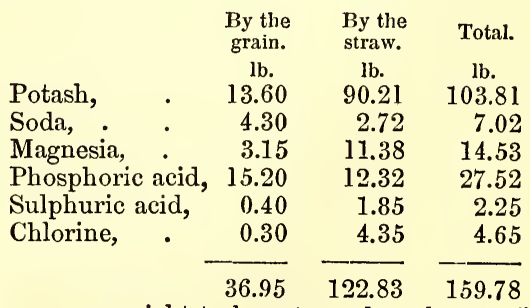

-or gross weight to be returned to the acre."

If the soil is to be maintained in possession of all its natural mineral ingredients, it follows, after such abstractions as this, 
that means must be employed for their restoration. This is to be effected by what are usually called mineral manures, or by the aid of such vegetable ones as may contain them all or in part.

Should, however, the recent discovery made by M. Barral, a French chemist of great respectability, prove to be correctand of that there appears to be little doubt-these ingredients, annually abstracted from the soil, are given back or provided again in a way and to an extent hitherto unsuspected. Chemists long ago had determined that the air we breathe consisted only of two distinct gases, oxygen and nitrogen, with a minute proportion of carbonic acid dispersed through a variable quantity of aqueous vapour. To these Liebig added carbonate of ammonia as constituting the essential parts of the whole, considering the minute traces of lime, potash, and common salt, as too insignificant to deserve notice. This celebrated chemist still further held as quite secondary and insignificant the presence of nitric acid, the action of which is so important in conveying nitrogen to the vegetable system, and declared the quantity as being too small to be even estimated in the rain of thunder-storms. M. Barral has, however, shown this in a different light. The following explanation of his experiments, with remarks by the editor, appears in a very recent number of "The Gardeners' Chronicle," 1853: "This eminent chemist was led, during the last six months of 1851 , to examine minutely the water collected in the rain-gauges of the Observatory at Paris. His mode of investigation is declared by Messrs Dumas, Boussingault, Gasparin, Regnault, and Arago, names foremost in French science, to be free from all objection, and to bear the most severe counter-trials to which they could expose it. M. Barral states, that although the quantities of the following substances varied in different months, yet the monthly average, from July to December inclusive, was as follows :-

\begin{tabular}{|c|c|c|c|c|}
\hline \multicolumn{5}{|c|}{ Substances in a cubic metre of Rain Water. } \\
\hline Vitrogen, & 8.36 & & -129 & \\
\hline Nitric acid, & 19.09 & " & 294. & \\
\hline Ammonia, & 3.61 & " & 55.7 & \\
\hline hlorine, & 2.27 & ” & 35. & \\
\hline Lime, & 6.48 & $”$ & 100. & \\
\hline Magnesia, & 2.12 & " & 32.7 & \\
\hline
\end{tabular}

" He did not ascertain whether all these substances are contained in rain-water collected at a distance from towns. But Mr Bence Jones found at least nitric acid in rain-water collected in London; at Kingston, Surrey; at Melbury, Dorsetshire; and, far from any town, at Clonakelly, in Ireland. If we assume that M. Barral's averages represent what occurs on an English acre, the quantity of such substances deposited on that extent of ground may be safely estimated as follows. The average depth of rain which falls in the neighbourhood of London is well ascertained to be about 24 inches per annum. This is at the rate of 87.120 cubic feet, or 2466 cubic metres of rainwater per acre; and this, according to the proportions per cubic metre in the preceding table, would afford annually of-

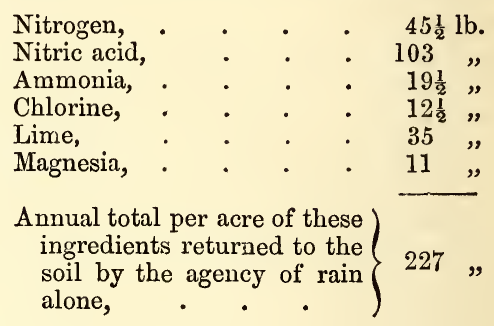

"Of these substances, the three first are of the utmost importance, on account of their entering so largely into the indispensable constitution of the food by which vegetable life is sustained. The quantity of ammonia thus ascertained to exist is about what is expected in $2 \mathrm{cwt}$. of Peruvian guano; and bountiful nature gives us, moreover, nearly $150 \mathrm{lb}$. of nitrogenous matter, also suited to the nutrition of our crops. Nature gives us food, and we improvidently waste it. What with shallow cultivation on the one hand, hard ill-tilled land, puddled furrow-trenches, and polished furrow-slices, rain-water, thus highly charged with the most nutritious ingredients, either runs off to ditches, or is so ill directed that it very imperfectly reaches the roots. On the other hand, by means of close cropping, that which is intended to bathe every part of a plant, and to be instantly absorbed by its verdant surface, is as completely turned aside as if two-thirds of the crop grew beneath a penthouse."

From this it will be seen that nature is 
constantly restoring to the soil a vast amount of those ingredients taken up by the crops, without the aid of man; but, that these valuable supplies be not wasted, it behoves man to keep the soil in a proper state to receive them. This can only be done by what we have so strongly urged elsewhere-deep cultivation and thin cropping-two important essentials in culture sadly neglected in most gardens, trenching for almost every crop being a thing scarcely thought of, notwithstanding we are well assured that very much of the success of the London market-gardener, in producing such enormous crops, depends on this operation alone. Were half the amount of the value of the manure which is yearly crammed into garden soils expended on trenching, and keeping the surface afterwards open, the advantages would be soon made apparent; and without that, all the manure, whether special or common, whether mineral or vegetable, and however applied, may be regarded as so much capital thrown away.

Again, besides the amount of matter restored to the soil, as shown above by $\mathrm{M}$. Barral, an additional supply is returned by the plants themselves, whose leaves are constantly decomposing carbonic acid, which they absorb from the atmosphere, liberating the oxygen, and appropriating the carbon to their own use; they derive supplies of nitrogen for the formation of their albuminous constituents from the volatile carbonate and nitrate of ammonia, and these they restore to the soil when they are buried in it. From the earliest ages certain crops have been grown for the express purpose of being returned to the soil for its enrichment, a practice probably of more ancient date than that of alchemy, and 3000 years earlier than modern chemistry. The exhaustion of the soil by crops is not so very alarmingly great, under good management, as some would have us to believe, and would be much less so if those parts of vegetables that are not to be directly consumed by man were returned to it at the time. Out of a crop of cauliflower, not one-fourth of the bulk of the crop is useable; hence, if the other three-fourths were immediately dug into the ground on which they were produced, they would return to it very nearly as much as they had taken from it during their growth, if not more. It is bad management that exhausts a soil ; and one of the worst parts of bad management is taking the whole vegetable produce off the ground, and either not returning it at all, or doing so after it has become so much decomposed and exposed to atmospheric action as to have nearly lost all its fertilising properties. In gardens much of this is daily carried on, too many believing that plants derive all their food from the soil. But such is not the case; the greater part of vegetation is derived from atmospheric sources, and when that is returned to the soil by digging it in, it in this way supplies it with more of the organic elements essential to future vegetable growth than the soil contained before the crop was sown or planted; in other words, it is enriched by the carbon, hydrogen, and nitrogen which the vegetable had obtained from sources entirely independent of the soil.

Plants have the power of converting the materials which constitute both common and special manures by a species of elaboration going on within them, so as to fit and appropriate the necessary quantity of each, and to dispose of them throughout the various parts of their structure, leaves, stems, seed, roots, \&c.; and not only that, but they are capable of supplying themselves at different times, and even in different parts of the same plant,according to theirrespective natures. "They all form," says Professor Johnston (in "Experimental Agriculture," p. 9), "more or less constantly and abundantly, a portion of the fixed and solid matter of the plant taken as a whole. They may not be found in any one part of the plant, when separated carefully from the rest, but, in the solid parts of the plant, taken as a whole, they are all, and always to be met with. When thus deposited, they become for the most part dormant, as it were, and for the time cease to perform active chemical functions in the general growth, though, as vessels or cells, they may still perform a mechanical function. They undergo various chemical changes in the intercourse, chiefly while circulating or contained in the sap, by which changes they are prepared and fitted for entering, when and where it is necessary, into the solid 
or fixed parts of plants. Thus the starch of the seed is changed into the soluble dextrin and sugar of the sap of the young plant, and then again into the insoluble cellular fibre of the stem or wood as the plant grows; and, finally, into the insoluble starch of the grain, as its seed fills and ripens. They each exercise a chemical action more or less distinct, decided, and intelligible, upon the other elementary bodies, and the compounds of them which they meet with in the sap of the plant. In regard to some substances, such as potash and soda, the sulphuric and phosphoric acids, this last function appears to be especially important. These substances influence all the chemical changes which go on in the interior of the plant, and which modify and cause its growth. The same is true of the nitrogen which the plant contains. This elementary body, in the form of albumen or some other of the numerous protein compounds which occur in the sap, presides over, or takes part in, almost every important transformation which the organic matter of the living plant undergoes. Thus it is always abundantly present where the starch of the seed or of the tuber is dissolved, and sent up to feed the young shoots; and again, when the soluble substances of the sap are converted into the starch of the grain of the tuber, or of the body or pith of the tree, one or other of the protein combinations is always found to be present on the spot where the chemical change in the transformation is going on. Besides these general functions, the several substances found in plants exercise also special functions in reference to vegetable life and growth. Thus-

"Nitrogen is most abundant in the sap of young plants, takes part in most of the changes of organic compounds which go on in the sap, and fixes itself, as the plant approaches maturity, in greatest abundance in the seeds and in the green leaves.

"Potash and soda circulate in the sap, influence chemical changes very much, and reside or fix themselves most abundantly in green and fleshy leaves, and in bulbous roots.

"Sulphuric acid is very influential in all chemical changes; is found, in most cases, in those parts of the plants in which potash and soda abound, and deposits a portion of its sulphur wherever the compounds of nitrogen form a notable part of the substance of the plant.

"Phosphoric acid exercises also much influence over the chemical changes of the sap, and finally fixes itself in greatest abundance in the seeds and other reproductive parts of the plant.

"Lime is very important to healthy vegetable growth, as practical experience has long testified. Among other duties, it appears to accompany the phosphoric acid in the sap of plants, and to deposit itself, in combination with organic acids, in the leaves and bark, and with phosphoric acid in some seeds and roots.

"Magnesia appears also to attach itself very much to phosphoric acid in the sap, and fixes itself, in combination with the acid, principally in the seed.

"Chlorine.-The chemical functions of this substance in the sap are less understood even than that of the other substances above mentioned. It exists chiefly in combination with soda, and is much more abundantly present in some plants, and in some parts of plants, than in others. Though, as I have said, its immediate chemical functions in the plant are not understood, it forms a most important constituent of the plant, in so far as the after uses of vegetables as 'articles of food' are concerned.

"Silica exists in the sap in a soluble form, and deposits itself chiefly in the exterior portions of the stems and leaves of plants. It is supposed there to serve as a defence to the plant against external injury, and to give strength to the stem, in the case of the grasses and corn-yielding plants; but what chemical functions it performs, if any, in directly promoting vegetable growth, we can scarcely as yet even venture to guess."

However extraordinary it may appear to the young cultivator, (and we know many of riper years who scout the idea that plants take up and are in part constituted of FLINT,) yet such is the case; and, moreover, all plants contain mineral matters, such as iron, copper, flint, sulphur, \&c.: if we may venture to hazard a supposition, their presence even in the leaves of the most delicate grass is necessary in the formation of what may be called the bone of the plant; and no 
doubt they answer a purpose analogous to the bones in animals, formed of lime, which is a mineral substance also.

Although, however, the presence of mineral substances in plants is necessary, yet they do not in general abound in large quantities. Their presence is not the result of accident, but of wise design, as beautifully shown by Professor Lindley in "Theory of Horticulture," p. 356 - "For although it may be asserted that the presence of iron, copper, or other substances, in plants, in minute quantities, is accidental and unimportant, yet such a supposition is gratuitous, if not altogether unfounded ; for I do not know what warrant we have for saying that any of the constant phenomena of nature, however minute they may seem to be, are accidental. This at least is certain, that where mineral substances occur abundantly in plants, they are part and parcel of their nature, just as much as iron and phosphate of lime are of our own bodies ; and we must no more suppose that grasses can dispense with silica in their food, or marine plants with common salt, than that we ourselves could dispense with vegetable and animal food.

"Flint is found on the exterior of the whole graminaceous order without exception. It forms the polished surface of the cane palm, the grittiness of many kinds of timber. Sulphur abounds in cruciferous plants, especially mustard; copper in coffee, wheat, and many other plants, (it is believed in the state of phosphate ;) iron as a peroxide in tobacco, \&c." In the fruit of the strawberry it has been found ; and the medicinal properties of that fruit are accounted for by many physicians by its presence. De Candolle, in "Physiologie Végétale," p. 389, asserts that 3650 kilogrammes of copper are consumed annually in France in the article of bread; and M. Sarzeau says that 560 kilogrammes of the same mineral are swallowed annually in France in the article coffee alone.

How these mineral substances are taken up by vegetables is no less curious than their presence in them. Most chemists believe they are in some way or other taken up by the roots. The experiments of John upon this matter, as quoted by Dr Lindley ("Theory of Horticulture," p. 357), would lead us to believe that VOL. II. they are supplied from the atmosphere as well ; for John "found that the Ramalina fraxinea and Borrera ciliaris, two lichens, contained a large quantity of the last metal, although he could not find a trace of it in the fir-tree, on the topmost branches of which the lichens grew. We cannot, therefore, suppose that such things are the result of accident, and that it is unimportant to the plants containing minerals thus constantly, whether such substances are present in their soil or not." We are afraid that some of our agricultural chemists have jumped at conclusions hurriedly, and drawn deductions which will not bear the test of close investigation. When they give us to understand, that because a crop of wheat, for example, abstracts $76.22 \mathrm{lb}$. of mineral substances from the soil per acre, per annum, consisting of potash, soda, magnesia, phosphoric acid, sulphuric acid, and chlorine, that the same weight (viz. $76.22 \mathrm{lb}$.) of these ingredients, contained in special manures, is the exact and proper return to be made for that abstracted by the crop, so as to leave the soil in the condition in which it was previous to the seed of the crops being sown.

Such calculations as these appear to have been made without duly considering atmospheric effects, because it appears there has been no allowance made for them. "Plants feed more by their leaves than by their roots."-LindLEY. "It is commonly supposed that plants derive the whole of their food from the soil, but this is a great error. It is a fact well ascertained by chemical experiments, that plants derive the greater part of their nourishment from the air, although the soil is equally essential to their growth."Solly, in Rural Chemistry,p. 96. "Plants pussibly draw from the atmosphere more than agriculturists commonly suppose; and the soil furnishes, independently of saline and earthy substances, a proportion of organic matter larger than certain physiologists admit."- Boussingault. "The leaves spread out their broad surfaces into the air for the same purpose, precisely, as that for which the roots diffuse their fibres through the soil; the only difference being, that, while the roots suck in chiefly liquid, the leaves inhale almost wholly gaseous food. In the daytime, whether in the sunshine or in the shade, the green 
leaves are constantly absorbing carbonic acid from the air, and giving off oxygen gas ; that is to say, they are continually appropriating carbon from the air. When night comes this process is reversed, and they begin to absorb oxygen, and give off carbonic acid. But the latter process does not go on so rapidly as the former; so that, on the whole, plants when growing gain a large portion of carbon from the air. The actual quantity, however, varies with the season, with the climate, and with the kind of plant. The proportion of its carbon which has been derived from the air is greatly modified, also, by the quality of the soil in which the plant grows, and by the comparative abundance of liquid food which happens to be within reach of its roots. It has been ascertained, however, that in our climate, on an average, not less than from one-third to fourfifths of the entire quantity of carbon contained in the crops we reap from land of average fertility is really obtained from the air."-J. F. W. Johnston, in Agricultural Chemistry and Geology, p. 40. And without quoting other authorities-of which there are many-we refer to the recent experiments made by M. Barral, given above, in which he shows that 227 lb. of the six elements of fertilisation are returned to the soil, per acre, per annum, by the rains which fall alone; while according to data, given above, by agricultural chemists, $76.22 \mathrm{lb}$. are all that is required per acre to re-establish the soil to its former condition, after a crop of wheat has been taken from it, so far as these mineral ingredients are concerned.

Nitrogen is required in great abundance in most plants, in some more than in others; and to obtain this from the atmosphere, the agency of saline substances may be necessary; for of such importance is their influence in this respect, that crops have been grown on soils destitute of organic matter, but containing saline substarices, which procured sufficient nitrogen from the atmosphere to cause the plants to flower, fruit, and yield ripe seed, notwithstanding they were only supplied with distilled water, which, of course, could convey to the plants none of those fertilising elements discovered by M. Barral in rain-water. Mr Stephens, in summing up the evidence he has so carefully collected, concludes by observing, in "Book of the Farm," vol. ii. p. 455-" Upon the whole subject of special manures, the rationale of their application may be based upon the certainty of the fact, that a large produce will be obtained, if we only return to the soil the mineral constituents of the crops we cultivate, in combination with nitrogenous substances, and the materials should be in a state to become fit for assimilation by plants." We are far from despising the use of special manures, but we would employ them as auxiliaries only, and advise the cultivator to consider as his sheet-anchor the contents of a well-managed dung-yard, which contain nitrogen in great abundance, which is known to excite the growth of vegetables, and also to render their products more valuable as food for man. It also contains much carbon, which, entering into their structure, imparts to them firmness of texture, and strength to maintain themselves in an upright position-the only position in which they could be placed, to derive the necessary advantages of atmospheric influence.

We look upon mineral or special manures, applied as restoratives in the routine of rotation, in the light of makeshifts; and whilst we admit their great value in agriculture, where, as things are managed, a sufficient quantity of stableyard manure cannot or is not to be hadand also their importance in elevated situations, to which it would be too expensive to transport it-still we would not have cultivators to place their dependence on them alone. Besides, there is more than mere manurial applications required. The soil must be prepared for their reception, so that the process of free and copious evaporation may go on ; and this cannot be expected to be completely realised, unless that soil be wrought to a considerable depth, and thoroughly amalgamated with those additions, whatever they may be, to insure a gaseous supply of food through the leaves, in addition to what is absorbed by the roots. Notwithstanding all this, and admitting that one species of crop may be grown for several continuous years on the same ground, it is, nevertheless (whether the cause rests in the exhaustion or excrementitious theory), wrong to crop the same ground, unless under peculiar cir- 
cumstances, two successive years with the same species of crop.

Pulverising and trenching may be regarded as a species of rotation, because by them the surface of the soil may be transposed, or the whole mass thoroughly incorporated together; thus presenting to a great extent new food to plants, both for facilitating the necessary chemical changes, and the admission of rain and air-the latter so important to the roots of plants. Deeply pulverised soils are increased in temperature, as well as rendered more uniform in that respect. The air admitted carries with it, during summer, heat from the sun, which is daily accumulating, and retained for a length of time, the earth being a bad conductor of caloric. "The free admission of atmospheric air to soil is also necessary for the decomposition of humus, or organic matter, by which carbonic acid is formed; and atmospheric air is also a great source of nitrogen, which has been lately found in all plants, and more especially in the spongioles of the roots. -Suburban Hort. p. 35. "And hence," says LIEBEG, p. 190, "the great value of animal manures to plants, all of which contain nitrogen, but especially those of carnivorous animals."

From the comparative uncertainty, as regards a correct analysis of plants, in which chemists have left us, we cannot see, not knowing the exact amount of the ingredients of the soil which plants are supposed to carry off with them (both in their growing state, and more especially when they have arrived at their fullest maturity), what amount of those ingredients should be added to the soil, to make up for its supposed loss, or even what these are. Professor Johnston, in "Lectures on Agricultural Chemistry," \&c., p. 528, admits "that we scarcely know as yet what any one entire plant, when fully ripe, carries off from the soil ;" and in another part of these "Lectures," he says "that our knowledge of the inorganic constituents of plants is yet in its infancy, and that our present opinion upon the subject ought, therefore, to be permitted to hang very loosely about us."

It appears, in connection with the rotation of crops, as well as with the application of manures, that too much stress has been laid upon vegetable chemistry, and by far too little regard paid to vegetable physiology ; the connection between both is so intimate and important as to render them, in the pursuit of true conclusions, inseparable. "Intimately connected with vegetable chemistry," says $\mathrm{Mr}$ Edward Solly (in Rural Chemistry, p. 121), " is the study of vegetable physiology : a knowledge of the one is essential to the perfect comprehension of the other, for it is impossible well to understand the chemical changes going on in the organs of plants, if we are wholly ignorant of the forms and structures of these organs; and, on the other hand, the most complete knowledge of the anatomy of vegetables could never lead any one to sound and correct conclusions respecting the nutrition of plants. It is rather to be regretted that both chemists and physiologists have appeared to avoid availing themselves of the advantages which both might have derived, by studying the results which the others had obtained. It is only by comparing together the observations of both that correct conclusions can be formed." The researches of Grew, Malpighi, and Duhamel, did much in elucidating the structure of plants, and the modes by which they derived sustenance; indeed, they may be said to have laid the foundation of vegetable physiology. In more recent times, Decaisne, De Candolle, Mirbel, Dutrochet, and Brongniart, in France; Meyen, Mohl, Link, and Schleiden, in Germany; Amici in Italy ; and Knight, Hooker, Henslow, Brown, Griffiths, and Lindley, in England, by careful observations, and the advantages of improved instruments, have reduced vegetable physiology from a series of vague and uncertain dogmas to a comparatively perfect system. Little had been done since the days of Priestley and Ingenhousz in the improvement of agricultural chemistry, save the labours of Sir Humphry Davy: these, however, an early death prevented him from prosecuting, for we dare scarcely say completing. It remained, therefore, for Liebig to do almost single-handed for vegetable chemistry what those we have named above, with many others, had been zealously engaged in doing for vegetable physiology. In his "Organic Chemistry," in tracing the sources by and from which plants derive their food, he has strongly 
drawn attention to the importance of physiology combined with chemistry. " $\mathrm{He}$ has exposed the fallacy of many of the theories which had been formed to explain them, and has established " (as $\mathrm{Mr}$ Solly remarks) "on good evidence the simple chemical rules which regulate the growth of plants. Although much has been done, and although chemists have laboured to remove the perplexities which encompassed the subject, there is still a very great deal that requires investigation; many important points are as yet imperfectly, or even not at all, explained; and many questions must be satisfactorily settled before a complete system of agricultural chemistry can be established. Till these difficulties are removed, it is premature to expect that chemistry can be of more than partial assistance to agriculture ; for whilst many of the fundamental laws of this section of chemistry are still scarcely understood, all attempts to apply them to practice must be incomplete and liable to error."

The rotation of crops in gardens is an important element in good management. They are, or ought to be, carried out upon two different systems-namely, the successional and simultaneous modes of cropping. The first has its chief feature in covering each piece of ground with only one species of crop at the same time, while simultaneous cropping is founded upon the practice of having several. The first presents the greatest appearance of order and system, and hence is that most generally followed in private gardens; while the latter, although less apparently systematic, is, to a certain extent, so in reality, and affords the largest return of produce, and is therefore often followed by commercial growers, who will, for example; sow with a crop of onions a thin crop of radish, lettuce, and sometimes a few carrots. The two former are removed early for use, leaving the onions to ripen off afterwards, and thus affording them more room as they increase in size. In the latter case, the carrots, sending down their roots to a greater depth, appropriate to themselves their own peculiar species of food, leaving the onions to extract theirs from nearer the surface; and, in some cases, carrots so grown are found to escape the grub. Simultaneous cropping is also carried on where the drill system is followed; and hence a piece of ground may be thus stocked:-tall growing pease, Jerusalem artichokes, scarlet runners, \&c., which attain a height of from 6 to 10 feet, may be planted at distances of 20 or 30 feet apart; garden beans, or low-growing pease, may be sown between these at a proper distance; and between these, cabbage, spinach, \&c., may be planted or sown, thus affording to all a full share of light and air. Another advantage arising from simultaneous cropping is, that crops will be progressing in different stages of growth, so that, as the most advanced is cleared off, the next in order will supply its place; or, when one crop is removed, another of a dissimilar kind may be immediately planted.

Successional cropping is best calculated for poor soils, and for gardens where the supply of manure is limited, as well as where the garden is small. The other cannot be so well carried out, unless the soil is in the highest possible state of cultivation ; and also in that order of things where the whole crop is removed almost at once, as in the case of sending it direct to the market; whereas in a smaller private garden it is only removed in small quantities at a time, according to the consumption, and therefore hangs longer upon the soil than is in accordance with this mode of cropping.

\section{$\S$ 5. TRANSPLANTING CULINARY ESCU- LENTS.}

There are, for the most part, only three objects in transplanting these (some of which, however, do not admit of the operation at all), as the common turnip, whether for crop or seed; while the Swedish turnip, radish, parsnip, beet, scorzonera, salsify, skirret, \&c., if not improved by the operation, suffer no injury by it if properly performed. Advantage is taken of this, and failures in portions of such crops are made up by thinning where they are too thick, and thus filling up deficiencies. In sowing for seed, transplanting has its advantages in this,-the roots can be selected, and misshapen ones rejected. All the Brassicæ-comprising the cabbage, sprouts, savoys, greens, cauliflower-are amazingly improved by transplanting from the seed-bed once or twice 
before their final planting out. It increases the formation of extra roots, enables the cultivator often to detect the symptoms of club, as well as gives opportunity for the rejection of malformed plants. The pea and all the beans are inproved in precocity. The process, however, would be unprofitable in the case of very large crops, and in the saving of seed. In the latter case, a removal of all weak and inferior varieties should be scrupulously attended to. Spinach does not admit, unless upon extraordinary occasions, transplantation ; nor do the whole tribe of small salads, such as cress, mustard, \&c. Lettuce admits of it freely, and so does endive: both may be grown to great advantage in small seed-beds, and when fit for removal placed to succeed crops which, while they (the latter) have been in a state of preparation, have been yielding their return. They, with celery, cardoons, \&c., are grown from seed first, to forward them for transplanting, and to economise room. Onions admit of it with impunity, leeks with singular advantage, while carrots will not submit to the ordeal. Potatoes transplant freely, if the roots are preserved; and all the perennial crops, such as asparagus, sea-kale, \&c., are benefited by the operation. "It is easy thus to see," Mr Loudon very justly observes (Sub. Hort. p. 621), "that by the transplanting system half the garden-ground will suffice that is requisite for the sowing system; and as a proof of the economy of this system generally, it may be observed that it is the one followed by all the market-gardeners in the neighbourhood of London. Another advantage attendant on the transplanting system--more especially in the case of esculents, the leaves of which are the parts used-is, that the plants, being deprived of part of their tap-root, throw out a greater number of lateral roots, in consequence of which the production of radical leaves is encouraged, and the tendency to run to flower is retarded; while a more succulent growth is induced, owing to the plants being placed in newly-prepared soil." Unnecessary cutting or lacerating of the roots should not, on these accounts, be tolerated. The only instance that strikes us at the moment, where a shortening of the roots by the knife is justifiable, is in the case of some of the cabbage tribe reared from seed in very poor soil : such, having a tendency to throw down tap-roots in search of food, may with great propriety be shortened at transplanting.

In every operation of transplanting the esculent productions of the kitchen-garden, regard must be paid to the preservation of the spongiolets, and this the more so when the operation is to be carried out without checking the growth or vigour of the plant, as in transplanting lettuces at any age. (For reasons, see Transplanting, Fruit-Garden). Nor should any curtailment take place in the foliage, unless of such parts as may be accidentally broken or bruised during the operation.

In regard to soil, some have gone so far as to recommend a different soil in the garden for its various products; this has long been proved to be not only unnecessary, but absurd. To carry out such views in the cultivation of the parsnip, for example, it would, according to them, be requisite to transport a section of one of the chalk hills of Kent, Sussex, or Hampshire, for their especial use ; and for sea-kale, to transport a sandbank from the sea-shore for a similar purpose. The chemical relationship seems to cease-that is to say, if it ever existed to the extent that plants growing on calcareous soils, or on siliceous ones either, will not grow in other soils deficient in, or actually devoid of, such chemical constituents-as soon as plants are taken under the fostering care of man; and few or none refuse to grow to far greater perfection in good garden soil, than they are anywhere to be found in their natural habitats. The samphire may be an exception to this rule; nevertheless we have had it growing amongst a collection of native plants in pots, in common soil, for many years. There has been a great deal too much attempted to be made of the relationship between plants and the chemical constituents of their native soil, by our flower-pot experimentalists, as well as of chalking out, according to the rules of latitude, longitude, and altitude, the prosperity of our fruit trees, and their periods of ripening their fruit. To this, reference will be made in its proper place. Meanwhile we say, in a good staple soil, 
on a dry bottom, highly manured and well trenched, and exposed to the weather, every esculent man has adopted may be grown, under proper management, to the highest degree of perfection. Fuller details on this subject will be given under each vegetable, \&c. discussed.

\section{$\S$ 6.-PROPOSED ARRANGEMENT.}

In treating on the culture and management of culinary vegetables, it would, no doubt, be desirable were a classification or systematic arrangement of them established, if for no other end than that of rendering reference to them more convenient. This has been attempted, but never satisfactorily accomplished; nor do we believe such an arrangement possible, from the circumstance of so many of the plants under this head being used for different purposes, and many having no point of union in culture, position in the garden, season of sowing, planting, use, \&c.

The following arrangement, so far as it goes, may be useful in this respect, viz. :-

Alliaceous plants, comprehending the shallot, garlic, rocambole, onion, leek, and chive.

Leguminous plants.-Pea, bean, French bean, and scarlet runner.

Brassicaceous plants.-White and red cabbage, Couve tronchuda or Portugal cabbage, cauliflower, broccoli, Brussels sprouts, savoys, boricoles or winter greens, and sea-kale.

Asparaginous plants.-A sparagus, alisanders, cardoon, globe artichoke.

Spinaceous plants.-Common spinach, New Zealand spinach, and white beet, \&c. Acetariacious plants.-Lettuce, endive, succory, dandelion, sorrel, tarragon, burnet, celery, mustard, curled and plain cress, water-cress, American cress, winter cress, Normandy cress, Indian cress, purslain, chervil, rape, corn salad, radish, \&c.

Esculent-rooted plants.-Carrot, turnip, potato, Jerusalem artichoke, red beet, skirret, scorzonera, salsify, parsnip, Hamburg parsley.

Oleraceous plants, comprehending parsley, dill, borage, thyme, sage, clary, balm, mint, costmary, tansy, basil, savory, marjoram, anise, caraway, coriander, angelica, \&c.

Medicinal plants.--Chamomile, elecampane, wormwood, rue, hyssop, and others.

Edible fungi.-Mushroom, truffle, and morel.

Miscellaneous, not referable to any of the above sections, but used in confectionary and domestic economy.-Rhubarb, love apple or tomato, egg plant, gourd, capsicum, marigold, liquorice, rosemary, lavender, horse-radish, \&c. 


\title{
C H A P T E R I.
}

\author{
ALLIACEOUS PLANTS.
}

\section{$\S 1 .-T H E$ SHALLOT.}

Natural History.-The shallot (Allium ascalonicum L.) belongs to the natural order Asphodeleæ, and the class Hexandria, and order Monogynia, in the Linnean arrangement. The order Asphodeleæ contains about five hundred and twenty species, the majority of which inhabit the temperate, and even the colder regions, few of them comparatively being found within the tropics. The genus Allium (derived from the Celtic all, hot or burning) comprises one hundred and twenty-six species, eleven only of which have as yet been found worth cultivation, and of those the subjects now to be treated of - namely, the shallot, garlic, rocambole, onion, leek, and chive-are in most repute. Many of the species are, however, very pretty, and, notwithstanding their unpleasant odour, have found a place in our flower borders. The roots of all the genus are eatable, and those of some-the onion, for example-rank among the most useful articles of food. None of the family are even suspected of possessing deleterious properties.

The shallot is indigenous to Palestine, abounding in the neighbourhood of Ascalon, from which circumstance the specific name, ascalonicum, has been given. It is often written eschalot; it was also formerly called scalion, evidently a corruption of Ascalon, from whence it came. It was introduced to Britain in 1548-by whom or how we have no record left.

$U$ ses.-On account of the mildness of its flavour, compared with that of our other cultivated Alliums, it is preferred in cookery as a seasoner in stews and soups. It is also much used in a raw state; the cloves or sections of the root, cut up into small pieces, form an ingredient in French salads, and are also sprinkled over steaks, chops, \&c. The true epicure, however, cuts a clove in two, and by rubbing the inside of the plate, secures the amount of relish to suit his palate. Shallot vinegar is made by putting six cloves into a quart bottle of that liquid; and when sealed down, it will keep for years. It also makes an excellent pickle. It has been in use since the days of Pliny, who says "the Ascalonian onions are proper for sauce."
Propagation. - The shallot is easily propagated, each bulb being formed of several parts, called cloves, which, when separated, form each a new plant, affording a peculiar instance of the mode which nature has adopted in fulfilling her laws for the renewal of the species. The shallot, being a native of the warmer climate of Syria, seldom if ever produces seed in Britain, and hence would ultimately become extinct, were this or some other viviparous process not devised, by which they are enabled to continue themselves.

Planting.-The proper season for planting is autumn, either during October or November, as much of the success depends on early planting - a practice recommended by Marshall nearly a century ago, and revived by Henderson, who, in a communication to the Caledonian Horticultural Society (vide vol. i. p. 199, of their "Memoirs"), says in reference to securing this crop from the attacks of maggots, "autumn planting is the whole secret." Subsequent experience has so far proved the correctness of this recommendation.

In light, well-pulverised soils, the cloves or sets should be planted in lines, twelve inches asunder, and the cloves four inches apart. In preparing the ground, after being trenched or very deeply dug, it should be gathered up in ridges of the above distance, and four inches in height. On the top of these the roots should be set, and merely slightly covered. In strong clayey soils, also, after being deeply trenched or dug, draw drills fifteen inches asunder and three inches deep; fill them up to the surface with light sandy compost, upon which set the bulbs, and cover them 
with the same, in a ridge-like form, to the depth of two inches. In medium soils, this preparation is unnecessary, as the plant is extremely hardy (at least no frost affects it in its dormant state). A. row twenty feet in length will be sufficient supply for a small family: this at the distance given will require eighty cloves, and so in proportion for greater breadths. No bulb should be planted deep; they should rest on the surface of the ground. Their roots proper (for a bulb is not a root) will penetrate the soil in quest of food; and many of them, unlike most other plants, send their roots down in a perpendicular direction (the hyacinth, grown in water-glasses, affords a familiar example), while the bulb is exposed to the influence of sun, light, and air. In shallow soils, planting on ridges has the advantage of affording greater depth than that naturally presented. Knight paid great attention to the cultivation of this plant, and strongly recommended surface, and even elevation planting, assigning as a reason the exposure of the bulbs to the warming influence of the sun during their growing season. By merely drawing a little soil around the bulbs at planting, to keep them steady in their places, the rains of winter, and the necessary use of the hoe, to keep down weeds and preserve the surface open, removes the soil gathered round them, when the bulbs will appear seated on the surface, which is their natural and proper position.

Subsequent cultivation. - Their subsequent management consists in repeated hoeing between the rows, to keep down weeds and maintain the soil open.

Soil and manures.-The soil natural, and consequently best fitted, for shallots, is a rich, deep, sandy alluvial deposit, of itself sufficiently rich without the addition of manure, to which most bulbous plants appear to have a great repugnance. If manure is to be added, in the absence of such conditions, that of the most decomposed description should be employed. It is better, therefore, to plant shallots on ground which has been manured for the previous crop, such as early celery, and in which the manure has been completely incorporated by the process, first of digging up the celery, and afterwards by trenching it two feet or more in depth. If the ground is so poor as actually to require manure for this crop, then it should be buried in not less than ten or twelve inches under where the bases of the bulbs are placed. The roots will reach it, and their spongiolets collect it at the very time the bulbs most require fertilising aid. Pigeon dung is an excellent manure to be applied to the roots of shallots; and guano and other modern fertilisers may with advantage be applied even on the surface, but not until the plants have made considerable progress in their growth, when they may be laid on during, or, what is better, immediately before rain, so that they may be carried down in a soluble state to the roots. A better way still is to apply them in a liquid state, which will assist in washing the soil from the bulbs, and invigorate the plants at the same time.

Taking the crop, and subsequent preservation. - When the leaves begin to assume a yellowish colour, and droop towards the ground, it indicates that the season of growth has reached its termination. Upon a dry day pull the crop, and arrange it thinly on boards placed so as to be partially shaded from the full sun, for too rapid drying would be injurious to it. While there, protect from wet; and when sufficiently dry for housing, remove the loose and most decayed leaves only, and place them on the shelves of the onion-loft, which, at that period, will be unoccupied. Look over them frequently, and remove all decaying bulbs, and the greater part of the now dried-up foliage. Part may be tied up in small bundles of a dozen or two of bulbs each, and suspended from the under side of the shelves, or otherwise if more convenient. Those from which the leaves have entirely separated may be placed thinly on the shelves, or put into nets suspended from the roof. In October or November following, those bulbs which have been best ripened should be selected for planting, and the remaining stock kept moderately cool, well ventilated, and not crowded together. The usual season of their ripening is in July and August: this depends, however, greatly on situation.

List of approved sorts, and their qualities.There appear to be in cultivation two varieties of shallots-the common and the Russian. The former keeps best; while the latter is considerably larger, and milder in flavour. A fair-sized bulb of the former, when ripe, should measure 
about three inches and a half in diameter, and the latter about an inch more. The Allium ascalonicum, var. majus of botanists, is, we believe, identical with the Russian. A long-keeping variety-said to keep for two years, is spoken of. We have had this sort, at least one so called, but could never see any difference in it, either as to keeping, or in size or form, from the Russian, with which we believe it to be identical.

Insects and diseases. - The principal, if not the only, disease the shallot is liable to is the attack of a maggot, generally found under the bulb, which soon becomes covered with a mouldy appearance, and speedily afterwards rots away. This is probably not different from Anthomyia ceparum, Meig., the common onion-fly, which see. Planting on the surface, or indeed on raised ridges, as recommended by Knight, had for its chief object the prevention of this disease. Imbedding the bulbs at planting in finely-sifted charcoal has also been recommended. In our present state of information regarding the habits of this insect, there can be no doubt that collecting the plants upon their indicating the first symptoms of the disease, and burning them, will have the effect of greatly reducing the number of insects in succeeding years. Spirits of tar dug into the ground at planting, and salt, soot, nitrate of soda, watering with lime-water, as soon as the leaves begin to flag during the heat of the sun, which is indicative of the first attack, as well as urine and guano water copiously applied, have all in their turn been repeatedly tried; and wherever a marked benefit was discovered, it in all cases depended on the remedies being applied upon the very first appearance of the disease.-(Vide art. ONION.)

Botrytis destructor (Berk) makes its first appearance as a white mould or mildew upon various kinds of Alliums, and often destroys the whole crop, particularly shallots. On its first appearance, the infected leaves should be cut off and burned : all other attempts at destroying it in a more perfect state appear to be hopeless. General Remarks.--Shallots, till within these few years, were little cultivated in Scotland, excepting in some of the first-rate gardens. In the markets, they were unknown, the onion and the leek being the only alliaceous esculents in demand. They have only to a limited extent found their way into the gardens of cottagers and small families even in England, a circumstance to be regretted, as they are exceedingly wholesome, communicating an agreeable flavour to many of our commonest dishes; and the supply for a family can be grown on a very limited extent of ground compared with the onion or the leek. All over Europe they are much more extensively cultivated and esteemed, and in some countries rank higher than the onion, their near ally. In France they are called Echalotte or Ail sterile; in Germany, Schalotte or Aschlauch ; in Holland, Chalot or Sjalotte; in Italy, Scalogni or Cipolle malige ; in Spain, Escalonia or Chalote; in Denmark, Scalotlogen; in Sweden, Chalottenlok.

A curious instance of the transmutation of the shallot into the onion has recently occurred VOL. II. in the grounds of Messrs Hardy and Sons, Maldon, Essex. "The transmuted shallots, or rather onions, raised by us from shallot-seed, did not produce potato-onions, as it was presumed they might, but numerous heads of seed varying from ten to twenty from a single bulb. They certainly possess the qualities of both onions and shallots in flavour, the size of the onions, and the fact of their being divisable like shallots; the blade is very narrow, partaking of both species; the seed is small." - Messrs Hardy's Letter. Plants have been raised from this transmutation, and grown in the garden of the Horticultural Society of London, and the following are the results, as given in the Journal of that Society:- "The seed was sown on the 20 th March, and produced a mixed race, varying in size, form, and colour. Some were regularly formed by concentric layers like an onion. Sometimes the formation consists of two closely conjoined, compressed, but separate bulbs, and frequently clusters are produced very like shallots. The colour, in some, approaches that of the silver-skinned onion, in others the blood-red, but the generality are reddish brown. They are strong flavoured, and have the appearance of being good keepers." They, lowever, want uniformity in size and colour, but, by judicious selection, a useful, sound, keeping variety may probably be produced. The circumstance is curious, and strongly favours opinions at present attracting considerable attention, and which may ere long overturn the long-established doctrine of the permanent distinctions supposed to constitute what is called species in plants.

Shallots are sold by weight. One lb. contains about twenty ordinary-sized bulbs.

\section{$\S$ 2.-GARLIC.}

Natural history.--Garlic, Allium sativum (from Sativum, cultivated), L., belongs to the same natural order, and class and order in the Linnæan arrangement, as the shallot. It is a native of Sicily, the south of France, and most of the south of Europe, being found growing in meadows, pastures, and waste places. It has been cultivated in this country since prior to 1548. Old Thomas Tusser notices it as cultivated in the time of Queen Mary. His twelfth verse for the month of November says, "Set garlic and beans at St Edmund the King."

Uses.- The roots of garlic (the only parts used) held a place in most of our early pharmacopœias, but, like many other of our vegetable medicines, have been little used by modern practitioners. Sydenham recommended its use in the first stages of dropsy, as being a warm, strengthening medicine. He also recommended it as a powerful resolvent, "for which purpose," Phillips informs us (vol. ii. p. 24), "he was led to make use of it in the confluent smallpox. His method was to cut the root in pieces, and apply it, tied in a linen cloth, to the soles of the feet, about the eighth day of the disease, after the face began to swell, renewing it once a-day till the danger was over." It is held a sovereign remedy 
against intestinal worms by many a good housewife at the present day, cloves of it being steeped in whisky, and administered in the morning to the patient fasting - a remedy probably founded on the experiments made by Rosentein and Tissot, who say that garlic "is capable of expelling worms, especially the tenia." From the earliest ages it has been employed in medicine as well as for culinary purposes. It is much used in foreign cookery, especially in the south of Europe, confirming what Haller says of it, "that the inhabitants of all hot countries are fond of garlic." In many parts of the Continent the peasantry eat their brown bread with slices of garlic, which imparts a flavour agreeable to them; but in the midst of a garlic-eating people, they are well off whose sense of smell is most slightly developed. In Britain it is seldom employed as a culinary ingredient, and then seldom served up in a solid state, the cloves being put in a small bag, and left in for a short time during cooking, and then taken out when the necessary amount of flavour has been communicated to the dish.

The mode of propagation, season of planting, subsequent culture, soil, manures, diseases, insects, taking up the crop and subsequent preservation, are all identically the same as for shallots.

Approved sorts and their qualities.-There is one sort only cultivated in Britain as a culinary plant-the common garlic. Phillips, however, says, in "History of Culinary Vegetables," vol. ii. p. 26, "Besides the common garlic, Allium sativum, the African garlic, Agracile, is now cultivated by our gardeners. This," he continues, "has been erroneously termed Jamaica garlic, from the circumstance of 'the seeds having been sent from Jamaica to England." It is not a native of the West Indies, but was brought from Africa to Jamaica, and is the same described by Pliny (book 19, chap. 6), which he says grows larger than the other garlic. $\mathrm{He}$ (Pliny) tells us that this kind of garlic was never planted in level ground, but on little hillocks like mole-hills, and that, as soon as they had shown their leaves, the mould was taken away from them; for the oftener they were laid bare, the larger the heads would grow. We make this reference, first, to show that what was regarded as a new feature in shallot-culture brought out by Knight, and published in the "Horticultural Society's Transactions," vol. ii. p. 98, was the usual mode of cultivating its near kinsman, the garlic, in the days of Pliny; and, secondly, to say that of this Jamaica garlic of large size we know nothing. Where attention is paid to culture, the common garlic will attain a size $7 \frac{1}{2}$ inches in circumference each bulb; whereas, if grown, as it usually is, in the most negligent manner possible, it does not attain half that size. More attention is paid to its cultivation on the Continent than with us, in consequence of its being so much more in demand; and hence imported bulbs are much larger than those of home growth. In Italy it is known by the name Aglio; in Holland, Knoflock or Look; in France, Ail; in Spain, Ajo; in Germany, Knoblauch; Alho in Portuguese, Tschesnok in Russian. In Wales, most of the alliaceous esculents have been and are more generally grown for domestic use than in any other part of Britain. Worlidge, writing early in the seventeenth century, speaking of that principality, says, "I have seen the greater part of a garden there stored with leeks, and a part of the remainder with onions and garlic."

Twenty ordinary-sized bulbs weigh one lb.

\section{§ 3.-ROCAMBOLE.}

Natural history.-Rocambole, Allium Scorodoprasum (from $\sigma \kappa o p o \delta o \nu$, garlic, and $\pi \rho a ́ \sigma o \nu$, leek, as if it combined the garlic and leek), L., belongs to the same natural order, and class and order in the Linnæan arrangement, as the last. It is a native of Denmark and many other parts of the north of Europe, and appears to have been early introduced to Britain, as we find it mentioned by Gerard in his "Herbal" as a cultivated plant in 1596.

Uses.-It is used for nearly the same culinary purposes as the shallot and garlic, is milder in flavour than either of them, but is employed to a very limited extent even in families of the highest order.

Mode of propagation, \&c.- Its mode of propagation is the same as that of garlic and shallot; only, as it sometimes produces cauline bulbs-that is, small bulbs upon the stem-they are, in cases of a deficiency of underground bulbs, used as a substitute both for use and planting. The whole routine of culture, preservation, \&c., is the same as for the two last.

Varieties, \&c.-There is only one sort cultivated in Britain.

It is to be met with in Covent Garden market in small quantities. In the Continental markets it is rather more abundant, and is, as a consequence, more employed in cooking. The French call it Ail d'Espagna; the Italians, Scorodopraso; the Germans, Rocambollem; in Holland, Wile Knoftook ; in Spain, Especie de Ajo dulce.

\section{$\S$ 4.-THE ONION.}

Natural history.-The Onion, Allium Cepa (from Celtic, Cep, head), L., belongs to the same natural order, and class and order in the Linnæan arrangement as the last three. Its native country is as unknown as is the birthplace of Homer. Spain has been named; that is, however, highly improbable, as the plant has been known and used as an article of food from the remotest ages of antiquity. Of one thing we are certain: it constituted an important article of food long before the exodus of the Israelites out of Egypt; for among the complaints made to Moses in the wilderness was, that they were deprived of the leeks, onions, and garlic, of which, said the murmurers, "we remember we did eat 
in Egypt freely."-Numb. xi. 5. Egypt is to this day famed for the superiority and sweetness of its onions, in comparison with those of Europe-a result, by the way, with which climate may have much to do, as those of Portugal are much more palatable than the same variety grown in Britain; and there is an evident difference, in respect to the mildness of flavour, between those grown in Cornwall and in the Highlands of Scotland. The onion must be a native of the East; and as mankind and civilisation travelled westward, so they would bring with them this ancient and popular pot herb. The compiler of the last edition of the "Hortus Britannicus," whose means and industry in research were undoubtedly great, neither gives the date of its introduction, nor states its native country. It has, however, been an inmate of our British gardens as long as they deserve the appellation. Pliny, who mentions, in book xx. chap. 5 , all the countries from whence the Greeks as well as the Romans procured different varieties of this plant, says he could not discover that they ever grew wild.

Uses.-The uses to which the onion is applied in domestic economy are so many, and of so varied a character, that a reference to a cookery-book would be required to discover probably one-half of them. Our present purpose may be served by stating that, when fully grown, they are used stewed alone, and also boiled and roasted. In their young state, from the time they are as large as an ordinary needle, until they attain the height of five or six inches, they are used in mixed salads, and for this purpose they are sown once a-week throughout the season. When about the size of a writing-quill, they are devoured in astonishing quantities by the humbler classes, who eat them raw, with bread and butter, or with bread and cheese. Even when fully grown, the labourer finds a wholesome relish in them, when cut into transverse slices and eaten in the same manner. In many parts of the Continent the humbler classes of artisans bring with them in the morning a small canvass-bag, containing a huge slice of brown bread, a large apple, or a moderate-sized onion. This homely fare forms his sustenance throughout the day; and he devours it with as much gusto and satisfaction as the higher-fed English labourer swallows his slice of homebaked bread, with its usual accompaniment, a rasher of raw bacon; while the Caledonian of the same status munches his piece of oat cake (without kitchen), and washes down the dry morsel with a draught of Adam's wine. The Scot is not a vegetarian, and cannot be persuaded to eat green meat; and here, with all his shrewdness and economy, he is at fault. We hope our countrymen have not been led away by what the author of the "Gryte Herbal" saith hereon: - "The onion being eaten, yea, though it be boiled, causeth headache, hurteth the eyes, and maketh a man dim-sighted; dulleth the senses, engendereth windiness, and provoketh overmuch sleep, especially being eaten rawe. Being rawe, they nourish not at all, and but a little though they be boiled."

The witty and satirical Dean Swift says-
"This is every cook's opinion,

No savoury dish without an onion

But lest your lissing should be spoiled,

Your onions should be thoroughly boiled."

Our immortal bard did not overlook their tearbringing property. He says-

\section{“ If the boy have not a woman's gift To rain a shower of commanded tears, An onion will do well.'}

Mode of propagation.-The general mode of propagating the onion is by seed. There are a few of the species, however, which do not ripen their seeds freely, and these are propagated by other means. These will be noticed in their rotation.

The onion being with us an annual crop, the seed system of propagation is followed, and those varieties which are propagated otherwise are, in general, dependent on the bulbs which form on the top of the stem, as in the case of what is called the tree-bearing onion, from its producing viviparous bulbs at the top of the stalk, or in that of the potatoonion, which produces offsets under grourid, rendering them capable of being continued without the aid of seed. Sometimes, again, the very small and imperfectly-formed bulbs are chosen for replanting, and from these fine crops of large well-formed bulbs have been produced. There are other modes of propagating the onion, but the processes of them are so truly scientific as not to come within the sphere of profitable culture. Although usually treated as an annual when grown for its bulb, or as a biennial when cultivated for seed, it is, in fact, naturally a perennial, and continues itself by offsets as well as by seed. The seed usually germinates in about a fortnight in ordinary mild weather.

Planting and sowing.-The season for sowing varies so much from the difference of the natural seasons, the wide differences of soils, climates, and situations, that no precise date can be given applicable to all places and circumstances. In our early days the $2 \mathrm{~d}$ of February was looked upon as the advent of onion-sowing; of later years it has been the middle of April before the operation could be commenced-a delayoccasioned solely in consequence of the ground being either frozen or too much saturated by wet. This delay, however, has less influence on the time at which the crop comes to maturity than might be at first 
supposed. Early sowing has, nevertheless, its advantages, one of which, and an important one too, is, that germination takes place slowly, the roots take the advance of the leaves, and, by their doing so, they are stronger and more numerous; and, consequently, when the invigorating effects of the advancing sun begin to act upon the foliage, they are in a condition to provide and throw into the system of the young plant a much greater quantity of nourishment than they could do if vegetation commenced in both simultaneously, which would be the case if not sown before the middle of April, when the air becomes warmed by solar influence. Another advantage has been stated as arising from early sowing, which is the greater strength communicated to the plant, and hence its greater ability to resist the attacks of insects. This view seems strengthened in the case of autumnal-sown onions, which seldom are attacked by the grub, because they are not only stronger, but have attained a greater degree of pungency before the season at which the insects are hatched. Late-sown onions have of late years often been cut off by spring frosts just as they were coming through the ground, or when they are about a couple of inches in height. The more rapid process of vegetation induced by those warm sunny days we now so often experience during the month of April, makes the young plants rush up rapidly, and in this tender state they fall a ready sacrifice to the sharp frosts which so often in the mornings succeed those warm sunny days. Where vegetation proceeds more slowly, and where it influences the roots sooner than the tops, the plants not only become better inured to the cold, but, should it even prove severe, the strength thrown into the plants by the roots enables them much better to resist its effects.

No positive date can be given as to the day or week, nay, even month, that the operation should be performed, suitable to all soils, situations, and circumstances; the condition of the soil should, however, never be disregarded. In this, as in all other cases of seed-sowing, the soil can never be too dry, nor too much pulverised; and this is more especially the case in all cold, damp, and strong clayey soils. In the neighbourhood of towns, where the soil is highly enriched, and contains a large amount of humus, and where, as in the case of commercial gardens, it is thoroughly wrought by repeated trenching and digging, the beginning of February may be taken as a good time for sowing, provided both the weather and soil are in a fit state. In all cold elevated situations, and where the soil is wet and in an imperfect state of culture, then the middle of March will be a more proper season, and in the worst situations of all, the beginning of April.

Autumnal sowing has not only the advantages stated above, but also that of affording during winter a supply of young onions for salad purposes, as well as for being used in French cookery, particularly in the artistic preparation of that not-to-be-despised viand, a properly cooked rump-steak, which is amazingly improved by being served up covered with young onions, cut up into the smallest possible fragments. Onions are also sown in autumn, say about the middle of August; and where they withstand the winter, they are transplanted in March or the beginning of April, according to the circumstances of soil, \&c., stated above, in lines a foot asunder, setting the plants six inches apart in the row. These, having the start of the spring-sown crop, bulb earlier, furnishing a supply after those from the onion-room have been consumed, and carrying on the supply till the spring-sown crop arrives at maturity. The best formed and best ripened bulbs of the autumn-sown crop are selected, and reserved for winter use, and fill the shelves of the onion-room long before the general crop is fit for storing. Many adopt the autumn sowing and spring transplanting, believing the onions so produced keep better than the others. This may be accounted for by their being more thoroughly ripened, during July and August, than the general crop, which ripens at a much later and more unfavourable season. We ought, however, to observe, that all autumn-sown crops of this plant, intended to arrive at maturity, should be transplanted.

The best mode of sowing the onion is in drills from nine to twelve, and even fifteen inches apart, according to the soil, and the size the crop is likely to arrive at. In poor soils, where the 
bulbs usually do not much exceed the size of a large walnut, the former distance will be sufficient; when they are to attain their fullest size, which they will do in rich, well-wrought ground, and in a medium climate, then the latter distance will not be too much, because the foliage requires room for development, and a full exposure on all sides to the influences of light and air, without which they could not elaborate the necessary amount of sap required for the perfect enlargement of the bulbs. This is clearly demonstrated in the case of onions sown thickly in a bed, and allowed to remain so, compared with the same sort grown in the drill manner, and allowed sufficient room. The latter will bulb, the former will not at all; but at the termination of their growing season, they will resemble more the character of the leek than the onion. In this state, they justly come under the denomination of sybo or scallion, or imperfect onion-that is, having leaves and stalk, but no bulb. In forming the drills, after the ground has been broken by being raked over deeply, and reduced to a pretty fine state of pulverisation, the line is stretched from end to end of the ground, and the drill opened with the corner of a sharp clean draw-hoe, the face of which is directed towards the line, the operator standing on the side still to be drilled, by which means the drills are left entire and open. Various contrivances have been employed to economise time in this operation. The drill-rake (fig. 1) is one of the simplest and most efficient, and is constructed of a headpiece, like that of a common rake, only double the size, into which broad flat wooden

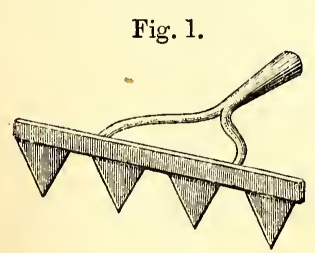

DRILL-RAKE. teeth are set, tapering towards the points, and at such distance apart as the drills are to be drawn. Sometimes the head is in two flat pieces, to admit of the teeth being set at different distances, to adapt it to different crops, according to the distance the rows are to be apart, these pieces being screwed together at each end; or if more than three drills are to be drawn at once, a third screw is placed in the middle. Others use a head perforated with small holes, one inch apart, into which the tops of the teeth are set, and fixed at any required distance. We use, attached to Sievewright's cultivator, which runs on a small wheel behind and another in front, a transverse iron bar perforated with holes, cut with femalescrews within, to receive the tops of the drill-forming teeth, which are cut with male-screws, so as to work in the others. By this means we can draw at once six or eight drills, and at any distance required: one man draws the machine, and another guides it behind. In either case it is necessary to set off the first drill with a line, afterwards the drill-tooth on the right-hand side should be made to travel over the last drill formed. This keeps the drills straight and equidistant. Such appliances work, however, best in light dry ground, and in such as are in a highly cultivated state, and free from stones.

Onion-seed is in general sown too deep ; $1 \frac{1}{2}$ inches is amply sufficient, and even one inch has been found by some growers to be better. Abercrombie, were it not that he recommends broadcast sowing (that is, promiscuously over the whole surface), is correct as to depth. He says, "sow broadcast equally over the rough surface, moderately thick, and rake in the seed in a regular manner." Some good cultivators, after the ground has been properly dug or trenched, lay very rotten manure over the surface, and on this they sow the seed, covering it slightly with earth from the alleys. This would be a very excellent plan, could the seed be disposed of in lines, and kept in them during the process of covering. All broadcast sowing, in the case of such crops, is far behind the present state of high cultivation : it is, however, not ill adapted to the limited scale of a manse or cotter's garden. The principle of deeply stirring the soil, and placing on its surface the necessary manurial application equally over it, is perfectly correct. It is thus in the most favourable position for its fertilising properties reaching the points of the roots, as it will be washed down to them by the rains; and they are the parts to which enrichment, to be useful, should be applied. The London market-gardeners sow their principal crop in February or the begin- 
ning of March, on beds five feet wide, and in the broadcast manner, covering the seed with soil from the alleys. They rake the ground, and draw a wooden roller over it.

The mode of planting depends on circumstances-namely, whether young plants of the current year, those of last autumn's sowing, or the smallest bulbs selected from the preceding crop, are to be operated on. In the case of the former, the seed should be sown about the beginning of February, in light, rich, sandy soil, placed over a hotbed of moderate temperature-say of $45^{\circ}$; or they may be sown in what is called a cold pit or frame-that is, a structure having no artificial bottom-heat, but depending entirely on solar heat transmitted through a glass covering. Towards the end of April, if the weather has set in favourable, the young plants should be carefully taken up, without injury being done to either their tender tops or equally tender fibres, and transferred to the open quarter of the garden-the ground being previously prepared by trenching or deep digging, and in a high state of enrichment from the manure applied to the preceding crop-say celery. The process of planting in most cases-in this one in particular-is most advantageously carried out by proceeding in the following manner; and as it is in the main applicable to the planting of all other crops, it deserves, for the benefit of amateurs, a clear explanation :-The ground being, it is presumed, already trenched and manured, commence at one side of the quarter or piece of ground to be planted; break down the surface with a wooden rake to a moderate evenness of surface, and freedom from clods and stones. Stretch the garden line from end to end perpendicular to the cross walks, or parallel with the adjoining crops or walk, so that the rows, while growing, shall appear as if laid down corresponding with other straight lines, as if they had been drawn by a parallel ruler; for nothing looks worse, when viewing the crops in a garden from the principal walks, than to see the lines of crops in the quarter or division nearest to the point of observation running five points to the east or south, while those in the piece adjoining are running as many points in an opposite direction. This being done, beat the sur- face of the ground, for about the breadth of nine inches, gently down with the back of a spade, so that the whole shall be smooth and level. Commence at the end of the line, or at different points, according to the number of men employed. The young plants are laid in shallow boxes, covered over with moss, or any other light covering sufficient to exclude the air while they are yet unplanted; each man having a box, and no more plants brought out than can be planted within an hour or so. They are then to be set in holes made by a small blunt dibber, about as thick as the little finger, and not above an eighth of an inch deeper than where they have been growing, and at the distance of 5 inches apart in the row. If, however, there is an apprehension of the maggot, or of other injuries befalling them, they may be set at double thickness, as it will be better to thin out in May every alternate plant, than to have to make up deficiencies. When the first row is planted, the line should be removed; and in doing this some caution is necessary, lest, in its removal, any of the plants be withdrawn from their places. If the line is gently drawn from the plants, rather than drawn upwards, little danger of this need be apprehended. A better way, however, is, when the process of beating the ground is finished, the mark of the line being still quite visible, to lift it at once, and set the plants along the marked line. There is an unfortunate pertinacity amongst a certain class of even young men to do nothing without a line. The man who cannot or will not plant a row of young onions as straight as an arrow by the mark of the line thus made, should be furnished with a pair of eye-protectors, and recommended to the nearest road-surveyor, to try his hand at macadamising. When the first row is finished, proceed in like manner with the next, and so on, watering, if the weather is dry, every three rows, as the work goes on. By this means the soil will remain untrodden, as it will be pointed over-that is, dug shallow as the operation proceeds. The only care to be taken during the operation is, that the plants be not set too deep (vide supra). The process of transplanting autumn-sown onions is precisely the same as above, except in so far as they, at the period of 
transplanting, are much larger-say from the size of a crow-quill to that of an ordinary writing one: the roots are proportionably larger also. The ground being prepared as above, and the line stretched out, a shallow notch in the back partthat is, that next the line-should be cut somewhat oblique, and no deeper than the exact length of the roots. The young plants should be carefuily taken up, with their fibres quite entire (on this much of the success depends) ; and to facilitate the operations, the ground in which they are growing should be loosened deeply with a three-pronged fork. They should also be placed in shallow boxes (trays), and protected from the sun and air while out of the ground. The whole thus far being in readiness, the planter arranges the plants in a slightly slanting direction against the oblique bank, taking care that no part of the plant is covered with soil save the roots ; and while the arrangement of the plants is going on, the roots are covered as they are placed by pushing a little of the finest soil against them with the hand. The plants should be allowed the same distance as those above. When they are all arranged in the first line, some more soil is placed over the roots by the spade, the line removed, and a fresh row marked out. We often, in our own practice, and operating on a very light soil, prepare the ground as we have described above; but instead of cutting out a slight notch, we lay the plants flat down on the smoothbeaten surface, and place over the roots a little well-pulverised soil. This is a good plan at an advanced period of spring, for should a warm shower or two fall soon after planting, the plants will rise themselves to a perpendicular position, having no part under the surface except the roots. And this is desirable, for if they were deep planted, their bulbing would be prevented, at least to a great extent.

The third mode of raising onions referred to above, is by small bulbs selected from the previous crop, and which may be about the size of a hazel-nut. Some sow expressly to secure such (vide infra). This practice is not noticed as a novelty, for it has been more or less adopted from time immemorial. Its true object is to obtain larger onions than the coldness of our climate enables us to do with springsown ones, and it, therefore, may be worth the attention of those who live in high and cold situations, even should they purchase the small ones to plant, which may readily be obtained from any respectable nursery or seedsman. The earliest written mention deserving much credit we have met with of this practice is recorded in "Systema Horticulturæ," by Worlidge, who practised towards the beginning of the seventeenth century. The late $\mathrm{Mr}$ Knight revived the practice, and his papers in "The Horticultural Transactions" led others to speculate in the same way. A quotation from that high authority will be quite sufficient for our present purpose. After some preliminary remarks on bulbous and other perennial plants having the property of accumulating in one season the material that composes the leaves and roots of the succeeding season, he proceeds : "This reserved sap is deposited in, and composes in a great measure the bulb; and the quantity accumulated, as well as the period required for its accumulation, varies greatly in the same species of plant, under more or less favourable circumstances. 'Thus the onion in the south of Europe acquires a much larger size during the long and warm summers of Spain and Portugal, in a single season, than in the colder climate of England; but, under the following mode of culture, which I have long practised, two summers in England produces nearly the effect of one in Spain and Portugal, and the onion assumes nearly the form and size of those thence imported. Seeds of the Spanish or Portugal onion are sown at the usual period in the spring, very thickly, and in poor soil, generally under the shade of a fruit tree; and in such situations the bulbs in the autumn are rarely found much to exceed the size of a large pea. These are then taken from the ground and preserved till the succeeding spring, when they are planted at equal distances from each other, and they afford plants which differ from those raised immediately from seed only in possessing much greater strength and vigour, owing to the quantity of previously generated sap being much greater in the bulb than in the seed. The bulbs thus raised often exceed considerably five inches in diameter; and, being more mature, they are with more certainty preserved in a state of perfect 
soundness through the winter than those raised from seed in a single season." Knight's mode of planting these was the same as our own-namely, to set them at ten inches from each other, or when in lines, twelve inches apart and eight inches in the line, the ground being prepared as above recommended for spring and autumn sown crops-setting the bulbs, however, on the surface, and drawing as much soil over them as will maintain them in their places until they attach themselves by their roots to the ground; after which, as in the case of shallots, \&c., already noticed, the earth is removed from them, and the bulbs are exposed to air and light. This practice, or that of saving all the very smallest from the previous crop, has been followed by some of the market-gardeners round London for ages. It is rarely practised in private gardens-why, we know not.

The fine large Portugal onions which we see in the shops are imported from that country annually. They are indispensable in the kitchens of the great, being much milder, as well as being of a much larger size, than can be produced in any quantity in this country. They are grown upon a somewhat similar principle to that noticed above; the method is sufficiently interesting to warrant our making the following quotation fiom "The Transactions of the Horticultural Society," vol. iii. p. 68: "Sow the seed very thinly in November or December on a moderate hotbed in a warm situation, with a few inches of rich light loarn upon it, and the plants protected from frost by mats and hoops. In April or May, when they are about the size of a large swan's quill, they are transplanted on a rich light loam well manured with well-rotten dung. The mode of transplanting is particular. The plants are laid flat, about nine inches asunder each way, in quincunx, the beard (fibres) of the root and part only of the plant covered with very rich mould, well mixed with twothirds of good old rotten dung. This compost is slightly pressed down on the plant; water is given, when the weather is dry, until the plants have taken root. Subsequently, the earth is occasionally broken around them by slight hoeing, in which operation care is taken not to wound the bulb. Weeding is diligently attended to, and the watering continued according to the state of the weather. In Portugal the means of irrigation are easy, the effects of which are particularly beneficial to the onion; for, by letting the water filter or pass through small heaps of dung placed in the alleys of the beds, a very rich liquid flows in upon the plants. The dung, as it is exhausted or washed away, should be renewed, and the water must be checked in its current, so that it may gently spread over the surface." This mode has been successfully tried in some parts of England upon very warm borders. In our present state of advancement we would apply liquid manure in a much more convenient manner, and there is no means of enrichment so beneficial to the alliaceous tribe as administering it in a liquid state.

We have already observed that the drill system is preferable to the broadcast order of sowing. This, however, applies more to private gardens than to commercial ones, or even to those whose onion-bed does not exceed two or three square yards. Although, in many of our first-rate market-gardens, the drill system is pursued, still by far the most adhere to the old plan of dividing the ground into three-feet-anda-half or four-feet beds, and sow promiscuously over them, and trust to handweeding for the suppression of weeds. In this they do not err, because, from the time the young plants are three or four inches in height (up to which time they do not injure each other), a constant system of thinning is going on-the thinnings meeting with a ready sale in the market; so that the crop is in due time sufficiently thinned to enable those left to attain their full size;-sowing in drills is therefore to them of less importance. We hold it essential in all well-kept private gardens. Treading the ground after the seed is sown is advisable in very light soils ; if dry at the time, it tends to consolidate it to a proper consistency. To do so, however, in strong soils, particularly when wet, is injurious. It cakes the surface, and render's it more difficult for the young plant to push its way through. To those who prefer the broadcast mode of sowing in beds, a better plan cannot be recommended them than that practised by Mr Smith of Pitfour. The ground being dug, the manure is laid 
on the surface in a very decomposed state. When levelled, the seeds are sown, and pressed down by the back of a rake; a little fine soil is thrown over from the alleys. When the plants are about an inch above ground, they are dressed every three or four weeks with a mixture of guano and charcoal-dust, to the extent of a handful to each square yard, one-third being guano, choosing moist days for applying it.

Before quitting the subject of sowing and planting onions, we may remark that, however sanctioned by the practice of ages, the custom of sowing other crops along with the onion, considered as a general one, is erroneous. The ancients believed that some plants had a sympathy with, or antipathy to each other. They also set great importance on sowing, planting, gathering the crops, \&c., at certain stages of the moon. Both these doctrines we have been taught from childhood to regard as absurdities ; but, strange to say, something about the latter has been very recently revived, and the greatest horticultural oracle of the age has thrown out a hint that we should henceforth desist from covering our cucumber beds at night, that the plants may receive the full influence of Luna's silvery beams. As regards the former also, while we must continue to consider the explanation utterly unfounded, the chemistry of plants has shown us that, in some cases at least, the practice might be less absurd than at first sight appears; for it is conceivable that two species of plants might draw essentially different nourishment from the soil, and that each might be withdrawing from it something, the excess of which was hurtful to the other-each thus indirectly aiding the healthy growth and full development of the other.

Returning, however, to the sowing of onions in conjunction with other plantsa little lettuce-seed may be thinly scattered over the ground after the onions are sown, as the plants will come off soon, and many of them may be transplanted; but the old practice of sowing leeks, parsley, or carrots along with onions, is doing justice to neither. It is, however, worth remarking that, in soils where carrots are destroyed by grub, they often, when sown along with onions or leeks, escape their attacks.

VOL. II.
The London market-gardeners sow in beds five feet wide, and in the broadcast manner. They cover with soil from the alleys, by throwing it over the seed. The beds are raked over with a wooden rake, and a light wooden roller is drawn over the surface, being more expeditious and proper than foot-treading, for which they have no time. Such ground as theirs is much benefited by rolling, as it is so soft, spongy, and full of humus, from the enormous quantity of manure which is at almost every change of crop trenched into it.

The number of roots required to plant any given space will be readily ascertained by referring to the distances given above.

The quantity of seed required to sow in the broadcast manner a bed 4 feet by 24 , when all or part of the crop is to be drawn young, is two ounces; but for the same space, if the crop is intended to come to maturity, half that quantity will be sufficient. According to the drill system of sowing, a third less in both cases will be ample. There is a great mistake in thick-sowing: the plants become weak and drawn up at first starting; and thinning, either in time or to a sufficient extent, is too seldom attended to. This should have been placed as one of the first items in the very sensible, but satirical, directions given by the author of a series of papers in "The Gardeners' Chronicle," entitled, "How to mismanage a garden." In stating, however, the quantities of seed necessary for sowing a given space, we may state, once for all, that our quantities are given on the presumption that the seed is good. For means of ascertaining this, vide article Propagation BY SeEd.

Subsequent cultivation.-The subsequent culture of this crop consists in keeping the ground between the drills perfectly clear of weeds by repeated hoeing-not waiting, however, as some do, till the weeds become the principal crop, requiring no small skill, and a vast amount of labour, to remove them, leaving the young tender onions exposed to the full rays of the sun, or perhaps to a frosty May morning, either of which they are in a very unfit state to withstand, having been hitherto snugly sheltered under the protecting shade of groundsel and chick- 
weed. The purpose of hoeing is twofold, in all cases save that of a gravel walk, whereon such an operation should, if possible, never be allowed. Hoeing keeps down weeds while in their cotyledon state, that being the most proper time to attack them; it keeps the surface of the ground open, and renders it pervious to the rays of heat and to air. Weeding only removes the evil after it has too long existed; in the process the ground is trodden to a hard surface, and unless immediately loosened by the hoe, it is rendered next to impervious to heat and air. The hoe, therefore, should be used at once, and one operation thus be made to do, instead of two. The best hoe, when deep-stirring the soil between drilled crops is performed, is the Spanish hoe, fig. 2, or the Vernon hoe, fig. 3; the former

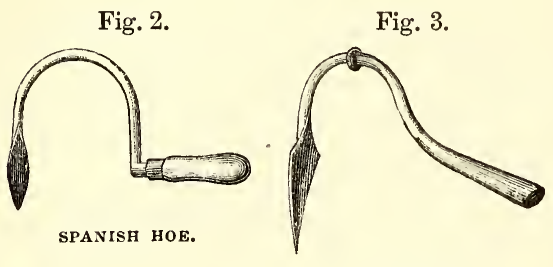

VERNON HOE.

an implement much employed in the West Indies for deep-stirring the sugar crops. The flat or common hoe is only useful for cutting down weeds; and as it is in general used, it does do little more ; whereas the deep-stirring of the soil is of the greatest importance, and can only be efficiently performed by such an implement as the hoe referred to. Here we see the great advantage of the drill system over the broadcast method of sowing. Thinning the crop should be attended to as a principle, and, unless in petty gardens, dependence should not be placed on the removal of superfluous plants for daily consumption; it is better to thin the general crop, and leave a row or two for daily use. The London market-gardeners thin their broadcast sown crops as soon as they appear above ground, by using small 2-inch hoes, kept exceedingly sharp and clean.

Soil and manures.-Onions require a deep, rich, mellow soil, always kept in a high state of enrichment by the application of such stimulants as guano, blood, salt, soot, the offal of a slaughterhouse, superphosphate of lime, at the time of sowing. But where the ground has been highly manured for the previous crop, such as celery, it is better to depend on that, with the addition of liquid manure where it can be applied, than on the recent application of stable-yard manure, unless in a state of great decomposition. The London growers, however, seem to put all danger from insects at defiance, and manure heavily with the strongest dungs, such as cow manure, nightsoil, \&c. They are seldom troubled with onion grubs, or, indeed, with any other; and this, they assert, is entirely owing to their ground being turned so frequently and so deeply over-thus, probably, preventing the insects from undergoing the various transformations which most of them have to pass through. Neither are they very particular as to the change of soil, or what is called the rotation of crops, for we have seen abundant crops of onions on ground from which three and four successive ones of that vegetable have been taken. To ordinary cultivators this may appear ano-malous, as it is also contrary to the principles of vegetable physiology: it does, nevertheless, occur in their practice, and they ascribe it simply to their ground being in the highest state of fertility, and in the highest state of cultivation; and they maintain that private gardens are neither the one nor the other.

Forcing.-The onion is forced during winter in many large gardens, being used in a very young state as an ingredient in salads. The seed is sown thickly in shallow boxes in light sandy soil: rich soil is not necessary, as the crop is gathered when the plants are little larger than an ordinary-sized needle. A very small quantity only is daily required, to give some idea of which we may mention that, when gathered and washed (for all salads should be prepared before leaving the garden-but of this more anon), with the leaves and fibres entire, the supply for the day will be a small bundle about the size of a man's finger. Boxes, therefore, or square earthenware pans (the latter of which we use), about 18 inches square and 4 inches deep, if sown every third or fourth day, and placed in a pit, or on a suspended shelf in a vinery where the temperature ranges from $45^{\circ}$ to $60^{\circ}$, will be a very fitting 
place for them. There are several modes of forcing onions, so as to have bulbs for use between the time those wintered in the store-room are finished, and that at which they ripen in the open air ; but the trouble and expense attending these methods might, we think, easily be spared by adopting the best modes of keeping the previous crop, and cultivating more largely that excellent sort known as the potato or underground onion (which see).

Taking the crop, and subsequent preservation.-The season of ripening, like that of all other crops, depends greatly on circumstances. This is first indicated by the leaves turning yellow and beginning to fade; and in the smaller bulbs, by the leaves dropping off altogether, which shows them to be perfectly ripe. The larger specimens, on account of their containing a much greater amount of elaborated sap thrown into them by their larger and more numerous leaves, do not indicate ripeness so soon, and a certain class, from over excitement, and grossness of stem, called thick necks, do not incline to ripen at all. These latter should be marked for immediate use, and left on the ground till required; or, if the ground is wanted for another crop, they may be taken up and laid in by the heels in some out-of-the-way place, till used up. The process of laying by the heels is thus performed : a trench is opened, and the plants, as they are taken up, are set in it pretty thickly, to economise space, but not so thickly as to cause them to rot or sustain injury from want of air. They are placed in a slanting direction against the back of the trench, and covered over as deep as they formerly were in the ground, the earth being laid over their roots and stems in the course of digging the ground to prepare it for another trench, which should be so far apart from the last that the leaves of the plants may not lie over those of the preceding row. The principal object, however, is to check vegetation and prevent the plants running to seed, hence the coldest situations should be chosen. The others should be gone over, and their stems bent gently over, or the strongest of them may have their necks twisted; this will check growth, and tend to their ripening. The sooner they are taken out of the ground after their growth has nearly ceased, the better; for, if left in, they are often attacked by maggots. As they are taken up, they should be placed in a dry airy place, but not in the full sun, to dry; and it will greatly facilitate that process if, instead of being left lying on the ground, they are spread thinly on boarding laid upon a gravel or paved walk or yard. As they dry, the roughest of the decaying leaves should be removed; and they should then be carried to the rootroom or onion-loft, and spread thinly upon the shelves, floor, \&c., to dry more completely. From this time until there is danger of frost entering the onion-room, the doors and windows should be kept constantly open, to afford them all the ventilation possible. The remainder may be removed into the house as fast as they dry sufficiently, and all should be at once taken up in the event of frost. After housing, they should be repeatedly examined, all decaying bulbs removed, and the whole frequently turned over.

The future management of the bulbs depends on the exclusion of frost; yet a low temperature must be preserved and ample ventilation given. When time permits, and wet days offer a good opportunity, a general dressing should take place. The smaller bulbs ought to be selected, and kept by themselves, for pickling, and many other uses which no being on earth could think of but a French or Italian cook. The next in size, which will also be the next best maturely ripened, should also be picked out: these are likely to keep longest, and should therefore be kept by themselves on the higher shelves, so as to be better out of the way. Those having a portion of the dried leaves still attached to them had better be strung up, and suspended from the roof part of the room. The process of stringing is thus performed: Three or four onions, having a portion of their tails still attached to them, are taken in one hand, while with the other a strand of matting or twine is to be tied round them. After placing three or four more bulbs, the twine or matting is wound round them pretty tightly_and so on, till the rope or bundle has attained a yard or so in length, when it is suspended by one end to the roof or other convenient part of the root-room. This is a good way of wintering onions, and takes up little space. The imported Portugal onions are secured in nearly the 
same manner, only a heated iron is applied to the base of the bulb where the fragments of the roots still exist; burning them off, and very slightly searing the base, tends to destroy vegetable life, and hence prevents the bulbs from sprouting during winter. And as we take some delight in showing that all things are not new under the sun, we may here just by the way mention that Pliny, to secure a similar end, adopted a somewhat similar means, as he recommends those who wish to keep their onions from sprouting to dip their heads in warm salt water.

Approved sorts, and their qualities. - The varieties of cultivated onions have long been, and still are numerous. Of those that are annually produced from seed, the following may be considered the best :-

1. Brown globe.-Large, globular, palish brown, slightly tinged with red; a very useful and hardy kind, of mild flavour, and keeps well.

2. New white globe.- Rather flatter in form than the last; of mild flavour; altogether a very handsome bulb; comparatively a new variety.

3. Blood red.-Known also as the Dutch and St Thomas's onion, of which there are also two sub-varieties,--viz., pale and very deep blood: a very old variety, esteemed by some for its extreme pungency, and also for its diuretic quality. Of middle size, flattish, and very hardy; deep red, or blood-coloured, keeps remarkably well; much grown amongst the Scottish and Welsh peasantry. Not greatly appreciated in genteel families. Perhaps one of the oldest cultivated sorts, as we find them noticed for their strong flavour so early as the days of Pliny.

4. Reading.-Supposed to be synonymous with (or so closely allied as to be scarce worth growing as distinct varieties) the Cambridge, white Spanish, Eversham, and white Portugal. It is much cultivated about Eversham, and also about Reading, and hence the origin of two of the names. An excellent sort for a general crop, but does not keep well; mild, large, flat, of a whitish colour tinged with green.

Strasburg.-Known also as the Dutch, Essex, Deptford, and Flanders onion. This is the most generally cultivated variety in Britain. A large oval bulb, reddish, tinged with green; extremely hardy, and hence generally sown for autumnal crops, and should be preferred in all cold and elevated localities : flavour rather pungent, no disqualification to palates vulgar, and has also the merit of keeping well.

6. Tripoli.-In seed-lists we find a round and a flat variety, both of which may be picked out of the same seed-bed; reddish, tinged with green and brown, soft and mild, does not keep well, but an excellent autumn sort. The largest onion grown.

7. Silver-skinned.-Of which there are the following sub-varieties, scarcely distinguishable except in name-viz., early silver-skinned, small silver-skinned, Nocera; flat, middle-sized, and shining when grown in rich ground. It is chiefly cultivated for pickling and dressed dishes, for which purposes it should not much exceed in size a large hazel-nut. It is usually sown in poor soil to prevent it becoming large.

8. Madeira large.-Known also as new giant: a new variety, the seed of which is annually imported from Madeira; a bulb of extraordinary size, but does not keep well.

9. True Portugal. - Large, flattish, globeshaped, exceedingly mild in flavour, but does not keep well, particularly if grown in Britain, as we have not climate to ripen it thoroughly. Genuine seed should be imported from Portugal. We have some doubts, however, as to this being different from the brown Portugal of the seed-shops.

10. James's keeping.-Evidently an English hybrid, and said to have originated with a $\mathrm{Mr}$ James, a commercial grower of Lambeth Marsh : of large pyramidal shape, brownish coloured, strong in flavour, and one of our best keepers, probably on account of its English origin, and consequent increased hardiness.

11. Lisbon, known also as Lisbon white.Genuine seed of this excellent variety is in general imported from the south of France. It is by no means hardy with us, and is slow in ripening; not to be recommended for a cold climate; of large size and globular form, skin whitish and very thin. Opinions differ as to the hardiness of this variety; it is described as hardy, and adapted for autumnal sowing by several good authorities: we have found it one of the tenderest.

12. Two-bladed, known also as the Welsh onion, from being much grown in that part of the kingdom.-This has a small, flat, brownish-green bulb, which ripens early, and keeps well. It is grown chiefly for its leaves, which are used in salads, and its small bulbs for pickling. The two-bladed early of some seed-catalogues is the same as this, which, by the way, ranks as a distinct species (Allium fistulosum, L.), and is a native of Siberia. Introduced in 1629. Some nursery catalogues enumerate a greater number of names, but their difference consists in the name only.

13. Tree, or bulb-producing onion.-This is a viviparous variety of the common onion; a native of Canada, the climate of which being too cold to enable the plant to produce seeds, nature employs one of those beautiful provisions she has in store for the fulfilment of the sacred command, " multiply and replenish," by the production of viviparous bulbs at the top of the stalk, which otherwise would have been graced with its head of flowers and umbel of seeds : they are sometimes produced on the sides of the flower-stem also. This occurrence is not uncommon in sub-alpine situations, where the temperature is too low for the ripening of seeds, and is well illustrated in many of the pasturegrasses; the Festuca vivipara offering one example, and one of the sub-varieties of the common rye-grass (Lolium perenne, var. vivipara) another. Why botanists have elevated the former into a species, and the latter not, appears 
anomalous. These cauline bulbs are planted in spring, and produce ground-onions of a very useful size; and, although smaller than the ordinary sorts, they make up for size in greater pungency of quality.

14. Potato, or underground onion.-For a somewhat similar purpose, this sort, which seldom ripens its seeds, increases itself by the formation of bulbs under the ground, as if determined to be the very antipodes of the last. This variety has been in cultivation just about half a century in the southern parts of England, where, on account of the crop ripening much sooner than that of the seed-produced sorts, a ready market has long been found for them in supplying ships leaving our ports in June and July, at which season they could not procure others fit for keeping. It has been stated to have been brought to this country from Egypt by our troops, on their return, after driving the French out of that country, about the beginning of the present century; and of this we entertain not a shadow of doubt, the land of Egypt being the land of onions since the days of the Pharaohs at least. This variety is cultivated to a very great extent in that country, and its singular mode of growth, apart from its excellent qualities, would no donbt attract the attention of our soldiers, who could hardly be supposed to be aware of the fact that it had been grown to a limited extent a few years only prior to the time to which we allude, in a London nursery. This onion is well adapted for cottage gardens, even in climates where the ordinary sorts do not ripen. It is cultivated to very great perfection in the parish of Currie, west of Edinburgh, where we have seen them as large and fine as in Devonshire. The mode of cultivation differs not from that of the shallot and garlic already disposed of, except that the individual bulbs do not admit of division; but in taking up the crop, numerous small bulbs will be found clustered among the larger ones, which are all attached to the main root after the manner of potatoes, and hence its trivial name of potatoonion. The plants growing stronger than either shallots or garlic, require more space both between the rows and also between each other in the line. Fifteen inches by ten may be given as an average distance. In one particular more they essentially differ; their bulbs are formed under ground: therefore, although the planted bulbs are best set on the surface, the earth should be gathered up around them in the way recommended (but with doubtful advantage) in the case of the potato. Their subsequent culture consists in keeping them clear of weeds, and a liberal supply of liquid manure during warm weather only. Planting should be attended to in November, although, in cold places, March may be with some advantage chosen. By the end of June, or beginning of July, they are fit, in most places, for taking up. Their curing and keeping are the same as we have already described for the others of the tribe. Much of the success, however, depends on the state of the ground. This is a root which we hold to be of importance in every garden, though it is too seldom found. In Devonshire, where they are much grown, there is a familiar saying, "Plant on the shortest day, and take up on the longest." This onion, like all the tribe of cultivated alliaceous plants, requires a rich deep soil, well manured, and dry at bottom. For this purpose the ground should be deeply trenched, and the manure (pigeons' dung, or the offal of a slaughter-house, if they can be obtained), wrought in during the process ; for, although it is perhaps-not generally known, the roots of this variety have been found extending to the depth of six feet under the surface. When the soil is naturally shallow, it should be gathered up in ridges, and upon these the sets should be planted. For cold situations, this has another advantage, as the solar rays will penetrate such ridges, and impart a greater degree of warmth to the soil around the roots than if they were planted on a flat surface. In establishments where fully ripened onions are required throughout the year, these may be brought forward at even a sufficiently early period to succeed the ordinary kinds that have been preserved during the winter. To effect this, the bulbs should be planted in pots in October or November, and kept in a cool pit through the winter, and turned carefully out into a well-prepared border at the bottom of a south wall (or equally warm place) early in March. The autumn-planted crop will require to be looked at during winter, and any bulbs that may have been thrown out, or displaced by the frost, reset; and in the coldest of all localities, a little mulching or rotten dung or leaves may, with advantage, be placed on the surface between the rows. This will exclude frost and the drought of spring at the same time.

The Welsh onion, or Ciboule, is seldom grown. It is an extremely hardy perennial of strong flavour, but does not produce bulbs. It is sown in August, to be drawn green in spring, for use in the manner of leeks, and in that state is not unfrequently termed scallion-an epithet applied by country people to all sorts of onions when they do not produce bulbs.

Onions for pickling are in demand in every family. For this purpose the silver-skinned varieties should be chosen, on account of their delicate white colour, slightly intermixed with greenish lines between the coatings. As they are usually preferred when small-about the size of a filbert-it is necessary they be sown on very poor soil. We have a border, the soil of which is soft alluvial sand: on this we have grown silver-skinned onions annually during the last thirteen years; trenching it as soon as the crop is gathered, two feet in depth, giving it no manure whatever; sowing thickly in the broadcast manner, in three feet beds-which are formed after the crop has come above ground, by running a hoe from side to side, and thus forming alleys fifteen inches broad, the seed having, to save time, been sown all over the piece. The situation is warm, which insures the ripening of the bulbs; the soil being very poor, and the crop thick, produces them of a small uniform size. In small gardens, and in those wherein the soil is of a very rich nature, such onions are difficult to procure. Sowing 
under the shade of trees has been recommended, but not judiciously. As a bed ten feet by four would produce a sufficient quantity for a small family, it were better to prepare such a space artificially, by removing the rich soil to the depth of eighteen inches, and replacing it with poor sandy or gravelly soil. We have grown pickling onions upon a bed of loamy gravel laid on the surface of the ordinary soil, to the thickness of twelve inches.

Diseases and insects.-The diseases and insects to which the cultivated alliaceous plants are liable, if not numerous, are sufficiently destructive. One of our greatest pests is

The common onion-fly (Anthomyia ceparum, Bouché). It is thus described by Mr Curtis, one of our highest entomological authorities, in the "Gardeners' Chronicle," 1841 :- "The male is of an ashy colour, roughish, with black bristles and hairs; the eyes are contiguous and reddish; the face silvery white; horns black; there are three obscure lines down the trunk, and a line of long blackish spots down the centre of the body, more or less visible in different lights; the wings are transparent, slightly irridescent, tinged with ochre at the base; the nervures palebrown; poiser's ochreous; legs ashy brown. The female is ochreous, or ashy grey, clothed with black bristles and hairs; the eyes are reddish and remote, with a light chestnut stripe between them, bifid and darkest at the base ; face yellowish white."

This insect attacks the plants in their young state, and continues feeding on them during the whole summer. Sometimes they attack the crop generally, and cause a total failure; at other times attacking them in patches only, the effects being most observable in dry weatherthe leaves turning yellowish, becoming flaccid, and the plant at last falling over and decaying. On removing the outer coating or skin of the plants destroyed, the cause will be discovered in the presence of a small grub, which eats its way into the very heart of the onion. This grub is white, smooth, and shining, and of a conical form, and nearly half an inch long. The eggs are deposited on the leaves when in a very young state, and close to the earth; as soon as the maggots are hatched, which takes place about the time the plants are about the size of a small quill, they bore their way through the outer leaf, and penetrate the onion at its base,

Fig. 5 .

Fig. 6 .

Fig. 4.

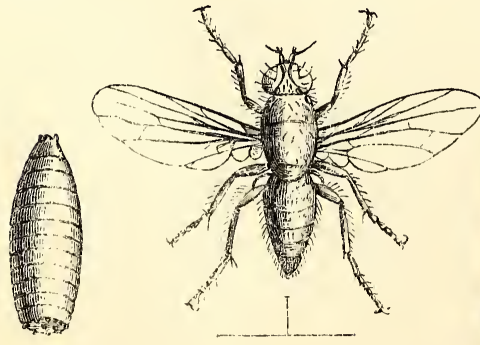

THE ONION-FLY, GRUB, AND PUPA. feeding chiefly on the bottom part of the bulb, causing it to separate from the roots, and occasioning a mass of mouldiness, familiar to every cultivator. These grubs, in general, attain their full size in about fourteen days, sooner in dry weather than in wet, and at that period descend into the earth, to undergo their transformations, when they become a reddish brown, indurated pupa, of an oval form. Fig. 4 shows the grub; fig. 5 , the pupa; and fig. 6 , the perfect insect.

Another enemy has recently appeared in the brassy onion-fly (Eumerus ceneus). This fly was discovered by Mr Curtis in 1842, and figured and described by him in the "Gardeners" Chronicle" for that year, (p. 252.) He detected the maggots in a box containing some rotten onions, which he found to be distinct from Anthomyia ceparum, the common onion-fly. "It is densely clothed with short hairs, thickly and distinctly punctured, and of an olive green, with brassy tinge; the antennæ are entirely black, the seta naked; the face is very hairy, simply convex, and silvery white; eyes darkbrown and slightly pubescent; rostrum very short; thorax with two whitish lines down the back; scutel semiorbicular, the margin thin, and somewhat denticulated and ciliated; abdomen linear in the males, the segments coarctate or constricted at the base-attenuated to the apex in the female, with six grey lunulate marks, three on each side, and nearly meeting on the back; wings transparent; the nervures piceous, the apical transverse, one somewhat bilobed; poisers yellowish white; legs rather short and stout, especially the hinder, the thighs being incrussated and serrated beneath with a double row of minute teeth; tibiæ bright rust colour at the base, the hinder curved; tarsi five-jointed and black, the hinder orange colour on the inside; the claws are small, and the suckers bilobed."

Specimens of this fly have been taken in the neighbourhood of London, flying about in June and July. The female has not as yet been observed depositing her eggs. The maggots do not confine themselves to the onion alone, but have been observed on cabbage-roots.

These may be considered the chief enemies to the onion tribe, attacking them all indiscriminately - the garlic, however, less than the others. The latter does not as yet appear to have spread, so as to be of the same importance as the former. The means hitherto employed for subduing these insects are these: Laying soot over the beds, or incorporating it with the soil ; applying salt in the same manner; watering with lime-water, gas-tar, stale soap-suds, soot-water, stale urine, old tobacco-water, \&c. It is evident, however, that it is not the smell which affects them-for few things can be more offensive than rotten onions, on which both these insects appear to luxuriate.

Their power of reproduction is so great, that unless they are destroyed the moment they are discovered to have attacked the crop (which will readily be known by the leaves drooping and turning yellow), their total eradication becomes next to impossible. All other means (except carefully pulling up every diseased 
plant, and committing them to the fire, collecting the soil around where they have been growing, and burning it also) can only be regarded as exceedingly superficial in their effects. Deep trenching and frequently turning over the soil are of great advantage : in the one case burying the pupa too deep for its again reaching the surface; and in the other, disturbing it during its transformation, and probably preventing that change from taking place. From what has been shown above of the economy of the onion$\mathrm{fly}$, it is clear that the most rational method of eradicating it is to capture the insects while in the grub state, and while they are entrapped within the onion. By doing so, their increase is greatly diminished; as it is probable that, for every grub so destroyed, hundreds of flies are prevented from appearing in spring, each of which may be the parent of hundreds of grubs during the following season. All other proposed remedies may be set down as comparatively worthless. Sowing onions year after year on the same ground is a very certain way of multiplying these insects, and might be carried to the extent of literally stocking the ground with them. Insects peculiar to any plant seldom attack the crop during the first year, and probably the second, after being planted in land not previously occupied with the same kind of crop, because the soil has not as yet become furnished with the pupæ of the insect peculiar to the plant. Hence some advantage arises from sowing onions after celery, and vice versâ, cabbage after potatoes, \&c. Spirits of tar is of great use, if applied in sufficient quantity to the soil immediately after the crop is removed; and it is a good plan to run it along (from the spout of a watering-pot) the lines of the rows where the onions had grown, because in that way, and at that exact time, it reaches the pupa in its most tender state; not, however, that it acts as an ordinary poison (the pupa not being in a feeding state), but it penetrates through the skin whenever it comes in contact with it; and no insect, in any of its stages, out of hundreds we have submitted to the ordeal, will exist after being brought into contact with this liquid. It is probable, also, that many other applications (turpentine, for example), if applied at this time, and in this way, might be found exceedingly efficacious.

Soot is not only an excellent manure for onions, but is also a safe precaution against the attacks of the grub. It may be sown broadcast all over the ground, previous to drilling, if in abundance; if scarce, sow it in the drills when formed. The following has been often applied to onion crops, even after the attack has commenced: Water the ground well (that is, give it a good soaking of water) in the following proportions, - add to 20 gallons of rain-water 1 peck of unslaked lime, $\frac{1}{2}$ peck of soot that has not been exposed to wet, 2 gallons of urine, $1 \mathrm{lb}$. of soft soap, and $2 \mathrm{lb}$. of flowers of sulphur; when the mixture is sufficiently settled to pass through the rose of a watering-pot, it may be applied. As a preventive of the grub in onions, it has been recommended to sow them on ground previously cropped with turnip. If advantage arise from this, it is founded on the rotation system.

The chemical action of soot, in this and in similar cases, as a fertiliser and preventive of grub, may depend on the elements of which it is constituted, which are carbonaceous matter, mixed with carbonated ammonia, giving out a strong pungent smell by the action of quicklime. It affords a brown extractive matter of a bitter taste, some ammoniacal salts, and an empyreumatic oil, either or all of which are disagreeable, if not really fatal, to insect life. Its chief basis, however, is charcoal in a state capable of being rendered soluble by the action of oxygen and water. Powdered charcoal has been found exceedingly efficacious in preserving the onion crop, but not to the same extent as soot, containing in itself few of the elements of which soot is composed. We were led to apply soot in a liquid form, as M. Branconnet has shown that "a watery infusion of soot is eminently antiseptic, and may be used for preserving animal matter from decomposition;" and antiseptics we find in practice greatly to preserve the parts of plants, to which they are applied, from rottenness, to which the onion is extremely liable when attacked by the grub.

The variety of silver-skinned onion known as Oignon de Nocera is sometimes attacked by a minute parasitic fungus (Vermicularia circinans). It appears in form of small round black spots, which, although not penetrating the substance of the bulb, greatly disfigure it, and render it unfit for use. This disease appears to be quite new, and was first detected in the gardens of the Horticultural Society of London in 1851.

Saving of seed, \&c. - The saving of onion seed is carried on to a very great extent in the neighbourhood of Reading, in various parts of Kent, to some extent in Cambridgeshire, Bedfordshire, and about Evesham. The great supply, however, is imported from France and Holland. The saving of any esculent vegetable seed, while it can be purchased genuine from the regular seedsmen, is the reverse of economy. Amateurs, however, often save various sorts of seed, as much for the satisfaction of presenting them to their friends, as on the mere score of economy to themselves. Gardeners are not unfrequently driven to this, as an expedient to preserve any particularly fine variety they may become possessed of, as well as to guard themselves against fraudulent dealers, who, it is to be regretted, are on the increase. There is no economy, however, in saving seeds with a view to evade paying a yearly seed-bill. Seeds so produced cost 100 per cent more than they can be purchased for. They are 
seldom so good, can never be calculated upon as pure, if more than one species be grown in the same garden, or indeed within a very considerable distance around.-For reasons, vide art. PropagaTION BY SEED.

To save onion seed, select some of the largest, best formed, and best ripened bulbs; prepare the ground as already directed, only choosing the warmest and most sheltered spot the garden affordsfor climate is the principal reason why we are compelled to import seed, which, until these few years, was subjected to an import duty almost equal to the prime cost. In November plant them, as we have advised for garlic and shallots. As the shoots advance in height, a line of stakes, three feet in height above the surface, should be driven in along each line of plants, and to these a double course of packthread or tarred cord should be fastened, by taking a turn of the cord around each stake, thus leaving the stems of the plants to grow up between the cords for their support; and, for greater security, these cords should be tied together at every twelve or fifteen inches in length. As the flower stems extend in height, add other courses of cord, till the height of nearly three feet is attained, at which height the heads of flowers will appear, and, if not broken by the wind, will become large heads of seed, which will ripen in August or September. The stems should be cut over then, and laid down for a day or two to dry; afterwards they may be tied up in bundles, further dried in the sun, and ultimately, if the crop is large, stacked by till thrashed out, or suspended in bundles from the roof, in a dry airy apartment, and rubbed out and winnowed at convenience. One ounce of seed contains 7636 seeds.

The European names of the onion are, Oignon in French; Zwiebel in German; Uijon in Dutch; Cipolla in Italian ; Cebolla in Spanish.

\section{§ 5.-THE LEEK.}

Natural history.-The Leek, Allium porrum, L. (from Celtic, Pori, to eat), belongs to the same natural order, and ranks in the same class and order in the Linnæan arrangement as the four last. It is said to be a native of Switzer- land; of this there are as great doubts as that the onion is a native of Spain. It is undoubtedly a native of the East, and was cultivated largely in Egypt in the days of the Pharaohs. Philips, in "History of Culinary Vegetables," vol. ii.p. 30, has thrown more light upon this dark subject than any other author we have read. He says, in speaking of this plant being adopted by the ancient Welsh as their badge, "The Welshmen still continue to wear leeks on St David's Day, in commemoration of a victory which they obtained over the Saxons in the sixth century, and which they attributed to the leeks they wore, by the order of St David, to distinguish them in the battle. The Welsh patron died about the year 544." Now, they must have been not only introduced, but much cultivated at this early period. That the leek is a native of Egypt is evident; for, besides the notice taken of it in the Bible, Pliny, in book xix. chap. 6, states " that the best leeks were brought from Egypt, and the next to them from Orthes, a town of Asia Minor," now called Guzelhizar. Aricia in Italy was celebrated for leeks in Pliny's time; for he says, "It is not long since leeks were brought into great notice through the Emperor Nero." Tusser sings their praise in verse, and says they were in common use in farm-houses long before his time. And Gerard, writing soon after Tusser's time, speaks of leeks in such a manner as to induce us to think them indigenous to our soil.

Its date of introduction is given in "Hortus Britannicus" as 1562 . In this case, as in several others in that work, when the date of a plant is not exactly known, the first year in which it is known to have existed in Britain, upon published authority, is given. That it was to some extent cultivated prior to that year is evident from the familiar way in which all our oldest gardening authors speak of it, and it is noticed by most of them.

Uses.-This plant has been more generally used as an esculent in Wales and Scotland than in England. It is now, however, cultivated much more generally in the gardens of the latter, and brought in greater quantities to their markets than formerly. The whole plant, except the roots, is used in soups and stews. The white stems, which are blanched by being planted deep for the purpose, are now largely used, boiled and served up with toasted bread and white sauce, and eaten like asparagus. They are much used in French cookery, and form an important ingredient in Scotch winter broth, particularly that national dish cock-a-leekie. But the most extraordinary use to which we have heard of their being applied is thus stated on the authority of no less a person than Lord Bacon, who says (in "Essay on Gardens," century ten), "I knew a gentleman that would fast (sometimes) three or four, yea, five days, without meat, bread, or drink; but the same man used to have continually a wisp of herbs that he smelt on, and amongst these herbs some of strong scent, as onions, garlic, leeks, and the like." The leek is a valuable vegetable for the cottager, a small piece of ground affording him a large and useful supply during winter and spring, when green 
food is scarce. It is easily cultivated and extremely hardy. The medicinal and dietary properties of the leek are similar to those of the onion, only in a milder degree.

Mode of propagation.-Leeks are propagated by seed sown for ordinary purposes at the same time as onions. Bulk of plant, in the case of the leek, being a primary object-and transplanting tends greatly to insure this-the seed is sown pretty thickly on a bed, and when of sufficient size, the plants are removed to where they are to come to perfection. Those who have not the convenience of a hotbed should sow the seed early in February, in as warm a spot as is at their disposal. Where, however, it is wished to have the leek in its fullest perfection, the seed should be sown thinly on a mild hotbed, such as of leaves in a state of fermentation, a material within the reach of most; while those near a tanyard may employ bark, or those in the vicinity of flax-mills the refuse flax-either of which, put up in the form of a cucumber-bed, will afford a mild and lasting heat. Without a glass frame, the purpose may be served by an occasional covering of tarpauling during frosty nights or heavy rains. Slight excitement only is required. The seed should be sown of a uniform thickness on rich compost, and covered to about the depth of a quarter of an inch, half an ounce being sufficient for a bed 2 feet by 4 , and so in like proportion for beds of larger size. Keep them clear of weeds, and water with tepid water occasionally until fit for transplanting. One ounce of seed contains 2924 .

Sowing and planting.--Sowing in the open ground may be performed from the beginning of February till the middle of April, the exact time depending entirely on the state of the weather and the condition of the soil. The earlier, however, the seed is sown the better, that the plants may attain a good size before final transplanting. If sown in beds of 4 feet in breadth, and in the broadcast manner, the same process should be followed as recommended for onions grown in that way. If sown as advised above, and which is much the best way, as soon as the young plants are of the height of 3 inches they should be carefully taken up, loosening the soil in the bed so that none of the fibres may be in the least injured. They VOL. II. should then be transplanted into another preparatory bed, having the same advantages of heat, \&c., as the first; but on this the heating material should be covered with completely rotten manure to the depth of 4 inches, and the plants set in it at the distance of 3 inches apart each way. This is to encourage their growth. When they have attained the height of 6 inches, another bed of equally rotten manure should be prepared for them, placed on the surface of the ground in a warm sheltered spot, but without bottom heat, and the plants a second time removed, with all their fibres entire, and planted in it at the distance of 6 inches apart each way; for here they are to remain till the season of final transplanting arrives, which will be from the beginning to the middle or end of May. The plants, having thus plenty of room, will attain the size of 9 inches or a foot, and be of goodly size of stem, which will be promoted by frequently watering them with liquid manure. Few people pay this attention to them, but transplant them at once from the seed-bed to where they are to grow to full size. In this they lose much, both in the size and delicacy of the produce, as the more rapidly all esculent plants are grown, the more tender they are. The ground being prepared for them, they are to be set in lines 18 inches apart, and the plants 10 inches from each other in the line-that is, if the largest size be wished for; otherwise, 12 inches by 7 will be sufficient. In planting, the dibber used should be $3 \frac{1}{2}$ inches in diameter, obtuse at the point ; but, if nearly cylindrical in form, so much the better, that the roots may have room in the bottom of the hole. The plants being taken carefully up, with as much of the rotten manure attached to them as possible, and carried to the spot on a shallow wooden tray, they are to be dropped carefully into each hole as it is made, and no soil whatever placed over them, as the rains and occasional waterings - probably one at the time of planting-will wash down sufficient to cover the roots to the extent required. The object of this large hole is, to allow the stem to swell out in size, without being compressed by the surrounding soil. The dibber should have a cross piece of wood fastened to it, form- 
ing a gauge as to the equality of the depth of each hole. The old and barbarous system long recorded in books, and practised by even the best gardeners, of cutting off a portion of the tops of the leaves, should be disregarded; and the curtailments of the roots, long held as a beneficial process, should be looked upon as an act of wanton madness. This is the usual mode of planting. A much better way is to stretch the line from end to end of the field, and, with a spade, to take out a trench a foot at least in depth, leaving the back of the trench immediately under the line as nearly perpendicular as possible. Along this trench set the plants, resting against the solid ground, placing their roots with the ball of manure around them in the bottom, and filling in the earth taken out of the trench carefully around them-taking care, however, not to let it reach so high as to cover the hearts of the plants. Water at the time of planting, if the soil be not already too damp, in which state it would be better to delay planting till it dry. However, by this drill mode of planting this crop, as well as most others, the ground is left quite open and loose, as the operator is always standing on the ground that is to be loosened up in the formation of the next drill. One sowing is, in general, sufficient for private families in Scotland; but in the south, where vegetation is so much more rapid, a second, or even a third sowing, may be found of advantage for continuing the crop till late in spring. Our own practice is to sow once, and, in the beginning of March, to dig up the crop, and lay it over in a cool shady place, covering the stems as high as they are blanched-a plan we have found much more suitable than that of sowing on heat for the early and main crop, and afterwards in April or May for late crops. Indeed, we have frequently found those sown in May run to seed at the very time those sown in February did. The only inducement for making successional sowings is, that some French cooks require them of a small size; and, to meet such demands, successional sowings should be made from February till July. Some plant in open trenches, after the manner of celery, and earth the plants as they proceed.

Subsequent cultivation.-The subsequent management consists in drawing a little earth to the stems as they elongate, keeping the ground clear of weeds, repeatedly stirring the surface between the rows, and of all things avoiding the practice of cropping the leaves-an old and nearly obsolete mode-which, however, has been recently recommended, with a view, it is asserted, of increasing the size of the stems.

Soil and Manures.-The soil, from what we have already stated, cannot be over rich, nor, indeed, need the manure be at all so decomposed as for onions. We believe the leek would luxuriate well on the top of a dunghill. It is important, however, that the ground be deeply trenched, and also that the manure be nearly as deeply buried; for as the roots are from one to two feet or more under the surface, so also should the manure be. Deep alluvial soils, if dry at bottom, are excellent for the leek; and next, those that are of a deep rich loamy nature : light shallow ones are the worst of all. In these latter, the plants should be set on the surface upon a bed of rich manure, and earthed up as they proceed, as is practised with celery.

Forcing.-The leek is never forced beyond the slight extent noticed above, to forward them for final planting.

Taking the crop, and subsequent preservation.-The crop is left in the ground till wanted for use. It is expedient, however, upon the appearance of frost, to take up a quantity to be laid in soil or sand in the root cellar, or other place of shelter, so as to be conveniently got at when wanted for use.

Approved sorts and their qualities.-The seedlists contain several names of sorts : of these, the Large Flag, London Flag, English Flag may be considered as identical. The Musselburgh is an early improvement on the Scotch Flag; and what is now called the Edinburgh Improved, appears to be only selected from the Musselburgh stock. The Dutch Flag, Erfurt, and Rouen, seem, as they are at present growing in alternate rows in the Dalkeith gardens, to be in nowise different from each other. They appear, however, to be disposed to attain a greater growth than any of the others. There is a sort of Allium grown in Pembrokeshire, and along the Welsh borders, called the hollow leek, producing roots in clusters like shallots. It is, however, scarcely worth the notice of the modern horticulturist, in the presence of so many sorts that are superior.

Saving of seed.-In saving seed, some of 
the largest and finest specimens should be selected; and as we in the North have scarcely climate for saving this or the onion, it has been found expedient to plant them at the bottom of a south wall, and to support the flower stems by it. In such a way, considerable quantities of good seed are annually produced about Musselburgh, and in other warm localities. In better climates, the same mode is followed as recommended for onions, (which see.)

The European names are-Porreau, or Ail ̀̀ tuniques, in French ; zahme Lauch, gemeiner Lauch, Porro-Zwiebel, or Spanische Lauch, in German ; Porro, or Porreta, in Italian; Puerro in Spanish; Alho Porro in Portuguese ; Purio in Swedish ; Pras in Russian; Prei, Look, or Porreye, in Dutch.

\section{$\S$ 6.-THE CHIVE.}

Natural history.-The chive (Allium Schoenoprasum, L.) belongs to the same natural order, and ranks in the same class and order in the Linnæan arrangement as the five last. It is indigenous to various parts of Britain, occasionally found in meadows and pastures, but at no great elevation.

Uses.-The young leaves are the parts used; the roots, although bulbs, are very small, and although partaking of the taste and properties of the rest of the family, have never, so far as we are aware of, been used as an article of food. The young leaves are used in mixed salads as a substitute for young onions, and by many are preferred, being milder in flavour. They are used in a young tender state ; and, to keep up a succession during spring, summer, and autumn, the plants are repeatedly cut over, whether the leaves are required or not, the intention being to have them always young. During winter they are obtained from plants taken up in autumn, and potted and kept in a mild temperature, say from 45 to 55 . They are very much used in soups, particularly in Scotch families, for seasoning various dishes, shred in small pieces, and served up with beefsteaks, being sprinkled over them just as they are taken from the fire. They are next to indispensable in omelets, and hence are much more used on the Continent, particularly in Roman Catholic countries, than in Britain. The process of gathering them is to cut them over near the ground, but so as to carry no earthy matter with them, for much of their flavour would be lost by washing. They are then tied up in small bunches, ready for the kitchen. The circumstance of their being gathered in this way, Loudon remarks, has caused them to be spoken of in the plural, Chives.

Mode of propagation, cultivation, \&c.They are propagated by division of the roots either in the spring or autumn. Their roots, growing in bundles, admit of this mode with great facility. They may also be singled out and planted individually, but this is seldom done. They grow in bunches often 6 or 8 inches in diameter. One of these may be divided into a dozen or more pieces, each of which will, in a few weeks, if planted in spring, form a compact patch. Each patch should stand clear of the other, so that in forming a new bed of them, they should be set a foot or 15 inches asunder. A plantation will last for many years, but it is well to renew them every third or fourth year. All the cultivation they require is to be kept clear of weeds, and the leaves frequently cut over. They will prosper in any ordinary garden ground, and there is only one variety in cultivation.

The European names are-Ciboulette, or Civette, in French; der Binsenlauch, or Schnittlauch, in German; Bieslook, or Suyprei, in Dutch; Cipoletta maligia in Italian; Cibollino de Inglaterra in Spanish; Cebolinha de Ingalaterra in Portuguese; Graslog in Danish; Luczer-lupny in Polish. 


\title{
CHAPTER II.
}

\author{
LEGUMINOUS PLANTS, OR THE PULSE TRIBE.
}

As has been elsewhere stated, plants of this natural order are recorded to have been amongst the first vegetables employed by man as articles of food. The legumes or pods, either in their earlier stages of growth, as in the case of the kidney bean, or in their more advanced growth, as the pea and bean, the seeds of which are eaten both in a green and ripened form, are the parts of the plant employed in culinary matters. This very extensive order contains no less than 244 genera, and upwards of 2630 species. Of these, the following, with their sub-varieties, hold a prominent position in garden culture, viz.:-The Pea, Garden Bean, Kidney, French or Haricot Bean.

\section{$\S 1$. -THE PEA.}

Natural history.-The pea (Pisum sativum, L.) belongs to the natural order Leguminosæe, subdivision Sarcolobæ; tribe Viciex; and to the class Diadelphia, and order Decandria, in the Linnæan arrangement. The genus Pisum contains seven species; three of which are cultivated for culinary purposes; besides the varieties and sub-varieties of Pisum sativum, whose name is legion-if faith is to be placed in the list of names found in seedsmen's catalogues. Pisum maritimum, a species which is indigenous to some parts of the east coast of England, has in former times been used as an article of food in times of scarcity. The name Pisum is derived from the Celtic pis, a pea ; or, according to Philips, from Pisa (a town of Elis), where pease anciently grew in great plenty. The Eng lish name appears to be a corruption of the Latin. Tusser, who wrote in the time of Queen Mary, and Gerard, soon after him, both wrote it "Peason ;" Dr Holland, writing in the time of Charles I., spells it Pease, since abbreviated into Pea.

The native country of the pea, like that of most of our cultivated esculents, is not now known. Modern catalogues refer it to the south of France, and Valmont Bomare distinctly says, " the garden pea was originally from France ;" and Mr Coles, in his History of Plants, says the Fulham pease, which came first out of France, is so called because the grounds about Fulham, "neere London, doe bring them forward soonest." Pease undoubtedly came originally into France, Italy, and Spain, from the East; and although we cannot identify the lentils used in the days of Jacob and Esau with the pea of later times, still, we know they were cultivated by both the Greeks and Romans in the time of Pliny, who informs us that the former sowed their pease in November, but the latter did not plant theirs till spring-and then only in warm places lying well to the sun. "For," says he, " of all things, pease cannot endure cold."

The time of their introduction into Britain is as uncertain as their native place. That they were cultivated to some extent in the time of Henry VIII. is more than probable, as one variety, the Rouncival-a name continued down to the present day-is mentioned by Tusser, in his "Five Hundred Points of Good Husbandry," who says,- -

" Dig garden, 'stroy mallow, now may you at ease, And set (as a daintie) thy runcival pease!"

And that they were then grown much as at present, would appear from the following line:-

" Stike plentie of bowes among runcival pease,

To clamber thereon, and branch at their ease."

In the early part of Good Queen Bess's reign, they were, however, less abundant than the above quotation would lead us to suppose. For, as Fuller remarks, they were seldom seen, except those brought from Holland, and "these were dainties for ladies-they came so far, and cost so dear."

Green pease appear to have been unknown to our Saxon ancestors; nor was it until after the Norman Conquest, and the establishment of monastic communities, that we read of such being used. Fosbrooke, in "Brit. Monasticon," says that, amongst other rarities, green pease were provided against midsummer, for the nunnery at Barking in Essex. And in "Archæologia," 13,373 , early pease are thus directed to be treated: "If one will have pease soone in the year following, such pease are to be sowen in the wane of the moone, at St Andrew's tide 
before Christmas." Bonnefonds, in his " Jardinier Français," (1651), describes the skinless pea as the Dutch pea, and remarks, that until lately they were exceedingly rare. They appear to have been introduced into France from Holland about 1600. Green pease became a popular delicacy in England soon after the Restoration of Charles II.; and, strange enough, even for late ones so early as 1769 , as it is a matter of history that on the 28th October of that year, a guinea a pottle-not quite half a dish-was given in Covent-Garden market; and as much as ten times that sum has been paid since in the same market, for a quart of green pease shelled.

Use.-Pease in their dried state are used in soups, either whole or split; in the latter form they are generally preferred. They are also made into puddings, and occasionally ground into meal : in either way they form an agreeable and nourishing food-not, however, well-suited to those of weak digestion. Old pease are often difficult to boil; indeed, sometimes no boiling will render them soft. This has been said to ensue when they have been kept more than one year; and also that those grown on land which has been manured with lime, marl, or gypsum, will even not boil at all, whatever their age may be. That such a circumstance sometimes occurs as their not boiling, is pretty well known to dealers; but that either of the causes assigned is the true one, seems to us to be doubtful. All the varieties of garden pea are esteemed for their seed while in a young state; indeed, for the tables of the great, they can hardly be either too small or too young. In their second state or size, they are used for green-pea soup; and, in their third state, when fully grown, but still quite green and soft, they form an ingredient in hodge-podge. In gathering, the young gardener should be informed for which of these purposes they are intended; for whether for the one or the other, the pease should be of a uniform size and age. We may here also observe, that several of our largest-growing varieties are objected to merely on account of size, and therefore it is unwise to indulge exclusively in such sorts; for although excellent, and most profitable to those who wish a good comfortable family dish of duck and green pease, they would be considered unfit for a first-rate table. The marrow-fats, although much prized by some on account of their peculiar fine flavour and delicacy, must give way, in this respect, to Sutton's early champion, Beck's morning star, early Frame, Prince Albert, Auvergne, and such like smaller sorts ; while Bishop's new long-podded, Thurstone's reliance, Hair's dwarf mammothall first-class pease for profitable culture-must not be calculated upon to afford a supply for mouths genteel. In a sanitary point of view, pease cannot be eaten too young, nor too soon after they are gathered; and hence people who have to depend on the public markets for their supply, seldom taste this very popular vegetable in perfection, and too often have it only when it is almost unfit for use. This is a formidable objection to imported pease. It is, of course, for the interest of the producer to keep back his pease till they are fully grown, because they mea sure better, and we believe with many take better, as they get greater bulk for their money. This may be so far excusable on the part of such; but it is inexcusable that a gentleman, having a private garden of his own, should be served with pease otherwise than in the very highest state of perfection-which they arenot, if allowed to become too old, or even too large. Mint is usually boiled with pease. It improves the flavour of them while young, and very much enhances the flavour of pea-soup ; it also corrects flatulency. A few sprigs of mint should accompany the pease to the kitchen.

There is one section of garden pease called sugar-pease, the pods of which have the inner film wanting, or much less tough than usual. The pods in this section are used with the young seed within them, and are cooked and eaten the same as French or kidney beans. This is a mode of using pease we would like to see much more generally in use: dressed in this way, they afford a delicious and economical dish.

Regarding the nutritive properties of the pea in a green state, we have never met with a satisfactory analysis. In a ripened state, the flour of pease is as three to two of bulk in grain ; and when dressed and split for soups, as four to two. According to Sir H. Davy, 1000 parts of peaflour afforded 574 parts of nutritive or soluble matter ; viz., 501 of mucilage, 22 of sugar, 35 of gluten, and 16 of extract, or matter rendered insoluble during the operation. The sugar-pease are much more used on the Continent than with us. The dwarf crooked sugar-pea (pois sans parchemin ou mange tout-zwerg zuckerchoutte), and the tall crooked sugar-pea (pois sans parchemin à grandes cosses-grosse schottige zuckererbse), are met with at every table d'hôte.

Yet how seldom do we see such a dish in our own country. This may be looked upon as the more singular, as Gerard, writing in 1597, in speaking of the different sorts of pease, or "peason," as he has it, says there are "some with tough skins, or membrances in the cods; and others have not at all, whose cods are to be eaten with the pease when they be yoong, as those of the yoong kidney-beane:" than this nothing can be clearer. Hoffman and Simon Paull treat largely on their medical properties ; and Lemery, "On Foods," remarks that "pease contain a viscous and thick juice, which causes flatulency, and produces gross humours ; and therefore they are not good for those that are troubled with gravel." Green pease may be preserved for winter use if dried in a cool oven, and afterwards placed in paper bags suspended from the roof in a dry room.

Propagation.-All the cultivated pease, being annuals, are propagated by seed.

Sowing.-The earliest crops to be produced in the open garden, without artificial aid, are obtained by a judicious selection of the most approved early varieties, choosing a favourable soil and situation, and committing the seed to the ground in 
the end of October, and throughout November, December, and January. It is of importance, also, in making this selection, that the hardier varieties should be chosen. Hamilton's November prolific is one of these. We have seen it, during the two last winters, standing in an elevated part of Dumfriesshire, when other early sorts alongside of it were much injured by the cold. In practice, we find it is not always the crop first sown, however, that is first fit for gathering, even within a few feet of the same altitude, and a quarter of a mile of the same line of latitude. Local circumstances, to a very great extent, set aside in such matters the dicta laid down by those who found the ripening of fruits or of garden crops upon what has been called the geographical distribution of plants. The London market-gardeners, to whom early pease are a remunerating crop, sow in December in rows, in borders under walls, or by the sides of hedges three feet and a half apart. The pease are never staked, and the sorts preferred are, early Frame, Bishop's new long-podded, Groom's superb dwarf blue. Mr Baker, in "Gardeners' Chronicle," 1848 , p. 365 , on this matter observes:- "I generally sow in November, by a wall in the garden, and the pease are trained to sticks in the usual way; but I have almost invariably picked first from the field crop, although not sown until February. It is very true that, having a large space to collect the first picking from, I gather a pod only here and there; but having found the earliest and best-filled pods nearest the ground, leads me to suppose that, by training on sticks, the exposure to the atmospheric air during night retards the growth, inasmuch as the heat given out by the earth during that period is prevented from assisting the growth of the pea by training,them above the ground; and, from the habit of the pea entirely covering the surface, but little circulation of the air takes place among them in the field. My land is a dry, siliceous, and strong soil, and radiates a considerable degree of heat during the day, which also tends to the early production of the crop."

Pease, like all other crops grown in rows or drills, wherever natural obstructions do not occur to prevent it, should be sown in a direction from south to north, unless in the case of very early crops transplanted at the bottom of a south wall, when, for obvious reasons, the direction should be reversed. The main intention of placing crops in the former direction is, that the sun may have free access to every part of the ground between the lines, and the plants themselves be placed in the most favourable position in this respect also. If placed in a counter direction-namely, from east to west-one side only would have the full advantage of the sun, while the other would derive little advantage whatever. Some draw their lines obliquely, fancying thereby to secure the early moruing and late afternoon sun in its greatest vigour, and calculating, also, that its influence is thus more equally diffused. In this, to a certain extent, they are right; but do they who follow this practice truly calculate the angle at which they place their rows, or do they reconcile the angle to the latitude of the place? or do they take into consideration that the sun is either constantly rising or falling in the horizon, so that whatever angle they may adopt is differently circumstanced as regards the sun's rays every day?

As to distance between the rows, when pease are sown in the usual manner-that is, row after row throughout the whole quarter-it may be taken as a general rule that as much space should be allowed between them as the sorts attain in height ; - thus, a pea of two feet in height should have two feet from row to row, and so on, up to Knight's tall marrow, which attains a height of from eight to ten feet-which latter should be placed at least ten feet asunder. A much better plan was, we believe, first recommended by the late Mr Cobbett, who is said, by those who seem to know, to have published the best English Grammar of the day; and we can safely say he wrote the best book on cottage gardens which has appeared before or since. His plan with pease was to sow in single rows, twenty, thirty, or fifty feet apart, by which every portion of the crop was fully exposed to the sun and air-the crop hanging, in consequence, profusely from bottom to top. By this means, also, he secured shelter to his other crops, as his pea crop served the purpose of so many temporary screens during the whole summer, at the 
very time they were of most importance. There is no loss of ground by this method, for other crops can be planted to within $1 \frac{1}{2}$ or 2 feet of the rows-which space is necessary for the purpose of gathering them. Any person of the least observation must have seen that the outside of the first or last row in a continuous piece always produces the earliest, finest, and most abundant crop. There is a great economy of space by sowing pease in the following manner, noticed in the "Gardeners' Magazine," vol. iv., p. 225 :- "If only two rows in one place, and two more in another, 15 or 20 feet distant, were sown, there would be four outsides; whereas, if they were all sown together, there would only be two outsides. Two rows in one place occupy 3 feet 6 inches in width, and two rows in another the same, making together 7 feet; but if four rows were sown together, they would take up between 11 and 12 feet of ground. Here there is a saving of nearly one half." Again, let us observe that, without infringing on the rules of rotation, the leguminous plants may nevertheless be grown on the same quarter or division, by sowing the tallest-growing sorts at great distances apart, placing the dwarf-growing ones between, interlining with kidney beans, which attain the height of 18 inches, and with the new dwarf cluster garden - bean of similar height : thus the object of full exposure would be attained, while the rotation would not be interfered with.

Sowing for principal crops should be attended to from the middle of March to the end of July, making a sowing every fortnight or three weeks at latest, where a constant supply is required daily. Some sow one crop just as the last appears above the ground. All this, so far as regards a constant and uninterrupted succession, depends far more upon the sorts sown than on the exact period, because some kinds come to perfection much sooner than others. (Vide List of sorts, \& c.)

For the latest crops of all, sow on the 1st, 10th, and 15th of August, and 1st of September, choosing dwarf early sorts, for the greater convenience of protecting them, should need be, during November and December, by spreading thin canvass over them on frosty nights.

There are few esculent crops that suffer so much from the malpractice of too thick sowing as the pea. The autumn-sown crops, of course, require more seed, as the chances they run of being injured by frost or devoured by mice are very great, and therefore that should be taken into account: if they escape these dangers, they can be thinned out, and the thinnings transplanted in spring. We would also remind amateurs, particularly those near towns, that the sparrows will take their tithe of them just as they come above ground, and that provision must be made for this in sowing. Fifty peas to the foot of line in such cases, and of such sorts as Warner's early emperor, Sutton's earlychampion, Fairbaird's champion of England, and Bishop's new long-podded, will be ample. For very small gardens, Bishop's early dwarf is well adapted, growing only 1 foot in height, and hence requiring no stakes. They should all be sown $3 \frac{1}{2}$ inches in depth. The fourth in the above list may be sown at half the above distance, as it sends out lateral branches, and hence requires space for them to spread. Pease are always, in England, sold by the pint or quart, peck, bushel, \&c.; in Scotland, often by the pound, when in small quantities. One quart is equal to about $2 \mathrm{lb}$., and contains of the largest sized peas 1298 , and of the smaller 2150. One pint of the small-seeded sorts, as the Frames, Charltons, \&c., will sow a row 20 yards in length; and the same quantity of larger-growing sorts will sow a row 33 yards long, on account of their being sown so much thinner. The old practice of treading down the pease, when sown in autumn, should be discontinued, and this the more especially if the ground be wet or the soil strong. The old Scotch practice of sowing in double rows is now seldom seen, at least where anything like good culture is exhibited.

Many cultivators, and ourselves amongst the number, set their pease for general crops singly, as is practised with beans, the larger-growing sorts from 3 to 4 inches apart ; and we also, in some cases, plant them in patches of five or six in each, allowing as much space from patch to patch as the pease attain feet in height, each patch assuming, when staked, a pyramidal form: by this means the whole surface is exposed to the sun and air. The 
usual allowance for seed is-for the frame section, which includes most of the early sorts, 36 peas per foot of line ; marrow-fats, dwarf variety, 24; do. tall, and all of similar habit, 12; blue Prussian, and those of the same size, 18, \&c. The depth for main crops should be, for the smallersized pease, $2 \frac{1}{2}$ inches ; for the larger kind, $3 \frac{1}{2}$ inches.

To obtain pease early, various methods have been tried: some sow in flat pots or boxes in January, placing them in a cool pit near to the glass, and transplant about the middle of March; others in small 60 -sized pots, so as to turn out the balls entire at planting; some follow Bishop's recommendation, and sow on thin narrow strips of turf, burying the turf under them at planting; while many sow in open drain-tiles filled with soil, some on narrow pieces of board, and many nail two boards together in form of a triangular trough about 3 inches deep, fill the same with mould, and sow thereon. At planting, drills are drawn; these troughs are set in them, the sides removed, and the row of pease left undisturbed. The following very excellent mode has been long practised by $\mathrm{Mr}$ Drummond, gardener at Blair-Drummond :-About the beginning of February, when he commences forcing peaches, "the border inside the house, on each side of the pathway, is covered to the depth of 3 or 4 inches with cow-dung, gathered from the park ; over this is laid 2 inches of decomposed tree-leaves, passed through a very wide sieve, raking level, and beating slightly with the back of a spade : upon this the peas are sprinkled as thick as they will lie together, and covered with sifted leaf-mould 2 inches thick. In the course of three weeks or so the pease are fit for planting out, being from 4 to 6 inches long. A border on the south aspect of a wall is chosen for the first planting; the ground being dunged, and dug deep and fine; furrows are taken out with the spade diagonally across the border; "the peaseare then raised from the border of the peach-house with a threepronged hand-fork," in large pieces, and carried to the prepared drill; "they are then divided by the hand into small patches, drawing each patch longitudinally, then placing it in the cut furrow in the manner of planting box-edgings, let- ting the roots hang as perpendicular as may be. By this operation very little of the dung and leaf-mould falls from the roots. The earth is then laid over and pressed firmly to the roots, and another furrow made, and drill-planted in the same manner, 9 inches from and parallel to the other, thus forming a double row. A little earth is drawn up about them with the draw-hoe, and they are staked pretty closely, and a few fir-twigs are stuck among the stakes to ward off sharp frosts; these are removed when the weather gets mild. Pease sown on the 1st of February, and transplanted as described above, are fit for gathering about the same time as, or even sooner than, those of the same sort sown on the 11th of November preceding, in the open air, along the bottom of a wall with a south aspect. Pease, by this process, have been gathered on the 26 th of May-certainly very early for such a locality; indeed, unusually early for any part of Scotland. Pease are readily transplanted while under 4 inches in height; they may therefore be sown on a warm border, or in a pit or frame covered with glass lights in severe weather, and transplanted in March. This, although not a very general practice, has been in use for above a century. Justice, in his "British Garden Calendar," published in $17 \check{9} 9$, recommends the practice, and reasons on the advantage of it. Bishop, in his excellent "Casual Botany," suggested sowing them in November on narrow strips of turf, and transplanting them undisturbed at a more advanced period of the season. Pease do not require this trouble ; indeed, it is questionable whether the operation of transplanting in the ordinary manner may not be an advantage, as the greater or less degree of injury to the long tap-roots causes them to put out lateral ones, which, taking a more horizontal direction, are nearer the surface, and consequently influenced somewhat by the sun-heat, besides having the effect of causing the plants to send out a greater abundance of roots, and hence enabling them to secure a greater amount of food. The London marketgardeners' practice is good, when they throw up triangular ridges in autumn, and sow a line of pease along the south side, and another along the opposite side, with a view to obtain an early crop 
on the south side, should they survive the frosts of winter and spring, on the side next the sun, the crop being accelerated by the radiated heat from the surface: should these fail, those on the northern side seldom do, and give a good crop, though somewhat later. In sowing the general crops during summer, if the ground be very dry, the drills should be well soaked with water previous to setting the pease: this moisture, being covered up, retains the seeds in good condition for a long time, and hastens their germination, which otherwise might as well be in the bag.

Subsequent cultivation.-When the crop has attained the height of about five inches, a little earth should be drawn around the stems, but not so close as to press upon them; it should form a sort of ridge, with a slight channel in the middle. The intention here is not, as in many other cases, to encourage the roots to diverge in a horizontal direction, for they have no disposition to do so; but rather to give a slight support to the plants, until they take hold of the stakes which are to support them. Those crops which are not to be staked require this support the most, and they should have the earth drawn up upon one side only, that the haulm may be thrown to one side, which will both facilitate the operation of gathering, and will keep the ground clean between them at the same time, while it supports the neck of the plants better than if the earth were drawn up on both sides.

The advantage of stopping pease is much greater than is generally admitted, as is also that of thin-planting the seed. If the seed of most kinds be planted 6 inches apart, instead of being sown, as they generally are, a saving of seed to the extent of five-sixths will be the consequence; and if the plants, even of the tall-growing kinds, are stopped when they have attained the height of 2 feet, and when they have made three joints after this stopping-if stopped again and again, after every three joints are formed, until the period when they are wished to produce their pods, an increase of crop, at least fourfold, will repay the trouble; and if several kinds were sown on the same day, this stopping will bring them into bearing in succession, and just at the periods when the crop is desired. Whenever stopping ceases, the pease will flower and set their pods. It vOL. II. will, however, be necessary to look over the crop occasionally, and remove any stray blossoms that may appear; for none should be allowed to set until the general crop is wished for. From this a good practical lesson may be learned : the stopping gives strength to the plants and encouragement to the roots, so that, when the crop is really wanted, both are in a much better condition to throw strength into the crop than if they were allowed to rush up into blossom at once. In the event, also, of a wish to retard the ripening of a crop to any particular period, timeous stopping. will effect this with greater certainty than repeated sowings made in the usual way.

Staking is the next operation in cul. ture, and it is of great importance it should be done early, before the stems fall over. Such an untoward circumstance often ruins an otherwise good crop. Newly transplanted pease, brought forward in a slight heat, require this attention at the time they are planted, on account of the shelter afforded them; and pease sown in autumn, as soon as they begin to break through the surface, or even before, demand the same attention. It is of consequence that the supports, whatever they may be, be equal in height to the height the variety attains. Many contrivances have been thought of for supporting this crop, with a view to do away with the not very elegant appearance of the stakes. Posts driven in the lines, having wide-meshed netting suspended from them, and hanging down both sides of the line, is one of the most feasible plans. Hexagon wire-netting, attached to iron props, has been suggested, and no doubt would do well were expense no consideration. Lines of cord stretched along the rows, and fixed to upright rods, are sometimes used; but by far the most general is branches of hazel, beech, or other deciduous trees. These, however, are very perishable. Branches of yew, lilac, or privet are the most durable, always excepting the larch, which makes the best of all. Whenever, therefore, the prevailing disease attacks young larches, of from 6 to 10 feet in height, to the extent of rendering their cutting down expedient, lose not the chance of securing them. They require little preparation farther than pointing the root end, topping them all to the required heights, and 
switching the side branches off on both sides, as if switching a hedge. We have had such in use for six years.

Whichever of these be emplayed, they should be stuck firmly in along both sides of the crop; and if placed in a slanting direction, so much the better for the tendrilled branches attaching themselves to them. They should be of equal height, and all straggling side twigs should be cut off for appearance sake.

Considerable advantage arises from topping the plants.when they have shown a sufficient number of blossoms. This stops the growth of the haulm, and diverts the whole energy of the plants to the formation and development of the pods. In dry weather, pease often require water; indeed, a good soaking to all crops in warm dry weather, whether they appear to require it or not, will be found of great advantage in preventing mildew and swelling out the crop. A humid climate suits the pea, and hence the longer continuance of our crops in Scotland than in the southern parts of England.

Soil and manure.-The pea comes earliest to maturity in light rich soils abounding in humus; hence the practice of adding decomposed leaves or vegetable mould to the roots at transplanting has the beneficial effects stated above. For general crops, a rich hazel loam, or deep rich alluvial soil, is next best; but for the most abundant of all, a strong loam, inclining to clay. For early crops, mild manure, such as leaf-mould, should be used, unless the soil is not exceedingly poor. If the soil is very poor, stronger manure should be employed. For general crops a good dressing may be given ; and for the dwarf kinds, such as Hair's mammoth, Bishop's new long pod, the soil can hardly be too rich. If poor, they do little good, particularly if, in addition to this, they be thickly planted.

The crop should be gathered as it becomes fit for use, for if even a few of them begin to ripen, young pods will not only cease to form, but those partly advanced will cease to enlarge.

Gypsum has been applied to pease as an auxiliary to farmyard manure, and the advantage is marked; and we have no doubt that, if it were applied as a top-dressing to the rows, at the rate of one cwt., and at a cost of about 3s., to a quarter of an acre, during the early stages of their growth, much benefit would result from its application in that way and at that period. It has much improved crops suffering from mildew, and those which have come up ill, or which have been injured by late spring frosts. It is more immediate in its effects when applied either before or during rain. Top-dressing with nitrate of soda has also been found of advantage under similar circumstances. The best manure, however, to be applied to pease, provided the ground stands in need of enrichment, is guano ; and we may once for all remark here, that, of all special manures, it is the best for almost all garden crops. It contains the ingredients required by most plants, and just in the proper state. We have used it with great advantage by following the practice of the Peruvians, who apply it to crops at three different times-viz., at the time of sowing, again when the plant is nearly half-grown, and a third time just previous to the ripening of the seed. With pease we have strewn a small quantity along the bottoms of the drills, which were drawn about 2 inches deeper than usual. This was mixed with soil, and about 2 inches of the common soil placed over it, upon which the pease were sown, and covered up in the usual manner. The quantity used was three pints of guano to a row 50 feet in length. The soil was dry at the time of sowing, and for a week afterwards, when genial showers fell, which we calculated would begin to render the guano soluble about the time the young rootlets had penetrated to its whereabouts. The plants grew stronger than those in the adjoining rows, which were not thus treated, and continued to keep the lead of them. When about a foot in height (the sort was Bishop's new long pod), $1 \frac{1}{2}$ pints of guano were dissolved in a tub of water containing 100 quarts: the row was watered with it. Just as we had gathered the first dish of pease, a similar quantity was applied. The pease continued to produce pods three weeks longer than the neighbouring rows, and the pods were much better filled and the pease larger, the joints of the straw much closer together, and the stems of the straw itself nearly half as thick again as under ordinary circumstances. Applying the guano dry under ground prevents the escape of 
its ammonia: the moisture in the soil renders it soluble; but in dry weather water should be applied to effect this the more speedily. The other rows had the same quantity of pure rain-water applied to them, and the soil was the same. A similar result followed the same experiment on beans, and on various other culinary crops. Our opinion is, that with guano, if the soil be in a proper state, and in the absence of stable manure, we are quite independent of all other so-called manures whatever; for although, for some purposes, they do some good, yet they can never be, like guano, of universal application.

Forcing.-Even in our present state of horticultural advancement, the pea is not very generally forced: it is, however, in some large establishments, grown in pots, boxes, and heated pits. Being a native of the more temperate regions, it does not prosper in much heat; that of a glasscase or late peach-house seems a proper medium. No doubt, ere long, pits will be constructed for the express purpose; and such structures as Mr Rivers' orchardhouses would be almost all that would be required. The very earliest and most dwarf varieties (vide Lists of approved sorts, \&c.) should be chosen. A rich vegetable soil should be employed. Pots with three or four seeds in each is the most probable means of attaining a crop. A temperature commencing at $40^{\circ}$ and $50^{\circ}$ during the day will be sufficient until the pods are beginning to form, rising gradually afterwards from $55^{\circ}$ at night to $70^{\circ}$ during sunshine. Forced pease should be invariably transplanted, to check luxuriance. If sown towards the end of October, with good management and in abundance of light, gathering may be expected by the 1st of March. After the pods are set, a little stimulant should be applied in the shape of liquid manure.

Approved sorts, and their qualities.-It is difficult, in attempting to do anything like justice to a subject so involved in confusion as the pea tribe has long been, and still is, to know whether it would be best to give a list of those names which have been sufficiently proved to be mere synonymes of one another, and of those kinds which are now quite unworthy of longer cultivation, or to confine ourselves to a notice of those which are really distinct, so far as their period of ripening, height, colour, and size can be considered as distinctive marks, and which are, on account of one or more of these characteristics, most deserving of cultivation. We think the latter mode will be the most generally useful.

As an instance of the deceptions carried on in the pea-trade, we may notice that of the Egyptian or mummy pea, said to have been raised from seeds taken out of a vase hermetically sealed, found in a mummy-pit in Egypt, computed to have been kept in that state about three thousand years, which has been found no other than the well-known branching marrow of our own gardens.

From the experiments made about two years ago in the gardens of the London Horticultural Society, under the direction of $\mathrm{Mr}$ Thomson, who sowed no less than 235 reputed sorts, all of which were at that time enumerated in seedlists, only twenty-seven of that number were selected as being really useful. This selection the editor of "The Gardeners' Chronicle" at that time thought might safely be reduced to half-a-dozen . During the years 1850-51, we sowed upwards of one hundred reputed sorts in the gardens at Dalkeith-fifty sorts in each of these years. They were in each case sown on the same day (25th March), in the same soil, and under the same circumstances. Out of that number we selected twelve as being truly distinct and useful; yet one half of these would be quite sufficient for even our use, who require them during the longest possible period. New sorts are yearly springing up, and therefore it would be injudicious not to give them a fair trial; for as we progress in pea-culture, as in everything else, we may naturally expect that improved sorts will arise and take the place of others that may be inferior. We have elsewhere stated that the height to which they grow regulates pretty nearly the distance at which they should be set apart, either between the rows, or individually in the line.

The following are the names of Mr Thompson's select list of eleven out of the above twenty-seven as determined at the above date; the heights and properties, \&c. we have added:-

1. Prince Albert.-From $2 \frac{1}{2}$ to 3 feet, according to soil; a white-seeded pea, forming with Kent's early, early hero, early Warwick, early May, and a lot of others, a section of which the true early frame is the type, and comprising our earliest sorts ; moderate croppers; pods contain from eight to ten peas each.

2. D'Auvergne. - 4 feet; seeds white ; remarkable for its long crooked pods, and the great number of moderate-sized peas each pod contains; one of our best for second or general crops. This is identical with Richardson's eclipse and Torwoodlea, two Scotch synonymes.

3. Dancer's monastery. $-4 \frac{1}{2}$ feet; seeds white; a good profitable sort for a second or general crop; peas of moderate size, rather above medium.

4. Bishop's new long pod. -2 feet; seeds white. A most abundant bearer, producing a succession of pods during most of the pea season. Like all dwarf peas of its class, it requires a rich soil, and from 4 to 6 inches between the seed in the line. We have had this pea producing a good supply for three months in succession. It is one of the most valuable sorts for small gardens, and for domestic use: its only 
fault in large establishments is the large size of the peas, but, although disliked by cooks on that account, it is much prized by them for many purposes. It originated with the late Mr David Bishop, author of "Casual Botany," and is a hybrid between Bishop's early dwarf, a pea of only 1 foot in height, and one of the marrowfats, carrying in itself the characters of both its parents.

5. Fairbeard's surprise. $-5 \frac{1}{2}$ feet; seed bluish; a profitable sort for a second or general crop. Pods thick, roundish, containing from seven to nine peas of excellent quality; the same as Fairbeard's early surprise.

6. Victoria marrow. $-5 \frac{1}{2}$ to 6 feet; seed bluish; an excellent sort for a general crop. Pods nearly 4 inches long, generally in pairs, containing from seven to eight large peas each; one of our very best peas.

7. Bedman's imperial.-3 feet; seed large; bluish green; called also Bedman's dwarf imperial; very similar to Flack's dwarf victory; perhaps scarcely worth growing as distinct sorts. Very prolific; peas large; excellent for greenpea soup.

8. Flack's new large victory. - Seed large; bluish green; 3 feet. Mr Thompson makes this distinct from the last. It seems to us to be a distinction without a difference. There would be, however, no loss in growing both these excellent peas under different names-much less, at least, than scores of others. Pease generally six in a pod, and of excellent quality.

9. Knight's tall marrow. -6 to 7 feet; seed large; wrinkled or indented when dry; white. Often called Knight's tall white marrow, to distinguish it from Knight's tall green marrow. Originated with the late Mr T. A. Knight (vide sect. PropAGATION BY SEED). Very much esteemed for its productiveness and fine flavour. Like all the tall marrows, only fit for a large garden, and then to be grown in single rows, at a great distance apart. Adapted for general crops. Pods large-from seven to nine peas in each; known also as Knight's late, Knight's tall green marrow, Knight's tall blue marrow.

10. Fairbeard's champion of England. -5 to 6 feet; seed large; wrinkled when dry; bluish green. One of the best of the wrinkled marrows, and well adapted for general crops, as well as early ones, being about as early as the Charlton. From seven to eight large peas in a pod.

11. Knight's dwarf marrow. -3 to 4 feet. There are two varieties of Knight's dwarf marrow, differing, we think, only in the colour of the dried seed, the one being white, the other greenish. However, either is valuable for a general crop. The bluish green variety appears to be preferred by Mr Thompson. Like all the marrows, the seeds are large. Pods large, containing six peas in each, and of excellent flavour; quite sugary.

To these we shall add from our own memoranda, resulting from our trial above referred to-

1. Hair's dwarfmammoth.-2 feet; seed large; wrinkly; bluish green when ripe. Equal in flavour to any of Knight's marrows, hitherto considered the best in this respect. A most productive pea, continuing, like Bishop's new long pod, in bearing for a long time, but somewhat later. Indeed, it is a truly second or general crop variety. It is larger in pod than Knight's dwarf marrow, and about seven days earlier. It requires highly enriched soil, and the peas to be planted from 4 to 6 inches apart in the line, as they branch out in the manner of Bishop's. One of the most valuable for small gardens and private families, and, like the latter, although large when sent to the table, if not too old, eats deliciously, having a great deal of the marrow property about it.

2. Lynn's prolific. -4 feet; seeds under medium size; wrinkled, and having a dark eye when ripe; seemingly a distinct variety of marrow; very productive, and stands drought well. Suited for a general crop.

3. Sutton's early Goliah. -4 feet; seed and pod large; in flavour resembling Knight's marrow-fats. Suited for a general crop, as it is an abundant bearer.

4. Early Charlton.-Too well known to require description, having been in cultivation for upwards of a century. We notice it here on account of its great hardiness and fitness for autumn sowing, to stand over the winter. How will those opposed to the doctrine of acclimatation account for this ? Originally it must have been as tender as the early frame, its constant attendant, which, like it, is also hardy; and both, with the exception of Hamilton's November prolific (which is no other than seed selected from the Charlton), stand the winter better than those of more recent origin.

5. The true early frame.-The type of the early Kent, of all the really early sorts, and probably of the next two.

6. Beck's morning star. -3 feet; seeds small; podding early and largely; pease a proper size for a first-rate table.

7. Sutton's earlychampion. -3 feet; resembling the last, only somewhat earlier.

8. Burbage's eclipse.-From 18 inches to 2 feet; seed large-from five to six in a pod; blue when ripe. An excellent bearer, having the young pease of a very proper size. Known also as Stubb's dwarf. Not so early as Bishop's new long pod; it is, however, a good dwarf pea for summer crops.

9. Groom's superb dwarf blue.-Under 2 feet; the most productive of its height; well adapted for small gardens, as a second cropper.

10. Woodford's dwarf. $-2 \frac{1}{2}$ feet; seed medium size; very dark green when ripe; a most abundant bearer, and well suited for small gardens as a principal crop.

The dwarfest of all peas are, the Spanish dwarf, Bishop's early dwarf, Thompson's early dwarf. These seldom exceed 1 foot in height, and in rich ground give fair returns.

11. Bellamy's early green marrow. -4 to 5 feet high; pods cylindrical, straight, containing generally six to seven peas; a good bearer and excellent pea.

12. Adamson's matchless marrow.-About 5 feet in height; pods curved, flattish, containing from six to seven peas. As early as the Charlton, and an excellent bearer. The following are 
so nearly related to it that it would be useless to grow them in the same garden-viz., tall Prussian, blue union, green nonpareil, tall imperial, tall blue imperial, tall green imperial, new tall imperial, Spanish patriot.

13. Blue Prussian.-A well-known excellent pea. We notice it here merely to give the synonymes - early Dutch green, fine longpodded dwarf, dwarf blue Prussian, royal Prussian blue, Prussian prolific, and green Prussian.

14. Woodford's green marrow. -3 feet in height; pods large, flat, containing six large well-flavoured peas, and an excellent bearer.

15. Dwarf imperial. - 4 feet in height; pods large, containing from eight to ten peas; a good bearer, and excellent for a late crop. Like all good sorts, has a host of names; viz., sabre, blue sabre, new sabre, dwarf sabre, imperial, blue imperial, dwarf green imperial, new improved imperial, new improved dwarf imperial, new dwarf imperial, new long-podded imperial, dwarf blue prolific, green nonpareil, blue scimitar, Sumatra.

16. Dwarf green marrow.-A good pea, but rather inferior to Knight's dwarf marrow. It is to be found in the seed-shops under the following names-New green nonpareil, Prince's superine summer, Wellington, extra green marrow, new green, early dwarf green, early green, new early green, royal dwarf marrow, Holloway marrow-fat, green rouncival.

17. British queen.-Height from 4 to 5 feet; pods large, containing seven very large peas in each; sometimes a single pea measuring $1 \frac{1}{2}$ inches in circumference. Hence too large for a firstrate table, but excellent for private family use.

18. Hair's defiance Knight's marrow. -4 feet high, remarkable for its strong habit, should be planted from 4 to 6 inches apart in the rows, and each row 4 feet distant. A remarkably profitable pea, of large size, and continuing long in a bearing state.

19. Tall crooked sugar.-Pois sans parchemin à grandes cosses-grosse schottige zuckererbse; a late rambling sort.

20. Dwarf crooked sugar.-Pois sans parchemin ou mange tout-zwerg zuckerschotte.

To those who intend growing this section of pease, we would specially recommend-

21. Dwarf sugar, or Ledman's dwarf--Growing about 3 feet high; pods long, cylindrical, and slightly curved; rather late, but a good bearer.

22. En eventail.-About 1 foot in height; assuming the habit of Bishop's long pod, and, like it, branching close to the ground; a moderate bearer.

23. Tamarind or late sugar-pea.-The best bearer, although the latest, in the section; nearly 4 feet high; pods from 4 to 6 inches long, proportionably broad, and slightly curved.

'The French grow many varieties of edible podded peas; and although suitable to their taste and climate, they are not so with us. The Dutch grow two sorts, and even these, for the most part, are found so tender, even in $\mathrm{Hol}$ land, that they are generally produced under glass.

Should, however, Messrs Weekes \& Co.'s new pea, recently brought into notice, turn out as represented, it will, as an edible podded sort, supersede these and all others of a like property, in bulk of produce at least, and may be found far more economical to cultivate, as an article of food, particularly for cottages, than any at present known. It is described as a new hybrid (the parents, however, are not stated), attaining the height of 4 feet; stem slender; great bearer, and exceedingly ornamental for its flowers and singular pods, which measure $5 \frac{1}{2}$ inches in length, and $1 \frac{1}{4}$ inches in breadth; of a glaucous green colour, each containing sevell seeds. It can either be cooked in the ordinary way (boiled, when young, whole, pod and all), or left to attain a more advanced growth, and then cut up and eaten like a French or kidney bean. Being so much hardier than either the kidney bean or scarlet runner, and yielding a produce even exceeding the latter (which at present is, we think, were it not for the short period of its existence, the most profitable vegetable that the cottager or small gardener can grow), this would even excel it for such a purpose. Should this new pea, upon further trial, equal the specimens grown last year, it will no doubt be considered the greatest novelty of the pea tribe that has yet appeared.

The following sorts stand in good estimation amongst growers :-

Early Warwick, $3 \frac{1}{2}$ feet-a sub-variety of early frame; Thurstone's reliance, 6 feet-one of the largest peas in cultivation; scimitar, 3 feet-an old variety, long podded, and fills well ; ne plus ultra, 6 feet-a green wrinkled marrow; old dwarf marrow, $3 \frac{1}{2}$ feet ; tall green mammoth, 6 feet-similar to Hair's dwarf mammoth in pod; matchless marrow, 5 feet; Melford marrow, $4 \frac{1}{2}$ feet.

“Warner's early emperor, Warner's early conqueror, early Bedalean, Essex champion, early railway (or Stevenson's railway), and early wonder, have been proved in the gardens of the London Horticultural Society to be all varieties of the old early frame, and possessing no one merit over the original. Danecroft rival, Danecroft early green, Farnes' conservative, green marrow, and the transparent pea, are by $\mathrm{Mr}$ Thompson considered to be all one variety. Clark's Lincoln green podded new early marrow - no marrow at all, but one in the way of the early frame. American dwarf, a good bearer, ripening about a week or ten days later than Bishop's new long pod-a very good dwarf variety. Early surprise, from a foot and a half to 2 feet in height; pods large, thick, containing generally six large blue peas, the plants having the strong stems and vigorous habits of the marrows. Early blue surprise identical with Fairbeard's early surprise. Queen of England, a sort of white marrow, inferior to the British queen. Waite's king of the marrows resembles the ne plus ultra. Great Britain similar in every respect to Knight's tall white marrow. Hunter's new marrow, about the same height as Knight's dwarf marrow; pods roundish or a little flattened, containing about six large peas ; larger than Knight's; of very sugary quality; when dry, indented; yellowish white; a good bearer."-Ex Jour. Hort. Soc., vol. v. p. 283. 
From experiments made in the London Hor. ticultural Society's garden on the following

peas, their period of coming to perfection was ascertained as follows :-

\begin{tabular}{|c|}
\hline k's Prince Albert, \\
\hline
\end{tabular}

-These are sub-varieties of the early frame, differing only in time of coming to bear.

Prince Albert, . . Sown March 28, 3 feet in height, Fit for use June 19. One of the earliest of peas.

Bishop's early dwarf, : 9 inches

$\begin{array}{llll}\text { Bishop's early dwarf, : } & 9 \text { inches } \\ \text { Early race-liorse, } & 3 \text { feet } & \text { Jor } & \text { June 29. An inferior var, of early frame. }\end{array}$

Shilling's grotto,

Dwarf green marrow, .

Blue Prussian,

Matchless marrow, -

Lynn's wrinkled marrow,

American marrow, .

Blue scimitar,

Bedman's blue imperial,

Flack's Victoria,

Victoria marrow,

Auvergne,

Groom's superb blue,

3 feet
3 feet
2 feet
3 f feet
4 feet
2 feet
3 feet
3 feet
$2 \frac{1}{2}$ feet
6 feet
4 feet
2 feet

June 29. An inferior var. of

waly 10. A good cropper.

July 10. A good cropper.

July 17. An excellent large pea, and productive.

Aug. 1. Good late sort.

July 17. Good pea, and abundant bearer.

July 25. A good bearer.

July 25. A good bearer.

July 17. Large pea, and good bearer.

July 25. Large pods.

July 17. An excellent bearer.

-The heights given above are those to which they attained in the Society's garden.

The following experiment regarding the precocity of the following peas was made in the garden of the Horticultural Society by $\mathrm{Mr}$ Thompson. Three varieties of early frame, amongst which was the true early frame from Paris, were sown in continuous rows on March 13. They proved to be the same, and were fit to gather June 9. Early Kent, fit June 2. Warner's early emperor, fit June 4. The two last are varieties allied to the early frame, the early Kent differing from it in being a week earlier, and not so strong-growing. "Warner's early emperor is not quite so early as the early Kent, and its growth is intermediate be. tween the early Kent and the early frame. Fairbeard's early surprise was sown April 23, and was fit to gather June 27 ; but the early frame, sown at the same time, was fit June 19. Fairbeard's is therefore eight days later than the early frame, and consequently, according to the foregoing statement, it would be a fortnight later than the early Kent." This pea, therefore, cannot rank amongst the earliest, but will hold a good place in the second early section along with the old Charlton, to which it seems related.

The following six varieties of dwarf pease will form a good succession, and will be found suitable for a small garden, or where ground is scarce:-The true early frame, Fairbeard's early surprise, Groom's superb dwarf blue, blue Prussian, Milford marrow, and Knight's dwarf marrow-sown in the order in which they stand.

Insects and diseases.-The greatest enemy to the pea tribe is the pea weevil. This destructive family of insects, the weevils, are enough of themselves to eat up the whole vegetation of the globe. M. Schonherr, a celebrated Swedish entomologist, spent thirty years in investigating their economy ; the results of his investigations have been published, and occupy no less than 7000 pages, octavo, in print. Of this formidable host we have between four and five hundred species existing in Britain. Two of these, Sitona lineata, the striped pea weevil, fig. 7 ; and Sitona crinita, the spotted pea weevil, may often be detected eating the young leaves and stems as soon as they appear above the ground, partiFig. 7. cularly in dry hot weather.

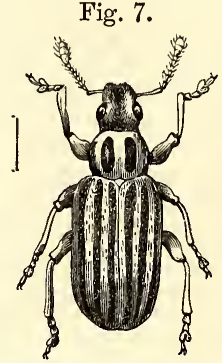
These pests will, in general, be found in full operation in June. The remedies suggested by the writer of a series of articles on entomology in "The Gardeners' Chronicle" are worthy of notice. "Any remedies, therefore," he says, "which we can suggest, must have for their object either the destruction of the perfect beetle, or the STRIPED PEA weEvil. protection of the plants-
neither of which is easy.
As to the former, we scarcely think that any STRIPED PEA WEEvil. protection of the plants-
neither of which is easy.
As to the former, we scarcely think that any As to the former, we scarcely think that any would creep at night (like damp grass, into which the wire-worm creeps; or bits of potatoes put into the ground, to which, as food, the same insect is enticed); possibly, however, dry hay laid along the rows might entice them into it as a retreat. Another means of destruction suggests itself, in connection with the habit of the insect of falling to the ground on being surprised. A bag-net about 2 feet long, and with one side flat, so as to allow of its being placed on the ground close to the sides of the rows of the pease, would, we think, be serviceable. This might be run along the rows, the plants being slightly swept over by a switch held in the right hand, the handles of the bag-net being held in the left hand; or, perhaps, by merely running the net along or across the rows, they might be jerked into it. As to the protection of the plants, soot and pounded lime have been suggested to be sprinkled over them, previously wetting them by a watering machine. In this respect the same kind of remedies must be used as have been proposed against the turnip flea-beetle, having for their object the rendering of the plant disagreeable to the insect by a coating of matter offensive to its taste ; or by forcing forward the growth of the plant as quickly as possible. We may also suggest the possibility of advantage resulting from drawing a cloth covered with pitch or tar 
over the rows of the pease : the insects might become fixed to the cloth, and might be easily destroyed."

Besides these there is a small beetle, Bruchus

Fig. 8.

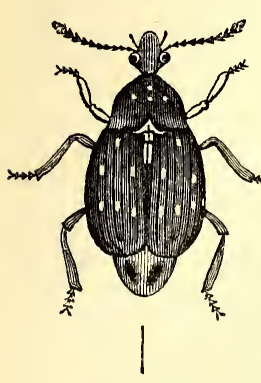

PEA BEETLE. pisi, L., fig. 8, which deposits its egg within the pea, which serves the grub for food, and is thus destroyed. It abounds most in dry seasons, and for it there seems no great chance of a remedy. It is, however, not very injurious to us in its attacks.

The description given by $\mathrm{Mr}$ Curtis, in the "Journal of the English Agricultural Society," vol. vii. p. 408 , of this insect is good:- "They pair in summer, whilst the pease are in flower, and producing pods ; the females then deposit an egg in almost every pea that has almost just formed. From the outside of these peas, when arrived at maturity, they do not appear damaged ; but, on opening them, one generally finds a very small larva, which, if left to repose, remains there all the winter and part of the following summer, consuming by degrees all the internal substance of the pea, so that in the spring the skin only remains; after which it is transformed into an insect, with scaly wing-cases, which pierces a hole in the skin of the pea, from whence it comes forth and resorts to the fields sowed with that pulse, in order to deposit its eggs in the new pods."

The American mode of destroying the pea bug, Bruchus pisi, is thus stated in "Hovey's Magazine of Horticulture:"-“Immediately after gathering the seed, it is sulojected to the action

Fig. 9 .

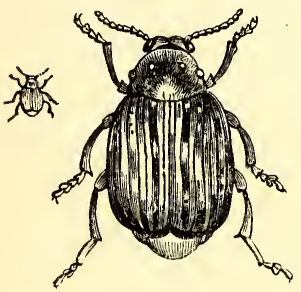

GRAIN BEETLE. of boiling water for one minute; by this means the grubs, or larvæ, which at this time are just below the integuments of the pea, are destroyed without injury to the vitality of the seed."

Another species, Bruchus granarius, fig. 9 , is often found in seed-rooms, in seed-peas; and the holes in the pea and bean

Fig. 10.

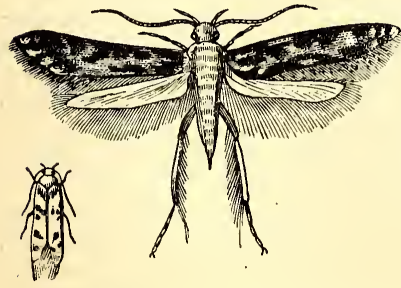

WHITE-SHOULDERED WOOL-MOTH. from which itissues after its transformation, are frequently occupied by the caterpillar of the white-shouldered woolmoth, Tinea sarcitella, fig. 10 , the well- known domestic pest, which lays its eggs on all manner of woollen stuffs, to the great annoyance of every thrifty housewife. They are often found in the seeds of pease and beans, and, in company with the Bruchus granarius, and the larvæ of a species of saw-fly, work sad destruction in the stock.

The Bruchus granarius is thus described in the " Cottage Gardener," vol. iii.p. 13:-“"These holes in the peas and beans are made by this beetle, which is produced from a grub or caterpillar, which has eaten away the vital parts of the seed; and when it has passed through the chrysalis state, and given birth to this beetle, the latter makes the hole in order to escape into the open air, there to perpetrate more mischief upon the growing crops. The body of the beetle is a dull brown, but the elytræ, or wingcovers, are black, dotted with white, but scarcely perceptibly so, unless magnified, as in our drawing. Naturally it is the size of the smaller figure, and is scarcely two lines long. The antennæ are eleven-jointed, black, and thinnest near the head, where they are also tinged with red. The head droops, the eyes are prominent, the fore-legs are rusty coloured. The female pierces through the pod of the pea or bean whilst very young, and often deposits an egg in each seed." This insect may be destroyed by the American mode of dipping the seed in boiling water, as noticed above.

The pupæ of the fly Phytomyza nigricornis, the black-horned leaf-miner, fig. 11, feed on the

Fig. 11.

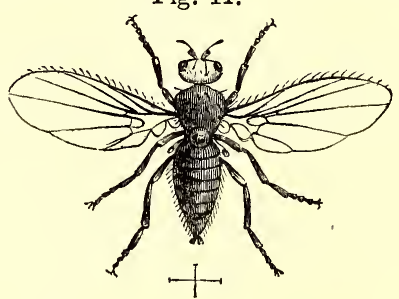

BLACK-HORNED LEAF-MINER.

parenchyma of the leaf, causing minute brown spots in it. The only mode of riddance appears to us to be picking off the infected leaves and burning them, to prevent the further breeding of the insect. The cross lines show the natural size of the insect.

The next serious enemies to the pea are mice and rats. As preventives, rubbing the pease with powdered resin, placing over them in the drills chopped furze, dusting them with lime, sowing charcoal dust along with them, have all been tried with more or less benefit. No plan is, however, so effective as catching the vermin in traps. Poison is dangerous, and seldom so carefully concealed but that some poor bird or other falls a victim to it. The best traps are the cage trap, baited with toasted cheese or broiled bacon; the next best, the old figure four trap, which has been used for this purpose above two centuries;; or the suspension trap, the most simple of any. It is constructed by soaking a few peas in warm water, and when they have 
begun to grow, a pretty strong thread is passed by a needle through the peas, leaving two of them on every 9 inches of thread, at which lengths the thread is cut off. Tie a knot at the end of each length ; take two pieces of straight wood, cut them into 1 -foot lengths, make a slit about an inch deep in the top end of each, and stick them in the ground near the line of pease. The thread is then drawn through the slit ends of the sticks, and a brick is placed with one end resting on the ground, and the other resting on the thread about 3 inches from its end, the two peas on the thread being near the middle of the brick, and 2 inches apart. The mouse, in attempting to eat or take away the peas, cuts the thread, and lets the brick down upon itself. It has been stated that mice will not take peas until they have begun to vegetate, and this is given as a reason for sprouting the bait: we do not think them so very fastidious; but if it is really so, they may be set the one way as well as the other. If people would only persevere with any of these traps, they would soon rid their garden of such pests, without the dangerous expedient of using poison. Phosphorus paste may be safely used in gardens for the destruction of both rats and mice. It is thus prepared: To 8 parts of phosphorus, liquified in 180 parts of lukewarm water, placed in a mortar, add immediately 180 parts of ryemeal (any other meal will do as well); when cold, mix them up with 180 parts of butter and 125 parts of sugar. These animals will greedily devour this mixture, after which they will swell out and die. It may be kept for many years without losing its efficacy, and can never injure human beings on account of the smell. This receipt was published some years ago by order of the Prussian government. Meal, butter, and sugar, mixed dry with plaster of Paris, and laid down where damp cannot affect it, will have a similar effect. The animals eat it readily. It soon afterwards causes in them a great thirst; as soon as they take water, the plaster of Paris powder swells out and bursts them. Pease, beans, or any other grain, steeped for several hours in water in which nux romica has been boiled, and placed in the way of mice, destroys them without even endangering the life of the cat that may afterwards eat them.

Amongst the feathered tribe, the sparrow, tomtit, chaffinch, and jackdaw are very destructive to pease at most seasons, but particularly so in spring, when their other kinds of food are less plentiful. The three first of these we can ill dispense with, because of the valuable services they render us in the destruction of myriads of even more formidable enemies during summer; as for the latter, we can hardly say a word in his favour, further than that we know God made nothing in vain; and even this "gentleman in black" may be of far greater service to us, in some less direct way, than we may be aware of at present. We never destroy birds, believing them far more useful than hurtful, which we hope to show more clearly when we come to speak of fruit and fruit-tree buds. To protect young pease in spring, we dust the rows over every morning, or every second one at furthest, with a little hot lime in powder, so as to render them quite white. Our mode of application is to have a stock of lime in a dry place, and to send a boy with a thin canvass bag in each hand, containing the lime in powder, and as he passes along between the rows, he shakes the bags over the pease, when the finer particles, passing through the canvass, powder the pease quite white, in which state these creatures will seldom touch them. This process answers another end -it greatly saves the crop from the pea weevil. The operation is best performed when the plants are dry, else the caustic property is soon destroyed. As the attacks of birds take place generally soon after daybreak, the dusting is in general done in the evening previous; but, no doubt, it would be more effectual if done soon after daylight in the morning. The jackdaw not only crops off the tops of the young plants, but, preferring the seed, it with great sagacity often begins at the end of a row, and abstracts every pea in the line. Liming the surface pretty thickly tends to keep them away, but no means are so thoroughly effectual as having wire cages, of a semicircular shape, and in lengths of from 6 to 8 feet, or more, to place over the drills, and these can most readily be thus constructed: Take a web of octagon wire-netting, 18 inches in breadth, cut it into convenient lengths, lay them flat over the garden roller or any other cylindrical body, bend them down at the edges, and by this mode a dozen of cages may be made in half an hour. Such cages are of vast importance in a garden : they not only protect pease from the attacks of birds, but they protect all other crops in like manner, and are valuable for laying over parsley, endive, young cauliflower plants, lettuce, \&c., during winter, when a little litter is laid over them, and frost thus repelled. The litter can be removed on fine days, and put on again when required, without injury to the crop. Such netting, 18 inches wide, costs $9 \mathrm{~d}$. per lineal yard; and cages so formed will last for years, if not wantonly injured when out of use.

In forming these cages, it will be of advantage, for giving them greater strength for moving about, as well as for keeping them in proper shape, if a bar of round $\frac{1}{2}$-inch iron be secured along their bottom sides-which can readily be done by a handy labourer, by splicing the edges of the netting to the bars along their whole length, and welding on a cross-bar at each end of the same sized bar, and one or two, according to the length of the cage, across from side to side. Such cages are next to invaluable, as means of protecting from cold and cutting winds all newly-planted crops in lines, as a few evergreen branches may be tied tightly to them, so as to prevent their being blown away; or waterproof canvass, or asphalt felt, may be employed when keeping the crop dry is an object. Two laths of wood may be used instead of the rods of iron; but they, of course, would be less durable.

We were driven to this expedient some years ago, when grievously persecuted by pheasants, and where to have killed one would have been accounted little less than a high misdemeanour. 
The jay is a sad plunderer of early pease, and where they abound, will have the first fruits of the crop in spite of fate. They are, however, becoming scarce in the country, and in some localities are rarely seen. The same may be said of the wood-pigeon, the most voracious of all birds. We hesitate not to shoot them. They were designed for food, and in no way are they better prepared than stewed with green pease.

Another enemy of the pea is

The mildew Erysiphe communis var. Leguminosarum, a parasitic fungus. The cause of its appearance was correctly enough determined by T. A. Knight forty years ago. He says"The secondary and immediate cause of this disease has long appeared to me to be the want of a sufficient supply of moisture from the soil, with excess of humidity in the air, particularly if plants be exposed to a temperature below that to which they have been accustomed. If damp and cloudy weather, in July, succeed that which has been warm and bright, without the intervention of sufficient rain to moisten the ground to some depth, the crop is generally much injured by mildew."-KNIGHT's Horticultural Papers, p. 206. While engaged in the production of those excellent peas which bear his name, he proved this theory by warding off mildew by copious waterings of the roots. The fashionable remedy at present is the use of one of the rival sulphurators. This no doubt subdues the disease, but it does not remove the cause.

General remarks.-Early crops of peas derive great benefit from reflected heat when planted at the bottom of a south wall. It is necessary, however, when warm sunshine follows cold frosty nights, to shade the pease from its influence an hour or two in the morning, or to sprinkle them with cold water if they have become at all frozen. Such precautions are also necessary when they are sown on ridges or sloping banks facing the south, which is often done, and with evident benefit in forwarding the crop. Now that glass is so cheap, we see little reason for those matter-of-chance practices, when a better end would be attained by covering the rows of pease with a narrow glass frame, made of a triangular form, and glazed on both sides, or on one only, according as they may be used, on rows running from north to south, or from east to west: in the latter case, such frames may have glass in the south side only.

The saving of seed is an important matter, and is confided to a class of cultivators known as seed-growers, many of whom will have from 10 to 40 or 50 acres under this crop. Some of the London trade have ground of their own, others rent ground; but by far the greater number contract with seed-growers, supplying them with the genuine varieties, visiting the crop from time to time, sending proper persons to rogue or pull up all spurious plants, and generally to report progress. The expense incurred for labour and rent, taxes, \&c., is great; so much so, indeed, that one wonders how a pint of pease could be purchased for such a trifle. Private growers do not economise by saving their own pease for seed, unless they have, by VOL. II. keen observation, detected one or more plants possessing more than ordinary merits; then they are right in carefully saving those, for in this way, for the most part, have all our finest peas been produced. Their being ripe will be readily discovered by the straw beginning to dry up, and the pods and peas showing evident symptoms that they are ready for housing. If the quantity be small, put the pods, with the peas in them, into canvass or paper bags, and suspend them to the roof of a dry and airy seedroom. If, however, the quantity be large, leave the pods attached to the haulm, and stack them by till the time of sowing or selling, when they may be thrashed out like other grain. Peas kept in the pod will retain their vegetative action for two or three years, and hence those sent to the colonies should be retained in the pods for greater preservation. When taken out of the pod, even when perfectly ripened, they rarely vegetate after eighteen months, and many not at the expiry of one year.

Pease in a green state are with difficulty sent to a distance, as, when packed closely together, fermentation speedily takes place. This is one of the causes why imported pease, and many of those brought from a distance to our markets, are discoloured, devoid of flavour, and, worst of all, very unwholesome to eat. Pease sent to a distance should be packed in open baskets, not in boxes, and laid in layers not more than 2 inches thick each, and, between such layers, a thin stratum of dry fern or straw should be placed.

The European names are, Pois, in French; Piselli, in Italian; Ervilhas, Portuguese; Erbse, German; Pesoles, Spanish; and Erwt, Dutch.

The quality of the garden-pea as a nourishing article of human food, compared with bread, butcher's meat, and some other vegetables, has been well ascertained to be in the following proportions :-

\begin{tabular}{|c|c|c|c|c|}
\hline \multicolumn{5}{|c|}{$100 \mathrm{lb}$. of pease contains of nourishing matter, $93 \mathrm{lb}$. } \\
\hline , & seed of Haricot kidney & beans, & - $\quad 92$ & \\
\hline , & seed of garden-beans, & . & 89 & , \\
\hline ," & wheaten bread, & $\dot{i}$ & 80 & ," \\
\hline ", & average of butcher's m & at, & 35 & \\
\hline , & potatoes, . & - & 20 & ", \\
\hline , & carrots, - $\cdot \dot{n}$ & • & - & \\
\hline & canoages and turmps, & - & & \\
\hline
\end{tabular}

The composition of the field-pea is thus given by Mr Stephens, in "Book of the Farm," and as little difference, if any, can exist between these and the garden varieties, it may be taken as a close approximation to the truth-

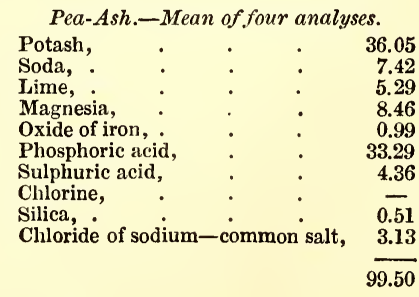

The chemical composition of pease, Mr Stephens observes, has not yet been carefully investigated. Let this statement suffice- 
Composition of the Grain. Water. Husk. Meal. $\begin{array}{lll}14.0 & 10.5 & 75.5\end{array}$

Composition of the Meal.

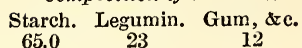

The inorganic constituents in the pea-

100,000 parts of seed contain 2464 , and 100,000 parts of the straw contain 4971 parts of inorganic matter, consisting of-

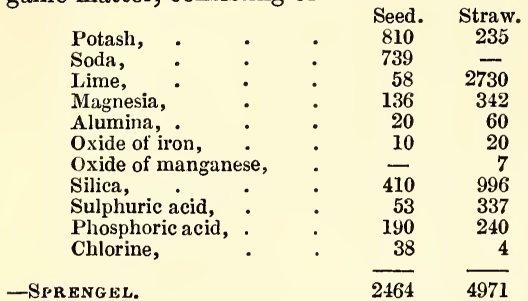

The per-centage of mineral ingredients taken from the soil is computed to be-pod, from 2.5 to $3 ; 7.1$ husk; 4.3 to 6.2 straw.

\section{§ 2.-THE BEAN.}

N'atural history.-The garden or broad bean (Vicia Faba L., Faba Tou., vulgaris Moen.) belongs to the natural order Leguminosæ, subdivision Sarcolobæ, tribe Vicieæ, and to the class Diadelphea, and order Decandria, in the Linnæan arrangement. The genus Faba contains only two species-the common garden-bean and the horse-bean, F. equina. The name Faba is derived, according to Isidorus, from phago, to eat; according to Martinius, from $p a b a$, to feed; while others will have it from haba-all of which are modifications of each other. The origin, however, is evidently Greek.

That the East, and probably Egypt, is the native country of the bean, is pretty generally admitted. This appears to be the most ancient of all our now cultivated esculents. The earliest notice we have of the bean is of those brought by the three loyal Israelites who followed King David, and presented him with beans when he fled across the Jordan from his rebellious son Absalom. One of the noblest families of Rome, the Fabii, derived its name from a circumstance connected with this plant. The Athenians used sodden beans in their feasts to Apollo. The Romans had a solemn feast called Fabaria, at which they offered beans in honour of Carna, the wife of Janus, whose palate, according to Lemprière, was gratified by the addition of bacon; hence the origin of bacon and beans is of far older standing than modern chawbacons suppose. Pythagoras enjoined his followers to abstain from beans, professing to believe that at the creation man was formed of them. The Romans believed at one time that the souls of such as had died resided in beans. Clemens Alexandrinus, Theophrastus, and even Cicero, entertained equally extravagant notions of them. The Egyptian priests held it a crime even to look at beans. The Flamen Dialis was not permitted to mention the name; and Lucian introduces a philosopher in hell saying, that to eat beans and to eat our father's head were equal crimes. A more rational use is stated by
Philips to have been made of them by the ancients, namely, "in gathering the votes of the people, and for electing the magistrates: a white bean signifying absolution, and a black one condemation;" and hence he supposes the practice was derived of black-balling obnoxious persons. Bean's grew wild in Morocco in Pliny's time; and he says some were so tough and hard that they could not be boiled tender. This is confirmed by the circumstance of one of our now most popular sorts, the Mazagan, having been introduced to us from a place of that name on the coast of Morocco.

At what period the first beans were introduced to Britain is not now known. It must, however, have been at a very early period. Some suppose they were introduced by the Romans : we can hardly suppose this, as they could not have been in any variety, which is tantamount to their not having been extensively grown, in Gerard's time, who says the gardenbean is the same as the field one, only improved by cultivation. One peculiarity in the bean is worth noting: the ancient authors mention only one bean, and Gerard appears only to have known two; and even at this day, they have not multiplied in varieties to any extent like the pea.

$U$ se.-The garden-bean is much less in estimation amongst the higher classes than the pea; and hence a much less proportionate breadth of it is required in first-class gardens than in those of less pretensions. It is, and always has been, the vegetable of the lower and middle classes, more used in England amongst them than in Scotland and Ireland, and by no means at all common on the Continent, even in vegetarian communities. It is used in its young state, in which state only it should be employed, as an article of luxury: the want of attention to this is one of the chief causes why it so seldom appears upon tables polite. Amongst the peasantry it is used in its full grown state, as an accompaniment to bacon. The white-blossomed bean, if gathered while quite young, makes an excellent dish, particularly if served with bacon or ham. The lower classes use them when full grown, at which time, although nutritious for strong constitutions, they are very unfit for persons of delicate digestion-for they are, particularly the inner skin, exceedingly indigestible.

Columella notices them, in his day, as food for the peasants only:-

"And herbs they mix with beans, for vulgar fare."

An English poet somewhere says, in allusion to the same,-

"And give them beans and bacon, till they burst."

It was a prevailing opinion among the ancients that beans were flatulent; and Hoffman and others among modern physicians assert the same; and not only that, but that the greener they are, the more they are so. Dr James says young beans are both a wholesome and nutritious food; but the now prevailing opinion is, that they are a flatulent and coarse food, better suited to the hard-working husbandman than to those of sedentary habits. Boyle, to ascertain the amount of air they afford-for on this their flatulency depends-treated them pneu- 
matically, and found that the expansion of a single bean while growing is capable of raising a plug loaded with one hundred pounds' weight. The beans are the usual part of the plant used as food; but Philips informs us that the green pods, boiled after the beans are removed, is a dish that many people prefer to the beans themselves, and that the pods should be served with parsley and butter, as boiled beans usually are. He adds also, that the young beans, boiled in broth, are esteemed highly emollient. Parboiled beans, boiled in a weak syrup of honey and musk, make an excellent bait for fish.

Propagation.-All the bean tribe, being annuals, are propagated by seed.

Planting.-Crops to come in earliest in the succeeding season are sown or planted (for beans are said to be planted, and not sown, as they are set at a greater distance from each other than pease and similar crops that are set thicker or sown) in the latter end of October, in November, December, January, and so on till May, sown after which they would scarcely come to perfection. Once a month for the early crops, and once a fortnight for the two general crops, is sufficiently frequent, few even sowing above three or four crops at most during the season. These periods apply to the open borders. They are also sown after the manner of pease (which see), under cover, and transplanted, as will be noticed below. Beans planted in March are fit for gathering in June, and ripen their seed in July.

The ground having been prepared by trenching or deep-digging, and well manured, drills are opened with the hoe (as has been described for pease) 3 inches deep; the drills for the Mazagan bean, which, on account of its hardiness, is preferred for autumnal planting, should be $2 \frac{1}{2}$ feet apart, the beans being set in the lines 3 inches distant from each other: the soil is drawn over them with the hoe or a coarse rake, and left quite rough on the surface. Finely raking the ground over such crops is an absurdity, but old habits are difficult to set aside. The opener the soil is left, the better it excludes frost; the smoother it is made, the more likely is it to cake, and become, in some soils, so hard that, were the beans not possessed of the extraordinary expansive power stated by Boyle, and noticed above, they could hardly force their way through it. True it is that the crust might be broken by drawing the rake over it, but in this operation there would be a great chance of breaking the necks of the beans at the same time. A crop of lettuce or early cabbage may be planted between the rows, either of which would come off while young, for use, before the beans arrive at a height to injure them. This is the practice of the London marketgardeners. We should here, however, state, for the information of that class of gardeners who think a cabbage is unfit for use until it be as hard as a cannonball, and almost as large as a drum, that early cabbage is in most estimation when quite young and tender, and just before they begin to turn in their leaves, or form a solid heart. This is an eligible situation for such a secondary crop, as the first planting of beans should be made on a warm well-exposed border. The same precautions will be required to save the crop from mice and other enemies as are recommended for pease. In cold damp soils and late situations, the rows of beans at planting may be covered with 2 inches of finely-sifted coal-ashes, rotten tan, or the like : either will exclude frost and counteract damp. Not, however, that frost is to be dreaded, for the bean will bear a considerable amount of it; but the principal intention is to keep the soil dry and prevent the escape of heat which it has absorbed during summer, and with which it has not by the end of October altogether parted.

In planting the secondary and principal crops, more open situations should be chosen ; and for them it is important that the ground be moderately enriched, and trenched at least 2 feet in depth. Trenching is an assistant to manure; in some cases it is even more than a substitute. In regard to distance, that depends, as has been shown in the case of pease, mainly on the sort of bean to be planted, and the height it is to attain. The new royal dwarf cluster is 1 foot in height; a distance of 18 or 20 inches should be given, as it branches out close to the ground-and even at that distance, in good ground, will nearly cover the whole surface. For the white blossomed, which attains the height of from 3 to 4 feet, that space, or rather more, should be allowed. Marshall's early prolific is about 18 or 20 inches high ; give it, therefore, a proportionate distance. Almost all the 
rest, with the exception of the dwarf tan or bog bean, which is not worth notice, grow about 3 feet or more in' height: they require, therefore, the largest space; and as has been, we hope, clearly enough stated in the case of pease, that there is great loss in crowding such crops, we would advise 4 feet apart for each of these. Regarding distance plant from plant, the new royal cluster branches out, forming a dense bush crowded with pods; it should be planted a foot apart in the line, and the seed placed $2 \frac{1}{2}$ inches deep; the white blossomed should be placed 3 inches apart in the line; Marshall's prolific as much, as it is a branching one to some extent, and the same depth as the last: the rest should not be nearer than 4 inches in the line, and all of them $3 \frac{1}{2}$ inches deep.

Beans forwarded for transplanting, as has been recommended for pease, should, as soon as they are 4 inches high, if the weather be favourable, be brought out and set in their permanent place. For this the best way is to stretch a line from end to end of the ground in a south and north direction (for reason, vide PEA). The surface is then smoothed down along the line, and a trench taken out with a spade, as in planting box-edgings, and to the depth of 5 inches, so that the roots may be the better arranged. Along the line set the plants at distances apart according to their kinds as specified above, taking them carefully out of the pots or boxes they have been growing in, separating them so that their roots receive no injury; cover the roots carefully, and proceed with the next row in like manner, pointing over the ground as the operation proceeds, and leaving it as rough as possible. A few branches of any deciduous tree should be stuck along each row, on the side the prevailing winds of the season blow from, avoiding evergreens, as, while they no doubt shelter the plants best, they are apt to become blown about by the wind themselves, and may do more harm than good. The wire cages we have spoken of in the article PEA will be found exceedingly useful for setting over the newly-planted beans, and to them a few branches of evergreens might be firmly tied. This would prevent their being blown about, and afford a much more efficient protection at the same time. Of all modes of protecting such crops, short of glass-cases, we believe there is none better than this. From the distances we have given, there will be no difficulty in calculating the quantity of seed required. The following is Abercromby's allowance, and his, as he was so minute in such matters, may be taken as the greater quantity-we, since his day, planting much thinner: For early crops, one pint of seed for every 80 feet of row ; for general crops, two quarts for every 240 feet; and for late crops, nearly the same as for the early ones. This is presuming the smaller kinds to be employed. In summer, it often happens that the ground is too dry to promote speedy germination in seeds so dry and hard as the bean. Some have recommended, in this case, steeping the beans for an hour or two in rain-water previous to planting. A much better way is to soak the ground with water as soon as the drills are formed, to plant the seed, and cover up immediately. Virgil says that soaking beans in lees, or dregs of oil and nitre, has the effect of causing a more rapid vegetation, and the production of a larger crop; and other ancient authors recommend their being steeped for three days in water mixed with urine.

It is a common practice in cottagegardening economy to plant beans along with crops of potatoes, and also with cabbage, setting a bean between every two plants in the line. Speechley, in "Practical Hints," p. 17, recommended this, and brought forward his beans first in a bed thickly sown, and in a warm place-when of a fit size transplanting them, setting a bean alternately with a potato or cabbage in the same row ; but in such cases he had his rows 3 feet apart, and his potatoes 18 inches apart, so that the bean is 9 inches from the potato on each side. Being advanced somewhat before planting, the beans have the start of the potatoes, and are matured and removed before they injure the potato crop. The propriety of this mode has been questioned; but we have seen excellent crops of both vegetables so produced. In garden-culture the process of transplanting beans is very advantageous: it moderates their growth, so that they do not incline to increasein height after their flowers are set, and induces precocity in the maturing of the crop. 
Instead of planting in continuous lines, a good way is to plant in detached patches of four or five seeds each, at distances agreeable to those given above.

Subsequent cultivation.-Beans, like all other crops, require to be kept clear of weeds, and to have the soil well stirred up between the rows during their growing season. When they have attained the height of 6 inches, it is proper to draw a little earth to the stems, merely to keep them steady, as the bean is not disposed to send out horizontal roots so near the surface as to derive any advantage from the soil thus gathered round them. Nor is it a usual practice to afford them any support, as in the case of the pea, although no valid reason can be given to the contrary. True, the bean is not a tendrilled climbing-plant like the pea, and hence could not attach itself to the supports presented to it; but we have seen the tall-growing sorts so often beaten down by strong winds, even in not very exposed places, that we often do, in defiance of usual practice, support them when in lines, by driving in, along the rows, stout stakes, 6 or 8 feet asunder, and running a line of tarred cord along both sides of the plants, at the distance of about 15 inches apart, the first one being that distance from the ground. Those grown in patches, as above, have three stakes to each, with two tiers of cord as above.

When the plants are fully in bloom, or rather when they have set their flowers, and the first series of beans have made two inches in growth, three inches of the tops of the plants should be pinched off, to throw that nourishment which would be expended in uselessly increasing the height of the plant into its general system, and consequently increase the bulk of crop, as well as advance its early maturity. This often-recommended operation, although disregarded by many, is of very signal importance. Some, to secure a very late crop, cut over a few rows of a progressing one just when the plants are in full flower. New shoots are formed at the bottom of the stem, which shoot up and produce a crop late in autumn. Did it not occur to him who first recommended this, that, by sowing a crop later in the season than general crops usually are, the same end would be arrived at, and in a far more business-like manner?
In gathering the crop, the first attack should be made on them when the beans are about the size of a marrow-fat pea. In such state only are they fit for a table where elegance in display and gastronomic taste are cared for. A disregard of this on the part of the purveyor has probably tended more than anything else to banish this excellent esculent from the tables of the great. The young gardener should attend to this; and, indeed, the same rule is applicable to every other article of garden produce. He may rest assured that his success in life depends to a great degree on the quality of his productions, not altogether on the quantity; and he may set it down as a pretty general rule, that all vegetables are most appreciated when young and delicate: and to none does this more strongly apply than to the Order of which we are now treating.

Soil and manures.--In a strong aluminous soil the bean luxuriates most; that soil must, however, be rich, and highly cultivated. In light soils they are earlier, but their produce is less, nor do they continue so long in bearing. It is the force of manure and high cultivation that enables the gardener to produce good crops of this plant when he has to contend with a light and gravelly soil. In soils of the latter description, it is scarcely possible to manure too highly; and of all fertilisers, that of the stable or cow yard is the best in such a case. Mr Stephens, in the "Book of the Farm" (vol. ii. p. 423), gives an excellent instance of the beneficial effects of gypsum being used as a topdressing to an acre of beans, and that at the very moderate cost of $6 \mathrm{~s}$., the quantity applied being $4 \mathrm{cwt}$. The balance in produce in favour of one acre so treated over another that received no top-dressing, was $11 \frac{1}{2}$ bushels of beans and 127 stones of straw, the cash profit in the one case over the other being $£ 3,4 \mathrm{~s} .4 \mathrm{~d}$. No other manure was used; the ground was, however, dressed with $2 \frac{3}{4}$ chaldrons of quicklime slaked in water, that held common salt in solution in the proportion of 1 cwt. to the chaldron of lime, before the beans were sown on the 6 th of March. The top-dressing was applied on the 6th of May, and the crop reaped on the 1st of September. The soil was partly moss, partly sand or gravelly loam, and had been trenched 16 inches deep, and 
well incorporated together. This is an interesting case to the gardener, supposing him to have the eighth of an acre under beans, which would be in his case a considerable breadth. He could, at the above rate, produce his crop at a cost of 1s. 6 d. for manure ; for we hold the previous lime-dressing, stated above, to have had immeasurably less effect on the crop than the already fertile state of ordinary garden-ground would. Another instance of the effect of a combination of special manures is given by the same accurate authority in the paragraph following that from which the above statement is taken: -2 cwt. animal charcoal (1 cwt. dissolved in sulphuric acid), $56 \mathrm{lb}$. sulphate of magnesia, 2 cwt. common salt, 1 cwt. nitrate of soda, at a cost of $£ 1,7 \mathrm{~s} .10 \mathrm{~d}$., used as a top-dressing to an acre of beans, produced $11 \frac{1}{2}$ bushels of beans, and 146 stones of straw, beyond that of an acre of the same kind of crop which was not topdressed at all, leaving a cash profit of $\stackrel{2}{ } 1,7$ s. 1d. in favour of the top-dressed acre. In this latter case the soil was a stiff loam resting on red sandstone. The top-dressing was applied on the 21st of May, and the crop reaped on the 8 th of October.

"Soluble manures, such as the nitrate and sulphate of soda and potash, and dissolved bones, are most commonly applied to the bean crop, in the form of topdressings, after the plants have made considerable progress above ground; and they can readily be brought within reach of the roots, and mixed with the soil, during the operations of hoeing. Gypsum and lime, as both of them require a long time for solution, should undoubtedly be applied to the land before sowing the seed. Gypsum may be very advantageously applied when sowing the seed, either in contact with it, or so near as to be within immediate reach of the roots. Common lime, in the caustic state, when laid on as manure for beans, does best when this operation is performed when preparing the land for the winter furrow. The organic composition of the straw and grain of beans, as determined by analysis, exhibits a larger amount of nitrogen than is to be found 'in any of the cultivated cerealia.' This fact proves the necessity for a soil being rich in decayed animal and vegetable matter, as well as in those mineral substances already spoken of. Organic manures must, however, be viewed only as subsidiary, in the cultivation of beans, to the more important mineral substances already referred to. Fortunately the two most important organic manures (farmyard dung and guano) also oontain important mineral matters. Farmyard manure is undoubtedly our best manure for beans, as it is for most other crops; yet its action can be greatly enhanced by the addition of purely mineral substances, because by the combination we supply all that is necessary for perfecting every part of the plant. Guano isrich in nitrogen(ammonia), but greatly deficient in alkaline mineral matter. Hence, when applied as a manure for beans, on soils different in potash and soda, its action is frequently feeble and unsatisfactory. On soils rich in vegetable matter," as almost all garden soils are, "it is advisable to use mineral manures, such as lime, magnesia, potash, and soda, either in one form or another; while on those that are deficient in decaying vegetable and animal matter, but abundantly supplied with mineral substances, an application of farmyard manure or guano would be preferable. While pointing out certain special manures as peculiarly adapted, by their composition, to the growth of beans, it should ever be kept in view, that the true art of cultivation consists in making the land support itself as much as possible; and this, on all soils of a medium character, or above it, can in a great measure be effected, irrespective of extraneous manures, by improving their physical condition, and eliminating, by deep and thorough cultivation, those stores of nutritious matters, which otherwise would remain locked up and unavailable. A thorough stirring, pulverisation, and clearing of the soil, during the growth of the bean crop, or indeed of any other crop whatsoever, is equivalent, and frequently superior, to adding a certain amount of manure where these operations are neglected, or performed in an inefficient manner; because, by the admission of air, oxygen, carbonic acid, and water, the great solvents of all mineral and vegetable matter contained in the soil are thus allowed to exert their peculiar action upon what would otherwise remain inert." -Monton's Cyclopedia of Agriculture. 
Sulphate of soda, and most other mineral manures, can only be beneficially applied to land in good condition and in careful hands, because if the land is poor, they will have no effect whatever. The stableyard manure-heap must ever be regarded as furnishing the principal part of the food of plants. What are called special manures are only the condiments, like pepper, salt, mustard, and the other contents of the cruet-stand.

Forcing.-The garden bean is not considered of sufficient importance to be ever forced in this country; if it were so, what has been said of pease under this head would be quite applicable to it.

Taking the crop.-Beans, to be eaten in proper condition, should be gathered young-indeed, when of the size of a large marrow-fat pea. They are gathered in succession, until the eye begins to turn black at the hilum or point of attachment to the pod, at which period they become exceedingly coarse food, flatulent, and indigestible.

Approved sorts and their qualities.-1. Early Mazagan.-From 2 to 3 feet in height; the hardest sort in cultivation, although originally a native of Mazagan on the African coast; stems slender; by no means productive, the pods containing seldom more than four beans. Its hardiness and precocity have secured it a place in our gardens for upwards of one hundred years. It is known as early Malta, early Aldridge, Stidolph's new early, and early Bromley. It is the Fève de Mazagan of the French. The weight of an average seed is about 15 grains, one pound containing 481 seeds.

2. Marshall's early dwarf prolific.-From 18 inches to 2 feet in height. This excellent bean originated about twenty years ago with $\mathrm{Mr}$ Marshall, a gardener, near Kingston, Surrey. It is fully a fortnight earlier than the Mazagan, much more productive, producing its pods in dense clusters near the ground; plant very much branched; beans larger than in the last, and producing from four to five in each pod. It is known as Marshall's prolific.

3. Royal dwarf cluster.-From 10 to 14 inches in height. Comparatively a new variety, the history of which is not very clearly known. We suspect, from its habit, that it is an accidental variety of dwarf habit of the last, or one of taller growth than the next. It produces its pods in clusters, three or four beans in each pod, which are smaller than the last. It branches considerably, and therefore should have not less than 10 or 12 inches in the line, which is nearly its proper distance between the rows. With Marshall's prolific it should have a place in every small garden. We grow it extensively on account of the delicacy and smallness of the beans while young.
4. Dwarf fan.-The smallest of all garden beans, seldom so tall as the last; pods small, round, containing in general three small oblong beans; pods produced in clusters close to the ground. Known also as the fan or bog, dwarf cluster, or bog bean. The Fève naine hâtive of the French. It is not in general cultivation, but is well suited for small gardens, yielding a moderate crop of weil-flavoured beans. Seeds weighing from 19 to 20 grains.

5. Whiteblossomed.-From 3 to 4 feet high; the most delicate flavoured of all beans, but by no means productive; pods long, nearly cylindrical, containing seldom more than four beans, which, when ripe, are of a blackish colour. Flowers white, unlike any of the family. It is known also as the white-blossomed long pod. An average-sized seed weighs $12 \frac{1}{2}$ grains; the smallest seeded of all the garden sorts.

6. Long pod.-From 3 to 4 feet in height; pods long and narrow, containing in general four beans of excellent quality. A most productive variety, and generally employed for the summer crops. One of the most popular of all the family, and has been long in cultivation, which accounts for the following synonymes. Lisbon, early Lisbon, Sandwich, Windsor long pod, Turkey long pod, common long pod, early long pod, large long pod, hang-down long pod, sword long pod, moon, Wrenche's early moon. To which Johnston's wonderful may, for all useful purposes, be added, as it differs only in the greater length of the pods, and slightly flatter form of the bean.

7. Dutch long pod.-From 3 to 4 feet high. An abundant bearer; will succeed the long pod even if sown on the same day. Pods long and broad, containing, on an average, from four to six large flat white beans.

8. Green long pod.-From 3 to 4 feet in height. Pods long, somewhat flattened, containing in general four rather small oblong beans, an excellent bearer, and only a few days later than the long pod. Esteemed on account of the fine green colour of the beans, which, if gathered at a proper time, retain their green colour when dressed. Known also as the green Genoa and green nonpareil. It is the Fève verte, Fève toujours verte, of the French. The long pods weigh from $23 \frac{1}{2}$ to $35 \frac{1}{2}$ grains, the lightest being Child's new early long pod, and the heaviest Sangster's imperial long pod.

9. Windsor. -3 to 4 feet high, pods short but very broad, containing seldom more than two beans, which are very large, flat, roundish ; esteemed excellent for a summer crop on account of their remaining longer fit for use than any other, excepting the green Windsor. Known also as Wrenche's improved Windsor, new Windsor, Kentish Windsor, broad Windsor, Taylor's Windsor, Taylor's large Windsor, Taylor's improved new Windsor, Mumford, and small Spanish. It is the Fève de Windsor of the French. Weight of seed, 47 grains (or $\frac{1}{9}$ of an ounce nearly), being the heaviest seeded of all beans.

10 Green Windsor.-Very much resembling the last, only the beans remain green after they are ripe, and hence are thrust into the market 
when most other beans are done. It is known as the Toker, and is the Fève de Windsor verte of the French. Weight of seed, 36 to. 37 grains.

11. Red Windsor.-In character similar to the last two, differing, however, in the beans boing of a light red colour while young, and dark red when ripe, which renders it seldom sought after. The French have a bean very similar, under the name of Fève violette. Weight of seed, $31 \frac{1}{2}$ grains.

12. Green China.-From 2 to $2 \frac{1}{2}$ feet in height. Not much cultivated, but is deserving of greater notice on account of its productiveness and coming in late ; pods long, cylindrical, containing three or four beans, which remain green when dry. Much cultivated on the Continent, and known as the Fève verte de la Chine.-Bon. Jard., edit. 1835.

13. Dwarf crimson seeded.-Esteemed only on account of its earliness and dwarf habit, in both of which it exceeds all the other beans, seldom attaining a foot in height. Pods produced in great abundance, about 2 inches long, well filled with beans of a crimson colour, and nearly as large as the long pod. Known as Vilmorin's dwarf red seeded. This is the Fève très naine rouge, nain rouge, of the French.

The varieties of beans have not increased by any means in the same ratio as pease, for which both cultivators and seedsmen ought to be thankful. As a selection from the above, we would recommend Nos. 2, 3, 6, 8, and 9; and No. 5, where delicacy of flavour, at the expense of a moderate return, is no object.

Gregory's early hang-down is merely seed selected from No. 6. The thick-seeded Windsor, Child's long pod, green long pod, Sangster's imperial long pod, green fan or cluster, and other names found in catalogues, are scarcely worth the attention of the cultivator.

Insects and Diseases.- The bean is liable,

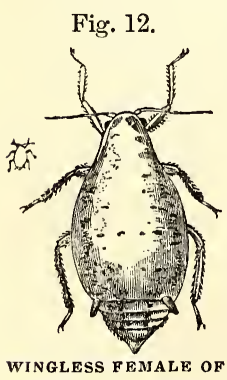

THE BEAN APHIS. particularly in dry seasons, to the attacks of the Aphis fabce, the black-fly collier, or, as it is in many places called, the dolphin. It is a species of plantlouse. It attacks the young stalks towards their tops, and the leaves also; and in a few days after their appearance, like all the Aphis family, it multiplies in prodigious numbers, rendering the top of the plants a mass of sooty blackness. Fig. 12 shows

Fig. 13.

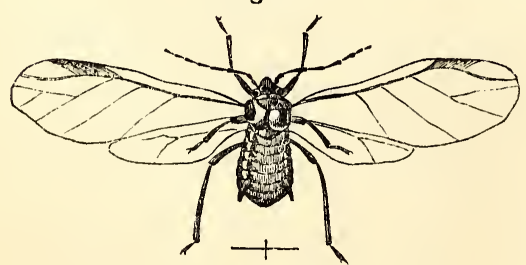

WINGED MALE OT THE BEAN APHIS. the female wingless insect; while fig. $13 \mathrm{ex}$ hibits the male. The ladybirds - of which there are two, the constant inhabitants of gardens, Coccinella bipunctata, fig. 14, $a$, the twospotted ladybird, and Coccinella septem-punctata,

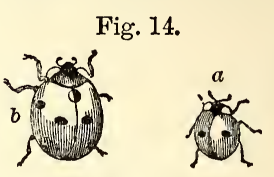
$b$, seven-spotted ladybird-are their greatest natural enemies, as they are to all the Aphis tribe. The grub of the ladybird is shown, fig. 15, $a$, LADYBIRDS. and the chrysalis $b$. They devour them in astonishing numbers ; and therefore, for this service, and from being harmless themselves, they rank amongst those insects which should be protected with the

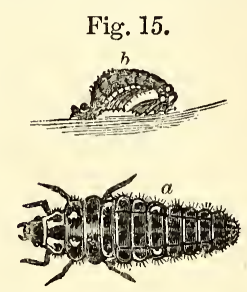

GRUB AND CHRYSALIS OF THE LADYBIRD. greatest care. The most ready means, and that generally followed, in ridding ourselves of the Aphis fabo, is to cut off the affected tops, put them in a bag, and consign them to the nearest fire. It is useless to cut off the tops and leave them on the ground; for even the wingless females will soon reascend the plants, and the winged males speedily regain their former station. The operation of topping beans lessens greatly the multiplication of these insects, as it is the young and most tender leaves

Fig. 16. and tops that they prefer to attack. Dust-

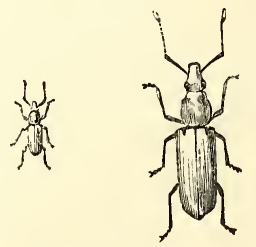

SITONA CANINA. ing the plants with caustic lime in powder, tobacco juice and fumigation, Scotch snuff, sulphur, hellebore dusted over them in a state of powder, and a variety of other means, have been adopted, all having more or less effect; but topping is the radical cure after all. The humble-bee (Bombus terrestris L., and $\boldsymbol{B}$. locorum), works considerable damage to the bean crop, by perforating the flowers on the upper Fig. 17. with its mandibles,

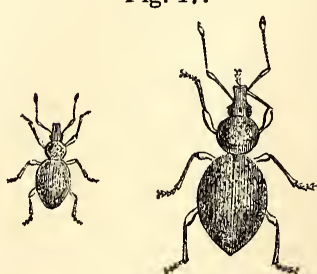

OTIORHYNCHUS PICIPES. and inserting its proboscis into the nectary to secure the honeycontained therein. This operation is necessarily of great injury to the crop, as the majority of the flowers so probed are not able to perfect their seed.

Several species of the extensive genus Sitona (weevils) are often very destructive, not only to 
the bean, but to most of the order Leguminosæ. These are, Sitona canina, fig. 16 ; S. lineata, fig. 7, and Otiorhynchus picipes, fig. 17. These attack the bean shortly after its appearance above ground, and continue to feed upon it during the whole progress of its growth. The same means must
Fig. 18.

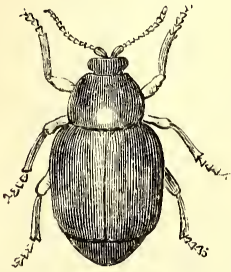

ELLOW-BOOTED BEAN BEETLE. be applied as noticed above. The little beetles, $\boldsymbol{B r}$ chus granarius, fig. 9 , and B. flavimanus, fig. 18, deposit their eggs in the blossom, to prey afterwards on the ripe seeds.

A parasitic fungus (Uredo fabce) abounds on the leaves of the bean towards the latter end of summer. Its effects do not seem to be of great consequence; were it even so, sulphur applied as has been recently recommended for the destruction of mildew, will lessen, if not totally destroy, this parasite.

The wire-worm (Cataphagus lineatus, Linn.), fig. 19, belongs to the order Coleoptera, family Elateridæ. The perfect insect is about one-

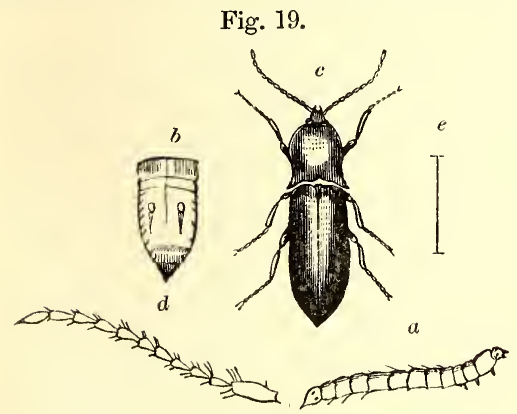

$a$, WIRE-WORM ; $b$, CAUDAL JOINT MAGNIFIED ; $c$, PERFECT BEETLE; $d$, ANTENNA.

third of an inch in length, and is described by Stephens as being "fuscous, with a griseous pubescence. Head and thorax blackish, the latter with the lateral branches nearly straight, and the posterior angles very acute; the disc very convex, and thickly punctate; scutellum fuscous ; elytra broad, a little attenuated, rounded at the apex, very convex; punctate striated; the striæ disposed in pairs, and united at the apex, griseous yellow, with the alternate narrowed interstices fuscous or dusky; margins and apex of the abdomen ferrugineous; antennæ and legs rufo-testaceous ; the femora sometimes dusky, rather variable in colour, being more or less pubescent or testaceous."

The true wire-worms are the produce of various species of click or skipping beetles, known as skipjacks and spring-beetles in parts of the country, or elaters, as-

Elater (Adrastus) acuminatus, the acuminated click beetle.

$E$. (Athous) longicollis, the long-necked clickbeetle.

VOL. II.
E. (Athous) niger, the black click-beetle.

E. (Agriotes) obscurus, the obscure click-beetle.

E. (Melanotus) fulvipes, the tawny-legged clickbeetle.

E. (Lepidotus) holosericeus, the satin-coated click-beetle.

$E$. (Agriotes) sputator, the spitting click-beetle.

E. (Athous) ruficandis, the red-tailed clickbeetle.

E. (Agrypnus) murinus, the mouse-coloured click-beetle.

$E$. (Dolopius) marginatus, the margined clickbeetle.

One of the natural enemies to these is the genera Fileria-slender worms, which live in wire-worms. Another is Steropus madidus, a ground-beetle or carabus, which feeds on them.

The millipedes and maggots of gnats are erroneously called wire-worms ; they are, however, destructive in their way. They belong to the genus Iulus.

I. Londonensis, the London snake millipede.

I. pilosus, the hairy snake millipede.

1. pulchellus, the beautiful snake millipede.

I. punctatus, the dotted snake millipede.

I. terrestris, the earth snake millipede.

I. latestnatus, the broad-lined snake millipede.

The larvæ of the Elater are of a yellowish-brown colour, slender, flat, smooth, and shining, slightly pubescent, resembling the meal-worm; the body, exclusive of the head, is composed of twelve scaly rings, the last of which is not notched at the tip, as is the case with some of the species; it has six very short legs, they have a series of spirals on either side; the under side of the end segment has a fleshy tubercle, employed as a leg, and, when not in use, concealed at the base of the segment. It remains five years in the grub state, in which it is most injurious, and commits great devastation among florists' flowers. These grubs are exceedingly destructive to carrots, potatoes, turnips, cabbages, \&c.; we have not unfrequently lost crops of beans also by them. Various have been the means employed to effect their destruction; the most simple, and probably the most effectual, is to place slices of potatoes on the point of a stick, and bury them about two or three inches under the surface, which should be examined every day, and the wire-worms collected and burned. By these means we succeeded in capturing in a border of carnations no less than 6360 at three takings. The number of traps set was 106, and the average number of wire-worms per trap was 20 : at one taking we captured 2120. By persevering in this manner for about a fortnight, examining the traps every third day, we so completely cleared the border, that it has been kept stocked with carnations for the last four years, and now we do no lose a plant. Some prefer laying the slices of potatoes on the surface of the ground, as also slices of turnip, and portions of lettucestalks, to which the worms are attracted, and may be collected in great numbers. Some recommend turning up the soil frequently, to expose them to the attacks of birds. Pheasants destroy them in vast numbers, but we are not certain but they do more harm in other ways 
than good in this. Rooks and moles are also very serviceable in keeping down this pest of the garden and the field. Spirit of tar, dug into the ground, at the rate of 1 gallon to 50 square yards, has been with us a satisfactory remedy ; and to render the liquid more divisible, we ab. sorb it in dry sand, or dry finely-sifted coalashes, and then sow the ground with it. The refuse lime of gas-works, which contains in general a considerable amount of impure sulphuret of lime, or lime combined loosely with sulphuretted hydrogen-a gas the most deleterious of all others to animal life-has been employed with singular effect also. The mole lives chiefly on the wire-worm for a great portion of the year. In cold wet seasons the wire-worm is most destructive. And, in fact, where ground is overrun with wire-worm, it is not a bad way of clearing it, to sow old seed of beans in drills, and to take them up after the first week once every two or three days, when the beans will be found thickly perforated by the insects, which may be destroyed and the beans re-sown again.

The gamma moth (Plusia gamma of some naturalists, Noctua gamma of others), fig, 20.

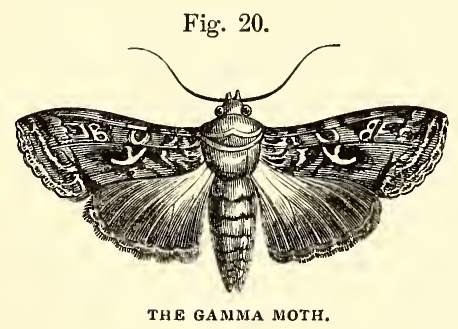

This very pretty moth may be described as having beautiful glossy greyish-coloured upper wings, marbled with brown, having a slight metallic shade; about the middle of each is a gold shining mark, resembling the Greek letter $\gamma$, from which circumstance it derives its name; the under wings are of a pale ash-colour with a brown edge; the head and collar purplishbrown, margined with grey lines; abdomen yellowish-grey, having elevated tufts of hairs.

The caterpillar is green, with several short single hairs interspersed over it; four small whitish-yellow lines down its back, and a broad yellow stripe along each side; head brownish green; furnished with twelve feet-two behind, four abdominal, and six fore-feet. The female deposits her eggs, which are hemispherical, on the under sides of the leaves, in a somewhat regular manner ; they are said to lay four hundred of these eggs at a time.

These caterpillars are found pretty abundant during the summer, feeding on lettuce, beans, pease, and even wormwood, thistles, and sage. They made considerable ravages in France in the year 1735, according to Reaumur, during the month of July, eating up all the leguminous vegetables, leaving nothing but the stalks. They are found all over Europe, in Siberia, China, and North America. They first make their appear-

ance about April, and continue in full vigour till October; when they deposit their eggs, which are hatched at various times from May to September, but chiefly during July. These caterpillars commit sad havoc in the south of England; rarely, however, in Scotland, where they are only found troublesome in dry warm summers. There are four generations of moths during the season, and when we consider that a single pair can produce 80,000 eggs, which, barring accident, might become the progenitors of $16,000,000$ of caterpillars in little more than twelve months, it is wonderful that they do not injure our gardens more than they usually do. Their attacks are pretty general, but, with the exception of lentils, they seem to prefer leguminous plants. The only means of subduing them appears to be capturing the insect in its moth state, hand-picking the caterpillars, or, as recommended for other insects which drop either from fear or from concussion, shaking the crop smartly, and receiving the caterpillars in a cloth spread along both sides of the rows. If merely shaken off, they would soon reascend the plant and renew their attacks upon it.

General remarks.-The European names of the garden-bean are-boon, Dutch ; fava, Italian; fère de marais, French; alver janas, Spanish ; and grosse bohne, German.

Where saving seed is an object, a row or two, according to their length, and the quantity of seed required, should be left ungathered; for it is a bad way to gather the best of the crop, and to save the last formed, and consequently the weakest and worst, for seed; a sure way to deteriorate the quality of the sort, and render a variety that a long succession of years' careful cultivating and selecting has been spent in producing, worthless, by the mismanagement of one season. Even then only the finest pods should be saved; and to prevent accident, the crop should be gone over just as the pods are fully swelled, and all the smallest removed. This will throw additional strength into the pods left for seed, and insure a pure stock. Beans, like pease, keep best in the straw, therefore they should, when properly winnowed, be laid up in bundles, and placed in a dry airy loft. The seeds will retain their vitality longer than the pea, and if well kept, will grow after four or five years.

The inorganic constituents of the bean are-

100,000 parts of seed contain 2136, and 100,000 parts of the straw contain 3121 parts of inorganic matter, consisting of :-

Potash :
Soda :
Lime
Magnesia
Alumina :
Oxide of iron :
Oxide of manganese
Silica
Sulphuric acid :
Phosphoric acid :
Chlorine.

-Sprengel.

\begin{tabular}{rr} 
Seed. & Straw. \\
415 & 1656 \\
816 & 50 \\
165 & 624 \\
158 & 209 \\
34 & 10 \\
- & 7 \\
126 & 5 \\
89 & 220 \\
292 & 34 \\
41 & 226 \\
\hline 2136 & 80 \\
\hline 3121
\end{tabular}

The composition of the bean is thus given in "The Book of the Farm," vol. i. p. 1300:- 
Bean-ash.

$\begin{array}{lccc}\text { Potash } & & \text { Mean of three analyses. } \\ \text { Soda : } & : & : & 33.56 \\ \text { Lime : } & : & : & 10.60 \\ \text { Magnesia } & : & : & 7.99 \\ \text { Oxide of iron : } & : & : & 0.56 \\ \text { Phosphoric acid } & : & : & 37.57 \\ \text { Sulphuric acid } & : & : & 1.00 \\ \text { Chlorine }: & : & : & \mathbf{0 . 7 3} \\ \text { Silica . } & \underline{1.15} \\ & & & \mathbf{9 8 . 9 3}\end{array}$

One quart of Mazagan beans (one of the smallest sized) contains 434 beans, and one quart of Windsor (one of the largest) contains 179 beans.

\section{§ 3.-THE KIDNEY OR FRENCH BEAN.}

Natural history.-The kidney or French bean (Phaseolus vulgaris, L.) belongs to the natural order Leguminosæ, subdivision Sarcolobæ, tribe Phaseoleæ; and to the class Diadelphia, and order Decandria, in the Linnæan arrangement. The genus Phaseolus contains 14 species of plants cultivated for the food of man, exclusive of the species multiflorus, which forms the next section. Of $P$. vulgaris there are many varieties in cultivation, but, like those of pease, they are at present in considerable confusion as to identity. The name Phaseolus is derived from Phaselus, a little boat, from the resemblance in its seed-pods.

The kidney bean is a native of India, introduced to Britain before 1597-some say so early as 1509, and that it was imported from the Netherlands, about which period gardening began to be attended to in England, the white kidney bean being the first variety known in this country. Phillips, in "History of Cultivated Vegetables," vol. i. p. 74, says, "this pulse is generally but improperly called French bean, for its old French name, Fève de Rome, evidently proves it not to have been a native of France. We also find that it was called the Roman bean in our language about the time of Queen Elizabeth. Gerard also gives it the name of Speragebean, and says it is called Faselles, or long peason. The Dutch at that time (1596) called them Turck's-boone-that is, Turk's-bean. From these facts, but more particularly from the account of the great Roman naturalist, we may conclude that this wholesome and excellent vegetable is a native of the eastern extremity of Europe, or that part of Asia now belonging to the Turks; for Pliny, in the 7th chap. of his 18th book, mentions these beans, and says those of Sesame and Iris are red, resembling blood. $\mathrm{He}$ also, in his 12th chap. of the same book, calls them Phaseoli, and says the pod is to be eaten with the seed. From this laconic notice we may assume they were but little esteemed at that time in Italy, where lupins were then so much admired for food. The French name of Haricot originated from their being much used by cooks in the composition of a dish so called. The English name of Kidney bean was given on account of the seed being somewhat of a kidney shape. Gerard mentions a consider- able variety that was cultivated in England in his time, and says, "The fruit and pods of kidney beans, boyled together before they be ripe, and buttered, and so eaten with their pods, are exceedingly delicate meate, and do not engender winde as the other pulse doe."

Kidney beans are amongst the most valuable of culinary vegetables, yielding a large return of crop, and continuing in use during the whole summer. The variety known as the scarlet-runner is a most productive sort, and although requiring the support of stakes in the manner of pease, yet it well repays the expense and the space it occupies. In Scotland this excellent vegetable is much less cultivated than in England; indeed, the cottager, and even many of a higher grade, do not appear to be aware of their merits. It is found in most cottage-gardens in the south, and made to add to the ornament of the garden, as well as used for culinary purposes. Philip Miller appears to have brought it into use as an article of food, for, prior to his time, we find it described as an ornamental annual, and placed in the flower border, and its flowers greatly sought after by ladies, to be put in their nosegays and cut-flower pots. The dried seeds of all the tribe are exceedingly nutritious as an article of food, constituting the haricot so much used on the Continent; indeed, the quantity of gluten contained in them nearly approaches that in wheat.

Uses.-Kidney beans, in their young state, are preserved in salt for winter use; they are also preserved as a pickle by themselves, and form an ingredient in mixed pickles. They are also used throughout the whole year as a legumen, and, being impatient of frost, a supply is kept up during winter and spring by growing them in hothouses. As an article of vegetable food, they are considered exceedingly wholesome, and much less flatulent than vegetables usually are. The ripe seeds are much used on the Continent in cooking under the name of haricots, which, as dishes, are as numerous as curries in Calcutta For this purpose, the ripe seeds are steeped a few hours in water, which swells them out, and causes the outer skin to separate freely from the seed. In this state they are nutritious, when simply boiled, and served up to be eaten with other meats, but much less palatable than when they have passed through the hands of a skilful cook. Haricots are much in use in Roman Catholic countries, forming the greater part of the food of the people during Lent. The ripened seeds also enter into a variety of soups and stews. As an article of domestic economy, they are by far too little attended to ; their produce is large; their cultivation during summer simple, and, in their green state, few vegetables are easier cooked. According to the analysis of Einhoff, 3840 parts of kidney bean afforáed 1805 parts of matter analogous to starch, 857 of vegetoanimal matter, and 779 parts of mucilage-clearly showing that it is the most nutritious of all legumens.

Propagation.-The whole of the dwarf varieties-that is, of Phaseolus vulgaris- 
are to be regarded as annuals in this country, and consequentlyoriginated from seed.

Sowing.-The kidney bean, being a native of India, is consequently a tender plant in Britain; therefore sowing in the open air, even under the most favourable circumstances, cannot be safely recommended before the beginning of April for England, and even then in dry light soils and warm places. The latter end of that month is sufficiently early for Scotland. After the first sowing, to keep up a proper succession during summer and autumn, other sowings should be made in May, June, and July. In cold situations, and in strong cold soils, it will be advisable to sow the seed in a prepared bed, in boxes or in pots, in light rich soil, and when the plants are about 4 inches high, to transplant them into their final spot. They bear transplanting well; and the process recommended in the case of pease is applicable to them also. To keep up a supply during winter, the process of sowing should commence about the middle of October, and be followed up every fortnight till the middle of March.

For early crops in the open air, a well-sheltered border with a southern exposure should be chosen, the ground thoroughly pulverised, and if not in high manurial condition, it should be made so by additions of semi-decomposed stable-yard manure dug in previous to sowing. (For special manures, vide Garden Bean, those recommended for it being applicable here also). The drills should be drawn across the border 2 feet apart, and about $1 \frac{1}{2}$ inch in depth; the seed should be set by the hand singly from 2 to 3 inches apart, according to the size of the variety, which distances apply to all the dwarf sorts, or kidney beans proper. The seed is sold by the pint, half a pint being sufficient to plant a drill 80 feet in length. It is very important that the drill be dry at the time of planting thus early, for, what with cold and excess of damp, the beans are very liable to rot as soon as they are buried in the ground. In sowing for transplanting, the seeds may be set as close to each other as nearly to touch; and, indeed, it is a good plan to sow one seed in a small 60 -sized pot, and when 3 inches high, if the weather be favourable, to plant them out with the ball entire. Even for secondary crops a warm spot is required; the distance as given above will be quite sufficient. We have, in general, found transplanting the first and second crops the most certain way of securing them early; and, indeed, for the former, have found much advantage by setting the lines from east to west, and placing along the northern side of each line a boarding 18 inches in height, which places the crop almost in the same condition as if it were planted at the bottom of a south wall, which seldom can be done with safety to the roots of the fruit-trees growing against it. The wire protecting-cages, alluded to in article GaRDEN BeAN, will be valuable in the case of the first and second crops of kidney beans; andindeed the triangular case with glass on one or both sides, described in article PEA, might be used with the greatest advantage in this case also.

Subsequentculture.-Top-dressing, should the plants appear weakly, with any of the special manures noticed in article GARDEN BEAN, in either a dry or dissolved statethe latter being much more instantaneous in its effects - keeping the ground clear of weeds, and frequently and deeply stirring the soil between the rows, constitute the essentials of good cultivation. Some of the taller-growing sorts, if the soil be rich, and the season mild and humid, will require topping-that is, merely cutting off those straggling top-shoots that, under such circumstances, often spindle up and rob the crop of much of its food, as well as causing an undue shade upon it.

Soil and manure.-A much lighter and more thoroughly pulverised soil is required for the kidney bean than for the garden bean, and where it is naturally strong the crop will be much improved, if sown or planted in light vegetable mould, placed in drills drawn 6 inches deep for its reception: this will give the crop a fair start; and deep hoeing through the summer, leaving the surface rough, will admit the heat of the sun, of which this crop, being of tropical origin, stands in need in our climate. The manures recommended, and the method of applying them, are similar to those described in article GARDEN BeAN ; but as the manurial effects should take place as soon after they are applied as possible, it is better that these should be in a liquid state. 
Forcing.--This esteemed legumen can be had in great perfection throughout the whole year. To afford a supply during winter, seeds of the most approved sorts, such as early six-weeks, Fulmer's early dwarf, Wilmot's forcing, \&c. should be sown about the latter end of September or 1st of October, presuming two distinct crops are already progressing in pits capable of being so heated as to not only repel frost, of which this plant is impatient, but also to afford a minimum temperature of $60^{\circ}$. Sow in small 60 -sized pots, one bean in each, in rather light, dry, rich soil. Place them in a glazed pit or frame, in a temperature maintained at not less than $60^{\circ}$ or $65^{\circ}$, keeping the pots near to the glass. When they have attained the height of 3 or 4 inches, transplant them into 7 -inch pots, placing 3 plants in each, having their roots and ball entire, so that they may sustain no check in their growth. At potting, place in the bottom of each pot, over the drainage, 2 inches of very rich but much decayed manure, and use a rich, light, rather turfy fresh soil for the roots to work in. Some, at potting, set the plants deep in the pots, with a view to add fresh soil around the stems afterwards. This is an absurd practice, as the roots of leguminous plants very rarely are emitted from the stem, and the consequence of this deep potting is to limit greatly the space for the roots to seek food in. As the natural temperature of the season declines, raise that in the pit or frame in which they are placed to $65^{\circ}$ as a night temperature, and $75^{\circ}$ as that of the day, allowing a rise of a few degrees in bright sunny days. In a pit heated by hot water, they will succeed better than elsewhere ; and, in default of such accommodation, set them on suspended shelves over the footpaths of the pine-stoves, but as close to the glass as will admit of their attaining the height of 10 or 12 inches. To lessen the labour of watering, place pans or feeders under the pots, and syringe the plants frequently, to keep down the thrip and red-spider, which are their greatest enemies. As the plants advance in growth, support them with small twigs to prevent their being broken by the force of syringing. Place a little liquid manure in the feeding-pans, to give additional food to their roots as soon as they appear to wish to escape through the holes in the bottom of the pots. For this purpose we use cow or horse urine, or liquid guano, as affording ammoniacal fumes, at least to such an extent as experience has led us to believe is very effective in keeping down both thrip and red-spider. Ventilate upon all fitting occasions, but avoid allowing cold draughts of air to blow on the plants at all times ; and, as a security against this, cause the air admitted to pass through a thin canvass screen, which will break its force, and so sift it into minute divisions that little injury need be apprehended. To maintain a regular succession, sow every ten days throughout the winter. So valuable a vegetable deserves a heated pit for its special accommodation; and by having one heated by hot water of 60 feet in length, divided into three compartments, a good supply may be kept up. For spring use, should they not be required throughout the winter, the 1st of January may be considered a very proper season to sow. Should the pit into which they are set have been occupied with melons, cucumbers, tomatos, or such plants as are subject to thrip and redspider, as a wise precaution, previous to arranging it for French beans, let it be cleared of all its internal contents, well brushed out with a birch broom, the lights put in, and every crevice and hole carefully stopped up, and burn within it half a pound of brimstone, keeping the fumes in from night till morning: remove the lights, and whitewash the walls with hot lime-water, and wash the whole of the wood-work with hot water, if painting be at the time considered unnecessary. This is a very necessary precaution to be taken against the insidious attacks of two of the greatest, although minutest, enemies the cultivator has to contend with.

French beans cannot be grown during winter in a temperature of less than $60^{\circ}-$ if ranging from that to $65^{\circ}$ and $70^{\circ}$, and $80^{\circ}$ during the day, so much the better; and where a bottom temperature a few degrees higher can be afforded, so much the more certain will be the success of the crop. They also, in common with all thin-leaved plants of tropical origin, require the fullest amount of light our gloomy atmosphere is capable of affording them; and hence they succeed best in 
low pits, or on shelves suspended near the glass. Moisture, accompanied with heat, is also essential ; without this, they would become the prey of thrip and red-spider. In addition to the sorts we have already named, may be mentioned the recentlydescribed variety, Newington wonder, which from its dwarf habit is well calculated for suspended shelves in hothouses, occupying less room than any of the other popular sorts. It does not appear to stand the process of transplanting, and should therefore be sown at once in the pots it is to be grown in, putting four or five beans in pots of 6 or 7 inches across. It does not require topping, as most others do ; nor, indeed, does it bear such a process with impunity. Give little water to the roots of any transplanted French beans, until the roots have pretty well filled the pots,-after which they will require it more or less, according to the size of the pots they are grown in ; those in small pots, of course, requiring the most. Water sparingly, if at all, after planting the seeds: it is time enough when they have begun to sprout, and when their roots are far enough advanced to be able to avail themselves of it. Topping the plants is practised by many, and no doubt those of rambling habits require it, to cause them to become more bushy, and hence to produce a greater number of pods. The time and manner of doing this is as soon as the cotyledons, or two first embryo leaves, are fully developed; and then the central shoot, which will also be making its appearance, should be carefully pinched or cut out. This process, however, somewhat retards the growth of the plant at first; but if time is not an object, the advantage will be apparent in a more abundant crop.

Approved sorts, and their qualities.-The varieties of kidney beans cultivated in Britain are in reality not nearly so numerous as the long list of names in some seed-lists would lead us to believe. In France, Spain, and other countries, where they are much more used as an article of food, and where the climate is favourable for their production in the open air, with as little trouble, and for as long a continuance, as our common broad bean, the varieties are endless, and, as is the case with peas with ourselves, continually increasing in number.

1. The early Dutch dwarf.-One of the oldest, if not the very oldest, cultivated variety. Pods long and narrow ; beans small, compressed, and, when ripe, of a white colour. A good early variety, and often employed for forcing, being of a dwarf habit. It is known as white long pod Dutch, early dwarf white, large white dwarf Dutch, dwarf Dutch, dwarf white Dutch. It is the nain hâtif de Hollande, nain de Hollande très hâtif of the French.

2. Early white.-Both pods and beans of moderate size ; plant very dwarf, from 9 to 11 inches ; very early, and on this account, and its small size, well adapted for forcing. The pods are eaten both while green and when the seeds are ripe. It is known as early Laon and dwarf white. It is the nain hâtif de Laon, or flageolet, of the French. The seeds when ripe are white.

3. Dwarf Canterbury.-This is a very old and esteemed variety, hardy and useful. Pods medium length, narrow; beans small, oblong, white when ripe, branching much near the ground; early and productive. It is so near akin to the dwarf Battersea, early white, and earliest white Battersea, as not to be worth cultivating as a separate sort; and its relations with what is called dwarf sabre, sabre nain, dwarf French white, are nearly, if not altogether, as close. It is probable two crops of dwarf Canterbury would afford all these supposed varieties. We think that dwarf Battersea ought to be the established name, in preference to dwarf Canterbury, as we find it has been cultivated above a century under the former name.

4. Fulmer's early dwarf. - An excellent forcing variety; pods long, narrow; beans small, dun when ripe. Known also as Fulmer's new dwarf, Fulmer's early, Fulmer's dwarf red, early dun, dwarf dun coloured, dwarf forcing dun coloured, earliest forcing, early frame. Mr R. Thompson, in his excellent papers in the "Gardeners' Chronicle," on the varieties of vegetables, remarks-"Close allied to the preceding is Fulmer's speckled dwarf, alias dwarf red speckled, dwarf light-red speckled, early dwarf-forcing speckled, large forcing dark-red speckled, Suisse rouge; and with beans a shade darker, we have also, very similar to the above, the early dwarf purple speckled, alias early purple, dwarf purple speckled, dwarf speckled. Allowing for another shade darker, and we may include with those the dwarf black speckled, alias dwarf black mottled."

5. Wilmot's forcing cream speckled.-An excellent forcing variety. Pods long, of uniform breadth; remarkable for crispness while green, and an excellent bearer. Not a great way, however, removed from the last, but sufficiently distant. Beans, when ripe, oblong, pale dun, speckled with dark chestnut.

6. Black Belgian.-Pods long, of uniform breadth ; crisp, very productive, and a good forcer. Dwarfer and earlier than the dwarf negro, which it somewhat resembles. This is much cultivated in Belgium, and there known as haricot noir de Belgique.

Mr Thompson thus notices the bean, in the "Journal of the Horticultural Society of London," vol. v. :- "Although the variety has been previously noticed" (in the Journal), "yet the present mention of it will doubtless be excused, 
for it may be usefully stated that it is the best both for early and late sowing. It is dwarf, and may be sown in pots before the open ground has acquired sufficient warmth; and if planted out when this is the case, it soon comes into bearing. Again, late in summer, when later sorts would not even blossom, if then sown, the sort in question would produce a good crop, that will keep in gathering condition till cut off by frost. This and the Newington wonder are considered the best varieties of dwarf kidney beans."

7. Dwarf speckled China.-An early dwarf variety, and abundant bearer, well suited for forcing. Pods smallish, uniform in size. Beans, when ripe, of a clear sulphur colour. Known also as early dwarf pink-spotted: why called pink-spotted, it is difficult to comprehend. Robin's egg, dwarf robin's egg (from the smali size of the bean), dwarf China, early China. It is the haricot de la Chine of the French.

8. Dwarf negro.-A popular variety ; pods of moderate size, uniform in size and breadth; exceedingly productive; dwarf and hardy; well suited for forcing. Beans, when ripe, quite black. Known as early negro, early black. It is the nain noir or negro of the French.

The dwarf negro is the favourite in the London market, on account of their being all green, and not marked or blotched, like some others. They are also all one breadth, very narrow and handsome; and they are very dwarf, and firstrate bearers.

9. Long-podded negro.-A new variety of the last, vastly superior to it; the pods being often nearly seven inches in length, and of very uniform breadth: rather a strong grower, and admirably suited for a general crop.

"These are often sown amongst lettuce, 6 feet apart, about the 1st of April; but by far the best plan is to sow in beds thickly, and transplant by means of the dibber. No plant lifts better, and the moving makes them show flower much sooner. The usual practice is to take the heads off the plants, leaving them a foot high, and to keep topping all the summer, which induces them to bear heavy crops. But the way to get them to bear earliest, is to save the roots in autumn, pack them away like dahlia roots, and to transplant them again in March, 6 inches root from root, in rows 5 feet apart. It should be borne in mind, that if beans are left to ripen, the roots will not be near so strong as they otherwise would be."CuTHILL in Market-Gardening around London, p. 24.

10. Long-podded China, an improvement on No. 7, having pods from 6 to 7 inches long; exceedingly crisp; of uniform size and breadth; very productive; and, like the last, good for a general crop.

11. Newington wonder.-Another recentlyproduced variety, of great excellence as a forcer, being of dwarf habit; large pods of good shape; does not bear transplanting well.

The following description of this excellent bean is extracted from the "Journal of the Horticultural Society" (vol. v.) :- "Very dwarf; about a foot high; early and productive. The pods are moderately long, not very broad; but having thick fleshy sides, within which the seeds form but slowly; and the pods remain long crisp ; their colour is dark green. It may be planted in rows 18 inches apart."

12. Early six-weeks.-A small early bean well suited for forcing. The Mohawk and Victoria speckled are early sorts, and well worth cultivation.

13. Sabre.-One of the largest, in point of size of pod, which is used for ordinary purposes, until it attains its full size continuing crisp and tender. In this state it is often cut into slices, and preserved by salting, in which state it will keep for a long time. The beans, in a dried state, are esteemed in haricots. It attains a height of from 2 to 3 feet, and therefore is benefited by being supported by short stakes like dwarf pease. It is a profitable sort where there is plenty of room.

14. Large running white-Another tallishgrowing sort, cultivated extensively on the Continent for its seeds, which form the large white bean or haricot, so very generally used in dishes of that name. It is seldom cultivated in Britain, nor could it be profitably cultivated for that purpose. It is known in our seed-lists as tender-podded bean, runner, long white, large white sugar, white long pod. It is the sabre à très grande cosse de Soissons - from Soissons, where it is cultivated to great perfection.

15. Bush haricot.-About 15 inches in height, branching close to the ground, and branching out in succession; not only continues to produce a long continuation of crop, but, by shading the ground, keeps it moist in dry seasons. Pods 5 inches long, and produced in great abundance ; being crisp, transparent, and excellent, comes early into use, and continues long in bearing. The dry seed is speckled red and white. It is the haricot solitaire of the French and Belgians: in both countries it is grown extensively.

There are many other names met with in seedlists, such as dwarf Canadian, liver-coloured, early yellow, large pearl, small do., magpie, grey, marbled Prague, round dwarf, \&c., which, if not identical with some of the above, are inferior to them in quality.

Amongst the newer sorts of kidney beans the following deserve notice-not, however, that they will ever supersede the dwarfer kinds already noticed for general crops, yet some of them may be found acceptable, under peculiar circumstances, in private gardens.

Haricot d'A lger is stated, in the "Bon Jardinier" for 1850, as being excellent and long cultivated in Lorraine. Pods of a palish-green colour, destitute of any tough lining, and exceedingly tender and excellent when cooked. The plants rise to the height of 2 or 3 feet, and therefore require support, but may be dwarfed by early topping.

Turkische bhone.-It is thus described in the "Bon Jardinier":- "This variety is perhaps the best of all ; it bears tolerably well; its pods are of an extraordinary length and breadth" (from 12 to 14 inches in length and upwards of an inch in breadth); "in a young state they make 
excellent haricot verts ; when nearly full-sized, they are still tender and fleshy, and may be used in this state, either fresh, being broken in small pieces; or, in winter, after being cut into narrow strips and preserved with salt." These were tried in the gardens of the London Horticultural Society, and, notwithstanding the cold state of the ground and unfavourable season, some of the pods attained the length of 10 inches, and nearly 1 inch in breadth. The expectation was that they would attain their full size in a good season. Mr R. Thompson thinks them, however, the same as the haricot sabre d'Allemagne of Noisette's "Manual du Jardinier," and the sabre of the "Bon Jardinier." They attain a considerable height, requiring stakes. The pods are large and crooked, and tender while the seeds are under half their growth; it is not so abundant a bearer, nor does it continue so long in use, as the haricot solitaire. It is, however, on account of the size of its pods, worth cultivation.

There are a number of French varieties now in the seed-shops, many of which, although very excellent in a better climate, would be unprofitable in such a climate as ours.

The following selection from the above may be considered sufficient for an ordinary garden : No. 1, 3, 4, 5, 9, 11, and 13 for particular purposes.

Shilling's new French bean, a very prolific sort; pods large, and remaining long tender. Wilmot's true forcing, excellent for that purpose. Black Belgian, negro, cream-coloured, and early Dutch. The last five are the sorts we usually grow.

Insects and diseases.-The most destructive of these, always in the forcing-houses, and often during dry warm weather in the open air, are the thrip and red-spider, both pretty numerous in species, and, as individuals, as incalculable as the sand on the sea-shore, while their minuteness renders them almost invisible to the naked eye. Indeed, they may be set down as among the worst enemies the cultivator has to contend with. Fortunately, however, our hothouses are infested with only two species of the former, both of which are considered of exotic origin. In the open air many of the species attack plants. The same means employed to destroy one species are fatal to the others also. The genus is thus described by the writer of the excellent articles on entomology in "The Gardeners' Chronicle,” 1841 (p. 228):-“They vary very considerably in colour, some species being black, others have the wings branded with white, but the general tint of the larvæ and pupæ is yellow-ochre. Their bodies are much depressed, and much broader than any other part in the female; the mouth is placed under, and at the hinder part of the face, and forms a short conical rostrum, lying when at rest close to the base of the fore-legs. The eyes are rather' large and coarsely granulated, and there are generally three ocelli or simple eyes on the crown of the head; the horns are eight or nine jointed, but the three last joints often appear to be united, when they look as if only six or seven jointed, especially in the larvæ state; they are either wingless, or they have four wings, which are narrow, and lie down the centre of the back, the edges being ciliated with long hairs; the legs are short, the feet being formed of two joints with a vesicle or little bladder at the apex, but not any claws. The larvæ resemble the perfect insect in form, but are often of a totally different colour; their bodies are soft, and they have no ocelli. The pupa is also similar, but the wings are sheathed, and the horns are generally thrown over the head. Some of the species are very active when they have arrived at their perfect state, running fast, skipping and flying well; and they are able to walk about in their previous stages. The two sorts most injurious to the gardener are a little ochreous species, which does not appear to be described, and the Thrips adonidum, fig. 21. The first I shall name T. ochraceus;

Fig. 21.

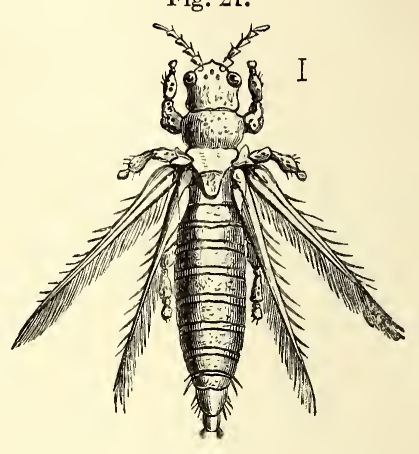

THE THRIP.

it is narrow and linear; of a bright and deep ochreous colour; the eyes are black; the horns appear to be only six-jointed, and brownish at the tips; it has three ocelli on the crown; the body is hairy; the tip pointed and bristly; the wings are shorter than the body in the male, lying parallel on the back when at rest; narrow, especially the under ones, and fringed; the hairs longest beneath and at the points; tips of feet dusky. There is so great variety in the form and structure of this family that it has been divided into several genera, to one of which belongs the other species called by $\mathrm{Mr}$ Halliday Heliothrips adonidum. The larvæ and pupæ are yellowish white, and the perfect insect is of a dull deep black, with the point and sometimes the whole abdomen of a rust colour; the wings are dirty white, the horns and legs yellowish, the extremity of the former black; it is a little larger than $T$. ochraceus, and is very troublesome in hothouses, attacking tropical plants by piercing the under side of the leaves; and one often sees at the top of the tail a globule of blackish fluid, which it soon deposits, and by innumerable spots of this glutinous matter the pores of the leaves are stopped up, and large portions of the surface become blotched. I have found specimens in October, but during March the full-grown larvæ and pupæ, which are as large as the perfect insect, are found in groups feeding on the under side 
of the leaves; and at this time the recentlyhatched and perfect insect either lies close under the ribs, or roves about in search of a mate. As this species is imported from some tropical region, it can endure great vapour-heat, and is consequently most difficult to extirpate."

The same means for their destruction have been employed that have been used against its equally tenacious neighbour the red spider, for they are generally found together. A dry high atmosphere is favourable for the production of both: their destruction may be completely effected by the means stated above-viz., the powerful fumes of sulphur; but this can only be employed to destroy such as lurk about the wood-work or building; or, in the case of deciduous plants like the vine and peach, while these are in a dormant state. With plants which retain their leaves, and with plants in a growing state, this application cannot be employed; for far less of such fumes that would destroy the insects would utterly kill the plants which the remedy was intended to cure.-(Vide Red Spider for other remedies.)

The Red Spider, fig. 22, is really no spider at all, but one of the mites, a very numerous and
Fig. 22 .

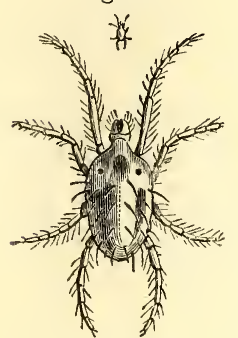

THE RED SPIDER. destructive race. It is doubtful if what is generally called the red spider is really only one species of insect, from the different appearances it assumes, the different positions it occupies, and the variety of plants which it attacks. It is in general considered to be the Acarus telarius of Linnæus, and has been a scourge to cultivators since his days. Like the thrip, it is of exotic origin, present always in hothouses where a sufficient temperature is maintained, and in greatest activity when that temperature is accompanied with an unusual degree of dryness-a fact pretty well established, as it never makes its appearance in a low temperature accompanied with moisture. In warm and dry summers it does great injury to vegetation, few plants escaping its attacks : to French or kidney beans it is most destructive, both in houses and in the open air. When very abundant, it has the faculty of spinning a web, and forming for itself a pretty secure retreat : from this circumstance it has obtained the name of spider, as well as the specific one telarius. Its mode of operation is to pierce the under side of the leaves, and to imbibe the juice, causing little yellow spots on the upper surface of the leaf at first, which soon spread, and give to the whole leaf an autumnal tint: as their attacks increase, discolouration goes on, until the tree or plant becomes so exhausted that it sheds its leaves, and smaller plants often actually die in consequence. The kidney bean is a familiar exaniple of this.

"The red spider, if magnified, looks like a crab of an oval form, with the legs so arranged VOL. II. that two pair are directed forward, and two pair incline backward: it has a few long scattered hairs, and is of a somewhat transparent yellowish white, more or less inclining to orange, with a blood-coloured dot or spot on either side of the thorax; the larger specimens, which appear to be females, have a bright chestnut-coloured body, the fore part of the thorax being ochreous, while the smaller ones have a lead-coloured patch on each side: unlike spiders, the thorax and body are so united that they form one mass; the head is narrowed and rounded, and from under the nose projects a short rostrum, composed, I believe, of two lateral valves, enclosing two fine bristles, which can be thrust out at the pleasure of the animal. Many of the acari have two feelers, like an additional pair of short legs, projecting from the head, but in this species they are very short and only two-jointed, and I cannot discover any eyes : the legs, which are nearly of equal size, are clothed with movable bristles, and seem to be composed of five joints, besides a minute vessel at the tip, from which proceeds a pair of bristly claws. The female is oviparous, and exceedingly prolific ; the eggs hatch in eight days, and it is very remarkable that, when first excluded, the young red spider has only six legs, the third pair being wanting; but this pair is attained when the insect changes its skin. A variety of sizes is apparent amongst them, independently of differences in the sexes, the females being the largest, with the oviduct slightly projecting; and quantities of their cast-off skins are scattered about the under side of the leaves which they inhabit." -RuRICOLA in Gardeners' Chronicle 1841, p. 164.

Syringing with considerable force has been of advantage in disturbing the insect, and no doubt driving many of them off the leaves: water at $150^{\circ}$ has been employed with beneficial effect. The means we have stated above are completely efficacious in ridding empty structures of them, but cannot be applied to living plants. A modification of this is to paint the flues or hot-water pipes slightly with a paint of sulphur and water, but the heated body must not exceed the temperature of $212^{\circ}$. Applying sulphur, either by burning it at a low temperature, syringing the trees with it mixed in water, or applying it in a dry powdered state, by means of the new invented sulphurators, and painting the branches with it, as well as, in the case of trees on the walls, the walls themselves-adding to it in any of these cases, except upon heated bodies, a small portion of soap, to make it adhere longer to the branches or leaves-are all efficacious. In any of these ways the insect may be subdued, if not completely destroyed, if taken in time and applied with vigour; but, like medicine, if put off too long, or taken in insufficient doses, and even in full doses not followed up according to prescription, all will be labour in vain. To crops in the open air, such as kidney beans, it may be readily applied by the sulphurators, or mixed with water poured over the leaves from the rose of a watering-pot. Ruricola, in "Gardeners' Chronicle," 1841 (p. 166), says, "A quarter of a pound of flour of sulphur - put into a watering-pot of water, and, when 
well stirred, poured through a rose along the flues when they are warm, or brushed over the steam pipes, but not after the foliage has expanded-produces the necessary effect, and in course of a week the plants should be well syringed." The same weight may be put to the same quantity of water when applied to kidney beans or other crops in the open air. The warmer the weather when the sulphur is applied, for obvious reasons, the better.

\section{§ 4.-THE SCARLET RUNNER.}

Natural history. - Scarlet runner (Phaseolus multiflorus, Wild.) belongs to the same natural order, and class and order in the Linnæan arrangement as the last. Although the scarlet runner is not so early as the kidney bean, it nevertheless produces a much larger crop of pods as excellent, and to some tasting better than the other. In Britain the green pods only are used; on the Contineut, the ripened seeds are as much an object of culture. "In Holland the runners are grown in every cottage-garden for both purposes; and in France and Switzerland it is grown chiefly for the ripened seeds : in the latter countries it is grown on very poor soil."-Encyclopodia of Plants, p. 616. The seeds are preserved in the pods attached to the straw, and are in winter thrashed out and boiled, and eaten with cream or butter, stewed in haricots or put into soups. The scarlet runner, although in general cultivated as, and considered to be, an annual like the kidney bean, is truly perennial. It is stated to be a native of South America, and was introduced into Britain in or before 1633. The French, now enthusiastically partial to this legume, at one time held it in utter detestation. Phillips relates an anecdote of a lady friend of his, who took some of the " seed of the scarlet runner to Jamaica, and by planting them in her garden they were brought to tolerable perfection; but her gardener, who was an old Frenchman, would not by any persuasion allow them to be eaten, on account of the scarlet or blood colour of the blossom." They occupy a place in most cottage-gardens in England, and are made both ornamental and useful. They cover arbours, are trained over pales and up the walls of cottages, which they enliven by the brightness of their blossom, while every day produces a supply of wholesome and nutritious food to the owner.

Uses.-The same as those of the kidney bean.

The mode of propagation is by sowing the seeds, or by planting the small tuberous roots saved from the last crop. These should be dug up in autumn, before the frost has killed the haulm, and be kept in boxes of sand in a cellar till the end of April, when they may be planted.

Sowing and planting. - Being rather more tender than the dwarf sorts of kid- ney bean, they do not admit of being planted earlier, nor should the tubers be planted sooner, as both may become rotten in the cold damp soil before vegetation can take place. In Scotland, one sowing or planting will be sufficient, as the plants will continue to bear as long as the season lasts. In England, where vegetation is more rapid, and the season longer, later sowing or planting will be necessary, making the difference in the times of sowing about ten or fifteen days. They should be sown in lines not nearer than 12 feet from each other, that being the height to which they will grow if in good soil, and supported with stakes so high. 9 feet may, however, be taken as an average height; and as that is more than can be conveniently reached from the ground, a greater height would be next to useless. The seed being larger than those of the kidney bean, the drills, whether for planting the seed or the tubers, should be not less than 3 inches deep, and the seed or roots placed 9 inches distant in the line.

Subsequent culture.-What has been said regarding the pea is quite applicable to this crop also: when the haulm has reached the height of 8 or 9 feet, it should be topped, for reasons given (vide KIDNeY Bean, Pea, \&c.) If the production of pods be greater than the consumption, the oldest should be picked off before the seed is much more than formed in them, as, if left on, the plants would be unnecessarily weakened, and the continuance of the crop much diminished. Where stakes are difficult to procure, the runners may be topped when 2 or $2 \frac{1}{2}$ feet high. This dwarfing, although it lessens the produce, nevertheless admits of good crops being obtained.

In staking runners, long slender rods are preferable to the branching sticks used for pease. These should be stuck along both sides of the row, as soon as the plants are 6 inches in height, and placed in a diagonal direction, reversed on each side, so that, when the row is finished, the supports will have the appearance of diamonded trellis-work. The intention of setting the stakes in this manner is to afford a more ready means to the stems of the plants to ascend by and twine round. These rods should meet at top, for, unlike the pea, which branches 
out as it ascends, and therefore requires to be kept more open at top, the runners branch most near the bottom, and are thinly furnished with side branches towards the top. As noticed for pease, strong wide-meshed netting, attached to poles driven in along the rows, may be very advantageously used where rods are scarce; and poles, furnished with cross pieces of lath, about a foot in length, nailed to them, will serve well for attaching tarred cord or common twine to their ends, and thus enclose the plants between three, four, or five lines of cord, according to their height. A very good way of training is to plant two rows 4 feet apart, to procure rods of sufficient length to admit of their thicker ends being thrust into the line of each row. The smaller ends may be brought together at the top, thus forming an arch or curvilinear trellis. These rods, if sufficiently strong, need not be nearer each other than 3 or 4 feet; and lines of cord should be stretched from one to the other, taking a turn round each in passing, and extending the length of the rows. Four or five lines of cord thus fastened will form a very excellent trellis for the runners to grow over, and form at the same time a nice shady arbour. Three larch poles, set in a triangular form, and fixed together at the summit, form a good pyramidal conductor for such plants. The London marketgardeners' practice is to top the plants when they begin to form pods; and when the object is to gather the crop in a green state, immense quantities are thus produced. Where, however, seed is to be ripened, in this country at least, stakes are found to be indispensable. Cottagers may attach cords to the wall under the eaves of their house, fastening the lower end to stumps of stick driven into the ground. If a prepared border is made along the bottom of the wall, and the beans set in it, they will entwine themselves around the cords, and thus improve the appearance of the cottage, and afford an excellent supply of a nutritious vegetable food.

Soil and manure.-A soil richer, and even deeper, than that suited to the pea is in this case required. In newly-broke-up soils, all the leguminosæ prosper well; and in older ones, if rich in humus, they produce wonderful crops. Light, poor, or gravelly land, although it hastens the maturity of the crop, is incapable of yielding such returns as those we have stated.

Forcing.-The scarlet runner, on account of its rambling growth, is unsuited for the forcing-house, and hence is never obtained earlier than the period of its natural growth.

Taking the crop, and subsequent preservation.-The young pods are gathered when from $2 \frac{1}{2}$ to $3 \frac{1}{2}$ inches in length, and before the seed begins to form within them. When rapidly grown, they may be used of a larger size, and in that case they are cut into long narrow slices when about to be dressed. When the seeds have fully formed in them, they are unfit for use in their green state. When grown for their seed, whether for future sowing or for haricots, they are pulled up when fully ripe, dried in the sun and stacked by, and afterwards separated from the pods for use, either by being thrashed out or by hand-picking, and the seed bagged or otherwise stored by till wanted.

Approved sorts, and their qualities. 1. Scarlet runner.-Attains the height of from 9 to 12 feet; flowers beautiful red, and abundant; pods rough on the outside; nevertheless they are, while young, as crisp and as well flavoured as any of the dwarf sorts; beans, when dry, dark red and spotted. Known also as the tall scarlet runner, a mere seed-list name. It is the Haricot d'Espagne, or écarlate, of the French.

2. White Dutch runner.-Pods rather longer and smoother than in the last; flowers and beans white; does not continue so long in a bearing state as the last; is known as the case-knife runner; scarcely attaining so great a height as the last.

3. Painted lady.--Resembling the last two in habit; the flowers, however, are variegated, being of a bright scarlet colour, intermingled with pure white, and hence very ornamental; not so productive as the last two, nor quite so delicate to eat. We believe this to be the same as the York and Lancaster runner of some seedcatalogues. It is the Haricot d'Espagne, à fleur bicolore, or panache, of the French.

These are the principal sorts in cultivation in Britain. The Americans possess many varieties of haricots, such as the Lima pole bean, but, like the Sieva and other sorts, they are by far too tender for our climate; and the same may be said of several European sorts, which, upon trial, are either too tender, or inferior to those named above. Of these we may name the Prague runner, or red pea, which, although related more to the true kidney bean than to the section in which the three above runners stand, has some merits also, the pods being tender, while the seed is round, and, when ripe, rather thick- 
skinned, mealy, and in flavour resembling the sweet chestnut. The Haricot d'Alger, Prague bicolor, Prague jaspé, are varieties of the Prague runner, the beans of all of which become soon unfit for use.

Insects and diseases.-These are the same as those attacking the order leguminosæ generally, and the kidney bean in particular (which see). The seed of all the runners cannot be depended upon to vegetate above a year. Besides the insects noticed in article KIDNeY BeAN, all the leguminosæ are liable to the attacks of slugs and snails, from the time they appear above ground until they attain the height of a foot or so, after which they seldom trouble them. Their destruction is simple: dusting the plants morning and evening with caustic lime in powder, as recommended for pease, will soon rid the plants of these pests.

General remarks. - There have been few substitutes found for any of the leguminous plants which are cultivated as articles of food. The white lupin, Lupinus albus, has from the earliest ages been extensively grown in Spain, Portugal, and Italy for the ripe seed, which is used in the same manner that haricots are by the French.

Several species of lentil are cultivated on the Continent also for soups and haricots: these are chiefly Ervum lens, the common lentil; $\boldsymbol{E}$. Ervilia, the winter lentil; Lathyrus sativus, the Spanish lentil; Cicer arietinum, the chick pea, \&c. Both lentils and lupins are cultivated in Italy now, as they were in the days of Pliny, as green manure, being ploughed or dug in when they had attained their greatest size, but before they had formed their seed, in which case it was supposed they would rob the ground of more than they restored to it. This remains a question for modern chemists to solve. Pliny states that beans also were cultivated in the vicinity of Macedonia and Thessaly for the avowed purpose of being dug into the ground, and that this was done just as they began to bloom.

One lb. of liver-coloured kidney beans (which sort is of medium size and weight) contains 792 beans. One lb. of scarlet runners contains 428 beans. 


\title{
C H A P TER III.
}

\author{
BRASSICACEOUS PLANTS, OR THE CABBAGE TRIBE.
}

The natural order to which the Brassicaceous plants belongs, contains plants of the greatest importance to man. The whole order is pre-eminently European : 166 species are found in the north and middle of Europe, and 178 on the shores of the Mediterranean. Dividing them into the two hemispherical divisions of the globe, it appears that while only about 100 species are natives of the southern, there are about 800 natives of the northern. "The useful qualities of the turnip, radish, the rape, and the cabbage, and all its multiform varieties, are all well known. The greater part of the order consists of plants possessing high anti-scorbutic powers. These appear to depend upon a certain acrid, volatile, oily principle, the chemical nature of which is imperfectly known. It is to be remarked that plants of this order are always eatable when their texture is succulent and watery, as in the roots of the radish and the turnip, and the leaves of the cabbage tribe. Cruciferæ are said to possess a greater share of azote than any other tribe of plants, as is apparent in their fetid smell when fermented."-Hortus Britannicus.

\section{§ 1.-THE CABBAGE.}

Natural history. - Brassica oleracea is the type of a numerous family, consisting of the white and red cabbage, cauliflower, broccoli, savoy, Brussels sprouts, and the borecoles, all of which are believed to have sprung from the wild cabbage (Brassica oleracea), specimens of which are to be found on the sea-shore at Dover, and at various other places in our own country, as well as in some other parts of Europe, but always in chalky or calcareous soil. Botanically, it ranks in the natural order Cruciferæ, sub-order Orthoploceæ (from orthos, upright; ploke, fold-cotyledons), and tribe Brassicæe (Orthoplocece siliquosce); and stands in class Tetradynamia and order Siliquosæ in the Linnæan arrangement.

The name is derived from Bresic, the Celtic name of the cabbage. Brassica was the name by which it was recognised by the ancient Romans, proceeding from prceseco, because it was cut off from the stalk. The Latins gave it the name of caulis, from which our modern names cole, colewort, are derived. The term cabbage is in general understood to mean those varieties which form a firm head, by reason of the leaves turning close over each other, in contradistinction to those open kinds, like the borecoles, \&c., which are called kales. Three varieties of cole are mentioned by the eldest Greek historians-the crisped, or ruffled, which they called Selinas or Selinoides, from its resemblance to parsley; the other, Lea; and the third, Corambe. Chrysippus and Dieuches, two learned Greek physicians, each wrote a book on the properties of this plant, and Cato and Pythagoras wrote in its praise. Apicius loathed them, and Drusus Cæsar held them in no esteem, while his father, the Emperor Tiberius, thought highly of them. The discovery of the art of distillation is attributed to the simple circumstance of an ancient physician having been called suddenly away from a mess of cabbage he was eating; he placed another plate over that in which his cabbage was, to keep them warm, and finding, on his return, the interior of the uppermost plate covered with moisture, he reasoned on the cause, and ultimately discovered the art of distillation.

The introduction of the cultivated cabbage into Britain was no doubt the work of the Romans ; and its dissemination afterwards may be attributed to the earliest ecclesiastic communities, who ever had an eye to the good things of this life. In all probability it would reach Scotland and Ireland by the same means, although it has been asserted that it was scarcely known in the former country till the time of Cromwell, whose soldiers are said to have brought it with them from England. Gerard is the oldest English author who has written fully on this useful vegetable; he mentions the white-cabbage cole, the red-cabbage cole, the curled gar- 
den cole: "the Savoie cole is," he says, "numbered amongst the headed coleworts or cabbages." He. notices the curled Savoy, but says, "the swollen colewort of all others is the strongest," and which he received from Master Nicholas Lete, who brought the seed out of France. The same author says, "Rape cole is another variety; they were called in Latin caulo-rapum and rapo-caulis-participating of two plants, the coleworts and turnips, from whence they derive their name. They grow in Italy and Spain, and some places in Germanie, from whence I have received seeds for my garden. ' They must,' he says, ' be carefully set and sown, as musk melons and cucumbers.' This variety has now become one of our hardiest field plants."-PHILLIPs.

There is scarcely an instance in the whole vegetable kingdom of a plant that has produced so great a number of varieties, differing so much in appearance and qualities, as the Brassica oleracea : few would suppose that the wild cabbage found on our sea-shore is the parent of such a progeny. In its natural state, with its green wavy leaves, without the appearance of a head, and, to superficial observers, scarcely distinguished from the wild mustard or charlock, it is as dissimilar to one of its own offspring, the drumhead cabbage, as it is to a Tarragonian grown cauliflower, which is said often to attain the weight of $40 \mathrm{lb}$.

Use.-In a variety of forms this esculent is in almost universal use throughout the whole civilised world. The Greeks and Romans used it in a raw state, to counteract the intoxicating effects of wine. One modern physician, at least, has recommended it for a like purpose. The Romans threw trefoil and nitre into the pot along with the cabbage, believing that such would cause them to boil green-a department in cookery in which many good housewives plume themselves not a little at this day. All vegetables, if fresh gathered, may be boiled without the least change in colour, if put into boiling water with a handful of salt, and allowed to boil in plenty of water, leaving the vessel uncovered. The uses to which it was applied by the ancients are given by Phillips, in his "History of Cultivated Vegetables," vol. ii. p. 99, summing up the whole with the observation that they believed it "light of digestion, and that it clarified the senses when moderately eaten."

The large drumhead cabbage is used extensively in Germany, and throughout the north of Europe, for making saur-kraut. The heads are cut into small shreds with a knife or plane made for the purpose, are packed in barrels or tubs along with vinegar, to which salt and cloves are sometimes added, and in this state they are preserved in excellent condition till late in spring: when used, it is stewed over a slow fire, and served up with most kinds of meat. In England they are pickled, and used for the same purposes as the red cabbage, which latter is grown principally in Britain for pickling. On the Continent it is much esteemed when slowly stewed in an earthen pan, along with a few slices of onion, and a larger propor- tion of apples, in which state it appears at most tables during winter, and is both excellent and wholesome. In all cases the cabbage is most wholesome when eaten young; and hence the English, and the Scotch now following their example, prefer them in the form of collards, cabbage-plants, or cabbage-sprouts, and like to see them brought to the table quite green and tender. In country places in England, and in most of our Scotch green-markets, they are only to be found when of a large size, thoroughly hearted, blanched white within ; in which case they are, particularly when long cut, exceedingly unwholesome, often tough, and in general flatulent and indigestible.

Propagation.-All the varieties of Brassicæ are raised from seed annually, but they may all be also propagated by cuttings-a method employed to preserve any particular variety from changing its character, which all are exceedingly liable to do when propagated by seed, on account of the natural disposition of the plants to shoot into hybrids. Hence our seed-lists are so crowded with names, and hence also the difficulty we often experience in getting seed which will produce plants identical with their parent.

Almost all the Brassicæ produce sprouts - the cauliflower and broccoli less freely than the others. By means of these, any choice kind can be increased by cuttings, and thus perpetuate the identical variety without the chances of its becoming deteriorated by rearing them from seed. However carefully seed of this family is saved, we are never certain in the results, and what we may have genuine and pure one year may become, by intercourse between neighbouring plants of the same family, next to useless another. The idea of propagating them by extensionthat is, by cuttings-did not, however, arise from this alone. It was introduced into England about thirty years ago from the Brazils, where the climate is too hot for the cabbage to perfect its seeds. The manner of proceeding is this:-The sprouts, when from 4 to 5 inches long, are taken from the stem of the plant, and, according to their state of succulency, are exposed to the atmosphere to cauterise the wounded part. In summer, a day and a night is in general sufficient; while in winter two or three days may be requisite. The cut end of the sprout is dipped in caustic lime or dry wood-ashes, to dry up the moisture. They are then 
planted like any other cutting-producing, in due time, plants fit for use, and cuttings for further propagating supplies. Amateurs might in this way keep up a succession of stock without the trouble of sowing seed. The great advantage, however, is in keeping the stock uncontaminated.

Sowing and planting.-The time of sowing for a principal crop, to come into use the May following, is by long experience fixed by the London market-gardeners to be from the 25th of August to the 1st of September. A fortnight earlier is expedient north of Newcastie, unless in very favourable situations, when the 25th of August may be taken. Much of the danger attending too early sowing is in the plants running to seed in spring without hearting. No doubt there is something in this; but seed of a good variety, and at least a year old, is not liable, under proper management, to do this. It is, however, well to make two sowings in all suspicious cases-the one during the first week, and the second during the last week in the month. Plants from this sowing should be planted out for good by the middle or latter end of October, the plants having been previously removed from the seed-bed as soon as they are fit to handle, and being transplanted into a nursery-bed in what is called the pricking-out manner. The largest of these should be planted first, and the smaller afterwards, which may extend the season of their final planting to the first or second week in November. This is to be considered as affording a general spring or early summer full crop. Towards the end of February or beginning of March, sow, to secure plants fit for planting out in May, June, and July, generally as the ground is cleared of early crops of pease ; this will give a supply fit for use during the latter part of summer and autumn. These are the two general sowings. For particular purposes, and in peculiar situations, a sowing will sometimes be required to be made by the beginning of February, to secure an early summer supply, should accidents have come over the crops of the August sowing, and also if it be apprehended that that crop should be insufficient for the supply.

In sowing seed of all the Brassicæ, and at whatever season, there are certain helpmates sent us in the feathered part of the creation, who thin our sowings. In so far they do good, but sometimes they overdo this act of kindness by thinning too much. Now as these are, according to our notions, friends we cannot dispense with, seeing the good services they render us, in a way and by means we could not do for ourselves, we must not, like barbarians, talk of their destruction. We must protect ourselves from their overofficiousness in this matter, by covering our seed-beds with netting closely secured at the edges, but elevated from 9 inches to a foot over the general surface, which is easily done by setting in a few slight branches to support the netting. It is a poor crop that will not repay the trifling trouble and expense of this ; and he who thinks the matter of so little consequence to himself as to disregard this precaution, is rightly served to find his seed entirely abstracted. Many other means have been proposed; we know of none so simple and so efficacious. Strewing the surface with lime, wood-ashes, soot, \&c., are all very well in their way; suspending half a score of egg-shells from lines run in all directions, stuffed cats, owls, hawks, \&c., are the vestiges of the dark ages of horticulture; and even our first published potatodanglers, fig. 23, stuck full of feathers, are but sorry preventives.

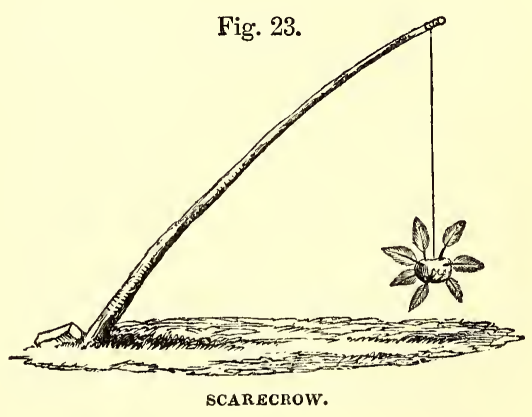

Seed for what is called cabbage coleworts-that is, young cabbage-plants, to be used while quite young, and, before they indicate hearting, a sort of produce much in use about London, and now becoming generalin private gardens-should be sown between the middle of June and the end of July, so as to be fit for final transplanting during August, September, 
and October, as ground becomes vacant for this purpose in particular.

Chappel's colewort, not much known beyond the London market, should be sown during July, August, and September, transplanted very close together, and upon all spare pieces of ground, under fruit-trees, \&c. They come into use early in winter or spring, and are much esteemed.

Late or drumhead cabbage is sown for general crops either in August, and kept over winter, being planted out in October or November, or early in spring, say February or beginning of March, and planted out in May or June. The former is the Scotch practice, the latter the English.

$\AA$ great deal depends as to the period of sowing, particularly for main spring crops, upon situation and circumstances. The rule laid down by the London growers in this respect may be taken as the latest period, as every circumstance is in their favour. Not so in cold climates and cold soils; and to meet the requirements of these, the seed should be sown earlier, from a week to a month, according to the unfavourableness of the place and circumstances. To sow too early would make the plants too strong, and certainly endanger their running to seed: to sow too late would be to have plants too weak to stand thewinter; and should they even do so, they will be late in coming to perfection. At the July sowing, it oftentimes appears that the ground is dry, in which case it will be of great advantage in securing a strong and rapid germination, to soak the ground with water some hours before putting in the seed; but, to prevent the soil from caking, it will be well to fork it over before sowing. The seed may with advantage, under such circumstances, be also soaked a few hours in tepid water. After sowing, if the weather continue hot and dry, shade the beds with branches or otherwise till the plants appear above ground, when the shading should be gradually removed. As soon as the plants are above ground, dust them over morning and evening with finely-pounded caustic lime, as a precaution against insects.

All the varieties of the Brassicæ, with the exception of the cauliflower and the CouveTronchuda, or Portugal cabbage, are exceedingly hardy, and therefore, in all ordinary cases, the most open and exposed situation should be fixed upon for the seed-beds. The exception to this is in cold places, where the early spring sowing will require the shelter of a warm south border, and in many cases the assistance of a slight dung-bed heat. Wherever the seed is sown, it should be freely exposed to the sun and air, and of all things shaded spots should be avoided. The seed should be sown thin. A bed 4 feet wide and 20 feet in length will require 2 ounces for the smallest-growing varieties of early cabbage, such as Aitken's matchless, \&c. For those of larger size, such as the vanack, the same quantity of seed will sow a bed 4 feet broad and 36 feet long. One ounce of seed will give from 2000 to 2500 plants. Cover to the depth of $\frac{1}{4}$ inch from the alleys, if the soil be light and well pulverised; if not, make the surface smooth with the back of a spade, and when the seed is sown, cover with a light compost to the above depth.

All the Brassicaceous tribe are very much improved by transplanting as soon as the young seedling plants are fit to handle, which will be when the young leaves have attained the size of about 2 inches in length. At this time they should be carefully removed from the seed-bed, and, if the weather be dry, the bed should receive a good watering, which will facilitate the operation of lifting without injury to the tender roots of the plants: the nursery-bed should be prepared by previous manuring and digging, and its surface, if dry, well watered previous to planting; and when the young plants are set therein, it should receive a gentle watering, to settle the soil about the roots. If the sun is powerful, a slight shading will be beneficial during the day until the roots have taken hold of the ground. This is more especially necessary for summer transplanted crops. In such nurserybeds, they should remain until well rooted, and, at planting, be set from 3 to 4 inches apart, according to the size of the variety. A second transplanting is often found beneficial to give greater strength to the plants, to encourage the multiplication of roots, \&c., but in this second removal they should have a third more space accorded them. By such means plants will attain a good size, and be fit for final 
transplanting the moment ground is cleared for them, thus economising both space and time. Those intended to be set in nursery-beds during winter should be planted from 4 to 5 inches apart each way, for too thick planting is injurious in two ways-it draws the plants up slender, and prevents the circulation of air amongst them, by which many damp off during winter. Great care ought to be taken that each sort is kept separate, and that all be correctly labelled to prevent mistakes in spring. The same attention should be paid to Brussels sprouts, savoys, and such kinds of borecoles or greens as may be required early in the summer. Transplanting all the cabbage tribe into nursery-beds is of great advantage to them; it renders them stocky and well rooted; and as this operation has for its object only the preservation of the plants during winter, it follows that a rather poor soil and open situation should be afforded them. It is of no use, however, to treat the Portugal cabbage in the above way; it is too tender to stand our climate, and therefore is better sown in spring in a moderate temperature, so as to be fit for final planting by the end of May. In cold, damp soils, we have found it of advantage to cover the surface between the rows of all newly-planted-out things, when the operation is delayed till too late a period, with finely-sifted coal-ashes to about an inch in thickness; they tend greatly to exclude the frost, and absorb a considerable quantity of humidity; besides, they render the ground comfortable and clean to walk upon. In many places where the crops are liable to be thrown out by winter frosts, tanners' bark is laid on the surface with beneficial effects. A good breadth should be planted by the first week in November, as little advantage would arise from planting again till the latter end of January. A supply of young plants for spring planting out should also, early in November, be removed from the seed-beds, and planted out in nursery-beds to stand the winter. In moist situations, the most open and exposed piece of ground should be chosen for this purpose, and, of all others, shaded and confined places should be avoided.

Under ordinary circumstances, the plants are set in their new locale by the dibber, of which figs 24,25 , and 26 are examples; but when the

plants are large, as it is the object to remove them

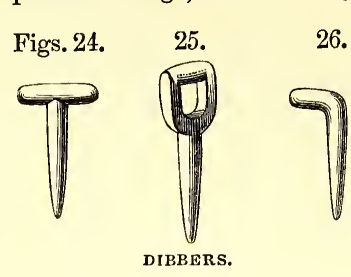
with as little check to their growth as possible, the planting - trowels, figs. 27 and 28, should be used. The dibber is too well known to require any description; the

broken handle of an old spade forms one of the best kinds, which only requires to be cut to the length of 10 or 12
inches, and to have its

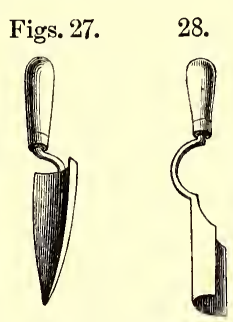

PLANTING-TROWELS. perforating end bluntly pointed. The trowel is a more perfect implement, and is employed in the removal of plants of a larger size than can be safely planted by the dibber. In using either the pointed or semicircular trowel, figs. 28 and 29 , the young plants may be taken up with a considerable ball of earth attached to the roots, while they suffer no injury by the process. A more perfect mode of transplanting by the use of the trowel is that by taking two of these, one in each hand, thrusting them down on opposite sides of the plant, at the same time drawing the handles slightly outwards; the faces of the trowels are thus made to collapse so much as to press the soil about the roots, and hence enable the operator to take the plant, with ball entire, from the seed-bed to its ultimate destination, and to place it in its

new abode without the least Fig. 29. check to its growth. As we

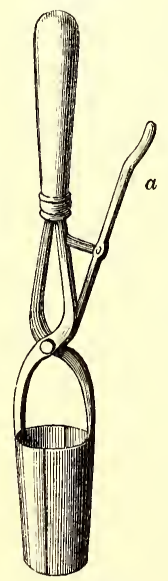

SAUL'S shall have to refer frequently to transplanting, we may as well here show the construction of transplants, which have been long in use, for transplanting such crops as the Brassicx, at present under our consideration. Fig. 29 is called Saul's Transplanter, because that intelligent horticulturist brought it into public notice many years ago in the pages of the "Gardeners' Magazine." It appears, however, to be an improvement on a similar implement invented by the Rev. Mr Thornhill, about 1820 , and used by him for transplanting turnips. It may be thus described: The blades are opened by pressing the lever $a$ towards the handle, when they open outwards, and in this TRANSPLANTER. State are thrust in the ground, having the plant within them; a counter pressure causes them to collapse, and to embrace the ball firmly, and in this state, the transplanter being drawn upwards brings with 
it the plant and ball entire. It is then taken to its new site, set in its place, when the handle $a$ is again pressed inwards, and the blades open and are withdrawn, leaving the plant and its ball entire, to be filled around with earth, and the

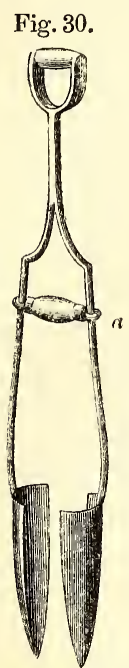

TRANSPLANTER.
Fig. 31.

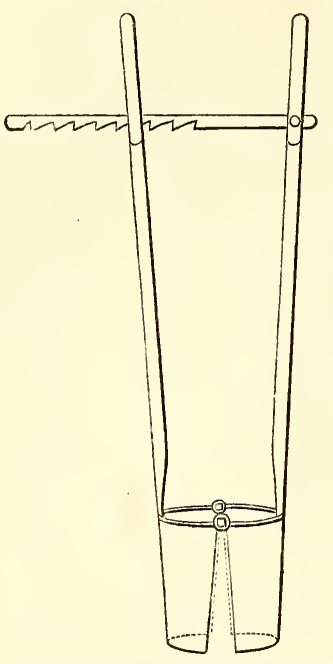

M'GLASHAN'S TRANSPLANTER. operation is repeated on another subject. Fig. 30 shows a modification of this implement, wherein the blades are opened by moving the slider $a$ upwards, and, when thrust down around the plant, the blades collapse by pressing the slider downwards. The operation afterwards is the same as the last. Upon exactly the same principle, but with much more mechanical ingenuity, is the transplanter of $\mathrm{Mr} \mathrm{M}$ 'Glashan constructed, which is admirably adapted to such operations. Fig. 31 will explain its form; its mode of opera-
Fig. 32 .

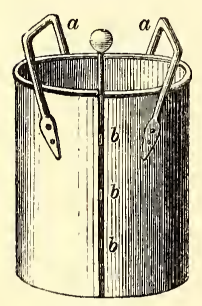

FLOWER TRANSPLANTER. tion will be at once understood. Fig. 32 represents another modification of transplanters ; it is constructed of two semicircular pieces of iron polished to a bright surface, which all transplanters should be, because we know practically they enter the ground better, and deliver the ball more entire than they would do if used in a rusty condition. These semicircular pieces are furnished with handles $a a$, riveted to them, and are inserted into the ground so as to enclose the plant between them. In this state they are attached to each other by two iron pins, passing through the eyelet-holes welded on their edges, $b \quad b \quad b$; on their being pulled up, the plant is elevated with its ball attached. Our objection to this implement is, that the radius of the blades, if we may so call them, is the same at bottom as at top, which renders it possible that they may be pulled up without bringing the ball with them; or if so, that it may drop out during its transportation to its new site. The others we have shown are different; they collapse the ball firmly - and not only that, but by their construc-

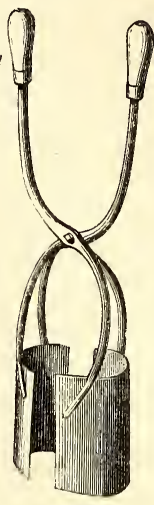

FRENCH

TRANSPLANTER. at bottom than at top, rendering it next to impossible that the ball shall be extracted. and also that it cannot slip out afterwards until relieved by the removal of the pressure upon it. Were the blades made in a tapering manner, then all the conditions of other similar transplanters would be fulfilled. All these transplanters are merely modification s of fig. 33 , long used in France for similar purposes. Its principle will be readily seen by our figure : the handles $a a$ are pulled outwards when the blades are thrust into the ground. They are pressed inwards when the operation of lifting upwards is desired.

In planting all the brassicaceous plants, drills should be drawn, 3 inches deep, at the distances apart which are to be allowed each variety; for amongst the tribe of early cabbage, to which most of our previous remarks apply, there are comparatively large-growing and small-growing sorts. The type of the former may be instanced in the vanack, and of the latter in Atkin's matchless. The ground should be prepared by heavy dunging and deep digging; but in this, as in all similar cases, trenching 2 or $2 \frac{1}{2}$ feet deep is better. The early sorts are set in lines from 15 to 18 inches apart, but Atkin's matchless, and one or two others, may safely be set at 12 inches only. The former should be from 1 foot to 15 inches distant in the line, while the latter may be set at from 10 to 12 inches. Plant, if possible, on moist days; if the soil is dry, run a good soaking of water into the drills previous to planting. Preserve the roots entire of those that have been transplanted into a nursery-bed, as every root is to them of importance. Those that have been drawn direct from the seedbed should have the tips of the taproot shortened, but none of the others.

The Scotch, late, or drumhead cabbage -for it is known by all these names-is seldom grown in gardens, unless where the bounty of the proprietor is, as it 
should be, directed to the wants of the poor, by providing them during winter with soup made from the scraps which fall from his own table--then the drumhead cabbage comes in well; and also when saur-kraut is made for winter use, this vegetable is indispensable. It attains a very large size, and therefore requires room. At planting, they should be set in rows 3 feet apart, and the plants 2 feet distant in the line. This also depends on circuinstances, for there are larger, taller, smaller, and dwarfer varieties of this kind. The above is the maximum distance.

Subsequent culture.-After planting, all that is required till they attain perfection is keeping the ground clear of weeds, stirring it frequently and deeply during summer, and drawing a little earth about the necks of the plants when about 9 or 10 inches high, which not only supports them in an upright direction, but offers opportunity for the roots to form near the surface, thereby increasing their means of collecting food, and greatly tending to encourage the more rapid growth of the plants. Some disapprove of the earthingup plan, and as a substitute draw the drills deeper at planting, which seems to amount to the same thing. White cabbage is grown for three purposes, the first of which is to procure heads fully formed and hard, the second is to draw for use before hearting, and the third to furnish sprouts from the old stems for a long time after the head has been cut off for use. The first of these are called fullhearted cabbages; the second, cabbage plants or collards; the third, cabbage sprouts. To have them in the former state, they must be sown four or five times a-year. To have them in the second state, oftener ; and in the third case, such as the vanack, an English variety, or M'Ewan's, a Scotch variety (the latter little known), should be selected. These, if planted in autumn or spring in good rich soil, will, after the first heads have been used, continue often for a couple of years sending up a fine and abundant supply of sprouts. When sprouts are not required, the old stems should be pulled up as soon as the heads are cut, and consigned to the rot heap. If left in, they greatly exhaust the soil for no end or purpose. Should any of the plants run to seed soon after planting, remove them without loss of time, and replace them with strong plants from the nursery-bed, using one or other of the transplanters noticed above. Early in spring, when it may be wished to have close-hearted cabbages, as soon as the leaves give indication of turning inwards, tie the whole of them loosely together with strings of matting or slender twigs of willow. During longcontinued droughts, water abundantly; and as all watering with pure water does little other than assist to render soluble the fertilising matter that may happen to be in the soil, and watering with springwater does little more than chill the ground, and merely sustains the existence of the plants, it is better, seeing that there is the same labour in both cases, to water all growing crops with liquid manure. Water, however applied, is thought to injure the flavour of culinary crops. With the cabbage tribe it is the reverse, as they seem to be improved in flavour by it. It also prevents the plants from becoming stunted in growth, which induces a disposition in them to favour the production of aphides on the leaves, and other insects on the roots. The roots left in the ground after the heads are cut off, when the intention is to have a succession of sprouts from them, should have the remaining leaves cut off, the ground between the rows forked up, and if a good manuring be applied at the same time, the benefit will be evident in the greater supply, and its longer continuance fit for use. To make the most of the ground when the breadth is planted at the distances directed above for the larger-growing sorts, strong early-sown coleworts may be planted, either one or two between each two permanent plants, according to the space accorded them. These coleworts or collards will be useful to pull up for early spring use, before the principal crop arrives at its full size. When cabbages are cut, the leaves should be all removed from the stem, and all the embryo sprouts, excepting five or six of the best placed and most promising, to produce sprouts for table use. If all but one be displaced, it will grow rapidly, and soon become as large and fine as the original head. Such cabbages as have been cut during May and June will, by this treatment, yield another crop in July.

Soil and manure.-The first cannot be 
too rich or too deeply wrought, and the latter can hardly be applied in too large a quantity. The whole tribe are gross feeders. The plant in its original state, as we have already shown, is a native of the sea-shore and of calcareous soils; it is consistent, therefore, with reason that lime and salt should be added to the natural constituents of the soil they are planted in. We have applied gypsum as a top-dressing to the surface between the rows, with the most beneficial effects, to all crops of the Brassica tribe, to which it seems admirably adapted. Its action appears to be directly and indirectly useful to such crops-directly as the food of plants, because, being soluble in water, it supplies sulphur and lime; and indirectly, because of its action on the volatile carbonate of ammonia, which becomes fixed when these substances meet. In the latter case, gypsum acts in the soil on the ammonia contained in rain water, in the same way as it does when applied to dunghills. A double action takes place between gypsum and carbonate of ammonia, when they meet in solution. Each of these salts being decomposed, their elements unite in the form of carbonate of lime and sulphate of ammonia, and the advantages from the change arise from the sulphate of ammonia not being volatile, as the carbonate of ammonia is. Stableyard dung, if applied with gypsum to fix or retain the ammonia evolved from the former during the process of turning it over, and during the fermentation of the mass, will, in the case of rich manure, require to be used in the proportion of one cwt. of the latter to a ton of the former. Superphosphate of lime has been found a useful assistant to cabbage crops, and where manure is scarce it may be sown in drills prepared for them at planting, or a little (say a dessert spoonful) put in each hole before the root is introduced.

Taking the crop, and subsequent preservation.-When the plants have hearted, the heads are cut off for use, just as they begin to show the first indication of blanching towards the centre, which is undoubtedly the proper time; some, however, cut them in a more advanced state, preferring quantity to quality. Cabbage plants, collards, or coleworts-that is, young plants which are not intended to heartare pulled up by the roots and tied up into bundles, the roots being either washed or cut off to keep the leaves cleaner. Sprouts are gathered by being cut off the stems at various sizes and ages, from the time when they have only five or six leaves in size, until they form into little cabbages of the size of a swan's egg. It is in the intermediate stages, however, that they are most esteemed. It is seldom in Britain that artificial shelter is required for any of the white or red cabbage tribe. In cold places, however, they may be laid over on their sides, and their whole stems buried under ground. (Vide Broccoli, section 5.) The full-hearted cabbage is often cut from the stems on the approach of severe frosts, but it is better to retain the stems attached, and these are buried entirely in dry soil, and so deeply covered that the frost cannot reach them. They should, however, be placed so far apart that the one may not touch the other. In peaty soils, cabbages thus buried will keep for months, that soil containing so great an amount of antiseptic properties.

Approved sorts of white cabbages (Brassica oleracea, var. Capitata) and their properties.-The improvement that has taken place of late years in the whole of the Brassica tribe is perhaps greater than in any other division of culinary vegetables. Such sorts as Atkin's matchless, Sut ton's imperial, Sutton's dwarf comb, Enfield market, Shilling's queen, \&c., have taken the place in most of our best gardens of the sugarloaf, early York, \&c. of bygone days. These sorts are more esteemed for their delicate flavour and tenderness, when cooked at a proper age, than for their largeness of size-a merit only fitting for the lowest grade of market purposes. These require, in consequence of their small size, less space to grow in than the larger and coarser kinds, and will, nevertheless, yield as good a return, of the same extent of surface, as their larger allies.

1. Sutton's dwarf comb.-When grown along with other sorts, we found it last year the earliest of any. It is small, hearts well in spring, andaffords a good supply of very delicate sprouts throughout most of the summer. It was planted 12 inches apart.

2. Sutton's imperial.-Another excellent early spring variety from the stock of Messrs Sutton and Sons, Reading; rather larger than the last, but equally early, tender, and useful, producing sprouts during the summer; planted 13 inches apart.

3. Atkin's matchless.-A small early cabbage of great excellence; conical when full grown; the leaves remarkably wrinkly, somewhat like a savoy; planted 12 inches asunder; stands the winter well; a variety of the early York, but much superior, if large cabbages are not required. 
4. Knight's early dwarf.-A nice little hardy sort, which may also be set at a foot apart each way.

5. Early Dutch twist.-An excellent cabbage of the smallest size, very delicate, and may be planted almost as close together as a crop of cabbage-lettuce.

6. Cattel's dwarf Barnes.-A small early sort of great merit.

The above are the smallest and earliest of cabbages, and exceedingly well suited for small gardens and private family use. They should be sown more frequently than the larger-growing sorts, so as to keep up a succession of young and delicate heads, much after the manner of sowing lettuce.

7. Early nonpareil, Shilling's queen, Shilling's superb dwarf, Tiley's early marrow, early emperor, early imperial, are all equally excellent for second earlies and second-sized cabbages.

8. Pomeranian cabbage is thus described by Mr Thompson in "Journal of Horticultural Society," vol. v. p. 280 :- "It is remarkable for its conical tapering form, very compact, and firm to the apex. It is very hardy, and may be cultivated like other hearting cabbages; but it may be interesting and useful to know that, at Macon, in France, the market-gardeners take it up out of the quarters when fully grown before winter, and lay it in the soil, to the neck, in a sloping direction. Thus treated, it withstood the severe winter of 1847 . The quarter thus cleared can be trenched and prepared for other crops-an important advantage in small gardens. It is likely to prove valuable in colder situations than is suitable for the Battersea and other cabbages grown in the neighbourhood of London."

King of the cabbages, London market, Wheeler's imperial, Vanack, Sprotboro, Paignton or Penton, $M$ 'Ewan's, are all admirably adapted for general crops, where large returns are required. The vanack has been cultivated above a century in the gardens of Colonel Wyndham at Petworth, Sussex, but it was only brought into general notice about thirty years ago. It has the merit of throwing out fine sprouts, which attain a large size for eighteen months or two year's after having been first cut. M'Ewan's is of similar character, but of Scotch origin; it is difficult to seed, as it goes on sprouting and growing for almost any length of time. The Paignton is of Devonshire origin, named from a village of that name, where it has been cultivated for ages. Its properties are much the same as those of the two last. 'The Sprotboro is a Yorkshire cabbage, also of great excellence, in habit and properties much resembling the last four. The Paignton is also known in seed-shops as the Cornish, Pentonville, and curled.

To this section we may usefully add Chappell's colewort, which is little known out of the London market, where it is brought in immense quantities as an open green cabbage, being pulled before it hearts, and is sold as a colewort, and much esteemed. Colewort, cabbage-plants, and collet are synonymous terms, used for cabbages cut for use before hearting, and to be eaten in a young state.

The early York is probably the type of all our best early cabbages, many of which, however, excel it in delicacy; no other sort is known to have the ribs and veins less prominent, which are its principal points. The vanack is again the type of the imperial, Fulham, and Battersea. Of the Portugal cabbage there are two varieties, a taller and a dwarfer sort. The Pomeranian is very distinct from all others, is a good late sort, of conical form, almost terminating in a point.

Approved sorts of Brassica oleracea capitata, var. Depressa, De C.-Large Drumhead.-Knowi also as the Scotch, cattle cabbage, flat pole, and Strasburg. Of this there are several subvarieties, differing chiefly in shortness of stem, concavity or flatness of head, \&c. Of these De Candolle has recognised two distinct forms; viz., Brassica oleracea depressa (depressed or flattened drumhead), and B. oleracea sphorica alba (spherical or great round Scotch cabbage). Those of dwarf habit are the best, as, being for winter use, they stand the frost better when close to the ground than when high above it. Unless, as we have already stated, a soupkitchen is to be supplied, or saur-kraut is to be made, a small plantation of either of these varieties will be sufficient even for a large garden. In small ones, unless a head or two be required for pickling (and even for this purpose the red cabbage is preferred), we consider it the least profitable of the family to grow, as it is of little use after the head is cut off. It still occupies a place in most farmhouse gardens and cottages in Scotland, and frequently appears in our markets - an evident symptom how far we are behind our southern neighbours in the art of vegetable cookery.

The seed should be sown, in most parts of Scotland, from the middle to the end of August, and a strong rich soil should be chosen. In the warmer parts, and generally throughout England, it is better to sow in March, to prevent the crop coming into use too early in the following autumn, for this is to be regarded as a winter esculent. The autumn-sown crop is sometimes, if the plants are forward, planted out in the early part of November, but in general about the beginning of April following. Those sown in February or March should be transplanted in May, June, or July. Their whole routine afterwards is the same as for savoys or other cabbage. To have heads of a large size, plant at the distance of 3 feet by $2 \frac{1}{2}$; give abundance of liquid manure during their growing season, or, in default of that, fork in a good dressing of half-decayed rich stable-manure, using a fork for the purpose, of which Dr Yellowlee's, fig. 34, is a good example.

Fig. 34 .

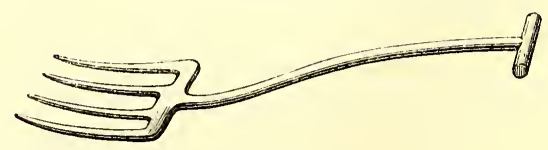

YELLOWLEE'S FORK.

Dr Yellowlee's fork.-Forks are preferable to spades for digging the ground, because, from their construction, the soil is much better 
broken and pulverised, and the operation as speedily performed. They are much better when the ground to be loosened is occupied with the roots of trees or other plants, because less injury is likely to befall them. The entire length of this fork is 3 feet $3 \frac{1}{2}$ inches, the length of the handle being 2 feet 2 inches; the prongs are 7 inches apart at top, and 6 inches at the point; the length of the prongs, which are three in number, is $13 \frac{1}{2}$ inches, and at the top $\frac{7}{8}$ of an inch square, tapering to a point. The straps fixing the head to the handle are 11 inches long and 2 inches broad, and $\frac{1}{2}$ an inch thick at the centre, tapering off at both sides.

\section{$\S$ 2.-THE PORTUGAL CABBAGE.}

The Portugal cabbage (Brassicce oleracea, var. Oblonga-Couve tronchuda, large-ribbed or Portugal cabbage, or Braganza) was introduced into England about 1821, from Trauxuda in Portugal, and is sometimes called Trauxuda kale. Of this there is a dwarf variety, much cultivated in Portugal, and known by the name of Murciana. This excellent vegetable, too little grown in Britain, is not exactly of the hearting kind. The centre leaves are deprived of their green or leafy part, leaving the ribs, which are boiled, and used much in the manner of sea-kale. It is exceedingly delicate, and in this respect different from the rest of the cabbage tribe. The dwarf variety Murciana (Brassica oleracea, var. costata, chou de Beauvais) is somewhat earlier, and throws out numerous suckers from the lower part of the stem, which the tall variety does not. It is much more tender than any of the others, and rarely stands our northern winters. For early crops, the plants should be sown in August, and kept under frames all winter, like cauliflower; or better, they should be, like it, sown on a slight bottom heat in February, and hardened off for transplanting in April or beginning of May. Its subsequent culture, \&c., resembles that of cauliflower (which see).

[The most extraordinary production in the cabbage tribe is Kerguelen's Land cabbage, the Pringea antiscorbutica of botanists, first discovered by Captain Cook, the circumnavigator, and subsequently observed by Dr Joseph Hooker as a native of Kerguelen's Land, or Island of Desolation, situated in the centre of the Southern Ocean-a cold, humid, barren, volcanic rock, on which that distinguished naturalist recognised only eighteen species of vegetation, but amongst them this brassicaceous production. It is described by him in the "Flora Antartica" as very abundant, particularly close to the sea. Its root-stocks are from 3 to 4 feet long, and lying close to the ground, bearing at their extremities large heads of leaves, sometimes 18 inches across, and so like those of the common cabbage, that if growing in a garden they would scarcely excite attention. They form a dense white heart, that tastes like mustard and cress, but much coarser. It abounds in an essential oil, which renders it more wholesome than the common cabbage. It may never be worth the attention of the British cultivator, but its existence in that desolate island, so far removed from civilisation as to be considered the most remote of all islands from any continent, suggests two important, although somewhat different, considerations. The first and most important is, that the Disposer of all that is good should have placed there a plant so valuable to those who traverse those little-visited seas, subject to one of the most fearful of all human diseases, scurvy, and that also presented to them the moment they put their foot on shore, where, from its luxuriance and abundance, it is likely, as Dr Hooker observes, to prove for ages to come an inestimable blessing to ships touching at this far distant isle. The next consideration is, how came it there? "The contemplation of a vegetable," he says, "very unlike any other in botanical affinity, so eminently fitted for the food of man, and yet inhabiting the most desolate and inhospitable spot on the surface of the globe, must equally fill the mind of the scientific inquirer and the common observer with wonder." A plant nowhere else recognised leads to the belief "that it was created, in all probability, near where it now grows-leads the mind back to an epoch far anterior to the present, when the Island of Desolation may have presented a fertility of which this is, perbaps, the only remaining trace." We know there is a theory recently promulgated, the adherents of which will account for the existence of this plant, in its unique form and isolated position, on the supposition that numerous centres of dispersion and new creations of developments exist, and that these do not in the slightest degree disturb the harmony of the general design of creation.

"One of the most mysterious of such phenomena is the change by which certain living entities seem to pass from the animal to the vegetable state, or vice versâ, without decomposition, or apparent disorganisation of fabric. Professor Von Esenbeck was the first to publish his opinions on the subject in 1814 . His observations were made chiefly upon the filamentous algæ, particularly the Oscillatoriæ. The animal state is inferred from the spontaneous movements of the individual, the vegetable state from its immobility. A monad or active molecule issues from the summit of the filament, and frolics in the fluid in which the plant vegetates. Ultimately a period arrives in which a complete metamorphosis ensues, the moving monad being gradually converted into a motionless vegetable." -The Rev. Patrick KeIth Clark's Botanical Lexicon, p. 264.]

\section{$\S$ 3.-RED CABBAGE.}

Red cabbage (Brassica oleracece, var. Capitata rubra, De C.) Of this there are several varieties, differing only in their size, height, and colour. Medium-sized varieties are to be preferred, as also are low-growing ones, on account of their being less liable to sustain injury from frost during winter; but colour is of all the most important, as its chief use is for stewing or pickling, when a fine red colour adds much to its appearance. The sorts, or rather names, in the seedshops, are, dwarf red, tall red, red pickling, early 
blood-red, large red, drumhead, Dutch, and the Aberdeen red-a coarse, open-hearted half cabbage, half borecole, much grown in the North of Scotland, in cottage and farm gardens, on account of its extreme hardiness.

The first and fourth are the best, if they can be procured genuine. They are sown, planted, and managed in the same way as the drumhead cabbage, noticed above, only, being of much smaller growth, they may at final planting be set at the distance of $2 \frac{1}{2}$ feet apart row from row, and $1 \frac{1}{2}$ feet in the line. On the approach of severe frost they should be taken up, and either buried in dry soil, or hung up by the roots in a dry airy shed, where they will keep for a long time. When the heads are cut, the roots should be immediately removed, as no longer of use, and to prevent exhausting the soil.

\section{§ 4.-BRUSSELS SPROUTS.}

Natural history.-Brussels sprouts (Brassica oleracea bullata, gemmifera, De C., bud-bearing cabbage). Of this much-esteemed vegetable, introduced into this country from the Continent only of late years, there are only two varieties, a taller and a dwarfer grower, varying in height from 1 to 4 feet. The former is the most productive, on account of its greater length of stem, along the whole length of which the sprouts, like little cabbages, are thickly set. The latter stands the winter best. It derives its name of chou de Bruxelles from having been extensively cultivated around Brussels from time immemorial.

Uses.-It is used much in the way other cabbages are, being sent to table stewed, and as a garnish for butcher-meat, and at the best tables is presented, from the size of a large marrow-fat pea, which they indeed somewhat resemble, to that of their natural full size, somewhat under that of a pigeon's egg. The outer leaves being carefully removed, the hard, compact, little sprout is presented whole. Sometimes they are boiled, which is done with great care, using plenty of water, and that at the boiling point, when they are thrown in, adding a handful of salt, and leaving off the lid of the vessel. With the view of preserving their delicate green colour, they are covered with a rich stock sauce, in which vinegar and nutmeg form a part. They are also served boiled with white sauce, and in private families with melted butter. The quantity grown throughout the north of France, Belgium, and Holland is truly astonishing, whole fields of them being seen all over the country.

Sowing and planting.-Regarding their cultivation, they are sown early in April, and transplanted in June, into rows 2 feet apart, and the plants set 18 inches distant in the line. Their sowing, transplanting, \&c., differ not from that of early cabbage: the same proportion of seed is also used. The soil, however, is in a different condition. If planted in soil as highly manured as that for other cabbage, the sprouts would be produced coarse, large, and open, whereas the smaller and more compact they are the better. In Belgium, where an almost constant succession of sprouts is kept up, they sow on a slight hotbed in February, prick out on a warm border, and transplant towards the end of April. They afterwards make two or three separate sowings, at the distance of three weeks from each.

In England, for private families, two sowings are necessary-one in February, on slight bottom-heat, and the other in April. In Scotland, they often sow in August, and keep the plants over winter for spring planting, and again in March for a secondary crop. In the former, the first-sown crop comes into use in September, in the latter in November, at which time vegetation is moderated in growth, and the crop lasts till March; the second following in April and part of May, if the crop be taken up in February, and replanted to give it a check. By early and late sowing, and the superior advantage of climate, the Brussels market is supplied from the end of July to the beginning of May following. "The London practice," says Mr Cuthill, a high authority in such matters, "is to sow in May; but it should," he remarks, "be March. The Scotch sow them in August. Sown in May, it is impossible for the plants to get so tall, stout, and cover the stems so well with fine large sprouts, as when sown in March. I have had them," he says, " 3 feet high, covered from top to bottom, each stem producing one peck of large close sprouts."

Subsequent culture.-The ground should be kept well stirred by frequent hoeing, not mere surface-scratching with a Dutch hoe, which, were it not for gravel walks, and that as seldom as possible, should be banished from gardens. The draw-hoe is better; and even that, beyond the mere cutting down of small weeds, which should not be allowed to appear, will be much better substituted by the Vernon hoe, fig. 3, or any of the modifications of it. When the plants are about a foot high, draw a little soil around their roots; and when about three parts grown, and while the sprouts are forming, the side leaves should be broken off, a few at a time, to give room to the sprouts to swell, and also that, by their removal, 
more of the energies of the plant may be thrown into them. It has been recommended to cut off the tops when the sprouts are fully formed, and this is a part of the Belgian practice. We prefer their retention, as from their form and position they protect the sprouts during winter from wet, frost, and snow. The top itself forms a useful vegetable after the sprouts are gathered, and, singular enough, possesses quite a different flavour from them. Many are deterred from cultivating this excellent vegetable, supposing it either too tender or less productive than a crop of cabbage, neither of which is the case. It is a plant calculated for simultaneous cropping, and may, therefore, be planted along with potatoes, placing the plants alternately in the rows.

"Select, if possible, a rich stiff loam for them; plant 18 inches plant from plant, and 2 feet row from row ; keep the ground well loosened by the hoe. Moulding up the stems is never practised, and as soon as the plants reach their height, which is known by the top beginning to cabbage, the latter ought to be cut out. This throws all the strength into the sprouts down the stem, making the bottom ones as good as those on the top."-CuTHILL, in MarketGardening around London, p. 24. We have given reasons above for not cutting the tops off where danger is expected from frost.

Taking the crop.-When the sprouts have nearly attained their full size, they should be gathered for use, taking the largest first, and so on in succession, cutting them off with a sharp knife. When taken to the vegetable house (an apartment in all good gardens, where the vegetables are carried to be washed and dressed fit for the cook), the outer leaves should be neatly cut off; and if moderate care has been taken they need not be washed, as that would spoil their flavour, particularly if to be sent to a distance. When dressed, they should be put into a small punnet basket, fig. 35, which is made of thin split laths, and of various dimensions-from 3 to 6 inches deep, and from 6 to 9 inches in diameter, according to the sort of vegetable or fruit to be placed in them, and with or without handles, to suit various purposes. In such baskets all the most choice vegetables are carried, such as forced potatoes, kidney beans, Brussels sprouts, mushrooms, \&c. This is a branch in garden refinement that has, nothwithFig. 35.

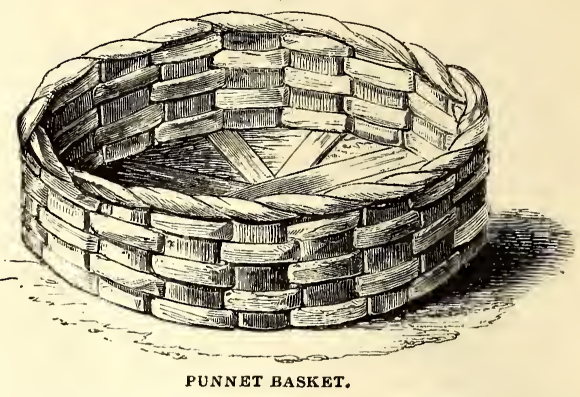

standing steam communication, not as yet reached Scotland. They can be purchased in Covent Garden for a mere trifle, and no garden should be without half a hundred of them at the least.

General remarks.-An unfounded prejudice exists against home-saved seed. Carelessly as much of this branch of horticulture in Britain is conducted, it is not much better on the Continent, at least where seed is saved for exportation. The case is different as regards that for home consumption. (Vide Saving Brassica SeEd, at the end of this chapter.) The safe way to save a pure stock, once obtained, is to propagate by planting the sprouts in spring after the manner of cuttings.

\section{$\S$ 5.-THE BROCCOLI.}

Natural history. -The broccoli (Brassica oleracea, Botrytis asparagoides, De C.) The broccoli is of more recent introduction to Britain than the cauliflower, from which they are supposed to have originated. In Miller's time, only two varieties were known, the white and purple, and from these it is more than probable that all our present varieties have emanated. The white varieties of broccoli resemble the cauliflower much, only they are much hardier and constitute a winter vegetable, while the former is adapted only for summer and early autumn. The white varieties are in all respects preferable to the purple or other coloured sorts.

Uses.-These are as a substitute for cauliflower during winter and spring, when the other cannot be had in perfection.

Mode of propagation.-The same as the cauliflower, \&c.

Sowing and planting.-The early varieties, such as the purple Cape, improved purple Cape,Walcheren, early white Cape, Grange's early cauliflower broccoli, and Gillespie's early, should be sown the first and second week in May for English practice, and a fortnight earlier for the Scotch climate, so as to have them in to succeed cauliflower, from the beginning of September 
and onward till Christmas. Another sowing of the same kind should be made during the second week of June for the south, and not later than the first week for the north. These will stand over winter, and come in early in spring. Plants from the first of these sowings should be transplanted for good about the first of June, having been previously pricked into a nursery-bed, to give them strength and abundance of roots. Other varieties, of which there is a great number, should be sown about the second and third week in May for the south, and ten days earlier in the north; and, if transplanted about the middle of June, will, from their difference in growth, produce their heads during March, April, and May. As a general rule, calculating upon the kinds, for an autumn supply sow in April, for a spring supply in May, making an allowance of a fortnight between sowing at London and at Edinburgh, unless slight artificial heat in the latter case is had recourse to. The mode of sowing, the quantity of seed, and the earliest management are the same as for early cabbage (which see). Transplanting has been objected to by some as having a tendency to cause such early sorts as the white and purple Cape to button, as it is technically called - that is, to run up prematurely to flower, before the plant is sufficiently strong to bring the flower to perfection. And the means employed is to trench and manure the ground in May, to tread it firmly down, and to sow the seeds in lines 2 feet apart, dropping three or four seeds into each hole, made at the distance of 2 feet apart from each other. When the plants comeup, they are all destroyed except the strongest, leaving one in each hole. The ground is kept deeply stirred during summer, and the plants are earthed up in the usual manner. The same mode has been recommended for early-sown cauliflower, lettuce, \&c. We think careful transplanting preferable.

Subsequent culture.-This is the same as for early cabbage. The distance apart must ever be governed by the size of the variety-thus, for example, Knight's dwarf protecting will require only 18 inches plant from plant; while the largergrowing kinds, such as Elletson's gigantic late white, should be set 3 feet distant row from row, and the plants 2 feet apart in the VOL. II. line. Much, however, in this depends on the soil and its state of cultivation. Deep stirring of the ground between the rows is all they require during the rest of the summer. It is only in very cold and damp localities, and in very inclement winters, that broccoli requires protection during winter; but it is advisable, with all the kinds intended to stand over till spring, to lay them over in November, which operation is performed by opening a trench at one end of the field, forming the back in a slanting direction, taking up the plants with as much earth about their roots as possible, removing two or three of the lower leaves, and setting them in the trench inclining towards the sloping bank, and covering up the roots and stems close to where the first pair of leaves issue. If the ground is much on the incline, begin at the lower part, so that the leaves of the plants may point downwards, to prevent the snow and rain settling in their hearts. This process not only prevents the weight of snow breaking down the leaves, but, by the check the plant sustains, causes the fibre of the leaves to assume a more tough and less succulent form by lessening the supply of food by the roots, and thus renders them less liable to injury from frost. In very unfavourable situations, the plants, when fully grown, may be carefully taken from the exposed part of the garden and replanted in the above manner in a more dry, warm, and sheltered place. It is, however, imprudent to set them in a damp or shaded situation.

When broccoli has attained its full size, which it will have done in most situations by the middle of November, the plants might be taken up carefully and disposed of as we have suggested (see post) for full-grown celery. This, in moderate climates, may be uncalled for, but in less favoured places it would secure their preservation, and at the same time clear the ground either for other crops, or for improvement by trenching, \&c. In such cases the ridges should run in an east and west direction, the earliest kinds being placed with their heads towards the south, while the later sorts should have theirs in the opposite direction. This position would tend to accelerate the one and retard the other. A bank of broccoli thus arranged could easily be 
protected on the top by a roofing of boarding, straw-thatched hurdles, or a tarpauling covering, which would ward off at once both wet and frost. Great care, however, must be taken in such a mode of protection that the plants be not crowded too closely together, else the want of a free circulation of air amongst them would be as disastrous to their safe keeping as if they had been left to take their chance where they were grown. As soon also as the heads are cut for use, the foliage should be instantly removed for the preservation of those that remain.

Laying in dry farm or stable litter between the rows, as high as the length of the stems, would protect the roots, but would afford no shelter to the leaves and hearts. Nor do we approve of Mr Knight's recommendation of transplanting in September, setting them deep in the ground with a view to encourage young roots from the stem to assist in swelling out the flower in spring. The late-flowering sorts will make roots when laid down as we have proposed, which will be in action during April and May, and no doubt will produce this effect. Those that flower before that period will have no such means of support, but must depend upon the sap already elaborated in the plant during the previous summer and autumn.

Soil and manure.-As we have said of cabbage, the soil can neither be too rich nor too deeply dug or trenched, the object in both cases being bulk of produce-unless, indeed, a very early supply is required, when a less luxuriant state in the plants may induce earlier maturity. All the Brassicæ are improved by lime, and to this may very safely be added a slight top-dressing of salt once or twice during their growth, the whole tribe being indigenous to calcareous soils, and in close proximity with the sea. Copious manuring with sea-weed, where it is readily procured, has produced excellent crops: a slight inquiry into the cause will lead us to view common salt as the stimulating ingredient. From experiments now in course of trial, we believe that flower of sulphur would be ultimately considered an excellent ingredient, not only as entering into the constitution of the plant, but as a preventive to the attacks of insects.

Taking the crop.-For the highest class tables broccoli should be cut when about the size of a goose's egg, three heads forming a genteel dish. When for ordinary purposes, it should be taken when nearly full-grown, and before what is technically termed the curd is brokenthat is, the flower opening-for on its firm and compact appearance much of its merit depends.

Approved sorts and their qualities.-1. Grange's early cauliflower broccoli is an old variety that, if procured true, still stands high in estimation, having a head nearly as large and as white as a cauliflower. The foot-stalks of the leaves are long and naked; the leaves somewhat ovate, slightly lobbed at the base, very slightly waverl, and incurving a little over the flower, defending it from frost and wet. Not a large grower, and, being upright in habit, may stand at 2 feet distance apart. If sown in April or May, it will flower from the end of September till the end of December. Successional sowings of it should be made from April till the end of June. The London market-gardeners sow only four sorts, of which this is the principal, the others being the Walcheren, late London white, and the early purple or sprouting broccoli.

2. Walcheren.-Comparatively new ; first produced by Mr Legg of Bishopsthorpe; so closely resembles cauliflower as to be scarcely distinguishable from it. The leaves are more curled than in the cauliflower, and its constitution is of a hardier nature, standing our ordinary winter in the open garden, and withstanding better the extreme droughts of summer. Mr Legg's practice in keeping up a constant succession is to sow the third week of April, middle and end of May, the middle and end of June, and the middle and end of July. For early spring use he sows about the 25th or 27 th of August, keeping the plants through the winter under hand-glasses in the usual manner, leaving, however, only three or four plants under each glass. Of itself, by the above timeous sowings, it will become a complete substitute for all the others, and be at any time scarcely distinguishable from cauliflower.

3. Gillespie's.-A fine white early autumn sort much grown about Edinburgh; does not appear to be as yet much known about London. Treatment the same as for Grange's early. The same distance also.

4. Early purple or sprouting, attaining the height of from 2 to 3 feet; somewhat spreading; requires to be set 3 feet apart. Much grown by the London market-gardeners, and is much prized by French cooks, who dress the little sprouts in a variety of ways. The flower is close-headed, and of a fine purple at first, if the seed is genuine. It branches into sprouts afterwards, but is apt to lose its colour and become greenish, as well as to produce numerous small green leaves intermixed with the flower, if set in too rich ground. The London growers sow it along with all their other broccolis in May, when it comes into use in November, and continues all the winter, as fresh sprouts of flowers are produced from the alce of 
the leaves after the head is cut. We sow in April to secure flowers in November. Sometimes a second sowing is made in June, which produces flowers the following April.

5. Purple cape.-A very early variety, the culture, \&c. of which we have already noticed. The last two are almost the only purple sorts worth growing, and the five described the best of the very early varieties.

6. Elletson's gigantic late white. - One of the largest as well as latest white broccolis; dwarf on the stem; but, as the leaves spread considerably, it should be accorded 3 feet distance each way.

7. Willcove late white.-As large as the last, but hardly so late; grows taller, and requires the same space.

8. Hammond's white cape.-An excellent pure. white broccoli, flowering about December.

9. Knight's protecting.--If not so pure a white as the others, it possesses the great merit of being exceedingly hardy; and, being of very dwarf growth and small size, it may be planted 15 inches asunder, thus yielding a great return of produce off a small space, and therefore excellent for small gardens. Its heads are larger than might be expected from so small a plant. It is known from all others by a bracteal leaf, which is produced on one side of the flower as if it were designed for protection from frost and wet. It appears to be an improvement on the small green Danish, from which it may possibly have originated.

10. Chapple's large cream. - A very large and excellent variety, to which may be added Dilston's bride and Snow's superb white, two first-rate sorts. The latter is a dwarf variety, with broad leaves and short petioles, in size and colour resembling a fine cauliflower. If sown in the neighbourhood of London in May, it will come into use in November. If sown in Scotland in April, it will come in at the same time.

To those who require the hardier varieties, we would recommend the Siberian, late green, or Danish, which are all the same. It has been proved to be the hardiest of any, coming in late in spring, when the slow increasing heat of the sun tends to swell it out to a fair size. The leaves are somewhat purplish, very much waved and indented; 2 feet apart is sufficient for it. The Russian dwarf is also equally hardy and small.

Besides, there are about thirty other varieties possessing less or more merit, and perhaps nearly half as many more names to be found in seed-lists, which are either worthless or possess similar or inferior merits to those in the above selection. They have been selected from about forty named sorts, which were grown within two years in the Dalkeith gardens.

The following broccolis are popular; their names, however, will suffice. Late dwarf purple Syrian, winter imperial, Dancer's pink cape, Adam's superb early white, Snow's winter white, impregnated white, Portsmouth cream-coloured, Sumner's late white, early Malta, Howden's purple, hardy green cape, American white, Tamworth white, Miller's dwarf, Stewart's early white, Addison's - the two latter much esteemed about Edinburgh. Indeed, Stewart's early white and Gillespie's white are more grown by the market-gardeners than any others, coming in early in winter of a good white colour, and lasting till spring.

'The true Walcheren, by successful sowings, may be had the whole year through, and, with Snow's superb white, Grange's early cauliflower, Knight's protecting, and Hammond's fine white cape, may be considered quite sufficient to afford a suppiy the whole season.

Gencral remarks.-The difference between the broccoli and cauliflower is very slight. The colour in some sorts of the former is no doubt sufficient to mark the distinction; but amongst the finer varieties of the white-flowering kinds of broccolis, this distinction almost vanishes. Professor de Candolle, who has taken more pains to describe and systematise the order Cruciferæ than any other botanist, has not forgotten to mark the differences which exist between them. He states, first, that both are varieties of each other, or rather varieties of the same race-Brassica oleracea botrytis, or cabbages producing heads or flowers of an eatable description, but of a very different organisation from the cabbage. From a very interesting paper upon this subject by that eminent botanist, published in the "Transactions of the London Horticultural Society," vol. v., the following brief extract is taken :- "The bunches of flowers, instead of being loosely spread into a pyramidal form, like those of a panicle, are close from their basis, and form a kind of a regular corymb; to which is added a second character, that may be considered as a natural consequence of the first: the pedicles, from being tightly kept together before their time of blossom, lose their shape, grow fleshy from adhering to each other, and in general produce nothing but the rudiments of abortive flowers; so that, contrary to all other varieties, where the leaves and stalks are alone taken for culinary purposes, in this the floral foot-stalk is the only part eaten. This race comprehends two varieties, viz., the cauliflower and the broccolis."

Their difference is thus shown:-"The Brassica cauliflora (cauliflower) has generally a short stem, white-ribbed oblong leaves, the pedicle uniting at the head of the primary branches into thick, short, irregular bundles, in the shape of a corymb; it appears to be a degeneration of the Brassica oleracea costata, or Portugal cabbage.

"The Brassica cymosa (broccoli); its stem is more elevated, the leaf-nerves less prominent, the pedicles altogether less thick and close; they are also longer, so that, on becoming fleshy, they resemble in shape the young shoots of asparagus: hence the name Asparagoides given by ancient botanists to broccoli. The broccoli seems to be a degeneration of some variety of the chou cavalier, tall or open cabbage. It is divided into two sub-varieties, the common or white broccoli, and the purple or Maltese broccoli ; and each of these is again divided into several kinds by the practical gardener."

Cultivation, by improving the finer kinds of white broccolis, is narrowing the aistinctive marks; but although so nearly alike, they must 
ever remain distinct, inasmuch as they derive their origin from two very distinct types, namely, the Portugal cabbage, and the tall curled kale. The cauliflower originated also in the south of Europe-some say the island of Cyprus-while the other originated in the north of Europe, but whether in Germany or Britain we have no certain means of knowing. Others think the broccoli of Italian origin; and hence the older authors, in describing the then two only known varieties, call them Brassica Italica alba, the white broccoli; and Brassica Italica purpurea, the purple broccoli.

To obtain seed:- "Such plants of each variety must be selected in March or April as most perfectly agree with their peculiar characteristics, and are not particularly forward in advancing for seed. As the stems run up, some growers recommend the leaves to be taken away; but this must be injurious. Mr Wood of Queensferry is particularly careful that no leaves appear on the surface of the head. He always lifts his plants, and plants them in another bed, watering them abundantly, as this, he finds, prevents their degenerating or producing proud seed; and when the head begins to open, he cuts out its centre, and leaves only four or five of the outside shoots for bearing. The sulphur-coloured he always finds the most difficult to obtain seed from. As the branches spread, four or six stakes should be placed at equal distances round each plant, and hooped round with string to support them, and prevent their breaking. When the pods begin to form, water should be given repeatedly, and occasionally some thrown over the whole plant, which tends to prevent mildew. Before the pods begin to change colour, those from the extremity of every shoot must be taken away, as these yield seed which produce plants very apt to run to seed without heading, and by an early removal the others are benefited. The branches ought to be gathered as soon as the pods upon them ripen. Different kinds must never be planted near each other, or they will reciprocally be crossed. The seed ripens in August or September; and it is often recommended to preserve it in the pod until wanted, but the general practice is to beat it out as soon as it is perfectly dry."-Cottage Gardeners' Dictionary.

\section{$\S$ 6.-THE SAVOY.}

Natural History.-The savoy (Brassica oleracea bullata major, De C.) has been known in Britain as a cultivated plant since the time of Gerard, who specially notices them, and ranks them amongst hearting or heading cabbages. It is sufficiently distinct from all these in the wrinkled leaves, which form its chief charac teristic. The Brussels sprout is considered a sub-variety of this, but it is much more removed from it, in appearance, than the savoy is from the cabbage. It forms an excellent, hardy, and productive winter esculent, and is divided into two pretty distinct classes, the green coloured and the yellow.

Its use is the same as the cabbage, being used, when fully headed, during winter, and as sprouts or collards, in some families, during most of the year.

Propagation.-It is propagated by seed sown annually, or by cuttings of the young sprouts in spring, after the head has been cut off.

Sowing and planting.-In many parts of Scotland these are sown in autumn, at the same time as cabbages, \&c. are, for next summer's consumption. This, however, can only hold good in very cold and late localities. In others, the crop would be too forward, for they are not required in a full-hearted state before November. There are circumstances, however, when this is different; namely, where green savoys are required during most of the year, in the form of collards, like young cabbages. Sowing, therefore, to meet these demands, must be made frequently. (Vide Early cabbage.) For a general autumn and winter crop, a sowing towards the end of February, and another towards the beginning of April, will be sufficient, so that the plants may be set in their permanent position in May, June, or July. Half an oz. of seed will be sufficient for a seed-bed of 36 square feet.

The distances, \&c. of the plants are in all respects the same as for cabbage, allowing those that are to be drawn as collards the same distance as cabbage collards, and those which are to remain to form perfect heads, from 24 to 26 inches between row and row, and 20 inches between plant and plant.

Subsequent culture.-An open and exposed situation is the best, beginning planting out as the young plants are ready in the nursery-beds; for it is important that they be removed from the seed-bed as soon as their leaves are about 2 inches in breadth. Choose the strongest plants for first planting, following up with the weaker in the course of a week or two, or as ground falls vacant from other crops. May and June is a good time for transplanting, when the crop is wanted during August and September, for southern practice; and the same time, or even towards the middle of July, for northern climates. The first, unless for market purposes, is perhaps too soon for English family use, as, during August and September, more desirable vegetables are in use. July and August are therefore a preferable transplanting season for 
English consumption. In planting savoys, as well as any other of the Brassica tribe, observe if any of the young plants are clubbed or have knotty protuberances on their roots; if so, cut them closely off; but it is better to throw away such plants entirely, as they never make good plants afterwards. Soil has much to do with this clubbing, and it is found to be far more prevalent in poor gravelly soils than in those that are deep and rich. Savoys may be planted in the simultaneous mode of cropping; as, for. example, between rows of previous standing crops, like pease, beans, early cauliflower, or the like, that are sufficiently advanced to be cleared off before the savoys will require the entire ground. The general crop will last in use from the beginning of November till the beginning of March, after which they will begin to run to seed, and should then be removed either to the rot-heap or the pigs, or be dug in as green manure for the succeeding crop.

Soil and manure.-These can hardly be too rich, and in a highly cultivated state. The same manures as for cabbage should be applied.

Taking the crop, and subsequent preservation.-In autumn, when the plants have attained their full size, and before they have become quite hard, they are fit for use, and in that state are more wholesome than when older; as all vegetable matter blanched white from deficiency of light is in a less fitting state as articles of food than when of their natural colour. In severe winters, the full-grown crops may be taken up and preserved as recommended for drumhead cabbage ; and for prolonging them in a useful state till late in spring, the same means may be employed as stated for broccoli.

Approved sorts and their qualities.-1. Dwarf green curled.-A useful, hardy, smallish sort, fit for small gardens, requiring only 18 or 20 inches space each way. Excellent for use before it becomes fully cabbaged. Known also as small dwarf green curled, pancalier de Touraine.

2. Cattel's green curled.-An improvement on the last.

3. Marcellin.-A new variety of excellent quality, growing much larger than either of the above, and hence requiring more room on the ground.

4. Cape, or drumhead. - The largest variety grown.
5. Globe.-Very finely curled, grows taller than most others, yet not spreading in proportion. Known also as large green curled, large green, large late green.

6. Dwarf yellow.-Curled; differing from the dwarf green only in colour, which we think no recommendation.

7. Feather-stemmed savoy.-This curious and useful variety has been in existence for several years, being raised by Mr Barnes of Bicton, and is a cross between the savoy and Brussels sprout. Notwithstanding, we do not remember having seen it noticed in any seed-catalogue. It is what may be called a sprouting savoy, producing numerous sprouts along the stem. A sowing of it should be made about the middle of April, and another about the 10th of May, planting out as the plants are of fit size, in the usual manner of savoys and other winter greens.

From an excellent article upon the Varieties of Savoy, by Mr R. Thompson, in "The Gardeners' Chronicle," 1850 , p. 244, being the results of experiments made in the gardens of the London Horticultural Society, we make the following additions:-

8. Early green curled, or new early.-Small outer leaves, rather plain, deep green; hearts readily, and is of excellent quality. It is considered the best for early use.

9. Early flat green curled.-Middle sized, very dwarf and flat headed, deep green, tender, and very good.

10. Early dwarf green curled.-Dwarf green, similar to the early green curled.

11. Large green German, or large late green. - "This," Mr Thompson says, "is the largest kind of savoy, and the best amongst the large. The leaves are plainer than in the other varieties; the head roundish, a little flattened, like a drumhead cabbage, which it also approaches in size. It is hardy, withstanding the frosts of ordinary winters well." This we apprehend to be the same as our cape or drumhead.

12. Conical savoy.-Rather small, pointed green, hardy, of very good quality, but affords much less weight of produce than several of the preceding sorts.

13. Early yellow savoy.-Middle-sized, roundish, yellow; hearts easily, of tender substance when cooked, and very good, but some object to the colour.

14. Early long yellow.-This, like the preceding, is an early variety, but it does not heart firmly.

15. Earliest Ulm saroy.-Described by M. Vilmorin, of Paris, in "Bon Jardinier," as being very dwarf, quickly forming a heart, which, though not large, is excellent. "It has been proved in the London Horticultural Society's garden, and stated by Mr Thompson as being the earliest in cultivation. It may be planted, in proportion to its size, considerably closer than the larger kinds." It is too small for market purposes, but in private gardens would no doubt be an acquisition.

It is somewhat singular that a long cultivated and popular vegetable like the savoy should have remained so long so true to its original character. Indeed, with the exception of the 
feather-stemmed, scarcely any change has taken place for years.

\section{§ 7.-THE CAULIFLOWER.}

Natural history.-The origin of the name cauliflower (Brassica oleracea Botrytis cauliflora, De C.) is from the Latin caulis, a stalk, and fero, to bear; its original name being coleflorie, or colieflorie. It is first mentioned by Gerard, who says, "The white cabbage is next best to the cole flourey; yet Cato doth cliefly commend the russed cole; but he knew neither the whites nor the cole flourey, for if he had, his censure would have been otherways." From this it appears not to have been known in his day ; subsequent Roman authors, however, mention it in such terms as to leave little doubt of its great antiquity, but of its origin we know nothing. Pierre Pompes, an old French author, says, "It comes to us in Paris, by way of Marseilles, from the isle of Cyprus, which is the only place I know of where it seeds." From this account Philips remarks, "It would appear that cauliflowers were not much cultivated in France in 1694, when his work was published; and the French have at present no distinct name for this vegetable, but call it chou fleur, or cabbage-flower."

Uses.-The heads or flowers are considered one of the greatest of vegetable delicacies when served up at the table, either plain boiled, to be eaten with meat, like other Brassicæ, or dressed with white sauce, after the French manner. It is much used as a pickle, either by itself or forming an ingredient in what is called mixed pickles. It may also be preserved a considerable time when pickled in the manner of saur kraut. It also forms an excellent addition in vegetable soups.

Propagation.-This is by seed sown at various times, for the purpose of keeping up a succession. It is scarcely capable of propagation by cuttings, and in our cold climate must ever be regarded as an annual plant ; for keeping autumn-sown plants under glass, or otherwise protected during winter, does not constitute a biennial.

Sowing and planting.-Three or four sowings annually is the usual practice; but the seasons of sowing depend somewhat on circumstances, of which climate is the principal. The most prevalent practice, however, is to sow between the 18 th and 24th of August; the plants of which sowing, when about 3 inches high, are transferred to a nursery-bed, and set about 4 inches apart. When sufficiently strong-which they will be by the middle of October-they are planted out in sheltered places, at the bottoms of walls, under hand-glasses, in cold pits or frames, in beds to be hooped over and covered with canvass, and sometimes they are potted and kept under glass till spring, when they are planted out for good in the warmest situation the garden offers. These plants flower during May and June following. The second sowing is made about the end of February or beginning of March, on a moderate hotbed, the plants being finally planted out in April; but they should have the advantage of a nursery-bed, if only for a few weeks, to strengthen them. These produce flowers during July and August. The third sowing is made about the beginning of April, in the open ground ; and the plants, after being pricked out in a nursery-bed, are finally transplanted in June, and will produce flowers from September until destroyed by frost. These are all important matters to attend to. Autumn-sown plants, if destroyed by frost during winter, may be replaced by others, forced upon hotbeds in spring, in time for the first planting; but a week's delay or advance in the third sowing may lead to the disappointment of either having the crop too early to supply the demand during October, November, and December, or it may be too late to flower at all. The above is the English practice, so far as dates are concerned. In most parts of Scotland, from the 1st to the 12th of August is the proper time for sowing the first crop, and a week in advance will be wise as regards the third. As for the second, as artificial means are employed, the exact date must ever be left to the discretion of the cultivator.

The London practice is to sow "the seed of spring cauliflower about the 20th of September, in open beds. Towards November, when the weather is beginning to get cold, frames and hooped beds are got ready in light rich land. The plants are pricked out not more than 4 inches apart each way: during the winter they are kept dry; no rain is allowed to fall upon them, but, whenever practicable, plenty of air is given to them. Frost has little effect on them under hoops ; but when excluded long from air, and kept in darkness, they sometimes suffer from damp to a considerable extent. They are planted out in the richest and earliest ground, in February or the beginning of March."-Market-Gardening round London, p. 22.

Some of our best English gardeners 
sow a fourth sowing about the middle of July, choosing a warm border, or throwing up ridges of soil and sowing the seed on the south side of the ridge, which ridges, of course, should run in an east and west direction. When the plants come up, they are thinned out to the distance of 9 inches or a foot apart, and allowed to remain without transplanting. In November nice little heads will be produced; and if not convenient to cover over the ridges with a roofing of boarding or thatched hurdles, as a protection from frost, the plants may be taken up and treated as noticed below. This late sowing seldom succeeds in Scotland, unless in warm, dry localities, such as along the shores of the Forth, where little rain or snow falls, and, from proximity to the sea, frost is seldom severe.

Half an ounce of seed is sufficient to sow a seed-bed of 36 square feet. Thick sowing should be avoided.

The method of sowing and planting is in all respects the same as for early cabbages (which see.)

The following ingenious method of keeping up a supply of cauliflower is communicated by Mr Henry Baily, of Nuneham Park, Oxfordshire, to "The Journal of the London Horticultural So, ciety," vol. v. p. 103. Mr Baily is one of our most enlightened horticulturists ; his method has both novelty and excellence to recommend it, and as his situation is not one of the warmest in England, similar success may attend those who follow his example. The true Walcheren sort only is used. "The first sowing for the spring crop is made about the 25th of August, and another, for smaller successional plants, a week later, upon an open border. As soon as the plants are large enough they are transplanted," that is, pricked out; "and as soon after that as they have made a few roots, they are again transplanted into small pots, called sixties: they are then placed in an open airy situation (either a frame, vinery, or peach-house, which is dormant), simply requiring protection from severe frosts; as they fill the pots with roots, larger ones are provided, and early in February the first crop, or handlight division, is planted out in a south border; the holes for their reception having received a barrowful of rotten dung, the mould is re-turned, form- ing a little hillock, on which these plants are placed, and covered with the glass till they begin to be established. The smaller plants are reserved for a successional crop, potted into larger-sized pots, and placed in temporary frames, covered with mats in severe nights, but fully exposed in fine genial weather. This crop is generally planted out in the alleys of the asparagus beds, completes its growth before the tops of the asparagus become too high, and then has its duration prolonged by the shade of its branches.

"For the next crop in succession I sow in pots, about the middle of February, subjecting the plants to the same routine of potting, \&c. Other sowings are made at intervals between this and the 20th of May, when the last crop is sown, which should be planted on a south border, for autumn use-extending up to Christmas, with protection.

"For the February supply, an early white broccoli, grown by Mr Wilmot, of Isleworth, is invaluable. It is sown the end of May, and should be taken up and protected in a cool vinery, as our winters will not admit of the production of cauliflower at that season, as the fine climate of Naples does.

"The roots should never be allowed to get matted in the pots, or the plants to suffer any check. It will readily be conceded that our object in the cultivation of those culinary vegetables, whose stems, leaves, or flowers are eaten, is to grow them in the most rapid and luxuriant manner, avoiding any check at any period of their growth : any curtailment of those resources of plants which have a tendency to increase their luxuriance, and consequently render them more tender, must therefore, be detrimental, and it is to avoid checking the growth of the plant that the practice of potting is adopted. In dry weather, when the plants are drawn out of the seed-bed, and planted with a common dibber, receiving daily dribblings of water, many will perish, and all will be materially injured. By the mode I have described this is avoided, and labour saved in the end. After planting out, a copious watering is given, either in the evenings of bright days or in dull and cloudy weather, when it is not rapidly evaporated." Such a mode as this is well adapted to our northern climate. 
Subsequent cultivation.-The safe preservation of the plants during winter deserves attention. They should be reared in an open, airy situation, and, when the leaves are about 2 inches in breadth, as we have stated above, should be invariably transferred to a nursery-bed, in an equally airy place, kept free from weeds and dead leaves, encouraged to make numerous roots, which transplanting considerably assists ; for the stockier and stouter they are got to be, without being drawn up tall and slender, or of too gross and succulent habit, the better they will stand the winter. It is not, therefore, expedient to have the nursery-bed too rich, as this would induce them to send down naked tap-roots, and assume a degree of grossness that would ill enable them to withstand the frost.

Hand-glass protection.-In October the hand-glasses should be filled, and those best fitted for the purpose are such as are constructed in pieces, so that the top may be removed entirely, or in part, on all favourable occasions, for the sake of ventilation, but more so to prevent the plants being drawn up or advanced farther than necessary. The glasses used for this purpose by the London market-gardeners are usually large bell-glasses, fig. 36,

Fig. 36.

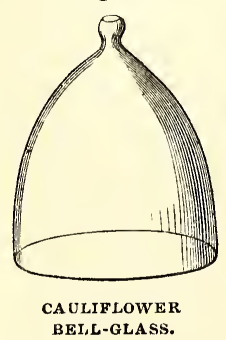

the corners, fitting into each other, and

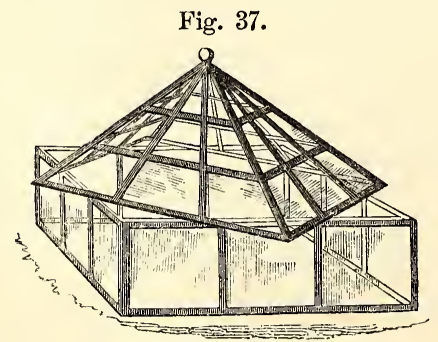

CAULIFLOWER HAND-GLASS. fastened by a wedge, and are each 20 inches square. Lead, zinc, wrought-iron, copper, \&c., are the worst possible materials to construct such utensils with. Some substitutes for these have been proposed, but their utility verifies the old saying-" Saving at the spigot, and losing at the bung-hole." The cast-iron handglasses will last for fifty years, the others not as many months. They are glazed with the fragments of glass which abound in all gardens where hothouses exist. The manner of planting under handglasses is this :-In the best exposed place of the garden, dig out holes in number agreeing with the number of bell or hand glasses at disposal. These holes should be $2 \frac{1}{2}$ feet square, filled with one-half rotted stable-manure, and rather more than the other half the soil taken from the hole, so that the place, when finished, may be 4 inches higher than the surrounding soil. The hand-glass should be set upon this preparation to mark its dimensions, and five plants placed in each space-one in the centre, and one within 4 inches of each corner of the space.

These are to be regarded as the permanent plants, but, to secure as many more as can be, with a view to their being taken up in spring and planted elsewhere, six or seven more plants may be pricked in between them. The glasses are to be set at first over them, supported on four bricks, one at each corner, and, as the winter draws on, these are to be removed, and the glasses set on the surface of the ground, or rather pressed about an inch under it, the better to exclude the cold. Ventilation must now be attended to by lifting the top off entirely, or partially as shown in our fig., and only closing it entirely down when severe frosts exist. The bell-glasses are to be managed in a similar manner, only on good days they may be elevated on one side an inch or two, or entirely removed. Here the advantage of the hand-glass in pieces will be apparent. These glasses may be placed at 3 feet apart, which will afford sufficient room for each patch of plants to develop themselves fully when the glasses are removed in April.

Wall protection.-Those transferred to the bottoms of walls will, in general seasons, stand well, and, if thinned out in spring, the superfluous plants being transplanted 
to an open part of the ground, will come in a fortnight or three weeks after those left to enjoy the reflected heat of the wall. In preparing the space for them at the bottoms of walls, which need not be more than 9 or 12 inches in breadth, no great fear need be apprehended of injuring the roots of the wall-trees, as these are, for the most part, progressing outwards. The soil may, therefore, be loosened up with a fork, and a good supply of stable-yard manure added, the plants set about 6 inches apart, to be afterwards thinned in spring to a foot distant. All the care required is to remove the leaves fallen from the trees, to dust the plants frequently for the suppression of slugs, and, in cold and damp localities, to cover the surface between them with finely-sifted coal-ashes to counteract damp.

Frame protection.-Plants for this purpose should be taken up from the nursery-beds : the taking up we consider essential, and much more than adequate to the saving of time which would result from placing the frames at once over the plants still growing in the beds. About the end of October or beginning of November transplant these into a bed of moderately conditioned soil, setting them 6 inches apart, so that the air may circulate freely through them. One precaution necessary in cold, damp, strong soils, is to elevate this bed 6 or 7 inches above the ground-level, to insure freedom from damp; cover with glass-lights at first till the plants take root, and afterwards, upon all occasions when frost or snow is apprehended, giving abundance of air at all other times. Cover the glass with canvass during severe frosts, keep the soil dry, and introduce between the plants, still further to insure this, an inch in thickness of finely-sifted coal-ashes; remove decaying leaves, pull up weeds, and leave till spring arrives, when more copious supplies of air must be given, and the plants gradually inured to stand the common atmosphere, when they may be transplanted out for good, which, according to climate, will be about the end of March or beginning of April, setting the plants in a warm place and in a highlyenriched soil, in rows $2 \frac{1}{2}$ feet asunder, and 18 inches plant from plant. In transplanting, remove with as much soil as possible about the roots, for which the vor. II. transplanters, figs. 29,30 , and 31 , will be found useful.

Wintering in pots.-Where very early crops are wanted, and where there is fit accommodation, a set may be planted in 48-sized pots in October, and kept under a glass frame, cool peach-house, or the like, till February, when they may be turned out of the pots with their balls entire, and planted in a pit covered with glass in a richly-prepared bed, and there allowed to produce their heads or flowers. Cauliflowers, at so early a period of the season, will only be regarded by those who profess to distinguish a superior quality in the cauliflower over a fine well-grown broccoli, which, at the same season, will be in high perfection in the open garden. This we, however, know to be the case with some persons, and therefore, to provide for such a taste, it will be advisable to adopt the potting system.

Spring-sown crop. - Plants originated from seeds sown in February or March should be also transferred to nursery-beds to harden and gain strength; in these they should be allowed 6 inches apart plant from plant, to facilitate the operation of lifting with balls by the transplanter. About the end of April or beginning of May (all, however, depending on the weather, which, of late years, seems to put all nice calculations of this kind completely out of the question) they may be transplanted into the open quarter, setting them in lines 3 feet apart, and the plants $2 \frac{1}{2}$ feet apart in the rows.

The summer-sown crop is to be treated in a similar way to the last, and transplanted about the middle of July at the same distance as the last.

Throughout their season of growth the same management is required as already detailed for other Brassicæ, taking care that every one that begins to flag or droop its leaves, showing very evident symptoms of being attacked at the root, be taken up, and without further ceremony consigned to the nearest hothouse furnace, and, in default of that, to the nearest fire; for by this means such insects as are the cause of these disasters may be greatly limited in their future operations.

Some excellent cultivators contend that the middle of August is not the proper season for sowing, and that the whole 
routine of wintering them, as usually is done, has a tendency to give unnecessary checks to the plants, and that it would be better to sow for early spring planting in the first week of October, on a very gentle bottom-heat, close to the glass, and to prick out the plants, as soon as they can be handled, into another bed, keeping them still close to the glass. This is the practice of Mr Barnes of Bicton, who says, in "Cottage Gardener," vol. vi. p. 309, "The last week in October and the first week in November they are potted into small 60 's, and plunged under frame or pit lights, still close to the glass, and in due season they are again shifted as required into larger pots. At the beginning of January they get their last shift into 7 -inch pots-that is, those intended to be turned out under hand-glasses the first week in February-but those plants intended to be grown on and forced in pots in some hothouse, are, of course, shifted into 10-inch or 12-inch pots; and those intended to be planted out into the borders and quarters are pricked into temporary shallow frames and turf-pits, in order to apply temporary shelter during the severe winter weather, byplacing over them spare-lights, thatched hurdles, \&c."

Soil and manure.-Much of the delicacy and excellency of the cauliflower depends on the quickness of its growth; therefore, to promote this, the soil cannot be too highly enriched, nor too deeply cultivated; and, as all the tribe thrive best in new soil, the deeper the ground is dug, and the more new or rested matter that is turned up for the roots, the better. Almost all highly-manured garden soils, if deeply trenched, will produce the cauliflower in great perfection; yet, in newlybroke-up soils, all the Brassicæ will luxuriate equally well even with a much more limited dependence on manurial applications.

Taking the crop and subsequent preservation.-The young heads or flowers are used when of the size of about 2 inches in diameter, and from that until they attain their greatest size, which may be taken as a maximum at 8 or 9 inches in diameter. It is not, however, size that constitutes the properties of a fine cauliflower, but its fine white or creamy colour, its compactness, and what is technically called its curdy appearance, from its resemblance to the curd of milk in its preparation for cheese. When the flower begins to open, or when it is of a warty or frosty-like appearance, it is less esteemed; and indeed, when exceeding the size of about 3 inches in diameter, it is only fit for secondary market purposes. Nor should it, during summer, be cut above a day before it is to be used.

Towards the middle of November, should the weather prove severe, both late cauliflower and early white cape broccoli, then coming into flower, should be carefully protected. To this end the plants may be taken carefully up by the roots, and three or four tied together, and suspended with their heads downwards from the roof of some cool shed or outhouse, where there is not too much air, yet which is free from damp : strong currents of air would exhaust the sap from the plants too rapidly, and the flowers would become tough and uneatable, because the roots cannot now make up the deficiency. The leaves, after they have been hung up for a few days, and have become somewhat dry, should be folded round the flowers and secured by a string.

The best way, however, to preserve them during winter, is to take them up with as much soil about their roots as possible, and to replant them in light, dry, sandy soil in an open shed, where the accommodation of a regular structure does not exist. Figs. 676,677 , vol. i. p. 437, may be referred to as examples of such. In cool pits, frames, \&c., they may be kept in a good state for many weeks ; and even divesting the flowers of their leaves and burying them in masses of peat-earth has, from the antiseptic nature of such a soil, a very beneficial effect on their keeping. In mild localities, the mere breaking down of a leaf or two over the flower is found sufficient protection, as it wards off both wet and frost so long as the temperature does not fall above $4^{\circ}$ or $5^{\circ}$ below freezing: but in colder places it is necessary to protect them as we have stated, or by taking up the plants and laying them in by the heels-that is, replanting them in a slanting direction, and covering the roots and stems fully up to the middle of the leaves, in a sheltered and northern border, and covering them with branches and straw laid over them to throw off the wet, yet admit air, 
or by any other means most convenient at hand. The drier, however, they are kept, under such circumstances, the better. They have also been kept for several weeks by being taken up when quite dry, the leaves folded over the flowers, and the whole buried in a trench in a dry soil. It is well in doing so to place them tops undermost, leaving a small portion of the roots above ground, which serves to draw them up by when wanted.

When the heads are cut and dressed for the kitchen, the stems should be cut off close under the flower, all the leaves removed, excepting two or three of the very young embryo leaves which are next to the head ; these are dressed along with the flower, and make a better appearance on the table. If caterpillars are troublesome, place the flowers in a pail of clean water with a handful of salt for a couple of hours in the vegetable-house, before sending them to the kitchen. This will dislodge any insects that may have taken shelter within the flower.

It often happens that one or other of these expedients may be useful even for prolonging summer crops, when the one crop does not follow immediately that preceding it, or during dry summers, when the principal crops become likely to be exhausted before the next comes in.

Approved sorts and their qualities.-The cauliflower, unlike its ally the broccoli, has, by some not easily understood cause, remained pretty constant to what we may suppose was its original state. For long we had only presented to us in seed-lists the early and late cauliflowers. These, however, have had some additions made to them-if even only nominal ones, we may be thankful, seeing how we cultivators are beset with such long lists of names, as a reference to the articles PEA, BEAN, and Broccoli will abundantly show.

1. Early London white; 2. Early Dutch; 3. London particular; 4. Fine late; 5. Large Asiatic; 6. Large late German; 7. Walcheren; 8. Mercer's new pearly; 9. New dwarf late Cyprian; 10. Epps's superb; 11. Early cauliflower; 12. EarlyLeyden - are all names to be found in seedlists. Concerning the merits of Nos. $3,5,7,8$, $9,10,11$, and $12, \mathrm{Mr}$ Thomson says Nos. 3, 8, 9,10 , and 11 appear to be all the same, and to these we have little fear in adding Nos. 1 and 2. He believes 1 and 12 to be identical with Legg's Walcheren broccoli. His conclusion is, that two varieties-namely, the large Asiatic and Walcheren - are found to be those most deserving of cultivation. The true Walcheren is distinguished from all others by its bluntly-rounded and broad leaves, and the closeness and almost snowy whiteness of its heads, even when grown to a large size. It is most difficult to procure genuine seed of this variety. Those who are fortunate enough to procure it once should, if possible, save the seed themselves. For manner of doing so, vide end of this chapter.

\section{$\S$ 8.-THE SEA-KALE.}

Natural history. - Sea-kale (Crambe maritima) belongs to the natural order Cruciferæ, and Linneæn class Tetradynia, and order Siliquosæ. The name Crambe is derived from the Greek name for sea-cabbage.

Although of comparatively modern cultivation in Britain, the date of the introduction of this vegetable into our gardens is not correctly known. Mr Curtis states, upon the authority of Jones of Chelsea, that he (Jones) saw bundles of it in a cultivated state exposed in 1753 in the Chichester market in Sussex. Mr Maher, in a communication in the "London Horticultural Society's Transactions," vol. i., asserts that seakale was known in this country above 240 years ago ; and that it was used by the inhabitants of the sea-coast as a common dish, is stated by both Parkinson and Bryant. The former flourished about 1629 , which is the date of the publication of his "Paradisi in Sole Paradisus Terrestris ;" the latter somewhere about 1783 , when he published " Flora Diætetica." Strange as it may appear, sea-kale plants were sent from this country by Lobel and Turner, 250 years ago, to the Continent, where, at this day, in many parts, gardeners scarcely know it by name. It is now pretty common in the Paris markets, but we have scarcely seen it in any other; nor have we met with it in a cultivated state in any way approaching to what is seen even in the neighbourhood of Edinburgh, where its cultivation was attended to as early as it was in the neighbourhood of London. Mr Gordon of Fountain Bridge gives sensible directions for its culture in his "Gardeners' Dictionary," published in 1774; and Dr Lettsom, it is well known, only brought it into notice around London in 1767. In 1853, a comparatively small breadth of it is cultivated around our northern metropolis, while around our southern one some bundreds of acres are covered with it. Professor Martyn and Mr Curtis, by their publications, brought it into notice in England. In the south of Europe it is scarcely known; in the north it is only beginning to be attended to. Several young German and Danish gardeners we have had in the gardens at Dalkeith only recognised it as a botanical plant, never having seen it in a cultivated state. The same taste prevails in America. Our oldest English authority for any knowledge of this plant is Gerard, who gives its native habitats, but says nothing regarding its cultivation. It was known in the time of Pliny to the Romans, who called it Halmyridia; and although they do not appear to have taken it into cultivation, they used it as a sea provision during long voyages; gathering it where it grew wild, and cutting it up, they put it in barrels where oil had recently been kept, and these they stopped closely up, so that no air could reach the contents. 
The sea-kale is a native of the sea-coast of Britain, as noticed by Gerard, who observes, in his "Herbal," that "the sea-colewort groweth naturally upon the beach and brim of the sea, where there is no earth to be seen, but sand and rolling pebble-stones." It is often found growing out of the crevices of the rocks even of our highest cliffs. When it grows lower down, it sometimes gets covered with sand, and thus becomes naturally blanched, which circumstance, no doubt, led to our blanching it artificially.

Use.-The young shoots and stalks, when from the length of 3 to 9 inches, are the parts used. These, however, unless blanched (etiolated), are no better than the coarser kinds of borecole, but when blanched they become exceedingly delicate, and much prized. The ribs of the leaves, even after they are nearly fully developed, are sometimes used, being peeled and eaten as asparagus. In either state they are tied up in small bundles and boiled, and served up with meat like cauliflower, covered with white sauce,melted butter, or on toasted bread. This kale cannot be too much boiled, so long as its form is not broken down; it should then be thoroughly drained, and set a few minutes before the fire, to allow more of the moisture to escape by evaporation, which renders it more crisp and better flavoured.

Propagation.-Being, unlike the majority of the Brassicæ, a hardy perennial, it is propagated by seed, and also by cuttings of the roots, which are replete with buds or eyes around the bottom of the crown, which are sufficiently visible; and also with dormant buds all over the surface of the roots, which are invisible, but will develop themselves if the roots are cut up into small pieces and planted. It is, however, in the estimation of some, best raised from seed, as such plants, at least for the first and second year, develop only one principal bud or eye on the top of the crown, throwing up a much stronger and better formed head than they do afterwards when several buds are allowed to expand. This, however, may be corrected by rubbing off the eyes when they have attained the size of large peas. The London market-garden practice is to propagate by the root in preference to the seed, and in the following manner:- "Towards spring, after the produce has been all cut, the roots are taken up; all the thongs," the long naked roots, "are cut off, and laid in large heaps ; and as soon as the cut part or upper portion becomes hardened, ground is prepared for its reception by manuring and trenching. The roots are then planted out a foot apart, in rows 18 inches asunder, and a crop of lettuces are planted between them: as soon as the buds become visible, all are cut out except the strongest one, the ground is kept clean, and nothing more is done till November, when they are ready again for forcing."

Sowing or planting.-Seed is sown in March; the roots planted at the same time. In cold damp soils, the beginning of April is better for the latter purpose ; but much in this respect depends on the wounded parts of the roots being well healed over, else there is danger of their rotting. The seed is best sown in drills 2 inches deep and 6 or 8 inches wide, scattering the seed equally over the bottom to give the young plants more room when vegetation takes place. Some sow in beds in the broadcast manner, either of which will do equally well, as the plants are only to remain there for one season, when they are to be taken up and transplanted for good. Two ounces of seed will sow a bed of 36 square feet, or if in drills a foot apart and 8 inches in breadth, the same quantity will suffice. The seed is large and light, very often either too old or imperfectly formed; but this is easily ascertained by cutting them through the middle: if sound, the seed will be found plump and solid. They should be covered to the depth of 2 inches. Sometimes they are sown where they are to remain, in which case the same quantity of seed will sow a piece of 75 square feet in drills 2 feet apart, as it must in this case be sown thin. Care must be taken, when the plants come up, that they be thinned to the distance of 15 inches. Some divide the ground into beds, and set the cuttings or young plants in rows in them 2 feet apart, and the plants 18 inches in the line: when three rows are thus planted, a space of 3 feet is left as an alley, and another bed of similar dimensions formed. Others, instead of thin-planting the beds, make shallow holes along the line, and drop three or four seeds in each, leaving one plant only to come to maturity. Some people think that in this way the plants are stronger than when subjected to transplantation. For ourselves, having practised all the ways, we could never see much difference in the results.

The seed usually vegetates in four weeks. Mr Martin's plan of increasing sea-kale is 
thus given by $\mathrm{Mr}$ Cuthill, in "MarketGardening round London," p. 20. " At taking-up time"-that is, when the established roots are taken up for forcing, beginning in November, and continuing till the middle or end of February-" all the thongs are cut off from the plants. The small prongs or end roots are at once cut into 4-inch pieces, and laid together in a heap for the winter. In February they are deposited thickly in beds, and covered with mould an inch deep; when sprouted, it is seen by the buds which is the top, and by the roots which is the bottom. The ground being prepared, they are planted" as described above. "This last plan makes finer plants, as the whole of the strength is in the roots when cut off in winter, which is not the case after they have produced a crop. After forcing, the pores of the thongs must be empty, and they take a long time in recruiting." When seed has been sown the previous season, the young plants should be carefully taken up the March following, preserving their roots with the utmost care ; they are then to be planted in richly-manured and deeply-trenched ground, in rows $2 \frac{1}{2}$ feet apart and 15 inches in the line, if the intention is to take them up the following winter for forcing. If, however, the crop is planted with a view to its remaining several years, either to be blanched where it grows, by covering with the sea-kale pot and the influence of the sun, or to be covered with leaves, dung, \&c., and excited into growth during winter, then the rows should be 3 feet apart, and the plants set 2 feet apart in the row, to allow a full development of the large foliage during summer, for on this much of the success of the future crops will depend.

Fig. 38 .

In planting seedlings, as

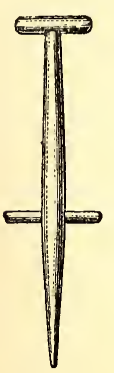

the roots have very few fibres, they may be set with the planter, fig. 38, which is a wooden dibber $3 \frac{1}{2}$ feet in length, with a crosshandle at top, and another cross-piece as a gauge within from 9 to 12 inches of its point, made so as to shift to suit the length of the roots to be planted, which will in general aversea. kale pibber. age from 9 inches to a foot.
The lower part, which is to perforate the soil, should be $3 \frac{1}{2}$ inches in diameter, and tapering to 2 inches at the point, which should be left obtuse. The holes being made by one man using the perforator, and being guided by a line, another should follow, having the roots arranged with their tops all in one direction; and as the holes arè made, he drops a plant into each, slightly filling in the soil about them with his foot as he progresses. From this it will be seen that propagation by the root is the most expeditious way, giving a saving of time equal to one season.

Subsequent cultivation. - Whether the plants are originated from seed, cuttings of the roots, or division of these, retaining the crowns entire, it is necessary that the ground around them should be kept clear of weeds, and also that it be repeatediy stirred up between the rows, and that not in the scuffling manner performed by the Dutch hoe, but well roused up by means of the Vernon hoe (fig. 3, page 38), care being taken that the extending roots be not mutilated. In spring, when the buds are beginning to break through the ground, and also once or twice during the growing season, a top-dressing of salt should be applied in quantity about $1 \mathrm{lb}$. to the square yard. The crop should be gone over in November in Scotland, and October in England, at which time the offices of most of the leaves will have ceased. Those that have changed to a yellowish hue may safely be removed as no longer useful, and this will admit light and air to assist the ripening of the buds. Where the beds are not intended to be destroyed, and the plants taken up for forcing, the ground between them should be forked up, and a good dressing of rich manure applied in which salt has been liberally incorporated. In this state the young crop, as well as those intended to be blanched without artificial heat, should remain till spring. Many recommend covering this crop with leaves or litter as a protection from frost-a recommendation wholly unnecessary, unless in some of the highest and coldest parts of Scotland, where such a precaution may be worth the trouble: for although the plant is a native of our own country, still its natural habitat is close to the sea, and never inland. What degree of frost 
it may stand without injury has never been accurately ascertained.

A portion, however, of the crops should be covered early in November, so that it may be got readily at during winter, to take up for forcing. Some of the London market-gardeners grow their sea-kale for permanent crops in rows from 4 to 6 feet apart, and in autumn, after the foliage has fallen off, they cover the crowns with soil dug from the alleys to the depth of a foot or 1.5 inches, by which means they procure it finely blanched in spring, and at little expense. As soon as this crop is cut, they level down the ridges, and crop with other things between the rows. Remove every flower-spike as it appears, for the production of seed is much harder upon the energies of the plant than taking a crop from it.

Soil and manure.-In a cultivated state, sea-kale does not appear to be very particular about either, succeeding in most, provided the former be sufficiently deep and dry at the bottom, and that the latter be rich and incorporated with saline matter. Deep trenching where the land will admit of it is important, and where that is not naturally the case it may be rendered so by excavating broad alleys between the beds, and elevating them with the material taken out.

Forcing.-From the middle of November till it comes into use in the open air, this vegetable may be had in great perfection, at a season when other choice vegetables are scarce, and this at little trouble or expense. In former practice it was recommended to force the crop in the ground on which it grows; than this there can scarcely be a more unsatisfactory, uncertain, and expensive process recommended. The objections we should state are, the waste of manure in producing the necessary heat by fermentation, the waste of time in carrying in and out, turning over and adding to the material, the uncertainty of the heat produced, the expense of blanching-pots, open wooden cages, or whatever other means may be used to separate the fermenting material from the crowns of the plants; the deterioration in flavour, the trouble in examining the state of the crop and of gathering it, and lastly, the injury inflicted on the plants by keeping them so long in a state of excitement. To avoid much of all this, and at the same time to insure a certain return within a given time, the best method is to adopt the lifting or taking-up process. To prepare the plants for this, forms, as in the case of taking up asparagus, an important element in the rotation of crops. Plants should be grown for the special purpose : a seed-bed should be sown annually, from which to transplant the young plants in March into lines, in ground deeply trenched and abundantly enriched, setting them 3 feet apart row from row, and 15 inches in the line. If these are stimulated abundantly during the season after planting, care being taken that they do not form flower-stalks, and that all lateral buds are displaced as they appear on the sides of the main stems, throwing the whole energy of the plant into the centre bud or crown, the roots will be in excellent condition for taking up for forcing as soon as their buds are fully matured, and the foliage has died away, which with us, in general, is about the beginning of November. The roots should then be carefully taken up, carrying with them as many of the small fibres as possible: soil is of little consequence, except in so far as it may be conducive to the preservation of the roots. For although these are not much required for the natural purpose of collecting food for the plant, under the treatment to which they are to be subjected, they are valuable, inasmuch as they, along with the bud and stem, contain the elaborated matter formed during the past summer, and hold it in store for the formation of the shoots and leaves, which the excitement of a moderate tem. perature and slight humidity will call into action. The best situation in which to place the roots is on the floor of a regular mushroom-house, or, in the absence of it, the floor of a cellar, outhouse, or enclosed shed, into either of which a slight temperature can be thrown by means of a hot-water pipe or otherwise. The advantage of such places is, that while the necessary heat can be applied, light may be sufficiently excluded to insure blanching, while air to a certain extent is admitted, which is of no small consequence to the crop. In such places also the progress of the crop can readily be ascertained, and the gathering effected with facility. It matters little what the soil is 
in which the roots are set; they derive no benefit from it further than exclusion from light and air, and being kept in a state of uniformity as regards moisture. This we apply pretty copiously by watering the roots and soil with tepid water, though others object to watering after the roots are arranged. In placing the roots, they are set closely together, and the soil is wrought regularly in amongst them, and up to within 2 inches of the tops of the crowns. Some, where the demand is not very great, place the roots in boxes or large flower-pots, which they place in any dark and out-of-the-way place ; and in this way we have seen as fine blanched sea-kale at Christmas, in a wine-cellar in the city of London, as we ever saw in any quarter. Under the stages in plant-houses, behind the flues in early vineries or peach-houses, or, indeed, wherever there is a vacant square yard of space, this vegetable may be produced in perfection. We state these simple means, because they are within the reach of most or of all, and also with a view to get rid of the waste of time and material by attempting to produce it in the open ground. From five to six weeks will be required between the time of setting in the roots and gathering a dish, in places without any artificial heat; in such as have a uniform temperature of from $50^{\circ}$ to $60^{\circ}$, the time will range from two, three, to four weeks, sometimes less. It is, however, best to allow excitement to go on very slowly, as the shoots will be much finer and stronger. When the crops are gathered, the old roots are thrown away. Those, however, which have been forced latest may be cut up into pieces of 3 to 4 inches in length, and planted in lines to produce a succeeding supply. Young seedling plants are, however, much preferable.

The practice of the London market-gardeners is thus described by $\mathrm{Mr}$ Cuthill : "When all the frames are removed, the dung and mould where cucumbers grew during summer are taken away, the trenches, which are 2 feet deep, are again filled with hot dung, and mould to the depth of 8 inches is put on the dung. The sea-kale roots are dug up, all the small buds round the main eye are pared off, leaving that by itself, which induces it to push stronger and finer. And now planting is commenced: a furrow is cut out by the spade across the bed, and they (the roots) are put in as thickly as possible. 5 feet across will hold from twentyfive to thirty roots; the next furrow is cut out at 4 inches from the last, and so on till the whole is finished; from 4 to 6 inches of straw is placed immediately on the crowns. The beds are hooped over, and straw is put over the hoops; and in this way I have seen 50,000 plants forced during the winter and spring, and that by one man alone." If we calculate that five heads are quite ample for an ordinary dish suitable to a large family, this grower must have himself provided no less than 10,000 dishes of this excellent vegetable by artificial means, and probably double that number from the open air. Such data will give some idea of the production and consumption of a London market-gardener.

Forcing the plants where they grow requires from six to seven weeks from the time they are covered up until the crop is fit for gathering. This, however, to some extent depends on the season and the quality of the fermenting material; and to effect this in the best manner, trim off all the decaying leaves from the plants, stir the surface up slightly around them, sprinkle about a teacupful of salt or caustic lime around the crowns, or water with lime-water to banish the earthworms, Lumbricus terrestris, L., which are apt, upon the application of heat, to throw up their casts about the young kale, and thus render it very dirty. The same means secure the buds from the attacks of snails, Helix aspersa, and slugs, Limax agrestis, L., the milky-slug, L. ater, L., the black slug; L. Maximus, the black striped slug; and Testacellus scutulum, the shield slug, which are all apt to feed upon the young buds, and certain to gather round them in consequence of the genial heat. Over either of these a spadeful of clean river or sea sand should be placed, or, in default of these, a spadeful of finelysifted coal-ashes, either of which keeps the young kale quite clean and fit for use.

The sea-kale pots should then be placed over them, one pot to a plant, and over these the fermenting material, to a depth sufficient to raise a fine genial temperature, not, however, exceeding around the pots $60^{\circ}$; less will do. 
The best kind of sea-kale pot is that represented by our fig. 39 ; it consists of two parts-a body, $a$, and a top, $b$ - which latter is necessary, as it can be taken off to examine the state of the crop, and also

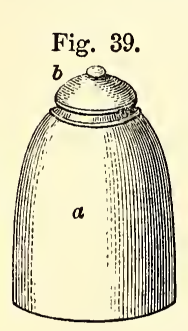

to gather it without having to remove the whole of the material. They are of various sizes-from 10 to 14 inches in diameter, and from a foot to 20 inches in height. There are other contrivances to effect a similar end, such as boxes perforated with holes, others made of 2 -inch laths nailed together, and about as far apart from each other, the top ones being left loose for lifting when the crop is to be gathered. Reason points out the defects of these, as they do not exclude the moisture, accompanied with various gases, which are evolved during fermentation of rank stable-yard litter, and which, in many cases, communicate a disagreeable flavour to the kale.

Amateurs may readily force sea-kale, if they have any dark warm cellar or outhouse, or one that can be heated to something like $55^{\circ}$, in the following manner:Procure an old cask or a large packingbox; perforate the sides of either with holes $2 \frac{1}{2}$ inches in diameter, and about a foot apart, making the first row of holes a foot from the bottom. Procure the roots; divest them, if large, of a few of their most extending roots; place them in the bottom of the cask or box, with their crowns exactly opposite the holes: when the first row is thus arranged, pack the roots around with sand or soil of any description, and work it well amongst them; over this arrange another set of roots in like manner; proceed till the box or cask is full. Give a gentle watering with tepid water as the process of packing proceeds. In the course of a fortnight, three weeks, or a month, according to the heat in the apartment, the young buds will begin to protrude through the holes, and in another week or so many of them will be fit to cut for use.

As a general rule, avoid a high temperature, whatever method is followed; from $55^{\circ}$ to $60^{\circ}$ is the proper heat to secure a certain return.

Taking the crop.-When the kale is from
3 to 6 inches in length, it is then in the most proper state for use. Each bud should be cut off with a sharp knife, taking about a quarter of an inch of the crown attached to its base to keep it together in compact form. This portion of the old crown is to be neatly pared down close to the part where the young sprout springs from, the kale dipped in clear water to remove any dust that may have attached itself to it in the process of gathering, and then be placed in a punnet-basket, of which fig. 35 is an example. If to be sent to a distance, it is best tied up in small bundles, which prevents the tops from being broken. From four to six buds make a dish. Three stout plants will produce about five dishes in a season when forced, and an ordinary managed plantation in the open air will continue in bearing about six weeks.

There is only one variety known.

\section{§ 9.-THE BORECOLE.}

Natural history.-Borecole (Brassicce oleracea sabellica, De C.), a family of the Brassicæ tribe both useful and numerous. The chief characteristic of the borecoles is that they are openheaded, not hearting like the cabbages, nor producing eatable flowers, like the cauliflower and broccoli. They are for the most part extremely hardy, and we entertain a strong notion that some of them are very early removes from the Brassica oleracea in its wild state. Some of the sorts are much cultivated in the north of Scotland, a circumstance arising from their hardy constitution, for, deprive them of that, and those varieties to which we allude have not a redeeming quality -ill-coloured, coarse, rambling-growing subjects, requiring long boiling and a strong digestion. Prejudice, we believe, continues the cultivation of those over that of one of the very best of the family, the German greens or Scotch curlies, which we believe to be equally hardy. They all belong to the same natural order, to the same class and order in the Linnæan arrangement as the rest of the esculents comprised in this chapter. Borecoles, in one shape or other, are cultivated in every country where attention has been paid to the rest of the Brassicaceous esculents.

$U$ ses.-The crown or centre of the plant is cut out towards the middle of November, and continues to be used throughout the whole winter, while in spring numerous small delicate sprouts are formed, which are acceptable at that season. When properly cooked, they are tender, sweet, and delicate, and are by some supposed to become intenerated after being exposed to the frost. The coarser sorts may be thus improved, the better sorts do not require 
it. The young tops of the Buda kale are sometimes, in spring, blanched by turning a flower-pot over it; or better, by placing a sea-kale pot on it ; or the roots may be taken up any time during winter, and planted in a bed of soil in a dark cellar, and treated as has been recommended for sea-kale. The bulbs, like turnips, found on the surface of the Egyptian kale or kohl-rabi, are stewed, boiled, and mashed like turnips, and sometimes sliced in some German salads. The tender tops of the others are served to table plain boiled, as a garnish for meat, and should assuredly accompany it in the popular Scotch winter dish "beef and greens." They enter largely into soups, and form an ingredient as essential in the national dish, "the kail-brose of old Scotland," as horse-radish does in that of "the roast-beef of old England." Scotch greens are often mashed with butter and pepper, and served in imitation of spinach, and, like it, garnished with hard-boiled eggs.

Propagation.-Most of the varieties, being annuals or biennials, are propagated by seed; those that are perennial or half shrubby, like the Woburn kale, are increased by cuttings, and some may be grafted on other sorts, (vide art. PropagaTION BY Grafting). One ounce of seed is sufficient to sow a bed of 40 square feet.

Sowing. - The latter end of March, in April, the first week in May, and lastly, about the 12th of August, are the seasons adapted to England; for Scotland, generally ten days earlier in each case. For the most part, however, those sown about the beginning of August, in the North, stand over winter, and are transplanted in spring. The English sow German greens, or, as they call them, Scotch kale, during the first week in April. In the last week in August a sowing is made of Buda kale, to be transplanted before the 1st of October, to furnish a late crop of greens in spring.

The method of sowing, \&c., is the same as for savoys (which see).

Subsequent culture.-The same as for savoys. In all cold exposed places, and where much snow is expected, it is expedient to lay them over in November, as recommended for broccoli, as their leaves are very liable to become broken by the weight of snow, particularly the tallergrowing kinds.

Soil and manure.-The borecoles being of less luxuriant habits, and it being also desirable that they should stand the winter, the soil need not be so highly manured as for the other varieties of Brassicœ. Where the ground is not revOL. II. quired for other crops or purposes, they may be made to succeed the summer pea crops, and, indeed, if ground be scarce, may be planted between the rows of late pease, or interlined with potatoes.

Approved sorts and their qualities.-Great confusion exists in this section of the Brassicæ, probably arising from the circumstance that the cultivation of them is for the most part local, those that are grown in one part of the kingdom being almost unknown in other parts. Although we have been engaged for some years proving various sections of culinary vegetables, we have not as yet had time to bestow the same attention on the present section; and rather than mislead, we shall quote the substance of an excellent paper by Mr Thompson, being the results of his experiments carried on in the garden of the Horticultural Society of London, where an immense number of sorts by name were grown together, to enable him to draw the conclusions regarding their nomenclature and merits; and certainly to no one better qualified could such an experiment have been intrusted. Mr Thompson begins by observing that "the varieties of these are endless : they ditfer in having stems dwarf or tall, leaves mole or less cut or curled; in colour, green, purple, or variegated with purple, red, green, and yellow. The transformations of all these render any attempt to give minute descriptions quite useless. It will be sufficient to point out the general characters of varieties that may be usefully distinguished as such.

"Dwarf green curled, or dwarf curled kale, dwarf German greens, very dwarf green curled, dwarf winter curled, Scotch kale, green Scotch kale, dwarf curlies, French dwarf curled, Canada dwarf curled, Labrador kale, green borecole, and dwarf green borecole. By one or other of the above names this is certainly known to every one. The Canada dwarf curled was found to represent exactly the finest dwarf curlies grown many years ago in many parts of Scotland, the plants being very dwarf and closely curled.

" Tall green curled, or tall German greens, tall Scotch kale, tall green borecole, and tall greens," with a host of French and German synonymes, for in both countries they are extensively cultivated. "Height usually from 2 to 3 feet, but 2 feet is the preferable growth. The plants are capable of bearing severe frost, and, like the preceding, it affords the best greens from the time when the first frost has mellowed its flavour, until the middle of February.

“Purple borecole, or purple or red borecole, tall purple kale, purple winter greens, brown kale, purple kale, curled brown kale, curled red kale. This in its formation and habits differs little from the tall green curled, but the colour is deep purple. As the leaves enlarge, they have an inclination to become green, but the veins still retain the purple hue.

"Variegated borecole, or variegated kale, variegated plumage kale," with various French and German synonymes. "A sub-variety of the purple borecole, having the leaves beautifully 
variegated, sometimesgreen and yellowish white, green and purple, bright red, purple and green. It is occasionally employed for garnishing, but it is very good cooked after frost. It is not quite so hardy as the purple borecole.

"Dwarf purple borecole and the dwarf variegated are merely sub-varieties of the two preceding, distinguished by their dwarfer habit of growth.

"Buda kale, or Prussian kale, Prussian or Buda kale, Buda greens, Russian kale, Hamburg kale, Anjou kale, Manchester kale, asparagus kale, Duke of York's kale, Camberwell kale; and so closely allied as not to be worth distinguishing from it are, the Jerusalem kale, Delaware greens, Delaware kale, ragged Jack, jagged kale, and the dwarf feathered kale. The Buda kale is not so tall as the purple borecole; very hardy; leaves purplish, somewhat glaucous; cut and fringed.

"Woburn perennial kale.-This is a tall variety of the purple borecole, with foliage very finely divided and fringed. The plant lasts many years, and may be propagated by cuttings, as it neither flowers readily nor perfects well its seeds. Its produce at Woburn is stated to have been more than four times greater than either that of the green or purple borecoles, on the same extent of ground. The weight of produce from 10 square yards was 144 lb. 10 oz. but some of the large kinds of cabbages and savoys will exceed this considerably, and prove of better quality. The Woburn perennial kale can, therefore, only be recommended where the climate is too severe for the more tender kinds of the cabbage tribe.

"Tree cabbage, or great cow cabbage, Cesarean borecole," with many French and German synonymes. "This grows to the beight of 6 feet, and in La Vendée and Jersey it is reported that it attains the height of 12 feet and upwards. The leaves are large, smooth, or but slightly curled; its sprouts are said to be good when cooked. Its merits have, however, been greatly overrated, for when tried [in England] against other cabbages, its produce was nothing extraordinary.

"The thousand-headed cabbage is allied to the preceding, but does not grow so tall, and sends out numerous side-shoots. On the whole, it is preferable to the tree cabbage.

"Flanders kale is a tall-growing kind, distinguished from the tree cabbage by its purplish foliage.

"Cockscomb kale produces sprouts along the ribs on the surface of the leaves, but it is of little value."

To these kales we may add the imperial hearting or cabbaging kale; and we believe the German cabbaging borecole of some seed-lists to be the same. It appears to be a sub-variety of the dwarf green curled, dwarf in growth, the leaves standing nearly upright, turning in slightly towards the centre, finely curled, and of great excellence as a small delicate variety.

The palm borecole is a tall rambling kale of no estimation in Britain. It is cultivated in many gardens in France under the name of Chou Palmier.

The Chinese cabbage (Brassica chinensis L.), although recognised as a species by botanists, has much the appearance of being connected with both the cabbage and the turnip. It is an annual of rapid growth, for, if sown at midsummer, it will ripen seed the same season. It is cultivated in some of the gardens around Paris, and has been tried in the Horticultural Society's garden at Chiswick: the results, however, lead to a belief that it is not suited to the climate of Britain.

The khol-rabi, or turnip-rooted cabbage (Brassica oleracea Caulo-rapa communis De C.)-Of this there are two varieties, the one having the turnip-shaped bulb, of a pale-greenish colour ; the other of a purplish-plum colour. The plant is of low growth. The part chiefly used is a turnip-looking bulb, formed by the swelling of the stem, which is short. It is extremely hardy, and much grown in the north of Europe, where the bulb is dressed whole, and eaten with sauce or with meat, as turnips usually are. The seed should be sown on a warm border in February or March, and planted out when the plants have attained the height of 6 or 7 inches. For successional crops, sow again in April and May; the latter will produce plants for winter use. It should be eaten while young, as it becomes hard and stringy, and does not boil soft if left to become old.

Diseases and insects.-Amongst the diseases that affect the brassicaceous tribe is the white rust, called by many the mildew, perhaps on account of its white appearance. The white rust is even more formidable than the mildew. Both are fungi, and although deriving their origin from nearly the same causes, are different in their botanical as well as their injurious distinctions. The common white rust (Cyspopus candidus of Greville, Uredo candida of Persoon) produces a white leprous appearance on the leaves of the plants. These white patches of parasitic fungi not only disfigure but materially injure the plants, as all parasites must do, whether of vegetable or animal origin, because they derive their very existence by exhausting the energies of the plant. Again, there is the Botrytis parasitica, which, in mild winters, sadly destroys the foliage of brassicaceous plants, and often attacks them while quite young. A third production of this kind, but happily of much rarer occurrence, has recently been discovered travelling, as it were, southward. This is Cylindrosporium concentricum, figured nearly thirty years ago by Dr Greville, and at that time abounding in the vicinity of Edinburgh, and detected by several botanists. Its first detection in England was only in 1850, and that no farther south than Northamptonshire. It spreads rapidly over the surface of the upper and under side of the leaves of most of the Brassicæ, the cauliflower in particular. These parasitics have been minutely examined by the Rev. M. J. Berkeley, A.M., F.L.S., an acute observer in these matters, and are pronounced by him to have no affinity with the genus $U$ redo, and still less with the parasitic moulds to which mildew properly belongs. These humble means at the disposal of a supreme Being, insignificant as they may appear even when viewed under the power of the best 
microscopes, are often a scourge to man. Whole crops of Brassicæ have often been completely destroyed by their united force. The only remedy at present known is caustic lime in fine powder, or clarified lime-water, administered to the plants upon the first appearance of the fungi, and continued vigorously until its disappearance. An interesting account of these parasites will be found in the third volume and also in the sixth volume of the "Journal of the London Horticultural Society," to which the cryptogamic reader is respectfully referred. The Erysiphe communis (Link, common mildew) is often found on the leaves, more especially in dry weather, for which an efficient remedy has been discovered in flowers of sulphur, thrown over the plants by one of the newly-invented sulphurators.

The most fatal disease, however, the Brassica family is liable to, is what is called the club in the root, from the many swellings or tubercles formed upon it, varying in size from half an inch in diameter to two or three. This is the production of the larvæ of Curculio contractus of Marsham, which, piercing the skin of the root, deposits its eggs in the hole, lives during a time on the sap of the plant, and then escapes and buries itself for a time in the soil. Frequent transplanting seems to be the first remedy, because by it the plants acquire numerous roots to throw strength into the plant; the next is to draw up every plant affected, and consign it to the flames; the third is next to useless, namely, cutting off the protuberances and retaining the plants. The first has this advantage, that while it greatly encourages lateral roots, the grub preferring those that are ramose, parts of these may be removed, while, at the same time, the others are throwing in support to the plant, enabling it to outgrow the disease. Renewal of soil is important, while the application of the whole list of mineral manures has not had the desired effect. Guano, placed in the holes at planting, has been found beneficial. In new soil this disease seldom appears.-(Vide section TURNIP.)

Snails and slugs are destructive to all the Brassica in a young state ; but as a good dusting of caustic lime so completely annihilates them, and a man can go over several acres per day sowing it broadcast, we do not see why we should occupy space with, or impose upon our readers the expense of any other remedy. Slugpicking and snail-hunting has been a stereotyped recommendation since the days of Mascall; it is not in accordance with the intelligence of the present day.

Tipula oleraceex, crane-fly, known in England as gaffer long-legs, and in Scotland ás daddy longlegs, is very destructive to most of the Brassicæ in its larva state. The larva is easily known by its long cylindrical body being destitute of feet. The cognomen of long-legs is taken from the insect in its more perfect state. It appears that the best way of capturing it is by setting traps of slices of turnip, potatoes, \&c., fixed to a wooden skewer, and sunk a few inches under the surface of the ground. The larvæ will be attracted by them, and if they are pulled up every other day, thousands of them may be found attached to the baits, and can be readily scraped off and burnt, while the same baits may be reset.

Fig. 40.

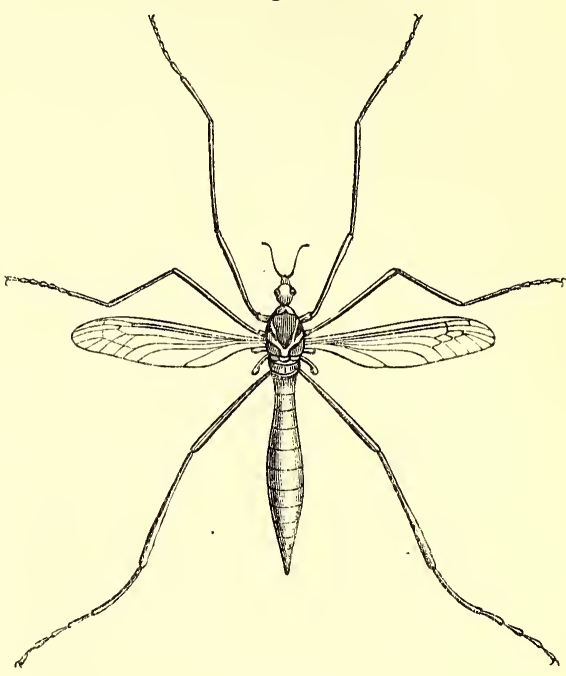

CRANE-FLY.

Against the attacks of such multitudes of obscure enemies-and those we have noticed as chiefly feeding upon the cabbage tribe are as nothing compared with the millions of millions that prey upon other productions of his careall the boasted intelligence of man would not enable him to contend, were it not that nature has so beautifully arranged it that one species of insect is made to feed on another, and thus keep up the balance in regard to their numbers and effects. Birds are great destroyers of insects at all times, but more especially in those stages of their existence when they are most destructive to vegetation; these, therefore, should rather be encouraged than destroyed. All crops can be easily protected from them during the time the seed is coming to maturity, or fruit ripening. Late frosts, hurtful in themselves to vegetation, are equally so to newlyhatched insects; and much of the damage laid to easterly winds and spring frosts may, upon strict inquiry, be found rather to arise from these minute enemies. To this we will have occasion to refer at some length in the fruitgarden department. Inundations during winter do much to thin insects in their subterranean retreats; and this points out to us the utility, where it can be applied, of irrigation, or indeed, laying the ground for a week during winter under water. Some of the most despised mammalia-the mole for example-are insectivorous, and destroy many of our enemies, the larvæ of wireworms being to that animal a savoury meal. So much satisfied are we of their assistance in this respect, that we rather encourage than destroy them. Amongst insects themselves, the genus Carabidæ, or ground beetles, destroy the pupæ of moths and butterflies while buried in the ground. Many of the winged insects actually 
lay their own eggs in the bodies of living caterpillars, and hence cause their destruction. But of all others, the very numerous family of Ichneumonidæ are the greatest destroyers, amounting in species to above 1300 , the females of all of which deposit their eggs in the bodies of other insects. Ants, not very troublesome in themselves, destroy aphides in vast numbers, and could we induce them to take up their abode in a border of cauliflower or early cabbage, the Aphis brassica would sustain a severe persecution.

Catching moths and butterflies is a wholesale mode of lessening the number of forthcoming caterpillars. Searching for chrysalides during winter, and dusting the crops infested with caterpillars, are the most rational means of ridding our kitchen-gardens of such pests; and to those means we may add cutting off the infested leaves, pulling up the roots which they have perforated, and consigning them to the flames, which is of vast importance, and, if persevered in, would almost clear the ground in a season or two. Trapping by placing slices of turnip, carrot, or potato attached to a skewer, and taking them up every other day and destroying the insects attached, is a most effectual method. But these or any other means, if not persevered in, will be as completely useless as all the nostrums recommended either in past or present times. Stimulants such as guano assist in rushing the plants forward, and hence enable them better to withstand the attacks of insects. Spirit of tar mixed with the soil destroys them by insinuating its penetrating poison through their hardest covering; and rape-cake has been found efficacious in a more extraordinary manner, the pupa feeding kinds devouring it until they die of repletion. Any or all of these may be tried with effect in the preservation of brassicaceous crops, but, we repeat, they must be applied in sufficient quantity and persevered in.

The cabbage moth (Mamestra brassica, or Noctua brassice of some entomologists.)-During the evenings about the latter part of May, the moth in its perfect state may be seen flying about in the neighbourhood of cabbage-beds, and in July, August, and September, in its caterpillar state, will be found committing sad havoc in the heart of the full-growing cabbages, as well as on the leaves of those less farther advanced. "The moth measures about $1 \frac{3}{4}$-inch from tip to tip of the fore-wings, which are dusky brown, clouded with darker shades, and marked with pairs of dark spots on their front edge ; from these spots proceed the streaks which mark the wings across; there are various spots on the wings, some yellowish, and those in the middle surrounded with white, the kidney-shaped one with a whitish grey crescent around it, and blackish beyond; the wings have a grey yellowish-striped fringe, and near this, at the point farthest from the body, they have a row of black triangular marks; the hind wings are light-brownish grey, with dark veins; the body and head are of various shades of blackish grey, with a darker stripe of the same colour down the centre of the back. The caterpillar is green, variously marked with grey or black, with a dark stripe down the back, and a dirty-yellow one down each side. The (spiracles) breathing-holes are white, surrounded with black, and

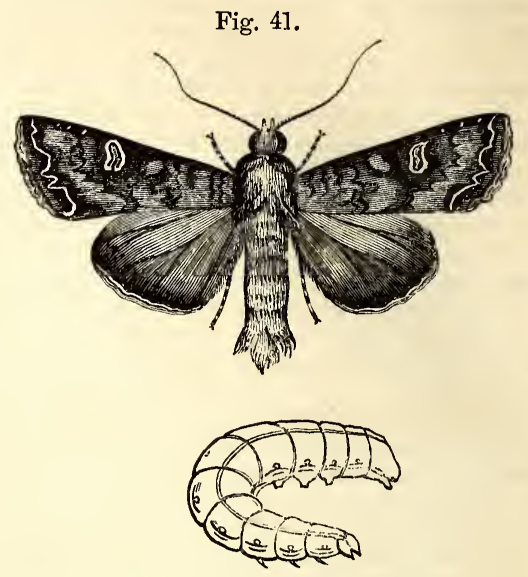

CABBAGE MOTH AND CATERPILLAR.

close above the yellow stripe. They bury themselves underground, and remain in the pupa or chrysalis state all winter."-Cottage Gardener, vol. ii. p. 83.

Fig. 41 shows the perfect moth and the grub.

The white line brown-eyed moth, Mamestra or Noctua oleracea, is another enemy of the cabbage tribe. For figure and description, see section TURNIP.

Aphis brassica (common cabbage-louse), fig. 42 , the winged male, and 43 , wingless female, is

Fig. 42.

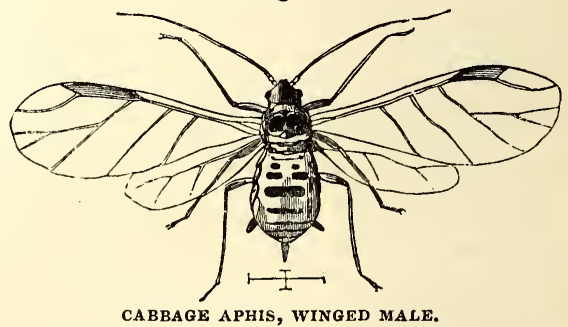

injurious in dry seasons. Dusting the plants with black hellebore, Scotch snuff, caustic lime in powder, are the usual remedies. They usually appear in greatest numbers from the middle of July to the end of November, and are found generally on the under sides of the leaves, the females being surrounded with their young broods, while the males are to be seen wandering about, no doubt in search of mates. The male is pea-green; the head, collar, and back of

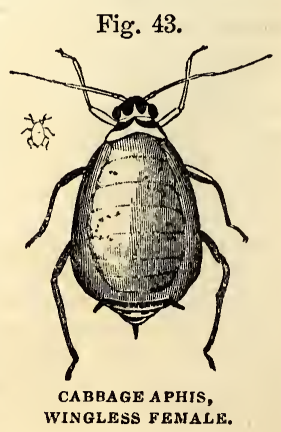


the body black; horns seven-jointed; trunk irregularly spotted with palish black; nervures of wings dark brownish-black; legs black; base of thighs greenish. The female is of a yellowishgreen and mealy white, spotted with black; body large and heavy; legs black; base of thighs green; horns shorter than in the male; the two first joints are green, while the third is ochreous; eyes, four in number, two large ones in the head, and two smaller on the collar.

The Pontia brassicce.-Well known as the large white garden butterfly, fig. 44, with black tips

Fig. 44 .

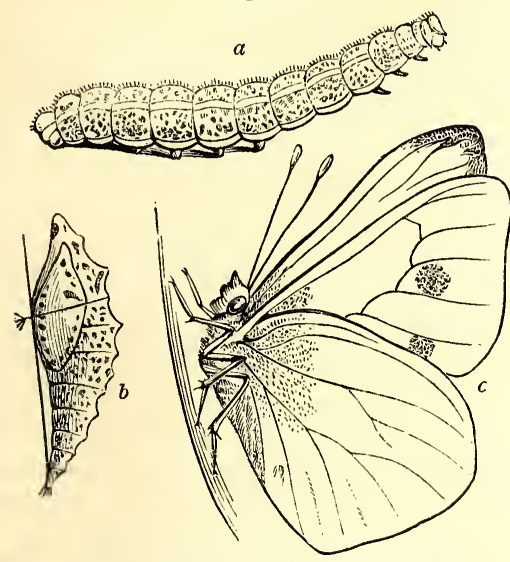

LARGE WHITE CABBAGE BUTTERFLY. $a$ caterpillar; $b$ chrysalis; $c$ butterfly.

to its wings; produces the equally well known green caterpillar, so often found upon the cabbage tribe in all stages of their growth. The caterpillars may readily be destroyed, and by doing so the increase of following years is greatly diminished, by dusting the plants over, twice a-day, as long as the insects appear, with finely-powdered caustic lime, shaken from a thin canvass bag, or by watering them over head with clarified lime-water.

And associated with it are Pontia napi, fig. 45, and Pontia rapce, fig. 46, the small white-and-

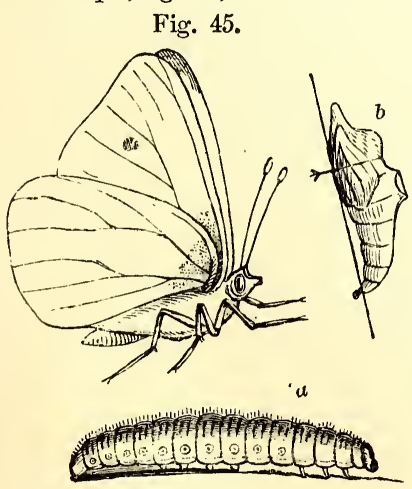

SMALL WHITE CABBAGE BUTTERFLY. $a$ caterpillar ; $b$ chrysalis ; $c$ butterfly.

green veined garden butterfly. The caterpillar of the first is greenish-yellow, with black spots; of the second, green, with small yellow rings on the sides of the body ; and of the third, green, but striped down the back and sides with orange.

Fig. 46.

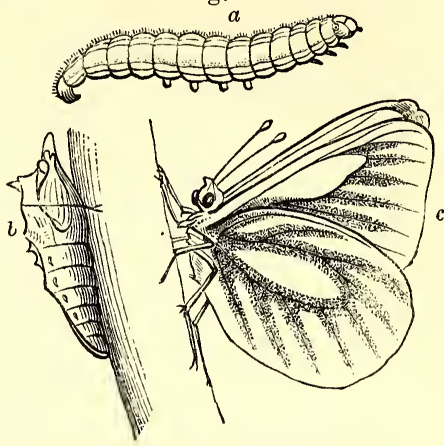

GREEN-VEINED CABBAGE BUTTERFLY. $a$ caterpillar; $b$ chrysalis; $c$ butterfly.

When fully grown, they retire to some sheltered dry place, and change into the chrysalis state, ready again in spring to change into their butterfly state, to lay their eggs, and produce a fresh breed of caterpillars.

The cabbage powder-winged moth (Aleyrodes proletella L.- the Aleyrodes chelidonii of Latreille) often commits sad havoc amongst the cabbage and broccoli crops in its fly state during the month of November. They begin to make their appearance in May, and live under the leaves where they are hatched, abounding during the months of June, July, and August. They are thus described in "Gardeners' Chronicle" for 1851 , p. 837 : "The aleyrodes is a minute fly, covered with white powder. The females have been observed about midsummer to remain quite quiet on a leaf for several days when about to lay their eggs; and when they had left the spot where they had rested, a small circular space covered with white powder was observable, around which were irregularly deposited from 9 to 14 eggs. These eggs are transparent, but afterwards turning to a yellowish tint. They hatch in about 12 days, and the young larvæ immediately run a short distance to spread-themselves more about the leaf, but in a few hours a scale is formed over them, so that they look like little tortoises or cocci, and exhibit no signs of life. The colour is almost white, with two yellow spots behind. They are not absolutely stationary, but only move very short distances as they increase in size, being furnished with six pectoral legs. The perfect insect is covered with white powder, the head and thorax black, variegated with yellow; the eyes divided and black; the antennæ nearly as long as the thorax, slender, and five-jointed; first basal joint stout, second very long, third and fourth shorter, the remainder slender ; the rostrum bent under the breast in repose; stout, biarticulate, with two very fine bristles passing through; the thorax sub-globose, the collar short, with three black spots; abdomen short, yellow or rosy ; the apex obtuse and dark ; wings forming a triangle in repose, and more or less deflexed, pure white, mealy; superior wings 
largest, with a single nervure curved at the centre, where there is an obscure black fascid, with a black spot at the extremity; inferior wings smaller, with a central longitudinal nervure; six legs, rather long, black, and powdered with white; feet long, and composed of two equal joints, terminated with two very fine curved claws, with a hook between them. These little creatures seem not only to withstand the cold, but even to multiply during the winter; for Reaumur says he found them in every state in December and January, as he had done in summer; and this will account for their extraordinary increase, which, from the small number of eggs laid by each female, appears at first to be inexplicable. Moreover, in less than a month the insects undergo all their changesfrom the deposition of the egg to the pairing of the perfect progeny ; it is therefore possible to have twelve generations in a year. Reaumur calculated that a single female might, in the course of a year, give origin to 200,000 descendants."

The best mode of lessening their numbers is to gather the infected leaves during winter and spring and burn them. Any choice plant attacked by them might be fumigated with tobacco smoke; and,indeed, small crops might be covered with canvass, and undergo the same operation; but such and all other means hitherto tried would be impossible with crops upon a large scale, as the brassicaceous tribe in general are.

In some seasons they are more destructive than in others, and would become a frightful scourge to man, were it not that they have their natural enemies in a species of Cynips, and one also of Acarus, which feed upon them.

Anthomyia brassica of Bouche (the cabbagefly).- The larvæ of this insect live underground, in the roots and stems of most of the Brassica tribe, eating passages through them, and causing them to rot. It is one of the most destructive of insects, and is thus described in "Gardeners" Chronicle," 1841, by the authority above cited: "'The male is darker, but of a brighter grey, with black bristles; there is a black stripe half-way down the middle of the thorax, and a curved one on each side; the body has a more decided black stripe down the centre, and the segments are marked by a line of the same colour; legs and antennæ blackish; wings a little smoky. The female is pale ashy grey; the eyes remote, with a dark chestnut-coloured stripe on the crown ; the wings are similar in tint to those of the
Fig. 47.

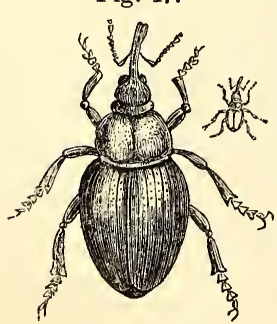
foregoing species, but the insect is considerably smaller, and this is the only striking difference between the females."

Ceutorhynchus sulcicollis of Gyllenhal, fig. 47, the Curculio pleurostigma of Marsham, deposits its eggs beneath the outer covering of the stems of cabbages, as may be discovered curculio pleurostigma. during winter and spring by the appearance of numerous galls or small excrescences covering the stems closeto the ground. Those are produced by the deposition of the eggs of this insect. On opening these galls, a small white maggot will be found within, without legs, the body being curved and fleshy, the head is palish orange, with chestnut-coloured jaws, the tips of which are black, as are also the two small eyelets, one on each side of the head. These larvæ, when fully grown, quit the galls and secret themselves in the soil, and remain there to undergo their transformation, first to the pupa state, and next to the perfect weevil, being about one-eighth of an inch long, of a black shining colour, slightly covered with greyish hair, the head and pro-thorax coarsely punctured; the cases of the wings have ten lines impressed on each, the interstices rough, the under side of the body covered with scales of a buff colour. The best remedy in the case of young plants is, as soon as the galls appear, to pull them up and burn them, by which means a riddance is made of the brood. To cut off these galls weakens the plants greatly. It is much the safest way to burn the plants at once.

Amongst other insect enemies that attack the cabbage tribe, we have reason to suspect one or
Fig. 48.

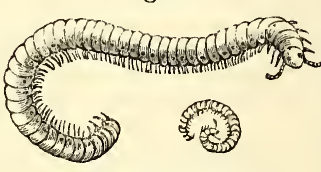

SNAKE MILIIPENE,

Natural size and magnified. two species of Iulus (snake millipedes), fig. 48, as they are often found in great numbers buried in and feeding upon the roots in a putrescent state. They may be detected in spring, in the roots of such cabbages as have suddenly died when about half-grown. When in that state, if the plants are pulled up, the roots will be found in a state of decay just under the surface of the soil, and in the decayed part multitudes of millipedes will be found. The editor of the "Cottage Gardener" queries their being the cause, and remarks, vol. ii. p. 139, "The question arises, Is this insect the cause of the disease by wounding and eating the bark of the plant? or does the parent wound the bark, depositing her eggs in the wound, and when wet, and the irritation produced by the larvæ, complete the fatal wounding? or does the decay first arise, and then this millipede comes to it to feed upon the putrid part, and the mites (Acari), which frequent the places where decaying vegetable matter occurs?" This question appears as yet undecided. Mr Johnston's opinion is quite in accordance with our own, namely, that this "Iulus does not attack the cabbage whilst this is healthy, but that the wound may be occasioned by the parent millipede, and that the young ones feed on the mites which frequent the decaying wound. The millipede, Iulus pulchellus," represented in our figure, "which is oftenest met with in these circumstances, is of a sandy-grey colour, having on each side a row of small crimson spots. The number of legs varies with the age of the insect, but the greatest number observed in Iulus pulchellus has 
been about 170. When disturbed, it coils itself round in the way represented in fig. 48. It has been found in decayed onions and pansy roots, as well as in cabbage stems. Quick-lime and gas-lime, incorporated with the soil, destroy or drive away these creatures."

The cabbage is also infested by the Altica consobrina, or blue cabbage-fly, or flea-beetle, attacking the leaves; Anthomyia trimaculata, destroying the roots; Altica concinua, the brassy cabbage-flea; Vanessa fluctuata, the caterpillars of which feed on cabbage-leaves.

Saving seed. - The whole of the Brassicæ are liable to change when grown from seed. They cross with each other so freely that it is scarcely possible to save the seed of any one variety with a certainty that seedlings from it will invariably come the same as the parent. Certain winged insects, such as bees, engaged in sucking the honey-like matter contained in the nectary, an appendage to the flower known to secret honey, and which is strongly exemplified in many cruciform flowers, to which tribe the Brassicæ belong, carry the pollen or fertilising dust from one flower to another, and thus become the agents of nature in the creation of cross breeds, for hybrids or mules we cannot call them. Nor is it only in the same garden that these causes of intermixture take place; they extend over much greater surfaces - often to the extent of a mile or more; and although we know that such do exist, we do not always know when and where they take place. It is impossible to save several kinds of brassicaceous seeds pure in the same garden, although Knight and others attempted this by covering over the flowers with fine gauze netting, and even by castrating the flowers when artificial impregnations of opposite plants were in course of experiment. It is, therefore, folly in people to save their own seed, unless their garden is far isolated from all others, as well as from fields where brassicaceous plants are cultivated. It would be, in a sense, foreign to our present purpose to follow this very interesting question further; we will direct our attention now to the best means of saving seed, presuming only one sort is saved in the same garden within the same year.

Cabbage-seed.--Select some of the best-formed specimens of the sort to be saved. They may either remain in the place where they have been growing, provided the climate is good, or the roots may be taken up and planted in the best situation the garden affords. In very cold and wet localities they should be planted at the bottom of a south wall, and when replanted should be set so deep in the ground that only a few inches of the stem may appear above ground. In spring they shoot up, and during summer the flower-stem is formed, and the flowers produced. The side branches of the stem should be cut away, as it has been proved by Bastien that the middle flower-stem produces the best seeds, and that plants produced from them are much earlier, and more perfect in character, than are those produced from the side or lateral branches of the flower-stem. No doubt it would be of use as a precaution to cover the flower-stem, as soon as the flowers began to open, with fine gauze netting, were it only to protect them from the operations of the bees and other winged insects. Flower-stems from the sprouts should not be allowed to exist. In a few days after the flowers begin to open, impregnation takes place; after that the covering may be removed, for no spurious impregnation can take place afterwards. Great care should be taken that the flower-stems are supported so as to prevent their being broken by wind or otherwise, and also that the seed is allowed to ripen thoroughly. To secure such, it will be necessary, as soon as the seed pods are formed, to cover them with netting, so as to exclude birds from them, and also that the stalks should be cut before the pods begin to open and shed their seed. All this may be considered trouble, but without such precautions no dependence can be placed on the purity of the stock. Common seed-growers do not bestow this attention, because, at the miserably low price at which all kinds of seeds are now sold, it would not remunerate them. They do their best, at least such of them as have a character to maintain. They visit their stock-farms frequently, and weed out all inferior plants, and do their best to secure a fair sample. They also avoid growing plants of the same natural order near to each other, unless it be such as do not flower at exactly the same time. The critical period may, in a general way, be embraced within the space of a week, and there are several of the Brassicæ that do not perfect their flowers within that period, and hence such may be grown side by side. Such, however, as experience has taught the seed-growers flower at the same time are grown on separate farms, or else they confine themselves to a less extended number of sorts, and occupy the ground with pease, beans, carrots, \&c., from which there is no fear of contamination. Some, upon a small scale, plant their varieties of Brassicæ on spots in the centre of corn or other grain fields, and this greatly lessens the chances of intermixture. Seed-saving in private gardens is by no means a profitable speculation; yet from the frequent disappointments we meet with, we are often driven to it as a measure of necessity. A superior stock of Brussel sprouts, or of Walcheren broccoli, or of a favourite cabbage, is of too much importance to lose wantonly. Here, however, there is the consolation that as the seeds of most of the Brassicæ will keep good for six or eight years, and even longer, we can save Brussels sprouts one year, Walcheren broccoli the next, and so on, including those other sorts that do not flower at the same time. The seed, when ripened, keeps best in the straw (using the phraseology of the trade), and where there is accommodation this may be done; otherwise, the seed may be thrashed out when ripe, and kept in paper or canvass bags in a dry airy seed-room. It is of advantage to the seed, and a precaution against weevils, to examine the seed three or four times during the year, and to turn it out into a seed-sieve which has been already rubbed over with salad oil, and to toss the seed about in it until the skin receive a slight coating of the oily matter, which will pre- 
vent the weevils from attacking it, and, by excluding the air, tend to prolong the vegetative properties of the seed. All the Brassicœ seed is better of being kept for a time, for reasons elsewhere given.

Brussels sprouts.-It is usual to save the seed indiscriminately from the flowers which proceed from the crown of the plant, as well as those which issue from the side sprouts. In both there is a difference. The experiments, in this respect, which we have been engaged in for some time, are not yet sufficiently matured to warrant us in offering an opinion, which may some day be considered rather novel. Dr Van Mons of Brussels held the opinion that seed saved from the crowns or tops was preferable to such as originated from the side sprouts.

Cauliflower and broccoli. - Both require a warm sheltered situation, yet one fully exposed to the sun-the former in particular; and hence its seed is seldom well ripened in Scotland; indeed, our best seed comes from Holland. The very finest and most perfectly formed heads should be selected, carefully taken up, and replanted. In spring, when the broccoli flowers begin to expand, the centre part should be cut out, leaving only five or six of the outside flower-shoots to come to seed. Transplanting the old stems from where they grew to where they are to produce their seed, is thought greatly to improve its quality. By the check the plant gets during the operation, it is so far weakened that it is not so apt to button, or even prematurely to flower; nor so likely otherwise to degenerate as it would be, if continued in its original place. The best remedy for either is seed well kept for several years. It was long thought that these seeds should be imported annually from Italy; but this, so far as the broccoli is concerned, is quite uncalled for, as we have finer varieties of English origin than are to be found in Italy or anywhere else. The only difference in saving cauliflower seed is, that the plants require a warmer situation to be placed in, and instead of planting the stems in spring, as with broccoli, the plants of the last August sowing, preserved during winter, should be chosen: and as they produce their flowers or heads during May and
June, the best specimens should be selected, and left where they are growing, that they may experience no check to delay the season of ripening their seed. The same precaution should be employed as noticed for cabbage, and every encouragement given to accelerate their period of ripening. Our best cauliflower seed is imported from Holland, and for its quality we have much greater reason to thank the better climate than the growers, who are not over particular in the matter, as Dutch cauliflower seed is sure to sell.

The European names of the common white cabbage are-Chou pomme, or Cabus blanc in French; Cavolo in Italian; Verca in Portuguese ; Wiss kopf kohl in German; Berza in Spanish; and Witte kool in Dutch. The Portugal cabbage, or Couve tronchuda, is the Chou vert à larges côtés of the French.

Red cabbage-Rood kool in Dutch; Berza colorada in Spanish ; Chou pomme-rouge in French ; Cavolo rosso in Italian; Roth kopfkohl in German.

Savoy-Herzkohl, or Wirsing, in German ; Cappucio in Italian; Chou de Milan, ou Pomme frise in French; Savooij kool in Dutch ; Berza de saboya in Spanish.

Brussels sprouts-Chou de Bruxelles, or àjets, in French ; Sprossen kohl in German; Spruit kool in Dutch : so little cultivated in the south of Europe as to have no other name than the French, Chou de Bruxelles.

Borecole-Chou verte, or non pomme, in French ; Cavolo aperto in Italian ; Col in Spanish; Grune kohl in German; Groen kool, Dutch; Kale, Saxon; Open kale, Scotch. The palm borecole is the Chou palmier, and turnip-cabbage Chou navet of the French.

Cauliflower-Chou-fleur in French ; Bloem kool in Dutch ; Berza florida in Spanish; Blumen kohl in German; Cavoli flori in Italian.

Broccoli-Broccoli in French; Broccoli in Italian; Broculi in Spanish; Scotsche kool in Dutch ; Italianische kohl in German.

Sea-kale-Chou marin in French; Col marina in Spanish; Meerkohl in German; Zeekool in Dutch ; Crambe marina in Italian. 


\title{
CHAPTER IV.
}

\author{
A S A R A G N O U S PLA N T .
}

THIs division comprises five sections of esculents, cultivated more as articles of luxury than of general utility; they nevertheless form a most important division in garden arrangement, often occupying an eighth part of the whole extent.

\section{$\S 1$-ASPARAGUS.}

Natural history.-Asparagus (Asparagus officinalis L.) belongs to the natural order Asphodeleæ, and to the class Hexandria, and order Monogynia, in the Linnæan arrangement. The name is derived from (A. intens) sparasso, to tear, on account of the strong prickles with which some of the species are armed. It is by no means a genus having much floral attraction, most of the species being rude climbers, with insignificant flowers; in general natives of warm countries, and of little value either in the arts or in domestic economy. One is a native of Siberia; and the common cultivated sort is a native of light sandy soils on some parts of our sea-shores, as well as of the salt-water steppes of Russia-where it becomes covered with sand and salt water during high tides-and other parts of the north of Europe.

It is said to attain an extraordinary size in a wild state on the banks of the Euphrates, and it has been suggested that roots of it should be imported to this country; we believe, however, that it owes its great size there to local circumstances, and that roots from these would with us become no larger than our own. The first notice taken of it as a cultivated plant is about 200 years B.c., in the time of Cato the elder, who lays down very sensible rules for its cultivation-much, in fact, as at present practised -and very wisely recommends that the beds should only be allowed to remain in a bearing state for nine years. Suetonius, in his Life of Augustus, informs us how partial that emperor was to asparagus; and Erasmus confirms this. Pliny, in book xix. chap. 4, tells us that it was cultivated in the gardens at Ravenna in his time, of an enormous size, so that three heads would weigh a pound. Mr Grayson, of Mortlake, near London, has produced 100 VOL. II. heads that weighed $42 \mathrm{lb}$., perhaps the largest ever grown in Britain. Gerard is the first English author who notices it, and calls it sperage, a name, within our own recollection, used by some old gardeners. He derives the name asparagus from the Latin asparagi, which he translates to signify "the first sprig or sprout of every plant, especially when it be tender." Gerard wrote in 1597. It is most extensively cultivated around London, many hundred acres being occupied with it-some individuals, such as Mr Grayson, having from 30 to 40 acres under this crop alone. Around Paris and Vienna it is extensively grown, and that to great perfection. Meager, in his "English Gardener," p. 188 , informs us that in his time (1670) the London market was well supplied with forced asparagus; and the means employed then were much the same as those at present in use, namely, placing the roots on warm dung-beds.

Uses.-Its delicate flavour is rather deteriorated than improved by those additions which a skilful chef de cuisine deems necessary in the preparation of most other vegetables. It is usually boiled and served alone, to be eaten with melted butter and salt. It is also served on toasted bread, with a plain white sauce. The small heads are cut into pieces of $\frac{3}{8}$ of an inch each, and served plain boiled as a substitute for green pease. In this latter way much of what is called the sprue, or small heads-or grass, as all asparagus is called-which reaches Covent-Garden Market is disposed of. Medically it is considered diuretic, and in Paris is much used by people of sedentary occupations, like tailors, weavers, authors, \&c., when they are troubled with symptoms of stone or gravel. It is said to promote appetite, and hence is served at an early period of the dinner. It is considered anti-scorbutic, and very good in dropsicai cases, but is to be avoided by those troubled with gout. The roots possess those healing properties more than the sprouts or buds; and, if we are to believe Galen, Hoffman, Dr James, \&c., it is because the roots contain more of the salt, from whence they derive that quality, than any of the parts grown above ground, which cannot imbibe it so copiously as the root itself receives it from the ground. It operates with much effect on the urinary organs, as all who eat it copiously know full well. But the most extra. 
ordinary virtue attributed to this plant is given by Antoine Mizold, "Cent. 7, Memorab. Aph.," 34, and Schenck, "Obs. Med. L.," i._- If the root is put upon a tooth that aches violently, it causes it to come out without pain." Modern dentists do not believe in this-nor do we. In Queen Elizabeth's time, asparagus was eaten "sodden in flesh-broth, or boiled in fair water, and seasoned with oil, vinegar, salt, and pepper, then served at table as a salad." It makes excellent soup, and is also used when ragoued. The part used is 3 or 4 inches of the young shoots or buds, taken just as they appear above the ground, which is generally in May and June. It is most esteemed when of a nice fresh green colour. The French blanch it, but they destroy the flavour peculiar to it by such means.

Propagation.-In the first instance, all asparagus is originated by seed. It is then replanted when of one or two years' growth : the first, if the roots are strong, is preferable.

Sowing and planting.-March is the best season for sowing. Some recommend sowing broadcast in beds; we have a penchant for the drill system, and in this case would recommend it. Prepare the ground by copious manuring, and deep-digging or trenching. Draw the drills a foot apart, and 2 inches deep. Sow thin: the seed is in general good, so there need be small allowance made on that account. One quart of seed will be quite sufficient to sow a bed in the broadcast manner of from 36 to 40 square feet. If sown to remain on the same ground (a practice seldom thought of in first-rate gardens), then a pint of seed will sow a bed $4 \frac{1}{2}$ feet broad by 30 feet in length. If the plantation is to be formed of roots from last spring's sowing, then 160 plants will be required for a bed $4 \frac{1}{2}$ feet wide and 10 yards in length. This is the age at which they should be planted. The above calculation is made presuming that the usual practice is followed of setting four rows in a $4 \frac{1}{2}$-feet bed, and the roots 9 inches apart. We would rather plant three rows in such beds, and give the plants 12 inches from plant to plant, which will reduce the number of roots required, and tend greatly to the future welfare of the crop. Plants of one year's growth we prefer; but much depends on the way they have been grown, for sometimes one-year-old plants are stronger and better than three-yearold ones, where little attention has been paid to them. In this case, as in most others, plants sustaining a check in their early growth from starvation are seldom so luxuriant or so long-lived as those which have been carefully nursed. In sowing for permanent crops, it is best to sow in $4 \frac{1}{2}$ feet beds (three rows say, 18 inches apart), and when they have advanced to the height of 6 or 7 inches, to thin them out to 12 inches in the line; or, as we have often practised, sow in single rows 3 feet apart all over the piece, and thin out the plants to a foot apart in the line. It is usual-and we find no fault with the practice-to sow a crop of onions along with the seed the first year: the onions, being sown broadcast, will not much injure the asparagus, while they will repay for the culture of the whole. For permanent crops we prefer the single-drill system; but for growing for two, three, or four years, for the purpose of taking up the roots for forcing, it is possible that the $4 \frac{1}{2}$-feet bed, with three rows in each, may be preferable. This is certainly the case in petty gardens, where a bed of the above breadth, and 10 yards in length, may be the extent to which the owner's means permit him to extend. This is, however, scarcely a crop for manse gardens; the small return only sets the mouth watering, and may lead to a breach of what is forbidden by the tenth commandment.

Whether the seed is sown broadcast or in drills, with a view to be transplanted afterwards, it will be requisite the spring following sowing to prepare the ground and beds for transplantation; and in this it matters little whether the roots are to be set in single lines, or two or three rows, in beds of the above breadth. The whole ground should be prepared alike, and the plants carefully removed to it.

In preparing the ground, we need hardly say it cannot be too highly enriched or too deeply trenched, the roots often extending 3 feet under the surface. It should be trenched to that depth, and a supply of manure placed in the bottom of each trench after it has been broken up with a pick-a foot more in depth, if the soil admits of it. As the process of trenching goes on, other layers of manures should be laid on each respectively, as it is from a foot to 3 feet in depth that the roots will be in search of food. The more lasting and permanent the manure applied thebetter, so that its decomposition maygo on slowly as the roots require it. Some, and 
with great advantage, retrench the ground again in an opposite direction, to insure a more thorough amalgamation of the soil and manure together. The operation should be, if possible, carried on during winter, but not when the ground is either wet, frozen, or covered with snow, having it ready for planting in March or April for Scotland. The London market-garden practice is as follows: - "The beds are prepared by putting on an immense quantity of manure, and trenching the ground 3 or 4 feet deep, mixing the manure as the work proceeds. In March the ground is measured out after the following manner: Suppose a fence runs north and south, or otherwise, 3 feet is allowed between it and the first row ; a drill is drawn about 2 inches deep, and the seed is sown thinly, say 6 inches or a foot apart, which gives choice of drawing out the weakest, in order that the permanent crop may stand 1 foot apart. The next row is sown 18 inches from the one just mentioned; then for the alley and two sides of the bed 5 feet are allowed; then another row of seeds, and so on, which gives two rows to each bed. The first year, onions are generally sown all over the ground; the second season, lettuce, or any dwarf-growing vegetable that will not choke the asparagus; and so on, until the third year, when the beds are formed out, and a few inches of mould dug out of the alleys, and put on the crowns. Only a few, however, of the finest heads are cut this year." - CutHILL in Market-Gardening round London, p. 18. We adopt another plan:-The ground is first thoroughly enriched, by laying with the stableyard manure a considerable portion of ground bones, say 2 inches in thickness, not very small, and about half an inch in thickness of rock-salt in the bottoms of the trenches, adding alternate layers of dung, with which salt is incorporated, and soil as the trench nears the top; and when the whole piece is thus trenched, we proceed to plant in the following manner: For single lines, trenches are taken out 1 foot in depth and 3 feet apart; in these the roots are placed, having been carefully taken from the seed-bed, with their most minute rootlets preserved, and carried to the spot in a shallow basket or planting-tray, and covered over with a little soil-for few plants suffer more than this by having their roots exposed to the air. The plants are examined to see that their buds are perfect, and are placed in the trench 14 inches apart, and the roots carefully spread out and covered with the soil, keeping the crowns about half an inch under the finished surface of the bed. When the bed system is followed, the ground is measured off in breadths of 5 feet and $2 \frac{1}{2}$ feet alternately: the former are the beds, the latter the alleys. Beginning at one end of a bed, a trench is taken out across the bed, 12 inches deep, or more if the roots exceed that length, for they should be laid in at their full length. One root is placed in the centre of the trench, and one on each side of it, 15 inches from each other. When these are planted, another trench is taken out, of the same dimensions, and 18 inches from the first, and planted in like manner. If the ground is dry, the whole gets a good watering of liquid manure-either diluted cow-urine, or water in which guano has been dissolved at the rate of $6 \mathrm{lb}$. to 50 gallons. The beds are left uncropped, and kept clear of weeds during their growth. Some have planted successfully in May, and even in June, after the plants have attained a considerable size. We mention this, more that advantage may be taken of it to make up any deficiencies that may arise from any of the plants having failed, than with a view of recommending it as a general principle. The seeds require about three weeks to germinate. The smallest asparagus bed that should be made should contain a rod (272 square feet, a little more than 30 square yards) of ground, as upon less than this a dish could scarcely be at any one time gathered. The extent of ground for an ordinary private family should not be less than 5 poles, which should yield about 125 heads daily for four weeks; but for large gardens, from one-eighth to a quarter of an acre will be required, exclusive of that which may be taken up yearly for forcing, which in many cases will require as much more.

In preparing ground for this crop, we have remarked that it cannot be too highly enriched, and that the manurial application should be kept near the bottom. This is because the crop may have to remain on the same ground for from seven to ten years, and therefore it 
would be difficult to apply manure to it afterwards ; besides, it is at the bottom of the soil that the spongiolets of the roots are chiefly placed, few being near to the surface. And although the asparagus is a native of poor drifting sand, still, as its chief merits rest on the size and tenderness of its shoots, it follows that, to insure this condition, they should be stimulated to the greatest extent possible, both by manure, and by being placed in the warmest part the garden affords. In sowing for the formation of a permanent plantation, some excellent cultivators sow in rows 2 feet apart in April, thinning out the plants to the distance of 1 foot from each other. Every alternate row is taken up the second year for forcing, thus leaving those that are to form the crop at the distance of 4 feet row from row, cropping the ground between them with summer cauliflower, which enjoys a partial shade from the asparagus. They also recommend, if the transplanting system is to be followed, that the operation be performed in April, when the young shoots are 3 or 4 inches long. The crowns are kept near the surface at planting; and in October, 2 or 3 inches of rich manure is laid over them, and is slightly forked in amongst the roots in the spring following ; but no soil is ever laid over them, and all the future care is an annual top-dressing as above, with liberal supplies of liquid manure, in which salt is mixed at the rate of 2 ounces to a gallon.

The Vienna mode of planting asparagus is to trench the ground 5 feet in depth, and to place a layer of bone, horn, chips of wood, or branches of trees, a foot in thickness, at the bottom of each trench; while the French practice is to excavate a trench 5 feet wide, and of the length the bed is intended to be. The best of the soil removed is reserved to be mixed in the following manner, viz. : -6 inches of best dunghill manure is laid along the bottom; over that, 8 inches of turf, 6 inches of dung, 6 inches of the best of the soil that had been removed and well sifted, 8 inches of turf, 6 inches of very rotten dung, and then 8 inches of the good earth removed at the excavation. The last two layers are then to be well incorporated together, and the beds formed 5 feet in breadth, with alleys 2 feet broad between them. The roots are then planted 18 inches asunder, placing a handful or two of mould under the centre of each, so that the roots may be spread out in regular order, and in form of an expanded umbrella, their crowns being kept $1 \frac{1}{2}$ inches under the surface. A small peg is then placed at each plant, and a spadeful of fine sand is placed over the crown, which finishes the operation. The plants should not be transplanted too early in spring, as there might be a possibility of some of the roots being overlooked, which might be deficient in healthy buds. To guard against that, defer planting until they have sprung an inch or two inches in length. Some defer it longer, and thus reason : Early in spring is the period in general recommended for making new plantations of this plant. Custom only sanctions the practice, while experience says custom is in this case a fallacy, and recommends the operation to be performed late in May, or even early in June, and that when the plants have attained the height of 10 or 12 inches.

In cold situations, this late planting is of the utmost consequence, and if attended to, would in a great degree remedy the defects, so often seen, of blanks in the beds, and not unfrequently the dwindling appearance of the whole crop during the first year. Many will no doubt object to this late spring planting, from a dread that the plants will suffer from removal while in a state of growth; and others will argue that, by following the "good old rule" of planting in March, the plants will have a longer season to grow in, and also that the roots will sustain less injury, because they have not then become excited into growth. All these fears are groundless: the young shoots, should they even perish, will be succeeded by much stronger ones from the latent buds, with which the crown of the plant is always furnished; and others may be consoled by the fact, that, the ground being much warmer in May or June than in March, and the juices of the plant being in motion at the time, it is prepared to encourage immediate growth; whereas the roots transplanted in March lie, till excited by the natural warmth of the soil, in a dormant state-the elaborated sap in them escaping at the injured parts, weakens them amazingly, as well as brings on a disposition to rot and decay. Keeping 
the beds well elevated, in cold soils and situations, enables the solar rays to reach the roots better than when planted on a level surface.

Subsequent cultivation.-We need hardly say that the beds should be kept free of weeds, as well as the alleys between them. The plants on seed-sown beds should be allowed three years to establish themselves, before any buds are gathered. Those on transplanted beds, if the roots were strong at planting, and have made good progress since, may have a few of the strongest buds gathered the second year ; the third, if all has gone on well, will admit of regular cutting. The practice of cropping the transplanted beds is injudicious, and that of cropping the alleys between the beds, although constantly done, is of questionable utility, because whatever crop is planted has a tendency to rob the beds; and the roots, which often extend towards them, run great risk of being either cut off or very materially injured. $\mathrm{Mr} \mathrm{Judd}$, in the "Transactions of the London Horticultural Society," vol. ii., thus very properly speaks on this point. Having dug out the alleys the first season, instead of repeating the operation the next, he lays on a coating of rich dung 3 inches thick, and carefully forks it into the beds and alleys - a process he continues in winter annually, "never digging out the alleys any more, as it is known the asparagus plant forms a fresh crown every season; and sometimes it happens that in a few years the crown will increase almost into the alley, so that in digging out this you must inevitably spoil that plant. If this is not the case when the beds are in a good condition, the roots will be sure to work out at the sides into the alleys; and by digging out the latter, these roots must be cut off, as you will often see them exposed all the winter, before dung can be got to fill the alleys up. Rather than be treated in this way, they had better be without anything all the winter, as asparagus does not suffer generally by frost." The practice of covering the beds in autumn with leaves or litter is sufficiently absurd, yet top-dressing them with rich manure is of great advantage; but this dressing, whatever it may be, should be covered with a couple of inches at least of soil, to prevent the escape of its gaseous matter into the air. The rains of winter will wash down to the roots much of its fertilising properties ; and in spring, part of it may be carefully raked off, and left to rot in the alleys. That which remains on the beds will prevent the crowns from rising above the surface, and tend to exclude the drought. It has been recommended to uncover the crowns during winter, and to cover them over again in spring-a very useless, if not an injudicious practice. The surface of the beds should be slightly stirred up with a fork in spring, and before the buds show above ground; and three times during the growing season of the plants, a thin sprinkling of salt should be applied to the surface, and, if possible, before rain. Upon the whole, disturbing the ground in the alleys, further than loosening it to the depth of 4 or 5 inches with a threepronged fork every spring, is reprehensible ; and still more so, digging them out in deep trenches, and piling the soil over the beds, as practised by many of the London market-gardeners, who do so to obtain stalks, or grass, as they term it, nearly a yard in length-all of which, excepting about 3 inches at the top, is perfectly useless, and well defined "drumsticks" in "The Gardeners' Chronicle"an immense expenditure of the energies of the plant, for no other purpose, that we could ever divine, than to encumber the dust-holes in London. The practice is still persisted in, notwithstanding the merited castigation the advocates of such an absurd practice received a year or two ago from $\mathrm{Mr}$ Cuthill and the editor of that journal.

Top-dressings, however, can be of far less advantage to the plants than laying a good foundation for them at the first making of the beds, because the spongiolets, or food-absorbing parts of the roots, are in all cases at their very extremities, however deep they may be ; and hence it would be excellent, had we the means of applying food to them by means of a sort of subterranean system of irrigation, so that the food might be presented to those parts of the roots which are designed expressly for its absorption. No doubt, much of the success of the noted asparagus grown along the banks of the Thames is owing to the food conveyed to the roots by the rise of the tide, supplying it at a 
depth of 3 or 4 feet at every flow-a depth that scarcely any manurial application, applied to the surface, could reach, or at least to the extent of being very beneficial.

The London market-garden practice is thus described by $\mathrm{Mr}$ Cuthill: " $\mathrm{Au}$ tumn arrives, and when the haulm is cut, the whole of the ground is forked over, and planted with cabbages, coleworts, or winter greens; then in spring the beds are largely supplied with mould out of the alleys, covering the crowns from 8 to 10 inches deep. The finishing of the cutting must be left to the grower. A fair crop of heads must be left, after four or five weeks' cutting, in order to grow the buds for the next year's growth, and to restore to the roots what has been taken from them in the shape of a crop; but not one head must be allowed to grow until you leave off cutting entirely, at the end of the fourth year. When the haulm gets ripe, it is to be cut down, and the mould thrown into the alleys, and there enriched; and the whole of the beds and alleys are planted again with cabbages, greens, \&c."

The exact season for cutting down the haulm must ever be governed by circumstances. In early soils and situations, the haulm will be ripe about the middle of October, and this is known by its becoming of a yellowish colour, and by other evident signs that vegetation has ceased for the season, at which time it may be safely cut down. In Scotland generally, in consequence of our late springs, and consequent late autumns, the plants drag out a weary existence to the middle of November, unless some early autumnal frost intervenes to put a stop to growth, which of itself is tantamount to cutting too early, because in both cases the supply of elaborated sap is cut off from the roots. Cut the haulm off in a dry day, and tie it up in bundles, to be kept dry till spring, when it will be found one of the best protections to dwarf standard and other fruit-trees, for which see FruIT-GARDEN.

In Holland, all the asparagus is blanched, which is effected by covering the beds early in spring with fine sand, which no doubt produces the shoots of a milky whiteness, and of a considerable size and tenderness, but totally devoid of its natural flavour. Not a single bud is to be seen above ground, the surface of which is kept as smooth as possible; and the state of the crop is ascertained by the buds pushing up little hillocks of the fine sand, when the owner, constantly on the watch, slips down his knife, and cuts off the shoot near its bottom, being guided as to its position by the upheaving of the sand. In defence of their practice, the Dutch say they have the whole shoot in a state fit for the table, while we have only a couple of inches.

Asparagus roots may be removed, with very good success, if carefully done, even at the age of eight or ten years, but it would be unadvisable to cut any of the buds during the following year.

Soil and Manure.-The asparagus is a native of sandy beaches often overflowed by the sea, having its roots watered by the spring-tides, yet not kept in a state of continual wetness, on account of the ready means the water has of escaping by filtration. This seems to point out certain principles in its cultivation-namely, a deep, pervious, light soil, perfectly dry at bottom, but capable of being subterraneously irrigated at times-the depth and lightness of the soil being favourable for the long delicate roots penetrating it in search of food. Such are the natural conditions of its growth. The artificial ones should in some degree assimilate to them, with the addition of abundance of manure to increase its size and expedite its growth ; for on its large size and rapidity of growth much of its delicacy and flavour depend. A deep light soil can in many cases be formed for it, and subterranean irrigation would even be a more simple affair. On these conditions much of the extraordinary success of the growers along the banks of the Thames depends; and were there even doubts of that, other evidence could be adduced-as that of the well-known fact that the finest asparagus grown in France is produced in a small sandy island in the Oise, where the surface of the beds is not more than 2 feet above the level of the river, the soil being a coarse gravelly sand, saturated with water at the depth of 3 feet from the surface. The banks of the Danube and Euphrates, as we have already stated, are celebrated for the size and quality of their asparagus. Many years ago we saw, for several consecutive seasons, very fine crops 
produced by Mr Foster, a market-gardener at Winchester, whose ground was liable to be overflowed by the river Itchen through the winter and spring; and so much did this affect the crop, that he used to cut heads of good size four weeks before it was produced in the neighbourhood of London, and five weeks earlier than we cut at Stratton Park, at a distance of only eight miles. The soil should not be less than 3 feet in depth; and where it is not naturally so, it were better to elevate the beds to that extent by adding to their surface, even by leaving the alleys wider, and taking a portion of the soil out of them for the purpose. A deep alluvial soil is excellent; a strong retentive clayey one the worst possible, and quite unsuitable if not rendered dry at bottom. As to manures, whatever they may be, salt should be incorporated with them, and they should be of the most lasting description, such as ground bones; and the proper mode of employing them so as to be of the most advantage, is by placing them as deep as the roots will penetrate; and as refreshers, copious applications of the strongest manures, in a liquid form, should be applied during the whole growing season.

When salt is applied as a top-dressing during the growing season, it may be laid on until the ground is perceptibly white with it; or if by rule, apply $16 \mathrm{lb}$. to 60 square yards of surface, which will be quite sufficient for one dressing.

Forcing.-By means of slight excitement, this excellent vegetable may be kept in successional fitness for the table from the middle of November until it comes in in the open ground. The conditions necessary to secure success are, strong healthy roots, from three years growth and upwards-carefully preserving the roots on taking them up-and a temperature not exceeding $50^{\circ}$ to $60^{\circ}$, with abundance of light and air, after the buds appear above ground. The means employed to effect these conditions are various. First, as to roots : these must be strong and healthy, and hence the supply ought to be grown in the same garden; and to secure them strong, they must be grown in highly-enriched soil. Some cultivators keep up a succession of roots by growing them for the express purpose, and in this case the buds are never cut, the plants being allowed to develop themselves fully for two, three, or more years, by which means the roots and buds become earlier ripened in autumn, and also have stored up a much greater amount of properly elaborated matter within themselves, upon which depends so much of the succeeding crop. Plants taken out of the ground in October or November, and placed in a state even of very moderate excitement, have not time to furnish themselves with fresh fibres, whereby to supply the roots with a sufficiency of food. The crop, therefore, depends on the amount of properly elaborated organisable matter provided for them during the previous season. This is the reason why a rich-manured soil is dispensed with, and a light sandy one preferred, in which to imbed the roots during the process of forcing, and also why the roots are afterwards thrown away. From this it follows that the greatest possible care should be taken to preserve every portion of the roots at lifting, as each, however small it may be, contains a certain amount of the material so essential in the development of the buds; and as they are prized according to their size, every curtailment of the roots is a certain loss to the crop.

By far the most usual mode of forcing asparagus is to place the roots on a moderate heat, either produced by fermenting material, such as dung, leaves, tan, \&c., or on beds heated by hot-water pipes, steam, smoke-flues, tanks, \&c. Of these the latter is decidedly the best mode, as affording a steady yet sufficient heat, combined with a greater share of humidity at the roots, as steam will find its way more readily through the joints of a stone or slate-covered tank, than through the closer joints of a hot-water or steam apparatus. It is true, any quantity of water can be supplied to the roots in the other cases, as described p. 447 , vol. i., in the case of the roots of cucumbers; still there is something so genial in the heat of a tank, when the roots are in proximity to it, that it seems to approach nearer to that of heat produced by fermenting material than any other.

Whichever of these ways may be adopted for securing heat, the surface on which the roots are to be placed should be prepared of light sandy soil, and on 
this the roots, as they are taken up, should be placed close together, and regularly spread out in a perpendicular direction, but not so crowded as that they shall touch each other; and as the process goes on, the soil should be carefully wrought in amongst them while dry; and when the bed is finished, it should be well watered with tepid water, to wash the soil more closely about them.

When the roots are old and very large, it may be found more expedient to place them in a horizontal direction; but, when young, there will be no difficulty in setting them upright, by which a greater number of crowns will be got into the same space. In regard to produce, it may be stated in a general way, that a three-light frame, or the same extent of pit, will produce, under good management, a dish of heads every other day for nearly three weeks. To maintain such a supply from the middle of November till the grass is fit to cut in the open ground, it will be requisite that a three-light frame or pit be planted every ten or twelve days from the last week in October till the second week in April, calculating for the climate of Scotland, and till the beginning of March for that of England. In large establishments, where this vegetable is required daily and in large quantities, double that extent at least should be planted. All that is required in the way of culture is frequent supplies of tepid water and abundance of light and ventilation, even to the extent of removing the glasses for an hour or two on all favourable occasions, and guarding against an excess of bottom-heat. On the Continent, the buds are often blanched with a view to render them more delicate; with us, a fine healthy green colour is a chief recommendation, and to secure this, as well as flavour, light and air are absolutely necessary. As a general rule as to temperature, the atmosphere of the bed or pit should range from $50^{\circ}$ to $60^{\circ}$, the former during night and the latter during the day, while the bottom-heat should approximate nearly, nor should it at any time fall below $48^{\circ}$. In severe weather it will be more expedient to retain the heat within by external coverings (for which vide fig. 789, vol. i.), than to increase it by an extra consumption of fuel.
The Dutch and German mode of forcing asparagus. - About the end of October deep trenches are dug between the beds in the open ground, which, for this purpose, seldom exceed $2 \frac{1}{2}$ feet in breadth, having one row of plants in the centre of each. These trenches are filled with leaves, rank stable-manure, or any other fermenting material capable of producing a strong heat. The surface of the beds is forked up and slightly covered with litter. The heat in the trenches stimulates the roots, and considerable success attends the operation, so far as quantity of heads goes, but they are without either colour or flavour. Some, however, have narrow frames covered with glass which they place over the beds, which is, no doubt, an improvement on the former practice. Both are modes attended with much labour and uncertainty as to time, and occasion an enormous waste of manure. Many Continental gardeners, however, adopt the taking-up system described above, and some also follow the methods to be noticed below.

Forcing in permanent beds.-_" Forcing in permanent beds has not been much practised in this country till of late years. There are now, however, many instances of this mode," under different modifications, "in many of our first-rate gardens. Beds to be so treated are built with pigeon-hole walls all round; they are of the breadth of an ordinary-sized garden frame, and of any required length; the depth of the linings or spaces between the beds is 3 feet, and their breadth $2 \frac{1}{2}$ feet. In these spaces dung, leaves, tan, or any other fermentable matter is placed, which heats moderately the body of the beds. Frames are placed over the beds, covered with glass-lights ;" all other coverings now should be looked upon as obsolete things. In such beds asparagus is forced year after year without renewal. "For later crops there is no objection to this plan, because, by the time the crop is gathered, the weather will be mild enough to allow the last set of stalks shooting up to their full size and development, and hence the roots will be supplied with proper nourishment to enable them to produce a crop of shoots the following season. But such roots as are forced early by this method, not having the same opportunity of acquiring their natu- 
ral supply of food, must reasonably be considered as placed in a very different position as regards their capability of production."-Practical Gardener.

Forcing in permanent pits, as practised in the Royal Gardens at Frogmore.-The plants while young are planted in brick pits 4 feet in depth, and in a rich loamy soil peculiar to the locality, and highly enriched with rotten stable-yard manure. These pits are 7 feet in breadth, and separated by trenches walled up on the sides in the pigeon-hole manner, and 20 inches across. In these spaces hot-water pipes are made to traverse (vide fig. 639, vol. i.), the bottom parts of the spaces being filled up with soil similar to the beds; and the upper part, also 2 feet in depth, is left open that the heat from the pipes may radiate freely and find its way into the pits, which are span-roofed and covered with boarding. The spaces between the pits in which the pipes are laid are covered with Yorkshire pavement to prevent the escape of the heat, as well as from its being comfortable to walk upon. The plants are excited early in December, that a supply may be obtained by the end of that month, the temperature ranging between $50^{\circ}$ and $60^{\circ}$. During the forcing period, the plants are assisted by occasional supplies of rich manure in a liquid form. For early forcing this plan is liable, to a certain extent, to the objections stated above-modified to some degree, however, inasmuch as the heat can be maintained at the roots till a late period of the season; and the latent or weak buds may thus be induced to spring and throw up stems, by which sufficient excitement is thrown into the roots to cause them to form a fresh series of buds for succeeding crop. The advantages of the system of permanent beds are held by many as important. It prevents, as they say, the great sacrifice of ground in the production of roots, which require at least two or more years' expensive culture to prepare them for a single return. We should, however, take into consideration the importance of what is called rotation in cropping; and for this the destruction of a few beds of asparagus yearly offers an excellent opportunity. In our own case, we have about an acre of ground under this crop for the purpose of forcing alone. This space we have in three sections, one VOI. II. of which is taken up every winter, and a corresponding quantity planted every spring from the seed-beds of the previous season.

The great supplies sent to the London markets are produced during winter by the taking-up mode, and are for the most part forced upon beds of fermenting material. Our own opinion is in favour of this plan, and next to it that of growing in pits heated upon the principle shown in our improved asparagus-pit, described p. 455 , vol. i., and covered with a glass roofing, to be kept on until the reserved stems become fully matured and the buds ripened, when it may be removed until required again the ensuing season.

The London market-gardeners' mode of forcing asparagus is by placing the fully matured roots on beds of fermenting dung, placed in trenches 2 feet deep, having 6 inches of mould to plant in. These are then hooped over, and covered with mats or otherwise to blanch them, and make them look fine and white, to suit the taste of the market. In private families, however, this white colour is no recommendation, a fine healthy green being preferred, and this can only be attained by growing it in the light; therefore the private gardener's practice is the best, and to attain it so, he has to employ glass sashes for protection, and to admit abundance of air during its growth. Mr Cuthill was the first, we believe, who attacked the old and absurd practice of blanching, and his views were aided by several sensible writers in "The Gardeners' Chronicle," and amongst them the talented editor of that paper.

Amateur forcing.-Forced asparagus is not, as is supposed, confined to the tables of the rich, and those only who have extensive forcing accommodation. Any amateur who has the command of a cartload or two of tree-leaves or stable-litter, or who is in the neighbourhood of a tanyard, where he may often get tan for taking away, may have as good asparagus in March, or even earlier, as his richer neighbour ; and with the aid of a one or two light frame, he may have it at his Christmas dinner. The way to proceed is, First get your roots, which can often, for so small a supply, be obtained from a neighbour, or bought from a nursery or market-garden. Many amateurs, how- 
ever, have their own asparagus-bed in their garden. To those, therefore, we say, Make up a bed in some warm sheltered corner of either of the above materials, and to the thickness of 3 feet or $3 \frac{1}{2}$, if leaves only be used; set the roots on the surface after the strong heat has declined, imbedded in the soil as stated above, or in leaf-mould or rotten tan; cover them at first only as high as the tops of the crowns, and after ten days lay on 3 or 4 inches more, keeping the surface within 9 or 10 inches of the glass : for, to secure a crop by Christmas, glass will be required for its protection. If for March use, the bed may be hooped over, and covered, first with semi-transparent canvass, which will admit light enough, and therefore may be kept on permanently; while during night and in cold wet days, and during frosts, a waterproof tarpaulin should be put over all, to throw off the wet and retain the internal heat. A very slight heat is all that is necessary. In regard to taking up the roots, amateurs will do well to commence on one side of the outer rows of the bed by digging out a trench, forking the earth as much as possible from underneath the plants, so that they may easily, and without straining or injuring their roots, move them out entirely by thrusting down the fork behind them. Be very careful, at the same time, that the buds about the crowns of the plants are not injured by the fork, or bruised in any way during their removal. Asparagus roots may be planted in boxes, and placed in a warm stable or cow-house, and when the buds have attained the height of 3 or 4 inches, they may be brought out daily into the open air when the weather is at all mild: this is with a view to give a greenish colour to the buds; but where colour is not a consideration, this removal will be unnecessary.

Asparagus is sometimes forced in the beds where it grows by covering the surface early in November with hot stablemanure to the depth of a foot, which starts the buds in about twelve or fourteen days ; the beds are divided by 3 -feet alleys, which are dug out, at the time of covering, to the depth of 2 feet, and filled with heated dung at the same time. In breadth the beds correspond with that of an ordinary garden-frame, which is placed over them as soon as the shoots have attained the height of from 2 to 3 inches, at which time the covering over the beds is carefully removed and added to the linings placed in the alleys, which raises them as high as the top of the frames all round. This heat, with a sufficient covering of mats or straw over the glass during the night, is found sufficient for the purpose ; and as the glasses are uncovered every day, the shoots become of a fine green colour, and are in a fit state for use by the latter end of the month, or beginning of December. The beds are covered in this way in succession, a three-light frame being placed to the end of the first, and so on, every ten days during the season of forcing. When the crop in the first frame is cut, the frame is carried forward, and the surface of the bed covered with dry litter or leaves to keep out the frost, but not to the extent to keep the roots in a state of excitement, as it is desirable they should remain at rest until spring, when the covering is removed, and they are allowed to grow in the open air. The portions of the beds earliest forced in this way are allowed a year to recover their strength, when they are again forced. Those that come in later in the season are forced the following year, and there are instances of this mode where the beds have been so treated for thirty years.

Asparagus may also be forwarded a fortnight or three weeks earlier than it will come into use in the open air, by merely covering the beds, about the end of February, with a frame and sashes, without any other artificial heat whatever; or the cloches, or small bell-glasses so much used by the French, may be advantageously employed: they are now being made at most of the large glass-works, and may be purchased at $1 \mathrm{~s}$. a piece, if taken in quantities of not less than 200 .

Taking the crop.-The buds are fit for cutting when from 3 or 4 inches above the surface, when of a fine green colour, and while they are firm and compact, terminating in a close obtuse point. They should never be blanched, although this has been strongly recommended by one who ought to have known better. The French, Dutch, and Germans almost invariably did so, and even put themselves to the trouble of placing an opaque tube over each bud, for the evident purpose of 
spoiling it. Were glass tubes used, the crop would be hastened forward, and probably increased in size, without doing injury to the colour or lessening its flavour. No shoots ought to be allowed to exceed 6 inches in length. In proceeding to cut the buds, remove with the side of the asparagus knife a little of the soil from around their base, push the knife gently downwards, keeping it quite parallel and close to the shoot to be cut, taking great care in doing so that none of the buds yet under ground are cut or injured: when the knife is as deep as the base of the shoot, give it a slight twist, pressing it at the same time close to the shoot, and draw it gently upwards, by which process the bud will be separated from the crown. The wound, being ragged, in consequence of being cut with a serrated edge rather than a smooth one like a knife, will not bleed so much, and hence be less weakening to the plant. Fig. 49 shows such an instrument: there are other forms in use,

Fig. 49.

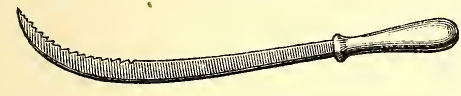

Asparagus CUTter.

but in no way superior to this. The blade should be thin, about 9 inches in length, with a wooden handle: by being slightly hooked at the point, it is less liable to cut the underground buds, while the serratures on the concave side more readily catch the shoot intended to be cut. Many use only a common pruningknife, especially when the shoots are to be cut about an inch under the surface; but the cleanness of the wound leaves the sap-vessels open, and a considerable waste of sap is the consequence. A shallow basket should be carried in the one hand to receive the shoots as they are severed by the other, and they should be laid with their buds pointing in one direction, and afterwards carried to the vegetablehouse to be washed, dressed, and tied up in bundles of about 3 inches in diameter each, the ends of the shoots cut all to an equal length and neatly placed together, so that no one bud projects beyond another. In this way they are ready for the cook, who, depending on the man who serves the kitchen having properly cleaned them, commits them at once to the saucepan of boiling water in bundles as they are. In cutting from established beds, every bud, large and small, is cut, and they are afterwards sorted into sizes, one, two, and three, the first being the largest and finest formed, the second of a less size, while all the small, or any that may' have the points of the buds damaged or broken off, are placed in the third, to be used for soups, imitation green pease, or the like. This general gathering will often continue for from four to five weeks, after which only a few of the larger should be taken, the rest being allowed to grow for the support of the roots and encouragement of buds for another season. The reason why the whole buds are cut away is, that if a portion of them were left and allowed to grow, they would draw too much upon the roots, and render them unable to continue sending up more; or if they do so, such shoots would be small and worthless. With young buds the case is different; they must not be so closely cut; only a few of the stronger buds should be taken, and even that not continued longer than about a fortnight. By the middle of June in England, and the latter end of it in Scotland, cutting should entirely cease if the future crop is to be regarded; or a bed or two may be gone on with ten days longer, and such beds have a respite the following season. Beds intended to be forced should have very few of the buds cut, if any at all. It often happens, in cold seasons, or in late situations, that the buds come slowly through the ground, and in such cases there is often a number of hard, dry, scaly appendages found attached to the shoots near their base. These should be carefully scraped off with a sharp knife in the vegetable-house before tying up, but on no account should the skin be peeled off. Some, however, prefer allowing the shoots to attain the height of 6 or 8 inches above ground, and then to cut over close to the surface. This is no doubt a very good way when it is growing rapidly, and in that way its full flavour will be attained.

Asparagus may be kept several days after cutting, if their ends be set into a shallow vessel of water, and placed in a dark cool cellar to prevent the buds opening.

Approved sorts and their qualities.-There is only one kind of asparagus, although seed-lists present us with a goodly number of names, 
such as Gravesend, Battersea, giant or Grayson's, true Reading giant, \&c. No doubt much depends on saving seed from the best plants, as is the case with everything else, instead of gathering it promiscuously, as is too frequently done; and while the prices of seeds are so low as they now are, there is little inducement for the seed-grower to do otherwise.

Insects and diseases.-Snails and slugs are often troublesome in mild springs, attacking the buds as they push through the soil: they often attack the stems of the plants when well grown, near to the ground; but they can be readily got rid of by dusting lime over the ground in the evenings. From its caustic property, on touching their bodies it speedily kills them. The wireworm is a much more to be dreaded enemy; it perforates the centre of the buds under ground, and totally destroys them. Piercing the ground amongst the roots with an obtuse-pointed dibber, and pouring about a table-spoonful of spirits of tar into each hole, will kill numbers of them; setting traps of slices of carrots, potatoes, or turnips, attached to a skewer, and burying them close to the tops of the crown, will attract them by thousands, and on drawing them up every other day they will be found attached to them, and may easily be destroyed. This may be considered a tedious process; it is nevertheless a most effectual one.

The asparagus beetle, Crioceris asparagi, fig. 50 , is often found during the early part of summer depositing its eggs on the stalks. It appears to abound much more in some seasons than in others; and while it is a grievous pest

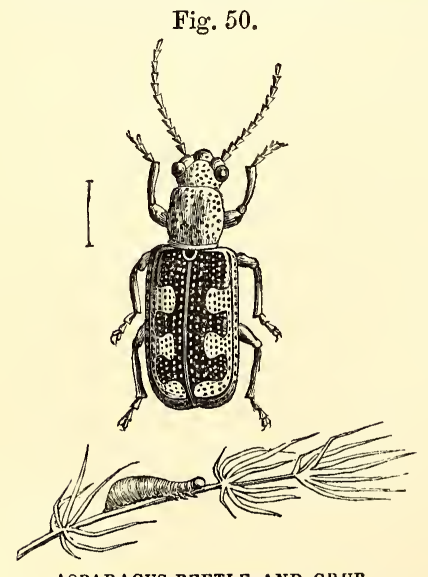

ASPARAGUS BEETLE AND GRUB.

in one locality, it is next to unknown in others. In the year 1836 it abounded in the gardens round London, the larvæ, beetles, and eggs being often detected on the same plant and at the same time. The larvæ feed on the leaves and stems, and are much more destructive to young plants than to older ones. The larvæ are fully grown about the end of June, and may readily be detected, appearing of a dirty olivegreen colour, fleshy and shining; in form somewhat cylindrical, narrower towards the head; tail recurved, holding fast to the plant by a fleshy foot; head small, black and shining; pectoral feet small, and six in number, and set at a considerable distance apart; sides plaited ; belly covered with fleshy protuberances. Their larva state is of short duration, continuing only about ten days : as it is only in this state they seem to feed, their numbers and activity must be great to commit much mischief, and hence, perhaps, so little is known of this insect or its habits. It then descends into the earth to undergo its changes. In its pupa state it assumes a curved position, and in the course of three weeks the perfect beetle is formed, when it ascends the plants to deposit its eggs. No very reasonable means has been as yet discovered for its destruction.

Asparagus copper-veb, or fungus. - This destructive fungus has recently been detected in Cambridgeshire, where vast quantities of asparagus are grown for the London market. It at first appears as a mould, spreading widely and rapidly amongst the plants, killing every one it attacks. It is also found to be destructive to carrots and potatoes. As yet this fungus is only known as a mycelium. It has not as yet been found in a perfect state of fructification, to enable botanists to determine its species. Saffron and lucerne crops have been long ago attacked by a similar, if not the same disease. The origin of this fungus is not known; its effects show themselves as a pale madder-coloured web, and although not visibly penetrating into the substance of the roots of the plants, yet it so completely impedes all communication of the tissues with the external air as completely to impair their vital powers. The only remedy hitherto found effective is to cut a trench round the infected plants, so as to stop the progress of its spreading, as, like most other fungi, it radiates from a centre; and it might be quicklime, if repeatedly applied, would arrest its progress, if not destroy it altogether-few of the fungi resisting its caustic effects.

General remarks. - The only certain way of improving this useful plant, is by carefully selecting seed from the finest specimens, growing them from their first stage to their last in highly enriched soil, preserving the roots from injury, and, above all, not cutting too severely. Support the seed-bearing stems during summer, and when the seed is ripe in autumn, cut over the stems and hang them up in a dry airy place till spring, when they may be rubbed or thrashed out of the berries and immediately sown. If for sale, pick off the berries and wash out the seed, allowing them to steep an hour or two in a tub of water, to soften the outer covering. When the seed is extracted, dry them thoroughly in the sun, and bag them till wanted. It is proper to remove the seed-bearing branches before any of the seeds fall to the ground, to prevent them taking possession of the beds and forming young plants amongst the old ones, which causes confusion and overcrowding. Asparagus seed will, if kept properly, retain its vegetative properties for fifteen or twenty years: it has been known to have been kept longer, but as no advantage can arise from this, seed of the preceding year's 
growth is greatly to be preferred. Under favourable circumstances, the seeds vegetate in ten or fourteen days.

Its European names are-Asperge in French; Aspergie in Dutch ; Sparagio in Italian; Spargel in German; Esparrago in Spanish.

\section{$\S$ 2.-THE ALISANDER.}

Natural history.-Alisander, or Alexanders (Smyrnium olusatrum L.), belongs to the natural order Umbelliferæ, and ranks in the class Pentandria, and order Digynia, in the Linnæan arrangement. The name of the genus is derived from Smyrna-myrrh ; being of similar qualities to that plant. It is a native of Britain, growing near the sea; and from its being often found near the sites of old buildings, it has been supposed that it was more generally cultivated formerly than at present. Smyrnium perfoliatum, a native of Spain and Italy, is thought by many superior to this sort.

Uses.-The leaf-stalks, when blanched, like celery or cardoons, are the parts used, and were, before the cultivation of the former, used for similar purposes. It is now rarely met with in British gardens, and has been introduced to those of the Continent only to a limited. extent.

Propagation.-Annually from seed, and its whole treatment is so similar to that of the cardoon as not to demand any general notice.

General remarks.-Its European names areMaceron in French; Macerone in Italian; Smyrner-kraut in German; and Cardo do coalho in Portuguese.

\section{$\S$ 3.-THE CARDOON.}

Natural history.-The cardoon, or chardoon (Cynara cardunculus L.), belongs to the natural order Compositæ, sub-order Carduaceæ, and to the class Syngenesia, and order Equalis, in the Linnæan arrangement. The generic name is derived from kyon, dog ; the spines of the involucrum resembling dogs' teeth. It is a native of Candia and the south of Europe, and was introduced into England in 1656. It does not appear to have been early or extensively cultivated, many of our older authors taking no notice of it. It is even still only in our best gardens that it is found to have a place. On the Continent it is different; and there not only are the midribs of the foliage used in culinary matters, but the corollas of several parts of the same natural order are employed in various departments of domestic economy.

Uses.-The foot-stalks as well as the midribs of the leaves are used for stewing, soups, and even salads in autumn and winter; but to prepare them for these purposes they require to be blanched, and the longer these parts are, and the more rapidly they are grown, the more are they esteemed on account of their greater crisp- ness, tenderness, and colour. The process of dressing them is thus given in "The Gardeners' Chronicle," vol. i. p. 143 :- "When a cardoon is to be cooked, the solid" not piped or hollow "stalks of the leaves are to be cut in pieces, about 6 inches long, and boiled like any other vegetable in pure water (not salt and water) till they are tender. They are then to be carefully deprived of the slime and strings which will be found to cover them; and having been thus thoroughly cleansed, are to be plunged in cold water, where they must remain until they are wanted for the table: they are then taken out and heated with white sauce, marrow, or any other of the adjuncts recommended in cookery-books. The process just described is for the purpose of rendering them white, and depriving them of a bitterness which is peculiar to them: if neglected, the cardoons will be black, not white, as well as disagreeable." They form, in the hands of a skilful cook, an excellent and wholesome dish, deserving far more general notice. One good plant will be sufficient for two or three dishes.

Sowing and planting.-Although a perennial in its native country, it is little better than an annual in Britain, and is therefore raised from seed annually, the first sowing being made about the beginning of March, on a very slight hotbed. The principal crop is sown about the middle of April. This is for an English climate; for a Scotch one they should be for both crops reared on a slight hotbed; and, to render their transplantation more certain and convenient, the seeds should be sown in 48-sized pots, one seed in each, and when 6 inches high transplanted at once to where they are to arrive at maturity. This rule is always necessary in Scotland, so as to get them ready for transplanting as soon as the danger of spring frost is over. The seed is pretty large, much after the form of that of sunflower or artichokes. In England it is expedient to sow again towards the end of June, for a late crop to come in during the following spring. Two ounces of seed will sow a seed-bed of 32 square feet. In Scotland it is best to sow always on a slight hotbed; in England, except for the first crop, they may be sown in the trenches where they are to come to perfection, making the ridges 5 feet apart, a foot deep, and the plants 14 inches apart in the line.

Method of sowing.-Trenches are dug as for celery; a moderate manuring only is necessary, and that manure should be in a decomposed state, as during the heat of summer copious supplies of water should 
be given, to prevent them running into flower, in which case the crop would be rendered useless. As we have before remarked, when water is to be used for such crops, it is no additional labour, and very little more expense, to have it enriched by a little guano. If the seeds are sown, two may be placed together, at the distance of 14 inches apart, one of the plants from which, should both vegetate, is to be removed when about 6 inches high. If transplanted from pots, or from a seedbed, set each plant at the above distance.

Subsequent cultivation. - Water during dry weather. When the plants have attained the height of 18 inches, place a stake 3 feet high to each, and tie the leaves loosely to them, the intention being to prevent their being broken by high winds. Earthing-up the stems should take place at the same time, putting a little often to them. Some do not earth up until the plants have nearly attained their full height, and earth up all at once; others do so progressively, believing that the stalks are, by this means, rendered more crisp, firm, and delicate; whereas, by the other method, they say the stalks are liable to become hard and stringy from exposure to the air; and there is, no doubt, some reason for thinking so.

Taking the crop, and subsequent preservation.-By September in England, October in Scotland, the early crop will be fit for use, and the successional crops will continue on till the end of March. The earth is carefully removed, the plant taken up by the roots, and carried to the vegetable-room. The roots are then cut off clean, as well as the points of the leaves as far down as to where they are found solid and well blanched. They are carefully washed, and the parts of the leaf-stalks left are carefully tied to the stem with a broad piece of clean fresh matting, in which state it is ready for the cook. When severe frosts are apprehended, a portion of the crop should be taken up, roots and leaves entire, and carried to the root-cellar and packed amongst sand, laying the plants down in rows and packing the sand around them, one course over another till the bin is full. In this way they keep well, and become more perfectly blanched. The remainder of the crops should also be taken up and placed on ridges, as will be found recommended for celery. The intention of taking up the plants and laying them on their sides with the points of their leaves inclining downwards, is to prevent the wet and snow getting into their hearts, which would soon rot them; for although naturally pretty hardy, from the luxuriant state in which they have been grown they are very susceptible of injury from frost, and equally so from wet.

Approved sorts and their qualities.-1. The common or plain cardoon without spines-the Cardon plein inerme of the French - is that most generally grown.

2. Cardon de Tours. - Cardon piquant; $C$. spinocissima of Prest.

3. Sparish cardoon.-Of large size, the midribs being very succulent and solid. The $C$. integrifolia of Vahl.

4. Red cardoon.-Cardon à côtés rouges of the French.

General remarks. - A plant saved over winter, and left unblanched, will afford a sufficient supply of seed for a moderate-sized garden. They ripen their seed in August; and seed well ripened and kept will retain its vegetative properties six or seven years. The cardoon of Tours is so formidable on account of its strong and numerous spines, that the workmen, in earthing it up, \&c., are said to be protected by wearing a leathern frock over their clothes. Some people, instead of earthing up the plants, envelop them by twisting hay-bands round them, and cover them up with earth only on the approach of winter.

Its European names are-Cardon in French and Italian; Kardonen in German.

\section{§ 4.-THE ARTICHOKE.}

Natural history. - The artichoke (Cynara scolymus Wild.) belongs to the natural order Compositæ, sub-order Carduaceæ, and the class Syngenesia, and order Aqualis, in the Linnæan arrangement. The origin of the generic name is given in last section. The English name, artichoke, is derived from the Celtic art, a spine, and chaulx, a cabbage. Loudon thought it more likely to come from carcioffo or kharchiof, its name in Arabic. The generic name is also supposed to be derived from Cinis, because Columella asserts the land in which it should be grown ought to be manured with ashes. Parkinson says it is so called from the ash colour of its leaves, a far more probable surmise. It is somewhat singular that this vegetable should bear the same name in English, French, German, and Dutch, with very little variation.-Vide General remarks. It was held in high estimation by the ancient Romans.-Vide Introduction. The Greeks and Romans appear to have procured it from the coast of Africa, as also from 
Sicily. It was brought to England in 1548 from Italy, as Phillips remarks, not Gerard, as erroneously stated in the "Encyclopædia of Plants ;" for although he has left us correct representations of both the French and the globe varieties, he makes no mention of their country or their introduction: we may therefore conclude that they were become common in 1596. The same excellent authority remarks, "By reason of the great moisture of our climate" compared with that of Italy, "and the attention which was paid to its cultivation, it soon became so much improved in size and flavour that the Italians sent for plants to England, deeming them to be of another kind, but they soon returned to their natural size when restored to that country." It grows wild in the open fields in Italy, and attains the height of 5 or 6 feet. On account of the great size of the roots, and their penetrating the soil so deep, they withstand the dry hot summers about Paris, where they are most extensively cultivated and most abundantly used.

Uses.-The parts used are the lower parts of the leaves or scales of the calyx, the fleshy receptacles of the flower freed from the bristles and seed-down, commonly called the choke, as they are very disagreeable when swallowed. Sometimes, particularly in France, the tender central leaf-stalk is blanched and eaten like cardoons. The bottom, which is the top of the receptacles, is fried in paste, and enters largely into fricassees and ragouts; they are sometimes pickled, and often used in a raw state as a salad. The French also cut them into thin slices, leaving one of the scales or calyx leaves attached, by which the slice is lifted and dipped in oil and vinegar before using. The English present the head whole, or cut into quarters upon a dry plate, the guest picking off the scales one by one, which have a fleshy substance at their base; these are dipped in oil and vinegar and eaten first, the bottom part afterwards with a knife and fork. What is called artichoke chard is the tender leaf-stalks blanched and cooked like cardoons. The Italians and French often eat the heads raw with vinegar, salt, oil, and pepper; but they are generally preferred when boiled, and in this state are sold in the streets of Paris as commonly as baked potatoes are in those of London. They are a favourite dish at a French breakfast, while in Britain we use them at supper. The young heads are pickled whole when about 2 inches in diameter. Medicinally, the stalks are considered aperient and diuretic; the leaves, in their natural state, boiled - in white wine whey, are thought beneficial in cases of jaundice, and, when cut into pieces and steeped in sherry wine, an excellent antibilious medicine. The roots were eaten by the ancient Romans sodden in water to enable them to drink to excess, as it excited a desire for liquor; and Athenian mothers desirous of having male children were recommended to eat of artichokes freely.

Propagation.-The plant may be propagated by seed, which in France ripens freely in September and October. It seldom comes to maturity in Britain. But this mode is seldom had recourse to ; it is, therefore, multiplied by suckers, slips, or by division of the roots. It is probably on account of our mode of propagation being on the extension principle, and the seed not ripening freely with us, that we have few or no new improved varieties of the plant.

Planting.-The suckers or slips are taken from the old roots in March or April, this depending on the season and situation; for it is better to delay a week or two, so that the young roots may be formed on the suckers before they are detached. In taking them off, a little of the earth is removed from the side of the plant, so that the position of the sucker may be seen; it is then cut from the old root with a knife, preserving as many of its young roots as possible. The slips or suckers should, however, not be taken off till they have attained the height of a foot or 15 inches, and be planted as soon after removal as possible. No curtailment of the leaves should be allowed, although a very general practice. They should be planted in rows 4 feet apart, and the plants set 2 feet distant in the line. It is usual to put them in patches of three plants each, but it is better to have one plant only. When the old roots are to be divided, the operation may take place earlier in the season, and when the young plant is only 5 or 6 inches sprung. This is, however, not so good a way as using the suckers without any portion of the old root being retained. Give water at planting, and continue it until they have taken root. The heads will be produced the first season, but later than those from established plants; which circumstance justifies the practice of making a new plantation every year, as it extends the season of the crop. Although artichokes will continue producing abundance of heads for a number of years, if in a good soil, and well attended to with manure, we think it better to plant annually, and so destroy half of the previous stock yearly, thus having strong young plants, and offering an opportunity for changing the soil.

Subsequent cultivation.-Keep the ground well stirred up between the plants, thin out all the suckers that arise in spring, 
except from three to four of the strongest and best placed. Cut the crop as it is fit for use, whether wanted or not, as allowing them to flower greatly weakens the plants, as does also allowing the stems that produced the heads to remain after the heads are cut off. No plantation should be allowed to continue on the same ground more than four or five years, and the biennial system is the best. When the crop is finished, which will sometimes occur about the end of October or beginning of November, the ground should be heavily manured, and the plants protected from frost by covering the ground around the roots with stable litter, leaves, fern, rotten tan, or finelysifted coal-ashes. The latter we prefer, as it excludes both the wet and frost better than either. Many dig out the soil from between the rows and bank it up over the roots, which is both a laborious and useless practice. Whichever of these modes is followed, it is essential that the leaves of the plants be not buried. In spring the covering is removed when all danger of frost is past, and before the plants begin to shoot.

Some recommend potting some of the small suckers in autumn, preserving them during winter in a pit, and planting them out in May. This is quite uncalled for, as abundance of small suckers may be obtained from the old plants in May naturally, if they have not been destroyed as they arise from the stools; or a few plants may be left only, moderately thinned for the purpose. Our May-planted crop, by such means, continues to bear till the middle of November. Protection is necessary, as, although it does not often happen in moderate climates that they are killed during winter, yet such accidents have occurred. It is recorded that once, during the seventeenth century, and again in 1739, almost all the artichokes in England were destroyed by the frost, and that they were again introduced from France. In 1814, in Scotland, they were much injured; and again in 1837-8, both in England and Scotland, unless where very carefully covered, they suffered very much. In all cold exposed situations covering is positively necessary, and in most others expedient.

Soil and manure.-The roots penetrate to a great depth, therefore the soil should be deep, well drained, and of a light loamy texture, highly enriched with good manure, and that deposited at the bottom of the trenches, as well as incorporated through the soil during the process of trenching. Few culinary plants, if we except the asparagus and sea-kale, like salt more than this; it should be supplied liberally, and repeated as a top-dressing twice during each growing season. Heads of very large size are produced in the Orkney islands, resulting, it is said, from the quantity of sea-weed employed in the culture. We incline to more than mere supposition, that the cause really is the saline matter carried up from the sea into the atmosphere, and in this way rendered beneficial to the plants. The larger the heads are, the more eatable matter will be formed; and not only that, but it will be more delicate and high-flavoured; therefore, to obtain large heads, strong plants must be produced, and this is only to be attained by allowing them plenty of room for their large foliage to develop itself fully, presenting the largest possible extent of their surface to the atmosphere, from which so very much of their actual food is derived. Young plants of two years' growth, and as rich a soil as can be afforded them for the roots to play their part in, are the conditions necessary to attain this end; and also, when the stalks show many heads, these should be reduced in number to two or three; and when the largest size of all is desired, only one head should be allowed to a stem, and all the small ones forming, on lateral branches, should be removed. A very dangerous recommendation has been given in "The Edinburgh Encyclopædia" -namely, to insure large lieads, "the ends of the leaves should be shortened." Than this no worse counsel could be given, inasmuch as the leaves perform so important a part in the collecting and elaborating the food of the plant. Abercrombie, the very best of our old gardening authors, but evidently no physiologist, errs much when he recommends cutting off all the large leaves previous to covering the plants in autumn. We say, let the leaves, large and small, remain and die off of their own accord ; for as long as a leaf remains green, or continues to be a living thing, it goes on supplying the plant with food until it becomes para- 
lysed by the frost, or dies off when its natural functions are completed. When we look back to the practice of the olden times, it is quite enough to make one shudder to think of the mutilations to which most plants were subjected. Celery and leeks were deprived of the half, and that half the best, of their leaves at planting : artichokes, cardoons, \&c., shared a similar fate. Ask the physiologist what is the use of leaves, and whether they should be wantonly destroyed? In deep peaty soils, when well limed and manured, artichokes have been grown to a very large size; and even the recommendation of Columella has, of late years, been revived-namely, to manure them with wood-ashes and salt.

Taking the crop, and subsequent preservation.-For pickling whole, and some other purposes, the heads should be cut when about 2 inches in diameter; for the other purposes, when they have attained nearly their full size, but before the scales of the calyx begin to open; for what is called bottoms, when they are at their largest size, and just as the scales begin to show symptoms of opening, which is an indication that the flowers are about to be formed, after which the heads are useless, unless it be for those who use the flowers to coagulate milk, which they have the property of doing; but very few, we believe, make use of them for this purpose. The heads should be cut close from the stalk, in which state they are fit for use. Three to five heads make a dish. If to be preserved for any time after cutting, they should be cut with 6 or 8 inches of the stalks attached to each head, carried to the dark root-cellar, and stuck into a bed of damp sand. They should be taken up from thence every third or fourth day, and a section about as thick as a pennypiece cut off the ends of each, and again replaced. This is to allow a free ascent of the moisture from the sand being carried up through the stalk for the nourishment of the head.

Approved sorts, and their qualities.-The English only recognise three sorts, viz., the oval, conical, or French, the large globe, and the dwarf globe. The French, however, possess other varieties; but these are not of sufficient merit to lay great claim on our attention. M. Jacques, formerly one of the royal gardeners at Neuilly, produced plants from seed a year or two ago, the heads of which weighed $2 \mathrm{lb}$., and VOL. II.

measured 2 feet in circumference, while the whole plant did not exceed 2 feet in height. We believe these varieties have not been received into this country. If this variety remains permanent, it would be a vast acquisition for certain purposes, but evidently too large for the way in which they are usually served up to our tables. The first has its heads oval and green, with the scales open; the second is somewhat larger, globular, and tinged with a purplish colour-the scales are also turned in, or inflexed, at the top; the third is in all respects smaller, but very prolific.

Insects and diseases.-The artichoke appears to have no diseases and few insect enemies; and in consequence of this, is considered, in rotation, to be an excellent crop preparatory for onions, carrots, \&c., which are so liable to their attacks.

Fig. 51.
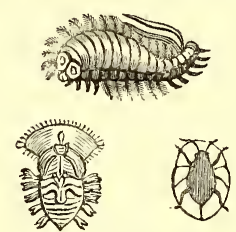

ARTICHOKE TORTOISE BEETLE.

giub, pupa, and beetle. body is black beneath, and the legs pale, with black thighs. The larva has a very flat body, with spines upon its edges; and it has the singular habit of covering itself with its own excrement, which it unites together in a mass, and carries on a kind of fork, attached to its tail. The pupa is also very flat, having thin toothed appendages at the sides of the body, with a broad thorax, prolonged forward into a rounded expansion, which covers its head." - Cottage Gardener, vol. iii. p. 317.

Generaī remarks.-The European names are, Artisjok in Dutch, Artischoke in German, Artichaut in French, Cinauco in Spanish and Portuguese, and Carciofo in Italian. The flower of the artichoke, when its florets, which form a noble purple crown, are expanded, have a fine appearance; at which time, if seed be desired, they should be partially broken over, and allowed to hang ten or twelve days with their crowns undermost, to prevent rain from entering, which, in such a mass, is liable to cause rottenness. After that period they may be cut off entirely, and hung upon a dry place, till the seed begins to drop out. It is rarely they ripen seed with us.

When artichokes are to be cultivated for chard, all the leaves are to be cut over in June to within 6 inches of the ground, and the stalks close to it-indeed, if broken over quite low down, the better. Young leaves will be produced in September and October; and when of the height of 2 feet, they should be tied together, but not very closely, and wound round with hay-bands to blanch them; and still farther to encourage etiolation, a little littering matter may be packed round them, secured from blowing away. Some, however, earth them up like 
celery or cardoons; and in wet seasons this is the better way, as the crop is less liable to suffer from damp. In six weeks the leaves and their stalks will become completely blanched: they are then fit for use, and should be gathered, and treated as has been recommended for cardoons. This is a process seldom attended to, because in its production the artichoke plants are destroyed, and therefore it should only be practised upon an old plantation, which may be intended to be destroyed at all events. It is, however, a good substitute, should any accident befall the cardoon crop, or in the event of their being neglected to be sown in time.

\section{§ 5.-THE HOP, ETC.}

As substitutes for the asparaginous plants already noticed, we may enumerate the following, which, although now not in general demand, have occasionally been used in times past, and may, under certain circumstances, be so again.

The Hop (Humulus lupulus Wild.) belongs to the natural order Urticeæ, and to the class Diœcia, and order Pentandria, in the Linnæan arrangement. The introduction of this valuable plant as an article of culture into Britain took place during the reign of Henry VIII.; it was brought from Flanders, where, and also in many parts of Europe, it has been cultivated from time immemorial. It is nevertheless indigenous to Britain : we have found it in the heart of the Grampians. The generic name is derived from humus, fresh earth, it preferring such soils. According to the "Encyclopedia of Plants," p. 835, "Lupulus is a contraction of Lupus salictarius, the name by which, according to Pliny, it was known because it grew among the willows, to which, by twining round them, and choking them up, it proved as destructive as the wolf to the flock." It seems to have been unknown to the ancient Greeks, as it is unnoticed by their authors; and Pliny is the first of the Roman authors who makes mention of this plant. A very interesting account of the introduction and early culture of the hop into England is given in Phillips' "History of Cultivated Vegetables," vol. ii. p. 233 .

As an article in the manufacture of malt liquors, it is well known. It is with its relation to culinary purposes we have at present to do. D. Rembert Dodoens is the first who has alluded to this plant as a kitchen herb. He says, "Before its tender shoots produce leaves they are eaten in salads, and are a good and wholesome meat." The young shoots, gathered in spring when about 3 or 4 inches above ground, were formerly used by country people as a substitute for asparagus, which in flavour at least they very much resemble. These sprouts are still to be found in Covent Garden Market, and in the green markets on the Continent are by no means unfrequent.

It is propagated by division of the root early in spring. It succeeds best in a deep soil, well enriched. The plants are set in what is called hills, or prepared mounds, at 7 or 8 feet apart, and three or four plants are set in each; but for garden culture, to procure the tops while young, the plants are set in lines 3 feet apart, and the plants 1 foot asunder in the row. They are very liable to the attacks of various species of aphis; hot lime, and, more recently, flowers of sulphur, are applied for their destruction.

The Milk Thistle (Carduus Marianus L., Silybum Marianus Gal.-- the Virgin Mary's milk or blessed thistle) belongs to the natural order Compositæ, sub-order Carduaceæ, and the class Syngenesia æqualis in the Linnæan arrangement. The generic name is derived from the name of a plant used by the old Greek writers, not now known; that of milk thistle, \&c., from an old dogma that the leaves of the plant, which are sprinkled with white blotches, were so marked by the milk of the Virgin Mary having accidentally fallen upon it. A native of Britain.

Uses.-It is not now cultivated, but rises up in most old gardens spontaneously, where no doubt it had formerly been grown, as the young leaves were once used in spring salads, or boiled as a substitute for spring greens. The young stalks, when peeled and soaked in water to extract the bitter taste from them, were cooked and eaten much in the way of sea-kale. The roots, when two years old, are used much in the way of salsify, which they very much resemble, and the receptacle of the flower is cooked and eaten like artichokes. There is little culture required beyond a good soil, in which it luxuriates very much.

The Cotton Thistle (Onopordum Acanthium) ranks in the same natural order as the last, and also in the same class and order in the Linnæan arrangement. The name is derived from onos, an ass, and perdo, to consume-because this plant is eaten by that animal: the specific name from its leaves, resembling those of the acanthus. It is erroneously called the Scottish thistle. It attains the height of from 6 to 10 feet, and is densely covered with a white cottony down. Indigenous to chalk pits and fertile lanes.

The receptacle was formerly used as a substitute for the artichoke, and its leaves and their foot-stalks as one for cardoons. It may be cultivated in the same way as cardoons.

The other substitutes for the plants in this chapter are-Silene inflate $\mathbf{H}$. K., the bladder campion, indigenous to Britain ; Phytolacea decandria L., the Virginian poke, a native of Virginia, the tops of whose shoots are eaten as a substitute for asparagus ; Ornithogalum pyrenaicum, or Bath asparagus-the flower-stems used for a like purpose; Tamus communis, or black bryony-also a substitute for the same; Arctium lappa, or the burdock-the young shoots also used for the same. The two last may be found abounding in moist rich soils, by the edges of woods, lanes, \&c. 


\title{
C H A P T E R.
}

\author{
S P I N A EOUS PLA N T S.
}

The principal of this class of plants are the varieties of the common spinach, and those of the sorrel. There are, however, several others of considerable importance in domestic economy, and to be found in gardens of the highest order, although rarely cultivated in those of minor note. They are less flatulent than many other vegetables, and therefore may, like the common spinach, be indulged in by those who are deterred from using brassicaceous plants, which are exceedingly so. As the chief merits of the tribe consist in the tenderness and succulency of the leaves, the richer the ground is, and the quicker the growth is promoted, the more likely are we to attain this in perfection.

\section{$\S 1 .-$ SPINACH.}

Natural history. - The spinach (Spinacea oleracea L.) belongs to the natural order Chenopodeæ, and ranks in the class Diœcia, and order Hexandria, in the Linnæan arrangement. The generic name is derived from spina, a prickle, from the prickly integument of the fruit, or seed, in some varieties. Spinach does not appear to have been known to the ancients, nor is its cultivation traced in Britain beyond 1568, when it is mentioned for the first time by Turner, in his "Herbal," who says, "Spinage, or spinech, is an herb lately found, and not long in use." Its native country is not known ; many, however, consider it a native of northern Asia. In spinach we have almost the only instance, among cultivated esculents, in which the male flowers are produced on one plant, and the female flowers on a distinct one.

Uses.-The leaves, which are the only part of the plant employed as food, are either boiled by themselves and served alone, or garnished with hard-boiled eggs: in either case it is mashed quite small. It is often used in soups. It is eaten with all sorts of meat; and is, when mashed with butter or rich gravy, much im- proved by the addition of a few sorrel leaves mashed with it. The expressed juice is often employed by cooks and confectioners for giving a green colour to some of their made dishes. When eaten freely it is mildly laxative, diuretic, and cooling. It of itself affords little nourishment. It should be boiled without the addition of water beyond what hangs to the leaves in rinsing them; and when cooked, the moisture which naturally comes from the leaves should be squeezed out before being sent to the table. The young leaves were used as a salad not only in the time of Queen Elizabeth, but even so late as the days of Charles I.

Propagation.-The varieties of spinach, being annuals, must be grown from seed.

Sowing.-The leaves are required during the whole year, therefore successional sowings become necessary. In England, a small sowing may be made in January if the weather is mild, a larger sowing in February, and a still larger one about the middle of March. In Scotland it is seldom necessary to sow sooner than the latter date, as the winter crop lasts longer with us before running to seed in spring than it does in the south. March may therefore be considered the best time for our earliest crop. Sow afterwards once in three weeks, till the beginning of May, then every week till the end of July. Three sowings should be made in August for winter and spring use, say during the first, second, and third week, each of which should cover a large space, as few winter vegetables are more useful. We always sow two crops during September, as at that period there is ground to spare; and if the crops be not required for other use, they will constitute an excellent green vegetable manure, if dug into the ground in spring. It will be found useful for pigs. The Londoll market-gardeners sow 
from the 12th of August to the 1st of September, for winter and spring use.

The seed vegetates in from 10 to 15 days, according to the season; therefore it may be advantageously sown between rows of newly planted pease, beans, cabbage, or the like, as it will be fit to cut off for use before they either injure it, or it prove an impediment to their growth. And when so sown, the drills should be made nine inches wide and the seed thinly sprinkled in them, to give the plants greater room than if sown in the ordinary manner. The germination of the seed may be hastened by its being steeped in water three or four hours previous to sowing; and in sowing during the heat of summer, when the ground is dry, the drills should be soaked with water before the seed is sown.

It should always be sown in drills, 2 inches deep and 18 inches or 2 feet apart for principal winter crops, the plants being, after they come up, thinned to a foot apart in the line. As the object is to have large succulent leaves, the ground cannot be too highly manured. The fullsized leaves should be 8 inches long and 4 broad. For summer crops it may advantageously be sown between rows of pease or similar crops, the slight shade afforded them preventing their running so soon to seed; but during summer the leaves will not attain the size stated above. If sown broadcast, 2 ounces of seed will sow a bed of 120 square feet. If sown in continuous rows, a foot between the summer crops will be sufficient. One ounce will sow 150 feet of a single drill.

Subsequent cultivation. - The summer crops should be abundantly supplied with water during dry weather, the plants only moderately thinned, as their duration is short. In November it will be well to thin the plants intended for a winter and spring crop to the above distance in the lines, to clear the ground completely of weeds, and to cover the spaces between the rows with finely-sifted coal-ashes, to counteract damp and to render the ground more comfortable to tread upon during the process of gathering the crop. This also saves the large lower leaves from being splashed with mud during heavy rains.

It is seldom the spinach crop is injured by frosts in Britain, particularly in mo- derately good situations. There are many places, however, in upland and cold districts, where it suffers much. In such the soil should be less enriched, as the less succulent the leaves are the better they will resist the frost. A more sheltered and warmer place should also be chosen for the winter crop. In some parts protection is necessary, and this, if even of a very temporary nature, will be found sufficient. Sticking the plantation pretty thickly with branches of broom, old pea-sticks, or indeed branches of any kind, so as to break the force of the wind and arrest perpendicular frosts, will, in most situations, be found sufficient; and in such 'situations, covering the ground between the rows with coalashes should be carefully attended to. Still such coverings should not be had recourse to unless in extreme cases, neither should they be applied too early or continued too late in the season. The ground should also be kept clear of weeds, and in gathering the crop the leaves should be pinched or cut off by the foot-stalk, and not plucked or lacerated as is so frequently done, as every leaf so torn and mutilated will almost certainly rot off.

Soil and manure.-In light sandy soils spring crops come into use soonest, but they equally soon shoot up to seed and become useless. In strong retentive soils they are later, and continue longer. In medium good garden soils, abundantly manured, the crops succeed best; and it is of importance, particularly for crops to come in during winter and to continue on till spring, that the situation chosen be open and well exposed. The ground cannot be too highly enriched for this crop, and much benefit has arisen from watering the plants with ammoniacal liquor from the gas-works. Pigeons' dung has been used with much advantage, and so also has guano in a liquid form.

Taking the crop.--The larger and lower leaves should be gathered first, and these should either be cut off with a knife or pinched off between the finger and thumb close to the bottom of their foot-stalks. It is wasteful and untidy to pluck the leaves off by the middle, and it is equally so to take the heart ones out. The summer crops, as they grow so rapidly, may be cut close to the ground with a knife. When the former is carried to the vege- 
table-house the foot-stalks of the leaves should be cut off and the leaves sorted, removing all the flower-stalks should any exist, and rinsing the whole in clean water, placing them afterwards in a clean basket to allow the water to drain from them.

Approved sorts and their qualities.-Notwithstanding the length of time spinach has been a very generally cultivated plant, but few varieties have appeared. Indeed, for long only two, the prickly and the round-seeded, were known, and it is only lately that a third has been added. Like the lettuce and endive, this vegetable is divided into two natural divisions -namely, the prickly and the round-seeded sorts.

Prickly spinach.-The leaves are much smaller, more round, and less succulent than any of the round-seeded varieties. It stands the winter rather better than they do, and is less liable to run to seed. Readily distinguished by the seeds being prickly, known as winter spinach, being chiefly sown in autumn to stand over winter. Bordeaux, the Epinard commun, of the French.

Lettuce-leaved spinach.-Of this variety $\mathrm{Mr}$ Thompson says: "This is an excellent new variety; the leaves are somewhat rounder than those of the Flanders spinach, of thick substance, and dark green colour. It is, perhaps, not quite so hardy as the Flanders, but it usually stands the winter well, and, from its superior quality, it deserves to occupy at least half the ground allotted to the winter crop of spinach. A variety called Epinard Gaudry, if not identical, is very similar to it." It is the Epinard d'Esquermes, ou à feuille de laitue, of the French. Seeds round.

Flanders spinach.-This is an improved variety of the next, with much larger and more succulent leaves often 8 inches in breadth. It is the Epinard de Flandre, Epinard de Flandre à très larges feuilles, of the French. It is also the principal sort grown in the Netherlands. Seeds round.

Round spinach.-This and the prickly-seeded were long the only two sorts grown. Leaves large, roundish, and thick. It is known also as summer spinach, thick-leaved round spinach, spring spinach, round spinach. It is the Epinard d'Hollande ou Epinard rond, of the French. Seeds round.

General remarks.-The European names are Epinard in French; Espinaca in Spanish; Spinagie in Dutch; Spinaci in Italian; Spinat in German. In Arabia it is called Hispane. From this latter name, and as it was formerly often called Olus hispanicum, it has been thought to be a native of Spain. Spinach contains a considerable amount of nitre-so much so that the water which is drained from it, after being boiled, makes as good match-paper as that made by a solution of nitre. In saving seed, as soon as the flowering is past, the male plants, which will have then performed their office, should be all removed to admit air and light to the female plants to enable them to perfect their seeds. The seed ripens in August and September, and, after being dried for a week or so in the straw, should be thrashed out. Birds are extremely fond of spinach seed, so much so as to render it expedient to cover the seed crop, if upon a limited scale, with netting, from the time the plants come into flower until the seed is ripened. Upon a large scale, boys should be stationed to scare them away. 'The seed retains its vegetative properties about four years.

\section{$\S$ 2.-WILD SPINACH.}

Natural history.-Wild or perennial spinach, (Chenopodium bonus Henricus L.) or, as it is called by country people, good king Henry, tota bona, and fat hen, names seemingly prevalent over a great part of Europe-the French peasantry, who use it much, calling it bon Henri, the Germans, guter Heinrich-belongs to the natural order Chenopodeæ, and to the class Pentandria and order Digynia in the Linnæan arrangement. It is indigenous to many parts of Britain, particularly in loamy soils in waste places. It was formerly grown in gardens for its leaves, which make an excellent substitute for spinach, and is so still in several parts of Lincolnshire. It would make an excellent spinaceous plant for cottagers, and is easily cultivated. The generic name is derived from Chen a goose, and Pous a foot, the leaves resembling the foot of that animal.

Uses.-The same as spinach.

Propagation.-Being a hardy perennial, it is increased by dividing the plant into pieces, each having a portion of the root, and a small bit of the crown which is thickly set with buds, which spring freely on being replanted. It may also be propagated by seed sown in March, and transplanted, when the plants are fit to handle, into a nursery-bed. In September following, they should be transplanted into lines a foot apart, and 10 inches in the line, in ground of a loamy nature, and trenched to the depth of 18 inches, as their roots penetrate to a considerable depth. The following spring the leaves are fit to gather for use, and should be picked as they advance, taking the largest first. In this way a bed will continue productive for several years. Most of the species of this genus, both indigenous and exotic, may be safely used as articles of food. It comes into use about the beginning of May, and continues all the summer. Its European names are, Ganzevoet in Dutch, Anserine in French, Meldenatrige in German, Anserino in Italian. 


\section{§ 3.-FRENCH OR MOUNTAIN SPINACH.}

Natural history.-French spinach or Orache (Atriplex hortensis) belongs to the natural order Chenopoder, and to the class Polygamia, and order Monœcia in the Linnæan arrangement. It is a native of Tartary, and was introduced into English gardens in 1548, and long used, as it still is in many countries abroad. It is now seldom seen in our gardens or markets; yet there are some people who prefer it to spinach, and in France it is greatly esteemed.

Use.-During the early part of summer, the stems are used; but after that the leaves, which are gathered in succession, and continue producing throughout the season. It is used as a substitute for spinach, having a pleasant slightly acid taste.

Propagation.-The seed is sown in drills in February or March, 2 feet apart, the plants being afterwards thinned out to 2 feet in the lines. Sow again for a succession for autumn use in June, choosing a rich, deep, and rather moist soil. There are several varieties, two of which only, the green and purple leaved, are usually cultivated. A plant or two left to produce seed will be sufficient for a large garden. The seed ripens in August.

The European names are, Arroche in French, Melde in Dutch, Armuelles in Spanish, Meldekraut in German, Atrepice in Italian.

\section{§ 4.--NEW ZEALAND SPINACH.}

Natural history. - New Zealand spinach (Tetragonia expansa, Hort. Kew) belongs to the natural order Ficoideæ, and to the class Icosandria Di-Pentagynia in the Linnæan arrangement. The name is derived from Tetra, four, gonia, an angle-from the angular form of the fruit or seed-vessel. It is a native of New Zealand, from whence it was introduced by Sir Joseph Banks in 1772. It does not appear to be at all used by the natives. It. was, for some years after its introduction, cultivated only as a greenhouse plant; but afterwards, through the recommendation of Sir J. Banks and the Horticultural Society, it began to be grown in private gardens as a substitute for spinach, and may be considered about as hardy as the kidneybean. It yields a large produce, but by no means equal to spinach; yet during dry warm weather in summer, it will be found in warm localities a useful substitute, as one sowing will keep up a supply, while several of the common spinach have to be made. Indeed, the warmer the weather, the better it succeeds.

Use, and its mode of cooking, are the same as the common spinach.

Propagation.-Being an annual, it is grown from seed annually.
Sowing.-Sow the seed thinly in a pot or pan early in March, which place in a moderate hot-bed till the plants are fit for pricking out; or, to save the latter process, sow in 60 -sized pots, three seeds in each, and thin out to one, which, when of sufficient size, and the weather sufficiently warm, plant out on a warm border, or, better, on a bed of soil placed over a slight hot-bed, where they may remain. About the 20th of May in England, or beginning of June in Scotland, will be a good time to plant out without shelter; but if on a mild hot-bed, they may be set out sooner, and protected during night by having the bed hooped over, and covered with tarpauling or Russian mats. Set the plants about 3 feet apart. In five or six weeks after planting, the leaves will be of sufficient size to admit of gathering. Protection will be required in autumn, should it be wished to continue the crop.

Gathering the crop.-Pick the leaves off singly, taking care not to injure the points of the leading shoots; they branch out and continue extending till killed by the frost. Half an ounce of seed will be sufficient for the largest garden, and from six to twelve plants enough for an ordinary-sized one. The seed is readily produced, and will retain its vegetative powers for two or three years, if retained in the capsule. It is usually known by its botanic name, and is not more subject to insects or diseases than other plants of the same order.

\section{§ 5.--GARDEN PATIENCE, ETC.}

Natural history.-Garden patience, or herb patience (Rumex Patientia L.), belongs to the natural order Polygonex, and to the class Hexandria, and order Trigynia in the Linnæan arrangement. The generic name is derived from Rumex, a spear-from the shape of its leaves; others say from Rumex, a name given by the Latins to a root of thorn; the specific name Patientia, from its slow operation in medicine. It is a native of Italy, and was introduced to England in 1573.

There are several species of the genus Rumex that have been or are still cultivated; and as their uses and culture are so nearly alike, we shall include them under one head.

The French round-leaved or Roman sorrel ( $R u$ mex scutatus L.), a native of France and Italy. Introduced to Britain in 1596, and Bloody-veined dock (Rumex sanguineus), a native of England, were both formerly cultivated in our gardens 
as substitutes for spinach. The former still is, and on the Continent it is grown very extensively.

The common sorrel (Rumex acetosa) has been cultivated from time immemorial as a spinach plant, as well as used in salads. In Ireland the leaves are eaten with fish and other alkalescent food.

The mild-leaved sorrel (Rumex montanus H.K., the Oxyria reniformis Smith). "The plant is one of those singular individuals which has the character of two distinct genera, and yet is referable to neither. Wahlenberg made it a Rheum, Linnæus a Rumex, and Mr R. Brown what it now is."-Ency. of Plants, p. 295. It is much used in France as a salad, and the male plant of this species is recommended in the Bon Jardinier to be planted as edgings. There are several varieties of the common sorrel; that called by the French l'oseille de Belleville is the most esteemed, on account of the largeness and succulent state of its foliage. We cultivate it in preference to all the other sorts; and in rich moist soil the leaves attain a large size and thickness. The sorrels are all of great importance in French cookery, and are both agreeable to eat and exceedingly wholesome; and could vulgar prejudice be got over, many an excellent dish might be gathered almost the whole year over from our roadsides. Phillips, in " Hist. of Cult. Vegetables," vol. ii. p. 214, very properly remarks: "Rarity being oftener coveted than excellence, it is not surprising that we should find this native vegetable discarded in an age in which novelty principally stimulates art to furnish our kitchens. The caprice of fashion extends even to our vegetable food; and zeal, which should emulate us to improve the virtues of our own plants, is often wasted in obtaining those of distant countries whose qualities are uncertain. Sorrel is scarcely known as a pot-herb in this country, except at fashionable tables, the small demand having now nearly banished it from the metropolitan markets."

The use of the sorrel is of great antiquity; Pliny observes that it renders meat more pleasant, and lighter of digestion. It is a valuable anti-scorbutic, and is used as an ingredient in salads, and when boiled, as a sauce for roast meat, particularly veal and pork-itgreatly assists their digestion. It is an excellent substitute for apple sauce for winter geese, and should, like spinach, be boiled without water. It makes a good substitute for spinach when beat up with butter, and is greatly improved by the addition of the yolk of eggs and cream. It enters into most of the soups and sauces for which French cookery is so famed, and they preserve it in quantities for winter use. It forms as prominent an article in the vegetable markets in Paris as green pease do in those of London. It was held in high repute in Britain about the time of Henry VIII. and Elizabeth. Its use has, however, greatly declined since that time; and yet, amongst all our modern additions, we have not one so wholesome, and of such easy cultivation, or one that would add so much to the sanitary condition of our peasantry, particularly those who live much upon salt provisions.
Propagation.-All the kinds may be propagated by seed, which should be sown in rows 18 inches apart, and the plants thinned to 12 inches distance in the line. Sow in March, in deeply-trenched soil, moderately enriched by manure, and rather inclining to damp. In autumn, the old plants may be divided and planted in rows at the above distance. Even fragments of the roots will, if planted, make good strong plants during the following spring and summer. As flowerstalks appear, cut them off. Keep the ground clear of weeds, and annually fork in some rich manure between the plants; or, still better, apply liquid manure without disturbing the roots.

General remarks.-The European names of the common sorrel are-Oseille in French; Acetosa in Italian ; Sauerampfer in German ; Acedera in Spanish; and Veldzuuring in Dutch. Those of the garden patience are-Rhubarbe des monies in French; Romice in Italian; Englische or winter spinat in German.

\section{$\S$ 6.-THE WHITE OR SICILIAN BEET.}

Natural history. - The white or Sicilian beet (Beta cicla $\mathrm{L}$.) belongs to the natural order Chenopodex, and to the class Pentandria, and order Digynia, in the Linnæan arrangement. The generic name is derived from Bett, red, in Celtic; the specific name, according to De Theis, is a corruption of sicula, under which name it is spoken of by Catullus. According to Phillips, beet is a name arising from the shape of its seedvessel, "which, when it swells with seed, has the form of the letter so called in the Greek alphabet." The white beet was introduced to England from Portugal in 1570, where, and also in Spain, it is an inhabitant of the sea-coasts.

Uses.-This species of beet-for it is considered botanically as a distinct species from beta vulgaris, the common, or red beet-is cultivated entirely for its leaves, whereas the red beet is grown for its roots. These leaves are boiled like spinach, and put into soups; and the midribs and stalks, which are separated from the lamina of the leaf, are stewed and eaten like asparagus, under the name of chard. As a spinaceous plant, the white beet might be grown to great advantage by cottagers and farmers, as it affords leaves fit for use during the whole summer;-but we require as great a diffusion of knowledge in the art of cooking amongst those classes as we do as regards the production of the article to be cooked. The great white or Swiss chard is a large variety of this species; the foot-stalks and ribs of its leaves are dressed like asparagus, and thought equal to that popular vegetable. There are other varieties, differing mainly in the colour of their midribs and stalks, each of which is, however, adapted to 
the same purposes. One ounce of seed is sufficient to sow a bed, for, transplanting, of 50 square feet.

Propagation.-It is best to sow the seed annually, first, in the beginning of March, and again in September-the latter to supply leaves late in autumn and early in the following spring.

Sowing and planting.-Always sow in drills, a foot apart for the smaller sorts, and from 15 to 18 inches for the larger. Should failures take place in the crop, the deficiences may be made good by thinning out where the plants have come up too thick, and transplanting them into the vacancies. The beet transplants well, and therefore, should ground be scarce at sowing time, the seed may be sown in beds, and the plants transplanted afterwards when ground is clear, taking care that in planting the roots be not doubled up.

Subsequent cultivation.-Thin the plants when about 6 inches in height to the distance of from 9 inches to a foot apart, according as the sort is large or small.

"In cultivating the Swiss chard, the plants are frequently watered during summer, to promote the succulency of the stalks; and in winter they are protected with litter, and sometimes earthed up, partly for this purpose, and partly to blanch the stalks. Fresh chards are thus obtained from August till May. When the garden sorts of white beet are transplanted, the proper time is during moist weather in May or June. The distance from plant to plant may be from 10 to 14 inches-much of the advantage of transplanting depending on the room thus afforded the plants, together with the general disposition of transplanted annuals, with fusiform roots, as the turnip, carrot, \&c. to throw out leaves and lateral radicles."-Encyc. of Gard., 841.

Soil and manure.-The soil requires to be deeply trenched, and of a fair staple quality; and as the quality of the crop depends on the size and fleshiness of the midribs when chard is intended, and on the breadth and succulency of the foliage when it is grown as a substitute for spinach, it follows that the soil should be rich, or rendered so by copious manuring.

Taking the crop.-The largest and fullest grown leaves should be gathered first; others will follow. If grown for spinach, the leaves should be rinsed in clean water, placed in a basket to drain dry, and so sent to the kitchen. If for chard, the foot-stalks should be carefully preserved, and their leaves tied up in bundles of 6 or 8 in each. Twelve stalks, with the leaves entire, will be sufficient for a dish.

Sorts and their qualities.-The common white has small roots ; the ribs, foot-stalks, and leaves of a delicate greenish-white colour.

The large white, or Swiss chard, has its leaves much larger and thicker; the foot-stalks and ribs are much larger and much whiter. In those parts of the Continent where this plant is much grown for chard, there are several sub-varieties in cultivation. The green beet, and yellowrooted varieties of Beta vulgaris, are also often grown for a similar purpose. The large Swiss chard is, however, the best. Another variety grown is Beta maritima (sea-beet), a plant indigenous to our sea-coast. In seed catalogues we find the following names-Golden-veined, small rooted, green-leaved, red-veined, silver or white veined, commonly called silver beet. Brazilian beet (Beta Braziliensis) has very large green leaves, and makes an excellent substitute for spinach, but is rather more tender than the green sort.

Insects and diseases.-The beets are not very subject to either the one or the other. The Haltica nemorum (the turnip flea-beetle), however, often attacks them, for which see section TURNIP.

General remarks. - The European names areBette or poirée in French ; Biet in Dutch ; Acelga in Spanish ; Mangold - kraut in German; Biettola in Italian. To save seed, a few of the best formed roots should be selected and kept out of the frost during winter, and planted in a warm well-exposed spot in spring. When the flower-stalks have attained the height of 2 or 3 feet, support them with stakes. The seeds will ripen in September. When dry, thrash them out; and keep them, when thoroughly dried, in paper bags till wanted. The roots of the varieties of white beet are not adapted for the table. 


\title{
C HAPTER VI.
}

\author{
ACETARIACEOUS OR SALAD PLANTS.
}

THE use of salads is of the greatest antiquity, and, in a sanitary point of view, of great importance, particularly in warm countries. In 110 country, however, are salad plants more cultivated or used than in France, where they form a very considerable item in the food of the people. The rich eat them as a luxury, the poor from necessity. In Britain, if we except the lettuce, endive, and celery, all of which find a ready sale in our markets-the former during the heat of summer, and the latter during winter-salads, in their greatest variety, are confined mainly to the gardens of the opulent. The artisans in our large manufacturing towns are yearly becoming greater consumers of lettuce, radishes, and celery, in particular, and find benefit from their use. Manchester has long been famed for its celery, and it has been grown to a larger size there than elsewhere. The varieties grown under the name of Manchester celery have long held a place in our seed-lists. The use of salads is only beginning to be appreciated amongst the middle classes in Scotland, and the natural prejudice against " eating green meat" is daily subsidinga prejudice, by the way, not altogether unfounded, from the coldness of our climate and habits of the people. Salads can only be grown in perfection where vegetation is of rapid growth ; and hence, even in England, we do not meet with the same excellent salads as are to be had at every table d'hôte in Holland, Belgium, and France.

\section{§ 1.-CELERY.}

Natural history.-Celery or smallage (Apium graviolens L.) belongs to the natural order UmvOL. II. belliferæ, and to the class Pentandria, and order Digynia, in the Linnæan arrangement. The generic name is derived from Apon, water (Celtic), from its growing in ditches and in watery places. Cultivation has transformed it from a coarse, rank, and even more than suspicious plant, to one of the most agreeable and wholesome of all our cultivated esculents. It is a native of several parts of Britain, especially near the sea, and known in its wild state as smallage, but never used by man, and not much relished by other animals. It appears to have been first cultivated in Italy, for in our oldest seed-lists it is called " Upright Italian Celery," and of this there was a red and white variety. Ray, a writer of older date than any seed-lists, observes " that, if neglected, it degenerates into its first unpalatable state." Phillips says, "Ache is the true English name for this vegetable."

Use.-The whole plant is used either in a green or blanched state, as well as its seeds. In the former, and also in the latter form, it is used to flavour soups. The seeds, at that season when the plant is scarce, are bruised, and put into a small bag to prevent their mixing with the soup; and old seed, that has lost its vegetating properties, is employed for the purpose. In its blanched form it is eaten raw, as a salad; generally served whole, sometimes cut into small pieces, but usually, particularly if large, cut into four quarters longitudinally, for greater convenience in serving it. It is also stewed in white sauce, and is sometimes made into an agreeable conserve. In Italy and the Levant it is seldom blanched, the green leaves and stalks being used either stewed by themselves or as an ingredient in soups. Nedically, it is considered diuretic, and a decoction of it has been found good in cases of gravel.

Propagation.-Although a hardy biennial in its wild state, it must be considered as little other than an annual in cultivation, unless when grown for its seeds, in which case it is treated as a biennial. It is invariably propagated from seed.

Sowing and Planting.-For the earliest crop the seed should be sown by the beginning or middle of February, in pans, 
placed on a moderate hot-bed. The seed will often be three weeks before it germinates. When the young plants of this sowing are about 2 inches high, they should be transplanted singly into small 60 -sized pots (in decomposed manure only), and plunged in a very slight heat, say $45^{\circ}$ to $50^{\circ}$. By the beginning of April they will require to be shifted into large 48-sized pots, and kept growing slowly till the end of that month or beginning of May, and then planted out in shallow trenches in the warmest part of the garden. Indeed, for those who wish the crop very early, it is well to dig the trenches 2 feet deep, and fill them to within 6 inches of the top with warm dung to stimulate the plants, and cover with handglasses, or, better, with longitudinal triangular frames, a foot in breadth at the base, which is open, and a foot in height, having one of the sides glazed. Others grow the plants in pits, shifting them from time to time into larger pots, and plant them out in trenches about the beginning of June, when from a foot to 18 inches in height, earthing them up at the same time, to prevent their leaves being broken. It is only in some families that this routine is necessary. The ordinary earliest sowing is made about the end of February, upon a slight hotbed, either covered with a regular frame and glass lights, or with hand-glasses. Plants from this sowing are once or twice transplanted on a slight heat, in very rotten dung, placed over the heating material about 4 or 5 inches thick, made into a state resembling thick mortar, and beaten firmly down, the plants being at their last removal set 4 or 5 inches apart. These will be fit for final transplanting into shallow trenches by the beginning of June, and will be fit for use in August. A second sowing should be made about the end of March, and if treated as above will be fit for transplanting into trenches by July, and come in for a general autumn crop. And a third sowing should be made about the middle of April, in the open border, or on an exhausted dung-bed, which, when twice transplanted, will be fit to plant out for good about the 10th of August; and as this may be considered the principal winter and spring crop, a much larger sowing should be made. We generally sow a fourth crop in May, to obtain plants for planting towards the end of September for our latest spring supply. Transplanting is of much importance for securing celery from shooting up to seed, and preventing it growing hollow in the stalks, or pipy, as it is technically termed. The check which the tap root sustains at each removal has a tendency to cause the production of lateral roots, and, as a consequence, the production of greater vigour and rapidity of growth in the plant, and hence a less disposition to run to flower. The object of transplanting on solid masses of rotten dung is to invigorate the plants, and render their future removal safe, as by cutting the dung into squares and lifting each piece entire, the roots are thereby secured from injury, and the plants from any sudden check to their growth. As to the quantity of seed, an ounce will be sufficient for most gardens; but as it is found to be expedient to grow several sorts, the quantities of each must be regulated by circumstances. The London market-gardeners sow for their first crop early in February, again in March, and last of all in April, for their latest crop ; the first only of these is sown on slight heat, and hooped over with mats.

Celery-seed should always be sown thin, to secure stocky plants, which never can be the case if they are crowded in their earliest stages. Their first transplanting is effected by using a small dibber or pricker, about half an inch in diameter. Their second removal should be with a dibber at least $1 \frac{1}{2}$ inch in diameter, and cut square across at the point, because at that time the roots will have ramified considerably, and if carefully removed will have small balls of earth or dung attached to them. Their final transplanting should be performed either with the transplanting trowels, figs. 27,28 , or the media they are growing in should be cut into square pieces with a sharp instrument, and, beginning at one end, a flat trowel or small spade should be introduced under the mass in such a way as to keep the ball perfectly entire about the roots. Water must be abundantly supplied during their whole preparatory growth.

Celery is grown in trenches, for the obvious purpose of enabling the cultivator to earth up the plants more conveniently, and hence insuring its more complete blanching. These trenches are 
either narrow, intended for a single row of plants, or 4 or 5 feet in breadth, for the reception of several rows of plants. The first is no doubt the best for crops to stand over the winter, where protection is not to be given, and where there is ground to spare. It is the plan adopted to secure very large specimens, and hence the Manchester growers practise it to attain the latter object; and the London market-gardeners grow it in the same way, because, as with asparagus, the larger it is the better it takes in the market. In private gardens, where large size is less a consideration than crisp, well-blanched, and high-flavoured stems, the broad trench, or Scotch mode, is greatly to be preferred, as well on account of its yielding a much greater number of moderately-sized heads upon the same extent of surface.

The London market-gardeners' mode of planting "is to dig out a trench two spades deep, banking the mould up on either side; then to fill in a foot of the strongest manure, such as cow-dung, and to cover this with 3 or 4 inches of mould for planting in. If the ground is very rich, half the quantity of manure is applied. The rows are generally from 4 to 6 feet apart. The plants are then taken from the seed-bed, and planted root and head entire-not trimmed in, a plan which ought to be discontinued in everything." To this we heartily say, Amen. "The plants are placed about 8 inches apart, the intermediate ground being planted with coleworts, lettuce, or any other light crops, which are likely to be off before the celery wants moulding up; but market-gardeners do not begin blanching until the plants are about 18 inches high, as it prevents rain and air acting on the roots."-CutHILL, Market-gardening around London, p. 23.

The Edinburgh market-gardeners' mode of planting.-Trenches 6 feet wide and 1 foot deep are dug out; a copious dunging is laid on the bottom, sometimes dug in, at other times spread over the bottom of the trench, trodden or beaten down pretty firmly, and 3 inches of soil laid over, into which the plants are set in rows across the bed, 14 inches asunder, and the plants 9 inches apart in the row. By this means space is economised, and the plants attain a fair average size and quality. The same plan is very often followed in private gardens; and where the new and improved sorts, such as Cole's, are grown, they arrive at the size most available for family use. This is decidedly the best way for amateurs to grow this crop. They should grow their plants in nursery-beds until they are 10 inches or a foot in height before final planting, giving plenty of water, and afterwards earthing up once a fortnight.

The Manchester growers dig their trenches 18 inches wide and a foot in depth, and from 4 to 5 feet distant from each other, and lay in 9 inches in thickness of compost composed of fresh strong soil and well-rotted hot-bed dung, in the proportion of three-fourths of the latter to onefourth of the former. The plants are taken from the nursery-beds with all their roots entire, and as much ball as will adhere to them. The side shoots or suckers are removed from the stems, and they are set in with a planting-trowel, 10 inches apart, the row being in the centre of the trench. They are watered liberally until fit for earthing up, but not afterwards.

Many plant on the surface-that is, marking out the size of the bed on ground that has been previously trenched, digging in at least 6 or 8 inches of rich halfdecayed manure, and planting either in single lines 4 feet apart, or making beds 6 feet broad, and planting across them, setting the rows 14 inches distant, and the plants in either way 8 inches apart in the lines. They may be earthed up as they advance, or not until they have attained the height of a foot. A mode of planting celery, which may be called the simultaneous manner, is practised in some parts, and with evident advantage where ground is scarce. The manner is as follows: Early potatoes are planted in spring (or we would recommend autumn), at about $2 \frac{1}{2}$ feet row from row; when they are earthed up, a hollow trench is thus of necessity formed, into which rotten dung is placed, and on that, covered to the depth of 2 inches with soil, celery plants are set. As the potatoes are taken up, a little earth is put to the celery, which goes on growing, so that when the potato crop is cleared, the celery one is well advanced, and treated in the usual manner. If the supposition be correct, that tubers increase in size after the haulm has been 
cut, this might be a profitable method of growing celery, by cutting off the haulm of the potatoes when it began to interfere with the celery plants, and thus giving them all the advantages of light and air, the potatoes to be taken up afterwards when ripe, to admit of the celery being earthed up.

It has been suggested to grow celery in water-tight trenches, with a view of supplying them with abundance of moisture at their roots, for there is no doubt that celery cannot be grown rapidly or of large size if kept very dry at its extremities; on the other hand, there is the danger to be apprehended of restoring to the plant its deleterious, nay, poisonous properties, which it, along with so many more of its near allies in the Umbelliferæ, is so well known to possess. It were better to adopt a system of subterranean irrigation with liquid manure, to be used only in very dry weather, and only when the plants are in their height of growth, and dispensing with it afterwards. We have adopted the following plan with advantage to the bulk of crop, and without any evident sign of lessening the wholesomeness of the plants : Along the centre of each single trench, imbedded in the manure, and over which the plants were set, we placed a course of drain-tiles laid on soles, having at the upper end (for the ground was considerably on the incline) an upright drain-pipe communicating with the tile-drain below. Into this liquid manure was poured, and allowed to escape through the openings between the tiles, thus moistening the dung and soil in which the roots were growing without at all wetting the soil around the stalks, which should be at all times kept as dry as possible.

Subsequent cultivation. - This consists chiefly in carefully removing any side shoots that may arise from the collar of the plants before earthing up, which is of considerable advantage in securing wellgrown heads. As to the time of earthing up, opinions of late years seem to differ widely; some advocate the old method of earthing up progressively, putting a little only at a time, taking care to keep the hearts of the plants clear of soil; others say that no earthing up should take place until the plants have nearly attained their full size, when the earth should be put to all at once; while others earth up when the plants are about 18 inches high for the first time, adding a little after. wards so long as the growing season continues.

Some, instead of using the natural soil of the garden, pack the plants around with fine sand, and others use finely-sifted coal-ashes for a like purpose. Mr Roberts, a year or two ago, recommended what he called his "celery-blanching tiles," which in principle were nothing other, except in price, than common drain-tiles, one of which he placed on each plant, with a view to blanch it, and at the same time to keep it from coming into contact with the damp soil. The idea struck us as sufficiently feasible, believing that etiolation would be effected, and that the protection afforded by the tiles would tend to preserve the plants during winter. We contented ourselves, however, with employing two common horse-shoe draintiles, placing one on each side of a plant, and thus forming as good a blanchingtile as the other. These were put on when the plants were about 18 inches high, and kept in their places by working earth in amongst them to keep them steady. Contrary, however, to expectation, we found the beds so treated the first to suffer from rot, and have since discontinued their use for this purpose, although we use them for another, which will be noticed below.

The grand secret, if we may make use of a word which is too often made use of as a cloak to imposture, is to earth up only when the plants and the soil are as dry as possible. To do so when either is damp, is entailing destruction on the crop.

The London market-garden practice is thus described by Mr Cuthill: "The operation of earthing up is all performed by the spade; no hand earthing is employed. Parallel lines are stretched on each side of the row 18 inches from the plants, and the mould is cut out of the alleys to form the blanching ridge. Late earthing up is effected in about three different times."

In earthing up the broad or Scotch form of beds, the plants being disencumbered of all side shoots or suckers, two pieces of board about 9 inches in width are placed one on one side of a row, and the 
other on the other side of the adjoining row, or, as "the knights of the whip" would say, one on the near side and the other on the off side of the space between two rows. These boards are placed inclining rather outwards at top; they are kept in their place by a triangular piece of board placed between them like a wedge at each end of the boards, while a man on each side of the bed breaks up the soil finely, which has been left between the beds on purpose, and throws it in between the boards as high as the present earthing up is intended to be carried, which, upon an average, is 6 inches. The boards are then lifted gently upwards, and the pulverised soil falls in between the plants. They are removed to the next space, and so on. This is an expeditious mode, and with very ordinary care none of the soil falls into the hearts of the plants. Others take the leaves of a plant in one hand, holding them together, while with the other they press the soil around their stems; but this is a timekilling process. Whichever process is followed, the soil should be kept open and loose around the plants, to prevent unnecessary pressure as well as exclusion of air.

The following mode of earthing up is detailed by $\mathrm{Mr}$ Duncan of Basing Park, in the "Journal of the Horticultural Society," vol. vi. p. 214 :- "The process is effected by three different operations. The first takes place when the plants have grown 9 or 10 inches in height. The small leaves immediately above the roots, and all embryo suckers, are carefully removed. After that the bed is completely saturated with liquid manure;" but subsequently to this period Mr Duncan "does not consider it requisite that any artificial watering should take place. The beds are then covered with about 4 inches of mould from the ridges, which helps to keep the plants in an erect position, and acts like a mulching on the roots, thereby preventing, in some measure, the evaporation of moisture from the bed. Some three weeks before the early crop is required for use, the second earthing takes place, which is performed in the following manner by two operators: Two boards, some 8 or 9 inches in depth, and equal in length to the width of the bed" (for Mr D. adopts the broad trench,or Scotch form), "are placed edgeways between the rows, each board resting against the plants in either row, so as to form at once space for the reception of the mould, and a protection to the leaves whilst the operation of earthing is being performed. When the required quantity of soil has been deposited, the boards are carefully withdrawn, and placed betwixt the next two rows; and so the work proceeds, until all has been completed. When the soil is of a very wet, tenacious character, dry ashes, fine mould, or other material, can readily be introduced next the plants, for which purpose double boards, properly adjusted and fixed to each other, form a ready medium by which to introduce the material. When sufficient blanching material has been deposited, the boards are carefully withdrawn, and placed in the opposite row; and it will be readily understood that the fine mould which has passed through the vacuum formed between the boards will be in immediate connection with the leaves of the plants, the common earth placed in the centre of the row enabling it to maintain that position. These boards can of course be set to any required distance apart, that distance being determined by the quantity of blanching material at command. This mode, whether applied to single rows or the more economical system of bed-culture, I consider to be at least equal in its effects to any advantage that can be derived from the use of tiles."

The third and final earthing of the early crops is accomplished at intervals of ten or twelve days before the celery is required for use, placing the mould high and close about the leaves. "I have, however," $\mathrm{Mr}$ Duncan observes, "found a different system to be advantageous with celery intended for winter use. Some time towards the latter part of October, when the weather is dry and favourable, the plants are fully earthed up ; but the soil is neither put so high, nor is it so closely packed to the leaves, as is recommended for the earlier crops, as I have found it to keep better under such conditions. After the winter has fairly set in, I have a sufficient quantity for a fortnight's consumption covered over with leaves which had previously been heated, from which cause they will readily remain in flakes of some 6 inches in thickness, and resist alike the action of 
frost and moisture. The covering over of a day's consumption is merely removed with a fork so much farther on to the bed, and this takes place from day to day. When, however, severe frost sets in, the whole of the ridges are covered over in the same manner; but the protection is removed on the recurrence of favourable weather, in order that the plants should not suffer from being too closely excluded from the atmosphere."

Soil and manure.-From the nature of this plant, the soil is of little consequence to it, except just that into which the roots are running; and this is in general prepared for them, as will be seen from what has been said above regarding the formation of the trenches, which, we repeat, cannot be made too rich with well-decayed manure. We avoid, however, rank dung, such as is used by the market-gardeners around London, which has a tendency, while it enables the plants to attain a large size, to render them, as they mostly are, tough and woolly. We use very decayed manure, and, until earthing up takes place, supply the roots with liquid manure poured in amongst them, and afterwards by the subterranean irrigation also noticed above; and the best manure we have found for this purpose is guano, with a little salt added, or water richly impregnated with soot or pigeons' dung.

Taking the crop and subsequent preservation.-Always begin at one end of a row or bed, taking the plants up by the roots, and carefully avoiding bruising the stems or breaking the leaves. Cut the roots off, and bury them in the trench, but remove the plant otherwise entire to the vegetablehouse. Remove the loose outer leaves, and lay them by themselves, to be washed clean, should they be required for soups. The best parts of them, being cut out and cleanly washed, are sufficiently good for the stock-pot, or for flavouring soup given in large quantities to the poora mode of disposing of the fragments of kitchen stuff we would like to see more generally practised. The centre and solid part of the head should be carefully examined, and every portion that appears to be discoloured removed; and when washed quite clean, they should be dipped in clean salt-water, to dislodge any small worms which, in mild winters, are apt to find their way into the very heart of the plant. Almost all vegetables should have a dip in the salt-water vat, if only for precaution's sake. The young gardener should recollect that in the selfsame state in which he carries the celery from the vegetable-house it is laid upon his employer's table, and therefore he should make himself acquainted with the form used by the family, for there are various ways of dressing it.

As frost sets in, a quantity of the crop for immediate use should be taken up carefully, retaining the roots and what soil may be attached to them, and tying the leaves together, carrying them on hand-barrows (which is the best way to prevent their leaves from getting broken) to the root-cellar, and laying them in amongst sand-not, however, too dry, else it would absorb the moisture too rapidly out of the leaves and stalks, and render them tough and ultimately useless for salads. From time to time during winter this should be attended to; and in the event of not having the convenience of a root-cellar, or, far better, of a vegetable-house (such as fig. 677, vol. i.), which we consider the best of all structures for the preservation of such crops during winter, supplies should be brought in; for, irrespective of preserving the crop from frost and wet, and being more comfortable for the man who serves the kitchen, it prevents the ground from being poached, and leaves it in a tidy state, which, according to the usual practice, it is not.

We have adopted two methods to insure its preservation during winter, either of which we have found to answer our utmost expectation. We grow most of our celery in what is called the Scotch fashion-that is, in trenches from 4 to 5 feet in breadth, setting the plants in rows across, and at such distances apart as accord with the size we wish the plants to attain. Following the London marketgardeners' practice, we do not earth up until they have attained nearly their full size, at which period we earth them up as high as can be done without burying their hearts. Should the weather continue mild, we give them a little more towards the middle of November, at which time, and when the leaves are perfectly dry, we tie them up loosely, and place a common drain-tile on each side of every 
plant, to protect the leaves above where they are covered with the soil, putting in only as much soil between the tiles as will keep them steady in an upright position. We then bank up the sides of the trench, as shown in the annexed cut, fig. 52, along thesides of which we drive in upright posts, 3 inches square, and of such a height as will carry a roofing of boards laid in an

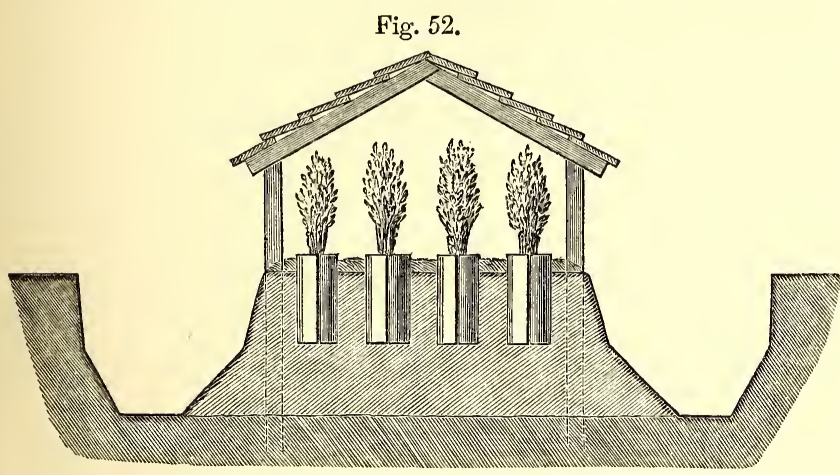

MODE OF WINTERING CELERY.

imbricated manner, a few inches over the top of the plants. These posts are set 10 feet asunder, and exactly opposite each other; and their tops are connected with coupling of lighter scantling. The roof boarding is then laid on, and nailed to the couples, which ties the whole roofing together. By this means the rain is thrown off, and falls into the deep trenches between the beds. Should the weather set in severe, we introduce a little light dry littering matter amongst the tiles, and nail a couple or three courses of boards along the sides; but this is seldom found necessary with us, although in many colder localities it will be of advantage. This side-boarding should only be used in very severe weather, as its absence permits a free circulation of air to blow through. The boarding which covers the roof is the portable wooden copings we use for our wall - trees, and which, during winter, would otherwise be laid past in the store-sheds. The posts and coupling are used for a variety of purposes during summer, such as supporting netting over single rows of gooseberries and currants, to preserve them from birds, temporary roofing over out-of-door summer mushroom-beds, \&c. Neither the posts, couples, nor boarding are ever cut, as we construct such temporary erections always of the same size.

The other mode of preserving celery to which we have alluded is even much simpler, and within the reach of every one. We first form a triangular embankment of soil, the sides sloping to something like an angle of $45^{\circ}$. These sides are made smooth, and are each in length somewhat more than the depth the celery plants have been already earthed up to. In November, when the growth has ceased, the plants are carefully taken up with balls about their roots, a few of the lower and coarser leaves are removed they are now of no furtheruse-and the plants are laid side by side along each of these sloping banks, their roots being placed at top, and their leaves pointing downwards, with the view of preventing rain or snow getting into their centres. When one course is laid along each side, soil is laid over and between them, to the extent of preventing any part of the one plant touching the other. The surface is again rendered even, and a second row laid on in like manner, taking care, however, to place the roots of this second course 6 or 7 inches higher than the last, or to select shorter plants; the intention being, in either case, to prevent the leaves of the top course resting on those of the lower. Indeed, it is a good plan to introduce a few branches between the two courses of leaves, should they appear too crowded, to admit a circulation of air to pass through amongst them. These are covered the same as the first, only to a greater depth, and the whole finished off much in the form of a potato pit. Should severe frost or much wet set in, we thatch the whole over with coarse littering straw, as far down only as where the leaves of the top course protrude through the soil. To thatch the leaves over would only tend to cause them to rot, for we have found in mild winters that they have continued to grow as well as if they had been allowed to remain in their ori- 
ginal position, if not better. The principle in either case is the same-keeping the plants dry, and preventing snow and rain from falling into their hearts. In cold wet soils, we think it would be an improvement to pack the plants, after being laid on the inclined banks, in finelysifted coal-ashes, or in sharp river-sand. The operation in both cases should be carried on when the plants and the soil are moderately dry.

Approved sorts and their qualities.-Of this vegetable there are two pretty distinct classes, namely, the red solid and the white solid.

Cole's superb red.-This is comparatively a new variety of great excellence, surpassing, we think, all others in solidity and flavour. Not that it is like the immense celery grown for the market or for exhibition purposes, which is too often more fit to look at than to eat; but it is of a size such as is required for a gentleman's table, averaging, if well grown, about $6 \mathrm{lb}$. per stick (for stock, head, and stem may be considered synonymous terms in speaking of the blanched part of this plant). It has also the remarkable property of not piping, or becoming hollow or stringy, and has been known to stand twelve months without running to seed. It is also extremely hardy. Mr Thompson, however, in estimating the comparative merits of this family, says that red solid, alias new large red, new large purple, new Russian, Cole's red, Cole's superb solid red, stripped solid, Violete de Tours, are the same thing, and so also is the Celeri violete, the seed of which has been for several years distributed by the London Horticultural Society as the very best red celery. The Manchester red is believed by him to be scarcely different, and may be considered the same variety, excepting that it has acquired a stronger habit and rounder stalks. Shepperd's red is now preferred to the Manchester red by the prize-growers, because of its flatter stems, and its consequently blanching better. In fact, he reduces all our red varieties to one, the type of which is the red solid.

Cole's superb white.-Differing only in colour from the last.

Manchester giant red.-One of the largest sorts grown, and excellent for soups and stewing, but far inferior to the last in point of flavour as a salad. It is grown largely for the market.

old solid red.-An excellent old sort, if it can be procured true. It used to be much grown by the market-gardeners about London; but they have since substituted another of far inferior quality, merely because it comes earlier into use.

Sutton's solid white.-A, very large yet solidgrowing sort, exceedingly white and crisp. We believe it originated a few years ago with the Messrs Suttons of Reading.

Lion's paw.-A short broad flat-stalked variety, of excellent quality, very crisp, and white.

Nutt's champion.-Originated with $\mathrm{Mr}$ Nutt of Sheffield within these few years. It attains, under good management, a very large size, and is, for its size, of excellent quality, very white, and not apt to run to seed.

Seymour's white champion.-This is an improvement of other white celeries raised by Mr Seymour. The number of years they have sustained their popularity is the best proof of their excellence. The stalks are broad, flat at the base, closely overlapping, and forming a compact, well-blanched crisp heart.

To the above sorts Mr R. Thompson adds-

"White solid, alias fine white solid, Celeri Turc, Celeri plein blanc.-Of strong and rather tall growth, blanches readily, and comes into use earlier than the red solid varieties.

"Italian, alias upright Italian, upright, large upright, giant, Patagonian.-Tall, strong, erect, deeply furrowed; not so crisp as Seymour's.

"Wall's white.-Is an improved variety of the Italian, which is esteemed by the growers for competition.

"Curled white, alias Nain frize.-Leaves much curled, resembling parsley, and, like those of the latter, might be employed for garnishing; it is dwarf, hardy, and crisp, but not fine flavoured.

" Early dwarf solid white, alias Court hatif, Celeri Turc of some-Dwarf, but very solid, and forms a compact heart; excellent for early use."

In estimating the difference between the eating qualities of red and white celery, it may be stated that the latter is preferred by most people of taste, on the plea that red celery is ranker in flavour, especially when cooked, than the white varieties; and, when served as a salad, the same opinion is entertained by many. Again, as to the difference between large and moderatelygrown celery, the case stands pretty much the same, large celery never being so crisp and tender, or so well-flavoured, as that of medium size, provided both have been equally speedily grown.

Diseases and insects.-In some soils, those abounding in iron in particular, celery is apt to canker, for which there seems no remedy except changing the constituents of the soil. A parasitic fungus has long been observed to attack the leaves when about half-grown, which is considered by botanists to be Puccinia $\mathrm{He}$ raclei Grev. Another has made its appearance within these three or four years, of which we have been unable to gain any information. So serious was it with us in 1851, that we had many hundred plants to throw away to prevent its spreading farther.

The celery or parsnip fly (Tephritis onopordinis Fab.), fig. 53, seems its chief enemy amongst insects. They breed in the leaves, and from the blotched appearance they cause, the leaves affected may readily be cut off and burned before the maggot is formed, for it is in that state that it is most to be dreaded. It is thus described in the "Cottage Gardener," vol. i. p. 73: "If the withered parts (of the leaves) are examined, and the cuticle or skin of the blisters is raised, there will be found beneath it some small green grubs, which have eaten away all the green pulp or parenchyma of the parts so withered. These 
grubs are the larva of Tepleritis onopordinis. The grubs may be found in the leaves of the

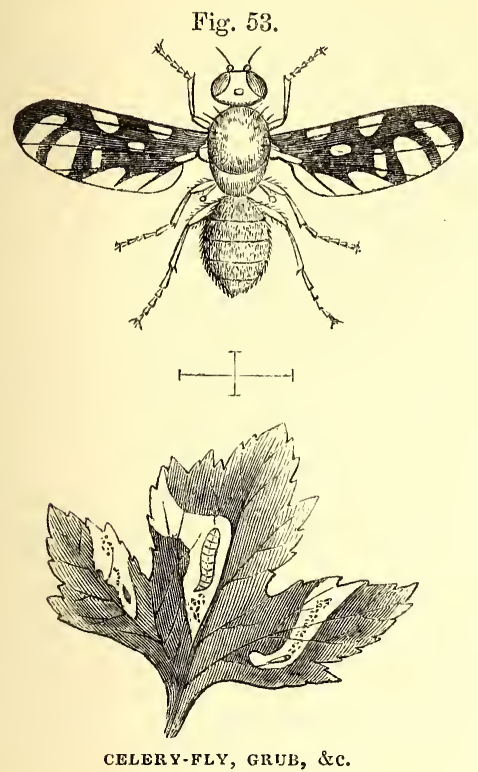

celery in June, July, August, September, October, and November, for there are two or more broods of them in the course of the year. The grubs, altliough less frequently, are found doing similar damage to the leaves of Alexanders and parsnips. When full grown, the grubs descend into the earth, and remain in the chrysalis state till the spring following, when they give birth to the fly. Then the celery-fly may usually be found upon the leaves of the laurel, hovering over flowers, and resting upon palings in the sunshine, from the middle of May to the end of July." Mr Westwood thus describes it: "The general colour of the body, which is five-jointed, varies from rusty brown to shining black; head buff, with black hairs; legs yellow; thorax (throat) sprinkled with long black hairs; wings black, with various pale spots; eyes green. The whole length of the insect is not more than onesixth of an inch, and its wings, when outspread, barely half an inch across." Mr Westwood sug gests that a string smeared with bird-lime, and stretched over the celery plants, might catch many of the parents. This is a good idca, and, if carried out, would no doubt entrap many moths and butterflies also, which would be a wholesale mode of lessening the numbers of grulus afterwards.

Piophila apii (the celery stem-fly), fig. 54.The discovery of this hitherto undescribed insect is due to the author of the article "Entomology," in the "Gardeners' Chronicle," 1848, p. 332, who thus describes it, and its mode of operation: "The larva burrows into the solid stem and fleshy stalks, working its way up the latter, its tract, as well as itsclf, being at first almost invisible, from its similarity in colour to VOL. II. the substance of the stem; so that we have no doubt portions of the stalks, although contain-

Fig. 54 .
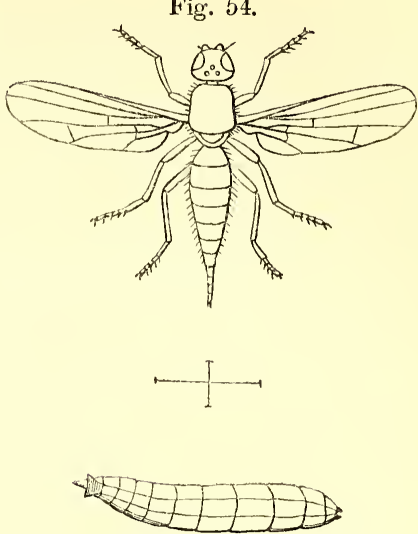

CELERY STEM-FLY AND GRUB.

ing the grub, are often eaten, owing to its presence not being suspected. The eye of the entomologist, however, especially if assisted by a moderate lens, easily detects the unwelcome visitor, which may indeed be expected when the solid part of the stem shows traces of being worm-eaten. It would seem, in fact, that it is in the solid part that the injury is commenced, the grubs eating upwards into the more succulent stalks of the leaves, leaving their traces in the former visible in their tracts, which become rusty red, owing to the action of the moisture and air upon the grooved surface which they have quitted. By careful examination and removal of the leaves, the authors of the mischief may be found in their burrows, in the shape of glossy white cylindrical grubs, with a slight yellow tinge, having the anterior part of the body pointed, and the hind part obtusely rounded, and marked with two black points, from whence proceed two delicate air-vessels, appearing like threads of gold beneath the transparent skin, and which run along the whole length of the body as far as the segment immediately behind the head, where they form two minute excreted appendages. The hind joints of the body are indistinct, but the fore ones are more distinctly to be traced. The mouth consists of a black horny apparatus, capable, as well as the head itself, of being withdrawn within the subsequent segment, as far as the two excreted lobes of the air-vessels above mentioned.

"The fly very closely resembles that reared from the cheese maggot. The thorax and abdomen are entirely jet black, and very glossy, with a very slight brassy tinge, and with fine golden grey hairs scattered over the body. The head is chestnut-coloured, paler near the mouth, and black in the middle above. The eyes and club of the antennæ are pitchy; the bristle of the latter luteous, or yellowish. The lcgs, including all the coxæ or joints by which they are attached to the body, are very pale straw yellow ; the tarsi, especially in the hind feet, some- 
what more dusky. The wings are entirely hyaline and colourless, with the veins very pale buff."

General remarks.-The European names of celery are-Appio in Italian; Sellerie in German; Apio hortensis in Spanish; Sellery in Dutch; and Celeri in French. The seed will keep for ten or twelve years. In saving seed, it is better to select some of the plants from the seed-bed, and to grow them without blanching, transplanting them again in spring, and placing them in a warm well-exposed part of the garden, growing if possible only one sort in a season. The side suckers should be removed, and seed saved only from the best umbels. The seed ripens in September, and, when thoroughly dried in the heads, should be thrashed or rubbed out, and bagged for use. A considerable prejudice exists with some people as to the colour of the heads-some preferring the red variety, while others will only have the white. The preponderance is, we think, in favour of the latter.

The management of Mr Cole, who raised the variety that heads our list, is to the following effect, and will be better understood in a separate paragraph, than had we divided his in structions into our different heads. His principal crop is thus produced: The trenches are dug out in the usual manner, 4 feet apart, and manured with the spent dung of an exhausted mushroom-bed. The seed is sown the second week of February upon a slight hot-bed. When the plants are strong enough, they are pricked out in rich soil under hand-glasses, and are removed, with balls of earth, into the trenches, in the first week of June, and set 9 inches apart in the row. At planting, as is usual, they received a copious watering, to prevent the possibility of a check. In earthing up, a medium course is adopted, neither too frequently nor too much at one time. About a month after planting, a slight earthing up is given them, they having been previously well watered with liquid manure or soot-water the day before this earthing takes place. Soot-water is given as a manure, and dry soot is sprinkled along the rows, to prevent the operations of worms. In regard to Mr Cole's success in growing celery, it should be stated that the garden is of a boggy subsoil, and below the level of an adjacent river, which accounts for the absence of watering, upon which most cultivators depend so much-he watering only once or twice after planting. He admits, however, that in dry or elevated situations it is almost impossible to give too much water, and would prefer giving a good soaking once every fortnight to watering more frequently and in smaller quantities. Very rich dung, he remarks, is not good for celery, and strong manure-water should be avoided. It has been imagined, pretty generally, that bad management rnade bad celery, and especially planting it in too rich soil, to induce extra luxuriance. This opinion Mr Cole dissents from so far; and we believe that he is right when he says-" The bad quality of celery is attributable to the bad kinds grown," and asserts that no person could grow this kind of celery, which has been named
" Cole's superb red," so as to make it either pipy or stringy, or inferior in flavour. In regard to the effect of culture, he says that " late earthing has more to do with making celery stringy than anything else, as it is quite certain that, if the leaves of celery are exposed to full light and dry air for a length of time, the tissue will become harder than if the leaves were grown in comparative darkness. We need no stronger proof of this than the acrid flavour of the outer as compared with the inner leaves of the same celery-a fact demonstrating that, if the leaves are exposed for a long time, they acquire an acrid flavour, which no blanching can wholly remove. For an early crop be sows in heat early in January, and pricks the plants out upon a slight hot-bed; for a second crop, in February, in heat, as before directed; and for a late crop, in March, in the open ground."-Jour. Hort. Soc.

$\mathrm{Mr}$ Cole believes that this celery could be grown to the weight of say twelve pounds, were it desirable to have it so; and for this purpose he would plant in trenches 18 inches deep, and the same in breadth, and use a compost of turfy loam, peat, and leaf mould, or thoroughly rotted cow-dung, in about equal quantities. He would also place the plants 18 inches apart in the row, and keep the soil well stirred between them during their early growth.

\section{$\S$ 2.-CELERIAC, OR TURNIP-ROOTED CELERY.}

Natural History.-Celeriac, a sub-variety of the last, is used for most purposes like the common celery, but is much hardier, and differs from it in having a considerable-sized swelling on the stem close to the surface of the ground, which is the part chiefly used, although the leaves, which are much smaller than those of celery, are also used in soups, \&c. It is much cultivated in the north of Europe, on account of its root, which may be kept in good condition for use throughout the winter: this the common celery cannot be with them, on account of the inclemency of the climate.

Use.-The bulbous-looking form which the root presents above ground is cut into slices, and used in German salads. This, as well as the leaves, is cooked like other celery, only they are less delicate to eat. It is not in very high estimation in Britain, although a few years ago the London markets were supplied with it to a considerable extent from the Continent.

Mode of propagation and season of sowing. - The same as for celery.

Planting.-The transplanted seedlings are removed from the nursery-beds, and planted on the surface (not in trenches) in moderately enriched soil. They should be set in rows 18 inches apart, and a foot from each other in the line. At planting, all the embryo suckers or side shoots should be rubbed off-a precaution to be 
kept in view throughout its growth, as the energies of the whole plant ought to be directed to the formation of the bulblike root.

Subsequent cultivation.-Keep the ground thoroughly stirred up between the rows; and when the bulbs have nearly attained their full size, they should be covered lightly with soil, to blanch them white, and to render the outer coating of their skin more tender. In warm dry weather give abundance of water, to encourage the swelling of the bulbs, for on their size and tenderness their merits depend.

Taking the crop, and subsequent preservation.-The crop will be fit for use in October; and in November, upon the appearance of severe frosts, they should be pulled up, with their leaves attached, and planted, in sand somewhat moist, in a cold pit covered with wooden shutters, where they will continue a sort of existence through the winter, and become somewhat intenerated by absence from light and moderate humidity. The usual practice is to cut off the leaves and bury the bulbs in pits under ground.

General remarks.-Seed is saved in the same way as celery, and may be employed for the same uses. It retains its vegetative property ten or twelve years. It is the Celeri-rave of the French, and the Knott-cellerie of the Germans. It is also called Knob-celery, Knoll-celery, Celeri navet. There is also a sub-variety veined with red, and another with curled leaves, or Celeri-rave à feuilles frisées.

Insects and diseases.-Celeriac is liable to be attacked by the celery-fly, Tephritis onopordinis, (which see.) We are not aware that the roots have hitherto been attacked by the Piophila apii, which has of late years been detected in the stems of celery.

\section{§ 3.-THE LETTUCE.}

Natural history.-Lettuce (Lactuca sativa L.) belongs to the natural order Compositæ, and sub-order Cichoraceæ, tribe Lactuceæ, and to the class Syngenesia, and order Aqualis, in the Linnæan arrangement. The generic name is derived from the Latin Lac, milk, from the milky juice which abounds in most of the genus, and has been of much importance in medicine. That of Lactuca verosa, for example, is highly narcotic, and has been even employed with great advantage as a substitute for opium. The production of the opium qualities, which exist in all this genus, is much lessened by cultivation, and especially by blanching; hence blanched endive is less poisonous than it would be if eaten in its natural state. It would be curious to know if this had anything to do with the process as a branch of culture. The lactucarium brought into notice some years ago by Dr Duncan of Edinburgh, who found it could be administered where poppy opium could not with safety, was prepared from the juice of lettuce.

The native country of the lettuce is unknown; it, however, appears to have been cultivated in Britain since 1562. According to Herodotus, it was in use 550 years before Christ; yet Pliny says the ancient Romans knew but one sort. In his time they were cultivated so as to be had at all seasons of the year, and even blanched to render them more tender. Gerard is the earliest English author who writes of them. He gives an account of eight sorts cultivated in his day. It is quite evident, from the names of many of our still cultivated sorts, that they must have reached us from the Greek islands - Cos, for example-and from various places in the Levant. Italy, Egypt, and the south of France have contributed their share.

Uses.-As one of our best salad herbs, the lettuce is well known-eaten raw in French salads, with cream, oil, vinegar, salt, hard-boiled eggs, \&c. It is also eaten by many with moist sugar and vinegar, and some prefer it with vinegar alone.

It is excellent when stewed, either alone or eaten with partridge; and in most vegetable soups it forms an ingredient, and is an excellent addition to Scotch broth and hodge-podge, as a substitute for cabbage. It is eaten at almost all meals by the French; by the English after dinner, if not served as adjuncts to dishes during the repast; and by many after supper, the time when the Romans first ate them-but in the time of Domitian they changed this order, and served them with their first entries at their feasts, much as is done in many parts of the Continent at this day. In a raw state lettuce is emollient, cooling, and in some degree laxative and aperient, easy of digestion, but containing little nourishment.

Propagation.-The only mode of propagation is by seed.

Sowing and planting.-As lettuce is required throughout the whole year, sowings must be made from the beginning of February to the end of September-say twice in February and March, and three times each other month. But where young lettuce is required when about 2 inches high, the seed will require to be sown once a-fortnight during winter, and once a-week during summer and autumn. The hardy kinds, to stand over winter for early spring use, should be sown in August and September. The first crop sown in February should be upon a slight hot-bed, and when about 2 inches high should be transferred to a colder bed covered with glass, and protected from frost. These may, in the beginning of April, be trans- 
planted to the bottom of a wall having a southern exposure, where they will be protected by the projecting coping, and by the awnings used to protect the blossoms of the trees. In default of such, plant in the warmest border the place affords, and protect by branches or other means most at hand. These may be set at 6 or 8 inches apart, and the smaller taken up with balls, and planted in the open border about the beginning of May, setting them 12 inches apart each way, as the smaller and hardier sorts should be chosen for this purpose. In February and March, seed should be sown on a warm border, and the plants protected until fit for planting in the more exposed borders or quarters of the garden. For a first general summer crop, sow about the end of March and beginning, middle, and end of April, in a wellexposed place, and continue sowing as above. During the heat of summer, sow on borders with a north aspect, and keep the ground moist by watering. Lettuce is much improved by careful transplanting; therefore every crop, as soon as the young plants will handle, should be set in nursery-beds sufficiently thin as not to touch each other. For this it is impossible to give exact dimensions, as so much depends on the varieties sownthe cos or upright-growing kinds requiring less space than the cabbage sorts; and even in each of these there exists a considerable difference in size. In all cases of nursery-bed transplanting, there is no error in planting thin, as the thinner the plants are the stockier they get, and the better rooted-the purposes aimed at in this stage of their growth. The beds prepared for them should be in high cultivation-that is, thoroughly enriched and completely pulverised. If a little half-decayed turfy soil, or half-decayed leaves, were dug into such beds, the roots would work into them, and lift with better balls, and especially so by using the transplanting trowel, fig. 27 .

In removing them from the seed-bed to the nursery plantation, the ground should be well watered if dry. Instead of pulling up the tender plants, as is usually done, the ground should be loosened by the transplanting fork, fig. 55 , which is a useful implement, and may be employed in taking up root-weeds too firmly fixed in the soil to be drawn up by the hand. Its principal use, however, is in loosening the soil about the roots of young seedling plants, such as cabbage, endive, lettuces, or the like, previous to their removal to the nursery-bed, and also in

Fig. 55.

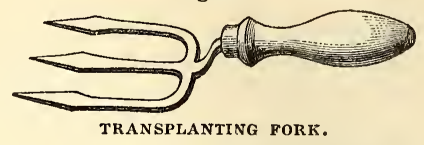

facilitating their removal from it to the place of final planting, when the transplanters, figs. 29, \&c. are not to be used, or the transplanting trowels, figs. 27 and 28. The use of all the latter implements is to remove with compact balls of earth around the roots; and they are of great service when plants of a large size are to be removed, particularly during dry weather, and when the least possible check is to be given to their growth. A saving of time and space is obtained by their use : for example, most of the Brassicæ, the lettuce, and endive, may be retained in nursery-beds, even should the former attain a height of 9 or 10 inches, and the two latter 4 or 5 , and until the ground intended for them shall be cleared of its present crop. The fork, fig. 55 , is, on the other hand, to be employed for lifting young crops of onions, leeks, lettuce, endive, and similar long and tender-rooted plants, so that, by loosening the soil around them, they may with greater safety be taken up than if pulled by the hand. These forks are of various sizes - the smallest is $3 \frac{1}{4}$ inches broad at the points of the prongs, 5 inches in length of prongs, and $10 \frac{1}{2}$ inches, including the handle. The prongs are flattened, and are half an inch broad at the broadest part, tapering to a sharp point. The fork is attached to the handle by a bent neck, the part of which that is attached to the prongs acts as a fulcrum, while the handle acts as a lever.

Fig. 56 is a modification of the last, the prongs of which are 6 inches in length, and which,

Fig. 56.

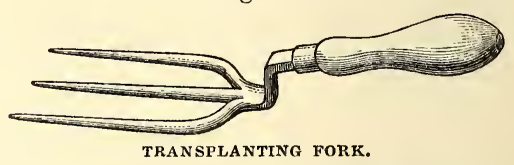

with the bending at the neck and handle, measures 13 inches in length. In this case the prongs are round, tapering to a point, are three in number, and form a fork 3 inches in breadth. It is used for similar purposes as the last, and also as a planting tool.

When transferred from the nursery-beds to the open quarters after the beginning of May, shallow drills should be drawn according to their size, and the plants set in them : being somewhat below the general surface, they will derive shelter; and should water be required, which will be the case in dry weather, it can be more advantageously applied. If in plan- 
tations by themselves, they should be accommodated according to their natural full size. 'Such sorts is the tennis-ball, or the Laitue grotte, two of the smaller sorts, will have plenty of room if 10 inches apart; while such as the drumhead will require nearly double. It is seldom, however, unless in large gardens, that plantations of lettuces are made to any extent by themselves; they form a sort of element in mixed planting, and therefore are often interlined with other crops, such as cauliflower, cabbage, broccoli, \&c., in which case a lettuce plant is very properly placed between each two of the others in the same line, that no interruption may take place in stirring the ground between the rows. In such places they are usually cut for use before they injure the regular crop.

The London market-garden practice is to sow the white cos variety from the 10th to the middle of October in frames, which are filled with soil to within 6 inches of the top, giving a slope of 1 foot in 4 the length of the lights, which always face the north. As soon as the seeds are sown the sashes are removed, and a man is placed in this department to keep off the sparrows, give air, and shut up in case of rain, the great secret being to keep them as dry as possible, and to let them have all the air they can get. This will appear strange to a non-eating salad reader, but it should be remembered that millions of this variety are so grown within a few miles of London, and the price obtained pays the expense. They are planted out in February or early in March, in rich well-trenched ground (after celery), 1 foot apart each way. Mr Cuthill, on whose high authority the above statement is made, observes, that "gentlemen's gardeners buy the same seed, but, owing to their mismanagement during winter, and the poor state of the ground, they cannot grow such fine lettuces as the market-gardener. Some of the latter," he continues, "are too greedy in regard to crops-for instance, they have often three crops on the ground, all coming forward one after another : but the best cultivators do not do this; it tramples the ground, and does not give any crop fair play. The rapid growth of a lettuce depends much upon hoeing or stirring the soil."
About the beginning of October an abundant supply of the hardier varieties should be planted out at the bottoms of garden-walls, on dry warm borders, and on raised banks, sloping both towards the sun and also from it. On these, in open places, lettuces often stand the winter well ; and should those on the sonthern side be cut off by strong sunshine succeeding severe frosts, those on the opposite side may escape, as the process of thawing will take place more gradually on them. In cold and elevated situations, pits and frames will be required to insure their safety. In such circumstances the plants should be kept moderately dry, and as much exposed to the light and air as possible: hence the lights should be kept off during good weather; and when on, should be tilted up both night and day, to admit full ventilation. If in frames, it will be of advantage in this respect if they be clear of the ground the thickness of a brick, so that air may pass freely through; in very severe weather they may be let close down to the ground. Covering the spaces between the plants with coal-ashes will be found of advantage.

In planting lettuce to stand over winter at the bottom of walls, every aspect should be made use of ; for it is often found that those set behind a north wall will succeed better than those having the protection of a south one.

Besides planting at the bottoms of walls for protection during winter, wherever there are pits or frames and glasses to spare for the purpose, these should in like manner be filled with young lettuceplants, to afford a spring supply should the others fail.

The seed of lettuces is small and light; half an ounce will sow a bed of 80 square feet, and will, under ordinary circumstances, produce eight hundred plants. The seed being small, it is necessary the ground should be well pulverised and made smooth before it is sown, and that it be not covered more than about the eighth of an inch.

Some recommend sowing lettuce on the ground where it is to remain, either broadcast or in rows, and, when the plants come up, to thin them to the distance of from 6 to 14 inches apart, according to their size or sort. This is a waste of 
ground ; for while they are in the seedbed or nursery plantation, they are progressing in growth, while other crops may be ripening off to make way for them. Besides, the process of transplanting greatly lessens their disposition to run to seed.

Subsequent cultivation.-During spring the young crops must be protected from frost, and during summer from drought by copious waterings of manure-water, and frequent stirring of the ground between the plants; and snails and slugs should be kept under by watering with lime-water when the ground is dry, or dusting with lime in powder when it is already too moist. During winter the plants should be kept dry, clear of weeds, dead leaves, and all matter likely to encourage damp amongst them. The ground should be frequently stirred amongst the plants, and abundance of air given to those under glass frames or hand-glasses. In the growing season every stimulant should be applied, for much of the excellence of the crop depends on its quickness of growth. Blanching being often desirable, the cos varieties will be hastened towards maturity by having their leaves loosely tied together with strands of matting: the cabbage sorts do not require this attention. For means of preserving during winter, see Endive; the means and method of proceeding are alike in both.

Forcing.-The lettuce is seldom forced in Britain; on the Continent there is a slight stimulus given to produce them fine during winter and early spring. In Holland and Belgium this is carried on with great care and success. The means adopted will be understood by what follows.

The Dutch and Belgian mode of obtaining fine lettuce throughout the winter is-About the beginning of October, not later, an exhausted hot-bed, on which melons or cucumbers have been grown, or, in default of this, a bed of fresh materials well fermented, and in which the excess of fermentation has ceased, is chosen, and formed into a bed for the purpose. These beds, we may observe, are for the most part either wholly or in part under the ground surface, a pit being dug out for their reception: this is rendered necessary from the intensity of their winters. After the heat has considerably declined, the surface is earthed over to the depth of a foot or more with light soil, chiefly decomposed leaf-mould with an admixture of sand, and filled up to within 9 inches of the under surface of the glass. The surface is rendered smooth and level by slightly beating it down with the back of a spade, and is then most correctly marked out into squares corresponding exactly with the size of the square of glass, and immediately under them. The plants, being previously sown very thin three weeks or a month before, are not excited by any extra means; on the contrary, they are kept exposed, and so become sturdy, stocky plants, so that when they have formed their fourth leaf they are judged fit for being transplanted into the prepared bed. One plant only is placed in each square, and that in its very centre. A few plants are put along the back and ends of the bed to serve as substitutes should any of the principal ones be eaten by the wireworm, which is very troublesome; if not required, these are thrown away. We do not recollect seeing any of the cos varieties so grown, but the cabbage kinds only: the Klein groen, or small green with black seed, very similar to our tennis-ball, or the Laitue grotte of the French, is used where the squares of glass are small; and the Groote geel or large white, similar to our drumhead or Silesian, where the squares exceed a square foot each. After planting a slight watering is given, and the whole surface of the bed is neatly covered with white sand, which is kept during the whole growth exceedingly clean and free of weeds and muscous matter. The lights are then put on, and made to fit exceedingly close all round the sides and ends of the frames, these being rebated for their reception; and no air is given for the first two or three days-that is, until the plants have taken root. Afterwards air is admitted, but with extreme caution, the lights being slightly elevated both at bottom and top, so that a complete change of air takes place almost instantaneously: this is found better in practice than a less efficient and more protracted mode of ventilation, and perhaps on this a great deal of their rapid growth and delicate crispness depends. In the early part of winter this ventilation is often left on during the day and night also; but the sashes are never completely removed, but kept on to keep the surface and the plants dry. When the lettuces have attained their full size, and have become conlpletely cabbaged, air is totally withdrawn; should the leaves touch the glass, the frame is carefully elevated a few inches to prevent their coming in contact with it, and running the chance of being injured by frost. When the frosty nights come on, the utmost vigilance is exercised to prevent the frost passing through the glass, for if it did so, the whole crop would inevitably perish: covering must therefore be attended to, as the exclusion of cold and damp are the principles of their preservation. During the whole process, no artificial heat must be applied; and water must also be withheld, as it is probable that the roots, having passed through, or nearly through, the bed of soil, abstract sufficient moisture from the dung-bed below, thus rendering surface-watering unnecessary. To do 
justice to the Belgian, German, and Dutch gardeners, we must say that their lettuce so grown is equal at Christmas to what we usually see in our own gardens in July and August. It should, however, be observed that with them a frame or two of such lettuce is considered as great a triumph of skill as a house full of fourpound queen pine-apples is with their brethren in Britain. To keep up a winter supply, two or three sowings are made, and consequently the same number of plantings. At this season frames are not required, as with us, for wintering planting-out plants, and other requirements in English practice, therefore every frame and glass sash at the command of the Continental gardener is employed in the production of his lettuce crops. The same practice has been repeatedly tried in England-as at Longleat, Bulstrode Park-but with very varied success. The late Mr Nieman, a celebrated Dutch gardener, brought over by the late Mr Labouchere, often acknowledged to us the great difficulty he had to contend with in the cloudy atmosphere at Hylands in Essex, even when compared with that of Holland. It is probable, however, that the more variable state of our climate had its share in his difficulties. A remark of this excellent cultivator is worth recording in this place-namcly, that the difference even of two days in plantations made in October not unfrequently caused a difference of from a month to six weeks of their attaining maturity towards spring. The plants are kept as close to the glass as is possible without their touching it, and the reason for placing each plant exactly under the centre of a pane of glass is to prevent the chance of drip falling into its heart, which would utterly destroy it. For this purpose, also, the glass should be kept in the best possible repair, and the laps should be leaded or otherwise secured.

Taking the crop.-As lettuce is gathered for use, the whole plant should be pulled up by the roots; but as there would be a great chance of the earth, particularly from amongst the fibres, getting in amongst the leaves in their transit to the vegetable-house, it is better to cut the roots off and bury them in the ground in which they grew. The outer leaves should be cut off, and the root part of the stem cut clear over with a sharp knife, the whole plant carefully washed and rinsed in clean water, after having been steeped a few minutes in the saltwater tun; any of the tips of the leaves injured by frost in winter, or by insects or drought in summer, should be carefully cut off, and the utmost vigilance exercised that aphides and other insects are not allowed to remain attached to the leaves, and that all sandy and earthy particles be carefully washed out; the lettuce should then be set on end, the top undermost, in a clean salad-basket, to allow the water to drain completely out; and it should be understood that it, as well as all other salads, receive no further cleansing after they are sent from the garden.

Approved sorts, and their qualities.-Lettuce is divided into two very distinct tribes or subfamilies - namely, the Cos and the Cabbage kinds. The former are of upright growth, hardy, and in general firm and crisp; the latter less hardy, and more soft and flaccid, and even on that account preferred by some. The same difference exists in regard to their merits for cooking, some preferring the one and some the other. French cooks usually choose the cabbage kinds.

Green Paris cos.-This is the best variety of cos lettuce at present grown, and although less hardy than the brown cos, it withstands our ordinary winters when planted at the bottom of walls. As a spring, summer, and autumnal lettuce, we think it unrivalled, growing to a large size, of a fine green colour, and, from the manner in which the outer leaves cove over the interior ones, becoming nicely blanched without having to be tied together. It is known as Sutton's superb green cos, Ady's fine large cos, and Kensington cos. It is the Romaine verte Maraichère, and Chicon, ou Romaine verte Maraichère, of the "Bon Jardinier."

Paris white cos.-This is the sort most generally grown by the London market-gardeners, millions of it being produced annually within a few miles of London alonc. Next to the green Paris $\cos$ this is the largest, the best, and the longest in running to seed of all the summer lettuces we have grown. It is less hardy than either the last or the following, and with them may be considered all of this class required in any ordinary garden. This opinion is, we observe, confirmed by Mr Thompson in the sixth volume of " The Journal of the Horticultural Society," p. 26, who remarks, "It was sown April 10th, and had not commenced to run July 27 th, while all the other cos lettuces sown on the same day were showing flower." Known also as London white cos, Sutton's superb white cos. It is the Romaine blonde Maraichère of the "Bon Jardinier," and is much cultivated, as well as the last, around Paris. Seeds white. The common white cos runs to seed sooner than this variety.

Brown cos.-This old and excellent sort still maintains its position as being the hardiest of all the class. It grows to a large size, blanches well, and is exceedingly crisp and tender. It is known as Bath cos, brown Bath cos, whiteseeded brown cos, Wood's improved Bath cos, hardy brown cos, and Sutton's Berkshire brown cos. This excellent variety is not much cultivated on the Continent; the outside leaves being of a brownish colour renders it with them objectionable. Seeds white.

Waite's white cos.-An excellent varicty, apparently intermediate between the Paris green cos and Paris white cos; not quite so dark or green as the formcr, yet somewhat greener than the 
latter. The three varieties are so excellent that either or all may be grown advantageously.

Bath green cos.- Has great merit as a hardy winter green sort, and nearly related to the old Bath cos, only less brown on the outer leaves, and while it has white seed this has black seed. Hence we have, in seed catalogues, black-seeded Bath cos and white-seeded Bath cos: the latter appears to be the hardiest, while the former seems to be the best.

Artichoke-leaved lettuce.-Comparatively a new variety of singular liabit, having the leaves long, upright, and very much cut at the edges; it is a hardy variety, blanches well, and does not speedily run to secd. It is the Romaine à feuilles d'artichaut of the "Bon Jardinier." The seeds are black, and should be sown in Junc and July for autumn and winter use.

The above may be considered the very best, and the following rank next to them in this respect: new crystal cos; early green cos, which is identical with the Brighton green cos; Brighton cos ; Egyptian cos, and Egyptian green cos; golden cos, the same as the Florence cos; Alphange cos (of this variety the French cultivate two sub-varieties, the one having black and the other white seed); red-spotted cos, the same as Aleppo, bloody, and the Sanguine ou panachée, Romaine panachée à grain blanche, of the French. Seeds white. Seed-lists contain such names as Bearfield cos, Victoria cos, white Brunoy (the latter said to be very large), and a variety of other names, which are either unknown to us, or which are identical with some of the above.

Of cabbage lettuce we have for summer cultivation-

The Neapolitan.-A large, white, crisp, and fir'm variety of great excellence-we think the best of all others for summer use, as it comes in carly, and is long in running to seed, blancling itself naturally; leaves somewhat curled, toothed at the cdges. Seeds white.

Large white.-Heads large, flat, compact, with smooth leaves and white seeds. Of medium earliness, and not inclined to run to seed during hot weather. A profitable sort. Known as the late cabbage, large mogul, Swcdish or sugar, Saxony, and princess, and is the Royal à graine blanche, Blonde Paresseuse, Blonde d'été ou Jaune d'cté of the Frcnch.

Malta.-Heads compact and flattish; leaves palish green; blanching naturally; of a fine pure white colour and tender consistency. An old tried variety. It is the Laitue de Maltc of the "Bon Jardinier."

Versailles.-Not much removed from the Neapolitan; the leaves are, however, of a paler green, of excellent quality, and cabbaging white and crisp. It is the Laitue de Versailles, Laitue de Versailles blonde, of the "Bon Jardinier."

Imperial.-An excellent large sort, but inferior to either of the above. It is known as the union, and is the Imperiale ou grosse Alemande of the French, and differs only from their Laitue 'Turque, or Turque à graine noire, in having white instead of black seed.

Black-seeded yellow. - Very similar to the large white cabbage lettuce, differing chiefly in the seeds of the present one being black. It is the Blonde de Berlin, Blonde à graine noirc, Royal à graine noire, of the French.

White Silesian. - One of the largest sizc. Leaves crumpled or wavy at their edges; palish green, slightly tinged with reddish brown on their outer surfaces. It is known as the drumhead, large drumhead, or cabbage, Spanish, imperial. A long-cultivated sort. It is the $\mathrm{Ba}$ tavia blonde ou Silesie of the "Bon Jardinier."

Of other summer cabbage lettuces the following rank next to the above: Asiatic, Belle bonne, Ice, Grand admirable, Victoria, Mogul, (which is identical with superfine new French), Laitue de Hollande, Grosse brune paresseuse, and Grosse grise. The Nonpareil of the English is synonymous with the Metrelle of the French.

Of cabbage lettuce we have, for winter and early spring use-

Tennis-ball.-A long-cultivated sort. Heads small, firm, white, and crisp ; very hardy, and not apt to run to seed in spring. Very much prized with French cooks, who dress it whole in a variety of ways. This is one of the sorts which should be sown about the 12th or 15th of August. 'The seed is black. It is known also as green ball or button, and capuchin. It requires little room in frames during winter, and yields a great return in spring, as almost the whole plant is eatable.

Black-seeded grotte.-An excellent winter lettuce, somewhat similar to the last. There is a black and white seeded variety, and also various other grotte lettuces, much more cultivated in France than with us, all of great merit. Where small, hard, compact, and delicate sorts are required, this class should be extensively grown; not that they differ much in appearance or quality, but by procuring several sorts by name, the chance of disappointment in procuring some that are good is lessened.

Brown Dutch.-An excellent hardy free-growing sort, attaining a pretty large size, cabbaging freely, and of good quality. The outside leaves are reddish brown, the centre ones white and delicate. It is less hardy than the others in this class; but as it is of much larger size, its cultivation is of consequence.

Hardy-green Hammersmith. - An excellent small hardy green variety, which has long been in cultivation. Leaves much wrinkled and concave; dark green in colour, and thicker than most of this class; seeds white; the hardiest sort in cultivation. It is known also as the early frame, early dwarf Dutch, Roman cabbage, hardy green, green Dutch, and Prussian cabbage. It is the Laitue verde of Continental gardens.

White Dutch.-Leaves yellowish green, sometimes tinged with reddish purple at their tips; seeds white ; cabbages freely, and of excellent quality; somewhat larger than the tennis-ball. Known also as the early yellow, early green forcing, and early cabbage. It is the Laitue à bord rouge, ou Cordon rouge, of the French.

There are four species of Lactuca cultivated besides $L$ sativa, the presumed type of the cultivated varieties described above. These are Lactuca crispa, palmata, intybacea, and quercina. 
Their merits have, however, attracted little attention in Britain. The Freneh profess to have several kinds in the seed-shops, which they eonsider adapted to the purpose of growing to be eut young, as we do mustard and cress. These they call Laitue ehicorée, Laitue à eouper, Laitue épinard. Any sort of the upright or eos varieties will do equally well.

Diseases and insects.-Among the latter are the lettuce-fly (Anthomyia lactuca Bouch.), the larvie of whieh, according to Ruricola, in " Gardeners' Chroniele," make their appearance in August, but more abundantly in September. They are not very readily distinguished from those of the eabbage and turnip, being of a yellowish-white eolour, tapering towards the head, which is pointed, and armed with two short blaek claws at the nose. These maggots live in the involucra of different varieties of lettuce, feeding upon the seed and receptacle ; and when they have devoured these, they push themselves out backwards, either to enter another seed-vessel, or fall to the ground to become pupæ. When the seed stems are gathered and dying, the larvæ ehange to pupæ, being bright chestnut coloured; oval cases, whieh are rough when examined under a lens, with two minute tubereles at the head, and two hooks and a few other tubereles at the tail. In May a few of the pupæ hatch, although they are sometimes found as early as April and as late as July. The male fly is intense black, elothed with short hairs and bristles; the eyes reddish brown, and meeting above; faee inelining to ehestnut eolour, with a bright spot of the same on the erown; the fore part of the trunk bears four varying whitish stripes; the body is ashy grey; the segments blackish, at the base a deep blaek ; wings, two, stained with black and beautifully irridescent; the base and poisers ochreous; the nervures of the wings pitelyy. The female is entirely ashy grey, and less bristly ; the eyes not meeting on the erown, with a bright ehestnut-coloured stripe between them; body oval ; the apex eone-shaped ; horns and legs blaekish; wings and nervures lighter than in the male, which it equals in size.

The Arctia caja-Euprepia caja of othersfig. 57 (the garden tiger-moth), appears in July, and often commits sad havoe upon lettuce erops, and also on strawberries. The caterpillar. is one of the most voraeious of all its elass, eating double its own weight per day. They do not eat the solid part of the leaf, but suek out the juice, and thereby destroy the plant as completely as others do who devour the leaves and stems. It has been ascertained that one of these caterpillars, weighing 36 grains, voided daily more than 15 grains' weight of excrement; yet its own weight only increased 2 grains daily. The caterpillar of this moth is to be found in June, and the perfect insect in July, so that its period of voracity is providentially not of long duration. It is well known in gardens in its perfeet state, and may be readily captured while on wing by using an entomologist's net. Hind wings bright red, with blue-blaek spots; fore legs of a reddish brown, marked with creamy white; thorax brown, with a red neek-band ; abdomen red, with blue-black bars. The caterpillars are vOL. II. dark brown, thickly eovered with reddish-brown hairs. They east their skin quite entire, and
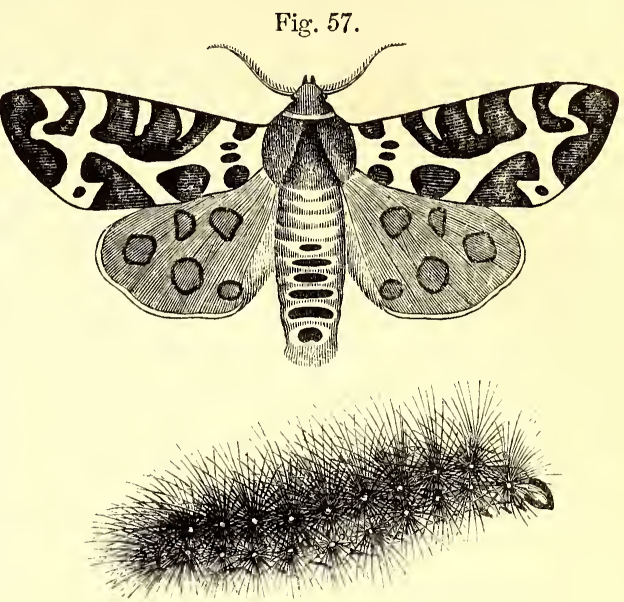

TIGER MOTH AND CATERPILLAR.

these may be frequently picked up in mistake for the living inseet.

Another enemy to the lettuce, as well as many of the produets of the garden, is the Tipula maculos $a$ of Hoffmansegg (the spotted garden-gnat),

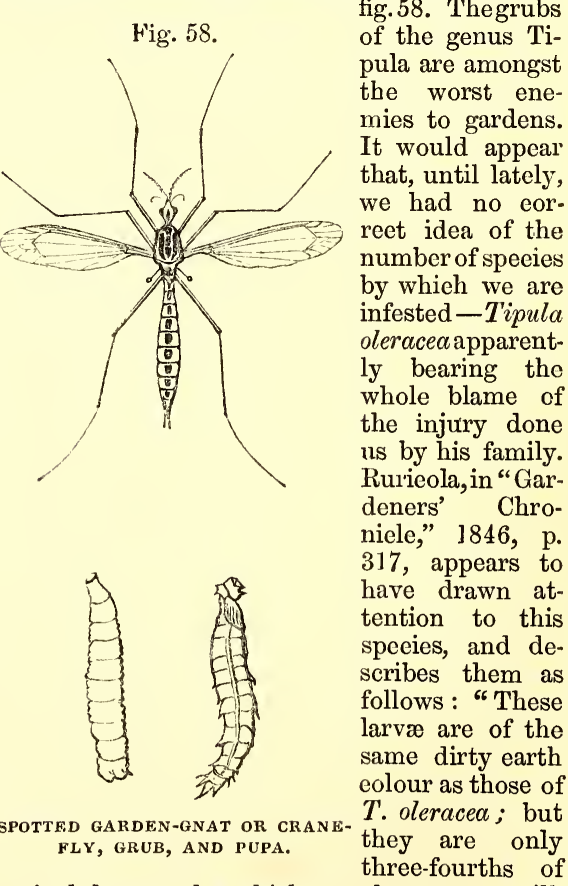
an inch long, and as thick as a large crow-quill. They are wrinkled, and when at rest contract themselves, drawing in the head and thoraeic segments, so that this portion looks more like the anal extremity: the animal, however, is able to thrust out its head and crawl along very 
well, although it is destitute of feet. The head is small, brown, and furnished with two black jaws, short antennæ, and, I believe, minute palpi. Two vessels of a pale colour are visible down each side of the back, and one in the centre; the tail is furnished with two divaricating hooks, and two short teeth between them; the stern being truncated, which will readily distinguish the larva from that of $T$. oleracea; it has two large spiracles, with two tubercles below, and two fleshy masses, which are capable of great dilatation, and assist it exceedingly in walking. In the spring these larvæ change to pupæ in the earth. They are about the same length as the larvæ, but scarcely so stout, and of the like dirty colour; the head and thorax are defined, the latter having a short slender horn projecting on each side ; the wings are small, but distinctly visible, as well as the legs, which are placed between them. There is a spiny elevated line on each side of the abdomen ; each segment having a transverse row of minute spines above, and five larger ones beneath; the penultimate joint is surrounded by six longer spines and two smaller ones ; and the apex produces a large conical process above, and a shorter one beneath. The flies are abundant in May and June in meadows, gardens, fields, hedges, and especially on the sea-coast. There must be either two or three broods in the year, or a constant succession of the flies, although the spring may be the season when the greatest number are hatched; but that will vary with the temperature." The gnat is "not quite half an inch long, but the wings expand one inch. The male is of a fine yellow colour; the black horns are longer than the thorax, and taper; they are 13-jointed; the first joint is elongated, the second small and cup-shaped; all the others are elliptical, with a few bristles at the base of each, excepting the apical one, which is very minute; head with a large black patch on the crown; forehead conical, with a little black dot on each side ; the face forming a cylindrical rostrum, with a hairy beak, bearing a black spot on the top. Palpi are longish and black; the eyes are hemispherical and black, but there are no ocelli; the thorax is marked with three black stripes down the back, the centre one the shortest, and the sides are spotted with black; the scutel has a black dorsal stripe; the abdomen is linear and obtuse, with a row of black spots down the back, and smaller brown ones on the sides; the wings divaricate or rest horizontally on the body; they are of a smoky, yellow tint; the costa is yellow; there are an areolet, two little stigmatic cells, and seven apical ones; six long black legs, very slender and tapering; base of thigh pale yellow, and of shanks yellowish brown. The female is rather larger; the horns shorter ; the abdomen is fusiform; the apex accuminated, and furnished with two fine sharp lateral valves, and a smaller centrical one. The eggs, which are scattered by the female, are intensely black, but dull, oval, and spoon-shaped. This, however, might arise in my specimens from their not being fertile.

"Some idea may be formed, from the following data, of the mischief committed by this insect.
On the 23d of April I found the grubs at the root of my pease; on the 29th some had eaten off trusses of flowers in the strawberry beds, close to the crown, retiring afterwards just beneath the surface of the earth; the first week in May they were not uncommon among the roots of lilacs and under tufts of grass ; on the 28th of the same month I observed some recently transplanted lettuce drooping, and, on examination, found the roots separated from the crown, a little below the surface, and close to where these grubs are difficult to detect, owing to their colour, and their remaining quite motionless when disturbed. The end of July they were eating the roots of dahlias, carnations, and various flowers; and the 7 th of August they infested some potato ground along with the $T$. oleracea ; after which I lost sight of them.

"Lime-water will not kill them; and the only mode I have been able to adopt with any success has been to search round sickly plants, and dig up all that have been killed by them, and destroy the culprits; but this must be done speedily, otherwise they will soon decamp to feed upon other plants. I should think watering with brine, nitrate of soda, or perhaps strong liquid manure, would keep them from our crops."

Brine applied sufficiently strong to kill or drive away these grubs would kill the plants themselves. Sulphur, tobacco or quassia water, or spirits of tar incorporated with the soil, we think, would be found more effectual. Besides these, a species of aphis has recently been discovered which commits great depredations on lettuce crops, by attacking the roots of the plants. This species does not appear to have been observed by entomologists prior to 1849 , and hence remains unnamed.

Birds are fond of the seeds of lettuce, therefore newly-sown crops should be protected by netting the beds over; and the seed, while ripening, requires a similar protection.

General remarks.-Select some of the bestformed plants that have stood over winter; thin them out where they stand, if the place is eligible; if not, transplant them in spring to a warm and sunny spot, setting them from 2 to 3 feet apart, according to the size of the kinds. Keep no two sorts near to each other; or, better, as the seed retains its vegetative properties for many years, grow in small gardens, only one sort for seed in one season. The seed will be ripe in August. Cut the plants when the flowers have faded, and leave them on a dry border for a day or two; the seeds will mature themselves in the seed-vessels, deriving nourishment from the sap in the plants; when dry, remove them under cover of a dry airy shed, and thrash out the seed when it begins to fall out. The seed ripens very irregularly if left standing, the top parts ripening and shedding before the lower parts and the side shoots are equally matured.

\section{$\S$ 4.-ENDIVE.}

Natural history.-Endive (Cichorium endivia L.) belongs to the natural order Compositæ, sub-order Cichoraceæ, and tribe Cinchoræ, and 
to the class Syngenesia and order Aqualis in the Linnæan arrangement. For derivation, vide Succory. It is said to be a native of the East Indies, China, and Japan, and to have been introduced in 1548. This Phillips disputes, and we think with good reason, for Ovid mentions it in his tale of "Philemon and Baucis." Columella also notices this vegetable as sufficiently common in his day; and Pliny tells us clearly that it was " eaten both as a pot-herb and salad by the Romans in his time;" and in book xx. chap. 8, he speaks of the endive or garden succory as being medicinal. Gerard gives an account " of the manner by which the garden endive was preserved for winter use in the time of Queen Elizabeth." His method may not be uninteresting to the horticulturist of the present day. He says - "Endive being sown in July, it remaineth till winter, at which time it is taken up by the roots, and laid in the sun or air for the space of two hours; then will the leaves be tough, and easily endure to be wrapped up in a heap, and buried in the ground with the roots uppermost, where no earth can get within it, which, if it did, would cause rottenness; and which, so covered, may be taken up at any time convenient, and used as salads all winter, as in London and all other places is to be seen ; and then it is called white endive." Taking a hint from this quotation, we have often, in taking up endive in November, planted it in an inverted position, by burying the leaves in dry peat earth (which is an excellent antiseptic), the roots uppermost, and exposed to the air, and have kept it in good condition from two to three months. From all we can learn, we are disposed to believe endive a native of Egypt, and that it was carried from thence to Italy, and from thence to Britain, along with many other of our horticultural productions.

Uses.-The leaves are the only parts used, and these only when blanched, to diminish the natural bitterness of taste. It is one of our best autumn, winter, and spring salads, and is also stewed much in the same way as lettuce.

Propagation.-In a cultivated state it can only be regarded as an annual, and is therefore propagated by seed, which is light, and vegetates freely: half an ounce of seed will sow a seed-bed of 40 square feet.

Sowing and planting.-The earliest crop should be sown in May, on a warm border, in rich and well-pulverised soil. In sowing, scatter the seed thinly, and cover to the depth of a quarter of an inch. If sown earlier, the plants are apt to run to seed in autumn; and if sown too thick, they come up slender, and if not timeously thinned, are much retarded in their growth. For principal crops, sow twice in June and twice in July; and for a late crop to stand over winter, if mild, and to come in early in spring, sow again towards the middle or end of August.
The seeds may be sown broadcast in beds 3 feet in breadth. In dry warm weather water freely, both while in the seed-bed and nursery plantation. As bulk of vegetable and tenderness in texture, as in the case of the lettuce, are the great requisites, every stimulus should be given to increase the rapidity of growth, and this will be accelerated by the application of liquid manure, such as dissolved guano, soot, or pigeons' dung, applying it either early in the morning or late in the afternoon. When the plants are about $2 \frac{1}{2}$ inches high, remove them carefully, and transplant them into another bed of equally enriched soil. Set the plants from 3 to 4 inches apart each way, water at planting, and afterwards, if the weather is dry. From this bed they may be transferred, when about 4 to 5 inches in height, to where they are to come to their full size. The early crop should be sparingly planted, unless the demand is great, as they are very apt to shoot up to seed, more especially if the seed is of last year's growth. A part of this early crop may be planted on a warm well-exposed border, and the remainder interlined with newly-planted-out broccoli or cabbage, or between rows of dwarf pease, the partial shade being of advantage to them, and in some degree preventing their running to seed prematurely. In taking up for final transplanting, great care should be taken that the roots are disturbed as little as possible, and that as much soil as will conveniently remain about them be also taken along with them-and for this purpose the planting trowel should be used, instead of the dibber. As the plants are taken up, set them closely together in the planting-tray, with their leaves upright, and on no account follow the barbarous practice of cutting the leaves off nearly by the middle. The planting-tray is a light shallow box, about 27 inches in length, 18 inches wide, and $3 \frac{1}{2}$ inches in depth. In the sides should be cut two slits sufficient to introduce the fingers, for greater convenience in carrying it to the place of planting. Such trays are much better than baskets, as they prevent the loose earth falling on the walks, protect the roots better from the air during their transport, and are more economical, on account of their greater durability. The ground for the principal crops should 
be open and well exposed to the sun, thoroughly manured, and dug or trenched to the depth of 2 feet. Draw drills from 12 to 15 inches apart, according to the kind of endive to be planted, as some, like the Batavian or broad-leaved sorts, require more room than the green-curled, and those to be used early in autumn may also be set closer together than such as are to remain till a later period. The drills should be 3 inches deep, which, by the earth falling into them during the process of hoeing, \&c., will assist in blanching the crop; they also render the process of watering more convenient, and economise the fluid, and afford, as in all cases of drilled crops, greater facility for cleaning the ground and stirring it up, upon which so much of the success of all crops depends. The plants, according to size, as stated above, should be planted with the trowel, at from 9 to 14 inches apart in the row : if the ground is in proper condition, this will not be found too great a distance; if otherwise, a less distance will suffice.

In all cold and late situations, the warmest spot in the garden should be set apart for this crop, as it is, with the exception of the kidney-bean and potato, the most tender of all our ordinary esculents. The shade of trees should be avoided; and for the latest crops of all, a dry place, and if possible sloping to the south, should be chosen.

Subsequent cultivation. - The summer crop will require nothing more than attention to watering, and keeping the ground clear of weeds, until it has nearly attained its full size, when a portion of the crop should be blanched; but this should not be done all at once, only in progressionsay from 20 to 50 plants about every third day. The methods of blanching are various. The Belgians, who are the best growers of this crop in the world, commence at the end of a row, and, taking the leaves carefully up with both hands first, hold them tight with the left hand, and with the right hand apply a small willow twig, and frequently a leaf of $\boldsymbol{J} u$. neus glaucus, which they use largely for all temporary tying purposes, round the leaves at top, thus causing the large outer leaves to blanch the more tender ones towards the heart of the plant. They are methodical in all their gardening opera- tions; and so, in this case, they thus tie up as many plants as will last for six days, at the end of which time they begin to gather the crop, which will be beginning to blanch, and will every day be improving. They take up as many as they require for the day's consumption, and with the twigs or rushes now disengaged, they proceed to where they left off tying at first, and tie up as many more as they have that day taken for use; and in this way they go on throughout the whole crop. They also sometimes draw a little earth about the plants as we do, but they prefer the former practice.

The English practice, in market-gardens, is to tie up in the same way, using strands of matting instead of twigs or rushes, performing the operation once in eight or ten days. This operation should in all cases be attended to in dry days, or when the leaves are completely free of damp from rain or dew. In private gardens the same plan is followed, though, in some cases, they draw earth around the plants when both are in a dry state, and thus effect the end, although not so completely. Indeed, when the earthingup system is to be followed, it will be found expedient to tie up the leaves first, and therefore, if this is done properly, earthing up must be superfluous. By tying up the plants while quite dry, drawing the leaves up in a conical form, and tying them tight about 3 inches under their tips, damp is prevented from getting to the hearts, and no deterioration can take place in the flavour; and therefore, we would say, for summer crops, this is the better way. For autumnal supply, when the weather is less favourable, and every means should be employed to prevent

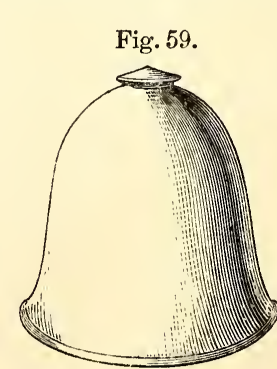

ENDIVE BLANCHING-POT. decay taking place, in consequence of damp, in privategardens at least, the endive blanching-pot, fig. 59, should be used. These are merely modifications of the sea-kale blanching-pot, fig. 39 , diminished in size ; and as there is no occasion for a portable top or lid to enable the cultivator to examine his crop, they are made 
all in one piece, having a knob at top to serve as a handle for lifting them off or on. They are from 9 to 12 inches in diameter, and the same in height; are placed over the plants when nearly full grown, the leaves being gathered up with one hand, while with the other the pot is placed over them, so as to enclose them completely, and thus insure their blanching, while they are protected from frost, snow, or rain. The curled-leaved varieties are much more readily blanched than the broad-leaved or Batavian sorts, therefore a corresponding degree of care is required in performing the process. As to the length of time required for blanching, much depends on the season. During summer, while the plants are growing vigorously, the process will be effected in a week; while towards autumn, and during winter, when vegetation is more sluggish, double or treble that time will be required. The other means employed are to invert empty flowerpots over the plants, taking care to stop up the holes in their bottoms, laying a slate or pan-tile over each plant, particularly the green curled sorts : the Batavian, from its difference in habit, does not admit of this process. Setting two long narrow boards along each side of the row, and bringing them together at top in form of a triangle, and afterwards drawing earth over them to keep them steady ; covering the dwarfgrowing sorts with half-decayed leaves, dry tanners' bark, sand (a method in use in the days of Gerard), coal-ashes, or even sawdust, are all had recourse to; but all of these, as will readily be seen, are far inferior to using the blanching-pot, or even the tying-up process.

For protection during winter, the London market-gardeners take up their latest crops, and set them thickly on sloping banks, by the sides of hedges, for the sake of shelter; while others throw up long narrow ridges, in an east and west direction, and plant both sides, which produces a succession-those on the southern side coming in first, while those on the opposite side, if later in arriving at perfection, have often the advantage over the others of withstanding the winter's cold better, being less influenced by the freezing during night, and rapid thawing during the day.

Partial shelter may be afforded the plants during winter, when planted in the open garden, by sticking the ground between and around them with old peastakes, branches of trees, furze or broom branches. This wards off cutting winds, and catches the perpendicular frost as it falls; but, in using such means, they should be stuck firmly in the ground, to prevent their being blown about so as to injure the plants by friction; neither should they be above 2 feet in height, as the lower they are the less effect the wind has upon them.

The best way, however, to secure fine endive during winter, is to take the fullgrown plants up in November, or before severe frosts set in, choosing a dry day, and when the leaves are also dry. Tie the leaves loosely together with matting, first removing a few of the largest and oldest outside leaves; take them up with good balls of earth attached to them, and carry them to the conservative-pit (fig. 676 , vol. i.), or the span-roofed vegetable-pit (fig. 677, vol. i.), and plant them in moderately dry sand, in half-decayed peat earth, if it can be procured, which, on account of its antiseptic properties, will resist decay longer. They should be placed closely together, but not so close as to touch each other. In the one case, they will be kept perfectly dry, in consequence of the permanency of the roof, while ample ventilation is secured by opening the sides, as shown in our figure. In such a structure they will enjoy almost as much air as if in the open ground, while they will be completely protected from damp and frost. The conservative-pit (fig. 676, vol. i.) offers also an excellent means of keeping endive, and all similar plants, if taken up with balls, and planted in it. The boarded roofing, which is in convenient pieces, keeps the interior dry, while light and air, when wanted, can be fully admitted, by propping it up as shown in our figure. All places for the purpose of keeping esculent vegetables during winter, should be placed in a dry airy situation, and with a northern aspect, so that the sun may rarely shine upon them: during their season of hibernal existence, every stimulus to growth should be guarded against. We have recently constructed a very useful pit for this purpose, 150 feet in length, and 7 feet wide. It is simply a brick wall, 15 inches in height, built parallel with an existing north wall, covered 
with standard or Ridder Morello cherries, the branches of which are 3 feet from the ground. A batten of wood, with notches cut out opposite the stems of the trees, is fastened to the wall by holdfasts at that height ; to this, and to the wooden wallplate on top of front wall, the rafters are fixed, at the distance of $3 \frac{1}{2}$ feet, which is the breadth of a great portion of our glass lights and felt shutters, that either may be employed if necessary. For protecting lettuce and endive the former are not required; and the latter, during winter, are mostly in use for covering over the glass lights of pits, \&c. during the night. As a covering for this pit, we employ the boarding used as portable coping to the garden-walls (vide fig. 40, vol. i.), which during winter is not required: these are laid upon the rafters in an imbricated manner, but not fixed to them, but they are secured to each other by cords every 6 feet, after the manner of Venetian blinds ; but instead of drawing up, like them, they are folded up the one over the other, when air is wished to be admitted, or when it is necessary to open them to take out the supply, remove dead leaves, \&c. When the plants are fully grown they are removed from the quarter, tied up, and planted in rotten tan within the pit; the boarding is put on, and the whole safely secured. We have never had so fine a supply of endive before as we have this season, and we are still cutting, on the 10th of March, that which was put in the first week in November.

Soil and manure.-The object being to produce the largest amount of vegetable matter, and in the shortest space of time, it follows that the soil cannot be too rich or in too high a state of cultivation for this crop. Beyond the application of stable-yard manure, as usually applied, we use no other manure, save that at every watering we enrich the liquid by the addition of guano, soot, or pigeons' dung, and occasionally ammoniacal liquor, or the water through which gas passes during the process of purification, which appears to be an excellent manure for most garden crops, but cannot everywhere be procured. It should, however, be understood that this liquor is not gas-tar, which of itself would have very opposite effects.

Taking the crop, and subsequent preservation.-Endive is always used in a blanched state, both for appearance sake and to get rid of a certain natural bitterness contained in the green leaves. It is divested of its outer, coarser, and unblanched leaves and roots, with any points of the remaining leaves that may have been injured, or have begun to decay. It is then thoroughly washed, rinsed afterwards in clear water, or in salt and water, allowed to drip dry, and placed in a clean basket fit for use. The preservation of the crop being so connected with its subsequent culture, for that the reader is referred to the last paragraph.

Forcing.-Endive is rarely forced in Britain, our chief dependence being placed on the preservation of the preceding year's growth over winter. The truth is, with all our affectation for French cookery and salad-eating, we are a vast stride behind our neighbours on the other side of the Channel in both. They force endive, and we do not. Their mode of proceeding is thus given in the "Bon Jardinier," which contains all that is new in French gardening: "For early-forced scarole (broadleaved or Batavian endive) the seed is sown in January, under glass, in a strong heat. After the seeds have vegetated, and the plants are fit to handle, which will, in general, be from twelve to eighteen days after sowing, they are pricked out on another hot-bed, at a lower temperature than the last, ventilation is attended to, and in course of the end of February and during March the crop is ready for use. The plants, of course, are small compared to those grown by us in the open air, but they are produced in great number on account of the extent of framing every garden contains, and the abundance of stable-yard manure procurable, by which almost all the forcing in the marketgardens about Paris is carried on. When the scarole has attained the height of 6 or 8 inches in the frames, it is tied up to blanch, which it does in a few days. Sometimes they sow the seed in October, in a bed with a mild bottom-heat, and afterwards prick out the plants into a similar bed, placing them at the distance of about 6 inches asunder, either under glazed sashes supported on frames, or under cloches or large bell-glasses, placed close together on the heated material. These they cover in severe weather with paillissons or straw mats, which they very 
properly prefer to Russian mats, the most indifferent of all protections, warding off neither cold nor wet in so efficient a manner. We have a third mode, by sowing about the middle of September in a cold frame: in about three weeks the plants are of fit size to prick out under glass to gain strength, after which they are transferred to a glass frame, set pretty thickly together, and protected from cold by ample coverings alone." It will be understood that these crops are not intended to attain the same size they do with us, but are cut for use while quite young and tender; and by such means we might obtain a better supply of young lettuce for winter use than we do by growing them in boxes, pots, or pans, in the high temperature usually done. This mode, however, imposes a great amount of labour in covering and uncovering; and from the high price of labour with us compared with that of France, it would hardly pay the commercial grower. In some few private gardens, such supplies, both of lettuce and endive, are kept up; but, in general, the demand for glass is so great with us for other purposes that few private families would afford the necessary means. Winter salad-growing is the beau ideal of the French gardener; his mind is, as it were, concentrated on it, and indeed he has little else to think of. Not so with those of Britain, whose winter operations are far more multifarious, besides the difference of climate.

Approved sorts and their qualities.-The endive, like the lettuce, is divided into two very distinct classes - the Batavian or broad-leaved (the Scaroles of the French, the Breitblattrige-endivie of the Germans), and the curled-leaved (the Chicorée of the French, the Endivien-cichorie of the Germans.) An excellent paper was published some years ago, in the 4th vol. of the "Transactions of the Horticultural Society of London," on the varieties of endives. The French seed-lists contain many names; those of Britain contain few, and of those, three or four are all that is in general inquired for.

The broad-leaved Batavian and small Batavian are the only two broad-leaved sorts worth the attention of the general cultivator. The former is known also as broad-leaved endive, common yellow, and double yellow; the latter is the Scarole petite, Scarole courte, Scarole ronde of the French. The former is that most usually grown, but from careless seed-saving is not always to be procured genuine. The latter has the following merits, which are worth notice, and are thus given by $\mathrm{Mr}$ Thompson, from spe- cimens grown in the London Horticultural Society's garden : "Leaves pale green, broad, of moderate length, slightly ragged at the edges; inner leaves hooked at the top, naturally forming a good heart; blanching with little trouble, and is mild and sweet compared with many others." Nearly allied to the broad-leaved, but inferior to it, is the curled Batavian, fine-curled and yellow-curled Batavian, which are all the same.

The large Batavian is merely a large variety of the small Batavian, inferior to it in not hearting so well. It is the Scarole grande, Scarole de Hollande, of the French.

Lettuce-leaved Batavian.-More tender than the other varieties, therefore not adapted for winter crops or cold localities, unless for early summer use; the leaves are large, blunt, and cut at the edges: it does not blanch without tying up. It is known as the white Batavian and new Batavian, and is the Scarole à feuille de laitue, Scariole blonde, of the French. The green and white Batavians are only seed-list names, referable to the common broad-leaved sort.

Large green curled.-An excellent sort, differing from the following only in being somewhat larger in size, and in having its outer leaves more upright. It is known as the green curled, yellow winter endive.

Small green curled.-With the last, the two best of their class, and most extensively grown. Leaves about 6 or 7 inches long, beautifully curled, the outer leaves lying close to the ground, the inner ones thickly set, forming a compact heart, easily blanched, very hardy, and, with the last, best adapted for winter use. It is the Chicorée frisée, Chicorée de Meaux, Chicorée endive, of the French.

White curled.-This sort is much used by the French for cutting young, as described in paragraph Forcing. The full-grown leaves are nearly 8 inches long, and when grown in the open air they seldom or ever form a heart, and are besides tough and bitter. It is the Chicorée blanche, Chicorée toujours blanche, of the French. Certainly not worth cultivating for a general crop.

Small French green curled.-This sort is much cultivated in France for the earliest crop, hearting early, and being less liable to run to seed than some others. It is so small and so prostrate that it is difficult to tie up. It is known as the fine-curled, and is the Chicorée fine d'été, Chicorée frisée fine d'Italie, and Chicorée d'été, of the French.

Dutch green curled.-So near in all respects to the large green curled as not to be worth growing as a separate sort. Like the other, it is hardy, and blanches well.

Long Italian green curled, or Endivia longa.Similar, if not inferior, to the following.

Italian green curled, or Endivia riccia.-So similar to the last as not to be worth cultivating as a distinct sort; indeed, neither is worth cultivation in a climate like ours.

Triple-curled moss.-A curious new sort, exceedingly well curled, and, although a variety of the following, is worth cultivating to a limited 
extent. It is the Chicorée mousse of the French.

Staghorn endive.-A variety of no long standing; leaves upright, much cut and jagged at their points, which accounts for the name. It hearts well, but is very tender, running to seed too soon, and being impatient of wet and cold. Useful as a summer endive, but useless for winter crops.

Insects and diseases.-Endive is pretty well exempt from both, but, like most tender esculents, is often attacked by slugs (Limax) and snails (Helix) ; but these can readily be subdued by dusting over with powdered lime, or watering with lime-water. Several species of the genus Iulus, or snake millepedes, attack the roots of endive. We have lately discovered Iulus pulchellus, fig. 48 , I. terrestris, and $I$. complinatus, abundant about the roots of endive plants in a cold pit. In the quarters where they grew, hundreds have been dug up during November and December, notwithstanding the plants were repeatedly watered with lime and soot water during their growth; and even nitrate of soda was pretty profusely applied at the time of digging the ground. In some seasons, however, lime and soot water have been found to save the plants; and a single experiment made this last season seems to argue in favour of flowers of sulphur being sown in the drills previous to the plants being planted out; and this, no doubt, would have acted better had the sulphur been buried deeper.

General remarks.-The seeds of endive are saved in the same way as those of chicory and lettuce (which see). The European names are Chicorée des Jardins in French; Endivia in Italian; and in German and Dutch the same; Endibia in Spanish.

\section{§.-SUCCORY.}

Natural history.-Succory, chicory, or wild endive (Cichorium intybus L.), belongs to the natural order Compositæ, sub-order Cichoraceæ, and tribe Cichoreæ, and to the class Syngenesia, and order Aqualis. The generic name is of Egyptian origin, adopted by the Greeks. The plant has been used by the Egyptians from the earliest ages, and it is most probable that they would communicate to the Greeks its name, as well as the manner of using it. Forskahl says the Egyptian name was Chikouryeh. They did, and still do, make their chicory of much consequence ; and it is well known that it, along with similar plants, constitutes half the food of the Egyptian peasantry at this very day. The specific name intybus is derived from the Arabic name Hendibeh; and Pliny informs us that in his day they called the wild endive (our chicory) Cichorium, and the cultivated sort (our endive) they call Seris. According to Phillips, the garden endive was cultivated in England in the reign of Edward VI.; "but the wild endive or succory," he adds, "intubus, being indigenous to the soil, was grown in all probability at a much earlier period, both as a pot herb and as a salad;" and Gerard informs us that " the leaves of these wild herbs are boiled in pottage, or broths, for sick and feeble persons ," and this generally correct authority adds that the wild endives (our chicory) "do grow wild in sundry places in England, upon wild and untilled barren ground, especially in chalk and stony places." There can be therefore no doubt that, cultivated or uncultivated, the chicory was in his day, 1548 , sufficiently well known.

The chicory is indigenous to many parts of the south of England, particularly in chalky soils, and it has been long cultivated in Italy, France, and other parts of the Continent as an agricultural plant, the leaves being used as green food for cattle, and the roots, when kiln-dried and ground, as a substitute for coffee. It has also been long used in these countries as a salad herb, the leaves and roots both being used; "yet in this country," says Loudon, " it had not attracted the notice of horticulturists till after the time of Miller." A revival of its uses was no doubt brought to England, from the Continent, after the general peace in 1814-15.

Use.-In other countries, as we have stated above, it has been extensively and profitably grown, particularly in Holland and Flanders, and has occupied a place in gardens for an unknown length of time, the leaves being blanched, and used as one of their most popular winter salads. The blanched leaves are called Barbe de Capucin, or Friar's beard. In Belgium the roots are scraped and boiled, and eaten along with potatoes, or with a sauce of butter and vinegar. The roots have been taken on board ship, and planted in boxes filled with sand, and in this way afforded wholesome salads for months together. Its principal use is as a substitute for endive, and it is employed for the same purposes.

The mode of propagation is by sowing the seed. A quarter of a pound will be sufficient for an ordinary private garden, as it is light, somewhat like endive, to which it is closely related.

Sowing and planting. - The seeds are sown, towards the end of June, in rich deeply-trenched ground. Sow thin, and cover about one quarter of an inch. When the plants corne up, thin them to the distance of 6 or 7 inches apart, transplanting those thinned out into another piece of ground equally manured and trenched, to allow the long fusiform roots to extend deep into it. Some sow, after the Flemish manner, in the broadcast way, and transplant on the same system. The drill mode is, however, better in both cases: the drills should be 9 inches apart, and the plants 6 inches distant in the row ; for the stronger the plants are, and the more room the foliage has for development, the more elaborated matter will be thrown into the roots; and it is this prepared matter, stored up in them during their 
growing season, that will supply the means for the young coming leaves during winter forcing, for they of themselves can collect little or nothing towards the support of the crop.

Subsequent culture. - About the beginning of October the roots should be lifted, the leaves being carefully cut off, but not so close to the crown as to endanger the hearts of the plants. Amateurs had better leave 3 inches of the base of the old leaves, to prevent accident. The roots are then planted as thick as they can well be done in a bed of sand on the floor of a mushroom-house, packing the sand closely about them, and, when finished, giving a good soaking of tepid water. Old boxes may be filled with them, and casks, as is usual on board of ship, having their sides perforated with holes $1 \frac{1}{2}$ inches in diameter, and 7 or 8 inches apart. Lay a few inches of sand in the bottom of the cask, and on that lay the roots horizontally, with their crowns in the centre of the holes; on this layer of roots lay more sand and roots until the cask is filled, watering each layer of roots as they are covered with the sand. Large flower-pots may also be used, or indeed anything that will keep the sand together. It may be convenient to fill many of these at once; and by placing them in a dry cellar or open cool shed, they will remain for a month or two stationary. For the first gathering, one or more of these should be removed to a warmer place, where a temperature of from $45^{\circ}, 50^{\circ}$, to $60^{\circ}$ can be maintained, and where light can be cornpletely excluded. In a week afterwards place another supply in, and so on during winter, or as long as the stock of roots lasts. They will seldom afford more than one gathering, and may be then thrown away, and the boxes or casks filled again with roots from the open ground. In this way a constant succession of salad may be kept up from the beginning or middle of November till April. Some recoinmend taking up all the crop of roots at once : this is unnecessary, until the beginning of February, when they will begin to become naturally excited into growth. It is expedient then to remove them to a bin of sand in the cellar, or to bury them deep in the ground, to prevent theirgrowth. It is also recommended by some to shorten the roots and remove the side fibres; but this is only cutting off the resources of the VOL. II. plant, and lessening its means of producing a crop of large succulent leaves.

This excellent and wholesome salad was, we believe, first brought into notice in Covent Garden market by the indefatigable Mr Cuthill, who, in 1839-40, carried the first sample of it which appeared there in his own hand. It was in consequence of a letter from that individual, published in "The Gardeners' Magazine," about fifteen years ago, that this plant was first grown as an article of field-culture, for the purpose of its roots being used for mixing with coffee. This is somewhat strange, seeing that it has been a common marketable article on the Continent time out of mind.

Mr Cuthill sows his chicory about the first of June, either broadcast or in drills. When the plants are up, they are thinned out to a foot apart, and the ground is kept free from weeds. The roots are taken up in November, and stored by exactly like beet. When endive becomes scarce, the chicory roots are planted in 16-sized pots, five roots in each. When the chicory begins to spring, invert 24-sized pots over those the roots are in ; exclude the air, and place them in a forcing house or frame: each pot will afford three or four cuttings. A dark mushroom-house, where a fire is kept, is an excellent place for chicory; and a cellar is good for spring crops, but seldom warm enough, if not artificially heated, to depend upon for a regular winter supply.

The Belgians and Dutch blanch immense quantities of chicory during winter and spring; indeed, it forms one of the most prominent articles in their vegetable markets for several months together. The roots are taken up in autumn, and all the larger ones selected; they are then placed in a bed, almost as close as they can stand together, with merely a little earth to fill up the spaces between them, experience having taught the Belgian gardener the important fact pointed out theoretically by Mr T. A. Knight, that the new annual supply of leaves of plants of this description is derived from the stock of sap elaborated in the preceding year, and requires nothing from the soil but moisture. Upon the bed of roots thus closely packed together, and defended from frost in winter, a slight hot-bed of manure is laid in spring, with 6 or 8 inches of earth interposed. Into this earth the leaves shoot, struggling 
for light and air, and becoming perfectly blanched and crisp, and losing most of their natural bitterness. With us who have greater convenience in the shape of heated cellars, or other places capable of having light completely shut out, we manage better, by planting the roots in beds on the floor, and thus produce the crop at less risk and greater certainty as to time, without half the labour. Planting in large pots or in boxes, and inverting pots or boxes of the same size over them, and setting them on the floor of a vinery, and behind the hot-water pipes or flues where they exist, or indeed in any out-of-the-way place where there is a temperature from $50^{\circ}$ to $55^{\circ}$, and exclusion from light, will secure this excellent winter and spring salad in great perfection, and with little trouble.

Those who desire it during spring may readily blanch it in the open ground, leaving some roots where they grew, and placing sea-kale or endive pots over them. They will continue to yield a crop until they begin to run to seed.

Taking the crop.-Each head of leaves is cut when of 7 or 8 inches long, taking with them a thin slice of the crown to keep them together, as in cutting sea-kale. When washed and tied up into small bundles of a handful each, they are fit for dressing. Three handfuls will make a good-sized salad, and may be eaten alone with oil and vinegar, or mixed with a little chervil and tarragon, or with young lettuce.

Sorts and their qualities. - The Continental growers profess to have three sorts- the common large-leaved, the Chicorée à navet, or Caféchicorée, and the variegated.

General remarks.-The European names are, Chicorée sauvage in French; Cicoria in Italian; Gemeine cichorie in German; Suikerei in Dutch; Achicoria in Spanish. In saving seed, select some of the strongest roots that have not been forced; support the flower-stalk as it advances, and cut them over when flowering is done, and treat them as recommended for lettuce. The seed will keep three or four years.

\section{§ 6.-DANDELION, TARRAGON, ETC.}

Dandelion (Leontodon Taraxacum L.) belongs to the natural order Compositæ, sub-order Cichoraceæ, and tribe Taraxaceæ, and to the class Syngenesia and order Equalis in the Linnæan arrangement. The generic name is derived from Leon, lion, Odon, a tooth, from the tooth-like divisions of the leaves. Indigenous to most parts of Britain, particularly in rich soils.
The young leaves, even in their green state, make an excellent ingredient in salads; and the leaves are an excellent substitute for succory, and for this purpose they are blanched during winter in a similar manner. (See Succory.) It is a neglected and despised plant, which might be made much use of by the poor, and those living in towns who have no gardens; for roots of it may be got for the digging up, and, if planted in sand in a dark cellar, or even in pots set on the window-sill, much wholesome matter might be obtained from it. The roots are as valuable as the leaves. It is produced in great quantities in the London markets, although we are not aware of its cultivation being tried upon a commensurate scale to meet the demand.

The amateur who may wish to grow the dandelion in his cellar for salad purposes, should possess the root-extractor, fig. 60 , an excellent implement for the purpose of taking up such

Fig. 60 .

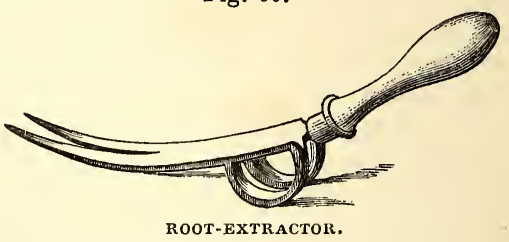

roots, which can be carried in the pocket. The mode of using it is to thrust it deeply into the ground, so placed that the root may be taken between the prongs. The bent part near the handle, acting as a fulcrum against the surface of the ground, greatly facilitates the withdrawal of the root without breaking it, when the handle is pressed towards the ground.

General remarks.-The European names are, Dents de lion, or Pisse-en-lit, in French ; Amargon in Spanish; Paardebloem in Dutch; Lowenzahn in German; Piscia in letto in Italian. Our own common English name bears a close resemblance to some of these.

Tarragon (Artemisia Dracunculus L.) belongs to the natural order Compositæ, sub-order Anthemideæ, and to the class Syngenesia and order Superflua in the Linnæan arrangement. The generic name is derived from Artemis, one of the names of Diana. Pliny, however, informs us that in his time there was an opinion that the plant was named after Artemisia, the queen of Mausolus, king of Caria. The specific name is said to have been given from the tortuous form of the roots resembling the sinuous tail of a dragon; others think it derived from Tarchon, the Arabic name of the plant. It is a native of the south of Europe, others say of Siberia, and was introduced into England in or before 1548.

It is cultivated for its leaves and the points of its young shoots, both of which are used as an ingredient in salads, soups, stews, pickles, and other compounds. Tarragon vinegar, so much esteemed as a fish-sauce, is made by infusion of the leaves in common vinegar. It is also added to most salads to correct their coldness. Three or four plants are sufficient for an ordinary family; but if required during winter in a forced 
state, twenty or more plants will be required. A very small portion is sufficient for a day's consumption, either for salads or soups, a small handful of the leaves or young shoots being quite sufficient. When used with salads, it should be cut up very small, and served on a plate by itself, so that every guest may season his salad to his own taste, as many have a dislike to its flavour.

Being a hardy perennial, it is increased by dividing the roots; every portion, however small, will grow, if only a bud be left at the top. It may also be propagated by seed, but this is seldom done.

The seed may be sown in March. The slips or roots should be planted at the same time, or in autumn. It may also be propagated by cuttings of the young shoots, taken off in July, when the plant is in full growth, and these will strike freely under a hand-glass in a sladed situation. It should be planted in a dry warm soil, as it is apt, when planted in cold damp soils, to die during winter.

If seed is sown, it should be in a small seedbed broadcast, and the plants removed, after they have attained the height of 6 inches, into rows in an open part of the garden, free of shade and damp, and where the soil is perfectly dry, in lines a foot and a half apart, and the plants a foot asunder in the lines. They will speedily increase, and become fine bushy plants the same year.

Their subsequent cultivation consists in keep. ing the ground around them clear of weeds; and when the plants are showing flower-stems, they should be cut off, unless a plant or two be left for seed. This is, however, seldom done, as the plant propagates sooner and better by dividing the root. Towards November, take up a few of the plants, and plant them in large flower-pots or boxes, to be placed in a mild heat to produce green leaves and shoots during the winter.

The plants potted in November may be from time to time, to suit the demand, placed on the floor of a vinery, or in a moderately-heated pit, in any temperature from $45 \circ$ to 600 .

A very small portion of the green leaves or tops of the young shoots should be picked off, and served with the salad daily; a somewhat larger quantity when it is to be used for stews or soups, and in still larger quantity when to be pickled, or for making tarragon vinegar; and for the latter purposes it should be supplied when the plants are at their fullest size.

General remarks. - No insects that we are aware of attack this plant. It should be planted in very dry soil, and in a sunny situation. The European names are, L'Estragon in French; Dragoncello in Italian; Dragun in German. A quarter of an ounce of seed will be sufficient for most gardens, but young plants are usually purchased from the nurseryman. Two dozen will be enough to make a plantation.

Burnet (Poterium Sanguisorba L.) belongs to the natural order Rosaceæ, and to the class Monœcia and order Polyandria in the Linnæan arrangement. The generic name is derived from Poterion, a cup, because it is used in cooling drinks.
Indigenous to Britain; generally found in calcareous soils which have long been under pasture.

The young leaves are often used in mixed salads, particularly when made in the Italian style, which, according to an old proverb, is good for nothing without them. The branches and leaves are also used in soups. It was formerly in higher repute than at present.

Propagation.-By seeds sown in March or in September, in any common garden-soil. Half an ounce of seed will be sufficient for any garden. It does not propagate so freely by division of the plant; and although this is recommended in books, it will be found better to transplant the young seedlings, when 3 inches in height, into lines a foot apart, and the plants 9 inches asunder in the line. A plantation thus made will last from six to ten years.

Subsequent cultivation.-All that is required is to keep the ground clear of weeds, to prevent the plants running to seed, by cutting over the flower-stalks as they appear. A few plants should be cut over by the ground occasionally, to keep up a succession of young and tender leaves. Twelve plants will be sufficient for an ordinary family. A small handful will be sufficient for a salad; for soups, double that quantity will be required.

The European names are-Pimperella in German; Pimprenella in French; Pimpinellain Italian.

\section{$\S$ 7.-WHITE AND BLACK MUSTARD.}

White mustard (Sinapis alba L.) belongs to the natural order Cruciferæ, and to the class Tetradynamia and order Siliquosa in the Linnæan arrangement. The name is derived from Sino, to hurt, Ops, the eye-as its pungency hurts the eyes; by others, from the Celtic $N a p$, applied to the cabbage tribe. It is indigenous to many parts of Britain - found growing in corn-fields. It was formerly called Senvia in English. Tusser mentions the use of mustard in Queen Mary's time; but it is uncertain whetler it was the white or the black mustard he meant. Gerard informs us that the gardenmustard, which produces the whitest seed, was not become common in Elizabeth's reign, but that he had distributed the seed into different parts of England to make it known. Mustard was not manufactured in his day, but was brought to table whole, or bruised in vinegar.

The principal use of both white and black mustard in gardens is as an indispensable ingredient in salads, the young leaves and stalks being cut close to the ground before the formation of the second series or rough leaves appear. Formerly the dry seed was pounded with vinegar, and, according to Gerard, "is an excellent sauce-good to be eaten with any gross meats, either fish or flesh, because it promotes digestion and sharpens the appetite."

Propagation always by seed. From a pint to ten quarts of seed will be annually required, according to the demand. Where a daily supply is required, seed should be sown every third, fourth, or fifth day throughout the year. $\mathrm{As}$ the crop is to be cut when in the cotyledon leaf 
state, it should be sown thick ; and, to prevent the earthy particles mixing with the leaves, it should never, if possible, be covered. Cotyledons, or seed-lobes, are appendages of the embryo, enclosing or accompanying the tender plantlet, and containing its first nutriment. Upon the principle of the presence or absence of cotyledons we have the grand and primary division of plants that are cotyledonous on the one hand, and plants that are acotyledonous on the other; as well as the subordinate divisions of monocotyledonous, dicotyledonous, and polycotyledonous plants also. These distinctions are deemed to be the true key to a natural system, and form the basis of the arrangements of Jussieu. The two first lobe-looking appendages, vulgarly called seed-leaves, as shown in this plant, exemplify the cotyledon. The London market-garden method is to sow the seed thickly on the surface of old tan made smooth and level, and laid over the floors of their vineries; for to such an extent is this branch of culture carried for the supply of the metropolitan demand, that one grower alone, Mr Chapman of Vauxhall, sows a whole house every other day. The tan is well wetted previous to sowing the seed, and no covering whatever is put on the seed beyond a damp mat, which is laid over to keep in the moisture. They vegetate according to the degree of temperature kept up, which should, however, not exceed $60^{\circ}$ or $65^{\circ}$. When the plants are about 2 inches high, the mats are removed during the day to admit light to give the necessary green colour to the young leaves, but are put on again at night to hasten the growth; for, as with all salads, the quicker the growth the better and more tender the produce. The crop is cut when from 4 to 5 inches in height, and each handful, as it is cut, is put into a clean punnet-basket, and is fit for use. If thus grown, no washing is required, excepting a slight rinse shortly before using. Steeping such salads in water, to rid them of sand and dust, destroys the flavour, and renders them flaccid and tough. They should be cut as soon previous to use as possible. The above may be called the winter culture upon a large scale. For private use, shallow boxes or pans are used, and these are placed in any spare corner of hothouses or pits, where the necessary temperature is kept up. The boxes should be 4 inches deep, and of a length and breadth suitable to the accommodation; and sowings should take place every third or fourth day, and the crop placed as near the glass as convenient, on suspended shelves or otherwise.

Summer culture must be conducted on the same principle, in spring and autumn covering the crop with hand-glasses ; and, during the heat of summer, sowing on cool borders behind a wall.

To save seed-a process scarcely worth the attention of the private grower, on account of its cheapness-a crop may be sown thin in an open part of the garden in March or April. The seed will ripen in August, and, when thoroughly dried, may be thrashed out for storing by. The European names are-Moutarde in French; Mostazo in Spanish ; Senapa in Italian; Senf in German; and Mosterd in Dutch.
Black mustard (Sinapis nigra L.) belongs to the same class and order as the last, and is, like it, a native of Britain, and found in similar situations; it is also a native of Italy. It is no doubt the mustard of the ancients, although there are great doubts of its being the mustard of Scripture, whose seed is referred to by our Saviour. It is by some cultivated as a salad plant, the same as the last, but the tender leaves are oftener used during spring as a substitute for, or as an addition to, spring greens. The uses of its seeds for medicinal and domestic purposes are well known. The ancients ate the young plants stewed, and the leaves of the older plants were boiled like other pot-herbs. The young leaves of both this and the white mustard are usually mixed with those of cress in salads. When grown as a spring green, the seed is sown in autumn, and the plants are thinned out to a foot or 18 inches each way. Like all oleiferous seeds, they greatly exhaust the ground, and retain their vegetative properties for an unknown length of time; so that, where it has once been grown, and the seed allowed to drop and become buried, they will come up for a century or more afterwards. This is the reason why charlock (Sinapis arvensis) is so difficult to eradicate out of land it has once been allowed to seed on. The black mustard has been recommended to be sown on soil infested with wireworm, the roots being so acrid that the larvæ will not eat it, and hence perish from hunger. The European names are the same as for white mustard.

\section{§ 8.-CRESSES.}

Garden cress (Lepidium sativum L.) belongs to the same class and orders as the last. The generic name is derived from Lepis, a scale, from the scaly form of its silicles, or parts of the pods to which the seed is attached. Its native country is unknown. Introduced to England before 1548 .

Of the same use as the white mustard (which see). Of the white mustard there is only one variety-of the garden cress there are several, all, however, used for the same purposes; but, being somewhat different in their cultivation, we shall notice them under the next head, viz.-

The common garden or plain-leaved cress, and the curled-leaved, are the two sorts most generally cultivated, and in this respect they differ not from that of the white mustard already detailed. In respect to merits, the former is the most delicate, as it is used younger; but the latter is preferable, particularly for garnishing.

Golden cress.-A variety of slower growth, and of a yellowish-green colour. Seldom grown.

Broad-leaved cress.-A coarser variety, with broad spatulate leaves, seldom forced, but usually grown for mixing along with rue, leektops, nettle-tops, and overgrown mustard, in rearing young turkeys and other poultry. For soups it answers very well.

Normandy curled cress. - A very valuable variety, by far too little cultivated. It was introduced to England about 1814, and in vol. vii., p. 38, of the "Gardeners' Magazine," we brought it before the notice of the public. Our 
practice, to secure a constant supply from the open air, as it is much hardier than any of the other varieties, is to sow at the bottom of a south wall in September and October; for Scotland, read beginning of September and middle of October. This will, with rery slight protection during severe frosts, afford a supply during winter and spring. Sowings made in an open border in March and April, and in a rather cool shaded place in May, produce crops in regular succession. In gathering it for use, the younger leaves should be picked off singly, rejecting the older and larger; but on no account cut the plants over as is done with common curled cress, as the Normandy variety will continue sending up fresh leaves for a long period. It is difficult to procure the seed true, the common curled being in general substituted for it. If, therefore, one is fortunate enough to obtain it genuine, it is better to save the seed for future use.

Broad-leaved Normandy-A sub-variety of the last, with broader and more succulent leaves. Its culture is the same as the last. To have either of these in perfection, they should be thinned out to 9 inches or a foot apart.

General remarks.-The European names are - Cresson Alenois in French; Tuinkers in Dutch; Mastinco in Portuguese; Gemenie garten kresse in German; Crescione in Italian; and Mastuerzo in Spanish.

Water cress (Nasturtium officinale H. K.) belongs to the same class and order as the former. The name is derived from Nasus, nose; Tortus, tormented-from the effects of most of the genera upon the muscles of the nose-a name given by Pliny. Indigenous in most parts of Britain, growing in small streams, generally where the water is pure, and having a slight motion.

One of the most wholesome of all our salad herbs, and one of the oldest in use. Its qualities are warm and stimulating - the very reverse, in some respects, to most other plants used in a green or uncooked state. Xenophon strongly recommended its use to the Persians; and the Romans recommended it to be eaten with vinegar, as a remedy for those whose minds were deranged, and hence the Greek proverb-" Eat cress, and learn more wit." The Dutch and English eat great quantities of this cress in spring, as an antiscorbutic. Gerard and Lord Bacon wrote strongly in its recommendation. The young shoots and leaves are eaten by themselves, often with bread and butter, and also used in spring soups and broths, as well as for garnishing cold meats. A salad so easily procured, and of so much importance to the health of townspeople and those of sedentary habits, cannot be too highly recommended. The supply of water-cresses brought daily to Covent Garden market alone has been calculated at 6000 bunches; and Mr Cuthill remarks that, "if 10,000 bunches more than they already received were brought every market morning, they would be all sold;" and this is perhaps not more than the half of the quantity sold in other parts of England daily.
Propagated by seed, and by planting rooted branches of the plants. Plants from seed are, however, to be preferred, as they do not run to seed so soon.

Sowing and planting.-Planting should be performed in spring or autumn, and sowing the seed in February, April, and June, by which fine young crops would be obtained.

Phillips recommends those having large pieces of water in their grounds to throw the plants on the surface of the water. They will mature their seed, and soon propagate an abundant supply. Cuthill says, "Few small places are without water, and nothing need be easier than to introduce it into a neatly-formed trench, 2 or 3 feet broad, and to plant the cresses," which may be gathered in a seedling state on the margins of brooks or streams, where they naturally abound. Plant three rows along the bottom of such a trench, setting the plants 2 feet apart in the line. Cover the surface between the plants with 2 inches of clean gravel or small stones, and allow the water to flow in to cover them to the depth of 3 inches at first, increasing it to 6 inches after they have taken root and begun to grow. Very excellent instructions are given for their cultivation in the 4 th vol. of the "Horticultural Society's Transactions," p. 540, and also in "The Gardeners' Magazine," vol. i. p. 151. The following is the substance of the former: The best place for forming a plantation is a clear-running stream, not more than an inch and a half deep, upon a sand or gravelly bottom; and if the water is supplied from a deep spring, so much the better, on account of the increased temperature of the water preventing it from becoming frozen during winter, and hence securing a constant supply of salad. The plants should be placed in rows parallel with the course of the stream. In shallow water, the plants should be set at the distance of 18 inches apart; in deep water, allow them from 5 to 7 feet. When the plants begin to grow in water one inch and a half deep, they soon check the current, so as to raise the water to the height of 3 inches about the plants, which is considered the most favourable circumstances they can be placed in. The cress will not grow freely in a muddy bottom, nor will the crop be so clean and well-flavoured. They should be planted in gravel or chalk. It is also absolutely necessary to have a constant current, for the plants will cease to prosper if the water is still or stagnant. They should be kept pretty thin by constant gathering, or pruning away occasionally superfluous shoots. In winter the water should be rather deeper than in summer, say 4 or 5 inches.

The beds should be cleaned out and replanted twice a-year, with a view of keeping them young and clearing away weeds, and any mud that may accumulate, as well as keeping the crop always clean and fit for use. Young plants are procured from the tops of the old ones, choosing those best furnished with roots. These are placed at the requisite distance on the bottom, with a stone on each to keep them in their places until they take root. The time of renewing the beds is in May or June, and from September to November. This renewal should be made progres- 
sively, so as to keep up the constant supply. Those replanted in May are fit to cut in August, and those planted in November are ready to cut in spring. It will be expedient to place planks in such a way, a few inches above the water, as to facilitate the operations of gathering.

The young shoots, to the length of from 4 to 6 inches, should be cut (not broken) off, and then carried to the vegetable-house, and thrown for two or three hours into a tub of clean saltwater, to rid them of insects or their larvæ. They should then be rinsed in clean water, and tied up in little bunches of about half a handful each.

Tipula repens (Linn.), T. sericea (Gmelin).-The larva of this insect fixes its cocoons very firmly to the under side of the leaves of water-cresses, and in this state is unconsciously eaten by thousands. The precaution stated above, of washing them in salt water, seems to be the only way of ridding the leaves of these insects.

The European names are-Cresson de fontaine in French; Berro in Spanish; Brunnenkresse in German; Agriao in Portuguese; Waterkers in Dutch ; Cressione di sorgenti in Italian. Many gardens have their water-cress beds; and no park in the kingdom, where water is to be had, even at the expense of an Artesian well, should be without one. The Parisians have formed water-cress plantations, and one or two exist in the neighbourhood of Edinburgh; and one existed a few years ago, and may do so still, at Mistley Hall, of 30 acres in extent.

American cress, or Belleisle cress (Barbarea pracos Dec.), belongs to the same class and order with the last. The narne is derived from its having anciently been called the herb of St Barbara. Indigenous to Britain, and found in watery places.

The Winter cress (Barbarea vulgaris H. K.) belongs to the same genus as the last, and is also indigenous to Britain, and found in moist shady situations.

Both are used as winter and spring salads. They are reared from seed; half an ounce will sow 20 feet of drill. Sow in drills a foot apart, and an inch deep, choosing a damp shady situation. Sow about the 20th of August or 1st of September for a winter and spring supply. In Scotland a warmer and better situation should be afforded them. For summer use sow once a fortnight from March to July. Water abundantly in dry seasons.

Pick the outside leaves for use, and cut down flower-stems as they appear. In November, afford the winter-standing crop a slight shelter of small branches stuck amongst them; and if too crowded, thin out to prevent injury from damp. The seed of both is produced in abundance by leaving a few plants to come into flower during the summer.

The European names of the American cress are-Cresson d'Amerique in French; Amerikanischer kresse in German. It is also called by some English seedsmen Black American cress, and also French cress. The winter cress is known as Cresson de Terre in French; Hierba de Santa Barbara in Spanish; Winter kresse in German; Erba di Santa Barbarea in Italian; and Winterkers in Dutch.
Indian cress, or Narsturtium, abbreviated to Sturtion by many (Tropceolum majus L.), belongs to the natural order Tropæolex, and to the class Octandria and order Monogynia in the Linnæan arrangement. This genus forms the whole of this order, which is remarkable as being the only natural order in which the peculiar acrid flavour of the Cruciferæ is found to exist. The generic name is derived from Tropaum, a trophy. The leaf resembles a buckler, and the flower an empty helmet, of which trophies were formed. It is a native of Peru, and was introduced to England in 1686 ; but the minor variety was first brought to Europe by the Spaniards in 1580. In the first edition of Gerard's "Herbal" we find it described by him as growing in his garden, where Holborn now stands. Both $T$. majus and $T$. minus are cultivated in our gardens-the former most generally. The French style the flower La grande Capuchin.

Both varieties are much cultivated as ornamental climbers; and the fruit, if gathered before it ripens, makes an agreeable pickle without the aid of spice, and is an excellent substitute for capers, which it much resembles. It is accounted a good antiscorbutic. The flowers as well as the young leaves are used in salads. The flowers are also used to garnish dishes, particularly by artificial light, and were in our early days much more employed for this purpose than at present. The seeds are pickled in salt and vinegar, when made into imitation capers.

Although both varieties are perennial in their native country, the coldness of Britain prevents their continuance through the winter; they are therefore treated as annuals, and sown every March or April. They are usually sown in single rows, one row of a few yards in length being sufficient for the culinary purposes of a private family. The seed, wlich is large, is deposited in drills $2 \frac{1}{2}$ inches deep. When grown for ornament, they are sown two or three seeds in a patch at the foot of a trellis or pyramidal support, and, if intermixed with sweat pease, have a very good effect. When grown by market-gardeners, or upon a large scale for their seed, they are sown in drills 3 feet apart, topped when about 2 feet in height, and treated the same as scarlet runners (which see). They transplant freely, and to have them early in flower particularly in Scotland, they are sown in March in pots or boxes placed in a mild frame or pit; when 3 or 4 inches in height, they are set in a shaded sheltered place for a few days to harden off, and are then transplanted to where they are finally to grow. This is the best way to manage them, as the seed, if too early sown in cold soil, is apt to rot; and if planted out too soon, is as apt to be killerl by the frost, more than $1^{\circ}$ of which it will not stand.

Soil.-This should not be too rich, else a gross disposition is induced, rendering them rambling in growth, and producing fewer flowers or seeds than if on a rather poor light soil.

Neither disease nor insects seem to attack the plants. The blossoms are endowed with the power of emitting electric sparks towards evening, a phenomena first observed by the daughter of Linnæus. It is most distinctly seen 
with the eye partly closed. The berries should be gathered for use while quite green. Abundance of seed will be found in a ripened state when the plants are pulled up upon the first attack of frost. They do not retain their vegetative powers above two years. Their European names are-Capucine in French; Mastruco do Peru in Portuguese; Kapuzinerblume in German ; Fior cappucino in Italian; Spaanche kers in Dutch; and Capuchinas in Spanish.

\section{§ 9.-PURSLANe, CHERVIL, ETC.}

Purslane (Portulaca sativa Haw, P. satixa, var. A urea Haw, and $P$. oleracea L.-all tlie three are cultivated in our gardens) belongs to the natural order Portulaceæ, and to the class Dodecandria and order Monogynia in the Linnæan arrangement. The generic name is derived from Porto to carry, Lac, milk, supposed medical quality. $P$. sativa is a native of South America, introduced in 1652 ; while $P$. oleracea is a native of Europe, introduced 1582.

Its young shoots and leaves, which are very fleshy or succulent, are used in summer salads, and are sometimes used in French and Italian soups, and also as pickles. Its existence is only to be looked for in gardens of the highest order.

Being annuals, they are yearly reared from seed. A quarter of an ounce will sow a bed of 32 square feet. For a first crop, sow in February or March upon a gentle hot-bed, for all the sorts are very tender, and this first crop must have the protection of a frame and glass lights. A two-light frame will be sufficient. As the plants reach the height of 2 inches, thin out to the distance of 4 inches apart; the soil should be of such light and rich consistency as that usually employed for cucumbers. The second crop should be sown upon a warm border in dry light soil in May, and successional sowings should be made, if a constant supply is demanded, every fortnight till the first week in August, after which recourse must be had to bottom-heat and the protection of glass coverings. It is best to grow them where they have been sown, but they may be transplanted in cases of emergency.

The shoots are fit for use when from 2 to 5 inches in length, and should then be cut off with a knife and slightly rinsed in clean water, when they are fit for use. The European names are-Pourpier in French; Porcellana in Italian; and Portulak in Dutch and German. The seed is not worth the trouble of saving.

Chervil (Charophyllum sativum Pers.) belongs to the natural order Umbelliferæ, and to the class Pentandria and order Digynia in the Linnæan arrangement. The generic name is derived from Chairo, to rejoice; Phyllon, leafthat is to say, a plant whose leaves have a pleasant smell. A native of several parts of Europe; by some considered indigenous to Britain.

Gerard cultivated it in his garden; and Parkinson says, "It is sown in gardens to serve as a salad herb." These are the earliest notices we have of its being cultivated in Britain. Pliny tells us that the Syrians cultivated it as a food, and they ate it both boiled and raw. It has long been cultivated by the French and Dutch, and they have still hardly a soup or salad but the leaves of chervil make a part of the composition.

The leaves are used in most salads and in many soups, and as a seasoner it is by many preferred to parsley, when used for these purposes.

Being an annual, it is propagated by seed. To maintain a constant supply, sow about the beginning of February, and make successional sowings every month till August, about the end of which a larger sowing should be made in a warm situation, to form a winter and early spring supply. Some only sow in spring and autumn, and trust to keeping the plants from seeding; this is, however, precarious. Sow in drills 10 inches apart and 1 inch deep. Transplant only in cases of emergency, unless it be in saving seed of the fine-curled variety, which will be improved in the fulness of its leaves, if transplanted once or twice before it shoots up for seed. An ounce of seed is sufficient to sow 24 square feet. It should be sown thin. A light soil, not over-manured, is the best for all odoriferous plants.

When the leaves are 2 inches in height they are fit for gathering; a small handful is sufficient for a large salad.

Plain chervil is the most common, but except that it is hardier than the curled varieties, it should be discarded.

Curled chervil.-This sort, on account of its delicately-curled leaves, is much used for garnishing, as well as for the ordinary purposes for which the plain sort is used. Being a larger grower, it requires more room for its development; indeed, the plants, to have justice, should stand a foot apart each way. When intended for winter use it should have the protection of hand-glasses, frames, or branches of trees stuck thickly around and amongst it. In very unfavourable situations it is well to pot a dozen or two plants, and shelter them under glass during winter.

Frizzled-leaved French chervil (Cerfeuil frise) is an improved variety of the last, even more beautiful, but also more tender. It is best adapted for summer crops.

Few insects attack the odoriferous plants. Seeds of all the varieties are easily saved. The plants of the two last varieties should be transplanted once or twice when intended for this purpose, and a somewhat richer soil will be of advantage. The European names are-Cerfeuil in French; Cerfoglio in Italian; and Gartenkerbel in German and Dutch.

\section{§ 10.-RAPE, CORN-SALAD, ETC.}

Rape (Brassica napus L.) belongs to the natural order Cruciferæ, and to the class Tetradynamia and order Siliquosæ. For derivation of the generic name, vide section CABBAGE. Indigenous to Britain.

It is cut when quite young, and before the second leaves are formed, and mixed with cress 
and mustard. Its culture for this purpose is the same as these. It is the Navette of the French; Mapo salvatico of the Italians; and the Rapskohl of the Germans.

Corn-salad (Valerianella olitoria Dec.) belongs to the natural order Valerianeæ, and to the class Triandria monogynia in the Linnæan arrangement. The generic name is a diminutive of Valerian. Indigenous to Britain. Another species is cultivated-viz., V. eriocarpa, or Italian corn-salad, of much larger growth.

Both are used as a substitute for lettuce in spring. The latter sort, besides being used for salads, is also used when grown in rich soil and of considerable size, as a substitute for spinach. They are both much used on the Continent, where they appear under the names of Salade de chanoine, Mache, Poule grasse, Doucette, \&c. Sow in August and September for winter and spring use, and in February and March for summer supply. The seed is small. Sow them, and cover about the eighth of an inch. An ounce of seed will be sufficient for an ordinary family.

Brook-lime (Veronica Beccabunga L.) belongs to the natural order Serophularinæ, and class Diandria and order Monogynia in the Linnæan arrangement. The derivation of the name is doubtful. Indigenous to most parts of Britain, growing in ditches and streams often along with water-cresses, and is used for the same purposes, and cultivated in the same manner.

Wood sorrel (Oxalis acetosella L.) belongs to the natural order Oxalider, and to the class Decandria and order Pentagynia in the Linnæan arrangement. The generic name is derived from Oxys, acid-from the acid taste of the leaves. Indigenous to most parts of Britain, existing in vast quantities in moist woods. It is used as an ingredient in spring salads; and, although quite capable of cultivation, it is found so plentifully in April and May, in a natural state, as to render its culture scarcely profitable. It is much used on the Continent, and is the Oseille of the French, Acetosa of the Italians, and Sauerampfer of the Germans.

Coriander (Coriandrum sativum L.) belongs to the natural order Umbelliferæ, and to the class Pentandria and order Digynia in the Linnæan arrangement. The generic name is derived from Koris, a bug, from the smell of the leaves. A native of England, in corn-fields. It is chiefly cultivated in gardens on account of the tender young leaves, which are used in soups and salads. It is raised by seed sown annually-in February and March for summer use, and again in August or September for winter supply. A very few plants are sufficient for an ordinary garden.

General remarks.- It delights in a light sandy soil. The plants should stand 18 inches apart. European names - Koriander, German and Dutch; Coriandre in French; and Coriandro in Italian.

\section{§ 11.-THE RADISH.}

Natural history.-Radish (Raphanus sativus L.) belongs to the natural order Cruciferæ, sub-order Orthoploceæ, and tribe Raphaneæ, and to the class Tetradynamia and order Siliculosæ. The generic name is derived from Rha, quickly; Phainomai, to appear, from its rapid germination. The name radish is supposed by Phillips to come from Radix, a root. The cultivated radish is thought to be a native of China, but it appears, by the writings of ancient naturalists, that its culture is of great antiquity in many parts of Europe, although it was not grown in England prior to 1548. They were very highly esteemed by the Greeks, and were grown largely in Egypt in the days of the Pharaohs, for the abundance of oil their seeds afforded; they are still grown to a great extent in that country at the present day. Pliny speaks of a turnip-rooted kind, and of a sweet and tender sort, and of one that continued good throughout the winter. Gerard cultivated four kinds of radishes in Queen Elizabeth's time.

$U$ ses. - In the south of France the roots are roasted in wood-ashes; they are also used there in soups, to which they give an agreeable flavour. The Roman physicians recommended them to be eaten raw in a morning with salt, and before taking any other food, a practice in use in some parts of England at present. Radishes abound in a penetrating nitrous juice, which makes them diuretic, and cleansing to the intestines and viscera. They are considered also as excellent antiscorbutics. At present they are usually eaten raw with salt as a salad, with butter and cheese. The leaves are sometimes boiled as greens, and the roots make an excellent dish, when rather too large for a salad, by being boiled and served to table as asparagus. Neither the roots nor leaves, however, afford much nourishment. The roots are often sliced and added to mixed salads, and are occasionally eaten alone with salt, vinegar, and other condiments. The young leaves are also eaten along with mustard and cress, and for this purpose the seed is often sown in the same manner as they are. The seed-pods, when nearly full-grown, but still green and tender, are added to mixed pickles, and pickled alone as a substitute for capers.

Propagation. - The radish, being an annual, is propagated from seed.

Sowing.-As this salad is in request throughout the year, successional sowings must be attended to. Where there is the accommodation of a tanked pit or dung-heated pits or frames, a sowing for winter supply should be made every ten days from the beginning of November to the beginning of March. Where such conveniences do not exist, then for winter use a sowing should be made at the end of October, another about the middle of November, one at the end of that month, and others twice during Decem- 
ber, January, and February; and from the end of that month sow every ten days, throughout the remainder of the season. These sowings have reference to the turnip-rooted and spindle-rooted varieties in ordinary use. For the black, white, purple, and brown Spanish sorts, which are hardy, and continue long fit for use after attaining their full size, these should be sown in August, and another crop in September, which will carry the supply through the winter, whether they be kept in the ground or taken up and pitted like potatoes. The seeds will in general germinate in less than a week, and in six weeks the plants will be fit for drawing.

The ordinary turnip and spindle-rooted sorts should be sown broadcast, whether in beds, in the open air, or in pits and frames. Avoid too thick sowing. Two ounces of seed will sow three lights of a pit or frame of the ordinary breadth, or the same quantity will sow a bed of 50 square feet in the open ground. They are often sown thinly between rows of potatoes in pits or frames, as they will be gathered off before the potatoes have attained a size to injure them. The hardy winter sorts should be sown in rows, as they occupy the ground much longer, and will require the surface to be frequently stirred up by the hoe. Cover the seed fully half an inch, and, in sowing in the open borders, avoid spilling any of the seed on the surface, as such will attract birds, which are very fond of them. Net the ground over to protect them; or, better, cover with wire-netting, which, although rather more expensive in the first instance, will be found the cheapest in the end. Both the tap-rooted and turnip-rooted kinds should be sown at the same time, as well as those differing in colour, to afford a greater variety in the salad. For late autumn, winter, and early spring crops, choose the warmest and driest border the garden affords; while for those for use during the heat of summer, a cool moist place should be selected.

The radish is one of the few exceptions to drill-sowing, which has for its chief principle the means afforded the cultivator for thinning his crops, so that each plant may have room to develop itself to the extent at which it should arrive, when vOL. II. in its fullest state of perfection. The radish does not require this care, as the strongest seeds vegetate first, and the plants produced from them are daily withdrawn for use, thus leaving room for those which follow in succession. It is good practice to sow at the same time with the radishes such seeds as lettuce, leeks, parsley, \&c., because these vegetate much more slowly than the radish, and as it is removed they come in in succession, and fill the space until such time as they are of such a size, and sufficiently hardened off, as to be fit for planting in the open border of the garden. Indeed, so great a difference exists in the germinating process of these seeds, that radishes, leeks, and parsley may be sown on the same day. When the first is withdrawn, the second takes its place; and when the plants are of a size to be transplanted to a nursery-bed, to fit them for final transplanting, the parsley comes in, which takes so long a time in germinating as to have given rise to the common but vulgar saying, "that parsley-seed goes nine times to the devil before it begins to grow." By following this rather unusual mode, time and space are economised; and as lettuce, leeks, and parsley plants are improved by transplanting, the advantages to them individually must be evident.

"The London market-garden practice for obtaining early radishes in the open air is to sow from the first to the last week in December, choosing as warm a situation for the purpose as possible. The ground being prepared, the seed is sown broadcast. The alleys are marked out, and the mould from them is thrown over the seed. A wooden rake is used to make all level; no iron rakes are used in market-gardens. After sowing, straw, which has been previously shaken out of the London stable-manure, is put over the beds 2 or 3 inches in thickness. When the plants come up, which depends upon the winter, the straw is removed every day, and put on every night, until all danger from frost is past. It takes $5^{\circ}$ of frost to hurt a radish after it is halfgrown."-CuthinL.

It is only in the most favourable situations that radishes, with the exception of the winter kinds, can be advantageously grown in the open air during winter. Did they even, by covering or otherwise, 
survive the frost, they would be hard and stringy compared with such as are grown rapidly on a mild bottom temperature; and, indeed, apart from their quality, what with covering and uncovering during winter, the cost in labour would be as much, if not more, than would attend their production in a heated pit or frame. Radishes grown in artificial heat require abundance of ventilation, and as little exclusion from the light by coverings as possible. The temperature should range from $48^{\circ}$ to $58^{\circ}$, but may be increased to $70^{\circ}$ with impunity; and tepid water should be frequently applied, for the radish is fond of moisture; but pouring cold water upon them, or any other forced vegetable, during winter, when the soil is already too cold, is extremely injurious.

Subsequent cultivation.-In the open air, radishes require protection during winter, either by the means practised by the London market-gardeners stated above, or by other means, such as hooping the beds over and covering with mats, \&c., all of which we think are far behind the conditions of the present age. If they are wanted during that period, glass as a covering should be employed; and where that is the case, either a tanked pit, or frames placed over vegetable matter in a state of fermentation, may be employed; the latter of itself, when broken down by decay, will, as a valuable manure, repay the expense of collecting and working.

Soil and manure.-The soil should be deep, light, and mellow, and thoroughly pulverised by deep digging or forking, without which radishes will ever be hard and unfit for use, and this is more especially the case with the long or spindlerooted kinds. Strachan, a writer in "The Horticultural Society's Transactions," vol. iii., p. 438, observes on this subject, "The character of a good long-rooted radish is to have its root straight, long, free from fibres, not tapering too suddenly, and especially to be fully formed on the top, or well shouldered as it is called, and without a long neck; the roots should be ready to draw while the leaves are quite small, whence the name of 'short-top radish ;' and if they soon attain a proper size, and also force well, they are then called early and frame radishes." To attain these properties the soil should be light and rich, but not made so by recent manuring, unless of a very decomposed description.

Taking the crop and subsequent preservation.-Radishes are gathered day by day, and hence serve the purpose of thinning; still they should on no account be sown thick; but, supposing the seed good, the plants at germinating should stand 1 inch or $1 \frac{1}{2}$ inches apart. The strongest-leaved ones, therefore, may be taken as the largest, but not always the best root; these should be drawn first. When collected and in the vegetable-house, they should be washed quite clean, the thready fibres, where they exist, cut close off, also a small part of the tips of the root, and all the leaves removed excepting two or three of the last-formed ones on the crown, which should be retained. Care must, however, be taken that the roots do not become too old and hard; a good criterion is to break them over, and if the parts separate freely, then they are fit for use; but if they do not, then they are too old for salad purposes. The winter or Spanish kinds should be dug up about the beginning of November, deprived of their tops, and pitted like potatoes, or buried in sand in the root-cellar. This sand, however, should not be too dry, for reasons given elsewhere.

Approved sorts and their qualities.-An excellent paper on the classification of radishes will be found in the 4th vol. of the "Transactions of the Horticultural Society," p. 13. By this arrangement they are divided into three classes, namely, Spring and Summer kinds, A utumn kinds, and Winter radishes.

The Spring and Summer kinds consist of scarlet or salmon-coloured-and its sub-varieties, short-topped scarlet, and early-frame scarlet; and to these we may add, as improvements on the originals, Wood's early frame, not very long, but very early, and well calculated for forcing; Beck's superb short-top, a long-rooted variety, of good shape and colour, with leaves particularly small; long white Naples, an improvement on the old long white cultivated in Gerard's time-avery good variety, and esteemed by some on account of its semi-transparent white colour. Purple-rooted only grown as affording a variety in point of colour.

Of turnip-rooted sorts we have the white and red; and of these some sub-varieties, particularly of the latter-such as the pink, rosecoloured, scarlet, and crimson, which are mere shades of difference in colour. The scarlet oliveshaped, a quick grower, tender, and considered rather milder than most other sorts. There is a grey turnip-rooted sort grown by some; we have found it to be merely a dirty white, and 
the large and small yellow turnip-rooted variety stand no higher in our estimation.

The Autumn kinds are the round brown, large in size, not very handsome in shape, nor of a decided colour. White Russian, a very large sort, of good form, somewhat resembling a wellformed white carrot; in flavour resembling rampion. Not in general cultivation in Britain, but much grown in the north of Europe. The red metz, a French variety esteemed in the Paris markets, in which it forms the principal supply; roots of moderate length, rose-coloured, with few leaves, and those very small.

Winter radishes.-Of these, the best is the large Spanish black-rooted. The skin is black, with numerous white dots; irregularly pearshaped; flesh white, firm, and solid; much hotter than any other kind. Esteemed on account of its hardiness, and as affording an agreeable winter salad when cut into slices. Large white Spanish; skin white, tinged with green; flesh firm, solid, and white; form oval; flavour hot and pungent. Purple Spanish is only a subvariety of the black; the skin is purplish ; flesh firm and white. Oblong brown, another subvariety, with brownish skin; shape pyriform; plant very hardy. The Chinese rose winter radish is also a sub-variety of the white Spanish.

Insects and diseases.-The plants, when in their seed-leaf state, are preyed upon by the aphides, and other insects, which are destructive to all plants of the order Cruciferæ : vide $\mathrm{CAB}$ BAGE and TURNIP.

General remarks.-Some of the best plants should be saved from the principal spring sowing, for seed. Take them carefully up in May, preserving the leaves entire; select the most perfectly formed roots, and transplant them into rows 3 feet asunder each way, inserting the roots with a large dibber so deep that their crowns will be just level with the surface of the ground. It is unwise to save seed from more than one variety flowering at the same time, as the plants are liable to hybridise with one another, and seed of the previous year's sowing should always be sown. To save seed of the winter radishes, some of the best-formed bulbs should be planted as above in March, and the pods gathered when they become brown and fully matured. Seed radishes must be covered with nets to protect them from birds ; and if the quantity of seed to be saved is great, the whole stalks should be cut over, and, when dried, stacked by until there is time for thrashing them out. If the quantity is small, the pods may be cut off individually as they ripen. "The seeds of the different varieties are easily distinguished by an experienced seedsman. Those of the long white radish are small, flat, and pale; of the scarlet and purple long-rooted, large; and of the first very light coloured, compared with those of the latter; of the white turnip, small, round, and brown; scarlet turnip rather larger, and somewhat darker; purple turnip larger and brown, being similar to the longrooted purple, except in size."-Cottage Gardeners' Dictionary, p. 762. The pods for pickling should be taken when nearly full grown, but still soft and green, which will in general be the case in August.

The European names are-Radis or Rave in French; Rabano in Spanish; Rettig in German; Rafano in Italian; and Tamme radijs in Dutch. 


\title{
CHA P TER VII.
}

\author{
ESCULENT-ROOTED PLANTS.
}

\section{§ 1.-THE CARROT.}

Natural history.-The carrot (Daucus carota L.) belongs to the natural order Umbelliferæ, and to the class Pentandria and order Digynia in the Linnæan arrangement. The generic name is derived from Daio, to separate, because it dispels flatulency; the specific name, Carota, is from the Celtic Car, red, the colour of the root. The carrot, in its wild state, is indigenous to many parts of Britain, generally in sandy or chalky soil ; when and how it became reclaimed, or elevated to its cultivated state, is not now known. In that state it was known to Dioscorides and Pliny; and the latter informs us, book xxv. chap. 9, that the best kinds came to Rome from Candia, and the next best from Achaia. 'Theophrastus, in the 9 th book of his "History of Plants," says that the best carrots are found in Sparta. Gerard calls these plants Daucus cretensis verus, or Candia carrots, and says that the true Daucus of Dioscorides does not grow in Candia only, but is found upon the mountains of Germany, and upon the hills and rocks of Jura, about Geneva. It is possible, therefore, that Britain received her first supply from the latter situation. Later authorities think that the carrot was introduced into this country by the Flemings, in the time of Queen Elizabeth, and that they were first sown about Sandwich, in Kent. Of one thing we are certain, that carrots, as well as several other culinary vegetables, were imported to London, from Holland, during Elizabeth's reign, and moreover that the leaves of carrots were used in the head-dresses of the ladies of her court, in imitation of those of previous date, who employed the leaves of the wild carrot for a similar purpose.

Uses.-Few vegetables are in greater demand for culinary purposes than the carrot. It is used in soups, in stews, as a vegetable dish, and even in puddings; and so much are young carrots in demand in good cookery, that they are supplied throughout the summer from the open ground by repeated sowings, and during winter by artificial heat. In some parts of England carrots are served up with fish, as parsnips have long been, during Lent. Both the seed and the roots are used in medicine, the seed of the wild carrot being considered one of the most valuable diuretics of native growth. The roots of carrots contain a large portion of saccharine matter, and have been used in the manufacture of sugar, and in distilling, all over the Continent, where their cultivation has been longer and more extensively pursued than in Britain. Excellent bread has been made of white carrots, when washed and boiled, and mixed with flour in equal proportions by weight: such bread will keep good for a week.

Propagation.-Although the carrot is a hardy biennial, the roots, which are the only part used in domestic economy, are fit for use the same year. Seed must therefore be sown annually, they admitting of no other mode of propagation.

Sowing.-A small sowing may be made about the beginning of February, choosing a warm dry border, with a view to have young carrots fit to draw for use by the beginning of May; and another should follow about the middle or end of the month, and again a larger sowing by the first week in March : the state of the weather must, however, regulate these. These periods are adapted to southern culture; in the north, it is seldom that seed sown previous to the 1st of March repays the trouble. Crops sown so early in the season will derive considerable benefit from the ground being covered with dry litter during the continuance of frosts. The early horn only should be employed for these early sowings. For general crops, the middle of March and during April is a proper season, taking advantage of the ground when it is dry. For a supply of young carrots during winter, sow on slight hot-beds in December, January, and February. The two later sowings will be the most satisfactory. The end of April, and even the middle of May, is preferred by many for getting in their principal. crop, they thinking thereby to escape the 
attack of the carrot maggot, Psila rosoe: this, however, depends to some extent on the season, for cold and late springs have their effect upon the insect as well as upon the germination of the plant. Many also defer till the beginning of May, from the apprehension that, if the seed is sown earlier, the plants will be liable to run to seed. Sow again in June and July, and lastly towards the end of August or beginning of September-the later crop to supply autumn demands. Some depend on this sowing for a winter supply, substituting them for young carrots, for which they are a poor representative, except in form, being devoid of the same flavour and colour; and although they may pass with some for such purposes, they will not escape the keen eye of a French cook. Forcing carrots during winter is no serious matter, if the conveniences of hotbeds or tanked pits be at command; and those who will not go to the expense of such conveniences must just go without, or be content with such as are produced by late sowing, and kept in the ground till they are wanted. One great advantage arising from not sowing too early, is that the seed has a much less time to lie in the ground before germinating, and hence brairds as soon as the weeds, and therefore admits of earlier hoeing ; whereas, if very early sown, the weeds get the start of the young carrots, and render the operation of cleaning them more tedious and less effective. The seed does not come up for four or five weeks in spring, and for three or four in summer and autumn. The seed of chickweed, and many other weeds, vegetates in much less time in the same temperature, and hence the propriety of bringing on an artificial germination, as afterwards to be noticed, reconciling as near as may be the germination of the carrots and the weeds.

The ground intended for this crop should be trenched not less than $2 \frac{1}{2}$ feet in depth, and well pulverised during the operation. It should be in a sufficient state of enrichment from the manuring of the previous crop ; hence the ground from which celery has been removed is deemed the best, on account of its having been well wrought during the previous summer and autumn, and also as having been sufficiently enriched. Notwithstanding this, trenching is desirable; and if this opera- tion has been carried on as the ground became vacant, and thrown up in rough ridges, so much the better, as all that will be required at the period of sowing the carrots will be to level down the ridges and break down the clods with a coarse rake, rendering the surface sufficiently level and smooth for the drawing of the drills; for carrots should, for principal crops, be always drill-sown. When the ground is dry and prepared as above, the drills may be drawn with the drill-rake in the way described under section ONION, p. 33 ; a very slight pressure will give them the proper depth-an inch and a half. The distance at which the teeth should be set depends on the distance the rows are to be apart from each other; 9 inches being the usual distance for horn carrots, and 1 foot for the larger-growing kinds. The head of the drill-rake being marked off in inches, the three screws are to be slackened, the teeth set at their respective distances, and the screws to be tightened up again.

Previous to sowing, the seed should be well separated by rubbing between the hands with an admixture of a little dry sand, or finely-sifted coal-ashes, without which preparation the seeds would not separate freely, and hence the crop would come up unequal and patchy, besides wasting much of the seed. A quiet still day should be chosen, as the seed is so very light that it would be liable to be blown away before it could be covered in. Sow thinly in the drills; and as the seed is committed to them it should be covered in immediately : by walking along each drill with a foot on each side of it, and by drawing the feet along, one after the other, the process of covering will easily be effected; or, if the ground is wet, by going down every fourth or fifth space, the same number of drills may be covered by the head of a wooden rake. In this state the ground should be left; all scratching and raking, after the seed is sown, is so much labour thrown away, and the ground anything but improved by the operation. In extremely light soils it may, however, be slightly footed over, or trod in, as it is technically termed, because such soils are improved mechanically by the process of compression; hence in old and longwrought market-gardens, where the soil is soft and spongy, a light wooden roller is 
employed; and indeed, in large private gardens, rolling is a more business-like way of performing the operation, and involves a less expenditure of time.

It is a common practice with carrotgrowers to assist the germination of the seed-a process by which several days are gained, and which is of advantage in late wet springs, and also in gardens, where the ground may not be prepared, or where the previous crop has scarcely been removed from it. This process is effected by mixing the seed with damp sand, and placing it in a warm situation, such as on the floor of a hothouse, or indeed anywhere else, if in a temperature from $10^{\circ}$ to $20^{\circ}$ higher than the soil into which it is to be sown. Others place the seed in a bag, and steep it in rain-water for about forty-eight hours, eight or ten days before sowing is to take place. If in quantity, and spread out on a floor to the thickness of 9 or 10 inches, it will of itself generate sufficient heat, by a slight fermentation which will take place in consequence of being thus wetted, to cause the seeds to chip or germinate; but if in small quantities, it had better be laid upon a floor, or in shallow boxes, in some warm room, until this takes place. In either case, great care must be taken that germination is not allowed to go too far, else, in the process of sowing, the germs of vegetation will be liable to be broken off, which will destroy the seed. In careful hands, this is a process in seed-sowing which might be advantageously applied to all seeds that are long in vegetating. On the principle of simultaneous cropping, many market-gardeners sow a thin crop of radishes broadcast along with their carrots; the former come off for use before any damage is done to the latter. The carrot-seed, when not prepared as we have stated, will, early in the season, lie in the ground for five or six weeks before they come up, while the radishes seldom lie above a fortnight. Others, particularly private gardeners, sow a thin sprinkling of leek-seed along with the carrot, which may be done in the same drill, to admit of early hoeing. Others sow onions, and instances have been known where cabbages, savoys, or Brussels sprouts have been planted in the same line, and at 4 or 5 feet apart, as well as where planted promiscuously over the ground, in which the carrot crop has escaped the attacks of insects, while crops adjoining, sown alone, have been completely destroyed. We have seen a case of this sort, in the very old garden of the Earl of Morton, which has probably existed as such for two centuries, on which as fine clean carrots were produced as could be wished, and not for one season only, but for several consecutive years ; and we were informed by $\mathrm{Mr}$ Smeal, the gardener, that before he practised planting brassicaceous plants along with his carrots, none had been produced previously in the garden, in the recollection of the oldest person living at Dalmahoy. The Brussels sprout is the best for this purpose, as it grows tall and slender, and shades the carrots less than any of the spreading sorts.

Carrot-seed is more liable to be bad than most other garden-seeds; it will, therefore, be a wise precaution to sow 50 or 100 seeds in a flower-pot, and place it in the heat of a mild hothouse to vegetate. By counting the number of plants which come up, the quality of the seed will be correctly ascertained. One ounce of seed, if good, will be sufficient for a bed of 130 square feet, if sown broadcast; and if sown in drills, the same quantity will be sufficient for 150 feet.

Subsequent culture.-When the broadcast-sown crop comes above the ground, it should be thinned out with the 2inch draw-hoe; this hoeing, however, is more to suppress weeds and keep the surface of the ground open ; the principal thinning should take place when the plants have attained the height of from 2 to 3 inches, and then they should be singled out to from 4 to 5 inches, clearing the ground at the same time of every weed. This will admit of after-thinning for immediate use ; and, should the demand not require them in sufficient time, then a third and final thinning should take place, leaving the horn sorts 6 or 7 inches apart, and the large-growing sorts 9 or 10 inches. Those of the former, in drills, may be left at 5 or 6 inches apart, and the latter at from 6 to 8 inches.

Much as we advocate deep-stirring the ground between the rows of crops generally, the carrot forms almost the only exception, as, by so doing, it encourages the lateral fibres to grow large, and produce what is technically called forked roots ; flat-hoeing, however, for the 
suppression of weeds, must be attended to.

Soil and manure.-A light deep sandy soil is natural to the carrot; in fresh loamy soils it also flourishes in great perfection; a cold stiff clayey one is the least of all adapted to it, and in such soils expedients like the following are sometimes had recourse to-namely, to thrust a long dibber, such as is used for planting horseradish, into the soil, filling up the perforation with sand, and dropping 3 or 4 seeds into it, to be thinned out to one, after they have attained the height of 2 inches. In this way, carrots of large size have been obtained. In well-drained peaty soil, they have been successfully grown, and in such are usually free of disease, or the attacks of underground insects. In strong soils, they are often difficult to get up : to aid them in this, deep drills are drawn, and filled with light soil, such as leaf-mould, in which clean sea or river sand has been incorporated; in this the seeds are sown, and vegetate freely, and establish themselves until of a size and strength to penetrate the stronger soil below. The manures best suited to this crop are those in a liquid state, applied during growth : stable-yard manure, unless in a very decomposed state, should not be applied; and to keep it until it is in a proper state for this crop is an evident waste of its fertilising powers, which had much better have been employed in the feeding of the previous crop. The application of fresh manure causes them to grow forked and misshapen, as well as to be attacked by insects : the only manure we apply, and even that is seldom, is a light dressing of pigeons' dung; guano may also be applied with advantage, especially if in a liquid form. The experiments made some years ago by $\mathrm{Mr}$ C. W. Johnston, on the application of salt to various crops, gave the following result in the case of carrots: Light sandy soil, manured with 80 bushels of salt, and 20 tons of stable-yard manure per English acre, yielded 23 tons 6 cwt. 1 qr. $18 \mathrm{lb}$; the same extent, with 20 tons of manure, only 22 tons 18 cwt. 0 qr. 26 lb. ; manured with 20 bushels of salt, only 18 tons 2 cwt.; without any manure or salt, 13 tons $4 \mathrm{cwt}$. Salt and soot is a favourite manure for carrots, and the quantity applied may be to the extent of 10 bushels of salt and
20 bushels of soot per acre. Turf-ashes, and the ashes of wood and garden-refuse, have been found beneficial when applied in a newly-formed state to carrot, onion, potato, and beet crops ; they contain silica, alumina, oxides of iron and manganese, sulphates of potash and lime, phosphates of lime, magnesia, common salt, and charcoal; they should not only be dug into the ground, but sown also on the surface, previous to drilling, by which means they come in immediate contact with the seed.

Forcing.-To obtain carrots in a young: state, fit for use during winter, artificial means must be resorted to. A mild hotbed of dung and leaves, or a tanked pit, is the proper accommodation for their seed; therefore, if the true early horn, which is the best suited for forcing, as well as most approved of by cooks, should be sown at the periods stated above, either the tank or bed should be covered, to the depth of 9 inches, with light rich sandy soil, and the seed sown in the broadcast manner, and covered about half an inch with sharp dry sand; a temperature ranging from $55^{\circ}$ to $65^{\circ}$ will be sufficient, and instead of raising this temperature by additional fire-heat, during frosty weather, rather cover up the glass roof to exclude the extra cold. Where a less abundant supply is wanted, seed may be sown in shallow pots, and these placed in a mildlyheated pit, close to the glass. Radishes and young onions may be obtained in the same manner.

The London market-gardeners' practice is to sow the early horn only, in frames and beds to be hooped over, and covered with mats. A sowing is made in November, and another in January. When the plants are up, they are thinned to 4 inches apart. The hooping-over and mat-covering practice is behind the intelligence of the present times. Private gardeners act better, and grow their winter carrots under the protection of glass coverings, either in pits or in frames.

Taking the crop, and subsequent preservation.-Carrots should be taken up on the approach of winter, and when their growing season is over. To leave them in the ground injures their flavour and colour; and if so left after the turn of the year; they begin to become hard, fibrous, and ultimately, when their spring growth commences, unfit for use. Besides, it is evi- 
dence of bad cultivation to allow any ground to be occupied with a crop during winter, which might as well be dug up and stored by, and so give an opportunity for the improvement of the soil by manuring, trenching digging, \&c. Dry weather should be chosen for taking up the crop, and the implement employed for loosening them in the soil should be a fork, and not a spade. When they are withdrawn from the ground, the tops should be cut off: on this part of their management opinions are somewhat at variance, some recommending cutting the tops off an inch from the crown, others close to it; while some say a slice about the thickness of a shilling of the crown of the root should be removed at the same time. Those who advocate the latter process, do so with a view to prevent any further vegetation; while others say that thus cutting into the root causes it to rot. Those who favour the former do so with a wish to preserve the capability of vegetation, though certainly not to encourage the tendency to grow. In either way they will keep equally well, if dried in the sun when taken up, and pitted, like potatoes, in long narrow pits, or packed in sand not over dry in the root-cellar, placing 3 or 4 inches of sand between each layer of roots; for both here and in pits, if laid in thick masses together, fermentation takes place, and in a short time the whole will become a mass of putrefaction. Their keeping till spring depends greatly on the low temperature they are kept in, and also on vegetation not being encouraged. When packed in sand, they can readily be examined during winter; and should any signs of vegetation appear, the embryo leaves can be rubbed off. Packing them in too dry sand has a tendency to absorb the natural juices in them, and cause them to become hard and tough, and indeed unfit for use. Although they will keep in the ground all winter unscathed by frost, yet, when taken up and exposed to it, they are very susceptible of injury from it. In storing carrots for winter and spring use, we may observe that all the small fibrous roots should be cut clean away, as, if left on, they are liable to decay, and cause the decay to spread through the whole bin. In cutting off the tops at lifting, remove a slice from the crown sufficiently thick to divest them of those latent buds which surround the crown, and which would burst out into leaves and begin to grow upon the least rise of temperature in the bin or cellar. Carrots so treated do not wither, owing to the evaporation from the wound; nor do they at all decay. If allowed to sprout into growth, the natural sap is drawn from them, and they become hard, dry, and destitute of flavour. If carrots are left in the ground all winter, they should, on the approach of severe frost, be covered over with litter ; for it not unfrequently happens, if left unprotected, that the frost will destroy them.

Approved sorts and their qualities.-Carrots are divided into two pretty distinct classesnamely, Horn carrots and Long carrots; the former are nearly cylindrical, terminating abruptly at the point: being considered of superior flavour and delicacy, they are preferred for garden culture. The latter have long tapering roots, are of larger size, and are more fitted for field culture than for domestic purposes.

Early or common horn.-The most esteemed of all garden carrots for general culinary purposes, on account of the smallness of its heart and tenderness of its fibre; form thick, short, and terminating abruptly. Crown hollow, with very small neck and short thin foliage. Known also as Dutch carrot, or Dutch horn. Average size from 6 to 7 inches in length. Is the same as the early scarlet horn.

Early forcing horn.--Root somewhat conical, seldom exceeding 3 inches in length; well adapted for forcing for winter use.

Early shont horn.-This appears intermediate between the early forcing and orange carrot in size, shape, and period of arriving at maturity; probably a hybrid between them. It appears to be little known in Britain, but is much grown on the Continent, where it is known as Carotte courte hative, and in our seed-shops as red horn, short red, and short orange.

White Belgian horn. - Resembling the last, except in colour. A delicate and excellent sort; but its colour is objected to by cooks. Is also known as transparent white.

Long red horn.-Merely a variety found in seed-lists, differing little from the first. Is known also as long red horn.

Of Long carrots, the following are most in estimation for culinary purposes-

Altringham.-" This sort is easily distinguished from the orange and long red by its roots growing more above ground, also by their having more convex or rounded heads, tapering rather more irregularly and terminating more abruptly at the point; in colour most resembling the orange, but having a smaller heart. This variety is more difficult to procure genuine than any of the others, from its being remarkably liable to sport, even although the roots grown for seed be selected with the utmost care." It is of Cheshire origin, having appeared at Altringham, a village in that county, about 
forty years ago. It is known also as greentopped and superb.

Long red.-Colour deep red; heart small ; roots long, not remarkable for their thickness. A popular sort in some parts of England, where it is known as long Surrey, Surrey, Chertsey, and Studley. It is the Carotte rouge longue of the French, and pretty extensively grown over the Continent.

Long orange.-A large variety, less esteemed for culinary purposes than the red sorts, on account of its colour. It was formerly much grown in gardens in Scotland. The roots are produced entirely under ground, and it is, therefore, less liable to the attacks of hares than those which grow partly above ground. Roots thick at the top, regularly tapering to a point. Heart large, hard, and not readily softening in boiling. It is known as the large red, large field or cattle carrot, Sandwich, and is the Carotte rouge pale de Flanders.

Purple-coloured.-Roots of medium size; deep reddish purple; heart large and yellow. It is better suited for wet soils than any other, and in such is often cultivated by the French. It is known also as red, deep red; and is the Carotte violette, La violette, \&c. of the French seed-lists.

White.-Seldom grown in gardens. Of it there are two varieties, the common white, not worth cultivating, and the large white greentop. Produces immense crops, and is delicate and well-flavoured when cooked, but does not keep well through the winter. The large white green-top has roots short, of large size and great thickness while under ground; greenish-coloured on the top, which rises considerably above the surface. On account of the shortness of its roots and their standing so high above the ground, it is better suited for shallow soils than any of the others. The French possess a much greater variety of carrots than we think worth cultivating in Britain.

Insects and diseases.- "Insects infest the carrot crop in the root, stem, and flower. The plant no sooner makes its appearance than it is attacked by aphides, which are scarcely larger than cheese-mites, of a uniform pale-green colour, with six legs, two horns, and no wings. Their presence is indicated by the yellow foliage, and in pulling up the plant the roots are sound and clean ; but the crown is not only discoloured, but, on opening the embryo leaves, number's of the aphides are found concealed. Dusting the crop on the appearance of the aphides with caustic lime is a good remedy.

"The root of the carrot is affected by a disease named the rust, in which the crop gradually dies off, loses its saccharine qualities, and, changing to a ferruginous colour, becomes of little value. This complaint is occasioned by the larvæ of the carrot-fly, Psila rosa, fig. 61, eating galleries along the roots, which they inhabit through the summer, when they become pupæ in the earth; but a new brood hatches in the summer every three or four weeks. This fly is 3 lines long, of a pitchy black; the wings lie horizontally along the back when at rest, and extend beyond the tail, and when expanded extend to 5 lines. The maggots are ochreous and VOL. II. shining, cylindrical, pointed at the head, and obtuse at the tail, resembling cheese-hoppers, though they cannot leap, and are exceedingly transparent, every internal part being visible.

Fig. 61.
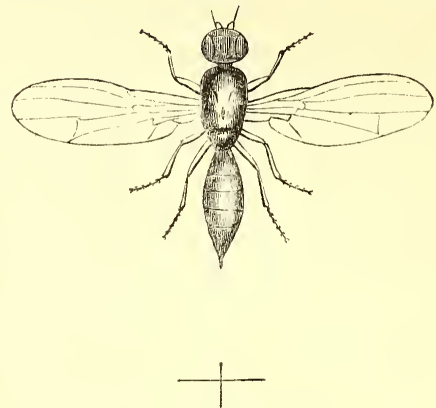

CARROT-FLY.

When the cavities have been opened by this maggot in the rest of the carrot, large numbers of the millipede (Polydesmus complanatus), and of the centipede (Scolopendra electrica), assist in extending the depredations. Another caterpillar, the larva of the ghost-moth, Hepialis humili, fig. 62 , also eats into the root of the carrot and injures it. The larvæ of the flat-body moths,

Fig. 62 .
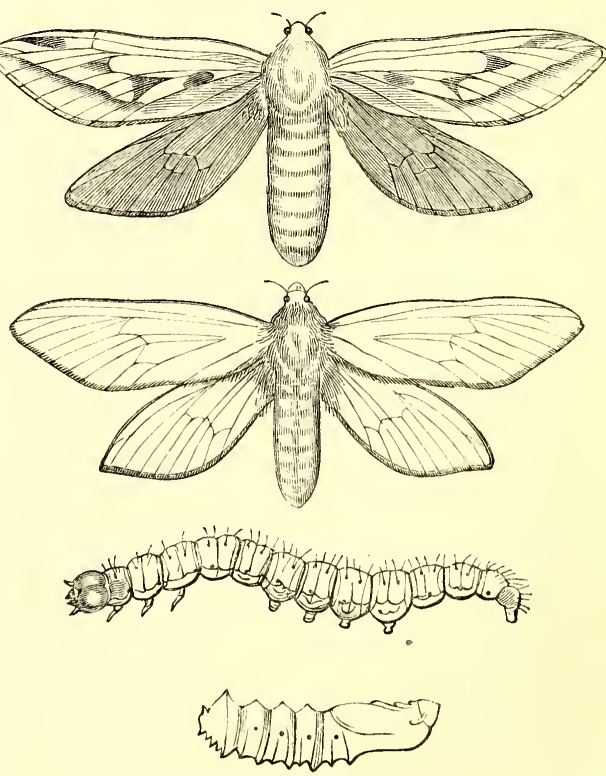

GHOST-MOTH (MALE AND FEMALE), CATERPILLAR, AND CHR YSALIS. Natural size.

Depressaria cicutella and depressella, bore into the stems of the carrot, causing the leaves to stint and decay; and the larvæ of the grey carrot blossom flat-body moth, Depressaria daucella, commit great havoc on the umbels of the flowers."-Book of the Farm, vol. ii. p. 96. 
Depressaria applana. - The common flatbodied moth, fig. 63 , is another of this genus of
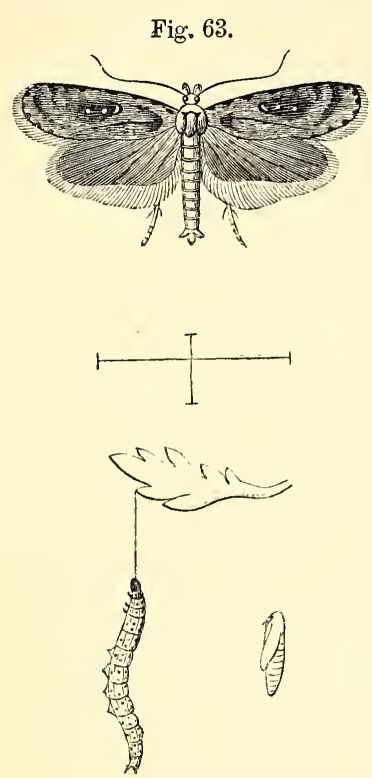

COMMON FLAT-BODIED MOTH, CATERPILLAR, AND CHRYSALIS. moths whose caterpillars do great mischief to seed - crops of carrots and parsnips, by devouring the whole umbels of flowers, and not unfrequently stripping the plants of their entire stem foliage. Their mode of operating is to draw the flowerheads together by many strong silken threads, which they afterwards extend into a network habitation, in which they remain consuming the seed-vessels, and after a time changing to pupæ, sometimes within the

web, at other times rolling themselves up in a leaf or within the stem, in which they remain secure.

The wire-worm (Elater) also attacks them under ground from their earliest stages. Copious applications of caustic lime are of much use in such cases; and highly-stimulating manures, such as guano or pigeons' dung, accompanied with deep-trenching, will be found beneficialthe former to stimulate the carrots into rapid growth, and the latter to derange the economy of the insects during their transformation.

Amongst other means of getting rid of the wire-worm is the rather novel one of feeding it with rape-cake to repletion. The rape-cake is broken up into small pieces about the size of marbles; and when the finer particles are removed by sifting, the larger pieces are sown in the drills at the same time as the seed; or a better way is to drill the ground from 3 to 4 inches deep, and in these drills to sow the rapecake, covering it up and drilling again at the usual depth for the carrot-seed. The month of March or beginning of April is the best time to sow the rape-cake, and which may be done weeks before the carrots are sown, because at that time the worm is resuming its depredations after awakening from its winter torpid state. The drier the season the more effective it will be in destroying the worm, as the cake will not so soon dissolve as it would in wet weather. The insect will devour it greedily, and continue to do so till it actually dies of repletion. Small pieces of rape-cake fastened to the end of a wooden skewer, and placed from 3 to 5 inches in the ground, form an excellent bait for catching this insect, managed as we have noticed at p. 69 .

old garden-soils have been long considered unfavourable for carrots, and as a remedy in such cases, it has been recommended to trench the ground 18 inches in depth, and to fork into the bottom of each trench quicklime laid over it to the thickness of an inch, applying it in autumn. In spring, at the period of sowing, a slight dressing of quicklime is laid on the surface, and mixed with the soil during the process of drilling and sowing. The rationale of this appears to be, that the lime acts chemically upon the inert matter contained in most soils in which a superabundance of humus exists, bringing it into a more active condition. It is possible that sharp river or sea sand would have much the same effect. Active manures, such as guano and pigeons' dung, act much in the same way, and excellent carrot crops have been produced when the latter has been laid on from 3 to 4 inches in depth, and thoroughly amalgamated with the soil.

To save seed, transplant the roots, if the weather will permit, about the beginning of January; set them in rows 3 feet apart, and the roots 2 feet distant in the line; and that they may have sufficient scope to work in, trenches at least $1 \frac{1}{2}$ feet in depth should be dug and the roots set in them, placing in the bottom of each trench a slight dressing of decomposed manure or a thin sowing of guano. If the ground on which the crop has been grown is in sufficient condition to mature the crop of seed without manure, it would be advisable to mark the carrots at the above distance and to dig up all the rest, leaving them where theygrew from seed. This may safely be done if the crop at taking-up indicates proper form and pureness as to variety; if otherwise, it is better to take them up entirely, and select the best-formed roots for replanting. The best seed will be produced where the plants are sufficiently apart to admit light and air amongst them. The first seed which ripens is also the best, and in moderate situations will begin to ripen about the lst of September, when care must be taken that all the heads as they ripen are cut off with a sharp knife and placed under covering to dry. The umbels of seed, if once wetted by rain when nearly ripe, will often rot altogether ; or if not, the seed will be of a bad colour and of inferior quality. As the seed dries it should be rubbed out and stored by for use ; it is injudicious to thrash it, unless the quantity be very great, and that with a very light flail, as the seed is apt to be injured by the process. One plant saved for seed will be sufficient for a small garden, and so on in proportion to size.

General remarks.-The European names areMohre, or Gelbe rube in German; Carota in Italian; Carotte in French; Geele wortel in Dutch; and Chirivia zanahor in Spanish.

" The nutritive matter contained in a crop of 25 tons, or 56,000 lb. per acre of carrots, consists of husk or woody fibre, $1680 \mathrm{lb}$; of starch, sugar, \&c., $5600 \mathrm{lb}$; of gluten, \&c., $840 \mathrm{lb}$; of oil or fat, $200 \mathrm{lb}$; and of saline matter, $800 \mathrm{lb}$. - Stephens ex Johnston's Lectures on Agriculturul Chemistry, second edition, p. 928. 


\section{§ 2.-THE TURNIP.}

Naturalhistory.-The turnip (Brassica rapa L.) belongs to the natural order Cruciferæ, sub-order Orthoploceæ, and tribe Brassicæ or Orthoploceæ siliquosæ, and to the class Tetradynamia and order Siliquosa. The generic name is derived from Bresic, Celtic name for the cabbage. It is found wild in various parts of Britain, chiefly in corn-fields and cultivated places. The ancient Greeks, to whom the turnip was well known, called it Gongyle, from the roundness of its root. At what period it became known in Britain, where it came from, or how its improvement from its native wild and useless state was brought about, is unknown. It appears to have been well known in Greece in the time of Democritus and Dionysius. Both the Greeks and Romans were well acquainted with its cultivation; and there are also faint traces in history that it was brought from Gaul and other northern provinces of the empire to Rome. It is also well known that, during the fifteenth century, the Flemings were considerably advanced in the art of cultivation, and that the turnip formed one of their important crops. From the remarks of Gerard, it would appear that turnips were not grown much in his time, except for domestic purposes. "It groweth," he says, " in fields and divers vineyards and hop-gardens in most parts of England. The small turnip groweth by a small village near London, called Hackney, in a sandy ground, and are brought to the Cross in Cheapside by the women of that village to be sold, and are the best I ever tasted. The bulbs, or knobbed root, which is properly called Rapum, or turnip, and hath given the name to the plant, is many times eaten raw, especially by the poor people in Wales, but most commonly boiled." The early Dutch white turnip is that noticed by our earliest horticultural writers. This, coupled with the well-known fact that, in Elizabeth's time, turnips, as well as other garden products, were quite common articles of importation to the English court, leads us to believe that our first garden varieties were introduced from Holland and Flanders, probably in the preceding reign. Those used for agricultural purposes were introduced long afterwards, mainly through Lord Townsend, who, when ambassador to the States-General in 1730 , seeing the importance of this plant as there cultivated, introduced both the plant and its cultivation into his own estate in Norfolk. In "Miller's Gardeners' Dictionary," 3d ed. fol. 1737, six varieties of garden turnips only are described, two of which, "the round turnip with a white root, and the round turnip with a purple root," he says, "are cultivated for the table in England." The introduction of the Swedish turnip, often grown in gardens in cold localities for winter and spring use, where the other sorts would perish, for the excellent greens or tops it produces, when other greens are scarce, and which are now also blanched and forced as a substitute for sea-kale, is more clearly traced; but whether it is of Lapland or Swedish origin, is not so satisfactorily determined. Sir John Sinclair says it was introduced to Scotland in 1781-2 from Gottenburg; others date its introduction from the same place at a somewhat earlier date. Miller, in the work quoted above, describes a yellow garden-turnip, and that it, as well as a long-rooted sort, "were formerly more cultivated than at present" (his time), "for it is now very rare to see either of these brought to the markets, though some years since they were sold in as great plenty as the common sort." The conjecture, therefore, by Professor Low, in "Elements of Practical Agriculture," p. 290, with regard to the yellow turnip, that it was a cross between the white turnip and the Swedish sort, admits of doubt. Yellow turnips being well known about the beginning of the eighteenth century, may have formed the type of most of those of the present day having globular roots, as, no doubt, the whites of the same period did of the majority of white globular ones as at present grown. Nor are we justified in presuming that the first removes towards improvement, in either case, took place in Britain, as at that time turnip cultivation was much more extensively pursued and understood in the Netherlands than it was with us in our then rude state of cultivation. In improved varieties, as well as in improved cultivation, Scotland, as far as regards this plant, stands pre-eminent at the present day. "Mr Morton, and the most eminent English authorities, now admit one turnip of Scotland to be equivalent to two of England; and that they cannot, in the latter country, raise turnips equal to those grown in Scotland. This superiority (of not only the Swedish, but other varieties) is not solely to be attributed to the soil and climate being more favourable to their growth, or to any improved method of cultivation adopted-these undoubtedly exercise considerable influence on the crop -but the principal cause of the superiority is to be traced, unquestionably, to the care bestowed in the growth of the seed."

Use.- "The use of the root in broths, soups, stews, or entire and mashed, is general in all temperate climates, and also the use of the tender radicle and stem-leaves, and the points of the shoots when the plant is coming into flower, as greens (or turnip-tops). The seedling-plants, when the rough leaf is beginning to appear, like those of all others of the Brassica family, are used in small salading. The earliest crop of turnips (without forcing) comes into use about the end of May or beginning of June" in the neighbourhood of London, and ten days or three weeks later about Edinburgh ; " and a succession is kept up throughout the summer by subsequent sowings ; and turnips may be had through the winter, partly from the open garden, and partly from roots stored up in the manner of potatoes. Hence a large portion of the kitchengarden is devoted to this crop. A well-grown turnip has a large smooth symmetrical bulb, a small neck, and a small root or tail, with few fibres, except nearest its lowest extremitv."-The Sub. Hort., p. 647. Boiled turnips, mashed or whole, is the orthodox accompaniment to a boiled leg of mutton. In the time of Henry VIII., turnips were used baked or roasted in the ashes, and the young shoots were used as a spring salad in those days, as well as boiled as a substitute 
for spinach. They make an excellent white soup. In France they are served as a sauce for ducks, and are much used for garnishing tongues, hams, stewed beef, \&c., being cut into roses and other devices. Turnip-tops, in spring, are the most wholesome of all green hardy vegetables, acting as a powerful antiscorbutic. The first settlers in Virginia were greatly afflicted with scurvy, until they were able to cure themselves by the turnips they cultivated. Turnips are nutritious and wholesome, and certainly ought to occupy a place on our tables more frequently than they do, particularly in Scotland, where the best turnips are grown and the fewest eaten; and this the more so, as potatoes are neither so nutritious, so wholesome, nor are they now so abundant. Turnips have also been converted into meal.

The French turnip, or navet, yellow or petit Berlin, is much cultivated all over the Continent for culinary purposes, and forms an important ingredient in almost all foreign soups. Stewed in gravy or stock, it is much prized; few dinners are served at which it does not in one way or other appear. The peculiar flavour is in the outer rind, so that, in using it, it should not be peeled. It was at one period much cultivated in Britain for cooking purposes, and is mentioned by Justice of Chichton as giving a higher flavour, if two or three are used, to dishes, than a dozen other turnips. It is occasionally met with in the London market, both of home growth, and also imported from Berlin and Altona. It is in demand in all families where high cooking is practised.

The bulbs, taken up in the beginning of winter, and planted in any light soil, rotten tan, or leaf-mould, and placed in dark cellars, the floor of a mushroom-house, or indeed anywhere, where a temperature of from $50^{\circ}$ to $60^{\circ}$ can be maintained, and light excluded, will send up their leaves in profusion, which, when from 6 to 9 inches high, and blanched white, form an excellent substitute for forced sea-kale-a luxury any one may enjoy by purchasing a few dozen roots in November, and growing them as above in a cellar, stable, or outhouse, in a moderate temperature, and in darkness.

Propagation.-By sowing the seed where it is to remain, excepting in the case of the Swedish or Rutabaga and Teltow, which readily admit of transplanting.

Sowing and planting.-For the earliest crop, seed of some of the most approved early varieties should be sown on slight hotbeds about the 1st of February, and treated as already recommended for radishes, $p$. 172. The first crop in the open air may be sown in the middle of March in England, the first week in April in Scotland, in both cases choosing the warmest border the garden affords. These will come into use, in the former case, in May and June, and in the latter about the end of May to the end of June-much, in all cases, depending on the locality, for there are some where it may be considered early if fit for use in July. A second sowing should be made about the middle of April in the one case, and the end of the month in the other. The first principal summer crop in both cases should be made from the middle to the end of May, for crops to come in from the beginning of July, and last throughout most of August. Sow again in June and July for the principal autumn crops; and indeed,in many places, it is sufficiently late to sow at the end of July for such as are to be used during winter. For nice small roots, to serve during winter and until spring, a sowing should be made on a well-exposed spot, both in the second and also in the third week in August; and in situations so favourable as around Edinburgh, and along the seacoast, a last sowing should be made about the middle or towards the end of September. This last crop we sow broadcast, and find them succeed very well, to be drawn for use through the winter, about the size of from a pigeon's egg to that of double that size. These also last much longer in spring, when taken up before vegetation commences, and buried in deep pits in a cold shaded place, to retard their growth. They serve for soups, and for that insatiable source of consumption, a French cook's stock-pot. Where very small young turnips are in constant demand, two or three intermediate summer sowings will be required; but these need not be so extensive as the general ones. It is unwise to depend for such a supply on the thinnings of other crops, because all turnips should receive their final thinning long before they are fit for such a purpose; and leaving them to attain the necessary size would only injure the principal crop.

The French turnip, or navet, should be sown during the first and third week in April, the first and third week in May, and again the second and last week in June, choosing the poorest light sandy soil.

The two earliest spring-sown crops should be sown broadcast in $3 \frac{1}{2}$-feet wide beds, for the convenience of affording temporary shelter in the event of late spring-frosts, and also because they are to be drawn young, and do not occupy the ground long after they are up. The latest crop of all may be sown in the same manner over the ground, without its being divided into beds; for, unless the 
ground is in a very bad state, they will require no further care after thinning with the turnip-hoe, fig. 64 , as the season
Fig. 64.

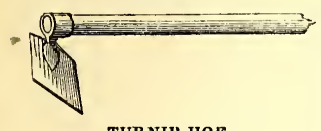

TURNIP-HOE. of weed-growing will have about ceased ; besides, their leaves covering the ground will tend to keep weeds down. All the other crops should be sown in drills, 1 inch in depth, and at a distance between according with the variety, as some have very small leaves, like the Maltese, while others have them larger, like the Robertson's yellow-stone, \&c. For the former, 1 foot is sufficient, thinning out the plants to 6 inches apart; while for the latter, 18 inches between the rows, and 10 inches from plant to plant, will be a proper allowance when thinned out for the last time. In all crops there is an evident mistake in too close cropping, and in few more so than in the turnip. These distances would be ill adapted for field cultivation, where bulk of crop is the object aimed at : the case, however, is different in garden culture, where no turnip should exceed the diameter of 4 inches; and from 2 to 3 inches is a better size. The ground should be in a highly-enriched state, that quick growth may be induced. It is of vast importance, in securing a good braird, and also a means of escaping the fly, that vegetation should be rapid. This naturally ensues when the seed is sown immediately before rain ; but as this cannot be at all times calculated upon, the drills, if the ground is very dry, may with advantage be well soaked with water before sowing, using soft rain or river water in preference to cold spring-water, that the temperature of the soil may not be lowered ; and for this purpose it is advisable to drill and water early in the morning, and to sow towards the afternoon, for, if the day be warm, the soil will have regained its former temperature. It is well also for this crop to mix guano and salt with the water, to the extent of a pound-weight of each to 25 gallons of liquid. The seed, also, may be steeped for six or eight hours in soft tepid water before sowing; and if soot or flowers of sulphur be mixed in the water, both will adhere to the seed, and render it less palatable to birds. In drilling, as for carrots, the drill-rake should be used for opening them equidistant, speedily, and of uniform depth ; and the drill sowing and covering machine (see section Potato) will be found a great economiser of labour. The ground, after sowing, should be left quite open, and by no means scratched over with a rake, as if it were a border of mignonette in a highly-dressed parterre. The agriculturist rolls his turnip-ridges down after sowing, to break down the hard clods of earth, and render the process of singling more easily effected, as well as more completely to cover the seed in the drills. The gardener has seldom the same reason for doing this, as his soil is presumed to be sufficiently pulverised by previous working; and it can only be where his soil is strong and lumpy, and, even then, when it is sufficiently dry that the roller may mellow down the clods, that he is justified in the operation. The evil of finely raking kitchen-garden ground is sufficiently obvious: it prevents the admission of heat and air to the roots or seeds ; in many cases renders it crusty and hard on the surface, so as to prevent the young plants breaking through it, and in fact completely stultifies every argument in favour of keeping the ground open and pervious to the elements. Many persist in raking garden ground from an idea of neatness and orderly appearance; but the best appearance such ground can have is a total absence of weeds, and a loose, open, pervious surface. There are no garden grounds kept in higher order than the market-gardens round London, in many of which a rake is scarcely to be found, and seldom employed unless in breaking in the ground previous to planting or sowing-rarely otherwise. In ground subject to the disease called Anbury, or Finger-and-toes, which is occasioned by a small species of Cynips (vide paragraph Diseases and insects), the usual means may be adopted at sowing; for, should they do no good, they can do no harm, and most of them will advantage the crop, as manures, sufficiently to repay the labour. These are, sowing the ground with soot, salt, guano, spirits of tar, lime, soapboilers' waste, or any cheap alkaline substance: these are best spread over the ground before drilling, as that operation greatly favours their thorough amalgamation with the soil. 
"The London market-gardeners sow principally the early stone or Dutch, it being in great demand in spring. Various ways of producing it are practised, such as growing it in frames; but the best plan is to raise it, like potatoes, in hooped bedsi. e., in trenches, dug out and filled with 2 feet of hot dung. Sow in February; hoop and cover with straw, and expose the plants daily; the quality of the turnip depends much upon quick growth and plenty of moisture."-CuthILL. This practice, however suitable it may be to the climate of London, would be found of little avail in most parts of Scotland, where, to insure success, glass coverings day and night must be had recourse to. We may, at the same time, take this opportunity of stating that, high as the system of culinary cropping is, as carried on by the London market-gardeners, all this hooping and covering with straw, mats, \&c., is neither profitable, nor up to the present state of horticultural science. Let them have pits miles in length if they will, 6 feet in width, supported on 10-inch piers, and sunk or elevated, below or above the surface, according to the dryness of the subsoil; and these not less than 4 feet in depth, with side walls of 10-inch brick-work, for durability and exclusion of cold, and covered with cheap glass. In spring, they could forward in these turnips, radishes, asparagus, lettuce, small salading, early cauliflower, early dwarf pease, French beans, \&c.; while tomatos, which come in altogether to the market, could be spread over three or four months. In summer, cucumbers, melons, \&c. could be produced; and in autumn, late cauliflower, full-grown lettuce, endive, \&c. could be protected, thus producing three crops per annum, and at (after the first cost of erection, which would last ten or fifteen years) little more expense of working than the present antiquated process, and certainty substituted for uncer. tainty, and constant success for frequent failures. It is quite notorious that a lettuce salad cannot be procured in Covent Garden market, after October, equal to what is quite common in the Paris market all the winter; and asparagus, before New Year's Day quite a novelty in London, is a common affair in Paris and Vienna by the 1st of December. Stable-manure, or heat produced by vegetable fermentation, must for long yet be the heating medium employed by the London growers, on account of its cheapness and abundance, and the comparative scarcity and expense of fuel. The Glasgow and Edinburgh growers are differently circumstanced, where a load of stable-manure costs nearly as much as a load of coal. Were the demand equal, and could the same prices be obtained in the latter case as in the former, no doubt heating by combustion would be adopted by the northern growers. In these days of cheap timber and cheap glass, there is no reason why Mr Solomon of Covent Garden market should exhibit in Regent Street asparagus purchased in Paris on the 1st of December, better than is shown in Covent Garden market on the 1st of February; and far less reason is there why we should be supplied with early potatoes and pease from Spain, Portugal, Holland, or even Cornwall, when these could be produced by every grower round London quite as early, of much better quality, being fresh, and as cheap, were they only to cast aside their dependence on the identical means employed a century and a half ago, which a reference to the writers of that period will evidently show. In open-air productions they surpass all Britain; in the production of forced vegetables and fruit they are immeasurably behind.

To insure a speedy germination of the seed, care must be taken that it is not buried too deep : 1 inch may be taken as the maximum depth in garden soils; if placed deeper, vegetation is considerably retarded; and indeed, if too deeply buried, it may not vegetate at all, until brought nearer the surface by some future operation of digging, \&c. Turnip-seed, if new, will germinate, and appear above the surface, in ordinary soils and situations, in the month of July, in about eight days ; but older seed, which should assuredly be employed, will take from ten to twelve days ; much of this, however, depends on the state of the weather.

The Swedish and Teltow turnips, as we havestated above, may be transplanted with every success. In gardens where the economy of ground is an object, it is well to sow both kinds in beds by themselves, in the broadcast manner, and, when the leaves are about from 3 to 4 inches in height, to transplant them to where they are to remain. By adopting this method, ground may be got cleared and prepared 
for their reception, which might otherwise be in full perfection of crop at the period when these turnips should be sown. In lifting them, care should be taken that the roots are got up entire : the operation of lifting them will be facilitated if the seedbed get a good soaking of water early in the morning of the day on which transplanting is to take place; it will cause the roots to part more freely with the soil, and they, at the same time, will absorb such a portion of water as will greatly make up for the evaporation to which their leaves will be exposed before the spongiolets are in a condition to throw in a sufficient supply for the support of the plants. A dibble of sufficient length and thickness must be used, so that the root may be set in the hole in such a manner that its natural position may be maintained, and the soil gently pressed around it, but not in the way practised by many, of thrusting the dibble into the ground as soon as the root is set in the hole, in an oblique direction, and giving it a twist, with a view, as they say, of firming it at the bottom. Such a mode of proceeding is of all others the most likely to push the root out of its perpendicular position, if not to break it entirely.

Besides the advantage of economising space, by following the transplanting principle in the case of Swedish turnips, we have the opportunity of rejecting forked or apparently malformed roots ; and even another, and to us rather unexpected, advantage has occurred in Wigtonshire, as reported by Mr Stephens, in "The Book of the Farm," vol. ii. p. 82, and obtained by $\mathrm{Mr}$ A. Johnstone, "on transplanting swedes on land which he had not got ready for sowing them at the proper season. He sowed some seeds of Skirving's swedes in a bed in April 1847, and transplanted the plants from them as late as the $22 \mathrm{~d}$ of June. From ten to twenty days afterwards, the transplanted plants running into flower, some of them were pulled up, and others cut over near the ground; when, in about fourteen days afterwards, bulbs began to form, and new stems and leaves were put forth luxuriantly. He then cut over others that had flowered, and the same results followed. Finding the green leaves succulent, he caused them to be pulled as green food, and continued to do so during the season, three times, never imagining that the bulbs would be of any value. Meanwhile, however, the bulbs enlarged until the end of October, when two were pulled up, and one weighed $18 \mathrm{lb}$. and the other $15 \mathrm{lb}$, with scanty stem and leaves, because the former ones had been cut down not long before." This appears so far to confirm the newly-started opinion that tubers and bulbs will increase in size after the leaves and stems have been removed from them.

"The question after such treatment of the bulbs" of the Swedish turnip "is, Are they deteriorated as food?" From an analysis made by Professor Johnston, it would appear they were not.

The Teltow may be set in rows 1 foot apart, and the plants 9 inches asunder in the row; while the Swedish should have 18 inches between the rows, and 12 between the plants in the line.

Thick-sowing can only be sanctioned on the plea of affording sufficient food for the Hattica nemorum (the flea or beetle), the Curculio contractus, Tenthredo (or sawfly), and other insect enemies, to satiate them during the period of their limited existence, and still leave a crop behind. Other means have been had recourse to, with more or less effect, such as that of mixing old and new seed in equal proportions, dividing the mixture, and steeping one half twenty-four hours in water; although sown at the same time, four distinct brairds will be insured, one of which has, so far as our experience goes, a fair chance of escaping. It has often happened, when one-year and three-year old seed have been mixed and sown together, that the insect completely ate up the braird from the new seed, and had disappeared before the other had come above ground. Radish seed, which germinates so much sooner than the turnip seed, has been sown for a like purpose ; for it should be borne in mind that these insects prey alike upon all cruciferous plants. No better way exists of ridding the crop of such intruders than that recommended by $\mathrm{Mr} \mathrm{A}$. Gorrie many years ago, which is to dust the young plants with caustic lime in powder, and the simplest way of applying it is to put it into a thin canvass bag, and to shake it over the plants twice a-day, when their leaves are quite dry; but this remedy, like most others, greatly depends on the quality of the medicine. 
Lime which has been slacked and laid by for some time is of little avail; it should be brought direct every three or four days from the kiln. Nor should it be laid on in large quantities, more especially when the leaves are wet, else it forms an incrustation on them, and shuts up their pores of respiration, making in such cases the cure as bad as the disease. We have no dread whatever of the turnip flea so long as a lime-bag is at hand, and provided those intrusted with the operation will do as they are desired. Heavy rains also discomfit the insects, and, as a substitute, water let fall from the rose of a watering-pot held 3 or 4 feet above them will produce a like effect. All blanks which occur in the drills, from whatever cause they may emanate, should be speedily re-sown ; but before doing so, fork up the soil in the patches, and drill and sow afresh ; and to keep the crop as equal as possible, steep the seed to be sown twentyfour hours in tepid water to forward its germination.

Subsequent cultivation. - Keeping the ground thoroughly stirred by deep hoeing with the Vernon hoe, or the grubbers attached to the hoeing, sowing, and drilling machine, which will not only promote growth in the plants, but prevent the appearance of weeds, are the principal operations required, if we except the important duty of thinning the crop as it advances. The distances we have stated above. The operation of thinning should be performed by the turnip-hoe, fig. 64 , which for this purpose should be kept clean, and as sharp as a knife. The 2 -inch or $2 \frac{1}{2}$-inch hoe is the implement best suited for this purpose, and a dexterous person, accustomed to such an implement, will hoe triple the quantity with it that an old woman will do with a 7 -inch tool, as recommended and used in the sister art. In very dry seasons, turnips, in all their stages of growth, are much improved by watering; it swells the bulbs out rapidly, and prevents their becoming hard, dry, and stringy, as well as running prematurely to seed. The first thinning by hoe should take place when the plants have made their first rough leaves-that is, those succeeding the radicle or cotyledon leaves, and at this time about 1 inch in breadth, if sown broadcast in beds, which the two earliest crops may be, and thinned to the distance of 2 inches apart, and in course of a week to the ultimate distance of 4 inches apart. Those of later crops in rows, when of the same size, should be thinned first to 3 inches, and in course of eight or ten days to 6 inches apart in the lines. This, again, depends on the size of the varieties, as well as the size at which they are to be drawn for use; so that in some cases the 3-inch distance may be sufficient, afterwards drawing every alternate one for consumption. In small gardens, where young turnips are in demand, this is sufficiently expedient; but otherwise it is better to give the second thinning, and to depend, as already said, for supply upon a small crop sown on purpose. Half that distance will be sufficient for the French or Teltow turnip, as neither its roots or foliage are large.

Soil and manure.-A turnip soil is almost a synonymous term with a light sandy or light loamy soil, for in such they succeed better than in stronger and more tenacious ground. In the former the crop will be much earlier, more easily managed, and the roots of better flavoured quality. In strong soils the crop is much later, more likely to shoot up to seed, particularly early in the season, and the roots are always deficient in flavour. New soils produce the best turnips, but all should be in an enriched state, either naturally or artificially. The richest manures may be applied, and, as has been shown practically in the case of one of the most powerful (pigeons' dung), disease as well as insects have been greatly kept under by it.

The turnip tribe differs from the potato in two most important points. First, The quantity of water they respectively contain. In the potato this forms three-fourths, but in the turnip ninetenths, of the whole weight, when taken from the ground; or they consist of-

$$
\begin{aligned}
& \text { Potato. Turnip. }
\end{aligned}
$$

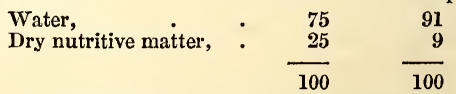

Second, In the presence of starch in the potato, while the turnip contains in its stead a substance called pectose, or pectic acid, which contains more oxygen than starch, but serves the same purpose in the nutrition of animals. "In fleshy fruits, such as the plum, peach, apricot, apple, pear, \&c., and in the bulbs or roots of the turnip, the carrot, the parsnip, \&c., there exists no starch, but in its stead pectose, or pectic acid. 
This substance is nearly as nutritious as starch, and serves the same purposes when eaten. It contains, however, less hydrogen and more oxygen than starch does, and changes more readily into other substances, both in the plant and in the stomach."-J. F. W. JoHnston, in Agricultural Chemistry and Geology, p. 46, 325.

Forcing.-This is seldom attempted in this country, excepting in gardens of the highest order. It forms an important part in Russian and German gardening, where otherwise it would be late in the spring before they could be obtained from the open ground. Slight beds of leaves, or of leaves and stable-yard litter combined, about 3 feet in height, are, for the purpose of forwarding an early crop in some British gardens, made up in February ; and when the heat has risen to the surface, they are earthed over, to the depth of 3 or 4 inches, with light rich soil. The seed is sown broadcast, and covered to the depth of half an inch, choosing the true early white Dutch, or early six-weeks, as coming soonest into use. Air must be freely admitted on all favourable occasions, and, in severe weather, the glasses covered during the night. When the plants have shown their first rough leaves, about the size of a shilling, they should be thinned to the distance of 3 inches apart; and when pulling commences, which will be when they have attained the size of a pigeon's egg, a regular system of thinning should take place by removing the largest first. Slight watering will be required, and that should be applied at from $60^{\circ}$ to $80^{\circ}$ of heat, with a view of adding to the warmth of the soil rather than abstracting heat from it. The turnip does not, however, require a temperature of more than from $50^{\circ}$ to $60^{\circ}$.

Taking the crop, and subsequent preservation.-From the time the bulbs are of the size of a pigeon's egg, until they attain a diameter of about 4 inches, they are fit for use, and are drawn progressively. When gathered and removed to the vegetable-house, they should be clean washed, the tops cut off close to the crown, and the tail-root close to the bottom of the bulb, in which state they are ready for the kitchen. Those that are intended for winter use may for the most part, in favourable situations, be left in the ground, securing a few for supply in the event of frost, which should be stored by in the root-cellar. In cold, wet, and uncongenial localities, it is quite necessary that vor. II. the crop be taken up and stored for winter and spring use, after the manner of potatoes (which see). To prepare them for this a dry day should be chosen, and also one when the ground is dry to pull up the roots, to divest them of their tops and tails, cutting both off close to the bulb. Some, however, object to this, and leave about an inch of the tops, and the whole tail or root entire, alleging that the sap is better retained in the bulb when these curtailments are not made.

Approved sorts and their qualities.-Garden turnips may be divided into two classes, the yellow and white bulbed sorts. The white varieties are most esteemed for table use in England; while the yellow, particularly during winter, hold the same rank in Scotland. French cooks rarely use the yellow sorts; they are, therefore, less generally grown in gardens, being consideredlessmild in flavour than the white ones, while many prefer them on this very account.

Yellow Malta. - This is a beautiful smallbulbed variety, from $1 \frac{1}{2}$ to 2 inches in diameter, of great symmetry in form, slightly flattened above, somewhat concave on the under side towards the tap root, which, as well as the neck, is remarkably small; skin very smooth, of a bright orange-yellow colour; leaves also small, admitting of their standing close on the ground. This is the very best, for summer use, of all the yellows, and, from its fine small size and form, is usually dressed whole. It is rather tender for winter use. Known also as the Maltese golden.

Robertson's golden stone.-A comparatively new and excellent variety, brought under our notice three years ago by Mr Robertson, Paisley. In shape it is nearly globular, and of a deep orangeyellow colour throughout, very slightly tinged with green on the top, often none; the best yellow for autumn and winter use, as it keeps well, and is exceedingly hardy. It attains a pretty large size.

Finland.-Is thus described in Messrs Peter Lawson and Sons' "Vegetable Products of Scotland," p. 13, div. iii. : "This is a beautiful little turmip, of a bright yellow throughout, even to the neck, somewhat similar to a small firm yellow Malta, but differing in the fine colour, and having the under part of the bulb singularly depressed, from which issues a small mouse-taillike root. It is also somewhat earlier than the yellow Malta." We believe it is also hardier. Our knowledge of it extends to having seen it growing in Messrs Lawsons' experimental grounds, and from its appearance we would judge it, along with the yellow Malta, well suited to small gardens.

Yellow globe.-Bulbs of medium size, globular, and always nearly under the surface of the ground; top greenish; leaves rather small and spreading. This is a most excellent turnip for garden culture, and, with Robertson's golden stone, the two most valuable where the larger-sized garden turnips are required. To these may be added the old and long-cultivated yellow Dutch and 
yellow stone, the last probably the type from which the third has been obtained. The yellow Preston, or Liverpool Preston, and the yellow Altringham, are both much grown in the northwest of England. We consider them good, but coarser in growth and delicacy of fibre than those we have described above..

White Dutch.-One of the oldest in cultivation, and most esteemed for early crops; round and much flattened; leaves medium-sized. While young, it is juicy and of excellent flavour, but apt to become spongy and dry when too old, particularly in dry seasons. In perfection when from $1 \frac{1}{2}$ to $2 \frac{1}{2}$ inches in diameter; after that size it is next to useless. It will not keep either in the ground or in pits through the winter, compared with the following.

White stone.-Shape of the bulb much more globular than the last, and firmer in texture and rather stronger in foliage. According to the Messrs Lawsons' description in " Vegetable Products of Scotland," div. iii. p. 14, "it is not, however, so well adapted for early spring-sowing, being more apt to run to seed, and has acquired the name of early from the circumstance of its arriving soon at maturity when sown at a late period of the season. A carefully selected and improved variety of this is known in some parts of England by the name of mouse-tail turnip; and, in addition to this, some seed-catalogues present us with the names of redtopped, mouse-tailed, and we think we may add the name of snowball also. It is sometimes also called the white garden stone.

Early six-weeks.-Bulbs of an irregular globular shape, produced for the most part above the surface of the ground. It arrives soon at perfection-hence the name; a good-tasted turnip, soft, and not adapted for winter use. We sow it as an intermediate crop during summer, for the supply of young tender turnips. It is known as the autumn stubble, early dwarf, and early ball.

Stone globe.-A well-shaped globular bulb, produced generally almost under the surface, which circumstance, together with its hardiness, renders it the best winter-keeping sort. Its leaves are stronger than any of the above, and of a much darker colour.

The French turnip or navet.-Of an oblong fusiform shape, from 3 to 6 inches in length, about 1 inch in diameter at the top; dull, semitransparent, whitish colour ; possessed of peculiar qualities, for which it is esteemed in French cookery. Vide Uses, p. 184.

Swedish turnips, we have remarked, p. 183, are grown in gardens, particularly in cold and elevated places, with a view to afford a supply during spring, when other sorts are consumed; as also for the purpose of placing the bulbs in a warm dark cellar in November, and successively in small lots till February, to produce a substitute for sea-kale, or to make an additional dish in resemblance of it. For the former purpose, a rather scarce and not often grown variety is the best-namely, Thorpland globe swede-on account of the symmetry of its form, which is of a beautiful globe shape, without the least appearance of neck, and also on account of its being smaller in size than any other variety in this section. We are aware that the prevailing opinion is that the larger the swede the more tender the fibre is, and altogether better for the purposes for which they are generally grown. This may in field culture be quite correct; but we have found this variety superior, in our estimation, for culinary purposes, when grown in garden soil, and not sown till the beginning of July.

Diseases and insects. - The turnip is attacked by a multitude of insect enemies, of which Athalia centifolia of some, A. spinarum of others (the turnip saw-fly, black caterpillar, blacks,

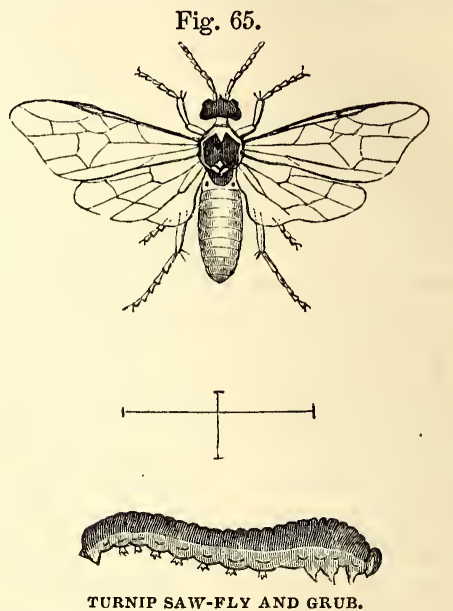

canker, black palmer, nigger or black grub), fig. 65 , is one of the worst. It is called saw$\mathrm{fly}$, from the use and appearance of the instrument with which it deposits its eggs. This is placed at the extremity of the abdomen of the female, on the under side, and is so constructed that it combines the properties of a saw and auger. The following excellent description is given by Mr Curtis, in "British Entomology," vol. xii. folio 617 , as quoted by Mr Stephens in the "Book of the Farm :"- "Head wider than long; deep black, with three ocelli in the centre; eyes oval ; antennæ black above, and for the most part dull yellow beneath; labrum and pulpi light yellow; thorax black above, with a triangular space in front; the scutellum, and a spot behind it, reddish orange ; the collar, which is rather long and slender, black on the sides and yellow in the middle; abdomen rather short, entirely orange yellow, inclining to red, with a small black spot on each side of the first segment; legs likewise orange yellow; the tarsi paler, approaching to whitish; the tip of the tibiæ, and of each of the tarsal joints, black; the tibiæ, with two spines at the apex and the joints of the tarsus, each with a very slender lobe beneath; extremity of the ovipositor black; wings yellowish at the base ; the costa and stigma black. Length, 3 to $3 \frac{1}{2}$ lines, exclusive of the antennæ. Antennæ short and somewhat club-shaped, nine or ten jointed in the male, but generally with the appearance of eleven joints in the female; the radical joint slightly thickened at the extremity; the second shorter and oval; the third as 
long, or longer, than any two of the other joints taken together; the remainder decreasing somewhat in length to the terminal one, which is large and oval. The flies, which appear in the early part of summer, and deposit their eggs on the young turnip plants, have probably survived the winter under ground in the pupa state, enveloped in their cocoon. Emerging from them, as soon as the milder weather is confirmed, in their winged state, the females immediately lay their eggs, after which they very soon die. The eggs appear, for the most part, to be placed round the outer margin of the rough leaves. In favourable weather they are hatched in a very short time, and the young larvæ immediately commence their attack on the plant. At first these larvæ are of a deep black colour, and, of course, small size; but they grow rapidly, and in course of a few weeks attain their full dimensions. In the course of their growth they change their skin several times, and most of their moultings are attended with a slight change in their colour. After casting their last skin, they are of a dark lead or slate-grey colour, paler beneath." $\mathrm{Mr}$ Curtis states that they are sometimes green, a colour which we never saw them assume, for in general they are not liable to much variation in this respect. Like most of the other larvæ of their tribe, when touched or in any way disturbed, they coil themselves up and remain motionless. When full grown, the larvæ cease to eat, and allow themselves to drop from the plant that nourished them to the ground, in which they usually bury themselves; or they take shelter among rotten leaves, moss, \&c. When examined after a short time, they are found to be completely enclosed in a cocoon composed of two distinct layers of silk. The inner layer is of a fine satin lustre; and when the cocoon is opened, it appears as if it had been washed with a solution of silver. When the fly is fully matured, it makes its exit by gnawing with its mandibles a hole in one end. The larvæ are known in different parts of the country by the names we have given above. They often destroy the crop entirely, and at other times very seriously injure it, destroying the leaves either wholly or in part. Some have asserted that they do not attack the Swedish turnip; but this opinion is not borne out by facts: nevertheless, they are less severe upon them than on the other varieties. Many remedies have been recommended for lessening their numbers: as to complete annihilation, that is only a thing to be wished for, without much expectation of its being realised.

Brushing them off the leaves, by drawing a light broom over the plants, has been recommended. This recommendation is offered on the presumption that they are unable to crawl on the ground and recover their position; but experience has shown us that they have the power of locomotion sufficiently strong to enable them to ascend again when brushed off. Besides, as they are produced in generations following each other in succession, from August till near November, a frequent repetition of brushing would be necessary during that period. Birds greatly assist us in reducing their numbers, and heavy showers of rain are of great service, while it points out to us a remedy in applying water from the rose of a watering-pot held somewhat elevated, that the force of the falling water may be the greater. A fire-engine, or a powerful garden-engine, may be brought to play upon them with great advantage; and, if wrought upon the walks, a boy may be employed to direct the nozzle, so that the water may not be unnecessarily wasted on the spaces between the drills. Lime-water is better than pure water; the trouble of applying both is the same, and the difference in expense next to nothing. The caustic property of the lime-water will destroy them in vast numbers; for falling upon them while young, and particularly when they have newly shed their skin, it is instantaneously fatal to them. Dusting the leaves with powdered hot lime once a day is very destructive to them, acting upon their tender bodies even with more effect than when applied in a liquid form. Catching the perfect fly before it has laid its eggs is the next most effectual plan; and this can readily be done by the aid of an entomologist's net, for they are slow flyers, and do not take long flights at a time. Each fly caught would prevent the coming into existence of from 250 to 300 caterpillars. Hand-picking the caterpillars would be very wholesome advice to give a Chinaman; but where labour is high, and large breadths have to be gone over, very unsuitable to the British cultivator. Old ducks and old fowls, as well as troops of young ones-remedies recommended by most writers upon rural affairs for more than a century-in most cases verify the old saying, "The cure is as bad as the disease."

Ceutorhynchus contractus, the turnip-weevil, closely resembling the Curculio pleurostigma, fig. 47 , is often found feeding upon young turnip leaves in company with Haltica nemorum, the turnip flea-beetle. It is a small insect, scarcely 1 line in length, of a uniform black colour, slightly tinted with metallic blue on the eiytra, the latter with punctured lines.

Haltica nemorum.-The turnip flea-beetle, fig. 66 , is thus described by $\mathrm{Mr}$ Stephens in

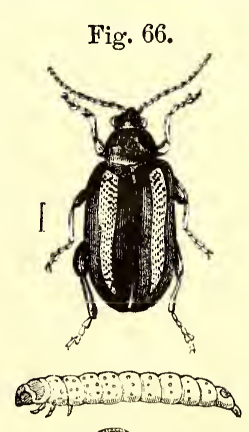

TURNIP FLEA-BEETLE AND GRUB, "The Book of the Farm," vol. ii. p. 73: "The insect which first infests the turnip plant, and attacks its seed-leaves, is the turnip flea-beetle, Haltica nemorum, fig. 66, usually, though improperly, designated the turnip-fly, which is a very different sort of insect. The flea-beetle is a coleopterous or hardshelled insect, capable of either penetrating the ground or bearing a considerable pressure." "It is a small insect," says Mr Duncan, "scarcely oneeighth of an inch in

length. It is smooth, shining, and of a brassy black colour, with a slight tinge of green, particularly on the wing-cases; the antenna black, with the second and third joints, and the apex of the first, of a pale colour. The thorax 
is convex above, and pretty deeply punctured; the wing-cases are much wider than the thorax, likewise thickly and irregularly punctured, each of them with a pale-yellow or slightly sulphurcoloured stripe running along the middle, curved inwards posteriorly, and not reaching quite to the extremity; the under side of the body and thighs black; all the tibiæ and tarsi of a pale hue. This little insect feeds on the turnip, which it attacks both in its perfect and larva states. When the plants have acquired some degree of strength, and the foliage is considerably developed, the injury done by it is insignificant; but, unfortunately, its favourite food is the young plant, just as it is beginning to unfold its cotyledon leaves. These it consumes with the utmost avidity, both as a larva and a full-grown insect; and where it abounds, the field is often wholly stripped of its crop in a very short time. Indeed, their powers of mastication are surprising for creatures of so small a size. They are found to attack the turnip plants as soon as the latter make their appearance; and one of the difficult points to determine is, how they are produced so speedily and so opportunely. In regard to the turnip saw-fly and lepidopterous insects the process is obvious, the eggs being laid upon the plant by the parent fly, and the larvæ evolved more or less speedily, but after the lapse of some considerable time. The appearance of the plant and insect being in the present case almost simultaneous, it has been thought difficult to conceive how the same process should be gone through."-Quarterly Journal of Agriculture, vol. viii. p. 353. Various conjectures have been formed to account for the early appearance of this insect on the turnip plant. "But these conjectures," continues Mr Duncan, "may now be referred to merely as matters connected with the past history of this insect, and as showing the difficulty that has been experienced in tracing it throughout its different forms and changes. This, however, has been recently done by $\mathrm{Mr} \mathrm{H}$. Le Keux, and we are no longer in doubt as to the points alluded to. This observer found that the sexes pair from April to September, during which period the eggs are deposited on the under side of the rough leaves of the turnip. The female insect does not apparently lay above one egg daily; in a week ten pair are found to lay only forty-three eggs. These eggs are very minute, smooth, and partak ing of the colour of the leaf. They are hatched in ten days; the maggots (fig. 66) are an eighth of an inch long, pale, fleshy, and cylindrical, with six pectoral feet; the eyes dark, and a dark patch on the first and last segments of the body; they immediately eat through the lower skin or cuticle of the leaf, and form winding burrows among the pulp, upon which they feed. The thickness of the leaf is sufficient to afford them ample scope for this, and they may be seen at work in their galleries by holding the leaf up to the light. These maggots or larvæ are full fed in sixteen days, when they bury themselves in the earth not quite 2 inches under the surface, selecting a spot near the bulb, where the turnip leaves protect them from wet and drought; they enter upon their chrysalis state in the earth, and the beetle emerges in about a fortnight. About thirty days carry the insect through all its different stages, and of these ten are passed in the egg state, six as a maggot, and fourteen in the chrysalis. There appear to be five or six broods in the season." - Transactions of the Entomological Society of London, vol. ii. p. 24.

On this subject Mr Stephens, in "Book of the Farm," vol. ii. p. 74, makes the following sensible remarks on the economy of such insects, and also on the remedial measures that might be adopted for their suppression: "In the case of those insects which feed on the foliage of plants in their larva state, and afterwards derive their aliment from other substances, the general law seems to be that a much longer duration is assigned to the larva than to the perfect insect; and it may be that this is not observed in regard to such as always consume vegetables, because in either of these conditions they serve the same purpose in the economy of nature, to which the prolonged existence of the larva bears reference in the other instance. Parallel examples are of frequent occurrence amongst insects. Unless the eggs of the common flesh-fly were hatched with extreme rapidity, the larvæ, when they appear, would neither obtain their food in perfection, nor fulfil the useful purposes for which they are now subservient. The remedies against the attacks of this insect," Mr Stephens fears, " are of a hopeless character; at least, it is better to prevent their appearance than to wage war against them when they do appear, as, even in the efforts to effect their destruction, the cultivator is the chief sufferer." The preventive measures, Mr Stephens thinks, are to keep the ground clear of weeds, particularly those of the cruciferous order, which are especial favourites with this beetle, to sow in drills instead of broadcast, "and to sow the seed thick and of the same age, for it is found the more rapidly the plants grow at first, they are the less often attacked; to put the seed for some time before it is sown amongst flowers of sulphur, and sow the sulphur amongst it." This latter is, we know from several years' experience, a very great check to this insect, and, indeed, to all others that attack the Cruciferæ. We have little faith in brushing the insects off the plants, as they have the means of taking possession of them very shortly afterwards: dusting with hot lime, and watering with lime-water, applying it with force, are the best remedies we have tried. For years we held a strong opinion on the advantages of sowing turnip-seed of several ages, believing that the insect would seize upon and devour the crop which first germinated, and which would be from the seed of the previous season's growth; and that by the time the older seeds had germinated, the brood would have changed into another state; and such, no doubt, might be the case, if the insects were all produced at once, or from one hatching of the eggs. Subsequent observation, however, has convinced us that this is not the case, but that brood after brood is hatched; and several germinations of 
seed would only be presenting each brood with a supply of food in about as regular a succession as they themselves are produced.

Burning the surface of the land is beneficial where it can be carried into effect, as by that means the chrysalides will be destroyed; and an equally certain way is to trench the ground deep. Indeed, the destruction of insects alone, were no other benefit to arise from trenching, would of itself be a sufficient recompense for the labour incurred. Sowing the surface of the soil with gas-lime two or three mornings after the seed has been sown, has been recommended on account of the disagreeable smell being so offensive to the insect as to drive it away. Spirits of tar, in the absence of gas-lime, would have the same effect.

Charcoal dust may also be sprinkled over the plants when they are either wet with rain or dew; and in small gardens the watering-pot may be had recourse to to wet the foliage, should neither rain nor dew fall. A temporary light-frame, stuck full of green alder-branches, and drawn over the crop, will discompose the enemy, and a stripe of light woollen cloth, 6 inches in width, and covered with bird-lime, tar, or any equally adhesive matter, and nailed to a wooden axle attached to two 12-inch wheels, and drawn over the crop, will greatly lessen their numbers, as the cloth brushing over the tops of the young plants will disturb the fleas, and cause them to leap from the leaves and become attached to the cloth; and, on account of its adhesiveness, they will be unable to disengage themselves. This operation, performed about twice a day, will in general secure a crop. In small gardens a piece of cloth so covered and attached to a handle like a small flag, and drawn over the plants backwards and forwards, will have the desired effect.

Aphis rapce Curtis, A. vastator Smee, A. dianthi Schrank, figs. 67 and 68, is thus well de-

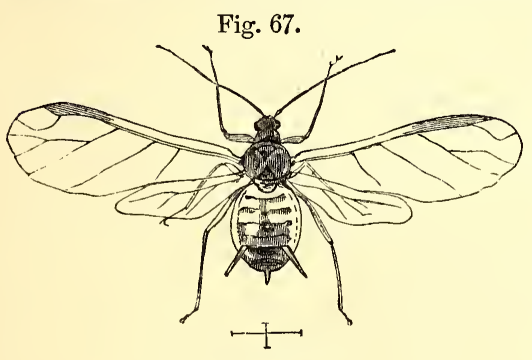

TURNIP APHIS, MALE.

scribed by Mr Stephens, in the "Book of the Farm," vol. ii. p. 77 : "Fig. 67 represents the winged male of the common turnip plant-louse, A phis rapce, magnified. Its characters are ochraceous; horns moderately long, setacious ; two first joints black, third ochraceous at the base; head blackish; collar ochraceous and brown; disc of shining black; abdomen greenish ; wings irridescent; the nervures light brown; tips of the thighs, shanks, feet, and claws black. Abundant beneath the leaves of the common turnip the whole of July, \&c. It is at once distin- guished from the other species by its long tubes and small apical cells of the wings. The cross
Fig. 68 .

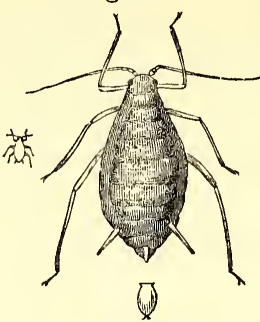

TURNIP APHIS, FEMALE. lines below represent the natural size of the body and of the expanse of the wings. Fig. 68 is the female of the same species magnified. It is bright green, shagreened ; horns fuscous, except at the base; eyes, tips of shanks, and feet black. The small figure on the left represents the aphis of the natural size; and the figure below represents one of the natural size just excluded."

Ruricola, in "Gardeners' Chronicle," 1847, p. 21 , of this insect says : "It is to be hoped that the species of the aphides will be determined, that it may be settled whether those found on the turnip (of which there are three species), the potato, the spinach, \&c., be identical or different. Another object will be to ascertain if the aphides actually feed upon the leaves and shoots of the later plants, and breed there." Of the Aphis rapce, he continues: "I do not remember ever observing it before midsummer. In July it becomes abundant, and in favourable seasons continues increasing until the end of October, or later. The first are hatched from eggs, and it is said are all females; these bring forth young until the autumn, when males are produced, as well as females furnished with wings. These lay eggs, which remain through the winter."

The injury done by the various species of aphides, or plant-lice, is incalculable, as may be well supposed when we consider that there is not a plant that would seem exempt from their attacks in one way or other; and it would also appear, from the investigations of some of our most eminent entomologists, that almost every plant has an aphis natural to it, and upon which it is designed to feed. Ruricola, in "Gardeners' Chronicle," 1842, p. 3, of this extensive genus thus speaks: "There is no family of insects which deviates in its habits more from the general laws of nature than the aphides; for whilst it is an almost universal rule that this class of animals should progress through the various transformations of egg, larva, pupa, and imago, the aphides have the power of evading three of these states by the faculty they possess of producing young; for, influenced by natural causes, they are both viviparous and oviparous-bringing forth young at mild seasons, when the temperature is not injurious to their tender offspring, and laying eggs in autumn, which are better calculated to resist the cold of winter. But this is not the most curious anomaly in their history, for a succession of young aphides can be produced without the presence of the male insects. For instance, as soon as an aphis is born, if it be taken away and placed upon a plant, under a glass, where nothing can possibly gain access to it, it will, notwithstanding, produce young; one of which, 
being also removed, and treated with the same care, will possess the same prolific faculty." Their power of reproduction, as observed by Bonnet and Reaumer, is such that the countless myriads of them which appear in our fields and gardens yearly can be accounted for without our having recourse to miraculous causes. "With such an inexhaustible power of fecundity, it is fortunate for man that no insects are subject to the attacks of such a variety of enemies. 1st, There are the lady-birds (Coccinella-figs. 14 and 15), which feed upon the aphides both in their larvæ and perfect states; 2dly, The aphidivorous Syrphidæ, the maggots of which are exceedingly useful in diminishing the number of the plant-lice. Then there are the Hemerobii; or golden-eyes, whose larvæ are called Aphis-lions (fig. 69), a ferocious family nearly related to the ant-lions. Another set of insects are parasitic, and deposit minute eggs in the old aphides, which then change to dull ochreous, horny objects, sticking to the leaves on which they had fed, frequently exhibiting a hole in their sides, from where the parasitic little ichneumon called Aphidius had issued." The earwig and ant wage constant war against them, and soft-billed birds work wonderful destruction amongst them; and to these we may add, as valuable to man-

The Aphis-lion, fig. 69, of which there are numerous species. They belong to the family

Fig. 69.
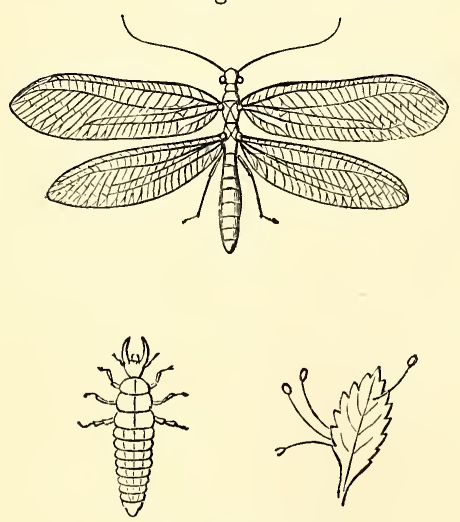

APHIS-LION, GRUB, AND EgGS.

Hemerobiidæ and order Neuroptera. The preservation of this family should be sedulously cared for, as they are bred, live amongst, and feed upon the plant-eating species of aphides, and are of the greatest importance to man. The aphis-lion makes its appearance in May, and may be observed walking about upon the leaves of plants, resembling more in appearance a small mass of cottony-like matter than a living insect. This covering is composed of the fragments of the skins of the aphides it has destroyed, and which, by a peculiar power invested in its jaws, as soon as it has sucked the vitals out of its prey, it throws upon its back, until, by repeated additions, it at last assumes the form of a large semi-globular mass, probably as a means of hiding it from its own peculiar enemies, or more probably as a covering of protection to its soft and fleshy body. When the larva is fully grown, it spins a cottony cocoon of a globular form, within which it is transformed into a short inactive pupa. Its duration in the pupa state varies with the season of the year : it, however, remains in this state throughout the winter, the fily being produced in the early part of summer. The flies are generally abroad in the evening, remaining quiet through the day ; they are by no means active on the wing. On being touched they give out a most disagreeable odour. The females deposit their eggs on the leaves of plants, especially such as are infested with aphides, attaching them to the leaf by a long slender arm or stalk, of a whitish colour-the eggs, when so placed, having somewhat the appearance of minute fungi. This stalk of attachment is composed of a glutinous matter the female discharges at the time of laying the egg, and this matter, hardening by exposure to the air, maintains the egg in a firm position. The insects, as soon as they are hatched, finding themselves in the midst of masses of aphides, commence warfare at once; and, as we have observed above, as the slaughter goes on, the skins, or fragments of them, are transferred to the back of the aphis-lion, until it becomes clothed as we have described.

And amongst parasitic insects which aid in the reduction of the aphides, may be instanced the

Aphidius avence.-This little ichneumon fly deposits its eggs in the apterous female aphides, and by this means reduces their numbers exceedingly. It is black and shining; horns long, and in the male having twenty joints: the body is brown, narrowing towards the base, which is rough and ochreous, as is also the margin and a patch on the back; legs rusty: hips and thighs, excepting the first pair, pitchy ; feet blackish; upper wings having a large smoky stigma, and a large central cell, the posterior ones entirely wanting.

Aphidius rapce, fig. 70.-Very similar in ap-

Fig. 70.

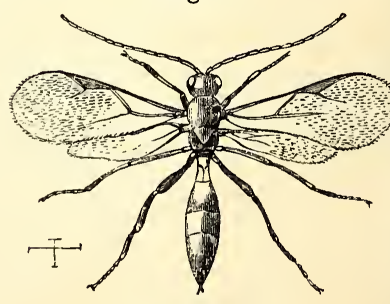

pearance and habits to the last ; the horns, however,are shorter, and only fourteen-jointed ; the underside of the base and the mouth ochreous; stigma of upper wings smoky ochre; APHIDIUS RAPE. legs bright ochreous, variegated with dark brown, and rusty.

Phytomyza nigricornis (the black-horned leafminer), fig. 11, often attacks turnip crops. They operate by feeding upon the parenchyma of the leaves, in which they form long irregular galleries on the inside of the lower cuticle; they are 
not visible on the upper side of the leaf. They often attack plants in greenhouses. The only way we know of is to pick off the leaves as soon as the insect appears to have attacked them, and to burn them. The larvæ of this insect are devoured in vast numbers by the Ceraphron niger and Microgaster virides.

Noctua exclamationis and $N$. segetum (the heart and dart moth, fig. 71, and common dart-moth, fig.
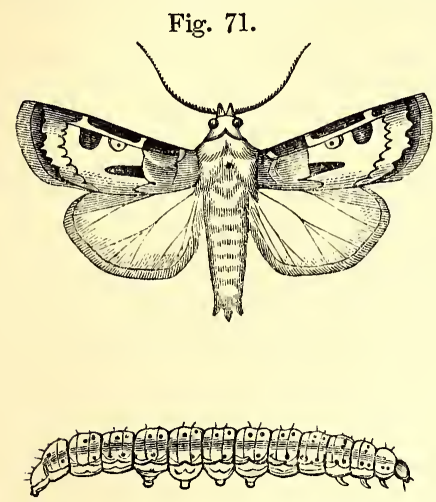

HEART AND DART MOTH AND CATKRPILLAR. Natural size.

72). These insects appear to be either increasing of late years, or more probably their economy is now better understood than formerly. They

Fig. 72.
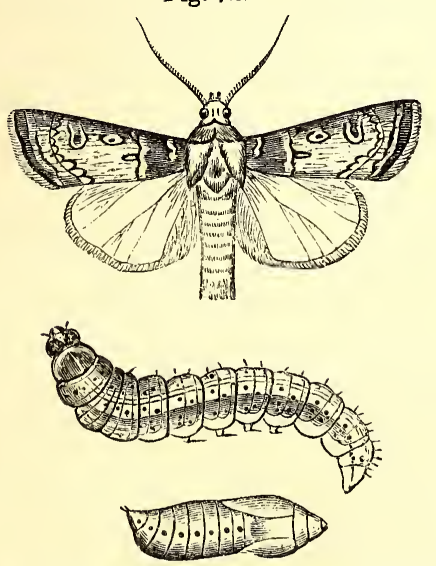

COMMON DART-MOTH, CATERPILLAR, AND CHRYSALIS. Natural size.

are of the class called surface caterpillars, or surface grubs, and are exceedingly destructive to various crops, particularly to turnips, potatoes, and mangold-wurzel. They commence hostilities upon the turnip in July, by eating off the crowns. They afterwards attack the bulbs, and render them unfit for use. In June they attack the mangold-wurzel by eating off the roots immediately under the surface of the ground, and they attack the potato when just pushing above ground. They seem to feed by night, and towards morning carry away portions of the leaves, which they drag into holes, so that they may feast upon them at leisure and in safety. Like rabbits, they seem to take a pleasure in cutting over the tops from mere wanton mischief, as they have been detected decapitating plant after plant, without waiting to eat the same.

"Where the eggs of $N$. exclamationis are laid has not been recorded; but it is stated that the moth of $N$. segetum deposits hers in the earth. The caterpillar of the former is somewhat cylindrical, but a little depressed above, having six pectoral, eight abdominal, and two anal feet. It is of a dull lilac colour, with a broad pale strip down the back, the margins of which form a darker line along each side, and there is a double dorsal line extending the whole length. The head is horny and brown; the minute eyes, and two curved lines, as well as the jaws, are black. The first thoracic segment is rather horny and dotted; the following segments have four little tubercles on each, producing hairs, as well as similar ones on the sides. The stigma is black. When full fed, it is an inch and a half in length, and buries itself, forming an oval cell of the earth, in which it changes to a shining rust-coloured chrysalis, to pass the winter in; and the following June, or perhaps earlier, the moth hatches." The moth "is of a clay colour, the wings reposing horizontally; the horns are like bristles, but slightly pectinated in the males; the tongue is long; on the front of the thorax is a transverse dark patch; on the upper wings there are two waved lines near the base ; to the second is attached a long, longitudinal, deep-brown streak; above it is a spot varying in size and form, and beyond it a large kidney-shaped one; then follows a transverse, pale, waved line; and near the cilia is a more irregular one. The under wings are white, the upper margin and nervures brown; but in the female the wings are entirely dark brown ; their expanse is $1 \frac{1}{4}$ inch. The caterpillar of N. segetum" (or Agrotis segetum of Curtis) " is more cylindrical than the foregoing species; the sides are greener, and there are three black dots on the stigma." An excellent description of it will be found in the "Royal Agric. Journ.," vol. iv. p. 106. Ruricola, in "Gardeners' Chron.," 1844, p. 619, from whose excellent paper the substance of the above is taken, suggests, as remedies, salt-water, lime-water, and an infusion of tobacco, as being distasteful to these grubs, and also soot applied to the surface early in the year, and laid on an inch in thickness. "One lb. of soap, dissolved in 16 gallons of soft water, and applied warm to the infested ground, especially round the roots, the surface caterpillars will dart out of their burrows, and may thus be readily collected; but no time must be lost, as they will retire under ground as soon as its effects have subsided." The use of hot water has been too little hitherto understood in the destruction of insects. It may be applied at nearly the boiling point to most plants without injury to them, but with manifest effect upon insects, particularly grubs, whose skins are thin 
and tender. We should not be surprised to see, ere long, a hot-water apparatus, somewhat similar to Mr Fleming's excellent machine for destroying weeds in walks, in use in every garden for the destruction of insects alone.

Bombyx tubricipeda (Linn.), the spotted buffmoth, fig. 73, is particularly destructive : no plant

Fig. 73.
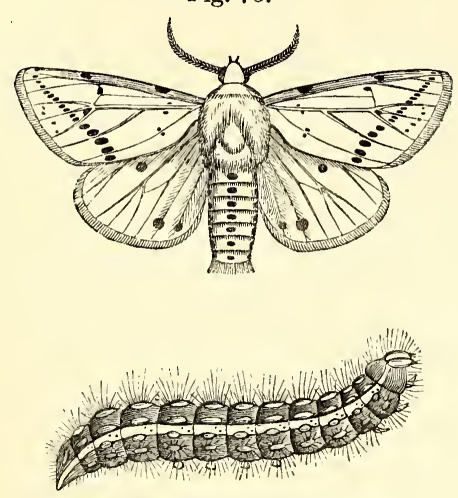

SPOTTED BUFF-MOTH AND CATERPILLAR. Natural size.

seems to come amiss to it; it feeds on the turnip, horse-radish, carrot, scarlet-runner, and even mint does not escape its ravages. In the months of May and June they are observed in pairs on walls, plants, \&c., when they should be destroyed. It is of a pale ochre or buff colour; antennæ black, bipectinate in the male ; the eyes, feelers, and legs black, with the exception of the thighs, which are orange ; tarsi and hinder tibia buff; body buff; the upper wings have one or more dots, with two black spots upon the margin ; it is, however, very variable in its markings, varying from whitish buff to deep ochre, with large black spots. The eggs, which the female deposits on the leaves of plants, are whitish, round, and smooth. The caterpillars, when first hatched, are of a yellowish white, with very few long hairs. When of full size, they are about an inch and a half long; they are then of a dark green, with a white line down each side. The stigmata white, and covered with reddish-brown hairs. It has six sharp-pointed pectoral feet, eight on the abdomen, and two hind ones, of a more fleshy nature.

Aphis brassica (figs. 42, 43), the Aphis florisrapæ of some entomologists, is also destructive to turnip crops, and indeed to most plants of the same natural order.

Aphis dubia (the black-spotted turnip-leaf plant-louse) is often found in company with $A$. rapce on the under sides of the leaves of turnips. Both these species resemble each other closely, only in $A$. dubia the colour is a dull green, shagreened; horns dusky at their extremities, as are also the tops of the thighs, shanks, and feet; the eyes and ocelli are black, as are two patches on the collar, and several transverse broken strips along the back.

Drosophila flava (the yellow turnip leaf-miner), fig. 74 , is a minute fly, which lays its eggs on the leaves. "The maggots produced from them eat into the pulp, and form large whitish blisters on Fig. 74 .

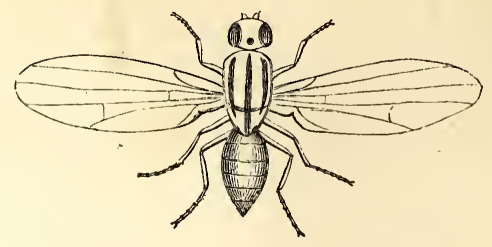

\footnotetext{
YELLOW-TURNIP LEAF-MINER,
}

the upper side. When full-grown, they are pale green, and change to chestnut-coloured pupæ, with two small horns at the head, and from these the flies are produced. These are ochreous, with black hairs, and two little feathered horns. The eyes are black; there are three ochreous strips down the trunk; the six legs and two balancers are yellowish and downy; the two wings are large and irridescent. The larvæ of this insect are destroyed by two little parasitic hymenopterous flies, the Ceraphron niger and the Microgaster virides.

Ceutorhynchus pleurostigma (the turnip gallweevil) effects the disfiguration, at least, of the turnip bulb, by "the female piercing a hole in the rind of the turnip with her proboscis, and depositing an egg in it ; and the young maggot, which is fat and whitish, often of a bright fleshcolour, lives on the substance of the bulb," causing those excrescences which are so often observed on turnips, particularly in dry seasons. The general appearance of this insect is that of a small black seed, and, excepting in colour, it resembles the turnip-seed weevil, which latter is of a grey colour.

Triphoena pronuba (the great yellow underwing), fig. 75 , in its caterpillar state, is one of
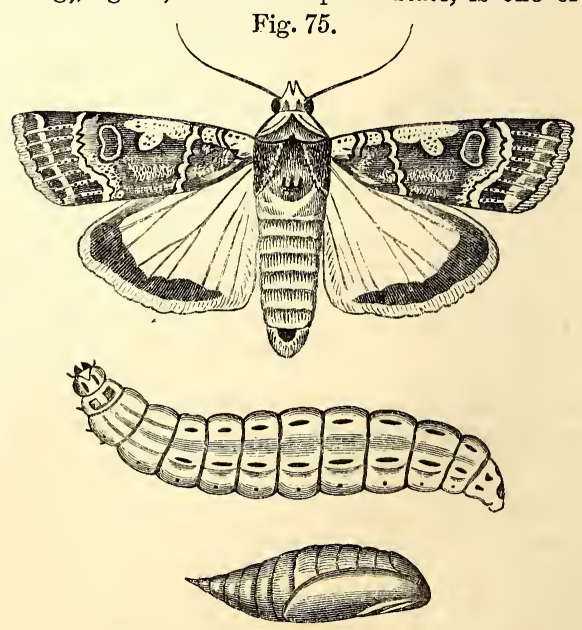

GREAT YFLLOW UNDERWING MOTH, CATERPILLAR, AND CHRYSALIS. Natural size. 
those enemies known as surface-grubs, and often attack the turnip-bulb, particularly in gardens where the plants are nearly full grown. These have also their natural enemies in the genus Cryp-

Fig. 76.

tops-Scolopendra of some(the centipedes),

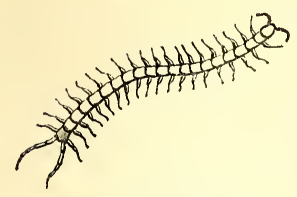

GARDEN CENTIPEDE. fig. 76. If the value of these were rightly understood, the vulgar prejudice against them would be removed. They are all of the carnivorous or flesh-eating section, and devour immense numbers of underground grubs, larvæ, \&c. They live entirely on the insects they find in the soil; they are of a rusty-red colour, more than an inch in length; antennæhairy, having seventeen joints; legs hairy, having twenty-one on each side. One species, Cryptops hortensis, is almost entirely confined to the southern counties of England; the rest are common everywhere.

Amongst lepidopterous insects-that is, those of the butterfly and moth kind-there are some species injurious to turnip crops, which are thus described by Mr Stephens in "The Book of the Farm," vol. ii. p. 79 :-

"Cerostoma xylostella (the turnip diamondback moth), fig. 77.-When at rest, the wings are

Fig. 77.

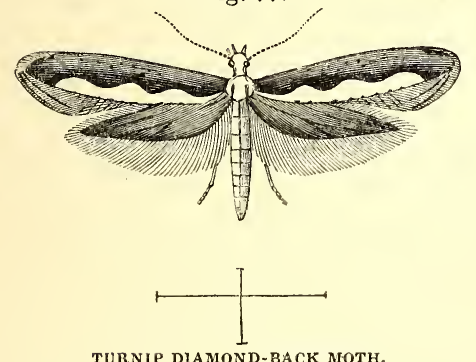

closed and deflexed, and the horns are projected forward in a straight line. It is more or less brown. The upper wings are long and narrow, and, when closed, form two or three diamonds upon the back. The inferior wings are lanceshaped, and of an ash colour, with a very long fringe. Its length is $2 \frac{1}{2}$ lines. The caterpillar is green, about half an inch in length, slender, and tapering to both ends. They are exceedingly active, and on the slightest touch wriggle themselves off the leaf they are feeding on, and let themselves down by a silken thread, and remain suspended till the cause of alarm subsides. As many as 240 have been counted on one leaf; and such is their avidity, that not the smallest vestige of a green leaf is left by them. This larvæ is destroyed by a black ichneumon, named Campoplex paniscus."

Mamestra brassicae (cabbage-moth), fig. 41, attacks the Swedish turnip.

Mamestra oleracea (white-line brown-eyed moth), fig. 78 (see section CABBAGE), is also an enemy to the turnip and lettuce, as well as the cabbage. This moth is of a dark rusty-brown VOL. II. colour. "The antennæ have white scales; the abdomen is ash grey, with brown tufts; the Fig. 78 ,

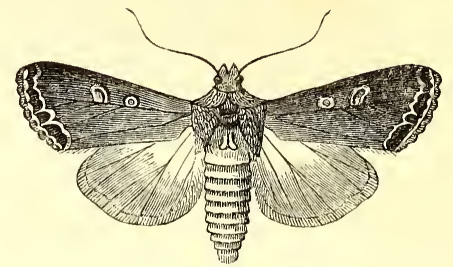

WHITE-LINE BROWN-EYED MOTH. Natural size.

feet are greyish brown, yellow-ringed below ; the fore wings have no connected cross lines; the round middle spot is surrounded with white; the kidney spot is orange colour in the shape of a lunule ; very delicate white and black dots are seen on the watered band; the fringes are deeply toothed, bordered by a faint white line; the under wings are ochre yellow, or dirty white, with darker shades towards the whitish fringes. The reddish or yellowish brown caterpillar has on the back, and on each side, a dark stripe, and a whitish one nearly over the feet; the under side and feet are light brown; it is dotted with black between the dark stripes. When young, and sometimes also when fully grown, the ground colour is green. The pupa is shining reddish brown, and remains in the earth during winter."-Kollar. It feeds upon the cabbage, turnip, and lettuce, and is best destroyed by sprinkling caustic lime over the plants as soon as the caterpillar appears, repeating the operation while they continue to appear.

Pontia brassica, fig. 44 ; Pontia rapce, fig. 46 ; and Chrysomela betula, are all more or less injurious to turnip crops, feeding on the leaves. They are, however, kept in check by the ichneumons Microgaster glomeratus and Pimpla instigator, and by the cynips Pteromatus brassice and $P$. pontia.

Anthomyia gnara, in its maggot state, attacks turnips during autumn, forming cavities in the bulbs. The female fly is ash-coloured, with a black line down the body, broadest at the base, and is nearly $\frac{1}{4}$ of an inch in length, and $\frac{1}{2}$ an inch in expanse. The male fly has a black trunk and legs; body linear, of the same colour as the female, with testaceous bands and black dorsal spots.

Anthomyia brassica (cabbage-fly), the $A$. radicum of some entomologists, fig. 79. When the maggots attack the roots of the cabbage, the roots " become enlarged and carious, the maggots revelling in the cavities. They are very similar to those of flesh-flies, being fat, yellowish white, tapering to the head, which has two black hooks. The blunt tail has two brown spiracles and some minute spines. Excepting in the depth of winter, they may be found feeding all the year. Heat, however, generates them most rapidly, for in May and June they exist at the same time as maggots, pupæ, and flies. The larvæ are transformed to bright rust-coloured horny pupæ in the roots, or they bury themselves in the earth. These cases have a few tubercles 
at the head, and minute teeth at the tail. The flies which hatch from them differ so much in Fig. 79 .
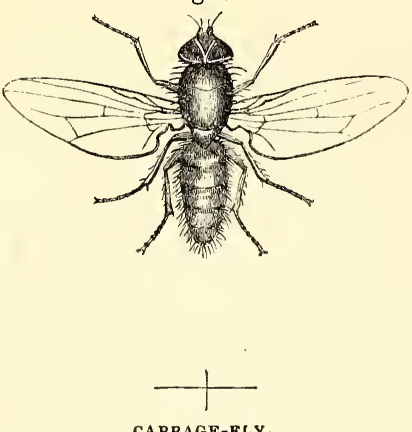

the sexes, that they do not look like the same species. The male is ashy grey and very bristly; the eyes nearly meet on the crown, and the face is silvery grey, with a long black streak on the forehead; the two horns are small, black, with a downy bristle; the trunk is grey, sides whitish, with three faint broken stripes down the back; body linear, shining grey, with a black stripe along the centre; the edges of the segments are also black; two wings transparent; two balancers ochreous; legs black and spiny. Female ash grey ; face silvery white; sides of the trunk pale; eyes distant, with a broad black stripe between them, of a chestnut colour in front; the body is conical at the tip; the wings are ochreous at the base; nearly $\frac{1}{4}$ of an inch long, and $\frac{1}{2}$ an inch in expanse.

"Anthomyia trimaculata is another species which attacks the roots of cabbages in summer and autumn. The male fly is light grey, reflecting white, with four black broken stripes down the trunk; three brown spots on the scutel; a black stripe on the body, which is checkered with brown; the legs are black. The female is a little larger and paler; tips of thighs and shanks reddish brown."

Regarding the diseases to which turnips are liable, the anbury and the finger-and-toes are the principal, if they are in reality distinct, which we believe they are not. The cause of these wellknown diseases seems little understood ; at least, opinions on the subject are at great variance one with another. Mr Marshall, in "Rural Economy of Norfolk," vol. ii. p. 33, ascribes it, according to the long-received opinion, to repeatedly cropping the same ground with the same crop, bad cultivation, and also the presence of an insect in the tap-root, by which the course of the sap is divided, and instead of the natural bulb an excrescence is produced. Mr Stephens, however, in "The Book of the Farm," vol. ii. p. 80, denies this, and we think with much better judgment. After stating the well-known fact that this disease has occurred on ground never before cropped with turnip, this high authority remarks: "If the disease were occasioned by the puncture of insects, better cultivation would not abate its virulence, but rather increase it, as the turnip would thereby be rendered much more palatable to them. The truth is, all such diseases arise from poverty of the soil-either from want of manure, when the soil is naturally poor, or rendered effete by over-cropping. Labour, clean, and manure the soil fully, according to the condition it presents, and no anbury will appear, unless it may happen in peculiar seasons, which always counteracts the effect of culture, and affects plants in a manner similar to the want of nourishment. Insects are invariably found in the buibs of turnips diseased with anbury, but they are the effect, and not the cause of the disease, as the habits of the insects clearly indicate." Repeatedly cropping the same ground would have analogous effect to poverty in the soil, as crop after crop would exhaust the soil of those principles necessary for the most perfect growth of the plant, and hence debility in the vegetable system would be the consequence, and a predisposition induced to the formation of these excrescences. On dissecting these swellings while in a growing state, no traces of insects are detected; but when decomposition takes place, several species of insects are then to be found feeding on the putrid mass, attracted no doubt to it by some peculiar instinct. These insects, however, will be found in the soil, and amongst the manure applied to the crop, and would doubtless remain there, and feed on other food, were the turnips free of this disease altogether.

Others, however, trace the cause of this disease to insect attacks, and attribute it to Curculio pleurostigma, and describe its operations thus:- The maggot found in those excrescences so frequently met with in the Brassica tribe, particularly in old gardens, where these crops have been long cultivated, and known by the names Ambury, Anbury, Fingers-andtoes, Club-root, \&c., is the larva of this weevil. It is described by Marsham as of a dusky black colour, having the breast spotted with white, the length of the body being one line and twothirds. Clubbing often makes its appearance on the roots even while in the seed-bed, and at first assumes the form of a small gall or wart; as the plant progresses, these become more numerous and increased in size; and within each, when cut open, is found the maggot, the larva of this insect. If left undisturbed, the maggot continues to feed upon the young woody part of the root, until it arrives at its pupa state, when it emancipates itself by eating a hole through the outer bark. While the maggot continues to feed on the alburnum, the excrescences enlarge; and as the alburnum becomes destroyed, the sap is prevented from ascending, and the plant languishes from want of support; and this becomes evident in dry weather, or in hot sultry days, when they will be seen flagging and ultimately dying. This disease is said to be more prevalent in old gardenground than in newly cultivated soils, and more especially where successive crops of Brassica have been grown year after year, and is accounted for from the circumstance of the eggs of successive generations being deposited in the soil, until at last it becomes completely replete with them. This disease is of more frequent occurrence in very dry seasons than otherwise, because such seasons are more favourable for 
the hatching of the eggs of insects deposited in the soil. The remedies proposed are-deeptrenching, change of crops, abundant supplies of water at the roots, charcoal-dust to the extent of half an inch laid over the surface, and slightly pointed in ; sulphur and also soot are applied with advantage, both of which are obnoxious to most insects; spirits of tar, dry gas-lime, the ammoniacal liquor from the gas-works, are also beneficial. Gas-lime has been applied in quantities varying from 8 to 12 bushels per acre, and, for turnips, is raked in just before sowing; while for other Brassicæ it is pointed in with a spade to the depth of from 6 to 8 inches, and applied in a larger quantity. In the case of transplantable Brassicæ, frequent removal has been attended with beneficial effects, but this is owing, probably, more to the care taken in removing the excrescences at each transplanting, than to any other cause in connection with the operation.

General remarks.-Saving turnip-seed, as well, indeed, as that of all brassicaceous plants, is a very peculiar branch of rural economy, requiring great circumspection in first selecting the specimens, which should always be the most perfect of their kind, and afterwards in preventing those intercourses to which all this natural order is so prone. Were cabbage, cauliflower, and turnip to be grown together, and did their period of flowering happen to occur at the same time, it is uncertain what might be the progeny from their seed. Certainly a great deterioration might be anticipated.-Vide Sect. Propagatron BY SEED. In saving, therefore, seed of any particular variety of turnip, no two sorts should be attempted in the same garden at the same time, nor should any other allied plant be suffered to flower at the same period. The finest formed bulbs should be selected in autumn, and transplanted into an open airy part of the garden, and placed, according to their respective size of growth, at from 2 to 3 feet apart each way, that the light and air may have free access to every part of the plant, while in flower, and while per. fecting its seed. In spring, as the flower-stems extend in height, if only a few plants be experimented upon, they should be tied to stakes for support; if many be grown together, they are less liable to sustain injury from wind. A judicious pruning away the smallest side-shoots, particularly such as flower latest, will be of advantage to those left; and besides this, little further care is necessary, beyond securing the crop from birds, which in all gardens can be readily excluded from the seed, by covering the plants with netting, elevated a foot or two above the topmost branches. If plenty of room is allowed, the seed will ripen pretty regularly; but the first-ripened pods should not be sacrificed by being allowed to shed their seed in waiting till the smaller and later ripen also. It is better to lose the last than the first. When the seed begins to change to a brownish colour in the pods, the whole crop should be cut over by the ground, and laid upon a dry place to mature fully. The accumulated sap remaining in the stems and branches will afford them sufficient nourishment, although separated from the root, to mature the seed. The precaution of covering with the net, while the crop is drying, must not be neglected; and when the whole is sufficiently dry, carry it to an open, airy, dry shed, and thrash it out, spreading over the floor a thick canvass cloth to receive the seed. The thrashing should not be done violently-a stout lath will be sufficient for the operation ; and when the first beating is over, gather up the seed from the cloth, pass it through a sieve, and lay what passes through upon another smaller cloth, still further to dry it. In a day or two afterwards, the same operation should be repeated, when most if not all the seed good for anything will be got out. Sift and dry the seed thus secured; and when thoroughly dry, bag it up for storing away. Great care must, however, be taken that the seed is thoroughly dry, and that, until it is so, it be spread out thinly; for, if laid in too great a thickness, or even bagged too soon, it is, of almost all other seeds, the most likely to get into a state of fermentation, which would destroy its vitality. Turnip-seed, like all the other varieties of Brassica, will keep for years ; and if well kept, will improve for the first four or five years; and, in some cases, such as early Dutch turnip, cauliflower, broccoli, \&c., we have had long enough experience to be satisfied that seeds of either, if, as we said before, well kept, are better at the end of the seventh or eighth year than they are the season following their saving.-Vide Sect. ProPAGATION BY SEED. The European names areNavet in French ; Steckrube, or Rube, in German; Nabo in Spanish; Navone in Italian; Raap in Dutch.

\section{§ 3.-THE POTATO.}

Natural history.-The potato (Solanum tuberosum L.) belongs to the natural order Solaneæ," and to the class Pentandria and order Monogynia in the Linnæan arrangement. The generic name is derived from Solor, to comfort, from some of the species soothing by stupifying. Some say from Sol, the sun; and others from Sulanum (from Sus), from its being serviceable in disorders of swine. The derivation may be possible, but theapplication is not very evident. The English name is probably derived from Batata, the Indian name of the sweet potato, which was known in this country thirty-three years before the introduction of the potato; or it may be from Papas, the name given it by the natives of Quito, where it grows wild. The order Solaneæ comprises upwards of 900 species, which are found distributed over a large portion of the globe, the genus Solanum alone furnishing about 170 of that number. All the tribe are suspected of possessing narcotic properties; but it is thought by many that this has been exaggerated, on account of the intense and deleterious properties of deadly nightshade (Atropa belladonna), the mandrake (Mandragora vernalis), and some others that belong to it. Some of them, like the potato, contain, however, very opposite properties in the same plant. An extract from the leaves and stems is a powerful narcotic, ranking between belladonna and conium, the 
poisonous hemlock; and the berries, although in a less degree, partake of the same nature also; while the tubers, when cooked, afford a wholesome and nutritive food. The water in which potatoes have been boiled is of a very suspicious character, and has been employed in the destruction of aphides on other plants-all these properties being, no doubt, known to the late Dr Neill, when he asks, "Who could, à priori, have expected to have found the most useful plant among the natural family of the Luridæ, several of which are deleterious, and all of which are forbidding in their aspect?" The people in Burgundy, long after its introduction into Europe, were interdicted from either cultivating or using the potato, as it was "deemed a poisonous and a mischievous root." Liebig concludes that the deleterious or poisonous alkaloid found in the leaves and stem of the plant is formed in and around the shoot, where it germinates in darkness ; but there is no evidence that the tubers are thereby rendered injurious or deleterious - or, if so, they are brought to a wholesome state by the process of boiling and roasting.

The native habitat of the potato has not been very correctly ascertained. According to Mr Darwin, it grows wild in the islands of the Chonos Archipelago, in great abundance in the sandy shelly soil near the sea-beach. Mr Low asserts it grew as far south as latitude $50^{\circ}$. Humboldt sought for it in vain in the mountains of Peru and New Granada. Ruiz and Pavon discovered it in a wild state at Chancay, on the Peruvian coast-a circumstance far more recently confirmed by $\mathrm{Mr}$ Caldcleugh, who sent native tubers to the Horticultural Society of London. Mr Cruikshanks, in letter to Sir W. J. Hooker, says, "This wild potato is very common at Valparaiso; it grows chiefly on the hills near the sea. It is often found in mountainous districts far from habitations, and never in the immediate vicinity of fields and gardens." $\mathrm{Mr}$ Meyer affirms that he found it not only in the mountains of Chili, but also in the Cordillera of Peru. This plant appears from other authorities to extend over a large extent of latitude, and hence it is by no means improbable that each of these travellers did find it in an indigenous state at the places mentioned. Indeed, from a remark of Mr Darwin's, this appears sufficiently probable, as he found the same plant on the sterile mountains of Central Chili, where not a drop of rain falls for more than six months, and within the damp forests of the islands in the Chonos Archipelago.

The date of the introduction of the potato into Britain is involved in nearly as much un. certainty as the exact spot of its nativity. It is generally believed that the first tubers were brought to England from Virginia by the colonists who were sent out by Sir Walter Raleigh in 1584, and returned in 1586. Phillips, in "History of Cultivated Vegetables," vol. ii. p. 80, however, thinks it uncertain whether Sir Walter or his people brought it, or whether it was not afterwards sent him by Sir Thomas Grenville, or by Mr Lane, who was the first governor of Virginia. Herrist, who went out with them, wrote an account which will be found in De Bry's " Collection of Voyages," vol. i., under the title "Roots." He describes a plant called openawk thus: "The roots are round, some large as a walnut, others much larger; they grow in damp soils, many hanging together as if fixed on ropes; they are good food either boiled or roasted." Gerard describes two sorts of potatoes, the one evidently the sweet potato (Ipomaca batatus), which was supposed to possess an invigorating property. The other sort was evidently our common sort, which he calls Batata Virginiana, sive Virginianorum. To the continent of Europe the potato had been introduced before its arrival in this country, and it is believed to have first found its way into Spain in the earìy part of the sixteenth century, from the mountainous parts of South America, where around Quito it is called papas. From Spain it reached Italy, and shortly after must have been brought into Belgium, for Clusius informs us that he received it at Vienna in 1598 from Mons, a city in Hainault, to which it had been carried from Italy by one of the attendants of the Pope's legate. It appears to have spread rapidly throughout Germany in Clusius's time. It reached Ireland in 1610, and not 1566, as asserted by some writers, who most probably mistook the common potato for the sweet sort procured either from Spain or Italy, as we have no account of its reaching Europe so early, with the exception of the surmise that Sir John Hawkins introduced it in 1563, a surmise not supported by historic evidence. It was certainly used as an article of food by the Irish long before its utility was generally known in England. In 1684 it was cultivated sparingly in Lancashire, from stock said to have been accidentally thrown ashore by a vessel wrecked on the coast; while others say it was carried over from Ireland, from the estates of Sir Walter Raleigh near Cork. Be this as it may, Lancashire has from about that period been famed for the abundance and quality of its potatoes, to which the mildness and humidity of the climate greatly tends. Gerard grew them in his garden in 1597, and recommends their "being eaten as a delicate dish, and not as common food." Parkinson appears to have paid more attention to their cooking, and recommends their being " roasted, and steeped in sack and sugar, or baked with marrow and spices, and even preserved and candied by the comfit-makers." Lord Bacon, who wrote his "Natural History" shortly after the publication of Gerard's "Herbal," calls them potadoroots, and gives a curious account of a method of growing them in pots. In the early part of the seventeenth century this root was grown in the gardens of the nobility as a curious exotic. Phillips, in " History of Cultivated Vegetables," vol. ii. p. 85 , says, "The potato appears to have been a great delicacy in the time of James the First; for in the year 1619 it is noticed among the different articles provided for the queen's household. The quantity supplied was extremely small, and the price high, being at that time one shilling per pound." 
The first instance we have met with of the potato being brought forward as an object of national importance is the letter of Mr Buckland read before the Royal Society in 1662-3, recommending the planting of potatoes in all parts of the kingdom to prevent famine. His contemporary, the celebrated John Evelyn, held them in small estimation. He says in "Sylva," writing in 1699, "Plant potatoes in your worst ground. Take them up in November for winter spending; there will enough remain for a stock, though ever so exactly gathered." London and Wise did not consider the potato worthy of notice in their " Complete Gardener," published in 1719. Bradley, a voluminous writer of the same date, says, after having described parsnips, carrots, onions, \&c., "Potatoes and Jerusalem artichokes are roots of less note than any I have yet mentioned." It was not until towards the middle of the last century that they were even generally known throughout England, and their cultivation even in gardens was scarcely understood in Scotland till about the year 1740, and it was not practised in fields till about 1760 . So little was their cultivation understood in 1725-6, that, as we are informed by "The General Report of Scotland," vol. ii. p. 111, the few potato plants then existing in gardens about Edinburgh were left in the same spot of ground from year to year, a few roots being taken up for use in autumn, and the remainder left in the ground covered with straw to protect them from the frost. In 1728, Thomas Prentice, a daylabourer at Kilsyth, showed the first example of planting them in the open fields; yet in no country in the world are they cultivated to so great a degree of perfection as they are in Scotland at this day.

Uses.-Most of these are so well known that it would be superfluous to recapitulate them all. Still there are many purposes to which they are applied not generally known; to these, therefore, we shall now only refer, premising, however, that those curious in the matter will find an interesting paper on the subject in the 1st vol. of the "Gardeners' Magazine," p. 438, in which thirty-one different uses of the potato will be found noticed. An agreeable brandy has been produced from the roots by distillation-an invention due, according to the "Memoirs of the Royal Academy of Sciences in Sweden for 1747," to Mr Chas. Skytes. He states that an acre of potatoes, compared to an acre of barley, will yield a spirit in the proportion of 566 to 156 , even admitting the potato to be planted on worse ground than the barley. Dr Anderson states in the "Bath Society," vol. iv. p. 50, that he had distilled from $72 \mathrm{lb}$. of potatoes that had been properly fermented (with no addition except yeast), an English gallon of pure spirit, considerably above proof, and about a quart more below proof. The tender tops are used, both in Canada and Kamtchatka, as spinach; the boiling, no doubt, freeing them of their narcotic property. The starch or fecula obtained from the roots is now extensively used to adulterate and even pass off as a substitute for sago, starch, arrow-root, tapioca, \&c.-a very harmless imposition, and a great boon to cultiva- tors, particularly since the appearance of the potato epidemic, as those judged unfit for the food of man in their natural state, are manufactured into representations of these supposed nourishing articles. Mrs Morris, a London lady, so early as 1807 discovered that the liquor obtained in making potato-starch would clean silk, cotton, or woollen goods, without damage to the texture or colour. Potato fecula is by many preferred for making soufflés, and is for that purpose, as well as others connected with domestic economy from a souffle to maccaroni, sold in the shops under the learned names of Fecule de Pomme de Terre, and others of more recent date, with which the public allows itself to be gulled. Asize is made from potatoes, which has great advantages over common size, for the purpose of white-washing, as it does not smell, and it has also a more durable whiteness. Yeast may also be made from the roots, fit for the use either of the baker or the brewer.

Potatoes are extensively employed by bakers to mix with their flour in making bread, and bread so made is considered lighter, and by many preferred to that made of flour alone. They are washed and peeled, and the pulp grated down, and in that state are mixed with the dough. Before potatoes became scarce in consequence of the disease, and their value approximated so near to that of wheaten flour as to render the operation scarcely remunerative, almost all cottagers, and indeed many families who baked their own bread, used them constantly. We know well that families of distinction, who keep their own baker, have potatoes mixed with most of their fancy bread, rolls, \&c. ; and this is done on their parts not for economy, but for improving the lightness of the bread, and rendering it more digestive.

Much has been said on the value of the potato as an article of food, and there can be little doubt that more dependence has been placed on it, as such, than it deserves. It is more than thirty years ago since $\mathrm{Mr}$ Cobbett stigmatised it as " the accursed root;" and that extraordinary man foretold then the disappointment that would in course of time arise from its too extensive cultivation. Without entering into the question of political economy, we may safely assert that much of the human misery that has taken place both in Ireland and the Highlands of Scotland may be traced to a too implicit reliance on this root as an article of human sustenance. Wherever the potato can be advantageously cultivated, there the cereal crops will prosper also; and if we analyse the produce of an acre of wheat, barley, or oats, with one of potatoes, the difference in the amount of nutritious matter will be sufficiently evident.

Man is said to be made up of a bundle of habits. Before the introduction of the potato, the Jerusalem artichoke was much appreciated, and were that plant sufficiently hardy to stand our climate, or rather to perfect its tubers within the same space of time as the potato, the loss of the latter would not be regretted, as the former is far more wholesome and much more nutritious. It maintained its place in public estimation even long after the potato was intro- 
duced. The potato having no peculiar taste, like bread it can be used long by the same individual without becoming unpalatable, and is no doubt a useful vegetable to be eaten along with butcher-meat upon the same principle that most other vegetables are. Long use has also habituated us to it, so that for such purposes its loss would be felt for a time; but as an article of direct food, on which thousands are to depend for sustenance, its total extinction would be no great loss. And this the less so, seeing we have substitutes for it as easily cultivated and as productive, could we only rid ourselves of a confirmed habit.

Propagation.-The usual and most expeditious way is to propagate by planting medium-sized tubers whole, or the larger ones cut into sections or sets, as they are technically termed, having one eye or bud to each. By this means a crop is obtained the same season, and the variety, if not subjected to a considerable change of climate, continued for years without much change in character or deterioration in quality. It is also propagated by seeds, when new or improved varieties are wished for, and by this means most of the sorts at present in cultivation have been obtained. The process has also been had recourse to since the appearance of the disease, thinking thereby, by getting a new breed either from seed home-grown, or imported from its native country, that a new progeny with increased vigour would be produced. These hopes have not been realised; seedling plants, from home-grown or imported seed, have suffered almost alike. Seed has, however, produced improved varieties ; but unfortunately the process has been so carelessly gone about, that, with very few exceptions, we do not find any satisfactory information as to the means employed. It is probable much of the success has been the result of chance, or the natural proneness of the plant to run into variety from seed, some of which may be good, others inferior to the parent plant. It is also propagated by layers and cuttings, modes that can only be considered expedient when sets are scarce, or when it is desirable to multiply a new or scarce variety.

Propagating the potato by cuttings has for several years been extensively employed by Messrs Hay and Sangster of London, with a view to restore vigour in the plant and arrest the progress of disease, by obtaining through this means a healthy progeny. In procuring cuttings, the potatoes are placed in spring on a moderate hotbed, as they think it important that the cuttings should be as short a time as possible on the old tubers. When the young shoots have attained the height of 4 or 5 inches, they are cut off much in the way dahlia cuttings are taken from the rootsthat is, taking no part of the old tuber along with them. They recommend that the planting of the cuttings in the open ground should be proceeded with before the 20th of May (that is, for the latitude of London), and, at planting, that 1 or 2 inches of their tops should be left above ground, and a slight watering given if the ground is dry. In this way they have secured a healthy crop over 5 acres of ground from the tubers produced by the plants originated by cuttings in the preceding year. "The only fault," they say, "in planting cuttings, is that the tubers grow too large, and some anything but handsome ; but this must be put up with, if growing any description of potatoes three years by that process is to bring the stock to a healthy state, which they have no doubt will be the result." By an analysis of the tubers grown in the ordinary manner by sets, the amount of fecula or starch was found to be only $11 \frac{3}{4}$ per cent, while those originated by cuttings gave $15 \frac{1}{2}$; and upon a subsequent analysis the latter were found to yield 17 per cent, which is nearly the maximum amount to be expected from healthy tubers. How far Messrs Hay and Sangster are correct in thinking that a deficiency of saccharine matter in the potato, "as it now exists with us, is the cause of the disease, may admit of doubt, as also that their process has the effect of restoring to the tubers that element; one thing, however, is certain, that potatoes struck by cuttings by ourselves have yielded an abundant, early, and healthy produce.

Layers of the young shoots will also root freely ; but the advantage of the latter mode is, we think, very questionable, except for the purpose of multiplying any rare or new sort expeditiously.

Propagation by seed is a very simple affair. All that is required, where the more nice and scientific process of intermarriage, or cross impregnation of the pistil of one sort with the pollen of another, has not been duly attended to, is to select the largest and best ripened apples 
(or plums, as they are called in some places) in September or October, and to wash out the seeds and keep them dry till spring, when they should be sown in drills in a warm situation; or the seed may be retained in the apples, and those buried in dry dust till spring, when the seed can be easily rubbed out. When the young plants have reached the height of 2 or 3 inches, they should be thinned out to 5 or 6 inches apart, and the thinnings may be transplanted at the same time. Towards the close of the season the tubers will have ripened, and, as they are small, select the largest, as they are the most promising, and keep them for planting in the February or March following, if circumstances prevent them being planted as soon as they are dug up and examined. The autumn following will determine their size and productiveness, and any old woman, after the first boiling, will give ample evidence as to their quality.

"A variety of the potato" originated from seed "is generally considered to continue about fourteen years in perfection, after which period it gradually loses its good qualities, becoming of inferior flavour and unproductive."-Cottage Gardeners' Dictionary, p. 739. To this rule there is, wethink, at least one exception, and that is in the case of the old and highlyapproved American early, which we have now known for about half a century, and see little difference in it in either of these respects. The case is very different with others, for those of our early days are now unknown even by name, and it is possible that the variety above named forms the exception to the above rule. May this not have been the original variety im. ported direct from America? The types of other varieties may have reached us from a more southern latitude, and been of larger size, more delicate, and of different qualities. The early varieties do not readily produce seed. This may be, however, effected, when desired, by planting the sets on little mounds of earth, and training the stem to a stake for support, and washing the soil from the roots, so that only the points of the fibres enter the ground. The plants will blossom and perfect seed.

Some sorts of potatoes produce seed, while others do not. Most of the late varieties produce seed freely; the early sorts rarely do so. This peculiarity was not unnoted by Mr Knight, who also discovered the cause, and accounts for it in this manner: "I suspected the cause to be the preternaturally early formation of the tuberous root, which draws off for its support that portion of the sap which, in other plants of the same species, affords nutriment to the blossoms and seeds; and experiments soon satisfied me that myconjectures were well founded." His experiments in connection with this matter will be found fully recorded in the "Philosophical Transactions for 1806," and also in the "Transactions of the Horticultural Society," vol. i. p. 188. Unless the object be to secure seed, experience has proved the advantage of cutting off the flowers before the fruit is set. Knight accounted for this, and states that the process will increase the gross weight per acre by about 1 ton in weight; subsequent experience has confirmed this opinion.

Planting.-The usual season for planting garden potatoes is from February to the end of April, the early varieties being planted first. Of late years autumnal planting has been resorted to as one of the many means recommended for saving the potato from the attacks of the mysterious epidemic, and, no doubt, with very beneficial effects, admitted by many as well as experienced by ourselves. Many, however, view this as a chimera. Autumn planting is by no means a new feature in cultivation; we have practised it for upwards of thirty years, founding our data upon the simple and indisputable fact that the soil is the natural matrix for the tuber to be preserved in during its season of repose. As regards precise seasons for planting, much depends on soil and situation. In all dry and warm soils, planting may commence in November; or, indeed, the tubers may be replanted the same day the crop is lifted: but this will not much hasten the season of their coming to maturity, only plants originated from this early planting will be stronger in spring on account of the tubers losing nothing of their elaborated sap, which all those taken up and kept, if not completely excluded from the air and kept in a low temperature, must inevitably do. With this extra strength, therefore, they push away stronger in spring, and not only arrive at a useful state 
sooner, but are better able to resist spring frosts than those which are so much weaker. Planting, therefore, may be gone on with from November to April, when the ground is dry and free from frost.

"Planting in the open ground is best done in October and November, and may thence be continued until the end of March. This last month is the latest in which any considerable plantation should be made. They will succeed if planted in May or even in June, yet it ought always to be kept in mind that the earliest planted, especially in dry soils, produce the finest, healthiest, and most abundant crops."-Cottage Gardeners' Dictionary, p. 738.

Dr Lindley, in an excellent article on the culture of the potato, "Gardeners' Chronicle," 1842 , p. 155 , remarks, "The period of planting should be as soon after the 1st of March as circumstances will permit." "I have uniformly found," $\mathrm{Mr}$ Knight says, "that, to obtain crops of potatoes of great weight and excellence, the period of planting should never be later than the beginning of March." This is in order to give the potato as long a summer as possible. From experiments made some years ago in the garden of the Horticultural Society, it appeared that a crop planted in the first week in March exceeded that planted in the first week of April by about $1 \frac{1}{4}$ tons per acre. It must be obvious, however, that the propriety of planting thus early will depend upon the nature of the soil, and that it is too early for wet heavy land, although it is the best season for light soils. In reality, land cannot be advantageously planted with potatoes until all the superfluous moisture has drained away or evaporated.

In some parts of England, sets for the earliest out-of-door crop are planted in October, choosing a dry sheltered situation and light soil. The sets are placed 8 or 9 inches deep, and the surface covered over with straw, fern, or dry litter, so as to exclude frost, retain the heat absorbed by the soil during summer, and effectually throw off rain; in fact, to retain the heat in the soil, and prevent its escape by radiation. The ground is generally set off into beds with deepish alleys between them, which receive the water thrown off by the covering. The plants appear above ground in March, and are protected from frost by hooping them over and covering with tarpauling. Early potatoes are in this way procured in May, or by the beginning of June, even in Lancashire.

Preparation of the ground for planting.Amongst the many instances of mismanagement to which this plant has in many cases been subjected, is a disregard to the thorough working of the soil in which it is planted; for although the tubers are produced at no great depth under the surface, still the food-collecting roots penetrate to a considerably greater extent. Trenching the ground, or, in absence of this, deep-digging, is an imperative condition to their most successful cultivation, and, in carrying out either of these operations, the manure, if such is to be applied, should be completely incorporated through the soil. The practice is absurd, although sanctioned by most writers on the subject, as well as followed by cultivators for ages, of placing it in immediate contact with the set or tuber in the trench or drill prepared for their reception. No doubt the slight fermentation which must of course take place when very rank stable-yard manure is employed, gives a certain degree of excitement to the tuber, and may, to some extent, cause an earlier development of roots; but no sooner are they produced than they begin to extend in length, and travel with a rapidity in proportion to their growth as far as they possibly can from the mass of manure placed by the planter for their special use. If the doctrine is to be considered valid which has been propounded by vegetable physiologists, that plants derive their chief rootsupport from the food collected from the soil by the spongiolets which are placed only at the extremities of the minute fibres, it appears pretty clear to us that the plant derives little if any advantage from the manure so placed, beyond the slight stimulus given during the short time fermentation is in operation, immediately after the tuber is placed either over it or under it (for they are placed in both ways); and that manure incapable of fermentation is not only absolutely useless but positively injurious, tending, as has been asserted by many of our most intelligent cultivators, to encourage 
greatly the disease under which the potato is now suffering.

Manure, to be beneficial to any plant, must be placed so that it is within the reach of the spongiolets; and as they ramify in all directions away from the seed, tuber, or whatever else may constitute the origin of the plant, it appears pretty clear that it should be as thoroughly mixed with the soil as possible, and hence confining it within the narrow limits of a drill is of all modes of application the most wasteful. In accordance with these views, they are right who recommend planting the potato in ground enriched by previous manuring, whether employed for the last crop, or applied to the ground without any crop at all, because they get rid of one of the evils stated above-namely, encouragement to the disease. Manure, to be useful to any crop, must become soluble, and this condition does not come immediately about in the case of such manures as rank stable-litter : its application, therefore, must be regarded as of little service to the present crop ; but that it will be so to the succeeding crop is to be expected.

The mode, therefore, of preparing ground for potatoes, as usually practised -viz., planting them in drills or trenches along with manure-must be regarded as erroneous; and, as we have already observed, if manure is to be applied, let it be so during the process of trenching or digging, and let it be as completely mixed with the soil as possible.

The methods of planting the potato are numerous. That of planting with the dibber, next to that of placing them in trenches amongst rank manure, is the worst. The rapidity with which the former is accomplished is an inducement with many, but it is a very imperfect one, unless the ground is very dry, and the dibber of a diameter to make a hole sufficiently wide to admit of the set or tuber falling to the bottom; and this is not easily done when the soil is light or gravelly, as portions of it will fall in before the set is introduced, and consequently some will be much deeper planted than others. The better way is, to open a drill with a hoe or spade, of an equal depth throughout, and to set the tubers in it, and afterwards draw the soil over them, so that in most cases there may vOL. II. be 6 inches of mould over them when finished. This insures their being all placed at a uniform depth, and leaves the mould quite open and soft both under and above them,-affording, in the first place, a proper state for the young and tender roots pushing their way into it; and, in the second, allowing the rains to sink in, and the solar heat and air to penetrate, and admitting of an uninterrupted process of evaporation taking place.

In regard to distance, much depends on the sort. The small-growing early kinds may be set in rows 15 inches apart, and from 8 to 10 inches in the line; but for the later and stronger-growing sorts, 20 inches to 2 feet should be allowed row from row, and from 12 to 15 inches in the line. There is no gain in too thick planting.

In ordinary garden-soils the sets may, with every propriety, be placed 6 inches under the general level of the surface. In damp wet land the sets should be placed on the surface of the dug ground, and the mould drawn over them, in form of a broad flat ridge, say 6 inches in height at the centre, and 10 inches at the base. Where the ground is very wet-but such is very unfit for the potato-the old lazybed manner may be followed, which is by dividing the ground into beds of 4 feet in breadth, leaving $2 \frac{1}{2}$-feet alleys between. Dig the bed deeply over, set the tubers in rows across, at the distances stated above, and cover them at first by laying 4 inches of soil, taken from the alleys, over them ; and in course of a few weeks afterwards, but before the shoots appear above ground, lay over that 3 inches more from the same. In such cases, ridges may be thrown up, from 1 to 2 feet in height, and from 2 to 3 feet apart; and on the top of these set the tubers, and cover them over as above. These are extreme cases; and when the soil is incapable of being thoroughly dried by draining; to admit of the process being carried on in a more business-like manner, it were better to crop the ground with some other crop.

In regard to the depth the sets should be put in the ground, much depends on its texture-as to whether it is light and pervious to solar heat and air, or whether it is retentive, and impenetrable to either. From experiments made in the London Horticultural Society's garden, the following results have been arrived at:2 D 
"In warm dry land we regard 9 inches as not too deep, provided the sets are large and strong; in cold stiff soil 4 inches would be better : 6 inches is a good depth in average soils, and indeed may be considered the best depth in most soils. Weak sets do not come up well at 9 inches deep ; but, on the contrary, 4 inches is too shallow, occasioning the tubers to be partially exposed to the light, and hence to become green. If, however, the land is so shallow as to admit of no greater depth, then more space must be allowed between the rows for earthing up. In one of the experiments above alluded to, different depths were also inquired into, when the rates of produce were nearly as follows: 3 inches deep gave 13 tons; 4 inches, 14 tons; 6 inches, $14 \frac{1}{2}$ tons ; and 9 inches, 13 tons. At so great a depth as 9 inches, sets are apt to perish, unless the soil is dry, light, and warm. The deeper, however, the sets can be safely inserted the better, for the following reason: Potatoes are formed on underground branches; the deeper set, the more branches will be formed before the shoots emerge from the soil, and consequently the more ample will be the means possessed by the potato plant for forming tubers. The important practice of earthing up is designed to effect the same end by compelling the potato stem to grow as much as possible underground." Subsequent experience has proved that earthing up is of littie consequence, if the potato has been planted from 6 to 8 inches deep in the first instance.

Size and choice of sets. - Since the prevailing malady has been so destructive to the potato, sets - that is, cut sections of the tuber, having one eye or bud each-are much seldomer used than formerly. The larger the set is, the stronger we would naturally expect the plant to be, on account of the greater amount of elaborated matter it contains compared with a smaller one; but this does not appear to be the case; no one set need have more than one eye or bud. Some have placed much importance on the end of the potato from which the set is cut, and recommend the rejection of the watery or less ripened end of the tuber, as well as the root end, or more ripened portion also, assigning as a reason that the former has too many small eyes, and that plants produced from it are apt to run too much to haulm; and to the root end they object, fancying that it is tardy in growth, and more liable to disease. They say, Make choice of the middle of the potato, which, if cut in two, will afford two sets, having one eye each. This appears to us to be merely the shadow of the well-known fact that fully-matured potatoes, fit for food, are less proper for seed than those that are much less perfectly ripened, and hence the demand for seed-potatoes from upland and late localities, where, in fact, the potato seldom ripens to full perfection. Physiologically, there is a difference between the two ends of a potato; practically viewed, it is so small that it may be little regarded. The late $\mathrm{Mr}$ Main was quite of this opinion, and set the whole down as a mere matter of speculation. In the present condition of the potato, whole tubers are preferred to cut sets, and these are usually selected from the medium-sized ones. The only objection that can be started against the employment of whole tubers is the number of eyes they have, compared with a mere section, which may be cut with only one. This objection is, however, easily remedied by the careful destruction, by the touch of a red-hot wire, of all the eyes but one; but this should be done with as little injury to the skin of the potato as possible, else the escape of its juices may be as great as in a section cut from a larger specimen. Mr George Lindley was of opinion that sets with only one eye in each, produced the crop ten days or a fortnight earlier than those having two or more eyes left on.

We have not planted sets for years, but use the medium-sized tubers whole. The late Mr Knight was of opinion that very small potatoes produced a much later crop than when large ones were used for seed: this is to a certain extent true, because they are too imperfectly matured, and therefore we never plant them. He also placed considerable importance on the position the sets are placed in at planting. In " Trans. Hort. Soc.," vol. iv. p. 448, he says, "When the planter is anxious to obtain a crop within the least possible time, he will find the position in which the tubers are placed to vegetate by no. means a point of indifference ; for these being shoots or branches which have grown thick instead of elongating, retain the disposition of branches to propel their sap to their leading buds, or points most 
distant from the stems of the plants of which they once formed parts. If the tubers be placed with their leading buds upwards, a few very strong and very early shoots will spring from them; but if their position be reversed, many weaker and later shoots will be produced, and not only the earliness but the quality of the produce, in size, will be much affected.

To the selection of sets some have attached great importance; for ourselves, we prefer, as stated above, medium-sized whole tubers, as these are not over-ripened, and because they do not require to be cut as larger ones do. Large tubers have been recommended, but have been shown to be of no advantage whatever. It has also been proved that, if the tubers are overripened, they are apt to produce disease. It is, therefore, better to take up the seedpotatoes before they become over-ripe; or, still better, to purchase seed from upland situations, where the potato seldom ripens to full maturity.

The argument of those who advocate the planting diseased tubers in preference to sound ones, is practically, that they soon rot away after the young shoot has begun to germinate, while sound sets are apt to remain till the ripening of the crop, retaining in themselves the germ of the disease-thus showing an effort, on the part of nature, to throw off the disease. Professor Lindley on this point remarks, "Gard. Chron.," 1850 : " It is possible that the morbid matter which causes disease may disappear in the general decay of the tuber, in the one case, and may remain unchanged in the other, prepared to seize upon the haulm as soon as it is ready to receive its influence." The experiments of Mr Smith, of Wokingham, Berkshire, appear to be very conclusive in this respect, and highly deserving of our attention. Mr Smith planted very much diseased sets on the 18th of March, the crop from which was ripe and taken up on the $2 \mathrm{~d}$ of $\mathrm{Au}$ gust, perfectly free of disease; while sound ones of the same variety, as well as others, were planted in February, and, being taken up during the August following, were found very much diseased. Very rotten late potatoes were planted by the same gentleman in November, $6 \frac{1}{2}$ inches ; over the sets were first laid 2 inches of the natural soil of his garden, then a layer of halfdecayed weeds, and over that a covering of the same earth. This crop was taken up also in the beginning of August, and was found without disease. These experiments were made from 1846 to 1849 ; and a person in the same town repeated the experiments in 1850, with exactly the same results. Whatever deductions may be drawn from these experiments theoretically, it will be admitted they are of great practical importance.

Subsequent cultivation.-As soon as the plants are fairly above the surface, hoeing and surface-stirring should be commenced, as few plants thrive better in an open and oft-disturbed soil. The very early crops should have the earth drawn around each plant in form of a cup, to serve as a shelter from the cold winds, which are a great enemy to them during early spring: a similar shelter will not be produced if earthed up in the usual form, which leaves them exposed on the top of the ridge. The draw-hoe, fig. 80 , is well adapted for this earthing-up purpose, while implements penetrating much deeper into the soil must be used for loosening the ground between the rows.

Of the implements used in potato culture, the following may be given as examples:-

Draw-hoes, fig. 80 , are amongst the most useful of all garden implements : they are employed

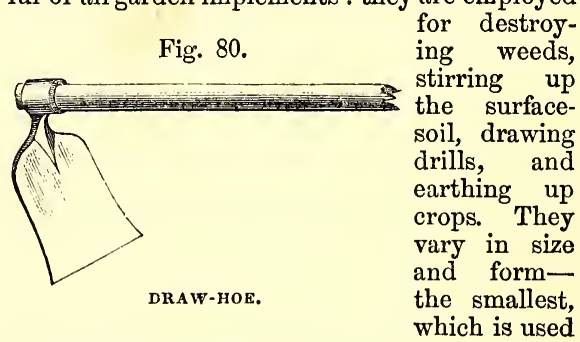

for thinning out seedling crops, being $1 \frac{1}{2}$ inches broad at the face or cutting part, and the same in depth of blade. There is attached to them a hose, 2 inches in length, for receiving the handle, which is straight, varying in length from 18 inches to 3 or 4 feet, according to the purposes for which they are intended. The shortest of these are very dexterously used by English labourers for thinning turnips which are sown broadcast, using one in each hand. The largest is 9 inches in the face and $3 \frac{1}{2}$ inches deep in the blade, and are almost all now made so that the hose part for receiving the handle is riveted on to the blade, as shown in our figure; formerly they were forged all in one piece, the hose part being on the top of the blade. The neck of the hose-piece should be slightly bent, so that when the operator is standing nearly upright, which is the easiest position for him, the face or cutting part of the blade may be at an 
angle of $68^{\circ}$, which is the best suited for entering the ground. From the smallest to the largest there are intermediate ones, differing in size, in general, to the extent of half an inch in length of face, and denominated $2 \frac{1}{2}$-inch, 3 -inch, $3 \frac{1}{2}$-inch, and so on upwards to 9 -inch hoes. Hoes in form and proportion to that of our fig. 80 are the most common in use. There are, Fig. 81. however, other 81 , which is triangular in shape, having each of its sides calculated for cut-

TRIANGULAR DRAW-HoE. ting up weeds and

TRIANGULAR DRAW-HoE. ting up weeds and the surface. It is used also for drawing drills, the corner points being well adapted for the purpose. The Vernon hoe and the Spanish hoe have been already described, page 38 .

Fine compound hoe, or draw-hoe and rake combined, is a useless affair. Wenoticeand figure it (fig. 82) to give us an opportunity of stating how much better it would be to have each as a -HOE AND RAK COMBINED. separate implement. Nearly twenty varieties of draw-hoes are figured in the "Encyclopædia of Gardening," all, with three or four exceptions, examples of those used in the rudest state of

Fig. 83. cultivation, and inferior to those of the present day.

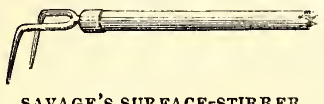

Savage's surfacestirrer, fig 83 , is a useful implement belonging to the hoe class. It may be described as a common hay-fork, of rather more than double the length of prongs, which are bent over about the middle, thus forming a two-pronged scarifier of considerable power and utility. The prongs are 3 inches apart, and 4 inches long from the bend to the points, but there is no reason why they should not be double that length. The operator, as in all cases with similar tools, walks backwards, while he presses the points of the implement into the soil, and draws it after him, stirring the ground as deep as the prongs will reach between rows of potatoes and other culinary crops. Such stirring implements are far more useful than the common draw-hoes, which, from their construction, cannot enter the ground
Fig. 84 .

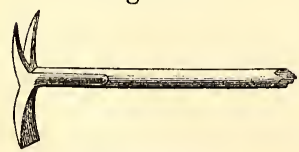

GUERNSEY PRONG. to a sufficient depth. Fig. 84 is an excellent implement in the section of hoes, and is known in the south of England as the Guernsey prong, being much used in that island. It is thus delineated and described in "The Gardeners' Chronicle" for 1851: "It is something in the shape of a hammer, the head flattened into a chisel an inch wide, and the fork the same. The whole length of this prong is 9 inches, and it is attached to a staff 5 feet long. Such an implement is light and easy to use ; it requires no stooping, and will tear up the deepest-rooted weeds." Its great utility, however, is to deeply

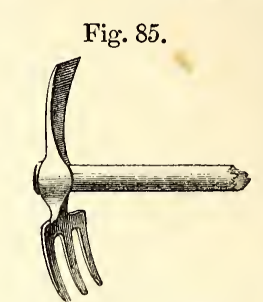
stir and pulverise the soil. Glendinning's pick-fork, fig. 85 , is a somewhat similar im. plement, and used by its inventor upon his strong and stubborn soil with great economy of time and advantage to the soil. The Bloomfield hoe, GLENDINNING'S PICK-FORK. fig. 86 , is much used in Norfolk. It is somewhat like the common draw-hoe, but having the advantage of keeping itself clear of
Fig. 86.

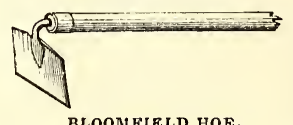

BL.OONFIELD HOE. mould at the neck, which the common hoe has not in consequence of the shortness of the neck, or that part which connects the blade with the handle. The specimen we have given, fig. 80 , is, however, less objectionable on this account, as will be seen by the manner the blade is attached to the handle.

Gidney's improved Prussian hoe, fig. 87. This is an excellent instrument, when cutting down

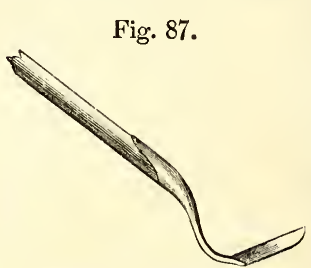
weeds only is the object, and where the soil is not to be deeply stirred. The blade is set at such an angle that it shaves down the weeds just below the surface, and leaves them uncovered; whereas the old hoe gIDNEY's PRUSSIAN нок. covers up the fallen weeds, and this aids them to root afresh. It is well calculated for hoeing flower-borders, and can be usefully employed in the kitchen-garden for hoeing footpaths between beds, or amongst young crops where seed-weeds are appearing. The face of the blade of the specimen our figure is taken from is 5 inches long, quite straight, the back rounding off towards the point, which is acute. The breadth of the blade is $1 \frac{1}{2}$ inches. They may, however, be made both larger and smaller, according to circumstances.

Dr Neuington's hand-cultivator, fig. 88, is of the hoe-stirring section. The frame or headpiece is 16 inches wide, of iron or heavy wood, to press the tines into the ground without much pressure being applied by the operator. It is fitted with three strong curved tines very sharp at the points, and which, when the implement is drawn forward, stir up the ground from 6 to 9 inches in depth. It is also fitted up with three shares for drawing drills for seedsowing. It is the type of fig. 89 , which $\mathrm{Dr}$ 
Newington calls his "Patent hand-row cultivator" and drill-hoe." The head and handle are much

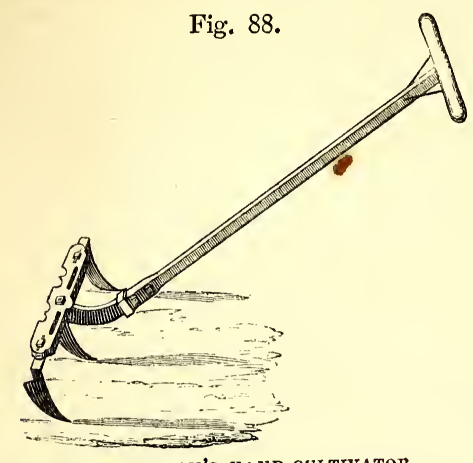

NEWINGTON'S HAND-CULTIVATOR.

the same as in the last, only the head is so constructed that when the tines (three) at present

Fig. 89.

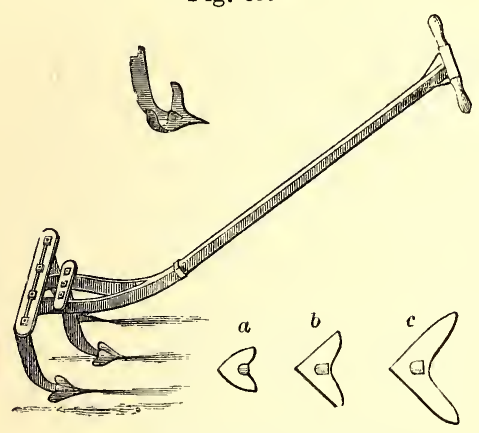

NEWINGTON'S HAND-ROW CULTIVATOR.

shown as affixed to it are removed, hoes of different sizes, $a, b$, and $c$, can be substituted, and in this form it acts as a weed-killing hoe; and these hoes are placed like the tines, one in the centre of the front bar or head, and two in the hind one. A moulding-board for earthing up has also been added to it, but we think with much less effect than in Sievewright's improvement upon it, which, although acting upon the same principle, is every way a superior implement.

Sievewright's improved cultivator is one of these contrivances for the economising of labour, and is thus described by him in " Gardeners' Chronicle," 1849 , p. 500 . After premising that the machine has been most effectually employed in hoeing between drills, grubbing up ground to the depth of from 5 to 6 inches, and earthing up between potatoes and cabbages, \&c., the intelligent improver proceeds: "When I use it for hoeing between drills, I use flat tines, which are five in number; the transverse bar at the back expands to the width of 21 inches, or contracts to 13 inches; and should even this latter width be greater than the drills will allow of, I take out the two hind tines. There is a weight which is fixed at pleasure on any part of the bar running through the centre, the position of which is regulated by the way in which you are using the machine, and which keeps it working steadily." This is a circular piece of cast-iron attached; and one, two, or more may be used, according to the weight required. "When I use the machine for grubbing up ground, or for working it into a fine state, I change the flat tines to a set which I have, similar in form to those of a common grubber: in this way I prepare the ground for the reception of a second crop, saving the expense of digging; and, when no manure is required to be buried in, doing the work far better than the spade. When earthing up potatoes or cabbages, thefront tine is left in, the four hinder ones taken out, the moulding-boards puton; andas the transverse bar expands or contracts, I am enabled to earth up potatoes 21 inches between the rows, or cabbages at 13 . The wheel in front regulates the depth to which the tines enter, and keeps them to a uniform depth in the soil; the hind wheel can be applied for the same purpose, and, where the ground is rough, has the additional effect of steadying the machine." In our own practice with this excellent machine, we place the weight referred to above here, finding in our light soil the hind wheel of little service. "The whole machine, which is of iron, is very light, and the work which I have described it as doing is quite within the power of a lad. The work a man could do with it would not be less than 2 acres per day, hoeing between turnips, at 2 feet apart; the grubbing he would do nearly

Fig. 90.

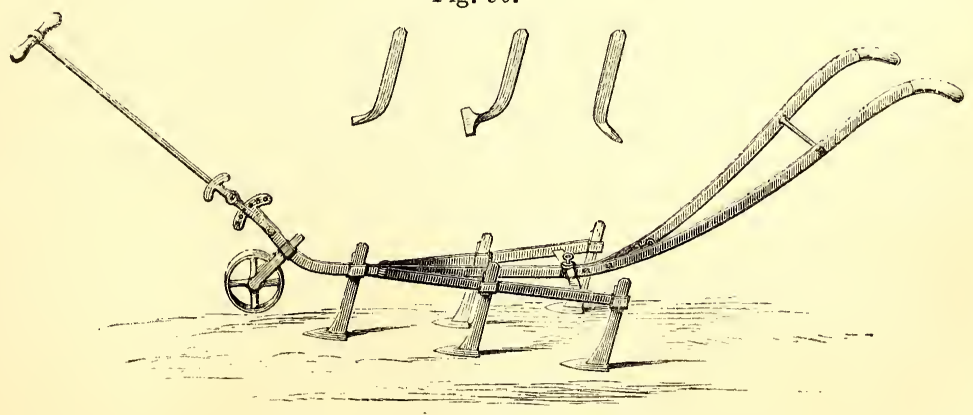

SIEVEWRIGHT'S CULTIVATOR IMPROVED. 
as quickly; and would certainly earth up potatoes at the rate of an acre per day." Through the courtesy of Mr Sievewright, who"early directed our attention to this machine, we have had two in operation during the last three years, and can corroborate all $\mathrm{Mr}$ Sievewright has said about it.
The modification of this implement, shown by figs. 90, 91, possesses, we think, important improvements on both $\mathrm{Dr}$ Newington's and $\mathrm{Mr}$ Sievewright's machines, both of whom had in view mainly its adaptability to the power of one man. Now we do not exactly see wherein there is any decided economy in this, and have thereFig. 91 .

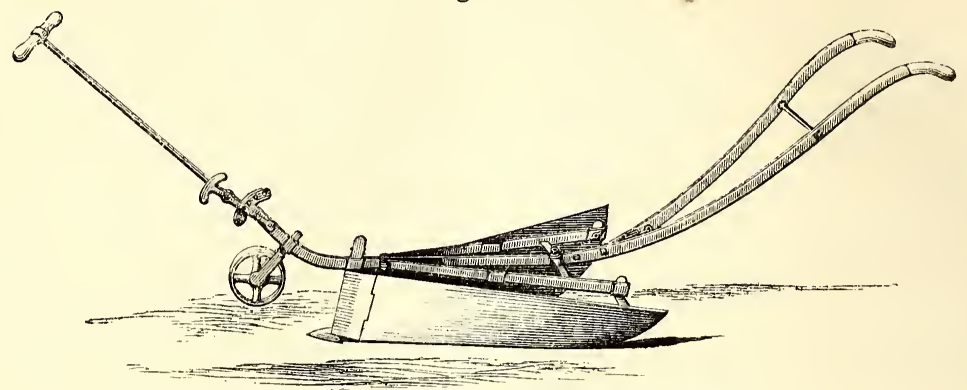

IMPROVED CULTIVATOR WITH EARTHING-UP BOARDS.

fore adapted ours to be wrought by a man and a boy. In both of the former the operator had to walk backwards - a position in which his physical power could not be exerted to its greatest advantage. Again, in stony and hard ground the machine was found ineffective; nor could the ground be stirred so deep, nor the other processes so satisfactorily accomplished, as when a man's whole power is directed to drawing it forward, while a boy guides it behind; and the two lift it more conveniently at the end of each row, and introduce it into the next, without disordering or dirtying the gravel walks at the ends of the quarters. Our other appendages will speak for themselves.

The grubbing-tines are the same, as are also the angular hoes, which fit into the same holes in the frame as in $\mathrm{Mr}$ Sievewright's; and the moulding boards are similarly attached. We have, however, added the stilts, which enable a boy to regulate the direction of the machine behind; while the man who draws it in front is left to exert his whole strength in pulling it through the ground, which he could not do to anything like the same advantage were he to walk backwards, and with the guiding of the machine to attend to at the same time. By gaining this additional force, the tines or grubbers can be let into the ground to double the depth they formerly could, which is an important matter, and to a certain extent supersedes the operation of digging in all loose and workable soils. It is true, weeds are not so completely buried by grubbing as by turning them in by digging; but garden-ground in a highlycultivated state should be clear of weeds; and even should they exist, the triangular hoes are to be fitted in, and the ground run over by the machine the day previous to the grubbing taking place. The expedition with which both these operations are accomplished, bears in no case anything like a relative proportion to the time occupied in digging ever so superficially the same breadth. The machine is so con- structed that the frame can be contracted or expanded to any extent to meet the difference in the breadths between all garden crops; and the same applies to the moulding boards also. With the improved machine which has been manufactured for us by Mr Slight, the eminent engineer, and which has been constructed to our entire satisfaction, we can grub to the depth of 9 inches, or hoe between the rows of crops, or earth up an acre of potatoes per day, at the cost of the wages of a man and a boy-say $3 \mathrm{~s}$. We may also state that we hold no exclusive right in this machine, it being a combination of former principles adapted by us to horticultural and nursery purposes with a view to the economy of time, and doing the work better than by the ordinary operations of shallow digging and hoeing, and the still more laborious operation of earthing up with the draw-hoe. These machines may be seen at the works, and procured of $\mathrm{Mr}$ Slight, engineer, Leith Walk, Edinburgh.

These are the parts embodied in both Dr Newington's and Mr Sievewright's implements; but to these we have added a drilling and sowing appendage, fig. 92, which not only draws the drills more equal in depth and distance than can be done by the ordinary process of using the garden-line and draw-hoe, but saves threefourths of the time, and lessens the evil of treading the ground in an equal proportion-a most important advantage when the soil is wet. The drilling and sowing appendage is fitted on the frame, and covers a space of $4 \frac{1}{2}$ feet in breadth, so constructed that the drills can be drawn to any distance apart, varying from 6 inches to 18 inches; and from 4 to 6 drills can be opened at the same time, and the seed deposited in them. The coulters that open the drills are placed on the front of the cross arm or bar; the boxes for containing the seed are placed on top of it; and the delivering tubes, for conveying the seed from them to the drills, are placed immediately behind and within these coulters, 
and reach to within 2 inches of the drill, by which the seed can be sown even in windy weather, when it could not be safely done by hand. The lower ends of the tubes are also sloped off, by which the seeds are readily seen as they fall. A set of similar coulters is placed behind the cross arm or bar, but somewhat wider than those in front, and reversed in their position, so

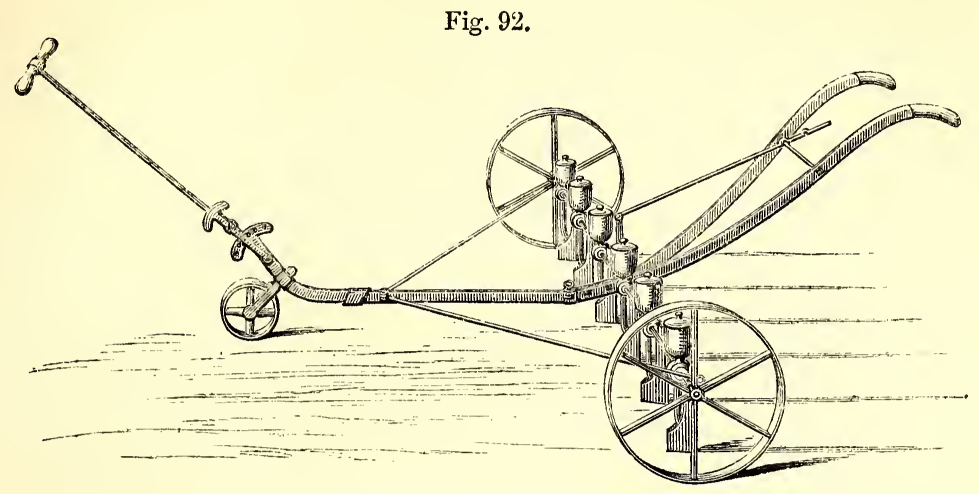

DRILLING AND SOWING MACHINE.

that as the seed is dropped in the drill, and the machine in motion, these latter cover the seed by drawing the soil, pushed aside by the opening coulters, over the seed, which finishes the operation.

The quantity of seed delivered is regulated by the extent of opening in the delivering-tubes, which can be graduated to any extent to suit such seeds as onions, turnips, \&c.; and can be entirely stopped at the ends of the rows, by the boy who guides the machine behind simply raising the iron rod shown between the stilts.

In commencing the operation of drilling and sowing, a line is stretched from one end of the ground to the other: along this line either the right-hand wheel, or the right-hand drill-coulter, according to circumstances, is to travel, so that the drills may be set off straight at starting. The machine is drawn along to the other end of the ground by the man, while the boy guides it along the line-thus opening, sowing, and covering in at once the number of drills the machine is set to. When the first series of drills is finished, the machine is turned, and proceeds in like manner to the other end of the ground, performing the same operation, the boy keeping in this case the left-hand wheel exactly upon the previous wheel-track. By this means, we believe, 2 acres of onions or turnips could be sown per day. Like all sowing-machines, this is best adapted for what are known as round seeds, such as rape, turnip, cabbage, onions, \&c.; but it is also adapted for sowing parsnips, carrots, \&c., provided the seeds of the latter are properly rubbed down and mixed with sand, as is usually done. The mouldboards being attached, deep drills can be drawn for potatoes; and when one of these is removed, short dung or any of the artificial manures can be ploughed in, or the weeds on the surface buried as in ordinary digging, and in one-third of the time. All such machines are, however, better adapted to light soils, or to ground in a high state of cultivation.

The later or principal crops should not be earthed up at all-or, if so, only to a limited extent; and for this purpose our improved cultivator with double mouldboards will be found far better than the ordinary hoe earthing-up system. If potatoes are planted 6 inches deep, they are rather injured than improved by earthing up, some say to the extent of diminishing the crop one-fourth. We have remarked in the article CARROT, $p$. 178 , that deep-hoeing between the rows is inexpedient, as it has the effect of encouraging the lateral fibres to extend, and to induce greater growth in them, producing side roots, or what are commonly called forks. Now, the very opposite course should be pursued in the case of the potato, and every encouragement given to the side roots to extend themselves, for nearly at their extremities the tubers are formed; so that deeply stirring the ground between them tends to their extension. This process, however, must not be carried beyond a certain stage in the growth of the plants, else the extremities of the roots, which it is our object to encourage, may become seriously injured if deepstirring takes place after they have nearly met in the spaces between the rows. This can only be ascertained upon examination. Some kinds of potatoes produce their tubers at a much greater distance 
from the stem than others. These are chiefly to be found amongst the late sorts. Most of the early ones produce theirs close to the stem, or at the extremity of very short runners, seldom more than 9 inches from the stem. The blossom should be cut off as soon as it appears, for most of the evil will be effected if the process is delayed until the fruit is formed. Whatever may be said of curtailing the stems, even when they encroach on other crops, nothing but dire necessity would induce us to do anything of the kind. Cutting the haulm off entirely as soon as the plants have produced their flowers, as recommended by M. Tombelle Lomba as a remedy against the disease, has by no means been satisfactorily proved to have that effect; but it has an effect which might naturally be supposed-that of very materially diminishing the crop. If the disease is purely atmospheric, it is natural to suppose the leaves and stems would be first affected, as their office is to collect and elaborate the matter they throw into the roots and tubers; and, in doing this, they would at the same time transmit the disease along with that matter. As a natural consequence, we are bound to believe that the leaves become affected by the disease before its effects become so evident in them as to be observed by man, and hence the transmission of it to the roots must be going on before any visible symptoms appear in the leaves themselves; and if they are allowed to remain on until the plants have perfected their flowers, the roots will be as much inoculated by that time as if the leaves and stems were left on altogether. So rapid is the transmission of the disease to the tubers, that we have traced symptoms of it in them before any visible signs of it appeared in the leaves, and to such an extent in one case as led us to inquire if the disease might not originate in the soil or manure, affecting the roots first, afterwards the tubers, and lastly the stalks and leaves -reversing the generally supposed order. If, therefore, any beneficial results are to arise from cutting off the haulm at all, it must be done much sooner than has hitherto been recommended; and by doing so, the supplies will be entirely cut off, and the tubers reduced to the identical circumstances of those that are sometimes kept over a year, and buried in sand in a dark cellar, and which are known to send out numerousimmaturely-formed potatoes from their sides, without the aid of haulm or leaves-a practice sometimes adopted to produce young potatoes during winter. No doubt the opinion laid down by $\mathrm{Dr}$ Lindley is quite correct, when speaking of the possibility of the tubers ripening after the haulm has been removed, after it has arrived at a certain condition; but before it has arrived at this condition (the season of flowering), has not the disease already been transmitted to the tubers? The rationale of his views is thus given by $\mathrm{Mr}$ Stephens in "The Book of the Farm," vol. ii. p. 258 : "That it may be that potato tubers, after having arrived at a certain condition, possess the power of continuing their growth by their own proper and unassisted vitality; and this is rendered the more probable by the well-known fact, that the flour which gives them their principal value does not descend directly from the leaves as flour, but is in the first instance of the nature of gum, or some othe fluid organisable matter, formed in the leaves and sent downwards into the tubers. Having reached the tubers, it undergoes its final change, and from a soluble substance is gradually converted by their vital force into insoluble flour. To that vital operation we have no reason to suppose that the leaves contribute: all that they do is to produce the matter out of which the tubers generate the flour. It must be observed that M. Tombelle Lomba does not cut off the stems till after flowering. It is possible that at that time the leaves of the potato have done their work as far as the tubers are concerned, and that their further duty is to nourish the tubers. If so, we have an explanation of the result of which M. Lomba so positively speaks." Not only, according to M. Lomba's assertion, is the disease arrested in its progress by cutting off the haulm, but the tubers suffer no diminution in size after the operation. This latter assertion has been by no means proved in practice, in this country at least, to be correct.

Soil and manure.-Potatoes succeed best on newly-reclaimed land, provided it be light and dry; and in newly-broken-up light rich loam they will succeed better without manure than with it, and, if less weighty in crop, will be of improved fla- 
vour and quality. We speak of flavour and quality, or fitness for the table, and not of their chemical constituents. $\mathrm{Mr}$ Johnston, in "Elements of Agricultural Chemistry and Geology," p. 327, has shown that the quantity of starch is larger in potatoes which are grown upon land long in arable culture, than upon such as are newly brought into cultivation or broken up from grass. One peck of potatoes, grown upon land near Paisley, which had been almost constantly under crop for the last thirty years, produced $7 \mathrm{lb}$. of starch; while another peck, grown on a bleachgreen, newly broken up, yielded only 4 lb. There is land in Cornwall, and also in Essex, that has yielded crops of potatoes annually for nearly as many years; but although the crops in both cases are both early and abundant, we have ever considered them inferior in flavour, at the table, to such as were grown from new land. In well-drained peaty soil they prosper, if not at too high an altitude. Clay soils produce waxy, and sandy soils mealy potatoes; and some soils have the property of changing the character of a variety entirely. Clay soils, in the present state of the potato disease, should be avoided. Out of 163 cases in England, 129 were returned as having suffered much by the disease. In Scotland, 16 cases escaped out of 27 ; but in Ireland and Wales there was scarcely an exemption from disease. Whereas in peaty soil, Mr Stephens, in "The Book of the Farm," vol. ii. p. 402, informs us, "out of 32 cases of the cultivation of the potato in moss in England, only 5 suffered much, and 17 little, while 10 escaped altogether; of 31 cases in Scotland, 9 were bad; of 41 Irish cases, only 2 suffered much, 24 little, and 15 escaped. The conclusion is-in pure, well-drained peat moss, potatoes suffer very little from disease."

In poor soils, manure must be applied, and this should be of the nature of halfdecayed leaves or littering matter, to keep the ground open and dry, particularly if the soil is retentive or the locality wet. Besides, manure in the state of either of these will undergo progressive decomposition along with the growth of the crop, and become reduced to a soluble condition, in which state only manurial applications can be of any use, about the same time that the tubers are forming and vol. II. swelling, at which period the plant stands most in need of increased nourishment. Very decomposed rich manure, applied at planting the sets, may be prejudicial in more ways than one, particularlyif brought into close contact with them. It cannot be of much benefit, because, by the time the plants really do require manurial assistance, this very rotten application will have become soluble and commingled with the soil. Not that in this way it is entirely lost; on the contrary, it is absorbed by the soil, and ready to be given out to the spongioles as they are formed: but unfortunately, as in general applied upon the drill-system, one-half of its fertilising ingredients descend, or are washed down perpendicularly to where it was placed; while the other half, and perhaps the most valuable-the gaseous portion-ascends upwards in a perpendicular direction also, leaving the broad spaces between the row's destitute of enrichment altogether. $\mathrm{Ne}$ cessity has long compelled the British agriculturists to adopt this mode of applying manure, because of the difficulty in obtaining a sufficient quantity to meet their extensive demand-and no doubt, for turnips and similar deep-rooting plants, the rule is unexceptionable; but the roots of potatoes take an entirely different direction in search of food from those of the turnip, carrot, mangold-wurzel, \&c., for these extend downwards, and occupy a narrow line in the direction of the row; and from the fusiform character of the two last, it would be inexpedient to encourage the enlargement of any lateral fibres they might send out, as each of these would either rob the principal root, or enlarge some of the lateral fibres into side roots, and cause in them that malformation known technically as "forking." Thus manure cannot be kept too close to the roots of such plants, and therefore laying it in the drills is perfectly correct. Not so, however, with the potato, whose roots ramify in all directions horizontally in search of food, and therefore require that the ground should be equally manured all over. This plant also requires the greatest quantity of azote at the later periods of its growth, when the tubers are swelling, because they contain a much greater amount of that substance than the leaves. Manures, therefore, to be beneficial to this crop, should 
be those of slow decomposition ; and it is equally clear that they should be distributed equally over the ground. Vide Preparation of the ground.

In these opinions we are supported by Dr Madden, who, in "Prize Essays of the Highland and Agricultural Society," vol. xiii. p. 359-70, observes, "that a manure, to suit well for the potato crop, should possess the following qualities: It must be spread equally through the soil, so that the spongioles, at the termination of the spreading fibres of its roots, may be supplied with nourishment. It must yield azote during the whole period of the growth of the plants-in fact, rather more is required during the later period than prior to the development of the tubers ; for, from M. Boussingault's analysis, it appears that they contain five per cent more of this substance than the leaves. In an economical point of view, therefore, the best manure for potatoes would be one which contained plenty of azote, but still did not decompose very rapidly - cow-dung, for example."

Peat ashes is an excellent manure for potatoes and turnips. Their constituents are as follow :-

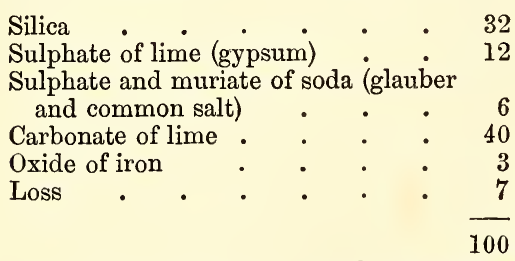

-the quantity applied, $1 \mathrm{lb}$. to the square yard, and applied in their most recent state.

Guano, if applied in a liquid state, and repeated frequently, will no doubt carry on the crop until its complete state of maturity has arrived; but the best security is to have the ground in high condition, either by large manuring for previous crops, or by trenching in plenty of cow or stable manure some time previous to planting the sets, and diffusing it equally over the whole. Salt has been beneficially applied to potato crops. On a soil of a light and sandy nature, without any manure, the return was 308 bushels of tubers per English acre; while the same extent adjoining, manured with 20 bushels of salt, gave a return of 584 bushels. Situation has also as much to do as either soil or manure in increasing the quantity of starchy matter or mealiness in the tuber, as well as securing the crop. "This starchy matter," Dr Lindley remarks ("Gard. Chron.," 1842, p. 155), "can only be formed abundantly by the action of light upon the leaves, which are the natural laboratory in which such secretions take place, and from which they are conducted by sure though hidden channels to the tubers, where they are stored up. To plant potatoes, therefore, in plantations or orchards, or under the shade of trees, is to prevent the formation of mealiness which renders this plant so nutritious, and to cause the tubers to be watery and worthless."

But the potato may suffer by its own shade as much as by the shade of other plants. When its sets are planted too close, the branches shoot upand choke each other, so that the more sets are planted the smaller will be the crop of this plant. Mr Knight was the first to point out this common error, and to show that there is a certain distance at which the sets of each variety of potato should be planted, so as to insure the greatest produce. By planting too close, the plants smother each other; by planting at too great a distance, land is uselessly wasted. Practice and well-conducted experiments demonstrate what theory suggested-that the true distance at which potatoes should be set is determined by the average length of the haulm. One kind of potato is dwarf, and only grows 6 inches high ; its rows should therefore only be 6 inches apart. Another kind grows 3 feet high, and its rows should be 3 feet asunder. The space from set to set in the row appears to be immaterial ; 6 or 8 inches is sufficient for those which grow 2 feet high. An experiment, made in the gardens of the Horticultural Society of London, "showed that the early champion, a sort whose stems are on an average 2 feet long, was planted in rows 2 feet 6 inches apart; the produce was 15 tons 19 cwt. $82 \mathrm{lb}$. net per acre," or, in round numbers, 16 tons ; "while, by reducing the distance between the rows to 2 feet, the produce was increased to 24 tons; but, by diminishing it still farther to 1 foot 6 inches, the produce was reduced to 22 tons $16 \mathrm{cwt} .102 \mathrm{lb}$; and where the rows were only 6 inches apart, the produce fell to 16 tons $17 \mathrm{cwt}$. $110 \mathrm{lb}$. Such an experiment seems conclusive."

As regards manure for potatoes, the 
inference is, that, under all circumstances, two crops, manured with guano, have been saved out of three; that, if applied to autumn and early spring-planted crops, it is advantageous, but that it is dangerous in late planting. The conclusion arrived at in the use of farmyard manure is, that if used abundantly in a very rank condition, and especially if applied in this state to late-planted crops, it is an extremely disadvantageous application. Ashes are a safe manure when applied by themselves, and crops have suffered little or nothing by disease throughout Britain when manured with them alone, in the proportion of 27 to 4 ; and, when mixed with farmyard dung, the success has been as 54 to $15-$ or that is, in the former case, 4 suffered much, while 27 suffered little or nothing; and in the latter, 15 suffered much, while 54 nearly escaped. Saline manures have not been productive of injury, and are perhaps beneficial. Without manure, the results are favourable as regards the disease. In England, 32 cases suffered much for 96 which escaped; in Scotland, 1 suffered for 11 that escaped; in Ireland, 1 for 7 ; and in Wales, 1 for 2-the whole giving 35 cases of suffering for 116 which escaped. Nothing is discernible in favour of salt; nor does sea-weed indicate a better effect. The conclusion of the whole matter may thus be summed up-That over-luxuriance, arising from whatever cause, was highly favourable to the progress of disease, and vice versa.

Forcing.-The true ash-leaved kidney, Jackson's improved kidney, British queen, early tenweeks, Soden's early Oxford, early Manly, golden dwarf, and others of a like early description, should be chosen for this purpose, selecting whole tubers of medium size, and placing them close together, either in shallow boxes filled with light rich sandy soil, or on the borders of an early peach-house or vinery ; or, better still, among half-decayed leaves placed on the surface of a moderate hot-bed, one light of which will be sufficient space to excite a sufficient number of sets to plant a pit of 15 or 18 sashes-that is, nearly 100 feet in length-of a 6 -feet pit. The genial heat from the leaves below will gradually excite the tubers (but on no account must the process be hurried, for too rapid excitement would cause an undue excitability in the sap, and greatly weaken the embryo shoots), while the humidity arising from the leaves below will prevent any loss taking place in the juices of the plant by unnecessary evaporation, the young roots of which will strike into the partially decayed leaves which will adhere to them, and render their removal a safe and easy matter. When the shoots have attained the height of 3 inches, they should be transplanted into a light soil, laid 4 inches in thickness on the surface of a bed of dung and leaves, in a slight state of fermentation. We do not usually, unless the heat is rather strong, cover the whole surface of the bed at once with soil, but only lay it in ridges across, about a foot apart, centre from centre, and on these set the plants, filling up the intervening spaces afterwards as the heat of the bed declines, and as the roots extend. By this means the heat from the bed has free means of ascending upwards to warm the atmosphere of the pit. The plants are set 4 inches apart in the lines. It should be remarked, that a very slight excess of bottom-heat is very prejudicial to this plant, causing them to become, in a few days, drawn up, slender, and etiolated in colour; and the same bad effect is produced by a deficiency of ventilation. At the same time, cold must be excluded, for few plants suffer sooner by frost than this; covering theglasses nightly with felt, or wooden shutters made to fit the sashes over which they are to be placed (vide fig. 789, and Sect. 5, ON COVERING THE ROOFS OF GLASS-HOUSES AND PITS, FOR THE EXCLUSION OF COLD OR THE RETENTION OF HEAT, \&c., vol. i. p. 551), is a much better precaution than employing extra heat in the bed.

Although we have mentioned a bed of fermenting material, we do so, not that it is better than, or even so convenient as, growing them in a tanked pit (of which figs. 629, 631, and 632, vol. i. pp. 450,451 , may be referred to as examples), which of all other modes is the best for all sorts of kitchen-garden productions required at an unseasonable period of the year. Indeed, for all such purposes we expect to see the day when long narrow borders shall be constructed, with vaults under them, and these heated with hotwater pipes, or having tanks of water circulating under them. In such cases, all that is required is an extent of common frames and sashes to be placed over them, to cover in the whole surface. In such borders, most of the productions of the kitchen-garden may be brought to great maturity. Beds of fermenting material can only be advantageously employed when abundance of leaves is conveniently to be had, or where tanners' bark can be got for little less than carting away. Either to purchase such material, or to employ stableyard manure, would be a much more extravagant process than even that of tanking or vaulting the border, as noticed above. The results never can be so satisfactory, on account of the uncertainty of heat, and the unnecessary amount of labour. Where there are extensive lawns to keep in order, or where the rotting down of tree-leaves is carried onfor the purpose either of converting them into manure, or employing them, when thoroughly reduced, and mixed with sharp river-sand, as a substitute for peat-earth for American plantsthen advantage may be taken of the process of fermentation, and potatoes, pease, asparagus, lettuces, \&c., all requiring very little heat, may be grown upon the masses. In this way we employ leaves to a great extent. As they are gathered. from the lawns and drives, they are 
carried to an enclosed place, well exposed to the sun, and shut out from general view, and made up into a mass 12 or 15 feet in breadth, 50 or more feet in length, and 4 feet in height. When hard trodden down, a fine genial fermentation takes place. This mass is covered with frames and sashes, and soil is laid on the surface of the leaves to the depth of 6 inches: on this the tubers of potatoes, when sprung a few inches high, are planted, pease sown, lettuce planted, and the full-grown roots of asparagus set. The lights are put on, and in cold weather are covered with felt shutters of a size corresponding to that of the glass sashes. In such large masses a pretty uniform heat is maintained without the aid of linings; and all the labour attending the process of culture consists in ventilating, on all favourable occasions, protecting in cold weather by covering, and planting or sowing for successional crops. Potatoes may be placed in a slight heat, in front of a vinery or similar place, when the young shoots are sprung to the length of 3 or 4 inches; or a better system is, when the young roots have made 2 or 3 inches' progress, to separate the plant from the tuber, and plant them in the beds as above. Examine the soil at the roots frequently, to see that they do not suffer from a deficiency of moisture, as leaves, during fermentation, give out a much less amount of vapour than stable-yard manure does; and if a proper degree of humidity is not kept up at the roots, the crop will be scanty and inferior in quality. When the potatoes have fairly established themselves, and their roots begin to appear on the surface, lay in between the rows 3 inches of dry fine soil, that has been kept for some time under cover, choosing a fine warm day for the purpose.

Forcing potatoes in pots was successfully practised by the late T. A. Knight, Esq., the essence of whose practice is to the following effect: At the season when the general crop of early potatoes is ripe, and in the process of being taken up, select tubers having perfect eyes, and plant then in 6-inch pots, one tuber in a pot. Bury the pots in the ground till they are required for planting ; during this period, the excitability of the plants is not at all expended on account of the low temperature at which they vegetate, and therefore, when plunged in the hot-bed, they instantly shoot with very great rapidity, and in a few days begin to generate tubers. One stem alone should be suffered to grow in each pot; for where more remain, the tubers are smaller, and the entire crop is not increased in weight. The embryo shoots should be rubbed off as they appear. When the plants grow in small pots, the gardener will have apparently the advantage of being able to take out the largest potatoes, by inverting the pots, without materially injuring the fibrous roots; but this will rarely be found possible in practice, because the plants, having the range of their roots confined to the limits of the pots, soon occupy the whole of their pasture, and therefore do not produce their tubers in succession, as they will under ordinary circumstances. Mr Knight confined his plants, (which were naturally of a very dwarfish growth), to small pots, because, under this mode of cul. ture, the tubers reach maturity sooner, and are superior in quality : but the crop is not so heavy as where the roots are permitted to extend more widely; and, therefore, where a larger but rather later crop is required, the best plan is to place the tubers in small pots to vegetate, and from these to remove them, with their roots and germs uninjured, to the hot-bed. "I tried," says this great experimentalist, "the effect of placing a few tubers on the floor of my cellar, disposing them just in contact with each other, and as soon as the germs were about 4 inches long, a hot-bed was made to receive them. This experiment succeeded perfectly; and as it is not attended with so much expense and trouble as either of the preceding methods, it will be found, in most cases, the most eligible. All that appears necessary to obtain an early crop is to advance the growth of the plant as much as convenient under a low temperature, so as to avoid all unnecessary expenditure of its excitability, and consequently to preserve its germs and roots, as much as possible, uninjured by transplantation." We have long grown the potato in pots, and with sufficient success; but we would always recommend the tubers being planted in the pots as soon as they are ripe. Bythis process, the slow and gradual vegetation is brought into action, and the pots can be placed in any out-of-theway place until the young shoots begin to appear above the ground, at which period, for reasons that require no explanation, they should be placed in a light and airy situation. Some will vegetate sooner than others, and those that do so should be first removed to the light; and a better place for them cannot be found than a cool pit covered with glass, or suspended shelves placed near the top of a vinery or peach-house, but always where frost cannot reach them. This slow process of germination is admirably adapted for those that are to be placed in a little higher temperature towards the end of January or middle of February, or even later; but for those intended to be excited by the end of November or middle of December, a little more heat will be required than that stated above. Of course, a crop thus early produced can never be expected to be so good as one produced later in the season. It is, however, a good plan to have all the tubers intended for forcing planted in pots, or laid on a floor, as Knight has recommended, and to draw from that stock crop after crop as they are to follow in succession.

The London market-garden practice for forcing early potatoes is thus detailed by Mr Cuthill in his work already quoted: "When potatoes are wanted early, a long bed 5 feet wide is dug out to the depth of 2 feet. This trench is filled with hot dung, on which 6 inches deep of the surrounding mould is put. Middle-sized whole potatoes are used for planting: they are placed in close succession along the bed, covered with 2 inches of mould, hooped, and covered over with mats and straw. In about a month they will have sprouted; frames are then got ready, placing 2 feet of hot manure along the whole line of framing, which is sometimes 100 yards in length; the mould is put on to the depth of 8 inches; the potatoes are carefully taken up 
from the striking-bed, all shoots are removed except the main one, and they are planted 4 inches deep. Radishes are then sown thinly over them, covering lightly with mould. When the haulm of the potato has grown to about 6 inches in height, the points are nipped off : this is done in order to give the radishes fair play; and although it may stop the growth for a few days, still the crop is always excellent. The plants are never moulded up, a plan which weakens the potato more than anything else. After planting, nothing more is required but to admit plenty of air, and give water," and, of course, exclude frost: "the crop is not dug up until it has come to maturity." Potatoes are also largely grown in hooped beds in the open ground, the tubers being sprouted as above described. "The beds or ridyes are dug out 2 feet deep in January, filled with hot dung, and covered with the surrounding mould to the depth of 10 inches. The potatoes are taken up and planted 5 inches deep, and above all radishes are sown. The ridges are then hooped over, allowing about 2 feet of space in the middle between the mould and the hoop. They are covered with mats and straw; but, as soon as the radish has come up, they are uncovered daily, and covered again every night. This is continued till the potatoes are ready for digging in May; for sometimes large losses are occasioned by a sudden change of weather on cold April nights." As regards the sorts grown for early forcing, our authority also states that $\mathrm{Mr}$ Fitch, a very extensive grower at Fulham, cultivates the true early shaw: many grow the ash-leaved kidney, because it always fetches the highest price; while $\mathrm{Mr}$ Cuthill himself prefers the lapstone kidney, as being the best flavoured, most mealy and productive. This variety is a great favourite with us; but we have not found it so good for forcing as the ash-leaved kidney and golden dwarf.

The following deception is common in the early-potato trade : The old tubers of the Cornwall kidneys are kept back till July, and then planted. "They grow fast, but the crop never ripens before frost sets in; as soon as the haulm is cut down, large quantities of straw are put on the ground, and towards Christmas the tubers are dug up, put into 1-lb. and 2-lb. baskets, and sold for new potatoes at from $6 \mathrm{~d}$. to $8 \mathrm{~d}$. per lb." They are full of water and tasteless, and the skin remains firmly attached; and, when cooked, they have very little resemblance to new potatoes, except in size and external appearance. The substitution of immaturely-ripened potatoes-of a variety known as the Dutch winter potatoes, for early-forced ones - is largely practised in Covent Garden market. Twentyfive years ago, we had these potatoes sent from Holland, with instructions to plant in autumn in the open ground, and statement that they would grow all winter, and be fit for use early in spring. The result was, that the tubers came into úse exactly at the same time as did those of the ash-leaved kidney and several others planted in March. This is a small round potato, with a very uniform outline and smooth skin, by no means uncommon in the vegetable mar. kets abroad as one of their ordinary sorts. If the old tubers be kept back till July and then planted, an abundant crop of immaturelyformed young tubers will be found at the roots; and, if kept in the ground till they are required, they have all the external appearance of fine forced potatoes, but not one of their qualities.

Young potatoes, during winter, are produced by selecting the largest tubers of almost any variety in spring, placing them in a cool cellar to retard vegetation. The embryo sprouts as they appear are rubbed off until the month of August or September, when they are removed to a warmer place-the floor of a mushroomhouse, for example. Here they are laid upon a bed of light sandy soil, about 10 inches apart from each other; they are then covered over with similar soil 4 or 5 inches deep, watered with tepid water, and allowed to remain so till December, at which time numbers of young potatoes will be found attached to the old tubers by a transformation of the matter in the latter into young potatoes, and this without the production of stems or leaves. They may, for the same purpose, be packed in soil in old casks, boxes, or, indeed, anything that will keep the soil together; and they may be placed in any out-of-the-way place, if in a temperature of from $50^{\circ}$ to $60^{\circ}$.

Taking the crop, and subsequent preservation.-The early varieties are taken up for use as they attain the required size, which, in warm exposures, will be about the beginning of June, and so on till the middle of July in less favourable places. Some remove the soil partially from about the roots, and gather the largest tubers, leaving the smaller ones for a week or so longer, in the expectation of their attaining a larger size. It is seldom, however, that this mode of proceeding realises the hopes of the grower, for the potato, if once disturbed at the roots, seldom recovers the check. It is, therefore, better to take up the crop as it is required, and immediately replant the ground with something else. For private use, only as many should be taken up daily as may be required for that day, as they will not keep good above a day or two; and hence the inferior quality of those young potatoes brought to the London markets from the Continent, compared with those grown in the vicinity. The end of May in England, and the beginning of June in favoured spots in Scotland, is the commencement of potato-taking; while, on less favoured situations, it is delayed till the beginning of July, even where partially protected. A week's delay in commencing on the crop is of much importance to the bulk and 
quality, for at those periods, particularly if the weather is warm, and accompanied with "May showers," the young tubers swell amazingly fast. In July, August, and September, they will be in fine condition for family use. Those intended for seed should be gone over, and if any spurious ones have crept in by accident, they had better be removed, as they will be readily distinguished by their haulm from the others. When the foliage of these begins to decay they may be lifted, so that the ground may be cropped with something else; and if they are buried in a deep pit, in a dry yet cool shady place, they will require no further care till the season of planting arrives. Care, however, must be taken that they are not laid in too great quantities together, for fear of their heating ; and also that each sort be kept separate and correctly named, to prevent confusion at planting time.

Late varieties constitute the great bulk of crop, and are those which require most care in taking up and storing. So long as the haulm continues green, the potato should be allowed to remain in the ground, as this is quite indicative that the tubers have not arrived at full ripened maturity, which again is indicated when the haulm begins to wither and die off. This is an important point in their management, so far, at least, as relates to those intended for the table, in which flouriness (or starch) cannot too much abound; and unless fully ripened, that conversion cannot properly take place. The art of keeping potatoes (if really free from the disease) is founded upon sufficiently correct principles, the leading point of which is exclusion from light; for if this is not attended to, they not only become injured in their nutritious qualities, but are actually rendered poisonous when allowed to become of a green colour, which they readily will do if exposed to light_-and this the sooner, if vegetation is in the least excited. Keeping them in potato-houses, therefore, unless so constructed as to exclude light, must be considered injurious to them. In this state of darkness they should be placed the very day they are taken out of the ground; and it were better that they were stored by in rather a damp state, than that they should be exposed for a day to the light with a view to dry them. Drying has a bad effect on the skin of the potato, for if subjected to this, the skin and part of the epidermis are made to part with their natural juices, which ever afterwards renders them incapable of absorbing moisture, even if presented to them. Fermentation is an important evil to guard against, as it changes the whole substance of the tuber, and, so far as seedpotatoes are concerned, destroys their vegetative principle. To guard against this, they should be placed in small quantities together, in long narrow ridges with partitions of earth between them, dividing the whole into compartments of a size such as will probably be sufficient to supply the wants of the owner for a week or so at a time. Potatoes once dried should never be again wetted until they are going to be used. Straw or any other littering matter is an objectionable covering for potatoes, for by its decomposition carburetted hydrogen is formed, and mixes more readily amongst the potatoes in the heap than it escapes through the soil laid over it. The greatest care should be taken that all tubers bruised or cut. during the operation of lifting should be kept by themselves for immediate use ; and were it not that potato-lifters would think us far too fastidious, we would say every one whose skin is broken should be rejected also, with the same care and upon the same principle that all apples bruised at gathering are kept apart from those sound ones carried to the fruit-room.

Keeping potatoes has the effect of diminishing the quantity of starch in them; those in October, according to Mr Johnston (in "Agricultural Chemistry and Geology," p. 329), which yielded readily 17 per cent of starch, gave in the following April only $14 \frac{1}{2}$ per cent. The effect of frost is also to lessen the quantity of starch. It acts chiefly upon the vascular and albuminous part, but it also converts a portion of the starch into sugar-hence the sweetish taste of frosted potatoes.

The housing of potatoes, unless such quantities as are required for immediate use, is exceedingly wrong in principle, as is also the manner in which many are carelessly pitted out of doors. The points to be kept in view are, a dry airy exposed situation, having a northern exposure and perfectly dry below, and piling the potatoes on a smooth level surface not lower 
than the surrounding soil, in long narrow ridges 3 feet on the base and 2 feet in the perpendicular, the sorts to be kept separate with earthen partitions as stated above. It is better to pile the whole stock in one or two long ridges than to have a dozen of small conical ones scattered about, and from which the frost and heat are seldom properly excluded. We prefer the floor of the pit to be level with the surface, to prevent the chance of damp; and also having the whole stock in one place for convenience of covering, turning over if need be, and for taking out as required. If the tubers are stored when very wet, and fears arise on this head, a row of drain-tiles may be laid along the floor under the potatoes open at both ends, but secured against the intrusion of mice or rats by placing a fine wire covering over their orifices. A tile-pipe or two may be placed with a like precaution, communicating with them, and passing like a small chimney through the top of the pit. During winter, these may be all left open for the circulation of air ; but by the beginning of February they should be completely stopped up, to prevent the entrance of heated air from without: at that time all danger from fermentation will have ceased. Where turf can be got conveniently, a course of it, with the green side uppermost, may be laid over the tubers, but no littering matter whatever; and if turf cannot be conveniently got, lay the soil on at once. The object of covering is twofold-first, to exclude frost and wet; and secondly, to exclude heat: now earth, although no bad non-conductor of heat or cold, is not sufficient of itself to answer our purpose; therefore, after 9 or 10 inches of soil are laid on, thatch the whole over $1 \frac{1}{2}$ feet in thickness with straw, fern, heath, or any similar non-conducting material. This is more particularly necessary with those that are to be kept till a late period in the spring, to last till new potatoes come in, which cannot be kept at too low a temperature. With the general stock, all that is required is to exclude wet and frost, therefore the thatching need not be so thick. In storing late potatoes for spring, use the principle of the ice-stack (figs. 720,721); and what has been said regarding the keeping of ice (vol. i., Section ICE-HOUSE) should be strictly kept in view.
Approved sorts and their qualities.-Potatoes are divided by cultivators into early and late sorts. The names, we should observe, are vcry arbitrary, most localities having their favourite sorts, and consequently their own names. An extended list would, therefore, be of little general utility, more especially in the present unhealthy state of the plant. We will, therefore, confine ourselves to the best of the early and second early varieties (which for the most part constitute the garden sorts), the names of which are more permanent.

Ash-leaved kidney.-One of the most popular sorts for forcing, and also for the earliest crop in the open borders. It fetches a better price in the market than any other, is exceedingly thin-skinned, of good form, and with few eyes. Should be planted whole, with the end containing the eyes uppermost; or, if cut, the incision should be made lengthways through the centre.

Jackson's improved ash-leaved kidney.-A selected variety from the last, of larger size, greater hardiness, and a more productive bearer. Not quite so early as the last, and, being better set with eyes, is much less liable to remain in the ground without springing: an excellent sort.

British queen.-A round potato of dwarf and prolific habit; one of the earliest.

Soden'searly Oxford.-A round potato of recent origin ; very early, and well adapted for forcing.

Fox's seedling.-A popular early potato about London, either for forcing, or for earliest crops in the open air. There appears little difference between this variety and that of early Manley and early Cockney. Tubers round, white, rather waxy.

Early frame and early ten-weeks are very similar. Indeed, any of the last five sorts may be grown with advantage, particularly for forcing, and the earliest border crops.

Jackson's golden drop.--Very dwarf in haulm, of good guality, and well adapted for forcing. Skin tender, and a productive cropper.

Rufford kidney. - A long-famed Lancashire variety, not in very general cultivation elsewhere. It is a very early variety, mealy, well-flavoured, and stands the frost better than any other early sort. It is also known as the lady's finger.

The most comprehensive as well as the best descriptive lists of potatoes, are those published by Messrs Peter Lawson and Son, the eminent nurserymen and seedsmen, Edinburgh, first in their "Agriculturist's Manual" some years ago, and more recently in their "Vegetable Products of Scotland," a work of much research and great accuracy, invaluable both to the gardener and farmer. These lists are rendered valuable, as they comprise all the known varieties, with clear descriptions " as to the habit of growth, nature and colour of foliage and flower; the shape and colour of the tubers, and peculiarities of the skin; with general remarks as to their use and comparative value for the table,"-forming a very safe guide, from which the cultivator may select suitable varieties, and reject such as may appear unadapted to his soil, or which, for other reasons, may seem unworthy of culture.

From the last of these works we have made the following selection, omitting, however, all 
such as are described as unhealthy, or of secondary merit :-

"Earliest garden sorts adapted for forcing, on account of their dwarf habit of growth. - Early frame, common.-Stem slightly reclined; about $1 \frac{1}{2}$ feet high ; tuber medium-sized, and round; colour whitish and roughish; mealy, good flavour, and healthy.

"Fox's early delight.-Stem growing upright; about $1 \frac{1}{2}$ feet high; tuber slightly hollow at the end, of a roundish shape; colour whitish, and slightly rough-netted like; mealy, superior flavour, and healthy.

"Fox's early globe.-Stem slender and reclining; about 1 foot high; tuber round, and feweyed ; colour whitish, slightly rough, and nettedlike; mealy, good flavour, and healthy.

“Fox's John Bull, or early kidney.-Stem slender and reclining; about $1 \frac{1}{4}$ feet high; tuber long, nearly uniform thickness; colour whitish, and smooth; pretty mealy, good flavour, and healthy.

"London dwarf kidney.-Stem slender and reclining; about 1 foot high; tuber flat, thickest near the point; eyes prominent; colour whitish, and smooth; waxy at small end; indifferent flavour, and healthy.

"Williamson's favourite. - Stem slender and reclining; about $1 \frac{1}{2}$ feet high ; tuber slightly elongated; colour whitish, and smooth; mealy, good flavour, and healthy.

"Earliest garden potatoes, not so well adapted for forcing as the preceding, on account of their taller habit of growth.-Ash-leaved early.-Stem upright and loose; about $1 \frac{3}{4}$ feet high; tuber roundish; colour whitish, and roughish; medium flavour, but healthy.

"Chapman's early kidney.-Medium between the ash-leaved and July kidney; resembles the latter in form.

" Early seedling. - Stem slender and straggling; about 2 feet high; tuber round, few-eyed; colour whitish, and smooth; mealy, superior flavour, and very healthy.

"Hopetoun early.-Stem strong and upright; about 2 feet high; tuber large, round, with few eyes ; colour dull white, rough, and netted-like; mealy, good flavour, and very healthy. Perhaps the most superior variety in the class to which it belongs.

"Invermay early. - Stem spreading; about $1 \frac{3}{4}$ feet high; tuber round; colour very white, and smooth; mealy, good flavoured, and very healthy.

"Kay's early American.-Stem slender and spreading; about $1 \frac{1}{2}$ feet high; tuber slightly oblong and flattish; colour light red, and roughish; mealy, medium flavour, and very bealthy.

"London particular. - Stem rather upright and compact; about 2 feet high; tuber round, or slightly elongated; colour whitish, and roughish; medium, good flavour, and very healthy.

"Main's particoloured seedling.- Resembles the ash-leaved kidney in form, but rather larger, and the round end of a deep purple colour. Raised in 1850.

"Musgrove's snow white.-Stem rather upright and compact, about 2 feet high; tuber round, hollow at the point; colour remarkably white, rough, and netted; medium, good flavour, and very healthy.

"Second early garden potatoes.-Caraccas.A late, prolific, and healthy growing sort; of superior quality; roundish, slightly flattened shape; reddish colour, and rather under the medium size. It was found wild in Caraccas. This variety is also known by the names of the American and the Prince-regent potato.

"Dwarf American early.-Stem pretty upright, about 1 foot high; tuber slightly flattened; colour white, and rough; mealy, good flavour, and pretty healthy.

"Early Wellington.-Stem slender and spreading, about $1 \frac{1}{4}$ feet high; tuber round; colour yellowish white, and very rough; mealy, good flavour, and very healthy.

"Gold-finder.-A very tall, luxuriant-growing, and productive variety, with white, slightly oblong, and somewhat flattened tubers; reputed as being of superior quality, and yielding a good crop, even on inferior soils, and with little manure.

"Lawhead early red.-Stem slender and spreading, about 2 feet high; tuber very round; colour dark red, and roughish; mealy, good flavour, and healthy.

"Lawhead early white.-Stem rather slender and spreading, about $1 \frac{1}{2}$ feet high; tuber rather large; colour whitish, very rough, and netted; mealy, good flavour, and pretty healthy.

"Lawson's conqueror.-Very similar to Peffermill prolific, but tubers generally of a larger size.

"Matchless kidney.-Stem upright and compact, about $1 \frac{1}{4}$ feet high; tuber oblong; eyes few and prominent; colour white, and smooth; mealy, superior flavour, and medium healthy.

"New early Windsor seedling.-Stem rather slender and spreading, about $1 \frac{1}{4}$ feet high; tuber round; colour white, and smoothish; mealy, good flavour, and very healthy; of excellent quality, being dry, mealy, and of an agreeable flavour, and very prolific.

"Painted lady, early. - Stem upright and compact, about $1 \frac{1}{2}$ feet high; tuber irregularly round; colour white and reddish; rather waxy, flavour medium, and healthy.

"Peffermill prolific.-Medium-sized ; skin roughish; eyes deep; roundish shape, inclining to a flattened oblong; colour buffish white.

"Prince of Wales' early.-Stem strong and upright, about 2 feet high; tuber large, slightly oblong, and flat; colour white, and smooth; rather mealy, good flavour, and very healthy.

"Prince Regent.-The commonest white potato in the Scotch market; similar as to quality, but rounder in shape than the Peffermill prolific.

" Ross's pigma kidney.-Stem spreading, about 1 foot high; tuber small, long, crooked, and feweyed; colour whitish, and roughish; mealy, superior flavour, and medium healthy.

"Rough Robs.-The potato to which this name has been applied is a healthy-growing and prolific second early, roundish shaped, slightly flattened, and reddish coloured; rough-skinned tubers; and is much esteemed in the lower part of Argyllshire and neighbouring districts, for the purpose of succeeding the earlier white sorts. 
"Seek-no-farther.-Stem compact and bushy, about $1 \frac{1}{2}$ feet high; tuber round; colour white, rough, and slightly netted; mealy, good flavour, and healthy.

"Smith's yellow Peruvian.-Tubers rather under the medium size; roundish, or slightly elongated, with numerous deeply-sunk eyes, and of a yellowish-white colour. This is a healthygrowing and superior late sort, having a peculiar yellow-coloured flesh; received from its native country, Peru.

"Tall American early. - Stem strong and pretty upright, about 2 feet high; tuber flattened; colour very white, and rough; mealy, good flavour, and very healthy.

"Taylor's forty-fold.-Stem slenderand spreading, about $1 \frac{1}{2}$ feet high; tuber oval, and much flattened; colour rough and dull reddish; mealy, superior flavour, and very healthy.

"White Sutherland kidney.-Stem very upright and compact, about $1 \frac{3}{4}$ feet high; tuber curved, flat, and small towards the stalk; colour whitish, rough, and netted; mealy, good flavour, and healthy."

The number of potatoes described in Messis Lawsons' work is 175; and as the whole have been grown in their extensive experimental grounds, and their conditions carefully recorded by competent persons, we consider their list as the best data in existence by which their respective merits may be calculated. The garden varieties extend to fifty-eight in number, and from these our selection has been made.

The nomenclature adopted above is that by which the varieties of potato are best known in Scotland; but an examination of the models of them, now in the Botanical Museum in the Royal Gardens at Kew, will enable those interested to recognise the sorts, although presented to them under new names.

Second earlies. - Early Shaw, or Shaw's early, a variety grown extensively by the London market-gardeners for forcing, to come to market in May. It is, for an early sort, a large, beautiful, oblong, white-skinned potato, whose only fault is its hollow eyes. It is very productive.

American early.-One of the most valuable potatoes ever grown. How long it has been in cultivation we have no knowledge, further than it was well known to us fifty years ago. It has always maintained a good position in the estirnation of cultivators, being very productive, of medium and uniform size, and of excellent quality from August till the following spring. It has resisted the epidemic better than any other sort. Round, yellowish white, very flowery at an early stage.

Fifty-fold. - A most extraordinary cropper; tubers not very large, of uniform size, and keep well.

Early champion. - Tubers large, round, white; very prolific, and in high estimation in the London market.

VOL. II.
Lapstone kidney.-Decidedly the best kidneypotato grown; an excellent cropper; tubers sometimes 7 inches in length and 3 in breadth. We have remarked of this sort, that it is longer in coming through the ground in spring than most others; and the stems at first appear weakly, but they soon lose this appearance, and grow most vigorously. It is a first-rate potato for the table in August and September, and will keep in excellent condition till May following, without losing either its mealiness or flavour. It is of Yorkshire origin, said to have been raised by a cobbler, and hence the name. Many of the late varieties, which were for a time popular, have disappeared, and others are yearly coming into notice-a circumstance easily accounted for, seeing how freely the potato is reared from seed. A catalogue of sorts was published in 1842 by Mr Chatwin, enumerating and describing above 150 varieties. On the Continent the sorts are innumerable, but chiefly small, as the potato is there regarded more as an article of food for man than for the inferior animals.

The last five sorts are chiefly of English origin, or cultivated more largely there than in Scotland. They are not included in Messrs Lawsons' descriptive lists.

Insects and diseases.-The Aphis vastator is frequently found on the leaves of the plants; but so far from its being the cause of the disease, as asserted by $\mathrm{Mr}$ Smee, it is seldom found upon them in sufficient numbers to produce much effect. The larva of Noctua exclamationis (the heart-and-dart moth, fig. 71) is far more destruc-

Fig. 93.
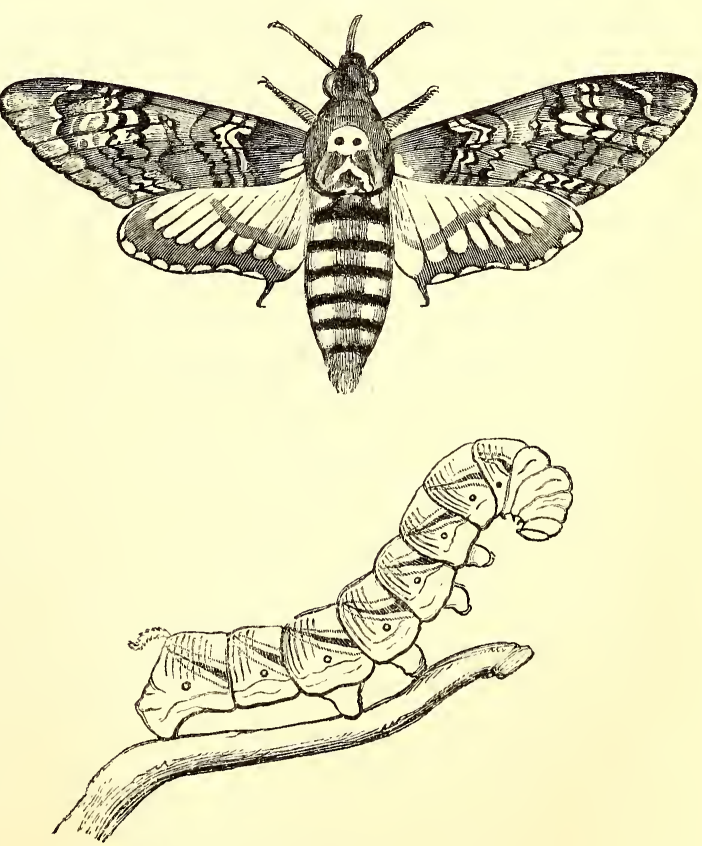

DEATH'S-HFAD MOTH AND CATERPILLAR. IIalf natural size. 
tive. It attacks the haulm just under the surface of the soil, and perforates right through it, and not unfrequently cuts it asunder. It also attacks the tubers, and may often be detected in them at taking-up time. The wire-worm or click-beetles, of which no fewer than sixty species occur in Britain, in their larva state, often attack the tubers; of the larvæ of Cataphagus lineatus, fig. 19, we have found as many as forty-seven attached to one moderate-sized potato. Their presence in soils cropped with autumn-planted potatoes we dread much more than frost, or any other evil that could befall the sets. C. obscurus is most abundant in Scotland, and although we are not deficient in any of the Elateridæ tribe, still it seems to be the most destructive. The caterpillar of that beautiful and rare insect, the death's-head hawk-moth (fig. 93), is sometimes found on the stems of potatoes, but not to such an extent as to inflict very great injury.

The caterpillar of Hepialus lupulinus (the garden swift) is often destructive to the potato.

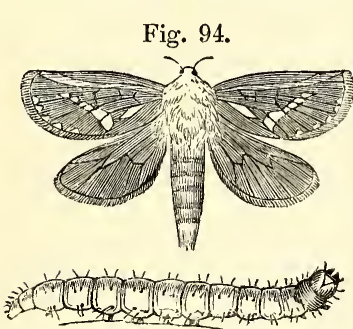
Natural size.
GARDEN SWIFT AND CATERPILLAR,

"The moth, usually, is chalky brown, head and thorax woolly, and its upper wings dark - bright brown, with a broad line of white; but sometimes this is absent, and at other times the upperwings are chalky

white. These moths appear about the end of May, and are very abundant in the evening in meadows and other grassy places. They deposit their eggs apparently indiscriminately, which soon hatch, and the caterpillars produced are cylindrical and yellowish white, with black dots and hairs on the upper part and sides of their segments. The caterpillar changes to an ochreous shining cylindrical pupa." - Gardeners' Chronicle. In the latter state it is often very destructive to potato roots.

The curl, which committed no small devastation on the potato crops some years ago, is now little heard of. Opinions as to its cause, and the remedies proposed, were both about as conflicting as those of the present more fatal epidemic. The cause of the scab or ulcers on the skin of the potatoes has been as little understood as either-some attributing it to the manures applied, and others to certain ingredients in the soil. It is now less heard of; superior cultivation has probably effected, so far, a cure in this case. The rook is gravely charged with making free with our seed-potatoes as soon as they are planted, and also helping himself to a few full-grown ones in autumn. We believe, if the matter were investigated by even a less enthusiastic ornithologist than Mr Charles Waterton, that it would be found the seed-potatoes purloined, in most cases, are infested with wireworms, which are attracted to the sets as soon as they are placed in the ground. We have long ago satisfied ourselves upon this point, and will never kill one of these birds ; but those that are sceptical may do so, and on dissecting the maw they will find abundant evidence as to this point in the numbers of wire-worms contained in it. It is possible that, during the short period of incubation, hunger may drive them to steal a few sets, but it is searching for the wire-worm more than the potato that leads them to the newly-planted fields. The important services the rook performs to man in the destruction of his more-to-be-dreaded enemies are so great, that they should be admitted as evidence to character when he is arraigned at the bar of vulgar prejudice.

Nothing like a correct opinion, we believe, can be offered regarding the cause of the disease which has for some years more or less been disastrous to the potato crop. All, therefore, that can be done, in our present state of knowledge on the subject, is to apply the best remedies we can. Our own opinion has all along been in favour of autumnal planting, not as aninfallible remedy by any means, but as one of very considerable mitigation. Nor are we alone in holding this opinion. Out of sixty-four returns furnished by various individuals throughout England and Wales of the state of the general crop of 1849, fifty-three were in favour of autumnal planting, while only eleven were against it. In England, those planted in autumn were by the same report stated to be better, in the proportion of thirteen to eleven, than those planted in January and February; and had these reports extended to May-for even to that late period do some people delay the finishing of their potato planting-the difference in favour of autumnal planting would have even been much greater.

General remarks. - The potato appears to change its character by change of climate or circumstances. Most of the Scotch and Irish varieties degenerate when removed to England, and often when removed from one district of the same country to another. They soon degenerate when taken to the West Indies; and although cultivated by our countrymen in many parts of the East Indies, where they yield good crops, yet are they deficient in that flavour peculiar to them in Britain. The most extraordinary change of character we recollect of is that recorded by Phillips, on the authority of Samuel Bate, Esq., who went with the first settlers to Van Diemen's Land. He took out about half a bushel of potatoes for seed, which were all of one kind; but to his great surprise, when they were dug up, he had five distinct varieties-viz., the white champion, the round red, the kidney, a small round potato, and the variety commonly called the miller's thumb. A sample of each of these was afterwards sent into a warmer climate, where, on being planted, they all degenerated into one, the original variety !

"The best rules to obtain and preserve sound potatoes and a good crop are-l. Grow none but those which ripen by August; 2. Plant whole middle-sized potatoes; 3. Plant on moderately-light soil, manured some months previously; 4. Apply no manure at the time of 
planting; 5. Plant in November, in light dry soils, but not until February in wet soils; 6 . Preserve seed-potatoes between layers of earth until required ; 7. Plant as you dig-that is, dig enough for one row, and then plant it with the dibber, so as to avoid trampling on the ground." Why not plant in the trench as the digging goes on? " 8. Let the tops of the sets be 6 inches below the surface; 9 . Do not earth up the stems; 10. Do not cut down the stems; 11. Take up the crop as soon as the leaves begin to look yellow, in July or early in August; 12. Store in a dry shed between layers of earth, sand, or coal-ashes."-Cottage Gardeners' Dictionary, p. 741.

The European names are, Pomme de Terre in French; Tartufi bianchi, or Pomo di Terra, in Italian; Kartoffel in German; Batata da Terra in Portuguese ; Aardappel in Dutch ; and Batatas Inglezas in Spanish.

\section{§ 4.-THE JERUSALEM ARTICHOKE.}

Natural history.-The Jerusalem artichoke (Helianthus tuberosus L.) belongs to the natural order Compositæ, sub-order Heliantheæ, and to the class Syngenesia and order Frustranea. The generic name is derived from Helios, sun, and Anthos, a flower, from the resemblance of the flowers of the genus to that luminary, which they are supposed to look towards while the earth is illuminated by his light. The popular name Jerusalem is merely a corruption of its Italian name Girasole, and artichoke is derived from the resemblance in flavour which the tubers have to the bottoms of artichokes. Parkinson, in whose time it was introduced, calls it Battatas de Canada. Coles describes them, forty years afterwards, as the Potatoes of Canada. In Martyn's edition of Miller's "Gardeners' Dictionary " we are informed that they were called so because the French brought them "first out of Canada to these parts; not that they are natives of that country, but had been introduced there from Brazil." A writer in the "Perny Cyclopædia," but upon what authority he does not mention, says this plant was introduced into Europe at the Farnese garden at Rome, from whence it was originally distributed. They were first planted in England in 1617, and so rapidly did their cultivation proceed that before 1629 they were so common in London "that even the most vulgar began to despise them." Parkinson remarks that in his time " they were baked in pies, with marrow, dates, ginger, raisins, sack, \&c.; but the too frequent use, especially being so plentiful and cheap, hath rather bred a loathing than a liking for them." We notice this plant thus largely because the advent of its restoration to cultivation has commenced, and also to show that they may yet be produced abundantly, which their long-neglected state induces some to doubt. "The potatoes of Canada," says Coles, "called by ignorant people Jerusalem artichokes, were of great account when they were first received amongst us, but, by reason of their great increase, they are become common, and consequently despicable, especially by those who think nothing good unless it be dear; but if any one please to put them into boiling water, they will quickly become tender, so that being peeled, sliced, and stewed with butter and a little wine, they will be as pleasant as the bottom of an artichoke."

The whole genus, except $S$. tuberosus, flowers most profusely, but, in consequence of its seldom producing flowers, few varieties have been produced. This is to be regretted, because if so, improved varieties might be expected, as has occurred in the case of the potato. Some varieties are said to have been produced in France, where it is much more extensively grown than with us, but of their merits little has hitherto transpired. The improvement wished for would be, increase in the size and number of the tubers, and a dwarfer habit in its growth. From the experiments made by Messrs Payen, Poinsot, and Terey, and published in the "Jour. de Pharm.," vol. xvi. p. 434, it appears that 100 parts of the tuber contain 23.96 parts of nutritious substance, or twice as much as is obtained from the potato, and something more than the cerealia contains. The tubers on which these experiments were made were grown on a sandy soil of moderate goodness, which had been manured with ammonicophosphate of magnesia. A state of flowering might be induced by removing the tubers as soon as formed, so as to throw the strength which would otherwise be consumed by them into the stem, on the principle pointed out by Mr Knight in regard to causing early potatoes to produce flowers. "I took," he says, "several methods of placing the plants to grow in such a situation as enabled me readily to prevent the formation of tuberous roots, but the following appearing the best, the others need not be referred to. Having fixed strong stakes in the ground, I raised the mould in a heap round the bases of them, and in contact with the stakes; on their south sides I planted the potatoes from which I wished to obtain seeds. When the young plants were about 4 inches high, they were secured to the stakes with shreds and nails, and the mould was then washed away by a strong current of water from the bases of their stems, so that the fibrous roots only of the plants entered into the soil. The fibrous roots of this plant are perfectly distinct organs from the runners which give existence, and subsequently convey nutriment, to the tuberous roots; and as the runners spring only from the stems of the plants, which are, in the mode of culture I have described, placed wholly out of the soil, the formation of tuberous roots is easily prevented: and whenever this is done, numerous blossoms will soon appear, and almost every blossom will afford fruit and seed." From this Mr Knight concluded " that the same fluid or sap gives existence alike to the tuber and the blossom and seeds, and that whenever a plant of the potato affords either seeds or blossoms, a diminution of the crop of tubers, or an increased expenditure of the riches of the soil, must necessarily take place." By these means he succeeded in producing varieties of sufficiently luxuriant growth and large produce for 
general culture, which never produced blossoms.-Horticultural Society's Transactions, vol. i. p. 188 .

If similar means were adopted in the case of the Jerusalem artichoke, there is little doubt that improved varieties would be produced; and in their case the want of blossoms may arise as much from their disposition to form tubers in an early stage of their growth, as from (as is generally supposed) a deficiency of climate.

Use.-The tubers may be used in every way like the potato, and, being more nourishing and less flatulent, may be eaten by people in delicate health, when debarred from the use of most other vegetables. They make one of our best white soups, "Soup de la Palestine." They are often planted in game-preserves as food for pheasants, and hares and rabbits are remarkably fond of them.

Propagation. - The small tubers are saved for planting whole, or the larger ones may be cut into one or two pieces the same as potatoes, only care must be taken that one eye or bud at least be retained in each set.

Planting.-Like potatoes, they may be planted the same day the crop is taken up in autumn, or at any other time when the ground is dry till the beginning of March.

They are planted in every respect as recommended for potatoes, only they require a greater distance between the rows on account of their great height, which often extends to from 6 to 10 feet, and also the large size of their foliage. The best method is to plant them in single lines at a considerable distance apart, for the same reason and in the same way as recommended for the lategrowing kinds of pease.

The usual mode of setting the tubers is to dibble them in, as is often done in the case of the potato-and in both cases improperly, because, the sets not being all of the same size, the smallest ones drop to the bottom, while the larger ones are often left suspended midway down, coming in contact with the sides of the hole, thus leaving them at unequal depths, and the latter in the worst possible position for enabling the radicles of the young roots to reach the soil. It is much better, in planting all tubers, and even very large seeds, to open drills with the hoe or spade, and to place the tubers in the bottom. Another good way is to place them in the bottom of the trench, either above or below the manure (when such is applied), as the process of digging goes on. This latter way is much the best when the land is wet or the soil strong, as treading upon it in either case, after being newly trenched or dug, would be injurious. When the sets are drilled in, they should be covered by the hoe or rake, and the soil placed over them left as loose as possible; and in either way the tubers should be kept as near as possible to 6 inches under the surface.

A piece of ground richly manured, and in an open situation, should be prepared, by being either trenched or deeply dug. The total neglect of these two conditions has long been the ruin of this esculent root, which, in general, is pushed away into some out-of-the-way corner, as if it were "branded with a mark of disgrace, and unworthy of being seen amongst the plants of the garden." In such situation it is allowed to remain for years neglected, and only visited when its tubers are sought for, which, in consequence, are small, and scarcely fit for the purposes of the cook. The tubers should be set 3 feet apart, if set in continuous rows, row from row, and 2 feet distant in the line. They will require no other attention till the following autumn, excepting keeping the ground clear of weeds. In regard to produce, they will yield as heavy a crop as ash-leaved kidney potatoes; and the nutriment contained in that quantity will be more than double. It has been recommended to top the stems when about 3 feet in height, in the expectation of throwing a greater amount of nourishment into the tubers : this has not, however, been found to be the result; while the shortening of the stems makes them send out numerous side branches, causing shade, of which the plant is very impatient. From experiments made in the extensive nurseries of Messrs P. Lawson and Son, it clearly appears that topping, instead of being beneficial, is really injurious. The produce of a row 60 yards in length of plants topped, yielded only 144 lb., while that of the adjoining row, of equal length, yielded $163 \mathrm{lb}$. Neither of these rows had guano or any other kind of manure applied to them. A third row had 8 oz. of Peruvian guano applied to the surface of the soil when the stems were 3 inches in height-it yielded 188 lb. ; while a fourth had a like quantity of guano applied at the same time, but 
buried 1 inch under the surface near the roots of the plants, in which case the produce was $210 \mathrm{lb}$. We have had on a poor peaty soil, manured as for potatoes, even much greater returns of crop.

Of all the substitutes recommended for the potato, no one is so likely to answer our anticipations as this. It is of as easy culture, equally hardy, affording twice as much nutriment, cooked in the same manner, and capable of being cultivated in the same soil.

Soil and manure.-A light, warm, sandy, loamy soil, or a peaty soil, with a sufficient amount of sand to sharpen it and keep it open, is the best for this crop. In overrich soils it is apt to grow too much to stem, and in close shaded places it is liable to be drawn up tall and slender ; in either case, with a less weight of tubers than in soils less enriched, and situations better exposed to the light and air.

Taking the crop, and subsequent preservation.-Taking the crop should be delayed so long as there is growth in the plants; for as long as vegetation goes on, the tubers will be increasing in size. Where the ground is not required, the stem may be cut over a foot from the ground, and the tubers left in the ground till wanted, as they are apt to turn blackish in cooking when exposed to the light. If the

ground is required, the tuFig. 95. bers may be dug up and

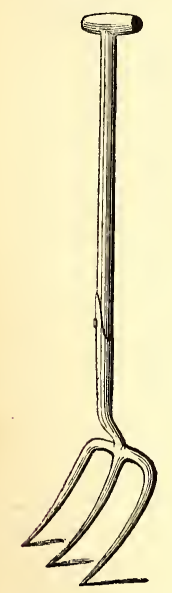

THREE-PRON stored by as recommended for potatoes, separating the smaller from the larger, and replanting the former in ground cleared of some of the brassicaceous or leguminous crops, but not in ground which has borne a crop of tuberous - rooted plants. Great care should be taken, in lifting the crop, that every tuber may be extracted; and the roots should also becleared off the ground, as every tuber, howeversmall, will grow, and prove troublesome to the succeeding crop. The best way to lift them is FORK. ${ }^{2}$ to introduce a three-pronged fork, fig. 95 , so deep in the ground as to reach below the tubers; to lift the mass as entire as possible; and to place it on the surface, when the tubers may readily be picked out. A few for immediate use may be buried in sand in the root-cellar, for it is a slovenly and time-wasting process to have to go to the plantation for a few roots as they may be required; and as the taking of them, in this way, is generally done in haste, there is a greater risk of leaving some of them behind.

List of approved sorts.-Generally it is considered that there is only one variety; this opinion, however, is incorrect, and arises out of the neglected state this excellent tuber is allowed to remain in. If the crop be carefully examined, as it should be, it will be found that there are some varieties better than others, both in size and form of tuber, and height of stem. Those, therefore, which show evidence of improvement in these respects should be saved for planting: and, in the absence of varieties being originated from seed, this is the only neans at present in our power of improving their quality.

In an excellent work recently published by Messrs P. Lawson \& Son, the eminent nurserymen in Edinburgh, entitled "Synopsis of the Vegetable Productions of Scotland," we find two varieties described - the "Common Jerusalem Artichoke," and the "Yellow Jerusalem Artichoke"-of which latter it is said, "The tubers of this variety are of a yellowish colour, and generally smaller and more irregularly shaped than the common sort; they are also said to be superior in quality, and of a more agreeable taste when cooked."

Rhizobius Helianthemi (the Jerusalem artichoke aphis, fig. 96). - Few cultivators, we sus-

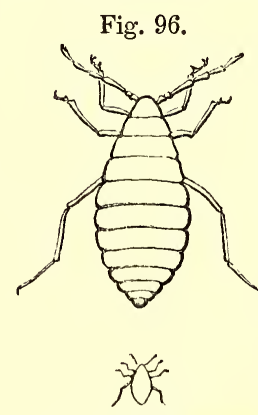

JERUSALEM ARTICHOKE A PHIS. pect, have any idea that this proverbially hardy plant is no more exempt than almost all others from the attacks of aphides. Such, however, is the case; and, stranger still, instead of feeding upon the foliage, as most other aphides do, this one feeds upon the tubers, and forms one of a section of this tribe whose habitations are subterranean. The species, however, to which we now refer, confines its operations entirely to the surface of the roots of this plant, thrusting its long proboscis into the tuber, and thereby deriving sustenance. The writer of the article Entomology in the "Gardeners' Chronicle " (1848, p. 399), thus lucidly describes it: "We have found these insects, in the middle of winter, on digging up the tubers of this plant, generally secreting themselves in the crevices formed by the juxtaposition of two or more of the tubers. Here they are to be found in small societies, consisting of one or two large individuals (females), and a few small and more slender ones (young). Of course, were they to occur in great numbers, 
they would deteriorate the quality of the vegetable; but we have seldom found above two or three dozen upon some of the scattered tubers. The full-grown insect is opaque, white, with a slight buffish tinge, finely serecious and setose ; the head and first segment of the body with a slightly greyish-brown tinge, and the legs and antennæ of a light buffish brown. The antennæ are moderately long, and apparently six-jointed, the third joint being the longest ; under a powerful lens, the extremity of the sixth joint exhibits some traces of articulation. The mouth is constructed in the same manner as all the Aphidæ, and other homopterous and hemipterous insects. The four anterior legs offer nothing peculiar; their tarsi are two-jointed, but the two hind legs are inserted quite at the sides of the body; they are long, with a long tarsus, composed of a single joint, armed at its extremity with two hooks; the peculiar lateral insertion of this pair of legs enables the insect to throw them upwards, backwards, and forwards, in the same manner as a dytiscus throws its hind legs about. We observed that they used these hind legs as feelers; the abdomen is destitute of the saccharine tube of the winged species of the family." The habits of this insect naturally point out to us a remedy against its attacks, by taking the tubers up, and washing them in lime or salt water, and afterwards storing them by for use ; and it is probable that the small ones used for seed, if served in the same way, and immediately planted in a different part of the garden from where they grew, would be secured against their attacks.

General remarks.- The European names areTopinambour and Poire de Terre in French; Erde Apfel in German; Aardpeeren in Dutch; Girasole in Italian; Girasol in Spanish.

\section{§ 5.-RED BEET:}

Natural History.-Red Beet (Beta vulgaris L., var. $r u b r a$ ) belongs to the natural order Chenopodeæ, and to the class Pentandria and order Digynia in the Linnæan arrangement. The generic name is derived from Bett, red, in Celtic, in allusion to the colour of its roots; others ascribe it to the second letter in the Greek alphabet, beta, from the fancied resemblance to it of the seed-vessel. The cultivated beets are natives of the south of Europe. The red beet was introduced into this country in 1546. One species, Beta maritima, or sea-beet, is a native of our southern sea-shores; its roots are not valued, but its leaves make a good substitute for spinach, and are used as such. It would appear from Pliny that Sicily is the native country of our cultivated beets, as, in his day, the Greeks were in possession of both a black and white sort: the latter they called Sicilian beet. Few esculent plants were held in greater estimation than this by the epicures of ancient Rome. Several varieties have been long, and are still, cultivated on the Continent, for being manufactured into sugar.

Use.-The roots are the only parts used, and are boiled and stewed, and eaten cold, with vine- gar and oil, in slices. They enter into mixed salads, and are much used for garnishing; and for all these purposes the deeper coloured they are the more they are appreciated. Some, however, it ought to be noticed, prefer them of a bright-red colour, but all must be of fine quality in fibre, solid, and of uniform colour. The roots are also eaten cut into thin slices, and baked in an oven; dried and ground, they are sometimes mixed with coffee, and are much employed as a pickle. The juice of the roots is sometimes used by young females in the Highlands as an economical rouge. Mixed with dough, it makes a wholesome bread, but for this purpose the white or yellow-rooted sorts are preferred. The roots of all the sorts are better baked than boiled.

Propagation. - All the varieties are produced from seed, which vegetates more rapidly if steeped, six or eight hours previous to sowing, in tepid water : when sown in dry soil, without this precaution, they are tardy in coming up-a circumstance noticed by Pliny, but rather overstretched by that historian. He says, the seed has "a strange and wonderful quality, for it will not all come up in one year, but some in the first, others in the second, and the rest in the third year." This slowness in vegetating should be taken into consideration, and the seed committed to the ground as recommended for carrots, else the weeds are apt to get ahead of the crop, and so render hoeing more tedious : one ounce of seed will sow a drill 150 feet in length.

Sowing and planting.-Three separate sowings should be made-namely, the first in the last week of March, the second in the second week of April, and the third at the end of that month. If the seed is new, and the spring cold, plants for an earlier sowing would be apt to run to seed, instead of forming bulbs ; yet, in families of distinction, where beet is required all the year, a small sowing should be made in a warm border, about the end of February, or, with much greater success, on the 1st of March, upon a very slight hot-bed. The London growers "are very particular about the time of sowing it, for one week too soon sown spoils it. It is generally put in about the first week in May; but even with the same seed, and same soil, it makes a great difference if it is transplanted, because, in removing the seedlings, all forked ones are thrown away. In this operation great care must be taken that the points of the roots are not broken off."'-CutHiLl, p. 26. 
Beet sown too early is liable to be killed by the late spring-frosts.

The superior varieties of beet for salad purposes do not grow very large nor long ; yet, from their fusiform shape, it is necessary that the ground should . be deeply trenched or dug, and finely pulverised, that the roots may meet with no obstruction in their descent, to induce their becoming forked or misshapen ; and, for the same reason, recent manure should not be applied, but ground employed which is in good condition, such as that from which celery has been removed. Although all the beets transplant well, if done while the plants are small, still it is better to sow in drills than broadcast-either to leave the crop in that way, or to transplant it entirely. These drills should be 18 inches apart, and 2 inches deep. Some pierce holes, 2 inches deep, with a blunt dibble, at the distances at which the plants should ultimately stand in the rows, and drop three or four seeds in each, thinning out the plants after they come up, so as to leave only one in each place. There can be no objection to this mode, more than that of sowing in the opened drills, provided care is taken to place the seeds at a proper and uniform depth. In strong retentive soils beet seldom grows so clean, nor will it vegetate so freely, as in light sandy soils. To obviate this defect, and secure well-formed roots, some drive a dibble 4 inches in diameter into the ground, a foot or 15 inches deep, at the distance of 9 or 10 inches apart, and fill these holes with sand, in which a little salt and guano have been mixed; in these the seeds are dropped, and thinned out as above. The roots, by this means, have less inducement to fork or send out side fibres, and in general are produced of good form and size. When blanks occur in the lines, at the period of thinning, the superfluous ones may be taken carefully up with the small planting-fork, fig. 55, as it is immaterial whether much soil is attached to the roots or not; and if planted in the blank spaces, on a moist day, they will succeed perfectly;-only care must be taken that the roots are not doubled up during the operation, but extended to their whole length, and the soil gently, and not too tightly, placed around them.

Subsequent cultivation.-Thinning the crop to the distances stated above, and keeping the ground clear of weeds, constitute all that is required till the roots are fit for taking up.

Soil and manure.-The soil in which the beet thrives best is that of a deep, light, sandy nature, rather dry than moist; and the best manure, if such be required, is guano, soot, and salt, in equal proportions, and applied when the seed is sown; -or, should the crop look weakly after it is 6 or 8 inches high, applied to the roots in a liquid state. Pigeon dung incorporated with the soil during the previous winter has been found beneficial; and spirits of tar, applied at the same time, has secured the roots from the attacks of wire-worm. Much of the sweetness and tenderness of the roots depends on the soil : on poor light soils, as also on heavy ones, the very best varieties will taste earthy and unpleasant. Salt is a beneficial application to this crop, as well as to the green and silver beets-one reason for which undoubtedly is, the beet being a native of the sea-shore.

Taking the crop, and subsequent preservation.-By the beginning of September in England, or the latter end of that month in Scotland, the roots will be of a good size for use, and by the end of October in both they may be taken out of the ground altogether. They are sometimes destroyed by frost, more especially in the north; but leaving them in the ground destroys their colour, for which reason it is better to take them up and place them amongst sand, along with other roots, in the rootcellar, or to store them after the manner of potatoes (vide p. 218). The London market-gardeners winter their " beet in large sheds stored in moderately damp mould, and banked up with straw. It is a mistake to pack it up in dry sand or earth for the winter; and the same may be said in regard to carrots, parsnips, salsify, scorzonera, and similar roots."-CutHILL in Market-gardening around London, p. 26. The object here is, that the moist soil may not draw the natural sap out of the roots so readily as dry sand or soil would do, and hence their tenderness; and possibly, in the case of the beet, the colour also may be preserved. In taking up the roots, the greatest care must be exercised that they are neither cut, broken, wounded on the skin, nor any of their fibres removed; and when the small-leaved varie- 
ties are grown, few if any of the leaves should be cut off. A dry day should be chosen, and when the roots have been exposed for a few hours, to dry the soil that may adhere to them, they should be packed by as noticed above. Some prefer packing the roots, for preservation during winter, in sifted coal-ashes, assigning as a reason that ashes are less likely to encourage decay; and others recommend placing them on shelves elevated a little above the floor in dampish cellars, believing that the air passing around them tends to their preservation. Both plans may be good in very damp cellars, but otherwise the roots would be liable to become shrivelled and dried up. The leaves should not be cut off closer to the crown of the root than 6 or 8 inches. The roots will keep till the July following.

List of approved sorts and their qualities.Whyte's black.-Judging from its appearance in a growing state, it would be by most pronounced a coarse sort, on account of the largeness and want of red colour in the leaves. It is, however, the darkest beet grown, but should not be sown before May.

Castlenaudari.-A good variety, brought into notice some years ago by the London Horticultural Society. It is much used in France, and prized on account of its flavour, which resembles that of the kernel of a nut. It is the Betterave rouge de Castlenaudri of the "Bon Jardinier."

Glen dwarf-red.-A very excellent crimson variety grown by Mr Handisyde of the Glen nurseries, Fisherrow; roots scarcely 9 inches in length, of uniform shape, not disposed to fork, with small thin dark-coloured leaves, scarcely 7 inches in length.

Cuttel's dwarf-red and Atkins' crimson.-Very similar to the last, all the three growing a good deal above ground.

Small deep-red.-We have grown this and the following for several years from the stock of Messrs P. Lawson and Son, who describe it in "Vegetable Products of Scotland," p. 24, thus : "Leaves rather small and spreading, of a very deep red colour; roots growing more above ground; smaller and more bluntly tapered than those of the common 'red beet;' both in skin and flesh of a much deeper blood-red colour. Known also as superb deep blood-red."

New blood-red or mulberry.- " This seems a sub-variety of the small deep-red, from which it differs chiefly in being of a much darker or almost blackish mulberry colour."

Barrot's new crimson.-This very excellent variety is less liable to fork than the Castlenaudari; colour very dark crimson, leaf-stalks tinged with yellow. Considered by Mr Thomson the best beet grown.

Insects and diseases.-The beet is not very liable to either, if in proper soil and under good cultivation.

General remarks.-The European names are Bette-rave in French; Barba-Biettola in Italian; Biet-wortel, or Karoot, in Dutch; Rothe Rube in German ; and Betarraga in Spanish. In saving seed, select the best formed roots, and try the colour by making a slight incision, which afterwards dry up with a little caustic lime. Both form and colour constitute their merits. Transplant them, and treat them as recommended for carrots and parsnips. Only in cold situations plant them in a warm sheltered place, against a south wall if there is room, or support them with stakes as the flower-stems extend, to prevent their being broken by the wind. One plant will produce seed enough for a small garden. No two varieties should be thrown together; and as the seed will retain its vegetative properties for nine or ten years, it is better to save one sort only annually.

\section{§ 6.-SALSIFY, SKIRRET, ETC.}

Salsify (Tragopogon porvifolius L. - leekleaved salsify) belongs to the natural order Compositæ, tribe Scorzonereæ, and to the class Syngenesia Equalis in the Linnæan arrangement. The generic name is derived from Tragos, goat, and pogon, beard, from the bearded appearance of the seeds-vulgarly goat's-beard ; the specific name from Porrium, the leek, on account of the resemblance of the leaves to those of the leek. Indigenous to England, but not very common. The roots, which are the only parts eaten, are long, white, and fleshy, tapering like the parsnip, but never attaining the same diameter. Tragopogon pratensis was cultivated in gardens in the time of Parkinson; but that spe. cies has been superseded by our present subject. It has been hitherto confined to gardens of the first order, but would be worth the attention of amateurs, as affording an additional dish of vegetable diet.

The roots are used simply boiled, like carrots and parsnips; the flavour is mild and sweetish. Dressed like asparagus, there is some resemblance in taste; and the flower-stalks, if cut in spring on the second year before they become hard, and dressed like asparagus, make an excellent dish.

It is propagated by seed sown in drills annually in March, April, and towards the latter end of May for succession. The drills should be 18 inches apart, and 2 inches deep. When the plants come up, thin to 6 inches in the lines, and keep them clear of weeds. In August and September, and thence through the winter, the roots will be fit for use; and in November, take up those remaining, and store them by for winter use in the same manner as parsnips. They are quite hardy, and may remain all winter in the ground, if it is not required for re-cropping or improving. One ounce of seed will sow a drill 40 feet in length. To increase the size of the roots, water with liquid manure during hot dry weather. The seed keeps good for four years. 
Save seed in the same manner as with carrots; one or two plants will afford a supply for a small garden. It is not subject to the attacks of insects, although hares and rabbits are exceedingly fond of it. It is, however, almost invariably attacked by Erysiphe cichoracearum, Link, a small parasitic fungus, one of the mildews ; but it has not the effect of injuring the growth of the plant much, as it is late in summer when it makes its appearance, in the form of numerous white blotches. This vegetable is much cultivated in France. The European names areSalsifis in French ; Sassifica in Italian ; Bocksbart in German; Barba cabruna in Spanish ; and Boksbaard in Dutch.

The Skirret (Sium sisarum L.) belongs to the natural order Umbelliferæ, sub-order Orthospermæ, and tribe Ammineæ, and to the class Pentandria and order Digynia in the Linnæan arrangement. The generic name is derived from Siw, water, in Celtic. The specific name is from Dgizer, the Arabic name, which signifies carrot. A native of China. Introduced before 1548 .

$U$ se.-The roots, which are composed of small fleshy tubers, joined together at the head or crown, are the parts eaten. When boiled, they are eaten with butter, and are sweet and very agreeable. They are much used in French cookery, and more cultivated on the Continent than with us, although there are few gardens of importance without them.

Their cultivation is similar to that of salsify, only they are propagated both by seeds and by off-sets taken from the old roots. The better way, however, is by seed.

The European names are, Chervis in French; Sisaro in Italian; Zuckerwortzel in German; Suckerwortel in Dutch ; and Chirira-tordesca in Spanish. In the north of Scotland, according to Loudon, it is cultivated under the name of Crummock.

Scorzonera (Scorzonera hispanica L., Spanish Scorzonera) belongs to the natural order Compositæ, sub-order Cichoraceæ, and tribe Scorzonereæ, and to the class Syngenesia and order Equalis in the Linnæan arrangement. The generic name is derived from Scurzon, the Catalonian name of the viper. The plants are esteemed in Spain as a certain remedy for the bite of a viper; but it is believed that the slender tortuous form of the roots has given rise to this belief, rather than any quality inherent in the plant; for it is a rule to which there are very few exceptions, that all plants used as food by man possess very inactive qualities. If their action was powerful, they would be unfit for food."-Encyc. of Plants, p. 668. It is a native of Spain, and was introduced to Britain before 1576 .

The roots, which are long, black, and tapering, about $\frac{3}{4}$ of an inch in diameter at top, are the parts used. They are boiled or stewed after the manner of parsnips; the outer rind is scraped off, and the roots placed to steep in cold water for several hours, to extract a part of their bitter flavour. It is also variously used in French VOL. II. and Italian cookery. It is found in all our best gardens, but, like salsify and skirret, occupies about one-fifth the space that parsnips do.

It is propagated by seeds; and its cultivation, in all respects, is similar to that of salsify and skirret, only requiring about 4 or 5 inches more space between the rows, and 3 inches more between the plants in the lines. An ounce of seed will sow a drill 40 feet in length. Few insects trouble it; but, like the salsify and skirret, the Erysiphe cichoracearum, or mildew fungus, sometimes attacks it. Its European names areScorzonere, or Salsafis d'Espagne, in French; Scorza-nera in Italian; Skorzonere in German; Escorzanera in Spanish; and Skorzoneere in Dutch.

Hamburg parsley (Apium petroselinum L., var. Tuberosum, "Bon Jardinier") belongs to the natural order Umbelliferæ, sub-order Orthospermæ, and tribe Ammineæ, and to the class Pentandria and order Digynia in the Linnæan arrangement. The generic name is derived from Apon, water, Celtic, from its place of growth. This refers more especially to A pium graveolens, the common celery. The Hamburg parsley is a variety of the common sort, Apium petroselinum, having large fleshy roots, and for these it is cultivated.

The roots as well as the leaves communicate an agreeable flavour to soups and stews. It is much grown in Germany, and was formerly so in England, and often cooked like the parsnip or the yellow turnip.

It is propagated by seed, which is sown at the same time as parsnips, and the routine of culture differs not from that of that plant.

\section{§ 7.-THE PARSNIP.}

Natural history. - The parsnip (Pastinacea sativa Tourn.) belongs to the natural order Umbelliferæ, sub-order Orthospermæ, tribe Peucedaneæ, and to the class Pentandria and order Digyuia in the Linnæan arrangement. The name is derived from Pastus, nourishment; or, according to others, from Pastinum, a dibber or dibble, the root resembling that implement in form. Indigenous to Britain; found generally in calcareous soils and in waste places. Like the carrot, it is difficult to say whether it is to cultivation or importation that we are indebted for this root in its present state-most probably to the latter-and that it may have been brought from Germany or Italy. Phillips asserts (but of this we have doubts) that if the wild parsnip is grown for two or three years successively in rich soil, it will assume all the characters of the cultivated sort. It appears to have been early reclaimed and cultivated on the banks of the Rhine, for Pliny says they were brought from thence to the Roman emperors' tables. Gerard speaks of a broad-leaved and narrow-leaved variety being grown in gardens in his time.

Use.-In former times the parsnip was more generally used as an article of food than at present, and was with us then, as in Roman Catholic $2 \mathrm{G}$ 
countries at present, used with salt fish during the season of Lent. It is a good substitute for the potato, yielding a large return of very nourishing food, and is often, by country people, eaten when mashed up with that root. Parsnips contain a very considerable portion of sugar, and from this cause they yield a very excellent spirit by distillation. In the north of Ireland the cottagers obtain a sort of beer by mashing the roots and boiling them with hops, and then fermenting the liquor. Wine closely approaching in quality the Malmsey of Madeira is made from the roots ; and marmalade made with parsnips and a small quantity of sugar is thought to excite appetite, and to be a very proper food for convalescents. Bread has also been made from the roots; and in Holland they are much used in soup. The present demand for them, however, is to be eaten with fish or other salt meats.

Propagation.-Always by seed, which is flat, thin, and light. Half an ounce of seed will sow a bed of 100 square feet, if sown broadcast, and the plants afterwards thinned to 8 or 9 inches distance. The seed will vegetate sooner if steeped in water, as recommended for carrot-seed.

Sowing and planting.-The seed should be sown as early in spring as the ground is in a proper state of dryness to receive it. As the roots extend to a considerable depth, the ground should be trenched at least 2 feet deep. The drill-system is the most proper to follow: the drills should be from 15 to 18 inches apart, and 2 inches deep; and when the plants attain the height of 3 or 4 inches, they should be thinned to 6 or 8 inches in the line. Where they come up too thick, they should at that stage be thinned; and as they transplant readily at that age, any blanks in the rows may be made up, or the plantation extended by additional rows.

Subsequent cultivation.-Keep the ground clear of weeds by hoeing, which should be shallow, for reasons given in article CARROT.

Soil and manure.-A light pervious deep soil is the best; and if in moderate condition, by the manuring of the previous crop, it will be better than applying manure at sowing. Should it be necessary to do so, let the manure be in the most decomposed state possible, or, if otherwise, lay it in the bottom of the trenches as the trenching proceeds. It will grow in a stronger soil than the carrot, and succeeds well in sand or in peat, if sufficiently manured. Soap-boilers' ashes have been advantageously employed as a manure for the parsnip, carrot, and potato; and where laid on to the extent of a pound-weight per square yard, have had good effect in resisting the attacks of insects at the roots.

Taking up the crop, and subsequent preservation.-By the end of September the most forward roots will be fit for use; but the general lifting should not take place till the leaves are killed by the frost. It is a much hardier plant than the carrot; and therefore, if the ground is not required, and the roots are free from disease, they may be left in the ground till spring with safety : otherwise they should be dug up, and their tops taken off as recommended for carrots, and either packed in sand in the root-cellar, or pitted as recommended for potatoes. In general it is best to take up all such crops, so that the ground they occupy may be manured and trenched during winter.

Approred sorts and their qualities.-Long Jersey.-An improved variety of the common sort, being much larger and thicker towards the top. This is the variety cultivated in the Channel Islands, where the cultivation of this plant has been long successfully carried on. The crown is somewhat concave or hollow-hence it is often known as the hollow-crowned, Guernsey, and hollow-headed. It is the Panais Lisbonnais of Guernsey. Roots 18 inches long, and often from 4 to 5 inches in diameter at the top. The best variety for cultivation.

Common long-rooted.-Scarcely so large as the last; crown or top slightly convex. This is the Panais longue of the French, the Panais coquine of Guernsey, Jersey parsnip, and Guernsey parsnip.

Turnip.rooted.-This variety is much smaller than either of the last, of good quality; and as its roots are short and somewhat turnip-shaped, it is adapted to thin soils. It is the Panais rond and Panais royal of the French. Root formed almost on the surface of the ground, and attaining the size of from 4 to 6 inches in diameter.

Early short-horn.-A recently introduced sort, of delicate flavour and appearance on the table. A smaller and shorter sort, if of improved flavour, would no doubt be valuable, as our present sorts are too large to appear whole.

The late Dr Neill, in "Edinburgh Encyclopædia," mentions the Le Batard ou de Siam yellow, apparently intermediate between the turnip-rooted and long-rooted varieties, and of more richness in flavour and tenderness of flesh than any of the others. It does not appear to be in our seed-lists, although mentioned by Noisette in "Manuel Complet du Jardinier." The introduction of this variety would, for garden purposes, be important.

Insects and diseases. - "The parsnip is subjected to the attacks of a few insects. The maggots of the parsnip-miner (T'ephritis onopordonis, fig. 53) 
are hatched from May to July, and feed upon the parenchyma or pulp of the leaf, causing large blisters upon them; and when two or three larvæ are feeding on the same leaf, the blisters unite and form large discoloured patches; but otherwise the mischief is not serious. The caterpillars of the flat-bodied moth (Depressaria pastinacella) infest the parsnips left for seed, and often much injure and diminish the yield. They prefer the parsnip to the carrot seed; and on this account the growers of carrot-seed sow some parsnips beside them, by which to lure the insect from the carrot crop. These caterpillars are greyish blue, with the head, thorax, and pectoral feet black; upon each of the segments are six distinct little black dots, producing single minute hairs; the sides and the belly are yellow, and the abdominal feet are dotted with black. They live in July upon the flowers and young seeds of the parsnip. There seems to be no better mode of ridding parsnip crops of these caterpillar pests than hand-picking, and shaking the umbels of the flowers over a vessel for them to fall into. The flower of the parsnip is not nearly so liable to be affected by insects, damp, or mildew as that of the carrot, on which account the crop of seed is a surer one, though it should be borne in mind that it will not retain its vitality beyond one year."-Jour. of the English Agr. Soc., vol. i. p. 419, as quoted in "Book of the Farm," vol. ii. p. 100. Besides these, the Depressaria applana, $D$. daucella (the carrotblossom moth), in the caterpillar state, make great havoc on the seed-crop of parsnips also.

General remarks.-The European names arePanais in French; Pastinaca in Italian; Charibaria in Spanish ; Pastinake in German; and Pinksternakel in Dutch. Seed is saved in the manner recommended for carrots. The quantity produced from one plant will be sufficient for a small garden; and, according to Mr Stephens, " from $1 \frac{1}{2} \mathrm{lb}$. to $4 \mathrm{lb}$. of seed, being very light, may be expected from a square rood of $16 \frac{1}{2}$ feet, dependent on the nature of the season, and escape from the attacks of insects. Birds do not injure it, though, if the seed is not gathered from the umbels as they ripen, they are very apt to be blown off by the wind." 


\title{
CHAPTER VIII.
}

\author{
OLERACEOUS PLANTS, OR POT-HERBS,
}

COMPRISING SUCH AS ARE USED FOR GARNISHING AND SEASONING.

THE plants which constitute this chapter, being chiefly aromatic, should be accommodated with a light poor soil, and be cultivated by themselves, on whatever scale the garden may be ; and if we except the common parsley, which requires to occupy a considerable space, a bed or two at most of the others will be found sufficient. The situation chosen for them should be some snug and convenient spot, sheltered and warm, as the majority of these herbs delight in a warm sunny spot; convenient, because some or other of them are in daily use, and if scattered over the garden, as is too often the case, much time is lost in collecting them. To prevent mistakes, every variety should be legibly named at the end of the bed next to the walk; and the smaller the garden is, the more necessity there is for this being attended to.

\section{$\S 1 .-P A R S L E Y$.}

Natural history.-Parsley (Apium petroselinum L.) belongs to the natural order Umbelliferæ, to the sub-order Orthospermæ, and tribe Ammineæ, and to the class Pentandria Digynia in the Linnæan arrangement. The generic name is derived from Apon, water, Celtic, from the place where the plant grows; and the specific name from petre, a stone, and selinum - stone selinum. It is a native of Sardinia, introduced about 1548. Gerard spells it parsele, parsely, and parsley; and says it " is delightful to the taste and agreeable to the stomach." Phillips asserts that "parsley easily resisteth the cold and the heat, if it be sown on a rich damp soil, or near a spring." This is a very different condition, as to situation, to what it enjoys in cultivation; and hence cultivators in general sow it in dry exposed places, to avoid its damping or rotting off during winter, which it is very apt to do in strong damp soils, be they ever so rich; and in much elevated situations it is with great difficulty preserved during winter and spring. That it would succeed better if planted near a spring is possible, as the water rising from a great depth is higher in temperature than the surrounding atmosphere, and hence springs seldom freeze. Few, however, have such springs at command, and so completely protected as to exclude hares, which are exceedingly fond of this plant. Indeed, it has been sown often in preserves, where it has been wished to encourage these destructive creatures. The seed of the common parsley has the property of remaining long in the ground after sowing before it vegetates-from forty to fifty days. Some fatal mistakes have occurred by people gathering Athusa cynapium (fool's parsley), a poisonous plant often found in old gardens as a weed, and so similar to parsley as to be readily mistaken for it; and instances are recorded where the leaves of Conium maculatum (hemlock) have been gathered for it in the same way. To avoid such, it would be well if amateurs, and those not sufficiently acquainted with these plants, were to cultivate the curled parsley only, which could not be mistaken for either. The plain-leaved sort, however, is hardier, and withstands the winter cold better, and therefore is often sown where the finer curled varieties would not survive.

Use.-Parsley is in great demand throughout the whole year for a variety of culinary purposes, and is the common garnish to all cold meats, and enters into many sauces, soups, broths, \&c. It should always be brought to table when any dish is introduced that is strongly seasoned with onions, as it takes off the smell and prevents the after-taste of that strong root. Forenoon tipplers chew a leaf of parsley to prevent their breath from bearing witness against them. The leaves are gathered when quite dry, and hung up in small bundles, in an apartment heated to from $60^{\circ}$ to $70^{\circ}$, to dry. In a day or two they may be pounded or rubbed down to a powdery state, and bottled for winter use. Hung up to the roof of a kitchen, or placed in a Dutch oven in the evening at a moderate distance from the fire, they will be in a fit state by morning for pounding. It retains its flavour long when dried, if kept excluded from air. In this state it is excellent for seasoning omelets and all similar dishes.

Propagation.-It is propagated by sowing the seed only. An ounce of seed will sow a drill 150 feet in length. 
Sowing.-Sow early in February, if the ground is dry; if not, from thence till the end of March. Another sowing should be made in May, and another in July, for young plants to stand over winter. To insure the finest curled varieties, the plants should be transplanted once at least; and if oftener, it is said, the more certain will be the result. Sow, therefore, either thinly broadcast or in drills, covering, in either case, to the depth of three-quarters of an inch. When the plants are fit to handle, take them carefully up, preserving their long taproots quite entire, and transplant them in lines 18 inches asunder; but where the ground is rich, and the variety extra curled, 2 feet will be a more proper distance. Set the plants carefully with the dibble, at first 3 inches apart; and after they have commenced growing for a week or so, thin out to 6 inches, making new lines with the thinnings; and in three weeks afterwards, finally thin to 12 inches, plant from plant, in all the rows. By this means, and in a highly-manured deeply-trenched soil, we have grown the plants so large as completely to cover the surface of the ground.

Some have recommended cutting over the old plants in autumn, to secure a supply in spring. It is much better to depend on an autumnal sowing, provided the seed is sown before the end of July. In small gardens, parsley may be sown as an edging to walks or footpaths, to economise space, as well as to be readily got at when wanted for use.

Subsequent cultivation.-Keeping the plants clear of weeds by deeply hoeing between them, and pulling up any that prematurely run up to seed, is their summer routine. On the approach of winter, cut off a supply of leaves for drying, as already noticed; remove decaying leaves, to prevent damp; and shelter the winter standing-crop by the best means at command - of which few are better than sticking the ground around them pretty thickly with short branches of deciduous trees or broom, stuck firmly in the ground, so as not to be blown about by the wind. Cover the surface between the rows with finely-sifted coal-ashes, to counteract damp, and to keep the leaves clean. In some situations it is necessary to take up a portion of the crop, and to pot it, or plant it in light dry soil under frames and sashes. In neither way does it do so well as protected where it has been grown. It is in such cases that drying the leaves and bottling them becomes necessary; but even in this way the dried leaves can only be used for seasoning: for garnishing they become entirely useless.

Soil and manure.-The first cannot be too rich, and the second should be the most potent that can be applied, particularly to summer crops; but both must be more sparingly employed in the case of crops to stand over winter.

Taking the crop, and subsequent preservation.-The leaves should be cut over by the foot-stalk, and not plucked over by the middle, as so often carelessly practised. Each leaf should be cut by itself, and the larger and more fully developed bottom ones taken first. When removed to the vegetable-house, they should be rinsed in clear water, and the foot-stalks of the leaves cut close off, and the leaves placed in a clean salad-basket ready for use.

Approved sorts and their qualities.-The original old plain-leaved is hardier than the fine curled sorts, on account of its being less succulent, and less liable to retain moisture. It is desirable to sow it in cold situations, particularly for winter and spring use; but for all other seasons and purposes,

The fine curled, or triple curled, should be preferred; and of these we have, amongst other names in seed-catalogues, the following: Myatt's, garnishing, very dwarf, and exceedingly curled.

Engelfield extra curled.-Very large and finely curled.

Reid's fine curled.-A variety we received some years ago from Mr Reid of Rise Park, Yorkshire, which is hardy, standing the damp cold of the Holderness, as well as that in many parts of Scotland which it has reached. By carefully selecting from any of the last three varieties, and by repeated transplanting and high cultivation, the finest curled parsley may be obtained.

Hambury parsley (Apium petroselinum, var. Tuberosum, "Bon Jardinier") is a variety of the common parsley cultivated for its large fusiform roots, as already described. It is used for all the purposes of the common parsley; and in addition, the roots are served at table as a separate dish, like the Feltow turnip or parsnip. The leaves are much larger than those of the common plane parsley, and fully as little curled.

The Naples parsley, or, as it is often called, celery parsley, appears to be a hybrid between the Hamburg parsley and the celery. In France and Italy the foot-stalks of the leaves are much used as a substitute for celery, and its cultivation is much the same. It is only in 
gardens of the highest order in Britain that it is found.

Insects and diseases seldom attack the parsley, unless in dry seasons, and in light ferruginous soils, where either an underground grub, or, we rather think, a disease of the canker description, destroys the roots, which causes the plant immediately to die. We have never been able to detect an insect at the roots, although the effects are exactly similar to those which affect carrots, when their roots are about as large as a quill. Saturating the ground with lime-water has arrested to some extent the disease; but whether this arises from the caustic effect, or from the mere addition of moisture, we cannot say.

General remarks.-The European names arePersil in French; Petersilie in German; Peterselie in Dutch ; Petroselino in Italian; Perejil in Spanish; Petruschka in Russian; Pietruszka in Polish.

\section{§ 2.-DILL, BORAGE, ETC.}

Dill (Anethum graveolens L.) belongs to the natural order Umbelliferæ, sub-order Orthospermæ and tribe Peucedaneæ, and to the class Pentandria and order Digynia in the Linnæan arrangement. A native of Spain, and introduced to Britain in 1570. The generic name is derived from Ano, upward, Theo, to run, on account of its quick growth. Others derive it from a Greek word signifying to burn, the plant being very heating.

A hardy biennial plant seldom used, and then only for the leaves, which communicate a slight degree of its powerful aromatic flavour to pickles, and also occasionally in soups and sauces. A dozen and a half good plants will be sufficient for a large family's use. It is little more than an annual with us, therefore requires to be sown annually, either in March or April, or as soon as its seeds ripen in autumn, and in favourable places will stand over the winter.

Like all warm and high-scented plants it requires a light poor soil, because such a soil is found most favourable for the preservation of their aromatic properties, such plants being only found naturally growing on such soils. It should be grown in drills 1 foot apart, and the plants thinned out to a like distance. Leave a plant or two to run up to seed, which ripens in autumn; and if grown, as all such plants should be, in a compartment by themselves, called the olitory or herb-garden, it will shed its seeds and come up abundantly in spring; which course may be followed for three or four successive years, at the end of which the situation should be changed, and this culture begun de novo. The European names are-Anith in French; Aneto in Italian; and Dill in German and Dutch.

Borage (Borago officinalis L.) belongs to the natural order Boragineæ, and to the class Pen. tandria Monogynia in the Linnæan arrangement. The generic name is taken from Cor, heart; $A g o$, to affect, on account of its cordial qualities. In- digenous to England, in waste places amongst rubbish. Pliny says that wine with this infused in it cheers the spirits; and hence the leaves are used, but more properly the flowers, as forming an ingredient in cool tankards. The Greeks called it Euphrosynon, for, when put into a cup of wine, it made those who drank it merry. It has been in use for these purposes since the days of Parkinson. Coles and M. Valmont Bomare say the flowers have no virtue when dry, and recommend using the roots during winter, which are said to possess the same properties. In Queen Elizabeth's time, both the leaves and flowers were eaten in salads. At present it is cultivated for cool tankards, and the young shoots and leaves for mixing in salads. The leaves contain so great an amount of nitre, that when dry they burn like match-paper. It is also used by some as a substitute for spinach.

Culture.-The seeds are large, so that two ounces will be required for an ordinary garden, which should be sown thin, 9 inches apart seed from seed; and a spring and August sowing will be sufficient, as, if the leaves only are to be used, if the flower-stems are cut out as soon as they form, the plants will continue forming leaves for three months in succession. Self-sown plants will arise in abundance, and therefore borage may be treated in this respect as noticed for dill. The young plants transplant freely, and as they rise from self-sown seed, it is allowed to ripen. If once introduced into a garden, it will not easily be lost. Cool tankard is a beverage composed of lime-water, lemon-juice, and sugar, and is exceedingly refreshing and exhilarating during summer.

The European names are-Bourrache in French; Borragine in Italian; and Borragen in German. The seed will keep for four years in the seed-room, and for an unknown period if deep in the ground. It makes an excellent green manure, and has been sown in spring for such, and dug in during autumn. In soils defcient in nitrous matter, the advantage of this is obvious.

Thyme-Common thyme (Thymus vulgaris L.), a native of Spain and Italy. Introduced to Britain long prior to 1548. Of this there is a broadleaved variety of altogether stronger growth, (Thymus citriodorus Pers.) lemon-scented thyme. These are the only two species cultivated; the latter is indigenous to Britain, belongs to the natural order Labiatæ and tribe Satureineæ, and to the class Didynamia and order Gymnospermia in the Linnæan arrangement. The generic name is derived from Thymos, courage, from its smell-reviving properties. Its use was well known to the ancient Greeks and Romans, and according to Evelyn it was cultivated to a great extent in the neighbourhood of Sandwich and Deal for its seed only, which was used in preference to the leaves, and also for medicinal purposes. Both are at present used, in either a green or dry state, in soups, stews, sauces, and stuffings, and the leaves of the lemon-scented kind are used for seasoning veal instead of lemon-peel. Before the introduction of the Eastern spices, both were more used than 
at present. The common sort is propagated by division of the plant, by cuttings, but best of all by sowing the seed, which should be sown early in the season, and transplanted out towards the end of summer, when the plants are about 2 inches in height. Where there is the convenience of sowing on a slight bottom-heat in February, the plants will be ready for transplanting in May, and will be fully established by the end of the season. The lemon sort is capable of increase by cuttings, and also by seed; but, being a low-creeping plant, it is usually done by division of the roots. Both should be planted a foot apart; and no soil can be too dry or almost too poor for either. Both are hardy; but for winter use the plants should be cut over when just coming into flower, tied up into small bundles, and suspended to the roof of a warm kitchen, so as to dry rapidly and retain their green colour. For the purpose of drying herbs, we have an apartment behind one of the pine-stoves, which can be heated to $60^{\circ}$ or $70^{\circ}$, from the ceiling of which we suspend cords; and as the herbs of this description are cut and tied up into bunches, which should not in any case exceed an inch in diameter where the ends of the branches are secured, they are fastened to the lines, and in three or four days are sufficiently dry to be packed by in air-tight boxes, and placed one on top of the other in a dry room. When the herbs are placed in the boxes, a false lid-that is, a piece of boarding exactly the size of the inside of the box-is placed over them, and a weight placed over that, to keep them quite tight together. The proper lid is then put on, and the name of the herb written on the side of each box. In this way they are secured from damp and dust, which are both alike destructive to them. For further on preserving herbs, vide Section MinT. It is important that all herbs be cut when perfectly dry, and just as they are coming into flower. A judicious plan is followed by those who grow this plant for the London marketnamely, by sowing the seed broadcast, and moderately thinning out the plants after they have attained the height of 2 inches. The rest are left upon the ground, and drawn up by the roots when required for use.

The European names are-Thym in French ; Timo in Italian; Tomillo in Spanish; Thimian in German; Tomilho in Portuguese; Gomeene thym in Dutch.

\section{§ 3.-SAGE, BALM, MINT, ETC.}

Sage.-Salvia officinalis L., the common sage, with its two varieties-variegata, the variegated, and tenuior, the slender; Salvia sclaren L., common clary; Salvia horminum L., hormium clary ; and the varieties, violacea, purple-topped, and rubra, red-topped-are all cultivated for culinary purposes. The common sage and its two varieties are, however, the most common. These are natives of the south of Europe, and appear to have been introduced about the same time (1597.) The common clary is a native of
Italy, and was introduced in 1562 ; the hormium clary is a native of the south of Europe, introduced in 1596. The sages belong to the natural order Labiatæ, and to the class Diandria and order Monogynia in the Linnæan arrangement. The generic name is derived from Salvus, safe; according to others, from Salvere, to save, on account of its supposed healing qualities. It is called sage in English, from the French word Sage, wise, from its supposed property of strengthening the memory, and making people sage or wise.

The French make an excellent pickle of the young leaves of common sage. In Britain it is commonly used as a seasoning for strong meats, sausages, ducks, goose, \&c., and was formerly much used in making sage cheese. The leaves of the narrow-leafed variety are employed in making sage tea. The Chinese use it for this purpose ; and at one time the Dutch carried on a profitable trade, by procuring the leaves from the south of France, drying them to resemble tea, and disposing of it in China, where for every lb. of sage they got in exchange four of tea.

All the sages are propagated by seed and by cuttings. The plantations should be renewed every third year, as they often suffer by the cold and damp of our winters. The seed should be sown early in March upon a slight bottom-heat; and if so, the plants will be fit to plant out in May, when they should be set in lines $\mathbf{3 5}$ inches apart, and a foot distant in the line. Cuttings are best put in (using the young wood of the present year's growth) about the latter end of May or beginning of June, planted in a partially -shaded place, and covered with handglasses. The cuttings may be taken off from 4 to 5 inches in length, cutting off (not stripping) the lower leaves as far as the cutting is inserted in the ground, and leaving all the other leaves entire. They will soon, at that season, form a small callosity at their base, from which the roots proceed. At this period, air must be admitted more freely to them, and when the roots have formed, the glasses should be removed entirely. By August they will be fit to plant in their permanent situations at the distances noticed above. Should any of them send up flower-stems the first season, these should be cut off, and, indeed, the tops of the shoots had better be all so served at planting, or soon afterwards, to cause them to become bushy and compact. In gathering green sage for use, cut off the tops and side-shoots neatly; and when gathering for drying, let the flowers be nearly expanded, and cut off the young shoots only, in a neat manner, that the old plants may sustain no injury. Keep the ground clear of weeds, and stir amongst the plants frequently, but apply no manure whatever.

The common clary sage is raised from seed, and also from cuttings, which are to be treated like the last; while the hormium clary, or annual sage, is to be propagated by seeds only. Sow in March in drills 1 inch deep, 15 inches row from row, thinning out the plants as they advance to a foot apart. Neither of the latter need be grown to any great extent; from 
a dozen to twenty plants will be sufficient for most families. The common sage, however, should be grown to about three times that extent. In very cold and damp situations, a very proper situation for common sage is an elevated rockwork, or the top of an old wall, where it may be quite dry, and fully exposed to the sun. In some situations it requires partial shelter during severe frosts, and for this purpose may be protected by covering it with branches or dry fern fronds. Protecting the roots, in most places, by a covering of rotten tan, leaves, or sifted coal-ashes, is very beneficial.

The European names of the common sage are -Sauge in French; Salvia in Italian and Spanish ; Salva in Portuguese; Schalweja in Russian; Szalwia in Polish; Salbey in German ; and Salie in Dutch. Those of common clary, Orvale in French; Schiarea in Italian; and Scharlachkraut in German.

Balm (Melissa officinalis L.) belongs to the natural order Labiatæ, and to the class Didynamia and order Gymnospermia in the Linnæan arrangement. The generic name is derived from Melissa, a bee, from the abundance of honey that bees gather from it. A native of the south of Europe, and introduced into Britain in 1573. The plant, in its green state, has an agreeable odour of lemons, and an austere and slightly aromatic taste, and hence is sometimes employed to flavour certain dishes in the absence of lemon-thyme.

"It has also been called apistrum, from apis, a bee, on the same account; and it is still the custom to rub the bee-hives with balm and sugar, or honey, previous to taking a swarma practice," as Phillips correctly enough observes, "which certainly appears to have the effect of attaching the colony to its new settlement." Pliny notices this method of securing the bees in his time, and says that, where there is plenty of balm in the garden, there is no fear of the swarms straying. He tells us, also, that it is a good remedy for the sting of bees and wasps, \&c.-a practice also still continued. The only other domestic use for which this plant is employed is in making cool tankards, balm wine, and balm tea. It is a perfectly hardy perennial plant, readily increased by division of the roots, either in autumn or spring. Set the plants a foot or 15 inches apart. They continue for several years, and adapt themselves to any ordinary garden-soil. Cut the crop when coming into flower, and dry as recommended for mint. The young leaves are the parts employed for making balm wine, and these are produced during the greater part of the summer. The dried plant loses nearly all that agreeable odour of lemons which the young green leaves possess.

The European names are-La melisse in French; Die melisse in German; Melisse in Dutch; Melissa in Italian, Portuguese, and Russian; Melisa in Spanish and Polish.

Mint. - Mertha viridis, green spearmint; Mentha piperita, peppermint ; and Mentha Pulegium, Penny-royal - are the sorts cultivated in gardens. They belong to the natural order Labiatæ, and to the ciass Didynamia and order Gymnospermia in the Linnæan arrangement. They are all natives of Britain. The generic name is derived from Mintha, daughter to Cocytus, who, according to the heathen mythology, was changed into this plant; the specific name, Pulegium, from Pulex, a louse, which animal it was thought to drive away. Mint and balm are said to be amongst the earliest medicinal plants selected by man. The young leaves of $M$. viridis are used in spring salads, and in acid sauce, with roasted lamb; it is boiled with green pease; it is generally used in pea-soup, and is relished by many in other soups also. As all do not, however, participate in the same taste for it, it should be served at table in a pounded state, so that each may help himself, according to taste. $M$. piperata is chiefly used for distillation; the leaves of $M$. pulegium are used in different branches of cookery.

It is propagated by division of the roots, the smallest portion of which will grow by removing off-sets or young shoots in spring, with a fragment of root attached to them - and in extremity, by cuttings of the stems. The first is the usual and best mode. Penny-royal is best increased by dividing the old plant into small pieces. It should be planted either in spring or autumn, before the plants begin to grow, or after vegetation has ceased, in drills drawn a foot asunder, and 2 inches deep. Select the youngest and strongest roots from amongst the old, and lay them lengthwise in the drills, and cover them over. When offsets are employed, remove them from the sides of the old plants in spring, when about 3 inches high, and set them in drills as above, 6 inches apart in the rows. Cuttings of the young stalks taken off in June, and planted like offsets, and at a like distance, will be established by autumn. Pennyroyal should be planted in spring, at the same distances as the above, preferring a dampish situation.

Young plants recently set will be improved in growth by occasional waterings, and especially penny-royal, particularly during dry warm weather. Keep the beds clear of weeds by hand-weeding, for, from the nature of the plants, their roots running in all directions near the surface, hoeing cannot be applied. Old beds should have a top-dressing of light soil or rotten $\tan$ laid over them every autumn, to the depth of 2 inches : in this the young roots seem to luxuriate. It also preserves the buds during winter, and causes them to spring earlier in spring. This should not, however, be applied to penny-royal. In some soils the mints will continue for years, in others it is well to make new plantations every second or third year. At the end of summer cut over all the remaining stems close to the ground before top-dressing. Cut the alleys off at the same time, and gather out all extending roots, so as to restrain the plants within their proper bounds.

A moist loose soil is the most proper for the spear and pepper mints, while a strong moderately-moist soil is more fitting for penny-royal; all of them naturally grow in situations which 
are occasionally irrigated or flooded by water. None of them require manure.

For medical purposes they should be gathered just as the flower appears; but for obtaining the essential oil, the flowering plant is preferred. In both cases, as well as for drying for culinary use, they should be cut when quite dry, and tied up into small bundles, and suspended in an apartment with a temperature of from $60^{\circ}$ to $65^{\circ}$. By this means the agreeable flavour and green colour are preserved. Some gardeners place this, as well as most other herbs, in strong wooden boxes, and subject the contents to considerable pressure by loading them with weights, or by the application of a screw, so as to compress them into solid masses. This is done with a view to prevent the air from acting upon them, and destroying their aroma. The operation is carried on in a high temperature, and the boxes kept afterwards in a dry room. Others again dry them quickly by suspending them in a very warm apartment; and when sufficiently dry, reduce them down to a fine powder, which they keep in wide-mouthed glass bottles with glass stoppers.

Forcing.-In most families green mint is in demand throughout the winter; and, to provide for this, roung healthy roots should be taken up and planted in shallow pans or boxes, in rotten tan, or light rich sandy soil, and placed in a pit or vinery, with a temperature of from $55^{\circ}$ to $65^{\circ}$. It is important that a sufficient stock for the contemplated demand should be potted in autumn, or a portion of the old beds covered with litter to keep out the frost, so that they can be readily got at when wanted. Place the roots pretty closely together, and when one gathering of the young shoots has taken place throw them away, and introduce others. To keep up a constant supply, set the first set of pans or boxes into heat by the 1st of November, in number or extent corresponding to the demand; and set in to a similar extent every fortnight, until the end of February, after which a supply may be expected from the beds, by placing a few hand-glasses over a portion of them. Supply the roots placed in heat with abundance of water, and keep the plants as close to the glass as possible.

The mints are seldom annoyed by insects; the Uredo labiatarum Dec., a parasitic fungus, a sort of mildew common to all the Labiatæ order of plants, often appears upon the leaves, but seldom to an extent to be injurious.

The European names of mint are-La menthe in French; Die munze in German; Munt in Dutch; Minta in Italian and Spanish; Miata in Russian; Mietka in Polish.

Costmary (Balsamita vulgaris W.) belongs to the natural order Compositæ, sub-order Anthemideæ, and to the class Syngenesia and order Superflua in the Linnæan arrangement. The generic name is derived from Balsamon, balm, from its strong balsamic smell; the English name Costmary from Costus, an aromatic shrub, and Mary-the Virgin Mary's costus. From its being put into ale, was derived the old English name of Alecost. A native of Italy, from whence it was introduced to Britain in 1568 . VOL. II.
It is sometimes put into salads, but seldom asked for. It is a hardy perennial creeping-rooted plant, easily propagated by division of the roots, and succeeds well in any ordinary garden-soil.

Its European names are-Coq-des-jardins in French ; Die frauenmunze in German; Tuinbalsam in Dutch ; Costo-ortense in Italian; Heirba de Santa in Spanish ; Balsmita in Portuguese Hanegræs in Danish; Svensk salvia in Swedish.

Tansy (Tanacetum vulgare L.) belongs to the natural order Compositæ, sub-order Anthemidæ, and to the class Syngenesia and order Superflua in the Linnæan arrangement. The generic name is.altered from Athanasia, itself a word which signifies a plant which does not perish, or an immortal plant. Indigenous to Britain, and an old inhabitant of our gardens. In Gerard's time, cakes were made of the young leaves in spring, mixed with eggs, and were called tansies. It was much used formerly during Lent, to correct the effects of eating salt fish; and cakes were made at Easter time and offered to visitors, a custom still kept up in many parts of England. At present it is chiefly used to flavour a peculiar pudding, called tansy pudding. The leaves are also used in omelets, and the curled variety for garnishing by those who like the smell. A hardy perennial plant of easy culture, spreading rapidly, and readily propagated by division of the roots. Like costmary, a plant or two is sufficient for a small garden. Roots of it potted in autumn and placed in slight heat, will afford a supply during winter; and for Lent use it should be so produced.

It holds a place in many cottage gardens, and is more used by that class than by any other. The European names are-Tanáisie in French ; Der rheinfarrn in German; Reinwaar in Dutch ; Tanaceto in Italian and Spanish ; Tanasia in Portuguese; Dikaja riabina in Russian; and Wrotecz in Polish.

\section{$\S$ 4.-BASIL, SAVORY, ETC.}

Basil.-Ocymum Basilicum L. (the sweet or larger basil), and the 0 . minimum $\mathrm{L}$. (the bush or least basil), are both cultivated in all firstrate gardens. They belong to the natural order Labiatæ, and to the class Didynamia and order Gymnospermia in the Linnæan arrangement. The generic name is derived from $O z o$, a smell, from their powerful scent. They are both natives of India : the former was introduced in or before 1548 , and the latter in 1573 . These are the two sorts cultivated in Britain, while $O$. gratissimum is cultivated in China as a culinary plant, and is also a native of the East Indies, and being a shrubby stove-plant, deserves our attention more than appears to have been bestowed upon it, more especially for winter use, when the annual sorts are so difficult to grow: a plant known from the earliest ages of Grecian history. "The leaves and bracteæ, or leafy tops, are the parts gathered; and on account of their strong flavour of cloves, they are often used in high-seasoned dishes, as well as in soups, stews, and sauces, and a leaf or two is sometimes introduced in salads."-Sub. Hort., 
p. 691. No turtle-soup is fit to present at a London civic feast without its due amount of basil seasoning. Phillips remarks that, during the reigns of Queen Mary and Elizabeth, farmers had learned to compliment their landladies when they visited their farms, and grew basil in pots to present to them. Tusser, in his "Five Hundred Points of Good Husbandry," has not failed to satirise this servile custom.

Being tropical annuals, they are raised annually from seed. In the southern parts of England the seed is sown on warm borders. In the north of England and Scotland, generally, it is sown in pots placed in a moderate temperature, and transplanted in May or June, according to the situation. In families of the highest grades, green basil is required throughout the year. For winter supply, seed should be sown in flat pots or boxes every fortnight, from the beginning of November to the end of March. From the first of April to the end of July, in England, it should be sown once a-month, and that upon the warmest and driest border the garden affords. In Scotland, it is better to sow at the same periods, with a view to transplant afterwards into the open garden; but in many cases it must be grown under glass.

The seed is small, therefore the surface should be rendered smooth, whether in pots or in the open borders. Sow thin, when the crop is to be grown without transplanting, which should be the case with such as are grown in pots, during winter; cover to the depth of a quarter of an inch; keep the soil rather dry than otherwise, and thin out if too crowded. The plants may be used when from 2 to 3 inches in height, therefore progressive thinnings may take place; and should the plants be thicker than is deemed expedient, a portion may be carefully taken up, and transplanted into other pots or boxes, where they will attain the height of 8 or 9 inches. Those grown in the open border, if the soil is light and rich, and the situation favourable, will, if set at the distance of a foot or 14 inches apart, cover the ground, and assume the appearance of dwarf stocky bushes. When the plants are just showing flower, they should be cut over by the ground, and dried as speedily as possible, either as recommended for mint, \&c., p. 237, or otherwise, so as to retain the green colour, as well as a portion of the agreeable aroma for which it is so much valued. When sufficiently dry, it should be rubbed down to a fineish powder, and kept in wide-mouthed bottles, with glass stoppers, for winter use ; but, like most other odoriferous plants, it loses much of its flavour when dried, and hence cooks always prefer it in the green state. The treatment of both sorts is the same.

The European names are-Basilie in French; Basilikum in German ; Basilicum in Dutch; Bassilico in Italian; Albahaca in Spanish; Alfavaca in Portuguese ; Wasilik in Russian; and Bazylika in Polish.

Savory.-Satureja montana L. (winter savory), S. hortensis L. (summer savory), S. capitata, (headed savory), S. viminea L. (twiggy pennyroyal savory). The two latter-the first a native of the Levant, introduced in 1596, and the second a native of Jamaica, introduced in 1783are seldom cultivated in Britain, although both are much used in their native countries. The two former are, or should be, found in every garden: they belong to the natural order Labiatæ, and to the class Didynamia and order Gymnospermia in the Linnæan arrangement; they are both natives of the south of Europe: the former was introduced in 1562, and the latter in 1652. The generic name is derived from Ss'atar, the Arabic name for all labiate plants. The winter savory is an aromatic annual plant. The winter savory grows to the size of a small shrub, when planted in a poor dry soil; but in rich earth its pores become too full of moisture to stand the severity of our winters: both these are noticed by Virgil, among the fragrant herbs and shrubs that should grow near the bee-hive. Although the above dates of their introduction are given, it would appear by Gerard that they had been in use long before ; and, with other Italian herbs used in Roman cookery, it is not improbable that they were introduced by the Romans themselves, and continued in the monastic gardens.

The perennial sort, S. montana, is propagated by cuttings taken from the young side-shoots in June, placed under a hand-glass on a shady border. The cuttings should be about 2 inches in length; and when they are rooted should be transferred to a nursery-bed, in a more warm and exposed place, to gain strength, so that they may be set out in their permanent place by the beginning of September. They should be planted in rows, 18 inches apart, and the plants set a foot apart in the line. 'They will continue for many years in a favourable climate and dry soil; but as they are apt to become straggling and unsightly, new plantations should be made every five or six years. In cold situations they demand slight protection during severe winters. It may also be propagated by seed, but the plants are longer in coming into use.

The annual sort $S$. hortensis is propagated by seed, which should be sown in warm localities in April ; but in colder places it is better to sow in pots placed in a gentle heat, and transplant in June. That sown in the open air need not be transplanted if sown in drills half an inch deep and a foot asunder, if thinned out to 6 or 8 inches in the rows.

The perennial sort should be cut for drying just as the flowers are opening, and the annual one when in the same state, and dried and preserved as noticed for other odoriferous herbs. Both are used during summer in a green state. They were both formerly used in the composition of cakes and puddings, but are now chiefly employed in seasoning sausages, force-meats, and sometimes in soups; occasionally in salads-a use the Romans were well aware of.

The European names are-Sarriette in French; Die saturei in German; Keul in Dutch; Santoreggia in Italian ; Ajedrea in Spanish; Segurelha in Portuguese ; Tschaber in Russian ; Ozabr in Polish; and Saer in Danish.

Marjoram-Origanum vulgare L. (common marjoram), 0 . heracleoticum L. (winter sweet marjoram), O. Marjorana L. (sweet or knot- 
ted marjoram)-belongs to the natural order Labiatæ, and to the class Didynamia and order Gymnospermia in the Linnæan arrangement. The first is indigenous to Britain, and common on dry chalky wastes; the second is a native of the south of Europe, and was introduced in 1640 ; the third is a native of Portugal, introduced in 1573 ; and a fourth, $O$. onites (pot marjoram), a native of Sicily, was introduced in 1759. The generic name is derived from Oros, a mountain, and ganos, joy -as if they were the joy of the places where they naturally grow. Common marjoram is a hardy perennial plant, readily propagated by division; and although it will continue for many years in most soils, a new plantation should be made every second or third year, setting the plants about 15 inches apart; and the operation may be performed in autumn or spring. Winter sweet marjoram is also a pretty hardy perennial, seldom, however, ripening its seed in this country; it is therefore, in general, propagated by cuttings, treated as we have recommended for savory: it requires a warm exposure and a dry soil. Siveet or knotted marjoram is a hardy biennial, rarely ripening its seed in this country; it should be treated as an annual, and sown every year; it should be sown in March, on a slight hot-bed in Scotland, but in England it is usually sown on a dry warm border in April. In the former case it should be transplanted, about the beginning of May, to a warm dry place, setting the plants in rows 15 inches asunder, and 9 inches apart in the line. The pot marjoram is a sufficiently hardy dwarf half-shrubby plant to stand our ordinary winters in the open border; it should, however, be propagated every third or fourth year by cuttings for new plantations, and treated like winter sweet marjoram.

The leaves and tender tops of all the marjorams are the parts used; they are in constant demand, being used in soups, made dishes, and other culinary preparations. Seeds of all the varieties may be obtained in the seed-shops; and plants, witl all other sweet herbs of a perennial character, may also be purchased-and where the quantity required is small, it is the most proper way. A quarter of an ounce of seed is sufficient for most ordinary gardens, and the same may be taken as the average quantity for most other herbs of the same description; they are all cut as they come into flower, and should be preserved as already recommended. When grown from seed, sow in drills, 9 inches apart and half an inch deep, in light finely-pulverised soil; from six to twelve plants of each of these will be sufficient for a small garden.

The leaves of common marjoram are used in many places as a substitute for tea, and also, along with those of $O$. Majorana, form ingredients in cephalic snuff. According to Forskahl (p. 59), the latter species derives its name from Marjamic (maryamych), its Arabic name. The European names of the marjorams are-La marjolaine in French; Der majoran in German; Mariolein in Dutch ; Maggiorana in Italian; Mejorana in Spanish; Macran in Russian ; Maicran in Polish.

Anise (Tragium anisum, Spr. - Pimpinella anisum L.) belongs to the natural order Umbelliferæ, sub-order Orthospermæ, and tribe Ammineæ, and to the class Pentandria and order Digynia in the Linnæan arrangement. The generic name is derived from Tragos, a goat, from the resemblance in odour to the smell of that animal. Linnæus's original name of Pinpinella has been altered, according to himself, from Bipennula-twice pinnate, in allusion to the leaves. Anisum is derived from Anysun, its Arabic name. It is a native of Egypt, introduced in 1551. Being an annual, it is sown yearly in April in drills, three quarters of an inch in depth and 15 inches asunder, and the plants thinned to a foot apart in the rows. A deep light soil is most proper. The seeds are used for distillation and expression, and imported in large quantities from Malta and Spain. They are also used in confectionary, and the green leaves in salads, and for seasoning and garnishing like fennel. The seed should be sown where the plants are to remain, as it does not succeed very well by transplanting. They are best raised on a slight bottom-heat for the climate of Scotland, two seeds in each pot of the 60 size, and in May turned out with the balls entire. In England this care is not requisite. Gather the crop for its seed when they are ripe, and during summer the young side-leaves will afford abundant supply for other purposes.

Its European names are-Anis or Boucage in French; Kleine bibernel in German; Kleine bevernel in Dutch; Pimpinella sassifraga in Italian; Pimpinella blanca in Spanish; Pimpinella branca in Portuguese; Bedvenez in Russian.

Angelica (Angelica archangelica L.) belongs to the natural order Umbelliferæ, sub-order Orthospermæ and tribe Angeliceæ, and to the class Pentandria and order Digynia in the Linnæan arrangement. The generic name is derived from its supposed angelic virtues; its specific name from $\mathrm{Arch}$, superior-an augmentative prefix: indigenous to England. Though only a biennial, it may be made to continue several years by cutting over the flower-stem before it ripens seeds, in which case it immediately pushes out below. Considerable quantities of this plant are grown by the London gardeners in moist situations, and along the banks of ditches. It is cultivated for its leaves and the tender flower-stalks, which were formerly blanched like celery. In the north of Europe the leaves and stalks are eaten raw, or boiled with meat and fish. In England and France the tender stems, stalks, and midribs of the leaves, cut in May, are candied by the confectioners, and a second crop will be produced the same season. If the stalks are allowed to flower, seed will be abundantly produced, which should be gathered as it ripens, as it soon sheds itself.

The seeds are sown in August, or indeed as soon as they ripen, to have the advantage of stronger plants in spring than if sowing were delayed to that time. Indeed, all biennials should be sown as soon as their seeds are perfected. When the plants are about 6 inches high, take them up carefully by loosening the ground about and under them, as they have 
long tap-roots ; plant in moist deep ground, for this plant is half an aquatic, and set, them in rows 3 feet apart and the plants 2 feet distant in the line, for they are tall and strong growers. They should not be cut the first year, but in the second they will be in fine condition; and if the first cutting take place in May, a second crop will be obtained; and thus on for several years, if the plants are prevented from running to seed, after which they must be cut down and a new plantation formed. Twelve plants will be enough for a small garden, and half an ounce of seed sufficient. They are sometimes increased by taking side-slips from the edges of the roots, but this is only a make-shift mode. The seed will keep for three or four years.

The European names are - Angelique in French; Die angelika in German; Engelwortel in Dutch; Angelica in Italian and Portuguese; Anjelica in Spanish; Angelika in Russian; Dziegiel ogrodny in Polish.

Caraway (Carum carui L.) belongs to the natural order Umbelliferæ, sub-order Orthospermæ, and tribe Ammineæ, and to the class Pentandria and order Digynia in the Linnæan arrangement. The generic name is derived from Caria, in Asia Minor, of which the plant is a native. It is now accounted a native of Britain, but the origin of its name, and the positive manner in which Pliny mentions from whence it sprang, refute this opinion. It is more than probable the Romans introduced it into Britain, as well as sundry other plants which modern British botanists have claimed as being indigenous, or absolute natives, without giving due consideration to the possibility of their having only escaped from gardens, and become so far naturalised as to grow without culture. That shrewd old botanist Gerard makes no mention of its being found growing wild in England, but says it grows abundantly in Germany and Bohemia "in fat and fruitful fields." Ray is the earliest author we have found who thought it a native, and states that "it grows wild in several places in Lincolnshire and Yorkshire;" but Ray flourished about a century after Gerard, who was born in 1545, while Ray was engaged in pomological matters so late as 1688 . This plant is cultivated in gardens for its under leaves, which are sometimes put into soups, and the young ones often into salads, and in former times the roots were eaten as parsnips. It is largely grown, particularly in Essex, for the seed, which is used for distillation and confectionary purposes. The seeds are used by cottagers to mix with their bread, and the Dutch introduced it into cheese. Unless for the seed, three or four plants are sufficient for a small garden.

It is propagated by seeds, which should be sown in autumn, as soon as they are ripe, in drills half an inch deep and 2 feet asunder, thinning out the plants to 18 inches in the lines. Like all aromatic plants, a poor light, but in this case a deep, soil should be chosen. A quarter of an ounce of seed is sufficient for most gardens.

'The European names are-Le carvi in French ; Der kummel in German; Karwey in Dutch; il Carvi in Italian; Alcaravea in Spanish; Alcaravia in Portuguese ; Timon in Russian; Karny in Polish ; and Kommen in Danish.

Coriander (Coriandrum satixum L.) belongs to the natural order Umbelliferæ, sub-order Coilospermæ, and tribe Coriandreæ, and to the class Pentandria and order Digynia in the Linnæan arrangement. The generic name is derived from Koris, a bug, from the smell of the leaves. Indigenous to England, in corn-fields. In gardens it is cultivated for the same purposes as chervil, the young tender leaves being used in soups and salads. It is propagated by seed, which should be sown in February, in light dry soil,in drills half an inch in depth and 15 inches apart, thinning out the plants to 9 or 10 inches in the lines. The plants soon run up to seed during summer; therefore, where a constant supply is required, sowings should be made every month from February to July. Sowings made during August and September should be on a warm dry border, and be protected during winter with a frame and glass.

The European names are-La coriandre in French; Der koriander in German, Dutch, and Russian ; Coriandro in Italian; Cilantro in Spanish; Coentro in Portuguese.

Marigold (Calendula officinalis L.-common pot-marigold) belongs to the natural order Compositæ, sub-order Heliantheæ, and the class Syngenesia, and order Necessaria, in the Linnæan arrangement. The generic name is derived from Calenda, first day of the month, on account of its flowering monthly, or rather from its being observed to flower most about the Calends of every month. It is a native of the south of Europe, and was cultivated in this country prior to 1573. The flowers are used in broths and soups; and in certain dairies they are put into the cream, before churning, to give the butter a better colour. In Holland, the flowers are dried for the purpose of being put into winter broths, and for this purpose are to be found in most grocers' shops throughout that country, packed tightly in barrels, and sold by the ounce, for few broths are made without them.

The common or pot marigold is a hardy annual plant, which should be sown in March or April, in any ordinary garden-soil, in drills 15 inches asunder and half an inch in depth. When the plants come up, thin them to 9 inches apart; but all this depends, as it does in all similar cases, on the richness or poverty of the soil. The flowers will appear in June, July, August, \&c., and should be gathered for use when expanded fully ; and for drying, when they are quite dry, and preserved as has been recommended for other herbs. As it is a showy flowery plant, and grown in most flower-gardens, for ordinary supplies, enough may be procured from that source-as the more the flowers are gathered, the longer the plant will continue in vigorous production.

The European names are-Le souci de jardin in French; Die ringelblume in German ; Goudblœm in Dutch; Calendula in Italian and Spanish; Nogotki in Russian; Nogietek in Polish. Two packets, each $3 d$., are sufficient for a medium-sized garden. 


\title{
CHAPTER IX.
}

\author{
ME D I I N A L P LA N T S.
}

In the olden times, when barbers exercised the art of phlebotomy, and gardeners were the " cullers of simples" and administrators of phy. sic, medical herbs were much more attended to in private gardens than at present. A few, however, are still found deserving cultivation even in these, while hundreds of acres are dedicated to this article of produce, for supplying the faculty with the majority of them. A considerable trade is also carried on by itinerant herbalists, who find a ready market for those of indigenous growth.

Chamomile (Anthemis nobilis L.) belongs to the natural order Compositæ, sub-order Anthemideæ, and to the class Syngenesia and order Superflua in the Linnæan arrangement. Indigenous to many parts of Britaill, growing generally in gravelly pastures. There is a doubleflowering variety, in general cultivated in gardens; it is much more ornamental than the single, but much less efficacious as a medicine. In many parts of England the peasantry have what they call a chamomile seat at the end of their little gardens, which is constructed by cutting out a bench in a bank of earth, and planting it thickly with the double-flowering variety of this plant, on which they delight to sit, and fancy it conducive to health. The flower is also sometimes used in the manufacture of bitter beer, and, along with wormwood, made to a certain extent a substitute for hops. The flowers, which are the only part used of the single-flowering variety, have long been in high repute, both in the popular and scientific Materia Medica, and give out their properties by infusion in either water or alcohol. This plant delights in a poor, light, sandy soil, fully exposed to the sun, and is increased by dividing the larger or older plants into small pieces, which will readily grow if small portions of the roots be taken with them. March, April, or May is the proper season for planting, although, with a little care, the operation may be performed at almost any other season. The young plants should be set in rows 8 or 10 inches asunder, giving a little water until they take root; or they may be planted as edgings to walks, or employed to cover a poor barren bank. One plantation will last from five to eight years in a productive state. The flowers should be gathered when fully expanded, choosing a dry day, and, when gathered, laid thinly on a piece of clean canvass, in a dry, airy, partially shaded place for a day or two; afterwards placed in a warm apartment to dry thoroughly; after which they should be placed in paper bags, or, better, in widemouthed glass bottles, tightly corked, and kept for use.

The French call it Camomille; the Germans and Dutch, Kamille; the Italians, Camomilla; the Spaniards, Manzanilla ; and the Portuguese, Macella.

Hyssop (Hyssopus officinalis L.) belongs to the natural order Labiatæ, tribe Satureineæ, and to the class Dydynamia and order Gymnospermia. It bears nearly the same name in most of the European languages, which signifies a holy herb, or herb for purifying holy places. From the frequent mention made of it in Scripture, we may infer that it grew wild in Syria and Egypt. It was long in considerable repute as a popular medicine, but its use now in this respect is chiefly confined to domestic administrators. In French and Italian cookery, the tops of the young shoots are sometimes used in soups, but its principal use is for medical purposes.

It is readily propagated by seed, which should be sown in March or April, upon a dry warm border - the plants to be afterwards transplanted in June or July, either as edgings in small gardens, or in rows a foot apart, setting the plants 8 inches asunder. It is in general, however, increased by cuttings or slips, which should be taken off in April or May, and placed under a hand-glass in a somewhat shaded place. By August or Septernber they will have struck root, and should then be planted out as above.

The plants bear cutting with advantage, and therefore should be pruned back as soon as the flower spikes, with a portion of the current year's growth, are cut off for drying; and this should be done when the flowers are abqut expanding. Tie them up in small bunches, and dry them as recommended for thyme, \&c. The plant delights in a dry soil, and is quite at home when established on top of an old wallwhich habitation can readily be secured for it by sowing the seeds between the joints of the stones or bricks. It is called Hysope in French; Isop in German ; Isopo in Italian; Hysop in Dutch; Hisopo in Spanish; and Hyssopo in Portuguese. 
Wormwood (Artemisia absinthium L.) belongs to the natural order Compositæ, sub-order Anthemider, and to the class Syngenesia and order Superflua in the Linnæan arrangement. Indigenous to Britain ; found in neglected places. Several of the genus are included in the Materia Medica, but, according to Dr Thomson, our present subject is the only one of them deserving to be retained. A long list of its virtues is given in "London Dispensatory," p. 152. The seed is used by the rectifiers of British spirits, and it is largely cultivated about Mitcham in Surrey for that purpose. The whole plant is used as a substitute for hops in brewing, and for this purpose it is cultivated in cottage gardens. It is also grown in most gardens for the purpose of being chopped up along with rue, cress, and similar plants, and mixed with the food of young turkeys. Plants of it should be set in every poultry-yard, as it is exceedingly beneficial to them. It is a hardy perennial plant, readily propagated by division of the root, as well as by seed, and may also be increased by cuttings, treated as already recommended for other herbs. An infusion of the seed makes an excellent and safe stomachic, taken to the extent of a wine-glassful every morning, the same as chamomile. Three or four flowers of the latter, and half a tea-spoonful of the seeds of the former, infused in a pint of warm water, and taken when cold, is the proper quantity.

The European names are-Absinthe in French; Wermuth in German; Alsem in Dutch; Ajenjo in Spanish ; Assenzio in Italian.

Rue (Ruta graveolens L.) belongs to the natural order Rutaceæ and tribe Ruteæ, and to the class Decandria and order Monogynia in the Linnæan arrangement. It long held a high place in medicine, but is now less regarded. It is grown in gardens, as stated in the last article, chiefly for the use of poultry, and is given them with advantage in cases of croup. A native of the south of Europe, and introduced prior to 1562. It is readily propagated by seed sown in March, and by cuttings planted in April or May. It delights in a poor, dry, calcareous soil, in which it will continue from eight to twelve years, if not cut down with frost, which sometimes happens, but seldom till the thermometer falls to $15^{\circ}$. In cold situations it should be planted in a sheltered place, at the bottom of a wall, or amongst large stones in a rockery. Formerly the English, as well as the Germans and Dutch, used the green leaves of rue in their ragouts. They were also used as a pickle, being first boiled and then preserved in vinegar, and were in that state esteemed a good sauce for meat. The celebrated antidote of Mithridates, king of Pontus, with which most medical students are familiar, and which has, with slight alterations, been in use for nearly nineteen centuries, is principally composed of this plant. The receipt will be found by a reference to "Phillips' Hist. of Fruits," p. 351. The European names are-Rue in French ; Raute in German; Ruta in Italian; Ruite in Dutch; Ruda in Spanish; Ruta in Russian; Rude in Danish; and Vinneta in Swedish.

Liquorice (Glycyrrhiza Tou ; Glabra L.; Liquiritia officinalis Monch.) belongs to the natural order Leguminosæ, sub-order Papilionacæ, tribe Loteæ, sub-tribe Galegeæ, and to the class Diadelphia and order Decandria in the Linnæan arrangement. The name is derived from Glykys, sweet; Rhiza, root. A native of the south of Europe, and introduced prior to 1562 . A hardy perennial deep-rooting plant, requiring a deep, sandy, loamy soil, trenched to $2 \frac{1}{2}$ or 3 feet in depth, and manured if necessary. Propagated by cuttings of the roots, which cuttings should be taken from the small horizontal roots of established plants. These should be cut into pieces 6 inches long, and planted in rows 3 feet asunder, setting the cuttings 18 inches apart in the line. During the first year after planting the ground may be cropped with lettuce, turnip, or any other shallowrooting, low-growing crop; and at the end of the first season cut down the stems close to the ground, and fork up the surface. The third or fourth year after planting, the roots will be fit for use. They should be dug up in autumn, taking care to open a deep trench along one side of the row, so as to get the roots out to their full length. When taken up, if grown for sale, they should be immediately disposed of to the brewers' druggists, as they are largely employed in porter brewing; or to medical druggists, who also use them in medicine. If for private use, they should be packed in sand in the root-cellar, like beet, parsnip, \&c., till wanted.

The European names are-Zoethout in Dutch; Reglisse in French; Regaliz in Spanish; Sussholz in German; Regolizia in Italian. It is chiefly grown in gardens for the saccharine juice obtained from the root by decoction, which is used as an emollient in cold and fevers.

Elecampane (Inula helenium L. ; Corvisartia helenium Merat.) belongs to the natural order Compositæ, sub-order Carduaceæ, division Vernoniaceæ, and to the class Syngenesia and order Superflua in the Linnæan arrangement. Inula is a corruption from Helenium. Indigenous to Britain, found in moist pastures in the south of England; one of our largest native plants, attaining a height of from 3 to, 5 feet. It long maintained a place in almost every garden in Europe, but of late it has been less regarded. The root is the part used; it is thick, fusiform, and aromatic, and is used medicinally in a candied state. Propagated by seed, but more generally by offsets taken off in autumn, when the plant is done flowering. These planted 3 feet apart in a deep, rather moist soil, in a partially shaded situation, will be fit for use the end of the second year. The European names are-Inule in French and German ; and Inulo in Italian. 


\title{
C H A P TER X.
}

\author{
EDIBLE FUNGI.
}

\section{$\S$ 1.-THE MUSHROOM.}

Natural history.-The mushroom (Agaricus campestris Linn.) belongs to the natural order Fungi, tribe Hymenomycetes, division Pileati, and class Cryptogamia, order Fungi, in the Linnæan arrangement. The generic name Agaricus is derived from Agaria, a region of Sarmatia. A vast number of species are indigenous to Britain, and many are natives of other parts of the globe, chiefly, however, in temperate climates. The English name Mushroom is probably a corruption of Mousseron, the French name of a variety of the fungus called Champignon. The genus Agaricus, like all cryptogamic plants, are produced by seeds, but these are so minute as to require glasses of the greatest power to bring them within the power of human vision. The seeds are placed between the gills or thin flat plates situated under the cap or pileus, and when in a ripened state are discharged spontaneously in immense numbers in form of small minute granules, by which the species is propagated. These falling to the ground, or being blown about by the wind, become attached to the leaves and stalks of grasses and other plants; are taken into the stomach of certain animals, such as the horse, and pass through the intestinal canal uninjured in their vegetative property ; or falling upon favourable media, become in time transformed into a white thready-like mucreous matter called spawn, which is capable of propagation; and ultimately, when placed in a proper degree of heat and humidity, of reproducing its species in the shape of perfect mushrooms.

Use.-Ignorance and prejudice are alike difficult to overcome, and this is perhaps in no case, in which the food of man is concerned, more clearly exemplified than in the case of the edible fungi with which our country abounds. Throughout the whole continent of Europe, profitable advantage is taken of them, so that in "France, Germany, and Italy they not only constitute the sole diet of thousands for weeks together, but the residue," Dr Badham remarks, " either fresh, dried, or variously preserved, in oil, vinegar, or brine, is sold by the poor, and forms a valuable source of income to many who have no other produce to bring into the market." So much importance is attached to the consumption of esculent fungi at Rome, that the government, in
1837, estabïished an official inspector (1spettore dei Funghi) to examine their qualities, and regulate their sale, which is conducted under the surveillance of the police, and a tax equal to a halfpenny sterling is imposed on every 10 pounds' weight.

In Poland and Russia above thirty sorts are eaten by the peasantry, not only in their fresh state, but dried in stoves, and preserved for winter consumption. The sorts generally used in Britain are, according to Sowerby, in his work on English Fungi, the truffle and morel, both of which are held in high estimation by epicures, and the true mushroom, the Agaricus campestris of botanists. The following species are occasionally eaten, and may be so with perfect safety-viz., A. violaceus, A. pratensis, A. deliciosus, A. cinnamoneus, A. lactifluns, A. solitarius, A. cantharellus, A. aurentiacus, A. procerus, A. virgineus, A. orcades, or fairy-ring mushroom, from their appearing in old pastures either in entire circles or parts of circles. To these Dr Badham adds, Agaricus vaginatus, A. rubescens, A. excoriatus, A. prunulus, A. personatus, A. heterophyllus, A. virescens, A. ruber, A. piperatus, A. nebularis, A. fusipes, A. ostreatus, A. ulmarius, A. orcella, A. castaneus, A. Georgii, A. comatus, A. atramentarius, A. cibarius, Polyporus frondosus, Boletus edulis, B. scaber, Fistulina hepatica, Hydnum repandum, Morchella esculenta, M. semilibera, (the esculenta and hybrida of Sowerby), Helvella crispa and lacunosa, Peziza acetabulum, Bovista plumbea, Lycoperdon plumbeum and bovista, all of the genus Clavaria. The above form the principal of the esculent fungi, and may all be eaten with perfect safety if taken while young or newly gathered. Hitherto the Agaricus campestris, of which, however, there are several sub-varieties, has been the species alone cultivated in Britain. That there are others deserving our attention, even in a cultural point of view, there is no doubt. Of these the following may be considered the most important.

Agaricus prunulus (Vitt.) is found only in spring, growing in rings on the borders of woodlands, at which time abundance of its spawn may be procured, and may be continued in the same way that the spawn of $A$. campestris is; namely, by transplanting it into bricks of loam and horse-dung, in which way it will keep for months. This mushroom is used both in its green and dried state, in which latter state it 
constitutes what is called "Funghi di Genoa," and is preserved by being simply cut into four pieces, and dried in the air for a few days, when it is strung up and kept for use. It is not a common species in Britain.

Agaricus procerus (Scop.), one of the most delicate of all the mushroom tribe. It is very common in England, and held in high esteem in France and Italy. It is an autumnal species, and makes excellent ketchup.

Agaricus exquisitus (Badham), A. Georgii (Withering), often attaining the weight of 5 or 6 lb. Considered less delicate than $A$. campestris in Britain, but looked upon in Hungary as a special gift from the saint whose name it bears. Persoon describes it as superior to $A$. campestris in smell, taste, and digestibility, on which account, he says, it is generally preferred in France. It is found abundantly in many places, generally growing in rings, and reappearing for many successive years on the same spot; and although sometimes found in old pastures, is in general to be met with in thickets under trees.

Agaricus deliciosus.-Found during September and October growing under Scotch fir-trees and other pines; of medium size, yellowish, zoned with deep orange on top, somewhat resembling $A$.torminosus, a deleterious species, but readily distinguished from it, as its juice is, when fresh cut, quite red, afterwards turning green, while that of the latter is white and unchangeable. Sir James Edward Smith, in his "Tour," says, "The market of Marseilles exhibited a prodigious quantity of $A$. deliciosus, which really deserves its name, being the most delicious mushroom known." And Mr Sowerby is equally high in its praise, remarking, "I had one dressed ; it was very luscious eating, full of rich gravy, with a little of the flavour of mussels."

Agaricus personatus.-One of our most common British mushrooms, appearing in October in circles, and sometimes growing in clusters amongst old pasture-grass. It has found its way into Covent Garden market under the name of Blewitts or Blue Hats.

Agaricus oreades (Bolt).-This is the wellknown Champignon, abounding in most old pastures, appearing in succession for several months, generally growing in circles, and greatly abounding after rain has succeeded dry warm weather, that being the most favourable condition for the growth and extension of the spawn, and the former the best condition for the production of the perfect mushroom. Dr Badham thus speaks of this species: "Independent of the excellent flavour of this little mushroom, which is as good as that of most funguses, two circumstances give it an additional value in a domestic point of view-viz. the facility with which it is dried, and its very extensive dissemination. When dried, (two or three days'exposure to the air is generally sufficient to effect this), the $A$. oreades may be kept for years without losing any of its aroma or goodness, which, on the contrary, becomes improved by the process, so as in fact to impart more flavour to the dish than would have been imparted by the fresh fungus; though it is not to be denied that the flesh then becomes coriaceous, and less easy of digestion."
Agaricus nebularis, reappearing for years in the same spot, generally in October, considered lighter of digestion than any other sort.

Agaricus comatus and $A$. atrimentarius are employed for making ketchup, but should both be used in a young state. The former is found abundantly growing on stumps of trees, appearing both in spring and autumn; while the latter is found amongst grass in meadows and pastures, from spring till late in autumn.

Agaricus virescens (Schoeff.), A. ruber (Schoeff.), and $A$.heterophyllus (Fries.), are all of the edible section. The former, indeed, has been recommended for cultivating by $M$. Thore. It is not very abundant in Britain. Vittadini describes it as excellent, and much used by the peasants about Milan, and asserts also that it is eaten in England. According to Roques, it is much used in France, both in a fresh and dried state. $A$. ruber appears in great abundance in many places from July till late in autumn, and is both delicate and easy of digestion. The latter is considered by Vittadini, Roques, and Dr Badham as not surpassed in flavour by any other of the mushroom tribe. It is found during the summer months, and often in abundance, under elm trees in dense woods.

Agaricus rubescens (Persoon).-Found during summer and autumn in woods, particularly in those of oak and chestnut. It is one of the most delicate as well as one of the most common of our British species, and eminently deserving the attention of collectors as well as of cultivators.

Agaricus orcella (Bull).-Appearing occasionally during summer, but far more usually during autumn, amongst grass, in woods and pastures, growing either solitary or in groups, and sometimes in circles, frequently succeeding a crop of $A$. oreades and $A$. prunulus : a most delicate and safe species.

Agaricus virgineus (Wullf).-Found abundantly in many places; in old pastures during autumn ; often in company with puff-ball, Lycoperdon plumbeum: indeed theyare often gathered and eaten together.

Agaricus piperatus (Scop).-Found in woods, during July and August, but not abundant; is very extensively used on the Continent, both in its fresh and dried state; it is also preserved, for winter use, in a pickle of salt and vinegar.

Agaricus fusipes (Bull).-An excellent fungus, found in great abundance at the roots of old oak trees, particularly after rain, throughout both summer and autumn.

Boletus edulis (Bull).-A prejudice exists in Britain generally against the whole of this genus ; nevertheless we have the testimony of ages that the ancient Romans were not only aequainted with this very delicious species, but also that they prized it exceedingly. Two species of boletus are eaten, and highly approved of, on the Continent, namely, B. edulus and scaber. The genus is extensively found in Britain, principally during autumn, but also occasionally during spring and summer, chiefly in woods of oaks, pines, and chestnuts. Of its merits as an esculent Mr Berkeley thus speaks: "Though much neglected in this country, it appears to be a most valuable article of food; it resembles much in taste the 
common mushroom, and is quite as delicate; it abounds in seasons when these are not to be found."

Boletus scaber (Fries.) - Found in similar situations to the last, but should be used in a fresh state, for when dried it loses much of its odour, and is both insipid and unfit for use.

Cantharellus cibarius (Fries.)-Found during summer in great abundance, and. continuing, although in less numbers, till towards autumn. It appears to be local in its habitats ; the neighbourhood of Chelmsford, in Essex, about Tunbridge Hills, and amongst moss under beechtrees in Burkhurst Park, are particularly famed for this highly-prized fungus : from these places the London demand is chiefly supplied.

Hydnum repandum (L.)-Found in woods, particularly those of oak or pine ; sometimes gregarious, or in rings, at other times solitary.

Fistulina hepatica (Bull.)-Grows upon old oak and chestnut trees, appearing throughout the summer often in great abundance, varying in size and shape, often, when fully grown, measuring 5 feet in circumference. Shœffer designates this "fungus pauperibus esculentus," or poor man's fungus, because it is a great acquisition to the poor where it grows in plenty.

Helvella lacunosa (Afy.), and H.crispa (Fries.) - Found in woods, not unfrequently growing on the stumps of old trees; in flavour somewhat resembling the morel, for which they are sometimes substituted.

Clavaria coralloides (L.), C. grisea; C. cristata (Pers.); C.rugosa (Bull.), are all esculent funguses indigenous to our woods and damp shady places.

Lycopordon plumbeum (Badham); L. Bovista (Pers).-Both are common, during summer and autumn, in dry exposed pastures, sometimes growing solitary, but for the most part in groups, and are known by the name of puff-balls; five species are recorded as natives of Britain, and all are found wholesome to eat.

The above enumeration of esculent funguses is given chiefly upon the authority of Dr Badham, whose excellent work, "Esculent Funguses of England," with coloured figures, should be perused by every gardener. M. Roques, Paulet, Persoon, and Cordier, have published excellent treatises on the kinds fit for human food, as used in France, and the no less valuable work by Vittadini gives the kinds in general use in Italy. The testimony of such authorities should shake the prevailing prejudices existing in Britain against a more general use of this valuable tribe of plants.

Much of the effects produced by indulging in the use of mushrooms has arisen from the place in which they have been produced, as it is a well-known fact in Covent Garden, that mushrooms gathered from the open fields, or even grown artificially in gardens, on open beds, are preferred to such as are produced in dark illventilated cellars. That mushrooms, when too old, and beginning to decay, become chemically changed, and may produce less agreeable sensations, is not to be questioned; but this in nowise affects the fact of their being as wholesome a diet as any other vegetable production used in its proper state. Steeping mushrooms in brine VOL. II. or acetic acid, before cooking, robs them of whatever deleterious principles they may possess. The residue left is pure fungine, which is perfectly innocuous, and the subsequent process of cooking renders them easy of digestion. Spirits of wine and vinegar extract some part of the deleterious properties, and tannin decomposes the greatest part of it.

Cultivation.-The Agaricus campestris, or common mushroom, is the only species that has as yet been subjected to successful cultivation, although there can be little doubt that all or most of the terrestrial-growing sorts would submit to the same process, if their natural habitats were sufficiently studied, and their spawn collected and propagated. In this way the common mushroom was first brought under the control of man. The morel is said to have been occasionally cultivated, and Lightfoot asserts that he succeeded in producing it from seed. The truffle has been cultivated in Germany and Italy to a limited extent, an account of which will be found in the "Gardeners' Magazine," vol. i. p. 320, and vol. $x$. Of the conditions necessary to the successful cultivation of fungi, little that is satisfactory is known. Moderate heat and moisture seem to be the principal agents that excite the seeds, in the first instance, and bring the plants to a state of perfection afterwards. Some species, however, are produced in a verylow temperature, but none without the necessary amount of humidity. That all funguses are originated from seed is incontrovertible. These seeds are called spores, and are quite invisible to the naked eye indeed, under an ordinary microscope, they appear as mere particles of dust; nor is it until we employ magnifiers of very great power that either their colours or shapes can be determined. The seeds of all cryptogamic plants are produced in enormous numbers, compared with those of phanogamous ones ; many, however, of them are abortive, and probably a greater number never reach a proper nidus on which they can establish themselves. The seed of the common mushroom, in falling from the gills when ripe, is no doubt wafted by the wind, and becomes attached to the stems, leaves, \&c. of grasses and other herbage, and, notwithstanding their being taken up by such animals as the horse, the deer, and the sheep, pass through their intestines without undergoing any material change in their vegetative existence; and hence, in the dung of these animals, when placed together, and kept moderately dry, and brought to a slight state of fermentation, we discover the first stage of existence of the future brood of mushrooms. This is practically called spawn, and consists of a white fibrous substance, running like broken threads through the mass of dung, which appears to be its only and proper nidus. The successive development of the seeds or spores is thus described by Dr Badham : "W When the spore ceases to be a spore, and to become a mushroom, the first thing it does is to send forth certain cotton-like filaments, whose interlacings entangle it completely, while they also serve to attach it to the place of its birth. These threads (like the spongioles attached to the roots of phanogamous plants) absorb, and bring 
nourishment to the quickened spore, which then maintains itself entirely by intus-susception. All this takes place before the germ has burst, or the embryo fungus begun to develop its organs. In some instances these elementary threads are, like the ordinary roots of plants, spread out to a considerable distance under ground, forming here and there in their course small bulbs or tubercles, each of which in turn becomes a new individual; in others, and more commonly, these spores are sprinkled about unconnectedly, affecting certain spots only, which become so many small matrices, whereof each furnishes a crop. The union of many germinating granules, together with these connecting threads, constitutes mushroom spawn, or, as it is technically called, carcytes. Examined a short time after quickening, the spore (or seed) is found to have swelled out into a fleshy kernel, which, in puffballs, truffles, and the uterine subterranean families generally, constitutes of itself the whole fungus: this only grows in size afterwards, the substance and original form remaining the same through the entire period of development. In those destined to live under the influence of air and light, this same rudimental nucleus gradually evolves new parts, and assumes a vast variety of forms (whereof each particular one is predetermined by the original bias imprinted upon every spore at its creation); and here there is a manifest analogy with the progressive development of new parts in the higher orders of plants. In such funguses as are wrapped up in a volva or bag during the earliest period of growth, this furnishes them not only with the means of protection, but of nourishment also. 'This volva, which is formed by the mere swelling out of the original fleshy bulb, when it has grown to a certain size, exhibits towards its centre the rudiments of the young fungus, of which the receptacle appears first, and all the other parts in succession. The embryo next taking to grow in its turn approaches the circumference of the volva, which, having by this time ceased to expand, is burst open, and sometimes with much violence, by the emerging amanite. As soon as the hymenium (or gills) has parted with its seed, which falls from it in the form of fine- dust, the fungus, collapsing, either withers on its stem, or else dissolves into a black liquid, and so escapes to the earth." This fine dust, whether it be regarded as seeds, germs, or sporules, is undoubtedly the means by which reproduction is effected. Little attention has hitherto been paid to the production of mushrooms directly from seed, although the circumstance of many sorts appearing, year after year, in nearly the same spot, would lead us to suppose that this is owing to the seed of the previous year's crop being shed, and retaining its vitality until the natural season of its again reappearing. The investigations made by Messrs Dutrochet and Turpin, in tracing the cause of the reappearance of the agaric in the familiar phenomena known as fairy-rings, evidently strengthen such an opinion; and we doubt not that, were even artificial mushroom-beds kept in a fitting state as to humidity and temperature, and a sufficient number of mushrooms allowed to come to full seed-ripening maturity, succeeding crops would be obtained for years by the same means, and on the same beds.

Nicol, writing practically on this subject, observes-"The cultivation of mushrooms is a process in gardening perhaps the most singular and curious of any. In the culture of any other vegetable, we either sow or plant something material-a seed, slip, or root, which we both see and handle; but, in the cultivation of the mushroom, we neither sow nor plant anything visible, at least to the naked eye. Yet it is certain that mushrooms are produced from seeds which naturally vegetate in the fields at certain seasons, and which may be made to vegetate artificially, at any season, by a certain process, and by a composition in which the dungs of certain animals form the chief ingredient.

"The droppings of horses are found to produce mushrooms more plentifully and with greater certainty than the dung of other animals," and the higher fed they are the more certain is the result; for it is notorious that the droppings of these animals while at grass, or when fed on green food, do not produce mushrooms to the same extent. Next to the horse, the droppings of the deer and sheep are found most suitable, probably on account of the seed of the fungus being less affected by moisture while passing through their intestines, than they would be in passing through those of the cow ; or, as probably, that they retain their vegetative powers better in the dry droppings of the former animals, after being voided, than they do in the moister ones of the latter. Or it may be that the seed passes through the stomachs of the former animals with less injury to their vegetative quality than through those of the latter, for assuredly all of them alike take into their stomachs the seeds in equal abundance along with their natural food. "The food of horses," as Nicol has suspected, " consisting mostly of corm and hay, may no doubt be more replete with the seeds of mushrooms than that of cows or other stock, which consists chiefly of green vegetables; but even the droppings of horses, when on grass or on tares, produce few or no mushrooms. This fact would seem to prove that either the seeds are collected in great numbers, and are better preserved by hay, or the straw and chaff of oats, than by green food; or that green food may have the effect of destroying them by its moistness in the stomach, or after passing through it." This appears to us to be the true rationale of the question, as the seeds of fungi will retain their vegetative power for months-nay, perhaps, for many years-if kept dry, as practically demonstrated by the care we take in keeping the spawn dry after it has been produced, and also that it is only in dry dunghills, or in dry sheds or places under cover, that we find it most abundant in a natural state. "It may further be observed," Nicol continues, "that animal matter seems necessary to the vegetation of those seeds, or the spawn of mushrooms. Hence, we find them produced plentifully in old pastures, and in cattle-sheds, whether these be frequented by horses, cows, sheep, or by all of them. But the common mushroom, Agaricus 
campestris, is never found in woods or fields from which cattle are completely excluded."

The production of spawn will be understood from the above;-it is to be looked for in a natural state during September in places where mushrooms are found indigenous, in the track of a mill worked by horses under cover, in ridinghouses, or sheds where horses are wintered or have long had access to, and in places where horse-droppings have accumulated and been kept dry. It is also sometimes found in half-decayed dung-hills, hot-beds, \&c.; but never in either of these, if very damp or saturated with water.

Preserving natural spawn.-The pieces should be retained as large as possible, and placed loosely in shallow hampers, or, if quite dry, in boxes kept in a dry place-here it will remain inactive for two or three years, or until it be required for spawning prepared beds; or broken into small pieces, and transplanted into properly prepared bricks or cakes - in which state again it may be kept for as long a period, or even for a greater length of time, if kept perfectly dry and in a moderate temperature.

Propagating or transplanting spawn.-For this purpose, atalmostanyseason of the year, but more especially from the end of May to the end of September, at which period the spawn is naturally most active, a quantity of dry horse-droppings, sheep or deer dung, should be collected, and placed under cover; to this a little finely-chopped fern fronds or dry littering dung should be added; and to this, when well mixed together, add as much rich mellow loam as will make the whole mass, after being wetted sufficiently and thoroughly incorporated, assume the consistence of thick mortar or grafting clay. The use of the fernfronds, or short litter, is to hold the material together; and of the loam, to give the bricks or cakes greater consistency. When the whole is well beat up together, spread it out upon a smooth level floor to the thickness of about 3 or 4 inches. When partially dried, cut it into pieces with a sharp spade in form of bricks 9 or 10 inches long, and 5 or 6 inches in breadth. Leave it in this condition for a few days to dry, so that the bricks may be handled without breaking; turn them over daily, still further to add to their solidity; and when nearly dry, perforate their upper edges with holes 1 inch in diameter, and place in each a piece of spawn about as large as a walnut, and cover the orifice of the holes with a little of the prepared mixture, to prevent the spawn from falling out. Then build the bricks or cakes in an open manner, as brickmakers do in drying bricks, and cover the whole with a coating of dry stable-manure in a slight state of fermentation, to cause the spawn to run through the bricks. Where there is the convenience of a dry warm place, or if space can be spared in the mushroom-house, this operation may be advantageously carried on in it, keeping the temperature at from $55^{\circ}$ to $60^{\circ}$. It will be readily ascertained when spawn has run through every part of the bricks, by breaking one or two through the middle, when, if in a good state, the spawn will appear like a fine white mould throughout the brick. They should then be laid by in a dry place till required, either for spawning the beds or for breaking down for future propagation. Various modes have been practised for propagating spawn, both by transplanting it into bricks and causing it to run in large massesthe materials used for the most part being the droppings of horses, cows, deer, and sheep, either alone or incorporated with loamy soil. Some, however, have added pigeons' dung, leaf-mould, rotten tan, and others coal-ashes : the advantage arising from any of which it is difficult to comprehend, more especially of the latter. The best materials, there can be no doubt, are the droppings of horses, deer, or sheep; and all or either of these will form a proper nidus for the germination, if we may so call it, of the seeds of the mushroom, for in each of these the spawn is naturally found: But as all of these require some adhesive matter to cement them together, none is better than an argillaceous loam: this, however, should not be used in too large a proportion, for we have invariably found the spawn to run best when the nidus prepared for it is not too hard or compact.

Procuring spawn artificially.-Early in March, while the cattle are on dry food, procure two parts of cow-dung, one of horse-droppings, and one of sheep or deer's dung. Dry them under cover, and break the whole down, so that it may pass through a half-inch sieve; mix the whole together, and lay the mass up in a conical heap. In forming the heap, tread or beat it closely together, which will prevent too rapid a state of fermentation. The heat during the process should not exceed $55^{\circ}$ or $60^{\circ}$-for the mushroom, in all its stages, is equally impatient of excess of heat and cold. If the process be carried on in a warm shed, possibly no covering need be applied, beyond that of an old mat or a little dry litter; if otherwise, a slight covering of warm littering dung should be laid over it, to promote and continue the necessary temperature above stated. In about four weeks the mass should be examined, to ascertain if the spawn has begun to run. This will be indicated by its appearance in the shape of white threadylike matter abounding in it. If this should appear not to be the case, then an additional covering of warm dung should be laid over it, to increase or revive the heat, which will, if the materials have been got together in proper condition, stimulate the spawn into one mass, ready for use. Sometimes the end is effected in three or four weeks, while at other times it will require double that time. Spawn thus produced is of excellent quality, but it will not keep so long as that made in the form of bricks.

Some spawn manufacturers, instead of planting the pieces in perforations made in the bricks, lay a course of them flat on the floor of a warm vinery, and sprinkle over them the-fragments of other spawn-bricks, broken down on purpose; and on this another course of bricks; and so on alternately, until the whole is built into a solid mass. - In a few weeks the spawn penetrates the whole mass, and may then be taken to pieces, and the bricks stored by in a dry place, where they will keep for years.

Producing mushrooms without transplanting spawn.-This is effected by making the bed a 
whole mass of spawn at once; and although by this means the crop is not always so soon produced, it has the merit of continuing to bear abundant crops for a much longer period than that of beds made in the ordinary way. Nicol, an old but excellent authority, followed this practice, and says the difference of time between beds coming into bearing made in this way, compared to the usual manner, will be three or four weeks; but the advantage will be, that in the one the crop will only last a few weeks or months, while in the other be has had them producing all the year over, and sometimes two years. His mode of proceeding was, after laying a foundation " of ashes, stone chips, gravel, or brickbats, so as to keep the bed quite dry and free from under-damp, to lay over it a course of horse-droppings, 6 inches thick. These should be new from the stable, and must not be broken, and the drier the better. They may be collected every day, until the first layer be formed to the above thickness, but they must not be allowed to ferment or heat. In the whole process of making up, the bed should be as much exposed to the air as possible, and it should be carefully defended from wet, if out of doors. When this course is quite dry, and judged to be past a state of fermentation, cover it to the thickness of 2 inches with light dry earth-if sandy, so much the better. It is immaterial whether it be rich or not, the only use of earth here being for the spawn to run and mass in. Now lay another course of droppings, and earth over, as above, when past a state of fermentation; then a third course, which earth over in like manner. This finishes the bed, which will be a very strong and productive one, if properly managed afterwards. Observe that in forming the bed it be a little rounded, in order that the centre may not be more wet or moist than the sides. If it be made up against a wall in a cellar, stable, or shed, it should have a slope of a few inches from the back to the front, less or more, according to its breadth. I have sometimes," he says, "been contented with two courses, as above, instead of three, and often, when materials are scarce, have made them up slighter, thus three 4-inch courses of droppings, with 1 inch of earth between each, and a 2 -inch covering at top. Such a bed as this I have had produce for ten or twelve months together; but very much depends on the state of the materials, and on the care taken in making it up, also on the after management. The droppings of hard-fed horses only are useful. Those of horses on green food will of themselves produce few or no mushrooms. This $I$ have proved in more than one instance, and I have moreover found that the richer the keep of the horses, the more productive are their droppings. I have made up beds from farm-horses fed partly on hard and partly on green food, and from carriage or saddle horses fed entirely on corn and hay-treated them in the same way in every respect-and have found, not once, but always, those made from the latter the most productive."

In any of these ways an abundance of spawn may be procured, the production of which is the first step in mushroom culture. To those, however, who have not ample means and proper material at command, we would say, procure the spawn ready made from a respectable nurseryman. It varies in price from $5 \mathrm{~s}$. to $8 \mathrm{~s}$. per bushel. That made by the London spawn-manufacturers we have always found to be the best. The demand is such, that the making of spawn bricks for the supply of the London trade is now carried on by parties who do little or nothing else.

The formation of the beds. - The modes of this are as different as the production of the spawn. A mere recapitulation of them would tend to little practical advantage, for all are aiming at the same end, although they make use of very different, and often very opposite means. The essential points to be regarded are, proper material, good spawn, moderate temperature, uniformity of humidity, according to the state of the beds or crop-as while the spawn is running, and until it is desired that the crops shall appear, they can hardly be kept too dry. Afterwards they require a greater amount of moisture, and this, particularly during winter, applied in a tepid state. Darkness has, by some, been deemed essential; light is admitted freely by others, and of the propriety of so doing there can be but one reasonable opinion.

The London market-gardeners, who grow largely for the supply of the market (even to the extent, as computed by Mr Cuthill, of not less than ten acres being continually under this crop in the immediate vicinity of London alone), form their beds in ridges in the open air, and in manner following: "As the stable-manure is brought home from the London stables, the short material is shaken out of it, and the long stringy part is kept for the purpose of covering, as well as for forming the interior of the ridges. The manure is not allowed to heat before it is put into the beds, if that can be prevented-for previously heated material does not produce so fine mushrooms. The fresher the horse-dung is, the longer will the crop last; and every one who makes up beds with unheated droppings knows how superior they are to fermented manure. If this is properly attended to, it does not signify what kind of mould is used for surfacing the beds with. When the interior temperature of the ridges gets down to $80^{\circ}$, it is spawned with pieces about 2 inches square, placed about 1 foot apart. The bed is then moulded over 2 inches thick, pressed with the feet, and afterwards beaten with the back of a spade. It is then watered and beaten again with the spade, and smoothed down. The more the mould is pressed, the finer the crop is, and the more solid the texture of the mushroom. This and green unheated manure constitute the two grand secrets in mushroom-growing. Beds made up in this way want but little watering, and when they are watered, it ought to be with liquid manure. The ridges are covered with straw and mats. Such ridges as these are continually being made, and when well formed they last for two or three months in good bearing."

Many think mushrooms produced on ridges, in the open air, superior to those grown in houses; but we think, when sufficient light and air are admitted, that they are as good in the 
one way as the other. Of olle thing there can be little doubt, that in properly constructed houses, and under good management, the crop may be calculated upon with much greater certainty than when exposed to the vicissitudes of open-air culture, unless it may be during the months of July, August, and September, their natural season of growth. Beds formed in the open air should always be constructed of open littering material, as recommended above as being the London practice; for if otherwise, the spawn would be extremely liable to be destroyed by excess of moisture. To guard against this in properly constructed mushroom-houses (as figs. 660 to 670 , vol. i.), the shelving should (as recommended, p. 467, vol. i.) be perforated with abundance of holes, if formed of iron plates, slate, or pavement; and if of wood, the boarding should be in narrow slips, at least 1 inch apart. These perforations and openings not only secure the beds against the bad effects of damp-to which they would be exposed, if laid in either material without such precautionbut they afford an opportunity for the mushrooms to protrude through them, and often yield nearly as good a crop from the bottom of the bed as from the top.

The German method of forming mushroom-beds on shelves, in houses constructed for the purpose (vide fig. 660 , vol. i.), was introduced into this country, about 1814, by the late Mr Oldacre, and may be considered the basis on which most subsequent forms have been founded. Prior to that period they were in general formed in shape of ridges set on the floor of a covered shed, and in no way differing from those built in the open air, or in form of sloping banks resting against a wall-in both ways occupying a great space, in proportion to the surface they presented, for the production of the crop. The material used by that excellent cultivator was the droppings of horses shaken out of the litter before it had been exposed to fermentation or wet, retaining with the droppings about onefourth in quantity of the shortest portion of the litter; to which he added about one-fourth dry turfy loam, to consolidate the mass, when beaten together, into a more solid and compact body than if only droppings and litter alone were used. On the solidity of such beds much of their success depends, as will be sufficiently apparent when we consider that they seldom exceed 7 or 8 inches in thickness. When a sufficient quantity is collected and mixed, as above, to form the bed, it is spread over the bottom of the shelf, or on the surface of the floor of the house (the lowest bed being placed under the first tier of shelves, and rendered perfectly dry below), to the thickness of 3 inches of the prepared compost. This is beaten with a wooden mallet into a close compact body, and over it another layer of the same thickness, which is in like manner beaten closely down; and so on, until the required height of bed is attained, which is in general 7 inches, leaving the surface as smooth and even as possible. "Reducing the beds into a very solid body," Mr Oldacre remarks (in "Horticultural Transactions," vol. ii.), " is a most essential point, for without it you cannot expect success; and the thickness of these must be particularly attended to, for where there is a much greater body, the beds will be subjected to a strong fermentation, and will be prevented by evaporation from retaining that consistency in the dung which is absolutely necessary for the production of a good and plentiful crop. On the contrary, if a much less quantity be laid together, the heat and fermentation will be insufficient to prepare the beds for the nourishment of the spawn; but the assistance of both, to the extent prescribed, cements the materials together, which, in addition to beating, increases greatly their solidity. The proper vegetation of the spawn, and the consequent crop of mushrooms, depend entirely upon a moderate genial heat and fermentation, neither too strong nor too slight." As soon as the temperature in the beds reaches from $80^{\circ}$ to $90^{\circ}$ Fahr., "the beds are beaten a second time, to render them more solid, if possible. Then make holes with a dibber 3 inches in diameter, and 9 inches asunder, through the compost in every part of the beds. These holes will be a means of cooling them, and preventing that excess of heat from taking place which would produce rottenness, and render them unproductive. If the beds do not attain the heat required in four or five days after they are put together, add another layer of compost, 2 inches thick, which will probably increase the heat sufficiently; if not, a part of the bed should be taken away, and the remainder mixed with fresh horse-droppings, and wrought together in the same way as before, in order to produce the proper degree of heat. Beds made after this manner readily generate natural spawn in summer, and frequently in the winter months." The materials for beds made upon this principle ought to be tolerably dry before being put together, as dryness and solidity form its leading feature, and is in reality the chief difference between this method and that of Nicol, already alluded to, whose plan, otherwise excellent, is to some extent defective in this respect. In planting the spawn, Oldacre examines the beds three or four days after the holes have been made; and when their sides are found to be dry, and the heat on the decline, it is introduced by being crammed into the holes, either in whole pieces, or into smaller fragments, and the whole bed beaten down, which finishes the operation. Care must, however, be taken that the heat has not declined too much, and also that it be not too strong at the time, as in either case the spawn would suffer alike, although from opposite causes. "About a fortnight after the spawn has been introduced," he remarks, "examine the holes, and if the spawn has suffered any damage froin over-heat or too much moisture in the beds, introduce fresh spawn as before. On the contrary, if the spawn be found good, and vegetating freely in the compost, such beds, if required for immediate production, may be covered with mould, agreeable to the rules hereafter laid down; and the beds intended for succession should remain unearthed, in the summer, till three weeks or a month before you wish them to produce-and in the winter, a 
month or five weeks. If the spawn be introduced in hot weather, air must be admitted as freely as possible into the house, till the spawn has spread itself through the beds; for if the place be kept too close, the beds will become soft and spongy, and then the crop will be neither abundant nor of good quality." In earthing over his beds, Mr Oldacre recommends rich maiden earth, wherein its turf is well reduced, laid on to the thickness of 2 inches, and beat as solid and level as possible. Care is to be taken that the soil be not too wet, as in that case it might chill the bed and injure the spawn; nor must it be too dry, because, if so, it could not be beaten so solid as is to be desired.

The subsequent management of the beds, according to the German method, is maintaining a temperature within the house of from $50^{\circ}$ to $55^{\circ}$, and the exclusion of light. "In watering the beds extreme caution is necessary, as well in the mode of application, as in the temperature of the water, which should be nearly $80^{\circ}$ or $90^{\circ}$, , and very lightly sprinkled with a syringe or a small watering-pot, otherwise the mushrooms are sure to sustain damage. If cold water be used, and given plentifully at one time, it will not only destroy the existing crop, but the spawn also, and render the beds so treated of no further utility. If the beds have been suffered to become very dry, it is better to give them several light waterings than one heavy supply. In gathering the inushrooms great care should be taken not to disturb the small ones that invariably, with good management, surround the stems of those which are more early matured. The best method is to twist them up very gently in all instances where it can be done ; but where it becomes necessary to cut them, great care should be taken to divest the beds of the stems of those that are cut, as they would rot, to the great injury of those that surround them."

The absence of light in the cultivation of the mushroom has been considered by many an almost necessary condition in securing success, and hence beds are made in dark houses, caves, \&c. ; while those made in the open air are as carefully deprived of light, by coverings of straw or litter, \&c., as if they were placed in the dark catacombs of Paris, where abundance of mushrooms are grown for the Parisian markets. These, however, are far inferior in flavour, and we may also add in wholesomeness, to such as are grown in the open air, in our old grassy pastures, fully exposed to both light and air. "The common mushroom," Dr Lindley observes, " unlike many of its kindred, is by no means a lover of shade; so far from it, the best and most beautiful mushrooms are found in exposed fields, where they delight in a rich soil, retentive of a certain portion of moisture, but well drained. The points, then, to secure in their cultivation are circumstances as nearly as possible similar to those which concur in the seasons most favourable to their growth in the natural ground-an even temperature, a welldrained and generous, but not a rank soil, kept moist, but by no means wet; a free access to light; and such an exposure to air as is consis- tent with the retention of a proper degree of humidity in the soil, and of temperature. The seasons in which mushrooms are most abundant are those in which a warm but showery autumn succeeds a hot summer - a circumstance towards which attention should be paid by those who would experimentally follow out the sub. ject." These remarks have reference to "A New Method of Growing the Mushroom," by Mr John Hawkin-for the construction of whose mushroom-house, vide figs. 663,664 , vol. i. In a communication in the "Gardeners' Chronicle" for 1844 (p. 85l), Mr Hawkin thus describes his method of culture : "The principal requisites for the successful culture of the mushroom are heat, light, air, and a damp atmosphere. In the first place, with me the dung is collected fresh from the stables, particularly from horses that are fed on dry food, such as corn and hay. It is thrown into a shed to dry, a little before it is made into beds. My boxes (beds) are trellised at the bottom, which allows the heated air to penetrate through the mass of dung easily; a little hay or rough litter is spread over the bottom of the box, in order to prevent the dung from passing through the trellis-work; and every 2 or 3 inches of dung that is added is beaten hard with a wooden mallet, until the layers reach within $1 \frac{1}{2}$ inches of the top of the box. As soon as the heat of the dung falls to a proper temperature, I insert large pieces of spawn into the bed, at the distance of about 8 inches apart. I rarely make use of spawn less than twelve months old, and the less that it is broken I find that it produces the better crops. About a week or ten days afterwards, I finish off the beds with green turf, $1 \frac{1}{2}$ inches in thickness, making the beds in my boxes in all about 9 inches in depth. I beat down the turf very firmly with the back of a spade in finishing; afterwards I have no further trouble, except in paying attention to the fire, and in admitting fresh air as it may be required. The house is heated by open tanks (vide fig. 664, vol. i.) which run through the centre of it, and which return again into the boiler, giving out a sufficient quantity of moisture for the necessary development and growth of the mushroom. During night the grassy turf becomes copiously loaded with moisture; and should the following day prove fine, I never omit giving abundance of fresh air by the doorway. The temperature of the house ranges from $60^{\circ}$ to $65^{\circ}$ during the day, and at night it is frequently allowed to fall as low as temperate. The great advantage of growing mushrooms upon fresh grassy turf is obvious to any one accustomed to their cultivation." Mr Hawkin, in allother respect, deviates even still further from ordinary practice. "I have been," he says, "in the habit of growing mushrooms, and with great success, upon coal refuse for the last two years; and at present I have two boxes at work, one covered with coal-dust, and the other with turf. The produce of these shows the relative advantages of the two methods, for although those from the coal-dust are large and of good flavour, they are decidedly inferior, in both respects, to those produced by the grass-covered beds; indeed, such is the superiority of the latter, that 
if the mushrooms from both beds were gathered and mixed indiscriminately, any one could without difficulty select those grown upon the turf from those raised on the beds covered with the small coal." In a later communication Mr Hawkin observes: "In my former paper I did not mention the shape in which the greensward is best adapted for the free spread of the spawn. I have since discovered that the turf is best after being partially dried in a shed, taking care to preserve the vitality of the grass, which I consider very essential in the growth of mushrooms. In all my experience, I have never seen them purely white on the crown, or the gills as red as the finest field mushrooms, and fully equal to the latter in flavour, but by this process." Mr Hawkin concludes by observing that he is confident that light and air, where an equal temperature can be preserved, are essential to the growth of the mushroom, and that they do not blanch by the exclusion of either; if they did this, the gill would also turn white proportionably-a dark confined atmosphere will never produce a healthy full-grown mushroom. One great advantage attends this method-namely, the labour and inconvenience of covering and uncovering the beds are dispensed with. Covering the beds with turf instead of loamy soil is a much nearer approach to their natural condition; besides, it retains the declining heat in the beds better; and should that heat fail, so as to render a top-dressing of dung necessary to increase the temperature, it can be more conveniently effected than if the bed was earthed up in the usual manner.

To secure abundance of mushrooms during winter is a matter of great importance to most people. The writer of the forcing department of the calendar of operations in the "Gardeners' Chronicle," 1845 , p. 612 , has given the following very rational directions: "The best way to secure good and lasting crops of mushrooms through the autumn and up to Christmas, is to make the beds in good time, and to give them plenty of time that they may work slowly, depending more on the warmth of the atmosphere than upon fermentation in the manure. The natural warmth in the last fortnight in August and the first three weeks in September will guarantee the safe working of the spawn, provided a proper medium is procured. Three things are requisite in the dung, to insure success in getting the spawn to work well-viz., what is technically called "sweetness," an open texture, and a medium degree of moisture. It is a good plan to make the beds in the mushroom-house at twice-that is to say, if the shelf be 1 foot in depth, which is not too much for substantial beds, 8 inches might be made at any time in July and August, and the remaining 4 inches as wanted. In this case, get new droppings from the stable-door, with a deal of fresh litter amongst them; and add one-third of strong loam at least, beating the whole into a solid mass. For the surface of such beds, well-fermented dung alone should be used, only made at least two months before the mushrooms are required, giving it plenty of time, without the hurry of fermentation, to establish itself all through the mass. It should be borne in mind that depth in the bed, and loam mixed with the dung, tend at all times to render the bed more durable. Remember that mushrooms are as fond of abundance of air and light as other plants. They grow most in the fields at night, not because they hate light, but because they can enjoy abundance of atmospheric moisture, which is so quickly dispersed by the returning day. Let, then, abundance of atmospheric moisture be secured at all times, as also an unfluctuating temperature of $55^{\circ}$ or $60^{\circ}$, and no fear need be entertained of injury from light or air, either day or night."

The practice of growing mushrooms in more light and air than was the practice formerly, is now greatly gaining ground; and as the principle is founded on corlect data, we cannot recommend it too strongly to our readers.

Besides producing mushrooms on beds made in the open air, as practised by the London market-gardeners, or in regular mushroomhouses, as is usually done by private gardeners, other means are often had recourse to, such as growing them in boxes, or even large flowerpots, placed in a proper temperature in warm cellars, offices, and spare places in hot-houses. Spawn is also often introduced into melon-pits and frames, as soon as the strong heat of the fermentation in the beds has subsided. The mushroom spawn, when broken into smallish pieces, is laid upon the soil which forms the hills on which the plants are set, and also over the surface of the bed. The earthing up and usual routine are followed, as regards the melon plants, the same as if no spawn was planted. In September or October, when the melon crop is finished, the haulm is removed, the glass lights kept on, and, as little water has been applied during the ripening of the melons, the spawn is in consequence encouraged to run over the whole surface. When this is found to be the case, gentle waterings with tepid water are given, and mushrooms are produced in great abundance. Should the weather become cold, linings of hot dung are applied, which warm the beds, and stimulate the spawn into renewed vigour. No darkened covering is put over the beds; while the spawn continues in a healthy state they receive no other covering than the glass, thus exposing them fully to the light : but if cold weather ensue, the glass is covered during night in the usual way. By this means the Rev. W. Williamson (who has detailed his practice in the 5th vol. of the "Horticultural Society's Transactions," p. 3) informs us he has gathered " 2 bushels from a frame 10 feet by 6 feet, at one gathering, some of the mushrooms weighing $2 \mathrm{lb}$. each. The mould being kept warm by the glasses, and properly watered, the mushrooms will continue to spring till the frost of winter prevents their further growth. I then," he continues, "leave the bed, frame, \&c., just as they are; and early in spring, as soon as the frost may be supposed to be over, I take off the frame and glasses, and cover the bed lightly with straw; when the warm enlivening showers of spring cause the mushrooms to be again produced in every part, till the drought of summer 
renders it difficult to keep the bed sufficiently moist for their growth. Sometimes I suffer the bed to remain, in order to produce a crop in the second autumn, but more generally take the bed to pieces for the sake of the dung, and also for the purpose of procuring and drying the spawn against the return of spring." Abundant crops of fine mushrooms are often produced by forming a small trench in the open garden, and filling it with the remains of an exhausted mushroom-bed, which is covered to the depth of 2 inches with soil; and on these are planted broccoli, cauliflower, \&c. The spawn often runs freely during the months of June, July, and August; and, being sheltered from too much rain by the leaves of the plants, mushrooms are produced throughout the autumn in vast quantities and with little trouble.

The cultivation of the mushroom in stables has long been practised by the Baron Joseph d'Hoogvorst, of Limnal, near Brussels, for whose arrangements see fig. 667 , vol. i. The beds are prepared much in the usual way of the droppings of high-fed horses, and beaten down in the boxes. One peculiarity, however, in the Baron's practice, and on which he places great importance, is the introduction of a small proportion of sal-ammoniac into his beds, a practice we have nowhere else seen adopted. The leading principles in successful mushroom culture may be comprised under the following heads: Procure proper material, such as highfed horse droppings; keep them moderately dry; never allow them to come to a high state of fermentation; keep the beds in an atmospheric temperature of from $55^{\circ}$ to $65^{\circ}$, and while the spawn is running, the temperature in the bed should not exceed those limits above from $5^{\circ}$ to $7^{\circ}$; plant the spawn when the beds are at a temperature of from $65^{\circ}$ to $75^{\circ}$; keep them dry while the spawn is running; cover, when it has sufficiently run, to the depth of 3 inches, with sound loamy soil, beat hard down; water, when the crop is wished to appear, and as may be necessary afterwards, with water at a temperature a few degrees above that of the air in the house ; admit abundance of light; moderate ventilation; never cover the beds with hay, straw, or litter; make up the beds in March, June, July, August, and September ;'apply water as nearly in the form of dew as possible; heavy waterings are injurious.

No doubt many of the edible fungi might be as successfully grown as the common mushroom, if sufficient attention were paid to their habits.

The insects most injurious to the mushroom are slugs, snails, and wood-lice. The presence of the two former pretty clearly indicates that the surface of the bed is rather too damp. They must be captured at night, by examining the beds with a candle. The latter is easily entrapped, as it seeks shelter during the day under pieces of slate, or any other similar thing laid on the floors or bed.

The European names for the mushroom areChampignon comestible in French; Hampernoclje in Dutch; Essbare blatterschamme in German; Pratajuolo in Italian; Seta in Spanish.

\section{§ 2. -THE MOREL.}

The Morel (Morchella esculenta Pers.), fig. 97, belongs to the same natural order, class, and order

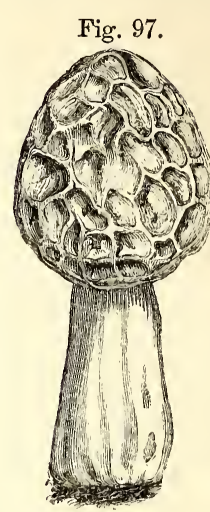

THE MOREL. in the Linnæan arrangement, as the last; it is a native of Britain, found in wet banks and woods, and in moist pastures; it is pretty abundant some seasons in Dalkeith Park, appearing in April, May, and June. In some parts of Dumfriesshire, as about Langholm, it is also abundant, but by no means a common fungus. It is used for the same purposes as the truffle, and if gathered when dry, may be kept for several months. Its cultivation has not, so far as we are aware of, been attempted; of its capability of submitting to culture we think there can be little doubt. If the spawn were collected from its natural habitats in June, and planted in beds differently formed, but approximating as nearly as possible to its natural conditions, a proper mode of cultivation would assuredly be in time arrived at. Lightfoot, the author of the first "Flora Scottica," says he reared the morchella from seed. The great value of the morel, which is one of the most expensive luxuries furnished by our Italian warehouses, and which is by no means met with in the same abundance as some others of the fungi, deserves to be better known than it is at present. The genus Morchella comprises very few species, and they are all good to eat: of these, according to Dr Badham, $M$. esculenta and $M$. semilibera only are indigenous to Britain. The "Hortus Britannicus," however, enumerates three species, viz. M. esculenta, $p a$ tula, and hybrida; as well as two varieties of the former, rotunda and vulgaris. Dr Badham remarks, "It is less generally known that this fungus, though by no means so common with us as some. others (a circumstance partly attributable to the prevailing ignorance as to when and where to look for it, or even of its being indigenous to Britain); occurs not unfrequently in orchards and woods towards the beginning of summer ;" and Persoon remarks that it prefers a chalky or argillaceous soil to one of a sandy nature; and adds that it not unfrequently springs up where charcoal has been burnt, or where cinders have been thrown. It is singular that, while the morel is held in such high estimation in Britain, it is little cared for in the Roman markets; and, as an anomalous case, we may mention, on the authority of Badham, that the common mushroom, Agaricus campestris, considered by most people in Britain as the only wholesome fungus, is " almost the only one condemned to be thrown into the Tiber by the inspector of the fungus market : indeed, in such dread is this held in the Papal States, that no one knowingly would touch it." 


\section{$\S$ 3.-THE TRUFFLE.}

The truffle (T'uber cibarium, common truffle, Sibthorp, fig.98; and Tuber albidum, white truffle,

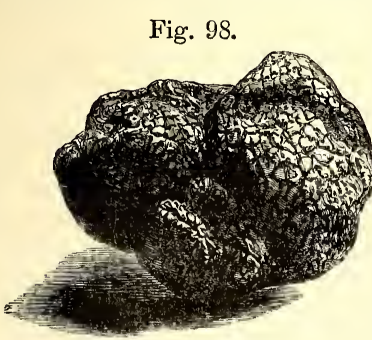

Cæs.) belongs to the same class and order as the last, and, like it, is exceedingly prized in the higher departments of cookery. They are indigenous to Britain, being found growing subterraneous-

THE TRUFFLE.

ly in the southern parts of England. The supply for the market is obtained from the trufflehunters, who teach dogs to scent them out, which they do with great sagacity, either pointing to the spot, or scratching the ground over where they are growing, when the truffle-hunter digs them up with a spade, rewarding his companion with a small piece of cheese. The truffle is of very irregular form, inclining to globular, without root or stem. The first-named variety (Tuber cibarium) is of a dirty black colour, the second ( $\boldsymbol{T}$ uber albidum) inclining to white, and both are very uneven and rough on the surface. Besides these, there are imported $T u$ ber magnatum, the Piedmontese truffle, and Tuber brochii, from Italy. The following British fungi are often substituted for the true truffle, viz. Hydrobolites tulasnei, found in Spyke Park, Wilts, and Melagonaster broomeianus, or red truffle, found in the neighbourhood of Bath. The truffle has never been satisfactorily produced artificially in Britain, though Bradley, writing in 1726, speaks of its cultivation as an easy matter. The Prussian gardeners have, however, grown it to some extent, and the Comte de Broch has done the same in Italy. The sort grown by de Broch is the Tuber magnatum, or Piedmontese truffle, and his modus operandi is as follows: In preparing an artificial soil he employs seven parts of garden-soil, two of well-pulverised clayey soil, and one of oak sawdust, thoroughly incorporated together. He also employs the soil where the truffle grows naturally, and this he trenches to the depth of 2 feet, picking out all the large stones, and adding oak sawdust, if necessary, and about one-tenth powdered shells if the soil is too stiff, for the truffle affects a calcareous soil. In preparing his bed, he selects a situation having a northern exposure, and thoroughly soaks his prepared compost with rain-water. After a day or two, when the bed is in a proper state of moisture, he makes holes 6 inches apart in it, in each of which he plants sound and good truffles, surrounding each with two or three handfuls of oak sawdust, covering the rows with a sufficiency of soil to prevent the truffles from being exposed to too much moisture. In this state the bed is left till the following autumn, keeping it in a state neither too wet nor. too dry. The truffles began to appear in October, and continued producing till VOL. II.
January. From the Comte's practice little practical information can be drawn, further than that truffles were planted in a situation and in a medium favourable for the seed which was contained in the planted tubers regetating at its natural season-a circumstance we think more simply accomplished, and with probably as much success, by the peasant truffle-hunter in our own country, who deposits again in the soil all very small and decaying truffles, unfit for market, in the spot from whence he dug them, or trans. plants them to situations where none grew before, choosing, however, a similar soil, and in general under shadow of a tree of the same species under which they are usually found. The oak and the beech appear to be the most favourable for the purpose. This would lead us to conclude that the truffle prefers a rather dry locality. Bradley's recommendation is, "that the truffle may be easily cultivated where there are woods or coppices of oak or hazel, and where the soil is not too stiff, or inclining to chalk. The soil where they are most found is a reddish sandy loam ; this, then, will be the best for our purpose, especially if it has lain long uncultivated. The soil should remain undisturbed till the months of October, November, or December, which is the proper season for planting, if the weather be open, for then the truffles are to be found in their full ripening; and then, likewise, one may find them in a state of putrefaction, which is the time when the seeds are prepared for vegetation. It is in the last state that we ought to gather truffles for planting, or at least they should be in perfect ripeness." He proceeds to plant as follows: "Open a spot of ground, of a convenient space, and take out the earth about 8 inches deep, and screen it, that it may be as fine as possible ; then lay about 2 or 3 inches thick of this fine earth at the bottom of the trench, and upon it lay some of the overripe truffles, about a foot and a half distance from one another; and as soon as possible prepare a thin mud, made of the screened earth and water, well stirred and mixed together, and pour it on the truffles till the open ground is quite filled up. By this means, in a few hours, the ground will be as closely settled about the truffles as if it had never been dug or disturbed at all, and you may expect a good crop in due time. You must, however, take care to choose your spots of ground in woods or coppices, or such places as are shaded with trees." As regards information on the culture of the truffle, we are in much the same state as were our forefathers ages ago, when it was gravely recommended to them by some of our earliest authorities, to collect the young mushrooms wherever they were found growing naturally, and, on taking them up, to transplant them on dungbeds in their gardens; or, as others recommended, to take full-grown mushrooms, and break them down in water, and water the beds with the infusion. To succeed in the culture of the morel or truffle, we should assuredly follow the course practised with the common mushroom, namely, secure and increase a supply of seed, preserve it in shape of spawn, and transplant it, in that state, into prepared 
beds, as nearly as possible resembling that in which they are found to exist naturally. Succeeding in this, more artificial means might successfully be adopted afterwards.

Little has been written on the cultivation of this valuable fungus in Britain. We believe the only works in English that treat on truffle culture are the translations, by Mr Francis Mascall, of M. Alex. de Bornholz's " Treatise on the Cultivation of Truffles," and that of Von V. F. Fisher, entitled "Instructions in Truffle-hunting." The essence of both will be found in the 13th volume of the "Gardeners' Magazine," p. 389-408, and is well worth the perusal of the English reader. The observations of Bradley, quoted above, will be found in his " Physical Observations on Gardening." No less than eleven treatises on this curious subject have appeared in France and Germany, the best of which is the article Truffle, by Drs Lippold and Funke, in " Lexicon of Nature and Art," part 3, and the splendid "History and Monograph of Truffles," by Dr Tulasne, Paris, 1851; and the plans proposed for their cultivation by Von Justi and Bulliard are worth consulting. They all agree that a soil should be chosen for the artificial cultivation of this fungi, as similar as possible to that in which truffles grow naturally; that it should be trenched to the depth of 2 or 3 feet; and that either pieces of the earth in which truffles grow naturally, or single truffles, fully grown, should be planted in it. Dr Klotzsch of Berlin recommends taking such truffles as are over-ripe, and nearly in a state of decomposition, breaking them in pieces, and placing them 2 or 3 inches deep in the soil, " in rather raised flat places," under coppices or underwood, and in a sheltered situation. No one appears to have tried its cultivation in a regular mushroom-house, although it at once presents many of the apparent essentials sought for by these authorities, under the shade of trees, coppices, \&c.; and we doubt not if those who live in the localities where they naturally abound were to collect the truffles when beginning to decay, at which time their seeds are fully matured, and to transfer them to a bed of earth composed of material similar to that in which they naturally grow, abundance of truffle-spawn would be secured, and might be multiplied as readily as that of the common mushroom : or why not gollect the truffle-spawn, and transfer it to artificial beds, as is often done in the case of the mushroom? The truffle being little known to cultivators generally, the following description by Mascall, in addition to our fig. 98, may enable them to detect it with certainty: "Of a round form, more or less approaching to that of a sphere, or of an egg, or sometimes kidney-shaped (for they assume all these forms), and somewhat rough with protuberances. The colour of the surface is, when it is young, whitish, but in those that are fullgrown it is either blackish or a deep black. The colour of the inside is whitish, with darkblue and. white, grey, reddish, light-brown, or dark-brown veins, of the thickness of a horsehair, which are usually variously entangled, and which form a kind of net-work or mat. Between the veins are numerous cavities, filled with a great deal of mucilage and small solid grains. These scarcely visible glands were formerly said to be the seeds or germs of the young truffles. The less the inside of the truffle is coloured with dark veins, the more tender and delicious is its flesh. The blackish external rind is hard and very rough, by means of fine fissures, grains, and protuberances, and forms, with its small facets, which are almost hexagonal, an appearance by which it somewhat resembles the firapples of the larch. Whilst the truffle is young, its smell resembles that of putrid plants, or of moist vegetable earth. When it first approaches the point of time at which it has attained its full growth, it diffuses an agreeable smell, which is peculiar to it, resembling that of musk, which lasts only a few days: it then becomes stronger, and the nearer the fungus is to its dissolution, which speedily ensues, so much the more unpleasant and urinous is the smell, till at last it is quite disagreeable and putrid. Whilst young, the flesh is watery, and its taste insipid: when fully formed, its firm flesh, which is like the kernel of the almond and the nut, has an extremely aromatic and delicious taste; but as soon as the fungus begins to decay, and worms and putrescence to attack it, its taste is bitter and disagreeable."

Wherever truffles are produced, they are to be found the whole year through, from the beginning of spring till late in autumn, but in the greatest plenty from towards the end of the month of August to the latter end of October. They thrive extremely, like all fungi, in warm moist autumns, and are then most delicious. After warm continuous showers, they are found nearer the surface of the soil, sometimes so high that they form little hemispherical mounds of earth, in which small clefts are produced by the sun's rays. If the soil is loose, and dry weather succeeds, the earth which was raised up falls down, and the truffle is seen half uncovered.

The favourite habitat of truffles is a somewhat moist, light, wood soil, which is defended from the immediate effect of the burning rays of the sun by large oak trees standing at a distance from each other, but is not deprived, by thick bushes, of the free access of the currents of air. Where, in woods, there are places bare of timber trees, and with but few bushes, or covered with pollard wood that does not stand thick, they thrive under an oak, beech, white thorn, and even under a fruit tree, and sometimes attain the weight of from a pound to a pound and a half. This unusual size, however, is only met with in moist warm grounds; here they lie nearer the surface of the soil. The drier the soil is, the deeper they are produced in it, but are usually so much the smaller: to this the vicinity of springs is the sole exception.

Truffles vary very much in size, form, and degree of roughness on the surface. Of all varieties those of a whitish colour are most highly prized, and hence the Piedmontese is the most in esteem. Truffles, being destitute of roots, draw their nourishment from the soil by absorbent vessels which cover their whole surface, and these vessels are in general in the form of small warts; and therefore the soil which surrounds 
them should be kept in a uniform degrce of moisture, for, if deprived of this, they soon wither away. On the other hand, if subjected to too great a degree of humidity, mould and other parasitic plants establish themselves on the surface of the tubers, and by degrees obstruct the absorbing vessels, causing putrefaction to ensue, and its concomitant attendants, insects, which prey upon the tubers, and ultimately destroy them altogether. The first cmbryo of the truffle is exceedingly small and tender, as is the case with all fungi ; and at that stage it is susceptible of injury, both from excess of drought and moisture. Their form is regulated much by the openness or looseness of the soil around them, and hence the necessity of the preparations by deep-trenching and sifting, as above alluded to. "If the soil is everywhere equally loose, the truffle assumes a globular form; but this is changed if there is on one side a greater opposition than on the other : hence the different forms of the tuber may be explained." As a rule in regard to their artificial cultivation, in connection with the state of the beds, we may, in quoting from the translation of Alex. von Bornholz's treatise, observe, "Where the soil is most moist, whether above or below the truffle, there it will either rise up or sink deeper. The degree of power of attraction of the moisture in the earth to that in the truffle, necessitates such a change of place. In moist summers and in wet winters we find the truffles near the surface, even projecting above it. In dry summers, upon poor dry places in woods, they have often to be dug from a depth of more than half a foot. Here is imposed upon them a greater pressure of superincumbent soil. If the earth is not very light, they cannot be fully developed, and therefore remain small. The largest are, consequently, in general, found not deep under the earth, and in shaded light soil-that is, somewhat moist; they are not, however, so well tasted as those of middling size. Vegetable mould from oak leaves and decayed oak appears to contribute to the production and growth of truffles, as advantageously as the dung of horses does to that of mushrooms. In the one case, a peculiar animal matter produces the effect; in the other, a peculiar vegetable matter. Perhaps the tannin, or some other yet undiscovered product of the oak tree, may be the principal cause. The more of this matter any species of tree contains, with the greater facility are truffles produced in its vicinity, and in greater numbers." In the formation of truffle beds, it would appear that they should be of considerable depth, say from 18 inches to 3 feet; the soil finely sifted, and composed of vegetable mould of oak-tree leaves, decayed oak sawdust, or probably half-decayed tanners' bark, as each contains traces of the tannin principle. Calcareous or ferruginous earth should be also employed; and the temperature, shade, and darkness of an ordinary mushroom-house form the fitting situation. In regard to soil, Bornbolz observes, that the greatest number of truffles, and the fincst, arc found in a light ferruginous calcareous soil. Such a one must, therefore, also be given to the plantations or beds. The soil is found naturally only in particular situations, for which reason truffles do not grow everywhere. The spawn of truffles appears to be extremely delicatc, and almost incapable, at least in its nat tural state, of being transported to any great distance; but this might be overcome, if those in whose vicinity truffles abound were to collect it and form it into spawn-bricks like that of mushrooms. Bornholz, in his treatise, confines himself entircly to their cultivation in woods and forests; all, therefore, that we can glean from his curious production is the medium suitable to the production and growth of this fungus in little other than a natural state.

From the review of Dr Tulasne's "History and Monograph of Truffles," in the "Gardeners Chronicle," 1852, p. 519, we learn that truffles and allied fungi are widely distributed over the globe. Some extend as far north as Upsal, while others are found to occur in Australia. "The black esculent truffles are by nature gregarious : they are found every year in the same spots. They occur exclusively in calcareous or calcareous and argillaceous soils, both on plains and declivities, and generally on ground more or less shaded by trees, but, as it appears, never on ground planted exclusively with conifers. Much has been written on the cultivation of truftles but, at present, experiments made in confirmation of published statements have not been at tended with success. The only fact which is certain is, that their production can easily be effected in certain calcareous soils, even where they may not have been observed before. The plan consists simply in sowing acorns on the soil, and when the oaks have attained the age of ten or twelve years, truffles begin to appear. The extensive truffle layers of Loudon at Vienna were actually produced in this manner. They consisted a few years since of naked uncultivated plains, where truffles were unknown. The plantations must not be too thick. They are cut down for the first time when they are about twelve years' growth, and then at intervals of from seven to nine years. They continue fertile for about twenty-five or thirty years, when at length scarcely any truffles are produced. From the facility with which the mycelium, or spawn, is developed in specimens which have been brought from a distance, Dr Tulasne conceives that it might not be impossible to trans plant the more valuable species into truffle layers where the more common sorts only occur. Their presence appears in general to depend more on the mineralogical charactcr of the soil than on particular temperature, limestone districts being certainly most favourable to their growth."

The European names are-Truffel in German; Tartufo nero in Italian; Truffe in French; and Aardnoot in Dutch. 


\title{
CHAPTER XI.
}

\author{
MISCELLANEOUS VEGETABLES.
}

\section{$\S$ 1.-RHUBARB.}

Natural history. - Rhubarb (Rheum Linn.) belongs to the natural order Polygoneæ, class Ennandria and order Trigynia in the Linnæan arrangement. There are fourteen species of rhubarb, all of which are cultivated either for culinary or medicinal purposes; none of them are indigenous to Britain. Rheum raponticum, a native of Asia, was the earliest introduced species, its date of introduction being 1573. The generic name Rheun is derived from $R h a$, the Wolga, from its being first brought from the banks of that river. It was well known to the ancient Greeks, who called it Rhabarbarum, from its being brought from the above river. The use of its roots for medical purposes is of the greatest antiquity, and we have authentic accounts of its being so used prior to the birth of Christ, as Dioscorides, who was physician to Antony and Cleopatra, wrote on its qualities, and recommended it for use. Paulus Egineta, who is said to have been the first who practised as an accoucheur, was also the first to use rhubarb as an opening medicine. The root has long been an important article of commerce, and was formerly brought from China, by Tartary, to Aleppo, Damascus, and Alexandria, and from thence it was sent to Vienna, and long maintained the distinction of Levant and Turkey rhubarb. In the time of Gerard, the leaves were used as a pot-herb, and considered superior to spinach or beet. The London Society of Arts, in 1792 and 1793, awarded a gold medal to Sir W. Fordice and $\mathrm{Mr}$ Jones for rearing rhubarb from seed; and in 1794 a like reward was adjudged to $\mathrm{Mr}$ Hayward for propagating it by offsets instead of seeds, for the purpose of bringing it to perfection in a shorter time.

$U$ se.-Theleaf-stalks of rhubarb have been long used in many parts of England in tarts and pies; in the manner of apples, they are also stewed alone or with rice, made into jellies and other preserves; and when fully grown, the expressed juice is made into an excellent wine. As an article of commercial importance in the London vegetable markets, it is of very recent date. About 1810 Mr Joseph Myatt of Deptford, long known for his successful culture of this plant, sent his two sons to the borough market with five bunches of rhubarb stalks, of which they could only sell three. The same cultivator now sends a waggon-load or two twice a-week to the London markets, besides many other growers, who supply the metropolitan markets with many tons of stalks weekly during the season.

Propagation, \&c.-All the varieties of rhubarb are reared from seed, which is the best way, but very generally they are increased by dividing the roots, retaining a bud or crown to each section. Whether originated from seed or increased by division, a deep rich soil is required to insure the full development of the foot-stalks, for upon their size, rapidity of growth, and consequently tenderness of fibre, much of their merit depends. The soil should be rich and mellow, but not damp, and should be trenched not less than 3 feet in depth. The seed should be sown in March, in drills a foot asunder, thinning the plants out, when a few inches high, to 9 inches apart. In autumn following they will be fit for transplanting in rows 3 feet asunder, and the plants set 3 feet apart. The same distance should be given to sets when propagation is effected by division of the roots. As, however, some of the sorts grow larger than others, a corresponding distance should be accorded them, extending to 5 feet between the rows, and 3 feet plant from plant. When manure is applied, it should be buried not less than from 2 to 3 feet, that the extremities of the roots may derive benefit therefrom. The plants should be set out singly, and not in threes, as is so often done. For the first year the ground between the rows may be cropped with lettuce, turnips, or similar low-growing crops; but after the second year the leaves will cover the whole space, and require it also for their full development. From the depth to which the roots extend, subsequent manurings will have little effect upon them; therefore, in preparing the ground for a new plantation, it should be enriched to the depth recommended above; and if a liberal supply of broken bones be incorporated with the manure, so much the better, as they consume slowly.

The after culture consists in keeping the ground clear of weeds, breaking over the flowerstalks as they appear, that their formation and seed-producing may not draw unnecessary supplies from the roots.

Gathering the crop.-During the second year 
after planting, a few of the largest and firstformed leaves, with their foot-stalks attached, may be gathered for use; but this gathering should not commence too early in the season, because in that casc the plants would be wcakened. From the third year, as long as the plantation lasts, it may be gathered with freedom. A plantation in a good soil, and not overmuch deprived of its foliage, will last from ten to fifteen years; but the former period is more consistent with good management, because plants under that agc will be more vigorous, and this will admit of a rotation taking place in the ground. When the leaves are about half expanded they may be taken off for use; but where the largest returns are expected, as in the case of market-gardens, they should be allowed to attain their full size. In removing them, they should be pulled off close to their base, and not cut, to prevent an unnecessary escape of sap, which in all succulent plants flows more copiously from a clean cut than from one slightly lacerated or torn; the foot-stalks should then be separatcd from the leaves, and tied up in bundles of from six to twelve in each, in which state they are fit for the kitchen. The London market-gardcners' practice of growing rhubarb is to manure the ground heavily; established plants are taken up and divided into as many pieces as they have eyes or buds; these are planted in March at 4 feet apart, and by autumn they will have produced roots from 6 to $12 \mathrm{lb}$. in weight. They also, in like manner, cut up the roots that have been taken up and forced during winter, and with them form new plantations in spring.

Forcing.-No plant is more easily forced than this, and in consequence it is had fit for use from the beginning of December until it comes into season in the natural ground. The London practice is to dig long pits, in any convenient part of the ground, to the depth of 2 or 3 feet, introducing 18 inches of hot dung. The roots, being taken carefully and entirely up, are set upon this dung closely packed together, having a sufficiency of mould worked in around them, to keep them excluded from the air. These pits are then hooped over and covered with mats, and over that 6 inches of straw. Wattled hurdles are also employed to place over them, and over these a covering of straw or dry litter in thickness more or less according to the state of the weather. $\mathrm{Mr}$ Cuthill remarks that in wet seasons rhubarb, when forced in the open ground, is deficient in flavour compared to what it is in dry and rather frosty seasons; and that the stalks are full of watery juices, which the roots takes up, and which the leaves cannot throw off or assimilate in consequence of being deprived of light and air, and that in such seasons the leaves often rot off' entirely. This has led some market-gardeners to adopt means of keeping the roots dry, and to this end they pack the roots in rather dry mould, and place them on the floors of long sheds, and cover the crowns to the depth of 2 feet with tree leaves. This is a much better method than forcing in the open air; but where such quantities are forced as are produced by the London cornmcrcial growers, an immense extent of shed room would be required. A much better way would be to place the roots upon a slight dung-bed, and to cover the whole with a portable roof constructed of boarding,laid in an imbricated manner, as shown at fig. 52, as used by us for protecting celcry. The predilection which the London growers have for covcrings of mats and straw has not one merit to recommend it, is sanctioned only by an antiquated and absurd prejudice of long standing, and entails a considerable expense in labour and material, which might be saved by a very limited amount of ingenuity. Forcing in private establishments is in most cases better done. Our own practice is to take up the first set of roots as soon as the leaves have died away, to remove them to the under beds of a mushroom-house, to a warm cellar, or to an apartment behind one of the pine-stoves, as already described under the article SEs-KaLE, p. 106; and those who have not these conveniences may set the roots on the floor of an early peachhouse or vinery, or even behind the flues or hotwater pipes, covering the roots with soil to exclude air and retain sufficient moisture around them, and to allow the leaves and foot-stalks to develop themselves in all the light such places afford. Some attach importance to growing rhubarb in the dark, with a view to blanch it. This process has no other effect than that of greatly deteriorating the flavour of the crop.

A temperature as low as that rccommended for sea-kale, p. 106, and similar treatment in every respect except that of blanching, is all that is required for producing rhubarb in its fullest perfection during the winter scason.

To sace seed.-One flower stem, if left on a single plant, will afford a sufficient quantity for maintaining a succession of young plants. In sects and diseases are unknown to the rhubarb.

Estimate of sorts.-The original species of Rheum undulatum, $R$. palmatum, R. Rhaponticum, \&c., have now nearly given place to hybrid varieties, possessing the merits of larger size, delicacy in texture, improvement in colour, or coming earlier into use: of these, the

Elford or Bucks early scarlet is, although a small-growing sort, esteemed for 'its brilliant scarlet colour, which it retains although forced in darkness.

Britannia rhubarb.-An early variety, and possessing the rather rare merit of scarcely ever throwing up flower-stcms. The leaf-stalks grow quite erect, and are both numerous and of large size, and of a beautiful dark pink colour.

Royal Albert.-Not so early as the last, nor so productive, on account of the energies of the roots being wasted in the support of the flowerstalks which it so readily sends up, and that in its earliest stage of growth.

Mitchell's grey eagle.-A new and excellent variety, originated with $\mathrm{Mr}$ Mitchell of Enfield.

Randall's early prolific. - Comparatively a new variety, of great merit and productiveness, as yet little known in the provinces; high coloured and well flavoured.

Myatt's Linnceus.-Of medium earliness, yielding large crops of leaves.

Myatt's Victoria.-The latest, with the excep- 
tion of $R$. Emodi, a sort seldom grown, and therefore valuable, as prolonging the season of this excellent vegetable.

Goliath. - Of medium earliness, and of large size. The European names for rhubarb are-Rhubarbe in French ; Ruibarbo in Spanish and Portuguese; Rabarber in German and Dutch ; Rabarbaro in Italian.

\section{$\S$ 2.-THE TOMATO AND EGG-PLANT.}

The tomato or love-apple (Lycopersicum esculentum, Tou, L.) belongs to the natural order Solanæ, section Pericarpum baccatum, and to the class Pentandria and order Monogynia in the Linnæan arrangement. All of the genus Lycopersicum, consisting of nine species and several varieties, are cultivated in their native countries (Peru and South America) as esculent fruits. The common tomato ( $L$. esculentum) was introduced to Britain in 1596, and with its varieties much cultivated, although, in most of Britain, requiring the protection of glass. The name is derived from Lykos, wolf, Persicon, a peach - in poetical allusion to the beautiful appearance and deceitful value of the fruit. The ripe fruit is in much request for making tomato sauce, one of the most delicious and wholesome of all sauces, both for meat and fish. Medicinally considered, it is an excellent substitute for calomel, and can be taken when that valuable medicine cannot, and with much less injury to the constitution. The ripe fruit is much used in soups in this country, and in France and Italy to such an extent that, to supply the demand, whole fields of the plants are cultivated. In a green state the fruit is pickled, and when fully ripened, the juice is expressed and kept for winter use in the form of ketchup. In the neighbourhood of London it is grown to a great extent, and finds a ready sale in Covent Garden market during September and October, and fetches a good price. It is singular that some of the enterprising growers for the metropolitan market have not thought of producing a supply throughout the year, as few fruits are more easily ripened. We believe we were the first to exhibit ripe tomatos in Covent Garden market-not for sale, but to show they could be produced in March and April, and, indeed, throughout the London season. The fruit seldom ripens in Scotland in the open air, unless in a few very favourable situations; nor is there much demand for them, unless in families of the highest order. It ripens in the vicinity of London and in the southern counties in August and September, and keeps in good condition throughout October.

The plants are usually propagated by seed, but will strike freely by cuttings taken off the terminating side-branches; and when their culture is to be extended throughout the year, the latter is the best mode, the plants so originated running less to haulm, and fruiting in greater abundance. They may be continued for several years, if kept in a sufficient temperature ; but when grown in the open air they perish annually, and, in fact, are annuals in their own countries. We have had plants in a constant state of bearing for three years, when grown in a pine-stove. The London practice is to sow the seed in seed-pans, in a genial heat, about the end of March, and, when the plants are about 2 inches high, to prick them off into large 60 sized pots, in light rich soil, and keep them near the glass in a growing state, admitting air on all favourable occasious, till the beginning of May, after which they are exposed more freely to harden them off, to be fit to plant out at the bottom of a south wall by the end of that month. They should, however, not be set too near the roots of the fruit-trees, as they exhaust the ground exceedingly. When first planted out, shelter them for a few days by sticking branches in front of them, to ward off cold windis and scorching sunshine. When fully established, they should be as much exposed as possible, as they require all the light and sunshine that even a London climate affords to ripen them tho roughly. Some form beds of warm dung, cover ing them with frames and sashes, and treat them the same as late cucumbers; others plant them at the base of sloping banks, covering the surface with slates to attract heat, and train the plants upon them. Some train the plant to a single stem, rubbing off all side shoots as they appear, which induces the flowers to issue from the base of the leaves attached to the main stem. Others train the main stem upwards against a wall or paling, and lay in the side branches in a horizontal direction; and others stop the leading shoot as soon as one or two branches of flowers are formed, and retain them in a very dwarf state, by which means a number. of plants may be grown in a small space. Our practice is to forward them in pots placed in pits or hot-houses, shifting into larger pots as they advance in growth, and in May to plant them in pits from which early potatoes and suchlike crops are removed, and to train them over the surface of the beds like cucumbers, placing slate under them for attracting a greater degree of solar heat. We also grow them in pots 14 inches in diameter, in very rich soil, arranged in a single row along the back of a pit, and train the shoots to the wall, by which means little space is occupied, so that the principal part of the pit can be used for other crops, such as late cucumbers and melons. For winter supply, we grow them in large pots placed along the front of a pine-stove, and train one leading shoot up under each rafter, as at that time the vines are withdrawn from the house. A cucumber or melon house is the best situation for them during winter, and a few plants will produce an ample supply for a large family. Those planted against walls, in the open air, produce abundant crops, in most places of moderate climate, in most seasons, but do not ripen well. If the fruit, when fully grown, be cut off, taking attached to it a foot or two in length of the stems, and suspended under the roof of a pine-stove or vinery, they will ripen to tolerable perfection.

Only two species are cultivated in Britain, the L. cerasiforme, or cherry-shaped, of which there is a red and yellow variety; and these are, for some culinary purposes, particularly for dress- 
ing whole, preferred to L. esculentum or common tomato, of which there are red-fruited, yellow-fruited, and white-fruited varieties, all of which are of various forms and sizes, according to the culture they are subjected to. It is highly probable that some, if not all the other species, deserve our attention, particularly $L$. peruvianum, which is perennial.

The red spider, fig. 22, and thrip, fig. 21, are exceeding apt to attack the foliage, when grown in a high temperature, and in too dry an atmosphere; and the Coccus adonidum, or mealy pinebug, infests them under similar circumstances.

One or two fruits, saved when fully ripe, will afford abundance of seed for a large garden. 'The seed should be washed out of the pulp, and, when thoroughly dried, kept in paper bags for future use. It retains its vegetative properties for two years.

The European names are-Tomate in French ; Tomates in Spanish; Appeltjes des liefde in Dutch ; Pomo d'oro in Italian ; and Liebes apfel in German.

The Egg-plant (Solanum Melongena Dunal) belongs to the same natural order and class, and order in the artificial system, as the last. The generic name is derived from Solor, to comfortsoothes by stupifying; the specific name, according to Forskahl, from Bydendjan, its name in Arabic-a name by which it is known in all the gardens in the West Indies, where it is much cultivated. It is a native of Africa, and,indeed, is found all over the tropics, where it is extensively cultivated, and used, both boiled and stewed, in sauces like the tomato. A correspondent in the "Gardeners' Magazine" asserts that the Chinese, on great occasions, cook the fruit, which very much resembles a hen's egg both in colour, shape, and size, while hanging on the plant, and serve up the entire plant to table in a pot. The plant was introduced into Britain in 1597. When fit for use, they part freely from the stalk.

Being an annual, it is reared yearly from seed, sown during March in seed-pans, placed in a temperature of $60^{\circ}$ to $65^{\circ}$. When the plants have attained the height of 2 inches, they should be set singly in pots of the large 60 -size, and still continued in the same degree of heat. As they advance in growth, repeated shiftings will be required; and when in pots 10 or 12 inches in diameter, they may so remain to perfect their fruit. A rich soil is beneficial, and as they seldom ripen in the open air of this country, they generally are accommodated with a place in a pit or greenhouse, after the plants have been removed out of doors for the summer season. Here they become both ormamental and useful. One fruit will afford sufficient seed for continuing the stock.

The varieties grown are the common or whitefruited, the violet-fruited, and yellow and red fruited: of these the French prefer the violetfruited, which they call Aubergine, and this sort is as common in the Paris markets as the tomato. Their favourite mode of dressing them is to scoop out the seeds, filling the cavity with sweet herbs, and then frying them whole. In Britain the plant is in general grown for its singular appearance, few people knowing that they are a wholesome and excellent food. Under the above management the fruit will ripen about the end of August.

The European names are-Molongene in French; Melanzana in Italian; Tollapfel in German and Dutch.

\section{$\$$ 3. THE GOURD AND PUMPKIN.}

The gourd and pumpkin (Cucurbita ovifera L., and $C$. pepo L.) belong to the natural order $\mathrm{Cu}-$ curbitaceæ, and to the class Monœcia and order Monadelphia in the Linnæan arrangement. All the varieties of gourds and pumpkins are eatable, as are also the species of the allied genus Lagenaria (bottle gourd), which last are natives of India, while the gourds and pumpkins are natives of Astracan, Chili, the Levant, and other Eastern countries; and although all tender annuals with us, most of them perfect their fruit in most seasons, and in nost parts of our coun. try. As the fruit of these plants is used in their green or unripe state, we use the term "perfect their fruit," because it attains its useful condition. The bottle gourds and pumpkins have been cultivated in this country since the latter end of the sixteenth century, but the gourds proper are of much more recent date. The pumpkin, $C$. pepo, has long been cultivated in England, where pumpkin-pies are much relished by the peasantry, who store by the fruit in autumn along with crab-apples for winter use. Many of the varieties are much grown on the Continent, and used in soups and stews, as well as boiled and fried. In America they are largely grown, and used not only for the above purposes, but are also put into pies and tarts.

The succade gourd, or vegetable marrow, is that of all others most cultivated in gardens, and is a profitable and wholesome vegetable. It is used for culinary purposes in all stages of its growth, dressed either whole while quite young, or cut into sections as it gets larger.

The $C$. melopepo maxima (the mammoth or large American gourd) is the largest of all the tribe, attaining the weight of from 160 to 200 lb., keeping for several months after it is ripe, and being used as a substitute for carrots and potatoes in soups and broths.

The majority of gourds are grown in this country for ornament; many of them, when trained to poles, are exceedingly curious; others are grown for use, but not to the extent they are either in America or on the continent of Europe.

As all of them are annuals, they are originated from seed, which should be sown in March upon a moderate bottom-heat, using rich soil, and covering the seed, which is large, to the depth of an inch. Where the number of plants required is not great, it is advisable to plant one seed in a large 60 -sized pot, and when about 3 inches high to re-pot into a 48-sized pot, which will be sufficient for the plants until they are to be planted out for good-which rarely can be, without protection, before the middle or end of May. The best situation for the vegetable marrow, or such as are to be grown for cooking, is the top of a compost-heap or dunghill, as they 
require a full supply of nutriment at their roots; and from the size of their leaves, \&c., they not only cover over compost-heaps or dunghills from sight, but also tend to decompose the latter. During summer they require liberal supplies of water, training the shoots to the ground, and reducing all superabundant side branches, and stopping leaders, after a sufficient crop of fruit has set.

The European names for the gourd and pumpkin are-Courge in French; Zucca in Italian; Abobaro in Portuguese; Kurbis in German; Calabaza in Spanish ; Kauwoerde in Dutch. The French distinguish the vegetable marrow by the name of Courge a la moelle, and theGermans call it Markige melonenkurbis. The large American gourd the French designate Potiron jaune; and the Germans, Melonenkurbis.

\section{\$ 4.-CAPSICUM.}

Capsicum (Cayenne or Guinea pepper) belongs to the natural order Solanex, and to the class Pentandria and order Monogynia in the Linuæan arrangement. The generic name is derived from Kapto, to bite, from its pungent qualities. They are chiefly natives of India, China, Egypt, and the Brazils, and are all cultivated and much used in the countries to which they are indigenous. The capsicum was first introduced into Europe by the Spaniards; and they are known to have been cultivated in Britain so early as the reign of Edward VI. Their varieties are almost without end, differing in size, colour, and form of their fruit. The commoner varieties are extensively grown by the market-gardeners about London, for the supply of the markets and for the Italian warehouses. They are much used in pickles, seasonings, and made dishes, as both the pod or capsule and seeds yield a warm acrid oil, the heat of which being imparted to the stomach is thought to promote digestion and correct the flatulency of vegetable aliments. The seed-pods of Capsicum frutescens furnish the Cayenne pepper of the shops. The expressed juice of the fresh pods affords liquid bird-pepper, so much used in soups in most warm climates. Both the green and ripe pods are used as pickles, and also for making Chili vinegar, which is done by simply putting a handful of the pods in a bottle, afterwards filled up with the best vinegar, and stopping it closely up. In a few weeks it is fit for use. Man-dram, so much used in warm countries to provoke a languid appetite, is prepared by gathering the pods while dry of Capsicum baccatum or bird-pepper, and mixing them with cucumbers cut into thin slices, with garlic and shallots cut also very small, to which a little lime-juice and Madeira wine are added, and the whole being mashed and mixed with liquid is fit for use. The pods, gathered fresh and eaten before meals, tend greatly to assist digestion.

The general mode of preparing Cayenne pepper is by gathering the pods when ripe. In India they are dried in the sun, but in Britain they should be dried on a slow hot-plate, or in a very moderately-heated oven; they are then pounded and sifted through a fine sieve, mixed with salt, and, when dried, put into close-corked bottles for the purpose of excluding the air. This article is subject to great adulteration, flour being often mixed with it, and, still worse, red lead, which is much of the same colour, and greatly increases the weight. The better way is to dry the pods in a slow oven, split them open, extracting the seeds, and pounding them down to a fine powder, or passing them through a coffee-mill, sifting the powder through a thin muslin sieve, and pounding down the parts which do not pass through, and sifting again until the whole is reduced to the finest possible state. Place the powder in air-tight glass bottles, but add no salt or other ingredient whatever. What is called Cayenne pepper-pot is prepared in the following manner, and is sold at a high price in the Italian warehouses: "Take the ripe bird-peppers, dry them well in the sun, then put them into an earthen or stone pot, mixing flour between every stratum of pods, and put them into an oven after the baking of bread, that they may be thoroughly dried; after which they must be well cleansed from the flour, and, if any stalks remain adhering to the pods, they should be taken off and the pods reduced to a fine powder: to every ounce of this add a pound of wheat flour, and as much leaven as is sufficient for the quantity intended. After this has been properly mixed and wrought, it should be made into small cakes, and baked in the same manner as common cakes of the same size; then cut them into small parts, and bake them again that they may be as dry and hard as biscuit, which, being powdered and sifted, is to be kept for use."

The annual capsicum and the larger sorts are usually grown for the supply of the markets more on account of their size than of their quality. We grow the capsicum largely for all culinary purposes, but prefer Capsicum frutescens, a shrubby perennial sort, producing small but very pungent pods. These we keep in bearing for from two to three years, when they are thrown away, and successional plants reared from seed.

The European names are-Piment in French; Spanischer pfeffer in German; Peberone in Italian.

\section{§ 5.-ROSEMARY AND LAVENDER.}

Rosemary (Rosmarinus officinalis L.) belongs to the natural order Labiatæ and tribe Monardeæ, and to the class Diandria and order Monogynia in the Linnæan arrangement. A native of the south of Europe, from whence it was introduced most probably by the monks in the dark ages. It has long been a cultivated plant in our gardens, and employed for the most opposite purposes. The sprigs are used as a garnish for some dishes; they are also by some stuck into roast-beef while roasting, and are supposed to communicate an agreeable flavour to it. They are in some parts presented to the company at funerals as tokens of remembiance, and are afterwards thrown into the grave. A 
decoction of the leaves is much used by ladies to wash their hair with, and it is not devoid of medicinal properties. It is often planted on graves by relatives both in England and on the Continent. The name is derived from Ros, dew; Marinus, of the sea-that is, dew of the sea. It is a half-hardy shrub, requiring to be planted in a dry poor soil, and in a warm sheltered situation. It is readily propagated by cuttings taken when the young wood is half ripened, and placed in a shaded place under a hand-glass. There are three varieties, the common green-leaved, the silver, and the gold striped-the former is the hardiest. It is also reared from seed, which is imported from France.

The European names are-Romarin in French; Romaro in Spanish; Rosmarino in Italian ; Rosmarin in German; Rosmarinho in Portuguese ; and Rosmaryn in Dutch.

Lavender (Lavendula spica L.) belongs to the natural order Labiatæ, tribe Nepeteæ, and and to the class Didynamia and order Gymnospermia. A native of the south of Europe. A plant of the greatest antiquity. It appears to be the Nardus stricta or Nardus indica of ancient writers, and by them was held in high esteem. A very interesting account of this plant is given by Phillips in his "History of Cultivated Vegetables," vol. i. p. 298.

The name is derived from Lavo, to wash ; and the plant is recorded in the "Hortus Britannicus" as being introduced in 1568, but it is highly probable it was cultivated in the monastic gardens at a much earlier period. There are two varieties cultivated, the common and the broad-leaved or French: the former is smaller in the spike, but much more aromatic; the latter is, however, the most extensively grown for distillation and other purposes. Alcohol extracts the virtues of the flowers completely, and gives off by distillation all their odorous parts; water acts less completely. According to the "London Dispensatory," "the oil on which its virtues depend is obtained separately in distillation with water, in the proportion of 1 ounce of oil to 60 ounces of the flowers."

Lavender is grown in most gardens, both for distillation and for being dried and placed amongst linen, as well as forming an ingredient in those repositories of perfumes called sweetpots. The flowers should be left on the spikes and gathered when quite dry, and just before they are fully expanded. When cut with about 6 inches of the stalks, and tied up in small bundles about an inch in thickness, and suspended from the roof of a dry chamber at a temperature of from $60^{\circ}$ to $70^{\circ}$, they will dry in twenty-four hours, and be fit for storing by for use. When the flowers fall from the spikes during drying, they should be gathered up and placed in paper bags, and in this state are as fit for use as if they had remained on the spikes. Lavender is grown to a great extent about Micham in Surrey, for the purpose of distillation ; and the lavender-water made of homegrown flowers is considered superior to that imported from France.

VOL. II.
It is readily propagated by seeds, cuttings, or slips-the former make the best plants. The seed is procured from France. It should be sown in March in poor light soil ; and when the plants are about 2 inches in height they should be transplanted to nursery-beds, in which they should remain till the following spring, when they may be planted out for good. Cuttings are struck in the same manner as with all other shrubby hardy plants, and when rooted should be treated as directed above for seedlings. A dry poor soil is the most favourable, and a warm situation fully exposed to the sun the best place for it, as well as for all other highly-aromatic plants.

The European names are-Lavande in French; Espliego in Spanish; Lavendula in Italian; Spiklavendel in German; Alfazema in Portuguese ; and Lavendel in Dutch.

\section{$\S$ 6.-THE HORSE-RADISH.}

The horse-radish (Cochlearia armoracea L.) belongs to the natural order Cruciferæ, tribe Alyssinex, and to the class Tetradynamia in the Linnæan arrangement. The plant is a native of England, found occasionally in damp watery places. The generic name is derived from Cochlear, a spoon, from the spoon-like or concave leaves of several of the genus. Most of the plants of this family, several of which are indigenous to Britain, are wholesome as salad plants, being powerful antiscorbutics, and capable of stimulating the digestive organs. The horseradish is cultivated in every garden, and is used as a condiment to roast-beef. The root is the part used, and is served at the table either finely scraped down or grated, which latter is the best and most elegant form. It is also used in winter salads and sauces.

Propagation is always effected by planting portions of the roots, which grow readily, and the soil most conducive to it is a deep, rich, light sand or alluvial deposit, free from stones or other obstructions, as the longer, thicker, and straighter the roots are the more they are valued. With the exception of the Jerusalem artichoke, there is scarcely another culinary vegetable of equal importance in which cultivation is in general so greatly neglected as in this. We find it often, like the former, planted in some obscure corner of the garden, where it may have existed for years, and only visited when the proprietor's table is about to be graced with old England's national dish. The operation of hastily extracting a root or two is too often all that is thought of, and the crop is left to fight its way amongst weeds and litter as best it may. The best directions laid down for improved culture are the papers by $\mathrm{Mr}$ Knight and Mr Judd, in the "Transactions of the Horticultural Society." The former, in vol. i. p. 207, says, "During winter, trench the ground 3 feet deep, and in the following February procure the sets, in the choice of which take the strongest crowns or leading buds from old plants, cutting them about 2 inches long. Mark out the ground in 4-feet beds and 1-foot alleys; 
then take from the first bed 9 inches of the top soil, laying it upon the adjoining bed; after which take out an opening at one end of the bed, in the common way of trenching, 15 inches deep from the present surface; then level the bottom, upon which plant a row of sets across the beds at 9 inches apart each way, with their crowns upright; afterwards dig the next trench the same width and depth, turning the earth into the first trench over the row of sets, thus proceeding, trench after trench, to the end. Where more than the produce of one bed is required for the supply of the family for twelve months, the third bed is next to be planted, which treat as directed for the first, only observing to lay the earth on the fourth, and so on for any number of beds. Upon every alternate bed which is not planted a dwarf annual crop may be grown. The plants must be kept clear of weeds during summer, and as soon as the leaves decay in autumn let them be carefully raked off; in the following February 18 inches of the earth of the unplanted bed must be laid as light as possible, and equally over the beds that are planted, then trench and plant the vacant beds exactly in the same manner as before directed. The following autumn the first planted horse-radish may be taken up by opening a trench at one end of the bed to the bottom of the roots, so that the sticks or roots of the crop may be taken up entire, which for size and quality will be such as have not generally been seen. The following February the one-year-old crop will require additional earth as before directed, which must, of course, be taken from those beds which are now vacant." $\mathrm{Mr}$ Judd, as described in the work last quoted, takes about 3 inches of the top part of the roots of the crop to be taken up, and those he cuts off, so as to leave only about a quarter of an inch under the crown. He trenches the ground 2 feet deep, and if he applies manure, puts it at the very bottom of the trench. "For if not done," he says, "the horse-radish, which always puts out side roots, would send out such large side roots from the main root, in search of the dung lying contiguous, as materially to injure the crop. In planting, holes are made 18 inches apart every way, and 16 or 18 inches deep. The root-cuttings are let down to the bottom of the holes, which are afterwards filled with finely-sifted coal-ashes, and the surface of the bed is raked over." We trench our ground for this crop 3 feet deep, laying on a good coat of manure in the bottom, which is well mixed with the soil 2 feet below the surface; the plant-sets, as recommended by Knight above, deprived of all their lateral small fibres, are dropped in holes made by a $2 \frac{1}{2}$-inch dibble, gauged to penetrate to the required depth; the holes are filled with the soil above, and the roots left to find their way to the surface, which they do in due season. We have also planted the roots in single lines in trenches dug out as for celery, and as the plants shot upwards, earthed them up during the first season. The winter following we remove all the soil from them, and cut off every fibre from the main stem or root, and earth them up again, by which means we have roots fit for use of a large size and uniform shape. In November we take up as much of the crop as is likely to be used during winter, and pack it by in moderately dry sand in the root-cellar, where it is easily got at when required.

The Scopula forficularis, the garden pebblemoth, is the only insect we know of that is injurious to the horse-radish. This insect, in its perfect state, measures rather more than an inch across the wings when fully expanded. The uppermost pair are hazel-coloured, with four stripes, two of which are very distinct, while the other two are faintly traced. The body and under wings are whitish, and on the latter near the middle there is a curved brownish streak, and another of a blackish colour on the margin. The first brood of caterpillars make their appearance in May, while the second do not appear until autumn. They are from 8 to 10 lines in length; head of a light-brown colour; body yellowish-green, with longitudinal black stripes. The means chiefly employed for their destruction are, dusting the plants with white hellebore powder in a dry state ; or caustic lime applied in their earliest state is equally effectual. A species of mildew often appears on the foliage, but, so far as we know, has little effect upon them. This is Erysiphe leguminosarum Link.

The European names are-Cranson, or Le grand raifort, in French; Ramolaccio in Italian; Rabano in Spanish; Merrettig in German; Loseelkraut in Dutch. 


\section{CHAPTER XII.}

S O I L S.

\section{$\S$ 1.-THEIR ORIGIN AND NOMEN- CLATURE.}

Av intimate knowledge of the nature, and even the nomenclature of soils, is of the utmost importance in every branch of cultural science,- to the horticulturist even more so than to the agriculturist, because the subjects under his care are more delicate, and of a much more varied character. The hardier fruit-trees will flourish in soils where the peach, apricot, and vine would refuse to live; and the same rule holds good amongst many of the productions of the culinary garden. It is, however, in connection with the thousands of plants of exotic origin that the nicest distinctions in regard to the nature and nomenclature of soils are called forth; for certain genera of plants-nay, even certain species of the same genus-require a very opposite soil from those brought from the same locality, ranking in the same natural order, and to all external appearances differing little from them. Those which naturally inhabit a silicious soil will seldom exist long in an argillaceous one; and those from peaty soils will not long live in calcareous ones. Seeds of most plants will germinate in a soil composed chiefly of vegetable matter; but after germination, those whose natural food is derived from an argillaceous soil, will soon linger and die of starvation.

There is no universal soil, wherein all the productions of a garden can be grown; while a rich loamy soil may be considered all that is desired by the agriculturist, for in such all his crops will flourish alike. The horticulturist has yearly to collect, from wherever they can be procured, soils of very opposite characters. To him, therefore, a knowledge of the nature of soils is of paramount importance; and that knowledge is only to be obtained by one or other of the three tests-which will be afterwards noticed-namely, botanical, chemical, or mechanical.

Some acquaintance with the nomenclature of soils is to him of equal importance. He may receive plants or seeds from Caffraria, described as having been growing in a silicious soil ; from New Zealand, as having been produced in an argillaceous soil; from Canada, in a peaty soil; from somewhere else, in a calcareous one, and so on. Therefore, without a sufficient knowledge of the difference between these, he would be liable to commit an error of almost daily occurrence, by sowing or planting in a soil perfectly unsuitable for the healthy development of the seed or plant.

A complete nomenclature of soils is still a desideratum, as was the case in every other department of natural science, until the various specimens in each were reduced to systematic arrangement. At present, the names of soils are scarcely even conventional, nor can it be otherwise until they are subjected to the rules of classification; and no doubt they will, through the agency of chemistry, be yet arranged into classes, orders, genera, and possibly species and varieties. The only feasible attempt we know of being made in this very important matter is that of M. de Gasparin, which, although far from perfect, is still a step in the right direction. He divides all soils into two classes-namely, inorganic and organic - and this will, we believe, always be recognised as the fundamental principle of all classification. His subdivisions we do not, however, think sufficiently clear to be of much practical use in their present state. The inquiring reader will find a translation of his arrangement, from the Comptes Rendues de l'Académie des Sciences, in the 27th volume of Jameson's Edinburgh New Philosophical Journal.

For every practical purpose of cultivation, at least with our present limited knowledge of the subject, soils may be divided into six different classes, and these, we think, will be found to express, sufficiently clear, an idea of the particular soil that is meant.

These are, argillaceous or clayey, containing above 50 per cent of clay; silicious or sandy, containing not more than 10 per cent of clay; calcareous or limey, containing more than 20 per cent of carbonate of lime; loamy, having from 20 to 50 per cent of clay; gravelly, composed of small stones, silica, a small portion of loam or of clay, and often a considerable percentage of oxide of iron, particularly in some pit gravels valued for their binding nature, and employed for walk-making, (in alluvial and river gravel this oxide is much less abundant); peaty, chiefly formed of vegetable matter, in which a large proportion is in an inert state-most so when it exists in deep masses, surcharged with 
water, and least so when in a thin surface stratum, resting on a dry base. Before, however, treating on these individually, we may offer a few brief remarks on the origin of soils generally.

"It is the generally received opinion of the formation of soils, that they have proceeded from the detrition of rocks by the force of external agencies; that the disintegrated materials have been carried by floods to the ocean, and, there accumulating, are formed into horizontal layers. But the decay of rocks themselves must be very gradual : in some dry countries, inscriptions are perfect, and their angular edges and shapes but little blunted or altered, after a lapse of twenty centuries. The formations that are taking place, in the present quiescent state of the globe, are made by the sea, fresh-water rivers, and by lacustrine deposits from substances collected and rolled along by the course of the waters over the earth. The depositions go on in many places visibly, though slowly: but we know little of the processes by which the vast alluvial deposits have been made, in a!l the different arrangements, mixtures, and combinations, to the depth of several hundred feet, and extending over vast tracts of various and discordant qualities; or of the means employed by nature in the process of alluvial deposition from the detritus accumulated at the foot of mountains, from the decomposition of rocks, and of the subsequent removal and consolidation, or even of the operations by which animal and vegetable exuviæ are converted into soil. But these petrifactions and organic remains would seem sufficient to place one fact beyond dispute, that the globe has undergone many extensive changes; and it is not a little curious that they are confined to particular formations, and are not found in contiguous strata, and that the remains of trees and vegetables are found only in some few similar situations. Soils are often found to contain substances that do not exist in the rocks on which they rest; and rocks frequently possess materials of which no vestiges can be detected in the upper soil, and which, being destructible, would be found in some degree or quantity, if soils were derived from the adjacent formations. These circumstances would appear to indicate that other agents than detrition have been at work in the production and creation of soils.

"The alluvial formation constitutes the greater mass of the surface of the earth, and is composed of rocky substances, formed of previously existing rocks, and supposed to be formed by the detrition and ruins of other formations, broken down by the gradual action of water. They are loose in texture, and are never covered with any rock or solid secondary strata, and may be reckoned a very recent deposition, the formation of which is still going on. 'The deposit is mostly composed of clay, sand, and gravel, which, in a great variety of combinations and modifications, constitute what is termed land or soil. They contain sands from quartz, and clays from the disintegration of slates, felspar, and micaceous rocks, mixed with other substances, and fill up hollow places, and form very extensive tracts. They also contain calcareous tuffs, common salt, and peat, subterranean and submarine forests, bogs, iron ore, metals, ores, and gems in grains."-DoNaLDson on Soils and Manures, p. 10.

This view appears also to have been taken by Mr Morton, who observes in his work on soils, that "the surface of the earth partakes of the nature and colour of the subsoil or rock on which it rests. The principal mineral of the soil of any district is that of the geological formation under it. Hence we find argillaceous soil resting on the various clay formations, calcareous soil over the chalk, and oolitic rocks and silicious soils over the various sandstones. On the chalk the soil is white; on the red sandstone it is red; and on the sands and clays, the surface has nearly the same shade of colour as the subsoil. The lime, potash, and iron, existing in various proportions in the rock, are acted upon by the atmosphere, and the rock is decomposed; some of it will form impalpable matter, some into sand, and some into coarse gravel or rubble. The surface is composed of the same materials as the subsoil, with the addition of vegetable and animal matter, in every state of decay, internally mixed with it; and we perceive a change in the external appearance of the surface whenever there is a change in the subsoil below."

Whitley, in "Application of Geology to Agriculture," dissents somewhat from these opinions, and asserts that "the ordinary effect of atmospheric influence does not appear sufficient to produce such changes. We are therefore led," he continues, "to the conclusion that some more powerful and effective agent has been at work; and the phenomena connected with the facts we have reviewed are only consistent with the theory of a vast body of water having, by its violent action, broken and comminuted the earth's surface to a considerable depth, thus holding in mechanical suspension the materials of which the soil and subsoil are composedthe coarser and heavier parts first subsiding, then the clays, and lastly the fine earthy matter. In endeavouring to establish this view of the formation of soil, the decomposition of rocks by atmospheric and chemical agencies must not be overlooked. These causes had probably produced extensive changes before that catastrophe, whose effects we have just been describing, and to the present time their operation tends to improve and deepen the soil. The crumbling down of rocks by decomposition may be regarded as conservative of the soil, by supplying fresh portions to replace those that are constantly washed away." Notwithstanding the attempt to establish this water theory, which is by no means new, Mr Whitley cannot altogether get rid of the theory laid down by Donaldson, Morton, and others. Ansted, in "Geology," vol. ii., follows, although in a more modified degree, the views of Whitley stated above.

In reviewing these opinions, Mr Henry Stephens, in "The Book of the Farm," we think very justly, draws the following conclusion: "It appears to me the origin of the soil is not so 
easily explained as the matter seems to be assumed by the authors just quoted; and that difficulty attends the explanation of their origin may be inferred from the fact of most geologists having hitherto paid little attention to the relations of the loose materials composing the surface of the globe. They are well acquainted, and coincide in opinion, with the relations of the indurated rocks which form the crust of the earth, but are far from being agreed as to the causes which have placed the enormous masses of incoherent matter, met with in every quarter of the globe, in their present positions. 'These masses of clay, sand, and gravel, bear no fixed relation to one another, like the indurated rocks, and, therefore, have not been placed by the operation of any law of order, but simply that of gravity; and it is this want of order in their position which baffles the ability of the geologist to ascribe the origin correctly."

The highly respectable authority last quoted further remarks: "Notwithstanding the possibility of the formation of mould upon the surface of hard rocks by means of atmospheric influences, there cannot, I think, a doubt exist that by far the largest proportion of the agricultural soil is based upon the incoherent, and not on the indurated rocks." The term rock, it should be observed, is used here as applied by geologists, " not only to the hard substance to which this name is commonly given, but also to those various sands, gravels, shales, marls, or clays which form beds, strata, or masses ;" and the conclusion arrived at by Mr Stephens is, " that the agricultural soils of the low part of a country are generally not derived from the hard rocks upon which they may happen to be placed, but have been brought to their present position from a distance by means of water." After stating various cases which have come under his own observation, he proceeds : " It is not denied that the chemical action of the air, and the physical force of rain, frost, and wind, produce visible effects upon the most indurated rocks; but of course they must have much greater effects upon incoherent ones. Combined in their action, they could only originate a mere coating of soil over the surface of indurated rock, if the rock were situated within the region of phanogamous vegetation, because it would then be constantly covered with plants. But the plants in their turn would protect the rocks against the action of external agencies; and although they could not entirely prevent, they would at least retard the accumulation of soil beyond what the supply of vegetation required. Some other agent, therefore, more powerful than the ordinary atmospherical elements, must be brought to bear on indurated rocks, before a satisfactory solution of the formation of soils can be accepted. That other agent is water; but the moment we assent to the agency of water being able, by its abrasive power and buoyant property, when in motion, to transport the abraded parts of rocks to a distance, and let them fall in coming in contact with some opposing barrier, that moment we must abandon the idea of the soils formed from abraded matter being derived from the indurated rock upon which they rest. Mr Buist draws these conclusions, after describing the relative positions of deposits to the rocks upon which they rest, in a large and important district of Perthshire, where he says "that the alluvial matters of these districts, in general, belong to periods much more remote than those originally assigned to them, and came into existence under circumstances prodigiously different from those which presently obtain ; that the present causes-that is, the action of our modern rivers, brooks, and torrents, and of the air and water on the surface now exposed to them-have had but little share in modifying our alluvial formations, or bringing them into their present form." Professor Johnston, in "Lectures on Agricultural Chemistry and Geology," sums up this matter in the following words: "Thus the general conclusion is fairly drawn, that the earthy matter of all soils has been produced by the gradual decay, degradation, or crumbling down of previously existing rocks. It is evident, therefore, that whenever a soil rests immediately upon the rock from which it has been derived, it may be expected to partake more or less of the composition and character of that rock; and that where the soil forms only the surface-layer of a considerable depth of transported materials, it may have no relation whatever, either in mineralogical character or in chemical constitution, to the immediately subjacent rocks. The soils of Great Britain are divisible into two such classes. In some countries an acquaintance with the prevailing rock of the district enables us to predict the general character and quality of the soil; in others-and nearly all our coal-fields are in this case-the general character and capabilities of the soil have no relation whatever to the rocks on which the loose materials immediately rest."

Sir H. Davy, in accounting for the transition from rock to soil, remarks that the operation may be easily conceived by referring to the instance of soft granite, or porcelain granite. This substance consists of three ingredients-quartz, feldspar, and mica. The quartz is almost pure silicious earth in a crystalline form; the feldspar and mica are very compounded substances: both contain silica, alumina, and oxide of iron. In the feldspar there is usually lime and potass; in the mica, lime and magnesia. When a granite rock of this kind has been long exposed to the influence of air and water, the lime and potass contained in its constituent parts are acted upon by water or carbonic acid ; and the oxide of iron, which is almost always in its least oxidised state, tends to combine with more oxygen. The consequence is, that the feldspar decomposes, and likewise the mica, but the first the most rapidly. The feldspar, which is, as it were, the cement of the stone, forms a fine clay; the mica, partially decomposed, mixes with it as sand ; and the undecomposed quartz appears as gravel, or sand of different degrees of fineness. As soon as the smallest layer of earth is formed on the surface of a rock, the seeds of lichens, mosses, and otlier imperfect vegetables which are constantly floating in the atmosphere, and which have made it their resting-place, begin to vegetate. Their death, decomposition, and decay afford a certain quantity of organisable matter, which mixes 
with the earthy materials of the rock. In this improved soil more perfect plants are capable of subsisting; these in their turn absorb nourishment from water and the atmosphere, and, after perishing, afford new materials to those already provided. The decomposition of the rock still continues; and at length, by such slow but gradual processes, a soil is formed on which even forest trees can fix their roots, and which is fitted to reward the labours of the cultivator. Such, therefore, being the opinions offered regarding the primary formation of soils, let us briefly consider them in the order in which they stand above.

Argillaceous or clayey soils derive their name from, and are formed of substances, the basis of which is argil or alumen, alumina or alumine, the oxide of aluminum, one of the newly discovered terrigenous metals, which unites with only one proportion of oxygen, and forms the wellknown base called alumina, which contains, in one hundred parts of "aluminum," about eight of oxygen. Sir H. Davy laid it down as a rule in practice, that the name clayey soil should not be applied to any soil unless it contained one-sixth of impalpable earthy matter, and did not effervesce to any great extent with acids. Argillaceous soils occur in extensive and often very thick beds in most alluvial districts, transported thither by the agency of water, and accumulating for ages. This soil is much mixed with silica and other matters, and is found of different colours, as oxide of iron in different states and combination is amalgamated with it. Clay enters into all good soils; in fertile ones it abounds to the extent of 9 to 15 per cent, whilst in barren lands it exists to from 20 to 40 per cent. "The absence of it," Donaldson observes, "forms a soil too dry and porous: a proper quantity forms the best clay lands and clayey loams, and a superabundance of it constitutes a soil too wet and cold for vegetable life, in a moist state, and hardening and contracting by heat into a state very hurtful to the growth of plants. The purest clay contains upwards of 60 per cent of sand, and is always mixed with mineral, animal, and vegetable substances." Stiff or strong clays, although they absorb the greatest quantity of water when it is poured upon them, or in the case of heavy rains, do not absorb most moisture from the atmosphere in dry weather. They then become caked on the surface, and evaporation from below is consequently interrupted, so that crops are burnt up on them sooner than on light porous sandy soils. Clayey soils are also much colder than lighter soils, because, from their closeness of texture and often superabundance of moisture, the rays of heat are prevented from penetrating them. They are, however, more uniform in temperature, as the same cause that prevents the absorption of heat prevents its radiation also. Clay has a strong affinity for water, on account of the alumina forming its basis retaining it with great tenacity; andit is this quality which constitutes its distinguishing characteristic. " It may be remarked," says Donaldson, " that this quality seems to exist in soils in a smaller proportion than is generally supposed, owing to the state of combination and exposure.
Clay soils of all colours and qualities are known by the property of holding water very tenaciously; and when once wetted, they are dried with very great difficulty. The most general colours of clayey soils are red and black, of various degrees of fertility and texture. The red soils are hardest; the black is softer, and in many cases very waxy and plastic, arising from the minuteness of the particles affording so many points of adhesion. In dry weather they are hard and lumpy, as the red-coloured; in moist weather they dissolve more easily and readily. Clay soils require much power and labour, and dry weather if possible, during the process of being wrought, and narrow ridging or thorough draining in order to carry away the excess of moisture. Clay soils, when well enriched with manures, are naturally well qualified for carrying good crops; but they require much care and industry, as well as a very considerable portion of practical skill in their management, in order to keep them in good condition; yet, when the natural adhesive toughness can be mastered, they generally yield the heaviest and most abundant crops. One grand requisite is, to keep the land in condition a poor worn-out clay being the most ungrateful of all soils, and hardly capable of repaying seed and labour." Clay soils, although, when in a high state of cultivation, capable of producing, during summer and autumn, most culinary vegetables in a high state of perfection, are very unfit for preserving them through the winter, or for producing them early in the season. If ameliorated by culture, and free from extra moisture, they are well adapted for most of the hardier sorts of fruit trees. The oldest specimens of these with which we are acquainted are growing in rich alluvial clayey soils.

The composition of pure clay is thus given by Donaldson, p. 15 :-

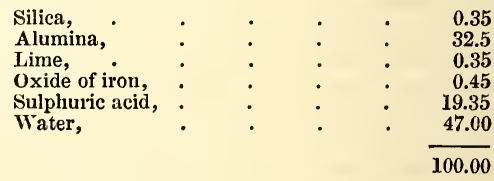

Silicious or sandy soils.-This term can only with strict propriety be applied to soils having at least seven-eighths of sand in their composition, and which do not effervesce when acids are applied. Calcareous sandy soils, which may be considered a variety of this soil, are readily distinguished from the strictly silicious by effervescing when acid tests are employed. "Sandy soils are composed of the rough, dry, and hard granulations of stones that have been disintegrated and reduced to particles by the action of the various agencies that are supposed to have contributed to the formation of the different substances that are found in the composition of the globe. Sands are much mixed with other substances, and accordingly vary very much in colour: white, red, yellow, brown, black, and green, with many varieties, as the chief colouring qualities are wanting or abundant. When more unequally reduced, sand forms gravel; when composed mostly of flints, it is called silicious; with mica it is micaceous; and when 
very finely reduced, it constitutes dust and quicksand. Silica is a most abundant earth, forming a great proportion of primitive rocks, and of the bases of the globe. In fertile sorts it averages 50 to 80 per cent; in barren lands, 40 to 70 ; but these proportions never mark with much precision the quality of the soil, as so very much depends on mixture and combination in a geographical position. Silica absorbs 0.25 , or one-fourth of its weight of water, without dropping, and evaporates twice as fast as chalk, and three times faster than alumen."DoNALDSON on Soils and Manures.

Silica forms the chief constituent in most soils, frequently forming nearly nine-tenths of its whole weight; "but this is by no means always the case, for in calcareous or limestone countries we frequently find soils containing a very large quantity of lime. These differences in the proportion of the earthy components of the soil give rise to the varieties of light or free, and stiff or clayey soils, which are also modified by a greater or less quantity of organic substances."-SoLLy's Rural Chemistry.

Silicious or sandy soils absorb heat from the sun readily, and hence are valuable for the production of early crops. They are also wrought with much greater ease, and at almost all seasons with equal propriety. They are incapable, however, of yielding the greatest weight of produce; and fruit trees neither live so long in them, nor are so exempt from various diseases. In homely phraseology, they are considered " hungry soils," and speedily decompose manure when applied to them. This is owing to their want of cohesion and incapability of retaining moisture. Air and moisture readily enter into them, and hence the putrification of organic matter goes on rapidly in them, and from want of body to retain its parts, it is soon washed down beyond the range of most roots, or its gaseous parts escape into the atmosphere. In the application of manures, therefore, to such a soil, no more should be applied at once than is sufficient for the sustenance of the crop of the current year. Increasing the depth by trenching will, to a certain extent, correct the former of these defects; and adding argillaceous matter to them in due proportions will transform them into a soil nearly approaching in texture and value to the alluvial.

Calcareous or limey soils.-These readily effervesce upon the application of acids, as both lime and magnesia have a powerful affinity for acids, and, in fact, are never present in the soil unless in combination with some of them, and that is most commonly the carbonic. Lime is also not unfrequently found combined with sulphuric acid, constituting gypsum or sulphate of lime. Calcareous soils are confined to the limestone and chalk formations; and in the latter case, when mixed with clay, become exceedingly difficult to work. Such soils, also, are late, and unfavourable for the brairding of seeds. However paradoxical it may appear, there are not unfrequent instances in the chalk countries where, although the substratum may be solid chalk to the depth of a hundred feet or more, there may be a great deficiency of it near the surface; and hence it is frequently brought up, by sinking shafts and tunnelling, for the purpose of being laid on as a top-dressing, and to make up what is found to be wanting in the surface-soil. Fruit trees do not long exist in a healthy condition where chalk forms the subsoil. In such a soil, according to Professor Johnston, there are $56 \mathrm{lb}$. of lime in $1000 \mathrm{lb}$. of calcareous soil; while barren soil contains only $4 \mathrm{lb}$. That is, if a soil contains more than 20 per cent of carbonate of lime, it is called a calcareous soil ; if it contains no more than 5 per cent, it is called a marly soil.

Loamy soils.-This term should be limited to soils containing at least one-third of impalpable earthy matter, and effervescing copiously with acids. Considerable discrepancy, however, appears to exist in regard to what loam really is. "Loam or mould is composed of decomposed animal and vegetable matters, is a principal material in soils, and differs greatly according to the quantity and quality of the matter itself, the state of reduction it has reached, and from the manner in which it is incorporated with the constituents of the soil. Such soils are almost invariably dry, and possess the happy medium of absorbing, retaining, and giving off moisture in the quantity most suitable to the growth of plants. Much of their fertility may be ascribed to the quality of the original subjacent formation being favourable to the growth of vegetation which grew and died upon it, and, along with animal remains, produced the quality and depth of the stratum. The property of depth generally bears a direct ratio to the fertility of the soil, or rather the latter to the former."DonalDson on Soils and Manures.

Sir H. Davy defines loam as "the impalpable part of the soil which is usually called clay or loam ;" and Reid, in "Chemistry of Nature," offers the following definition: "The term loam is applied to soils which consist of about one-third of finely-divided earthy matter, containing much carbonate of lime." Mr Stephens, in "The Book of the Farm," says-"Loam, in my opinion, consists of any kind of earth containing a sensible admixture of decomposed vegetable matter. I say," he remarks, " a sensible admixture, since no soil under cultivation, whether composed chiefly of clay or of sand, but what contains some decomposed vegetable matter. Unless, therefore, the decomposed vegetable matter of the soil so preponderates as to greatly modify the usual properties of the constituent earths, the soil cannot, in truth, be called by any other name than a clayey or sandy soil ; but when it does so prevail, a clay loam or a sandy loam is formed. But if loam is almost synonymous with clay, then a sandy loam must be a contradiction of terms. Again, a soil of purely vegetable origin-such as crude peat or leaf mould-cannot be called loam, as admixture of an earth of some sort with vegetable matter is required to make loam, under every recorded definition of that term. Thus, then, all soils have the property of clay and sand; and a considerable admixture of decomposed vegetable matter converts them into loam. Hence it is possible for husbandry to convert 
any earthy soil into a loam, as is clearly exemplified in the vicinity of large towns."

Loamy soils are by far the most valuable of any, and are also the most numerous. Hence we have clay loams and sandy loams, which are synonymous terms for strong loams and light loams - the former containing a larger proportion of clay, and the latter of sand. Closely allied to these are gravelly loams, which, with sandy loams, are exceedingly valuable, and when unaccompanied with iron are amongst the more useful of all soils. They neither retain too much water, nor do they exclude the sun's heat. They are evidently of alluvial formation, and exist most generally by the margins of rivers, or in the bottoms of valleys. We have also fibrous loams, much sought after by the plant cultivator; as well as virgin or maiden loam, equally in request. The former of these is valued for the abundance of the fibrous roots of plants it contains, which, as they decompose, afford food to the living plant; while the latter is procured from ground that has never been subjected to cultivation; and although a small portion only of organisable matter is found in it, still it is perhaps of all others the best suited to horticultural purposes.

Gravelly soils vary considerably in degree-that is, fromabsolute sand combined with small stones, to gravelly loam. The former and its nearest allies consist almost entirely of small stones and particles of silicious matter, and are barren and unproductive ; the latter of fragments of rock or water-worn stones, varying from the size of a large pea to a hazel-nut, or even larger, intermixed with sand and with more or less of loam or vegetable matter. Most of the soils in this section are open and porous, and give off moisture very quickly; and on this account, together with the heat they absorb, are apt to burn in warm dry seasons. Such soils seldom suffer from wet, unless when very shallow and on a retentive subsoil. If unmixed with iron, and containing a sufficient quantity of loam or vegetable matter, gravelly soils are valuable in the garden, yielding early spring crops, and tending, from their dryness, to maintain them during the frosts of winter. They are not, however, in general adapted for fruit trees or fruit-bearing plants. The small stones of which gravelly soils are so much composed tend greatly to increase their temperature, and act as better conductors of heat than the finer particles of sand; but although such soils are more easily heated than sands, they are cooled also in the same proportion. Gravelly soils, when loam and vegetable matter predominate, are, next to light or moderately light loams, amongst the very best garden soils.

Peaty or mossy soils.-These are evidently of vegetable origin, and as they contain a superabundance of vegetable matter, often in an inert state, they require the agency of other matter to break them down and fit them for the growth of plants. Peaty soils in any of their modifications are seldom employed in the formation of garden soils, although, in the state of that known amongst plant cultivators as " heath mould " or " sandy peat," they are indispensable in good culture. Such peat is found, for the most part, on the sides of mountain ranges, and near the tops of secondary hills, and is composed of a basis of black soil, the result of deeayed vegetation of the lowest order of plants, intermixed with the remains of heath, Caluna vulgaris, \&c., and abounding in small white sparkling particles of silica, or fragments of primitive rocks, and sometimes also of small fragments of the more recently formed white sandstone. It is found in shallow beds, seldom many inches deep, evidently the accumulation of ages, partly transported thither by rain and melting suow from a higher altitude, and partly by the decomposition of vegetation. To be useful for plants, this soil must be free of oxide of iron, which often abounds in such formations. Turfy peat is the result of semi-decayed vegetables, various species of Musci, Juncece, Cyperacece, \&c. ; and when dried moderately in the sun and broken down, is valuable for most plants, and may be modified by the addition of sand or loam to suit the majority in cultivation. Black and inert peat is in its natural state totally useless, and can only be rendered useful by burning, charring, roasting, draining, reducing in volume and shape, and by a large admixture of sharp silicious sand. That from the sea-beach is valuable, as carrying with it a considerable amount of saline matter. Peat bogs, which have been drained and cultivated, contain often 80 to 90 per cent of organic matter.

The origin of peat, when in masses, opens up a broad page in the historical changes which a country has undergone. Often under 10 or 15 feet of this substance, and extending over hundreds of acres, may be not only discovered a rich argillaceous soil, but also the marks of former cultivation; in other cases the same soil, in which are still to be found the roots of oaks and other trees, while remains of their trunks and branches are scattered through the superincumbent mass, and these of a size with which our present largest trees are as mere saplings, and, strange to say, often at altitudes where the whole art of man could not get the same species to grow at the present day. Such magazines of almost neglected matter might be advantageously employed by being transported to light sandy or gravelly soils, in which there is a great deficiency of vegetable matter. Their ashes also form valuable stimulants, and assist in the germination of seeds on all, but more especially on strong clayey soils. Celery, potatoes, and carrots are almost the only garden crops that seem to prefer an improved peaty soil. Fruit trees seldom succeed in such, although many shrubs and American trees do.

Subsoils exercise a considerable influence on the soil above them. Thus a clay subsoil, being impervious to water, renders the surface soil, of whatever nature it may be, wet and cold. A gravelly or porous subsoil tends greatly to dry, and of course render warmer, the surface-soil, even should it be clay; and when a porous soil rests upon a gravelly subsoil, scarcely any degree of humidity can injure it. A rocky subsoil may be either drying or retentive, according to its structure; "a massive subsoil keeping 
the soil above it habitually wet; while a stratified one, if the lines of stratification dip downwards from the soil, will preserve even a retentive soil above it in a comparative dry state." Much, therefore, in the formation of garden soils depends on the subsoil, and to this too little attention has been in general paid. All efforts to improve, or even to make a new garden soil, by the collection of the best materials, will be unsatisfactory, if the subsoil is left impervious to the free passing downward of water, or if left in its natural state, when containing a superabundance of mineral matter unprofitable or deleterious to the roots of trees or plants to be cultivated over it. Where subsoils are retentive of water, they should be thoroughly drained; where they contain mineral matter unfit for the roots of plants, their character should be changed by the application of counteracting agents.

All soils consist of two parts-organic and inorganic; the former chiefly owes its origin to the remains of vegetables and animals, "which," as Professor Johnston observes, "have lived or died in or upon the soil, which have been spread over it by rivers and rains, or which have been applied by the hands of man, for the purpose of increasing its natural fertility. This organic part varies very much in quantity in different soils. In some, as in peaty soils, it forms from 50 to 70 per cent of their whole weight; and even in rich long-cultivated soils it has been found, in a few rare cases, to amount to as much as 25 per cent. In general, however, it is present in much smaller proportion, even in our best arable lands. The rich alluvial soil of the valley of the Nile contains only 5 per cent of dry organic matter. In stiff and very clayey soils, 10 to 12 per cent is sometimes found; in very old pasture-lands, and in gardens, vegetable matter occasionally accumulates so as to overload the upper soil. To this organic matter the name of humus has been given by some writers. It contains, or yields to the plant, the ulmic, humic, and other acids. It supplies also, by its decay in contact with the air which penetrates the soil, much carbonic acid, which is supposed to enter the roots, and thus to assist the growth of living vegetables. During the same decay, ammonia is likewise produced, and this in large quantity, if animal matter be present in considerable abundance. Other substances more or less nutritious are also formed from the organic matter in the soil. These enter by the roots, and contribute to nourish the growing plant, though the extent to which it is fed from this source is dependent both upon the abundance with which these substances are supplied, and upon the nature of the plant itself, and of the climate in which it grows.

"Another influence of this organic portion of the soil whether naturally formed in it, or added to it as manure, is not to be neglected. It contains-as all vegetable substances do-a considerable quantity of inorganic, that is, of saline and earthy matter, which is liberated as the organic part decays. Thus living plants derive from the remains of former races, buried VOL. II. beneath the surface, a portion of that inorganic food which can only be obtained from the soil, and which, if not thus directly supplied, must be sought for by the slow extension of their roots through a greater depth and breadth of the earth in which they grow. The addition of manure to the soil, therefore, places within the easy reach of the roots not only organic, but also inorganic food."

The inorganic part of soils is that which remains behind when the organic part is destroyed or abstracted by burning the whole to a red heat in the open air, and consists entirely of earthy and saline substances. The former are insoluble in water, while the latter are soluble. The amount of soluble matter contained in the majority of soils is not great, "and consists of common salt, gypsum, sulphate of soda, sulphate of magnesia, with traces of the chlorides of calcium, magnesium, and potassium; and of soda, potash, lime, and magnesia, in combination with nitric and phosphoric, and with the humic and other organic acids. It is from these soluble substances that the plants derive the greater portion of the saline ingredients contained in the ash they leave when burned. In hot weather, the evaporation on the surface causes the water to ascend from the porous subsoil; and as this water always brings with it a quantity of saline matter, which it leaves behind when it rises in vapour, it is evident that the longer the dry weather, and consequent evaporation from the surface, continues, the thicker the incrustations will be, or the greater the accumulations of saline matter on the surface. When rain falls, the saline matter is dissolved, and descends again to the subsoil. In dry weather it reascends; hence the surface-soil of any field will contain a larger proportion of soluble inorganic matter in the middle of a hot dry season, than in one of even ordinary rain : hence also the fine dry weather, which, in early summer, hastens the growth of crops, and later in the season favours their ripening, does so probably, among its other modes of action, by bringing up to the roots, from beneath, a more ready supply of those saline compounds which the crop requires for its healthful growth.

"The earthy or insoluble portion of soils rarely constitutes less than $95 \mathrm{lb}$. in 100 of the whole weight. It consists chiefly of silica, in the form of sand ; of alumina, mixed or combined with silica, in the form of clay; and of lime, in the form of carbonate of lime. It is rarely free, however, from 2 or 3 per cent of oxide of iron; and, where the soil is of a red colour, this oxide is often present in still larger proportion. A trace of magnesia also may be almost always detected, and a minute quantity of phosphate of lime. The principal ingredients, however, of the earthy part of all soils are sand, clay, and lime; and soils are named or classified according to the quantity of each of these three they may happen to contain."

The following simple rule has been laid down by $\mathrm{Mr}$ Johnston, by which the practical cultivator may determine sufficiently accurately the nature of an ordinary soil : "If an ounce of soil be intimately mixed with a pint of water 
till it is perfectly softened and diffused through it, and if, after shaking the heavy parts, it be allowed to settle for a few minutes, the sand will subside, while the clay-which is in finer particles, and less heavy-will still remain floating. If the water and fine floating clay be now poured into another vessel, and be allowed to stand till the water has become clear, the sandy part of the soil will be found on the bottom of the first vessel, and the clayey part on that of the second, and they may be dried and weighed separately. If 100 parts of dry soil, not peaty or unusually rich in vegetable matter, leave no more than 10 of clay when treated in this manner, it is called a sandy soil ; if from 10 to 40 , a sandy loam; if from 40 to 70 , a loamy soil ; if from $\bar{\tau} 0$ to 85 , a clay loam; from 85 to 95 , a strong clay soil ; and when no sand is separated at all by this process, it is a pure ayricultural clay." Such a soil is not of very frequent occurrence; and were it even so, it would be one of the worst for garden purposes.

"If a soil contain more than 5 per cent of carbonate of lime, it is called a marl; if more than 20 per cent, it is a calcareous soil. Peaty soils, of course, are those in which the vegetable matter predominates very much. The quantity of vegetable or other organic matter is determined by drying the soil well upon paper in an oven, until it ceases to lose weight-taking care that the heat is not so great as to char the paperand then burning in the open air a weighed quantity of the dried soil: the loss by burning is nearly all organic matter. In stiff clays this loss will include also a portion of water, which is not wholly driven off from such soils by drying upon paper in the way described. To estimate the lime, a quantity of the soil should be heated in the air till the organic matter is burned away. A weighed portion (200 or 300 grains) should then be diffused through half a pint of cold water, mixed with half a wine-glassful of spirit of salt (muriatic acid), and allowed to stand for a few hours, with occasional stirring. When minute bubbles of gas cease to rise from the soil, the water is poured off, the soil dried, heated to redness as before, and weighed: the loss is nearly all lime."-JoHnston's Agricultural Chemistry and Geology, pp. 81, 86.

\section{$\S$ 2.-DETERMINATION OF THEIR NATURE, ETC.}

This is only to be arrived at, with any degree of certainty, by botanical, chemical, or mechanical tests-that is, by observing the plants that grow on them; by chemical analysis; and by their roughness, smoothness, taste, smell, or fracture.

For almost all practical purposes the former will be found a safe guide, but for strictly scientific determination a chemical analysis is requisite, while many who are ignorant of both botanical and chemical science arrive at pretty correct conclusions by the last of these. The qualities of a soil may be determined with considerable accuracy, by a good botanist, from the plants which grow upon it, so far as the actual state of the soil has reference to culture; they do not, however, always indicate the improvement of which it is susceptible, although they do so to a very considerable extent. The Tussilago farfara, or common colt's-foot, is as certain an inhabitant of the blue clay as its existence. It has appeared in the clay brought from the bottom of the London basin when boring for water, and exposed to the air, in places where it existed not before ; and the accidental deposit of a single cartload of blue clay upon a barren sandy waste has been soon followed by the appearance of this plant. The Salicornia herbacea is only found in saline soils; the Vaccinium uliginosum in peaty ones; the Rumex acetosa in ferruginous; the Arenaria rubra in sandy; and the Caltha palustris in marshy soils. Marshy soils are indicated with considerable certainty by the plants which grow in them, and the same may be said of very dry soils; but the earths of fertile soils cannot be inferred with so much certainty, as cultivation, manures, and circumstances have so much changed their primitive character. Chemical analysis is not always to be depended upon either, for testing the soil of a large field for example, as there may be a very considerable difference in various parts of it; so that, in fact, the result of the analysis is that of the identical spot from which the specimen was taken, and may be very different from that of other parts-nay, probably from the greater part of the whole field. Loudon thought that " by far the greater number of plants only indicate the state of a soil relatively to water and organic matter. In short," he observes, " nature may be said to have only three kinds of soil relatively to plants-the dry, the moist, and the fertile." "Such a mode of estimating the comparative properties of soils," says Mr Stephens, "might be correct enough were their products constant; but when these change with the circumstances in which the soil is placed, the test scarcely admits of general application." The very circumstance of changing the condition of soils, whether by adding to or diminishing their natural constituent parts, as by manuring, burning, or draining, no doubt has the effect of causing the disappearance of many plants and the appearance of others; but these latter indicate, by their presence, the change produced, and form a new series of tests by which the soil, in its improved state, is to be again recognised. A copious application of lime will produce plants affecting a calcareous soil, their appearance indicating the addition of a new matter that did not previously exist in it. Draining produces similar results - namely, the disappearance of bog or aquatic plants, and the vegetation of moderately dry soils appearing. High cultivation is productive of a still greater change in vegetable products; for with the manure the seeds of plants are brought in, the produce of more fertile soils-and even with the crops sown-the seeds of plants not even indigenous to our country, although now erroneously considered as such, will be introduced. The Chrysanthemum segetsm may be offered as an example. Nothing but a complete 
change in the constituent parts of a soil will cause the plants of one soil naturally to affect another, a circumstance every plant cultivator knows from experience.

Taking soils in the order in which we have already placed them, we find that in

Argillaceous soils, Saponaria officinalis, Orobus tuberosus, Lotus major, Carex (many species), Juncus (many species), Tussilago farfara, Potentilla reptans, P. anserina, P. argentea, Thalictrum flavum, Spiræa ulmaria, Aira cæspitosa, Equisetum arvense, \&c., exist.

In Calcareous soils, Onobrychis sativa, Clematis vitalba, Veronica spicata, Verbascum lychnitis, Lithospermum officinale, Campanula glomerata, Phyteuma orbiculare, Helianthemum vulgare, abound.

In Silicious soils, Veronica verna and most of the annual speedwells, Silene Anglica, Echium vulgare, Herniaria glabra and H. hirsuta, Arenaria rubra, Spergula arvensis, Papaver argemone, P. hybridum, Thymus serpyllum, Rumex acetosella, Acynos vulgaris, Trifolium arvense, Lamium purpureum, abound.

Loamy soils produce Anagallis arvensis, Sherardia arvensis, Valerianella olitoria, Lithospermum arrense, Lapsana communis, Sonchus oleraceus, Fumaria officinalis, Gnaphalium margratelia, Triticum repens, Chrysanthemum leucanthemum.

Gravelly and peaty.-In the former chiefly Polygonum convolvulus, P. aviculare, Aira caryophyllea, Agrostis vulgaris, Lolium perenue, Papaver dubium; and on the latter Vaccineum (various species), Spergula subulata, Tormentilla officinalis, Calluna vulgaris, Erica tetralix, Galium aparine, present themselves.

Rumex acetosella indicates the presence of iron, Equisetum arvense a cold and retentive subsoil, Alchemilla aphanes is indicative of a most unproductive soil; while Cirsium arvense and Senecio Jacobea, although both affecting light and strong loams, indicate in both cases a fertile one, and the little Draba verna and Scleranthus annuus are found on the most sterile of all.

This list might be greatly extended, but we have confined it to such plants as are likely to be met with on soils intended for gardening operations. Stellaria media and Fumaria officinalis are indicative of a rich condition of soil.

Chemical analysis.-Most of the authorities we have consulted seem to hold out little hope that the majority of cultivators, in their present state of chemical knowledge, can derive much advantage from attempting to analyse soils themselves, and therefore recommend the employment of professed chemists, who have both the requisite conveniences and a thorough knowledge of the subject, founded on scientific principles.

The quantity of soil best adapted to a perfect analysis is stated to be 400 grains; it should be collected in dry weather, and exposed to the air till it becomes dry to the touch. Discretion must be used in selecting specimens for examination, as soils differ much even within a very limited space. It should therefore be taken from different places, and from 2 to 3 inches below the surface. It is here that the first and principal difficulty arises in the true analysis of soils; and if due caution be not exercised, the chemical result may be as far from the truth, as regards the whole field, as if we trusted to its nature from examining the plants which grow upon it. Soils, when collected for this purpose, if not immediately submitted to the chemist, should be kept in close-stopped phials until wanted.

All soils, as may be supposed, are variable in composition: their nature is generally a good deal dependent on the subsoil and the stony matters beneath the surface, many of which are slowly decomposing or crumbling away, and adding to the soil the substances of which they consisted.

We have already stated that a soil, to be useful for the purpose of cultivation, consists of silica, potash, soda, alumina, lime, magnesia, oxide of iron, oxide of manganese, phosphoric acid, sulphuric acid, chlorine, and organic matter. We shall now glance at the relative importance of these constituents to plants, and their amount in the soil, upon the authority of Dr Henry Madden of Brighton, as quoted in "T'he Book of the Farm."

"Silica.-This is the pure matter of sand, and also constitutes on an average about 60 per cent of the various clays, so that in a soil it generally amounts to from 75 to 95 per cent. In its uncombined state it has no direct influence upon plants, beyond its mechanical action in supporting the roots, \&c.; but as it possesses the property of an acid, it unites with various alkaline matters in the soil, and produces compounds which are required in greater or less quantity by every plant. The chief of these are the silicates of potash and soda, by which expression is meant the compounds of silica, or more properly silicic acid, with the alkalies potass and soda.

"Alumina.-This substance never exists pure in soil. It is the characteristic ingredient of clay, although it exists in that compound to the extent of only 30 or 40 per cent. It exerts no direct chemical influence on vegetation, and is scarcely ever found in the ashes of plants. Its chief value in soil, therefore, is owing to its effects in rendering soil more retentive of moisture. Its amount varies from $\frac{1}{2}$ per cent to 13 per cent.

"Oxide of iron.-There are two oxides of iron found in soils-namely, the peroxide and protoxide; one of which, the protoxide, is frequently very injurious to vegetation-indeed, so much so, that $\frac{1}{2}$ per cent of a soluble salt of this oxide is sufficient to render soil almost barren. The peroxide, however, is often found in small quantities in the ashes of plants. The two oxides together constitute from $\frac{1}{2}$ to 10 per cent of soil. The blue, yellow, red, and brown colours of soil are more or less dependent upon the presence of iron.

"Oxide of manganese.-This oxide exists in nearly all soils, and is occasionally found in plants. It does not, however, appear to exert any important influence either mechanically or chemically. Its amount varies from a mere 
trace to about $1 \frac{1}{2}$ per cent. It assists in giving the black colour to soil."

These four substances constitute by far the greatest bulk of every soil, except the chalky and peaty varieties, but nevertheless, chemically speaking, they are of trifling importance to plants, whereas the remaining eight are so absolutely essential that no soil can be cultivated with any success, unless provided with them, either naturally or artificially. And yet, when it is considered that scarcely any of them constitute 1 per cent of the soil, their value will no doubt excite surprise. The sole cause of their utility lies in the fact that they constitute the ashes of the plants; and as no plant can by possibility thrive without its inorganic constituents (its ashes), hence no soil can be fertile which does not contain the ingredients of which these are made up. The very small per-centage of these ingredients in any soil necessitates a minute analysis of every soil before it can be ascertained whether or not it contains any or what propor- tion of these ingredients. But the reason for such minuteness in analysis becomes obvious when we consider the immense weights which have to be dealt with in practical agriculture; for example, every imperial acre of soil, considered as only 8 inches deep, will weigh 1884 tons, so that 0.002 per cent-that is, only a twothousandth per cent, the amount of sulphuric acid in a barren soil-amounts to $80.64 \mathrm{lb}$. in the imperial acre !

"Potash and soda exist in variable quantities in many of the more abundant minerals, and hence it follows that their proportion in soil will vary according to the mineral which produced it. For the sake of reference, I have subjoined the following table, which shows the amount per cent of alkalies in some of these minerals, and likewise a rough calculation of the whole amount per imperial acre, on the supposition of a soil composed solely of these rocks, and of a depth of 10 inches, and the amount is abundant beyond conjecture.

\begin{tabular}{|c|c|c|c|c|c|c|c|c|c|c|c|}
\hline \multicolumn{2}{|c|}{$\begin{array}{l}\text { Name of } \\
\text { Mineral. }\end{array}$} & $\frac{\begin{array}{c}\text { Amount per } \\
\text { cent of Alkali. }\end{array}}{17.75}$ & Name of Alkali. & \multicolumn{8}{|c|}{$\begin{array}{l}\text { Amount per imperial acre in a soil } \\
10 \text { inches deep. }\end{array}$} \\
\hline $\begin{array}{l}\text { Felspar, } \\
\text { Clinkstone, } \\
\text { Clayslate, } \\
\text { Basalt, }\end{array}$ & $\dot{\text {. }}$ & $\begin{array}{l} \\
3.31 \text { to } 6.62 \\
2.75 \text { to } 3.31 \\
5.75 \text { to } 10 .\end{array}$ & $\begin{array}{l}\text { Potash, } \\
\text { Potashand soda, } \\
\text { Potash, } \\
\text { Potasl and soda, }\end{array}$ & $\begin{array}{r}\text { tons } \\
422 \\
71 \\
35 \\
17\end{array}$ & $\begin{array}{r}\text { cwt } \\
18 \\
17 \\
18 \\
0\end{array}$ & $\begin{array}{cc}\text { qrs } & \text { lb } \\
2 & 8 \\
2 & 0 \\
3 & 0 \\
0 & 0\end{array}$ & $\begin{array}{ll}8 & \\
8 & \\
0 & \text { to } \\
0 & \text { to } \\
0 & \text { to }\end{array}$ & $\begin{array}{r}\text { tons } \\
143 \\
71 \\
25\end{array}$ & $\begin{array}{r}\text { cwt } \\
15 \\
17 \\
7\end{array}$ & $\begin{array}{l}\text { qrs } \\
0 \\
2 \\
3\end{array}$ & $\begin{array}{l}\mathrm{lb} \\
0 \\
0 \\
7\end{array}$ \\
\hline
\end{tabular}

"One unacquainted with chemistry will naturally ask the question-How is it that these alkalies have not been long ago washed away by the rain, since they are both so very soluble in water? The reason of their not being dissolved is the following, and it may in justice be taken as an example of those wise provisions of nature, whereby what is useful is never wasted, and yet is at all times ready to be abundantly supplied.

"These alkalies exist in combination with the various other ingredients of the rock in which they occur, and in this way have such a powerful attraction for those ingredients that they are capable of completely resisting the solvent action of water as long as the integrity of the mass is sustained. When, however, it is reduced to a perfectly impalpable powder, this attraction is diminished to a considerable extent, and then the alkali is much more easily dissolved. Now this is the case in soil, and consequently while the stony portions of the soil contain a vast supply of these valuable ingredients in a condition in which water can do them no injury, the impalpable powder is supplied with them in a soluble state, and hence in a condition available to the wants of vegetation.

"In the rocks which we have mentioned the alkalies are always associated with clay, and it is to this substance that they have the greatest attraction: it follows, therefore, that the more clay a soil contains, the more alkalies will it have; but at the same time it will yield them less easily to water, and through its medium to plants."

The following analysis of a soil, drawn up by Baumhauer of Utrecht, from a tract in North Holland gained by embankment from the sea, without reference to its barrenness or fertility, is given by Mr Stephens, in order to show the great variety of substances contained in it, and also how important they are in the growth of plants :-

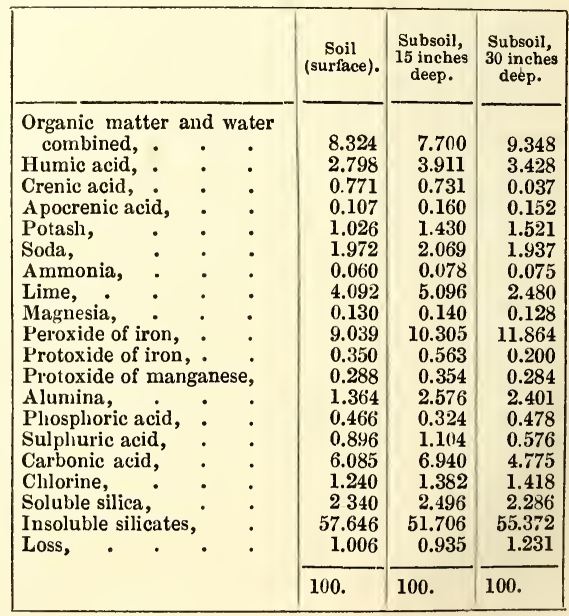

" On comparing the constituents of' such a soil as the above with the mineral ingredients obtained by incineration from the ashes of plants, it is found that plants withdraw from the soil chiefly its alkaline, mineral, aciủ, and earthy ingredients; and if all these were not essential to the very existence of the plants, they would 
not of course be taken up by them; and as the plants constituting our cultivated crops withdraw those ingredients in a varied amount, it follows that unless the soils we cultivate contain them in ample amount and variety, it will be impossible for the plants placed above them to arrive at a perfect state of development of all their parts; for, chemically speaking, and rationally speaking too, soils cannot be expected to produce crops abundantly, unless they contain a sufficient supply of every ingredient which all the crops we wish to raise require from them.

"The practical purpose of all analyses of plants and soils should, therefore, be to make us acquainted with the constituents of every variety of cultivated crop at their different stages of growth, and to ascertain whether or not each soil in use contains a sufficient supply of such ingredients. The analysis of plants should have thus a twofold object-namely, to guide the cultivator in the treatment of the plants at the various stages of their growth, and to instruct him as to what quantity the ripe plant in its healthy state finally carries off of those ingredients from the soil."

Professor Johnston, in "Lectures on Agricultural Chemistry and Geology," observes, " The latter only of these two applications of such knowledge has hitherto been kept in view by chemists; and so little has been done in reference to it, that we scarcely know as yet what any one entire plant, when fully ripe, carries off from the soil. In reference to the former application, the few imperfect researches detailed in the preceding sections (of the second edition of his Lectures) contain all that we yet know. We may well say, therefore," he concludes, "that our knowledge of the inorganic constituents of plants is yet in its infancy, and that our present opinions upon the subject ought, therefore, to be permitted to hang very loosely about us."

'The following table, showing the per-centage of mineral ingredients taken from the cultivated soil by some plants, is given by Mr Stephens :-

\begin{tabular}{|c|c|c|c|c|}
\hline \multirow{2}{*}{$100 \mathrm{lb}$. of } & \multicolumn{2}{|c|}{ ROOT OR TUBer. } & \multicolumn{2}{|c|}{ Leaves. } \\
\hline & Undried. & Dried. & Undried. & Dried. \\
\hline Potato, . & 0.8 to 1.1 & 3.2 to 4.6 & 1.8 to 2.5 & 18 to 25 \\
\hline Turnip, : & 0.6 to 0.8 & 6.0 to 8.0 & 1.5 to 2.9 & 14 to 20 \\
\hline Beet, . & - & 6.3 & - & - \\
\hline $\left.\begin{array}{l}\text { Jerusalem } \\
\text { artichoke, }\end{array}\right\}$ & & 6.0 & 一 & - \\
\hline Carrot,. & 0.7 & 5.1 & - & 16.42 \\
\hline Parsnip, ; & 0.8 & 4.3 & 一 & 15.76 \\
\hline $\left.\begin{array}{l}\text { Mangold- } \\
\text { wurzel, }\end{array}\right\}$ & 1.2 & 7.0 & 0.53 & 7.55 \\
\hline Cabbage, . & - & - & 一 & 18.25 \\
\hline
\end{tabular}

On the results given in the above table, together with three others of a similar nature by $\mathrm{Mr}$ Stephens, Professor Johnston makes the following observations: "The quantity of inorganic matter contained in the same weight of the different crops we raise, or of the different kinds of vegetable food we eat, or with which our cattle are fed, is very unlike; and the quan. tity contained in different parts of the same plant is equally unlike. These results cannot be the effect of accident. They are constant on every soil, and in every climate; they must, therefore, have their origin in some natural law. Plants of different species must draw from the soil that proportion of inor ranic matter which is adapted to the constitution, and is fitted to supply the wants of each; while of that which has been admitted by the roots into the general circulation of the plant, so much must proceed to and be appropriated by each part as is suited to the functions it is destined to discharge. And as from the same soil different plants select different quantities of saline and earthy matter, so from the same common sap do the bark, the leaf, the wood, and the seed select and retain that proportion which the healthy growth and development of each requires. It is with the inorganic as with the organic food of plants-some draw more from the soil, some less; and of that which circulates in the sap only a small portion is expended in the production of the flower, though much is employed in forming the stem and leaves." Of the great utility of analysis Dr Ure observes, that "no system can be devised for the improvement of land independently of experiment; but there are few cases in which the labour of analytic trials will not be amply repaid by the certainty with which they denote the best methods of melioration, and this will particularly happen when the defects of composition are found in the proportions of the primitive earths. In supplying organic matter a temporary food only is provided for plants, which is in all cases exhausted by means of a certain number of crops; but when a soil is rendered of the best possible constitution and texture with regard to its earthy parts, its fertility may be considered as permanently established. It becomes capable of attracting a very large portion of vegetable nourishment from the atmosphere, and of producing its crops with comparatively little labour or expense."

Determining the quality of soils mechanically and empirically.-The difficulties which present themselves to most cultivators in carrying out a scientific chemical analysis of soils have been noticed: we shall now briefly state more simple means by which the most important constituents of soils may be determined, so far, at least, as is applicable to cultivation.

The best practical directions on this head are those published in the "Encyclopedia of Gardening," of which the following is an abbreviated extract:-

"The specific gravity of a soil may be ascertained, by introducing into a phial which will contain a known quantity of water equal volumes of water and of soil; and this may easily be done by pouring in water till it is half full, and then adding the soil till the fluid rise to the mouth. The difference between the weight of the soil and that of the water will give the result. Thus, if the bottle contains 400 grains of water, and gains 200 grains, when half filled with water and half with soil, the specific gravity will be 2 - that is, it will be twice as heavy as water; 
and if it gained 165 grains, its specific gravity would be 1825 , water being 1000 .

"The presence of clay and sand in any soil is known - the first by its tenacity, the other by its roughness to the touch, and by scratching glass when rubbed upon it.

"The presence of calcareous matter in soil may be ascertained by simply pouring any acid upon it, and observing if it effervesces freely. Muriatic acid is the best for this purpose. Cal careous soils, magnesian soils and clays, are, for the most part, softer to the touch than arenarious soils. To ascertain the quantity of calcareous earth present, dry soil thoroughly, and weigh 100 grains of it, which gradually add to 1 drachm of muriatic acid, diluted with 2 drachms of water, in a phial poised in a balance; the loss of weight will indicate the escape of carbonic acid, which will be 44 per cent of the quantity of calcareous earth in the soil.

" The presence of organised matter in any soil may be ascertained very satisfactorily by weighing it after it is thoroughly dried, then subjecting it to a red heat, and weighing it again-the weight so found will be the proportion of organic matter, and carbonic acid gas, if there should be any. The same object may also be attained by ascertaining the specific gravity of the soil, but with less accuracy.

"The presence of metallic oxides in a soil may generally be known by their colour. Ferruginous soils are red or yellow; cupreous soils interspersed with greenish streaks, \&c. Cupreous impregnations of soils are rare, and the usual green matter in such soils as the greensand of English geologists appears to be coloured by iron, which is almost the only metallic impregnation in considerable quantity in any soil.

"The presence of salt, sulphur, coal, \&c., may be known by the absence or peculiarity of vegetation, as well as by colour, and the appearance of the water of such soils. Saline soils may be distinguished by the taste; sulphureous soils by their smell when thrown on a hot iron ; and the presence of coal by its fragments, which will be left after the soluble matters are removed by water and muriatic acid.

" The capacity of a soil for retaining water may be thus ascertained: an equal portion of two soils, perfectly dry, may be introduced into two tall cylindrical glass vessels, in the middle of each of which a glass tube has been previously placed. The soils should be put into each in the same manner, not compressed very hard, but so as to receive a solidity approaching to that which they possessed when first obtained for trial. If, after this preparation, a quantity of water be poured into the glass tubes, it will subside, and the capillary attraction of the soils will conduct it up the cylinders towards the top of the vessels. That which conducts it most rapidly, provided it does not rise from the weight of the incumbent column of water in the tube, may be pronounced to be the better soil.".

" Soils, considered scientifically," says Dr Madden, " may be described to be essentially a mixture of an impalpable powder, with a greater or smaller quantity of visible particles, of all sizes and shapes. Careful examination will prove to us that, although the visible particles have several indirect effects of so great importance that they are absolutely necessary to soil, still the impalpable powder is the only portion which directly exerts any influence upon vegetation. This impalpable powder consists of two distinct classes of substances; viz., inorganic or mineral matters, and animal and vegetable substances, in all the various stages of decomposition. A very simple method may be employed to separate these two classes of particles from each other ; viz., the impalpable powder and the visible particles ; and in so doing, we obtain a very useful index to the real value of the soil. Indeed, all soils, except stiff clays, can be discriminated in this manner. The greater the proportion of the impalpable matter, the greater, coeteris paribus, will be the fertility of the soil. To effect this separation, the following easy experiment may be performed: Take a glass tube, 2 feet long, closed at one end ; fill it about half full of water, and shake into it a sufficient quantity of the soil to be examined to fill the tube about 2 inches from the bottom, then put in a cork, and, having shaken the tube well to mix the earth and water thoroughly, set the tube in an upright position for the soil to settle down. Now, as the largest particles are of course the heaviest, they fall first, and form the undermost layer of the deposit, and so on in regular gradation, the impalpable powder being the last to subside, and hence occupying the uppermost portion. Then, by examining the relative thickness of the various layers, and calculating their proportions, a very accurate mechanical analysis of the soil may be made."

Colour of soils.-Soils vary in colour from black to dirty white, the intermediate ones being red, blue, brown, light yellowish brown, \&c. They derive these colours, for the most part, from the oxides of iron contained in them, and also from decomposing vegetable substances, which all surface-soils contain in a greater or less degree: some black soils may be totally devoid of iron altogether, but these are few. All very black soils, such as peat and deep deposits of vegetable mould, are inert; and hence so is old garden mould, long under cultivation and gorged with organic manure. The protoxide of iron is a powerful agent in communicating a black colour, sometimes, in stiff clays. White soils are chiefly over the chalk formation, and the sandy deposits found near the sea-shore are often of a yellowish white, both, however, darkened by the addition of organic manure.

Red soils, or rather dull brownish red, derive their colour from peroxide of iron.

Blue soils are found in the basins of stagnant water, and in deposits of great depth, such as the London basin of blue clay; they change colour to a darkish brown by exposure and cultivation.

Brown and light-yellowish brown-coloured soils are by far the most common as well as the most valuable; and although this colour is derived from oxide of iron, it does not often exist to a very injurious extent, excepting in gravelly soils of a binding nature. A greater variety of colour exists in subsoils; and the brighter these 
are, the more injurious they are to the soil over them.

The effects of colour on soils are various, and have a considerable influence in regulating the quantities of heat absorbed from the sun's rays. The darker-coloured soils absorb most heat, while the lighter-coloured ones reflect most of that element. Much, however, of the absorbent property depends on the nature of the soil, whether it be free of superabundant water or the reverse ; and hence a great difference exists in this respect between water-logged black peat and dry black soil containing abundance of organic matter, although the latter facilitates the preservation of temperature, in consequence of the vegetable and animal matter it contains, when heated to the same degree, cooling more slowly than a wet pale soil entirely composed of earthy matter'; while stiff clay is with difficulty heated, and soon parts with the heat it acquires. Hence it may be considered the coldest of all soils. Chalks are heated with difficulty, but, being drier, when once heated they retain it longer, less being consumed in causing the evaporation of their moisture. Soils containing much carbonaceous or ferruginous matter acquire a much higher temperature from the sun than pale soils. From the experiments made by Schuibler with the thermometer in the shade, sand of a natural colour indicated a temperature of $112 \frac{1}{2}^{\circ}$, black sand, $123 \frac{1}{2}$, and white sand, $110^{\circ}$, giving a difference of $13^{\circ}$ in favour of a black colour.

Sir H. Davy ascertained that a black-coloured soil containing one-fourth of vegetable matter increased in temperature in one hour $23^{\circ}$ by exposure to the sunshine; while a white chalk soil only gained $4^{\circ}$ in the same time, and under the same circumstances. But the black soil, when removed into a shaded place, cooled $15^{\circ}$ in half an hour; while the chalky soil only lost $4^{\circ}$ in the same time, and under the same circumstances.

Most physiologists agree that the pure soil, without organised matter and water, is of no other use to plants than merely affording them a fixed abode, and a medium by which they may fix themselves to the globe. Soil is also necessary to plants by enabling them to obey those laws of nature by which their roots are kept below the surface, and their leaves exposed to the free atmosphere.

"It is commonly supposed that plants derive the whole of their food from the soil, but this is a great error. It is a fact well ascertained by chemical experiments, that plants derive the greater part of their nourishment from the air, although the soil is equally essential to their growth."-SoLLY in Rural Chemistry.

In general, much more depends on the texture of a soil, and its capacity for retaining or parting with water and heat, than on its chemical composition.

Subsoils are of vast importance, because on them depends, in a great measure, the capacity of the surface-soil for retaining or parting with water and heat. Of these, the worst is clay, kept wet with subterraneous water, and the best are those of clay resting on gravel or porous rock. The latter of these is the best of all for a garden, because, while the water finds a ready means of escape through the fissures in the rock, the roots of the trees are at the same time prevented from extending too deep, and made to take a horizontal direction nearer the surface, and also prevented from extending into a cold and uncongenial soil, causing late excitement of the sap in spring, immature ripening of the wood and buds in autumn, and consequent paucity of fruit and premature decay in the trees. The soil, for reasons already stated, is, under such circumstances, much increased in temperature, producing earlier crops of culinary vegetables, and those of much better quality, on account of the greater rapidity of their growth. Gravelly subsoils are not always to be depended upon, for these are sometimes placed over basins of impervious clay, from which water cannot escape until it rises as high as the margin of the basin itself, leaving the gravel thus saturated with water. Again, gravel abounding in oxide of iron is a bad subsoil, and often occurs. Next, therefore, to a rocky bottom, that of clean alluvial gravel is to be preferred; and in such all the productions of a garden will prosper, and that often when the climate is indifferent. Notwithstanding these advantages, how seldom, in practice, is this attended to. Much labour and expense are gone into in the improvement of the upper soil, while the lower and more important is left in its natural state. Artificial subsoils are seldom thought of, although of their advantages no one can doubt; and as nature has pointed out to us that an open porous one is the best, our first attempt at garden-making should be to imitate her in this respect. We have shown in vol. $i .$, p. 29,31 , how an artificial subsoil can be formed for fruit-tree borders; and as experience has proved the great utility of these, the enlightened horticulturist will at once see the feasibility of extending the same process under the whole of his garden. We may here only state, that where sufficient drainage material may be difficult to procure, recourse may be had to concreting, taking care that a sufficient number of drain-tiles be laid underneath for the free escape of the water, which should find its way into them through apertures left in the concrete floor. It must, however, be admitted that such excessive drainage is not applicable to all situations alike, so far as the removal of water alone is concerned; but so far as keeping the roots near the surface, and preventing their extending into a badsubsoil, the rule is absolute. Along the eastern coast of Britain, where the annual fall of rain is less than half of what it is on the western, this excessive drainage is of much less importance, unless in low situations where subterranean water abounds, and where it cannot be conveniently drained off.

Mineralogical quality of soil.-The temperature and proper amount of moisture in a soil is of far greater importance to the successful culture of plants than its mineralogical structure, and this has reference to hardy as well as to tender plants. "I entertain little doubt," Dr Lindley observes (in "Theory of Horticulture"), "that the time is at hand when it will be considered 
quite as necessary to furnish heat for the soil as for the air ; not, however, heat without moisture, for that would evidently produce much greater evils than it was intended to cure, as has indeed been found by inconsiderate experimenters. I quite agree," continues the doctor, "with Mr Writgen in believing that it is the temperature and moisture of a soil, much more than its mineralogical quality, that determine its influence upon vegetation."

Moisture in the soil.- "Water," continues the same authority, " is one of the most important elements in the food of plants, partly from their having the power of decomposing it, and partly because it is the vehicle through which the soluble matters found in the earth are conveyed into the general system of vegetation. Its importance depends, however, essentially upon its quantity. We know, on the one hand, that plants will not live in soil which, without being chemically dry, contains so little moisture as to appear dry; and, on the other hand, an excessive quantity of moisture is, in many cases, equally prejudicial. The great point to determine is, the amount which is most congenial to the constitution of the plant. The effect of continuing to make plants grow in a soil more wet than suits them, is well known to be not only a production of leaves and ill-formed shoots, instead of flowers and fruit, but, if the water is in great excess, of a general yellowness of appearance, owing, as some chemists think, to the destruction by the water of a blue matter, which, by its mixture with yellow, forms the verdure of vegetation. If this condition is prolonged, the vegetable tissue enters into a state of decomposition, and death ensues. In some cases the joints of the stem separate, in others the plant rots off at the ground; and all such results are increased in proportion to the weakness of light and the lowness of temperature. Decandolle considers that the collection of stagnant water about the neck of plants prevents the free access of the oxygen of the air to the roots ; but it seems to me," says Dr Lindley, "that much more mischief is produced by the coldness of the soil in which water is allowed to accumulate. It seems also probable that the extrication of carburetted hydrogen gas is one cause of the injury sustained by plants whose roots are surrounded by stagnant water; but upon this point we want much more satisfactory evidence than we yet possess."

Stones are valuable in all soils, as they, in general, are composed of the same material as the soil itself, and, indeed, may be considered as the soil in a previous state. By their gradually crumbling down by the action of air, frost, and moisture, they are continually adding new impalpable matter to the soil. As a considerable portion of this impalpable matter is annually consumed by plants, were it not for the presence of stones the soil would ultimately become sterility itself. The practice, therefore, of raking off stones is just removing so much of the soil itself; and the ill effects of this have been long ago observed by cultivators, while they were ignorant of the cause-so much so, it has been stated that some farmers, finding their land so much deteriorated after the removal of the stones, returned them again, after which the soil regained its former fertility.

Impalpable matter in the soil-that is, matter not to be perceived by touch-is of vast importance to vegetation, and is a product of both organic and inorganic matters. Its functions are exceedingly complicated, and are thus spoken of by Dr Madden: " In this portion of the soil, the mineral and organic matter are so completely united, that it is quite impossible to separate them from each other; indeed, there are weighty reasons for believing that they are chemically combined. It is from this portion of the soil that plants obtain all their mineral ingredients, and likewise all their organic portions, in so far as these are obtained by the roots; in fact, plants receive nothing from the soil except water, which has been associated with that portion which is at present engaging our attention.

The particles forming the impalpable matter are in such close apposition that the whole acts in the same way as a sponge, and is hence capable of absorbing liquids and retaining them. It is in this way that soil remains moist so near the surface, even after a long-continued drought; and I need not say how valuable this property must be to the plants, since by this means they are supplied with moisture from below, brought up by the capillary action of the soil during the heat of summer, when otherwise, unless artificially watered, they would very soon perish.

Another most useful function of this impalpable matter, is its power of separating organic matter from water in which it has been dissolved. Thus for example, if the dark-brown liquid which flows from a dunghill is taken and poured on the surface of some earth in a flower-pot, and a sufficient quantity added to soak the whole earth, so that a portion flows out at the bottom of the pot, this latter liquid will be found much lighter in colour than before it was poured upon the earth; and this effect will be increased, the nearer the soil approaches in its nature to the subsoil. Now, as the colour was entirely owing to the organic matter dissolved in it, it follows that the loss of colour is dependent upon an equivalent loss of organic matter ; or, in other words, a portion of the organic matter has entered into chemical combination with the impalpable mineral matter, and has thus become soluble in water. The advantage of this is, that when soluble organic matter is applied to soil, it does not all soak through with the water, and escape beyond the reach of the roots of the plants, but is retained by the impalpable portions in a condition not liable to injury from rain, but still capable of becoming food for plants when it is required." Hence liquid manure may be applied to the soil at any period of the yearan opinion that has been disputed by those who have not fully considered the matter.

The surface of soils is of vast importance, because on its inclination greatly depends the proper amount of moisture retained by the soil for the growth of plants. If too steep, it throws off the rain too rapidly; and if too flat, it often retains 
too much moisture, and occasions a loss of heat by evaporation. But, on the other hand, surfaces with a considerable inclination towards the south are much more highly heated by the sun's rays than those that are flat, because they are placed at a better angle of elevation for action of the sun's rays upon them. "If the actual increase of temperature produced by the perpendicular rays of the sun beyond the temperature in the shade be between $45^{\circ}$ and $65^{\circ}$," as has been computed by Schübler to be often the case in clear summer days, this increase would be only half as great if the same light spread itself in a more slanting direction over a surface twice as large. Hence it is sufficiently explained why, even in our own climate, the heat so frequently increases on the slopes of mountains and rocks which have an inclination towards the south.

\section{§ 3.-THEIR IMPROVEMENT.}

All soils are capable of improvement, so as to render them more fit for the purposes of remunerative cultivation. The principal operations in connection with this are draining, pulverisation, consolidation, alteration of their constituent parts, exposure to atmospheric influence, \&c.

Draining is the first and principal means of improving a soil, and without it all other attempts will prove of no avail. The number and form of drains will depend on local circumstances, of which the texture of the soil is by no means the least worthy of careful study. The leading or main drains in gardens should invariably be placed under the walks, and the overflow drain carried beyond the enclosed boundary. With this drain all the others should communicate. The depth of drains will depend on local circumstances, but it is better that they be deep than otherwise, so that they may not be disturbed by trenching or other excavations. Those in connection with the cellars, stoke-holes, \&c., should be sufficiently deep to thoroughly clear them of water; and in their first formation they should be laid even to a greater depth, to provide against the chance of sinking either the one or the other at a future time.

The principal drains in a garden, as well as in all dressed grounds, should be laid down in the most substantial manner possible, in the first instance, and their lines correctly marked on a plan upon a large scale, that they may be easily got at in case of accident, and their direction should be such as to come under permanent buildings, \&c. as seldom as possible. Without due attention to these points, much trouble and expense may be incurred, besides the derangement attendant on breaking up the ground afterwards. Main drains often offer a convenient entrance for rats and other vermin; their mouths should be guarded with efficient iron gratings; and as a farther precaution, drain-traps should be placed in them at convenient distances.-(Vide vol. i., Sect. Tanks.)

There is no operation in the whole catalogue of improvements in which so much gross ignoVOL. II. rance has been displayed as in that of draining; and, in many cases, strict adherence to the dicta laid down by professed drain-doctors has amounted to time and capital thrown away. A universal system of draining in all soils, and under all circumstances, is about as unreasonable as a universal medicine to be administered for all diseases, and to all ages, sexes, and constitutions.

The advantage of draining is twofold: the first is the elevating the temperature of the soil by the abstraction of superfluous moisture, and hence admitting the rays of heat to occupy those spaces formerly charged with water;- the soil thus becomes warmed by conduction; and the atmosphere above, by a diminution of evaporation. Air, from which the roots of plants derive so much advantage, is admitted to them, the soil and atmosphere above it becoming materially changed for the better. So important a matter is this, in relation to horticulture, that many now form their borders for their finest fruittrees upon the top of vaults kept constantly filled with air; and few exotic fruit-houses are now built without abundance of subterranean ventilation being provided. In wet districts, and where the subsoil is a close tenacious clay, the process of vaulting might be carried over the surface of a large garden, and with the most beneficial effects. Next to that would be the formation of a bed of loose stones, scoriæ, or other similar material, between the soil and subsoil, not only to act as a general drainage, but also as a means of admitting air to the roots of plants. In such a stratum draintiles should be laid in lines 6 or 8 feet asunder, and made to communicate with cross ones, which latter should have a free communication with the external air. On wet clayey retentive subsoils, this would, next to vaulting over the whole area, be the most effectual means of thoroughly improving the soil, so far as the admission of air and the abstraction of water are concerned. The temperature of a heavy soil in a garden in Hampshire has been raised $15^{\circ}$ by drains $4 \frac{1}{2}$ feet deep. Drainage not only permits the heat to penetrate deeper into the soil, but it also enables it to retain it a longer time. $\mathrm{Mr}$ Shearer has clearly shown the effect of vaulting, which is drainage carried to its fullest extent, in the case of the fruit-tree borders in the gardens at Yester-a cold locality, at the base of the Lammermuir range - to be equal to $9^{\circ}$ of increased temperature-(Vide vol. i. p. 31.)

Now, in all naturally cold countries, whatever tends to increase the temperature of the air or of the soil must be regarded as being of the utmost importance to vegetation; and as draining produces this effect, its importance must be of the greatest consequence to man, and that importance increases as we recede from the equator. For, although exactly the same depth of rain should fall annually in lat. $1^{\circ}$ south that falls in lat. $50^{\circ}$ north, the soil in the latter will be many times wetter than in the former, on account of a greatly diminished amount of evaporation, in consequence of our greater distance from the sun. The importance of draining has of late years been fully acknowledged; and 
although the modus operandi has been treated by some as a science, still by far the greater number of operators have acted upon the most incorrect principles.

Few superficial observers are perhaps aware of the great difference in temperature between thoroughly-drained soil and that in which superfluous water is allowed to lodge. Dr Lindley asserts-and, we believe, from very accurately made experiments-that the difference in summer is from $10^{\circ}$ to $20^{\circ}$. The reason for this is, that heat cannot be transmitted downwards through water. "Thus neither the heat of the sun nor the warm rain can penetrate to the roots of plants in ill-drained land; even boiling water poured on the surface would have no effect in warming soil on which cold stagnant water naturally stands. Air also cannot reach the roots of plants grown in soil of this nature. It is as important to admit air to the roots of plants as to their leaves-both extremities of plants feed on air, the roots more than the leaves." -Lindley. Place a plant in a situation where air can get no access to its leaves, and they fall off, to be followed by the decay of the stem: roots, under the same circumstances, will gradually shrink and die. The same high authority elsewhere observes, that " many believe that the whole advantage (of draining) consists in removing water; but water is not in itself an evil-on the contrary, it is the food of plants, and its absence is attended with fatal results. It is the excess of water which injures plants, just as an excess of food injures animals-with this difference, that animals can refuse what is hurtful to them, while plants have no choice, but must take into their systems whatever is in contact with the spongioles of their roots. The latter are, therefore, more readily gorged than the former. But undrained land is not merely wet; it is water-logged. All the interstices between the particles of earth being filled with water, air is necessarily absent, except that small quantity which is dissolved in the water. In this way plants are deprived of the most essential part of their food; but when the water is removed, the air takes its place, and holds in suspension as much water as the roots can thrive upon; for it is not water in a fluid state that plants prefer: it is when it has assumed a state of vapour that they feed upon it best; so that the removal of water permits air, and air-borne vapour, the best of all food for roots, to take place."

As to the depth and the distance between the drains, opinions are still, and, we believe, ever will be, at variance; the case must therefore be as it has been-left greatly to the judgment of the operator. The following very sensible remark has been made by Mr Stephens, in "The Book of the Farm," vol. ii. p. 617 , on this subject: "With our present experience, however, I do not see the utility of cutting drains as deep as 6 or 8 feet, merely to extend the space between them, when perhaps the same effect might be obtained by 3 or even 4 feet drains at narrower intervals, unless very deep drains at very wide intervals can be executed with more economy. Experience has yet much to elicit in regard to the distance that should be between sub-drains of different depths in different soils, to effect the best results."

Pulverisation.-Next in importance to rendering the soil dry by draining, is the process of pulverising, which is effected by trenching, digging, grubbing, stirring, \&c. The depth to which this operation should be carried, must of necessity depend on the soil and subsoil. Sir $\mathbf{H}$. Davy observes, "In rich clayey soils it can scarcely be too deep; and even in sands, unless the subsoil contains some principles noxious to vegetation, deep comminution should be practised. When the roots are deep, they are less liable to be injured, either by excess of rain or drought." Trenching has a most beneficial effect on all soils of a depth consistent with the best principles of horticultural culture, by reversing the surface, and mixing and transposing the whole mass operated on. It improves the temperature of the soil, renders it capable of allowing the rain-water to filter through it, and enables the roots of plants to travel freely in search of food. "By changing the surface, fresh soil is exposed to the action of the weather; by chang. ing the position of all the parts, new facilities for chemical changes are produced; and by loosening the whole mass of the soil, air and rain are more readily admitted. By loosening soil, the air is admitted among its particles, and confined there; and hence it becomes a nonconductor of heat, and is consequently warmer in winter, and cooler in summer, than if it were in one firm mass. By the confinement of air in the soil, the heat imparted to it by the sun during the day is retained, and accumulates in all free open soils to such a degree as to raise their temperature over that of the air, especially during night. From thermometrical observations made at different places, it appears that the mean temperature of the soil, at about one foot below the surface, is somewhat higher naturally than the mean temperature of the atmosphere on the same spot; and hence we may reasonably suppose that, by draining and pulverisation, the temperature of the soil may be permanently increased, as well as that of the atmosphere."-Sub. Hort., p. 55. The atmospheric air which becomes buried in the soil during the process of trenching and deep-digging -in the latter case in a less degree-becomes decomposed by the moisture in the soil, and the following chemical changes take place: "Ammonia is formed by the union of the hydrogen of the water with the nitrogen of the atmosphere, and nitre by the union of the oxygen and nitrogen. The oxygen may also unite with the carbon contained in the soil, and form carbonic acid gas and carburetted hydrogen." Heat is given out during these processes ; "and hence," as Dr Darwin, in "Phytologia," sect. 2, remarks, " the great propriety of cropping lands immediately after they have been comminuted and turned over; and this the more especially if manure has been added at the same time, as the process of fermentation will go on faster when the soil is loose and the interstices filled with air than afterwards, when it becomes compressed by its own gravity, the relaxing influence of rains, and the repletion of the partial vacuums 
formed by the decomposition of the enclosed air. The advantages of the heat thus obtained in exciting vegetation, whether in a seed or root, especially in spring, when the soil is cold, must be very considerable." By a judicious system of trenching, fresh soil will be brought into im. mediate contact with the roots of plants-a circumstance of so much importance in culture, and so well known, as to require no further comment. "The free admission of atmospheric air to soil is also necessary for the decomposition of humus, or organic matter, by which carbonic acid is formed. The atmospheric air is also a great source of nitrogen, which has been lately found in all plants, and more especially in the spongioles of the roots. The soil also, when loosened, becomes a rapid conductor of water; and supposing the texture of the soil to be suitable for culture, it will retain a sufficient quantity of moisture for the purpose of vegetation, and allow the escape of what is superfluous by filtration into the subsoil, or into the underground drains which have been formed as a substitute for a porous substratum. The mere act of pulverising any soil has a tendency to improve its texture, more especially if the operation be frequently repeated. In summer, by exposure of a soil to the air, the particles are separated by the evaporation of the water in their interstices by heat; and by exposing a soil to the frosts of winter, the particles are separated by the expansion of the water in the act of freezing. Clayey soils containing iron are in an especial manner improved by exposure to the atmosphere, the iron being still further oxidised, and thus acting like sand in separating the particles, as well as being less likely to be rendered soluble by the addition of saline matters."-Sub. Hort., p. 55 .

Jethro Tull, one of our earliest writers on improved husbandry, estimated the process of pulverisation so highly, that he fancied no other assistance was required in the preparation of the soil for crops; in this, however, he overrated this important operation. Du Hamel, entertaining somewhat similar views with Tull, decidedly proved that the multiplication of the fibres of plants is as the inter-pulverisation of the soil ; but the advantages thus gained by vegetables, by the increased number of their fibres, must depend a good deal on the quantity of food within their reach. However much the two authorities last quoted may have overrated pulverisation, one thing is certain, that where it is carried out to its fullest extent, the quantity of manure required is very materially lessened. Nevertheless, as garden soils require to be loosened to a considerable depth, to allow the roots of many plants to descend, it also follows that enrichment should be carried to the same extent, and the more so when we consider that the roots of all plants collect their nourishment from their extreme points, and that many of them, such as beet, parsnip, \&c., derive their food at a depth of 2 or 3 feet below the surface.

Digging, ridging, and trenching.-These are important operations, performed, in general, only by commercial growers, who have to make the most of their ground; and as they carry out these to a great extent, it is quite evident that they see the propriety of so doing. In private gardens they are much neglected; and hence one of the causes why the productions in them are so inferior to the others. In ordinary digging, the spades used in the market-gardens around London are $13 \frac{1}{2}$ inches long and 10 inches broad, while those used in private gardens are seldom more than 10 inches by $7 \frac{1}{2}$ inches, the former loosening the ground to a greater depth. Nor is even this deemed sufficient; almost every portion of ground is single or double trenched annually.

Garden spades are of various forms, and adapted to various purposes; but, until of late years, little improvement has been made in them. For all purposes of lifting the soil, or digging pits, they are better than forks; but for the mere purpose of pulverising by loosening, and for digging up roots, and digging amongst plants and trees, broad-pronged forks are preferable.

Lindon's cast-steel spade is the best: the blade is thinner, yet stronger, than the garden spade in ordinary use, thus constituting an important point in its favour, because one-third less power is required to force this thin-bladed tool into the ground than the thick and clumsy spade hitherto used. The handle, also, in Lindon's spade is improved by being curved a little forward, giving it a better leverage than if it were straight, and rendering less stooping necessary in using it. The blade is highly tempered, which renders it an excellent cutter of roots, working clean, and wearing well. It is known also as the Birmingham spade.

Black's spade is in much repute in Scotland, as being of excellent material. It is very similar to Lindon's in form-scarcely, however, so light, and probably less highly tempered. They are manufactured at Ford Forge, Ford, Northumberland.

The sizes of the above spades are,

No. 2. $-7 \frac{1}{4}$ in. bd. at bottom, $7 \frac{1}{2}$ in. at top, and 12 in. deep.

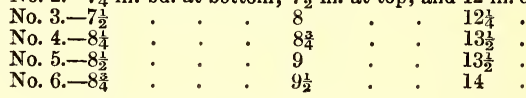

The prong spade.-This implement is much used in some parts of Kent, and also in Cheshire, where it is called a "mule," from the cir cumstance of its combining, as it were, the common three-pronged fork and the common spade. In form it is a three-pronged fork with a spade handle, the points of the prongs being united together by a slip or strap, about $1 \frac{1}{2}$ inches wide, of steel welded to them, which forms the face or cutting part. It is lighter than the common spade, and well adapted for digging purposes.

It has been remarked, as one advantage arising from this deep-digging and trenching, that slugs and other insects are comparatively of rare occurrence in such grounds, possibly from their eggs and larvæ being buried too deep to become hatched by the solar heat near the surface. There can be no doubt, the deeper the soil is dug, the more fitted it is for productive crops; evaporation is promoted, a free and more extended sphere is offered to the roots, which en. ables them to range farther in search of food, and the soil becomes much less exhausted by 
even succeeding crops of the same kind being grown upon it, an effect which must ensue when the depth is limited to 9 or 10 inches. Rotation of crops, so much insisted on by most cultivators, is, under this deep-trenching system, a matter of very unimportant consideration. No doubt, when the soil is naturally thin, and rests on a bad subsoil, it would be injudicious to trench so deep, at least at the commencement of its cultivation; because that portion which is really sufficiently fertile to produce moderate crops would be entirely buried, and a soil totally unfit for most plants would be brought to the surface. The improvement of such a subsoil, if sufficiently dry, can, however, be progressively accomplished by bringing up a portion of it at each digging, and incorporating it with that already formed, so that, in course of time, most soils may be rendered deep and fertile to any reasonable extent. Thin or shallow soils are greatly improved by a system of trenching much used by us, which, without bringing the bad subsoil up, yet loosens it to a considerable depth, and that without the expenditure of much manual labour. The process is as follows: At one end of the piece, open a trench not less than 3 feet in width, and as deep as the soil is already made, removing the earth taken out to the opposite side, for filling up the last trench. When the first trench is opened-say to the depth of a foot or more, according to circumstances-commence picking up the bottom, allowing it, however, to remain where it is. For this purpose we use picks made on purpose, the prongs of which are 20 inches in length with these the subsoil is torn up and loosened to the depth of 18 inches, if the operation is not interrupted by rock. This, with the 12 inches of surface-soil already removed, gives us a depth of $2 \frac{1}{2}$ feet at the expense of actually removing 1 foot. It is a good plan to lay over the subsoil thus broken up a good coating of undecayed dung, which, in itself, improves the soil beneath, and affords nourishment to the roots of the crops when they descend to that depth. One of the great evils arising from a rich surface and an impoverished subsoil is, that the roots are pampered, and probably, in some cases over-fed, in their earliest stage of growth, and while the plants are small and require less nourishment: when they have attained, say half their size, and consequently stand in need of a proportionate amount of food, their roots, having passed through the rich soil, enter into that in which little or no nourishment for them is to be found. The consequence is, the plants sustain a sudden check, from which they never afterwards recover, but begin to assume a sickly worn-out appearance, run up to seed, or cease to increase in size, and ultimately die of sheer starvation.

During winter, when the ground is unfrozen, and when neither too wet or covered with snow, digging, ridging, and trenching should be proceeded with; it is the most profitable employment men can be engaged in. Every piece of ground, as soon as it is cleared of its crop, should be operated upon in one or other of these ways; and whichever be adopted, the rougher the surface can be thrown up, the better, so as to expose the largest possible extent to the action of the weather. All manurings, without such operations as these, are of little avail ; and these, even with a very limited supply of manure, will secure excellent crops. Ridging is a species of deep-digging or trenching, where the soil is thrown up in parallel ridges; it is found valuable in strong tenacious soils, as it presents the ground so ridged up to the action of frost better than when the surface is left more level.

The purpose of trenching is to deepen the soil by breaking into the subsoil, and improving that, as well as bringing part of it at each operation near to the surface, and amalgamating it with the soil, which has already been probably over-cropped. It promotes filtration and the descent of solar heat; it gives scope to the roots, and affords them a broader pasturage; it supplies rest to an already over-wrought soil, and brings into action one that has not probably as yet contributed the least share towards the sustenance of plants. In fact, it is cultivation upon a grand and high-culture principle. The more a soil is disturbed, and the deeper that disturbance is carried, the better, and the more manifest will its improved state be, even in the general acceptation of soil management. A very sensible practical writer, J. B., in the "Cottage Gardener," vol. i. p. 6, observes, "A soil would never get exhausted, if managed with skill, but would continue to improve in depth and fertility in proportion to the industry bestowed upon it. The food of plants, it is true, may be exhausted from the soil by a repetition of cropping with any one family of plants, if we neglect the application of such fertilisers as may have been taken from the soil by that family; but no part of the growing season is required for the soil to rest, or lie fallow, if judiciously managed by a successional varying of the crops, or supplying to them such food as may be a compensation for what has been taken off by the previous crops. The first object to be attained for securing a certain and profitable return of produce from the soil must be thorough drainage; the next object is breaking into the subsoil to the desired depth-not without first considering whether it is properand profitable to shift and turn up the subsoil at once to the influence of the atmosphere, or whether it is best to break into it well first, by shifting the surface-soil, and allowing the subsoil to remain, to receive, first, the beneficial influence of the atmosphere ; and then, at the next trenching, a portion of the subsoil may be safely stirred up and mixed with the surfacesoil: this practice, continued for every succeeding crop, will establish a healthy fertilising surfacesoil to any desired depth."

Bastard trenching is an excellent plan where the soil is too thin to admit of thorough trenching. In performing the operation, a trench is dug about a yard in breadth ; indeed, no trenching should be of a smaller breadth, as, the wider the trenches are, the less chance is left to careless operatives of leaving dykes, or undisturbed portions of soil, between each trench. One full spit and a shovelling is then taken out, and car- 
ried to the end where the operation is to finish. Lay a good dressing of dung on the bottom of the opened trench and dig it deeply in, then fill in with the top spit and shovelling of the next trench, which serve in the same manner, and so on till the whole is finished. By this mode the good soil is kept still on the top, and the subsoil is enriched and loosened at the same time, providing nourishment to the roots, and inducing them to descend in search of it. In time, this improves the soil to a sufficient depth for ordinary purposes, allows the rain to penetrate downward, and prevents the crops from suffering from drought. The only objection to it is that little change takes place in the soil, the top remaining at the top, and bottom at the bottom. Strong soils are rendered more open by it, the capacity of absorbing moisture is increased, and the surface is rendered drier.

In trenching, besides the amelioration of the soil by the mechanical operation of loosening, mixing, and turning over, other advantages should be aimed at; as, for instance, where it is of a strong retentive description, river or any sharp sand, lime-rubbish, the refuse chippings of soft sandstone incorporated with it, will be found of vast importance. In soils of an opposite character, or such as are denominated light, hot, dry, and hungry, abounding to an extreme in sand, and deficient in vegetable matter, clay, turfy peat, vegetable mould, marl loam, \&c., should be added. Indeed, anything that can be procured of a diametrically opposite character to either of these soils, if added, will improve them. Clay, if very strong, may be advantageously laid on the surface in autumn, and when broken down by the winter frosts, will be in excellent condition to incorporate with the soil. Changes thus effected will be far more lasting and beneficial in their effects than all the manure that can be applied without them.

Consolidation is a species of soil-improvement the very opposite to pulverisation. It nevertheless sometimes becomes necessary, as in the case of light spongy peat-soil and very light sand. Neither of these, however, are often met with in garden culture. Compression is employed when seed is sown in dry weather upon light soils, and is effected by treading or rolling. Deepdigging and trenching, where the soil admits of it, should not, however, be disregarded, the operation of compression being confined to the surface only.

Alteration of the constituent parts of soils.This is a species of improvement of vast importance, and consists in adding or subtracting those ingredients in which they are deficient or possess in superabundance. This species of improvement, in the present case, we will consider with reference only to an alteration of its texture, and observe that sandy soils will best be improved by the addition of alumina or clay, and strong clayey soils by an admixture of sand and in both cases lime should be added, as no soil can be permanently fertile without alkaline matter. A much smaller quantity of clay applied to even a drifting sand will consolidate it, and convert it into a good working loamy soil, than of sand to produce a like effect upon strong clay. Peat or other vegetable matter added to sand will enrich it, by supplying organic or vegetable matter in which the sand is deficient: but although this increases its fertility, it adds little to its texture ; it may, therefore, be added as a compound mixed with clay and lime. Light sand, on a subsoil of loam or clay, will be improved by trenching so deep as to bring up a sufficient proportion of the clay to the surface ; and peaty soils will be improved by the addition of sand, as well as of clay and loam. The quantity of one soil to be added to another, so as to render its texture perfect, can only be determined by experiment. The following is given as a practical example: "If we take an extreme case, and suppose that any given soil is so sandy as to require the addition of one-sixth its bulk of clay, or so clayed as to require one-sixth of its bulk of sand, then, in the case of a kitchen-garden, when the soil is 3 feet deep, every square foot of the clayey surface will require the addition of half a cubic foot of sand; and, in the case of a lawn, where the soil is 1 foot in depth, every square foot of sand will require the sixth of a cubic foot of clay. To cover a statute acre with soil to the depth of 1 inch, will require 121 cubic yards. Hence, to add 2 inches to the soil of a garden of 1 acre, exclusive of the space occupied by the walks, would require 242 cubic yards or cartloads. It frequently happens that a sandy or gravelly soil is incumbent on a bed of clay, and the contrary. In either of these cases, the supply of the required soil may be obtained by digging pits, or sometimes even by deep-trenching. The earth thus obtained will generally be without organic matter, but that can be supplied afterwards by manuring. Where the soil required for the improvement of another soil can be obtained in the state of surface-soil, the effects produced will be the more immediate for the organic matter that such soil contains; but even when it is obtained from the subsoil, the change in the condition of the soil to which the new soil is applied will soon be rendered obvious, though not so much the first year as it will be in two or three years afterwards, when the amalgamation of the two soils is more complete. Much of the effect of adding one soil to another will depend on their intimate mixture, and this can be best effected by repeated trenchings or diggings in dry weather, and when both soils are as nearly as possible in a state of dry powder. This point is of great importance, particularly when the soils mixed together contain a good deal of organic matter, because, if a very intimate mixture of both soils is not effected, they will, from the difference of their specific gravities, in a few years separate into two different strata. There is, indeed, a constant tendency to do this in all soils under culture, and more especially in all such as have been improved by admixture. This takes place in consequence of the softening of the soil by rains, by which the particles are, in a manner, held for a time in suspension, and the heaviest gradually take a lower place than those which are lighter. Hence the necessity of digging or trenching such soils 
occasionally to the depth to which they have originally been improved."-Sub. Hort., p. 52 .

Exposure to atmospheric influence.-The action of the atmosphere effects important changes on soils. Indeed, as has been already stated, some trace the very origin of soils to atmospheric causes. Some of the advantages soils attain from exposure to the atmosphere have been noticed under the head Pulverisation. The others are effected by altering the position of their surface, by throwing it up into narrow ridges, deep-digging, and leaving the surface as rough as possible, frequently stirring it with the hoe, and tearing it up, where the soil is hard or tenacious, with the pick, or, still better, with Glendinning's pick-fork, vide fig. 85 . The effects of all these are to render the surface open and pervious to solar heat, frost, and rain, which, acting by expansion, contraction, \&c. of its parts, break it down, and render it more fitting for the growth of plants. "The influence of the sun's rays upon any plane are demonstrated to be as their number and perpendicularity to that plane, the effects of the atmosphere being excepted. Hence one advantage of ridging land, providing the ridges run from north to south; for on such surfaces the rays of the morning sun will take effect sooner on the east side, and those of the afternoon will remain longer in operation on the west side; whilst at mid-day his elevation will compensate, in some degree, for the obliquity of his rays on both sides of the ridge."-Encyc. of Gard., p. 477. Another advantage such an operation on surfaces has is, that the soil will dry sooner than if left undisturbed, on account of a freer evaporation going on. The air, upon which so much of the success of cultivation depends, will be admitted more readily to the roots of plants ; and when we consider that they derive so much of their support from that element, the necessity of admitting it into the ground largely will be the more apparent.

Burning soils, with a view to their improvement, is an important matter in preparing land for planting, laying down as lawns, \&c., and has of late been strongly recommended for almost all garden purposes.

The theory of burning soils rests entirely on chemical doctrines, of which Sir H. Davy and others have given clear elucidations. The advantages of burning "are, that it renders the soil less compact, less tenacious, and less retentive of moisture ; and when properly applied, may convert a matter which was stiff, damp, and in consequence cold, into one powdery, dry, and warm, and much more proper for a bed for vegetable life."-Encyclopedia of Gardening, p. 477. The chemical change which takes place in soils subjected to the process of torrefaction, is stated by Sir H. Davy to be as follows : "The bases of all common soils are mixtures of the primitive earths and oxide of iron; and these earths have a certain degree of attraction for each other. To regard this attraction in its proper point of view, it is only necessary to consider the composition of any common silicious stone. Felspar, for instance, contains silicious, aluminous, and calcareous earths, fixed alkali and oxide of iron, which exist in one com- pound, in consequence of their chemical attraction for each other. Let this stone be ground into impalpable powder, it then becomes a substance like clay; if the powder is heated very strongly, it fuses, and in cooling forms a coherent mass similar to the original stone ; the parts separated by mechanical division adhere again in consequence of chemical attraction. If the powder be heated less strongly, the particles only superficially combine with each other and form a gritty mass, which, when broken into pieces, has the character of sand. If the power of the powdered felspar to absorb water from the atmosphere before and after the application of the heat is estimated, it is found much less in the latter case. The same effect takes place when the powder of other silicious and aluminous stones is made the subject of experiment ; and two equal portions of basalt ground into impalpable powder, of which one-half had been strongly ignited, and the other only exposed to a temperature equal to that of boiling water, gained very different weights in the same time when exposed to air. In four hours the one had gained only two grains, while the other had gained seven grains. When clay or tenacious soils are burnt, the effect is of the same kind; they are brought nearer to a state analogous to that of sand."

There is no doubt something startling to the mind of the practical cultivator in the idea of burning soil, not taking, at the same time, into consideration that the process is not recommended as applicable to all soils, but only to some; and these are such as contain a superabundance of vegetable matter in a dead inert state like most peat soils, and those requiring more powerful agencies than that of mere working with the spade to bring them to a state more useful to plants, such as strong clays and tilly soils. Upon both of these the operation of burning has been attended with marked advantage. In connection with horticulture, there is another class of soils that are benefited by such an operation : these"are old garden soils, glutted with organic matter by the yearly application of manure-dung, the lapse of ages, and at the same time replete with destructive insects, in all their various states of transformation from the egg to the perfect state. Such soils are benefited by burning, or perhaps by the less torrefying operation of charring or half-burning, a process highly recommended by many of our most enlightened horticulturists. This we shall now proceed to notice; but before doing so, we may remark that both burning and charring the soil have been carried to a much greater extent in England than in Scotland, but why, we must leave our readers to speculate.

Charring is a process for correcting the tenacity of strong soils, accumulating manure, and converting materials, in themselves next to useless, into excellent fertilisers, such as sawdust, prunings of bushes and hedges, coarse turfy sods, the parings of road-sides, tanners' bark, shavings, and similar vegetable matters. $\mathrm{Mr}$ Barnes of Bicton was amongst the first to bring this material before the notice of the gardening public; his mode of operation, therefore, may 
be sufficiently explicit. In the "Cottage Gardener," vol. i. p. 83, he thus writes: "To char turfy soil, the best method is to cut it into sods of about a spade's width, and 2 or 3 inches thick, when the soil is not over-saturated with moisture." Summer would, therefore, be the best season, as the sods would be drier; but summer brings with it its own multifarious duties: charring in winter only requires more time, and a greater amount of woody matter to mix with the sods, and cause them to burn. "Burning any kind of material, and allowing it to be consumed, producing nothing but smoke and ashes, is a real waste of valuable property, which could be turned to valuable account for the improvement of the soil. To char sods or surface-soil of any kind, proceed by placing a small quantity of combustible stuff-such as dry weeds, shavings, hedge-trimmings, furze, heath, brushwood, or bushes, or any kind of dry vegetable refuse the place produces near at hand; then commence packing the sods, no matter how or what thickness, as any thickness may be charred by placing amongst them, as the heap or kiln is proceeded with, some one of the fore-mentioned materials, or old tan or sawdust, just to keep the materials ignited. It is no matter how large or small the kiln may be formed, for this may be regulated by the convenience of the material at hand, and the quantity of charred materials required;-only, when the kiln is formed, it should be slightly covered or cased with fineish earth, to prevent the fire from flaring, and to maintain a steady smouldering, charring, or roasting. Much smoke will escape for a time, and when the smoke begins to subside, it is a sign that the materials are charred enough : the fire should then be smothered out by casing up the outside of the kiln quite close to the earth; no large quantity of fuel is required; if too much is employed, it is more likely to consume the sods away into mere smoke and ashes." Charred soil thus produced has been found exceedingly beneficial to many crops; and although, at first sight, the idea of consuming all the organisable matter in the soil subjected to this ordeal seems like reducing it to sterility, yet certain chemical changes are produced which vastly more than make up for this supposed loss. We prefer winter for carrying out such operations, as there is less interruption caused to the general routine, and also because we have a hundred or two of cartloads of useless material to get rid of at all events, such as the pruning of trees, the rotten branches blown down by the autumnal gales, and immense quantities of the thinnings and prunings of banks of evergreens, and the general vegetable rubbish collected over many acres of pleasure-ground.

It follows, therefore, that winter is the most convenient time for this operation. We need hardly remark that the material charred or roasted should be kept dry till wanted for use, and this is easily effected by forming the mass into a conical form, covering it with a foot of garden earth, and then thatching the whole with straw.

Charring or roasting the soil for the destruction of insects is easily effected : after the mass has been for a day or so in operation, about 3 or 4 inches of the surface upon which crops have grown that have been much injured by insects, along the bottoms of wall fruit-trees, under espaliers, and that under gooseberry bushes and standard fruit-trees, about as far as the branches extend, is carefully scraped off and carried to the charring kiln and thrown upon the mass of ignited matter, mixing with it any combustible material, so that a slow and steady combustion may be maintained. The object of this is to destroy the larvæ of the insects, which take shelter in the soil during winter, as well as to effect that chemical change on the soil itself that the action of fire is known to produce.

Preparing the ground for the next season's crop. -In all well-regulated culinary gardens, certain systems are acted upon, without which all would become confusion and disorder. One of these is the preparation of the soil, during the previous autumn or winter, so that, when the season of sowing and planting arrives in spring, all is ready for the operation. The expert cultivator has a place for everything, and a thing for every place; and these are so proportioned and arranged that each, as it were, prepares for its successor. In reference to such preparation of the ground, we have the following excellent digest, given by a first-rate practical cultivator in the calendar department of the "Gard. Chron.," 1850, p. 808, which is in such perfect accordance with our own views that we shall transcribe it.here: "Before the general manuring and trenching of vacant ground, it is necessary to decide on the rotation of crops which are to be cultivated upon it during the ensuing summer, that these operations may be ordered with reference thereto. This arrangement should be made, not for one year only, but for a series or cycle of years. One of the first principles to be attended to is, that no annual crop be grown for two successive years in the same plot of ground. Another variation should be made, by taking care that those crops which immediately succeed each other are not such as are liable to be preyed upon by the same kind of insects, as their increase is encouraged to a fearful extent by thus putting into their way the species of food which they are most fond of. It is important that both the manuring and the depth of the trenching be regulated by the requirements of the crops; and their rotation should be so arranged that the ground be not trenched to the same depth for two successive seasons, but so that different portions of the soil be brought to the surface in turns. The perennial or permanent crops will, of course, form a class of themselves, as they do not require a change of situation for many years; but when this is necessary with any portion of them, the ground from which they are removed will fall into the ordinary rotation in the way of a regular exchange." Confining ourselves, for the present, to the ordinary or annual crops : "By cultivating the celery and cardoon in the Scotch or wide-bed system, a large ridge of soil is thrown up between the trenches, the surface of which is excellently adapted for the cultivation of dwarf pease, beans, spinach, turnips, and similar 
vegetables; observing, however, that only the earlier sowings of these several crops should be made on these ridges, that they may be removed in due course before the ground is required for earthing up the principal crop. As a matter of course, this arrangement must not interfere with the very earliest crops of pease, \&c., which are generally allowed a place on the side borders near the walls. For the latest crops of pease, \&c., a portion of ground must be allotted which, with that occupied by the celery and cardoon ridges, will generally amount to about one-third of the ground occupied with annual vegetables. The extensive turning over which the celery and cardoon soil undergoes, acts admirably in preparing the ground for deep-rooting plants, such as turnips, carrots, onions, \&c., and in the following year may be devoted to the cultivation of the cabbage family, whose roots are confined more nearly to the surface. If the vegetable garden is so large as to allow of the cultivation of a portion of the general potato-crop within the walls, it will form a fourth course in the rotation, and will come in after the Brassica tribe. Working upon this system, cabbages have been planted during the autumn on ground which had been occupied by late summer turnips; and the ground which now requires trenching is that from whence the later turnips, onions, carrots, beet, \&c., have been removed, and which will next spring be planted with cauliflower, broccoli, \&c. Preparation for these should be made by trenching in a very heavy dressing of manure, which shall serve for that and the succeeding crop." To effect this with certainty and satisfaction, a plan of the ground should be made, and the system accurately marked upon it; indeed, without such a plan nothing like correctness of system can be carried out. Such is the preparation of the ground for future crops, as practised in private gardens. The routine of culture and preparing the ground in a marketgarden is widely different. "If we take a fiveacre piece of ground," says Mr Cuthill, in "MarketGardening around London,"-_"say in November, we find it full of cabbages, which, being planted out about the 25th of October, will be strong healthy plants. The moment these are off, the land is again trenched and cropped with early celery, in well-dunged trenches 6 feet apart, with two or three rows of lettuce or coleworts between them; for market-gardeners do not mould up their celery unless it is very large (often 18 inches high), so there is plenty of time for a crop of coleworts or lettuces to come to maturity. In November, Mr Fitch of Fulham (a very successful grower in a garden of 150 acres) has often upwards of 20 acres of cabbages, every hole and corner, under trees, and all spare places, being full. In March it is again dunged and trenched, and sown with onions; and very often lettuces are planted in the beds, as well as in the alleys. When the onions are off, the ground is trenched and planted with cabbages and coleworts, \&c. Next spring a crop of cauliflower, gherkin cucumbers, French beans, or scarlet runners is taken off; but the grand point in the course of rotation is to be continually sowing, and, whatever plants are ready when the ground is empty, to plant these. The land can well sustain so much cropping on account of the heavy dungings, trenchings, and hoeings which it receives. If you ask a market-gardener what is to succeed this or that crop, the answer is, 'Don't know ; it depends on what is ready for planting.' Continued trenching, two spades deep, for any crop, seems expensive; but a strong Irish labourer will turn over from 12 to 14 rods a-day with comparative ease. Marketgardeners know that, after an active crop, the top soil for several inches deep is entirely exhausted, and hence the reason for continual trenching, in order to bring up the top soil that but a few months before had been turned down, with a large proportion of dung to enrich it and fit it for active use, along with the halfdecayed manure." Here we have two excellent modes of preparing the ground, but the order of rotation is different. The private gardener has to calculate almost to a day when this or that crop is required to come into use, and must be guided accordingly. The market-gardener is not so tied down; his only care is to see that not a bit of his ground remains empty for a day: he crops it with something, well knowing that the day the crop is fit for use he has a demand for it in the market. By preparing the ground in either way, the maximum of produce may be expected.

\section{§ 4.-WATERING.}

Watering is an important operation in gardening, and often very inefficiently performed. Were it always as convenient as it is practicable, we would say that water should not be applied to any plant at a lower temperature than that of the earth that surrounds the roots, because in such cases the roots sustain a sudden chill, and the soil is reduced in temperature to the extent often of suspending growth until it has again become restored to its former heat. This is the case more especially in spring, when the first energies of the plant are being called into action, and when it requires most the aid of heat to increase its excitability. Cold water poured on the roots of plants, even during summer, has a most paralysing effect for the time, but this is of shorter duration, as the increased heat in the soil counteracts the effects. In autumn it is equally injurious as in spring, because it lowers the temperature at a season when every particle of heat should be allowed to accumulate to maintain the roots in a proper condition during the winter. The application of cold water to plants growing in a high artificial atmosphere is equally injurious; but no excuse can be made why it should not be applied at even several degrees of temperature above that of the atmosphere of the house, and water applied to the foliage under such circumstances should be equal at least to the air in the house. Wall trees suffer much from want of watering over their branches and leaves, and it is probable that one-half at least of all the disasters they suffer arise from a total neglect of this. Evaporation or perspiration must go on more 
rapidly in the foliage of trees trained against a wall, from the increased heat by which they are surrounded, than from the foliage of trees growing as open standards. Added to this, a more limited amount of rain falls on such trees, from the position their branches are placed in, and the projection of the copings, than on trees exposed to rain on all sides. We have elsewhere stated that trees derive food from water through their leaves; if, then, wall trees are not syringed, and that often, this supply of food is denied them. Yet how much do we expect from such trees, both in their healthy appearance, and in the size, quantity, and excellence of their fruit. In dry soils, and in seasons of drought, much advantage has been found from watering at the roots also ; yet this is seldom thought of, or, if it is, more seldom practised. Water, however, to be useful, must be applied copiously in all out-of-door culture; mere dribblings do more harm than good; and water applied to the roots of plants in the open air, in particular, should be manurial in a slightly diluted form. A few cwts. of guano or pigeons' dung, or other fertilising matter, in the absence of liquid-manure tanks, can easily be incorporated with the water used, and its effects will be sufficiently evident. Watering such crops as celery, cauliflower, asparagus, or such others as require abundance of enrichment, with water unenriched, is next to labour thrown away; and next to this is even enriched water, when not sufficiently largely applied.

The way in which water is applied to even our most common crops is a matter of consideration, so that the quantity used may be applied to the greatest advantage. Now, this cannot be done when it is thrown upon the ground with great force as from the spout, or even very coarsely perforated rose of a wateringpot. In this way it acts much in the way of a thunder-storm shower, battering the ground quite hard, running on the surface, escaping in waste, and, when let fall on young and tender plants, beating them down and half-smothering them with mud. How different, and how much more beneficial, is the gentle evening or morning shower, which falls lightly and equally over the surface! Such we should endeavour to imitate, in the application of water artificially. In light porous soils, heavy showers and heavy waterings have the effect of carrying off, either on the surface or washing downwards, the fertile particles of the soil, leaving the heavy silicious portions behind, and, when long continued, reduces the soil to a state almost of sterility; and were it not for the amount of fertilising matter added to the soil by the fall of genial showers (referred to, p. 18), the rains alone would render the earth almost sterile. It is not, therefore, by the quantity, but by the way in which water is applied, that it is beneficial or injurious to plants. Plants in pots suffer much from careless watering. The soil in a pot, already too moist, is, by the application of more water, rendered waterlogged-that is, containing more water than the roots can take up-and, by its occupying the spaces between the particles of soil which should be occupied with air, it actually chokes the plant VOL. II. for want of that necessary element. This state of matters may readily be detected by feeling the weight of the pot, which will be found much heavier than one of the same size in a proper state as regards water. When the soil around the roots of pot-grown plants is allowed to become too dry, it is not, in such cases, applying water in quantity all at once that is to restore matters to their proper state ; it is by giving a little, and repeating that every few minutes, until the whole ball of earth becomes equally restored to its proper state of humidity. Pouring it on in quantity all at once prevents its penetrating through the ball, and causes it to escape between the sides of the ball and the pot, leaving the centre as dry as ever. More plants are killed by injudicious watering than by almost any other point in bad management. In the application of water, to whatever plant or crop, the nearer we imitate nature, the more satisfactory will the results be.

On this subject the following excellent remarks are made by Dr Lindley in "The Theory of Horticulture," p. 125: "When plants are watered naturally, the whole air is saturated with humidity at the same time as the soil is penetrated by the rain; and in this case the aqueous particles mingled with the earth are very gradually introduced into the circulating system, for the moisture in the air prevents a rapid perspiration. Not so when plants in the open air are artificially watered. This operation is usually performed in hot dry weather, and must necessarily be very limited in its effects : it can have little if any influence upon the atmosphere; then the parched air robs the leaves rapidly of their moisture. So long as the latter is abundant, the roots are suddenly and violently excited, and after a short time the exciting cause is suddenly withdrawn by the momentary supply of water being cut off by evaporation, and by filtration through the bibulous substances of which the soil usually consists. Then again, the rapid evaporation from the soil in dry weather has the effect of lowering the temperature of the earth. Such a lowering, from such a cause, does not take place when plants are refreshed by showers, because at that time the dampness of the air prevents evaporation from the soil, just as it prevents perspiration from the leaves. It is therefore doubtful whether artificial watering of plants in the open air is advantageous, unless in particular cases ; and most assuredly, if it is done at all, it ought to be much more copious than usual." To the vast utility of copious watering we have the testimony of ages; of which the natural overflowing of the Nile, the irrigation practised artificially in India and China, and other warm countries, as well as the application of water in an enriched state in the melon gardens of Persia, are examples; and wherever irrigation has been carried on upon correct principles, even in the colder climate of Britain, the good effects have been shown. So far, however, as gardens are concerned, the water should be in an enriched state, more especially for annual crops-perennial ones seldom requiring such aid. However much we may recommend 
copious watering, or even irrigation, in gardens, one thing is quite obvious, that in most gardens, as at present constructed, little or no provision has been made for securing a supply, and still less for its economical distribution; and therefore, as long as the gardener has his water to carry about in watering-pots, we may expect to see this operation either entirely neglected, or very superficially performed. We have shown how such full supply and proper distribution could be secured (vide vol. i., articles WATER, Tanks and Cisterns, \&c.) There would be no great difficulty even in applying enriched water in a tepid state to our most common crops, thus securing two important points-namely, enrichment in a form ready for the use of plants, and this without lowering the temperature of the soil about the roots; indeed, it might be made to increase it.

From all this it will be seen that watering in the open air is of sufficient advantage, if properly applied; but even in our present advanced state of horticultural science, we seem to have little control over it. With plants in glasshouses the case is different, and there a more correct system may be pursued. There are two conditions in the existence of plants that, as concerns watering, are widely different; those are, the season when they are at rest, and that when they are in the vigour of growth. In the former state they require but little water, because they lose little by perspiration, and hence the draught upon the root for food is greatly diminished; while in the latter case they require an abundant supply, on account of the increasing perspiration, which begins to take place as soon as the plant is excited, and increases as the foliage develops itself. During the younger state of the leaves, their draughts on the roots are most powerful, on account of their perspiratory action being most rapid at that stage, which points out to us the necessity of watering abundantly, and affording them all the light in our power. This supply should be gradually diminished as the tree or plant arrives at a full development of its parts, be the object of their cultivation fruit, flowers, or wood; the only exceptions being oleraceous annuals. Watering while the fruit is arriving at maturity tends, no doubt, to increase its size, but this is obtained at the sacrifice of a great amount of its flavour; and watering when the wood is about nearly formed, causes it to continue growing till too late a period of the season; and, as a consequence arising from its increased size, and the quantity of fluid contained within it, it is prevented from ripening, and is therefore liable to be killed by the frosts of winter.

Plants with large leaves require more water than those having smaller ones; and hence in nature it will be observed, that many aquatic plants, and those growing by the banks of rivers and lakes, have leaves above the ordinary size. It may be taken, however, for granted, that such plants as the Victoria regia, the Nelumbiums, Nymphæas, \&c., have extraordinary perspiratory power on account of the very large size of their leaves; and to aid them in decomposing the fluid they absorb, a high temperature and bright sun are necessary. The melon and the cucumber, although by no means aquatic, having large leaves, perspire enormously, and hence require a very large supply of water, if placed in a high temperature ; but in a low temperature, and equally watered, gum, canker, and spot take place, and ultimately death - the heat not being sufficient to decompose the water taken up by their roots. Plants in metallic hot-houses, when glazed with large panes of glass, require much more water than plants of the same species grown in houses with small glass, and much crowded with astragals and rafters. Practically, plants in pots or tubs should be watered often, and not too much given at a time. If water be given in large quantities, it passes through the soil in the pot, provided it be properly supplied with drainage, carrying with it much of the richness of the soil ; and if imperfectly drained, causing the soil to become water-logged, cold, and impervious to the air, so necessary to the welfare of the roots.

Watering is the mainstay of gardening in warm climates; it is particularly so in artificiallyheated climates, and by no means to be disregarded even in our naturally cold and humid country. The utility of watering newly-planted trees, shrubs, and plants is admitted, but the proper application of it little understood. Many conceive that the water for their plants, like what they use themselves, cannot be too fresh and cold. Than this, a greater mistake does not occur in the whole art horticultural. Rain water is best of all ; and dirty stagnant water, and at a high temperature, the most congenial to the plants. Cold spring water is the worst of all. It is not going too far to say, that pure water should seldorn or never be applied, but that it should be impregnated with some enriching matter, of which our modern artificial manures offer abundant choice. Those who have found, if they have bestowed a passing thought on the matter, how little service their continued watering has done to their plants in a dry summer, would do well to think of this.

The fertilising matter conveyed to the soil by rain water, compared with spring water, must exercise a most important and beneficial influence on plants. The researches of M. Barral, of Paris, show that, in six months, the rain which fell upon an area, equal in extent to one English acre, contained, as near as possible,

$$
\begin{aligned}
7.75 \mathrm{lb} . \text { of ammonia. } \\
36.50 \text { - nitric acid. } \\
5.56 \text { - chlorine. } \\
12.60 \text { - lime. } \\
4.81 \text { - magnesia. }
\end{aligned}
$$

A writer in the "Critic," referring to M. Barral's experiments and statement above, says, "From July to December is usually the drier half of the year, as well as that in which the less fuel is consumed, so that we may safely double these quantities in estimating the annual supply per acre of nitrogenous compounds gradually distributed over a country by the rain. For the sake of illustration, I have calculated the amount of the solid constituents of the rain falling on an area equal in extent to Great Britain; and, balancing the various causes likely to lessen or 
to increase the quantity of these matters which would so fall on this island, we may venture to set the one against the other, and apply the above statement to our own country, as the basis of an estimate which singularly manifests the 'power of littles,' as well as the grand scale on which even the minutest of natural phenomena proceed. Thus, on the Parisian data, the weights of these fertilising materials, annually supplied to the soil of this island by the rain, amount to about

$\begin{aligned} 400,000 & \text { tons of ammonia. } \\ 1,850,000 & -\quad \text { nitric acid. } \\ 279,000 & -\quad \text { chlorine. } \\ 640,000 & -\quad \text { lime. } \\ 244,000 & - \text { magnesia." }\end{aligned}$

It is probable no other water in a natural state contains so great an amount of fertilising matter; and it is quite certain that the waters of many springs, should they peradventure contain no material injurious to vegetation, contain little or no matter which can properly be called the food of plants. It therefore is a matter of the deepest importance to the horticulturist to endeavour to secure rain water; and this is by no means so difficult a matter, if properly gone about.-(Vide sect. TANKs, \&c.) Next to rain water is that which is, by chemical analysis, proved to contain the largest portions of the above constituents, and the least amount of those matters which are either useless or injurious to vegetation. Those who have to depend on springs for their supply of water for garden purposes, had need ascertain the chemical properties of them before going to the expense of forming wells, pumping the water up, or bringing it from a distance; for as the water may be deficient in the above matters, so is their labour in vain, and the expense incurred next to thrown away. Plants require something more than pure water. It is the quality and not the quantity of water that is beneficial to them. Some interesting experiments have recently been made by Chevandier and Salvetat, to solve the pro: blem, whether it is the quantity of water applied or put in motion, or the presence of matter held in solution - which water, acting as a vehicle, conveys to the roots of plants - that is of greatest benefit to them. In carrying out their experiments, the water of two different springs was made use of, which, for clearness, we shall call No. 1 and No.2. 255,744 cubic metres of water,
No. 1, was applied per hectare, and 164,281 cubic metres from No. 2, to the same extent of surface. The produce in the first case was 2312 kilogrammes per hectare, while in the other it was 7896 kilogrammes. The second year's experiments produced the following results: 126,273 cubic metres were applied per hectare fromspring No. 1 ; the weight of crop produced was 2749 kilogrammes. 130,311 cubic metres from No. 2 produced, in weight of crop, 10,469 kilogrammes. "We thus see," say these gentlemen, " that with equal quantities of water, and in conditions in other respects perfectly comparable, or even with larger quantities of water from the bad spring (No. 1), the crop of the meadow watered by this spring has only been one-third or onefourth of the crop produced under the influence of the water from the good spring (No. 2). It is then in the quality of the waters, and not in their quantity, that we should seek for the causes of these very considerable differences in the crops."

The gases and organic matters held in suspension in both these springs were nearly the same. Messrs Chevandier and Salvetat therefore sought the solution of the problem in the organic matters dissolved in these waters. According to the centesimal composition of these matters in the springs No. 1 and No. 2, taken as types in this extract, oxygen and hydrogen were found in the same proportion; but these matters were more rich in carbon in spring No. 1 , and more rich in nitrogen in spring No. 2. The nitrogen in No. 2 was to that of No. 1 as 100 to 42 , whilst the carbon in No. 2 was to that of No. 1 as 100 to 94. The two propositions here laid down do not sufficiently explain the different fertilising powers; but the following will do so: "If instead," say these gentlemen, "of considering merely the absolute quantities, either of organic matters or of nitrogen contained in these matters, we seek the relative proportions of the nitrogen and carbon which enter into their composition, we find that 100 of carbon correspond in the good spring to at least 11 of nitrogen, and, for the bad spring, to 4 of nitrogen at the most; whence we see, that the fertilising properties of our good springs correspond completely to a proportion three times stronger, considered relatively to the carbon."-(Vide The Chemist, and Comptes Rendus of Feb. 1852.) 


\title{
CHAPTER XIII.
}

\author{
M A N U R E S.
}

\section{§ 1.-KINDS OF THEM, ETC.}

VARIOUs materials, under the general denomination of manures, have from the earliest ages been applied to the soil, with a view to increase its fertility, or, in other words, to supply plants with those elements necessary for their healthy development, in which the soil may be either naturally deficient, or which have been extracted from it by previous crops. Hence the great importance of ascertaining, first, what elements the soil is really deficient in; and, next, what the material added contains necessary for the crop to be reared. All plants do not require the same food, and hence, without a knowledge of these important facts, very useless, if not injurious additions, may possibly be made. It is to arrive at a correct conclusion as to this, that the chemistry of soils and of plants, as well as a practical knowledge of their various natures, is of so much importance; and without some knowledge of this, all manurial applications whatever must be regarded with suspicion as to the effects they will produce.

Manures are usually divided into two classesviz., organic and inorganic ; but more naturally into three - namely, animal, vegetable, and mineral. The first of these comprehends those substances that are derived from animals, either in the shape of excrements or from their dead bodies; the second includes the vegetables so applied, either singly or mixed; while the third is composed of minerals, fossils, earths, or earthy substances, either applied in their natural state, calcined, or otherwise reduced in form, or mixed with other materials. Organic manures consist of carbon, hydrogen, and nitrogen, and are undoubtedly the most valuable. Inorganic manures, or the substances employed as such, are very numerous, but the mode in which they act is still very imperfectly understood. Were they as important as some would lead us to believe, they of all others would be the most valuable to the horticulturist, on account of the convenience of their application. Considerable difference of opinion exists as to organic or inorganic matter being the best fertiliser of the soil, Mr Lawes, and with him the majority of practical experimenters, contending for the former, while Liebig, heading the chemical host, as strongly advocates the latter. We are inclined to follow the former, so far as the principal supply of the food of plants is concerned, and admit the theory of the latter only in so far as a proportion of certain mineral matters is necessary for the proper action of the organic matter in its preparation as the food of plants, when reduced to an aqueous or gaseous state; and also that certain minerals enter into the structure of plants, giving them greater strength of stem, and thereby enabling them better to maintain an upright position-a position of all others the most favourable for the action of atmospheric influences upon their respective organs. The real object of a plant is to reproduce its kind, and when it has done so, as in the case of annuals, it ceases to live-decomposes, and becomes resolved into that matter upon which it had existed, and becomes the food of plants of its own, or probably of other species. The decay of the annual parts of plants, such as their leaves, \&c., in the case of trees, and the higher grades of vegetable life, aids the nourishment of themselves while in life, and fertilises the soil after their removal for other crops. Such is the natural production of organic manure. On the other hand, the advocates for mineral or inorganic manures say: Plants, by means of their roots and leaves, perform the various functions necessary for their existence; the former penetrate the soil in search of moisture, and of mineral ingredients dissolved by moisture, and essential to the plant; and these materials are taken up by the sap and passed into the leaves, where, by the influence of the sun, the leaves acquire the power of acting on various gases contained in the atmosphere, of absorbing them, and of changing them into materials adapted to their growth. Without the mineral ingredients from the soil, plants could not obtain them otherwise, as none exists in the air. "If we consider," says Mr Nesbit, in "Lectures on Agricultural Chemistry," "the nature of plants, in their wild state especially, we shall find, if they be supplied with an adequate quantity of mineral matter according to their varieties, and according to their circumstances, they will obtain all the rest from the air without any assistance." Mr Nesbit appears to overlook the amount of nourishment plants, even in their natural state, derive from the decomposed remains of their predecessors.

No doubt, all plants derive a great part of 
their food from the atmosphere; and the lower they are in the scale of creation, the more evident this appears. The first traces of vegetation on the cooled-down masses of lava in volcanic regions are various species of cryptogamic plants, which have scarcely such appendages as roots; there, however, they thrive, deriving their whole sustenance from the atmosphere: by their dissolution they leave organic matter in the fissures, which, in turn, supports a higher order of vegetation to perform the same important office, until a sufficient body is at last accumulated to give support to the largest shrubs and trees; thus showing, as we rise in the scale of vegetable life, the vast importance-nay, the positive necessity-of organic matter being present to secure healthy development. The operation of rendering the most sterile soils in course of time fertile, may be instanced in the case of planting with trees soils naturally possessed of little or no organic matter; yet on such soils do trees not only grow, but in time produce thousands of tons of valuable timber, leaving the soil much richer in vegetable mutter than it was prior to their being planted. During the first few years, trees so circumstanced make little progress; but as they increase in size, and as their annual exfoliation increases, so do they in bulk and magnitude. These facts are quite sufficient to prove that vegetables have the power of feeding largely on the atmosphere, and deriving from it the great bulk of their sustenance. According to some, more than three-fourths of the solid matter found in plants is derived from the air, and not from the soil. Seeing, therefore, that plants derive so much of their food from the air, it may not be unimportant to inquire from whence and how their food is provided. We believe that it arises from the soil in the shape of gaseous matter disengaged by the great chemical operations constantly going on in the great laboratory of nature, and mixing in the atmosphere, and fed upon by the leaves of plants. The supply necessary to keep up this enormous demand is almost provided by plants themselves, even when in a state of nature, by the annual shedding of their foliage and decaying parts; but when in an artificial state, where greater development is required, then it becomes necessary for man to add to these sources such materials, or what are called manures, as he deems best fitted for their wants and circumstances.

Before noticing those manures individually, which experience has proved to be of most consequence to the horticulturist, we may observe that, according to our highest authorities on vegetable chemistry, all plants are composed of oxygen, hydrogen, carbon, and nitrogen, or azote, with a small proportion of saline bodies. All manures, therefore, should include these elements, as a deficiency of either may prevent the formation of those parts in the vegetable, for which its peculiar organisation is contrived, and on which much of its healthy existence depends.

All manures must be rendered soluble, as no solid substance can enter into a plant. Hence, manures deposited in the soil must undergo a species of dissolution, and become watery or gaseous before they are capable of being absorbed either by the roots or leaves of plants. Sir H. Davy thought that such parts as became gaseous, and passed into the atmosphere, afforded little benefit to plants, as the gases soon become diffused through the mass of surrounding air. More recent authorities think otherwise, and say that plants feed as much by the leaves as by the roots, and that their food is supplied by the gases which evolve around them, and which of necessity must arise from the soil.

The application of manure to the soil no doubt acts in two very different ways, yet both indispensable to vegetable existence; first, by being taken up by the roots, after it has assumed an aqueous form; and, secondly, by being taken up by the leaves, when it becomes resolved into a gaseous state. The first of these has been long admitted, the other has more recently been recognised. To insure that effect in the former, the manure is buried in the soil near to where the roots are situated. To secure the latter, it may be thought that the manure should be spread upon the surface, or probably applied to the leaves in a more direct manner. Neither of these are absolutely necessary, although of the effect there can be little doubt. A certain portion of the manure, when reduced to a proper state, is taken up by the spongioles at the termination of the roots, while another portion, becoming disengaged from the soil, escapes into the air which surrounds the plant, and is fed upon by the leaves. Hence one of the great advantages of frequently stirring the soil between rows of crops, and which is done by most people for the suppression of weeds; by others, as they say, to promote evaporation, and for the admission of the rays of heat to the roots-few thinking that this very necessary operation tends to facilitate the escape of fertilising gases from the soil, to be fed upon by the leaves, which would be prevented from escaping if the surface was allowed to remain hard and unbroken. No doubt, a great amount of these gases is dissipated into the atmosphere, and may be lost; or they may be returned, for aught we know, by various atmospheric operations - such as becoming mixed with rain, dew, \&c., and in this state be brought to act upon the foliage in a way unknown to man. Nor is it necessary that these gases should become amalgamated with either rain or dew; they may remain in a gaseous form, and be carried to the foliage, if not of the plant from under which they arose, of others equally important, by the action of the air itself. The effects of gaseous food taken up by the leaves must of course be much less apparent in the open air than it is within the more limited atmospheres of pits and hot-houses; and that they are actually less is admitted. The principle, however, remains the same. The heat and moisture afforded by the tank system of heating is, so far as these two elements go, quite satisfactory; but plants do not thrive, by any means, so well in houses so heated, as in others heated by the use of stable manure in a state of fermentation; nor do plants, in pits heated by leaves, tanners' bark, flax refuse, or any 
other vegetable matter subjected to the same amount of fermentation, either prosper so well, or recover from a sickly state so soon as they do in pits heated by stable manure, all other conditions being the same. Practically we know this, and account for the difference in the almost total absence of ammoniacal gases, or other fertilising matter, in the former when compared with the latter. So apparent is this, that some of our best cultivators-Mr Fleming of Trentham, for example-have recommended pigeons' dung, or other highly-enriched manures, to be mixed with the water in the tanks, to bring about this invigorating state in the atmosphere of pine-stoves, which are deficient in it-although heat and moisture are abundantly supplied by the tanks. Sickly plants, in pots, plunged in a common dung-bed, rapidly recover; and pines grown in such material, althorgh their roots are also confined within pots, attain a larger size and greater vigour. Melons grown in pots suspended by their rims, and placed over a vault heated by dung linings, are found to prosper as well as when planted in soil laid immediately over the hotbed. These and many other instances could be given of the healthy condition of plants, when grown in an atmosphere highly charged with gases arising from dung in a state of fermentation, and while their roots are almost precluded from being affected by them. That the plants so circumstanced must derive no ordinary share of their nourishment in a gaseous form is pretty evident, and it is equally so that that nourishment is not conveyed to them by their roots. It follows, therefore, that the leaves are the organs through which it is supplied. If, therefore, such an operation goes on in pits and hothouses, it necessarily follows that the same action is going on in the open air-with this difference only, that, in the former case, the atmosphere is more limited and the supply more abundant than in the latter. These effects have been discovered and accounted for by men of observation and intelligence--such as those who practise in the highest grades of artificial cultivation; while the more superficial go-by-rote practitioners scarcely bestow a thought upon the matter.

Absorption by the root is the process by which plants take up their food from the soil, or from those ingredients which are artificially added to it, called manures. The investigations of Hedwig and Decandolle have long ago set this matter at rest, at least to the satisfaction of vegetable physiologists. For the satisfaction of others, we may state that the food of all vegetables must be reduced, by some means or other, to an aqueous form, and in that state it is absorbed by the spongioles, or sponge-like appendages situated at the points of the fine fibres or rootterminators, and from them conveyed through the roots to the stem, branches, and leaves of the plant.

Absorption by the leaves was first elucidated by Duhamel and Marriotte, and by the experiments made soon afterwards by M. Bonnet of Geneva, chiefly with a view to ascertain whether the absorbing power was alike on both surfaces of the leaf. The deductions drawn by him were, that the leaves of herbs absorb moisture chiefly by the upper surface, and the leaves of trees by the under surface. The cause of this disparity between the absorbing surfaces of the leaves of trees and herbs was not very clearly shown by Bonnet. Keith conjectures that the physical cause might be the existence of a greater or smaller number of pores in the leaves of the herb and tree respectively. The chemical cause would be the peculiar degree of affinity existing between the absorbing organs and the fluid absorbed. Duhamel, looking to the physical cause merely, regarded the lower surface of the leaf of the tree as being endowed with the greater capacity of absorbing moisture, chiefly for the purpose of catching the ascending exhalations which must necessarily come in contact with it as they rise, but which might possibly have escaped it if absorbable only by the upper surface, owing to the increased rapidity of their ascent at an increased elevation; and regarded the upper surface of the leaf of the herb as being endowed with the greater absorbing power, owing to its low stature, and to the slow ascent of exhalations near the earth. "This," says Keith, "does not throw much light upon the subject; and the experiments were still deemed insufficient, as not representing to us the actual phenomena of vegetation, though the fact of the absorption of moisture by the surface of the leaf is fully confirmed by such phenomena." The leaves absorb both moisture and gaseous food by means of the stomata, or, in the absence of pores or stomata, by means of the absorbent power of the epidermis, not only of the root and leaf, but often, as it may be believed, by the other parts of the plant also-at least when they are soft and fleshy, as must be the case in succulent plants, which are for the most part devoid of leaves altogether.

\section{§ 2.-ORGANIC MANURES.}

Stable-yard manure.-The most important, and hence the most general in use of these, is stable-yard manure, composed of vegetable matter, such as straw, hay, and other material employed either as food or litter to such animals as the ox, the horse, the cow, the hog, \&c. It is valued in proportion to the quantity of their excrements mixed with it, the amount of urine with which it is saturated, and the manner in which it is kept previous to its application to the soil. The quality of the food, and the peculiar construction of the digestive organs of the animal, affect it also in some degree; and hence the dung of one animal is of higher value than that of others. "For," as Donaldson (On Soils and Manures) lemarks, "the same kind of food given to animals of a different genus will yield an excrement of a very different quality; and even when given to those of the same species, and under the same treatment, a great difference will often be found, which can only be attributed to the construction and action of the constitutional organs. The dung of swine is of a cold nature, inclining to form a saponaceous 
mass, and constitutes a manure of great power and duration. The dung of cattle contains matter soluble in water, and gives in fermentation nearly the same products as vegetables, absorbing oxygen, and producing carbonic acid gas. The insoluble part seems to be mere woody fibre, and analogous to the residuum of the vegetables that constitute their food, after being deprived of all the soluble materials. The process of rumination imparts a richness of quality from the juices of the saliva, necessary for, and produced by, the additional chewing which the food undergoes." The great value "of stableyard manure arises from its containing both animal and vegetable substances; the former abounding with molecules of the body itself from fatty matters, the latter yielding an aliment to plants from saccharine and extractive matters, but exerting no action on the soil or its contents; the former possessing substances more active and energetic, which also afford a direct aliment, and act on the vegetable matters, and decompose and stimulate the humus in the soil, which becomes exhausted by supplying the extractive matter and carbonic acid, and requires a regular renovation." As regards the state in which the nutritive matter of stable-yard manure should be, to be at once ready to enter into the composition of plants as an aliment of food, there can be but one opinion-it must be aqueous or gaseous; and hence recently-applied manure, in a solid state, can be of no use to them until it is reduced to a state of solution and suspension. Water is the vehicle by which fertilising matters are conveyed to the plant, and these must be in a very comminuted state to be capable of being suspended in it. "Chemists are much divided," Dr Madden observes, " as to what precise amount of decomposition is requisite to render organic matter in a proper state to become food for plants. All agree that decomposition must have commenced; some maintain that it must be completed. My own belief, founded on extensive observations, and not a few experiments, is, that all the products of decomposition, in every stage, are available as food for plants, provided they are either liquid, or capable of dissolving in water." In reference to applying manure to the soil immediately before planting or sowing, Mr Donaldson very justly observes, "A mass of dung, cold or warm, lying in a drill, must be in too gross a form to present and afford ready and palatable aliment to the tender fibres of plants, and a further reduction and mixing is necessary to produce that matrix of comminuted and finely-blended substances in which plants delight so very much to grow. The influence of air and moisture will reduce dry substances to a manure by blending with the soil. Much time, however, is required, and a great quantity of moisture, and frequent stirring of the land. It is reasonable to suppose that stable-yard dung, and all substances that are applied to land as manure, should be in a reduced state; and in the case of the former, it would require an application to the land at an early season, that it may be broken and mixed by the subsequent working of the implements ;" or, in other words, that it may be changed and rendered soluble by the time the plants require its assistance. The best compost-heap is the soil itself, and the best system of manure management is to bury it in the soil before it loses any of its fertilising properties. The changes necessary for its reduction to a proper food-supplying state will take place better in the soil than in the exposed midden. Of all manures, stable or farmyard dung is the most effective fertiliser yet known ; and although other's may be equally quick in their effects, yet none of them are so lasting. Compared with inorganic fertilisers, and many of the modern artificial compounds, it may be set down as the real substantial food of plants; while they can only be regarded as provocatives of appetite-mere stimulants, producing a sudden and ephemeral effect-leaving the plant much in the condition of an animal pampered with rich food in youth, and left to shift for itself before it has arrived at a state of puberty - that is, the period of existence, in both animals and vegetables, when they require the greatest amount of nourishment.

Notwithstanding the vast importance of this material as a fertiliser, few things within the whole range of rural economy are so shamefully wasted and misused. Unnecessary exposure to the weather, excessive fermentation, and a slovenly or ill-timed application of it to the soil, are of every-day occurrence. To avoid these (the two former in particular, for it is in these states that the greatest loss is sustained), the manure should be committed to the soil as soon after it is made as convenient, and the process of fermentation and decomposition allowed to go on there. Gardens are differently circum stanced in this respect from farms, because, in the former, crop after crop succeeds each other in such rapid succession, that almost daily, if not weekly, opportunities offer of ground being cleared of one crop, and under preparation for another. These opportunities should not be lost sight of ; and as garden ground can hardly be too much enriched, manure in greater or less quantity should be applied to almost every crop. By this means the ground becomes enriched by the very essence of the manure, which would otherwise be allowed to escape in a gaseous state during excessive fermentation, deprived of much of its best properties while undergoing unmecessary decomposition, whether on the dunghill or cucumber-bed, leaving it at last, when in the form of what is called spit dung, so highly prized by some gardeners, in a state little better than that of a mass of inert peaty matter.

The application of stable-manure, for the most part, has hitherto been considered as beneficial only when applied immediately previous to the sowing or planting of the crop. To this longreceived opinion we by no means subscribe, and therefore in practice apply such manure generally in autumn, through the winter, or, indeed, whenever the ground requiring enrichment is cleared of its crop. This is new neither in theory nor in practice; it has been acted upon by excellent cultivators with the best results, and was first recommended by Sir Humphry Davy, who has clearly enough explained the principle in his papers on "Agricultural Chemistry." When 
the manure is used in a fresh state, and dug into the ground in autumn or during winter, the soil has sufficient time to become completely impregnated with the elements of fertility required for the succeeding spring crop ; at sowing or planting, a slight dressing of guano, bone-dust, nitrate of soda, \&c., is given, to act as stimulants in furthering the early growth of the crop: as the roots descend into the soil, they come into immediate contact with the store of food previously buried in it, and now reduced to that state in which only it can be serviceable for the purpose of nutrition. Much of the fertilising properties of manure is lost by over fermentation, the effects of rain, snow, and exposure to the weather; and by the time the process, as generally followed, of turning, fermenting, and preparing the dung, has been accomplished, little of its best properties is left; whereas, when the manure is applied to the ground soon after it is made, and dug in, a slow process of fermentation takes place, during which the ammonia is absorbed by the alumina as fast as it is formed. This will be found to be more strikingly the case in strong soils than in light unes, as the former contain a greater portion of alumina and humus than the latter; besides which, a mechanical improvement takes place during the decomposition of the littering matter, it keeping the soil open and more pervious to air. Another advantage attends autumn or winter manuring; namely, it is more convenient to bring the manure in then than during summer, when the process of carting or wheeling would be destructive to the walks. And as garden ground can scarcely be rendered too rich, it matters not how long before the crop is to be sown or planted the manure is applied, as it is enriching the soil all the while, and fitting it for cropping when the time for so doing arrives. Stable-yard manure loses more than is generally imagined by the process of making, as usually performed. Direct experiments have shown, as stated in Morton's " Cyclopædia of Agriculture," that " $100 \mathrm{cwt}$. of fresh farmyard manure are reduced to $80 \mathrm{cwt}$., if allowed to lie till the straw is half-rotten; 100 cwt. of fresh farmyard manure are reduced to $60 \mathrm{cwt}$., if allowed to ferment until it becomes 'fat and cheesy ;' 100 cwt. of fresh far'myard manure are reduced to 40 or $50 \mathrm{cwt}$., if completely decomposed. This loss not only affects the water, and other less valuable constituents of farmyard manure, but also its most fertilising ingredient, nitrogen. Chemical analyses have shown that 100 cwt. of common farmyard manure contain about $40 \mathrm{lb}$. of nitrogen; and that, by fermentation during the first period, $5 \mathrm{lb}$. of nitrogen is dissipated in the form of the volatile ammonia; in the second, $10 \mathrm{lb}$; in the third, $20 \mathrm{lb}$. Completely decomposed manure has thus lost about one-half of its most valuable constituent. The practical deduction which appears to be warranted by these experiments is, where it is possible, to apply farmyard manure in a fresh state to the land." Rank and entirely unfermented manure may be less valuable to thin light soils than to strong heavy ones, but even much of this depends on the kind of crop which is immediately to follow. Our own experience satisfies us as to the advantage of manuring at these times, and in this way, and we have only to look to the highest cultivated garden-ground in Britain, the London market-gardens, for a corroboration of the fact. They lay on the manure as they receive it, not with a view so much to benefit the succeeding crop, as to keep the ground in a high state of enrichment for all succeeding ones, the manure undergoing all the while the necessary changes from a solid to a soluble state.

Green vegetable manure is an important and much-neglected fertiliser in garden culture. It requires no previous preparation: all plants contain much saccharine or mucilaginous inatter, and therefore cannot be used too soon after their death. Green vegetable manure consists of the sweepings of lawns, annual weeds before they have formed their seed, pond weeds, fresh turf, the refuse of vegetable crops, \&c. A great waste occurs in most gardens by carrying all refuse vegetable matter to the rot-heap, there to decompose and be returned again in the shape of vegetable mould, while no small portion of it is sent down the nearest river, or buried in the most convenient pit. In its application it should not be buried to too great a depth, otherwise the necessary fermentation would be prevented by compression. At a proper depth decomposition proceeds slowly, the soluble matters are gradually dissolved, and the slight fermentation that goes on, checked by want of a free communication with the air, tends to render the woody fibre soluble, without occasioning the rapid dissipation of elastic matter. The use of green vegetable manure is of the greatest antiquity it was practised by the ancient Romans, and still largely employed in Italy to this day. It is probable, however, that this mode of enriching the soil is better adapted to warm climates than to colder ones, because vegetation is more rapid and luxuriant in the former than in the latter. This opinion, sound or unsound, bears lightly on gardens, where the climate is supposed to be warmer, and the crops far more luxuriant than on farms. We think highly of green vegetable manures; and, operating upon a light soil and good climate, we commit all such matter not eaten by animals to the soil, and indeed grow spinach, spurrey, and buck-wheat, and other rapid-growing crops, for the express purpose of being dug into the ground immediately before the formation of their seed.

Sea-ueed ranks amongst green vegetable manures, and its application as such in places where it abounds is so far commendable. It abounds in soda, or the mixed mineral alkali, and contains somewhere about $\frac{1}{8}$ th of a gelatinous subtance similar to mucilage, and $\frac{4}{5}$ ths of its weight of water, but no ammonia. Like all green vegetable manures, it should be applied to the land as soon as gathered, as it loses both bulk and quality by keeping. Its effect on land is transient; and it is probable that, if the expense of collecting and applying were expended on the purchase of salt, applied in rainy weather, the effects would be equal.

Rape-cake contains carbon and hydrogen, and hence is an effective manure, better fitted, how- 
ever, for damp soils than for light and sandy ones. Applied as manure, it has been found destructive to that intolerable pest the wireworm (vide p. 182). The rate at which it is applied is about 5 cwt. per acre.

Leaves of trees.-These, from necessity, are collected during autumn and winter in large quantities where extensive pleasure-grounds exist, and, when rotted down, afford an excellent manure for most soils. Leaves of trees, when decayed, as well as the wood itself when so reduced, form what is termed vegetable mould, containing more charcoal but less oxygen, weight for weight, than the plants that produced it. It yields more ammonia, and contains more azote, on account of the different circumstances under which it has been formed. Vegetable mould is an indispensable manure in garden culture.

Tanners' bark.-Both bark and leaves of trees, according to Donaldson (in "Treatise on Soils and Manures," p. 15), "require long time, and much mixing and preparation, to reduce them to mould: hot lime will be the quickest solvent for fresh bark, and the destruction of the fibre being effected, earths and dung may be added, which will bring the whole mass into a soluble and putrescent state. Hot stable-dung has been used in the first application, in order to reduce the woody fibre; but caustic lime is stronger and quicker, and, after the dissolution has been effected, the mild materials may be added, and the mixture completed. Time will effect a dissolution of the fibre without any mixture with the bark; but a long period will elapse, and it is usually preferred to break it up by hot applications. The reduced mixture may be used as a manure for any purpose, but chiefly for topdressings, arising from its finely divided and comminuted state." With decayed leaves and a copious admixture of sharp sand, it makes an excellent compost for growing American plants in, many of which, particularly azaleas and rhododendrons, thrive as well in it as in natural peatearth. It is a good manure to light sandy soils deficient in vegetable matter, and, in a less decomposed state, valuable for keeping open soils of the closest texture.

Sawdust is much of the nature of tanners' bark ; but, unless entirely of oak wood, it contains much less of the tannin principle, that of coniferous trees containing a considerable amount of resinous matter; but when both are extracted, it forms with sand a good substitute for peat, for hardy shrubs and trees. Both, however, should be broken down without the use of caustic lime, when intended for such purposes, as all American plants have a dislike to calcareous matter even in small quantities. Both tanners' bark and sawdust, when charred, constitute a tolerable manure, and are both in that state valuable, when incorporated with nightsoil, urine, and similar potent manures.

Horns and hoofs of animals are similar in con. stitution, containing very little earthy matter, and only 0.023 of phosphate of lime; the chips and shavings of both, procured of the combmakers and turners, have been strongly recommended by some. Repeated trials have not led us to a like conclusion.

VOL. II.
Oyster shells, like those of both sea and freshwater animals, are constituted chiefly of carbonate of lime combined with soft animal matter; they differ chiefly from animal bones in having a much greater proportion of carbonate of lime, affording sometimes almost pure lime when burned.

Bones ground to powder are extensively used in stimulating the first efforts of vegetable life, being sown immediately before, or along with, the seed. In a crushed state they are employed in the formation of vine border's, as they are slow in decomposing, and, in their decay, afford food to the plants long after all other manures applied at the same time have become exhausted. We have found them excellent as drainage for plants intended to be kept long in the same pot. The action of bone-dust upon the soil appears to be little understood. "Up to a certain quantity used," Mr Stephens remarks, "this manure has an evidently beneficial effect, but beyond that quantity no apparent benefit is derived from its use, in so far, at least, as the crop is concerned. I have tried to raise turnips," he says, "with different quantities of bone-dust, varying from $12,16,20$, to 24 bushels per acre, and found the crop improved up to 16 bushels ; but the quantities beyond that, even to 24 bushels, produced no greater effect on the crop in the same field, and on the same sort of soil, than 16 bushels. It is therefore unnecessary," he concludes, "in so far as the crop of turnips is concerned" (that being the crop he experimented upon), "to sow more than 16 bushels of bone-dust alone, or 8 bushels with coal-ashes or street manure." Where the ground has been previously manured with farmyard dung, 8 bushels of bone-dust is deemed amply sufficient for any annual crop. Bone-dust, in combination with sulphuric acid, has of late years been greatly recommended, and in this state it has been found to have greater effect in raising crops on strong land than bone-dust alone. The manner of preparing the sulphurated bones is to mix a given quantity of sulphuric acid with twice its bulk of water, and to place twice the weight of bone-dust as of the acid in a tub or trough, and pour over the bones the prepared liquid gradually, and by times; the bones will become entirely dissolved, and form a mass with the acid and water. When the mass is dried, it will assume the appearance of granulated powder, and is then fit for use. Onecwt. of bones, with $56 \mathrm{lb}$. of sulphuric acid, will be sufficient bonemanure for an acre of strong garden-ground, previously manured with stable-dung; for it should never be overlooked that bone-dust, like most others of the modern fertilisers, should only be regarded as an auxiliary, and not as a general manure. Bones are often broken down by fermentation with sifted coal-ashes, and even with pure sand, and their value considerably increased, probably on account of their being disintegrated to the smallest possible degree, and thereby mixing more readily with the soil. The method of fermenting bone-dust in this manner is thus described by Mr Stephens: "Mix four cart-loads of bones with as many of sand, or mould, or sawdust, in a flat-topped heap. The bones should be thoroughly drenched with 
water, and the other materials moistened. In a few days such a heat will be generated in the heap as to render it unbearable by the hand. As the wet side of the heap will not be heated so much, it should be covered with sand. Whether the heat should be allowed to die out before the heap is used is a point still unascertained, but a large heap makes better manure than a small one; so do unboiled bones and fresh ones than boiled and stale ones. Large bones may be reduced by fermentation in this way, by turning the heap over at the end of a fortnight and watering it afresh, and at the end of a month very few whole bones will remain." This is important to those living remote from bone-mills, and might be taken advantage of where dog-kennels are maintained, for the preparation of the bones of dead carcases used for the dogs' food.

Blood is a powerful manure, and has been beneficially applied to fruit trees, particularly the mulberry and vine. In general, it is formed into a compost with earthy or vegetable matter.

Animal bodies.-Of all manures, animal substances, when well prepared, are the most powerful- promoters of vegetation. "In that state," Mr Donaldson remarks, "almost the whole body becomes volatile, and so far attenuated, subtilised, and refined, as to be rendered capable of entering the vessels of the minutest plants. So soon as the parts are sufficiently divided to be mixed with the earthy materials, the process of preparation must go on quickly; and as soon as incorporation has been effected, the application must not be long delayed, in order to prevent the great loss of bulk that will ensue from the rapid decay of the many complex elementary substances which compose the organisation of the bodies." A few years ago, Mr Roberts, author of an excellent treatise on the vine, brought the use of carrion, or animal manures, before the notice of the public, and employed it largely in the preparation of fruittree borders. Of its advantages as a powerful fertiliser there can be no doubt. There is, however, something loathsome in the application. When formed into a compost, slaked lime may be mixed with the heap just before it is to be used, and, by combining with the other ingredients, will quicken their action, and add a soapy and very valuable quality to the compost.

The dung of birds, either domesticated or wild, affords one of the most powerful of all manures. That of pigeons is the most common in use, merely because it can be procured in the greater quantity. That of domestic poultry is equally efficacious. All are excellent for root crops; and carrots grown with no other manure than pigeons' dung have yielded heavy crops, and free from the attacks of insects. It is an excellent manure for pot plants, but requires to be used sparingly. We use it extensively in pine culture, for camellias, oranges, \&c.; and for most rapid-growing plants we use it in a liquid state. Pigeons' dung is hot and stimulating, abounding in the volatile alkaline principle. The fresher it is applied the better, as long keeping, and especially fermentation, of which it is very susceptible, diminishes the amount of soluble matter. Its principal part is carbonate of ammonia, leaving a consider able residue of carbonate of lime and common salt. The dung of domestic poultry differs only from it in containing a greater portion of silica.

Rabbit's and deer's dung is considered even superior to that of pigeons and poultry, and like that should be used while fresh, and in small quantities, when employed as a simple; and when used as a compound, mixed with equal parts of earth to correct its fiery and corrosive fermentation. It forms an exeellent top-dressing or mulching for all sorts of rapid-growing pot plants, and may be laid on the surface of the soil in pots to the thickness of half an inch.

Guano is the accumulation of the excrements of sea-fowl, found in great quantities in situations where these birds congregate. It has been used by the Peruvians for ages. It was first made known to Europeans in 1804 by specimens sent over by Baron Humboldt. It was afterwards mentioned by Sir H. Davy and Sir Joseph Banks; and about thirty years from its first importation by Humboldt, it began to attract considerable notice in Europe. Various analyses of it have been published; but, in consequence of the extent to which adulteration has been carried, these tests can be of little general advantage. The component soluble parts of genuine guano are, muriate of ammonia, phosphate of ammonia, sulphate of ammonia, sesqui-carbonate of ammonia, oxalate of ammonia, with about equal quantities of water, and soluble organic matter, and urea. The constituent insoluble matters rather exceed the soluble, and of these urate of ammonia and sub-phosphate of lime form by far the largest portion. This manure, before using, should be kept as dry and as little exposed to the air as possible; hence, for horticultural purposes, where no large quantities are required at a time, but the applications are frequent, it is best kept in casks. It is an excellent stimulating manure, beneficial to almost all garden crops, and is found of much service when mixed with composts for pot plants. Water is necessary to bring its fertilising powers into action; a circumstance which appears to be well understood by the Peruvians, who never omit giving water whenever it is applied to the roots of plants. In dry soils, during dry weather, it is not active in its effects; but when applied to growing crops in a liquid state, its effects are speedy and obvious. Indeed, we seldom water any plant or crop, which we wish to stimulate rapidly, without mixing guano with the water. Like bone-dust, it is better adapted to light soils than to strong clays; and its effects are much more obvious in dry and warm climates than in cold and wet ones. In the present adulterated state of guano, it is absurd to lay down rules as to the quantity to be applied to a given space. Such rules have been promulgated with apparently great care ; but, we fear, in many cases, not taking into account that not two samples of guano are found to agree in their constituents. Colour is no test, and even weight is not to be depended upon. The following simple test was published by "The Times' Cor- 
respondent," and is worth the attention of purchasers: "Burn 200 grains of guano in an open fire, in a common iron ladle; it must be frequently stirred, and after keeping it at a strong red heat for ten minutes, and allowing it to become cold, if the ashes weigh more than 72 grains it is not genuine Peruvian guano."

In applying guano in a dry state, it is advisable to keep it somewhat apart from the seed or roots, because, if of good quality and applied liberally, it is apt to injure them; so it is also, when applied in a liquid form to growing crops, if applied too strong, and allowed to fall on their leaves or tender stems. Like all other potent manures-the dung of birds, rabbits, deer, \&c. -it is better to apply a little at a time, and repeat the application. In its liquid state we have found it very beneficial to all the Brassica tribe, to asparagus, rhubarb, and sea-kale.

Nightsoil is an excellent manure, computed in value at five times that of horse-dung. It is, however, transient in its effects, and leaves no residuum or earthy matter for decomposition. It is usually mixed with other materials, such as peat, garden soil, vegetable mould, \&c., in the proportion of one part of its bulk to five of the others. It is, however, most conveniently managed when in a liquid state; and when reduced by water in the proportion of 1 to 5 , is in a fit state to be applied to the soil over the roots of plants, but never over their leaves.

Liquid manure is composed of the urine of animals, dissolved excrementitious matter, the draining of dunghills, wash-houses, sculleries, \&c. From such sources it is easy to conceive that it is of variable value as a fertiliser, depending upon the proportions which each of these contribute to the manure-tank. Its importance in culture is daily becoming more valued and understood. Its effects are of the most active description, because it is, as it were, the already prepared food of plants. The Chinese and Flemings, who have employed it for ages, and place great reliance upon it, allow it to putrefy in large tanks, and dilute it with water previous to using. Chemists assert that putrefaction is not only useless but actually injurious, causing the soluble matter to dissipate; and also, that in an unmixed state it would contain too much animal matter to form a fluid fit for the roots of plants to take up by absorption. On the other hand, they admit that urine, in a putrid state, abounds in ammoniacal salts, and if less active than when in a fresh state, is nevertheless a very powerful fertiliser. From the way in which it is generally collected -namely, conveyed to a reservoir or tank-it follows that putrefaction can scarcely be prevented, as it has often to remain for days, or even weeks, accumulating before it is required for use. Opinions as to the relative value of solid and liquid manures are as yet greatly at variance; the cost of its application, when applied upon a large scale, is no doubt a strong argument against its use, and this the more so when it has to be conveyed to a considerable distance, and where steam or mechanical power, and great extent of pipes, are required for its transport. According to the calculations made by Mr Stephens on the sewerage water of Lon- don, it is stated that 560 tons of water only afford one ton of fertilising ingredients; and if this estimate is correct, then would we say sewerage water would be one of the most expensive applications that could be applied to any land. But the sewerage water of London is very different from liquid manure collected in private establishments, because it is so much attenuated in consequence of the extraordinary supply of water brought into London for domestic and other purposes, and the enormous quantity of rain water which falls on the general surface, all of which, excepting what little is lost by evaporation, finds its way into the common sewers, and is mixed with the materials which constitute the whole mass, and thereby greatly reduces its manurial value. From this it is quite evident, that, in the management of a liquidmanure tank, care should be taken that a limited quantity only of rain or other water be admitted into it-perhaps not more than sufficient to flush the drains occasionally out. The arguments brought forward against liquid manure, based on the analysis of the sewerage water of towns, scarcely apply to the liquid manure procured from private dwellings, or even well regulated farmyards; and it is from these sources that liquid manure applied to horticultural purposes is derived.

The urine of animals possesses greater value than solid dung, but is liable to great loss when not properly treated. The ammonia, which is one of its principal ingredients, is found in greatest quantity when putrefaction has taken place; but as ammonia is a highly volatile substance, it evaporates rapidly from the watery solution; and when this evaporation is allowed to take place, then liquid manure ceases to be so valuable as it otherwise would be. With little trouble this evaporation may be prevented. Sulphuric acid and sulphate of iron, both cheap articles, if mixed with the liquid in the collecting tank, will fix the ammonia ; for liquid manure containing an average amount of ammonia, $1 \mathrm{lb}$. of acid to $150 \mathrm{lb}$. of liquid will be sufficient; and where a greater or a lesser amount of ammonia is contained, a greater or lesser quantity of acid will be required.

The acid should be diluted with water before throwing into the tank. Sulphuric acid in no way affects the value of liquid manure as a fertiliser; it only prevents the loss of ammonia, as the compound it forms with it does not become volatile under the temperature of boiling water. Indeed, experience has proved that its value is much enhanced by the addition of the acid. From the experiments of Mr Kinninmonth, quoted in "Morton's Cyclopedia," " 3000 gallons of cows' urine, to which sulphuric acid has been added, are equal in fertilising value to $6 \mathrm{cwt}$. of Peruvian guano, and superior to 20 cwt. of farmyard dung. To 160 gallons of urine he adds $3 \mathrm{lb}$. of acid diluted with water." However little value some chemists and many agriculturists may place on the drainings of the dunghill, or on liquid manure properly collected and prepared, gardeners place great reliance on it, and agree with Nicol, who emphatically declared it to be "the nectar of 
vegetation." The analysis of Professor Johnston has shown that liquid manure contains, in a soluble state, every element necessary to the growth of plants; and also that the drainings of the dunghill are more valuable than urine alone, containing as it does a considerable portion of phosphate of lime, as well as an appreciable amount of silica in a soluble state, neither of which are found in urine alone.

Liquid manure is most beneficially applied to the roots of plants while in a vigorous growing state. It can be applied to them also when solid manures cannot, as in the case of vines and fruittrees, plants in pots, and to culinary vegetables, at any time during their active existence. To prevent unnecessary saturation, it should be as little attenuated as possible; and; to prevent chilling the roots of tender plants, it should be heated to from $60^{\circ}$ to $100^{\circ}$. With these precautions it may be applied to all plants with beneficial effects.

Ammoniacal liquor is a solution of ammonia, partly caustic and partly combined with carbonic acid and sulphuretted hydrogen. This is a powerful fertiliser, but is much too strong to be applied to crops in an unmixed state: it requires to be attenuated by the addition of from four to six times its bulk of water. In this state it may be advantageously applied to the roots of growing crops, such as all the Brassicæ, spinach, asparagus, \&c. Of this excellent fertiliser Mr Solly remarks, "In consequence of the volatility and caustic nature of free ammonia, it is found advisable for most purposes to fix the ammonia of gas liquor. This may either be done with an acid, or with gypsum or sulphate of iron : a cheap acid like the sulphuric is, generally speaking, the most convenient."

Fish, blubber, and the residue of many manufactured materials composed of animal or vegetable substances, have been used with considerable effect to most soils and to most crops.

Charcoal and the ashes of trees, shrubs, and all vegetables, whether in a living or dead state, afford a considerable amount of fertilising material ; but all those, to be useful, must be burned under a slow smouldering fire. When slowly consumed they contain the fixed alkaline salt called potash, which is extracted by lixiviation and crystallisation. Wood ashes contain soluble salts, earthy phosphates, and carbonates, silica, and metallic oxides, differing in quantity and composition in different plants. Vegetable ashes contain lime, potash, soda, silica, magnesia, and the sulphuric, carbonic, phosphoric, and muriatic acids, with oxide of iron and magnesia; but the products differ very much in different plants. These ashes should be kept perfectly dry after being obtained, as water washes out the alkali, leaving only the insoluble and earthy parts behind. Burned clay and charred peat stand in near relation to these.

A long list of organic manures might be given, but the above are those of greatest importance, and, if rightly prepared and applied, may be considered sufficient for all practical uses. It is not in the deficiency in point of numbers, but in the preparation and application of manures, that we are so far behind.

\section{$\S$ 3.-INORGANIC MANURES.}

Of all inorganic or mineral manures lime is the most important. It is found existing in the composition of most plants. In one form or other it is found in most soils-in calcareous ones abundantly, in silicious soils sparingly, and in fertile loams in moderate yet sufficient quantities, suitable to the plants produced on them. Lime, considered as a manure applicable to horticultural purposes, ranks under the five following heads:-

Quick or hot lime is valued for rendering soluble organic matters, such as vegetable or animal manures already in the soil, and is in that state when it is taken from the kiln, after the water and carbonic acid are driven off during the process of burning. In this state it has a powerful attraction for water, and when applied to the soil, greatly assists in converting the woody fibre and other organic matters into humus, forming what is called humate of lime. The carbonic acid gas which may exist in the soil, or is supplied by water or the atmosphere, reduces the humate of lime to a soluble state, and converts it into a fit food for plants. Quick or caustic lime is especially useful in soils rich in humus, as most all old garden-soils are. It is also useful in soils containing sulphate of iron, by decomposing that salt which is so hurtful to vegetation. It also renders inert matter, such as dead peat, nutritive and useful to plants.

Mild lime is lime which has been exposed to the action of the atmosphere for some time after burning. Its action upon vegetable substances is altogether different from that of quick or hot lime, inasmuch as it prevents the too rapid decomposition of substances already dissolved, acting in this respect similarly to chalk.

Quick lime is most advantageously applied to soils abounding in inert vegetable matter ; mild lime, to soils naturally deficient in a proper amount of calcareous matter, which may be determined by their not effervescing when acids are applied to them. "When a soil deficient in calcareous matter contains much soluble vegetable manure, the application of quick lime should always be avoided, as it either tends to decompose the soluble matters by uniting to these carbon and oxygen, so as to become mild lime, or combines with the soluble matters, and forms compounds having less attraction for water than the pure vegetable substance. The case is the same with respect to most animal manures, but the operation of the lime is different in different cases, and depends on the nature of the animal matter to which it is applied. Lime should never be applied along with animal manures, unless they are too rich, or for the purpose of preventing noxious effluvia."-Encyc. of Gard., p. 490. In regard to the season of application, Mr Donaldson remarks, "Autumn is not to be recommended, owing to the solubility of lime in water, and the want of the summer heats, to develop the action of the lime on the substances in the soil. Caloric must be held as one chief spring of chemical affinity; it dilates bodies, separates the particles, diminishes the attraction for each other, and proportionably augments the attraction of 
the particles of adjacent bodies, and consequently produces combinations, and facilitates reciprocal unions. The winter will chill the operations, and at that time there is no growing crop to derive any present benefit." And in regard to the condition of the soil, when lime is to be applied, the same authority remarks, "Lime being reduced to a finely pulverised state by calcination, it requires the soil to be, if possible, equally well prepared with itself; for between a variety of finely-blended ingredients there will be produced a number of reciprocal actions and affinities of the different parts, that would not happen in a smaller quantity of these substances in a more aggregated and cohesive state." Besides, as a manure, lime is useful in all garden soils which contain, to a greater or less extent, snails, slugs, and insects, constantly preying on the crops cultivated.

An opinion has long existed against the use of lime containing magnesia. "Such lime," says Mr Edward Solly, "is in general objectionable as a manure. It is probable that the tendency which magnesia has to remain caustic for a long time, absorbing carbonic acid but slowly from the air, is the cause of this." Magnesian limestone, though it has been found to injure crops, has yet been used with good effect in some cases. "When a magnesian limestone is burned, the magnesia is deprived of carbonic acid much sooner than the lime; and if there is not much vegetable or animal matter in the soil, to supply by its decomposition carbonic acid, the magnesia will remain for a long while in the caustie state, in which state it acts as a poison to certain vegetables."-Encyc. of Gard., p. 490. On the other hand, Mr Donaldson, from his own practical experience, says, "On one field a double allowance, or 400 bushels to an acre, was applied as an experiment to test the noxious quality of the lime. In every case the application was attended with the very best success, and for several years the green and culmiferous crops were excellent; and on the ground where the double allowance was applied, the crops showed a great superiority. Here was no damage from magnesian lime, but very great benefit." Different kinds of lime no doubt produce different effects, but that none produce the injurious effect noted above is, we think, more than probable. Limestone rocks containing alumina and silica produce a poorer lime than pure limestone, but it is only their poverty in calcareous matter that renders them less valuable, and certainly no noxious quality to affect vegetation exists in them.

Chalk is a calcareous formation, supposed to be composed, according to recent microscopical observations, of animalcules so exceedingly minute that one cubic inch of it contains upwards of a million of them. It is of three kinds-hard chalk, soft chalk, and chalk marl. Hard chalk is burned, and used in the same way as lime; soft chalk is dug from pits, and when spread on the surface of the ground falls into a fine powder, and is then readily incorporated with the soil. Chalk marl is not very abundant, and when found it is used in much the same way as the last. As a manure, chalk acts much in the same way as lime, only it is considered much less powerful in its effects. It also acts mechanically on soils, rendering strong clays more easily worked, correcting acidity in sour lands, and retaining moisture in such as are very light, burning, or sandy. Like shells and shell sand, it is useful to soils naturally deficient in calcareous matter.

Sulphate of lime or gypsum is also a calcareous formation, or limestone in combination with sulphuric acid. It is useful in soils deficient in calcareous matter, and to some extent absorbs the ammonia from the air, and yields it to plants in greater abundance than they could otherwise obtain it.

Phosphate of lime forms a constituent in almost all plants, therefore its presence in any matter, or in its native pure state, is useful as a manure. It is present in excrementitious substances, and to a considerable extent in the straw forming farmyard dung, and is a combination of phosphoric acid and lime.

Nitrate of potass, which is the chemical name of nitre or saltpetre, when reduced to a powder by bruising, and applied during wet weather as a top-dressing at the rate of one or two cwt. to the acre, appears to act beneficially on loams and clays, and the reverse on light sandy soils.

Nitrate of soda, a compound of fossil alkali, is soda combined with nitric acid. It is used in the same manner as the last, and thought by some equal to it in affording nourishment to plants.

It has been remarked by Mr Solly, in "Rural Chemistry," p. 146, that "the nature of the influence which nitrates exert in vegetation is but little understood. Some plants, such as the sunflower, tobacco, lettuce, and many others, always contain more or less of these salts. Others do not contain them, but, when supplied with nitrates, are subsequently found to contain the base without the acid. The soda, potash, or lime is combined with some organic acid, whilst the nitric acid has disappeared. It is probable that, in these cases, the nitrogen of the acid is assimilated by the plant, or that it assists in the formation of gluten and alburnum. Nitrates can have but very little value as manures on the soils which naturally contain salts of nitric acid, or which, in consequence of the substances they contain, are constantly forming nitrates. On soils containing neither nitrates nor other alkaline salts, they appear to produce very beneficial effects. These remarks apply equally to nitrate of soda and nitrate of potash ; at least similar effects are produced by the two salts as far as regards the increased formation of gluten and alburnum."

Common salt is a combination of soda with muriatic acid. This, with several other saline substances, has been long employed as a manure, and the results have been as various as the applications themselves. It is employed, no doubt, with marked advantage to plants, natives of the sea-coast, such as asparagus, sea-kale, \&c., and in such cases is applied by sowing it on the surface previous to rain, and at the rate of from 16 to 20 bushels per acre, this being repeated two or three times during the year. Salt is found in every animal and vegetable manure. It is detected in many plants, and consequently is of advantage in stimulating vegetable growth, as 
well as in the destruction of numerous insects. Notwithstanding, Mr Solly remarks of saline matter, "Nearly all that can at present be said respecting saline manure is, that the addition to the soil, in moderate quantity, of those substances which plants are found to contain, is always good. The mode in which they act, and the office they perform in the nutrition of plants, is as yet but very imperfectly understood."

Ashes constitute an important class in manures, differing, however, in their effects, according to the substance which has undergone the process of burning, and the manner in which the process has been accomplished. The ashes of all vegetable substances consist principally of those substances which plants require, as charcoal, lime, phosphoric acid, and alkaline salts. Of these, charcoal or carbon is the most valuable; and hence, to secure it in the greatest quantity, the process of burning should be carried on as slowly as possible; and this is best effected by covering up the mass while burning, and admitting no more air than just sufficient to keep up a smouldering fire. The ashes of all vegetables contain almost the same constituent parts, and are found useful in all soils, and to the majority of crops. They should always be applied while newly burned, as they lose much of their value by keeping, even although kept under cover. A medium quantity of vegetable askes may be taken at $1 \mathrm{lb}$. weight to the square yard. Coalashes are less valuable as a manure ; but as they are to be had in much greater abundance than any other, their use should not be disregarded. The finer parts only should be employed, as the half-consumed coal and the vitrified cinder can be of little use. The finer parts, separated by sifting, contain alumina, carbon, silica, carbonate of lime, oxide of iron, sulphate of lime, and potash. Their chief value appears to consist in the carbon and lime they contain.

Soot consists principally of carbon, with a considerable portion of salts of ammonia, which it obtains during the combustion of the coal : these are, however, extremely volatile, and hence much of them is lost. Soot is one of the best of all manures for garden ground; its effects, however, last only during one crop, and its application should coincide with the time of sowing or plant- ing. It is beneficial also as being destructive to insects: the quantity applied may be from 40 to 50 bushels per acre.

It is not from any want of manures that any land need be left in a state of comparative sterility, their value depends on their proper accumulation, preservation, and application; yet, for all that has been written upon the subject, we see in all directions the very essence of fertility allowed to run to unprofitable waste. The following paragraph from "Donaldson on Soils and Manures," p. 110, is deserving the especial attention of cultivators: "The effects of manures of all sorts depend much on the quality of the land to which they are applied, and also to the state of preparation of the soil, at the time when the substances come in contact with each other. Finely reduced and pulverised bodies cannot mix with those of a grosser form. Masses, clods, and lumps, either of homogeneous or heterogeneous substances, will lie together, and remain in the original state of cohesion or aggregation; but no affinity or composition takes place at sensible distances, and consequently no results follow from the combined influences of the bodies in union. The contact of a pulverised substance with a mass of gross formation cannot produce the effects of combination; the finer particles of the former touch only the external surface of the latter, the interior parts remaining unaffected and unavailable for the purposes and effects of alteration." The profitable application, therefore, of manures, depends on deeply working the soil, reducing it, during the operation of turning, to a pulverised state, blending the manurial application thoroughly with it, and never allowing it to lie long undisturbed, or to become exhausted by heavy cropping and scanty applications of manure. The land must be kept in constant possession of decomposing animal and vegetable substances, by the frequent application of putrescent manures; and if so done, the mineraling redients we have referred to may then be applied with a certainty of remunerating the cultivator for his expense and labour, but no direct dependence should be placed on mineral manures alone, and far less on the compounds sold as artificial fertilisers. 


\title{
HARDY FRUIT GARDEN.
}

\author{
I N T R 0 D U C T I 0 N.
}

\section{§ 1.-SELECTION OF KINDS, ETC.}

IN no department of horticulture has greater progress been made within this last quarter of a century, than in the production of improved varieties of fruits, and the superior management of the trees and plants that produce them. In both cases there was ample room for amendment. For the former, we are mainly indebted to our Continental neighbours, both for the numerous fine varieties we have received from them, and also for the impetus the experiments of Dr Van Mons, and other Flemish pomologists, gave to those of this country, in rearing new varieties from seed. For the latter, not only we, but they also, are indebted to the perseverance, skill, and superior intelligence of such men as Rivers, Thompson, Errington, Fleming, Spencer, Harrison, \&c., who, carrying out the theories laid down by Lindley, Knight, Hayward, \&c., and even establishing new ones of their own, have reduced the science of fruit-tree management to principles founded on reason and a correct physiological basis. Equally great has been the advance in the no less important part of the science, the establishment of a correct nomenclature, for which we were at first under eminent obligations to the Horticultural Society of London, by the publication of their fruit catalogue, in which the names of a vast number of fruits have been determined, by comparisons drawn from trees grown in their own garden. One of the many names previously given to the same variety in different parts of the kingdom has been selected as the standard, and the others given merely as synonyms. This was a great boon to fruit-growers, and, to a certain extent, raised up a spirit of observation and inquiry amongst them, the advantages of which are so obvious as to require hardly any comment from us. The task was herculean; and, without the means at command of so important a society, could not have been satisfactorily carried out. That such a first effort should haye attained a perfect result, it would be unreasonable to expect. It, however, so far paved the way for others to complete a task so creditably begun. The publication of the "Pomological Magazine," under the auspices of that Society, the "Guide to the Orchard," by Mr G. Lindley, and "The Orchard" by ourselves, subsequently followed; and these three works have done more than all the others that preceded them to render the nomenelature of fruits intelligible to general readers.

The pomological work projected by $\mathrm{Mr} \mathrm{R}$. Hogg, if equal to the excellent specimen he has given us in his "British Pomology," which includes the apple only - and we have no doubt it will be so-will, from the published material at his command, collected by others, and his own thorough practical knowledge of the subject, place pomological nomenclature in Britain on a basis superior to that of any other country in the world.

Following up such examples, we have given copious descriptive lists of fruits. Our object, however, has been directed more to a selection of the best kinds suited to the variable climate of Britain, and also to its different localities, than to any attempt at a general description, which, in a work like the present, would be quite out of place. Our descriptions are brief, but, we hope, sufficiently clear for all purposes of practical cultivation ; and our recommendations only given where experience, during a long and extensive practice in various parts of the kingdom, justifies them. We have throughout steadily kept in view the exclusion of bad and inferior sorts, and passed unnoticed some few new varieties, with regard to the merits and defects of which we have not as yet had an opportunity of satisfying ourselves. The advantage of giving the synonyms must be apparent to most, as on looking over nursery catalogues, where too often synonyms are disregarded, a person about to form a selection of fruits has to wade through the list of names, and probably, after fixing on 
fifty, as he supposes, different sorts, finds, upon consulting a list similar to our own, where the synonyms are given, that he has not more than one-third of the number of really distinct varieties in his selection, and that the remainder are only different names for the few he finds to be really distinct. And well it is for him who has consulted such a list, for, had he not, it is probable his misfortune would not be discovered until the trees had arrived at a bearing state-a loss both of time and of hope. In illustration of this, we may state that a person not well acquainted with such matters, in purchasing twenty peach trees, might find, when they fruited, he had in reality got only one sort, namely, the Grosse mignonne, which has no less than twenty-three English synonyms besides several French ones, and all, it must be admitted, very taking and popular names too.

In pears, apples, cherries, \&c., similar instances, although not to the same extent, occur ; and this confusion exists (as in the case of the peach referred to) more generally with the very best sorts than with those of inferior quality.

In former times, not only our commercial orchards, but many of our best private gardens, were encumbered with fruit trees, few of which had much claim on the attention of the cultivator-a fact which a reference to the nursery catalogues of the early part of the present century, or a glance back at our early days, will sufficiently prove. These things are now greatly altered, so far as the leading nursery catalogues and the best private gardens are concerned; but we regret to see little improvement taking place in the cider orchards of England, and the large commercial orchards in Scotland. The former, in a commercial point of view, require great amendment, and the attention of cider-makers should be directed more to chemistry than to "use and wont;" the latter, if not speedily remodelled, had better be utterly destroyed, as no one will use such inferior fruit as is grown in them, while they can obtain a superior article at the same cost, brought from Holland, France, or Germany. Not that we think the fruits imported from either of these countries, although they possess greater natural advantages in regard to climate than our own, are in general equal to what might be produced at home, were we to discard our inferior sorts, and cultivate such improved sorts as are quite suitable to our climate. If this is not done, there is no doubt that the land at present occupied as orchard ground would be far more profitably employed in the production of agricultural crops. We believe there are few situations, either in England or Scotland, dedicated to the production of fruit, that, under good management and a judicious selection of sorts, might not produce fruit equal, and in many cases superior, to that imported. Both remain nearly stationary, as regards culture and selection; and while they remain in their present state, they must continue to be unprofitable to their owners.

In market-gardening considerable improvement has taken place-a result arising from competition from abroad; and the day is not far distant when the trashy fruits which at present cumber and greatly deteriorate other crops in such gardens, both in England and Scotland, will be removed, and their places occupied with better sorts. This was done almost simultaneously by most of the large commercial growers about London, as soon as the superior Flemish and other pears were made known to them. Apples and all other fruits underwent a similar change ; so that the kinds found in the market thirty years ago, with the exception of a few good and standard sorts, are now almost unknown even by name. A similar improvement has taken place in most private gardens by the introduction of sorts of the very highest character, possessing the properties of ripening early and keeping late, thus spreading the fruit season over the greater part of the year, instead of its covering, as formerly, scarcely half of that period.

We would remind those (and we know there are many such) who, from a mistaken notion of economy, still allow their gardens to be crowded with old and worthless sorts and worn-out trees, producing precarious crops-and those on walls in general towards the extremities of their branches, and of very inferior qualityhow much they lose, both in quantity and quality, and how much they would gain by rooting such trees out, and planting those of higher merit. There is no profit in very large fruittrees: if standards, they scarcely repay the value of the ground they occupy; and if on walls, as we have stated above, scarcely onesixth of the surface is made available for the production of fruit.

Trees of moderate size afford a greater variety, because a greater number can be planted in a given space, and, if managed as recommended by the best writers of the present day, they will come into bearing a year or two after planting, will be most productive, and continue in health and productiveness, if rightly treated, for a quarter of a century-quite long enough for any fruit-bearing tree, exposed to our variable climate, to be allowed to exist. No doubt there are some exceptions to this rule, but they are very few indeed. In the selection of hardy fruits much discrimination is required, so as to adapt the sorts to the requirements of the owner, as well as to the situation, soil, and climate where they are to grow. Over soil we have complete control; over situation and climate we have comparatively little, and here it is that the judgment of the planter is called into action. Latitude should weigh with him much less than altitude; for experience teaches us that many excellent fruits will arrive at great perfection at 100 feet of altitude, which would scarcely ripen at all at double that height in the same latitude. Some localities are, however, from natural causes, little understood, better adapted to the ripening of fruit than others, even in the same latitude, and at the same elevation. The green gage plum we may instance as an extraordinary example of this : it is found to ripen, as a standard, to greater perfection at Traquair, from 400 to 500 feet above the level of the sea, than it does at Dalkeith, which is only 100 feet-the two places being within half a degree of each other. The gar- 
dens at Airthrey, at 150 feet altitude, situated at the base of the Ochil hills, have long been noted for the size and excellence of their peaches and pears; indeed, those that we have seen of the latter equal any we ever saw grown in the vicinity of London; while in gardens at the same altitude, on the southern banks of the Forth, with equal skill and attention, the same results do not occur. Figs ripen against the wall at Preston Hall, 412 feet above the level of the sea, better than they do at Dalkeith or Melville Castle, only a quarter of that height. The peach, the apricot, and many of our finer apples and pears, ripen in some parts of Ross-shire better than they do in many parts of Northumberland, even at the same height above the sea. The apricot ripens to perfection in many places on the eastern coast of Scotland, at an altitude where it is scarcely possible for them even to exist on the western shores.

Altitude, or elevation above the level of the sea, is considered by most writer's on climate, $\& c$. , equivalent, as regards the warmth or coldness of a locality, to receding whether north or south from the line- 600 feet of altitude being thought equal to a degree. These calculations, however correct they may be as general principles, are in reality of little importance to the British horticulturist. From the insular position of our country, its hilly and diversified surface, as well as its being situated between the parallels of $50^{\circ}$ and $58^{\circ} 40^{\prime}$ of north latitude, great and marked inequalities of heat, cold, and moisture occur over its surface, all of which operate powerfully on the productions of his care. The geographical position has more influence on the cultivation of fruits, \&c., than the difference in altitude below that point at which fruits cease to ripen. Cultivation has also a great effect on this : drainage, shelter, and exposure, will elevate the temperature, and improve the climate in one situation over that of another at the same altitude, and render the soil capable of raising a higher class of vegetable products, as well as mitigate the effects of certain baneful atmospheric influences. The geological series, also, produces a very considerable influence, both physical and chemical, upon the superincumbent soil, irrespective of their exact altitude. Professor Johnston, struck with this fact in travelling through Wigtownshire, remarks, in "Letters on Scottish Agriculture," that, in passing an " imaginary line, the husbandry changes, the soil seems more barren, the people more indolent, and their methods more primitive. You ask the reason, and you are told that the climate is unfavourable to profitable culture. But if, in the midst of this broad tract of country, a patch of limestone, or trap, or old red sandstone appear, all at once the difficulties appear to vanish, and heaven and earth seem to conspire in rewarding the diligent cultivator of the soil." The same holds good in the cultivation of fruit trees, which are so often found to prosper upon such formations, while they linger out an unprofitable existence 100 or 200 feet lower. The influence of elevation above the level of the sea is little understood, nor has it been sufficiently attended to. Sir Thomas Brisbane and Mr Gal- braith, in " Edinburgh New Philosophical Journal," have calculated the decrease in temperature to be $1^{\circ}$ for every 212 feet of altitude-in latitude, $55^{\circ}-57^{\circ}$; and Mr Hewitt Watson, in "Magazine of Natural History," vol. i. p. 444, raises it to 239 feet, between $53^{\circ}$ and $59^{\circ}$; admitting, at the same time, that neither of these estimates are worthy of implicit reliance. Others state the decrease of temperature at $1^{\circ}$ for 300 feet in perpendicular elevation. With such uncertain data regarding altitude, little advantage can be taken in selecting a situation either for a garden or orchard. We have, in vol. i. p. 20, spoken of the advantages of rather elevated situations for gardens in general; and, in addition to the opinions there offered, we may add, that fruittree gardens and orchards should never be established in low-lying places, because they are of all others the most subject to the baneful effects of rimes and frosts.

The primary object, therefore, to be kept in view in the selection of fruits for any particular locality, is to ascertain its climate and circumstances, to draw conclusions either from those fruits which prosper in such places, or from those nearest approaching thereto. Perfection is not to be arrived at at first; for, from what has been briefly stated above, there are certain circumstances of which man remains as yet comparatively ignorant, which may lead to satisfactory results, or the reverse.

We have, in various instances, noted those sorts not in general cultivation which are capable of arriving at perfection in northern latitudes, with a view to their being substituted for the inferior sorts supposed alone fit for such localities. In good climates, good fruit, under any ordinary management, may be expected. It is in indifferent or bad climates that we wish to see superior fruits introduced, to the exclusion of the bad ones now grown, and to stimulate growers in carrying out our views.

"We hope to live to see the day," says a sensible writer, in the " Cottage Gardener," No. 221, "when such pears as the Glout morceau, Passe colmar, Ne plus meuris, and Beurre rance, will be offered in Covent Garden market during the winter and spring months, in quantities as great as the Lammas, Williams' Bon chretien, and Bergamots, are during the early autumn. There is no reason why this should not be so, if commercial growers could only be brought to see what their own interest is. The greatest London market-gardeners have long found out the value of such crops, and it is by them that the market and fruiterers are mainly supplied; but the country orchardists are wholly ignorant of, or perfectly easy on the subject." And we as earnestly wish to see the day when our Scotch markets and street venders' stalls shall be swept of the veriest trash which is hawked about under the name of apples and pears-when the Elshenhalfts, the Pow Megs, and the Grey goodwives, shall give place to the Dunmore, Louis bonne (of Jersey), Urbaniste, \&c.

Next to judicious selection is the judicious management of the trees themselves; and amongst the modern improvements in this department is that of keeping a proper balance 
between the roots and the branches-by operating more upcn the former and less on the latter than has been the practice in bygone days. ${ }_{2}$ Not, however, that root-pruning is altogether a new idea, for it was, to a certain extent, recommended by Bradley and others of our older authorities, to be employed upon extraordinary occasions. It has now been reduced to a regular system, and those enlightened individuals who have adopted the practice place much greater reliance upon its due administration than upon all the systems of branch-pruning recommended by former practitioners.

With a view to convey something like a correct idea of fruits, it has been suggested that nurserymen should keep specimens of their fruits, modelled in wax, for the guidance of purchasers. A more economical way would be for the nurserymen of Britain to join together in the publication of a pomological work with coloured figures, somewhat similar to Hooker's "Pomona Londonensis," the "Pomological Magazine," or the "Pyrus-Malus Brentfordensis," the colouring to be under the direction of a competent person, so that uniformity might be insured. We are aware of the cost of such works, and also that neither of the first met with such encouragement as to induce their proprietors to continue them; but in the case to which we refer, a share of the expense ought to be borne by the purchasers as well as by the nurserymen, by a slight additional price on each tree. Both would be equally benefited, and therefore both should bear their share of the expense. The late Messrs Ronalds of Brentford published their beautiful "Pyrus-Malus Brentfordensis" at their own cost; and we have their authority for stating that the expense was covered in a few years by the increase in their sales of apple-trees, these being the subjects illustrated. It is not at all unusual for nurserymen to go to the expense of figuring new fruits, but the public have no great confidence in these representations. Some have also had many of their fruits modelled in wax, and very beautifully coloured,- - the Messrs Lawson of Edinburgh are doing so at the present time; and, beautifully as these specimens are executed, the misfortune is that the next nurseryman who follows their example, by employing different artists, and procuring the specimens from either better or worse localities, will produce models as different as the fruits themselves would be in these circumstances. It would be otherwise with coloured figures; they would be all engraved from the same plates, and as the colouring is done in general by hand, a great difference might occur here, if not under the direction of some competent individual. No doubt they might be coloured by a process employed in France for several years, and exemplified in the coloured plates in vol. $i$. of this work; but this process, although much more correct, is attended with much greater expense. Collections of fruits, engraved and coloured, are much more portable, and far less liable to injury than specimens of them modelled in wax, and coloured ever so correctly. A short letterpress description, somewhat in the tabular form, as adopted by the Horticultural Society in their catalogue of fruits, would not add much to the expense of such a work ; and if so executed, sufficient confidence would be placed in it by the public, as it would render both description and figure, as it were, stereotyped, and completely remove the impression formed, that little confidence can be placed in nursery descriptive catalogues in general.

One of our very best pomological writers $(\mathrm{Mr}$ Errington) has very lately remarked in "The Cottage Gardener,"-- "Since the million will have gardens, and possess the desire, without the practical knowledge, to avail themselves of superior fruit, some means should be placed within their reach, of knowing at a glimpse what will suit their purpose. Catalogues they understand not; neither have they time nor inclination to collect facts by a slow and tiresome detail, which would put them in a position with the really practical man, in adapting kinds to their necessities, or as subordinate to their schemes." No doubt, even the best executed coloured figures of fruits would only satisfy so far as actual appearance goes; but this goes a great way with fiuit, as well as with a section of humanity it might be deemed rudeness to name. Size, colour, and form would be identified; but flavour, quality, and season of maturity, would only be obtained from a short letterpress description.

Of such importance is the settlement of the nomenclature of fruits, which, notwithstanding all that has been done of late years, is still in a state of very great confusion, that the Belgian government has very lecently issued a royal commission, not only with a view of settling the nomenclature, by reducing the names by which they are at present known to a common standard, but also to collect and publish all existing information concerning their merits and cultivation. Much gratified we are that our hints thrown out above have already been thus anticipated, and that to the extent of being carried out under the auspices of one of the most enlightened sovereigns in Europe. We are also glad that the interests of Great Britain in this matter are intrusted to the care of two of her most accomplished pomologists, $\mathrm{Mr}$ Rivers and Mr Thompson, who, we have no doubt, will see that the publication is made available to our climate and circumstances-both of which are so different irom those of Belgium, that a work adapted to the one country, however creditably executed, would be of little value to the other.

In regard to the extent to which we have carried our select lists of fruits, it may be considered by some that they are too exclusive, while others may think them by far too much extended to come directly under the denomination of selections. Our object has been to bring together as many of the best varieties in each class as we know to be worthy of general cultivation, bearing in mind, at the same time, that many of them that are adapted to one purpose, or to one locality, are by no means fitted for others; and also, that the tastes and requirements of different individuals are nearly as vari- 
ous as the kinds of fruits themselves. In forming selections, however, much depends on the space to be planted, the season when the fruit is most required by the owner, whether he is curious in possessing a great variety, or content with a few sorts that may suit his taste and circumstances. As a general rule, we would say, in reference to apples and pears, Plant few of those which ripen early and do not keep long; of other fruits, a just proportion of those that ripen early and ripen late-in both cases with the view of prolonging the season to its utmost limits. Those who are short of room, yet anxious for variety, may accomplish this by working three, four, or more sorts on the same tree. One advantage attending the cultivation of a number of sorts is, that some kinds in some seasons, from a variety of causes, fail altogether, whereas others escape, probably to fail in their turn the succeeding season. From these lists there will be no difficulty in selecting sorts to meet all these emergencies, their adaptation to circumstances, quality, time of ripening, and duration, being all given.

\section{§ 2. THE ORIGIN OF NEW FRUITS.}

Concerning the origin of new varieties of fruits, little is satisfactorily known. One thing is certain, that under favourable circumstances this process has been going on probably since the creation of fruit trees themselves. That the type of our cultivated apples, for example, is really the crab apple in something like its present state, seems confirmed by the circumstance, that by sowing seeds of any, even of our most highly-improved sorts, a certain disposition is manifested by them to return to their natural or wilding state. Mr Robert Thompson, one of our highest pomological authorities, appears to be of opinion that seedlings of some of our most approved varieties are found only to partake in some degree or other of the properties of their direct parents, but that the greater part of such seedlings more or less affect a wild and inferior character, in no marked manner resembling their parent, in form, colour, nature, time of arriving at maturity, \&c. This opinion is borne out by the observations made by some of the best American pomologists, one of whom asserts, that " a hundred seeds of the golden pippin will produce large-leaved apple-trees, bearing fruit of considerable size ; but the tastes and colours of each will be different, and none will be the same in kind with the pippin-some will be sweet, some bitter, some sour, some maukish, some aromatic, some yellow, others green, red, or streaked." The same rule also seems to hold good in all countries where the apple is cultivated. Seeing, therefore, that fruits produced from trees not only originated from seeds taken from the same parent tree, but also from seeds taken from the same fruit, possess qualities essentially different, the mystery attending the origin of new varieties of fruits appears the more extraordinary and unaccountable. With these considerations in view, we need not be surprised at the few varieties of superior fruits, which make their appearance, like angels" visits, " few and far between,"nor at the apparent apathy with which experienced growers regard new varieties, however highly recommended, until they have "seen, felt, and tasted," and hence are enabled to judge for themselves. Mr Thompson asserts, and his opinion is borne out by most of our leading vegetable physiologists, "that there is a strong tendency in plants from seeds of cultivated fruit-trees of high character to revert immediately to the state of wildings." The late $\mathrm{Mr}$ T. A. Knight, who experimented much in such matters, and with the best results, proved the fact that the wild crab-apple, fertilised by the pollen of a cultivated apple of merit, produces better varieties from seed than can possibly be obtained from seeds taken from the most approved fruits themselves. Seeds of cultivated sorts, or, what are deemed preferable, seeds of wildings originated from cultivated varieties, have been chosen by the Flemish pomologists to sow with a view to obtain improved kinds. These seedling plants they nourish and get into a fruit-bearing state as soon as possible, and from the seeds of these they raise a second generation; thus they go on sowing the seeds of generation after generation, and begin to look out for improved sorts from the second and third generation upwards. This, however, is too early to expect a complete fulfilment of their wishes, although a very perceptible amelioration ensues in each of these generations, and by continuing the process the highest degree of excellence is often at length obtained. For the consolation of experimentalists, we may mention that Dr Van Mons found that good varieties are sometimes obtained from the third generation of apples, in others it may be the fourth or fifth; and in the case of pears, upon which this excellent observer experimented most, the sixth or seventh. An interesting paper on this subject was published some time ago in the "Annales Soc. de Hort. de Paris," by M. Poiteau, headed, "Considerations on the Processes employed by Nurserymen for obtaining better Sorts of Fruits, and on the means by which Nature appears to accomplish the same effect." M. Poiteau commences by observing that improved varieties of our best fruits seldom originate with nurserymen, but owe their origin more to the chance productions found in woods, hedgerows, or in obscure places where the finer sorts are scarcely known. In this opinion he is strengthened by what has occurred in our own country. Our nurserymen and pomologists have acted on the very natural principle that good varieties might be expected from good sorts. In this they have, strange as it may appear, been most signally disappointed; and Duhamel, and other leading French pomologists, failed in the same way. From these failures M. Poiteau concludes that experimentalists are wrong in expecting to obtain at once what can only be produced in time. He seems to infer that seedling apples and pears, for instance, require some years and some cultivation before they can arrive at their fullest state of perfection; thus so far agreeing with the doctrine laid down by Dr Van Mons and others. He may be correct enough in the theory he has formed as to the facility with which the 
Americans in the United States so readily procure superior fruits, and at so little trouble : this he attributes to the ignorance or neglect of grafting shown by the earlier settlers, who, in the anxiety to establish a country, neglected to perpetuate their fruit trees by grafting or budding, and had at last recourse to rearing their trees from seeds. From the fruit trees imported into America now about three hundred years ago, seedlings have been originated both by accident and design; and these, having undergone the ameliorating influence of successive generations, have now arrived at that period of their existence which has developed their true and useful character. Climate has, no doubt, much to do in regard to the superiority of American apples; but the breaking up of new lands, as conjectured by M. Poiteau, has little to do with this, or with the production of new or improved fruits; for although such circumstances may improve the quality of the fruit, it cannot alter its physical nature or constitution.

Most of our present esteemed fruits were brought by slow degrees to the West from the East, and principally from Asia and Egypt. Gibbon, in "History of the Decline and Fall of the Roman Empire," vol. i. p. 52, remarks: "In the more remote ages of antiquity, the world was unequally divided. The East was in the immemorial possession of arts and luxury, whilst the West was inhabited. by rude and warlike barbarians, who either disdained horticulture, or to whom it was totally unknown. Under the protection of an established government, the productions of happier climates, and the industry of more civilised nations, were gradually introduced into the western countries of Europe ; and the natives were encouraged, by an open and profitable commerce, to multiply the former as well as to improve the latter. Almost all the flowers, the herbs, and the fruits that grow in our European gardens are of foreign extraction, which, in many cases, is betrayed even by their names. The apple was a native of Italy; and when the Romans had tasted the richer flavour of the apricot, the peach, the pomegranate, the citron, and the orange, they contented themselves with applying to all their new fruit the common denomination of apple, discriminating them from each other by the additional epithet of their country." This progress, in a westerly direction, is going on at the present day; and the fruits of the East are sought for with considerable avidity by the tribes inhabiting the western shores of America. Indeed, as we have elsewhere stated, no very long time has elapsed since neither the grape, the apple, nor the pear existed in that great and progressing continent.

Improved varieties of fruits accidentally or scientifically produced.-Accident no doubt gave rise to the first improvements in fruits, and the same operation is still daily going on in nature, almost unheeded by man, until the changes are made manifest to him by their welcome results. The reason why such changes should take place, or why the character first stamped by nature on what has been botanically called species, or highly-marked variety, should have a tendency to disappear in the progeny, has been "a stum- blingblock to the systematic botanist and to the superficial student in physiological science." But that such a tendency does exist is well known even to very superficial observers ; and it has been remarked by those who have looked more closely into the matter, that this tendency is more remarkably developed in some species than in others.

It would be out of place here to attempt to account for this deviation from what has been considered an unalterable law of nature. Taking it, therefore, as an admitted fact that such a disposition does exist, let us consider briefly the share that accident and design have in the matter.

That the scientific process of hybridising is no doubt the most certain mode of procuring improved varieties of fruits is quite clear, yet many varieties, including some of our very best fruits, have been originated by accident. This is exemplified in the case of the Claygate pearmain, scarlet nonpareil, and Hawthornden apples ; the two first having been found growing wild in the neighbourhood of Esher, Surrey-the former in a hedgerow, and the other in the garden of a petty public-house ; and the remains of the last still exist in the grounds of Sir J. W. Drummond of Hawthornden. Many others have had a similar origin. Of our best pears, Williams' Bon chretien sprung up from seed in the garden of a schoolmaster at Aldermaston in Berkshire ; the Elton originated in an orchard in a parish of that name in Herefordshire; while the excellent Chaumontelle was found wild at Chaumontelle in the department of the Oise in France, and the Duchesse d'Angoulême in a similar manner in the forest of Armaille, near Angers. The Elruge, which is one of the best, if not the very best of our nectarines, owes its origin to a nurseryman of the name of Gurle or Gourle, at Hoddesdon in Hertfordshire, in the reign of King Charles II., before the doctrine of the sexual intercourse between plants was much known in Britain, from which we may naturally infer that artificial impregnation had not been had recourse to. The Claremont nectarine-a variety of the last-was raised by $\mathrm{Mr} \mathrm{John}$ Greening, gardener to the Duke of Newcastle, who owned Claremont about the year 1750 ; and Fairchild's nectarine was raised from seed by a gardener of that name, so long back as previous to 1722 -both of these, in all probability, without the aid of artificial fecundation.

In America the same accidental originations have been going on ever since the apple was introduced into that country; and, as accidental varieties of the very highest order there, may be named the Newtown pippin, Baldwin, Swaar, and the Spitzenburghs, as well as many others. Many of their peaches and plums-the Washington, for example-are also of accidental origin. The same thing might be instanced as occurring in all countries, and not only as regards fruits, but also very generally as regards plants.

At what period artificial impregnation, or the crossing one variety with another, began to be practised, with a view to improve either plants or fruits, has not been at all satisfactorily recorded. The process was unknown to the an- 
cients, therefore the means of obtaining crossbred varieties artificially could not have existed prior to the discovery of the sexes in plants, nor in all probability for many years afterwards. Among the first to promulgate the theory of successfully raising fruits from seeds, and the advantage of raising successive generations of the same seedling, was Dr Van Mons of Brussels, who published his views thereon in the "Journal d'Agriculture des Pays Bas," in which he recommends sowing only the seeds of existing varieties of the highest merit, and asserts that, by doing so, varieties will be produced with a much greater chance of improvement than if parents of much less merit were employed. In this view he is supported by the late Mr G. Lindley, who says, in "Guide to the Orchard,"- " In sowing seeds for the purpose of procuring improved varieties, care should be had, not only that the seeds be taken from the finest existing kinds, but also that the most handsome, the largest, and the most perfectly ripened specimens, should be those that supply the seed. A seedling plant will always partake more or less of the character of its parent, the qualities of which are concentrated in the embryo when it has arrived at full maturity. How this concentration takes place, we are as ignorant as why certain constitutional peculiarities are in man transferred from father to son, and from generation to generation; but we know that it does take place. Now, if the general qualities of a given variety are concentrated in the embryo under any circumstances, it is reasonable to suppose that they will be most especially concentrated in a seed taken from that part of a tree in which its peculiar good qualities reside in the highest degree. For instance, in the fruit of an apple growing upon a north wall there is a smaller formation of sugar than in the same variety growing on a south wall; and it can be easily understood that the seed of that fruit which is itself least capable of forming saccharine secretions, will acquire from its parent smaller power of the same nature than if it had been formed within a fruit in which the saccharine principle was abundant. It should, therefore, be always an object with the gardener, in selecting a variety to become the parent of a new sort, to stimulate that variety by every means in his power, to produce the largest and the most fully-ripened fruit it is capable of bearing." The correctness of these opinions is, however, denied by others. Many of our finest varieties of fruits have been no doubt produced by means similar to the above; but by far the greater number are of accidental origin. The writings of $\mathrm{Mr}$ Knight, the late Dean of Manchester, Dr Lindley, and a host of others, have thrown much light on this subject in this country; and the physiological authorities on the Continent have contributed abundantly also.

The following excellent fruits may be instanced as a few of the many that have been originated by artificial means: the Napoleon pear was raised by Dr Van Mons at Louvain; the Marie Louise, by the Abbé Duquesne in Belgium; our English Hacon's incomparable, by Mr Hacon of Downham Market, Norfolk; the highly esteemed Gansel bergamot, by Lieut.-General Gansel near Colchester, from a seed of the autumn bergamot; Coe's golden drop plum was produced by a market-gardener of that name at Bury St Edmunds, from a stone of the green gage, the blossom of which had been impregnated by the white magnum bonum, the two trees growing near to each other in his garden; the Downton imperatrice was raised by $\mathrm{Mr}$ Knight of Downton Castle, from a seed of the white magnum bonum, fertilised by the pollen of the blue imperatrice; the Pitmaston orange nectarine was raised from a seed of the common Elruge by Mr Williams of Pitmaston ; the Acton Scott peach was raised by Mr Knight, and is the offspring of the noblesse impregnated with the red nutmeg; the almond peach was raised by the same gentleman from a seed of the sweet almond, the blossom of which had been impreg. nated by the blossom of a peach. The male parent of the Downton strawberry was the old black, while the female was a kind of scarlet; and in the case of the Elton cherry, the father was the White heart, while the mother was the Graffion, \&c.

The spontaneous change of character in fruit.Mr Knight states in the second volume of the "Hort. Trans.," p. 160, that he had a tree of the white magnum bonum plum forty years old, which one year produced on one of its branches red fruit perfectly like the red magnum bonum. He also mentions one of his May Duke cherrytrees, which some years previously had constantly on one of its branches oblong fruit, ripened later, and of greater weight than those on the other parts of the tree. But the most extraordinary instance we know of spontaneous production is that of the excellent and wellknown Hunt's tawny nectarine, the origin of which is stated by Mr George Lindley, in "Guide to the Orchard," p. 282, to have been, not through the seminal process, but, as it appears, by a spontaneous effort in nature to enlarge the parts of fructification of the flower of Fairchild's nectarine, by which was produced this admirable variety, so superior to that from which its origin is dated. $\mathrm{Mr}$ Lindley remarks, "There appears to me a great singularity in this accidental change of character, and to some it may appear incredible ; but I state it as a fact that has happened under my own inspection, being perfectly satisfied that it had never been observed previously by any other person." After enumerating several similar instances, Mr Lindley proceeds, "These, with many other instances of spontaneous production which might be enumerated, lead me to conclude that we may possibly have other varieties, both of plants and fruits, which have not originated from seed."

Peach and nectarine fruit has been frequently produced on the same tree, and even on the same branch. The first instance of this extraordinary freak is recorded in the correspondence between Peter Collinson, an eminent English botanist, and Linnæus ; and the last, which we had an opportunity of seeing some years ago, was in the extensive fruit-gardens of the late $\mathrm{Mr}^{\circ}$ John Wilmot of Isleworth. The tree was of the Royal George variety, and seldom failed in pro- 
ducing fruit of the peach and nectarine on the same branch.-(Vide art. The Peach.)

The mountaineer peach comes almost as often smooth as downy, and was raised between the red nutmeg peach and the violette hâtive nectarine, conclusive enough evidence of their common origin.

Native locality of fruits.-All fruits appear to thrive best, with the least amount of care, in the locality in which they originated. This is instanced in many cases in our own country, and even more strikingly so in America, where we find that the Newtown pippin and the Esopus Spitzenburgh are the apples of New York; Peck's pleasant, and the Seek-no-farther, are those of Connecticut; the Baldwin, and the Roxbury russet, the leading kinds in Massachusetts, \&c.

\section{§ 3. ON THE DURATION, IN A HEALTHY STATE, OF FRUIT TREES.}

The late eminent Mr T. A. Knight about half a century ago started an opinion that every variety of fruit tree had assigned to it a limited period of existence, and, as the end of that period approached, unmistakable evidence would be given, by the declining health and vigour of the trees, and also of the quality of the fruit they produced, of its approaching end. Speechly, Williamson, and others, refer to a deterioration which appeared to them to be taking place in the case of the fruit trees of their time; but this they attributed to a change of climate in our country, and anticipated a restoration upon the return of more genial seasons. Our own opinion is strongly in favour of the theory that our seasons, for many years past, have been less genial and less favourable for the growth of fruit than they were formerly; and, however little the mean temperature for the year, or for a series of years, may differ, still we believe our summers are less warm, and our springs much colder, accompanied with a considerable amount of frost, and this at a much later period than heretofore. Beforethe general employment of the thermometer, which is of comparatively recent date, we had no other means of arriving at anything like correct data as to the temperature of our climate but the historical facts of early harvests, notwithstanding the rude system of agriculture followed, the enormous oaks and other timber trees, the remains of which are abundantly found in bogs, and in altitudes and situations where the oak can scarcely, with all our boasted improvements in arboriculture, now be got to grow at all ; for, with the exception of those in Hamilton Park, and a few solitary specimens in other places, we have no oaks in Scotland now bearing any comparison with those of former ages. More pertinent to our present subject, however, are the data furnished by Langley in his "Pomona," who has given us, in the case of almost every fruit cultivated by him at Twickenham near London, the date of the very day when they ripened-dates by no means corresponding with the ripening of the very same sorts, and in the very same locality, at the present time. No doubt tender fruit-trees suffer much from a succession of cold frosty springs occurring late in the season, and more especially when following a cold wet autumn, when the wood becomes only imperfectly ripened, and also when the latter end of February and March has been sufficiently warm to set the sap in motion, causing the buds to burst forth, and the young shoots and foliage to expand, as they often do thus early, and are as often cut off by frost in April and May. Thus far the healthy duration of fruit trees is seriously affected, and they may no doubt be killed outright.

Returning, however, to Knight's theory-a doctrine, we confess, we were at one time almost a convert to. His views will be best explained by using his own words : "I think I am justified in the conclusion, that all plants of this species" (the apple being that on which his experiments were ehiefly made), "propagated from the same stock, partake in some degree of the same life, and will attend the progress of that life, in the habits of its youth, its maturity, and its decay, though they will not be any way affected by any incidental injuries the parent tree may" sustain after they are detached from it."-KNIGHT's Treatise on the Apple and the Pear, p. 15.

This opinion received countenance from several eminent pomologists, not only in this country, but on the Continent and America also. Kendrick, in his "New American Orchardist," declares his assent to it. Of the Doyenne pear he says, "This most eminent of all pears has now become (in America) an outcast, intolerable even to sight;" of the excellent brown beurre, "once the best of all pears, now become an outcast." The St Germain "has long since become an abandoned variety," \&c. Downing, Hovey, and other American authors, deny that such a fate has befallen these fine fruits; and the healthy existence of them in France, where they have existed from their very origin, as well as their present state in Britain, where they have all been cultivated for a much longer period than they have been in America, proves to us the unsoundness of the doctrine, and the danger of confounding causes and effects. On this Downing very sensibly remarks, in "Fruits and Fruittrees of America," p. 552: "While we admit that, in the common mode of propagation, varieties are constantly liable to decay or become comparatively worthless, we believe this is owing, not to natural limits set upon the duration of a variety - that it does not depend on the longevity of the parent tree, but upon the care with which the sort is propagated, and the nature of the climate and the soil where the tree is grown. It is," he continues, "a well-established fact, that a seedling tree, if allowed to grow on its own root, is always much longer lived, and often more vigorous, than the same variety when grafted upon another stock; and experience has also proved that, in proportion to the likeness or close relation between the stock and the graft, is the long life of the grafted tree. Thus, a variety of pear, grafted on a healthy pear seedling, lasts almost as long as upon its own roots. Upon a thorn stock it does not endure so long; upon a mountain ash rather less; upon a quince stock still less, until the average life of the pear 
tree, when grafted on the quince, is reduced from fifty years-its ordinary duration on the pear stock - to about a dozen years. This," Mr Downing says, "is well known to every practical gardener, and it arises from want of affinity between the quince stock and the pear graft. The latter is rendered dwarf in its habits, bears early, and perishes equally soon." We do not quite agree with $\mathrm{Mr}$ Downing as to this very great limitation; but, admitting it even to be so to a certain extent, we would, for many purposes and reasons, retain the quince stock; for longevity, under certain conditions, is of secondary consideration, more especially as pears upon quince stocks produce their fruit so soon after grafting, that there would not be much greater trouble in keeping up a succession of young trees than in doing the same with gooseberries and currants. Following $\mathrm{Mr}$ Downing, who says, "Next to this, the apparent decay of a variety is often caused by grafting on unhealthy stocks; for although grafts of very vigorous habit have frequently the power of renovating in some measure, or for a time, the health of the stock, yet the tree, when it arrives at a bearing state, will, sooner or later, suffer from the diseased or feeble nature of the stock.

"Carelessness in selecting scions for grafting is another fertile source of degeneracy in varieties. Every good cultivator is aware that if grafts are cut from the ends of old bearing branches, exhausted by over-bearing, the same feebleness of habit will, in a great degree, be shared by the young graft; and, on the contrary, if the thrifty straight shoots that are thrown out by the upright extremities, or the strong limb sprouts, are selected for grafting, they insure vigorous growth and healthy habit in the graft. Finally, unfavourable soil and climate are powerful agents in deteriorating varieties of fruit trees. Certain sorts that have originated in a cold climate are often shortlived and unproductive when taken to warmer ones, and the reverse. This arises from a want of constitutional fitness for a climate different from its natural one. For this reason the Spitzenburgh apple soon degenerates if planted in the colder parts of New England; and almost all northern sorts, if transplanted to Georgia." And upon the same principle, many fine apples of America will not thrive in England, much less in Scotland. "But this only proves that it is impossible to pass certain natural limits of fitness for climate, and not that the existence of the variety itself is in any way affected by these local failures."

Knight's doctrine was denied by Decandolle, Mr George Lindley, and by many of the most eminent vegetable physiologists. The former of these says, "Varieties will last as long, and remain unchanged as long, as man chooses to take care of them;" and of this we have sufficient evidence in the case of the pearmain, which is the oldest apple on record, and still showing no symptoms of decay; and the same may be said of the Cat's-head, London pippin, Winter queening-or quoining, as written by some, and probably more correctly. The latter observes, in "Guide to the Orchard," p. 16, in reference to the supposed degeneracy of the golden pip- pin, "I cannot for a moment agree to such an opinion, because we have facts annually before our eyes completely at variance with such an assertion." Mr Knight even set a limit to the existence of a variety, and observes, "that no variety of apple will continue to exist more than two hundred year's." Nearly two hundred varieties could be named that have continued thus long, and without those discouraging symptoms he anticipated. Downing remarks, and with great good reason, "that the hardihood of any variety depends greatly upon the circumstances of its origin. When a new variety springs up accidentally from a healthy seed in a semi-natural manner, like the Seckel, the Dix, and other American native sorts, it will usually prove the hardiest. It is as if it were an effort of nature to produce a new individual out of the materials in a progressive state, which garden culture has afforded. Cross-bred seedlings-one parent being of a hardy nature, and both healthy - such as Knight's own seedlings, the monarch and Dunmore pears-are next in hardiness. Lastly, we rank varieties reared by Van Mons' method - that of continually repeating reproductions. This, as Van Mons distinctly states, is an enfeebling process, without any compensatory element of vigour. Hence it follows, as a matter of course, that seedlings of the fifth and sixth generations, as are some of his varieties, must in their origin be of feeble habit. Van Mons himself was fully aware of this, and, therefore, resorted to grafting by copulation"-in fact, root-grafting, as Downing remarks-"well knowing that on common stocks these new varieties would in light soils soon become enfeebled and decayed." Mr Downing seems very averse to grafting on quince stocks, and we believe we can clearly see through this, which, at first sight, may seem prejudice on his part. The climate of America is favourable (that is, around New York, the sphere of Mr D.'s operations) to the production of fruits, at a much earlier age of the trees than with us in Britain; and therefore those means recommended by Mr Rivers, and others of our best fruit-cultivators, are uncalled for. With such a climate we would probably also use the pear stocks; but in a climate such as ours in Britain, and in garden-ground highly manured, we would decidedly, for certain purposes, give the preference to quince stocks. Downing has clearly taken Knight upon the weak side of his theory when he says, "In Knight's original essay on the decay of varieties, he clearly states that the local decline of a variety is mainly owing to neglect, and to grafting on a bad stock. We allude to the fact, repeatedly verified, that healthy young shoots taken from the roots of an old variety in apparent decline, produce trees which are vigorous and healthy. The decay," Knight says, "of the power of life in the roots of seedling trees is exceedingly slow comparatively with that in the branches. Scions obtained from the roots of pear-trees two hundred years old, afford grafts which grow with great vigour, and which are often covered with thorns like young seedling stocks; whilst other grafts, taken at the same time from the extremities of the branches of 
such trees, present a totally different character, and a very slow and unhealthy growth. Although Mr Downing's remarks are more of a practical nature than of a physiological one, they bear the stamp of keen observation. Physiologists may, nevertheless, differ from him in certain deductions he has drawn.

Knight's theory does not, however, appear to be new, for we find Mortimer, author of some tracts on religious education, and also on husbandry, in or about 1707 , setting forth in great grief the constitutional decay of the Kentish pippin-a variety at the present day as healthy as it was at any period of its existence. No doubt, many of the apples and pears of the fifteenth and sixteenth centuries have disappeared, as will not a few originated from seed even in the present century before it has closed. But this very naturally arises from the circumstance of their being superseded by better sorts, or being found inferior to many old ones still in cultivation. Many of the trees in the Herefordshire orchards, to which Mr Knight alludes as being the diseased progeny of others long since dead, having outlived their prescribed period of existence, which he limits to two hundred years, may ere this, as has been justly remarked by Mr Hogg ("British Pomology," p. 97), have disappeared also, "having performed their part, and fulfilled the end of their existence." The golden pippin, amongst others which Mr Knight names as being now worn out, appears to be in as thriving a condition, when not maltreated, as it was two hundred years ago. In the Brompton Park nursery, Mr Hogg assures us, (p. 97,) the same golden pippin has been cultivated for nearly two centuries, and continued from year to year by grafts taken from the young trees in the nursery quarters. "I never," he says, "saw the least disposition to disease, canker, or decay of any kind; but, on the contrary, a free, vigorous, and healthy growth." The vine, the peach, and the apricot have been propagated by extension since the earliest ages, yet we never hear of a wearing out of any of the varieties of these trees; and this possibly arises from their being treated in a more natural manner, or more care being bestowed on their cultivation, than has been the case with the apple, which, we know from ocular demonstration, is nowhere worse managed than in the very Herefordshire orchards to which $\mathrm{Mr}$ Knight refers. 


\title{
CHAPTER I.
}

\author{
PROPAGATION.
}

Notwithstanding the close analogy that for the most part exists between the natural economy of animals and vegetables, there are some important points in which they widely differ, and in none more so than in the means of their propagation. All vegetables were created for the sustenance of animals; and hence, wherever the latter exist, the former are to be found. The whole surface of the globe, whether covered with water or not, is replete with vegetation, and that vegetation exists not without its use.

To supply the whole animal creation, it follows that vegetables must be not only exceedingly numerous, but also readily produced-at least, their means of production must be much greater than that of animals themselves. Plants not only produce immense quantities of seeds, but they extend themselves by shoots which run on or under the ground; they are capable of multiplying themselves by roots, tubers, and bulbs, both formed under ground, and in some cases on the leaves and stems; and they produce innumerable buds, each of which, by human art, can be rendered equivalent to a seed, and hence the great facility by which plants are multiplied both by nature and art.

It is probable that something like one hundred thousand species of plants exist; the herbaria of some botanists contain nearly that number in dried specimens. Thirty thousand seven hundred species are enumerated as indigenous or cultivated in Britain alone; and if we include the botanical varieties, and those of cultivated vegetables, fruits, roses, and florists' flowers, eleven or twelve thousand may be added.

\section{$\S$ 1. PROPAGATION BY SEED.}

Propagation by seed is the primary and most general process by which plants are multiplied and perpetuated. All plants are so produced, although in many, such as ferns, mosses, algæ, and fungi, the seeds are so small as to be invisible to the naked eye; yet these latter are amongst the most widely diffused of all vegetables. The seeds of the latter are dispersed by a variety of means, by atmospheric and other causes, their minuteness and buoyancy adapting them well for aerial distribution. There is scarcely a point on the globe's surface, or even in the ocean's depths, VOL. II. where they are not found to exist: the whole atmosphere seems to be replete with them; and when they are brought into contact with solid bodies forming a proper nidus for them, there they take root and flourish. Nor are the plants originated from such apparently slender means proportionably minute; many of them attain an almost gigantic size, as exemplified in many of the Algæ, whose ramified branches extend to the length of hundreds of feet, while the tree ferns rank amongst the monarchs in the tropical forests.

The powers of reproduction by seed are truly immense : a single capsule of the tobacco plant contains about one thousand seeds; one of the common medicinal poppy, eight thousand; while the vanilla plant has been computed to contain from ten to fifteen thousand. Each of these, upon a very moderate calculation, produces from twenty to thirty capsules on each plant. To give some idea of the powers of reproduction amongst cryptogamous plants, we may state that a single frond or leaf of the common spleenwort is estimated to produce one million of seeds.

Most plants originated from seeds resemble their parents in a very striking degree, although, occasionally, deviations of greater or lesser importance occur ; hence many of our most valuable plants, and especially fruits and culinary vegetables, have so originated, and when sufficiently marked by symptoms of improvement, are denominated varieties.

All vegetable life commences from seed. Its first stage and after progress are thus described by Dr Lindley in "Introduction to Botany:" "If we place a seed in earth at the temperature of $32^{\circ}$ Fahr., it will remain inactive till it finally decays; but if it is placed in moist earth above the temperature of $32^{\circ}$, and screened from the action of light, its integument gradually imbibes moisture and swells, oxygen is absorbed, carbonic acid expelled, and the vital action of the embryo commences. It elongates downwards by the radicle, and upwards by the cotyledons, the former penetrating the soil, the latter elevating themselves above it, acquiring a green colour by the deposition of carbon absorbed from the atmosphere in the light, and unfolding in the form of two opposite roundish leaves. This is the first stage of vegetation. The young plant consists of little more than cellular tissue, only an 
imperfect development of vascular and fibrous tissue being discoverable in the form of a sort of cylinder of bundles lying just in the centre. The part within the cylinder, at its upper end, is now the medulla, without it the bark ; while the cylinder itself is the preparation for the medullary sheath, and consists of vertical fibres passing through cellular tissue, and separates them horizontally in every direction.

"The young root is now absorbing from the earth its nutriment, which passes up to the summit of the plant by the cellular substance of the medulla, and is thence impelled into the cotyledons, where it is aerated and evaporated : such of it as is not fixed to the cotyledons passes down through the bark into the root. The plumula, forced onwards by the current of sap, which is continually impelled upwards from the root, next ascends in the form of a little twig, at the same time sending roots in the form of fibres downwards in the centre of the radicle, which become the earliest portion of wood that is deposited: these fibres by their action now compel the root to emit little ramifications. Previously to the elongation of the plumula, its apex has acquired the rudimentary state of a leaf; this continues to develop as the plumula elongates, until, when the first internodium of the latter ceases to lengthen, the leaf has actually arrived at its complete formation. When fully grown, it repeats in a much more perfect manner the functions previously performed by the cotyledons; it aerates the sap that it receives, and returns the superfluous portion of it downwards through the bark to the root; it also sends fibres down between the medullary sheath and the bark, thus forming the first stratum of wood in the new stem. During these operations, while the plumula is ascending, its leaf forming and acting, and the woody matter created by it descending, the cellular tissue of the stem is forming and expanding horizontally to make room for the new matter forced into it; so that development is going on simultaneously, both in a horizontal and perpendicular direction. This process may not inaptly be compared to that of weaving, the warp being the perpendicular, and the weft the horizontal formation. In order to enable the leaf to perform its functions of aeration completely, it is traversed by veins originating in the medulla, and has delicate evaporatory pores (stomata), which communicate with a highly complex pneumatic system, that extends to almost every part of the plant.

"The plumula having produced its first leaf, others are successfully produced around the axis at its elongating point, all constructed alike, connected with the stem or axis in the same manner, and performing precisely the same functions as have been just described. At last the axis ceases to elongate ; the old leaves gradually fall off; the new leaves, instead of expanding after their formation, retain their rudimentary condition, harden, and fold over one another, so as to be a protection to the delicate point of elongation, or, in other words, become the scales of a bud. We have now a shoot with a woody axis, and a distinct pith and bark, and of a more or less conical figure. At the axilla of every leaf, a bud had been generated during the growth of the axis, so that the shoot, when deprived of its leaves, is covered from end to end with little symmetrically-arranged projecting points, which are the buds. The cause of the perfect shoot being conical is, that, as the wood originates from the base of the leaves, the lower end of the shoot, which has the greatest number of strata, because it has the greatest number of leaves above it, will be the thickest; and the upper end, which has the fewest leaves to distend it by their deposit, will have the least diameter. Thus that part of the stem which has two leaves above it will have wood formed by two successive deposits; that which has nine leaves above it will have wood formed by nine successive deposits, and so on; while the extreme vital point, as it can have no deposit of matter from above, will have no wood, the extremity being merely covered by the rudiments of leaves hereafter to be developed. If at this time a cross section be examined, it will be found that the interior is no longer imperfectly divided into two portions-namely, medulla and skin-as it was when first examined in the same way, but that it has distinctly two internal, perfect, concentric lines, the outer indicating a separation of the bark from the wood, and the inner, a separation of the wood from the medulla: the latter, too, which in the first observation was fleshy, and saturated with humidity, is become distinctly cellular, and altogether or nearly dry." Such is the first progress of a plant from seed.

Air, moisture, darkness, and heat are all indispensable in promoting germination; the absence of them is the cause why seeds found at greater depths in the soil than air penetrates to, vegetate when brought within its influence, and why they remained inactive during the period of their being so deeply immured. What degree of credence may be given to the assertions of those who say they have caused to vegetate the seeds of wheat taken from the tomb of an exhumed mummy, or of raspberry tarts being made of fruit the produce of plants raised from seed taken from a Roman barrow, we shall not stop to investigate further, than by observing that, if these facts are true, an absence of air, or rather oxygen, was the cause of this long-suspended vitality. That seeds do remain for a number of years in a sound state when placed so deep in the ground as to be beyond the influence of oxygen, and probably an insufficiency of moisture, is highly probable; but there is a vast difference between a score or two of years and a few thousands.

When seeds are sown too deep, the effect is that they germinate much slower than if placed nearer the surface, and often, particularly in damp soils and wet seasons, they perish altogether. The following table was drawn up by Petri : Of seed sown at the depth of $\frac{1}{2}$ an inch, $\frac{7}{8}$ ths came above ground in 11 days; at 1 inch in depth, all came up in 12 days; at 2 inches, $\frac{7}{8}$ ths came up in 18 days; at 3 inches, $\frac{6}{8}$ ths came up in 20 days; at 4 inches, $\frac{4}{8}$ ths came up in 21 days; at 5 inches, $\frac{3}{8}$ ths came up in 22 days; at 6 inches, $\frac{1}{8}$ th came up in 23 days,-showing, therefore, that shallow sowing is the best. 
The larger seeds are, such as the acorn and walnut, the deeper they should be placed; while very small seeds, such as rhododendron, \&c., should be sown upon a smooth surface, and scarcely covered at all. It is necessary, however, that light be excluded until germination has taken place. Moisture is necessary to germination, and without it seeds would ever remain inactive. The quantity of moisture requisite to cause germination in some seeds is much greater than in others; and hence pease, beans, \&c., when hasty vegetation is desired, are often soaked in water for some hours before sowing; and in dry states of the soil, the drills are watered before the seed is committed to them. Decandolle found that a French bean, weighing 544 milligrammes, absorbed 756 of water. The grand secret of keeping seeds in a good state in our seed warehouses is their being kept dry; and if they could be at the same time excluded from air, no doubt their period of vitality would be considerably lengthened. Absence of light is another condition, as has been shown by M. Boitard and others, almost indispensable to germination. In the case of very small seeds which do not admit of being covered with soil, germination will be much assisted if they be covered with bell glasses, either painted black or having a thick piece of cloth laid over them. According to the recent experiments by Mr Hunt, the luminous or lightgiving rays, and those nearest the yellow, have a marked effect in impeding germination; while the red or heat-giving rays are favourable to the process, if abundance of water be present; while the blue rays, or those concerned in chemical action or actinism, accelerate the process and cause rapid growth.

The temperature necessary for the germination of seeds extends over a considerable range in the scale of the thermometer. We have stated that $32^{\circ} \mathrm{Fahr}$. is the lowest with which we are acquainted at which seeds will germinate, because below that degree water freezes, and cannot, therefore, be absorbed by the tissue of the seed. It is possible, however, that some of the less perfect plants, such as some of the Algæ and Fuci in the arctic regions, may vegetate even below that point. The general range, however, is from $45^{\circ}$ to $80^{\circ}$ or $90^{\circ}$. Some seeds, such as the rose-acacia, Robinia, pseudo-acacia, and some of the Australian acacias, have been immersed for some minutes in boiling water without their vitality being destroyed. In cultivation, some require the heat of the tropics, while others vegetate freely in the heat of our springs. The highest limit of prolonged temperature which cereal grains can bear in water is stated by Edwards and Colin to be $95^{\circ}$, and the highest they can bear in sand or earth, $113^{\circ}$ Fahr. Dr Lindley records a case of the seeds of the raspberry having germinated after being subjected to the boiling point of syrup, which is $230^{\circ}$. The seeds of the annual poa, Poa annua, chickweed, Alsine media, and various others, will germinate at very little above $32^{\circ}$. These points may therefore be taken as the maximum and minimum of vegetation.

One seed producing twin plants.-In the ordinary course of nature one seed produces one plant as a general rule; but to this there are exceptions, one of which is stated by $\mathrm{Mr}$ Thwaites, in "Annals of Natural History," as occurring in the case of a single seed of a hybrid fuchsia producing two plants "extremely different in appearance and character, one of them partaking of the character of $F$. fulgens, and the other of $F$. coccinea," these being the two parents. "It cannot be doubted," he says, "that these very dissimilar structures were the produce of one seed, since they were closely coherent, below the two pairs of cotyledon leaves, into a single cylindrical stem, so that they had subsequently the appearance of being branches of one trunk." It is not improbable but the same thing frequently occurs in the case of forest trees of natural growth, where two apparently distinct trees rise from one root-a circumstance accounted for, but we believe erroneously, by supposing two seeds to have sprung up together, and that, from the closeness of their stems, a species of natural engrafting had taken place while they were very young.

Vitality of seeds.-We have stated above, that some seeds are supposed to retain their vitality for two thousand years or more. There are others, if placed under the ordinary conditions natural to them, that lose that power almost immediately after they have arrived at full maturity; many continue sound one year, others two, and some few not only for a greater number of years, but also being improved by keeping. We are not aware that this latter fact has ever been physiologically accounted for, but practically it is known to be the case. The following common instances may be given. Melon and cucumber seeds are improved by age, in so far that they are found to be more productive of fruit, and less prone to grow to branches than when new; cauliflower and most of the brassica tribe are less liable to button or run prematurely into flower; while turnips bulb better, produce less top, and are less liable to run to seed in spring, when the seed is several years old, than if the produce of the preceding season. If such seeds, therefore, be well kept, we would say that they were better when four years old than when one year; and, if this theory be correct-and we think the experience of years goes far to prove it so-that they are in a very fitting state for sowing while under ten years. Gardeners have long seen the importance of this, and hence it is no unusual thing for many to carry about seeds of the two first mentioned in their pocket, that the necessary maturation may be accelerated by the warmth of their bodies. We state this latter case, not as an example to be followed, but as an evidence of the principle. The object aimed at in the cultivation of the plants we have named is not bulk of gross vegetable matter, but to insure an end; and that end is to produce those fruits and vegetables in the highest state of perfection they are capable of arriving at, and most fitted for the purpose for which they are cultivated.

A still more extraordinary instance than those of the mummy wheat, \&c., of the protracted vitality of seeds, is to be found in a paper by $\mathrm{Mr}$ Kemp, published in the "Annals of Nat. 
Hist.," vol. xiii. p. 89. It relates to the discovery of the seeds of plants which upon vegetating were found to be those of Polygonum, Convolvulus, Rumen acetosella, and a species of Atriplex. They were found at the bottom of a sand-pit 25 feet deep, about a quarter of a mile west of Melrose. These seeds were im. bedded amongst decayed vegetable fibres, resting on a stratum, 8 inches in thickness, of fine sandy clay. Under this stratum was a mass of gravel resting on a mound of the boulder formation 90 feet in thickness, and which $\mathrm{Mr}$ Kemp supposes was formed by the action of glaciers. The stratification of fine sand over the seeds was alternated with thin seams of impalpable clay, in both of which traces of carbonaceous decayed wood and minute pebbles were found, resembling much the foundation of a river, as seen when a section is cut through its bed to some depth. These and other circumstances led Mr Kemp to the conclusion that the river Tweed had anciently run in this direction, or that a lake of considerable size had at some very remote period existed here, but which is at present at a considerable distance, and between 50 and 60 feet below the level of the sand quarry. "It is certain," Mr Kemp concludes, "that in the time of the Romans, about two thousand years ago, no lake existed here" and when we reflect on the time necessary to have worn down the barrier of trap rock, and to have drained so large a lake, which must have stood at its highest level whilst the thin layers of sand were deposited over the bed with the vegetable remains, the antiquity of these seeds is truly astonishing, and it is most wonderful that they should have retained their power of germination."

Oily seeds lose their vitality speedily, probably from their power of absorbing oxygen, and the chemical changes thus induced. Some seeds retain their vitality much longer than others: thus the seeds of melons have been kept for forty-one years, maize thirty years, rye forty years, Mimosa sensitiva sixty years; and, stranger than all, those of the kidney bean, after being kept for a century, have germinated, while gardeners in general believe that they are of one year's duration only.

Seeds will germinate more speedily, if sown fresh, when nearly ripe, than if they were fully ripe; and in the latter case, if sown immediately, than if kept for some time-say for some months, or a year or two.

Intimately connected with the nature of seeds is the condition of the soil into which they are sown; and hence gardeners bestow both much more labour and care in its preparation than farmers do. All soils must be thoroughly pulverised to render them fit for the reception of the seed, and hence the great necessity of trench. ing, digging, forking, raking, \&c., to reduce the soil to a fit state of fineness. These operations must not, however, be carried on when the soil is wet, else all the labour will be worse than in vain. All soils must be comparatively dry before the seeds are committed to it. The soil, to be in a fit state, upon examination should be found to be composed of small particles sepa- rated from each other by interstitial spaces or cavities. If wet, these cavities will be full of water instead of air; but if in a proper state, the particles will be sufficiently moist, while the cavities will be dry and charged with air. Nor is it the presence of air alone, although one of the essential conditions of certain germination, that is here required; these cavities admit the solar heat, without which, in early spring, germination would be slowly enforced-so slow, indeed, that many seeds might perish before vegetation could take place.

In light sandy soils, abounding in carbonaceous matter, seeds germinate rapidly ; in stiff clayey soils, more tardily; and if imbedded in peatmoss fully charged with water, they would either perish, or remain probably for ages in a dormant state. Most seeds vegetate freely in decayed vegetable mould, with a slight addition of fine sand; others, such as some of the Coniferæ, would speedily rot in such a preparation, and succeed best when placed in a more primitive soil.

The following table was drawn up by Adam. son, founded on experiments made by him in France, as to the period seeds remain in the ground, after sowing, before germination takes place : "Strawberry blite, beans, mustard, kidney beans, turnips, radishes, 3 days; lettuce and aniseed, 4 days; melon, cucumber, gourd, and cress, 5 days; horse-radish and leek, 6 days; orache, 8 days; purslane, 9 days ; cabbage, 10 days; hyssop, 30 days; parsley, 40 to 50 days ; almond, chestnut, peach, and peony, 1 year ; rose, hawthorn, hazel-nut, and cornel, 2 years."-(Fam. des Plantes, vol. i. p. 84.)

The following table was drawn up from experiments made in the botanic garden at Geneva; the seeds were watered similarly, and the temperature to which they were exposed was $53^{\circ} \mathrm{Fahr}$. as a medium : Amaranthaceæ, 9 days ; Cruciferæ, 10 days; Boragineæ, Malvaceæ, Caryophylleæ, 11 days; Compositæ, Convolvulaceæ, Plantagineæ, 12 days; Polygoneæ, 13 days; Campanulaceæ, Leguminosæ, Valerinaceæ, 14 days; Gramineæ, Labiatæ, Solaneæ, 15 days; Rosaceæ, 17 days; Ranunculaceæ, 20 days; Onagrariæ, 22 days; Umbelliferæ, 23 days.

The order in which seeds germinate appears often to be very capricious. Many, when sown in spring, vegetate in course of a few days, which, if sown in autumn, would not come up the same year. The seeds of biennials, for the most part, are committed to the ground as soon as they are ripe, vegetate, and become strong plants before winter, and flower the ensuing summer. Heat, moisture, and absence from light, are the conditions necessary to insure germination. The former is a main agent in accelerating this process, and hence seeds are sown, in general, in spring, instead of during winter, although the latter practice is often resorted to in the case of garden pease and beans, with a view to insure their early ripening. This is, however, a questionable practice. Seeds of tender plants are sown in hot-beds, in pots plunged in a mild bottom-heat; and sometimes imperfectly ripened ones are sown, and this has been found to promote their early vegeta- 
tion. Steeping seeds for a few hours in warm water has the desired effect, more especially when the soil into which they are to be sown is dry, as often occurs during the heat of summer. Hard seeds, such as walnuts, almonds, \&c., are often buried for a day or more in matter in a moderate state of fermentation; and at other times the shell is paired, cut, or filed thin, so as to assist the embryo plant's escape from its hard encasement. The more scientific operations are, watering with chlorine, as first suggested by Baron Humboldt ; the application of iodine and bromine, in conjunction with hydrogen, as proposed by Goppert : both these, as well as oxalic and other acids, have no doubt the effect, when frequently applied, of hastening the process of assimilation, and accelerating vegetation. These have, however, the effect of causing the plants thus hastily brought into a state of existence to assume a sickly appearance through overexcitement, and not unfrequently many of them die. Dr Hamilton and M. Otto of Berlin made seeds to vegetate, notwithstanding they were from twenty to forty years old. On the use of these, as well as of a diluted solution of chlorine, Dr Lindley, in "Theory of Hort.," remarks, "Theoretically, it would seem that these effects ought to be produced, but general experience does not confirm them; and it may be conceived that the rapid abstraction of carbon, by the presence of an unnaturally large quantity of oxygen, may produce effects as injurious to the health of the seed as the too slow destruction of carbon, in consequence of the languor of the vital principle."

The natural season for sowing seeds is unquestionably as soon as they are ripe, but for the purposes of cultivation it is often necessary to defer this process for a few weeks or months. This is pointed out to us by nature; and, indeed, some seeds are not capable of retaining their vitality many days after they are removed from the plant. Seeds which naturally fall from the plant, either by their weight or by some other means not easily accounted for, secrete themselves in the ground, or amongst moss or leaves, \&c., so as to be ready in spring to start into new life. Many vegetate during autumn, and survive the winter, as it were, in an infant state, and are prepared, upon the approach of the warmth of spring, to perform the offices they are destined for. This is remarkably exemplified in the case of most of the plants from North America and California; and these, according to the most approved mode of culture, should be sown as soon as they are ripe; while, again, Mexican and Chilian seeds succeed best when sown in spring. According to $\mathrm{Mr}$ Gordon of the Chiswick gardens, the seeds of the trees and shrubs of Europe and of India "should be sown in autumn, and annuals or perennials in the spring. All seeds, of whatever kind, should be sown in dry soil, and not wetted till they begin to vegetate. In the case of old or sickly seeds, to water them at the time of sowing is to insure their destruction by rotting. Shading is to be preferred to watering; and one of the best constructions for the purpose is a pit glazed with double sashes. Finally, all seed- lings should be potted or transplanted as soon as possible, except bulbs."

Experiments in seed-sowing are and have been numerous: that of sowing them in snow, as exemplified by M. Lucas, and detailed in the "Garten Zeitung," is one of the most extraordinary, and least in accordance with the laws of vegetable physiology, as snow must be nearly at the temperature of $32^{\circ}$; hence they are placed at the very lowest point of temperature at which germination can take place. For what reason M. Lucas follows this unusual course does not very clearly appear. The majority of seeds experimented upon by him were those of alpine plants, which of necessity in nature vegetate at the lowest temperatures. The idea of sowing seeds in new-fallen snow, or in that which has been frozen in ice-houses, and immediately placing them in a hot-house with a temperature of $60^{\circ}$ or $65^{\circ} \mathrm{Fahr}$., can amount to nothing other than sowing the seeds in the usual way, and drenching them with the coldest water; and that, with a view to accelerate their vegetation, is contrary, in the fullest extreme, to what has been considered the highest principle of good culture. In the Munich botanic garden, experiments were first tried, and frequently since in Britain, of sowing seeds in powdered charcoal, the results being an advance of one day in the process of germination. Minute seeds are often sown on the surface of charcoal blocks, on pieces of sandstone, and woollen cloth kept moist, but with effects of small importance to the general cultivator, although valuable to the botanical gardener, as, by this means, seeds of mosses, ferns, \&c. are found to vegetate freely; while, if sown in soil, they might, from their minuteness, be liable to be too deeply buried.

According to Mr Limburn, electricity has a powerful influence in accelerating the vegetation of seeds, on account of its connection with all transformations of organic substances, either as cause or effect. Dr Carpenter, entertaining the same views, remarks that, "when chemical decomposition takes place, electricity is always developed," but suspects that in some instances it may be absorbed again by the new state of the compound. The influence of different coloured glass on the germination of seeds has been successfully experimented upon by Dr Horner of Hull, M. Payen, and others, who arrived at the conclusion that the violet or deoxidising colour produces similar chemical ef fects to the negative or alkaline pole, while red produced the acid or opposite effect by the retention of the oxygen. Alkalies were successfully employed by M. Maltuen, who found that seeds vegetated sooner when placed at the negative pole of a galvanic battery than at the positive or acidised pole. Seeds enclosed by him in phials of acids and alkalies were found to germinate much quicker in the latter; while in the former they vegetated much slower, and sometimes not at all.

\section{$\S$ 2.-CROSSING OR HYBRIDISING.}

We have elsewhere stated that most plants originated from seeds resemble their parents in 
a very striking degree, although occasionally deviations of greater or less importance occursometimes, no doubt, naturally, and of late years much oftener by design. The true cause of such departures from legitimacy in nature, is, we believe, enveloped in obscurity. Man has it, however, in his power, under certain conditions, to create varieties ad infinitum, by the process of cross-breeding or hybridising - a process requiring great nicety in the performance, and a thorough knowledge of the physiological structure of plants. The old philosophers had some slight idea of sexuality in plants, and Pliny somewhere says, "All trees and herbs are furnished with both sexes ;" but nothing definite appears to have been known until Sir Thos. Millington and Nehemiah Grew, in or about 1676 , clearly established the fact. It is true, Malpighi, Cæsalpinus, and others, laid claim to priority in the discovery ; but, in alluding to this circumstance, Dr Lindley remarks, "I see nothing so precise in their works as we find in the declaration of Grew, 'that the attire (meaning stamens) do serve as the male for the generation of the seed." " It would also seem, from a very curious passage in the Book of Deuteronomy, that the Jewish lawgiver was well acquainted with the phenomena of hybridisation, and with the danger of deterioration attendant on its being allowed to proceed indiscriminately among cultivated esculents : "Thou shalt not sow thy vineyard with divers kinds of seeds; lest the fruit of thy seed which thou hast sown, and the fruit of thy vineyard, be defiled."-Deut. xxii. 9. Camerarius, so early as 1694 , threw out some conjectures upon this subject. It was afterwards taken up by Bradley, in 1717, who proved the fact by producing hybrids artificially; by Linnæus, in 1744; and fully established by the experiments of Koelreuter, and since by a host of others. It is not a little strange that the great founder of the sexual system should have written a dissertation on the subject; and although he endeavoured to establish a doctrine which was true, the facts upon which he grounded his doctrine, in the estimation of modern botanists, were false. He even went so far as to allege that hybrid productions may occur between species belonging to different genera, and even to different families-a case, says the systematic botanist, of which no example that oan at all be relied upon has ever yet been met with. That natural hybrids do exist is not denied by the most strenuous advocates of the purity of the sexes in plants, but they say that they are few in proportion to the number of vegetable species, and none exist which are the certain production of a union of species belonging to distinct families.

There are some who think otherwise, and contend that, if man can by art produce hybrids at will, by the connection between two species of the same genus, why should not nature have done the same over and over again since the creation of the world, and that by the very instruments which are the grievous marplots of every gardener who wishes to keep his favourite variety of melon, or his select early cabbage, pure and unadulterated? None knows the dif- ficulty of preventing a species of adulterous intercourse from taking place amongst certain families of plants better than the enthusiastic hybridiser; for, with all his precautions, such things will happen. The fact is admitted in the case of diæcious plants, and conspicuously so in the numerous so-called species of willows. The doctrine held by some of the older botanists was recently almost revived by the hints thrown out by a learned gentleman at one of our scientific meetings in Edinburgh, much to the alarm of the younger, and perhaps more ardent than prudent students in botanical science, that there were originally only a few species formed, and that the rest of the vegetable world had arisen by hybridisation. The willow and the carex have been sore stumblingblocks to botanists : either of them may ask the first botanist that comes his way, "Pray, sir, what is a species?"

"Plants agree," says the author of the "Suburban Horticulturist," "with animals in the offspring when it is reared from seed bearing a general resemblance to the parent; but as in every family the children of the same parent differ individually in features, temper, disposition, \&c., so among seedling plants, from the same seedpod no two plants will be found exactly alike, and some will occasionally differ considerably from all the rest. Nevertheless, it is an undoubted fact that all seedling plants not only possess the character of the species from which they have sprung, but even, in by far the greater number of cases, some of the peculiarities of the individual. The seeds of any kind of cultivated apple, for example, will produce plants, the fruit of all of which will more or less resemble that of the parent, though, perhaps, some one or two among a hundred may be considerably different. Hence, by selecting from beds of seedling plants those which are in any way remarkably different from the rest, new varieties are procured; and till within the last half-century, when crossbreeding began to be practised by gardeners, this was the only way in which an improved variety of any species of plant was procured. If the seeds of varieties did not produce plants closely resembling their parents, how could all the improved varieties of culinary, agricultural, and floricultural plants be perpetuated? That the same law which governs herbaceous plants holds good in trees and shrubs cannot be doubted; and if the seeds of a variegated tulip are more likely to produce plants which shall have variegated flowers than those of a tulip with only one colour, so, we should say, the berries of a variegated holly are more likely to produce plants with variegated leaves than those of a green-leaved holly. If this law did not hold good in ligneous as well as in herbaceous plants, how are we to account for the different varieties of Hibiscus syriacus coming true from seed?"

Adrantages of hybridisation.-By this process man is enabled to change the character and quality of flowers, and of fruits. The colours and symmetry of the former may be changed, or made more perfect, and the size, form, and flavour of the latter may be much improved, by a judicious selection of the parents called into action. Indeed, by this process man 
possesses the power of modifying the works of nature, and rendering them better adapted to his wants and wishes. What would our gardens now be, were it not for the hundreds of beautiful flowers produced by this means, as well as the many superior fruits produced in a similar manner? The families of roses, azaleas, rhododendrons, pelargoniums, fuchsias, and hosts of others, bear evidence of this ; while the improved kinds of pears, apples, strawberries, peaches, \&c., give a still more conclusive corroboration. By this process the races of fruits and of culinary vegetables have been brought as near to perfection as it is, perhaps, possible to bring them; and it is more than probable that wheat, corn, barley, \&c. are capable of being yet greatly improved if sufficient pains were taken with them. Nay, even the timber trees of our forests, which are subject to the same laws, are capable of a like improvement.

The laws of hybridisation, or cross-breeding."The species to be experimented on must be nearly related. The apple will not cross with the pear, nor the gooseberry with the currant." -Keith.

M. Decandolle says, "that hybrids resemble the female parent chiefly in the leaf and stem, and the male parent chiefly in the flower and organs of fructification." Professor Lindley reverses this order, which has led Keith to remark that the resemblance may have been found to be sometimes the one way, and sometimes the other. The Hon. and Rev. W. Herbert thinks, from what he saw in Amaryllides, that in hybrids the flowers and organs of reproduction partake of the character of the female parent; while the foliage and habit, or the organs of vegetation, resemble the male. "Hybrids may be fertilised, however, by the pollen taken from one of the parents, and then the offspring assumes the character of that parent."-Balfour.

Further, on this very interesting subject $\mathrm{Dl}^{\circ}$ Lindley remarks, in "Theory of Horticulture," that "if the pistil of one species be fertilised by the pollen of another species, which may take place in the same genus, or if two distinct varieties of the same species be in like manner intermixed, the seeds which result from the operation will be intermediate between its parents, partaking of the qualities of both father and mother. In the first case, the progeny is hybrid, or mule; in the second, it is simply cross-bred. In general, cross-breeds are capable of producing fertile seed, and thus of perpetuating one of the species from which they sprang. Hybrids, on the contrary, are often sterile, and therefore incapable of yielding seed. Reasoning from a few facts, and from the analogy of the higher orders in the animal kingdom, it has been believed that all vegetable hybrids are sterile; and when sterility is not the consequence of the intermixture of two species, it has been thought that such species are not naturally distinct, however different their appearance. But facts prove that undoubted hybrids may be fertile; and when we consider that plants are not analogous to the higher orders of animals, but to the lowest, concerning whose habits we know nothing whatever, it is obvious that no analogical inferences can be safely established." -(Vide Mr Anderson's views at the end of the article.)

Duration of hybrids. - "Hybrids are fertile only till the third or fourth generation, when they revert ultimately to the type of one of the parents."-Keith. "When cross-breeding is effected between what are considered different species, the offspring is a mule, or hybrid, which, in most cases, is incapable of maturing seeds, and generally, in the course of a few years, degenerates, or reverts to its original parentage. There are, however, instances of mules, or true hybrids, producing fertile seeds, from which plants have been raised, partaking of all the characters of the parent hybrid plant," as in the case of some of the Cacti.-Sub. Hort.

Uncertainty in propagating hybrids even otherwise than by seed.- "It would appear, from the case of the Cytisus adami, or purple laburnum, that a true hybrid or mule cannot always be propagated with certainty, even by portions of the plant, or by what is called extension, since it never can be certain whether the portion taken off for propagation will produce the mule or one of the parents. As it is uncertain what are and what are not very distinct species, many of the plants originated by cross-breeding, and considered mules, may in reality not be so, and may consequently prove permanent and improved varieties."-Idem. In this extraordinary tree the foliage is sometimes that of the common laburnum, sometimes of the purple cytisus (C. purpureus), and sometimes intermediate between the two. The origin of this anomalous tree is not believed to have been from seed produced by the ordinary process of crossbreeding, but by some accidental mixture of the cellular tissue.

The difference between propagating by seed and propagating by any other mode is very great.In propagating by seed, the species or variety in the abstract is propagated; while by all the other modes practised by gardeners the species or variety is continued without alteration in character, by an extension of the parts of the original tree or plant. Thus, a Hawthornden apple of 1852 is not a new plant, but only a portion of the original tree continued by the process of extension, carrying with it its habits and properties. Were we to sow the seeds of variegated plants, or of weeping oaks, ashes, or elms, few of the progeny, if any, taking it as a general rule, would exhibit the variegated or pendulous characters of their parents; and hence the necessity of continuing these varieties by budding, grafting, \&c. On the same principle, were we to sow the seeds of any of our best apples, pears, plums, \&c., the chances of obtaining from their seeds improved varieties, or even such as are equal to the parents, would be few indeed; we, therefore, are compelled to continue them by other means than from seeds. There are, however, exceptions to this rule, as in the case of the pendulous or weeping oak at Moccas Court, many of the acorns of which, when sown, produce plants very similar to the original; and we recollect having pointed out to us by the Duc d'Aremberg a splendid purple or copper-coloured 
beech in his grounds at Enghien, which produced a considerable proportion of plants from seeds closely resembling the parent, while other trees of the same age and size in the same grounds produced invariably plants having green leaves, and in all respects like the common beech. In regard to the aptitude of fruit trees to produce plants from seed slightly removed in quality from the tree from which the seed was taken, we may mention the golden pippin apple and the green-gage plum, which often do produce plants whose fruit is as little different from the originals as that of the progeny of the wild plum or crab apple is different from the trees from whence the seeds were gathered. It should, however, be remarked, that as these two fruits are amongst the very best of their class, and may have taken many generations to have brought them to their high condition, it is possible that it may take as many more to reduce them in quality to their original or natural state. The circumstance also strongly favours the theory laid down by Dr Van Mons in the "Journal d'Agriculture des Pays Bas," of Mr G. Lindley in "Guide to the Orchard," both elsewhere referred to, as well as that of most cultivators who have made the propagation of improved fruits and plants their study. The practice of our best seed-savers is to save seed only from plants in the highest state of perfection, and in the highest state of culture; and by this means they continue to us not only what are called species, but varieties also, little changed from the plants from which the seeds were taken; and by this means only can we continue annual and biennial plants, as well as many of greater longevity, although we cannot depend on this mode in all cases with the same degree of certainty that we can on the various modes of propagating by extension-namely, by cuttings, division of the plant, grafting, budding, \&c.

However much the systematic botanist may be discomposed by the production of hybrids, or of cross-bred plants, the florist, pomologist, and gardener have much cause to rejoice ; for by this process they all have gained a triumph over nature, which, according to her greatest advocates, she could hardly have effected herself. The happy results of the late Mr Knight, in the production of some of our very finest fruits, and of our best pease, and other culinary vegetables, is of itself sufficient proof of the great utility of the process. We are told by botanists that hybrids are fertile only to the third or fourth generation. How can this doctrine be reconciled with the case of Knight's marrowfat pea, which has continued to reproduce itself over the whole kingdom for upwards of thirty generations, and is likely to continue as long? For the interesting experiments of $\mathrm{Mr}$ Knight, vide the "Philosophical Transactions from 1789 to 1820 ," and various papers in "Horticultural Society's Transactions, first series," \&c.

"The power of hybridising," Dr Lindley remarks, "appears to be far more common in plants than in animals. There is scarcely a genus of domesticated plants in which this effect cannot be produced by the assistance of man.
It is, however, in general, only between nearly allied species that this intercourse can take place, those which are widely different in structure and constitution not being capable of any artificial union. The cause of the sterility of mule plants is at present unknown. Sometimes, indeed, a deficiency of pollen may be assigned; but in many cases there is no perceptible difference in the healthiness of the structure of the fertilising organs of a mule plant and of its parents." Even the very comprehensive anatomical observations of Professor Henslow have thrown no light upon the subject, for he could detect no appreciable difference between the condition of the stamens and pistil of the hybrid or its parents. Amongst the instances of fertile hybrids found wild in nature, Dr Lindley states the following as remarkable cases: "The Cistus ledon constantly produced between C. monspeliensis and laurifolius, and Cistus longifolius between C. monspeliensis and populifolius," observed by Mr Bentham in a wood near Narbonne. "The same acute botanist ascertained that Saxifraga luteo purpurea of Lap., and S. ambigua of Decandolle, are only wild accidental hybrids between $S$. aretioides and culyciflora. Gentianas, having a similar origin, have also been remarked upon the mountains of Europe. It is difficult not to believe that a great number of the reputed species of Salix, Rosa, Rubus, Aquilegia, and other intricate genera, have also had a hybrid origin."

It has been stated above, as the opinion of some, that hybrid varieties cease to reproduce themselves after the third or fourth generation. We have already instanced Knight's marrowfat pea, \&c., as an exception to this rule; and on looking over some old seed-lists-that of Gordon, for example, one of the earliest seedsmen established in Edinburgh-we find in his "Dictionary," published in 1774, the names of many of our kitchen-garden seeds identical with those of seed-lists of 1852 : that any change for the worse has taken place in them, we have no right to suppose. True species, in the strict sense of the word, they cannot be ; hybrids, therefore, they must be. The same may be said of our cereal plants, some of which have, no doubt, existed for centuries. It appears, therefore, pretty clear that hybrids may continue in a fertile state for ages, without much, if any, deterioration. Had hybrids in a state of nature been as long and as closely watched, it is probable that our list of recognised individuals would be much larger than it is at present. The following explanation of the law which governs this case is given by Dr Lindley: "It is a general law of nature that seeds will perpetuate a species, but not a variety; and this is no doubt true, if rightly considered: and yet it may be urged, if this be so, how have the varieties, well known to gardeners and agriculturists for many years, been unceasingly carried on from generation to generation without change? The long red and round white radishes of the markets, for instance, have been known from time immemorial in the same state in which they now exist. The answer is thus : A species will perpetuate itself from seed for ever under any cir- 
cumstance, and left to the simple aid of nature ; but accidental varieties cannot be so perpetuated -if suffered to become wild, they very soon revert to the form from which they originally sprung. It is necessary that they should be cultivated with the utmost care; that seed should be saved from those individuals only in which the marks of the variety are most distinctly traced; and all plants that indicate any disposition to cast off their peculiar characteristics should be rejected. If this is carefully done, the existence of any variety of annual or perennial plant may be undoubtedly prolonged through many generations."

The following concise and excellent rules are given in the "Cottage Gardeners' Dictionary," regarding the process and effects of hybridising : " Hybridising, strictly speaking, is obtaining a progeny between two different species, by fertilising the stigma of one with the pollen of another ; and cross-breeding is obtaining a progeny between varieties of the same species. The progeny of hybrids cannot produce seed, but crossbreeds are fertile." The following may act as a guide to the raiser of varieties: "The seedvessel is not altered in appearance by impregnation from another plant; therefore, no hasty conclusion of failure is justified by that want of change.

"The colour of the future seed out of that first hybridised seems to be most influenced by the male plant, if its seeds and flowers are darker than those of the female. Captain Thurtell, from his experiments on the pelargonium, found thecolour and spot of the petals to be more influenced by the male than by the female. Indeed, all experience proves that the progeny usually, though not invariably, most resembles in colour the male parent. Large stature and robustness are transmitted to the offspring by either parent, but $\mathrm{Mr}$ Knight generally found the most robust female parent producing the finest offspring. Captain Thurtell, from lengthened observation, has ascertained that the form of the petals follows most closely that of the female parent. $\mathrm{Mr}$ Knight says that the largest seed, from the finest fruit that has ripened earliest and most perfectly, should always be selected. In stone fruit, if two kernels are in one stone, these give birth to inferior plants. The most successful mode of obtaining good and very distinct varieties is to employ the pollen from a male flower grown on another plant than that bearing the female parent. To avoid previous and undesired impregnation, the anthers of the female parent, if they are produced in the same flower with the pistils, must be removed by a sharppointed pair of scissors, and the flower enclosed in a gauze bag to exclude insects, until the desired pollen is ripe. Another effectual mode of avoiding undesired impregnation, is bringing the female parent into flower a little earlier than its congeners, and removing the anthers as above described: the stigma will remain a long time vigorous if unimpregnated. When double flowers are desired, if a double flower should chance to have a fertile anther or two, these should be employed for fertilisation, as their offspring are almost sure to be double."

VOL. II.
Besides the opinions already stated, others believe the sterility of hybrids to arise from a deficiency of pollen or fertilising dust; while the highest authority we can quote (the late Dean of Manchester) was of opinion that it arises from the juices of each individual type being inadequately adapted to yield the exact proportion of what is wanted for the pollen of its kind; and that, wherever that adaptation is perfect, a perfect offspring is produced. "Where it is not perfect," he says, "an inadequate or a weak fertilisation takes place. It is further to be observed that there is frequently an imperfect hybrid fertilisation, which can give life, but not sustain it well. There are several crosses which I have frequently obtained, but could not raise the plants to live for any length of time." $\mathrm{He}$ states various crosses which he effected, but lost the plants so produced while they were yet young, "on account," as he says, "of his soil being very uncongenial to them," but which, he believed, undermorefortunate circumstances, would have been saved. Such crosses sometimes are a hundred times more delicate in their first stage than natural seedlings. "In these cases, I apprehend," says the same authority, "that, although the affinity of the juices is sufficient to enable the pollen to fertilise the ovule, the stimulus is insufficient, the operation languid, and the fertilisation weak, and inadequate to give a healthy constitution. It has been generally observed that hybrid fertilisation is slower than natural fertilisation, and that often a much smaller number of ovules are vivified. The same cause probably operates in that respect; the affinity not being perfect, the necessary ingredients are attracted by the pollen less readily and insufficiently, and by many of the grains not at all."

Seedlings originated by unknown causes, or sports of nature.-The laws of nature are, upon the whole, uniform, and seldom are they violated. In endeavouring to trace the first origin of our longest established cultivated culinary vegetables and fruits, a degree of obscurity appears to envelop the subject. If all our apples and pears originated from the Pyrus malus and Pyrus communis, and our cabbages and pease from Brassica oleracea and Pisum sativum, as we are taught to believe, when, and by what means, it may be asked, did the first remove from the originals to an improved variety take place? This is a problem we have never met with any satisfactory solution of. The original plant in either case could not be a hybrid, for there was no other to hybridise it with. High cultivation was very unlikely to be the cause either ; for, at the period of their first appearance, we may presume, cultivation was little attended to. Were they accidental sports, as the weeping ash and weeping oak and various others are said to have been? If so, this process must have been going on from a very remote period, as we are informed that twentytwo sorts of apples, and thirty-six kinds of pears, were known to the Romans in the days of Pliny. The Romans knew nothing of hybridising, nor was it well known to the moderns till about the beginning of the present century. We can readily 
admit that natural hybridisation may have been going on since the first tree in each of these cases came into existence ; but how came they to exist in the first instance? If nature has been sporting in this way for so long a period, she will, no doubt, continue to do so till the end of time. If she has done so in the case of our fruits and culinary vegetables, may she not have been doing so in the case of all other plants and trees? and, therefore, may not hundreds of these, recognised now as species at least, have been so produced? Is not the same law going on yearly in the case of nine-tenths of what are called our improved vegetables? for it is certain that even that proportion is not produced by actual artificial cross-breeding; and the same thing is also going on in the case of our improved fruits. By what other means were the Hawthornden, Claygate pearmain, scarlet-nonpareil apples, \&c., produced? The former, now in the last stage of decay, was a foundling in the garden from whence it takes its name; and of the two latterthe one was found wild, in a neglected hedgerow, at Claygate, near Claremont, and the other in the garden of a petty public-house in the village of Esher, and within a mile and a half of each other. Many other instances could be given in support of this sportive freak of Dame Nature's. As these fruits are amongst the most valuable of their kind, we need not despair of being provided with still more improved fruits to the end of time, and that without the intervention of artificial means.

The weeping ash, set down as a sport of this kind, appears to be cursed with that sterility which some attribute to mules and hybrids, as it produces no seed capable of reproducing itself in its pendant form. But the truth is, the original weeping ash, from which we presume every plant of the kind in Europe has been derived, is a female tree, so that, when the original or any of its progeny has produced seeds, it has been in consequence of their flowers having been fecundated by those of some male tree in its vicinity. The Moccas Court weeping oak is more fortunate, as it produces from seeds almost all the young plants with drooping branches. Whatever may have been the origin of the golden pippin apple and green gage plum, they, as already remarked, produce plants from seed in general differing as slightly from themselves as the produce of a crab-apple or wild plum differs from the trees that produced them. The seeds of the cultivated cabbages and pease are well known to produce a progeny in all respects like their parents. Well may we say with the Psalmist, "O Lord, how wondrous are thy works ! in wisdom hast thou made them all."

However diversified opinions may be upon the subject of hybridising, or cross-breeding among plants, of the vast importance of it none can doubt. Those interested in the matter should consult the valuable papers by the late Dean of Manchester, the Hon. and Rev. W. Herbert; Mr Thwaites' interesting remarks in the "Annals of Natural History," i. N. S., p. 168 ; or Lindley's works, \&c. \&c.

The former of these talented writers spent a long lifetime in the study of this particular department of vegetable physiology, and arrived at conclusions of themselves original, if not substantially correct; but as to their col'rectness, for ourselves, we have not a shadow of doubt. This opinion is greatly strengthened by the declaration of Professor Lindley, who says of the paper from which the following quotation is taken, "that it $i$ s the best paper we possess upon the practical value of the facts elicited by hybridising." "I will therefore state briefly and humbly," says the Dean, "what is the general basis of my surmises as to the diversification of vegetables, to which that of animals must be in a certain degree analogous. We know that four races of men have branched out of one stock-the white, the black or African, the brown or Asiatic, and the red, with various subdivisions of aspect amongst them; and we know nothing of the mode or time in which those diversities arose. Revelation and history are equally silent on those facts. They must have occurred very early. We are equally in the dark as to the races of dogs. Old writers allude to different kinds of dogs, and we do not know when or how any one of those we possess originated; and the same may be said in respect to the origin of languages. From these facts I draw this inference, which seems to me incontrovertible, that a course of change was in operation in the early ages after the Deluge, which had ceased, or was greatly diminished, before the era at which our knowledge of events began to be more precise, and handed down by writing. I shall be told that these different races of men breed freely together, and that these dogs intermix and produce mongrels also, and we see thereby that they are only varieties of one kind. Granted: I entertain no doubt of their having respectively descended from one pair of created individuals. But how do you prove to me that the cat, lynx, tiger, panther, lion, \&c., did not descend from one created pair? I am rather inclined to think that they did (but this is only surmise), and even the hor'se and the ass from one created pair ; and I am quite unable to believe that the several sylviæ of the wren family, some of which can with difficulty be distinguished except by the proportions of their quills, and which have, nevertheless, very diverse habits, notes, and nests, were created separately and specially; and when I look to the vegetable races, I am still more unwilling to assent to the assertion, that every plant which this or that botanist has called a distinct species, or even a distinct genus, had a special creation in the period before the sun and moon shone upon this world, when God created vegetables. Upon what authority is such an assertion made? Upon none but the dictum of those who are pleased to inculcate it. Upon what ground is it made? Upon none that will bear investigation ;-upon a rash assumption that everything cross-bred is sterile, and that, if the offspring is sterile, the parents are thereby proved to have been descended severally from the Creator. In the first place, the fact is even false as to animals. Buffon records an instance of the fertility of a mule. I have seen that which I am satisfied was a hybrid between a bitch and a fox which was the father of 
many puppies. But if the fact were positively true, how is it to be proved that the constitution and frame may not have undergone such changes in the diversification as to prevent intermixture? If I can show that in one genus of plants crossbreeding is not only easy, but more easily obtained than fertility by the plants' own pollen, and that in others, so closely allied to it as to make it a question whether they are not sections of one genus, cross-breeding cannot be effected generally, and in no case easily; that in some genera of plants many or all the cross-bred varieties are fertile, and in others nearly allied thereto, all, or almost all, are sterile,- the assertion that the races of canis or dog must have had one origin because their crossed produce is fertile, and the races of felis, from the cat to the tiger, must have had separate origin because their. crossed produce is sterile (supposing the fact to be true, which is not ascertained), must fall to the ground. The only thing certain is, that we are ignorant of the origin of races, that God has revealed nothing to us on the subject; and that we may amuse ourselves with speculating thereon, but we cannot obtain negative proofthat is, proof that two creatures or vegetables of the same family did not descend from one source. But we can prove the affirmative, and that is the use of hybridising experiments, which I have invariably suggested; for if I can produce a fertile offspring between two plants that botanists have reckoned fundamentally distinct, I consider that I have shown them to be one kind; and indeed I am inclined to think that, if a well-formed and healthy offspring proceeds at all from their union, it would be rash to hold them of distinct origin. We see every day the wide range of seminal diversities in our gardens. We have known the dahlia, from a poor single dull-coloured flower, break into superior forms and brilliant colours ; we have seen a carnation, by the reduplication of its calyx, acquire almost the appearance of an ear of wheat, and look like a glumaceous plant; we have seen hollyhocks in their generations branch into a variety of colours, which are reproduced by the several descendants with tolerable certainty. We cannot, therefore, say that the order to multiply after their kind meant that the produce should be precisely similar to the original type; and if the type was allowed to reproduce itself with variation, who can pretend to say how much variation the Almighty allowed? Who can say that His glorious scheme for peopling and clothing the earth was not the creation of a certain number of original animals and vegetables, predestined by Him, in their reproduction, to exhibit certain variations which should hereafter become fixed characters, as well as those variations which even now frequently arise, and are nearly fixed characters, but not absolutely so, and those which are more variable, and very subject to relapse in reproduction?"-Jour. of Hort. Soc.

For the following remarks on this very interesting subject we are indebted to our excellent friend Isaac Anderson, Esq., S.S.C., one of the most scientific, energetic, and successful hybridisers of the present day :-

"To go fully into the theory and practice of hybridising, a volume might be devoted to the subject, and still leave it unexhausted. To start with the beginning would be to start with creation itself-in fact, nature, as conjectured by Linnæus, was occupied by but few original types of the innumerable vegetable forms which have been transmitted to us. How these few first types, if that great authority was right in that belief, have become varied and multiplied, from classes to tribes, from tribes to genera, and from genera to species and endless varieties, belongs to those mysteries of Divine agency which set all inquiry at nought, and upon which it were equally unprofitable and presumptious at the present time to speculate. For who, in treating of such a science, dare invade a field where the Omnipotent invoked no aid from man -ere yet, indeed, man was; while the sun and skyey influences, and the whole host of insectivorous races, now extinct, were perhaps but parts of the agencies and instrumentalities by which,

With herbs, and plants, and fruitful trees,

The new-formed globe He crowned,

and made it fit for man's use and habitation? Who can speculatenow on those atmospheric properties, 'instinct with life,' under whose influence man grew and increased in strength, till the span of his existence extended to near a thousand years-when there were giants on the earth-

'When man was in stature as tow'rs in our time,

The first-born of Nature, and, like her, sublime?'

A life-giving and life-sustaining Spirit breathed the will, and effected the purposes of the Creator. Perhaps a larger portion and a more genial form of electricity than now obtains, may have imparted a principle of higher vitality to the air, and through that medium have communicated a stronger impulse, and more enduring energies, to both animal and vegetable life. This may or may not have been; certain ic is that a change has taken place. Since the Deluge, the vital forces have been greatly weakened. Man, since then, has scarce lived a tithe of his former term; and the vast exhumations of fossil flora bespeak an exuberance and variety of vegetation, in temperate zones, that have no parallel at the present day even in tropical regions.

"Why do we now see natural families with gener $\alpha$ whose affinities are indisputable, and yet in their extreme links so dissimilar? Perhaps no one of the larger family of plants has its intermediate connections better filled up than the Ericacea, yet how many links are awanting in the chain between the Rhododendron arboreum of India, of 40 feet high, and the Chamceledon (Azalea) procumbens of our own Highland mountains, of only 4 inches, or some of the smaller heaths! Though all allied, how many links are there between the various tribes, and even genera, of this most interesting family; e. $g$., between the Vacciniece and the Pyrolece as separate tribes, or between the Rhododendron and the Menziesia, as separate genera. The scarcelyknown elevated plateaus and ridges of the Andes have already yielded up some kindred races in the Bejaria and Thibaudia; and who can tell if, when better explored, many more links awanting may not be supplied? Travel- 
lers have observed examples of the Rhodoreæ on the high mountains of the Hawaian group of isles in the Pacific, and in Manilla, Malacca, and other islands of the Indian Ocean, as well as in North-west America, yet unknown to gardens. Thirteen species of this family have been observed on one hill in Borneo alone, and at least half that number in Java. Dr Hooker has reclaimed about thirty species from the Sikkim ranges of the Himalaya, and of such varied growth and aspect, that our thymes may represent the one group, and our oaks the otherone tiny thing ( $R$. nivale) creeping on the ground at 18,000 feet above the sea, and another $(R$. barbatum) attaining a stature of 60 feet.

"All these discoveries have done much to fill up many gaps in this widely-distributed family, and the discoveries of future travellers may fill up many more.

" Dut how many tribes, genera, and their species, are for ever lost! Man inhabits but the disjecta membra of a former world. With continents, now beneath the ocean, are buriedperhaps for ever lost-genera which, if restored, might supply all those gaps which leave existing races so wide apart. But nature has left materials to work upon, and the art and ingenuity of man may do much to fill up the blanks.

"A very eminent nurseryman-the late $\mathrm{Mr}$ Cunningham of Comely Bank-so far filled up one link of this order, by hybridising the Phyllodoce (Menziesia) carulea with the Rhodothamnus chamcecistus, and producing therefrom the beautiful (so-called) Brianthus erectus. But the parents were too far remote, and the progenya proper mule-is consequently barren. It is somewhat singular, that while the $M$. ccerulea will cross with the Rhodothamnus chamcecistus, the latter will not be crossed with it. It was the Menziesia that bore the seed from which the socalled Brianthus was raised. I speak from my own experience in this matter, having, before Mr Cunningham's experiment was known, attempted unsuccessfully to cross the Rhodothamnus with the $M$. Ccerulea, though I have since succeeded in ripening seeds and raising plants from the cross inverted. These I sowed on 18 th June 1850, and on 10th September four young plants had come through.

"To those who would attempt the hybridising or cross-breeding of plants, I will now offer some suggestions for their guidance.

"It is an essential element to success that the operator be possessed of indomitable $p a$ tience, watchfulness, and perseverance. Having determined on the subjects on which he is to operate, if the plants are in the open ground, he will have them put into pots, and removed under glass, so as to escape the accidents of variable temperature-of wind, rain, and dust, and, above all, of insects. A greenhouse fully exposed to the sun is best adapted for the purpose, at least as regards hardy and proper greenhouse plants.

"Having got them housed, secure a corner" where they are least likely to be visited by bees or other insects. The plants which are to yield the pollen, and the plants which are to bear the seed, should be both kept in the same temperature; but where this cannot be managed, pollen from an outside plant, in genial summer weather, may be used, provided it can be got; for there is a class of insects which live exclusively on pollen, and devour it so fast after the pollen vessels open, that, unless the plant is under a hand-glass (which I would recommend), it is scarcely possible to get any pollen for the required purpose. To secure against chances of this nature, a sprig with opening bloom may be taken and kept in a phial and water inside, where it will get sufficient sun to ripen the pollen. But here, too, insects must be watched, and destroyed if they intrude. An insect like, but smaller, than the common hive bee, which flits about by fits and starts, on expanded wings, after the manner of the dragon-fly, is the greatest pest, and seems to feed exclusively on pollen. The hive bee, the humble bee, and wasp give the next greatest annoyance. All these may be excluded by netting fixed over apertures from open sashes or the like. Too much care cannot be bestowed on excluding these intruders, whose single touch, in many cases, might neutralise the intended result; for the slightest application of pollen native to the parent plant is said by physiologists to supersede all foreign agency, unless, perhaps, in the crossing of mere varieties; and the truth of this observation consists with my own experience. Without due precaution now, the labour, anxiety, and watchfulness of years may issue in vexation and disappointment.

"As a further precaution still, and to prevent self-fertilisation, divest the blooms to be operated on not only of their anthers, but also of their corollas. Remove also all contiguous blooms upon the plant, lest the syringe incautiously directed, or some sudden draft of air, convey the native pollen, and anticipate the intended operation. The corolla appears to be the means by which insects are attracted; and though, when it is removed, the honey on which they feed is still present, they seem puzzled or indifferent about collecting it; or if, haply, they should alight on the dismantled flower (which I never have detected), the stigma is in most cases safe from their contact.

"It will be some days-probably a week or more, if the weather be not sunny-erethe stigma is in a fit condition for fertilisation. This is indicated in many families, such as ericacece, rosacece, scrophularinece, aurantiacece, \&c., by a viscous exudation in the sutures (where these exist) of the stigma, but generally covering the entire surface of that organ. In this condition the stigma may remain many days, during which fertilisation may be performed; and this period will be longer or shorter as the weather is sunny, or damp or overcast.

"In certain families, such as the Malvacece, Geraniacece, \&c., where the stigma divides itself into feathery parts, and where the viscous process is either absent or inappreciable by the eye, the separation of these parts, the bursting of the pollen, the maturity of the stigma, and all which a little experience will detect, indicate the proper time for the operation, sunny or cloudy weather always affecting the duration of the period during which it may be successfully performed.

"As to the proper time and season best adapted 
for such experiments, a treatise might be written; but here a few remarks must suffice.

"As for the season of the year, from early spring to midsummer I would account the best period; but, as I have just observed, I regard all cold, damp, cloudy, and ungenial weather as unfavourable. On the other hand, when the weather is genial, not so much from sun heat as at times occurs from the atmosphere being moderately charged with electricity, when there is an elasticity, so to speak, in the balmy air, and all nature seems joyous and instinct with life, this, of all others, is the season which the hybridist should improve, and above all if he attempt muling.

"The hybridist should be provided with a pocket lens, a pair of wire pincers, and various coloured silk threads.

"With the lens he will observe the maturity of the pollen and the condition of the stigma, whether the former has attained its powdery, and the latter (if such is its nature) its viscous condition. If he find both the pollen and the stiyma in a fit state, he will, with the pincers, apply an anther with ripened pollen, and by the gentlest touch distribute it very thinly over the summit of the stigma. The operation performed, he will mark it by tying round the flower-stalk a bit of that particular coloured silk thread which he wishes to indicate the particular plant which bore the pollen, and at same time tie a bit of the same silk round the stem of the latter, which will serve till recorded in a note-book, which should be kept by every one trying experiments on a large scale.

"It would be out of place here to give even a general outline of the parts of flowers, to show how these differ the one from the other in various tribes of plants. The experimenter, if he is not a botanist, and even though he is partially acquainted with the science, must, from books and observation, make himself familiar with the various organs, male and female, of each separate family of plants on which he means to work, otherwise he will be often puzzled where to find them, or even to distinguish the one from the other:

"As for the time of the day, it may be done almost any hour from 9 A.M. till 4 o'clock P.M., and with equal success. My other avocations have often limited me to earlier and later hours; but I would suggest from ten till two o'clock as the best time of day, always preferring fair, genial, and sunny, to chill, damp, or cloudy days.

"On recurring to my note-book for 1850 , I find a very favourable state of atmosphere occurred in the beginning of March of that year, when I crossed the Phyllodoce (Menziesia) cerrulea with the Rhodothamnus (Rhododendron) chamacistus, sowed on 18th June that year, as above noticed. At this time, too, I succeeded in crossing the above rloodothamnus with a large-leaved whiteflowered Nepal species of rhododendron, the blooms of which were 2 inches across the limb. But though I ripened that season three or four pods of this last cross, each pod of seed beautifully ripened, all of which I sowed, I cannot assert that any one seed vegetated; and though it is now nearly three years since the seeds were sown, I still preserve the seed-pot. And I may remark here, from my own experience, that two years is not too soon to despair of vegetation even of seeds from abroad, on which, of course, no cross had been effected.

"Few seasons have occurred so favourable for the hybridist as the short interval in the beginning of March 1850, above alluded to. Singularly enough, happening to visit Lord Rosslyn's gardens at Dysart House, on the 1st of June that year, with the late Professor Dunbar, $\mathrm{Mr}$ M'Intosh (the author), and Mr Sprott, I observed the above rhodothamnus marked as crossed. I found it had been crossed at the above period, and with $R h_{0}$ dodendron arboreum! The seed-pods were then fully swollen, and approaching maturity ; but I have not heard that anything has come of them.

"It is quite unnecessary to offer any directions as to the results to be effected. If it is desired to reproduce the larger, finer formed, or higher coloured bloom of a plant having a tall, straggling, or too robust a growth, or having too large or too coarse foliage in a plant without these drawbacks, I need not suggest to select, in another species of the same family, a plant of an opposite character and properties-say of dwarf compact growth, handsome foliage, and free flowering habit; and if such can be obtained, work with it, making the latter the seed-bearer. Or, if it be desirable to impart the fragrance of a less handsome kind to another more handsome, I would make the cross upon the latter. I cannot speak with certainty from my own experiments how far perfume may be so communicated; but I have some things far advanced to maturity to test it ; and I entertain the hope that fragrance may not only be so imparted, but even heightened, varied, and improved. Or if it be desired to transfer all, or any valuable property or quality, from a tender exotic species to a native or hardy kind, work upon the latter; for so far as constitution goes, I agree with those who hold that the female overrules in this particular. I would offer this caution to those who wish to preserve the purity of certain flowers for exhibition, especially those having white grounds, not to cross such with high-coloured sorts. I once spoiled a pure white bloomed Calceolaria for exhibition by crossing it with a crimson sort; all the blooms on those branches where the operation had been performed, being stained red, and not the few flowers merely on which the cross was effected.

"In this note, already too long, I cannot further illustrate my remarks, by recorded experiments in the various tribes upon which 1 have tried my hand; but I cannot leave the subject without inculcating, in the strongest manner, the observance of the rules I have laid down to prevent vexatious disappointments. If any doubts arise about the cross being genuine or effectually secured, let not the seeds be sown. Three, four, five, and even six years, must oftentimes elapse with trees and shrubby things ere the result can be judged of ; and if eventually it prove a failure, or even doubtful, it is worse than labour lost, inasmuch as it may mislead. If there is no great departure from the female parent, the issue is to be mistrusted. It is singular, if well accomplished, how much of both parents is blended 
in the progeny. Gentlemen eminent as physiologists have read nature's laws in these matters a little differently from what my own humble experience has taught me, and assigned to the progeny the constitution and general aspect of the one parent, while they gave the inflorescence and fruit to the other. I have crossed and inverted the cross, and can venture to give no evidence on the point, except, perhaps, as to constitution, to which the seed-bearer, I think, contributes most. A well-managed hybrid should and will blend both parents into a distinct intermediate, insomuch so as to produce often what might pass for a new species. If the leaning be to one more than another, it is probably to the female, though this will not always be the case.

"Again, it is asserted that a proper hybrid$i$. e., one species which is crossed with another species, which is separate and distinct from itwill produce no fertile seeds. This does not accord with my observations. Dr Lindley has remarked very justly ('Theory of Horticulture,' p. 69), 'But facts prove that undoubted hybrids may be fertile.' My hybrid, Veronica Balfouriana (an intermediate between $V$. saxatilis and $V$. fruticulosa), seeds, I would say, more abundantly than either parent; and the progeny from its self-sown seeds I find to be of various shades of blue, violet, and red, rising in my garden, some having actually larger, finer, and higher-coloured blooms than the parent bearing the seed; and I am familiar with the same result in other things. Yet I am far from asserting fertility in the produce between two members of allied but distinct genera-such, for example, as in the Brianthus, which I have found to be unproductive, whether employed as the male or female parent. As above conjectured, its parents were far too remote in nature's own arrangement. The hybridist has a field before him ever suggestive of new modes of acting. He may try, as I have done, what may be effected under various tinted glass. My persuasion is, that I effected from a pale yellow a pure white-grounded calceolaria, by placing the plants under blue-shaded glass, by which the sun's rays were much suladued. He may also apply chemicalsolutions to plants with ripening seeds. Nature, in producing, as it sometimes does, plants with blooms of colours opposite to those of the parent, must be governed by some law. Why may not this law be found out? For example, under what influences was the first white fuchsia, the F. Venus Victrix, produced, the purest yet of all the race, and the source from which all the whites have been derived?

"While I have necessarily confined the above remarks to things proper to the flower-garden, a wide and still more important field lies beyond. The late lamented Mr Knight of Downtown did much in this way to improve our garden fruits and other esculents, and with a success that none else-so far as I am awarehas since attained. Why should not these efforts be extended to the improvement of agricultural as well as horticultural productions? Why not carry them into field and forest, to the creation of new, more useful, and more elegant forms? Nature is boundless, and its objects are endless, and this subject, of all others connected with plants, the most engrossing and exciting. Rich results await the intelligent experimenter ; but I would advise none to embark in the pursuit who has not sufficient leisure to devote to it, and, as I said before, who is not possessed of indomitable patience, watchfulness, and perseverance, with a fixed determination not to be fretted or discouraged by frequent failures.

$$
\text { "MARYFIELD, June } 1853 .
$$

\section{§ 3.-PROPAGATION BY GRAFTING.}

The art of grafting is so ancient, that anything connected with its origin is lost in the obscurity of ages. Theophrastus and other Greek authors speak of grafting as if it had been well known in their day; and Varro, a Roman writer, notices upwards of twenty modes as practised in his time. The late Professor Thouin enumerates above forty methods; and M. Louis Noisette some years ago published a description, with figures, of one hundred and thirty-seven. The most probable conjecture as to the origin of that variety of it known as inarching, is the accidental meeting of two branches from neighbouring trees, which, first by friction against each other till the bark of both branches became displaced, and afterwards by uniting, formed so perfect a junction, that when either was separated from its fellow, it became supported by the other. Of the origin of grafting proper, as well as of budding, we know little or nothing.

We have stated above that the varieties of grafting are very numerous. In this country these are divided into what may be called three distinct species-namely, grafting, budding, and inarching ; while on the Continent no such distinction is observed. The French, for example, have no proper word to represent either, but use the general term graffe for all of them. Hence, graffes par gemmes-grafting by buds, or budding, \&c.

The theory of grafting has been explained by Decandolle, in his "Physiologie Végétale ;" by Mr G. Lindley, in "Guide to the Orchard;" Keith, in "Physiol. Botany ;" Knight, Loudon, and others. On this subject the former says: "The shoots springing from the buds of the scion are united to the stock by the young growing alburnum, and once united, they determine the ascent of the sap rising from the stock ; and they elaborate a true or proper juice, which appears evidently to reascend in the inner bark. This sap appears to be sufficiently homogeneous, in plants of the same family, to be, in course of its passage, absorbed by the growing cellules near which it passes, and each cellule elaborates it according to its nature. The cellules of the alburnum of the plum elaborate the coloured wood of the plum; those of the alburnum of the almond, the coloured wood of the almond. If the descending sap has only an incomplete analogy with the wants of the stock, the latter does not thrive, though the organic union between it and the scion may have taken place; and if the analogy between the alburnum of the scion and that of the stock is wanting, the organic union does not operate; and as the scion cannot absorb 
the sap of the stock, the graft does not succeed." Mr G. Lindley, after referring to the circumstance that, in the stems of grafted trees or plants, the cellular tissue is found alive in the liber and medullary rays only, and that it is essential that those parts both in the scion and stock should be brought into contact, proceeds: "In regard to the medullary rays, these are so numerous, and so closely placed, that it is scarcely possible that a portion of one stem should be applied to another, without the medullary rays of both touching each other in many points. No care, therefore, is required to insure this, which may be safely left to chance. But in regard to the liber (or bark), as this is confined to a narrow strip in both stock and scion, great care must be taken that they are both placed as exactly in contact with each other as possible, so that the line of separation of the wood and bark should, in both stock and scion, be accurately adjusted. The success of grafting depends very much upon attention to this. But there are other reasons why this accuracy in adjusting the line between the bark and wood of the stock and the scion is so important. It is at that part that the roots of the latter pass downwards over the former; and it is also there that the substance called cambium, which serves as food for the young descending fibres, is secreted. It is obvious that the more accurate the adjustment of the line separating the wood from the bark, the more ready will be the transmission of young fibres from the one to the other; and that the less the accuracy that may be observed in this respect, the greater the difficulty in this transmission will be. Provided the stock and scion be of exactly the same size, the adjustment can scarcely fail to be accurate in the most unskilful hands : it is in the more common case of the scion being much smaller than the stock that this is to be more particularly attended to." Keith's remarks are even more clearly given : he says, "The whole of the art of artificial grafting is founded upon the capacity inherent in plants of uniting together by the stems in given circumstances and in a given mode. But the natural graft is always effected by means of the union of the liber of the respective stems composing it; so that the perfection of the art of grafting consists in applying the liber of the graft and stock together in such a manner as will most facilitate their incorporation; and hence the graft will not succeed, unless the two libers are brought into contact, and closely bound together. Nor will it succeed well, unless the plants engrafted have some natural affinity to one another, as that subsisting between the plum and cherry; in which, and in all other cases, the union is effected by means of a granular and herbaceous substance exuding from between the wood and bark, and binding and cementing together the stock and graft, though not uniting the former layers of wood."

Knight, in reference to the same subject, says: "It will be necessary that I describe the motion of the sap, as I conceive it to be at the period when grafts are most advantageously inserted. The graft first begins its efforts to unite itself to the stock just at the period when the formation of a new internal layer of bark commences in the spring ; and the fluid which generates this layer of bark, and which also feeds the inserted graft, radiates in every direction from the vicinity of the medulla to the external surface of the alburnum. The graft is of course most advantageously placed when it presents the largest surface to receive such fluid, and when the fluid itself is made to deviate least from its natural course. This takes place most efficiently when a graft of nearly equal size with the stock is divided at its base, and made to stand astride the stock;" as in a variety of saddle-grafting invented by this eminent physiologist.

The principle on which all the operations in grafting, budding, and inarching are founded, according to Loudon, is dependent on the phenomena of the union of newly generated tissues, when in the act of being generated. "No union can take place between the parts of plants previously formed, but only when these parts are in the act of forming. Thus two shoots or branches may be selected, and by means of similar sections be most accurately joined, and placed under the most favourable circumstances for uniting; yet when the two are bound together, though a union do ultimately take place, not one particle of the existing tissue, at the time of grafting, becomes united with similar tissue brought in contact with it. Close contact is all that takes place with regard to these surfaces of the scion and stock, but a vital union only occurs when nascent tissues meet. The alburnums of the preceding year never unite. The vital union is formed solely by the coalition of newly generated tissues, thrown out by such parts as have the power of generating them. This power does not exist in the heart-wood, nor in the outer bark, but only in the alburnum, or rather in the substance imbedded between it and the inner bark, constituting the cambium. If the sections are placed against each other so as the inner barks coincide, the scion may perhaps derive an immediate supply of moisture ; but it does so only in a mechanical way, and a piece of dry sponge might as truly be said to have formed a connection from its absorbing moisture, in consequence of its being placed on top of a stock, as the scion, that only takes up moisture as above mentioned. When, however, new tissue is formed by the parts of the respective sections, then, when the portions so formed protrude so as to meet, they immediately coalesce, forming a connecting chain of vessels between the buds of the scion and the roots of the stock. If an old grafted tree is cut down, and all the wood cut away to the original portions which existed at the time of grafting, it will be found that the sections made by the grafting-knife are only mechanically pressed together, and may be easily taken asunder. Instances frequently occur of the inner bark of the scion being placed out of contact with that of the stock, and a union nevertheless ensues; but this takes place in consequence of the cellular substance protruding from the respective alburnums over the surface of the old wood, 
which it only covers: as soon as the new-formed tissue of stock and scion touch each other, a union is then formed."

The objects of grafting are manifold: these, however, may be divided into three distinct heads-namely, to continue in their original purity, as well as to multiply, by extension of their parts, trees and plants which could not conveniently be otherwise increased; to accelerate the production of fruit and flowers; and to induce fertility.

Grafting with a view to continue the purity of a variety of fruit or flower.-Were we dependent entirely for a reproduction of our finest fruits and flowers upon the progeny arising from their seed, our disappointments would be great and frequent, if botanists are correct in their theory that all varieties, however improved they may be compared with the parents from which they originated, whether the intercourse between the flowers was brought about by the agency of man or from accidental causes, have a direct tendency to revert to the type from which they originated: then would all our most choice fruits and flowers revert backwards, if their propagation were continued by sowing their seeds. The quality of many seeds is liable to be affected by impregnation, occasioned by the casual introduction of the pollen of congenerous plants into the blossom; and notwithstanding the greatest care that man can possibly take, a species of accidental intercourse is perpetually going on : so that even were plants capable, in a high-bred state, of continuing themselves in a state of purity, and of reproducing themselves undeteriorated, and supposing such a law did not exist of varieties returning, after a time, to their original type, this promiscuous intercourse would be of itself quite sufficient to warrant the adoption of propagation by extension, if the object be to maintain the improved variety. Grafting makes us independent of any such chances, as if the graft of a favourite variety or species of either a flowering shrub or fruit-bearing tree be placed on a proper stock, and the usual culture bestowed upon it, it may be continued for ages without showing signs of deterioration. Nay, even many herbaceous plants may be, thanks to M. Tschoudi of Metz, the inventor of this variety of the art, continued in the same manner. The possibility of continuing a variety beyond the natural limit of life allowed to the tree from which it was taken, was doubted by $\mathrm{Mr}$ Knight and some others. The majority, however, of botanists, and our highest physiological authorities, deny the truth of Knight's views, and contend that a variety may be continued by extension to an almost indefinite period. Many trees and plants may be readily multiplied by extension, that could not be otherwise increased. By this means, also, we multiply such plants as will scarcely root by cuttings or layers, and rarely produce seeds (if ever) in this country, as well as almost all other trees which contain in themselves only one sex. Willows, poplars, \&c. of this class, for the most part root freely by cuttings. All our esteemed fruits are increased by extension in some of its various modifications, as are the numerous varieties of variegated leaved, weeping or pendent-branched trees and shrubs, \&c.

Grafting with a view to accelerate the production of flowers or fruit.-Plants originated directly from seeds are apt to grow luxuriant, and to attain both a considerable size and age before they show a disposition to produce flower-buds. This habit is corrected by removing scions of them, and working them on stocks of the same, or of some nearly allied species, by which process flowering is accelerated by impeding the descent of the properly elaborated juices of the tree, and inducing the principle of maturation instead of encouraging that of growth. The plant itself may be cut down and grafted with its own branches to produce a like effect. Grafting a young twig upon an old stock has the effect of making it flower earlier than it would otherwise do, in consequence of the accumulation of sap in the old stock becoming beneficial to the twig, and giving a check, at the same time, to its tendency to produce leaves. As an example, if a seedling apple be grafted the second year of its growth on the extremities of a full-grown tree, or even on a stock of five or six year's from seed, it will show blossom. buds the second or third year ; whereas, if it had remained ungrafted, it might not have shown such buds for ten or twenty years. It is a usual practice in gardens to take shoots off seedling camellias, rhododendrons, and similar plants, and to graft them on established plants of the same species, and thus we prove their merits several years before the original plants would show symptoms of flowering.

Grafting with a view to induce fertility.-The laws which govern this part of the vegetable economy are almost identical with those of the preceding. Mr Knight observes, that the effects produced by grafting "are similar to those which occur when the descent of the sap is impeded by a ligature, or by the destruction of a circle of bark"-ringing, as it is practically called. "The disposition in the young trees to produce and nourish blossom-buds and fruit is increased by this apparent obstruction of the descending sap, and the fruit of such young trees ripens, I think," he says, "somewhat earlier than upon young trees of the same age, which grow upon stocks of their own species: but the growth and vigour of the tree, and its power to nourish a succession of heavy crops, are diminished, apparently by the stagnation in the branches and stock of a portion of that sap, which in a tree growing upon its own stem, or upon a stock of its own species, would descend to nourish and promote the extension of the roots."

Grafting with a view to renew the lead.-This is often found to be expedient, particularly in the case of old and diseased trees, and also those which have ceased to be productive. If such trees were cut down either to within a few inches of the point where they were formerly grafted, or were the larger branches amputated close to or at any distance from the main stem or trunk, abundance of young shoots would be produced, arising from innumerable 
latent buds with which their bark is everywhere replete. From these, provided the stem be not cut over below the original graft, abundance of material would be provided for the future formation of the tree. But if care be not early taken to rub off all the buds as they begin to develop themselves, leaving only the proper numbers, and thosc well placed, the one would rob the other; and before a selection is made, the season of growth may be nearly terminated. Besides, in this case, if the tree be large, or of an indifferent variety, no change for the better takes place-only the rcnewal of the tree, which will be some years in coming into a bearing state. It were better, under such circumstances, to graft the tree with more choice kinds, which would not only give an improved quality of fruit, but that fruit would be produced within a much shorter space of time. The grafts could also be put on where the branches are required to be; and the sap being directed at once into proper channels, the shoots would attain a size and maturity, before autumn sets in, which would enable them to elaborate the nccessary sap for the formation of fruit-buds within a very short space of time. The process of heading down and grafting old fruit-trees is a much neglected part of fruit-tree culture ; and we need not point to commercial orchards only, when we see so many cases where a different course might be expected.

One of the advantages of this mode of grafting is, that the organisable matter deposited in the roots and the part of the trunk left, is thrown with great force into the scions, causing them to make strong and vigorous shoots ; but as they cannot take up all this organisable matter at once, it is stored up for them in the interior of the tree, and supplied to the branches, in future years, as it is wanted: whereas, if the tree operated upon be not cut down entirely to one stem, but its branches only partially shortened, and part of them only furnished witl scions, these branches will attract the juices to themselves, and greatly weaken the scions, if indeed they do not entirely perish.

Grafting several sorts on one tree.-In heading down old trees of large size, particularly in small gardens where only a few trees can be accommodated, this process may be advantageously adopted, and a variety procured. Even on one apple or pear tree, both early-ripening and late-kecping sorts may be produced, thus affording a long succession of fruit; whereas, if only one kind be grown, that season is greatly diminished. Many varieties of camellias may, in like manner, be produced from one plant, affording a greater variety as well as a long succession of blooms.

Grafting to produce a reduction in size.-This is effected by working the apple on the paradise or doucin stock, the pear on the medlar or quince ; while, to produce a contrary effect, the former is grafted on the crab, and the latter on the wild-pear stock. Dwarf fruit-trees are valuable in small gardens; and indeed some nurserymen, such as Mr Rivers, have planted whole miniature orchards, the trees being much the size of ordinary gooseberry bushes.

VOL. II.
Choice of grafts.-These should be chosen from the young shoots of the previous summer growth, if indeed the operation be not performed by using scions of the same season's growth, as practised by Knight, the Baron Tschudy, and others; but for general practice the outside lateral shoots are preferred, because they are less robust, and less apt to run to wood, than those from the centre or top of the tree, and not so weak as those at its base, and under the shade and drip of the rest. Besides this, they are better ripened, and are found from experience to produce the truest specimens of the fruit of the tree from which they are taken. "An exception to this rule is to be found when the trees are in a sickly state, when, of course, the grafts should be taken from the strong shoots in the centre, or near the top of the tree. The ends of each scion should be cut off, and the middle part only used, rejecting both the top and the base of the shoot. The grafts should be cut off the parent tree some time previous to their being used, experience having shown that it is necessary to allow the stock to have the advantage over the graft in forwardness of vegetation. The sap of the stock should be in active motion at the time of grafting, which would also be the case with the scion, if left on the parent tree; whereas the grafts being taken off some time before, the buds will be unavoidably kept back, and ready only to swell when placed upon the stock. Grafts should be collected any time in January or the beginning of February, and kept at their whole length, laid in dry mould, in a situation where they are not exposed to either frost or much sunshine, until they are to be used."-Practical Gardener.

Choice of stocks. - Such stocks must always be chosen as have a near affinity to the scion to be put upon them. There is, however, another reason why a judicious choice of stocks should bc made - namely, that they may be adapted to the soil they are to be planted in. Hence, where the soil is damp, and subject to be overflowed occasionally with water, such as meadows by river sides, \&c., the quince will be found the best stock for the pear; whereas in dry sandy soils it would be the very worst. Again, the white-beam tree is the most proper stock for the pear in chalky soils; in such soils even the pear stock would scarcely live. On properly adapting the stock to the soil, and the variety to the climate, much of the success in fruit-tree culture depends.

The influence of the stock upon the graft.-On this subject opinions have long been at variance, and it appears also to have attracted notice at an early period. The following may be regarded as the leading opinions offered: "Crab stocks cause apples to be firmer, to keep longer, and to have a sharper flavour. If the breaking pears be grafted on a quince stock, the fruit is rendered gritty or strong, while the melting pears are much improved on such stocks."-Miller. "The scion overruleth the graft quite, the stock being passive only."-Lord Bacon. "This, as a general proposition, remains true, it being evident that the scion, bud, or inarched shoot, is endowed with the power of drawing or forming from the stock that peculiar kind of nour- 
ishment which is adapted to its nature, and that the specific character of the engrafted plant remains unchanged, although its qualities may be partially affected."-Neill. "The influence exercised by the stock is very marked, and it is of great importance to select good stocks on which to graft slips. In this way the fruit is often much improved by a process of ennobling, as it is called. The scion, also, in some cases seems to exercise a remarkable effect on the stock. Slips taken from varieties with variegated leaves, grafted on unvariegated, have caused the leaves of the latter to assume variegation; and the effect, when once established, has continued even after the slip was removed. The effects of grafting are well seen in the case of the red laburnum, when united to the yellow species. The red laburnum is a hybrid between the common yellow laburnum and Cytisus purpureus, or the purple laburnum. The branches below the graft produce the ordinary yellow laburnum flowers of large size; those above often exhibit the small purple laburnum flower, as well as reddish flowers, intermediate between the two in size and colour. Occasionally the same cluster has some flowers yellow and some purple."-Balfour. "Since, then, the developments of the graft are proved to be, in fact, altogether uninfluenced by the stock, it may be safely asserted that the latter ought to be considered as a medium only, or vehicle, through which the vascular organs of the former pass, and are conveyed into the soil, whence their spongioles and rootlets, by the aid of electric agency, affect the intro-susception of the nutritive sap. It may, however, be admitted, that in the first instance the cellular system and juices of the stock exert an attractive energy upon those of the graft; and that while the former affords an appropriate matrix or bed, wherein the granular excisions from the scion may affix themselves and be secured, the latter keeps up that supply of moisture which is required till the union is completely effected. A graft or scion, therefore, can be viewed in no other light than as a cutting deposited in a more congenisl medium of nutrition than the soil, all the former developments of which cutting are completely its own, and wholly independent of any physical agency or influence exerted by the stock." Tower's. "In proportion as the scion and stock approach each other closely in constitution, the less effect is produced by the latter; and on the contrary, in proportion to the constitutional difference between the stock and the scion, is the effect of the former important. Thus when pears are grafted or budded on the wild species, apples upon crabs, plums upon plums, and peaches upon peaches or almonds, the scion is, in regard to fertility, exactly in the same state as if it had not been grafted at all; while, on the other hand, a great increase of fertility is the result of grafting pearsupon quinces, peaches upon plums, apples upon white thorn, and the like. In these latter cases, the food absorbed from the earth by the root of the stock is communicated slowly and unwillingly to the scion; under no circumstance is the communication between the one and the other as free and per- fect as if their natures had been more nearly the same : the sap is impeded in its ascent, and the proper juices are impeded in their descent; whence arises that accumulation of secretion which is sure to be attended by increased fertility. No other influence than this can be exercised by the scion upon the stock. Those who fancy that the contrary takes place-that the quince, for instance, communicates some of its austerity to the pear-can scarcely have considered the question physiologically, or they would have seen that the whole of the food commutated from the alburnum of the quince to that of the pear is in nearly the same state as when it entered the roots of the former. Whatever elaboration it undergoes must necessarily take place in the foliage of the pear, where, far from the influence of the quince, secretions natural to the variety go on with no more interruption than if the quince formed no part of the system of the individual."-G. Lindley.

The influence of the graft upon the stock appears scarcely to extend beyond the power of communicating disease, as shown by the difficulty of inducing health and vigour in a tree that has been grafted from another in an unhealthy state, even although grafted upon a healthy stock for many times in repeated succession. Disease is certainly communicated to the stock when the infection is conveyed by scions taken from a tree in which inherent or hereditary disease exists.

In connection with the influence the stock may be supposed to have upon the graft, we may observe, that while it is perfectly possible to have a hundred sorts or more of apples upon one tree, and of pears upon another tree, each after its kind, yet no difference occurs in one of them, either in size, colour, flavour, or form, but each grows on as if it were upon a distinct stock of its own. And, again, we have white, red, and the intermediate variety of currant called the champagne by some, all growing on the same bush, and all retaining their individual characters for years. The peach, nectarine, and apricot have been wrought all on one tree, each retaining its own marks of peculiar distinction. The sap which supplies nourishment to all these is only a simple fluid collected by the roots of the stock, passing upwards through it to the fruit and to the leaves, which latter have the power of digesting and modifying it, and forming it into a proper juice, which re-descends in the inner bark; and even every bud and leaf upon the whole tree maintains its individuality, and prepares its own proper food or organising matter out of that general aliment the sap, which was conveyed, a mere simple fluid, alike to all: the leaves, therefore, have the power of preparing the food for the plant, and of conveying it to the fruit. If, therefore, any change takes place, it would be in the leaves, which are the vegetable laboratory, and not in the fruit. The sap, which ascends year after year in form of a mere simple fluid, and returns again year after year in an elaborated form, gives bulk to the branches, stem, and even to the stock; still the latter, although increased in size, is not altered in constitution, and will no more throw 
out branches similar to those of the engrafted part than the latter would branches similar to those of the stock. The stock and graft remain ever distinct, although forming one and the same living subject. But while grafting or budding never effects any alteration in the identity of the variety or species of fruit, still it is not to be denied that the stock does exert certain influences over the habits of the graft. These have, however, been already noticed in a general way.

The Americans and French graft and bud their fruit trees on much taller stocks than we do, and they believe that this tends to the durability of the tree. The late Mr Wilmot was of opinion that the stock, becoming buried by the accumulation of soil in gardens, was in time transferred into a perfect root; and when this takes place the trees begin to decline in vigour, and soon after die.

$G$ General remarks on stocks for fruit trees.-The late Professor Thouin, who studied this matter thoroughly, observes that the historians and poets of antiquity have written, and the moderns have repeated on the faith of others, that every scion will take on any sort of stock, provided there be any resemblance in their barks! Thus Pliny, Varro, Columella, \&c., speak of apples and vines grafted on elms and poplars, and of different fruits being borne on the same stock; and some of later date state even more absurd anomalies. Even to this day, if such extravagances be not really believed by many gardeners on the Continent, there are some of those who do their best to persuade us to believe that such things are; and many ingenious contrivances we have seen by which such deceptions are carried on. One of these is produced by what the French call Graffe des Charlatans, and is often constructed by selecting an old orange stem, with a few miserable branches on top, into which they drill holes from the bottom of the stump upwards, through which they ingeniously introduce slender shoots of roses, jasmines, and like flexible plants, the roots being planted in the same soil as the orange, the shoots being introduced below the surface of the soil, and the points of the tops brought out at various heights of the orange stem.

The classical reader will no doubt recollect Pliny's account of the tree in the garden of Lucullus, grafted in such a manner as to bear apples, pears, plums, almonds, olives, figs, and grapes. Similar ingenious deceptions are practised in Italy to this day.

Mr Rivers, a highly respectable nurseryman, has long paid great attention to the subject of stocks, as well as several others, who have given us the benefit of their experience in various forms. One of the most experienced writers on fruit trees-Mr Errington-in a valuable article in the "Journal of the Horticultural Society," after remarking that other causes must be at work, beyond the mere question of a deficiency of heat in our climate, to prevent the finest Flemish pears arriving at full maturity, observes, "If the habit of the quince was duly considered, and the soil adapted accordingly, the result would be a much higher amount of success than is at present attained. I do not wish it here to be inferred," he says, "that I would advocate the total banishment of the pear stock from our gardens ; not by any means. What I would urge is, that most of the Flemish kinds which are not found to succeed in a satisfactory way on the wall, would probably be much improved if grown as dwarf standards or pyramidal trees, providing proper means are taken to subdue, and continue under control, their tendency to produce superfluous shoots. The production of such shoots constitutes the principal reason why the fruit becomes unsatisfactory. The tree is in exciting soil, perhaps, with unlimited power to range in quest of food; the necessary consequence is, that abundance of breastwood is formed. Now, it so happens that the amount of foliage on such breastwood is not an iota more than is requisite to elaborate the great amount of watery juices thrown into the system. No: nature, true to her principles, produces no more foliage than is necessary for a due amount of reciprocation between the root and the branches. The tree is half stripped of its elaborative powers at the moment they are most needed, and the consequence is large fruit filled with half-elaborated juices. The gardener, to be sure, is obliged to cut away the breastwood, or general barenness would be the result." This refers to the practice of what is called summer pruning. If a proper dwarfing system, Mr Errington believes, were adopted, there would be little occasion for disbudding. "There are three ways," he observes, "of arriving at this end : the one by using the quince stock; the next, by the free stock in a limited amount of soil; and a third, by rootpruning. The free stock, with a very limited amount of soil, offers a chance of striking at the root of the evil. If, however, the limitation is very severe, and the soil too light, there arises another source of failure; the fruit, in a hot dry summer, may become stunted, and crack. To be sure, top-dressings will obviate these things, but it would be preferable so to establish pear-trees as that they would require little or no assistance in the way of labour. We come now to the quince stock; and to this I would beg to draw particular attention. I think there can be no doubt of the general success of the quince, provided those who adopt it could be prevailed upon to prepare the stations for its reception with some regard to the habits of the quince. It will be at once admitted that the great object here is to provide a congenial soil for the stock ; this done, the success of the graft will follow as a matter of course." With a view to modify the luxuriance of the free stock, this high authority suggests a system of root-pruning, to commence even while the stocks are in theseedbed, by means of frequent transplanting, to check the tendency in them of forming strong and forked roots, and to induce them to form plenty of fibrous roots near the surface. By this means, and placing the roots afterwards under proper control, it is probable that the pear upon free stocks may cometo assume a dwarfish habit-and if so, it would be desirable, as any ordinary garden soil would suit it; whereas, if the quince is used, a soil and other conditions must be artificially prepared for it. Some pears succeed bet- 
ter on the quince stock than others, but all are similarly affected in assuming a diminished habit, and most of them an increase in fertility.

Pear stocks are obtained by sowing the seeds of the cultivated pear, and are, to distinguish them from those produced by sowing the seeds of the wild pear, called free pear stocks, while the others are called wild pear stocks. They are both more hardy and more durable, and hence adapted for standard trees in the formation of orchards. On the Continent great attention is paid to the different kinds of stocks, which nurserymen form into three classes to suit intending purchasers. The quince stock is propagated from layers, which no doubt adds to its property of dwarfing the trees for which it is used.

Most cultivators bud such pears as are intended for standards or for walls, and graft those intended to be grown as dwarfs, as in the latter case the tree divides into branches the first year, and is at the end of that period almost as far advanced in its formation as a budded tree will be at the end of the second year.

Apple stocks. - The stocks employed for the apple are the wild crab, which is procured by sowing the seeds of the most healthy wildings found in the woods. It is the most durable, and least subject to canker. The free stock is the produce of seeds of the cultivated apple, and ranks next to the former in durability and hardihood. The doucin, or paradise stock, is preferred for dwarf standard trees and espaliers. It is less durable than the crab stock, is reared by layers, and succeeds better in a rather rich moist soil than in a poor dry one. The French stock is also reared by laying, and is the most diminutive of all stocks-used principally for trees to be grown in pots, or planted as miniature orchards. Budding is preferred for apples intended for full standards, the bud being inserted within 9 inches of the ground; while those intended for dwarfs, espalier's, and most of the delicate-growing sorts, are grafted for reasons already given.

The Paradise stock, like the quince, has the natural property of emitting numerous fibrous roots near the surface of the ground, and also of controlling the vigour of the graft, and inducing an earlier state of bearing in the tree. There are three varieties of Paradise stocks used on the Continent-viz., the Doucin, Pomme de Paradise, and the Pomme de St Jean. The two former are used for different purposes - the first for dwarf trees, and the second for pyranidal ones. It is, however, rather too delicate for our climate, unless in the most favoured situations, though of all others the best for trees to be grown in pots. The Pomme de St Jean is of a hardier character than the true Paradise, although not quite so dwarf. The Doucin is much more vigorous, and, with the English Paradise stock, the most worthy of our attention for the production of fruitful pyramids.

Apricot stocks.-The stocks usually employed for this purpose are, the common plum, the muscle plum, the pear plum, the common apricot, and the Brussels or St Julian stock, which latter is preferred for standard or rider trees. The first of these is obtained by sowing the stones of that plum, and is regarded as one of the best for the purpose, and hence very extensively employed; the second from seeds of the pear plum, so called from the shape of the fruit; the third from stones of any of the cultivated sorts, but it is considered inferior to stocks from various species of plums; the last has been long used in this country, and is well adapted for the apricot, but not for peaches, being too full of sap at the budding season. The late Mr Knight anticipates that, by budding the Moorpark apricot upon the apricot stock, it would become hardier, and less subject to that disease or state of debility which is supposed to be the cause of its branches dying off during summer. Rogers, who is an excellent practical authority, says there are two stocks the Moorpark may be wrought on-viz., the muscle for dwarf trees, and the Brussels for riders and standards.

Plum stocks.-The plum is wrought upon the Brussels stock and common plum stock, and apparently with equal advantage.

Peach and nectarine stocks. - "These are usually propagated by budding only, and the stocks used are either the Brompton stock, muscle plum, or pear plum. The latter is the best, and therefore used for French peaches and others of our finer sorts. It is said that peaches wrought upon the pear plum stock are much less liable to mildew than those upon the common muscle stock; the muscle is the best for English peaches. The Brompton stock is strongly disapproved of by $\mathrm{Mr}$ G. Lindley, who says : 'Where trees of a permanent character are required, the substitution of such a stock cannot be too strongly condemned.' On the other hand, Rogers-by no means a slight authorityas well as the most eminent nurserymen, are of a different opinion. It may have happened that a dislike to the Brompton stock arose from seeing the fate of apricots budded upon it, for which fruit it is ill adapted and unsuitable. The late Mr Ronalds says: 'I have no reason to think that the Brompton stock will not bear as fine fruit, and last as long, as either the muscle or pear plum, if budded low for dwarfs." "

Quince stocks.-The quince is generally propagated by layers, where the object is to procure them for stocks for pears. If laid during winter, the plants will be rooted by the following autumn, when they should be transplanted into nursery lines, when in two years they will be fit for grafting. They are also propagated by cuttings, put in during winter; and the better varieties, intended to be cultivated for their fruit, are grafted, the stem being trained to a clean rod to the height of 4 or 5 feet.

Almond stocks.-The plum stock is preferred for cold and wet soils to those of the peach or the almond itself, being much hardier than they. The peach stock is thought by Mr R. Thomson to be less durable than the almond, and more apt to die off in this case, as they are apt to do when used as a stock for the peach and nectarine. Almond stocks are procured by sowing the seeds when the object is to grow them for stocks, as well as for obtaining new varieties.

Cherry stocks.-The small black or wild red cherry of our woods is generally chosen for this purpose. The Morello cherry has been recom- 
mended with a view to render the trees of a dwarf habit; and the Mahalab cherry has been suggested for a similar purpose. These are all obtained by sowing their seeds, which, if sown in spring, will come up the same season, and should not be transplanted till the second season after sowing. They will be in fit condition to bud the season following, if intended for dwarfs, but not until the second, third, or even fourtl year, if intended for standards ; though as regards this much depends on the strength they attain. Standards should be budded or grafted 6 feet from the ground-the former process being performed during summer and the latter in spring, and also in all cases where budding may have failed. The cherry does not succeed equally well in all soils, and we believe that much of this depends on the kind of stock employed. The wild cherry stock is best adapted for strong damp or clayey soils, and the Mahalab for soils of a light sandy or chalky nature. Stocks originated by sowing the stones of the cultivated varieties are of short duration, and subject to gum, and shed their blossoms without setting their fruit; on these accounts, therefore, they should never be employed.

Medlar stocks.-The Whitetorn stock has been, but very improperly, used as a stock for this fruit. The common pear stock is the most natural, and therefore the most proper, for the purpose of either budding or grafting.

Rose stocks. - The rose is propagated by budding, from cuttings, layers, suckers, and seeds. The more valuable varieties are increased by the former method, as, if the operation be properly performed, every bud will form a distinct plant. Budding is also had recourse to in the formation of standard roses-a taste of Dutch origin, imported into France, and from thence, in 1803, brought into England. The stocks in general use have been the tree rose, Rosa villosa, $R$. canina, $R$. rubigenosa, $R$. tormentosa, $R$. pomifera, \&c. These have been collected from the woods in a wild state, planted in nurseries, and budded the same year or the following spring. Notwithstanding the great demand for rose stocks annually, little has been done by cultivators to improve or render more certain the supply. Of late the Manettii, crimson Boursault, and some other strong-growing kinds, have been employed; and for perpetuals, Bourbons, Noisette, Chinas, Teas, \&c., the Manettii stock is recommended. But budding must be performed in September ; if earlier, the buds are apt to perish in consequence of the watery nature of the stocks. July and August is the proper time to bud, when the dog rose is used as a stock.

Stocks in general, physiologically and anatomically considered.-There are certain relationships existing between plants, beyond which it would be impossible to produce a union by the process of grafting or budding-somewhat analogous to that limitation which prevents cross fecundation from taking place in the flowers of plants of dissimilar species. Neither process would be successful by employing plants of such remote relationship as the melon and the vine, the potato and the cabbage, \&c. These limits, so far as grafting or budding is concerned, de- pend anatomically on the organic structure of the tissue, and physiologically on the vital functions of the plant. The anatorny of plants is yet so little known, even to botanists, as well as to general cultivators, that they are unable to determine by dissection the plants which bear sufficient analogy to each other to cause them to produce either of those unions to which we have referred, and therefore all we at present know of the matter is what is taught us by practical experience. "From this it is found that as plants of the same natural family have an analogous organisation, they alone can be grafted on one another with any prospect of success; though the success of the operation, even within this limit, will not always be complete, partly perhaps from some difference in organic structure, as in the case of the apple and pear, which can only be united for a few years, but chiefly on account of the physiological differences which may and do frequently exist. Decandolle succeeded in grafting the lilac and the fringe tree on the ash, the fringe tree on the lilac, and the lilac on the phillyrea, and the olive on the ash and the privet; and although these grafts did not live a long time, on account of the physiological differences of the species, yet their having succeeded at all sufficiently proves the anatomical analogy of plants within the same natural order. This analogy is greater between plants of the same genus; more so still between individuals of the same species; and most so between branches of the same individual." Sub. Hort.

Dr Van Mons considers grafting upon small pieces of the roots taken out of the ground, and planting the same as soon as grafted, to be of all others the best mode with regard to the condition of the grafted sort-first, because the smallest quantity of the stock is used; and, second, because the lower part of the scion being thus placed in the ground, after a time it throws out fibres from that portion, and so at last is actually growing on its own roots.

There are other conditions in which a union by grafting cannot take place-viz., plants with milky sap, as most species of Euphorbia, will not unite with those having thin, watery juices. Thus Acer platanoides, for example, being the only species of that genus having milky juice, cannot be grafted on any others of that family. Any two plants whose sap is not in motion at the same period, cannot be successfully grafted. Although in the case of the common laurel and cherry an adhesion takes place, their union cannot be calculated upon beyond three or four years. Soft-wooded trees or plants will not readily associate with hard-wooded ones; nor woody plants with such as are herbaceous ; nor will annuals with perennials.

Season of grafting.-The number of plants upon which this operation is performed is so great, and their constitutions and circumstances so variable, that no definite period can be fixed upon as a general rule. From what has been said of the phenomena of the art, it will have been understood that the period of operation is to be entirely guided by the state of the ascent of the sap. The spring, therefore, is the proper 
season for apples, pears, and all similar hardy trees, in our climate. There is, however, an exception to this otherwise very general rule practised both in Germany and in North America, where, the stocks having been taken up in autumn, are kept in sheds or cellars, and grafted during the winter. The whole stock of a nursery may thus be done when no other work could be carried on out of doors; and when spring returns, the stocks, with their grafts tied and clayed in the usual manner, are taken out and planted. The same practice is often followed in Britain in the case of roses and pears, particularly in the latter case, when root-grafting is employed. The vine is best grafted when in leaf ; if done sooner, it is apt to bleed to the extent of preventing the union from taking place. The general rule, however, is, to let the sap in the stock be in full motion, while the sap in the scion is only beginning to flow at the time of operating. "This mode of reasoning," says Mr Towers, "appears to be founded upon the old theory of the exclusive agency of the ascending sap; and it implies that the scion is in a dry and thirsty condition, eager to imbibe the ascending fluid. The electrical theory considers the flow of the sap as an operation of induction-that the buds of the twigs are the immediate instruments by which that induction operates-and therefore, that just in proportion to the identity of condition subsisting between the scion and the stock, will be the speed and certainty with which the union between the two is effected. What experience may finally determine," says this authority, "I know not; but I am prepared to say, that I have instances now before me-all of successful grafting-wherein the scions taken off the tree at the moment of 'grafting in the crown,' were more speedily knitted to the stock than others that had been cut off and kept in mould for some weeks before they were wanted."

Rhododendrons and azaleas, and most similar plants, succeed by grafting in March, using scions of last season's growth ; or they may with equal success be wrought in autumn, using scions of the same year's growth. Plants growing in hot-houses, and subject to artificial excitement, may be grafted at almost any season, if the wood be in proper condition.

Hard-wooded deciduous trees, such as the oak, elm, \&c., should be grafted in April, cutting the stocks down to within 6 inches of the ground, using scions of the previous year's growth, and, when clayed over, earthing up all round.

Succulent plants, such as Cacti, \&c., should be grafted in August; they succeed best when the scion is cut in form of a wedge, and inserted in the crown or along the angular sides of the stock: no claying is necessary, only a little soft moss or cottony matter wound round the part of union to prevent the entrance of moisture. Partial shade is necessary until the union has taken place.

The operation of grafting will be readily understood by a glance at fig. $99, a$ being the stock, and $b$ the graft or scion; as will be seen, a smooth sloping cut is made in an upward direction on the stock, while a similar cut is made in the scion, but, of course, in an opposite or downward direction. These two cuts are

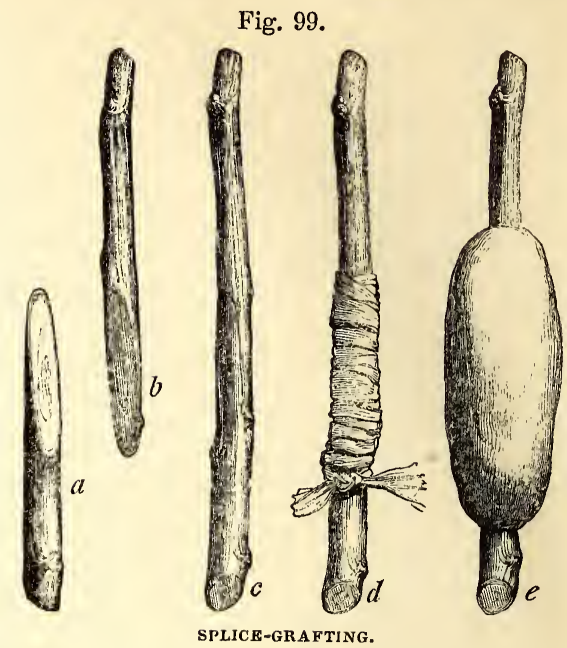

made so that the inner bark of the one corresponds with the inner bark of the other; they are then placed together $(c)$, and secured by matting $(d)$, to keep them in their places, and covered with clay or other adhesive matter $(e)$, to exclude air, \&c. The operation is most simple when both stock and scion are of exactly the same size; when otherwise, the operation must be varied to suit the circumstances: the chief point, however, is so to shape the incisions that the inner bark of the one may fit the inner bark of the other; and the more these are brought into immediate contact together, the greater certainty attends the operation.

Whip, splice, or tongue grafting, fig. 100.-This is the mode adopted by most nurserymen, as be-

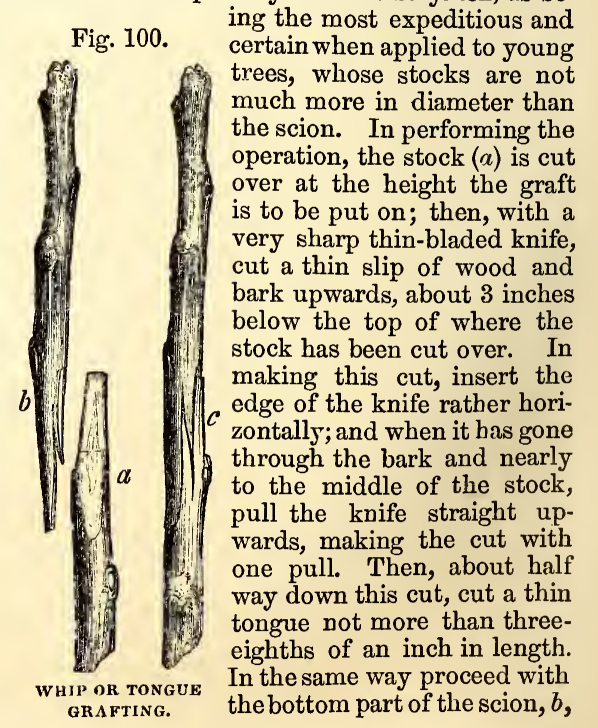


making a sloping cut of about the same length as the cut in the stock; then make a tongue to correspond with that in the stock, but cut upward instead of downward. The scion is then to be placed on the stock, inserting the tongue of the former into the tongue of the latter $(c)$; bring the two edges of the cut in the top of the stock and the two edges of the cut in bottom of the scion to meet exactly; or, if the graft be larger than the stock, so that both edges of the bark of the former cannot meet the corresponding edges of the latter, then let one of the edges meet-but this must be done with great precision. The stock and scion, being thus joined, must be bound closely together by soft matting, beginning to wind it round the stock an inch or two below the part grafted, and continued upward to the top of the stock; and this, if well done, will in many cases be sufficient to secure success even without the application of clay. A ball of well-prepared clay (some use graftingwax) is then formed round the stock and scion, when the operation may be considered finished. The use of the clay or wax is in order more effectually to exclude the air until the union takes place; and both this, and the chance of the clay falling off during dry weather, will be secured by drawing up earth around the ball so as nearly to reach its top-that is to say, when the graft is put on near the ground. When it is placed at a greater height, such as in the case of standard trees, then a little moss or soft hay tied over it will be equally efficient as a nonconductor of heat and moisture. The clay or wax envelop is intended also to preserve the graft in a uniform temperature, and so prevent the rising sap from being checked by cold days or nights. When the scion and stock are both of kinds that do not unite freely, the tongue is sometimes omitted, but in that case more care is required in tying. In the case of shoots having much pith, such as those of the rose, the graft is often put on the stock without being tongued into it.

In the French mode of whip-grafting there is no more pared off the stock than exactly the breadth of the scion; and in the early days of grafting in this country it was performed without a tongue, the addition of which gave rise to the term, as that of whip-grafting owes its name to the manner in which the scion and stock are cut so as to fit each other when secured in the manner of a whip-thong to the shaft or handle. The term splice is borrowed, evidently, from nautical phraseology.

Sometimes, in this mode of grafting, the stock is not shortened down to the scion, but a portion of it is left with a bud at its extremity, the better to secure the rising of the sap to the graft. Some operators cut a notch in the scion immediately under a bud, which notch is made to rest on the top of the stock; and if both stock and scion are of the same size, the top of the latter is certain of being healed over the first season.

A sub-variety of splice-grafting, fig. 101, is often practised in the case of peaches, "nectarines, apricots, and other tender shoots with large pith. It is found of advantage to have a quarter of an inch of two-year-old wood at the lower extremity of the scion, and to have the

stock cut with a dovetail notch.

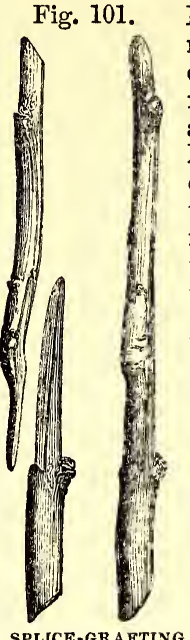

In the case of the fruit trees mentioned, the buds of the scion on the back and front are removed, leaving two on each side and a leader; and when these have grown 6 or 8 inches, their extremities are pinched off with the finger and thumb, by which means each shoot will throw out two others: this produces in autumn a finely-shaped tree with ten branches. Such trees will bear two or three fruits the second year from the graft." Gardeners' Magazine.

Downing describes American whip or tongue grafting as follows: Having chosen the stock, " cut it off at the point where it appears best to fix the graft. If the stock is quite small, it may be within 3 or 4 inches of the ground. Then, with a very sharp THE PEACH. knife, make a smooth cut upwards about 2 inches in length. Next make a slit from the top of this cut about one-fourth of the way downwards, taking out a thin tongue of wood. Cut the scion 4 or 5 inches long, or so as to have three buds; then shape the lower end with a single smooth sloping cut about the same length as that on the stock, and make the tongue upward to fit in the downward slit of the stock. Now apply the scion accurately to the stock, making the inner bark of the scion fit exactly the inner bark of the stock, at least on one side. Without changing their position, tie them together carefully with a piece of best matting or tape, and finally cover the wound with well-prepared grafting clay or wax. This ball of clay should more than cover the union by an inch above and below, and should be about an inch thick. If grafting-wax be used, it need not be above half an inch thick. About the middle of July," for plants in the open air, "after a rainy day, remove the ball of clay, and if the graft is securely united, also the bandage; and the angle left at the top of the stock should now be cut off smoothly, in order to allow the bark of the stock and the scion to heal nearly over the whole wound." Mr Downing further remarks, "Though it is little attended to in common practice, the amateur will be glad to know that the success of a graft is always greatly insured by choosing the parts so that a bud is left near the top of the stock, and another near the bottom of the scion. These buds attract the rising sap to the portions where they are placed from woody matter, and greatly facilitate the union of the parts near them, the upper part of the stock and the lower part of the scion being the portions soonest liable to perish from a want of nourishment."

Saddle-grafting (fig. 102) is practised only where the stock is of moderate dimensions. The stock $(a)$ is cut into a wedge-like form, and 
the scion $(b)$ slit up the middle so as to adapt it that it shall be seated across or ride upon the

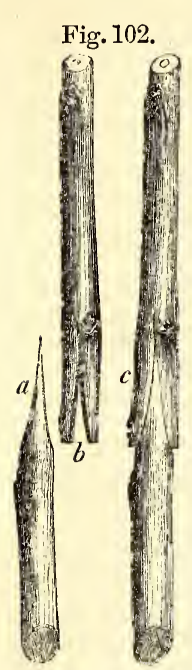
former $(c)$. This mode was invented by Mr Knight, and used by him chiefly upon cherry trees; and he states that even where the wood has been so succulent and imperfectly ripened as to preclude every hope of success by any other mode, he found this most successful. The advantage of this mode consists in offering the largest surface for the junction of the scion and stock, but, as in whip-grafting, the bark must, at least on one side, be neatly fitted to the bark on the other. Fig. 102 will show the principle.

A variety of saddle-grafting is much used in the Herefordshire orchards, of which fig. 103 is a representation. In this case the scion must be much smaller than the stock, and is split up between 2 and 3 inches from its lower end, so as to have one
SADDLE-grafting.
side stronger than the other.
This strongest side is introduced its lower end, so as to have one
SADDLE-GRAFTING.
side stronger than the other.
This strongest side is introduced Fig. 103. between the bark and wood,

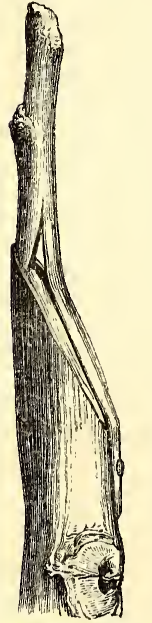
while the thinner division is fitted to the opposite side of the stock. Mr Knight, who described this mode in the "Horticultural Society's Transactions," says that the apple and pear grafted in this manner seldom fail, and that it may be practised with success either in spring upon last year's scions, or in July with the young wood of the current year. He attributes one advantage to saddlegrafting which must appear very clear-namely, that the sap is not driven from its natural course, but ascends from the stock to the scion freely, which is not the case when the scion is put on one side of the stock only, as in almost all other methods.

HEREFORDSHIRE Cleft-grafting appears to have SADDLE-GRAFTING. been one of, if not the most primitive of all modes, which may be naturally inferred from its great simplicity. Although easily performed, it has a clumsy appearance, but is yet very useful in the case of old trees requiring to be cut down from old age, disease, or other causes. Fig. 104 will show the principle. $\alpha$, the stock, of almost any diameter. Two grafts $(b c)$ are here shown, but there may be several, according to the size of the stock. The operation is thus performed: The head of the stock is first sawn over, and afterwards smoothed with a knife. A cleft is then made in the stock with a hammer and splitting knife, or as often with a carpenter's chisel. The scion is prepared as shown in our figure, and pushed into the cleft as the knife or chisel is withdrawn, taking care that the inner bark of the scion fits to that of the inner

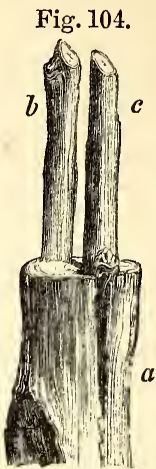
bark of the stock. When the number of grafts desired is inserted the whole is bound round with matting, and clayed in the usual manner.

"Apple stocks in many American nurseries," according to Downing, " are grafted in great quantities in this mode, the stocks being previously taken out of the ground, headed down very near the root, cleft-grafted with a single scion, sloping off with an oblique cut the side of the stock opposite that where the graft is placed, and then planted at once into rows, so as to allow CLEFT-GRAFTING. Only a couple of buds of the scion to appear above ground. It is not usual with many either to tie or clay the grafts in this case, as the wound is placed below the surface; but when this plan is adopted, the grafts must be set and the trees planted at once, drawing the well-pulverised soil with great care around the graft. Another way of grafting apple stocks common in some western nurseries consists in tongue-grafting on seedling stocks of very small size, cut back almost to the root. This is performed in winter by the fireside !- the grafts carefully tied, and the roots placed in the cellar in sand till spring, when they are planted, the top of the graft just above the ground."-Fruits and Fruit Trees of America. Cleft-grafting with one scion is in general not a good mode, because if the split has been made right through the stock, it is in danger of being injured by the weather before it is covered by wood by the scion. If the cleft is made only on one side of the stock, the evil is mitigated;another objection arises when only one scion is used-the tree becomes, as a matter of course, attached, as it were, to one side of the root only.

Cleft-grafting the vine.-This is sometimes done, and in the following manner: A spur is cleft, commencing the incision exactly above the second eye; the scion, when chosen, is cut in a wedge-shape form at its base, commencing the slope of the wedge immediately below a bud, so that when the scion is inserted into the cleft, the bud of the latter and that of the stock may be placed opposite each other, which is considered the most favourable position for success. The process of tying and claying goes on in the usual manner, with this exception, that a small hole is left in the clay opposite to the bud of the scion, to allow that bud to develop itself freely. When the scion has grown fourteen or fifteen days, it is then headed back to one bud, which is left to draw up the sap until the union has fairly taken place between the stock and scion. The proper season for grafting the vine in this way is when the shoots have made four or five leaves; if done sooner, there is a danger of the sap flowing so fast as to prevent a union taking place by what is called bleeding.

This process is often adopted in grafting suc- 
culent plants; and some Continental growers employ it in the propagation of roses and camellias. For the latter purpose it is of considerable importance, as each individual bud is made to form a plant, the scion being cut so that only one bud is attached to each.

Crown-grafting is merely a variety of cleft-grafting, and is sometimes called rind-grafting. It is practised upon old trees, either for their total renewal, or upon large amputated branches, to renew by degrees. It is, upon the whole, a better mode than cleft-grafting, because the stock, if old, is

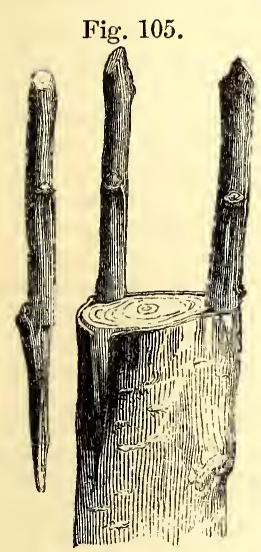
not subjected to the chance of being split, the scions in this case being placed between the bark and the wood. Fig. 105 will illustrate the process. It is performed later in the season than cleft-grafting - that is, from the end of March to the end of April -because then the bark separates more freely from the wood than at an earlier period. In rind or crown grafting, great care must be taken that the bark of the stock be not bruised during the process of opening the bark for the reception of the scion, and for this purpose a proper spatula or grafting-knife

CROWN-GRAFTING. should be used. Some use a piece of hard
wood, fashioned in form of a scion, and others one of bone or ivory ; the use of these is to separate the bark from the wood without injuring the former. The scion is prepared without a tongue, and is placed in the stock so that its wood may be in contact with the alburnum of the stock. Sometimes it is cut with a sort of shoulder at the top of the cut, that the graft may rest on the wood of the stock, and in this case it is called shoulder-grafting.

Fig. 106. The graft being prepared in either

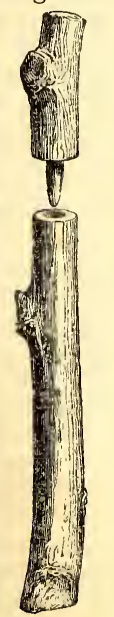
way, the bark of the stock is opened to the extent of 2 inches, and the scion is made to pass down between the wood and the bark. If the bark of the stock is hard and dry, and does not yield readily, it may be scored or cut perpendicularly downward, to enable the operator to open it, so that he may thrust the scion into its place with its cut side next to the wood: this being done, tying and claying is followed as in former cases.

Peg-grafting. - This is one of the oldest varieties of this mode of propagation, although now seldom used. The annexed cut, fig. 106 , and a brief description, will sufficiently explain its principles. The stock is cut over horizontally at the desired height, and a peg-arafting. hole is drilled in the centre to VOL. II. receive the end of the scion, and this hole must be in proportion to the size of the tree to be operated upon: if for a small tree (for it should be understood that the scion in this case may comprise the whole tree) or plant, a $\frac{1}{4}$-inch gimlet will be sufficient; but for one of large dimensions and spreading head an augur of 2 or 3 inches may be used. The depth to which this perforation should be made will, for obvious reasons, be determined by the size of the scion. The scion should also be of the same diameter with the stock, and so fashioned that a portion of its lower end is reduced, leaving only so much of the centre as will form a peg (from thence the name) or dowel, in the phraseology of carpenters, to fit exactly into the perforation, so that, when the surface of the section of the stock and scion come together, the bark and alburnum of each shall be exactly opposite to each other, which leads at once to the conclusion that the diameter of both stock and scion should be equal. When the scion is thus fitted on top of the stock, the graft or top of the tree is supported firmly in its upright position by props, to secure it against displacement by winds, \&c. The place of junction is carefully clayed round ; and if the operation is performed close to the ground (which, although not in all cases necessary, is in most instances expedient), is covered over with soil, the better to exclude the air. By this means trees of almost any size may be grafted, so long as we have mechanical power to elevate them, and keep them in their proper place. This was accomplished prior to 1817 by Dr Van Mons of Brussels, who inserted an entire tree on the stump of another. This rather uncommon effort in the art of grafting was shown to the late Dr Neill in 1817, who describes it as follows in "The Horticultural Tour," p. 310 : "A neighbour having, in the spring season, cut down an appletree about 15 feet high, which Dr Van Mons considered as a desirable kind, and a good healthy tree, he immediately selected Figs. 107, 108. a stock of similar dimensions,

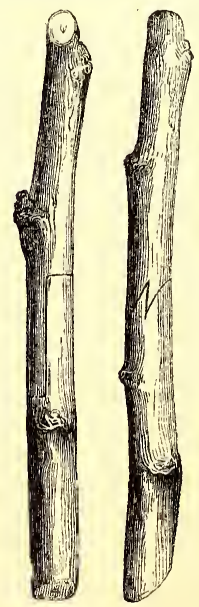
and, cutting it over near the ground, placed on it, by the mode of peg-grafting, the foster tree, supporting the tree by stakes, and excluding the air as we have noticed above." The experiment succeeded perfectly, the tree becoming, in course of the second summer, nearly as vigorous as ever.

The same tree we saw in 1832 , in as vigorous a state of health as any in the garden.

Whip-grafting in the French manner.-The French propagatorsnever pare more off the stock, whatever its size may be, than exactly the width of the scion.

Shoulder or chink grafting will readily be understood by the annexed figs. 107, 108. It SHOULDER-GRAFTING, is best adapted for trees or $2 \mathrm{U}$ 
branches of which the stock and graft are of the same diameter.

Side-grafting is merely a modification of whip or splice grafting, and is performed on a stock whose head is not cut off, or on a branch without its being shortened. The great utility of this mode is the facility it offers of supplying branches to parts of trees where they may have become too thin, or making a branch in case of accidents. It is usefully employed upon wall or espalier trees that have become naked of fruit-buds towards the centre, while they may have abundance towards their extremities. Branches of the same kind, or of other kinds if desired, may be by this mode put on at the base of the old branches, and the young shoots trained over them as they advance, thus providing young and bud-producing wood at those parts of the trees which must have otherwise remained in a state of sterility. The same may be done upon standard fruit-trees with a like effect, and ornamental trees and shrubs may be again clothed with young wood at those parts where, from mismanagement or otherwise, they may have become naked. There is only one disadvantage attending this mode, which is, as the branches on which the operation is performed have not been cut back, the scions are less likely to take: as the flow of the sap is not materially interrupted, it is apt to pass without forming the union between the graft and the branch. This may, however, be so far remedied by bending down the branch to as low an angle as possible, which will tend to moderate the rapidity of the sap's flow; or a tight ligature may be placed on the branch a little above the graft. The French have a variety of side-grafting, which they call veneergrafting, and which they employ in working young oranges and camellias. For this purpose they form theincision in the stock by taking a very thin slice out of it, and leaving a sort of shoulder at the lower extremity, upon which the scion is made to rest. This is one of the neatest modes in use, as, if well done, the union becomes so complete as scarcely to be detected afterwards. Another variety of side-grafting is practised on the vine in its dormant state. An opening is cut out of the branch to be operated upon from 2 to 3 inches in length, and to the depth of about half the diameter of the branch, the ends being cut obliquely, something in the dovetail form. The scion is cut so as to fit this space, tied round, but not clayed, as the branches should be laid down so that the grafted parts may be covered with moist soil. About a month thereafter the plants are plunged in a mild bottomheat, and in about the same length of time the young shoots will begin to show themselves. Wedge-grafting is also a modification of sidegrafting, and is advantageously employed in the propagation of the Deodora and other Coniferæ. We believe this method owes its origin to $\mathrm{Mr}$ Barron, who describes his plan in "The Gardeners' Magazine," and from which we learn that both the scions and stocks are of the wood of the preceding season's growth; that the slit in the stock is cut through the pith, and is from 1 to $1 \frac{1}{2}$ inches in length. The scions used are from 3 to 5 inches long; they are inserted in the usual manner, and grafting-wax is used instead of clay, as being lighter, and therefore less liable to bend down the heads of the stocks.

Root-grafting.- This method is often practised in nurseries, in cases where stocks of the species intended to be increased are scarce; and at other times, as in America and Germany, as has been already noticed, to economise time, as the operation can be performed during winter and under protection, so that, when spring arrives, they may be taken out and planted in nursery lines. One precaution ought to be taken in this modenamely, washing the top parts, at least of the root stocks, to prevent the possibility of earthy matter getting between the scion and stock. The mode of attachment may be any of those already described, as the whole difference consists in forming a union between a scion and a root instead of a stem. When the attachment has taken place, and planting is completed, draw up the earth around the neck of the plants so as to cover the point of union. The rarer species of oaks, for example, may be grafted upon the roots of the common sorts; and the otherwise useless roots left on the ground, upon removing old thorn hedges, may be used as excellent stocks upon which to attach pear-grafts.

Herbaceous grafting.-This is comparatively a modern invention, discovered by Baron de Tschudy, and consists in attaching the solid parts of herbaceous plants, or of woody plants when in an herbaceous state, to others of the same or sufficiently nearly allied species, while in a similar state. The tree Peony has been successfully grafted upon the fleshy roots of the herbaceous kinds of the same genus; the melon and cucumber, the potato and love-apple, have been frequently grafted upon each other; and almost the whole of the cultivated Brassicæ have been or are capable of being grafted on each other. These latter are, however, to be regarded more in the light of experiments than of useful

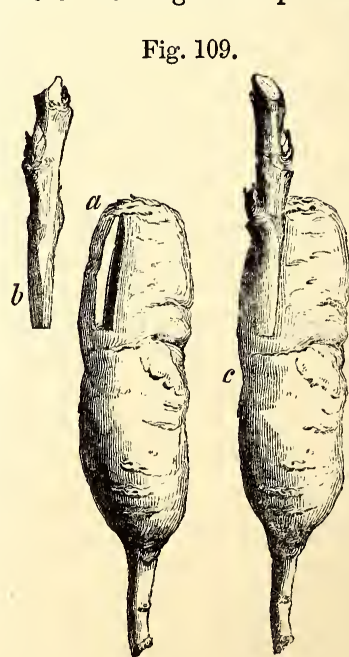
adaptations. In the case of the tree Peony, fig. 109 , the operation isperformed from the middle of July to the middle of August. The tubers throw out roots in autumn, and are then taken up and potted, and preserved under cover during winter. The operation is exceedingly simple, and consists in selecting single tubers of the plant, in which a triangularnotch, $a$, is cut near its top; to this notch ROOT-GRAFTING THE TREE PEONY. a scion of the tree sort, $b$, is made to fit, having two or three buds upon 
it; when placed, $c$, it is tied with soft matting, and clayed or waxed over in the usual manner. If the plant which supplies the scions be scarce, then one bud may be used instead of three. In selecting the tubers for this purpose, barren ones-that is, those having no visible eyes-are as good, if not possibly better, than those which have them. By this means dahlias may be multiplied largely, as every tuber is suitable for a stock, while only the crown of the whole root produces cuttings.

Grafting annual and perennial plants is possible ; we have yet, however, we confess, to learn its utility.

Expeditious grafting.-In nurseries and large plant establishments every means to secure stock of a new or rare plant is of vast importance ; and hence many ways have been tried to effect this end. Heat, moisture, shade, and slow excitement are the necessary conditions, and hence the operation is going on at all seasons, and the success attending it, in judicious hands, is truly astonishing. For as soon as a new plant is procured, an almost certain calculation is made according to the number of leaves or buds on it, that in a few weeks as many plants will be produced as there are leaves or buds on the original. The French adopt a very appropriate name for this species of propagation; they designate it Greffe étouffée, or stifled graft, from the process being carried on under closely-covered bellglasses to exclude the air. The plants, being small, are in pots plunged in bottom heat, and in this state they are kept until a union takes place. Almost every first-rate propagator has his own way of putting on his grafts, or rather buds; for, as we have said, every bud is made available, and expected to produce a perfect plant. The cleft form is often adopted, but almost any of the others, under judicious modifications, are equally applicable. Great care is, however, taken that the scion or bud be placed on the stock near to a bud, and this bud must be scrupulously preserved, as on that much of the success depends. When the bud or scion is placed on the stock, it is secured with a soft worsted, cotton, or other thread, and the place of union, as well as the top of the stock not covered with the scion, is covered with a thin coating of grafting-wax. Sometimes, however, a little moistened cotton or fine moss is used as a substitute. As the moisture arising under the bell-glass would soon become too great for the plant in its yet tender state, that covering is frequently removed, and wiped clear of the condensed steam; airis admitted, after the first three or four days, by little and little at a time, until the union has taken place, when it is more abundantly supplied, and ultimately the glass is removed altogether. The principal thing required in this delicate operation is neatness in execution, and rigid attention to the conditions we have named above.

Grafting Coniferce.-Somewhere about 30 years ago, the Baron Tschudy practised the grafting of the Pinus laricio on the common Scotch fir in the forest of Fontainebleau ; a description of his method has been published in "The Gardeners' Magazine." Various modes have since been adopted in Britain, in consequence of the increasing popularity of that natural order of trees, the difficulty of procuring seeds of many of them, and the increasing demand for their possession. Engrafting the Coniferæ, which a very few years ago was looked upon as requiring skill little short of necromancy, has now become an every-day affair in every respectable nursery establishment. The rationale, however, of Tschudy's theory is no doubt the foundation of most of our modern manipulations. The pine and fir tribe is successfully grafted about the middle of June, at which time the young shoots will have attained somewhat more than half their year's growth. An inch or two of the top of the shoot, the leader being preferred, should be cut off; the leaves to the extent of 3 inches should be cut off, leaving, however, a pair of

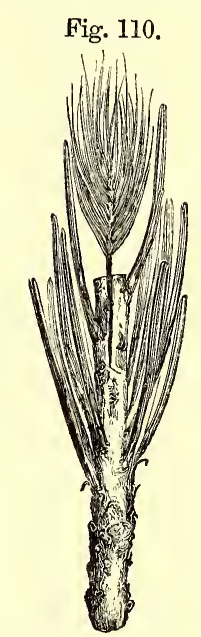

GRAFTING CONIFERS.

Fig. 111. leaves, fig. 110, opposite and close to the section or point where the shoot was cut over : these, being retained, will draw nourishment to the extremity of the stock before the scion becomes fully attached. The shoot is then split down between the pair of leaves to the depth of 2 inches, and the scion, which,in our example, is that of Abies morinda (the stock being Pinus cembra), having its lower leaves cut off, and a thin portion of its sides pared off, is inserted to the. full depth of the incision in the stock, in the same manner as in cleft-grafting. When the scion is much smaller than the stock, as in our example, the scion is kept close to the side of the stock, so that the bark of both may unite. Two scions, one on each side, may be put on, one of which may be cut away afterwards, should both take-the two giving a double chance of success.

The usual process of tying the graft and claying completes the operation, or, in the case of feeble subjects, grafting-wax may be employed as a substitute for clay.

Fig. 111 shows a variety of wedge or side grafting, we believe first practised by Mr Barron at Elveston, and successfully employed in the case of coniferous trees. The scion in our example, fig. 112 , is the Deodar cedar, and the stock the Pinus cembra. $\mathrm{Mr}$ Barron used the cedar of Lebanon as a stock. He also used scions of 
the preceding year's wood, and inserted them in wood of one or two years' growth. We have

found, when the stock was Fig. 112. strong, as the leading shoot of Pinus cembra, or other similar growing Conifera, that the current year's shoot, when about three parts grown, is admirably suited for the purpose; and also, as in our illustration, when the scion has grown to about the length of 2 inches, and with 2 inches of last year's woodwhich latter is inserted in the young wood of the stock, cutting the latter quite through the pith to the depth of $1 \frac{1}{2}$ or 2 inches, and securing it in the usual manner. The Pinus cembra may be objected to by some, on account of its less robust growth; but the same success has followed when

SCION OF CONIFER PREPARED FOR GRAFTING.

the common spruce, and even the Scotch fir, have been used.

Grafting coniferous trees is a process had recourse to mainly to multiply the rarer species and varieties of which seeds are difficult to procure. So far as perpetuating some of the rarer species to fill up the blanks in botanical and systematic collections, until others originated from seed can be obtained, we see no great harm in having recourse to this mode of propagation; but here the limits of that species of increase should cease. Some have endeavoured to promulgate the theory that grafting has the effect of rendering certain species-those from Mexico, for example, which are too tender to stand our northern climate-more hardy, if wrought on hardy and free-growing stocks; a doctrine we cannot by any means subscribe to, more than that the same opcration would have the effect of causing trees of this order to assume the longevity, size, and full development of those originated from seeds. Such, however, is at present the rage for pines, that a glance at the stocks most fitting for the purpose of grafting may not be considered out of place here. The larch has been long used as a stock for the deodar, on which it takes readily; but seeds have been so abundantly imported of that magnificent tree, as to render both the process of grafting, and striking it from cuttings, obsolete matters. The cedar of Lebanon has been also employed for the same purpose, but the stocks in this case are as valuable as the species to be wrought upon them. The Scotch fir (Pinus sylvestris) makes a good stock for Pinus Banksiana and its congeners. Pinus austriaca (the Austrian pine) is probably the best stock for the majority of the tribe; it is a free grower, and transplants well, even when of a considerable size. The Weymouth pine (Pinus strobus) has been recommended as a stock for Pinus Lambertiana, $\boldsymbol{P}$. monticola, and those allied to them; but who would think of choosing as a stock a tree that is by no means adaptcd to our climate? -indeed, so much so, that few good specimens of it are to be even met with. The pinaster (Pinus pinaster) is a stock on which Pinus Lemoniana and its allies will take freely and grow rapidly for a time ; but the days of this, as of all other grafted pines, are few, compared with those originated from seed. The spruce fir forms the general stock for the section of Abies; while Juniperus Virginiana (the red cedar) is suitable to the whole of the free-growing junipers, and many of the cypresses also ; and the common yew to the whole genus Taxus: the more delicate species, however, will succeed better on stocks of the upright or Irish variety.

Grafting soft-wooded or succulent plants.-The object here is more of an ornamental than of a useful character. The beautiful Epiphyllums truncatum, Russellianum, \&c., are slow-growing plants on their own roots, but become exceedingly beautiful when grafted on the tall and stronger-growing species of Cacti, such as Pereskia, Opuntia, Cereus, \&c. The operation is simple, and requires the scions to be taken just as they are beginning their growth: they are inserted in the stocks by simple incision, and secured, for a short time, from falling off by a slight bandage of matting. They are found to succeed best when placed in a rather shady place till adhesion takes place.

Many varieties of pelargonium may also be wrought on the same stock, which, for conservatory specimen plants, has a pleasing and rather novel appearance. The cleft mode of grafting is adopted generally on the Continent, choosing the stock and scion of nearly the same size, and placing them under a glass shade ; or if large, in a close somewhat shaded place for a few days. About fifteen days will in general effect a union of the parts. According to a paper in the "Révue Horticole," it would appear that grafted pelargoniums have continued healthy for many years.

Inarching, or grafting by approach, differs only from most kinds of grafting we have referred to, in having the scion still attached to the parent tree, from which it is deriving support until the union with another takes place, when it is separated entirely. Professor Thouin enumerates thirty-seven varieties of this mode of propagation. These, however, may practically be reduced to two-namely, crown inarching and side inarching, the former having the head cut off at the time of operating, and the latter retaining the head for a time. The spring is the season for performing this operation generally, but any season when the sap is in proper condition, as may be the case in hot-houses, is equally proper. This species of grafting is the only one to be met with in nature, and that only in the case of plants of near propinquity ; it may also have given the first idea of grafting to man. Inarching is productive of the same effects as grafting, in inducing an early disposition to fruit-bearing; it is also applicable to plants that are difficult to propagate by other means.

Method of performance.-For this purpose it is necessary that the plants to form both the stock and scion should be either growing near to each other, or, if in a portable state, placed so that their branches may meet. A portion of 
the bark is then removed from each at theintended point of union, say from an inch to three inches in length, according to the size and strength of the subjects; these parts are fitted together so that the inner barks of both coincide, and the process of tying and claying or waxing follows as in common grafting. Some, however, tongue the scion and stock in the same manner as is done in tongue-grafting. In either way the cambium shortly becomes developed, the alburnum of the scion and stock becomes united; and when this is accomplished, the scion may be cut off a little below where it is united to the stock, either at one operation, or only partially cut through first, and in a few days completely severed. A week or two after the union has taken place, the remaining portion of the scion, if any was left on when separated from the parent plant, should be cut off close to the stock, that the wound may heal over, and leave the stem as perfect as possible. Side inarching without tonguing is well adapted for young shoots of camellias, oranges, \&c. ; and when the wood of both scion and stock is soft, and full of sap, a union speedily takes place. Tongue inarching is useful when both or either stock and scion are more fully matured, and are somewhat hard and dry.

Inarching with partially-nourished scions only differs from the modes we have described above, in having the lower end of the scion inserted in a phial or bottle of water. This water should be changed from time to time, and at each renewal a very thin slice should be removed from the scion, by which its vessels will be kept open, and be able to draw up sufficient support to the graft until adhesion has taken place. Sometimes the end of the shoot is placed in a pot of damp soil, and at others stuck into a potato or turnip.

Grafting-clay is prepared by mixing three parts of strong clay with one of horse or cow dung, separated from straw or litter, but having a small quantity of hair, such as is used in preparing plaster lime, mixed with it, to render it more adhesive and less liable to fall off. This mixture should be well incorporated and spread out on a hard flooring, and beaten with mallets or clubs, turning it frequently and giving a little water, and beating again until reduced to a very strong plaster, and fit for being put on with the hands, and for remaining on both in wet and dry weather without crumbling or cracking. The addition of the hair will be found better than using straw or hay, as is usually done. Some recommend the addition of a little salt, with a view to preserve the moisture, which, if not too abundantly applied, may have its advantages.

Grafting-wax.-Of this there are many forms, the principal of which are wax and rosin, wax and pitch, tallow and rosin or pitch melted together, and used in a melted but not in a very hot state. Sometimes these are applied in the form of dough or putty; at other times they are spread on paper, cotton, linen, \&c., and cut into narrow strips. For very delicate plants the latter forms are the best, and we believe that, for ordinary purposes, for exotic plants, cobblers' wax is better than any, as it can be applied in a cold state; whereas the heat at which most of the others will be of sufficient consistency and ductility for putting on must be too great for very tender plants, more especially when in a soft and herbaceous state. One of the best preparations for this purpose is that recommended by Mr Powell-viz., 1 lb. of pitch, $1 \mathrm{lb}$. of rosin, $\frac{1}{2}$ lb. bees'wax, $\frac{1}{4}$ lb. hogs' lard, $\frac{1}{4}$ lb. turpentine, melted and mixed well together ; this composition to be, while in a fluid state, spread upon sheets of moderately thin brown paper, which, when cold, is to be cut into slips about three quarters of an inch in breadth. The scion, when fitted to the stock, is to be wound round with one of these slips, which, when breathed upon, becomes soft and limp, and when cold, sufficiently adhesive to remain on until purposely taken off. Plastic wax, which is as pliable as the last, is thus prepared: Common sealingwax, any colour except green, one part; white wax, one part ; mutton fat, one part ; and honey, one-eighth of a part. Melt the fat and white wax first, add the sealing-wax gradually in small pieces, and lastly, put in the honey just before taking it off the fire; pour it into tin moulds, and keep shaking it till it begins to congeal. When breathed upon, or similarly slightly hcated, it is fit for use.

In grafting, there are certain important requisites which must be attended to, whatever mode of performing the operation may be adopted. These requisites are thus noticed by Professor Balfour, in his recently published excellent "Class Book of Botany," p. 659: "In the case of dicotyledonous trees, carc must be taken to bring the growing parts into contactthe two alburnums and the two libers. We cannot expect that the old wood of trees, in which the active processes of plant life have ceased, will unite. The plants on which grafting is practised must be botanically allied, or, at all events, there must be a similarity in the composition of their sap. Union may take place between plants which, in their natural state, require the same chemical ingredients in the same proportions. This is generally the case with varieties of the same species, more rarely with plants of different species, and least frequently with such as belong to different genera. The lemon may be grafted on the orange, because, as Lindley says, the sap of the latter contains all the earthy and saline substances which the former requires, and can supply them in sufficient quantity to the engrafted twig. But the fig or the grape would not flourish and ripen fruit on the same stock, because these fruits require other substances than the root of the orange extracts from the soil, or in greater quantity than the sap of the orange can supply them. The sap of the stock, in order that grafting may be successful, must contain all that the engrafted bud or shoot requires in every stage of its growth. If the potash or lime required by the grape be not taken up, and in sufficient quantity, by the root of the orange, it will be in vain to engraft the former upon the latter, with the hope of its coming to maturity or yielding perfect fruit. Grafting of varieties on the stem of the species whence they are derived is very common, and is con- 
stantly practised with roses, camellias, apples, \&c. Species of the same genera are also frequently united. The peach may be grafted on the almond, the apricot on the plum, the plum on the cherry, and the pavia on the horse-chestnut; but the operation will not succeed between the horse-chestnut and the almond. Genera of the same natural order also may sometimes be united : thus, the pear may be grafted on the quince, or on the thorn, or on the amelanchier, all of these belonging to the natural order of Rosaceæ. The lilac is said to adhere to the ash and to Phillyrea latifolia; the olive to the ash; the chionanthus to the ash and lilac-all belonging to the natural order Oleaceæ. The chestnut may be grafted on the oak, in the family Amentiferæ; and Bignonia radicans on Catapala, in Bignoniaceæ. There are marked instances of plants which seem to be allied, and yet which cannot be grafted on each other. Thus chestnuts will not graft on beeches, nor apples on pears. As regards the persistence of leaves, it is generally necessary that the plants should correspond ; but to this there are exceptions. Some evergreens can be grafted on deciduous plants. Prunus laurocerasus and $\boldsymbol{P}$. lusitanica (the Portugal and common laurel), both evergreens, live for some time grafted on the birdcherry, and are less sensible to cold than those growing on their own roots. Eriobotrya japonica and $E$. glabra, also evergreens, live for a very long time grafted on the hawthorn. The cedar of Lebanon, grafted on the common larch, lives upwards of ten years, but it remains stunted and dwarf." The instances of parasitic plants, like the loranthus, mistletoe, \&c., adhering to other plants of widely different genera, cannot be fairly stated as exceptions, as the process by which they adhere is not common grafting. "The seeds, and not the buds or slips, are applied to the stems on which they grow, and certain root-like processes are sent inwards." Mr Beaton, Mr Moss, and others, have stated that buds or slips of mistletoe have been made to adhere.

Grafting has the effect of causing some plants to become more hardy and others more tender, and also of causing plants to produce their flowers and fruit at an earlier period of life than were they at once originated from seed; and Knight thought that the fruit upon grafted trees ripened earlier in the season than on trees of the same age, and of the same kind, not grafted, or even grafted upon stocks of their own species; and that the growth and vigour of a tree, and its power to produce successive heavy crops, are diminished apparently by the stagnation in the branches and stock of a portion of that sap, which in a tree growing upon its own stem, or upon a stock of its own species, would descend to nourish and promote the extension of the roots. When the stock is by nature weaker than the scion, or incapable of supplying it with as great an amount of sap as if the supply was derived from its own roots, without any such interruption, then the tree grafted will be limited in the period of its existence in proportion to the scanty supply of sap; and on the other hand, the scion of a weakly variety will gain strength and increased longevity when worked upon a stock capable of supplying it with as much or a greater quantity of sap than its roots would supply. But much of all this depends on the accuracy of the operation, for a weakly scion may be inaccurately fitted to a stronger stock, and hence an interruption to the ascent of the sap will take place, and consequences directly opposite to what is anticipated are certain to ensue. It is, therefore, probable that it is more owing to the bungling way in which the operation is performed that grafted plants and trees so frequently die a year or two after they have been grafted, and that some others, although they may continue living for a greater number of years, fail in assuming a robust and healthy appearance. The nature of the sap, in some trees, may have its share in this ; and it is probable that to either or both of these may be attributed the repeated failures in securing healthy and robust young trees in the order Coniferæ.

Monocotyledonous plants were long considered as incapable of being grafted. Signor Calderini of Milan published, in the "Annales des Sciences Naturelles," an account of some experiments he made on grasses of different species. This experiment was made with a view to improve the vigour of delicate and tender varieties, by grafting them on more robust stocks; and in this he succeeded. The modus operandi was from having observed that these plants have at each knot a shoot enclosed in the sheath of the leaf, which can be easily drawn out while the plant is young. He introduced some of these into plants of the same species, having previously removed their young shoots, and found that half of them succeeded. The experiment, we fear, goes no further than to resolve a physiological problem, which is in itself sufficiently interesting: the practical advantage appears to us to be very slight.

\section{$\S$ 4.-PROPAGATION BY BUDDING.}

Budding is the operation of transferring the buds of one tree to the branches of another; and its use is the propagation of plants which could not be effected at all, or much less conveniently, by the other modes of extension, such as striking by cuttings, grafting, \&c., as well as multiplying a species or variety more expeditiously than by either of the other modes of propagation-as one bud in this case is sufficient in itself to form the future plant, when planted on the alburnum of another of the same or very nearly allied species, while several buds are required in all other methods of propagation. In transferring the bud of one plant to the wood of another, they become vitally united together, as in the case of grafting, which can only be regarded as budding in another form. This process is chiefly employed on woody plants and trees, although it is sometimes performed upon herbaceous plants also, but never on annuals. The varieties of budding are numerous, - much more so than useful. Professor Thouin enumerates twenty-three methods by which the operation is performed; and it is probable that 
ten or a dozen more have been added since he wrote. These for the most part owe their origin to foreign cultivators, who are not only expert in performing the operation, but attach considerable importance to some or other of the various modes. Few comparatively of these are practised by the British gardener, who very judiciously calculates more on success than on manipulations from which apparently little is to be gained. All the modes are, however, based upon the same principle-viz., the union between the bud and stock, which takes place, in the first instance, in consequence of the exudation of organisable matter from the soft wood or alburnum of the stock; and that union is rendered permanent and complete by the returning sap from the leaves of the stock, or from those of the shoots made by the bud.

The rationale of budding has been thus laid down by Mr George Lindley, in "Guide to the Orchard :"- "Budding differs from grafting in this, that a portion of a stem is not made to strike root on another stem, but that, on the contrary, a bud deprived of all trace of the woody part of a stem is introduced beneath the bark of the stock, and there induced to strike root. In this operation no care is requisite in securing the exact contact of similar parts, and a free channel for the transmission of the roots of the bud between the bark and wood of the stock; for, from the very nature of the operation of budding, this must of necessity be insured. The bark of the bud readily coheres with the wood of the stock, and secures the bud itself from all accidents or injury. But if precautions of the same nature as in grafting are not requisite in budding, others are of no less moment. It is indispensable that the bud which is employed be fully formed, or what gardeners call ripe. If it is imperfectly formed, or unripe, it may not be capable of that subsequent elongation upwards and downwards upon which the whole success of the practice depends. Secondly, great care should be taken, in raising the bark of the stock for the insertion of the bud, that the cambium be not disturbed or injured. The cambium is a secretion between the wood and bark, not only destined to support the descending fibres of the buds, but also to generate that new cellular substance within which the descending fibres are finally found imbedded.

"If, in the preparation of the bark for receiving the bud, this cambium be injured or disturbed, it becomes much less capable of effecting the cohesion that is necessary than if uninjured. In budding, therefore, the bark should be carefully lifted $u p$, and not forced from the wood with a bone or metal blade, as is usually done; for although it is no doubt true that an operation clumsily performed will often succeed, yet it should be remembered that, if skilfully managed, it would be attended with much more success; and that a habit of continually operating with delicacy will enable the gardener to succeed with certainty, in cases in which a bungling practitioner would be sure to fail."

Besides the mere purpose of propagation, budding induces important changes in the condition of plants; as, for example, buds trans- ferred from a seedling tree or shrub, and placed on one of greater age, will produce fruit and flowers sooner than if left on the parent plant. Weak-growing plants, budded on stocks of a stronger character, become strengthened; and hence the weaker-growing vines attain greater strength when budded on the Nice or Syrian. The double yellow rose, which seldom flowers naturally, will do so if budded on some of the stronger-growing China varieties; and almost all weak-growing plants, if wrought upon strongergrowing ones of the same species, will be equally strengthened.

Almost all buds are formed at the axle of the leaves or extremity of the branches, and contain the rudiments of future leaves, branches, or fruit, as well as in many cases the means of propagation. Buds do not spontaneously disengage themselves from the plant to form new individuals, but they may be taken off by man, and artificially made the means of producing future plants. When buds are situated as above, they. are called normal or irregular buds, to distinguish them from those that are placed around the collar of the plant, upon the leaves or stem, or otherwise not being axillary or terminal, and which are called adventitious. Some plants do not produce buds, such as annuals; and even many trees in tropical countries are equally destitute of them, while in cold countries they universally exist.

The season for performing the operation of budding is in spring, when the sap is rising, and in the end of summer-say July or Augustwhen the sap is descending. In the former case the inserted bud develops itself immediately, while in the latter this development does not take place till the spring following. At other seasons, also, the operation has been successfully accomplished, much depending on the kind of tree, and also on whether it be an early or late season. In budding at an early season, it is of importance to insert the bud with the whole of its wood attached; but as the season advances, and the buds have attained their full maturity, the less of this woody matter that is retained the better. "Budding in spring or early summer is generally accompanied, it may be presumed, by a copious current of sap. Not so, however, late summer-budding on all occasions; for the season may have been unusually warm and dry, the stock may be short of sap-or, in other words, be beset with a paralysed root action. All these are impediments. A copious watering the evening previous to the process will, however, promote the free rising of the bark, on which so much depends. In addition to this, a cloudy day is preferable to a sunny one." - Cott. Gard. Dict. Mild cloudy weather is in general preferred for this operation, because the viscous surfaces exposed to the air are more liable to be dried up by evaporation, by which the healing process is retarded, if not entirely prevented; besides, in dry windy weather the bark will not rise so well. This opinion has, however, been of late questioned by $\mathrm{Mr}$ Saul of Bristol, who says, in the "Gard. Mag. of Botany," "that in warm weather the sap is more gelatinous; and the bud, on being extracted and inserted in the 
stock quickly, properly tied, \&c., adheres more readily. On the contrary, in wet cloudy weather the sap is more thin and watery, and the bud will not unite so freely." To this he adds, " that a fall of rain-likely in such weather-after the buds are inserted, will fill up the incisions, and thereby rot and perish the buds before they have time to unite with the stock. Not only is clear weather best for the experienced budder, but likewise for the amateur and tyro."

Much of the success depends on the state of the bud. "The mature bud," Mr T. A. Knight, in "Transactions of the Horticultural Society," vol. iii. p. 136, observes, " takes immediately with more certainty, under the same external circumstances; it is much less liable to perish during winter, and it possesses the valuable property of rarely or never vegetating prematurely in the summer, though it be inserted before the usual period, and in the season when the sap of the stock is most abundant. I have," he says, "in different years, removed some hundred buds of the peach-tree from the forcing-house to luxuriant shoots upon the open wall; and I have never seen an instance in which any of such buds have broken and vegetated during the summer and autumn. But when I have had occasion to reverse this process, and to insert immature buds from the open wall on the branches of trees growing in a peach-house, many of these, and in some seasons all, have broken soon after being inserted, though, at the period of insertion, the trees in the peach-house had nearly ceased to grow." The season of budding, or rather the state of the plant, is of primary importance in securing success. "Buds should either be inserted," says Dr Lindley, in "Theory of Horticulture," p. 244, " when the vegetation of a plant is languid, or growth above the place of insertion should be arrested by pinching the terminal bud; otherwise the sap which should be directed into the bud, in order to assist in its adhesion, is conveyed to other places, and the bud perishes from starvation. For similar reasons, when a bud begins to grow, having firmly fixed itself upon the stock, the latter should be headed back nearly as far as the bud, so as to compel all the current of sap to flow towards it, otherwise the buds of the stock itself will obtain that food which the stranger bud should be supplied with."

Much of the success in budding depends on the condition of the bud at the time of its insertion; yet even as to that condition opinions are by no means agreed. We have stated that such buds as appear to have lost their root or heart by the operation of extracting the woody matter under them, are useless, and should be discarded. This is denied by Mr Lymburn, who asserts that blind buds owe their origin to the bud itself being diseased, more than to the supposed injury they sustain in the removal of the piece of wood connecting the bud with the alburnum. A writerin "The Gardeners' Chronicle," 1842, on this matter remarks: "The bud will sometimes take although the heart appears to be gone ; it will sometimes adhere and start quickly, although more wood be left in the shield than will allow the sides to lie flat down; but the evident desideratum is, that no more of the wood adjacent to the eye be left than will secure its close attachment to the shoot upon which it may be placed, nor any less than may suffice to retain the growing principle, the limit of which must be defined by a combination of theory and practice, and at present remains a problem to be solved. Many buds have I inserted, in which the eyes have not been sufficiently swollen, and no produce has come forth; and many a bud have I inserted, in the hope that the cambium would fill the vacant hole, which fear told me was too large, yet which a scanty supply of buds induced me to retain-but all in vain; for though the bark adhered the eye was lost, and many a wood bud inserted thus has become dry before it could adhere. I believe the great secret to be, taking the bud in its proper state-i. e., full formed (not too near the base of the stock, from which it will part with difficulty, nor too near the top, because insufficiently ripe)-and toinsert it when the receiving plant and the weather are in a favourable state to continue the elaboration of those juices necessary to form a junction. The period of the year is, comparatively speaking, immaterial. I have inserted buds at all times, and have now in my possession a plant that was worked on the 21 st October, ten years ago. Shoots that grow angularly, and are nearly the same size all the way up, afford better buds than such as are produced on long rapid-growing branches: the buds on the latter are seldom well defined, and if inserted at all hollow in the centre, are sure to go blind."

Peculiar advantages of budding.-To those noticed above we may add the facility that the process affords of producing a shoot in almost any part of the tree, at the will of the operator, where, without this transplanting of a bud, no branch could be induced to form. Many varieties may by this mode be made to grow on a single tree, which no other species of grafting could effect so completely. Many stone-fruits are multiplied by this means, which could not be so successfully accomplished by the other species of grafting. And, lastly, the buds of young fruit-trees, when transplanted to the branches of full-grown ones, will be induced to produce their fruit, which, if left on the parent stem, might not do so for years. Mr Knight, by transferring the blossom buds of an abundant fruit-bud-producing tree to the branches of those which do not show these organs so profusely, or even sparingly, caused excellent crops of fruit thus to be produced on the latter.

Natural advantages of budding.-Trees propagated by this means are thought to be of longer duration than those that are grafted; they also are in general two years longer in producing fruit than grafted ones of the same species. The process is employed on most stone-bearing fruit trees, as in their case grafting is apt to bring about a disposition in them to throw out gum at the point of union. Trees, also, which may have failed to take by grafting in spring, may be budded in July or August - an important matter in fruit-tree nurseries. Most woody plants may be multiplied by budding, many of which could not be operated on by grafting. 
Preliminary process.-The state of the bark, both in the bud and in the stock, must be attended to, as the bark in both cases must separate freely from the wood beneath; for if either be unfit in this respect, there will be little chance of success in the operation. This is best ascertained by making trial incisions, and if both are found in a proper state, operations should be at once commenced. If the bark do not rise freely, the process should be delayed for a few days; and this state will often be accelerated if copious supplies of water be given at the roots, more especially in hot dry weather. The buds are then to be cut from the parent tree, not individually, but the shoots of the current year on which they are growing are to be removed, their leaves cut off, leaving a part of the leafstalk to each, to serve as a handle in inserting the bud, as well as to act as a protection to it. The reason for cutting off the leaves is to prevent the loss of sap by evaporation; and branches so treated may be sent to a great distance without injury to the vitality of the buds, if they are carefully packed in an air-tight case. While the operation of making the incision for the bud in the stem of the stock is going on, the cuttings should be placed handy, kept under shade, and with their lower ends in a pan of water. As each incision is made a bud is cut from the shoot, and when prepared, is inserted as quickly as possible, and fixed by coiling a strand of fresh matting, \&c. around the bud and stock - which matting should be kept in a moist state, and handy to the operator. Such arrangements are necessary in most sorts of budding.

Shield-budding, in one or other of its modifications, is that most generally practised in Britain, and, with flute-budding, may be said to embrace all the modes of the operation really useful.

Shield-budding in spring is much practised by the Continental cultivators, and is found expedient at other seasons, in the case of roses, camellias, and many other flowering plants. For Diduous trees like the

$$
\text { Fig. 113. Fig. 114. apple and pear, the buds }
$$

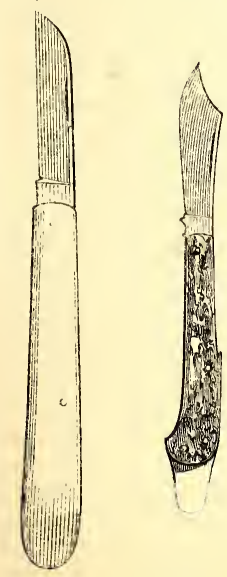

COMMON AND FRENC BUDDING-KNIVES. VOL. II. a portion of the wood is removed with the bark in which the bud is situated. The bark of the stock is then cut in manner resembling an inverted T; the greatest care should, however, be taken that the edges of both the cuts in the stock and bud be cut clean, so that they shall, when brought Fig. 115. and uniform union. In the

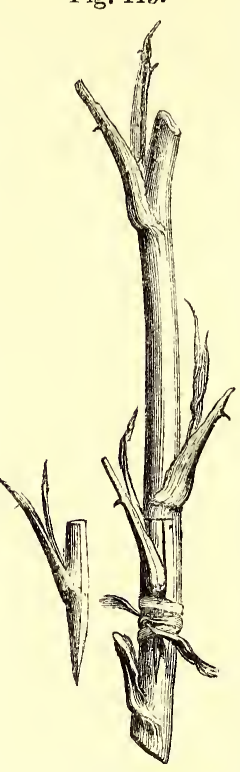

SHIFLD-BUDDING THF BOSE. case of the rose, the stock should be pruned down about eight days after the bud is inserted to the shoot above it on the opposite side of the stock, which shoot should be shortened by being pinched back to two or three eyes, removing at the same time all the side shoots as they are produced; and when the shoot formed from the bud has extended to its fifth leaf, the point should be pinched off, which will cause it to branch out, and, in all probability, induce it to flower in September of the same year. The rose is also budded in spring, even before the sap rises in the stock, in the following manner: A niche is cut out of the stock, say an inch or so in length, as in fig. 116. A bud is taken of the same size and form, retaining a small portion of the wood; the bud is then nicely fitted to the niche, and secured by coiling a strand of soft

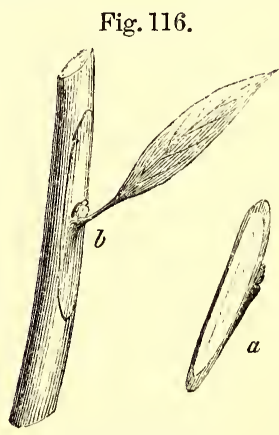

SHIELD-BUDDINA THE CAMELLIA. matting round it. In the same manner the camellia, orange, and rhododendron may be budded, only in their cases the entire leaf should be left attached to the bud. A modifcation of this manner is often resorted to in the case of the latter plants - namely, by taking a bud, $a$, with a portion of the wood attached to it, and cutting out a similar piece from the stock, $b$, and substituting the onefor the other.

In the case of some very delicate plants, cotton wool or very soft moss is placed round the bud, and secured by passing a strong thread round it, to keep it firmly in its place. In ordinary cases, however, the expert budder uses nothing but fine strands of bast matting, and the success as well as the expedition with which this nice operation is performed in our British nurseries is truly wonderful.

The rationale of budding is thus clearly 
explained by Dr Lindley, in "Theory of Hort.," p. 223: "By these means we gain the important end of bringing in close contact a considerable surface of organising matter. The organisation of wood takes place on its exterior, and that of the bark on its interior surface, and these are the parts which are applied to each other in the operation of budding; in addition to which the stronger bud finds itself in its new position as freely in communication with alimentary matter, or more so, than on its parent branch. A union takes place of the cellular faces, or horizontal system of the stock and bark of the bud; while the latter, as soon as it begins to grow, sends down the woody matter or vertical system through the cellular substance. In consequence of the horizontal incision, the returning sap of the scion is arrested in its course, and accumulates just a little above the new bud, to which it is gradually supplied as it is required. Sometimes the whole of the wood of the bud below the bark is allowed to remain; and in that case contact between the organising surfaces of the stock and scion does not take place, and the union of the two is much less certain. As it is, however, usually practised with tender shoots before the wood is consolidated, the contact spoken of is of less moment. In all cases, a portion of the wood of the bud must be left adhering to it, or the bud will perish ; because its most essential part is the young woody matter in its centre, and not the external surface, which is a mere coating of bark." This shows the necessity, in carefully removing the woody part of the bud, of preserving the root, as it were, of the bud itself, as has been already said.

The Americans do not extract the wood from the bud, but leave it in as cut from the shoot, and this may possibly suit their hot, dry climate; but from experiments made by Mr Saul of Bristol, who has practised both methods in the extensive nurseries at Durdum Downs, his conclusions are in favour of the English modenamely, of extracting the wood from the bud. Sometimes, he says, there may be an advantage in putting in the wood-namely, when the shoot has become firm, and the wood and bark will not freely separate ; then, if inserted in a freegrowing stock, it will in all probability take. The evidence of both writers and practitioners in both countries is so divided upon this subject, that we shall here transcribe the American practice, as given by Mr Downing, one of their best authorities, in his useful work, "Fruits and Fruit Trees of America."

American shield-budding. - "Having your stock of buds ready, choose a smooth portion of the stock. When the latter is small, let it be near the ground, and, if equally convenient, select also the north side of the stock, as least exposed to the sun. Make an upright incision in the bark from an inch to an inch and a half long, and at the top of this make a cross cut, so that the whole shall form a $\mathrm{T}$; from the stick of your buds (your knife being very sharp) cut a thin smooth slice of wood and bark containing a bud; with the ivory heft of your buddingknife now raise the bark on each side of the incision just wide enough to admit easily the prepared bud. Taking hold of the foot-stalk of the leaf, insert the bud under the bark, pushing it gently down to the bottom of the incision. If the upper portion of the bud projects above the horizontal part of the T, cut it smoothly off now, so that it may completely fit. A bandage of soft matting is now tied pretty firmly over the whole wound, commencing at the bottom, and leaving the bud and the foot-stalk of the leaf only exposed to the light and air." $\mathrm{Mr}$ Downing continues: "The American variety is found greatly preferable to the European mode, at least for this climate. Many sorts of fruit trees, especially plums and cherries, nearly mature their growth, and require to be budded in the hottest part of our summer. In the old method, the bud having only a shield of bark, with but a particle of wood in the heart of the bud, is much more liable to be destroyed by heat or dryness than when the slice of wood is left behind, in the American way. Taking out this wood is always an operation requiring sonie dexterity and practice, as few buds grow when their eye or heart wood is damaged. The American method, therefore, requires less skill, can be done earlier in the season with younger wood, is performed in much less time, and is uniformly more successful. It has been very fairly tested upon hundreds of thousands of fruit trees for the last twenty years; and although practised English budders coming here are at first greatly prejudiced against it, as being in direct opposition to one of the most essential features in the old mode, yet a fair trial has never failed to convince them of the superiority of the new."

Saul's new method of shield-budding.- "In budding," says this cultivator, "the top bud on the shoot should be commenced with, cutting from about an eighth of an inch below the bud or eye, to from half an inch to an inch above it, in order that there may be a sufficiency of bark to hold with the fingers without rubbing the portion which is to be inserted; take out the wood as already recommended; next make an incision in the shoot of the stock close to the main stem, and about half an inch long-this is long enough; cut across at top, raise the bark with the end of the budding-knife without bruising it, and insert the bud. It is now to be tied with a little worsted or cotton. One tie below the eye and two above will be ample, as its entire length will not be more than half an inch, and a quarter of that below the eye. The most inexperienced amateur need not give more than two rounds below the eye and three above; but the practical workman will find the number I have mentioned sufficient."

Shield-budding reversed, fig. 117, is performed by having the transverse cut at the bottom of the perpendicular slit instead of at its top, and of course the shield is reversed in position. This mode is preferred by those who think that the sap rises in the bark equally with the wood; but as this opinion is not fully entertained, the process is seldom used in this country, although very generally in the south of Europe, particularly in the propagation of orange-trees. It is admitted to be suitable for trees having gummy sap. For the same purpose budding with the 
eye turned downwards has been recommended. But its most important use is to induce

a state of productiveness in Fig. 117. fruit trees. In consequence

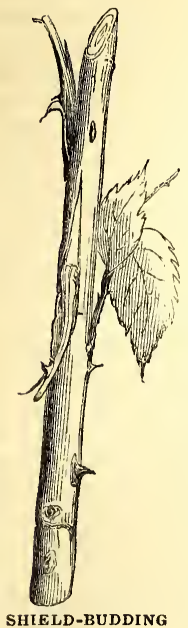

REVERSED. with a similar accident. of the bud being placed with its point downwards, an interruption takes place in the flow of the sap. This effect is denied by many, who say the shoots after a time regain their natural position, and hence no such interruption takes place. The shoot, no doubt, the second year, takes its natural upright direction; but the bend, which is always abrupt at the point where it changes from the pendant to the upright, has much the same effect on the sap's flow as that of bending an upright branch downwards, but probably not to the same extent.

Shield-budding with a terminal bud.-This mode is sometimes employed in the case , or of a branch meeting

Shield-budding with a view to repair injury done to the bark.-This is done by paring the edges of the wound smooth, and selecting a piece of healthy bark from the same or from another tree of the same species, of the same shape and size, and covering the wound so exactly that a union takes place and the blemish is repaired. It is difficult to cause adhesion in the case of stone-fruits by this means. It is, however, seldom resorted to.

Shield-budding the rose is thus described by Mr Paul, in his beautiful work, "The Rose Garden," p. 131; and as few have succeeded more in the propagation of "the queen of flowers," we present his modus operandi in nearly his own words: "The piece of bark is called the bud ( $d$, fig. 118), and the tree in which it is inserted is called the stock. In the first place, obtain a shoot from the tree whose identity we wish to perpetuate; from this the leaves are cut off, leaving, however, about half an inch of the leaf-stalk to every bud. Before proceeding farther, the prickles should be rubbed off both stock and scion, that they may not interfere with the operation, or annoy the fingers of the operator. Now take the buddingknife in the right hand, and make a longitudinal cut, $a \alpha$, about an inch in length, terminated at the top end, $b$, with a cross cut. In using the knife, take care not to cut too deep: through the bark is all that is necessary; deeper is, indeed, injurious. Now turn the handle of the knife to the incision, running it up and down the cut $a a$, twisting it slightly on either side, to raise the bark. All is now ready for the reception of the stranger bud. Take the shoot which is to furnish it in the left hand, with the thicker part towards the finger-ends; with the knife in the right hand, commence cutting about half an inch behind the bud, passing the knife up- wards under the bud, and to about the same distance beyond it. The knife should have a

Fig. 118 .

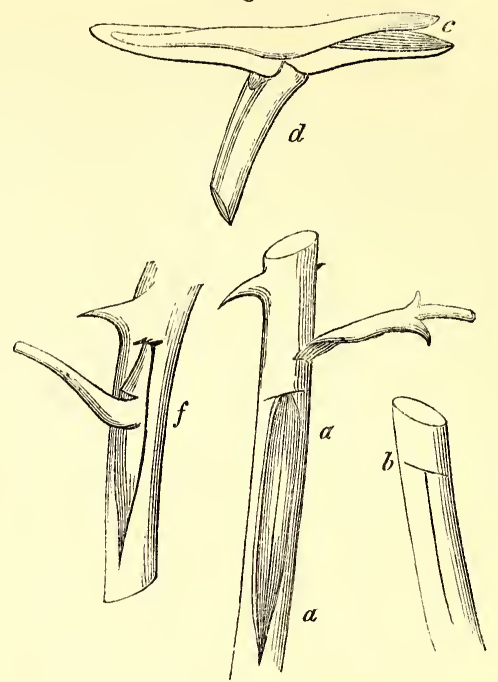

NR PAUL'S NETHOD OF SHIELD-BUDDING.

keen edge, that the bark may not be ruffled in the operation. In cutting out the bud, the knife should pass through almost level; it may, however, in some cases dip a trifle, when passing directly under the bud, as the wood before and behind it is not always on the same level. If the bud be cut ever so skilfully, there will be a little of the wood adhering thereto. This some advise the removal of ; others say, let it remain. Much depends on circumstances. If the shoot is not fully ripe, or if, from the nature of the variety, the wood is soft when taken, cut the bud as shallow as possible, and place it with the wood in the stock; but the shoot is usually ripe and firm, and then the wood should be withdrawn. To do this easily, place the bud between the fore-finger and thumb of the left hand, with the cut uppermost, and with the upper end pointing from the hand. Insert the point of the knife just beneath the wood, cthat is, between the wood and the bark-and by a skilful twist of the knife (which can only be acquired by practice) the wood may be jerked out. Now with the same hand place the bud on the bark of the stock, parallel with the longitudinal incision, and with the upper end towards the top of the shoot; then with the handle of the knife raise the bark on the side opposite to that on which the bud is placed, pushing two-thirds of the bud beneath the bark with the thumb. Now raise the bark on the opposite side, and the bud may be gently pushed under with the handle of the knife, or will probably drop in. When properly placed, the eye of the bud should be directly under the opening caused by the raising of the edges of the bark of the longitudinal incision $f$; if it be not so, the handle of the budding-knife should be inserted beneath the bark, to push it to a right 
position. But if the bud be not deprived of the leaf-stalk, if that is allowed to protrude from the opening, the eye will be secured in the best site. After being inserted, the bud should be drawn upwards to the cross cut, and the upper end cut at the same angle, that its bark may abut against the bark of the stock laid open by the cross cut $b$. The bud then is inserted, and it now remains to bind it in. For this purpose, take worsted or bast-the former is generally preferred; commence tying at the bottom of the cut, passing upwards till the whole length of the incision is bound over. Where the buds are feeble, or where success is deemed important, it is customary to tie a little damp moss or a leaf over the bud after the operation is completed, which is in no case objectionable, except on account of the additional time it occupies.

"About three weeks after the operation has been performed the worsted may be removed. If the bud is not well united, let it be tied up loosely again; if it is, leave it untied, and there is an end of care till the following spring. In February, the wild shoot may be cut away 2 inches beyond the bud, when the latter will break, and soon form a tree. It is often said that it is unnecessary for the bud to remain so long dormant, and that it may be made to break, and, if an autumnal rose, even to flower, the same year." Mr Paul admits the truth of this statement, but condemns the practice. "It is accomplished by cutting off the wild shoot a few inches above the bud, or by tying a ligature tightly round it at the same distance. The object sought is to cut off certain channels, through which the sap naturally flows, that it may become concentrated in the vicinity of the bud. The results are usually premature development and an unsound plant. If the buds break soon after being inserted, the shoots are puny and weakly, evidently suffering from want of nourishment. If allowed to lie dormant till spring, they have a rich store of food at their command, and grow with surprising vigour. When a bud has shot a few inches, and formed two or three good leaves, the heart of the shoot may be pinched out, when from the axle of each leaf an eye will in time push forth. In May the stock may be headed down close to where the bud has been inserted; and if the growth be vigorous, the wound made in so doing will quickly cicatrise, and a perfect plant is the result. If the time for budding be demanded, we should give July. It does not, however, require great penetration to see that this point depends in some measure on the season. The practised hand will cut and raise the bark, to ascertain when it parts freely from the wood; the tyro will find a criterion in the prickles. If slight pressure cause them to separate from the bark, the stocks are in good order, and the fitness of the shoots or scions may be ascertained by the same test. If the weather be hot and sunny, morning and evening are the best periods for budding. A July sun pouring down his rays upon the operator is not altogether pleasant, and in regard to the operation, does not increase the chances of success. The shoots from which the buds are to be taken should not be cut from the tree till we are ready for action, and the less time that elapses between the different branches of the work the better."

Scallop-budding may be performed at almost any season of the year, and also upon trees hav. ing thick or corky bark. It is accomplished by taking a thin tongue-shaped section of bark from the side of the stock, and a similar section from the shoot containing the buds, but in neither case removing the wood. The section containing the bud is then laid on the corresponding scallop in the stock, its upper edge exactly fitted as in shield-budding, and at least one of its edges as in whip-grafting. After this it is secured in the usual way.

Annular or ring budding is performed either. at the period of the first ascent, or at that of the principal movement of the sap in August. The stock and scion are formed as in fig. 119 ; but in either case

Fig. 119. the top of the stock is not to be

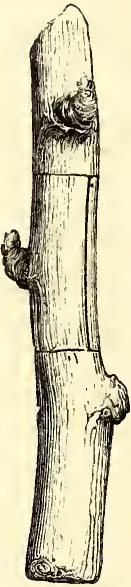

ANNULAR

BUDDING. interfered with. This is a valuable mode of propagating trees or shrubs with hard wood and thick bark, or those which, like the walnut, have buds so large as to render it difficult to bud them in the common way. This mode is also of great value, and may be turned to good account, where hares or other vermin have destroyed the bark near the ground, or even for fractured bark upon any part of a tree. Recourse is frequently had to such a process in America, and it is thus described by Downing: "In snowy winters, fruit trees in orchards are sometimes girdled at the ground by field-mice, and a growth of twenty years is thus destroyed in a single day, should the girdle extend quite round the tree. To save such a tree it is only necessary, as soon as the sap rises vigorously in the spring, to apply a new ring of bark, in the annular mode, taken from a branch of proper size ; tying it firmly, covering it with grafting clay to exclude the air, and finally drawing up the earth so as to cover the wound completely. When the tree is too large to apply a single ring, separate pieces, carefully fitted, will answer; and it is well to reduce the top somewhat by pruning, that it may not make too large a demand on the root for a supply of food."

Flute or tube budding.-Of this there are four distinct varieties, with several modifications; none are much practised in Britain. Common flute-budding, as will be seen by our figure of annular or ring budding, consists in removing a ring of bark from the stock, and procuring a similar ring of equal size, having a bud or buds, and which is to be fitted to the un. barked part of the stock, making the two barks fit exactly at their point of union. Should the ring to be attached be of rather too large diameter, it may be slit down the middle, and a por- 
tion removed, so that, when placed on the stock, it may fit closely ; and if too small, it is in like manner slit up longitudinally, to admit of its being put round the stock, and, if properly performed, a piece may be added to make good the deficiency. It is then tied on, and the slit covered with clay or grafting-wax, to exclude the air till adhesion takes place. The operation is generally performed in spring, and considered by the Continental cultivators of advantage in the case of plants difficult to bud by other means. In the south of France, terminal flutebudding is chiefly had recourse to in the case of figs, mulberries, walnuts, chestnuts, \&c.

T'erminal flute or tube budding, fig. 120.-Of this there are several modifications. It is em. ployed in cases of trees that are Fig. 120. thought not to take readily by

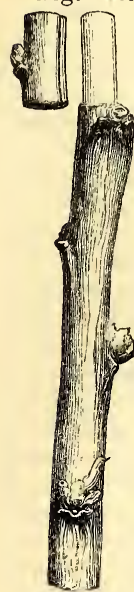
other means, such as the white mulberry, the walnut, \&c. The operation is usually performed in spring, but may also be done in autumn. In the former case, the scions are taken off in autumn, aud stuck in the ground in a cool shady place till spring. The scion and stock should be as nearly of the same size as possible, at least the stock should not exceed in diameter the scion, as it is desirable that the ring of bark taken off the latter should completely surround the former. When, however, the ring of bark is larger than the stock to be covered, a small portion of it may be cut out, so as to make the joining of its edges as complete as possible; for it is important that they do join.

TERMINAL portant Budding with the pushing eye offers very considerable advantages in the process of rapid propagation. M. Carrière, in " Révue Horticole," October 1852, throws the following light upon this interesting subject. "Let us only see," he says, " how a rose-grower proceeds when he wishes to propagate speedily some new or choice variety. He encourages, by every means in his power, the perfect development of the first young shoots the plant pushes out; and in this he succeeds in obtaining in June, and even sometimes in May, eyes with which he can bud fresh stocks - these eyes, of course, being ready to push into shoots. The plants so budded will supply him, towards the end of summer, with other young shoots furnished with dormant eyes, such as will only push next spring, and with these he can bud more stocks, and obtain plants that will flower in the following season ; and by this course, in the space of a year he finds himself in possession of a considerable stock of a variety of which he had only a single plant at the outset." By the same process, M. Carrière observes, other plants can be as speedily propagated, and instances a new variety of lilac, which he had received on the 24th of May, on which day he took off a small shoot from which five buds were wrought. Four of them took, and one of them is now (October) 28 inches long, affording 22 eyes fit for budding, which, if budded now, would produce next spring a score of plants, the produce of one of the buds wrought last May. " Admitting," he continues, " that the success was not always so great, and that each of the four first buds produced shoots having only 16 eyes, still I should next spring find myself in possession of 64 plants."

Of all the varieties of budding, that termed shield-budding, fig. 115, is the most useful, and, therefore, the mode followed most generally in our nurseries. It is now considered pretty generally, amongst practical propagators, to be the most judicious way of multiplying most of the varieties of fruit and ornamental trees and shrubs, and to a very considerable extent has superseded propagation by grafting. The season for performing the operation, as has already been remarked, extends over a considerable period, depending much on the kind of tree and condition of the wood; and the only rule that can be laid down in this case is, that the buds should appear plump, fully and perfectly formed in the axles of the leaves, and the bark rising freely in the shoot into which the bud is to be inserted. This state depends upon the free ascent of the sap; and hence, as has been sug. gested by Mr Saul, mild and warm weather is to be preferred to a dull and cloudy state of the sky for performing the operation. Hardy evergreens take freely if budded in June, selecting buds from shoots of the preceding year's growth, and inserting them in the stock without removing the wood, as is usually practised with deciduous trees. Towards the end of June and beginning of July, the most forward in growth of deciduous ornamental trees and shrubs should be proceeded with, taking the buds from the most advanced shoots of the present year's growth. From the beginning of July to the middle of August, stone-fruits and pears, with the exception of the peach and apricot, may be successfully wrought; and during the latter end of August and beginning of September, peaches, apricots, and apples will be in a fit condition for budding.

Many other modes of budding might be described-the above maybe taken as the principal.

The part of the stock into which the bud is to be inserted demands consideration. In a leading article in "The Gardeners' Chronicle," 1842 , this matter is very clearly explained. "When first inserted, the bud is mainly nourished by matter sent down from the leaves of the stock; it should therefore be introduced near the lower end of a shoot, and not near the point. The number of buds to be put into the same branch is no further material, than that many incisions in the same branch will have the effect of impairing its general health. As the young bud is to be nourished at first by the leaves above it on the stock, the best place to insert it is close beneath some leaf in full activity; it is not, therefore, the most open and smooth part of the stock that is to be selected (although we see this daily done) when a choice can be made. For the same reason, it might appear injudicious to shorten the branch into 
which a bud is inserted; but if the shoot is not stopped, the rising sap will be attracted into the youngest leaves, and expended in their increase; while, on the other hand, if the shoot is stopped, the sap will be forced laterally into the buds already forming on its sides, and the new bud will participate in this advantage. It is therefore, upon the whole, advantageous to cut off a part of each shoot into which a bud is introduced. The removal of a quarter of it is enough to answer the intended purpose. As it is important in every way that the vigour of the budded branch should be preserved for the buds which are forming, all flowers or fruit should be cut off it, and from the twigs in its vicinity, otherwise these parts will consume the organisable matter which should be applied to the service of the new buds."

\section{§ 5.-PROPAGATION BY CUTTINGS.}

The accidental sticking into the ground of a willow twig, or other equally ready-rooting plant, may have taught man the art of increasing by cuttings. Propagation by cuttings, like grafting, by no means constitutes a new plant, but only an extension of the parent from which it was taken, differing in no respect in habit, nature, or property, from it. Many plants originated from cuttings, such as the poplar, vine, elder, willow, \&c., attain as large a size and as great an age as if originated from seed; and the first and last of these are almost invariably propagated by this means, and that often when the cutting or truncheon is of large size and considerable age. The oak and beech amongst trees are, however, exceptions to this rule, and it was long thought that coniferous trees were incapable of this mode of increase. Subsequent experience has, however, proved this opinion to be incorrect, for with the exception of Araucaria excelsa, Pinus nobilis, and a few others, our expert nursery propagators increase this popular family with seemingly little difficulty; and there is no doubt but that plants of this order so multiplied will make more healthy and more durable trees than those propagated otherwise than by seeds. Instances have occasionally occurred of some coniferous trees propagating themselves somewhat in the way of layers or cuttings, as where their lower branches have come into contact with the ground, and been partially by accident covered with soil, they have rooted, and their points taken an upright direction, and when afterwards separated from the parent, have become tolerable trees. These instances are of rare occurrence, but they no doubt first led the observing cultivator to adopt, artificially, a more certain process than was thus pointed out to him by nature.

That portion of a plant or tree selected for a cutting must of necessity contain buds or eyes on its surface to secure its growth, and hence that portion of the stem or branches called the irternode-that is, the spaces which intervene between leaf and leaf, or bud and bud, as exemplified in the vine, fig. 121, and which have no buds, as from $a$ to $a$-is of all parts the most improper for the purpose. The vine, and some other plants, when subjected to a humid atmosphere, throw out roots even into Fig. 121. the air, but these are always W $b$ bund to proceed from the buds, Dr Lindley observes, "that the roots of a plant are as much productions of buds as branches are, and that the stem is nothing more than a collection of such roots held together, under the form of wood and bark." This is the reason why cuttings should be cut close below a leaf bud; if cut above it on the internode, roots could not be produced. This opinion seems confirmed by the circumstance of the old trunks and larger branches of pear-trees, when blown down, often striking roots at those parts where they come in contact with the ground, as instanced in the celebrated old pear-tree at Holme Lacy, in Herefordshire, and in the case of many others of far less note. Upon carefully examining such trees, it will be found that the roots have emanated from latent buds which, had they not come in contact with the soil, would for ever have remained dormant. And in the very same way, roots were produced CUTTING OF VINE.

from the under side of the ancient mulberrytree described by Dr Neill, in "Horticultural Tour," p. 13, as then existing in a garden at Canterbury, and which had been prostrated above a century. Another well-authenticated fact is recorded by Mr Livingstone in the fourth volume of " The Horticultural Society's Transactions," in the case of the Petrocarpus marsupium, one of the largest trees of the East Indies, which is " readily propagated by cuttings of all sizes, if planted even after the pieces have been cut for many months, notwithstanding they appear quite dry, and fit only for the fire. I have witnessed," says Mr Livingstone, "some three, four, five, six, or seven inches in diameter, and ten or twelve feet long, come to be fine trees in a few years. While watching the transformation of the $\log$ into the tree, I have been able to trace the progress of the radicles from the buds, which began to shoot from the upper part of the stump a few days after it had been placed in the ground, and marked their progress till they reached the earth. By elevating the bark, minute fibres are seen to descend contemporaneously as the bud shoots into a branch." And even chips of such trees as the poplar, having a portion of the bark remaining on them when buried in the ground, have been known to produce roots, and ultimately to become trees - a circumstance noticed by some of our oldest horticultural writers. Of fruit-bearing hardy trees, the vine, fig, mulberry, gooseberry, and currant are for the most part propagated by cuttings. Almost every variety of apple may be propagated by cuttings; the pear less readily; and all other 
sorts are so by grafting, budding, suckers, or layers.

Cuttings of hardy fruit trees should be taken off any time between the shedding of the leaves in autumn and the first swelling of the buds in spring. The earlier, however, they are taken off and planted after the ripening of the wood the better, as in this case the callus will form at their base during winter, and the roots commence to push out early in spring. A somewhat shaded and cool place, such as behind a north wall, is the most proper; and one exposed much to the sun is the worst that could be chosen. During April and May, while their roots are forming, they will derive considerable benefit from the partial shade of the wall, if not planted nearer it than 3 or 4 feet; while by the end of June, and through July and August, they will enjoy a sufficient amount of sunshine. By the end of October they will be ready for transplanting into nursery lines in the open garden. For the various modes of performing the operation applicable to hardy fruit trees, vide articles Vine, Fig, Mulberry, Gooseberry, and APPLE.

Cuttings of hardy deciduous trees and shrubs are for the most part taken off in autumn, when the wood becomes ripe, and may be either planted at the same time, or laid in pretty thickly in the soil, having one-third of their lower ends covered with earth. In this way space is economised; and as the callosity will form at their root ends, if brought in close contact with the earth, as well in this position as if planted out, they may afterwards be planted when time permits, only the earlier in spring the better.

Cuttings of Coniferce. - Many of these are now found to propagate freely by this means, and many fine plants are already in our collections, even of the very recently introduced sorts, such as Taxodium sempervirens, Cryptomeria japonica, and the funebral cypress. The hardier and more common sorts, as the Arbor vitce, Irish or upright yew, Swedish juniper, and many of the small-leaved kinds of pines, if the cuttings are taken off as soon as they have completed their annual growth, and planted in a shady border, or in a pit with a northern exposure, will root freely, and make as good plants as if originated from seed. The following very judicious directions have recently been given by $\mathrm{Mr} \mathrm{Ap}$ pleby, in the ninth volume of "The Cottage Gardener," p. 242 : "The way to manage Coniferæ is first to prepare a pot to put them in; drain it well, and cover the drainage with a little moss ; then fill the pot to within an inch of the top with a compost of loam and sandy peat, mixing it freely with sand ; upon this place an inch of pure sand, watering it gently, to settle it and make it firm. Then prepare the cuttings - take them off the tree just at the point where the last made wood joins to the wood made the previous year; trim off, without wounding the bark, the lower leaves and branches, if any, and insert the cuttings in rows across the pot till it is full. The best time to do this is about the month of October, though they will do pretty well up to the March following. Water the cuttings gently, and allow the tops to dry, then place them in a gentle hot-bed, just warm enough to cause them to form a swelling at the base, but not so hot as to induce shoots, unless the operator has the convenience of a greenhouse to harden them off. They do not require bell-glasses, or at least will root very well without them. In putting in cuttings of Coniferæ in the above manner, I refer more especially to the more rare kinds, such as the Cryptomarias, some Cupressuses, Taxodium sempervirens, Arbor vitce, and some of the genus Taxus. Others that are more common may be successfully propagated by cuttings put in early in the autumn, under hand-glasses, on a northwest border, where the sun leaves them about ten o'clock. For such the ground should be prepared in a similar way to that in the cuttingpots, and the cuttings put in by the same method. The hand-glasses should be kept on through the winter, and removed as soon as they begin to grow in spring." This excellent cultivator recommends taking up the cuttings and potting them, and placing them under a cold frame to encourage their free growth, and most wisely recommends their being planted out into nursery-rows before the roots become matted round the sides of the pots. In the case of Araucaria excelsa, which is rarely propagated successfully by cuttings, he recommends taking out the central shoot, or main leader, which will induce several other leaders to push up, any of which may be taken off as soon as formed, and put in as cuttings. This will no doubt secure young trees of perfect form, keeping the original as a stock to provide future supplies.

Cuttings of partially ripened wood.-In this state most hardy evergreen shrubs are propagated, as well as roses, particularly the China, tea-scented, and most climbing sorts, which, if put in towards the end of summer in a sheltered somewhat shaded place, will form their callosity during winter, and root freely in spring. Of greenhouse plants, the camellia, oleander, and similar plants, may be given as examples. These should be allowed to form their callosity in a low temperature, which should be increased as the roots begin to appear.

Cuttings of young wood.-The majority of hardy soft-wooded flowering plants will readily root by this means. Pinks, carnations, double wallflowers, and most double-flowering herbaceous plants, penstemons, salvias, verbenas, dwarf lobelias, snap-dragon, lychnis, mimulus, \&c., may be given as examples. And of soft-wooded greenhouse plants, pelargoniums, fuchsias, cuphia, heliotropium, calceolaria, selago, tropæolum, maurandia, \&c. These should be cut immediately under an eye or bud, as shown in fig. 122, their lower leaves removed so far only as the cutting is to be planted in the soil or sand, the leaves above that being left entire. They should also be planted as soon after they are removed from the parent plant as possible, to prevent their suffering from unnecessary evaporation. They should be placed in pots well-drained, and half-filled with the soil congenial to the species; orer that should be laid a covering of pure white sand, in which there are few or no traces of iron or earthy matter, of a thickness somewhat more thall 
the depth to which the cutting is to be set, say from one-half to an inch in depth. It is of much
Fig. 122.

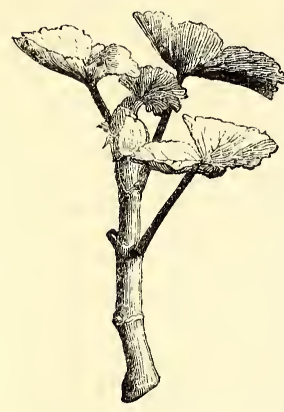
importance that the cuttings be planted before the leaves flag, and it is of equal consequence that they be arranged as near to the edge of the pot as possible; those so placed will root sooner than such as are set nearer the centre. In striking soft-wooded plants, no suspension to their energies should be allowed to take place if possible; hence their leaves should not be permitcutring of pelargonium. ted to flag, the operation should be carried on in the shade; and when planted they should be gently watered, and the pots plunged in a slightly-heated hot-bed, and air excluded by covering with bell or hand glasses, if the convenience of a properly-constructed propagating pit is not at command. Shading from bright sun, keeping the atmosphere damp around them, and maintaining a uniform mild heat, are the conditions most favourable for their speedily striking root. Care must, however, be taken that they suffer not from damp, and that air be admitted to them gradually, and increased as the process of rooting advances. This requires not daily, but almost hourly attention. Softwooded stove-plants require a bottom temperature of from $70^{\circ}$ to $80^{\circ}$. Greenhouse softwooded plants, on the other hand, require it in a less degree, say from $50^{\circ}$ to $60^{\circ}$, particularly until the callus is formed, after which, and when the roots are growing, it may be increased $10^{\circ}$ more. Some of these, however, should have no extra bottom-heat beyond that of the temperature of the house or pit they are placed in until their callosity is formed, after which an increase as above will stimulate both the roots and young shoots. No leaves should be cut off or reduced in size unless they are numerous and large, and even this can only be sanctioned to the extent of preventing a greater degree of evaporation taking place in them than can be supplied by the cutting or the humidity which surrounds it; and shading should only be resorted to with a view to secure the same end. Too close an atmosphere, accompanied with the necessary moisture around soft-wooded cuttings, tends greatly to destroy them, by causing them to damp off ; and this is more to be guarded against when they are closely covered with bell or hand glasses than when grown in a properly-constructed close pit, where the glass roof can be slightly obscured or covered with thin canvass, thus modifying both the amount of light and air. All soft-wooded plants are readily increased by cuttings, if the above conditions be complied with. As soon as the cuttings begin to grow, more air and light should be admitted to them; and such as begin to push up weakly shoots should be topped, and, when fairly rooted, transplanted from sand into small pots in their proper soil.

The hard-wooded plants that strike best from young shoots or tops are Erica, Epacris, Chinese azaleas, Perium, Chironia, the majority of Australian plants, Cytisus, *Begonia, Boronia, Burchellia, Calothamnus, Calycanthus, Ceanothus, Chamæcistus, Cistus, Chimonathus, Helichrysum, Astelma, *itron, clethra, clianthus, colletia, *combretum, coronilla, corræa, cotoneaster, *croton, daphne, deutzia, diosma, adenandria, dracophyllum, *echites, Edwardsia, elæagnus, empetrum, enkianthus, ephedra, escallonia, fontanesia, *Franciscea, *gardenia, gardoquia, gnidia, gravellia, Hudsonia, hyssopus, Ilex, Illicium, *Ixora, *Jatropha, Lambertia, Laurus, Lechenaultia, Ledum, Luculia, Medicago, Metrosideros, *Murraya, Myrtus, Phylica, Pimelea, Plumbago, Polygala, Psoralea, *Quisqualis, Solea, Weigelia, \&c.

These, with the exception of those marked *, will strike, if placed in a moderate bottom-heat, say of $55^{\circ}$ to $60^{\circ}$, until the callus is formed, when it may be increased to $65^{\circ}$ or $70^{\circ}$. Those marked * will require from the first a bottom temperature of from $70^{\circ}$ to $85^{\circ}$, accompanied by the conditions stated above.

Many plants, however, are difficult to strike by cuttings by any of these means; other modes are therefore resorted to, as in the case of the orange and lemon, the cuttings of which should be cut off under a bud, and their base made to rest on the bottom of the pot, without the intervention of any soil whatever-" the object of which seems," as observed by Dr Lindley, " to be to place the absorbent or root end of the cutting in a situation where, while it is completely drained of water, it may, nevertheless, be in the vicinity of a never-failing supply of aqueous vapour. If it were surrounded by earth, water would readily collect about it in a condensed state, and the vessels being all open in consequence of being cut through, would rise at once into the interior; but the application of the root end immediately to the earthen bottom of the pot, with which it is so cut as to be nearly parallel, necessarily prevents any such accumulation and introduction of water, unless over-watering is allowed." In following out this principle, the intelligent reader will naturally discover that shallow pots or pans are better for the purpose than those that are of the usual depth, unless they be only half filled with soil, so that the base of the cutting may touch the bottom without being of too great a length, which would disfigure, in many cases, the future plant. By only half-filling the pot, the sides above the sand or soil afford a partial shade; and where bell-glasses are not at hand, a piece of glass may be laid over the mouth of the pot as a very good substitute.

$\mathrm{Mr}$ Alexander Forsyth, one of our most intelligent gardeners, many years ago published in "The Gardeners' Magazine" (vol. ii. p. 564) an improvement on this mode. Fig. 123 will show his principle. A 48-sized pot was rendered water-tight at the bottom, by pressing a handful of clay, $a$, over the drainage holes, above which he placed broken crocks, $b$, 
so as to raise the bottom of a small 60 -sized pot placed within the other, so that the tops
Fig. 123.

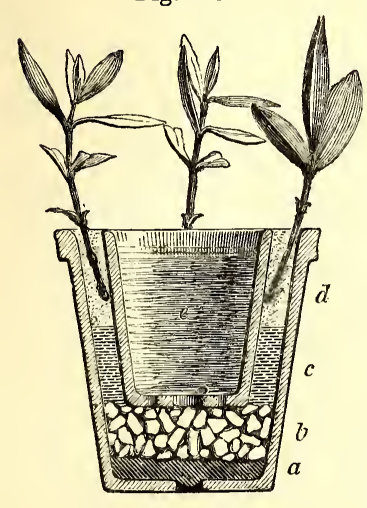

STRIKING CUTTINGS. of both should be level. The spaces between the inner and outer pots he filled up with soil, $c$, proper for the plant to root in, and above this white sand, $d$, placing the cuttings so that their bases should rest against the sides of the inner pot-which latter is filled with water, $e$, which, passing through the

sides of the pot, reaches the cuttings. Others have, to effect the same end, placed a flat piece of crock under the base of each cutting, at such a depth in the soil that the cutting shall rest upon it; and others insert a smaller pot, turned bottom upwards, within a larger one, and insert the cuttings round the sides of the inverted pot. This is a good plan where bottom heat is applied, as it ascends within the inverted pot, and reaches the roots better than if it had to ascend through the soil.

Cuttings of plants that are slow in forming a callus are sometimes half severed from the parent plant, and allowed to remain so until the wound be healed and the callosity begin to form, a circumstance which leads to the belief that air is a necessary agent in its formation. Plants containing a large pith are often difficult to strike, and those with hollow stems are equally so: in such cases the joint or node should invariably be cut through; and in this, as in all other cases, the cut should be made with a clean sharp knife. Regarding the choice of cuttings, where strong and upright plants are required, the tops of the leading shoots, or the strongest of the side ones nearest to them, should be chosen; and where less robust and bushy plants are aimed at, then the more slender side-shoots, and those nearest the bottom of the plant, should be taken. These latter shoots, if taken off with what is technically called a heel-that is, a small portion of the older branch at the junction between the two-will strike more readily, as the portion of heel removed is in general well supplied with very minute incipient buds.

The best time for taking off cuttings of greenhouse and stove plants is in spring, or early in summer, that the young plants may be established before next winter. There are, however, circumstances which set this rule aside, and in such cases the process must be carried on at other seasons. Many hard-wooded plants require to be slightly excited into growth early in spring, to cause them to push out shoots fit for the purpose; by this means many heaths and VOL. II. other plants which do not strike readily, or take a long time to root, are pushed on, so as to be established before the close of the growing season.

Bottom heat.-The importance of bottom heat, accompanied with sufficient light and moisture, is thus spoken of by Professor Balfour, in "Class Book of Botany," p. 658 : “ In causing cuttings to strike, we require a somewhat higher temperature than that of the climate in which they naturally grow. A willow-cutting stuck in the open ground will strike root; but it does so much faster, and more vigorously, when placed in a hot-bed. A white-thorn cutting in the open ground will not root at all; in a warm propagating-house it will do so readily. It is not the temperature of the atmosphere, but the temperature of the soil that requires to be raised. We must first obtain roots, and then leaves will follow. The cellular tissue of roots is first produced by a local process, and the production of this tissue is kept up by the heat of the soil: hence the necessity for bottom heat, in order to secure good roots in the first instance; and without them there will be no vigorous leafbuds." This has reference more directly to cuttings of soft-wooded plants, which, immediately after being inserted in the soil or sand, begin to form roots, often in the course of a few days. Others require longer time; and some require months, before the stimulating effects of heat should be applied. On the advantage of bottom heat applied at the proper time, Dr Lindley remarks, in "Theory of Horticulture," p. 213 : "This is for the purpose of giving them a stimulus at exactly that time when they are most ready to receive it. Had they been forced at first in bottom heat, the stimulus would have been applied to cuttings whose excitability had not been renovated, and the consequence would have been a development of the powers of growth so languid that they probably would not have survived the coming winter; but the stimulus being withheld till the cuttings are quite ready for growth, it tells with the utmost possible effect."

Many plants, like the oleander, will strike roots if their ends be placed in water, and, when sufficiently rooted, may be planted in ordinary soil. Others, such as pelargoniums and verbenas, will root readily if planted in damp sphagnum; and many shrubs, such as the rose, will root readily in half-decayed flax-dressers' refuse. Most plants will root, if set in the soil natural to them; but the universal medium in which all valuable plants, and such also as are difficult to root, succeed best in, is clean silver sand laid over the soil in which the plant delights, so that when the roots pass through the sand they find their natural supply of food awaiting them. Many plants, although they will root freely in sand, refuse to grow in it, and in such cases the cuttings should be removed from it as soon as their roots are formed, and potted in soil congenial to them.

Cuttings of heaths, and similar hard-wooded plants, may be safely sent to a great distance, if placed loosely in a tin case, with from a teaspoonful to a table-spoonful of water, according 
to the size of the case, and that hermetically closed. We have known them so sent from Edinburgh to Vienna; and further, Dr Falconer, in reference to deciduous cuttings, directs that they be cut early in November, choosing oldish and firm wood, with the greatest number of buds; cover their ends with wax, and roll them up separately in cotton; make them up in bundles of from six to ten cuttings in each, and envelop them again in cotton covered with paper, and finish with a wrapper of stout silk or cloth, coated on the inside with India-rubber in the liquid or adhesive state, so that the side and end folds may adhere together for the exclusion of both air and wet. In this way Professor Balfour states, in "Class Book of Botany," p. 658, "slips have been sent to Saharunpoor via Bombay (a distance of 7000 miles from Falmouth), in a state fit for planting when transmitted, in less than two months; and cuttings to be grafted will continue good double that period. Sometimes ringing is had recourse to, in the case of cuttings of plants which are difficult to strike. The interruption of the sap caused by this process has the effect of forming a callosity, which, if inserted in the ground after being separated from the plant, sends out roots freely.

Piping is a species of propagation differing not in principle from striking by cuttings. It is applied to pinks, carnations, and similar herbaceous plants having jointed or tubular stems. Pipings should be put in as early as they can be got fit for the purpose, that the plants may become strong before winter, which in England is about the end of May or beginning of June. They are taken from the young shoots of the current year's growth. The lower leaves, in the case of pinks, are stripped off to within two or three joints of the top of the cutting. The lower part is cut off, close under a joint, with a clean and sharp knife. "When prepared, throw them into a pan of water, for from five to seven minutes, to stiffen. The piping-bed being ready, press them into the soil about three quarters of an inch, and the same distance apart: give a good sprinkling of water, and as soon as the foliage is dry put the glasses over them. In about three weeks they will have rooted : at this period the glasses may be removed by degrees, first lifting them half an inch on one side for a day or two, and then all around; about the fourth day they may be taken off. Pinks will root as freely under a north wall as anywhere, and require less attention in shading when in such a situation. Prepare a layer of spit dung, let it be put together thoroughly wet, and beat down with a spade to a level surface: if 8 inches deep, it will be quite sufficient to keep the worms from disturbing the young plants. On this must be placed the compost for the plants to root in, 3 inches deep. This should also be in a moist state, but not wet, and be moderately pressed to a smooth surface. The compost for the purpose must be of a sandy quality-leaf-mould or decayed vegetable mould, and silver sand sifted fine, equal parts of each. In the absence of the above, any sweet and sandy soil, proportioned as above, will answer; but we give the preference to leaf-mould from its cool and retentive nature."-Mr Neville in Beck's Florist.

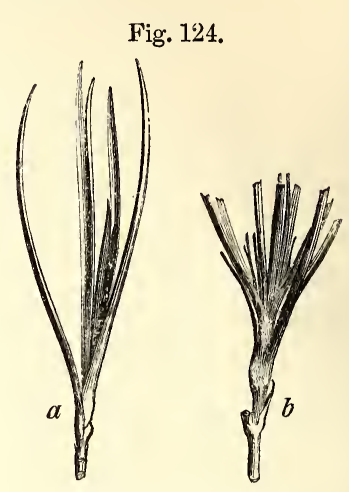

This may be considered the English practice. In the north, where the growth is later, slight bottom - heat is often required to make up by slight excitement what we want in warmth of climate and time to secure strong plants before the season of growth terminates. The best florists have long abandoned the absurd practice of shortening the leaves of their pipings, and prepare them as shown, fig. 124, $a ; b$ exhibits the mutilated piping of bygone times.

Propagation by cuttings of the root.-Many plants may be multiplied by cuttings of the roots, instead of the more usual way of using portions of the branches or young shoots. Mr Knight has shown that the energies of a variety, artificially produced (a hybrid), exist longer in the system of the root than in that of the stem; so that it is more expedient to employ the roots of old varieties of fruit trees for cuttings than to use the stems. Many rare trees, shrubs, and plants are propagated by this means, and excellent thorn-hedges are formed by planting cuttings of the roots-that is, pieces of them about a foot in length; and even chips, having some portion of the bark on them, of the poplar, if planted, will produce trees. In both cases adventitious buds exist, and these, when called into action, form the future plants. Horseradish, sea-kale, \&c., if their roots be cut into pieces of only a few inches in length, and laid horizontally in the soil and slightly covered, will form roots at the one end and leaves at the other. The Moutan pæony is most successfully multiplied by cuttings of the roots. The common plum-trees and Pyrus japonica are readily propagated in a similar manner, plums having an extraordinary power of forming buds on their roots. That beautiful and not long introduced plant, Anemone japonica, bas this property in an eminent degree all over the surface of its roots, so that they may be chopped into very small pieces, yet each of these is capable of forming a new plant. And amongst the equally interesting family of Enothera the two species $C E$. macrocarpa and $Q E$. coespitosa are scarcely, excepting by seed, capable of being otherwise multiplied. Acacia pubescens amongst greenhouse plants, and some of the Ardisias, Clerodendrons, Dracanas, \&c., amongst stove plants, are only by this means increased with certainty.

Cuttings of the flower-stems are found to be the best mode of propagating the double-flowering varieties of the scarlet lychnis, double rockets, and many similar border-plants; these, if cut 
into lengths before flowering, and placed under a hand-glass in a shaded border, root freely.

Cuttings of leaves.-Some idea of this mode of reproduction was entertained about the beginning of the last century, as in a work in Dutch by Agricola we find copperplate illustrations showing whole fields covered with orange leaves represented as growing into trees. Although this is sufficiently absurd, still a knowledge of the fact that some plants will strike roots from their leaves must have been possessed. The leaves of the orange, aucuba, and the fig, being furnished with adventitious buds, will multiply in this manner, as will also most of the gencra Achimenes, Gloxinia, Gesnera, and many other plants of like habit. Some of the Begonias, and many succulents, do the same; and the old and much
Fig. 125.

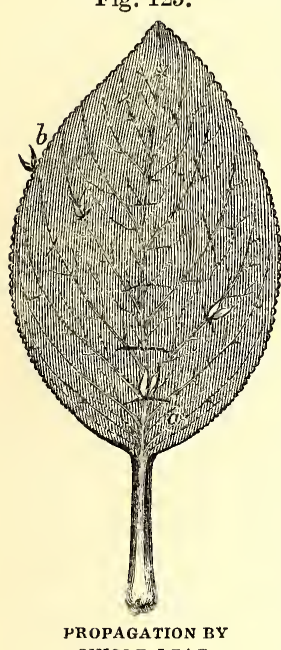

SINGLE LEAF.

Fig. 126.

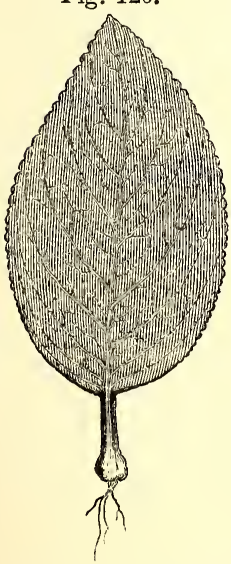

PROPAGATION BY LEAF, neglected Bryophyllum produces abundance of young plants along the margins of the leaves. The process is as simple as the result is certain, and all that is required is to lay the leaves flat down on the surface of silver sand, keeping them in their place by pegging them down; or, better, laying a small stone upon them, placing them in heat, with a due amount of moisture and shade, as in other cuttings: from the veins $a$ and crenate notches $b$ around their edges, roots will speedily issue, as shown in fig. 125 ; and when formed, the leaf, if it has not rotted away, may be torn up into pieces. Sometimes the footstalk of the leaf is inserted in the sand as if it were a cutting proper, in which case an excessive development of cellular tissue takes place at the base; a callus is formed, from which roots proceed, fig. 126, and afterwards a leaf-bud, which forms the future plant. "It is not surprising," Dr Lindley remarks, " that leaves should possess this quality, when we remember that every leaf does the same thing naturally while attached to the plant that bears it; that is to say, forms at its base a bud which is constantly axillary to itself."
Besides the plants we have named above as capable of propagating themselves by this means, the following are given as examples by Professor Balfour, in "Class Book of Botany:" "Some leaves naturally produce buds on their surface, as may be observed in Malaxis, Aspidium bulbiferum, and Nymphwa micrantha. Other leaves, when placed in particular circumstances, give rise to leaf-buds at their margin. Thus the leaves of Bryophyllum callycinum, when placed on the surface of damp soil, exhibit little roots all round their edge. 'The leaves of Dioncea muscipula can also be made to produce buds, and so can those species of Gesnera, Gloxinia, and Achimenes. Occasionally leaves take root and form plants, as was observed by Knight in Mentha piperita. Buds are formed accidentally on the leaves of Drosera, Portulaca, Cardamine pratensis, and Nepeta glechoma. They are also formed on fleshy detached leaves, as on those of different species of Crassula, Aloe, on the bulb scales of Eucomis regia, Lilium candidum, Hyacinth and Squill, and on the leaves of Ornithogalum thyr'soides. The leathery leaves of Ficus elastica, Hoya carnosa, and of specics of Citrus, Aucuba, and Theophrasta, are occasionally bulb-bearing."

Bell-glasses and hand-glasses are placed over newly-planted cuttings, with a view to preserve a uniform degree of humidity in the atmosphere by which they are surrounded, and to prevent, by the almost exclusion of air, that draught on the energies of the foliage which would take place were they not protected from its action. An excess of heat, cold, air, water, and even light, are alike injurious to tender cuttings; and, therefore, to guard against these extremes such coverings have been adopted.

\section{§ 6.-PROPAGATION BY LAYERS.}

Layering is one of the simplest, and probably one of the most primitive of all modes of propagation. It consists generally in bending down the branches of shrubs or trees which are produced nearest the ground, and sufficiently flexible for the purpose, so that a portion of their length near to the middle may be covered with earth, and so bent that the point of the branch may be placed in a perpendicular direction. The object of layering is to multiply the tree or plant without alteration in its character. The result, however, often is that a tree originated by layering does not always assume the robust and upright character of its parent, but assumes that of a rooted branch, as is often exemplified in the case of coniferous trecs. This may, however, be corrected by cutting over the layer, after it is fairly rooted, near to the ground, where some latent bud may spring, and so send up a proper leader. It is also employed in the case of plants which are difficult to graft or bud, and which do not strike freely by cuttings, or produce seeds in this country. Herbaceous plants, such as the carnation, strike root readily from layers, and make as good plants as if propagated by any other means. Hardy shrubs with long trailing branches, like 
the clematis, honeysuckle, Wistaria, \&c., propagate readily by this means: the branches being trained along the surface of the ground, strike root at every eye or joint, but are made to do so usually at every alternate joint; and some of them, like the bramble, will emit roots from the growing point, if inserted in the soil to the depth of an inch; and the same mode has been practised with the common gooseberry. Roses, for the most part, are multiplied by layering, the operation being performed during spring or autumn, and sometimes at both seasons in the same year. The supply of plum and paradise stocks for grafting or budding fruit-trees on is procured by a species of layering, consisting merely in spreading out the branches on the surface of the ground, pegging them down early in spring, and covering them entirely, excepting the very points, with soil to the depth of half an inch. During summer, every bud sends up a shoot and produces roots at its base, forming by autumn a perfect plant, which is then cut off and transferred to nursery lines. The vine is often, in nurseries, propagated by bending down the shoots, and laying them in pots plunged in the ground; but this is by no means a process to be recommended, as vine-plants so produced are seldom well rooted.-(Vide art. Vine.) Many rare plants, difficult to increase otherwise, are laid in pots suspended so as to admit of their being either bent within the circumference of the pot, or introduced through the drainage-hole in its bottom; and sometimes the Chinese practice is followed, of forming balls of earth, covered with moss, around the branch, and supporting it there until roots are formed in the mass.

The season for performing the operation extends nearly over the whole year. Hardy trees and shrubs are usually operated upon in spring, before the ascent of the sap, or delayed until the sap is pretty well up, towards the middle of summer. In nurseries where propagation is carried on to a large extent, very many of the hardy trees and shrubs are layered during autumn and winter. With plants in the artificial atmosphere of a hot-house the case is different, and hence the operation may be performed at such times as the plant is found to be in a fit condition, irrespective of the above seasons. The best condition for the shoot to be in, is when that part of it where the sap is to be interrupted, whether by tonguing, ringing, notching, twisting, slitting, piercing, or bending, is somewhat mature, or what is called halfripened. The shoots most proper to select are those in a healthy vigorous state of growth; and the most improper, such as are stunted, diseased, or imperfectly formed. Old wood will root in time, but neither so soon nor with the same certainty as half-ripened wood, because the flow of the sap is more sluggish in the former than in the latter.

The operation of layering is thus performed in general practice: The ground around the stool or parent plant, presuming it to be growing in the open ground, is loosened carefully, and if dry, moderately watered; the shoots intended to be laid are then selected individually. If, as in the case of the rose or similar shrubs, only each shoot is to become a new plant, an incision is made on the under side of the shoot, and a few inches from its base; the top of the shoot is gently bent upwards, both with a view to give the future plant an upright direction in growth, and also to keep open the incision, particularly when tongued. In this position it is let into a slit formed in the soil, the part where the incision is made being the point of connection between the descending portion of the layer (which is to be removed after rooting has taken place), and the upright portion of the layer which is to constitute the future plant; the earth is then firmed about the shoot, which so far completes the operation. In layering, a great error is often committed by inserting the part to be rooted too deeply in the ground, and this is often done to save the trouble of pegging them down with a hooked stick. From 3 to 6 inches is the proper depth for layers of trees or shrubs; but even this must be regulated by their size and age. The shoots of the previous season's growth are the most proper for this purpose, although older branches, particularly of soft-wooded trees and shrubs, in extremecases, may be used : but these require a longer time to root, and often have to be examined; and when indications of the formation of granulous matter do not appear, fresh incisions should be made to induce this state. Or the suggestion thrown out by Mr Knight may be followed of taking up the layer entirely, and removing a ring of its bark below where the former incision was made, so as completely to prevent the descent of the sap, and thereby force the shoot to employ it in the formation of roots. The part of the layer at which the incision is to be made, should be immediately under a bud or joint, which is the point most favourable for the emission of roots. The depth to which the incision should be made depends much on the nature of the plant, but in ordinary cases it should only penetrate into the alburnum, and not through it, as in the latter case the ascent of the sap would be arrested, and the buds and leaves above it prevented from performing their proper functions; whereas, in the former case, the effect desired will be accomplished, which is to form an impediment to the descending sap, causing it to exude at the wound, and form that granulous matter which is the certain indication of the first formation of roots. There are various means of causing this interruption to the descending sap-namely, ringing, or removing a ring of bark, wholly or in part, from the layer; twisting it by bending it at a very acute angle ; cutting a notch out of it; forming a slit or tongue, kept open by placing in it a small piece of chip or stone; twisting a piece of wire tightly round it; boring a hole with a broad awl or gimblet, or driving a nail or peg through it. Several of these practices are sufficiently barbarous as well as uncalled for : ringing in particular cases, and notching, tonguing, and twisting in ordinary ones, are all that is necessary ; and often a thin slice of bark paired off the side of the layer is found sufficient. The fact of the sap ascending chiefly through the 
alburnum to the leaves and buds, and descending in an elaborated form by the inner bark, forming in its downward progress a new layer of alburnum, shows sufficiently that even ringing does not interrupt the rising of the sap in spring, so long as the alburnum is not cut through ; yet its descent is interrupted in consequence of the incision made during the operation of ringing, and which, although it merely cuts into the alburnum, in no way affects the inner bark. A granulous callosity is formed at the point of interruption, from which roots will in due time protrude. In the case of some trees, if the incision in the layer does not extend half-way through, no granulous matter will be formed the first year ; but, on the other hand, if the incision is made much deeper than half the thickness of the layer, then the ascent of the sap is interrupted, and the shoot naturally dies.

The manipulations of this mode of propagation are exceedingly simple; the following will explain the routine. In ordinary cases of nursery layering, the plant to be propagated is called the stool, and hence all nurserymen have their stool ground, in which is planted every kind of tree or shrub to be thus increased. Stools are cultivated only for the production of shoots proper for layering, hence they are cut close down to facilitate the operation. The stool occupies the centre, the young healthy wood is reserved, and the slender and unhealthy is cut out. The ground around being loosened up, shoot after shoot is gently drawn down from the stool; a notch, tongue, or other incision is made on its under side, and from 6 to 10 inches

Fig. 127.

from its base the earth

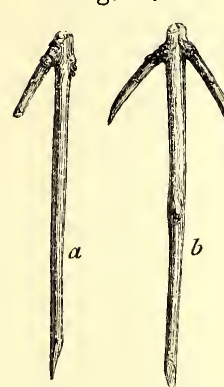

is opened, and the layer is fitted into the soil, of a depth according to its nature and strength. It is then secured in the desired position by a hooked peg, fig. 127, $a$ single, $b$ double, or by a shoot of flexible nature, such as willow, which is twisted at the middle, and the two ends thrust into the ground, one on each side of the layer ; the soil is laid over it, the point of the shoot cut off, leaving one or two PEGS FOR LAYERING. eyes above the ground surface. The incision for the most part consists in simply entering the knife below a bud, and cutting to such a depth as has already been noticed, and drawing the knife upwards, leaving what is in common phraseology called a tongue; sometimes a simple notch is cut out: in the former case, a bit of tile, thin stone, or chip of wood, is inserted to keep the tongue open or the wound from collapsing. Some plants will root freely without any incision being made, others if only a small portion of the bark be pared off ; some if they are slightly twisted or fractured, while others will not, unless a ring of bark be taken off or tightly bound round with a piece of wire. The effects of all these are the same-namely, the obstruction offered to the descending sap, and the consequent formation of granulous matter, and the after protrusion of roots. In the case of trees which are too strong or too brittle to bend down, so that their side branches may be laid in the ground, the process of plashing them-or of elevating the soil in pots, boxes, or raised banks-must be had recourse to. In the former case the branch may be plashed-that is, cut in its under sidecausing the direction of the fracture to run towards the main stem, cutting and proceeding until the branch will readily bend as low as required, taking care, however, that a sufficient portion of the bark remains unbroken on the upper side. The side branches may then be laid in the usual manner.

The time required for layers becoming sufficiently rooted to be fit for separation from the stool, depends on a variety of circumstances. Some trees and a few shrubs require two years; roses, and the majority of similar shrubs, if their present year's wood be operated upon when about half ripened, will be fit for removal the same autumn; if laid in spring or the winter preceding, they will be ready about the same time; while herbaceous and soft-wooded plants will produce their roots in a few days or weeks : and the same will occur in the case of many plants under artificial excitement.

Layering herbaceous plants is had recourse to in the case of rare or valuable plants, as being attended with much less risk of losing the plant than if the more ordinary process of making cuttings were adopted: it is also had recourse to with a view of getting stronger plants in a less space of time. The process is commenced when the shoots are of sufficient lengths and have attained some degree of consistency, which state usually occurs about the time the
Fig. 128.

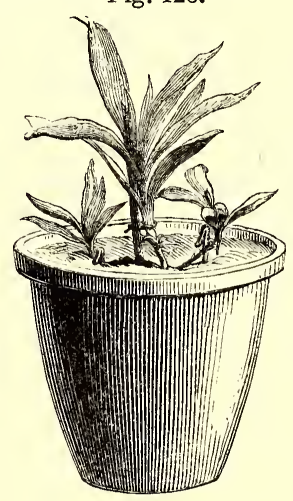

LAY ERS. plant is coming into flower. The annexed cut, fig. 128, shows the principle in the case of a double sweetwilliam, dianthus barbatus, flo. pleno. The lower leaves of the bestformed shoots being cut off, a thin buddingknife is inserted below a joint or bud, and is passed half through the branch, and continued about threequarters of an inch upwards, the bending of which upwards keeps the cut open, while the shoot is pegged down, and covered with soil, somewhat sandier than that in which the plants are growing. The form of the peg used in laying is as represented in fig. $127 a, b$; but often, and perhaps more conveniently, a small twig of willow may be used, cut to the length of 6 or 8 inches, and bent over, both ends of which are thrust into the ground, and keep the layer in its proper place. And in some extreme cases, where a shoot is sent from a distance, or accidentally separated 
from the original plant, the root end is placed in a phial of water, and the top end laid in a pot. The intention of the end being kept in the phial of water, is to supply it with moisture while the rooting is taking place.

Propagating by cuttings of single eyes or buds. -Next to the seed, the eye or bud is the part of a plant by which its propagation is best effected. This has already been shown in the cases of budding, grafting, layering, and striking by cuttings, in neither of which cases can roots be formed or growth be effected without the presence of a bud. Buds thus containing within themselves the elements necessary for the production of a new plant are taken advantage of by cultivators, although not to the extent in practice that might be, seeing that, theoretically speaking, almost all plants are capable of being so increased. The potato, Jerusalem artichoke, \&c., ever since their introduction, have been propagated by eyes; the vine, since the time of Speechley, by the same means.-(Vide art. Vine.) Many exotics, both woody and herbaceous, as the bignonia, hollyhock, \&c., are readily multiplied by single eyes. In deciduous plants, like the vine and bignonia, it is only necessary that the eye should be dormant, and that a portion of the wood on which it is produced be taken with it. This portion of wood is in general not more than an inch in length; and often when buds are placed opposite to each other, this piece of wood is split through the middle, each eye forming a plant. There is no obvious reason why we should be so sparing of this piece of wood, seeing the larger it is the more organisable matter it must contain, and consequently support the bud with a larger amount of nutriment, until it forms roots for its own support. It therefore follows that buds having 6 or 7 inches of the wood on which they are produced, instead of one inch, left attached to them, and covered with soil, must afford a proportionably greater supply of strength to the nascent branch. The late Mr Knight appears to have been of this opinion, and in the $2 d$ vol. of the "Hort. Soc. Trans." offers the following explanation: "Every leafbud is well known to be capable of extending itself into a branch, and of becoming the stem of a future tree; but it does not contain, nor is it at all able to prepare and assimilate, the organisable matter required for its extension and development. This must be derived from a different source - the alburnous substance of the tree, which appears the reservoir in all this tribe of plants in which such matter is deposited. I found a very few grains of alburnum to be sufficient to support a bud of the vine, and to occasion the formation of minute leaves and roots; but the early growth of such plants was extremely slender and feeble, as if they had sprung from small seeds; and the buds of the same plant, wholly detached from the alburnum, were incapable of retaining life. The quantity of alburnum being increased, the growth of the buds increased in the same proportion; and when cuttings of a foot long, and composed chiefly of two-year-old wood, were employed, the first growth of the buds was nearly as strong as it would havo been if the cuttings had not been detached from the tree. The quantity of alburnum in every young and thriving tree, exclusive of the palm tribe, is proportionate to the number of its buds; and if the number of these were, in any instance, ascertained and compared with the quantity of alburnous matter in the branches, stem, and roots, it would be found that nature has always formed a reservoir sufficiently extensive to supply every bud. But those of a cutting, under the most favourable circumstances, must derive their nutriment from a more limited and precarious source, and it is therefore expedient that the gardener should, in the first instance, make the most ample provision conveniently within his power for their maintenance, and that he should subsequently attend very closely to the economical expenditure of such provision." This explanation applies also to cuttings set in the usual manner, but in such cases a considerable portion of the cutting is exposed to the air, and subject to lose much more of its organisable matter than when laid flat and covered with soil in the way that a cutting of a single eye cut to the length of an inch would be. We have invariably found, in propagating by this means, that when 3 or 4 inches on each side of the bud or eye have been retained, and when their ends have been covered with sealing-wax, and the whole cutting, except the eye, covered with soil, the young shoots produced have been much stronger than when the cutting has been planted erect, or when it has been divested of its wood, as done in general when single eyes are used. Plants having large buds strike readier by eyes than those having small buds-no doubt for the reason conjectured by Lindley, " that such eyes are not sufficiently excitable, and that consequently they decay before their vital energies are roused; and, in addition, they do not contain within themselves a sufficient quantity of organisable matter upon which to exist until new roots are formed."

Knight's explanation, and the sound reason last given, perhaps led to the process of propagating the vine by what has been called the coiling system already referred to, for which see fully art. VINE.

The rarer and better kinds of holyhocks and many similar plants can only be increased, with a certainty of continuing the already improved varieties, by an extension of themselves by cuttings, and this is usually done by cutting down the principal stem so soon as it shows its merits on flowering. The young shoots which, after this treatment, spring from the crown, are usually selected for cuttings; and as they are not very profusely produced, the progress of increase is necessarily slow. A more expeditious mode of increase will be found by dividing the weaker shoots which rise round the main one, after they have attained the length of 18 inches or 2 feet, into cuttings of one bud each, much in the way vines are propagated. These budcuttings, when planted in light rich soil, will each produce a plant under ordinary good management. The main stem may also be used if cut in lengths of 2 or 3 inches, dividing the stem longitudinally, taking care not to injure the 
buds, and placing the cuttings thus formed slightly under the soil, but not so deep as to cover the top of the bud; granulation will take place round the edges of the bark, and roots will emit themselves freely. When these have become sufficiently formed, they must be taken up and planted in a prepared bed, to gain sufficient strength for planting out permanently. By this process, from fifty to a hundred plants may be produced from one. Holyhocks raised from seed, however carefully saved, can never be depended upon for perpetuating the identical variety.

The importance of buds in the propagation of plants, although well known to botanists, has hitherto been comparatively little attended to by propagators in general. Their universality is much greater than is usually thought; for as has been stated by Professor Balfour, in "Class Book of Botany," the higher classes of plants may be considered as consisting of numerous buds united on a common axis. These possess a certain amount of independent vitality, and they may be separated from the parent stem in such a way as to give origin to new individuals. In some instances buds are produced, which are detached spontaneously at a certain period of a plant's life, as instanced in stem buds of Lilium bulbiferum, L. tigrinum, lxia bulbifera, \&c. "The cloves formed in the axles of the scales of bulbs are gemmæ, or buds which can be detached so as to form new plants. Such is also the case with the corms of Colchicum. In these instances buds are developed in the usual way in the axles of leaves or scales-that is to say, at the points where they join the stem." Besides the true or visible buds, there are embryo buds contained in the bark of many, nay, probably of most trees. Of these are the excrescences called uovoli, found in the bark of old olive-trees, and, according to Signor Manetti, used by the Italian gardeners for propagating that tree. These are supposed by Professor Lindley to have been " adventitious buds developed in the bark, and by the pressure of the surrounding parts forced into those tortuous woody masses in the shape of which we find them." It does not appear that advantage has hitherto been taken in this country of these as a means of increasing the trees on which they are found, although there is little doubt they might be employed in cases of emergency. 


\title{
CHA PTER II.
}

\author{
P L A N T I N G.
}

Planting, or rather transplanting, which is a much more correct and definite term, is a process in horticulture regarded by many as attended with no small degree of difficulty, more especially when the subjects to be operated upon are of large size and of considerable age, we believe because the causes of success or failure are by them little understood. Others, and by far too great a number, stop not to consider causes or effects, but treat the matter as a mere mechanical operation, and consider only the most expeditious and least troublesome mode of tearing the plant from the ground, and sticking it in another situation. That want of success should follow such views is to be expected.

The earlier writers on this subject have for the most part confined their remarks to practice; more recent ones have directed their attention to the theory. Amongst these, the late $\mathrm{Mr}$ George Lindley considered the cause of success, in transplanting, to depend upon two circumstances-namely, the preservation of the spongioles of the roots, and the prevention of excessive evaporation. These views are also entertained by the most successful planters of the present day. The rationale of transplanting is founded on a general law in nature, by which plants are enabled to renew, within a certain time, those parts which may have been lost or injured during the process of removal, and their requiring a season of repose annually. Now, to aid them in the accomplishment of the first, is to lessen the causes of those losses and derangements, by careful lifting, transplanting, and placing them in circumstances the most favourable for their condition; the next important point is to choose that season when the plant, according to its kind, is either closing its state of excitement, in a state of repose, or just before excitement commences for another season. This extends the period of transplanting over a considerable space of time. The question, however, is, even when reduced to these limits, whether the first, second, or third states is the most proper for the operation? Each has had its advocates; and it is possible that, under certain circumstances, plants may be successfully removed during any of these stages.

Every tree, even of the same species, will not admit of being transplanted with equal success at the same time. It will, therefore, depend much upon the discrimination of the operator, and his judgment will no doubt be regulated by the state of the roots. It will afterwards be seen that the majority of opinions are in favour of early autumn planting; but this has reference to the state of the plant as much as to the state of the season. The autumnal state-that is, the commencement of the season of repose-begins in some plants, even of the same species, sooner than in others. Small plants may always be removed with less chance of failure than large ones, because their fibres and spongioles are less liable to injury during the operation, and hence their growth suffers less interruption. Large plants, such as trees and shrubs, can never be removed without a certain amount of injury being done to their roots, and that at the points - the very parts of most importance to them, because there the spongioles are situated; and these, if once destroyed, must be reproduced before the plant can derive nourishment for its future support. Now plants such as trees and shrubs, in the open air, require that support most early in spring, before the state of foliation takes place: it follows, therefore, that plants removed during autumn must have these organs soonest produced; those during winter, next in rotation; and those late in spring, sparingly, imperfectly, if at all; and hence, in the latter case, the trees are left without support at the very time they require it most. Plants require nourishment, although in a much less degree, even when they are in what is called a state of repose, which is that season when deciduous trees are without their leaves. "When plants are in an active state of growth, a constant perspiration is taking place from their leaves, which is supplied by the absorption of the moisture in the soil by the spongioles of the roots; and when this supply through the roots is cut off by the destruction of the spongioles, the leaves wither, the plant dies, or becomes greatly injured; but there is a period in the growth of every plant in which the leaves either drop off, as in deciduous plants, or cease to be in a state of activity, as in evergreens; and it is only in this state that the operation of transplanting can be successfully undertaken with large plants. Even when trees are without leaves, perspiration is going on to a certain extent through the bark, and absorption to supply 
this waste must necessarily be taking place at the same time through the spongioles; for though the functions of all plants are annually in a dormant state, yet they are never wholly inactive; and hence, even in transplanting trees without their leaves, the effects of more perspiration by the bark than the roots can supply must be guarded against."-Sub. Hort.

The state of the weather has very much to do with the successful removal of all plants, whether large or small. Dry, windy, and frosty weather, as well as powerful sunshine, are the most unfavourable of all. They all draw upon the vital energies of the plant, without restoring any support to it. Perspiration and evaporation go on more rapidly in such states of the weather than in any other, and this more when plants are in leaf than when they are bare, because the process is going on both by the leaves and the bark ; and this is one of the principal causes why we cannot remove large trees in leaf so safely as when the leaves are off. A mild"damp atmosphere is the most fitting for the operation of transplanting, and this will be greatly enhanced if genial showers of rain fall during the night. This theory has, however, to a certain extent, its opponents, but the arguments adduced by them are so weak, and so devoid of true physiological reasoning, as to be scarcely worth refuting. A sample, however, may be given, in those who assert that the Brassica tribe, for example, grow faster, when at transplanting they have been kept sufficiently long out of the soil to cause their leaves to fade. Decandolle appears to have favoured this view, and observes that it causes them to pump up moisture rapidly, in proportion to the degree in which their interior tissue has been deprived of it.

Mr Barron-no mean authority in such matters-on the other hand, believes that much of the success in transplanting large trees depends on the quantity of soil taken along with the roots-that is, that the size of the ball should be consistent with the size of the plant or tree to be removed; and that if this is attended to, trees may be removed any month in the year (as they have been at Elveston) with almost uniform success ; although, he continues, spring, summer, and autumn are to be preferred to winter, for this reason-" When plants are removed in a growing state, the moisture surrounding the roots is immediately absorbed, and at once assimilated as food for the plant; the wounds where the plants have been cut across or injured speedily heal over, and fresh rootlets form, all going on in a natural process without much apparent check : but in winter, when the ground is cold, and the plant in a dormant state, the moisture which surrounds the roots, instead of being taken up by the plant, remains there in a state of inactivity, and has more or less a tendency to rot and decompose certain portions of the roots where severed. Hitherto the planting of trees has been confined to that period of the year when they have ceased growing, and are in an inactive state ; and had not necessity compelled me," says Mr Barron, " to step out of the ordinary track, I might still VOL. II. have been in the dark as to what can be done successfully. Had I not ventured on some bold experiments, and prosecuted my work at all seasons, but waited for the usual time of planting, in sixty years we would not have accomplished what has been done in twenty.

"Trees of large size have been moved in the middle of summer, during hot weather, with perfect safety; and I find that when an operation can be performed under favourable circumstances, the more active the state of the plant is at the time of removal, the better it will succeed." Mr Barron "has moved spruce firs in June from 30 to 40 feet in height, when the leading shoots had grown from a foot to 18 inches; and during the operation all the young shoots, and even the leader, became flaccid and drooping; but after being planted a day and well watered, all got right again; the leader became erect, and prospered as if nothing had happened. It must be understood that summer planting is only applicable to evergreens, and such as can be depended upon for lifting with large balls of earth. It is possible, nevertheless, to transplant deciduous trees when in leaf, but only under peculiar circumstances should it be attempted."

Transplanting is an operation in connection with fruit-tree culture, that ceases not on the first formation of a garden or orchard. Deaths are occasionally taking place, and changes of opinion as to the merits of certain kinds of fruit are coustantly occurring. Where apples and pears are on healthy stocks, should the kinds not meet the approbation of the owner, these may be changed by heading them down in spring, and grafting more approved sorts on them, with much less loss of time than by replanting. This rule does not, however, apply to all the fruit trees growing in a garden, and therefore they may have to be cleared out entirely, and new plantations made in their stead. Hence transplanting is almost a daily operation in gardening. This is the case generally with strawberries, gooseberries, currants, and raspberries. The former of these require, according to the highest authorities, to be replanted once in two or three years, and some advocate the principle of replanting some varieties of them annually. Although the three next, in some very favourable localities, continue to bear well for many years, the short time required to bring them into a bearing state induces many to change their sorts, discarding such as they esteem more lightly, and replacing them with those of a higher character. Some very excellent cultivators think that the period of their useful existence is limited to a few years, and consider the fruit from plants of three or four years' growth superior in quality and quantity to those that have been in a bearing state a quarter of a century. Others make plantations every fifth or sixth year, with a view to carry out the rotation of other crops upon high cultivation principles. With these latter we perfectly agree, and this the more cordially if the ground is naturally poor, and not eminently qualified for such crops. Renewing the plantation of gooseberries has the advantage of lessening the attacks of insects, which seldom attack 
them for the first two or three years after being set in a new soil-that is, one where they have not been for years cultivated. Taking up the same trees and replanting them again becomes necessary also when the roots, as in the case of the peach, the nectarine, apricot, plum, and pear, have descended into a bad subsoil, or where the branches have extended beyond their proper limits, and interfere with the neighbouring trees, and also where, from the accumulation of soil over their roots, they have got too deep to be within the power of solar influence. These and many other causes render the replanting of fruit trees so often necessary as to make this operation a subject of annual consideration.

Many good cultivators take up a portion of their fruit trees annually, shortening the strongest roots, removing a portion of the soil, and supplying its place with fresh mould, more especially towards the extremities of the roots, and placing the roots so that they may be near the surface. In most cases this is a commendable practice; and although it involves somewhat more labour than merely root-pruning the trees, the labour is well expended; because the roots are kept within proper bounds, and induced to produce numerous food-collecting fibres, instead of strong tap and extended roots, which merely act as conductors of the sap collected by the spongiolets, and often, when they extend into a bad subsoil, throw into the system of the tree too great a supply (and that often of deleterious quality) of sap, inducing coarse and over-luxuriant wood, from which fruit need hardly be expected.

The season of transplanting established trees, in consequence of the still undecided state of opinions, extends in practice over a period beginning in ordinary cases in October and ending in April. Our own opinion is in favour of early autumn transplanting; and we would rather do so in October than in March, unless under very peculiar circumstances. We are aware, however, that many cases occur wherein the operation has to be carried on throughout the whole winter. Transplanting evergreens may, however, be safely done in April, early in May, and also during the latter end of August and throughout September; and they may be planted at other seasons also. Planting, when the term implies very young plants, seedlings, \&c., may be safely done at various other seasons, according to their circumstances and kind. The dark months of winter may, however, be regarded as the least favourable.

Under all these circumstances, we have thought it better to treat the subject of transplanting generally, and under one head, offering first a few remarks on the preparation of the ground, as on this as much of the success depends as on either the season or manner in which the operation is performed.

Preparing the ground for planting.-The nature of the tree to be planted should always regulate the extent and manner of the preparation. If we except the quarters of a well-made garden, and the borders for fruit trees-and even these are not always exceptions-we will find that far too little attention has been paid to the sub- ject. In reference to ornamental trees and shrubs, where bulk and full development of form are the objects sought, we may here observe, that care in preparing the ground for them is as essentially necessary as for the most delicate fruit-tree. But how seldom is this attended to, and how frequently is the practice followed of digging pits large or small, according to the size of the tree or plant, setting the tree in it, and filling in the soil-be it what it may-around the roots, while the ground between the plants is left wholly undisturbed. Should the tree live, and the roots extend as far as the limits of the pit, they are in general arrested there in their progress by the formidable obstruction of impervious undisturbed soil. The first consequences are, that the roots are limited to the circumscribed pasturage of the original pit, which in time becomes exhausted, and unable to afford nourishment to them. If the soil is clayey or damp, the tree may be considered as placed for half the period of its existence in a state of puddle; the roots, being prevented from extending themselves in proportion to its size, become easily overturned by wind, and, in fact, it is reduced to the condition of a tree grown in a large flower-pot, the soil within which cannot long continue to supply food to it, and hence it languishes and dies of starvation; whereas, if the soil be trenched previous to planting, these evils are avoided. The more we comminute the soil, the more nourishment will be absorbed by the roots, and the more vigorous and healthy will the plant become. "Trees, irrespective of fruit-bearing ones, far more than agricultural crops, require depth of soil to raise them to perfection; the effect of climate appears much less necessary in giving them their greatest magnitude. If, in transplanting, we must often increase the cold, and other circumstances adverse to trees, it becomes us the more diligently to study that the soil be rendered as rich and deep as possible, in order in some sort to counterbalance those disadvantages. Let it be observed, also, that an open soil, besides being favourable to the transmission of nutriment to the roots of plants, is likewise favourable to their extension, and thereby enlarges the field whence nutriment is derived. Nor are these the only benefits resulting from a friable soil; for, in addition to its being adapted to supply vegetables with food, it is always most suitable for effecting those changes in the soil itself which are equally necessary to the preparation of such food."-Mr Withers' letter to Sir Henry Steuart.

There are other reasons why ground intended to be planted should be prepared both by draining and deep trenching, the principle of which is, "that the roots near the stem become in time inactive, and have little or nothing to do as preservatives of life, except by acting as conduits, while the functions of absorption go on through the spongioles, which, being at the extremities of the roots, extend wherever food and moisture are to be found. This property prevents a plant from exhausting the earth in which it grows; for, as the roots are always spreading farther and farther from the main stem, they are continually 
entering new soil, the nutritious properties of which are unexhausted."-Lindley's Introduction to Botany, vol. ii. p. 180.

In former times, fruit-tree borders were made as deep and as rich as the means within the reach of the maker could accomplish, just on the very principles best fitted for the growth of timber, and perfectly in accordance with $\mathrm{Mr}$ Withers' views, and, consequently, sterility in the trees (if fruit-bearing ones) was the consequence. From this very cause also proceed late and immature growths, and that in a climate where every ray of heat from the sun is of so much importance. Modern practitioners, sensible of these errors, have adopted a different and more rational course in the formation of shallow borders, and those elevated above the general surface, or placed at the most favourable angle of inclination, so as to expose the roots to the influence of the solar rays. This elevates the temperature of the soil so as to bring it in advance of the atmosphere; or, in other words, renders it so much warmer that the roots become excited before the branches. The first effects of this is a healthy and moderate growth, and early maturity of the wood in fruit trees. The same rule should be followed in planting standard fruit-trees, because the same results are wished to be attained. In the case, however, of trees planted with a view to obtain the greatest bulk of timber within the shortest period of time, as well as of those planted for ornament, the case is widely different: for them the ground cannot be trenched or prepared too deep; and the same holds good in planting shrubberies. Single specimens, on the lawns or in the park, require equal care, the pits for which should not be less than three times the diameter of the ball, or of the extent of the roots. Many tender shrubs and trees should be planted on slightly raised mounds, so that their roots may be within the reach of solar influence, to assist in exciting them to earlier summer growth, and, consequently, enabling them to mature their wood more completely early in autumn, and hence escape the chance of being killed by frost during winter.

Preparing the ground preparatory to planting fruit trees is a subject of very great importance, whether the operation extends to the planting a new garden, or embraces only the making up deficiencies in one already existing. On the formation of fruit-tree borders we have expressed our own views as well as those of others, vol. $i$. pp. 27-32. The principles there laid down we consider quite sufficient to meet the generality of cases. These principles are-a dry bottom, proper breadth and depth, a congenial soil, and leaving the surface over the roots uncropped with flowers or vegetables. These identical principles apply also to orchard trees, or such as are grown without the protective shelter of walls, and in a modified degree also to individual trees planted to make good deficiencies, from whatever cause they may arise. In connection with the latter case we may here briefly remark, that the soil, for a very considerable distance around where a tree has been removed from, has become almost completely exhausted of those elements necessary for entering into the constitution of its successor ; and it may contain the seeds of disease, and abundance of the eggs of insects that have played their part in hastening the decay of the former. Surely we cannot be blind to the necessity of removing the whole, or a considerable part at the least, and supplying its place with fresh soil suitable to its kind. All plants have a strong affinity for new soil, and fruit trees in an especial degree. The soil of a long-cultivated garden, be it ever so good for the purposes of ordinary cropping, is very unfit for the sustenance of fruit trees, and this the more so according as it abounds in humus. Turfy loam from a field or common which has been long in a state of pasturage, and on which no trees of any kind have grown, is of all the most proper, and the fresher from the field it can be applied the better, and also the nearer it is taken from the surface, carrying in with it any fibrous vegetable matter that may be growing upon it. In this there is, however, some discrimination required, light friable loam being most suitable for the peach, strong clayey loam for the pear, plum, and cherry ; while the apple, fig, and apricot will be quite at home in a soil intermediate between these. Where fruit-tree planting is to be carried on upon an extensive scale, the preparation of the ground should be carried on for at least during two or three months previous to planting, so that the ground may be completely drained, trenched, and exposed to the action of the weather. Where blanks only are to be filled up, and the trees to be removed are only recently cleared of their fruit, there is less time for such preparation. The spot upon which the young tree is to be set, as well as that of the border, to the extent of 4 or 5 feet from the stem, should, however, be prepared to that extent at the time of planting. 'This will afford sufficient scope for the roots of most trees during the first, and probably the second season; and additions to this can be made from time to time afterwards.

We are of those who believe that this yearly adding new soil to the roots of fruit trees is infinitely better than making the border, unless in the case of new gardens, all at once. A prepared space, with a radius of 4 feet, is amply sufficient for the roots of any tree for the first year after planting; that space to be yearly extended by removing a zone of 18 inches or 2 feet in breadth, and filling it up with prepared new soil. It gives the operator, at the same time, an opportunity of regulating the extension of the roots, either by curtailing them, should a too luxuriant habit be indicated, or to encourage them with generous food, should an opposite state be apparent. Under some circumstances it is more convenient also, as requiring neither an extraordinary quantity of material nor an extra amount of labour at one time. This indulgence, however, should not be allowed to extend so far as, after a year or two's compliance with the rule, to neglect it altogether. The subject of concreting, vaulting, ventilating, \&c., has been already explained in vol. i. pp. 27-32; and as further notice of these improvements will be found in the forcing-garden department, 
art. VINE, a detail of them is here unnecessary. The soils also for each kind of fruit tree will be described under each.

Early autumn transplanting has long been recognised as a leading feature in good management. It is founded on correct physiological principles, and practical experience has confirmed the soundness of it. Miller, and since his time most of the leading horticulturists of this country, have been of opinion that immediately after the fall of the leaf in autumn is the best season for the operation, in all situations where the soil is dry; but others, differing in this respect, recommend that the end of February, or indeed until the sap is beginning to rise, is to be preferred. This latter is an extreme case, and if the soil is so wet as to render this delay within even the verge of probability, this indicates a very unfit situation for trees not actual aquatics. Many excellent gardeners recommend transplanting so early in autumn that even a considerable quantity of the leaves in a mature and active state should still remain on the trees ; for, they say, by the action of such leaves the injuries which the roots may sustain during the operation will be speedily repaired, new roots will be produced, and the plant will thereby become established before winter, and be in a condition to start into growth in spring, little, if at all, affected by its removal. "Early in autumn is undoubtedly the best time, considered physiologically, because then, whether the plants are with or without some of their leaves, the wounds made in their roots will begin to cicatrise, and to protrude granulous matter, and in many cases even spongioles immediately; and by the time spring arrives, the plant, if it has been taken up with most of its roots, will grow with as much vigour as if it had not been transplanted. For obvious reasons, the next best season to that immediately following the fall of the leaf is the remainder of the autumn, and the winter months during open weather. There may be local reasons why the beginning of spring may be preferable to autumn, but such reasons can never apply generally. A second argument in favour of autumn planting is the dampness of the atmosphere which prevails at that season and during winter, by which the perspiration through the bark is lessened, and the demand made on the roots to supply the waste is consequently diminished. In spring, not only is the sun more powerful, but drying winds generally prevail, which have a constant tendency to drain the young branches of their moisture. These drying winds are much more injurious to newly-transplanted evergreens than to deciduous trees, as will afterwards appear."-Sub. Hort. In early autumn the temperature of the soil is much higher than it is in winter or spring, on account of the solar heat absorbed by it during summer being still retained in it; and this warmth is of all things the most favourable for the production of young roots, a circumstance well known to gardeners. This of itself is, in our opinion, sufficient ground for insisting on early autumn planting. The season of transplanting, as has been already remarked, extends from thelatter end of September till the middle of February - that is, from the period when the leaves begin to change colour until the young fibres of the roots begin to form in the spring. No doubt the operation may, and often is, performed much later in spring, and also sometimes, under peculiar circumstances, even throughout the summer. In reference to the former we may remark, that trees transplanted after the middle of March have not justice done them. Many are afraid of even October planting, because the leaves are still on the trees. Let no such fears be entertained, for if trees be planted then, they will push new roots before the severe weather sets in, and will so far have established themselves before the first movement of the sap in spring. If trees are not transplanted sufficiently early in autumn to make new roots that season, it is much the same as if they had not been planted till early in spring; for new roots - the formation of which is the primary effort of every transplanted treewill not and can not be formed during the dead months of winter, the temperature in the soil being much too low to enable them to be so. If transplanted in the end of September, or during the month of October, in ordinary soils, the solar heat absorbed during summer being still retained, will have much the same effect upon them, only in a somewhat less degree, as if they were plunged in the genial heat of a moderate hot-bed. The whole rationale of early autumn transplanting is the warmth of the soil, and the humidity then existing in the atmosphere.

Downing bears similar testimony in favour of autumn planting, and says, "that the best season for transplanting all deciduous trees is in autumn, directly after the fall of the leaf. The tree is then in a comparatively dormant state. Transplanted at this early season, whatever wounds may have been made in the roots commence healing at once, as a deposit directly takes place of granulous matter from the wound; and when the spring arrives, the tree is already somewhat established, and ready to commence its growth. Autumn planting is for this reason greatly to be preferred in all mild climates and dry soils, and even for very hardy trees like the apple, in cold latitudes, as the fixed position in the ground which trees planted then get by the autumnal and early spring rains gives them an advantage at the next season of growth over newly moved trees. Evergreens may also be planted at the same season; but with them, as with deciduous ones also, the operation should be suspended during frost, and, if possible, advantage taken of mild damp weather."

Reasons for very early autumn transplanting.Winter and early spring are considered by many experienced planters to be the very worst seasons for transplanting large trees, whether evergreen or deciduous, because at that period their roots are in a comparatively inactive state, and consequently must suffer from having to remain long dormant, and unable to take hold of the new soil so as to draw nourishment from it for the support of the tree. Hence, such say that summer, when they are in their most active state, would be the proper season for this operation, provided we had such a command over 
the atmosphere as to prevent too great a drain on their mutilated roots, for the supply of the leaves in the process of perspiration caused by the powerful influence of the sun's rays on their porous tissues. Shading, to prevent evaporation, and creating an artificial atmosphere around them, would do much to secure success, but the difficulties attending the accomplishment of these conditions are not easily overcome.

"It behoves us, then," says Mr Middlemas (in "Magazine of Botany"), " to look for a period when the tree is neither in a torpid state nor in its most active growth, nor when the sun's rays are so destructively powerful on trees unprepared with a sufficient supply of evaporative matter for the roots to satisfy the demands made upon them by a hot dry atmosphere; and we shall find that from the middle of August to the end of September is the best period, as providing for the circumstances of our case."

Reasons against spring transplanting.-We find Dr Lindley exposing the evils of spring planting both in his excellent " Theory of Horticulture," and elsewhere in his voluminous and useful writings. In a leading article in "The Gard. Chron." he observes, in directing attention to late spring planting, "If we search for solid reasons why spring planting should be adopted, we fail to discover the least resemblance to them ; or if resemblances to reason do appear, they vanish the moment they are examined. The great argument in its favour is its occasional success, but this is of all reasons the most unsatisfactory. The cause of death, when trees are removed, is almost entirely that they lose the fluid contained within them faster than it can be renewed, the end being the drying up of their vessels, which is immediately followed up by a loss of vital force. If we inquire whether the circumstances to which spring-planted trees are exposed are favourable or unfavourable to this fatal loss of fluid, we find them to be the former in an enormous degree. The air is peculiarly dry in the spring, and frequently in rapid motion at the same time : all objects exposed to a current of dry air must part with their moisture rapidly, and consequently such a state of things is most unfavourable to plants which require to retain their moisture. At first their young bark is the channel through which the moisture flies off; but as soon as young leaves appear, should the trees live long enough, and the perspiring surface is thus extended, this loss goes on with far greater rapidity, and life is soon extinguished. Evergreens, which have always a very large perspiring surface, are on that account exposed to much more danger, and consequently the losses among them are much greater. That the excessive loss of fluid from the interior is the true cause of death in newly-planted trees was proved by the late Mr Knight, who surrounded their stems with damp moss, and thus preserved them. Established trees suffer nothing from the dry air of spring, because their roots are unmutilated, and restore the moisture as fast as it flies off from their surface. But newly-planted trees, whose roots are necessarily much mutilated, suffer, because they can- not obtain fluid from the soil until the roots are renewed, and before that can happen they are dead; for the feebleness of the stem actually diminishes the capability of the plant to renew its roots. But it is said, if a tree is just budding when planted, it is in the most favourable state, because it will immediately make fresh root, the act of vegetation upwards being simultaneous with growth in a downward direction. There is here, however, a fallacy : it is assumed that the upward and downward vegetation will go on when a plant is transplanted, as well as if it had been left in its former place: that, however, depends on the external conditions to which it is exposed. If the surrounding air is damp, and remains so, evaporation being thus prevented for a sufficiently long time, roots will be quickly formed, and the plant will go on growing; on the other hand, if the air is dry, and exhausts the branches of their moisture, new roots cannot be formed, and the plant will die. Life in such a case is staked upon the chance of the atmosphere being in a very favourable state, and the chances are ten to one against its being so. These considerations abundantly prove the spring to be the worst of all seasons for a planter."

Other seasons for transplanting.-There have been many instances of successful transplanting at all seasons except midsummer; and there are many, as Downing remarks, "who, from having been once or twice successful in transplanting, when trees were nearly in leaf, avow that to be the best season, not taking into account that their success was probably entirely owing to a fortunately damp state of the atmosphere at the time, and abundant rains after the experiment was performed."

The theory of transplanting by the preservation of the spongioles of the roots. - This theory, although long recognised by physiologists, was first brought into general notice by $\mathrm{Mr}$ George Lindley, in the "Guide to the Orchard." "It is well known," he remarks, "that plants feed upon fluid contained in the soil, and that their roots are the mouths through which the food is conveyed into the body. But the absorption of fluid does not take place either by all the surfaces of their roots, nor even of their fibres, but only by the extremities of the latter, consisting of bundles of vessels surrounded by cellular tissue in a very lax spongy state, whence those extremities are called spongioles. It is only through the spongioles that absorption to any amount takes place. These spongioles are exceedingly delicate in their organisation, and a very slight degree of violence destroys them. It is scarcely possible to remove the soil from the roots without injuring them in some degree; and if transplantation is effected violently or carelessly, they are in a great measure destroyed. In proportion to the size or age of a tree is the difficulty of preserving them increased, and hence, at the same time, the difficulty of transplantation is augmented. If by any method the spongioles could be preserved unharmed, there would be no reason whatever why the largest forest trees should not be removed as easily as 
the young plants in a nursery ; but their preservation in such a case is impossible, and therefore the transplantation of trees of great magnitude cannot be effected. It is because of the security of the spongioles from injury when the earth is undisturbed, that plants reared in pots are transplanted with so much more success than if taken immediately from the soil. Hence, also, when earth is frozen in a huge ball around the root of a plant, transplantation is effected with the same kind of certainty. The practice of cutting the roots of large trees the year previous to removing them, is attended with success for a similar reason; wherever the roots are cut through, the new fibres which are emitted, provided the plant is in health, in short tufts, and each terminated by a spongiole, are much more easily taken out of the ground without injury than if they were longer and more scattered among the soil. When destroyed, the spongioles are often speedily replaced, provided a slight degree of growth continues to be maintained. This is one of the reasons why trees removed in October succeed better than if transplanted at any other time. The growth of a tree at that season is not quite over; and the first impulse of nature, when the tree finds itself in a new situation, is to create new mouths by which to feed when the season for growing again returns."

The importance of preserving the spongioles has been denied by Rogers, a practical writer of credit, and little regarded by planters in general; while it has been admitted by Downing, Loudon, Sir Henry Steuart, Decandolle, Dutrochet, Bonnet, Papin, Sennebier, Lindley, and others. Rogers gives as a reason for disregarding the preservation of the spongioles, that they die annually as soon as they have performed their allotted functions. "In this the man of science," he remarks, "is at fault, because the practical man knows that leaves on the head of a tree are produced at the same time as the fibres are on the roots, and in many cases both FALL OFF together. The root fibres (or spongioles, if the learned must have it so) of the apple do not, indeed, all fall off, but certainly all become inert and torpid during winter. Besides, it is not altogether to the preservation of the last year's fibres that the planter looks for success; nor can the transplanted tree be much benefited by saving those organs which have already done their duty. Both the one and the other must rely on the new vigorous fibres which will be produced in the new station; and if a removed tree be planted in soil which is fine, moist, and warm enough, no fears need be entertained but that new fibres will quickly come forth to carry on the growth of the plant. In transplanting a tree, however, there is no necessity for needlessly exposing the roots to the sun and drying air; this might enfeeble the main roots, which would be of worse consequence to the plant than the mere loss of the spongioles-a majority of which would certainly have died, even if the tree had been allowed to stand in its former place." The Rev. Patrick Keith seems to be of nearly the same opinion; while the author of "The Domestic Gardener's Manual" thinks " that it appears unphilosophical to consider any parts of the root to be fugacious and perishable which have been propelled by the permanent members of the tree: such are the radicles or rootlets attached to the young shoots of the past year."

This office of the spongiolets has been disputed by Link, who observes, "Many physiologists assert that the root points suck up the nutritive juices from the soil, and through the roots convey it to the plant, and hence Decandolle called them spongioles. Ohlert, an acute observer, proved that this is not at all the case, adducing in evidence of his opinion, that plants whose root points or spongiolets hang in the air continue to grow even when these points are cut off and closed over with sealing-wax. In that case, however, he admits that it is necessary the roots themselves should be in water, or in very damp earth. It is worthy of remark, that precisely at the point where the absorbing surface commences, there also the vessels with their accompanying cellular tissue commence."

As all plants draw a considerable proportion of their food from the soil, it follows as a fact doubted by no sane person, that that food, to enter into the structure of the plant, must needs be in a liquid state. No doubt, plants also derive much support from the atmosphere, which is drawn up by them in a gaseous form through the pores of the leaves as well as through the bark of the trunk and branches. These latter views are entertained by Duhamel, Marriotte, Bonnet, Papin, Priestley, Greer, Saussure, Dutrochet, Keith, \&c.

The ancients had clear enough perceptions of the absorbent power of the roots; and Anacreon, in one of his little poems in honour of drinking, makes the very trees of the forest drink. Two lines in ode 19 may be thus translated:-

$$
\begin{gathered}
\text { "The black earth drinks, } \\
\text { And the trees drink it;" }
\end{gathered}
$$

meaning the moisture the earth contains.

To reach the plant, this moisture must pass through their roots; and if we examine how this is done, it will clearly enough appear that the liquid food is drawn into the roots by those innumerable little absorbent bibulous sponges (spongiolæ) with which even the finest fibre of the root is furnished. "This is the grand apparatus," says Keith, "that nature has destined to the office of the absorption of vegetable nutriment; and it is owing to the powerful absorbent property of the spongiolæ of which it consists that the scientific gardener, in the transplanting of his young trees, or the scientific and ornamental planter, in the transplanting of his trees of full growth, is so extremely careful to preserve entire even the minutest fibre and extremities of the roots."

The experiments made by Hales, many years ago, being made chiefly on the sections of roots laid bare and merely immersed in water, show only to a limited extent the absorbent power of roots, but in no way illustrate the natural action of the spongiolæ, collecting nourishment at ten thousand different points, acting simultaneously together in taking in moisture from the soil. In this respect his experiments are defective. "The absorption of juices by the 
roots takes place solely by the extremity of each radicle fibril, or, which is to say the same thing, by the spongioles which terminate each of their ramifications." Decandolle had some idea of this when he remarked that young trees exhaust the earth very near their trunks, while the old ones with horizontal roots produce this exhaustion at a distance from their trunks, the more considerable in proportion as they are larger.

Modern physiologists have confirmed this opinion, in remarking the longitudinal direction of the fibres, and the thickness of the cellular envelop, which prevents the juices from reaching them laterally. Sennebier has also shown this by very conclusive experiments. "It is certain, then," he says, "that the absorption of roots only takes place by their extremities: we may here mention that it is, then, at the extremities of the roots, and not at the base of the trunk, that we ought to apply water, manures, and, in general, all the substances which we wish plants to absorb."

If preserving uninjured the spongioles, and consequently the roots of trees, even to their very extremities, be of the importance stated by the authorities above, then it would appear that, in removing a plant or tree, far less of the success depends on the compactness and size of the ball, providing every root is not contained within it, than on the preservation of the roots, which can only be preserved to a very limited extent, as all beyond the ball must of necessity be cut off. As trees increase in age and size, so do their roots diverge from their stem-no doubt in search of food, and also to enable them to maintain their natural upright position; and it is no uncommon thing for shallow-rooting trees - the Abies tribe, for example-to extend their roots horizontally to the extent of 40 or 50 feet, that is, giving a diameter to the field they occupy of from 80 to 100 feet. Now, according to the doctrine maintained by the advocates for the spongiolet system, the whole of the foodabsorbing portions of the roots must occupy a zone forming the circumference of a circle whose diameter is equal to that given above. For Decandolle, if we mistake not, stated the theory, and almost every physiological writer of eminence since his time has confirmed his views, that the absorption of the food takes place solely at the extremity of each radicle fibre, by the spongiolets which terminate each of the ramifications. If these opinions be correct-and they have not been as yet controverted-it would follow that it were better to dispense with a ball altogether, and direct our whole attention to the preservation of the roots, even to their most minute points; and hence we apprehend that washing the soil entirely from the roots, tying them up in bundles, enveloping them in moss, and using every other possible means for preserving them uninjured to their full extent, will yet be considered the most likely means to insure the successful removal of very large trees.

The ball system of removal, however long sanctioned by practice, must be defective in every case in which the ball does not include within it every rootlet and spongiolet; and this can only be in the case of very small and young plants, whose roots have not extended beyond a radius of 4 or 5 feet from the trunk; and although the rule is not without exceptions, it may be set down as pretty near the average, that the spread of the tree may be taken as nearly that of the horizontal extent of the roots. "Roots, in their lateral extension, bear usually a relation to the horizontal spreading of the branches, so as to fix the plant firmly, and to allow fluid nutritive substances to reach the spongiolets more easily."-BALFOUR in Manual of Botany, p. 66.

The prevention of excessive evaporation at and after the process of transplanting. - "Evaporation takes place in plants to an inconceivable degree in certain circumstances. In damp or wet weather this evaporation is least; in hot dry weather it is greatest. This loss has all to be supplied by the moisture introduced into the system by the spongioles; and hence, if the spongioles are destroyed, and evaporation takes place before they can be replaced, a plant must necessarily die. This is the reason why deciduous trees cannot be transplanted while in leaf: it is impossible to remove them without injuring their spongioles, and it is equally impossible to hinder the evaporation by their leaves; but if they are kept in pots, it matters not at what season their removal takes place, because, as their spongioles are then uninjured, even excessive evaporation would be made good by their action. It is well known that certain evergreens can be transplanted in almost all months ; this arises from their perspiration being much less copious than in deciduous trees, wherefore the spongioles have less difficulty in supplying the loss occasioned by it ; yet even evergreens cannot be removed in the hottest months of the year, because then the action of such spongioles as may be saved in the operation would not be sufficient to supply the waste by evaporation. Plants first beginning to grow in the spring, with their leaves just turning green, are in a most unfit state to remove; for, when transplanted, their roots will not have time to form a sufficient number of new spongioles to supply the loss to which the rapid perspiration by the leaves at that season will give rise." George Lindley. In these views Mr Lindley is supported by Dr Hales, Guettard, Knight, and others.

Excessive evaporation may be greatly counteracted by shading, and copious applications of water thrown over the foliage and branches by a fine syringe or garden engine. We say a fine syringe, because the water so applied should be as much in the form of dew as possible, and applied night and morning until the roots have made sufficient progress to be able to supply the trees by natural means.

Preparing the places for transplanted trees.Digging pits is the usual term applied to this operation, and in too many cases the term is not misapplied ; for thousands of trees annually have their roots thrust into pits from which few of them ever escape. He who prepares a space sufficiently large, so that the roots maybe spread out to their fullest extent in all directions, 
loosening the ground below, applying, if need be, fresh soil, either in whole or in part, and taking care that the roots be not placed too deep, may be considered as one who looks upon a tree as a living subject, whose life is to be rendered long and vigorous by a good supply of food, and a soil mellow and easily penetrated by the smallest fibre. He, on the other hand, who thrusts the roots of his trees into the smallest possible hole, and supplies them with the least possible quantity of proper soil-trusting to what he seems to believe the inextinguishable powers of nature to make roots and branches under any circumstances-is starting in a very false direction.

"There is an unhappy propensity prevalent to consider a tree as destitute of the wants of plants in general, and to believe that if it is provided with sufficient soil to cover its rootsno matter what the quality may be-it cannot possibly fail to thrive ; but, on the contrary, care and attention are as imperative in preparing the soil for trees, and will be followed with equally satisfactory results, as in the treatment of any other of our cultivated plants."-STANDIsH, in Practical Hints on Planting Ornamental Trees, p. 6.

The size of the pits for the reception of the roots depends much upon the circumstances whether the ground has been thoroughly prepared by draining, trenching, and the addition of proper soil, or whether the tree is to be planted where other trees formerly stood. In the first case, an opening twice the diameter of the roots, as we have formerly stated, when fully spread out, will be sufficient; while, in the latter case, the space should be at least double that size, having the whole of the old soil removed, and replaced with new.

Filling in the soil at transplanting.-This is an important matter in securing success. When the roots are spread out to their full extent on the prepared bed-which should not be the hard and impervious subsoil, but a bed of prepared compost, into which the roots may penetrate and find nourishment-the soil, which should be well pulverised, must be worked in, so that every part of the surface of the roots may be covered. Much of the success in transplanting depends on this ; and more fully to accomplish it, water in moderate quantities may be given, particularly in light soils, to wash the finer particles among the roots, so that no cavities may be left about them. Watering at autumn planting is, however, of much less importance than in spring, particularly if the planting has been delayed to a late period. Where the soil can be wrought properly around the roots in a dryish state, so much the better, as the rains during winter will consolidate it, and supply the roots with the humidity they require. Although we here recommend filling in around the roots with finely-pulverised soil, we mean that only so far as to cover them. The compost beyond this can hardly be too fresh, and should contain a large portion of fibrous matter, such as turf taken from a rich field cut up into pieces by the spade, which is a very necessary precaution in the case of trees planted in what are called prepared borders.
"In filling in the soil, avoid the absurd, although very general practice, of moving the tree backwards and forwards to get the soil down among the roots. This I have called an absurd practice, for every pull you give the top draws the roots out of their places; and when the tree goes back into its proper place, the roots, being unable to push themselves back again, remain doubled up under the tree, thus thwarting the object you had in view, of spreading the roots carefully out at the commencement."-MIDDLEMas in Magazine of Botany.

In planting fruit-trees where the subsoil is of indifferent quality, it is advisable to place under each a piece of pavement 3 feet square and about a foot or 15 inches under the surface, that the descending roots, when they come in contact with it, may be made to take a horizontal direction instead of a perpendicular one. This is one great means of keeping them near the surface, and has been found of much advantage in this respect, even when the soil is of the very best quality, and whether the bottom of the border has been concreted, vaulted, or resting on a drainage of broken stones or not. Where these improved modes have not been attended to, such pieces of pavement may be of larger size; and where the subsoil is wet, cold, or bad, they should be laid hollow underneath, that dryness may be secured. This is a practice of old standing, many of the oldest fruit-trees still existing in monastic gardens being found so provided. In planting where the ground has not been thoroughly prepared, instead of filling in the soil only taken from the pit, it is of advantage, after the rootlets are slightly covered with fine compost, or the best of that taken out of the hole, to break down the sides of the pit all around, and to use it also for working in amongst the roots, as by this means the size of the pit is increased, and the scope of loosened soil for the roots considerably extended. In planting rare or ornamental trees in parks, the surface turf should be broken down and placed in the bottom of the pit; and where it can be spared, a ring of turf should be taken off all around, and wrought up along with the soil used in covering the roots.

Treading the soil about the roots of trees at transplanting is a dangerous operation, more honoured in the breach than the observance. The soil should be carefully wrought in about the roots by the hand or with the end of a blunt stick; for the pressure of the foot, unless carefully applied, is apt to break off the principal roots unobserved.

Manuring trees at transplanting.-This is an old and barbarous custom, practised only by those who know nothing at all about the matter. Naturally rich and fresh loam is all the manure they require. Trees require manuring only when, by reason of old age, or by having exhausted the food natural to them within the range of their roots, they become exhausted from want of sufficient food.

Mulching trees at transplanting is an old but useful practice. It means covering the surface above where the roots are, immediately after planting, with light littery matter-moss, decayed leaves, or the like-with a view to exclude 
frost, or rather to prevent the escape of heat, and lessen the evaporation of the moisture from the soil, and hence to preserve the roots from suffering from drought. Mulching with rich manure is injurious, unless in the case of vines or trees requiring greater stimulus than the soil affords.

Shallow transplanting.-Deep planting is one of the greatest evils to which trees, particularly fruit-bearing ones, are subject. No tree should be planted deeper than it formerly grew, as its roots are stifled from the want of air, or starved by the poverty of the soil at the depth at which they are placed. It is much the better and more natural process, in fact, to plant the tree so that it shall, when the whole is complete, appear just as deep as before, but standing on a little mound 2 or 3 inches higher than the level of the ground about. This, when the ground settles, will leave it nearly level with the previous surface. "I advise," says Harrison, "that the roots of fruit trees against a wall be not planted more than 6 inches deep, and plant nearer the surface than 6 inches in wet heavy soils, and deeper in those that are lighter."

"Nothing is more common than too deep planting, and the temptation to it is greater, because deep-planted plants, from having their roots more accessible to moisture, are more cer. tain of growing the first year, and are less in want of mulching to exclude the heat and drought, and of staking to prevent them from being moved by the wind." Deep planting, by placing the roots almost beyond the reach of solar influence, retards the growth in spring, and continues it so late in autumn that the wood and buds are seldom properly ripened : therefore in cold and wet climates deep planting should be avoided, as tending greatly to increase the evils such climates are known to possess. There are some plants of a herbaceous character which do not suffer from deep planting, of which the horse-radish may be given as an example; the majority, however, do suffer, and shrubs and trees in an especial degree. This fact, although long known, appears never to have been physiologically or satisfactorily explained. In tracing the analogy which exists between vegetables and animals, the evil of deep planting has its counterpart in the injury that may be inflicted upon the latter by wantonly or accidentally causing damage to be done their most vital parts. All vertebrate animals may be deprived of existence, if serious injury be done to that part at the back of the neck between the spinal marrow and the brain; and the same fatal consequences will follow in a plant if injured at that part which connects the root and stem, which is the part of all others the most susceptible to injury, and is called the neck or collar of the plant. If a seedling plant be cut over at that point, the root immediately dies; but when a tree and most herbaceous plants have attained a certain age, this is less likely to happen, in consequence of their being then furnished at that point with adventitious buds, which, should the tree be cut over, are called into action, and throw up shoots or suckers; but if the collar be cut through, no such action can take place. Deep VOL. II. planting produces a similar fatal result, although not so instantaneously; and hence the unhealthy and ultimate death of all trees either planted too deep or having earth heaped over the roots, and thus burying the collar or vital part. This part also is so sensitive as to be more readily injured by cold than any other part of the plant; hence the necessity of protecting half-hardy plants by covering it during winter. To this reference will be often made in speaking of the protection of half-hardy plants, trees, and shrubs.

Transplanting on raised hillocks.-Many excellent cultivators, with a view to keep the roots of their trees, more especially fruit-bearing ones, near the surface, that they may be within the reach of solar influence, and often to prevent their entering into a bad subsoil, plant on raised mounds or hillocks. In this, so far as fruit trees are concerned, and also where the soil is damp naturally, or rendered so by a wet climate, they do well, because it is the most economical way of finding a good substitute for a bad soil, and in many situations it is wise to have recourse to it. This need not, however, be carried any further than to set the roots upon the natural level of the surface, covering them sufficiently with good soil, which will, for all ordinary purposes, be found sufficient.

No man has had greater experience, or has succeeded better, in the removal of trees, than $\mathrm{Mr}$ Barron of Elveston. On the advantages of mound or hillock planting he says, in "The British Winter Garden," p. 37- " Much advantage would be gained in every way by placing the tree on the surface of the ground, and by adding soil for the roots to be planted in. By this means the roots, instead of being cramped in a pit, and arriving speedily at the subsoil, have the depth of the natural soil to sport in, besides the free access of the air (so essential) being secured. The trees, when planted, have on this plan the appearance of being placed on raised bases, and in this way height is gained." This opinion is founded on sound practical experience. The physiological reasoning by Dr Lindley, in "Introduction to Botany," vol. ii. p. 181, given below, confirms the above, and proves to us the advantage of mound planting, by reason of its enabling us to add with so little trouble to the extent of the pasturage of the roots, if the object aimed at be bulk of timber or size of shrub, as well as circumscribing the range of the roots, keeping them from penetrating a bad subsoil, and above all elevating the temperature around them, if the object be healthy and productive fruit-trees. "It is generally believed that roots increase only by their extremities, and that, once formed, they never undergo any subsequent elongation. This was first noticed by Duhamel. Variations in this experiment, which has also been repeated by knight, produced the same result; and the whole phenomena appears to be one of those beautiful evidences of design which are so common in the vegetable kingdom. If plants, growing in a medium of unequal resistance, lengthened by an extension of their whole surface, the nature of the medium in which they grow 
would be in most cases such as the mere force of their elongation would be unable to overcome; and the consequence would be, that they would have a twisted, knotted form, that would be unfavourable to the rapid transmission of fluid, which is their peculiar office. Lengthening, however, only at the extremities, and then by the continual formation of new matter at their advancing point, they insinuate themselves with the greatest facility between the crevices of the soil. Once insinuated, the force of horizontal expansion speedily enlarges the cavity; and if they encounter any obstacle which is absolutely insurmountable, they simply stop, cease growing in that particular direction, and follow the surface of opposing matter, till they again find themselves in a soft medium."

Replanting the ground formerly occupied with trees. - Never plant trees in the same spot where an old tree stood before, as the ground has become exhausted of that very food upon which only the young tree would have to depend for its support. The ground, however, in such cases as that of wall-tree borders, may be removed, and fresh soil brought to replace it. Other reasons have also been assigned for this, the principal of which are those of Brugmans and $\mathrm{Ma}$ caire, who believed that the roots of plants return a portion of their peculiar secretions back ayain into the soil. More recent and more satisfactory experiments have not strengthened their opinions, but so far reversed them, that the root secretions are now regarded as unimportant, if not altogether apocryphal, except in cases where the roots have been wounded.

Supporting trees after transplanting.-This is an important operation, and deserves much more attention than has hitherto been paid to the subject. When a tree or shrub is removed to a new place, the operation of lifting, be it ever so carefully done, destroys many of the extreme points of the roots, all of which act as subterranean guys, maintaining the tree in an upright position, and resisting the severest gales that blow. They in this case exemplify one of those many and wonderful provisions made by the Creator of all that is good and perfect, even in the economy of the vegetable kingdom. The roots here perform a compound office-they collect and prepare the necessary food for the plant, and at the same time, by their united strength, support it in that position in which only it could exist. These supports, therefore, being cut off, artificial ones must be provided, until the trees are re-established again. In planting fruit trees against walls, the branches should be immediately attached to them, to prevent their being broken, or the bark injured by friction (the necessary pruning to be attended to in spring). Standard trees of whatever size, as well as shrubs, demand attention in this respect, as they are more exposed to the action of the wind. If they are small, single stakes will be sufficient ; but if large, stronger poles, and of greater length, will be required, and these should be placed in a triangular form, let into the ground at a proper distance from the roots, and brought together at the tops, in such a way and at such a point as shall best act by there oppos- ing resistance in maintaining the perpendicular position of the tree. Care must, however, be taken that a collar of some elastic or soft material, such as straw, shavings, moss, gutta-percha, $\& c$. , be placed at the point of pressure, to prevent the bark being injured by friction, or indeed extreme pressure during high winds.

There are many ways of supporting newlytransplanted trees and shrubs. Some make use of strong wire made fast to them at a considerable height, and fastened to stout pegs driven into the ground, forming with the surface an angle of $45^{\circ}$; while others use cords or ropes of various strengths, according to the size and exposure of the trees. No doubt either of the latter are more light and elegant, so far as appearance goes, and therefore well fitted for use in the ornamental parts of the grounds, but neither are so strong or so steady in their support as larch or other poles set against them in a triangular form. The injury done to newlytransplanted trees, for want of such precautions, is very great: the strain upon the roots is considerable, and in spring, when our severe winds occur, the first efforts made by them in the formation of new roots are often completely destroyed; and from the constant waving about of the head of an unsupported tree, a cavity is formed in the soil at the base of the stems, which, increasing in size, admits both water and too much air to the roots, both of which are injurious to them; but the great mischief is the derangement of the roots.

Change of climate injurious to newly-transplanted trees.-Much of the want of success in forming new plantations arises from the circumstance of the sudden transition from the climate of a snug well-sheltered nursery to the open bleak exposure of an open park or unsheltered plain. "The comparatively dry state of the atmosphere in the latter is not the least prominent source of the injury. Wherever vegetation is scanty, there will the atmosphere be deficient in moisture-a subject of great importance, when considered in relation to the progress of young trees. And as vegetation not only participates in the benefits, but materially augments the atmospheric moisture of a district, it follows that, by employing other trees as shelter to those we are most solicitous about, we combine several essentials to success-viz., breaking the force of the winds, affording a gentle shelter, and condensing and retaining a large amount of moisture."-Hints on Planting Ornamental Trees, \&c., p. 12. The same effects apply to transplanting trees of any size from thickly-planted woods, and placing them as single specimens or in small groups in open parks, exposed to more air, cold, and evaporation than in the atmosphere in which they were accustomed to grow. This is a prevalent source of failure in transplanting, and seldom attributed to the real cause.

Some trees are more difficult to transplant than others.-Of fruit-bearing trees, the cherry, particularly when large, is the least successfully removed; and next to that the apricot, nectarine, peach, pear, apple, and quince, their facility in transplanting being in the order in which we have placed them. Of ornamental trees and 
shrubs, the whole order of Conifers, the Almond, Broom, Aralia, Arbutus, Baeeharis, Bireh, Beeeh, Caragana, Cistus, Colutea, Cratægus, Cytisus, Genista, Holly, with the whole genus Ilex, the Juniper,Lonicera, Mespilus, Mulberry, Phyllerea, Pyraeantha, Quereus, Rhus, Rosa, Robinia, Sweet Bay, Ulex, Walnut, \&e., are mueh less impatient of transplanting when of a large size than are the Poplar, Willow, Alder, Ash, Azalea, Rhododendron, Kalmia, Ledum, Aueuba, Ligustrum, Buxus, Portugal and common Laurel, Cornus, Tilia, Syringa, Ribes, Spiræa, Laurustinus, \&c. \&e. Ameriean plants in general transplant well, on aecount of their numerous fine hair-like roots, whieh cause the soil to adhere to them in large masses. Without balls, however, they would rank amongst the most diffieult of all. Plants with long tap-roots, with few fibres, are very diffieult to move; and others whose soft spongy roots, when broken or cut, do not readily heal, are equally so.

The proper size for transplanting.-This varies with the sort of tree as well as with its age. It is, however, a maxim settled both amongst theorists and praetieal men, that health, immediate vigour, and duration, are all greatly promoted by transplanting all trees of a small size, as well as while they are eomparatively young. It often happens, however, from a variety of eauses, that large trees must be transplanted to attain eertain ends. This indeed may be done, and is done, but the suceess depends mueh on the way the operation has been performed. But transplanting large trees, even with the greatest eare and judgment, affects them more or less, and a long period of rest or feeble growth must be expected to follow; while in planting young trees, they eommence a vigorous state of growth, and often attain a great size long before the large trees have fairly arrived again at a healthy condition. "The small tree, transplanted with its system of roots and branehes entire, suffers little or no cheek; the older and larger tree, losing part of its roots, requires several years to resume its former vigour. The constitution of the smaller tree is healthy and unimpaired, that of the large is frequently enfeebled."Downing.

The first suecessful attempt at removing very large trees of which we have any eertain knowledge, was that of the Duke of Saxe-Coburg, the grandfather of His Royal Highness Prince Albert, who transplanted ehestnut trees of 30 or 40 feet in height. Capability Brown, Sir Henry Steuart, and others in our own eountry, have sinee performed great feats in this way; but by far the most sueeessful transplanter of trees of extraordinary dimensions is certainly $\mathrm{Mr}$ Barron of Elveston near Derby. In his reeently published work, "The British Winter Garden," he informs us that his first attempt in this way was upon three eedars of Lebanon, "varying in height from 28 to 35 feet, and the diameter of their branehes from 25 to 30 feet, with trunks varying from 3 to 4 feet in eircumferenee." That these trees suffered not by removal is evident from the following table of dimensions, taken when transplanted, and again in 1852:-

\begin{tabular}{|c|c|c|c|}
\hline $\begin{array}{l}\text { Height of } \\
\text { trees in } \\
1831 .\end{array}$ & $\begin{array}{c}\text { Cireumference } \\
\text { of trunk in } \\
1831 .\end{array}$ & $\begin{array}{l}\text { Height of same } \\
\text { trees in } \\
1852 .\end{array}$ & $\begin{array}{c}\text { Circumference } \\
\text { of trunks in } \\
1852 .\end{array}$ \\
\hline $\begin{array}{l}28 \text { feet } \\
32, \\
35 \%\end{array}$ & $\begin{array}{l}4 \text { feet } \\
3 \\
3,\end{array}$ & $\begin{array}{l}46 \text { feet } \\
55,6 \text { in. } \\
58,6,6\end{array}$ & $\begin{array}{l}6 \text { feet } 1 \text { in. } \\
6,3,3, \\
6,,\end{array}$ \\
\hline
\end{tabular}

" The success," Mr Barron observes, "which attended these operations, led to a bolder attempt the following November. A large eedar of Lebanon, measuring in height 33 feet, diameter of branehes 48 feet, and eireumferenee of stem (one foot from the ground) 6 feet, was removed to the distanee of between two and three hundred yards ; and with due attention to both braneh and root watering the following summer, the result was satisfaetory, as its comparative dimensions now show : viz., when planted in 1831 its height was 33 feet, and eireumferenee of stem 6 feet; it is now (1852) 44 feet in height, and 7 feet 10 inches in girth of stem. Yews of a very large size, some of whieh are supposed to liave been several eenturies old when purehased by the late Earl of Harrington, have been removed a distanee of 10,12 , and 25 miles. The dimensions of two of them are given by $\mathrm{Mr}$ Barron as follows: First, "Trunk at 3 feet from the ground, 7 feet 3 inches in eircumferenee ; height 33 feet." Second, "An old yew, whieh forms an arbour 14 feet 2 inehes high, and 59 feet 9 inches in circumference" round the branches, the girth of the trunk being 7 feet 4 inches. These are perhaps the oldest if not the largest trees that have ever been successfully transplanted in the world.

In planting fruit trees most judieious gardeners prefer what are called maiden fruit-treesthat is, one year after being grafted-while others prefer trees two years from the graft. Much, however, here depends on the kind of tree and the mode of training to be adopted. Fruit trees of greater age are often planted-indeed, when eoming into a bearing state, sueh trees will produee fruit sooner; but there ean be no doubt, taking into eonsideration health, duration, and the ease with whieh a maiden or even a oneyear-trained tree ean be made to grow into any form, that either of these is preferable. Fruit trees, however, like all others, may be suceessfully transplanted almost of any age and size, if the requisite preeautions be taken.

Preparing trees for transplanting.-Trees or shrubs taken from the nursery in a young state require no other preparation beyond being carefully taken out of the ground with their roots as little eurtailed or injured as possible. The case is wholly different when trees or shrubs of a large size and age are to be transferred from one place to another. The late Sir Henry Steuart wrote a whole book almost upon this subjeet, without, it would appear, having been aware that the very rules he endeavoured to ineulcate as new had been praetised for more than a century before. The whole rationale of the system consists in shortening the leading roots at a proper distanee from the stem, whieh distanee must ever be governed by the size of the tree to be operated upon; and this is done 
by digging a trench round the whole mass of roots, undermining them, and cutting off all the roots projecting beyond this line. "The trench should be dug at such a distance from the tree as will include all the large and sufficient ball of roots; and it should be done in the spring, or before midsummer, when it is desirable to remove the tree next year. After all the roots that extend to this circular trench are cut off, the earth is replaced, and the season following an abundance of small fibres is sent out by the amputated roots, which, when the whole is now removed, will insure the success and speedy growth of the tree. This is more completely the case when the tree is prepared two years before transplanting. A variation of this mode, which has been found quite as successful and less laborious, consists in leaving the trench open, and covering it with boards only, or boards and a top layer of turf. The tree is then somewhat checked in its growth, it throws out an abundance of small fibres into the ball of earth containing the roots, and is the next season transplanted with great ease and safety."Downing.

If the air is prevented from reaching the roots by a sufficient covering being placed over this trench, there can be no doubt but that this latter niode is to be preferred.

When prepared compost, as recommended by some, or even the natural soil of the place, is thrown again into trenches thus formed, the certain consequences are, that the young fibres and still more delicate spongioles will form themselves in this soft and easily-penetrated medium, and consequently when the removal takes place, notwithstanding all the care that may be bestowed during the operation, those important organs are more or less injured or destroyed-in fact, placing the tree in about the very same condition that it would be had it been removed the preceding season, when the cutting of the roots took place. Those who advocate this plan, and profess to have studied its effects so closely, must surely have observed the condition of the spongiolets upon opening the trench at the period of the intended removal. Leaving the trench empty, and covering it over to partially exclude the air, as recommended by Downing, must certainly be considered of the two modes the most likely to be of advantage to the tree. And when the trench is opened, if the earth of the ball intended to be left were carefully removed from around the roots to be amputated, and were they cut back a few inches within the circumference of the ball, the young fibres and spongiolets would form within it, instead of without it, and at the time of removal would be very considerably protected. Mr Barron, although apparently a convert to Sir Henry Steuart's mode of preparation in early life, set it afterwards entirely aside, and chalked out a course of his own, the success of which has been sufficiently demonstrated. He says, in "The British Winter Garden," p. 27: "The thought occurred to me that if it were possible to remove a tree with a large mass of earth, something similar to that containing the roots after Sir Henry Steuart's pre- paration, I should be stealing a march upon him, and be as forward at once as he would be in two years.

"I then set about conquering the mechanical difficulty, which, when once accomplished, I saw that success would be certain, and many of those mystified physiological difficulties removed to which Sir Henry Steuart seemed to attach so much importance, and which, if not properly understood, would result in failure." Mr B. remarks that he would have been glad to have brought to bear upon the undertaking which he had encountered any portion of Sir Henry's system, but this he found impracticable. Having constructed his machine, which will be afterwards noticed, he proceeded in manner following to prepare a tree for removal: "A tree being about to be lifted, say from 40 to 50 feet high, the diameter of the branches being of the same dimensious, the groundwork operations would commence on the outside of a circle of from 20 to 25 feet in diameter, more or less, judging from the kind of tree how far the roots would extend horizontally; the soil would be then cleared from the roots, carefully preserving every fibre, as far as practicable, up to within a given space of the stem, where the remaining mass of earth and roots must be left undisturbed." Mr B. deviates from general practice in forming his ball nearly a parallelogram instead of a circle; and for such a tree as that noticed above, the dimensions of the ball are 9 feet 6 inches by 8 feet. During the operation of removing the earth from amongst the roots, the greatest care is taken that none of the roots that extend beyond the ball of earth be in any way injured, for on their preservation so much of the success of the plan depends. The rationale of $\mathrm{Mr}$ Barron's principle amounts to this, that instead of cutting the roots two years before planting, with a view to cause them to send out numerous fibres and spongiolets, many of which will inevitably be destroyed at removal, and have to be replaced by others before the tree can be supplied with its requisite amount of food, he removes at once, preserving the roots as much as possible, and allowing them to form new fibres and spongiolets in the place where they are to remain undisturbed. By his process, also, he reduces the weight of the ball of earth considerably, and hence renders the transport more easy. Heavy balls must have an immense strain on the roots, however carefully the operation may be performed; and the injury roots sustain from this alone, but which is not discernible to the eye, is more than is generally supposed, and in many cases more than even the loss of a portion of the roots. Some erroneously suppose that by shortening the extremities of the roots they form multitudes of others within the ball, throwing them out laterally from their sides. Such is not the case, for roots elongate only from their points; so that, in fact, after a time, no food-collecting roots exist for a considerable distance from around the stem of the tree, as they are always extending in a direction from it.

Shortening the roots, as recommended by Sir Henry Steuart and others, would be of advan- 
tage, if, after the ball was formed and the roots cut, it was enclosed within a casing of very strong planking, or staves strongly hooped round, and a few inches larger than the size of the ball, to admit of fine fresh mould being rammed in all round for the fibres and spongiolets to form in for a season. Trees so prepared might be very safely removed almost at any period; and when set in their new situations and the casing removed, the roots would extend in quest of nourishment in the surrounding soil, and bear their removal without injury.

Could the roots be secured in any way, there is no fear of removal in these days of improved mechanical appliances, so far as mere weight is concerned. But there are other considerations to be kept in view, of which the situation the tree to be removed is placed in, the condition of the ground, which is in general too soft to sustain heavy carriages, the obstacles which bridges under railways, tunnels, acute turnings in rides, drives, the branches of other trees, \&c., present, have all to be taken into consideration, all of which present serious obstacles to the transplantation of large trees. With trees standing isolated in an open park the case is very different, and it is those, and such as grow on the outskirts of plantations, that the planter should choose for his experiments in gigantic transplantation.

Thinning the branches at the time of removing large trees. - In planting large trees whose lieads are crowded with branches, it will be expedient to partially thin them ; the quantity of branches to be removed will partly depend on the nature of the tree and the intention of the planter. The beech and the hornbeam are impatient of pruning when transplanted, and have been known to be killed by an injudicious use of the knife; other trees, such as the yew, holly, lime, elm, and all the Acer tribe, require little pruning at planting, because they have an abundant supply of fibres around and near the main stem. Apple and pear trees, if of a large size, are improved by a moderate curtailment of their branches. The intention of thinning the tops of trees during this operation is twofold; first, it prevents the wind from having too much effect upon them; and, secondly and principally, it lessens the draught upon the roots, by lessening transpiration by a superabundance of leaves, and reduces them to the amount of moisture which can be absorbed by the roots, which as yet are diminished in num. ber and action.

Transplanting from pots.-Many rare trees and shrubs are grown in pots in nurseries, to render them more portable, and better fitted for transportation to a distance. Of these, most of the rare Conifers, Arbutus, red cedars, junipers, the rarer species of oaks, \&c., may be given as examples. The circumscribed space allotted to the roots within the narrow limits of a pot from 5 to 9 inches in diameter, produce a tendency in them, for want of room to extend themselves horizontally, to assume a spiral form; the whole ball will also become a confused mass of roots, searching in all directions for food. To turn plants so circumstanced out of the pots into pits ever so large, or into soil ever so well prepared, with their balls entire, and their roots in their constrained state, would be much the same, so far as the progress of the plant's growth is concerned, as if it continued to remain in the pot in which it had been growing. Such balls should be carefully broken up, but this ought to be done without the roots being broken during the operation. To facilitate this, if the balls be steeped in water, the soil will separate more freely; and indeed, in extreme cases, it will be well to wash the whole of the soil away, so that the roots may be carefully singled out and spread in their new bed in their natural position. When plants are kept too long in pots-and this is a case of very frequent occurrence-their roots become so tortuous that they cannot be straightened out, and this is the more difficult the older and stronger they are.

Mr Barron, in " The British Winter Garden," has clearly illustrated the evils of growing plants in pots intended to be afterwards planted out, and expected to become in time goodly trees. "The contorted form which the roots are compelled to take while imprisoned within the limits of a small pot, prevents the free passage of the sap food collected by the spongioles from reaching the stem in sufficient time and quantity to support the leaves and branches while they are exposed to the powerful action of solar heat, as well as the evaporating action of dry parching winds. The first consequence of this mistreatment in youth is sickliness and deformity, and afterwards premature death. This is very generally observable in coniferous plants, and of itself quite enough to account for the very few healthy cedars of Lebanon, for example, to be found in Britain of any age, and the multitudes of them which everywhere abound in a distorted, enfeebled, and diseased state. No plants which are expected to attain a large size should ever be grown in pots, but should be treated upon the same principle as our common forest trees, with this difference, that they never should be allowed to remain more

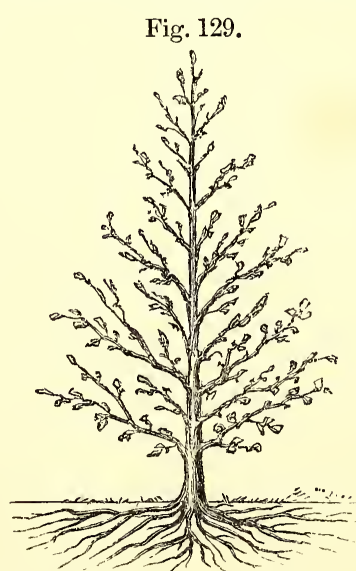

than two years, and in some instances not so much, without being transplanted. By this means the main roots would radiate from the common centre in a natural manner, and the roots become sufficiently numerous to insure a ball of earth to adhere to them. Plants would thus be furnished of a very superior quality, EXAMPLE OF WELL-FONMED Roots. and at a much 
cheaper rate than those mutilated and expensive deformities nurtured in pots."

Fig. 129 shows the state of the roots of a tree that has never been confined to the circum-

Fig. 130.

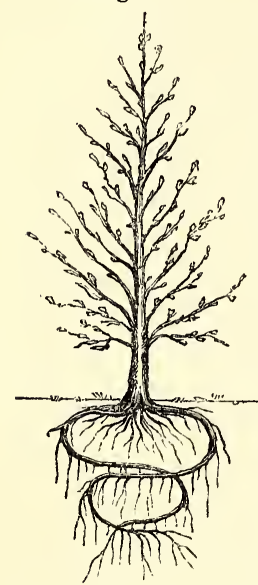

EFFECTS OF POT CULTUR ON ROOTS. scribed limits of pot culture, fig. 130 that of one which has. In the former, the roots are extending in all directions near the surface in search of food; in the latter, their free produce has a direct tendency downwards, where they neither can derive food, nor, from their position, have the same effect as the former in maintaining the perpendicular position of the tree.

In such a condition, and much worse, will be found the roots of camellias, and other plants grown in pots, where care has not been taken at shifting to disentangle them, and afford them more space for their horizontal extension. Fig. 131 shows the state of a fruit or forest tree subjected in early life to pot culture; and the same effect will be produced on the roots of many trees when planted in pits

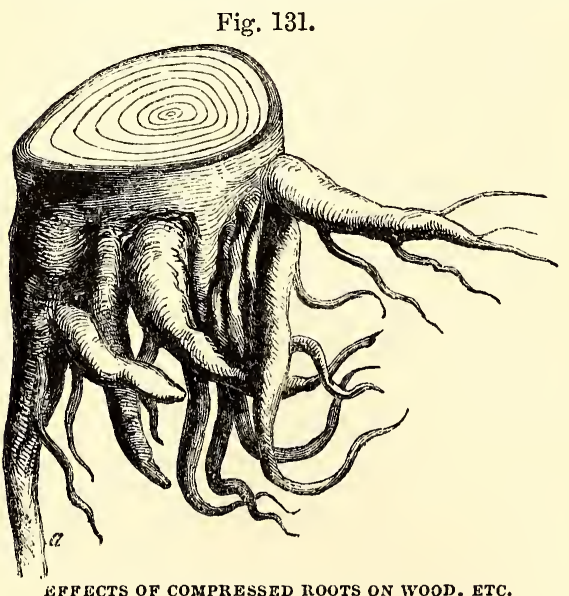

with their sides walled round with undisturbed soil, as referred to page 358 , when speaking of the necessity of preparing the soil previous to planting. In fig. 131 it will be seen that, where the roots have been most confined and contorted, the supply of sap thrown into the side of the tree immediately above them has been limited and irregular, as seen by the smaller and irregular portions of the annular rings in the transverse section of the trunk; whereas, again, where the root $a$ has had the means of penetrating deeper into the soil, and consequently been enabled to collect a greater amount

of sap food, the annular rings above it are larger and more uniform in size.

Transplanting fruit trees with a riew to correct over-luxuriancy of growth, and to induce them to form blossom-buds. - This operation mas be regarded as a species of root-pruning, and the effects will be found to be similar. Taking up a tree entirely enables the operator not only to regulate the state of the roots better than merely root-pruning, as usually performed, but it also enables him to bring the roots nearer to the surface, should they have been over-deep before, and also affords an excellent opportunity of removing the exhausted or bad subsoil, replacing it with better, or of draining or vaulting under it, if deemed necessary. Root-pruning and taking up and replanting are the very opposites to severe branch-pruning. All fruit trees may be thus treated with much advantage, and those which, by reason of their great age, have ceased to bear fruit, and even make little or no wood, in consequence of the flow of the sap becoming sluggish, will, when root-pruned and replanted in fresh soil, assume a renewed vigour, become fruitful and healthy. We lately saw two apricot trees which had been planted thirty years, and had ceased to produce fruit, and appeared to be in the last stage of decay ; by being taken up and replanted in fresh soil, they became as vigorous and productive as they were when only twelve years old.-(Vide Rootpruning.)

Taking up and replanting fruit trees, without any curtailment of their roots beyond those that may be broken or injured during the operation, is somewhat different from mere rootpruning, both in the operation and in its effects. The intention in the latter case is a curtailment of the stronger roots, which are known to be the chief collectors of clude and superabundant food, which for the most part goes to the formation of coarse watery shoots, themselves the very emblems of sterility. The lifting and replanting process affects this also, and besides, if performed every two or three years, according to the state of the trees, brings about a state of productiveness in them, by transforming the coarse, watery, barren shoots into short, firm, well-matured wood, fully set with flowerbuds. Another advantage attends the lifting process, which root-pruning does not so completely effect : the trees are prevented in spring from pushing too early, as the food is longer of being collected, and hence the ascent of the sap is proportionably delayed. We all know that the later in spring vegetation is excited, the greater chance there is of the blossoms escaping our destructive late spring frosts, and the greater will be the chance of a good set of fruit. $\mathrm{Mr}$ Marnock, in referring to this process, observes: "But this is not all; for just as the greatest demand is being made upon the tree by the swelling and ripening of the fruit, the roots are becoming stronger and more numerous, and entering into the recently disturbed, and it may be new earth. They are in this way enabled to swell off and ripen the fruit in a manner superior to what they would if merely root-pruned or not lifted at all. It is not difficult," he 
continues, "to believe the effects which new maiden soil would be likely to have in such cases; for it must be remembered that trees thus accustomed to be lifted do not become unbealthy, the roots being all carefully tied and relaid in newly prepared fresh loain."

Transplanting coniferous plants.-These for the most part do not affect a deep or rich soil; the thin soils formed over primitive formations appear to be their natural element. Some, however, seem to luxuriate more than others in deeper soils, such as a few of the Abies tribe; but although we find them occasionally indigenous in such soils, we do not find that they avail themselves greatly of them, as their roots for the most part spread out horizontally very little under the surface. In cultivation, however, they do not refuse to thrive in soils of medium quality and of considerable depth; but in one thing they seem to have a choice-namely, a new soil which has not been long, if at all, under cultivation. The season of transplanting has usually extended, as in the case of deciduous trees, throughout the later end of autumn, all winter, and the early part of spring. We believe, for ordinary purposes, those seasons may be all very well; but for the removal of coniferous plants of great value and rarity we would decidedly prefer the month of June, when the young shoots had made considerable progress. Mr Barron moved spruce firs in June from 30 to 40 feet in height. We have ourselves removed many rare and valuable conifera during June, and would, from our previous success, do so again at that time rather than at any other. There has, in general, been a kind of dread in the minds of cultivators in transplanting coniferous plants, particularly when they have attained some age and size. We see no more difficulty in doing so in their case than in that of any deciduous tree whatever. All depends on performing the operation properly, and choosing a right season.

One of the greatest evils in planting the rarer coniferous plants is the absurd practice persisted in of choosing plants grown in the nurseries in pots. A greater mistake cannot be fallen into. It imposes an unnecessary expense on the nurseryman which the purchaser has in general to pay, while he also receives a very inferior article for his money. Nurserymen, in nine cases out of ten, receive orders for coniferous plants accompanied with positive instructions that if the plants are not in pots, on no account are they to be sent. There are few nurserymen nowadays but are aware of the absurdity of this, but they have no choice. With some of their customers they may remonstrate, and point out how much more it would be to their advantage to have plants out of pots; with others they cannot do so-for in plant buying, as in everything else, there are people who pride themselves on fancying they know the value of the article sold better than the seller himself. But let a nursery. man speak for himself: "A prejudice exists against plants removed from the open border; in numerous instances it is a just one. It has arisen from the fact that many growers do not transplant often enough. The consequences are the plants become coarse-rooted, and, being removed in an indifferent condition, are often lost before they can recover the check consequent on their change of situation. But the remedy for this is in the hands of the purchasers; if coarse-rooted plants cannot be sold, growers will cease to bring them into the market. But the disadvantages of pot-grown plants are, as a general rule, of equal magnitude with the worst condition of those badly managed in the open borders; yet purchasers patronise the former to a large extent, and to the exclusion, in a great measure, of the latter. As it becomes a matter of great importance to nurserymen that their stock of pot-grown plants should occupy as small a space as possible, pot-bound specimens are the rule rather than the exception. And for plants intended to be removed to the open ground as permanent specimens, scarcely a worse condition could exist: it is superlatively bad." This quotation is so truly practical that we are inclined to give it at length. "For the purpose of illustration, we will suppose a person to have purchased a plant in the condition above described, and intends immediately to plant it out. On removing the pot, he is delighted to find coil upon coil of fine healthy roots. He knows very well that to commit them to their new situation in an uncoiled state will be highly improper, and so, with great care, he proceeds to disentangle them. However careful he may be, the loss of many valuable roots will result, and damage to the remainder will be equally certain. Those in the interior of the ball, from their size and woody texture, will not yield at all; and he closes his half-finished labour with the conviction that he has seriously damaged his plant. But this mutilation, great as it is, is far preferable to having planted it with its matted roots undisturbed. A plant which has once been thoroughly pot-bound never gets so firm a hold on the soil as one whose roots have never been confined. Instances are numerous where valuable specimens,'after years of growth, have been blown down in consequence of the very slight manner in which they retained their position. The main roots, when young, had from pot culture acquired a coil-like arrangement, which during all their subsequent growth they adhered to-enlarging, but not spreading increasing in bulk, but contributing little to the mechanical support of the tree: in fact, they often destroy each other; and in every instance of a plant which in its young state has been subject to the confinement of a pot, these conditions will be evident."-Hints on Planting Ornamental Trees, \&ic., p. 311, by STANDISH and NobLe. Plants also grown for several years in pots become, from sheer starvation, debilitated in constitution, deformed in structure, and rarely, if ever, even should the roots become untrammelled, assume the same portly appearance as those whose roots have not been subjected to the same restraint.

Transplanting evergreens.-Regarding the best season for performing this operation opinions are greatly divided. We have removed evergreens at all seasons with apparently equal success. The late excellent $\mathrm{Mr} \mathrm{M}^{\prime} \mathrm{Nab}$, in his 
"Treatise on transplanting Evergreens," says: " Evergreens may be planted with much greater safety in mild weather in autumn or winter, than at any other period of the year." Another authority remarks that evergreens may be transplanted in autumn, or at any time in open moist weather during autumn, winter, or early spring. In dry or frosty weather it is always dangerous to remove them, because the sap in an evergreen is more or less in motion at every season of the year, and the plant is never so completely dormant as deciduous trees are. In regard to the season of performing the operation, we have the following reasoning by $\mathrm{Mr}$ Glendinning, in the "Journal of the Horticultural Society of London," vol. iv. p. 41 : "This I consider to be a much more important matter than many seem to imagine. The winter months-that is, from the end of October to the beginning of Aprilhave been generally recommended; indeed, almost universally so. From these opinions $I$ entirely dissent, how eminent soever the authorities may be who have laid them down. I quite admit that a qualified success may have attended their operations; but I deny, independently of this, that it is the right season for the execution of such work, and the slightest acquaintance with vegetable physiology will conclusively demonstrate the truth of this assertion. Let it be clearly understood that I am alluding to plants of from 6 to 30 feet and upwards in height, and not to mere nursery stock," which are frequently removed to keep them in a transportable state. Mr Glendinning, passing over the details of the ascent and descent of the fluids in plants, and the formation and deposition annually of new wood in all ligneous vegetation, proceeds : "It will be sufficient for my purpose to state that this extension and formation takes place chiefly after midsummer, and principally in evergreens during autumn, when the young shoots begin to attain a certain degree of consistency. It is during this downward tendency of the fluids, and when the solar action is in some measure on the decline, that I should seize and conduct with all rapidity the operatious of transplanting; and if this is intended to be conducted extensively, I should recommend the end of August as a good time to begin, September being the safest month in the year, selecting such plants to commence with as have matured their shoots. Another and very important reason remains to be stated why autumn is to be preferred, for undertakings of this kind, in preference to winter. The force of the sun during summer, although now on the decline, has warmed the earth to a considerable degree and depth, so that the mutilated roots are comparatively situated on a gentle bottomheat, which rapidly promotes cicatrisation, and frequently aids the emission of young spongiolets during the current autumn. The season which I have here ventured to urge for the performance of the work under consideration as undeniably theoretically the right period, appears beyond all question; and being no mean experimentalist in rural embellishment with plants of considerable magnitude, I can attest also that it is practically the season to be preferred be- yond all others. I had occasion to superintend the removal of upwards of two thousand trees and shrubs, all evergreens, and varying in size from 6 to 40 feet high, during one autumn." The trees were prepared by cutting in the roots the previous spring, "and as the undertaking was rather gigantic, the work was begun in August, and finished with the year. The result was, of course, watched with some interest; and the following summer, when an examination took place, I found that those trees which were transplanted early in the season indicated little change from their removal; but the contrary was the case with those which had undergone similar transplantation during December. In fact, the gradual diminution of the motion of the sap, accompanied with declining atmospheric action, which tended, in conjunction with the usual autumnal precipitations, to cool and saturate the earth, clearly and progressively exhibited our comparative success. The exact period to commence these operations must be determined by the nature of the season, and the state of maturity the current year's growth has attained. In some seasons an earlier beginning may be made than in others; some kinds of plants also ripen their wood much earlier than others: these, therefore, should receive the earliest attention. In hot and dry autumns the foliage of some of the larger specimens of certain species and varieties, especially such as have large and succulent leaves, will flag and droop. To guard against any injury arising from this, it will be advisable to well water the roots at planting-not with cold water from the well, but from the pond, where it has been exposed to the sun. With specimens of great rarity and value it would amply repay the additional trouble to occasionally syringe the foliage in the evening for a short period after planting. This, however, will not be required should the weather be cloudy or moist." Here we have not only good practical rules laid down, but physiological reasons given for them. In Mr Glendinning's views we quite concur as to the season of planting. It happens, however, unfortunately, that at this particular period, August and September, our flower-gardens and dressed grounds are expected to be in the highest state of keeping, because it is at that time that proprietors most generally reside at their country residences. The necessary confusion caused by the removal of a great number of evergreens at that time would be highly inconvenient. This, however, does not affect the principle of the reasoning given above; nor should it make us lose the opportunity of transplanting evergreens in woods and plantations, and in these cases the proper season should not be lost sight of ; and again, if the operation is to be carried out upon a large scale, it is much better even to sacrifice the appearance of the grounds for one autumn than to lose the chance of a favourable tirne for performing the operation.

The formation of roots is the first effort of every tree after transplanting; and hence the importance of August, September, or October planting, because there is sufficient solar heat in the soil to aid them in this effort, which 
there would not be during the winter months, but which is beginning again to be the case in April. "Add to this, that about the end of April and the beginning of May, we have frequently warm showers, which, to shrubs of this description, are extremely beneficial, and are always much better for vegetation than water, however well it may be artificially applied. The reason assigned for planting evergreens in August and September is, that they have made their shoots, and, if carefully done, will make fresh roots and become tolerably established before winter. Experience proves these seasons to be the best for transplanting these plants, and upon an extensive scale, and in exposed situations, we would advise the adoption of it. At the same time, circumstances may warrant their removal at almost any day in the year. Evergreens are extremely fond of shelter and of moderate shade ; and it is probably the want of these that is the principal cause of the failure of these plants when removed at seasons when there is a long continuance of cutting winds, without any rain. In our practice at Stratton Park [Hampshire] we implanted 50,000 evergreens in one season, beginning in November and ending in February. Circumstances led to our planting at this season. The soil, for the most part, was of a strong clayey nature, and by no means favourable for plants in general ; still, the success here was complete, inasmuch as out of that number not 200 plants died. The plants were brought a distance of twenty miles, and were from 2 to 3 feet high. We attribute the success in this instance to the shade which the plants enjoyed in summer and the shelter during the first winter after planting, as they were planted partly in very old plantations and partly in young ones, in both cases well sheltered and shaded."

The majority of sensible planters prefer the months of April, May, September, October, and November for transplanting evergreens, and this latitude is given because one month, from the state of the weather, may be better one year than in another. The ground temperature, in properly drained soil-say in the vicinity of London-is calculated to be in November $47^{\circ}$, and this is a very good root temperature for such plants to be placed in, and in such they will speedily strike root, which is the great object to secure.

Of all trees and shrubs requiring support from the effects of wind, \&c., none require it more than evergreens that have been recently transplanted, as they present so large a surface of foliage at all seasons, and are consequently more liable to constant oscillation than those that are deciduous. When this is not guarded against, the young roots are prevented from taking hold of the soil around them, and the ball which accompanied them becomes separated from the surrounding earth; interstices are formed, into which rain first and frost afterwards enters, both equally injurious to the young and tender fibres; while in spring the parching winds would penetrate and prove equally injurious: they therefore require particular attention in this respect. Many VOL. II. modes have been recommended, but the particular mode is of far less consequence than that it is properly done by one or other ; for much of the success of transplanting depends on this being effectually accomplished.

Next in importance to supporting evergreens after transplanting, is that of watering them; and if the operation be carried on late in spring or during summer, the equally important precaution of shading them, not only from the effects of bright sunshine, but also from the still more evaporating effects of dry, cold, cutting winds, should not be disregarded. It is not the quantity of water actually given a newly planted tree, but the way in which it is applied, that is the chief point to be considered. Indeed, we believe that more harm is done by excessive watering at the roots by some, than by a deficiency of it in others; and this the more so if the water is taken from wells, which, if not of more than ordinary depth, are always much colder than the soil surrounding the roots. River or pond water should be chosen, and that applied frequently, and in small quantities at a time. If large doses of cold spring water are applied to the roots of newly planted trees, the temperature of the soil is lowered, the vital energies of the roots are paralysed at the very time they require the utmost amount of excitement, to enable them to recover the injuries sustained during the process of removal by the rapid formation of new roots and spongiolets, which is their first effort ; and everything, therefore, to assist them in the formation of these should be carefully attended to. Indeed, it is by no means going too far to say, that it were far better to apply water at the roots under such circumstances at $100^{\circ}$ than at $35^{\circ}$ - a temperature by no means uncommon in spring water during winter and early spring. Syringing over the tops has a most beneficial effect upon all trees, particularly evergreen ones, and this should be applied two or three times during the day. Shading from the sun in bright days lessens the evaporation, which would otherwise be going on in the foliage; and shelter from drying winds not only counteracts this in an eminent degree, but also maintains an increased temperature around them; and increase of temperature to the foliage, although less exciting than at the roots, has much to do with the successful results of planting. The best shading for such purposes is thin canvass, called by manufacturers screen-cloth, because, while it breaks the force of the wind, it admits light in a modified form to the plants ; for exclusion of light, at a period when every aid man has at his command should be given to afford increased excitement to the roots, would be as bad as leaving them exposed altogether. In giving water at the roots, it is of little use to pour it around the stem, although we every day see due preparation inade for this, by the formation of basins scooped out of the soil, and close to the trunk of the tree: whereas, to be of any utility, whatever precaution is made for preventing the waste of water, it should be immediately over where the young roots and spongioles are forming, which will only be around the circumference of the ball removed, 
where what is called the preparation system has been followed; or immediately above that zone in which the roots are placed when trees are either planted without balls altogether, or having their most important ones beyond the ball, if managed as recommended by Mr Barron.

Fig. 140 will explain his method, and will clearly enough show that, by adopting this course, a far greater amount of roots can and will be removed, attached to a tree, than could be by any process of ball preparation. As the process of separating the roots from the soil is carried into effect, they should be tied up in small bundles; and if the weather be hot or very dry, they would be greatly benefited if enveloped in soft hay, damp moss, \&c.- - and this the more so, if their removal is to a great distance, or if they are to be subject to lengthened exposure. The same number of roots, as here shown, could not by any possibility within the power of man be so safely removed if enclosed within a ball of earth. The preparation system may no doubt be of advantage in the case of very rare or valuable trees, naturally difficult of removal; but we think it requires to be carried farther than any description we have read of it, or any example we have hitherto seen reduced to practice.

The transplantation of trees of great age and size has long been a weighty consideration with ornamental planters. The greatest difficulty, however, which for long presented itself, was the means of lifting them to the surface, and of conveying them to their new abode. Brown, the most noted transplanter of his day, employed the common janker. Sir Henry Steuart and others improved upon that; but still his machine was defective. It is only, therefore, within these few years that machines at all adapted to the purpose have been constructed upon correct principles, and these, as a consequence arising from the importance of the case, have of late multiplied exceedingly. Mr Saul and the late $\mathrm{Mr} \mathrm{M}^{\circ} \mathrm{Nab}$ were amongst the first who applied mechanical aid to the removal of large trees in an upright position, and the success of the latter was most complete. Mr Barron of Elveston, who, we believe, has removed the largest trees hitherto attempted in Britain, has also, as a mechanical inventor and most successful planter, given sufficient evidence of his ingenuity, as has Mr Holmes of Amport House, and $\mathrm{Mr}$ Mackay, the enterprising gardener to Edward Strutt, Esq: of Kingston Hall, near Derby ; and last, although by no means least, Mr S. M'Glashan, a respectable sculptor in Edinburgh, who, treating the matter more mechanically than physiologically, has taken planters by surprise in the performances he has already exhibited.

$M r$ Saul's tree and shrub lifting machine, fig. 132 , is of very simple contrivance, and exceedingly well calculated for the removal of subjects under half a ton in weight. It may be described as a low two-wheeled truck, with strong hooks attached to the hinder part, to which the cradle in which the plant is placed is suspended; in front is a long pole, which acts as a lever in upraising the plant, as well as securing the ball when loaded, and also as the

Fig. 132.

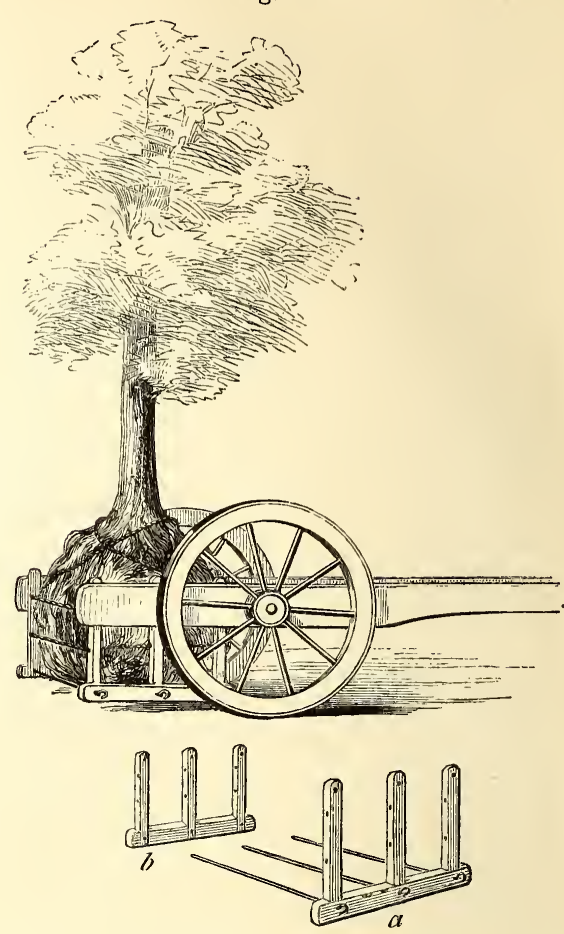

SAUL'S TRANSPLANTING MACHINE.

means of draught, as at its farthest end a cross bar is fixed (not shown in the cut), by which men drag it along, or to which a horse may be yoked. The ball is prepared in the usual manner, and when the trench around it is opened, one of the iron sides $a$, which forms the cradle, is set upright in the trench, close to the ball, and three iron rods are passed through it, under the ball, and also through the corresponding holes in the other iron side $b$, which, for the purpose, is placed in the trench exactly opposite the first. These three iron rods (there may be more if the ball is large or the ground sandy, but for each rod there must be corresponding holes in each of the sides) are furnished at one end, as shown in the cut, with eyes, and at the other with screws, so that, when they are all fitted in their places, they may be screwed up tightly so as to keep the opposite sides of the ball together. Those we use are somewhat different from Mr Saul's, which has only one upright arm to each of the two sides, while ours has three, as shown in the cut. By our three upright arms we not only secure the under side of the ball (which is all Mr Saul did), but we also secure the sides, and if need be the ball all round, by running iron rods through the arms, which it will be seen by the sketch are perforated on purpose. These sides and rods being all 
screwed up tight, the plant may be removed at once; or, if there be any apprehension of danger in consequence of the roots being cut, the soil may be filled in, and the whole allowed to remain until the root wounds are healed, and the spongioles again formed, when all that is required is to remove the soil from the trench carefully, and proceed as if the lifting had taken place at once. When all is prepared, the truck is run back, the wheels kept on two planks laid over the sides of the trench, the draught-pole is elevated until the two strong hooks in the hinder part of the trench catch into the top holes of the centre arm of each of the sides, which, for that purpose, are about 6 inches higher than the others. These being hooked on, the draughtpole is drawn down, the tree and its ball are drawn up from the pit, as by a lever, the ball secured to the truck, and if the tree is tall it may be attached to the draught-pole by a rope. A rope is also taken round the stem of the tree, quite at its base, and carried once or twice round the ball, and then secured to the axle. The tree is then removed to its destination, and when placed over the centre of the pit-the wheels at the same time being supported by two planks laid across the sides of the hole, and blocked to keep them steady-the rope is removed, and the draught-pole is elevated so as to let the ball rest on the bottom of the pit prepared for it. The truck is then disengaged from the ball by unhooking it from the cradle, and removed; the iron rods below and around the ball are unscrewed and drawn out, which is readily accomplished by cutting back the side of the pit to allow their being pulled out in that direction. This finishes the operation.

The advantage of placing the cradle under the ball some months previous to final removal is considerable in the case of very rare or choice plants, as then the conditions of the roots and spongiolets will be seen in their natural condition according to the season of the year they are to be examined. If it is deemed expedient upon sight to tighten up the screws of the cradle, it can readily be done, and the whole mass of ball and plant can then be raised from the pit, and may be placed on whatever machine may be at command, in the absence of the truck here represented, to convey it to its new abode. Should the young fibres and spongiolets have even somewhat extended beyond the sides or ends of the cradle, no injury need befall them, as, in the process of lifting up the ball, the whole pressure will be upon its bottom, where few, if any, roots of consequence will be found; and should there be some, their loss will be a matter of little consideration compared with the side ones. The holes in the tops of the two frames being from 2 to 3 inches in diameter, a couple of strong poles may be passed through them, when smaller plants are to be removed, by which four, six, eight men, or more, may readily lift the ball out of the hole, and place it upon a low truck or carriage. Should, however, the tree be large and the ball very weighty, two iron bars may be employed instead of the wooden ones, and various mechanical powers may be used for lifting. The rationale of this mode is, that the roots and ball can be removed with as little injury as if they were growing in a box. It will readily be seen from this, that a separate cradle will be required for each tree removed, assuming the preparation of the root is deemed necessary; but when we consider the trifling expense of each, and that they will last for years, and insure the safe removal of the tree, their cost, we think, will not be grudged. The cradle we have just described we think has advantages over the boarded platform recommended by both $\mathrm{Mr}$ Barron and Mr Mackay, on account of its being more readily placed under the ball, and also as it gives protection to the sides and ends of the ball, which these do not. We may also add, that it is well adapted for being attached to their machines; and when the tree is small, we would say no means of elevating it for the purpose of being placed on a truck is better than that of M'Glashan's levers, having for their.fulcrum two trestles, one on each side of the tree.

Standish and Noble's crate.-These enterprising cultivators, in preparing ornamental trees in the nursery preparatory to their being permanently removed to their ultimate place of growth, particularly in exposed situations, propose to grow them for a season or two in skeleton boxes or cradles. " Procure," say they, " some pieces of elm plank, about $1 \frac{1}{4}$-inch square, 9 inches long; also a quantity of larch stakes, about $1 \frac{1}{2}$ inches in diameter, and 9 inches long; split them longitudinally; take four pieces of the elm, one for each corner, and nail them to the pieces of larch, leaving spaces of about threefourths of an inch between each two, leaving the top open. You have now the skeleton of a box or crate for the reception of the plant. The spaces between the bars are to allow free egress to the roots. Place a plant in each, in a compost of good turfy loam, with a little leaf-mould. At first they should be placed in sheltered situations, but removal should take place twice a-year, in spring and autumn; and at each remove a less sheltered situation should be chosen, till they at last occupy a tolerably exposed locality. They should always be kept planted as deep as the top of the crates. At the close of the second season they will be in a suitable condition to be placed in their permanent places. They need not be removed from their crates, as they will be quite rotten before the roots are of sufficient size to be obstructed by them. By adopting such a course, success will be obtained where every other means have failed." The practice of growing plants in coarse baskets for a time before removal has long been in use. The crate system is, however, we think, preferable, as being more durable, and better fitted for transport.

$M r M^{\prime} N a b$ 's apparatus for removing trees.Great success has for years attended the removal of trees, both deciduous and evergreen, in the Royal Botanic Garden, Edinburgh. The thorough knowledge the late $\mathrm{Mr} \mathrm{M}^{\top} \mathrm{Nab}$ had of vegetable physiology, and his long experience as a most excellent cultivator, led, no doubt, in the first instance, to his finally determining not only the most fitting season, but also the most judicious mode of performing the operation. With trees or shrubs of small growth, little difficulty pre- 
sents itself; but when they are of extraordinary size, other appliances must be had recourse to than that of merely carrying them by the hand, or on an ordinary handbarrow. Such appliances have been constructed in these gardens; and we are indebted to $\mathrm{Mr}$ James M'Nab, the present intelligent curator, for being enabled to delineate, by the following woodcuts, the apparatus employed by him for this important purpose. We may, however, premise, by saying that they are simple and easily constructed, which is not the least of their merits. The ball of the tree intended to be removed is carefully separated from the surrounding soil, with as many of its roots preserved as possible, the stronger being cut off close to the surface of the ball, while the more flexible are tied up in bundles, enveloped in soft hay or straw, and covered with a double mat, to keep the whole together. A piece of cord is then placed loosely round it, between which and the cord are set upright pieces of thin boarding, from 2 to 3 inches broad, of equal length, and 3 or 4 inches apart all round, the cord keeping them in their proper places. These boards being adjusted, a strong half-inch rope doubled is put round the upper part of the ball,
Fig. 133.

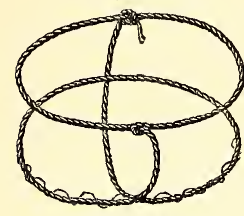

$M$ 'NAB'S MODE

OF TRANSPLANTING. making it fast in front, but not too light; the remaining portion of the rope is taken down the front, as in fig. 133, and is made to surround the ball again near the bottom, after which the ropes are to be tightened up by means of a rack-pin, so that the whole may bekept tightly together. The ball is then to be undermined on one side, as near to the centre as possible, and a piece of strong board, say 8 or 9 inches broad, is to be introduced under it, and the tree drawn gently over to the side under which the board is placed, while the operation of undermining the opposite side is going on; and when a similar board is placed under that side, the tree is to be brought to the perpendicular again, resting on the two boards, which may be called the lifting boards. Two strong ropes are then brought under the lifting boards, as shown in fig. 134. The ends

Fig. 134.

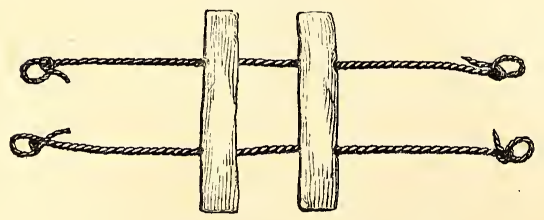

M'NAB'S MODE OF TRANSPLANTING.

of these ropes are then brought up and secured to the handspikes or bearers, as shown in fig. 135; and, to prevent the ropes from slipping off the lifting boards, notches are cut in them into which the ropes fit. For plants which two, six, or ten men can easily carry, this apparatus is quite sufficient, if the distance be not great to which the tree is to be removed. When ten men are employed, two additional handspikes are placed across the others at right angles, which will Fig. 135.

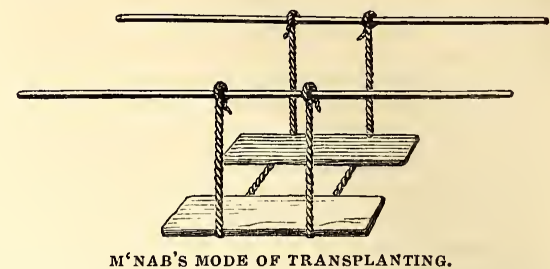

afford lifting power for four men more, six being employed upon the others, in the way in which masons carry large stones upon their hand-

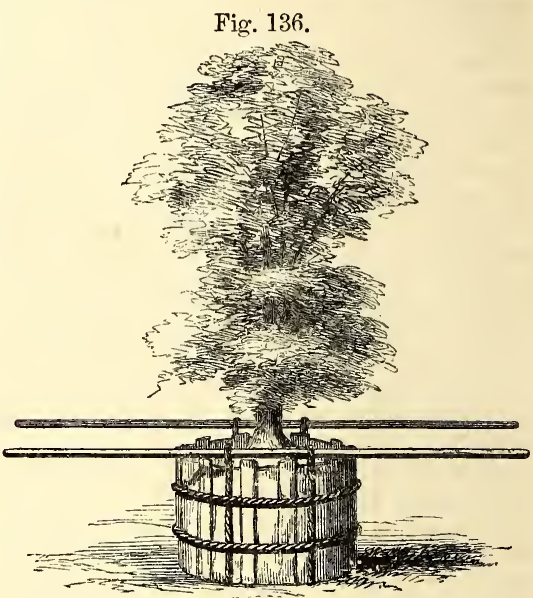

M'NAB'S HAND-TRANSPLANTER.

barrows. Fig. 136 shows the tree ready for removal. If the tree be too large for six men to

Fig. 137.

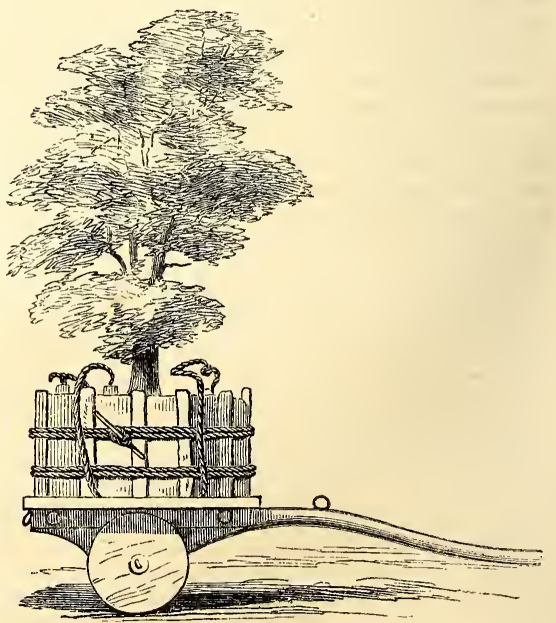

M'NAB'S TRANSFLANTING TRUCK. 
carry with perfect ease, it will be better to employ a wheeled machine, which $\mathrm{Mr} \mathrm{M}$ 'Nab has also provided, and which will be understood by the annexed engraving, fig. 137. An inclined plane is formed in the side of the pit, the machine is pushed down close to the ball, and a plank placed under each wheel, to prevent them sinking in the soft earth, and so lessen the resistance in pulling it up again.

The ball is lifted on the machine, or often slid upon it by sinking the machine lower than the ball, and placing it as far under as possible. The machine must be strongly built, mounted on low broad wheels, and having its top or floor projecting a little over the wheels to prevent the loose earth falling down and impeding the operation of the axles. Fig. 137 shows the tree mounted on the carriage and ready for removal. When so placed, the ball is firmly secured to the machine, and if the tree be of considerable height guide-ropes are used, so that it may be kept in an upright position.

One of the most difficult operations in the process of transplanting large trees or shrubs is lifting them from their original position to the machine by which they are to be transported. We have shown, by fig. 138, one process whereby a plant of considerable weight maybe so elevated, that the transporting machine may be set under the ball with great ease, and that without injuring the plant or straining its roots. The ball being prepared as before recommended, two chains are drawn under two flat pieces of iron, previously placed under the ball, from 6 to 12 inches in breadth, and of a thickness equal to the weight they are likely to have to sustain, and also in length corresponding toits diameter. The chains having a strong ring each at one end, through which the other end is made to pass, this, being furnished with a hook, enables the planter to tighten them up as close as possible. These chains are kept in their places by another smaller chain, which is passed round the ball, and, taking a turn round each of the upright chains, is fastened by a hook in manner as above. The ends of two strong levers are then introduced under the chain, on the top of the ball, and a fulcrum of stone, wood, or some other resisting ma- terial is placed quite levelat each side of the bank, as shown in our cut. The ordinary operation of

Fig. 138.

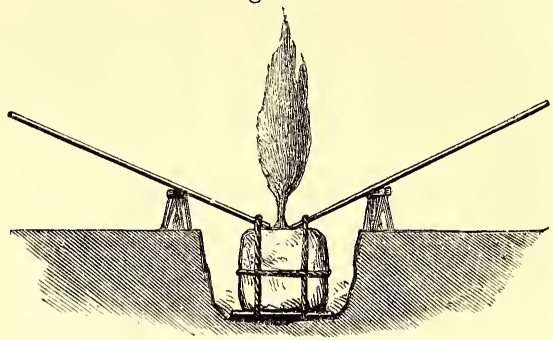

RAISING-POWER IN TRANSPLANTING.

the lever will elevate the ball, if the fulcrums be high enough, sufficiently to allow a low truck or transplanting machine being pushed under it. Should, however, the machine be mounted too high, two strong wooden trestles can be used. If the tree be high, guide-ropes should be attached to keep it in a perpendicular position. Where the convenience of a tree-transplanting machine is not at hand, the plant may thus be easily set on a strong handbarrow, and that, with the plant upon it, may be placed on a common cart, and so removed to its destination. For plants of moderate size, a handbarrow will be found useful both for placing it in a cart, and also for taking it from thence to the pit prepared for it.

Fig. 139.

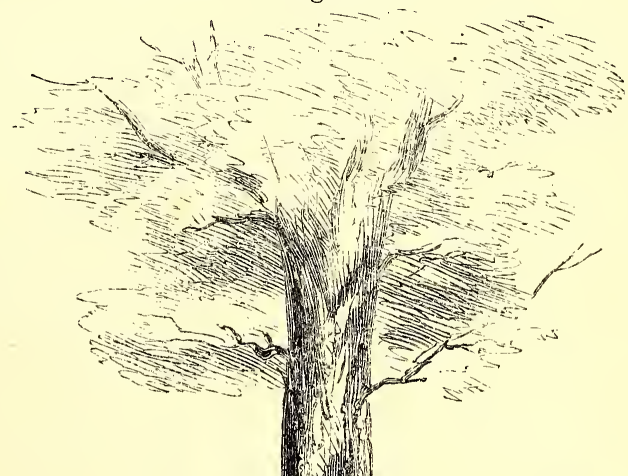


$M r \cdot M^{\prime} N a b$ 's tree-lifting machine, fig. 139.-Two wheels, much stronger, and about a foot higher than those of an ordinary icart, are fixed to a pair of strong shafts similar to those in a brewer's dray, kept together by a bar of timber 6 inches by 3 , placed about 18 inches in front of the wheels. Under the bar, and along under those parts of the shafts forming the body of the machine, is bolted on a bar of iron, 3 inches by 1 inch, and at the centre of the body of the machine are forged on to this bar two sockets in which the axles of the wheels work, for there is no axle extending across from wheel to wheel as in ordinary two-wheeled carriages. A similar bar of iron is bolted on to the inside and outside of the frame for greater strength. At front, and also at back of the carriage, so as to be clear of the wheels, are placed two wooden cylinders or rollers, 6 inches in diameter ; the outside ones are wrought with short iron hand-spikes, and the two inside ones by a winch at each end, and they are also furnished with a rack-and-pinion catch to keep them in their place when fully wound up. At the back part of the carriage is a double iron bolt, which ties the back ends of the carriage together, and whieh can be tightened up by a screw at each end. This bolt answers the purpose of an ordinary cross-axle, which latter could not be used, as it would cross the carriage at the exact point where the stem of the tree is to be placed. In loading the tree or shrub, the ball is prepared as in ordinary cases ; two strong planks are laid from side to side of the trench which surrounds the ball, the two hind rollers and the double iron bolt are removed, the carriage is then pushed backwards, the wheels running on the two planks, until the trunk of the tree or centre of the shrub is, as near as can be calculated, in the exact centre of the carriage. The hind rollers and double iron bar are then put in their places, the latter being screwed up as tight as possible to keep the wheels from spreading, which they have little chance of doing, on account of the great strength of the framework. Several narrow pieces of stout plank, and of equal lengths, are then pushed as far under the ball from the two opposite sides as can conveniently be done, and from the two opposite sides are pushed in as near to one-third of the diameter of the ball, each, as can be, two strong $2 \frac{1}{2}$-inch cable ropes, the ends of which are brought up to the rollers, and introduced under the one and over the other, and so made fast to the outer ones. The two rollers at each end of the carriage are then set in motion, the outer ones by the short iron handspikes, and the two inner ones by the crank-handles. By continuing the process of winding-up, the cable is, of course, shortened, and as it is, it carries up with it the ball in a very easy and gradual manner, the thick pieces of plank introduced under it preventing it from sinking into the ball, or injuring the roots. When the ball is elevated sufficiently high to be free from the ground, the hole may be filled in, and horses put to the machine, one in the shafts, and two abreast as leaders, if the tree is so large as to require them ; one, however, or in general two, are found sufficient to remove a tree weighing, includ- ing the ball, from two to three tons. Horsepower, however, depends a good deal on the ground the machine has to pass over. If the tree is very high, guide-ropes are necessary to keep it in a perpendicular direction, to assist in passing through amongst other trees, and more especially in assisting to plant it in a proper state when placed in its new situation. When the tree has arrived at the place of its destination, all that is required is to place the two strong planks formerly used along the two sides of the pit,and to draw the machine overit, stopping when the ball is exactly over the spot where it is to beplaced. The wheels being blocked, and the horses removed, all that is to be done is to begin uncoiling the cable by reversing the motion of the rollers. When the ball is set down, the short planks placed under it are to be removed, and the cable pulled out. Our machine, made of the very best materials and workmanship, cost $£ 25$. It has been in use eleven years, and has not required the least repair. It is capable of removing trees to any distance: we once brought a tree to Dalkeith from the neighbourhood of Glasgow, 46 miles, with one horse, with the greatest ease. Twowheeled carriages are much more convenient for transplanting trees than four-wheeled ones, on account of the less space required for turning, and hence are better adapted for being taken into thick plantations, and for traversing drives, walks, and rides, which often have very acute curves.

$\mathrm{Mr}$ Barron's large tree-lifting machine.-Although illustrated by three very good woodcuts in " The British Winter Garden," the letterpress description of this machine is rather too brief to be clearly understood. This ponderous machine consists of two parts, each mounted on a pair of massive broad-tired wheels. The front part is furnished with shafts, constructed so as to give the greatest degree of strength, being without mortice or tenon. The hinder part has a short pole behind, to which horses may be attached to draw it backwards when detached from the front part. Both these parts are furnished above the axle with thick bolsters or bearers, and are connected together by two strong oak beams trussed with iron rods, 21 feet long, which serve for bearers to support the tree in a pelpendicular position. The carriage is allowed as much lock as possible, and the whole fitted together with bolts and nuts, which in a few minutes can be taken to pieces at any part, separating the front and hind wheels in order to place the carriage with the greatest facility in a proper position for placing the tree upon it, as well as for lowering it in its new situation. The platform upon which the ball of the tree rests, and which is portable, and placed under the ball before it is removed from its original place, is suspended from the two long bearers by ten strong chains, the whole being raised by a screw-jack, and suspended sufficiently high for travelling; six of the ten chains are stronger than the others, the links being $\frac{7}{8}$-inch iron. "Each of the chains must have a strong ring at one end, and a hook at the other: two of these are termed liftingchains, one at each end of the poles for the screw-jack to lift by; the other four strong 
chains are termed sling-chains, and are placed on the beams of the carriage. When the tree is raised at one end, the two sling-chains are hooked into each other under the poles, then the four smaller or corner chains are placed on the beams the same as the sling-chains. The end of the poles being raised, the corner chain is then fastened round the ends of planks which form the platform under the ball; the poles at the other end must now be raised, and the sling and corner chain fastened as at the other end. Two screw-jacks have been tried, one at each end, which, at first sight, might appear to expedite the operation, but this," Mr Barron thinks, "is not the case, as it is necessary for the corner chains to be fast at one end whilst being raised, in order to keep the tree in an upright position. In the majority of cases, (except where the tree is very tall, or top-heavy, or the ground uneven over which the carriage has to pass), it will be found that the weight of the immense mass of earth, when properly balanced in loading, and the corner chains all properly fastened, will be sufficient ballast for maintaining the tree in an upright position, and that the guide-ropes are seldom used except when the tree is loading or unloading." The carriage with its load is drawn over the spot where the tree is intended to be placed, the hind and fore parts of the machine are separated, the tree let down by the same means by which it was elevated, and withdrawn from both sides ; the operation of covering up the roots after they have been regularly spread out is proceeded with, and the whole routine completed. The platform and mode of introducing it under the roots, as well as Mr Barron's mode of preparing the ball and preserving the roots, will be best understood by an extract from his recently published "British Winter Garden," p. 31. "A tree being about to be lifted, say from 40 to 50 feet high, the diameter of the branches being of the same dimensions; the groundwork operations would commence on the outside of a circle of from 20 to 25 feet in diameter, more or less, judging from the kind of tree how far the roots would extend horizontally. The soil would be then cleared from the roots, carefully preserving every fibre as far as practicable, up to within a given space of the stem, where the remaining mass of earth and roots must be left undis-

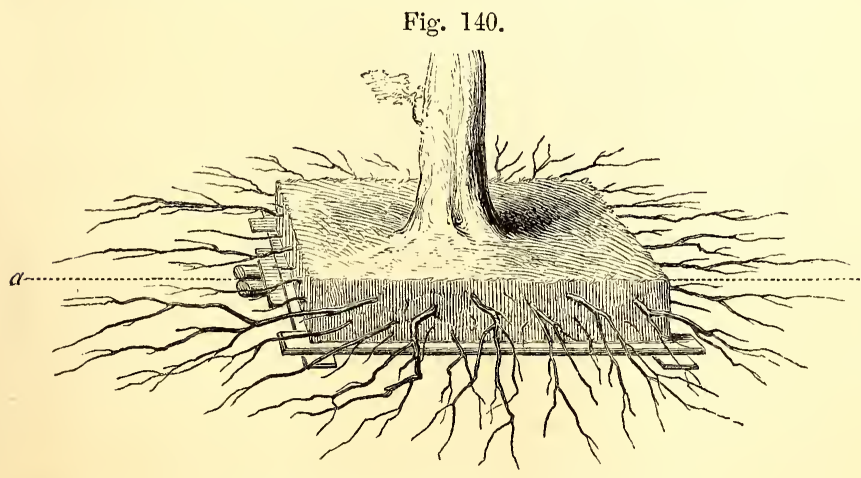

PREPARATION OF ROOTS FOR TRANSPLANTING. turbed, as shown in fig. 140, forming a parallelogram, say 9 feet 6 inches by 8 feet. A channel is then excavated about 2 feet square, in the direction of the line $a b$; this being done, two strong poles (of ash, oak, or Spanish chestnut) of equal thickness are then passed under the centre of the tree, and on them a strong 3 -inch ash or oak plank, 20 or 22 inches wide, hooped at the ends to prevent it from splitting. A strong chain is then laid under the poles at each end, by which the mass is raised by a screw-jack. The ball is slightly hollowed out below all round, to admit of bevelled-edged planks being placed under it, the two end ones resting on the centre broad plank, and the two side planks resting on the ends of the two cnd ones. Some litter must then be stuffed firmly in, between the planks and the soil, with a spade, by which means every part will stand to its bearing when raised up." The cut will show the care $\mathrm{Mr}$ Barron bestows on the preservation of the roots ; and in this he is right. His mode of lifting is also favourable to this, as there is no pressure upon them, the whole mass being placed on a platform under the ball, where there are few roots, and even these in most cases of no great importance. There are difficulties, however, to contend with even in this excellent mode of taking up large trees, the chief of which is getting the platform placed under the ball, as well as in its removal after the tree is set in its place; and we suspect that in such hands as Mr Barron's the operation would be more completely accomplished without any ball whatever, as it is quite evident that there is scarcely a useful food-collecting fibre within the compass of the ball here exhibited, and this the more so the older and larger the tree is. In our opinion the ball here preserved is of more importance in supporting, or rather in preventing the main roots from being broken entirely off, in consequence of their great length, during the operation of removal, than in containing within it a single root capable of supplying the tree with food, and that the tree must remain in a state of perfect inactivity until the formation of fresh spongiolets at the points of the fibres preserved, or depending for the time entirely on those which may have escaped injury during the operation. For there is no doubt that those that have been preserved will speedily become most active in providing a supply to the tree as soon as they are again imbedded in their natural element.

Mr Barron's smaller tree-lifting machine. The machine we have described above, as will readily be understood, is for the removal of trees of a very large size. The one we are about to notice is for transporting trees and shrubs of 
much smaller dimensions; and as these are by far more generally operated upon, we think this latter machine deserving of especial notice. The framework or platform to be placed under the ball is somewhat similar to, and introduced under the ball much in the same way as the last, only the centre poles are in this case dispensed with, "as a 3 or 4 inch oak plant is found sufficient, if free of knots, to carry a tree from 1 to 2 tons weight. By this machine, when the tree is ready for being lifted, it can be raised in a few minutes by a windlass at each end; and when taken to the place of destination, it can be lowered from it in the same short space of time." This carriage is like the first in two parts, a hind and fore part-a circumstance rendered necessary to facilitate the process of loading and unloading. It differs also in having three wheels instead of four, and in the lifting power being given by a windlass at each end, over which the cable passes, which is secured to the platform under the ball. As the windlass is turned round by means of levers, as in weighing an anchor, the cable is wound round it; and as it becomes at each turn more and more shortened, the platform and ball upon it are elevated until sufficiently clear of the ground. By these simple mechanical appliances the great transplanting operations carried on at Elveston during the last twenty-five years have been executed.

Mr Holmes' machine for transplanting trees of large size.-Trees of large size have been very successfully transplanted by Mr Holmes, Amport House, Hants, as described by him in the sixth volume of the "Journal of the Horticultural Society," p. 14. The ball is prepared in the usual way by excavating a trench around it, preserving the roots with as much care as possible. A common four-wheeled timber carriage is made use of, and an inclined roadway formed on one side of the hole for the fore wheels to run in. "The fore wheels are separated from the hind ones; the former run backwards down the roadway towards the tree, and the hind wheels on the opposite side (a more abrupt way having been cut for them, as it is of no further use as a roadway), so as to place the tree to be removed in the middle, and so that the ball and roots clear the wheels. Instead of the pole belonging to the carriage when used for timber being retained, a pole with a slight curve in the centre, so as not to come in contact with the stem of the tree, is used, which, together with two strong oak or ash beams, about 1 foot square by about 12 feet long, serve to connect the wheels and sustain the weight of the tree to be lifted; and two short cross pieces, about 3 feet in length and from 2 to 3 inches thick, are placed at right angles with the beams running lengthways, the stem of the tree being in the centre of the hole. These cross beams have each a hole cut through, about 6 inches by $2 \frac{1}{2}$ inches, in order to let the eye of the screw through." These screws are each 2 feet long. "The top of thiese cross beams sustains the plate in connection with the screws, which plate is 6 inches square. Connected with the plate is the box or nut through which the screw passes. The screws are worked by levers inserted in their heads horizontally, and there is an eye at the bottom of each screw, made very strong, for the chain to pass through. The chains are then braced completely round the ball of the tree, so as to injure the roots as little as possible, and care is taken that each chain has its due share of the ball or weight of the tree, and also that an old mat is placed next the ball, then a piece of plank, and next to this the chain, so that neither ball nor roots may be harmed. A man is then placed to work each screw, which he does by a small pin or lever 2 feet long. When the men have screwed up as high as the screws will allow, the tree is wedged so as to retain what has been obtained by the screw. For this purpose a few wedges 2 or 3 feet long are required, and a few flat boards for the wedges to rest upon firmly. When the wedges are found to sustain the weight, unscrewing is commenced and the chain tightened. In this way the process of raising goes on, alternately screwing and wedging." If the screws were of a sufficient length, we think that wedging would not be required, and that the ball would be lifted in much less time. "If things are well managed the tree will generally swing at the second screwing up; and, in raising, a little tact and care is necessary. Care should be especially taken that the wedges are securely placed before unscrewing; and when the bottom of the ball is high enough to clear the surface of the ground in travelling, the horses are put to ; before which it is well to lay a few planks down for the wheels of the carriage to pass over until upon firm ground. No tying of anything is required, as the pressure upon the screws prevents everything from slipping, and the tree continues perfectly upright during the whole process of removal. A guide-rope is attached to the tree by way of precaution, but its greatest use is in placing the tree upright during the operation of transplanting.

"When the tree has arrived at its destination the fore wheels are driven down the roadway through the hole till they arrive at an abrupt termination, which is left in order that the tree shall be exactly in the centre of the hole. The ball is then unscrewed until it touches the ground; the chains are loosened; the screws and cross-beams are taken down; the hind wheels separated from the fore ones by pulling out the hind pins or bolts which fasten the beams to the carriage; and lifting the beams one on each side, and one end only on the ground, the fore wheels are then pulled out, then the hind wheels, leaving the tree standing upright as at first, which concludes the operation as far as removal is concerned."

There can be no doubt that keeping trees in a vertical position during the process of removal is the most rational, because there is no strain placed on either the stem or the roots. Whereas that of janker-moving is attended with both these evils, as well as an almost certain displacement of the bark at the part where it comes in contact with the axie; and even if the bark is not displaced entirely, the whole of its vessels 
as well as those of the trunk at that part, become bruised to an extent from which the tree seldom recovers. Throwing over trees with large balls and strong roots on their sides to effect their removal in a horizontal direction, is most destructive to the roots on the side of the ball which is undermost. Difficulties will present themselves, however, in the case of trees to be removed in a perpendicular state when they are to be taken out of thickets, or where surrounded by other trees, on account of the branches coming in contact with each other. Trees so situated are not the most fit to be taken out of sheltered plantations and planted in open parks. It were better, therefore, to choose such as are growing on the outskirts of plantations as being better suited to stand the exposure of the open park, as well as on account of their being free from the entanglement of the branches of others. It is worthy of remark that Mr Holmes' season of operation commenced in September, and was finished in December. The number of trees transplanted in 1847 was 204, of which 199 were in a thriving condition in November 1850. They averaged from 48 feet in height to 13 feet; and in girth of stem, at 2 feet from the ground, from
43 inches to 7 inches. The kinds were -7 yews, 18 oaks, 59 beech, 1 purple beech, 3 birch, 22 elms, 5 limes, 36 hornbeam, 25 horse-chestnuts, 28 sycamores. We have in this an excellent specimen of successful transplanting; and no doubt much of the success depended on the early period at which the operation was performed.

Mr Mackay's large tree-lifting machine.The vast improvements of late years carried on at Kingston Hall by the spirited proprietor, Edward Strutt, Esq., by which a barren knoll has been transformed into a richly-wooded park, owes much of its present effect to the skill and perseverance of Mr Mackay, Mr Strutt's intelligent gardener, under whose directions the improvements were carried on. Mr Mackay, improving on Mr Barron's large machine noticed above, constructed one more perfect in some of its details, although not essentially differing in principle. A description and figure of this machine was given in "The Gardeners' and Farmers' Journal." It has since been figured and described very lucidly by $\mathrm{Mr}$ Brown of Arniston in "The Forester," second edition, p. 474, which description and illustrations, figs. 141 to 144 , will show its principle. "It consists of two pairs of

Fig. 141.

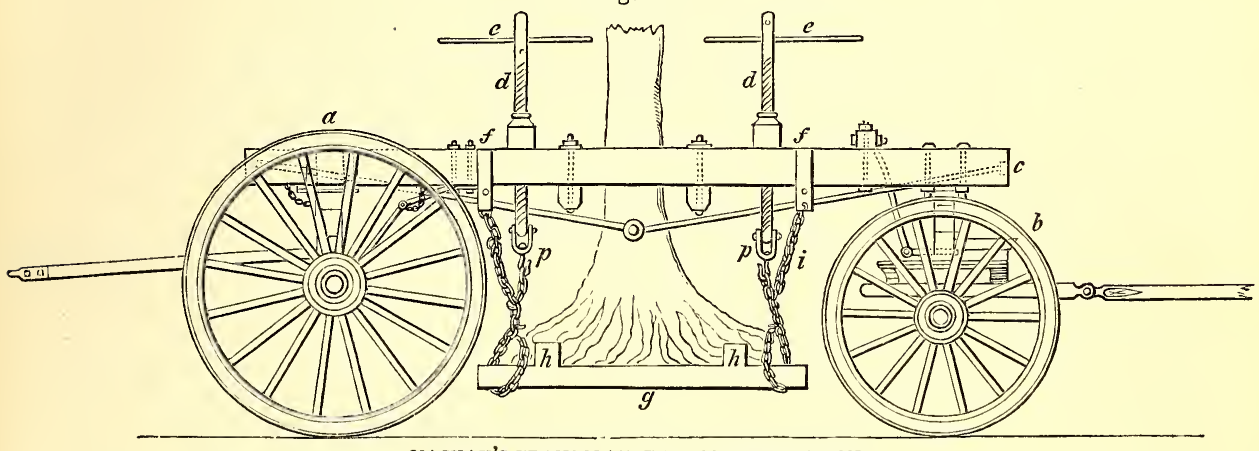

MACKAY'S TRANSPLANTER. SIDE ELEVATION.

Fig. 142 .

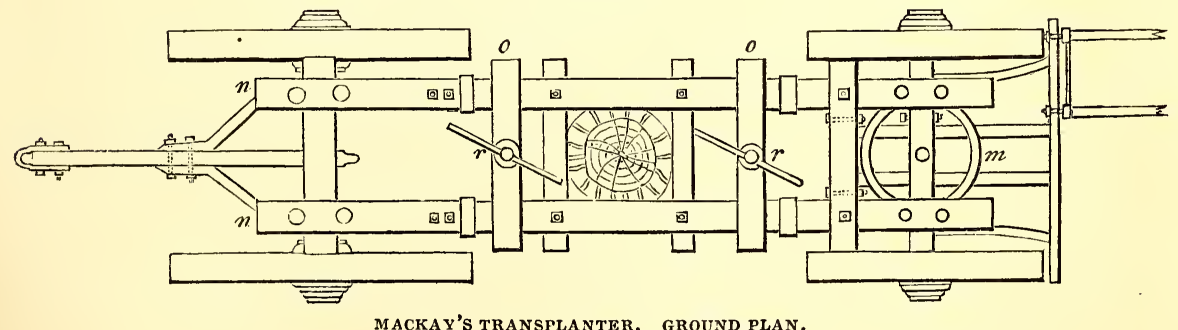

wheels, each pair working upon a strong axle of superior quality. Each pair of wheels is about 9 inches broad in the rings. The pair situated on the front part (see engraving at $b$ ) are about $5 \frac{1}{2}$ feet in diameter, and the pair situated on the hinder part (see engraving at $a$ ) are about 7 feet in diameter, and distant from each other about 15 feet, thus making the length of the VOL. II machine, from one axle to the other, 15 feet. On each of the axles is placed a strong frame, in order to raise the horizontal bearers to a convenient height, and at the same time with the view of making the whole machine move in a workable manner (see section of front frame $k$, and section of back frame $l$ ). The front frame, I may also state, is made to turn upon a hori- 
zontal wheel, in the same manner as in the case of a carriage, for the easy turning about of the

Fig. 143.

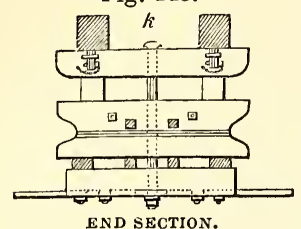
apart, are two strong beams of wood about 10 inches deep by 6 inches broad (see side elevation
Fig. 144.

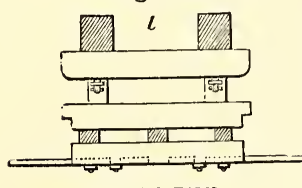

END SECTION. machine in any required direction, as is represented in the ground plan of the tree-lifter at $m$. Resting upon the frames, as is shown in the front and back sections, and parallel to each other at a dis. tance of about 2 of tree-lifter at $c$, and also on the ground plan at $n n$ ). Those beams are fastened to the frames placed above the fore and aft axles by means of strong iron bolts, which can be un. screwed as occasion

may require ; and placed across upon those horizontal beams again are two others (see ground plan at 00 ), into which the jack-screws are placed. The screws are also shown in their working state in side elevation $d d$, with their cross handles for turning, $e e$. On the under part of these jack-screws, as placed through the cross-beams, are attached the strong chains by means of which the tree is principally taken out of its place (see $p p$ ) ; and as an assistant to them, there are side-chains attached to strong iron rings fixed upon the beams (see $f f$ ). Those side-chains are made to act along with the others attached to the screws, and are also found necessary to retain the weight of the tree, and its ball of earth during the reversing of the screws for a new hold. The horizontal planks upon which the ball of earth rests, supported by the chains, are shown on side elevation at $g$; and the two cross planks which are placed upon the horizontal ones, for the better support of the ball, are also shown at $h h$. Those planks require to be made of the best oak, not less than 4 inches thick." To render this description of the machine more perspicuous, it will be necessary to refer to the mode of loading and unloading, and as this is inseparable from the process of taking up and replanting the tree, we shall give it as described by Mr Marnoch, in "The Gardeners' and Farmers' Journal," who was present to witness the operation of removing trees 40 feet in height, weighing each, including the ball of earth and the machine, upwards of ten tons, and drawn by nine horses. The trees to which Mr Marnoch alludes "were growing on the outskirt of a wood. The ground around the tree was cleared, and at $4 \frac{1}{2}$ feet from the stem of the tree a circular cutting was made to the depth of about $3 \frac{1}{2}$ or 4 feet, and about $2 \frac{1}{2}$ feet in width. This done, then, on the most open side of the tree, a sloped cutting was made from the surface of the ground to the bottom of what may now be called the ball of earth, and a similar sloping opening was made on the opposite side of the tree. The first of these sloped cuttings was made for the purpose of drawing out the tree, up this slight incline, to the surface of the surrounding ground. The circular opening in the earth around the tree, and the two sloping roads on each side being now prepared, the workmen commenced to undermine the ball of earth : this being done all round, four stout oak planks, long enough, were placed under this ball of earth in front and behind-that is, crossways to the direction of the machine; and under these two others were placed lengthways, with their ends in the direction to back and front of the machine. It will now be understood that these four oak planks under the ball of earth cross each other at their four extremities; and around their extremities, at each of the four corners, four sets of strong iron chains are fastened." A reference to our plate will explain this. "The first sct of chains brought into play were those attached to the bottom of the jack-screws. Being made as tight as possible over the ball of earth, they were then hooked on to the end of the jack; the two jacks were then turned by two or four men each, as the case may require, until the tree and the ball of earth were raised from the ground about 6 or 10 inches. The jacks were then permitted to rest, and what are called the sidechains were put in requisition. These, as we have already stated, are fastened to the planks under the ball of earth, at the same point as the others, the opposite ends being made fast to the side-beams, as shown in the engraving. This being done at the four corners, the jacks are then reversed, and the first set of chains slackened; the weight of the earth and the tree is then sustained by the side-chains. The first set of chains are again adjusted, and made as tight over the ball of earth as manual power can effect. The jack is again run down, and again attached to the chain at the lowest point it will reach. The jacks are once more applied, and run up to the end of the screw, which has been completely worked up to the under side of the crossbeam on which it rests. By this second lift the jacks have probably gained upon the 6 or 10 inches which they made at the first lifting, and the bottom of the ball is now 15 or 20 inches from the ground. The side-chains, which are now quite slackened by the second lift of the jacks, are again made as tight as possible; this accomplished, and all securely and equally fastened to the side-beams again, the jacks are gently reversed, till the weight is equally divided betwixt the side-chains and the jack-chains. The tree is now in a condition to be drawn out, unless it should be necessary to give it a third lift with the jacks, so as to raise it still higher from the ground; and if so, the side-chains must always be attended to as already described. They may properly be called the guard-chains, as they prevent accidents in the event of breakage of any of the jack-chains, when the former would take the weight of the tree, and prevent its falling. About 10 or 15 feet up the stem of the tree, four ropes extend from this point to the two front and the two back cormers of the machine; and this is all that experience has 
found necessary to preserve the tree in a perpendicular position during its transport on the machine to its future place of growth. The perpendicular position of the tree is, however, greatly secured by the four side or guard chains. We have now to describe the mode of introducing the stem of the tree within the machine. This is effected in the following manner: the machine is drawn as near to the tree as circumstances will allow; it is then taken to pieces by unscrewing the different bolts, - that is, the main horizontal beams are unfastened and thrown over the wheels on either side. The larger hindwheels are then placed in their proper position on the sloped cutting behind the tree, and the smaller fore-wheels on that before the tree; the large beams are then lifted on to their places, one on either side of the tree, and made secure with the iron bolts and the requisite fastenings. The tree then stands with its stem betwixt the side-beams, and with a pair of wheels behind and another before. The chains and jacks are then applied as already described, and thus the process is complete. The next and only other point deserving further allusion, is that of planting or placing the tree where it is ultimately to grow, and this is effected in the following way: The hole, sufficiently large to receive the ball of earth, is dug the necessary depth ; then, on the opposite sides of the hole, a sloped cutting, wide enough to admit the machine to be drawn down and through it, is also provided. Into this cutting, therefore, the machine and tree are drawn, and through it the team of horses first pass. When the tree has reached the proper point, the machine is permitted to rest. Props of brick or stones are then raised at the four corners immediately under the ends of the crossplanks. These props may be three or four bricks in height; and when all is prepared in this way, the jacks are reversed, and the ball of earth gradually lowered down, till the ends of the cross-planks rest upon the corner props, and the tree has taken its proper perpendicular position; and this is effected by raising or lowering of these corner props. All being adjusted, any opening that may remain betwixt the bottom of the ball of earth and the bottom of the hole provided for the tree is filled up with earththe whole being made firm around and under the roots of the tree. The brick or stone props are then struck out, and the planks removed-a process easily effected, as the tree now rests upon the earth which has been placed under and about it. These planks are, however, well ironed at each end, that, in case of any difficulty in removal, a horse or horses may be readily yoked, and the planks withdrawn." The whole of this routine differs little from that followed by $\mathrm{Mr}$ Barron, excepting in one particular, which we think a very important one, namely, setting the newly-removed tree in a pit, instcad of planting it on, or nearly on, the surface, as practised by Mr Barron. In good deep soils we would admit of an excavation to the depth of 18 inches being formed, on which the tree, if of large size, is to be set, and the earth gathered up around it in form of a flat-topped mound. The operation of supporting the tree after planting would be by this considerably facilitated, and the roots would be placed in an equally favourable condition.

M. Vallet's tree-lifting machine.-However excellent both these machines may be, there is little doubt that both must be regarded more as improvements upon the machine invented by $\mathbf{M}$. Vallet, a French nurseryman in the time of Louis XIV., than as original inventions. M. Vallet's was invented to facilitate the taking out and in the immense orange-trees in tubs, for which the French gardens are and were celebrated. It consisted of a four-wheeled machine, without an axle to connect the two hinder wheels, which was left out to allow the machine to be pushed back, so that the orange-tub could be enclosed between tlie framework forming the sides. When the tree was so placed, a strong bar of timber was secured to the two ends of the beams which formed the sides of the carriage, and answered the end of a portable axle. On the top of four strong upright posts, one at nearly each corner of the machine, was placed two rollers, the dowels at their ends working in iron sockets fixed in the upright posts, the hindermost of which was made so as to be taken out and put in conveniently; over these rollers a cable-rope was wound, so that by unwinding them the two cables fell towards the ground, and was made to pass under the bottom of the orange-tub, and when re-wound again by a man at each corner working a short lever, the tub was elevated to any height required to clear it of the ground, while being removed to the allotted place. The bar of timber connecting the hinder part of the sides of the machine, as well as the hinder roller, being removed, the machine was drawn forward, leaving the tree in the position allotted to it. With a similar machine we have removed hundreds of large trees, by introducing a platform of strong planking under their balls, and passing two cable-ropes under it, as here described; and with another with two wheels, very similarly constructed, we have moved many of great size and weight.

The most powerful and perfect of all such machines, is, however, undoubtedly that invented and patented by Mr M'Glashan, who has favoured us with the following description and drawings of it, figs. 145, 146.

"The first part of Mr M'Glashan's process is to lay down a square frame of $\mathrm{T}$ iron $a$, in size equal to that of the ball to be removed. $\mathrm{He}$ then takes cutters $b$, made of malleable iron, and 1 foot broad, and 3 feet deep, or with head and neck $4 \frac{1}{2}$ feet, and with holes at different heights for a pin, which regulates their depth to be screwed in, so that they can be made, at very little trouble, to lift any depth of ball from 1 to 3 feet. These cutters are driven with wooden mallets into the soil to the depth required all round, and being inserted sloping inwards, they give to the enclosed mass the form of a square blunted wedge. A bar of angular iron $c$ is then laid along the top of the four rows of cutters, and extension-rods $d$, going across the frame, force the heads of the cutters apart as far as necessary, and consequently cause the points to converge at the bottom. Two parallel beams $e$ are then laid 
across the frame, upon which are laid two crossbeams. A clasp or collar $f$ lying hard on these two cross-beams is thus put round the trunk of the tree, which being made tight by screws, as shown in figure, serves to steady the tree and bear a portion of the weight when the lift is taken. The means of raising the mass are a car- riage, which also serves the purpose of transplantation. It consists of two long beams $g$, which have an open space for wheels to run in, as shown in figure; the length of the axle is the same as the thickness of the beam, and is set in a plumber box, with a cast-metal seat $h$, that is bolted to the long beams which rest on it, and

Fig. 145.

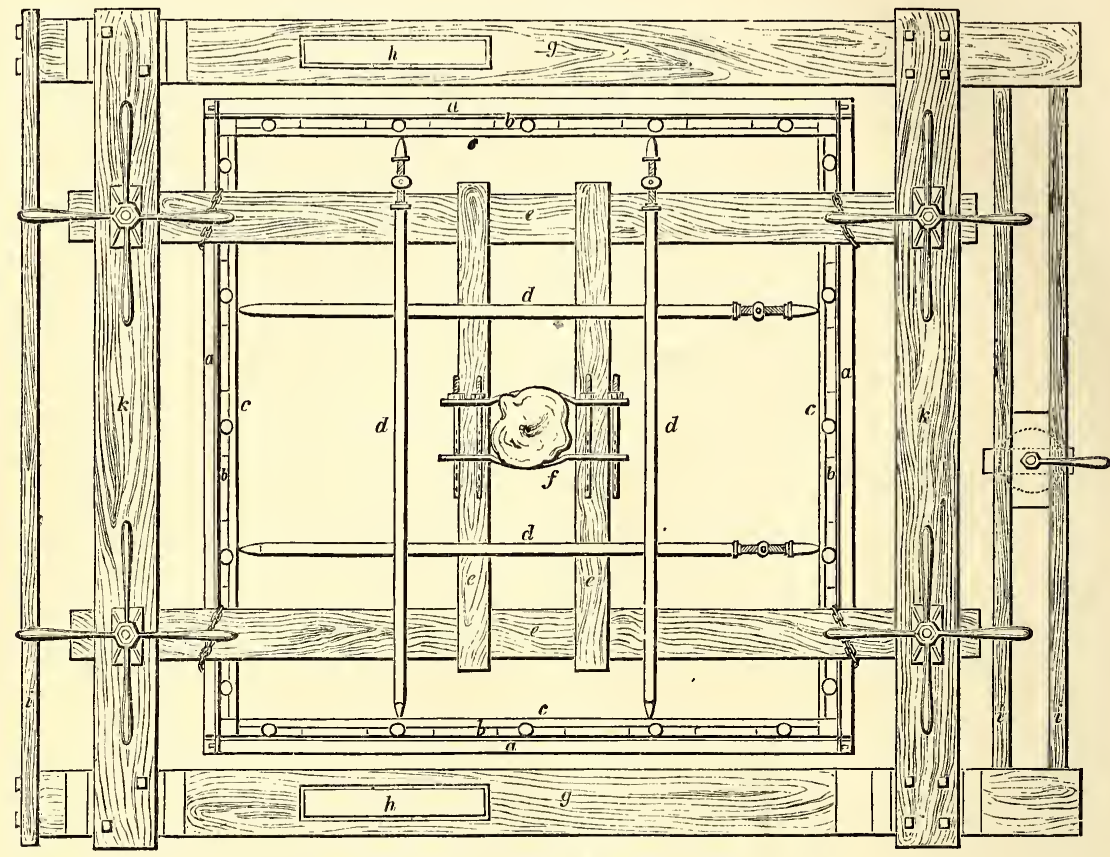

M'GLASHAN'S TRANSPLANTING MACHINE. GROUND YLAN,

of course adds to the strength of them. The two long beams are bound together fore and aft by bars $i$, and cross-lifting beams, which are firmly bolted to them. There is a third wheel in front, which carries a portion of the weight, and, having a handle on the top, acts as a helm does to a ship, so that the carriage can be turned very quickly round a sharp corner, and easily governed through amongst trees. There are two bolsters $j$, raised up on the long beams at each end of the carriage, across which are laid two beams $k$ (already alluded to in describing the binding of the long beams), for the screws to work upon; these serve to give height enough for the ball being raised to clear the ground. Horses can work at any end of the carriage by simply affixing the shafts to either of these extremities. The beauty of the construction of this carriage is, that it is as applicable for removing large shrubs as it is for forest trees.

The end bar $i$ and cross-lifting beam $k$ are taken off, and the carriage is moved back, enclosing the tree until it is exactly over the lift; the end bar and cross-lifting beams, which were taken off, are replaced and screwed on. The process of raising the mass is accomplished by screw power; the screws are two or four in number, as the case may require, and so arranged as to make the lift equal. They are made fast to the beams of the frame, and are worked by men. When the screws are put in motion, the frame and enclosed mass rise erectly upwards. In ordinary circumstances, a tree with a ball of 10 feet, in about 20 minutes' working of the screws, should be completely raised from the pit. The propelling power, when the ground is soft, and horses cannot be used, or when it may be inconvenient to use them, is by a winch attached to the front of the carriage, and block and tackle made fast to some neighbouring tree, piles driven into the ground, or any other hold most convenient; but when the way is clear, and the road good, horses will do the work more expeditiously. It will be found necessary, when the ground is soft, to lay planks to prevent the wheels sinking, until they get on hard ground. The pit being already dug, the mass is moved directly over it, and the tree and ball are lowered into it on the same principle as it was raised; the earth is then filled in all round about, and the apparatus removed, and the operation is completed. The reader at first sight may be apt to think that the cutters which were put round the ball would sever the large roots; but if the operator has any desire to 
preserve them, which, $\mathrm{Mr} \mathrm{M}^{6} \mathrm{G}$ lashan remarks, is still a very undecided question, it can be easily done by finding out their position with piercers, and driving a cutter on either side of them, and thus preserving them entire. Although 10 feet is the size described here (as it has already been done), it can either be reduced or enlarged as the tree or operator may require.

Fig. 146.

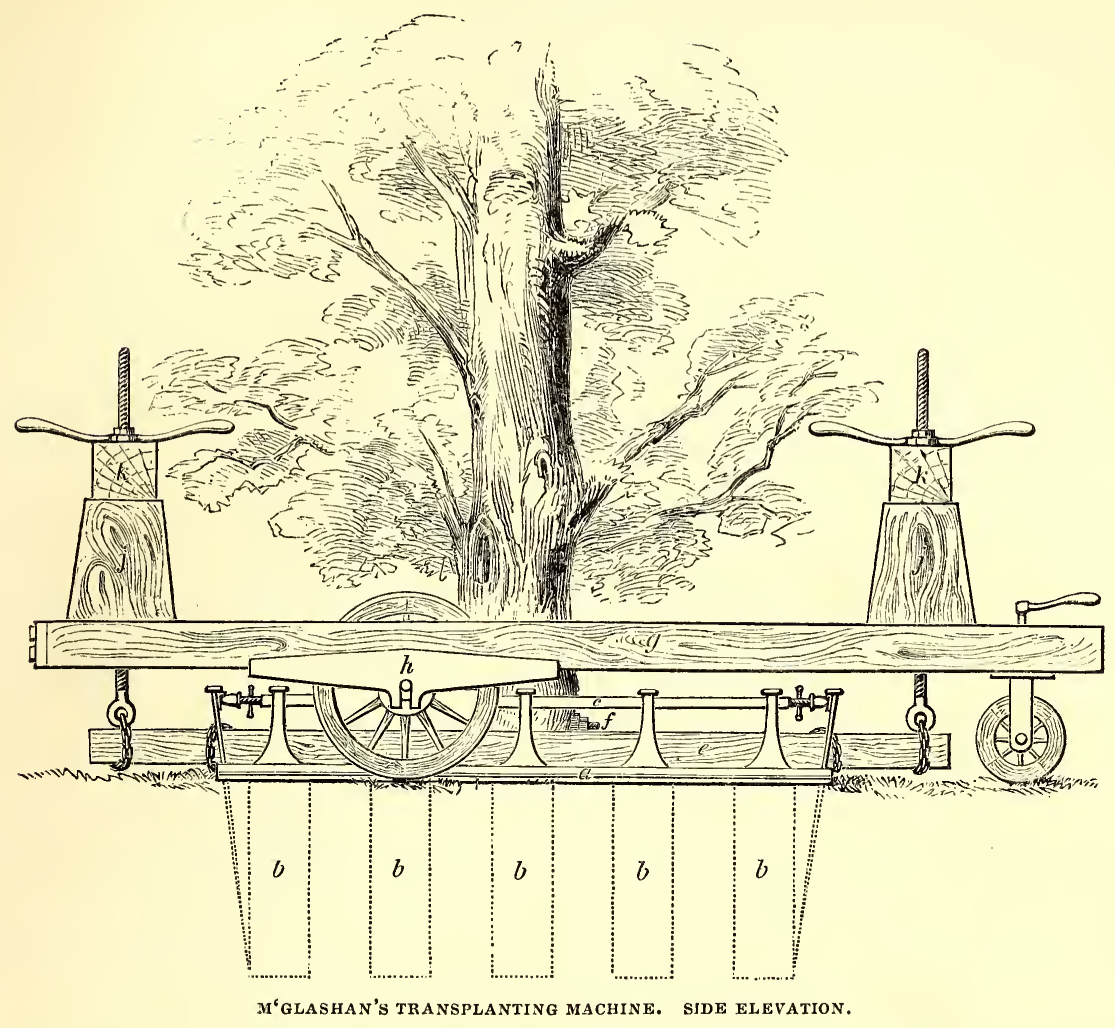

We think this machine would be much improved, and rendered more generally useful, if the frame were extended in length, so that, instead of the ball being square, it might be oblong in shape. To render the machine wider than 4 feet would be disadvantageous, because it could not be made to travel on walks or narrow roads, and would be difficult to introduce amongst bushes and other trees. Lengthening it out to the extent of 4 feet more would present no difficulty in those respects; while by doing so, instead of a ball 4 feet square, we could have one 4 feet (the present breadth) by 8 feet in length, securing upon two sides a much greater length of uninjured roots. The spade-shaped cutters are as good as anything that can be devised when the intended ball is of a size to include all or at least a sufficient number of roots, according to the size of the tree to be removed; but to remove a tree with a greater extent of roots, the ball should be cleared all round with spades and picks in the usual manner, saving as many roots as possible, tying them together in bundles, and disposing of them in such a way that they would be secured against accident during the operation. This being done, the ball should be undermined, and the tap or downward-growing roots cut off with a long chiselshaped instrument, instead of tearing them up and breaking them by main force.

When the ball is thus prepared, if broad grippers, in form like the letter $\mathrm{L}$, were introduced along the sides of the ball, just as $\mathrm{Mr}$ M'Glashan's cutters are, but not quite so close together as to prevent the roots which extend beyond them from passing between them, the turned in ends of the grippers made to pass under the ball on all sides, the extension-rods attached to them, and the $T$ iron placed as in his method, the ball would be kept together not only at the sides, but under the bottom likewise. The operation of lifting should be exactly as recommended by the patentee; for of all modes hitherto employed none are so simple and effective as his. The turning in the ends of the grippers below the ball would prevent the soil from falling away from below, and the ball being prepared as in ordinary cases, this transplanter could be employed in all soils, however stony or gravelly, which at present it cannot be, on 
account of the moral impossibility of driving in the cutter's amongst stones and gravels. This slight modification does not in the least detract from the usefulness of this valuable machine, nor alter its most important principle, which is the lifting power it possesses above all others. In the case of very large trees, it would be well to have an extra set of wheels, from 8 to 9 feet in diameter, which would greatly lighten the draught, and admit of a better counterpoise to the top of the tree, the weight of the ball being more under the axle. Another improvement in the case of very tall trees, with a view to maintain them in a perpendicular position, would be to place a soft collar round the trunk, 10 feet or so above the ball, and to secure to it and the four corners of the machine four diagonal poles, which would act as stays or fulcra, and prevent the tree from falling over. As at present constructed, the weight of the ball is the only counterpoise. Low wheels are used when large shrubs or trees, branched to the ground, are to be removed, because the machine, in that case, is better got under the branches. These alterations suggested themselves to us while Mr M'Glashan was lifting a large tree lately in Dalkeith Park, and he at once acceded to them; for we have seldom inet with an inventor who is more open to conviction in all matters relating to the improvement of his invention. In the experiment referred to, he took up, with a most perfect ball of 4 feet on the side and 3 feet deep, a large holly tree, and had it ready for attaching the horses, in one hour and five minutes, with no other assistance than one of his own men and three labourers to assist in driving in the cutters and working the screws by which it was lifted out of the ground.

Beyond a certain size, this, as well as all other machines of a similar character, is incapable of removing trees with safety, not, in the case of Mr M'Glashan's machine, from want of mechanical power, but on account of the amputation to which the roots are exposed, unless we go at once into the principle laid down some years ago by the late Sir Henry Steuart. If weadmit his theory to be correct as to cutting back the roots the season previous to removal, and allowing the wounds to heal over, and the roots to send out a new supply of fibres with their necessary spongiolets, then the cutting back and removal at once, as proposed by Mr M'Glashan, is no doubt preferable ; because, with all the care with which trees can possibly be removed upon Sir Henry's plan, the spongiolets must be seriously injured, particularly by his method of transporting them (namely, upon a rude kind of janker); whereas, by Mr M'Glashan's plan, the same amputations take place at the period of removal which take place at the commencement of Sir Henry's, and the formation of new fibres and their necessary spongiolets goes on after removal, and these are not afterwards interfered with: lessening the risk of removal in the first instance one-half in favour of $\mathrm{Mr}$ M'Glashan's machine, and much more if we take into consideration the injury the bark sustains when thrown over the axle of the janker, and the no less injury done to the roots during their transport, and placing them in the pit prepared for them. For trees or shrubs requiring a ball of 4 feet on the side, or 64 cubic feet, and from that downward, within which the whole roots are enclosed, Mr M'Glashan's is the best we have seen.

The circular form of ball is no doubt the most natural, and therefore must be the best, because the roots of all trees, where no obstruction prevents their extension, do radiate from one centre, which is immediately under the trunk. This being the case, all deviations from the circular form must be erroneous in principle, and very seldom followed in practice.

It is seldom that trees of extraordinary size are removed, unless under very peculiar circumstances ; and if so, they are seldom conveyed to any great distance. When such cases occur, it were better to tunnel under the balls; and after placing a strong flooring of planks under them, secured to each other at the ends by a plank laid transversely, and to cross-planks laid over the top of the ball, and screwed firmly to these below, to prop them up in that position until proper rails be introduced under the whole, with rollers between the rails and plank flooring, laid at an easy incline from the bottom of the ball to the surface, and so continued to the new pit, into which the ball could be slid down by a similar incline. It would signify little whether the bottom flooring were left in the new pit or taken out ; the loss would only be the material, which in time would rot away. But even if this were a matter of consideration, their removal could easily be effected by drawing them out at either end after they were disengaged from each other. Trees of any size may be removed in these days of mechanical ingenuity ; to do so most successfully is merely a question of expense.

We shall now describe the smaller apparatus for removing shrubs and young trees, fig. 147 .

Fig. 147.

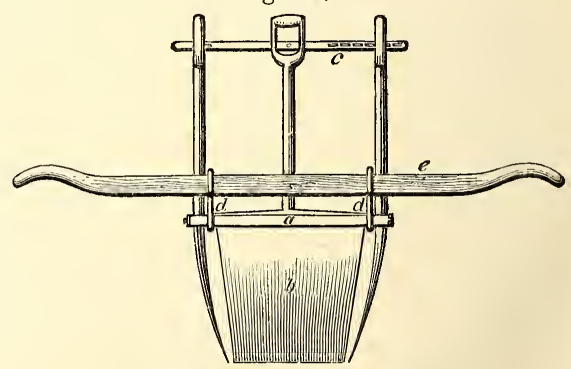

M'GLASHAN'S SMALL TRANSPLANTER.

The first thing done is to open the frame $a$, (which has a joint at one corner, and a fastening at the other), and put it round the tree, and again fasten it; four spades $b$, with wooden handles, are then driven with the foot in the inside of the frame (or if the ground is hard, with a mallet striking on the head or tramp), as deep as required. 'I'wo extension-rods $c$ are then passed through the opposing handles of the spades, the handles are then drawn apart, and holes being in the extension-rods, a pin is 
put in which keeps the spades in that position. Two hooks, $d$ are then put on two opposite sides of the frame, through which a handspike $e$ is passed; all things are now ready for the lift, which is taken by four men, two at each end of the handspikes, who take hold of them and lift up shrub and ball. (It will be found that lifting a ball of earth 2 feet square on this principle will not occupy more time than five or six minutes). It may then be carried on the handspikes, or, if the distance is great, on a wheelbarrow, to the place where it is to be transplanted; the hole being dug, the ball and plant are placed in it, and the earth filled in round about, and the apparatus removed, which completes the operation. The sizes of this machine vary from 14 inches to 4 feet 6 inches, but of course a 4 -feet 6 ball will require four spades entirely of malleable iron, and the extensionrods require a screw at one end (instead of a pin), to facilitate their application.

The janker tree-lifter.-This, if not the best of such machines, is evidently that in most common use. It is, however, only calculated for carrying them in a horizontal position, while all those we have noticed are calculated to carry them perpendicularly. No doubt the latter is the best, because there is no strain on any part of the stem, and also because the branches are less liable to sustain injury during their tranport. One objection to the janker is in its construction: the wheels should be much higher than they generally are, and, in addition to this, the bolstering over the axle should be much greater and the pole longer. These things complied with, the branches would be carried higher, and hence escape the injury they sustain by trailing on the ground. Neither in loading nor in the act of transport can the janker be much objected to ; it is in the unloading and placing the tree perpendicularly on its new site that the defects of this machine become most obvious, and this is increased in proportion to the size of the tree. The raising the height of the wheels and bolstering over the axle, as we have suggested, will, however, to some extent modify this defect. Many hundred trees of from twenty to thirty feet in height have been transplanted by this machine in Dalkeith Park within these few years, and a success has attended the operation more than usually satisfactory. These trees were in no way previously prepared, but great care has been taken in preserving the roots, performing the work in fitting weather, and above all, giving sufficient scope to the new roots to extend in prepared soil.

Transplanting herbaceous plants.-These for the most part are easily transplanted, as well as multiplied, and the season for performing the operation is either before they begin to grow, in spring, or immediately after they have ceased flowering. Indeed the rule should be, to divide for propagation, and to transplant as soon as they have done flowering, and when the stems are beginning to decay. So extensive is this assemblage of our gardens, that the processes of transplanting and subdivision will require to be going on at almost all seasons of the year. For general purposes, however, the autumn and spring are the most proper. Herbaceous plants are easily transplanted with few exceptions, and some of these are, Dictannus, Potentilla, Dianthus, Acanthus, Astragalus, Coronilla, Lathyrus, Linum, Iberis, Lupinus, Lythrum, Medicago, Nepeta, Papaver, Pisum, Santolina, Statice, Trillium, Helonias, and several others.

Transplanting by mudding-in, or puddle-planting.-This mode is much in use in Belgium and Germany, and over most parts of the Continent where the soil is light; it is, however, seldom practised in Britain. The operation is thus performed: When the pit is dug, water is poured in, and the mould is stirred about until it becomes a complete mass of mud ; mould and water is added until the pit is about lialf full. 'The tree is then inserted, and the roots wrought up and down, so as to spread them as equally through the mass as possible. The remaining soil, after being converted into mud, is then thrown in until the pit is full, when the whole is covered with dry soil, and raised round the stem, leaving a hollow basin for future supply of water; the whole is then mulched over with litter. Councillor Diel, a scientific German author and amateur, asserts that trees thus planted in spring prosper better in cold situations than those planted in autumn in the ordinary manner. Ponty, in " Rural Improver," observes of this practice, that the soil becomes speedily too firm and too hard, and prevents the roots of the plant from spreading, and also that water is completely prevented from reaching to the roots. In this opinion every sensible planter will concur.

Transplanting by fixing with water.-This is merely a modification of the last method, and is sufficiently useful in dry soils, in dry weather, and when the tree is to be planted without a ball. Ponty, in the work last quoted, approves of this plan, and describes it as follows: "The hole being made, and the tree placed in it in the usual manner, the root is then slightly covered with the finer part of the soil, the tree being at the same time shaken, as is common, to settle the earth about its roots. Water is then applied with a common garden wateringpot, by pouring it upon the soil with some force, in order to wash it close to and among the roots of the plants. But this can only be done efficiently by elevating the pot as high in the hand as can conveniently be used, after first taking off the rose. It will be obvious that for such purposes a large pan with a wide spout is to be preferred. The hole is then filled up with the remainder of the soil, and that again consolidated with water as before, which usually finishes the business." The late $\mathrm{Mr}$ M'Nab, a high authority, recommended the soil to be wet enough to be almost like thick paint.

Placing the tree according to its former aspect. - Some cultivators attach considerable importance to placing the tree at planting so that it shall stand in the same position with respect to the cardinal points that it did previous to removal; believing that, in the case of trees having young and tender bark, the bad effects of sunstrokes are thus guarded against. The majority of planters, however, pay little attention to the 
subject as regards trees in general. There can be little doubt that, in the case of a tree that has been for some years trained to a wall, in the event of its being transplanted, the surface exposed to the sun should be again so placed; the back of its stem and branches being inured to exposure; while that which has been shaded and placed next the wall would be liable, from its greater tenderness, to suffer not only from sun-strokes, but from the effects of severe frost also. Whether this precaution be absolutely indispensable, as regards trees in general, admits of some doubt: one thing is evident, that an adherence to the rule can in no case be injurious, and may be easily effected by marking the side of the tree that faced the south with a piece of chalk, or by tying a strand of matting round it, the knot of which will indicate the particular side of the tree.

Transplanting turf.-Turf is transplanted, or, as it is in general called, laid, for the purpose of forming grass verges, and for covering whole lawns, and often for repairing blanks in both. The whole art of turf-laying consists in rendering the ground upon which it is to be placed both level and of equal solidity, that it may not settle into holes or inequalities. Much of the success in both these depends on the quality of the turf procurable; that from old sheep-pastures composed of the dense and low-growing perennial grasses, and completely free of all other plants, and from a rich loamy soil, is to be preferred. The turf should be cut, for the former purpose, of a breadth somewhat more than the breadth of the finished verge, unless that verge exceed 18 inches in breadth, when it will be better to cut it in two breadths for the convenience of removal. For the latter purpose a width of 12 inches will be sufficient. It should be cut as nearly of an equal thickness as possible, having the sides cut perpendicular, and the ends of each piece obliquely. Each piece may be of the length of from 3 to 4 feet, and be rolled up to prevent its breaking in the transport. The ground should be carefully and firmly trodden and beat down. When the whole is laid, it should be again beaten down by the implement called the turf-beater, formed of a piece of oak plant, 11 inches by 8 inches, and 22 inches thick, with a handle projecting in a slanting direction, and the sides neatly cut straight by a line with another implement called the edging-iron, fig. 148,-an implement invented by us many years ago, and for utility not as yet superseded. In the case of large lawns, sowing with the approved lawn seeds is the most economical mode, but it requires a year or two before it becomes what is called a firm sward, or that is fit to walk upon. Sometimes, where parterres are to be laid down on the lawn, their forms are defined with turf verges, and the intermediate spaces sown with grass seeds. With-

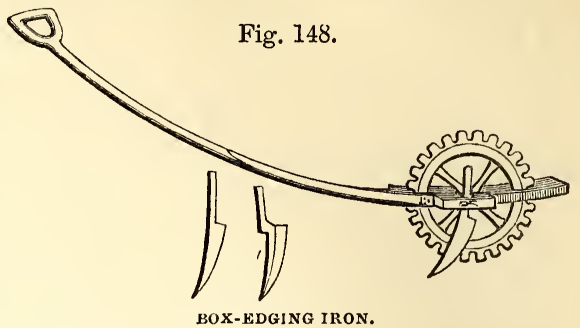

out this, it would be next to impossible to define the lines of intricate patterns correctly,-the only alternative being sowing down the whole space, and, after the second year, cutting the figures out of the entire piece. The same remarks are also applicable to the formation of grass terraces. For a list of lawn grasses, vide Flower Garden. In preparing the ground for a lawn, whether to be sown or turfed, it is requisite to have the whole completely cleaned of root weeds, trenched 2 feet deep, completely levelled, and afterwards repeatedly rolled until it becomes of a uniform even surface. The turf is then to be laid on, fitting it closely, and beat and rolled until it be solid and even.

It is of some importance in the formation of lawns, whether they be turfed or sown, to consider the nature of the soil upon which they are formed. If too rich, the grass, in either case, will grow too quickly, be apt to become coarse, as well as difficult to keep in good order by the scythe or mowing-machine. This evil, however, time will correct, as the repeated cutting will in time reduce the surface to a proper condition. If the soil, upon the other hand, be too poor, then the sward never becomes compact and uniform, dying away in patches, and those occupied with such plants as are the well-known emblems of sterility. A moderately rich soil is the best ; it will produce grass of a better colour, more uniform in surface, and continue longer ere overrun with moss. The proper season for laying or planting turf is after or during autumnal rains, and during winter in the absence of frost. Late in spring and during summer is the worst season, as the heat and drought are equally unfavourable to the grasses taking root, and the turfs shrinking or contracting in size leave inequalities between them which it is difficult to rectify.

Transplanting in the Quinqunx manner.-This mode consists in setting the plants so that those in one row may be opposite to the blank in the other, so that, whichever way the plants are looked at, they will appear to be in direct lines. 


\title{
CHAPTER III.
}

\author{
PRUNING AND TRAINING.
}

Pruning and Training are two important operations in horticulture, and so closely connected together, that we shall, to prevent repetition, consider them under one head. The principles upon which both are founded vary according to the object in view. The latter depends more or less on these facts-namely, that as the sap in all trees has a natural tendency to flow in an upward direction, it follows that the buds at the highest points above the roots will be the strongest, and more disposed to produce leaves and shoots; and therefore, when the formation of wood is desired, the nearer to the perpendicular a tree is trained the better; whereas if the formation of blossom-buds be the object in view, the very opposite direction should be given them. In fact, training is the power which governs the flow of the sap in trees and plants. Hence our best nurserymen train their weakly-growing young plants nearly in a vertical direction, while they place the strong-growing kinds nearly horizontally, and even in some cases nearly pendulous. During the growing season they have an eye to the progress each is making, and elevate or depress the shoot according to circumstances. The same rule should be attended to by gardeners, even with trees in a bearing state. Pruning, on the other hand, consists in regulating the number and bulk of the various parts of a plant by the reduction of its branches, leaves, buds, bark, or roots, so as to produce different effects on the parts which remain. The principles upon which pruning is mainly founded are thus clearly given in the "Suburban Horticulturist:" "The nutriment of plants is absorbed from the soil by their roots, and formed into leaves, branches, flowers, and fruit by their buds; by operating on the buds and roots, therefore, we can regulate what is produced by them. If the stem and branches of a plant contain a hundred buds, by removing half of these the shoots or fruit produced by the remainder will be supplied with double their former supply of nourishment; and if all the buds be removed but one, the whole of the sap sent up by the roots will be modified by that single bud, provided care be taken to remove other buds as they appear. On the other hand, when the whole of the buds on a tree are so abundantly supplied with sap from the roots as to produce chiefly leaves and shoots without VOL. II. blossoms, then, by cutting off a portion of the roots, the supply of sap is lessened, a moderate degree of vigour is produced, and instead of barren shoots blossom-buds appear. By these means the growth of plants is controlled by pruning. Pruning has not the power to increase the vigour of an entire tree, because it cannot increase the quantity of food taken up by the roots, but it can diminish the vigour of the entire tree by cutting off part of the roots, or it can increase the vigour of particular parts of the tree by amputating the branches, or taking off the buds at other parts. Pruning can prevent trees from producing flowers, and hence increase their general vigour and longevity. It can modify the general form of trees, as well as increase particular parts of them, and it can add to the vigour of stunted or diseased trees, by concentrating their sap, or directing it to a few buds instead of a great many. One of the most useful effects of pruning is to cause the development of dormant or adventitious buds, which is effected by amputating the shoot, branch, or stem close above any point where visible buds are usually situated, though they may now be wanting there."

Volumes have been written on the practice of training and pruning fruit trees, while really little that is useful is to be found relative to the theory of it. Among the former are the writings of Errington, Seymour, George Lindley, Hill, Knight, Forsyth, Fleming, Spencer, Beaton, Harrison, Hayward, \&c. ; and the Continental writers have been equally numerous, amongst the most celebrated of whom may be numbered Pro. fessor Thouin, Quinteney, Arand d'Andilly, \&c. \&c., all of whose works may be consulted with great advantage; while, on the latter subject, d'Albert, Cours Theorique et Pratique de la taille des Arbres Fruitiers, edit. 6, should be studied: a translation of this work would be valuable to the English reader. Of late years, however, the theory of pruning and training has been occupying the attention of some of our most eminent practical horticulturists.

The principal objects of pruning are the modification of trees to be trained to walls or espaliers, the promoting the formation of blossom-buds, the promoting growth and bulk, the curing disease, the enlargement of the fruit, renewal of the head, proportioning the head to the roots, 
\&c. \&c. Pruning is also performed upon the roots to induce fruitfulness, and to check overluxuriance in growth.

In pruning, the following practical rules should be attended to: Commence the operation upon hardy trees, as soon after the beginning of October as the gathering of the fruit will allow. Avoid frosty weather, or when it appears to be approaching. In cutting, always draw the knife in an upward direction, and leave the wound smooth to prevent the lodgment of water. In removing young wood, leave about an inch of the branch above the last bud, and make the cut on the side opposite to it. But, in removing an old branch, cut it as close to the stem or branch left as possible, in order that the bark of the latter may cover the wound the sooner. Use a sharp knifc, a due share of consideration, and be not in too great a hurry, lest you rcmove the branch which ought to have been left.

Fig. 149 shows the leading methods of training wall and espalier trees, with the names by which they are designated.

Fig. 149.

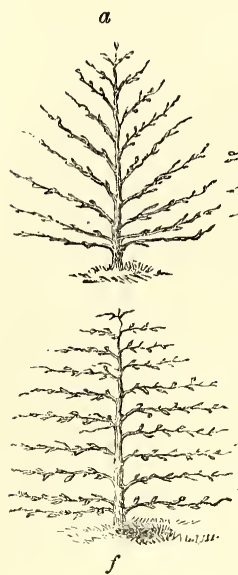

$f$

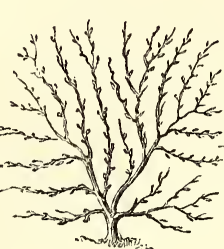

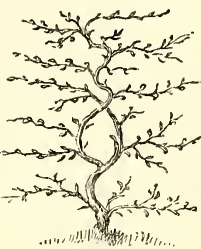

$g$
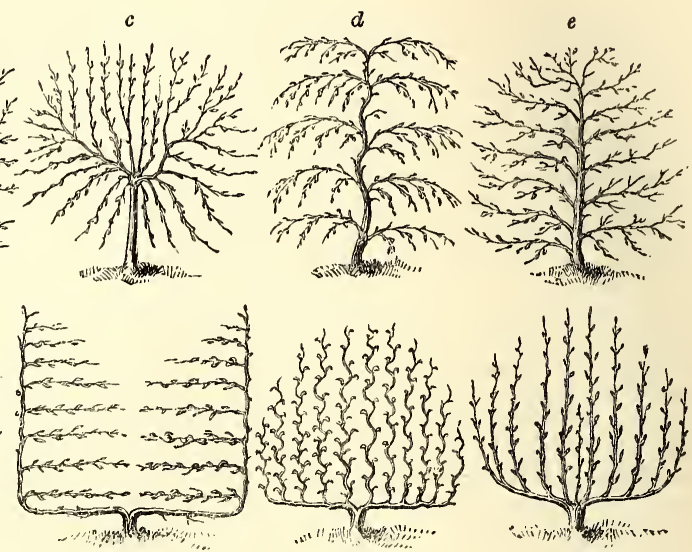

$h$

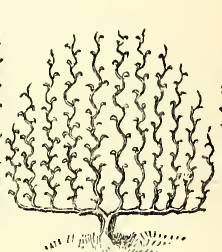

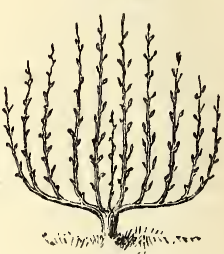

$k$

VARIOUS MODES OF TRAINING.

$a$, the herring-bone fan; $b$, the irregular fan; $c$, the stellate fan ; $d$, the drooping fan ; $e$, the wavy fan ; $f$, the horizontal; $g$, the horizontal with screw stem; $h$, the horizontal with double stem; $i$, the vertical with screw shoots; $k$, the vertical with upright shoots.

Besides these, espaliers are trained horizontally, or in form of a tablc, the stem rising through the centre of the trellis, and the branches being trained in a radiating form. Sometimes the espalier is placed vertically, sometimes placed at one or other angle of elevation, either according to the latitude of the place or the whim of the owner. They are also now often trained in the domical and curvilinear manner, of which examples will be found in sect. Espalier RaILS, vol. i. p. 556 .

Sometimes necessity occasions the trees to be planted on one side of the wall, having their branches brought over the top, and trained pendulous on the other; while others have planted the trees on one side of the wall whose branches were to cover the other, depending on the check the sap would meet with where the branches come in contact with the sides of the coping ; and one old and exccllent fruit cultivator, $\mathrm{Mr}$ Mearnes, trained the stems of his trees, bare of branches, to the top of the wall ; and, by carrying a leader horizontally, trained the shoots from them in a downward direction, reversing the position of the vertical with upright form. The varieties of training now in use are less numerous than formerly, and other modes of inducing fruitfulness are practised, and with evident effect.
In regard to training dwarf standards within a limited space, in addition to the examples we have given, and the notice of others, we may add that of them all there appears none superior to that of training in the spiral form, fig. 154, \&c., p. 395 ; that is, having the branches trained spirally round stakes set in a circular form, which stakes are to be removed when the branches have attained a sufficient size to retain their spiral form; and next to that, Hayward's mode, fig. 151, \&c., p. 395. All pruning and training, however, must be considered subordinate to a proper selection of stock, and to operations on the roots, much more than on the branches. The true balancing of the power of the roots to that of the branches is most important.

The season for pruning.-The season of pruning, in the general acceptation of the term, extends over the whole year. The process is, however, divided into two general sections, namely, winter and summer pruning. Many do not begin to prune till spring, commencing with the apricot, and taking in close succession the peach, plum, pear, cherry, and last of all the apple and fig, the sap of which is not properly in motion till April. Autumn and winter pruning, so far as concerns fruit trees, is objected to 
by some as being injurious, and causing a dryirf and hardening of a portion of the wood close to the part cut, and hence the granulous matter does not so easily protrude between the bark and the wood as when the trees are furnished with sap. Hence, say they, all pruning of fruit trees should be pcrformed at the period at which the rising of the sap takes place. We should, however, observe that the vine is an exception to this rule. "To prune in autumn strengthens a plant, and will bring the blossombuds more forward; to cut the wood late in spring tends to check a plant, and is one of the remedies for over-luxuriance. At the opening of spring, the blossom-buds can be certainly distinguished, which is a great guide to the judgment in many critical cases; but on the other hand, if the blossom buds get much swelled, they are liable to get bruised or knocked off, in the various operations of untacking, cutting, and re-nailing the branches. Supposing the winter course of pruning to be divided into three periods-autumn, the cold months of winter, and the beginning of spring-the plants to be exccpted from the two first are uniformly the fig, when not in a forcing-house; the vine, for the most part, because the autumn is seldom hot and fine sufficiently long to ripen the year's shoots. Some except the peach and nectarine from the middle period, but not from the first; because, they say, if a severe frost happen immediately after the pruning, the points of the unripened shoots, and particularly the wood-bud next to the cut, are generally so much hurt that there must be a second shortell. ing farther in than was intended, to furnish those shoots with leaders."-Encyclopoedia of Gardening. Theoretically speaking, autumn, soon after the fall of the leaf, is the best season for pruning, when the object is to promote strength in the tree; and next to this, winter, performing the operation in mild weather: for until the soil becomes lowcred in temperature, the roots are collecting a certain stock of nutriment, which they will throw into the branches retained after pruning; whereas, if the pruning be deferred till spring, all the nourishment which has been taken up by the rejected branches will be lost in consequence of their removal.

Dr Lindley, in reference to this subject, gives the following reasons why pruning in early autumn should have this effect: "During the

Fig. 150.

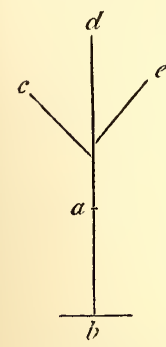

DISTRIBUTION OF SAP. exact proportion which that part bears to the part abstracted. When, however, early or autumnal pruning is employed, $a$ to $c, d$, and $e$ are removed before the sap has accumulated in them, and then all which the roots are capable of collecting during the period of repose will be deposited in the space from $b$ to $a$, and consequently branches from that part will necessarily push with excessive vigour."-Theory of Horticulture, p. 259.

Winter pruning. - Some trees may, undoubtedly, be operated upon during the month of October, and some ought to be so even sooner, as in the case of the raspberry, the old wood of which should be removed as soon as the fruit is gathered, bccause whatever amount of food such wood may draw from the roots after that time, is so much unnecessarily drawn from the soil : besides, its removal admits more light and air to the young wood left, on which the succeeding crop depends. Trecs which are weakly, and such as have their wood well ripened, may also be pruned thus early. Yet for the generality of fruit trees and bushes, the beginning of November is a good time (and this even depends a good deal on the locality, as to whether it be early or late); and the process may extend over the following three months. Early pruning has, however, like early autumnal planting, its advantages - the principal of which is, that the wounds may begin to heal up before severe frosts sct in. Cultivators, for convenience, often defer this operation till the frost is too severe for operating upon the soil; and to this too many are driven, from not having a sufficient number of men to do everything in its proper season. Hardy trees and shrubs may, with few exceptions, be pruncd during autumn and winter; but such as are tender should be left till spring, as, besides the injury which may be done them by frost, in consequence of their wounds not bcing healed, many of their branches may be killed or injured during winter; and the extent of this cannot be ascertained till even late in spring, and after they have begun to grow. Tea-scented and all similar tender roses offer examples of this.

Summer-pruning fruit trees.-Could this operation be dispensed with, and rendered unnecessary by root-pruning, limiting the supply of food, and attention to training, it would be most desirable. While, however, rich, deep, and broad borders are indulged in, there can be little hope of this being secured. To plant in rich borders and deep soils has as great a tendency to increase the size of the rubbish-heap as the size or quantity of the fruit. A continuance of this practice will produce those annual crops of breastwood which must be removed and consigned to the faggot-pile, for removed they must assuredly be to prevent a general barrenness.

It is worth while to inquire when and how this is to be best accomplished, and it is equally worth while to study how their formation may be, if not entirely, at least in a great measure prevented.

Bearing on this point, we find $\mathrm{Mr}$ Errington, in "Journal of the Horticultural Society," thus 
writes: "I hold it good pear-culture to make a practice annually of selecting the shortestjointed and most mature annual shoots, and tying them down to the principal leaders in the June and July summer-pruning. The old and mechanical spurring system is nearly exploded by this time : it has proved a most fallacious course of practice for centuries, and the gardener who adheres yet tenaciously to it must be much attached to mere prescription. I do not say, Spur not at all. No; when natural spurs continue to form and advance into blossom-buds, by all means preserve them, but do not entirely rely upon them." The more we prune, the more the trees will grow to wood; the more we cut off the extra supply of food, whether by circumscribed borders, root-pruning, ringing the branches, \&c., the more we conduce to fruitfulness, and the less to the formation of superfluous wood. Branch-pruning is, therefore, a secondary consideration, unless we do so to increase the bulk or model the form of the tree.

The following opinions bearing on this case deserve the careful consideration of the pomologist. "If we take into consideration the physiological fact," says a writer in "The Gardeners' Chronicle," " that by the agency of leaves the gases extracted from the atmosphere by those organs, and the juices drawn from the earth by the roots, are mixed, assimilated, and rendered subservient to the tree, thereby increasing its growth and perfecting its fruit, the question arises, Is summer-pruning beneficial or injurious? Admitting, then, that the leaves are the agents by which the juices of the tree are prepared to enter into its system, to take part in the various economy of life and the reproduction of its species, it is clear that by removing those leaves we diminish the resources of the tree. Now, in summer-pruning, we remove at least one-third of the leaves of the tree subjected to the operation; and nature never clothes a tree with more leaves than is necessary for its resources at the roots; a proper harmony is thereby kept up between those two organs, and perfect health of the tree is the result. In summer-pruning we deprive the tree of its digestive organs without at all diminishing the absorption at the roots; the juices, consequently, not meeting with a proper surface of exposure, which, if no summer-pruning had been performed, they would have done, are returned to the tree in a crude and unprepared state; the wood thereby is not of a natural texture, and the fruit is deprived of its proper flavour. That the over-abundant juices of a tree so treated make a vigorous effort to repair the injury is manifest by the quick appearance of succulency from all parts of the tree, at once establishing the fact that it is overcharged with sap in a crude state. The growth of the tree at that period is not of the character of one slowly and gradually increasing in stature, but a rapid and simultaneous outbreak of the sap from all parts, even where no appearance of buds had previously existed. That trees left untouched till winter-pruning, with a whole summer's growth, are both unsightly and untidy, cannot be denied; and to leave them in that state to be broken by the winds of autumn, would evince carelessness, whatever good intentions there may have been in so doing, and would be highly censurable. But the tree may be rendered an object by no means unsightly without cutting away half its branches, as is the common practice : the latter may be stopped, and when practicable secured to the wall, or what ever substance they may be trained upon; and when the period for general pruning comes, all superfluous branches may be removed. As the greater abundance of leaves is at the base of the shoots, the loss in topping, and the occasional removal of a whole shoot, in comparison to the general practice, would be but trifling." Another writer in the same paper says, "So far" as I can judge from my own observations, the summer-pruning of fruit trees is productive of nothing but leaves to the trees and disappointment to their owners. I have pruned at all times, but I can safely say that I never yet saw a blossom-bud upon the spur left for its production, if the tree was at the same time growing vigorously. It is true that, by root-pruning or ringing the bark, I have made these spurs form blossom-buds, but these practices are not always desirable." "Never cut away a twig in summer," says another authority, "that can by any possibility be got to the wall. Prune freely in the winter, and keep the trees well balanced; nail in at full length all young shoots intended for bearing; keep up a succession of young shoots, to supply the places of those that are worn out and exhausted in bearing, and ring the trees when the growth of them is too luxuriant." Opposed to these opinions are two other writers in the work last quoted, the first of whom remarks: "Many, however, do not so much deserve blame for pruning as for not pruning. The wall trees in many places grow as they may, and rows of fruit trees in the quarters are never pruned during the summer; all are generally left for the winter-pruning. The luxuriant growth of wood in summer robs the fruit of nourishment, and increases the extension of roots to supply the increased extent of branches in the following season. During winter the knife removes a great portion of the branches, and the equilibrium that nature has established between them and the roots is partly destroyed, and a luxuriance of fruitless branches continues to be produced on account of the increased powers of the roots. I imagine that summer-pruning does diminish the absorption at the roots, and that it establishes a uniformity of circulation between roots and branches. There is such a mutual action existing between these, that whatever is done to curtail the one will affect the other. A judicious summer-pruning of crowded and luxuriant young shoots will allow a free circulation of air, and the genial influence of the sun will better mature the wood that remains; it will also moderate the action of the roots, and the reciprocity between them and the branches will thereby be better established without the necessity of pruning much in winter. The use of a strong pair of scissors is all that is necessary for pruning fruit trees in summer, when the wood 
is soft. By this practice the juices supplied from the roots, and the gases received from the atmosphere, will be concentrated in the branches, and better fruit be obtained." The second, in following up a somewhat similar principle, observes: "If pruning commenced with the growing season, and was judiciously carried on, the result would be very different from what arises from the bad practice of allowing a tree at midsummer to present the appearance of an unclipped hedge. The tree might well make a rapid and simultaneous outbreak from all parts; and even if the superfluous wood was left on the tree until the winter-pruning, the rapid outbreak in spring, although not to such an extent as it had been in summer, will yet be much greater than is consistent with the permanent wellbeing or fruitfulness of the tree; for as sure as these strong growths are cut away after having become strong, so sure will the tree make vigorous efforts to replace them, unless the supply from the roots is interrupted. Hence the great good of root-pruning, or, what is perhaps better with young trees during the first three or four years after planting out, when they are so very apt to make strong wood, of lifting and replanting them. If pruning begins as soon as shoots appear where they are not wanted, a great deal of wall may be gone over in a short time. The trees by this method will present a neat and healthy appearance; and the young wood, by bcing freely exposed to the action of sun and air, will be well ripened. Respecting leaves, when they are produced in great quantity, they are generally small, and small leaves are by no means so efficient as larger ones are. Take the vine for example. If the foliage is good, and has been properly exposed to the influence of light, \&c., the wood will be strong, the eyes plump, and, under favourable circumstances, a good crop will be produced. Now, the reason of all this is very obvious-it is breadth and health of foliage, and not numerical quantity, that is requisite to elaborate the juices of a plant; two or three large and healthy leaves are better than a dozen small ones. Physiologists are in error when they forbid us to prune a forest tree because a few of its leaves would be lost. This remark is equally applicable to fruit trces; but still I am no advocate for putting the axe-and-saw mode into general use. Pruning, to be efficacious, whether on fruit or forest trees, should be pcrformed as soon as the part to be rejected is capable of removal."

The object of summer pruning seems to be twofold,-namely, the production of artificial spurs, on which fruit-buds are expected to form, and relieving the tree of superabundant growth, as well as admitting sun and light to insure the ripening of the fruit. The encouragement of artificial spurs is wholly unnecessary; their existence is a scourge on the trees, drawing that nourishment from the soil by the roots for their useless support, that by better management would be directed to the enlargement of the fruit and the healthy condition of the trees. No doubt fruit-buds are sometimes formed upon these spurs, and in some varieties of trees more than on others ; they, however, in course of time, as the spur enlarges, become situated so distant from the wall as to derive little more benefit from it than if they were growing on open standards. All fruit trees, such as the apple, pear, \&c., have, within their wood, up to a considerable age, abundance of latent buds ready to be called into action, and these are called into action when the necessary conditions are complied with, and one of these conditions is a total removal of all artificial spurs during the winter-pruning. This undeniable fact shows the absurdity of summer-pruning with a view to the production of fruit-buds, as these will be abundantly produced wherever the artificial spurs are discouraged, and being set close on the branches, and near the wall, derive that benefit from radiated heat for which walls are especially constructed.

Pruning standard fruit-trees.-The principal object here is to modify the head, and to thin the branches so that the sun and air may penetrate to every part, so that the fruit may ripen throughout the whole tree in an equal manner. All standard fruit-trces should be allowed to assume their own natural habit of growth, and those habits are various-some throwing out their branches horizontally, others almost upright or fastigate, some drooping, and others very irregular. Such habits should not be interfered with; a reduction of redundant or misplaced branches, together with all useless spray and dead spurs and shoots, should only be effected. All fantastically formed heads in standard fruit-trees only tend to defeat the real object for which they were planted; and although very copious directions have been laid down by most writers on the subject, from the days of Mascall downwards, model'n pormologists agree with us in allowing every tree toassume its own natural habit. "If the tree is to be left to its natural shape, which is by far the best, it will, in the apple, pear, cherry, and most other fruit trees, assume something of the conical shape, at least for some years; but whatever shape it has a tendency to assume, that shape must not be counteracted by the pruner, whose operations must be chiefly negative, or directed to thimning out the weak and crowded shoots, and preserving an equal volume of branclies on one side of the tree or on the other,--in technical language, preserving its balance."-Encyc. of Gard. The directions for this mode of pruning, laid down by Thomas A. Knight, in his "Treatise on the Apple and the Pear," as also in various of his interesting papers in the "Trans. of the Hort. Soc.," are of great practical utility. The essence of them is, that the points of the external branches should be everywhere rendered thin and pervious to the light, so that the internal parts of the tree may not be wholly shaded by the external parts: the light should penetrate deeply into the tree on every side, but not anywhere through it. When the prumer has judiciously performed his work, every part of the tree, internal as well as external, will be productive of fruit; and the intermal part, in unfavourable sensons, will rather receive protection than injury from the external. A tree thus pruned will not only produce much 
more fruit, but will be also able to support a much heavier load of it without danger of being broken; for any given weight will depress the branch, not simply in proportion to its quantity, but in the compound proportion of its quantity, and of its horizontal distance from the point of suspension, by a mode of action similar to that of the weight on the beam of the steelyard; and hence $150 \mathrm{lb}$., suspended at 1 foot distance from the trunk, will depress the branch which supports it no more then $10 \mathrm{lb}$. at 15 feet distance would do. Every tree will therefore support a larger weight of fruit, without danger of being broken, in proportion as the parts of such weight are made to approach nearer to its centre. We have gone to some length on this head, as we know little attention is in general. paid to standard fruit-trees, and more especially in orchards of high repute, and from which a considerable profit is expected to arise. If there be any department in gardening in a more deplorable state than another, of a surety it is our commercial orchards. Indeed, even where better things might be expected, standard fruit-trees are allowed to grow as if they had no legitimate owner.

Coxe, an American pomologist of high repute, remarks very truly, "when orchard trees are much pruned, they are apt to throw out numerous (superfluous) suckers from the boughs in the following summer; these should be rubbed off when they first appear, or they may be easily broken off while young and brittle,-cutting is apt to increase their number." This chiefly takes place when trees have been long allowed to grow wild, and afterwards been subjected to unnecessarily severe pruning.

Our principal orchard as well as full standard fruit-trees are the apple, pear, plum, and cherry. The above remarks have reference chiefly to the two first of these, and the following to the two last. The muscle plum, orleans, and damson, are the principal plums fitted for standard or orchard culture in the northern counties of England and most of Scotland. There are, however, some varieties of other plums of inferior quality grown in some of our commercial orchards, whose total extirpation would be no serious loss. In the south the case is different, and there the green gage and other first-rate plums are met with ripening in great perfection. Plums, when grown as full standards, require but little pruning, and that consists of moderate thinning out of the branches when the trees have passed the meridian of their growth. The plum is liable to suffer from spring frosts on account of its early blossoming; it is therefore wise not to thin their branches too much, as often, when the frost has destroyed all the blossom on the outer branches of the trees, those that are towards the centre will escape, on account of the protection afforded them by the external ones. Indeed, the same rule holds good even in more favourable climates, and therefore a self-protecting power like that, although, as Mr Errington observes, it may cause " the tree to look somewhat confused amongst its sprucely pruned neighbours, must not be despised, more especially as it brings such fruit to perfection despite of its unsystematic appearance." The cherry, with the exception of the May duke and Kentish, are not so productive in our northern orchards as in those of the south; but we have several varieties of geans which rival either in flavour and productiveness. In the south the case is different, the greatest drawback in their culture being the difficulty to preserve them from birds, and hence we would suggest the propriety of growing them as espalicrs or dwarf standards, in which case they could be readily protected by nets. Standard cherries require little pruning, except shortening back the young shoots for a year or two after planting. They submit unwillingly to pruning when of a large size, and are apt to dwindle away after the removal of many of their large branches. The case is rather different when trained as espaliers or dwarf standards. In both cases they will continue productive for years under a judicious use of the pruning-knife.

Pruning dwarf standards.-Much of what has been said above is applicable to these also. They are, however, more generally planted in gardens, and require a greater degree of nicety in their management. Here also the kind of tree should be allowed to assume its own natural character without restraint, and the branches so disposed that the sun and air may penetrate to all parts of it. At the winter-pruning every shoot of the present season's growth should be cut clean away, excepting the terminal ones at the points of the branches, which should be shortened back to half their length, or more, according to their strength, and the size the tree is intended to be grown to. Natural spurs or fruit-buds will protrude from the main branches all over the tree in most kinds of apples and pears, and others will arise from those parts where the young shoots were cut from, without the encouragement of artificial spurs, as is sc often practised. Some sorts of apples are better adapted to this mode of pruning than others, and there are some few to which it is wholly inapplicable.

Spring pruning, although highly recommended by some, is equally condemned by others. Downing says, "We should especially avoid pruning at that period in spring when the buds are swelling, and the sap is in full flow, as the loss of sap by bleeding is very injurious to most trees, and in some brings on a serious and incurable canker in the limbs."

Hayward in his "Inquiry into the Causes of Fruitfulness and Barrenness in Plants and Trees," and also in his "Science of Horticulture," has strongly advocated constraining fruit trees within limited bounds. In the former of these works, page 235, he observes: "If it be desired to train them so as to fill a circular space, like bushes, they are best trained with their branches reversed, as by this mode they are not only brought to bear a great deal of fruit in a small compass, but are confined and protected against injury from high winds without stakes, which saves a great deal of trouble and expense. To follow this plan, plants must be obtained to furnish one upright stem, of from 3 to 4 feet in height, and at this height be made to throw out 
from four to six branches, 3 or 4 feet long, like this figure-fig. 151. At the winter-pruning the
Fig. 151 .

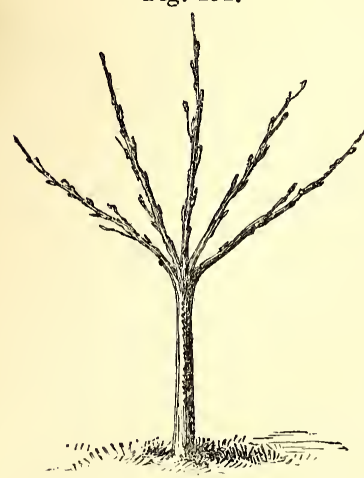

HAYWARD'S MODE OF TRAINING. branches must be brought down and fixed to the stem with small willow twigs or twine - thus, fig. 152. When they are fixed in this manner the sap will not flow in sufficient quantity down the branches to form wood branches at their ends, but the buds will more readily form for blossoms and fruit. The wood shoots will be thrown out on the upper sides of the reversed
Fig. 152.

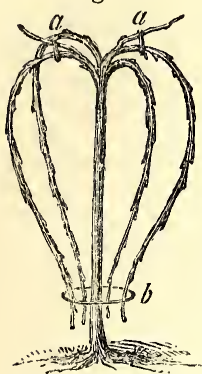

HAYWARD'S MODE OF TRAINING. branches, where they may be permitted to grow their full length during summer, and in the winter they may be either cut out, or brought down the same as the first branches, either to fill up any vacant space, or to take the place of the old branches if cut out. Or, if it be desirable, the sten may be allowed to advance to form another tier of drooping branches for bearers, as in fig. 153 . Indeed, on the same principle, it may be carried to a greater height. The general management of pruning, \&c., of trees trained in this manner, must be conducted and regulated
Fig. 153.

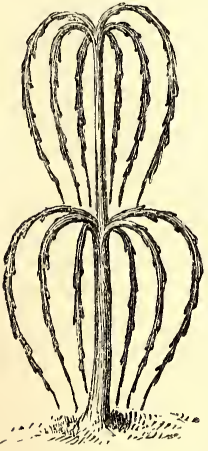

HAYWARD'S MODE OF TRAINING. by the same principles as when trained to any other shape. All collaterals thrown out where they are not wanted must be removed as soon as they are discovered; and as the reversed branches or bearers are worn out, they must be cut away, and fresh ones brought down in their places." To prevent crowding, when the ends of the branches are brought down towards the stem of the tree, instead of being tied to the stem they should be tied to a hoop, b, fig. 152, fixed to the stem, about 18 inches from the ground; and as shoots are most likely to be sent out from the curvature given to the branches, as at $a$, fig. 152, those best placed to admit of being bent down to supply the place of the parent branch when worn out, should be selected, and all the rest rubbed off. It would not be of advantage to have more than two tiers of pendant branches, as in such a case the trees would become too tall, and be liable to suffer from the wind; besides, the topmost tier of branches would rob those of the undermost, upon the principle already explained. Much of the success of this method of training lies in attention being paid to disbudding, or the removal of all such shoots as are not wanted to carry on and extend the tree, or to succeed and occupy the place of the worn-out bearers. Mr Hayward remarks that this mode of training is well adapted to apple trees on paradise stocks, pear trees on quince stocks, cherry trees, \&c., and also to peach trees in pots. It has also the advantage of requiring no stakes for support, which of itself is no small recommendation. Hayward's quenouille mode of training is even better adapted to small gardens than that practised by the French-(vide Pruning and Training the Pear.) The spiral-cylinder mode of training recommended by Hayward is well adapted to small gardens, and is thus described by him-" In-

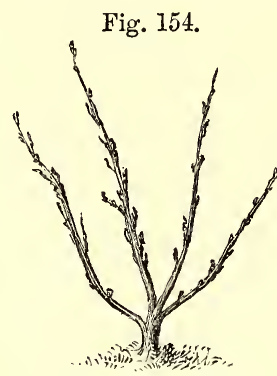

SPIRAL TRAINING.

Fig. 155.

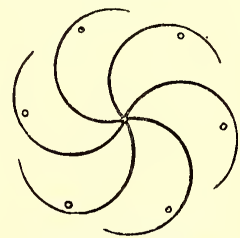

SPIRAL TRAINING.

Fig. 156.

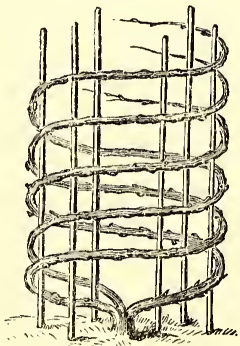

SHIRAL TRAINING. quiry into the Fruitfulness and Barrenness of Plants and Trees," p. 238- "Prune and manage the tree so as it shall form from three to six branches, of as nearly equal size as possible, within about 6 or 8 inches of the ground, as in fig. 154 ; and as soon as the branches are grown from 3 to 5 feet long, fix six rods or stakes into the earth for supporting them, in a circle about the root, as in fig. 155, the centre dot marking the root, and the others the rods. Each branch is then to be brought down, and being fixed to the rod near its base, the branch is to be carried round in a spiral manner, on such an elevation as will form an inclination of about $15^{\circ}$, and each branch is to be fixed in the same manner, one after another; thus all will move in the same direction, one above the other, like so many cork-screws following in the same course, as shown in fig. 156 . As, from the position of the branches, the fruit-bud of each leader will present the most vertical 
channel for the sap, the strongest shoot will form there, and thus afford the meaus of continuing the leaders to a great height, and for a great length of time, without crossing or obstructing each other, or throwing out useless collaterals. At the same time, by the depressed position of the leading branches, enough sap will be pushed out on their sides to form and maintain vigorous fruiting spurs. As trees trained in this manner need never exceed the bounds allotted them on a border or bed, a greater number of trees may be planted, and a greater quantity of fruit produced in a given space, than can be the case when they are trained in any other manner. But as pear and apple trees on free stocks may be found to grow too rude and large after a few years, those best answer which are grafted on dwarf-growing stocks-that is, pears on quince stocks, and apples on paradise stocks. However, to keep dwarf trees from growing too luxuriant and rude, it is a good practice to take them up and replant them every three or four years. If this is done with due care as soon as the leaves are off the trees in the fall of the year, it will not injure them, nor prevent them bearing a full crop of fruit the following year." 'Three to four branches we have found to succeed much better than six, which, unless the circle be large, causes crowding and want of light and air to the inner side of the trees. We do not anticipate that trees trained in this form will continue long productive towards the base of the branches; but, on the other hand, successive ones might be brought in - the old spurs being cut clean away, and the branch made a conductor for a young shoot procured near its base, or grafted on it by the side-grafting process.

The horizontal mode of training may be said to be entirely confined to the gardens of Britain. Neither the French, Dutch, Germans, or Americans practise it ; and they, with ourselves, may be considered the only nations where training becomes necessary, or is cared for in the culture of fruit. Hitt, if not the inventor of this method, was certainly the first to reduce it to system and practice. He employed it in various forms, and subsequent cultivators have added to or varied it also. It is the mode which, with the exception of the pendulous, places the tree under most constraint, and removes it farthest from its natural position. There are two objections to this mode; namely, the length of time required for a tree to attain the height of the wall or espalier, as in general it only makes an increase in height of from 9 to 12 inches yearly that is, the distance between each horizontal branch. The other is: should one of the three eyes, at which we are directed to cut back the perpendicular leader, get injured, or not break, we must then lose one of the side branches, for a leader cannot be dispensed with ; and, consequently, a blank is left on one side or the other which is not readily rectified. To obviate this defect, it has been proposed to combine the fan form with the horizontal. Of these proposals the most novel is that suggested by Mr Green, and published in " The Gardeners' Magazine," vol. viii. p. 539. For a wall under 20 feet in length, he would plant a pear tree, for example, at one end of the wall, at $a$, fig. 157, and striking a line from $b$ to $c$, the one near the top,

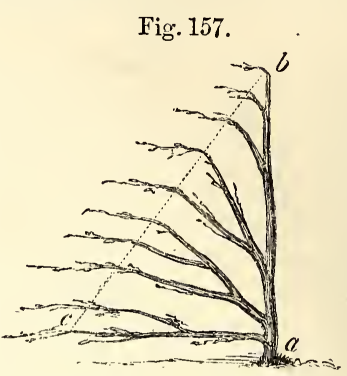

GREEN'S MODE OF TRAINING.

Fig. 158.

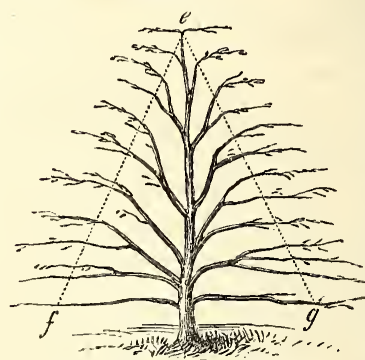

GREEN'S MODE OF TRAINING. and the other near the bottom of the wall, he would then train all the shoots to one side after the fan manner, bending the shoots where they intersect the line, as shown in our figure, into the horizontal direction. In the case of walls of greater length, he would plant the tree as in ordinary cases, striking two lines from the top to the bottom of the wall, as indicated in fig. 158, by e $f g$, training the tree in the fan manner as before, and giving the branches a horizontal direction at the points where they severally intersect the two lines $e g$ and $e \hat{f}$. It is easy to suppose how, upon the same principle, trees could be trained taking a perpendicular direction. We notice this mode more on account of its novelty than its utility or beauty.

The principal objects in training fruit trees are, to place the branches so that the sap may be under the control of the cultivator ; to place the leaves so that they may be presented with greatest advantage to light and air; to expose the fruit to the same influence ; to place them, when grown, against walls, so that they may enjoy a greater degree of heat, by its being radiated from the wall, than were they grown as standards or open espaliers, on which no radi ated heat can be expected. The constraint imposed upon the tree by its branches being fastened to a wall or espalier tends to moderate its growth, and hence, instead of making much wood, a disposition is brought on for the formation of fruit-buds. Branches which are loose, and capable of being moved by the wind, grow more luxuriantly than such as are fixed to another body, and hence, all other circumstances being equally favourable, trees so trained are more productive of fruit than such as are left in their natural position. Training in a perpendicular direction is more favourable for the luxuriant growth of trees, than when they are trained horizontally, and much more so than when they are caused to grow in a pendant form. This accounts for the practice of elevating the direction of one branch, and depressing that of another, when they are of unequal 
strength, and when it is desired that they should be in that respect equal. The branches of every tree which are produced nearest the root beeome weaker year after year, and are the first to decay, because of the tendency of the sap to rise rapidly towards the topmost, and hence leave them without their due share. It is for this reason that, in training all trees, it is recommended to secure wood there for the after supply of the tree, which, if neglected, would soon cause them to assume a naked and worn-out appearance. Great care must, therefore, be taken to keep up a supply of wood there, and that can be readily effected by cutting the young wood well in, by training the young shoots vertically instead of horizontally, by not leaving too many on, and by stopping them when they have attained about a foot or so in length ; these lower buds will thus be strengthened, and when cut back to a healthy bud the following year, wood of a proper strength will be produced. The shoots produced on the upper side of all branches, trained either horizontally or inclined, will be the strongest. It follows, therefore, in keeping up a proper equilibrium, that the weakest shoots of the former, and the stronger ones of the latter, should at the proper pruning season be retained. The best wood-buds will be placed at or near the base of the shoots, where proper attention is paid to stopping during summer; therefore, in peach training, for example, a due share of young wood should be secured from these, and the more so if the tree is weak or requires an increase of branches.

Pruning to increase the vigour of the tree.This process applies to old fruit-trees whose heads have become diseased and unproductive. It is also practised upon young trees which are in an unhealthy or weakly state, and eonsists in cutting over the entire stem a few inches above the graft, and at other times merely cutting back the larger branches to near the stem or trunk. This operation has the power of increasing the vigour of the tree in two ways: " 1 st, If we assume that a certain amount of nourishment is supplied by the roots to all the branches and buds of a tree, by cutting off onehalf of the branches on it at the proper season, we direct the whole supply of nourishment to the remaining portion, which will consequently grow with nearly double their former luxuriance. $2 \mathrm{~d}$, Again, when a tree becomes stunted or enfeebled in its growth, the thinness of its inner bark, with its eonsequent small sap-vessels (which, it must be remembered, are the principal channels for the passage of the ascending supply of food), renders the upward and downward circulation tardy, and the growth is small. By heading back or pruning judiciously, all the force of the nourishing fluid is thrown into a smaller number of buds, which make new and luxuriant shoots with larger sap-vessels, and which afford a ready passage to the fluids; and the tree, with these renewed energies, will continue in vigour for a long time. This treatment is especially valuable in the case of small trees of feeble or stunted growth, whieh are frequently cut back to a single bud, and a new shoot or shoots full of vigour gives a healthy habit to the tree."-DownING.

VOL. II.
Pruning newly transplanted trees.-Maiden trees, when planted against walls or espaliers, require pruning in order to furnish them with a head. The received opinion is, that towards the end of March or beginning of April, as the wood-buds begin to swell, one of three courses must be taken; either shorten the shoots of the previous year, or eut down the tree to two, three, or four eyes, according to the strength of the tree, eutting the weakest always most in. This course is most frequently practised, particularly in the peach, nectarine, and apricot. If the shoots be well placed for beginning the form of the tree, instead of heading down the stem, shorten these back to two, three, or four eyes, according to the strength of the tree, and at the same time, for wall or espalier trees, rub off the fore and back wood-buds. Downing on this subject takes an opposite view: "Pruning the heads of transplanted trees at the season of removal," he thinks, "is generally an injurious practice. It is eertainly needless and hurtful in the case of small trees, or those of such a size as will allow the roots to be taken up nearly entire; for as the aetion of the branches and the roots is precisely reciprocal, and as new roots are rapidly formed just in proportion to the healthy action of the leaves, it follows that by needlessly cutting off branches we lessen the vital action of the whole tree. At the same time, where trees are transplanted of so large a size that some of the roots are lost in removing them, it is necessary to cut back or shorten a few of the branches, as many as will restore the balance of the system, otherwise the perspiration of the leaves may be so great as to exhaust the supply of sap faster than the roots can collect it. A little judgment only is necessary to see at a glance how much of the top must be pruned away before planting the tree, to equalise the loss between the branches and the roots." Many of the best French cultivators do not head back their trees at planting, more especially the peach, but lay in the two original or principal branches at their full length, and only disbud them late in autumn, by which the vigour of the tree is greatly promoted. These branches are trained to an angle of from $60^{\circ}$ to $65^{\circ}$, and in autumn all the buds are neatly removed by a penknife, excepting four on each branch. Late in the autumn of the second year, the four lateral shoots from the two principal branches are eut back to one-third of their length. In the following year, the lateral branches are disbudded to the extent of onehalf ; and in the future winter management, disbudding takes the place of our usual custom of pruning, a practice at one time strenuously recommended by Walter Nicol.

The following reasons are given in "Theory of Horticulture," p, 260, against this very general practice: "With regard to pruning plants when transplanted, there can be no doubt but it is more frequently injurious than beneficial. It is supposed, or seems to be, that when the branches of a transplanted tree are headed back, the remaining buds will break with more force than if the pruning had not been performed; but it is to be remembered that a transplanted 
tree is not in the state of one which has not been removed. Its roots are not fully in action, but from the injuries sustained in removing, they are capable of exercising but little influence on the branches. The great point to attain, in the first instance, is the renovation of the roots, and that will happen only in proportion to the healthy action of the leaves and buds: if, therefore, the branches of a plant are removed by the pruning-knife, a great obstacle is opposed to this renovation; but if they remain, new roots will be formed in proportion to their healthy action. The danger to be feared is, that the perspiration of the leaves may be so great as to exhaust the system of its fluid contents faster than the roots can restore them, and in careless transplanting this may doubtless happen: in such cases it is certainly requisite that some part of the branches should be pruned away, but no more should be taken off than the exigency of the case obviously requires; and if the operation of transplanting has been well performed, there will be no necessity whatever. In the case of the transplantation of large trees, it is alleged that branches must be removed, in order to reduce the head, so that it may not be acted upon by the wind; but in general it is easy to prevent this by artificial means. In the nurseries it is a universal practice to prune the roots of transplanted trees; in gardens this is seldom performed. Which is right? If a wounded or bruised root is allowed to remain upon a transplanted tree, it is apt to decay, and this disease may spread to neighbouring parts, which would otherwise be healthy; to remove the wounded parts of roots is therefore desirable. But the case is different with healthy roots. We must remember that every healthy and unmutilated root which is removed is a loss of nutriment to the plant, and that too at a time when it is least able to spare it. There cannot be any advantage in the removal. The nursery practice is probably intended to render the operation of transplanting large numbers of trees less troublesome; and as it is chiefly applied to seedlings and young plants with a superabundance of roots, the loss in their case is, therefore, not so much felt. If performed at all, it should take place in autumn, for at that time the roots, like other parts of a plant, are comparatively empty of fluid; but if deferred till the spring, then the roots are all distended with fluid, which has been collecting in them during winter, and every part taken away carries with it a portion of that nurture which the plant had been laying up as the store upon which to commence its renewed growth." The practice of cutting off the whole tops as well as a considerable portion of the roots of young quicks or thorns, when planted for the formation of hedges-a practice almost invariably followed in Scotland-is radically wrong, and hence there can be no doubt that the opposite practice, followed in the north of England, of planting them of a much larger size and without any curtailment whatever, is much preferable, leaving whatever pruning may be necessary for forming the fence till after the plants have become thoroughly established; and were the hedges in the latter case as well managed afterwards as they are in the former, the advantage of the superior mode of planting would be more evident.

Cutting back or shortening the branches of treesat transplanting is considered seldom necessary by Hayward, who says, in "Enquiry into the Causes of the Fruitfulness and Barrenness of Plants and Fruit Trees," \&c., p. 210: "When the plants and trees are of a proper form or figure, and are removed with care at the proper season of the year, it is better to observe such care in removing and taking up as to do it without injury to the roots, and to take them up sufficiently early in the season to allow them full time to recover and establish themselves, so as to furnish the sap required to sustain the head and branches undiminished. But if it be late in the season when trees are taken up, and the roots are so much diminished and injured that the following season the trees cannot be furnished with sufficient sap to sustain the head and branches, or to prevent the bark and vessels from becoming inflexible, it is better to head them back; for as the sap-vessels of the branches which form the old head will be in capable of expanding, so as to receive all the sap furnished by the roots, the second season after removal, when the roots are restored, the sap will force its way out and form new branches near the root: the better plan, therefore, in such cases, must be to reduce the head and branches just at the time the buds open in the spring after planting, and to shorten them to such lengths that the roots may be able to fill and support them."

Much, however, in a practical point of view, depends on circumstances, such as the care with which the trees have been taken up and removed, their healthy and robust state, and the suitableness of the soil they are planted in, as well as the early season at which they have been transplanted. If these be as they ought to be, then in few cases is shortening back the branches necessary, as, if planted sufficiently early in autumn, the roots will have placed themselves in a condition to supply in spring all the nutriment required by the branches, providing the trees are not old and of very large size. Whereas, in opposite cases, when the trees are carelessly taken up, and their roots much broken and exposed during their transit from one place to another, if they are naturally weakly, or rendered so by improper management previously, the soil into which they are to be placed uncongenial, and the planting delayed till late in spring, then either a curtailment of the branches must take place, or the tree be allowed to stand over for a season to become established at the roots, and heading back performed the following autumn. Wherever fruit trees can be transplanted so as to render heading back unnecessary, it is of great advantage, as such trees come much sooner into a bearing state, and seldom after, unless from unnecessary excitement at the roots, require very much pruning; whereas young trees once established at the roots, and headed hard back, begin to grow to wood vigorously, are much longer in coming 
into a fruit-bearing state, and continue long of robust habit, increased rather than diminished by severe pruning, and causing the mutilating practices of root-pruning, ringing, \&c., to be had recourse to.

Leaf-pruning consists in removing a portion of the foliage while the tree or plant is in a growing state ; an operation in tree management, we believe, first described, and its effects explained, by $\mathrm{Mr}$ Beaton, one of the most scientific gardeners of the present day. The object is to reduce the strength in over-luxuriant trees, and the means employed to pinch the leaves off as fast as they are unfolded from the bud, thus preventing the formation of buds at their axils, and the return of the sap to the root. "When a tree fills the space allotted to it against a wall. and shows a disposition to still further growth, by throwing up strong vertical shoots above the wall, and luxuriant breastwood on the main boughs, instead of checking this disposition by any of the ordinary modes of pruning, $\mathrm{Mr}$ Beaton assists the tree to throw off the superabundant sap by disleafing the breastwood and vertical shoots, and in the winter-pruning he displaces all the buds on such shoots, even those on the points, after which they die off by degrees, and are cut out. If trees are not very luxuriant, indeed, one jear of this treatment will reduce them to a moderate degree of strength. As buds are only formed in the axils of the leaves, probably much disbudding and pruning might be saved by disleafing as soon as the leaves are developed; but it must always be borne in mind that every leaf has not only the particular office to perform of nourishing the bud in its axil, but the general one of contributing to the nourishment of all that part of the tree which is between it and the farthest extremities of the roots. Hence, in particular cases, where it is desirable to give additional vigour to the roots, instead of disleafing or disbudding a weak tree, all the leaves and shoots it produces, even the breastwood and upright shoots, which the French call gourmands, ought to be encouraged within certain limits. Disleafing is frequently practised with fruitbearing plants, both ligneous and herbaceous, with the view to admit the sun and air to the fruit, and sometimes also to assist in ripening wood by stopping growth."-Sub. Hort., p. 344 . Of the ingeniousness of this practice there can be no doubt, and in skilful hands the desired effect will be accomplished. We would, however, depend more on taking up and replanting, or on root-pruning, in all cases where these operations can be conveniently carried into effect. The removal of leaves while plants are in a growing state has the most debilitating effect on them of any operation that can be applied, and if carried too far would certainly terminate their existence. To a limited extent the practice has advantages ; and in the case of strong-growing trees, the removal of the leaves from such shoots as the experienced pruner foresees are to be removed at all events, may be done with the greatest safety, and, if done in proportion to the strength of the tree, will be attended with great advantage.
Root-pruning is an operation often rendered necessary to check over-luxuriance in the tree, and to induce in it a disposition to become fruitful. It is, when properly and timeously performed, perhaps one of the most important modes of pruning. There is no doubt, seeing, how much the tree depends on its roots, that they should demand as much of our attention as the branches; for unless a proper balance is kept up between them, no satisfactory results need be looked for. The best cultivators of fruit trees, fully alive to this, have the roots of their trees as much under their control as the tree itself, and are no more afraid of laying them bare, and using the pruning-knife upon them, than they are at operating on the branches. By a proper adjustment of the roots, the sterility of trees, whether proceeding from want or superabundance of food supplied from the soil, is corrected, fresh and proper food is secured to them, the roots placed in the best position to take up this food, which is in general when near the surface, where they have their necessary share of solar heat, \&c. Where excessive vigour exists in the tree, and consequently an absence of fruit, this is remedied by curtailing, or even totally removing, so many of the strong roots as may be thought necessary. Autumn is in general preferred for this operation, although many have executed it with good effect even when the trees were in full growth. The operation need seldom be repeated oftener than once in three years, and sometimes less frequently suffices, much depending on circumstances. The operation is generally performed by opening a trench so wide that the operator may easily reach the roots, and to such a depth as to get completely under them ; for it is often those which strike downward which most require to be operated upon. The distance also from the stem of the tree will depend on its size, and the extent to which the operation is to be carried. The larger and stronger roots should be cut off, leaving the wounds clean and smooth, to enable them to heal over speedily, and form callosities from which the new and more valuable roots will proceed. Root-pruning during summer was, we believe, first brought into notice by the late Mr Beattie, of Scone Palace, who operated upon a whole wall of pears with remarkable effect; and we find Mr Errington extending the operation over a much greater extent of the season. "It will appear," he says, "strange to some persons to talk of root-pruning when trees are in full growth; but in all probability a better period could not be chosen than the end of July, for to delay longer with subjects requiring the operation, especially of trees of tender habit, is to lose a good chance of facilitating the ripening of the wood. Late growths are antagonistic in all cases to the latter process ;" and this holds good most in rich deep soils which encourage growth, and cold late autumns, incapable, in consequence of want of heat, of ripening the wood. Root-pruning has received a fresh impetus by the example shown by $\mathrm{Mr}$ Rivers, the respectable nurseryman at Sawbridge Worth, who may be said to have reduced 
periodical prunings of this kind to a perfect system; and with this, and the use of less luxuriant stocks, has secured a new character in the habit and productiveness of many of our best fruits.

Root-pruning is often very carelessly and inefficiently performed; and hence, like many other excellent things not properly done, the result falls short of the operator's expectations, and he becomes loud in condemning the principle. It is not to be done by merely thrusting down a blunt spade or other instrument all round the tree, and mangling such roots as the instrument comes in contact with-nor even by digging a trench all round, and cutting off the stronger horizontal roots that may come in the way, leaving them ragged and lacerated at the points of amputation. The evil sought to be remedied much seldomer rests with such roots than with those which descend in a perpendicular direction under the centre of the tree, which such a mode of operating cannot reach. The best directions we have met with on this subject are in a leading article in "The Gardeners' Chronicle" for 1841 , p. 763 , as the practice of $\mathrm{Mr}$ Crace, as applied to the roots of pear trees which he wished to keep of small size. He root-prunes annually, leaving the roots each time about an inch longer than before. "He does not leave the roots with their ends wounded, as they would be if chopped through with a spade; but he cuts all the larger roots obliquely with a sharp knife, so as to leave a clean standing wound, 3 or 4 inches long, with its face downwards. The effect of this," he says, "is to cause the wound to send forth a fan of fine fibres from its whole circumference. The young fibrous roots of a plant proceed in all cases from the surface of the wood, and not from the bark; they only pierce the bark when they seem to grow from it. When the root is crushed by the blow of a blunt tool, all the part exposed to the blow is killed, and soon decays. That decay may either proceed no farther than the vicinity of the injury, or, as will happen more frequently than we suppose, it will spread and infect the sound parts in contact with it. In either case the production of young fibrous roots can only take place by forcing them through the bark which lies over the wood from which they have to spring. But when the wound at the end of a root is clean, decay will not take place; and the surface of the wood will produce fibres from that part which is in contact with the earth. No resistance is offered to this process; on the contrary, from the moment that the fibre begins to form, it finds itself in contact with the earth, where its food resides, and there, imbibing vigour from the soil, it immediately contributes to the general system something of that organisable matter out of which more fibres are to be produced."

Many advocates for root-pruning recommend the operation to be performed annually, "for," as Mr Rivers remarks, "if a tree is suffered to grow two, three, or more years, and then rootpruned, it will receive a check if the spring be dry, and the crop of fruit for one season will be jeopardised; therefore, those who are disin- clined to the annual operation, and yet wish to confine the growth of their trees within limited bounds by root-pruning - say once in three years-should only operate upon one-third of their trees in one season; they will thus have two-thirds in an unchecked bearing state." The operation is usually thus performed: A trench 18 inches wide or so is opened all round the tree, and about 2 feet distant from the stem; but this distance depends greatly on the size and age of the tree, the object being to ascertain the state and position of the roots, so that those growing in a perpendicular direction, or those of strong growth growing in a horizontal direction, may be amputated to a proper length. This is a necessary operation in most soils and situations, but more especially so in cold and wet soils, and in northern climates, because it has the effect of causing the trees to make less luxuriant growth, and hence enables the wood to become more perfectly ripened in autumn. Every fruit-bearing tree, under certain conditions, may be advantageously operated upon from the period of the fruit's ripening until the sap begins to flow in the roots. These conditions appear to be favourable soils and warm situations, where not only the fruit but the wood also ripens early. Under circumstances of an opposite character, where the trees grow late in autumn and ripen their wood imperfectly, it is often necessary to reverse the operation, and root-prune during summer. There is also some danger to be apprehended when root-pruning is performed during winter, particularly in wet seasons and in damp soils, as the roots that have been cut at that time are liable to rot, and the wounds are long in healing.

Mr Errington has shown that he has induced fertility and moderate growth in pears which had been long perfectly barren, and which had put forth nothing but wood of unproductive luxuriance, by root-pruning, even when the operation of ringing had failed to produce a similar effect. The order in which this cultivator places the various kinds of fruit trees to which this process has been beneficially extended is-Pears, apples, plums, peaches, and nectarines, and then the cherry and apricot.

The pear, when upon the pear or free stock, is peculiarly liable to form tap-roots, and when these are growing luxuriantly without bearing, it is almost, he says, impossible to root-prune them too severely, at least the deeper roots. "If in an orchard, and the trees are of some size, they may be curtailed all round; the amount of root removed bearing, of course, $=$ a direct ratio to the amount of luxuriance. In the espalier border we must be content to get at them how we can, remembering that even the cutting of one side will assuredly affect the whole system of the tree, although not, perhaps, in an equal degree; at least we dare not, in the present state of gardening science, affirm it."

Root-pruning the apple.-The apple, even on the crab stock, is much less liable to send down tap-roots than the pear on the free or pear stock; it therefore requires this operation to be performed with some degree of caution. If too severely limited at the roots, the tree is apt 
to become greatly paralysed; small leaves and short slender shoots will be produced the first year afterwards, and that inveterate enemy the red spider will grievously attack the foliagc. "We introduce this caution," says Mr Errington, "to show that some degree of moderation becomes necessary, and that although root-pruning is correct in principle, yet it may be prejudicial in rash hands."

Root-pruning the plum.-The majority of plums are little liable to assume those gross habits which render other trees unproductive; still cases frequently occur where an examination of the roots, and a slight curtailment of some of the stronger ones, may become necessary. In youth, and in over-rich soil, the plum is apt to make strong wood, and in such cases root-pruning should be attended to; but, like the peach and nectarine, much may be done by topping the strongest shoots early in their growth, which will cause them to send out laterals of a more useful size. Old plum-trees, when once in a bearing state, seldom require such attention. When, however, it is intcnded to grow the plum in the pyramidal standard form, root-pruning becomes an almost necessary condition, and even performing the operation during summer has been found to have its advantages. As the pyramidal form is so well adapted to small gardens, on account of the small space the trecs occupy, and the little shade they occasion, every means should be employed to bring them into the most perfect state possible. The French have not in general succeeded in growing the plum in this way, although in the case of most other hardy fruits they have long excelled: indeed, it is to them we are indebted for our first knowledge of this mode of training. Of rootpruning they know little-at least, if they do, they seldom practise it; and it is to this we attribute their want of success in regard to the plum, when trained even in their favourite fashion.

Root-pruning the peach and nectarine. - The great object in root-pruning these is to keep the roots near the surface to insure early growth, moderately strong wood, and that thoroughly ripened in autumn. If the roots are deep, vegetation is late in spring before it is brought into action, crude and excessive juices are drawn from the soil ; and although the wood grows vigorously during summer, it is so watery and gross, and continues growing so late, that our ordinary scasons are not sufficiently warm to perfect its elaboration. Much, therefore, depends on stopping strong shoots to causc a supply of laterals to be formed, and thesc, if properly managed, will be found preferable to the others. When the peach attains a bearing state, unless the roots be too deep, or too highly stimulated with manure, they seldom produce wood of a character that will render an interference with their roots necessary.

Root-pruning the peach, nectarine, and apricot is practised by Mr Fleming at Trentham with the most beneficial effects, to counteract late growth in a climate proverbial for its bumidity, and by no means warm. By this means this excellent cultivator keeps his trees in a fine bcaring state, by limiting thcir growth within proper bounds, and thus insuring well-ripened wood and buds, and as a consequeicc abundance of fruit. As soon as the fruit is gathered from the trees a general examination of their roots takes place, and such as require it are shortened back more or less according to their strength. This checks their growth sufficiently early, and facilitates the ripening of the wood, which, without this cutting off the supply at the roots, would continue to grow on to so late a period that ripening, under the circumstances of the situation, would be impossible. In all late situations this plan should be followed, whether they be wet or dry ; and there are few places, in the north in particular, whcre it should be neglected, for it is almost midsummer before we have anything like free and vigorous growth; and in consequence of our usual mild autumns, our trees continue growing on, till their progress is all of a sudden arrestcd by frost, the shoots being full of sap, and of so gross a character that no art of man can afterwards save them from destruction. This is the foundation of nine-tenths of all the mishaps and diseases to which our wall-trees are so liable.

The middle of September, or as soon as the crop is gathered, is the proper season for root-pruning the peach, with a view to induce productiveness the following year. 'The operation will check the growth and hasten the ripening of the wood, which, from that period till the end of the season, will have sufincient time to become matured.

Root-pruning the cherry.-Unless for the reasons, above given, this is found to be seldom necessary. The stronger-growing sorts will sometimes, for the first few years, make very strong wood, and in such cascs the operation may be had recourse to ; and if not, where the branches run off, bare of fruit-buds to a considerable distance from the trunk, such wellplaced young wood as may arise from them may be trained, using the naked branches as a trellis on which to train them. We have in some cases side-grafted young branches on such branches, and so covered the centre of the trees with young and fertile wood. Mr Rivers, with a view to correct the gross habit of some of these sorts, has wrought them upon Mahaleb stocks.

Root-pruning the apricot.--In some soils rootpruning is advantageously pcrformed on this tree, and we have seen it done on trees of considerable size and age, with cvident success. Some pomologists, however, object to root-pruning this tree, but upon what grounds we never could clearly see.

Root-pruning the fig and the vine.-In open-air culture these seldom arc thus operated upon, it having been found better to plant them in shallow borders, with impervious bettoms, and to confine the roots of the former within proper bounds by walling them round. The most thorough system of root-pruning is, however, taking up the trees in early autumn, regulating their roots, and immediately replanting them again ; and this, if properly done, may be performed with great advantage upon every kind of fruit-bearing tree. In the case of amateurs, and even where large trees are to be operated 
upon, without sufficient manual power to effect the entire taking up and replanting, and also in eases where local obstructions eome in the way, we would say, be content with opening a trench round the roots, as far as may be convenient; and after removing the soil carefully from the roots within reach, reduce them according as they can be got at, remembering, however, that the fewer roots reached the more severely they may be dealt with.

Ringing is a species of pruning practised often on the bianches of fruit trees, and occasionally on the larger roots also. The operation consists in removing one or more rings of the bark, by which the return of the sap is obstructed, and it is thus obliged to accumulate above the part operated upon, instead of taking its otherwise natural course downwards. The practice has been long in use on the Continent; but until Mr Knight explained the physiological nature of the operation, little notice was taken of it in Britain. The following excellent explanation of the theory has been given by that eminent man in "The Trans. of the Hort. Soc.," vol.iv. p. 229 : "The true sap of trees is wholly generated in their leaves, through which it descends through their bark to the extremities of their roots, depositing in its course the matter which is successively added to the tree; whilst whatever portion of such sap is not thus expended sinks into the alburnum and joins the ascending current, to which it communicates powers not possessed by the recently absorbed fluid. When the course of the descending current is intercepted, that naturally stagnates and accumulates above the decorticated space, whence it is repulsed and carried upwards, to be expended in an increased production of blossoms and of fruit ; and, consistently with these conclusions, I have found that part of the alburnum which is situated above the decorticated space to exceed in specific gravity very considerably that which lies below it. The repulsion of the descending fluid, therefore, accounts, I conceive, satisfactorily for the increased production of blossoms and more rapid growth of the fruit upon the decorticated branch; but there are causes which operate in promoting its more early maturity. The part of the branch which is below the decorticated space is ill supplied with nutriment, and ceases almost to grow ; it in consequence operates less actively in impelling the ascending current of sap, which must also be impeded in its progress through the decorticated space. The parts which are above it must, therefore, be less abundantly supplied with moisture, and drought in such cases always operates very powerfully in accelerating maturity. When the branch is small, or the space from which the bark has been taken off considerable, it almost always operates in excess, a morbid state of early maturity is induced, and the fruit is worthless. If this view of the effects of partial decortication, or ringing, be a just one, it follows that much of the success of the operation must be dependent upon the selection of proper seasons, and upon the mode of performing it being well adapted to the object of the operator. If that be the production of blossoms, or the means of making the blossoms set more freely, the ring of bark should be taken off early in the summer preceding the period at which the blossoms are required; but if the enlargement and more early maturity of the fruit be the objects, the operation should be delayed till the bark will readily part from the alburnum in the spring. The breadth of the decorticated space must be adapted to the size of the branch; but I have never witnessed any except injurious effects whenever the experiment has been made upon very small or very young branches, for such become debilitated and sickly long before the fruit can acquire a proper state of maturity." The operation of ringing, although for certain purposes valuable enough, should not be carried too far; and if it could be so managed that decortication should be performed only on such branehes as could be afterwards entirely removed without disfigurement to the tree, then it might be more generally adopted. Its effects in all cases are to render those portions of a tree operated upon unhealthy, and ultimately to make them perish. There is a modification of ringing less injurious to the tree, but also less effective in its results, namely-

Spiral ringing.-This mode of ringing has beell suggested as being less likely to be injurious to fruit trees, particularly peaches, apricots, plums, and cherries, which are much less patient of the operation of common ringing than apples and pears; but the evil ought not in any case, for such purposes, to be extended farther than the edges are capable of healing in some degree, or the life of the subject might be endangered by the process.

Ringing by ligature, \&c.-Instead of removing a ring of bark, a piece of wire, of strength proportioned to the size of the branch, is placed firmly round the part, which has the effect of obstructing the sap, but to a much less degree ; and a weight, such as a heavy stone, \&c., is used in Malta and elsewhere, by being placed in the fork of a branch, and made fast, so as not to be shaken down. The pressure it exerts on the part it touches to some extent obstructs the circulation of the sap, and produces, in proportion to its compression, the effect desired.

A girdle of Roman cement, put on in the manner of claying a graft, has been recommended, and a piece of rope tightened round the branch has also been tried. In either case, the compression caused has had the same effect as that of cutting a ring of the bark out altogether, and with far less serious effect on the branch, as both are removed when the effect is produced. In regard to the season of ringing by cutting the bark, as well as the breadth of the ring removed, that must be guided by circumstances. The former, some assert, may be performed at any season, but its effects will only be obvious when the sap is on the ascent. In regard to the latter, the breadth of ring removed should bear a proportion to the size of the branch operated upon. It may vary from 1-16th of an inch to an inch, which latter will answer the purpose as well as if of greater breadth, and has the advantage of healing over during the same, or, in general, the succeeding year; and the depth to 
which such an incision should go should not exceed the thickness of the outer and inner bark. Its effectis are more obvious on the apple and pear than upon other trees, some of which, such as stone-fruits, seem to be scarcely affected by it at all; yet, curious enough, it affects the gooseberry, while the currant is unaffected by it, so far as our observation goes. It has a marked effect on the setting of the fruit, if performed just before the blossoms begin to expand.

With root-pruning so thoroughly understood as it appears now to be, we do not think that, except in extreme cases, the mutilating practice of ringing should be much resorted to. It is rather a dangerous operation in unskilful hands, and only answers the purpose of root-pruning to the extent of the branch or branches it may be applied to, while it is generally detrimental, if not fatal, to the parts above where the operation is performed; whereas, root-pruning acts upon every part of the tree alike, and more or less at the will of the operator, and at the same time,instead of being hurtful in its consequences, is extremely beneficial, as it prevents the roots from penetrating too deep, and getting into a bad subsoil, which is one of the principal causes of unhealthiness and unproductiveness in them; it limits the supply of sap collected by them to the just requirements of the tree; it increases the number of proper food-collecting fibres, directs them to where the proper food is to be obtained, and keeps them near the surface, so that they may enjoy the influence of both warmth and air, two essential elements, of which they would be deprived if allowed to penetrate deeply into the soil ; it admits also of placing fresh soil for the roots to work in, and of the renewal of that from which they have extracted all that is useful to them.

Close pruning is chiefly performed both on forest and fruit trees when the shoot or branch to be removed is in a ripened or mature state, and when the part to be amputated is to be cut out from the branch or stem from which it has sprung. Although this is done in both cases, and upon branches of considerable size, the intention being chiefly directed to the removal of ill-placed or superfluous growths, still one object must in neither case be overlookednamely, to cut so that the surrounding bark may cover, in the least possible time, the whole of the wounded part; in both cases, to prevent decay taking place in the main stem or larger branch; and in fruit-bearing trees, to leave no appearance of a wound or blemish on the bark. The smaller the branch removed, the sooner will the wound be healed overa circumstance which points directly to timeous pruning, and to the evil of allowing superfluous or ill-placed branches to continue on the tree. This is particularly the case as regards stone-fruits, whose wounds, if large, heal very tardily, and often not at all. In cutting, the wound should not be made larger than the size of the section of the part removed, that the process of healing by the bark's covering it over again may speedily take place. Yet, at the same time, the branch should be cut close in to the stem, to prevent the development of latent buds, which are placed at the base of such branches, and if not removed along with it, would become excited in spring, and have to be removed afterwards, besides preventing the operation of healing over from taking place, at least in so complete a manner as could be desired. There is, however, an evil, on the other hand, in cutting too closely in-that is to say, when an unnecessary portion of the bark of the stem or branch, from which the other has been cut, is displaced, because a much longer time will be required before the healing process is accomplished. In pruning fruit trees, on the principle of encouraging the production of natural spurs or flower-buds, instead of that of creating artificial ones, the removal of the young wood at the winter's pruning demands attention in this respect, in so far as, if the young wood be not cut close in to the branch from which it issues, the latent buds left at its base will develop a mass of useless foliage, and in time form crowded clusters of spurs, most of which will be productive of small spray and leaves, with few or no flower-buds ; while, if cut close in, natural flower-buds will be protruded from the edges of the wound, and along the smooth clear branch, both below and above where the young shoot was removed from.

Spur pruning.-The creation of artificial spurs on fruit trees was the invariable rule of former practitioners. 'The best cultivators now depend upon the inducement of natural spurs. "It must be confessed," says Loudon, "however, that pruning has but little to do with the production of spurs that are prolific in blossomsthat depends far more on adjusting the nourishment supplied by the root to the demands of the fruit-bearing branches, to the mode of training, the kind of tree, and other particulars, which, when attended to, spurs are produced naturally." The means of producing these spurs have been sufficiently explained in various parts of this work.

Pruning by stopping, or pinching back.-This is a species of pruning performed on trees during the growing season of their young wood, and is important in the case of many trees. When a shoot is stopped or pinched back while in a growing state, the sap which was impelled towards the point of the shoot - as the sap always is, in proportion to the vertical or horizontal direction of the branches and young shoots - is now taken up in adding to the size or substance of the leaves or fruit which may be upon it, or in developing the buds at the axil of the leaves; or, in other cases, converting them from leaf-buds to flower-buds. In the case of the vine, this has been known since the days of Pliny; and, according to a paper in the "Ann. Hort. Soc. Paris," three crops of grapes have been obtained in one season from the same vine by adopting this process - a process, however, no one expecting a large crop the following year would, if wise, run the risk of following. Mr T. A. Knight, in following the stopping system in the case of the fig (his plants being kept in excitement by means of artificial temperature), obtained three crops 
of fruit from the same plants within the same year. "The principal use of stopping, however," as is sensibly remarked by Mr Loudon, "is to promote the setting and swelling of fruit either on the shoot of the current year, as in the case of the vine and the melon, or at its base, as in the case of the peach. Much of the winter-pruning of trees might be prevented by stopping the shoots early in summer, provided the state of the tree did not require that the shoots should be allowed to grow their full length, in order to send down nutriment to the increase of the roots, in consequence of which, greater vigour is in turn imparted to the stem and branches. In this case of pruning, as in every other, the state of the tree, and a variety of circumstances connected with it, require to be taken into consideration."

Finger-pruning or disbudding is performed in the spring, as well as during most of the summer, and has for its object the reduction of the number of young shoots to proper limits, by displacing them when about an inch or two in length, thereby economising the energies of the tree, and directing the sap-which would go to their formation, and afterwards be, along with them, cut off and thrown away-into those that remain. Indeed, finger-pruning or disbudding, if properly attended to, would, in most cases of trained trees, render the pruning-knife unnecessary, unless for the removal of dead, diseased, or superabundant shoots. The removal also of superabundant fruit-buds during early spring, although seldom practised, is deserving of attention. To allow all to expand, greatly weakens the tree, and is one of the causes why we so often have a bad crop of fruit notwithstanding an amazing display of blossom. It would be perhaps going too far to say that every flower-bud should be removed excepting those only from which fruit is to be expected; yet, could we be guaranteed against accidents, there is no doubt that the tree would gain greatly in strength, and the setting of the fruit be more completely insured.

Without, however, going so far as this, the operation of disbudding, or reducing the number of blossom-buds to something like an approximation to double the number of fruit required, would, if carried into effect, be attended with very marked results. This operation may be performed during winter, at or soon after winterpruning, or it may be delayed until the buds begin to swell in spring. In inexperienced hands the latter period would be the safest, as the wood and blossom-buds could then be better distinguished.

Bending down the branclues of standard fruittrees is done with a view to moderate the flow of the sap, and answers the same purpose as training wall-trees horizontally, or in the pendulous form. In the case of full standard trees, strings are attached to the points of the extending branches, and fastened to the stem or trunk: weights suspended from the extremities of the branches have been suggested, but this is neither so business-like nor so safe a course as that of forming a single rail, elevated a few inches above the surface of the ground, and of a diameter corresponding with the spread of the branches; their points being fastened to the rail, depresses the branches to any required degree, and prevents their being broken by the wind. The top of the tree may by this means be modelled into what may be called the domical form, similar to fig. 800 , vol. i. The time for performing the operation is soon after midsummer, and the effect produced is the interruption of the flow of the sap, and the consequent production of abundance of blossom-buds.

Laying bare the roots of fruit trees is an old practice, mentioned by Evelyn as common in his day. It is still very generally practised on the Continent, the soil being removed around the stem to such a depth as to expose the principal roots to the action of the atmosphere. Of its utility there are great doubts. It can only be regarded as a species of mutilation - such as cutting notches in the stem and larger branches, stripping off portions of the bark, as recommended by Arnaud d'Andilly about the middle of the seventeenth century in France, and towards the latter end of the last century, and beginning of the present, in Britain, by Lyon, King, Forsyth, and others. Every species of artificial mutilation, whether of plants or animals, has the effect of bringing about a sort of prematurity, and until that state is arrived at, reproduction does not take place. But these should only be had recourse to as expedients when the more natural modes of providing a proper soil and situation, and judicious pruning and training, have failed.

For further on pruning and training, vide articles Apple, Pear, Vine, \&c.

Pruning is to be regarded as an art, and a very important one, when conducted upon correct principles, which it is not always, and that more especially in its application to the growth of timber. The remarks previously made, and all those which directly apply to fruit trees, have for their object the very reverse of accumulating bulk of woody matter; the operations of the pruner must, therefore, be guided accordingly. If straightness of stem and bulk of timber be the object, as may be said to be the case in all coniferous trees growh for profit, then, as a general rule, it may be stated that the leading shoot should be preserved with the greatest care. No doubt such plants will, like most others, replace such losses, when they accidentally occur, by sending up one or more leaders; but if these are left to themselves, a striving for the lead will enfeeble the whole. All should be removed save the strongest one, if well placed. The sap in all plants has a natural tendency to flow to the highest points, and hence we find the leading shoots always the strongest, the buds farthest from the root, if nearly vertical to it, the plumpest and best formed; while both lateral branches and their buds, toward the bottom of the tree, are found invariably to be the weakest, and in course of time die away altogether. Hence, we think, nature teaches us our first lesson in timber-tree pruning; she does so, beginning near the ground, and proceeds upwards progressively, but slowly; and so should we, if increasing tho bulk of the 
stem, or of the main branches, of ramifying trees, such as the oak, chestnut, \&c., whose main limbs for certain purposes are as valuable as the trunk itself, be our object. Nevertheless, this is a business requiring caution and carly attention, for if lateral branches be allowed to form near the bottom of the stem, and permitted to continue, their amputation afterwards has a serious effect not only on the constitution of the tree, but on the quality of the timber also. "When a large branch is cut off iminediately from the body or trunk of a large tree, the usual sap which supplied it in its ascent from the roots will be stopped short, and for a time will ooze out from the cut part. In a short time, however, the sap, as it rises in those vessels of the trunk which formerly supplied the branch taken off, becomes stagnated, and causes rot in that part, which can never be the case while the branch remains to draw up and prepare the sap in its leaves; and this is the case in all instances of large branches, as they are cut from large trees. But in the case of a branch being thus cut from a young sapling in a rapidly growing state, the tree is not injured but improved, the sap of the plant being in such a vigorous state that rot cannot take place. Now the practical deduction to be drawn from this is, that the amputation of a large branch immediately from the body of a large tree, instead of being favour. able to its health and value as timber, is the reverse. I say immediately from the body of the tree, because the cutting off of a part of a branch is by no means injurious to the health of a tree; but, on the contrary, when part of a large tree is cut off-i.e. foreshortened-the flow of the sap to that part is checked, and the body or trunk of the tree is in proportion enlarged."

Such are the views entertained by a very good practical forester, Mr Brown of Arniston. Similar views have been entertained for many years,
Fig. 159.

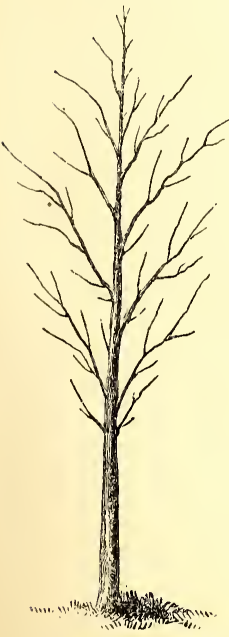

PROPERLY PRUNED TREE. and much discussion has taken place on the subject, particularly in the earlier volumes of "The Gardeners' Magazine,"and since in the two principal horticultural newspapers. "The Forest Pruner," by Ponty, and " Nicol's Planter's Kalendar," edited by Mr Sang, are de. serving of perusal in connection with this matter. The annexed woodcuts, from the last of these works, will explain the effects of judicious and injudicious pruning better than a lengthened letterpress description. Fig. 159 represents a trec of thirty years' growth, which has been regularly and properly pruned. Fig. 160 , a tree of the same age, which has been neglected as to pruning during its early growth, and has now been pruned in a way too frequently practised-namely, by

Fig. 160.

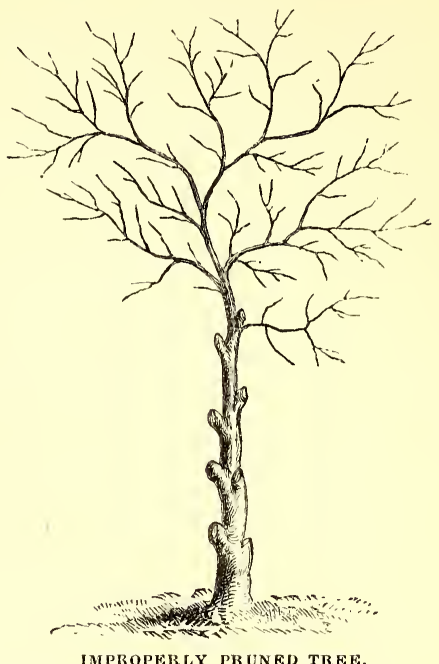

sawing and lopping off the branches after they have attained a large size. Fig. 161 shows the bad
Fig. 161.

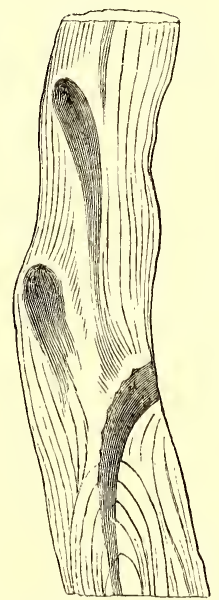

EFFECTS OF PAD PRUNING ON WOOD. consequences of neglecting early pruning, in the case of a plank cut from an ash tree, which had been pruned by lopping off the large branches many years before it was felled. "The cuts in this case," says the very intelligent and venerable editor, " had been made several inches from the bole, and the branches being very large, the stumps left had become rotten. The enlargement of the truuk had not, however, bcen stopped, for tlie new wood had covered over all the haggled parts, in some places to several inches thick. Yet the effects of the previous exposure to the action of the weather, by injudicious pruning, is strikingly marked by the decayed state of the partsconnected with the branches which had been amputated." From this it will clearly appear, that, if pruning is to be practised on deciduous trees at all, it should be commenced while they are young, and carried on progressively; and if so, no such blemishes will be found in the timber when cut up. Yet it does sometimes happen that young plantations under twenty years' growth are to be pruned (and we wish we could say that this was of more frequent occurrence). In such cases, where the ill-placed branches, or those intended to be 
removed, exceed in diameter 2 inches, it is better to commence at their extremities, and shorten them back yearly. By thus cutting off their supplies, by reducing the number of leaves, the base of the branch will not increase much in size, but as it is yearly lessened more and more of its nourishment, it will become sickly, and ultimately die away altogether; and as this process goes on simultaneously with the increasing growth of the trunk, the core or dead fragment left in the trunk will be very small, and in most cases not traceable in the wood when the tree is felled. Besides, this foreshortening of the lower branches will cause the sap which was destined to support them to take another direction, and as none is so natural to it as to ascend through the trunk, it will take that course; for it is an invariable law in the vegetable economy, that when a branch of a healthy plant is cut off, all that sap which would have been expended in supporting the part removed is directed into the parts which remain. However much pruning may be insisted upon, witl the view of increasing bulk of timber, an even more important matter is allowing the trees plenty of room, so that they may both have a sufficient supply of food by the roots, and also an opportunity of properly elaborating the same in the leaves, by a full exposure to light and air.

Pruning Conifers. - As regards the season of pruning coniferous and ornamental hardy trees, be they evergreen or deciduous, winter has in general been the time chosen. This is, however, a mistake-the latter end of spring and throughout the whole summer being the most proper time, because at that season the wounds made speedily heal up, on account of the trees being in the full vigour of growth. The considerations of leisure time and antiquated habits should be disregarded. Coniferous plants were, until within these few years, almost exempted from the pruning-knife ; indeed, so far was this prejudice carried, that it was held little short of murder to remove a branch from them. One noble example we have, however, who foresaw (now rather more than a century ago) the propriety of pruning this section of trees. The third Earl of Haddington, who planted Tyningham, the greatest planter of his day, and author of a work on arboriculture, thus says: "I was once a great enemy to the pruning of firs, because what came from Norway never had met with that treatment; but now I think, when the side branches are taken away when they are young, there can be no harm in it, for the bark will soon grow over the wound, and so no knot can be within that part, for the fir never puts out side branches after they have been once cut off. What I purpose is, that after it has been planted for three years to begin and cut away two tiers of branches, and every year afterwards cutting away one." As the great object is altitude in the pine tribe, the removal of the lower tier's of branches has a positive tendency to effect this, although it would be hazardous to amputate branches of a large size. Coniferous pruning should be performed only during summer; and when the trees assume flat spreading heads (we do not mean those whose natural habit is to be so, but such as the larch, silver fir, \&c.), the extreme points of two or three tiers of branches even above that which is to be removed may with great advantage be foreshortened. This should, however, be done so as not to disfigure the tree, or show the points of the amputated shoots. That most elegant and likely to be most valuable of all our recently introduced conifers, the Cedrus deodar, is a case in point. It naturally, while young, sends out branches in a horizontal and afterwards a drooping manner, and these extend themselves to a great distance, often reclining on the ground by reason of their own weight: this disposition is the cause why the leading shoot of this tree seems so weak, and so seldom takes a perpendicular direction. Prune the lower branches as stated above, and this apparent debility in the leader will disappear, and it will assume its proper habit, and shoot with great vigour in a perpendicular direction. All the young plants of this species, while yet in the nursery, should have their lower branches taken off, and those above, to the extent of two tiers, foreshortened also. The Abies canadensis and Cedrus libani are familiar instances of sad mismanagement in this respect. The former is oftener found assuming the character of a flat or bushy shrub than that of a tall tree; and the latter very often a scrubby meagre-looking tree, branching out within a few feet of the ground, and ramifying into innumerable branches, either vertical or horizontal. Now, we consider (apart from the erroneous practice of keeping such plants in pots till they are finally planted out) that want of judicious early pruning is the cause why the former seldom attains the character of tree, and the latter becomes a caricature of its natural habit. No doubt, in the case of both there are seminal varieties occasionally occurring, which may in some few instances account for such forms; but, in general, it is a thorough neglect of pruning in youth which is the principal cause.

Coniferous plants will bear pruning with as much impunity, and with as great advantage, as other trees or plants, if the operation is performed at the proper season and at a proper age. None, however, suffer more from an opposite course.

Pruning ornamental trees.-These require the least care on the part of the cultivator in this respect of all other trees, as there is no inducement to alter their natural forms, or to reduce their bulk, the fullest development of their natural character being the object for which they are grown.

Pruning ornamental shrubs.-Here as much science is required as in the pruning of fruit trees. The chief object to be kept in view is the reduction of all redundant wood, and an encouragement of those parts from which the flowers are to come. Some require to be spurred in, like the vine; some will admit of little curtailment of the young wood further than judicious thinning, as many of them flower on the terminal points; while others, like the rose, flower on the young wood, which, with a view to throw greater strength into it, requires to be left moderately thin, so that the energies of the 
plant may be thrown into the flower-buds and the shoots that produce them. Shrubs are, however, much neglected in this respect, and by far too little care is taken to thin them of superfluous wood, to remove that which becomes old and decayed, and of no other use than to overcrowd the plant, and prevent a free circulation of air from passing through it. Flowering shrubs should be deprived of their decayed flowers as soon as they fade, if the production of seed be not an object. A want of precaution in this respect exhausts the plants more, in their exertions to perfect their seed, than that of the production of the flowers themselves. Stopping or pinching off flower-bearing shoots after they have shown their flower-buds, as is often practised on the rose, retards their season of blossoming; while stopping the shoots around them, on which no flower-buds appear, has just an opposite effect.

The barbarous practice of using the hedgeshears-justified only by those who are too lazy to use the pruning-knife, or too ignorant to know how to use it-cannot be too severely condemned. Yet nothing is more common than to see fine and rare shrubs, which, if left to themselves, would become the ornaments of the lawn, metamorphosed into so many shaven and shorn dumpy. haycock-looking monstrosities of various sizes and shapes, presenting not one single feature of their natural character or form. One great plea is used in defence of the practice, namely, the keeping them within prescribed limits-few indeed, in these days, venturing to assert that they are improved in appearance. Root-pruning would be a far more justifiable operation, and judicious hand-pruning, taking care that, while extending branches are foreshortened, and some removed where they are too crowded, the natural habit of the tree or shrub be not destroyed. The shears, as a pruning instrument, can only be usefully employed in cutting ornamental hedges and box-edgings, or in the formation of verdant architectural subjects in the tonsile style.

Cree's system of pruning forest trees.-This system deserves the serious attention of all who wish to grow forest trees with straight stems and sound timber. The operation of foreshortening the branches, which forms the characteristic of this mode of pruning, is begun while the tree is young-indeed, before it is removed from the nursery-and is continued until the trunk of the tree has attained that height which the cultivator deems the most proper for the purpose for which the timber is intended. The philosophy of this mode of pruning is twofold-namely, the production of timber clean and free from knots; and this most desirable end is attained, because the side branches are shortened annually, whenever they extend beyond three or four feet from the main stem, and, as a consequence, they seldom attain a greater diameter close to the stem than about an inch; and as these side branches are not removed until the tree has attained the height of 16 to 20 feet, the wound caused by their removal speedily heals up, without leaving a trace of their existence on the bark, or the vestige of a knot in the timber when cut up for use-quite the antipodes to the effects of removing large branches, as illustrated as by figs. 160 and 161. If these branches be removed close to the trunk, but without disturbing the bark of the latter, the wound in all cases will heal over within two years. The first removal of branches close to the stem does not take place until the tree has, as we have said, attained the height of from 16 to 20 feet, with a stem, close to the ground, of nearly as many inches in circumference; and the mode of removal is by taking one tier of branches off annually, commencing with the tier nearest the ground, and performing the operation a little before midsummer, while the sap is in motion so that in its downward flow so much of it will be arrested around the sides of the wound, and matter deposited sufficient for the formation of new bark, to cover over the part where amputation took place.

By this process of foreshortening the side branches, the tree is made to assume a narrow conical form; and as the trees to which we refer are supposed to be growing in a close plantation, no other form would present so great a surface of foliage to the action of light and air within the same space, and it is to the presence of these elements we must look for the accumulation of bulk in timber. The same practice may be extended to trees growing in open exposed places; but while such may not produce the same cubic contents of timber within the same space of time that a tree would growing alongside, and allowed to take its natural course of growth, the timber of the pruned tree, on cutting up, will be the superior. After the trunk is in this way cleared of branches to the height the cultivator desires, all pruning ceases, and the top is allowed to take its own course, so long as it is allowed to grow. Mr Cree's mode is exceedingly well adapted to coniferous trees, not only for the production of superior timber, but also when planted for shelter to other trees, as the operation of shortening the side branches throws strength into the leader, inducing an upright growth, while the shortening prevents their spreadingout tendency, and allows a much freer circulation of air to the trees around them : if properly followed out, it would admit of those planted as nurses to remain longer, and to attain a more useful size, than is usually the case, before their total removal. This process should be strictly followed in all cases where height to trees is a consideration.

Pruning and training will be found further detailed under the heading of the various subjects to which they are to be applied.

Implements employed in pruning.-Many fanciful implements have been from time to time invented and recommended for this purpose. Ingenious although some of these are, and amusing as they may be to amateurs, they are regarded for the most part with indifference by the practical operator. The following we consider the most useful, and with them every operation of pruning may be advantageously accomplished.

of pruning-knives the peach-pruner $a$, fig. 
162 , is in very universal use. That of Saynor of Sheffield is deservedly popular.

The London peach-pruner, $b$, of Barns of Sheffield, is also an excellent implement, tapering to a narrower point than the former, and there-

Fig. 162.

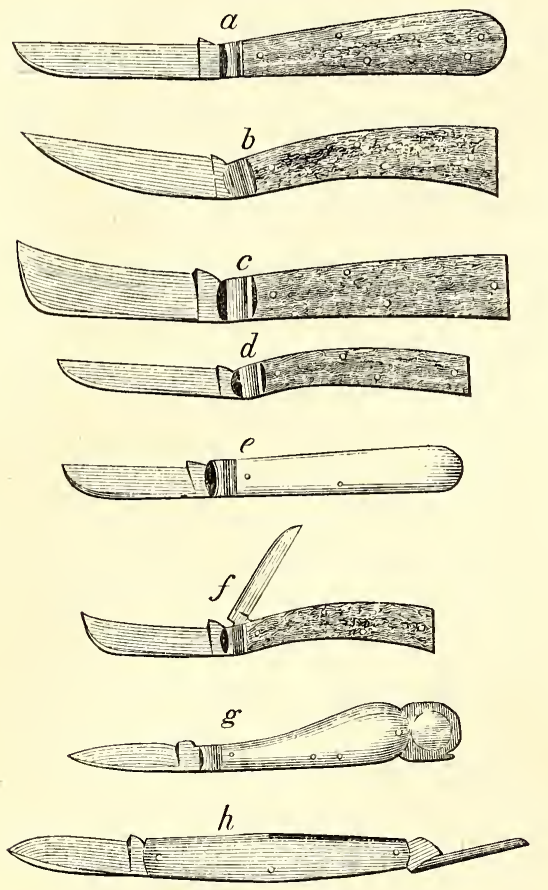

PRUNING-KNIVES.

fore, for the purpose of pruning, either when the shoots are close together or when they have not been disengaged from the wall, superior to it. They are the two best in use for the purpose. Either of these is well adapted for grafting purposes, the blades being thin.

T'he strong pruner, $c$. of Saynor of Sheffield, is well adapted for all ordinary pruning where the branches to be removed are pretty strong. Some prefer the blade when more curved towards the point; this, however, makes little difference in the working of the implement in proper liands. This may be considered the common garden-knife, and is a vast improvement over that of former times, which consisted of a blade fixed to the handle with. out a joint, enclosed in a sheath of leather or pasteboard (most generally the latter), and carried in a side pocket on the thigh of the operator. The present is a clasp or folding knife, and may be carried with much greater safety to the person. The former is now nearly out of use. The blade of a knife cuts on the same principle as a saw; a hooked-pointed blade, therefore, is injurious when used in cutting woody shoots, and can never leave so clean a section as a blade with a straight edge.

$d$ and $e$ are small peach-pruners, the one having a horn handle nearly cylindrical, but slightly curved, the other a bone or ivory handle somewhat ovate, tapering in a wedge form, much in the way of the common buddingknife, but less thin at the point. In cases of emergency it may, however, be used for budding also. They are both well adapted for peach-pruning, particularly when operating upon the young wood. Those we use are also of Saynor's manufacture.

$f$ is a small pocket-pruner having two blades, the one larger than the other. Its merits consist in its lightness and small bulk, as well as being useful for pruning, making cuttings, or cutting flowers.

$g$ is a convenient pruner when soft or small branches are to be operated upon. It is furnished at one end of the handle with a small botanical glass, useful in searching out insects or other minute objects. It is known also as Saynor's botanical-knife. A useful knife for amateurs, being small and readily carried in the waistcoat pocket.

$h$, Saynor's new budding-knife. This is not only a convenient pruner, but is well adapted for the purpose for which it is intended, having a folding gouge-like blade at one end of the handle for lifting the bud or bark after the necessary incisions have been made.

Pruning-chisels are nearly as various as pruning-knives. The best, however, are in shape of a carpenter's chisel, but with a handle of greater or less length and strength, according to the height and size of the branch to be amputated. They vary in breadth of cutting face from 1 to 3 inches, and are wrought by placing the face of the chisel upon the part of the branch where the cut is to be made, and being held there (when the handle exceeds 2 feet in length) by one man, while another, with a wooden mallet, striking upwards, drives the chisel through the branch. Thus branches of almost any size may be cut off at from 7 to 20 feet from the ground. Branches nearer the ground may be cut off with chisels with shorter handles, but in Fig. 163. most such cases it will be better to sever them with the saw and smooth

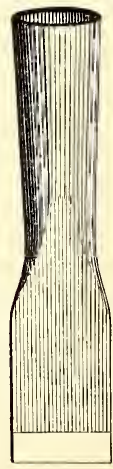
the wound afterwards with the knife. Fig. 163 shows this chisel without its handle. Another modification of it is sometimes used in orchard and ornamental tree-pruning, differing only from the former in having a guard or plate placed behind the blade to prevent its entering too far into the trunk from which the branch is to be removed. Careful workmen need no such precaution. 'The advantage of the pruning-chisel in all cases over the pruning-saw, is its saving the trouble of ascending the tree, and the damage that may be done to the branches by a man PRUNing- going up to cut off the branch. An cHisec. excellent substitute for all pruningchisels is found in the American or Indian pole-saw, fig. $164 a$, which has a blade about 4 inches broad and from 18 inches to 2 feet in length, fixed to a pole-handle of any 
required length so as to reach the branch to be removed. This saw differs from the common saw in operating by pulling instead of thrusting,

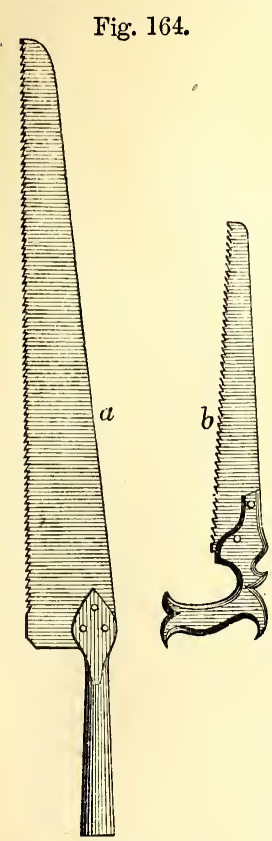

so that a person standing on the ground can work the saw to every advantage, while it is sufficiently stiff not to break while passing through the wood. The common pruning-saw, fig. $164 b$, is about 2 feet in length, and varies from 1 inch to 3 inches in breadth. It is used on branches within reach and of ordinary dimensions, and also where the chisel could not be conveniently applied. The cuts made by saws, of whatever description, should have the wound smoothed by the knife or small plane, and in most cases be painted over with some mild paint, to exclude the air and moisture.

Pruning-bills may be described as largeknives, and in the hands of an expert workman are valuable in cutting off branches larger than the

PRUNING-SAWS. pruning-knife could sever. The stroke should
always be given in an upright direction, and, if possible, one blow should perform the operation. They are very useful in pruning thick and overgrown shrubbery, as they can be wrought with greater effect in thick jungles than almost any other cutting implement. Sometimes they have only one cutting face, and that is in general somewhat crooked towards the point; at other
Fig. 165.

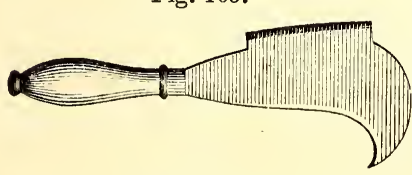

PRUNING-BILL. times they have an axelike face of several inches in length upon their back - see fig. $165-$ useful in cutting large branches; and at others they have, instead of a cutting face upon their back, a set of teeth or serratures, by which the operation of sawing may be performed.

Of averuncators-from the Latin averunco, to prune-there is a considerable variety, differing little in principle, however they may in form. Their use is to remove branches at a considerable height from the ground without the aid of ladders or the trouble of ascending the tree. Fig. 166 exhibits one of the best of them. It may be described as a cutting implement, with a compound blade attached to a handle of greater or less lengtlı, and operating by means of a lever acted upon by a cord passing over a pulley. The branch, which should rarely exceed the diameter of $1 \frac{1}{2}$ inches, is taken between the cutting faces; the cord, on being pulled smartly down, acts upon the lever so as to bring these cutting faces together, severing the branch at the same time.

Fig. 166.

Fig. 167.

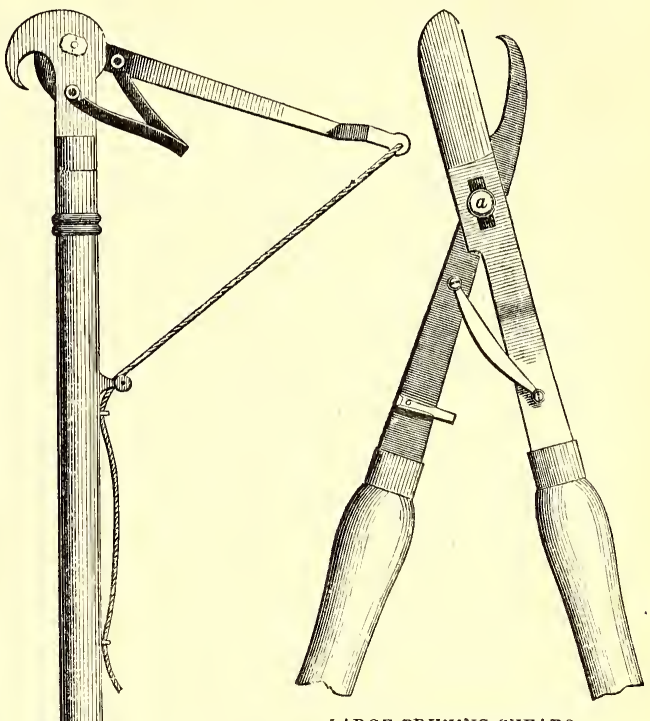

LARGE PRUNING-SHEARS.

AVERUNCATOR.

Pruning-shears are also still greater in variety. Fig. 167 shows one of the most powerful. The blades or cutting faces move on a centre at $a$, by which one of them opens and encloses the branch to be removed, which it does by making a clean draw-cut, leaving the section of the portion remaining on the tree as smooth and as little lacerated as if it had been removed by a knife or a chisel. They are very usefully employed in reducing the size of shrubs or ornamental trees, less frequently, however, in the case of fruit trees. The subject before us is capable of severing a branch from 1 to 2 inches

Fig. 168. in diameter. An implement

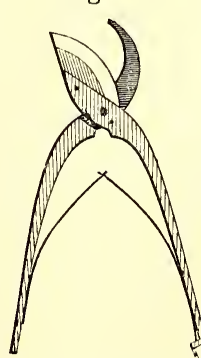
is made smaller in size, and therefore adapted more for smaller amputations. Fig. 168 is a newly-invented pruning implement, by Mr Underwood, Haymarket, London, Cutler to the Government Offices, \&c. It is powerful in its action, and of excellent material, the best of its kind. $a$, fig. 169 , is one of the smallest of its kind, and useful for pruning off tender shoots. It UNEERWOOn's is, however, more an instruPRUNING-SHEA RS. ment for theamateur than for the practical pruner. $b$ and $c$ are of similar use. $d$ is in operation somewhat 
similar to the averuncator, fig. 166. It is for cutting off leaves, bunches of grapes, flowers, \&c., that may not be readily reached by hand; and while it severs the leaf, \&c., it still holds it until it may be taken in the hand. The wire $x$, passing round the handle and lever in form of a ring, when pulled downwards by the cord $y$, draws the leaves towards the handle, and causes

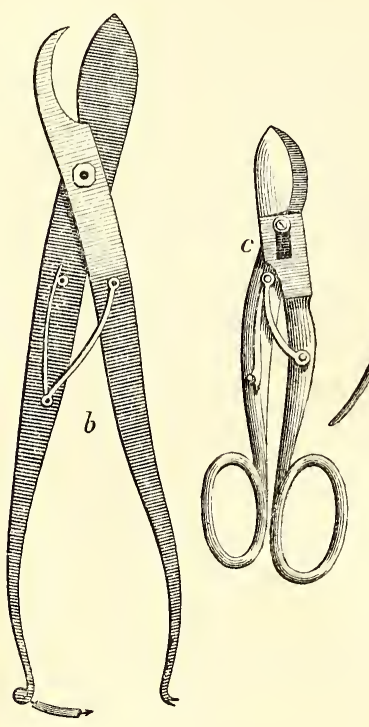

Fig. 169.

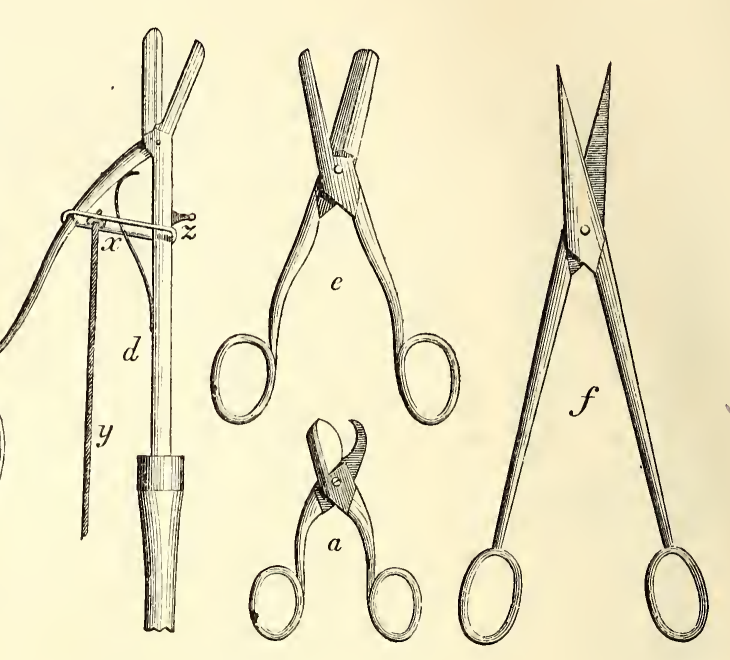

PRUNING SCISSORS.

the shear-like faces to meet: these, instead of being sharp at their edges, meet in what may be called the half-check form, bruising rather than cutting asunder the footstalk of the flower or leaf, and thus preventing its falling. The stud $z$ above the ring prevents it from slipping upwards, and the spring between the lever and handle keeps the shears open until acted upon by the cord. $e$ cuts in the same manner, and is useful in gathering roses or other flowers, or pruning soft branches when it is desirable that they shall not fall to the ground as they are cut. $f$, the grape-thinning scissors, may be regarded as a pruning implement, because they are everywhere employed for thinning or pruning out the superfluous berries from the bunches of grapes. They differ no otherwise from common scissors than in being much longer, and having their cutting points much more pointed that they may be worked among the berries with less danger of pricking those that are to be left.

The gooseberry pruner, fig. 170, is useful in removing the superfluous young wood from the

Fig. 170.

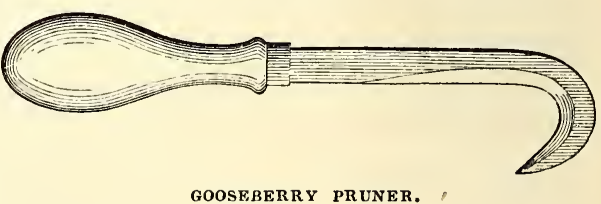

gooseberry and currant, and similar trees, and is considered by many to facilitate the operation, when compared with the pruning-knife. 


\title{
CHAPTER IV.
}

\author{
THE APPLE.
}

IN its natural state the apple is indigenous to most parts of Europe, and known in common parlance as the Crab-tree, botanically as PyrusMalus, Linn.: Malus communis, Dec. As a fruit-bearing tree it is cultivated in all civilised countries, but more especially in those of temperate climates. In a natural state it is unknown in America; but when it was introduced there is as unknown to us as the period when the first remove took place from the crab or wild state to the useful or cultivated. "It is probable," Hogg, in his excellent work on the Apple, remarks, "that it has existed as an indigenous tree throughout all ages, and that the most ancient varieties were accidental variations of the original species, with which the forests abounded. These being cultivated, and subjected to the art and industry of man, would give rise to other varieties, and thus a gradual amelioration of the fruit would be obtained. That such a change might take place naturally in Britain, as well as in other parts of Europe, where it existed indigenously, is highly probable; and that some improvement in this respect had taken place prior to the Roman invasion is natural to suppose; but of this we have no certain record, because the history of our country extends no farther back. That a similar amelioration had taken place at a much earlier pcriod in other countries is certain, as the apple is spoken of as existing for ages before. History, however, informs us that apples were not only brought to England by the Romans, but that in the days of Pliny they possessed two-and-twenty varieties." $\mathrm{Mr}$ Hogg is of opinion that the apple existed in this country at a very early period, and remarks, "that it was known to the ancient Britons before the arrival of the Romans, is evident from their language. In Celtic it is called Abhall or Abhal; in Welsh, Aval; in Armoric, Afall and Avall; in Cornish, Aval and Avel. The word is derived from the pure Celtic, Ball, signifying any round body. The ancient Glastonbury was called by the Britons Ynys Arallac, and Ynys Avallon, which signifies an apple orchard, and from this its Roman name avallonia was derived." The apple must therefore have been known in Britain before the arrival of the Romans, and that it continued to exist after they had left the island, and before the Norman conquest, is certified by William of
Malmesbury, who says " that King Edgar, in 973 , lay down under the shade of a wild appletree." In a bull of Pope Alexander III., in the year 1175 , confirming the property belonging to the monastery of Winchcombe, in Gloucestershire, is mentioned "the town of Twining, with all the lands, orchards, mcadows," \&c. And in a charter of King John, granting property to the priory of Lanthony, near Gloucester, is mentioned "the church of Herdesley, with 12 acres of land and an orchard." That any of these early varieties, or even of the aboriginals, now exist is highly improbable, as the introduced kinds in all probability were lost between the time when the Romans quitted Britain and that when the ecclesiastical power began to be established. Many seedlings might, however, during this period, have been originated naturally from the seeds of the early imported sorts, as well as from those of our earliest varieties. From such sources, and the importations afterwards by the monks of Norman varieties, it is probable that the stock of apples was first raised in England. The same causes would lead in time to thcir dispersion into Scotland and Ircland. As the priesthood in those days combined with their own profession various others, of which horticulture, we are informed by their historians, was one, it is quite reasonable to suppose, as they spread over the kingdom, that they carried the apple with them. If any of the originally imported sorts do really exist, which some think probable, it must have been by the process of engrafting, by which process any variety may be continued for ages upon the principle of extension of its actual parts, while the tree from which the scions have been taken may have long ceased in itself to exist. In its wilding state, the apple is a tree of considerable longevity, while in its improved or cultivated state it is much less so; the hardier kinds being supposed by some to extend only to from two to three centuries, many unquestionably not nearly so long. Much of this, however, depends on soil and situation. Like all other trees, the apple accommodates itself to the soil and situation in which it may be placed to a greater or less degree, and many varieties attain a much greater degree of perfection in some localities than others. The apple succeeds best within a certain range of latitude, of which the 
southern parts of England and the northern provinces of France may be taken as the latitudinal range. Exceptions, however, may be made to this rule, in the case of the apples of Astrachan in Russia, and the Malo di Carlo from the north of Italy. The transportation of the former south improves its condition, while the removal of the latter northward has an opposite tendency. The apples of Normandy, fine in their own country, are deteriorated much when transplanted even to England, and still more so if carried into Scotland.

A similar process of amelioration seems to have been going on in America, where, as we have observed, the apple does not appear to be indigenous; the change effected naturally has been from seedlings raised either by accident or design, from the earlier introduced sorts, and also by importations from Europe. May not the former circumstance in some degree account for the supposed superiority of many of the American apples?-and if so, may we not suppose that the apple trees of America are, if thus from a cultivated origin, of shorter duration as to longevity than those of Europe, which have at some period been raised from the tree in its natural state?

Many of the varieties enumerated in the following list are as yet little known in Scotland, and even in many parts of England they are equally unknown, if we except the gardens of the higher classes. These lists might have been greatly extended, but we think the selections made will be found of some advantage to those who have gardens and orchards, and who may not have made the subject of pomology a branch of their study.

The selections are also made with a view both to insure quality and abundance of produce, as well as to extend the season of perfection to as great an extent as possible, and the variety is sufficiently extensive to meet, we think, the wants of most. America may be called the land of the apple, as the Netherlands is of the pear; we have, therefore, selected a few from their long catalogues of fruits, which we think likely to suit the climate of Britain. Few of the finer sorts of American apples have had a fair chance in Britain. To assign a reason for this would be difficult. We cannot lay it entirely to the difference of climate. We may do so, to some extent, to a want of knowing that many of their varieties are far superior to many of our own, and that they are produced under circumstances, so far as climate is concerned, as adverse as that of many parts of Britain. While the Pomme de Neige, Straat, Gloria mundi, and other excellent American apples, thrive so well with us, it is surely worth making the experiment with many others.

Of the number of sorts cultivated prior to the time of Parkinson we have no certain record, but that they were numerous is evident from the following data. Parkinson enumerates no less than 59 sorts. Hartlib mentions 200 , and was of opinion that 500 existed. Rea, in his "Pomona," names 20 varieties, 16 of which are not mentioned by Parkinson. Meager gives a list of 83 as cultivated in the London nurseries in his day, of which 51 are not noticed in the lists of Parkinson or Rea. Worledge gives a list of 92 , chiefly cider apples. But the most complete enumerations are those of the London Horticultural Society, extending to 1400 varieties, and Mr Hogg's descriptive catalogue of 942 sorts, many of which are noticed for the first time.

Several attempts have been made to establish a classification of apples founded on characters at once permanent and well defined; of these, the systems propounded by Sickler, Christ, Manger, Diel, and Dochnahl, particularly the two latter, are the most perfect. Those interested in this important department of pomology should consult Mr Hogg's work on "British Pomology" from page 7 to 15 , in which also will be found an ingenious classification of British apples, by himself. The three principal divisions into which this author arranges this fruit are: 1st, Summer apples, "consisting of such as either ripen on the tree or shortly after being gathered, and which generally do not last longer than the beginning of October." 2d, Autumnal apples, "including such as are in use from the time of gathering to Christmas." $3 \mathrm{~d}$, Winter apples, "including such as are in use during the whole of the winter and spring." These are again divided into sections founded on the form of the fruit, and these again into sub-sections founded upon their colour.

This classification is not, however, considered perfect by Mr Hogg himself, as we find by a letter addressed by that enthusiastic pomologist to the editor of the "Cottage Gardener." $\mathrm{He}$ says, "It is not such a classification as I should like to have introduced, but it is sufficient for all ordinary purposes. I am engaged on a system which will reduce apples to a more natural arrangement; but as I have not been able to bring the numerous varieties into their proper positions as yet, I shall defer its publication till a future time." In justice to Mr Hogg, we ought to state that in his private grounds at Werth, in Sussex, he has in cultivation a greater collection of hardy fruits than has hitherto been congregated in any other garden, or for a similar purpose. Those, therefore, who are engaged in the science of pomology should put themselves in communication with him, as he is still adding to his already extensive collection, and informs us, in the letter above referred to, that for the purpose of adding to his stock-which he grows principally for the purpose of enabling him to study their characters thoroughly previously to, we trust, giving the world the benefit of his acute researches into every hardy fruit, from the almond to the strawberry-he purposes to exchange grafts with those adding to their collections, and to supply those who may not be in a position to exchange grafts at a small cost.

Size of the apple tree.-The apple tree seldom, in this country, attains a large size, although in some localities it attains a considerable age. The case is otherwise in America, as Downing mentions two of enormous dimensions, which, ten years before he wrote, were 130 years old, and one of these, at 1 foot from the ground, measured 13 feet 2 inches in circumference of trunk, the 
other 12 feet 2 inches. These trees bore that season about 30 or 40 bushels of fruit, but in the year 1780 they together bore 10 bushels. Another apple tree is also recorded by the same authority, which measures in girth 12 feet 5 inches, and which has yielded in a single season $121 \frac{1}{2}$ bushels.

Use of the apple.-In France and great part of Germany the apple constitutes a considerable portion of the food of the people of all grades, and is extensively manufactured into cider, brandy, \&c. But it is in America that its value is fully appreciated. The finest sorts are, as with us, esteemed for the dessert, but they are there eaten at meals to a much greater extent. "As the earliest sorts ripen about the last of June, and the latest can be preserved till that season, it may be considered as a fruit in perfection the whole year. Besides its merits for the dessert, the value of the apple is still greater for the kitchen ; and in sauces, pies, tarts, preserves, and jellies, and roasted and boiled, this fruit is the constant and invaluable recourse of the culinary department. Apple butter, made by stewing pared and sliced sweet apples in new cider until the whole is soft and pulpy, is a common and excellent article of food in many farmers' families, and is frequently made by the barrel in Connecticut. The juice of the apple unfermented is in some parts of the country boiled down till it becomes molasses. When fermented, it forms cider; and if this is carefully made from the best cider apples, it is nearly equal to wine; in fact, many hundreds of barrels of the cider of New Jersey have been manufactured in a single year into an imitation champagne, which is scarcely distinguished by many from that made from the grape.

"Dried apples are also a considerable article of commerce. Farmers usually pare and quarter them by hand, and dry them in the sun; but those who pursue it as a matter of trade pare them by machinery, and dry them slowly in ovens. They are then packed in bags or barrels, and are used either at home, in sea stores, or are exported."-DownING's Fruits and Fruit Trees of America.

Planting the apple.-Mr Hogg, in introduction to "British Pomology," says: "The apple may be grown on almost any description of soil, providing it is not absolutely wet. That on which it succeeds best is a humid sandy loam, or a well-drained strong clay, which, if it possess a calcareous or gravelly subsoil, will be still more advantageous. It is not requisite that it should be of so great a depth as for pears, as the apple, having no tap.root, does not penetrate so far into the soil. From 18 inches to 2 feet will be found a good depth; but where the soil is good, and the subsoil sufficiently humid, without being literall $\because \ddot{y}$ wet, even 1 foot or 18 inches will answer every purpose." Rogers, we think, writes sensibly on this subject in "The Fruit Cultivator," p. 8. He says, "The size and flavour of the fruit, the general health and duration of the trees, is most commonly the result of good or bad soil. Climate and situation also affect both trees and fruit, but not in the degree in which the same are affected by the qualities preVOL. II. dominant in the land. Of all soils to be met with, that of a soft hazel loam, containing a small portion of sand, seems to be the most congenial to the apple generally. In such soils the tree is seen to flourish longest, is most productive, and remains freest from disease or attacks of insects." He agrees with $\mathrm{Mr}$ Hogg as to depth of soil, and adds, "If the bottom be wet, the trees should be planted high, and every means taken to drain the ground. A wet bottom of gravelly clay should be avoided if possible, no kind of apple thriving long if the roots once enter into such a cold substratum."

In planting pyramidal trained apples on the paradise stock, they may be set at 5 feet distance apart; and if root-pruned every third or fourth year, should they show symptoms of over-vigorous growth, this will be found ample space for them. The age of such trees, when planted, should be from two to three years, and those sliould be chosen which have a straight leading stem, well furnished with buds and branches from the stalk upwards. If the soil be shallow, wet, or of indifferent quality, it will be advisable to plant them on little mounds, by gathering the surface-soil together; or, better, by laying down a wheelbarrow-load or two of fresh fibrous turfy soil, and planting upon it.

Deep-rooted soils in sheltered situations are not the most proper for the apple, although such have been most erroneously recommended; for it is often seen that apple trees succeed well in any kind of loam, though it be not more than 1 foot in depth, so as the bottom is sound and dry; the roots take an extensive horizontal range, the young wood is always of more moderate growth, and better ripened than when the roots strike deep into the ground. For all kinds of training, young trees two years from the graft should be chosen. Apples intended for dwarf standards are worked low upon paradise stocks, to correct luxuriance in growth. Those intended for half or full standards are wrought high on crab or free stocks. The varieties of apples are now so great that it may reasonably be expected, and it is so, that one variety succeeds in one sort of soil, and in one situation, better than in others; for this reason we have given an extended list, so as to meet the greatest variety of soils and circumstances.

Criterion of a good apple-Dessert sorts.-Elegance in form and colouring is no doubt a high recommendation, and such produce the best effects when on the table. It does not, however, by any means follow that the handsomest apples are the best flavoured-often quite the reverse. The Pomme d'Api may be given as an example. Medium or small-sized apples, such as the golden pippin and nonpareils, afford a good criterion; while Adam's pearmain and Alexander, although exceedingly handsome, are too large to dish well, as confectioners and tabledressers phrase it. In flavour they should be sugary, rich; and if highly aromatic, so much more are they esteemed by connoisseurs. The flesh should be firm, crisp, and juicy-properties which may in general be looked for in yellowish-fleshed apples, and those having a brownish russety skin marked with dull yellow and 
red; and seldom in pale yellows, light greens, or bright reds, if all of a uniform colour.

Culinary sorts.-For the generality of purposes these cannot be over large. Their great merit consists in their becoming equally soft throughout, when boiled, stewed, or roasted, of which the Hawthornden, Carlisle codlin, and Beauty of Kent furnish examples; while the Yorkshire greening, Normandy rennet, and several russets, are the types of those which fall soft in cooking, and still retain their form when in their ripe state, a quality much prized by cooks. The Norfolk biffin is of ali the best for baking whole in a coolish over, and then flattening by pressure into the form they appear in the shops. The Hawthornden and Keswick codlin are preferred for making apple-jelly, affording a clear and transparent preserve. The Keswick, Carlisle, and most other codlins, fall in cooking while yet green, and as they are early, are much valued on this account.
Cider sorts.-The more astringent they are, without regard to richness in flavour, as the Siberian Harvey, the better they are fitted for this purpose, although, strange to say, many of the highest-flavoured apples, such as the golden Harvey or brandy apple, are often used for this purpose. Mr Knight states that the specific gravity of the juice of any apple recently expressed, indicates with very considerable accuracy the strength of the cider it will produce.

In the descriptive list of fruits reference is frequently made to their size. The annexed diagram is intended as a scale by which those we describe as large, medium, or small sized may be understood. No. 1. Alfriston, large ; No. 2. Dutch mignonne, medium sized; No. 3. Golden pippin, small sized.

Fig. 172 represents the forms of apples. Their designations are as follows :- $a$, conical; $b$, irregular conical; $c$, pearmain shaped; $d$, oblate ; $e$, roundish ovate; $f$, oblato-ovate.

Fig. 171.

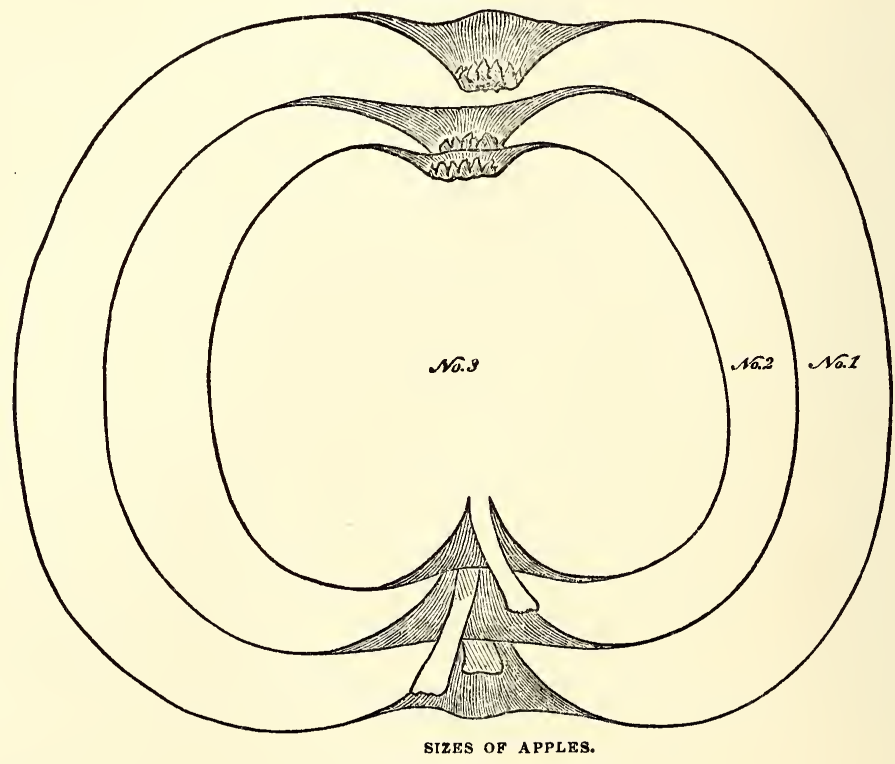

Fig. 172.

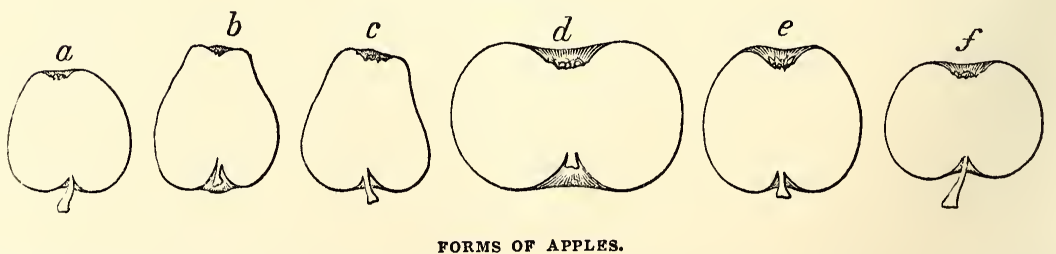

LIST OF SORTS AND THEIR QUALITIES. DESSERT APPLES.

Acklam's russet.-Colour pale yellow, tinged with green, greyish russet on the side most exposed to the sun; form round, slightly flat- tened; below medium size. In use from November till March. Quality first-rate. Tree very hardy and an excellent bearer. Of Yorkshire origin. Succeeds best in a dry soil. Syno. nym, Norfolk pippin.

Annat scarlet pippin. - An excellent apple 
of Scotch origin. We have received excellent specimens from $\mathrm{Mr}$ Archibald Gorrie, the raiser. Size medium, quality good, exceedingly beautiful, and in condition for the dessert during January and February. Tree perfectly hardy, and a good bearer.

Ashmead's kernel.-Colour yellow, covered with dots of russet; form oblate; size medium; quality excellent. In use from November to May. Tree hardy, a good bearer, but most of all esteemed for its long keeping. Possessing so much of the richness of the nonpareil as to lead to the belief that it is a seedling from that variety.

Baddow pippin.-Colour deep lively green, changing as it ripens to yellowish green on the shaded side, dull red on the side next the sun, changing to orange; form roundish or oblate; size medium. In use in November, keeping till April and May. Quality first-rate, partaking somewhat of the Ribston and nonpareil. Comparatively of modern origin, being only introduced to public notice in 1848. Synonym, Spring Ribston.

Barton's incomparable. - Colour yellowish green, covered with patches of pale-brown russet; form ovate or conical; size below medium. In use from October to February. Quality of the highest excellence. Tree healthy, attaining a large size, and an excellent bearer. "This variety," Mr Hogg observes, "seems to be but little known, and, considering its excellence, rarely cultivated. I am not aware that it exists in any of the nurseries, or that it was at any period extensively propagated. The only place where I ever met with it was in the private garden of the late Mr Lee of Hammersmith, whence I procured grafts from a tree in the last stage of decay." It is probable that trees of it may exist in the nurseries of Messrs J. and C. Lee at Hammersmith, or in the Brompton Nursery. It is a variety that should be rescued from oblivion.

Beachamwell.-Colour yellow; form ovate ; size smallish ; quality first-rate. In use from December to March. Synonyms-Motteux seedling, Beachamvell seedling. Tree very hardy, but does not attain a large size, and is by no means so extensively cultivated as its merits deserve.

Borsdorffer.-Colour yellow and red; form oval; size smallish; quality first-rate. In use from November till March. This is a German apple of great merit, but rather tender for the north of England, and most of Scotland.

Breedon pippin.-Colour dull yellow, tinged with orange, inclining to red on the side most exposed to the sun; form roundish; size small. In use during October and November. Quality one of our best dessert apples, somewhat resembling and exceeding in flavour the Court of Wick. Tree hardy, not attaining a large size, and an abundant bearer.

Christie's pippin.-Colour yellow, tinged with green on the shaded side, mottled with russet and red spots next the sun; form oblate and very handsome; size medium. In use from December to February. Tree constitutionally weak, subject to canker and mildew. When wrought on the paradise stock, it forms a compact and handsome little pyramid. Quality first-rate for dessert, and withal a very abundant bearer.

Clara pippin.-A new dessert apple of considerable merit. It is a seedling from a pip of the Court of Wick. Fruit about the size of the parent; flesh firm, orange; flavour rich and sugary ; keeps till the end of May. Skin rather tough, orange yellow on the one side, tinged with brownish red on the other side. Originated with F. J. Graham, Esq., prior to 1848.

Cockle pippin.-Colour brown and yellow; form ovate; size medium; quality first-rate. In use from December till April. Slender-growing tree, but an excellent bearer, and very hardy. Ripens well as a standard at Dalkeith. Known also as Nutmeg cockle pippin, Nutmeg pippin, White cockle pippin, and Brown cockle pippin.

Colonel Vaughan's. - Colour waxen yellow, streaked with crimson on the shaded side, bright crimson streaked with very dark crimson, thickly strewed with greyish-white dots on the side next the sun; form oblato-conical, or conical; size below medium. In use in September and October. Quality very excellent for the dessert. Not met with in general cultivation, but is very extensively grown in Kent for the supply of Covent Garden market.

Cornish aromatic.-Colour russety red; form roundish ; size large ; quality first-rate. In use from October to January. Tree a free grower, and an excellent bearer. Synonym, Aromatic pippin.

Court-pendu Plat.-Colour dark-red; form oblate; size medium; quality first-rate. In use from December till April. This is evidently of French origin, and is one of the most popular apples on the Continent. Too tender for Scotland and the north of England, unless planted against a wall, where it ripens to perfection, but seldom attains its fine colour. Its foreign synonyms are numerous. In England it is sometimes called Wollaton pippin, Garnon's apple, at which latter place we saw some years ago many trees of it in great luxuriance as open standards, and learned from the proprietor, the late Sir John G. Cotterall, that his father had brought them from France. It has the peculiarity of blossoming late, and hence often escapes the spring frosts; and accordingly Mr R. Thomson says it has been called the wise apple.

Court of Wick.-Colour yellow ; form oblate; size under medium; quality first-rate. In use from October till March. Tree slender in growth; good bearer; one of our best dessert fruits. It does not appear to be much cultivated in Scotland. At Dalkeith it ripens well on dwarf standards. As is the case with most good fruits, it has a host of synonyms - as Golden drop, Wick's pippin, Fry's pippin, Phillip's reinette, Kingswick pippin, Wood's new transparent, Wood's Huntington. There is a scarlet Court of Wick, but that is inferior, and should be guarded against.

Crofton scarlet.-Colour brownish red; form oblate; size medium ; quality good. In use from October to December. Synonym-Red Crofton.

Crofton white.-Similar to the last, only of a paler colour. Keeping only till the beginning of November, and somewhat inferior in quality. 
Cornish gilliflower.-Colour dull-green on the shaded side, brownish-red streakcd with brighter red, and slightly marked with russet on the side most exposed to the sun. Form ovate, angular on the sides, and ribbed round the eye. Size large. In use from December to May. Quality one of our best dessert apples, remarkable for its rich and aromatic flavour. Tree hardy, attaining the medium size, but not a great bearer. Mr Hogg remarks, that it produces its fruit at the extremities of the last year's wood, and great care should therefore be taken to preserve the bearing shoots. It succeeds well grafted on the paradise stock, and grown as an espalier and open dwarf

Creed's marygold.-Colour deep rich yellow on the shaded side, deep orange next the sun, and covercd with beautiful red, striped with darker red on a ground of delicate russet, and thickly strewed with dark russety dots. Form roundish; size medium. In use during October and November. Quality excellent as a dessert apple. Tree hardy, and of slender growth. Raised by Mr Creed, gardener, at Norton Court, Kent, a few years ago, from a seed of the scarlet nonpareil.

Downton pippin.-Colour fine lemon-yellow, with a slight tinge of red next the sun, marked with a few traces of delicate russet, and strewed with numerous pale-brown dots. Form somewhat cylindrical; size small. In use from November to January. Quality good as a dessert fruit, greatly rcsembling the golden pippin, but less rich in flavour. Tree healthy, and a vigorous grower and a most abundant bcarer. Raised by Mr Knight of Downton Castle from the seed of the Isle of Wight orange pippin, impregnated with the pollen of the golden pippin. Synonyms - Elton pixpin, Elton golden pippin, Knight's pippin, Knight's golden pippin, $S t$ Mary's pippin, Downton's pippin of Diel.

Dredge's fair maid of Wishford.-Colour yellow, covered with large patches of thin brown russet tinged with orange, and a few red streaks on the side next the sun. Form oblate-cylindrical. Size medium. In use from December to March. Quality fit for the dessert, and excellent for culinary purposes. Tree middle-sized, a free grower, and abundant bearer.

Dredge's fame.-Colour dingy yellow, with a tinge of green, covered with patches of thin russet, mottled with pale red on the side exposed to the sun. Form roundish, inclining to ovate; size above medium. In use from December to March. Tree hardy, coming early into fruit, and an abundant bearer. This and the last originated with a nurseryman namcd Dredge, of Wishford, near Salisbury. We have grown both these on the chalky soils of Hampshire with great satisfaction, but have found them less healthy and productive in Scotland.

Early harvest.-Colour pale yellowish-green, becoming clear pale yellow as it ripens. Form round. Size medium. In use in the end of July and beginning of August. Quality one of our best vcry early apples. Tree healthy, but not a vigorous grower, and a most abundant bearer. This is of American origin, but succeeds admirably in this country, ripening at
Dalkeith as a dwarf standard about the middle of August. Synonyms-Early French reinette, Early July pippin, Large white Juneating, Yellow harvest, Prince's yellow harvest.

Early Julien.-Colour pale yellow, with an orange tinge next the sun strewed over with numerous minute whitish dots; form roundish, slightly flattened, and prominently ribbed from the eye downwards to the base; size medium. In use about the second or third week in August. Tree healthy, hardy, but not a strong growcr; an excellent bearer, rivalling in this respect the Hawthornden, to which it bears some resemblance, and in point of quality is quite equal to it. It is thought to be of Scotch origin, and if so, is one of the best early apples produced in the north.

Elford pippin. - Colour yellowish green, marked with russet on the shaded side ; red, and striped with darker red on the side most exposed to the sun; form roundish, inclining to ovate; size medium. In use from October to January. Tree healthy, a vigorous grower, and excellent bearer. Quality first-rate as a winter dessert apple. Brought recently into notice by Mr Maund, and conjectured to have originated at Elford, near Lichfield, to which neighbourhood it is as yet chiefly confincd.

Esopus Spitzenberg.-Colour almost entirely clear bright red, marked with fawn-coloured dots on the side next the sun ; yellow tinged, and streaked with red on the shaded side; form ovate; size large. In use from Novem. ber to February. Of its merits under English cultivation, Mr Hogg remarks: "Along with the Newtown pippen it ranks as one of the most productive and profitable orchard fruits, but like many, and indeed almost all the best American varieties, it does not attain to that degree of perfection in this country that it does in its native soil. The tree is tender and subject to canker, and the fruit lacks that high flavour and peculiar richness which characterises the imported specimens." We think, however, if this excellent fruit, along with many other of these American varieties, were planted against southern walls, that they would succeed. Since the adoption of hollow walls heated by hot water, and the more recent improvement of glass walls, we see no reason why such apples as this and the Newtown pippin should not be so accommodated. It would be more profitable than planting peaches, plums, \&c., in them. Mr Hogg, in describing the Flushing Spitzenberg, which is an American apple of little value, says, "In the Horticultural Society Catalogue this latter is made synonymous with the Esopus Spitzenberg, but it is quite a different variety."

Essex pippin.-Colour yellow; form round; size under medium; quality good. In use from October till February. Allied to the golden pippin, but much hardier.

Farleigh pippin. - Colour yellowish green; form oblong-ovate ; size medium; quality good. In use from January till May; much esteemed on account of its long keeping. Tree hardy and a fair bearer.

Fearn's pippin.-Colour reddish; form oblate; size medium ; quality, both for dessert and culin- 
ary purposes, first-rate. In use from October till March. Tree of slender growth and an abundant bearer. Succeeds well as an open standard at Dalkeith. Synonyms-Clifton nonsuch, Ferris pippin, and Florence pippin, in Covent Garden market.

Foreman's Crew.-Colour pale yellowish green, with reddish brown on the sunny side, thinly covered with yellowish-brown russet; form conical, flattened at both ends ; size below medium. In use from November till April. Quality first-rate. Tree rather tender, and subject to canker; well adapted, if wrought on the paradise stock, for dwarf-training. It is an abundant bearer. Originated about thirty years ago with T. S. Foreman, Esq., Pennydarron, South Wales.

Gilliflower, Cornish.-Colour dark green and yellow; form ovate; size medium; quality firstrate. In use from November till May. Were this not a rather shy bearer, it might be set down as one of the very best apples. Rather tender for the north of England and the most of Scotland. Known also as the Cornish July flower, an evident corruption of the proper name.

Gilliflower, summer. - Colour striped; form roundish; size large; quality good. In use in September. This is a very abundant bearer, but best suited to the climate of England.

Gogar pippin.-Colour pale green;form oblate; size medium; quality fair. We introduce it on account of its hardiness being suitable to most of Scotland, of which it is a native, having originated at Gogar near Edinburgh. It is often sold as the stone pippin, from which it is perfectly distinct. In use from November till April.

Golden drop, Coe's. - Colour yellow ; form ovate; size under medium; quality first-rate. In use from December till June. This is an excellent apple, and highly deserving extended cultivation.

Golden Harvey.-Colour russet and vellow; form roundish; size under medium; quality first-rate. In use from November till June. One of our best dessert apples, having a peculiar flavour of brandy, hence often known as the brandy apple. It is much cultivated in the west of England, even in elevated localities, for the purpose of making the best quality of cider, as well as for the dessert. It is, however, by no means a hardy tree, yet succeeds well at Dalkeith as a dwarf standard.

Golden pippin.-Colour yellow; form roundish ; size under medium; quality first-rate. In use from November till April. An old favourite, possessing all the properties of a first-rate apple. Requires the protection of a wall even in the best situations in Britain; and under such circumstances it ripens to great perfection in many parts of the north of Scotland. The varieties of the golden pippin, with their synonyms, are so numerous, that we shall only give the leading sorts, leaving the reader to take for granted that all other names are merely synonyms of one or the other, or that they are of inferior quality to those we shall notice. The golden pippin was noticed by Evelyn in 1660 as common in England.
Golden pippin, Frankland's.-Colour yellow ; form oval; size under medium; quality excellent. In use from October to January. Apparently a hardy variety, as two trees of large size and considerable age fruited most abundantly in a neglected part of the Claremont Gardens, seldom failing. This appears to be of American origin, in which country of apples the golden pippin seems to be lightly esteemed. Downing, one of the highest pomological authorities in that country, observes of them that they have "never become popular, either because the taste here does not run in favour of small apples, with the high and sub-acid flavour of the golden pippin and other favourite English sorts, or because our Newtown pippins, Swaars, and Spitzenbergs, \&c., are still higher flavoured, and of a size more admired in this country. There are half-a-dozen sorts of apples which are improperly called American golden pippins, but we have never yet been able to find a distinct and new variety of this name. What are so termed are usually the Fall, or the yellow Newtown pippin." Synonym-Sudlow's fall.

Golden pippin, Hughes's.-Colour yellow; form roundish; size under medium; quality first-rate, deserving high recommendation. In use from November till March.

Golden pippin, new.-Colour yellowish ; form roundish ; size under medium; quality inferior to the two foregoing, although good. In use from November till April. Tree scarcely so hardy as the preceding, but, like it, a most abundant bearer.

Golden pippin, scarlet.-Colour yellowish russet, slightly dashed with scarlet streaks; form semi-conical; size under medium ; quality rather inferior to the preceding, yet good. In use from November till January. Tree hardy, and suitable for a dwarf standard in most gardens. It is an excellent bearer, rather deficient in juice.

Golden pippin, summer.-Colour yellow; form ovate; size under medium; quality first-rate. In use during August and September. A very valuable summer apple, and a good bearer.

The cluster golden pippin, and Screveton golden pippin, are inferior to those named above.

Golden russet.-Colour dingy, yellow russet on the shaded side, sometimes with a little bright red on the side next the sun ; form ovate; size medium. In use from December to March. Quality excellent-indeed, one of our best. Tree healthy and a good bearer, if planted in a warm situation, in which only it will ripen thoroughly. This is a very old English variety, and is thus quaintly described by Worledge "It has no compeer, it being of a gold-coloured coat, under a russet hair, with some warts on it. It lives over the winter, and is, without dispute, the most pleasant apple that grows, having a most delicate aromatick hautgust, and melting in the mouth." Synonym-Aromatic.

Golden pearmain, winter.-Colour deep rich golden yellow, paler on the shaded side than on that exposed to the sun, where it is of a deep orange, with streaks and mottles of crimson, and covered with russety dots; form abrupt 
pearmain shaped; size medium. In use from October to January. Quality first-rate, and withal a beautiful fruit. Tree healthy, hardy, and will grow in almost any situation, and is very productive. It appears that some confusion has existed between this excellent apple and the no less excellent variety now, according to Mr Hogg, to be known as King of the pippins. It appears that the late Mr Joseph Kiri, of Bromptoll, a most respectable pomologist, brought out this apple under the latter name, whereas Diel had previously possessed and described it under the name of the golden winter pearmain. Acting on the recognised principle of priority of description and of naming, Mr Hogg has very properly withdrawn the name of King of the pippins, and substituted Diel's narne of golden winter pearmain; the name of King of the pippins belonging, according to him, to another and very different apple. Synonyms-King of the pippins, Hampshire yellow, Hampshire yellow golden pippin, Jones's Southampton pippin.

Ingestrie's yellow.-Colour yellow; form oval ; size under medium; quality fair. In use during the latter end of August till October. We notice it as a great bearer, and excellent substitute for some of the golden pippins, in situations. where they may not succeed. It ripens to full perfection in September as a dwarf standard in the Dalkeith gardens, and we find it a valuable dessert fruit. Both this and the red Ingestrie are cross-bred seedlings raised by Mr Thos. A. Knight from the golden pippin.

Irish peach apple.-Colour pale yellowish green, tinged with reddish brown, thickly marked with green dots on the side most shaded, but on the other side of a fine red, speckled with yellow spots; form roundish, slightly flattened and somewhat ribbed ; size medium. In use in the vicinity of London by the first week in August. One of the very best, as it is one of the very earliest apples. Tree hardy and an abundant bearer. Synonym-Early Crofton.

Juneating, white.-Colour pale yellow ; form roundish; size small; quality good; ripens in July, and valued as an excellent early fruit, particularly when grown against a wall, in which case it ripens in the end of June. The synonyms are many, and can only be, for the most part, regarded as corruptions of either Ginetting, or Juniting, by both of which it has been known for ages. Mr Hogg has, however, altered the orthography in his "British Pomology" to Joanneting, and has adduced reasons which, to us at least, are very satisfactory. Tree slender grower, but healthy, and a most extraordinary bearer. One of the very best to be worked on the paradise stock for pot culture. So excellent do we consider this little early apple, that to those who are building orchard-houses we would recommend them not to omit having a tree or two of this in them.

Kerry pippin.-Colour red and yellow ; form oval; size medium; quality first-rate. In use during September and October. Tree slender in growth, but a very abundant bearer. Synonym-Edmonton's aromatic pippin.

King of the pippins (Hogg's). - Colour greenish yellow, with a blush of red next the sun, slightly marked with light-brown russet; form oval, or conical ; size medium. In use in the end of August to beginning of September. The very circumstance of its early ripening proves this to be a very different fruit from the golden winter pearmain, so often called King of the pippins. Quality one of the richest-flavoured early dessert apples.

Large yellow bough.-Colour pale greenish yellow ; form oblong-oval ; size above medium. In use in the beginning of August (in Britain). Quality as a dessert fruit first-rate. Tree vigorous, and an excellent bearer. Synonyms-Large early yellow bough, Sueet bough, Early bough, Bough, Sweet harvest. Of American origin.

Leyden pippin.-Colour pale green and red ; form roundish : size medium; quality first-rate. In use during August and September. Tree moderately hardy, a very great bearer, somewhat resembling the white Astrakan.

Lord Nelson, Kirk's. - Colour red; form roundish; size large; quality good. In use from November till March. Tree not robust, a good bearer, and very showy fruit.

Lucombe's pine-apple.-Colour uniform clear pale yellow, with a slight orange tinge on the side next the sun, the whole surface covered with pale-brown russet dots; form between ovate and conical, slightly ribbed near the apex; size rather below medium. In use from October till January. Quality first-rate. Tree hardy, and an excellent bearer. Originated in the highly respectable nursery establishment of Lucombe, Pince, \& Co. of Exeter. Synonym-Pine-apple pippin.

Maclean's favourite. - Colour yellow; form roundish ; size medium; quality excellent. In use from October till March. Tree hardy ; moderate bearer. According to $\mathrm{Mr}$ Thomson, of the London Horticultural Society, a fruit " of the highest excellence."

Margil. - Colour yellow and red; form ovate ; size under medium; quality first-rate. In use from October till March. Tree a slender grower, but very hardy, and an abundant bearer. In flavour this apple somewhat resembles the Ribston pippin, which is no slight recommendation. Synonyms-Munch's pippin, Never fail.

Morris's Court of Wick.-Colour pale green where shaded, with light red next the sun; form roundish oblate; size small, resembling its parent, the old Court of Wick. In use from October to February. As a dessert fruit excelling in quality even its much-prized parent. Tree slender in growth, hardy, and a good bearer.

Morris's russet. - Colour brownish russet where shaded, but where exposed to the sun in good seasons becoming bright red on one side; size below medium. In use from October to February. Quality of the highest degree of excellence as a dessert apple. Both these excellent apples originated with a $\mathrm{Mr}$ Morris, a market-gardener, near Brentford. Of their merits as regards a Scotch climate we have no information as yet, our trees of them not having yet fruited.

Newtoun pippin.-Colour greenish yellow ; form roundish; size medium ; quality first-rate. In use from December till May. Of American 
origin, and is one of the very best of their apples. Downing, a high American authority, thus speaks of this excellent apple in his very interesting work on "The Fruits and Fruit Trees of America," p. 118: "The Newtown pippin stands at the head of all apples, and is, when in perfection, acknowledged to be unrivalled in all the qualities which constitute a high-flavoured dessert apple, to which it combines the quality of long keeping without the least shrivelling, retaining its high flavour to the last. It is very largely raised in New York and New Jersey for exportation, and commands the highest price in Covent Garden market, London. This variety is a native of Newtown, Long Island, and it requires a pretty strong, deep, warm soil to attain its full perfection. The tree is of rather slender and slow growth, and even while young is always remarkable for its rough bark." It is too delicate to be grown as an open standard in Britain, but is richly worth the protection of a wall, more especially in Scotland. Fine specimens have been so produced in the Dalkeith gardens. Synonyms-Green Newtown pippin, American Newtown pippin, Petersburgh pippin, Large Newtown pippin, Green winter pippin, Hunt's Newtown pippin.

Newtown pippin, yellow.-Colour yellow; form roundish ; size large ; quality first-rate. In use during December till April. A much hardier tree than the last, and ripens in inferior situations. Downing, already quoted, speaks highly of this American apple. He says that it "strongly resembles the foregoing, and it is difficult to say which is the superior fruit. The yellow is handsomer, and has a higher perfume than the green, and its flesh is rather firmer, and equally high flavoured; while the green is more juicy, crisp, and tender. When fully ripe, it is yellow, with a rather lively red cheek and a smooth skin ; few or none of the spots on the green variety, but with the same russet marks at the stalk. It is also more highly fragrant before and after it is cut than the green. Both the Newtown pippins grow alike, and they are both excellent bearers. This variety is rather hardier, and succeeds best in the eastern States. We have kept the fruit until the 4th of July." Both these varieties are much better worth growing on south walls than many of our peaches and plums, and on heated walls would ripen equally well as on standards in America.

New rock pippin.-Colour green; form round; size medium; quality first-rate. In use from December till June. A valuable dessert apple on account of its long keeping.

Nonpareil apples present a formidable list in most catalogues. In that of the Catalogue of the fruits cultivated in the gardens of the Horticultural Society of London, no fewer than 30 varieties, exclusive of synonyms, are enumerated. The following may be taken as the best.

Nonpareil, old.--Colour greenish yellow; form roundish; size under medium; quality first-rate. In use from December till June. In the south of England this tree, in favourable situations, bears well as a standard, while in most other parts, the north of England and Scotland in particular, it does not succeed without the pro- tection of a wall. The whole section possess a peculiar and excellent flavour, and are all good keepers. The synonyms, exclusive of foreign ones, are-Hunt's nonpareil, English nonpareil, Loveden's pippin.

Nonpareil, Braddick's.-Colour green; form roundish; size under medium; quality first-rate; one of our very best winter dessert apples. In use from December to the end of April. An abundant bearer, but requires a wall. Synonym, Ditton nonpareii.

Nonpareil, early.-Colour greenish yellow; form oblate; size under medium; quality firstrate. In use from September till January. Its synonyms are-New nonpareil, Stagg's nonpareil, Hick's fancy, Summer nonpareil, Lacy's nonpareil.

Nonpareil, Stubton.-Colour greenish yellow; form roundish; size under medium; quality firstrate. In use from December till April. Flesh peculiarly rich and sugary.

Nonpareil, Downton.--Colour greenish russet; form roundish; size under medium; quality firstrate. In use from November till April.

Nonpareil, golden.-Colour yellowish russet; form round; size under medium; quality firstrate. In use from November till March. This is one of the handsomest of the section.

Nonpareil, Pitmaston.-Colour pale green and russet; form roundish; size under medium; quality first-rate. In use from November till February. Synonyms-Pitmaston russet nonpareil, St John's nonpareil.

Nonpareil, Pitmaston.- "One of the greatest bearers," says Mr Errington, "with which we are acquainted. This we call everybody's apple; no cottage garden should be without a couple. We have a tree which we have never known fail of a good crop for eighteen years; this can be said of few apples."

Nonparcil, Ross. - Colour slightly russety, tinged with red on the side next the sun; form roundish, tapering a little towards the eye; size medium. In use from November to February. Quality one of the very best of its section. Tree hardy, succeeding in most soils in a healthy state, and withal a great bearer. Certainly the best Irish apple in cultivation.

Nonpareil, scarlet.-Colour red; form roundish; size under medium; quality first-rate. In use from December till March. Tree hardy compared with the rest of the section, found wild in the garden of a public-house at Esher, Surrey. This, however, with all the section, requires the protection of a wall, to which they are well entitled, as forming one of the most valuable tribe of the apple kind. Much as we esteem the nonpareils in Britain, in America they are less thought of. Mr Downing describes only three, and observes of the old nonpareil that it "is a favourite apple in England, but it is little esteemed in this country."

Nonsuch park apple.-Colour yellow; form roundish; size under medium; quality excellent. In use from November till March. Somewhat resembling the golden pippin.

Ord's apple. - Colour deep grassy green, freckled with grey specks and brown russety dots on the shaded side; brownish red, with 
patches of darker red on a russety ground on the side exposed to the sun; form somewhat conical, but very irregular in outline, on account of the prominency and unequal size of the ribs; size medium. In use from January till May. Quality first-rate, deserving more general cultivation. By no means a common fruit in nurseries, but one which should be found in all. It originated from a pip of a Newtown pippin imported in 1777. Synonyms-Simpson's pippin, but not Simpson's seedling, which is an inferior fruit.

Osterley pippin.-Colour yellowish green, with many russety dots on the shaded side; palish red, with russety specks on the side exposed to the sun; form orbicular; size rather below medium. In use from October to February. Quality excellent, as may be supposed, seeing that it was raised from a seed of the Ribston pippin at Osterley Park, Middlesex. Synonym -Osterley apple.

Pearmain. *-This is an extensive section. The following may be considered the best.

Pearmain, Adam's.-Colour yellowish red; form pearmain shaped-that is, of the form of a truncated cone, to which the whole section al most bears a striking resemblance; size medium; quality first-rate. In use from October till February. Tree moderately hardy, and an abundant bearer. Synonym-Norfolk pippin.

Pearmain, Claygate.-Colour yellowish red; form similar to the last; size medium; quality first-rate. In use from November till the end of March. This excellent dessert apple was first discovered in a hedgerow at Claygate, near Esher, Surrey. Tree hardy; an abundant bearer, requiring a rather strong soil.

Pearmain, Herefordshire.-Colour yellowish green and red; form same as the last; size large; quality first-rate. In use from November till March. A very old and excellent Herefordshire variety. Tree hardy and a good bearer. It is known also as the Old pearmain, Parmain, and Royal pearmain of some. So highly is it esteemed in America, that Downing says of it, "this delicious old variety, generally known here (America) as the winter or autumn pearmain, is one of the finest of all winter dessert fruits; and its mild and agreeable flavour renders it here, as abroad, a universal favourite, both as a dessert apple and for cooking."

Pearmain, Mannington's.-Colour rich golden yellow, covered with thin. brownish russet on the shaded side, with dull brownish red on the side exposed to the sun; form pearmain shaped; size medium. In use from the latter end of October till the beginning of March. Tree hardy, an early and abundant bearer, frequently producing fruit the second and third year from the graft; in habit rather a smallish tree, and well adapted for small gardens; quality first-rate, out by far too little known. It is somewhat singular that this excellent apple, although known to have originated so early as about 1770 , was not made generally known till 1847, through the London Horticultural Society. "The original tree grew up at the root of a hedge where the refuse from a cider-press had been thrown; it never attained any very great size, but continued to preserve a stunted and diminutive habit of growth till it died about the year 1820."-Hogg.

Pearmain, Lamb Abbey.-Colour green, yellow, and red; form same as thelast; sizemedium; quality first-rate. In use during January, and continuing till April, keeping well without shrivelling. Tree healthy, a free grower, and an excellent bearer. Originated from a seed of an imported Newtown pippin, sown in 1804 by the lady of Neil Malcolm, Esq., of Lamb Abbey, Kent.

This is an extraordinary kind. "If the Pitmaston has a rival," says Errington, "in point of sure bearing, this is the fruit. It grows compact, and the fruit generally in clusters like bunches of nuts."

Pearmain, scarlet.-Colour yellow; form as the last; size medium; quality first-rate. In use from September till January. Tree hardy and a good bearer. Synonyms-Bell's scarlet pearmain, Oxford peach-apple.

Pearmain, Rushock-Colour deep yellow, almost entirely covered with cinnamon-coloured russet; form conical, very handsome; size rather under medium. In use from December to April. Quality first-rate. First brought into public notice by Mr B. Maund, and figured by him in his interesting work on "Flowers and Fruits." This excellent apple is of Worcestershire origin, raised about the year 1821 .

Pearmain, Hubbard's. - Colour, generally covered with pale-brown russet, yellowish green where shaded, and brownish red where most exposed to the sun. In hot seasons the russety ground disappears, and then the yellowish green becomes predominant, mottled with orange or pale red next the sun. Form more ovate than is general in this section; size small. In use from November to April. Quality one of the richest flavoured of dessert apples. Tree hardy and healthy, although of slender growth, and an excellent bearer. Requires a warm situation in Scotland, and in many places is well deserving a place on an east or west wall. It is a real Norfolk apple, and largely grown for the Norwich market.

Pearmain, royal.-Colour dark green while on the tree, changing after gathering, first to a greenish yellow, and afterwards to a clear crimson strewed with russety specks; form pearmain-shaped, having a very prominent rib on one side; size large; in use from November to March; tree vigorous, hardy, and an abundant bearer. A very old English apple, and must not be confounded with the summer pearmain, which in some nurseries is sold for this. Herefordshire pearmain of some.

* "Much doubt has existed," Mr Hogg remarks, " as to the origin of this word, and in a communication to "The Gardeners' Chronicle "for 1848, I there stated what I conceived to be its meaning. The early forms in which it was written were Pearemaine and Peare-maine. In some early historical works of the same period " (it should be understood that the pearmain has been in cultivation in Norfolk since the year 1200) " I have seen Charlemagne written Charlemaine, the last portion of the word having the same termination as pearemaine. Now, Charlemagne being derived from Carolus magnus, there is every probability that pearemaine is derived from Pyrus magnus. The signification, therefore, of pearmain is the Great pear-apple - in allusion, no doubt, to the varieties known by that name bearing a resemblance to the form of a pear." 
Pearson's plate.-Colour yellowish green and red; form oblate ; size under medium ; quality first-rate. In use from November till April. Tree hardy. A very handsome and excellent dessert apple, less cultivated than it deserves.

Pennington's seedling.-Colour green, changing to yellowish green, with many large russety spots on the shaded side; rough brownish russet, with a slight tinge of brown on the side exposed to the sun; size medium; form oblato-ovate. In use from November to March; quality a dessert apple of the highest order.

Pine-apple russet. - Colour greenish yellow, with numerous white specks on one part, and rough thick yellowish russet on the other; form roundish ovate, with obtuse angles; size medium. In use during September and October; quality excellent, and abounding in juice more than most others. Tree hardy, and a good bearer. Synonym-Hardingham's russet.

Pomme grise.-Colour green in the shade, covered with thick scaly russet, deep orange on the side next the sun; form roundish; size small. In use from October to February ; quality first-rate. Of Canadian origin, but has been long introduced into England. Tree rather a slender grower, but an abundant bearer. Synonym-Grey apple.

Ravelston pippin.-Colour greenish yellow, nearly covered with red streaks, and strewed with russety dots; form roundish, somewhat irregular, in consequence of the prominent obtuse ribs; size medium. In use during August. Quality in Scotland deemed excellent as a dessert fruit, and is so much prized as to be in general grown against a wall. In the better climate of England, and in competition with so many superior fruits, it sinks into a second or third rate scale. It is, however, a valuable table apple for the colder parts of Scotland, where the finer kinds will not succeed.

Rzinette, Franche-Colour greenish yellow, changing as it ripens to pale yellow, always tinged with carmine red when fully exposed to the sun; form roundish oblate, slightly angular on the sides; size above medium; in use from November to April ; quality first-rate. Tree a free grower and an abundant bearer; requires a dry light soil and warm situation. A very old French variety, and there esteemed as much over most other varieties as the Ribston or golden pippin is with us. Synonym-French reinette.

Reinette, golden.-Colour red and yellow; form oblate; size medium; quality first-rate. In use from October till January. Tree hardy, and an excellent bearer, requiring a light and warm soil ; fruit also handsome, and of great excellence. This variety is much prized on the Continent, and flourishes under the following synonyms in Britain - Wyker pippin, Kirk's golden reinette, Dundee, Yellow German reinette, Megginch favourite.

Reinette grise. - Colour yellow russet; form oblate; size medium; quality first-rate. In use from November till March; of French origin; hardy, and a good bearer.

Ribston pippin.-Colour greenish yellow and red; form roundish; size medium; quality firstVOI. II. rate. In use from November till March; originated at Ribston Hall, Yorkshire. Tree hardy; good bearer; requires a good, rich, deep soil: in light poor soils it is apt to canker, and the fruit to become spotted. Its excellence claims for it a place in every garden. Synonyms-Glory of York, Travers's apple, Formosa pippin.

Russet, Powell's.-Colour pale-brown russet upon a greenish-yellow ground, which is rarely visible unless shaded closely by a leaf, tinged with brown on the sunny side; form roundish, flattened somewhat at the base; size small. In use from November to February; quality firstrate. Tree slender, but an abundant bearer, ripening as a standard in the Dalkeith gardens.

Russet, Boston.-Colour russet; form roundish; size medium; quality first-rate. In use from December till April. This excellent apple is of American origin, a native of Massachusetts, and is one of the most popular market fruits of the country. It is a prodigious bearer, and in every way highly deserving extensive cultivation. Synonyms-Boxbury russet, Shippen's russet of some. It succeeds well in Britain, and deserves to be more generally known.

Russet, keeping.-Colour pale yellowish-brown russet, with a bright fiery red cheek on the side most exposed to the sun; form roundish; size medium. In use from October to January, or even March; quality of the highest rank. This is another excellent apple brought into notice by Mr Hogg, who remarks, in " British Pomology," "that it is an apple which is very little known, and does not seem at all to be in general cultivation."

Russet, rosemary.-Colour yellow, tinged with green on the shaded side, pale red on the side exposed to the sun, slightly covered with palebrown russet; form ovate; size below medium. In use from December to February; quality of the very first class.

Russet, Syke House.-Colour russet; form oblate; size under medium; quality first-rate. In use from November till March. Tree slender grower, a free bearer, and in every way deserving extensive cultivation. It ripens well on dwarf standards at Dalkeith.

Sam Young.-Colour russet; form oblate; size under medium; quality first-rate. In use from November till March. Originated at Kilkenny in Ireland, hence the synonym Irish russet. Well adapted to the climate of most of Scotland.

Straat.-Colour greenish yellow ; form roundish; size medium; quality excellent. In use from November till May. This fine apple somewhat resembles the Newtown pippin, but is apt to canker in cold situations. Synonym-Straat of Floy and Kenrick, American pomologists. An apple in high esteem among the Dutch settlers in Albany, where the original tree grew in a street (stroat, Dutch), and hence the name.

Thorle, summer.-Colour pale yellow on one side, bright red on the other; form oblate; size rather under medium; quality first-rate, the flesh being very white and delicate. In use during August and September. This excellent hardy fruit succeeds well in most ordinary situations as a standard; is improved when grown as an espalier, with a southern exposure; one tree may 
with advantage be planted against a wall in a large garden. It is known also as Watson's Nonsuch, Whorl pippin.

Wanstall.-Colour deep golden yellow on the shaded side, red striped, and mottled with darker red on the side next the sun ; form roundish, and tapering a little towards the apex; size medium. In use till May and June. Tree hardy, and an excellent bearer; quality firstrate, being equal in flavour to the Ribston pippin; originated with a tailor at Sittingbourne, in Kent, somewhere about 1810.

American apples of high merit not much cultivated in Britain :-

American summer pearmain.-Colour yellow, covered with patches of light russety brown, and slightly streaked with red; form oblong; size medium; quality very tender, and rich flavoured. In use during September, and fit for either the kitchen or dessert. Tree healthy, great bearer, and succeeds well on light soils.

Baldwin.-Colour yellow in the shade, striped with crimson, red, and orange, where exposed to the sun ; form roundish ; size large ; quality first-rate. In use from November till March, but is in highest perfection in January. "The Baldwin," says Mr Downing, "stands at the head of all New England apples, and is unquestionably a first-rate fruit in all respects. It is more largely cultivated for the Boston market than any other, and bears most abundantly, while in flavour and general characteristics it evidently belongs to the same family as the American Esopus Spitzenberg, and deserves a like popularity.

Belle-Fleur, white. - Colour pale yellowish white, marked with small brown dots; form pearmain-shaped; size above medium ; quality good. In use from October till April. This is one of the most widely disseminated and popular apples in the Western States; grows strong, and bears abundantly. According to Downing, identical with the Cumberland Spiceof-Coxe, which may be considered as one of its many synonyms - always a good sign of a valuable fruit - to which the following may be added -White bellflower, Green bellflower, Detroit, White Detroit, Monstrous bellflower of Coxe; Ohio favourite, Hollow-cored pippin of some.

Chandler.-Colour greenish yellow, streaked and overspread with dull-red; form roundish; size large; quality good. In use from November till March. Tree of moderate growth, and a great bearer. A great favourite in Connecticut.

Early strawberry apple. - Colour yellowish white, striped with bright and dark red; form roundish; size rather under medium; quality excellent, and described by Downing, in "Fruits and Fruit Trees of America," as being amongst the very finest of their apples. In use at New York, in the vicinity of which it originated, in July.

Fall pippin.-Colour yellowish green, becoming a fine yellow when fully ripe, with often a tinge of brownish blush on one side; form roundish ; size large; quality first-rate. In use from October till December. On the authority of $\mathrm{Mr}$ Downing, this is said to be a veritable
American apple, although thought otherwise by Dr Lindley and Mr R. Thomson, who make it syuonymous with Reinette Blanche d'Espagne. The former authority observes, "It is very probably a seedling raised in this country (America) from the white Spanish reinette or the Holland pippin, both of which it so much resembles, and from which it in fact differs most strongly in the season of maturity." It is considered the first of autumn apples in the middle States, where its beauty, large size, and delicious flavour for the table, or for cooking, render it very popular. Unlike the majority of American apples, this comes to great perfection in Britain ; a first-rate culinary or dessert fruit.

Jonathan.-Colour light yellow in the shade, nearly covered with red stripes, deepening into dark red where exposed to the sun ; form roundish ovate; size medium; quality excellent. In use from November till March. This is a fine apple, of great beauty and good flavour; grows vigorously, and is very productive.

Mouse apple.-Colour dull green, becoming yellowish when fully ripe; form roundish oblong; size large ; quality excellent. In use from November till March. This is one of the most popular American winter apples, considered by some superior to the Rhode Island greening, and deserves extensive trial everywhere.

Northern spy.-Colour yellowish in the shade, nearly covered with rich dark red, marked with crimson and purplish streaks where exposed to the sun ; form conical, much ribbed ; size large ; quality excellent. In use from December till May. This is comparatively a new American fruit, of the Spitzenberg family, and has of late years attracted a good deal of notice. Tree hardy, and bears well.

Peach pond sueet.-Colour light red, somewhat striped ; form somewhat flat, and slightly one-sided and angular in shape; size medium; quality excellent. In use during September and November.

Peck's pleasant.-Colour green when first gathered, turning, when ripe, a beautiful clear yellow, with bright blush on the sunny side; form roundish, somewhat angular; size above medium; quality first-rate. In use from November till April. This excellent fruit belongs to the Newtown pippin class, which is sufficient recommendation to it.

Porter.-Colour bright yellow on the shaded side, with a dull blush next the sun ; form oblong, narrowing towards the eye ; size large; quality good. In use during September. This is a great favourite in the Boston market. The fruit is remarkable for its beauty, and the tree for its productiveness.

Pumpkin russet. - Colour pale yellowish green, slightly covered with russet; form round; size large ; quality excellent, considered as one of the most valuable of the large sweet apples of New England. In use from September till February.

Russet, Putman. - Colour yellow, blotched with russet; form flattened at the ends; size above medium; quality excellent. In use in March and April. It is considered decidedly the most valuable keeping apple in the West, 
not inferior to the Newtown pippin. Tree healthy, luxuriant, and a good bearer.

Swaar.-Colour greenish yellow, turning to dead gold when ripe, with many distinct brown spots, and slightly marbled with russet on the sunny side; form roundish ; size large; quality first-rate. In use from December till March. A truly noble American fruit. According to Downing, " produced by the Dutch settlers on the Hudson," and named Swaar, meaning heavy, on account of its unusual weight; requires a deep rich sandy loam to bring it to perfection. It is one of the finest-flavoured apples in America. Does not succeed in damp or cold soils.

Sweeting, ladies'.-Colour red in the sun, pale yellow in the shade; form roundish ovate; size large ; quality first-rate. In use from December till May. Mr Downing considers this the finest winter sweet apple for the dessert yet known or cultivated in America. Its handsome appearance, sprightly flavour, and the long time it remains in perfection, render it universally admired. No garden should be without it. Tree healthy, and bears abundantly.

\section{KITCHEN APPLES.}

Alfreston.-Colour greyish yellow; form roundish ; large. In use from November till April ; quality first-rate. This excellent apple is of English origin, and often called Lord G'xyder's Newtown pippin,Oldacre's new, Shepherd's pippin, Baltimore and Newtown pippin. Such names must, therefore, be regarded as mere synonyms. One of the largest and best culinary apples; tree hardy; strong grower, and abundant bearer.

Astrakan white-Colour pale yellow; form conical; medium size. In use during August and September; quality first-rate ; of Russian origin. An excellent apple, and with the following well adapted even to the climate of Scotland:-

Astrukan red.-Differing merely in colour from the last, and possessing all its merits. Colour bright red. These two excellent apples, although arranged under the head of kitchen fruit, are, from their beauty, delicacy in flavour, and early ripening, amongst the very best sorts grown for the dessert. We have placed them under the head of kitchen apples, because they are for culinary purposes exceedingly valuable, and being very great bearers, although of short duration, will be found a valuable addition in most gardens to our summer fruits. Neither are cultivated so extensively as they ought to be.

Bain's.-Colour striped; form oblate; medium size; adapted to both table and culinary use; quality second-rate, nearly approaching the Ribston pippin.

Beaufin, Norfolk.-Colour very dark red; form oblate; medium size; quality first-rate for culinary purposes ; grown very extensively in Norfolk for the purpose of drying, in which state they are much prized during winter; keep in their natural state in fine condition from January till June. We have rarely seen this fine fruit growing in Scotland, and from the state of our own trees at Dalkeith, think them rather tender for our northern climate. In the southern counties of England it is a remarkably abundant bearer. Like most other good apples, it is known in various parts under various names-viz., Read's baker, Catshead, Catshead beaufin.

Beauty of Kent.-Colour deep yellow, slightly tinged with green, slightly marked with red on the sunny side, almost entirely covered with deep red on the side next the sun; form roundish ovate; size large; in use from October till February. When well grown, this is perhaps the most magnificent apple in cultivation. Tree strong, and a vigorous grower, and excellent bearer, subject, however, to canker when wrought on the paradise stock, and when planted in moist and heavy soils ; of Kentish origin, not probably of older date than the beginning of the present century. Synonym-Kentish pippin.

Bedfordshire foundling.-Colour yellow; form roundish oblate; large size; quality first-rate; in use from November till March. This very handsome and excellent apple is of Bedfordshire origin, and in many English nursery catalogues is called the Cambridge pippin; hardy, and a good bearer.

Belle bonne.-Colour pale greenish yellow, marked with reddish streaks on the sunny side; form ovato-conical ; size above medium. In use from October till January. Tree very hardy; strong and vigorous grower, and abundant bearer; quality most valuable for culinary purposes. This very old English variety was known to Parkinson in 1629 , and also to Ray and Worledge, although, according to Hogg, " not noticed by any subsequent author, or enumerated in any of the nursery catalogues of the last century, until discovered by Mr G. Lindley growing in a garden at Gatton, near Norwich."

Bess Pool.-Colour yellow, slightly shaded with red on the shaded side; almost entirely washed and striped with clear red on the side exposed to the sun; form conical; size above medium. In use from November till March. Qualityexcellent either for desscrt or for culinary purposes. Tree hardy, and a vigorous grower, and abundant bearer. This variety has the valuable property of flowering late in spring, and hence often escaping our late spring frosts.

Bland's jubilee.-Colour dull yellow, tinged with green, changing to yellow as it ripens, and marked all over the surface with large russety dots ; form round, slightly ribbed; size large. In use from October till January. Quality excellent either for culinary purposes or the dessert. Synonym-Jubilee pippin, from the circumstance of the seed from which it sprang being sown on the day of the jubilee of George III., in 1809. The first fruit was produced in 1818. Mr Hogg remarks : "It is a variety which is not met with in general cultivation, but deserves to be more extensively known." The only notice we have met with of this excellent apple is in vol. v. p. 400 of the Horticultural Society's Transactions, and in Hogg's "British Pomology."

Brabant bellefleur.-Colour greenish yellow, changing to lemon as it attains ripeness, slightly striped with red on the side next the sun; form roundish ovate, inclining to oblong or conical; size large. In use from November to April. Quality of the finest for culinary purposes. 
Much grown in Brabant, and introduced to this country by the Messrs Booth of Hamburg. Tree hardy, middle-sized, and an excellent bearer. Synonyms-Glory of Flanders, Iron apple.

Blenheim pippin.-Colour yellow ; form roundish ; size large ; quality excellent both for table and kitchen use. In use from November till February. Of English origin, as the name implies, where it is found to be an excellent bearer and most valuable market fruit, both on account of its showy appearance and excellent quality. Synonyms-Northwick pippin, Blenheim orange, Woodstock pippin.

Buckland, Devonshire.-Colour pale yellow ; form oblate; medium size. In use from December till March. Quality first-rate, and an excellent bearer. Known in the west of England as Dredge's white lily, Buckland lily, White lily. Rather delicate for the climate of Scotland.

Burn's seedling. - Colour yellow, slightly marked with russet, and red on the side next the sun; form roundish, flattened at the base, sometimes inclining to conical; size medium. In use from October to January. Quality excellent for culinary purposes. Originated with Mr Burn at Tottenham Park, Wiltshire.

Calville Blanche d'Ete.-Colour pale yellow; form like all the Calvilles, having the angles or ribs of the fruit particularly prominent; size above medium. In use during August and September. An excellent kitchen apple, and most extensively cultivated in France. Rather delicate for our Scottish climate, although in good situations we have seen it arrive at great perfection. The White Calville, in our British fruitcatalogues, is the same fruit. A very old French variety, mentioned in the "Jardinier Français" so early as 1653. Distinguished from almost all other apples by the large size of its foliage, being often $4 \frac{1}{2}$ inches long, and $3 \frac{1}{4}$ broad.

Calville Blanche d'Hiver.-Very similar to the last-differing, however, from it, as its name denotes, by being a winter instead of a summer fruit. In use from January till April. The Calvilles are all of French origin, and extend in varieties to about twenty described sorts, inclusive of synonyms. The Calville form is peculiar to the whole section, and the majority of them are of a showy red colour, and the whole possess a rather delicate consistency and agreeable flavour. Of these the Calville malingre is the best bearer, and, with the Calville Normande, is in use from January till April ; while the Calville rouge d'été and Calville rouge precoce are in use during July and August. An excellent bearer. Tree liable to canker in damp situations. Well adapted for dwarf standards, and, when wrought upon the paradise stock, the appearance of the fruit is much improved.

Cambusnethan pippin.-Colour yellowish red ; form oblate; medium size. In use from October till January. Quality first-rate, good bearer, and exceedingly hardy. Originated in the parish of Cambusnethan, near Wishaw, Lanarkshire. Its synonyms are - Winter red streak, Watch apple.

Cat's-head.-Colour pale green; form oblong; above medium size. In use from October till January. Quality first-rate. Tree hardy, and a moderate bearer. In England it is known as the Costard and Coustard apple.

Chalmers's Allan Bank seedling.-This is a native of Scotland; form roundish; size and quality first-rate. Tree hardy, and a good bearer. It is stated in Messrs Lawson's descriptive list as a dessert fruit. We think it, however, better adapted for culinary purposes, and have found it esteemed by the confectioners. In use from December till May.

Cillini.-Colour greenish red; form oblate; above medium size. In use from October till December. An excellent apple, much resembling the Nonsuch, from which it is probable it originated, in the pomological establishment of $\mathrm{Mr}$ Leonard Phillips, of Vauxball, who about 1814-20 paid great attention to the culture of apples.

Cobham.-Colour greenish red; form roundish ; above medium size. In use from November till January. An excellent kitchen and table fruit, somewhat allied to the Ribston pippin. An excellent apple, and very hardy. Of English origin.

Cockpit.-Colour yellow; form roundish ; about medium size. In use from December to March. Quality first-rate. A Yorkshire apple, very hardy, and an excellent bearer.

Codlin, Keswick. - Colour greenish yellow; form conical; about the medium size. In use during August and September. A Cumberland apple of great merit, being very hardy ; a very great bearer. Tree of moderate growth, being fit for use almost before any other. The codlins are all good kitchen apples; but with the exception of the winter codlin, which keeps till February, are of short duration. This latter is an excellent, bearer, and by far too little cultivated.

Codlin, Carlisle.-Colour pale yellow, slightly specked with russet; form ovate, irregular, and angular at the sides; size above medium. In use from August to December. Quality of the first order as a culinary fruit. Tree very hardy, and a most abundant bearer. As it is of dwarfish growth, it is well suited for small gardens. Mr Hogg says, "It is a dwarf variety of the old English codlin."

Codlin, Manx. - Colour pale yellow ; form conical ; quality first-rate. In use during August and October. A very hardy variety, and great bearer, without attaining the size of a large tree. Supposed to have originated in the Isle of Man. Synonyms-Irish codlin, Irish pitcher, Eve apple of Scotland, Frith pippin.

Dumelow's seedling. - Colour yellowish red; form roundish; quality first-rate. In use from November to March. Less liable to shrivel by keeping than most others, and also retains its flavour to the last. Synonyms-Wellington, Duke of Wellington, Normanton wonder.

De eighteen ounces.-Size very large; quality good; form roundish ; colour greenish yellow ; a remarkably handsome fruit and good bearer. In use during December and January.

Devonshire quarrenden.-Colour deep purplish red, except where it is accidentally covered with a leaf, and then it is of a delicate yellowish 
green; form oblate ; size rather below medium. In use in August and September. Quality valueable as an early dessert apple, and also for culinary purposes. Tree hardy, attaining rather more than medium size, and a most abundant bearer, well adapted to cottage gardens and market purposes. Mr Hogg remarks of it, that " it succeeds well in almost every soil and situation, and is admirably adapted for orchard planting. In almost every latitude in Great Britain, from Devonshire to the Moray Firth, I have observed it in perfect health and luxuriance, producing an abundance of well-ripened fruit, which, though not so large nor so early in the northern parts, still possessed the same richness of flavour as in the south." Synonyms - Sack apple, Red quarrenden.

Dickson's empereur.-Size large ; quality good ; form oblong; colour pale green; tree hardy and productive; fit for both culinary and dessert purposes. In use from October to the end of January.

Dutch mignonne.-Colour yellowish red; form roundish; quality first-rate. In use from December till April. Of foreign origin, probably the north of Germany. Tree of moderate growth, and an abundant bearer. SynonymStettin pippin.

Easter pippin. - Colour green ; form roundish; size under medium; quality first-rate ; an abundant bearer. Tree very robust while young, and seldom productive until they have attained ten or twelve years of age. Its greatest merit is its property of keeping better than almost any other apple. We have had them in excellent condition the second year, by being merely kept in a pit similar to potatoes. It does not appear, from what we have seen, to be adapted to the climate of Scotland. Its synonyms areClaremont pippin, French crab, Ironstone pippin, Young's long keeping, Winter greening.

Ecclenville seedling.-An Irish apple of great merit. It originated at a place of that name about fifteen years ago. Tree healthy. Fruit large, somewhat resembling the Hawthornden. Keeps till January, and in high estimation as a kitchen fruit. Ripens well as a standard in the Dalkeith gardens.

Emperor Alexander.-Colour greenish yellow, with a few faint streaks of red on the shaded side, bright crimson upon an orange ground on the side exposed to the sun; form ovate; size above medium. In use from September to December. Tree hardy, making strong wood, and a good bearer. Quality excellent for culinary purposes; its great beauty and size render it valuable for the market. Of Russian origin ; and although brought into notice in 1817 by the late $\mathrm{Mr}$ Lee of Hammersmith, is supposed to have been introduced many years before, as there can be little doubt of its being identical with the Phonix apple figured by Brookshaw in 1808. Synonyms-Russian emperor, Aporta.

Fill-basket (Hogg).-Colour pale dull greenish yellow on the shaded side, streaked with patches and pencilings of pale red on the sunny side, the whole covered with russety dots; form conical, round at the base, flattened at the top, and angular at the sides; size medium. In use from October to January. Quality excellent for culinary purposes. Tree hardy, and a great bearer. It is much cultivated in the vicinity of Lancaster, and is consequently hardy in constitution. To distinguish this from the Kentish fill-basket, it has been suggested to denominate our present subject Lancashire fill-basket.

Flower of Kent.-Colour greenish yellow, with many green dots on the shaded side ; dull red, marked with streaks of brighter red, and dotted with light grey dots on the side exposed to the sun; form roundish, considerably flattened, with obtuse angles extending into the eye where they form knots on the apex; size large. In use from November to January. Quality, a culinary fruit of the highest order. Tree strong in growth, and hence better adapted for orchard culture than for private gardens. It is a showy fruit, and a good bearer. One of our oldest English varieties, being mentioned by Parkinson.

Forge.-Colour of a fine golden yellow, mottled with crimson on the shaded side; dark red, marked with patches of deep crimson on the side exposed to the sun; form roundish, slightly ribbed; size medium. In use from October to January. Quality excellent, either for the dessert, kitchen, or cider. Tree hardy, healthy, and quite free from canker and disease, and withal an abundant bearer. Mr Hogg has recently brought this excellent apple into notice, and expresses surprise that so beautiful " a fruit should have hitherto escaped the notice of pomologists, it being so universally grown, and generally popular in the district to which it belongs. In the north-eastern parts of Sussex and the adjoining county of Surrey it is extensively cultivated ; and I believe," he says, "there is scarcely a cottager's garden where it is not to be met with, it being considered to supply all the qualifications that a valuable apple is supposed to possess."

Gilliflower. - Colour streaked with yellow; form roundish ; quality second-rate; size medium. In use from October till February.

Gloria mundi.-Colour pale yellow; form roundish; quality first-rate; size very large. In use from November till January. This most valuable apple deserves more general cultivation. It appears to be of American origin, but on this Mr Hogg has thrown some doubts, and hence the synonyms - Baltimore, American gloria mundi, New York gloria mundi, American mammoth. The English synonyms areGlazenwood gloria mundi, Monstrous pippin, $O x$ apple, Josephine.

Glory of England.-Another valuable culinary apple brought into notice by $\mathrm{Mr}$ Hogg, who describes it as follows: "Fruit large, $3 \frac{1}{2}$ inches wide, and over $2 \frac{1}{2}$ inches high ; ovate, somewhat of the shape of the Emperor Alexander; ribbed on the sides, and terminated at the eye by a number of puckered-like knobs; skin dull greenish yellow, with numerous whitish specks, particularly round the eye, and covered with large dark russety dots, and linear marks of russet; but on the side exposed to the sun it is of a deeper yellow, with a few broken streaks and dots of crimson. Eye small and slightly closed, 
set in a shallow and puckered basin. Stalk short and fleshy, inserted in a wide, deep, and russety cavity ; flesh greenish yellow ; tender, soft, juicy, sprightly, and slightly perfumed. An excellent culinary apple, in use from October to January."

Golden knob.-Colour pale green, becoming yellowish green as it attains ripeness, covered with russet round the base, and on the shaded side yellow, marked with reddish streaks and many crimson dots on the side next the sun, the whole covered with russet freckles ; form roundish ovate; size below medium. In use from December to March. Quality excellent for orchardists, on account of the healthiness of the trees and the abundance of their crops. It is largely grown in Kent for the London markets. It is also well adapted to cottage gardens.

Golden noble,-Colour yellow; form round ; size large ; quality first-rate. Very handsome in appearance. In use in September and Decem. ber. A moderu variety of Norfolk origin.

Gooseberry.-Colour deep green, with a brownish tinge where exposed to the sun, marked profusely with minute russety dots ; form roundish-ovate; size very large. In use from October to January. Quality as a culinary fruit surpassed by none. Most extensively cultivated in Kent for the London market, which is a good criterion of its merits. This is not the apple which is described under the same name in the "Pyrus Malus Brentfordensis."

Green tiffing. - Colour at first pale green, changing to yellowish green on the shaded side; quite yellow, profusely marked with minute russety dots on the sunny side; form conical, somewhat angular, and ribbed at the sides; size medium. In use from September to December. Tree hardy, and an excellent bearer. Extensively grown in Lancashire, where it is held in high repute. Synonym-Mage's Johnny.

Greennup's pippin.-Colour pale straw-colour, tinged with green where shaded, beautiful bright red, and marked with several patches of delicate russet on the side exposed to the sun; form roundish, broadest at the base, having a prominent rib on one side extending from the base to the apex; size above medium. In use from October to December; quality excellent either for kitchen or dessert. Tree healthy and hardy, and an abundant bearer. Originated at Keswick, and made known about fifty years since. According to Mr Hogg, to whose pomological zeal we are indebted for the publication of both this and the last fine apples, "it is much cultivated throughout the Border counties, and is a valuable fruit where the more choice varieties do not attain perfection." The same authority states that, "when grown against a wall, as it sometimes is in the north of England and Border counties, the fruit attains a large size, and is particularly handsome and beautiful."

Harvey's Wiltshire defiance. - Colour deep sulphur-yellow, even on the shaded side; deepercoloured, profusely covered with minute russety dots, with occasional patches of russet on the sunny one; form conical, having five prominent acute angles descending from the eye to the base; size very large. In use from October to
January. Quality first-rate; a handsome and desirable apple. Mr Hogg, to whom we are indebted for a knowledge of it, says, "It is comparatively little known, but it is well deserving theattention of thefruit-gardener and orchardist; to the latter particularly so, as its size, fine appearance, and handsome shape, make it attractive at market, and its solid and weighty flesh gives it an advantage over many apples of its size."

Hauthornden.-Colour greenish yellow where shaded, beautifully tinged with bright pink where fully exposed to the sun, particularly if grown against a wall or espalier; form roundish; size large, frequently 15 inches round; quality firstrate. In use during October, November, and December. An abundant and early bearer. Succeeds best in a strong clayey soil; in light soils apt to canker and die away. The remains of the original tree still exist at Hawthornden, a few miles from Edinburgh. Synonyms White Hauthornden, Red Hawthornden. It resembles somewhat the Maiden's blush of America, which latter fruit is, however, of higher flavour and much greater beauty. The Hawthornden, like its excellent rival the Ribston pippin, is very subject, as we have stated, to canker. Mr Errington, in remarking on this, observes that both "will frequently become renewed if grafted on old stocks, which, although not successful with other sorts, have notwithstanding a sound and clear stem. We have a Hawthornden branch of considerable size, which was some years since grafted on the lower part of a French crab (a remarkably healthy kind of apple), and which is totally free from canker, and produces abundantly every year."

New Hawthornden.-A seedling from the original apple of this name; superior to it in its present state, and probably very similar to it when in its earliest stages of existence. The fruit of the new sort is not only larger, but keeps better, and the tree is more rubust, and less liable to canker.

Hoary morning.-Colourstriated; form roundish; size large ; quality excellent. In use from October till March. Tree hardy, and a good bearer. The synonyms are-Downy, Sam Rawlings, Dainty apple.

Holland pippin. - Colour greenish yellow with a slight tinge of light brown, and marked with large green dots ; form roundish and flattened, with ribs on the sides; size large. In use from November to March. Quality first-rate. Tree vigorous, healthy, and a good bearer. Originated in the Holland district of Lincolnshire, hence its name. Synonyms-Pie pippin, and Summer pippin of the Americans.

Hollandburg.-Colour red and yellow ; form roundish; size large; quality good. In use from November till January. Tree hardy; a good bearer, producing large and handsome fruit. The English synonyms are-Kirk's scarlet admirable, Horsley pippin, Haxberry pippin; while in France it is cultivated under the name of Bonne rouge, he beau rouge.

Hunthouse.-Colour pale yellow; form oblong; size medium; quality good. In use from December till March. Of Yorkshire origin. Tree hardy and a great bearer, never attaining a large size; 
branches slender and rather pendulous; succeeds in situations where many other sorts would not grow.

Hutton square.-Colour dull greenish yellow where shaded, and strewed with very small russet dots; where exposed to the sun it is of a dull red, dotted with black spots; form roundish; ovate outline very irregular; size large. In use from November till March. Quality first-rate for culinary purposes, and very passable as a dessert fruit. Said to have originated at the village of Hutton, near Lancaster, where it is extensively grown. This is another valuable addition to our list of apples brought into notice by $\mathrm{Mr} \mathrm{Hogg.}$

Jansen Van Welter.--Size large; quality good; an excellent bearer, and keeps in excellent condition both for the dessert and culinary uses from December to June.

Kentish fill-basket.-Colour yellow, green, and brown; form roundish; size large; quality firstrate. In use from November till January. A valuable Kentish fruit, and deserving of general cultivation. Its synonyms are-Kentish pippin (which, by the way, is a quite different apple), Lady de Gray's, Potter's large. Hogg remarks, " This is not the Kentish fill-basket of Miller or Forsyth, nor yet of Rogers, the variety described under this name by these authors being evidently the Kentish codlin."

Kentish pippin.-Colour pink, yellow, and brown; form conical; size medium. In use from October till January. Altogether an excellent fruit. This is also frequently called Kentish fill-basket, Beauty of Kert. Along with the last, deserving a place in most gardens. Tree hardy, healthy, and a good bearer. This is a very old variety, having been cultivated in the Lowdon nurseries prior to 1670 . Synonym-Red Kentish pippin.

Lady's finger.-Colour dull greenish yellow, interspersed with small russety dots, slightly tinged on the side next the sun with dull blush, with numerous spots of deep red; form pyramidal, distinctly five-sided, somewhat flattened at the eye, where it is terminated in five prominent knobs, with a smaller one between each ; size below medium. In use from November to April. Quality excellent ; tree hardy and a good bearer. The white Paradise pippin, often called the lady's finger, is a very different fruit.

Leadington monstrous. - Colour green; form oblong; size very large; quality first-rate. In use froin October till January. A hardy tree, and well suited to the climate of Scotland. Synonym-Green codlin.

Lemon pippin.-Colour yellowish green; form oval ; size medium; quality first-rate. In use from October till April. Tree hardy and a good bearer, and suitable to the climate of most of Scotland. Synonyms-Kirk's lemon pippin; Quince of Rogers. A variety of great antiquity, and noticed by most of the early writers on pomology.

London pippin.--Colour yellowish; form oblate; size medium; quality first-rate. In use from November till April. Tree hardy and an excellent bearer; keeps well, and is not liable to shrivel. An esteemed apple in Covent Garden market, which is a sufficient recommendation. Known also as Royal Somerset, Neu London pippin, Fine crown pippin, White pippin. Well known in Solnelsetshire prior to 1580.

Lucomb's seedling.-Colour pale greenish yellow on the shaded side, bright red on the side next the sun; form roundish and angular; size large; quality first-rate. In use from October till February. Tree of strong and vigorous growth, and a good and early bearer. Originated in the nursery of Lucomb and Pince of Exeter.

Melrose.-Colour pale yellow, tinged with green on the shaded side, yellow tinged with orange, and dotted over with crimson spots, where exposed to the sun; form roundish ovate, inclining to conical, but very irregular in outline; size large. In use from October to January. Tree healthy, hardy, and an abundant and free bearer. Tọ Mr Hogg, who has in his work on "British Pomology" brought forward many excellent apples which were previously little known, we are indebted for a knowledge of this excellent fruit. It appears to have been long cultivated in the Border counties, and to have been almost confined to them. It is, however, doubtful if it be of Scotch origin, and may probably have been introduced by the monks of Melrose. Mr Hogg considers it to be "without doubt the largest and one of the most useful apples of which Scotland can boast, and requires only to be more generally known to be cultivated throughout the length and breadth of that country." Synonym-White Melrose.

Minchall crab.-Colour yellow; form oblate; size medium; quality good. In use from December till February. Tree very hardy and an excellent bearer; of Lancasterian origin. Synonym -Lancaster crab. Extremely well suited to the climate of Scotland.

Mitchelson's seedling.-Colour deep yellow, slightly mottled with carnine on a delicate russet ground on the side exposed to the sun; form somewhat ovate; size above medium. In use from December to February. Originated by a market-gardener formerly of Kingston-uponThames. Valuable either for the table or culinary purposes.

Velson.-Colour greenish yellow on the shaded side, deep yellow, interspersed with largeish dark spots, often encircled with a fine crimson ring on the side exposed to the sun; form conical; size large. In use from September to January. Tree strong and of vigorous growth, very hardy, and a most abundant bearer; quality first-rate for culinary purposes. Of Yorkshire origin, in which country it is a great fevourite. Synonyms-Backhouse's Lord Nelson, Nelson codlin.

Nonsuch.-Colour greenish brown; form oblate; size medium; quality first-rate. In use in September, and if well ripened will keep till November. Tree moderate in growth, hardy, and an excellent bearer.

Nonsuch, round winter.-Colour striated; form round; size large; quality first-rate. In use from November till March. Tree hardy, not subject to canker, moderate in growth, an excellent bearer.

Northern greening. - Colour green; form oval; size medium; quality first-rate. In use from 
November till April. Tree hardy, good bearer ; keeps well, without shrivelling; called by some Cowarne queening, by others John apple, and others Walmer Court. "We have," says $\mathrm{Mr}$ Errington (writing, be it observed, from a cold part of Cheshire), "no apple in cultivation superior to this for kitchen purposes. A hardy and healthy tree, a good bearer, the fruit of a good size, and remaining in use from Norember till May. It is a capital kind for the hedgerow also-that is, to be planted in hedgerows between fields. We are inclined to think that it loves a humid atmosphere, for it is extensively cultivated in our northern counties, more especially Lancashire and Cheshire. We wish some of our friends would try it in the West Highlands, where such an addition would be a great acquisition."

Pearmain, autumn.-Colour yellow and red form pearmain-shaped; size medium; quality good. In use during September and October. Synonyms - American pearmain, Royal pearmain of some, Summer pearmain of others. Flesh yellow, with a peculiar rich flavour. Tree hardy, and a good bearer.

Pearmain, Baxter's.-Colour yellow and red; form pearmain-shaped; size medium; quality good. In use from December till March. Tree hardy, and an excellent bearer. Probably a seedling from the Herefordshire pearmain, to which it bears a pretty close resemblance.

Pearmain, Hormead.-Colour uniform clear yellow, slightly dotted with brown russety spots; form of the regular pearmain shape; size medium. In use from October to March. Quality first-rate for culinary purposes, and fit for the dessert also. Tree hardy and a good bearer; of Sussex origin. Synonyms-Hormead pippin, Arundel pearmain.

Pearmain, winter.-Colour greenish yellow; while growing, slightly marked with red streaks on the sunny side, very deep red on the side exposed to the sun. After keeping some time, the shaded side changes to deep yellow, streaked with flesh colour, and the sunny side to a deep red or crimson; form true pearmain-shaped; size large. In use from December to the end of April. Tree healthy, hardy, and an abundant bearer. One of the oldest English apples on record, having been cultivated in Norfolk since 1200. Synonyms-Old pearmain, Great pearmain.

Pomme, St Germain.-Size medium ; quality excellent; form oblong; colour pale yellow; tree healthy, and an excellent bearer. In use for the kitchen during November and December.

Pope's apple.-Colour clear yellow, tinged with greenish patches, interspersed with dark dots, slightly marked with streaks of crimson on the side exposed to the sun; form ovate; size large. In use from November to March. Quality valuable, either for the dessert or for culinary purposes. This apple is extensively grown for the London markets in some parts of Kent, of which county it appears to be a native. According to Hogg, "this apple has all the properties of the Bleuheim pippin, and is much superior to it, keeps longer, and has the great advantage of being an early and abundant bearer." It appears to be little known out of the district where it originated.

Queening, crimson.-Colour red; form Calville-shaped; size medium. In use from September to November. A much-esteemed Herefordshire fruit. Synonyms - Red gueening, Summer queening, Scarlet queening, Herefordshire queening. Tree hardy, and a good bearer. We have not observed this variety in Scotland.

Queening, winter.-Colour red; form conical; size medium; quality good. In use from December to March. Somewhat resembling the Cornish gilliflower both in appearance and flavour. Probably of foreign origin, as it is much cultivated and esteemed abroad.

Red streak, Keeping.-Colour striped red and green; form roundish; medium size ; quality good. In use from December to April. Tree hardy; a good bearer; very handsome fruit.

Reinette, Blanche d'Espagne. - Colour palegreenish white, slightly tinged with red; form roundish ovate; size very large; quality, both for dessert and kitchen, first-rate. In use from November to March. It is difficult to trace the history of this apple-from its synonyms, it would appear to be either of Spanish or American origin. In the former country it is known as the Reinette d'Espagne, and in the latter as Fall pippin, Large fall pippin, and in England often as Cobbett's fall pippin, having been introduced to Britain by that extraordinary man. It is evidently too tender for most of Scotland, but thrives well in the soutl of England, cankering only when planted in too cold situations.

Reinette de Canada.-Colour brown on one side, yellowish green on the other; form flat conical; size very large; quality, either for the dessert or kitchen, first-rate. In use from November to April. It is stated by $\mathrm{Mr} \mathrm{R}$. Thomson, of the London Horticultural Society's Gardens, as being " a good bearer, and, though large, of excellent quality even as a dessert fruit. Is probably the best apple of its size, and surpassed by few of those that are smaller. It therefore deserves extensive cultivation." Of American origin, and somewhat hardier than the last; still would require a wall in most parts of Scotland and the north of England.

Reinette, Franche Grauwe-Colour brownish russet; form roundish; size medium; quality first-rate. In use from December to April. Of French origin, therefore requiring a favourable situation in this country.

Reinette, royal.-Colour striped with yellow and red; form conical ; size large; quality good both for dessert and culinary purposes. In use from December till April. By no means a delicate tree, and well suited to most parts of Scotland and the north of England, in both of which it has proved an excellent bearer.

Rostocker. - Size large ; quality excellent; form oblate ; colour red. In form and colour somewhat resembling the old Norfolk beaufin; very showy ; an excellent bearer. In use for culinary purposes from November to June.

Russet, golden.-Colour russet; form ovate; size medium; quality, both as a dessert and kitchen apple, first-rate. In use from December 
till March. Tree rather tender, and deserving a good situation, even in the south of England. T'his has been by some called Golden mundi.

Russet, Pile's.-Colour dull green, covered with pale-brown russet, interspersed with greyish-white dots on the shaded side; dull olive mixed with orange, intermixed with dots of darkish russet on the side next the sun; size medium. In use from October to March. Form roundish oblate; quality excellent. Tree healthy and vigorous, and an excellent bearer.

Russet, royal.-Colour, as the name of the section implies, russety; form conical ; size large ; quality first-rate. In use from November till May. Tree hardy, and an excellent bearer. Synonym-Leathercoat.

Rymer.-Colour pale green and red; form roundish; size large; quality first-rate. In use from December till April. Tree hardy, and an excellent bearer. Synonyms-Newbold's Tuke of York, Nexbold's Admiral Duncan, Calduell, Green gossings. A valuable apple, too little cultivated.

Sack and sugar.-Colour yellow ; form roundish ; sizeunder medium; quality good. In use during August. Tree hardy, and an excellent bearer.

Stone pippin, Norfolk. - Colour pale green and yellow; form oblong; size medium; quality good, either for kitchen or table. In use from November till July. Tree hardy, and an abundant bearer, and valued for its long keeping. Synonyms-White stone pippin, Winter stone pippin, White pippin. Often confounded with the Gogar pippin, which is a superior table-fruit.

Stonyroyd pippin. - Colour yellow ; form roundish; size medium; quality first-rate. In use from January till April. Tree hardy, and a good bearer.

Striped beaufin.-Colour bright green, very much marked with streaks and patches of deep red, and thickly strewed with russety dots; form roundish, somewhat depressed ; size very large. In use from October till May. Tree very hardy, and a great bearer; of Norfolk origin. Appeared prior to 1794, and latterly brought into notice by Mr Hogg.

Strumer pippin.-Colour greenish yellow and brown; form conical; size medium; quality first-rate. In use from February till June.

Sugar-loaf pippin.-Colour green; form oblong; size medium; quality first-rate. In use in August; one of our best early apples. Tree hardy, and a good bearer. Known also as Hutching's seedling.

Superintendant.-Size large; quality good; an abundant bearer, and one of the best culinary apples in cultivation. In use during November and January.

Tarvey codlin.-Colour olive green, slightly yellowish on the shaded side; yellowish red, spotted with large red dots, next the sun; form conical; size large. In use during November and December. Quality good for a cold climate, which is our principal reason for introducing it here. It appears to be a seedling raised by the late Sir G. S. M'Kenzie of Coul, in Ross-shire, from a seed of Manx codlin, impregnated with some variety of nonpareil.

Tower of Glammis.-Colour greenish yellow; VOL. II. form conical ; size large ; quality first-rate. In use from November till February. A Scotch apple of very great merit. Synonyms-Late Carse of Gourie, Glammis Castle.

T'riomphante.-Size above medium; quality excellent; form oblong; colour red; an excellent bearer, and very beautiful fruit. In use for the dessert or kitchen during November and January.

True yellow Settin.-Size large; quality excellent; form somewhat flattened; colour yellowish; a great bearer, and fit for the kitchen or dessert from December to June.

Wadhurst pippin.-Colour yellowish brown, marked with red; form roundish; size large; quality first-rate for culinary purposes. In use from October till February. 'I'ree hardy, and a good bearer.

Waltham Abbey seedling.-Colour yellow ; form roundish; size large; quality first-rate for culinary purposes. In use from September till January. Tree hardy, and an excellent bearer ; like the last, of English origin. Synonym-Dr Harvey.

Warner's king.-Size very large; quality excellent; in excellent condition for culinary purposes from December to April.

Winter majetin. - Colour greenish brown striped with red; form roundish; size large; quality first-rate. In use from January to May. T'ree hardy, and an excellent bearer.

Wood's greening.-Colour green; form conical; size medium; quality good. In use from January to May. Tree hardy, and a moderate bearer; esteemed for its long keeping.

Wormsley pippin.-Colour pale green; form roundish; size medium; quality, for the table or culinary purposes, first-rate. In use during September and October. Tree hardy, and an excellent bearer. Known also as Knight's codlin.

Yorkshire greening.-Colour dark green; form oblate; size large; quality first-rate. In use from October till February. One of our most esteemed English apples. Tree hardy, and, when wrought on a paradise stock, inclined to spread horizontally near the ground.

\section{APPLES FOR ORNAMENT OR PRESERVING.}

Lady apple.-Colour-ground colour at first greenish, turning, when ripe, to light lemoncolour on one side, with brilliant deep red on the other; form round and flattened at both extremities; size very small; quality, valuable as an ornament to the dessert through winter and spring, and to the orchardist on account of the high price obtained for it in Covent Garden market, which is supplied largely with this fruit from France and America, where it is now abundantly grown. In use from December till May. This beautiful little apple is of French origin, but sufficiently hardy to ripen its fruit upon ordinary stone walls and espaliers in Scotland. An abundant bearer, producing its fruit in bunches; tree subject to canker in cold soils. Its synonyms are-Api Petit, Pomme d'Api, Pomme Rose. Well adapted for pot culture when wrought on the paradise stock. One of the oldest apples in cultivation, and is asserted to have been brought from the Peloponnesus to 
Rome by Appius Claudius. Supposed to have been introduced to Britain towards the end of the seventeenth century; ripens on a stone wall at Drumlanrig Castle and elsewhere in Scotland.

Lady apple, black.-Differ's from the former only in colour, which is nearly black; hence the synonym, Api noir. Cultivated on account of its extraordinary colour, contrasting oddly with the last. Tree hardy, slender in growth; ripens at Drummond Castle and other parts of Scotland.

Siberian crab, scarlet.-Colour scarlet, over a clear yellow ground; form regular, somewhat flattened at the ends; size about three-fourths of an inch in diameter; stalk about 2 inches long, very slender; tree very hardy, and an abundant bearer. Highly ormamental when planted in shrubberies, \&c. Fit for preserving in September and October. Its fruit, when ripe, has at a little distance much the appearance of cherries.

Siberian crab, yellow.-Very similar to the last in all respects excepting in colour. Fit for use in September and October.

Siberian crab, large scarlet. - Fruit about double the size of the two former; colour paler red on a yellow ground. Fit for use in September. The Siberian crabs are much esteemed for preserving and for making jelly. " No garden should be without them.

The Norfolk beafin is largely cultivated for drying 'and confectionary purposes; it is, however, noticed elsewhere.

\section{SELECT LISTS OF APPLES FOR PECULIAR} LOCALITIES

A pples of fine quality suited to a cold northern climate in America.- "Fameuse, Canada Reinette, Pomme de Neige, Rhode Island greening, Boston russet, Porter, Baldwin, Swaar, Red Astrachan, Ladies' sweeting, Northern spy, Golden ball."-Fruits and Fruit-Trees of America. 'This list affords some data as to the hardiness of some of the best American sorts.

Apples of excellent quality for a small garden in Britain, where twelve trees are only required, the climate being moderately good. - Keswick codlin, Hawthornden, Ribston pippin, Blenheim pippin, King of pippins, Strumer pippin, Downton nonpareil, Reinette du Canada, Waltham Abbey seedling, Herefordshire pearmain, Bedfordshire foundling, Wormsley pippin.

A similar list of twelve sorts, where the climate is bad or indifferent. - Keswick codlin, Paradise pippin, Tower of Glammis, Oslin pippin, Red Astrachan, Cockpit, Coul Blush, Cambusnethan pippin, Manx codlin, Melrose, Spice apple, Annat seedling.

List of asples on walls at Dalkeitli.-American Newtown pippin, Court-pendu Plat, Scarlet nonpareil, Court of Wick, Hughes' golden pippin, Kirk's golden pippin, Nonpareil old, Nonpareil (Braddick's), Nonpareil Downton, Golden pippin old, Reinette du Canada, Reinette Grisse, Martin nonpareil, Flat nonpareil.

List of apples at Dalkeith on dwarf standards, chiefly on paradise stocks, that have fruited in the new gardens, and seem suitable to the locality.Alexander, Alfreston, Ashmead's kernel, Astrachan white, Astrachan red, Beachamwell, Beauty of Kent, Brabant belle fleur, Blenheim pippin,
Cambusnethan pippin, Cellini, Cobham, Cockpit, Keswick codlin, Cockle pippin, Manx codlin, Cornish aromatic, Court of Wick, Scarlet Crofton, White Crofton, Devonshire Quarrenden, Doonside, Duchess of Oldenburg, Dumelow's seedling, Dutch mignonne, Early harvest, Elton pippin, Fearn's pippin, Golden Harvey, Golden noble, Hawthornden (soon cankers in our light soil), Hollandbury, Hunthouse, Yellow Ingestrie, Kentish pippin, Kerry pippin, King of pippins, Lemon pippin, Margil, Nonsuch, Northern greening, Oslin, Paradise pippin, Claygate pearmain, Lamb Abbey pearmain, Scarlet pearmain, Golden reinette, Golden russet, Royal russet (has died of canker), Skyehouse russet, Rymer, Sam Young, Waltham Abbey seedling, Wormsley pippin, Ribston pippin, seldom attaining its full size, Wykin pippin,'Thorl pippin, Summer golden pippin, Downton pippin, Early nonpareil, Yorkshire greening, Red Ingestrie, Court-pendu Plat, Kentish fill-basket, Adam's pearmain, Braddick's nonpareil, Scarlet nonpareil, Hughes' golden pippin, Ross nonpareil, Royal pearmain, Pile's russet, Scarlet golden pippin, Summer pearmain, Sweeney nompareil.

Select list of apples suitable to the north of Scotland, and other cold and exposed climates.-Hawthornden, Yorkshire greening, Tower of Glammis, Oslin, Red Margaret, Keswick codlin, Devonshire Quarrenden, Nonsuch, Winter strawberry, Winter greening, Coul blush, Carlisle codlin, Grey Leadington, Gogar pippin, Kerry pippin, Summer Leadington, Summer strawberry, Summer queening. It must, however, be taken into consideration, that latitude alone does not prevent the ripening of many other finer fruits. Altitude and shelter have also much to do in the case; and hence along the coast, where protected from the sea spray and boisterous winds, there are many situations in Argyllshire, Aberdeenshire, \&c., where the apples of the south of England will ripen in full perfection. The misfortune is, they have in few instances been tried. There appears to be a sort of stereotyped feeling amongst fruit-growers too generally to rest content with those sorts that have been grown in their locality for ages, the majority of which, compared with many of far more recent date, are nearly as useless as the uncultivated crab; and acres are allowed to be cumbered with trees whose fruit is not half as valuable as the pasture would be under their branches. In many gardens the walls are covered, the quarters shaded, and the crops ruined, by trees long past the age of profitable production, and of sorts now considered by the best pomologists of the day absolutely worthless. Were fruit trees an expensive article to purchase, some excuse might be found for this ; but when we consider that trees of the finest kinds in cultivation can be purchased for one shilling a-piece, this excuse ceases to hold good.

Without allowing what may be supposed a spirit of nationality to influence our reason, we must state that many of the districts north of the Forth or the Tay are better adapted for the cultivation of the finer kinds of apples than a great extent of the Border counties on both sides of the "marches." Peaches and apricots 
ripen better at Dunkeld in Perthshire, and even at Brahan Castle in Ross-shire, than they do in many gardens in Cumberland and Northumberland, although several degrees farther south. If this is the case as regards these exotic fruits, surely it holds equally strong in regard to apples, pears, \&c., which are next to indigenous to our climate.

Mr Hogg, who has paid greater attention to the Border fruits than any other pomologist of equal intelligence, and who, himself a native of those districts, has had ample opportunity of drawing fair conclusions, gives, in his excellent work on British Pomology, the following list for the Border counties of England and Scotland, and the warm and sheltered situations in other parts of Scotland: 1st, Summer and Autumn apples fit for the dessert. - "Blenheim pippin, Cambusnethan pippin, Devonshire quarrenden, Greennup's pippin, Grey Leadington, Irish peach, Kerry pippin, Margaret, Oslin, Ravelston pippin, Red Ingestrie, Summer pearmain, Summer strawberry, T'am Montgomrie, White paradise, Whorle, Wormsley pippin, Yellow Ingestrie." For kitchen purposes.- "Carlisle codlin, Dutch codlin, Early Julian, Hawthornden, Hill's seedling, Melrose, Keswick codlin, Manx codlin, Spring Grove codlin, Tarvey codlin." Winter apples for same localities, (those marked * require a wall).-Dessert sorts - "Balmanno pippin, ${ }^{*}$ Barcelona pearmain, *Braddick's nonpareil, Baxter's pearmain, Bogmiln favourite, Contin reinette, ${ }^{*}$ Court of Wick, Doonside, Gogar pippin, *Golden pippin, *Margil, Green longlast, *Nonpareil, *Pearson's plate, Pitmaston nonpareil, Pow captain, *Ribston pippin, ${ }^{*}$ Scarlet nonpareil, *Sturmer pippin." Kitchen winter-apples. - "Bedfordshire foundling, Brabant belle fleur, Dumelow's seedling, Green virgin, Pile's russet, Red Fulwood, Royal russet, Rymer, Sir Walter Blackett's favourite, Tower of Glammis, Waltham Abbey seedling, Winter strawberry, White Fulwood." All the apples in our foregoing lists are known to succeed in the southern parts of the island; particular selections, therefore, need not be made. Our wish is to direct the attention of cultivators to the necessity of grubbing up useless kinds, casting aside all associations of antiquity, and substituting those that are more valuable, and equally adapted to their climate.

The following is a list of approved sorts suited for espaliers or dwarfs, succeeding well when grafted on the paradise or doucin stock, and adapted to the northern parts of Scotland, and other exposed situations, given by $\mathrm{Mr} \mathrm{Hogg}$ : "Adam's pearmain, Aslimead's kernel, Barcelona pearmain, Boston russet, Braddick's nonpareil, Breedon pippin, Bringewood pippin, Christie's pippin, Claygate pearmain, Coe's golden drop, Cornish gilliflower, Court of Wick, Court-pendu Plat, Downton pippin, Dutch mignonne, Early harvest, Early nonpareil, Franklin's golden pippin, Golden Harvey, Golden pippin, Golden reinette, Hawthornden, Hubbard's pearmain, Joanneting, Kerry pippin, Keswick codlin, Manx codlin, Margaret, Margil, Nonpareil, Oslin, Padley's pippin, Pearson's plate, Robin. son's pippin, Scarlet pearmain, Sturmer pippin, Summer golden pippin, Summer pearmain,
Taunton golden pippin, Wyken pippin." In how few gardens, in the situations referred to, do we find even a tithe of these excellent sorts cultivated, while the walls are covered, and the quarters crowded with trees of unnecessary size and age, not only unproductive, but were they even otherwise, the crops produced are of an inferior quality.

List of apples rather delicate to ripen generally in Britain, and which $\mathrm{Mr}$ Rivers proposes to grow in his orchard-house: Green Newtown pippin, Yellow Newtown pippin, Northern spy, Melon apple, and the Male Carle, a favourite Italian apple of great delicacy and beauty, the other being American apples of the highest character. It is to us, however, questionable whether a glass structure might not be more usefully employed than in growing fruits which we can import from their native country in the fullest perfection, and at infinitely less expense and trouble.

Select list of apples which ripen at Culzean Castle, Ayrshire.-Alexander, Blenheim pippin, Court of Wick, Codlin white, Duke of Wellington, Early Julien, Eve apple, French reinette, Ganges pippin, Gravenstein pippin, Hawthornden, Kerry pippin, King of pippins, Margil, Nonpareil scarlet, Ribston pippin, Royal russet, Wareham russet, Stirling Castle, Spitzenberg, Yorkshire greening, Wormsley pippin, Hughes' golden pippin, Reinette Dorée, London pippin, Early Margaret, Summer queening, Ord's apple, Herefordshire pearmain, Paradise pippin.

Select list of apples which ripen at Drumlanrig

Castle, Dumfriesshire, (S. and W. indicate Standard or Wall).-Alexander, S.; Alfreston, S.; Astrachan white, S. ; Blenheim pippin, S. ; Cambusnethan pippin, S.; Codlin Keswick, S.; Codlin Manx, S.; Downton, S.; Dumelow's seedling, S.; Golden pippin, W.; Golden cluster, S.; Scarlet golden pippin, S.; Gravenstein, W.; Hawthornden, S., does best in strong soil, cankers and dies off in light soil; Kerry pippin, W.; King of pippins, S., one of our hardiest fruits, resisting spring frosts; Melville pippin, S.; Nonpareil, several varieties, W.; Nonpareil scarlet, W.; Oslin, S.; Paradise pippin, S.; Pearmain Lamb Abbey, S.; Pencaitland pippin, S.; Quarrenden Devonshire, S.; Ribston pippin, W.; Russet royal, W.; Pomme d'Api, or Lady apple, W.

The following apples are all excellent, and may be given as a list suitable for a garden in the southern counties of England, to be grown as espaliers or dwarf standards, arranged in the order of their ripening :-

Dessert sorts.-Early harvest, Early red Margaret, Summer golden pippin, Kerry pippin; Wormsley pippin, King of the pippins, Wyker pippin, Claygate pearmain, Maclean's favourite, Court of Wick, Ribston pippin, Pitmaston nonpareil, Hughes' golden pippin, Golden Harvey, Braddick's nonpareil, Herefordshire pearmain, Court-pendu Plat, Reinette du Canada, Downton nonpareil, Scarlet nonpareil, Sturmer pippin.

Culinary sorts.-Keswick codlin, Dutch codlin, Nonsuch, Hawthornden, Waltham Abbey seedling, Blenheim pippin, Bedfordshire foundling, Dumelow's seedling, Alfreston, Gloria mundi, Northern greening, Brabant belle fleur, Royal russet, Norfolk beaufin, Easter pippin. 
Mr Thomas Rivers, in his excellent pamphlet on the culture of "Pyramidal Fruit Trees," gives the following list of twenty-four dessert, and the same number of kitchen apples, ripening from July to June the following year, placed in the order of their ripening:-

Dessert apples.- "Juneating White, Early red Margaret, Red Astrachan, Early harvest, Irish peach, Kerry pippin, Pine-apple russet, Margil, Ribston pippin, Golden reinette, Maclean's favourite, Golden drop (Coe's), Ashmead's kernel, Nonpareil (old), Reinette Van Mons, Newtown pippin (yellow), Sykehouse russet, Pearson's plate, Reinette du Canada, Golden Harvey, Winter peach-apple, Spring Ribston pippin, Victoria (Hulbert's), Sturmer pippin."

Culinary sorts. - " Hawthornden, Nonsuch, Large yellow bough, New Hawthornden, Selina, Emperor Alexander, Gravenstein, Blenheim pippin, South Carolina pippin, Monstrous Leadington, Wadhurst pippin, Waltham Abbey seedling, Round winter nonsuch, Herefordshire pearmain, Winter Colman, Bedfordshire foundling, Greave's pippin, Dumelow's seedling, New Bess Poole, Pomme royal, Baxter's pearmain, Brabant belle fleur, Rostocker, Gooseberry apple."

\section{LIST OF CIDER APPLES.}

The earlier writers on pomology have left us a scanty list of cider apples; even Mr Knight, in "Treatise on the Apple and Pear, and the Manufacture of Cider and Perry," says, "I do not think Herefordshire so much indebted for its fame as a cider country to any peculiarity in the soil, as to the possession of a few very valuable varieties of fruits." Rogers considerably extends the list of cider apples, and remarks of the following two popular sorts, the Styre and Red-must, that the former produces the best cider from light soils, while the latter yields the best from strong heavy land. Mr Knight was of opinion that the Siberian Harvey and Foxley are superior to all others for the purpase of the press. Mr Lindley, in " Guide to the Orchard," describes several ; and the "Fruit Catalogue" of the London Horticultural Society enumerates even more. The most complete list, however, is that given by $\mathrm{Mr}$ Hogg in "British Pomology," and, we believe, contains all that are worth cultivating for the purpose (the specific gravities we have added to Mr Hogg's list)-viz. :

Alban, Bennet apple (specific gravity of juice, 1073) ; Best bache, Brainton seedling, Brierly's seedling, Bringewood, Bovey red streak, Cadbury, Coccagee, (gravity same as the last) ; Cowarne red, Devonshire red streak, Devonshire wilding, (spec. grav., 1069); Downton pippin, Dymmock red, Forge, (spec. grav., 1080); Forest styre, (spec. grav., from 1076 to 1081); Foxley, (spec. grav., 1080); Fox whelp, (spec. grav., 1076 to 1080) ; Friar, (spec. grav., 1073 ); Garter, (spec. grav., 1066) ; Golden Harvey, (spec. grav., 1085); Golden pippin, Golden Worcester, (spec. grav., 1078); Grange, (spec. grav., 1079) ; Hagloc crab, Hogshead-a fruit of remarkable value, (spec.grav., 1081),-requires a dry calcareous soil to bring the fruit to perfection. So highly is the cider from this apple prized, that sixty guineas have been offered and refused for a hogs- head (about 110 gallons). Isle of Wight pippin, Kingston black, Minchall crab, Monkton, (spec. grav., 1074) ; Pawsan, Red Ingestrie, (spec. grav., 1076); Red-must, (spec. grav., 1064); Red streak, Royal wilding, (spec. grav. 1079); Siberian Bitter sweet, (spec. grav., 1091) ; Siberian Harvey, Sops in wine,(spec. grav., 1091); Steade's kernel,Sweet Lading, Winter Lading, (spec. grav., 1074); Woodcock, Winter pearmain, (spec. grav., 1073) ; Yellow Elliot, Yellow Ingestrie, (spec. grav., 1076.)

Many of our best apples are of French origin. The following key to the pronunciation of their names may be useful :-

Court-Pendu Plat.-Coor pahn-du plah.

Drap d'Or.-Drap dor.

Fenouillet Gris.-Fen-nool-yai gree.

Male Carie.-Mal carle.

Pomme de Neige.-Pum de naije.

Reinette Blanche d'Espagne.-Ren-ett blansh d'Espagn.

Reinette Triomphant.-Ren-ett tre-ome-fant.

\section{DISEASES.}

The diseases to which the apple tree in a natural state is subject are few. In an artificial state they are many: some occasioned by being planted in an improper soil, brought on by injudicious management, late spring-frosts, unsuitable climate, accidental injuries, and the growth upon them of parasitic plants, such as the mistletoe, fungi, various species of lichens, \&c. The first of these are to be removed by pulling the plants out of the branches during winter, and the two latter by washing with caustic lime-water, and painting the old wood over during winter with vegetable spirits of tar. Draining the ground, thinning the branches, and promoting a free circulation of air through the trees, greatly prevents cryptogamic plants from establishing themselves on the trees.

Canker has been variously accounted for ; the chief cause, however, may be stated to be uncongenial soil, cold climate, accidental wounds, arising often from careless pruning; and some have considered it a hereditary disease. $\mathrm{Mr}$ Rivers, who has long studied the cultivation of fruit trees, is inclined to think that planting on mounds, examining the roots annually in autumn, so that they may be carefully pruned, and prevented from penetrating into a bad subsoil, keeping the roots near the surface, and supplying them with fresh soil at their extremities, \&c. will be found a complete remedy. "I am not quite satisfied," he says, "on that head, but am strongly inclined to think that canker may be entirely prevented by this annual attention to the roots, so that if the soil be unfavourable and apt to induce a too vigorous growth in apple trees, followed by canker, the roots should be annually pruned; but if the trees make shoots of only moderate vigour, and are healthy and fruitful, their roots may remain undisturbed; and pinching their roots in summer, and training them in a proper direction, if required, is all that the trees will want."

There is no doubt that canker in fruit trees, if not entirely, is greatly owing to the cold subsoil into which their roots often descend. This was pointed out long ago by Mr Reid of 
Balcarras, Fifeshire, and quoted by Dr Lindley in "Theory of Horticulture," to this effect: "He found that in a cankered orchard the roots of the trees had entered the earth to the depth of 3 feet; and he also ascertained that, during the summer months, the average heat of the soil at 6 inches below the surface was $61^{\circ}$; at 9 inches, $57^{\circ}$; at 18 inches, $50^{\circ}$; and at 3 feet, $44^{\circ}$. He took measures to confine the roots to the soil near the surface, and the consequence was the disappearance of canker and ripening of the fruit." It may be remarked at the same time that too deeply-planted trees, or such as send their roots to too great a depth, are late in their foliation, and seldom bring their fruit to full perfection.

Some varieties are more subject to the attacks of canker than others; and even the same sorts, when planted in different soils, are attacked in one while they escape in others. The Hawthornden apple, for example, is seldom troubled with canker in a strong loamy soil, while in a light sandy one it suffers exceedingly. The larva of Semasia Waberana has been accused of laying the foundation of canker in fullgrown apple trees.

Parasitic fungi of the mildew tribe frequently in dry seasons attack the foliage; but as it appears now to be an established fact that the application of sulphur in a dry powdery state, thrown upon the trees by any of the ingeniously-

Fig. 173.

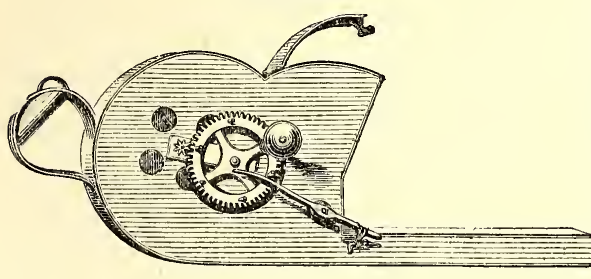

SULPHURATOR.

contrived sulphurating machines-fig. 173, for example-affords a complete remedy, their effects are less to be dreaded.

The evaporating effects of long-continued easterly winds during spring are exceedingly injurious to the young expanding leaves of the apple; frequent syringing, to keep up the necessary humidity, has been resorted to with good effect; as has also protecting the opening buds by covering them with semi-transparent canvass made in form of balloons, enclosing the whole tree, and secured round the stem to prevent the wind tearing them to pieces. The dried haulm of asparagus, the dried fronds of fern, or similar dry and light material, tied to the branches in small handfuls, break the force of the wind, ward off late spring-frosts, and tend greatly to the preservation of the opening leaves and expanding blossom.

Insects injurious to the apple.-Of these the American blight, woolly aphis, or apple bug (Aphis lanigera Linn., the Eriosoma mali of Leach, E. laniger $a$ of others), fig. 174, is amongst the most serious. This insect appears to be indigenous rather to France and the north of
Germany than to America, from which country it has been erroneously supposed to have reached us. The American pomologists repudiate its nativity to their country, and assert that
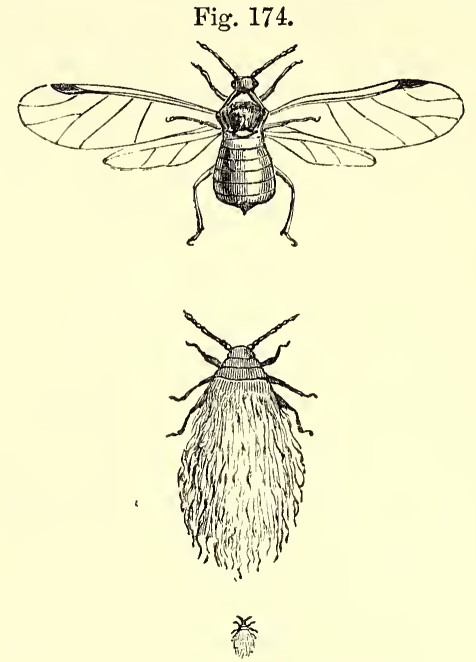

AMERICAN BLIGHT INSECT.

Winged male and wingless female.

it was imported there along with trees from Europe. This insect frequently perforates the stem and branches of the apple, and causes those excrescences which are often seen on branches even of considerable age and size. Externally its existence is readily discovered by the appearance of a fine white down in the crevices of the older branches, and in some nurseries abounding on the wood of one or two years' growth, within which downy covering a great number of minute woolly insects will be found, yielding, when bruised, a reddish matter somewhat similar to cochineal, which is the produce of another species of the same family. During summer this pest may be easily got rid of by washing the parts affected with a solution of sulphuric acid, in the proportions of $\frac{3}{4} \mathrm{oz}$. by measure to $7 \frac{1}{2} \mathrm{oz}$. of water, applying the liquid with a piece of sponge tied to a piece of stick, or by a small soft painter's brush. The first rain that follows will wash the mixture into the most minute crevice of the bark or part affected. In winter these insects quit the trees and bury themselves underground to feed on the roots-thus never ceasing their attacks until the tree is completely killed, which will certainly be the result if they are not subdued. The trees infected should be taken up during winter, their roots washed clean with water, and left exposed for a day or two to the action of cold before replanting; and at that operation a new station should if possible be allotted to the tree, or the soil in which it formerly grew completely removed, and fresh soil substituted. When the trees are so large as to render their lifting difficult, the soil should be removed from them, and that about the roots well saturated with ammoniacal liquor. If the period of 
their descent be closely watched, and the ground under the tree be covered with 2 or 3 inches of soil, and that removed every second or third day, and the operation repeated as long as their descent continues, the greater part of the insects may thus be secured and carried away, and so disposed of that they cannot again return to their former place. Spirit of turpentine, strong whisky, or gin, applied to the patches infested with the insect, is an excellent remedy, and may be applied on its first appearance, and if persevered in will destroy them. Some sorts of apples are exceedingly subject to its attacks, such as the codlins and old juneating; while the russets, so far as our observation goes, are seldom attacked by it. During winter, but taking care that none fall on the buds, spirits of vegetable tar applied will be found of advantage; for although at that period the insects have descended from the branches and attacked the roots, the disagreeable odour of the tar will prevent their returning to the branches in spring. If either of these remedies be applied in a proper manner, this insect will soon cease to appear.

The winter moth, (Geometra brumata of Linn., the Cheimatobia brumata of Stephens), fig. 175,
Fig. 175 .
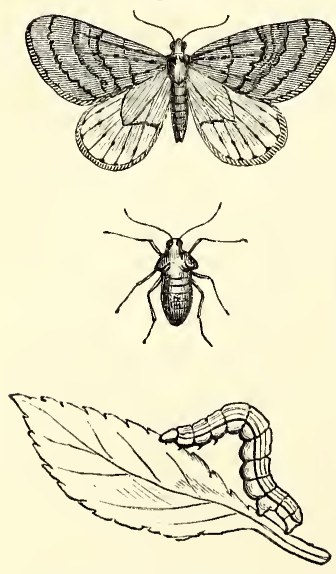

WINTER MOTH.

Caterpillar, winced male, and wingless female. the caterpillar of which is the green looper, is a very destructive enemy to most fruit trees, the apple amongst the rest. This moth makes its appearance in November, hence the name. It proceeds from a lightish-brown pupa which lies buried a few inches under ground from June to the end of $\mathrm{Oc}$ tober. The male is winged; the female has only sliort rudiments of wings, not adapted for flying. The female is found almost stationary on the

stem of the tree, awaiting the male, which may be seen flitting about towards evening, at which time pairing takes place. The female then ascends the tree, and deposits her eggs singly on the buds, and partly on the ripened wood and twigs. The number of eggs deposited by one female amounts to above two hundred, and they are so firmly glued to the bark as to resist being washed off by rain, and so hardy that no frost destroys their vitality. As the warmth of spring advances, and vegetation progresses, the eggs are hatched. The young caterpillar is at first exceedingly small, and hence aeldom seen; but on closely examining the blossom-buds their fine web will be discovered, and as soon as the petals begin to develop, their attacks become more visible, and the gnawed appearance of these indicates the presence of the enemy. Others also at the same time attack the leaf-buds, which, as well as the petals, they glue together in such a way that they are prevented from expanding properly. As the blossom begins to expand, these caterpillars descend and find their way into the receptacle, on which they feed; and when a fruit is formed, they commence their attacks on it, and quit it not until they have nearly devoured it. When they have accomplished the destruction of the fruit, they then attack the tender foliage, and as they increase in size and strength, proceed to the larger leaves, which, if the insects are numerous, they soon strip from the trees. The wood-buds do not escape them; and when they have denuded the tree of its foliage, they bury themselves in the earth, where they repose in a pupa state, as already noticed. Ants are the natural enemy to the caterpillar of this insect, and may be seen devouring them in great numbers. Deep hoeing and disturbing the soil under the trees is a mode of getting rid of these pests, and copious waterings after May around the roots destroy many more, especially if lime-water is employed. Amongst birds who aid us against these enemies, the titmouse is one of the most industrious. Means have been resorted to, such as collars of woollen thread, wool, \&c., dipped in turpentine, oil, tar, \&c., fastened to the stem of the tree, to prevent the ascent of the female in spring, and a band of bird-lime has been found very efficacious in not only preventing their ascent, but in catching them by hundreds. Hot lime, in a very fine state of powder, dusted over the trees, as the caterpillars come into existence, is an excellent remedy.

The mottled umber moth, or lime looper, (Geometra defoliaria of Linnæus, Hybernia defoliaria), fig. 176, although a parasite of

Fig. 176.

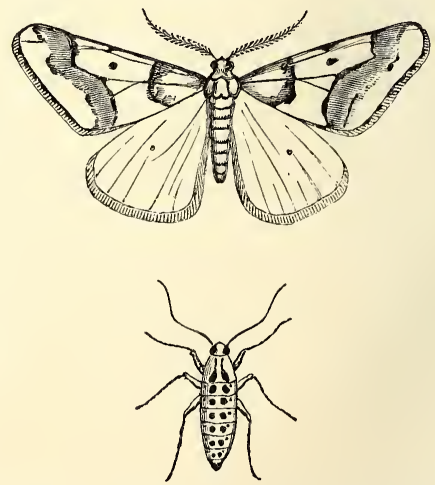

MOTTLED UMBER NOTH. MALE AND FEMALE.

the lime tree, is found on the apple also. In its caterpillar state, it is readily distinguished from the caterpillars of the other loopers by the brimstone-yellow stripe on each 
side of the body, which otherwise is of a reddish colour. In its pupa state, it is of a brownishred colour, and found in the earth from the end of May to the beginning of November. The male has wings, the female none. Although its appearance is not strictly periodical, there are some years when it by no means abounds, while in others it is found in vast numbers, doing much injury to apple trees as well as others. The female moth quits the ground in November, and, being without wings, creeps up the stem of the trees. As soon as pairing has taken place, she ascends to the very top, and lays about two hundred eggs, not in masses, but scattered over the tree. The ant is here again actively employed in their destruction, and sinall birds also play their part; while man must have recourse to the bird-lime bandage already recorded, as well as any of the other precautions noticed above,-and none are better and of easier application than dusting the trees over with finely-powdered hot lime, the finer particles, while dry, finding their way amongst the scales of the buds, and into the embryo flower, and by their caustic property destroying the caterpillar in its young and most tender state, and so preventing it from even commencing its destructive operations.

The caterpillar of the small ermine moth, Tinea (Y Yonomeuta) padella of Hubner, is most destructive to the shoots of the apple tree, and still more so to the hawthorn, whole hedges being entirely denuded of their foliage by it. The female lays from twenty to thirty eggs about the end of June or beginning of July, choosing for a nidus either a blossom or a fruit-bud, and depositing her eggs in a mass, and not singly, as in some other cases. The larvæ are hatched in the autumn, but do not quit the hard gummy substance which the female forms for the protection of her eggs until the following spring, when the caterpillars are found in a mass in the centre of either the fruit or wood buds. They then commence their ravages with an extraordinary degree of voracity, devouring all before them; and by throwing finely-spun threads from their stationary residence, which is by this time completely covered with a very fine but strong network of webbing, over the adjoining shoots, they secure them also for a continuance of food; so that by the middle of June, when they have attained their full size, they have not only devoured all the leaves within their reach, but covered the twigs also with an unsightly webbing. As soon as they are fully grown, and have at that time attained the length of four or five lines, and assumed a dirty-yellowish colour, with a black head, and a longish black spot on the side of each abdominal segment, they draw near together, and each spins for itself a small web, in which it changes to a brownish-yellow pupa, remaining in this state only a few days, when it is transformed into the moth state. From the circumstance of their living in communities, and enveloping themselves in these conspicuous webs, they are easily detected, and destroyed by hundreds.

The codlin moth, Tortrix (Carpocapsa) po- monana of Linnæus, fig. 177 , has become a sad
Fig. 177.
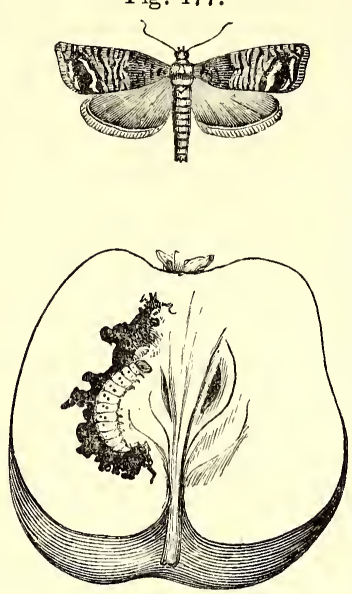

CODLIN HOTH AND GRUB. pest to the American orchardists, and by Downing is said to have been introduced to that land of apples from Europe. It appears in the early wormeaten apples and pears in the form of a reddish-white grub, and causes the fruit to fall prematurely from the tree. It is equally destructive in Europe, and is very gene. rally distributed; perhaps there is no garden in which its appearance has not been recognised. The following description of it is from Kollar's work on insects, translated from the German by J. and M. Loudon : "This moth is to be seen in the evening, usually in the beginning of May, on the apple and pear trees, busily engaged depositing its eggs either on the calyx, or in the hollow part of the fruit at the stalk end. It appears to prefer apples to pears - at least more grubs are found in the former than in the latter, when both sorts of fruit are plentiful. It invariably selects the finer sorts of this fruit, knowing instinctively that they will be the most palatable to its future progeny. In favourable weather the little grubs are hatched in a few days, so that in May apples and pears are found infested by them. At first the grub is white, with a black head and collar, and black slanting double dots, which run in four rows from the head to the abdomen. It afterwards becomes more of a flesh colour, the head and collar turning brown, the dots grey and indistinct. It is fully grown in three or four weeks, as its food never fails. It now leaves the fruit, whether it be still hanging on the tree or has fallen off, and selects for itself a secure place on the stem of the tree to spin its cocoon and become a pupa. It usually chooses the rents and seams of the loose bark, hollows itself out a chamber, and spins a white web over itself, intermixing some of the loose bark with it. The little grub becomes a pupa immediately in the web, and in a few days the moth comes out, which shortly afterwards pairs, and deposits eggs on the fruit. In this way, in July and August, and partly in September, much sound fruit will again be pierced and infected with the caterpillar of this moth, which is then numerous in proportion to the number of eggs of the first generation which were laid and hatched in May. In the year 1822, which was warm and dry, more than the half, particularly of the choice fruit, was grubeaten, and moths were still seen laying their eggs till the end of September. Fortunately 
those caterpillars which are so late in laying their eggs seldom arrive at maturity, as the fruit is taken off the tree before that time." To dininish this insect, the fallen apples, as well as all those on the trees which show symptoms of having grubs in them, should be collected and given to the pigs, or otherwise disposed of. The loose bark should also be yearly removed from the stems and branches of the trees; for under this the moths have deposited many of their eggs, and under it the pupa spins its cocoon from which the young moths emerge in the spring. A good plan with such trees as are badly affected by this insect, is to paint them over, during winter, with spirits of tar. Dusting the tree with powdered hot lime, and burning straw or other refuse, so as to cause a considerable smoke to windward of the trees, will also greatly lessen the number of these insects.

The small bark-beetle, or apple-tree scolytus (Scolytus homorrhous of Megerle), fig. 178,
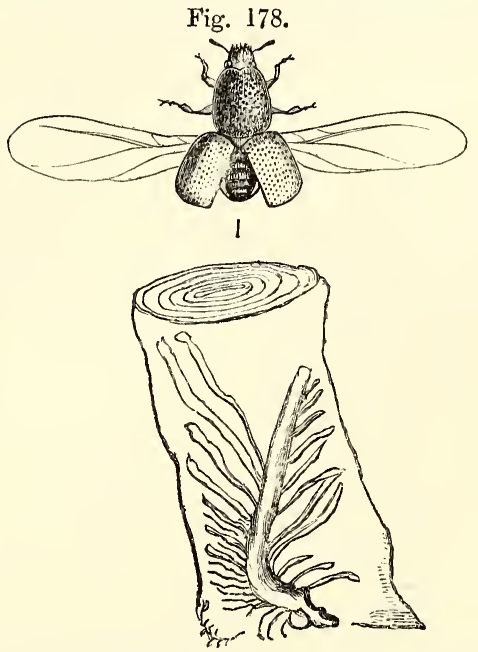

SMALL BARE-BEETLE, AND SECTION OF WOOD SHOWING ITS RAVAGES.

is one of the borers, or those which perforate the bark and often the woody trunks of trees, causing destruction where it abounds. Schmidberger is, however, of opinion, that it only attacks diseased or unhealthy trees; but of this there are doubts. It appears to multiply very fast; and no other remedy has apparently been fallen upon, except cutting down the infested trees, or removing them altogether. Towards the end of May they form numerous furrows in the inner bark, and in each furrow so formed deposit a white egg, which shortly afterwards produces a white larva, which lives upon the bark until the autumn, occasionally feeding upon the sap wood, and leaving the outer bark as a protection from the weather, and to conceal itself from its natural enemies. It is fortunately not very cornmon in Britain.

The apple weevil, Curculio (Anthonomus) pomorum of Fabricius, fig. 179, which, exclusive of the proboscis, is scarcely a line and a half long, is often the cause of the total

loss of the apple-crop. Its wing-cases are of a dark-brown colour, marked with whitish-

Fig. 179.

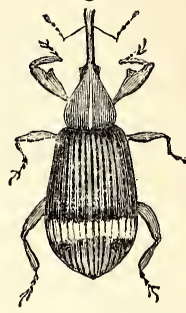

grey stripes; its rostrum, eyes, and the under part of the abdomen are black. It makes its appearance as soon as the blossom-buds are pretty full of sap, and soon after begins to lay its eggs, which, according to the state of the weather, are hatched from the beginning to the end of April. The female bores a hole with her proboscis in the centre of the blossom, and therein deposits her egg, proceeding in the same manner until every blosAPPLE WEEvil. som in the bud she has chosen has been perforated and tenanted by an egg each. She travels from bud to bud until she has laid her complete complement, which, although not correctly known as to numbers, must yet be large, from the devastation committed. The warmer the weather is, the sooner the eggs are hatched; and hence in seasons that are late, and the fine weather setting rapidly in, we find much less injury done to the blossoms than in others when the progress of vegetation is more slow. As soon as the grub or larva is hatched, it commences its attacks on the fructification, and devours it entirely. The appearance of the blossom now is as if it were scorched, and if cut open, a small white grub, with a black head, will be found, which by degrees turns to a yellowish colour, and in a few days afterwards will be found in the perfect beetle state. During summer these beetles remain on the trees, and feed on the foliage, and in autumn ensconce themselves under the loose bark on the stem and larger branches. The application of vegetable spirits of tar to the trunks and branches, during winter, is by far the most effectual way to lessen their numbers, if not totally rid our trees of them.

The oblong weevil, Curculio (Polydrusus) oblongus of Linnæus; Nemoicus oblongus of Stephens, fig. 180, is both a numerous and destruc-
Fig. 180
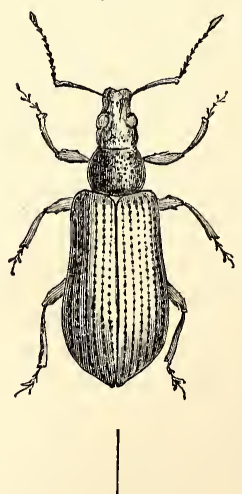

OBLONG WEEVIL. tive insect, in size only two lines long, and rather more than a line in breadth. Its antenuæ and feet are reddish, its ely. tra furrowed, and of a reddish-brown or blackish colour: its body, head, and thorax quite black. It seems to feed on all fruit trees alike, and on the peach and apple in a particular degree, preferring the young and tender leaves to all others. Pairing takes place in spring, and in June the female buries herself in the earth, and lays her eggs there, and the grub, when hatched, lives on the 
roots of plants, remains under ground all winter, and appears in spring in the transformed state of a perfect beetle. They are more injurious to young trees than to old ones, and commit sad havoc often amongst nursery stock. Watering the ground with lime-water, about the roots of the trees, during winter, applying spirits of tar, or ammoniacal gas-water, will do much to lessen their numbers.

Melolontha (Anisoplia) horticola of Fabr., Phyliopertha horticola of Kirby and Stephens, fig. 181 , is a leaf-eating beetle, and is particularly injurious to the apple,

Fig. 181. although it attacks other fruit

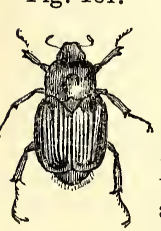
trees also. Its body, head, and thorax are dark green ; its antennæ reddish, with a strong terminal club; the wing-cases reddish brown, somewhat shining, and not extending to the extreme point of the body. They attack the foliage of trees, which they perforate with holes both large and numerous. They inhabit the trees during summer, pair in early autumn, when the female drops to the ground, and deposits her eggs two or three inches under the surface. The larvæ, when hatched, continue in the ground, feeding on the roots of plants, until transformed in spring into beetles, when they

ANISOPLIA Horticola AND again ascend the trees in search
LARVA. be taken for their destruction as recommended for the last.

Aphis pyri mali; the apple aphis or plantlouse.-This extensive genus, generally known as the green-fly, is sufficiently well known. It has been asserted that almost every genus of plants has a species of the family almost peculiar to itself. For mode of destruction, \&c., vide Peach Tree. It is singular that Kollar, in his "Treatise on Insects injurious to Gardens," \&c., should not mention the Aphis larigera, an insect, according to Downing, common in France and Germany, and which he asserts was transported from those countries to America.

The black-veined white butterfly, or hawthorn pontia (Papilio cratcegi Linn.; Pieris cratagi, Stephens and Curtis), fig. 182. This insect, which only flies by day, is thus described by Kollar: "It is of considerable size, and quite white, except that the ribs or veins of the wings, and a short oblique stripe from the second to the third vein of the upper wings, are black. This distinguishes it from the cabbage butterfly, Pontia brassica (vide fig. 44), so destructive in kitchen-gardens, which is also for the most part white, and almost of the same size, having a broadish black border at the tips of the upper wings. Besides this, the under side of the posterior wings of the cabbage butterfly are yellow, and its fore wings have always two black dots on the under side, which are seldom seen on the upper one." It is necessary to mark these distinctions, as the two insects are often confounded together. To this we may also add that the vol. II. caterpillars of the first attack trees, those of the latter the various kinds of Brassica only. Our

Fig. 182.
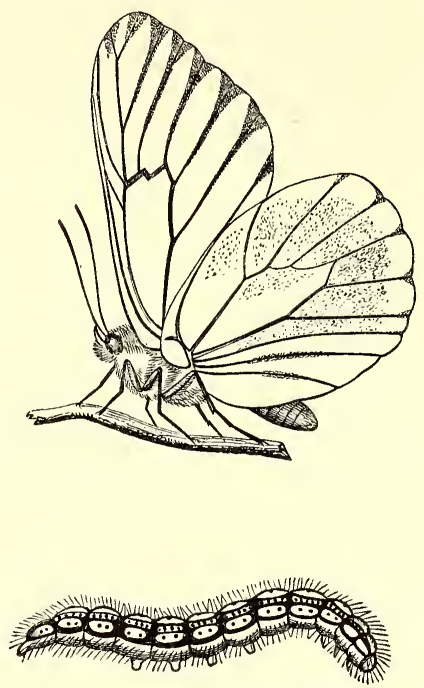

BLACK-VEINED WHITE BUTTERFLY AND CATERPILLAR.

present subject appears in the butterfly state from the end of May till about the middle of June, laying its eggs about the beginning of July, which are of a shining yellow colour, cylindrical, and longitudinally ribbed. They are deposited on the surface of the leaf to about the number of 150. About the 18th of the month the eggs are hatched, and the young caterpillars come forth of a dirty yellow colour, covered with hair. "The head," Kollar remarks, "is black, and there is a black ring round the neck, and a brownish-red stripe on both sides. As soon as it begins to rain, they immediately draw the leaf together over them by means of a web; and on the 21 st of July a covering was ready under which they were quite sheltered during continued rain. In the mean time they enjoyed the food which the epidermis of the leaf afforded, and they gnawed it entirely off, so that nothing remained but the veins." They appear to be impatient of wet, and retire into their nest during rain. Birds, rain, and other insects destroy them to a great extent-indeed, so much so, that it has been computed that rarely 20 or 30 out of a family of 150 survive to occupy their winter abode, which they begin to construct so early in autumn as September, at which time they cease to feed on the trees. They remain in the caterpillar state during winter; and upon the approach of warm weather in spring they quit their winter abode, and commence operations about the beginning of April upon both the young leaves and blossom-buds, devouring the latter first, and afterwards attacking the former.

Bombyx (Liparis) auriflua (Porthesia auriflua), Stephens, fig. 183, the yellow-tailed moth, is one of the most destructive insects infesting 
fruit trees, often prevailing to such an extent Fig. 183.
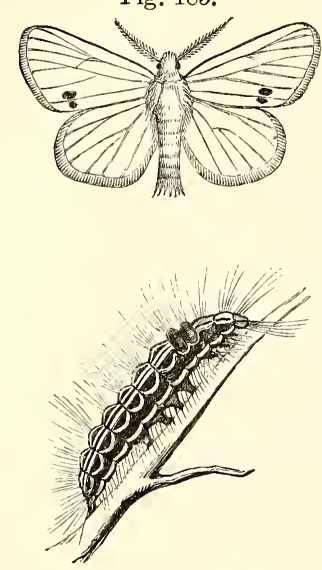

YELLOW-TAILED MOTH AND CATERPILLAR.

that not a leaf or fruit is left uninjured. "The yellowtailed moth," Kollar observes, "is a nocturnal lepidopterous insect, as it only flies about and propagates its species by night. In the daytime it sits quietly on a leaf, or on a wall, and suffers itself to be cauglit in the hand. Its fore wings are of a dazzling whiteness, as is also the greater part of its body ; only the principal vein of the fore wing of the male is brown on the under side, and it has also sometimes a few black

dots on its wings. The male is distinguished from the female by its more slender abdomen, terminating almost in a point, by a smaller tuft of hair on the tail, and by its strongly pectinated antennæ, the rays of which are yellowish brown." This moth usually appears about the beginning of July. The female, in general, lays her eggs on the under surface of the leaf, depositing them in small masses, and covering them with hair from her tail. Each mass of eggs contains from 200 to 300 , and when they are deposited the female dies. About the end of July, according to Kollar, the caterpillars are hatched, when they appear of a dirty yellow, "with a black head, and a black ring round the neck. They are thickly covered with hair, and have four rows of blackish dots along the back. Of these dots, two pairs on the anterior part of the back, and two pairs on the posterior part, are larger than the rest. By these dots the young caterpillars of the yellow-tailed moth are strikingly distinguished from those of the hawthorn butterfly." The caterpillars of our present subject, like those of the last, feed on the epidermis of the leaf, and in like manner devour them, and weave a web over themselves for protection. After devouring the leaf they first attack, they remove to the next, and construct a shelter, within which they can feed at their ease. In August they change their skins, and by the middle of September they cease feeding, and in November become perfectly inactive, and remain so during the most severe winters, being unaffected by $60^{\circ}$ below zero of Fahrenheit. They commence their attacks on the trees early in spring, even before the buds have begun to unfold. About the end of April they change their skins for the second time, and about the end of May they change for the third time, at which period they appear of a reddish brown, marked on the sides with whitish spots. After this change they disperse themselves in all directions, and are not fastidious as to food, for they attack alike the apple, the pear, the plum, the oak, and the beech, \&c. In June, pupation takes place, when several caterpillars unite together, and, rolling leaves together in form of a ball, form for themselves a common web, and become dark-brown pupæ. Kollar says, "They prefer the damson tree for pupation, to which they repair from the neighbouring apple and pear trees, and undergo their change, to the number of from five to twelve in a ball." About the end of June the moths begin to renew their species. Its effects are readily distinguished from those of the winter moth; the former only eating the leaf, whereas the latter devour's both leaf and foot-stalk, leaving the trees perfectly denuded. Heavy rains naturally destroy vast numbers of the females, should those occur during the period of their laying their eggs ; for during the process they sit upon the surface of the leaves completely unprotected, and are consequently washed off. Water thrown on the leaves by a powerful garden-engine will have a similar effect. Birds are less useful to us in the case of this insect than in that of most others, on account of the caterpillars being covered with thick hairs on their backs, which produce a burning and itching sensation when handled without gloves, and hence are supposed to have a sinilar effect on the throat or stomach of birds when they eat them. Its natural enemy, however, is a small hymenopterous fly, which lays its eggs in those of the yellow-tailed moth, making use of the egg for its food, and of the empty shell afterwards for its habitation. Other minute insects, chiefly of the field-bug tribe, devour their eggs, and last of all its insect enemies is the ichneumon, which lays its eggs in the body of the full-grown caterpillar. The nests of eggs are readily seen enveloped in a brownish covering or web, and should be removed and destroyed at any time during winter. While in the pupa state they enclose themselves within small balls, attached generally to the lower branches. and may be in this state captured at the end of June.

Bombyx chrysorrhcea Fabr., Bombyx phceorrhcea Curtis, Porthesia chrysorrhad Stephens (the

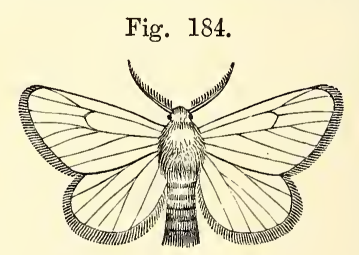
brown - tailed moth), fig. 184 has occasionally been very destructive. In 1782 such were its ravages that Mr W. Curtis wrote a treatise in quarto on it, and prayers were offered up in

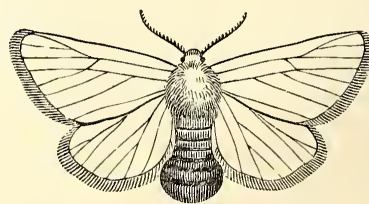

BROWN-TAILED NOTH. Male and female. some churches for deliverance from such a scourge. The poor were employed to collect the webs at 1s. per bushel, and so abundant were they in the parish of Clapham, that 
eighty bushels were collected in one day. It is of almost annual appearance in our apple and pear orchards, and attacks both foliage and blossom. Its habits are very similar to those of the last, and the same means must be resorted to for its destruction. Concerning its effects on fruit trees, for it attacks almost all sorts, Mr Curtis remarks, "The injuries they sustain are most serious, as, in destroying the blossoins as yet in the bud, they also destroy the fruit in embryo. 'The owners of orchards, therefore, have great reason to be alarmed."

Bombyx (Gastropacha) neustria, Clisiocampa neustria of Curtis and Stephens (the lackey, or barred-tree lackey moth), fig. 185, in its cater-

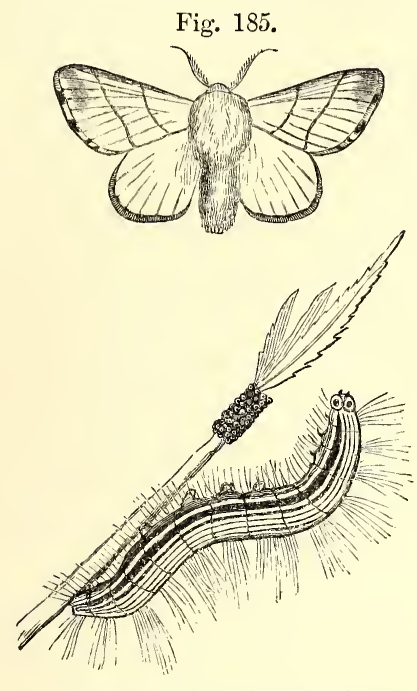

LACKEY MOTH AND CATERPILLAR.

pillar state is one of the greatest insect enemies the cultivator has to contend with. Its attacks are not confined to fruit trees alone; it is equally destructive to almost every deciduous and evergreen tree and shrub. The moth is seldom seen by day, as it conceals itself beneath the leaves, and amongst whatever herbage is near. It is thus described by Kollar: "The moth is of the middle size. The male, which is somewhat smaller than the female, measures with expanded wings, from tip to tip, from 1 inch to 15 lines. The ground colour of the whole insect is either light yellow or reddish-yellow ochre. The upper wings have always a darker band in the middle, which is bordered with two lighter cross lines ; the fringes are whitish and brown spotted. The under wings are always of a uniform colour, either light yellow or brownish, according as one of these colours predominates in the whole insect. The antennæ are strongly pectinated in the male, but less so in the female; the latter has a thicker abdomen." This moth appears in the month of July, and immediately after pairing the female lays her eggs, which she attaches to the young twigs in the form of a ring. The number of eggs laid is from 200 to 350 , of the same colour as the bark of the tree, and so hard as to set both frost and insect enemies at defiance. The caterpillars appear about the first of May. In June they are full grown, and are often an inch in length, slightly covered with hair, having blue, yellow, and red stripes down the sides, and a whitish line down the back. They may often be detected in wet days taking shelter in the forks of the tree, congregated to the number of from 50 to 200, and enclosed under a silky substance or nest. In this state they are readily destroyed. During June and July they may be captured in their cocoons, which may be found attached to the brauches of the trees. During winter the rings of eggs are perceptible after a little practice, and should be scraped off with a knife and burned. The ichneumon fly destroys thousands of them in their caterpillar state, and the beautiful beetles, Calosoma, Inquisitor, and Sycophanta greatly reduce their numbers.

The caterpillars of the gipsy moth, Bombyx (Liparis) dispar Linn., Hypogymna dispar Stephens and Hubner, fig. 186, are readily
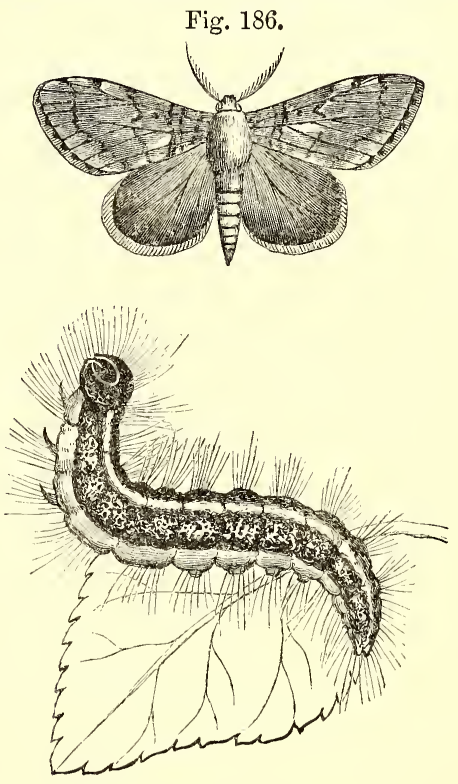

GIPSY MоTн.

Male and larva.

distinguished from those of other insects by their large spotted yellow head, and by the six pair of red dots on the hinder part of the back. The back is thinly covered with hair, and tufts of it appear on the sides of their bodies. The eggs are hatched early in spring, and the caterpillars spread rapidly over the tree, and attack the buds as they begin to open. They attain their full size about the end of June, and soon after form their cocoons, in which they become transformed into their pupa state. In August the moth appears, and towards the end of that month, and during September, the female lays her eggs, depositing them chiefly on the trunks of the apple and other fruit trees, and to the number of from 200 to 500 in a mass, 
covered with a yellowish web, to protect them during winter. The female moth is much larger than the male, and of a whitish grey, having the fore wings marked by brownish stripes. The male is dark brown, with three or four blackish stripes on the fore wings. This is one of the most voracious of caterpillars, particularly destructive to apple and other trees in town gardens. In autumn, as soon as the leaves fall, the trees should be examined, as at that time the masses of eggs may be readily discovered under their yellow covering, which they do not seem to conceal, as they are in general attached to the bare trunk or larger branches. The female may be easily caught in August, sitting on the trunks of the trees; and the caterpillars, towards the end of June, will be found basking on the exposed trunks of the trees, and may easily be destroyed.

The lunar spotted-pinion moth, Noctua (Cosmia) pyralina, appears in June and July. Fore wings brownish red, almost copper-coloured, marked with several dark-brown transverse lines. Under-wings lightish grey, darker towards the edges, which are yellowish. The caterpillars are thick, fleshy, and green, with ten dark lines along the sides, and a white stripe along the back. They make their appearance in April, and continue growing till the end of May, when they become transformed into a bluish-coloured pupa. They are exceedingly destructive to the foliage and flower-buds of the apple, and are often found on other fruit trees also. Both in their moth and caterpillar state they are easily captured, by shaking the tree suddenly, which causes them to drop to the ground. A cloth should be spread under the tree to receive them, for in both states, if not carried off and destroyed, they would reascend and commence their destructive operations again.

The pale brindled beauty - moth, Geometra (Amplidasis) pilosaria, is occasionally found on the apple tree, but more generally on the pear, which see.

The grubs of the Bacchus, or purple-apple weevil, Curculio (Rhynchites) Bacchus Schonh., fig. 187, like those of the codlin moth, come

Fig. 187.
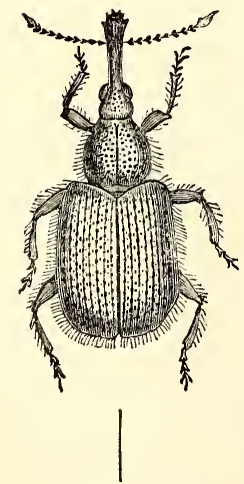

PUR PLF-APPLE WEEVIL. into existence within the fruit of the apple. Kollar thus describes its habits: "It is found early in spring on the fruit trees, and only begins the work of reproduction about midsummer. To effect this, it selects the smooth side of the apple, bores a hole in it with its proboscis, and proceeds to form a roomy chamber in the heart of the fruit. It then lays an egg at the entrance, pushes it in with its proboscis, and closes the opening; at the same time arranging the epidermis partly with its proboscis, and partly with the extremity of its body, so as to cover the hole,

of which, by means of a glutinous moisture which issues from its mouth, it finishes securing the entrance. This operation is no sooner over than it bores a second hole near this chamber, in which to deposit another egg. It sometimes happens that it deposits three or four eggs in one apple before it leaves it for another. The grub, which is whitish, with a black head, is hatched from the egg in the course of a few days, and immediately begins to eat the food that is so near it, making its apartment larger and larger by eating deeper into the apple. It generally advances as far as the core ; and, like the caterpillar of the codlin moth, it makes itself a passage to the surface of the apple, probably to throw out its excrement, or to admit more air. The larva attains its full size in three or four weeks, and resembles the caterpillar of the codlin moth, but is not so much of a flesh colour, although about the same size. It leaves the apple when it is ready for transformation, and hides itself in the earth, from which, in the following spring, it reappears as the Bacchus weevil." Although attacking the fruit of the apple in very much the same way as the codlin moth, it is readily distinguished from it, as its larva, like that of all weevils, is destitute of feet. The trees should be gone over before the fruit is half grown, and every one removed that has the appearance of being perforated. In the beetle state they may be greatly reduced by shaking the trees suddenly, during June and July, when the beetles will fall from the leaves, and may be collected and destroyed, few of them escaping if a canvass cloth be spread under the tree for them to fall upon.

Curculio (Rhynchites) alliaria of Fabricius, although attacking other fruit trees as well as the apple, may be here noticed as a small beetle, scarcely 1 line in breadth, and $1 \frac{1}{2}$ lines in length, of a steel-blue colour, attacking the young shoots, and often seriously injuring recently-put-on grafts. When the young shoot of the tree or graft is about 9 inches long, the female begins operations in a very curious and instructive manner. Kollar, who appears to have watched her proceedings, thus describes them : "As soon as she has reached the most suitable part of the shoot, she marks the place by a prick, or by a small cut, where she intends to cut off the bud or shoot. She then recedes about a line upwards, and begins (with her head turned downwards), on the side that is not next the tree, to bore it with her proboscis, till she reaches the middle of the shoot. With it she also widens the chamber, and prepares it for her offspring. She then places herself over the entrance, and lays an egg, which is pushed in by the proboscis, and conveyed to the proper place. This operation lasts an hour. Immediately after, the female returns to the former place, to cut off the shoot, moving it from the one side to the other with her proboscis until she has cut it a certain depth; she then gives some decided thrusts, which she continues without fatigue till the shoot only hangs by the under part. When she observes this, she gets up on the point of the twig to make it fall over by her own weight. It not unfrequently happens that it falls imme- 
diately, the shoot having been previously so cut as to remain attached to the stem only by the bark. If the beetle, however, finds that the pierced shoot does not fall, she turns back to labour again at the same place, and cuts still deeper through the branch; and if she is not able to divide it, she gets up once more to the farthest extremity, by which means she generally succeeds in bringing the separated branch to the ground. When this labour is over, she feeds upon a leaf, scraping off the epidermis, which serves her as food. After the beetle has rested about an hour, she goes again to work; and if there be still a place for the reception of a second egg on the shoot she has divided from the stem, she bores a second hole with her proboscis near the first, and, laying another egg, pushes it into its proper place. When the twilight comes on, she reposes under a leaf for the night. Next morning, as soon as the sun is up, the female beetle again begins her day's work, and often continues this employment until after the end of June, so as, by this means, to leave a numerous offspring behind her. The egg in the shoot is hatched in the course of eight days, and a white grub, with a black head, then makes its appearance. It feeds on the pith of the shoot, and if the shoot falls off, it arrives at its full size in four weeks. It then leaves its dwelling, and buries itself some inches deep in the earth. It there prepares itself a roomy chamber, in which it remains till spring, when it again appears as a steel-blue coloured weevil." The whole economy of this insect is extraordinary; and besides it there are many others of very similar habits, many of them laying their eggs in the pith of the petiole or foot-stalk of the leaf ; and instead of cutting off the leaf at once, they perforate the foot-stalk at both sides, and deposit their eggs in the orifices, causing an interruption to the flow of the sap which leads to the withering up and falling off of the leaf, from which the grub escapes, and enters the earth, and by spring is transformed into a perfect beetle. Both the shoots in the former case, and the leaves in the latter, must fall to the ground, that new weevils may be produced, because their transformation takes place in the earth, and not in the branch or leaf. A knowledge, therefore, of the habits of insects, closely studied, points out, in most cases, the means for their reduction. In the present case, the bitten-off shoots and the fallen leaves should be carefully collected as soon as they have fallen, and consigned to the nearest fire, because in them are enclosed the eggs of the insects, which, if left to lie on the ground, would only afford means for the grubs reaching the earth, which is their natural and necessary winter abode.

Several species of the genus Yponomeuta are exceedingly destructive to garden productions. $Y$. padella of most authors, changed by Zeller to $Y$. variabilis, feeds on the sloe and white thorn, often completely denuding them of their foliage. It also attacks the bird-cherry (Prunus padus), although to a much less injurious extent. $Y$. cognatella of some, Y. euonymi of Zeller, feeds on the leaves of Euonymus Europaeus. Y.padi of Zeller is exceedingly destructive to Prunus padus - chiefly, however, attacking old trees. Y. mali- vorella (of Stainton), fig. 188, is the species of this genus most destructive to the apple tree.

Fig. 188.
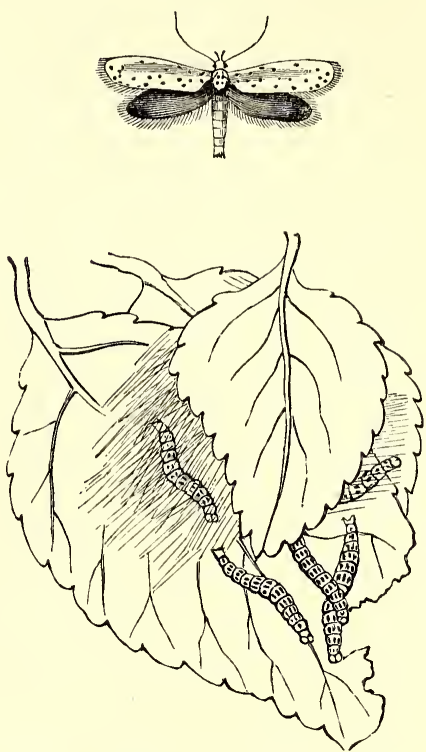

YPONONEUTA IALIVORELLA AND CATERFILLARS.

It differs from $Y$. padella in the pure bright white colour of its upper wings, marked with numerous small black dots: the cocoon is also different from that of $Y$. padella, being also white, but so opaque that it is impossible to see the enclosed chrysalis.

The caterpillars of $Y$. malivorella commit sad devastation in most seasons upon the leaves of apple trees, devouring them as fast as they develop themselves. Nor is it by feeding on the young foliage alone that they destroy it; they also spin a strong white shining web, with which they so completely cover the young shoots, and even oftentimes the older branches, as to prevent the free development of the leaves, while the web, hanging in festoons from branch to branch, offers a ready means of communication to every part of the tree.

Very great confusion appears to exist amongst entomologists regarding this insect; most of those we have consulted appear to confound $Y$. malivorella with $Y$. padella. Kollar, Hubner, and M. Guerin Meneville, who has been employed by the French government in investigating the insects injurious to cultivators, have all fallen into this mistake; while Treitsche seems to confound it with $Y$. cognatella, which is also a distinct species. We have been unable to meet with any very satisfactory description of $Y$. malivorella, and therefore beg to refer the interested reader to Mr Lewis's history of its earlier stages, in the "Transactions of the Entomological Society," vol. i., and to Mr Stainton's "Systematic Catalogue of British Tincidæ and Pterophoridæ." 
The natural enemy to this insect is stated by $\mathrm{Mr}$ Westwood, in "The Gardeners' Chronicle, 1849," p. 60 , to be a very minute hymenopterous parasite, belonging to the genus Encyrtus, which breed in such vast numbers in the body of a single caterpillar, that its skin, instead of shrivelling up, is distended and dried so as to retain its proper form. He also suggests, as a mode of destroying the perfect insect, to attack them as soon as they have made their appearance, and before they have deposited their eggs. "The general simultaneous appearance of the whole brood in the winged state, together with the very conspicuous appearance of the moths, will render this easy. A sheet may be laid beneath the branches in the daytime, which should then be sharply struck with a stick, when the motlis, which are at that time sluggish, will fall into the sheet, and may be easily destroyed. The destruction of one moth thus prevents the injuries which would otherwise arise from one, if not several, colonies of caterpillars in the following season."

The apple-bark beetle (Bostrichus dispar Autor, Apate dispar Fabricius, Xyloterus dispar Erichson). This is one of a tribe often called borers, on account of their boring into the trunks and branches of fruit trees. Uur present subject seems to prefer the apple to all other trees, but, fortunately, it is not very abundant in Britain; neither is it of yearly appearance, some years appearing, and for several others scarcely seen. The female beetles have the head and thorax black; the male is chestnut brown, with reddish thighs; the points of the antennæ and palpi reddish in both; the cases of the wings long, blackish, and slightly hairy ; thighs black ; feet reddish yellow. The female is about as large again as the male, and also of different form (from thence the name dispar, or unlike). They seldom lay their eggs near the ground, or on trees of less than $\frac{1}{2}$ inch in diameter, preferring the principal stem or larger branches of established trees. When the female has selected a place to her mind, she begins boring through the bark and wood in a somewhat oblique direction, often till she reaches nearly the centre of the stem or brauch, but more generally in the alburnum or young wood. These perforations are not in direct lines, but often very crooked, and branching off in different directions, forming various chambers in which the young are reared. The presence of the insect within a tree is readily detected either by the exudation of the sap at the openings of the perforations, or by the appearance of minute particles of wood, somewhat like fine meal, pushed outside the entrance of the hole. For the reception of her eggs the female makes the mouth of the hole considerably wider than it is within. In this she lays her eggs to the number of from seven to ten at a time, and may lay from thirty to forty in all during the season, of a pure white colour, somewhat pointed at the ends. The first brood of larvæ is liatched about the end of May, and they appear to remain enclosed in their wooden chambers almost immovable until they have assumed the perfect beetle state. They are very destructive to the apple in Ame. rica and the north of Germany, in which latter country the general remedy has been to cut off the branches infested by them; and if the whole tree is attacked, to cut it down, and remove it from the garden. We have little doubt that spirits of tar applied to the stems and larger branches would be found a complete remedy, acting both by destroying the larvæ, and also by deterring the female from boring into the tree, on account of the offensive smell of the tar.

The apple chermes, fig. 189 (Chermes mali Schmidt, Psylla mali of others), resembles con-

Fig. 189.
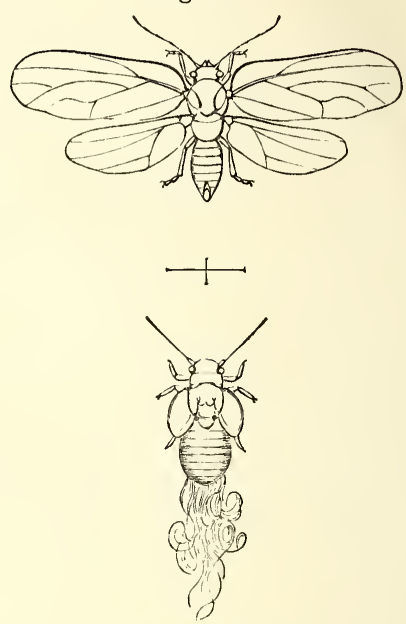

APPLE CHERMES. MALE.

Perfect and preparatory state.

siderably the Chermes pyri in size and appearance, on account of which it has been by some thought identical. The former differs greatly in its economy from the latter. Chermes mali pair and lay their egors in September, whereas $C$. pyri do so early in spring. The eggs are said by Kollar to be "perfectly formed in the body of the mother before pairing takes place, as is the case with Aphis mali (the apple aphis). Before they pair, five or six of them assemble on an apple leaf, and indeed usually on one that has become somewhat yellow, and each male selects his female. The males are either of a lively green, striped on the back with yellow, a dark yellow with brown stripes, or almost completely green, with dark-yellow dots; the abdomen is entirely yellow. The females are of a prettier colour, and somewhat larger ; the entire back, reaching as far down as the forceps, at the extremity of the abdomen, is red, striped with greenish yellow and brown, and the abdomen is orange-yellow and green ; they are green or greenish yellow on other occasions." The eggs are laid on the twigs of the trees both of the current and last year's growth, and frequently in the knots and about the base of the 
spurs. They are in general arranged in regular order, in rows lying close together. When laid on the shoots of the previous year, they are placed singly, or in heaps, without order, and in both cases are so firmly glued to the bark as to be with difficulty rubbed off. They are provided with a covering, which protects them greatly from the attacks of other insects. The eggs are hatched about the beginning of April, and in four weeks' time the insect has arrived at its perfect state. It is fortunate that this insect is not very destructive, nor does it every year appear in the same numbers; for, on account of its small size, it is difficult to capture in any of its stages of existence. It is more injurious to the blossom than to the foliage.

The figure of 8 moth, fig. 190 (Episema cceruleocephala, Bombyx ceruleocephala Linnæus,
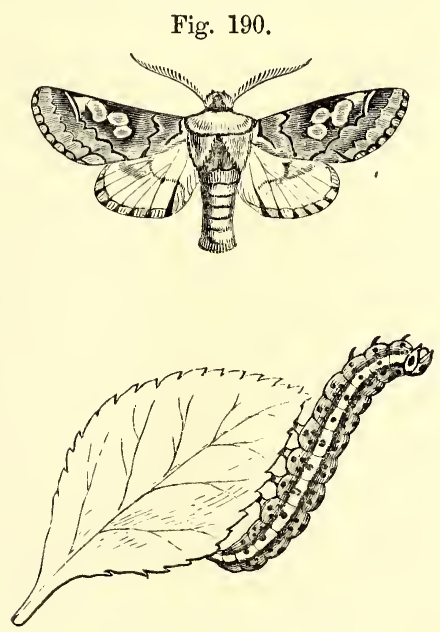

FIGURE OF EIGHT MOTH,

Male and larva.

Noctua caruleocephala of others), was denominated by Linnæus the pest of Pomona, and the destroyer of the blossoms of the apple, pear, and cherry. The moth in its perfect state exceeds 1 inch in length between the tips of its extended wings. The fore wings are bluish grey, with evanescent cloudy spots, having a lighter-coloured spot in their centre, on which there is a peculiar mark, somewhat resembling the figure 8 . The under wings are of an ashy-greyish colour, with a dull spot on their middle. The antennæ of the male are pectinated, those of the female filiform, and in both are of a rusty-brown colour. The thorax is of the same colour as the fore wings; the abdomen, with the extremity of the body, is similar in colour to the hind wings. Pairing takes place either in autumn or in the following spring, immediately after which the female lays her eggs singly on the trees. The caterpillars have thick fleshy bodies, and are much sought after by birds, which seem to luxuriate upon them. They are exceedingly voracious, and speedily strip a tree of its foliage. They attain their full size in
June, at which time they are nearly 2 inches in length, and nearly $\frac{2}{8}$ ths of an inch in thickness, of a yellowish-green colour, with three pale-yellow stripes running longitudinally, the centre one being somewhat broader than the others. The head is small, of a bluish colour, having two black circular spots upon it; on the body are many black tubercles, each bearing a single hair. Previous to entering the pupa state, they repair to the stems of trees, where they construct a nest of moss, small chips of wood-dust, \&c., in which they abide for three weeks before the pupa transformation takes place. The pupa is small, dull, reddish brown, and cylindrical in form. In this state it usually remains three months, more or less. The nıth appears in October and November, and sometimes not till April or May. Were it not for the assistance rendered by birds, ichneumon flies, and other insects, this pest would be more serious in its effects. In their caterpillar state they are difficult to detect, the colour resembling so much that of the trees they live upon, and also because they ensconce themselves behind the leaves when apprehensive of danger. Shaking the trees or the larger branches dislodges many, which may be received on a canvass cloth spread under them. We have known them dislodged when a gun has been discharged amongst the branches, and also when a powerful water-engine has been made to play with force upon the tree.

Tinea corticella attacks the apple tree under the bark.

Curculio vastator Marsham, Otiorhynchus picipes (see fig. 17), a short-snouted weevil, is very injurious to the blossom of the apple, appearing in March and April.

The apple-tree mussel scale (Aspidiotus con-

Fig. 191.
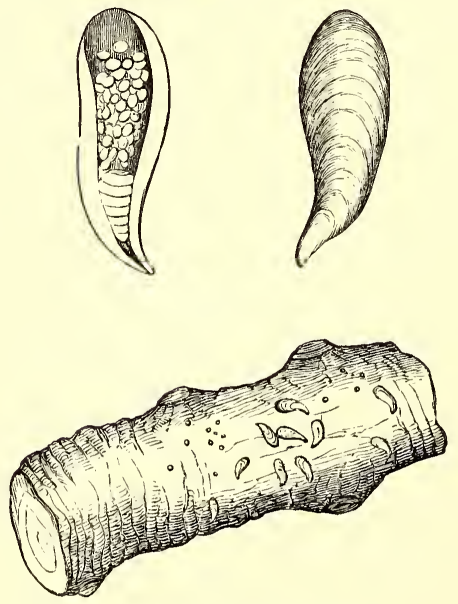

APPIE-TREE MUSSEL SCALE.

chiformis), fig. 191, attacks both the trunks and branches of apple and pear trees; and though so exceedingly small, and of the same colour as the bark it is attached to, it is nevertheless very 
destructive. The scales are hard, dry, and shining, and often found in large numbers crowded together, even to the extent of frequently lying one above another. When they find their way to the skin of the fruit, which they sometimes do, more especially on wall trees, they destroy its beauty, and give it a disgusting appearance. An excellent fig. is given of it in "The Gardeners' Chronicle," 1843, p. 735, and also a lucid description by Ruricola, who says, " The scales are hard, dark, and shining, exceedingly like a minute mussel-shell, but rather more elongated. They are slightly curved, transversely wrinkled, roundish at the tail, and attenuated at the head, which is semi-cylindrical, less opaque, and of a rusty colour. They adhere firmly to the bark, having the margins broad beneath and woolly; and when dislodged, the space they had covered appears white. Within the shell is found a fleshy green female, occupying part of the cavity towards the tapering extremity, the hinder space being entirely filled with white oval eggs, amounting sometimes to fifty or more. 'They are rather larger than in most species, I think, and produce little white fat Cocci, with two antennæ and six legs. They are lively, and run about for several days; but having fixed themselves, then grow, and by degrees become very different creatures from what they were immediately after their birth." Syringing the trees during winter with boiling water, boiling chamber-ley, lime-water laid on with a brush, and dusting the branches with powdered lime while they are wet, scraping the bark with a wooden knife, so as to destroy the females without bruising the tree, and even painting the branches with train and linseed oil, have all been tried with little apparent effect. We have found spirits of tar laid on during winter most effective; and could it be applied with safety during May, when the young ones are creeping from under the shell, there is no doubt that it would destroy every one of them. The branches have also been painted over with thick coats both of clay and lime-water, thinking to suffocate the insects; and some with a like view have recommended painting them over with glue.

The apple saw-fly (T'enthredo testudinea Stephens and Klug), fig. 192, may be detected about the end of June and beginning of July, when the apple fruit is about one-fourth its full size, and is found in such fruit as has fallen from the trees. "By collecting some," says J. O. W., in "The Gardeners' Chronicle," 1847, p. 852 , "which had only recently fallen, we dis. covered the insect before it had time to escape ; thus learning one point in its economy-namely, that the insect does not eat its way out of the apple whilst the fruit is upon the tree, and then crawl down the branches and stem of the tree to go into the earth, or to make a cocoon upon the bark; but that, on the contrary, it waits patiently until the fruit falls from the want of support afforded by the more vital portion of the apple which it has consumed. And here we may observe that this is perhaps the most important fact for the cultivator, as it lets him into the secret of the locality in which the insect passes its inactive and helpless state, and there- fore is that in which it may be most advantageously combated. These caterpillars are rather pale, dirty, buff-coloured; the head tawny, and with a very slender pinkish line down the back; the body is very much wrinkled transversely, and is furnished with three ordinary pairs of thoracic legs, with six pairs of very short ventral prolegs, and with another pair of the latter at the extremity of the body, thus making twenty legs in the whole. These larvæ, having eaten their way out of the apples, descend into the earth, where they form their cocoons, and remain in-

APPLE SAW-FLY AND GRUB. active until the following year. In the middle of the month of May we again directed our attention to the same apple trees which had afforded us these larvæ in the preceding summer, and which were now coming into full blossom. We now observed many specimens of the perfect saw-fly, produced from the last year's larvæ, flying about the blossoms, within which they settled; and we distinctly saw one of the females bend down the extremity of the body in the act of depositing an egg within the blossom; but they are at this time exceedingly timid, so as not to allow of our approach. The upper surface of the body is shining black, the front and sides of the head, and the shoulders, antennæ, legs, and under side of the body, being pale orange-coloured, and the wings are slightly stained with brown." As the apples attacked by this insect fall to the ground before ripening, the remedy presents itself to us-namely, gathering the fallen fruit, and consigning it to the nearest fire.

The American orchardists are greatly annoyed by several species of insects apparently not indigenous to Europe. As, however, the Aphis lanigera, or American blight, as it is usually called, was imported to the New World from the Old, and the almost equally destructive codlin moth, Carpocapsa pomonana, has also been transported along with apple trees from Europe, it is not at all improbable that some of these insects may be introduced to Britain, now that many of the best sorts of American apples are yearly brought over for planting in our gardens. Of these the apple-borer (Saperda bivittata) of American entomologists, Clisiocampa Americana, a species of lackey moth, different from those of the same family that are troublesome in Europe ; Anisopteryx pometaria 
(of Harris), the cankerworm, are the most formidable.

The borer attacks the stems of the apple and quince. It assumes the form of a white fleshy grub, entering the tree at the collar, just above the surface of the ground, where the bark is tender, and either girdles the tree or perforates it through every part of the stem, finally causing death. This grub is described by Downing as the larva of a brown-and-white striped beetle, $\frac{1}{2}$ inch long, remaining in its grub state two or three years, coming out of the tree in the perfect form early in June-flying in the night only from tree to tree, and finally depositing its eggs during June and July in the collar of the tree. For its destruction, thrusting a flexible piece of wire as far as possible into the perforation made by the insect, washing the stems in May quite down to the ground with a solution of $2 \mathrm{lb}$. of potash in 8 quarts of water, plugging up the holes with soft wood, after having placed a bit of camphor in the aperture, have all been had recourse to. But the most effectual of all wholesale remedies for the suppression of nocturnal-flying moths, is, placing a little fatty matter, refuse tallow, or common whale-oil, in flats or earthenware pans, such as are used for setting flower-pots in, and placing in each about 1 inch of cotton wick. These should be arranged along the tops of the garden walls, or laid upon the walks or open spaces in the orchard, and lighted in the twilight, and allowed to burn till towards daylight in the morning. The moths are attracted by the light, as other species are to the flare of a candle, and are thus consumed by hundreds at little trouble or expense. This is the most feasible way of getting rid of all nocturnal-flying insects.

A French entomologist has recently discovered a simple mode of enticing nocturnal-flying moths, by hanging festoons of string or rope, daubed with honey water, from tree to tree. The moths are attracted to the festoons, and may be captured in nets such as are used by entomologists. And a still better plan was discovered by Victor Adouin, of France, some years ago-namely, placing a flat vessel on the ground, partially filled with oil or tallow, in which a cotton wick was placed, and set light to ; over this was placed a bell glass, besmeared with oil or similar glutinous matter. The moths, being attracted to the light, fly towards it, and in attempting to get at it are caught by the glutinous matter on the surface of the glass, or fall into the oil beneath, and in either case are certain to perish.

The American caterpillar (Clisiocampa Americana), in its moth state, is of a reddish-brown colour, measuring about $1 \frac{1}{2}$ inch from tip to tip of the expanded wings. They appear in greatest abundance about midsummer, laying their eggs chiefly on the apple and the cherry, in the forks, and on the extremities of the branches. The eggs are hatched about the middle of May, when the young caterpillars come forth in thousands, and often completely strip the trees of an orchard of their foliage. They live for six or seven weeks in their caterpillar state, feeding most voraciously upon the leaves of the trees. They spin their cocoons about the middle of June, and towards the beginning of July the VOL. II. moths come forth. They are also nocturnal fliers, and may be kept under by the means recommended above.

The canker-worm (Anisopteryx pometaria of Harris). This insect has already reached Britain, and has for several years been most destructive to apple trees in the Dalkeith gardens, where it probably came along with a collection of American trees. This season (1853) it has been rarely detected ; indeed, so rarely, that specimens could not be procured to admit of their thorough examination. The account given of them by Downing, in "Fruits and Fruit Trees of America," is to the following effect: "The male is a moth with pale ash-coloured wings, with a black dot a little more than an inch across. The female is wingless, oval, dark ashcoloured above, and grey beneath. The insect rises out of the ground early in spring, chiefly in March. The females have no wings, climb slowly up the trunks of the trees, while the winged males hover about to pair with them. Very soon after pairing they lay their eggs, to the number of from 60 to 100 each, which they arrange closely in rows in the forks of the branches and on the young twigs. About the 20 th of May the eggs are hatched, and the canker-worms, dusky brown, or ash-coloured, with a yellow stripe, make their appearance, and commence preying on the foliage. After feeding about four weeks they descend into the ground 3 or 4 inches, where they remain in a chrysalis form, to emerge again the next season." This much being known of the economy of this insect, the remedy at once suggests itself ; namely, capturing the female in her ascent of the trunk, or preventing her ascending altogether. The American cultivators surround the trunk of the tree with a bandage of canvass 4 or 5 inches wide, which is thickly smeared with tar, and in order to prevent the tar from becoming dry and hard, they mix a little coarse train-oil with it. This belt catches and detains all the females on their upward journey, preventing them from ascending to lay their eggs, and probably causes them to die of starvation, seeing they cannot reach the trees to feed on the leaves, their natural food. Various other means have been employed to effect the same end-such as a mixture of common tar and coal tar, perhaps superior to the former from its being so offensive in smell, and less liable to become dry by exposure to the weather. A circular leaden trough filled with oil, and secured to the trunk of the tree, has also been used, and even patented in America. But it must be evident that, to prevent the female from creeping up between the trough and the stem, the trough should be fastened very tightly around the boll of the tree, the pressure of which might prevent the free ascent of the sap, and thus the remedy become worse than the disease. We have used a collar of coarse flannel saturated with spirits of tar, with which black pepper was incorporated, and attribute greatly to this the absence of the insect during the present season. We also, as a preventative against this as well as other insects which hybernate in the soil under the trees, remove in autumn 4 or 5 inches of soil in a cir- 
cular form as far as the branches extend, and convey it to the charring-heap, or dispose of it on parts of the garden far removed from the apple trees; thus carrying the insects in their chrysalis state to such a distance as prevents their reaching their favourite trees, exposing them to become the prey of birds, or otherwise disorganising their economy, so that few of them have the means of perpetuating their species. Mr T. A. Knight was strong in the belief that insects did more harm to our fruit trees in spring than all the late frosts and easterly winds, which in general have the credit of stripping our trees of their blossom and foilage. We are very much of the same opinion; and those who take the trouble to examine into the matter will be of the same opinion also. In the suppression of insects, as in the cure of diseases, excellent remedies may be prescribed; but if not taken in time, and followed up, little benefit need be expected. Amongst precautions, we may state that all fruit which falls from the trees before arriving at maturity should be carefully gathered up and permanently disposed of ; for their dropping, in many cases, is occasioned by insects having laid their eggs in them ; and if allowed to remain on the ground, the insect, when in a fit state for the change, will quit the fallen fruit and bury itself in the ground, or remain in the decomposing mass, and undergo its necessary transformation. Instructing children to search for and destroy the cocoons and nests formed for hatching the young, is an important and much neglected affair in fruit-tree management; for each of these destroyed lessens the number of enemies almost incredibly. Birds devour immense numbers of insects in their caterpillar state - some, indeed, nearly live upon such food during their continuance in that state; and they not only devour those that are lying on the exposed leaves and shoots, but also hunt those out whose nature it is to eat their way into the bud, or which have been hatched from eggs laid in the embryo bud. Therefore birds should not be destroyed, when performing for us so important an office, under the mistaken idea that they are eating the buds themselves. Whatever injury may be done to the buds by their endeavours to extract the insect, cannot be in any sense more destructive to the crop than if the insects had been allowed to remain in it. Birds should, therefore, be encouraged in gardens rather than destroyed or scared away. The only injury they do us is taking a small portion of the ripe crop, as if in repayment of the essential services they have done us; and if we are to deny them this small recompense, let it be by netting the fruit over when it begins to ripen; for surely that crop must be of small value in our estimation when it is not deemed worth the trifling expense and small labour of being covered for a few weeks with netting. Birds attack the apple only when nearly ripe, and there is no doubt they select the very best specimens. In large orchards the fruit they destroy in this state is as nothing compared to the service they have rendered us during spring. Insectivorous birds, during winter, search for and destroy multitudes of the eggs of insects ; and however firmly they may glue their eggs to the stems and branches of the trees-however strongly they may cover them with webs-still these birds will find them out; and, as has been stated by Reaumur, the greenfinch tears open the strong nest of the browntailed moth (Bombyx chrysorrhoea), and devours the caterpillars in their infant state. The red and green woodpeckers (Picus major and veridis), the nut-hatch (Silla cossia), the tree-creeper (Certhia familiaris), not only devour great numbers of caterpillars, but also of the beetle tribes, which greatly infest our fruit trees. The chaffinch is one of the greatest destroyers of insects in their pupa and larva states, yet no bird is so persecuted; and the same may be said of the titmouse, and the ox-eye, tomtit, redstart, redbreast, goldfinch, and wagtail, all of which are consumers of caterpillars and the eggs of moths. Amongst other animals, the common toad and the bat are serviceable to us in this way. The bat lives almost entirely upon insects, keeping up a constant attack upon them during night, when birds have gone to repose. They greatly serve us in attacking nocturnal-flying insects, which, from their habit of concealing themselves during the day, would escape the attacks of day-flying birds. But the greatest enemies insects have are insects themselves. We have in several instances shown this in the preceding pages, certain genera being created by nature as if for the express purpose of keeping in check insects of other genera, to restore or maintain the equilibrium among her creatures, and particularly to prevent the preponderance of some sorts, which, without this wise provision, would multiply to such an extent as to produce a general desolation in the vegetable kingdom. To effect this, the groundbeetles (Curabidæ), for example, destroy the pupæ of moths while deposited in the earth. Many flies deposit their eggs in the living caterpillars, and so destroy them. But the most serviceable of all are the Ichneumonidæ family, extending to 1300 European species alone, all of which lay their eggs in the bodies of other insects. Nature has provided other means for the preservation of our fruit trees from the attacks of insects, such as heavy rains, late spring-frosts, storms, and cold ungenial weather, happening at the.period when pairing is taking place; and by these means, although in themselves sometimes destructive even to the fruit crop, the numbers of insects are so reduced that our gardens and orchards for several years afterwards escape their ravages. The pupa of the destructive small winter-moth (Geometra brumata) for example, is often destroyed while lying in the earth during wet autumns and winters-a circumstance which would lead us to believe that irrigating the ground round the roots of our fruit trees with liquid manure, during autumn or winter, would have a most beneficial effect in ridding us of this destructive enemy.

The European names of the apple are-Pommier, in French ; Der-apfelbaum, in German ; Appelboom, in Dutch ; Melo, in Italian ; Manzano, in Spanish; Maceira, in Portuguese; Jabion, in Russian. Tgffah, in Arabic. 


\title{
CHAPTER V.
}

\author{
THE PEAR.
}

THE pear, like the apple, is indigenous to most parts of Europe, and is known botanically as Pyrus communis Wild. As a fruit-bearing tree, it stands next in popularity to the apple ; and although less valued for culinary purposes, and also for the press, it is much more esteemed for the dessert; and in consequence of the improvements brought about in modern times, it now ranks as the most valuable of all our hardy fruit-bearing trees, continuing in perfection throughout most of the year; and being more easy of digestion than the apple, it may be eaten with safety by invalids, when the apple cannot. As a press-fruit, it is valued in the manufacture of perry, by many preferred to cider, and by some (ourselves amongst the number) considered superior to much of the trash sold at enormous prices, under the name of Rhenish wines. The pear is met with in highest perfection in climates superior to Britain, and hence the fruit produced in the north of Italy, France, and the Channel Islands, ranks higher than that grown in our best gardens, even against walls. The chaumontelles, bergamots, and beurrés rank high, and of these, excellent varieties have been long in cultivation. An extraordinary impetus was given to the improvement of this fruit about the beginning of the present century; and, with very few exceptions, it would matter little were all of a preceding age swept from our fruit catalogues. To the cultivators in Belgium are we mainly indebted for most of our finest varieties ; and this will be understood when we state that two eminent amateur cultivators, whose friendship we had the pleasure to enjoy, fruited no less than eight thousand varieties, originated from seed, in the course of a very few years. These were the Abbé Duquesne of Mons, and Professor Van Mons of Brussels. Van Mons alone raised eighty thousand seedling pears, and spent the greater part of his life in carrying out this his favourite study. Above seven hundred kinds of pears have been collected and proved in the garden of the Horticultural Society of London (vide descriptive catalogue of fruits published by that Society). These gentlemen procured by this means several hundred varieties of superior merit, the most of which are in cultivation in the Netherlands, and many of them in our British gardens, ranking amongst our finest fruits. The pear, historically speaking, is not so ancient as the apple. At what period it became ameliorated, or removed from its wilding state, we know not. It is more than probable that this, as in the case of the apple, arose from accident. In regard to longevity and hardihood it is greatly superior to the apple; and it is not improbable but that there are trees still existing, and in a fruitful condition, whose age must be computed by centuries. The Romans cultivated thirty-six varieties in the days of Pliny; and in this country, so early as the time of Parkinson, we were in possession of sixty-four sorts. Our present nursery catalogues enumerate nearly four hundred, while those of the Continent greatly exceed this number. From the oldest trees in Scotland, taking their fruit as a criterion of merit, it will appear that little advance had been made in this country; and that, notwithstanding our early political intercourse with France, few even of their then good pears had been introduced; and until within the last thirty or forty years we had few pears possessing the most moderate qualities. The pear.tree is not only hardier than the apple, but will succeed in soils where the latter will not ; but, again, it requires a much more genial climate to bring its fruit to perfection. The history of the cultivated pear is involved in great obscurity. That it originated in milder climates than even those of France and Belgium is highly probable. It was at an early period common in Syria, Egypt, and Greece ; and from the latter country was imported into Italy, from whence it would spread into France, Germany, and ultimately to Britain. It is reasonable however to suppose, that, as the tree in its wild state existed here, some varieties, possessing greater merits than the original type, arose naturally; but that the better sorts were introduced from Italy in monastic times is equally probable. Theophrastus and Virgil speak of the productiveness of old pear-trees, which evidently shows that the tree had even then been long in cultivation. Pliny, in his fifteenth book, observes, "of all pears, the Costumine is the most delicate and agreeable; the Falerian, esteemed for its juice; and the Tibernian, because it was preferred by the Emperor Tiberius." But even these could bear no comparison with those of modern times; for the same authority says, "All pears whatsoever are but a heavy meat, unless they are well boiled or baked." The 
pear is not indigenous to America, but an introduced tree ; and since its period of introduction, hundreds of varieties have been originated, and many, such as the sickle-pear, of very great merit, the soil and climate both being congenial to the growth of this tree and the complete maturisation of its fruit. Mr Downing, in his work on "The Fruits and Fruit Trees of America," mentions two trees of great size and productiveness, one of which is growing in Illinois, believed not to be more than forty years old, the girth of whose trunk, 1 foot above the ground, is 10 feet, and at 9 feet from the ground, is $6 \frac{1}{2}$ feet: in 1834, it yielded 184 bushels of pears; in 1840, it yielded 140 bushels. The other to which he alludes, and calculates as being perhaps the oldest in the country, was planted more than two hundred years ago. From this we may premise that the pear was introduced somewhere about two centuries and a half ago to that important fruit-growing country. In England, the pear tree attains a large size and great age. In 1829, we visited an extraordinary tree of this sort, growing in what was once the garden of the parsonage-house adjoining the park of Sir E. Stanhope, at Home Lacy, Herefordshire. It then covered nearly half an acre of ground; the larger branches, each of which was in size equal to a large tree, had, during the course of ages, fallen down and taken root at the parts where they came in contact with the ground, and shot up in form of distinct trees, yet all more or less connected with the main trunk. This tree has been known to produce in one year thirty hogsheads of perry. Both Loudon, and others quoting from him, have stated the produce as only fifteen hogsheads. We were informed by the proprietor that the quantity was double. 'This variety is a true perry pear, very hardy and productive, having an austere juice.

Pears,like apples, are divided into three general sections; namely, summer, autumn, and winter keeping sorts. The two latter are the most esteemed, affording as they do our principal dessert-fruit at a season when all others are past and gone. Mr Rivers justly observes, "The pear seems to require a warm moist climate. Jersey is probably the most favourable seat for pears in Europe, and next to that fertile spot the low moist situations around London, particularly in the neighbourhood of Rotherhithe. In that deep alluvial soil, the jargonelle and other fine pears may be said to attain the highest possible degree of perfection. In many parts of France the climate is too warm. The neighbourhoods of Cheltenham and Worcester are very favourable for the culture of pears. Many of the Flemish and French varieties ripen their fruit well, and are first-rate in size and flavour." At Pinkie and Luffness, on the margin of the Firth of Forth, they also ripen well, and attain a large size, on account of the deep alluvial soil they grow in, and the humidity of the atmosphere, from its proximity to the sea. It has been set down as a general rule that pears grown against a wall will have their fruit much larger in size than the same kinds trained as standards, but that they are, more especially in dry warm seasous, deficient in flavour, nor do they keep so well. But we think somewhat of this depends on cultivation, as the temperature is increased by the radiated heat from the wall. These consequences would follow if a corresponding stimulus is not given to the roots, and this wo think is accomplished by copious waterings with enriched liquids during the time of the swelling of the fruit. We water copiously with guanowater, and we find Mr Rivers does the same, applying it with an unsparing hand.

Planting pears. - The pear requires a good, naturally rich loamy soil ; not, however, enriched by artificial means, as that would only have the effect of producing a luxuriance of growth that would require much skill and labour to overcome, by training, root pruning, and other manipulations, all of which it were better to avoid. The pear is cultivated in a variety of forms, according to the hardiness or tenderness of the kind; and the sorts are chosen most suitable to the purposes for which they are intended. And hence we have full standards, half standards, espaliers, wall-trained, dwarf standards-and of all these some one or more varieties. To arrive at certain ends, two stocks are also chosen for working the pear uponnamely, the pear stock and quince stock: the former, where longevity and strong trees are required, generally for orchard purposes; and the latter, where small trees of greater precocity in coming into a bearing state, occupying less room, \&c., should the period of their existence even be of shorter duration.

An opinion has been entertained by some, to the effect that the pear wrought upon the quince stock is of short duration. Of course, we have no means of drawing a parallel in this respect between them and such as have been wrought upon the pear stock, because the former is of modern adoption; whereas the oldest fruit-trees in Britain are pears wrought upon the latter, and no doubt have existed for centuries. In France, where the quince stock was first employed, thousands of healthy and productive trees may be found of considerably more than half a century's growth; and the fine wall of pears in the garden of the Horticultural Society of London, upon quince stocks, are in the highest state of vigour, although they are now about twenty-six years old, and were amongst the first trees of the sort planted in Britain. Admitting that they are of shorter duration than those upon pear stocks, the small space they occupy, and the abundance of fruit produced, and that from the second year in general after planting, are, in our estimation, of far greater consequence than if they were to grow as large as the oak, and, like it, live for a thousand years (vide article STocks). In planting the pear on quince stocks, Mr Rivers observes that it is necessary that the stock should be covered up to its junction with the graft; and "if the soil is not excessively wet, the tree may be planted in the usual manner, so that the upper roots are on a level with the surface of the soil. But with pear trees on the quince something more is required than the ordinary mode of planting." This is simply to form a mound of compost, above half-rotten manure and earth, mixed in equal quantitics, 
which must cover the stock up to the junction of the graft, to the letter $a$ (fig. 193), "and this is made of rich compost," in order to encourage it to emit roots into the surface-soil, and to keep it from becoming hard and "bark-bound." To make this emission of roots more certain, the stem may be tongued as used in layering; $i$.e., the bark must be cut through upwards from the
Fig. 193.

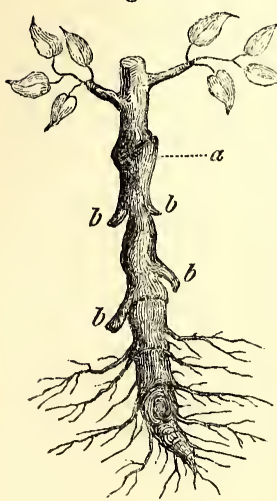

PLANTING PEARS ON QUINCE STOCKS. root, and a slip about one inch in length raised, $b, b, b, b$, (which are the raised pieces of bark), and these raised pieces of bark must be kept open by inserting a piece of broken flowerpot or slate. Several of these tongues may be made, and by the end of the first year after planting, every incision will have emitted roots; the stock, owing to its being kept continually moist, will swell and keep pace with the graft, and the tree will flourish and remain healthy. As the mound will subside by the heavy rains of winter, presuming the trees have been planted in autumn, fresh compost of the same nature must be added in spring, and again every succeeding autumn. The great object in the culture of the pear upon quince stocks, is to encourage the growth of its very fibrous roots at the surface, so that they may feel the full influence of the sun and air. These mounds may be made ornamental, if required, by placing pieces of rock or flint on them, which will also prevent the birds scratching at them for worms; but the stones selected must not be large and heavy. In light friable soils, they may be from 3 to 4 inches above the surface of the surrounding soil : in heavy, retentive, wet soils, from 6 to 8 inches will not be found too high."

The soil for the pear, although moderately moist, must, however, not be wet; and to secure this, efficient drainage should be provided. And as it is an object of vast consequence, in a cold climate, that the roots of all fruit-bearing trees be kept as near the surface as possible, the trees should be planted high, and the prepared ground, whether wall borders or otherwise, prepared in breadth of surface instead of in depth, that the roots may be induced to extend in a horizontal direction, instead of going down beyond the range of solar influence.

The soil for pears upon quince stocks should be somewhat tenacious, and rich in humus; not, however, so stiff as to retain water, but only to the extent of being free from dryness. The quince prefers a soil in which, if we may so speak, a kind of subterranean irrigation is going on, but not constantly, and in which no stagnation can take place. It appears to luxuriate most when planted by the sides of streams that rise and fall frequently, such as those in connection with water-power mills, or when the influence of tides throws back the water in the under strata for a few hours, and from whence it naturally again recedes. To obtain for the quince, or rather for pears wrought upon stocks of that kind, a border soil in gardens so circumstanced is quite possible, but not very convenient. The nearest approach, therefore, will be to avoid light sandy soils, and to employ only those of a sound loamy nature, or what is in general called strong loam, or the nearest approach to rich fribbly clay. No doubt watering artificially during the period of the fruit's swelling will go far to increase its size ; and this we have found in practice to be attended with good results, more especially in dry seasons. Pears on quince stocks succeed better in wet localities than in those that are remarkable for their drought; but in such cases complete drainage should be provided underneath.

Within the last half-century the pear has undergone, not only in character, but also in culture, a most important revolution; few of those, even of that recent date, that were then considered excellent are now to be met with in our best gardens, their places being taken by others of superior merit. The number of varieties has during the same period increased amazingly; and, as a natural consequence, as these differ from each other in habit, so must, to a very considerable extent, the proper management of them differ also,-for the stock, climate, soil, mode of pruning, \&c., suitable to one variety, may be unsuitable to another. It is here our great difficulty arises, and without a due consideration of these points all hope of general success must be abandoned. So much are even the Belgian fruit-growers aware of this, that one of their most eminent members, M. J. de Jonghe of Brussels (from the introduction of whose descriptive Pear Catalogue the following abbreviated translation is made), thus remarks : "It is very difficult to lay down general rules for the proper culture of the pear, and its numerous interesting varieties, without a special monograph, in which the character and circumstances of every variety, in different soils, different climates, under different modes of pruning, whether trained as espaliers, pyramids, or standards, as well as the situation they are planted in, and the stock on which they are wrought, were fully established or ascertained. Such a work would require very many years to complete. At present the practical man looks upon the question in a point of view appropriate only to his own garden or nursery. Several treatises have been published in which the authors have described the results of their own experiments, often founded on facts observed in certain localities; but if these directions were afterwards acted upon in other climates, and on different soils, the expectations of the cultivator would often be found far from successful. Before establishing a plantation of pears, it is first requisite to examine the nature of the soil, its depth, the situation of the garden, and, finally, the climate in which they are to be placed. If the intention is to plant pears either as standards, espaliers, 
or pyramids, where the soil consists chiefly of clay, it is certain that trees grafted on pear stocks will in general be found to possess too much vigour, and will be long before they come into a bearing state. For such a soil trees must be chosen that are grafted on the quince stock; and this offers no inconvenience for espaliers, though it will not answer so well for pyramids. The quince stock forms no strong roots in the soil, and hence the tree cannot resist strong winds if it is not supported by stakes, and the inconvenience of employing these supports is well known. The bark of the trunk becomes bruised by rubbing against them, and the branches are liable to be broken. To avoid these inconveniences, we recommend giving preference to pyramid trees that have been grafted on pear stocks. At the same time, attention must be paid that for a strong and rich soil only those varieties should be chosen, the wood of which is not too vigorous in its growth. There is a great number of that kind, in the varieties with delicate fruit. For standards, those varieties are chosen that grow strongest, and form short and thick wood.

"In a light and deep soil, with an airy situation, it is certain that, nearly without an exception, all varieties will succeed if on pear stocks, pyramids as well as standards. If a tree grafted on a pear stock, and trained as an espalier, grows too strong, the roots must be pruned to keep up the equilibrium, and to force the tree into a bearing state.

"In pruning, care must be taken that in those varieties of vigorous growth the fruiting branches be left somewhat longer, while those of a middling growth should have them a little shorter. It is an error, though it has been stated in a much diffused and much consulted work, that fruit obtained from trees grafted on the quince stock are superior to fruit obtained from trees on pear stocks. It is known in general, and we have observed it more than once, that several varieties of pears cultivated on quince stocks and trained as espaliers, produce finer fruit than pyramids or standards on pear or free stocks, but it has not as yet been proved in Belgium that the fruit has been of better quality." The above remarks are, according to M. J. de Jonghe, applicable to the climate of Belgium, and, with the exception of climate, to Britain also; their summer months-June, July, and August-being warmer than ours, which favours greatly the swelling of the fruit and maturity of the wood. Their winters are much colder than ours, and their springs and autumns much the same. The pears of Belgium are much hardier than those of Jersey or of France, and consequently better adapted to our climate. Unfortunately, however, a proper distinction has not, in general, been made between those of the one country and the other. The French and Jersey pears require both a fine warm light soil and good climate, and therefore must be very precarious with us, even upon walls, unless in the very warmest localities; nor are they adapted to the strong cold clay soils in which we often see them planted, and as often recommended as the most fitting for them. We err, also, in growing all kinds, both of French, Jersey, and Belgian origin, indiscriminately on quince stocks, and grafting from such a source for our future supply of trees. There is great discrimination also required as to the time of the fruit remaining on the trees, and on its proper treatment in the fruitroom. In gardens in moderately sheltered places both in England and most of Scotland, the hardier strong-growing sorts of Flemish pears will succeed almost as well as pyramids as they do in most parts of Belgium; and fruit so produced, if not so large, is better in quality than the same sorts grown against walls. The Beurré Diel, for example, although not considered a first-rate pear in Belgium, ripens at Dalkeith on pyramids and standards abundantly, and forms one of our next to best sections of pears. Much confusion has also arisen in the nomenclature; most of them having French names cannot well be distinguished in catalogues from those of France and Jersey, as the authorities are seldom given.

Although the Flemish or Belgian pears, even of the best varieties, succeed admirably in such a climate when wrought on pear or free stocks, still in most parts of Britain, where our soil is colder and often stronger, the same sorts will not ripen with us so well as if wrought on quince stocks. This forms an important element in their success, and should guide the purchaser in making selections from the Belgian nurseries. Mr Rivers, who has studied the culture of the pear both on the Continent and in Britain, advocates greatly the use of quince stocks for all the finer varieties ; and even for the hardier kinds, if they are to be planted in strong soils and cold situations. "In recommending pears on quince stocks," he remarks, "for all cold soils and situations, even in the far north, I may appear theoretical, but from my own experience in some very cold and clayey soils in this neighbourhood [Sawbridgeworth], I feel sanguine as to the result; for I have observed, in my frequent visits to the pear gardens of France, that many sorts are often too ripe. Now this is just the tendency we require. In our cold and moist climate, most certainly pears will not get too ripe, more especially in the north of England and in Scotland." In selecting pears it is of no small importance to choose those that will succeed each other, so as to keep up a supply throughout the year. During the latter end of summer and autumn we have abundance of good pears to meet the demand; besides, at that time we have other fruits in high perfection. It is therefore from Christmas until forced grapes, peaches, \&c., come in, that pears form of themselves the principal part of the dessert. The varieties of Belgian pears raised at Malines by the late Major Esperen are deserving of especial consideration for this purpose ; and of those, Josephine de Malines, Suzette de Bavay, Beurré Bretonneau, Cassanthe de Mars, and Begamote Esperen, may be offered as examples.

Regarding the proper management of the pear, Mr Rivers observes, in his recently published " Catalogue of Fruits for 1853-1854," after stating the great importance of biennial remov- 
THE PEAR.

451

ing the trees, "It is the most simple of all methods of root-pruning : it consists of merely digging a trench round the tree early in Novembes, and lifting it out of the ground carefully, with all the earth possible attached to the roots, shortening with the knife any that are strapgling. If the soil be rich, so that trees unremoved are inclined to grow too vigorously, no fresh compost will be required, and it will merely be necessary to shovel into the hole some of the earth from the surface around it to 2 or 3 inches in depth; this will prevent the tree settling down too deeply. If the soil be poor, some rotten dung, at least six months old, and loam, or any light earth, equal parts, or moor earth, may be placed at the bottom in the same manner, and some of the same compost, say half a wheelbarrowful to each tree, may be thrown over its roots when replanting it." The following treatment he finds equally beneficial to the pear, whether on the pear or quince stock: "In low situations, near brooks and rivers, a black moor earth is generally found. This unprepared is unfit for horticultural purposes, but if dug out and laid in a ridge, and one-eighth part of unstacked lime spread over it, turning it immediately and mixing the lime with it, it will become in the course of five or six weeks an excellent compost for pear trees. I have," he says, " in some instances added half a bushel of burnt earth to a barrowful of this moor earth ful to a tree will be enough. The only method to cultivate successfully pyramidal pears, on

Fig. 194.

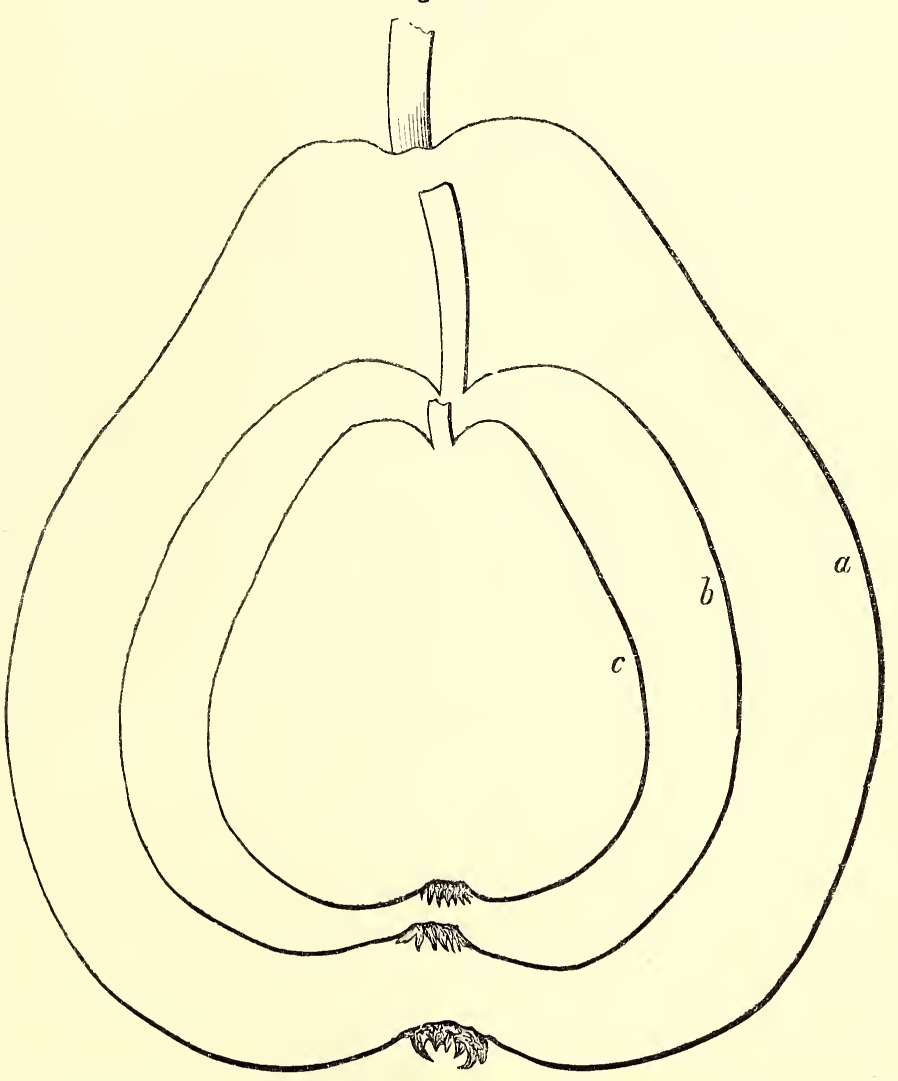

SIzEs or pears. $a$ large (Beurré Diet); $b$ medium (White Doyenne); $c$ small (Seckel).

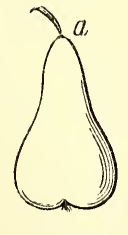

Fig. 195.
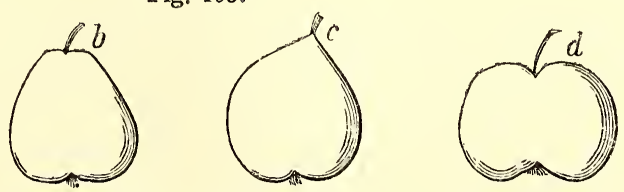

FOR MS OF PEARs, $a$ pyriform ; $b$ obovate ; $c$ turbinate ; $a$ roundish. 
pear stocks, is by biennial removal,-in this way they become nearly as prolific as those on quince stocks; and what is of great importance, in light gravelly or chalky soils they will to a certainty succeed where pears on quince stocks will as certainly fail."

Protecting the blossom of the pear in spring.Many varieties of the pear blossom early, and consequently are exceedingly liable, especially the Jersey and French sorts, to sustain injury from spring frosts. The protection of the blossom is therefore a matter of great importance. Those on the walls can readily be secured from injury by the use of portable wooden copings, as elsewhere described, and securing to them thin seni-transparent canvass, as recommended for the protection of the blossom of the peach and apricot. Pears trained as pyramids, particularly when wrought on the quince stock, are readily protected also ; and we have found no way better than driving in three larch poles, a foot higher than the tree, and fastening three hoops to them-one near the bottom, another at half the height of the tree, and the third quite at the top. These hoops form cylindershaped frames, and to them thin canvass should be secured, which will not only ward off any ordinary degree of frost, but also protect the blossom and embryo leaves from the parching effects of our cold easterly winds.

Criterion of a good pear-Dessert sorts.-Dessert pears should be of medium size, or larger, of a sugary aromatic flavour; the flesh soft, sub-liquid or melting, as in the case of most of the Beurrés or butter pears; or of a firm and crisp consistence, or breaking, as in the winter bergamots. High colouring seldom accompanies high flavour in this fruit. The Glout Morceau, Seckel, and Chaumontelle offer examples of this.

Culinary sorts should be large, falling when stewed, the flesh firm, and rather austere than sweet. Firm-fleshed sorts are preferred for compotes, and the falling sorts for marmalade.

\section{SELECT LIST OF DESSERT PEARS.}

Aston Town-Colour yellowish green ; form roundish; size under medium; quality firstrate. In use in October and November. An abundant bearer, and excellent standard pear in the south, requiring, and fully meriting, a wall in the coldest parts of Scotland. This excellent hardy pear originated near the village of Aston in Cheshire. The tree is characterised by its long slender branches, which have a halftwisted dangling appearance. It is especially worthy of notice in an unfavourable soil and cold climate. In the midland districts of Scotland it succeeds well as a standard.

Andrew's.-Colour pale yellowish green, with a dull red cheek, and a few scattered dots; size rather above medium; form pyriform, somewhat one-sided; quality excellent. In use in September. Of American origin, and there held in high repute. Downing describes it as of most excellent flavour, a certain and regular bearer, even while young; and the tree, which is very hardy, never suffers from blight.
Beadnell's seedling. - Originated from seed with Mr Beadnell of Tottenham; described by Mr Thomson as a middle-sized fruit ; form turbinate; colour pale yellowish green on the shaded side, red next the sun, much speckled with whitish-grey dots. In use at Chiswick from the middle of September to the middle of October. Tree vigorous, and an abundant bearer ; flesh melting ; flavour exceedingly rich. In $\mathrm{Mr}$ Thomson's description of this pear in the "Journal of the Horticultural Society," he says, "It is not buttery, but so melting, when used in its full perfection, that one can scarcely say whether he is not rather drinking than eating. This variety cannot fail to be very acceptable on account of its rich, abundant, and highly-refreshing juice."

Belle et bonne.-Colour yellowish green; form roundish ; size large ; quality good. In use in September. An abundant bearer; requiring a wall in most places. Of Belgian origin. Synonym-Gracieuse.

Bloodgood.-Colour yellow, sprinkled with russet dots; form turbinate; size medium; quality one of the highest flavoured of all early pears. In use during August. Of American origin ; brought into notice in 1835. So highly is it prized in America that Downing says of it, that it surpasses every European variety of the same season; and together with Dearborn's seedling, another New-World sort, will supplant in all their gardens even the jargonelle and all inferior early pear's.

Bergamot, autumn.-Colour greenish brown; form round; size under medium; quality excellent, the flavour being peculiar and rich. In use during October. One of our oldest varieties, and bearing best on aged trees. Succeeds in most situations as a standard. SynonymsCommon bergamot, English bergamot, York bergamot. This is one of the oldest pears known, and supposed by pomologists to have been in England since the Roman invasion. Mangor conjectures it to be of Turkish origin, and to have been originally called Begarmoud (princely pear), from the Turkish beg or bey, and armoud a pear.

Bergamot, Gansal's.-Colour yellowish brown; size above medium, sometimes large; form flatly obovate. In use in October and November. Quality excellent. Of English origin, from a seed of the autumn bergamot, but very superior to it. It is well ascertained that this excellent pear originated about 1768 with General Gansal, of Donneland Hall, Essex. From some defect in the fructification, it does not set well, although it produces a sufficiency of blossom, and hence it has been suggested to work every other branch with some other variety that flowers at the same period, with a view that the one sort may impregnate the other. Indeed, so excellent a pear merits the little trouble that would arise, were the blossoms to be impregnated artificially by the hand. Requires a wall in all situations. Rather a shy bearer. Synonyms-Bonne rouge, Ive's bergamot, Brocas bergamot, Diamant, Staunton, Gurle's beurré.

Bergamot, Gansal's, late.-Raised by Mr Williams of Pitmaston; ripens in December, and 
bears well as a standard, setting freer than the old Gansal.

Bergamotte cadette.-Colour pale grcen; form obovate ; size medium; quality good. In use from October to January. The fruit ripens very unequally, some ripening early, while others from the same tree remain for months - a peculiarity not without its advantages. SynonymsBeauchamps, Beurré Beauchamps, De cadet.

Bergamotte de Hollande.-Colour brownish green; form roundish; size medium; quality good, but chiefly esteemed for its long keeping. In use from March to June. Synonyms-Lord Cheney's, A moselle, Bergamotte d'Alençon, Bergamotte de Fougère, Beurré d'Alencon. Requires a wall in all situations to grow it to perfection.

Beurré, winter.-A seedling from the Easter beurvé. Originated with $\mathrm{Mr}$ Rivers. In use during February and March. A good bearer, and quite hardy as a standard tree around London.

Beurré d'Amalis.-Colour greenish brown; form obovate; size above medium; quality excellent. In use in September. Somewhat similar to the Brown beurré, but much hardier. Succeeds as a standard in good situations, but requires a wall in Scotland. Synonym-Beurré d'Amalis gros. Of Belgian origin.

Beurré d'Aremberg.-Colour pale green and brown ; form obovate ; size medium; quality excellent. In use in December and January. Succeeds in the south of England as a standard, requiring a wall in the north, and throughout Scotland. A Belgian variety. SynonymsDuc d'Aremberg, Deschamps, Colmar deschamps, D'Aremberg parfait, L'Orpheline, Beurré des Orphalines. This very excellent pear was raised by the Abbe Deschamps, in the garden of the Hospice des Orphalines, at Enghien in Belgium.

Beurré Bosc.-Colour russet; form pyramidal; size large; quality excellent. In use in October and November. In warm situations will succeed as a pyramid, double worked on the quince; requires both a good soil and situation. Synonyms-Marianne nouville, Calebasse Bosc, Bosc's flaskhenbirne. Raised by M. Van Mons, of Brussels, in 1807. One of the finest-flavoured pears when fully ripened.

Beurré Duval.-Colour pale green; form pyriform; size medium; quality good. In use in October and November. This variety is of Belgian origin, raised by M. Duval; a great bearer, and deserving general cultivation. Succeeds as a standard in favourable places, but requires a wall in Scotland. Succeeds best when on the quince stock.

Beurré, brown.-Colour brown, tinged with dashes of red ; form obovate; size large; quality first-rate, but varying much according to soil and situation. In use in October. An old French varicty, long considered the best pear in cultivation. Like most good fruits its synonyms are numerous-viz., Beurré rouge, Beurré gris, Beurré dore, Beurré vort, Beurré du roi, Beurré d'Anjou, Beurré d'A mbleuse, Beurré d'Ambroise, Golden beurré, Red beurré, Badhams, Isambert, Isambert le bon. Requires, and justly deserves, a wall.

Beurré Gris d'Hiver noureau.--Size large; quaVOL. II. lity first-rate. In use during February. Very productive; ripening well in favourable places as a standard. One of the best pears in cultivation.

Beurré de Capiaumont.-Colour brownish red; form obovate; size medium; quality excellent. In use in October and November. Tree healthy, and a great bearer, withstanding late spring frosts better than most others. Succeeds as a standard in the south, requiring a very favourable situation to succeed so in the north of England or in Scotland, where it is generally grown against a wall. The branches have a tendency to become pendant when grown as a standard; forms a handsome tree when wrought on the quince. Synonyms-Calebasse rass, Capiaumont. Of Flemish origin. A profitable orchard pear.

Beurré Diel.-Colour yellowish brown; form obovate; size large; quality excellent, as regards the fruit and hardihood, as well as the productiveness of the tree; deserving most extensive cultivation. In use during October and November. A fine standard pear around London, and has produced good fruit as such in the gardens at Dalkeith, but in general requires a wall in Scotland. An old Belgian fruit, raised by Dr Van Mons in 1805, and by him named after Dr Diel, a distinguished German pomologist. Synonyms-Beurré magnifique, Beurré incomparable, Beurré royal, Diel's Butterbirne, Dorothée royal, Gros Dillen, De Melon, Beurré de Gelle, Poire de Melon, \&c.

Beurré Easter.-Colour greenish brown; form obovate; size large; quality excellent. Tree hardy, and a good bearer; altogether one of the most valuable keeping pears in cultivation. Succeeds well around Loridon as a standard, and is of better quality whell so grown than when against a wall, as in the latter case it is apt to become mealy and dry, and does not keep so well. Mr Thomson recommends this fruit to be gathered rather earlier than usual, and ripened off in a warm place. In use from January to March. The synonyms are-Bergamotte de la Pentecôte, Beurré Roupe, Beurré de Paques, Beurré d'Hiver de Bruxelles, Beurré de la Pentecôte, Bezi Chaumontel tres gros, Doyenne d'Hiver, Doyenne d'Hiver nouveau, Doyenne du Printemps, Cannirg, Du Patre, Philippe de Paques. Always second-rate when on standards or on pear stocks; on the quince it is much bettcr.

Beurré Knox. - Colour pale green; form obovate; size large ; quality good, although not firstrate. In use in October. A most abundant bearer, and large and handsome fruit. Subject to rot at the heart when fully ripe. Of Flemish origin.

Beurré Kenrick.-Colour greenish yellow, with indistinct russet spots; form flat at the eye, and tapering towards the foot-stalk; size medium; quality excellent. In use in September. A Flemish seedling raised by Dr Van Mons, and sent to America, and named by Mr Manning, the American pomologist.

Beurvé Langelier.-Size large ; quality firstrate; form obovate. In use in October and November. One of the very best autumn pears. Succeeds well on the quincc, bears well as a pyramid, and in good situations will do as a standard. 
Beurré, Mollett's Guernsey.-Colour yellowish green, nearly covered on one side with darkbrown russet in stripes and tracings; size medium; form oval-pyriform, often having a remarkable extension, or prolonged neck of the fruit, where it unites with the stalk; quality excellent. In use in December. Originated with Charles Mollett, Esq., of Guernsey, and brought into notice by $\mathrm{Mr}$ Thomson in 1842 . Synonym-Mollett's Guervsey Chaumontelle.

Beurré Preble. - Size large; form oblongovate; colour greenish yellow, mottled with russet. In use in October and November. A large and excellent American pear.

Beurré Rance.-Colour brownish green; form obovate pyriform; size above medium; quality first-rate. In use from March to May. Bears well as a standard around London, and has attained a fair size as such in the gardens at Dalkeith; its great merits, however, claim for it a place on a south wall. The best late pear yet known, and its late keeping renders it truly invaluable. Synonyms-Beurré de Ranz, Beurré de Flandre, Beurré Epine, Hardenpont du Printemps. A Flemish variety, raised by M. Hardenpont, of Mons. Mr Downing remarks : "It must be called Beurré de Rans, or Ranz, from the name of the commune in which it was raised, and not rance (rancid)."

Beurré Spence.-Regarding this pear there appears no small confusion. All the light Mr R. Thomson, in the third edition of the "Catalogue of Fruit in the Horticultural Society's Garden," throws on it, is, "B. Spence, said to be excellent; but the varieties received under this name have hitherto proved synonymous with other sorts." Downing, in "Fruits and Fruit Trees of America," says, "It is probable there may be a true Beurré Spence, since Dr Van Mons claims to have raised one, and has pronounced it the finest of all pears. But it is certain that neither the pomologists of England or America have yet been able to obtain it correct." Mr Rivers, in sending a tree as of this sort to Mr Downing, says, "This is the Beurré Spence of the Parisians. I ate it there in October, and thought it simply a good pear, scarcely deserving the high encomiums given by Van Mons to Mr Braddick." Van Mons' original description runs thus: "Wood short-jointed; leaves small ; branches horizontal or declining. The fruit is of the shape and size of the Brown beurré; skin green, handsomely sprinkled, and marked with reddish brown and reddish purple ; flesh tender, juicy, sugary, and perfumed. It ripens about the last of September."-Revue des Rerues, 1830, p. 180. Mr Rivers, describing the Beurré Spence in his possession in his catalogue, merely says it is of first size; first quality; melting ripe in October very productive as a standard; and, in a footnote, repeats the quotation above. In the "Fruit Catalogue" of Peter Lawson \& Son, we have the Beur'ré Spence of Van Mons thus described: "First size; first quality; obovate; melting. A fruit that has been much confused; but this is the right sort, according to Van Mons' original description ; ripens in September and October." From the same excellent authority we learn that there is a Beurré Spence cul- tivated in the gardens of the Caledonian Horticultural Society, which the author thus describes: "First size; first quality; long pyriform; melting. This variety ripens its fruit as a standard. Habit of tree vigorous; young shoots dark and rather upright; fruit of a brownish colour; flesh very rich; ripe in November and December." No notice is taken of this variety in Lindley's "Guide to the Orchard." If, therefore, the Messrs Lawson's sort be the correct one, and if it is so valuable a variety as Van Mons would make it appear to be, it will be weli for cultivators to apply to that respectable firm for trees. From the intercourse between the house of Lawson \& Son and foreign pomologists, we have no doubt theirs is the veritable sort. Be it good, bad, or indifferent, it would be a pity to lose sight of a pear Van Mons thought so highly of, for whose opinion we have so high a respect.

Bezide La Motte.-Colour pale yellowish green, thickly sprinkled with russety dots ; form roundish, flattened at the eye ; size medium; quality good. In use in October. An admirable old French variety. Tree exceedingly vigorous and productive, deserving a place in every garden. Synonyms - Bein Armudi, Beurré blanc of Jersey.

Bleekers meadow.-Size medium; form roundish; colour bright clear yellow, occasionally sprinkled with crimson dots. In use during October and November. Tree hardy, and bears great crops. Of American origin.

Bonchretien fondant.-Colour greenish brown; form oblong; size large; quality excellent, has a cool refreshing juice, somewhat like the St Germain. The tree is hardy and an excellent bearer. In use in October and November. Succeeds well as a dwarf standard when wrought on the quince stock.

Brougham.-Colouryellow, agood deal covered with russet; form roundish obovate; size large; quality melting, and of excellent flavour. In use in November. Tree hardy and very productive; succeeds in most places as a standard.

Buffam.-Size medium; form oblong obovate; colour deep yellow, turning somewhat reddish towards ripening. In use in September. Originated in Rhode Island, apparently from a seed of the Doyenne. An excellent American orchard pear of the first quality.

Calebasse.-Colour yellowish russet ; form oblong; size medium ; quality good; flesh crisp and juice sugary. Tree hardy and an abundant bearer. In use in October. Synonyms-Beurré de Payence, Calebassed' Hollande, Calebasse double extra, Calebasse Musque.

Chaptal.-Colour brownish; form obovate; size large; quality excellent. In use from December to April. Tree hardy as a standard in most places, and an excellent bearer.

Chaumontel.-Colour yellowish brown; form oblong; size large; quality first-rate. In use from November to March. Succeeds as a standard in the south of England, but requires a wall, which its great merits entitle it to, in the north, and generally throughout Scotland. It has fruited several years as a standard-trained quenouille, in the garden at Dalkeith; but although high in flavour, and keeping longer than 
such as we grow against walls, was very much smaller, and less perfect in form. Jersey and Guernsey are famous for this pear, there attaining a larger size and finer quality than we ever recollect to have found them on the Continent. This is of French origin, and has been long in cultivation, and was about the earliest introduced really good pear from that country. As a wall-tree on a west exposure it ripens well in the north of Perthshire. Synonyms-Oxford Chaumontel, Jersey Chaumontel, Bezi de Chaumontel, Peurré d'Hiver, Winter beurré.

Citron des Carmés.-Colour yellowish green; form obovate; size medium; quality one of the best early pears. Tree hardy and an abundant bearer. Succeeds in most places as a standard. To secure its early ripening, it will require the aid of an east or west wall. Synonym-Magdalene, Madeleine.

Colmar.-Colour greenish yellow and brown; form obtuse pyriform; size above medium; quality excellent. In use from November till February. Tree rather tender, requiring a wall in most parts of Britain. A very old French variety, and now long cultivated in our gardens. Synonyms-D'Auch, de Manne, Colmar Dore, Incomparable, Poire Manne, Berqamotte Tardife.

Colmar Neil.-Colour pale yellow; form obovate; size large; quality excellent. Succeeds in many favourable places as a standard; an excellent bearer. It has ripened as a pyramidal standard in the gardens at Dalkeith.

Columbia.-Colour pale green, turning, when ripe, to a fine golden yellow; form obovate, somewhat elongated; size large; quality first-rate. In use from November to January. Of American origin, and there highly esteemed as a valuable market and dessert fruit. Tree hardy, an abundant bearer, and fruits while young. Synonyms-Colombian Virgalieu, Colombia Virgalouse.

Comte de Lamy.-Colour pale-greenish brown; form roundish ovate; size rather under medium; quality first-rate. In use in October. Succeeds well in the south as a standard, and has as such ripened in the gardens at Dalkeith; but highly deserving a wall in every garden in the north of England, and generally in Scotland. Of Flemish origin. Synonyms-Beurré curte, Dingler, Marie Louise nova, Marie Louise the Second.

Copea.-Colour yellow, with specks of russet; form broad turbinate; size large; quality good. In use during September and October. Somewhat resembling the Beurré diel in flavour. Tree hardy, and an abundant bearer. Originated a few years ago at Philadelphia.

Crassane. - Colour greenish brown; form roundish; size large, when not overcropped; quality first-rate when in a favourable climate ; gritty when in too cold a situation. In use in November and December. Requires a wall in most parts of Britain. Synonyms-Beurré Plat, Bergamotte crassane, Cresane. An old French variety, long cultivated in Britain.

Crassane, Althorp.-Colour greenish brown ; form roundish ovate; size rather under medium; quality excellent. In use in October and November. Of English origin, raised by the late T. A. Knight, Esq. Tree much hardier than the former, and succeeding well as a standard in most favourable places. Well worthy of a wall in the colder parts of Scotland and north of England.

Crassane, winter.-Colour greenish yellow and brown ; form turbinate ; size large ; quality excellent. In use in January. Tree hardy and an abundant bearer, suitable to similar situations as the last.

Cushing.-Colour light-greenish yellow, sprinkled with small grey dots; form obovate ; size large; quality excellent. In use about the middle of September. Another excellent pear of American origin.

Dearborn's seedling. - Colour pale yellow; form obovate ; size medium; quality excellent. In use in September and October. Tree hardy and an abundant bearer, succeeding well as a standard in most places. Of American origin, in which country it is held in high repute. It is somewhat allied to the White Doyenne, and probably sprang from a seed of that variety.

De Louvain.-Colour light yellow, slightly marked with russet, taking a ruddy tinge next the sun; form obovate, slightly tapering towards the stalk; size medium ; quality excellent. In use from September till the beginning of December. Raised by Dr Van Mons of Louvain in 1827. Synonym-Poire de Louvain.

Dix.-Colour deep yellow, marked with russet dots ; form oblong; size large ; quality excellent. In use in October and November. Another American pear of very great excellence, originated near Boston, about forty years since. It is described by Mr Downing as "a fruit of the highest excellence, and well deserves the attention of all planters." It is one of the hardiest of pear trees, and although the tree does not come into bearing until it has attained a considerable size, yet it produces abundantly, and from its habit will undoubtedly prove remarkably long lived and free from disease.

Downton. - Colour yellowish brown; form pyriform; size rather under medium; quality good. In use in January and February. Of English origin, having been raised by the late T. A. Knight, Esq. Tree hardy and an abundant bearer, succeeding well in most places as a standard.

Doyenne blanc.-Colour pale yellow ; in most respects resembling the next, except in colour. In use in September and October. An excellent bearer, and where it succeeds as a standard in the southern countries its fruit is superior to that grown on a wall. It has no less than twenty-five synonyms-a proof of its excellence, and the extent to which it is cultivated, as well as of the great confusion that exists in pomological nomenclature, and the necessity of synonyms being strictly attached to all fruit-tree lists. These are-White beurré, White autumn beurré, Beurré blanc, Beurré du Roi of some, Beurré Anglaise, White Doyenne, Dean's, Snow pear, Poire neige, Poire de lemon, Poire de seign. eur, Pine pear, Warwick Bergamot St Michel, Bonne-ente, $A$ courte quesse, Monsieur Citron, de September, Passe Colmar d'automne of some, Valencia.

Doyenne gris.-Colour russety red; form obovate; size rather under medium; quality 
excellent. In use during October and November. Keeps better than the preceding, which it much resembles in all respects except in colour. An excellent standard pear in a favourable climate, and deserving a wall in the north of England and most of Scotland. Synonyms-Doyenne grey, D. red, D. d'Automne, D. rouge, D. galeux, St Michael Dore, Grey Dean's.

Duchesse d'Angoulême.-Colour brownish yellow ; size very large, sometimes weighing $1 \frac{1}{4} \mathrm{lb}$; form obtuse-oblong; quality, when grown as a standard, as it is in the south of England, excellent; apt to become dry and devoid of fine flavour whenever ripened against a wall. It is a splendid pear. In use in October and November ; if standard-grown, it will last much longer. Of French origin, being discovered accidentally in a hedge near Angers. This variety appears to succeed better on the quince stock than on any other.

Dundas. - Colour brilliant clear yellow, slightly sprinkled with greenish-black dots, bright red towards the sun; altogether a very beautiful pear; form obovate; size medium; quality very good. In use from the beginning of October till December. Another of Dr Van Mons' rearing. We have not seen it growing as a standard in Britain ; it is, however, on account of its great beauty, worth a place on an east or west wall.

Dunmore. - Colour green-brownish russet; form obovate; size large; quality first-rate, in flavour equal to the Brown beurré. Of English origin, having been raised by the late $\mathrm{T}$. $\mathrm{A}$. Knight, Esq. Tree hardy, and an excellent bearer. In use in September. Succeeds in most places as a standard. Its blossoms are less liable to be injured by spring frosts than most others - a most important quality, particularly for the climate of Scotland.

Elton.-Colour brownish red; form oval ; size rather under medium; quality excellent. In use in September. Fruit generally without cells or seeds. Succeeds in favourable situations as a standard. Of English origin : found by T. A. Knight, Esq., in an orchard in the parish of Elton, Herefordshire. The original tree in 1812 was supposed to be at least 170 years old.

Emerald.-Colour pale-greenish brown ; form obovate; size medium; quality excellent. In use in November and December. Succeeds as a standard in all favourable situations. Tree hardy, and an excellent bearer. Bears some resemblance to the Glout morceau, but better adapted to our climate. Of English origin.

Eyewood.-Colour russet ; form oblate ; size under medium; quality excellent. In use during October and November. Tree vigorous and hardy, and an excellent bearer, succeeding well as a standard in most places. Another excellent pear of English origin, raised by T. A. Knight, Esq.

Flemish beauty.-Colour russet red; form obovate ; size large ; quality excellent. In use in September and October. Tree hardy, and succeeding as a standard in all ordinary situations. The fruit should be gathered before it becomes too ripe, else it is apt to become dry and musky. This pear is held in high repute in America, where, according to Downing, it attains a size of 12 inches in circumference. Synonyms-Imperatrice de France, Josephine of some French catalogues, La belle de Flanders, Bosch, Bosc sine, Bouche nouvelle, Fondante du Bois of some, Brilliant.

Fondante d'Automne.-Colour pale green; form obovate ; size rather under medium ; quality excellent. So highly is this pear esteemed in America, that Downing says, "If we were asked which are the two highest-flavoured pears known in this country [America], we should not hesitate to name the Seckle and the Fondante d'Automne (autumn-melting)." In use in September and October. Tree hardy, and succeeds well as a standard in most gardens, and where not, it deserves a wall. This variety should not be confounded with the Fondante du Bois nouvelle, which is an inferior fruit.

Fondante du Bois.-Colour greenish yellow ; form obovate; size rather under medium; quality excellent. In use from December till March. Tree hardy, and an excellent bearer, even as a standard in moderate situations. A fruit altogether equal to the Passe Colmar in quality, and keeps longer.

Forelle.-Colour yellowish green and russet, beautifully speckled with red; form obovate; size rather under medium; quality excellent. In use from November till January, or later. A German fruit of great merit. Requires a warm soil and exposure, and well deserves to be trained as an espalier, and in colder localities against a wall. Synonyms-Poire truite, Forellenbirne - both meaning trout-pear, because the skin is speckled like a trout. It has been remarked that pears like this, having so brilliant a colour, are rarely possessed of such merits and of so rich a flavour. They are in general astringent and coarse. This tree blossoms early, and has often been remarked to have its fruit so far advanced as to have withstood the late spring frosts that have frequently destroyed the blossoms of all others.

Frederic de Wurtemberg. - Colour yellow when ripe, with a fine red or rather rich crimson side next the sun ; form irregular pyriform ; size large. In use in September. Quality very good. Tree moderately hardy and a good bearer. Originated with Dr Van Mons in 1812. A very different fruit from the Beurré de Capiamont, with which it is often confounded.

Fulton.-Colour at first grey russet, changing as it ripens to a fine cinnamon russet; form roundish, flattened; size below medium. In use in October and November. Quality excellent, if gathered before fully ripe, but if allowed to ripen on the tree of much less value. Tree hardy. Very great bearer. An American variety ; a native of Maine-likely to become a good market-fruit in this country.

Glout morceau.-Colour pale green ; size large ; form obtuse elliptic ; quality first-rate. In use from November to January. One of the finest pears grown. An excellent bearer, but requires a wall in most situations. Ripens well as a standard in the neighbourhood of London. A Flemish pear of the highest merit. Synonyms-Cal- 
mar d'Hiver, Beurré Hardenpont, Beurré d'Hiver nouvelle, Beurré de Cambron, Roi de Wurtemberg, Gloux morceaux, Hardenpont d'Hiver, Got luc de Cambron, Linden d'Automne. This excellent pear has got into disrepute in some localities, on account of its not ripening. In cold climates this is the case when wrought upon the pear stock ; on the quince it ripens well in most parts of Scotland-a circumstance pointed out by $\mathrm{Mr}$ Rivers, even in the superior climate of Hertfordshire. On the quince stock we have little doubt that this pear would ripen against a wall even in the Highlands.

Groom's Princess-Royal. - Colour greenish brown, slightly traced with russet ; form roundish ; size medium; quality excellent. In use during January and February. Tree hardy and a good bearer as a standard. This is a very recent addition to our English pears, having been raised by $\mathrm{Mr}$ Groom, the well-known florist.

Hacon's incomparable. - Colour brownish green ; size above medium ; form roundish. In use from November to January, or later. Quality excellent. Tree very hardy, and an excellent bearer, succeeding in most places as a standard. Synonym-Downhain seedling. Originated with Mr Hacon of Downham Market, Norfolk. On the pear stock it requires from seven to ten years before it comes into a bearing state, fruiting on the quince stock much sooner. Blossoms seldom injured by spring-frosts.

Harrard.-Colour russety olive yellow, with a brownish-red side next the sun ; form oblong pyriform ; size rather above medium ; quality " one of the best and most profitable orchard pears to plant in quantity for market purposes. It produces enormous crops of fine-looking fruit, which is of fair quality, and commands the best prices. The tree is remarkably hardy and vigorous, its upright shoots forming a fine head."(Downing.) It is of American origin, and well worth the attention of orchardists in this country as a substitute to the trashy sorts so generally grown, its only fault being its not keeping. Synonyms-Boston Espurne, Cambridge sugar pear.

Hessel.-Colour brown ; size below medium; form obovate; of value not so much for high flavour as for its wonderful productiveness, for which reason $t$ is extensively grown for the market, and it of all early autumn pears is the best adapted for cottage culture, and this the more so as the trees are of moderate growth. Very hardy, and fitted for all situations. Originated at the village of Hessel near Hull.

Jargonelle (of the English.)-Colour yellowish brown; size large; form pyramidal ; qua lity the best of its season. In use in August and September. Tree hardy in pear soils ; apt to canker when grown as a standard in light soils. Ripens as a standard in most parts of Scotland, and attains a high flavour, but to grow it to its full size and beauty a west or east wall is required. We have two jargonelles in cultivation - the one called the English and the other the French. It is highly probable that both were introduced here from France. On this Mr Downing remarks, "Although called by 'Thomson the English jargonelle, to distinguish it from the fruit more common under that name on the Continent, there is no doubt that it was introduced originally from France. Antiquarians derive its name from Gergon, Italian, a corruption of Gracum, whence Merlet supposes it to be the Numidianum Grocum of Pliny, and the Groculum of Macrobius. This, if correct, would prove it to be a very ancient sort." Synonyms-Gross cuisse madame, Sweet summer, Espargne, Saint Sampson, Beau present. Saint Lambert, Poire des tables des princes Frauenschenkel. Tree of strong yet pendulous growth.

Jargonelle (of the French). - Colour light green, becoming lemon-colour, with a tinge of red on the sunny side when ripe; form obovate; size medium; quality very inferior to the last, and decaying soon at the heart. In use during August. Tree of strong upright growth; much cultivated in France, and there recognised as the Cuisse Madame. Synonyms-Sabine d'Eté, Supreme, Vermillion d'Eté, Bellissime d'Eté, Bellissime Supreme, Bellissime Jargonelle, Red Muscadel.

Jean de Witte.-Colour brownish green; form obovate; size under medium. In use in January and February. Quality excellent, something in the way of the Glout Morceau, but keeps longer, although not quite so rich. Tree hardy; an excellent bearer as a standard tree around London ; does not appear to succeed so well in the north, unless planted against a wall.

Lawrence. - Colour pale yellowish green, slightly marked with palish brown; form obovate, tapering to an obtuse end; size above medium; quality excellent. In use from October to March, ripening regularly in succession, and not liable to rot. This is another American pear of considerable merit. Downing informs us that it rose from seed in Flushing, Long lsland, in the neighbourhood of two other pear trees only, the St Germain and the White Doynne, and bears some proofs, in its qualities, of being a natural cross between the two.

Louise Bonne (of Jersey).-Colour greenishbrown and red; form pyramidal; size above medium, when not overloaded; quality good. In use in October. This excellent pear is claimed by the English as a Jersey fruit, and by the French for the neighbourhood of Avranches. It was first brought into notice in 1820, when specimens of the fruit were exhibited at the London Horticultural Society Rooms, from the garden of General Gordon of Jersey. It is now in very general cultivation, and succeeds well as a stand. ard in most gardens. Synonyms-William the Fourth, Louise Bonné d'Avranclies, Beurre ou Bonne Louise d'Araudore. We have adopted the synonyms given by $\mathrm{Mr}$ Thomson in the "Fruit Catalogue of the Horticultural Society," and the description from fruit grown for several years in the Dalkeith gardens. The Louise Bonne of Jersey of Mr Rivers appears by his description to be different. In a foot-note in his Descriptive Catalogue of Pears, he says, "This is (that is, his variety) the Louise Bonne d'Avranches of the French; the true Louise Bonne of Jersey is an inferior variety, ripening earlier in the season." We have trees of both varieties, 
but that of Mr Rivers not having as yet fruited with us, we are unable to say whether in reality a difference exists.

Mansuette.-Colour pale green and brown; size large; form oblong obovate; quality excellent. In use in September and October. Mr Thomson remarks, "This is one of the few good French pears that will bear as a standard, and as such this requires a favourable soil and situation."

Marie Louise.-Colour brown and yellow; form oblong; size above medium, often large; quality first-rate. In use in October and November. This truly fine pear was raised from seed by the Abbé Duquesne, of Belgium, in 1809 ; introduced to England by Dr Van Mons in 1816. Tree hardy, and succeeds well as a standard in favourable situations, where it bears abundantly, and the fruit so produced is superior to that grown against walls, in which latter condition it must ever be grown in most parts of Scotland. Synonyms-Braddick's field standard, Forme de Marie Louise, Princesse de Parma, Marie Chretienne, readily distinguished from the next by its crooked and declining branches, with narrow leaves.

Marie Louise (Van Mons).-Colour yellow, brownish red on the side next the sun; form pyriform; size above medium; quality secondrate, but in good seasons, when not overcropped, of excellent quality; apt to produce more fruit than the tree can bring to full maturity. Tree hardy, erect, with strong dark-coloured shoots. In use in September and October, and does not keep well. An inferior fruit to the last. The Marie Delcourt of $\mathrm{Mr}$ Thomson in "Fruit Catalogue of the Horticultural Society," No. 306, is probably the Marie Louise (Delcourt) of Mr Rivers, No. 90 in his descriptive catalogue, and also of the French gardens, said by the last authority to be superior to the old Marie Louise.

Moccas.-Colour pale brown; form obovate; size medium; quality excellent. In use in December. A hardy tree, bearing well as a standard, and almost resembling the following in point of merit. Originated at Moccas Court, Herefordshire. One of our best standard pears.

Monarch, Knight's. - Colour yellowish brown; form obovate; size medium; quality excellent. In use in January. Tree hardy and a great bearer, even in a standard form. Originated with T. A. Knight, Esq., and with the last two valuable acquisitions to our standard-growing pears. They are both well worthy of the notice of Scottish orchardists. Knight called this the Monarch, because he conceived it superior to all others, and on account of its perfecting its fruit in the first year of the reign of William the Fourth.

Moorfowl eg., Galston's.-Colour yellowish brown; form roundish; size medium; quality good. This appears to be a rather rare sort; we have only seen two or three specimens in cultivation, and these grown against walls in Perthshire. In use in October and November. A very different fruit from the Old moorfowl eg. an old hardy Scotch variety not worth growing where better sorts will live.
Napoleon.-Colour pale green, turning yellowish by keeping; form obtuse pyramidal; size large; quality excellent. In use in November and December. Tree healthy, and ripens as a standard around London, but requires the aid of a wall in Scotland. It was raised by $\mathbf{M}$. Liart, a gardener at Mons. According to Downing, he received a medal from the Mons Horticultural Society for the fruit, and hence the synonym Medaille; the original tree was purchased for thirty-three francs by the Abbé Duquesne, who bestowed on it the name of Napoleon. Synonyms-Roi de Rome, Medaille, Charles d'Autrich, Sucrée Dorée of some, Wurtemberg.

Neil.-Colour pale yellow; form obovate; size large; quality good. In use in September and October. Tree very hardy, succeeding in many places as a standard; a great bearer, and producing beautiful fruit. Raised by Dr Van Mons from seed sown in 1815, and named by him in honour of that excellent man and enthusiastic horticulturist, the late Dr Neil of Edinburgh. Is apt to overbear itself, and should be, therefore, moderately thinned of wood, and even that shortened so as to insure a limited supply of young shoots.

Nelis, winter.-Colour yellowish-brown; form obovate; size under medium; quality excellent. In use in December and January. Produces excellent although not full-sized fruit as a standard around London; requires a wall in the north of England and most of Scotland. This is another excellent Belgian variety. Synonyms-Beurré de Malives, Milanaise, Cuvelier, Nelis d'Hiver, Etrounieau, Bonne de Malines.

Ne plus Meuris.-Colour brownish russet; form roundish, usually very irregular; size medium; quality excellent. In use from November to March. "One of the best late pears, although not so handsome as some."-(Тномson). Of Belgian origin, from the stock of Dr Van Mons.

Passe Colmar.-Colour brownish yellow; form obovate; size large; quality excellent. In use during December and January, and with good management much longer. This very excellent pear is of Belgian origin, and being raised by the Counsellor Hardenpont. It is one of our most popular winter pears, on account of its excellent flavour, vigorous growth, and abundant bearing. In too rich soils it is apt to grow too Iuxuriantly, and therefore root-pruning has often to be had recourse to, to keep it of moderate size, and to induce fruitfulness. This is a much more hardy tree than the old Colmar, and is exceedingly free from canker and disease. To increase the size of the fruit, they should be regularly thinned after setting, and some recommend, to attain this end, the removal of half the fruit-bearing spurs in the month of March. Synonyms-Chapmans, Colmar d'Or, Colmar Preul, Colmar Hardenpont, Colmar Gris, Colmar Epineux, Passe Colmar Epineux, Passe Colmar Gris, Passe Colmar Gris dit Precel, Beurre Colmar Gris dit Precel, Fondante de Mons, Fondante de Panisel, d'Anans, Present de Malines, Marotte Sucrée Jaune, Souveraine, Gambier, Colmar Souverain, Cellite, Beaurre d'Argenson, Regentin.

The Passe Colmar d'Or is given by $\mathrm{Mr}$ Thom. 
son, in "Fruit Cat. Hort. Soc.," No. 339, as merely a synonym of the common Passe Colmar. Mr Rivers says, in " Descriptive List of Pears," p. 4, "It is the better variety of the two, quite distinct. Those fruits which are exposed are of a rather bright red on the sunny side. The $\mathrm{Col}$ mar gris, unripe, is of a uniform grey, and, when ripe, not so yellow as the preceding. Both varieties bear abundantly as standards on the pear stock, but do not ripen their fruit kindly at Sawbridgeworth. On the quince they succeed well." This is by no means an uncommon result in the case of many other pears, and the more unfavourable the situation, the more obvious is the case.

Petre.-Colour pale yellow, slightly marked with russet towards the eye ; form obovate size about medium; quality excellent. In use in October, and if gathered before fully ripe will keep some weeks. This is an American pear of the highest excellence. The original tree, Downing informs us, is still growing in the old Bertram Botanic Garden, near Philadelphia. It appears to have originated from a seed received by the elder John Bertram from Lord Petre of Essex, in 1735, who, in twenty-five years afterwards, received fruit of this tree, which he pronounced superior to the tree from whence the seed had been taken. Tree moderate in growth, and an excellent bearer.

Prince's Saint Germains.-Colour greenish, covered with a brownish russet, the side next the sun turning dull red as it approaches maturity; form obovate, somewhat inclining to oval ; size medium. In use from November till March. An American pear, originated from a seed of Saint Germain panachée, or Striped Saint Germains, but much superior to it. It is a very hardy tree, and an excellent bearer ; keeps well, and esteemed superior to the old St Germain. Synonym-New St Germain.

Saint Germain.-Colour yellowish green and brown; form pyramidal ; size large; quality excellent. In use from November to January. An old French variety, long cultivated in our gardens; requires a wall in most situations, and is a healthy tree, and abundant bearer. Synonym - Saint Germain Gris, Saint Germain Jaune-names evidently arising from the colour of the fruit in different stages of its existence.

Seckel.-Colour brownish red; form obovate; size small ; quality excellent. In use in October. Another American pear of great merit, well adapted to European culture ; an excellent bearer, having a peculiarly rich aroma, with a honied sweetness. Synonym-New York red-cheek, Red cheeked Seckel. A very curious account of the supposed origin of this pear is given by Downing, page 415 of his interesting work on the "Fruits and Fruit Trees of America." Grafted on the apple, this excellent pear has been much improved in size, but not equally improved in flavour. If it is one of the very best of the American pears, it is, we should think, one of the most ugly in appearance. Although it succeeds well in many parts of Britain, it has never attained maturity at Dalkeith.

Swan's egg.-Colour greenish brown; form obovate; size under medium; quality good ; the best of our hardy standard pears, ripening in most parts of Scotland, and an abundant bearer. Trees apt to grow very tall. In use in October.

Thomson's.-Colour pale yellow; form obovate ; size medium ; quality excellent. In use in November. Flavour resembling the Passe Colmar, exceedingly rich. Tree hardy, and bears well as a standard in most gardens.

Vicar of Winkfield.-Colour greyish brown and red ; form pyramidal ; size very large ; quality good. In use from November to January. Tree hardy, and a good bearer, but most esteemed on account of its large size. Of French origin, being discovered a few years since growing naturally in the woods of Clion by a French curate, whence it obtained the names of Le Curé, or Monsieur le Curé. It was soon thereafter imported into England by the late Rev. Mr Rham, vicar of Winkfield in Berkshire, and hence that name also. The other synonyms are-Dumas, Bourgermester, incorrectly of Boston; and Kenrick, an American pomologist, has called it Clion.

Van Mons' Leon le Clerc.-Colour yellowish, nearly covered with a brownish russet; form oblong ovate; size large ; quality very good. In use in October and November. This fine pear originated with M. Leon le Clerc, an amateur cultivator of Laval, in France. This must not be confounded with Leon le Clerk, described in our list of culinary pears.

Comparatively new or recently introduced dessert pears.-Abele de St Denis.-Size large ; quality excellent; succeeds well on the quince stock in forming a pyramidal tree, and where there is room to spare deserves a wall.

Beau Present d'Artois. - Size large ; quality good, half melting. In use during September. Tree very hardy, and succeeds well on the quince, either as a standard or pyramid, in either case bearing abundantly.

Belle Hugevine. - Size large; quality excellent, and fruit handsome. In use during March and April. Tree very hardy, succeeding well on the quince stock.

Belle apres Noel (Esperen).-About the size, and something of the shape, of a moderatesized cressane; colour bright yellow, red on the sunny side; very handsome; rich and highflavoured. Tree rather of slender growth, yet an abundant bearer. In use from the middle of December to middle of January. Lately brought into notice by $\mathrm{Mr}$ Rivers.

Beryamotte d'Esperen.-Size small, somewhat resembling the Autumn Bergamotte; quality excellent. In use during April and May. Succeeds well on the quince stock.

Beurré Andusson. - Size medium; quality good, and very handsome. In use in November. Tree hardy, and succeeds well, and bear's abundantly as a pyramid on the quince.

Beurré Bretonneau.-Size large; quality excellent. Tree hardy, and succeeds well on the quince, double-worked, and is very prolific as a pyramid. In use during May and June.

Beurré Brissic.-Dize medium; quality excellent; form obtuse. In use in March and April. 
Tree hardy, succeeding well as a standard on the quince stock. Deserving general cultivation.

Beurré Colmar (Van Mons).-Size medium; quality excellent; form obovate ; colour yellowish when ripe; a first-rate autumn pear. In use during October and November. Originated from seed with Dr Van Mons. Altogether a different pear from the Autumn Colmar, which it somewhat resembles.

Beurré Daviss.-Size medium ; form obovate; quality good. In use during January and February. Somewhat resembling the Passe Colmar, but keeps better. Tree hardy, succeeding well as a standard.

Beurré de Benoist. - Size large; quality excellent, slightly perfumed, and has an agreeable flavour. In use during September and October. Adapted to standard culture, and succeeds well on the quince. Synonym-Beurré A uguste Benoist, Benoist.

Beurré d'Esperen.--Size large; quality good. Tree hardy, healthy, and succeeds well on the quince stock. In use in January.

Beurré de Streckman (Van Mons).--Size medium; quality good. Tree hardy, and bears well as a dwarf standard, more especially on the quince stock. In use in January and February.

Beurvé Dumortier. - Size medium; quality good, and of high flavour - the fruit we tasted was remarkably so ; form obovate ; colour dullish yellow. In use in September. Tree hardy, and succeeds as a standard in Mid-Lothian.

Beurré Giffara.-Size medium; quality excellent; ripe in August. Tree hardy, and a great bearer, succeeding as a pyramid or standard.

Beurré Gris d'Hiver nouveau, or Beurré de Luçon.-Of this pear Mr Rivers remarks :"This fine large pear is, I think, calculated to form an important feature in our winter desserts. It seems to be of the race of the old Brown beurré-Beurré Gris of the French. Its fruit has nearly the same shape, but it is larger, and generally covered with a thick coat of russet. It differs, however, widely from its type, in the great merit of being fit for the table for two or three months after Brown beurrés are gone. It is in season generally from the end of January till the middle or end of March. Its flavour is peculiarly high, its flesh melting and very juicy, but liable in some soils to be a little gritty. It does not grow freely on the quince unless under glass, and is not hardy enough for a pyramid on the pear stock, unless in very warm and sheltered situations; but it amply deserves a wall (a south-east, south, or west aspect, would be the most eligible). It ought to be in every good collection of pears." It must, from the above, be considered a delicate sort, and only fitted for the best situations.

Beurré Langelier's. - Size large ; quality excellent; one of the very best winter pears; succeeds well on the quince, and bears as a pyramid in most ordinary situations. In use during October and November. Synonym - Langelier's Beurré.

Beurré Lucratif.--Size medium; quality excellent; colour pale yellowish green; form obovato. In use during September and October.
Tree hardy, succeeding either as a standard or on the wall. Of Flemish origin.

Beurré Magnifique.-Size large; quality excellent; form obovate. In use in November and December. Tree hardy, and an excellent bearer, either as a wall or standard-the former in most parts of Scotland. This name has been given as a synonym to the Beurré Diel by $\mathrm{Mr}$ Thomson in third edition of "Catalogue of Fruits," \&c., but subsequent experience has proved this to be a very different fruit.

Beurré Montfontaine.-Size medium; quality excellent; form obovate; flesh crisp. In use during September and October. Tree hardy, a good bearer, and ripens well as a standard.

Beurré Navez (Van Mons).--Size large; quality excellent; said by M. Van Mons to be the sweetest of all pears. In use during September.

Beurré Robin.-Size large ; quality good. In use during October and November. Tree very hardy, succeeding on the quince, and equally adapted for standard, pyramid, or wall.

Beurré Seutin.--Size large; quality good. In use in January. Succeeds well as a pyramid on the quince stock, suitable also for walls or standards.

Beurré superfin.-Size large; quality excellent. Tree healthy, succeeding well on the quince, and makes a handsome pyramid. In use during December and January.

Beurré supreme.-Size medium; quality good; form roundish, resembling that excellent pear, Comte de Lamy. Tree hardy, succeeding well as a standard, and is in all cases an excellent bearer. In use during October and November.

Beurré winter (Rivers).-Size large; quality excellent, flavour very rich and vinous. Tree hardy, and succeeds well on the quince stock double worked, either as a wall or standard. This excellent pear was raised by Mr Rivers of Sawbridgeworth, from the seed of the Easter beurré. In use during February and March. Must not be mistaken with either the Black Achan or Chaumontel, both of which are sometimes called Winter beurré.

Bezi d'Esperen.-Size large; quality good. In use during December and January. Tree very hardy, succeeding well on the quince stock either as a pyramid, standard, or wall tree. This excellent pear was raised from seed supposed to be that of the Winter Nelis, by the late Major Esperen of Malines.

Bonne Gustave (Esperen).--Size large; quality good. In use during December. Tree hardy, and bears abundantly wrought on the quince, whether trained as a pyramid or standard.

Canning.-Size large; quality excellent. In fruit resembling the Easter beurré, but differing in the habit of the tree, which is more robust and hardy. Succeeds as a pyramid on the quince, but deserves a wall in most places. In use during January and February. This is given by Mr Thomson as a synonym of Easter beurré ; it appears, however, to be different, more especially in respect to hardiness.

Columbia.-Size large ; quality excellent, juicy and melting; form obovate ; colour, when ripe, a fine golden yellow tinged with orange. In 
use from November to January. Of American origin, and, so far as it has been tried in Britain, likely to become a great acquisition, as it is both handsome and productive.

Compte de Flandre.-Size very large; quality excellent, melting; of Belgian origin. In use during December. Tree hardy, succeeding well on the quince stock, and trained either as a standard or pyramid, but in most places deserving a wall.

Delice de Jodoigne (Bouvier). - Size large; quality good; an excellent autumn pear. In use during November. Tree very hardy, succeeding well on the quince, and trained as a pyramid.

De Spoelberg.-Size medium; quality good; flavour rich and melting. In use in November. Tree hardy, succeeding on the quince double wrought, either as a standard or wall. Synonym - Vicomte de Spoelberg.

Dr Bouvier (Van Mons).-Size large; quality excellent. In use in February and March. 'Tree hardy; inclined to put out thorns; succeeds well as a standard, and if double wrought on the quince.

Doyenne d'Effay.-Size medium; quality excellent; melting; form obovate. In use in November. This excellent pear succeeds on the quince, and makes a handsome pyramid.

Doyenne, Goubault.-Size large; quality excellent, as are all the Doyennes. In use from January to April. Tree very hardy, succeeding well on the quince as a standard in most places.

Doyenne nouveau.--Size medium; quality excellent; flesh tender and juicy; form obovate. In use in April. Very productive when wrought on the quince and trained as a pyramid.

Drapiez. - Size medium; quality excellent; form obovate. In use in October. A very excellent autumn fruit, especially as it succeeds as a dwarf standard on the quince stock.

Eliza d'Heyst (Esperen).--Size medium; quality excellent. In use in March and April. Tree tender, requiring a warm soil and situation. On the quince, if double wrought, it succeeds well, but requires the protection of a wall in Britain.

Fondante de Charmensier.-Size large; quality excellent. In use in December. Remarkable for its productiveness as well as fine quality.

Frederick de Wurtemberg. - Size large; quality excellent; flesh juicy and melting; colour deep yellow when ripe, with dashes of red crimson next the sun. In use in September and October. Tree hardy, and bears early, even as a standard. One ô̂ Dr Van Mons' excellent pears, deserving of general cultivation.

Grand soleil (Esperen).-Size large; quality excellent; flesh half-melting. In use from December to March. Tree tender, requiring a wall in most situations. Not adapted to the generality of Scotland.

Josephine de Malines (Esperen).-Of this pear $\mathrm{Mr}$ Rivers gives the following high description: "This very fine late pear seems to be of the Passe Colmar race, for it has much resemblance to that well-known variety in its pointed leaves, the size and form of its fruit, and general habit. It grows freely on the quince, and VOL. II. bears well as a pyramid, even when grafted on the pear stock, but deserves a wall in the cool and moist parts of England. Its season [but who, $\mathrm{Mr}$ Rivers very justly remarks, can tell the ripening season of pears, when $N e$ plus meuris ripened this season in November ?] is from March to the end of April, and its flavour is, I think, the highest and most agreeable of any pear known, being, at the same time, perfectly melting and juicy."

Paquency.-Size medium; quality excellent; flesh inelting; sweet, with a rich perfume; form obovate; colour dull yellow, slightly marked with russet. An excellent French pear, succeeding on the quince and trained as a pyramid. In use during October and November.

Saint Aubin sur Riga.-Size large; quality excellent; flesh melting, tender; flavour rich. A New Jersey pear of much excellence either as a wall or standard. In use in January and February.

Suffolk thorn.-Size medium; quality good; flesh melting; flavour partaking of Gansal's Bergamotte, from which it is a seedling; form roundish. In use in October. Forms a hardy tree when wrought on the quince stock.

\section{KITCHEN PEARS.}

Bellisime d'Hiver.-Colour brown and red; form roundish; size large. In use from November till April. Quality first-rate. This is one of our best stewing-pears. An abundant bearer, and succeeds in favourable situations as a standard. Synonyms-Teton de Denus, De Bur, Belle Noisette.

Belmont. - Colour pale yellowish brown; form obovate; size large. In use during November. Quality good. An excellent bearer, and succeeds in most gardens as a standard.

Bergamot, Easter.-Colour pale green; form obovate; size medium. In use during March and April. One of our best culinary pears. Keeps well, retains its juice, and if properly ripened is not liable to shrivel. Succeeds as a standard only in favourable situations; requires a wall in most parts of Scotland and the nortl of England. Synonyms-Robert's keeping, Pad. dington, Bergamotte d'Hiver, Bergamotte de Bugi, Bergamotte de Paques, Bergamotte de Toulouse, Winter Bergamot, Terling, Royal Tairlon.

Bezi d'Heri._Colour greenish yellow; form roundish; size medium. In use from October to January. An excellent bearer, and much esteemed for culinary purposes. Succeeds as a standard in ordinary good situations, but requires a wall in cold upland places. Synonyms -De Bordeaux, Bezi royal.

Bon Chretien, Flerish. - Colour vellowish brown; form obovate; size medium. In use from November to March. A great bearer as a standard in the southern counties of England, rarely so succeeding in most of Scotland. Synonyms-Bon Chretien T'urc, Bon Chretien nourelle.

Catillac.-Colour brownish yellow and red; form turbinate; size large. In use from December to April. Succeeds well as a dwarf standard trained quenouille in the south of England, requiring a wall in the north, and throughout Scotland generally. Much esteemed as a culi- 
nary fruit. Synonyms-Pound pear, Bell pear, Groote mogul, Grand monarque. An old French variety.

Franc real d'Hiver.-Colour brown and yellow; form roundish ovate; size medium. In use from December to March. Tree hardy, and a good bearer. Stews tender, and of a bright light purple colour. Synonyms-Franc real, rin or d'Hiver.

Gilogil.-Colour russet; form roundish; size very large. In use from November to February. Quality good, and very handsome. Synonyms-Garde d'Ecosse, Poir gobert, and various corruptions of Gilogil.

Leon le Clerc.-Colour yellowish russet; form obovate; size largc. In use from December to April. Not identical with Van Mons' Leon le Clerr. Quality good for culinary purposes.

Uredale's St Germain.-Colour greenish brown; form pyramidal; size very large, sometimes attaining the weight of $3 \mathrm{lb}$; long esteemed as one of our most valuable culinary fruits; requires the aid of a wall in most situations. In use from January to April. Synonyms-Lent St Germains, Chambers' large, Union, Uvedale's warlen, Piper, Pickering's pear', Pickering's warden, Germain baker.

\section{SELECT LISTS OF PEARS FOR PAKTICULAR LOCALITIES, ETC.}

The following selection is considered by $\mathrm{Mr}$ Errington to be the best twenty sorts in the country :-1, Citron de Carmes ; 2, Jargonelle ; 3, Dunmore; 4, Williams' Bon Chretien ; 5, Beurré d'Amalis ; 6, Fondante d'Automne ; 7, Louis Bonne of Jersey ; 8, Aston Town ; 9 , Beurré d'Capiamont; 10, Marie Louise; 11, Althorp Cresane ; 12, Beurré Diel ; 13, Passe Colmar ; 14, Hacon's Incomparable ; 15, Glout Morceau ; 16, Winter Nelis ; 17, Knight's Monarch ; 18, Easter Beurré ; 19, Beurré Ranz; 20 , Ne Plus Meuris. Of these the following are adapted to a southern orchard-Nos. 1, 2, 3, $4,5,6,7,8,9,10,11,14$; for a northern orchard-Nos. 1, 2, 3, 4, 5, 8, 14 ; for east and west walls in the north, or for dwarf standards in the south-Nos. $2,3,10,11,12,13,15,16$, $17,19,20$; sorts fitted for cottages, being particularly profitable-Nos. $2,3,4,7,9,12,14,17$.

Mr Rivers recommends the following twelve varieties as pyramidal pears on the quince stock, placed in the order of their ripening. Those marked * may be chosen by those who require only a few trees. This selection is made, however, for the southern and midland counties:-Doyenne d'Eté, July ; *Jargonelle, August; Bon Chretien (Williams), September ; ${ }^{*}$ Beurré d'Amalis, September ; Van Mons' Leon le Clerc, October ; * Louis Bonne of Jersey, October ; Beurré Diel, November ; Belle de Neol, December; *Yasse Colmar, December ; ${ }^{*}$ Orpheline d'Enghien, January and February ; Josephine de Malines, March ; ${ }^{*}$ Susette de Bavay, April and May.

For twenty-four sorts add-Citron des Carmes, July ; Beurré Giffard, August ; Beurré Andusson, Septernber; Doyenne, white, October; Doycnne gris, October ; Duchesse d'Angoulême,
November ; Urbaniste, November ; Winter Nelis, December; Beurré Langelier, January ; Beurré Sterckman, February ; Beurré, Easter, March; Beurré Ranz, April and May.

N.B.-The Orpheline d'Enghien of Belgium is synonymous with the Soldat Laboreur of France and the Beurré d'Aremberg of most of the collections in Britain.

The following are given by the same authority as suitable for wall-culture :-

For south or south-west walls. - ${ }^{*}$ Crassane, ${ }^{*} \mathrm{Col}$ mar, St Germain, *Doyenne d'Eté, Chaumontelle, Passe Colmar, Jargonelle, * Glout Morceau, Brown Beurré, Fortunée, Van Mons' Leon le Cler'c, Gansal's Bergamot.

For west or north-west walls.-Beurré Diel, Beurré d'Amalis, *Beurré Langelier, Beurré Ranz, *Beurré Gris d'Hiver nouveau, *Duchesse d'Orleans, Grosse Calebasse, Marie Louise, Napoleon, Louise Bonne of Jersey, *Josephinc de Malines, *'Triomphe de Jodoigne.

For enst or south-east walls.- ${ }^{*}$ Beurré Easter, Citron des Carmes, Orphcline d'Enghien, Bergamot d'Esperen, Duchesse d'Angoulême, Crassane d'Hiver, *Winter Nelis, *Doyenne Gris, Susette de Bavay, Urbaniste, *Triomphe de Jodoigne, Doyenne Goubault.

For nortl walls.-Catillac, Bellisime d'Hiver, Passe Tardive, Leon le Clerc de Laval, and Vicar of Winkfield, all good baking or stewing pears. These are, however, only adapted to north walls in the south, or more favourable situations in the north. These are almost without exception unsuitable for either standards or north-aspected wall-cultivation in cold northerly places; for although many of them will arrive at a pretty good size, still they will not ripen even when grafted on quince stocks.

Select li:t of pears which ripen at Culzean Castle, Ayrshive.-Beurré Langelier, B. d'Capiaumont, B. d'Amalis, B. Rance, Leon le Clerc, B. d'Aremberg, B. Easter, B. Brown, B. Gracioli of Jersey (?) Hacon's Incomparable, Louise Bonne of Jersey, Marie Louise, Napoleon, Winter Nelis, Fondante d'Automne, Thomson's, Gansal's Bergamot, Autumn Bergamot, Beurré Diel, Duchesse d'Angoulĉme, Glout Morceau, Jargonelle, Knight's Monarch, Passe Colmar, Williams' Bon Chretien, Urbaniste, Compte de Lamy, Dunmore, Chaumontelle, and Catillac.

Select list of pears which ripen well at Drumlamig Castle, Dumfriesshire. (W. \& S. indicate their being grown on walls or standards).-Bergamot autumn, W.; B. Gansal's, on a quince stock, S.; Beurré d'Aremberg, W.; B. brown, W.; B. Rance, W. ; B. Easter, W.; Bon Chretien, summer, W.; B. Williams', W.; Citron des Carmes, W. ; Cressane, W.; Doyenne, white, very fine on standards; Duchesse d'Angoulême, W.; Dunmore, W.; Glout Morceau, W. ; Green pear of Yair, S. ; Hessel, S. ; Jargonelle on W. and S., grafted on the quince stock, on the latter producing large crops, but the fruit is rather gritty; Louise Bonne of Jersey, W.; Marie Louise, W.; Moorfowl Egg, S. ; Napoleon, W.; Winter Nelis, W.; St Clair? W.; Urbaniste, W.; Van Mons' Leon le Clerc.

Pears which ripen at Dalkeith Park, MidLothian. (S. and W. indicate Standard and Wall.) 
-Aston Town, S.; Bergamot autumn, S.; B. Gansal's, S. and W.; Beurré Ananas, S. and W.; B. Bosc, W.; B. Brown, W. and S.; B. d'Aremberg, S. and W.; B. d'Capiaumont, S. and W.; B. Diel, S. and W.; B. Duval, S. and W.; B. Easter, S. and W.; B. Rance, S. and W.; Chaumontelle, S. and W.; Citron des Carmes, W., (for an early supply, would no doubt ripen also as a standard, if tried); Colmar, W.; Compte de Lamy, W.; Crassane, W.; Crassane Althorp, W.; Doyenne Gris, IV.; Duchesse d'Angoulême, W. and S.; Glout Morceau, IV. and S.; Hacon's Incomparable, W.; Hessel, S.; Jargonelle, W. and S.; Louise Bonne of Jersey, W.; Marie Louise, W. and S.; Moorfowl Egg, Galston's, W.; Napoleon, W. and S.; Winter Nelis, W.; Passe Colmar, W.; St Germains, W.; Seckel, S., indifferently; Swan's Egg, S.; Vergouleuse, W.; Doyenne Blanc, W.; Auchan, W. and S. Many other sorts recently planted have not as yet fruited.

List of perry pears.-Barland, an old Herefordshire pear, where it is much cultivated and esteemed; Foxley, also esteemed for the same purpose, of Herefordshire origin, (specific gravity, 1070); Huffeap brown, Huffcap red, Huffcap yellow, Hampton rough, Courel, Moorcroft, Oldfield, (spec. grav., 1067); Squash, (spec. grav., 1060); Teinton, Squash new meadow, Stumpfling, Wolf's, Holemore, (spec. grav., 1066) ; Longland, (spec. grav., 1063.) Of these the Barland, Teinton, Squash, Oldfield, Longland, and Holemore, are most in repute in Herefordshire, Worcestershire, and other perry-making districts.

Pears suited to the north of Scotland. Those marked * require a wall, unless the situation is remarkably sheltered and warm.-Alexander de Russe, Autumn Bergamot, Beurré d'Aremberg, Beurré Diel, *Beurré Rance, * Chaumontelle, *D'Auch, Gilogil, Grey Doyenne, Hassel, *Jargonelle, London Sugar, Marie Louise, Moorfowl Egg, Madeleine, Poire Neil, Prince's pear, Passe Colmar, Saint Germain, Swan's Egg, Summer Bergamot, White Doyenne, Winter Nelis, Yat. Auchan, Gratioli (of Jersey), *Althorp Crassane, * Knight's Monarch.

Pears fur kitchen or steving purposes, (not included in our descriptive list, p. 461, recommended by Mr Rivers).-Belle Angevine, ripe in April, very large; Black Pear of Worcester, February, fine red when stewed ; Chaptel, April, good bearer; Ramilies, February, large, a great bearer; St Lezin, October, large and handsome; Summer Compote, August, an abundant bearer.

Pears, many of which are of recent introduction, (recommended by Mr Rivers, and not included in our descriptive list, many of which we have in cultivation, but as they have not as yet produced fruit with us, we give them on Mr Rivers' authority).--Archduke Charles, large size, first quality, season November to December, productive, standard; Bergamotte Bernard, medium size, second quality, December to May, productive, standard; Bergamotte Fievée, small size, first quality, September, productive, standard ; Beurré Golden, (Rivers), a very old variety of the Doyenne family, very productive, standard; Beurré de Beaumont, large size, first quality, September, very productive, standard; Beurréde Bordeaux, medium size, first quality, October, very productive, standard; Beurré de Mortgeron, medium size, first quality, September-like Beurré Rance, but seems to do better on the quince stock than that variety-very productive, standard; Beurré de Noirchain, medium size, first quality, April, very productive, standard; Beurré Moire, large size, first quality, October, very productive, standard; Beurré Van Marum, large size, first quality, November, productive, standard; Bonne d'Eté, medium size, first quality, November, productive, standard-this is given as a synonym of the White Doyenne in the "Fruit Catalogue of the Horticultural Society." Mr Rivers says, in France this is a well-known and distinct variety. Bomne des Zoes, medium size, first quality, September, productive, standard; Caemnais, medium size, first quality, November, productive, standard; Captif de St Hélène, small size, first quality, December, very productive, standard; Delice de la Cour, large size, first quality, November, productive, standard; De Lepine, medium size, first quality, January, productive, standard; Duchesse d'Orleans, large size, first quality, October, very productive, standard : Fpiscopal, medium size, first quality, May to June, productive, standard ; Inconnue (Van Mons), medium size, first quality, February, very productive, standard; Miel de Waterloo, large size, first quality, November, very productive, standard; St Marc, large size, first quality, November, very productive, standard.

Amongst the many new pears enumerated in Mr Rivers' Catalogue for 1853-54, the following winter varieties, adapted to quince stocks, deserve the attention of planters: Susette de Bavay, in use from March to May; Fondante de Malines, January and February; Belle de Noel, or Belle après Noel, January; Alexınder Bivort, January ; Zephirin Gregoire, January to March; Duchesse de Mars, December and January ; Soldat d'Esperen, December.

The following distances may be given at which pear trees should be planted, according to the sort of stock employed and mode of training adopted: viz., Standards on pear stocks, 20 feet apart; espaliers or walls trained horizontally on the pear stock, 24 feet; espaliers or walls trained vertically on the quince stock, 4 to 6 feet; espaliers or walls trained horizontally on the quince stock, 15 feet; pyramidal trained on quince stocks, and not root-pruned, 6 feet; pyramidal trained on quince stocks, root-pruned, 4 feet ; pyramidal trained on pear stocks, not root-pruned, 10 feet; pyramidal trained on pear stocks, root-pruned, 6 feet.

Grouring the finer varieties of the year in pots. - This appears to be a Russian practice, rendered necessary on account of the deficiency of climate to cultivate the best French and Belgian varieties without the aid of glass covering during the severity of their winters. They are, therefore, kept in cool glass-houses during winter, where they blossom and form their fruit; and as soon as the Russian summer sets in, which is so much more warmer than ours, the trees are set out in tlie open air, wliere they ripen to great perfection. This circumstance appears to have led Mr Rivers to adopt pot culture and the construction of orchard-houses, 
reversing, however, the Russian practice, as more suitable to the climate of Britain; and hence his practice is to winter his trees in the open air, plunging the pots in rotten tan or sawdust, for the equal preservation of the roots and pots. In February he removes his potted trees into very economical glass structures, which he calls glass-roofed sheds, or rather wooden pits with glass sashes as a covering. No fire heat is applied. An abundance of air is admitted by sliding shutters placed in front and back of his structures-solar heat transmitted through the glass roof being found sufficient to ripen the most delicate and choice. "The trees," he says, "for this purpose, should be dwarf bushes, grafted on the quince stock, and the pots 15 inches deep and the same over; liquid manure and surface-dressing in summer will enable them to carry a good crop. I potted some pears," he informs us, "on quince stocks, in the beginning of April 1847, and placed them in front of a (glass) shed facing the south. Several now (1848) are full of fruit, the pots used being 12 inches wide and the same in depth. They have stood in the south front of the shed all the summer, the surface of each pot being covered with moss, and the plants are in fine liealth. Apples on the paradise stock may be grown in pots with the same facility." The principle, in the hands of Mr Rivers, who is an excellent cultivator, has been attended with the most satisfactory results. It is, however, by no means a new feature in fruit-growing in this country, for we recollect seeing apples growing in pots in the nursery of Messrs Gibbs at Brompton, in 1815; and very soon afterwards the late Earl of Lauderdale erected a very large glasshouse at his seat at Dunbar, in which apples and pears were grown for several years in pots arranged upon stages like greenhouse plants.

The most prolific and eligible sorts of pears for pot culture, Mr Rivers remarks, are the "Brown Beurré ; Beurré Gris d'Hiver nouveau; Doyenne d'Hiver Nouveau; Easter Beurré; Glout Morceau; Bergamotte d'Esperen; Gansal's Bergamot; Duchesse d'Orleans ; Beurré d'Aremberg.; Beurré Rance; Crassane d'Hiver ; Doyenne Goubault ; Marie Louise ; Passe Colmar; St Germain, and Van Mons' Leon le Clerc. The above are all autumn and winter pears. If summer pears are desired, Doyenne d'Eté, Jargonelle, Citron des Carmes, and Colmar d'Eté may be potted."

So many of our best pears being of French or Flemish origin, the following key to the pronunciation of their names may be useful :Ananas d'Eté-An-an-ah Da-tay; Angleterre - Ahn-glet-are ; Beurré - Bur-ray ; Belle de Bruxelles-Bel de Broos-ell; Belle et BonneBel-a-Bun ; Belle-Lucrative - Bel-lu-crah-teve ; Beurrê de Capiumont-Bur-ray de Cap-u-mohn; Beurré d'Amalis-Bur-ray Dah-mah-lee; Beurré Gris d'Hiver nouveau-Bur-ray Gree dee-vair noo-vo; Beurré Diel-Bur-ray De-ell ; Beurré Bronzée-Bur-ray Brone-zay; Bezi dé HeriBa-zee Daree ; Bezi Vaet-Bazee Vah-ai ; Beurré Crapaud - Bur-ray Crah-po ; Bezi de Montigny - Ba-zee de Mon-teen-gnee ; Bon Chretien Fondante-Bone Cray-te-an Fone- donte ; Boucquia-Boo-kiah; Calebasse GrosseCal-bass Groce ; Capucin-Cap-u-san ; Chaumontel très Gros - Sho-mone-tell tray Gro Compte de Lamy-Conte de Lah-me; Colmar Epine-Col-mar A-peen; Crassanne-Cras-sahn; Cuise Madame-Kuees Mah-dam; D'AmoursDam-oor ; De Louvain - Dul-oo-van; Délices d'Hardempont - Day-lece Dar-dahn-pone; Doyenne d'Eté-Dwoy-on-nay Day-tay; Doyenne Panaché - Dwoy-on-nay Pan-ah-shay ; Dumortier - Du-mor-te-ay ; Duchesse d'Angoulême - Du-shess Don-goo-lame; Duchesse d'Orléans-Du-shess Dor-lay-on; Enfant ProdigeOn-font Pro-deeje; Epine d'Eté-A-peen daytay; Figue de Naples-Feeg de Nah-pl; Fondante du Bois-Fone-dont du Bwoi; Fondante d'Automne - Fone-dont du-tonn; Forme de Délices-Form de Day-lece; Forelle-Fo-rel; Fortunée-For-tu-nay ; Franc Réal d'HiverFronk Ray-ahl Dee-vair; Glout Morceau-Gloo Mor-so ; Héricart-Hay-re-car ; Jalousie-Jaloo-zee; Jalousie de Fontenay Vendée-Jal-oozay de Fone-ten-ai Von-day; Léon le ClercLay-on le Clair; Limon-Lee-mohn; Louise Bonne-Loo-eze Bun; Madeleine, or Citron des Carmes - Mad-lane, or Cee-trone day Carm; Marie Louise-Mah-re Loo-eze ; Michaux-Mesho; Passans de Portugal-Pah-sahn de Por-tugal; Pailleau-Pahl-yo; Paradise d'AutomnePar-ah-deze du-tonn; Passe Colmar-Pas Colmar; Quilletette-Keel-tet; Reine CarolineRane Car-o-lene ; Reine des Poires-Rane day Pwore; Rousselet Hâtif-Roos-lay Hat-eef Senspeau-Sahn-po ; Sieulle-Se-ull ; Sucrée de Hoyerswarda-Seu-cray de Hoyerswarda-Surpasse Virgalieu-Seur-pass Vere-gal-yu ; St Germain-San Jare-man ; Sylvange-Seel-vonje Vallée Franche-Vol-lay Fronch; Verte Longue -Vairt Longh; Verte Longue Panachée-Vairt Longh Pan-ah-shay; Virgouleuse - Ver-gooleuz; Wilelmine-Wil-el-meen.

European names. - Pear, English - Poire, French-Pero, Italian - Pera, Spanish-Birnbaum, German-Peer, Dutch and Flemish.

In regard to any systematic mode of classifying the pear beyond that of arranging them as summer, autumn, winter, and baking pears, however inuch such may be desired, we know not of any satisfactory attempt even having been made by British pomologists. Should Mr Hogg proceed with a general work on British pomology, which it is to be hoped he will, we may then expect to see this interesting and difficult subject handled with that talent and success he has given a foretaste of in his excellent work upon apples.

It may be remarked that we have retained few of the older pears in our descriptive list : these have become now obsolete in all good collections, as their places have been taken up with infinitely better sorts. These, for the most part, have been obtained from Belgium since the general European peace. They are found, by the experience of thirty years or more, to be well adapted to our climate, either as wall, espalier, or standard fruit in general, and as adapted to the former in many very in. different localities, as well if not better than many of those formerly so cultivated. In regard 
to our estimation of their respective qualities, we cannot do better than quote what $\mathrm{Mr} \mathrm{R}$. 'Thomson says on the subject in the third edition of the "Catalogue of Fruits in the London Horticultural Society's Gardens." "It is necessary to observe, that varieties esteemed of the highest excellence by some are deemed inferior by others. In general, this diversity of opinion is owing to difference of soil, situation, or climate; for if these are unfavourable, a variety which proves to be first-rate, when produced under favourable circumstances, is often found inferior to one that, although second-rate, is more hardy."

The number of varieties now in cultivation is very great. The Romans, in Pliny's time, possessed 36 varieties. Parkinson enumerates 64 ; Miller, 80. The "Luxembourg Nursery Catalogue" at Paris contained 189 select sorts about the beginning of the present century. The "Catalogue of the Horticultural Society" contains 442 described sorts. G. Lindley, in "Guide to the Orchard," 162 ; Rogers, in "Fruit Cultivator," 50 ; Downing, an American author, 233, many of which are of American origin; P. Lawson and Sons' " Fruit Catalogue," 267 ; Mr Rivers, in "Descriptive Catalogue of Pears" for 1843-4, enumerates 156 sorts of dessert pear's and 14 baking and stewing sorts, of all which, Mr Rivers informs us, he has specimens in a bearing state, and from which his grafts are taken, which insures correctness of nomenclature.

\section{DISEASES.}

The pear is much less liable to disease than the apple, at least in Britain. In America, and also in France, it is otherwise. In the former country, two diseases often assume a decided character, the one called The Insect Blight, and the other The Frozen Sap Blight, appearing sometimes in succeeding seasons, and again only after the lapse of several years. The insect blight is thus described by $\mathrm{Mr}$ Downing, in his excellent work, "The Fruits and Fruit Trees of America:"-" In the month of June or July, when the tree is in full luxuriance or growth, shoots at the extremities of the branches, and often extending down two seasons' growth, are observed suddenly to turn brown. In two or three days the leaves become quite black and dry, and the wood so shrivelled and hard as to be cut with difficulty with a knife. If the branch is allowed to remain, the disease sometimes extends a short distance farther down the stem, but usually not much farther than the point where the insect had made his lodgment." This insect has been described by Professor Peck under the name of Scolytus pyri. "It is very minute, being scarcely one-tenth of an inch long; and it escapes from the branch almost as soon as, by the withering of the leaves, we are aware of its attack: hence it is so rarely seen by careless observers. In the perfect state it is a very small beetle, deep brown, with legs of a paler colour. Its thorax is short, convex, rough in front, and studded with erect bristles. The wing-covers are marked with rows of punctured points, between which are two rows of bristles, and they appear cut off very obliquely behind.
This insect deposits its eggs some time in July or August, either behind or below a bud. Whether the egg hatches at once we are not aware, but the following spring the small grub or larva gnaws through the sap-wood, or tender alburnum, beginning at the root of the bud, and burrows towards the centre of the stem. Around this centre or pith it forms a circular passage, sometimes devouring it altogether. By thus perforating, sawing off, or girdling internally, a considerable portion of the vessels which convey the ascending sap, at the very period when the rapid growth of the leaves calls for the largest supply of fluid from the roots, the growth and the vitality of the branch is checked and finally extinguished. The larva, about this time, completes both its transformation and its passage out, and, in the beetle form, emerges with wings into the air, to seek out new positions for laying its eggs and continuing its species." The remedy pursued for the destruction of this insect consists in cutting off the branches about a foot below the point where it has fixed its abode, which is readily discovered by the discoloration in the bark, and this operation should be carried into effect upon the very first indication of its appearance.

'The frozen sap blight is occasioned by the trees continuing to grow late in autumn, and being attacked by early autumnal frosts while the sap-vessels are full of fluid matter. By alternate freezing and thawing of the sap it loses its vitality, and becomes dark and discoloured; and in some cases, Downing remarks, it becomes so poisonous as to destroy the leaves of other plants when applied to them. So serious is this disease in some parts of America, as to create great alarm amongst the cultivators of fruits. "To distinguish the blight of the frozen sap from that caused by the attacks of the Scolytus pyri," Mr Downing remarks, " is not difficult. The effects of the latter cease below the spot where the insect has perforated and eaten its burrow in the branch, the former spreads gradually down the branch, which, when dissected, shows the marks of the poison in the discoloration of the inner bark and the pith, extending down some distance below the external marks of injury. If the poison becomes largely diffused in the tree, it will sometimes die outright in a day or two; but if it is only slightly present, it will often entirely recover. The presence of black, dry, shrivelled spots of bark on the branches, or soft sappy spots, as well as the appearance of thick clainmy sap in winter or spring pruning, are the infallible signs of the frozen-sap blight." We are not aware that such a disease has yet made its appearance in Europe, although others may exist of less virulence, arising from similar causes in certain seasons. Duhamel, in "Traité des Arbres Fruitiers," mentions a diseased state in the sap, arising from excess of manure, somewhat analogous to this American disease. He says, "The sap, corrupted by putrid water or the excess of manure, bursts the cellular membranes in some places, extends itself between the wood and the bark, which it separates, and carries its poisonous acrid influence to all the neighbouring parts like a gangrene." "In a soil over-moist 
or too rich," Downing remarks, "the pear is always liable to make late second growths, and its wood will often be caught unripened by an early winter. For this reason, this form of blight is vastly more extensive and destructive in the deep rich soils of the western states than in the dryer and poorer soils of the east. And this will always be the case in over-rich soils, unless the trees are planted on raised hillocks, or their luxuriance checked by root-pruning."

Although the frozen-sap blight occurs to a much less extent in Britain, still its existence may be traced in situations where the tenderer pears are attempted to be cultivated, and especially so when a sudden and early winter succeeds a damp and warm autumn, and less so when the summer has been dry, and the growth of the trees has been completed early. When the trees continue to grow to a late period in the autumn, which they often do in cold situations, where they are induced to make a second growth, and where, in consequence of being planted in too deep and too rich a soil, vegetation is prolonged beyond its natural limits, strong and watery shoots are produced; and when in this state, the pear-shoots in many parts of Europe are as subject to this disease as they are in America. The varieties of pears which naturally ripen their wood early in the autumn are rarely attacked by this disease, and hardy ones, at least in Brịtain, are more certain to escape than the finer or more delicate sorts.

Insects.- The pear is attacked by most of the insects that infest the apple tree. The Aplis lanigera Illig. Eriosoma lanigera of more recent entomologists, howcver, rarely attacks it. Several of the insects we have described as destructive to the apple tree are more or less to be found on the pear tree also. The following may be regarded as the principal enemies almost peculiar to the pear.

The red-bud caterpillar, (Tortrix ocellana, Penthina ocellana Fr., Pyralis luscana Fabr.,; - the moth of which is given, fig. 196-is very destructive to the buds of the pear, and is also found on the apple. The moth is thus

Kollar: "A white broad transverse band, studded with grey spots, e $x \mathrm{t}$ e $\mathrm{n} d \mathrm{~s}$ through the middle of the forewings from

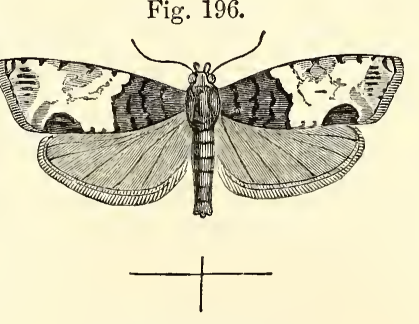

one edge to the other, and occupies more than onethird part of their whole surface, thus distinguishing this moth from every other. The other parts of the fore wings are grey. This moth is found on fruit trees towards the end of May. Its wings are closed, and lie slanting on its body. It is very timid, and can only be caught in cold rainy weather. Throughout the month of June it lays its eggs singly, sometimes on the fruit-buds, and sometimes on the leaf-buds only, where they remain all winter, and only come to life the following spring. As soon as the sap is in motion, and the bud somewhat expanded, thelittle caterpillar creeps out of its hiding-place, and begins to gnaw the bud. A honey-drop is not unfrequently seen on the bud, which, issuing from the wound made by the insect, serves as a sign to assure us that the fate of the bud is decided, and that it will never expand farther. The bud is prevented from growing, and from attaining its full size, by the honey-drop, the points of the calyx of the flower thus becoming so closely glued together that their unfolding is necessarily prevented ; and the caterpillar takes advantage of the opportunity to devour one blossom after another, until it arrivesat its full size. Should the caterpillar come out rather later, when the buds are already unfolded, some of the blossoms are spared, and consequently some of the fruit. As soon, however, as the caterpillar finds a fruit near it, it immediately takes possession, and feeds upon it. It attains its full size in about four or five weeks ; it then spins itself a white cocoon, in which it changes to a lightbrown pupa, and makes its appearance again in May as a moth." Dusting the trecs, just as the buds are expanding, with finely-powdered hot lime, we have found the most effectual remedy. 'The operation should be performed two or three times during the period of the buds' expanding, and, if possible, in still weather. The finer particles of the caustic lime, finding their way into the heart of the buds, destroy the insect in its most tender state, and just as it is hatched. The moths are slothful, and seldom quit the tree, so that their capture while on the wing is seldom accomplished.

The pear-weevil, Curculio (Anthonomus) pyri of Kollar, is as destructive to the pear as the Curculio pomorum is to the apple. In their perfect state, both insects are so much alike that they are scarcely distinguishable; in their larva state, on the contrary, they differ exceedingly both in their form and mode of living. "While the apple-weevil," Kollar remarks, "contents itself with only single blossoms for the abode of its offspring, the pear-weevil attacks all the blossoms, and even the blossom-buds and leaf-buds together. If a pear tree is examined at the time of blossoming, it will be seen that many buds are brown at the points, as if affected by the hoar-frost. If these buds are examined closely, there will be found a dirty-white rugose maggot, with a dark-brown head, which in time is changed to a small weevil. It is scarcely more than 3 lines long, brown, with a white uneven band, almost in the middle of the ely. tra, and two black shoulder-spots, therefore perfectly like the apple-weevil. This insect probably passes the winter under the bark, or in the earth near the stem. Early in spring, when the pear tree begins to bud forth, the female lays her eggs in the buds, which causes them to become brown by degrees, and to fall off when the insect has attained its perfect state." The principal remedies against this insect are cutting off the buds that assume the brown colour referred to above, and burning them, as 
they eertainly contain the inseet; and if not eut off for this purpose, would only serve as a habitation, wherein it would become perfeet, and so continue the propagation of its species without a chance of fruit being produced. If, as is sus. pected by some, the female shelters herself in the ground and not on the tree, then the applieation of a collar, covered with some adhesive matter, as already referred to, would prevent her ascent by the stem of the tree.

The red-footed beetle, fig. 197, (Luperus rufipes, Fabr.,) a small but destructive beetle, infests the foliage of fruit trees very generally.

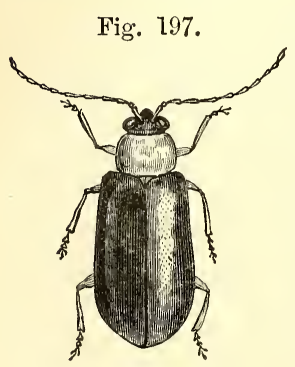

Last year, 1852 , it appeared in great numbers during May on the leaves of pear trees, perforating them into innumerable holes, and continuing its depredations throughout the greater part of the summer. Its name is derived from the colour of its feet, which are brightish red, while the whole body is of a shining black colour. The tips of the antennæ are also black, the basal part being red-

RED-FOOTED BEETLE.

ish. It deposits its eggs in the ground round the base of the tree, and when the beetles are hatched, they ascend the tree by climbing up the stem. Their ascent may be prevented by the means we have already suggested, and the eggs removed or destroyed as also already reeommended in similar cases.
Fig. 198.

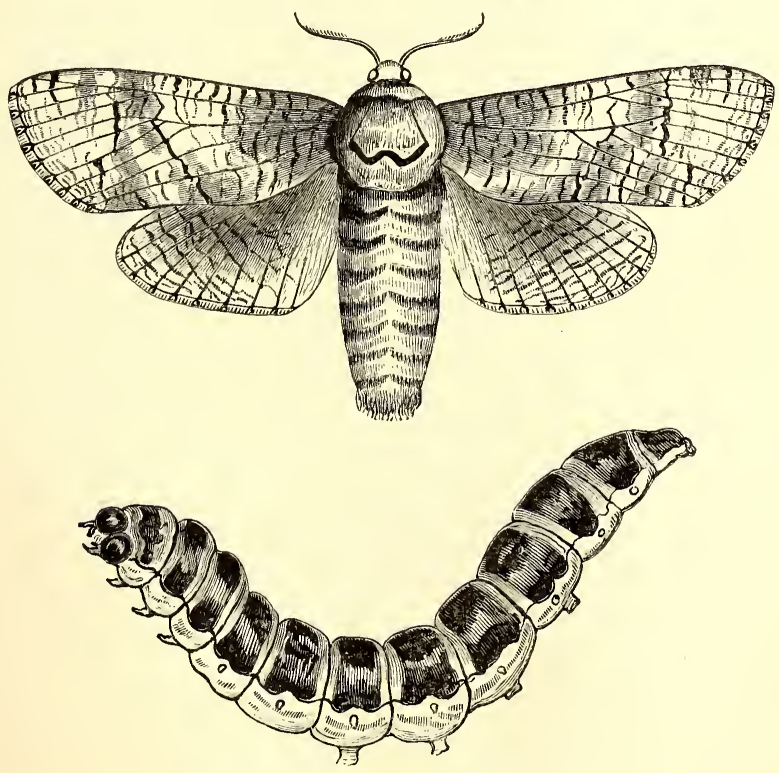

The goat moth, fig. 198, (Bombyx ligniperda, Colsus ligniperda Linnæus, Fabricius, and others,) is not only one of the largest native species belonging to the Lepidopterous order, but also one of the most destructive to some of cur timber and fruit trees, and is not unfrequently discovered in the interior of old pear-trees, where it excavates for itself a habitation in the solid timber. It is a nocturnal-flying moth, and from its great size is easily captured in a gauze net. As many as one thousand eggs have been discovered in the body of a single female, which leads to the conclusion that its natural enemies must be both powerful and many; for so destructive an insect, if allowed to multiply unrestrained, would soon spread desolation in our fruit gardens and forests. The perfect insect measures from $2 \frac{3}{4}$ to nearly 3 inches from point to point of its fore wings, which are of an ashy-brown colour, shaded with dark brown, especially across the middle, marked with many irregular transverse streaks in the form of irregular network. The hind wings are brown, the reticulations being marked with somewhat obscure lines. The thorax is ochrecoloured in front, palish in the middle, with a black bar behind. The female is larger than the male. It sits quietly during the day, and is with difficulty discovered on account of its general colour resembling the bark of the tree. The autennæ have a light-grey shaft and black rays, and are more strongly feathered in the male than in the female. The caterpillar, when fully grown, is nearly 4 inches long, and as thick as a man's finger, of a dull yellowish hue, with dark chestnut-coloured scales on the back of each ring of the body. The body is smooth and shining, having only a few short scattered hairs upon it. It is dark red on the back, and the spiracles at the sides are of the same colour. The head is black. The caterpillar emits a strong goat-like smell, from whieh its Englisl name is derived. Its organs of manducation are so powerful as to enable it to masticate the hardest wood, and to bore deeply into the timber, in which it forms a burrow for shelter and the propagation of its species. When they attack young trees, they so completely exeavate the interior as to cause them to be broken over by even very slight winds. They live entirely on the wood, and, unlike most other moths, do not attack the foliage. They live chiefly in the same tree they have chosen for their abode; and their presence in it is only discovered by the decaying appearance in the tree itself, or by their exerement being observed on the trunk, and known by the name of worm meal - the digested portion of the wood they have 
subsisted upon. They remain two years and upwards in their larva state, and during that period cast their skin eight times. Pupation usually takes place in spring, and shortly before that the caterpillar becomes of a light ochre-yellow colour. The abdomen of the pupa is yellow. The back is furnished with strong spines, sometimes black, and at other times of a reddish-brown colour. The cocoon is constructed of chips of wood, gnawed off and cemented together with a glutinous secretion, and lined with silk. It is placed immediately within the opening of the tree, which enables the pupa to press itself partially out of the hole when it arrives at that state of maturity at which the shell bursts, and when the moth is in a state to emerge into the air, which is usually in June and July. The female is furnished with a strong ovipositor, by means of which she introduces her eggs into the bark of the tree, the caterpillars, while young, living between the outer and inner bark, and as they get older and stronger they penetrate into the solid wood. The means most likely to keep this formidable insect in check are to endeavour to capture the moth during night, either by catching it while on the wing in a gauze net, or by decoying it to its destruction by placing burning lights near to where it abounds; fumigating it while in the caterpillar state within the tree, by blowing fumes of sulphur or tobacco into the holes; or mechanically, by thrusting iron wires into the holes, or immuring them within their wooden prison by stopping up the holes in such a way as to prevent their escaping, or the entrance of the limited amount of air necessary for their existence.

The pear saw-fly, Tenthredo homorrhoidalis of Fabricius, attacks the fruit of the pear, within which she deposits her eggs. The fly appears towards the latter end of May and begiuning of June. The female chooses the under side of the leaves of the pear tree, where she lays her eggs to the number of from forty to sixty. The eggs are merely attached to the leaf, and are arranged with extraordinary precision, one row being quite regular, and the next so placed that the eggs in it cover the spaces between those in the row immediately in front of it. In form they are longish, in colour yel lowish, and have the appearance as if besmeared with mucous matter. In a few days the young caterpillars are hatched, and are at first of a whitish yellow, changing gradually to a darker colour. Kollar describes the caterpillar as being hatched within a few days after the eggs are laid, and "as soon as it is exposed to the light, it spins a web over itself, the threads of which proceed from its mouth. The caterpillar never appears out of this web, and when it has partly eaten a leaf, it spins itself a web on another, and always in company with other caterpillars. It has a black head, and immediately under the throat two black dots. The other parts of the body are ochre-coloured and transparent, without hairs. It measures from 9 to 10 lines long. The two fore feet, and the two upon the last segment, are finely tapered, so that they rather resemble claws than feet, because they do not use thẹm for crawling, but to draw themselves out of and into their webs. These caterpillars attain their full size in five weeks; they then leave the trees, and bury themselves deep in the ground." Here again we have a remedy presented to us, by destroying the caterpillar in the soil, as has already been suggested. But man has here also excellent assistants in keeping in check this destructive insect. The caterpillar or larva of Ophion mercator, an ichneumon fly, lays its eggs in the body of the pear saw-fiy, just as the latter has attained its full size, and just before it drops from the tree to the ground, living upon its vitals, and making use of its empty skin for a winter shelter.

The pear chermes, (Chermes pyri Schmidberger, Psylla pyri Stephens.) The chermes is allied to the aphis, and, like them, extremely destructive to such fruit trees as each species prefers. The chermes of the pear infests the young shoots mainly, and by insinuating its long and delicately-formed tongue into the tender bark, it not only sucks up the juices, but defiles the leaves and shoots with its disagreeable and abundant excrement. When full grown, this insect is somewhat larger than a large aphis, having a broad head, terminating in front in two cone-like protuberances. The mouth, instead of being situated in the head, is placed in the middle of the breast, behind the hind and fore feet. The male is somewhat of a crimson colour, shaded with dark brown or black. The female is without shading, and is entirely crimson. The wings in both sexes are pure white. They make their appearance in spring in their winged state, and immediately pair, after which the female lays her eggs in great numbers on the young leaves and blossoms, as well as on the young shoots and embryo fruit. Hatching is completed in a few days, the young chermes resembling plant-lice, but being of a dark yellow colour, and furnished with six feet. Kollar remarks, "They are called nymphs or larve in this state, according to the extent of their development. After a few days they change their skins, and become darker, and somewhat reddish on the breast. After changing their skins, they quit the leaves, blossoms, and fruit, and proceed more downwards to the bearing wood and the shoots of last year, on which they fix themselves securely one after the other in rows, and remain there till their last transformation. When the nymphs have moulted for the last time, and have attained their full size, the body swells out by degrees, and becomes cylindrical. They then leave their associates; and before they lay aside their nymphlike covering, they search out a leaf, to which they fasten theniselves firmly, and appear as if they were lifeless. After a few minutes, the skin splits on the upper part of the covering, and the winged insect proceeds from it." During summer it flies about, and towards autumn selects a place of shelter, and remains there till early in spring, when it again begins its work of regeneration. The natural enemy to this chermes is the ant, which is attracted to the twigs infested by the copious fluid-like excrement emitted by the chermes, which they devour as well as many of the insects that produce it. The 
young chermes may be easily brushed off the leaves by means of a painter's brush, and, as they fall to the ground, destroyed by treading upon them. The winged insect may be captured during May, their red colour rendering them distinguishable to the eye.

Aphis pyri-mali, the pear-and-apple aphis. That the pear and the apple have each a distinct species of aphis peculiar to themselves is believed by some; a large majority, however, of entomologists consider the aphis found on both these trees to be identical, and hence the name Aphis pyri-mali. However this may be settled by men of science, it is quite enough for all practical purposes to consider them the same. Those species of the genus Aphis, plant-louse, grecn-fly, black-fly, \&c., which are destructive to fruit trees, are too well known to require a lengthened description of their forms and transformations. It may be sufficient for our purpose to glance at their mode of attack, and offer the best methods for their suppression or destruction. They do not eat up the leaves of trees and plants like many caterpillars, but they insert their rostrums into the leaves, and suck up the sap, causing the leaves to wither and fall off, and, consequently, greatly enfeebling the tree, if they do not kill it altogether. They appear as early in spring as the first development of the tender leaves, being produced from eggs laid on the branches the previous autumn, and consist entirely of females, destitute of wings; and these are no sooner brought into existence than they begin their work of destruction. In about ten or twelve days this earliest brood begins to produce young, which appear to be females also, and, like the former, destitute of wings. In about ten days afterwards, this second brood produces. a third brood; but, unlike the former, this latter has in it both winged and wingless females, and the same production of both. winged and wingless insects takes place in several succeeding generations to the extent of about sixteen, some say twenty, generations annually. The insects of the third generation that have wings quit the tree on which they were hatched when of mature size, and seek out for themselves other trees on which to feed and propagate their species, the wingless ones remaining on the tree on which they were bred. Towards the midale of September, males and females are produced, at which time the last generation for the season is produced. The apple-andpear aphis, Kollar remarks, produces the males without wings, while the peach aphis, Aphis persica, produces them with wings: he also observes, "When these newly-born males and females have attained their full size, pairing takes place. The females then no longer produce living young ones, but lay eggs, from which the mothers of the forthcoming generations proceed. They do not, however, lay their eggs on the leaves, because these would fall off, and the eggs lying on the ground till spring would be destroyed; but they lay them on the twig or shoot itself, and either all around it, like the apple aphis, or on the buds, or near them, like the plum-and-peach aphides, the latter someVOL. II. times laying their eggs on the matting with which the twigs are fastened to an espalier in summer. The females, having thus provided for their future spring progeny, die off by degrees in the autumn. The eggs have now only the winter to endure, which never kills them, however severe and changeable it may be." The natural enemies of the aphis are the larvæ of syrphideous flies, or those of the ladybird beetles, figs. 14 and 15, which are nourished chiefly on the aphides. The ant is said by some to be attracted to the locality of the aphis by the sweet fluid ejected by the latter, and that they feed only on it; our own observation is, that they not only feed on this ejected excrementitious matter, but that they devour the aphides themselves in inconceivable numbers. Birds also aid in devouring them. The eggs of the apple-and-pear aphis may readily be rubbed off the shoots on which they are laid during autumn and winter; they are easily seen lying close together like grains of gunpowder. Washing or syringing the infested shoots with a strong decoction of tobacco, tobacco liquor, wetting and dusting with Scotch snuff, are amongst the prevailing remedies. Fumigating with tobacco, where the trees can be covered so as to retain the smoke, is also destructive to the aphis in every state but in that of the egg, and dusting the trees with finely-pulverised caustic lime is also efficacious. Trees that have been painted over during winter with spirits of tar, have been found exempt from the attacks of aphides for several years after. The following mixture, applied every year, or every other year, has been found efficacious in the case of fruit trees generally,-viz. 1 lb. quicklime, 1 lb. Scotch snuff, $1 \mathrm{lb}$. sulphur vivum, $\frac{1}{2}$ lb. lamp-black, $1 \mathrm{lb}$. soft soap, mixed together in water until the whole form into the consistency of thick paint, applied by a painter's brush to the branches in February, taking care that every part of the tree is covered with the mixture. In preparing tobacco-water for this and similar purposes, $\frac{1}{4} \mathrm{lb}$. of strong tobacco, steeped in 1 gallon of softi water for six or eight days, occasionally stirring it, and squeezing the tobacco to disengage the juice, will make a liquid sufficiently strong for the purpose of washing the shoots; and if reduced by adding another gallon of water, will be in a fit state for syringing over the trees.

The wood leopard-moth (Zeuzera osculi), fig. 199 , is in its habits similar to the goat-moth, fig. 198, living in the trunks of various trees, the apple and pear amongst others. The caterpillar of this moth is $1 \frac{1}{2}$ inches long, of a yellowish colour, with four black spots on each side of the segments, with the exception of the thoracic and apical ones. The head is marked with two black spots, and its feet consist of two anal, six pectoral, and eight abdominal ones. The males are hatched about the end of June, and are much smaller than the female, which measures about $2 \frac{1}{4}$ inches when the wings are expanded. The male is white, with semi-transparent wings. The nervures are ochreous, with many large black spots, having a green tint on the superior, much smaller and paler in the 
interior. The antennæ are black, shorter than the thorax, setaceous. The head and thorax are

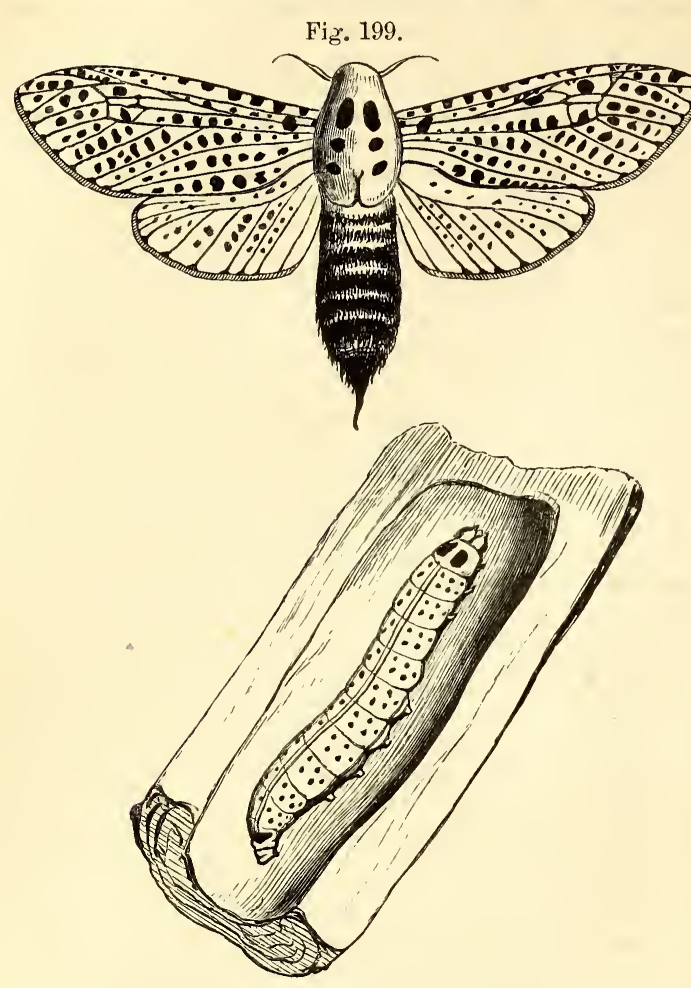

WOOD LEOPARU-MOTH AND CATERPILLAR

densely clothed with fine wool. The thorax is long and oval, with six black spots in two lines down the back. The legs and eyes are also black, the abdomen branded with grey and black. The antennæ in the female are simply setaceous, the basal joints woolly. The abdomen is larger and blacker than in the male. The females make their appearance later than the males, and are found till about the end of $\mathrm{Au}$ gust. The eggs are laid in July, and the larvæ issue from them in September, but do not arrive at maturity till the following June. The moths are nocturnal flyers, the females far more abundant than the males. The former deposits her eggs in holes made in the bark by means of her strong horny ovipositor, to the number sometimes of three hundred or more. The larva feeds at first on the bark of the tree, but soon afterwards gaining strength, proceeds to form for itself a tunnel, in which it resides, in the wood of the tree. Like that of other tree-borers, its presence in a tree may be detected by the excrementitious matter, like sawdust, protruding from the surface of the bark; and when such is perceived, tobacco or sulphureous smoke should be injected, or the larva crushed by the introduction of a piece of wire. It has been recommended as a preservative to fruit trees to paint their bark over with oil. A far more effectual mode would be to paint them with vegetable spirits of tar.

The caterpillar of the pear-mothTortrix (Pcedisca) angustiorana Haworth-fig. 200, although most injurious to the fruit of the pear,

Fig. 200 .

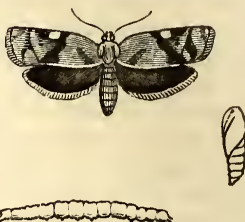

PEAR-MOTH, GRUB, AND CHRYSALIS.

appears to have long escaped the observation of entomologists. An excellent description, with figures, is given of it in " 'The Gardeners' Chronicle," 1850 , p. 20 , from which we learn that the caterpillar is bred in the blossom, and commences its attack on the fruit immediately under the crown or eye. It is thus described: "It was a quarter of an inch long, of a dirty greenish ochreous colour, and reddish brown down the back. The head was ochreous, with a square labrum and two minute antennæ, having two brightish brown pointed spots at the base, and a line of the same colour on each side, with minute black eyes. The thoracic segment is ample, horny, shining, and ochreous, variegated with brown. There are eight or ten minute tubercles on each segment, from which arise longish hairs : the six pectoral legs are spotted with black, and it has eight abdominal and two anal feet." As means for destroying this insect, it has been suggested to search for the maggots in the eyes of the fruit, where any of the web by which it covers itself is observed; but in extracting them it is probable that injury would be done to the fruit itself. The more rational way is to collect the leaves in autumn, and to burn them, as it is conjectured that the eggs are laid upon them, or that the caterpillars spin webs amongst the withered leaves, or in chinks in the bark, to undergo their transformations. If they hybernate in the latter, nothing can be better than painting the trees with spirits of tar.

The small pear-midge (Sciara pyri Schmidberger) is injurious to the pear by laying its eggs in the blossom, and after attaining its larva state it feeds on the fruit, which, according to Kollar, drops off in great numbers. The perfect insect is described by him as having "clubshaped halteres, the club dark brown and the stem whitish. When alive, the abdomen is of a lead colour, with black wings. The head and thorax are black, as are also the antennæ; the palpi are of a pale yellow, the feet whitish, and the tarsi black. The veining of the wings is the same as in the other species of Molobrus" or Sciara.

The large pear-midge (Sciara pyri major 
Schmidberger, Sciara Schmidbergeri Kollar) is similar in its effects to the last, differing little from it except in size. We are not aware of any means being taken for the suppression of either.

The slug-worm, or slimy caterpillar (Selandria atra Stephens, not Tenthredo cerasi of Linn., nor T'. oethiops of Fab.), fig. 201, is a well-kuown

Fig. 201.

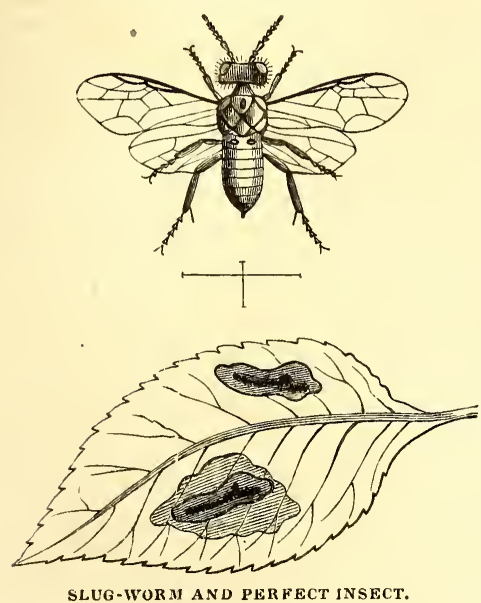

insect, so far as its effects are concerned, to those who cultivate the pear and the cherry, for it is equally injurious to both, often stripping the trees of their foliage. In their caterpillar state they are both singular and inactive, reposing upon the foliage more like black slugs or young tadpoles than the larvæ of a sawfly. Their being also covered with a moist slimy matter gives them the appearance of the excrements of birds. The caterpillar has six pectoral, and fourteen abdominal, but no anal feet; the whole body is of a deep bottle-green colour, thorax very much dilated, nearly concealing the whole head. They feed entirely on the upper sides of the foliage, retreating beneath the leaves during the day, and wandering about during the night in search of fresh food. They cast off their bottle-green skins when about a month old, and afterwards appear in a coat of a buff colour, which has led to their being considered, in this state, as a distinct insect. Shortly after this change they quit the trees, and retreat under the earth, where they repose till about the end of July the following year, when they emerge in their fly state, and commence the process of reproduction. Ruricola, in " The Gardeners' Chronicle," observes, in describing this insect, that he is inclined to believe that there may be two sorts of this genus which feed upon pear-leaves; for towards the end of July he had some dull ochreous larvæ sent to him, eating the leaves in the usual way, with black heads only; and when they changed their skins, they became entirely of a deep ochreous colour. The natural enemy to the slug-worm appears to be a minute very hairy species of Acarus, or spider. The artificial means for their suppression is syringing with lime-water in which soft soap is incorpor- ated, with a view to make it more adhesive; and tobacco-water has also been employed, applying it both early in the morning andlate in the afternoon. We think, however, the application would be more effective if used during the night, at which time the insect is traversing the foliage, and unprotected by it. Dusting the trees late in the evening with caustic lime is a much more effectual remedy, and of more easy application.

The large pear astyages-Astyages (Coleophora) hemerobiella-fig. 202, although very

Fig. 202.
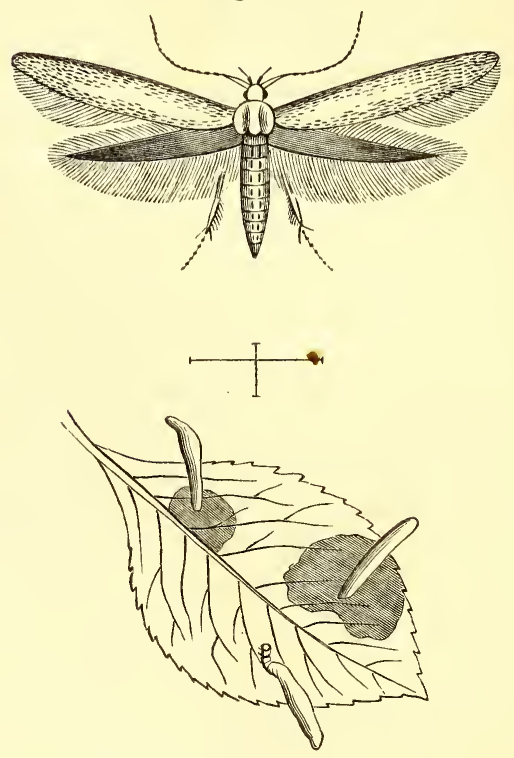

LARge PEAR astyages aNd gRUB.

destructive to the foilage of the pear in some seasons, seems to be of periodical development, appearing in vast numbers in one season, and scarcely to be found in others. The caterpillars are enclosed in a dark-coloured, nearly cylindrical case, about half an inch in length, having both ends open; out of the lowermost end the head of the caterpillar is from time to time protruded; while from the upper end, which is somewhat elastic, the insect discharges its excrement. The caterpillar is of a palebrown colour ; the head small, and deep black; the sides are flesh-coloured, with small dark spots; the segment immediately behind the head is black, hard, and glossy, with a palish line down the middle ; the two next segments have a small black triangular spot liear the margin, and a smaller dark dot on each side, above the legs. The remaining segments, with the exception of the last, are destitute of reflexed hooks; while the last has a pair of prolegs on the under side, the whole of this segment being covered with a black scaly patch. This caterpillar's mode of feeding is different from that of most others, its mouth being brought into close contact with the under surface of the leaf: the insect, ensconced in its cylindrical covering, seems to stand erect, or at right angles with 
the plane of the leaf. It forms a slight opening in the cuticle of the leaf, merely cutting through the surface, but not piercing through the whole leaf, applying its mouth to the edges of the opening thus made, and feeding upon the parenchyma alone. It then by degrees introduces the fore-part of its body between the two surfaces of the leaf, and mines the leaf round the spot where it made its first perforation, to the extent of half an inch or more in diameter, leaving the surface of the leaf marked with a broadish blotch. It moves from leaf to leaf, and proceeds in each case exactly as above. When it has attained its full size it ceases feeding, and begins to attach itself to the branches or trunk of the tree, and there undergoes its transformations, appearing again in its moth state in the July following. The body and fore wings of the perfect moth are of a dirty white colour, speckled over with very minute darkcoloured dots. The hind wings are of a brownish colour, the legs being hairy. In size it is somewhat more than half an inch from tip to tip of the wings. The best way of ridding the trees of this pest, is by watching for the insect when it first commences its attack on the foliage ; it can then readily be seen standing on end, as it were, on the under surface, and from its position and size can be easily picked off.

The pear-tree oyster-scale (Aspidiotis ostreceformis), fig. 203, is much less frequently met with

Fig. 203.
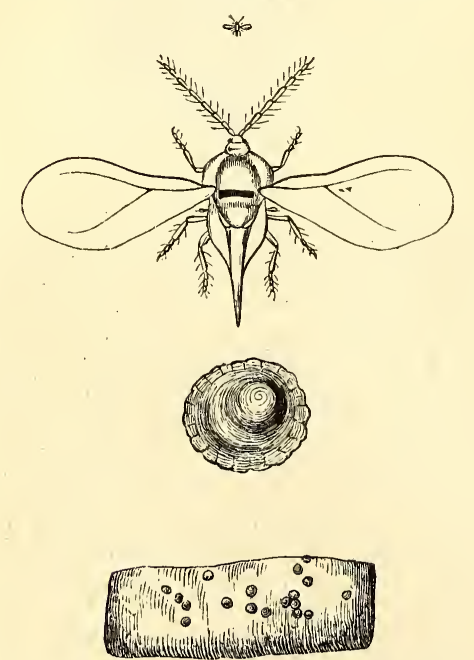

PEAR-TREE OYSTER-SCALE AND PERFECT INSECT.

than the apple mussel-scale, fig. 191; or, from its external appearance being so similar to the colour of the bark of the pear tree, may be either overlooked by cultivators, or mistaken for the bark. Its effects are similar to those of the apple mussel-scale, and the means of destruction are no doubt the same. The reader is referred to our notice of that insect.

The Paradoxical pear platygaster (Psilus Boscii Turine, Platygaster Boscii Latreille, and Inostemma Boscii of Haliday), is a very minute hymenopterous insect, scarcely a line long, yet very destructive to the fruit of the pear. Like the large and small pear-midges, the blackgall midges, \&c., they lay their eggs in the blossom, and the larvæ exist within the fruit. Such pears as are attacked are, according to Kollar, easily known, "because their growth far exceeds that of the other pears, and they (the insects) have attained a considerable size when the petals have scarcely fallen off. When this is seen, it is certain that one sort of larva or other is to be found in the core. When a pear thus infected is permitted to grow, it increases in size for some time, loses its roundness and bright green colour, and becomes longer, as if pressed inwards all round the middle part. This is the period when the larva begins to attain its full size, and therefore the best time for taking them from the tree; for if rainy weather ensue, cracks are found in the pears, by which the larvæ find a convenient outlet to precipitate themselves to the ground. It is seldom that the larvæ are found in the core of those pears lying on the ground, if the rainy weather is of long continuance; and when this is the case, most of the pears remain on the tree till they are almost completely rotten. If the weather be dry, they usually fall off sooner; and in that case those pears that are lying on the ground should be picked up and destroyed, because, when in that state, the larvæ often remain long in them." This is no doubt the most judicious way of thinning these destructive creatures, and those allied to them. They have, besides, their natural enemies, which feed upon and destroy them, particularly a gold-shining hymenopterous insect which makes its appearance exactly at the same period as the platy. gaster and pear midges.

The Black-gall midge (Cecidomyia nigra, Meigen) is very destructive to the blossom of the pear, and to a great extent produces those failures in the crop which are usually set down as caused by easterly winds, late frosts, \&c. They lay their eggs in the blossom as soon as the petals begin to show themselves out of the calyx, by piercing through the petals with their long ovipositor, and depositing their eggs on the anthers, to the number generally of ten or twelve, often more, for as many as twenty larvæ have been found in one pear. The eggs, if the weather is warm, will be hatched in four or five days; and before the blossom has fully expanded, they descend to the core of the firuit, where they separate, and begin to devour the fruit in all directions. By the time they have eaten up all the pulp they have attained their full size, and only wait an opportunity of escaping from their feeding-place and find their way to the ground, which they do by availing themselves of cracks in the fruit, or when it becomes partly decayed, so that they can reach the surface, from whence they spring to the ground and bury themselves. Sometimes they eat their way out of the fruit to effect their escape, and at other times remain for a considerable time within the pear, even when it has fallen from the tree. The larvæ of so many insects are found within the prematurely fallen apples and pears, that it is well to gather all such up, and 
thoroughly destroy them. The gall and pear midges usually attain their full size during the second and third week in May, much depending on the weather whether it be cold or hot.

The common elm-destroying scolytus (Scolytus destructor Oliv., Hylesinus scolytus Fabr.), fig. 204, although called elm scolytus from its
Fig. 204.

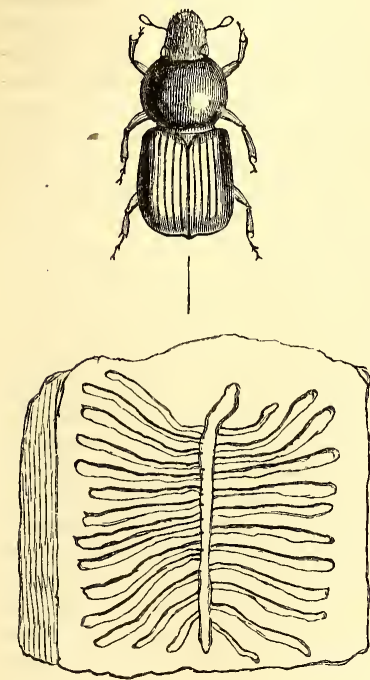

ELM-DESTROYING SCOLYTUS, AND SECTION OF WOOD SHOWING ITS RAVAGES. attacks on that tree, is nevertheless as injurious to other treesalso-the pear and apple in a particular degree; and although nearly related to the Scolytus hoemorrhous of Mey, fig. 178 , it is evi dentlydistinct. Dr Hammerschmidt has, however, endeavoured to establish another species, viz. Scolytus pruni, or plum scolytus; this other entomologists regard as identical with S.destruc tor, found also on the plum as well as on the apple and pear. The perfect insect, or beetle, has the head and thorax black and glossy, very thickly dotted with very minute dots. The antennæ are of a light blackish brown, terminating in a sort of knob. In size the beetle varies from two lines in length, and half a line in breadth, to nearly a third less. In form it is nearly cylindrical. The wing-cases are cut off somewhat obliquely behind, and are somewhat hollowed near their base, are of a brownish-black colour, marked with dots in very fine lines. The legs are of a reddish brown, the second joint being pretty broad. The larva is yellowish white, with a yellowish shining head and brown mouth, from one to two lines in length. They confine their operations entirely to the inner bark of the tree, which they often so completely destroy as to cause the death of the whole branch, and often of the tree itself, when they attack the main trunk. Their destruction is difficult, for their presence is seldom discovered until their effects give unmistakable evidence of it, when the branch had better be removed entirely, or that portion of the bark removed under which they burrow, and the inner bark with the insect scraped off and destroyed. Painting the external bark over where they reside with spirits of tar will probably be found the most effectual remedy with the least injury to the tree.

The pale brindled beauty-moth-Phigalia
(Geometra amphidasis) pilosaria Autor-fig. 205, appears about the middle of March. Fore wings Fig. 205.
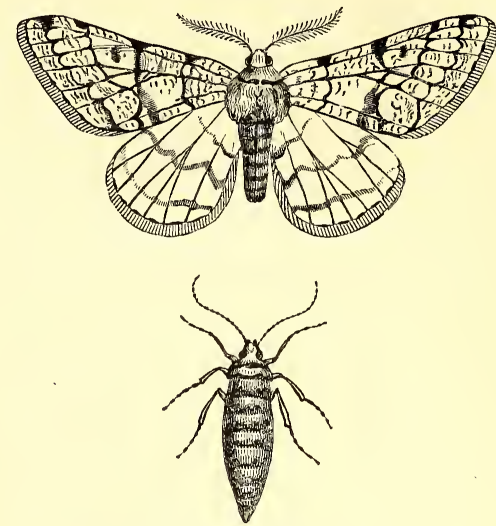

PALE BRINDIED BEAUTY-MOTH. Male and Female.

of the male are brownish grey, thickly covered with dots of the same colour, with a few white ones interspersed, and traversed by greyish cross stripes. Hind wings whitish, with a brownish undulating stripe running through their middle. The margin of the wings are fringed, having behind the fringe a dark grey line. The female is without wings, much smaller than the male, brownish, with many angular tufts of very soft hair. The body of the male is greyish green, with many long soft hairs. The sexes pair in March, and immediately after the female begins to lay her eggs, which she disposes in rows upon the small twigs of the pear tree, to the number of upwards of one hundred and fifty, which occupies her several days. The caterpillars are of a green colour, and appear just as their food, the young leaves, begin to expand. Their natural enemies are the ants, and to escape from them the female deposits her eggs generally on lofty pear-trees. The eggs being deposited on young small twigs only, and covered with a web of long grey hairs, may be readily detected, and should be gathered and destroyed.

The pear-leaf miner-Argyromyges Scitella (Mentz in Isis), Argyromyges Clerkella Stephens, Curtis, Knight, \&c., but not of Linnæus, (perhaps not pear-tree blister-moth, Tinea Clerkella often confounded with this insect, but twice as large)-_"measures about $\frac{1}{4}$ of an inch in the expansion of the fore wings, which are of a glossy silvery-white colour, the terminal portion being orange with white fringe, but varied on the fore margin near the tip with two white triangular spots edged with black lines, and with a trident-like black mark at the tip, and with the margin terminated by a black spot glossed with purple. The perfect insect generally appears at the end of May, when the female deposits her eggs on the under surface of the leaves, the young larvæ penetrating the under cuticle, and feeding on the parenchyma, leaving the two surfaces of the leaf untouched, and 
thus forming large oval or roundish patches, several of which often unite together, and thus

Fig. 206.
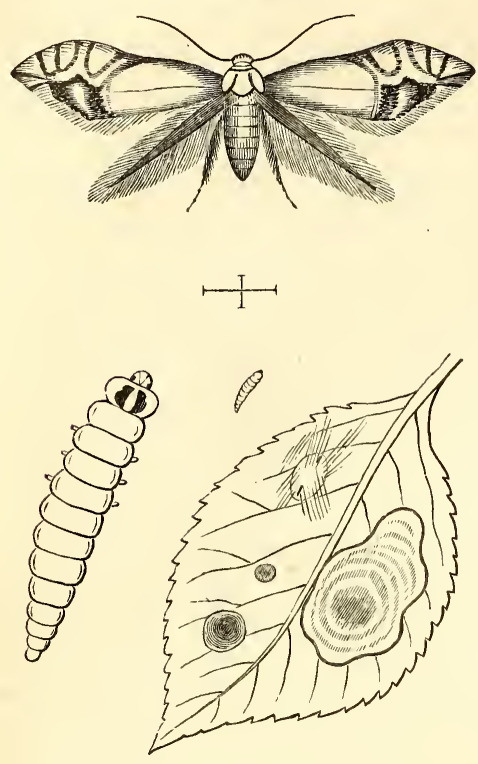

PEAR-LEAF MINER AND GRUB.

the greater part of the leaf assumes a blistered appearance. It is in the beginning of autumn that the leaves appear to be most affected by the ravages of these larvæ, which are fleshy, yellowish white, hairy, with six pectoral, eight abdominal, and two anal feet depressed. When full grown, the larva pierces the upper cuticle of the leaf, and comes into the open air ; it then spins some longitudinal threads close together on the upper surface of the leaf, and beneath these forms its cocoon, which Mr Curtis describes as boat-shaped, with the keel upwards, of a very close texture, and with a slit at each end, the cast-off larva skin being thrust through one of these slits, and the-moth escaping by the other. Sometimes these insects abound to such an extent as seriously to affect the growth of the trees, and the size and flavour of the fruit." We have more than once remarked that the most radical mode of subduing such insects as deposit their eggs on the leaves, or in the ground under the trees, is in the first case to collect all the leaves when they fall in autumn, and consign them to the flames; and in the second to gather up the soil at the bottom of the walls for a foot in breadth, and the whole surface as far as the branches of standard trees extend, and carry it to the charing-pile, or wheel it away to those spaces in the garden that are not planted with fruit trees. This is a wholesaie disposal of the insects while in their chrysalis state ; and one hour thus employed will be equal to days in picking them off the leaves, and pounds of tobacco and other remedies thrown on the trees, which might nearly be as usefully thrown into the sea.

\section{§ 2.-PRUNING AND TRAINING THE APPLE AND PEAR.}

The apple and pear are grown as orchard or full-sized standard trees, as dwarf-standards in various forms, as espaliers, and, in the case of the more choice kinds, trained against walls, particularly in cold situations, and in various other ways, as noticed below. Trees intended to be grown as full-sized or orchard trees should have a stem not less than 6 feet in height, and that stem encouraged to attain the greatest possible strength. Such trees are often rendered useless for this purpose by mismanagement in the nursery before they are planted out for good, by the absurd practice of training up the stems as if they were intended for whip handles; by which they become attenuated and slender, and long after require the support of stakes to keep them in a perpendicular position, and even to carry the weight of their own heads. The following hints may be the means of remedying this defect. In purchasing the trees, choose those that have their summer-shoots upon their stems ; but to secure this, the selection must be made before the nurseryman has trimmed his trees in the usual manner. Allow these to remain till the time of planting, and then shorten them to the length of about 3 inches, leaving them in form of spurs to produce shoots and leaves in the ensuing summer. Shorten the leading shoot to three buds above the intended height of the future trunk or stem. About the beginning of August (sooner in the south) these stem-shoots should have the tips of their points pinched off ; but the shoots above the contemplated height of the stem, and which are to form the branches of the future tree, should be left entire. In autumn, as soon as the young wood is ripe, cut close off to the stem the lower half of the stem-shoots, and top the upper half of them to about the length of $\mathbf{3}$ inches as before, that they may, during the following summer, draw strength to the stem. This practice is, we are aware, by no means generally followed, as most people cut off every vestige of shoot from the stem, considering them not only unnecessary, but actually injurious to the tree. They serve, however, an important purpose, as they elaborate sap which is deposited in the stem in the formation of layers of wood, strengthening the stem by increasing its size, and acting with the shoots now forming the head in the production of roots. In autumn, or early in winter, the shoots above the stem, which are now to be considered the foundation of the structure of the future tree, should be cut back to about 9 inches in length, and two shoots from each of these should be encouraged during summer. If any of them, however, show a much greater robustness of character than the others, they should have their tops pinched off towards the end of July, so that all may be said to have a fair start the ensuing spring. The direction of the shoots forming the head of the new tree should now be attended to, and made to diverge in the directions most proper for forming a uniform and equally-balanced top. From want of attention to this, many trees 
become misshapen at the outset, which all pruning afterwards will not be able to correct. The side shoots on the stem should be shortened in July as in the previous year, but in autumn they are to be cleared off entirely, having performed their allotted office; and in cutting them off, do so close into the stem, that the wounds may heal over, and the stem ultimately be as clear of blotches as if such shoots had been removed in the nursery, or as if they had never existed. Should the stem, however, be still apparently too weak to sustain the head without support, a few of those side branches immediately under the top may be left for another season, when they may be finally removed. The head may now be allowed to take much of its natural growth, for trees differ from each other in the habits they assume ; the only care taken being to cut out any shoots that cross each other, io pinch back during summer those likely to overgrow the rest, and at next winterpruning to shorten, where necessary, for the production of branches where they may be required for the perfect formation of the tree.

Established orchard trees should not be left, as is too often done, to run wild. The principles of pruning them are simple. Thin out the branches, that every part may alike enjoy the influence of light and air ; remove all dead, diseased, or misplaced branches, as well as the young spray which is apt to arise towards the hearts of the trees, particularly after severe pruning has taken place. 'The most superficial observer must have reinarked how superior the fruit of the apple and pear is, both in size, flavour, and colour, when produced on the outside of the tree, or towards the extremities of the branches. This is entirely owing to the greater portion of light, sun, and air it has compared with that growing towards the centre of the tree, almost deprived of these elements. This of itself should be sufficient reason for thinning all standard fruit-trees. Thinning out old and exhausted branches, and encouraging those that are younger, has also its advantages, because the sap becomes more elaborated and concentrated in wood of three or four years' growth than in such as is older; and the older the tree grows, the more sluggish is the circulation of the sap in it, that reciprocity which should exist between the roots and the branches goes on declining, and hence the tree begins to die by piecemeal, and the fruit becomes inferior, and at last worthless. From this it becomes quite apparent, that, as the tree, from age or bad soil and climate, begins to show such evident signs of decay, amputation of even large limbs should take place, and that young wood should be encouraged. This principle is carried to its fullest extent in the operation of heading down the tree entirely, and allowing it to renew itself by the formation of a complete set of new branches. After this, the knife will be brought into requisition until the tree again assumes a size to require the use of the saw in the removal of its branches.

Pruning the apple and pear as a half standard is conducted exactly as above, so far as the modification of the head is concerned. The branches, however, never attain so large a size, and are, therefore, constantly under the control of the knife. The stem, in this case, averages about 3 feet in height.

Pruning the apple and pear as an open duarf (en gobelet, or en tonnoir, Fr.)-This mode is well adapted to small gardens; and as the trees are not intended to be of a large size, they are wrought on the doucin or paradise stock; and the sorts best adapted are those of slender growth, making short wood, and requiring little pruning to keep them within the prescribed bounds. Trees for this purpose should be planted as maiden plants, but should not be cut down when planted, but allowed to stand a year to become established, and then be headed down to 4 or 6 inches, according to their strength: from three to four shoots will be produced from each of the cut-down branches, and these will be sufficient to form the future head. "At the end of the second year," Mr George Lindley directs, (in "Guide to the Orchard," p. 119,) "two or three of the best placed from each branch should be selected, and shortened back to 9,12 , or 15 inches each, according to their strength, taking care to keep the head perfectly balanced, so that one side shall not be higher nor more numerous in its branches than the other, and all must be kept as near as may be at an equal distance from each other. If this regularity in forming the head be attended to and effected at first, there will be no difficulty in keeping it so afterwards, by observing either to prune to that bud immediately on the inside next to the centre of the tree, or that immediately on the outside. By this means, viewing it from the centre, the branches will be produced in a perpendicular line from the eye; whereas, if pruned to a bud on the right or left side of the branch, the young shoot will be produced in the same direction, so that if the branches formed round a circle be not thus pruned to the eyes on the right successively, or on the left successively, a very material difference will be found, and the regularity of the tree will be destroyed in one single year's pruning, which may be readily illustrated thus:Fix four branclies, either in a direct line or to a circular hoop, at the distance of 8 inches from each other; let the first branch on the left be called $a$, the second $b$, the third $c$, the fourth $d$; head down $a$ to the left-hand bud, $b$ to the right, $c$ to the left, and $d$ to the right. When these have grown a year, those between $b$ and $c$ will be only 6 inches apart, while those between $a$ and $b$, and between $c$ and $d$, will be 10 inches; thus the distances now are not as 8 to 8 , but as 6 to 10 , which would require two years' pruning in a contrary direction to restore the head to its former regularity; and it must not be forgotten that this system of pruning will hold good in every other case. What has just been said has reference only to the leading shoots, which are always produced from the terminal buds when pruned, and which alone form the figure and beauty of the tree. The intermediate space must, of course, be provided for at the same time, having a regard to the number of branches thus employed, that they do not crowd each other. On the contrary, they must 
be kept thin and perfectly open, so as to admit plenty of sun and air, without which the fruit produced will be small, and good for little: the middle of the tree, indeed, must be kept quite open from the first to the last, taking care that all the surrounding branches lead outwards, and preserve a regular distance from each other. In pruning the supernumerary shoots, they should be cut down to within an inch of the bottom, which will generally' cause the surrounding eyes to form natural blossom spurs; but where the tree is in a vigorous state of growth, branches will probably be produced instead of spurs; if so, they must all be cut out close except one, which must be shortened as before. In all the winter prunings care must be taken to keep the spurs short and close, none of which should at any time exceed 3 inches-cutting out clean all blank spurs, which have produced fruit the previous summer, to the next perfect bud below."

In pruning the apple and pear, every means should be taken to encourage the formation of natural spurs in preference to artificial ones; and it may be here remarked that some kinds are naturally disposed to produce the former at an earlier age and in greater abundance than others, and these should have a preference in the formation of a selection, particularly in small gardens. The Keswick codlin may be given as an example of the one, and the Claremont or Easter pippin of the other. The former of these produces natural spurs profusely at an early age, while the latter rarely produces them until the tree has attained a considerable size and age, and even then but sparingly.

This mode of training occupies more surface space, certainly, than that of espaliers, about to be noticed; but, on the other hand, it gives a much greater surface of exposure to the sun and light to the leaves and fruit. We rarely encourage artificial spurs, but cut out all lateral shoots close in to the wood from whence they arise; and it is curious to see that young natural spurs will protrude from the smooth clean bark on the wood of one, two, or more years' growth, and especially so from the little callosity formed at the places from which the laterals were cut. The varieties which do not send out a sufficient number of natural spurs from the surface of the main shoots rendered bare by this system of pruning, are easily made to be clothed with fruit-bearing spurs by selecting young wood well placed, and laying it in upon the naked branches referred to. And again, by following out Mr Fleming of Trentham's practice, we side-graft shoots near the base of those naturally devoid of fruit-buds, and by either of these simple plans have fruit (the attacks of insects in the blossoms excepted) from near the surface of the ground to near the top of the trees. By this mode of pruning the fruit is much less liable to be blown from the trees by winds than if they were produced from buds formed on spurs often a foot or more in length.

Pruning the apple and pear in the conical or pyramidal form. - When the trees are so pruned, and the horizontal branches form stages above one another, they are termed en girandole, or chandelier-like, a form in much repute on the Continent. These are, in general, produced by cutting in the side branches, as shown at fig. 207 , which shows the tree in three successive stages. Fig. 208 shows such a tree when mo-

Fig. 207.

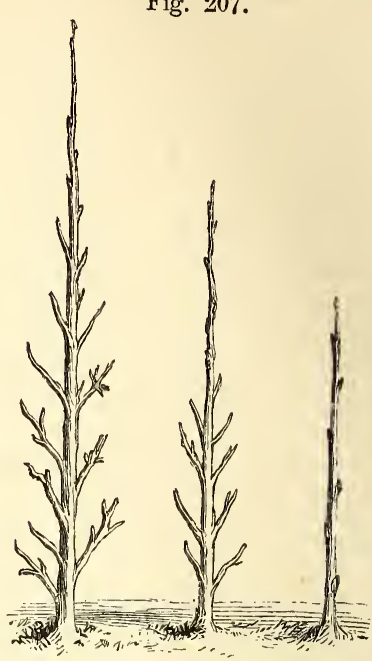

GIRANDOLE TRAINING.

First stages.

delled to the desired shape, and fig. 209 the same tree with the branches of the current year's growth tied down in the quenouille man-
Fig. 208.

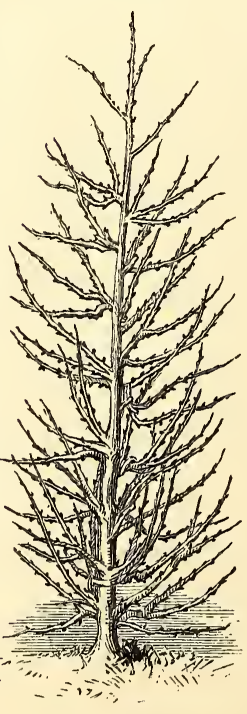

GIRANDOLE TRAINING Complete.
Fig. 209.

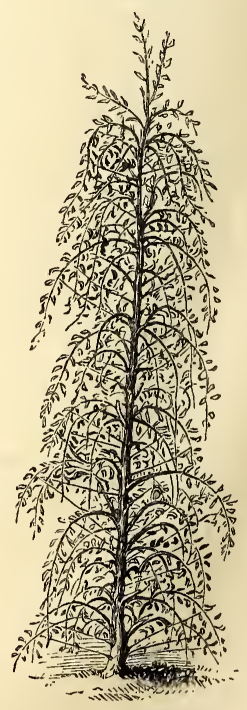

QUENOUILLE TRAINING. ner. Trees may be trained in this manner to the height of 12 or 15 feet, but for small gardens from 6 to 10 feet will be found a preferable size. The effect of bending down the branches 
in this manner is to cover the shoots with blossom-buds, and to produce most excellent crops. Although this tying-down mode of training is productive of large crops of fruit, still the restraint imposed upon the energies of the trees has the effect of rendering them comparatively shortlived. The French gardeners themselves admit that trees trained in the en quenouille manner do not last longer than ten or twelve years, and many of them are giving up the practice. This, however, may be in a great degree attributed to overcropping and too severe rootpruning; for a French or Belgian gardener is everlastingly operating on either the roots or branches of his trees. Trees managed in this manner in Britain are not found to be so shortlived. The process of pruning may be thus described: Shorten the upright or leading shoot to a foot above the ground. Let the shoot from the uppermost bud be trained upright in summer, and those below outwards. It must be understood that we are speaking of a young tree commencing to be so trained, allowing all to grow without stopping. At autumn or winter pruning, cut back the upright leading shoot to 15 inches above where it was previously cut. The buds, of which several will be found situated immediately below where a vigorous shoot has been shortened at the winter-pruning, are certain to break into shoots in spring; continue to train the uppermost or leading shoot in a perpendicular direction as before, and the others spreading. Thus proceed till the tree has attained the desired height, observing most particularly never to allow the upper tiers of branches to extend as far as those below them. The form which the tree should ultimately assume is that of a pyramid or cone, broad at the base, and gradually tapering towards the top. If the top branches overhang the bottom ones, the latter will become weak and useless for fruit; they will linger for a time, and then die off. At the autumn or winter pruning the tree should be cut so as to liave a regular tapering outline ; and during summer, should any of the upper shoots threaten to extend beyond the lower ones, such shoots should have their points pinched off. Shoots not required to form branches should also have their points pinched in summer. If the branches are disposed to grow too upright, they should be tied down to nearly a horizontal position, and secured to small stakes, or to the next lower ticr. If kept in a right position for two or three weeks in the growing season, they will afterwards retain it. This is a very good mode of growing both apples and pears, particularly in small gardens, as they occupy much less space, and cause less shade than if grown as open standards. Indeed, fruit trees do not producc fruit in exact proportion to their size, but rather to the mode of pruning and training they are subjected to, as well as the stocks they are wrought upon. Mr Rivers has shown many exccllent examples of this, and in his "Miniature Fruit-Garden" has exemplified his principles as regards this mode of training the pear and apple. A perfectly-managed pear-tree, trained as a pyramid, he illustrates by the annexed cut, fig. 210 , as it ought to appear VOL. II. in July, before its leading side-shoots and perpendicular leaders are shortened, " which is best

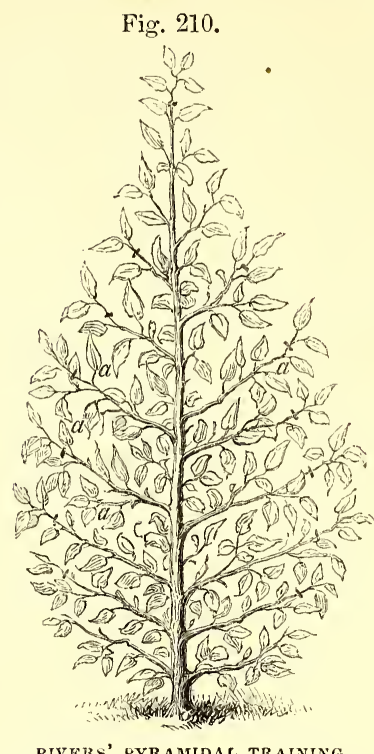

done towards the end of August. This shortening must be made at the marks shown on our figurc, and all the side shoots shortened in the same manner, as well as the leading shoot. The spurs $a$ are the bases of the shoots that have been pinched in June." After referring to autumnal or early winter planting, Mr Rivers proceeds "Care should be taken in selecting trees that are furnished with buds and branches from bottom to top; but if a young gardener intends to plant, and wishes to train up his trees so that they will become quite perfect in shape, he should select plants one year old from the bud or graft ; these will, of course, have good buds down to the junction of the graft with the stock. The first year, a tree of this description should be headed down so as to leave the shoot about 18 inches long. If the soil is rich, from five to six or seven shoots will be produced; one of thesc must be made the leader, and if not inclined to be quite perpendicular, it must be fastened to a stake. As soon in summer as the leading shoot is 10 inches long, its end must be pinched off; and if it pushes forth two or more shoots, pinch all off but one, to about 2 inches, leaving the topmost for a leader : the side shoots will in most cases assume a regular shape; if not, they may be this first season tied to slight stakes, to make them grow in the proper dircction. This is best done by bringing down and fastening the end of each shoot to a slight stake, so that an open pyramid may be formed; for if it is too close and cypress-like, enough air is not admitted to the fruit. They may remain unpruned to the end of August, when each shoot must be shortened to within eight buds of the stem; the abortive buds, of which there are generally from three to four at the base of each shoot, 
should not be included in that number. This will leave the tree like the annexed fig. 211,
Fig. 211.

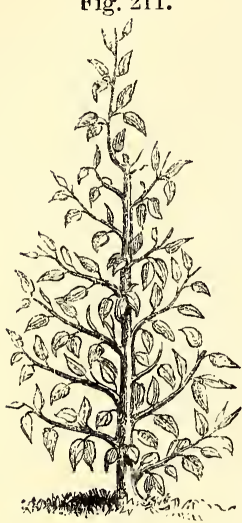

RIVERS' and no pruning in winter will be required. The second season the trees will make vigorous growth. The side shoots which were topped last August will each put forth three, four, or more shoots. As soon as these are 4 inches long, they must be pinched off to within 3 inches, all but the leading shoot on each side branch; this must be left on, to exhaust the tree of its superabundant sap till the end of August. The perpendicular leader must be topped once or twice: in short, as soon as it has grown 10 inches, pinch off its top; and if it breaks into two or three shoots, pinch them all but

the leader, as directed for the first season. In a few years most symmetrical trees may be formed. When they have attained the height of 6 or 8 feet, and are still in a vigorous state, it will be necessary to commence root-pruning to bring them into a fruitful state." It should be remembered that Mr Rivers places great importance on root-pruning and the use of quince stocks for the pear.

Amongst both apples and pears certain sorts assume naturally very different forms of growth; some grow close and compact, some horizontal and crooked, while others are slender and thin in their growth, and are indisposed to put forth lateral shoots. "The lower part of every branch," it is observed by Mr Rivers, "will then generally be furnished with dormant buds, so that to form a good pyramid of these slendergrowing varieties it is necessary to begin the first year with a graft, and to pinch the leader as soon as it is 6 inches long. If by any neglect the lower part of the pyramid be not furnished with shoots, but have dormant buds, or buds with only two or three leaves attached, a notch must be cut about half an inch in width just above the bud from which a shoot is required. This notch must be cut through the outer and inner bark, and the cambium or first layer of wood ; and if the shoot or stem is young, say from 2 to 4 inches in girth, it may be cut round half its circumference. If this is done in spring or summer, the following season a shoot will generally make its appearance-sometimes even the first season, if the stem or branch is notched early in spring. Varieties of apples inclined to be compact and close in their growth, form very handsome pyramids; but they are apt to be unfruitful, as the air is not admitted enough to the interior of the tree. This may be easily avoided by bringing the lateral shoots down to a horizontal position for a year or two, and fastening the end of each shoot to a stake ; an open pyramid shape will thus be attained, which the tree will keep. Other varieties put forth their laterals horizontally, and some are even pendulous. The leading perpendicular shoot of varieties of this description must be supported by a stake till the tree is of mature age."

The winter-pruning of pyramid pear-trees is almost reduced to a mechanical operation, when the summer management has been properly attended to. Keeping the tapering form in view, it consists in cutting each shoot a little shorter than the one immediately below it, taking care to cut to a bud situated on the side of the shoot towards that direction in which it would be most desirable the prolongation should proceed. Shoots that are too vigorous for the rest are not cut to a bud on the upper side, but to one situated below.

M. Cappe has introduced another feature of regularity in training besides that of a merely tapering contour. This is effected by what is called a tutor - that is, a straight perpendicular stake, to which the stem is trained; and from the top of this stake five wires are stretched to as many equidistant points on a hoop near the ground, or to five short stakes set at equal distances from each other, as well as from the base of the stem. These wires represent the angles of a five-sided pyramid - a pyramid apparently 15 feet high on a pentagonal base. Branches are trained directly from the stem to each of the wires. In order to convey some idea of this mode of training, one may imagine five upright trellises or screens projecting from a central upright, forming the partitions of as many equal recesses widening outwards. The branches form such partitions with good effect, as regards regularity; and when they become ornamented with leaves and fruit, the whole will prove an interesting sight.

It has been suggested that, instead of branches radiating from the stem in five directions, it might be found more convenient to train them out to four points-say east, west, north, and south. It may, however, be observed that, in the pear tree, five buds form a spiral once round the shoot or stem; or, in other words, supposing the leading shoot to be perpendicular, every fifth, tenth, fifteenth, \&c. bud will be in the same vertical plane. Therefore, if the number of buds between one branch and that next above it be always a multiple of five, the branches radiating in each of the five directions will originate exactly above each other.

Espaliers.-Apples and pears are very successfully grown when trained as espaliers. The advantages are, the little space which they occupy compared with standards, the protection of the fruit from the effects of high winds, and their capability of being protected in spring from late frosts, and when the fruit is ripe from the attacks of birds. The best mode of constructing espalier rails will be found described and illustrated in Sect. "Espalier Rails," vol. i. p. 556. In regard to the management of the trees themselves, supposing them to have been planted in autumn, as soon as the leaves have fallen, they are then in a fit state for commencing training. But, first, we should observe that espalier training admits of all the modifications practised when similar trees are grown against walls. 
The two leading modes, however, are the open fan and horizontal. In the former of these the centre is left open, in consequence of the curvature given to the inner branches, which gives the tree an awkward and vacant appearance, and on this account it is, therefore, seldom adopted. In proceeding to form a young tree upon the horizontal principle, the following is the method adopted: Let a plant one year old from the graft be taken with three equal even shoots, if this can be secured: fix five short stakes in the line of the proposed espalier-one in the centre, and two on each side-about 12 or 15 inches distant; train the centre shoot perpendicularly to the centre stake, and the two side ones horizontally to the four others, keeping these at their full length till the plant has been a year established. This is by no means a general practice, but one worthy of due consideration (vide article, Pruning newly-planted fruit trees, p. 379). If the tree appears healthy and vigorous, cut back the branches, the two side ones to 6 inches, and the centre to 9 or 10 . When the young shoots are produced from these, train the extreme or strongest one from each of the side branches horizontally. The centre shoot will have proauced three shoots at least, the uppermost of which must be continued perpendicularly, and the two next beneath trained horizontally, one on each side. This process must be continued from year to year, till the tree has arrived at its intended height, which is generally about 5 feet. If the centre shoot produces three others annually, when cut down to 9 inches, it will require seven years to complete the seven series of horizontal branches ; but sometimes it happens that the centre shoot produces two series, or five branches, by shortening it to 18 inches instead of 9 ; if so, this advantage may be seized. Should the tree, after having been planted a year, not possess sufficient vigour to throw out three shoots from the centre branch, all the three centre branches must be cut back to two or three eyes, and a single shoot trained from each; theyear following, proceed as directed at first. In this mode of training, the horizontal branches must be laid in at their whole length, without shortening any of them until the extreme branches of the two adjoining trees meet each other.

The following directions on the formation of horizontal espaliers are given by $\mathrm{Mr}$ Robert Thompson in "Morton's Cyclopædia," and as no higher authority in such matters can be quoted, as well as because his method differs in some essential points from that just given, we are induced to give it at length :- First season. $\mathrm{Mr}$ Thompson plants in autumn, and commences pruning and training the spring following, instead of giving the tree a year to establish itself, as recommended by Mr Lindley. "The tree being headed down to a foot above the ground. train the shoot which pushes from the uppermost bud upright in summer; and the shoots which push immediately below this, incline one to the right and another to the left, for the commencement of the two lower horizontals : but these must not be trained horizontally in the first instance ; they must be elevated to form an angle of about $45^{\circ}$, or like the legs of a wide $\mathrm{V}$, taking care to bend them down gradually if they are likely to become too strong. If one of them grow stronger than the other, depress the strong and elevate the weak." This, to a certain extent, acts as a substitute for allowing the tree to have a season for establishing itself previous to the horizontal training of the branches. "Second season.-Cut back the upright leading shoot to a foot above where it was shortened in the preceding season. If the side shoots are strong, bind them to nearly a horizontal position, and train them at full length ; if weak, shorten them a little, and allow them to retain nearly their former elevated position, in order to encourage their growth. Lateral shoots may be permitted to grow till July, and then stopped by cutting or pinching off their points. Third season.-Cut back the leading upright shoot to a foot, as before, and encourage side shoots from it to furnish the third course of horizontal branches. The tree being well established, these will be stronger than the first two produced, and therefore need not be so much elevated. The others may now be brought to a horizontal position, but with a gentle curve upwards from the stem. They should not come directly out from the latter, as the rounds of a ladder are inserted in its sides. If they spring from the stem 10 or 11 inches from the ground, they should ascend in the first instance, and then be trained along in a horizontal direction, at the height of 13 inches. The next course of branches, originating a foot above the former-that is, at the height of 22 or 23 inches from the ground-must be trained at the height of 25 inches, thus leaving a foot between the courses. In this manner the tree is formed; and the directions here given, being applicable to subsequent seasons, need not be repeated. When the stem, however, has attained the height to which the upright shoot must be shortened in autumn, two shoots only for horizontals will be required; and those afterwards springing from it, being apt to grow too strong, must be kept in check. Winter pruning.-The portions of shoots left at greatest length in the summer-that is, the breastwood-must now be cut in more closely than the others. Many of those which were shortened to 3 inches need not be touched; but where there are several of such too near each other (causing crowding in the spurs), some of them may be cut to one eye, or even quite close to the old wood. Fruit spurs that have extended too far should be shortened, and before they get too old they should be cut back to their lowest buds, which will then push others afresh."

Rivers' upright mode of triining the apple and the pear.-With a view to economise space, and at the same time to be enabled to plant a number of sorts of new and esteemed pears on quince stocks, so that they would quickly come into bearing, Mr Rivers, finding the horizontal method would take up too much space, adopted the expedient of transforming his horizontallytrained espaliers into the form represented by the annexed fig. 212. As will be readily seen, all the horizontal branches were removed excepting the lowermost pair, and from them ver- 
tical ones were laid in. One great objection to horizontally-trained apple and pear trees, both on walls and espaliers, as in general managed,

\section{Fig. 212.}

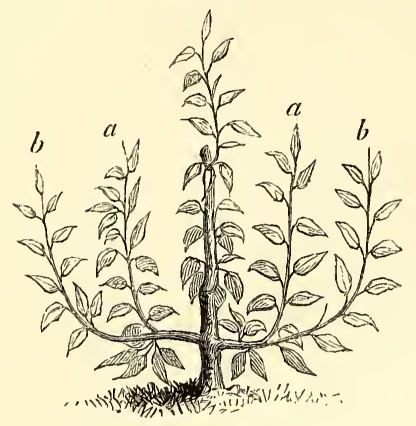

RIVERS' UPRIGHT TRAINING.

is, that the fruit they produce is usually towards their extremities, and these, extending to a great length, cause an amazing waste of space, while the centre parts are comparatively barren of fruit. The following will, in connection with the figure, explain his views: "The shoots $a$ a should be 8 inches from the central stem, and those marked $b \quad b$ the same distance from those marked $a a$. Thus a tree with five branches will occupy 32 inches, say 3 feet, of wall room ; a tree with seven branches will require 4 fcet, but as some space ought to be allowed for the spurs on the outside branches, say 5 feet. If the wall is of a moderate height- 8 feet, for instance-a tree with seven branches will produce quite fruit enough of one sort. This method offers a strong contrast to espaliers on pear stocks, planted in the usual manner, 24 feet apart, and trained horizontally: nearly five trees for one will give so many additional chances to the cultivator. A single tree may fail, or its fruit may become imperfect, owing to an adverse season, but out of five trees there will in every season bc a good chance of having some good pears." The first proceeding, as regards the vertical shoots, after they are ripened, is to shorten each to from 10 inches to a foot in length, and the lcading shoot must be shortened at the same time, and to the same extent. In May, each of these shortened branches will have put forth two or three shoots, which, as soon as they have attained the length of 4 inches, are to be pinched back to 2 inches, leaving the topmost one on each shoot, $a a$ and $b b$, as shown in the cut. The leader is to be served in the same manner. "You will then, if the tree is five-branched, have five young leading shoots. As soon in June as they have attained to 8 inches in length, pinch off the end of each, and when they break into two or three shoots as before, pinch all but one to each branch: this may be repeated, if the soil be rich, two, three, or four times in the summer. The tree will soon reach the top of the wall, and every bud in the five branches will bc perfect, either a blossom-bud or one in embryo. When every branch has reached the top of the wall; commence root-pruning in autumn." (Vide Rootpruning, p. 399). If larger trees are wished, so as to give more fruit of each sort, "trees with nine upright branches may be planted 7 feet apart, or trees with eleven upright branches 9 feet apart. Trees, however, can seldom be purchased with shoots so numerous; young trees must, therefore, be planted and cut back annually for two or three years, till the proper number of perpendicular shoots are supplied."

This is an excellent plan for amateurs, or those having only a limited extent of walling, yet desirous of growing a considerable number of sorts, either for variety, or for proving new kinds. The vertical position, however, of the branches will induce a disposition in the trees to grow strongest near the top of the wall or espalier, but this can readily be corrected by judicious root-pruning, and pinching back the young wood. Reversing the position of the tree-that is, allowing the main stem to reach the top of the wall or espalier, and carrying a branch horizontally under the coping to the right hand and to the left, and from these training the wood produced by them in a pendant form-would moderate the flow of the sap, and produce flower-buds in even greater abundance.

Pruning and training the apple and pear against walls. - The finer varieties of these, especially the latter, can only be expected to ripen in full perfection in the northern counties of England and in most of Scotland when trained against walls, and those in the coldest localities must have a southern exposure. There are a few situations, however, even in Scotland, where many of the finest apples and pears ripcn tolerably well when trained as espaliers and dwarf standards, and many others where they ripen on walls with an east or west exposure. On walls heated by hot water, as at Dalkeith, most of the French and Flemish pears ripen to full perfection. The general modes of training adopted are the horizontal and fan forms; other forms are, however, often met with. In regard to the quality of the fruit and its capability of keeping, there is no doubt that the same varieties ripened on open standards around London, and in the southern counties, are far superior to those ripened on our best unheated walls in the north. On account of the natural luxuriance of growth, more especially of pears, the horizontal mode of training has long been the favourite form, and more especially in the case of walls of less than 8 or 9 feet in height. Mr Rivers, however, and others, have adopted other forms (vide fig. 212). For the mode of setting offthat is, giving the first formation to horizontaltrained trees-vide article Espaliers, p. 556. The same rule is applicable to wall trees also. Much of the health of all fruit-trained trees depends on judicious summer-pruning. (See our remarks on that head also.) Harrison, an excellent practical authority, has, in his "Treatise on Fruit Trees," laid down his views on the summer and winter pruning of the apple and pear in a very clear and luminous manner, carrying the subject through twelve consecutive years, with useful illustrations. His principle is to 
keep the fruit-spurs very short, never allowing one spur to have more than three or four fruitbuds. He also cuts off the spurs entirely, or cuts them down for renewal every fourth or fifth year. By this means he has much larger fruit, and each spur is allowed to bear only once, when it is cut out, and succeeded by an embryo bud at its base. As an illustration of this part of his judicious practice, we subjoin fig. 213 from his useful work, which exhibits the condition of a portion of a branch of a pear tree during the twelfth year of his routine. "The spurs No. 1 must now be cut down to two fruitful buds, as a $a$ fig. 213, which will

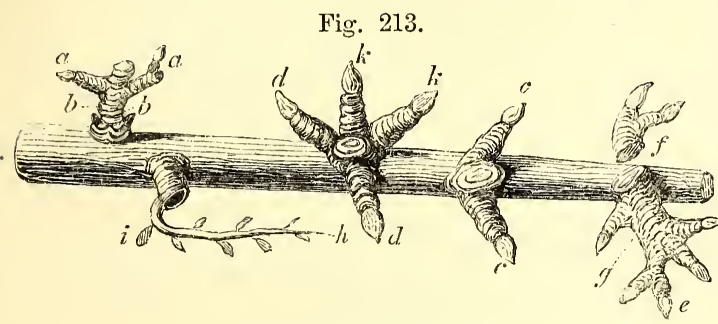

HARRISON'S MODE OF TRAINING.

eause an embryo or more, as $b b$, either to push the following summer, or to swell considerably, so as certainly to push after the old part of the spur has been pruned away. At the next winter-pruning it must be cut down to the lowest fruitful or growing bud, if there be such situated about an inch from the branch which supports the spur,otherwise the spur must be cut down to about half an inch from its origin. It will sometimes happen that when one of the spurs is cut down, three or four fruitful buds or shoots will arise around that part which is left, as $d d, k k$. If they are fruitful buds, they must all be allowed to remain until the next winter-pruning, when they will generally be in the condition described by $d k$. In thinning them, all must be taken away except two, which two should be the strongest and best matured; and if they be situated at the opposite side of the old spur, as c c, they must be preferred to those that are closer together, as $k k$; for when that is the case they interfere with and injure each other. When those spurs which remain come to have lateral spurs, as spur $e$, one of the main spurs must be cut away, $g$, the spur to the left. When shoots are produced instead of fruitful buds, as represented by $d d, k k$, they must be pruned down once, or more if required, during summer, and at the winter-pruning they must be regulated agreeably to the foregoing directions. It will frequently be the case that a fruitful bud will be formed at the lower part of such a shoot. If two shoots, situated in the manner of the buds $c c$, have each a fruitful bud at its base, both of them may be left, but otherwise only one. If none of the shoots should have a fruitful bud, then two of them, situated as before described, must be left, and be cut down to the lowest-growing bud upon them; and when a shoot pushes the next summer, it must be nailed down in the direction described by $l$, which will cause it to form a fruit-bud at its origin, as $i$, and at the next winter-pruning the shoot must be cut off just above such fruitful bud. This method of nailing down shoots during the summer, in order to make them productive of fruitful buds, may be practised upon any part of the tree when circumstances require it. When the spurs thus cut down and regulated have borne fruit a proper length of time, they must then be cut down to the lowest bud, or entirely cut away, as the case requires. These instructions must also be attended to in every other spur, upon every part of the tree, at a similar age and state."

of the great advantage of laying in young wood as practised by Mr Harrison, there can be but one opinion-it keeps up a succession of young wood on the trees, from which only fine fruit can be expected, and gets at once rid of these cumbersome anduseless masses of old spurs which long were allowed to disfigure the wall and espalier trees in even our best gardens.

Fig. 214 exhibits the appearance of a branch of

Fig. 214.

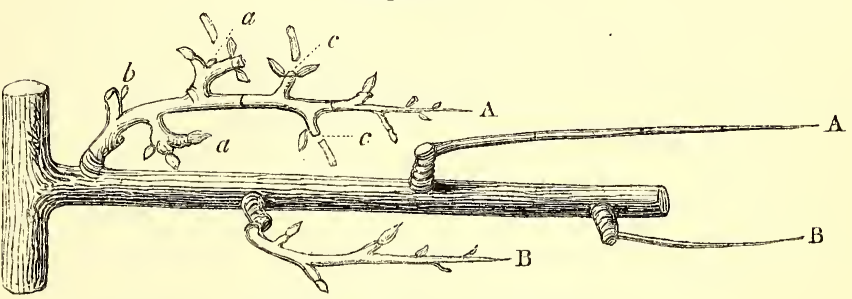

HARRISON'S MODE OF TRAINING.

a tree so treated during the tenth and eleventh years of Mr Harrison's routine, and fig. 215 its appearance in the twelfth year. "The spurs upon that part of each shoot which were produced" by being nailed or laid in previous seasons, "will now have borne fruit one season; they must be allowed to retain all the fruitful buds there are upon them; there will generally be two or three, as at $a a, c c$. If the shoots (which pushed from the spurs) that were shortened during last summer should now have a fruitful bud at the bottom of them, they must be cut off just above each bud. If there should not be a fruitful bud, let such shoots be cut down to half an inch in length." If the young shoots laid in "should extend so as to crowd each other, let their ends be prumed back so far as they interfere, at which they must afterwards be kept, by cutting them back to that part every summer and winter pruning.

"All the spurs upon the shoots, fig. 214, A A, 
must now be allowed to retain whatever fruitful buds there are upon them, as $a \alpha$, with the exception of the first spur upon the shoot, as $b$, which must now be cut down to the lowest bud, or entirely away. This is done in order to obtain a new shoot. Those spurs upon that part of the shoot produced the second year after being trained in, as $c c$, will generally have two or three fruit-buds each; they must be all retained. The shoot B will now be furnished with fruitful buds," but as it will sometimes happen that a shoot or two will push instead of fruitful buds being formed, such shoots must be pruned back during summer to 2 inches, once or more if required, which will cause them to form fruitful buds at their lower parts. "The shoots A A, fig. 215," which is the state of mat-

Fig. 215 .

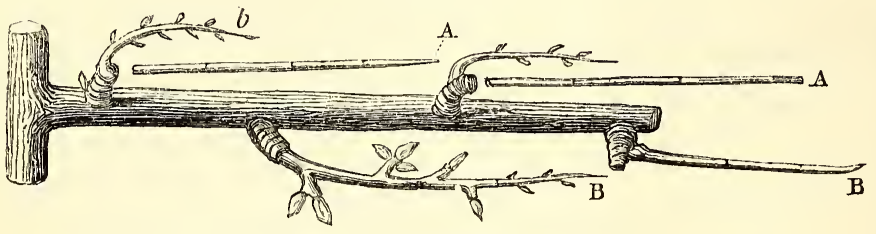

HARRISON'S MODE OF TRAINING. young wood, \&c. very rank on the branches, and hence a different mode of treatment is necessary, which leads to the second part of our quotation bearing upon the necessity of laying, in a succession of

Spur-pruning the apple and pear.-That both these trees produce their fruit upon spurs is true, and wherever natural spurs or fruit-buds are produced, they should be carefully retained, if not produced in too great numbers, in which case they should be thinned out at the winterpruning. The creation of artificial spurs was long the sheet-anchor of the cultivators of bygone days, and consisted of annually, about midsummer, cutting off every young shoot formed upon the main branches to within about half an inch of their base: some, however, were not very particular as to distance; and hence, in the course of a very few years, immense bundles of spray projected from the face of their wall and espalier trees a foot or more in length, placing what few fruit might by accident be produced àt such a distance from the wall as to derive no ter's at the tivelfth year, "must now be cut away so far back as to the origin of the shoot $b$, which shoot must be trained forward in order to supply the place of the shoot A cut away. The spurs upon the shoots B B will now generally be well supplied with fruitful buds; they must be permitted to retain three each, and next year four each, except the first upon the shoot, which must at that time be cut down in order to produce a new shoot for training in, as was done to the shoots A A. The spurs which afterwards proceed from these shoots, obtained by thus cutting down spurs, must have the same proportion of fruitful buds left upon them at every winter-pruming, and also be treated in every respect as already laid down for the spurs which the preceding shoots supported. This practice of cutting back the shoot A up to the spur $b$ must be done for the first renewal; but when a new one is desired the second time, the old shoot must be cut so far back as only to leave about half an inch remaining, unless there be a growing or fruitful bud situated near its origin, when it must, in that case, be pruned off just above it; and a shoot must be nailed in from some of the intermediate spurs, in order to supply the vacancy caused by the removal of the old shoot."

Harrison very justly remarks, that pear trees produce their fruit on spurs and buds similar to apples; the first part of his practice, which we have quoted above, is applicable to them also, but, he continues, "one mode of pruning is not alike suitable to all kinds of pear trees." The St Germains, Brown beurré, Bergamots, Swan's egg, \&c., amongst pears, have their spurs as far distant from each other as apples have; while some other sorts, such as the Crasane, Chaumontelle, \&c., have their spurs more benefit from it than if it had been produced on a standard tree. The case is now different, and this spur-producing system is considered obsolete by every cultivator of ordinary understanding. The pear and the apple both will produce better and more abundant crops of fruit from natural buds formed on shoots of two years' growth, if of a proper character, and duly exposed to light during the growing season, than upon all the artificial spurs that can be created by the mismanagement of man. To secure a supply of such wood, all that is required is to select, at the July disbudding, or summer-pruning as it is called, such a number of the shoots of the current year as can be conveniently laid in, either between the permanent branches or tied down over them, choosing such as are short-jointed and changing towards a brownish colour, which latter indicates that they have nearly arrived at their full maturity, and only require a short period longer to complete the elaboration of their juices and the formation of embryo fruit-buds. Green and luxuriant shoots should be rejected and removed at their base, either at the time of selection, or, better, at the winter-pruning. These selected shoots should be laid in regularly, but not too thickly, all over the tree. The practice of Harrison, already given, bears upon this subjectforcibly, and the no less high authority of Errington bears similar testimony. On pear-pruning and the tying-down system he says, "By this we mean the reserving many of the annual shoots at the winter-pruning, and tying or otherwise fastening them down on the old wood. Pears, say they, bear best on the two-year-old wood. Be it so; then let us take care that some of the annual shoots reach two years. We have before advised that all the shortest-jointed and early-ripened 
wood should be left; and now, when the knife must of necessity be passed over the trees, in order to correct the omissions of the past summer, let us again advise that every short-jointed shoot with plump eyes or buds be tied down to the old wood-not more, however, than one, or at most two, at a given point-and thus proceeding from the root-stock to the extremities to clothe the wood from end to end. When this plan is adopted, all barren-looking spurs may at once be cut clean away, unless they give promise of blossom-buds. The hobby, however, must not be ridden too hard; still preserve the genuine fruit-spurs with the utmost care. Under this system let there be no spurring back, as it is called, of young and green shoots, in order to create spurs. Such, we know, may sometimes be generated, but it is the exception to the rule. By this tying-down mode, when established, plenty of natural spurs will be produced; and, moreover, the tendency to produce 'breastwood' will be lessened exceedingly."-Cottage Gardener.

'Training the apple and pear in a pendant form, referred to before, has the effect of producing fruitfulness without having recourse to much pruning or mutilation. For this purpose trees are sometimes planted on one side of a wall or espalier, and trained to a single stem to the top, and are there headed back for the purpose of producing shoots to be trained in a downward direction on the opposite side. A modification of this is to train a single stem to the top of the wall or espalier, and from thence to carry two leading shoots in a horizontal direction to the right hand, and also to the left, as recommended by Mearns, and from these to train the shoots proceeding from them downwards on the same side of the wall or espalier. Another modification is to train two similar horizontal branches along nearly to the bottom of the wall or espalier, as recommended by Rivers, and to train the shoots from them upwards, in either a perpendicular or sometimes in a diagonal or spiral direction. The flow of the sap is considerably checked by having to travel in a horizontal line; but the evil in the latter case is, that the upright or diagonal branches nearest the centre of the tree become much stronger than those towards its extremities.

Training to horizontal trellises forming a plane with the horizon, and also on inclined walls, has no doubt the effect of moderating the rapidity of the flow of the sap, but both place the blossoms in a position more liable to sustain injury from frosts than where the trees are trained vertically. They are, nevertheless, under such circumstances, more readily protected by artificial coverings.

Summer-pruning the apple and pear when trained as espaliers.-Mr Robert Thompson thus speaks of this important operation : "Unless summer-pruning be duly attended to, fruitfulness need scarcely be looked for. If neglected in summer, a mass of shoots will give the espalier the appearance of an untrimmed hedge; and the question is, What is to be done with these superabundant shoots? If they are left to the winter- pruning, and then cut close to the horizontal branches, the latter will be rendered naked, and must remain weak, for they cannot increase in thickness if they are not furnished with sufficient foliage. If, on the other hand, the shoots are shortened back to within a few buds of their bases, almost every one of these buds will push, and a greater thicket than that produced in the preceding summer will be the result. The alternative is summer-pruning. The leading shoots must not be meddled with in summer, at least not until the branches of one tree meet those of the adjoining; but all other shoots produced along the branches must be checked. When the most forward of these have pushed about 6 inches, shorten them to 3 inches. The rest may be allowed to go on a little longer; but in the first fortnight of June, all of them should have their points cut off. About midsummer commence at the upper part of the tree, and shorten more than half the shoots to within 3 inches of their bases. About a week after, treat nearly half of those which have pushed from the lower branches in the same manner; and a few more of the shoots on the upper part may be also shortened to 3 inches. Reducing thus the superfluous shoots by degrees, the tree does not experience a shock like that which is the consequence of a sudden and an extensive privation of foliage. If trees have been neglected till midsummer, the shoots must not then be swept off close, or nearly so, at once. In such a case, shorten them all a little, and then keep on reducing them in a gradual manner. No entire shoots should be left throughout the summer, but a sprinkling of those shortened to stubs of 6 or 8 inches in length may remain till the winter-pruning. Their leaves will elaborate sap for the benefit of the tree, whilst, from their shortened condition, they occasion no injurious shade as regards the adjoining parts where fruitspurs are being formed." This mode of summermanaging espalier pear and apple trees has a direct reference also to such trees trained against walls, and to a very great extent to such as are grown as standards. It is a part of practice which merits the attention of every fruit cultivator.

Thinning the crops.-It is common with most people to remark, that the apple and pear bear well and the reverse in alternate years. The cause of this, for the most part, is allowing the trees to exhaust themselves by carrying more fruit in one season than they can well bring to full maturity. The trees become greatly weakened, and the extraordinary draught made by their roots upon the soil in which they grow extracts from it all, or nearly all, the food it contains suitable to their existence. The organisable matter stored up by the tree for its natural support is also exhausted in its efforts to perfect the crop; and hence in the succeeding season, with a deficiency of elaborated sap in the tree itself, and a deficiency of food in the soil within reach of the roots, we need not wonder that a season of sterility results. Fruit cannot be expected unless the buds are stored with the necessary amount of elaborated sap for their full development, the production of blossom, and much 
more so the several parts of the fructification. No doubt the application of stimulating food would go far to remedy this, by applying it both to the roots and leaves; and hence trees under glass continue to bear large crops for many successive years. But these stimulants are not so easily applied to trees in the open air. Were the fruit judiciously thinned as soon as it is fairly set, with something of a like care we bestow on our peaches and vines under glass, our apples and other hardy fruits would not only yield us year after year an ample supply of fruit, but that fruit would be of a very superior quality. Every deformed or under-sized fruit that a tree is allowed to ripen is like a robber preying upon the energies of the tree; and even when gathered they are found to be of little or no use to the owner. It frequently happens that when apples are set too thick, nature takes the case into her own hands, and points out to man what he should have done to ease her of her superfluous burden, and throws them off herself by thousands. To have fine fruit and yearly crops, judicious thinning should be early attended to. All fruit trees and fruit-bearing plants should be thinned of their fruit as soon as it is fairly set, and for this purpose finepointed scissors should be used. It may be considered by many as too high a refinement in fruit culture to say that even strawberries, gooseberries, currants, \&c., should be subjected to the same rule. Whatever these may think, one thing is quite certain, that if the finest specimens of these fruits be desired, that end can only be attained by judicious thinning. There is, no doubt, a considerable amount of labour attending such a proceeding, and he who will not bestow that labour must be content to put up with a very inferior quality of fruit, and also with diseased and shortlived trees, and the usual failure of crops. There is no garden in Britain where this principle is carried out to the full extent it ought to be. This, however, in many cases, rests not so much with the gardener as with the employer; for there are few gardens in which nearly double the amount of manual labour bestowed on them would not require to be employed, in order to carry out high culture to its fullest extent. Some attention, in this respect, is bestowed on wall fruit-trees, and all our best cultivators, awake to the importance of the matter, see its propriety, and act accordingly. But there are too many who have never thought (or, if such a thought ever crossed their mind, they have not acted upon it) of thinning the apples and pears, cherries and plums, with which now and then their wall trees are excessively loaded; the consequence is, that for several years perhaps, afterwards, they are saved the trouble, and, until the trees naturally regain their wonted vigour, their walls are devoid of fruit; or if there is any, it is limited in quantity and inferior in quality. Peaches and apricots on walls are in general better attended to in this respect, and the consequences are manifest; as the same rule applies to every fruit-bearing tree, the same practice should be followed. By early thinning fruit, another important end is gained, narnely, the destruction of thousands of insects which deposit their eggs or entomb themselves in the embryo fruit. 


\title{
CHAPTER VI.
}

\author{
THE PEACH AND NECTARINE.
}

THE peach and nectarine, although in cultivation spoken of as two distinct fruits, owe their origin to one and the same parent, Amygdalus Persica L., Persica vulgaris of Miller; and some botanists consider the peach, nectarine, and almond as only one species. The only difference between the two former is, that the one has a downy skin, and the other a smooth one. Their jdentity has been again and again confirmed by fruit of both sorts being produced, not only on the same tree, but on the same branch; and one instance is recorded of the same occurring in one fruit, one side of which was downy, like the peach, and the other smooth, like the nectarine; and the Boston nectarine originated from a peach-stone. The French have always considered them the same, and designate them as downy and smooth peaches. Persia is considered the native country of the peach, and its introduction into Italy is conjectured to have occurred so early as the time of the Emperor Claudius. It was first received into England about the middle of the sixteenth century, and in all probability was imported from France, where it had been long cultivated prior to that period. It was introduced into America by the earlier settlers, somewhere about 1680 .

So great a resemblance exists between many kinds of peaches and nectarines, that sufficient marks of distinction would be difficult to fix upon, so as to know the one from the other, had we no other characters to assist us except the form, size, colour, texture, \&c. of the fruit alone, as in the case of the apple and the pear. There are happily other and more permanent characters, and these have been made use of by several ingenious pomologists, with a view to establish a classification, the importance of which in a family of fruits so numerous as the peach has now become will be sufficiently obvious. Poiteau and the Count Lelieur in France, and Mr Robertson of Kilkenny, were the first to turn their attention to this matter; but it remained for the late $\mathrm{Mr}$ G. Lindley, and $\mathrm{Mr}$ Thompson, of the London Horticultural Society Gardens, to establish, upon a clear and satisfactory basis, a mode of classification which for the purpose will be regarded as sufficiently correct. Mr Thompson divides the varietics of this fruit into two grand divisions, namely, peaches and nectarines; these, again, into two classes, melters or freeVOL. II. stones, or such as have the flesh parting freely from the stone when ripe ; and pavies or clingstones, or such as have the flesh adhering to the stone when ripe. Clingstone peaches with us are termed Pavies by the French, and our clingstone nectarines are their Brugnons. Mr Thompson classes all peaches and nectarines into three other divisions, founded on the leaves. First, Leaves serrated and glandless; second, Leaves crenated or serrulated, with globose glands; third, Leaves crenated or serrulated with reniform or kidney-shaped glands. These glands are placed on the foot-stalk of the leaf, close to its junction with the leaf itself. Some little discrimination is required to distinguish the two forms of glands from each other, and the cxamination should take place when the leaves are fully developed, and not from one leaf alone, but from several on the same branch or tree.

The flowers, again, afford two subdivisions; namely, First, Large flowers, as in the Noblesse peach: Second, Small flowers, as in the Elruge nectarine.

The varieties of peaches and nectarines are now numerous. In 1573 , Tusser merely mentions peaches red and white. By 1629 they had increased considerably, as Parkinson enumerates 21 sorts ; Miller, in 1750, 31 varieties; Longley, in Pomona, 1729, describes and figures 39; Rogers, 43; Forsyth, in 1806, describes 40, and gives the names of 27 more, which he says may be added. This long list, however, requires to be purged of many which are mere synonyms. The Horticultural Society Fruit Catalogue contains 47, 19 of which are nectarines; and Downing, in "Fruits and Fruit Trees of America," describes no less than 79 peaches, and in addition 18 nectarines. Some of the American nurserymen's catalogues enumerate above 100 kinds, all originated in that country; and the Fruit Catalogue of Messrs Lawson \& Son describes sorts.

The peach will not succeed either in England or France as an open standard, but in China and the United States it attains a high degree of perfection when so grown. The peaches of Pekin are celebrated as being amongst the finest in the world, and of a very large size. In no country in the world is the peach grown in such quantities as in the United States. In the eastern states they do not succeed well, but in all the 
middle, southern, and western States they grow, and produce the heaviest crops in every garden and orchard. " Thousands of acres," Downing observes, " in New Jersey, Delaware, and Maryland, are devoted to this crop for the supply of the markets of New York and Philadelphia; and we have seen, in seasons of great abundance, whole sloop-loads of fruit of second quality, or slightly decayed, thrown into the North River in a single morning. Many growers in New Jersey have orchards of from 10,000 to 20,000 trees of different ages, and send to market, in good seasons, as many bushels of fruit from the bearing trees." Of the peaches of China we have received several-the flat peach, remarkable for its singular shape, and the more recently introduced Shanghae, for its enormous size.

Propagation.-The peach and nectarine are propagated in Europe, like all other fruit-bearing trees, by seed, when the object sought for is new or improved varieties ; in America, often by seed for general planting, although their best pomologists condemn the practice. In the middle, southern, and western States the peach flourishes with little care, many growers having peach orchards containing from ten thousand to twenty thousand trees, many of them from seed; and thousands of acres in Maryland, Delaware, and New Jersey are occupied with peach orchards for the supply of the Philadelphia and New York markets alone. They also raise the peach from seed, for the purpose of stocks, because, as $\mathrm{Mr}$ Downing, an American author, remarks, "The peach is the most easily propagated of all fruit trees. A stone planted in the autumn will vegetate the ensuing spring, grow 3 or 4 feet high, and may be budded in August or September. Two years from this time, if left undisturbed, it will usually produce a small crop of fruit, and the next season bear most abundantly, unless the growth is over-luxuriant." In Britain the case is otherwise, the peach-stock being in much less repute-the plum, which is a much hardier stock, and better fitted for a cold and variable climate, being preferred. The stocks, therefore, employed for the peach and nectarine in Britain are the Brompton stock, mussel-plum, pear-plum, apricot stock, and the almond ; and, when very dwarf trees are required, the Mirabelle plum is sometimes chosen. Of these the pear-plum stock is preferred for the French peaches, and most of our finer sorts ; and next to it the mussel-plum is in general preferred for English peaches (vide p. 328). In France, the almond stock is preferred by some growers with a view to check the over-luxuriance of the peach; and Knight recommends its use for the finer varieties of peaches and apricots, and also as a preventive of mildew. The plum stock is preferred for clayey soils, and the almond for such as are light, shallow, or chalky.

In regard to rearing the peach and nectarine from seed, at least in Britain, little attention has been paid to the subject, although several excellent sorts have been so produced (vide Select List). The late Thos. A. Kuight held the opinion that the peach, if reared from seed, would in course of successive generations become so hardy, or naturalised, as to succeed as an open standard in many parts of Britain. This opinion has not, however, been borne out by experience, nor has much attention been bestowed on the subject. He also observes, that it may always be made to produce fruit, when three years old, from seed, and thinks it possible to bring it to that state at the end of a single year. In rearing the peach from seed, the process is to bury the stones in autumn in thick layers covered with earth. Early in spring the stones are taken out of the earth, cracked, and the kernels sown in rows in a mellow loamy soil, to the depth of about an inch. In September following they are fit for budding, which is performed within 6 inches of the ground. Some English cultivators sow the seeds of the peach and apricot at the bottom of walls in the places where they are to remain, and bud them in July of the same year, or graft them in March the spring following. In grafting the peach a sub-variety of splice-grafting (vide p. 331, fig. 101) is very successfully followed, although, in the hands of an expert operator, almost any of the methods noticed under the head Propagation by Grafting, p. 330 to 333, may be adopted. Budding is the mode of propagation most generally followed in our British nurseries, and is performed in July or August, inserting the bud within 6 inches of the bottom of the stock for dwarf-trained trees, and from 3 to 5 feet high for standards or riders. The bud will shoot the following spring, and under favourable circumstances produce a shoot from 3 to 4 feet in length during the first summer. In spring the top of the stock should be cut off close above the bud, and the wound smoothed, that the bark may heal over it. These shoots are, however, when strong, often stopped in June the season after budding, or in the March following, to four, five, or six eyes, from each of which a lateral shoot is produced, which forms the first part of the structure of the future tree. The shoots of the second and third years' growth are shortened by pinching their tops off to three or four eyes, according to their strength, in June, to cause the production of another set of lateral branches, or headed back with the knife in March following to from four to six eyes. The number of eyes left, in both cases, must ever depend on the state of the trees, whether they are growing luxuriantly or the reverse. In the former case, more buds should be left than in the latter. Some, however, pinch off the point of the shoot produced by the bud when it has attained the length of 6 or 8 inches. Five buds are then allowed to extend, and these again have their points pinched off, leaving from 5 to 6 inches of shoot, each of which is disbudded, leaving only two shoots from each. Under favourable circumstances and vigorous growth, a fan-shaped tree is thus produced in one season.

Situation and soil.-The peach and nectarine not only require the best situation our gardens afford, but, in the greater part of Britain, require the protection of walls also. As a general rule, they should occupy stations on those walls having a southern exposure, a few points to east or west of south making little difference. In many parts of England they are grown to great perfection on direct east and west walls, but seldom 
with profitable success either as standards or trained to open espaliers. In few places in the north of England or Scotland will they prosper otherwise than against south walls. In certain upland and cold districts it is imprudent to plant the peach at all, as pears would yield a far more profitable and certain return. Latitude has much less to do with this than altitude; for excellent peaches and nectarines are produced in some parts of Ross-shire and Sutherland, while in many of the localities along the English and Scotch border they would not do much more than drag out a miserable existence, and to attempt to grow them for their fruit would be hopeless. One cause why the peach succeeds so well in the far north, and one which has, so far as we know, been unnoticed in books on horticulture, is the lateness of the season when the flowers expand, and the warmth of summer and fine autumns which in general occur in the warm sheltered spots chosen for gardens so far north, and which are often at the base of hills or rocks, enjoying the reflected heat therefrom.

Some lay great stress on the quality of the soil in which the peach is planted, and no doubt on this much of their future prosperity depends; but, on the other hand, much depends on situation as regards local climate and circumstances. We have had very healthy trees growing in a cold stiff clayey soil upon a chalk subsoil, as at Stratton Park, Hampshire ; on a rich loamy soil on a gravel substratum at Abercairney, Perthshire ; on a poor light sandy soil (ground originally reclaimed from a heath-clad common), at Claremont, in Surrey; and on an equally light and poor sandy soil at Taymouth Castle, in Breadalbane, and on a made soil of intermediate quality at Dalkeith. In the deep rich alluvial soils of the Carse of Gowrie, splendid peaches are produced from very healthy trees, and also in the deep alluvial sandy soils in Flanders. In all these cases; however, climate, either naturally or artificially, is favourable to the growth of the peach. These, and various other circumstances coupled with climate, lead us to the belief that much of the ultimate success depends on judicious pruning and training, reducing or encouraging the strength of the trees, as the case may be; adapting these operations to the circumstances of both soil and climate; and, above all, selecting those varieties which are suited in constitution to the locality. The nomenclature of soils is still so vague that it is almost next to useless to recapitulate those recommended in books. As a specimen, however, we may make the following quotation from the "Encyclopædia of Gardening," p. 913 : “A good soil for peach trees," according to Abercrombie, " is composed of three parts mellow unexhausted loam, and one part drift-sand, moderately enriched with leaf-mould, or the cooler dungs." Forsyth says, "Peaches require a lighter soil than pears and plums, and a light mellow loam is best." Harrison prefers "a loamy soil, somewhat adhesive," as do most of the experienced gardeners of the present day. From even these high authorities little can be gleaned useful to the tyro in horticulture. A rich, mellow, somewhat adhesive loam, taken from near the surface of an old pasture, without the aid of artificial enrichment, together with what vegetable matter may exist on it, is no doubt the best for all moderately-good climates. A lighter soil may be more advisable in a cold or damp climate or locality. There are other circumstances that have too often been very much overlooked, namely, that there are certain soils naturally, and certain localities having nothing very particular in their general character, that are much better suited to the peach than others contiguous to them, and between which it would be difficult to draw a visible line of distinction. In good climates, even with an indifferent soil, peaches often thrive well. In bad climates, be the soil what it may, they do not. It should, however, be our aim in culture to improve both, and so far as possible to make each act as auxiliary to the other.

In making prepared borders for the peach, we would avoid too light a soil, and approach a pretty rich mellow alluvial or loamy deposit, taking special care that the bottom is rendered thoroughly dry, and the depth graduated to the coldness or dampness of the locality; and no means are better to insure this than vaulting them underneath, as recommended, vol. i. p. 31 . Thorough drainage is not only necessary to the welfare of the roots of the peach and all other tender trees-and this the more so, as the situation is cold, late, or damp-but subterranean aeration, or underground ventilation, is equally essential; and next to vaulting or chambering the borders underneath, is the system of draining and aeration recommended, vol.i. p. 27, and illustrated by fig. 11. Indeed, were arrangements made by which the air in such vaults could be heated during spring and summer as exemplified in the gardens of the Marquis of Tweeddale, vol. i., p. 31 , the roots of such trees as the peach and nectarine would be placed in a condition nearer to that of their native country, Persia, and made to reciprocate with the atmospheric heat their leaves are placed in during the warm days of our springs and summers. With this increased temperature and aeration below, a stronger and more congenial soil could be employed for them, thus not only insuring healthy development in youth, but also extending the period of theix productive existence. Under such conditions the peach and nectarine would flourish in situations where at present they can scarcely be maintained alive.

In cold and damp situations, peach-tree borders can hardly be made too shallow, if resting on a solid subsoil. We have them, in some cases, only 14 inches deep, resting on an alluvial gravel; but in warm and dry places a greater depth may be indulged in. We have more than once, in vol. i., pointed out that, as all borders are reduced in depth, they should be proportionally increased in breadth, to allow ample scope for the roots, while at the same time they are kept near to the surface, that they may enjoy the benefit of sunheat and air. We may also here state as an unconditional rule, that no peach-borders should be cropped, and that they should be as little disturbed as possible. Leaving the borders of fruit trees uncropped, or admitting only crops of 
the lightest nature to grow upon them, was re. commended by Justice nearly a century ago. Gardeners, in general, look upon this practice as a loss of ground, which no doubt it is; but if a sacrifice is to be made, it is certainly better to choose the least evil, and rather leave the borders entirely uncropped than injure the fruit trees, for which both they and the walls were constructed. Too deep, too damp, and too rich borders have been more disastrous to the peach than all the other ills that befall it, as under these circumstances the wood can never ripen, but will continue growing on till the winter frosts arrest its progress; and without properly ripened wood all our other efforts are in vain. The more the border slopes from the wall towards the walk in front the better, as the surface is presented at a better angle of elevation to the sun than were it flat. Again, all peach-borders should be elevated above the general surface, and this the more so as the situation is cold, damp, and late. No manure whatever should be dug into peach-borders, nor mixed with the soil on their first formation, unless, in the latter case, the soil be poor indeed, and the nianure very much decomposed. Enrichment is better applied by mulching during the warm months of summer with pretty good manure, the essence of whicl is washed down by rains, and the evaporation and drought counteracted while it is lying on the surface. Rank manure is highly injurious to all fruit trees. Better have a 6 -feet border uncropped, than an 18-feet one cropped in the usual manner.

Planting.-From the remarks offered in the article Transplanting, p. 356, it will be seen how important a matter early autumnal planting is; and if it is so as regards trees in general, it must be especially so in the case of our most valuable and tender ones, amongst which the peach and nectarine stand so high. The importance of shallow planting is there also enforced, p. 365. In regard to arrangement, peaches and nectarines should be placed together on the same wall. Such an arrangement gives the appearance of system ; it offers great facilities in the formation of the borders, and in covering the fruit from the attacks of wasps and flies, the blossom in spring from frosts, and admits of the walls being heated with hot-water pipes, \&c. In planting, regard should be had to keeping the early-ripening kinds together, the medium-ripening sorts by themselves, and the late ones next each other. Weak and vigorous growing sorts, in each of the sections, should be planted alternately; and riders or standards of the same sort as those immediately under them, as dwarfs are to be considered the permanent trees; so that, when the former are removed to make way for the latter, no confusion may arise in the collection, and no one sort be entirely removed. The permanent trees should be planted not less than 20 feet apart from stem to stem, and the riders placed exactly between them, on all walls from 10 to 12 feet in height, the intention of the riders being to cover the top part of the wall while the dwarfs are filling up below. On walls of less height the distance should be greater to allow the trees to extend horizontally. A good deal, however, as to the distance depends on the soil and climate. If these be congenial, a peach tree will cover double the distance, as they often live to a considerable age, and attain a large size. In regard to the age of the trees at final planting, some cultivators prefer maiden plants, while others give a preference to those which have been trained for two, three, or four years in the nursery. Those who are good trainers, and particular about their trees, will choose the former; while many, and by far the most, prefer the latter, especially when it is desirable to have the walls filled speedily, or to make up blanks occasioned by deaths, \&c. Maiden trees are those of the season next after budding, and have only one shoot. The season after, they are called trained trees, and have several shoots. Healthy and moderately vigorous young trees only should be selected for planting.

Shallow-planting is of much importance in peach culture,-indeed, what is called surfaceplanting, in most soils, is to be recommended; that is, spreading out the roots on the prepared soil no deeper than the general level of the border, and laying over them 6 or 8 inches of soil, leaving it in form of a slightly raised mound.

Taking up and replanting trees of ordinary dimensions is often requisite, more especially when they either have got into a too vigorous state of growth, making too strong shoots, and showing a deficiency of flower-buds; or when, in an opposite condition, making small wood, and showing symptoms of debility -in the one case indicating that the roots are throwing into the system too large a supply of sap, and in the other a deficiency. Taking up in autumn, and replanting, is a remedy in both cases : in the former, the strongest roots should be shortened back, and in the latter fresh and suitable soil should be afforded them, and that of a richer nature than that in which they have formerly been growing. Judicious replanting and rootpruning, adding or diminishing the supply of food by the roots, will in a great degree obviate the necessity of branch-pruning; for if a reciprocity be not maintained between the roots and branches, by placing the roots in the condition we have here described, pruning will assuredly, in most cases, rather aggravate the complaint than alleviate it.

Pruning and training on the open walls.-So much has been written on pruning and training these trees, and so little remains to be added to the excellent rules laid down, that it appears to us to be mere egotism to profess to lay down rules which may have novelty only to recommend them. We deem it, therefore, far more useful to recapitulate the substance of what has been already written, especially of late years, than to confine our observations to our own practice alone ; for, in truth, we have been acting for the last forty years upon one or other of the systems pursued with the utmost degree of success by those who have made the culture of the peach their particular study. The peach is also cultivated under such a variety of circumstances that no one method recommended can be wisely considered universal in its application. 
And we may here also remark, that much of what will be said regarding pruning and training these trees in the open air, is applicable to those under glass also.

Season of pruning. - Some advocate early autumn-pruning, some midwinter, and others early in spring, when the buds begin to swell. 'The earlier authorities say little of early autumnal pruning, but many of them advocate winter for performing the operation, chiefly December and January. The most prominent of these are Hill, Abercrombie, Kennedy, Nicol, and, latterly, Harrison. The advocates for spring-pruning are Forsyth, Rogers, Switzer, \&c. Amongst more recent writers opinions on this matter are similarly divided. Mr Errington, practising in a cold part of Cheshire, and one of our most celebrated fruit-tree managers, says, "Many persons do not like to prune their peaches in the dead of winter : for our own part, we have pruned at all seasons for many years, and could never find any injury resulting from midwinter pruning of the peach. Where such has occurred, it must have been in the case of bloated and badly-ripened wood, and this is liable to such injuries under any circumstances." The prevailing opinion is, we believe, in favour of spring-pruning, because, say its advocates, the wood-buds are at that time more easily distinguished, and the wounds heal more rapidly. Our own opinion is, if they arc not pruned by the end of October or beginning of Novembcr, particularly in cold climates, it is better to defer the operation till spring. Much of this, however, depends on the ripened state of the wood, andalso if the locality is subject to severe winters or to late spring-frosts. The best thing under adverse circumstances, such as cold situations, \&c., is to depend on summer-pruning mainly, leaving little to do afterwards, but the removal of such wood as may have sustained injury during winter, thinning out where too thick, and shortening back shoots either imperfectly ripened, or such as have been left at too great a length previously. Harrison prunes as early in the season (that is, in autumn) as the state of the trees will permit, beginning his general winter-pruning by operating on the peach and nectarine first. "I have," he says, "noticed some persons prune peach and nectarine trees that were in a bearing state as late as April, when the bloom-buds were just bursting. The effects that followed such a system were in numerous instances very evident, for soon after the blossoms had expanded, many of those situated nearest to the end of the shoots withered and dropped off, and the tree was considerably weakened. Young trees, however, I uniformly prune in spring for the first two years after being planted, being careful that it is performed before the rising of the sap." As we have noticed in the case of the vine, early autumn-pruning strengthens a tree, while late spring-pruning greatly weakens it. Both Forsyth and Rogers disagree with Harrison, both recommending spring-pruning, the latter remarking that sometimes young shoots or old branches receive injury or die during the winter, and which cannot be seen till the spring-growth commences: "another thing," he says, "the later a peach tree is pruned, the sooner are the wounds healed." The principal object to be aimed at, in pruning and training the peach and nectarine, is to keep up a constant succession of young wood in every part of the tree ; for unless this be accomplished, the crop of fruit must be partial and defective, and the trees themselves become naked and worn out. With this object before us, it behoves us to adopt that mode of pruning and training most likely to insure success ; and as some of the many methods laid down are better adapted to some situations, soils, and circumstances than others, it is necessary that we not only make ourselves acquainted with these modes, but also choose from amongst them that which is best suited to the circumstances with which we have to deal. In connection with this, $\mathrm{Mr}$ George Lindley, in "Guide to the Orchard," p. 301, remarks, "To effect this (that is, keeping up a succession of young wood), the annual shortening of the young wood is perfectly calculated; but the manner in which this ought to be performed has by no means been fixed upon one certain principle : the various methods laid down and insisted upon by writers being greatly at variance with each other, they leave the inexperienced gardener in a dilemma as to which course he should pursue." A supply of young wood throughout every part of the trec is to be effected by pruning alone, and a judicious distribution of its young wood. "Commencing with the winter-pruning," Mr Lindley continues, "the first rule to be laid down as a basis 'for' all the rest, is to shorten every shoot in proportion to its strength, and to prune to where the wood is firm and well ripened; this will cause all the pithy and unripened wood to be removed, thence causing a supply of that which is better ripened for the ensuing year. But in order to give every facility to the ripening of this wood, it must be trained thin, not in profusion, according to the general custom, but such shoots only as may be required for the following ycar. Trees which have arrived at a bearing state should have their strongest bearing shoots shortened to 12 or 14 inches, those next in strength to 8 or 10 , and the weaker ones to 4 or 6 inches, pruning each to what is termed a triple eye, or that where there is a blossom-bud on each side of a wood-bud. Where branches are not in a bearing state, these triple eyes will not be found. They must, therefore, be pruned to a wood-bud alone, which is always known by its sharp point. When the tree has been pruned once in this manner, the shoots must be trained neatly, nearly parallel to each other, so that a line continued in that dircction would lead itself clearly out to the extremity of the tree."

Mr R. Errington, in " Cottage Gardencr," vol. i. p. 116, lays down the following very explicit rules: "To understand this operation the better, it may be well to state what are the prime objects ; namely, First, To thin out or remove superfluous shoots, in order to insure sufficient light and a due circulation of air to the remainder ; Secondly, To shorten back, for the twofold purpose of removing unripe or immature portions, and of inducing plenty of successive 
shoots lower down the tree. For illustration, we will suppose an established tree, which has been planted five or six years. The nails being all unloosened, excepting a few to hold the principal shoots, operations should commence at the bottom of the tree near the collar. Here it is that a watchful eye must be keenly exercised, at each returning pruning season, in order to preserve and continue a due succession of rising shoots from the lowest portion of the tree. It is evident that if the young shoots at this point are not taken care of, the lowest part of the tree will become barren, and a part of the wall wasted, besides which the tree will not be so ornamental. It frequently happens that some of the young shoots at this point are inferior in character ; and very frequently shoots which spring from the collar, and reach a yard or so, possess a fine young shoot lower down, which is fitter to become the leading shoot of that portion of the tree than the one already existing. When such is the case, it becomes necessary to cut away the older portion. This must be done with a clean cut, and nearly close to the point from which the future leader comes. However, the first point is to cut away any cankered or diseased shoots, and then to shorten judiciously these at the lowest level that can be obtained. It may here be observed that no fruit should ever be permitted to grow for the space of a foot from the top of the collar on any given shoot. If fruit is produced at these lower extremities they are always inferior, and they only serve to oppress a portion of the tree which ought always to be kept as a nursery for young shoots to keep the tree well furnished. By 'shortening judiciously' we mean cutting back the lowest-placed young shoots as lou as possible, provided a few good eyes or buds are left, and that such shoots are not required to fill existing blanks in the walls. Such, then, when pruned back, may be considered a guarantee against the tree becoming naked. We come now to bearing wood, and here the main point is selection, supposing there are more young shoots than are required. In making a choice, it is necessary to distinguish between the different kinds of young shoots ; for, strange to say, there are at least three kinds of young shoots on many peach and nectarine trees, and on most there are two." These Mr E. characterises as-first, Perfect bearing shoots; second, Exhausted shoots; third, Barren shoots, and these refer to the young spray. First, " Perfect bearing shoots.-These, in general, may be known by the majority of the eyes or buds seated on them being threefoldthat is to say, in threes. When such is the case, the two outer ones are almost always blossombuds, and the central one a wood-bud. All such is deemed wood of first-rate character, and it is the aim of the experienced cultivator to secure as much of this as possible. Second, Exhausted shoots. - We are not assured that this is the most proper title to give this class of shoots ; one thing, however, we do know, that when such shoots do begin to prevail generally over a tree, it is a pretty sure sign of a 'breaking up of the constitution of the tree.' These have, for the most part, a single bud at a joint, and that bud a solitary blossom-bud. Such could not be readily distinguished, in early autumn-pruning, by a novice from the next class ; and this is a reason why amateurs who prune for themselves had better defer it till the early part of February. Wood of this character, if left on the tree, has seldom vigour enough to produce fine fruit. Indeed, such wood not unfrequently 'sets' its blossoms more freely than the preceding class ; and it is by no means unusual, at the thinning period at the end of May, to meet with shoots of this class with a score of fruit on, yet no leading bud or growing shoot. These fruits exhaust the tree much, and eventually fall off. Third, Barren shoots.-These, again, possess solitary buds. They are, however, usually of late growth, and may readily be known by their pale and unripe character; or they are the production of over-luxuriant trees, and serve to denote a tree of gross constitution or vigour misplaced. The difference between these and the preceding section is at once apparent at spring-pruning. The solitary buds of the former become very plump of a sudden, whilst these do not appear to increase at all. Wood of this character, if ripened, is frequently of eminent service, inasmuch as it serves to keep up the main fabric of the tree; and although not bearing wood itself, it is capable of producing fine bearing shoots for the ensuing year. Some of this must, therefore, be occasionally reserved, especially if a blank or space bare of shoots in any part be anticipated; for be it understood, much in peach-pruning depends on a far-seeing eye or skilful anticipation." The above may be taken as a description as to the condition of the tree, while the following may be considered as the rationale of Mr Errington's management of it, so far as regards its pruning : "The main business is to reserve a series of shoots all over the tree of the class No. 1, and where this class cannot be obtained, to secure enough of No. 3 ; the No. 2 class may, in most cases, be considered a last resource. So much for selection. We must now advert to the shortening of the young spray. Only two reasons exist for shortening at all : the one is where shoots overtake each other, in which case some must be made to retreat, or the tree would be soon all confusion; the other is founded on the necessity of removing unripe portions. The first case any mere tyro can judge for himself, the second requires some care. A little practice will, however, teach the uninitiated the difference between the two. The principal criterion is hardness. A practical man would soon distinguish them, although blindfolded, by means of his knife. Colour has something to do in this matter. Ripe wood is generally of a brownish colour-unripe, of a pale and delicate green. In ordinary cases about one-third has to be cut away ; nevertheless it is not easy to lay down a general maxim as to shortening, for it becomes necessary, for the sake of successional wood, to shorten more severely at the lower parts of the tree,decreasing in its amount progressively upwards." In regard to the removal of large branches, Mr E. observes: "We never take these away unless compelled by sheer necessity. The 
peach and nectarine are very impatient under such violent operations. Sometimes, however, actual decay of a branch takes place, and then such operations must be performed. The main business is to secure the wound afterwards. The admission of air and wet to the wound is ruinous. A kind of grafting-mixture, composed of cow-dung and lime, is very good. This should befastened down with some waterproof material, and the whole tied tight. Upon all wounds on two or three year old wood we make a point of applying a good coating of thick white-lead."

Harrison, who practised in a high cold climate in Yorkshire, has very differently laid down his practice in his "Treatise on Fruit Trees." After having recommended, as strongly as the authorities we have already quoted, the necessity of keeping up a sufficient supply of young wood from towards the bottom of the tree, and also retaining as much wood as near to the origin of each branch as possible, to prevent nakedness below, he proceeds : "The mode of treatment recommended and practised bysome persons, of leaving wholly unshortened, at the winter regulation of the trees, all the wood which is retained for bearing the following year, very much promotes the naked state of the branches" towards the bottom and centre of the tree. "I have tried," he says, "such a system of management, and with all the efforts I could make I never could keep the trees perfectly furnished with bearing wood for many successive years, because the leading bud always pushed a shoot first, and then three or four more near the top; and by the sap finding such ready means of being employed in these new shoots, the lower part was always nearly destitute of them. Not only is particular attention required to the obtaining and preserving bearing-wood in the situations described, but also in retaining a proper supply for bearing fruit the following year. The distance which I consider best is to have all the young wood trained at 4 inches apart, for when they are much nearer than this the shoots crowd each other, because in summer one or two shoots are required to be retained for supporting the fruit which is upon the tree that season, as well as for a supply of wood to bear fruit the ensuing year." In regard to shortening the wood, \&c., $\mathrm{Mr}$ H. scarcely differs from the opinions already given above, but in addition to their observations, he says : "If there be any short spurs formed upon the two or three year old wood which is retained, let such be preserved, as they will be productive the following year." This is in accordance with Knight's views, afterwards to be noticed, and is stated by that great man as being valuable in cold climates, where theyoung wood of the peach does not always ripen. "It will sometimes happen," Mr Harrison continues, "that short wood will be found to have only single and double blooming buds upon them, with a growing ' or wood-bud' at the end, but no intermediate growing ones. In this case, if wood be not wanted at that part, the shoot must be left its entire length; but if wood be required in order properly to furnish the tree, let such a shoot be cut down so as to leave about half an inch of it remaining; there are generally a few embryo buds around the base of the part from which a new shoot will afterwards push. When a tree happens to have a great number of shoots so supplied with blooming buds, one-half, one-third, or one quarter of them must be cut down as directed, according to the quantity the tree has, or the portion of new wood required. The necessity of cutting to a growing or wood bud is, that at the ensuing spring a shoot may push at the top of that one shortened at winter-pruning, so that whatever fruit there may be below it the following summer, it may have its required support from the tree, otherwise it will drop off or be defective in size and flavour. If a fruit of particular importance happen to be so circumstanced, a bud may be inserted artificially above the fruit, by which nourishment will be derived to it, and it will be matured. With the view of having a leading shoot to every branch of last year's wood, some persons do not prune their trees till late in spring, when they can more certainly discover which bud will push, and which will not. But so many injurious effects result from this practice, that it is advisable entirely to give it up. When it is found expedient to cut back part of a main branch, let it be done very cautiously. Always prune close up to a good lateral shoot for a supply, and do not prune farther back than 3 feet from the end of the branch; for when a large portion of a main branch is taken away, the superabundant sap accumulates at the entrance into the lateral shoots, and the bad effects resulting from it will soon be apparent."

Mr Knight's method of managing the peach upon a species of spur pruning is, as was recommended by him, exceedingly well adapted for cold climates, and where the trees do not thoroughly ripen their young wood. "Instead of taking off so large a portion of the young shoots, and training in a few only to a considerable length, as is usually done, and as I should myself do to a great extent in the vicinity of London, and in every favourable situation, I preserve a large number of young shoots which are emitted in a proper direction in early spring by the yearling wood, shortening each where necessary, by pinching off the minute succulent points generally to the length of 2 or 3 inches. Spurs which lie close to the wall are thus made, upon which numerous blossom-buds form rery early in the ensuing summer; and upon such, after the last most unfavourable season, and in a situation so high and cold that the peach tree in the most favourable seasons had usually produced only a few feeble blossoms, I observed as strong and vigorous blossoms in the present spring as I had usually seen in the best seasons and situations; and I am quite confident that if the peach trees in the gardens round the metropolis had been pruned in the manner above described in the last season, abundant and vigorous blossoms would have appeared in the present spring. I do not, however, mean to recommend to the gardener to trust wholly in any situation for his crop of fruit to the spurs produced by the above-mentioned mode of pruning and training the peach tree. In every 
warm and favourable situation I would advise him to train the larger part of his young wood according to the ordinary method; and in cold and late situations only, to adopt to a great extent the mode of management above suggested. A mixture of both modes in every situation will be generally found to multiply the chances of success, and therefore neither ought to be exclusively adopted or wholly rejected in any situation. The spurs must not be shortened in the winter or spring, till it can be ascertained what parts of them are provided with leaf-buds." This very sensible paper of Mr Knight's is well worth the attention of peach-growers in all situations, even in those whose climate is sufficient to mature the young wood to perfection, and especially so in all late and cold localities.

$M r$ Seymour's mode of management is characterised for neatness, regularity, and almost mathematical precision in the disposal of his wood. It is, however, we apprehend, in the most favourable situations for peach-culture that this excellent plan can be fully carried out. The annexed cut, fig. 216, will explain the principle upon which Seymour works, better

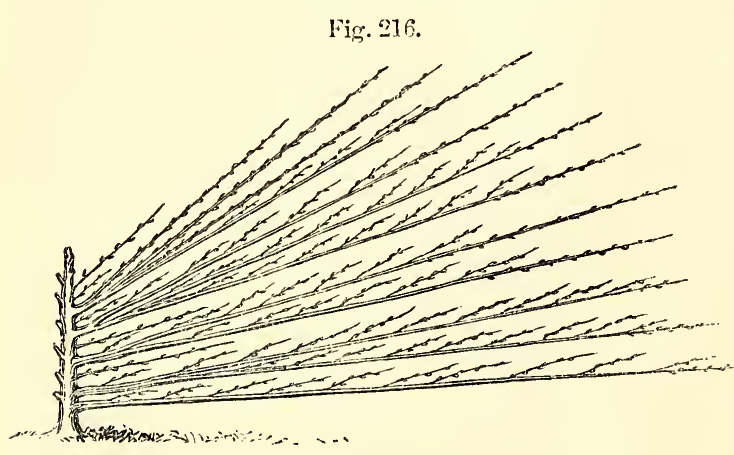

SEYMOUR'S MODF OF PEACH-TRAINING.

than a lengthened description without it, and will show how capable the plan is of being carried out, as it were, mechanically. It will also be seen that every side shoot and every lateral proceeding from it is placed exactly where it should be; and to understand the principle more clearly, it will be necessary to refer to the commencement of the process immediately after the tree is planted. We may, however, first remark, that from the base of every lateral in spring a second one is produced to take the place of the other at the winter-pruning. And further, no laterals are allowed to be more than one year old, and are cut off close to the leading shoot on which they are produced, with the view to prevent the formation of spurs. In course of time the leading shoots become worn out, and are then removed, their place being supplied from the best-placed laterals near to the main stem. The first step in starting a newly-planted maiden tree, upon Seymour's principle, is to head the plant down to three eyes, each of which eyes will produce a shoot during summer. In autumn or in spring, whichever is adopted for winter-pruning, head down the centre shoot of these to three eyes, to produce in the following summer three more shoots as before, leaving the side shoots always at full length. In spring, all the buds on the lower sides of these side branches are rubbed off, leaving those only which proceed from the upper side of the branch, and these from 9 to 12 inches asunder. When the young wood has extended to the length of 5 or 6 inches they are stopped; but the leading branches, as we have stated, are never interfered with. Every year will produce a side shoot on each side of the tree, and the laterals which proceed from them, at the distance we have stated, are at first laid in between them; but the following spring these are removed from the wall, and trained up in the main side-branches. In shortening what may here be called the upright leading-shoot, it is necessary to observe that, although we have stated three buds as being sufficient to be left, this depends greatly on the health of the tree. If weak, or of moderate growth, three buds will be sufficient; but if strong and vigorous, then five may be safely left, which will thus give two pairs of side shoots instead of one, and thereby fill the wall sooner. By the autumn of the third year, the number of laterals will be doubled on the two sidebranches first laid in, as a new lateral is sure to spring from the base of the one laid in the previous season, as well as one from its point. As to winter-pruning in the fourth year, all the laterals of two years' growth, and which have already produced a crop of fruit, are to be removed entirely; and those of the previous summer's formation are to be unfastened from the wall, and laid upon the main leading sidebranches in their place. Here it is that the beauty and symmetry of Seymour's mode of training become apparent.

So methodically has the author of this mode of training laid down his principle, that, taking a radius of 10 feet, making the middle of the trunk near the ground the centre, he divides the curved line described into 10 -inch parts, which are the points to which the leading side-branches are all directed. Should it at any time appear to the cultivator that these side leading-branches are too far apart, or if it is thought that more laterals can be advantageously laid in between them than those already produced, then some of these may be shortened, and so increase the number. This will seldom be necessary with strong-growing trees, although it may be so in the case of weak-growing ones.

Hayward's mode.-This author writes on the subject of fruit trees both practically and physiologically. How far he is correct in all the deductions he has drawn may admit of some doubt; he has, however, treated his subject so differently from most other writers, that we deem his theory deserving a place amongst those of others who have made the training of the peach their peculiar study. On our present 
subject he says: "As the fructification of plants depends much upon the state and condition of the branches, and the surface of the trunk, branches, and leaves exposed to the sun and the air, the proper adjustment and arrangement of these must be an important object of our consideration. Experience proves that very fine fruit is seldom produced on very strong or on very weak branches, but generally on branches of a middle growth; therefore, to render a tree permanently fruitful, it must be necessary to manage and train it in such a manner that all the sustenance furnished by the roots shall be appropriated to the production of branches of a proper and equal growth, and that these be so arranged as to present the needful surface of leaves to the required influence of the sun and air. In determining the form and figure of a tree, as few persons are so devoid of taste as to prefer deformity to symmetry, or to be indifferent whether their plants and trees exhibit beauty or ugliness in their forms and figures, we may take it for granted that it must be desirable, as far as possible, to blend beauty with prolificacy in training them, which may be done in great perfection. But the raising and training a tree is like the building of a house, or the raising any regular structure; for if the plan be not first arranged and understood, and a proper foundation laid to sustain it, disorder and confusion must pervade the structure, and it can never be rendered desirable, commodious, or elegant. Then as the first stem and branch of a plant must form the foundation of the future tree, before we fix on a plan, or begin to train, we must first determine the space it is intended to occupy, and next, the form we wish it to assume. Every plant and tree is appointed by nature to attain a certain comparative height, and fill a certain comparative space, before it fructifies. Then in determining the heights to which plants and trees shall grow, we may consider the objects of nature to be two-the one to place it above the obstruction of inferior plants, the other to afford a certain surface of bark, or such a space of trunk and stem for the sap to pass over and through, as is necessary to prepare that which is required for fructification." To the second of these our subject is more especially, at present directed. "Then how to attain the required surface of bark, or of stem or trunk, within 1 foot or 2 of the earth, with a tree that nature has appointed to grow 8 or 10 feet high before it forms its head for fructification (which is the case with the peach tree), must be the first or grand object of training; and, as has been observed, as the peach tree bears its fruit on shoots of the last year, and when the branches are either too strong or too weak they will not bear fine fruit, it must be necessary to train this tree in such a manner and form that its sap shall be so equally divided as to form shoots of a medium growth, and that they be so placed as uniformly and constantly to cover the same space with fruit branches every successive year. The sap in all erect young trees, of which the peach is one, will flow into and through those channels that occupy the most vertical position next the root; VOL. II. and it is not only in this respect evident that the flow of the sap is impelled by a principle directly opposite to that which impels the
Fig. 217.

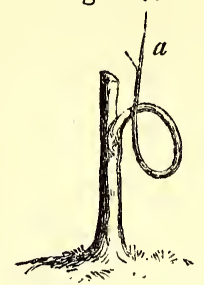

HA YWARD'S MODE OF PEACH-TRAINING.

Fig. 218.

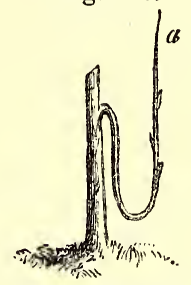

HAYWARD'S MODE OF PEACH-TRAINING.

Fig. 219.

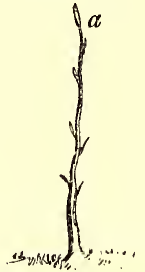

HAYWARD'S MODE OF PEACH-TRAINING.

Fig. 220.

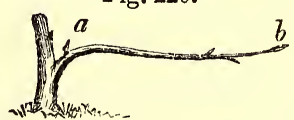

HAYWARD'S MODE OF PEACH-TRAINING. flow of water, but as water will flow over a great height above its level, with equal rapidity through a syphon as through a tube of the same size placed in a declining position, and leading into an open space below its level; so will the sap flow through a branch so disposed as to form an inverted syphon in equal quantity as through " the same branch had it been fixed in a vertical position. Thus the strongest shoot will form at the point-bud $a$, fig. 217 ; or as in figs. 218 and 219. But if a branch be fixed in a horizontal position"as in fig. 220 - " the strongest shoot will be produced in the most vertical bud nearest the base $a$, and the pointbud $b$ will form the weakest shoot : it must, then, be obvious, that if it be desired to train the branches in a horizontal position, and still to extend them as much as possible in length, the point of the branch $b$ must be turned up," as in fig. 221. "The point-bud $b$ will then form nearly as strong a 3 shoot as if the branch had been fixed in a vertical position; and the bud at $a$, from its vertical position, and being nearest the root, will take a large share of sap, and form a strong shoot also ; if therefore it be desired to direct the full supply of the sap to the point-bud $b$, and from that to form

Fig. 221. the strong-

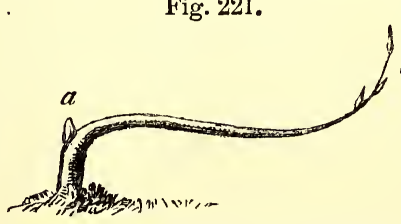
est shoot the root will $b$ supply, the buds at $a$, with all intervening buds, must be removed: when HAYWARD'S NODE OF PEACH-TRAINING. all intervening buds are removed, as habit soon reconciles a plant to the position of its trunk, its sap will be passed with 
equal facility through it when in a depressed position as when in an erect one, after a year or two."

The above may be considered as the rationale of $\mathrm{Mr}$ Hayward's theory of the circulation of the sap; and, in accordance with these views, he has laid down a principle of training the peach -of which fig. 222 will give a very correct idea

Fig. 222.

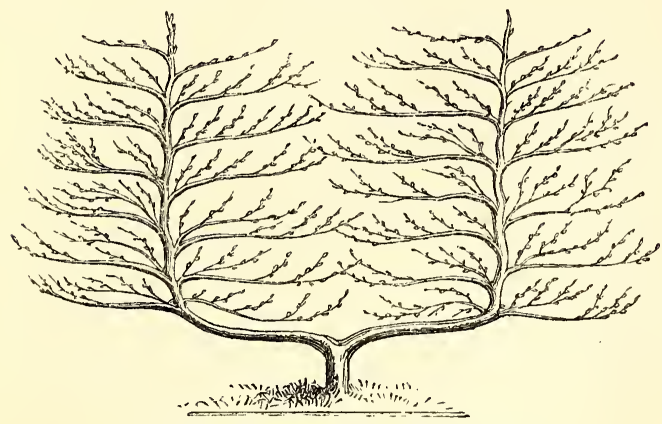

HAYWARD'S MODE OF PEACH-TRAINING.

Fig. 223.

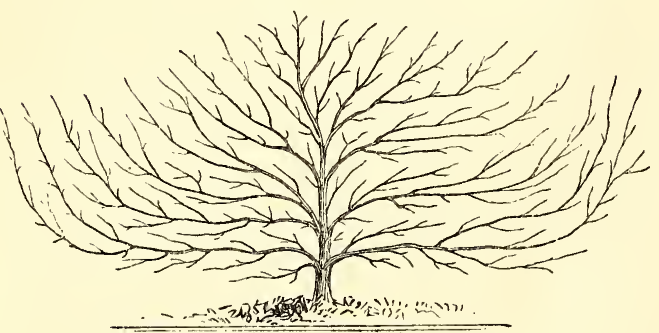

CALLOW'S MODE OF PEACH-TRAINING.

-when the tree has arrived at its full size and form. Some attention is required at starting upon this principle. From our figure it will be seen that two branches start from the main stem, and are laid in in nearly a horizontal direction. To secure this without loss of time, it becomes desirable that these shoots should be produced the first year from budding. "This may be done," Mr Hayward observes, "by either inserting the buds opposite to each other in the stock, or, when performing the operation of budding, those buds only should be inserted which have three leaves appended to them, as from such buds three shoots will be produced. Then, as soon as the buds begin to swell in the spring, the centre shoot should be picked out with the point of a pen-knife: the two lesser shoots will then push out sufficiently strong to form two equal stems; and these, if carefully guarded, will produce a plant of proper growth the first year from the budding. But if plants with two equal stems are not to be had, plants with one good stem only may be made to throw out a second one without cutting back. By this mode one stem will appear to be a year behind the other in growth; but as the division of sap will be equal in a few years, the youngest will attain the size of the other, and in the end both stems will be equal in their appearance and in their produce." Hayward's theory has been rather severely treated by theoretical critics, many of whom find it easier to discover faults than to remedy them. His writings, although not in all cases agreeing with the generallyreceived physiological opinions, may nevertheless, as a whole, be perused with advantage.

Callow's method of training the peach, fig. 223, shows the main branches in a position more horizontal than that in modes in general use ; the ends, however, of all the leading branches are elevated, forming a curvilinear inclination with the horizon, giving the trees a very elegant although not a constrained appearance. The old wood is not overcrowded, and the spaces between are very regularly filled up with the young bearing-wood, which is shortened yearly in spring, according to its strength, while the leading shoots are allowed to extend with little shortening at all. This mode is better adapted to low than to high walls.

The fan mode of training, in one or other of its various modifications, is that most usually followed, both with the peach and apricot. It is by far the most natural, and admits of remedying the defects arising from the loss of a branch, which is almost impracticable in forms so truly symmetrical as those of Hayward or Seymour, and to which both these trees are oftentimes subject. In one or other of its forms it is most generally followed in Britain, and is deserving of our attention-first, because no peach trees in the world are trained so well ; secondly, because, on account of climate and other circumstances, Britain is not the most favourable for the successful culture of the peach, nectarine, and apricot, to all of which this mode is applicable ; and where the greatest deviation from it has been practised, there the superiority of its principles is most clearly exhibited.

A method of fan-training was recommended some years ago by $\mathrm{Mr}$ Kendall in the second volume of "The Gardeners' Magazine," p. 142, which has so much system and simplicity in its whole arrangement that we have often been induced to follow it in training these trees on the
Fig. 224.

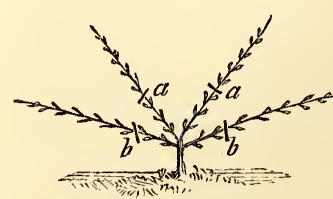

KENDALL'S MODE OF PEACH-TRAINING. open walls. A maiden tree is selected and planted. In spring it is headed down to four eyes, placedin such a manner as to throw out two shoots on each side, thus presenting the appearance 
of fig. 224. At the following pruning season the two uppermost shoots are cut back to their eyes, $a$ a, placed in such a manner as to throw out one leading shoot, and one shoot on each side; the two lowermost shoots are cut back to two eyes, $b b$, so as to throw out one leading shoot, and one shoot on the uppermost side. The tree has then the appearance of fig. 225 , having five leading shoots on each side, and systems to which this class of trees is subjected, consists in preserving a sufficient quantity of young wood at the bottom of the tree ; and on no account must the pruner cut clean away any shoots so placed without well considering if it will be wanted, not only for the present, but for the future good appearance of the tree." Fig. 226 is a figure of a tree so managed in a more advanced state, in which it will be observed that the five

Fig. 226.

Fig. 225.
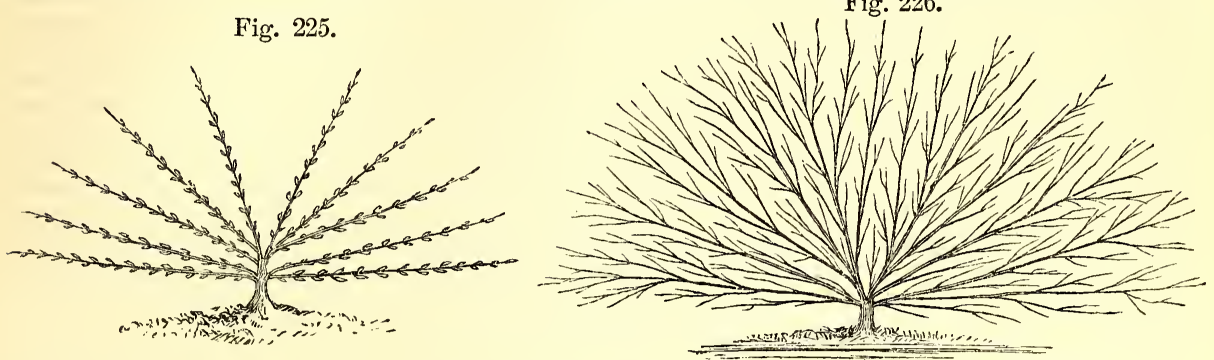

KENDALL'S MODE OF PEACH-TRAINING.

which are to form the first stage of the future tree. These ten shoots must be placed in the exact position in which they are to remain, and none of them are to be shortened. The third year, each of the ten leading shoots must be allowed to produce two other shoots on the uppermost side-one of those near the bottom, and the other about the middle of the shoot, and a third shoot from the under side of each-about half-way between those left on the upper side. The leading shoots should be allowed to extend without shortening. All shoots except those enumerated should be removed by disbudding. From the commencement of the fourth year fruit may be allowed to be produced, in quantity according to the strength of the tree, for nothing is so injurious to the peach as to allow it to produce fruit during its second and third years, if the object be to have healthy and longlived trees. From the end of the third year, all the shoots excepting the leading ones must be shortened at the season of pruning, but to what length will depend on the vigour of the tree and judgment of the operator, bearing in mind that the stronger the tree the shoots should be laid in at greater length than when the tree is weakly ; and care should also be taken that the young wood be not retained too thick. In shortening the shoots at the autumn or winter pruning, Mr Kendall recommends "cutting close to the bud, so that the wound may heal the following season." This operation has been questioned by $\mathrm{Mr}$ Beaton and others, who say the cut should be made at some distance above the bud. To this we will have afterwards to refer. The following season each shoot at the extremity of the leading branches should produce, besides the leading shoot, one on the upper, and two on the under part, more or less, according to the vigour of the tree: whilst each of the secondary branches should produce, besides the leading shoot, one other, placed near to the bottom; " for the grand art of pruning," $\mathrm{Mr}$ Kendall observes, "in all leading shoots laid in at full length when the tree was as is represented by fig. 224 , are quite traceable in the advanced state of the tree, fig. 226, and form its chief ramifications, the secondary and young yearling wood filling up the spaces between them. The general equilibrium of the tree is maintained by laying a greater quantity of wood, during summer, where the leading or secondary branches show too much vigour, as well as allowing a greater quantity of fruit to be produced on them also. Where debility appearsin the case of others, a completely opposite course is adopted.

"Whatever system of training," Mr Kendall observes, "is to be pursued, the leading branches should be laid in in the exact position they are to remain; for whenever a large branch is brought down to fill the lower part of the wall, the free ascent of the sap is obstructed by the extension of the upper and contraction of the lower parts of the branch. It is thus robbed of part of its former vigour, whilst it seldom fails to throw out, immediately behind the part most bent, one or more vigorous shoots. To assist the young practitioner in laying in the leading branches of the tree, the following method may be acceptable: Drive a nail into the wall, exactly where the centre of the tree is to be, then with a string and chalk describe a semicircle of any diameter ; divide the quadrant into $90^{\circ}$; the lower branch will then take an elevation of about $12^{\circ}$, the second of about $27 \frac{1}{2}^{\circ}$, the third about $43^{\circ}$, the fourth $58 \frac{1}{2}^{\circ}$, and the fifth about $74 \frac{1}{2}^{\circ}$. A nail should be then driven into each of these points, and the chalk rubbed off."

In very unfavourable situations, perhaps no systematic mode of training can be completely carried out, at least to the extent shown in our previous examples. In such cases, therefore, the cultivator must adopt that which may be characterised as the irregular mode-namely, to secure a supply of young wood from such parts of the tree as produce it, and often, instead 
of placing it in the direction in which it would be placed in ordinary cases, he must lay it in wherever there is wall space convenient to the part from whence the shoot arises, and this without regard to whether the shoot is placed in a direction towards the root or top, and also to whether it is placed vertically or pendulously; the chief care being to cover the wall, as well as the naked branches which, under such circumstances, are almost sure to present themselves. It must be admitted that excellent crops are thus produced; but the trees will ever want that trim orderly appearance which those present which may be trained upon any of the foregoing principles. One important point in peach-pruning in Britain is the yearly shortening of the young wood, for without such a precaution in such a climate the tree would cease to exist in vigour for any length of time. The above may be considered the leading features of peach pruning and training as practised in Britain.

American practice.-How strange it may appear to us, who take such trouble with our peach trees, to read in Downing's " Fruits and Fruit Trees of America,"- "It has always been the prevailing doctrine in this country that the peach requires no pruning. It has been allowed to grow, to bear heavy crops, and to die pretty much in its own way." The bad effects of this neglect have been discovered, and although most of their peach trees are grown in open orchards as our apples are, experience has led the most enlightened American cultivators to adopt pruning upon the shortening-in principle, and the advantage of this alteration has become sufficiently obvious. "At the end of February," says Downing, "or as early in the spring as may be, we commence pruning. This consists only of shortening-in, $-i$. e., cutting off half the last year's growth over the whole outside of the head of the tree, and also upon the inner branches. By reducing the young wood one-half, we at the same moment reduce the coming crop one-half in number ; the remaining half, receiving all the sustenance of the tree, are of double the size." Training the peach against walls or espaliers is but little practised in America, the climate being quite sufficient to ripen the fruit without artificial assistance. In the neighbourhood of Boston, espalier training is occasionally practised, and in the colder parts, such as New England, it is followed with great advantage. Mr Downing, one of the highest pomological authorities in America, strongly urged peach-pruning on the attention of his countrymen, and the best cultivators there are now following his judicious directions.

French practice.-The climate of France, like that of America, is extremely favourable for the cultivation of the peach. They, however, do not ripen so well on standards as they do in the latter country; but, in consequence of their warm summers, they attain very great perfection, often on espaliers, but always on walls, and those even of the very humblest description, as those at Montreuil, where the finest peaches in Europe are produced. From these circumstances it will readily appear that much less pains is taken of the peach tree by the French gardeners than by the English, so far as pruning and training are concerned. In some few particulars of culture, however, they differ from us,-as, for instance, using the almond as a stock for the peach, which they find to answer better in the light dry soil of Montreuil than any other; but they at the same time give a preference to the plum stock where the soil is strong and of a rich black humus. The mode of training in general adopted is a species of open-fan training, and great care is taken to elevate the points of the weaker branches, and to depress those that are too strong. In the general or winter pruning, all shoots that show fruit-buds only are cut clean away. "This will appear," Dr Neill observes (in "Horticultural Tour,") " absurd to any one not a horticulturist ; but if such branches do exist, their excision is quite prudent; for wood-buds, or shoots, are like pumps to draw sap toward the branchlets, and, if they be wanting, the blossom on the twig commonly fails to set; or if the fruit form, it soon falls off, or at all events is deficient in size and flavour." From four to eight flower-buds are left on each twig, according to its strength, much in the same way as with ourselves.

The Montreuil mode of training is a modification of the open-fan manner, in which two main branches are laid in to the right and left of the centre at an angle of $45^{\circ}$; the wall is afterwards covered with subordinate branches from these, and the best laterals they produce. The great advantage of this mode of training is, that, whenever the wall gets naked below, it can be covered by bringing down the two main branches and their subordinates. Lelieur describes an important modification of this mode of training by Dumoutier; and M. F. Malot, a Montreuil peach-grower, has also improved upon it, by first covering the lower portion of the wall, by preventing any shoots being produced from the upper sides of the two main branches till the part of the wall below them is covered. Upon the whole, we have little faith in French training, and cannot refer to a wall of peach trees we ever saw approaching mediocrity. In such a climate the peach will grow without much care ; and wherever that is the case, the less restraint that the branches are subjected to the better.

A mode of training the peach in the open air, somewhat approximating to the Montreuil system, has been recommended by Professor $\mathrm{Du}$ Breuil. It consists of training two main branches laid in to the right and to the left of the stem, and much about the same angle as in that referred to, as are also the branches from the under side of these ; but the branches for filling the centre are reversed till they are brought to an angle of $45^{\circ}$, thus forming right angles with the branches from which they proceed. With the view to maintain a proper balance of force in the flow of the sap, and to lessen the necessity for using the knife, all the branches occupying the centre of the tree are arranged nearly to one angle of elevation $\left(45^{\circ}\right)$, while those produced on the under side of the main branches have an elevation of 
only $20^{\circ}$, and are allowed two years' growth before those occupying the centre are allowed to spring: the branches on the upper side, and having the greater elevation, are thus prevented preponderating over those on the lower side, as the sap has to turn at an angle of $90^{\circ}$ in course of its passage from the main branches to those on the upper side, while the lower branches diverging at an angle of not more than $35^{\circ}$, the sap consequently meets with little or no obstruction compared with the others where it has to turn at a right angle. This mode of training is excellent in principle, although, we fear, not readily reduced to practice, because it places the branches in a position the most likely to insure an equal distribution of the sap, which is the end aimed at in every kind of pruning and training. Trees under this mode of management have for some time been in training in the gardens of the London Horticultural Society. Sufficient time has, however, not as yet elapsed to enable us to speak with certainty as to the result. Of one thing we are, however, certain,--the experiment could not be in better hands than those of $\mathrm{Mr}$ Thompson, who, we believe, some years ago tried some experiments of a somewhat similar kind. Amongst others were the following interesting ones, which are thus reported by Mr Thompson:-

"Amongst various other forms for training the peach, some trees were set off with two stems, like the letter $U$, from the outsides of which branches are trained horizontally. In one of these, the two lowest horizontals are continuations of the two main stems; and from the upper side of these, about 18 inches apart, shoots are trained upright and parallel, from which all the other horizontal branches proceed; also, from both sides of these horizontals, bearing shoots, about 18 inches apart, are trained with an inclination forwards. By this mode it is sometimes difficult to maintain an equal degree of vigour in both sides of the tree.

"Another tree is intended to be trained with a wavy central stem. When in the state of a young shoot, this stem is bent first to the left, then to the right; and where it commences to turn from left to right, a shoot is encouraged on the outside of the bend, and trained straight to the left, in a direction a little above the horizontal. Where the stem shoot is again turned from right to left, another branch is extended to the right; and so branches are intended to proceed alternately, one on either side, from every outward bend of the stem, till the wall is covered.

"Trees were trained in the Society's garden with wavy stems, according to the systems of Hitt and of Hayward,- the former having strictly horizontal branches from the bends, with perpendicular bearing shoots; the latter with gently curved branches, with the bearing shoots inclined. But each tree, in both systems, had two straight naked stems, elevated at an angle of $45^{\circ}$, and $4 \frac{1}{2}$ feet in length, before they took an upright wavy direction. These naked stems invariably became scorched on the sides exposed to the sun, and the upper portions of the bends were also more or less affected in the same way.
"Professor Du Breuil's mode is unquestionably far preferable to either of the above, inasmuch as the tree has only a single stem, from which the wall is furnished from bottom to top, instead of being unfurnished to the height of 4 feet, above which the naked stems only begin to branch, according to the systems of Hitt and Hayward. In Professor Du Breuil's tree, there are no long naked stems exposed to the scorching influence of the sun's rays, and even the bends can be protected by foliage. On the whole, this mode is considered highly deserving of a trial in Britain."

When winter-pruning has been finished, the trees, previous to their being again fastened to the wall or trellis, should, if only for precaution's sake, be dressed with the following composition-namely, to one gallon of warm water add one pound of flour of sulphur and four ounces of soft soap; incorporate the whole together, add some strong clay, and beat up the whole until the mass assumes the consistency of thick paint; and, finally, before using, add two quarts of hot or unslaked lime : with this cover the whole of the branches, young and old, laying it on with a painter's brush. This composition will soon dry on the trees, when the process of tying or nailing them to the wall or trellis may be proceeded with. Some add a little soap, which, if it does no good, can evidently do no harm. All these properties are very good for young wood, but for all wood of more than two years' growth we prefer vegetable spirits of tar as the universal panacea. Apricots, plums, and cherries, if they need it, may be painted over with either of the above compositions.

Various modifications in training the peach and nectarine have been recommended, and tried with more or less success-that of the horizontal way with the least of all success; and this has been accounted for, because the peach has a seeming disposition to grow by fits, if such a phrase may be used; that is, a preponderance often occurs in the vital action in one part of the tree more fully than in another; and as every means should be employed to correct this, the methodical principle of horizontal training is consequently less fitted for it than any of the modifications of fan-training, because, by the latter, too strong young shoots may be corrected by depressing their direction, while those that are weak may be strengtheried by placing them in a more elevated position. Indeed, thus depressing or elevating the shoots has much the same control over the flow of the sap as root-pruning and weakened stocks have on most other fruit trees in affording supplies.

Regarding the longexity of the peach and nectarine.-The peach, even in its native country, Persia, is not a tree of great longevity; and in its transplantation to a climate so inferior as that of Britain, it is natural to suppose its nature in this respect is not improved. Soil, situation, and judicious management have much to do in this matter; and hence we find a few specimens which have continued in a pretty healthy state, in favourable situations, for nearly half a century. Perhaps twenty-five years may 
be taken as a pretty fair average of its profitable existence in this country. When the peach begins to indicate decided decay by the larger branches dying off one after another, it is much better to root it out at once, and remake the border for the reception of its successor. In strong loamy soils it flourishes best; in light sandy soils it often declines in ten or twelve years. Such is the case at Dalkeith ; and to keep up a supply, we plant a certain number of young trees annually, placing them in the spaces between the old ones, but always preparing a fresh body of soil for them. As these young trees advance in growth, the older ones are reduced in size year after year until they are totally removed. There is no advantage in retaining diseased or worn-out peach trees, and that the less so as the finest fruit is produced on those that are young.

Protecting the blossom is an important part of peach-culture ; so much so, indeed, that in few places in Britain can a crop be safely calculated upon without some sort of protection during the period of the trees coming into blossom, and until the fruit is fairly set. There are, however, judicious and injudicious modes of protection employed; the bad effects of the latter have led some, of late years, to question the propriety of covering at all. Any covering, excepting glass, put on at the period of the buds beginning to swell, and allowed to remain on until the fruit is set, must rank in the category of the latter; while such as is capable of being conveniently placed and replaced, according to the state of the weather, will rank with the former, and the more so, in proportion to the amount of light which is admitted to the trees through it. Therefore, sticking in branches of trees and fronds of fern amongst the branches, covering with bast mats, thick canvass, two or three courses of netting, of whatever kind or quality, must be bad, as excluding both light and air, and preventing the exhilarating effects of sun, light, and heat from acting on the young and tender buds. If put on sufficiently thick to ward off a sharp spring-frost, they, in the same ratio, prevent the heat of the sun from acting on the trees. They exclude light and air, and, therefore, the young buds and blossoms forced into development under such conditions become attenuated and weak; and when the covering is removed, which is in general all at once, neither the one nor the other are in a condition to stand unscathed either the powerful sun during the day, or, peradventure, $5^{\circ}$ or $6^{\circ}$ of frost during the night. The tender leaves and young shoots of the peach and nectarine require protection from late springfrosts, and also from the cold evaporating effects of an east wind, which we believe to be one of the most serious evils those trees are subjected to when grown on the open walls; and this the more so in exposed situations, and along the eastern coasts of Britain, which are so much subject to east and north-easterly winds. The most effective, and at the same time the most economic protection we have found for all fruit trees on open walls, is 9-inch wooden projecting portable coping, secured to permanent iron brackets, built in the walls close under the stone coping (vide vol. i. p. 70, figs. 40 and 41). This coping is of inch deal, and in 21 -feet lengths, or the lengths of ordinary battens; the brackets are placed so that the ends of the boarding shall meet exactly over them, and two other brackets are placed at equal distances-viz., 7 feet between-to stiffen and support them towards the middle. Each bracket is perforated with two holes, through each of which an inch screw is introduced, and screwed upwards into the boarding. When the season of spring protection is over, these screws are withdrawn, and the boarding removed and placed under cover until again required. Were we operating on the west coast of Scotland, with its 50 or 60 inches of rain per annum, we would assuredly allow these copings to remain on peach and apricot walls during the whole summer and autumn ; but in a locality where the annual fall of rain rarely reaches 20 inches, we remove them during the growing season of the trees, that they may enjoy both the rain and dew which fall upon them, and which we have no permanent projecting copings of stone to intercept. As soon as the fruit of the peach and nectarine begins to change colour toward ripening, these wooden copings are again put on to keep the trees, and particularly the fruit, dry, and also to prevent its being driven off by the heavy rains we periodically experience during August and September, as well as to assist in ripening the wood. The screen covering we use is thin semi-transparent canvass, manufactured at Dunfermline under the name scrim cloth, 40 inches in breadth. Three courses of this sewed together, forming a sheet 10 feet wide, is quite sufficient for a 12-feet wall, as the fruit within 2 feet of the bottom is of less consequence, and even that is greatly protected by the canvass above it. Keeping it this distance from the ground secures the canvass from damp from the soil, and renders its fastening to the wall more convenient. Around the edges and ends of our sheets of canvass is sewed on a strong tape, which greatly prevents tearing: and they are in pieces in length equal to that of the wall they are to cover. These sheets are fastened to the front edge of the wooden coping with tin tacks, with very broad heads, and these are driven in 7 inches apart. Along the bottom and up the ends are sewed on pieces of twine 10 inches in length and 10 feet apart, by which the screen is secured (when down) to the bottom part of the wall, to the eyed studs we use for fastening the branches of the trees to the wall by. When the wall is to be uncovered, a boy goes along the bottom and unties the cords, while a man walks along the top of the wall and draws the canvass up, which, with a slight turn of the hand, he throws into a loose roll, and as he proceeds he fastens it to the top of the wooden coping by means of twine nailed to the boarding. It may be thought that this covering and uncovering is attended with the loss of much time; such, however, is not the case. A wall 1100 feet in length is usually covered and uncovered on both sides in half an hour. In cold windy days this covering is often left on for days together; but when the weather 
is mild and humid the covering is removed by eight A.M., and replaced at half-past five in the afternoon. From the thinness of the scrim cloth very little more light is intercepted than would be with indifferent glass. As the season advances the covering is removed earlier in the morning, and often left off during the night, by which means the foliage and young wood become gradually hardened to stand both the sunshine and night air without injury.

Many complicated and expensive modes of covering have been recorded; we find none, however, answer our purpose better than the above. The lightness of the canvass prevents it injuring the trees when blown against them, and as it is secured at bottom and ends, it is not displaced by the wind. Protecting the ripe fruit of the peach and nectarine is best effected by using Haythorn of Nottingham's hexagon gauze netting, which should be fixed to the front of the portable coping boarding, and secured to the bottom of the wall in the same manner as the canvass screens above referred to, and put on just as the fruit begins to ripen, and kept on till the crop is gathered. The best protection for both ripe fruit and expanding blossom is undoubtedly glass: that material is now cheap, and an economical way of applying it is, by having a portable structure of rafters set against the wall, and the spaces between filled with glazed sashes, which, when not in use, may advantageously be employed covering forcing-pits and protecting plants during winter.

Glass screens can be constructed in a variety of ways, and at no very extravagant expense. Figs. 107 to 109, vol. i., exhibit one mode of effecting this protection, fig. 479 another; and the narrow peach-houses, figs. 468 and 474 , show how such crops can be with certainty secured even in the most unfavourable localities in Britain. We apprehend, however, the day is not far distant when, instead of building walls of the ordinary construction, or orchard-houses upon the scale recommended by $\mathrm{Mr}$ Rivers, whole fruit gardens, from one quarter of an acre to several acres in extent, will be covered with glass, in which all our very finest fruits may be ripened to the highest state of perfection, and that without the aid of artificial heat altogether. Such structures should not be surrounded by walls, on account of the shade which south, east, and west walls produce, but should be glass all round, or upon three sides- at least, extending to within from 6 to 9 inches of the ground. The whole roof should be a fixture, with the necessary ventilation at the apexes of the ridges, and constructed on the ridge-andfurrow principle. The side sashes should be movable, running on low wheels fitted to iron rails, and made to pass each other, so that ample ventilation may be secured, and means afforded for taking in and out the trees. In no case should structures of this kind exceed 15 feet in height. The trees should all be in a portable state for the convenience of removing the hardier kinds - such as the apple and the pear-to a sheltered place during winter. The roots being confined within slate boxes-made to take to pieces, for the convenience of examining into their state of health, as well as for removing exhausted soil and the replacement of fresh matter-will also be completely under the control of the cultivator, and those evils completely remedied which ever must attend trees planted permanently in prepared borders. Trees so grown must be retained in the standard form, which is that most natural to them.

Thinning the fruit.-The peach, when in a healthy state, is apt to set more fruit than the trees can bring to full perfection without serious injury to themselves. Thinning should be performed at three times: first, when the fruit is fully set, which it often does in clusters of several close together,--these should be thinned out with the point of a sharp knife when of the size of ordinary-sized peas; next, when the fruit is about the size of a marble, at which time all malformed and very small ones should be taken off, leaving none in pairs, or on shoots devoid of a leading wood-bud at their points; and lastly, when the stoning is completed, which will be about the beginning or middle of July. Indeed, this is the most important thinning, as up to this time the crop is unsafe, and not unfrequently, from a variety of causes, the majority of the fruit may drop off of their own accord. So much depends on the state of the trees, and also on the sort, that no general rule can be safely laid down as to the distance the fruit should stand apart from each other. From 6 to 8 inches may be given as pretty near the average distance; and where fine specimens are wished for, and the varieties large, as in the case of the Grosse mignonne, 10 inches will be a proper distance.

Principles in connection with the successful cultivation of the peach as a wall tree in the general climate of Britain.-First, and most important, as we have elsewhere noticed, are shallow, welldrained borders of properly prepared soil, and shallow planting. To carry out these we would advise, and that more especially in cold damp soils and elevated situations, that the borders be only from 18 inches to 2 feet in depth, resting on a foundation naturally dry, or artificially made so; that the soil be renewed at each period of planting, and composed of the turfy top-spit of good, strong, and rather adhesive loam, of a mellow texture, and that without any manure whatever. In such a soil the peach luxuriates, and if planted shallow will long continue healthy, and yield abundance of fruit. On the other hand, deep rich soils are the bane of the peach, the very nursery of disease, and the foundation of premature decay; and these are aggravated when uncalled-for enrichments are added, more especially towards the end of summer. Light sandy soils are equally uncongenial to this tree, and in such it seldom attains age or sufficient stamina to produce fine fruit, or remain exempt from disease and the attacks of insects. Where shallow borders and high planting are carried out, the roots, being kept near the surface, are greatly benefited by solar influences-indeed so much so, as to be in a condition equivalent to several 
degrees of southern latitude. During the droughts and heats of summer, such borders should have repeated waterings of diluted liquid-manure; and if the surface be mulched with half-rotten dung, to exclude drought and prevent excessive evaporation, so much the better. The borders should neither be cropped nor deeply dug; the surface, however, to the depth of 3 or 4 inches, should be kept open by repeated hoeings. Such may be considered the proper conditions for the roots.

Overcropping trees, be they ever so healthy, exhausts the energy of the peach, shortens its life, predisposes it to the attacks of insects, and greatly lessens the value of the fruit both in size and flavour.

On the proper regulation of the branches much of the successful culture of this tree depends. Although much stress has been laid upon certain systems of winter pruning and training, as already noticed, these are as nothing compared to the management of the young wood during its growing season. This requires constant examination as to thinning, stopping, and laying in, so that there may be, as it were, a regular equalisation of the sap throughout the whole tree. Strong watery shoots should be kept in check, else they act the part of robbers, draining the sap from the profitable and less luxuriant wood, and causing a superfluous action of root; and should they ever be cut out at the winter pruning, they leave a preponderating power in the root, leading to a necessity for root-pruning - a circumstance greatly to be avoided in the case of all stone-fruits. On the judicious disbudding of the trees much of their future welfare depends; but this must not be mistaken for what some people call summer pruning, which deserves rather to be called summer murdering; for more peach trees are killed by this than by almost all other ills besides. Instead, therefore, of allowing the trees to exhaust their energies in the production of numberless shoots, to be, after they have wellnigh pumped the whole sap out of the tree, cut out and cast away, let disbudding commence early in the season, and be carried on progressively, so that by the end of May or beginning of June, according to situation and season, at which time the final thinning should take place, no more young wood shall be left on the tree than is sufficient for the next year. The judicious peach-pruner will ever have before his eyes the maxim, "to thin little and often;" for, as Mr Errington has very properly remarked, ${ }^{66}$ Nature does not like to be taken by surprise ; and by doing this with a light hand, she is able in the interval to restore the reciprocal and necessary balance between the root and branches. I make no doubt," he continues, "that gum is often excited, certainly increased, by a wholesale robbery of this description, soon after the tree has budded." Every shoot intended to be retained should be carefully fastened to the wall as it advances, and laid in between the older branches; and upon all occasions when these are naked, the young shoots should be laid in upon them, by which the action of the sun upon the bare bark will be modified, and the young shoots better exposed to the light and air. "All young and growing shoots," says the authority last quoted, "except the weak ones, and those on the lower portions of the tree, should be stopped the moment the fruit begins to change for ripening. By this time the true bearing wood of the next year will be well formed. All after this may be regarded as superfluous, and tending to keep up a late action in the root, which will be of no service to trees in health, or of sufficient vigour. Stopping also tends to concentrate the energies of the tree at the very time that concentration is most required; for at this period all that is wanted is abundant elaboration preparatory to the ripening of the wood and the flavouring of the fruit. However, at this juncture all the lower and weaker parts of the tree should be excepted. This is the commencement of their harvest; and this, with the stopping of the luxuriant wood through the summer, will tend more to equalise the sap than all the systems of pruning which have ever possessed undue importance."

Disbudding-or summer pruning, as it is erroneously termed, because the operation should be performed when the young shoots are little more than in their bud state-should commence early, be performed by degrees, and completed about midsummer. The first time, in going over the trees, those shoots or buds termed foreright or breastwood, and which generally project at nearly right angles from the wall, should be rubbed off when from 1 to 2 inches in length, as should also be such as are produced between the wall and branch. In about ten days afterwards the trees should be again examined, and those buds of the description noticed above, which may have been overlooked or produced since, should be removed, as should those which appear too crowded, or produced in bundles too closely together, leaving only the most promising and best placed of them. From this time a weekly examination should take place, and due regard should be paid to the thinning out of all such as have hitherto been spared. At this time the greatest attention will be required, because now the selection is to be made of those required to produce fruit next season, as well as to fill up the wall and model the tree. The lowest placed young shoots all over the tree should have a preference amongst those to be retained, and the others left should not be closer together at any given time than from 4 to 5 inches, choosing always the best formed and best placed; and all leading shoots should be encouraged to their full length, unless it may be desirable to cause them to produce side shoots in thin parts of the tree, in which case they may, if strong, be pinched back. No two shoots, if possible, should be allowed to proceed side by side from any given point. Avoid the evil of laying in too many shoots, and secure them to the wall or upon the old and naked branches of the tree, as they extend in growth. Throughout the whole growing season all gross watery shoots should be displaced; and no good shoot, unless there be sufficient evidence of its being required, should be suffered to remain. The thinner the 
young wood is laid in, the more it will be exposed to light and air, and it is on these that its ripening to full maturity depends.

As soon as the crop is ripe and gathered, the leaves will begin to assume their autumn hue, at which time such shoots as may have been preserved merely until the fruit upon them has been ripened, and are no longer required, should be cut out, to admit light and air to those retained for future bearing. As the leaves begin to drop of their own accord, their removal should be assisted by drawing the hand upwards along the shoots, or by using a small handbroom, drawing it upwards in like manner always in the direction of the buds, and never the reverse ; the ripened leaves will by this means be gently brushed off, and light and air admitted to the young wood: all superfluous and watery shoots should be removed at the same time. When the fruit is ripe it should be examined daily, and all that is fit gathered carefully by the hand, and carried in shallow baskets to the fruit-room, taking care never to place two fruits on top of each other. Various fruitgatherers have been invented; none are equal to the experienced hand. However carefully the fruit is gone over, some will fall from the trees; and therefore, to prevent such from injury, it is well to suspend long strips of hexagon netting, so that the fruit may fall into it. Contrivances under the denomination of " fruit-protectors" have been also invented, but, like the fruit-gatherers, they are held in small estimation by good cultivators. Those of Halliman are ingenious enough; they are of little importance as accelerators of the ripening of the fruit, but are found of some advantage in preventing it from falling when ripe, and also improving the flavour somewhat if allowed to remain a day or so after it has parted from the tree. These advantages, however, bear no proportion to their expense.

Peaches on heated walls should not be stimu. lated in spring: the later vegetation commences, the greater the chance of escaping the spring frosts ; and, indeed, so much importance do some attach to this, that they unloose their trees early in February, and withdraw the branches some inches from the wall, to retard vegetation till a later and more congenial season. The use of heated walls, as has been shown in vol. $i$. , is not to force forward the trees in spring, but to assist them during the end of summer in ripening their fruit, and in early autumn in ripening the wood and buds. The beginning of July and through August and September is the time that heated walls should be brought into action; and the intention is to place the trees in a somewhat similar condition, as regards temperature, to what they naturally experience in the middle or peach-growing States of America, the warmer parts of France, \&c., where, although the heat of summer and autumn is much greater than with us, their winters are much colder. Many good cultivators cover their peach trees early in spring with thick canvass, and thus, by excluding the air, retard their vegetation considerably. The absence, however, of light in this case is very injurious to the trees.

VOL. II.

\section{§ 2.-FORCING THE PEACH AND NECTARINE.}

By the beginning of November, the wood in even the latest peach-houses will have ripened. The operation of pruning and preparing for forcing will, from that period to the begiuning of January, demand attention. Ventilate all the houses freely day and night, unless the frost sets in severely, when it will be expedient to shut them up during the night, and to admit less air during the day. The borders should be carefully but slightly forked over (not dug with a spade) within and also without the house, in all cases where the roots extend beyond the walls. In the latter case the borders should be covered so as to exclude excess of wet, and also frost. If not concreted on the surface, they should have a foot or 15 inches of dry leaves laid over them, and this thatched with long littering straw, or otherwise secured against displacement by wind. The object is not to throw heat into the soil, but to retain the natural heat already in it, as well as to exclude cold and damp. Indeed, where very early forcing is carried on, it were better to have the roots entirely confined within the house, and this the more so in wet localities. The interior borders will be dry if the roof be in a proper state of repair, and will therefore require two or three rather copious waterings ; and if these be done with weak liquidmanure, so much the better. The trees will require a supply of food to carry them through the season; and this is more effectually done by using it in a liquid state. The old and barbarous system of digging in rich manure in a solid state cannot be too much condemned. The surface-soil of the borders within, to the depth of 2 inches at least, should be carefully scraped off before forking commences, and removed; and this may be done to a somewhat greater depth, provided the roots are not interfered with. This portion of the soil, from repeated waterings and other causes, becomes annually exhausted of its organic parts, and becomes in time entirely useless as regards affording food to the trees. The surface should then be filled up again with a compound of turfy loam, chopped up, but not reduced too fine, and enriched with sheep or deer dung, the essence of which will be progressively carried down to the roots in the process of watering. All water applied to the roots from the time the house is shut up, till the natural heat of the summer renders the precaution unnecessary, should be at a temperature of from $80^{\circ}$ to $90^{\circ}$. When the house is heated by smoke-flues, these should be completely cleaned of soot, repointed, and whitewashed with hot lime-water, for the purpose of preventing the escape of smoke and sulphureous gases through the joints of the mortar. It is necessary for appearance sake also. The back wall or other parts that may be of brickwork should have a similar colouring, and the woodwork, if possible, should also be painted at least every second year. If hot water is used, the boiler and pipes should be emptied, cleaned inside, and then refilled with soft rain. water. 
For Pruning the Peach and Nectarine, vide general article Pruning, also Pruning and training the peach on the open walls, p. 488.

Previous to tying the wood to the trellis, the branches should be carefully gone over, the oldest wood and main stem painted with vegetable spirits of tar; while the younger wood and smaller branches should be carefully laid over with a thin paste composed of 2 ounces of soft soap, 3 handfuls of flour of sulphur, and double the quantity of hot lime, mixed together in about a gallon of soft rain-water. Care must be taken, in laying on this latter dressing, that the brush be always drawn upwards, and never downwards, for fear of displacing the buds. For some time after this is applied, avoid watering with the syringe or engine, and keep up the necessary humidity by throwing a little water on the floor-pipes or flues twice a-day. At this period of peach-forcing much benefit would be derived by the trees, were a quantity of rank stable-dung placed in a ridge-like form along the centre of the house, and this frequently turned over. The vapour arising from the mass, accompanied as it always is, more or less according to the richness of the dung, by ammoniacal gases, will be very beneficial to the trees, and tend to the suppression of insects. This cannot at all times, however, be done, on account of the litteriness of its appearance ; and sometimes it is objected to on account of the labour attending bringing it in and taking it out again. Urine from the cow-house or stable, placed in evaporating pans on the top of the flues or hotwater pipes, is a good substitute, and attended with less trouble and disagreeable appearance. Either of these methods may be had recourse to until the flower-buds begin to show the colour of their petals, after which this operation must cease, as well as syringing over the trees. The temperature at first must only amount to that obtained by shutting up the house, and may range during the day at from $45^{\circ}$ to $55^{\circ}$, decreasing to from $40^{\circ}$ to $45^{\circ}$ during the night. This very first step is a critical one in peachforcing at this season of the year, when the temperature is so varied, more especially in Scotland. Some days no artificial heat will be needed, while there may be others when a slight degree of heat will require to be thrown into the pipes or flues. This is a point depending entirely on circumstances, and nothing but strict vigilance can regulate it. The middle of January is a very proper time to commence peach-forcing, if the object be to have the fruit ripe by the beginning of June; and this is quite as early as the climate of this country warrants us in attempting it, if the flavour of the fruit is to be considered more than a week or two's time in its production. Some begin to force much earlier, even towards the middle of November, and throughout December ; and although success often attends this early excitement, neither the size nor flavour of the fruit is to be compared to that which ripens at the period we have stated. To insure anything like success in this early forcing - that is, having the fruit ripe in March or April-it were better to adopt pot-culture, by which means the roots of the trees could be stimulated by being plunged in fermenting matter, or placed over a tank, on the top of flues, or otherwise, where the roots would derive that amount of heat that would stimulate them into activity even before the sap rose in the tree. Vaulted borders capable of being warmed would be valuable in such cases of early peach-forcing. Those who wish to have their fruit ripe at a later period of the season, have much fewer difficulties to contend with, and consequently their cares are fewer, and their chances of success much greater. The general management of the trees, however, in all these cases, is governed by the same laws. Recurring again to the state of the peach-house set agoing towards the middle of January, and with which we commenced these remarks: About twelve or fifteen days after shutting up, the blossoms will be beginning to expand. At this period the humidity of the house, hitherto caused by copious steaming and slight syringing, should be lessened, and a slight increase of temperature afforded. This increase of heat should not exceed $5^{\circ}$ or $7^{\circ}$, for to hurry on the expansion of the blossom and setting of the fruit might endanger the whole crop. The more slowly the buds swell, and go through the stage of forming the parts of fructification, and the important offices depending upon it, so much the more certain will the success be. When the flowers are fully expanded, and the pollen fit for dispersion, a more abundant ventilation will be necessary ; and this must not be withheld, even though it becomes necessary to throw a little additional heat in every morning, and continue it through the early part of the day, to make up for the extra supply of air admitted. At this time the 'greatest care must be taken that ventilation be properly administered, and that no draughts of cold frosty air be allowed to blow in directly upon the trees. Where none of the various modes of ventilation we have given (vide vol. i., article VentiLATION) are employed, it will be well to place at this early period thin canvass or gauze netting screens over the air-openings, that the cold air may be sifted through them, and its full force broken ere it reach the trees. The peach-forcer will at this time see, and we hope be convinced, of the great importance of bringing in the air towards the bottom of the house, as well as of admitting it under the flues or hot-water pipes, so that the "raw chill" may be taken off it before it comes in contact with the blossom. The temperature now should range from $55^{\circ}$ to $60^{\circ}$ through the day, but accompanied with a free circulation of air. $5^{\circ}$ of sun-heat may be allowed in addition to the above, but the night temperature should not exceed $45^{\circ}$, which should ever be accompanied with an increase of moisture as the fruit is setting. During the flowering period no syringing can possibly be permitted, but the necessary humidity should be kept up by slight steaming and frequently watering the surface of the floors, \&c. As soon as the flowers begin to decay, the young fruit will be seen seated between the fragments of the petals. At this time a peculiar condition is traceable in the embryo fruit, and this condition has been pointed out by one of the best 
pomological writers of the day as indicative of the true condition of the action of the root. "If they (that is, the embryo fruit) are many days struggling to get free of the decaying blossom, it is a sure symptom of a sluggish action of the root; on the contrary, fine fresh trees in their prime will soon enable their progeny to escape."

This sentence is worth the attention of the cultivator; it will indicate to him sooner than the after state of the foliage whether all is right at the roots or not. When the fruit has arrived at this stage, less ventilation must be given, and consequently less artificial heat also. But these changes in ventilation and temperature must not be effected suddenly, but progressively. All sudden changes, in regard to stone-fruits in particular, are dangerous. Let the temperature now be maintained at from $55^{\circ}$ to $60^{\circ}$ during the day, and from $45^{\circ}$ to $50^{\circ}$ during the night. We speak now of artificial heat. The sun-heat through the day may rise not only with impunity, but with advantage, to $75^{\circ}$; but it should always be borne in mind that there is a vast difference in connection with vegetable life between the heat produced by fire and that produced by the sun. Also let it be kept in view, that with this increase of sun-heat, ventilation must bear a relative proportion. At this stage of the fruit's existence moisture must be increased, not at the roots, but over the tree and its young leavesfirst to refresh and nourish them, and also to keep down the appearance of both thrip and red-spider, the bane of peach trees, and often confounded together. The existence of both may be traced to a high temperature, unaccompanied with a due share of humidity. Syringe freely after this state; it frees the tree of the decaying remnants of its blossom, keeps the surface of the foliage clean and fit for the necessary operations of respiration, and greatly tends to prevent the appearance of the two enemies just named. In regard to syringing, little and often ought to be the rule. In bright sunny weather, syringe morning and evening only. In dull cloudy weather it may be done throughout the day, but of course more sparingly. After the fruit has fairly set, the trying season of peach-forcing may be said to be over, as all that follows is judicious thinning the fruit, stimulating what is left by all legitimate means; and in this respect nothing tends more to the health of the tree and swelling of the fruit than a proper adjustment in the quantity of young wood that is to be left, and the first step in this direction is judicious disbudding. This is a species of pruning differing from most other kinds, because it very early removes the superfluous or ill-placed shoots that would otherwise rob the tree and fruit also of a very large portion of their energies, if left to assume their full size, or even a portion of that size, and have to be removed afterwards, carrying with them the whole material of which they are composed to the dunghill, instead of being retained in the tree. A neglect of disbudding in time is like winking at petty thefts until the culprit is at last convicted of open highway robbery. Besides a neglect of timeous disbudding, another serious evil is likely to arise at this period, and that is founded upon the rules laid down by the older writers, who advise copious and severe syringing to take place at this time, to clear the trees of the decayed blossoms, to suppress insects, and to do many other kind offices to the foliage, without thinking that nearly the whole of the water so applied falls to the ground and saturates the soil - reducing by this means the soil in the interior to the condition of an open border, subject to the heavy rains that fall from the heavens in the wettest localities, causing not only many of the fruit to drop, but inducing a condition in the trees whereby they put forth strong watery shoots devoid of flowerbuds, and produced only for the apparent purpose of being cut out and cast away. Disbudding is an imperfect operation, and scientifically it might be carried into effect, both as regards blossom and wood-buds, before either had developed themselves. Practical experience has not fully recognised this doctrine, and waits until the young shoots have attained the length of 2 inches, when the operation commences, and is carried on progressively until the tree is disburdened of its superfluous wood, and left in a condition to perfect that which is left. The process of disbudding should be carried on progressively during four or five weeks, taking a little off at a time, and going over the trees frequently. For this very important operation no definite rules can be laid down, as so much depends on the state of the trees, whether they are young and full of vigour, or old, and arrived at a state of forming fruitbuds more abundantly than wood-buds. During the process of disbudding, two different kinds or forms of shoots will be observed-the one strong robust shoots, which in general spring from towards the centre of the tree, and often, from emanating from the centre or strongest bud of a group, proceed in an outward direction instead of a lateral one, and hence, even if required to be laid in in arranging the form of the tree, liable to be broken in consequence of want of flexibility. These strong or watery shoots, for the most part, aree to be removed, reserving only such of them as may be required for filling up any vacant space in the centre of the tree. The other wood to which we have referred is of moderate, and often even of slender growth, but it is the wood destined to produce the flower-buds for another season. This should be moderate in growth, not drawn out to a great length, and very shortjointed-that is, thickly set with buds. Deficiency in ventilation, too high a temperature, and want of attention to disbudding, all tend to cause this unnecessary elongation. A contrary course tends to the formation of short, stout, well-formed shoots, abounding .with bold and well-swelled buds.

The period of stoning is considered a precarious time with all stone-fruit. If all be not right at the roots, if sudden changes of temperature or inefficient ventilation be allowed to exist, then many of the fruit will fall off. The former of these evils 
will only indicate itself, and give warning of what ought to be seen to, in autumn; the others can only be guarded against by close vigilance. The temperature during this period may be elevated to the extent of $5^{\circ}$ or $6^{\circ}$ during the day by sun heat, but no alteration should take place in the artificial temperature, more especially during night. Nature requires time in perfecting her works; and it is to violating this law, by forcing her to go on faster than she wills, that most of the disasters in forcing-houses are to be traced. Indeed, in the hands of many, much of our cultivation under glass may well be called forced in the true sense of the word, which, according to our dictionaries, means "to compel, to overpower, to drive by violence," \&c. To accelerate would be a word much nearer the proper meaning. Syringing over the leaves may be now more freely indulged in for the prevention, not the suppression, as too often recommended, of the red-spider. The borders must at this time be frequently examined; either too much moisture or too little will be equally fatal. $\mathrm{Mr} \mathrm{R}$. Errington concludes a very sensible article with the following maxims, which we hesitate not to call the golden rules of peach-forcing: ".Heat.-Through every part of the process only use artificial heat as necessary. Let all real advances be made under the influence of increased light. Beware of high night-temperatures; we have had a thermometer at from $38^{\circ}$ to $45^{\circ}$ during the first swelling without any perceptible harm. Air and moisture.-Never permit the atmosphere to become dry at any time. A somewhat dry and mellow state of air is requisite at blooming time, and more especially when the fruit is ripening. A dry air, long continued, will be almost certain to produce red-spider. Beware, however, of much moisture with very low night-temperatures. Disbudding. - Not only disbud frequently, but at all times pinch off the points of shoots which are growing too luxuriantly. Disbudding should be completed by the time the stoning process commences, if possible. Ripening process.The slower peaches are ripened, the finer and higher flavoured will be the fruit. Those who think to increase the size and appearance of their fruit by a close course of treatment will find themselves miserably mistaken."

Ventilation in all the growing stages of peach culture is of the greatest importance. In former times it was thought of only during the day, and admitted not only directly upon the trees, but in a few places only, and those of considerable size, and often so ill arranged that, while one part of the house was chilled, and the trees subjected to a strong current of cold air blowing with force upon them, other parts were scarcely affected, and that purity of atmosphere so essential to success was in a very great degree denied them. The case is different now in all well-constructed peach-houses; and not only is air admitted night and day, but it is let into the house by means of many small apertures, and those placed either close to the ground or brought entirely upwards through the flooring, thus diffusing a uniformity of ventilation in all parts, and during the night as well as during the day. Examples of ventilation upon this principle will be seen illustrated in vol. i. When the fruit has fully swelled, and is beginning to change colour prior to ripening, too much air cannot be admitted. Indeed, of so much importance was this thought by the late Mr Knight and others of his day, that they practised and recommended the total removal of the roof-sashes during a great part of the day, so that no interruption should prevent the fruit enjoying the full force of the sun; and in this way the flavour and colour of their fruit was made to approximate nearly to that grown on the open walls. Neither peaches nor nectarines acquire such perfection, either in richness or in flavour, when grown under glass, as they do when grown in the open air. No doubt, in consequence of our modern hothouses being much more transparent than those of other days, the fruit produced in them are much better in this respect than formerly; and although the operation of removing part of the roof is impracticable in the case of houses with fixed roofs, still the greater admission of light through large plates of glass, and thorough ventilation day and night, renders such removal unnecessary. One great objection to the removal of a portion of the roof is the sudden transition to which the foliage is exposed, from the modified and often insufficient amount of light to its brightest rays direct from the sun. Some also entertained the opinion that the roofs of all peach-houses should be removed during the period of the trees' rest-that is, from the time the wood and buds were ripened until the time when they were to be brought into excitement again. 'This also is now looked upon as unnecessary ; the amount of ventilation, when properly applied, being found quite sufficient to prevent the trees becoming excited before the desired time. During the period of rest, the trees should be kept at a low temperature, and water only applied should the soil become very dry. Plants are often kept in the peach-house during winter, and lettuce, endive, and cauliflower are as often planted, when fully grown, in the borders, to insure their preservation from frost. This should not be-the peach withstanding, even in its native country, a degree of frost during winter which would kill any of these intruders; and trees subjected to such a low temperature are known to break much stronger and freer when excited, than such as are wintered at a higher temperature. When the fruit begins to ripen, the same precaution should be taken regarding gathering it, and preventing it from falling to the ground, as noticed for trees on the open walls. The French generally, and some pomologists of our own country, assert that the fruit should be plucked from twenty-four hours to two days before it is to be eaten, and during that time recommend its being placed in the fruit-room. This may be very judicious in the case of fruit grown in the open air in wet seasons, but as regards fruit ripened under glass it is wholly unnecessary. One grand feature in peachforcing is to insure the proper ripening of the wood in autumn. In general, the glass is found 
sufficient for this, but in the case of very late sorts, grown in peach-houses to supply the table in September, a little fire-heat may be in some seasons required, but its application should be after the fruit has ripened.

Besides ripening the peach in regular peachhouses, other means are often had recourse to, such as growing them in pits heated by solar influence, by fermentation-a practice much in use on the Continent-and growing them in pots, as practised by some few in this country. Figs. 466,467 , vol. i. p. 347 , are examples of pits used for the former process ; and figs. 405, 407, and 408 , vol. i. p. 302 , although described as for the vine, are equally applicable to the peach. The peach being more difficult to bring to perfection at a very early season than the vine, we hold all such contrivances as far behind the age we live in. The culture of them in pots is a different affair, and requires both skill and attention. The best directions given on this head are those of Mr Hutchison of Eatington Park, published in "The Gardeners' Chronicle," Mr Hutchison having succeeded, so far as we know, in ripening peaches earlier and with greater certainty than any other person. His practice is to select trees of three years' growth, planting them in 12-inch pots, cutting back the wood to four buds, and shifting them yearly until the trees have filled with roots an 18-inch pot, which is the largest size he believes to be required. The soil he uses is good turfy loam, mixed with decayed portions of wood from the bottom of a wood pile. When the trees are established to his mind, he " brings them into the house in mild weather during November, a little earlier or later according to the state of the weather. Do not start them all, however, at once; the last lot need not be put in until the first of January. Any later than this would not answer, as the weather, if clear, is then hot through the day. Commence forcing at $55^{\circ}$ at night, allowing the thermometer to fall to $50^{\circ}$ in the morning, if cold; but if the weather is mild, never to fall below $55^{\circ}$, and from that to $60^{\circ}$ is the usual temperature kept up throughout the period of forcing during the night: during the day I make up for low night-temperature when I have the chance of sun-heat. Do not be fastidious about a few degrees; to get it high enough is the main point-say from $70^{\circ}$ to $85^{\circ}$ and $90^{\circ}$ - until the fruit is stoned, then keep them very hot during the day, viz., from $95^{\circ}$ to $105^{\circ}$, and sometimes even as high as $110^{\circ}$. Of course, a great deal of moisture is required with this high temperature; syringe over head twice a-day, and sometimes oftener when the air is dry, and you will scarcely ever be troubled with green-fly or red-spider. Watering at the root must be carefully attended to : very little is wanted until the trees get covered with leaves, but after the fruit is stoned they should be watered plentifully. Of course the water must be gradually withdrawn as the fruit approaches maturity, in order to increase their flavour." By these means excellent ripe peaches have been produced for several years so early as the first week in April, and Mr Hutchison anticipates doing so even in March. They are grown in pots in the open air, and transferred to the pine-stove to produce their fruit. Fumigation is studiously avoided, and insects kept under by a free use of the syringe.

Much advantage has resulted from assisting the impregnation of the flowers in early forcing, particularly when the trees are young and of robust habit, and where they are deprived of the natural agencies-a sufficiency of air, and the operation of bees and other insects. The pollen may in such cases be applied from the stamens to the pistil by means of a fine camel-hair pencil. In cases of trees of the most fructiferous description, which often produce flower-buds in abundance but no wood, except at their termination, should even the blossoms set their fruit, it almost invariably drops off soon afterwards for want of the terminating bud to draw up sap for their support. Knight has suggested in such cases inarching a small branch with leaf-buds immediately above the fruit-buds, and has succeeded in causing such fruit to ripen to great maturity. The same authority transferred fruit-buds from one tree to the barren branches of another, and produced fruit by the process- of course, of the same variety as the tree from which the fruitbuds were taken.

Peaches, nectarines, and apricots have been most successfully grown in pots by Mr Rivers of Sawbridgeworth, in his orchard-houses or deep pits. For the principles of his practice, see article APRICOT.

Training the peach and nectarine under glass.The fan mode is usually adopted, and the trees planted to suit the structure they are grown in. When the peach-house is a mere narrow passage, of some 5 or 6 feet in breadth (see figs. 468 , 474 , vol. i. p. 348,350 ), the trees are usually planted against the back wall, which they are made to cover, being trained to a wire trellis from 3 to 4 inches distant from it. For very early forcing this is a very eligible way, as the roots are for the most part confined under cover of the house, and exempt from excessive cold or excessive wet. When the houses are of greater width, the trees are otherwise arrangedviz., one set covering the back wall, as in figs. $469,472,473,477$, vol. i., with a curvilinear trellis in front, as there also exhibited, the trees on which are usually planted just within the front parapet-wall, their roots finding their way into the exterior prepared border; while others are planted nearer the centre of the house, as in fig. 472, vol. i., and trained partly to gable trellises, suspended from under every alternate rafter, and partly up three wires, placed a foot under the top part of the rafter, as shown in fig. 472 ; and sometimes the gable trellis is continued over the footpath, forming a series of arches, as in the case of one of the peach-houses in the gardens at Dalkeith, of which the annexed fig. 227 is an interior view. This last we consider to give the greatest extent of surface for training, with the least amount of shade. Another mode of training, often met with in old houses, is to cover the whole lean-to roof with a trellis, and to train the trees over it, these being planted near the front of the house. By this means the fruit is placed near the glass, and sufficiently well exposed; but much of the interest is lost 
to the owner, as few of the fruit are seen, the foliage intervening between it and the eye of the observer. It affords also less training surface than several others; and however well it may

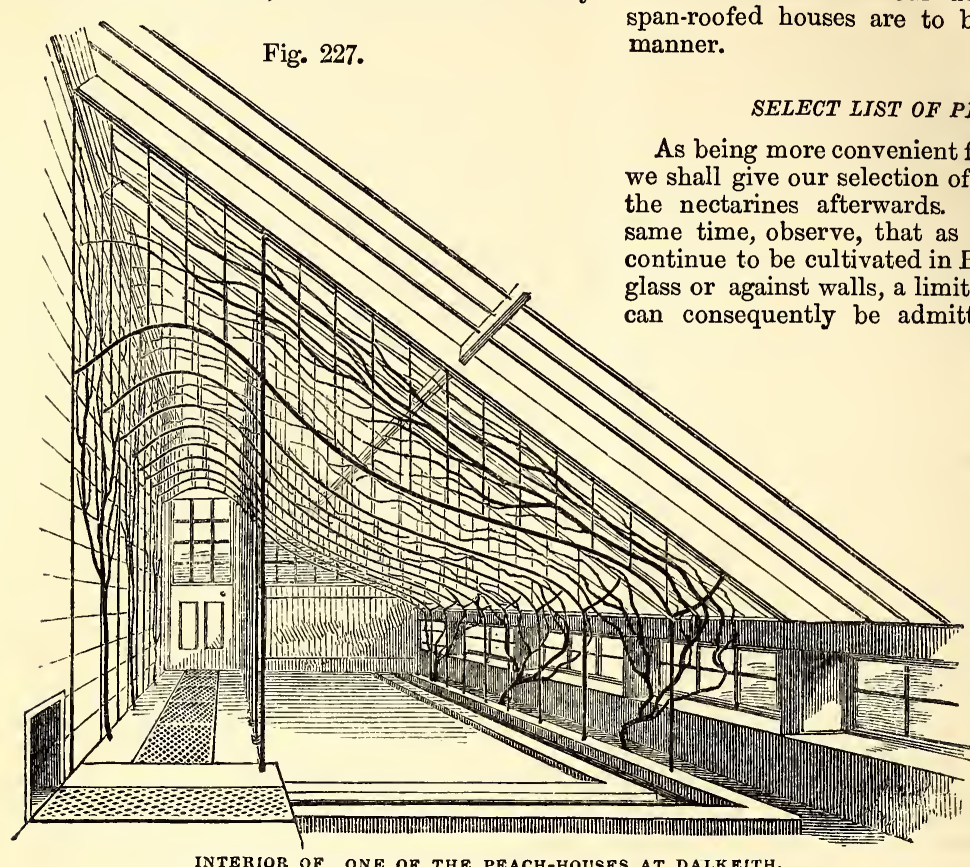
manner. is of all forms the best calculated for the admission of light and air to all parts of the tree. In the new gardens at present completing for Lord Panmure from our designs, three large span-roofed houses are to be planted in this

As being more convenient for ready reference, we shall give our selection of peaches first, and the nectarines afterwards. We may, at the time, observe, that as both are and will in Britain either under glass or against walls, a limited number of trees into one garWe shall, although with some degree of reluctance, omit those of American origin, because we have had so little opportunity of ascertaining their merits when grown in Britain, and those we have ourselves cultivated have in no case equalled the best peaches already in this country. We will, however, append a list of American peaches, marking those most highly suoken of in such works on American pomology as we have had an opportunity of consulting. One of their authors very sensibly remarks: "It is very desirable to reduce the collection of peaches to reasonable limits, because as this fruit neither offers the same variety of flavour or the extent of season as the apple and the pear, a moderate number of the choicest kinds, ripening from the earliest to the latest, is in every respect better than a great variety, many of which must necessarily be second-rate." In regard to the effect of climate on the peach, when grown in France or England, and those grown in the United States, the same very impartial authority says : "It is worthy of remark that most of our American varieties of the first quality have proved second-rate in England. This is owing to the comparative want of sun and heat in their climate. Indeed, our finest late peaches will not ripen at all except under glass, and the early varieties are much later than with us. On the other hand, many of the best European sorts are finer here than in England, and we have lately endeavoured to introduce all of the foreign sorts of high quality, both with the view of improving our collection, and because we believe they are generally purer and healthier in constitution than many of our own native kinds." The time of ripening is that of the open air in the vicinity of London, unless where otherwise expressed. 
Acton Scot.--Leaves crenate, having globose glands; flowers large; flesh melting; colour pale yellowish white, slightly marbled with red next the sun; size medium ; quality excellent ; ripens the end of August; suture shallow. Of English origin. Raised by Mr Knight between the Noblesse and Red nutmeg. It is an early and excellent fruit, and ripens well with us at Dalkeith. Brought into notice 1814. A freestone. The Early Downton, one of Knight's seedlings, may almost be considered synonymous with this, at least so near to it as not to be worth growing as a distinct sort; and the same may almost be said of Knight's early.

Admirable early.-Leaves crenate, with globose glands; flowers medium, between large and small, so that it is difficult to determine to which it belongs; and here the propriety of Mr G. Lindley's third subdivision, in regard to the size of the flowers, occurs; still we think Mr Thompson right in confining the size to large and small. Under good cultivation the present would rank amongst the large flowering sorts. Flesh white and melting; colour yellowish white on the shaded side, red on the side next the sun; size above medium; quality good; ripens the middle of September. A very excellent French peach, but not early, as the name implies; but having been given in the time of Miller, it is retained, and also to distinguish it from the following. Synonyms - Admirable, $l$ 'Admirable, Belle de Vitry of the "Bon Jardinier," but not of Duhamel.

Admirable late.-Leaves crenated, with globose glands; flowers small; flesh melting, greenish white, but red at the stone; colour pale yellowish green, with a palish red on the side next the sun; size large; quality firstrate; suture dividing the fruit pretty nearly all round; ripe from the middle to the end of September. Of this peach Mr Thompson says : "One of the very best peaches, and ought to be in every collection ; is very proper for the peachhouse, to succeed the earlier sorts." SynonymsLate purple of some, Teton de Venus, Bourdine, Boudine, Royal, La royale, Pêche royale, Narbonne, Judd's melting, French Bourdine, Belle bausse or bauce of some, Motteux's, Pourprée tardive of some. In giving the synonyms above we have followed Thompson. Mr G. Lindley says, in describing the Bourdine, which he will not admit to be a synonym of the Late admirable, "That there is some resemblance between this (i.e. the Bourdine) and the T'eton de Venus, and the Royale, will not be denied; but that they are identically the same is what I cannot admit. Duhamel, who has always been regarded as the highest authority in what regards the fruits of his own country, would have discovered this, had it been the case, long before he published his book. In addition to this, where is the nurseryman, I would ask, who has ever successfully budded the Bourdine upon the mussel-stock?" Mr L. concludes by determining to consider the Bourdine, Teton de Venus, and the Royale, as three distinct varieties. The question is, Are they sufficiently distinct in quality as to be worth growing all three in the same garden or peach-house? We think not.
Alberge, yellow.-Leaves crenate ; glands globose; flowers small ; flesh melting, deep yellow, reddish next the stone; colour yellow in the shade, deep red or purple on the exposed side ; size medium; quality good ; suture extending from the base to the apex ; ripe end of August and beginning of September. A very neat and hardy peach; has ripened occasionally as a standard near London. Hardy, and suited to cold localities. Synonyms-Alherge jaune, Red alberge, Purple alberge, Peche jaune, Goldfleshed, Golden mignonne. Often confounded with the Rosanna. An examination of the glands will show the difference, the one being reniform, the other globose.

Barrington.-Leaves crenate, with globose glands; flowers large ; flesh melting, yellowish white, slightly reddish towards the stone; colour pale yellowish green in the shade, deep red next the sun; size above medium; quality excellent; suture slightly marked along one side ; ripe middle and end of September; stone very rugged, ovate, terminating in a sharpish point; tree hardy. Originated at Burwood in Surrey. Synonyms-Colonel Ansley's, Buckingham mignonne.

Bellegarde.-Leaves crenated; glands globose; flowers small ; flesh melting, pale yellowish, slightly red towards the stone; colour pale greenish yellow on the shaded side, rich deep red, with dark purple dashes, on the side next the sun ; size above medium; quality excellent; suture shallow ; ripe from beginning to middle of September; stone rather large, slightly pointed, separating freely from the pulp. An excellent and handsome peach. Forces well, and keeps after ripening better than most. Synonyms-French Royal George, Noir de Montreuil, Early galande of some, Galande, Brentford mignonne, Violette hative of the English, Violette hative grosse of do., Large violet, Ronald's Brentford mignonne, Smooth-leaved Royal George of some.

Catherine.-Leaves crenate, with reniform glands; flowers small ; flesh firm, yellowish white, adhering closely to the stone, which is of medium size, roundish ovate, and slightly pointed; colour pale yellowish green on the shaded side, beautiful red where fully exposed to the sun; size large; form ovatish, often somewhat swelled on one side, terminating at the apex in a fleshy nipple ; ripe end of September and beginning of October. One of the best late clingstone peaches. Mr Lindley remarks : "There is no doubt as to this being an English peach." There is a variety in the nurseries under the name of William's Catherine, so very similar in every respect as to be almost identical with this, and no doubt a seedling from it.

Chancellor.-Leaves crenate; glands reniform; flowers small ; flesh melting, and separat ing freely from the stone, which is oblong, tapering towards the points; size large; suture very distinct ; form oval ; flavour rich and vinous; colour pale yellow where shaded, dark crimson where exposed to the sun; less downy than most peaches; ripe about the middle of September. This, although a French peach, is 
not the true Chancellière of Duhamel, which latter has large flowers. An excellent peach, although less delicate in texture than many others. Synonyms-Stewart's late galande, Late chancellor, Noisette, Edgar's late melting. The Late purple (Pourprée tardive) is also closely allied.

Early Anne.-Leaves doubly serrated; glands none; flowers large, almost white; flesh melting, very white, parting freely from the stone; colour very pale, almost white; flavour excellent; size rather below medium ; form round. An old inhabitant of our gardens. Of English origin. Ripe the middle of August. The best of our extra early sorts, as the Red and White nutmegs, although equally early, are deficient in size and flavour.

Flat peach of China-Cultivated more for the singularity of its form than for anything else. It is, however, well adapted for forcing in pots. As Chinese peaches are now being introduced, we may, with a view to prevent confusion so ar, observe that the Java peach (Peen-to), and Chinese peach of some catalogues, are synonyms of the Flat peach of China.

George the Fourth.-We notice this sort, which is said to be the most popular peach for garden culture in the United States, and has been for some years grown in Britain, where it has not turned out to the expectations of any one, either as a house or open wall tree; thus corroborating the opinion so honestly given by Mr Downing (vide p. 506). We were led to try it from the high character given it by $\mathrm{Mr}$ Lindley, "Guide to the Orchard," p. 258. Both in England and Scotland, in the peach-house and on the open wall, did it alike disappoint us.

Late Warburton.-Glands globose; size large; form roundish oval ; colour pale yellow where shaded, bright red where fully exposed to the sun ; flesh melting, yellowish white, and of very superior:flavour. This excellent new peach promises to equal if not excel all our late sorts, ripening about the middle or end of September. Synonyms-Warburton seedling, Warburton $A d$ mirable.

Madeleine de Courson.-Leaves doubly serrated; glands none; flowers large; flesh very pale white, with a tinge of red next the stone, from which it separates freely ; flavour rich and vinous ; size rather under medium ; form round; colour pale yellowish where shaded, beautiful red where exposed to the sun; stone rather large in proportion to the size of the fruit, but not so much so as to be objectionable, the excellence of the fruit overbalancing this apparent defect. Ripe end of August and beginning of September. Synonyms-French Magdalen, $M a$ deleine rouge, Rouge paysanne. Of this peach $\mathrm{Mr}$ Lindley observes: "This is a very excellent peach, and ought to be found in every good collection of fruit. It is the True red Magdalen of Miller, and as such should never have given way to the one now cultivated under that name; but, like the Elruge and Red Roman nectarines of that author, the ignorance of some and the indolence of others have allowed far inferior fruits to usurp their names." This is a very old and favourite French fruit.
Malta. - Leaves doubly serrated; glands none; flowers large; flesh melting; greenish yellow, slightly stained with purple next the stone, from which it parts freely ; size medium; suture broad and shallow along one side, slightly marked on the other; colour pale greenish yellow on the shaded side, marked with blotches of dull purplish red where fully exposed. Ripe end of August and beginning of September. Fruit bears carriage, and keeps well after being gathered. An excellent hardy sort, well calculated for the coldest situations peaches are attempted to be grown in. Italian of some.

Mignonnegrosse.-Theking of peaches. Leaves crenate; glands globose ; flowers large ; flesh melting, pale yellow, reddish next the stone, from which it readily separates; size large; form roundish-depressed; suture moderately deep ; flavour of the highest order ; ripe about the middle of September ; colour yellowish in the shade, very deep red where fully exposed to the sun. An excellent bearer, whether in the peach-house or on the open wall; less subject to mildew than most other French sorts. It is also one of our best forcers. From the delicate texture of the fruit, it requires careful packing when subject to long carriage. Producing varieties from its kernels very similar to itself, which may partly, as well as its great excellence, account for the long list of synonyms ; for, as we have remarked in the case of the apple and pear, no surer criterion can be given of the superior quality of a fruit than the number of names it is cultivated under. English synonyms.-Early May, Early French, Neil's early purple, Neal's early purple, Ronald's early galande, Ronald's seedling galande, Foster's, Foster's early, Early vineyard, Superb royal, Transparent, Kensington, Royal Kensington, Royal sovereign, Johnston's early purple, Johnston's purple avant, Padley's early purple, Purple avant, Early purple avant, Purple hative, Swiss mignonne, Large French mignonne, Mignonne, Grimwood's Royal George, Grimwood's new Royal George. French synonyms-Vineuse, Veloutée, Veloutée de Merlet, Pourprée hâtive of some, Avant, Belle bausse, Belle bauce, Belle beauté, La Royal of some, Vineuse de Fromentin, \&c. Of this fine peach Downing remarks: "The Grosse mignonne is certainly the 'world-renowned' of peaches. In France (its native country), in England, in America - in short, everywhere, it is esteemed as one of the most delicious of varieties."

Mignonne petite. - Leaves crenate; glands kidney-shaped; flowers small ; flesh white, reddish towards the stone, from which it separates freely; size below medium; form roundish, compressed near the stalk; suture deep, extending the whole length of the fruit, ending in an obtuse nipple; flavour excellent; colour yellowish white where shaded, fine red where fully exposed to the sun. Ripe beginning to middle of August. A very desirable early fruit. Synonyms-Early mignonne, Small mignonne, Mignonnette, Pêche de Troyes, Double de Troyes.

Noblesse.-A peach next to the Grosse mignonne in deserved popularity. Leaves doubly serrated; glands none; flowers large; flesh 
melting, greenish yellow quite to the stone, from which it separates freely ; size large ; form roundish, having an acute nipple at the apex; flavour of the very highest character; ripe the end of August; colour pale yellowish green where shaded, marbled with dull red where exposed to the full sun; a beautiful delicate-looking fruit; one of the very best either for the peach-house or the open wall. It is also one of the hardiest, and perhaps the most common in cultivation. In regard to this sort some discrepancy appears to exist between Mr G. Lindley and Mr R. Thompson, the latter making the Vanguard a synonym of the Noblesse, while the other asserts they are distinct. Our own opinion is, that they are so near alike, although both ranking in the highest class as to merit, as to be scarcely worth cultivating in a private garden as distinct sorts. They are, however, so excellent that a tree or two under each name will cause no disappointment. The characters of the fruit given by both these excellent authorities are very much the same. Mr Lindley, however, remarks: "Nurserymen need not be at any loss to distinguish the two sorts, when maiden plants, in the nursery, the lateral shoots of the Noblesse being nearly as long as the main leader, those of the Vanguard being less numerous, and exceeded considerably by the main shoot; besides, the plants of the Vanguard are of a taller growth than those of the Noblesse. Indeed, so obvious and invariable have I found these characters, that, should the two kinds become inadvertently mixed in the nursery rows, the most inexperienced foreman would be enabled to separate them without any fear of mistake." Synonyms - Mellish's favourite, Vanguard of Thompson, Lord Montague's noblesse.

Pourprée hâtive. - Leaves crenate; glands kidney-shaped; flowers large ; flesh melting, pale yellowish white, becoming very red towards the stone, from which it parts freely; size as large as the Grosse mignonne; form roundish, flattened at the apex; suture deep, extending over the apex; flavour excellent; ripe towards the end of August; colour pale whitish yellow where shaded, deep red or purple where fully exposed to the sun; stone rugged, obtuse at the ends. Synonyms - Early avant of some, Pourprée hative à grandes fleurs of the French gardens, Avant rouge of some. The Pourprée hâtive, Veritable, Du vin, Early purple, and True early purple, are probably nothing different from the Grosse mignonne, and may be seedlings from it. If different, the shade is so small as not to warrant their being propagated for sale under the two names.

Rosanna.-Leaves crenate; glands kidneyshaped ; flowers small ; flesh melting, deep yellow, but very red near the stone, from which it parts easily; size medium; form roundish, somewhat flattened; suture reaching to the apex, terminating in a sharp-pointed nipple; colour yellowish white in the shade, deep red or purple where fully exposed ; flavour good; ripe about the beginning of September. Often confounded with the Purple alberge. An examination of the glands will determine the difference ; for while in the Rosanna they are reniVOL II. form or kidney-shaped, they are, in the Purple alberge, globular or round. In this we have a good instance of the importance of making the glands a character of distinction. Of French origin. Synonyms-Petite rosanue, Alberge jaune, Peche jaune, St Laurent jaune of "Bon Jardinier." This is a very hardy peach, and has occasionally produced ripe fruit as a standard in good seasons and favourable places--indications of its suitableness for the open walls in cold localities.

Royal George. - Leaves doubly serrated; glands none; flowers small; flesh melting, juicy, yellowish white, very red towards the stone, and separating freely from it; size large ; form round; suture deep, extending beyond the apex; flavour excellent; ripe towards the end of August ; colour yellowish white where shaded, deep red where fully exposed to the sun. One of our best peaches, and one extensively cultivated both under glass and in the open air. An excellent forcer, but both in the peach-house and on the open walls subject to mildew, which all serrated-leaved varieties in general are. In consequence of its popularity, there are several sorts sold under this name; and we believe the humblest nursery in Britain would be found, upon inquiry, competent to supply a considerable number of trees of the Royal George. Synonyms - Early Royal George, Superb, Red Magdalen, Millet's mignonne, Lockyer's mignonne, Griffin's mignonne, French chancellor of some, Early bourdine of some, Madeleine rouge a petites fleurs, Double swalsh of some. The Royal George mignonne, and New Royal George mignonne of the nurseries, are so closely allied to the Royal George that their cultivation in the same collection may be regarded as superflu. ous. The smooth-leaved Royal George has been identified as synonymous with the Bellegarde (which see.)

Royal Charlotte.-Leaves deeply and coarsely serrated; glands none; flowers small; flesh melting, white, but very red at the stone; size above medium ; ripe beginning of September ; colour pale greenish white where shaded, marbled with deep red on the exposed side; form somewhat ovate ; suture of moderate delineation. A peach of great excellence, and may be almost known from most others by the coarse and deeply-serrated character of its leaves. Synonyms-New Royal Charlotte, Grimwood's Royal Charlotte, Early purple of Kew, Lord Nelson's, Lord Fauconberg's, Lord Fauconberg's mignonne, Madeleine rouge à moyennes fleurs, Madeleine à petites fleurs. According to Mr G. Lindley, this is made to be somewhat different from what he calls the Old Royal Charlotte, ("Horticultural Society's Transactions," vol. v. p. 540, and "Guide to the Orchard," p. 250.) If different, as in the case of many others equally nearly related, they are not worth growing in private gardens as distinct sorts. Both, if they must be considered distinct, are very excellent, and very closely allied to the Royal George, the chief difference being in its deeply and coarsely serrated leaves; and Williams' Early purple and Williams' seedling are scarcely different from the Royal Charlotte.

Of American peaches of high character we may $3 \mathrm{~T}$ 
enumeratethe following : Freestones- ${ }^{*}$ Brevoort, Cooledge's favourite, Druid Hill, * Early Tillotson, Farly York, Early sweet water, Haine's early red, La Grange, Morris's red rarevipe, Morris's white rareripe, President, Red rareripe, (different from Morris's rareripe,) Rareripe late red, * Snow, Van Zandt's superb, Scott's early red, Strawberry, Washington, Walter's early, White imperial, Bergen's yellow, Baltimore beauty, Crawford's early Melocoton, Crawford's late Melocoton, Columbia, Pool's large yellow, Red cheek Melocoton, Yellow rareripe. Clingstones-Blood clingstone, Heath, Large white clingstone, Lemon clingstone, Oldmixun clingstone, Orange clingstone, Tippecanoe, Washington clingstone. These are all highly recommended in "Fruits and Fruit Trees of America;" several have been introduced into Britain : we offer the list here as a guide to those who may wish to import peach trees from America. Those marked ${ }^{*}$ are cultivated by $\mathbf{P}$. Lawson and Sons, Edinburgh.

We have omitted many names of peaches which are to be found in our nursery catalogues, because so many are merely synonyms, several not worth growing, and a few represented better by those we have included in our list.

Shanghae peach.-Discovered by Mr Fortune in the north of China, and sent by him to the London Horticultural Society, in whose gardens it has fruited. Buds of this variety sent to Paris by the Society have produced fruit of a very large size and of exceeding high flavour. One fruit, ripened in a late peach-house in the Dalkeith gardens without fire-heat, measured 10 inches in circumference. Flowers large; petals deeply coloured ; glands reniform ; leaves crenated; colour pale yellowish where shaded, crimson red next the sun; flesh pale yellow, very deep red next the stone, to which it is somewhat firmly attached, but not to the extent to be ranked as a clingstone. Tree apparently healthy and a good bearer. Ripens about the same time as the Bellegarde. We apprehend that it must be regarded as a forcing peach in Scotland ; its great merits, however, justly entitle it to such accommodation.

The following selection, adapted for pot culture, is given by Mr Rivers, viz., Red nutmeg, ripening in July; Early Anne, Early Tillotson, Acton Scot, Grosse mignonne, Galande, Noblesse, Royal George, Pourprée hâtive, Reine des Vergers, a beautiful and hardy new peach; Barrington, Chancellor, Walburton admirable, Late admirable. These ripen nearly in succession, are all melting peaches, and produce fruit from July till the middle of October. With such of the French clingstones, or Pavies, as the Pavie de pomponne, Sanguine grosse admirable, \&c., which seldom ripen in our country, he thinks the peach season may be extended till the middle or end of November, by removing these late varieties in fruit from his orchardhouse in October to a warm forcing-house, where they may enjoy a gentle dry heat.

His selection of nectarines is as follows : Fairchild's early, Hunt's tawny, Elruge, Hardwicke seedling, Pitmaston orange, Violette hâtive, New white, Roman, Early Newington, Newington, and Late melting,
The names of the following peaches are pronounced as under: Abricotée-Ab-re-co-tay; Belle de Vitry - Bell-de Ve-tree ; Grosse Mignonne-Groce Mene-yon; Madeleine de Courson-Mad-lane de Coor-son ; Pavie de Pompone -Pah-vee de Pom-pone ; Pourprée hâtive Poor-pray hat-eve.

\section{SELECT LIST OF NECTARINES.}

Duc du Telliers.-Leaves crenated; glands kidney-shaped; flowers small; flesh melting, greenish white, reddish towards the stone, from which it parts freely ; size above medium ; form somewhat oblong and slightly compressed. Ripe towards the end of August. Colour pale greenish where shaded, deep red or purple where fully exposed to the sun; flavour excellent. Synonyms-Du Tilly's, Du Telliers, Duc de Tello. of French origin, and long cultivated in this country, but not prior to 1729 , as it is not mentioned in Langley's “ Pomona." . According to Rogers, introduced by M. Dutilly Gerrardet, a Dutch merchant, who settled at Putney in Surrey. Its delicacy in growth, and tendency to weaken itself by producing a profusion of bloom in the manner of Fairchild's, has prevented its being so generally cultivated as its merits deserve.

Elruge.-Leaves crenated; glands kidneyshaped; flowers small ; flesh almost white to the stone, from which it freely separates; size medium; form ovate; suture shallow at the base, deepening towards the apex. Ripe the beginning of September. Flavour excellent; stone ovate, pale, in which it is distinguishable from the Violette hative, whose stone is of a deep red colour ; colour palish in the shade, almost blood colour where fully exposed to the sun. One of the best, if not really the very best nectarine in cultivation; an excellent bearer, and one that forces well. It is an English variety, the origin and even the name of which is enveloped in great obscurity. The description we have given above refers to the Elruge figured in "The Pomological Magazine," t. 49, buit not to the Elruge described by Mr G. Lindley in "Guide to the Orchard," p. 288; and which latter evidently applies to the Elruge of Miller, Ed. 8, No. 2, and of Langley, in "Pomona," p. 102 , t. 29 , f. 3 , and the Elrouge of Switzer, p. 92. This latter Mr Lindley calls Miller's elruge, to distinguish it from the modern ones, and describes it as having "leaves doubly serrated, without glands; flowers small; fruit middle-sized, rather more long than broad; skin greenish yellow on the shaded side, but when exposed to the sun, of a dark red or purple colour ; flesh greenish yellow, melting and juicy, of very excellent flavour, and separates from the stone. Ripe the beginning or middle of August." This variety appears to be lost; and a comparison of the two descriptions will show that they are different fruit, more especially as in the one the glands are reniform, and in the other these characters are entirely wanting. Of the last variety we of course know nothing ; the other we hold to be the best of nectarines. The original appears to have been of English origin, raised from seed in the time of Charles II. by a 
person of the name of Gurle or Gourle, on whose name the name of the sort is a kind of anagram. The synonyms of the Elruge of our day areClaremont, Oatland's, T'emple's of some, Common elruge, Spring grove, Anderson's of some. The Downton is a cross between the Elruge and Violette hative, and Hoy's new seedling closely resembles the Elruge.

Fairchild's, or Fairchild's early, is so truly different from all others as to require no specific description. It is the earliest, although the smallest of its kind. Its bright yellow and scarlet colour, the abundance with which it is produced, especially if grown as a dwarf stan. dard, or even in large pots, renders it valuable, particularly in large establishments, where a long continuance of fruit is required. In such a structure as River's' orchard-house this prolific little fruit, as well as Hunt's tawny, which might be grown in the same manner, would both arrive at the greatest perfection. As both force well, they might be advantageously grown in pots, and fruited in a low span-roofed pit.

Golden.-We introduce this as, at least to us, a very scarce variety; and although not ranking in merit with the Elruge, it is of such a beautiful colour, and so different from all others, that in all large establishments a tree of it should be planted. Leaves crenated; glands kidneyshaped; flowers small; flesh yellow, firm, somewhat reddish towards the stone, to which it closely adheres; size above middle ; form somewhat oval, having a smallish acute nipple at the apex. Ripe the middle of September. Colour bright yellow or orange, to which fruit it has at first sight a much greater resemblance than to any of its natural family ; flavour, although not first-rate, pleasant and agreeable. It was formerly much cultivated, and is mentioned both by Miller and Langley. It is, as we have said above, now seldom met with. SynonymsOrange, Fine goid-fleshed. Pince's Golden nectarine is allied to this, but is rather larger, and about a week later in ripening, and has large instead of small leaves. Tree healthy, although not vigorous, remaining freer from insects and mildew than most others.

Hardwick seedling. - Glands reniform; size large; form roundish, inclining to oval; flesh melting, palish green, slightly red towards the stone, from which it separates freely. Ripe beginning of September. Colour palish green where shaded, deep violet-red where fully exposed to the sun. An English variety raised a few years ago at Hardwick House, in Suffolk, and has been described by Mr Rivers "as one of the best and hardiest of nectarines, and a very excellent bearer."

Hunt's tawny.-Leaves doubly serrated; glands none ; flowers large; flesh melting, deep orange, separating freely from the stone; size rather under medium; form oval, somewhat conpressed on one side. Ripe middle of August. Colour pale orange on the shaded side, coloured with deep red on the side most exposed to the sun. Synonyms-Hunt's early tawny, Hurt's small tawny, and probably Hunt's large tawny of the nursery catalogues may be the same also. This very desirable sort is deserving cultivation for its hardiness and early ripening. The origin of this variety is curious (vide article SponTaNEOUS REPRODUCTION, \&c., page 305.)

Newington.-Leaves doubly serrated; glands none; flowers large ; flesh yellowish white, very red towards the stone, to which it closely adheres ; a true clingstone; size rather above medium; form nearly globular; flavour of the highest character, and inuch esteemed by some when beginning to shrivel. Keeps well after becoming ripe, and bears carriage better than any other, even of the clingstone tribe. Ripe beginning of September. Colour palish amber where shaded, bright red, or almost scarlet, on the side most exposed to the sun, and often, in good seasons, interspersed with fine r'usset. An excellent fruit, and a good bearer. A very old variety mentioned by Miller, Hitt, and Switzer, and evidently from the synonyms of English origin. Synonyms-Smith's Newington, French Newington, Old Newington, Scarlet Newington, Sion Hill, Round, North's large, Rough Roman, Red Roman of some, Anderdon's, Anderdon's round, Anderson's of some; the only French synonyms being Brugnon, de Newington d'Angleterre.

Newington early.-Leaves doubly serrated; glands none; flowers large ; flesh greenish white, becoming exceedingly red towards the stone, to which it adheres closely; size rather under medium; form somewhat oval; slightly compressed on one side, furnished with a small nipple at the apex. Ripe latter end of August. Colour palish green; on the side most shaded bright red, approaching to scarlet, and mottled over with a darker shade of colour where fully exposed to the sun. Like the last, of English origin. Synonyms-New early Nexington, New dark Newington, Early black Newington, Lucombe's black, Lucombe's seedling, Black, Early black. This is also an excellent fruit, and preferable to the last in point of flavour, as well as ripening somewhat earlier.

Pitmaston orange.-Leaves crenate; glands globose ; flowers large; flesh melting, deep orange, with a circle of bright crimson next the stone, from which it parts freely. Size above medium; form almost heart-shaped, the apex terminating in an acute nipple. Kipe beginning of September. Colour rich yellow on the shaded side, dark brownish red where most exposed to the sun. Of English origin, having sprung from a kernel of the Elruge, sown by J. Williams, Esq., of Pitmaston. Tree very hardy, and an excellent bearer, well suited for the coldest localities nectarines are grown in. Synonyms -Williams' seedling, Williams' orange.

Red Roman.-Leaves crenate ; glands kidneyshaped; flowers large ; flesh greenish yellow, very red towards the stone, to which it closely adheres. Size very large for a nectarine ; form roundish, somewhat compressed. Ripe middle of September. Colour greenish yellow where shaded, deep red or purple where most exposed; flavour excellent; one of our very best, largest, and longest cultivated sorts (supposed above two hundred and fifty years). Notwithstanding this sort has been described as a clingstone by almost every pomological writer since the days of Parkinson, it is strange that in most 
collections a freestone is found under this name. Synonyms-Roman,Old Roman,Brugnon musqué, Brugnon violette musqué of the French, from which country it was first imported.

Violette hâtive.-Leaves crenate; glands kidney-shaped; flowers small; flesh melting, pale yellowish green, tinged with red near the stone, from which it parts freely. Size medium; form slightly conical. Ripe beginning of September. Colour yellowish green where shaded, dark purplish red where fully exposed to the sun ; flavour excellent. One of our very best nectarines, either for forcing or the open wall. Of French origin, but long cultivated in this country. English synonyms - Large scarlet, New scarlet, Lord Selsey's elruge, Hampton Court, Aromatic, Vermash of some, Violet, Early violet, Violet musk, Violet red at the stone, Early Brugnon, Brugnon red at the stone. French synonyms-Brugnon hâtive, Petite violette hâtive, Violette d'Angervillieres, Violette musqué.

White, new.-Leaves crenate; glands kidneyshaped; flowers large; flesh greenish white, separating freely from the stone; size medium; form globose; ripe beginning of September; colour very pale yellowish green, becoming almost white where much shaded; flavour excellent. Of English origin, said to be from a seed of the old white nectarine, an inferior sort sown by the Rev. Mr Neate of Whitstone, near London. One striking peculiarity between the old and new white nectarine is stated by $\mathrm{Mr}$ G. Lindley, that the former "could never be made to take on the mussel stock, while the latter requires no other." Mr Thompson states in "Fruit Cat. of Hort. Society," p. 108: "In a favourable soil and warm exposure both sorts of white nectarines acquire excellent flavour, under other circumstances only indifferent;" almost implying that they are delicate sorts. We have found both extremely hardy and healthy, both in England and Scotland ; the New White, Red Roman, and Catherine peach having withstood the severe spring frost of 1849,1850 , which not only stripped one whole wall in the gardens at Dalkeith, but very many both in England and Scotland. We have remarked also, over a period of thirty years, that the white nectarine is less subject to insects and diseases than any other.

Amongst the newly-introduced nectarines, the following hold a prominent position. Their merits, however, before they can be confidentially recommended, will require a longer period of trial.

The Barker nectarine. - Fruit large ; form obovate ; colour dark red next the sun, palish yellow green on the shaded side; flavour rich, but less aromatic than the Violette hative; glands of the leaves kidney-shaped; flowers small. Introduced by Mr Barker from Snedia, a town in Syria, about eleven years ago. The same gentleman also introduced the much-talked-of Stanwick nectarine from the same country. Both varieties are, however, very distinct, the former having bitter kernels, while those of the latter are sweet. Mr R. Thompson, in "Journal of the Horticultural Society," vol. vi. p. 25, relates the following curious circumstance connected with the original tree received at Chiswick: "It was noted as producing leaves with globose glands, large flowers, and peaches of little merit. Subsequently, one small twig was observed having leaves with reniform glands. Buds from this were taken, and worked on a tree against the south wall. Fruit of it has this year (1851) been obtained for the first time, and proven to be the nectarine!

Stanwick nectarine.-This fine fruit has turned out, after a very fair trial, to be completely unsuited to the climate of Britain; for we find that even in the Royal Gardens at Frogmore, and when planted in a good situation, it has not ripened even in the unusually fine season of 1852. The same want of success has occurred at other places in the neighbourhood of London. When planted by Mr Rivers in one of his spanroofed orchard-houses, alongside of the Elruge and Violette hative, neither particularly early sorts, it did not ripen along with them in the first and second week of September, their usual season ; but the fruit remained hard and green till past the middle of October, when it cracked, and did not ripen at all. It must, therefore, now be regarded as a forcing-house fruit, to which its great merits as to flavour entitle it. As it appears to be a very late variety, and of delicious flavour, it may, if crossed with such as the two varieties named above, become the parent of a race of high-flavoured late nectarines, and, although requiring fire-heat to ripen them, if planted in a house by themselves, extend the season of the fruit to a much later period of the year than at present.

American varieties of nectarines of high reputation, which it might be advantageous to introduce to our forcing-houses. On our open walls, the want of climate will ever preclude them from general cultivation in Britain :-

Boston.-According to Downing, "the largest and most beautiful of all nectarines." Raised from a peach-stone. Fruited at Downton Castle, Herefordshire, by Mr Knight, in 1823, and in the collection of the London Horticultural Society's gardens.

Bloomfield-Glands obscurely reniform ; size large; form roundish; flesh yellow, and adhering closely to the stone. Ripe end of September. Colour dull yellow where shaded, dingy red where fully exposed to the sun. Flavour second-rate.

The Americans do not seem to excel in nectarines as they do in peaches. Those described by them are chiefly our English and French sorts.

The names of the following nectarines are pronounced as under :-Brugnon Violet Musqué -Brune-yon Ve-o-lay Meus-kay ; Brugnon Musqué - Brune-yon Meus-kay; D'Angleterre Dahn - glet - are ; Duc du Tellier - Deuk - du Tel-yay.

Diseases.-The peach and nectarine, as may be supposed, when transplanted from the genial climate of Persia to the variable one of Britain are liable to various diseases, the effect of climate, and wholly unknown in their native country. Of these the most serious are-canker, mildew, gum, honey dew, wrinkling of the foliage, and blotches on the young wood, as if 
seared with adhesion. For gum and canker abundance of remedies have been prescribed; as both are, however, generally occasioned by being bruised or wounded in some way or other, and not unfrequently by injudicious pruning, and probably often by being planted in a wet uncongenial soil, the best remedy is to avoid as much as possible these causes. In regard to mildew, some sorts are more liable to its attacks than others, such as the Royal George, Royal Charlotte, \&c. The appearance of this inveterate enemy, and also of its congeners, was attributed by Mr Knight to a derangement in the foliage, caused by the leaves absorbing an excess of moisture from the atmosphere under certain conditions. "It has long appeared to me," he says (in "Horticultural Transactions," vol. i. p. 86), "to be the want of a sufficient supply of moisture from the soil, with excess of humidity in the air, particularly if the plants be exposed to a temperature below that to which they have been accustomed. If damp and cold weather in July succeed that which has been warm and bright, without the intervention of sufficient rain to moisten the ground to some depth, the wheat crop is in general much injured by mildew. I suspect," he says, "that in such cases an injurious absorption of moisture by the leaves and stems of the plants takes place ; and I have proved that, under similar circumstances, much water will be absorbed by the leaves of trees, and carried downwards through their alburnous substance, though it is certainly through this substance that the sap rises under ordinary circumstances. If a branch be taken from a tree when its leaves are mature, and one leaf be kept constantly wet, that leaf will absorb moisture, and supply another leaf below it upon the branch, even although all communication between them through the bark be intercepted; and if a similar absorption takes place in the straws of wheat, or the stems of other plants, and a retrograde motion of the fluids be produced, I conceive that the ascent of the true sap or organisable matter into the seed-vessels must be retarded, and that it may become the food of the parasitical plants, which then orily may grow luxuriant and injurious." Few plants are exempt from its attacks, although somesuch as the peach, vine, rose, pea, hop, \&c.are more liable to be seriously injured by it than others. It is not at all improbable but that every species of plant has its own peculiar species of both vegetable and animal parasites, although the same species may not unfrequently be found on different plants; but in general they are found in greater abundance on the plants to which they appear to have some affinity. Thus we have the Erysiphe communis, the mildew of the pea, the Botrytis effusa on the spinach, the Acrosporium monilioides and Botrytis destructor on the onion, Ecidium berberides on the berberry, Cylindrosporium concentricurn on the cabbage, and the recently discovered Oidium Tuckeri, so destructive to the vine, and Vidium crysiphoides, the mildew of the peach. That the various species of mildew are composed of innumerable myriads of very minute fungi is, we believe, admitted on all hands; there appears, however, to be as yet some diversity of opinion as to their origin. Desaisne and Leveille, eminent French naturalists, assert that mildew always originates on the external surface of the plants, and that only on subjects whose tissue is in a previously diseased state. The majority of British cryptogamists equally assert that it is first developed within the tissues, and that it afterwards makes its way through the stomata. The roots of the minute species of fungi, which constitute what is usually called mildew, penetrate the pores of the epidermis of the leaves, and sometimes the very young wood of plants, as exemplified in the case of the peach ; to support themselves they exhaust the plant on which they grow, by robbing it of its juices and intercepting its respiration. "Every specimen of these fungi," says the compiler of "The Cottage Gardener's Dictionary," "emits annually myriads of minute seeds, and these are wafted over the soil by every wind, vegetating and reproducing seed, if they have happered to be deposited in a favourable place, or remaining until the following spring without germinating. These fungi have the power of spreading also, by stooling or throwing out offsets. They are never absent from a soil, and at some period of its growth are annually to be found upon the plants liable to their inroads." There are no doubt particular states of the atmosphere more favourable to the appearance of mildew than others ; for it has been generally observed to make its appearance more in a dry than in a moist state, which leads some to suppose that the dry air, acting upon the delicate surface of vegetable tissue, is favourable to its existence. Practically, we find its attacks on the peach and other fruit trees more severe in dry springs than in those that are more genial and humid-on turnips, in dry summers and autumns, than in those that may be considered even wet-in dry localities more than in those where abundance of rain falls. These opinions are somewhat strengthened by the well-known fact, that on wall-trees kept thoroughly syringed with clear water, and on crops of pease and turnips repeatedly watered, mildew seldom makes its appearance. Applying clear water copiously during dry weather, and with considerable force, by a syringe or small garden-engine, will, in a great degree, prevent its appearance ; should it, however, make head, powdered sulphur applied upon its first appearance will completely subdue it; and as a precautionary measure the peach trees should be sulphurated even before its appearance. How the sulphur acts has not as yet been very satisfactorily explained. As it is insoluble in water, there can be no advantage in following the general rule of syringing with water in which sulphur has been steeped, unless the crude particles are held in suspension, so as to be distributed equally over the leaves and young shoots which are the parts affected. The fumes of black sulphur are found a complete remedy, but require to be most carefully applied, else the remedy would become worse than the disease. Mons. Grison has suggested the application of hydro-sulphate of lime, which is obtained simply by boiling $1 \mathrm{lb}$. of flour of 
sulphur and an equal volume of quicklime in a glazed earthen vessel, with five pints of water: it should be allowed to boil ten minutes, and kept constantly stirred, after which it should be allowed to settle, and the clear liquid to cool; and after diluting with a hundred pints of water, it should be applied to the trees by the syringe or engine. In peach-houses, dry sulphurating is the safest, as there is danger in washing the flues or hot-water pipes with either sulphur alone, or combined in this way with lime; but in either case the heated surface must not exceed the temperature of $212^{\circ}$.

The origin and nature of honey dew are even more undetermined than of mildew, some believing it to be an exudation from the leaves, caused by the puncture of aphides; others, that it is a species of excrement passed by them; and many, that it is an exudation of superabundant saccharine matter, caused by a heated and dry state of the atmosphere. Whatever its cause may be, it is highly injurious, as it not only covers the surface of the leaves with a thick glutinous substance, but its adhesiveness causes dust and other filth to accumulate upon them, till at last their pores of respiration are completely sealed up, and their vital functions become suspended. Copious syringing is the best remedy.

Blistering, wrinkling, or curling up of the leaves, is seldom met with in peach-houses; on the open walls it is of not unfrequent occurrence. The very circumstance here referred to certainly points pretty nearly to the cause; namely, a want of reciprocity between the action of the roots and leaves, the latter drawing more rapidly on the former than they are adequate to. Blistering usually takes place when the leaves are from half to nearly fully grown, at which time their sap-vessels are enlarging rapidly to contain the supply expected from the roots, and which enlargement of vessels is encouraged in consequence of their being placed in front of a brick wall, and stimulated by the reflected heat from it and the warm shelter it affords, particularly in bright sunshine. In fact, the leaves at that time are growing rapidly, while the roots, on the other hand, are fighting their way in a cold, nay, probably also a wet substratum, almost paralysed from want of heat, and hence unable to send into the leaves that supply of sap they require to enable them to develop so rapidly as the position they are placed in would otherwise enable them to do. Their sap-vessels, not being charged with sap so rapidly as they are forming, and themselves acted upon by the heat of the wall, perhaps not less than $80^{\circ}$, while the roots are not above $45^{\circ}$, begin to collapse and pucker up, and assume the appearance so well known as blister, curl, \&c. This, like many other diseases, is laid to the charge of the east wind, while, in fact, it has nothing to do in the matter. Trees in properly prepared borders, either thoroughly drained or vaulted, the roots kept near the surface, and the soil unpolluted with doses of manure, very seldom show a curled or blistered leaf.

Blotches on the young wood may be traced partly to the same cause, and also to the borders being made too rich. As soon as the shoot appears affected, it should be cut out without loss of time somewhat below where the disease has appeared; for on cutting the shoot through, even where the bark may appear sound, the disease will be found to have extended downward, and may readily be seen by the discolouration in the wood.

Insects.-The red spider, Acarus telarius, fig.
Fig. 228.
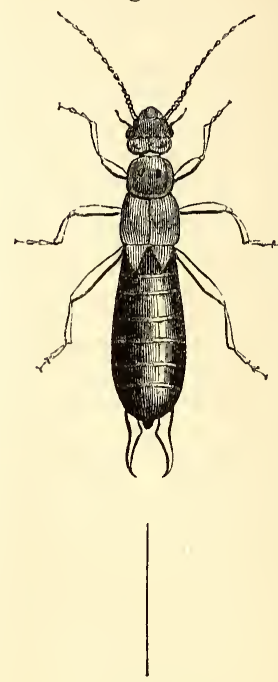

THE FARWIG. 22 , is always destructive in peach-houses when a high dry temperature is maintained. On the open walls it is less troublesome, unless in dry hot summers. Its appearance, in both cases, indicates sufficiently that the trees are sadly neglected as regards syringing. For description and means of destruction, vide p. 77.

Two species of thrips, Thrips adonidum and T. ochraceus, are often injurious to the peach. For description and means of destruction, vide fig. 21, p. 76 .

The earwig, Forficula auricularis Linn., fig. 228, is very injurious, not only to many flowering plants, but to fruit trees also. Amongst the latter, the peach and apricot are often attacked when the fruit is ripening; and so also, in some localities, are the pearand apple. Its habitation is usually under the old bark of fruit trees, in the hollow stems of decayed ones, and under stones and elsewhere, wherever there is a dry and dark abode to be found. Its attacks are upon the ripening fruit, which it devours internally, often leaving the empty skin seemingly as perfect as if its pulp were left entire. It is too well known to require a lengthened description. When fully grown it measures from eight to ten lines in length of body, including the forceps-like appendage at the end of the abdomen. It, however, varies according to age and sex. The body has very short wingcases, which conceal the wings, whicl are folded up both length and breadth ways. The body is destitute of hair, of a light brown colour, and about two lines in breadth; the only practicable mode of suppression is catching the insect in traps, of which the following are the most effcient: small empty flower-pots stuck inverted amongst the branches of the trees, or elevated on the poles or supports used for dahlias, to the flowers of which they are very destructive, having a little dry moss placed within them, in which the insect takes shelter during the day. If these are examined daily, a number of the enemy will be discovered, and should be shaken out into a watering-pot half filled with water, 
and afterwards destroyed. Any sort of hollow tubes, such as bean-stalks or the hollow stems of Jerusalem artichokes, are excellent traps for them, if stuck amongst the branches ; the sweet pith entices them into these, as well as the obtaining shelter from the sun: from these they may be blown into a pot of water. They also take shelter between the folds of paper, in moss, under rags, or, indeed, in any dry covering. There is no easy mode of preventing them from ascending the walls or espaliers to feed upon the fruit, but several ingenious contrivances are in use, fitted with water, oil, or tar, placed round the stem of the tree, which effectually prevents their ascent by creeping up (vide Dahlia in FLower GarDen); but as they are furnished with wings, none of the latter prevent their ascent by flying. The trapping system is the best, and, if persevered in, will soon rid the trees of their intrusion. The earwig often attacks the blossoms of the peach tree as well as those of flowering plants; they have been destroyed in vast numbers by mixing oxide of arsenic with finelypounded loaf-sugar and wheaten bread, rubbed finely down in equal parts, and placed in hollow tubes distributed over the trees.

The figure of 8 moth, Noctua (Episema) cceruleocephala Autor, Bombyx cceruleocephala Linn., of all trees prefers the peach, almond, and apricot. For figure, description, \&c., of this garden pest, see page 443 .

The peach or poplar saw-fly (Tenthredo populi Linn.), in general appearance and at a little distance is not unlike the common house-fly, but somewhat larger, and, like all hymenopterous insects, has four wings. The greater part of the body is black, but towards the abdomen whitish square incisions appear, extending from both sides towards the middle in almost parallel lines. The palpi and tibiæ are yellow, as are also the feet; thighs black; antennæ simple and jointed. The fly makes its appearance in April, about the time the leaves first come forth. The female lays her eggs at different times, to the number of thirty or forty, which she arranges in rows on the surface of the leaf. The process of hatching occupies only a few days if the weather is fine, and from them a greenish-white grub is produced, and these shortly after spin themselves a web, under which they take shelter. They soon after their birth commence devouring that portion of leaf after leaf which best suits their palate, for, like some other insects, they never consume the entire leaf. Their period of grub existence extends to five or six weeks; they present the appearance of lightgreen grubs with black heads, having three pairs of fore-feet and one of hind ones. When full grown they drop to the ground and form for themselves a dark-brown covering or case, and in this they remain an inch or two under the surface of the earth until spring. The best mode of subduing this injurious insect is to gather off the surface-soil under the trees, particularly along the bottom of the walls, and have it charred or burnt. This should invariably be done as soon as the leaves have fallen. The pale yellow eggs may also be easily detected in spring, immediately after they are laid on the points of the young leaves; as they are laid pretty closely together, great numbers of them may be thus destroyed. The trees, when much infested by this insect, present the appearance as if they were covered with cobwebs. The specific name Populi is not very appropriately given to this insect, which has led Mr Westwood to remark, that there must either be considerable diversity in its habits, feeding as it does at one time on the poplar and at another on the peach and apricot, or the specific name must have been applied improperly - and if so, it should be changed to prevent confusion.

More than one species of Aphis attack the peach-tree foliage, whether in the peach-house or on the open wall. A. rumicis, one of the commonest of the genus, was last autumn (1853) unusually numerous and destructive, and, in consequence of its appearing at the same time as the cholera, has been supposed by some to have an intimate connection with that disease, and hence has been denominated the cholera-fly. The Entomological Society has, however, lately decided that the hot, moist, stagnant state of the atmosphere which prevailed during the end of September and beginning of October, and which was favourable to the spreading of that disease, induced also an extraordinarily rapid development of the Axhis rumicis, as well as of other species of the genus, the winged females of which attacked the leaves of the peach to a much more than usual extent, stripping the trees of every leaf even before the fruit was ripe. Some entomologists, however, assign one particular species $(A$. persicce) as peculiar to the peach and nectarine. Fumigating with tobacco is the usual and most effectual mode of clearing the trees or houses of them. This remedy should not be delayed until the insects make their appearance, nor should fumigation take place during the flowering of the peach. A slight application administered once or twice before the flowers begin to expand has been often known to keep the trees free of them during the season. T'The aphides which appear on the peach, like all others of the tribe, are produced from eggs laid during the previous autumn; the succeeding broods, as we have elsewhere remarked, are viviparous, and are produced with inconceivable rapidity. The old practice, therefore, is good, of washing the young wood, and scrubbing the old branches during winter with water, as by that means many of the eggs are removed. It appears to be of little consequence whether clean water be used, or that into which soft-soap, tobacco liquor, \&c., has been incorporated; for neither can have any effect upon the vitality of the eggs. Friction alone removes them. As they take shelter about the material by which the trees are secured to the trellis or wall, it is well to remove and burn these annually. They also find shelter in the trellises, and about the chinks in the walls; it is well, therefore, to paint the one and colour the other with hot limewash before forcing commences. If laying on a mixture of sulphur, snuff, hot lime, \&c., making them adhere by means of soft-soap to the branches, be of advantage, it must be attributed 
more to their effects upon the aphides as they emerge from the egg than to any effect they have on the egg itself. However, as prevention is better than cure, such an operation should not be neglected. In fumigating, the leaves of the trees should be quite dry: when wet, many of the insects will be found secreted under them, and so escape the tobacco fumes. A still evening should be chosen for the operation, and every opening in the peach-house securely closed, to prevent the wasteful escape of the smoke. Of fumigating instruments there are several. Brown's is probably the best. That most in use, however, is simply a hard-burned flower-pot of 6 or 8 inches diameter, into which a few live embers are put, and over them a handful of damp unrolled coarse tobacco or of tobacco-paper. A small hole being cut in the side of the pot near its bottom, the nozzle of a pair of common bellows is applied; and by blowing the air in, slow combustion takes place, accompanied with a large quantity of smoke. A little damp hay, old dried pot-herbs, or moss, is often added along with the tobacco, to increase the quantity and density of the smoke. Care must be taken that flames do not proceed from the pot, else the foliage might be injured. Where the house or pit can be conveniently covered with canvass during the operation of fumigating, and this allowed to remain on all night, few of the aphides will escape. In the morning the house may be freely ventilated, and the trees subjected to a copious syringing, directing the water with force both to the right hand and also to the left. Some excellent cultivators never fumigate at all, but depend on copious syringing for the prevention of aphides.

Peach trees on the open walls are much less capable of being fumigated, unless at a very great waste of tobacco, however well they may be covered over during the operation. Other means are consequently had recourse to, such as burning gas-tar, on a still day, in vessels arranged along the bottom of the wall, the fœtid black smoke proceeding from it destroying the insects; wetting the leaves, and dusting them over with Scotch snuff or finely-powdered dust of tobacco : the damp on the leaves acts on the snuff or tobacco, and is instantly fatal to the tender aphides. Finely powdered quicklime dusted over the trees, when dry, is attended with beneficial effects, and may be applied by Curtis's lime-duster, or any of the sulphurators now in common use ; or, in default of these, place the lime in a small canvass bag of thin material, and dust it over the trees. Strong tobaccowater applied with the syringe is also very efficacious. Four ounces of tobacco to a gallon of water, allowed to stand for a week, and frequently stirred and squeezed, the better to extract the narcotic quality, will be strong enough should half a gallon of water be added at the time of using.

The scale or turtle scale (Aspidiotus conchi- formis), fig. 181, frequently found on the wood, and sometimes on the leaves of the peach, belongs to the numerous and apparently little un. derstood family of Coccus, of which further notice will be taken under the articles GRAPEvine, Pine-APPLe, \&c. English entomologists appear as yet to have made no great progress in the examination, nomenclature, and identification of this troublesomefamily. Bouche in his " Garten Insecten," and " Naturgeschichte der Insecten," has thrown a good deal of light on those injurious to the gardens in Germany; and as we suspect them to be all of exotic origin, it is probable that those he has described may be identical with such as are the pests of our own gardens. Neither of Bouche's works has as yet been translated, and therefore they are unavailable to the English reader. Regarding their suppression, as they are comparatively large, and attached to the bark of the peach tree, they are easily recognised during winter, and more especially in spring, when the trees begin to become stimulated into growth. At that time, and for some weeks afterwards, they emit a sweet sort of matter, which Bouche takes to be their excrement, and which, falling on the foliage, produces, effects similar to that of the honey dew (which see). This matter, he also remarks, attracts ants, which not only feed upon it when discharged, but also squeeze the cocci to obtain a larger amount of it, and thus aid us by killing numbers of the scale. They are easily rubbed off by the hand; but even in removing the adult insect the eggs may be left attached to the bark, or hidden on various parts of the trees. We have found spirits of vegetable tar, when applied during winter, a complete remedy against those secreted on the older branches; but as this penetrating liquor cannot be safely applied to the young wood of last year's growth, many consequently escaped. It is uncertain at what time their eggs are laid; therefore, presuming that this does not take place till they have nearly arrived at their full size, it will be well to remove them during winter, or as early in spring as they can be discovered by the eye.

The plum-tree tortrix, Tortrix (Carpocapsa) waberiana Autor, is often injurious to the peach on the open walls. - Vide art. Plum.

The oblong weevil (Curculio oblongus) appears in May, and often attacks the leaves of the peach.-Vide page 436 .

The black vine-weevil (Curculio sulcatus) attacks the peach as well as the vine.-Vide art. Grape-vine.

Hylobius abietis, one of the pine-tree weevils, often also attacks the leaves and young shoots of the peach.-Vide art. PLUM.

The European and Asiatic names of the peach and nectarine are-Le pecher, in French ; Der pfirschenbaum, in German ; Persikboom, in Dutch ; Persico, or Pesco, in Italian; El melocoton, in Spanish ; Scheptals, in Russian; Baratsk fa, in Hungarian. 


\title{
CHAPTER VII.
}

\author{
THE APRICOT.
}

THe native country of the Apricot is unknown. It is found wild in countries remote from each other, but whether introduced to any of them, or whether indigenous to all, is a matter we are entirely ignorant of. Thunberg states it to be a low-spreading Japanese tree; Grossier, that it covers the mountains to the west of Pekin; Professor Pallas, as indigenous to the mountain range of the Caucasus ; while Regnier and Sickler assign it a parallel extending between the Niger and the Atlas, and others give Armenia for its place of birth - a circumstance which Linnæus availed himself of, by naming this species of prunus Armeniaca. Lamark, in "Illustrations des Genres," has founded a genus under the title of Armeniaca, and dubbed the apricot Armeniaca vulgaris. The native country given in the "Hortus Britannicus" is the Levant, and the date of its introduction into Britain, 1548. It appears to have found its way into Italy at an early period, and from thence was brought into England some time prior to 1560 , as we find it noticed as being cultivated in 1562 on the authority of 'Turner, in his "Herbal;" and again, Hakluyt asserts that it was brought into England from Italy by Wolfe, a French priest, and gardener to Henry VIII.

The apricot is evidently a native of a warm country as well as its near ally the peach, and hence requires the warmest situations we can give it, so as to bring its fruit to full perfection. Daily experience proves this, as several sorts of peaches will thrive in very cold parts of our island, where the apricot, although it will live, seldom ripens its fruit. In connection with want of sufficient climate, we may state that few varieties have been raised from seed in Europe, compared with even the peach ; while we know the Chinese possess many, few of which have been introduced liere; and it is probable that in such countries as we have named above many, and possibly some very superior sorts, do exist, which may have escaped the eye of travellers. Were we, however, to reason à priori, the introduced Musch-Musch from Upper Egypt has not excelled our old and excellent Moorpark; and it remains yet to be proved whether the far more recently received Early sweet-kernelled Kaisha, and some others from Syria, may come up to the anticipations formed of them. It may, however, prove with them as with the far-famed VOL. II.
Stanwick nectarine-deficiency of climate may unfit them for open-air culture here.

In regard to the number of sorts, the following data may be more amusing than instructive. Parkinson names six as grown in his time, 1629 ; Rea, seven in 1720; Gordon of Fountainbridge, near Edinburgh, seven in 1774 ; the Luxemburg Fruit Catalogue, fifteen in 1800 ; the Fruit Catalogue of the Horticultural Society in 1842 , seventeen ; many of the nursery catalogues fourteen; while that of the Messis Lawson of Edinburgh extends to seventeen. In "The Fruits and Fruit 'Trees of America" Downing describes sixteen, and Mr Rivers gives fifty-nine.

The best classification we have seen is that of Thompson, who divides the whole into two classes-viz. 1st, those with bitter kernels ; $2 \mathrm{~d}$, those with sweet kernels; and these again into sections - viz. fruit small ; fruit large; flesh parting from the stone; flesh adhering to the stone; channel of the stone closed up; channel of the stone pervious.

Propagation.-The apricot, like the peach, is propagated by seed when the object is to obtain new or improved varieties; and the same process is followed in both. It is occasionally increased by grafting, but more generally by budding, on the mussel or plum stock in the case of ordinary varieties; but as regards the Moorpark, an apricot stock is preferred by many, who say, that trees so wrought are exempt from a peculiar disease to which that fine variety is liable-a dying away of whole branches, for which no very satisfactory cause has been as yet assigned. Apricots intended to be planted as standards or riders are usually budded on the St Julian plum-stock, because that stock, being strong, produces excellent stems. The operation of budding should be performed from the middle of June to the latter end of July; later in the season they do not succeed so well. The buds of apricots should, if dwarf trees are intended, be inserted 6 or 8 inches from the ground; riders, or dwarf standards, at the height of from 4 to 5 feet. It is often the practice to rebud-that is to say, when one variety is budded on a stock, to bud the same or another variety upon the shoot so produced. The intention of this is to have dwarf trees; but its advantage is not very apparent, unless it be with a view to produce trees for pot-culture. 
Planting the apricot.-Much of what has been said of the peach and nectarine is applicable to the apricot also. The Brussels and Breda, believed to be the same, are the hardiest of this class, and are sometimes planted in the south of England as standards. One of the latter existed some years ago in a garden in the village of Esher, Surrey, which had attained a very large size, and produced enormous crops annually. This, we would say, is adapted to walls in the coldest localities, where other and finer kinds would not succeed. On east and west aspects the others succeed well in the southern localities of England, but in most of Scotland they require, and are justly entitled to, a southern aspect. A light rich loamy soil is most suitable for the apricot, if it is natural, or only slightly aided with well-decayed manure; if too rich, it causes too great a production of young wood, which in cold situations, in particular, is disadvantageous, as such wood would seldom ripen, so as to become of itself productive of fruit. A less exciting soil will induce the production of innumerable fruit spurs and buds, and on them fine fruit close to the wall is produced, and on such the Scotch gardener should in the majority of cases rest his hopes. In selecting trees for planting, those of from two to three years trained in the nursery are the best, and those should be selected which have two branches on each side with a leading one in the centre; and it is desirable that the side branches on each side be of equal strength, and that the stock be free from canker or disease. In regard to the season of planting, Forsyth advocates early planting, even to an extent beyond what we have endeavoured to inculcate, in our advocacy for performing that operation even earlier than the majority of writers have hitherto done. He says August is the best season for planting, as soon as the leaves fall from the trees. It should, however, be understood that Forsyth was writing for the neighbourhood of London, he at the time being superintendent of the Royal Gardens at Kensington, the site of which is now to be sought for amidst a locality of Italian palaces. In good soils, permanent trees should be planted 20 feet apart.

Pruning and training.- The apricot is for the most part trained in the fan manner, and such we think the best form, and most suitable to the nature of the tree. Harrison and Forsyth recommend the horizontal mode; the former, however, recommending an elevation of $20^{\circ}$ at the extremities of the branches, which angle is to be elevated or depressed according to the luxuriance or weakness of the tree. Hayward trains the apricot as he does the peach, fig. 222, in what may be almost called the horizontal manner, arising from two main stems laid in at first nearly horizontally, and afterwards turned up to a more vertical position towards the points; and from these the branches are trained in a similar direction, choosing the shoots which proceed from the buds on the upper side of the branch, and not those arising from the under. Inclination and depression are recommended by him too, with a view to regulate the flow of sap, for on this much of his theory is founded. The season for pruning the apricot may be considered identical with that of the peach. Most of the varieties, with the exception of the Moorpark, bear their finest fruit on the young shoots of the preceding year, and for this reason a yearly stock of the bestformed and best-placed shoots should be laid in. This is necessary also to keep up a healthy state in the trees. They also produce fruit on natural spurs, but these should be retiined only when placed close to the wall; for although the fruit will set even on those of several inches distant from it, they seldom ripen in cold situations. The Moorpark mainly produces its fruit on short spurs of from two to three years' growth; these, therefore, should be encouraged. Many excellent pomologists prune and train their apricots as they do their peaches-that is, by laying in annually a store of young wellplaced wood, shortening them more or less according to their strength; they at the same time encourage the growth of spurs close to the wall on the older branches, which latter mode is advisable in cold climates, for reasons given by Knight (ride Peach).

In shortening the young wood, care must be taken to cut at a wood-bud, or at such as are triple, so that a leader may be secured to draw nourishment to the fruit behind it. Where, as is often the case, the whole young shoot is covered with fruit-buds, and has only one, and that the terminal one, a wood-bud, then such shoots are not to be shortened at all, unless there be urgent necessity for having young wood in the exact place: when this is the case, the shoot should be cut back beyond all the fruitbuds, as at its base dormant wood-buds may be situated, which, if cut back to, will push and fill the place with one or more shoots according to its strength. In securing a supply of young wood, great care should be taken to obtain a shoot as near to the origin of the branch from which it proceeds as possible, both in summer and winter pruning. In regard to shortening, the most vigorous may have one-fourth of their length reduced; if of medium strengtl, one-third; and if very weak, one-half or even more; and in cutting, make the slope in the same direction as the bud, and about a quarter of an inch above it. Spurs should be carefully examined to prevent their elongating; if they consist of three or four fruit-buds, cut them back to two. If the spur consists of only two buds (a wood-bud and a fruit-bud, the former being the lowest), cut the spur close to the latter; if there be only one wood-bud, and it be the lowest, cut the spur down to that; but if it be the uppermost, let it remain its whole length, and the second bud will push a shoot next year, whilst the lower one will form a fruit-bud, which, at the following winter pruning, is to be cut off entirely. In regard to the production and regulation of such buds, Harrison justly remarks : "Sucl buds will be produced at the lower part of those shoots that afterwards push, and are shortened to three or four buds in summer pruning, and to two in winter pruming, also upon slort lateral spurs, placed close to the old wood. When there is a sufficient supply of such lateral spurs, all the shoots which have been produced and 
shortened, as before described, may be cut clean away at winter pruning, with the exception of a leading one. After those spurs have borne fruit three years, let each be cut down to the lowest bud or to an embryo, for a new succession." The young gardener can scarcely consult a better authority on the management of the spurs of trees than Harrison's "Treatise on Fruit Trees," $2 \mathrm{~d}$ edition : the descriptions are truly practical, and the illustrations - which, by the way, are not remarkable for artistic taste-convey the ideas of the author even better than his letter-press descriptions. Under even good management, the apricot is apt to become in time crowded with worn-out branches; these must be removed at the winter pruning. This state of things is most likely to occur where too great dependence has been placed on the spurring system, and where little attention has been directed to laying in a constant supply of young wood.For Forcing the apricot, see post.

Protecting the blossoms. - The apricot blossoms early in the spring, and often in the end of February and beginning of March, and is often subjected to $10^{\circ}$ or $15^{\circ}$ of frost; it therefore requires protection. The means recommended for the peach are applicable to it.

Thinning the fruit.-As soon as the fruit is fully set, and about the size of a small marble, thinning should commerice, as they are apt to set in groups. These should be thinned so that not more than two or three are left in each cluster. They are in this state excellent for tarts, and should be appropriated to that use. After the stones are fairly formed, another thinning should take place, when the crop should be left at frum 6 to 10 inches fruit from fruit, according to its natural size, and the purpose it is intended for when ripe. The Masculines, and other small-growing sorts, which are chiefly used for preserving, may stand at the lesser distance, while the Moorpark, and similar large varieties, should stand at the greater. To over-crop the trees is destructive to their heaith, and, besides, the fruit can never attain its full size, flavour, or excellence.

Gathering and protecting the ripe fruit.-Apricots should be gathered before they are dead ripe, else they are apt to become dry and mealy. To enhance their flavour in wet seasons, portable projecting wooden copings should be placed over them, to keep the trees and fruit dry. To protect them from wasps and flies, Hawthorn's octagon netting should be suspended from the coping, and kept on during the last stage of ripening. Sometimes one side of the fruit becomes soft and ripe before the other; in such cases it is well to gather it, and remove it on trays to a warm dry pine-stove, turning the unripened side uppermost, and exposed freely to the sun and light. A day or two in this position will ripen the hard side, and make the whole in fit condition for the table.

Summer pruning. - Early in May the trees should be gone over, rubbing off with the finger and thumb all foreright, irregular, or misplaced shoots when in a young state, retaining only those that are of medium growth, and are required to be trained in for next year's bearing. These shoots should be secured to the wall as they advance, placing them in the spaces between the older branches, and where these are close together, training the young wood over the naked branches. Shoots which are left on for filling the wall, particularly in the case of young trees, should, when they are growing too luxuriantly, be topped early in June, which will cause them to throw out lateral or side shoots, which, if the topping be done sufficiently early, will ripen and become fruit-bearing ones. A judicious attention to disbudding, topping, and regulating the quantity and position of the young wood during summer, will leave little to be done at the winter examination beyond the removal of dead wornout branches, and spurs which have exceeded their proper size, and the points probably of such young wood as may not liave ripened, or have been injured by severe frost. All superfluous spray and very weak wood should be cut out, and no more left on the trees than is absolutely required. The shoots, being laid in moderately thin, will, by being exposed to the sun and air, ripen properly, and form plump and perfect buds, which they would not do if left too crowded.

Root-pruning should be attended to when the trees assume too gross a character; and taking up and replanting may be resorted to when they show indications of debility, or a habit of growing too late in the autumn-a certain proof that their roots have got too deep, and require to be brought nearer the surface.

\section{SELECT LIST.}

Blenheim.-Flowers large; fruit large; form oval; colour orange, tinged with deep yellow; flesh moderately rich ; stone roundish and impervious ; kernel bitter ; a good bearer, but inferior to the Moorpark, although somewhat earlier, indicative of its hardier origin ; one of the few raised from seed in England. Synonyms-Shipley's, Shipley's large, Miss Shipiey's, from the lady's name with whom this variety originated.

Breda.-Flowers large, tinged with deep red before they expand; fruit rather small ; form roundish; colour orange, becoming darker when fully ripe, if exposed to the sun; flesh deep orange, rich, parting freely from the stone, which is small and impervious; suture distinctly marked; kernel sweet. This nice little apricot, although supposed to have come originally from Africa, is sufficiently hardy to ripen well as a standard in most seasons in the southern and warmer parts of England, producing immense crops of fruit on walls in most situations. Excellent for preserving. Adapted to the walls of most parts of Scotland, where the larger and better kinds do not ripen. Synonyms - Ananas, Amande Areline, Brussels, De Hollande, Persique, De Nancy, Hasselnussmandel.

Hemskirk.-Flowers large; fruit large; form roundish, somewhat compressed on the sides; flesh bright orange, and tender; flavour rich and luscious; stone rather small, pervious; kernel bitter. In general character resembling the Moorpark, but differing from it in ripening earlier, indicative of its English origin. It has 
this merit over the latter, that it will ripen fully in seasons when the Moorpark does so only imperfectly, and hence is better adapted to colder localities. Brought into notice by the late $\mathrm{Mr}$ Lee of the Hammersmith nursery.

Kaisha, new early. - Of the merits of this new fruit we can from experience say nothing, as it has not as yet fruited with us. It was introduced by John Barker, Esq., from his garden at Betias near Seudia, in the Pachalik of Aleppo, and first fruited in 1848 by $\mathrm{Mr}$ Warmington of Kensington. It is certainly earlier than any of our established sorts, having ripened in $\mathrm{Mr}$ Warmington's garden on the 12th of July, on the open wall, alongside of trees of the Moorpark and Turkey apricots, which at the same time were quite hard and green. The following description of the fruit exhibited before the London Horticultural Society is thus given in the Journal of the Society, vol. iv., p. 189 : "The fruit is roundish, $5 \frac{1}{2}$ inches in circumference; rather deeply and acutely channelled on one side near the base, the channel becoming less, till only like a shallow indented line as it approaches the summit, where it terminates in a slight depression formed round the base of the style. The fruit is semi-transparent; skin slightly downy, pale citron-coloured where shaded, tinged and marbled with red next the sun; flesh tender, juicy, of a clear citron colour, parting freely from the stone, sugary, and delicious, like well-refined lump-sugar, combined with apricot flavour; stone small, roundish; kernel sweet like a nut. A valuable early variety for the dessert, and probably excellent for preserving; if it be employed for this purpose, the transparency of its flesh will exhibit a new feature amongst apricot preserves. By its clear citron-coloured flesh it may be distinguished from the orange-fleshed varieties hitherto known in this country, some of which have likewise sweet kernels, such as the Breda, Musch-Musch, and Turkey. It is earlier than either of these.

Large early. - Flowers large; fruit medium size; form oblong, somewhat compressed; colour pale orange where shaded, bright orange with a few reddish spots next the sun; suture deep; kernel bitter; stone impervious; flesh of the same colour as the skin, parting freely from the stone; flavour rich and juicy. Ripens next after the Masculine, and hence our third earliest, admitting the Kaisha to be the earliest of all. Synonyms-Gros Precoce, Gros d'Alexandrie, Grosse Fruhe, De St Jean, De St Jean rouge.

Masculine red.-We notice this sort on account of its earliness. It possesses no other merit. It is no doubt hardy, but its fruit is very small and indifferent in flavour. Where it will succeed as a standard it may be planted for culinary uses; but where requiring a wall, it should give place to the Kaisha or the Large early.

Masculine white.-Scarcely differing from the last, except in colour, and being a day or two earlier; our remarks above are equally applicable to this sort.

Moorpark.-Flowers large; fruit large, about $7 \frac{1}{2}$ inches in circumference each way; form roundish, compressed on the sides; colour orange where shaded, brownish red marked with many dark specks on the side next the sun; flesh bright orange, separating freely from the stone; flavour rich and luscious; kernel bitter; stone singularly perforated and rugged. "This pervious passage," Mr G. Lindley remarks, "has not been noticed by any of our writers till lately, nor is it readily discovered; its aperture is in a small groove on the thin side near its base, a pin inserted into which and pushed forward will open its farther orifice, and thus effect a passage through the stone." The best apricot in cultivation. Mr G. Lindley asserts that this fruit was introduced and cultivated by Sir W. Temple, in his garden at Moorpark, prior to 1700 , for in that year that great man died. Hooker, in his "Pomona Londonensis," claims the merit of its introduction for Lord Anson, by whose name it is sometimes called. Rogers, we think with more reason, doubts its introduction by either, or even by Lord Dunmore, who also at one time resided at Moorpark, and whose name it also sometimes bears, giving in support of his opinion the fact that this fruit is not mentioned by Miller in his folio edition of 1748 ; nor is it mentioned by Grey of the Fulham nursery, who published his catalogue ten years afterwards. And he (Grey) had at that time one of the finest collections of fruit trees in the kingdom, and was not likely to overlook or remain ignorant of such a fine fruit as the Moorpark apricot. Another account says, and we think with some probability, that the original tree was brought from the Netherlands about the middle of the last century by Sir Thomas Moore, and planted at Moorpark, then the residence of Lord Dunmore. A difficulty of far greater consequence than that of its mere introduction presents itself in drawing a line of distinction between this variety and that cultivated under the name of the Peach apricot. So small is the shade between them that we may venture to call it a "distinction without a difference." The tree introduced under the name of the Peach apricot is distinctly stated by Forsyth, in his " Treatise on Fruit Trees," to have been brought from Paris by the Duke of Northumberland in 1767 , where it is still known as the Abricot pêche, and described as such by Duhamel and all the highest pomological authorities in France, where the Moorpark is only known as a name in nursery catalogues. Mr G. Lindley says, in reference to this difference, "The Peuch apricot is supposed by some to be the same as the Moorpark, and indeed it has all its leading characters; but an extensive cultivation of it for more than twenty years has convinced me to the contrary. Its wood is similar, but more gross, less firm, and the tree more tender." Rogers denies the difference in toto. Hooker, in "Pomona Londonensis," t. ix., and Loudon, in "Encyclopædia of Gardening," makes them synonymous. Forsyth, p. 4, says : "It is generally thought to be the same as the Moorpark, but upon a minute examination the leaves will be found to differ." Downing, in " Fruit Trees of America," p. 157, says: "It very much resembles the Moorpark, but the two are readily distinguished by the eye when standing near eacl other." But our greatest of all pomological authorities 
Mr Thompson, says, opposite the Moorpark (in "Hort. Soc. Fruit Cat.," p. 48): "Like the Abricot pêche, from which, if it at all, it scarcely differs;" and in remarks opposite the Pêche, p. 49 , he says: "In all probability there would be no error in identifying this with the Moorpark." We need quote no further evidence in support of our own opinion that they are identically the same. Synonyms of both-Anson's, Dunmore's, Dunmore's Breda, Temple's, Iunt's Moorpark, Oldacre's Moorpark, Sudlow's Moorpark, Walton Moorpark, De Nancy, Anson's imperial, Peach, Peach royal, Wurtemberg, De Tour's, Pêche grosse, De Luxembourg. Abricot pêche of the French.

Musch-Musch.-Fruit small, only about $1 \frac{1}{2}$ inches in diameter; form round; colour orange red; flesh yellow, tender, and rich; kemel sweet; stone impervious; fruit smaller, otherwise somewhat resembling the Breda, and appreciated, notwithstanding its small size, for the richness of its flavour. It appears to be a native of Asia, and takes its name from the city of Musch on the frontiers of Turkey. It is much grown in Egypt, particularly about Alexandria, and also in Upper Egypt, where the fruit is dried as an article of commerce. It is by no means a liardy tree, and therefore requires to be placed in dry warm situations. It seems to prosper healthily at Dalkeith, but has not as yet produced fruit with us. Synonym-D'Alexandrie.

Orange. - Flowers large; fruit of medium size; form spherical; colour orange, slightly marked with a ruddy tinge where exposed to the sun; flesh moderately juicy, and darkish orange, adhering to the stone; kernel sweet; stone roundish, small, and impervious. Tree rather hardy; a good bearer. Only fit for preserving. Symonyms - Royal orange, Royal George, Royal Persian, Early orange, Royal orange. Has been long in our gardens, being mentioned by London and Wise, who wrote in the time of Queen Anne.

Roman.--Flowers large; fruit medium-sized; form oblong compressed; colour pale yellow; suture indistinct; kernel very bitter; stone oblong, impervious; flavour indifferent, unless ripened a day or two in the house, and even then only fit for preserving. Tree hardy, perhaps the most so excepting the Masculine, therefore adapted for cold localities, where the finer sorts will not succeed. The blossoms also withstand the spring frosts better than most. Synonyms - Transparent (a very ridiculous name), Abricot commun, Grosse Gemeine, Gemeine, Brussels of some, Turkey of some, Algiers.

Royal.-Flowers large ; fruit large ; form spherical-ovate; colour dullish yellow, faintly tinged with red; stone pervious; kernel bitter; flesh pale orange; flavour rich and vinous; ripens rather before the Moorpark, and approaches it nearly in size. The leaves are larger than in that sort, therefore the wood requires to be laid thinner in to the wall or espalier. Of French origin, having been raised some years ago in the royal Luxembourg gardens.

Turkey.-Flowers large; fruit medium size; form spherical, not at all compressed; colour deep yellow in the shade, brownish orange, slightly mottled where exposed to the sun; kernel sweet, nearly as much so as the almond. This peculiarly, as well its form and colour, identifies it as different from the Roman. The latest ripening sort we have; stone impervious. Synonyms-De Nancy of some, Large Turkey.

\section{SELECTIONS OF APRICOTS FOR PARTICULAR LOCALITIES.}

For a small garden in a farourable climate.Large early, Breda, Moorpark.

For a small garden in a cold climate-Red masculine, Breda, Roman.

Grown at Dalkeith Palace.-Breda, Moorpark, Large early, Hemskirk, Muscl-Musch, Royal, Turkey, Orange, Kaisha (the latter not yet fruited).

From sixteen returns received from gardeners in Scotland we find only the Red masculine, Moorpark, Breda, and Orange cultivated.

From ten returns from gardeners in England we find the Moorpark, Breda, Turkey, MuschMuscl,, Orange, Masculine, Large early, Hemskirk, and Roman, in general grown. And upon an average we find the Moorpark and Roman the most generally cultivated.

Mr G. Lindley gives the following as a selection for a small garden in the southern and midland counties of England : Breda, Brussels, which he considers distinct; Hemskirk, Large early, Moorpark, Peach apricot (which is the same), Red masculine, Roman, and Turkey.

For the northern counties of England and southern of Scotland.-Brussels, Hemskirk, Large early, Moorpark, Orange, Roman, and Royal.

For the Highlands of Scotland.-Breda, and Red masculine.

The principal addition to the $\Lambda$ pricot of late years is-

The new red-fleshed late Frogmore, a variety that promises well on account of its lateness, prolonging the season of this fine fruit to a greater length than formerly. Well adapted to be grown in an orchard-house or in a late plum and apricot house, in the manuer of standards, along with Jersey seedling and Tardive d'Orleans.

Jersey seedling. - A very late variety, but rather of small size, raised at Petit Menage by James Robin, Esq. Esteemed chiefly for its late ripening.

The following new rarieties of the Moorpark race are recommended by Mr Rivers as possessing sufficient merits to insure them a space in most gardens. "They seem more hardy than that sort; their branches do not die off suddenly, as is so often the case with trees of that favourite variety, and yet their fruit is as large, or larger, and of equal excellence:"-

Alsace, Large red, or Gros rouge. - "Tree hardier than the Moorpark; skin and flesh of fruit deep orange red; an excellent variety."St Ambroise. A highly-esteemed variety from France.

Tardive dOrleans.-Stated to ripen two or three weeks after the Moorpark.

Precoce d'Esperen.-Fruit melting; medium size ; ripens the middle of July; "' a variety, say Knight and Perry, "of first-rate excellence."

The following selection is given by Mr Rivers of Apricots adapted for pot-culture in the 
orchard-house: viz., Early red masculine, ripe in June; Large early, ripe in July; MuschMusch, equally early ; Blenheim, Moorpark, Peach, which we think the same; St Ambroise, Royal, and 'Tardive d'Orleans. These ripen in succession, and nearly at the same time as those on the open walls; "for it must be understood that fruits in thoroughly ventilated orchardhouses are not much accelerated unless the season happens to be very sunny. It is not an early but a certain crop that must be expected from such houses."

Diseases.-The apricot is liable to the same diseases and attacks of insects as the peach and nectarine, although in a less degree. 'The Moorpark, in particular, is subject to the dying off of large limbs and branches during summer without any apparent cause, and as sudden in its effects as its cause is unknown. When budded on the plum stock it is less liable to this diseasc, and considered hardier than when upon its own loots. Mr Knight, to avert this disease, recommended budding this variety on the apricot stock ; subsequent experience does not, however, confirm this opinion. Some have supposed the disease to arise from the effect of the sun shining too powerfully on the uncovered branch, and to provide against this recommend training the young adjacent shoots over the naked branches. This also does not in all cases prove a remedy. This disease is much less prevalent in Scotland than in England-a circumstance that would seem to favour the cause assigned above, as the sun is less powerful with us, and sun-strokes of rarer occurrence. The best precaution is to keep up a succession of young wood near the bottom of the trec, to make up for the loss of such branches as die off in this manner.

Insects.-Curculio (Otiorhynchus) tenebricosus, fig. 229 , one of those destructive beetles known

Fig. 229.

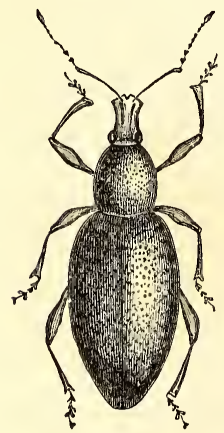
generally as weevils, is very injurious to the apricot, and is often found in great numbers secreted in nail holes and other crevices, in old and dilapidated garden walls. It also attacks the vine and the peach in hothouses. Wall--trees may be cleared of them by filling up the loles where they take shelter during winter, and washing the wall with a thick paint of Roman cement and water. The loose bark on the trees should be removed, as with it numbers of the insects will be cleared away; the stems as wcll as the branches of the peach trees should be washed with strong decoctions of tobacco; quassia, amara, and aloes have also been usefully employed. It also attacks the peach and plum.

OTIORHYNCHUS TENEBRICOSUS.

The oblong weevil (Curculio oblongus), Fig. 180, appcaring in May, often commits sad havoc upon the leaves of apricots, peaches, plums, pears, and apples. A similar remedy should be applied.

The Podisca angustiorana, Ditula angustiorana, Tortrix angustiorana of some, Fig. 200, is very destructive to apricot trees. The eggs may be detected during winter attached to the old stems of the trees in oval-like patches. The caterpillars appear during May and June, and when full grown are about half an inch in length, of a pale yellowish-green body and brownish-yellow head. They appear along with the young leaves, which they immediately begin to gnaw, and roll themselves up in them, so as to be with difficulty discovered. The grub afterwards passes into the state of a brown shining chrysalis rolled up in the leaves, coming forth in its moth state in July. In this state it is small, little more than one-fourth of an inch in length. The fore-wings are reddish brown in bands, varying in their shades of darkness ; the hind-wings are dusky. The best remedy we have found was picking off the leaves into which the caterpillar had rolled itself, and carefully destroying them. With us they do not appear annually, at least to the extent they did in 1843 , 1850. There is no doubt searching for the patches of eggs during winter would be a better and more certain riddance, but it requircs a sharp eye to detect them, and a full amount of perseverance to prosecute the search.

Tortrix (Carpocapsa) Waberiana, although called the plum-tree tortrix, is equally destructive to the almond, peach, and apricot.

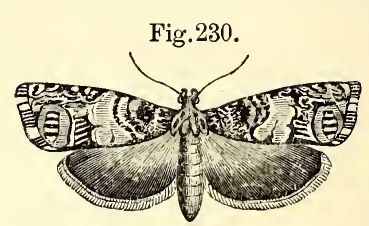

Its presence is readily detected by the appearance of small conical heaps of a red brown on the bark of the stem and branches of these trees. Under each of these

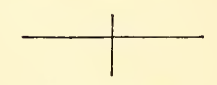
hcaps is a minute hole leading to a furrow under the bark,

PLUM-TLEE TORTRIX. in which a small larva is seen, which feeds on the the sap and the exudation of gum, from which other diseases arise, terminating at last with the total decay of the tree. The larva, when full grown, is only three or four lines long, of a dirty green colour, with red head. It has three pairs of true feet, and five pairs of fleshy prolegs, the body sprinkled with a few small hairs. During nearly the whole year the larva remains under the bark. The moth, which is very beautiful, of a brown colour, resembling that of the bark of the apricot and peach trees, which renders its capture difficult, makes its appearance in May and again in August, for two generations are produced annually. The moth is only from five to six lines long. Fore-wings dark brown and yellow, with several silvery lines and yellow dots; the hind-wings are brownish. The pupa is brown, remaining under the bark until near 
the time of its becoming a moth, when it screws itself nearly out of its hiding-place, being often seen with half its body beyond the orifice. The female deposits her eggs on the outer bark, and when the young caterpillar is fairly formed, it begins to burrow into the inner bark. The remedy to be applied is, upon the appearance of the reddish dust on the surface of the bark, to thrust a strong needle into the hole under the dust, and so destroy the grub in its habitation. As it is in the stems and larger branches that these insects take up their abode, the surface may be laid over with a solution of lime both in May and September. The most effectual means, however, would be to paint the stems and branches where the insect is enclosed with vegetable spirits of tar, which would completely destroy them.

The mussel, or dry scale (Aspidiotus concliformis), Fig. 191, is occasionally found on the one and two years' wood.

European names. - Abricotier, French, although in some of the provinces it is pretty generally called Alberge, being the native name of a variety common in the interior of France, and propagated from seed, indicating little variation during successive generations; Albarico queira, Spanish ; Abirkoos, Dutch ; Aprikosenbaum, German; Albercocco, Italian. The names by which it is known in various European countries all seem to be corruptions of the original Arabic term Berkoche. 


\title{
CHAPTER VIII.
}

\author{
THE PLU M.
}

The domesticated or cultivated plum is supposed to derive its origin from a species indigenous to Britain, the Prunus domesticus L. It is also a native of Asia Minor; in both cases found growing naturally in hedges and the sides of woods. At what period the plum became known and appreciated by man, in a state of improvement beyond that of its wilding state, is uncertain. Some have set it down as originating in Asia Minor, and, if we are to credit Pliny, it was brought from Syria into Greece, and thence into Italy; from thence improved varieties crept into France, and from thence are received inany of our most esteemed varieties. The plums of merit that have originated in this country are few in number, yet some of them rank along with those of the highest order of excellence : of these, Coe's golden drop, Coe's late red, Downton imperatrice, Standard of England, Chapman's Prince of Wales, Angelina Burdett, Woolston's black gage, Knight's large green drying, Lucombe's nonsuch, Goliath, Isabella, Ickworth imperatrice, Nectarine, Rivers' early, Sharp's Empereur, claim an English origin; while the Tay Bank, Late green, Menette, New apricot plum, Topaz, and Russet, seedlings of great merit, raised by Charles Guthrie, Esq., Dundee, and Lawson's Golden gage, raised by that indefatigable horticulturist, Mr Arch. Gorrie, include the best of those of Scottish origin. France and Germany have produced their share; and, strange enough to say, America, with only three species of the genus Prunus, $\boldsymbol{P}$. maritima and pubescens, from neither of which a cultivated plum has been reared, has nevertheless produced more excellent plums than any other country whatever. The original parent of all cultivated plums, Prunus domesticus, is not indigenous to that country, but has been introduced. The late Mr Downing, in speaking of the excellence and number of plums raised from seed in America, says, "That the soil and climate of the middle States are admirably suited to this fruit is sufficiently proved by the almost spontaneous production of such varieties as the Washington, Jefferson, Laurence's Favourite, \&c., sorts which equal or surpass, in beauty and flavour, the most celebrated plums of France and England." Yet, notwithstanding the great success attending American culture, one of their highest pomological authorities admits that, even with the important additions made to this family in America, "the Green gage, an old French variety, still stands at the head of the list for high flavour, though several sorts are nearly equal, or quite equal to it." England has, at last, done even more than this ; for we have now, on the high authority of Mr Thompson, this declaration: "The Woolston black gage retains all the properties of that plum (the Green gage) with more sugary sweetness. I have," he adds, " no fear of my judgment being called in question." Seeing, therefore, the progress making in improved varieties of the plum, none need be alarmed at our recommendation to set about purging their gardens of old and valueless sorts, and to replace them with those that are so far superior. The same space, labour, and expense that are next to thrown away on the one produce the other.

The earliest data we have in our possession of the number of cultivated plums is that of Tusser, who enumerates ten varieties, Parkinson sixty, Miller thirty-two, Gordon of Fountainbridge twenty-five sorts as useful, exclusive of eight used as stocks or for ornament, concluding by adding, "There are many more kinds of plums imposed upon the public, but those here mentioned are the principal." Peter Lawson and Son, whose fruit catalogue is at once the most comprehensive and useful of all its predecessors, describe with great minuteness no less than seventy-six different sorts, Rivers eighty-eight, Rogers thirty-five. In the $3 d$ edition of the "Catalogue of Fruits in the Horticultural Society of London Gardens," one hundred and twenty-seven sorts are described; and to that number about twenty new sorts, exclusive of American ones of recent date, may be safely added.

The uses to which the plum is applied are too well known to require any especial remark here, further than that much more might be made of it even as a hedgerow tree, producing little shade and yielding abundant crops. The hardier sorts, planted under such circumstances, would pay better than the miserable, maltreated timbertrees we see used for that purpose, as the wood is of great value in the finest kinds of cabinet-work and in making musical instruments. In many parts of the Continent the tree is so cultivated 
for its fruit, which for nine months of the year appears at the tables of the most humble in one state of preparation or another. Medicinally, the fruit, when fully ripened, is mildly laxative; and one variety, the Saint Catharine, is grown extensively about Tours, in the south of France, and when prepared by drying in the sun, and afterwards in a spent oven, they form the well-known prunes, and are in that state exported to every part of the world. The highest class prunes, however, are chiefly made in Provence of the Perdrigon blanc, Brignole, and the Prune d'Ast, being more fleshy and having a better bloom. In the north of France, Belgium, and Germany, the Quetsche plum is largely cultivated for the purpose of drying, forming in commerce the German prune of the shops. The Damson is much used in some parts of England as a preserving plum, but, unfortunately for ourselves in Scotland, we treat all these good things with neglect. The apple, pear, and plum may be called the poor man's fruits, and, with proper management, he may enjoy them all during three parts of the year, greatly to his health's benefit and the saving of his purse : these fruits constitute a very considerable portion of the food of the lower classes all over the Continent.

A prejudice exists in the minds of many that the plum is an unwholesome fruit, and apt to bring on diarrhœa or British cholera; such is not the case if the fruit be fully ripe, of good quality, newly gathered, and eaten in moderation. The skin is, however, indigestible, and should be always removed. In a cooked state, and more especially when preserved in the shape of prunes, few fruits are more wholesome; it is often prescribed by the faculty as a mild laxative, in cases even of the most delicate constitutions. The French always remove the stones before making the plum into compotes, pies, or puddings, a practice highly to be recommended.

Were plums planted as hedgerow trees, or were steep banks, and other places equally difficult to cultivate, occupied with them, a profitable return might be expected, by the simple process of converting them into prunes for home consumption. We have abundance of situations at present unoccupied with any useful crop, and that in situations where the best prune plums, the Saint Catharine, and the White Perdrigon and Brignole, would ripen perfectly. There are, amongst even our Scotch seedlings, those which would answer the purpose quite as well, and, being naturally hardy, they might be planted in very many parts of Scotland. 'The process of preparing prunes is simple, but not in general known to the peasantry of this country, nor, indeed, to professed confectioners. The following is the French mode: The fruit is collected when just ripe enough to fall from the trees when gently shaken; when they are not growing on grass, a few mats or coarse cloths are spread under them, partly to save the fruit from being too much bruised, and also to prevent its being dirtied by falling on the ground. The plums are then laid thinly on sieves or shallow baskets, and left exposed to the sun for several days, or until they become quite soft; they are then put into a spent oven and shut quite closely up, and VOL. II. allowed to remain there for twenty-four hours ; they are then withdrawn, and when the oven is slightly reheated, they are again replaced, and allowed to remain in it for a like length of time. The following day they are again taken out, and the sieves gently shaken so as to turn the fruit, and returned again to the oven a third time, slightly increasing the heat at each operation. After remaining another twenty-four hours they are taken out and allowed to cool. The operation of rounding is then performed-that is, turning the stone in the fruit without breaking the skin, and pressing the two ends together between the thumb and finger. After this they are placed on the sieves, and put into the oven for the fourth time, at a temperature such as when bread is withdrawn. 'The door is closed, and luted round the edges with clay, to prevent the entrance of air, and the plums are allowed to remain in for one hour, when they are again removed. The oven is again reheated and shut up, and when a cup of water placed within it is heated to the extent of just being able to bear the finger in it, the fruit is again placed within and allowed to remain for twenty-four hours, which completes the preparation of the prunes. They are then packed in small boxes for sale. Somewhat greater care is taken in preparing the best French prunes. The fruit is gathered by hand, taking it by the stalk, and handling it as little as possible-it is recommended, but not always practised, to gather the fruit before sullrise in the morning-it is then laid upon vine leaves placed in shallow baskets, and taken to the fruit-room, where it remains several days exposed fully to sun and air, after which the same process is employed as described above. The greatest care is taken to preserve the fine bloom on the fruit, even when quite dry. This is not so easily managed, and manufacturers scruple not to give their fruit an artificial bloom, by using indigo in fine powder and other means, which the domestic manufacturers would do as well to avoid.

In describing plums, Downing justly remarks: "The surface of the young wood, when just ripened, is an important character, as it is snooth in some varieties, and downy or covered with soft hairs in others. In some varieties the flesh parts from the stone, while in others it adheres. And finally, the depressed line or channel which runs down one side of the exterior surface of the plum is called the suture, and the prominence or absence of this feature enables us to distinguish many kinds at first sight." These, where remarkable, will be noticed in our descriptions.

Propagation.-Like other fruit trees, the approved varieties are propagated by extensionthat is, by budding or grafting, chiefly on the mussel, St Julian, pear plum, Brussels, Magnum bonum, or similar free-growing plums, these stocks being procured by sowing their seeds, by layers, or by suckers. The former of these make by far the best and longest-lived trees. The Mirabelle plum is used as a stock when dwarf trees are desired; and more recently the sloe (Prunus spinosus) has been employed, with the view to produce the dwarfest of all. Some 
sorts of plums, such as the Damson, Wentworth, Bullace, \&c., are propagated by suckers or layers without grafting at all. The Damson reproduces itself by seed, little changed from the parent; but it makes the worst of all stocks, and, besides, the buds are difficult to take on it. To obtain new or improved varieties, seeds of the finest kinds are sown, sometimes promiscuously, at other times after a careful fecundation of the flowers of one variety with those of another. This is the most certain way of obtaining varieties partaking more or less of the qualities of the parents. The Americans use for stocks such as the Blue gage, Horse-plum, \&c., but originate a great number annually from seed, and by this means have obtained many excellent varieties.

The seed should be sown as soon as they are collected, placing them in drills an inch and a half in depth. In good soil the seedlings will attain a height of 18 inches or more the first season. In the autumn or ensuing spring they are taken up, their tap-roots shortened, and the strongest selected and planted in nursery lines, where they remain one year, and are then cut down to the ground, when they will throw up straight handsome shoots 6 feet high the first year. Apricots or plums, intended for standards or riders, are budded on them the following summer. The Brussels stock, on account of its vigorous growth, is often preferred for this purpose, although it is less durable than the common stock. Many nurserymen, and with great good reason, use the common stock, and insert the bud about 9 inches from the ground, and find, if the stocks are strong and soil good, that they will send up shoots standard high the first year: the less vigorous will attain that height the second year. The common stock makes the most durable trees. Plums to be trained as dwarfs are grafted instead of budded.

Soil.- The plum in its wilding state affects a rather light and dry soil, and in such it is found to succeed in many gardens. In one of a loamy nature, and of moderate texture, it appears to prosper, but best of all in a good strong loamy soil, provided it be dry at bottom. In light sandy soils the fruit does not attain so large a size, nor does it set so freely upon the trees. That the plum prefers a strong soil is evident from what occurs in the richer and stronger loams of England, of which the Royal Gardens at Frogmore offer an excellent example, where the finest plums we have seen are yearly produced. Downing, in speaking of soil, says of American experience : "The plum will grow vigorously in almost every part of this country (America), but it only bears its finest and most abundant crops in heavy loams, or in soils in which there is a considerable mixture of clay. In sandy soils the trees blossom and set plentiful crops, but they are rarely perfected, falling a prey to the curculis, an insect which harbours in the soil, and seems to find it difficult to penetrate or live in any one of a heavy texture, while a light warm sandy soil is exceedingly favourable to its propagation." The comparative absence of insects on the plum in strong soils is conspicuous enough in our own country. However, besides the favourable condition of light soils to the propagation of insects, the trees growing on these strong ones are much more vigorous, and better able to resist their attacks. Regarding depth of soil, that should not exceed $2 \frac{1}{2}$ feet; and should it naturally exceed that depth, then it were better to lay pavement supported on piers a foot or so in height, so as to leave a shallow vault under the roots. A solid flooring of pavement, or even concreting, which would be much cheaper, has the disadvantage, particularly in strong soils, of retaining water, whereas vaulting them prevents such an evil. The intention of vaulting is to prevent the roots taking a downward direction, and it greatly facilitates the operation of rootpruning when it has to be had recourse to, as a circular trench opened all round presents all the roots to view, and obviates the difficulty of undermining to reach the roots that take a downward direction.

Situation and planting.-Many of the finer plums require to be planted against a wall, as the Reine Claude de Bavay, Coe's golden drop, \&c. ; and in elevated situations and cold climates, these, to attain perfection, require a southern exposure; whereas, in warmer localities, they, as well as most of the other superior varieties, will be found to ripen perfectly on walls of east or west aspects, and in the most favourable situations of all, even as dwarf standards. Much, however, depends, where the soil and climate are both cold and humid, on the depth of the border they are planted in. Vaulting has not only the property of securing a sufficient degree of dryness to the roots, but where carried sufficiently out, and the external air admitted into the vault, of elevating the temperature of the soil about the roots from $7^{\circ}$ to $9^{\circ}$.-(Vide Vine-Borders.) It may be taken as a rule that, in all cold soils and situations, every fruit tree will derive important advantage from being so treated. The generality of plums succeed in most places when trained as espaliers, in which case the horizontal mode of laying in the branches should be adopted. A great many do as well, if not better, when planted as dwarf standards as when planted against walls. By judicious attention to their roots, they will require very little branch-pruning, and produce abundant crops even when wrought upon the ordinary stocks. In former times, before root-pruning was attended to, all sorts of trees were planted at a very great distance apart ; for example, Harrison directs for a wall 12 feet high the plums trained horizontally to be set 20 feet asunder, "a greater or less distance according to the height of the wall." Now, under judicious root-management, half that distance will be sufficient, accommodating double the number of trees. Full standard plums should never be planted, unless it be the Damson, and those of its kind used for preserving, \&c.; and these, if planted in grass, will suffer less loss of crop than were the fruit to drop off and fall on the naked soil. They in this way also occupy more space, and cause more shade and interruption of the air to other trees or crops around them, than 
their fruit is worth. They are also difficult to gather, protcct, or prune, and never produce in the same proportion so much fruit as well-regulated dwarf standards do. In the latter case the fruit can be hand-gathered, they can be readily protected by nets, or grown under a wire covering, as recommended for cherries; and pruning, \&c., can be accomplished while the operator stands upon the ground. It is not size of tree but productive habit that ought to be aimed at in fruit-tree culture. Indeed, the larger such trees become, the less fruit they will produce, compared with their size, and the space of valuable ground they cover; nor is the fruit itself nearly so good-as is clearly exemplified in the plum.

East and west aspected walls are suitable for the main crops, although a tree or two of some of the earliest sorts should be placed on a south wall, and one or two of the hardier kinds even on a north one, with a view, in both cases, of lengthening out the season to a greater extent.

'The Green gage, one of the finest and most useful of all plums, seems to be exceedingly capricious in regard to situation. It is an excellent dwarf standard or even orchard fruit in the south, requiring, however, a wall in the most of the north of England and Scotland, and indced in many places a southern aspect. As an exception to this rule, we may observe that there still exists the remains of an old orchard of standard trees of this sort, of great age, in the park of the Earl of Traquair, at Traquair House, in the upper parts of Peeblesshire, which produces abundance of fruit, and that of as good quality as is to be in general seen in the neighbourhood of London. The plum requires the same space to spread on the wall as the peach and apricot, already mentioned, when on its ordinary stock, and properly rootpruned-much less, however, when on the sloe or Mirabelle. As the leaves of the plum tribe vary greatly in size-say from the Blue imperatrice to the Washington, nearly the two extremes-allowance should be made for this in the distance the branches are placed apart in training. A very general mistake has been fallen into in planting fruit trees, more especially those planted against walls: insufficient room has been afforded them. This is easily accounted for-all men are anxious to make as much of their walls as they can, and hence crowding them with trees is of very frequent occurrence. Trees occupy little space when first planted, and if placed at their proper distances, the walls look for a long time naked. It were better, therefore, to plant intermediate trees of sorts that would come into bearing early, were it even gooseberries, currants, and suchlike, than to set the permanent trees so close together, that when they come to their full size the branches of the one run into those of the other, and lience cause great confusion, ending in shortening the terminal shoots, and in many cases causing the trees to throw out innumerable masses of useless spray, and disturbing the natural organisation of fruitful buds. But there is, on the other hand, even a greater error committed when they are planted at too great a distance apart, and encouraged to cover too great an area of wall surface; for when so encouraged and treated, as too frequently is the case, they produce fruit only at and near the extremity of the branches, while the centre and greater part of the tree is perfectly barren. By repeated transplanting, and by judicious root-pruning, the plum, even upon its strongest stocks, may be kept within very moderate bounds, and continued long in health and productiveness. Now, instead of planting plums at 20 or 24 feet distance apart on the walls, half that distance will be found sufficient under ordinary circumstances, thereby affording space for a greater variety. There arc, however, soils and situations so favourably circumstanced that the plum really requires from 18 to 20 feet distance when trained as a wall tree, as in the Royal Gardens at Frogmore and some others; but these are the exceptions, not the rule. In general, smaller trees will be found the most profitable. A good deal, however, in this, depends on the height of the walls and mode of training. On walls under 8 or 9 feet in height, with horizontal training, 16 or 18 feet will be a good distance in ordinary cases ; and for walls of greater height, and the trees fan-trained, from 12 to 15 feet will be sufficient, provided the trees are to be root-pruned, and triennially transplanted. The autumn is a good season for taking up such plum trees as are making too much wood, and showing too few fruit-buds. The plum lifts easily, or rather safely, and lifting and replanting is one of the very best means of lessening over-luxuriance, and inducing greater fertility.

Pruning and training.-The majority of the older horticultural authorities recommend training the plum in the horizontal manner; modern authorities recommend the fan or more natural form. Amongst plums there is a greater difference in respect to habit than even in the case of cherries. The Imperatrice and Washington, or the Magnum bonum, may be taken as the extremes in regard to habit. In the latter cases somc excuse may be found for horizontal training, as it has a considerable effect in modifying the luxuriance of growth; while in the former, which is a weak grower, a more vertical direction given to the branches would induce greater strength in the tree. Miller recommended horizontal training to modify the growth, and was adverse to shortening the branches, because the more these trees are pruned, the more they grow to wood. Modern practitioners have, by operating on the roots, planting in shallow borders, and other manipulations, rendered both horizontal training and severe branch-pruning almost unnecessary. Among these, Mr Rivers holds a prominent position. He recommends summer-pinching the young wood, annual root-pruning, and surface-dressing, in preference to any other mode of culture. $\mathrm{He}$ is also experimenting on the use of the sloe as a stock to work them on, with a view to check excessive growth. The plum is a very hardy tree, and well fitted for training as an espalier, and as such most sorts ripen their fruit in ordi- 
narily good situations; and if protected with portable board copings while the trees are in bloom, it will be found a pretty certain security against late spring frosts. The fundamental rules are, allowing the shoots plenty of room on the wall or espalier by thinning out the older branches that may have become exhausted, or can be readily spared, and laying in young wood as required, either by being directly attached to the wall, or trained upon the older and barren branches. There are often gross watery shoots which arise towards the lower part of the tree near the stem, which, if not rubbed off during summer, must be totally cut off at winter pruning; but it is better to prevent their appearance altogether, either by finger-pruning in summer, or root-pruning in autumn. In regard to distance, the smallergrowing sorts should have their main branches laid in about 6 or 7 inches apart, and the largergrowing ones at 9 or 10 inches; and on these, and between them, the young wood is to be trained. The plum bears the knife better than the cherry, but the less knifing on any tree the better; for if the roots be under proper control, very little winter pruning will be found necessary. All the sorts bear their fruit on small natural spurs, which form on the ends and along the sides of the bearing shoots, of from one to three years' growth; in most sorts, however, it is two years before the spurs on these branches bear fruit. By great neglect, spurs will extend to a considerable distance from the wall ; these should be cut back to the bud nearest their origin, or removed entirely, and the branch on which they grow covered with such young shoots as can be most conveniently made available.

Summer pruning should commence about the beginning of May, and consists chiefly in disbudding, or rubbing off with the finger and thumb, all foreright shoots and back shoots (that is, those which protrude from the front or back surface of the branches), excepting where there may be a vacancy to fill up, where a young shoot from the side of the branch cannot be brought in for the purpose, for it is better to have a somewhat ill-placed shoot than no shoot at all. This pruning, or disbudding, should be gradually gone on with, the first operation being a removal of foreright and back wood. Towards the end of May, if the trees are strong, gross shoots will show themselves, and these, when about half a foot in length, should have their points pinched off. In three or four weeks after, a more general going over and disbudding should take place, because by that time it is readily seen what shoots are fit for being finally retained; and these should be immediately trained to the wall or espalier, or tied down upon the naked old branches of the tree, and all the others removed. Avoid laying in too much wood, and regulate the distance, shoot from shoot, according to the size of the leaves of the sort.

Winter pruning. - Dwarf-standard trees, if root-pruned, will require little attention at this time further than thinning out and reducing the spurs, and snags or stumps of shoots pinched back at summer pruning. The young short- jointed shoots, tied down upon the older branches during summer, will become furnished with blossom-buds during the next summer, and these should not be interfered with; only such as are not required should be cut off entirely, excepting in cases where young wood is wanted to fill up blanks, then such as are best placed for the purpose should be cut back to one or two buds from their base, which will induce the production of young shoots to fill the vacant spaces.

The following diagram shows the comparative sizes of plums.

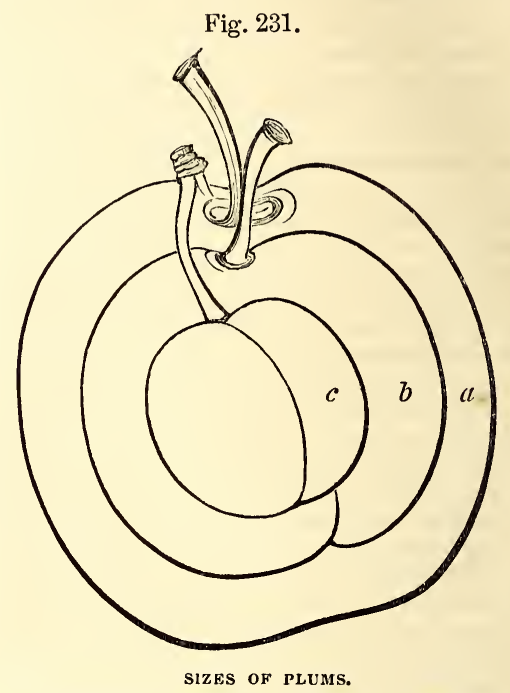

$a$ Waslington, large; $b$ Coe's late red, medium; $c$ Mirabelle, small.

SELECT LIST.

Abricotée rouge.-Size medium; quality second-rate; colour clear red in the shade, violet in the sun, covered with a fine blue bloom; form oval; separates from the stone. Ripe end of August. Of French origin. Known in England as Red apricot plum on account of its apricot flavour. Young wood smooth.

Apricot.-Size above medium ; quality good ; flesh yellow, melting, juicy, and high flavoured; colour yellow, tinged with red; form roundish. Ripe in September. Branches quite downy, nearly white; suture deep. Dessert fruit. This is the true Apricot plum of Duhamel, not that of Thompson, which is much inferior, a clingstone, which this is not, also having smooth branches, and an oval fruit.

Angelina Burdett.-Size medium; form somewhat oblong; colour rich purple, copiously covered with an azure bloom; flesh amber green, parting freely from the stone; flavour delicious; ripens upon an open standard about the middle of August. Against a wall it would no doubt ripen sooner. One of three excellent plums originated from seed by Mr Henry Dowling, of Woolston, near Southampton, about ten years ago. 
Ashridge black.-Size medium; quality secondrate; colour purple; firm, round. Ripe in August. Tree hardy, and a good bearer. Excellent for kitclien use.

Bingham's.-Size large, and handsome; quality excellent ; flesh yellow, adhering to the stone ; colour deep yellow, slightly spotted with red on the sunny side; form oval. Ripe September. Branches downy; of American origin. A fine dessert fruit in a warm climate.

Bleecker's gage.-Size medium; quality excellent; flesh yellow, sweet and luscious in flavour, fit for dessert, separating freely from the stone ; form roundish oval. Ripe September. Branches downy; suture scarcely perceptible. Distinguished from the Yellow gage, to which it is somewhat allied, by its longer and stouter stalk. Of American origin. SynonymGerman gage.

Blue imperatrice.-Size medium; quality firstrate; flesh greenish yellow, rather dry, but quite rich and sugary, adhering closely to the stone; colour deep purple, with a thick blue bloom; form obovate. Ripe in October. Will hang, if protected from frost, till the end of November, or may be kept in the fruit-room even much longer, the fruit being covered singly with soft tissue-paper, and laid in an air-tight drawer or shallow box; branches long, smooth, and very slender. An abundant bearer, and one of our very best late plums. Synonyms-1mperatrice, Veritable imperatrice, Violette, Imperatrice violette.

Buel's favourite.-Size above medium ; quality good; fleslı yellowish green, juicy, rich, and high flavoured; colour pale green; form ovate. Ripe beginning of September. Suture quite distinct for half the circumference ; free stone; branches smooth and reddish; of American origin.

Chapman's Prince of Wales.-A seedling from the Orleans, but differing from it in being larger in size, more inclining to oval, and of a bright purplish pink, with more bloom, and superior flavour to the original parent. Unlike the Orleans, it never cracks, is quite smootl in the young shoots, whilst the Orlears is downy. Leaves large and roundish, easily distinguished from any other plum. Trees vigorous in growth, and most abundant bearers while yet the tree is young. The blossom is protected in a remarkable manner by the large foliage ; and the fruit is thickly set all up the stem, which at an early age is completely furnished with fruit-bearing spurs. Succeeds excellently as a standard or pyramid. Originated at Brentford End with $\mathrm{Mr}$ Chapman, an extensive market-gardener there, in 1830. An excellent kitchen fruit. Tree very hardy, and adapted to a Scotch climate, even as a pyramidal standard.

Cheston.-Size medium; quality good; form ovate; skin dark purple, with a fine blue bloom; stalk quite short; flesh yellow, firm, sweet, separating freely from the stone; branches downy ; ripens about the beginning of August. Tree hardy, and an abundant bearer. SynonymsMatchless, Diaprée riolette.

Coe's fine late red.-Size medium; quality excellent ; flesh yellowish, firm, crisp, and juicy, with a rich vinous flavour, parting freely from the stone; form nearly round; suture well marked, running along one side; colour purplish red, with a fine blue bloom. Ripe October and November, and, with protection, will hang on the tree till the end of the latter month, and in the fruit-room may be preserved even later. One of our best late plums. Synonyms-St Martin, St Martin rouge, Prune de St Martin of the French. Excellent for dessert purposes.

Coe's golden drop.-Size very large, indeed one of the largest ; quality, as a dessert fruit, of the highest character ; form oval ; colour light yellow, with a number of dark rich spots on the side next the sun; suture well marked on one side, on which it is a little more swollen than on the other; flesh yellow, adhering closely to the stone ; flavour rich and sweet. Of English origin; raised in Suffolk by a gentleman of the name of Coe. Ripe by the end of September, keeping through October, and also November, with slight protection, and even up to Christmas in the fruit-room. One of our most excellent late plums. Branches smooth and pretty strong. Synonyms - Coe's imperial, Golden gage, Bury seedling, New golden drop, Fair's golden drop, Coe's.

Columbian.-Size very large, six or seven inches in circumference; quality excellent; flesh orange, very rich and sugary, separating pretty freely from the stone; form nearly globular colour brownish purple, with numerous fawncoloured specks. Ripe in September. Of American origin; raised from a seed of the Green gage. A very attractive dessert fruit. Tree remarkable for its strong growtl and productiveness. Branches and upper side of the foliage downy.

Cooper's large, Cooper's large red, Cooper's large American of Cox, La delicieuse of Lindley. - Some difficulty appears to arise in the identification of these names, first, we think, mooted by $\mathrm{Mr}$ Downing. Our own opinion is that they are identically the same, difference of climate causing the apparent difference, both as to time of ripening and the property of hanging on the tree. Cox, an American pomologist, describes this plum as being raised by $\mathrm{Mr}$ Cooper of New Jersey from a stone of the Orleans. He adds, that, although it is a fine large plum, it is exceedingly liable to rot upon the tree. This opinion is strengthened by that given by $\mathrm{Mr}$ lves of Salem, and more so by Downing, who says it is scarcely known now in that country. Cox describes it as ripening in August, and that it is of the largest size. Lindley and Thompson consider it the same as the La delicieuse, and describes the latter" as having its "branches long and smooth; fruit oval ; suture rather broad, shallow, swelled a little more on one side than on the other; stalk an inch long, slender, slightly inserted; skin pale yellow on the shaded side, but deep purple on the other ; flesh yellow, and separates from the stone; juice peculiarly rich and abundant. Ripe in October with the $1 \mathrm{~m}$ peratrice." Such we have found Cooper's large to be. The difference in time of ripening, and also the American disposition of rotting on the tree, may safely be traced to climate. Of American origin. 
Corse's field-marshal and Corse's admiral.Two very good American purple plums, of rather large size and fair pretensions, but inferior to

Corse's nota bene.-Size rather above medium ; form round; quality good; colour pale lilac, or pale brown, somewhat green on the shaded side; flesh greenish, firm, juicy, sweet, rich, and parting freely from the stone. Tree very hardy, and a very abundant bearer. Originated near Montreal-a sufficient guarantee for its hardihood.

Cruger's scarlet.-Size above medium; colour red where exposed, with numerous golden dots, pale fawn-coloured in the shade; form roundish ovate; flesh deep orange, with a mild agreeable flavour. Ripe in September, and hangs long afterwards on the tree. Branches downy; suture rather obscure.

Damson.-This plum is too well known to require any further description than to state that there are three varieties in cultivationnamely, the Common, the Shropshire, and the White, all of which are exceedingly hardy and productive, and much valued for preserving. They are too little cultivated in Scotland, in most parts of which they would succeed as standard trees, and where the garden is limited might be advantageously grown in shrubberies or the edges of plantations, as hinted at for geans. The Shropshire is the true prune or round damson, and is decidedly the best. Synonyms in addition to the above-Early damson, Shailer's uhite damson. Damsons are frequently raised from the stone, so that varieties may be expected.

Damson, winter.-A valuable sort on account of its extreme lateness, hanging on the tree till the middle of November, six weeks after the common damson has disappeared. It is also an abundant bearer, and of hardy constitution. By far too little cultivated. Similar in colour, size, and form to the common damson.

Dean's Jedburgh seedling.-Colour purple; form roundish ; size medium; separating freely from the stone; of first-rate quality as a dessert fruit or for kitchen purposes; ripe in September. This excellent variety was raised a few years ago by $\mathrm{Mr}$ Deans, a respectable nurseryman at Jedburgh. It is quite hardy, and eminently fitted for standard culture.

Denniston's superb. - Size above medium; colour pale yellowish green, marked with a few large purple blotches; form round ; suture distinct, often extending quite round the fruit; flesh very thick (stone very small); flavour rich and vinous, approaching the Green gage, from which it is probably a seedling; branches downy; ripe in September; of American origin. Tree hardy and productive.

Diamond.-Size very large; colour purple; form oval ; flesh deep yellow; an excellent culinary fruit, but rather too coarse for dessert; ripe beginning of September ; of Kentish origin.

Diaprée rouge.-Size large; colour reddish purple; form obovate; flesh pale green, juicy, melting, and rich, parting freely from the stone, which is small; ripe in September; fit for either the kitchen or dessert. Tree hardy, and a good bearer, but slow grower; branches almost smooth. Synonyms - Roche corbon, Mimms, Imperial diadem of Thompson. Downing thinks, however, that it is different from the two latter, which are esteemed excellent sorts. The Diaprée rouge is of French origin, while the Mimms, according to the "Pomological Magazine," No. 2, p. 6, was raised from a stone of the Blue perdrigon, at Mimms Place in Hertfordshire. The same authority states that the Imperial diadem, which in no respect differs from it, and is much cultivated about Manchester, but of whose origin little is known, may probably be the same as the Mimms plum. Mr Thompson considers them the same.

Double bearing. - A small and indifferent plum, which we notice here to prevent planters being attracted by the name; for although it lipens fruit in succession during August and September, we have many superior sorts, both early and late, to afford a sufficient supply for a much longer period.

Drap d'Or.-Size small ; colour yellow ; form round, very much resembling the Green gage, except in colour, and in ripening a week earlier; flesh yellow, sugary, and rich, separating freely from the stone; branches slightly downy; ripe by the middle of August; of French origin.

Downton imperatrice.-Size under medium; colour pale yellow; form oval; flesh yêllow, melting, and sweet, adhering to the stone; branches long, slender, and smooth; ripens beginning of Uctober, and, like its class, hangs long on the tree, and will keep a considerable time afterwards. A hybrid raised by $\mathrm{Mr}$ Knight.

Early favourite (Rivers).- Size medium; ripe middle of July; said by Mr Rivers to be the best early plum in cultivation. We have not seen this fruit, and give it and the following on the authority of that gentleman.

Early prolific (Rivers).- Size smallish; early, hardy, and an abundant bearer, and, like the last, of excellent quality; ripe the end of June.

Emerald drop (Downing). - Size medium; colour pale yellowish green; form long, oval; flesh greenish yellow, very juicy and rich, slightly adhering to the stone; ripe in September; suture strongly marked; branches long and smooth; raised in America from a stone of the excellent Washington.

Fotheringham.-Size medium; colour deep red on the exposed side, bright red where most shaded; form obovate; flesh greenish yellow, separating freely from the stone; branches smooth; ripe in August. A very hardy sort, long cultivated, and in all probability of English origin. Synonyms-Sheen, Grove House purple. Well known so early as 1700 .

Gage, green.-One of the best known and most valued of plums, requiring no description further than giving a list of synonyms, and the following useful quotation from Rogers' "Fruit Cultivator," p. 351: "There are some spurious sorts of the Green gage crept into the English nurseries which should be guarded against; and great numbers of the Little Queen Claude are imported from France, and disposed of in this country as the True green gage. But these 
fruits are easily detected by judges: they have not that fine red and brown mottled cheek which the true sort have, neither is their flavour equal. The only fault of the True green gage is its liability to crack in wet weather." Synonyms - Bruyn gage, Schuyler's gage, Burgnon gage, Wilmot's green, New green, and Late green gree, Bradford gage, Isleworth green gage. 'The following are the French synonyms: Abricot vert, Damas vert, Sucrin vert, Vert bonne, Dauphine, Reine Claude, Grosse Reine Claude, Grosse Reine, Drap d'Or of some. Of early origin; introduced to England by Lord Gage from the Charteuse monastery near Paris. Said to have been introduced to France by Quecn Claude, wife of Francis I., but from whence does not very clearly appear. The best of all plums, if Woolston's black gage be excepted.

Gage, imperial.-Sizc above medium; form oval; suture very distinct; colour pale green tinged with yellow, marbled with dull green strips; flesh greenish, juicy, melting, and rich, generally separating from the stone-not always so, appearing to be affected by soil and climate; shoots slightly downy; of American origin, from a stone of the Green gage. It lias long held a high position amongst the plums of that country. Tree hardy, and a great and regular bearer, and has the peculiarity of succeeding better on light soils than any other of the plum tribe. In strong soils Downing asserts that the fruit is often insipid. Ripens about a week later than the Washington. Synonyms-Flushing gage, White gage of Boston, Prince's imperial gage, Superior green gage.

Gage, Lawson's golden.-In size and quality very much resembling the Green gage, from which it is a seedling crossed with the excellent Coe's golden drop. Originated by $\mathrm{Mr}$ Archibald Gorrie, a celebrated Scoitish pomologist. In habit this tree resembles the Golden drop parent, carrying also with it the fine golden colour, beautifully marked with purple spots. Flesh rich and melting, separating freely from the stone. The original tree is in Mr Gorrie's possession. Healthy, liardy, and an abundant bearer, even as a standard in an elevated situation in the Carse of Gowrie, a rather singular and happy circumstance, as neither of its parents is so hardy. Named in compliment to Charles Lawson, Esq., the most eminent of our Scotch nurserymen.

Gage, purple.-Size medium; form similar to the Green gage; colour purple or violet, dotted with pale yellow; suture shallow, but distinct; ripe in September, and hangs long on the trec; and although shrivelling, is not apt to crack, hence a useful fruit of its kind. Branches smooth; flesh rich and high-flavoured, separating freely from the stone. Altogether one of the best purple plums. Synonyms - Violet Queen Claude, Reine Claude violette.

Gage, red (of America).-In size and form resembling the Green gage, from which it is a seedling, raised by $\mathrm{Mr}$ Prince, senior, of the Flushing nurseries, in 1790. Branches smooth, ripening about the end of August.

Gage, Woolston black.-Size medium; form round; colour jet black, powdered with light blue; flesh yellowish green, parting from the stone; flavour equal in every respect to the Gieen gage, with this superiority, that while the latter is apt to crack in a wet summer, and will not keep after being gatliered, this, on the contrary, will endure, if the climate be dry, through August and September. This excellent plum deserves an east or west wall, although it ripens well on an open standard. Mr R. Thompson speaks of Mr Dowling's three plums in this manner: "Your three plums are first-rateviz., Angelin, Burdett, Standard of Englandand I should recommend their extensive cultivation. As regards Woolston black gage, I consider you have thrown the Green gage into the shade, for it retains all the properties of that plum with more sugary sweetness. I have no fear of my judgment being called in question."

Gage, yellow.-Size medium; colour pale yellow; form round; branches smooth; flesh separating freely from the stone; ripe beginning of August; somewhat rescmbling the Green gage, but not so rich in flavour. Synonyms-Keine Claude blanche, Conne's green gage, White gage.

Gisborne's.-Size medium; form oblong; colour yellow; branches downy; ripe in September; much esteemed for culinary uses. Tree hardy as a standard, and an abundant bearer.

Goliath.-Size large; quality excellent for culinary and market purposes ; showy, but only fit for secondary desserts, and inferior to the Colombia, an American sort of even larger dimensions, form roundish ; colour purple, dusted with blue bloom; flesh dull greenish yellow, a little coarse-grained, adhering partially to the stone. Ripe end of August. Tree hardy, strong grower, and an excellent bearer ; shoots somewhat downy, straight, and strong in growth. A very excellent culinary fruit. Of English origin. Synonyms-Caledonian of somc, Nectarine of others, Wilmot's late Orleans, St Cloud, Steer's emperor.

Guthrie's Scotch seedling plums.-If Scotland has been behind Belgium, England, and America in the production of new and improved apples and pears, she has so far redeemed her pomological character in the production of some very excellent plums, carrying with them an extraordinary degree of hardiness and productiveness, qualities essentially requisite in an unfavourable climate. 'The first of these, Larson's golden gage, we have described. The following are the production of Charles Guthrie, Esq. of Tay Bank, Dundee. Our descriptions were taken this season. We have only to remark that the Green gage appears, from Mr Guthrie's answer to our inquiries, to have beell largely concerned in their production. "The plums I formerly sent you I can give you na account of," says Mr Guthrie, "except that in my earliest attempts to raise new plums I had only the Green gage, Yellow magnum, and Old Orleans in my garden; and from the Green gage I almost exclusively saved sceds, the first seedling I ever fruited being the Tay Bank."

Guthrie's new apricot pium.-Circumference 4 inches by $4 \frac{3}{4}$; bright apricot colour all over ; shaded near the fruit stalk with broad dashes of russety red; flesh yellowish ; slightly adhering 
to the stone, which itself bears a striking resemblanee to that of an aprieot; juicy, rich in flavour ; ripe in September; an exeellent dessert plum. Tree hardy, and bearing abundantly as a standard.

Guthrie's minette plum. - In size much the same as the last; colour yellowish green on the shaded side, marked with irregular dashes and spots of russety red; form somewhat oblique ; stone moderate in size; flesh firm, juicy, and of excellent flavour. An excellent hardy plum ; ripe in September.

Guthrie's T'ay Bank plum. - $\frac{1}{2}$ inches in greatest circumference ; form orate; colour greenish yellow, with slight spots of russety red seattered over the sunny side; flesh juicy ; flavour approaching the Green gage, whieh we would suppose to be one of the parents. Through the kindness of $\mathrm{Mr}$ Guthrie we were favoured with specimens of this plum two years ago, and consider that the fruit is in progress of improvement since that time. This excellent fruit has been awarded the C. H. S. silver medal. A very valuable fruit for the climate of Scotland ; ripe also in September.

Guthrie's topaz.-Another of Mr Guthrie's seedlings, but one we have not seen in perfection, the specimen sent being injured in the carriage. It is thus described by Mr Rivers of Sawbridgeworth, who is growing it for sale : "Size first; quality first ; use, table ; season, October; colour green; inclined to shrivel on the tree."

Imperatrice, Dounton, vide Downton imperatrice.

Imperatrice, Ickworth. - Size rather under medium; colour purple ; form obovate; flesh greenish yellow, sweet and rieh, adhering to the stone ; branehes smooth ; ripe in September; hangs long on the tree if protected from autumnal frosts, and if wrapped in soft paper will keep several weeks in the fruit-room - a property all this class possess, rendering them, with Coe's golden drop and the Reine Claude de Bavay, the most valuable of all late plums. Raised by $\mathrm{Mr}$ Knight, and a hybrid between the Blue imperatrice and Coe's golden drop.

Imperatrice blue, vide Blue imperatrice.

Imperatrice white.-Size rather under medium form obovate ; colour white; ripe in September; branehes smooth; equally valuable for the table and preserving.

Jaune hative. - Only worth cultivating on account of its earliness; ripening about the middle of July if planted against a wall, and thus ushering in the plum season. Synonyms -Catalonian, Amber Primordian, White Primordian, Early yellow, Sainte Barnabe.

Jefferson.-Size very large; colour golden yellow, purplish on the side next the sun; form oval; flesh deep orange, parting freely from the stone ; flavour exceedingly high and luscious ; branehes slightly downy; ripe in September; will hang ten days on the tree; suture slightly marked. This is a comparatively new and a very highly esteemed American fruit. Tree hardy, and an abundant bearer. This is probably the best of all American plums, and only surpassed with us by the Green gage, Angelina
Burdett, and Woolston's black gage. It should find a place in every garden. It will require a wall in most parts of scotland to ripen it in perfeetion.

Kirl's.-Size above medium; form round; colour dark purple; flesh firm and very rich ripe in September; branehes smooth. 'Tree hardy, and an excellent bearer. The history of this excellent plum is not vcry well known. The late Mr Kirk of Brompton deteeted the fruit as being new to him on a fruit-stall in London, and after much search obtained grafts of it, whieh he propagated and sold extensively. An excellent sort for dwarf-standard culture.

Laurence's favourite. - Size large; form round; colour yellowish green, slightly clouded with a darker shade ; ripe end of August ; flesh resembling the Green gage, and nearly approaehing it in flavour; when ripe parting freely from the stone. A remarkably handsome and excellent fruit. Branches downy. Of Ameriean origin, from a stone of the Green gage. In general appearance a Green gage, only two or three times as large. Synonyn-Laurence's gage.

Lucombe's nonsuch. - Size above medium; form roundish; colour somewliat resembling the Green gage, only more distinctly marked with a yellow orange tinge; flesh rieh, sweet, adhering to the stone; ripe in September. Tree hardy, and an exeellent bearer. Originated in the highly respectable establishment of Lucombe and Pince of Exeter. Branches smooth. Lucombe's seedling of Thompson is probably a synonym of this excellent plum.

Magnum bonum, red-Magnum bonum, white. -Two plums sufficiently well known to require no deseription, further than to enumerate their synonyms, with a view to prevent imposition and confusion. Synonyms of the former-Imperiale, Red imperial, Florence, Purple egg, Purple magnum bor.um. Synonyms of the latter-Yellow magnum bonum, White mogul, Wentworth, White Holland, Egg plum. Both valuable for preserving. The latter, when fully ripened, of pleasant flavour. Downing remarks that the Red magnum bonum has frequently been produced from seed on the North River with little differenee of eharacter, exeept that some were clingstones and others freestones.

Morocco. - Size medium; form roundish ; colour very dark purple; flesh yellowish green, adhering partially to the stone; flavour rich, becoming very sweet when fully ripened; branches downy. Tree hardy, a moderate Dearer ; ripe beginning of August. Synonyms -Early damask, Black damask, Black morocco, Early morocco, Early black morocco.

Orange.-Size very large; form ovate; colour bronzy yellow, with many roughish white dots, slightly purplish towards the fruit stalk; ripens about the lst of September ; branches smooth ; flesh deep yellow, rather coarse-grained, adhering to the stone. This is recommended only on account of its large size, being probably the largest of all plums, and its fitness for market or culinary purposes. Tree hardy, and a great bearer. Of American origin. Synonym Orange gage.

Orleans. - This popular culinary plum has 
become numerous in varieties, between which it would be difficult to define the difference ; any or all may be cultivated with about the same amount of satisfaction. They are hardy and good bearers, and admirably adapted for standard culture.

Pond's seedling.-Size above medium ; form roundish ; colour purple; ripe beginning of September ; branches smooth ; flesh yellowish, separating freely from the stone; flavour secondrate. We introduce it here on account of its productiveness, and property of hanging a long time on the tree.

Reine Claude de Bavay.-Size largish ; form roundish oval ; colour yellowish white ; ripens end of September, and keeps long on the tree, and on this account is at present, perhaps, the most popular late plum of its class. A very fitting sort for the orchard-house or late plumhouse.

Royal.-Size medium; form round, sometimes slightly ovate; colour reddish purple, covered with a remarkably thick pale bloom; flesh dullish yellow, parting freely from the stone; flavour exceedingly rich and vinous; shoots downy. Tree hardy, and a most abundant bearer ; ripe towards the middle of August, but will keep hanging on till the middle of September. Of French origin, but long cultivated in this country. Synonym-La royale, excellent for dwarf-standard culture.

Royale de Tours.- - Size large; form roundish; colour red in ihe shade, deep violet next the sun, sprinkled with numerous yellow dots; flesh greenish and firm; flavour exceedingly rich and agreeable; suture deep, extending more than half round; branches smooth ; ripe during August. Originated or introduced from Tours in France. An excellent early variety.

Royale hâtive.-Size medium ; form roundish; colour light purple; branches very downy; ripens beginning of August ; flavour exceedingly rich; in general appearance and flavour resembling the Purple gage. Of French origin.

Standard of England. - Size rather large; form oval; colour crimson red next the sun, covered with violet bloom, bright red on the shaded side; flesh slightly yellow, separating from the stone; flavour exquisite; ripens the latter end of August. Originated from seed about ten years ago by Mr Dowling, Woolston, near Southampton.

Victoria (Denyer's).--Size large ; form ovate ; colour brightish red ; ripens about the middle of September. Of English origin within these late years. This is a large and very showy plum, but of no particular merit as to flavour. It is, however, for culinary purposes, highly deserving of cultivation. It appears to us to approach so closely to Sharp's emperor, and Duane's purple French of America, as to lead us to believe them identical, or if different, scarcely so much so as to deserve a place each in the same garden. Denyer's Victoria has the merit of suffering less from spring frosts while in blossom than any other.

Washington.--Size large ; form roundish oval; colour dull yellow, marbled with green, and where exposed to the sun slightly dotted with VOL. II. pale crimson ; suture obscure, except near the fruit-stalk ; flesh firm, yellowisl, parting freely from the stone; flavour sweet and luscious; wood strong and downy; tree remarkable for its strong growth and the large size of its leaves, which are crumpled and glossy. Ripe towards the end of August. This splendid plum is a native of New York, where, and over most of America, it stands higher in general estimation than any other. "Although," says Downing, "not equal to the Green gage and two or three others in high flavour, yet its great size, its beauty, and the vigour and hardiness of the tree, are qualities which have brought this noble fruit into notice everywhere." From America it reached England in 1821. It does not appear even in its native country to attain its fullest perfection, if planted in a sandy soil, until it has attained some age.

The majority of the plums we have described above are dessert sorts, and of the highest class; from the number some will be found adapted to most situations and requirements. We have omitted many well-known sorts, but all or most of those we would consider no loss if expunged from our gardens entirely. No argument can convince us that it is expedient to retain an inferior fruit tree in any garden, more especially if occupying wall-room, nowadays, when we have such a variety of first-rate sorts to replace them with, merely because they have been long residenters, and we have been content with the fruit, inferior although it may be. We have included a number of American sorts, because that country is making great progress in the rearing of superior kinds, the climate and the soil, particularly along the banks of the Hudson, which is for the most part a stiff clay, being so congenial towards bringing the plum to its greatest perfection. We have noticed one or two to which a light soil is apparently better adapted.

The following list includes those most esteemed for preserving and drying purposes-viz. :

Autumn compote.-A fine preserving plum and abundant bearer, raised from a stone of $L a$ delicieuse by $\mathrm{Mr}$ Rivers. It hangs on the tree till the middle of October.

Stoneless.-This curious sort was brought to our notice many years ago by the late Mr Kirk of Brompton. We have found it much prized by confectioners. The kernel is destitute of stone, and is enclosed in a roomy cavity by the pulp alone.

Wine sour, or Rotherham.-A most abundant bearer and hardy tree. The flavour is a peculiar acid, somewhat like tart claret; hence the name.

Saint Catluerine.-A good bearer, producing the most delicate kind of prunes.

Quetsche.-This forms a considerable section of preserving plums, cultivated to a great extent over most of the continent of Europe for the purpose of drying. The German prune of the shops is made from them.

Perdrigon blue.-This and the white variety furnish the Brignole prunes of commerce.

Mirabelle.-Much esteemed for preserving, and exceedingly hardy.

Muscle, also used for preserving.-It is inferior to most of the others, but is grown largely for stocks, and will succeed as a productive $3 \mathrm{Y}$ 
bearer in climates where the other better preserving sorts would not.

Damson.-One of our most popular preserving plums, deserving of extensive cultivation as a standard tree.

Bullace.-Black, white, and yellow, excellent for culinary purposes, and of extreme hardihood.

Alibuchari.-A sort of Quietsche, well adapted for drying. Tree very hardy, and an abundant bearer.

The following are chiefly grown for stocks.Brompton, Brussels, Muscle, Pear plum; Cerisette, Damas noir, the latter much grown by the French, Mirabelle for dwarfing purposes; and Mr Rivers has lately turned his attention to the common sloe (Prunus spinosus) for a similar purpose. Stocks are also obtained by sowing the seeds of St Julian, Magnum bonum, and such free-growing sorts, and layers and suckers of the same are often taken. The stock from seed is by far the best where a long duration of the tree is desired. The Damson is not a good stock to bud on.

Select list for particular situations and purposes.-We have been favoured with the following list as growing in one garden within twenty miles of London: Kirk's plum, Royale hâtive, Reine Claude Violette, Précoce de Tours, Early and Ickworth imperatrice, Green gage, Reine Claude de Bavay, Coe's golden drop, Jefferson, Washington, Denyer's Victoria, Reine Claude d'Octobre, Coe's late red, Columbia, White magnum bonum, Goliath, Early Orleans, Shropshire darnson, and St Martin's Quetsche, the two last for preserving.

Sorts grown at Drumlanrig Castle, Dumfriesshire, on a cold retentive clay soil.-Coe's golden drop, Coe's fine late red, Kirk's, Green gage, Blue gage, Jaune hâtive, Magnum bonum white, Magnum bonum red, Nectarine (or Caledonian), Orleans, Wilmot's new Orleans, Washingtonall as wall-trees, Damson only as standards.

Sorts grown at Culzean Castle gardens, Ayrshive, close to the sea.-Coe's imperial (synonym of Coe's golden drop), Diamond, Diaprée rouge, Magnum bonum white, Green gage, Annat green gage (synonym-Lawson's golden gage), Imperatrice blue, Imperatrice white, Inperatrice Ickworth, Jefferson's, Kirk's, Orleans early, Orleans Wilmot's, Reine Claude violette, Reine Claude d'Octobre, Denyer's Victoria, Nectarine (or Caledonian) on walls.

Sorts grown at Dalkeith, Mid-Lothian, on a dry light made soil, one hundred feet above the sea, and within three miles of it.-Coe's fine late red, Coe's golden drop, Cooper's large, Damson, Shropshire (as standards), Diaprée rouge, Drap d'Or, Yellow gage, Ghiston's early, Goliath, Green gage, Imperatrice Downton, Imperatrice blue, Imperatrice Ickworth, Jefferson, Kirk's, Magnum bonum red, Magnum bonum white, Morocco, Nectarine, Lucombe's nonsuch, Orleans early, Orleans, Wilmot's, Reine Claude de Bavay (not yet fruited), Reine Claude violette, Victoria, Denyer's, Washington, on walls, believing, however, that most of them would succeed as well as dwarf standards or espaliers.

Sorts recommended by $M r G$. Lindley for the Highlands of Scotland. - Coe's golden drop,
Diaper, Early Orleans, Fotheringham, Goliath, Green gage, Imperatrice, Kirk's, Lucombe's nonsuch, Morocco, Orleans, Prune Swisse, Purple gage, Red magnum bonum, Violette hâtive, Wentworth, which latter is synonymous with White magnum bonum.

The same authority gives the following, exclusive of those in the last list, as being adapted to the northern counties of England and southern of Scotland: Early amber, Nectarine, Précoce de Tours, Imperial diadem, Mimms, Prune damson, Washington, La delicieuse, Saint Catherine, White bullace.

It would be of little use giving lists of fruits ripening in the southern counties, as it is climate we require; the great object to be kept in mind in forming such lists is to ascertain those good sorts that will ripen in ordinary or bad situations. In good situations all we have described will assuredly succeed.

The following lists have been published by Mr Rivers, in his "Miniature Fruit Garden," of the sorts of plums adapted to be grown as pyramids, according to his ingenious mode of rootpruning, and using the sloe as a stock, with a view to curb the over-luxuriance of the plum, as well as from its being a stock of great hardihood, and whose roots do not appear to be much affected by soil, provided it be dry. We should, however, here remark, that the object in view is to produce trees of very moderate growth, and of early and great productivenessthe very reverse of what has been hitherto recommended for orchard trees, but the mode, with very few exceptions, that should be regarded as the best for garden culture.

Hardy dessert piums adipted for pyramids.Early favourite, Early Orleans, Royale hâtive, Monsieur Gross Surpass, Perdrigon, Violette hâtive, Green gage, Purple gage, Washington, Jefferson, Reine Claude de Bavay, Coe's golden drop, Imperial de Milan, Fellenberg, Reine Claude d'Octobre, Saint Martin Quetsche, Coe's late red.

Hardy kitchen plums adapted for pyramids.Early prolific, Goliath or Caledonian, Prince of Wales, Denniston's superb, Nectarine, Mimms or Diaprée rouge, Royal de Tours, Denyer's Victoria, Yellow magnum bonum, White mag. num bonum, Blecker's scarlet, Orange, Smith's Orleans, Autumn compote, Downton imperatrice, Frost gage. The latter, we fear, will be found too late in ripening in the north.

Diseases and insects.-The plum, in proper soils, and in moderately good climates, is comparatively a healthy, hardy tree, but not remarkable for attaining a great age. Canker and gum attack it in uncongenial situations, and follow accidental wounds, as well as the removal of large branches, if not very neatly performed. The green and black aphides, red-spider, and thrip, are its common insect-enemies in Britain. In America their attacks are so formidable as almost to discourage its cultivation in some parts of the United States. The curculio or plum-weevil (Rhynchønus nenuphar') is, Downing observes, "the uncompromising foe of all smooth stone-fruits. The cultivator of the plum, the nectarine, and the apricot, in 
many parts of the country," after the most flattering appearance, "has the frequent mortification of seeing nearly all, or, indeed, often the whole crop fall from the trees when half or twothirds grown;" and this is the effect of this insect alone. The attacks of insects in America are of a far more serious character than with us; for really, with the exception of the aphides, our trees seldom sustain much damage.

Hylobius abietis, fig. 232, a large beetle often found on the coniferous trees, sometimes
Fig. 232.
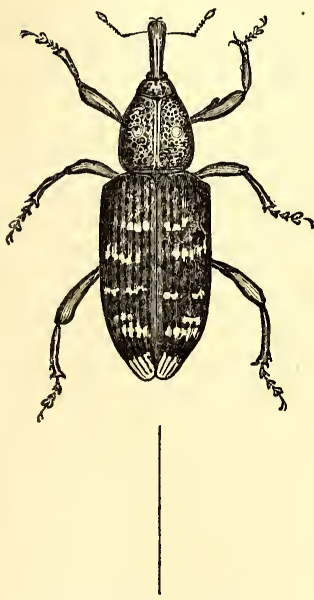

HYLOBIUS ABIETIS. attacks the leaves and young shoots of the plum, as well as occasionally the peach also. From its size it. is readily picked off and destroyed.

Curculiotenebricosus, fig. 229, a member of the beetle family, sometimes attacks the plum as well as the apricot -(which see, p. 522). Thrips ochraceus infests the fruit of the plum when it is ripe, and in dry seasons. Theleaves suffer from its attacks also. Copious syringing the trees previous to the fruits beginning to ripen is apparently the only means of defending our crops from it.

The Red plum grub, Tortrix (Carpocapsa) nigricana Schmidb., fig. 233, is most destructive to the fruit, in which the grub, while yet quite

Fig. 233.

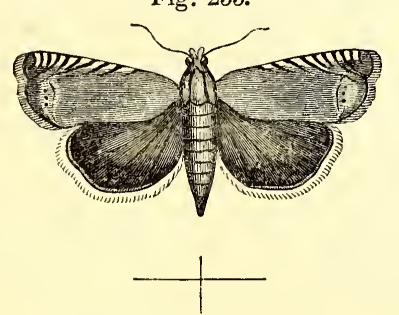

FED PLUM GRUB, MOTH.

small, buries itself, penetrating to near the stone, and feeding on the pulp, causing the fruit to drop off. Having attained its full grub size within the fruit, it creeps out and penetrates the loose bark of the tree, forming a habitation in which it resides during winter. It changes into a light-brown pupa early in spring, and emerges in its moth state about June. The size of the moth is not much more than that of the common house-fly ; wings nearly black, and, when the sun shines upon them, presenting a remarkable metallic lustre. The fruit which falls in consequence of the attack of this insect should be gathered up, and so disposed of as to prevent the caterpillars from reaching the tree. The bark of the old branches and stem, on which it finds a sufficient protection for the winter, should be painted over with spirits of tar The best way, however, is to carry away every fruit before the caterpillar has time to quit them.

Tortrix Wreberana, fig. 230 , in its larva state, feeds upon the inner bark of the plum, as well as on that of the peach and apricot. Its mode of attack, \&c., will be found described, p. 522 .

The copper-coloured weevil, Curculio (Rhynchitis) cupreus Schonl, fig. 234, according to Kollar, is called the Pfaumenbohrer, or plum-

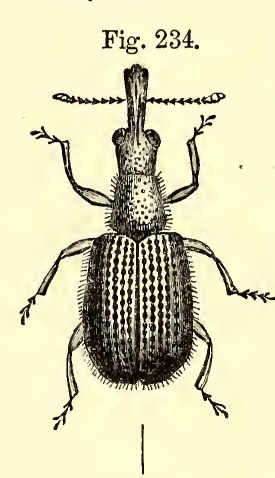
borer, in Germany, because the plum is selected for the reception of its eggs, and for the nourishment of the little larva proceeding from them. In default of plums, they make use of the soft spring shoots of the plum and apricot tree. "When the plums have attained the size of large almonds, the female weevil selects one in which to deposit her eggs. As the larva can only be transformed COPPER-COLOURED WEEVIL. into a beetle when buried in the earth, and as, when

buried in the plum,it cannot come down of itself from the tree, the parent beetle obviates the difficulty by cutting through the stalk of the plum with her proboscis as soon as shehas deposited her egg. As if the operation of cutting through the stalk were too great a fatigue to be accomplished at once, she divides the labour, and first cuts the stalk only about half through, leaving the other half to be divided after the egg has been laid. She then gets on the plum, and, fixing her abdomen on the stem, begins cutting with her proboscis the epidermis of the fruit into arched cuts; she then raises up the skin, bores a hole beneath it in the pulp, and forms a roomy chamber for her offspring. When this is accomplished, she lays her egg at the entrance of this apartment, and pushes it in with her proboscis. She then turns the epidermis down, closing the entrance to it so effectually that not the smallest drop of water can reach the egg. She then cuts the fruit-stalk completely through, causing the plum to drop off. Laying the egg and cutting through the fruit-stalk occupies her two or three hours, from which it will be understood that but few eggs are deposited in the course of one day, and the more so as only one egg is intrusted to each plum. The egg does not remain long dormant in the plum on the ground. If the weather be favourable, the larva will have consumed the interior of the plum in five or six weeks, and having attained its full size, it will be ready for its final change. It then buries itself into the earth, where it awaits its transformation. This takes place the following 
spring, when it appears as a beetle, and again begins the work of regeneration." We have given this long extract because it shows the habits of this and similar insects, and points out pretty clearly how we are to rid ourselves of them. As the fruit continues to be cut off day by day, and as a few weeks are necessary to accomplish this and the hatching of the grub, during that time every plum found on the ground should be carefully gathered up and destroyed before the grub quits them for its earthy abode. The eggs are laid from the latter end of June to the end of July. It is only by a knowledge of the economy of insects that we can ever expect to find out the proper means for their destruction, and in this case it is exceedingly well exemplified.

The brindled beauty-moth (Biston hirtarius), fig. 235, appears often in March, as soon as the leaves upon which it is to feed begin to ex-

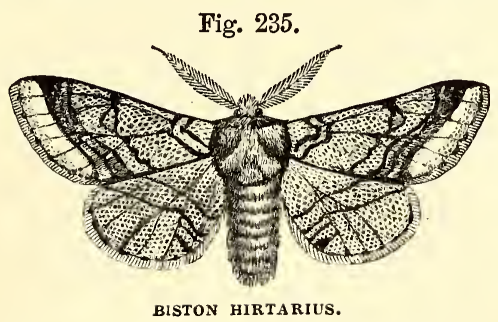

pand themselves. More generally its appearance occurs in April, in which latter case it is most destructive. Those appearing earlier are often killed by the frost. The caterpillars appear in May, and feed on the leaves of the plum, as well as on those of other fruit trees. They are of a greenish-brown colour, with greyish marks and numerous tubercles. The moths measure from an inch and a half to two inches from tip to tip of the wings. They are of a dark yellowish grey, or light brown, thickly spotted with dusky atoms, and three or four dark equidistant streaks on the hind margin. The posterior wings are similar in colour, having generally three streaks, which in general are nearly imperceptible. The wings of the female are more transparent; their transverse bands are nearly indistinct, and the colouring less distinct. The caterpillars change to the chrysalis state early in July, and remain in that state till spring.

The larva of the plum saw-fly (Tenthredo morio Fabr.) is one of those which attack the fruit in its young state, instead of the leaves. The cause of the fruit of the plum tree often falling off when scarcely the size of a pea is well ascertained to be owing to the female of this insect making choice of the newly-expanded blossom as a nucleus in which to deposit her eggs. It is said by some that she prefers the blossoms of the largest kinds of plums, and seems to pass over the Damson, as unfitted for her purpose. The eggs are laid between the teeth of the calyx of the flower, in a hole in the most fleshy part, which she saws out in an oblique direction, but not extending completely through. In each of these she deposits an egg, which is very small, whitish green, and transparent. Hatching is completed in a few days thereafter, when a delicate whitish larva makes its appearance, with a dark-brôwn head, six pairs of middle feet, three pairs of fore feet, and one pair of anal feet. Kollar says that it then leaves its birthplace, where there is no suitable food for it, and hastens to the plum growing near it, which is then scarcely the size of a hemp-seed, and attacks it. It fixes itself in the centre of the plum-stone, which, being tender and milky, affords it the best nourishment: the plum in the mean time continues to grow, and the larva with it, so that the latter never wants for food. It has attained its full size in six weeks, when it is ready for its final destination. The plum then drops to the ground, when the insect buries itself in the earth, and surrounds itself with a covering. It remains in this state till the forthcoming spring, when it undergoes its last transformation, and appears again as a perfect saw-fly, which again ascends the plum tree and continues its species. "The destruction of the fallen plums in this case also should be attended to with care; and it is highly probable that syringing the tree about mid-day with clean water, during the time of the trees blossoming, would be equally beneficial; for it is a well-known fact that the female is rarely seen before 8 A.M., or after 4 or 5 P.M., but that during the middle of the day, particularly in fine weather, she is very busily engaged in depositing her eggs, but on wet days she is seldom seen so engaged. The Germans have a proverb, 'If the plum crop is abundant, it must have rained during the time of blossoming."

The European names of the plum : Prunier, French - Pflaumenbaum, German - Prugno, Italian-Ciruelo, Spanish-Prüimboom, Dutch - Amexieria, Portuguese-Sliwnik, RussianSliwina, Polish. Barkuk, Arabic.

The names of the following plums are pronounced as under: Abricotée Rouge - Ab-reco-tay Rooje; Diaprée Rouge-De-ah-pray Rooje; Drap d'Or-Drah-dor; Jaune HâtiveJaun Hat-eve; Mirabelle-Me-rah-bell ; Précoce de Tours-Pray-cose de Toor; Prune SuissePrune Su-ece; Royale Hâtive-Rwoy-al Hat-eve. 


\title{
CHAPTER IX.
}

\author{
THE CHERRY.
}

THe cherry, in a cultivated state, was introduced to Britain, from Italy, about two hundred years after Christ. Of its earlier history or origin we know little or nothing. The majority of botanists, from the time of Linnæus to that of Decandolle, refer the cherries of our gardens to Prunus avium L., and Prunus cerasus L.; the former colresponding with our small wild black cherry of the woods, and the latter with our common red sour cherry. Decandolle, however, has referred the Guigniers, or geans, to Cerasus Juliana, and the Bigarreaus, or hard cherries, to Cerasus duracina. Under these four species, Seringe, in Decandolle's "Prodromus," has attempted to form a classification, which does not appear to be very satisfactory. A more simple and better arrangement was afterwards formed in the "Nouveau Du Hamel," in which all the cultivated varieties there enumerated are referred to the same species as Linnæus assigned them to, substituting, however, Cerasus sylvestris of Ray for Prunus avium L., and Cerasus vulgaris for Prunus Cerasus L. The French pomologists usually arrange their cherries into five classes-viz., Merisiers, Guigniers, Bigarreautiers, Cerisiers, and Groittiers. The first of these includes the wild cherries of our woods; the second, the geans and part of the Hearts; the third, Bigarreaus and remainder of the Hearts; the fourth, such as the May duke, Kentish, and Flemish; and the fifth, the Morello tribe.

Mr R. Thompson some years ago drew up a classification of cherries, which is the most perfect we are at present aware of, and will be found in the "Transactions of the Horticultural Society," $2 \mathrm{~d}$ series, vol. i., p. 248. It is somewhat singular that the wild cherry of Europe has only been comparatively lately naturalised in America. Downing says, "It is the original species from which nearly all the fine varieties of hearts and other sweet cherries have sprung. It is chiefly valued in America for the manufacture of cherry brandy. It affords also the most valuable seedling stocks on which to bud and graft finer varieties." Seventy-two sorts are described by Downing, besides several grown for ornament. So early as the Roman invasion eight kinds were known. In 1415 the fruit was publicly sold in the streets of London. In 1573, Tusser mentions red and black cherries;
Parkinson, thirty-four sorts; Ray, twenty-four ; Miller, eighteen; the Luxembourg garden, about the beginning of the present century, possessed forty-two sorts. Messrs Lawson's Catalogue gives forty-four sorts, Rivers fiftynine, and Rogers twenty-five ; while the "Fruit Catalogue of the Horticultural Society," published in 1842, enumerates eighty sorts, and to this number considerable additions have been made, originated either in Europe or America. The Americans classify cherries under four heads; namely, Heart cherries, Bigarreau cherries, Duke cherries, and Morello cherries. For ordinary purposes this arrangement has its advantages.

$U$ ses.-The cherry is not only highly prized when ripe as a dessert fruit, but is extensively employed in domestic economy for pies, tarts, \&c.; the fruit is also dried and used as a conserve, and, when ripe, for making cherry brandy. The celebrated liqueurs, Maraschino, Ratifia, and Kirschwasser, are also made from this fruit. The first is manufactured chiefly in Italy, and is distilled from the leaves and kernels of a small gean or mazard, pounded to cake in a mortar, mixed with honey, and slightly fermented. The last is a distillation of the juice of the common black mazard or gean, in which the stones are ground up with the juice, the whole being fermented before distillation takes place. The gum is nearly equal in quality to gumarabic, and the wood is hard and durable, taking a fine polish. It is greatly planted as a roadside tree in Switzerland and Germany, forming delightful avenues, both beautiful and useful. One of these in Moravia extends the length of six ty miles-namely, from Brunn to Olmutz; and there are others of equal extent between Strasburg and Munich. 'I'hese avenues are planted by the government, and all persons are allowed to help themselves to the fruit, the only condition being that they do not mutilate the trees. The Morello is preferred for cherry brandy, and the Kentish, from its peculiar property of slipping from the stone, for drying.

Propagation. - Like all other fruit-bearing trees, the cherry is propagated by seed, when new or improved varieties are desired. Knight has long since asserted that no other fruit tree sports so much into varieties as the cherry, when originated from seed; and he further 
observes that new varieties are much wanted, as according to his views all the old sorts are hasting to decay (a favourite doctrine of his regarding all fruit-bearing trees, but one with which we cannot concur). Still we think the production of new varieties from seed may peradventure put us in possession of kinds superior to those we at present have; indeed we have sufficient proofs of that in the many new varieties produced of late years. The stones of cherries are sown in light sandy soil in autumn, whether with a view to obtain new varieties, or merely for obtaining stocks on which to bud or graft already known kinds. The plants remain in the seed-beds for two years, when they are taken up and planted in nursery lines; 3 feet apart, and 1 foot distant in the line. The season following they are fit to bud, if ordinary dwarf trees are desired; but if standards, they require from three to four years' growth, by which time they have attained strong stems. The grafts or buds of full standards are placed nearly 6 feet from the ground, for half standards 4 feet, and for dwarfs from 6 to 9 inches from the ground. Budding is performed in summer, and such as fail to take may be grafted the spring following. Those who grow cherry-stocks for the trade ought only to employ the seed of the small black or red wild cherry for that purpose. Dwarf cherries are preferred when grafted, as they make the better plants. Regarding the proper stock for very dwarf cherries, to be grown in pots for orchardhouse culture, or for growing in the open air in what may be called a miniature cherry-orchard, Mr Rivers observes: "The Cerasus mahalab, or perfumed cherry, is most eligible as a stock for dwarf cherries. It will grow well in calcareous and shallow soils, which are unfavourable to the common cherry-stock; it is, however, better adapted for that tribe of cherries of which the May duke is the type, and for that of which the Morello is the representative, than for the Bigarreau and Heart cherries, which are apt to gum and grow too rapidly in proportion to the stock."-The stones of the common cultivated cherry are often employed by nurserymen on account of their being more readily procured. They make the worst of all stocks, and trees so produced are liable to disease, and are shortlived. Stocks are also propagated by layers and cuttings from the roots, as well as by removing the suckers which are often thrown up from the roots. Neither of these, however, equal in health and longevity those originated from seed of the wild sorts. The Morello is also used for a dwarfing stock.

Planting and soil.-The cherry seems to be rather capricious as regards soil. We have seen the best crops, and the healthiest trees we ever saw, lately growing in a deep strong clay, while we remember well that the oldest cherryorchards in England are planted on deep sandy loams, often resting on a rocky bottom. Generally speaking, however, this tree thrives best on a good deep loam, and worst of all on thin gravelly soils. The Morellos, Bigarreaus, and Hearts prefer a stronger soil than the Dukes and their kindred. In regard to situation, a dry airy one is the best, as in close, damp, and shaded places the tree seldom prospers. The majority of cherries succeed well in all moderate climates, when planted as dwarf standards not exceeding 8 or 9 feet in height. In this way they are readily protected from birds by netting over each tree, when they stand far apart from each other, or by netting over a row or plantation when they are planted close together. Some years ago an admirable specimen of a cherry orchard existed at Hylands, in Essex, and "may do so still, completely enclosed within a close wire-covering, sufficiently high to admit of trees 9 feet in height being grown within it, the wire-work being close enough to exclude birds. A cherry orchard of sufficient size to supply a large family, if planted with dwarf trees, and those judiciously selected, so that the very earliest, intermediate, and very latest ripening sorts are introduced, would give a certain supply, and the season of this fruit would be greatly prolonged-say from the beginning of June till the middle of October-taking for example the Early May and May duke for the earliest, and ending with Coe's late carnation, and the different varieties of Morellos (vide SELECT LIST). which latter hang long on the tree, and improve in flavour after they are ripe. Were such an orchard covered with galvanised octagon wirenetting, supported on neat metallic posts and rails, with gates of entrance and exit, it would be a valuable appendage to a first-rate garden. To plant cherries or plums on walls, unless it be a few of the earliest sorts of both, and such as the Reine Claude de Bavay, Reine Claude d'Octobre, the Imperatrice, and Coe's golden drop among the last, which would not, in all situations, ripen without the aid of a wall, is useless; and where walls are expressly built for such a purpose, it is little short of wasteful extravagance. In most parts of Britain the May duke and many others would come to as great perfection if grown as dwarf standards as they do on walls. If protected from birds, they keep better as standards. It is of importance that this fruit be fully ripened, for, like the grape, the cherry can hardly be over-ripened, and both are in general used before they arrive at full maturity. The Kentish cherry is a valuable sort much neglected in the North, where it succeeds well as an open standard. The Morello also succeeds in many places better as a standard than as a wall tree, and, being later in coming into blossom, has a better chance of escaping our destructive spring frosts. The cherry is impatient of much pruning, therefore when planted in a not over-rich soil, and treated as a dwarf standard, little pruning will be required, especially so if attention be paid to the roots, by a curtailment of them when too gross a habit begins to be manifested in the tree. 'To grow standard cherries to the height of 20 or 30 feet is the height of absurdity. On such as these a greater weight of fruit will not be produced than on those not much more than a quarter the height. While speaking of this class of fruits, we should remark that there is a section of them called Geans, much better known in Scotland than in England, generally of a very hardy, high-flavoured, and productive descrip- 
tion, which deserve more general attention on the part of cultivators (vide SELECT LIST of Cherries.) The finer varieties of them should find a place in every cherry orchard. These may be stigmatised as the progeny of our wild cherry, or Merry of the English ; but what, let us ask, is the parentage of our Dukes, Hearts, Bigarreaus, \&c? - they are the offspring of the wild cherry, wherever they have come from. As to soil, Mr Errington says, "Less preparation is necessary for the cherry, in regard to soil, than for any other fruit tree, as it is not so impatient of indifferent subsoils as some of our other fruits, neither is it so liable to disease, gumming generally through accidental wounds-being the evil cherry trees are most liable to." This, we think, admits of some qualification, else why, in the same garden, in different soils, do cherries differ so much in their health and productiveness? The cherry, we think, as we have stated above, is exceedingly capricious as regards both soil and situation. To prolong the season of this delightful fruit, a tree or more, according to the contemplated demand, of the earliest sorts, should be planted on a southern wall to begin the season with, and probably follow sharp on the heels of the crop produced by artificial means in the cherry-house or covered cherrywall; for it should be borne in mind, that from the time cherries or any other fruit make their appearance on the dessert-table a supply should be kept up; and hence one of the many advantages of not producing such fruits as cherries, strawberries, peaches, \&c., earlier than a supply can be continued. With our orchard systen of growing the cherry, it may be expedient to have a tree or two on east or west aspects, to fill up the lapse between those on the south wall and those in the open orchard. Again, there is an excellent cherry called Buttner's October Morello, which is one of the latest of all; a good breadth of a north wall should be planted with it, which by netting, and afterwards by covering with canvass during night, may be continued up to November, frost only putting an end to its season. There is an excellent variety of Morello known as Sir Herbert Taylor's Morello, which, by the way, we think is identical with Ronald's large Morello, a variety grown some years ago in Hampton Court gardens. But really these royal gardens, in the olden time, caused vast confusion in the nomenclature of fruits ; everything extra good in them had the word royal, or the name of some great person, prefixed to it. The variety we allude to is a good one, and may be heard of through the Messrs Jacksons of Kingston, the lessees of the royal gardens at present. This cherry should be planted to some extent on a south wall, for when ripened thoroughly it is a most agreeable addition to our dessert sorts, which those on north aspects seldom are. Those who are disposed to adopt the dwarf-tree cherry-orchard principle should consult what Mr Rivers, of Sawbridgeworth, says and does upon the subject. He is a great man in the dwarfing way, and acts upon the principle of beginning at the beginning. By root-pruning he limits the amount of sap to suit the circumstances he has in view, or, what is by far a more natural course, by adapting the stock to the purposes sought. The cherry, like most other fruit-bearing trees, when grown in the fine climate of the south of England, succeeds in borders and soils of a very considerable depth. We would not, however, recommend the same latitude to be given to their roots in cold, and more especially damp and wet localities. In these, we say, let the soil be shallow, and thoroughly drained below. Let the roots take rather a horizontal direction than a perpendicular one, and make up in length and breadth what you deny them in depth.

Pruning and training the cherry.-The cherry may be very successfully grown in most parts of Scotland as a standard tree, or trained to an espalier. Several of the cherries, and amongst these the Morello, are well adapted to this mode of training, and would be much more becoming tenants to it than many of the hardy and coarse apples we often see indulged with a place upon it. In regard to training on walls, the horizontal mode has been recommended by Harrison, Forsyth, and others, while the fan form is that usually adopted and in the highest repute amongst our best cultivators. Pruning and training the cherry in the horizontal manner are thus performed: The young tree is headed down in spring, the first year after being planted, to seven buds. The second year the upright leading shoot is cut down to about a foot, and all the side branches are laid in horizontally and never shortened. This process goes on year after year until the tree has attained the height of the wall or espalier, and covered the extent laterally which it is intended to occupy. The third year natural spurs will begin to be formed, and artificial ones may be secured by cutting back the young side-branches to two eyes or buds, which buds will produce fruit the following season. The regulation of the spurs afterwards is much the same as that recommended by Harrison for apples and pears (vide p. 481). The Morello, although recommended by some to be trained in the horizontal or half-fan manner, is by others treated in the same manner as recommended by Seymour for the peach (vide fig. 216), which tree in its habits it somewhat resembles. Like it, it bears its finest fruit on the young wood of the preceding year's growth, and sometimes, but with less certainty, on spurs formed on the two-year-old wood, and scarcely ever on wood of a greater age : therefore a sufficient supply of young wood should be left, and as equally distributed over the tree as possible, attending to secure a constant supply near the bottom, as, if this were neglected, it would render the lower parts completely barren. Worn-out or illplaced branches must be removed at every winter pruning, to make way for the young wood to be laid in. Morellos are often ruined from laying in the wood too close; and in consequence of this the wood is too weak to produce healthy, strong, fully-developed flower-buds, and those it does produce, should they ever expand into blossom, will upon examination be found defective in the parts of fructification from their debilitated state. Mr Underwood, on examining the blossoms produced on such 
crowded shoots, found that in fifty flowers there was not above two styles; of course, under such circumstances no fruit could be expected. The blossom-buds in Morellos for several years at first are weak, and do not set their fruit so freely as after they have attained a greater age.

As cherries vary in habit, so do the modes of pruning and training differ also. To render this more perspicuous we will suppose cherries divided into three classes-viz., the small-leaved kinds, of which the Morello is one; the mediumleaved sorts, to which the Dukes belong; and the large-leaved ones, such as the Hearts, Bigarreaus, \&c. The Morello leaf is somewhere about 3 inches long, the young wood should therefore be laid in about that distance shoot from shoot, the Dukes about 5 inches, while the Hearts, \&c., should not be closer than 6 or 7 inches. These dimensions have no reference to the distance at which the old branches should be trained, as these, producing no leaves, cause no shade, or rather interruption, to the light; upon their bare branches, however, young shoots may be trained by being tied down upon them, but not to the extent of otherwise crowding the tree. In winter pruning the Morello, care should be taken not to shorten the young wood, unless it be for the purpose of creating young shoots to fill up blanks toward the bottom or centre of the tree, for such shoots have in general their only good wood-bud at the very point.

The following excellent directions as to the training and pruning the other kinds of cherries are given by Mr Errington in "Cottage Gardener :"- "Fan-training is what we must beg to recommend for the cherry in general; and it is obvious, that with the grosser-growing kinds with large leaves, either the first-trained shoots in the young tree must be placed at a great distance apart, in order that the young shoots ultimately produced may be nailed between, or, that the first shoots being put at the ordinary thickness, much sacrifice of young wood must be made, or they must be tied down on the succession plan. We advise those, therefore, who have the largeleaved cherries laid in too thickly, to re-arrange them, so that most of the young shoots they may have produced may be trained in or tied down to the older and barren wood, as the case may be; for assuredly cherries of this habit do not produce many surplus young shoots, providing that they are anticipated, and a provision made for their future training. Under such circumstances most of the young shoots may be tied down or otherwise encouraged; no two, however, should be permitted to lie abreast of each other: where two shoots are produced in a parallel direction, within about 4 inches of each other, one of necessity must be spurred back, leaving about $1 \mathrm{inch}$ at the lower end, which will prove a nucleus for future blossom-buds. No shortening back is required with the cherry in general. The only cases which can justify the practice are, on the one part, crippled or distorted points, and the necessity that exists in young trees to prune for wood, which signifies an attempt, by pruning, to cause one healthy shoot to subside into some three or four, for by such means is the desired form of the tree uliimately completed."

The cherry, when trained as a standard, requires very little pruning; all that is in general required is to keep the branches moderately thin, so that a full share of light and air may penetrate to every part.

As the cherry, when intended for standard training, is multiplied more generally by the process of budding than by grafting, and requires to be headed down the first year, it follows that early autumn-planting should be attended to with a view to their making fresh roots before spring. In April, or as soon as the buds spring, they should be cut down to within 3 or 4 inches of the place where the bud has been inserted. If, therefore, planted by the end of October, and if the trees are good, there will, by this process, be as many eyes as will produce the necessary number of shoots wherewith to form the future tree. About four shoots will be sufficient to form a standard head, the rest, therefore, should be displaced. These shoots must be allowed to extend without shortening, and all superfluous ones removed that may not be required for forming the head. If this be attended to during the first three or four years they will rarely become confused afterwards, and all future care required will be to secure a young shoot conveniently situated to replace any branch that may die or be broken by accident.

For espalier and wall-trained cherries grafted trees are to be preferred, and, according to $\mathrm{Mr}$ George Lindley, "must be trained horizontally instead of obliquely, and always continued at their full length." "In Dukes and Hearts he recommends that the branches should be 8 or 9 inches apart, beginning at the bottom of the tree, and continuing each additional shoot in a parallel direction till the number the wall will permit be completed. This mode of training will give a curved direction, more or less, after the first two or three on each side have been formed, to every additional shoot before it gains its horizontal direction, in consequence of which lateral shoots must be secured for the last series in their ascent, in order to fill up the middle of the tree." The Morello cherries, being weaker-growing sorts, are better to be trained in the fan manner; and as the fruit is in general produced on the young wood of the preceding year, as we have elsewhere remarked, it should be laid in at its full length, and all spurs which may have been formed should be removed when of two years' standing. As to the distance at which the shoots of the Morello should be laid in, Mr Lindley gives the following judicious directions: "That none of the branches should be trained nearer to each other than 3 inches, and from that to 4 or 5 , continuing the out-leaders at full length, as also those that follow at different distances, securing at intervals, in every part of the tree, a supply of young wood to succeed the leaders. When the trees have attained their full size, these leaders should be cut out annually, in the winter pruning, in order to make room for the next succeeding branches." 


\section{SELECT LIST.}

Archduke.-Size of fruit about one-fourth larger than the May duke, of which it is evidently a variety, ripening, according to Downing, a fortnight later, and, he thinks, inferior in flavour. Colour at first red, changing almost to black. Tree hardy, and more vigorous in growth than the May duke; somewhat pendulous in habit when grown as a standard. Synonyms-Portugal duke, Groitte de Portugal, Late archduke, Late duke of some, but not the Late duke or Anglaise tardive, which is a different cherry.

Belle de Choisy.-Size large; colour red; form round; quality first-rate. A good and handsome fruit, but not a great bearer. Originated in 1760 in the village of Choisy, near Paris. Ripens from the beginning to the middle of July.

Bigarreau.-Size large; colour pale yellowish red; form oblate heart-shaped; quality of the highest character. "This noble fruit," says Downing, "is the Bigarreau par excellence, and is unquestionably one of the largest, most beautiful, and delicious of cherries." Generally a good bearer, either against a wall or as a standard. Ripens about the end of July. The synonyms are, as in the case of all first-rate fruits, very numerous - viz., Graffion, Yellow Spanish of most American gardeners, White bigarreau of Manning and Kendrick, Amber or Imperial of Cox, Turkey bigarreau, Bigarreau royal, Italian heart, Harrison's heart of Thompson, West's white heart, Transparent of some, Bigarreau gros, Bigarreau tardive, Bigarreau de Holland of Pince.

Bigarreau, white.-Size very large ; colour yellowish white at first, changing to marbled red ; form heart-shaped, rather irregular in outline. Downing remarks, "It does not appear to be known at this time in England, though it is probably identical with the Harrison heart of Forsyth, and the Bigarreau of Hooker." It is inferior to the Bigarreau or Graffion in hardiness, and is a very poor bearer while the tree is young, though it bears fine crops when it has arrived at from twelve to fifteen years' growth. The trees of this and the former sort may be readily distinguished even when very small, as the Bigarreau has broad flat foliage, while the White bigarreau has narrow waved leaves. Synonyms - White ox heart (of the middle States), White bigarreau (Thomp.), Large white bigarreau, Tradescant (Cox), Ox heart (Cox), Harrison's heart, Turkey bigarreau, Bigarreau blanc. Mr Rivers states that a tree of this excellent cherry exists in his nursery at Sawbridgeworth, the scion of which, tradition says, was brought from Russia, and presented to his grandfather by Baron Dimsdale, when he returned from Russia after inoculating the Empress Catherine.

Bigarreau, Holland, (of Downing). - Size very large ; form heart-shaped, rather pointed ; colour very pale yellowish, white in the shade, beautifully mottled on the sunny side with bright carmine red; leaves very large and broad. This is certainly one of the most beautiful of all cherries. Of French origin, and appears to be identical with the Bigarreau d' $H o l$.

VOL. II. lande, figured in the $2 \mathrm{~d}$ edition of Noisette's "Jardin Fruitier," and there stated to have been received from Holland in 1828. Of this fine fruit Downing says: "A cherry so large, fine, and beautiful, and so productive even when young, and which is of rapid and hardy growth, cannot fail soon to become a general favourite in our fruit gardens. It ripens about a week earlier than the Bigarreau, and the branches, which are spreading, or even drooping, are literally loaded with heavy bunches of fruit." Synonyms - Bigarreau d'Hollande (Noisette), Spotted bigarreau, Armstrong's bigarreau. Mr R. Thompson makes this synonymous with the Bigarreau. Mr Downing thinks this "is undoubtedly an error." Mr G. Lindley only notices one Biyarreau in "Guide to the Orchard ;" Rogers only one in " Fruit Cultivator," but gives us the following interesting information: "Miller does not mention this cherry in the 4th edition of his Dictionary, nor does it appear in English catalogues till some time after the middle of the last century. It was, for some time after its introduction here, called the Turkey heart, because it was first received from that country into France. The Dutch call it the Graffion, but it is best known by its French name of Bigarreau. It is a curious circumstance that this cherry must have been in this country for many years before the merits of its fruit were known. It might, perhaps, have been considered as only Harrison's heart, and therefore neglected." There was no such cherry in the Royal Gardens at Richmond in 1770 , but $\mathrm{Mr}$ Rogers found two fine trees of it in the Surrendon Gardens in 1780.

Bigarreau couleur de chair.-Size large; form oblong, heart-shaped; colour pale yellowish red; ripens the beginning of July; leaves not very large. Tree moderately vigorous. Mr R. Thompson thinks it inferior to the Bigarreau, "flesh tender, but not rich." Downing, again, says, "It is a beautiful and excellent variety, much more tender in its flesh than most of its class." So much for climate. Our own opinion is, that it deserves a place in every garden. Synonyms - Flesh - coloured bigarreau, Large heart-shaped bigarreau of Manning, with various French synonyms.

Bigarreau, Napoleon.-Size large; form oblate heart-shaped; colour pale yellowish red; flesh very firm, almost too much so; ripens end of July and beginning of August; good bearer. Tree hardy.

Bigarreau tardif de Hildesheim.-Size above medium ; form heart-shaped ; colour yellowish red; of excellent quality, and has the great merit of being the latest sweet cherry known. Ripens towards the end of August, and keeps on the trees till the middle of October. Tree hardy, and a good bearer. Of German origin.

Bigarreau, black, of Savoy (Kendrick).--Size large; form regularly heart-shaped; colour glossy black when ripe ; hangs long on the tree after being ripe. We have not met with any notice of this variety in any English work. It was received in the neighbourhood of Boston, United States, from Savoy, and although even in America not considered as a first-rate cherry, it is appreciated on account of its keeping. 
Bigarreau, new large black.-Size large ; form obtuse heart-shaped; colour quite black and glossy at maturity; flavour rich and luscious, more juicy than the rest of the Black bigarreaus. The fruit is quite as large as the Black Tartarian, and ripens among the latest varieties.

Black eagle.-Size above medium; form oblate heart-shaped ; colour glossy black; quality first-rate. Ripens beginning of July. Tree hardy and a good bearer. This very excellent cherry was raised by the daughter of Mr Knight, of Downton Castle, in 1806, from the seed of the Bigarreau, fertilised by the May duke.

Black heart.-Size rather above medium; form heart-shaped; colour glossy black. Ripens the beginning of July. Tree hardy, very liable to the attacks of aphides just as the fruit is ripening. This very old variety is an abundant bearer, and well known. Synonyms-Early black, a title to which it has no claim, Black caroon of some, Black caroon gean, Spanish black heart, Ansell's fine black, Black Russian of some American gardens.

Black heart, Manning's early.-In no respect different from the last, except in its ripening ten days earlier, about the same time with, or even a little before, the Mayduke, which to some may be a merit. A seedling from the Black heart, raised by Mr Manning, a celebrated American pomological writer.

Black heart, Buttner's.-Size medium ; form heart-shaped; colour glossyblack. Ripensin July. A very abundant bearer, and although marked by Mr Thompson as a second-rate fruit, is worthy of extensive cultivation. Of German origin.

Black heart, Werder's early.-Size large ; form heart-shaped; colour black. Ripens beginning of June. Tree hardy and a good bearer. A good cherry, and valuable for its earliness. Synonym-Werder's Early black. Also of German origin.

Bowyer's early heart. - Size rather below medium; form heart-shaped; colour amber, mottled with red. Ripens early in July. An excellent middle-sized early cherry.

Buttner's October morello.-Size medium; form round; colour dark red. Ripens in October, and hangs on the tree during November. The latest of all cherries. Tree hardy and an excellent bearer. Raised at Halle, in Germany, and worthy of extensive cultivation.

Buttner's yellow.-Size medium ; form roundish; colour pale yellow. Ripens in July. Tree hardy and an excellent bearer. Also of German origin, and one of the few cherries entirely yellow.

Downton.-Size above medium; form round heart-shaped; colour pale yellowish red, nearly semi-transparent. Ripens beginning of July. An excellent bearer, and in richness scarcely inferior to the Elton. Raised by Mr Knight, of Downton Castle, from the seed, it is believed, of the Elton.

Downer's late.-Size medium ; form roundish, inclining to oval; colour soft, but lively red, mottled with a little amber in the shade. Ripens beginning of August. Tree hardy, a regular and great bearer, the fruit hanging on long after it is ripe. Raised in the neighbourhood of Boston, United States. A very valuable late cherry.
Duke, late.-Size large; form obtuse heartshaped; colour dark red. Ripens end of August. Tree hardy; great bearer as a standard ; valuable for its late ripening, and hangs very long on the tree; juice very abundant, but not so sweet as the May duke. Must not be mistaken for the Archduke (which see). Branches slender, more resembling in growth the Morello than any of the Duke family. Synonym-Anglaise tardive.

Elton.-Size large; form heart-shaped; colour pale yellowish red. Ripens beginning of July. Tree hardy, and a great bearer, esteemed the richest of all cherries. Raised in 1806 by $\mathrm{Mr}$ Knight, of Downton Castle, between the Bigarreau and the White heart for its male parent.

Florence. - Size large; form heart-shaped; colour pale yellowish red. Ripens end of July. An excellent cherry like the Bigarreau, but ripens considerably later, and liangs much longer on the tree. Introduced originally from Florence, and succeeds well on the Mahaleb stock.

Jeffrey's duke.-Size medium; form round; colour red. Kipens beginning of July. Tree hardy, compact grower, not very vigorous in growth; an excellent bearer. Synonyms Jeffrey's royal, Cherry duke, Royale, Royale ordinaire, or Cerise royale of the French gardens, Jeffrey's royal caronn. This is probably the Royal Kensington duke of Rogers, cultivated by Jeffrey, the proprietor at one time of the Brompton Nursery, and successor to London \& Wise, who at that early period introduced it from France.

Kentish.-Size medium; form round; colour red. Ripens end of July. Tree extremely hardy, even as a standard in most parts of Scotland. "Good bearer; the stone may be drawn out by the stalk, so strongly are these attached; the fruit may then be dried in the sun or in an oven." One of the most valuable of kitchen or preserving cherries. Thought to have been introduced from Flanders in the time of Henry VIII. Very extensively grown in Kent for the London markets, and largely imported from the Continent. Synonyms-Common red, Flemish of some, Kentish red, Sussex, Virginian May, Pie cherry, Early Richmond, De Montmorency, Muscat de Prague.

Kentish late.-Very similar to the last in appearance, but possessing the merit of ripening two weeks later. Of its history little appears to be known, farther than it is believed to be a seedling from the Kentish raised in America. It is better known and more abundantly cultivated on the Hudson and about New York than any other sort. It is there grown along the fences and road-sides, propagating itself by seeds and suckers.

Knight's early black.- Size large ; form oblate heart-shaped; colour black. Ripens early in June; quality excellent; fruit early and fine. Tree hardy and an excellent bearer, somewhat resembling the Black Tartarian, but more obtuse in form. Raised by Mr Knight between the Bigarreau and the May duke somewhere about 1810 .

May duke.-Size medium ; form oblate heartshaped; colour red; ripens end of June. This 
is the most popular and most universally cultivated cherry in existence, thriving almost equally well in all countries, situations, and soils. "May duke" is said to be a corruption of Medoc, a district in France where this variety (the type of all the class now called Dukes) is believed to have originated. Synonyms-Early duke, Early May duke, Large May duke, Morris's duke, Morris's early duke, Benham's fine early duke, Thompson's duke, Portugal duke, Buchanan's early duke, Millet's late heart duke, Anglaise, Royale hâtive, Cherry duke of some, and various other Continental names, as may be supposed to be the case with a cherry of such universal culture.

Morello. - Size large; form oblate heartshaped; colour dark red; ripens July and August. A most valuable and well-known cherry, extremely hardy, and valuable for preserving purposes. Its name is said to be derived from the dark purple colour of its juice, which resembles the morus or mulberry, or, as others will have it, from the French movelle (a negress), from the dark and shining skin. In America the true Morello has, according to Downing, not been extensively grown, theirs being a small and inferior variety. Synonyms-Dutch morello, Milan, Large morello, Black morello, Late morello, (not Rumsey's late morello, which see), Ronald's morello, Sir Herbert Taylor's morello, which, by the way, is no doubt an improved variety of this, at one time grown in the royal gardens at Hampton Court.

Morello, Frogmore new.-This new and excellent variety has recently been brought into notice on account of the very great perfection it has been brought to in the royal gardens at Frogmore, in which we believe it originated. So far as we are aware, the stock is confined to Mr Small's nursery, Colonbrook, near Windsor. For productiveness and size it far surpasses the old Morello, and should, on these merits alone, find a place in every garden.

Morello, Rumsey's late.-Size large, often producing its fruit in pairs ; form roundish; colour, before fully ripe, of a light yellowish red colour, becoming at maturity a rich lively red, with a distinct suture line on one side. "A few of the fruit commence ripening about the first of August, even in America, while many on the tree are yet small and green, and they continue ripening gradually until the first frosts."-Fruits and Fruit Trees of America, p.199. Originated about fifteen years ago on the banks of the Hudson, U.S.

Royal duke.-Size large ; form oblate ; colour dark red; ripens middle of July. Tree hardy and a good bearer, succeeding in ripening to the May duke. It is readily known from the Late duke and $A v^{\prime} c h d u k e$ by its upright growth, which is similar to that of the May duke. SynonymsAnqlaise, Anglaise tardive.

Tartarian, black.-Size large; form oblate heart-shaped; colour black; ripens end of June; quality first-rate. A very excellent cherry. Introduced to Britain about 1796, from Russia, by Mr John Fraser. The tree is remarkable for its rapid growth and the large size of its leaves. Synonyms - Tartarian, Fraser's black heart, Fraser's black, Fraser's Tartarische, Fraser's black Tartarian, Ronald's heart, Ronald's large black heart, Black Circassian, Superb Circassian.

Tartarian, white.-Size medium; form oblate heart-shaped; colour pale yellow; ripens beginning of July. Tree rapid in growth; leaves large. Introduced in 1794 by Mr John Fraser, an enterprising botanist and traveller in Russia, and afterwards a nurseryman at Chelsea. Plants of both this variety and the last got into the Brentford nursery, and for reasons which may not be difficult to comprehend, but difficult to reconcile with honourable transactions, became speedily disseminated under the cognomen of the head of the Brentford establishment. Synonyms-Fraser's white Tartarian, Fraser's white transparent, Amber à petit fruit.

They are both probably natives of Circassia, although it has been stated that the Black Tartarian originated in Spain, from whence it was transmitted to the gardens of Russia. In wall and espalier training the Tartarian cherries, from the size of their foliage and strength of shoots, require to be laid in at a greater distance from each other than any other section, the Bigarreaus excepted.

Waterloo.-Size large; form oblate heartshaped; colour black; ripens beginning of July. Tree of irregular growth; moderate bearer. Originated with $\mathrm{Mr}$ Knight by fertilising the Bigarreau with the pollen of the May duke. Named in consequence of its having produced its first fruit about the time of the battle of Waterloo.

White heart. - Size medium; form heartshaped; colour pale yellowish red, somewhat resembling the Bigareau; ripens end of July. Fruit growing in pairs, and often in threes. An old and much esteemed fruit, which, together with the Black corone and Kentish cherries, were cried about the streets of London a century ago, and sold for one penny per lb. This, like most of our best cherries, is of French origin. Tree, when planted as a standard, rather straggling in growth; on walls or espaliers, the branches being slender, and leaves, although long, neither very broad nor closely set, they may be laid in closer than most others, the Morellos excepted. Synonyms - Amber heart, Dredge's early white heart, White transparent. There is an Early white heart described by Downing, which would appear to be different from this, although introduced to America from France. Of its form he says, "The tree grows rather erect, with a distaff-like head when young," which does not agree with the habit of the one under consideration, although it does in the following, "In the nursery the young trees are easily known by their long and slender shoots, with few branches."

Geans.-That section of the cherry tribe which includes the Guignes or Geans is extremely neglected, especially in England and America. This remark may be met by asserting the superior climate they enjoy, which enables them to cultivate the higher grades of cherries. Yet on the continent of Furope, with an equal climate, we find them cultivated extensively, and in great variety. In Scotland more attention has been paid to this section, and they 
are probably the first cherries our ancestors possessed, and hence we have them regularly cultivated for sale. From the well-arranged Fruit Catalogue of Dickson and Turnbull, Perth, we take the following, as grown by them: Amber, Black Dutch, Castle Menzies, and Hungarian, all great bearers, of excellent quality, and of extreme hardiness. They make fine ornamental trees when planted in parks and pleasure-grounds, cheering us in spring with the abundance of snow-white blossoms with which they are covered, and again, in autumn, giving those fine red tints so much admired by painters. We have found them valuable when planted in the screen plantations around gardens, as affording food for birds, and hence saving the fruits of the garden from their attacks. The black gean or dwarf cherry of Castle Menzies, and the cream-coloured gean of Ardvorlich, are both highly worthy of cultivation.

New or recently introduced Cherries.-These we have not as yet fruited at Dalkeith. The descriptions are taken from the very correct Fruit Catalogue of Peter Lawson and Son :-

"Belle d'Orleans.-First size, first quality; roundish; one of the earliest and very best of cherries; ripens the middle of June.

"Carnation, Coe's late.-First size, first quality; round; excellent; raised at Bury in Suffolk; very useful for preserving, being a little acid; skin bright red, slightly marbled; flesh tender, rather firm but juicy, and when fully ripe of an excellent sub-acid flavour. Standard or wall. End of July or beginning of August. Dessert or preserves.

"Donna Maria.-First size, first quality; new variety, and said to be of the finest quality.

"Jaune de Prusse.-First size, first quality; heart-shaped. New and excellent variety lately introduced from France; skin golden yellow; flesh yellowish and very sweet. Wall or standard. Beginning of July. Dessert. This, with Buttner's yellow, may be considered as two truly yellow cherries. Whether this may add to their popularity remains to be seen. Synonym Prussian yellow.

"Reine Hortense.-First size, first quality; new, large, and excellent variety. Recently introduced from France. Like most of our best fruits, this has already a number of synonyms, as - Hybride de Laeken, Belle de Laeken, Monstreuse de Bavay, \&c. \&c.

" Rivers' early amber.-First size, first quality; heart-shaped. Large and very beautiful variety, raised by Mr Rivers, Sawbridgeworth, Herts. Skin amber-coloured, tinged with red on the sunny side; flesh yellowish, juicy, melting, and rich; it is also hardy and very prolific. Standard or wall. Middle of June. Dessert.

"Tardive de Mons. - Second size, first quality. New variety from France; said to be very sweet; hangs long on the tree."

\section{SELECT LIST OF CHERRIES FOR CERTAIN LOCALITIES.}

At Drumlanrig Castle, Dumfriesshire.-The May duke, Morello, Kentish, and Black Tartarian, are all grown on walls.

At Dalkeith Palace, Mid-Lothian.-Arch- duke, Belle de Choisy, Bigarreau, Black eagle, Black heart, Downton, Elton, Florence, May duke, Morello, Tartarian black, Tartarian white, Waterloo, White heart, and Knight's early black, are grown against walls, chiefly north-east and west, all of which would succeed almost equally well as dwarf-standards, an experiment now in course of trial. The lists we have received from various gardens in Scotland show that few sorts of cherries, comparatively, are planted, and those chiefly against walls. It will be for us now to look to the very early and the very late good sorts; of the intermediate ones we have sufficient variety.

Mr Rivers' list of good cherries, planted as dwarf-standards, and wrought on the Mahaleb stock, is somewhat numerous; yet we believe that this style of growing cherries is all that can be required in the southern and midland counties of England, and even in all the better localities in Scotland. In colder and more unfavourable places they may be planted against walls. This enlightened fruit-cultivator divides cherries into the three following sections, viz:-

Section 1, the May duke tribe--Archduke, May duke, Royal duke, Jeffrey's duke, Belle de Choisy, and Cerise Indulle, or Early May.

Section 2, the Morello tribe.-Carnation, Carnation (Coe's late), Kentish, Late duke, Griotte de chaux, Morello, Reine Hortense, Louis Philippe, Belle de Sceaux, and Belle magnifique.

Section 3, Bigarreau and Heart cherry tribe. -Bauman's May, Belle d'Orleans, Bigarreau de Hildersheim, Bigarreau gros cœuret, Bigarreau Napoleon, Black eagle, Black Tartarian, Buttner's black heart, Buttner's yellow, Downer's late red, Downton, Early amber heart, Elton, Florence, Holland bigarreau (Downing), Knight's early black, Knight's late black, Manning's early white, Tradiscant's black, Trempe précoce, Werder's early black heart.

Selection of cherries for a small garden in the southern and midland counties of England is thus given by $\mathrm{Mr}$ G. Lindley (Guide to the Orchard): Archduke, Belle de Choisy, Bigarreau, Black eagle, Black Tartarian, Downton, Elton, Florence, Holman's duke, Kentish, Knight's early black, May duke, Morello, Waterloo.

Northern counties of England and southern of Scolland.-The same as above, with the exceptions of Bigarreau and Florence, which are considered tender.

For the Highlands of Scotland he proposes Black Tartarian, Kentish, May duke, and Waterloo, as being hardy. Much, however, as we have already stated, depends on situation, for many localities in the Highlands are better adapted for fruits than some in the border counties of England and Scotland.

Diseases.-The chief, and apparently the only, disease to which the cherry is subject is the gum, or exudation of gummy matter on the stem or larger branches. This is attributed to wounds, too highly manured soil, and probably sometimes to the difference between the tree and the stock it is wrought upon. When the bark of the cherry is wounded in spring before the foliage is developed, whether by contusion or severe cutting, water is apt to find its way into 
the injured part, and prevents the natural healing at the time of the sap's ascent; extravasated sap exudes from the part, and forms that gummy appearance known as the gum. When the soil is too rich, the sap is thrown into the stem and larger branches faster than the circulating vessels can carry it away; some part or other of these becomes ruptured by the force of the sap within, and as it exudes through the ruptured part of the barkit assumes the gurnmy appearance. Root-pruning and replanting in a less highlyenriched soil are the only remedies in this case, for the cherry, of all fruit trees, is the most impatient of a rich soil. The difference between the tree and stock acts much in the same way as an over rich soil: when the stock is of a nature to throw more sap into the tree than it requires, the same consequences follow; and when it sends up an insufficient supply of sap in spring, in consequence of the sap-vessels being too small to admit of its free ascent, the tree would show evidence of its diminished supply by the smallness of its shoots and foliage, but this is made up by the leaves, which draw a considerable share of their nourishment from the atmosphere. The returning sap, however, is impeded in its descent in autumn at the point of union of the stock and graft, the vessels become there often greatly distended, producing those procuberances so frequently seen on cherry trees; the weaker vessels then often become ruptured, extravasated sap exudes, and forms into a gummy substance. The cherry on its own roots-that is, directly originated from seed-lives to a considerable age, and often attains a large size, but seldom when budded or grafted, more especially if on stocks originated from seed of the cultivated sorts.

Insects.-Tenthredo cerasi L. is often exceedingly injurious to the foliage of the cherry, rolling itself up in the leaves to undergo its transmutations, at which time it should be sought for and destroyed.

The Morello cherry louse (Aphis cerasi) infests the under sides of the leaves, and often the young shoots also. Tobacco, either applied by fumigation or syringing, is one way of getting rid of this as well as most others of the family. It is said to be more prevalent on trees in damp soils than otherwise. When the young shoots are attacked during summer, dip their points in strong tobacco-water, or syringe them with water, and immediately dust them over with Scotch snuff.

Nasmyth recommends, as a remedy against the black-fly, "a mixture of pitch with one-sixteenth part of powdered orpiment, one-sixteenth part of sulphur, dissolved over a slow fire until they are well incorporated; when cold, divide it into small pieces about the size of a hen's egg, and burn it under the trees, with damp straw, directing the smoke as much as possible where the insects are most numerous." We have found common coal-tar, and also naph tha, placed in earthenware pans, such as are used for setting under flower-pots, burnt under the trees, have a most beneficial effect. The operation should be carried on on a quiet still day; in either case, the day following the trees should be syringed over with clear water, directing it against them with sufficient force to rid the leaves and shoots of the dead and dying. Burning either in an iron ladle, such as plumbers melt their lead in, held under the trees, and carried from tree to tree, is an expeditious mode of ridding young trees in nursery lines of these intruders.

The red-spider (Acarus telarius), fig. 22, is often destructive to the cherry foliage, particularly in dry seasons, and more especially in the cherry-house.

The European names. - Cerisier, French Kirschbaum, German-Kersenboom, DutchCiriegio, Italian-Cerezo, Spanish-Wischnaija, Russian-Wisnia, Polish.

The names of the following cherries are pronounced thus: Belle de Choisy-Bel-de Schwoi-sey; Belle Magnifique-Bel Man-guefeek ; Bigarreau-Be-gar-ro ; Bigarreau RougeBe-gar-ro Rooje ; Bigarreau Couleur de ChairBe-gar-ro Coo-lur de Shair; Bigarreau Gros Cœuret-Be-gar-ro Gro Keur-ai ; Bigarreau Tardif de Hildesheim-Be-gar-ro Tarduf de Hildesheim; Gros Bigarreau Rouge-Gro Be-gar-ro Rooje; Griotte d'Espagne-Gre-ote Des-pan. 


\section{H A P T ER X.}

\section{FORCING THE APRICOT, PLUM, AND CHERRY.}

Tre plum and cherry have been long subjected to a species of forcing, the success of which depended greatly on the means used to effect the end - the most primitive mode being that of placing glass sashes in front of walls, heated by flues or by solar action, on which the trees were permanently grown. Switzer states that these fruits were also forced by the London gardeners by means of dung-heat, and probably under frames or pits, similar to many of those still in use in many parts of the Continent (vide vol. i. fig. 405). In this way (although we have no record of the fact) it is most probable that those cherries were produced, mentioned by Daines Barrington as appearing on the table of Charles II. at his installation-dinner, given at Windsor on the $23 \mathrm{~d}$ of April 1667, and at which, he also informs us, forced strawberries and icecreams were also produced.

Forcing cherries, until within these few years, was carried on upon a very limited scale in Britain, and, with the exception of one or two commercial growers, was almost confined to the royal gardens. Plums began to be afterwards forced, and last of all the apricot. It would be difficult to assign a sufficient reason why these three excellent fruits should have been so long disregarded, seeing the peach and nectarine have been so long and so very generally forced in all our best gardens; and the more so as regards the apricot, as it approaches in its nature and character so closely to the peach and nectarine, and is a native of almost the same country. A prejudice long existed, but upon no rational ground, that they were more difficult to manage under the influence of a glass structure than the others. Experience has now completely overcome that prejudice; and hence we have our cherryhouses, plum-houses, and apricot-houses, constructed in some cases in no way different from the ordinary peach-house, as exemplified, No. 4, in Ground Plan of the Royal Gardens at Frogmore, plate 10 , vol. i., and at other times upon the span-roofed principle, as shown, No. 11, in the same plate, and in elevation, fig. 495, vol. $i$. Figs. 493, 494 exhibit cross sections of structures which we think admirably fitted for the purpose of producing these fruits in the highest degree of perfection. Mr Ewing's glass walls, plate 2, vol. i., those of Mr Spencer of Bowood, fig. 79 , and the novel glass erections contrived by Mr Fleming at Trentham, described at p. 353 , 355 , vol. i., are all eminently adapted for this purpose; and with such structures as these in the hands of a skilful gardener, no more diffculty need be apprehended in producing early crops of the one class of fruits than of the other.

Where the trees are planted out as permanent occupants of such structures, and where early forcing is an object, by the latter end of November they should be pruned, tied to the trellis, the borders slightly forked up and top-dressed as recommended for the peach-house, and all things prepared for commencing slight excitement by the beginning of January. Where the trees are grown in large pots or tubs, with a view to their being set out of doors after their fruit is gathered and their wood ripened, or where, as is frequently done with great advantage, they are turned out of the pots and planted in the open ground, and allowed to remain there every alternate season, to recover from the effects of their season of being forced, a selection of them should be removed into the structure during November; and as those for potculture should be trained as standards or dwarfs, and of various heights, to suit the construction of the house, the tallest, if a span-roof, should occupy the centre of the floor; if a lean-to house, the side next the back wall, the lower ones in either case being placed in front. By judicious annual root-pruning, and planting in fresh turfy soil, plums and cherries may for this purpose be grown in the open air, and if carefully taken up as early in autumn as the wood and buds are ripened, and planted in the borders of the forcing-house, they will fruit as well as if they were permanently established. Early planting has, however, much to do with this; and we need hardly remark that the situation they are placed in when in the open air should be the warmest and most sheltered the garden affords, to insure an early maturation of their buds. Apricots, from being a much tenderer tree, should be grown against a south wall, and when transferred to the house, either trained to a trellis or trained in the espalier fashion. They may, however, be grown in pots as standards, but in such a case should remain permanently under the glass covering, because, if treated otherwise, their wood and buds would not become sufficiently matured without artificial 
assistance. One object in growing the plum and cherry in pots is, that, in the event of any of them not setting well, or becoming unhealthy, they can be more readily removed, and their places supplied with others from the reserve stock, for such a stock must always be maintained. The plum is liable to grow much to wood, which the confinement of its roots in a pot would greatly check. Again, after their fruit is gathered, they can be removed to the open air, and the house used for other purposes, such as ripening late crops of melons, figs, \&c.

$\mathrm{Mr}$ Rivers, who has carried the pot-culture of fruit trees to a very great extent, says, in defence of his system over that of growing the trees planted out in the border of the orchard-house, that "they cannot, when grown out of doors, be kept in control, even with annual root-pruning. I have some peach trees which have been planted in the raised border of one of our orchard-houses four years: they bear admirably; but in spite of root-pruning they will grow too rapidly. Now in pots the size and growth of the trees may be regulated with the greatest nicety : the annual root-pruning can be done with much facility, and there is no occasion to dig or disturb the borders, which must be done to a great extent to root-prune properly trees planted in them. In fine, as far as my experience has gone, I can imagine nothing so eligible as pots or vases for fruit trees in orchardhouses, or fruit conservatories." As these trees require abundance of air and light, the oldfashioned lean-to houses and pits are the very worst they can be placed in, the best being span-roofed houses with large glass, as little opaque surface in the roof as possible, and their sides glazed to within a foot or 18 inches of the ground. The whole length of the ridge should be capable of opening at least to the extent of 18 inches in breadth, and the side ventilation placed in the parapet walls, with glass ventilators a foot in breadth of opening, and extending the whole length of the structure, with the exception of 12-inch pieces only between them, to sustain the superstructure. Thus ventilation can be abundantly secured close to the ground; and the sides being fixed, the cold air, instead of rushing in directly upon the trees, will have to pass over and under the hot-water pipes, and be by this means of equal termperature with the air in the house before it reaches the foliage. Want of light and ventilation has hitherto been the cause of failure in the early production of these fruits.

Early in October plums and cherries should be potted, to keep up a proper succession of trees fit for forcing, because, under the best management, some may be expected to die or become sickly yearly. Trees for this purpose, although healthy, should not be of luxuriant growth, and those in which a predisposition to form abundance of fruit-buds has been induced by root-pruning should be preferred; the confinement of their roots by the pots afterwards will maintain this disposition. Cherries and plums are often to be met with in nurseries which, from some cause or other, have ceased to make much wood, and have become thickly set with short fruit-bearing spurs; these are very eligible for this purpose, but to secure a supply within the same garden it is better to purchase maiden trees, and to attend to root-pruning and pinching back the young wood during summer, to save the risk of transporting them from a distance when they are required for potting.

The soil for potted plants should be good rich loam of a sound firm texture, and the size of the pots for the first season should not be less than 12 inches in diameter, and 18 inches when shifted the second year.

In regard to sorts of cherries adapted for forciny, the May duke is in general preferred, although Nicol, and others since his time, have recommended the Morello. Mr Rivers, in his interesting work, "The Orchard-House," recommends a great many, the earliest-ripening sorts of which are the Early May, the May duke, the Archduke, Jeffrey's duke, the Belle de Choisy, and the Royal duke. He proposes, as the proper stock for dwarf cherries and for such as are to be potted, the Cerasus mahaleb, or perfumed cherry, as trees grafted or budded on it form beautiful dwarf-bushes - remarking, however, that it is better adapted to cherries of which the May duke is the type, and for that of which the Morello is the representative, than for the Bigarreau and Heart cherries.

The sorts of plums hitherto in general estimation for forcing are-The Green gage, Orleans, Drap d'Or, Yellow gage, and Early Orleans. The Reine Claude de Bavay, an excellent Belgian variety, has more recently been recommended for this purpose, as lias also the Jefferson, a first-rate American plum; and it is not at all improbable that Angelina Burdett, Standard of England, and Woolston black gage, may be found deserving of the notice of the plum-forcer. $\mathrm{Mr}$ Rivers gives a select list of sorts adapted to orchardhouse culture, the earlier of which are-Early favourite, Early prolific, Jaune hâtive, Early Orleans, Royale hâtive, De Montfort, Peach, Imperial Ottoman, Purple favourite, Mamelonne, Isabella, Green gage, Columbia, Reine Claude de Bavay, Tay Bank, Lawrence's gage, Jefferson, \&c. This intelligent cultivator recommends plums intended for potting to be grafted on the sloe (Prunus spinosus). "If," he remarks, "they have been removed the year previous to potting, they will be full of bloom-buds, and will bear a good crop the first season; if they can be procured already established in pots, the crop will be better and the fruit larger. The sloe stock is not, however, absolutely essential ; for I have," he says, "some grafted on the common plum-stock which are abundantly fruitful."

The soil and root and branch management for plums are essentially the same as for cherries.

The apricots hitherto suljected to early forcing have been the much-esteemed Moorpark and the Early red masculine; the latter esteemed only on account of its ripening somewhat earlier. The more recently introduced MuschMusch and Kaisha, both early sorts, may be also confidently employed. Apricots have hitherto been considered more difficult to manage under glass than either plums or cherries. They, under 
all circumstances, require a free ventilation and pure air. Why this should not be afforded them, in such structures as those we have elsewhere described, is strange, as ventilation and light are so abundantlyprovided, that under good management otherwise they enjoy as much of both as if grown against an open wall. In the economical orchard-houses constructed by $\mathrm{Mr}$ Rivers, possessing by no means the same advantages of light and ventilation as those we have recommended, that cultivator has produced excellent crops for several years past; and although his structures are intended for ripening the fruit by solar influence only, the whole routine of his practice in selecting and preparing his trees, is just that required for trees to be forced into fruit at an earlier season, and by the agency of artificial heat. With this conviction on our mind, we cannot do better than give his practice as detailed by him in his "Orchard-House," pp. 11 to 14 : "The most eligible trees for pot-culture-i.e. apricots, plums, and cherries-are those that have been in pots one or two years : if these can be purchased, so much the better; if not, trees that have been removed and cut down one year in the nursery; if neither of these descriptions can be found, dwarf maiden-trees will do. Trees that have not been in pots must not be potted till the end of October; if omitted then, in November or December they may be repotted into pots of the size selected for this description of culture. I have named 11-inch pots, because they are portable; but 15 or 18.inch pots may be employed if larger bushes are required, and they may be shifted into these large pots as they advance in growth: 11-inch pots will at any rate do well to commence with. I have named three months for potting trees; they may, indeed, be potted till March, but no fruit must be expected the first season. If fruit-bearing trees that have been in pots can be procured, they cannot be potted too early ir. October." The compost recommended by $\mathrm{Mr}$ Rivers for stone fruits is "two-thirds turfy loam and one-third decomposed manure, to which some road or pit sand should be added." In no case should the materials be sifted, for the rougher and more porous the better. Drain thoroughly, and in doing so break away the greater portion of the bottom of the pot, or have them made with an opening in the centre 6 inches across; lay large pieces of broken pot over this opening, and place on it some of the most turfy of the compost, upon which to set the tree, and fill round the sides in the usual manner. To illustrate his principle, $\mathrm{Mr}$ Rivers takes an apricot tree from its commencement, " as its treatment will serve, with slight modification, for nearly every fruit tree under pot-culture in orchardhouses. Well, then, if it is a bush with four, five, or six shoots, and if it has been in a pot, these will be short and well ripened: they may be shortened when the plant is potted, whether in autumn or spring, to about 8 or 9 inches, and the shoots, whether three, five, or seven, left so as to form the foundation of a nice regularly-shaped bush. The first season after potting, the tree will in May put forth from each shoot three or four; all these but one on each shoot, the leader, must be pinched off with the finger and thumb to within an inch of their base; they will then in time form fruit-bearing spurs. Your tree will probably, if it has been potted and grown under glass previously, bear fruit the first year ; it will to a certainty make a fine and healthy growth. Let us suppose the summer over and October arrived; the leaves are yellow and falling, the tree is going to rest - let us assist it as follows: Gently lift up one side of the pot so that you can see the roots that have made their way into the border during summer; take a sharp knife, begin oll the side next to you, and gradually sever every fibre and root close to the bottom of the pot, then shorten each leading shoot of this summer's growth to about 9 inches. All the trees must be operated upon in this manner; they may then be placed close together for the winter; water must be withheld, and the trees suffered to remain dry and completely at rest during the winter. "The severing the roots from below the bottom of the pot shows a sufficient reason why the bottom should be broken away; or if made on purpose, a much larger opening made than is usually the case : the roots escape during their season of growth, and collect nourishment which they throw into the tree; and at this season, when their energies are stopped, they are cut away altogether." Although, as we have already stated, Mr Rivers' object is more to secure with certainty a crop of fruit at a later period of the season, employing solar heat only, than to ripen them so early as April or May, still his routine is so replete with excellent practical instruction that we cannot do better than follow him out in his second year's treatment, as the only difference between late and early forcing consists in greater care being taken in respect of ventilation and the application of artificial heat in the latter case than in the former. "I may now," he says, "carry their treatment through the second year. February is with us, and if the season is mild, buds are beginning to swell and flowers to expand; the trees in the orchardhouse, are, however, dry, dusty, and stagnant: place them in their stations; give each a small quantity, say a pint of water; let them rest three days, then give them a quart each; in short, gradually saturate the earth in the pots, and afterwards water them regularly as required by the state of the weather. The shoots, if the weather is mild"-and the same will be the case with slight artificial excitement-" will soon begin to swell, and in March, or early in April, if the season is late, they will be in full bloom." By artificial excitement, the same state will be brought about in January or February. "If the weather is sunny, with sharp frosts at night, as is often the case in early spring, the shutters (ventilators) both back and front may be open all day, and closed at night. During wind, frost, and cloudy weather, they may be closed day and night ; the ventilation through the crevices where the boards join will be amply sufficient." It should be remarked, that Mr Rivers' orchardhouses are formed of boarding all round; in better houses, a diminution of ventilation will 
answer the same end. "With this treatment nearly every blossom will set. As soon as the fruit becomes the size of a large pea, commence syringing the trees both morning and evening with soft water, and continue till they begin to change colour." In houses heated by smoke-flues or hot-water pipes, syringing will have to be earlier commenced, and carried to the extent only to counteract the drying effects of artificial heat. Mr Rivers commences thinning the apricot when of the size of a horsebean, "leaving at first, upon a bush that has been two years in a pot, about three dozen, which, when the fruit has attained the size of a small nutmeg, must be reduced to two dozen ; the third year, a tree, if it has prospered, will be able to bear three dozen; it is, however, better to have a few finely-grown fruit than many that are small." Plums and cherries will require similar treatment, and a judicious thinning of the fruit also ; but in all cases it is safest to delay the final thinning until the kernel is fairly formed in the stone, and the fruit has begun to swell in size. If it be desired to have the trees removed, and placed on the table laden with their ripe fruit, this can readily be done by cutting the roots below the pot as before recommended. This will do little injury to the trees, as the chief functions of the roots have begun to cease when the fruit is ripened, and it will have the effect of only putting the trees to rest a little prematurely. They should in such cases be returned to their former places, and be supplied with water till October. "In pruning the second season," Mr Rivers observes, "summer-pinching in May and June must be continued, as before recommended; and in October, when the trees are put to rest, the leading young shoot of the summer on each branch must be shortened to 6 inches. The third season these shoots may be reduced to 4 inches. As the tree becomes fruitful, but very short annual shoots will be produced; these, if longer than 4 inches, may be shortened to 1 or 2 inches, till ultimately pinching off the tops of the young shoots to 1 or 2 inches will be all that is required.

"To sustain the tree in its confined state, something more must be done than allowing its roots to go into the border. Annually in March," but as soon as they are excited artificially, be it December or January, "every tree must have a top-dressing of some strong stimulant. I have applied," Mr Rivers informs us, "with much success, horse-droppings saturated with nightsoil, exposed to the air two or three months previously, placed on the surface of the pots, previously stirring the soil, and taking out a portion of it with a pointed stick to 2 or 3 inches in depth. Liquid manure not too powerful must be applied once a-week during the summer," and in the case of early forcing, from the time the foliage forms till shortly before the fruit ripens. "Weak guano-water, one pound to thirty gallons, is perhaps as good as any; a weak soaking of this once a-week is better than using it too frequently." An important part of forcing fruit-trees in pots is to avoid the extremes of wet and drought at the roots, and this is VOL. II. the more important on account of the thorough drainage below, and the constant evaporation going on over the whole surface of the pots. Some, to counteract this, envelop the pots in a covering of hay-bands, or cover them with moss. We believe that want of proper attention to the roots in this very respect has been the cause of the frequent failures in forcing stone-fruits in pots, and hence the unpopularity of the practice. "The treatment for the second year," Mr Rivers continues, "may be continued every season without variation, except as regards pruning; and every spring the pots should have a fresh station on the border, and after two or three years the soil of the border may be removed 2 or 3 inches in depth, and filled up with a compost of burnt earth, 1-inch bones, and turfy loam; and it will be necessary in very dry winters to watch the trees to see if their roots shrink: if so, they must have a small quantity of water, but not in severe frosts; and if the winter is excessively severe, some dry hay or litter may be laid on and around the pots."

Time of beginning to force.-This of course must be regulated by the time the fruit is required to be ripened, as well as the varieties to be grown ; for some of these, particularly in the cases of the cherry and the plum, will ripen a month or more sooner than others. From the beginning of December to the beginning of February may be taken as the periods of commencement. But as these are fugacious fruits, it is better to commence by introducing a few at a time, and bringing in others in succession; yet were it not that the dark months of winter are the most unfavourable for the setting of the fruit, their successional bringing in might be avoided by filling the house at once with a portion of those which ripen earliest, those which naturally succeed them, and also of those, like Coe's golden drop, which ripen the latest. If forcing is begun by the first of February, the earlier, varieties of cherries and plums will, under ordinary circumstances, be ripe by the first week in May, the later sorts by the second week, and apricots by the first week in June : much, however, depends on the season, whether it is cold, cloudy, or otherwise. Beginning at the first of January, the earlier plums and cherries may be expected to ripen by the last week in April, and so of the others. No doubt both cherries and plums may be ripened in less time, but it will be with less certainty as to crop and the health of the trees. During the time the trees are in blossom, avoid syringing over their tops and the application of tobacco smoke for the suppression of the green and black fly, and admit of extra ventilation, applying a slight increase of fire-heat to maintain the temperature at the regulated point, but upon no account allow it to exceed that point, for the process of flowering should not be excited by such means. If the house is properly fumigated just as the wood-buds are bursting, there need be little cause to fear the appearance of the fly. Fumigation is extremely dangerous during the period of the development of the blossom.

Temperature.-Merely shutting up the structure during the night-say from four in the 
afternoon to eight o'clock in the morning-for the first week will be sufficient; that is, ranging to about $40^{\circ}$, gradually raising the temperature during the three following weeks to $42^{\circ}$ as a minimum, and in warm sunshine allowing it to rise during the middle of the day to $52^{\circ}$, admitting air freely all the while. Shut up early in the afternoon, so as to enclose as much solar heat as possible, thereby lessening the demand upon the furnace, which should be regulated with great precision, particularly during the night, for it were better the night temperature fall a degree or even two under the above minimum than exceed it to that extent. During the fifth and sixth week the minimum by fireheat may be elevated to $45^{\circ}$, but the maximum of such heat should, during the day, be kept at or rather under $48^{\circ}$, while that of solar effect should be by ventilation restrained to $55^{\circ}$, and so kept until the trees are in bloom. During the period while the blossom is fully expanded, and while the fruit is setting gradually, raise the fire-heat to $48^{\circ}$ and $52^{\circ}$, as the maximum and ninimum, admitting all the while a free and genial ventilation, but avoiding cold drafts of air; and for preventing accidents arising from this cause, if subterranean ventilation is not provided for, as so abundantly shown in vol. $i$. then the air admitted through the front or side windows should be made to pass through thin canvass screens, to break its force and cause a more equal diffusion of it throughout the house. Small openings, and many of them, will be found of advantage at this stage, which is the most precarious in forcing these fruits. During the setting of the fruit, air should be sensibly felt, not in gusts, but in gentle zephyrs throughout every part of the house; and if this is attended to with a steady night and day temperature, as stated above, with the necessary amount of moisture at the roots, and a proper hygrometric state of atmosphere, there will be no fear of success even with these reputed ill-setting fruits.

Water, when applied either to the roots or branches, should never be less than equal to the temperature of the house, and indeed that applied to the former should be $5^{\circ}$ or $10^{\circ}$ warmer. Air at so early a season can very seldom be admitted with safety direct from the external atmosphere; hence the great importance of bringing it in in underground drains, causing it to pass over the tanks or hot-water pipes, or sifting it through canvass screens, so as to break its force or elevate it to the internal temperature.

Watering. - From the day the trees are brought into the house, or should they be permanent occupants of it, syringing should be daily attended to until the blossoms begin to expand, at which period it should be suspended until the fruit is set. After this it should be resumed with a view to clear the trees of the decaying blossoms, refresh the foliage, and suppress insects. Avoid too much water at the roots during the period of the fruit's setting, but at the same time avoid an opposite course, either of which would be equally detrimental. After the fruit has stoned and the flesh begins to swell, give more copious root-waterings, and if of a fertilising nature so much the better.

There is no doubt that the day is not far distant when we will have orchard or fruit houses upon a very large scale, and it is probable that such hardy trees as the plum and cherry will be grown in a portable state, to admit of their being placed in the open air after their fruit is gathered. The vine, peach, and apricot may be found to require such protection at all times, to enable them in cold countries to ripen their wood, and the fig may remain a permanent tenant on account of its producing more than one crop per annum. Mr Rivers has shown very satisfactorily what can be done on a small scale and in very economical structures; and his arguments and success in pot-culture strengthen an opinion we have long entertained, that the control we have over the roots by his process will lead to results that planting out and annual root-pruning can never realise in the case of such trees as these, when subjected to artificial excitement. 


\section{CHAPTER XI.}

\section{THE F I G.}

The Fig (Ficus carica L.), as a fruit-producing tree, is of great antiquity, indigenous to a great part of Asia, introduced from there and naturalised in Italy, from whence it was carried into France and Britain. At what precise time its introduction took place is involved in obscurity, for we hear nothing of its culture in England prior to 1562, when Dr Turner wrote his "Herbal:" that it was, however, imported by the Romans along with the vine is more than probable; and as vineyards are well known to have been established in England as early as 280 A.D., and that the vines were brought from Italy, it is but natural to suppose that the same inducement that led to the importation of the vine would lead to that of the fig also; and this the more especially, as the fig constituted then as large a share of the sustenance of man as the vine did, or at present does. Hart observes "that the religious fraternities of the dark ages spread out from Italy in all directions, carrying with them the knowledge of agriculture and gardening;" and there is little doubt that they disseminated the fruits of Italy into every country where the climate was favourable for them. That both the vine and the fig disappeared for a time from Britain is highly probable. 'The fig was much cultivated by the Romans, who were possessed of no less than twenty-nine sorts prior to the Christian era, all of which have been described by the naturalist Pliny. The second introduction of the fig appears to have taken place in 1525 , when Cardinal Pole brought from Italy those identical fig-trees which still exist in the archbishopric gardens at Lambeth Palace; and Dr Pocock, the Oriental traveller, first brought the fig to Oxford, and planted a tree in 1648 in Oxford college garden, of which tree the following anecdote is told: Dr Kennicott, the celebrated Hebrew scholar and compiler of the Polyglot Bible, was passionately fond of this fruit, and seeing a very fine fig on this tree that he wanted to preserve, wrote on a label "Dr Kennicott's fig," which he tied to the fruit. An Oxonian wag, who had observed the transaction, watched the fruit daily, and when ripe gathered it, and exchanged the label for one thus worded, "A fig for Dr Kennicott."

In the southern parts of England, about Arundel and Tarring, the fig grows as an open standard, and there, as in the Isle of Wight, it attains a great size and age, and produces fruit not much inferior to those of Italy. In the midland and northern counties of England, as well as over almost all Scotland, the fig requires the protection of a wall, and even with that there are many parts where it can scarcely exist. The fig is one of our most wholesome fruits, and although, in its natural state, it is by no means popular with persons in general in this country, yet those who have been accustomed to them abroad, or who have acquired a taste for them at home, become passionately fond of them. The fig has a peculiar quality of intenerating animal fibre. Philips (in "Pomona Britannica," p. 169) relates the circumstance of a gentleman "that hung up a haunch of venison, lately killed, in a fig tree when the leaves were on, at about ten o'clock in the evening, and which was removed before sunrise in the morning, when it was found in a perfect state for cooking, and that in a few hours more it would have been in a state of putrefaction." The fig did not reach the United States till 1790, and, singular enough, they have not produced varieties of this fruit, notwithstanding the great progress made in most others.

Gerard and Parkinson speak of the fig as if little cultivated in their day. Miller appears to have been the first to collect varieties, as we find by his "Gardeners' Dictionary" that he introduced above a dozen new sorts from Italy. The Catalogue of the Horticultural Society enumerates forty-two sorts, Downing fifteen, the Catalogue of P. Lawson and Son sixteen, Forsyth fifteen, Rogers nine, which, he remarks, are the only ones worth the British grower's attention; while Langley, in "Pomona," says, "The several kinds of figs that are worth our cultivating in England are the White, the Blue, and the Black." Rivers gives twelve, George Lindley, in "Guide to the Orchard," twenty-seven.

Propagation.--The fig is readily propagated by seed, cuttings, layers, suckers, roots, grafting, occasionally, but rarely, by budding. Seed taken from the ripe fruit of home growth, or from those that are imported, vegetate readily; and by this means plants will be obtained differing little from the tree the seed was taken from. When, however, new or improved varieties are sought for, the process of crossing one variety 
with another must be had recourse to; and this is not accomplished without skill and care on the part of the operator, in consequence of the parts of fructification being placed within the fruit, and not developed prior to its formation, as in the case of the other fruits we have described. The fruit of the fig is an excellent example of what botanists call an aggregated fruit-that is, a fruit formed by an intimate or apparent union, proceeding in reality from different flowers. In the case of the fig it is either a hollow pedicle, or rather a kind of fleshy involucrum, formed of a great number of thick bracts intimately united at the base, either with each other, or with the top of the pedicle, and very slightly free at their extreme apex. The flowers are very numerous within this involucrum, "or what in the fig we call the fruit ; the female ones, which are the most numerous, and more central, are transformed into as many caryopses as there seems to be seeds."-Vegetable Organography. The flowers, therefore, being enclosed within this fleshy involucrum or fruit, cannot easily be reached so as to apply the ordinary process of crossing, with a view to produce cross-bred varieties, and hence few if any are artificially produced. With the fecundating operations in the fig we practically know very little. This subject many years ago attracted the attention of Sir Charles Monck, who, after many interesting experiments, came to the following conclusions : first, That fig trees never bear figs which contain both kinds of florets in an efficient state; secondly, That figs in which the anther-bearing florets only are perfect never come to be eatable fruit; thirdly, That you may pronounce, from the external shape of a fig, which kind of floret prevails-the stigma-bearing in the pear-shaped, the anther-bearing in the squat-shaped figs; and, fourthly, That fig trees which put forth crops of figs, and cast them, most probably do so from defect of setting. (For more of his experiments, see "Hort. Trans.," vol. v.) Cuttings, however, strike root freely, and these are chosen in autumn from the best-ripened wood of the same season's growth, selecting those that are from 8 to 10 inches in length, retaining about an inch of the older wood at the base, and planting them at once in light sandy soil, and without taking anything off their tops. They should be planted in pots, and these plunged in a dry warm sheltered place, and protected from frost during winter. In spring they should be placed in a more open and airy situation, and by the autumn following they will be fit for shifting into larger pots, or, if upon a large scale, into nursery rows. The young plants require little pruning, only training them to one stem to the height of a foot, if for dwarf standards, or to 3 or 4 feet if for training in the stellate form, or as elsewhere noticed, after which the branches should ramify into their natural habit, thinning out only in cases where they may become too thick. If the trees are intended for training against walls or espaliers, training for the first and second year is necessary. If properly attended to, figs from cuttings will begin to produce fruit the second and third year. The nursery prac- tice is to have stools-that is, established figtrees, planted out-and from these they obtain stock by laying the young branches into pots sunk in the ground, and often into the ground itself, removing them, the autumn following, either into nursery rows, or into pots, to facilitate their being sent to a distance during their growing season. It is better, however, when they are sent out while in a dormant state, to send them without pots altogether, which lessens the expense of carriage, and in no way injures the roots if they are properly packed. When fig plants are required to meet any pressing demand, branches of two, three, or more years' growth may be made use of for layers. They root readily, and in ten months will be ready for separating from the stool, and planting in their permanent position. Figs propagated by suckers are apt to send up suckers ever after, and, besides this, they seldom make such short-jointed well-formed wood as those originated from cuttings. It is a process, therefore, which should not be encouraged. The fig is grafted in the same way as the vine, that variety called inarching being justly preferred, and it is equally readily but rarely budded. Indeed, unless it be in the case of receiving grafts or buds from a distance, where it might be inconvenient to transport a whole plant, these latter modes of propagation are scarcely worth attention. The fig may also be struck from single eyes the same as the vine (which see), and it is probable in the one case, as it is well known to be so in the other, that better and shorter-jointed wood might be produced.

Soil.- The fig is not difficult to accommodate with a soil, provided it be dry at bottom; but this must be considered as an essential condition for securing success in its cultivation. In all dry light sandy loams, without the addition of manure, the fig prospers; and if there be a preference to any other, it is certainly one of a calcareous nature. With figs grown in pots, with their roots circumscribed, and subjected to the excitement of forcing, a richer soil is necessary, and such will derive much advantage if supplied occasionally with liquid manure. For such, however, as are to be grown in the open air no such stimulants are required, as they would have the effect of causing the trees to make too much wood, and that long-jointed and too gross to insure its ripening in our shortgrowing and cold seasons.

Planting.-In all cases where the fig is grown as a dwarf standard or espalier in the open air, unless it be in the southern and warmer parts of England, such as the Isle of Wight, along the Sussex coast, about Arundel, Worthing, \&c. where they attain a large size, and ripen in great perfection, they should be confined at the roots by walling them in, allowing each tree from 16 to 20 cubic feet of soil to grow in. When growing against walls, they may be allowed from one-third to a half more space for root room. There are few places, however, in Britain where the fig ripens satisfactorily unless against walls; and in the majority of cases they require, particularly in Scotland, a southern exposure. The fig bears transplant- 
ing well, and it is much better to confine the roots to a limited space, and to take them up once in two or three years, reducing their roots, and affording them fresh soil, than to plant them in rich deep broad borders at 15 or 20 feet apart from each other. In the latter way one-third of the space will be amply sufficient. The fig succeeds better on low walls than on high ones, the former being warmer, and the mode of training more natural to it, as the fig is naturally a low-spreading tree. In all temperate climates exceeding the mean of that of Edinburgh, the fig thrives upon walls, and exceeding that of London as an open dwarf standard. No doubt this fruit has and will ripen in peculiar situations, where the mean temperature may be somewhat under these points, but not with the same certainty and satisfaction. One of the best walls of figs we know of in Scotland is that of Preston Hall, 400 feet above the sea, and a naturally cold situation, but upon a dry subsoil. They ripen also on walls one hundred miles farther north, clearly showing the natural advantages of certain localities, over which, apparently, neither latitude nor altitude appear to have much influence; for there are many situations near the sea, and much farther south, where the fig refuses to ripen as a standard, and seldom does so even on walls; while at New Tarbet, seven hundred miles north of London, and exposed to the blasts of the German Ocean, they ripen admirably on the open walls, as do also apricots, almonds, mulberries, peaches, and many of the finer pears. Vaulted borders will be found exceedingly valuable for the fig, because they secure dryness and increased heat at the roots at the same time.

Pruning and training.-The principal consideration to be kept in view, lefore any system of pruning or training be adopted, is to make ourselves thoroughly acquainted with the mode of bearing natural to the tree to be operated on. A very excellent paper, by the Hon. William Wickham, will be found in "The Transactions of the Horticultural Society," in reference to this subject. "The fig tree is distinguished from most if not from all other trees by this extraordinary property, that it bears, and in warm climates brings to maturity, in every year, two successive and distinct crops of fruit, each crop being produced on a distinct set of shoots. The shoots formed by the first or spring sap put forth figs at every eye, as soon as the sap begins to flow again in July and August. Thesefigs (which form the second crop of the year) ripen in their native climate during the course of the autumn, but rarely, if ever, come to perfection in England, where, though they cover the branches in great abundance at the end of that season, they perish and fall off with the first severe frosts of winter. The shoots formed by the second flow of sap, commonly called midsummer shoots, put forth figs in like manner at every eye, but not until the first flow of sap in the following spring. These last-mentioned figs, which form the first crop of each year, ripen in warmer climates during the months of June and July, but not in this country before September or October. In warmer climates, indeed, very little attention is given to this crop, because the midsummer shoots, on which it is born, are commonly in proportion only of one to six or eight in length, when compared with the shoots of the spring, which produce the second crop; and the crop itself is always small in the same proportion; but in England it is the reverse, as no care or skill of the gardener can ever insure a second crop of ripe figs in the open air." With the view to increase the proportion which the midsummer shoots should bear to the spring shoots, both in number and length, this intelligent amateur cultivator purposed breaking off the spring shoots, at the time they. nearly attain their full growth, about from 6 to 15 inches from their base, according to their strength, leaving enough of each shoot to admit of its being bent back and fastened to the wall at the ensuing winter pruning, taking care that one eye at least be left uninjured, and besides leaving a sufficient number of shoots unbroken, to secure a supply of wood for the proper filling up of the wall. These shoots should be broken off and not cut; and it has even been found in practice to be better, although less sightly, to break them only partially at first, leaving them slightly attached to the tree, and to cut them off afterwards when the sap has ceased to flow. The rationale of fracturing them, instead of cutting them off at once with a knife, is, that they would produce at their extremities only one single midsummer shoot, whereas, if fractured, it generally happens that, on the second flow of the sap in July, two or three more shoots are pushed from the fractured part instead of one, and each of these, according to its length, will produce several figs in the ensuing spring, and their fruit is thus rendered capable of ripening during our ordinary summer and autumn heats. "A sufficient supply of midsummer shoots being thus secured during the summer, room must be made for them at the succeeding winterpruning, by cutting away as much of the old wood as will admit of their being all trained in at full length, and nailed close to the wall, which should always be done before the first severe frosts. Keeping this object in view, the knife cannot well be used too freely in cutting away the old wood, nor is there any reason to fear that its free use will either injure the future crop, or deprive the tree of its regular supply of branches. The midsummer shoots being trained in, each of them will produce, in the following year, one spring shoot, at least, at its extremity, whilst another will rise from each eye of the remnants of the old spring shoots that had been preserved in the manner above described, when these shoots were broken in the preceding month of June. From this fresh supply, by pursuing the system here explained, either fruit, wood, or both, may be obtained for the succeeding year, at the discretion of the gardener. When he wishes for wood, he must suffer these new shoots to grow to their full length; when fruit, and not wood, is desired, he must break them in the month of June, in the manner and with the precautions that have been minutely explained."-Hort. Trans., vol. iii. p. 74, \&c. 
The horizontal mode of training the fig is recommended by $\mathrm{Mr}$ G. Lindley, who says, "Horizontal training appears the most eligible for the fig, as it checks its luxuriance, and by this means adds materially to the ripening of its wood; for unless this be accomplished, it will be in vain to look for fruit."-Guide to the Orchard, p. 171. Harrison approves of the horizontal manner of training, and observes, "The trees will thus be more fruitful than if trained more erect; but in those cases where the trees trained horizontally are still too luxuriant, let the branches be trained in a pendulous manner, and the more luxuriant the tree the greater must be the declination. In pruning and training the great object must be to keep a regular supply of young wood in every part; the shoots must be trained so far apart that the sun and air will be properly admitted to every part. In those kinds which have very large leaves - the Brumswick, for example-the distance must be 12 or 14 inches at least. The principal crop of figs is produced upon those shoots that are made after midsummer; and there," Harrison says, " care should be taken to preserve the fruit through the winter, and they will come to perfection in June or July following. When a sufficiency of lateral bearing shoots is not produced, they may be attained by attending to the following directions: When the spring shoots have done growing, which will generally be by the end of May or beginning of June, let each shoot, where wood is required, be stopped by pinching the end betwixt the finger and thumb so hard as to feel that the shoot gives way to the pressure, but not so as to break it. This will cause shoots to push below where it was stopped. If a shoot thus desired to be stopped be a long one, let it be bruised or broken nearly in two about the middle, or a piece of string be twisted very tight round it, and the end of the shoot above the string be brought nearly parallel down by the side of the remaining part of the shoot, and this will cause shoots to push where desired. These lateral shoots generally show plenty of fruit during the months of August, September, and October, but appearing late in the year they will not ripen in this country the same season, but some of the fruit will attain to half their size or more. Such as attain to the size referred to, or even much larger than a large marrowfat pea, will very seldom survive the severity of the winter." This being usually the case, "it is a very general practice, at the end of autumn, to take away all figs that are of the size described. The removing them is certainly proper, but they ought to be taken away at a much earlier period, even as soon as they are discovered to be figs. By doing so, the sap is diverted to the formation of one, or occasionally two, embryo figs at the side of the fruit removed, and which will be so small as to escape injury by covering them during winter, but will ripen well the following summer." Differing in opinion from Harrison, the Rev. George Swayne, in "Horticultural Transactions," p. 430, recommends displacing, as soon as they can be recognised by the eye, all em- bryo figs produced after midsummer on the same year's wood; his object being to prevent unnecessary exhaustion to the tree by allowing it to produce ernbryo fruit, which would never ripen without artificial heat in our climate, but rather to employ the energies of the tree in the preparation of new embryo figs for the following year. His words are: "If this operation be performed in due time, it will not fail to prepare on one, and often on both sides of almost every fig so displaced, such embryos. For this purpose the trees should be examined once a-week from the beginning of August, at which time the figs of this second crop usually begin to show themselves, and this examination must be repeated as long as they continue to make their appearance. Most gardeners omit removing these late figs at all, or delay the practice till October or November, when no benefit is derived from it." Like Harrison, he approves of horizontal training, and defers pruning till late in spring, when the leaf-buds and fruit-buds can be clearly distinguished.

Knight's mode of training differs from most others, and no doubt has much of the advantages expected, in moderating the flow of the sap. He says, in the work last quoted, vol. iii., p. 307 : "Let the stems, if there be, as usual, many within a narrow space, be gradually reduced to one only; and from the top and parts near it of this let lateral branches be trained horizontally and pendantly in close contact with the wall. Under such treatment all troublesome luxuriance of growth will soon disappear. The pendant shoots will not annually extend more than a few inches, and few or no more leaves will be produced than those which the buds contain before they expand. The young wood consequently ceases to elongate very early in the season, and hence acquires perfect maturity, while, by being trained close to the wall, it is secure, or nearly so, from injury by the severest frost. The quantity of mature and productive wood thus necessarily becomes very great, relatively to the size of the tree; and the fruit being in contact with the wall, and not shaded by excess of foliage, acquires an early and perfect maturity." In fact, Knight's theory amounts to this: Train the fig to one stem until it reaches the top of the wall or espalier, lay in a shoot horizontally on both sides, and train the shoots they produce in a downward direction; or, slightly modified, train the fig in the stellate form (vide fig. 149.) Much of all this, however, may be accomplished at less trouble by root-pruning, limiting the range of the roots, and using a soil rather poor than over rich. The fig seems to draw a large share of its nourishment from its leaves. They present a much larger surface to the atmosphere than those of most of our fruit-bearing trees do, decidedly more so than any cultivated in the open air in this country; hence less dependence is placed on the roots, which, if placed in rich soil, and allowed to range to a great distance, throw into the system of the tree more nourishment than it absolutely requires, and in disposing of this extra food the tree is induced to form wood and leaves in proportion, and hence the strong and 
luxuriant shoots produced, which may be called shoots of sterility, for it is not on them that fruit is to be looked for. The fig, when pampered in even moderately rich soil, and protected by the shelter of a wall, will be found, upon even a very superficial examination, to consist of three kinds of wood; one of an over-luxuriant character, long in the joints, or spaces between the nodes: such wood is also usually thick and succulent in substance. Another kind of wood will be found attenuated, long-jointed, spongy, and pale coloured ; while a third kind will be found short-jointed, stiff, and strong, with the buds or nodes set thickly upon it. This last is the proper bearing wood, and therefore should be retained at the general pruning. A large portion of the second sort should be removed, and all of the first, unless it be where wood is required for the extension of the tree, when the best situated shoots should be selected. The trees should be gone over in May, and a species of disbudding attended to, at which time an eye should be kept to these three kinds of wood while yet in their young state ; and such as are not required for keeping up the necessary supply of young wood should be removed from the first and second of the above kinds; and even should the last be overcrowded in places, some of them should be removed also. All suckers from the root should be removed as they appear, unless a shoot or two be occasionally required from amongst them to fill up any vacancy on the wall. Where the tree is trained to one clear stem, of course all the suckers must be removed, and advantage taken of the suckers only when the tree is trained in the ordinary regular manner. In September another general examination should be attended to, at which time all superfluous shoots should be removed to admit light and air to the ripening fruit and wood intended for future bearing. The shortest-jointed wood only should be at this time retained, and such of them as can be conveniently tied down upon the older and naked branches should be so done: indeed, they should be completely clothed with young wood to protect their bark from excessive sun, as well as to admit of the young wood being so disposed of that portions of the wall may be fully exposed to the sun that it may absorb heat from its rays ; for it should be borne in mind that a wall, be it ever so well exposed, if completely covered with branches and foliage so as to prevent the free action of the sun upon it, is, so far as heat is concerned, of little more use to the trees than if it did not exist at all. Stopping the young shoots about the end of August or beginning of September is an important part of fig-tree management. The very short spur-like shoots with short joints referred to above do not require this, but there are others which do, and they are such as are retained and selected for their firmness of texture, moderate growth, and proper position, after all the superfluous ones have been cleared away. The stopping of such at this time should consist of merely squeezing flat the terminal point, which will have the effect of inducing the formation of embryo fruit for the succeeding season. This stopping, Mr Errington very properly remarks, "is a matter of some nicety, and the period of performing it must be determined both by the kind and its condition and habit. A too early stopping with some figs, which are not very difficult to fruit, would cause them to develop the fruit for the ensuing season too early; for if they become as large even as a black-currant berry, they will be almost sure to perish with severe weather during the ensuing winter. Stopping is, therefore, a matter of some nicety, and had better be performed over late than early."

Protecting during winter.-No fruit tree requires protection in this country unless in the most unfavourable situations, except the fig, and these require it to some extent in most places, more especially in very cold seasons, for there are instances of almost every fig tree in England having been killed to the ground during intense frosts. Various means have been adopted to prevent such casualties. On the Continent, where the winters are more severe than in Britain, the branches are detached from the walls, and those of dwarf-standard trees are bent down and partially buried under ground; others envelop the branches in straw or hay bands, and leave them fastened to the wall. In general, however, those that are grown as dwarf standards have their branches tied together and thatched over with straw or reeds. A covering of light dry fern fronds or branches of evergreen trees is found, in most seasons and most situations, sufficient for them in the greater part of Britain. Covering the surface over the roots with littering matter, coal-ashes, or rotten tan, and also winding hay-bands round the collar of the tree, is also of vast importance to them. Figs grown in cold localities would de. rive great protection were the portable wooden copings already referred to placed over them towards the end of autumn, and allowed to remain on until late in spring. These would tend to keep the trees dry, and the drier the wall and the wood of the trees are kept during winter, the less likely are they to suffer from cold. The best of all protections next to glass is undoubtedly canvass, suspended from the wooden coping, and secured near the bottom of the wall. By this means air and a modified amount of light are admitted, which are both denied them when they are too thickly thatched with branches, and still more so when under these branches hay or straw is packed, offering an excellent winter asylum for field-mice, which not only often devour the embryo fruit and buds, but also occasionally strip the bark also. Where the fig will withstand the winter without covering at all, it is in every sense better for the trees; for when very thickly covered during winter, the branches are so tender that when it is removed in spring the check they receive is very injurious. The covering used must be in proportion to the coldness or warmness of the situation.

Accelerating the ripening of the fig artificially. -The only rational, and therefore the best means of hastening the ripening of the fig in the open air in this country, is decidedly covering them with a portable wooden structure fitted 
with glass sashes. Many extraordinary means have been adopted to effect this, and merit our notice more to point out their absurdity than their utility. Of these the process of caprification, as long practised in the Levant and other fig-growing countries, is the chief. It consists of collecting the spring figs in which a species of gnat has already deposited its eggs, and these figs are placed upon the trees so that when the gnats are hatched they in their turn deposit their eggs in the figs constituting the autumn crop, effecting, as it is asserted, the process of fecundation in the flowers within the fruit during their passage to the centre of the fig. Ringing the branches was recommended by Sir Charles Monck; and the same intelligent and ardent horticulturist found, by splitting a fig from end to end, that it ripened six weeks before others that were left untouched. In Italy the fruit is often wounded with a knife to produce a similar effect; and in the same country a drop of brandy or other spirits is let fall into the eye of the fruit, while at other times a bodkin dipped in spirits is thrust into the side of the fig. At Argenteuil in France women are sometimes employed in dropping oil into the eye of the fig, which they adroitly do with a piece of hollow rye-straw, dipping it into the oil and forcing it into the eye of the fruit alternately. These absurdities merited the rebuke of M. Olivier, who said of them that it was " a tribute which man pays to ignorance and prejudice."

That mutilation brings on a sort of ripeness in fruits is undeniable, but the flavour and quality are lessened in proportion. Touching the eye of the fruit with a drop of sweet oil is the least objectionable of the means employed. In our own practice, however, we have not found the ripening sensibly affected. The late Mr Downing thought otherwise, and says, "In an unfavourable soil and climate the ripening of the fig is undoubtedly rendered more certain and speedy by touching the eye of the fruit with a little oil. We have ourselves," he says, "frequently tried the experiment of touching the end of the fig with the finger dipped in oil, and have always found the fruits so treated to ripen much more certainly and specdily, and swell to a larger size, than those left untouched."

Forcing. - The fig submits with greater impunity to earlier excitement than either the peach or the vine. They admit of being forced in pots or boxes, and indeed this appears to be the preferable way. Plants for this purpose should be low and bushy, having the wood perfectly ripened and short-jointed. At two years from being taken from the parent plant (for figs are in general propagated by laying, although they succeed equally well by cuttings), if kept in pots and abundantly supplied with air, light, and water, in a glazed pit or house, they will be in fit condition for forcing. In the south of England, where the fig thrives so well in the open air, the protection of glass during the plant's growth is uncalled for, but in all situations where it does not succeed as an open standard that protection is necessary. The Nerii and Lee's perpetual are best adapted for forcing, on account of their less luxuriant habits. There are other sorts, however, that are found to do well also. Like all plants intended for early forcing, it is a primary object to have the plants well rooted, and their wood stored with a sufficiency of properly elaborated sap; and this can hardly be expected unless the plants are grown in pots, sufficiently stimulated, and reared in a fitting temperature. Early in November (for earliest crops) the plants should be examined to see that all is right at their roots, and especially that the drainage is complete: all that are deficient in these points should be re-potted and reserved for successional crops. If the pots are full of healthy roots, we would not advise shifting, but to remove them at once into a pit or fig-house, and to arrange them so that their shoots may be placed as near to the light as possible. If a slight degree of bottom heat can be applied to their roots, so as to place them in a temperature from $5^{\circ}$ to $10^{\circ}$ greater than that of the branches, it will be of great importance to them. By putting the roots in action first, the embryo fruit is greatly stimulated, and much less likely to turn yellow and drop off, which they are apt to do early in the season if this want of stimulant be denied them. It is a good practice to place them first in a pit, plunged in leavcs or other matter in a slight state of fermentation, and when the fruit has shown itself to remove them to the fig-house, where they may enjoy a greater amount of air and light. That a check, however, may not be felt, the temperature of the fig-house should be kept nearly at that point, whatever it may have been, that the roots enjoyed while in their plunged state. As the young leaves expand, abundance of tepid water should be given the roots, and syringing attended to at least once a-day. The thrip and red-spider are grievous enemies to the fig in high temperatures, and their presence is invited when the atmosphere is anything like dry. The fig is also more likely to suffer from a deficiency of water at the roots than by an excess of it in a forced state, however different its condition may be when growing in the open air. Large spanroofed pits having a morning and afternoon exposure, and where a humid and moderate bottom-heat can be regularly maintained, are, in our opinion, the best of all structures for the extra early forcing of the fig. For principal crops the plants may be of larger-growing sorts than those named above, and they may also be planted out in prepared borders, only so arranged that their roots are not allowed to ramble at large in search of food. For to this they have so great a predilection that they become gross in habit, making long-jointed coarse wood instead of moderately-sized shoots thickly set with fruit-buds. Various means have been suggested to check this over-luxuriance of habit, among others that of chambering them in by building walls closely jointed so as to form compartments for the roots of each plant, varying, according to the sort, from 4 feet to 16 superficial feet each compartment-that is, supposing them squares, from 2 to 4 feet on the side each square, and from 2 to $2 \frac{1}{2}$ feet in depth, laying in a deep drainage of broken 
limestone or chalk lumps where they are to be had. In lean-to houses the back wall may be covered entirely, training the trees to a wire trellis, and the front may be planted in the standard manner. It is of great importance in forcing the fig, even when the trees are planted out, that means be employed whereby a slight degree of bottom heat may be applied to them when necessary, and this can best be done when a substratum of stones or brickbats is laid 2 feet over the whole bottom of the borders, and hotwater pipes made to traverse through the mass; or the borders may be vaulted and heated below, as exemplified in the vineries at Yester, vide vol. i., fig. 445.

The Nerii and Lee's perpetual are well adapted for standard-growing, while the Brown Ischia, White Genoa, \&c., are adapted to the back trellis. As to temperature, almost under any circumstance the starting-point may be taken at $50^{\circ}$ for atmospheric heat, and $60^{\circ}$ for bottom heat, and when the fruit begins to grow, raising progressively to $55^{\circ}, 60^{\circ}$, and so on progressively much the same as for vines. Water less abundantly until the fruit has fairly begun to swell, after which it must be applied more abundantly to aid in its swelling, and syringing must be attended to, using for both purposes water as near the temperature of the house as possible. Manurial stimulants are not so congenial to the fig as to most other fruits; it should therefore be supplied more sparingly, and not at all if the trees assume a strong habit of growth. Ventilation is, as in all similar cases, important; a neglect of it is one of the causes of the fruit's dropping prematurely. As to pruning in early forcing, little is required beyond thinning the branches should they have become crowded, or displacing any ill-placed or awkward shoot previous to their being put into a state of excitement. What is usually called summer pruning, but which, in the case of figs forced from November to May, may be called spring pruning, consists in pinching out the top of the young wood when it has attained the length of 4 or 5 inches, the portion left producing the second crop of fruit; and this, under favourable circumstances, may be repeated a second time, so as to secure something like a third crop. It is better, however, when the plants are in a portable state, to be content with two crops, and to let the plants go to rest after perfecting the second crop; and where there is a sufficient stock of plants, which should always be the case where a long continuance of fruit is desired, set after set should be brought in. It is less difficult to produce ripe figs throughout the year by these means than any other fruit. Forcing the fig at later periods of the year is to be considered a repetition of the above practice. To grow the fig in perfection, it should have accommodation especially set apart for itself; and if the trees be well managed, few fruits will equal it in duration, quality, and quantity of its production. To ripen off the late crop, say in October and beginning of November, a temperature will be required of at least $60^{\circ}$, accompanied with a free circulation of air to prevent dampness affecting the fruit.

VOL. II.
The varieties best adapted for pot-culture, Mr Rivers says, are "the White Ischia, the Saint Jean, both most abundant bearers, the White Marseilles, the White Genoa, and the Brown Turkey. If more varieties are required, the Nerii and the Pregussata may be added." The two latter are held high in our estimation, both on account of their productiveness and their delicacy of flavour.

The fig is well adapted for growing in pots in pits, and still better in such structures as $\mathrm{Mr}$ 'Rivers' orchard-houses, but in such limited spaces the quantity produced must ever be small compared to what may hereafter be confidently expected, not only as regards figs, but all other fruits, where structures covering an acre or more will be erected.

\section{SELECT LIST.}

Angelique.-Colour pale greenish yellow; form obovate; size small. This little fig is somewhat like the Marseilles, but longer. It is a very abundant bearer, and of excellent quality. In the case of the fig we will omit the French synonyms, considering the English ones sufficient for every practical purpose.

Brunswick.-Colour violet brown ; form pyramidal; size one of the very largest; flavour rich and excellent, although by no means so delicate and tender as most others. It is one of the hardiest, a remarkably strong grower, which nothing but root-pruning or walling in the roots can check. It is of too robust a character for forcing. Synonyms-Madonna, Hanover, Black Naples, Red, Brown Hambury, Bayswater, Clementine.

Genoa, large white-Colour whitish yellow; form roundish, rather lengthened towards the stalk; size large; skin thin; flavour excellent.

Ischia, black.-Colour dark violet, almost black when ripe; form round, somewhat flattened at the apex ; size medium; pulp deep red ; flavour excellent. Synonyms-Blue 1schia, Early forcing. An excellent bearer, and comparatively hardy, as are all the Ischias. Of moderate growth.

Ischia, brown.-Colour brown; form obovate; size medium; flesh tender and of excellent quality. Synonym-Chestnut-coloured Ischia.

Ischia, white.-Colour pale yellowish green; form roundish obovate; size small. One of the hardiest of the pale-coloured. A moderate grower and good bearer.

Malta.-Colour light brown; form much compressed at the apex, and tapering very much towards the stalk; flesh pale brown, and of a very sweet rich flavour. Hangs long on the tree after becoming ripe, and if left till shrivelled it becomes a fine sweetmeat. Synonym-Small brown. This is the Malta of Lindley. Thompson, No. 21, in "Hort. Soc. Fruit Cat.," has it Malta white.

Marseilles. - Colour white, or rather pale greenish yellow; form roundish obovate, slightly ribbed; flesh rather dry, but very sweet and rich. Tree hardy and an excellent bearer. One of the very best for forcing. SynonymsFord's seedling, Pocock's, White standard, White 4 B 
Marseilles, White Naples. The oldest variety in cultivation.

Nerii.-Colour pale greenish yellow: form roundish obovate; size small; flesh similar in colour to a pomegranate. Lindley observes, "It is much the richest of its species, and there is in its juice a slight degree of very delicate acid, which renders it peculiarly agreeable to most palates." From the experiments made by Knight, it would appear not to be adapted for forcing. He says, "It offers fruit very abundantly, but the whole falls off alike in the stove and in the open air, and it succeeds only in low temperature under glass. I have obtained it in high perfection by bringing the fruit forward till it was about one-third grown in the stove, and then removing the pots in which the plants grow to the conservatory." Experience does not confirm this, and we find it forced with great success by many good gardeners.
Pregussata.-Colour purplish brown; form roundish, somewhat flattened; size medium; flesh deep red, with a luscious high flavour; seeds unusually small. Introduced from the Ionian Islands. An excellent forcing sort, and tolerably hardy on the open wall.

Turkey, brown.-Colour brown, covered with rich blue bloom; form pear-shaped; size large; flesh red; flavour exceedingly luscious. Synonyms-Lee's perpetual, Walton, Italian, Jerusalem, Murray, Brown Naples, Brown Italian, Early, Howick, Blue, Ashridge forcing, Blue Ischia of some, Large blue, Purple, Biue Burgundy, Small blue, Common purple.

Diseases and insects.-From these the fig is almost exempt, unless in forcing, when few trees are more subject to the attacks of redspider and thrip.

European names. - Fico, Italian - Figuier, French-Viggenboom, Dutch-Figenbaum, German-Higuera, Spanish. 


\title{
CHAPTER XII.
}

\author{
THE ALMOND, QUINCE, MEDLAR, \&C.
}

\section{$\S$ 1.-THE ALMOND.}

THE Almond is a native of Barbary, China, and Persia, and most Eastern countries. From Persia it has long been introduced into all the countries westward, and certainly into England in 1548. This early introduction is, however, doubted by some. Dr Turner, who wrote his " Herbal " in 1645, nearly a century afterwards, says, "Almond trees grow much in hygte Germany beside Sypre, in a cytie called Newstat, and great plentye in Italye, and some growe in England, but I have hearde of no greate store of the fruyte of them that growe in England." It has always been cultivated largely in Asia, and is mentioned in Scripture as one of the charms of the fertile land of Canaan. And at even an earlier period it is spoken of by Jacob, when he sent his sons the second time to Egypt to buy corn: "Take of the best fruits of the land, and carry down the man a present ; a little balm, and a little honey; spices and myrrh, nuts and almonds." As a fruit-bearing tree in this country it is of little value, ripening only scanty crops in the most favourable situations. As, however, it is sometimes cultivated for its fruit, it demands a place here. As an early spring flowering ornamental tree, few equal it in beauty, particularly in the neighbourhood of London, where it forms one of our most interesting town trees, enlivening the cheerless aspect of early spring with its peachcoloured blossoms almost earlier than any other tree of equal beauty. There are only a very few places in Scotland where it exists as a standard tree, much less producing its blossoms. There are two distinct varieties-nay, botanically speaking, species of the almond,-the common, or sweet almona, Amygdalus communis, and the bitter almond, Amygdalus amara. The former is sometimes treated as a wall tree, and pruned and trained as peaches are, to which it is naturally closely allied (vide PEACH.) "From its tender habit," Rogers remarks, " and the liability of its flowers being killed by frost, it seldom bears ; and even when it does, the produce is far inferior to imported fruit. The tree should be planted on a south aspect, and requires a good rich loamy soil fully 18 inches deep, and on a dry subsoil. As the tree approaches a bearing state, it will be observed to produce numerous spurs, which, as they are generally thickly set with blossom-buds, should be carefully preserved. Those spurs bear the best fruit; but as the latter are liable to be too much crowded together, they must be timeously thinned." The almond not only resembles the peach in its general habit, as to manner of growth, form and colour of flower, and foliage; it is also subject to the same diseases and attacks of insects, which are to be prevented by the same means as are advised for the peach. The oil of almonds has some repute in medicine ; in the arts it is often employed, and in the manufacturing of nostrums, oils for the hair, lotions for the face, \&c., it cuts a conspicuous figure. Perhaps the inventors of those popular and fashionable cosmetics are indebted to Pliny for the first idea, as he states that a decoction of the roots of the bitter almond tree supples the skin, prevents wrinkles, and gives a fresh and cheerful colour to the countenance. Another very important virtue is ascribed to the fruit of the bitter almond-namely, the preventing or relieving intoxication. Plutarch relates that Drussas' physician, who was the greatest bacchanalian of his age, took at every cup five bitter almonds to allay the heat and the fumes of the wine, owing to which he never became intoxicated.

The varieties cultivated in Britain are the sweet and the bitter almond; of the former there are several sub-varieties, the most esteemed being the Sweet Jordan, having a tender shell and large sweet kernel. As an ornamental tree, the variety Macrocarpa is in greater repute, on account of its splendid and very large blossoms. The French cultivate eight varieties, the climate being congenial to the tree. The almond is largely cultivated in our nurseries for stocks on which to work the peach and nectarine, the late Mr Knight and others being of opinion that the finer kinds of nectarines, when budded on the almond stock, are less liable to be attacked by mildew than when on the stocks in ordinary use. The French nurserymen use the sweet hard-shelled variety (Douce $\grave{a}$ coque dure) as a stock for the peach on dry soils. Several other varieties of almonds are noticed in works on fruits, but as none can be regarded as valuable additions to our home-grown desserts, we think them undeserving of further notice here. Many botanists are of opinion that the almond 
and peach were originally the same speciesthat the latter is the effect of accidental variation produced by high cultivation, the stone being covered with a much larger amount of rich luscious flesh, while the former retains its original character, consisting of merely a stone covered with a thick dry woolly skin, the flesh of the one being the eatable part, while it is the kernel in the other. Indeed, Knight has distinctly stated his belief "that the peach was only a swollen almond." The best stocks are produced by sowing the kernels, and the same rule should be observed as noticed for the peach.

The fruit should be gathered early in November, stripped of their outer shell or covering, and laid out to dry for a few days before placing on the fruit-room shelves, to undergo a still greater state of dryness; they should then be packed in boxes amongst dry sand till wanted. Damp is greatly to be avoided in their preservation, as they are not only apt to decay, but also to lose their aromatic flavour.

The European names of the almond areL'amandier, French-Mandelbaum, GermanAmandelboom, Dutch - Mandorlo, Italian Almendro, Spanish-Mindalnoe derevo, Russian. Him ho gin, Chinese.

\section{§ 2.-THE QUINCE.}

The Quince (Pyrus cydonia L., Cydonia vulgaris Willd.) is a native of Austria and various other parts of central Europe. The generic name Cydonia is given this tree because the fruit is said to have first attracted attention in the city of Cydon, in Candia or Crete. It was well known to the Romans, for Columella says, "Quinces not only yield pleasure but health." Introduced to Britain previous to 1597 , according to Gerard's "Herbal." Tusser mentions it as being well known in his time, and Langley in "Pomona," 1729, says " the best kind is the Portugal pear quince, next to which is the Portugal apple quince, and lastly, the very worst of all is the English quince. The varieties in present estimation are the common quince, the appleshaped quince, the pear-shaped quince, and the Portugal quince. Of these Mr Thompson observes of the three former, in "Hort. Soc. Fruit Cat." :- "These are often confounded with each other. It is probable that from seeds of either sort varieties have been and still may be obtained, some of which would produce apple-shaped and some pear-shaped fruit." The Portugal quince he describes " as distinct from the preceding sorts ; it does not, however, become, except in very favourable seasons, of so deep an orange; its leaves are broader, and it grows less contracted; consequently it is the best sort for grafting pears upon." There are some other names to be found in nursery catalogues, but the fruit bearing them differ little from sub. varieties that may be found arising from seed taken from any of the family. The Portugal quince is rather a shy bearer. The fruit is unfit for eating in its raw state. It is, however, highly esteemed for making excellent marmalade, for stewing, and a few thin slices of it greatly improve the flavour of apple pies. The fruit is also dried, and wine is made from the juice mixed with sugar and water. Of late years it is cultivated largely for stocks on which to graft the pear, when dwarf trees and early fruiting are desired.

Propagation.-By layers, both for pear stocks and for obtaining trees afterwards to bear fruit, occasionally by cuttings, and rarely by grafting. Layers root the first season, and are afterwards removed to nursery-rows; those intended for fruit-bearing are trained to clean stems from 4 to 5 feet in height; those for stocks are cut down and wrought near the ground.

Pruning.-The head is modelled by shortening in the first-produced branches to obtain a sufficient number; afterwards its management is similar to that of standard pears.

Soil and situation.-A deep moist soil is what it luxuriates most in ; in light sandy dry soils it is shortlived, and the fruit it produces are few and small. The edges of streams or ditches seem to be its favourite site; and where the soil is rich and loamy approaching to clay, its fruit attains its largest size. It does not appear to be naturally a very long-lived tree, and hence, perhaps, a good deal of the prejudice against it as a stock for the pear has arisen. Two or three trees are sufficient for a large family.

Diseases and insects.-Beyond the Rhizoctonia mali, a subterranean fungus which is sometimes found on the roots, we are aware of none of material consequence, although it is probable that some of the many insects which prey upon its near ally the pear may occasionally feed upon it also.

The European names are-Membrillero, Spanish-Coignossier, French-Cotogno, ItalianQuittenbaum, German-Kweeboom, Dutch.

\section{$\S$ 3.-THE MEDLAR.}

The Medlar (Mespilus germanica L.) is indigenous to various parts of the south of Europe, and appears to be now naturalised in some parts of England, where it has either been sown by birds, or in some way or other has escaped from gardens or nurseries. The tree attains no great height, is of a peculiar spreading habit, often assuming a grotesque character, and that more of a strong shrub than of a tree.

There are five cultivated sorts, and of those three only are worth the attention of the profitable fruit-grower, viz. :-

The Nottingham medlar is the best, being sharper and of higher flavour than the rest. Synonyms - Common, Narrow-leaved Dutch, Small-fruited.

The Dutch medlar is the next in estimation, The fruit of which is larger than the last, and somewhat resembling the apple in form; the leaves large and entire, and downy on the under side. Synonyms-Large-fruited, Large German, Common of some, Broad-leaved Dutch.

Monstrous medlar. - Is quite equal to the Nottingham in flavour, in size equal to the Dutch, and bears abundantly. Synonym Neffe monstrouse.

A tree of each is quite sufficient for a large family.

Propagation is effected by seed, by layers, by 
cuttings, and by grafting on any of the arborescent species of Mespiles, Cratagus, or Cydonia, the mode of proceeding the same as for the mulberry (which see).

Soil and situation the same as for the apple.

Pruning and training.-The medlar is best trained to a single stem from 4 to 6 feet in height, allowing the branches to ramify out in their natural direction, which is something between the horizontal and drooping. Sometimes they are grown as shrubs, many shoots being allowed to arise from the roots, and at other times trained as espaliers. The former is the most natural and best way. In either case the pruning is the same as for the quince. To get both, however, in the first instance, to form good heads, the shoots should be shortened for a year or two after planting; after which, especially in the case of the medlar, pruning (except the cutting away dead or misplaced branches) should cease, as the flowers are terminal, or produced at the points of the shoots. The fruit should be gathered early in November, choosing a dry day, bruising them as little as possible, and then, placing them thinly on the shelves of the fruit-room, or in any other cool place, examine them frequently, and remove all such as appear beginning to decay, for they are subject to be attacked by a minute fungus, which would speedily spread over the whole stock.

Insects or diseases seldom attack them.

The European names are, Nespolo, ItalianNeflier, French-Mispelboom, Dutch-Nespero, Spanish-Mispelbaum, German.

\section{$\S 4$. THE WALNUT.}

The common Walnut (Juglans regia L.) is a native of Persia, and conjectured to have been introduced from France into England in, or prior to, 1562. The Romans held this tree in high estimation, and hence the generic name Juglans, from Jovis glans, the nut of Jove, the specific name regia, the queen, from its pre-eminence among nuts. Walnut, its vernacular name, is said to be derived from Gaul-nut, the nut of Gaul, from whence we received it. The leaves and the oil have been employed in destroying intestinal worms; the unripe fruit is used in medicine for the same purpose, and the practice seems to be as old as the days of Pliny, who says, "The more walnuts one eats, with the more ease will he drive worms out of the stomach." In good soils the tree will endure for a century, and be productive to the last. It usually begins to produce fruit when about twenty years old. In some light soils, and probably when the roots have been confined, they have been known to fruit when under ten years old; but by budding, as has been shown by Knight, they will fruit in three or four years. The fruit should be allowed to ripen on the tree, and to fall of its own accord, when the nuts may be gathered, deprived of their husks, dried and preserved in sand till wanted for use. Green walnuts make an excellent pickle, and should be gathered before the stone becomes so hard as to be felt when pricked with a needle.

Propagation, for general purposes, by seed sown in spring in nursery-beds or broad drills, the seedlings being transplanted the following autumn, but more generally the second autumn. The strongest plants might be drawn out the first autumn for transplanting, leaving the smaller, which would then have plenty of room, to the next planting season. This is the most eligible way when the trees are required for timber; but for fruit, the best varieties, of which there are several, should be taken from fruit-bearing trees, and inarched or budded on young healthy seedling stocks. Boutcher, an eccentric but clever nurseryman of Edinburgh, towards the beginning of the last century, suggested this mode of propagation ; and it would appear that Abercrombie practised it, and the late $\mathrm{Mr}$ Knight, about 1814, suggested the practice of budding, and detailed his views of it in the "Transactions of the Horticultural Society." To the usual mode of inserting the bud in shoots of the same year's growth, this enlightened horticulturist found that the walnut formed an exception-" possibly," he says, "in some measure, because its buds contain within themselves, in the spring, all the leaves which the tree bears the following summer, whence its annual shoots wholly cease to elongate soon after its buds unfold: all its buds for each season are also consequently very nearly of the same age, and long before any have acquired the proper degree of maturity for being removed, the annual branches have ceased to grow longer, or to produce new foliage." To obviate these advantages he contrived to retard the vegetation of the stocks comparatively with that of the bearing tree. " There are at the base of the annual shoots of the walnut and other trees, where those join the year-old wood, many minute buds, which are almost concealed in the bark, and which rarely or never vegetate, but in the event of the destruction of the large prominent buds which occupy the middle and opposite ends of the annual wood. By inserting in each stock one of these minute buds, and one of the large and prominent kind, I had," he says, " the pleasure to find that the minute buds took freely, whilst the large all failed without a single exception. This experiment was repeated upon two yearling stocks which grew in pots, and had been placed during spring and the early part of summer in a shady situation under a north wall" (this was his mode of retarding vegetation), "whence they were removed late in July to a forcing-house and instantly budded. Those being suffered to remain in the house during the following summer produced, from the small buds, shoots nearly 3 feet long, terminating in large and perfect female blossoms, which necessarily proved abortive, as no male blossoms were procurable at the early period in which the female blossoms appeared; but the early formation of such blossoms sufficiently proves that the habits of a bearing branch of the walnut tree may be transferred to a young tree by budding, as well as by engrafting by approach. The most eligible situation for the insertion of the buds of this species of tree (and possibly of others of similar habits) is near the summit of the wood of the preceding year, and, of course, very near the base of the annual 
shoot; and if buds of the kind above mentioned be skilfully inserted in such parts of the branches of rapid growth, they will be found to succeed with nearly as much certainty as those of other fruit trees, provided such buds be in a more mature state than those of the stock into which they are inserted." The fruit of the walnut being of much importance in climates suitable to their ripening, and as there are so many superior sorts to be met with, and so many more inferior ones growing, it would be well if nurserymen would bestow more attention on the matter. Layers make inferior plants, and grafting is not very successful ; but inarching may be readily performed during February or March. Budding is, however, by far the best method, for it would be often found impossible to inarch shoots of a superior kind, the branches of which might be 20 feet from the ground, upon a stock of 6 or 8 feet in height.

Soil and situation.-The walnut will succeed in any ordinary soil, if on a dry subsoil, and the best situation is in a warm sheltered part of the park, where they will become both ornamental and useful. They are rather large for garden culture, unless attention be paid to root and branch pruning. One objection to them in gardens is, the space they would occupy, and the shade they would produce.

The best varieties of walnuts are-the Large double-kerneled French, the Tender or thinshelled, a very fine variety, both of which are grown about the village of Worthy, near Winchester; the Highflyer of Thetford, described in the "Transactions of the Horticultural Society," vol. iv. p. 517; and the new Dwarf prolific, or Noyer fertile, recently raised from seed at Chalons. Mr Rivers says he saw plants of this sort in Paris only 2 feet in height and full of fruit. Mrs Harvey, in her book recently published, "The Adventures of a Lady in Tartary," \&c., speaks of a walnut cultivated in the valley of Kashmir with a shell as thin as paper, and easily broken with the hand. The Highflyer of Thetford has a shell nearly as thin, and is, on that account, a variety deserving of more general cultivation.

Diseases and insects.-The walnut is proverbially exempt from these. Late spring frosts, however, in some situations, often injure the young expanding shoots, and recurrence of such disasters brings on a debility in the tree often ending in its death.

The European names are-Noce, Italian-Noyer, French-Walnoot and Ockernootenboom, Dutch -Walnussbaum, German-Nogal, Spanish.

\section{$\S$ 5.-CHESTNUT, OR SPANISH OR SWEET CHESTNUT.}

The Chestnut (Castanea vesca Willd.) is indigenous to the territory of Castanea, a town of Thessaly, where magnificent trees of it are still found. Some of the oldest trees in the world are of this kind, as those on Mount Etra, and the celebrated one at Totworth in Gloucestershire, recorded as being a large tree in "Doomsday Book." It is supposed to have been brought from Sardis to Italy, and no doubt was introduced to Britain by the monks. It was long thought that the roof of Westminster Hall was constructed of the timber of this tree, but the general opinion is in favour of its being of oak. Why it should be called Spanish chestnut does not clearly appear; most likely a nurseryman gave it this name to distinguish it from the Horse chestnut (AEsculus Hippocastanum), which has no connection whatever with our present subject. The chestnut, but whether as a fruit-bearing or ornamental tree is not now known, must have been early planted in Scotland, as trees of great magnitude and age exist at Castle Menzies, Newbattle Abbey, and other places.

Propagation.-This is effected for all general purposes by sowing the seed in broad drills, and transplanting the trees, when one year old and upwards, into nursery lines, to fit them for final planting in woods and forests. When grown for their fruit the process of grafting is had recourse to, the scions being taken from approved varieties, of which there are several, and grafted on stocks of those promiscuously reared from seed. They take readily by any of the ordinary modes of grafting, and when scions are taken from bearing branches, they often produce flowers the succeeding year. "I am much inclined," Mr Knight remarks, "to think that, by selecting those varieties which ripen their fruit early in autumn, and by propagating with grafts or buds from young and vigorous trees of that kind which have just attained the age necessary to enable them to bear fruit, it might be cultivated with much advantage in this country both for its fruit, \&c."

Soil and situation.-The chestnut will thrive in lighter soils than the oak, but better in a loamy soil than in a poor sandy one. Indeed, there are few soils, if not wet, in which this tree will not prosper. It is of too large a size for admission within the limits of a garden, but makes an excellent shelter tree in belts or plantations surrounding it.

Pruning.-Little attention has been paid to this matter, the trees being usually allowed to grow pretty much in their own way; and where their natural development is wished to be brought out, and where they stand sufficiently apart from other trees, no doubt nature should have her way. But with such as are to be cultivated for their fruit, root-pruning would have, combined with grafting or budding, the effect of bringing them into a much earlier habit of fruit-bearing, and limit their size to that of an ordinary apple-tree. The fruit should be allowed to fall of its own accord, and when the nuts are freed from the husk or outer capsule, they should be well dried and laid by in drawers, or on the shelves of the fruit-room, or packed in clean sharp sand till wanted.

Select list. - The varieties in cultivation in the south of France and north of Italy are numerous. In Britain only a few are known to exist; of these the Devonshire prolific and Knight's prolific are the best, the former being also known as the Prolific, New prolific, and the latter as the Downton. The best of the numerous French varieties is called Marron. The chestnut, like 
the walnut, can only be profitably grown for its fruit in moderately good situations, and unless that superior kinds be procured, even there the fruit would be inferior to that which is imported.

The European names are, Castagno, ItalianChataignniere, French-Kakstanjeboom, Dutch -Castano, Spanish-Vastanienbaum, German.

\section{$\S 6$. -THE FILBERT.}

The common hazel-nut of our woods (Corylus Avellana L.) is the type of the filbert and its allies in a wild or unreclaimed state. The improved varieties of the common hazel are comparatively of modern date, but the original was no doubt well known to our Anglo-Saxon ancestors, in whose language hazel signifies a headdress, and that it was, in its wild state, indigenous to Britain before their time is a question not doubted. The Romans at an early period cultivated this fruit, and the specific name A vellana is derived from Avellino, a city in the kingdom of Naples, near which these trees grew in such abundance that, according to Swinbourne, the produce yielded in his time twelve thousand pounds sterling annually. Some historians assert that they were imported into Italy from Pontus, and known to the Romans by the appellation of $N u x$ Pontice, and after their establishment at Avellino were called $N u x$ Avellana, and now in botanical nomenclature Corylus, from the Greek, signifying a bonnet, to which the singular calyx may be well compared, and Avellana as already explained. Formerly nuts were divided into short-bearded and long or full bearded; from the latter filbert seems to have been derived.

Propagation.-New and improved varieties can only be expected from seed, and therefore nuts of the most approved varieties should be sown in October or November in light rich soil, covering them to the depth of $\mathbf{2}$ inches. Care, however, must be taken that rats and mice be prevented from attacking them, by careful trapping, or rubbing the nuts at sowing with arsenic mixed with tallow or any similar greasy matter. Of course, the most likely way to secure new and improved varieties would be to adopt the necessary precaution of crossing one variety of merit with another. Some advantage would be gained in securing strong plants were the seed sown on a mild heat. When the plants are one year old, transplant into nursery lines about 2 feet apart, and 1 foot plant from plant in the line. This process is, however, seldom followed, and stock is procured from suckers, which are better than layers, always making the best trees, as it is important the future tree should be upon a single stem, which plants from layers do not produce in like perfection. Seedlings or suckers afford excellent stocks for grafting proved and esteemed sorts upon, but these must be trained to single stems. The usual nursery practice is, however, to grow them from layers or suckers as most convenient, which latter are produced in abundance. Grafting superior sorts on stocks of the common filbert or hazel-nut in March, and more especially on the Spanish nut, has the effect of inducing a much earlier state of bearing; besides, grafted filberts are less subject to be encumbered afterwards by suckers from the root. Mr G. Lindley, however, approves of propagation by layers, and observes: "If the laying them down has been properly performed, the layers will be well rooted by the end of the year, when they should be taken up and planted into nursery rows 3 feet apart, and 1 foot from each other in the rows. Previous to their being planted they should be pruned, leaving only one, and that the best shoot, shortening it to 1 foot or 18 inches, according to its strength. As the plants grow up, they should be trained with single stems of 18 inches or 2 feet high, which will allow room to clear away any suckers the plants may afterwards produce. Some, however, very judiciously train them to stems 3 feet in height." Where ground is not to spare in gardens, the filbert may be successfully grown along the sides of plantations, and in sunny places in open woods and copses.

Soil and situation.-A good hazel loam, rather light than otherwise, resting on a dry substratum, is the best of all for this tree. It, however, prospers in the majority of soils, if of a loamy nature, abounding in vegetable matter, such as decayed leaves of trees, grasses, \&c. The slips or outer enclosures of the garden are the best places to plant them in, provided they are fully exposed to the sun and air; for it is a great mistake to plant them under the drip or shadow of other trees, from the mere presumption, because the common hazel-nut is found in such situations occasionally, that it should be equally proper to plant cultivated filberts in the same condition. The boundary of an orchard is a very proper situation for a plantation of filberts, and if well managed will give an abun. dant and useful return; much, however, depends on a proper selection of sorts, and on a judicious mode of cultivation, two matters very seldom thought of.

In strong soils they do not succeed, and in such as are wet not at all. Avoid using dung at planting, and if they assume too luxuriant a habit after the third year, root-prune them pretty hard in. The filbert does not become very productive in a young state; it requires to have its strength moderated by age. In the fifth or sixth year they should bear abundantly. The distance at which the plants should be set apart is 10 feet by 8 , and if trained to 3 -feet stems, they may be interlined with gooseberries or currants to economise space, while the partial shelter of the nut trees above would greatly aid in protecting the others from the effects of late spring frosts.

Pruning and training.-The filbert, and all the rest of the hazel-nut tribe, to be remuneratively productive, requires as much care in their culture as the apple or any other hardy fruitbearing tree. They should, in all cases, be trained to a single stem, varying from 1 to 3 feet in height, and all suckers carefully removed upon their first appearance. They do not require too rich a soil, for that only induces a habit of wood-making, which will require root- 
pruning to counteract. The fruit is produced mostly on the extremities of the shoots, and where they are fully exposed to light and air. The branches should be kept moderately thin, and the centre of the plant open. The head is formed much in the same way as the red or white currant, and is accomplished by selecting four or five shoots, which are well placed, and these may be obtained in any number by heading back those first produced, and this heading back and training must be continued until the bushes have attained the height of 6 or 7 feet, which is quite sufficient within the limits of a garden. In winter pruning, some discrimination must be used, for the filbert, unlike the gooseberry, currant, \&c., is what is called by botanists moncecious ; that is, having the male and female flowers separately, but on the same bush. The male blossoms are readily distinguished in spring hanging in catkins 2 or 3 inches in length, profusely covered with a fine yellow dust, which is the fertilising pollen. On the other hand, the female blossoms are rarely seen without a careful search being made for them. When in bloom, they are of a brightish pink colour, very small, and closely set on the sides of the mature wood. They do not appear until three or four days after the male flowers have opened. "Now, it so happens," Mr Errington observes, "that trees at a certain age, or under certain conditions of culture, will sometimes produce either almost entirely male blossoms, or otherwise females. Those with the males alone must of necessity be barren for that year; but if only female blossoms appear, branches should be cut bearing catkins of male flowers, and suspended or tied amongst those possessing female blossoms only. Occasionally, too, on a sunny day, a branch of the dusty catkins may be carried in the hand like a rod, and brushed lightly over the tips of the female-bearing bushes. Many good crops of nuts have been lost for want of this precaution: it is vain to think of the female blossom yielding fruit, without the catkins have been near them in February; then the blossoms may be readily distinguished, and then it is that pruning may be successfully carried out. The fruit is produced principally on the former year's wood, and generally form compact side-shoots, the produce of leaders of a short-jointed and mature appearance. Such lateral fruit-bearing branches may be induced in greater abundance, by shortening back strong shoots of this character. Thinning out is, however, one of the principal matters, for unless this be duly attended to, the bush will become crowded with spray worse than useless. A great deal of small spray will be produced on the inner portions of the branches; and these, although of the character of bearing-wood, are generally unfruitful : most of these must be pruned away. Any one who observes the habit of the nut closely, will soon perceive that the shrubs are most disposed to bear at the extremity of the branches, thus evincing their partiality to plenty of light and air. These, then, are the portions of the tree where the eye must be directed as to fruit-bearing properties. Such leaders, however, must not be encouraged so thickly as to cross each other ; and in order to prevent the lower portion of the head from becoming naked, a good, strong, wellplaced shoot may be occasionally encouraged, heading it back in due time, in order to keep it producing side branches. After duly thinning away superfluous shoots, the principal leaders should be all shortened. As a general rule, wo would say, remove about a quarter of the length; this, as before observed, will cause the tree to produce abundance of side spray, from which, in the future spring, the fruiting shoots may be selected."

The filbert is in no part of Britain grown to the same extent, or in the same degree of perfection, as in the county of Kent. The Kentish peasantry practically understood the management of this tree long before its habits were studied or known to gardeners. The principle of the Maidstone pruners appears to be to "check and control the natural growth, and thereby bring forth the fruit-bearing principle in greater force and energy." Each plant should have one strong upright shoot, of not less than 3 feet in height, this being necessary in order to the future form of the head; and this, early in the spring, after the trees have been put out in their final stations, is cut down to about 18 inches from the ground. This height will admit of a clear stem of 12 inches below, and which part must be at first, and ever afterwards, kept free from shoots, as well as suckers from the roots. This deprivation of shoots and suckers will cause the buds left at the top to push with greater vigour. If eight strong shoots be produced in the first summer, they must be carefully preserved, as that number is required to form the head; but if less than this number come forth, then two or three of the strongest (or the whole if necessary) must be shortened back to half their length at the next pruning, in order to obtain the requisite number. These branches are to be carefully preserved and trained outwards and upwards, at first nearly horizontally, but curving gradually upwards at the point. The easiest mode of doing this is by using a hoop of the proper size, placed within the shoots, and to which the latter are tied in a star-like order, and at equal 12-inch distances. Such a laterally curving position may be much assisted by the pruner always cutting at an out side bud, which, when grown sufficiently far outwards, naturally turns up to form the permanent branches. The points of the branches are allowed to rise to the height of 6 feet, but never higher; and the middle of the tree is always kept free from shoots and branches, so that a well-trained head resembles a large bowl. The subsequent management of the trees, both while gaining the desired form, and after having gained it, consists in preserving all the short spurs which will be produced on the branches, and cutting away or shortening the laterals, which rise every year from the same. The management of these laterals is of great consequence. If they exceed the length of 6 inches, they may be cut back to a few buds ; but if less, they should be preserved, as their points are generally fruitful. The grand object with the 
pruner is to have the branches thickly beset with fruitful spurs, and which are only reduced in length when, after a few years' growth, they become too distant from the branch, when they are cut back to a healthy spur behind."-Rogers, p. 157.

The following sorts are the most valuable:-

Cob nut.-Fruit short ovate, and slightly compressed; size large; husk hispid; shell thick and hard. Trees upright in growth. Synonyms -Barcelona of some, Prolific, Dwarf prolific, Glasgow prolific, Pearson's prolific, Downton large, Large cob of some, Great cob of others. A profitable and useful sort.

Cosford.-Fruit oblong; size large; husk hispid; shell remarkably thin, and striated longitudinally; of excellent quality, and a great bearer. Synonyms-Thin-shelled, Miss Young's. Originated at Cosford in Suffolk.

Frizzled.-Fruit oval and compressed; size medium; husk hispid; shell thick. A very great and early bearer, and distinguished from all other filberts or nuts by its much laciniated husk, from which it has obtained the name of Frizzled. Synonyms-Cape nut, Frizzled nut. Originated in a garden at Hoveton, near Norwich. Northamptonshire prolific.-Fruit oblong; size medium; husk hispid; shell thick. Esteemed chiefly for its earliness. This, however, should not be confounded with the Northampton filbert, which is of inferior quality, and readily distinguished from this by the smoothness of its husk.

Spanish. - Fruit oblong and very large; husk smooth; shell thick. A variety well known in the market and largely imported. By no means productive in Britain, but excellent as a stock on which to graft the Cosford, Frizzled, \&c. Synonyms-Toker, Large bond nut, Lambert's large, Lambert's, Cob of some, Great cob, Large cob also of some, Sir John Aubrey's. It is different from Barr's Spanish, which has ovate fruit of medium size; husk hispid and extremely short; shell very thick; and altogether inferior in quality.

Bond nut.-Fruit oblong ovate ; size medium; husk hispid; shell thin; of excellent quality. Different from the Large bond nut, given as a synonym to the last.

Large round cob.-Fruit round; size large; husk smooth; shell thick; a good bearer and of excellent quality.

Downton large square. - Fruit short and obtusely four-sided; size large; husk smooth; shell thick; above average quality, and, with the next, originated with Mr Knight at Downton Castle.

Downton long.-Fruit oblong; size medium; husk smooth; shell thick; much the same in quality with the last.

White filbert.-Fruit ovate; size medium; husk hispid; shell thick; a profuse bearer and of excellent quality. Synonym-Wrotham park.

Red filbert.-Fruit ovate; size medium; husk hispid; shell thick; resembling the last in every particular, excepting in the pellicle or skin of the kernel, which in this variety is red, while in the last it is of a pale colour. In both the husk is long and tubular, contracting so much beyond the point of the fruit as to prevent its falling out, and on this account was constiVOL. II. tuted into a species by Willdenow under the name of Corylus tubulosus. Synonym-Red hazel.

This, as well as all the cultivated fruit-bearing sorts, is a variety of Corylus avellana, and not of Corylus tubulosus, as stated in Lawson's Catalogue, and also in Loudon's "Encyclopædia of Gardening." The "Hortus Britanricus" makes the red filbert, the var. Rubra of Corylus acellana of Linn., a native of Britain, while C. tubulosus of Willd., a native of the south of Europe, was not introduced till 1759, thirty years after the publication of Langley's " Pomona."

Diseases and insects.-We are aware of no disease peculiar to this tree. Several insects attack the foliage, but rarely to the extent to produce injury. It is otherwise, however, with Balaninus nucum of Germar, Curculio nucum L., the nut-weevil, fig. 236. The maggot of this
Fig. 236.

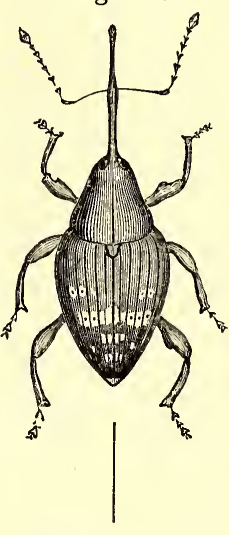

THE NUT-WEEVIL. insect is that which we so frequently meet with in the kernel of the nut, and is the offspring of a beetle found abundantly, if looked for, upon the hazel and filbert trees, from the end of May to the end of August. Having chosen a nut, the female commences cutting a hole through the yet soft and tender shell, which she does with apparent ease with her jaws, which are placed at the point of her long slender rostrum; this having completed, she turns round and deposits a single egg in each cavity. The nut continues to grow, seemingly unaffected by its inmate. In about eight days after the egg has been laid, hatching is completed, and the young maggot finds food already prepared for it in the kernel, upon which it continues to feed until it has nearly consumed it. At this time, as if aware that its supply of food is nearly exhausted, it begins eating a hole in the side of the shell with its jaws, of sufficient size to admit of its liberation, sometimes while the nut is attached to the tree, but oftener, it is believed, after it has fallen to the ground. It then buries itself in the earth in an elliptical cavity, which it forms for itself, and remains there during winter, changing in the following spring, or even later, to a whitish chrysalis.

The best remedy for their suppression is to examine the crop, and gather and destroy all the fruit that indicates the presence of the maggot, which may be ascertained by the marks on the shell caused by the female having perforated it, as described above; and also by collecting the soil around the bushes to the depth of 2 inches, and burning or burying it in pits 2 feet deep, using the soil taken from the pits to replace that taken away.

The European names are-Noisetier, FrenchHaselstraude, German-Hazelaar, Dutch-Nocciuolo, Italian--Avellano, Spanish-Avelleira, Portuguese - Frandik, Turkish - Oreschnik, Russian. 


\section{CHAPTER XIII.}

\section{THE CURRANT, RASPBERRY, GOOSEBERRY, STRAWBERRY, \&c.}

\section{$\S$ 1.-THE RED, WHITE, AND BLACK CURRANT.}

THE Black Currant (Ribis nigrum L.) is a native of most parts of Europe, abounding, however, in greatest plenty in the northern parts of Russia, Siberia, \&c., and being frequently found in Britain in moist woods, by the sides of swamps, rivers, \&c., indicating pretty clearly its predilection for a moist deep soil when in a cultivated state. The red currant (Ribis rubrum L.), and its variety, the white currant (Ribis album L.), are both indigenous to the north of Europe, and are also found in woods and hedges in many parts of Britain. The currant does not appear to have been known to the ancient Greeks or Romans. The English name is evidently derived from the similarity of the fruit to the small grapes of Zante, which, dried, form the coranths or currants of the shops. The currant has been long cultivated in this country, and of late years has become greatly improved in size by cultivation.

The first mention we find made of the currant is by Bacon, who says, "The earliest fruits are strawberries, gooseberries, corans; and after them, early apples and early pears." Worlidge, in his "Vinetum Britannicum," published in 1675, speaks slightly of them : "The English curran, once in esteem, but now cast out of all good gardens, as is the black, which was never worth anything. The white curran became native to our soil, which is also improved in some rich moist grounds that it hath gained a higher name of the greatest red Dutch curran. These are the only fruits that are fit to be planted and propagated for wine." Currants are quite unnoticed by Tusser, and Gerard considered them a kind of gooseberry, if we are to understand from the following allusion that he meant them. When describing the gooseberry, "We have also," he says, " in our London gardens another sort, altogether without prickles, whose fruit is very small, lesser by much than the common kind, but of a perfect red colour, wherein it differeth from the rest of its kind;" evidently referring to our red currant.

To the Dutch we are indebted for the first endeavours to improve this fruit by cultivation; while the French, Germans, and Americans have paid little attention to it. Nor is it to be expected that in countries where the grape-vine flourishes, and where the summers are so dry and warm, and as favourable for the vine as unfavourable to the currant, it should be otherwise. The currant, therefore, does not succeed in the middle and southern states. The natural colour of the currant, in an indigenous state, is red : cultivation has produced the white and pale-coloured varieties. The black currant is of a blackish cast, even in its native wilds, in the woods of the north of Russia, and in medium elevations in Siberia, where the fruit, although very large, is very insipid. Its natural habitat in Britain is by the sides of swamps, or in damp woods, and chiefly in cold strong soils, which accounts for the circumstance of its not succeeding so well in dry exposed gardens as in damp partially shaded borders.

Langley, in his "Pomona," published 1729, speaks only of red and white Dutch; of the black currant he says nothing. G. Lindley, in "Guide to the Orchard," p. 160, describes six sorts, and very properly remarks, "There are several worthless varieties of the red currant to be found in gardens, which ought to be rooted up, and replaced with the larger fruited." To this circumstance nurserymen should pay attention, and propagate only the best varieties, of which there are now several. It does not appear that, until within these very few years, any attempt had been made in procuring new or improved kinds, although so much had been done in this respect in the case of gooseberries ; for we have, time out of mind, heard of no other than the Large red, or Large Dutch, White Dutch, and Champagne, and of one Black currant only.

Propagation.-By seed, when new varieties are wished for, but this is seldom practised. The seed should be sown in light rich soil as soon as the fruit is completely ripened. The autumn following, the plants will be fit for planting out into nursery lines. The most careful experiments made in producing varieties from seed by the indefatigable Mr Knight only yielded five plants - three red and two whiteout of two hundred seedlings, possessing greater merits than their parents, and these were scarcely worth continuing. Since that time, how. ever, some few superior varieties have been obtained, which will be found in the Select List; but how they originated no very satisfactory 
account has been furnished. The best and most general mode is by cuttings of the ripened shoots of the current year's growth, treated in all respects as the gooseberry,--namely, the most vigorous and straight young shoots should be chosen and collected before the autumn pruning commences ; the tops being cut off, the remainder of the shoot, from 1 foot to 14 inches in length, is retained for the cutting. All the eyes or buds, as far as the cutting is to be inserted in the ground, should be rubbed off, to prevent the appearance of suckers afterwards. If the cutting is a foot long, one-half of it should be inserted in the ground, and the soil made firm about it at planting, which operation should be performed as soon as the cuttings are removed from the tree. The best situation for them is a shady border, where they should be set in rows 18 inches apart, and the cuttings 8 inches in the line. We need hardly remark, after what has been said in article Propagation by Cuttings, that all cuttings should be cut off immediately under and close to a bud or joint ; for the internodes, or spaces between the joints, are incapable of emitting roots. Some lay in the cuttings till spring, and then plant them. There call be no advantage from following this practice, if time and space admit of their being planted at once. Others attribute much of the success of rooting to the placing a little bundle of moss at the base of the cutting at planting, removing it again when they are transplanted the following year. Unless it be to retain a little moisture at their base, and in ordinary soils this will not be required, this practice may be disregarded.

Soil and situation. - The red and white currant attain the largest size in a strong, rich, rather moist soil, although excellent crops are produced in most ordinary good garden-ground. 'The fruit ripens earlier, if it is not of so large a size, in light rich soils, and seems to prosper rather better when somewhat shaded than when exposed to the full sun. Like the gooseberry, they are best planted in quarters by themselves, and in rows 5 feet asunder, and the same distance plant from plant in the line. They also do well trained as espaliers, either vertically or in the domical or curvilinear manner, and in the former of these ways occupy the least room. Like the gooseberry, the plantations should be renewed every six or seven years; but in good soils they will continue productive for twelve or fifteen years, or more, extending in size until they become too large and encumber each other, unless great pains are taken to reduce them by cutting out the old branches and encouraging the young wood. The proper season for transplanting is October and very early in November. The usual practice of scattering them over the garden by the sides of walks, \&c., is erroneous ; for when in a compartment by themselves their roots are allowed to remain unmolested by digging amongst them, and manure can be easily applied by top-dressing, or using it in a liquid form. They are also much more conveniently protected from birds by netting. In this way we have them till the end of November-longer, indeed, than they remain good when trained against northern walls. Previous to planting, the ground should be trenched two feet and a half deep, adding a heavy dressing of rank manure at the same time. Deep rich soils are most beneficial to the gooseberry, currant, and raspberry, because, although the roots descend in search of food, from their hardy constitution they always ripen their wood perfectly, which is not the case with more tender trees, such as even the apple, pear, peach, \&c.; and hence, in their cases, shallow soils with less enrichment are found to be essential to their health. To insure fruit earlier than that produced in the open quarters, a few trees should be planted against a south wall, and this accommodation may even be formed between the more permanent trees, but in no case should the latter be sacrificed to the former; rather set apart a small portion of the wall to them entirely. The black currant differs not in the means of propagation from the red and white, but it luxuriates more than they in a deep, rich, rather humid soil. In light, warm, shallow ground it produces scanty crops, and the berries are very small; whereas in soils the reverse it attains its fullest size and excellence.

Pruningand training.-Training in the standard form is comparatively of modern date, and certainly possesses the merit of occupying less space than if grown as low-spreading bushes. The fruit is better exposed to the influence of light and heat, and hence increased in flavour, if even diminished in size. The stems are trained to about 3 or 4 feet in height, and the head is closely pruned in every year soon after the fruit is gathered. Many advocate the practice of planting red and white currants in shady situations, thinking thereby to increase the size of the fruit. This, no doubt, to some extent is true, but always at a sacrifice of flavour. The same end would be attained by supplying the plants during the swelling of the fruit with copious supplies of liquid manure, and even during very bright sunshine shading them during a few hours daily by means of thin canvass screens. When currants are trained against walls, the best form is to carry a branch to the right hand and another to the left of the main stem, about a foot from the ground, and to train the side shoots about 9 or 10 inches apart in a vertical direction. The black currant requires little pruning. A moderate thinning out of the branches, removing old and exhausted ones, to be replaced by young ones selected at the autumn pruning, is almost all that is required. The red and white currant should be treated as recommended for gooseberries, only shortening more or less all the young wood left on according to its strength. Suckers arising from the roots should be carefully displaced, and that at the part from whence they issue. Where the spurs, particularly on old plants, have become crowded, thin them out, retaining the youngest and best situated. The white currant is much more slender in growth than the red variety, and does not produce nearly so much young wood; less pruning, therefore, is necessary, and it may even be of advantage to leave the side branches at pruning somewhat longer than those of the red variety, to encourage the prin- 
ciple of growth laterally, by which additional strength may possibly be thrown into the spurs, and indeed induce a stronger habit in the whole plant. In regard to young plants both of currants and gooseberries, it may be observed that red and white currants struck from cuttings the preceding season usually produce three or four weakly shoots of a few inches only in length. Three of the best of these should be selected, and if nearly equidistant so much the better. Remove the tips of the shoots, to induce them to throw out stronger ones the following season, from which the future form of the plant is to be moulded. For dwarf plants to be grown as bushes, the circular form is that most usually adopted. The best way of securing this form is to place a strong hoop, about 30 inches in diameter, supported on neat stakes driven into the ground, and to this hoop to train the young shoots. Some carry this further, and by using stakes of greater length place another hoop above the former a foot or so, and continue to train the branches as they advance. The branches should not be trained closer than about 12 inches asunder. Should the leading ones, the first year after planting, be not of sufficient length to reach the hoop, conductors of small twigs should be employed to lead them towards them, and also preserve them from being broken in their young state. A good deal in this depends on the size of the plants when first planted in their permanent situation; and, therefore, plants partially trained in this way in the nursery for two years are to be preferred, and at that age, if carefully removed early in autumn, will not fail to succeed. These, if they reach the hoops at planting, will require little pruning the following year, as the operation of transplanting, acting as a species of root-pruning, will induce them to throw out spurs or fruit-buds rather than to elongate from their points. The following season, as the leading shoots extend, they should be conducted vertically towards the upper hoop, and all superfluous side-shoots removed as in ordinary bushtraining. The advantage of this cup or cylindrical mode of training is, admitting abundance of light and air to every part of the plant, leaves and fruit alike enjoying their influence. The plants occupy less space, and have a more business-like appearance. In common bush-pruning, the leading branches should have a portion of their points cut off annually at the winter pruning, to cause them to send out side spurs. If this topping is neglected, the consequence will be that, if the plants are growing feebly, a great portion of the young leading shoots will be devoid of spurs, whereas they ought to be clothed with them in moderate profusion along their whole length. In regard to the extent of terminal shoots removed, 7 inches may be taken as the average; but the strength of the wood and condition of the plant should govern this, bearing in mind, in all such cases of shortening, the stronger the shoot the less should be cut off ; whereas, in proportion to the weakness of the plant the more should the reduction be, even often, in extreme cases, to leaving only an inch or two.
As to side pruning or spurring in-that is, cutting off at winter pruning those numerous young shoots that are produced during the preceding summer-" the business assigned these," Mr Errington thinks, and no doubt with much good reason, " is doubtless to elaborate matter of an accretive character for the due encouragement of the growth of spurs which otherwise would consume more of the accretive material than they create, and thereby draw too heavily on the system of the tree. They are thus, as it were, rendered self-supporting; and although we cannot speak from experience, yet we have little doubt that if any one would persist in disbudding all these shoots the moment they appear, the spurs at their base would soon be troubled with a sort of vegetable atrophy, and dwindle away in a very short period. Good pruners, therefore, cut all these back to within about $\frac{1}{2}$ inch of their base, which gives the spring blossoms plenty of room for growth, and at the same time leaves an eye or two of wood-buds, which in a few weeks produce the same character of spray as their progenitors; thus a permanent provision is made for the stability and long endurance of the spurs." The leading branches should be carried up as nearly perpendicular as possible, and these at proper distances, shortening the terminal shoots to cause them to furnish the whole branch with spurs, and these to be thinned when too much crowded, shortened back when they attain too great a length; but this is seldom, under good management, found to be the case. In pruning the red and white currant, six main shoots are considered by many as quite sufficient to be retained as leaders, when the plants are to be grown in the bush form. The terminal shoots on these should be, at the winter pruning, shortened back to 4 or 6 inches according to their strength; but when they have extended to nearly the intended height of the plant, these terminals should be cut back yearly to one or two buds. All the lateral shoots are to be cut back to within an inch of their base at every winter pruning for the production of spurs. The pinching back the young shoots during summer has no doubt the effect of admitting more light and air to the centre of the bushes, but it may have, to a certain extent, the effect of lessening the amount of elaborated sap formed by the leaves, and through these shoots conveyed to the spurs. However this may be regarded by physiologists, one thing we think is quite certain, that a large portion of the weakest of the young spray, and where it is most crowded, should be removed, if only to allow light and air to act fully on the best formed shoots and leaves that are left. What has been said above in reference to the red and white currant is very applicable also to the gooseberry, their near ally. Summer pruning the currant is no doubt of great advantage both to the fruit and health of the tree. This should, however, be cautiously performed, and indeed a sort of early disbudding should take place, by removing all weak in shape, or superfluous young shoots, because the matter destined for their support will be directed into the parts left.

Forcing.-Both the red and white currant are 
readily forced, so as to ripen their fruit in April and May. Young plants of three years' growth well set with buds, and of moderate size, should be potted into 12 -inch pots the season previous to forcing; if carefully done, they may be potted even in October, and set at ollce in a somewhat close pit, to induce them to make young roots. The slightest artificial excitement should be applied until the buds have all broken ; after that, a temperature, accompanied with abundance of light and ventilation, ranging from $58^{\circ}$ to $63^{\circ}$, will be sufficient until the period of the fruit's changing colour, when an addition of $5^{\circ}$ more may be given. The plants which have borne one crop, if as early as April, should be thrown away, and another set provided for the following year. If ripened in May, they may be removed to a lower temperature for a few days, and finally plunged out in some sheltered place till the returning season of forcing arrives. The black currant does not force so well, and even should the fruit ripen it soon falls off, which neither of the others do, and hence they are valuable for being placed on the table in the pots in which they have been growing.

\section{SELECT LIST.}

Red currants.-Dutch red.-Fruit nearly twice the size of the common currant; bunches from $2 \frac{1}{2}$ to 3 inches long, and somewhat less acid than the common; leaves slightly downy; a very abundant bearer; should take the place of the common red sort. Synonyms-Large-bunched red, Long-bunched red, New red Dutch, Large red Dutch, Red grape, Morgan's red, and probably not different from the Pitmaston prolific.

Raby Castle.-One of the newly-raised sorts of very great excellence, bunches from 5 to 6 inches in length ; fruit bright red, hanging on the tree a month longer than most other sorts. Synonyms-May's Victoria, Goliah, Houghton Castle, Lauder's large red.

Wilmot's large red.- - Resembles the last in size; berries hanging loosely on the bunch, which in length and size of berries is very similar to the last.

Knight's sweet red.-Considerably less acid than even the White Dutch, and hence preferred for the dessert.

Knight's early red.-The only merit of this variety is its ripening ten days earlier than any other, and hence desirable for forcing or planting on a south wall.

Woolly-leafed Dutch. - Remarkable for the downiness of its leaves; an immense bearer, and hangs long on the tree, which is strong and vigorous in growth.

Goodwin's red.-An excellent currant of large size, introduced a few years ago from France; adapted on account of its strong growth for standard training, and in that way continues late in perfection.

Champagne, or Pheasant's eye.-Seemingly a hybrid between the old red and white currant; a most abundant bearer, and of excellent quality. The colour, which has a reddish-white tinge, disqualifies it for making either white or red jelly; but for all other purposes it is equal to any of the others, and from its peculiarity of colour it makes an addition to the dessert.
Whitecurrants.-Dutch white.-Of fair size and good quality, considerably less acid than the Red Dutch, and hence preferred for the dessert. Synonyms-Morgan's white, White crystal, New white Dutch, Reeve's white, White Leghorn.

Wilmot's new white.-Bunches from 4 to 6 inches in length ; berries large, hanging loosely on the stalk, remarkable for the deeply-cut margins of the leaves.

Victoria white, Speary's white, and Pearl white, appear to us to be one and the same. Not so long in the bunch as the last, nor are the berries quite so large.

Black currants.-Common black.-Of this there are undoubtedly several varieties, as situation or soil can hardly cause the great difference that exists. In some the bunches have only three or four berries, while in others they average six or seven; and in some the fruit only at the shoulder of the bunch sets, while all below is either entirely fruitless, or the berries imperfect and very small.

Black Naples.-Fruitlarge, often three-fourths of an inch in circumference; the bunches are also large and well formed. The blossoms and leaves expand earlier than any other sort, which is an excellent mark of distinction. The fruit is, however, later in ripening than the Common black, thus prolonging the season of the fruit's utility.

Ogden's black grape.-This, with the last, is the best of the family, and both should be extensively grown. The bunches and berries are both large, and of excellent quality; rather earlier than the last.

Diseases aud insects. - The magpie moth, Abraxas grossulariata, is often very destructive to the foliage of all sorts of currants (see art. GooseberRy). The Acarus, or red-spider, fig. 22, during warm dry seasons, particularly in light soils, also attacks them.

The triple-spotted currant-moth (Tinea capitella L., Lampronia capitella Curtis), fig. 237,

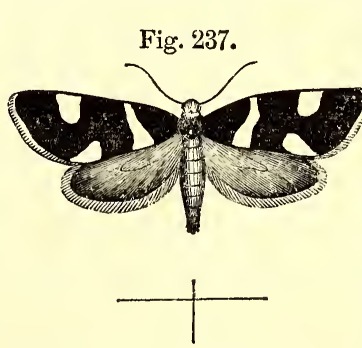
is another enemy to the currant. The larvæ, instead of attacking the foliage, feed upon the interior of the young wood, which they often so destroy as to leave the trees leafless by May. All shoots at-

TRIPLE-SPOTTED CURRANT-Moth. tacked should be cut off and burnt, which seems the only practicable way of ridding us of this pest. We believe it has not yet been discovered where the female deposits her eggs, nor does it appear that the larva has been figured or described in any of our works on entomology, but the moths have frequently been captured about the middle of May. The body of this moth is described as being of a fuscous brown, the head covered with a crest of bright ochreous hairs; the antennæ are like bristles in both the male and female ; the upper wings are bronzed and freckled with purple and 
yellow ; the under wings have a purple shade ; the wings measure 8 lines from tip to tip, and the length of the body is 3 lines. There is a palish-yellow transverse band near the base of the upper wings, which tapers towards the margin, forming a somewhat lengthened triangle. There are two spots of the same colour, one on the costa, and the other opposite and near the lower angle.

The currant sphynx-moth (AEgeria tipuliformis, Sphinx tipuliformis, Trochilum tipuliformis), fig. 238, is very destructive to these

Fig. 238.
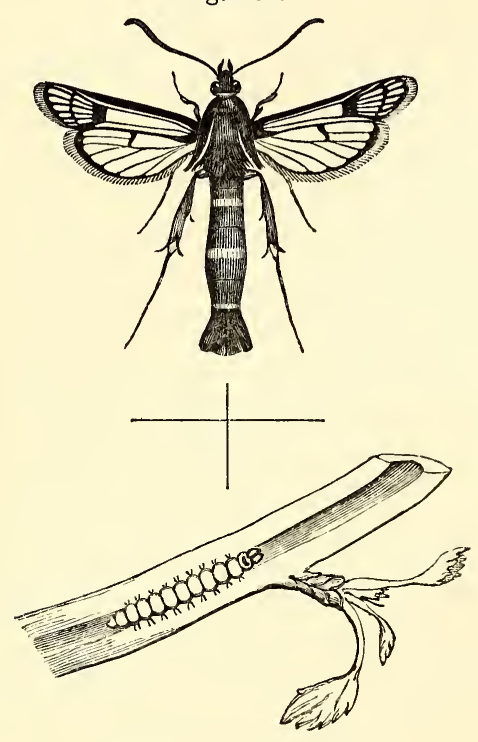

THE CURRANT SPHYNX-MOTH.

trees, particularly to the black currant, which soon indicates the presence of the insect by the withering of the foliage and branches. Towards the end of May, and in June, the female lays her eggs, choosing the crevices of the twigs, and as soon as the larva is produced, it penetrates to the centre to feed upon the pith, eating its way downwards until it has attained its full size. It then changes to its pupa state, and becomes furnished with serrated short spines, which enable it to ascend to an opening in the side of the shoot previously prepared by the larva. From this opening the moth escapes. The caterpillar is fleshy, of a dirty-white colour, with a scarcely traceable dorsal line; it has sixteen feet, two anal, six pectoral, and eight abdominal; the head and four horny spots are bright brown. The moth is brilliant black, inclining to purplish, which contrasts well with the yellow lines encircling the body, one of which forms a collar to the neck; and two longitudinal strips run across the thorax, while there are three similar strips upon the abdomen of the female, and four on the male. The wings are transparent, with dark nervures, and surrounded with a purple margin, sprinkled with golden scales; the supe- rior wings toward their apex are of a golden colour, uniting with a dark lunate spot on the disc, enclosing a transparent ovate spot, across which four dark nervures are visible. The only remedy appears to be the removal of the affected shoots, and burning them to prevent the further spread of so annoying an enemy.

The Aphis ribes also sometimes attacks the currant; fumigation or syringing with tobacco liquor, or dusting the shoots with Scotch snuff after well wetting them with the syringe, or after a copious shower of rain, is the only effectual remedy, ex. cepting cutting off the parts affected, which can readily be done, and without injury to the trees, as the aphis attacks the points of the shoots only.

A species of coccus very much resembling Coccus vites, which attacks the vine, is said, on the authority of Mr Kirby, to attack the currant also. This insect we have never observed. That eminent entomologist is not likely to have been mistaken. He describes its eggs as of a beautiful pink colour, concealed in a mass of cotton-like web. Removing the loose bark, and rubbing the branches with a hard brush, will displace both eggs and full-grown coccuses, unless they take refuge amongst the spurs, in which case they will not easily be dislodged. Earwigs sometimes also attack them, but these are easily captured if perseverance be exercised by the means indicated at page 514 .

The European names of the red, white, and black currants are all the same-viz., Grosseiller commun, French - Johannisbeere, GermanAalbezie, Dutch-Ribes rosso, Italian-Ribes rojo, Spanish - Groselheira vermelha, Portuguese-Smorodina Krasnaja, Russian.

\section{§ 2.-THE RASPBERRY.}

The Raspberry, in its indigenous state, is the Rubus idceus of botanists, and is found often in great abundance in shady woods and moist situations in various parts of Britain, and over most of the north of Europe. The Dutch, who reclaimed the currant, most likely transferred the wild raspberry also to their gardens. All the fine varieties of which our gardens now boast appear to have originated from the long-cultivated Rubus idceus, or Mount Ida bramble; and this is asserted to have been first introduced into the gardens of the south of Europe from Mount Ida: the name raspberry (Raspo, Italian) is supposed to be derived from the rasping roughness of its wood; and the Scotch designation (raspis) is evidently from the same root. From the circumstance of the red and yellow Antwerp varieties being the first recorded as cultivated in Britain, we are inclined to believe that these two excellent sorts were imported from the Netherlands. Although these two sorts still maintain a good position with fruit-growers, some of the newer sorts originated from seed fully equal them in flavour, and excel them in size. From a quotation already made from Tusser's "Five Hundred Points of Good Husbandry," it is evident that the plant was culti: vated in his time. Langley in "Pomona," 1729, says, "We' have only three raspberries in England, the red, the white, and the purple. The 
Dutch have recently added three varieties to our former list, namely, the Long-fruited Antwerp, Round-fruited Antwerp, and the Yellowfruited Antwerp. The French seem to have contributed none until we recently received the Franconia, and Belle de Fontenay, and the Yellow French of Lawson's Catalogue. There is not even a French synonym given to any raspberry in the "Fruit Catalogue of the Horticultural Society" excepting two, sufficiently conclusive that little progress is making in that country in the improvement of this fruit. England has been active of late years in their production, as the names in the following list will show. It may be here remarked as something rather strange, that considering with what facility both currants and raspberries are produced from seed, so few new or improved sorts should have been raised, and this the more so as so many gooseberries are annually produced. A curious hybrid has been produced by Mr Rivers, which he calls the Black raspberry, obtained by crossing the raspberry with the common bramble. The fruit produced is a dark purple. No doubt the bramble might be much improved if crossed with the raspberry; we do not think, however, that the raspberry would be improved to the same extent by the same means. This recalls to mind a reminiscence of our early days-the humble petition of the Rubus chcememones to the members of the Caledonian Horticultural Society, praying that it might be transplanted from its dreary mountain abode and taken under their fostering care, or that it might be allowed to enjoy the nuptial embrace of the raspberry, its near kinsman, with the view of elevating its condition and admitting the progeny (if any) to appear amongst the fruits of the garden. The petition is written in the Ossianic style, and highly creditable to the author, $\mathrm{Mr}$ Archibald Gorrie, both as a poet and early hybridiser; for in those days little was known of the mysterious working of nature, in changing the conditions of fruits particularly.

The Americans have originated none from our common origin, but they have taken into cultivation three of their indigenous species of Rubus-namely, Rubus strigosus of Micheaux, their American red or common currant, the Ohio Everbearing, and the American black (Rubus occidentalis Lin.), all of which are probably better fitted for their climate than any of ours. Mr G. Lindley, in "Guide to the Orchard," enumerates twenty-two sorts, the "Fruit Cat. of the Hort. Soc." only fourteen as worthy of cultivation; and the more recently-published fruit-catalogues of Peter Lawson and Son, and Dickson and Turnbull, Perth, present us with fourteen and eight sorts respectively.

Propagation.-By seed, when new varieties are desired, which should be sown soon after the fruit is fully ripened, washing away the pulpy matter, drying the seeds, and sowing them in pans or pots, in light rich soil, placed in a cool frame or pit. In spring they will vegetate in the ordinary temperature of the climate, or they may be placed in a mild heat to further their growth. When about 4 inches high, transplant them into a nursery-bed, about 8 inches apart: the season following they may be planted out in lines in rich moist soil, and many of them will produce fruit the same season. They do not propagate readily by cuttings, but as they send up an abundant supply of suckers from the roots during summer, these, when stock is required, should be preserved, and taken early in autumn and placed in their permanent situation. The strongest and bestformed suckers should be selected for immediate planting, the smaller reserved and headed back to 6 inches and planted in nursery lines, where, by October following, they will be sufficiently strong for planting out. Either way they will produce some fruit the first year after planting, and will be in great perfection the season following.

Soil and situation.-The raspberry requires a deep, rich, and humid soil, abounding in decayed vegetable or humic matter. An enriched peatbog, thoroughly drained, is the best soil of any, and next to that a deep, well-trenched, rich garden soil : if it can be irrigated during the growing season of the plant, so much the better. In light, shallow, dry soils the plants are weak, the fruit scanty and small. Partial shade is suitable also, and hence many plant them in the borders behind northern walls; but this must ever be at a sacrifice to the trees growing against them, as the raspberry roots descend deep and spread widely, and would rob the other trees of their due share of nourishment. They also require shelter, as the young wood is liable to be broken by high winds. An outer slip, if well sheltered, even should it be partially shaded by trees, provided they do not hang over them, is the best position for them in a garden. Let the soil be at least 3 feet deep, and at trenching bury in the bottom a copious supply of undecayed manure. The ground between the rows should never afterwards be dug by the spade during the existence of the plantation : the surface may be slightly loosened annually with a fork, and all manurial applications applied by top-dressing or in a liquid form. There are two sorts of roots to the raspberry, the one going down to a considerable length in search of food or moisture, the other running horizontally very little below the surface. It is from these latter the young plants or suckers. proceed. When young plants are not required, all these suckers should be destroyed; the nutriment necessary for their formation will then be thrown into the fruit of the present year, and also into the canes which are to produce the succeeding crop. When their surface roots are deprived of their suckers, they then become food-collecting roots, and that is the reason why they should not be disturbed.

Planting.-Raspberries, like all other hardy fruit-bearing plants, should be planted in October or early in November. If in single rows, the plants should be set 5 feet plant from plant, that there may be room for training the shoots sufficiently apart to admit sun and light to the fruit while ripening; for although the raspberry thrives and grows luxuriantly in shady situations, still, during the ripening of the fruit, air and sunlight are highly important, thus leading to the conclusion that portable 
shading during growth, and a full exposure to the sun during the ripening period, are an important consideration, if fine, large, and highflavoured fruit is a thing cared for. If planted in quarters by themselves, which certainly offers some important advantages, they may be set in rows 6 feet asunder, and the plants 5 feet apart in the rows. This admits of occasional irrigation, as well as of covering the crop, when ripe, with netting. The plants, in favourable soils, will continue productive for many years, but as they greatly exhaust the soil, and may, as has been stated by a writer in the "Gardeners' Magazine," vol. x., p. 14, poison the ground they are long grown in by the excretions exuding from their roots, repeated replanting is a matter of consideration; and our own opinion is, that it is the exhausting principle, and not the excretions they give off, that renders change of soil necessary. New plantations should be made every third or fourth year, and this is the more necessary in proportion to the lightness, dryness, and poverty of the soil. In the generality of soils, the finest fruit is produced the second and third year after planting; and where fruit of a large size is required, no doubt timely thinning should be practised, and a stimulating effect given to the roots by copious applications of liquid manure. Besides this, all suckers should be removed as soon as they appear above ground; and no more than four or five canes or young shoots should be permitted to grow, which will be sufficient for producing wood for bearing the following ycar. This, however, destroys the chance of a succession of young plants, which in private gardens is a matter of little consequence, as a supply can be porcured from the nurseries; or if deemed important, a portion of the plantation set apart for dessert purposes can be so treated, while in that from whence the supply for preserving purposes is taken, a supply of suckers may be allowed to grow.

Winter pruning and training.-Early in Scptember (that is, as soon as the crop is gathered) cut away all the old bearing-wood (that is, the wood that has produced the crop for the season) close to the ground, leaving from four to five of the strongest and best of the young shoots, or canes, as they are usually called,- that is to say, if the plants are very strong; if weakly, half the number left will be sufficient. If pruned so early as the middle of September, the whole of the shoots left should be shortened; but if the pruning be delayed till the middle of November, defer the shortening of the shoots till the end of February, as they are sometimes in cold climates liable to be injured by frost. It is the usual practice to shorten the whole of the shoots to the same length, but some with greater propriety shorten them at different lengths, according to their strength-say two of the strongest at 4 feet in height, two at 3 feet, and one or two at 2 feet. This depends, however, a good deal upon the kind, some growing to a much greater height than others, but the above will form a scale. By this means the young bearing-wood of next year is equally divided over the plant, and prevents that crowding and confusion which occurs when they are all cut to the same length.
Their height depends also on the strength of the plants; the stronger they are, the longer they should be left. In regard to training, some tie the canes merely to an upright stake; others dispense with stakes, and bring the tops of the two nearest plants together, having them in form of an arch. Many of the London growers run long rods or laths, supported by poles, from end to end of the rows, and train the canes to them in a sort of open-fan manner; while some very orderly persons train them to a regular espalier, which latter is certainly the neatest, and with the last exposes the fruit better to the sun and air. Indeed, either of these modes is equally proper, and any one of them may be adopted to suit peculiar circumstances. Although we have stated above that four or five canes should be left on each plant, this applies only to such as are very strong, and in soils highly favourable. Weak plants should not have more than two or three rods retained, and when, from accident or other causes, the plants are very weakly, the canes should be cut down altogether in order to strengthen the plants for the ensuing season. No canes should, however, be left longer than 6 feet, at which height we have often had the Felstof, which is one of the strongest growers. No fruit-bearing plant disagrees more with having its roots disturbed than this; therefore digging about them should be carefully avoided, and enrichment given to the roots by the application of liquid manure and top-dressings of the richest kind; and in the removal of root-suckers, it is better to pull them up than to dig them up, as in the latter case the roots may sustain injury from the spade.

A late crop of fruit is often obtained by cutting down all the canes early in spring to within 6 inches of the ground; they will then shoot up new wood, and produce fruit in September and early part of October, and this more especially with the variety called Double-bearing; or these latter may have each alternate plant cut down annually, which will cause a continuance of the ripe fruit till the end of September. By this process more light and air is admitted to the plants that produce the fruit, or the same end may be effected by cutting down each alternate row.

Summer pruning.-This chiefly consists in the removal, while they are quite young, of all superfluous young shoots arising from the root of the plants, leaving three or four for the bearing wood of next season. Much depends on the size and strength of these, and consequently a plant will be better enabled to produce that number than a greater. The light and air also are permitted to act upon them, and hence the sap in them must become much better elaborated, and the buds from which the fruit of the succeeding year is to be expected will be more fully matured. When the fruit is gathered, the wood which produced it should be cut down close to the ground as no further beneficial to the plant. The shoots left should be carefully preserved, and secured to whatever supports may be used, nor should the terminating points be at present interfered with. It is a 
great expenditure of the energies of the plant, as well as robbing the soil, to allow the bearingwood to remain one day after the fruit has been gathered.

Forcing the raspberry.-This is done pretty extensively on the Continent, where they are planted in the front of pits (vide figs. 405, 407, vol. i.), the fruit-bearing wood introduced in December, under the glass roof, the young wood allowed to rise and ripen trained to a trellis outside, to be in turn introduced the succeeding season, when the old wood is cut away altogether. The same end could be better effected by growing the plants in large pots plunged in the open garden, and the plants shaken out carefully, and planted in front of the pits or houses annually. When the day arrives when we shall have properly constructed hothouses of sufficient capacity, instead of the mouse-trap lean - to things at present dignified with the name, rarely exceeding 30 feet in length and 12 feet in breadth, we may then calculate upon having the raspberry and its compeers growing in pots or tubs in all the luxuriance they at present assume in the open air.

\section{SELECT LIST.}

Barnet. - Fruit very large, conical, bright purplish red; ripens early, and is very productive. Canes long, yellowish green, branching much more than the Red Antwerp, particularly near the ground. Bearing-shoots almost smooth. Inferior, however, to the Red Antwerp, in respect of its not bearing carriage so well. Synonyms - Large red, Lord Exmouth's, Cornwall's prolific, Cornwall's red, Cornwall's seedling.

Belle de Fontenay.-A new variety of French origin, described by Messrs Henderson of the Wellington nursery, St John's Wood, as being " a great improvement over other varieties in cultivation, producing racemes of fruit at every leaf ; it is also a remarkably prolific bearer from the spring to the autumn, being a perpetual fruiting variety of large size, good colour, and fine flavour; very dwarf, not more than from 3 to 4 feet in height, and branching off so as to form quite a bush." We have introduced this and the White globe on account of their dwarf habit; the descriptions are quoted from the catalogues of the respective firms. Not having seen them in cultivation ourselves, we cannot speak from personal knowledge of them; the respectability of the parties, however, is a sufficient guarantee for the correctness of the descriptions.

Cox's honey.-We notice this variety, being a yellow or whitish fruiter, as there is such a paucity of that colour, the Yellow or White Antwerp, White monarch, White globe, and Old white, a worthless variety, being the only others not of a red colour. Cox's honey has the peculiarity of bearing its fruit in clusters along the stems, and is otherwise of good quality, but by no means in general cultivation.

Double-bearing.-The fruit is small though abundant; its merit consists in its bearing late in the season, often till destroyed by the frost; and to encourage this habit, the shoots are cut down in spring to within a few inches of the VOL. II. ground. Since the appearance, however, of Rivers' large-fruiting monthly, this variety is now nearly neglected, the other being superior in every respect. Synonyms-Late cane, Siberian, Red double-bearing, Perpetual bearing.

Falstoff-Colour red; size very large; quality excellent, and an abundant bearer. The original plant was found growing naturally from seed under a Portugal laurel in the grounds of the Rev. - Lucas of Filby, in Norfolk, and by him made known under the name of the Filby raspberry, and to him we are indebted for the first of our stock. The name of Falstoff was given it by a nurseryman in the same neighbourhood, and by him it was brought to the general knowledge of the trade. Not only is the fruit of large size and rich flavour, but it has the property of continuing to bear successional crops for a greater length of time than any other of its class. Canes strong, branching, light yellowish brown, with pretty long bristles. Being a large grower, it should be allowed plenty of room-certainly not nearer than 6 feet plant from plant.

Franconia.-Fruit very large, obtusely conical, dark red, flavour more acid and tart than that of most others. Canes strong, branching ; leaves rather narrow and long, and of a very deep green colour. This is reputed the hardiest of raspberries, notwithstanding its Parisian origin. Downing says, "It flourishes admirably in New England, and north of us (New York), where the climate is too cold for the Antwerp, and is by far the finest variety for a norther'n latitude." It bears carriage well, and ripens a week or ten days later than the Antwerp.

Red Antwerp.-Fruit large, conical ; flavour rich and sweet. Canes of moderate growth, becoming pale brown early in autumn, their lower parts covered with strongish dark-brown bristles, the upper parts nearly smooth. Leaves large and plaited. There are, unfortunately, some inferior varieties of this excellent sort in the nurseries; these, when detected in fruit, should be grubbed up. It is the most general in cultivation, being of good size, shape, and flavour, and withal most productive. It is of Dutch origin, and was introduced to Britain many years ago from Antwerp. SynonymsKnevett's Antwerp, Howland's red Antwerp, Burley, New red Antwerp, True red Antwerp. Of this sort Mr Thompson remarks in Supplement to "Hort. Soc. Cat.:"-_" Amongst all the cultivated sorts of raspberries, none are known to possess the rich aroma of the Red Antwerp; but it has been so long grown in this country that the stock appears to want renewing. The three reputed best sorts of Antwerp raspberries have accordingly been recently introduced for trial."

Rivers' large-fruited monthly.-“A free grower, abundant bearer, and valuable for producing late crops; very different, however, from the weak-growing old double-bearing."-'Tномгson.

Rogers' Victoria.- Size of fruit medium; form conical; colour bright red; canes smooth, brownish red, growing erect. This is a long-bearing sort, and continues to produce fruit in pretty good succession till November. 
Victoria.-Another English seedling of recent date. It fully equals the Red Antwerp in size and flavour, and excels it in the long continuance of its production, yielding fruit from July to the beginning of November. Canes of moderate height and strength, sending out numerous branches from 18 inches to 2 feet in length. Synonyms-Cornwall's Victoria, Superbe d'Angleterre.

White globe.-Size of fruit medium; form roundish; described by the Messrs Lawson as "very prolific, bearing its fruit in large clusters; canes strong, with thickly-set bristles, but of a dwarf habit, and needs no stakes; very distinct."

White monarch.-Fruit large ; form roundish or conical ; colour pale yellow ; canes strong, with many side-brauches; flavour excellent; producing fruit in succession for several weeks.

Yellow Artwerp.-Fruit large, nearly conical, whitish yellow; flavour sweet and excellent; canes strong, light yellow, with many bristles or spines. Synonyms - Double - bearing yellow, White Antwerp.

The varieties cultivated in the Dalkeith gardens are-Falstoff, Red and yellow Antwerp, Franconia, Rivers' large-fruited monthly, Rogers' Victoria, White globe, and Double-bearing.

From ten out of fifteen returns from various parts of Britain, the Red and Yellow Antwerp, Falstoff, Double-bearing, and Cornwall's red or Barnet, only are grown.

Insects and diseases.-The raspberry is seldom seriously attacked by either. The fruit, when ripe, is frequently found to contain the grub of, so far as we are aware, an undetermined insect, and this occurs more frequently when the plants are growing in too poor, too shallow, or too dry a situation.

The raspberry leaf-miner (Fcenusa pumila), Fig. 239.
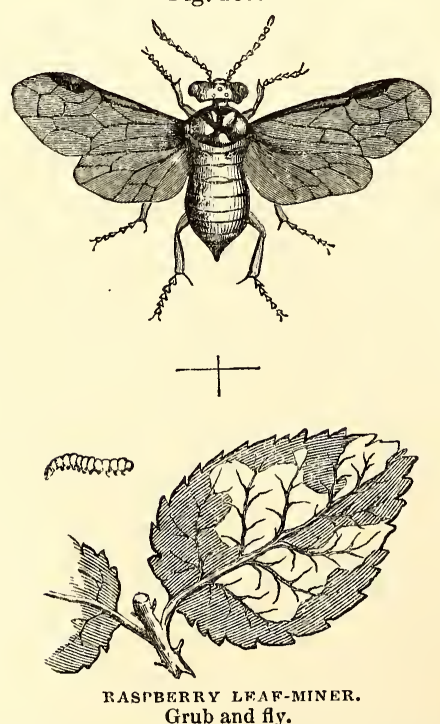

fig. 239 , is sometimes found on the foliage about the beginning of July. Its presence will be in- dicated by the appearance of large blotches on the leaves, which upon examination will be found to contain, between the two surfaces, small dirty-green caterpillars, having three pairs of legs attached to the segments following the head, and six pairs of ventral and one pair of anal prolegs. During the growth of the caterpillar they shed their skins several times, leaving the exuvia within the blotch; these blotches often extend over the greater part of the leaf, and when they have eaten up all the substance, and have attained their full size, which is about three-eighths of an inch in length, they eat their way out and fall to the ground, where they undergo their transformation. The perfect insect appears about the beginning of August, about an eighth of an inch in length and rather more than a quarter of an inch from the points of its fore wings. It is of a shining black colour, the edge of the abdomen polished as far as the second joint. The legs and thighs testaceous and palish, tibiæ occasionally white at the base; the wings, with the front margin and stigma, black. "Some specimens," Mr Westwood conjectures, "must appear in the winged state in the following spring, in order to deposit their eggs in the new raspberry leaves. These are either a fresh brood, or, as appears more likely, are the survivors of those hatched in August." The only remedy appears to be cutting off every leaf infested, and consigning them to the flames.

The grub of the raspberry bud (Tinea corticella of Linnæus, Haworth, Stephens, and Westwood, Tinea rubiella of Swedish entomologists, Alucita variella of many German entomologists, Alucita multipunctella of Duponchel, Glyphypteryx variella of Stainton, Lampronia corticella of others), is found committing sad havoc on the buds of the raspberry from the middle of April to the end of May. In its caterpillar state it is about a quarter of an inch long, of a scarlet colour, which renders its detection easy, seated in the interior of the bud, which it devours entirely, and betraying itself by the dying away of the blossom-buds and young shoots. The body is thickest at the middle, tapering away towards the hinder extremity; head black; the segment immediately behind has two black double spots; the anal segment is also black, as are also its three pairs of jointed legs, which are attached to the three segments following the head, and four pairs of vertical and one pair of anal prolegs, which are of a fleshy colour. About the beginning of June the caterpillar spins itself a web, attached to the withering leaves, in which it changes to a chrysalis of a light chestnut colour, and continues in that state about a fortnight. The moth, which is very beautiful, has shining brownish upper wings, varied with gold-coloured spots, two of which are larger than the rest, and are placed on the upper edge of the wings. Hind wings dark brown; the head thickly clothed with gold-coloured hairs. The eggs are laid in July, and are hatched in August, and immediately commence feeding on the foliage. Where they secrete themselves during winter is not very well known, but they begin their attacks on the buds the following spring, which appears 
to be the best time for attempting their destruction, and that by carefully examining the buds, and squeezing the tender insects within them. Should they deposit their eggs on the old bearing-wood, those will of necessity be cleared away at the winter pruning, as all that wood is at that time cut away, and we can scarcely believe them endowed with sufficient sagacity to choose the young wood which is to be left.

Raspberries are often attacked by the omnivorous weevil Otiorhynchus sulcatus (see VINE); they attack the stems in June, and often do much mischief. No means have been as yet found out of preventing or even destroying them with any great degree of success.

\section{§ 3.-THE GOOSEBERRY.}

The smooth-fruited gooseberry (Ribes uvacrispa), and the rough-fruited kinds (Ribes grossularia), so called because its berries resemble half-ripe figs (grosse), are the types from which all our cultivated sorts have sprung. These are indigenous to many parts of Britain, inhabiting the edges of thin sunny copses, and where the ruins of ancient buildings are found. In its wild and uncultivated state the fruit is small, but of a rich and agreeable flavour, and, unlike most other fruits, becomes deteriorated in flavour as it increases in size by high cultivation. At what period our forefathers directed their attention to the improvement of this fruit is unknown. The earliest notice taken of it that we have met with is in the writings of Gerard, Culpepper, and Tusser. The latter, in his "Five Hundred Points of good Husbandry," says-

\footnotetext{
66 The barbery, respis, and gooseberry too,

Look now to be planted, as other t!ıings do

The gooseberry, respis, and roses, all three,

With strawberries under tiem, trimly agree."
}

And strange enough this ancient arrangement, with the exception of the roses, is much the same as is practised in market-gardens at this day. The gooseberry is almost the only fruit wc can claim as in reality our own, for with the exception of a pale-coloured sort imported from Flanders in the time of Henry VIII., we have met with no other instance of any of this fruit having been imported before or since. The Dutch have several varieties differing from ours, but they possess no extra merit.

The locality in which the gooseberry has attained its highest cultural excellence is Lancashire, a circumstance attributed to the taste of its manufacturing population.

It may be called the fruit of the poor, as every cottager having a garden has the means of cultivating it. In the warmer countries it is almost unknown, and, excepting in some parts of Holland and Germany, where the climate is moderate and somewhat humid, it is greatly disregarded, the grape-vine taking its place. "The Manchester Gooseberry Book," published annually, contains the names of all the leading varieties and their weights, some of which are so great as to be almost incredible, size and weight being the objects aimed at by the Lancashire growers, and curious expedients are called into action to effect this. The situation chosen, say they, should not be shaded, nor should it be exposed to the full rays of the sun, because, under such circumstances, the fruit would ripen too rapidly without giving sufficient time for the swelling of the berries. To effect a partial screen from the sun without creating shade, it has been recommended by some to plant rows of Jcrusalcm artichokes between the lines of bushes, or to place them on the sunny side; others have suggested growing lines of scarlet runners between them for a like purpose. Neither of these expedients come up to the mark of high culture. It were better, therefore, to screen the bushes while the fruit is swelling with such portable screens as coarse paper, thin canvass, or the like, which could be used or disused according to circumstances. The process of suckling consists in applying rich and stimulating manure to the roots, by forking it in, or better, by laying it on the surface to keep the roots moist, and permitting its fertilising parts to be washed down by the rains. No method, however, is so good and so conveniently applied as using liquid manure, poured repeatedly on the surface, and extending to the farthest ramifications of the roots, that the spongiolets may pump it into the system of the plant. Besides feeding by the roots, "those high in the fancy" place a shallow vessel of water immediately under each berry, which is partially submerged in it, leaving only tliree or four fruit on the tree, according to its strength. They also water the plants frequently over the tops, particularly in dry weather; and with a view still farther to throw the whole energies of the plant into the fruit, they remove almost every young shoot that is not absolutely required for the formation of the bush. These operations are not gone into in private gardens, but in all rich soil liquid manure, in preference to solid, is or should be applied. The ground should be as little disturbed beyond hoeing as possible, as deep digging destroys the best of the roots.

Where high-flavoured and small fruit is desired, a warm sunny spot should be chosen, the soil light, and not much enriclied by manure. The richest-flavoured gooseberries we ever tasted were grown accidentally on the ruined walls of Cawdor Castle, insinuating their roots between the fragments of stone and mortar-a pretty good hint that the debris of a limestone quarry, if wcll exposed to the sun, may not be a bad situation where high flavour is aimed at. Amongst the earlier ripening is the Yellow sulphur, and amongst the latest the Red Warrington. If the former be planted in a warm sunny exposure, or on a south wall, they will ripen early in June; while the latter, planted against a northern wall, or in a cold northerly border, and protected by a net, may be had in good condition to the end of October.

Our earliest writers give little information regarding this fruit. Tusser, Mascall, Turner, Parkinson, Ray, Rea, and Philip Miller, all mention it; the latter observes, "There are several varieties obtained from seed, but as there are frequently new ones obtained, it is needless to enumerate them." Forsyth, in 1800 , 
mentions ten sorts as common, besides fortythree new sorts grown in Manchester. The Horticultural Society of London published an account of seventy varieties, as cultivated in their garden for the purpose of identification, vide "Horticultural Society's Transactions," second series, vol. i. p. 218. In this collection many of the Manchester sorts have been omitted, as being below the standard of merit in regard to flavour, notwithstanding their great size. Gordon of Fountainbridge, near Edinburgh, writing in 1774, enumerates twenty sorts, amongst which are the still popular ones, Hedgehog, Champagne, Rambouillon, Chrystal, Ironmonger, \&c. Mr Thompson, in " Horticultural Society's Catalogue," third edition, enumerates one hundred and forty-nine sorts; and in the more recent "Fruit Catalogue," published by Messrs Peter Lawson \& Son, one hundred and ninety-five sorts are described, and there are no doubt a much greater number in cultivation, three-fourths of which might be dispensed with. Langley, in "Pomona," 1729, says, "We have several kinds of gooseberries; the following five are, however, the most valuable: Champagne, White Dutch, Amber, Walnut, Rambouillon, and the Damson berry." The largest enumeration of sorts is that, however, of Mr G. Lindley, in "Guide to the Orchard," who extends his list to no less than seven hundred kinds. The gooseberry is not a fruit of the United States, notwithstanding the pains that have been taken to introduce it there. Downing assigns as a reason want of a correct knowledge of its culture, and the very inferior sorts operated upon. This is, we think, mere presumption. The true reason is, the heat of the climate during summer, which places the middle and southern States of America upon a par with the middle and south of Europe, where, as we have already said, this fruit does not succeed. The varieties are increasing rapidly, and many fine-flavoured, although not very large ones have originated in Scotland, of which Gorrie's Robina deserves especial notice, as do also a white-fruited variety of the old Ironmonger, originated with $\mathrm{Mr}$ Archibald Gorrie, of Annat Cottage ; and a still more singular and equally valuable variety of this best of gooseberries, a variety striped with red and white, brought to our notice by his son, Mr William Gorrie, of Preston Hall, being, so far as we are aware, the only striped gooseberry in existence. Of other Scotch varieties, little known out of the localities where they originated, we may mention as especially worthy of cultivation, Kippenross seedling, Delvin mignonne, Delvin porcupine, Scotch rough red, \&c.

Propagation.- New varieties are obtained by sowing the seeds of superior kinds, which seed should be sown in light rich soil soon after the fruit has ripened. If to be sent to a distance, the seed should be washed out of the pulp, and when dried packed up in paper bags for transportation. Sow the seed shallow; by next spring they will regetate, and by the following autumn be fit for planting into nursery lines. This mode is, however, seldom had recourse to, the usual and best method being to propagate by cuttings. The gooseberry may, in extraordinary cases, be also propagated by layers, and also by grafting and budding, but these means are seldom resorted to.

Propagation by cuttings.-The best cuttings are got from the strongest and straightest wellformed shoots of the current year, taken off about the end of October and planted immediately. As far as the cutting is to be placed in the ground--say from 6 to 8 inches-the buds should be cut out to prevent suckers arising from them. Plant the cuttings in a deep rich soil, in a shady situation, such as a border with a northern exposure. The top of the cutting should be cut off, so that only about 6 or 8 inches remain above ground, and they should be set in rows 12 inches apart and 6 inches distant in the line. By the following autumn they will be well rooted, and fit for transplanting into nursery lines for a season, after which they will be fit for being permanently planted out. Some recommend putting in cuttings in July, while the fruit is on the tree, to prevent mistakes as to kinds; but if a correct list is kept of the sorts, which should be the case, this departure from ordinary practice is uncalled for. They will, however, strike root very well at that season, if attention be paid to water and shade them till the roots are formed.

Soil and situation.-Where high-flavoured fruit is wished for, and a judicious selection made, a rich light soil, dry at bottom and fully exposed to the sun, should be chosen. Where large fruit is the object in view, then the soil cannot be too rich or deep, and the situation should be partially shaded from the meridian sun during the period of the fruit's swelling. It should be withal rather moist than dry. It is usual to plant gooseberry bushes along the sides of walks, between and under the shade of apple and pear trees, \&c. Such is the very worst situation for them, because their roots are perpetually exposed to be disturbed by the spade; being so scattered about, they are difficult to preserve from birds, and the space they cover must ever bear consideration where ground is scarce. A few of the earlier ripening sorts should be planted against any vacant parts of a well-exposed wall, and trained to it as wall-trees usually are. Some of the very late ripening kinds should be planted against a northern wall, to retard their ripening, and enable them to be protected from birds by nets, and from frost with canvass covering. The general plantation should be in an open quarter of the garden; and whether they are trained as espaliers or in the open - bush form, they should be in rows not less than 5 feet apart, and if grown in the latter manner, 4 feet apart in the line; if as espaliers, the same distance line from line, and to a 3 -feet trellis; they should have 5 feet plant from plant. The early and late sorts should be kept apart to render covering with nets more convenient, and the same distinction should be made with the large and small growing sorts. Beside the three varieties of jam berries, a good number of the Warrington and Pitmaston green gage should be planted, as hanging long on the bushes after they are ripe, and if protected with nets, will last as long grown as 
espaliers or open bushes as if trained against a wall. The season for planting is October and beginning of November. The ground should be deeply trenched and highly manured previous to planting, keeping the manure well down in the trenches. All future enrichment should be in shape of top-dressing, slightly forked, but not dug in; or better, liberal supplies of liquid manure during the period of their growth. The space generally recommended for gooseberries is from 8 to 10 feet between the rows, and 6 feet plant from plant. This distance is on the presumption that the bushes are to grow to the usual absurd size we see them so often permitted to do. Much smaller plants, and these often renewed, occupy much less space, and yield greater crops in proportion.

Pruning and training.- Young plants receiving their first pruning require care, for on this the proper form the bush is afterwards to assume depends. At this time select five or six of the best-placed shoots; if equidistant or nearly so, so much the better, cutting all the others away, and shortening the remaining shoots about half their length. The centre should always be kept open like a vase, so that the air and light may find their way freely to every part of the plant. The season following they will have made many shoots, some drooping outwards, some nearly horizontal, some nearly growing upright, for the habit of the gooseberry varies much according to the variety. The majority of the large Lancashire sorts assume a pendant or creeping habit, and therefore require to be supported upon a hoop or horizontal trellis. In November established plants should be pruned if the attacks of birds on the buds during winter and spring be not apprehended, and then all branches that cross each other should be removed, still keeping the middle of the bush somewhat open, shortening the pendulous shoots a little above the point where they touch the ground to prevent their coming in contact with it. The main branches should be kept at least 7 or 8 inches apart; all the young wood not required for modelling the plant is removed by some by cutting it close to the old stem, and the terminal shoots shortened to nearly half their length, and even more if the wood is weak, although some allow them to remain their full length; and this practice answers very well where moderate-sized fruit is desired, because it will be produced almost along the whole length of the shoot. Where large fruit is an object, then shortening the young wood should be followed; those who adopt the former practice calculate upon a supply of young wood being produced in succession from the base of the former shoots, or from the main stem itself. Those who follow the latter mode procure their supply of young wood from a bud near to where the amputation took place. Some depend on the spurs, some on the two-year-old wood, and others on the young shoots of the previous season, for their fruit, and no doubt the finest fruit is produced on the latter. Winter pruning should be finished by the beginning of November.

As the bushes begin to get worn out by age the knife must be used more freely, and whole limbs removed to make way for a fresh supply, to be selected from the most promising and best placed young wood.

We are no advocates for large overgrown old bushes, and would rather keep up a stock of young plants, and grub out the old ones progressively; not, however, planting the same ground, but beginning the plantation in another part of the garden, thus affording a change of soil as well as insuring finer fruit, for the largest berries will be found on plants of three or four years' growth, carrying out, so far, the principle of rotation. Another advantage attends a renewal of gooseberry plantations-that the caterpillar seldom attacks them for the first year or two after planting: a matter easily accounted for, as the ground up to about that time has not become the nidus of the insect in its chrysalis state, which, to exist there, must first fall from the trees. Gooseberries may be trained with great advantage in the espalier manner, both as regards facilitating the gathering of the crop, and exposing the fruit more fully to the sun (for to have high-flavoured gooseberries, like all other fruit, they should not be grown in the shade), as well as economising space. The trellises should be placed in parallel lines, and 5 feet apart; nor need they exceed 3 feet in height. Curvilinear trellises are also an excellent mode of training both the gooseberry and the currant; and when these are of sufficient height to form arcades over walks, they offer considerable training-space without causing much waste of ground. The fruit amateur might cover all his walks in this way with various kinds of fruit trees, making the space which would otherwise be occupied with the walk the border for the roots, and covering it with castiron grating laid on rails, to render it comfortable for walking on, and that without injury to the roots. For specimens of such trellises, vide vol. i., figs. 795,797 .

In removing superfluous young wood, cut close into the old branches, for the gooseberry will produce natural spurs abundantly, and all attempts at creating artificial ones only tend to increase the formation of an unnecessary quantity of leaves and small spray. The finest fruit is produced on young wood, thcrefore in winterpruning reserve a supply of this, and cut out old branches progressively to make room for it. It is quite possible to continue the gooseberry for years if attention be paid to this.

The gooseberry is trained in the Trentham gardens by being planted between two lines of espaliers about 4 feet in height and 9 inches apart; the branches are trained inside of the rails, which keeps them in a limited space, admitting of a larger supply being obtained on the same space of ground than in the ordinary manner of bush-growing. They are also much easier protected from frosts in spring, and from the attacks of birds on the buds and fruit, and are much easier gathered. It is also trained as a standard 3 or 4 feet in height of stem, and in this way produces high-flavoured fruit; and if those of drooping habits, such as many of the large Lancashire sorts, were trained in this manner, and their branches brought over a hoop at that height, and trained pendant in a way similar 
to that recommended by Hayward for apples and pears (vide figs. 152 and 153), the advantages would be great, and the appearance novel. Such late-keeping sorts as the Warrington might be kept on bushes thus trained till destroyed by the frost; and although that sort is considered a coarse thick-skinned fruit, still if thoroughly ripened, which it is seldom allowed to be, it becomes not only highly flavoured, but the skin softens into an agreeable pulpy-like conserve.

Summer pruning.- In proportion to the health of the plants and richness of the soil will be the quantity and strength of the young wood produced. Were the removal of this young wood from the centre of the bushes in particular of no other advantage than admitting light and air to the fruit and buds, it would amply repay the trouble. It renders also the operation of gathering the fruit much more easily effected, and must improve its flavour. The terminating young shoots must not, however, be shortened; and hence the barbarous method proposed by some, of using the hedge-shears in performing the operation, must be considered as opposed to good culture. The centre of these plants should always be kept open and clear, and therefore such shoots as arise there may with every propriety be removed, and that close to the old wood from which they spring. Shortening the lateral shoots about the period of the fruit ripening, by nipping off a few inches of their points, will have a tendency to arrest the rapid ascent of the sap, and throw more of it into the buds at their base, which, according to the spurring system, will strengthen the buds which are to form the future bearing spurs. Some object to this, and, indeed, to much of what is called summer-pruning, on the ground that in proportion to the number of leaves so will the action of the roots be; but we see little advantage in causing the roots to draw nourishment from the soil for no other purpose than that of the formation of wood that is certain to be cut out at the winter pruning and thrown away. Severe summer pruning, when done all at once, may be injurious to the functions of the plant; but for this there is 10 absolute necessity, as these shoots may be removed progressively, and if so, before they have attained any considerable size. A species, therefore, of disbudding would be the most judicious plan.

\section{SELECT LIST.}

Several attempts at a classification of this fruit have been attempted ; that of the Manchester list is perhaps sufficient for all useful purposes. The principal divisions are founded on colour-red, green, white, and yellow; and the subdivisions as to the skin being smooth, hairy, or downy. In the Hort. Soc. classification, two other subdivisions are added, founded on the form of the fruit, as to whether it is round or oblong.

Those marked thus * are the Lancashire prize, or extra large sorts.

Skin whitish.- * Abraham Newland, * Bonny lass, Bright Venus, Caldwell white, * Captain (white), Champagne white, * Cheshire lass, Crystal, * Eagle, * Fleur-de-lis, * Lady of the manor, Lion white, *Lion, ${ }^{*}$ Manchester white, ${ }^{*}$ Nichol's seedling, Orleans white, Ostrich, * Queen of Sheba, Queen Caroline, * Radical, *Wellington's glory, * White Briton, White rose, * White wine, * Whitesmith, Blunderbuss, Calderbank's white, Crawford's seedling, Dreadnought, Early white, Gascoigne white, Leader, Old Briton, Royal white, White eagle, White rasp.

Skin greenish white. - * Governess, Hedgehog, * Princess-royal, Queen Charlotte, *Thrasher, Large early white, Platt's white, Queen Anne, Speedwell.

Skin yellow and smooth.--Amber Ball, Yellow, * Bonny Roger, * Bunker's Hill, * Cheshire cheese, * Duckwing, * Golden chain (Forbes), * Smiling beauty, Braid’s glory.

Skin yellou. - Andrew Fairservice, * Birdlime, * English yellow, Globe yellow, Golden chain, Golden orange, * Leader, * Leader (Piggot's), Pilot, Yellow (Davis).

Skin yellow and downy. - ${ }^{*}$ Britannia, Golden drop, * Prince of Orange, Rumbullion, Battle of the Nile.

Skin yellow and hispid.-Champagne yellow, * Cottage girl, Golden lion, Goodall's seedling, * Jolly gunner, * Jolly Nailor, * Rookwood, Sulphur or rough yellow, Yellow Smith, Sulphur, Early, or Chedle's golden lion, or Golden ball, or Moss's seedling, Aston, Atlas, Golden sovereign.

Skin greenish yellow. - * Conqueror, * Tim Bobbin, * Viper, Nelson's waves.

Skin red.- * Bloodhound, * British crown, * Guido, * Wonder.

Skin red and smooth.- - British queen, * Emperor Napoleon, Globe small red, Old England, * Prince Regent, Red Turkey, * Roaring lion, * Sportsman, Wilmot's early red, one of the earliest in ripening.

Skin red and douny.-Black Prince, * Farmers glory, * Magistrate, Miss Bold, Walnut red, Warrior, Admiral Rodney, Bloodhound, British hero, Nonsuch.

Skin red and hispid. - * Cæsar, * Captain Red, Champagne red, Cheshire lady, ${ }^{*}$ Crown Bob, Hole in the wall, *Huntsman, Irish plum, Ironmonger, Ironmonger striped, Ironmonger (Delvin), Jam or Raspberry, Jam Gogar, Jam (Fletcher's) - of the Jams a considerable number should be planted, for, as their name implies, that conserve is made from them-Keen's seedling, * Lancashire lad, Prize Porterfield, Raspberry, * Rifleman, Rob Roy, Rough red (Pitmaston), Rough red (Scotch), Rough red (small dark), Shakespeare, * Sir John Cotgrave, * Top-sawyer, * Triumphant, * Warrington red, Yaxley hero, Scotch best jam, Atlas, British lion, Conqueror, Bell's fancy, Marquis of Stafford, Mogul, Overall, Rifleman, Royal oak, Sappho, Shakespeare, Sir Francis Burdett, Starling, Supreme, Victory.

Skin green and smooth. - * Bumper, * Green Providence, * Peacock, Porcupine, *Hedgehog, Shaws, Green walnut, or Belmont green, or Smooth green, or Nonpareil, Rumbullion, Hebburn, Green prolific.

Skin green and smooth.-Favourite, Glory of Rateliff, Green gage, Green gage (Pitmaston), * Greenwood, * Heart of oak, *Hopley's Shannon, *Jolly farmer, * Jolly tar, Nonpareil, * Northern hero, * No bribery, Nutmeg Scotch, 
Ocean queen, * Peover, Pecher, Royal George, Smooth, Green, Walnut, Bangor, Beauty, Derby ram, Diogenes, Fair-play, Favourite, Glory of Rateliff, Green globe, Independent.

Skin green and hispid.-Gillie's late, Glenton green, Green Gascoigne, * Langley green, * Lord Crew, * Troubler, * Wistaston hero, Globe.

Skin green and downy.- - Jolly Anglers, * Perfection, Chance.

Holland of Manchester's List of Lancashire Show Gooseberries for 1853 :-

Red. - Alderman, Atlas, Ashton-on-Mersey, Briton, Bangor, Conductor, Commander, Crownbob, Companion, Conquering hero, Defiance, Dan's mistake, Don John, Echo, Flixtonia, Guido, General Moore, Give it a name, Highlander, Ironsides, King Cole, King William, Lion's provider, Lion, Lurnper, Lancashire lad, Lord Middleton, London rival, Lincoln, London, Monarch, Magnet, Manchester, Magistrate, Napoleon le Grand, Old England, Plumper, Red Robin, Ricardo, Red Jack, Red champagne, Sam Hunter,Slaughterman, Speculator,Stewart,Twigem, 'Top-gallant, Top-sawyer, Useful, Wonderful, Wallace, Wistaston, Warrington, Wilmslow.

Yellow.-Atilla, Birdlime, Broom-girl, Comet, Catherine, Creeping Jane, Columbia, Captain Cooke, Duckwing, Drill, Dublin, Forget-me-not, Great western, Game-cock, Go-bye, Gunner, Goldfinder, Two to one, Glory, Jubilee, Leader, Lord Valentia, Lightning, Lord Rancliffe, Morton hero, Marygold, Oakmere, Oldham, Pilot, Peru, Perfection, Railway, Rockwood, Shuttle, 'Teaser, The chief, Yellow companion, Yellow boy.

Green.-Away she goes, Botheram, Banksman, Bumper, Dean Swift, Favourite, Green wonderful, Green ocean, Green snake, Great Britain, Green prince, Green river, Greenock, General, Gretna green, Hollin's green, Invincible, Keepsake, Launcelot, Liberty, Little wonder, Myrtle, Moorcock, Overall, Providence, Peover ranger, Peacock, Queen Victoria, Rough green, Random green, Turkey cock, Tom joiner, Thunder, Telegraph, Thumper, Turnout, Weathercock.

White.-Alice Hawthorn, Antagonist, Ardsley beauty, Chorister, Bee's-wing, British Queen, Cossack, Competitor, Coppice lass, Dauntless, Eagle, Eagle's daughter, Eliza, Fleur-de-lis, Fair start, Freedom, Flora, Fair Helen, Foley, Glory of Ounsdale, Harkaway, Jenny Lind, Lucy Long, Lady Leicester, Lady Stanley, Mrs Scarisbrick, Mary Yates, Mayor of Oldham, Miss Hammond, Miss Walton, Nonpareil, Ostridge, Philip the

First, Snowball, Snowdrop, Queen dowager, Queen of trumps, Tally-ho, White cockade, White hart, White lily, White hare.

List of Lancashire Show Gooseberries, taken from the "Lancashire Gooseberry Register" of 1852, with their weights.

\begin{tabular}{|c|c|c|c|c|c|}
\hline & \multicolumn{2}{|c|}{ RED. } & & \multicolumn{2}{|c|}{$\begin{array}{l}\text { Weights. } \\
\text { dwts. }\end{array}$} \\
\hline London, . & - & . & - & 37 & 7 \\
\hline Companion, & . & - & - & 31 & 11 \\
\hline Wonderful, & . & . & . & 31 & 0 \\
\hline Slaughterman, & . & . & . & 30 & 0 \\
\hline Conquering hero, & & . & • & 28 & 9 \\
\hline Napoleon le Gran & nd, & . & . & 26 & 19 \\
\hline Lion's provider, & . & . & . & 26 & 22 \\
\hline Roaring lion, & . & . & . & 26 & 2 \\
\hline
\end{tabular}

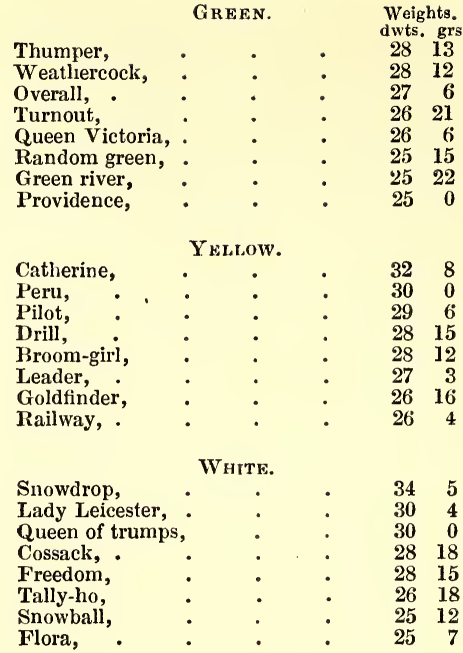

Diseases and insects. - Of the former there are few, old age and starvation in too poor soils only excepted; of the latter there are many, of which the following are the most destructive :-

The gooseberry and currant saw-fly (Nematus trimaculatus, N. Ribesii, Tenthredo ventricosa, T. Grossularia, for by all these names this leafdestroying insect is known) fig. 240, is equally

Fig. 240 .
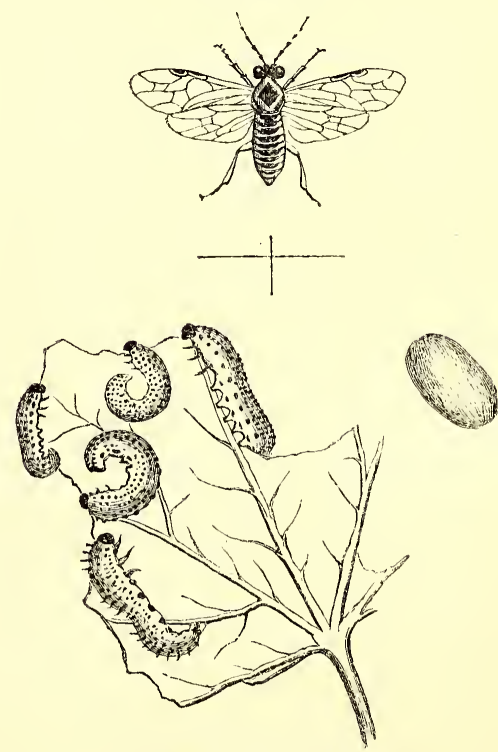

GOOSEBERRY SAW-FLY.

Grub, cocoon, and perfect insect.

destructive to the gooseberry and currant. About the beginning of April the flies emerge from their winter abode, and soon after the female begins to deposit her eggs on the under side of the 
newly-expanded leaves, choosing the sides of the veins or nervures as a fitting place. With the saw-like appendage for which this family is remarkable, the female commences cutting into the leaves and stalks, and in the openings deposits her egg between the cuticles or under the bark. The larva becomes hatched in about seven or eight days, and commences feeding on the leaf, which it speedily anatomises, increasing in size, and frequently changing its skin, until it is about three-quarters of an inch in length. It is now of a dull pale-green colour, the first thoracic segment being deep yellow, the penultimate being also of the same colour; the feet, tail, and head are black, and each segment is dotted black also, some having as many as twenty-four spots arranged in lines down the back, while those on the sides are more irregular, with one large one at the base of each foot. They have six pectoral sharp horny feet; the fourth segment appears destitute of feet, but the six following are each furnished with a pair of legs, which assists them in walking; they have also a pair of feet at the extremity of the last segment. In the fly state it assumes an ochreous colour; the body is orange, sometimes bright; the wings are irridescent, and, when expanded, are about two-thirds of an inch in length; the antennæ are almost as long as the body, bristly, brownish above, and nine-jointed; the crown of the head and eyes are black, as are also three large confluent spots in the centre of the trunk, and also a large patch on the breast or sternum. The broods of caterpillars appear in succession occasionally from March till October, but in greatest numbers in May and the early part of June. Sometimes they severely attack the gooseberry in July and August, and after denuding the bushes of their foliage, they descend into the earth, spinning themselves a yellowish cocoon of an elliptical form, and remain in their pupa state till the following spring. Those of the early summer brood descend in like manner, but in course of three weeks, or even less, undergo their transformation, and again appear as perfect flies. Syringing the bushes on the first appearance of the caterpillars until the foliage is well wetted, and then dusting them all over with powdered caustic lime, or powdered hellebore, is effective; the operation should be performed at least two or three times to secure their destruction, and the earlier it is done the better. Watering with lime-water has much the same effect, and covering the ground under the bushes with fresh tanner's bark is also advantageous. The most radical cure, however, is to remove the soil under the bushes to the depth of 3 inches, and to dig pits 2 feet deep and bury the soil in them, substituting that taken from the pits for that which has been removed. Water heated to $140^{\circ}$, and applied by the syringe or garden engine, has been found destructive to the caterpillars, while the foliage has sustained no injury. A strong infusion of digitalis has also been found useful when syringed over the bushes while the caterpillar is in a young state. The barbarous practice of striking the stem of the bush suddenly with a mallet, so as to cause the caterpillars to fall to the ground, where they may be bruised to death with the back of a spade or trodden under foot, is attended with much injury to the stem and bark of the tree.

The magpie - moth (Phalana grossulariata Linn., Abraxas grossulariata Leach), fig. 241,

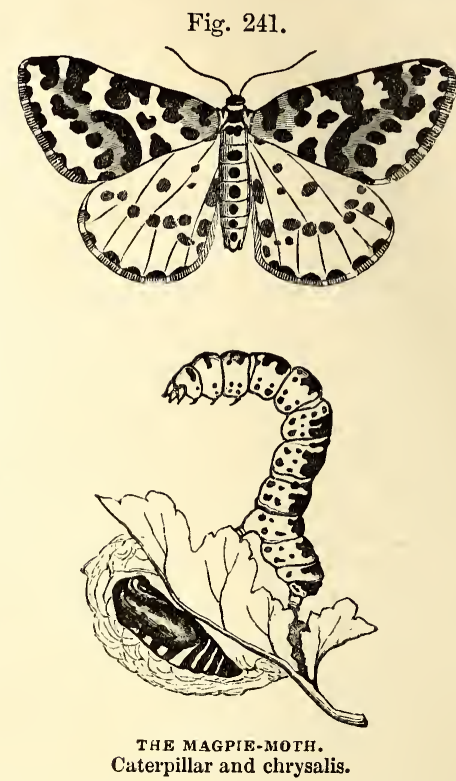

is a very pretty moth, which may be seen flying about in great quantities during the evening about the end of July. In colour it is a creamy white, covered with black spots; the thorax and abdomen, a space near the base of the upper wing, are of a fine orange colour ; the horns are slightly thicker in the males. The females, after pairing, deposit their eggs on a leaf, which are hatched about September ; they are not full grown till the end of May, and sometimes they do not become pupæ until the end of June, in three weeks from which time they are transformed into moths. In the caterpillar state they are exceedingly destructive, frequently stripping the gooseberry and red currant bushes of their leaves, leaving nothing but the foot-stalk; and sometimes the peach and almond are attacked by them. The caterpillar is of a cream colour, spotted with black, with orange spots," is slightly hairy when it arrives at maturity; it spins a web constructed so loosely that the pupa sometimes falls out. The chrysalis is of a black colour, having a few orange rings round the segments of the body. The eggs being laid on the foliage, and the young larva feeding on them, a remedy is suggested by burning the leaves when they fall off in autumn.

The small magpie-moth (Phalcena vanaria Linn., $P$. halias Godart and Curtis), fig. 242 , is equally abundant and destructive to gooseberry and currant bushes as the last. The caterpillars of this moth appear as early in spring as the leaves on which they are to feed begin to unfold 
themselves. Unlike the last, it undergoes its transformation on the surface of the earth, and

Fig. 242.
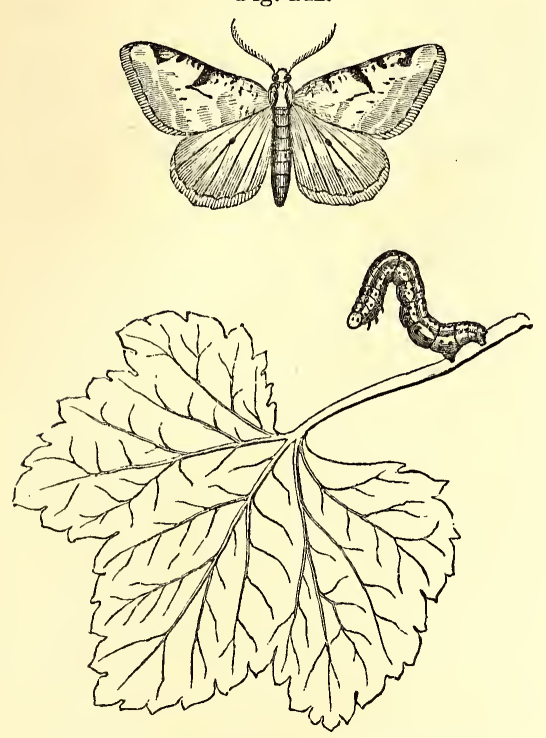

SMALL MAGPIE-MOTH AND CATERPILLAR.

hence is much more easily destroyed, as, if towards the end of May or beginning of June, at which time they quit the tree for the ground, this surface is removed to the depth of an inch and burned, or buried to the depth of a foot and a half, the insect, in its chrysalis state, will be completely subdued. This, indeed, seems to be the only radical remedy. In its moth state, the wings are of an ash colour and freckled, the upper ones having four brownish marks on the superior margin, the second crossing the middle of the wing, forming a V or L. The larva is about an inch in length, of a bluish green, with two white dorsal and two yellowish lateral lines; it is dotted with small blackish tubercles, which produce short black hair.

A strong decoction of the roots of white hellebore (Veratrum album), applied to the leaves with a syringe on a dry day, has been found efficacious; the liquid drying on the leaves acts as poison on the caterpillars, they being compelled to eat it along with the leaves or perish of hunger. Hellebore powder applied to the bushes, taking care that it reaches the under side of the leaves, acts in the same manner. In applying it, the branches should be held up by one person while another applies the powder by means of a sulphurating machine, fig. 173. Flour of sulphur, lime dust, and Scotch snuff, applied in the same way, have had a like effect.

The chaffinch, sparrow, bulfinch, and some other birds, often attack the buds early in spring, and commit sad havock amongst them, often to the destruction of the entire crop. It is doubtful what induces them to do this; it cannot be want of food, as in some seasons and VOL. II. in some situations, even where these birds abound, they entirely escape. It appears most probable that in such seasons the buds contain the eggs of insects, to devour which the birds are led by instinct; and as it is well known that some insects abound more in one season than in others, and also that some make their appearance periodically, it may be that the sagacity of the birds discovers this when man is totally ignorant of the fact. The only means of securing them from the birds is by netting the bushes over; but then the insects may be as destructive on their part - a matter that can only be ascertained by closer observation than appears as yet to have been instituted.

Much advantage has been found, in the suppression of insects, from covering the ground under the bushes in March with fresh tanner's bark: whether the remains of the tannin principle has the effect of destroying the chrysalis, or whether the thin covering of an inch and $\mathbf{a}$ half prevents their ascent, is not very clearly understood; the effect, however, has in many cases been satisfactory. Another advantage of covering the ground with tan is, that weeds are greatly prevented from rising, and there is a clean and comfortable surface to tread upon in summer-pruning and gathering the fruit; besides, it prevents the mud from splashing over the fruit on such bushes as are trained close to the ground.

Forcing the gooseberry is seldom practised; why, it is not easy to say, for a dish of ripe sulphurs in April is, at least to our taste, infinitely preferable to a dish of May-duke cherries at the same season. The former may with greater certainty be obtained than the latter, and those having an airy pit may produce the one, where, with the same means, they would likely fail with the latter. The plants intended to be forced should be potted the season previous into 12inch pots early in October, choosing nice small plants well furnished with buds. Some succeed well by potting in October previous to placing them in the pit. Abundance of light and air are the essential points to attend to, and a temperature at no time exceeding, by artificial means, $55^{\circ}$ or $60^{\circ}$. Towards ripening a slight increase of heat may safely be given, and throughout the whole process a sufficiency of moisture should be administered at the roots. When so produced, they are usually placed on the table attached to the plants. The early-ripening sorts are best adapted for this purpose.

European names of the gooseberry.-Grosella and Uva-espina, Spanish-Groselpeira, Portuguese-Uva-spina, Italian-Groseiller épineux, French - Krischownik, Russian - Kruisbezie, Dutch - Stachelbeeretrauch, German - Grielle, Piedmont-Feaberry and Feverberry in some parts of England-Grozer and Grozet in some parts of Scotland.

\section{$\S$ 4.-THE STRAWBERRY.}

Of the Strawberry (Fragaria L.) the cultivated sorts, now exceedingly numerous, have sprung from the following types-viz., the Wood strawberries and Alpines from Fragaria vesca, which is indigenous to most parts of Europe; the Pines from Fragaria grandiflora, indigenous to 
Surinam; the Scarlets from Fragaria Virginiana, a native of North America; the Chiles from Fragaria chilensis, from South America. The last three were, however, believed by Knight to be varieties of only one species, as he found them to breed together indiscriminately. The Hautbois is derived from Fragaria elatior, a native of woods in Britain; the Green pine from Fragaria collina, a native of Germany. The varieties of cultivated strawberries, up to the beginning of the present century, were meagre in the extreme to those of the present day. Langley, in his "Pomona," published in 1729, enumerates only three sorts, and says, "The several kinds of strawberries worth our notice are the Scarlet, the Hautbois, and the Wood strawberry." In 1842 the London Horticultural Society published a list in their "Fruit Catalogue," extending to thirty-one recognised sorts, of sufficient merit to justify their cultivation, besides about as many considered worthless, with an immense host of synonyms. A supplement has recently been published to that Catalogue, wherein twenty-nine new sorts of merit are enumerated, making somewhere about sixty sorts in cultivation. The strawberry, which is the most delicious and wholesome of all berries, is only to be found in perfection in northern climates. It appears to have been unknown to the ancient Greeks and Romans, although they were acquainted with most of the fruits we now possess. We have no recollection of its being even alluded to by either their poets or historians; it has been left to a modern bard to sing its praise in true pastoral style-

\section{"A dish of ripe strawberries, smothered in cream."}

Downing, the American pomologist, in speaking of the excellence of this fruit, remarks, "We have heard of individuals who really did not, by nature, relish strawberries, but we confess that we have always had the same doubts of their existence as we have of that of the unicorn." The botanical name Fragaria is in allusion to its fragrant or perfumed fruit, and not, as some have it, from fragaris, to break, from a supposed property in the fruit of dissolving or breaking the stone in the bladder. The Scarlet, or Virginian, was the earliest introduced to Britain, and the Chile succeeded it in 1727, either of which must have been the strawberries referred to by our older writers. It is singular that Langley makes no mention of this last sort, seeing it was introduced two years before his "Pomona" was published. The fruit, or rather more properly the fleshy receptacle usually called the fruit, with its seeds placed on the surface of the pulp, and not, as in general in the case of berries, within it, is held in deservedly high estimation, both as a luxury, and also for its medical properties. The fruit soon becomes almost entirely soluble when taken into the stomach, and neither there, nor when left in heaps to rot, does it undergo the acetous fermentation, which accounts for its being eaten in moderate quantities in perfect safety even by the most delicate persons.

Propagation.-Strawberries are reared from seed, and also more generally by young plants formed on the runners at almost every joint, and sometimes, but rarely, by suckers taken from the sides of the established plants. The advantage of this mode of increase is not very apparent. The two former are the most natural, and unquestionably the best.

Propagation by seed is adopted when new or improved varieties are wished for, although with some kinds - the Alpines and Wood strawberries, for example-it should be followed in preference to any other. Some excellent cultivators also rear other early-bearing sorts from seed, with a view to produce a late crop the autumn following; they being sown in spring, and no doubt, under favourable circumstances, that result is effected, although it has been asserted by the late Mr Williams that such fruit is very deficient in flavour, probably owing to the diminished temperature and decreased sunshine. The process about to be described for Alpines is applicable to the other sorts also, the first process being sowing the seed, which should be taken from the finest specimens of the fruit when it has attained its fullest degree of ripeness. The pulp should be bruised down with the hand into a vessel containing water, and as the pulpy matter is reduced the seeds will separate from it; and thus, by repeated washing, and the seed falling to the bottom, they may be collected, dried, and preserved in paper bags till spring. About the middle of March a slight hotbed should be prepared of leaves in a state of moderate fermentation, over which a bed of rich soil should be laid to the depth of 9 inches, rendered as level and smooth as possible, upon which the seeds are to be thinly sown, and covered with very firm fine mould to the depth of an eighth of an inch; the frames and glass-lights should then be placed over the whole ; very slight waterings should be administered until vegetation commences, and the water applied in a tepid state. When the young plants appear above ground, ventilation must be attended to; and if they come up too thick, they should be moderately thinned; for it is of great importance to obtain stout stocky plants, which never can be the case with any seedlings, if crowded too thickly at first. In the south the hotbed is seldom em. ployed, the seed being sown on a well-exposed border, and in some cases even on one with a northern exposure. We have found it most successful in the North to sow on heat as above. From the end of June to the middle of August the plants will be in a proper state for trans. planting, and the situation best adapted to them is a northern border, with a rich and moist soil. They should be planted in rows 2 feet asunder, and a foot distant in the line. The Alpines come into bearing at a much earlier age than most others, nor is it found that they continue to be productive so long; and hence this rear. ing from seed is exceedingly well adapted to them, besides continuing the season of the fruit to a much later period. The Wood strawberries are in character very -similar to the Alpines, and, like them, are best reared from seed, only the process of sowing should take place as soon as the fruit is ripe, choosing a bed of rich soil; and when the plants are so large as to be fit to 
handle, they should be transplanted into another bed, to gain strength and stand over the winter. The March following they should be transferred to the ground where they are to come to maturity, and be set in rows 2 feet apart, and 15 inches asunder in the line, thus having three rows in a bed, with a 3 -feet alley between, to admit of the crop being gathered without treading on the fruit. The soil should be trenched $2 \frac{1}{2}$ feet deep, placing manure near the bottom of the trenches, for their roots will descend to that depth, and derive nourishment from it. Strawberries should never be planted on the same ground that has been cleared of a similar crop; depth of new soil and abundance of room is of the utmost importance in securing success with every description of this fruit.

Propagation by runners.-The young plants which spring from the joints of the runners or stolons should be carefully preserved from the time they make their appearance, until they become rooted and fit for removal from the parent plant. It is during the process of gathering the fruit, and watering, as advocated by most, and extensively practised by the London market-gardeners, that the young plants are most exposed to injury by being trodden upon. On this account the importance of allowing plenty of room between the rows or beds must be sufficiently apparent. On securing well-rooted plants as early in the season as possible depends much of our success in the cultivation of this fruit; and this has even led some good cultivators to peg down the runners as they advance along the edges of the rows, leaving a space clear in the centre for walking upon. As soon as the young plants are sufficiently rooted they are cut off from the parent plant, the roots carefully extracted by loosening the soil with a fork. They are then transferred to nursery-beds, prepared by deep digging, and 4 feet broad, the plants being set at distances apart according to the size of the foliage-thus Cuthill's Black Prince, which is the smallest in foliage of modern varieties, is set 6 inches apart plant from plant, while the British Queen and similar strong-growing kinds are allowed 9 inches. These remain in such beds till the month of October, when ground is prepared for them, in which they are to remain to perfect their fruit. This ground is prepared by trenching from $2 \frac{1}{2}$ to 3 feet in depth, placing undecayed manure in the bottom of the trench, which bottom is previously torn up by a pick ; manure is again applied when the trench is about half full, and again when within 9 inches of the surface. The plants of the largegrowing sorts are carefully taken up with a trowel, fig. 27, retaining as much of the soil as possible about the roots, and are planted in lines 2 feet apart, and 18 inches in the line, leaving a 3 -feet alley between each three rows. Those of less size, such as Cuthill's Black Prince and the Grove End seedling, the only small-growing sorts we grow, are set at the distance of 18 inches between the rows, and 12 in the line, with alleys 3 feet wide between every three rows. By this process we have abundant crops the following season. This may appear a waste of space, but it is not so if sufficient justice is to be done the plants. Even a greater distance than this is given in the Royal Gardens at Frogmore, where the finest strawberries we have ever seen are yearly produced. Some'growers sink small pots in the spaces between the rows, and allow the runners to root in them, and, when this is effected, they are turned out of the pots, with their roots and ball entire, into the ground, where they are to remain to produce their fruit. Many plant in beds containing two rows each, 18 inches apart, and the same distance plant from plant. These narrow beds are made with a view to render the fruit more easily gathered, and to prevent treading on the bed, all the operations being carried on from the broad alleys between the beds.

Soil and situation.-In old garden-soils the strawberry is seldom found to do well ; indeed, in some it refuses to grow, while in land newly broken up into cultivation, if of sufficient depth and staple, and moderately enriched, they as invariably do well. As such conditions, however, cannot often be provided, recourse must be had to deep-trenching, or burying the old soil, and bringing towards the surface that which has either not produced strawberry crops at all, or has been for some years lying undisturbed. In light sandy soils, strawberries are shortlived, and produce small fruit; while in rich mellow loams they live longer, and produce the best fruit. In strong clays some varieties refuse to grow, while some others, like the Grove End, thrive amazingly. Depth of soil is an essential point, for their roots will descend to the depth of $2 \frac{1}{2}$ or 3 feet; manure, therefore, should be deeply buried, so as to be placed within reach of the spongiolets. In regard to situation, although many sorts will grow under the shade of trees and bushes, still the fruit is never of the flavour it would be if planted in an open place fully exposed to the air and sunshine. Where the inclination is considerably towards the south, the fruit will be earlier produced; and not only are such situations frequently chosen, but artificial banks have been thrown up, presenting an angle of even more than $45^{\circ}$ towards themeridian. Strawberry walls, and prepared bedswalled up with brickwork or masonry, to form reservoirs for water, as well as alleys between the beds, sloping banks covered with stones placed between the plants, and various other contrivances, have been recommended, involving considerable expense in their erection, and producing little advantage over well-prepared plantations in the opcn garden. No doubt the reflected heat from stones, when so placed, has some effect on the ripening of the fruit, and their covering the surface tends greatly to retain moisture in the ground by lessening evaporation. But when we consider that our best cultivators now only allow the plants to occupy the ground for one crop, and seldom more than two, and that new stations are afforded every change of plants, it surely cannot be worth the expense of forming such erections, to be pulled down and reconstructed every alternate year. The same effect can be much easier secured by placing along the sides of the rows a course of tiles or narrow slates 
just as the blossom is beginning to appear, and on these the trusses of fruit will lie quite dry, secured from the splashing of mud during heavy rains, the moisture will be retained at the roots at the time it is most required for the plants, and all the advantages of increased heat will be secured to the fruit which more cumbrous and expensive means can afford. As soon as the crop is gathered, these tiles or slates should be removed, as being no longer useful. A refinement on this mode of preserving the fruit was proposed a few years ago by Mr Roberts, who described his principle in a pamphlet. Of the utility of his plan there can be little doubt, were the expense of tiles employed not considerably greater than the value of ten crops of fruit. Fig. 243 represents Mr Roberts' plan : $a$ a bed

Fig. 243.

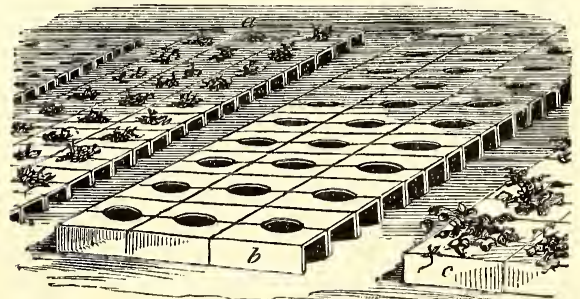

ROBERTS' MODE OF GROWING STRAWBERRIES

of young strawberry plants, with the tiles placed around them; $b$ shows a bed with tiles placed down without the plants, $c$ shows the end of a strawberry bed, with the fruit and foliage upon the tiles. In this latter circumstance, the utility, in our estimation, of Mr Roberts'principle only consists. To place them around the young plants must be obviously injurious, as they would deprive the roots of a considerable quantity of rain during their growing season. But to place them around the established plants, just as the blossom is appearing, is as obviously an advantage, as the fruit would lie dry and clean on their upper surfaces. These tiles, as shown at $b$, have a semicircular hole cut out of their inner sides respectively, so that in placing them around the plant, the leaves being carefully held up, one tile is placed on one side, and another on the other. Another advantage these tiles have over paving tiles or slates, as noticed above, is, that they stand on flange-like edges, thus allowing a circulation of air to act under them, and prevent ing the cold and damp from affecting them, as to some extent would be the case were they laid flat on the ground. Glass tiles have also been recommended, but their expense precludes them from general use, as reflectors of heat only. They might be employed, as well as circular earthenware tiles, with great advantage, if made the base on which to set large bell-glasses (fig. 36), now dignified with the French designation of Cloches, and within these few months brought into notice by the greatest horticultural oracle of the age as a new and excellent improvement, forgetting, or probably not knowing, that similar glasses were in uso amongst the
London market-gardeners by the hundred more than a century ago. Were each strawberry-plant provided with either an earthenware or glass tile, constructed as shown above by Mr Roberts, and covered with a cloche, if we must call it so, the advantage of accelerating the ripening of the fruit, as well as securing its preservation, and, no doubt, adding both to its size and flavour, must be apparent. We do not, however, mean to recommend the whole strawberry plantation to be covered in this way, but only such sorts, or such number of plants, as may be wished to ripen earlier. There are some of the finest new sorts that will not prosper unless in the warmest and most favourable localities. With such means, we believe, even these would ripen to perfection in the coldest parts of Britain. Cloches are offered, suitable for this purpose, at $£ 10$ per 100 . Nor is it only for this purpose that they are useful ; it is by means of them, in a great measure, that the Parisian gardeners are enabled to produce such fine lettuces, \&c., both late in autumn and early in spring.

The main crops of strawberries should be planted in the open quarters of the garden, where they may be fully exposed to the sun, and apart from the shade of trees or walls, unless in the case of a plantation of the Ellon pine, Comte de Paris, Myatt's Eleanor, or other late sorts, as also the Alpines, plantations of which should be formed in northern borders, or in as cool and somewhat shaded situations as the garden affords. The great advantage of forming the plantation in the open quarters is the rotation the change of site offers, the convenience of covering the crop with nets, and also the appearance of order and system in the arrangement. There are other modes recommended for securing late crops-that of Williams is to cut off all the blossoms of the Alpines early in May. This is again repeated at the end of the month. Towards the end of June more blossoms appear, and continue to be produced, yielding fruit until cut off by the autumnal frosts. Others have recommended late planting, say during the month of May, and at the same time choosing late-ripening sorts. Flavour is, however, greatly sacrificed by all these means, if we except the Alpines and Wood strawberries, which maintain their flavour little impaired until killed by the frost. These we have more control over than any other sorts; for, being reared from seed annually, the process of sowing may be deferred to a later period than we have stated above. It is also a good and simple way of prolonging the season of this fruit, particularly with the Alpines and Woods, to plant them in 6-inch pots when removed from the seed-bed, and to plunge them in a cold north border, taking them up in September, and placing them in pits or frames covered with glass. In this way we have gathered Alpines nearly up to Christmas.

Further, in regard to the preservation of the fruit during its growth and ripening, some have recommended laying tiles painted black around the plants ; this, however, can have little effect in increasing heat, as the foliage will cover the tiles and prevent the direct rays of the sun from falling on them. Many lay straw between the 
rows with a view to keep the fruit clean, and this practice has been almost invariably followed for an unknown length of time, and, indeed, is by some supposed to have given rise to the name strawberry, to distinguish it from gooseberry, the young fruit of which is used for sauce for young geese, and raspberry from the rasplike appearance of its wood. Others spread long littering-dung between them, and many the sweepings of grass lawns. These, however, are all objectionable, on account of the harbour they afford to snails and slugs. In wet seasons they tend more to encourage mouldiness and damp than any presumed good they effect. New tanner's bark is less objectionable in these respects, and has the effect, which we believe has not been very satisfactorily accounted for, of preventing the growth of weeds. The refuse of flax-mills has also been used, but, like the sweepings of lawns, the short fragments of the woody structure of the flax plant, and the short leaves of the grass, are apt to adhere to the fruit, and often to spoil it. Long narrow boards are sometimes laid along the rows; this has its advantages, as has also laying twigs of birch, branches of furze (whin), and heath. These, while they support the fruit from the ground, afford less shelter to snails and slugs, and keep the fruit drier. Some support the trusses of fruit by sticking in a row of small twigs on each side of the row, keeping the fruit-stalks in an upright position ; - this is a direct violation of the natural habit of the plant, the fruit of which, if we except the bush and common Alpines, lies close to or reclining towards the ground. The bush Alpine, of which there is both a red and white variety, grows upright, and throws out few or no runners, and the fruit is borne on upright fruit-stalks, showing above the leaves.

General culture. - Renew the plantations yearly, or every other year; avoid the barbarous custom of cutting off all the leaves in autumn, as well as of digging between the rows. If manurial applications be absolutely needed, apply them in a liquid state, and that so early in the season as to precede the appearance of the blossom. Watering, upon which so many rest much dependence during the swelling of the fruit, should also be avoided, as being wholly uncalled for in a properly prepared soil of 3 feet in depth. All the water applied can scarcely reach the roots at their feeding points, therefore it is useless; but in very dry soils, if subterranean irrigation could be applied during the growing season of the plant, the advantages would be great. The injury the swelling fruit sustains by the operation of watering, unless it could be thrown over the whole plantation by a powerful engine, falling on the plants like a general shower of rain, is tenfold greater than all the benefit. Keep the ground quite clear of weeds during summer by hand-weeding and the occasional use of the hoe, and the shallower this operation is performed the better. The American mode of culture is thus described by Downing, and as it differs essentially from the general methods followed in Europe, it may be proper for us to notice it here. "Early in April, or in August, being provided with a good stock of young plants, select a suitable piece of good deep soil. Dig in a heavy coat of stable manure, pulverising well, and raking the top soil. Strike out the rows 3 feet apart with a line ; the plants should now be planted along each line, about a foot apart in the row. They will soon send out runners, and these runners should be allowed to take possession of every alternate strip of 3 feet, the other strip being kept bare by continually destroying all runners upon it, the whole patch being kept clear of all weeds. The occupied strip, or bed of runners, will now give a heavy crop of strawberries, and the open strip of 3 feet will serve as an alley from which to gather the fruit. After the crop is over, dig and prepare this alley, or strip, for the occupancy of the new runners for the next season's crop. The runners from the old strip will now speedily cover the new space allotted to them, and will perhaps require a partial thinning-out to have them evenly distributed. As soon as this is the case- say about the middle of August-dig under the whole of the old plants with a light coat of manure. The surface may then be sown with turnips or spinach, which will come off before the next season of fruits. In this way the strips or beds occupied by the plants are reversed every season, and the same plot of ground may thus be continued in a productive state for many years."

Gathering the fruit.-This should be performed when the plants and fruit are dry, more especially such fruit as is intended for preserving, and these should have the fruit gathered without any portion of the calyx or foot-stalk being left attached; it very much saves the bruising of the berries when sent to the confectioner. Those best adapted for jam and similar preserves are Cuthill's Black Prince, when a rich dark conserve is wished for; and the Grove End scarlet when a bright carmine colour is, as it is most generally, wished for. For these purposes we grow no other sort. For preserving whole, Keen's seedling is in high estimation, being both of a large size and of a good colour. These should be picked also without the stalk, and the best formed and finest specimens only selected. With such fruit as are to be sent for dessert the case is different, as these should have the foot-stalks scrupulously preserved, and cut off with a pair of scissors to the length of an inch or an inch and a quarter, according to the size of the variety. We are glad to see this mode of gathering the fruit becoming more general in Scotland than heretofore; and although the stalks and calyx somewhat tell to the advantage of the grower, the consumer has the satisfaction of believing that the fruit has not been handled by the gatherer. The stalks also admit of their being eaten without soiling the gloves. Those who prefer eating them with port-wine, or sugar and cream, can prepare them for themselves by holding the foot-stalk between the finger and thumb of one hand, and disengaging the fruit from it by using the dessert fork with the other. Strawberries are now so large that they are often eaten with the knife and fork. Those who gather them should put gloves on their hands, and if not, the hands ought to be washed clean. 
Forcing.-The choice of suitable sorts, and potting them as early in the season as young plants can be got, forms the first feature in this operation. The strawberry, being a native of temperate and even cold climates, submits un. willingly to a high temperature; and when they are suddenly subjected to such, the effects become apparent by the slender appearance of the leaves and foot-stalks, and absence of flowers; or should the petals even develop themselves, they are usually unaccompanied by healthy stamens or pistils, and consequently produce no fruit. The sorts we generally force are Cuthill's Black Prince, Grove End scarlet, Keen's seedling, and British queen. They are put into a state of slow excitement about the beginning of December, in the order in which we have placed them above. As early in summer as the young plants begin to show themselves upon the runners, we plunge 3-inch pots in the soil between the rows up to their brim; the runners are drawn over these pots so that the young plant shall be placed in the centre of the pot, and kept in their places by means of small hooked pegs, and sometimes by placing a small stone upon them. The pots are filled with strong rich turfy loam, but not drained. They soon begin to make roots, and in about ten or fifteen days the pot will be full of them, at which time the plants are cut from the runners, and with the pots are removed to an open warm space, where they are shifted into the fruitingsized pots, turning them out with their balls entire. In the case of Cuthill's Black Prince, which is a very small grower, the pots used are $4 \frac{1}{2}$ inches in diameter; with the Grove End scarlet 5-inch pots are used; and with Keen's seedling and British queen, which are both strong growers, $6 \frac{1}{2}$-inch pots are used. One plant only is placed in each pot. The soil used is fresh turfy loam, as strong as can be procured, but not entirely clay. The pots are well drained, and the soil rammed pretty tightly around the roots. We use no manure in the soil unless it is poor, but place in the bottom of each pot above the drainings 2 inches of rotten cow-dung, and water frequently with liquid manure. When the plants are potted, which they will be progressively, as young ones are procured, though no time should be lost, as the earlier they are potted the better, they are set upon a dry flooring of sifted coalashes, well soaked with lime-water for the destruction of worms, in beds of a convenient breadth, the pots standing quite close together, and each size arranged byitself. They are watered freely overhead with clear water applied through the rose of a watering-pot, and kept in this position till the frosts begin to set in, when they are placed in cold pits and covered with glass. As all have not the extent of pits that we have, other expedients must be adopted, our object being to preserve the pots from being broken by the frost, as well as to keep the soil in them dry and the roots safe from injury. Triangular banks may be thrown up, and the pots plunged lying on their sides, with their mouths outwards; or the pots may be built up against a wall, facing any aspect but the north, the uppermost course being protected by boards laid along. The usual way, however, is to plunge the pots in a bed of soil; but we have invariably found the plants to root less freely in this way than in the others, and as abundance of roots is an important consideration, every means to encourage them should be followed. If plunged at all, nothing is better than sifted coal-ashes.

In former times it was advised to pot the runners in July or August, and to nurse them in pots for two seasons, pinhcing off all blossoms as they appeared. Three plants were also directed to be set in each pot. The former is labour thrown away, and the latter erroneous in principle. It is better to use smaller pots, and to place one plant only in each ; for it frequently happens, from one cause or another, that one if not two plants out of the three may become blind,- that is, they may not show bloom at all, or perhaps only in a very weak or imperfect state, leaving only one useful plant in each large pot. Plants, also, of two years' standing were not unfrequently used, taking them up in October, and potting them with large balls into 9 or 10 inch pots. With Alpines a more rational mode was followed; namely, to sow the seed in pots in January upon a gentle heat, hardening them after they had attained the height of an inch and a half by placing them in a cooler situation, and then potting them in May into 6-inch pots. In October they were in flower, when they were placed under the shelter of a cold glazed pit, and about the latter end of November placed in a forcing-house or pinery. These and similar plans have now given way to that of plants rooted as we have described, grown freely during the summer and autumn, and forced the following winter. We also take the strongest runners as early in the season as they are pretty well rooted in the soil between the rows, and plant them in small pots, placing them in close pits or frames until the roots have filled the pots, and then shifting them into pots of a size suitable to the variety. Old plants may, however, be taken up carefully with their roots entire, and placed in a tanked pit pretty closely together, working in soil between the balls. The operation should be performed early in October, and a very slight degree of bottom heat applied till the end of November, when they will be found well rooted, and in a condition to commence growth upon the application of an increased degree of warmth from the tank. The object of this is to give the roots the start of the leaves; and to insure this while the roots are enjoying a temperature of $55^{\circ}$ to $60^{\circ}$, the tops should be kept at $40^{\circ}$ or $45^{\circ}$, and this can readily be done by abundant ventilation, even to the extent of keeping the lights off altogether during the mild weather, and only putting them on when the atmospheric temperature falls below these points. Where the convenience of tanked pits, or those having pipes in a vault below, is not at hand, then the pots may with great propriety be plunged in some material, such as tan, or leaves undergoing a steady fermentation. Indeed, a bed of any fermenting material, or of leaves, constructed about the middle of October, and the strawberry plants in pots plunged in it without any covering whatever, would be found 
of vast advantage, particularly for the earliest crops. To take the plants at once from the open air, or even from a cold pit, and place them on shelves in a structure where the atmospheric heat is $60^{\circ}$ or $70^{\circ}$, is as unnatural as anything that can be devised. When they have been root-excited as above, they may then be removed to the structure in which they are to fruit, beginning with a temperature by day of $55^{\circ}$, and at night falling to $45^{\circ}$, by fire-heat, admitting of a rise during sunshine of $10^{\circ}$ or $15^{\circ}$ for an hour or two only. In such a temperature, and under the root-exciting principle, the plants will push strongly, and throw up strong flower-stalks, if kept sufficiently near the glass. As one set of plants is removed to the fruiting structure, another should take their place on the bottom heat, to be preparing for their final removal.

Some have recommended retaining the plants that were forced at the most advanced period of the season, turning them out of the pots, and planting them in the open air, and in autumn following to take them up and re-pot them for forcing during a second winter. We have tried this method repeatedly, but with very indifferent success. The usual time for bringing the strawberry into the forcing-house is from the beginning of January to the end of February; but where ripe fruit is required early, the first set is brought in about the end of November.

Strawberries, forced so as to ripen in April, and when the fruit has been gathered planted out, not unfrequently produce a fair crop late in the autumn; and, should they not begin to show their flower-buds till so late as to prevent a chance of the fruit coming to perfection if carefully lifted and planted in large pots, and brought progressively into a top temperature of from $45^{\circ}$ to $55^{\circ}$, will often yield an excellent crop of fruit through the early part of winter, and before those treated in the usual way could be got sufficiently advanced. A bottom heat of $60^{\circ}$ should be maintained, and the glasses kept shut close down until the roots have begun to grow, when air should be more abundantly supplied, particularly during their flowering period, at which time the atmospheric temperature should be gradually raised to $60^{\circ}$ during the day by fire-heat, falling during the night to $55^{\circ}$. When the fruit is fairly set and swelling, a degree or two more should be allowed, and $10^{\circ}$ or $12^{\circ}$ during sunshine for a few hours daily. As soon as they begin to throw up their flowertrusses, remove all the weakest, and also such plants as show no flower-buds at all. If plants be thus carefully taken up, and not subjected to too high a temperature, or deprived of sufficient air, they will produce excellent crops at much less expenditure of labour in watering, \&c., than when grown on suspended shelves in the usual manner. The elaborated sap contained in such plants must be much greater than that found in young plants of only a few months' growth, which of itself must tend greatly to the development of both flowers and fruit. Great care must, however, be taken to preserve the roots as entire as possible, for at the lifting season they are charged with organisable matter for the nutri- tion of the plant when its growing season arrives; if, therefore, they are destroyed to any extent, so much of this nutrition is withdrawn from them.

In placing strawberries in forcing-houses, they should be set upon shelves suspended from the roof, and as near the glass as possible, even should their leaves almost touch it. The feeders under the pots should be half filled once a-week or so with weak liquid manure, and as soon as the fruit is set, the trusses should be supported erect by sticking small twigs in the soil for the fruit to recline upon ; without this support the weight of the fruit would cause the fruit-stalks to bend downwards over the edges of the pots, and hence greatly interrupt the free ascent of the sap at a time when the plants require it in greatest abundance. Strawberries on suspended shelves require abundance of water, but this must not be applied in excess. The smallest and misformed fruit should be carefully cut away with a pair of sharp-pointed scissors as soon as they show themselves; the nutriment which would be wastefully taken up by them will thus be directed to the larger and more perfect specimens.

Regarding the most appropriate structure for forcing this fruit, pits have advantages over larger houses, as the plants may be left undisturbed from the time they are finally potted until the fruit is gathered, as bottom heat can be applied to the roots, while ventilation, or a removal of the sashes, will place the tops in the condition stated above. Fig. 673, vol. i., offers a good example of this sort of pit; a reference to it will show that it can be heated either by hot-water pipes or dung linings. Fig. 649 exhibits a tanked pit, with supplementary hotwater pipes to afford atmospheric heat, after the plants are fully established at the roots by the heat from the tank alone. Fig. 638 shows a strawberry-house or pit exceedingly well adapted for fruiting them in, the plants being set on suspended shelves close to the glass, after they have been removed from the bottom heat recommended; and an equally good example is offered at figs. 629 and 632 , where both bottom and top heat is completely at the command of the cultivator.

\section{SELECT LIST.}

After the establishment of the London Horticultural Society, the numbers of varieties sent for examination, and many of these under a dozen of names, induced the directors to draw up a classification, which was at first done by Mr Barnet, and afterwards improved and completed by Mr Thompson, his successor. That arrangement divided them into seven classes, as follows :-

Class 1, Scarlet strawberries-Class 2, Black strawberries-Class 3, Pine strawberries-Class 4, Chile strawberries-Class 5, Hautbois-Class 6, Green strawberries - Class 7, Alpine and Wood strawberries.

Of Class 1 the following may be considered the best:-

Grove End scarlet.-An abundant bearer; fine 
vermilion colour; much esteemed for preserving; size large for a scarlet; somewhat spherical and depressed; flesh palish scarlet; of an agreeable sub-acid flavour; in perfection during the end of May and towards the middle of June, somewhat later in Scotland, continuing to bear during three weeks or more in succession; leaf more than usually wide in the serratures. Raised from seed by the late W. Atkinson, Esq., in his garden at Grove End, about the year 1820. Synonym-Atkinson's scarlet.

Cuthill's Black Prince.-This is unquestionably the earliest strawberry in cultivation; an excellent bearer, and, so far as our experience goes, of all others of its class the best for early forcing. Fruit conical; colour rich and darkish ; flavour excellent. The habit of the plant is dwarf and slender; leaves small, and slightly glaucous beneath; foot-stalks of the leaves and stolons of a peculiar reddish colour; trusses of fruit often as large as the whole plant. The flowers remain often attached to the calyx till the fruit is ripe; many of the petals tinged with red. Synonym - St Andrew's pine.

Cuthill's Prince of Wales.-We have some doubts whether this should be classed as a Scarlet or Pine; this, however, in no way affects its excellent merit of being one of, if not the latest in ripening, which renders it equally valuable with the last. If planted in a northern border in the neighbourhood of London, it will bear fine fruit up to the beginning of September, and a fortnight later in most parts of Scotland, continuing much longer in use than the Elton pine, hitherto considered amongst the latest of our strawberries. Colour scarlet; form conical; flavour excellent; size nearly equal to that of Keen's seedling.

Coul late scarlet.-Form ovate; size large for its class; quality excellent, and an abundant bearer; ripening, in the neighbourhood of London, about the middle or end of July, consequently later in Scotland. Synonym-Sir Geo. $M$ 'Kenzie's late scarlet, with whom it originated at Coul, in Ross-shire.

old scarlet. - Form roundish; size medium for its class; flavour excellent; ripening from the middle to the end of June, and much sought after by confectioners on account of its fine colour, which, however, is in this respect rather inferior to the Grove End. Synonyms-Scarlet Virginian, Virginian, Scarlet, Early scarlet, Original scarlet, Uld Bath searlet of some. Disappearing rapidly from cultivation, and, with the other Scarlets, will soon be found only in private gardens, the large manufacturing confectioners preferring the Keen's seedling, a very inferior fruit for the purpose.

Roseberry.-Fruit conical ; large for its class; flavour excellent; plant of slender growth; an abundant bearer, continuing long in succession; ripe about the end of June and beginning of July. Also valuable as a preserving fruit, and forces well. It was raised from seed, at Aberdeen, about thirty-five years ago. SynonymsScotch scarlet, Aberdeen, Aberdeen seedling, Rose (from the original plant being found under a rose-bush), Prolific pine, Brickley scarlet.

Blacle strawberries.-Myatt's pine.-Fruit oval; size large; quality excellent; ripe about the end of June; flavour exceedingly rich, and, notwithstanding all that has been said, very good bearer, particularly during its second year.

Elton.-Form ovate; often depressed or cock'scomb shaped; size very large; quality excellent ; one of our very best late sorts, ripening about the end of July and beginning of August in the neighbourhood of London; in perfection during August and early part of September in Scotland, particularly when planted on a northern border; colour dark shining red when exposed to the sun, palish where shaded by leaves; a very great bearer. It requires to be thoroughly ripened, else the natural acidity will be predominant. Synonym-Elton seedling.

Downton.-Form oval ; size large. "The best of its class," Mr Thompson remarks, "all its properties taken into consideration." One of Knight's numerous seedlings, ripening about London during July, in Scotland during the first fortnight of August. Readily distinguished from any other strawberry by the form and colour of its leaves. Synonyms - Knight's strawberry, Knight's seedling, not often now met with in gardens.

Pine strawberries.-Myatt's British queen.One of our most popular strawberries, and deservedly so, being high-flavoured, of large size, and an immense bearer, as well as very handsome fruit, weighing often from one to two ounces each, and in some cases measuring 5 inches in circumference. The habit of the plant is strong, therefore requiring plenty of room, even to the extent of $2 \frac{1}{2}$ feet between the rows, and 18 inches plant from plant in the line. It is, nevertheless, a rather tender variety, requiring to be grown in a good climate or on a warm border, and in cold situations should be protected during winter by laying littering-dung between the rows and around the plants. It forces remarkably well, but for this purpose is better adapted for the later crops than the very early. It is not improbable that already good and inferior varieties exist of this excellent fruit, a circumstance not at all unfrequent amongst strawberries, when left for more than two years in the same place, as seedlings may spring up amongst the older plants unobserved. And if seedlings are designedly raised, this may to some extent account for this strawberry succeeding well in some places and not in others. That keen observer of such matters, Mr Cuthill, of Camberwell, has rather more than hinted at this, in his remarks on the superior crops produced by Mr Beach, of Worton. "I am not certain," he says, "that Mr Beach's Queens are not altogether different from those of others, as I myself have often found my beds sport." The finest crops of this variety are produced in newlycultivated, deep, alluvial, loamy soil, such as that in the Royal Gardens at Frogmore, where this strawberry prospers amazingly. It, however, does not dislike a light soil, provided it is rich and trenched very deep; in such it produces excellent crops of very large fruit in the Dalkeith gardens.

Myatt's Eliza.-Form oval; size medium for its class; quality good. Ripens about the middle 
of June around London, and the beginning of July in Scotland. A good variety.

Myatt's Deptford pine.-Fruit roundish ovate; size large, and quality excellent. Ripens about the end of June about London, and toward the middle of July in Scotland. The leaves are very downy on the under side.

Myatt's Eleanor.-Fruit ovate, often compressed; size large; quality good; an excellent bearer; and although ripening during July about London, is found by experience to be the best and latest of all the large sorts cultivated in Scotland, the fruit occasionally attaining the weight of $2 \frac{1}{2}$ ounces, and bearing during the end of August and part of September.

Myatt's mammoth.-Fruit irregular roundish; size very large, which is its chief merit. Ripens about the beginning of July about London, towards the end of that month in Scotland.

Keen's seedling.-Fruit large, round, or ovate, often compressed on the sides, and assuming a cock's-comb appearance; colour dark purplish scarlet; flesh scarlet, firm, with a pleasant flavour. It is not only an early variety, but a most abundant bearer and excellent forcer, and at present more extensively grown in commercial gardens than any other. It has been the most useful strawberry of its date of origin, but even with all its excellent qualities, in favourable climates it will likely give place to the British queen, or others of more recent date. Synonyms-Keen's black pine, Keen's new seedling, Keen's new pine, Murphy's child.

The old pine, or Carolina.-Fruit large, oval, or conical, occasionally cock's-comb shaped; colour bright scarlet; flesh palish, firm, and juicy, with a very rich flavour; excelled only, in our estimation, by the British queen in the class to which it belongs. It is described in the "Pomological Magazine" "as a good bearer on suitable soils and in proper situations. It is so firm, and at the same time so abundant in rich juice, that its greater weight is almost sufficient to distinguish it when compared with an equal quantity of any other sort. Its leaves are also of a darker green than those perhaps of any other kind of strawberry. It ripens later than the preceding sort, and although not so very productive as the one just alluded to, yet its superior richness renders it highly deserving of cultivation." In this description we perfectly concur, and regret to add that so truly excel. lent a variety should be neglected for others of far less merit. We have forced this strawberry most successfully. This variety is believed to have been introduced to Britain many years ago from Carolina, and nearly all the class of Pines have been directly or indirectly raised from it. Thus it has become the parent of some of the very finest strawberries we have. Synonyms-Scarlet pine, Old scarlet pine, Old Carolina, Common Carolina, Carolina pine, Large Carolina, Carolina, Blood pine, Pyne hill, Pine, Grandiflora, Black pine, Borsdorf, Surinam or Scarlet-fleshed of some (but not the True Surinam), Superior, Shew's scarlet, Kew pine, Windsor pine, Nortl's seedling, Devonshire scarlet pine, Varnished, Barham pine, Regent's favourite, Cock's-comb pine, Miss Gunning's pine.

VOL. II.
Ingram's Prince of Wales.-Fruit of large size, fine shape, and of a beautiful glossy red colour; flesh light red, solid, very juicy, and of exquisite flavour. Ripens ten days earlier than the British queen. The fruit bears carriage well, and consequently it will be a useful market variety. The plant is of free growth, compact habit, hardy, and very prolific. Raised by Mr Ingram of the Royal Gardens at Frogmore in 1849, and there extensively cultivated for forcing, openair culture, and also for autumnal bearing.

Comte de Paris.-Fruit roundish; size large; quality good. Ripens about London from the beginning to the middle of July; ten days later at Dalkeith. An excellent variety, nearly allied to the Elton.

Kittley's Goliath.-Fruit roundish ovate; size large; quality good. Ripens about the beginning of July; ten days later in Scotland, where it bears abundantly.

Horey's seedling.-Fruit very large, roundish oval, or slightly conical; deep shining scarlet; seeds slightly imbedded; flesh firm, with a rich agreeable flavour. This is an American variety, raised by $\mathrm{Mr}$ Hovey, an enterprising nurseryman of Boston. The plants are unusually vigorous and hardy, producing very large crops, and the fruit is always of the largest size and finely flavoured. The leaves are large, rather light green, and the fruit-stalk long and erect. Considered in the States to be the finest of all their large strawberries, and succeeds well in Britain wherever it has been tried. It is nearly allied to Keen's seedling, which is no bad recommendation of it.

Ross's phonix.-Fruit very large, generally cock's-comb shaped or compressed; colour very dark purplish red, with a shining surface ; flesh firm, of the richest pine-flavour. This is another American strawberry of great merit. It is described by Downing as having " all the good qualities of Keen's seedling, with the great additional ones of being perfectly hardy, and always bearing most abundant crops." It appears to be a very hardy variety, and might be suitable in climates where the large new kinds would do little good. It was raised from seed of Keen's seedling in 1837, by a Mr Ross, of Hudson, New York. It resembles the parent, but the leaves are broader, very dark green, with very coarse serratures, and lie close to the ground.

Princess Alice Maud.-Fruit roundish; size large; quality excellent. Ripe from the middle to the end of June; in many respects resembles Keen's seedling.

Trollop's Victoria.-A first-class fruit of great size, great bearer, and well adapted for forcing.

Nicholson's Ajax.-A first-rate fruit, of large size and excellent flavour. Mr Cuthill, in bearing testimony to its merits, says, that nine fruit of this variety, after two days' travelling, weighed 16 ounces.

Princess-royal.-Fruit ovate; size large; quality good. Ripens about the end of June in the neighbourhood of London.

Prince Albert.-Size very large; form ovate; colour rich scarlet; flesh rather soft, and not so bigh flavoured as the British queen, and some others of the recently produced large sorts. It 
has the merit of being a good bearer, which, with its being a large showy fruit, renders it well fitted for market purposes.

Chili strawberries.-Of the varieties in this class Mr Thompson observes, "It may be said of the Chilis generally that they are tender, bad bearers, and of indifferent quality." We think rather otherwise, and consider the true Chili to have been the best of all the large strawberries of its day, and by no means a bad bearer. No doubt they are superseded now in size and flavour by more modern varieties, such as the British queen, \&c. There are, however, some extraordinary strawberries reported as indigenous to Chili, which would be worth introducing to our gardens. Wilmot's superb, a coarse and woolly variety, originated from this class, was the first variety of extraordinary size produced in this country. It is probably now lost in cultivation, as well as most of the varieties of Chilis.

Hautbois strawberries. - Haut-bois, literally High-wood-that is, Woodstrawberries, with high leaves and foot-stalks. The highest-flavoured strawberries belong to this class; they have, nevertheless, of late fallen into disrepute from a supposed defect in their parts of fructification, by reason of which many plants appear sterile; or, should they set their fruit, it, from some rather obscure cause, does not swell, and, consequently, comes not to its natural maturity. From whatever cause this defect arises, it is quite certain that, in former times, they were noted for their abundant crops. Mr Thompson, in "Catalogue of the Fruits in the Horticultural Society's Gardens," thus remarks of them: "Of all strawberries the Hautbois are the most variable. They certainly retain a general character, from which they naturally do not depart; but constancy in character in varieties of this class, denominated as distinct, is but little to be depended on. The fruit will occasionally change from globular to ovate, and the contrary; while fertile plantations will produce runners that may perhaps be sterile, and seedlings, many of which will certainly prove so. The latter ought to be carefully looked after and extirpated, which can only be effectually done in new plantations whilst the plants are single, and at the time they are in blossom. It will also be necessary to observe, that in all sorts of Hautbois here enumerated there exist both the prolific, and also those sterile plants commonly called males, which have long stamens. They may be distinguished from the prolific by their flowers being scarcely so large, with the receptacle small and imperfect. The plants producing such should in all cases be destroyed." The same authority further states: "I believe there is no such thing as distinct plants of male and female Hautbois. Stamens and pistillums are to be found in either a perfect or imperfect state in every individual flower. Imperfection generally takes place in the pistillum, together with the receptacle; to see that these are sound is all that is necessary to be attended to." If Hautbois had been pro pagated by seed more than any other variety of strawberry-but of this we have no evidencethe belief might be entertained that generation after generation of them were travelling back to their original state, in which the same defects may exist. Downing, in speaking of barren and fertile plants, observes, and we think with much truth: "A great deal has been said and written lately respecting male and female strawberry plants, and the absolute necessity of planting a due proportion of both, in order to have a crop of fruit. There is some practical value in this notion, but, like most hobbies, it has galloped considerably beyond the boundaries of sober truth." The whole of the matter lies in selecting the young plants produced by old ones which have perfect flowers - that is, blossom, containing both stamens and pistils or both male and female organs of generation, which is easily done by any person of common observation while the plants are in bloom, or when they are in fruit, and to reject all young plants produced from those either defective in their organs or having no fruit. Sterile plants have a natural disposition to propagate themselves by runners, much more so than those which are producing fruit ; they have not the same draught upon their energies that fruit-bearing plants have, as the latter have to supply nutriment to both the fruit and its new progeny. These views were entertained by Downing and George Lindley, the former of whom says: "Carefully select, when there is a tendency to sterility in the plantation, only runners from the most fruitful perfect plants. This is easily done, by choosing such plants when in blossom as have a natural proportion of stamens and pistils, or such as, when in fruit, show large well-developed trusses of fruit. Set a little stake down by these, and take runners only from them for your new plantation. In this way (and we have seen it repeatedly proved) good plantations will be secured, with every plant productive." If people, therefore, will, from sheer carelessness, continue to propagate from runners indiscriminately, they have themselves to blame if this excellent class of strawberries prove unproductive.

Large flat hautbois.-Form roundish, or approaching to hemispherical ; size large, palish red next the sun, very pale where shaded; flesh firm, greenish white, highly perfumed; an abundant bearer; retains its character better than the other sorts. This is indeed one of the finest of all strawberries; but as some people have a dislike to the musky flavour of the Muscat grape, while most others like it, so with this class of strawberries. Synonyms-Bath, Formosa, Weymouth, White, Salter's, Lowder's.

Prolific, or Conical.-Fruit medium for this class, conical ; dullish purple; flesh firm, rich, and highly perfumed; ripening about the beginning of July ; an abundant bearer; flowers the largest of its class ; stamens long, continuing round the base of the fruit even to the period of its ripening. Synonyms-Musk, Hermaphrodite, Spring grove, Double-bearing, Dwarf, Hudson's Bay of some, Regent's Sacombe, Sir Joseph Banks', the Caperon royal and Caperon hermaphrodite of the French.

Green strawberries.-In this class there are none worth particular attention.

Alpine or Wood strawberries.-This class is 
remarkable for the abundance of their produce, although the fruit individually is small, for a peculiarity of flavour, and for continuing to bear till killed by the frost. Mr Thompson justly observes : "The best of these are the Red and White wood, and the Red and White alpine. The latter are apt to degenerate, and assume the characters of the Wood varieties." The Cisalpine or Napoleon, spoken highly of by $\mathrm{Mr}$ Cobbett in his "English Gardener," is merely the Red alpine under another name.

Red alpine.-Fruit conical and red; the largest of its class; an abundant bearer, and exceedingly high flavoured. Like all of this class, they prefer a pretty strong soil, and will prosper in a soil less deep than the larger-sized strawberries in former classes. SynonymsScarlet alpine, Prolific alpine, with a long list of French synonyms, in which country, and over the most of the continent of Europe, this class is very popular ; for it is comparatively only within these last few years that our large English strawberries were known or cultivated there.

White alpine.-Similar in character, except in colour, and being sometimes, when large, more compressed at the ends than the last, and like all the class continuing to bear fruit from June to November.

Red wood.-Fruit roundish, otherwise very similar to the first, only bearing fruit, however, during June and July.

White wood.-Similar in all respects to the last, differing only in colour.

Red bush alpine, or Russian.-Fruit roundish ovate; in size and colour resembling the Red alpine, only distinguished, and that prominently, by the plants growing like small bushes, sending out no runners, and carrying the fruit upon strongish upright fruit-stalks, the fruit appearing over the leaves, and not lying prostrate like the Alpines.

White bush alpine.-In all respects resembling the last, differing only in colour.

Several varieties of strawberries are in cultivation besides those described above. We have selected them as being the best, both from experience and observation.

Insects and diseases.-The aphis sometimes attacks the plants in the open garden, but more frequently in the forcing-house. In the former case, dusting the crop over with finely. powdered hot lime before the bloom appears is the remedy; in the latter, the usual recourse must be had to tobacco fumigation, but this should not be attempted after the blossom begins to expand. The red-spider and thrip also attack them in the forcing-house-a pretty clear indication of too high a temperature, and too limited an amount of humidity in the atmosphere of the house. The remedies already noticed should be attended to. The slug and snail are, however, their great enemies in the open garden, but a good watering with lime-water, or dressing the ground between the rows with hot lime just before the flowers begin to open, will in most cases secure the crop from their attacks.

The larvæ of St Mark's fly (Bibio marci, Tipula marci Linn.), fig. 244, like many of the allied species, are gregarious, and are sometimes found in strawberry-beds to the amount of a

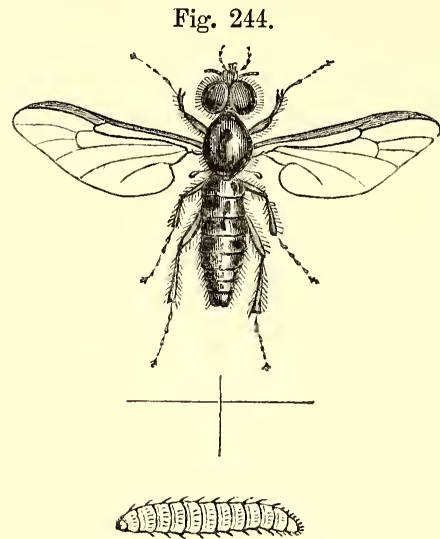

\section{Bif7275 \\ ST MARK'S FLY, GRUB, AND CHRYSALIS.}

hundred or upwards in one group. They seem, however, to prefer long-undisturbed spots; hence their unwelcome appearance is much less to be dreaded when the one or two year system of planting is adopted, than when the plantation is allowed to stand for several years. The larva, which is of a dark-brown colour, is to be found in October, in form somewhat cylindrical, flattened underneath, and nearly linear; head small, deep brown, and occasionally chestnut or light brown, very shining. It has no feet. The mouth is furnished with tridental jaws, palpijointed, as are also the lips and maxilla. The length of the full-grown larva is about three quarters of an inch. It changes in March to a pupa of a pale ochreous colour. The face is heart-shaped ; the antennæ short, brown, and curved; thorax gibbous; abdomen cylindrical, terminated by two small spines. They remain in the pupa state for nearly five weeks, the males appearing about the end of April, and the females towards the middle or end of May. The perfect insect, throughout the whole of this extensive genus, as regards males and females, is very dissimilar, both as to size and colour. The head is larger in the male than in the female, and the wings are much lighter and smaller. The male is black, shining, covered with long soft hairs; the head and eyes large ; antennæ short and nine-jointed; thorax round; scutel half orbicular ; abdomen long, tapering, the point blunt, armed with forceps; legs long, especially the hinder ones, as well as the thighs, which are also broad and compressed. Wings of the male white and transparent ; costal margin brown, as are also the stigma and surrounding nervures. Female larger and darker than the male; head small and oval; eyes small, lateral, and remote; abdomen large, tapering towards the base; apex trigonate; wings very ample, brownish; stigma and costal nervures 
darker; legs stout, the hinder ones being the longest; interior thighs inflated. The eggs are deposited in May, but are not hatched before August. The eggs are laid in the earth, and also in the dung of horses and cows, in which latter the larvæ seem to delight. They perforate the earth in manner similar to a honeycomb, and in this state live on the roots of the strawberry. It is not, however, confined to this plant, but attacks others also. Above where the colony in its larva state is located, fine earth will be found turned up to the surface. When such is observed, the nest should be dug up, and the larvæ searched for and destroyed.

The European names are-Fraisier, French -Erdbeerpflanzè, German-Aardbezie, Dutch -Fragaria, Italian-Fresera, Spanish-Morangueiro, Portuguese-Semljaniza, Russian.

\section{$\S$ 5.-THE MULBERRY.}

The black-fruited Mulberry (Morus nigra Linn.), a native of Persia, is the only one of the family cultivated in Britain for its fruit. It is supposed to have been brought to Europe by the Romans, and most probably to England by the monks, as it was cultivated in the gardens about London at a very early period. Tusser, writing in 1573, and Gerard in 1596, speak of it as a tree well known. It attains, like most slow-growing trees, a very great age, many still existing in some of the oldest gardens in England which must be a century or more in age. One in the grounds of the Duke of Northumberland at Sion House is well known to be above three hundred years old. Some of the old mulberry-trees in the gardens near London are no doubt the remains of those planted in the time of James I., who as unsuccessfully attempted to set up a silk manufacture in England, as certain wiseacres did towards the latter end of the reign of George III., both in England and Ireland. It thrives well in the southern counties as a standard tree, producing enormous crops of fruit, which are used for all the purposes to which the raspberry is applied, and to which fruit it has a considerable resemblance. In the warmer parts of Scotland it attains a respectable size, but is less fruitful than in warmer climates. Like the strawberry, the fruit does not undergo the acetous fermentation, and hence with that excellent fruit it may be safely eaten by those who are constitutionally prevented indulging in fruit generally.

Propagation.-The Black mulberry may be propagated by seeds, which should be washed out of the pulp when fully ripe, dried, and kept till spring, when it may be sown in pots or pans in light rich soil, and plunged in a mild bottomheat to hasten germination. When the plants have attained 3 or 4 inches in height, they should be transplanted into nursery lines 18 inches apart, and the plants 12 inches in the row. The site should be warm and sheltered, and the soil highly enriched. They are also propagated readily by layers, cuttings, suckers, and grafting. The former is the mode usually practised by nurserymen, who have their mulberry stools, and propagate them by layers, training them to a single shoot. They generally root the first year, and should then be planted out in nursery lines 3 feet apart, and 18 inches in the row, where their first training will be effected. The fourth year, if in rich soil, they will be fit for sending out for permanent planting. Cuttings of the previous year's growth, having a small portion of older wood at their base, if planted in autumn in rows a foot apart, and 6 inches in the line, leaving only two buds above the ground, will strike freely; and when the two buds have advanced a few inches, retain the strongest and best placed to form the stem of the future tree, rubbing the other off, that all the energies of the roots may be thrown into the new stem. When rooted, and the foliage begins to drop, they should be transplanted to nursery rows, at the same distance, and treated as above, for layers. Cuttings of the same year's growth will root freely in a close warm frame, and in either of these ways better stems will be obtained than by the layering process. Cuttings, even of the wood of several years' growth, will also root and make excellent trees, and such, it has been remarked, will come into a bearing state sooner than by the other processes. For this purpose straight clean branches, if planted even where they are to remain permanently, will root, and sometimes in four or five years begin to produce fruit. Suckers are long in coming into a bearing state, and the process of grafting orinarching may be:looked upon as more a matter of experiment than of utility, because we have no improved varieties to perpetuate, and the stock would produce a better tree and fruit as soon if left to its own resources.

Planting, soil, and situation.-Like all other deciduous trees, the mulberry should be planted by the end of October or beginning of November. The soil cannot well be too rich, and enrichment applied to the surface over the roots, either by top-dressing, mulching, or in a liquid form, should be attended to during its growing season. One tree is sufficient for a large family, as they bear abundant crops; and as the fruit does not keep above a day after it is ripe, a greater number would be superfluous. It is best planted in the standard form, and on a grass plot, or the ground under the branches covered with clean straw, hay, or similar soft material, for the fruit to fall upon without sustaining injury by bruising, or being soiled by falling on the bare ground. The American practice is, where the tree is growing on dug ground, to sow the space as far as the branches extend " thickly with cress-seed six weeks previous to the ripening of the fruit, which will form a carpet of soft verdure." Clean moss may also be used, or better, Haythorn's octagon netting suspended to the lower branches, into which the fruit would fall when ripe, and be less likely to suffer either by bruising or mud, while it could be much more easily collected.

The mulberry is sometimes planted against walls with a southern exposure, and in this way produces large supplies. Two enormous trees exist thus treated, one in the gardens at Harewood House, near Leeds, and the other at Old Alesfoord in Hampshire. Both are trained in 
the horizontal manner, and must be little short of a century old. The mulberry, until it is moderated in growth by age, rarely fruits, and hence, when treated as a wall fruit, it has to be subjected to various species of mutilation, such as ringing the branches, root-pruning, cutting out half sections of the larger branches, \&c. The true reason, however, is that, like most trees of the monœcious class, the young trees often produce male blossom only for some years after they are planted, yet afterwards become exceedingly fruitful. Another peculiarity attends this tree - the older it is, the more it increases in productiveness, and on aged trees the fruit is much larger and better flavoured than on young ones. In regard to soil, much was said about thirty years ago, and rich soils were not only recommended, but the most powerful of manures applied to its roots. One authority only, but with him we do not agree, says, "No tree, perhaps, receives more benefit from the spade and the dunghill than the mulberry; it ought, therefore, to be frequently dug about the roots, and occasionally assisted with manure."-WILLIaMs, in Horticultural Transactions, vol. ii. p. 92. As an ornamental tree on the lawn it is eminently deserving a place; but to cover a south wall with a tree of this sort to the extent of 100 feet or more, is, we think, a great waste of space, that would be far better occupied with Flemish pears.

Pruning and training.-All the pruning standard mulberry-trees require is to keep the branches moderately thin, and never to shorten the young wood. When trained against a wall, the horizontal mode is usually and very properly adopted. As the fruit is produced chiefly on the shoots of the same year's growth which spring from the wood of the previous year, and sometimes on spurs formed on the two-year-old wood, care must be taken to encourage these, and as they are formed train them on the main leaders, unless these are of a large diameter, and so place the young wood too far from the radiated heat from the wall, in which case the fruit would derive little or no more benefit from it than if the tree was grown as an open standard. But this state of things may be improved by training the young wood close to the wall between the larger branches, if there be room for them; if not, train them to the upper and under sides of the main branches, instead of to the front or outer side of them. The best season for pruning the mulberry is when the flowerbuds become visible in spring, for until that time they cannot be readily distinguished from the wood-buds. The mulberry is the latest of all trees in coming into leaf in the spring; and it has been remarked that, after the leaves have attained the size of a sixpence, frost seldom afterwards occurs.

Insects and diseases.-The Black mulberry may be said to be exempt from these in Britain. The White mulberry (Morus alba) is cultivated for the purpose of feeding silkworms with the leaves. In Spain and Persia they prefer the black variety, and in China both are grown extensively for this purpose.

The European names are-Moral, Spanish-
Murier, French-Moerbezieboom, Dutch-Moro, Italian-Maulbeerbaum, German-Schekowiza, Russian. Tut, Persian-Tatai-iba, Brazilian.

\section{§ 6.-THE CRANBERRY.}

Of this excellent fruit there are two distinct species, both of which have been more or less cultivated. Vaccinium oxycoccus Linn., Oxycoccus palustris Pursh., is a native of many of our upland peat-bogs ; Oxycoccus macrocarpus is a native of similar situations in North America. Both have been cultivated in several gardens most successfully during the last forty years, about which period the late eminent Sir Joseph Banks brought their cultivation into notice in his garden at Smallberry Green, near Hounslow. The American species was the one cultivated by him, and the situation a small circular pond in his garden, which we well remember having seen in 1814 ; it yielded the previous year, upon an area of 326 superficial feet, no less than $3 \frac{1}{2}$ bushels of berries. The culture of both sorts is identically the same; some, however, growing them in dry soil, while others grow them in a soil that is very damp. The American sort is preferred, as yielding the larger crop, and the common or European for a supposed superiority in flavour. Either may be successfully grown if planted in a border of peat-earth 18 inches deep, and situated at the north side of a wall. The plants can readily be purchased in the nurseries, and if planted $1 \frac{1}{2}$ feet apart, will in a few months become a matted surface of branches interwoven together, and in that state yield amazing crops of fruit. A bed once planted will continue in bearing for almost an indefinite length of time. In this way a very productive border existed in the gardens of Charles Barclay, Esq., Burryhill, Surrey. The margin of a pond is an excellent situation for either sort, and in such a situation a long narrow bed existed in the gardens of the late C. Labouchere, at Hylands in Essex. A marginal border about 2 feet broad was formed round the edge of the pond, and nearly level with its surface, so that the roots were kept very moist without the water covering the plants. Both sorts were grown, and continued for years to yield abundant crops without any renewal of the firstformed peaty soil. Immense quantities of cranberries are annually imported to Britain, both from America and Russia, a disbursement on our part which might be saved, and the amount earned by the peasantry around where the British kind grows in a natural state. So long ago as the days of Lightfoot, the author of the first "Flora Scotica," we find that from twenty to thirty pounds' worth of the fruit was sold each market-day for five or six consecutive weeks in the small town of Langton, on the Cumberland border, thus throwing a considerable amount of capital into a poor and needy locality.

If such, therefore, were the demand for this fruit in a petty town like this, situated in a poor and thinly-peopled locality, what might not be made of it in many other places, in particular those convenient to railway transit, as by that means the large manufacturing towns of 
England, and even the metropolis itself, would open up a ready market for all that would be collected. If would-be philanthropists would direct the attention of their poor neighbours to this branch of remunerative occupation, and assist them at first in finding the best market for their industry, it would do far more good than half the visionary schemes they entertain, amounting, in short, to transportation for ever from their fatherland, or, which is even worse, immuring them within the walls of rural bastiles, and rendering them consumers at the expense of the community instead of industrious producers, earning their bread in independence by the force of their own moral and physical energies.

The cranberry, unless where a natural formation of peaty soil abounds, can never be profitably cultivated, yet for private consumption a cranberry-bed is worth having in every firstrate garden.

Those who are fond of adding to their desserts will find several species of Vaccinium well worth cultivation, and the large-fruited Gaultherea shallon may also be included.

We believe, from observation, that a hundred pounds' worth of this fruit might be collected by the poor yearly in Strathbran, a poor and neglected tract extending from Dunkeld on the east to Glenquoich on the west, which would almost maintain the thinly-scattered population still existing in that dreary tract. Were such resources pointed out to these poor people (for of the natural products of their locality they are ignorant), it would be their own fault if our shops in Edinburgh, Glasgow, \&c., were not supplied by them instead of importing them from Russia, where the boors collect them for the export merchants, who send them over to us in barrels in the state in which we see them in all our Italian warehouses.

The Scotch cranberry, or Blae-berry (Vaccinium vites idoea $\mathrm{L}$.), is most abundantly distributed over most of our mountain tracts. Its fruit makes as good tarts as either of the cranberries above named, and is even more submissive of cultivation. From this also important resources could be derived, wereattention bestowed in pointing out its value to the peasantry who live in localities where it abounds.

European names.-Airelle, French - Heidelbeer, German-Blaauwbessen, Dutch-Mirtillo, Italian-Mirtilo, Spanish-Myrtillo, Portuguese -Tscherniza, Russian-Borrowkiczarne, Polish.

The common Bramble (Rubus fruticosus L.) -Of this there are many acknowledged wellmarked varieties, some of which, by the aid of cultivation, might be turned to good account; and some of these we have seen, more especially at Brockelsbury, Lincolnshire, some years ago, trained to a south wall, by which the fruit was not only considerably increased in size, but in flavour also. The Cloudberry (Rubus chamamones L.) seems to bid defiance to the cultivator, otherwise it is a fine fruit, and by far the most grateful of all our Scotch indigenous fruits. It is produced on a low creeping plant in peatbogs at considerable altitudes, and in appearance bears a striking resemblance in form to the raspberry. It has been suggested to cross this plant with the latter, or even with the common bramble, with a view to render it more capable of cultivation.

The European names are-Ronce, FrenchBrombeerstrauch, German-Braamen, DutchRovo, Italian - Zarza, Spanish - Jaschewika, Russian-Jezyny, Polish. 


\title{
CHAPTER XIV.
}

\author{
THE GRAPE VINE.
}

THE Vine (Vitis vinifera L.) is the oldest fruitbearing plant we read of, with the exception of the apple and the fig. That it was cultivated extensively in the antediluvian world we have pretty good evidence; for Noah had no sooner escaped from the general destruction of mankind by the Deluge than he set about planting a vinevard and made wine, showing that he was not ignorant of the plant, its culture, and its use. It is, however, strange that the veritable native country of the vine is still by no means satisfactorily known. It is probable that it existed in an indigenous state over a vast portion of Asia, where the climate was suited to its growth. An interesting account of its introduction into Egypt, Greece, and Sicily is given by Dr Sickler, and how it found its way into Spain, Italy, and France, in which latter country it is believed that it has been cultivated since the second century. At what period it was first received into Britain is our present inquiry, and in search of that we must go back to the entrance of the Roman armies into this country. Some determine its date of introduction to be about ten years after the Christian era, the Romans having at that time full possession of this island; others think it was not till about the year 280, when Probus, a great encourager of agriculture in all the Roman provinces, was emperor. That it was introduced prior to that date is confirmed by the historic fact, that at this time license was granted to the inhabitants of the various provinces to plant vineyards; and the Britons are expressly said by Vopiscus to have been included among the number who partook of this decree. Some have attributed its introduction to the Phœnicians, who, it is well known, traded to the southern coasts of Britain for tin so early as the days of Solomon; and as they are said to have planted the vine, which they brought from Palestine, in the isles of the Mediterranean Sea, it is not unlikely they brought it to Britain also. The venerable Bede says that vineyards were growing in several places in his time (731). They are particularly noticed in "Doomsday Book," and William of Malmesbury, in the twelfth century, commends the county of Gloucester as excelling all other parts of England in the quantity and quality of its wines.

The vine was at one time cultivated for the purpose of wine-making in Britain with almost as much success as in France. The Duke of Norfolk had, at Arundel Castle, in Sussex, a vineyard, from which an immense quantity of Burgundy was made; and the celebrated vineyard at Pain's Hill, in Surrey, was noted for many years for the excellence of its champagne. It is noticed by Miller and Langley, and more particularly by Barry, in his "History of Wines." Switzer gives us the names of many who grew the grape for wine-making in his day, amongst which he particularly mentions Rocque of Walham Green, Warner of Rotherhithe, the Duke of Rutland at Belvoir Castle, and others, who grew the grape and made excellent wine; certainly a proof that the climate was more favourable in Britain than it has since been.

In America, the grape-vines cultivated in Europe are much sought after. In the milder states vineyards are established, and vineries under glass rapidly increasing, and most of our known sorts are already cultivated there. The native grape of America is Vitis labrusca. It is described by one of their best pomological writers as "usually stronger in their growth than those of the Old World, with larger and more entire foliage, and, in their native state, with a peculiar foxy odour or flavour, and more or less hardness of pulp." Although the native vine of America does not possess the same excellent properties as the Asiatic or European cultivated sorts, still it makes good wine, and attains a prodigious magnitude, "several having been measured on the banks of the Ohio, the stems of which were 3 feet in circumference, and the branches 200 feet long." The extent to which the vine might be trained in a favourable climate is unknown; the two largest ever seen in England were, the vine grown against a house in the public street in Northallerton, long since dead, which covered a space of 137 square yards, and that at Valentines, in Essex, about 147 square yards. The celebrated Hampton Court vine covers the roof of a glass-house, about 116 square yards in area; that at Cumberland Lodge, near Windsor, somewhat more; and yet these latter vines are impatient of restraint, and would extend much farther if allowed; they are computed to produce annually 2200 bunches each, averaging $1 \mathrm{lb}$. each, or nearly a ton weight. The vine at Northallerton, when cut down, had a trunk of no less than 4 feet in circumference near the ground. 
These dimensions are as nothing when compared to the vine trees of antiquity. That enthusiastic author, Evelyn, in his "Sylva," speaks of the vine attaining a size from which a statue of Jupiter and columns in Juno's temple were made. In his day it was discovered that the great doors of the cathedral at Ravenna were made of vine planks, some of which were 12 feet long and 15 inches broad. Strabo speaks of a vine that was 12 feet in circumference; Pliny the historian mentions one that was six hundred years old in his time, and remarks that the ancients classed vines amongst trees on account of their magnitude, and various extraordinary dimensions are given in Lib. xiv., chap. 1. The name vine is indicative of its arborescent growth, being derived from Gwyd, pronounced Vid, a tree; Celtic, the best of trees.

The size to which bunches of grapes have been grown, especially in countries favourable to the natural culture of the vine, is still more extraordinary than the size of the vines themselves. Strabo, who lived in the reign of Augustus, testifies "that the vines in Margiana and other places were so big that two men could scarcely compass them with their arms, and that they produced bunches of grapes 2 cubits or a yard long." Huetius asserts that in his time Crete, Chios, and other islands in the Archipelago, afforded bunches of grapes, sometimes thirty-six and forty pounds in weight. The large bunch produced by Mr Speechley, at Welbeck, of the Syrian grape, and which was considered the wonder of the age, weighed nine. teen pounds and a half. If we are to believe Egidius, Van Egmont, and John Heyman, who travelled through, and published their observations on the then state of Asia Minor, they speak of grapes being produced near Damascus, the berries of which were as large as a pigeon's egg, and of very exquisite taste. Cultivators of the present day desire more to have large berries than very large bunches.

Propagation. - The vine is propagated by seeds, layers, cuttings of 9 or 10 inches in length, by coiling, sometimes, but rarely, by budding, and also by single eyes or buds. Established plants are often grafted when it becomes desirable to substitute one sort for another. Propagation by seed is chiefly called into use when the object is to originate new or improved varieties; and to effect this with the greater certainty, the pistils of one sort should be impreg. nated by the pollen of another. The conditions necessary for this have been explained, (vide Crossing and hybridising). In former times layering was a popular method of increasing the vine amongst nurserymen ; it is, however, a practice now nearly obsolete, and we may almost say the same of propagating by cuttings of considerable length. Coiling is a mode brought into considerable notoriety by Mr Mearns, the rationale of which will be given below.

Propagating by single eyes.-A mode discovered by the Rev. Mr Mitchell, about 1777, is that approved of by all good cultivators, as affording better-rooted plants, and those very short-jointed in the wood. The practice of $\mathrm{Mr}$ Errington is excellent, and is to the following effect : "Well-ripened wood from healthy established vines is the best, and that with very large joints or buds, removed with a small portion of the last year's old wood, grows the strongest. About 1 inch of the shoot above, and 1 below the bud will suffice ; the cutting will thus be 2 inches in length. Some persons cut the shoot through longitudinally, removing three-fourths of the wood on the bud side and most of the pith ; we, however, never found any decided advantage in the practice. Single eyes may be put in pots about 4 or 5 inches in diameter; soil a rich and mellow loam, or a very good garden-mould. Care must be taken to secure excellent drainage, and the eye must be placed an inch at least below the surface of the soil. And now a bottom heat, although by no means indispensable, will be of immense service; it will, indeed, rear them in half the time otherwise required. From $70^{\circ}$ to $80^{\circ}$ will be proper; and if it can be secured, an atmospheric warmth of $50^{\circ}$ to $60^{\circ}$ will soon produce shoots. If they are plunged, means must be taken to prevent worms getting into the pots; 3 inches of coal-ashes under the pots will secure this. After potting, they will require little attention till they have made shoots above the soil ; a little water will be requisite occasionally. In a month they will be nice plants about 6 or 8 inches in height, and their pots will be full of roots; and those who wish to obtain large plants must give them a shift, and such may be a final one for the season. Seven or eight inch pots will now be necessary, and a more generous soil still. Nothing can exceed old turf which has lain in the compost-yard for a twelvemonth, with one-third its bulk of old leaf-soil and good manure, adding a little sand and charcoal to the mixture, which must not be too fine. They should again receive bottom warmth until the pots are nearly full of roots; and if the eyes were started in the beginning of February, such will be the case about midsummer, when, if necessary, the pots may be removed from the plunging medium: caution must be exercised in so doing. It must be understood that there is no absolute necessity for taking them out of the plunging medium; we merely removed it in order to get them as near to the light as possible, this :being an all-important affair." Many set the eyes at planting closely together in large pots, and as soon as they have made some progress they transplant them singly into other pots, but this is both unnecessary and detrimental to the young roots, many of which will be broken during the operation, let it be ever so carefully performed. Others set the eyes in sixty sized pots to start them in, but in such small pots the roots are liable to suffer from drought, and if not early repotted, will also suffer from want of room. Exciting the eyes so early in the season as recommended above, is applicable rather to vines to be afterwards grown and fruited in pots ; for ordinary supply they need not be planted earlier than the beginning of March. The cuttings from which the eyes are to be taken should be selected at the season of pruning, choosing the best ripened wood with bold plump eyes. These cuttings should be 
stuck in the ground, and so kept till the end of February or first of March. Some writers have attached great importance to the selection of cuttings from whence the eyes are to be taken, and their reasoning is sufficiently good. $\mathrm{Mr}$ Robertson chooses buds from vines that for several years previously were started in Decem. ber, and concludes that the young vine, inheriting to a considerable extent the habit of the parent, will hence be better adapted for starting at so early a period. Mr Shortland chooses such shoots as are upon the most productive part of the vine, from a conviction that the young plant will partake of a similar state of fertility. The usual length of shoots produced from a single eye the first season is about 4 or 5 feet; there have, however, been instances where they have grown to the length of 30 feet.

Coiling is a modification of propagating by long cuttings; but instead of using a piece of wood 9 or 10 inches in length as in ordinary cuttings, or of employing only little more than 2 inches of wood as in buds, here several feet in length are chosen. According to Mr Mearns' practice, if single rods be desired, then 4 or 5 feet only of coiled shoot is used, and from this all the buds are removed excepting the uppermost one. Narrow deep pots are used, and within them the shoot is wound round in form of a coil of rope, whence the name-the lowermost coil being kept several inches above the bottom of the pot, to provide room for the roots to extend themselves. The bud and upper end of the shoot are kept 2 inches below the surface of the soil in the pot. The operation is recommended to commence between the middle of January and the end of March, and as soon as placed in the pots they are plunged in a temperature between $90^{\circ}$ to $100^{\circ}$, where they remain until they require shifting into larger pots, after which they are placed in a suitable situation until November or December, when they are again excited into growth. As the shoot extends, it is trained upright until it attains the height of from seven to ten joints, at which time it is topped; after this, laterals will begin to make their appearance, but these must be displaced as they appear. If all has gone on well, several buds will become excited at the same time, in which case the shoots must be cut down to the lowest excited eye, one shoot only being allowed to remain, and that must be carefully preserved, and divested of all laterals and tendrils. When these shoots have attained the height of 5 or 6 feet, which is the maximum height required, they must be topped, leaving the uppermost lateral, after being stopped above its first joint, to carry off the remaining sap. Towards the end of summer the plants are removed to the open air, and set in a warm sheltered place; and when the wood is ripe, the shoots are headed down to one, two, or three eyes, according to their strength; they are then placed in a cool northern exposure until the weather becomes cold, when they are again removed to a southern exposure like that they occupied.previous to heading down; and here the pots will require to be plunged, or, better, well mulched with litter to exclude too VOL. II. much cold, rain, and frost. The plants may be brought into a pit or vinery from the middle of December to the first of April, according to the time the fruit is wished to be ripened. Those first brought in had better have their shoots coiled round three or four stakes placed in the pots for the purpose, or a piece of strong wire may be bent in form of an elliptical hoop, and the shoots trained to it; the intention being, by bending the shoots, to cause the buds to break more regularly, which they would not do very early in the season if placed in a perpendicular position. From the time the plant is first excited until the fruit is changing colour, a slight degree of bottom heat will be highly advantageous to them.

Propagating by layers. - This mode of propagation is now nearly obsolete, although some nurserymen still continue to propagate in this manner, as attended with much less expense and trouble. Another circumstance, we think, has its influence also. Many gardeners are not content with small plants originated from single eyes, but will have what they suppose to be fine strong plants of 5 or 6 feet in length, without considering that such plants, depending for their existence mainly on the parent, and not on roots entirely of their own, are found to be, when turned out of the pots in which they have been laid, almost devoid of roots altogether; and should they, peradventure, have any, they consist of two or three strong roots without fibres, often extending beyond the limits of the pot, and as often broken entirely off when the pots are removed from the soil. Another reason in favour of vines from layers is, they are somewhat cheaper than those from single eyes; and this even has its weight with some people. The difference between vines propagated the one way and the other is observable for years after planting, and he must be a tyro indeed in vineculture who cannot discern it. The following brief description will explain the process : A strong shoot of the preceding year's growth is taken from the wall, and bent so as to reach about 3 inches under the surface of the border; the last eye, before it reaches the ground, is destroyed, and the eye next above the surface beyond the part buried in the ground is carefully left, as from it the young shoot is to proceed. The soil being cleared away to the depth of 3 inches as above, the shoot is brought down to that point and then bent upwards, and is kept in its position by a pretty strong peg, and the compressed soil over and around it. The top of the shoot is cut away, leaving the bud we have alluded to just above the ground-line. The vine roots pretty freely in this way, but the great objection to it is the injury the young roots sustain in autumn, when the layered plants are dug up to be sold, potted, or otherwise disposed of. Nurserymen, however, to avoid this evil, plunge pots in front of the wall, or around their vine stools, if they are grown in the open quarters, and lay the shoots in them. In this way the young plants may be removed when rooted with much greater safety. Vines are also layered as follows, especially when the shoot selected to be operated upon is so inconveniently placed as to be with 
difficulty brought to the ground: The shoot is brought through the hole in the bottom of the pot filled with soil, and which for the purpose must be secured to the wall, and the soil kept at a proper point of moisture during the summer. The bud which is nearest the centre of the pot is cut away, while two buds are left on the point of the shoot above the level of the pot, from one of which the young shoot springs: the weakest of them, should both grow, is rubbed off. In August, or early in September, the layer is cut from the parent plant, and either planted out at once, or shifted into a larger pot, and placed in a warm sheitered place till the wood ripens. It is of importance to have the layers removed from the parent plant before the first of September ; for it has been found, where this has not been attended to, that the roots die off, which may of itself explain, in a great measure, why vines, from layers planted in spring, do so frequently die. In general, the operation of layering commences early in March, which is believed by Green of Stepney to be much too early ; for he has stated in "Gardeners' Magazine," vol. iii. p. 24, that if vines be layered ever so early in spring, they will make no roots before the middle of July; thus circumscribing the rooting period for layers to something like six weeks-a very short time indeed, particularly in a soil and climate such as that of Britain. The case might be somewhat different were the operation of rooting carried on in bottom heat.

Layering the shoots of the current year.-A case of this kind was, so far as we recollect, first noticed by the late Dr Neill, in the "Edinburgh Encyclopædia," as being brought under his notice by our predecessor, the late Mr M'Donald, in the old gardens at Dalkeith. The practice is, however, we may remark, founded on what has been done in nurseries in rooting several exotic plants, difficult to root otherwise, and for ages practised in China, both on young and old wood. In the end of June or beginning of July, when the vines have made shoots 10 or 12 feet long, any spare shoots are selected, and bent down so as to form a double in a pot filled with earth, taking care that a portion of last year's wood containing a joint pass into the soil in the pot. Within ten days roots will be found to have proceeded plentifully from the joint of last year's wood, at which time the layer may be separated from the vine. By this method a vinery might be furnished in three months as completely as it would be by the ordinary process in two (the Doctor says three) years. "Supposing the layers to be made on the 1st of July, they might be cut and removed to the new house on the 9 th; by the 9 th of October the roof would be completely covered with shoots, and next season the house would yield a full crop of grapes." Not that it would be prudent to do so if permanent plants be desired, as the few roots made would be unable to provide the necessary supply for such large plants. It is a remarkable circumstance that nurserymen, such as the highly respectable house of the Lees of Hammersmith, who have very extensive vineries, have not adopted this expeditious mode of propagating the vine. There can be no doubt that such a range of vineries as that alluded to would yield a much greater profit from young plants than from the most abundant crop of fruit that could be produced in them. Those eminent cultivators, no doubt aware of the great superiority of vines propagated by the more expensive process of single eyes, adopt that course in preference to a more expeditious and less costly method, thereby supplying a much better article, though at a less remunerative profit.

Propagating by seed.-We have stated above the object generally had in view of propagating by this process. We may here remark that little success has attended the labours of even the most enthusiastic in this important department of pomological science. Speechley obtained many seedlings, but none of extraordinary merit and those who have succeeded him have not been much more fortunate. This is the more extraordinary considering the importance of the subject, and the eminent success that has attended the creation of so many excellent varieties of apples, pears, strawberries, \&c. The rearing from seed is a simple operation. Select the seed from the best specimens of the fruit, separate them from the pulp, and keep them in a dry cool place till spring. March is a very good time to sow in light rich soil, in a moderate temperature. Sow the seeds singly in small pots, and when the plants have attained the height of 6 inches, shift into larger pots, and stimulate them throughout the growing season with abundance of light, air, warmth, and weakened liquidmanure; keep them growing till the end of summer; place them then where their wood may become ripened, and during winter where severe frost cannot reach them. Head them down in December to one eye, shift early in spring, and give another summer growth, with all the stimulants necessary. The third or fourth year they will fruit, and their merits, if any, may be ascertained; or they may be inarched in their second year's growth upon established plants, and thus expedite their bearing fruit.

Propagating by cuttings.-In regard to the selection of cuttings, the same rule should be acted upon as recommended for eyes. The older practitioners in this country, copying, no doubt, from the Continental growers, used, as is still done in France at the present day, cuttings a foot and a half in length. For years past a reduction to about half that size has been adopted by British gardeners. The cuttings, being selected at the autumnal pruning, are to be preserved by being stuck half their length in dry light soil in a sheltered situation. In March they should be deprived of their two lowermost buds, and planted in pots in light rich soil, and placed in a mild bottom-heat. The general rule appears to be to plant them in the open air, generally at the foot of a wall, in a warm sheltered place. This may be all very well in the south of France, or even in some few parts of the south of England, but would be anything but well in the greater part of England and most of Scotland. If Mr Green, already quoted, be correct in his theory, that vines, when laid, do not make roots in the open 
air before the middle of July, we have no reason to suppose that cuttings root any earlier, if so soon. If vines are to be propagated in this way (but for which we see no good reason), then we say let them have the comfortable accommodation of a slight bottom-heat, and be covered with a frame and glass lights.

Relative merits of propagating the vine by long cuttings, layers, and single eyes.-Hayward, in estimating the merits of these modes of propagating the vine, after approving of the single-eye method, remarks, in "Science of Horticulture," p. 187: "When cuttings are planted, of 10 or 12 inches in length, in the usual way, they remain a year or two before a quantity of sap is supplied, and consequently the old bark and vessels become fixed; and notwithstanding they may throw out some strong shoots after this time, when taken up for transplanting the roots are unavoidably reduced, and the supply of sap again lessened, and the vessels contracted. And when plants are raised as layers, in pots or in borders, they are suffered to remain connected with the parent plant a long time after they strike root, and being thus nurtured by both furnish very strong branches; but these, on being separated from the old branch, are thereby deprived of half their supply of sap, and in consequence the vessels contract, become inflexible, and incapable of extension like cuttings. When plants are raised from a single eye, the roots form immediately around the eye, and the young stem striking directly from them is without old bark or old vessels, and being raised in a pot, is readily turned out with all its roots entire and uninjured, and immediately takes to the soil; the shoot extends in proportion to the sap supplied, and, thus proceeding unchecked, will, the first year after transplanting, often form a shoot strong enough to produce fruit the following year; whilst the strongest plant that may be raised from a layer or cutting in the usual manner will not produce a shoot of half the strength the first year, and is seldom in a state to produce fruit in a shorter time than three years." In confirmation of these views, we find most gardeners giving a decided preference to plants originated from single eyes, and all those who force the vine in pots entertain a similar opinion.

Propagating by grafting or inarching.-The one of these operations is just a slight modification of the other, and is seldom had recourse to in the case of the vine, unless when it may be desirable to prove the quality of a seedling of promising appearance, which may, by being grafted or inarched on an established plant, produce fruit sooner and in greater perfection ; or where, as is often the case, some worthless sort is planted, and it is desirable to substitute it by another, which otherwise could only be done by planting: this is not always convenient, nor is it proper to plant a young vine in a border already crowded by the roots of others. It is sometimes, however, practised in cold late soils, in which some of the more delicate kinds do not prosper. In such cases they may be advantageously grafted on some of the hardier sorts that are established. But even in this case it would be better to prepare the border properly for them, by vaulting, heating, covering, or any of the many ways elsewhere pointed out. This is an operation that can only with propriety be accomplished when the trees are in foliage and the sap in circulation. At other times the incision would cause the vine to bleed to the extent of preventing the necessary adhesion taking place between the stock and scion. Grafting the vine, although practised in the days of Speechley, remained little understood until the late T. A. Knight explained the rationale, and exemplified various modes in practice. He selected his scions having one joint of the previous year's wood at their base ; these he fitted to branches of nearly the same size but of older growth, and employed clay in so damp a state that its moisture supplied the scion with nourishment until adhesion had taken place. The scions used by Mr Knight had about 2 inches of old wood and 5 inches of young wood, by which means the junction of the new and old wood, at which point cuttings most readily emit shoots and receive nutriment, was placed close to the head of the stock, and a single bud only was exposed to vegetate. Speechley preferred cleftgrafting when the stock did not exceed 1 inch in diameter, and whip-grafting when of a larger size. Gowan, in " Gardeners' Gazette," recommends wedge-grafting, and selects his scion with one eye, which he forms in shape of a wedge. The stock he prefers is a shoot of the previous year's growth, and nearly of the same size as the scion, and this he cuts over a little above the second eye from the old wood, and slits down nearly the whole length of the young wood. He then pares with a penknife as much as is necessary of that half of the stock which is opposite to the eye or bud as to make it fit exactly the sides of the scion. The scion is then inserted, with its eye opposite to that left on the side of the stock; it is then tied up and clayed in the usual manner, only covering the whole with clay, excepting just immediately over the eye. Hovey, in " Magazine of Horticulture," an American publication of great merit, recommends heading the stock over, below the surface of the ground, in autumn or late in spring, after the sap-leaves are fully expanded. He inserts the scion as in cleft-grafting, but where the stock is sufficiently large he places a graft on each side in order to avoid accident; and when they are bound round, he draws the soil around the whole, leaving the top bud of the scion just even with the surface.

Braddick adopted whip-grafting with great success, using cuttings of the previous year, and choosing that state of the vine when the sap ceases to flow thinly and rapidly. His success in this case led him to suppose that vines might be grafted with certainty by employing the young wood of the preceding year's growth from the time the shoots of the stocks had made four or five eyes until midsummer. "He also found that vines growing on the open walls might be grafted with shoots of the same summer's growth, worked into the rind of the young wood, from the time that young bunches of grapes become visible until July, while those under 
glass might be graifted a month later. In ncither case, however, should the operation be deferred after the periods mentioned, on account of the time required for ripening the grafts before winter."-Gardeners' Monthly Volume.

There are various other modes of grafting the vinc, particularly those practised on the Continont, (vide Chaptal on the Vine, and others), but those we have noticed are quite sufficient for all practical purposes.

Propagating by budding.-This operation, although quite practicable, has been seldom had recourse to. The season most suitable is just as the sap begins to risc. The bud to be chosen should be a plump well-formed one, taken from thoroughly well-ripened wood. Cut the eye out about 3 inches in length, taking as much wood with it as can conveniently be got at each end of the eye; cut off about a quarter of an inch of the upper bark, making the ends quite thin. Next, measure off the exact length of the bud on the bark of the vine intended to be budded, and make a notch slanting upwards at the upper part, and another slanting downwards at the bottom; then take the piece neatly out, so that the bud may fit nicely in, and by making the notch, as stated above, each end of the bud is covered with the bark of the shoot. Bind the bud firmly round with matting, and clay it, taking care, however, that the clay does not cover the eye of the bud; then tie it round with moss, and keep it constantly damp, and as the sap rises the bud will begin to swell. When the vine commences to push out young shoots, take the top ones off, in order to throw a little more sap into the bud, and, as it is perccived to be getting stronger, take off more young shoots, and so continue until they are all removed. Budding can only be performed where the long-rod system is practised, as in that case you have the power of confining the sap to the bud, which will grow vigorously. As soon as this is perceived, cut the vine down to the bud. Budding has the advantage over grafting of not leaving an unsightly appearance where the bud is inserted. A bud likewise grows more luxuriantly. A shoot from a bud has been known to extend, in one season, to the extent of 30 feet."-F. L. S. in Gardeners' Chronicle.

Formation of vine borders.-Upon the subject of breadth and depth of vine borders there has been a variety of opinion, only to be equalled by the diversity of opinion as to the proper soil.

Speechley, the very father of vine-growing, says, "One-fourth part of garden mould (a strong loam); one-fourth of the swarth of turf from a pasture where the soil is a sandy loam; onefourth of the sweepings of pavements and hard roads; one-eighth of rotten cow and stable-yard dung mixed; and one-eighth of vegetable mould from decayed oak leaves. The swarth should be laid in a heap till the grass-roots are in a state of decay, and then turned over and broken with a spade; afterwards it should be put to the other materials, and the whole should be worked well together."

Griffin recommends good loamy turfy soil, one half; rich solid dung, one quarter; bricks and lime rubbish, one quarter. This he lays on a well-draincd bottom, covered with brick, stone, or lime rubbish, about 6 inches in depth.

M'Phail preferred earth of a loamy nature, and for early forcing a strong deep loam, not destitute of a mixture of sand, and well manured with rotten dung, on a dry bottom of hard clay.

Abercrombie directs the top-spit of sandy loam from an upland pasture, one-third part; unexhausted brown loam from a garden, onefourth part; scrapings of roads free from clay, one-sixth part; vegetable mould, or old tan, or rotten stable-dung, one-eighth part; shell-marl, or mild lime, one-twelfth part. His borders he recommends to be from 3 to 5 feet in depth, and, where practicable, not less than 4 feet wide within the house, and not less than 10 feet wide without.

Vine borders at Wishaw House, Lanarkshire, in a cold and wet locality, are thus formed: Breadth 12 feet, depth of soil 18 inches, under which is laid a foot of hard clinkers, by way of drainage. The soil used is that natural to the garden, which had for years been under pasture, and is a remarkably strong, rich, brick-clayey loam, with no other preparation than the addition of a moderate supply of stable-manure. In this soil the best grapes ever produced in Scotland have been grown for the last three years.

Judd recommends one-half rich gritty loam from a common, one quarter of rich old dung, and a quarter of lime rubbish, tan, and leaf mould, mixed together. "These materials were kept separate, frequently turned over during winter, and afterwards mixed and laid upon a prepared bottom to the depth of 3 feet. $\mathrm{Mr}$ Judd does not recommend the use of much manure, believing that, although the vine will bear an extraordinary quantity of manure, yet its growth is thereby retarded, especially when young."-Horticultural Trarsactions.

Harrison: "The border outside the house ought to be at least 21 feet broad, 5 feet deep at the back, and 2 feet 6 inches or 3 feet at the front." The border is rendered perfectly dry at bottom with draining, the bottom having an inclination from the house towards the walk of at least 1 foot. One drain should run along close to the front of the house, and another at the front of the border, with cross drains, filled with stones, and 3 inches above the substratum. If the bottom be wet, lay 6 inches of broken stones, brickbats, \&c., besides the drains, and over these 2 inches of smaller stones or gravel, rolled down to an even surface. This he prefers to paving the bottom, as the water will filter down to the drains. The soil he recommends is, one-half good gritty loam; the top-spit of a pasture-field which has not been lately cultivated; one quarter well-rotted manure from hotbeds; and one quarter of lime rubbish, bone dust, old spent bark, and pigeon or fowls' dung. The whole to be procured twelve months before wanted for use, and frequently turned and chopped to pieces, but always in dry weather." -Treatise on Fruit Trees.

Appleby, an excellent cultivator, says, in "The Gardeners' Chronicle:"- "The width of the border need not be more than 20 feet, 
and should not be less than 15. It is a good general rule to have the border the same width as the houses. The width of the border being determined upon, and the soil removed, the top-spit, if it be good, may be mixed with the compost; then slope the bottom with a gentle descent from the houses, making it pretty firm. This is the floor of the border. At the extreme edge build an open drain, extending 6 inches below the level of the floor, and 6 inches above it, and 1 foot wide; the sides to be built in the pigeon-hole manner, to allow a ready passage for the superfluous water. Have this floor paved with strong slates, or thin flags, and then covered all over with broken stones or brick ends, about the size of a man's fist. When these are levelled, cover them either with small twigs, bean straw, or thin turf, laid with the grassy side downward. This is to prevent the soil from falling amongst the stones below. It is now ready for the compost. 'The best compost for the vine is the thin top-spit from an old pasture, chopped, and thoroughly mixed with onefourth rotten 'horse-dung, and one-fourth rotten leaves, or one-third dung when leaves cannot be had. Bones are excellent when broken small, and mixed in the compost, but not indispensable. The border should be 2 feet deep in a low damp situation, or 3 feet in a high dry one."

Mr Roberts, in his excellent work on the cultivation of "The Vine under Glass," says : "The width of the borders outside the house ought to be 24 feet, cleared out to the depth of 3 feet 6 inches, upon a bottom of retentive clay, well prepared, with 1 foot of fall from back to front. A main drain ought to run along the extremity of the border, 1 foot 6 inches deep, with cross drains, in an oblique direction, leading into it, so as to have a perfect command in draining off superfluous water, in order to keep them dry. Place upon the bottom thus formed broken stones and lime rubbish to the depth of 1 foot, leaving a depth for compost of 2 feet 6 inches. Upon the broken stones, every 6 or 8 feet square, place limestones, of the same nature as the far-famed Skipton rock, which contributes to retain moisture in a dry season, and facilitates drainage in a wet one. In autumn, when all is ready, and the weather favourably dry, proceed at one end of your border, wheeling in and mixing the materials in proportion as they stand to each other, on no account breaking the materials in mixing, but turn them in as rough as possible." To those who may not have perused Mr Roberts' book, we may say that he places great reliance, in the formation: of his borders, on the carcasses of animals buried in the compost.

Vine borders, according to $\mathrm{Mr}$ Saunders, should be 16 feet in width at the least, 3 feet 6 inches deep, including.drainage, \&c. " And, first, the higher side of it which abuts the vinery should, when the border is entirely finished, be exactly on a level with the interior flooring; and that being the criterion as to the height, the necessary calculations for the depth required to render it so must be made before the foundation of the border is commenced, and the ground should be prepared accordingly, always preserv- ing from the beginning the proper declivity, not less than 18 inches from the house to the front of the border, and to have the surface of the ground quite smooth before laying the foundation over it, which must be done as follows: Spread concrete to the depth of 4 inches, and when sufficiently hard, upon that put 8 inches of brickbats ; and next form a drain, extending along the front edge of the border, to receive and carry off all superfluous water." Having proceeded thus far, the bottom of the border is to be made up of rich loamy fibrous turf, cut from an old pasture, not too retentive of moisture, but yet of that adhesive nature termed "strong loam." The remainder of the border may be filled up with the following compost: two parts of rough turfy loam in sods, cut in pieces 2 or 3 inches square; one part of well decomposed farmyard manure, and the remaining fourth part composed of equal quantities of droppings from the sheepfold and leaf-mould: let all be well incorporated, adding a little sand if the loam be deficient in it; the whole to be performed in dry weather. When it is thus prepared, suffer it to lie in a heap for a month or two, turning it occasionally, so that it may become completely amalgamated. The operation of thus mixing the soil should, if possible, be carried on in an open shed or otherwise, so as to be protected from heavy rain, but at the same time fully exposed to the air.

A great mistake, we believe, has very generally been fallen into, by making vine borders both too rich and too light. Such may be all very well for open-air culture, but where such draughts are made upon the vine, as is the case in general in hothouses, we think a stronger soil would be preferable. As to making very rich borders in the first instance, we can see little advantage, as they can be enriched ad libitum by the application of liquid manures whenever it may be required. If a pure rich maiden loam can be procured, little else is needed; and "we know gardens where such was natural to the spot, whose vine borders have continued for many years without further enrichment than what has been washed downwards by the rains from the mulching laid over them during winter. In the formation of vine borders in damp localities, we prefer placing them on the surface of general ground-level altogether. For examples, vide vol. i., figs. 111, 435, 436, $437,445,447$.

In regard to the depth of vine borders, very much depends on soil, situation, and climate, as well as the nature of the subsoil. In light soils and dry localities, as at Dalkeith and its vicinity, where the soil is naturally light, resting on an alluvial bed of gravel, with a depth of rain averaging about 20 inches only, borders require to be made deeper, and assisted with a mixture of strong loamy soil ; whereas at Drumlanrig, and many parts of the west of Scotland, where the soil is naturally strong and retentive of moisture, and where the depth of rain averages according to locality from 40 to 60 inches per annum, the depth should be much less, and thoroughly drained or vaulted below, as well as rendered lighter and more 
pervious by the addition of other material. Again, where the climate is cold, the soil should be lighter because it is more readily penetrated by solar heat; whereas, in the warmer climates of the south of France and Italy, its depth may be indefinite. It is, however, better to increase the breadth than to increase the depth in most cases, because the roots of the vine travel far in search of food, and it is always desirable to have them near the surface.

In cold wet districts it is advisable to form borders within the house for the preservation of the roots of the plants, as well as for the safety of the main stem, through which the whole supply of sap flows upwards to the other parts of the trees. In such cases, however, it is proper to build the walls on piers or arches, to admit of a free escape of the roots outwards, if they are disposed to go in that direction. A reference to vol. i., sect. VINERIEs, will afford abundant examples. In the vineries in the new gardens, from designs furnished by us, and at present forming for Lord Panmure, which are upon the span-roofed principle, and glass all round to within 2 feet of the ground, the vines are to be planted inside of the house. The parapets are built on piers 4 feet apart, and the borders are prepared both inside and out. A 9 -inch air-drain is carried along their ends, which necessarily front the south, with openings to the surface 6 inches square and 6 feet distant from each other; to this 4 -inch tile air-drains are connected 3 feet apart, and run along the whole length of each house, terminating in a heated chamber under the corridor, which extends the whole length of this extensive range, and connects all the houses together. In this chamber are placed the mains of hot-water pipes, which supply the houses as they pass along, the water being admitted into one or all by means of valves. The heat in this chamber causes an indraught of cold air from without, and as the drains are laid open in the joints, it diffuses itself through a mass of broken stones 18 inches deep, which forms the drainage under the whole of the borders. The air is regulated by metallic traps placed along the front of the houses close to the terrace-walk, which are opened or shut according to circumstances. The borders are formed on top of this drainage to the depth of 2 feet, the roots having the range of the whole breadth of the houses within, which are 18 feet wide, with a 12 -foot border between each two houses. The soil is of a rich old-pasture, turfy nature, and probably has never been in a cultivated state; a slight addition of rotted stable-yard manure and broken bones is all the enrichment added. The houses stand upon a terrace 3 feet above the level of the garden, the ground in front sloping considerably towards the south. In a range of vineries designed by us, and also at present erecting for Sir James Carnegie, Bart., we have placed the whole borders above the garden-level, the situation being low and damp; the superstructure stands 3 feet above the terrace-walk in front. Indeed, we hold it a most essential point to have every hothouse elevated at from 2 to 3 feet above the surrounding ground, as it secures efficient drainage in the case of fruit-tree houses, and gives an expression of importance by the increased elevation.

No vinery can be considered complete, if not elevated, drained, and ventilated underground as here described, or chambered below, as shown with so much success by the Marquis of Tweeddale at Yester (vide vol. i., figs. 445, 447). Shallow borders, and those not glutted with rich manure, are much to be desired. Stimulating the roots of the vine before the buds and branches, is now admitted to be a leading feature in good forcing management. With this view, we have a range at Dalkeith of lean-to houses, drained and ventilated as noticed above; and at every 3 feet the air - drains pass through the front parapet-wall immediately under the hot-water pipes, and as these are opened and shut, heat can be withdrawn from the interior of the house and made to circulate through the drainage, and consequently to rise through the border above, and so excite the roots into active growth before the vines themselves are taken into the house; thus giving the roots a proper advance of the buds, the very reverse of what takes place in ordinary cases, where the vines are exposed to a temperature of $55^{\circ}$ or $60^{\circ}$, while the roots are below $40^{\circ}$.

Nor is it only with affording, by" the means we have suggested, abundance of drainage and moderate subterranean warmth to the roots of vines growing in borders without the house, that we are to rest contented-particularly in the case of vines excited into growth during December and January, or such as are retarded to produce fruit from November to January. We must also have the surface of the external border protected against frost, excessive rains, and melting snow. The means formerly adopted, such as covering with leaves, straw, or littering dung, afforded little of the advantages required; and laying vegetable matter in a state of fermentation over them, with a view to stimulate the roots into action by the heat descending from it, is next to labour and fertilising material thrown away. Concreting the surface has its advantages, no doubt, but a portable roofing of earthenware, or glass tiles, or large slates, laid on rafters elevated 4 inches above the ground, with longitudinal laths nailed to them, to which such tiles or slates can be secured, would be found the cheapest and most efficient in the end. During summer these could be removed to admit of the heat of the sun entering freely into the border; and in very wet and cold localities, external borders should be entirely abandoned. And if grapes are to be produced in cold damp climates, houses of sufficient capacity as to length and breadth must be erected for them, so that the roots may be kept entirely under their protection, and outside borders abandoned.

Concreting the bottom of vine borders, and indeed those of other fruit trees, has long been practised, the object being, by the formation of an impervious flooring, to prevent the descending roots from extending below the prepared soil, and from entering a bad subsoil. It also prevents the damp from below, in low damp 
situations and wet soils, rising upwards and rendering the borders too wet, particularly those in which the vine is to be grown. Vaulting answers the same ends, but with greater advantages-namely, rendering the border not only drier, but also warmer, which is of so muchimportance, by the ascent of atmospheric air, which, warmed by the sun, is admitted to the vaulting below, and from it, passing upwards through the soil, carries with it a considerable amount of solar heat, which becomes, during its ascent, absorbed by the soil. Another advantage arises from this process, perhaps of as great importance to the roots as the warmth-namely, air, upon which, says a great physiological writer, "the roots feed as much as the leaves." Vaulting admits of copious application of liquid manure during the growing season of the trees, and that without rendering the border in any part or at any time too damp. In vaulting hothouse borders-and we hold that all should be so-it is important that they be amply constructed, so that a free circulation of air may pass under them; and to this end a sufficient number of apertures should be left, with the means of regulating them by convenient stoppers, that air may be either admitted from within the house or from the external atmosphere. Besides, they admit of hot-water pipes being carried under them, if deemed necessary. Next in importance to actual vaulting must be forming the border upon a mass of stones laid in as open a manner as possible 18 inches or 2 feet in depth, thickly interspersed from front to back with large drain-tiles, having a communication capable of being opened and shut both with the external air and also with the air of the house, or with the vicinity of the furmace, for the admission of heated or cold air, as may be deemed expedient. This, where pavement is expensive, will be an excellent substitute for vaulting, and constructed at much less cost.

Concreting the surface of borders, whether those in front of vineries or those of the open wall, is of far more recent introduction. We have already, in vol. i., stated some of the advantages. The objections stated are principally the exclusion of air and solar heat, in consequence of the impenetrable covering of concrete on the surface, the exclusion of rain, their liability to become too hot during summer, and too dry in winter. At first sight these objections seem to be strengthened by the generally received opinion that the surface of all cultivated soils cannot be kept too open and pervious to genial rains, air, and solar heat, and hence the admitted utility of frequently stirring the surface between the crops in the open garden. Under the latter circumstances the openness of the surface is the only means by which air, solar heat, and sufficient moisture can by any possibility reach the roots; and when the soil is not thoroughly drained, or resting on a dry pervious subsoil, evil may arise from too great a quantity of the latter being admitted, which, being prevented from escaping downwards, saturates the soil and induces sterility, or serious injury to the roots, and also, by lowering the temperature, retards the crops from arriving at maturity. Concreting unvaulted borders may be subject to some of those charges, but certainly not when the proper precaution of vaulting is attended to. The evidence of Messrs Spencer, Fleming, Beaton, Hutchinson, and indeed most of the best gardeners in England, is to our mind most conclusive as to the advantages of these combined processes. They say, and say truly, that concreting has the effect of detaining moisture in the soil when most wanted -namely, during summer-and excluding it, when least required, during winter; it causes heat to be absorbed abundantly and progressively, as it is required during spring, summer, and autumn, and excludes cold as well as retains heat during winter, which, without such a covering, would, in the first case, be abstracted by the penetration of frost, and in the second would allow the heat accumulated during summer to escape by radiation from the surface. It not only maintains a degree of temperature and humidity exceedingly congenial to the roots of plants, but secures them from mutilation by digging on the surface, as well as prevents the soil from becoming too compact in consequence of treading upon the borders.

Vaulting is of great importance whether the ground is concreted or not, and this will be found to be the more apparent in the coldest and dampest localities. There are many favourable situations, where all sorts of fruit trees prosper to the maximum of perfection, where such precautions are not absolutely necessary, but these are few in proportion.

For further details on concreting, vaulting, heating, and aërating borders, vide vol. i. pp. 322 to 330 , and figs. 435 to 447 ; also pp. 27 to 31, fig. 11.

Cropping vine borders.-Not only should vine borders be left entirely uncropped, but digging and forking should also be avoided. Handweeding and shallow-hoeing are all that is required.

Renovating vine borders.-However well they may be formed in the first instance, in the generality of cases they will require amendment in some shape or other. Those that have been made too deep and too rich in the first instance will be the first to require assistance. Every artificially-formed border sinks more or less according to its depth, and the proportion of decomposable matter put into it. The first evil arising from its sinking is taking the roots down with it, unless they are unfortunate enough to have become suspended in consequence of the main stem and branches being secured to the house or trellis within. When such sinking takes place, it is too much the practice to topdress the surface of the border to keep it up to its original level; by this means the roots become buried too deep to derive much benefit from the sun, or whatever covering may be placed over them to excite them into action, as already referred to. The second evil arising is consolidation, and consequently stagnation, by the derangement of the texture of the soil; than this a greater misfortune can hardly occur. The third evil may be traced to a stoppage of the drainage, in consequence of the drains becom- 
ing filled with those particles in the soil which may find their way into them, either by excessive rains, heavy waterings, or other causes; and hence the border becomes waterlogged, and quite impervious to heat or air. The fourth evil, and a certain concomitant of a border made over-rich, is that the whole body becomes a mass of black fatty humus, containing in itself neither sufficient food for the roots nor a sufficiency of sand to insure a proper transmission of moisture or permeability to the atmosphere. When any of these cases occur, and they are of daily occurrence, it is of no use to stand still and look on; a complete renovation must take place, either of the whole border at once or in part, as the supply of fruit may be excused for a season. In either case the border must be opened from back to front, beginning at one end, and carefully taking up the vines one by one, as early in the season as the fruit is cut. When one trench is cleared out to the bottom, arrange the floor and drainage, and fill up the trench again with new soil, replanting the vines as the process goes on-that is to say, if they are worth the trouble; if not, young ones can be planted next summer, as already recommended (vide Planting vines). Where one season's fruit can be dispensed with, no doubt the better way is to remake the border entirely in autumn, and to replant as above suggested. Where it is desirable to continue the old vines, they may be taken up, if carefully done, and replanted with every chance of success. A full crop, however, must not be expected the following year.

For further on the formation of vinery borders, vide vol. i. p. 320 , \&c.

Planting vines.-The season at which vines, whether for growing against walls in the open air or to be kept under glass, are most advantageously planted, is a point in culture by no means settled; some advocate planting while the vine is in a dormant state, either in autumn or spring; while others, and ourselves amongst the rest, prefer planting after the sap is up, and indeed after the young wood has made several feet in length, should the season even be as far advanced as the middle of June. Our reason for preferring this mode is, that the plants may have the advantage of a much longer season of growth, being excited slowly in a mild heat under glass, and stimulated to the fullest extent by frequent repotting, rich soil, abundance of light and air. By the season we have named, the soil in the border they are to be planted in will have acquired a considerable amount of heat from the sun, the roots will be in full vigour of growth, and the shoot (we prefer leaving one only) will have made considerable progress. If carefully turned out of the pot, and the roots arranged so that none of the spongiolets are injured, the plant will scarcely sustain any check, but will commence growing with increased vigour, care being taken that the operation be performed in a dull cloudy day, or that the foliage be partially shaded for several days after planting. The shoot will reach the top of the house early in autumn, and in sufficient time to ripen its buds and wood before winter sets in. Others prefer planting in autumn and spring, and some cut down the young vine to a single eye at the time; while others-and that excellent cultivator, Mr Saunders of Tedworth, is one-plant in autumn without heading down at all: in this they may be correct with strong-grown plants, but we doubt the propriety of the practice when the plants are weak.-(Vide Saunders' mode of Pruning, afterwards to be noticed, and also our fig. 425 , p. 313, vol. i., from which it will be seen that, instead of his vines breaking at the first or second bud above the surface of the border, as is generally practised, they, from the construction of the double wall in front of his vinery, break immediately under the rafter.)

Mr Appleby, like us, approves of summer planting; he says, "The young vines should be placed in a forcing-house early in spring, planted in baskets lined with thin turf, and filled up round the balls with leaf-mould. The two topbuds only are to be allowed to push, one of which is to be removed when the chances of accident are over. As soon as the warm weather of May or June sets in is the best season for planting, when the vines are removed still in the baskets, which are allowed to rot away by degrees. By this process the roots are secured against accidents, and the plant sustains no check.

"When it is thus placed, and whilst the stem is bare, let a slit be made below each bud, beginning about an inch from them, and making it up to the buds: the buds which are to be treated thus are those three that are at the lower side of the stem, but the two remaining ones at the upper side must not be meddled with. In making the slit, cut into the shoot about one-third of its thickness. When the three tongues are cut, let a little of the soil be put between them and the other part of the wood, so that the tongue will form an angle of $45^{\circ}$ with the stem. The advantage of slitting the stem in the manner described is, that a great number of roots will be produced from each tongue, which consequently increases the number of feeders to the plant, and will contribute in a great measure to promote the vigour of the vine." After the vines are planted, the temperature should be kept at about $55^{\circ}$ during the night, ventilating freely during the day. When they have struck root in the border, increase the temperature to $68^{\circ}$ during the night, with a rise of $7^{\circ}$ or $8^{\circ}$ during the day. Continue the fireheat till about the beginning of July, after which it may be discontinued. Vines so managed should be allowed to extend until they reach the top of the louse, and a few feet in a horizontal direction, after which they should be cut back, but so as to leave four or five buds upon the horizontally-trained part, which will tend to strengthen the upright shoot considerably. All laterals that are produced should be taken off when they have grown about 6 inches. In doing this, $\mathrm{Mr}$ Harrison recommends to "pull them off backwards from the bud. The reason for taking away the laterals is, that the natural fruit-bcaring buds are frequently injured by their being left, and pinched back once or twice during summer. The reason of laterals being retained and pinched back, as alluded to, is to prevent 
the natural fruit-buds from prematurely pushing; but if the leading shoot be kept growing forward as directed,"the buds will not prematurely break or be injured-on the contrary, will be a great deal bolder, and produce much finer fruit the ensuing year."

Harrison recommends planting at this season also, but suggests taking the vines from the pit and placing them in a greenhouse, or some cooler apartment than they were previously in, with a view to prevent them receiving a check by their removal. "After they have been thus prepared for two or three weeks," he says, "they may be planted." In planting, he recommends that the ball be placed so far from the front wall that five eyes of the new shoot, lying horizontally upon the earth in the hole, may be outside, the top of the vine being introduced into the house through the opening in the wall. The vine must be placed so deep in the hole that the upper side of the ball may be 6 inches below the surface, allowing 2 or 3 inches for settling. This we think planting too deep. Others plant by placing the roots 2 feet or more from the wall, laying that length of the stem of the plant in the ground, and tonguing it underneath with a view to cause it to send out roots between the ball and front wall of the house, a process in summer planting we think quite uncalled for.

Mr G. W. Jolnnston, in "The Gardeners' Monthly Volume," vol. ii. p. 50, remarks on vine planting: "A very fine loose soil should be promoted, containing a considerable portion of lively sand, which is well known to facilitate quick rooting. Bencath each vine should be placed a barrowful of chopped turf and of sandy loam, which have been lying together for several months: their being in lumpy masses, not disintegrated, will at once facilitate the escape of moisture and promote a rapid extension of root. To prevent sudden droughts, owing to extreme porosity, the looser compost may be strewn through and over the fibres. The best time to plant is when the plants are about emerging from their torpidity, whether naturally or induced. The ball of earth should be carefully separated, and the roots trained as carefully out as an exhibition-flower on a trellis. When merely covered with a fine compost, a layer of the same turfy matter may be placed over it, and over that a mulching of such fermented droppings as are used for making a mushroom-bed. If indoors, thèy require skilful applications of water for the first four months, after which they may have it more frequently in the heat of summer, especially if they have made much progress. Those planted outside are liable to suffer in the first four months from drenching rains, which must be guarded against by some kind of covering." Our own practice is to remove the soil to the depth of 3 or 4 inches, and to a sufficient extent to admit of the roots being spread thinly and carefully over the space. The pot being laid upon its side, presuming the roots are to be outside the house, and the young shoot placed in a proper position so as to pass over the top of the parapet-wall, care being taken that neither the stem or any of the leaves are injured, the pot is then broken to pieces, VOL. II. the ball carefully broken down, and the roots singled out to their full extent; a little light rich soil is shaken over them so as completely to cover them, and over this 3 or 4 inches of the prepared border-soil is placed on top. The surface is then mulched over to prevent excessive evaporation, and a gentle watering of tepid water is applied. The shoot within is then brought up by degrees to the end of the rafter, and trained to it very loosely, to admit of the plant following the roots, should they sink an inch or so into the ground. Such shallow planting requires water to be frequently given to the roots should the weather be dry, and during the first season the ground over them should be kept covered to prevent excessive evaporation.

It will be necessary to have previously pro. cured young plants from eyes-others are not worth planting-and of one year's growtl. Towards the latter end of February or beginning of March, these plants should be headed down to one bud, and placed in a mild dung-pit, but not plunged for the first few days. They should be repotted into 10 -inch pots in light rich vegetable soil, and placed near to the glass. As the young shoots advance, care must be taken that they be properly supported to prevent their being broken; and when from 4 to 6 feet in length, which they will be by the middle of May, they are in a fit condition for planting; but to attain this height they must not be excited too much, and abundantly supplied with air and liquid manure during their growth.

Vines intended to be grown against walls in the open air should be planted about the first of May, presuming they have hitherto been grown in pots. It would be useless to plant earlier, as the soil is cold, and there is in general little growth in the atmosphere. Where vine borders are vaulted and heated below, which, to have the vine in perfection, must eventually be generally done, we would advise that the plants be slowly excited under glass without the aid of fire-heat, but the pots plunged in a mild bottom temperature for the encouragement of the roots. Under such circumstances the vines may be forwarded to the extent of a foot of young wood, or more, previous to planting out. For a few days the bottom temperature should be attended to, to prevent any check to the roots; and with a similar intent towards the young shoots, they should have the temporary protection of a glasslight for ten days or a fortnight, or, in default of these, a shading of fine semi-transparent canvass during the day, and a thicker covering during the night; for if vines sustain a check at the first outset of growth they seldom recover it. If this is properly attended to, by the first of June they will have established themselves, and thus be considerably in advance of those planted in the ordinary manner. Where the advantages of heated borders do not exist, a somewhat different course should be pursued; namely, to plant just as the buds begin to swell. In the case of vineries, there are two cases to be considered; namely, whether the plants are to be planted inside the house or outside. If the former, great advantage will be obtained by having the vines brought forward in pits or otherwise, 
so that by the first of May they may have extended to the length of from 5 to 7 or 8 feet, with their roots proportionably advanced. A dull cloudy day should be chosen ; the plants, still in their pots, set in the places intended for them for a day or two, to accustom them to the change from a close pit to an open airy vinery. The same process of planting should be followed as recommended for those planted outside. The shoots should then be secured to the trellis or rafter, a slight watering given to the roots with tepid water; and if the sun is likely to come out strong, shade the glass over them for a few days, until they have taken root in the fresh soil. With vines to be planted in the external border we would pursue the sane course, only deferring the planting out to the first week in June. This, however, depends a good deal upon locality, for a difference exists of at least a fortnight or three weeks between the south of England and that of Scotland, except in the case of vaulted and heated borders, where they are much nearer upon a par. Young vines planted within the house will require great care during their first season as to a sufficient supply of water at the roots; in the open air, that must be administered with greater caution, and that always in a tepid state. Should heavy and cold rains succeed the planting in the external borders, means must be used to prevent it frorn saturating the soil or chilling the roots.

Distance at which the vines should be placed.The usual distance is to place a vine to each rafter, although some place them under the middle of each sash. Either of these distances is somewhere from 3 feet 9 inches to 4 feet apart. Some advocate planting them much closer, while a few think one or two vines sufficient for a large house. From the natural fertility of the vine, it is questionable if one plant will not produce as great a weight of fruit as twenty. The chief objections to this would be want of variety, and the impracticability of withdrawing them, either in part or in whole, from the house during their season of rest.

Arrangement.-Experience has long taught us the impropriety of planting strong-growing and weak-growing sorts promiscuously in the same border. The evil of this is, the strong-growing kinds would rob the weaker sorts, and engross the whole border to themselves. To remedy this there are two ways. In large establishments, different kinds may be grown in different houses. 'Thus there may be a Muscat house, a Hamburg house, a Frontignan house; \&c.; and this is a very commendable arrangement, as these not only differ in the luxuriance of their roots, but at certain seasons require a somewhat different treatment within. For small places, where there is not the accommodation of separate houses, and where variety may be required, it will be judicious to divide the borders by cross walls of masonry, setting apart a division to each. By adopting this plan, a soil may be prepared for each section more consistent with its habits than were they all planted in one, as some require a strong, while others prefer a much lighter soil and shallower border. It should not escape the attention of the planter, that where variety is required within a limited space, the early-ripening sorts should be planted at the end nearest to where the flues or hotwater pipes enter, and the later-ripening sorts at the opposite end. This is done with a view to prolong the season of the fruit. There is much less danger of not securing a crop in the mixed style, unless the forcing be very early or very late; but the great evil arises from the difference of time each requires to ripen in-the Sweetwaters and Frontignans ripening early; the St Peters, Tripoli, Syrian, White Nice, \&c., ripening later-indeed, being not nearly arrived at maturity after the former have been cut, and the wood is in a fit state for going into repose, which implies a cool temperature, which could not be afforded them if the ripening of the others is at all a consideration. It would be folly to force the late sorts of grapes early, and as bad to attempt to retard the early sorts till February or March. Some sorts, such as the Chasselas musque, or St Albans, as it has been called by some, require to ripen in a very dry atmosphere, and, if kept in such, will hang on the vines from their ripening in September to the beginning of January. Such a sort planted along with late-growing kinds, which require a greater degree of atmospheric moisture to enable them to attain maturity, would crack and rot before it had fully attained its utmost degree of ripeness, without which it is little better than a common Muscadine.

Autumn or winter pruning.-It is often asked, When is the proper season to prune the vine? To answer this in a general way, it may be said that this depends entirely on circumstances. Vines, to be forced early, must be pruned early; yet it does not follow that vines to be forced late should be pruned late, for late pruning is bad for the vine; yet it is late before the fruit is cut, and the vines cannot be pruned before, excepting by one process, and that is the destruction of every bud not required for next season's crop. It has been asserted by some writer, whose name we do not at the moment recollect, that in the case of vines growing in the open air, early-pruned plants produced their fruit earlier the following season than those near them that were not pruned till much later in the season. This may have been owing to excessive bleeding, which is well known to retard growth. As a general rule, we may say that vines should be pruned at least six weeks before they are to be put into'a state of excitement. Whatever effect this may have on the ripening of the crop may be left for speculation; but one certain advantage arises from the practice, that the wounds have time to heal before the ascent of the sap, and consequently bleeding is prevented. The vine, like most other fruit-bearing trees, is trained in a variety of forms, and consequently the modes of pruning will vary accordingly. The most usual modes, however, in hothouses are the spur, long rod, and irregular forms.

Spur-pruning. - The spur mode of pruning will be understood by the following remarks, commencing the first season after planting: Train one shoot to each rafter, the plants, it being presumed, being planted opposite to them. 
During this season allow the shoot to extend to as great a length as possible without stopping; and should it reach farther than the top end of the rafter, train it along the back wall in a horizontal direction. As the laterals proceed from the main shoot, pinch them off at the first joint, and also remove all the tendrils. Some content themselves with this extent of pruning, while others begin to cut off the lowermost laterals entirely, about the time the main shoot reaches to within a fourth of the length of the roof or rafter, and so continue removing them all the way up as the wood hardens. The second autumn, or beginning of winter, the vine should be cut down to three eyes from the bottom of the rafter; and after it becomes excited, train in the shoot from the top eye to the wire under the rafter, and throughout the season manage it exactly as the shoot of last year, only stopping it at the top of the house to throw more strength into the eyes below. The other two shootsthat is, those from the first and second budsare to be stopped when they attain the length of three or four joints. On the lowermost of these a bunch may be left to prove the sort, and both these should be laid in at nearly right angles, and be stopped again and again as they push fresh shoots, always pinching them off at the first joint. The third autumn, if all has gone on well, the wood will be strong and well ripened; and at this time cut back the leading shoot to about one-third the length of the rafter. The following excellent directions have been given by $\mathrm{Mr}$ Appleby for proceeding after this period: "Train the top shoot up the rafter again, repeating the same operations of removing tendrils, stopping laterals, \&c., stopping it at the top. The side shoots or spurs stop at the joint which shows fruit, and only leave one bunch on each spur. This is a general rule for every year afterwards. Tie each spur at right angles from the centre, so that there are two rows of bunches, one on each side of the rafter. The fourth season bring up the spurs to twothirds of the intended length of the vine, following the system as during the third season, with this difference, that if any of the spurs are weak, and show poor bunches of fruit, nip off the bunch to strengthen the spur. The fifth season the shoot extends the length of the rafter, so that in four year's the whole rafter will be clothed with fruit-bearing spurs, after which all they require is to cut through the second bud every pruning season. Only leave one bud to each spur, though some cultivators leave two; but in that case the spur will soon become long and unsightly, and the farther the bud is from the main stem, the weaker it will be. Even with one bud each year, the spur will in time require renewal. To effect this, train up a young shoot from the bottom, and the year after cut off the old shoot with all its spurs, and manage the young one exactly in the same way as a young vine. Do this to every other vine, as the house would be without fruit one or two years were they all cut down at once."

Seaton and Griffin-the former a horticultural amateur of considerable eminence, and the latter a successful cultivator-have laid down theories of pruning and training the vine. The former, with a view to equalise the production of fruit over all parts of the tree, and also to arrange them so that the early and late kinds shall ripen in the same house at the same time, trains the early sorts nearest the bottom, the medium kinds towards the middle of the house, and the later sorts towards the top; and when it is desired to have some early and others late, let the order of arrangement be reversed, \&c. The latter leads only a single shoot up under each rafter. At planting, the vine is cut down to one eye, the shoot proceeding from which is about Christmas following cut down to 2 or 3 feet. The second year after planting, one shoot only is trained from the extremity, and it is again headed down in winter, so that the united length of the two years' wood is from 10 to 15 feet. At the Christmas following, the shoot is cut off at the end of the rafter. From the spurs proceeding from this main shoot the fruit is produced. The spurs are cut back to single eyes every winter, till the main stem becomes coarse and unsightly, when it is entirely removed, precaution having been taken to provide during the two preceding seasons a successor to it. "As soon as the plants become sufficiently strong to furnish wood, from the point where they enter the house, for a second and third branch, then a proper number must be fixed upon as permanent plants, and their side branches brought successively forward and trained to the contiguous rafters, one bearing-branch being applied to each rafter, and the plants which originally belonged to these rafters taken away entirely. I also contrive," Mr Griffin continues, "to spread the branches, when in bearing, on either side of the rafters under the glass, but so as not to occupy the whole space under the glass with the foliage ; for I consider," he says, "that a very great advantage arises to the fruit from giving free admission to the sun from the centre of each light."-Hort. Soc. Trans.

Saunders' system of spur-pruning--Mr Saunders, in "Practical Treatise on the Vine," treats his vines upon this principle. As we have elsewhere noticed, he plants in autumn, using plants 3 or 4 feet in length. "Towards the end of March or beginning of April the sap will, without artificial heat, begin to circulate through the newly-planted vines, and the buds will then, of course, be immediately put forth, all of which must be removed with the exception of the two leading ones ; and the safest method of doing it is by pressure between the finger and thumb, as the use of the knife would at this early stage cause them to bleed." "When the two reserved buds have broken, and grown a foot or rather more in lengtl, cut off the weakest, thus leaving only one shoot to each vine. And here it may be as well to remark, for the information of the learner, that this shoot will in due time become a part of the bearing stem ; consequently, as it progresses, great attention must be paid to tie it in regularly and carefully, and every precaution must be taken to prevent its being bruised or broken, either by handling it roughly, or tying it too tightly. In the latter case, be sure to give plenty of room for expansion." The usual 
allowance is to give as much room as will admit the finger freely within the folds of the matting along with the shoot. "The matting used for this purpose should never be drawn tight, as, by so doing, if the brittle shoot is not broken by it, the circulation of the sap will be obstructed, which is an evil carefully to be avoided. When the shoot has grown long enough to reach the top of the house, it will be necessary to stop its further progress by pinching it off; and, provided it is in as vigorous a state as it ought to be at this period, it will very shortly throw out a lateral branch at the bud nearest the extreme end, where it has so recently been stopped, which lateral branch must be allowed to grow until it has attained the length of 12 or 14 inches, when it should be stopped, by taking a joint off at the point, as recommended in the case of the principal shoot." The object for doing this is, that "the crop for the next season entirely depends upon the buds along the rod remaining in a quiescent state; and the only means of keeping them so is the encouragement given to the sap to flow freely to the part to which the lateral branches or buds are proceeding, and thus preventing the buds from being prematurely excited. There will also be either a direct or lateral shoot thrown out from the base of these important buds; but they must not on any account be permitted to make much increase; and in order to prevent it, stop them beyond the first joint - and this must be repeatedly done if their growth should rendcr it necessary ; and the same rule must be observed with the lateral shoots at the end of the vine also." The first stage of spring pruning, commonly called disbudding, or the removal of superfluous buds before they have developed into shoots of any size, is a species of pruning all trees that cannot be too much insisted upon, as it regulates the proper number of branches that should be left before they have in any way drawn upon the energies of the tree. All removals after this stage, unless in extraordinary cases, are much to be guarded against, for every branch allowed to extend beyond the length of 2 or 3 inches when removed, robs the tree of that amount of strength which was required for the shoots' formation; and when we come to consider the great injustice done to a tree by allowing it to form perhaps a hundred shoots a foot or two long, and then to cut two-thirds of them off at once, which with inexperienced and careless people is often the case, we need not wonder at scanty crops or sickly and diseased trees. This early disbudding is of the greatest importance to all trees. When, therefore, the vine is pruned upon the spur system, and the young shoots have grown to the length of about 2 inches, at which time also the bunches will be readily distinguished, the number of shoots should be selected, choosing those which have the most promising share of fruit, if they are properly placed at as regular distances apart as possible, leaving not more than twelve or thirteen on each vine. The others should be all cut away close to the main stem. In the November following, planting when the wood is fully matured and ripened, Mr Saunders recommends that the vine should be pruned; and in doing so, it is, he observes, "the practice of many persons to cut the young rods down to within a bud or two of the original stem, thus deferring the time of bearing for another year, with a view of invigorating and establishing the vines." This practice Mr S. dissents from, and states that if his practice is followed they will be in a fit state to carry a good crop the next season-that is, the season after planting. $\mathrm{Mr}$ S.'s system of winter - pruning is as follows : "Shorten the young shoots 2 feet from the top downwards; by doing this, the vines in the next season will have room to shoot forth and bear their fruit without coming in contact with the top or back of the house. The lateral shoots should be also cut off to within half an inch of the bud from the base of which they proceed."

Harrison approves of spur-pruning, and says: "When the vines are pruned, the lateral shoots or spurs must be cut down to two buds, as fig. 245, $a$ a $a$. By shortening the spurs so much,

Fig. 245. the vine is kept in a vigorous state, considerably larger than if they were left longer. But it is necessary to leave two buds upon eacl spur, for it sometimes happens that one of the buds will not show fruit well, or may be damaged; but by reserving two, a supply is more certain." At the winter-pruning, the second rear after planting, "the leading shoot must be pruned back so as to leave 5 or 6 feet in length of the last summer's wood. 'This must be rcgulated, however,
according to the length of the $a$ 'This must be rcgulated, however,
according to the length of the "swe resters. If the vines are to be HARRISON's trained to the length of 14 feet, it SPUR VINE- must now be pruned to 5 feet, and TRAINING, the year following to 5 feet more, tent." If the vines are to be trained to the extent of 17 or 18 feet, which is the case in some wide houses, the leading shoot must be cut so as to leave a regular proportion of young

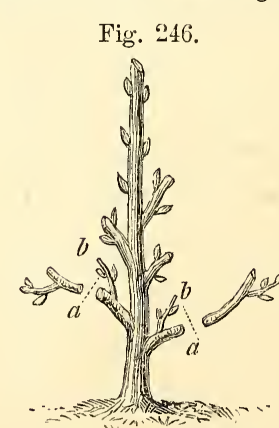

HARRISON'S SPUR VINETRAINING, THIRD YEAR。 wood each year, until they reach the top of the house. "IVhen the spurs or buds $a \quad a \quad a$ liave broken, and show good bunches of fruit, one only must be left on each shoot. If the uppermost bud do not show fruit, let it be taken entirely away; but if the uppermost buds show fruit, and the lowest do not, both of them must be retained."

The same authority at the third years' pruning remarks : "Those spurs which had two shoots retained during the last summer, must now have only the lowest retained, and each spur must be pruned off as fig. 246, $a$ a. The 
shoot $b$ must also be cut down to two buds. This attention to keep each spur supplied with bearing-wood as near to the main stem as possible, must in every future pruning be strictly pursued."

Paxton's method of spur-pruning.-This intelligent cultivator details in "Gardeners' Chronicle," 1842, pp. 757 and 758, another mode of spur-pruning, which we think admirably adapted to out-of-door culture, and, like the Thomery mode, it reduces the whole operation to something like system. "The sketch" (fig. 247), he

Fig. 247.

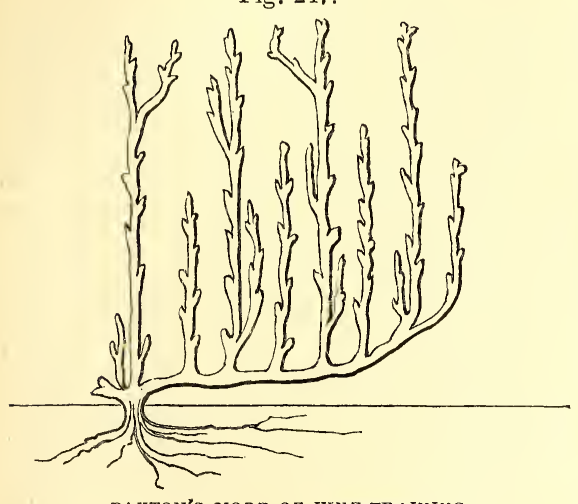

PAXTON'S MODE OF VINE-TRAINING.

says, "represents a portion of the vine when pruned in autumn on the spur system, with short rods of five or six eyes each left at convenient intervals on the oldest branches throughout the vine. The perpendicular main shoots should not be less than 2 feet apart, and when pruning them no useless eyes should be leftthat is, no eye should be allowed to remain but where a shoot is desired in the following season. By attending to this the vine will not have to develop (as is usually the case) an immense quantity of superfluous branches; and although this operation may appear a tedious one at the time of pruning, an immense saving of labour and time will be effected at a busier period in spring, and the quantity of fruit may be easier regulated in proportion to the strength of the vine. If this is attended to, nothing will be required in summer but securing the young fruit-bearing shoots to the wall, and shortening them at one eye above the bunch as soon as the fruit is set, excepting the leading shoots, which should not be stopped until the lower part is ripened, otherwise the main eyes for the next season may be induced to grow prematurely. In autumn the young wood from the spur is shortened back to one or at most twc eyes, and the terminal shoots in proportion to their strength; but, for the strongest wood, from eight to twelve eyes will be found as many as will break well. When commencing to train a young vine in this manner, the side branches should not be brought to the horizontal position at first, but be lowered gradually, as the number of suitable branches for upright stems is ob- tained; by this means they acquire strength faster than if trained horizontally at first."

Fleming's system of spur-pruning.-Amongst the various modes of managing the vine practised at Trentham, is a mode of pruning of which the following is the substance: "When a vine has reached the top of the house, and is of sufficient age and strength to maintain a crop, it is allowed to throw out a lateral shoot at every 10 inches, or nearly so; every altermate lateral is allowed to bear the first season; the non-bearing shoots are only permitted to ripen their wood, which is stopped on the spur system. At the pruning season those laterals which have produced the crop are cut clean back, while those which have produced no fruit are cut back to a spur having about two eyes on it. These spurs are designed to produce the following year's crop, whereas the shoots which are thrown out from the pre: ceding year's bearing-spurs are permitted to form well-ripened spurs only, and which are destined to produce the crop of the succeeding year ; and as this plan of alternate fruitful and barren is followed up, it is found to answer better than any other system of pruning, either for moderately early or late culture; and, judging from the remarkably abundant and beautifully-coloured late crops which are produced, no doubts as to the system can be entertained."-Gardeners' Chronicle.

Advantages of the spur system.-The chief merits of this system are, regularity, absence of excessive shade under the foliage, a greater admission of light and air to the fruit, and; prin- cipally, that no more wood is grown than is absolutely necessary for the production of the present crop, and at the same time securing a supply for the ensuing year. This is a very popular mode of training, and practised by many of our first-rate grape-cultivators. Mr Saunders thus states its advantages over that of growing a fresh supply of rods annually, that is long shoots, by which, he justly says, "the quality of the present crop is noi only materially deteriorated, but by the growth of the new rods the house becomes crowded, and, consequently, that free circulation of air, so essential to the grape in all its stages, is considerably lessened by the superabundance of foliage intercepting to a great extent both light and air. Another advantage also arises from the spur system : the double demand made upon the vine, by bearing its fruit and feeding the rods at one and the same time, greatly accelerates its exhaustion and decay."

In the spur system a greater number of bunches are produced than upon the long-rod principle, but in the opinion of many they are neither so fine nor so large.

Renewal of vines pruned on the spur system.Vines treated in this way will continue, if in a proper soil, in good condition for ten or twelve years, after which the spurs will become rugged and unsightly, hence a removal will be found expedient. This is best effected by cutting the vine down to within a few inches of where it enters the house. There are at such parts abundance of latent buds ready to start as soon as the head is removed. The young shoot produced by this 
operation must be carefully attended to during summer, and at the next winter-pruning it should be shortened back to the length of 6 or 7 feet; the next winter-pruning in a proportionate degree, and so on until it gains the top of the house. Of course this method will involve the loss of a crop for a season; but when this cannot be done conveniently, every alternate vine may be thus cut down, leaving the others to produce fruit, which in this case may be left on in greater number, to make up for the loss of the others.

Some vine-growers renew their vines by training up a young shoot from some convenient part near the bottom, and by this means, in a couple of years, have a shoot prepared to form the future leader. This mode is not to be recommended, for in effecting it both shoot and crop suffer.

Mearns' long or succession mode of pruning is fully described and illustrated in the fourth volume of "Transactions of the Horticultural Society ;" the following is the substance of it. The plants are set within the house, close to the front wall, at the distance of $2 \frac{1}{2}$ feet apart, and are headed down to within a foot of the ground at the time of planting. Only one shoot is allowed to proceed from each plant, which, at the termination of the first season, is cut down to the second or third eye. The year following, two leading shoots are allowed to proceed, the strongest of which is stopped when it has extended three or four joints beyond the middle of the roof, and the weaker after it has grown 3 or 4 feet. In autumn following, the leading shoots are shortened back, the principal one to the length of the middle of the roof, and the lower one to the third eye. At the third season's pruning, one leading shoot is trained in from the principal leader, and from the bearing-wood immediately below the last fruit-bearing side-shoots are produced, one branch is left on each, and the shoot stopped at one or two joints above it. No side shoots are allowed to proceed from the shoot coming up in succession, the leading shoot of which is to become the bearing-wood of the next year. By this process the lower part of the house in the third season is furnished with a crop of fruit from shoots proceeding from wood of the preceding year, and parallel to this bearing-wood on each vine is the young wood for the next year's crop. At next winter's pruning the shoot from the extremity of the bearing branch is cut off to within a few inches of the top of the rafter, and the shoot following in succession, and parallel to the last, is shortened back to the middle of the roof, and all the spurs which had borne fruit upon the other are cut clean away. At this period of management each vine is furnished with two shoots of bearing-wood, that portion of old wood that had produced fruit during the past summer, and also a spur within a few inches of the ground, from which a shoot for the following year is to be produced. During the fourth season a full crop of fruit is produced on the upper and lower half of the house, the longest shoot bearing on the upper half of its length, and the shorter on its whole length, while two leading or successional shoots are encouraged, the one from the short shoot, and the other from the spur near the ground. The centre shoot, which had now borne two crops of fruit, and been allowed the whole length of the rafter, is cut out at the winter-pruning of the fourth year, and the side shoot growing parallel to it is substituted in its place, which has now reached the top of the house; and this shoot is in its turn cut out and replaced by the next in succession, and so on-thus constituting the successional system, which might be continued for any length of time.

Main's long-shoot pruning. - The following judicious remarks on winter-pruning the vine, especially those grown in pine stoves, were given some years ago by the late $\mathrm{Mr}$ J. Main, in a note whicl appeared in "The Gardeners' Magazine," vol. ii. p. 413. Of the advantages of the plan we have had several years' experience. "In the culture of the vine it is sometimes necessary to lay in shoots of great length. In such cases much care is required that a regular and sufficient number of the fruit-buds should break from the bottom to the top, and prevent the lower part of such shoots from being quite naked and barren. To avoid this, let the pruner, after cutting the shoot to the required length, and finding, from the firm texture of the wood, that it is sufficiently ripened, proceed to thin the buds as follows: viz., leave the uppermost bud, which may be called 1 ; cut out 2 and 3 , leave 4 ; cut 5 and 6 , leave 7 ; and displace 8 and 9 , and so on to the bottorn of the shoot. This thinning of the eyes will cause all those that are left to break regularly, and so alternately with each other, that the disposition, whether for the sake of superior fruit or facilitating the future management of the tree, will be found exactly what the manager would wish, he taking care to stop all the young shoots in their progress, immediately beyond the fruit, except the lowest, which must be trained to its full length for similar management the following year." This is a mode of proceeding that deserves much more attention than appears to be bestowed upon it.

The illustrations given by Harrison show the long-rod principle more clearly than any description can convey. Fig. 248 represents a

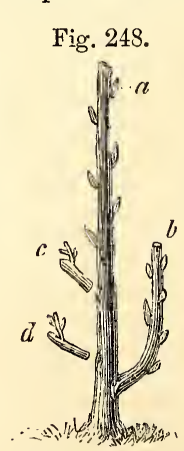
vine at the second winter-pruning; the leading shoot $a$, proceeding from that which produced fruit the previous summer, is cut back, leaving 6 feet only of that year's growth; the lowest shoot $b$ must be cut back to the length of 4 feet from its base. The intention of this cutting back is to retain no more buds than the plant will be fully able to sustain, both in the formation of wood and production of fruit. That part of the main stem which produced fruit is to be completely deprived of its buds, HARRISON'S HONG- as shown "at $d$ c. Fig. 249 , SECOND YRAR. Shows the "vine at the third 
year's winter-pruning. Here the leading shoot $a$ is to be cut off to 6 feet in length; the second

Fig. 249 .

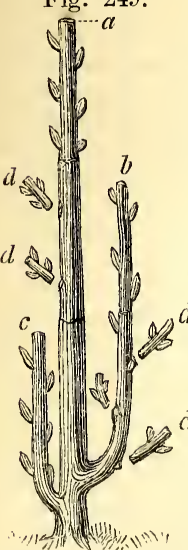
shoot $b$ is to be cut back to 6 feet, and the third shoot $c$ to 4 feet in length. All the lateral shoots, whether they bore fruit or not, are to be cut clean away, as shown at $d d d d$. When the vines break the ensuing spring they will have three distinct bearing-shoots, as $a b c$. The uppermost must the ensuing summer be allowed to bear two bunches of fruit. This is done with a view to prevent its growing too vigorously, and drawing too much upon the energies of the lower parts of the tree. The top buds of the shoot $b c$ should not be allowed to produce any fruit, as it is from them that the young shoots HARRISON'S LONG- are to be produced. At this IUDVINE-TRAINING, period it will be desirable to THIRD YEAR.

procure a young shoot from the bottom of the vine, from the old wood, if possible; if not, as low down on the others as it can be got; and also it would be desirable, if it proceeded from the opposite side of the vine to that from which the last shoot $c$ was trained in.

Fig. 250 shows the vine in its fourth year's

state, which is merely a repetition

of the two previous cuts, except. 0 ing that the main stem, which had

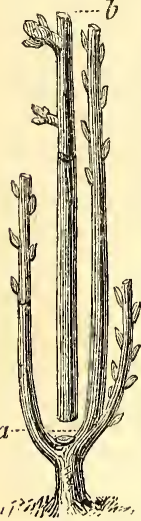

HARRISON's LONG-ROD

VINE-TRAINING,

FOURTH YEAR. reached the top of the house, has now to be entirely cut out at its base $a$, the shoot $b$ then becoming the leader. This is the simple rotation, and mav be continued for years. There is one principle to be observed in this mode of pruning - the constant succession of young wood, and upon that alone the fruit is to be produced, which, according to many, will be larger and finer than when grown upon even the spur or any other mode. This opinion, however, may admit of some qualification.

Renewal of the vine pruned on the long-rod principle.-Although vines treated in this way, if properly managed, will last for many years without renewal, as that it may be that, from exhaustion from overcropping or insufficient food, they may show symptoms of weakness. In such cases, therefore, they should be cut entirely down to one or two buds at the base of the lowermost or lastformed shoot, and be afterwards treated as young plants.

Pruning the vine when grown in the open air.Here a greater variety of forms may be indulged in, and circumstances often dictate why one method of pruning and training-for these are inseparable operations-should be preferred to another. In gelleral a small amount of young wood is considered of advantage, on account of its being more likely to become well ripened, particularly in a climate like Britain, which is not naturally congenial to the vine. Objections, however, have been stated against this doctrine; those by Mr Clement Hoare are certainly the strongest, which he has detailed in his interesting "Treatise on the Vine."

Hoare's method.-Instead of the spur system, or that of employing young wood in short lengths, he prefers shoots trained to their full length, and to produce those he cuts down to very short spurs, at the winter-pruning, the bearing-wood of the preceding season, keeping up a constant supply pf young bearing-shoots at their full length, and so on in alternate succession. This is reducing the process to an extremely simple principle, which mainly consists in getting rid annually of the old wood, and keeping up a constant succession of young bearing-wood. He gives the following simple directions: "In pruning, always cut upwards, and in a sloping direction. Always leave an inch of blank wood beyond the terminal bud, and let the cut be on the opposite side of the bud. Prune so as to leave as few wounds as possible, and let the surface of every cut be perfectly smooth. In cutting out an old branch, prune it evell with the parent limb, that the wound may quickly heal. Prune so as to obtain the quantity of fruit desired, on the smallest number of shoots possible. Never prune in frosty weather, nor when a frost is expected. Never prune in the months of March, April, or May. Pruning in either of these months causes bleeding, and occasions thereby a wasteful and injurious expenditure of sap. Let the general autumnal pruning take place as soon after October as the gathering of the fruit will admit. Lastly, use a pruning-knife of the best description, and let it be, if possible, as sharp as a razor." For the first four years of the vine's management he recommends: In the spring next after planting, two buds only having been left, the one which is produced most weakly is to be removed, and all the others are to be rubbed off excepting the one selected to remain, and this one must be preserved with the greatest care. In November following head down this shoot so as to leave only two buds, and during the following spring manage as before, and in the November following cut down to three buds. The third spring retain two shoots, treating them as before, but in September pinch off their tops, and in November prune them so as to retain seven buds. In February of the fourth spring, bend the two shoots already produced down into a horizontal position parallel with the ground; destroy the first, second, fourth, fifth, and sixth buds on the horizontal shoots, and allow the shoots from buds three and seven to extend, and train them to the wall in an upright although tortuous direction, somewhat similar to the plan recommended by Forsyth. During the succeeding summer prune and train as already directed, removing all superfluous shoots as they appear. In the November following cut back the outermost shoot on one side, and the innermost on 
the other side of the shoots already recommended to be laid in, to about eight or twelve eyes, according to the strength of the vine in the one case, and to one bud each in the other. In the fifth spring train in the shoots from these single buds in the same tortuous manner as before. The plant will now have assumed the form which it is permanently to retain, and the manner in which it is trained may be considered as the commencement of a system of alternately fruiting two shoots and training two at full length for bearing-wood in the following year, which method may be continued every year without any alteration, until the capacity of the vine is equal to the maturation of more fruit than can possibly be borne by two single shoots, which on an average may be estimated at sixty pounds' weight annually. Several years must elapse before this will be the case; but when it is, the arms or "horizontal branches" may be lengthened by the training-in of a shoot at their extremities, and managing it in the same manner as when the arms were first formed. It is very advisable, however, that the vine should not be suffered to extend itself farther on the wall, for in such case the bearingshoots emitted from the centre are sure to decline in strength, whereas, by confining the dimensions of the vine to a single arm on each side of the stem and each arm to the support and nourishment of two branches only, the very best description of bearing-shoots will never fail to be generated close at home, and these, as the vine advances in age, will become prolific almost beyond conception. Mr Hoare says he has "often ripened as many as seven full-sized bunches of grapes off two shoots which have pushed from a single bud on vines managed in this manner."

Mr Hoare prunes in winter, leaving on each vine no greater number of buds than appears, on an average calculation, to be sufficient to produce as much fruit as the vine is allowed to mature. "Vines thus pruned, with the bearing-wood annually adjusted to their respective powers of maturation, being kept within a small compass of the surface of the wall, are easily managed throughout the summer. With respect to the number of buds necessary to be left at the autumnal pruning to produce any given weight of fruit, I have found it to be a good general rule, and applicable to all those sorts of grapes usually cultivated on the open wall, to consider every bud (rejecting the two bottom ones on each shoot) as equal to the production of half a pound's weight of fruit-that is, if the stem of a vine measures 5 inches in girth, its capability is equal to the maturation of twenty-five pounds' weight of grapes, and therefore the number of buds to be retained after pruning will be fifty." In connection with this part of his theory, he says that, until the stem of the vine measures 3 inches in circumference, no fruit should be taken off it; and as a rule for the guidance of the pruner, he recommends that the stem of the vine be measured at the autumnal pruning, and no more buds retained than are supposed necessary to produce the given weight of fruit which corresponds to its girth.
The proportion above stated, he remarks, would be too great, even in the strongest-bearing sorts; but as accidents frequently happen to the bunches during their early growth, and as there will be in general some buds that will not burst, provision must be made against these casualties, by reserving a greater number of buds than would otherwise be required. The proportionate number, therefore, above mentioned, $\mathrm{Mr}$ Hoare "has found to answer well, and be sufficient to meet all contingencies."

Mr Hoare has seemingly a great aversion to old wood in any part of the vine, and in his attempt to establish this point, he says: "The old wood of a vine, or that which has previously borne fruit, is not only of no further use at any subsequent period, but is a positive injury to the fertility of the plant. The truth of this remark," he continues, "depends on the fact that every branch of a vine that produces no foliage, appropriates for its own support a portion of the juices of the plant that is generated by those branches that do produce foliage." The arguments here adduced, in support of the process by which the life of a vine is sustained, are not exactly in accordance with received opinions; but from them he draws the conclusion, "that every naked branch of a vine, or one that does not directly produce foliage, diminishes the capacity of the plant for the production of young bearing-shoots, inasmuch as it contributes nothing to the growth of the vine, but, on the contrary, requires to be fed annually with a certain portion of the elaborated juice of the plant, which would otherwise be expended in the enlargement of the diameter of its stem, and thereby the increase of its capacity to mature fruit, and in the extension and multiplication of its roots." Naked branches are, therefore, according to this doctrine, consumers and not producers; consequently, the great object to be kept in view will be to procure a sufficient quantity of young bearing-wood, with the very least possible quantity of old wood.

Mr Hoare's notions of vine management are, for the most part, greatly at variance with general practice, and some of his views are not in accordance with physiological doctrines. In regard to the size of the vine, or the quantity of wood that should be allowed to be produced, he says : "It is a very common notion, but an erroneous one, and one that has no doubt arisen from the universally defective method of pruning and managing that plant, whereby the wood is suffered, and indeed encouraged, to extend itself disproportionately beyond the capability of its fruit-bearing power. I scarcely ever allot more than 40 or 50 square feet of surface for one vine; and unless the soil and situation be very superior indeed, a single vine will require a space of time, not less than twenty years at least, before it will possess a sufficient degree of strength to enable it to mature annually a greater quantity of grapes than can be trained on the last-mentioned extent of surface." After alluding to the extent of surface usually allotted to the vine, he continues: "And this seems to be done under the idea that the more wood there is in the vine, the more grapes it will produce, 
or that the one will be in proportion to the other. It happens, however, that the fact is precisely the reverse. If a vine be suffered to make a large quantity of wood, it will bear but little fruit; if it produce good crops of fruit, it will make but little wood: the one checks the other. To permit a vine, therefore, to make a great quantity of wood, under the idea of getting thereby a great quantity of grapes, is completely grasping at the substance and catching the shadow."

On the Continent, where the vine is grown with advantage on the open walls, various modes of pruning and training are resorted to. That practised at Thomery, near Fontainebleau, a locality famed for the excellence of its grapes, especially the Chasselas de Fontainebleau, which, when ripe, is sent in presents over most of the European continent, is the most systematic, if not the best. The walls against which they are grown are of clay, and about 8 feet in height. The vines are planted several feet from the wall, but with what advantage we could not even learn on the spot. The spur system is followed, and two branches are trained from each stem, as will be seen by our cut, fig. $25 \mathrm{l}$, and to these three

Fig. 251.

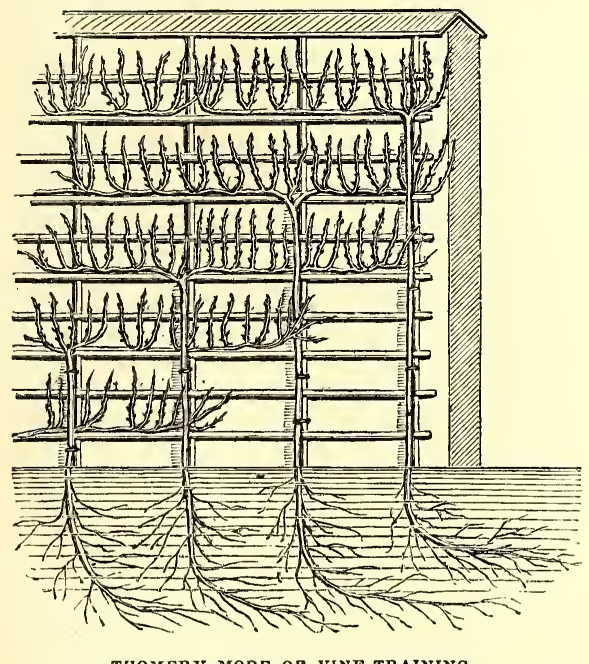

THOMERY MODE OF VINE-TRAINING.

peculiarities the most of the success is attributed. The following perspicuous description of this peculiar practice is thus given from the "Gardeners' Monthly Volume," vol. i. p. 139, which is an abbreviated quotation from the "Horticultural Society's Transactions," vol. i., and "The Gardeners' Magazine," vol. v.: "The main branches of each particular vine-plant assume above ground the form of the letter $\mathrm{T}$, each arm being 4 feet long, the spurs 7 inches apart, and the upright stem being shorter or longer according as the two arms, or horizontal branches, are higher or lower on the wall. The horizontal branches are placed 18 inches apart, the lowest being 6 inches from the ground, so that a wall VOL. II.
8 feet high will contain five lines of mother branches. If the plants are all planted on one side, their stems at the base of the wall will be 18 inches apart, but in very poor situations they are planted on both sides of north walls, and the stems of those on the north side brought through holes in the wall to the south side. During the formation of the cordons, the spurs on their arms will successively come into bearing, and each, when pruned down at the season to two or three eyes, will produce as many shoots with fruit. Of these, at the next winter's pruning, only the lowest shoot is suffered to remain, and that, at the same time, is to be cut back to one, two, or three eyes, according to its strength. The eyes at the bottom of the spurs are very small, and very much crowded; there are at least six within the space of one-sixth part of an inch. When the spurs are cut to the length of 1 or 2 inches, these small eyes are robbed by those above them; but when the spurs are cut short immediately above these eyes, they then break, develop themselves, and produce good bunches. Of this the vignerons of Thomery are well aware: they never leave their spurs more than an inch long, and sometimes less, by which means they always keep the bearing-wood at home; and, extraordinary as it may appear, spurs that have borne for twenty years are no more than an inch long. Should more than two shoots break from a spur, all above that number are suppressed, and not more than two bunches are left on each side of these; for a moderate crop of good grapes proves of greater value than a more abundant crop of inferior quality. When the space of walling allotted to the five cordons is completely occupied, about 8 feet square, or 64 square feet, are filled, and the produce calculated on is three hundred and twenty bunches; for each arm being 4 feet long, and furnished with spurs 6 inches apart, the two arms will carry sixteen spurs of two eyes each; and, allowing two bunches to every eye, each tier or cordon will bear sixtyfour bunches: the number on five cordons will, consequently, amount to three hundred and twenty. This precise length of 4 feet to each arm has been determined by experience to be the fittest. The vignerons found that when the arms were left of a greater length, the spurs in the centre gradually declined, and good bunches were produced only at the extremities of the cordon; but when reduced to 4 feet, the spurs on the whole length were perfect, their eyes well filled, and the bunches of fruit fine and well swelled. Training in cordons after this manner affords these additional advantages ; every portion of the wall is equally filled with bearing-wood, and when once the cordons are completed, the pruning and training becomes so uniform and simple that it may be intrusted to any intelligent workman. But what renders this practice of still greater value in this country is, that the fruit on these small spurs always ripens earlier than on the stronger wood."

The vine produces its fruit on the young wood of the same season, and this is procured from the buds which are placed on the wood made during the previous year, whether it has 
produced fruit or not, whether it has been reduced to the condition of a short spur by pinching back during summer, or is a shoot of greater length. Hitherto it has been considered expedient, and as suitable to our climate, to encourage the growth of young wood only to a limited extent, because the smaller the volume the more likely is it to become matured. The older or leading branches, however, have been and are carried to a considerable length, some holding an opinion that the fruit produced farthest from the root is the best. This, we may, however, remark, is different from the views introduced by the managers of the vineyards on the Rhine, and indeed in all cold vinegrowing countries, who train their vines much as we do our red and white currants. In accordance with the former of these views, vines are often trained as in the annexed figs., 252, 253,

Fig. 252.

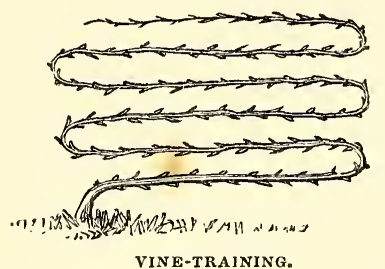

254, which undoubtedly answers the end in view. Others again plant their vines at considerable distances apart, and, training the main

Fig. 253.

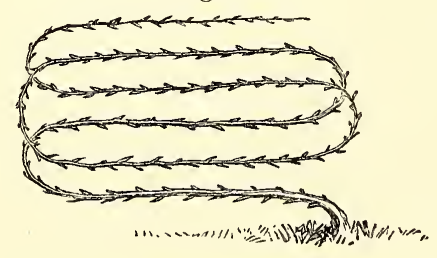

VINE-TRAINING.

single stem upright to the height of the wall, conduct a main leading shoot along under the coping to the right hand and to the left, and

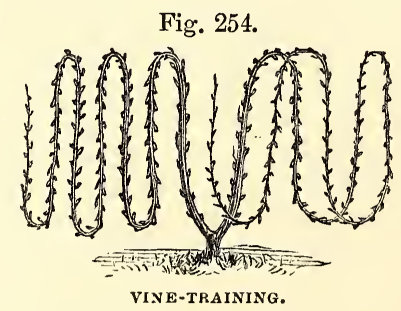

these they carry to a very great distance, and, by inverting the bearing shoots, fill up the spaces on the wall not as yet covered with other fruit trees. As the vine may be carried to 100 feet or more in each of these directions, various sorts might be grafted on them to afford variety, while the root of the main stem would not interfere with the roots of the other trees, as one plant would be sufficient for a long wall.

The vine is also trained on the open wall in the vertical manner, the most opposite in principle from that to which we have alluded. It is thus managed: The plants are set at from 3 to 5 feet apart, their shoots consisting of three series; viz., the oldest, which has also attained the greatest length, or reached nearly to the top of the wall, will produce its fruit upon the upper third of its length. At the winter-pruning this shoot is cut out close to the ground, to make way for another young shoot, which will rise from an eye in the shoot adjoining. The next oldest branch bears fruit on the second or middle third of its length, and in course of the following season gains the top of the wall, and is cut out in its turn. The next or lowest branch produces its fruit on the lower third of the wall, each of these succeeding one another in regular succession. This mode is often met with in English gardens.

Mr Saunders, of Tedworth Park, has shown, in his "Treatise on the Culture of the Vine," $p$. 23 , a much more systematic method of training on the open wall. He plants his vines 2 feet apart, either in autumn, or, if the border is not prepared, not later than the month of March, so as to enable them to strike young fibres before the buds begin to swell. At pruning leave only two buds at a foot and a half from the ground; those under them should be cut off with a sharp knife after they are grown to the length of a foot or so. Select the strongest and remove the others. When the shoot has reached to the top of the wall or building pinch it off, and follow the directions given for vines planted in vineries. It will, however, require some degree of care and attention in keeping the lateral shoots removed so as to admit the sun and air to the wood, in order to get it well ripened before the cold weather sets in, as much of the success

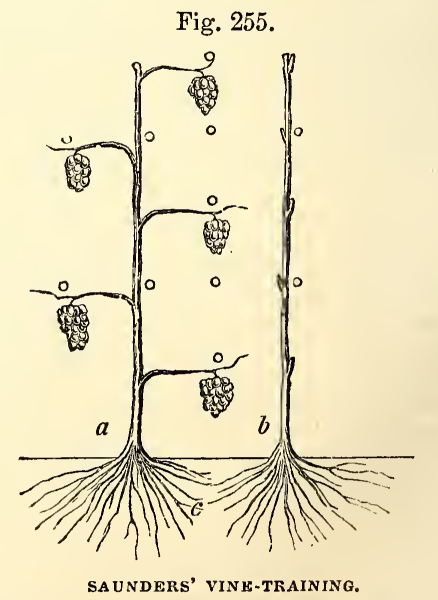

depends on the wood and eyes being well matured. When this end is fully accomplished, 
prune off the stems of the lateral shoots nearly close to the eye from whence they proceed. As the spring advances they will begin to bud forth, when care must be taken to select the strongest shoots, leaving them at regular distances, as shown in the sketch, fig. 255 . In pruning, cut each shoot or spur back to two eyes from the main stem, one of which only is to be left to produce fruit. The object of leaving two is to guard against accident, in case of one being injured previously to their budding forth: the same system is to be pursued year after year. The main stem will of course increase in size, but it will be many years before the spurs or side branches assume an unsightly appearance, or occupy much space, provided the "spur system" of pruning is carefully carried out. This is the most simple, and still the most systematic mode of pruning that could be devised for amateurs and others who cultivate the vine against the walls of their houses or in their gardens.

Hayward's mode of pruning and training.This author held the opinion that the greater length the sap had to travel through the body of the vine, the more abundant, fine, and highflavoured will the fruit be. One of his modes of training will be understood by our cut, fig.

Fig. 256.

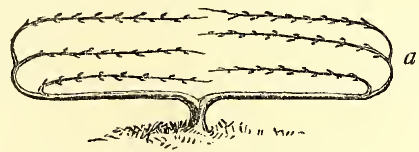

HAYWARD'S VINE-TRAINING.

256 , and another form is exemplified by fig. 257. These are so clear as to require no further description. We may, however, remark

Fig. 257.

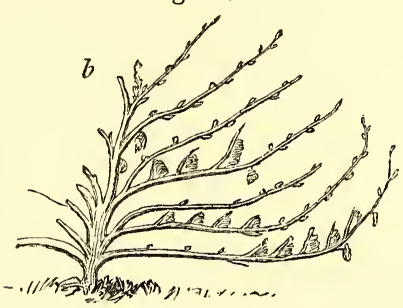

HAYWARD'S VINE-TRAINING.

that he carried his theory so far as to recommend that one vine only should be planted in a vinery, and, if variety were wanted, to graft other sorts on the solitary plant. Neither views are consonant with rational experience.

The old or fruit-tree manner of pruning the vine.-This is the method perhaps most generally practised in the case of vines on open walls, and is not unfrequently followed by gardeners under various impressions as to its utility. Vines so trained differ little in form from fan-trained peaches or Morello cherries, and of necessity contain a great proportion of old wood, between the branches of which the young shoots are laid in.

Renewal of the vine under any mode of pruning, dec.-The causes of debility in the vine may be traced to injudicious pruning, too heavy crops, impoverished soil, and occasionally, but not frequently, in this country, attacks of frost. Cutting down to within a short distance of the root, as regards vines of no extraordinary dimensions, and to within a short distance of the main or larger branches in the case of such as those at Hampton Court, Cumberland Lodge, \&c., carefully smoothing the wounds, and performing the operation early in winter, that the amputations may be healed before the ascent of the sap, are the means of renewal. It is, however, probable that some defect at the roots may be the principal cause of debility in the vines, and this no heading down can remedy. Recourse must therefore be had to a careful examination of the roots and border, and if these are found to be in a bad condition, lifting and pruning them, as well as a partial or complete renovation of the soil, drainage, \&c., must be attended to. When, however, these are in a bad state, it will be the most judicious way to remove the whole of the plants, soil, \&c., and commence de novo.

Summer-pruning the vine.-This is a very important operation, and in proportion to its importance do we find that our best authorities greatly differ in opinion. The superficial observer will sum up the whole into the removal of superfluous and ill-placed shoots, and shortening those on which the fruit is produced. Others go so far as to say that not a shoot should be allowed to form unless such as have fruit upon them, and those actually necessary for producing the fruit-buds of the succeeding season; and that every fruit-bearing shoot should be pinched off close to the fruit, as more than this only tends to exhaust the vine. Others say, " Let the young vines ramble freely, training the wood so as to expose every available surface of the leaf to the light. The complete elaboration of a few select buds is as nothing compared with the ultimate advantage arising from a border well filled with roots ; for rest assured the volume of roots will be in direct proportion to that of the top, although unseen."-Gardeners' Monthly Volume. The writer of the Calendar of Operations in the "Gardeners' Chronicle," 1846, p. 359, on the subject of stopping vines, offers the following excellent remarks: "Although much stress is continually laid on the stopping of vines during the growing season, yet this important process has its limits, the passing of which will lead to weakness in the constitution of the vine. Two reasons seem to exist in favour of the process: the one, concentration of the powers of the vine for a period in the immediate neighbourhood of the fruit, thereby increasing its size ; and the other, the prevention of the secondary shoots of the vine from overlapping and smothering the principal leaves. After these points are duly accomplished, vines, especially young ones, may be allowed to ramble freely, more especially in the period between the first and last swelling, or during what is called the stoning process. It 
is by no means uncommon to see young vines nearly destroyed by over-bearing, especially the Muscats. These "show" in an extraordinary way on strong young canes in newly-made borders; but if the fruit be allowed to remain, and close stopping be resorted to, the constitution of the Muscat will be completely broken up. Let such be, however, allowed to make as much wood as they please," and a different result will be the consequence. The usual practice is to stop each shoot at the second joint above the bunch, carefully preserving the two leaves to draw nourishment to the fruit, and to enable the sap to become properly elaborated. Many excellent cultivators pursue a different course, and stop each shoot at the joint next to the bunch, and assert that the fruit does equally well, and that a much greater amount of light is allowed to enter the house, and on this account probably one leaf performs the functions of two. Be this as it may, no doubt that both more light and a better circulation of air is admitted to the fruit in consequence of this short stopping; but the question is, Are these, beyond a certain extent, actually essential to the maturity of the fruit, or are they an equivalent for the greater amount of nutriment which would be supplied by an increase of foliage? $\mathrm{Mr}$ Mearns, an old and expert grape-grower, says in "Horticultural Society's Transactions:"-"Blind all the eyes on each fruit-spur as soon as they push above a joint or two before pinching them back, always cautiously retaining one eye ; and be particularly cautious that nothing should happen to injure the leaf that accompanies the bunch, for if that is lost, the fruit comes to nothing." In pruning on the close-spur system, no doubt depriving the spur of the leaves beyond the fruit is decidedly objectionable, for it is the leaves beyond the fruit that draw nourishment to it, and not those behind it, unless in a very limited degree.

The preservation of the leaves on vines, as indeed of all other trees and plants, is of vast importance-indeed, so much so, that the removal of a single leaf tends to lessen the vigour and energies of the tree : much more so must that be the case when they are removed in numbers, under the mistaken notion of admitting light to the fruit, and the still more barbarous practice of denuding the vines almost entirely, as some ignorant persons do in the case of vines in the open air, with a view to accelerate their ripening. The leaves in plants perform a somewhat similar office to that of the lungs in animals. An animal cannot exist without its lungs more than it can without its stomach, neither can a plant live or grow without its leaves. The use of the lungs in the former is to expose the chyle to the action of the air, which they decompose, so that its oxygen may unite with the chyle, and thus change it into blood. In plants, the leaves act the part of lungs, and not only decompose air, but act on light also, in the process of elaborating the sap; and hence plants can no more live without light than without air or food, as light is necessary to turn their food into sap, or to bring it into the proper state for affording them nourishment.
The rules in general laid down for thinning the wood and stopping the shoots, held by some necessary, as they think, to throw a greater supply of strength into the remaining buds, and by others to prevent an unnecessary expenditure of the energies of the tree in the formation of wood to be afterwards cut off, seem to admit of some debate; at least they are not reconcilable with the views entertained by some eminent physiologists. In a leading article in "The Gardeners' Chronicle," 1843, p. 443, we find the following reasoning. After submitting six propositions to the notice of cultivators, the author proceeds: "If this be so, the system of rigorous pruning of the vine must be wrong; on the contrary, its leaves should be allowed to form in abundance, and that destruction of laterals at an early period which is so much recommended must be injurious, because all those laterals, if allowed to grow, would by the end of the season have contributed somewhat to the matter stored in the stem for the nutrition of the fruit; because the preparation of such matter would have been more rapid, and because the ripening of the fruit which depends on the presence of such matter would have been in proportion to the rapidity of its formation. If we are not greatly mistaken, the early maturity and excellence of the grapes obtained out of doors by Dr M'Lean of Colchester, is entirely owing to the large quantity of wood which he lays in during the summer, as early as he can possibly persuade his vines to produce it. It is a mistake to suppose that the sun must shine on the bunches of grapes in order to ripen them; nature intended no such thing, when heavy clusters were caused to grow on slender stalks, and to hang below the foliage of branches attached to trees by their strong and numerous tendrils. On the contrary, it is evident that vines naturally bear their fruit in such a way as to screen it from the sun; and man is most unwise when he rashly interferes with this intention. What is wanted is the full exposure of the leaves to the sun : they will prepare the nutriment of the grape; they will feed it, and nurse it, and eventually rear it up into succulency and lusciousness. We therefore submit to our horticultural readers that rigorous summer-pruning is wrong. Let us not, however, be misunderstood: we do not suggest that a vine should form all the wood it likes ; that should be provided against by good winterpruning, and by rubbing off such buds as are not to grow into shoots. What we contend for is, that those shoots which are selected to remain should be permitted to produce as much foliage as possible; and that as the destruction of laterals is the diminution of foliage, that destruction should be discontinued. When, however, branches have grown for many weeks, and are in the autumn beginning to slacken in their power of lengthening, theory says it is then right to stop the shoots by pinching off their ends, because after that season newlyformed leaves have little time to do more than organise themselves, which must take place at the expense of matter forming in the other leaves. Autumn stopping of the vine shoots should, therefore, be not only unobjectionable 
but advantageous, for the leaves which remain after that operation will then direct all their energies to the perfection of the grapes." Few, we believe, who have studied the economy of vegetable life, will dispute the correctness of the views here stated. For ourselves, we believe that excessive summer-pruning is most injurious to the health of the vine as well as of other fruit-bearing trees. We would, however, submit for consideration, whether the present limited size of vineries in particular has not much to do in the matter, and renders summerpruning a measure of necessity often against reason and conviction; and added to that, the number of vines planted, and the too crowded manner in which the old wood is trained in. To admit of the full development of the vine, our houses should be differently constructed; the small, low, narrow lean-to's should give place to the span-roofed form, and these should be glass to within from 9 inches to 2 feet from the ground. By this means a much greater amount of light would be admitted, and, by increasing the width and height, room would be provided for a much greater development of both wood and leaves. ( $V$ ide figs. 431,432 , vol. i., as examples.)

Forcing.-It has now become no very un. common thing for grapes to be seen on the table every month in the year. The month of March is that in which they are most difficult to be produced, because the retarded fruit is kept with difficulty till that period, and never without serious injury to the vines, and the earliest forced fruit is with equal difficulty brought so early to perfection. Some, to get ripe grapes in March, begin to put the vines in motion in August, and to secure this with the greatest amount of success it will be best to adhere to the culture of them in pots. To have grapes ripe in April is sharp work, and even under the very best management the fruit will neither be so abundant nor so high-flavoured. Long observation led former cultivators to draw the following conclusions as to the length of time which will ensue between the time of beginning to force and the ripening of the crop-viz., five months when the course of forcing coincides nearly with the natural growing season; when short days compose a third part of the course, rather less than six months; and when the course includes full half of the winter it will require seven months,-and even then the crop can hardly be considered equivalent to the expense and labour. Vines started early in November have been ripened by $\mathrm{Mr}$ Mitchell, of Brighton, between the 14th of March and 17th of April: being rested till the latter end of May, and then pruned and put into action again, they produced a second crop of ripe grapes by the lst of December. This is practicable, but of its utility there are great doubts. Ripe grapes, of excellent flavour and colour, are produced by Mr Fleming, at Trentham, so early as March, by allowing the vines to break naturally in autumn; no artificial heat is applied to them until they are nearly in blossom, when a higher temperature is applied, and progressively increased until they are ripe. 'There are some peculiarities in Mr Fleming's practice that deserve especial notice; and as a summary of that practice has been given in "The Gardeners" Chronicle," we cannot do better than transcribe it. "By midsummer the young wood is perfectly matured, the foliage drops, and the vines are pruned. These early vines are never allowed to bear upon the old wood: a young shoot is brought up from nearly the bottom of the bearing branch and is carefully trained, being the source from whence next year's crop is to be obtained." To obtain a crop of grapes moderately early and of good quality, forcing may commence on the lst of January, at which period we propose to commence our remarks. These, however, are applicable in a great degree to those started about the end of November and any time during December. It has been recommended, when very early grapes are desired, to begin forcing in September in preference to the middle of December, as by the former course the fruit will be set before the middle of November, and have more assistance from the sun at that time than it would have in January, about the beginning of which month it would be setting if the vines were excited in November. The first operation in forcing is "to set your house in order," by examining the heating, whether flues or hot water, both of which should be put in a condition to go on throughout the season, as repairs during that period would be attended with danger (vide Forcing peaches). At or soon after pruning, all the old and loose bark should be removed from the stem and branches, but not to the extent of wounding the inner bark or liber; nor should a knife be employed, lest incisions may inadvertently or carelessly be inflicted. This frees the trees of a useless encumbrance, presents the young and useful bark to the sun's influence, and removes multitudes of insects and their eggs. Supposing the vines to have been pruned in November, they should now be washed, or rather painted with the composition recommended for peaches, or with some of those noticed under the head of Diseases and insects. The same care should be taken of the borders; indeed, in all such cases the whole surface-soil should be annually removed so long as the roots are not interfered with; for such additions to the soil, however much they may be slighted by men of ordinary observation, are of vast consequence to the roots, and consequently to the vines, during the whole season of growth. In the formation of the compost for thus top-dressing the vinery borders, a greater quantity of manure should be added; for the vine is a generous tree, and will give a return of fruit to its owner much greater than any other tree in cultivation. It is also a more gross feeder, and can digest a greater amount of manure than any other. The roots of vines travel far in search of food; and although, for very early forcing, it is well to have them under the protection of the roof during winter, it is seldom that they can be so circumscribed as to be kept within such bounds. When such is the case, liquid manure should be applied to the roots, but never at a lower temperature than $90^{\circ}$, or even more; for before it can reach the roots its temperature will be reduced to $70^{\circ}$, or 
even lower. Care should at all times be taken that cold water be not applied during early forcing; this, instead of benefiting the vines, does the reverse, by chilling the temperature around the roots, and so paralysing their energies that the spongiolets will not take it in; and, which is worse, if applied in excess, they, as well as the fine young fibrelets, may be destroyed altogether. Where the borders without the house are not vaulted, as described vol. i. p. 329, figs. 445,446 , or heated by some of the means abundantly there laid down, other means must be employed to ward off the excess of damp by rains or melting snow during winter, and also for the exclusion of frost. Both are readily guarded against by covering the external border with leaves, fern, dry rank stable-litter, \&c., and coal-ashes have been used by us with considerable advantage. The chief advantage of all or any of these, it will be readily understood, is to exclude frost, and to absorb extra moisture; hence they should be frequently turned over, removed when too damp, and replaced with dry material.

For more effectually insuring this, vide vol. i. pp. $328,377$.

For the first week after starting keep the house at that temperature caused by the action of the feeble rays of the sun on the glass only, giving slight ventilation during the day, but shutting up carefully every night. Water the surface of the borders, floors, and all with liquid manure heated to the extent previously mentioned. During the second week throw a slight degree of heat during the day into the flues or hot-water pipes, and shut up early in the afternoon, so as to enclose as much of the solar heat as possible.

Before proceeding further in this direction, we must say a word or two in behalf of vines differently situated. We have been speaking of such as are presumed to be planted within the house, and whose main stems are guarded against the effects of cold. There are manyand we would say by far too many-particularly in old gardens, where the roots are not only wholly in the borders without the house, but also where the main stem is without the house also, and often to the length of 2 or 3 feet, as the distance from the surface of the border may be from the point where the vine enters the house. Vines so situated require to have their stems most carefully protected from cold, the most primitive mode being to envelop the part exposed with hay-bands, or to cover them with dry littering matter, so secured as to prevent its being blown away. Others, and with better effect and much neater appearance, enclose them within wooden boxes stuffed with dry moss. The ingenious mode in use in the Royal Gardens at Frogmore, and also that used by $\mathrm{Mr}$ Saunders at Tedworth (vide vol. i. p. 315, fig. 428 , p. 313 , fig. 425), are worthy of imitation, the former being the best mode of securing the stems hitherto devised. In all such cases, however, there is danger of the cold reaching them, which leads to the conclusion that it is better to plant them within the house near the front, so that the roots may have a free passage through openings left in the parapet-wall into the external border; for it should be remembered that the part called the collar or neckthat is, the part which connects the stem and roots together-is the most tender part of the whole plant, and that most liable to suffer from undue exposure. Where vines are not intended to be taken out during winter, planting inside is most judicious. In very early forcing it is well to envelop the greater part of the main stem in a bandage of moss, to prevent too rapid evapora. tion in the surface of the larger branches, and also to act as a reservoir of moisture to counteract the effects of fire-heat. Another precaution at this time is deserving notice-that is, placing the whole of the vine within the house in a horizontal position, to secure regularity in the buds breaking. If the vines are trained at once up the rafters, the flow of sap upwards will be so rapid that the eyes at the extremities of the shoots will break first and strongest, and hence deprive those towards the bottom of the plants of their due share : many of the buds will thus not break at all, and if they do, they will be weak and often devoid of fruit. The case is very different when the vines are laid in horizontally at first, every bud breaking strongly, and showing fruit nearly to the ground. We follow this practice, and allow the young shoots to attain the length of from 3 to 4 inches before they are placed in a more perpendicular position (vide vol. i. p. 339). The vine, like all other trees, requires a season of rest, this season commencing after the fruit is gathered and the wood fully matured. This season is longer or shorter, according to circumstances, but whatever may be its length, it must be accompanied with a low temperature : still, however, the vines must not be exposed to severe frosts, which have in many instances killed them altogether; and this is more particularly the case with vines grown in the high temperature of a pine-stove. The elastic powers of the wood are restored by a few weeks' exposure to a low temperature, which it never fails to lose when long confined to a dry and warm atmosphere. The buds are found also to break stronger and more uniformly. Some cultivators disregard this, and keep their vines in their pine-stoves for many successive years together; but these in general allow the temperature to fall so low during autumn as not to greatly injure the pines, and reason that at that season, the vines being trained close under the glass, the temperature of the house is low on account of the diminution of solar heat, and also that near the roof the house is coldest, on account of the radiation of heat from the glass. This, however, is a plan not to be recommended, and can never be so beneficial to the vines as when they are, after being pruned, laid down along the front of the house, and separated from it by removing the front sashes 1 foot or 18 inches within the house, and excluding the vines altogether.

For various modes of wintering vines, vide vol. i., art. VINERIES.

Vines, during the early period of forcing, in consequence of the use of an unnatural application of fire-heat, and too often a scanty supply 
of water over the foliage, are very liable to be seriously infested with thrip, red-spider, and other insect enemies, and if precautions are not taken in time, these are productive of the worst consequences. (For remedies, \&c., vide Diseases and insects). A liberal supply of tepid water by the syringe tends to soften the bark, and causes the buds to burst more freely. Indeed, the water, during early forcing, should not be applied under $70^{\circ}$ or $80^{\circ}$ of heat.

During this period-that is to say, November, December, and January, and more especially during the two former months-forcing must be carried on with great caution, as at this season natural circumstances are adverse; and at this period the term forcing should only properly be employed,-at all other times acceleration would be a far more appropriate term. Every advantage should be taken of admitting air on all fine days; and where ventilation is placed in the front of vineries in the usual manner, the opening parts should be covered with thin canvass blinds, or Haythorn's octagon netting, through which the cold air will be admitted, but so broken in force that its dispersion through the house will be regular and uniform. At this season the advantages of the various methods of ventilation described and figured in vol. i. (vide articles VINERIEs, VENTILATION, \&c.) will become practically manifest. Nocturnal ventilation in mild weather will also be of great advantage. On fine days allow the internal temperature to rise to from $15^{\circ}$ to $20^{\circ}$ above that at which the house is kept during the night. This matter is seldom thought of, nay, too often reversed; consequently, where such a violation of natural laws is allowed to exist, the highest expectations of the cultivator cannot be expected to be realised. Until the buds have all fully broken, the vines should be syringed three times a-day in clear weather, and once only in dull cloudy days, using water at from $70^{\circ}$ to $80^{\circ}$ of temperature. After the buds have fairly broken, administer the water $10^{\circ}$ warmer. We have already alluded to the matter of heated borders, and also to the advantage of covered or concreted surfaces. Where neither of these has been provided, the laborious process of covering and uncovering the external borders must be submitted to, and it is at this season that sceptics in such matters are most likely to become converts. Be this as it may, the external borders must now be kept dry, and frost must be excluded: if the soil around the roots be slightly warmed, so much the better, that the action of the roots may be put in advance of that of the buds. Roots of vines in unprotected borders, and as near the surface as they ought to be, with the thermometer in the open air at zero, while the atmosphere around the plants within is at from $60^{\circ}$ to $70^{\circ}$, and often higher, cannot be expected to be in a condition for supplying the foliage and fruit with that share of food which they require.

Maintain a humid atmosphere, either regulated by the hygrometer, or, as is more usual, by what may be called practical sensation. In dry bright days give plenty of water, but lessen this in cloudy, and more especially in rainy or snowy weather. When the vines begin to open their flowers a drier atmosphere is required; but this transition must not be effected abruptly, as all sudden changes are extremely dangerous. Syringing over the plants should be abandoned during the time the vines are in flower, but as soon as they have set their fruit the process should be resumed. Some excellent vine-growers doubt the propriety of lessening the hygrometric condition of the vinery at this stage, and some continue to syringe, using the finest cap, even while the flowers are fully expanded. Were it not that we have seen excellent crops of even Muscats, which are considered shy setters, follow this course, and that in the practice of first-rate grape-growers, we would hesitate to countenance the process, as it appears contrary to the laws of vegetable physiology. Our earliest crops we set in a drier atmosphere; but as regards those setting at a later period of the season, when the sun is more powerful, we sometimes use the syringe to a moderate extent.

An excess of heat after the buds have all fairly broken, and during the early formation of the young shoots, will have the effect of giving undue excitement to the trees, without a corresponding excitement at the roots; weak shoots and small malformed bunches will be the consequence. Every bud that does not show fruit should be displaced, unless where a shoot is wanted for the formation of the vine. Vines in a high state of vigour will likely break at every bud, and each of these may show one and many of them two bunches. Now, as this is much more than any vine can bring to maturity, an early selection should be made not only of the buds showing the finest bunches, but even these should be reduced in number in proportion to the present strength of the vine and its future condition. In no case should two bunches be allowed to remain on one spur, nor should more spurs be left on than is requisite for the present and future crop. Without due regard to this, a superfluity of spurs would be produced, and in consequence a superfluity of leaves also, which, however useful they are in their own proper proportion, would be so crowded as to prevent their being all fully exposed to the influence of the sun's rays, without which exposure their functions would be greatly lessened.

Summary of practice as regards Hamburgs and other ordinary vines upon the spur principle. -The vines being started, as already noticed, syringing with warm water should be continued, although some excellent growers say no, and depend for a sufficiency of humidity, after all the buds have started, upon the evaporation which rises from the paths, which they keep moderately moist by repeated waterings, and in houses having no stone paths by judicious watering the surface of the border within. This, however, must be done with sufficient caution to prevent the soil in the borders becoming at any time saturated with water, and no water should be employed under $70^{\circ}$ of temperature. As soon as the young shoots proceeding from the spurs have attained the length of about 2 
inches, the embryo bunches will begin to appear, at which time a careful selection of the most promising and best placed should be made, and the others rubbed off. As far as practicable the bunches retained should be at equal distances from each other, and, as has been elsewhere noticed, in regulating the spurs, the bunches will stand alternately on each side of the main shoot, leaving of Hamburgs, \&c., not more than twelve or thirteen bunches on each vine; in the case of Frontignans, and other smallgrowing kinds, fourteen or fifteen may be left, if the vines be in a vigorous state, and the roof sufficiently long. After the selection is made, all the spurs on which fruit is not left should be cut clean away, cutting close into the main stem, which will induce a speedy healing of the wounds. When the young shoots on which the fruit is growing have extended three joints beyond the bunch, they should then be stopped, by pinching their tops off either at one or at most two buds above the fruit. Some, however, allow these shoots to extend several joints further before stopping, to produce a greater number of leaves, which they think materially assists the swelling of the fruit; while others assert that they only rob it of a share of its nourishment. Both under certain circumstances are right. The leaves no doubt contribute greatly to the fruit, for without them it would come to nothing; still, valuable as they are, if left in such numbers as to cause confusion and crowding, their presence does more harm than good; for it is always best to have every leaf left of as large a size as possible, and this they could not be if they have not room to develop themselves fully, so as to present their entire surface to the light. Our own practice is to stop according to circumstances, such as the size of the leaves of the variety, the space they have to grow in, \&c., so that an intermediate course between the two extremes may be deemed the safest in practice. One rule, however, is imperative-namely, leave one bunch only on each spur, if fine bunches are desired. As soon as the bunches are in flower, which is indicated by their appearance, and also by the agreeable odour diffused through the house, the atmosphere should be kept something closer, and also somewhat more humid, giving air when the temperature exceeds $76^{\circ}$. As soon as the berries are set thinning should commence, clipping out the smallest and inside berries first (vide Thinning the fruit). Remove lateral shoots as they appear, unless there is ample space for their leaves, and unless the leaves left on the fruitbearing shoot be considered insufficient for their nourishment. Those who wholly remove the laterals do so, believing that they draw from rather than assist in supplying the fruit with food, and also that sun and air may be admitted more freely to the crop. That air and light are highly important to the maturation of fruit is undeniable, yet the vine is naturally a low creeping plant; and even when it attains, as it does in some countries, the character of a tree, still the fruit in all cases is produced and matured under the shade of its leaves. In cultivation we see as well-coloured and as highly-matured grapes growing under the shade of their own leaves in vineries, where the whole surface of the roof is covered, as we do in those where they are confined to the rafters only, deprived of laterals, and fully exposed to the sun and light. Laterals are, therefore, only to be considered useful as contributing to the support of the fruit in cases of a deficiency of proper leaves, and where the latter are deemed sufficient the former should certainly be removed. During the whole process of forcing, air must be freely but judiciously admitted : a reference to vol. i., articles Ventilation, VinERIES, \&c., will show how this can best be accomplished. The temperature should be continued, as stated above, until the berries have perfected their seed; during the period of its formation they remain almost stationary in size. After this critical period vegetation goes on more rapidly, and consequently a slight increase of heat should be given; this may safely be done if it is wanted to have the fruit ripe at a particular season, otherwise it should be allowed to go on slowly and progressively. From the time the berries begin to change colour, and during the whole ripening period, air should be abundantly admitted, and under proper management during the night as well as the day, maintaining the heat, however, at not less than $60^{\circ}$. When the fruit has fully ripened ventilation must ever be increased, watering at the root nearly suspended, the atmosphere kept dry, and the bunches frequently examined, and all decaying berries removed. A partial thinning of such laterals as were left should take place after the fruit has ripened, and the whole of them should be removed when the wood begins to indicate ripeness by turning brownish, and the leaves beginning to fall.

Regarding the management of Muscats, they in no way differ as to pruning from other vines. We would, however, recommend their being planted within the house in most situations, although there are some places where they are found to do well when planted in the ordinary manner. Mr. Saunders, in his "Treatise on the Vine," observes, and we think justly-at least his theory is borne out in his own practice-that " much good is derived from planting them thickly; it enables the grower to train every alternate vine up and the other down" (for it should be observed he plarts in a prepared border within the house, setting the plants in a row along the middle of the vinery), "thereby entirely covering every inch of glass surface. Allowing each vine to carry ten or twelve bunches, you thereby secure a much greater weight of fruit than by any other plan, and at the same time each individual vine is not overcropped." No vine we know of suffers more from overcropping than the Muscat. Many err in keeping their Muscat-house at too high a temperature: of course where they are grown, as they often are, over pines, this control of heat is not so much in our power; where they are grown in a house by themselves, a uniform heat, as recommended for other sorts, is all that is required. During their blooming a closer atmosphere than was previously required 
is essential. Some recommend artificially impregnating the blossom to secure a regular set of berries; and in the case of weakly plants and badly constructed houses this may be necessary - not so, however, if the plants are vigorous, and the general treatment judicious. Never apply water unless in a tepid state to the roots, and the colder the soil the warmer the water should be; for cold water, or even an excess of it at any temperature, is exceedingly injurious to this class of grapes. As we have already observed, some of our best cultivators do not syringe their Muscats after the buds have advanced to the length of 2 inches : we do not in in all cases do so; nevertheless, it may be expedient in dark atmospheres-that is to say, in situations where the sun shines feebly upon them. On the other hand, where plenty of light is admitted, we would syringe with tepid water until the berries had attained nearly their full size. The temperature in which we have found Muscats prosper best is about $65^{\circ}$ by night, and from $75^{\circ}$ to $85^{\circ}$ by day, from the time they are in bloom until they are ripe. Of the importance of this difference between day and night heat, see page 629 . Root-watering and ventilation as for other grapes already noticed. When the fruit has fully ripened, a diminished temperature is expedient, even lowering it, but gradually, to $45^{\circ}$ or $55^{\circ}$ as the minimum: this lower temperature will cause the grapes to keep longer, and this without shrivelling. Muscats ripened by the middle of November-if the ends of their stalks, or a piece of a few inches in length of the branch on which they have been produced, if it can be taken with them, be sealed at the ends, and the bunches suspended in a dry cool room, so as not to touch each othermay be kept in good perfection till March; indeed, much better than if left hanging on the trees. The vines are also greatly relieved by the fruit being removed, and in a fit state for pruning, \&c.-Vide vol. i., figs. 421, 423.

Forcing vines in pots. - When the vine began to be first grown in pots and forced for early crops, the same plants were continued for years. Mr Stafford, one of the first cultivators upon this principle, says, "In respect to the treatment of the plants, I never throw them out on account of old age; I always renovate them, and have plants ten or twelve years of age as perfectly young, to all appearance, as though they were raised last year in the pot." $\mathrm{Mr}$ Stafford plants in pots 13 inches wide at the top and 15 inches deep. The soil used is rich vegetable mould, and the sorts chosen those that are naturally prolific, and not the largegrowing kinds. "The more delicate sorts," he observes, " are superior, when grown in pots, to any grown on rafters; and I have often proved that a pot placed in the house on the first of January, and the same variety trained up the rafter and subjected to the same heat, the former will ripen its fruit at least a month earlier than the latter." This is no doubt owing to the temperature at the roots of those in pots being much nearer to the temperature of the atmosphere of the house than of those growing in a common border. Here is one pretty eviVOL. II. dent proof of the advantage of heated borders, or of having the roots of the plants placed in a temperature approximating to that in which the fruit and foliage grow. Opinions have differed much as to the age of vines to be grown in pots since Mr Stafford gave his ideas publicity, and many gardeners fruit the vine the second year from its being struck from an eye, and afterwards throw them away altogether. Of the propriety of this there may be doubts, as if the vine, after ripening its fruit, be cut down to the ground, and allowed the following season to recover itself, we see no reason why they may not be continued for years.-Vide $M r$ Spencer's practice, \&c.

The following is the substance of $\mathrm{Mr}$ Errington's practice, as given in "Cottage Gardener," vol. v. p. 211: "The plants are struck from single eyes, and during the first season's growth stimulated to the fullest extent with a view to obtain strong plants. By October the leaves will be all fallen, and the plants may be instantly pruned back to about 9 inches in length, when they may be plunged in any sheltered and dry spot for the winter, laying the pots on one side to keep out the rain, and throwing some litter over them in severe weather to keep out frost. In the course of January in the succeeding year they may be again introduced to heat as before, and must receive the final shift; the size of the pot must be in part dictated by the position they are to occupy; a pot, however, at least a foot in diameter must be used. And now, again, the most perfect drainage must be employed, and both it and the lumpy turfy material must increase in the size of their component parts in a just ratio to the increase of pot-room. When the buds commence to grow, a selection must be made of the eyes or shoots to be reserved; and here practices differ-some retain four or five shoots, others only one or two. These points must be ruled principally by the position they are to occupy. We may merely observe that most good cultivators reserve only one cane, and this is trained carefully as in the preceding season, and is usually stopped when about 6 feet in length. This throws extra strength for a while into the principal leaves, and through them into the fruit-buds at their base. The stopped shoot will shortly push another leader, and this may be permitted to produce another foot or so of shoot, when it will be well to practise a second stopping. All this while the laterals are regularly stopped, as in the first year's culture. In August or September they will sink to rest, and may be pruned immediately; the length left to bear must be entirely determined by the size of the pots. About 3 feet, or nearly so, may be left to a 12-inch pot, and about 4 or 5 feet if in 15-inch pots; regard must, however, be had to the space overhead, as to height, \&c. They must now again be plunged for their period of rest; and the best place is a shed or outhouse facing the north, taking care to preserve the roots well. If required to be forced early, they may be introduced to heat soon after Christmas; and now a bottom-heat of $75^{\circ}$ will be of great service, if only for a couple of months. They will 
require no re-potting. A little of the powdery surface-soil may be removed from the top of the balls, and replaced with a rich and turfy topdressing. It is a good practice, at the first introduction to heat, to form the cane into a curve, in order to make it develop the buds with more equality. As soon as the young shoots show the bunch a selection must be made; three shoots with bunches will be plenty for a 12-inch pot, one more bunch to every inch of increase of size in the pot. Thus, a 15-inch pot would carry six bunches, one on each shoot. Not a shoot must be left on but those carrying bunches." All the subsequent management recommended by Mr Errington is the same as for vines in ordinary vineries; each shoot is stopped at a single eye or two beyond the bunch, just before the bunch blossoms. As soon as the last swelling of the berries commences, stopping may again take place, and all new growths be kept in check, as such would rob the system of the plant, "for all now becomes concentration and elaboration. Throughout the whole process as great a surface of foliage must be presented to the light as possible, and the smaller leaves of laterals must not be permitted to shade the principal leaves." Water must be rather sparingly applied when the plants are beginning to be excited, and increased gradually as the foliage increases in size, to be again decreased as the fruit ripens. When the fruit is cut, encourage the ripening of the wood by all possible means; and when it is fully matured, plunge the pots again as during the preceding autumn. The soil should be turfy and rich, the pots filled to one-sixth of their depth with boiled bones, charcoal, and potsherds. As to bottomheat, let this be kept a few degrees above that of the temperature of the house, and when the pots are not placed in plunging material, cover their surfaces so as to prevent the roots from being injured by drought or sunshine. If plunged in fermenting matter, let $80^{\circ}$ be the maximum: as to atmospheric heat, commence with $55^{\circ}$ during the day, rising gradually to $60^{\circ}$, and further to $75^{\circ}$, when the flowers are expanding. The night heat should not exceed $45^{\circ}$ at starting, rising to $55^{\circ}$ by the blooming period, and never afterwards exceeding $60^{\circ}$. On very dull days, and during severe weather, descend to the night heat during the day. The sorts recommended by $\mathrm{Mr}$ Errington for potculture are the Black Hamburg, which he seems to prefer, admitting that others succeed well with the Muscat of Alexandria, and that Muscadines and Frontignans answer equally well.

$M r$ Spencer's practice.-The practice of $\mathrm{Mr}$ Spencer of Bowood, one of our very first authorities in such matters, is given in "The Gardeners' Chronicle," 1844, pp. 195 and 213, of which the following is the substance: "If the vines are intended to be fruited the next season, the eyes should be potted in 32 -sized pots, placing them 1 inch below the surface, and using soil of a light turfy nature; or if stiff, using a portion of half-decayed leaves : only one eye must be planted in each pot. This should be done early in February, and when finished, the pots containing the eyes may be plunged in any pit or frame that may be at work, where a bottom-heat may be maintained at $90^{\circ}$ or thereabouts: they may remain there until growth has commenced, when, sunlight being indispensable to the welfare of the young plants, they should be placed (if they were not previously) as near the glass as possible, sinking the pots as the plants reach the glass, but still keeping a steady bottom-heat, and supplying them with air every day if possible. It is supposed that the heat of the pit or frame varies from $60^{\circ}$ to $90^{\circ}$ in sunshine. When the pots are filled with roots, which will be some time in April, they may be transferred at once to their fruiting-pots, which should be $2 \mathrm{~s}$. or $4 \mathrm{~s}$. (that is, the price of the pots), according to the strength the vines are wished to attain, bearing in mind that those in the smaller size will ripen their wood earlier, and consequently be available for forcing at an earlier period than the others." The compost Mr Spencer uses is " two-thirds turfy loam, from a down having a chalky bottom, and onethird decomposed nightsoil. Should the loam be strong, I use the same proportion of halfrotten horse-droppings. The turfy loam should be only half decayed, and it should be used as rough as possible. After potting the plants should be placed in some house or pit where a temperature of from $60^{\circ}$ to $80^{\circ}$ or $85^{\circ}$ is maintained. They should also be so arranged that the shoots, as they advance, can be trained immediately under the glass, and be exposed as much as possible to the light. The front kerbs and back shelves of pine-pits are suitable places, and the partial shade that the vines afford benefits the pines during three or four of the summer months. Where there is only a vinery, they may be trained between the permanent vines, or in any other place where the cultivator can make room for them. As the shoots advance train them carefully, and stop the laterals as they appear. When first potted the plants will want but little water, but it must be gradually increased as the pots become filled with roots; they will then require it regularly during their growth, and manure-water may occasionally be given, although the quantity they demand the first season is small in comparison to what they require afterwards. The most suitable length of cane for pots is from 4 to 6 feet; but if from any peculiarity in the house in which they are to be fruited a longer length may be required, they should be left accordingly, as the vine will grow strong enough for fruiting 8 or even 10 feet long. After it has grown a foot more than the length required the next season, it should be stopped; three or four of the upper laterals, however, may be allowed to grow at a few joints, to prevent the topmost eyes breaking. Manure-water may be now applied to cause the buds to swell, and care must be taken to preserve the principal leaves, as they are now performing a most important part in regard to the crop next season. When the wood appears to be turning brown (or ripening), water should only be applied to prevent the vines flagging; the laterals should be taken off, and every means should be employed to ripen 
the wood perfectly. Indeed, if it is not convenient to allow a large admission of air when they are growing, it would benefit them much to be removed to a cool house, where they would have the benefit of more air and a lower temperature at night. By the beginning of September, if the following directions have been followed, the vines will be ripe enough to place out of doors. The north side of a wall is the best place, and the pots should be laid on their sides, and every means taken to throw the plants into a state of rest; the cultivator will thus find himself in possession of vines which, for strength and vigorous habits, may justly be mistaken for older plants. If the above mode of obtaining fruiting vines for one year should be thought too troublesome, from the plants requiring bottom-heat during their first stage, the eyes may be planted singly as before in 48-sized pots, and set in any house or pit where there is a little heat; they will be longer by this method of developing their roots, and may not want shifting into larger pots before May or June, when the plants may be shifted into 24 sized pots, in which they may remain through the season in any house or pit in which room can be found for them, paying attention to watering, tying up, \&c. They may be stopped when 2 or 3 feet high, and, when the wood is fully ripened, removed out of the house, and plunged in any material out of doors that is a non-conductor of heat. In February or March cut these plants down to two or three eyes, shake them entirely out of the pots, and place them in similar sized pots to fruit in as the former ones, taking care to spread their roots (in potting) regularly through the soil, that when growth commences each spongiolet may be in immediate contact with food: this is a much better practice than placing them in a pot without disturbing the ball, as is often done. The same routine of management must be followed with these through the season as recommended for the others. From the larger amount of organisable matter the vines possess by this mode of treatment, they will generally be found stronger than those raised the same year, and they possess the advantage of ripening their wood earlier in the summer." The article from which the above quotation is taken is continued at p. 228 of the work last quoted; and as we deem this the very best we have met with on the culture of the vine in pots, we consider it would be very imperfect were we to withhold the following excellent remarks. "The next consideration," says Mr Spencer, " is the time you wish your grapes to ripen; this being ascertained, it is quite easily known when to commence. It may be stated that vines under the above-mentioned treatment will be ready for forcing early in November, and consequently will ripen their crop by the end of March. As the principal use of vines in pots here (Bowood) is to occupy the houses (where vines are planted on the outside) during the period when they are inactivesay from November to May-those in pots are generally forwarded in their first stage in any pit or house whose temperature may happen to suit them; and finally, when the wood of the permanent vines is sufficiently ripened to allow of their being placed outside, the pots are taken in, and arranged in their places on shelves put up for the purpose; by these means the houses are of far more use than if they remained empty half the year. However, the precise mode in which the vines are to be fruited depends on the kind of houses the cultivator has at his command; a flued pit answers well, but the best description of houses is that which admits the rays of sun to pass through it in the winter at as near right angles as can be. Such a house, admitting considerably more light during the winter months, is much more suitable for such a plant as the vine than low flat houses." Hence the great advantage of span-roofed houses. "Whatever the house is, if not perfectly ready for the vines when you wish to begin forcing, get them placed in a dung frame, where you can give them a moist heat of $55^{\circ}$. This will cause their buds to swell regularly, and prepare them for removal to the fruiting-house when ready, without losing time. Previous to losing their leaves in autumn, they may, if thought advisable, be disbudded on Roberts' system, leaving a few more buds than you want bunches; but one objection to this system is, that if by any accident through the winter the bud should get injured, it leaves a blank which, had the next buds remained, might easily have been supplied." The following, as regards the weight of fruit to be produced and the after-management of the vines, merits attention. "The number of bunches that may be left on each vine will depend on the soil, size of the pot, \&c. When the vines are strong, and No. 2 pots are used, I usually leave six or seven bunches on the Hamburg, the same on the Sweet water, and one or two more on the Muscadine. If the vines are not so strong, four or five bunches on the Hamburg will be sufficient. It is much better to have rather fewer bunches, and the berries fine and well coloured, than ill-coloured puny bunches, which always is the case when too many are left on the vine. The vines, from being placed in the house, presuming their buds to be swelled, must have their temperatures raised to $55^{\circ}$ fire-heat, $65^{\circ}$ when in bloom, and it will be better if this heat, by night is never exceeded : of course, on all days when there is no likelihood of sun-heat, the heat of the house should be raised $5^{\circ}$ or $10^{\circ}$ by artificial means. Air should be admitted by some means or other every day early; this is of consequence, or the leaves are apt to get damp, and their texture being so extremely thin when the hot sun and the drying winds of March act on the foliage, they often burn and shrivel, and consequently are unable to swell off the fruit or give it colour. During all the time the vines are in a fruiting state, manure-water in some shape or other must be frequently given; but this should be in a pure state, and at a temperature equal at least to that of the house." Mr Spencer uses "manure-water made by pouring nearly boiling water on equal parts of sheep or deer dung and fresh horse-droppings; this is fined by a lump of hot lime, is drawn off clear, and when used is diluted with equal parts of rain water. A weak solution of guano is beneficial, 
but great caution is required in using it. It is astonishing, during the period of active growth, what an immense quantity of dung-water vines will take. I have frequently watered them twice a-day with it, and this I prefer to placing bottom-pans or feeders under the pots. If dung-water is properly cleared and diluted, it may be given twice for fresh water once. When the grapes are fully swelled and beginning to colour, water must be more sparingly applied, using clean water only. When the fruit is ripe, if the house is wanted for other purposes, the plants may be removed to any dry house or room, where the grapes will keep until wanted." We have kept grapes on plants in pots for six weeks after they were ripe, by placing them in a dry dark room. The leaves by that time having performed their necessary functions, fall off, and the plants are put into a complete state of rest, suffering in no way from the darkness to which they have been subjected. Upon the whole, the pot system is much to be preferred to that of subjecting established plants, whose roots are not under the sarne control, to the process of extremely early forcing. Although success has attended fruiting the vine in pots the season after they have been originated from the bud, yet a greater degree of certainty will follow the practice of fruiting them when not less than two years old, and resting the plants every alternate year.

The best structures for pot-culture are shown in vol. i. figs. $421,423,424$.

$M r$ Saunders' trough-culture, which is merely a modification of pot-culture (vide rol. i., fig. 424 ), is detailed in his "Treatise on the Culture of the Vine;" and we may here remark that we have never seen finer grapes grown in pots than those we have seen under this judicious cultivator's management. If, he says, the vines are required for early bearing the following season, the eyes-for it should be observed, he grows his young vines from eyes-should be put in before the middle of January. The pots in which the eyes are put are plunged in a pit or frame in not less than $80^{\circ}$, nor to exceed $90^{\circ}$, the surface-heat being maintained at $70^{\circ}$. When they have attained the height of 2 or 3 inches, they are taken from the pots in which they were placed at first, and planted singly in 60-sized pots. The soil used at this stage is loam, leafmould, and sand, with a little well-decomposed stable manure, which, he says, tends much, at this early stage, to strengthen and give vigour both to the roots and shoots. They are again placed in bottom-heat, and a sweet and pure atmosphere maintained around them, keeping them rather dry than otherwise. "As soon as the pots are filled with roots, re-pot them into 32 -sized pots, using at this potting a greater portion of decomposed manure, and so much the less of vegetable mould. Put them, when potted, into a vinery or other convenient house, where a temperature of from $70^{\circ}$ to $80^{\circ}$ is maintained. Place the pots on shelves, and as near the glass as practicable. The great object to be observed is to keep them in a free-growing state, air being a most essential point, which ought to be admitted freely, but of course not depressing the internal heat below the previous given standard." When these pots are well filled with roots; shift again into No. 8-sized pots, using a stronger compost; drain thoroughly, and place them in a house where they are to complete their first year's growth, keeping them at least 16 inches from the glass, and increasing the temperature from $5^{\circ}$ to $10^{\circ}$. "When the vines have grown to the length of 8 or 12 feet, it will be necessary to stop them; and if they are as healthy and strong as they ought to be, they will, immediately after being pinched off, throw out lateral shoots from the buds at the extreme ends; and as it is of great importance to prevent the other principal buds from breaking"-that is, those upon which the crop is depending-" allow the top laterals to grow to the length of 6 or 8 inches before pinching; but the lateral shoots, which are emitted from the base of the fruiting-buds, should be stopped beyond the first joint. Water freely at the roots, and occasionally with liquid manure. When the wood is fully ripened, remove the plants to a south wall, protecting the roots from frost, and the rods from being broken, until the season arrives when the plants are to be again excited into growth. All the pruning necessary is merely to shorten the rods to the required length, which of course will be regulated greatly by their strength, and this shortening should be done as soon as the leaves fall off. The period of taking them into the house again will depend on the season the fruit is required to be ripened. Mr Saunders begins forcing Muscats in December, and has the fruit ripe in the first week in May. The temperature, during the first fortnight, he maintains at from $50^{\circ}$ to $55^{\circ}$, and gradually increases it to $70^{\circ}$. Until the vines have pushed into bud, the atmosphere is kept genially moist by frequent syringing with tepid water; and if dry at the root, a little liquid manure is applied. To prevent the roots becoming at this stage too damp, the pots are slightly elevated, by placing pieces of wood or brickbats under the edges of their bottoms. This also favours the circulation of the heat of the house around them, and admits air to the roots through the openings in the pots. "When the buds are regularly broken, and each shoot advanced to the length of 6 inches," Mr Saunders then plants the vines in troughs, which run along his vineries near the front, and also towards the middle of the house, the former elevated about midway between the floor and the glass roof, and the latter on top of the parapet wall, which forms one side of the passage behind. The vines in the former case are trained up the roof to the middle of the rafter, while the latter are trained to the top of the house. Mr Saunders gives the following reasons for transferring his vines from the pots into troughs: "The advantage," he says, "to be derived is immense, by securing to them all the benefit of a border, independent of giving every root and branch an equal temperature, which is so essential to promote their perfection." His mode of transferring them to the troughs he thus describes: "First, place upon the laths forming the bottom 
of the trough a layer of pieces of turf of about 3 inches square, to prevent the fine mould from being washed down; next, put a layer of 2 or 3 inches deep of the following compost-viz., equal quantities of rich turfy loam and rotted manure from the farmyard, with the addition of a little sand. Proceed to remove the vines from the pots by turning the ball upside down on the palm of the hand, having a convenient bench on which to rest the hand and ball while cautiously removing the crocks, and any objectionable soil, or worms, and taking the utmost care in doing so not to injure the roots. When so prepared, place the ball on the surface of the soil in the bottom of the trough. Proceed in like manner till the whole are done, leaving a space of about 3 inches between the balls of each, to be filled up with the same compost as used under them." The roots are covered from 1 to 2 inches deep with soil previously brought to the temperature of the house; they are then tied to their proper places, well syringed, but no water given to the roots for a few days unless absolutely necessary, and then it is to be done by pouring it immediately on the balls, and not over the whole soil in the troughs. The plants will soon show the benefit they derive from the change. "When sufficiently advanced, stop each shoot one joint beyond the fruit, and leave only one bunch to each, and from eight to twelve to every vine, according to their strength. A heavier crop would deteriorate both from the size and colour of the berries. A plentiful supply of water will now be necessary, which can be given abundantly without fear of injury from stagnation, provided the directions given for the formation of the trough have been strictly attended to." $\mathrm{Mr}$ Saunders produces two crops annually in this way. Indeed, he says, in his "Treatise on the Vine," p. 31, "the trough system might justly be said to possess all the requisites of a vine border -viz., drained, heated, and covered with glass. With these advantages the grower is enabled to produce Muscats with as great success as any other kind; and by commencing forcing them in the beginning of December, ripe fruit of this much-esteemed variety can be had by the first week in May. Another most material point is gained by producing the Muscat in troughs: you are thus enabled to keep a constant supply of fruit for the table without forcing the permanentlyplanted vines, which is an object of great importance, as it enables us to give them a longer season of rest, and by so doing the vines will become stronger and better every year, and consequently the fruit will be of a superior quality, and can be well ripened in time to succeed the early forced ones. In this way it is quite possible to have ripe Muscat grapes throughout the year, a desideratum which many have pronounced unattainable."

When the vines thus grown at Tedworth have ripened their fruit, and when it is cut for use, the vines are carefully removed from the troughs and placed out of doors against a wall, protecting the roots from the sun, \&c. Vines thus treated will yield a fair crop very early the following season, but not equal to what they do the first year. They ought to be removed when the fruit is gathered, to make way for another successional set of vines.

Mr Saunders' great forte is in the cultivation of the Muscat of Alexandria; yet he grows other varieties also with equal success.

Both pot and trough culture must be admitted to have advantages in early, or rather winter forcing, over forcing vines where the roots are in external borders, subject to the extremes of cold and wet, and where the roots are so little under our control. Next to this, as respects insuring success, must be regarded the system of planting within the house, or in vaulted borders without, heated by hot-water pipes, and protected above either by concreting or such other means as will exclude both frost and an excess of humidity. Pot and trough culture entail a considerable amount of labour and expense; but again, under such management as has been year after year exemplified by the authorities we have quoted, there is no doubt that the return is equal to the trouble and expense incurred.

Retarding crops of ripe grapes.-Late crops of grapes demand the utmost vigilance to secure a supply till February. The grape has the property of remaining on the tree after it is ripe for a longer period than any other of our cultivated fruits. Much of this depends, however, on the kinds grown. Of these, West's St Peter's, Black Barbarossa, Hamburg, White Nice, Syrian, Charlesworth Tokay, Muscat of Alexandria, and its varieties, are best adapted for long keeping. For this purpose a house, or houses, should be set apart, and in such houses no other plant should be allowed to enter; for it is impossible to keep greenhouse plants in a healthy state, and preserve the grapes at the same time. The conditions next to these essential to the long keeping of the grape are, that they are fully ripened before the growing season closes, that the atmosphere of the house after that period be kept low but not cold, uniform and dry, a free amount of ventilation at times, and, so far as can be without injury to the vines, a very limited amount of light: indeed, when the grapes and wood are fully ripened, light should be entirely excluded. These conditions are, however, unfavourable to the vine, and hence the great advantage of growing late grapes in pots, as they may, when the fruit is fully ripened, be removed to a dry dark apartment: as the plants, according to some of our most eminent growers, produce only one crop of fruit, the preservation of the plants afterwards is a matter of no consideration. Grapes are often kept a long time when removed altogether from the vine, and this is effected by taking with each bunch a piece of the shoot on which it was produced; and the larger this piece of wood is the better, so long as its removal does not interfere with the regular pruning of the vine. Both ends of such shoots are sealed over, and they are then suspended in a dry, dark, cool room. They, however, require repeated examination, so that all decaying berries may be removed as soon as they show symptoms of decay. Under ordinary circumstances, grapes 
ripened late in autumn are kept on the vines till after Christmas, and in order to secure this, fires must be used occasionally during the day, when ventilation should at the same time be given, in orderto carry awayallaccumulating damps. "Too great a depression of heat," says Mr G. W. Johnstone, (in "The Gardeners' Monthly Volume,") "will induce a state of rest; too much heat will hurry them through this stage." $\mathrm{He}^{\text {" has found }}$ by experience that an average of $45^{\circ}$ to $55^{\circ}$ will suit better than either a higher or a lower temperature, unless, indeed, as sometimes happens, the leaves have ripened and are shed; then, indeed, $35^{\circ}$ will suffice, provided damp can be expelled by such a temperature. Fires should be kept going early in the morning, accompanied with a free circulation of air, more especially if somewhat dry and free from fogs. The house may be closed towards two or three o'clock, P.M., with the exception of a very little back air, to permit the egress of steam through the night. The bunches should be examined with the scissors at least once a-week, and berries removed the moment that the slightest decay is visible." After the middle of November the house should be darkened by day by a covering of canvass, which, however, had better be removed towards dark, to admit of night ventilation through the top of the glass or otherwise. Drip must be guarded against, and where the vinery is badly glazed, a waterproof covering will be found advantageous.

Ripe grapes are often kept a month or more after being removed from the vine, by placing each bunch within paper or cotton bags, and suspending them by the stalks to cords run across a dry airy room. Speechley preserved his grapes by removing the bunches attached to portions of the shoots on which they grew, having two or three joints above the branch, and a sufficient length of it below to reach to the bottom of a quart bottle filled with water, and changed every twelve or fourteen days. The lower end of the shoots were placed in the bottle, and at every time the water was changed a thin slice was cut off the end of the part of the shoot immersed. Grapes should always be suspended; but it has been questioned whether by the stalk in their natural position, or reversed, as recommended by Mr Lavanchy, in "Cottage Gardener," vol. i., p. 82., who says: "Instead of tying the bunches by the stalks when hanging them up, take a piece of worsted 2 feet long, tie the two ends in a knot, make a noose, and insert two or three of the berries of the point of the bunch in it: do the same thing at the other end of the loop: hang the two bunches on a nail or a rod, putting one of them higher than the other that they may not come in contact. The advantage of this method is obvious. When the bunch hangs in its natural position, the berries rest on each other; and if one decays, the contusion spreads so rapidly that the whole bunch is soon destroyed. Reverse the position of the bunch, and almost every berry is separated from the others, and disease is far less likely to spread than in the former case. The grapes should be kept in a cool dry room."-Vide Management of the fruit-room.
The floors of late vineries should be kept covered with dry coal-ashes, removing them when they become damp, and replacing them with dry ones. A few lumps of unslaked lime, placed in shallow boxes, have also the effect of drying the atmosphere, in consequence of its capability of absorbing moisture; lighting the fires by day, and ventilating freely at the same time, has a similar effect, the moisture being carried off in vapour; night-fire, on the contrary, with the house shut up, has the very opposite effect. Avoid ventilation in damp and rainy weather, as the air will carry moisture in along with it. With these, and a low uniform temperature, and, if possible, absence of light, grapes may be kept till the end of February. It is, however, most essential that the roof of the house be in the best possible state of repair, so as completely to exclude rain. While recommending the vinery to be kept dry, care must be taken at the same time that this is not carried to excess, else the fruit will shrivel, and become unsightly and almost useless.

Produce of the vine.-This must vary very much according to good management, size of the house, season of the year, whether the variety produce large or small bunches, \&c. As a pretty general rule, a single stem of the vine trained under a rafter of an ordinary-sized vinery, and pruned on the spur principle, will yield upon an average from fifteen to twenty pounds weight yearly; a vine on the long-rod principle about twenty or twenty-two pounds; and if trained over the house, the same vine, in either case, will yield a third more; that is to say, if each is allowed the same extent of roof surface. Mr Clement Hoare, in his "Treatise on the Vine in the Open Air," has laid down a rule, taking for his data the circumference of the stem of the plant close to the ground.

"Scale of the greatest quantity of grapes which any vine can perfectly mature, in proportion to the circumference of its stem, measured just above the ground:-

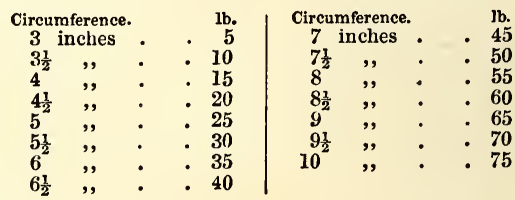

"It will be seen, that if $2 \frac{1}{2}$ inches be deducted from the circumference of the stem of any vine, the capability of it will be equal to the maturation of ten pounds of grapes for every remaining inch of girth. The proportional quantity for fractional parts of an inch may be easily calculated."

And another writer on the vine says, no vine should be allowed to produce fruit until its girth near the ground measures 3 inches, when it may ripen five pounds, and for every half-inch increase five pounds may be added. It is always wrong to overcrop any tree, a young vine in particular.

Training the vine.-The vine is trained under 
the rafter, over the whole surface of the roof, upon gable or suspended trellises, sometimes 12 inches from the glass, and from that to 20 , and in some few instances at even a much greater distance (vide vol. i., fig. 403). The object of training under the rafter is to allow the light to have full influence on the foliage and fruit, which no doubt it has much more than when the whole roof surface is entirely covered. How far, however, this is of advantage to the fruit has been questioned by some, as elsewhere noticed. Eighteen or even twelve inches may be sufficient for small-leaved vines with short foot-stalks, but twenty is a much better distance for large-growing leaves and vines full of vigour; and this is the more necessary where large panes of glass are used, because at that distance the leaves have room to develop themselves fully without coming in contact with the glass; and as air is more freely admitted around them, the leaves are much less liable to be scorched by inferior glass, or lenses forming on that of even good quality.

Producing a long supply by successional houses. - It has been suggested, as the most certain plan of securing this end, to have six houses, beginning to force, the first at the end of October, the second on the first of December, the others on the first of January, February, March, and April; while others have attempted the same thing with half the number, beginning the first in November, the second in February, and the third in April.

Syringing is considered a most essential operation in vine culture by some cultivators, while others scarcely recognise it as of the least importance after the buds have broken. It is perhaps best to take an intermediate course, and consider that both are acting on the extreme. $\mathrm{Mr}$ Johnstone, in "The Gardeners' Monthly Volume," remarks : "If sufficient atmospheric moisture is provided by means of a permanent character, little syringing will be needed. It may be practised with benefit, during the time the buds are swelling, two or three times a-day. When blossoming comes, it must be altogether omitted. It may be resumed again until the berries be as large as peas, when it will be better entirely dispensed with, taking care to provide plenty of atmospheric moisture in lieu of it. The desirable amount of moisture in the air of the vinery is by no means arbitrary, but ought to be regulated according to the rule pointed out by nature. Her law is that the moisture of atmospheric air, unaffected by accidental causes, is proportional to its temperature, the moisture increasing with the heat. The exceptions offered by the sirocco and other hot dry winds have their accidental origin from passing over arid torrid plains, incapable of affording moisture to the passing air. The consequences such winds bring upon vegetation are well known to be destruction of its foliage, and in many instances death. Air at rest, as in a hothouse, does not absorb moisture so rapidly as air in motion; therefore, if the whole area of its floor was a tank of water, the air confined within the house would never be so saturated with moisture as air at the same temperature passing over water, moist earth, and vegetation, as in the ordinary course of nature. To obviate this, gardeners promote the diffusion of watery vapour through the air of their hothouses by placing pans of water upon the flues, by having open gutters of hot water cast upon the hot-water pipes, and by even admitting jets of steam. The difficulty attending all these processes is to have the amount of vapour in a natural proportion to the temperature of the house. A little inequality is not of much consequence, but, other treatment being correct, the nearer to the dictate of nature so much the nearer will the plants be to a state of best vigour."

"A consequence of air being duly impregnated with moisture is, that evaporation of water from a given surface exposed to that air proceeds slowly, shown by its causing but little cold."

"The easiest mode of ascertaining the difference between the temperature of the air and an evaporating surface is by having two equally graduated thermometers hanging in the hothouse, with the bulb of one enclosed in a piece of thin muslin. Upon moistening this with water of the house's temperature-made so by keeping it in the house-the number of degrees this causes the mercury to sink will be the difference between the air's temperature and that of an evaporating surface. In the winter months the gardener may be satisfied that the air has the desirable moistness, if that difference be not more than $6^{\circ}$, and during the summer months $3^{\circ}$. This is much less than the difference found by observation in a tropical climate, but there the evaporation was promoted by exposure to a free circulation of the air."

"Although syringing and steaming, duly regulated, are unexceptionable practices occasionally, yet, as before observed, they are not to be depended upon solely as the means of keeping the air of the hothouse in a proper state of moisture, in conformity with the natural laws already pointed out. Gardeners know this from their experience, and have adopted, consequently, several modes of keeping the moisture of the air always accordant with its temperature."

Many depend on damping the floor, walls, and foot-paths of their vineries, for insuring the requisite amount of atmospheric humidity, and some have combined with this the diffusion of fertilising gases at the same time. This has been effected by watering with highly enliched liquid, by setting vessels of it in various parts of the house, by sprinkling the foot-paths with ammoniacal liquor-but this is objectionable on account of the disagreeable appearance; and hence $\mathrm{Mr}$ Milne, an intelligent cultivator, has placed it in three tin cans, in a house 42 feet long, and found that in two days the liquid had wasted one-third of its bulk, the residue changing to a thick substance like coal-tar. It might be injurious to apply much of this to the roots of the vines; besides, it has been found that its fumes did not become disengaged so readily when sprinkled on the soil as when thrown upon hard non-absorbing borders. The advocates for non-syringing should bear in mind that the foliage of plants, in even the best regulated houses, is liable to beconie covered with dust, 
which nothing but syringing can remove; besides, there may be something advantageous in the movement caused in the foliage by the force with which water is thrown upon them. In regard to the maintenance of a due amount of moisture in vineries, vide vol. i., p. 350.

Why water is to be withheld from grape-vines immediately befor'e the fruit ripens.-In dry summers and autumns our most hardy fruits are found to be much higher in flavour than when these seasons are rainy. This circumstance, no doubt, gave the first idea of withholding water from fruit-bearing plants prior to their beginning to ripen. Vegetable physiologists account for this on the ground that at that period of the fruit's growth, decomposition, or dissipation of the water that they have been draining from the stem under favourable circumstances, such as a dry season, takes place; and hence, by diminishing the quantity of water given artificially, we accelerate maturation, because less time will be required to dissipate that element, and greater opportunities offered for the formation of saccharine matter, and the acquirement of certain flavours for which any peculiar fruit may be prized; while an excessive supply of water would not only retard the process of ripening, but would also prevent the formation of saccharine matter, and render the fruit acid and tasteless, by the proper proportion of carbon and water which constitutes the saccharine matter in the grape being destroyed, and water formed in them in excess. It also chills the roots, and, by consolidating the soil around them, prevents the admission of air, so beneficial to the roots of all plants. Most cultivators recommend maintaining a dry atmosphere while the grapes are ripening, calculating thereby to improve their flavour; and such is a very generally received opinion, and seems to accord pretty well with the natural condition of the vine in most countries where it is grown in the open air. We fear, however, that this dry state of the atmosphere is carried too far, and the transition brought about too suddenly. To avoid destroying the bloom on the fruit, syringing should be discontinued before colouring begins to take place ; but, on the other hand, a general humidity should be kept up until the fruit is fully ripened, and the foliage begins to change, indicating that the growing season is past; and this can be easily effected by sprinkling the walls, floors, \&c., which will cause a fine genial evaporation to take place similar to natural dew. At Cabul and Candahar, where the finest grapes in the world are produced in the open air, dew falls heavily at the season of the grapes ripening. There is no doubt, however, that there the sun's influence during the day is much greater than with us, even in our best vineries; but still there is reason to believe that our house grapes would be greatly improved, both in size and flavour, if so important a part of their nourishment were not so suddenly and so completely cut off as is the case in very general practice.

$A$ deficiency of heat and light injurious to the vine.-No plant will grow in a temperature below the freezing point, although some will exist under that point for a time. More or less heat is required to cause the germination of every seed, and also to continue the future plant in a healthy condition. This amount of heat varies according to the nature of the species of plant subjected to it. If the heat in which a plant is placed be sufficient to rouse its vital forces, and to bring them into action, under such circumstances a plant will flourish. If, however, the temperature be reduced to that point which is just sufficient to prevent the extinction of its existence, then it becomes incapable of assimilating the food it absorbs from the earth and air; its tissue, no doubt, grows, but does not become solidified by the incorporation of assimilated matter; watery particles form in its structure, and a sickly yellowness takes the place of its healthy green colour. If a fruit-bearing plant, flavour and fruit become diminished; and if a flowering plant only, it ceases to produce even flowers. A deficiency of heat is the cause of the inferiority of many of our fruits compared with other countries; and a diminished amount of it in our hothouses, whether caused by accident or want of care, is readily discovered by the want of flavour in our fruits, and the absence of flowers on many of our plants. It is deficiency of heat that prevents the finer sorts of grape-vines from arriving at perfection in the open air of this country, and of the inferior quality of such even of the hardier sorts grown on our open walls, compared with those produced in more congenial climates. Allowing the temperature of our vineries to fall too low even for a very short period is as injurious to the plants as an excess of it would be; for although these causes are the antipodes of each other, the bad effects in either case are equally apparent. The following paragraph will explain pretty clearly how this is:-

"The maturation of the fruit is dependent upon the action of the leaves and roots, and the secretions that it forms are principally derived from light. It is, however, certain that the juices furnished by the leaves undergo a further alteration by the vital forces of the fruit itself, which alteration varies according to species. Thus the fruit of the peach is sweet, but there is no perceptible sweetness in its leaves; and the fruit of the fig is sweet and nutritious, while the leaves of that plant are acrid and deleterious. Anıong the immediate causes of the peculiar changes that occur in the secretions of fruits are heat and light, without which the peculiar qualities of fruits are imperfectly formed, especially of species that are natives of countries enjoying a high summer temperature. It is found that among the effects of a high temperature and an exposure to bright light, is the production of sugar and of certain flavours, and that under opposite circumstances acidity prevails. As sugar is more rich in carbon than vegetable acids, and has no free oxygen as they have, the sweetness of pulpy fruits ripened under bright sunshine may be understood to arise from the decomposition of carbonic gas, and the expulsion of oxygen being greater under sunshine than in the shade. Another cause may be, the greater facility by which vegetable acids enter into combination with gum and starch, and so form 
sugar at a high than at a low temperature." Theory of Hort., p. 76.

Day and night temperature in our forcinghouses.-Nature has sufficiently pointed out to us that she has for the wisest of purposes established a difference between the temperature of the day and of the night. She has given man the day to carry on his avocations, and night to take that repose necessary to enable him to renew his vigour for the duties of the succeeding day. With similar wisdom, the difference between the temperature of the day and that of the night has been established, that the inferior animals, as well as vegetables, should have their necessary rest also. In constant excitement plants could not exist, therefore they have their diurnal season of action and repose. "In the day, when light is strongest, and its evaporating and decomposing powers most energetic, temperature rises and stimulates the vitality of plants, so as to meet the demand thus made upon them; then, as light diminishes, and with it the necessity for excessive stimulus, temperature falls, and reaches its minimum at night, the time when there is the least demand upon the vital forces of vegetation; so that plants, like animals, have their diurnal season of action and repose. During the day, the system of a plant is exhausted of fluid by the aqueous exhalations that take place under the influence of sunlight; at night, when little or no perspiration occurs, the waste of the day is made good by the attrac. tion of the roots, and by morning the system is again filled with liquid matter, ready to meet the demand to be made upon it on the ensuing day." - Theory of Hort., p. 90 . Such being the order of nature's operations, the forcing-gardener will do well to follow her example; for he may rest assured that all his other efforts will be in vain if these simple conditions be not complied with. He bas it, however, in his power to guard against extremes of temperatures under his glassy coverings which he has not in the open air. The natural extreme of heat in these latitudes is seldom if ever too great for vegetation, the natural extreme of cold often is. The range, therefore, of temperature in his artificial climate must not extend to the maximum difference, but nearer to the minimum, because, during winter, when the former takes place, vegetation has sunk into comparative repose; while, during summer, a part of spring and of autumn, when vegetation is in full play, the difference in temperature should be more nearly approximated. The prevailing system hitherto followed by many in the regulation of their hothouses has been the very reverse of this. High night-temperatures have been kept up by the most unnatural of all means-namely, fire-heat ; a forced and unnatural excitement has been imposed upon plants, and the consequences have been more or less fatal to them, in nearly the exact proportion to which this fallacious practice has been carried. An obvious analogy exists between the difference of temperature during the day and night, and that between summer and winter; the former may not inaptly be regarded as one long day, and the latter as a night of similar duration. During the former period, abounding with VOL. II. light and heat, vegetation is pushed on to its fullest extent, declining as autumn advances, and as light and heat decrease, until winter nearly, but not entirely, arrests the vital action of vegetation. During winter, the vital action of plants ceases not entirely, but is only reduced in intensity. "The roots continue to absorb from the soil food, which is slowly impelled into the system, whence it finds no exit ; it therefore gradually accumulates, and in the course of time refills all those parts which the previous season's expenditure had emptied. In the meanwhile the excitability of the plant is recovered by rest, and may be even conceived to accumulate with the food that the absorbent system of the roots is storing up. At lengtb, when the temperature of the season has reached the requisite amount, excitability is once more aroused, an abundance of liquid food is ready to maintain it, and growth recommences, rapidly or slowly in proportion to the amount of excitement, to the length of previous repose, and to the quantity of food which had been accumulated."-Theory of Hort., p. 92.

A series of experiments on the progress of the growth of plants during night and day was made some years ago in the garden of the Horticultural Society of London, and the following results appear. The supposed average of the night temperature to which they were exposed was $69^{\circ}$, and the following is the rate of growth in inches :-

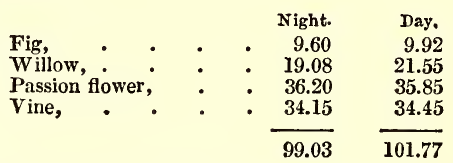

From this it appears that they grew as fast by night as by day, because they were unnaturally stimulated, for the apparent difference is obviously unimportant. A different result followed growing these and other plants in the open air, and fully exposed to the cold night-temperature of England, as the following table will show:-

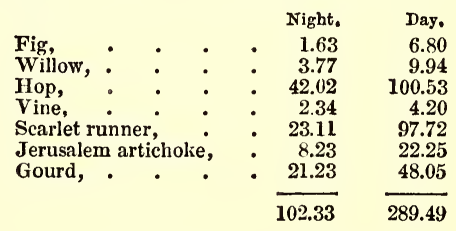

In carrying out this latter experiment during a part of another month, the total growth by night showed 119.07, and by day 337.16, showing the natural growth of plants to be, during the night, only 1 inch, while during the day it was 3 inches; while by bad artificial treatment, as will be seen from the first table, their growth was equal during both day and night. "The inevitable consequences of this inversion of natural growth is immature or unripe wood, with imperfect ill-constructed buds and a feeble constitution, entirely incapable of bearing the shock of great falls of temperature. More especially, $4 \mathrm{~L}$ 
water accumulates in the system, and is never decomposed or removed by perspiration in the requisite degree. In short, plants growing fast by night can neither ripen their wood nor form their inner structure well ; and, therefore, they are incapable of developing their natural beauty, or of resisting those extremes of temperature which are natural to them."-Gard. Chron.

Excess of heat injurious to plants.-With inexperienced people having the means of heating a glass-house in their power, much greater injury is done by keeping up an unnecessary degree of heat than the reverse. They think, if they have plenty of heat, that all that is necessary in forcing or plant-culture is in their hands. A few years ago we had recommended to us, by the pine-growers of the day, roasters, Calcutta pits, and all sorts of places that could be heated little short of a fiery furnace; the consequence was, burning of the roots, scorching the tops, abundant armies of scale and white bug, and ultimately the loss of the plants themselves. To this extraordinary heat humidity bore no proportion, and ventilation was abandoned to prevent the escape of the heat. These matters are better managed now. A necessary temperature, with a corresponding degree of humidity, and a perpetual change of air by day and night ventilation, is now the practice of our best growers. The effects of too high a temperature in our forcing-houses are thus sensibly described by Dr Lindley: "When, in the case of a given plant, the temperature is permanently maintained at a much higher degree than the species requires, it is over-excited. If the atmosphere is in a proportionate state of humidity, the tissue grows faster than the vital forces of the plant are capable of solidifying it, by the decomposition of carbonic acid and by other means; its excitability is gradually expended, the whole of its organisation becomes enfeebled, the vital functions are deranged, and a state of general debility is brought on. Such plants are soft and watery, with thin leaves, long joints, slender stems, and no disposition to produce flowers. A slight lowering of temperature affects them more than a much greater lowering would have done under other circumstances; and a permanent abstraction of light readily destroys them. Their inability to decompose carbonic acid, and to assimilate their food in proportion to their rate of growth, prevents their becoming so green as is natural to them, and gives them a pallid hue; and if it is their property to secrete other colouring matter, that, like all their other colouring secretions, is greatly diminished. But if, with a preternatural elevation of temperature, there is a proportionate abstraction of moisture, the loss of fluid by perspiration and evaporation goes on faster than the roots can make it good or the tissue transmit it; the secretions of the spurs are elaborated faster than the parts to receive them can be formed; the old leaves burn and dry up, and young leaves perish in like manner as fast as they are formed. Such being the result of preternatural high temperatures in dryness and in moisture, it is easy to conceive that, although such extremes cannot but be prejudicial, yet that they may be approached for particular purposes with advantage. A high temperature and dryness will be favourable to the formation of secretions of whatever kind; while a high temperature, with moisture, will lead to the production of leaves and branches only."-Theory of Horticulture, p. 83. A high and moist temperature in the case of pine-culture will grow young plants rapidly; with fruiting plants the consequence would be the production of innumerable suckers and crowns much larger than the fruit. A curious circumstance in connection with this is recorded by $\mathrm{Mr}$ Knight in the third volume of "The Horticultural Transactions," who says that the effects of an exceedingly high temperature caused unisexual plants to produce male flowers only, while a very low temperature produced a contrary effect; and he further observes that he had many years previously succeeded, by longcontinued very low temperature, in making cucumber plants produce female flowers only.

Season of rest.-The vine, like all other plants, requires a season of rest, that season commencing at the fall of the leaf. Vines, therefore, grown in pine-stoves at that period should be withdrawn, and their wood exposed to the open air on the outside of the house. This is deemed immaterial by some, and there have been instances where they have been left exposed to such high temperatures for several successive years; yet in the end these vines will show the bad effects of such unnatural treatment, and finally dwindle away and die. Perpetual excitement is as fatal to them as uninterrupted exertion would be to animals. Perpetual excitement exists nowhere in nature, although annuals might at first sight be stated as an exception to the rule. Their period of existence is short, scarcely exceeding the growing season of a perennial or ligneous plant. When they perfect their seed they cease to exist, and the seed, the material by which they are to be reproduced, rests in the soil till the returning season of growth. When plants are in a dormant state the majority of them lose their foliage, and in consequence of the want of leaves they are unable to make use of the nourishment applied to their roots, and hence it is wrong to stimulate them with nourishment or warmth, at least to the extent of causing them to grow when they ought to be at rest. In vineries where the plants cannot be withdrawn, the fullest amount of ventilation should be given during day and night. Notwithstanding the advantages the vine enjoys by being kept during its natural winter in a low temperature, care must be taken that they do not suffer from severe frosts, for instances have occurred where vines exposed on the exterior of a vinery have been killed; but this only happens in the most intense winters.

The best modes for withdrawing the vines at this season are exemplified, vol. i., fig. 425, as practised by Mr Saunders, and fig. 428, as in use in the Royal Gardens at Frogmore, by either of which the stems can be very conveniently withdrawn without the usual danger of twisting them near the root. Our own practice, whether in pine-stoves or regular vineries, is, as soon as 
the plants are pruned, which is immediately after the fruit is gathered and the wood properly ripened, to remove them from the roof of the house, and to train them horizontally along the bottom of the front; for, as will be seen by a glance at fig. 414, vol. $i$, the vines are planted in a narrow border between the cavity in which the hot-water pipes are placed and the front parapet wall, which latter being built on piers, the roots have free egress to the outer border. The front sashes are then removed and fitted to a portable framework resting on the 4-inch brick wall $c c$, excluding the vines, and placing the house in a fit state for forcing asparagus upon a tank which in one house covers the centre pit $g$, and in the others suspended shelves are attached to the roof and occupied with strawberries. The vines remain in this horizontal position until every bud has broken and attained the length of 1 or 2 inches, when they are again taken up to the roof, but for the first ten days trained loosely in a diagonal manner along the roof, after which they are taken up the trellis in the usual way. In our late vineries we allow them to remain in the horizontal direction till the buds begin naturally to swell in spring, at which time we replace the front sashes in their former place to secure them from frost, but at the same time we exclude them from the heat of the house within by placing felt shutters in the spaces they have just been removed from, thus retarding the breaking of the buds till the end of March or middle of April. Those we wish to bring in sooner we treat much in the same way, only replacing the front sashes in their proper place at an earlier period. By this means the stems are not in any way strained, and from the flow of the sap being moderated on account of the vines being placed in a horizontal position, every eye breaks strong, those at the bottom as strong as those at the top.

Bleeding of the vine.-The judicious cultivator will avoid late pruning of the vine to prevent its loss of energy by bleeding, and where this is avoided no doubt it is the best course. Duhamel treats the matter lightly, and even asserts that vines, after a copious bleeding from severe late pruning, grow as vigorously and yield as plentifully as others which were pruned at the usual season. All plants bleed most freely about the time of the opening of the bud; but in proportion as the leaves expand the sap flows less copiously, and when they are fully expanded it nearly entirely ceases. At such times, therefore, amputations may be made with impunity. Many remedies have been proposed to prevent the bleeding of the vine; the best assuredly is to prune in autumn or early in winter, before the sap begins to flow. Should bleeding occur, we have found nothing so effectual as selecting small potatoes about the size of a hen's egg, with their skin perfectly entive, and sticking one firmly on the end of each shoot cut.

Colouring grapes. - Erroneous notions are often entertained regarding this process, many exposing the fruit to the fullest light and sun, while others, following more closely the example of nature, allow the necessary shade which the leaves are calculated to produce. Hence grapes, ripened where the vines are trained well over the roof, colour in the fullest degree of excellence, even when the wood and foliage are between them and the light. Abundance of air, a total suspension of syringing, and a steady moderate temperature after the berries begin to change colour, is all that is required, provided the vines are in a healthy state and the roots in a congenial soil. Lowering the temperature at the period of changing colour is bad; it should be maintained during the day, and so increased as to admit of the fullest ventilation being given.

Thinning the fruit. - The vine, when in a vigorous state, frequently shows double and sometimes triple the number of bunches it is able to ripen in perfection, without evident injury to itself, and a lessening of the crop during succeeding years. This should be attended to as soon as they have little more than made their appearance. The smaller and most crowded bunches should be removed first, as well as those on weak shoots; and in the course of a week a second examination should take place, and a further reduction be made. The number of bunches left on a vine, or the distance they should stand from each other, is dependent on the sort, whether it be a strong or weak grower; whether its bunches are naturally large or small; whether the vine is in a vigorous state of growth or the reverse; and also whether fruit of the greatest excellence be desired, or a heavy crop, although individually the bunches and berries be of a less perfect character. No two bunches should be left on a spur unless the vine is unusually vigorous, and the variety of small size. Indeed, there is no real gain in having too many bunches, as the weight of twelve, at proper distances, will often be greater than that of sixteen on the same space. The same holds good in regard to thinning the berries, which process should commence as soon as it is ascertained that they are all perfectly set. Some sorts set their fruit much better than others, and only such as indicate that seeds are in them should be retained, removing all the small ones, which would only disfigure the bunch, and never arrive at maturity: the removal of these should form the first thinning. After the berries have attained the size of small peas a second thinning should take place, and at this time the shoulders should be gently elevated, and suspended by threads to the trellis above, so as to admit air and light into the thickest part of the bunch: should any of the points of the shoulders or bottom of the bunch appear thinly set with berries, these poiuts may be removed. When thinning is completed, every berry should hang clear of another, to admit of their swelling to their proper size. In thinning, it requires care not to prick any of the berries, for if this is done they in general speedily decay.

Gathering the fruit. - The berries towards the point of the bunch are the latest in ripening; these should be taken as a test of the maturity of the rest, and this can only be determined by tasting them. It more frequently happens that grapes are cut before they are fully ripe 
than the reverse; indeed, the grape, like the currant, can scarcely be over-ripened. Muscats, for example, should not be cut until after they have changed from a greenish white to a yellowish colour; and the deeper coloured all the black varieties are, the more certain are they of having attained perfection.

Various modes of cultivating the vine have been adopted; the aim in most cases has been their production at the least amount of labour and expense. Of these, attempting to grow the vine in greenhouses over other plants is a favourite mode with amateurs. This can never be attended with a satisfactory result, as both the vines and plants must be less or more sacrificed in their turn. Growing them in wooden structures heated with hot dung, although a favourite practice in many parts of the Continent (vide vol. i., figs. 405, 407, 408), is neither very satisfactory nor profitable, and bespeaks paucity in invention and means, and in this country carries us back to the days of Switzer, Laurence, Justice, \&c. Mr French some years ago revived the practice, but his vinery was large, and warmth was communicated to it by placing a huge ridge of warm dung along the centre, which was wheeled out when fermentation had nearly ceased, and replenished by wheeling more in from the stable-yard, which was adjoining. More recently, covering the inside of the roof of cow-stables with vines, the roof being either composed of more glass than usual, or being entirely covered with glass tiles, has been tried, the animal heat generated within being the method of heating adopted. A better way, and less expensive, would be to train vines to a trellis over the tanks which receive the waste steam or hot water from manufactories, and covering them over during cold weather, during night, with thin canvass. The heat ascending from the water below would, at the height of 18 inches or so, be quite sufficient to ripen many of the hardier sorts of grapes. The last and best method is no doubt the orchard-house recommended by Mr Rivers, where the operation is carried on mainly by solar heat only. Ripening the grape, when grown against walls in the open air, by enclosing each bunch within a glass vessel constructed for the purpose, no doubt accelerates maturity, but the expense of these glasses is nearly equal to the fruit produced. It has also been recommended to train the vine over a bed of brickbats, flints, scoria, or other heat-absorbing material, and to place common hand-glasses over the parts where the fruit is produced. All these and similar contrivances are but sorry representatives of a wellarranged vinery, which, in the long run, will certainly prove to be the cheapest and the best.

\section{SELECT LIST OF VARIETIES.}

Of the grapes of early days we have little information handed down as to their merits; the only representative we have of them is the Miller's Burgundy or Miller grape-the Le Meunier of the French, according to Chaptal, "Treat. sur le Vigne," vol. i. p. 169-a vine of which existed in the Horticultural Society's
Gardens, obtained from the remains of an ancient vineyard at Tortworth, in Gloucestershire, where there have been discovered many Roman antiquities, and, among others, evident traces of vineyards possibly first planted by that people.

The increase of varieties that has taken place in Britain is somewhere in the following order : Tusser, in 1560, speaks only of red and white grapes. Parkinson, in 1627, enumerates twenty-three sorts. Ray, in 1688, speaks of twelve sorts being most in repute. Rea, in 1702, mentions seventeen. Langley, in 1729, describes nineteen. Speechley, in 1789, describes seventy, all of which wore growing in the gardens of Welbeck at the same time. Forsyth, in 1806, describes fifty-three. Lindley, in 1831, sixty-two. Loudon, in "Encyclopedia of Gardening," edition 1835, forty-nine. Rogers in 1834, twenty-eight. Thompson, in 1842, ninety-nine, and to these about a dozen have been added since. Judicious cultivators, however, do not much care for extensive collections, but wisely content themselves with a selection of the best sorts, of which the following may be considered such: White Muscat of Alexandria, Black Hamburg, Black Prince, Chasselas musqué, Black, White, and Grizzly Frontignans, Lombardy, Royal muscadine, Black St Peter's, White sweetwater, Charlsworth, Tokay, and White Nice.

The foreign lists of grapes are very extensive. The catalogue published by Harvey, in 1802, of the varieties of grapes in the Luxembourg, contained no less than two hundred and sixty-seven sorts; and D. Simon Roxas Clemente of Madrid describes one hundred and twenty, and enumerates thirty-six authors, who, up to his time (about 1810), had written on the vine since the days of Columella; to these, half a score of names might be added up to the present time.

The only grapes for out - of - door culture in the climate of Britain, are-Early black July, Royal muscadine, Esperion, Black Hamburg, Black Prince (Lombardy or Black Portugal), Black cluster (Miller's Burgundy); and of these the Royal muscadine is the best.

Black Prince.-Bunches long, and generally without shoulders; leaves thick and fleshy; lobes slightly divided, but broadly serrated round their edges ; slightly tinged with red, becoming of a pale red or dark purplish hue towards autumn. Berries elliptical ; size, when well. grown and sufficiently thinned, above medium; colour dark purple, with a rich blue bloom; seeds large; flavour sweet, and abounding in juice. A very hardy vine, a good bearer, and in general colours well. A very showy grape, and ripens well in a cool vinery, and in the south on the open wall. Synonyms-Stewart's Black Prince, Alicant, Black Lisbon, Lombardy of some, Black Spanish, Black Valericia, Cambridge Botanic Garden grape, Boston, Sir Abraham Pytche's black, Pocock's Damascus.

Chasselas musqué. - Bunches medium, long, and rather loose; leaves small, of a fine healthy green colour; berries globular; size medium; skin thin, and very apt to crack just before ripening, if kept in a damp atmosphere. If 
kept dry, they will remain in perfect condition from the beginning of October till the end of January. This very excellent fruit has been much condemned on account of its cracking, and of course rotting inmediately after; but if kept in a dry atmosphere it will keep as long as the Hamburg or Muscat of Alexandria-at least such we have found to be the case. One of the very best grapes in cultivation. Colour yellowish white; flavour rich and musky; in short, one of the highest flavoured grapes grown. An excellent early forcer, and sets best in a moderate heat. Synonyms-Le Cour, Musk chasselas, St Albans, Josling's St Albans, Golden drop, Wilmot's Muscat muscadine.

Damascus, black. - Bunches large; berries globular; very black; skin thin; of excellent quality; when thoroughly ripened, requiring the same temperature as the Muscat of Alexandria; valuable as a late-keeping grape, but rather a shy bearer; many of the blooms do not set. Synonym-Worksop Manor grape.

Esperion.-Bunches large, resembling in size and shape the Black Hamburg; leaves a good deal cut, changing to an orange colour when the wood becomes ripened; berries medium size; form various, often round, sometimes flattened, or indented with a groove on one side, or both; colour deep purple or black ; flavour pleasant, although not remarkable for sweetness. A very hardy, luxuriant, and most prolific grape, equally adapted for forcing or the open wall. Synonyms - Hardy blue Windsor, Cumberland Lodge, Turner's black, Red port of some.

Frontignan, white.-Bunches of medium size, long, tapering, without shoulders; leaves small; berries medium sized; form globular ; colour a muddy white, or greenish yellow; skin thin; flavour rich and musky. All the Frontignans are excellent, alike as regards flavour, productiveness, and their adaptation to the forcing-house or open wall, wherever the grape ripens. Synonyms-White Frontignac, as it is usually pronounced, Muscat blanc of Duhamel (a very improper name) Muscat blanc de Jura, Moschata blanca, White Constantia, Nepean's Constantia, Raisin de Frontignan, Muscateller, Muscado Bianco, Moscatel commun, besides scveral German synonyms. Of this grape $\mathrm{Mr}$ Thompson remarks: "By many esteemed the best; however, the Black, Grizzly, and White Frontignans differ little, except as regards colour; but the Blue Frontignan is very different, being somewhat oval, with scarcely any Muscat flavour." The Constantia grapes from the Cape of Good Hope are almost invariably confounded with the Frontignaus: they are one and the same thing.

Frontignan, grizzly. - Bunches of medium size, somewhat long and tapering, shoulders smallish ; berries of medium size ; form round; colour grizzly, or palish brown; flavour rich and musky, and when left on the vine to become somewhat shrivelled are exceedingly agreeable. An old inhabitant of our gardens, being introduced by Sir W. Temple prior to 1654. All the Frontignans require a dry soil, and should, where possible, have their roots separated by cross walls from the stronger-growing kinds; for although they are most prolific in fruit-indeed, so much so as very frequently to show double or triple the number of bunches which they should be allowed to carry - they are what may be called indifferent rooters. Synonyms - Red Frontignan, Red Constantia, Grizeline, Muscat gris, Muscat rouge, and a number of other French and German synonyms. Lindley, Downing, and others, think the Red Frontignan different: we are inclined to attribute the seeming difference to circumstances of soil, culture, \&c.

Frontignan, black.-Bunches small, and much less tapering than in the two last; size of berry medium; form round; colour dead black. An excellent bearer and of high flavour, equally suited for the stove, cool vinery, or open wall. The Frontignans were early introduced to England; this, for example, by Sir W. Temple, prior to 1654 , being brought from the neighbourhood of Frontignan, a town in France, long celebrated for the excellence of its muscadine wine, generally called Frontignan. The Constantia wine of the Cape of Good Hope, so much prized and so scarce, is from this class of grapes, there called Constantias-Rogers says, from the Muscat of Alexandria. Forsyth more correctly says, "From the Red and White Constantia is made the famous Constantia wine, so called from a place near the Cape of Good Hope," p. 177. Synonyms-Red Frontignan of some, Black Constantia of some, Purple Constantia, Purple Frontignan, Sir W. Rouley's black, with various other French and German synonyms.

Hamburg, black.-Bunches large, often very large; 8 to 10 inches deep; shoulder large, often 7 to 8 or 9 inches across the top of the bunch. Size of berries large; form globular or oval, for they assume both forms, particularly in the older varieties, for there are now many; skin thickish, tending to the long keeping of the fruit after it is ripe; colour in general of a brownish black, but when not over-cropped, and properly ripened with sufficient light, quite black, and covered with a thick bloom; flavour sugary and rich. The best of all black grapes, and deservedly the most popular. Introduced from Hamburg in 1724 by a Mr Warner. An excellent forcing grape, as it is also for late crops, to keep through the winter. It seldom ripens against walls in England, at least not to the same perfection some other kinds do. Synonyms-Black Gibraltar, Gibraltar, Black Portugal of some, Black Teneriffe, Valentine's, Hampton Court vine, Admiral, Victoria, Salisbury violet, Warner's, Warner's black Hamburg, Purple Hamburg, Red Hamburg, Brown Hamburg, Dutch Hamburg, Frankendale, Frankenthaler, and a host of French, German, and Dutch synonyms besides.

The Black Hamburg is described by Knoop in 1771, one of the best German writers on pomology, under the name of Franckenthal, and it is in general known all over the Continent as the Frankenthal, from a place of that name on the Rhine, in Bavaria. It is nowhere there identified as either the Red or Brown Hamburg, although we have seen as many instances of those colours there as we have seen in Britain. Colour entirely depends on circumstances, as 
we have often proved in our own practice; the same vine producing black fruit one year, while in others it produced red or brown coloured berries; and we have observed the same occur in the case of the large vine in Hampton Court gardens, as well as in that of one of its progeny, the still larger vine at Cumberland Lodge. The principal causes which lead to this difference in colour are, over-cropping in either the present or previous season, cold damp soil, insufficient light, or rather where the fruit is grown at too great a distance from the glass, difference of temperature between the roots and foliage, want of sufficient ventilation, the roots being too deeply buried, the loss of, or injury done to the foliage, \&c. A plant of the Black Hamburg, exposed to one or more of these conditions, will assuredly produce red or brown berries, or, in other words, they will not colour well, a circum. stance of such frequent occurrence that most intelligent gardeners are aware of it; and plants of what are called the red or brown varieties, if wholly exempted from these causes, will with equal certainty produce dark-coloured fruit.

Theword Hamburg is usually written Hambro'. Various seedling varieties of this excellent grape have been raised, the principal of which are Wilmot's new black Hamburg, called also

Wilmot's Dutch Hamburg.-With us generally larger in the berry, and very black in colour, even when growing in the same house with the other varieties. It is a good variety, and worth cultivation. Fruit globose, rather earlier, and of higher flavour.

Judson's Richmond Villa black Hamburg.Evidently a seedling variety of the Black Hamburg; the berries are, however, more oval and elongated, ripening of a fine deep black colour, profusely covered with a rich bloom, like an Orleans plum, having a fine rich flavour, and very juicy. Is an abundant bearer and good setter, colouring full ten days earlier than the common Hamburg.

Millhill Hamburg. - This is an excellent variety, somewhat later in ripening than the common Hamburg, and having a tougher skin, although by no means thick, is adapted for keeping longer than any other variety of this section. The berries are also larger, more globose, and not always so well coloured as the ordinary sort. It has a singular propensity to send its roots deep into the soil, and hence, when it gets into a cold damp subsoil, the leaves become sickly in colour, wasted on the surface, and apt to flag under the influence of bright sunshine. It appears to be a cross between the Hamburg and Black Damascus, partaking, however, most of the former.

Pope's Hamburg.-In many respects similar to the last.

Braddick's seedling Hamburg.-Scarcely differing from the common sort.

Knevett's black Hamburg.-With no greater claim to difference than the last.

Hubshee.-One of the choicest of the Deccan grapes. Bunches large; berries oblong, brownish black; flesh dry and firm; skin thick; flavour not high, yet agreeable; requires as much heat to ripen as the Muscat of Alexandria, and has the merit of hanging long on the vine after it is ripe. Much has been said by travellers regarding the extraordinary merits of the Indian grapes. Few of them have as yet reached this country, and those that have are not held in very high estimation. Synonym-Kalee.

Lashmar's seedling grape.-Supposed to have been raised from seed of some foreign fruit by a Mr Lashmar of Brighton. Fruit white, medium size, oval form, and an abundant bearer. Its chief merit, however, is its hardiness, ripening a week or ten days in the open air before any other.

Lombardy, black.-Bunches large, and well shouldered, long and loose; size of berries large; form roundish ovate; colour black, when properly ripened, previous to ripening of a reddish purple; skin thin; of a sweet and pleasant flavour. A very late grape, quite unfitted for the open wall, but exceedingly valuable in the vinery, on account of its late ripening, productiveness, and property of keeping through the winter in good preservation. Leaves small, turning purplish as the fruit ripens. Succeeds well if planted along with the White Muscat of Alexandria, the same temperature being required for both, and also as they are late ripeners, and the best of keepers. Synonyms-West's St Peter's, Money's West's St Peter's, Raisin de Carmes, Raisin de Cuba, Lombardy of some, Poonah,not the common Lombardy, or Flame-coloured Tokay, which is a different and inferior grape. This grape, under the name of Poonah, was imported in 1817 from Bombay by Sir Joseph Banks. It is extensively cultivated over India.

Muscat of Alexandria, white-Bunches very large, well shouldered, rather loose, and irregular in form; berries large; form oval; colour at first whitish green, but when fully ripened fine pale amber ; skin thick; stones small, and often absent in the largest berries; flesh firm and hard, and of a delicious musky flavour. This is evidently by far the best of all white or palecoloured grapes. Like the last, it requires the highest temperature the vine is capable of bearing, and sets its fruit in such temperature better than in the ordinary temperature of vineries in general. When thoroughly ripened, it will hang on the vines during the winter, and that without losing any of its fine musky flavour. Indeed, it is a grape that cannot be over-ripened. Synonyms-Round Muscat of Alexandria,Totten ham Park Muscat, Malaga, Lunel, White Tokay of some, White Muscat of Lunel, Muscat of Jerusalem, Alexandrian Frontignan, Muscat Eschcolota, Zebibo of Sicily, Passe musqué, White passe musqué, Passe-longue musqué of the French. Speechley, in "Treatise on the Vine," written in 1789, says: "This grape is in high estimation, and is at present more generally planted in hothouses than any other sort." From a very interesting series of papers recently published in "The Gardeners' Journal," on the varieties of fruits cultivated in some of our very best gardens, we find the Muscat of Alexandria ranking with the Black Hamburg and St Peter's, as the most general in cultivation, more especially for late autumn and winter use. Various seedling varieties of this excellent grape exist. The most 
remarkable success in this species of improvement has followed the persevering skill of $\mathrm{Mr}$ Burn at Tottenham Park, and Mr Saunders at Tedworth Court, Wiltshire, who have each reared several varieties of great merit. Whatever opinion may be entertained regarding the identity of the Tottenham Park Muscat with the original sort, is perhaps now unimportant; and hence we have followed $\mathrm{Mr}$ Thompson in giving it above as a synonym only. Of one thing we are certain, that both these eminent horticulturists have in cultivation varieties peculiarly their own. We have examined them minutely in both gardens, and strongly as we hold thatsoiland management have the effect of greatly altering the general characters of fruits, we believe that neither has produced the change unmistakably obvious in certain vines grown by these individuals. Indeed, we have their own acknowledgment that they were originated by them from seed. Of the origin of the Cannon Hall Muscat we have no certain account; in all probability it arose accidentally from seed. We, through the kindness of Colonel Stanhope, and his very intelligent gardener, $\mathrm{Mr}$ Wilson, received two plants of this variety in 1841 . They were planted in a pine-stove at Dalkeith along with other Muscats, but from that day to this we have been unable to distinguish any difference whatever between the Cannon Hall, the Tottenham Park, and other Muscats growing together. This, we know, is contrary to received opinions; and had we not received the plants from Cannon Hall direct, and seen them planted, we would have naturally set it down to some mistake or other. Mr Thompson, in "The Fruit Cat. of the Hort. Soc.," states the difference between the Cannon Hall and the common Muscat consists merely in these words, "Scarcely so highflavoured as the White Muscat of Alexandria."

The Queen Muscat grape is described in the "Journ. of the Lond. Hort. Soc." as being well adapted to pot culture, and to be somewhat larger than the Royal Muscadine. "The berries are also fully as large as that variety, but perfectly distinct, being oval. They are yellowish white, semi-transparent, so that the one seed which each berry contains can be seen through the skin. The flesh is firmer than that of the Sweetwater, but much more tender than that of the White Muscat of Alexandria, rich and sugary. It is an early grape, and, as far as can be judged of it," grown in a pot, "it appears highly deserving of cultivation."

Muscadine royal.-Bunches of medium size, sometimes, however, exceeding that standard: berries globular; size medium; colour at first greenish white, but when thoroughly ripened in sufficient light turning to an amber colour, somewhat tanned with a brownish tinge; skin thin; flavour excellent. One of our hardiest grapes, ripening well in the south on the open wall. We remember seeing a number of plants of this sort trained as low standards, much in the way the Rhenish vine-growers train theirs, and loaded with ripe fruit, growing in a warm sheltered border in the late Mr Kirk's nursery at Brompton. It also forces well, and is very productive. This is the Chasselas par excellence of the French, who cultivate it to a large extent at Fontainebleau near Paris. Synonyms-Common muscadine, Amber muscadine, D'Arbois, D'Arboyce, Pearl of some, Early white Teneriffe, Amiens, Raisin de champagne, White chasselas, Chasselas, Chasselas doré, Chasselas de Fontaineblcau.

Major Moray's grape, or Abercairney grape.The history of this excellent variety is rather obscure; all that is known of it is, that it was first noticed in the garden of the gentleman in Perthshire whose name it bears. It is a fine handsome medium-sized grape, of quality somewhat similar to a well-perfected Black Hamburg. It is probably a cross between that grape and the St Peter's, evidently the result of accident.

Nice.-Bunches of the largest size, loose, with extending shoulders; berries medium sized; form globular; skin rather tough; colour greenish white, changing, when fully ripened in the sun, to a yellowish green; leaves of large size, very downy underneath; whole plant robust and strong; sets freely, and is a very great bearer. It is not a grape of high flavour, but its enormous bunches, and the great length of time the fruit keeps after ripening, claim for it a place in every large establishment. Unsuited for the open wall, and requires about the same temperature as the Syrian and most other large-growing sorts. It is singular Mr Lindley takes no notice of this grape, nor does his cotemporary Rogers. We believe the first notice of it is to be found in the "Transactions of the Horticultural Society," "Fruit Catalogue of Horticultural Society," and in our " Orchard," p. 383, " Practical Gardener," p. 439, "Encyclopædia of Gardening," p. 950, "Downing," p. 245. It must therefore be of comparatively modern introduction.

Reeve's muscadine.-Berries oval; skin yellowish white, rather thick; flavour rich ; flesh melting and juicy; bunches large and broadshouldered; stalk of the berries short and stiff. A good grape, ripening as early as the Black Hamburg, and an excellent bearer.

Sahibee. - Esteemed the best grape at Kermanshah in Persia. Several sorts from that land of fruits have been tried in Britain, but none of them rivals the Muscat or Black Hamburg. Bunches large ; berries oval, yellow, white, and red; skin thick ; flavour good. Requires, as do all Persian grapes, a high temperature to ripen it thoroughly. This variety breaks early into leaf, and appears adapted to very early forcing. It prospers best in a dry atmosphere, and should not be syringed over the foliage after the fruit is set.

St Peter's, black.-Bunches above medium size, loose, very generally without shoulders; colour very black when properly ripened; berries about medium size; form round; skin thin; flavour excellent, but its greatest merit is in its keeping properties. If ripened by the beginning of October, it will hang on the vines till March. Mr Lindley says in "Guide to the Orchard," p. 198: "The berries, when subjected to a high temperature, are very apt to crack, on which account it is not advisable to plant it in the forcing-house ; but for the vinery it is a most excellent grape." We do not find this to be the case, although ours are planted in a pine stove. 
The temperature of our late vineries we find insufficient to ripen it properly. Synonyms-St Peter's, Black Palestine, Oldacre's West's St Peter's.

Sweetwater. - Bunches medium sized, loose, the foot-stalks of the fruit stiff, and the berries set apart from each other more than in most grapes; berries large; form globular; colour greenish white while ripening; when perfectly so they become more white, and shaded with a slight russety tinge if well exposed to the sun; flesh watery and sweet, but not high flavoured. An excellent forcer, and ripens even earlier than the Royal muscadine, whether on the open wall or in the forcing-house. Its greatest fault is that it does not set well, particularly if the vines are old. Synonyms-Stillward's sweetwater, Dutch sweetwater, Chasselas royal, Chasselas precose, Water zoete blanc of the Dutch. They also call it Parel druyf, and force it in large quantities, often by dung-heat, for the public markets.

Tokay, white. - Bunches compact, medium sized; berries oval; colour white; skin thin. An excellent grape, either for the vinery or open wall. In both situations it is an abundant bearer. From this variety the fine Tokay wine of Hungary is made. It appears to be less cultivated in Britain than it deserves.

Tokay, Charlsworth.-Bunches compact, medium sized; berries oval; colour white; skin thick; flavour somewhat resembling the Muscat of Alexandria. A modern grape of great excellence, and an abundant bearer, requiring the temperature of the vinery. Rogers considers this inferior to the White tokay in point of bearing. Our own experience does not lead us to think so. It has been long known in Cheshire, and was cultivated, Mr Rogers says, many years ago in the gardens of the Earl of Stamford.

Trebbiana grape.-Very similar to the Tokay in growth, size, period of ripening, flesh, and flavour, somewhat shorter in the berry, less liable to shrivel after being ripe than either the Muscat of Alexandria or its nearer ally the Tokay. Like these grapes, it requires heat to ripen it, and has been pronounced by Mr Beaton, one of our highest authorities, as the next best white grape to the Muscat. And Mr Tillery of Welbeck adds his highly respectable testimony to its merits, describing it as a fine-shouldered bunch, in colour resembling a well-ripened Muscat, and capable of keeping unshrivelled until the end of February or beginning of March. Mr Beaton says it is of Italian origin, and that it was introduced into Britain by the late Earl Powis about twenty-seven years ago. $\mathrm{Mr}$ Tillery gives Portugal as its native country, and conjectures it to be a seedling between the White muscat and the White Portugal grape of the shops. In culture it should be planted along with the Muscats and Black St Peter's, all of which require much heat to ripen well.

Verdal.-Bunches loose; berries round and white; skin thin. Of excellent quality, and very early; well adapted for pot culture.

Wortley Hall grape--Bunches large, well shouldered, much in the way of the Black Hamburg; berries large ; form roundish ovate; colour glossy dark purple; flavour sweet, pleasant, and slightly musky ; seeds large, only one generally in each berry. An excellent late grape, which mainly induces us to add it to our list, as all late and good-keeping grapes are desirable, because they carry the grape season through the winter, when such fruit is most acceptable, and also obviate the necessity of forcing at the most unnatural season. According to Lindley, this variety "sprang up from seed in the stove at Wortley Hall, in Yorkshire, and first bore fruit in 1819."

To these the following names of new or littleknown sorts may be added : Black Barbarossa and White Barbarossa, noted for their late keeping; bunches long; berries oval; skin thick; flavour excellent when ripe. De Cheras, Gros Coulard, a white grape of good quality, with very large berries, fitted for the vinery only. Noir d'Espagne, a round purple grape of good quality, having in general but one seed in each berry. Of modern introduction.

Raisin de Calabre.-Bunches long; berries white, round, with a thin skin, adapted to the vinery ; quality second-rate, but having the property of hanging long on the vine after it is ripe without shrivelling, rendering it an acquisition to those who wish for grapes during the winter. Synonym-Calabrian raisin.

The Isabella and Catawba, American varieties, long since proved in England and found wanting, are at present in course of being thrust upon the attention of fruit-collectors by some of the London trade. Foxey-tasted grapes will not suit English palates. Indeed, from the native grapes of America, which have originated from Vitis labrusca, and not from Vitis vinifera, we have nothing good to expect, for, as one of their own best pomological writers observes, "The varieties of grapes at present grown are chiefly the finer sorts of the wild species, or, which is most generally the case, they are accidentally improved varieties that have sprung up in woods and fields from wild vines. They are therefore but one remove from a wild state ; and as extensive trials are now being made by various cultivators to produce new varieties from these, there is little doubt that in a few years we shall have many new sorts, combining the good qualities of the best foreign grapes with the hardiness of the indigenous ones." Such, therefore, being the case with the grapes of America, we need not look forward to any important addition to our own present stock from that quarter. The Isabella and Catawba are at present amongst the most valuable of American grapes, yet in Britain no one cares to eat them.

The following Persian grapes are in cultivation to a limited extent in this country. Their merits, however, have not as yet been sufficiently determined : - Faskbee, Hoolookee, Kamadanee, Keshmushee or Kishmish, Kohlauree, Lauree, Yek-yendee, and some others.

The names of the following grapes are pronounced as under: Chasselas musqué-Shahslah meus-kay; Chasselas de FontainebleauShah-slah de Fon-tan-blo; Ciotat-Se-o-tah ; Lenoir-Lun-war.

Diseases and insects. - The vine may be said to be naturally almost exempt from disease; the 
principal to which it is liable may be considered debility, brought on by bleeding, occasioned by late spring-pruning, the remedies for which have been noticed at p. 631 ; derangement in the circulation of the sap in spring, occasioned by severe frost succeeding warm weather; and the occasional loss of its young shoots in spring arising from a similar cause. The most fatal, however, of all is mildew, which, to some extent, in certain places, has been observed for years; nor was much attention paid to it until within these few years, when mildew of a most virulent description made its appearance, and has been determined to be a distinct fungus from Erysiphe communis Dec., or common mildew, which probably was that to which we have referred. The new mildew has been denominated Oidium Tuckeri. The devastation it has already made in Madeira, and indeed most wineproducing countries, is too well known to require any notice here. It is enough to say that it has established itself in the grape-houses of many parts of Britain. Its appearance, character, habits, \&c., are apparently as mysterious as those of the potato disease, and, like it, it has caused no small consternation amongst horticulturists. Mr Harris, in an interesting pamphlet lately published, describes the attack of the Oidium as follows: "The grapes, when blighted (attacked), are covered with what appears to be a white powder like lime, a little darkened with brown and yellow. These fungi send forth laterally, in all directions, thread-like filaments, which become so completely interwoven with one another as entirely to cover and enclose the skin of the grape in a compact and firm network, and on each are seen the eggshaped capsule or seed-pod. The pips and juice go on swelling, and at times, when struck late in the season, the grapes become partially ripe and coloured; but very soon expansion from within going on, checked with this network, the grape bursts; but as it cannot burst as it should do were it unconfined, the edges of the part where it bursts turn inward in place of outward, and the pips are exposed to view as the teeth of a man when the lips are drawn back. If the grapes are attacked in an early stage of their growth, they dry up, fall off, and become very offensive, but do not split."

Various remedies have been proposed, the basis of all of them being sulphur; indeed, that mineral in fine powder has been employed successfully by blowing it over and through amongst the berries by means of a sulphurator, commencing the application as soon as the disease makes its appearance; and many wisely do so even before it has time to manifest itself. Dr Price has recently, in "The Chemical Gazette," proposed, as even a more effectual and at the same time more durable remedy, pentasulphide of calcium, having observed the effects of the disease in three vineries at Margate, in Kent, near to where it first appeared in England, these houses " having been for five consecutive years infested with the disease; and notwithstanding the employment of sulphur as flour of sulphur, no abatement of its ravages could be detected. I was," he says, "induced to emVOL. II. ploy a solution of pentasulphide of calcium, a diluted solution of which, having been found in no way to act injuriously to the young and delicate shoots of several plants, was applied to the vines; the object in view being that the pentasulphide should be decomposed by carbonic acid, and that four atoms of sulphur, together with the carbonate of lime formed, should be deposited in a uniform and durable covering on the stems and branches of the vines affected. Although but few applications were made, the stems became coated with a protective deposit of sulphur, and the disease gradually but effectually disappeared, insomuch that the houses have been, and now are, entirely free from any disease or symptoms of infection. The young wood is in no way affected by its application; the vines operated upon were in 1851 covered with the disease, but since the autumn of 1852 they have received no further treatment. The vines in the immediate neighbourhood, and adjoining one of the houses, are covered with the disease ; but notwithstanding their close proximity, no indication of the disease has at present been detected in any of the three houses." The following directions are given for its preparation, but in most cases we would suggest to put the matter into the hands of a chemist to insure success; and this the more so, as its preparation is not a costly affair. Boil thirty parts by weight of caustic lime, with eighty parts by weight of flour of sulphur, suspended in a sufficient quantity of water; heat is applied until the solution has acquired a darkred colour, and the excess of sulphur ceases to dissolve. The clear solution is drawn off, and, after being diluted with water, may be applied to the vines by means of either a sponge, brush, or syringe. A saturated solution of pentasulphide of calcium may be diluted with from twelve to twenty times its volume of water previous to its being employed.

The Thrips (Thrips adonidum), fig. 21, and Thrips ochraceus, both described p. 76, are oftentimes very destructive to the vine, attacking the foliage particularly in its young state. And next to them is the family of Acarus, or redspider, of which the following are found in vineries and hothouses, but by casual observers confounded as the same :-

Acarus telarius Linn., fig. 22, the common red-spider, or garden-mite.

Acarus holosericeus, another species nearly as destructive as the last. It is, however, more readily detected than the last on account of its bright vermilion colour.

Acarus geniculatus is less destructive in the vinery than the others, but is often found infesting the bark of the plum and other fruit trees during spring. They present themselves congregated in great numbers at the base of the spurs and small branches, resembling a gummy exudation. They are also exceedingly minute, and of a brownish-red shining colour. The appearance of both Thrips and Acarus in vineries, as well as in all other planthouses, is indicative of too high a temperature and too dry an atmosphere-conditions which bring these greatest of pests speedily into existence. 
The old adage, "that prevention is better than cure," is in few cases better exemplified than as regards thrip, red-spider, mildew, \&c., which are so annoying in most forcing-houses. Peaches, vines, and all deciduous plants mav be in a great degree protected from them by the use of sulphur in a volatilised state; in no other are its effects so completely accomplished. It must be obvious to every experienced cultivator that a wholesale riddance of such pests is of vast importance, compared with the bit-by-bit and often superficial modes so frequently practised, and those only after the evil has done its worst. No doubt, applied either in a dry or pasty state, sulphur is sufficient for the destruction of mildew; and in this state it can be safely applied even when the trees are in full foliage: but for the destruction of insects of such tenacity as the thrip and red-spider, it is of little more use than any other covering would be that would exclude the air and imprison them until it is washed off again by repeated syringings, except in so far as it may become partially volatilised by the heat of the sun or the temperature of the house. Mr Robert Fish, one of the conductors of " The Cottage Gardener," on this very subject throws out the following useful suggestions: "If, then," he says, "the fumes of sulphur are so useful to the gardener, how can he most safely apply them? A most important question-no law of mediums here will do. If you err, let it be on the safe side of low temperature. Whenever sulphur burns, it will destroy, not only animal life, but everything vegetable that is growing and green. It will burn spontaneously when exposed to a temperature of $302^{\circ}$; at a higher temperature still the very fumes will ignite. In putting a paint of flour of sulphur on the warm end of a flue there is always this great danger. It should always be put on at some yards from the furnace. It fuses or melts, but does not burn, at $220^{\circ}$. Long before that heat, it gives off volatile fumes. Hence, for hardy things, smearing hot-water pipes, unless close to the boiler, will never be dangerous, because the water will scarcely ever reach $200^{\circ}$. But at that heat the fumes given off would be ruinous to foliage just forming, and many flowers would immediately drop. In all cases where leaves are young and deficient in organisable matter, I should not like to use a higher temperature to obtain fumes than from $170^{\circ}$ to $180^{\circ}$." Many. tender plants in leaf will survive the fumes so produced with impunity, but the utmost care must be taken that the heat do not exceed the first of these limits. Some plants will not bear. them at all ; therefore such should be removed during the operation. It is not, however, to plants in leaf that we are at present referring; it is to the peach-house and vinery, and such deciduous fruit trees as are in a state of rest; and the object is, by giving them the full amount of fumigation that they will bear, to rid them of the insects which lie ensconced in crevices of the bark, under loose fragments of it, and at the base, and even within the scales of the buds, as well as those taking shelter in the woodwork or masonry of the structure.

The intelligent authority we have quoted. above, not content with the volatilised fumes, goes still farther, and actually burns the sulphur, thereby producing sulphurous acid, burning, in the case of French bean, melon, cucumber pits, \&c., a handful or two of sulphur upon the removal of one crop, and before a succeeding one is put in, removing a little of the surface-soil, and giving air freely for several hours after the operation is completed. That more disagreeable than dangerous insect, the scale, which sometimes attacks both peach and vine trees, was completely subdued by the same means. In regard to the mode of burning and the necessary conditions, the following is the substance : A few bits of dry straw are laid over red embers in a pot, and on that the flour of sulphur is placed, and the top of the pot covered with dry moss. The embers and the sulphur are quite effectual of themselves, as combustion proceeds slowly. Whatever is mixed with the sulphur must be dry. "Use no other noxious matter with it. To make assurance doubly sure," Mr Fish " had burned tobacco and bruised laurel-leaves with the sulphur; but in every case the painting was affected, being rendered brown or black-a matter of no great moment in a pit, but of great consequence in an ornamental house." Regarding the state of the trees, "the growth must be finished, and the wood hard and firm. If not well ripened, the bark will be injured in places, as if burned with a hot iron. The dryness of the wood is indispensable. Equally indispensable is the dryness of the house, especially on all parts covered with paint. If wet, a grey sulphuret of lead is apt to be formed, and covers the paint with a dark powdery substance, if there is enough of moisture for sulphurated hydrogen to be formed." In regard to quantity, "in this Mr Fish has never been particular; a small handful, or two table-spoonfuls, to a house 12 feet by 8 , repeated according to size, is an average quantity." Time and mode of application, "a quiet day or night, every cranny in the house stopped, and no door or window opened for at least a dozen of hours after it is seen that combustion has fairly commenced. Used with caution, sulphur, more than it has been, will be found a great auxiliary; used carelessly, it will bring disappointment."

The vine scale (Coccus vitis Schr.) is found on the branches of the vine as well as on other fruit-bearing trees. It is, while young, somewhat long and of a brownish colour, changing with age to a blackish brown, and in shape hemispherical and wrinkled. This is a true coccus, characterised by the female having a scale or covering inseparable from its body. In their young state both sexes are alike, but the male larva produces insects with two wings, with two tail-threads, while the females have no wings, and afford shelter under their scales. for their young. The females are shield-like, somewhat concave below; they have six small legs, changing, as the insects become old, into part of their bodies. The insect is furnished with a sucker underneath, with which it pierces the cuticle of the plant and extracts their juices. The female dies soon after impregnation, affording by her body a protection to her eggs, which. 
are covered with long white wool, and this wool sometimes completely envelopes the shoots of the vine. They propagate rapidly, and where they become numerous they are difficult to subdue. The remedies are to paint over the whole wood and the under sides of such leaves as they have settled upon with a weak solution of gumarabic; allow it to remain on for a week, and then wash it off. Another method is to brush them off as they appear, and also to wet the leaves and branches with spirits of turpentine twice or three times during the early stage of growth. As a preventive, the old wood during the early part of winter should have all its loose bark removed, and afterwards be painted over with the following composition-viz., half a pound of soft soap, quarter an ounce of black pepper, and one pound of sulphur, to four gallons of water, boiling the whole for twenty minutes, and when cold laying it on about the consistency of thick cream. A little lime and soot may be added to give it a body. The exact proportions are of little consequence, the object being to cause it to adhere to the bark, to prevent the eggs of the larvæ from coming into life. Painting the old wood with spirits of tar is a complete remedy.

The mealy bug (Coccus adonidum Linn.) attacks both the vine and the pine. The males of all this family have wings, and are capable of locomotion; the females are stationary, being fixed to the old wood of the plants they infest, or on the leaves of the pines. 'The males are so small as to require a microscope to distinguish them clearly. The females are much larger, and not unlike a common house-bug in shape, but with a scaly skin, and while hatching they envelop themselves in a white cottony-like covering. Spirits of turpentine applied to every part infected will, upon the first or second application, rid the plants of them. As they are not all hatched at the same time, and as even this spirit has little or no effect upon them in the egg state, their escape from the egg should be carefully watched, and the spirits applied at that time. During the application the house should be kept pretty warm for a few hours, to cause the spirit to vaporise. This is also a good remedy for the vine scale (Coccus vitis). The following preparation is efficacious in the case of the mealy bug (Coccus adonidum)-viz., two pounds of flour of sulphur, one pound of tobacco, two pounds of soft soap, and a wineglassful of spirits of turpentine. The sulphur, turpentine, and soap should be wrought up to a pretty thick lather with warm water, and the tobacco boiled for an hour, and the pure liquor added. The above quantities will make five gallons. Sponging or syringing the leaves and branches with water at a temperature of $115^{\circ}$, if frequently applied, will also prove destructive to this insect. The vapour or steam from rank stable-yard manure in a high state of fermentation is also of advantage, and hence the use of this in vineries during the first proeess of forcing.

The genus Aspidiotus is so near akin to Coccus that they are often confounded together; their effects are the same, and means of suppression as noticed above.
The caterpillar, Tortrix vitisana of Linn., Cochylis vitisana Jacquin, Cochylis reliquana Treitsch, the vine tortrix, is found on the vine in April and May, at which time the female lays her eggs. The caterpillars appear as the buds begin to expand, which they cover with a web of white threads. It is less destructive in vineries than on the open walls. Their mode of attack is to eat the inner parts of the blossom, and having finished one embryo flower they repair to another, and so on until they have covered the whole branch with their web, and destroyed the complete flower.

When fully grown, the caterpillar measures from 3 to 4 lines, of a dirty-green colour, beset with small whitish warts, from which stiff hairs arise. "The head and first segment of the body are yellowish brown, the six fore-feet blackish, and the others of the same colour as the body. They enter the pupa state towards the end of June, and appear as moths twelve days afterwards. Pupation takes place either in the cocoon or in a curled-up leaf. The pupa is brown, with rough points. The moth is 3 or 4 lines long, and with the wings extended 6 lines broad. The head is yellowish brown; the antennæ, which are half as long as the whole insect, are black and annulated. The upper wings appear marbled with rust colour and bluish grey, having two incomplete cross bands of the latter colour, or whitish, in the middle of the first of which is a dark rusty dot. The under wings are white, with brownish veins and snow-white fringes."-KoLLAR.

A second generation of caterpillars appears towards the end of August from the eggs of the first. These caterpillars penetrate into the berry, and feed on the unripe pulp. The full-grown caterpillar leaves the bunch of grapes to undergo pupation in some suitable place, often at the root of the vine. They remain in this state till April, when the moths appear. There are other species of Tortricidæ which attack the vine on the Continent, but as these probably never appear in this country, we need not further notice them. The remedies given for the destruction of the coccus are applicable to the tortrix also.

The common wasp (Vespa vulgaris) is exceedingly destructive to the ripe fruit. There are two most efficient ways to guard the crop against their attacks- namely, destroying every nest to be found within a mile of the garden (for they will travel thus far). If boys are em. ployed during the day in discovering the nests and marking them, they may be blown up at night after dark, when they are congregated together, by firing a squib of gunpowder within the hole, afterwards digging up the nest, and saturating it with water; or half a pint of spirits of tar poured into the hole, and the opening closed up by placing a piece of turf over it, or pouring a quart of common tar into it, smearing the surface round the entrance, will be found effective. The ventilators may be cov: ered with fine gauze netting for the admission of air and exclusion of the enemy; and if the glazing be in a proper state of repair, no wasp can enter. Capturing the females early in the season, before they have begun to breed, is also an excellent 
plan for ridding us of one of our most voracious and destructive enemies. The wasp-catcher, or entomological forceps, fig. 258, the invention of Mr Underwood, cutler, HayFig. 258.

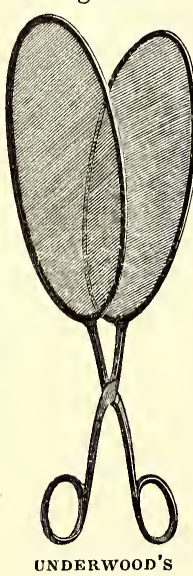
market, London, is well adapted for catching such stray wasps as may find their way into the vinery after the openings have been covered with gauze netting - of which, by the way, that of Haythorn of Nottingham is the best-or such female wasps as appear early in the season, for the capture of one of these is tantamount to the destruction of a whole nest in July or August. We have used them for sixteen years, and consider them a valuable and indispensable implement in every garden.

Curculio betuleti, Rhynchites betuleti Schonh., has apparently been confounded with Rhynchites bacchus, which has also been considered as the peculiar enemy to the vine, whereas it is never found on that plant, but on pears and other fruit trees. It is the $\boldsymbol{R}$. betuleti, or vine-weevil, that infests the vinery. This is a small weevil, of a greenish or steelblue colour, 4 lines long, including the rostrum, which is nearly a third of the whole length. On the thorax of the male, towards the front, on both sides, are observed short spines. The abdomen is almost quadrangular. The spines are wanting on the thorax of the female, and her rostrum is shorter. The beetle appears in spring as soon as the trees are in full foliage, and begins its work of destruction in May. It makes use of the vine partly for a dwelling, and partly for the food of its young. She commences operations by cutting the petiole of the leaf about half-way through, so as to cause it to fall down. She then begins to roll the leaf together, generally alone, but sometimes assisted by the male. While this operation is going forward, she also lays her eggs; that is, she pierces the roll, lays an egg in the opening, and pushes it in with her rostrum in such a manner that it remains on the inner surface of the leaf. When she has introduced five or: six eggs in this manner, between the different folds, she rolls the remaining part of the leaf entirely together. In a few days the eggs are hatched in the rolls, and a whitish small worm comes out of each egg, with black oblique stripes over the back, and a reddish head. The young worm subsists on the leaf, and as only a few eggs are laid in a roll, each maggot finds sufficient nourishment to enable it to attain its full size. In four or five weeks it is fully grown. In the mean time the leaf has become so dry that they are easily blown off by the wind, and by this means the worm falls to the earth. If this does not take place, the worm leaves the partly-consumed leaf when fully grown, and reaches the earth, where it buries itself, to reappear in spring as a weevil.

The black-vine weevil (Curculio sulcatus Fab.), fig. 259, is another vine weevil sometimes de-

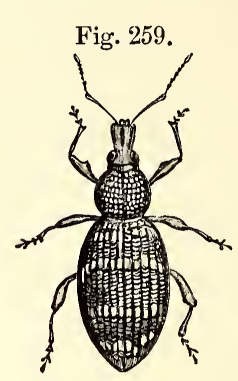
structive to vines in hothouses, appearing in the open air from May to June, and in hothouses much earlier. Their appearance in the vineries at Studley Castle is recorded by $\mathrm{Mr}$ Edwards, and they are stated to have made sad havoc amongst the young shoots and foliage, appearing there about January, from which period till the end of April they feed on the buds and leaves, and this probably during night, as they are seldom seen during the day. In their maggot state they live on the roots of ELACK viNE-we Evir. the vines, which they greatly weaken. About June the maggots change to pupæ, according to the statements of some; while others say that they live in the ground all winter, and are metamorphosed in spring.

The clay-coloured vine-weevil (Curculio picipis Fab.), another species nearly allied to the last, is equally destructive in the vinery. Like the last, this feeds by night, and may then be found gnawing the young wood, or feeding on the tips of the shoots. Both are supposed to be brought into vineries along with the leaves used to fill the pits. They lurk under the old rugged bark of the vine, which should be removed during winter, and the wood washed or scrubbed with a hard brush. Ruricola, in "Gardeners' Chronicle," suggests the following mode of capture, which we think an excellent expedient; namely, "As soon as the beetles appear, sieves should be held at night under the branches and leaves, when, by shaking, the beetles will readily fall into the sieves; but as they drop down when approached, this operation must be proceeded with gently and quietly. Multitudes may be thus collected both in and out of doors, and if the person who carries the light has a pail of water, the sieves can be emptied into it." This will prevent their escape; and when the operation is completed, the whole should be cast into boiling water, or the water into which they have been already thrown should be boiled, or the whole thrown into the nearest furnace; because, on account of their horny cover, they are capable of resisting uninjured any amount of heat under the boiling point.

The European names of the grape-vine areVite, Italian - Vigne, French-Wyngaard, Dutch -Weinstock, German - Vid, Spanish - Winograd, Russian. 


\title{
CHAPTER XV.
}

\author{
THE PINE-APPLE.
}

The Pine-apple, Bromelia ananas Linn., $A n$ anassa Thun., Sativa B. R., is undoubtedly a native of the New World, although it has been found in such great abundance as to appear to be indigenous in Africa, which has led Afzelius, a learned botanist of the seventeenth century, $\mathrm{Mr}$ Geo. Don, a botanical traveller in that country, the late Mr Sabine, and others, to express opinions to that effect. It was cultivated in China long ere its culture was attempted in Europe; and Father Athanasius Kircher describes it as growing there, and producing fruit twice a-year, and known to the Chinese by the name of Tampolo-Mic. They, however, acknowledge having received it from South America. It is also described by Loureiro as occurring in Cochin China, and that one variety of it continues green when ripe, having a white flesh; from which we may suppose that it had even in his time sported into varieties, for Kircher clearly describes it as of a yellow colour. It is now naturalised in most of the West India Islands, and in many parts of the East Indies, in Surinam, New Spain, \&c.; yet to all these, according to the highest botanical authorities, it had at one time or another been introduced from South America. It is quite evident that it is not an original native of Africa; for the fruits of that country were so well known to the ancients, and most of them have been described by the authors who wrote on the plants of India with so much accuracy, that had so conspicuous a plant existed it could not have escaped their observation.

Its early history, and the date of its introduction into Europe, have now been pretty accurately ascertained. The following account of these we gave some years ago in "The Orchard," and to that little further information has been added since. "It was first discovered by Europeans in Peru, where it is called Nanas, and was by them first introduced into the West, and afterwards into the East Indies, where it has been long successfully cultivated." Parkinson, writing in 1640 , says, "It was first brought from Santa Cruz, in Brazil, where it is natural, both into the West and East Indies, being not natural to either of them, but is only manured there, and not growing plentifully." The earliest account we have of the pine is in "The Universal History of India," printed at Seville by Gonzalo Hernandez de Oviedo, who went to America in 1513. In this work he mentions three kinds of pine-apples as being then known. These the Spaniards designated by the general name of Pinus, and often tried to send the fruit, both in a ripe and green state, to Spain, but without success. They also attempted its introduction by sending the crowns, but these also perished during the voyage.

The next account we have met with of the pine is in a "History of the New World," by Geronimo Benzona, an Italian, who resided in Mexico from 1541 to 1555 , and published his history in 1568. André Thevet, a monk, has described this fruit under the name of Nanas, and also informs us that in his time (1555-6) it was often preserved in sugar. The year following, Jean de Lery, a Huguenot priest, makes frequent mention of it, and describes it as a fruit of such excellence that the gods might luxuriate upon it, and which should only be gathered by the hand of a Venus. F. Hernandez being sent by Philip II. of Spain to Mexico, amongst other valuable information relating to the natural history of that country, transmitted to Europe drawings of this fruit, which he designates Pinea indica.

The introduction of the pine into Europe appears to have taken place about the middle of the seventeenth century, some say direct from South America, but more probably from the West Indies; for at that period M. Le Cour of Leyden, in Holland, had them in cultivation in his garden at Driehock, where he attempted to grow them without bottom-heat, treating them as ordinary dry stove-plants. He afterwards, however, had recourse to low pits and tanner's bark, and has left us a detail of his mode of culture, as well as plans of his pits, in a small work in the Dutch language. It might appear from the remark of the celebrated Lady Mary Montague, while on her journey to Constantinople in 1716, that the pine was not cultivated in England at that date; for she says she saw pineapples served at the Electoral table at Hanover, which she had never seen or heard of before. This would almost lead us to doubt the MS. note written by Mr Peter Collinson on the plate Ananas in the "Hortus Medicus Amstelodamus," saying, "Sir Matthew Decker first brought the Ananas or pine-apple into England, to his garden at Richmond, where I saw them about the 
year 1712 ;" for it is scarcely probable that such a fruit could have been known in England, and unknown to that extraordinary woman. This opinion is somewhat strengthened by the assertions of those who say that the pine was introduced to the United Provinces by their East India ships direct from China.

Lord Bacon does not allude to its being cultivated in his time, although he particularly mentions it in his "Essay on Plantations and Colonies." The first account of their appearance in England, without referenee to their having been there grown, is of a present of pines received by the Protector Cromwell about 1651, but these were probably from the West Indies or from Holland. And Evelyn, in his Diary, 9th August 1661, says, "I first saw the famous Queen pine, brought from Barbadoes, and presented to his majesty (Chärles II.); but the first that were seen in England were those sent to Cromwell four years since." In the same Diary, July 19, 1668, he says: "I was at a banquet which the king gave to the French ambassador. Standing by his majesty at dinner in the presence, there was of that rare fruit called the King pine, growing in Barbadoes, in the West Indies. The fruit of them I had never seen. His majesty, cutting it up, was pleased to give me a piece from his own plate to taste of; but in my opinion it falls far short of those ravishing varieties of deliciousness described in Capt. Ligon's history, and others; but possibly it might, or certainly was, much impaired in coming so far." Evidently a fruit not produced in this country. There appears to be some mistake in describing this as the King pine, as that variety was raised from seed in this country, from a seed taken from a rotten fruit which came from the West Indies to Henry Heathcote, Esq. The foliage would have determined the question, as the King pine is smooth, without prickles. Philips, in his "History of Fruits," thinks it probable that it was from the crowns of these pines that Mr Rose, the royal gardener, is said to have raiscd the first plants that fruited in England. We have met with no account to lead us to believe that Rose fruited the pine prior to.Sir Matthew Decker; on the contrary, there is prettygood evidence that Decker's plants had been received from Holland. Gouge, the historian, distinctly says, " Sir M. Decker first introduced the culture of the Ananas;" and a work was published in 1726, entitled "An Account of the Ananas, or West Indian Pine-Apple, as it now flourishes in Sir M. Decker's garden at Richmond, under the care of Henry Telende." To those who have not an opportunity of referring to the above pamphlet, published in 1726 , we can refer them to a well-known work, "A. General Treatise on Agriculture, Husbandry, and Gardening," by Professor Bradley, 1757, page 433 , where a full account of Telende's practice will be found. Abercrombie, who wrote so soon afterwards, distinctly says, " Holland was the first European country where the culture of the pine was attempted, from which country we in England were supplied with the first plants." The only proof which at all identifies Rose with the early production of the pine rests on two paintings, both of which we have seen, one the original, it is supposed, in the collection in Kensington Palace, and the other sold at the dispersion of the valuable collection of articles of taste and vertu which long constituted the Waldegrove collection at Strawberry Hill, representing Rose on his knees, presenting a pine-apple to King Charles II. We have, however, no positive proof that this fruit was produced in the Royal Gardens. The Sloanean MSS., in the British Museum, inform us that the pine was not introduced until the year 1690, when the Earl of Portland procured plants from Holland. In the Fitzwilliam $\mathrm{Mu}$ seum at Cambridge there is a painting by Netscher, a landscape, in which is introduced a pineapple, stated to have been first fruited in Britain in the garden of Sir M. Decker. The paintings to which we have referred of Rose and his royal master have, we think, after the above evidence, no reference whatever to his being the first cultivator, as the pine he is represented to be in the act of presenting in all probability came from Holland. In the "Hortus Kewensis" the pine is recorded to have been introduced to that establishment in 1690 by $\mathrm{Mr}$ Bentick, but it can only be considered in this case as a botanical plant. Professor Bradley informs us that in 1730 pine-stoves were to be met with in the principal gardens in England. The Chelsea Botanic Garden; W. Parker, Esq., near Croydon, Surrey; John Blackburn, Esq., Lancashire; Mr Fairchild, at Hoxton, \&c., are especially noticed as cultivators. In 1744, James Justice of Crichton informs us that pine-stoves had been then erected in Scotland; and in his "British Gardeners' Directory," published in 1744 , he gives plans of his own pine-stoves at Crichton, near Dalkeith, in which he fruited the pine for the first time in North Britain.

Propagation.-The pine is occasionally, but rarely, propagated by seed; indeed, were it not with a view of obtaining new or improved sorts, this means would never be resorted to, at least in Britain. Those fruits which ripen during August and September in this country produce more seed than such as ripen either at an earlier or later period, no doubt on account of their flowers being more perfectly formed, and the process of impregnation taking place under the favourable circumstances of extra vigour in the plant, and the abundance of light which they enjoy at the season of flowering, compared with those which bloom during winter or later in the year-which latter have also the disadvantage of swelling during the dark months of winter: Speechley raised more pines from seed than pcrhaps any other cultivator in Britain, few of which had much merit, and not one of them is, if we except the Welbeck seedling (a coarse illflavoured sort), to be met with in cultivation at this time. Seed of the pine-apple is usually brought from the West Indies, but even there the seed is not abundant. When raised, they should be kept (if they arrive in autumn or winter) in a warm room, enclosed in paper bags till March, when they should be sown in rich soil, in small pots plunged in a warm hotbed. The seed should be sown 1 or 2 inches apart, or 
one in each 60-sized pot, and covered about a quarter of an inch. After germination has taken place they ought to be fully exposed to the light, and their small tender grass blade-like leaves protected from wood-lice, which, if not prevented, would soon eat them up. By the end of August the plants will have attained a fit state for transplanting, after which they should be treated like other young pine plants. In the third year they may be expected to produce fruit.

The other modes of propagation are by gills (small secondary suckers produced at the base of the fruit), crowns which surmount every perfect fruit, suckers which arise from towards the middle of the stem of the plant. Latent buds are often found to exist on the stem, and these, as will be noticcả below, are excited into growth after the plant has completed all its other functions. Gills are rarely cared for, unless it be in cases of new or rare sorts which it may be desirable to increase ; they are long in coming to a state of fruitfulness. When used they are treated exactly as common suckers.

Crowns were formerly in more repute than they are at present, and unless of large size, or in the case of new or rare sorts, we rarely plant them, believing that large suckers produce fruit sooner. Their culture, however, is in all respects the same as that of suckers.

Suckers are produced from the stem of the plant, having their origin in buds thickly set in some sorts, as the Muscow queen, more sparingly in others, like the Providence, Cayenne, \&c. During the growth of the plant all superfluous suckers should be destroyed upon their first appearance, which can readily be done by thrusting a small gouge-like instrument, set in a long handle, into their centres, and by giving the hand a slight twist the centre or lieart of the young sucker will be destroyed. From ona to two suckers is quite enough to be left on any single plant, unless an increase of stock is more an object than the fruit itself. Allowing more to remain reduces their individual strength, as well as draws considerable nourishment from the fruit, to develop which in perfection requires all the strength of the plant. When the fruit is cut, the suckers are usually separated from the parent plant, and as practised by some, laid in any out-of-the-way place for a week or more to dry, as they please to term it, and afterwards potted, although often planted in the tan or leaf bed in which they strike root. Both these modes are contrary to the laws of good culture. Suckers grow amazingly fast while kept on the old plants, and when they can be allowed to remain so for a few weeks, their size and strength is improved. This is, however, not always convenient, as the old plants have often to be removed to give place to others; and with some the suckers are taken off even before the fruit has attained nearly its full size, with a view to throw all the strength of the plant into it. This is attended with danger both to the sucker and swelling fruit: the former is apt to be broken during the operation, as they do not separate freely from the old plant until they become hard, and of a chesnut-brown colour at their base, at which time they may be removed easily and without danger. Their removal under any circumstance before the fruit is cut, requires great care to prevent any undue shaking or twisting of the fruit-stalk; for if this takes place, the growth of the fruit is greatly impeded, the fruit-stalk often withering up, and a premature ripening is the consequence. Indeed, to avoid this, if it can be avoided, no plant should be disturbed from the period of the embryo fruit showing until it is fully matured. This is one great advantage attending the planting-out system.

Allowing suckers to remain unpotted a day after they are removed from the plant, tends only to weaken them, as they part with their sap rapidly, and this the more so if placed in a dry situation ; indeed, it may be safely inferred that every day they are exposed to this evaporating treatment is tantamount to a loss of three or four in their growth. They should be potted as soon as they are removed from the parent plant, setting one sucker in a pot of about 6 inches in diameter, less or mole, according to their strength, and immediately placing them in a mild bottom-heat, with little ventilation and partial shade, giving no water at the roots, and only a little by syringing overhead every other day, continuing the same until they are fairly rooted, when more air and water, and less shade, should be afforded them. The strongest and best-formed suckers should be retained, and all root ones discarded; indeed, these last should be destroyed as soon as they appear, as they seldom produce good plants, and, if left on, only rob the fruit and proper suckers of their due share of nourishment. In removing suckers from the parent plant, great caution is required, that they be not twisted or broken during the operation. The stem of the old plant should be held firmly by one person, while another is separating the sucker from it; and this separation should not be persevered in until the sucker is a foot at least in height, and fully ripened at the base, and can be removed easily; and without injury either to itself or the old plant. There is no advantage gained by removing the suckers too soon, for they make much greater progress in growth while attached to the plant than if they were taken off prematurely, and ever so well attended to. Some retain one or two suckers on the plant after the fruit is cut, and allow them to produce fruit, which they do within a short space of time; but such fruit is seldom of a large size, nor does a house or pit of pines ever look so well as wher each plant is on its own bottom. Speechley, in 1796, practised this ; and Knight, many years afterwards, followed so far out this system, and planted the old root with the sucker attached to it, shaking off all the soil from the roots, and replanting them in fresh compost. An almost similar practice was followed by $\mathrm{Mr}$ Dale and others. Mr Hamilton of Thornfield has more recently revived this method, and detailed his practice in his "Treatise on the Culture of the Pine-Apple."

The following is our mode of treating the pine in its first stages of growth : Having a larger 
stock of plants than is to be found in any other private establishment, and having fruit at all seasons, it follows that the removal of suckers is of very frequent occurrence; and to provide accommodation for them we have a long narrow pit, portions of which we prepare from time to time for them, by filling it with leaves or tan, as is most convenient at the time. The suckers are potted into 6 or 7 inch pots, according to their size, in light pretty rich turfy soil, as soon as possible after their separation from the old plants. They are allowed plenty of room, for if crowded too much at this period, they never afterwards make such good plants. The pots are plunged into a bottom heat of from $85^{\circ}$ to $90^{\circ}$, which conduces to the rapid formation of roots: undue evaporation of their sap is prevented by partial shading and diminished ventilation, but no water is given to the soil until the roots have made some progress, but during the while the plants are occasionally sprinkled overhead with the syringe, using water $3^{\circ}$ or $4^{\circ}$ warmer than the temperature of the air in the pit. During winter this sprinkling must be cautiously applied, as a considerable evaporation is rising from the $\tan$ or leaves; while, during summer, it is administered every afternoon, except in very cloudy weather, and that before the sun's rays are much diminished, or have ceased to shine on the glass, thus producing a fine genial vapour, and converting the circumambient atmosphere into a state of humidity, which the plants appear to delight in, and to inhale by their respiratory organs. This respiration and perspiration of fluids, being going on day and night, tends greatly to keep the plants in a vigorous growing state. Pits heated entirely by fermentation require much less water at all times, and more especially during winter, than such as have that kind of bottom heat only while the atmospheric temperature is regulated by hotwater pipes, as ours all are, and still less than such as are heated by hot-water pipes, both for bottom and top heat. This overhead watering of the pine, except while it is in flower, is more congenial to its nature than only watering at the root. By the former practice the leaves are all moistened by the water, which holds in solution a portion of the food of plants, is absorbed by the epidermis, and assists the assimilation and augmentation of true sap in its descent to the roots. Care, however, must be taken that individual plants may not be supplied to excess; this will readily be seen by the appearance of the leaves; and when such cases occur, such plants should be removed, their roots examined, and, if need be, re-potted into drier and fresh soil. When the roots have filled the pots, or nearly so, but not sooner, the plants should be shifted into larger pots, which will offer at the same time an opportunity of turning over the bed, and adding fresh material to it, should the heat be on the decline. At the same time, the utmost care must be taken that too much new matter be not added at a time; for the pine, although a native of the warmest parts of the tropics, is more readily injured by an excess of heat at its roots than even by a diminished temperature, and this the more so when the plants are grow: ing rapidly, and the pots filled or filling with large, soft, and delicate roots. From $90^{\circ}$ to $100^{\circ}$ may be taken as the maximum of bottom heat, $70^{\circ}$ to $85^{\circ}$ as the medium, and under that even to $50^{\circ}$, according to the state of the plants, but below that it is well not to allow them to fall. After re-potting, a pretty brisk bottomheat is required for two or three weeks; it creates a fresh stimulus in the action of their fluids, and encourages the descent of assimilated matter to the trunk, which it should be our great object to increase in size, for it is upon its strength, and not on the length or breadth of the leaves, that the production of fine fruit en tirely depends. A fine genial heat at that period also causes fresh roots to form, and their feeding on the new compost causes the growth of the plants to be redoubled, particularly if attention is paid to the keeping up of a warm humid atmosphere.

Except during the time the suckers are making their first roots, and for a week or so after every re-shifting, the pits should be kept pretty close, air should be admitted daily, less or more, of course according to the season of the year, and in some degree according to the construction of the pit or house they are grown in. Indeed, air should be admitted freely, should even a little extra heat be thrown in during the time, for it is a due proportion of this element that gives the pine plant strength, hardens its texture, causing the plants to grow with broad, thick, short leaves, and a strong stem. A deficiency of it, as well as of light, and placing them too far from the glass, invariably produces tall, longleaved, lanky plants, unable to support themselves without artificial aid. For a few days after re-potting, a slight shading should be placed over the glass.

The size of the pots for the second shifting, if all has gone on well, should be from 9 to 10 inches in top diameter, using rather a stronger compost than that into which they were first planted. Their fruiting-pots should be from 11 to 12 inches in diameter, unless it be in the case of the Providence or similar strong-growing sorts, or where the intention is to grow other kinds to a greater age, when pots of 13 or 14 inches may be used. We have seldom, however, found much advantage from using over large pots. At each shifting the plants should be set farther and farther apart, that the light and air may act freely upon their whole surface.

We grow all our young pines in pots plunged in leaf or tan beds, greatly preferring the former, in low pits of various sizes, and transfer them from the smaller to the larger pits as they advance in growth, and from the largest section of these we fill up all blanks as they occur from the fruit being cut in our fruiting-houses; nevertheless, from the immense number grown at Dalkeith, many are ripened off in the larger pits also. Some we plant out, and others we keep in large pots to produce their fruit, with very little apparent difference in success. All our fruiting-houses are heated by hot water placed under the beds, which with us, where coal is cheap, is found to be a vast saving in labour. Indeed, we would 
heat all our growing-pits also in this way, were it not that, in consequence of the floors having to be at a permanent distance from the glass, the smaller plants would be placed too far from the light when quite young; and as they extend in size, their leaves would be broken by coming in contact with it, both of which we can readily avoid by lowering or filling up the beds to the required height. Indeed, were it not that pineries form a feature in an extensive establishment, we would prefer fruiting them all in low, well-lighted, and ventilated pits. In the winter arrangement of the plants, care should be taken that they be not set too crowded together, and also that they be kept as near to the glass as the safety of their foliage will admit of. In plunging in the fermenting material, care also should be taken that the pots be not set too deep, or that fermentation be not allowed to become too great; for during autumn, under a strong sun-heat, the plants in general make great progress, and their roots being in full action, notwithstanding the bed they are in is only of a moderate temperature, they will be plentifully furnished with those indispensable foodsuckers, the spongioles; it becomes, therefore, a measure of great consequence that they are not injured during the process of re-potting, and still more so that they are not injured by excess of bottom heat. The latter would act in two ways extremely injurious to the plants, either by checking their growth till the formation of fresh spongioles, or starting them into fruit at a very unfavourable season. Not but that, where pines are required throughout the year, as is the case here, a considerable number should start at this time for early spring use; but if many do so, it will occasion dissatisfaction in the supply late in spring, when they are equally wanted. During winter, excess of damp must be guarded against in low pits, more especially those heated by dung linings; and should a deficiency of heat occur during cold weather, it will be much safer to exclude the cold by adequate roof-coverings, than to attempt to keep it out by the application of strong linings.

A high and arid temperature, often greater during the night than the day, was one of the greatest errors of former cultivators; and stimulating the plants into a forced perpetual growth, at all seasons of the year alike, one of their next; and another was, roasting the roots when the tanbeds were first made up, and allowing them to perish from cold and damp in water-logged pots, caused by excessive watering, and allowing the tan-bed to become completely exhausted. These and many minor errors have been sufficiently pointed out, and now all good cultivators avoid them. The pine during winter requires much greater care than during summer, for during winter the vital energies of plants are weakened, and, therefore, more easily injured by excess of heat, moisture, \&c., than in summer, when they are in a different condition, and able to digest their food better; for every element by which they are surrounded is more or less to be considered as their natural aliment. During the months of October and November, a general VOL. II. regulation of the stock should take place; and nothing should hinder this being set about at that time, unless it be a deficiency of tree-leaves (where they are used) for making up the beds with, or in localities where the autumn is in general fine, and the spring cold and late. In such cases it may be expedient to defer the general arrangement a little later, that the beds may hold out longer in spring. Where pine-beds are heated by hot water as a bottom heat, this becomes less a matter of consideration. Nothing, therefore, should prevent this being set about, except it be that the pits or houses may not be as yet cleared of the plants which are ripening or swelling fruit, to disturb which we hold to be exceedingly injurious to them. Indeed, it is only necessity that can induce us to move a pine after it has started.

Soil.-That there are proper and improper soils for most plants is a matter granted. The pine, however, in this respect, appears to be far less fastidious than many other of our fruit-bearing plants; we have grown it in many and very opposite ones, and with apparently very similar results. In its earliest stages, a rich light soil abounding in vegetable matter, with a small proportion of sharp sand, is favourable for the production of roots; in its secondary stage, a rich friable loamy soil appears favourable for insuring robustness of growth; while in its last or fruiting stage a rich aluminous clay is productive of large fruit; and this appears to be the soil in which it naturally grows. These are, however, not without exceptions. For several years we grew the plants, in all their stages, in a light, somewhat sandy marly loam, dug from a common in Surrey, at a depth of from 10 to 15 feet under the surface, without even a trace of vegetable matter in its composition. At present we are growing them in semi-decayed vegetable matter alone, consisting of sphagnum and other mosses, with abundance of phanerogamous vegetable remains dug from a bog in Crichton Moss, used when only a few months carted home, and during the time fully exposed to the sun and air. In both these cases, a little sheep or deer dung was the only addition.

The French, to the best of our knowledge, have never grown their pines in other than sandy peat, such as plant cultivators in this country use for heaths, New Holland plants, \&c.; and yet in such a soil, containing a large portion of silvery sand, the extraordinary pines at Meudon, near Paris, have been grown. Many pine-growers in this country have successfully adopted the same sort of soil-merely dug fróm a hillside and used immediately, after being cut into large pieces, a foot square and 2 or 3 inches in thickness when the plants are planted out, and from 3 to 4 inches square when used for potting, and without any enrichment whatever.

In Trinidad, where some of the largest pines naturally growing are found, in the Bahamas, and almost all over the West Indies, the pine is in general found luxuriating most in a light brown-coloured alumina approaching to a perfect clay. And strange enough to say, according to the analysis of Professor Jolly, iron in excess is the characteristic of the pine-apple land of New 
Providence, to which that large variety, the Providence pine, is indigenous. As it were the very antipodes to this, Mr Hamilton informs us, in "Treatise on the Culture of the Pine," p. 2, that his compost for planted-out pines consists of "the refuse from the garden, such as cabbage, tree, pine, and vine leaves, sweepings of walks, \&c., to which is added a little old mortar and a few broken sticks to add to its porosity, with some old pea-wood at the bottom as drainage. This constitutes the compost for the pines in which they appear to delight, and they are intended to remain for years undisturbed, and perfect a succession of fruit." He afterwards, however, says, (p. 31): "The soil I have found most suitable for the plant in all its stages is a sound loam, which has been several years under grass. Let the turf be stripped off to the depth of 2 or 3 inches, and add one-third of well-decomposed dung from the stable-yard, or from an old hot-bed; to this may be added one-tenth part of wood-ashes. Let the whole be piled up in a ridge, and in a few weeks it will be fit for use." It should, however, be recollected that he turns his plants out with the ball entire into a bed of new tan, in which he fruits them, thus placing them, in their last stage, in a great measure depending on the food they can collect from the tan in a state of fermentation. The soil used by Mr Fleming of Trentham, who has succeeded in growing pines of first-rate excellency, is composed of two-thirds maiden loam from an old pasture, and one-third peat in a rough state. The loam is enriched, and believed to produce better swelled fruit from smaller plants than either an entirely peat or lighter soil would do. The peat and loam are mixed together, and laid under a wooden platform upon which sheep are fed. This platform is constructed of narrow strips of wood, through which the dung and urine of the sheep fall. After the soil under the platform has become thoroughly saturated with the liquid, it is removed, and placed under a covered shed, where it lies till wanted for use. A fresh supply is placed under the platform to undergo a similar fertilising operation. Before using the compost, if the loam happens to be of too stiff a nature, clean pit-sand is added to reduce it to the required degree of tenacity. Mr Glendinning, the only other authority we shall quote, himself an excellent pine-grower, states, in his "Treatise on the Culture of the Pine-Apple," p. 16, "All plants under artificial care, but more particularly those of a fruit-bearing kind, assume peculiar habits according to the nature of the soil in which they are planted, and their tendency to fruitfulness is governed in an extraordinary degree by the texture and composition of this fundamental element of vegetable life. This observation will more particularly apply to the pine plant than to any other cultivated for its fruit; with soil and treatment calculated to produce such a disposition, the healthy yet inflexible appearance of such plants is singularly imposing. The under leaves take a horizontal direction, and the others to the centre gradually less so: they will, according to their strength, be proportionably broad and sufficiently robust to resist injury from carrying and potting; the foliage will radiate from the surface of the soil, neither showing a naked stem nor too much sunk in the pot, with a stem of relative strength, the general character rigid, and of a dark green powdery hue; therefore much depends on the compost employed. Having experimented on various kinds of soil, from very light friable loam to that approaching nearly to a clay, my opinion is decidedly favourable to a stiff soil ; and in forming a proper composition, my experiments with manures have been equally varied. After numerous trials I use the following compost, because it is easily procured and soon prepared; and after years of experience, it has been accompanied with the most satisfactory results, which no consecutive experiment has induced me to forego.

"The soil which prevails in this locality (Bicton, Devonshire) is of a very free sandy nature, hence the difficulty of procuring that of sufficient tenacity in which to cultivate the pine plant. 'The most unctuous spots on the common are preferred, and the surface, about 2 inches thick, only is taken, and this is either covered with long grass, furze, or heath." Mineral impregnations abound in the locality, but these, to the extent usually found in earths, Mr Glendinning thinks of less importance than is generally supposed, and attributes the injury said to arise from soils taken from low wet situations more to the effects of stagnant water with which they have been saturated during winter than to mineral oxides. "In consequence of this winter saturation," he says, "the grass grows long, and the heat of the succeeding season, from this accumulation and combination of vegetable matter on argillaceous land, produces putrescent acetification, which can only be addulced and purified by being fully exposed to the action of the atmosphere in a healthy situation. Where soii can be procured sufficiently retentive from healthy situations, such as old pastures, the addulcing process may be dispensed with, and it may therefore be brought into immediate contact with the enriching material. Having brought into the compost-ground a sufficient quantity of this turfy loam of the proper temperament, it will be necessary next to collect a quantity of fresh sheep or deer dung, and begin forming a square heap in layers, putting three barrows of dung to six of loam and one of leaf or vegetable mould, and continue putting one after the other until the heap is 3 feet high. No other preparation will be necessary, as it will be fit for use in three or four months, when with a sharp spade it must be cut through the heap perpendicularly, and the largest pieces again chopped. Notwithstanding the coarseness of the material, it will be found to suit in an admirable and superior manner; the strong roots will pass unobstructed through the mass of clods into the drainage. It will also give a constitution of inflexibility to the plants contradistinct from those grown in a lighter and mellower soil, which the practical cultivator knows full well; they will generally exceed his most sanguine expectations." "If this plant is kept moist and warm at the root," Hamilton observes, "it will 
flourish without the agency of rich composts. I have tried as an experiment to grow it in moss mixed with broken pots; the plant made quite as much progress as those in rich compost, which is an evident proof that water and air constitute the principal food of the pine-apple." From what has been said above, it will be seen how conflicting are the opinions of even our best cultivators as regards the texture of the soil : in one point they mainly agree, namely, that it should be fresh and unexhausted, and the coarser or more pervious it is the better.

Potting.-This is an important operation in pine-culture, commencing when the suckers are taken off, and continued as the plants progress, until they are placed in the pots in which they are to produce their fruit. In all cases, if the pots are not new, they should be thoroughly washed and dried for a day or two previous to using. The soil should also be comparatively dry - that is to say, it should have been under cover some time before the operation is to take place; and when it becomes necessary to re-pot during winter, it should be brought nearly to the temperature of the soil already about the roots. Some writers have laid down particular seasons when this should be done, a rule which should be totally disregarded. All plants do not grow alike rapidly even in the same pit or house, and there is a great difference between one sort of pine and another in this respect also. Potting or transplanting must be governed by the state of the plants, whether they are growing fast or the reverse, whether the pot is full of roots or not-and this, even with very expert growers, can only be ascertained by examination. It is as injurious to re-pot a plant when its roots have not occupied the whole ball in which it is growing, as to delay until they have become completely matted all over its surface. The operation should always be performed with great caution, as the roots of the pine, when in a healthy state, are full of sap, and easily injured by being bruised or broken; and the food-collecting points, the spongiolets, are extremely brittle, and exceedingly liable to be broken off. For this reason, shifting or potting should not take place while the spongiolets are just pushing their way through the ball, as it is next to impossible to preserve them when in that state. When the roots have passed through the ball, and begun to traverse its outer circumference, is the proper time for the operation; and even then great care is required in turning them out of the pot and placing them in another, so as to maintain the ball of earth around them entire. Disturbing the roots or reducing the ball of a healthy vigorous-growing plant, gives it a check in proportion to the extent either of these is interfered with. The only cases when a departure from this rule is warranted, is when the soil has become sodden by excess of moisture or deficiency of drainage, or when the roots have been injured by too much bottom heat, or where, from other causes, they have become unhealthy, or almost disappeared altogether. In such cases the soil should be removed, often entirely, the roots examined, all that are dead or unhealthy cut away, even should the plant be reduced to the state of a rootless sucker. It was for years recommended, but upon what solid ground we are at a loss to guess, to shake away most of, if not the whole soil, and to cut off the lower roots of the plants; and some went so far as to cut them off entirely. Others, at potting, strip off a number of the lower leaves, with, we suppose, the idea of allowing the embryo roots to escape more freely from the stem than they could by forcing their way through or between the leaves. There is more propriety in this than in the disrooting process; for, on examining the bottom of the stalk of the pine, it frequently happens that the young roots have twisted themselves around the stem, and cannot readily be separated from it without the danger of breaking them. This would indicate that, if the leaves had been sooner removed, those roots would have extended themselves into the soil. The removal of such leaves is opposed by some, but we think their removal, under such circumstances, highly proper; but, unless to meet such a case, we would not remove one healthy leaf, either at potting or at any other time. Over-potting is injurious to the pine, the plant remaining long stationary in growth afterwards. It would be desirable were it possible, which it is not, to give a scale of sizes of the pots adapted to each potting, from the planting of the sucker to the ripening of the fruit. This, as in many other cases in horticulture, must be left to the discretion of the operator; but, in a general way, we may remark, that the pot into which the plant is to be set should seldom be more than 3 inches greater in diameter than that from which it has been taken. It is also equally impossible to state how often the plant should be shifted, as also the exact size of the pot in which it is to produce its fruit. The former must be regulated by the progress the roots have made, and the latter by the variety, whether a strong or medium grower, as well as its size, age, and weight of fruit desired. We never re.pot a pine after it has shown fruit; indeed, if we can avoid it, we never remove them from the spot after the embryo fruit is indicated by the plant throwing up narrow leaves in its centre.

As general data for the size of pots, we may state that we usually plant our strong suckers in 5 or 6 -inch pots, smaller ones in 4 -inch ones, at each shifting increasing the size, if the plants are vigorous, 3 inches, and fruit them in pots of 12 or 13 inches in diameter, according to their size. As our practice is to fruit the plants comparatively young, say from eighteen months to two years, we encourage their growth; and by shifting with the balls entire, they never sustain a check from the day they are first planted until the fruit is ripe. Of course, they are not over-stimulated to growth during the dark months of winter.

Drainage is an important part of this process, and to secure this completely we place over the holes in the bottom of the pots-for we have from three to five in the bottom, and in largersized ones as many round the bottom part of the sides-large pieces of broken pots with their concave side undermost; above these, fragments of pots in smaller pieces, technically called crocks, to the depth of from 1 or 2 inches; over these 
a thin sprinkling of moss (Hypnum), to prevent the finer particles of the soil being washed into the drainage. We have frequently substituted rough charcoal, ground bones, broken shells, small stones, \&c., for crocks, but with no perceptible advantage. Over the crocks is placed as much soil, choosing the rougher portion, as will elevate the ball, when set upon it, to within an inch and a half of the top of the pot. When the plant is set in its place, the soil is then filled in all round. When we have used strong loamy soil, we do not pot hard-that is, press it down forcibly; but when we use light sojl, we press it pretty tight with a blunt wedge-shaped piece of wood, and this more or less according to the degree of humidity in the soil at the time, avoiding always using wet earth.

To facilitate the operation of potting, it is usual, as the plants are taken out from the pit or house, to tie their leaves up with matting. This, no doubt, in some cases, saves the leaves from being broken; it is, however, often more injurious than beneficial, and this the more so if the plants be vigorous and their leaves strong, particularly if tied too tightly up. Bringing them from nearly a horizontal position to a perpendicular one cracks them near their base; for the leaf of the pine, when in robust health, is by no means flexible, and this cracking of the leaves is, in such cases, most injurious to them, while plants whose leaves are so flexible as to require it are not in the condition they should be in. This want of rigidity in the foliage is the certain consequence of the plants being kept too crowded, too far from the glass, or inadequately supplied with ventilation.

Watering.-An excess or deficiency of this element is equally injurious; for although the pine, like most other succulent plants, will survive the entire want of water, even to the extent of six months at a time, nevertheless, when in a proper temperature and good health, it requires a liberal supply. On this subject $\mathrm{Mr}$ Glendinning very justly remarks, in "Treatise on the Culture of the Pine," (p. 33): "Any attempt to determine the requisite quantity of water to keep the pine plant in a healthy state, and the times of its application, must be evidently absurd; the practical cultivator, and indeed every one who aspires at the cultivation of this or any other plant, must be guided exclusively by his own personal observation of circumstances; for a little application of the mind will soon supersede all the rules that could be prescribed for his direction, and enable him to supply the proper quantity of water with some degree of precision. Much at all times must depend on the state of the atmosphere and the season of the year. During the summer months, when evaporation and transpiration are at the maximum, a corresponding degree of moisture must, by artificial means, be created, to secure a growing atmosphere for the plants. Solar heat and light, and moisture, must be relatively and equably supplied; the one without its due proportion of the others will soon produce disease, and ultimately death. The pine plant ought never to be soaked with water, although, if the soil recommended and the manner of potting previously detailed were adopted, little injury comparatively would result for some time; but to all plants in a highly-manured compost, continual drenching would soon wash away its strength and exhaust its capabilities. Independently, however, of this weakening and reducing that fundamental principle of the plant's existence, the soil, by continual percolation, another serious effect would arise, as the absorbent powers would, from being fed to satiety, soon become debilitated, thus at one and the same time changing the colour and the constitution of the plant. It would be an important improvement in watering, if it could be calculated in its administration to give no more than just enough to moisten the soil in the pot without any escaping at the bottom." Water should be given in small quantities and often, and much of this depends on whether the plants are growing in close pits heated by fermentation, from which great evaporation arises, and as it condenses on the inner surface of the glass, falls back again in form of dew; or whether they are grown in large well-lighted houses heated by fire-heat, and planted out on a bed of prepared soil. In this latter case the evaporation is limited unless water be sprinkled over the bed, on the walls or floors of the house; and even much of this is carried off by the air through the ventilators. We seldom water pines at the roots during winter when grown in pits on tan or leaf beds, but slightly syringe the plants overhead once a-week in clear weather, and much seldomer when it is cloudy and dark. During summer, however, syringing is performed once or twice a-week, but much more copiously than during winter; and root-watering, according to the size and vigour of the plants, is applied as often. In large houses a humid atmosphere is maintained, according to the period of the year and state of the atmosphere, by syringing the plants overhead, wetting the walls, and sprinkling water on the floors, but never intentionally on the hot-water pipes.

Experience has convinced Mr Hamilton " of the necessity of maintaining a moist atmosphere, and keeping the roots continually moist (not wet), by watering over the whole plant, which has a tendency to invigorate it by the absorption of the immense surface of its leaves. To the deficiency and wrong application of this element" he "ascribes many failures in its culture. If the plant is sufficiently supplied with moisture, the leaves will absorb till every channel and cell in their structure is expanded with the accumulation of vapour. The plant will be benefited if kept continually moist before the fruit has swelled much, after which the leaves ought to be kept drier, in order to bring out its sweet secretions."

At whatever time or in whatever quantity the water is applied, it should always be several degrees warmer than the atmosphere of the house. Indeed, from $70^{\circ}$ to $90^{\circ}$ is not too much for the water. It is always better that the pine be rather dryat the roots than that it should be deficient of moisture on the leaves. This seems to be its natural condition, for in the West Indies and other pine-growing countries, where 
rain does not fall for months at a time, the dews during night fall copiously. Syringing, therefore, in the after part of the day, is preferable to the morning. Young suckers without roots, and such plants as may from one cause or other have had their roots cut off, should be very sparingly watered at the root, but maintained in health chiefly by syringing overhead. In regard to this mode of applying water, we may observe that it is not the quantity thrown over the plants or over the house, but its equal diffusion, that is most advantageous, and therefore a very fine cap should be put on the syringe, so as to deliver the water almost in form of a copious dew. Plants newly shifted, and such as are in large pots, do not require so much water as those whose pots are full of roots; the former are easily injured by an excess, the latter seldom. Plants swelling their fruit and nourishing suckers require most of all, and such as are ripening their fruit should be deprived of it nearly altogether.

The application of water in an enriched form is beneficial to the pine in all its stages of growth, more particularly so when it is about to start into fruit. Nevertheless, strong liquidmanures should be used with caution. Injurious effects may be produced by an injudicious application of liquid manure, especially such as contain salts and alkalies in solution, as they have a tendency to cause excitement, and if applied freely may cause the plants to start prematurely into fruit. Many modes of preparing a simple and yet beneficial liquid manure for the pine have been proposed. The following may be considered as the most simple and useful: Where the stock of pines is large, two tanks (vide art. TANKs, vol. i.) will be required, each of a capacity to hold from one to three hogsheads. Into one of these throw about a hogshead in quantity of sheep or deer droppings, to which add about one peck of unslaked lime, and the same quantity of soot; fill up the tank with rain water, and stir it up occasionally for three or four weeks, at the end of which it will be fit for use; then fill another in like manner, to undergo a similar process of fermentation. When the liquid from the first tank is wanted, stir it well up and draw off the water into a tub, causing it to pass through a fine meshed sieve. This even then will be rather strong, and should be attenuated by the addition of rain water to the extent of one-third of the whole. In this state it is fit for being applied to the roots of the plants; but that none of it may fall on the foliage, it should be applied by a long tube with a funnel end, into which the liquid is to be poured; and if the smaller end be laid close to the pot, it will be conveyed to the roots without any of it falling on the leaves of the plants, which should be carefully avoided. Many, however, question the utility of liquid manure altogether.

Discrimination is required in watering pines; some sorts, as the Queens, Providence, and Envilles, will require three times as much water as the Black Jamaica, Montserrat, Sugar-loaves, and indeed all the Black pines. During December and January very little water will be required by such plants as are growing in pits heated by fermentation, but in houses heated by fire-heat the atmosphere must be kept moist, not by repeated syringing over the plants so much as syringing upon the walls and floors of the house, that the dry air caused by the fireheat may be counteracted by moisure rising in shape of vapour. Condensed moisture should be prevented from lying above twenty-four hours at a time in the axils of the leaves. A little additional temperature, with due ventilation at the same time, will correct this. The whole section of Black pines require very little water at their roots during winter; indeed, it is better they be kept rather dry at the root than otherwise.

Ventilation.-Too much air can scarcely be admitted to the pine, from the time the suckers are fully rooted until the ripening of the fruit; indeed, at that period they require it to a much larger amount than is in general accorded them, so long as the temperature is kept up. The want of sufficient air causes the plants to elongate in their foliage, produce narrow leaves, and throw up weakly fruit. This is one of the objections to growing them in pits, compared with larger and better ventilated and more transparent houses. It is possible, nevertheless, in close pits, to swell the fruit to a larger size, but they are always of very inferior quality compared with those grown in houses where more light and air are admitted to them. The only time when ventilation should be limited is while the suckers are rooting, and immediately after potting or shifting.

Shading is deemed necessary by many good growers, not only after re-potting, but also during the hot months of summer; for it is remarked that pines do not make the same progress then which they do in the early months of spring and the later months of summer and beginning of autumn, particularly the latter. This has led many to shade with thin canvass screens, during an hour or two in the middle of the day, during the hottest days of summer, and others to train vines over the plants grown in pine-stoves. In both these plans we accord, and would not think of growing pines in a large pine-stove without vines being trained up the rafters. No doubt pines, like all other fruits, require the full influence of the sun and air to perfect their flavour and colour; and to effect this, the plants (if not planted out) may be removed to one end of the house, and that separated from the other part by a glass partition, the plants in their pots set on a bed of sand and fully exposed to the sun's influence, without water, in a high temperature, and abundance of air. Excess of shading causes the plants to produce long attenuated leaves and long slender fruit-stalks, two conditions in which the pine can never develop its fruit in perfection.

Atmospheric temperature.-All living things in nature have a season of growth and another of repose; and in the case of the pine, although it sheds not its leaves annually as the vine, still it must have its season of rest. That season is during the dark months of winter; and not only that, but during the night throughout the year, 
at which times a diminished temperature, less water, and even less air, should be admitted. Speechley was correct when he thus wrote: "There is nothing so prejudicial to the pine plant (insects and an over bottom-heat excepted) as forcing them to grow by making large fires, and keeping the hothouse warm at an improper season, which is injudiciously done in many hothouses. It is inconsistent with reason, and against nature, to force a tropical plant in this climate in a cold dark season, such as generally happens here in the months of November and December, and plants so treated will in time show the injury done them. If large plants for fruiting, they generally show very small fruitbuds with weak stems; and if small plants, they seldom make much progress in the beginning of the next summer." Nature points out to us the difference between day and night temperature, and what better guide can we have? Tables of day, night, and bottom temperatures may be thought valuable by some, even were they to extend to every day and night in the year. Such would, however, really be found of little use to the practical cultivator, on account of the sudden transitions of the external atmosphere. And, again, the very best cultivators do not confine themselves to a few degrees up or down; $40^{\circ}$ may be taken as the minimum temperature to which the pine should be subjected; and it is better that it should fall even thus low for a few days during winter than that it should be kept at a much higher degree by artificial means.

The following table of night temperature is given by Glendinning, in "Treatise," \&c., p. 53.

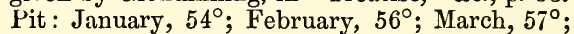
April, $59^{\circ}$; May, $60^{\circ}$; June, $61^{\circ}$; July, $62^{\circ}$; August, $62^{\circ}$; September, $60^{\circ}$; October, $58^{\circ}$; November $56^{\circ}$; December, $54^{\circ}$. Stove temperature: January, $65^{\circ}$; February, $68^{\circ}$; March, $71^{\circ}$; April, $73^{\circ}$; May, $75^{\circ}$; June, $76^{\circ}$; July, $78^{\circ}$; August, $78^{\circ}$; September, $76^{\circ}$; October, $73^{\circ}$; November, $69^{\circ}$; and December, $65^{\circ}$. And Hamilton, on atmospheric temperature suited to the pine, observes: "The temperature for succession plants in the winter from $55^{\circ}$ to $60^{\circ}$ at night, and $65^{\circ}$ to $75^{\circ}$ during the day. In autumn, winter, and spring, if fruit are to be swelled, they will require from $60^{\circ}$ to $70^{\circ}$ at night, and $70^{\circ}$ to $80^{\circ}$ during the day. In summer, the maximum under the effect of strong sunshine may rise to $90^{\circ}$, and may be allowed to drop as low as $70^{\circ}$ in the morning. In very bright sunny weather the plants in fruit had better be shaded than to admit too much air at any time through the day."-Query? "In order to swell this fruit to a large size, no air ought to be given until the thermometer reaches $80^{\circ}$ or $85^{\circ}$, which will generally reach that point in the morning by nine or half-past nine. To keep it down to this, give it the benefit of air until halfpast ten, then close the house, shade the plants, and water them over their leaves; thus let them remain until half-past two or three in the afternoon; then unshade, and let them have all the benefit of the light and sun; give a little air, which must remain until half-past four; then close the house, syringe again over the leaves of the plants, which will keep them moist during the whole of the night." Regarding bottom heat he observes: "Newly-potted plants will be benefited by a heat of about $90^{\circ}$ for two or three weeks, after which time it may fall to $85^{\circ}$ maximum, and $80^{\circ}$ minimum, but in the winter $75^{\circ}$ will be sufficient for the younger stock. The bottom heat required for those plants which are to produce several fruit from the same plant ought to be as equal as possible at a medium of about $80^{\circ}$, and not to fluctuate more than $3^{\circ}$ above or below."

The following table of temperature as to artificial heat only is given in the " Cottage Gardeners' Dictionary," differing a little from that of Glendinning, given above: an intermediate course between the two will be a very safe guide.

\begin{tabular}{|c|c|c|c|c|c|}
\hline & & & $\begin{array}{l}\text { Day. } \\
64^{\circ}\end{array}$ & $\begin{array}{c}\text { Night. } \\
60^{\circ}\end{array}$ & $\begin{array}{c}\text { Rise } \\
\text { in sunshine } \\
6^{\circ}\end{array}$ \\
\hline $\begin{array}{l}\text { January } \\
\text { February }\end{array}$ & $\dot{.}$ & $\dot{.}$ & $\begin{array}{l}04 \\
66\end{array}$ & 60 & 6 \\
\hline March & . & . & 70 & 62 & 6 \\
\hline A pril & . & . & 74 & 64 & 8 \\
\hline May & - & . & 76 & 65 & 10 \\
\hline June & . & . & 78 & 66 & 10 \\
\hline July & - & . & 80 & 68 & 10 \\
\hline August & . & . & 80 & 68 & 10 \\
\hline September & . & . & 76 & 63 & 10 \\
\hline October & . & . & 73 & 62 & 8 \\
\hline November & - & • & 70 & 62 & 8 \\
\hline December & . & . & 64 & 60 & 6 \\
\hline
\end{tabular}

"For bottom heat, by adding $5^{\circ}$ to every one of the above artificial day temperatures, we shall be as correct as by any tedious detail. Thus July and August should have a bottom heat of $85^{\circ}$, which ought never to be exceeded in pine-culture." According to Dr Watson's Essays, the lowest temperature of the air averages $70^{\circ}$ in the morning in countries where the pine grows naturally, rising during the day from $85^{\circ}$ to $90^{\circ}$ in the shade. According to the "Philosophical Transactions," the greatest heat in Bengal, where pines are grown, is from $98^{\circ}$ to $\mathrm{I} 20^{\circ}$ during summer in the shade, rising sometimes to $140^{\circ}$ in the sun. In countries where the former temperature ranges the pine is found in greatest perfection, and such temperatures would suit British cultivators had we an equal amount of light, and were all our heat derived from the sun.

The main elements necessary for the most perfect culture of the pine are heat, air, light, and moisture; these constitute the atmosphere in which the pine delights to grow. A few brief remarks on the atmosphere of hothouses may not be out of place here, the same being, under slight modifications, applicable to all other forcing-houses as well as to the pinestove.

The atmosphere of hothouses, more especially in connection with humidity. - Light and heat appear for long to have been the principal elements thought necessary in hothouse culture. About thirty or forty years ago, inquiries began to be made in regard to humidity, and these being founded on meteorological and chemical principles, as well as by ascertaining the operation of nature in the open air, showed most distinctly that with increase of temperature an increase of moisture was indispensably necessary. An imitation of these conditions was commenced by 
most of the best cultivators of exotic plants, and practical experience has long ago proved the correctness of the principle. Professor Daniell, whose opinions on these matters are entitled to the utrnost respect, observes, in "Transactions of the Horticultural Society," vol. vi. p. 1, "If the hygrometer were consulted in hothouses as commonly managed, it would be no uncommon thing to find in them a difference of $20^{\circ}$ between the point of condensation and the air, or a degree of moisture falling short of $500^{\circ} . "$ Dryness in the atmosphere of hothouses, although accompanied with sufficient heat, has as disastrous effects upon the plants as the evaporating east winds in spring, accom. panied by their coldness, has upon tender trees in the open air. To provide against these effects, Dr Daniell says : "The danger of over-watering most of the plants, especially at particular periods of their growth, is in geueral very justly appreciated, and in consequence the earth at their roots is kept in a state comparatively dry: the only supply of moisture, being commonly derived from the pots and the exhalations of the leaves, is not enough to saturate the air, and the consequence is a prodigious power of evaporation. This is injurious to the plants in two ways: in the first place, if the pots are at all moist, and not protected by tan or other litter, it produces a considerable degree of cold upon their surface, and communicates a chill to the tender fibres with which they are lined. The danger of such a chill is carefully guarded against in the case of watering, for it is one of the commonest precautions not to use any water at a temperature at all inferior to that of the hot air of the house : inattention to this point is quickly followed by disastrous consequences. The danger is quite as great from a moist flowerpot placed in a very dry atmosphere. The custom of lowering the temperature of fluids in hot climates by placing them in coolers of wet porous earthenware is well known, and the common garden-pot is as good a cooler for this purpose as can be made. Under the common circumstances of the atmosphere of a hothouse, a depression of temperature, amounting to $15^{\circ}$ or $20^{\circ}$, may easily be produced upon such an evaporating surface. But the greatest mischief will arise from the increased exhalations of the plants so circumstanced, and the consequent exhaustion of the powers of vegetation. Some idea may be formed of the prodigiously increased drain upon the functions of a plant arising from an increase of dryness in the air, from the following consideration: If we suppose the amount of its perspiration in a given time to be 57 grains, the temperature of the air being $75^{\circ}$, and the dew point $70^{\circ}$, or the saturation of the air being 849 , the amount would be increased to 120 grains in the same time if the dew point were to remain stationary, and the temperature were to rise to $80^{\circ}$, or, in other words, if the saturation of the air were to fall to 726 .

"The cause why plants in living-rooms do not thrive so well as those that are kept in plant structures, is chiefly owing to the extreme dryness of the air, while a constant drain upon the leaves and the soil of the pots is maintained.
Hence the fibres in the inside of the pots are alternately moistened and dried, and cooled and heated, and the leaves are deprived of their water by evaporation instead of by perspiration. Besides the power of transpiration, the leaves of vegetables exercise also an absorbent function, which must be no less disarranged by any deficiency of moisture. Some plants derive the greatest portion of their nutriment from the vaporous atmosphere, and all are more or less dependent upon the same source. These considerations must be sufficient to place in a strong light the necessity of a strict attention to the atmosphere of vapour in our artificial climates, and to enforce as absolute an imitation as possible of the example of nature."

Professor Lindley, in "Theory of Horticulture" says: "The skilful balancing of the temperature and moisture of the air, in cultivating different kinds of plants, and the just adaptation of them to the various seasons of growth, constitute the most complicated and difficult part of a gardener's art. There is some danger in laying down any general rules with respect to this subject, so much depends upon the peculiar habits of species, of which the modifications are endless. It may, however, I think, be safely stated, that the following rules deserve especial attention: Most moisture in the air is demanded by plants when they first begin to grow, and least when their periodical growth is completed. The quantity of atmospheric moisture required by plants is, cateris paribus, in inverse proportion to the distance from the equator of the countries which they naturally inhabit. Plants witl annual stems require more than those with ligneous stems. The amount of moisture in the air most suitable to plants at rest, is in inverse proportion to the quantity of aqueous matter they at that time contain. Hence the dryness of the air required by succulent plants when at rest."

John Rogers, Esq., on the same subject, observes in "The Gardeners' Magazine" that "the causes, whose constant operation renders our artificial climates unnaturally $d r y$, are principally two-the condensation of moisture on the glass, and the escape of heated and damp air through the crevices of the building, the space which it occupied being constantly supplied by dry external air. A third drain of moisture formerly existed in the absorbing surface of brick flues, which drank up the moisture of the air in contact with them, and carried it off with the smoke into the outer air. The very general use of hot water in iron pipes has removed this nuisance, and we have now only to contend with the two first mentioned. Some idea of the drain of moisture, by the escape of heated air, may be formed from the following considerations. The capacity of air for moisture-that is to say, the quantity of water which a cubic foot of air will hold in invisible solution-depends upon its temperature, and increases with it in a rapid ratio. It is doubled between $44^{\circ}$ and $66^{\circ}$. The consequence is, that every cubic foot of air which escapes at the latter temperature carries off with it twice as much moisture as it brought in. Where the difference of temperature is greater, 
the drain becomes greater also: air entering at $44^{\circ}$, and escaping at $80^{\circ}$, carries off three times as much as it brought in; escaping at $90^{\circ}$, four times. Now the escape of air from our best glazed buildings is considerable at all times, even when the lights are closed; and if the glazing be defective, and the laps be not puttied, it is very great indeed. The amount of moisture thus abstracted cannot be very easily estimated, varying exceedingly according to the height and construction of the building heated. There exists, however, another drain of moisture, constantly affecting all hothouses, however perfectly constructed, and however cautiously ventilated-viz., the condensation on the glass. In this case the expenditure is capable of pretty accurate calculation. It has been ascertained by experiment that each square foot of glass will cool $1 \frac{1}{4}$ cubic feet of air as many degrees per minute as the temperature of the inner air exceeds that of the outer air - that is to say, if the temperature of the outer air be $44^{\circ}$, and of the house $66^{\circ}$, for every square foot of glass $1 \frac{1}{4}$ cubic feet of air will be cooled $22^{\circ}$ per minute; and the moisture which this air held in solution, in virtue of its $22^{\circ}$ of heat, will be deposited on the glass, and will either drain away out of the house or fall in drip. The greater the difference between the temperature of the internal and external air, the greater will be the amount of condensation; and be it observed, that the capacity of air for moisture does not increase simply in the arithmetical ratio of its temperature, but by a scale considerably more rapid, so that the expenditure of moisture at high tem. peratures is much greater than at low temperatures, for equal differences between internal and external air."

We stated at the beginning of this subject; that in the olden time heat and light only were thought of as necessary elements in hothouse management, and hence the miserable appearance of the productions in them, compared with those met with in our improved state of cultivation. The skilful balancing of the temperature and moisture of the air, as Dr Lindley has so correctly designated it, is the grand secret. Unless that balance be maintained, every other part of cultural skill goes for nothing. The means of effecting this deserve inquiry. The water required for the actual support of the plants at their roots is quite insufficient for this other purpose; recourse is therefore had to the application of water, so disposed of throughout the house as to create moisture in the atmosphere of it. This is, howcver, too hap-hazard a way: by pouring water on the floor until it becomes nearly sodden, if of earth; on the flues or hot-water pipes, when probably at their greatest heat, until the interior is reeking with half-boiling vapour as thick as a London fog; or if steam be employed, the steaming-valve is opened, and the house is suddenly charged with scalding vapour, to an extent to which an Indian jungle is purity itself, while a few minutes before all was nearly as dry as an Arabian harmattan ;while we want only an increase of humidity analogous to what occurs in nature, a humidity which there is governed by the temperature- an atmosphere possessing great elasticity. There is a danger, however, of overdoing this artificial endeavour to establish the balance above alluded to; and when this extreme is fallen into, the remedy is as bad as the disease, and the process of transpiration is prevented from proceeding in its proper course. We have shown, vol. i. p. 248 , and elsewhere, various means for effecting this increase of moisture; but no certain results can ever be expected in practice, until the hygrometer becomes as permanent a piece of furniture in every hothouse as the thermometer is at present. The neglect of this instrument is notorious; we do not recollect of having seen halfa-dozen in use in hothouses during our horticultural existence. The one instrument is as useful as the other, and until both be equally consulted, that balance on which so much of our success depends can never be accurately attained. Intimately connected with this subject is another, which has of late (by some means or other, probably imaginary, or not previously inquired into) caused great alarm amongst some cultivators, who have attributed their disasters to a scorching influence of the glass during the night. When we first heard of this, we began to quake for sheet and all other glass of modern introduction, as the evil appeared to be directed against that material. Day-scorching we have had some experience of, but the idea of nocturnal scorching was rather astounding. A reference to the excellent papers of Mr Daniell, which we have already, and we hope with some degree of usefulness, quoted, set our mind at rest upon this rather alarming subject. "In a well ventilated hothouse," says that authority, "by watering the floor in summer we may bring the dewpoint within four or five degrees of the temperature of the air, and the glass will be perfectly free from moisture; by closing the ventilators, we shall probably raise the heat ten or fifteen degrees, but the degree of saturation will remain nearly the same, and a copious dew will quickly form upon the glass, and will shortly run down in streams. A process of distillation is thus established, which prevents the vapour from attaining the full elasticity of the temperature. This action is beneficial within certain limits, and at particular seasons of the year; but when the external air is very cold, or radiation proceeds very rapidly, it may become excessive and prejudicial. It is a well-known fact, but one which, I believe, has never yet been properly explained, that by attempting to keep up in a hothouse the same degree of heat at night as during the day, the plants become scorched. From what has been premised, it will be evident that this is owing to the low temperature of the glass, and the consequent low dew-point in the house, which occasions a degree of dryness that quickly exhausts the juices."

A great amount of evaporation, as we have already stated, takes place during night, and is greatly increased when a cold frosty night is succeeded by bright sunshine during the early part of the following day. This must be provided against, particularly during winter-forcing; at other times its effects are less palpable. How this is to be counteracted is deserving of 
attention. It is certaiuly neither the most natural, efficient, or least dangerous, to have recourse to the too common practice of pouring pailfuls of cold water on the surfaces of overheated flues or hot-water pipes. Such a process is far more likely to scald the foliage and bark of the trees which are in direct proximity with the heated bodies, than to restore that necessary equilibrium in the atmosphere of the house which has been deranged by excessive evaporation. To this process there are other objections besides scalding. It is too transient in its effects, and this the more so the hotter the heating bodies happen to be. Far more natural and effective modes of supplying this diminished humidity have been detailed (vol. i. pp. 350, 422); and in addition to what we have there stated, we may here add that, if sufficient care be taken to keep the footpaths damp, and the borders in a proper state of surface moisture, at temperatures from $60^{\circ}$ to $65^{\circ}$, abundance of evaporation will arise from them to replace that which is lost by condensation on the inner surface of the glass, and the escape of moisture so produced through the laps or fractures in the glass. In the case of small houses and pits, where the roof can be conveniently covered, as also recommended vol. i. p. 551 , the loss of heat by radiation from the exterior surface of the glass will be greatly lessened, and consequently loss of moisture by condensation on the interior will be in like manner diminished. There are many plants that experience little inconvenience from even a considerable variation in the state of the humidity. Others do so, and more especially those that, like peaches and vines, are forced into growth at an unnatural season; and this decidedly so during their period of flowering, setting their fruit, and also while it is ripening. 'To regulate these matters with anything like certainty, the hygrometer should be consulted, and those of Daniel or of Simmons may be considered the most proper for the purpose.

To those who do not use the hygrometer we may say that, in trusting to appearances only, they should bear in mind that there is a great difference in the quantity of artificial humidity required between a house heated by smokeflues, or even hot-water pipes, and one heated by fermentation; evaporation and absorption are rapidily going on in the former, while in the latter the heat produced is supplied with a considerable amount of moisture at the same time. Notwithstanding what we have said in favour of a humid atmosphere for the pine, this must not be carried to excess, else the most injurious effects would result. "No more," Glendinning wisely remarks, "should be given in steaming or syringing at one time than just enough to moisten the foliage, otherwise the soil will become glutted, the plants will remain stationary, and assume an unhealthy appearance, indicated by the yellow sickly appearance of their foliage. Moisture, notwithstanding, if properly and judiciously supplied, is calculated to be of infinite advantage in moderating the temperature and supplying a material element in demand during an excess of evaporation; and which at the same time being a VOL. II. primary article of food for the plants, is always in requisition in proportion to the supply of solar heat and light." The pine should never be watered overhead while it is in blossom, and at that period abundance of air should be given, but not so as to reduce the temperature. This is, indeed, the most critical period of its existence; and if checked by being removed or repotted, or if water be allowed to fall into the flowers, deformed and small imperfect fruit will be the certain consequence.

Bottom heat. - It seems to be an axiom in horticulture that all plants in a growing state should have the temperature of the soil in which they grow higher than that of the air by which their leaves are surrounded. "Warmth," says Dr Lindley (in "Theory of Horticulture"), "acts as a stimulus to the vital forces, and its operation is in proportion to its amount within certain limits. If, then, the branches and leaves of a plant are stimulated by warmth to a greater degree than the roots, they will consume the sap of the stem faster than the roots can renew it; and therefore nature takes care to provide against this, by giving to the roots a medium permanently more stimulating - that is, warmer-than to the branches and leaves."

The vine succeeds best in countries such as Bordeaux and Madeira, the soil temperature in the former being $75^{\circ}$ and that of the latter $80^{\circ}$. The pine-apple requires a still greater temperature during its period of growth. We believe that the vine might be brought to great perfection in the south of England, if planted against a southern wall, and in a vaulted border subterraneously heated, as has been exemplified at Yester by the Marquis of Tweeddale, and the ripening of the pine in the garden of Lady Rolle at Bicton, near Weymouth, which, however, must be regarded as an experiment, the success of which depended upon the heat applied to its roots in the bottom of the trench in which it was set. Some experiments made by the late $\mathbf{T}$. A. Knight have been misrepresented or misunderstood, and for a time led some to suppose that bottom heat to the pine was injurious rather than the reverse. That great horticulturist repeatedly disavowed entertaining any such sentiments. He certainly did express a belief "that the bark bed was worse than useless;" and in this he was perfectly correct, because its temperature is constantly subject to excess and defect, and that more especially as bark beds were in general managed at the time he so wrote, and it must be confessed are so with many even at this day. But while Mr Knight was experimenting on the pine and the melon, the temperature of his stoves was kept up to from $70^{\circ}$ to $85^{\circ}$; and he found, by placing thermometers plunged in the soil in the pots in which his plants grew, that the heat at their roots was very nearly the same, being surrounded by air at that high temperature, which the soil acquired and retained, so that it was found less subject to variation than had they been plunged in a bed of fermenting material. He further says, in "Horticultural Transactions," vol. vii. p. 255, that the temperature of the air was varied in his stove generally from about $70^{\circ}$ to $85^{\circ}$, and that the temperature of 
the mould in the pots varied very considerably less than that of the air of the stove, the mould being in the morning generally some degrees warmer than the air of the house, and in the middle of the day and early part of the evening some degrees cooler. "It is therefore clear," as Dr Lindley observes (in "Theory of Horticulture," p. 115), " that he considered a high temperature necessary for the roots of his pineapple plants;" and we find from one of his papers ("Horticultural Transactions," vol. iv. p. 544), " that he considered it better to obtain the required temperature from the atmosphere than from a bark bed, the usual source of bottom heat, because its temperature is constantly subject to excess and defect; and he even admitted that, if the bark bed could be made to give a steady temperature of about $10^{\circ}$ below that of the day temperature of the air in the stove, pine plants would thrive better in a compost at that temperature than in a colder. It is therefore plain," Dr Lindley continues, " that the dispute about bottom heat was not as to the necessity of it, but as to the manner of obtaining it. We have, doubtless, much to learn as to the proper manner of applying bottom heat to plants, and as to the arnount they will bear under particular circumstances. It is, in particular, probable that, in hothouses, plants will not bear the same quantity of bottom heat as they receive in nature, because we cannot give them the sarne amount of light and atmospheric warmth; and it is necessary that we should ascertain experimentally whether it is not a certain proportion between the heat of the air and earth that we must secure, rather than any absolute amount of bottom heat." It is not the use, but the abuse, of bottom heat that we should guard against; we should endeavour to secure its uniformity and proportion to the heat of the atmosphere, and its application to plants at proper seasons of their growth; for there can be no doubt many plants require a much higher degree of heat at one season than at another, and also that an excess of it, particularly when the plants are not in a state of growth, is worse than would be even a slight deficiency. In the case of the pine-apple, we find, when the plants are in high health, and fully stimulated onwards by moisture and heat, that the fruit swells best, attains its largest size, full amount of juice, and highest flavour, when these stimulants are gradually reduced towards the period of their ripening; a strong bottom heat at that time is apt to cause a premature ripening, which would not be the case were the plant less strongly excited.

Another evil to be guarded against from too much bottom heat is, "if the roots are placed in a warmer medium than the branches, and are thus induced to absorb fluid faster than the slower action of the leaves can consume it, the superfluous sap will burst through the stem, and distend its tissue till the excitability is impaired or destroyed. Or if, on the other hand, a branch is caused to grow in a warm medium while the roots remain in a very cold one, the former will consume the liquid sap faster than the latter can supply it, and the consequence will be that the leaves will die, or the fruit will fall off, or the flowers will be unable to set their fruit, from want of a constant supply of food. Not that it is necessary for the temperature of the earth and air to be equal, for this does not happen in nature, but it is requisite that they should have some near relation to each other." -Theory of Horticulture, p. 50.

The age of the pine.- Why the pine has been denominated a triennial is hard to say, because neither in its native country, nor always with us, does it require three years' growth before producing its fruit, which it may be said terminates its existence. In the tropics they produce their fruit often under one year, and some of our best cultivators cause them to do so in an almost equally short time; some sorts, like the Queen fruit, when from twelve to fifteen or eighteen months, while others, such as the Providence, require from two to three years. Some continue them much longer, and obtain larger fruit; but largeness of size, although much prized by some, does not in this case, as well as in many others, constitute excellence of flavour; nor is size at all times to be measured by the age of the plant. Much of the time occupied in growing the plant depends on the size of the sucker when first planted, and much also on the mode of culture. The most profitable return is undoubtedly from plants from eighteen months to two years' growth ; and the most useful size is from 4 to $8 \mathrm{lb}$. weight. No doubt a 12-1b. pine is a showy affair, and may grace, as they often do, the same table for a dozen nights together, merely to be looked at, while a well-modelled plaster-of-Paris cast would answer the purpose nearly as well. They are seldom eaten while in anything like perfection, the preference being given to fruit of a much smaller size, and of higher flavour, cut at the side-table, and handed round.

Growing the pine in all its stages without fireheat has been long successfully accomplished; the means used are fermentation from leaves, tanner's bark, flax refuse, and stable-yard manure. Taking into account labour, and the waste of the last material, we do not see that there is any economy in the practice, at least except for amateurs growing pines on a small scale. The structures used are pits (vide sect. Pirs, vol. i.) or deep frames. No doubt, in such structures, and with either of the three first, for affording bottom heat by fermentation, young plants can be grown to a great degree of excellence, and in pits heated by leaves or tan we grow several hundreds annually; but we always prefer the aid of a hot-water apparatus for affording atmospheric heat, particularly during autumn and winter. One objection to pits, as in general constructed, deserves to be stated. They are, for the most part, too narrow, and hence surrounded with opaque walls of bricks or masonry; and the plants are set too deep in them, and hence deprived of sufficient light and ventilation, except at the points of the leaves. Certainly young pine-plants grow exceedingly fast in such pits, and fruit attains a large size in them, even when compared with more light and better ventilated structures, as may be instanced in the 
monstrous Queens grown by M. Pelvilain, at Meudon, near Paris, vide figs. 625, 626, 627, vol. i., and held up by "Mirabile Dictu," in the columns of "The Gardeners' Chronicle," as something that English growers might hear of, but never equal. These large pines were, however, admitted, after the changes had been rung on them for several weeks, by the editor of the same journal, as being in point of flavour worse than the worst Providence-watery and tasteless ; "we could not have believed a Queen to be so deteriorated. If then," he adds, " the French have triumphed in weight, the English may console themselves that they are still unrivalled for quality." These Meudon Queens, grown in close, damp, ill-ventilated dung-pits, weighed $8 \mathrm{lb}$. $1 \frac{1}{2} \mathrm{oz}$. only, some say from 7 to $10 \mathrm{lb}$., and were unfit to eat, while several English growers have produced the same variety $6 \mathrm{lb} .11 \mathrm{oz} ., 6 \mathrm{lb} .4 \mathrm{oz}$., $6 \mathrm{lb} .15 \mathrm{oz}$; and Mr Barnes of Bicton is believed to have produced the same sort upwards of $8 \mathrm{lb}$. in weight, all of which were of the highest flavour, and grown under the influence of light and air, and in structures the atmosphere of which was as pure as that of the open garden. Although young plants increase rapidly in bulk when grown in low, close pits, heated alone by fermentation, they do not possess the same strength that plants do when grown in low-roofed houses, exposed to light and air on all sides. The former show long, narrow, thin, flabby leaves, while the latter are short, thick, and broad in the leaf, each of these being as stiff and unbendable as the leaf of an aloe. The former also send up long slender fruit-stalks, seldom fit to support the fruit that surmounts them without the aid of stakes and guy cords; while the other have a short stout fruit-stalk, with a small crown, the top of which is seldom equal to even half the height of the fruit. The former is a watery, tasteless fruit; the latter is full of luscious sweetness. Every pine-grower of ordinary experience knows full well that he can swell his pines in close humid pits to a large size, even from plants eighteen months old. He also knows that the same variety grown in a light airy house gives much the better fruit. Such a pine-pit as we have described, p. 343 , fig. 462 , vol. $i$., is much better adapted for growing the young plants in than any of the numerous pits we have described, being 17 feet wide within; and our fig. 464 is what we call the beau ideal of a perfect fruiting-house. It will be understood that both these, being span-roofed structures, present their ends to the south and north; and it will be seen that light and air have as much effect upon the plants as if they were growing in the open air, there being only 4 feet from the top of the bed to the ridge of the roof (the rims of the pots, in fig. 464, being level with the side walls, instead of several feet under them, as is usually the case). The next pit in merit to those of our own design is, in our opinion, that of $\mathrm{Mr}$ Flem. ing of Trentham, fig. 463 , which is also on the span-roofed principle, only having its direction from east to west, the southern side of the roof being longer than the northern by one third, so as to admit of the sun's rays falling on every plant within. It will also be observed that, like our own; the rims of the pots are nearly level with the side walls. Several pine-stoves and pits, at present in course of erection from our designs, are upon nearly the same principle.

It may be worthy of remark, that the pit employed by $\mathrm{Mr}$ Adam Taylor in 1769, who first grew and fruited the pine in Britain by means of fermentation alone, is superior to nine-tenths of those that have been constructed since. A figure and description of it will be found in his "Treatise on the Culture of the Pine-Apple," in which we find a frame of glass resting on a brick wall 12 inches high above ground, the front of the pit above that being $2 \frac{1}{2}$ feet high, made of movable glazed sashes to admit light and air. The ends also are of glass, the back or northern side only being opaque, and constructed of 2-inch planking. In describing his frame for young plants, Mr Taylor advises it to be " $3 \frac{1}{2}$ feet deep at back, and $2 \frac{1}{2}$ feet deep in front." And he afterwards observes: "It is also necessary that they should have a sufficient share of the sun. If therefore a border of glass, to the height of 15 inches, was fixed in the fore part of this frame, it would be of singular service to the plants; whereas if the perpendicular front should be composed entirely of wood, they would be too much shadowed." Here we have, eighty-four years ago, an excellent model of a pine-pit, which, if heated by hot water, would be superior to most of those in use at the present time. As a general rule, we would say that no pine plant should be grown in a pit where the surface of soil in the pot is 1 inch under the level of the line of glass; and supposing the surface of the tan-bed to be exactly on a level with the exterior ground-line, the sides and ends should be composed of glass to within 9 inches of that level, the superstructure resting on a stone or brick plinth of the above depth, merely to preserve the woodwork from decay. Pits so constructed should have their back and front sides capable of being opened for ventilation. The culture of the pine in pits is already well understood, but our pits themselves are for the most part exceeding badly contrived.

Growing the pine planted out in beds.-This, although by no means a new feature in pine culture, having been occasionally resorted to, has nevertheless, within these few years past, been carried out upon a much larger scale than heretofore, and with more or less success. It would appear to be the most natural mode of treating the plant; and there can be no objection started against it, were it not that young plants, during the first six or nine months of their growth, are small, and require less room to grow them in than is required when they attain a fruiting state; and hence smaller structures are provided for them during this period, in which they are brought forward to take their place in the fruiting-house bed, when those which have alrcady occupied it are thrown out after having produced their fruit. Having them in a portable state during their preparation, enables the cultivator to add to the soil at their roots progressively, to remove them from one structure to another as they increase in height, to remove such as are defective or that start prematurely 
into fruit, \&c. To plant the suckers in a prepared bed as soon as they are removed from the old plant, and to allow them that space which they would afterwards require, would be a waste of space that few can afford, and a wasteful expenditure of fuel equal to that which would be required to ripen off a houseful of fruiting plants. The soil in the bed, however well prepared, would become exhausted of its best parts, and become consolidated before the plants come to an age to require their greatest nourishment from itnamely, while swelling their fruit. Upon the whole, therefore, it is most expedient to grow the young stock of plants in pots, and to plant them out when they have nearly attained a fruiting state. Some, however, grow their plants, even from the first stage, planted out in beds of tan, half-decayed leaves, or soil placed over a heating material below; and when any alteration becomes necessary in their arrangement, or for securing additional heat to the roots, take them carefully up, injuring the roots as little as possible, and immediately transplant them into another bed which has been prepared for them. This, however, requires great care in the operation, as well as having a spare bed into which to place them, for it would be highly injurious to the roots to leave them exposed while that bed was preparing. Of those who grow them in something like this manner, M. Pelvilain of Meudon claims our notice. His frames, as we have described, vol. i., are boxes of old shiptimber, supported on pieces of wood $2 \frac{1}{2}$ feet above the ground. In this box are placed 16 or 18 inches of peat soil, containing a considerable quantity of silver sand-just such a soil as heaths are grown in. He sticks his plants at once into this soil, setting them as far apart as he judges will afford them room to grow without being crowded during the first season's growth. This planting takes place at no stated period, but just when the crowns or suckers are at his disposal. Those planted in the spring, say of 1853 , would in the summer of 1854 produce fruit such as is reported to have been grown by him. During their growth, clear water only is administered to them, and the heat obtained from fresh stableyard manure (vide figs. 625, 626, vol. i.) thrown in under the box, which is so closely fitted that no steam is allowed to enter the frame. The removal of the young plants at the end of the first season to the fruiting-pit, is done by taking them out of the bed of soil in which they have been growing, and transplanting them, with their roots entire, into the bed in which they are to perfect their fruit. In summing up the merits of this plan, "Mirabile Dictu" observes: "Independently of the economy attending the arrangement of cultivating the young plants separate from those in a fruiting state, their removal has a tendency to throw them more rapidly into fruit. As they are, however, transplanted with the whole of their roots, the constitutional vigour of the plant is not thereby impaired; on the contrary, when they begin to require greater support, the roots have penetrated into tlie fresh soil; hence they are shortly in a position to supply a larger portion of nutriment than if their removal had not taken place." No more plants can with equal propriety be grown in a planted-out bed than in pots; and the roots of plants turned out of pots into a prepared bed will be in as good a state, if not much better, for finding their way into the fresh soil, and as capable, if not more so, of supplying as large a share of nutriment to the plant, as by this mode of rude transplantation.

Mr Hamilton, in his excellent little "Treatise on the Culture of the Pine," in reference to the planting-out system, observes: "When the pine is cultivated on the common bark-bed, the following method, I have experienced, answers better than growing them in pots, and will save a great deal of trouble, particularly if the old stools are preserved. Suppose the bed will hold five rows, three of the middle rows are turned out, first placing a sod of about 14 inches diameter, green side downwards, under each ball, then add 3 or 4 inches of chopped turf all round them. The interstices between each plant are then filled to the height of the ball with new tan, which will give a gentle humid warmth to the roots, and cause them to strike freely into the fresh turf, and lastly into the tan, in which they will be preserved in a healthy state for a great length of time. The two outside rows are to be kept in pots, which will have to be removed when the bottom heat declines; and some of the old tan will have to be taken out, and fresh added along the side of the bed. This will keep the roots of those planted out sufficiently warm for five or six months. The plants so treated may remain for years, and perfect a succession of fruit. After each is cut, a little fresh turf ought to be added round the trunk, and filled up as before with new tan; and thus a slight surface-heat is obtained at each process of barking up, which will encourage the emission of new roots without injuring the old ones.

"This compost answers well where bottom heat is supplied by hot-water pipes, \&c. However, I would give a preference to chopped turf, no matter of what texture, provided it be well stored with vegetable fibre : such compost will require no mixture, except it be heavy, and deficient of grass roots, then mix with it some brickbats, chopped wood, or charcoal. The latter will be the best sweetener of the compost, and will equally preserve its porosity." Mr Hamilton does not recommend "turning out plants where the old system is practised, because the plants will frequently make a long growth before they fruit, which is a great sacrifice of time and room, with no adequate advantage in the size of the fruit, to say nothing of the quantity of compost required to prepare the bed for another stock of plants. If they are planted out with the intention of adopting my system-viz., of fruiting from the old stools-the most beneficial results may then be expected, as the plants may remain undisturbed for years, and so produce one fruit annually. No destroying or shortening healthy leaves, no reducing the ball, and consequently injuring if not destroying the original fibrous roots, is in accordance to my experience. Destroy the original fibres, and if the growth of the plant be not materially affected, the period of its fruiting will at least be much 
protracted. Preserve them undisturbed, and in due time they will complete the full development of both plant and fruit." This careful turning out is widely different from M. Pelvilain's method of transplanting, and more in accordance with good culture. Upon the whole, planting out should only be attempted in beds heated by hot water underneath, either by tanks or pipes laid in a vault below, the plants carefully turned out of pots when they have attained, or nearly so, their fruiting size.

The cultivation of the pine in prepared beds, instead of growing them in pots, has its advantages, and although long ago suggested, and even practised by Justice and others, has only become prevalent since means have been so efficiently discovered of maintaining the necessary bottom heat without being dependent on heat produced by fermentation. The majority of cultivators grow their young plants in pots, and when about to start into fruit turn them out in the prepared beds. Mr Fleming of Trentham, one of the best pine-growers in Britain, adopts a different practice, and grows them in all stages upon the planted-out principle; he finds they neither sustain injury nor check by being transplanted, which he does when he finds it necessary to economise space. As the fruit is cut he removes the old roots and fills up their places with young plants, adding at the same time a supply of fresh soil. As pines are produced at Trentham at all seasons of the year, it follows that transplanting is not confined to stated periods.

One of the excellent parts of Mr Flcming's practice is, that he does not roast his pine plants, nor does he water them during their growing season out of a gill measure; he abides by the laws of nature in both cases, and keeps his fruiting pines in a night temperature in fine weather at $65^{\circ}$, but in frosty weather allowed to fall to $57^{\circ}$. His younger plants enjoy an atmosphere during winter of $55^{\circ}$, and air is admitted to them all night and day, increased or decreased according to the state of the weather, but never entirely withheld. This day and night ventilation has very much to do with the success which follows so common-sense a practice. Indeed, the low top and bottom temperature employed there is such as would have frightened the pine-growcrs of the early part of the present century into fits. Water is sparingly applied during winter, but during the growing season it is administered freely.

Pines, to be fruited during winter and in early spring, will require a night temperature of something like from $65^{\circ}$ to $70^{\circ}$. The succession and younger stock will be better in a temperature of which $40^{\circ}$ may be taken for the minimum. All pine-growers admit that some care is required to swell off pines to anything like a respectable size during the dark months of winter, and this holds good to a very considerable extent more in Scotland than in the clearer sky of the south of England. Many believe that the Queen pine is perfectly unadapted for winter fruiting, while some few think otherwise. Amongst these is Mr Barnes of Bicton, near Sidmouth, Devon, a most successful cultivator, who a few years ago frightened half the pine-growers in Britain by his declaration that a row of well-swelled pineapples might be grown amongst the kitchengarden crops easily in any locality which will grow Gurken cucumbers, dablias, heliotropes, \&c. In his communication to "The Gardeners' Clironicle" on fruiting pines in the open air, he gives the following very sensible view of his opinion on fruiting pines during winter in the pine-stove: "It was at one time considered impossible to swell off a pine in winter; consequently, if a plant showed fruit late in the season, or in winter, it was cast to the rubbishhcap as useless. The same ideas are still entertained by some growers with respect to the Queen pine, which they say cannot be swelled off in winter. I, however, find no difficulty in the matter; but there will always exist some difficulty at all scasons in swelling pines freely in a strong fire-heat, or in any kind of artificial heat, when there is but little light. If a high night temperature is maintained, more especially, fruit of good quality will never be obtained in winter and early spring. Such conditions of growth-viz., high night-temperature and much bottom heat-will only have the effect of producing small fruit with withered stalks and gawky crowns. It is best not to be tied to any fixed degree of heat, either by day or night; a healthy, sweet, and humid temperature is my aim at all times, regulating the temperature at all seasons by light. In the short dark winter days, our main top-heat is produced by linings of fermenting materials placed at the summit of our succession-pits, allowing the old dry linings at the base to remain all winter undisturbed. By adopting this method we have at command surface heat to dry the interior air, and abundance of air, day and night, is admitted, which maintains a healthy sweet atmosphere. For the last two months," October and November, "my pines have been from $3^{\circ}$ to $10^{\circ}$ above the exterior atmosphere. In very dark sunless weather no excitable temperature is maintained, but more heat is given as the light becomes stronger." Although Mr Barnes does not here connect his practice with physiological reasons, it is quite clear that his practice of regulating temperature by the amount of light is founded upon the most correct principles. We have elsewhere shown the intimate connection between light and heat, and upon thcir proper application much, if not nearly all, of successful exotic culture depends.

On the propriety of this planting-out mode of culture many of our best pine-growers differ greatly in opinion. For ourselves, we think that if the plants are placed in the prepared bed at the time they would otherwise receive their last shift, so that they may make roots in the soil, as they would do in the fresh soil in the fruiting-pots, advantages do arise from the practice, because their roots, having much more scope to range in search of food, will produce larger fruit. Nevertheless, this planting out will retard their starting into fruit for a greater or less period of time, and hence is not to be recommended for expeditious culture. It may generally be 
remarked, that, if the plants have sustained no check, they will rarely show fruit until the pot is full of roots, till they have ceased to make strong roots, and have provided themselves with a network of very fine white fibres, completely covering the surface of the sides of the ball. Or if they are planted out when even in a much younger state, their roots will ramify through the soil, and often do produce those fine small roots, which is a certain sign of their having arrived at a state ready to show their fruit. Some argue, and we think rightly, that if plants are not turned out until they have completed their root system, it matters little whether they are planted out or retained in the pots, and that it is quite immaterial into what soil they may be placed, as the roots will extend no farther than the original ball. In regard to the process of planting out, it is of importance, if the plants are young and have not completed their full growth, that ample space be afforded them in the bed, so that their leaves may have plenty of room to admit of their taking their natural curvature, and thus expose the greatest amount of their surface to the direct influence of the sun's rays; and this distance must vary according to the natural size of the variety. Globes require the least space on account of their erect habit of growth, and Providences the greatest, as they are the largest of all pines in natural habit. During the process of planting the soil should not be compressed, and therefore it is better to begin with the back row, if in pits, or the front row, if in houses; the smallest plants going into the latter, while the larger ones should stand in the former. Set whichever of these rows is first placed at proper distances apart, and pack the soil lightly about it before beginning with the next. When the whole is planted, surface the bed over with tan to the depth of 2 inches, which will prevent the surface of the compost from caking by the water falling upon it during the process of rootwatering, or of syringing the plants overhead. It also prevents excessive evaporation, and, owing to the tannin principle it contains, tends to keep the atmosphere of the house or pit in a pure and wholesome state. "If the plants have been previously grown in pots," Mr Fleming remarks, "the roots should be carefully unwound, and spread out into the new soil; but we generally prefer plants which have been grown without pots from their infancy. With this object in view, we devote a space along the front of each pit for the growth of suckers, and this does away with the necessity of succession-pits. This system enables the pine-grower of limited extent to keep a succession of fruiting pines and plants in a single house or pit; and it is not less useful where larger quantities are cultivated, as, by growing young plants in the front row, it allows the entire surface of the bed to be kept much nearer the glass than would be admissible if the pits were filled exclusively with fruiting plants. In the soil for the suckers we mix a small portion of rough new tan, which the roots delight in, and it facilitates their being moved with good balls. In this manner," he says, "we have not the slightest difficulty; indeed, the plants grow with the greatest vigour immediately after being shifted; and those which are moved with a ball of earth have a decided advantage over those which have been previously grown in pots, unless, indeed, the pots are much larger than those which are ordinarily used for this purpose. Those which are lifted do certainly get a few of their points shortened, but they are always furnished with a supply of young feeders, which are ready on every side to strike into the new compost. To keep up a constant successional supply, we plant at all seasons of the year. As soon as a fruit is cut the plant is pulled up, and after removing a basketful of the old exhausted compost, its place is supplied with an equal quantity of new material. In this the finest plant, carefully selected from the front row, is planted. This practice of filling up vacancies as they occur may be continued for several years, till it is advisable to renew the soil in the bed entirely. In such a case we choose a period immediately after a rather large proportion of plants have ripened their fruits; and during the time that has been occupied by these in completing their maturity, we allow the vacancies to remain unreplenished, in order that at the time of clearing there may be fewer plants in the way. Of the latter, we carefully lift those plants which have not yet thrown up their fruits, and set them in a close shed; but those whose fruit is in progress we allow to remain, carefully clearing away the soil from around them, as far as it can be done without injury to their roots. After the old soil and leaves are cleared out, they are replaced with new materials in the same order as at first; namely, the hot-water pipes are laid in a bed of ashes or gravel, about 18 inches below the surface of the soil, and over the pipes is a ridge of gravel, the crown of which is 12 inches from the surface; the intervals between should be filled with oak leaves to the same level, thus having 12 inches of soil. In replanting, of course, those which were lifted with balls are planted in the back rows, and the remaining rows of fruiting plants are supplied with the finest young plants from the fronts of the pits. After planting, at any time, the atmosphere for a few days afterwards should be kept rather moister than usual, and the shading more freely used than at other times, till the plants have commenced to make roots into the new soil." These excellent directions differ in no respect from our own practice, except that we grow our young plants in pots in separate pits, and plant them out as vacancies occur in the two fruiting-houses in which the planting-out system is followed. Were we even more in favour of the planting-out system than we are, we could not do so to a greater extent, as our other fruiting-houses are planted with Muscat grapes, and hence it is desirable to have our plants in a portable state, to enable us to remove them when the grapes are ripe. Not that we recommend this removal of the plants, as they cannot be lifted after they have attained their full size without evident injury being done to them, and it is only necessity that induces us to do so. At first sight it would appear to many that a house of pines planted out at the same 
time would ripen simultaneously or faster than might be desired; this is, however, not the case, some sorts requiring a longer time to start into fruit than others, and even some plants of the very same variety. This fear of a glut of fruit at one time need not be apprehended, for as soon as one fruit is cut the plant is immediately taken out, and its place supplied by another taken from the growing-pits. We greatly prefer turn. ing out our plants from pots to transplanting them, as practised by our excellent friend $\mathrm{Mr}$ Fleming; for there are only two things which make us nervous in pine culture-namely, the least injury done to the roots, and breaking or bruising the leaves even at their very points.

Growing the pine from suckers attached to the old stem.-This was attempted by Speechley, and others, as already noticed, since his time; and more recently by Mr Hamilton of Thornfield, who has described his practice in his "Treatise on the Culture of the Pine-Apple," p. 40, which amounts to this: He sets his plants, when fit for their last shift, into pots, of which the dimensions are not precisely given, but we presume them to be from 10 to 12 inches in top diameter; in these the first fruit is to be perfected. After this these pots have to be placed in others much larger and deeper, having three or four pieces of broken potsherds placed between their bottoms, and the space between the inner side of the one and outer side of the other filled with broken brickbats. The bottom leaves of the sucker which produced the last fruit are removed, to admit of the embryo roots placed between the old stem and leaves finding a ready escape into the soil, at which time the old compost in the pots, as far down as the roots, is also removed, and replaced as high as the embryo roots appear, with "sods of the lumpy part of the compost; and the fresher the better, providing the vegetable fibre is dead." His reason "for using rough soil is in order to keep the surface open, so that the atmosphere may have full access to the bottom of the roots." "By this process," Mr Hamilton says, "the third fruit is invariably larger than the second, and considerably larger than the first, and will, generally speaking, be produced in an equally short period." One sucker only is in general allowed to remain on a plant. But Mr H. adds, "I have frequently allowed two suckers to remain on the plant till the first showed fruit; I have then carefully taken the other off, and planted it in a small pot, leaving the former to perfect its fruit on the old stool; but supposing both suckers to show fruit at the same time on one plant, as they frequently do, then they ought to remain, and to perfect their fruit thereon; and the weight of each, I have found by experience, will be nearly as heavy as though the plant had only produced one, particularly if both should fruit at the same time. The only possible cause that I can assign for this result is, that the plant with two fruit has twice the number of leaves, and has therefore the advantage of absorbing a greater quantity of nutriment from the atmosphere, especially if moisture and ventilation be attended to." When the second fruit has been cut, the soil is again removed from the surface of the pots as low down as the roots; a few of the bottom leaves are removed, as noticed above, in preparing for the second fruit; and earthing up is performed in the same manner, the same routine being continued till the fourth fruit has been cut; he then cuts "off the sucker which produced the last fruit, with from 6 to 8 inches of the old trunk attached to its bottom. The leaves are all dressed off that portion of the old trunk, and from the bottom of the sucker, which will have a quantity of roots in the axil of the leaves. It is then planted in a pot 9 or 10 inches diameter at the top, placing the attached trunk at the bottom of the pot, before the drainage is put in, in order that the sucker may be sufficiently deep to be covered with the soil, and thus it is intended to perpetuate a successive progeny of suckers and fruit. Thus may the routine of potting and re-potting be so far dispensed with, that once in two or three years is all that is required; and if fresh turf be used, its strength will not be exhausted in that time."

We have tried this mode of culture, but consider it very inferior to starting the sucker at once upon its own roots, growing the plant rapidly, taking one fruit from it, and throwing the old plant away. No doubt much handsomer plants are produced in this way, and the trouble of shifting can in no way exceed that of stripping off the leaves and soiling the plants up, as practised by Mr Hamilton.

Expeditious culture.-That mode of culture by which Queen pines of 6 pounds' weight and upwards can be produced on plants fifteen months old must be considered super-excellent. This has been effected by Mr Hewitt, gardener to J. Purdy, Esq., at Bayswater, and simply consists in the following details. The structures are as unpretending as the routine of practice is simple yet complete. They consist of a fruiting-pit 18 feet long and 10 feet wide, 6 feet in height at the back and 4 feet in front, and two succession-pits of rather smaller dimensions. They are heated by 4-inch hot-water pipes; a flow and return pipe passes round the pit for surface heat; while six rows of pipes, of the same dimensions, are buried amongst tan for supplying bottom heat; for no dependence is placed on it for affording heat, but it is used only as a plunging material for the pots to be placed in. This bottom heat is regulated by valves, so that it can be decreased or withdrawn entirely. The plants all grow on their own roots, the suckers being taken off when in a proper state of firmness at their base, and are potted the same day, so that the embryo roots receive no check, but push immediately into the soil, and become at once available for the support of the plant. The plants are from this moment kept constantly growing without any interruption until the fruit is perfected. Air is admitted through tubes placed under ground, and communicating with openings by the side of a gravel walk at some distance in front of the pit, which openings are fitted with stoppers for the regulation of the stream of pure air which enters the front of the pits immediately over 
the hot.water pipes. Thus, having the plants close to the glass, supplying them with pure air, moderated in temperature before it reaches them, an equable and moderate bottom heat, and the plants being kept constantly in a growing state, constitute the sum of Mr Hewitt's practice.

\section{SELECT LIST.}

A very excellent classification of pines was drawn up, by order of the London Horticultural Society, by the late Mr Munro, and published vol. i., part 2, second series of their Transactions. As many of the sorts there enumerated are not now considered deserving of cultivation, and as some new and superior sorts have since appeared, we do not think it worth further reference.

To show, however, how varieties have multiplied, we may observe that Taylor, in his "Treatise on the Ananas," published 1769, describes five sorts; Philip Miller, in 1737, describes the same number; Speechley, in 1796, fourteen; Nicol, in 1822, ten; G. Lindley, in 1831, thirty-seven; Rogers, in 1834, nine ; the " Encyclopædia of Gardening," 1835, eleven; the Systematic Table of Mr Munro, fifty-two.

Anson's queen.-Spines medium size; form of the fruit cylindrical; colour of the flowers purple; average weight of fruit 4 to $5 \mathrm{lb}$.; quality second-rate. Synonym-Lemon $A n$ tigua.

Antigua, black.-Spines large; form of the fruit cylindrical; colour of the flowers lilac; average weight of fruit $E$ to $7 \mathrm{lb}$.; quality excellent; flesh pale yellow; should be cut just on the point of changing from a green to a yellow colour, at which period it abounds with a quick lively juice of exquisite flavour; leaves rather narrow, long, of a dark green colour, tinged with a purplish brown. SynonymsJagged-leaved Antigua, Brown Antigua, Wortley's West Indian.

Smooth-leaved Antigua.-Spines none; form of the fruit roundish; colour of the flowers pale lilac; average weight of fruit 5 to $6 \mathrm{lb}$; quality second-rate. Both this and the last apt to throw out gills or secondary suckers at the base of the fruit. Leaves palish green, exceedingly brittle; colour of the fruit palish yellow; should be cut before it becomes too ripe. Synonyms-Green Antigua, Smooth green Havannah of some, from which it is totally different.

Blood.-Spines very large and distant; form of the fruit cylindrical; colour of the flowers lilac; average weight of fruit 4 to $5 \mathrm{lb}$.; quality inferior, its singularity only warranting it a place in large collections; colour of the fruit dark blood; flesh palish yellow. Synonyms-Bloodred, Claret.

Buck's seedling.-Spines medium; form of the fruit pyramidal; colour of the flowers lilac; average weight of fruit 6 to $8 \mathrm{lb}$; quality second-rate.

Demerara.-Size of the spines small, thickly set; form of the fruit roundish; colour of the flowers lilac ; average weight 4 to $6 \mathrm{lb}$; q quality good. Synonyms - New Demerara, Harrison's new.

Enville.-Size of the spines medium; form of the fruit pyramidal; colour of the flowers lilac; average weight 5 to $8 \mathrm{lb}$; quality good during summer and autumn, apt to become dry and stringy during winter and spring ; not adapted for winter or spring ripening. Leaves strong, covered with a slight downy meal on the under side. Of this there are two varieties, the one a well-formed pyramidal fruit with a small crown, the other cock's-comb-shaped, with an immense number of very small useless crowns, somewhat in form of a helmet, and hence distinguished by many as the Trooper's helmet, the last a very inferior variety. Synonyms-old Enville, Sugar-loaf Enville, Cock's-comb. To these the Spring Grove Enville and New Enville may be added.

Globe.-Size of the spines medium; form of the fruit globular ; colour of the flowers lilac ; average weight 4 to $6 \mathrm{lb}$.; quality good; fruit apt to decay if not cut when just ripe; colour of fruit bronzy yellow; leaves very upright, readily distinguished in this respect from all other pines. An excellent summer and autumn fruit.

Globe, Russian.-Size of the spines large; form of the fruit roundish; colour of the flowers lilac; average weight of fruit 4 to $6 \mathrm{lb}$; q quality excellent as a summer or autumn fruit; less erect in habit than the last.

Havannah.-Spines none; form of the fruit cylindrical or conical; colour of the flowers purple; average weight of the fruit 4 to $6 \mathrm{lb}$.; quality second-rate ; leaves smooth, palish green, and much spreading; colour of the fruit bronzy yellow; swells tolerably well during winter. Synonyms-Smootl-leaved Antigua, Ripley, Old king, with neither of which it is identical; Brown Havannah, nor do we think it different from Smooth Havannal or Green Havannah.

Jamaica, old. - Size of spines small; form of the fruit oblong; colour of the flowers purple; average weight of the fruit 4 to $6 \mathrm{lb}$; q quality excellent; one of our very best winter fruitingpines; leaves somewhat narrow, long, marbled with dullish brown. Synonyms-Black Jamaica, Black Barbadoes, Tawny, St Vincent's sugarloaf, Copper-coloured, Montserrat of some.

Jamaica, nev. - Size of spines small; form of the fruit pyramidal; colour of the flowers lilac; average weight of fruit 4 to $6 \mathrm{lb}$;; quality during summer excellent, but not adapted for winter ripening. Synonyms-St Kitt's, English globe, Brown Antigua, New black Jamaica, Montserrat of some.

Montserrat.-Size of spines medium; form of fruit cylindrical; colour of flowers purple; average weight of fruit 4 to $7 \mathrm{lb}$.; quality excellent for a winter pine, and, like the Uld Jamaica, swells its fruit better during winter than most others. Great confusion exists between this and the Black Jamaica, the Montserrat in one part of the kingdom being called the Black Jamaica, and rice versâ. Synonyms-Brazil, Red Ripley, New Ripley, Copper, Cape Coast, Bogwarp, Copper-coloured Antigua, Cochineal, Indian Crevle, Malacca, Red Antigua, Sumatra.

Otaheite.-Spines medium size; form of fruit somewhat globular, inclining to longish; colour of the flowers lilac; average weight of fruit 4 to $7 \mathrm{lb}$; quality excellent as a winter fruit, 
swelling well during that season; leaves Iong, rather upright, slightly marbled with purple; crown of medium size; altogether a handsome fruit. Synonym-Anson's.

Providence, new.-Size of spines small; form of the fruit oblong, sometimes rather conical, and often irregular, particularly when topped with many small useless crowns, growing closely together in a cock's-comb form; colour of the flowers dark purple; average weight of fruit from 7 to $12 \mathrm{lb}$; quality third-rate, but grown pretty extensively on account of its large size; leaves broad, thick, somewhat spreading, marbled with purplish shades; seldom producing above one or two suckers on the stem. SynonymsWhite Providence, Mealy-leared Providence, Woolaton green Providence, Woolaton Providence.

Queen.-Size of the spines large; form of fruit cylindrical; colour of the flowers lilac; average weight of the fruit 3 to $6 \mathrm{lb}$.; quality excellent; one of the best and most useful of all pines, and in very general cultivation; leaves short and stout. There are several inferior varieties of Queens grown which ought to be expelled from gardens. Synonyms-Broad-leaved Queen, Common queen.

Queen, Antigua.-Size of spines large; form of fruit cylindrical; colour of the flowers dark lilac; average weight of the fruit 4 to $6 \mathrm{lb}$; quality good; pips large and flat; apt to produce secondary suckers or gills at the base of the fruit. Synonyms-Yellow Caraile, Black Caraile, Lord Effingham's.

Queen, Moscow.-Size of spines large ; form of fruit cylindrical; colour of the flowers lilac; average weight of fruit 3 to $6 \mathrm{lb}$.; quality excellent; leaves short, stout, marked with slight traces of longitudinal lines on their back near their base ; plant apt to send up many suckers; an excellent and useful pine, and from its small size well adapted for low-pit culture.

Queen, Lemon.-Size of spines medium; form of fruit cylindrical; colour of flowers lilac; average weight of fruit 4 to $7 \mathrm{lb}$; q quality good, but inferior to the Common queen. SynonymsRipley's new queen, Lemon-coloured Barbadoes, White Burbadoes, Barbadoes queen.

Ripley.-Size of spines large; form of fruit roundish; colour of the flowers purple; average weight of fruit 3 to $5 \mathrm{lb}$; quality excellent. Synonyms-Heaton House, Montserrat, Old Ripley, Ripley's.

St Vincent's.-Size of spines medium ; form of fruit pyramidal; colour of the flowers purple; average weight of fruit 3 to $5 \mathrm{lb}$.; quality excellent; valuable for ripening during winter, as the fruit seeds well at that season. SynonymsGreen olive, St Thomas's, Stubton seedling, Green St Vincent's, Bahama sugar-loaf.

Sierra Leone.-Size of spines medium; form of fruit cylindrical; colour of the flowers purple; average weight of the fruit 4 to $6 \mathrm{lb}$; q quality excellent.

Sugar-loaf, brown-leaved.-Size of spines medium; form of fruit cylindrical; colour of flowers lilac; average weight of fruit 4 to $6 \mathrm{lb}$. quality excellent; leaves long, brittle, tinged with brown. Synonyms-Antigua sugar-loaf, Striped brown sugar-loaf, Mocho.

VOL. II.
Sugar-loaf, orange.-Size of spines small; form of fruit cylindrical ; colour of the flowers palish purple; average weight of fruit 3 to $5 \mathrm{lb}$; quality excellent.

Sugar-loaf, striped.-Size of spines large; form of fruit cylindrical; colour of the flowers lilac ; average weight of the fruit 3 to $5 \mathrm{lb}$; quality good; leaves striped with purple lines. Synonyms-Green sugar-loaf, Green striped sugar-loaf, Brown striped sugar-loaf, Purple-striped queen, Prickly striped sugar-loaf, Bird's-eye Bahama, White sugar-loaf of some.

Trinidad.-Size of spines medium; form of fruit cylindrical; colour of flowers lilac ; average weight of fruit 8 to $12 \mathrm{lb}$. In Trinidad it is said to attain the weight of $26 \mathrm{lb}$. A very large showy fruit, but of very inferior flavour. Synonym-Pitch lake.

The following varieties are of more recent date than those described above; viz.-

Smooth and Prickly Cayennes.-The first has few or no spines on the leaves; fruit large, cylindrical; average weight 4 to $8 \mathrm{lb}$; f flavour excellent; of rapid growth, and comes early into a fruiting state, swelling its fruit well during winter. The second has much the same appearance, excepting in being furnished with spines, and possesses most of the properties of the other, except in flavour, in which it is considerably inferior.

Prince Albert.-Much in the way of the $E_{n}$ ville, from which we believe it to be a seedling variety, superior, however, to it in swelling its fruit better during winter. A large and showy fruit.

Black Prince.-Fruit cylindrical; pips many, but with us not particularly well swelled; flavour inferior, although a handsome well-shaped fruit.

Comte de Paris.-One of several varieties of the Queen pine originated in France within these few years. Like the rest of its section, its merits depend on its cultivation.

Diseases and insects.-The pine may be said to be exempt from the former, and the latter are few in number compared with those of other fruit-bearing plants; and of these the Coccus bromelia Bouche (the pine-apple scale), and the Coccus adonidum Linn. (the mealy bug), are the most formidable. They are both too well known to require description. The remedies proposed for their destruction are many. The following, however, if properly applied, are the most effectual : $2 \mathrm{lb}$. of sulphur, $2 \mathrm{lb}$. soft-soap, $\frac{1}{2} \mathrm{lb}$. of tobacco, $2 \mathrm{oz}$. nux vomica, $1 \mathrm{oz}$. camphor dissolved in a wine-glass of spirit of turpentine, boiled together in 8 gallons of soft water for the space of one hour, and when the liquid has fallen to $120^{\circ}$ of heat, not lower, immerse each plant separately in it, roots and all, having previously shaken the soil entirely from them. After the plants have somewhat dried, re-pot them in fresh soil, and re-plunge them in entirely new fermenting material. The pit in which they are to be placed should have been previously emptied of any material in which they formerly had grown, and the walls have been whitewashed at least twice with caustic lime, the woodwork repainted; and previous to the 
pit being refilled with tan or leaves, pans of sulphur should be ignited in it, and the fumes kept closely shut up in it for twenty-four hours, which will completely destroy such of the insects as may have taken shelter in crevices of the walls, \&c. Syringing the plants with clean water, heated to $130^{\circ}$, has been found efficacious. At these temperatures the insect will be killed without the plants sustaining any apparent injury - a fact experimentally proving that a degree of heat and moisture sufficient to destroy animal life will not have a like effect on that of vegetables of so robust a nature as the pine. old plants infested with insects should, as soon as the fruit is cut, be thrown away; and if necessity compels the retention of suckers from them, let them be immersed, first in the morning and again in the afternoon, in the above mixture. The females of this genus are much larger than the males; they differ from them also in being stationary, as well as by far more numerous. When she once fixes upon a spot on the surface of the leaf, she seems disposed there to remain ; and when removed from the leaf, a number of eggs and young larvæ will be found immediately under her; for in this way she produces her young, being supposed to be both oviparous and viviparous. When the young are of a proper age they creep out from under her, and begin operations similar to those of their parent. It is highly proper, previous to immersing the young plants, to examine them carefully, and to displace all the scale found on them; for if once displaced their power of action is destroyed, and the young larvæ will not withstand the liquid in which they are placed, and the eggs will at the same time be washed away. A high and dry temperature is most favourable for their production. A high and humid temperature is that in which they most rarely appear.

The European names of the pine-apple areL'ananas, French-Ananas in Dutch, German, Italian, and Portuguese-Pina de Indias in Spanish. 


\section{CHAPTER XVI.}

\section{THE CUCUMBER AND MELON.}

\section{§ 1. -THE CUCUMBER.}

THE common or cultivated Cucumber (Cucumis sativus) has been cultivated in warm climates for upwards of 3000 years. It was largely cultivated in Egypt long before the exodus of the Israelites, as we find the want of it in the wilderness one of the grievances complained of to Moses. It is mentioned in a particular manner by several of the earliest Greek writers on plants, some of whom recommend that the seed should be steeped in milk and honey two days before sowing, that a sweeter and more pleasant flavour may be communicated to the fruit. Similar opinions, although sufficiently absurd, were held at a much more recent date, and even the great Lord Bacon countenanced such. Pliny informs us that they were much grown in Africa as well as in Italy in his time, and also that the Emperor Tiberius had them served at his table every day in the year. The means employed to effect this, he states, was to grow the plants in beds mounted on wheels, so that they might be removed from the open air into covered buildings during winter and on cold days. Columella, however, throws more light upon their early cultivation. He says: "Those who wish for them early should plant the seeds in well-dunged earth, put into osier baskets, that they may be carried out of the house and placed in warm situations when the weather is cold; and as soon as the season is advanced, the plants may be sunk in the earth with the baskets, or wheels may be put upon large vases, that they may be brought out and in with less labour." From an observation of the same writer we learn that they were grown in frames, covered with specularia, probably talc, or some transparent mineral which the Romans, even in his time, knew well how to split into thin laminæ, so that light might be transmitted through it. From the knowledge they also had of heating, (ride vol. i., INTRODUCTION), not only by flues but by hot water also, we are led to the belief that this fruit was pretty extensively forced during the days of Roman splendour. And coming nearer the point, he expressly says: "It was for Tiberius to show that cucumbers might be grown fere toto anno, which was done in frames filled with hot dung." Their introduction to Britain is stated, in the
"Hortus Britannicus," to have been in 1573 ; but, in "Gough's British Topography," cucumbers are stated to have been common in the time of Edward III.; and during the wars of the houses of York and Lancaster the plant was lost by neglect, and was re-introduced during the reign of Henry VIII. The first successful forcer of the cucumber in England was Fowler, gardener to Sir Nicholas Gould, who presented a brace of the fruit to George I. on the lst of January 1721.

Propagation. - The most usual mode is by seed, and also often by cuttings. The seed is often sown in shallow pans, and sometimes we sow it in pots, to avoid the necessity of shifting the plants when they have completed their cotyledon leaves, and in such cases the pots are crocked and filled about half full of soil; three or four seeds are dropped in, and covered about half an inch in depth. When the plants are up, they are thinned to one or two in each pot, and as they advance in height the pots are filled up with soil around them, and kept as near to the glass as possible, yet plunged in a genial bottomheat. During autumn and winter the glass of the roofs, whether of pits or frames, should be kept as clean as possible by frequent washing, so as to admit the greatest amount of light; for on this very much of our success must depend. Those who have not the convenience of pits or houses heated by hot water must have recourse to the old dung-bed process. The seed-bed, under such circumstances, need not exceed in size a one-light frame, and this should be prepared of well-fermented material by the first of January or beginning of February for ordinary spring and summer crops. If for cucumbers to fruit during winter, this bed should be prepared by the end of August, so that the plants may become established before the days shorten. By rearing the young plants in such a frame, they can be kept nearer the glass, while at the same time their roots are placed in a good bottom-heat, which is not always so readily effected in structures of a larger size. Indeed, it is questionable whether this is not the best plan for rearing both the melon and cucumber from seed at any season of the year, and until they are fit for planting out where they are to produce their fruit. After that period, however, no structure is worse adapted to them than the 
dung-bed in general use. Many propagate the cucumber by cuttings, and although the melon is capable of being so increased, it is more rarely practised. Cuttings of cucumbers are taken off from the tops of vigorous-bearing shoots; if for winter bearing, this operation commences about the middle or end of September, planting the cuttings, which should be from 5 to 6 inches in length, and always cut over close below a joint, in pots filled with rich mould, and plunged into a genial bottom-heat. If shaded and occasionally watered, they will strike root in a week, and be fit for planting out where they are to produce their fruit in six or eight days longer, and begin to produce fruit by the beginning or middle of December. The advantage of cuttings over plants reared from seed for winter work is, that they are much less succulent, and less liable to damp off during cloudy and damp weather. By this means also a favourite variety may be continued for years, whereas by seed they are liable to degenerate; and if more than one sort is grown in the same house or pit, it is scarcely possible to continue a variety pure, on account of their liability to sport by the one impregnating the other. Some object to plants originated by cuttings, and assert that they do not continue so long in a bearing state as those derived from seed; but if such plants be cut back when they begin to decline, and allowed to make a fresh supply of young wood, they may be continued in a bearing state for many successive months. When cucumbers are desired during December and January, sow the seed or put in cuttings during September; if in February and March, sow the seed or plant the cuttings in October; for spring and summer bearing, originate the plants in January.

Cultivation.-To secure a crop of cucumbers during winter, plants should be originated by the middle or end of August, continuing their propagation up to the middle of September, either by sowing seeds of approved sorts, for there are some that succeed better through the winter than others, or by cuttings taken off such sorts as may be still in a state of bearing, the tops of the shoots being chosen for the purpose. Plants from cuttings are less liable to grow so much to branches and leaves, and are also firmer in texture, and better able to endure the dark and damp of winter. In either case, the main object is to have the plants well rooted and short in the stem, and that of considerable thickness, or what are practically known as stout stocky plants. During their young state they should be kept near to the glass, which will prevent their being drawn up tall and slender. Under the head MELon we have endeavoured to point out the great advantage of cucumber-houses or capacious pits where the plants may be trained to a trellis about 15 inches under the glass roof, the fruit being allowed to grow in a suspended manner. A reference to vol. i., sect. MELon aNd Cucumber Houses, will show several structures adapted for this purpose. We carry on this operation in a tanked pit, the tank being of brick and cement, and 3 feet wide, divided in the centre by a row of bricks, set in cement, on edge, which forms a flow and return trough, the water being from 3 to 4 inches deep. This affords bottom heat, and atmosphere or top heat is obtained from a flow and return 4-inch iron pipe, communicating with the same boiler, with a valve so constructed that either top or bottom heat may be applied at pleasure, the top one of which is exactly level with the top of the water in the tank, which prevents the latter from overflowing. These pipes are placed between the tank and front parapet wall. The tank is covered with 2-inch Caithness pavement, closely jointed to prevent earthy matter getting into the water; and on top of this pavement are set wooden boxes, each 20 inches square and 10 inches deep, having no bottoms, as these would prevent the free ascent of the heat of the tank to the soil in which the plants grow. Over the pavement and within each box is placed about $1 \frac{1}{2}$ inches of broken crocks as drainage, and over that 3 inches of decayed cow-dung, and upon that the soil, which is light rich loam, vegetable mould, with a little sharp sand. When the soil is first placed in the boxes, it is gathered up in form of a mound, and on the top of this the plants are set, one generally in each box. As the roots extend through the sides of the hill or mound, they are covered to the depth of an inch or so with similar soil, kept within the pit to be of a proper temperature; as the roots extend, more and more soil is added until the box is full. The plants are not topped till they reach the trellis, at which time they are stopped to cause them to throw out lateral shoots. The boxes are placed about 18 inches apart, which admits of the heat in the tank rising into the pit, which is found of advantage in very cold weather. If more heat is desired at the roots, $1 \frac{1}{2}$-inch boards of the size of the spaces between the boxes can be placed over those parts of the tank, which prevents the ascent of heat at those parts, and consequently increases the heat in the tank, and also in the soil in which the plants are growing. We have, however, seldom to have recourse to this expedient, as we have abundance of bottom heat without. Before putting the soil in the boxes, we place in each a 2-inch drain-pipe, the bottom end of which rests on the crocks under the soil, and through this pipe the greater part of the water used during winter is poured, which waters the roots without wetting the soil on the surface, this being usually kept sufficiently damp by the condensation that falls from the glass roof, and directs the water to the points of the roots, the part where they naturally absorb it.

We prefer such boxes to covering the whole surface of the tank for winter forcing, as each of them is quite sufficient to maintain a plant as long as it continues in a fruit-bearing state, after which time it is thrown out, the box again refilled and planted; and this process goes on throughout the season, keeping up a supply of plants by sowing seed, or striking them from cuttings. These, however, planted out in October, continue till January, after which period young plants are readily produced to keep up a regular supply. Tepid water is alone used, both for watering at the roots and syringing over the 
foliage; but this latter is seldom required, as the atmosphere is kept sufficiently moist without; and were it otherwise, it would be better to throw the water upon the walls or floor to increase evaporation. Air is given as abundantly as the state of the weather will admit of, and where it can be brought in through the front wall, so as to blow upon the pipes, to raise its temperature before reaching the plants, so much the better. Air is a most important element in winter-forcing, a deficiency of it, next to a want of heat and light, the greatest of all evils; and it is in a great degree to the abundance of air and greater amount of light that cucumbers and melons enjoy in our modern structures over those low, dark, ill-ventilated pits or frames in former use, that our present success is due. During severe frosts, the apertures for the admission of air should be somewhat contracted, as the great difference between the external and internal temperatures causes a rapid circulation, as the cold air forces its way through every creek or cranny; for however tight the glass roofs may be made, still there are ways and means by which cold air will find its way in, and more so during very low temperatures, as the pressure from without becomes greater.

In former times, great importance was attached to the process of fertilising the female flowers. When seed is the object of culture, this no doubt is a very indispensable matter; but as it happens that cucumbers are prized more when devoid of seed than when they are full of them, the process has become questionable, particularly in the case of winter-grown fruit, or that produced by early forcing. This opinion is entertained by Mr W. P. Ayres, who, in his "Treatise on the Cucumber," p. 16, entirely dissents from the practice, which he declares to be not only unnecessary, but even injurious. "It is well known," he says, "that cucumbers having seed in them are much less valued than those which have not; and hence the long-growing kinds, which seldom produce seed even for their own propagation, are preferred." He offers the following reasons on this subject: "For my part, I am disposed to think, where long fruit is desired, impregnation is positively injurious, because, if seeds are the result of impregnation, the energy of the plant will be expended in perfecting the seed, instead of in the production of the fruit, as every practical man knows that the production of one seed from it will weaken the plant more than a dozen of fruit fit for the table." Cucumbers having seed in them are more apt to become crooked and deformed than those which have none, particularly such as have a few seeds formed near their lower ends. In the case of the melon it is different, as its fruit is useless until it has become fully ripened; and to acquire that maturity, it is necessary that it should be perfect in all respects, and this it would not be were seed awanting. The melon does not set its fruit with the same freedom as the cucumber; and hence impregnation is a necessary operation, either naturally or artificially. The cucumber, on the other hand, is useless for the pur- poses for which it is grown when it arrives at anything near its state of ripeness, and is most fit for use, comparatively speaking, when it is only a few days old.

Much of the success attending the production of the melon and cucumber depends on the structure in which they are grown: for ourselves, we greatly prefer large span-roofed pits or houses, training the plants all over the roof in the manner of vines; and such pits or houses, when so occupied, have a very novel and interesting appearance. The fruit also is produced in far greater perfection, and the plants will not only bear more profusely, but will continue a much greater length of time in a bearing state, which is, no doubt, owing to the greater circulation of air and exposure to light than when grown in pits in the usual manner.

The practice of Mr Cuthill, one of the most successful growers for the London market, is deserving of imitation, and is within the reach of every one having no more accommodation than a three-light cucumber pit or frame; we will give the substance of his practice, which is equally applicable during winter to pits or houses of the largest size. The seed of the cucumber is sown in a small pit sufficiently heated in pars 2 inches deep, the seed being previously steeped twenty-four hours in tepid water. When the cotyledon or seed-leaves have separated, the plants are potted off into 4 -inch pots. Two plants are put into each pot, and no water is given for three or four days, in order to prevent the stems from damping off. "Air is given day and night at the back of the pits, and the heat is kept up at night to $65^{\circ}$, and by day to $70^{\circ}$. 'The plants are shifted round every three or four days, and are watered, when required, with soft tepid water. As the plants only remain in the seedbed," which, in small establishments, may be a one-light box, or in default of this they may be reared in the pit in which they are afterwards to be grown, "for about three weeks, the larger pit should be prepared for their reception by the expiration of that time. This is effected by lime-washing their insides for the destruction of insects, \&c.; the hot-water pipes which traverse the middle of each pit at the bottom, for bottom heat, are covered with a layer of oak faggots, putting some rough dung or straw over them to keep the mould from mixing with the faggots. The mould, rough from the fields, is put in so as to form a ridge in the centre of the pit to the depth of 16 inches, leaving the top of the mould about 2 feet from the trellis. The fire is lighted at least six days before the plants are put out, there being forty-eight lights to one fire, consequently much cold water and mould to heat. As soon as the mould is heated through, the plants are planted out immediately under the centre of each light, inserting them in the mould up to the seed-leaf, and placing a stick to each plant reaching the trellis. The latter is formed by a strong stick under each rafter, and by hazel rods about 4 feet long placed 5 or 6 inches apart." In such pits we screw iron studs 12 inches long into the rafters, and by running twine through their eyes construct a convenient trellis, creating less shade, and capable of being 
cut in pieces, should such become necessary in arranging the branches while pruning. "As soon as the plants reach the trellis their heads are nipped off. The plants are now frequently watered with diluted manure-water in a tepid state, and the atmosphere is kept humid by placing zinc saddles filled with water on the top of the hot-water pipes" that heat the atmosphere of the pits. "Plenty of air is given, and the heat is kept up by day to $70^{\circ}$, and by night to $65^{\circ}$." The shoot is, in Mr Cuthill's practice, " pinched off at the fruit, and not at the joint above it, as is the practice with many; and by this mode the frame does not get so full of useless branches. The male blossoms are kept off at all times, and after the plants come into hard bearing, every encouragement must be given them, administering liquid manure freely." By this process $\mathrm{Mr}$ Cuthill keeps his plants in a healthy bearing state till September, cutting fruit during the whole summer, and afterwards ripening the seed; for the seed of cucumbers is in great demand, some seed-houses holding a stock of from half a ton to a ton; besides, Mr Cuthill grows for sale a peculiar black-spined variety, which we have found a most productive bearer. Water is given liberally during summer, particularly during bright sunshine and while the plants are in full bearing, to the extent, he informs us, of about four gallons per week to each light. "I never," he says, " in the height of summer, use cold water, for I am persuaded that half the diseases in cucumbers arise from watering with cold water from tanks not sufficiently open to the sun and air." He places much importance on the admission of air, but remarks : "Never give air at the front of the pits, for this reason, the moment you admit air in front and back a dry hot current is produced, which, in cucumbergrowing, above all things, should be avoided; but if air be given at back only, circulation of heated air will be maintained. And be it remembered, the older the plants the more heat they require." These excellent directions are admirably adapted to cucumbers grown in large houses as well as in pits, and if followed cannot fail of producing abundant crops.

In former times, when the cucumber was trained close to the soil in which it was grown, picking out the eye or stopping at the first joint, as practically understood, was held as good practice; and so, no doubt, it was, when lateral branches were desired, and those proceeding from near the root or centre of the plant were deemed the best, and most capable of being trained equally over the bed in which the roots were placed. And so, no doubt, it is still with cucumbers grown on ridges or under handglasses, in what may be called out-of-door culture; for, as Abercrombie remarks, it " is necessary to promote a strong stocky growth and an emission of fruitful laterals, and from these other prolific runners will be successively produced. The vines," he continues, "without the process of stopping, would generally be both weaker and so deficient in fertile runners that they would sometimes extend 2 or 3 feet without showing fruit." The case is now different, and this primary stopping is disregarded, the leading shoot being trained upwards until it reach the trellis to which its lateral branches are to be trained. At such time stopping becomes imperative for the production of lateral shoots proceeding in all directions.

Nicol was amongst the first to point out the difficulty of cultivating the cucumber during winter in pits heated by fermentation. "Those who would have cucumbers at Christmas," he says (a thing sometimes attempted), "will find it more practicable and less troublesome if the plants be grown in a flued pit than if they grew on a common hotbed." And M'Phail, writing about the same time, observes, "To cultivate cucumbers on a dung-bed, the fruit is sometimes watery and ill-tasted." And the same authority afterwards remarks, "Cucumber plants will grow in a hothouse where the pine-apple is cultivated, but they will not be very long-lived there." He recommends to sow the seed in August, in boxes filled with vegetable or other light earth, and to place them on shelves in the back side of the hothouse, where the sun may shine on them in the short days. They may, perhaps, produce a few fruit in the months of December or January. In the absence of a regular cucumber pit or house, the pine-stove is still the most suitable place for them; and if placed as described above, but much better if over a fiue or hot-water pipe, by which their roots would be kept at a proper temperature, good crops may fairly be expected. With a properly constructed cucumber - house, cucumbers may be obtained in great perfection during the whole winter. The same cannot be expected at anything like a remunerative cost by continuing their cultivation in dung pits and frames. We are quite aware that such things have been accomplished, but the utmost amount of success bore no proportion to the cost and risk compared with the certainty and economy of growing them in a well-appointed cucumberhouse.

Open-air culture.-The cucumber is grown to a large extent in the south of England as an open-air crop, very rarely so in Scotland, both on account of want of climate and a want of taste amongst her people for so refreshing a vegetable. The usual practice is to raise the plants from seed sown in a cucumber or melon pit about the middle of April. When the plants have made their cotyledon or seed leaves, they are potted off into 4-inch pots, setting three or four plants in each. By the middle of May a pit is dug 2 feet deep, 3 feet broad, and of a length corresponding with the number of plants to be planted out. The warmest situation in the garden is selected for this purpose, and the plants have their tops shortened at the second or third joint. The trench is then filled with warm stable-yard manure in a high state of fermentation, and covered over to the depth of 10 or 12 inches with rich light mould, into which they are planted as soon as the soil has become sufficiently warmed, and are then covered with hand-glasses set about a yard asunder from each other. After they have begun to grow, the glasses are raised a little every fine day for the admission of air, and as the weather gets warmer 
more air is admitted, and at last the shoots are allowed to extend from under the glasses, and trained horizontally close to the ground. The glasses are, however, kept over the centre of the plant until they are fully established, and the warmth of the weather renders their protection no longer necessary. Should the branches appear too thin, their tops are pinched out, which causes the emission of numerous others; yet care must be taken that these become not too crowded, so as to cause confusion and undue shade. In this way the plants in good seasons will continue to produce for the space of about two months. Were they trained over flints, slates, bricks, or other heat-absorbing bodies, no doubt their productiveness would be greater, and their season prolonged. The heat reflected from such bodies would warm the air about the plants, and keep them at the same timefrom being in close contact with the cold ground. Temporary frames covered with glasses would still further aid their growth, and insure a better crop.

The minimum temperature the cucumber will bear without injury is $55^{\circ}$, the medium $75^{\circ}$, the heat in the mould being $80^{\circ}$; but in clear sunshine, with ample ventilation, an atmospheric temperature of from $80^{\circ}$ to $90^{\circ}$ will not be too much.

Saving seed.-For the production of cucumber-seed a fruit or two of the earliest forced crop should be left, as seed from these is found to vegetate and produce fruit in much less time than those that are produced later in the season, and much more so than those that are grown under hand-glasses or in the open air. Some also attach importance to the part of the plant that produces the fruit, preferring the fruit that is grown nearest the root. The finest specimens seldom produce seed, while inferior ones do; and fruit, it is asserted, produced on the first, second, or third joints, do produce seed, while in nineteen cases out of twenty, fruit produced on joints beyond these limits produce no seed in the higher-bred varieties, and hence seed of them is often difficult to procure in the shops. This, however, does not apply to the ordinary shortgrowing sorts, which seed abundantly on all parts of the plant. Some high in the cucumber fancy save seed from the first good fruit set on the plant. They should remain on the plant until thoroughly ripened, which will be indicated by the fruit-stalk withering, and the skin of the fruit attaining its full yellow colour. When cut, keep them still in a warm place until they begin to decay, when the seed should be scraped out of them into a vessel of water, and kept there until the pulp attached to the seed begins to separate from it, after which they should be washed in clean water, during which process the lighter and abortive seeds will float on the surface, and may be thrown away. After five or six days' drying in the sun they are fit for packing by for future use. A good deal of importance is placed on the age of both melon and cucumber seed, those under two or three years being apt to grow too luxuriantly, requiring much pruning, and being less productive of flowers and fruit than those of a greater age. Indeed, seeds of both vegetate freely, if properly kept, at the end of four, six, or even twenty years. For the production of melon-seed, those from the finest specimens and of highest flavour should be kept, and treated the same as above.

\section{SELECT LIST}

Cucumbers, from their natural proneness to impregnate each other when grown together, are exceedingly difficult to keep true to their original points of merit; and as they are so liable to sport into varieties, we need not be surprised at the long lists of names with which our seed-lists abound. The leading varieties in present estimation are the following :-

Cuthill's black spine, 6 to 9 inches long, hardy, early, and productive.

Early short green prickly, 4 inches; hardy, productive, and excellent for pickling ; quick in coming into production.

Early long green prickly, 7 inches; hardy and productive; a good market sort.

Victory of Bath, 17 in.

Tiley's captivation, 18 in.

Tiley's phenomenon, 18 in.

Good for general crops.

Hunter's prolific, 18 in.; very crisp and sweet, requiring more heat than most other sorts; spines white, covered with a good bloom, and not apt to turn yellow at the base.

Lord Kenyon's favourite, 12 to 18 in. long; excellent for winter cultivation.

Early green cluster, 6 in.; hardy, prolific, and comes early into bearing. It is chiefly characterised by its fruit growing in clusters; well adapted for hand-glasses.

Flannigan's, 15 inches; a good forcing variety, of upwards of thirty years' standing.

*Brownston's hybrid, 15 in.

*Victory of England, 21 in., excellent for early forcing.

Kilway's victory, 18 in.

Conqueror of the west, 18 to 20 in.

Holm Pierpoint wonder, 18 in.

Snow's prize, 18 in.

Allen's victory of Suffolk, 24 in.

Prize-fighter, 16 in.

Duncan's victory, 28 in.

* Ringleader, 15 in.

*Pratt's hybrid, 18 in.

*Sion House, 9 in.; an old and well-tried winter forcing variety. Of this there is an improved variety.

Sussex hero, 18 in.

Roman emperor, 12 to 15 in.

Hamilton's black spine, 12 to 15 in.

Gordon's white spine, 12 to 14 in.

It would be to little purpose to describe cucumbers further than as we have done above, on account of the great uncertainty which must ever exist in maintaining any one variety perfectly true for two consecutive years. No doubt, if people were to cultivate only one variety in a garden, there might be some chance of continuing it true for a longer period ; but when several, and often many, are grown in the same garden, and often in the same house or pit, the thing 
seems impossible. If only really good varieties were to be grown, this crossing might be of less consequence, as the chances are that varieties may appear equal to the parents. It is, however, most desirable to exclude all doubtful sorts, for one bad variety may greatly deteriorate the whole stock.

Insects and diseases.--The same insects infest the cucumber as the melon (which see). The diseases are mildew, canker, gumming, and malformation of the fruit. The fruit, when grown in too low a temperature, often becomes so bitter as to be uneatable; and such is the case in cold seasons with many that are grown in the open fields and gardens. This bitterness arises from an imperfect elaboration of the juices; those in the neck part of the fruit being least digested, it is always the most bitter part. Deformity in the fruit depends on insufficient nourishment or over-cropping, and points out its own remedy. Gumming and canker arise often from a deficiency of heat in the beds, with excess of water, particularly if applied in a cold state. It is one of the most fatal, and if not taken in time destroys the fruit, and often the plant also. It is believed by some to be infectious, but this is not the case ; a drier atmosphereand increased temperature are the principal remedies; and if taken in time the ulceration may be greatly arrested by applying caustic lime or charcoal in a powdered state to the parts as soon as the spot appears. Mildew is mainly caused by too dry an atmosphere, and may be subdued by frequent applications of sulphur applied by the sulphurating machine in a dry state. Neither the melon nor cucumber is at all liable to disease, if kept in a vigorous-growing state; but when, from injudicious treatment, disease of any kind appears, an alteration of that treatment must be instantly set about. It is seldom that either of these diseases attacks the plants when grown in properly constructed houses or pits, having the branches trained to a trellis under the glass; but when they are grown in close, damp, ill-ventilated pits or frames, and their roots permitted to extend into the dungbed below, either, if not both, of these diseases is Jikely to ensue.

The European names are-Concombre in French-Gurke in German-Komkommer in Dutch-Pepino in Spanish and PortugueseCetriuolo in Italian-Kira, Indian-Ogorek in Polish-Ogurzi in Russian.

\section{§ 2.-THE MEION.}

The Melon (Cucumis melo), or Musk melon, and Cucumis citrullus, the Water melon, have been cultivated from the earliest ages; and although the former is stated in the "Hortus Britannica" to be a native of the East Indies, and the latter of the south of Europe, both have been so long in cultivation in all warm climates that it is quite doubtful from what country either of them originally came. The date of the introduction of the Musk melon into Britain is set down at 1570 , and of the water melon 1597. The Musk melon, a name now nearly obsolete, was brought to this country from
Jamaica, the Cantaleups from the neighbourhood of Rome, the Romanas from Italy-also differing from the Cantaleups in being larger, oval-shaped, netted on the surface, with larger foliage, and more abundant bearers. From these two sections most of the varieties long cultivated in Britain originated, in consequence of their being often grown together in the same frame, and hence the one became crossed with the other, producing almost innumerable varieties, many of them for a time maintaining a degree of popularity; but, like all annual fruits in general cultivation, they have been continually changing, so that the sorts that were held in the greatest esteem, even within our own recollection, are now only historically known. Melons are at present divided into four pretty distinct sections; namely, Cantaleups and Romanaswhich includes the Rocks, the Scarlet and Green fleshed varieties, the type of all of which was probably the old Musk melon - the Winter melons, Persian melons, and, lastly, the Water melons, which latter is a distinct species.

Cultivation.-The melon was long cultivated, like its near congener the cucumber, on beds of fermenting material, a process attended with vast labour, care, expense, and waste of manure. Although many gardeners managed, by these rude and very uncertain means, to cut cucumbers through the winter and very early in spring, few, if any, attempted the ripening of the melon till a more advanced period of the season. A great improvement took place in the culture of this esteemed fruit, when men began to think of growing them trained to trellises at a considerable distance from the soil they grow in, so that the fruit might hang suspended, and derive a full share of sun, light, and heat, placing the fruit in a far better position to ripen on all sides alike than when lying on the damp ground with probably the indulgence of a piece of slate or broken flower-pot under it-a position the worst of all others for allowing full maturity to take place on all sides; and hence that portion which lay undermost was always deficient in colour, shape, or flavour. Not so with melons grown suspended from the roof of a house or pit; and hence capacious houses have been since erected for the growth of both melons and cucumbers, and pits variously modified and enlarged for a like purpose. In vol. i., section MELON and CUCUMBER Houses, several examples will be seen. Fig. 640, which is one of those in use in the fine gardens at Trentham, and in which Mr Fleming has so successfully grown his celebrated hybrid Ispahan varieties, may be referred to as an economical and good example. This melon is well known and esteemed, not only for its superior flavour, but also for its productiveness, one set of plants being sufficient to produce fruit throughout the season. From this pit ripe fruit has been cut at the unusually early period of the middle of April, and the last at the end of October, a period of nearly six months, the same plants continuing all that time in bearing. The cucumber and melon houses at Poltalloch, fig. 652, afford a specimen of our own ideas of what such houses should be, and, together with the excellent houses in the. Royal Gardens at Frogmore, 
fig. 658, may be considered all that can be desired upon a large scale. Davidson's cucumber-house, fig. 655, and Ayres', fig. 657, will meet the requirements of smaller establishments. Whoever has seen the beautiful narrow curvilinear melon-houses in the Viceroyal Gardens at Dublin, where the roofs are literally covered with fruit of great excellence, and in all stages of growth, will at once admit the great advance made in this department of culture. Melons are seldom as yet cultivated so as to fruit during the winter months; the reason hitherto has been, the want of proper accommodation, and the almost universal cultivation of thick-skinned varieties, like the Rocks, \&c., requiring the full effects of our " $\mathrm{dog}$-days' sun," to penetrate a skin so formidable, and to render the pulp within it eatable. Both these obstacles are now greatly overcome-viz., eligible structures are prepared for them, and varieties whose skin is not much thicker than that of a russet-apple are in very great repute, and are not now uncommon. Of the possibility, therefore, of producing the melon during winter, or at least continuing it much later, and producing it much earlier in the season, there is no doubt. That the melon, from want of sufficient sun during the dark months of autumn and early spring, will ever be inferior in flavour to those ripened during the bright days of summer, is sufficiently obvious; the difference, however, would not be much greater than that between a pineapple ripened at Christmas and another in $\mathrm{Au}$ gust; yet a pine at the former period is, apart from direct flavour, as valuable and as much prized as one at the later season. There is a useful section of this fruit called Winter melons, of which the Valentia, a well-known sort, is one, and the Dampsha is another. These will keep a long time after they are cut-say from the end of October to the middle of December-if only hung up by the stalk in a net in a dark cool room. And these might be grown so as to ripen at even a later period, while Fleming's hybrid Ispahan varieties could be ripened by the middle of March.

Propagation.-This is most usually by sowing seed, although some do so by cuttings; and certainly, when several sorts are grown in the same structure, and there is a desire to continue the variety pure and unchanged, the latter mode is the best. In propagating by seed, a bottom heat of from $75^{\circ}$ to $85^{\circ}$ is essential; and seed of more than two or three years' keeping is preferred, as the plants from this do not grow so much to wood as plants from younger seed do. The seed should be sown in shallow pans instead of in ordinary pots, as the roots coming in contact with the bottom of the pan extend horizontally instead of perpendicularly, and hence become better furnished with fibres. The soil should be one-half rich loam and one-half vegetable mould, and the atmospheric temperature of the seed-bed should range from $70^{\circ}$ to $80^{\circ}$, which must be kept up, notwithstanding there is a little ventilation during day and night. When potting takes place, which should be when the two cotyledon leaves are nearly formed, two plants should be placed in each 5-inch pot, VOL. II. and the soil should be dry, and brought up to the temperature of that the seedling plants have been growing in. Do not set the plants deep in the soil, but when the pot is about three parts filled, set the roots upon it, and merely cover them at first, allowing them to recline on the inner edges of the pots: in a day or two afterwards, add a little more dry warm soil over the roots, and so on till the pot be filled, giving water in moderation, for the melon in its young state is very liable to damp off, particularly if much vapour exist in the pit at an early period of the season. Melons sown on the first of January, which is early, under the dung-bed system, will require six weeks' nursing in the seed-bed before planting out. Those sown about the first of February will require five weeks, and those about the same period in March from three to four weeks, the nursing period decreasing with the season. Those who would attempt fruiting melons during winter, should have their plants established in the fruiting house or pit by the middle of August, or cultivate some of the sorts so successfully grown by Mr Fleming, and referred to at p. 672, which, under good management, may produce fruit during winter. Mr Cuthill, in speaking of melons sown on the first of February, which is a good time for an early crop, remarks in his "Practical Treatise on the Melon," p. 38 : "Your plants have by this time been once stopped, say 5th March, and the fruiting pit or frame got ready; the hills ought to be 15 or 18 inches from the glass; the plants thrive much better on account of the great body of air ; the first hills ought to be 1 foot high, composed of half loam, the other half leaf or any other light mould; no dung mixed with the hills. In planting out, let one plant take the back, the other the front of the pit or frame; watering depends on the state of things at the time. In the course of a short time they will want another stopping. As the roots appear, lead them down the hills by covering them with a little mould, adding at the same time about 2 inches of mould over the surface of the bed; and as the roots cross the bed, when they make their appearance they must be covered with mould, kept in pots in the pit on purpose, and continue doing so until the fruit be all set, but after that time they must not be disturbed, neither must the bed be over-moulded. When finished, the hills ought to be 4 inches higher than the surrounding mould; 6 or 8 inches," Mr C. consider's, "is quite enough for very early melons. Allow three or four runners from each plant, and when they grow to within 6 or 8 inches of the sides of the frame, stop them. They in a few days put out their side shoots with fruit in abundance. Stop them one joint beyond the fruit upon each plant. If one or two be wanted very early, cover the fruit with sand or dry mould. After it gets the size of a walnut, you may plunge a thermometer if you want to know the heat, when it will be found the fruit is swelling rapidly in a bottom heat of about $90^{\circ}$, or $15^{\circ}$ more than the atmosphere of the pit ought to be kept at. Syringe a great deal over head and round the flues with diluted 
dung-water; but as soon as the melons are nearly full grown, water should be withheld altogether." Mr Cuthill is no great advocate for much dung in his melon soil, neither does he like it over stiff, or the mould to be hard trodden. This is quite in accordance with the practice of the best melon-growers on the Continent, and diametrically opposite to that very generally practised in Britain. The variety grown by this excellent cultivator is one called Cuthill's early scarlet flesh, by far, he says, the hardiest and best for an amateur, and the only one now grown for the first crops for Covent Garden. By following the above rather unique practice, he tells us that he has cut melons for four years from the 5th to the 10th of May, and has set fruit on the 5th April, and cut the melons on the 5 th of May.

Melons produced by the 5 th of May is exceedingly good work; nevertheless, as we have already stated, $\mathrm{Mr}$ Fleming has produced them by the middle of April, and we have no doubt that in properly-constructed light and airy houses they may be produced even earlier. Heat, air, and moisture we have now completely under our control in all properly constructed plant-houses; but the fourth great and indispensable element, light, is much less at our command; and it is the absence of light that renders the cultivation of both the melon and cucumber so difficult a matter during winter and early spring. Their being annuals, and, therefore, unlike the peach or the vine, capable of being originated from seed at the will of the cultivator, and, so far as the first process of germination goes, of being made to vegetate at any day in the year, the absence of sufficient light alone prevents these plants from attaining their full development, and consequently the fruit also. Propagating the melon by cuttings is had recourse to when new seed has been sown, the plants from which, having a tendency to grow too vigorous, are restrained if their tops be taken off and converted into cuttings. Cuttings are also used when any peculiarly fine sort is wished to be continued without the risk of contamination with adjoining sorts. Melon plants thus produced require little stopping or pruning, but they also require a more generous and rich soil when planted out.

Impregnation of the blossoms is an imperative process in melon-growing; the female flowers are distinguishable from the males by the appearance of the embryo fruit at their base. The best situated of those should be selected, and by carefully divesting the male blossom of its corolla, its farina should be brought into contact with the centre of the female flower, and that from twelve to two o'clock in the day, when the flowers are quite dry and the sun at its greatest power. The operation should commence when from six to ten female flowers are formed on the plant; and when it is perceived that four fruit is secured on each plant, which will be known by the rapid swelling of the embryo fruit, the whole of the blossoms, male and female, should be cut off as they appear, that the whole strength of the plant may be directed entirely to the fruit. The production of un- necessary blossoms weakens the plant as much as the production of the fruit. The shoots on which the fruit is set and swelling, should be pinched off three or four eyes above the fruit, stopping all lateral shoots from time to time to prevent confusion, as well as cutting out all coarse shoots which are not wanted. The greatest care should be taken of the foliage, exposing it as much as possible to the sun, for no plant dislikes shade more than the melon. Watering is an important matter, both as regards the roots and foliage; weak liquidmanure should be applied to the former, and that more especially when the plants are grown in pots, boxes, or over tanks or vaults heated by hot water. In the case of such as are grown on dung-beds this is less necessary, as the roots, passing through the bed of soil, enter into and luxuriate amongst the manure the bed is composed of. The foliage should be watered with clear rain-water administered at all times from $3^{\circ}$ to $5^{\circ}$ higher than the temperature of the air in the pit or house; for although the melon does not require so humid an atmosphere to grow in as the cucumber, still the great drain made on the plant by the fruit renders it necessary that sufficient aqueous food be supplied it. Water at the roots is most required during hot sunny weather, and while the fruit is swelling. In watering, let it be applied towards the extremities of the roots, for there the absorbing spongiolets exist, and keep the centre of the plant rather dry than otherwise. Over much water there, particularly in the case of the weaker and often finer varieties, has a tendency, particularly very early or very late in the season, to induce a species of damp, which, if not guarded against, often rots off the main stems. When water is applied to the foliage, it should be by means of a very fine syringe, more resembling a fine copious dew than the heavy shower-baths often inflicted upon them with a coarse watering-pot rose. In properly constructed melon-houses, the requisite amount of humidity can be supplied to the foliage without the use of the syringe at all. This holds good in a very essential degree in the case of the Persian melons, which are not only our best sorts, but also our most tender ones. They are found to require a much higher temperature, a much drier atmosphere, and a much more humid soil, than those of other countries. In Persia, where they are grown as an open-air crop, the melon-grounds approximate nearly to our English water-meadows: water in a highly enriched state is carried along the higher levels, and made to irrigate the soil copiously, while the plants themselves, elevated on raised beds, are exposed to the full influence of a powerful sun,-from which those who wish to cultivate these fine melons will understand, that a rich soil, dry on the surface, but rather moist below, a high atmospheric temperature, with abundant ventilation day and night, are important essentials ; and therefore, to secure this in artificial culture, we must have structures covered with the brightest glass, so as to admit every possible ray of sunlight, a competent bottom heat, and a surface heat of from $85^{\circ}$ to $95^{\circ}$. Such cannot 
be provided in pits or dung-bed frames of the ordinary construction, and can only be secured in span-roofed houses, or pits covered with glass to within a foot or two of the ground surface. It is also of importance, in the case of Persian melons, that they be grown in the suspended manner, so that the light and sunshine may act to their fullest extent on both foliage and fruit. They differ, also, in a remarkable manner from those generally grown in Europe, being destitute of the thick rind which renders one-half of each fruit useless, while they have their skin so thin and delicate that they are subject to injury from causes that would not affect those in ordinary cultivation. Their flesh is also extremely tender, rich, and sweet, abounding in high-flavoured juice, exceedingly grateful and refreshing. For the most part they are abundant bearers, as are also the hybrids originated in Britain by crossing one variety with another; and their beauty when ripe is as pleasing as their flavour is agreeable. Water melons form another section: although believed to be those mentioned in Sacred History, and constituting almost both the food and drink of the Egyptians for several months in the year, they are little regarded in Britain. In Italy, America, and the south of Europe, they are much grown and esteemed; their culture differs little from those in ordinary use, excepting that they require more space for the extension of their branches, and the greatest amount of light that our means will enable us to give them.

Where the practice is followed of growing the melon on the surface of the soil, care should be taken that a piece of tile or slate be placed under every fruit as soon as it has begun to swell, to prevent its damping off; and as the fruit increases in size, it should be elevated so high as to be above the leaves, that it may fully enjoy the effects of light and air. Until the full complement of fruit is fairly set, and beginning to swell, the branches should be kept thin, and the plants not over-excited; but after that stage they should be allowed a greater latitude, and a greater amount of exciternent. Those grown suspended from trellises, in properly constructed houses, need not be placed above the foliage, but allowed to hang under it, because, in this latter case, abundance of air and light will reach them. The period which elapses between the fecundation of the blossom and the ripening of the fruit varies in different sorts; from five to six weeks may be taken as an average, although some cultivators require less time. This also depends on the season of the year at which the process is going on, and also on the state of the plants; for if they be stinted from mismanagement, or from an over-crop, the fruit will ripen prematurely in less time than stated above, and before it has attained its full size. The ripening of the fruit may be known by the following criteria: by the base of the foot-stalk cracking away from the rind, by the agreeable and powerful perfume of the fruit, and by its turning of a yellowish colour. The first, however, of these is most to be depended on, as it not only indicates ripeness, but also that the fruit has been grown to its most perfect state, for such indication is not found in fruit ripened prematurely through mismanagement. The Winter melons do not, however, show this cracking away of the footstalk ; they are cut when they begin to give out a slight perfume, and when they have attained their full size.

Growing the melon in the open air is seldom practised with success in Britain, although some of the hardiest and quickest-growing sorts are sometimes ripened in the south of England under the protection of hand-glasses like cucumbers, and under glass coverings in pits or frames, with a slight degree of bottom heat placed under them at planting. The plants are treated as in other cases as to transplanting, \&c., and should be grown to a pretty large size before being finally planted out. Sometimes they are planted in small wicker baskets, made on purpose; and as a considerable number of plants may in this way be kept in a heated two or three light frame until they have begun to form their flowers, the plan is worth the attention of those who have no better accommodation. The plants are set in the place where they are to perfect their fruit, their roots finding a ready means of escape through the bottom and sides of the baskets, and that without experiencing any material check in their growth; thus, as it were, gaining three or four weeks in their growth, and enabling us to delay their final planting out until the season has become sufficiently advanced to secure them from being injured by late spring-frosts. The practice of many of the London market-gardeners is to prepare the beds on which early potatoes, \&c. have been produced, by digging out holes along the centre of the beds, each sufficiently large to hold a wheel-barrowful of well-fermented manure; and as soon as the mould over it is moderately heated, the plants are set, and closely covered with hand-glasses for a few days. After the branches have extended as far as the limits of such glasses, they are removed, and covered with any spare sashes that may have been in use for forwarding other crops, now either cut or fit to stand the weather without such protection. In the cultivation of melons with diminished heat, little water should be given them, and that always in a tepid state, and only applied towards the extremities of the roots, keeping the crown of the plants rather dry than otherwise.

\section{SELECT LIST.}

Beechwood,green-fleshed.-Of excellent flavour, and an abundant bearer; 2 to $3 \mathrm{lb}$. in weight.

Bromham Hall.-A comparatively new and excellent green-fleshed sort; 2 to $3 \mathrm{lb}$.

Trentham hybrid, green-fleshed. - Equally worthy of cultivation, and one of the easiest culture.

Cuthill's early cantaleup. - Hardy, and an early bearer; 2 to $3 \mathrm{lb}$.

Green-fleshed Masulipatam.-Skin thin, green, early, and of excellent quality; 1 to $2 \mathrm{lb}$.

Green-fleshed Egyptian.-Skin thin, white, early, and of excellent quality; from 2 to $3 \mathrm{lb}$. 
Hardy ridge.-Skin thick, yellow; flesh red; a good bearer, although late in ripening; from 3 to $4 \mathrm{lb}$.

The above are of moderate size, and quite sufficient in variety for any family. Those who wish for very large fruit, may grow the old black rock, which often attains the weight of $14 \mathrm{lb}$, three parts of which is composed of uneatable skin, carbuncles, and seeds, and of no particular good flavour; or if even larger be desired, the French and some of the Italian sorts may be grown, whose properties, in this country, are little better than gourds.

Of Winter melons, the

Green Valencia, although not rich in flavour, is firm, saccharine, and juicy; and upon the whole, if fully ripened, better than many of the summer varieties grown.

Dampsha.-The flesh of which is dark-green near the skin, rather whitish towards the centre, quite melting, and of excellent flavour. The first-produced fruit in the season is somewhat cylindrical, bluntly pointed at both ends, the whole surface being prominently netted, and of a pale yellow or dark olive colour. The secondary crop has the fruit more pointed and less netted, and the skin becomes much darker. The great merit of this section is, that they keep for a month or two if suspended in a net in a dry room; 4 to $5 \mathrm{lb}$.

De la Moree.-Skin thin, yellow; flesh greenish white; a winter melon of great merit; from 4 to $5 \mathrm{lb}$.

The Persian melons are with us fewer in number; but of those, hybrids are now produced of great excellence, the principal of which is the Dampsha, already noticed; and the following ought to be cultivated in preference to many others :-

The Sweet melon of Ispahan. - With ovate fruit from 8 to 12 inches in length; skin of a deep sulphur colour, nearly smooth; flesh white, crisp, sugary, and very rich. Of this excellent sort some interesting varieties have been raised by $\mathrm{Mr}$ Fleming, possessing merits deserving of very general cultivation; 6 to $10 \mathrm{lb}$.

Geree, or Ostrich Egg.-A green fruit of an elliptical form, in general 8 inches long, and $2 \frac{1}{2}$ inches in diameter; skin mottled with dark seagreen upon a pale ground, sometimes netted, at others not. The closeness of the reticulations in a great measure distinguishes this from the Darce. Flesh nearly 2 inches thick, bright green, melting, very sweet, and high flavoured; bears abundantly, but is rather tender. An excellent sort for a melon-house of the description already noticed; 4 to $5 \mathrm{lb}$.

Cassabar.-This is also one of the tender Persian varieties, which requires a brighter sun than we often have in England, much less in gloomy Scotland, in which latter country it seldom does well; 4 to $5 \mathrm{lb}$.

Melon of Keiseng. - A very beautiful eggshaped fruit, 8 inches long and 5 inches in diameter; skin pale-lenıon colour, regularly netted all over, with a few cracks lengthways; flesh about 2 inches thick, nearly white, abounding in a cool, delicate, and highly flavoured juice; 4 to $5 \mathrm{lb}$.
Green Hoosainee.-Fruit egg-shaped, in general 5 inches long, and 4 inches in diameter; skin, when matured, light green, with regularly netted surface, yellowish on the side most exposed to the sun; flesh pale greenish white, full of a pleasant sweet juice; skin thin; seeds unusually large. One of the hardiest of the Persian melons, except the next, and withal a free and abundant bearer. Of this there are several varieties, but all of superior merit; 3 to $4 \mathrm{lb}$.

Large Germek.-A large, handsome, ribbed fruit, from 7 to 9 inches in length, and usually 6 inches in diameter, having the distinguishing mark of a corona or circular scar from 1 to 2 inches in diameter at the apex; skin sea-green, closely netted; flesh from $1 \frac{1}{2}$ to 2 inches thick, clear green, firm, juicy, and high flavoured; an abundant bearer, larger than the majority of melons of its class, ripening early, and soon arriving at a bearing state, generally producing a second crop spontaneously; $6 \mathrm{lb}$.

Darce.-Fruit about 9 inches long and 6 inches in diameter; skin mottled with dark seagreen upon a pale ground, coarsely netted, although sometimes nearly smooth; flesh white, thick, crisp, and melting, very sweet when fully matured; 4 to $5 \mathrm{lb}$.

This class of melons deserves peculiar attention, and although requiring a high temperature, will fully repay the connoisseur, as of all others, including the hybrids produced from them, the highest flavoured of all the family.

Of Water melons there are several varieties; the following are the best:-

Imperial.-Fruit of medium size, nearly globular; skin pale green and white, remarkably thin; flesh solid to the centre, reddish, crisp, and high flavoured; a native of the Mediterranean coast; seeds light brown and very small.

Carolina. - Fruit oblong, very large; skin dark green, and marbled with white, thick; flesh deep red, and sweet, although often hollow at the centre; flavour good; seed large, and black.

Spanish.-Fruit oblong, large; skin dark green, slightly marbled, moderately thick; flesh red, rich, and very sweet; one of the best hardy Water melons.

Insects and diseases.-The principal diseases of the melon are gum and canker, usually brought on by accidental bruises on the stems, and sometimes by over-watering near the centre of the plant, and also by an insufficiency of heat. Quicklime or charcoal, in a fine, dry, powdery state, applied to the parts, and repeated as either becomes damp, is the best remedy; and this will be considerably aided if the part of the shoot affected be kept clear of the damp ground. Additional heat and less water should also be attended to. This disease is much more prevalent in plants grown on dung-beds than in such as are trained to trellises in airy well-ventilated structures. The insects which infest the melon are chiefly the red-spider, aphides, and thrips, and the same remedies should be applied as recommended for forced French or kidney beans, vide p. 76.

The European names are-Melon, FrenchMelona, German-Melean, Dutch-Melone, Italian-and Melon, Spanish. 


\title{
CHAPTER XVII.
}

\author{
MANAGEMENT OF THE FRUIT-ROOM.
}

In vol. i., section FroIt-Room, we have given examples and thrown out hints as to gathering the fruit, and its disposal for winter keeping. It remains for us now to state the importance of following up the rules therein laid down, and to remind the pomologist that an almost daily examination, particularly during gloomy Novem. ber, is absolutely necessary. This visitation, however, should not be put off, as is too often the case, till some wet day, when nothing advantageous can be done out of doors. During this procrastination the whole contents may be seriously injured. Attend, therefore, assiduously to pick out every fruit that shows symtoms of decay. Exclude light and heat from late-ripening sorts; ventilate to the extent only of drying up superfluous moisture when it indicates dampness, for too dry an atmosphere will cause the fruit to shrivel; and take the fruit for use in the rotation of its ripening. Bring forward sorts that may be tardy in coming to maturity, by placing them in greater warmth, lest a lapse takes place between them and those already in use.

In the Revue Horticole, Jan. 1851, the following conditions are stated to be essential to the keeping of fruit and management of the fruitroom: " 1 . It must have an equable temperature. It is more than anything by change of temperature that the fermentation of the juices is occasioned that destroys the fruit. 2. The temperature should range from $46^{\circ}$ to $50^{\circ} \mathrm{Fahr}$. A more elevated temperature favours fermentation too much, and hastens decay, while a lower degree of heat prevents it entirely; and thus, as a certain degree of fermentation is necessary to the ripening of the fruit, it will continue in the same state as when gathered from the tree. 3 . The fruitroom should be completely inaccessible to light. Light accelerates the maturation of the fruit, by facilitating those chemical changes on which it depends. 4. It should be so constructed as to preserve all the carbonic acid generated by the fruit. This gas appears, from the experiments of Couverchel, to tend powerfully to the preservation of the fruit. 5 . The atmosphere of the room should be rather dry than moist. Humidity is a necessary condition to fermentation; it diminishes the resistance of the tissues of the fruits, and favours the expansion of their fluids; it is therefore desirable to avoid its excess. But, on the other hand, the room should be by no means completely dry ; for the fruit, losing in the meanwhile much aqueous fluid by evaporation, becomes too much dried, and will not ripen. 6. The fruit should be so arranged as to prevent it as much as possible from being crushed and pressed. In order to realise these conditions, a dry soil must be chosen for building the room; the site should be a little elevated, having a north exposure and entirely shaded from the sun by a plantation of evergreens." From this it would appear that, at a temperature below $46^{\circ}$, fruit would remain in a state of vegetable suspension; and it is probable that from that point to $40^{\circ}$ is the minimum temperature at which the house should be kept while the slow and gradual process of ripening is going on.

The third condition stated above (namely, darkness) has been long acknowledged, but seldom practised. It is of vast importance. The fourth condition (namely, the preservation of the carbonic acid generated by the fruit) is, we confess, new to us, although, on reflection, we have no doubt of the correctness of Couverchel's theory : it, however, leads us to the conclusion, that the less ventilation given the better. We need hardly remark, were it not that there are too many instances to the contrary, that cleanliness in the fruit-room is as essential as freedom from excessive damp and dryness is to the well keeping of fruits. Before the season of fruit-gathering commences, the walls of the fruit-room should be white-washed with hot lime-water; or if of wood, washed with chloride of lime; the shelving and floor thoroughly scrubbed with soap and water; and as it has been most happily remarked, by that excellent pomologist, Mr Errington, a liberal expenditure of elbow grease should be applied. We often sprinkle the floor of our fruit-room, which is of wood, with chloride of zinc, the material employed by Sir William Burnett for the preservation of timber, canvass, and a variety of sanitary purposes. As this preparation is known to prevent the growth of parasitic fungi on dead vegetable matter, we see no reason why it should not do so on that which has about ceased to exist. Much of the success of keeping fruit depends on the state it was in when taken from the tree. On this subject Mr Thomas Rivers, in reference to pears, which must also hold 
good in the case of apples, observes in his useful pamphlet on "The Culture of Pyramidal Fruit Trees," p. 17 : “The fruit of pears, more especially those on quince stocks, should not be suffered to ripen on the tree: the summer and autumn varieties should be gathered before they are quite ripe, and left to ripen in the fruit-room. The late pears should be gathered before the leaves take their autumnal tints; if suffered to remain too long on the trees, they frequently never ripen, but continue hard till they rot. In most seasons, the first and second week in October is a good time, but much depends on soil and climate." This is the season when we gather the majority of our winter pears at Dalkeith; but it should be remembered we enjoy an autumnal climate almost equal to that of London. In other parts, not only of Scotland, but even of this same county, should frosts not occur, the latter end of the month may be in quite sufficient time.

Mr Downing describes the American practice of gathering and keeping apples as follows: "In order to secure soundness and preservation, it is indispensably necessary that the fruit should be gathered by hand. For winter fruit the gathering is delayed as long as possible, avoiding severe frosts; and the most successful practice with our extensive orchardists is to place the good fruit directly, in a careful manner, in new tight flour-barrels, as soon as gathered from the tree. These barrels should be gently shaken whilst filling, and the head closely pressed in; they are then placed in a cool shady place, as under a shed open to the air, or on the north side of a building, protected by a covering of boards over the top, where they remain for a fortnight, or until the cold becomes too severe, when they are carefully transferred to a cool dry cellar, in which air can be admitted occasionally in brisk weather. Some persons place a layer of clean rye-straw between every layer of apples when packing them in barrels. When apples are exported, each fruit in the barrel should be wrapped in clean coarse paper, and the barrels should be placed in a dry airy place between decks."

In regard to gathering apples and pears, we may here remark, as a pretty general rule, that both pears and apples should be gathered when they will scarcely hang any longer on the tree: this is the true criterion of their being ripe. There are, however, exceptions to this rule, both amongst apples and pears: amongst the latter we may instance Williams' Bon Chretien and the Flemish beauty, both of which must be gathered before they readily fall from the tree, otherwise they become too musky to be pleasant; they, with the Jargonelle, when the object is to prolong their season, should be partly gathered before they become ripe, and placed in the coolest and darkest part of the fruit-room, with a view to retard their ripening. Those left on the tree to ripen will come into use first, and be followed by those soonest pulled. In some seasons that excellent pear, the Glout morceau, will, and may remain on the tree till after the leaves have fallen.

All fruits undergo considerable changes during their growth and maturation. At first they are tasteless or slightly bitter; when more advanced, they acquire a sour taste from the production of acids : in the apple and gooseberry is found malic acid; in the grape, tartaric acid; in the red currant, citric acid. As maturation approaches, the acids diminish in quantity, becoming at first neutralised, and afterwards partially decomposed by the alkalies present in the fruit; the cellulose forming the sides of the cells and vessels is transformed, and, together with the gum, is changed into grape-sugar, which is the keeping material in fruits. Apples or pears gathered before they have arrived at maturity do not keep because of the absence of their due share of sugar; and grapes, if not perfectly ripened before their season of growth ceases, will not keep, but shrivel and continue sour, on account of the absence of saccharine matter, and presence of tartaric acid.

The changes which take place during ripening are thus shown by Professor Balfour in "Class Book of Botany," p. 606, to consist chiefly of a diminution of the quantity of water and of ligneous matter, and an increase of the quantity of sugar. The changes in these ingredients are thus tabulated :-

\begin{tabular}{|c|c|c|c|c|c|c|c|c|}
\hline \multirow{2}{*}{\multicolumn{3}{|c|}{ NAMES of Fruits. }} & \multicolumn{2}{|c|}{ WATER. } & \multicolumn{2}{|c|}{ Sugar. } & \multicolumn{2}{|c|}{ Ligneous Matter. } \\
\hline & & & Unripe. & Ripe. & Unripe. & Ripe. & Unripe. & Ripe. \\
\hline $\begin{array}{l}\text { Melting peaches, } \\
\text { Duke cherries, } \\
\text { Red currants, }\end{array}$ & : $\quad$ : & & $\begin{array}{l}90.31 \\
88.28 \\
86.41\end{array}$ & $\begin{array}{l}80.24 \\
74.85 \\
81.10\end{array}$ & $\begin{array}{l}0.63 \\
1.12 \\
0.52\end{array}$ & $\begin{array}{r}11.61 \\
18.12 \\
6.24\end{array}$ & $\begin{array}{l}3.01 \\
2.44 \\
8.45\end{array}$ & $\begin{array}{l}1.21 \\
1.12 \\
8.01\end{array}$ \\
\hline Green-gage plums, & , & & 74.57 & 71.10 & 17.71 & 24.81 & $\begin{array}{c}\text { Includin } \\
1.26\end{array}$ & $\begin{array}{l}\text { seeds. } \\
1 . J i\end{array}$ \\
\hline $\begin{array}{l}\text { Apricots, } \\
\text { Jargonelle pears, }\end{array}$ & . & - & $\begin{array}{l}89.39 \\
86.28\end{array}$ & $\begin{array}{l}84.49 \\
83.88\end{array}$ & $\left\{\begin{array}{l}\text { trace \& } \\
\text { then } \\
6.64 \\
6.45\end{array}\right.$ & $\begin{array}{l}16.48 \\
11.52\end{array}$ & $\begin{array}{l}3.61 \\
3.80\end{array}$ & $\begin{array}{l}1.86 \\
2.19\end{array}$ \\
\hline
\end{tabular}

Berard thinks that these changes in fruits depend essentially on the action of the oxygen of the air. Fleshy fruits, he says, may be preserved with little alteration for many weeks in vacuo, in nitrogen, and in hydrogen gas. Peaches, plums, and apricots may be kept for twenty or thirty days, and pears and apples for three months, in a sealed bottle containing a 
little sulphate of iron, lime, and water, which remove the oxygen of the air. Fremy found that the ripening of the fruit was arrested by covering it with varnish, which he supposes to act partly by preventing the access of air, and partly by stopping the transpiration, and thus checking the flow of sap from the fruit. This we have found to be the case, both in retarding the ripening of the Juneating, one of our earliest apples, while on the tree, and also for prolonging the keeping of the Ribston, one of our medium keeping apples, by coating them, in both cases, over with gum-arabic, a gum easily dissolved and removed from the fruit.

Besides the keeping of apples and pears, there are other fruits that require the aid of a wellregulated fruit-room. When pines come in more rapidly than desired, or when it may be desirable to retard their ripening for several weeks, which is frequently the case, the plants should be removed carefully while in the pots, after they have attained their full size and are about to change colour, their leaves tied up, both to economise space, and also to prevent their being broken; and if set in the fruit-room, and kept moderately dry and dark, they will keep in excellent order three or four weeks. When it is desirable that they should ripen, they should be again transferred to the fruitinghouse, and plunged and tended as before their removal; or, still better, as practised at one time by $\mathrm{Mr}$ Barron, be placed at one end of the stove, which might be separated from the general part of the house by a glass partition, and in which a high temperature, depending, however, chiefly on solar heat, might be kept up during the day, and the plants so placed as to be as close to the glass roof as possible. They also should be freely exposed to the sun and light, and neither water nor bottom heat whatever applied to them; the natural sap in the plant supplies the scanty amount of nutriment required, the warmth and light of the sun giving the fruit its fullest amount of flavour.

Melons cut a day or two before becoming ripe will keep a week in a cool dark fruit-room, and will have both colour and flavour imparted to them if suspended in a netting bag for a day or two, placed close under the glass in a dry warm atmosphere. There are sorts, however, that may be kept for weeks with little trouble or care, but the majority of the finer sorts should be eaten the day they are removed from the plant, unless when grown on damp dung-beds, imperfectly exposed to light, and as imperfectly ventilated, a mode of growing this excellent fruit we wish to see completely exploded.

Grapes.-Under the head Retarding crops of ripe grapes we have alluded to Speecliley's mode of preserving this fruit when cut from the vines, and also given the ingenious method pursued by Mr Lavanchy, as stated by him in "Cottage Gardener" (which see). Forsyth, and sundry writers since Speechley's time, recommend removing the bunch having attached to it a portion of the shoot on which it has been produced, to the extent of a joint or more below each bunch, all, however, with his exception, recommending, and very properly, that the fruit be thoroughly ripened before its removal. The lower end of the shoot should be cut smooth, and hot sealing-wax or pitch applied to it, so as to seal the wood closely that no air can enter the tissues connecting with the bunch. If the shoot, for convenience sake, be cut over above the fruit, then the same process of sealing should take place. The bunches thus prepared are suspended from laths or lines run across the fruitroom, taking care that the bunches do not touch each other, and also that they be frequently examined, and any decaying berries instantly removed. A cool moderately-ventilated atmosphere is the best, and darkness, for reasons already given, is a necessary condition towards success. Grapes are often preserved by sealing the end of the fruit-stalk when cut, and enclosing the bunch in paper bags, and sometimes in bags made of fine cotton, tying the mouths of the bags tightly, and suspending them from the roof of the room. In the former of these evaporation is checked, and the carbonic acid which is generated by the fruit is prevented from escaping. In using bags, they should be large enough to slip easily off and on, as it is necessary to examine the fruit frequently. The general fruit-room, stocked with apples and pears, is much too damp an atmosphere for keeping grapes; it is, therefore, better to place them in a separate apartment, but at the same time in one where the temperature is uniform, and neither too hot nor too cold. Grapes are also kept in jars, being first enveloped in fine soft paper, and afterwards packed round with dry sand, bran, or paper shavings. These jars, if carefully stopped at top, may be placed on the floor of the fruit-room or otherwise, as most convenient.

Vines grown in pots readily present the means of keeping the fruit in a very perfect state much longer than any of the means hitherto recommended. When the grapes are thoroughly ripened, the plants should be removed from the vinery, and placed in a dry well-ventilated room, with a minimum temperature of $55^{\circ}$, and maximum of $60^{\circ}$. It is also important, as in the case of all other fruits, that light be completely excluded; the leaves wiil soon fall off, and the fruit will draw its limited requirement of support from the remaining sap contained in the plant: this, with the diminished evaporation from the surface of the berries, will maintain them in a fine plump state for two months or more.

In America, the grape is preserved in great quantities for winter use, by packing them away, as soon as ripe, in jars, boxes, or barrels, between layers of cotton wadding, in which way they are kept plump and fresh till March.

Peaches, nectarines, and apricots, from their nature, are not capable of being long kept without losing much of their flavour, as is the case with much of the fruit imported, and even sent from distant parts of the country to the Lcndon or other markets. To insure safety during transit, they are gathered before they are ripe; and although they soften and colour somewhat approaching their natural state, still their ripening, or rather their softening, taking place in the 
absence of sunlight and air, flavour, under such circumstances, cannot be expected. The case is somewhat different in private gardens, where, to suit a particular demand, they may be gathered when they are what is called bone-ripe, kept for a week in a cool fruit-room, and a day or two before they are required for use, if placed close to the glass in a moderate temperature, and fully exposed to light and air, their flavour will be considerably improved. Attempts have been made to keep them in ice, and a case of nectarines, transported from America, consigned to the London Horticultural Society, came perfectly safe and sound to hand; but their flavour was gone, in consequence of the extremely low temperature to which the fruit had been exposed. Some connoisseurs in fruits recommend keeping the peach and nectarine a day or two in the fruit-room previous to its being used, and consider that the flavour is by this means improved. Such an opinion prevails pretty generally in France, where the finest peaches are produced. Mr Williams of Pitmaston long ago stated in "Horticultural Transactions," vol. ii. p. 115, that should the season prove wet when the peaches are ripe, they should be gathered and placed for about two days in a drying-room before they are eaten. In this that excellent pomologist was right; and it would be well if cultivators paid more attention to his recommendations.

Plums of most sorts may be kept for several days on the table of the fruit-room without deterioration, and, like peaches, may be improved by a day or two's keeping, particularly in wet seasons. It is a sort of received opinion that each sort should be brought to table as soon as they are gathered. There are, however, exceptions to this rule; for the Blue imperatrice and Coe's golden drop will keep long after they have attained all the ripening which our climate affords them. Both, particularly the latter, will keep for several weeks, if wrapped in soft paper individually, and placed in air-tight drawers or cases in a dry airy fruit-room. We have fre- quently kept Coe's golden drop till Christmas. The fruit-room also becomes the depository of other fruits, which, although less difficult to keep in a useful state, are nevertheless not the less important to the gardener.

The almond is seldom cultivated in Britain on account of its fruit, because, unless in the favoured climate of the south of England, it is seldom brought to perfection, and also because of the reasonable charge made for imported much better samples. Those, however, who ripen the almond may have it, in perfection, in a fresh state, from September to the end of October, after which the outer coating or pulp should be removed, and the stones thoroughly dried and imbedded in dry sand in boxes, and if placed in the fruit-room they will keep through the winter.

The chestnut seldom ripens in Scotland unless in very favourable situations; in England it does, but not equal to those imported from France and the south of Europe. With us, we select the finest and best ripened, and after removing the husks we dry them, and afterwards deposit them in layers in boxes amongst very dry sand. These keep till March, but for winter use we lay the dried specimens on shelves similar to those used for our apples. If laid in large quantities together, a slight fermentation takes place, a premature decay ensues, and the season of their usefulness is thereby shortened.

The filbert, and all the hazel-nut tribe, should be gathered when the cup or covering of the fruit turns brown, and when the nuts begin to drop of their own accord. When gathered with the husks attached to them, they may be laid on the shelves of the fruit-room like any other fruit, and by this means they will keep good till Christmas. For later keeping, remove the husky covering, and pack the nuts in boxes in dry sand, which will exclude the air, and prevent the kernels from shrivelling. The late $\mathrm{Mr}$ Braddick kept filberts thus packed in air-tight barrels for two years in a cool cellar. 


\title{
THE F L O W E R GAR D EN.
}

\author{
CHAPTER I.
}

\section{PLANT-HOUSES.}

IN order to simplify the arrangement of this department, and at the same time to render our remarks on the whole as perspicuous and connected as possible, we propose to divide the subject into two chapters, namely :-

The management of exotic plants in houses and pits, and the flower garden proper, in which hardy plants only, or such as are capable of developing themselves in the open borders, will be treated on, as well as those requiring slight protection during winter, and which are now cultivated with so much advantage for the purpose of furnishing our parterres during the summer and autumnal months.

The first division will be arranged in the following order, viz. : The Camellia-House, OrchidHouse, Bulb-House, Orangery, Heath-House, Aquarium, Stove or Tropical Plant House, Succulent-House, Conservatory, Green-House, Flower-Forcing House and Pits, Cold or Conservatory Pits, \&c.

The second division will be arranged as follows, viz.: American Garden, Rosary, Lawn, Shrubbery, Florists' Flowers, Conservative Wall and Border, Alpine Garden and Rockery, Perennial Plant Garden, Parterre, and Reserve Garden.

Many of the plants which belong to each of these subdivisions, although widely differing from each other in their botanical affinities, have, nevertheless, so close a connection, so far as their culture is concerned, that the same mode of treatment, in the majority of cases, is applicable to them all. Where, however, a difference of management occurs, it will be particularly noticed.

Our Select Lists of plants in each division will be limited to those only which possess the highest degree of merit for the abundance and brilliancy of their flowers, or the beauty or singularity of their foliage, but without regard to their date of introduction or origin, unless in such cases where we deem them worthy of general cultivation.

As even very condensed lists, were we to give VOL. II. both the generic and specific name to each plant individually of the thousands we mean to treat on, would occupy much space, we propose to give, in all cases where the genus is sufficiently important, the generic name only, intending that to imply that the majority, if not the whole, of the genus is deserving of cultivation. For example, Miltonia and Odontoglossum (in Orchids) indicate that all the species of both these genera are highly deserving of culture in every collection.

In cases where the genus is very extensive, and where many of the species constituting it have little to recommend them beyond their strict botanical value, a selection of the best sorts, as exemplified in Acacia, Daviesia, \&c., and in the case of mere varieties, as in Camellia, Azalea, \&c., only will be given.

Experience has long ago led, and most of our best cultivators now adopt the rule, to grow only the most choice and select plants; and indeed many confine themselves to a very few genera, and these they grow to the highest degree of perfection. This can never be accomplished where a general collection is attempted, on account of the vast difference which exists in their natures, particularly in their seasons of growth and rest, which in itself renders the most perfect cultivation impossible, if attempted in the same house.

This latter circumstance has had its weight in inducing us to adopt the above subdivisions, as well as the appearance it gives of design, order, and effect, produced by associating plants either of one country or climate, or of those having some natural affinity, with each other.

Suppose an amateur, for example, were to choose any one of the above subdivisions, instead of making his plant-house a receptacle for every plant thrown in his way, he would find by this method far more satisfaction than he wouldin the incongruous massing of plants of discordant habits, with which nine-tenths of our plant-houses are at present stored. To attempt in private estab- 
lishments a general collection is as absurd as it is impossible. The case is otherwise if our ambition be to possess a very complete collection of one genus or order, because this is completely within our power; and he who possesses the best collection of Ericas, Orchids, Camellias, \&c., stands far higher in the scale of merit in the floricultural world than he who, attempting to have everything, has nothing in perfection.

By our arrangements the followers of the mixed style will find they have not been overlooked; for the mode of propagation, general management, \&c., given of each division, if taken collectively, will afford, in less space, more information than had we treated the subject as is in general done-namely, as greenhouse and stove plants only. The same remarks are equally applicable to the flower garden also.

\section{$\S 1$.-CAMELLIA-HOUSE.}

It is not in every establishment that an entire house is dedicated to this deservedly popular genus; plants, therefore, approximating to them in constitution, and admitting of almost the same treatment, may be grown along with them with every degree of success, while their flowering at different periods of the year maintains a degree of interest throughout the whole season, which the camellias would only produce during a portion of that time.

Of plants which may be admitted into the camellia-house, the following are the most important: viz., Chinese azaleas, of which there is now a great variety-Daphne Fortuni, hybrida, japonica, indica, alba, and rubra; Illicium floridanum; Magnolia fuscata, pumila, fragrantissima; and, indeed, where it will not flower in the open air, the magnificent Magnolia grandiflora also; Nerium oleander, of which nursery catalogues furnish long lists of varieties; Olea fragrans, Rhododendron javanicum, Gibsonii, arborea ; Arborea alba, and the majority of the Indian sorts, as well as many of the more tender hybrid varieties; Luculia gratissima, Pinciana; Enkianthus reticulata, quinqueflora, \&c.

The camellia, in its native country, Japan, is a hardy, lofty-growing tree, where, as Thumberg assures us, the cold is intense, snow falling, water freezing, and the thermometer falling to many degrees below the freezing-point; and in speaking of the hardihood of the camellia, Dr Lindley observes : "If we look at the plants which we have in our gardens from the country of the camellia, they will be found to be amongst the most hardy exotics which we possess. Cydonia japonica, Pyrus japonica, Wisteria, Weigela, Forsythia, Chimonanthus, Moutan, \&c.who has seen them hurt with English frost? Thus it appears that the companions of the camellia in its own woods have no such tenderness of constitution as to demand a greenhouse." At Dalkeith, in the Botanic Gardens of Edinburgh, these and many other plants from the same locality have stood our winters for years without the slightest injury. During the extraordinary severity of the frost in 1837-8 the camellia stood, without protection, in several places in England without damage, enduring $6^{\circ}$, $9^{\circ}, 12^{\circ}$, and $14^{\circ}$ of frost. We direct attention to this matter, to show amateurs particularly how liable they are, in their care of their pet plants, to injure them more by too much heat during winter than the reverse. We must, at the same time, consider the conditions the plants were placed in to enable them to withstand so great a depression of temperature; and this will be best explained by stating that it is the want of heat during our summers, and not, unless in extreme cases, the cold of our winters, that prevents the camellia, azalea, \&c., from growing in the open air in all moderately temperate situations in Britain.

The hardiness of a plant is not, however, to be determined by its abstract power of withstanding cold, but by the treatment it has received to enable it to do so. The camellia, in its native country, is exposed during summer to a heat equal to that of Bengal, while in winter the cold is nearly as intense as it is at Moscow. There is little doubt that if camellias were grown under glass, and exposed to such high temperatures during summer, they would stand our ordinary winters in the open air with impunity; and if not, they certainly would be quite safe under glass without the aid of fireheat altogether. Heat is necessary during their growing season, to enable them not only to make wood, but also to ripen it thoroughly; and this can be accomplished by attending to the following conditions-namely, that they shall not begin to grow till the very end of spring, and that they shall cease to grow at the very beginning of autumn. Their growth must be limited to our period of summer. If they are allowed to become excited into growth early in spring, they are certain to be cut off by late frosts; and if allowed to grow till late in autumn, their wood can never be sufficiently matured to stand the cold of winter. If their growth in the open air, therefore, terminates by the beginning of August, it has the heat and dryness of that month and part of the following to complete the ripening process. By these means only can the camellia be grown as a wall plant or as an open shrub in the open air of Britain.

It is also worthy of remark that some varieties are much hardier than others, and, therefore, better fitted for open-wall culture. The single white and the blush pæony flowered have been found to be the hardiest, and C. reticulata has stood with very slight protection in the vicinity of London since prior to 1837.

The camellia is decidedly impatient of heat, unless during the period when it is making its wood; and when attempts are made to get them into flower early in winter by introducing them into houses where a high temperature is maintained, their flower-buds are almost certain to drop off; and should they even remain on and expand their flowers, these will be of short duration and small size. From this it is quite certain that the proper temperature of the camellia-house should be high during the period of the wood's growth, and during its attaining maturity, gradually allowing it to fall, even to the extent of having $2^{\circ}$ or $3^{\circ}$ of frost during its period of rest. When vegetation 
commences again in spring, a little more than mere solar influence is occasionally required, particularly in dull cold weather, until the flowering season is past ; and then, as the woodbuds begin to swell, it should be gradually increased to from $65^{\circ}$ to $70^{\circ}$, but, at the same time, accompanied with abundant ventilation, and continued at this, with a few degrees more of sunheat in bright clear days, until the wood is fairly formed and beginning to ripen, after which a gradual decline should take place until the temperature falls even to $33^{\circ}$ or $34^{\circ}$. If the wood is properly matured, as will be seen from what has been stated above, no harm will befall the plants should $2^{\circ}$ or $3^{\circ}$ of frost enter the house. The practice of shutting up the house close during the period of the wood's formation is as erroneous as that of keeping the plants in a perpetual state of excitement. Although we cannot assert that all the plants we have named above, as inhabitants of the same house with the camellia, are equally hardy, yet we know all of them to be capable of withstanding several degrees of frost with impunity : our remarks on them will be made seriatim.

Propagation of the camellia by seed.-To obtain new varieties, and also for stocks, seed, being frequently produced in Britain particularly from single or semi-double varieties, should be sown in March, in light sandy loam, covered to the depth of half an inch, and plunged in a mild bottom-heat. When the plants are 4 inches in height, they are fit for transplanting into pots 3 inches in diameter, and are afterwards treated as other young camellia plants are. Those who grow camellias from seed with the expectation of obtaining new varieties, should adopt the process of cross impregnation; by this means, by far the most beautiful of our present varieties have been obtained.

By layers.-Stools of the common single red sort are planted in pits, where they may be slightly protected during winter. The young shoots are laid around the mother plant as other evergreens are, and the season for doing so is autumn, when the young shoots have attained some consistency; by the following autumn they will have formed roots, when they should be separated from the parent plant, potted, and grafted or budded the following spring. By this means great numbers of young plants are obtained annually.

By cuttings.-This is the general mode of propagation for the production of stocks; and although it requires a longer period to grow them to a proper size, still the facility with which they are produced, and the means of obtaining cuttings from established plants, are so great, that many propagators prefer this mode to that of layers. The cuttings are selected from the new wood when it has become nearly ripe, which generally happens about July or August. Cuttings from the single-flowered varieties are preferred; for although cuttings taken from the double or finer sorts will strike root freely, still they do not make such good plants as those that are budded or grafted on stocks of the single-flowering kinds. Make the cuttings 4 or 5 inches long, cutting them over smoothly just under a bud; displace the two lowermost leaves, leaving the two uppermost and the top of the cutting perfectly entire. Set them in the pot thickly in sandy loam, and place them in a cold frame or pit; in eight or ten weeks most of them will be rooted, when they should be transplanted singly into 3 -inch pots, in light sandy loam, and returned to the pit till the approach of winter, at which time they should be removed to a pit or house where the frost may be excluded. Give them a slight excitement early in spring by additional temperature, which will render them fit for grafting or budding the following season. Some propagators reduce the length of the cutting to one bud only, cutting them over a little above and a little more below the bud, taking a thin slice off the wood on the side opposite to the bud, thus making each cutting consist of the bud, its leaf, and a piece of wood above, and somewhat more below; they are then inserted in silver sand placed over light sandy loam in well-drained pots, the leaf being retained entire. If put in during autumn, many of them will be rooted before spring, if kept covered close in a cool pit. Towards spring, a slight bottom-heat will be required to cause a free development of the shoot, and also the increase of roots: cuttings so treated as early in the autumn as their wood is fully ripened, will root before the beginning of winter if plunged in a mild bottom-heat, and kept warm and close. If planted in August, they will root before the middle of November sufficiently to carry them through the winter in a dormant state; and in spring, if moderately excited, will grow rapidly, and be in advance of those treated as above.

The Messrs Chandlers, the most extensive growers of the camellia round London, advise taking off the cuttings in July and August, or as soon as the young shoots are sufficiently ripe at the base; cut them over smoothly with a sharp knife at a joint, and divest them of one or two leaves at the bottom; plant them firmly about 2 inches deep in pots filled with compost, and the upper half with fine white sand; water them well, and plunge the pots in a tanbed, and keep them closely shaded for three or four months, by which time short fibres, or a calosity from which new roots will issue, will be produced, after which they are to be potted in small pots, one plant in each.

Propagating by grafting and inarching.-The former operation may be performed from the beginning of September to the end of February; and the tongue method, fig. 106, is in general preferred. As the plants are operated upon, they should be set on a bed of coal-ashes or sand in a cool pit, and covered with hand-glasses, and partially shaded until the union takes place between the scion and stock, which will be determined by the former beginning to grow, at which time shading should be discontinued, and air progressively admitted to the plants. The grafts should be taken from plants whose sap has not commenced to flow, or they may be cut off a few days before using. Inarching, on the other hand, should be done either just before the growing season terminates, or before it 
commences. The plants should be placed in a moderately warm place a week or so before the operation commences, to set the sap in motion: for mode of proceeding, vide article INARCHING, p. 336. Although this is a more certain method of securing a union, the plants are seldom so well shaped as those increased by grafting.

Propagation by budding.-This is a popular mode of increasing stock much practised by nurserymen, particularly in regard to new or rare varieties, as by this means every bud may be made to produce a plant, which by ordinary grafting or inarching could not be done. This operation may be performed at almost any season of the year, but spring, summer, and autumn are preferred, on account of the sap being then in a state of motion. The process has been described, p. 338 . It remains for us here only to state, that in the case of the camellia the operation should be carried on in a close, rather moist, pit; or if the process is limited to a few plants only at a time, they should be placed under a bell or hand-glass until the union takes place, and be all the while partially shaded. This, however, can only be done when the stocks are small; and, indeed, it is upon these generally this mode is practised. After the bud is inserted, it is expedient to retain the top on the stock until the bud has grown some inches, as, if removed sooner, the full flow of sap from the stock would be thrown in the direction of the bud before it was sufficiently established to receive it without injury. After the bud has begun to grow freely, the top of the stock should be pinched off two or three buds above where the bud is placed. Buds succeed with greater certainty than grafts; but the method is less expeditious than either grafting or inarching in the formation of a saleable plant.

General culture.-During the period while the young wood is making the plants require the largest supply of water, with an increased temperature-indeed, they can hardly have too much, provided the drainage is complete. When the flower-buds are formed, water must be less abundantly supplied; and this must continue until the growing season again arrives. Nevertheless, the soil must not be allowed to become dry; and care must be taken that the water finds its way to the very centre of the ball. The atmosphere must also be kept rather moist than otherwise, and the leaves frequently syringed overhead. In most parts of England, the plants may with advantage be set out of doors in a sheltered situation as soon as the flower-buds are fairly formed; and that worms may be prevented from entering the ball, they should be set upon a flooring of coal-ashes, frequently watered with lime-water. In such a situation they may remain until the nights begin to become cold, when they should be removed into the camelliahouse for the winter. Their roots, for the most part, keep growing all winter, and therefore they will require to be kept judiciously watered all the season through. Pruning should be attended to by shortening the shoots which may be growing too fast, removing dead and weakly diseased branches, and keeping the heads suffi- ciently thin, that the summer air may penetrate freely. The camellia, like all other woody plants, requires a season of rest, and that season should commence as soon as it has done flowering. When again excited for the formation of its young wood and flower-buds, a little extra heat and a moister atmosphere will be required. If flowered during February and March, a slight degree of fire-heat will tend to cause the flower to develop fully; but this must be applied with due caution, for the least excess of heat will not only prevent the flowers from expanding fully, but will render them of short duration. Such as do not flower before April and May, will perfect their blooms fully by solar influence alone. The summer or growing temperature may be taken at from $60^{\circ}$ to $70^{\circ}$; that of winter, or the season of rest, at from $33^{\circ}$ to $40^{\circ}$. Notwithstanding the hardiness of the camellia, and its withstanding the ordinary frosts of our climate with impunity when planted in the open air, it is only to be found in its greatest perfection when grown under glass, in a light, well-ventilated structure. As the season of flowering, where a large stock is kept, does not apply to every plant in the collection alike, nor should it do so, as it is desirable to continue the production of bloom for as long a period as possible, such as have done flowering should be removed to a cool deep pit, and be followed by others as they cease to flower. In this pit, if the frost is little more than excluded, so much the better ; and here they should remain, very slightly watered, and as much exposed to the air as the weather will permit, until every bud has started into leaf. This simultaneous starting of the buds is an important part of good culture, for if they are kept too close and warm, the strong buds toward the extremities of the branches will push away, leaving those that are below them, and which are the weakest, deprived of a sufficient flow of sap to enable them to break at all; or if they do so, they produce shoots too weak either to produce flowers or to keep up the proper form of the tree. This is one of the principal causes of so many camellias being found divested of branches toward their bottoms. During this season of rest, the balls should be examined, the drainage repaired if required, and the surface-soil scraped off as deep as the topmost roots, and replaced with fresh material. When the buds begin to break, water them more liberally at the roots, and syringe occasionally over the tops, keeping the atmosphere pretty close for the accumulation of solar heat, but at the same time admit fresh air in the middle of every good day, that the buds be not drawn up weakly. When they have begun to develop their young leaves, shading during bright sun must be rigidly attended to, as the young expanding foliage is exceedingly liable to be scorched by too much sun-heat. This treatment should be continued for about five or six weeks, of course less or more according to the season, when most of them will have completed their growth, when more air should be admitted, but not to the extent of lowering the temperature too much, until their flower-buds be also perfected. As soon as the young wood and 
flower-buds are thus far perfected, care must be taken that the same degree of excitement by solar heat be not continued further, else the consequence may be that the flower-buds will become transformed into wood-buds, and elongate into branches instead of bloom. More air and less water should thus be given to bring about their season of repose, which will take place in about a month after the above change of treatment. Potting such as require it should then be attended to, which, with plants done flowering about the end of April or beginning of May, will begin their season of rest during July and part of August, provided they have hitherto been in a healthy state. Those plants that have flowered from November to Christmas in the preceding year, will have, it is presumed, undergone the above treatment; these will be fit to re-pot much earlier, and the others in succession, thus giving a long continuance of flowering. No plant, however, should be re-potted unless it absolutely requires it, but such should be turned out, to see the state of the roots, drainage, \&c., and be replaced and surface-dressed instead. In regard to the application of liquid manure, our best cultivators say that not a drop should be given while they are forming their young wood and flower-buds; but after the latter are all set, they should have it once a-week until their blooming season is again past.

The camellia, like most other plants, is sometimes liable to become unhealthy; and this arises from a variety of causes-derangement at the roots, too many flowers being allowed to be produced, and excessive heat. The former of these is the most frequent, and must be determined by careful examination; the two latter point out their own remedy. When the roots are at fault, reduce the ball even to the extent of removing the soil altogether; prune away all the black and diseased ones, re-pot in fresh compost, using pots or tubs much smaller than those they had been growing in, setting the plants, after the operation, in a close cool pit with a moist atmosphere, keeping the soil at the roots, however, rather dry until the new ones are formed, thinning the flower-buds, which often set in clusters as thick as hazel-nuts, with a pair of fine-pointed scissors, leaving, in healthy plants, not more than two close together. In the case of sickly plants making little or no wood, the whole of the flower-buds should be removed, that the energies of the plant may be thrown into the system for the production of wood, instead of being directed to the production of bloom. Plants so circumstanced often shed their flower-buds; and where even a slight disposition is shown to this, it is quite time the thinning process, or complete disbudding, take place. Indeed, thinning the flower-buds, even in healthy trees, should be attended to; and if neglected, the flowers will never arrive at that perfection they would do if a proper proportion, according to the strength of the tree, only be left on.

Potting.-Much difference of opinion exists as to the proper season for this operation, some recommending the spring, just after the plants have ceased flowering, while others prefer the month of August. Our own practice is to examine the state of the roots, and to perform the operation before the growing action has commenced. To do so afterwards would be highly injurious, as, however carefully the work is executed, the young tender spongiolets would be not only greatly injured, but many of them entirely destroyed. Where a large collection is grown, or even a number of plants of the same sort, all will not be alike fit to pot at the same time, and this can only be determined by root examination. It is also the best time to supply new food, as, when the young roots are produced, they have it to feed upon; whereas, if potted long before root-action commences, much of the value of the soil is lost. The state of the roots can be readily ascertained, in the case of small plants grown in pots, by simply turning them out; and in the case of large ones growing in boxes, by adopting our orange-box, fig. 260 , the sides of which are made to open, so that the state of both the roots and soil can be easily determined. Although the camellia requires an abundant supply of water during its growing period, few plants of so robust a character suffer more from saturation at the roots, and hence great care must be taken to provide abundant drainage in the pots or tubs. Many large camellias require re-potting only once in two or three years, and some remain in excellent health for even a longer period. This greatly depends on the quality of the soil they are planted in, the efficiency of the drainage, and the proper attention to watering. Indeed, it is not desirable to cause large camellias to make much wood, which would be the conseguence if annually encouraged at their roots. When camellias have arrived at the contemplated size, they should be treated much in the same way as fruit treesnamely, made to produce abundance of flowerbuds on short well-formed shoots, rather than making yearly wood, like willows; and when once they get into a state of flowering, if not allowed to produce too many flower-buds, and at the same time fed by a judicious application of liquid manure, they will remain for years without much additional soil being applied to their roots. A yearly growth of 3 or 4 inches is quite sufficient, and in this state they may be maintained for years.

Small plants, of course, are to be considered as best grown in pots; and when these require root-room exceeding 15 or 18 inches in diameter, it is much better to transfer them to slate tubs or boxes at once. Wooden boxes and tubs are by far the most expensive in the end. Our attention has been long directed to this subject; and, so long ago as 1826, we contributed an article to " The Gardeners' Mag.," vol. i. p. 140, with a sketch of a plant-tub or box suitable to the orange, camellia, and similar large specimens of exotic plants, which, for convenience and practical utility, has not been yet superseded. These boxes are of wood, pitched within for durability; but their utility lay in their formation. Two of the sides are kept in their proper place by two iron studs, which are let into the bottom framework, and their upper part is secured to the other two sides by the iron bands, which 
being lifted up, allow the two opposite sides to fold down, (as partly shown in the sketch), these being hinged, at their lower parts, to the

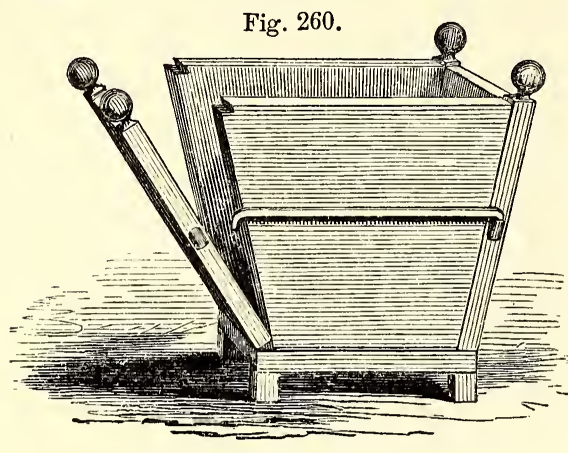

ORANGE OR CAMELLia Box.

bottom frame of the tub. The form is somewhat tapered for appearance' sake, and they are elevated from the floor of the house upon wooden blocks, which, while it preserves them from decay, admits air to the roots through the perforations in the bottom, as well as secures sufficient drainage.

Soil.-The French and Germans very generally grow their camellias in peat; the English as generally in peat and loam; while many, and ourselves amongst the number, prefer light rich mellow loam, reduced in tenacity by a portion of white silvery sand. Peat varies in its nature so much, that while in some kinds of it the camellia will grow, in others it will not long continue to exist. Fresh maiden light loamy soil is, as a general rule, that most to be depended upon; and, as in the case of the majority of flowering plants, no enrichment should be added at the time of potting, unless the soil is very poor indeed.

Planting out the camellia in prepared borders. - This is often done in conservatory borders where the camellia is planted indiscriminately along with other plants, having no natural affinity with each other either in character or treatment. If planted out at all, they should be placed in a house by themselves, or along with such plants as are somewhat allied to them, such as those named at p. 678. A span-roofed structure is the best, because the plants are better exposed to light and air ; a lean-to house the worst, because they are certain to become one-sided, the branches always drawing towards the light. Where such absurdities exist as a house with a dead wall or opaque side, the camellia may be planted against it, and trained to a trellis; but in such a case the prepared borders should be elevated above the floor of the house at least to the height of 2 feet, and if rendered hollow below, so much the better. If planted in the centre bed of the house, it should assuredly be vaulted underneath, to insure warmth and freedom from damp at the roots. In either case, however, the display of bloom will come almost at the same time, and therefore shorten the season of their display.
It is better, as a general rule, to grow them in a portable state, by which they can be retarded or advanced in flowering as already noticed. Their roots are under the control of observation, which they are not when planted permanently out; and as most of the ailments which befall them arise from derangement at the roots, the remedies are better applied. Planted-out camellias are often apt to grow, for the first few years, too much to wood, and too little to bloom; for a few years after, their growth becomes moderated; they flower pretty well, but in time the soil becomes exhausted, and they commence a rapid decline, and ultimately perish. The plants, and the structure they are grown in, are both of too artificial a character for us to attempt giving a natural appearance to their arrangement.

Camellias, like oranges, should be, when of a large size, planted in highly artistic vases or boxes, of which figs. 548, 549, vol. i., offer excellent examples; and a greater variety may be seen in the establishment of Messrs Minton at Stoke-upon-Trent.

The slate tubs manufactured by $\mathrm{Mr}$ Beck of Isleworth (vide ORANGERT), or our own orangetub, fig. 260 , are so constructed that they may be taken to pieces without disturbing the ball, and admit of the roots being examined, and fresh soil applied with the greatest facility.

The varieties of camellias are now so numerous as far to exceed the bounds of utility. New varieties are yearly appearing; not one out of many are equal to some long in cultivation; and although some very fine ones are occasionally produced, they are few compared to the many not worth the attention of the cultivator, who prefers a selection of fine blooming sorts to a long catalogue of names. A few well-selected varieties will ever be found the most satisfactory.

\section{SELECT LIST OF CAMELLIAS.}

Whites.-Alba pleno, Old double white-of this there are two or three excellent varieties; Alba pleno, var. Fimbriata, and of this the variety Insignis is no doubt an improvement; Candidissima, Decus Italicum, Edita, Innocenza, Magnifica, Martha, Myrtifolia alba, Reine des vierges, Low's imbricata, Ochroleuca.

Pink or rose-coloured. - Lefebvriana, Triumphans amabilis, Woodsia, Apollinea d'Italie, Hendersonii, Caroline, Chandlerii elegans, Vexillo di Flore, Felecite, Pictorium superbum roseum, Rubini, Pulasky, Imbricata, Bella di pistola, Berenice, Palmerii, Paride, Roi Leopold, Storyii, Wilderi.

Salmon-coloured.-Palmer's superba, Cooperii, Fordii, Brownii, Henri Favre, Rubescens striata, Globosa coccinea.

Deep red or scarlet.-Zeffiro, Sophia d'Italie, Sylvie d'Italie, Strombio, Vauxii, Chandler's eximia, Gretry, Bealii, Mathotiana, Mazeppa.

Blotched or striped.-Marchioness of Exeter, Duchess d'Orleans, Queen Victoria, Archduchess Augusta, Baltimoreana, Prince Albert, Camellia de la Reine, Craswelliana, Duc de Bre- 
tagne, Emilia Gavazzi, Emperor, Grand-duchess d'Etruria, Eastii, Guillaume Tell, Lady Hill, Landrethii, Madonna, Perigrina, Chalmers' perfecta, Cunningham's perfection, Princess Adelaide de Carigana, Teutonia, Princess Baciocchi, Spectabilis, Alexina, Dunlop's Americana, Aristo d'Italie, Low's caryophylloides, Countess of Orkney, Henri Favre, Imbricata tricolor, Juliana, Maculata perfecta, Madonna, Marie Teresa, Negri, Parini, Pirzio (Gruneberg's), Teutonia, Tricolor, Tommasini, Variegata (Casoretti's), Visconti nova (Castoretti's).

Diseases and insects. - The plant appears exempt from any natural disease; young plants no doubt frequently die off suddenly, but if the roots be examined, the cause will in general be found in their diseased state, caused by stagnant water being allowed to accumulate in the soil from imperfect drainage. The black and green aphides sometimes attack the young leaves and shoots; these are subdued by tobacco fumigation; and the white and brown scale which attack the mature leaves, and often the bark also, are to be removed by washing them off with soap and water.

Camellias are often much injured when taken into the drawing-room while in bloom. This chiefly arises from the plants being kept too dry at the root, and decidedly so for the leaves; for the atmosphere of any sitting-room is much too dry for any plant to exist long in, more especially a plant like this, that requires to breathe in a moist rather than a dry air.-(See what we have said on this subject, vol. i. p. 424.) They suffer much also from over-heat, which should not, in rooms, exceed $40^{\circ}$; and, again, when placed near the windows, they suffer from the cold draughts of air let in upon them when the windows are partially opened in the morn. ings. Heaths and camellias suffer more from these causes than almost any other plants. Although camellias suffer from want of humidity in the sitting-room, the other extreme of over-watering must be equally avoided; and setting the pots in pans of water must not be looked to as a remedy, but the very reverse.

The Chinese or Indian Azaleas require the same temperature under glass as the camellia ; they are, however, scarcely so hardy when planted in the open air. The soil for them differs also, and should be sandy peat, such as Ericas are grown in. They are propagated by seed freely, both for stock and new varieties, and many beautiful ones have by this means been produced. As in all other cases when improved varieties are desired, impregnating the best-shaped flowers of one variety with highcoloured varieties of another is the most likely process. The seed should be sown in February, in shallow pans filled with peat soil, rendered very smooth on the surface and slightly covered with fine white sand, over which a little light moss (Hypnum) should be placed, to retain moisture, and obviate the necessity of much watering, which might disturb the seed. The pans should be set on a gentle bottom-heat in a close pit or frame. When the young plants have attained two or three leaves, transplant them into store pots thickly in fresh sandy peat; for if left longer in the seed-pans, like seedling rhododendrons, they are apt to damp off. By the spring following, they will be fit for transplanting singly into pots $2 \frac{1}{2}$ inches in diameter; shift as they proceed in growth until they flower, when the good varieties should be selected and the worthless ones thrown away. They propagate readily by side-grafting, the scions being from 1 inch to $1 \frac{1}{2}$ inches long, and secured to the stock with a soft worsted thread, placing them in a close pit or frame in a mild temperature, or, if in a hothouse, under bell or hand glasses until a union takes place. Grafted plants are propagated largely in nurseries, but they never make such permanent plants as those propagated by cuttings. Cuttings are prepared by taking the tops of the young shoots when about 3 inches long, cutting off the lower leaves, leaving the upper ones entire. They should be set thickly in a pot filled about three parts up with peat earth well drained below, having about $1 \frac{1}{2}$ inches of fine silvery sand on top for the reception of the cuttings. Water gently at planting, and cover closely with a bell-glass, and plunge the pots in a temperature of from $55^{\circ}$ to $60^{\circ}$, wiping the condensed steam out of the glasses daily, and shading till roots are formed. Afterwards keep them in the pit with the glasses removed, at first only during the night, but afterwards both during day and night, to harden them off for potting singly into pots $2 \frac{1}{2}$ inches in diameter, keeping them in a close place till they have fairly taken root; after which they may be gradually exposed to the air and light, and treated like other hard-wooded plants. Much of what we have said regarding the camellia is equally applicable to the Chinese azaleas, the chief difference being that of soil.

As to the best time for potting Chinese azaleas, opinions are greatly at variance, some advocating spring, and others autumn : for ourselves, we consider that no precise rule can be laid down in this case, further than to be guided by circumstances - such as the state of the plants, and the time they have produced their flowers. We prefer to have the operation performed immediately before the root action commences, for reasons given under the camellia. It therefore follows, presuming those views to be correct, that shifting or re-potting should immediately follow the season of flowering, be that when it may. Where, however, the soil is either exhausted or sodden from imperfect drainage, and more especially where the roots are diseased or defective, then a reduction of the ball becomes absolutely necessary, and the roots require singling out and pruning, which cannot be done with safety, if root action, however feeble, has once taken place. As the flowering season, however, extends from the beginning of January to the beginning of June - for during that long period both camellias and azaleas are now to be found in flower-so should the season of re-potting be extended also.

Camellias and azaleas, which are forced into bloom during November, December, and January, although re-potted when their flowers have passed away, should not be excited into too early growth : the process of root-making and 
wood-making should be allowed to go on slowly and gradually; a contrary course will cause them to make long budless shoots, which, to maintain symmetry in the plants, will require to be cut back, and to a certainty a very linited formation of flower-buds. Excessive shifting will be productive of the same evil. Camellias and azaleas intended to bloom late should be kept in a cool place, and by the middle of February or earlier, according to their state, be placed in a cold pit merely protected from frost and excess of moisture ; the roots must, however, be prevented from suffering from drought, to prevent the shedding of their flower-buds.

The azalea bears pruning freely, and therefore all strong over-luxuriant shoots should be pinched back when about 6 inches in length, to cause them to send out lateral shoots of more moderate growth. It is important to keep them moderately thin of branches, and to order the shape of their heads so as to present the aspect of symmetry. They are sometimes grown as standards trained to single stems, but their natural habit is that of low-spreading bushes.

Diseases and insects.-The chief disease to which they are liable is one not very easily accounted for-namely, a sudden dying off close to the surface of the soil; and some varieties are more liable to this than others, which, to counteract this, it has been recommended to graft on the stronger-growing kinds, such as A. phœnicea; but, on the other hand, grafted plants of most of the delicate kinds are liable to die just above the stock in an equally sudden manner. The thrip often attacks the whole tribe. The best remedy we have found is, to prepare a strong lather of soap and flour of sulphur in a large tub, and to immerse the plants two or three times in it, setting them again upon the stages, and shutting up the house or pit close for two or three days. The green aphides attack the young shoots, but this is easily subdued by fumigation.

The Oleander (Nerium oleander) is propagated readily by cuttings of the half-ripened wood, set in sand in a moderately warm pit. Cuttings of older wood strike roots rapidly, if their ends are immersed in a phial of water. The soil best for these is peat and loam, enriched with cow manure, with a little leaf-mould. Their growth is easy, but their flowering is not so; and this is more to be regretted, seeing they are exceedingly beautiful, and, according to nursery catalogues, exceedingly numerous in varieties. The oleander naturally occupies a vast geographical range, being abundant in many parts of Europe, Africa, and Asia; nevertheless, it is found in very similar situations in them all-namely, the margins of brooks, lakes, and rivers, enjoying a hot dry summer, inundated to the depth of a foot or two while coming into flower, and with a comparatively cool winter. Its natural soil is a rich, deep, alluvial deposit. Under such conditions oleanders have three extremes-soil extremely rich, extreme drought during five or six months in the year, and saturated with water after the blossom-buds are formed. These are the really true guides to the proper cultivation of the oleander. They delight in strong rich loam; and as soon as the flower-buds appear, the pots ought to stand in saucers of water till the bloom is over; and with an occasional syringing on warm afternoons, we imitate so far their natural climate. This beautiful plant, although many years introduced to our gardens, is rarely seen in perfection of bloom, arising from improper treatment-such as the want of sufficient light, heat, and air, while it is making its annual growth, and a deficiency of water at the roots at the time of its growth and setting its flower-buds. When it has made its summer's growth, it should be kept in the camellia-house and exposed to the full sun, and very little water given from the end of September till the beginning of March. It is apt, without judicious pruning, to become straggling and overgrown. Mr Beaton remarks on this, as the flowers are produced " on the ends of the shoots made last year, we must not cut off their points in order to get a bushy head, for if we do, we cut away the flowering parts. Therefore, to keep a large plant in good flowering order, one-half of the flowering branches must be cut down every year to the last joint from the old wood as soon as the flowering is over for the season. Now, as the young shoots start off in threes round the flowers, and begin to lengthen long before the flowers expand, such of the shoots as are intended to be cut down ought to have the three points of the shoots round the flowers stopped as soon as they appear. This will throw the whole strength of the branch into the flowers, and will also cause the bottom eyes to push out three strong shoots as soon as the flowering branches are cut down." The oleander is often cultivated and flowers well in a dwarf statethat is to say, when only about a foot in height; and these, when crowned with from one to five or six flowers, are exceedingly pretty. To effect this, cuttings should be put in about the lst of April, placing each cutting in a small pot, and plunging them in a mild bottom-heat and in a high humid atmosphere. When rooted, shift into a 48-sized pot in a light rich compost. Grow the plants for the first season to one stem only, supplying them with abundance of water and air, and keep them near the glass until the end of August; after which, less water must be supplied, and keep them in a moderately cool pit till the end of September, and during winter when the frostis to be barely excluded. About the beginning of the following April cut them down to the height they are desired to flower at, but do not re-pot them; encourage the growth all summer as before; three shoots only should be allowed to proceed from where they were cut down to, and, if well managed, each of these will be of an equal length. Winter them as above, when the following summer they will produce from two to three flower-buds each, according to their strength. Pinch back the three young shoots which arise around the flower, leaving only one joint to each. When their blooming season is over, place them in a pit where they may be fully exposed to the sun. The plants by this plan will flower only once in two years, but a succession should be kept up, which will afford a fine bloom every 
year. And to effect this, Mr Beaton, who, we believe, was the first to promulgate this mode of flowering dwarf oleanders, directs to cut down "the plants every year about the end of March ; but when there is room, some may be cut down as early as February and forced, others in March, and a third lot at the end of April, and they will come in next year in succession for bloom." Under this system the cut plants are potted annually about the end of May, or as soon as the young shoots are about two or three joints long; and the flowering ones are potted in April, when they have made a little growth. When the pots are too large for the size of the plants, as they will be about every three years, the roots are reduced " by cutting off the outside and part of the bottom of the ball with a sharp knife, so as to enable them to be put into a 5 or 6 -inch pot. The best time for this operation is just after flowering After re-potting, keep the plants in a close pit, and in four or five weeks the new roots will be formed. In following out this system, never top a growing shoot, unless it be those that arise around the flowers, as already adverted to; and never omit cutting down the plants every spring after flowering.

Insects. - The white and brown scale are the only enemies the oleander is subject to : their removal is best effected by washing them off with soap and water.

\section{SELECT LIST OF INDIAN AZALEAS.}

Agertonii, alba, alba striata, alba magna, arborea purpurea, ardens, Apollo, Aurora, amœna, ascendens, carnosa elegans, Chelsonii, Californica, candidissima, cruenta elegans, Duc de Brabant, Duke of Devonshire, exquisite, Extranii, Edmondsii, Fielder's white, Frostii, fulgens, Gledstanesii candidissima, Hebe, incomparable, Iveryana, lateritia, lateritia alba, lateritia grandiflora, magnifica, magnifica alba, magniflora, modesta, Mrs Fry, Marie, Murryana, optima, obtiesa, optabilis, pallida, præstantissima, purpurea pleno, Perryana, refulgens, ramentacea, rubra pleno, rosa punctata, rubens, sanguinea, splendens, speciosessima, triumphans, variegata, Vesta, violacea superba, vittata, vittata punctata, vittata Fortunii, vittata rosea.

The other plants we have named as associates to the camellia require almost the same treatment, soil, and temperature with it, except the Indian rhododendrons, which prefer a turfy, sandy, peat soil. They are all propagated by cuttings, and the rhododendrons by grafting, laying, and budding. They are also readily propagated by seed, treated as recommended for the azalea; but they require a greater supply of water during the formation of their young wood and flower buds than the others. The rhododendrons are impatient of heat, and require a partial degree of heat and abundance of water during the period they are making their growth. The Daphnes are increased freely by cuttings, or by grafting on the common stocks of their own genera, the Magnolias by layers, the Olive, Luculia, and Enkianthus, by cuttings VOL II. of the half-ripened wood, planted in sand in a mild temperature, and covered with a bell-glass.

The varieties and even species of rhododendron have been greatly increased of late, both by the introduction of that section of Indian species known as the Sikkim rhododendrons, chiefly discovered by Dr Joseph Hooker, and by numerous hybrids, originated in Europe. Some of the former, being found at an altitude of 14,000 feet, are no doubt hardy; the majority of them, however, cannot be so regarded, and, like Arboreum and its varieties, should have the protection of a glass structure, to which the splendour of their flowers and singularity of their foliage justly entitle them. Many of the finer varieties of the latter flowering too early, even when planted in the open borders, to escape the late spring-frosts, deserve a similar protection, at least during the period of their flowering. These may be grown during the summer months in a sheltered border in the garden, and repotted during winter, and kept in the camelliahouse during spring, and afterwards turned out to perfect their growth, which they do in our climate, if not too early excited and afterwards exposed to late spring-frosts.

\section{SELECT LIST OF RATHER TENDER HYBRID} RHODODENDRONS.

Yellow.- * Aureum punctatum, *aureum superbum, *Bakerianum, *Burlingtonii, *carneum elegantissimum, *carneum versicolor, *conjestum aureum, *crystallinum, *cuperum, *macranthum flavum, *ornatum, *primulum formosum (*Smith's), flavum.

Amongst Scarlets and Crimsons the following merit attention: Adele, atro coccinium, atro rubens, Blandyanum, boquet de flore, Bryanum, Broughtonii, Charles de Loose, Comte de Bobrinsky, Comte de Rambuteau, eximium giganteum, Gontran, Hendersonii, illuminator, invictum, Jacksonii, Lady Warrender, magnificum novum, Mars, Prince Albert, præclarum, princeps tigrinum, Reine d'Angleterre, Rembrandt, rubrum spectabile, triumphans.

White or White-spotted, with other colours. -Abelianum, album elegans, album flavum, Alexandria, Bianca, campanulatum superbum (Jackson's), coriaceum, delectum (Chandler's), delicatissimum (Waterer's), eburneum, luciferum, superbissimum album.

Indian Rhododendrons (species).-Alpinum, argenteum, barbatum, Boothii, calycatum, Campbelliæ, campanulatum, campylocarpum, ciliatum (lilacinum), cinnabarinum, Dalhousiæ, Edgeworthii, Falconerii, formosum (Gibsonii), fulgens, glaucum, Griffithii (Aucklandii), Hodgsonii, Javanicum, jasminiflorum, lanatum, lepidotum, Maddeni, Nilagereanum, niveum, nobile, Paleii, pubescens, robustum, roseum, setosum, Thomsonii, Wallichii, Wightii.

\section{$\S 2$.-ORCHID-HOUSE.}

In vol. i. we have given seven examples of orchid-houses, figs. 556 to 565 , any one of which is well adapted for the purpose. It only remains for us now to speak of the cultivation and 
management of the plants themselves. An extensive natural order of plants such as this is, and found occupying so large a geographical range, sufficiently points out the impossibility of growing them successfully in one house at all seasons of the year, and, indeed, of growing many of those from the same country at all times together, as the seasons of rest do not take place with all the species of any one country at the same time. The temperature of India being much greater than that of China, Japan, and the Brazils, from which many of our finest species come, demonstrates pretty clearly that a considerable difference should exist in culture in this very respect. Where a large collection is cultivated, it will be best to have either three distinct houses, or, if one only, that it be divided into three compartments, each to be capable of being heated more or less, irrespective of the others. The successful cultivation of these plants depends mainly on heat and moisture; yet there is a season when both must be greatly mitigated. Orchids are divided into two distinct groups as regards their habits of growth, some being tcrrestrial, or growing upon the earth; while others, and by far the greater number, are epiphytical, or growing upon trees, \&c. It is necessary that this difference in their habits be understood, so that their position in culture may not be transposed.

Propagation.-This is effected by division of the plant, each piece having either one or more pseudo-bulbs, and if with portions of roots attached, so much the better excmplified in Stanhopea; by division of the plant having fleshy, underground, spreading roots, like Goodyrea; by portions of the plant having aërial roots, as in Renanthera, \&c. It is possible, but it is rarely practised, to propagate them from seed.

Season and soil for potting orchids.-No particular season can be recommended for potting orchids, all depending upon their state, whether theyare healthy or the reverse. If in good health, the majority may be potted soon after their flowering has ceased; for in the majority of cases they begin then to make fresh roots, and commence their season of growth. Now, as their roots are fleshy and tender, and liable to sustain injury while in their growing state, it is better to anticipate this by potting before the new roots have begun to form. Those that are unhealthy should be potted as soon as any indication is observed that derangement has taken place at the roots; and this holds good whether they be grown in pots, baskets, or on logs. A yearly examination, if not a regular potting, should take place, and oftener if the soil appears to be sodden, or if the plants have lost their roots, which many of the more delicate ones are apt to do, both from excess of moisture and the reverse. In either of the latter cases, the whole of the soil should be removed with care, and after re-potting little water should be given at the roots until they have again begun to grow. Regarding soil, the consideration ought to be its capability of retaining or parting with moisture, and neither its richness nor poorness, as orchids depend little on either, so far as their nutriment is concerned.
The most proper soil is that which is not retentive of moisture, or liable to become stagnant, as some peaty soils are, and also that it be capable of resisting decomposition for the longest possible period. The compost we employ is simple in the extreme, and consists of dry turfy peat, beaten upon the potting-bench with a wooden mallet, and passed through a half-inch sieve to subtract the finer earthy particles; for the turfier, light, and more open it is, the better. Some lay great stress on charcoal, in small pieces, being incorporated with the turfy matter; for ourselves, we believe that soft sandstone, reduced to the size of from a hazel-nut to a walnut, or even broken fragments of pots, is equally good. In addition to the above, a portion of silvery sand and of white bog-moss (sphagnum), chopped up into short fragments, is all that is required. This is a good compost for such as are to be set in pots or baskets, and of habits similar to Cymbidium, Cryptopodium, Zygopetalum, \&c. But such as Bletia,Corysanthes, Neottia, \&c. require a greater proportion of the finer portions of peaty matter and silvery sand incorporated with the compost. The great mass, however, seem to prefer being attached to blocks of wood, or planted in baskets, and suspended from the roof. Some recommend these blocks to be charred, but we have found no advantage from this. The whole natural order, with the exception of the true terrestrial genera, Orchis, Habenaria, Dissa, Caladenia, \&c., and those named above, appenr to derive their nourishment more from the atmosphere than from the body they are set upon or planted in.

Pots, baskets, and blocks.-The ordinary garden pot is very generally employed, and used of a size somewhat larger than ordinary plants of the same bulk are accommodated with. Such pots are in general filled with drainage from one-third to one-half of their whole depth. Pots thickly perforated with holes along the sides, others with slits cut out of them, have frequently been used, the object being to admit a free escape to the water, and the admittance of air

Fig. 261.

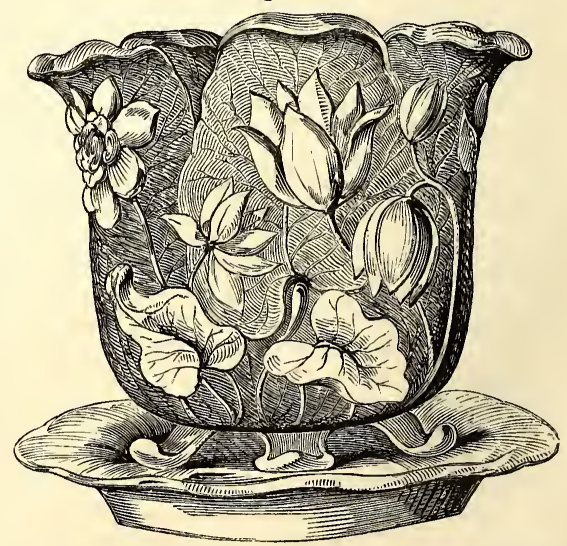

to the roots. More recently, highly ornamental pots (Figs. 261,262), or shallow suspended vases 
(Fig. 263), have been also employed, and with much better effect, more especially when they

Fig. 262.

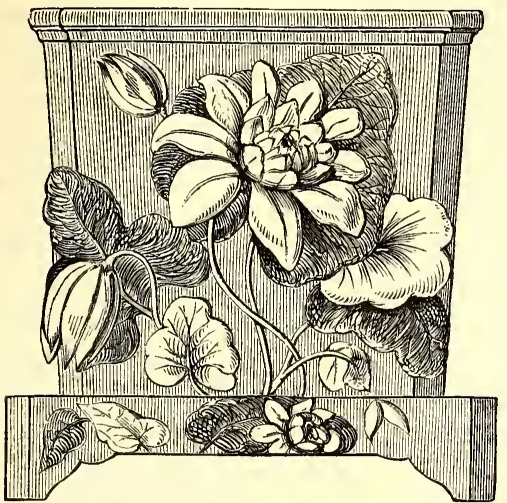

are to be suspended and exposed to view. These and others, in great variety of pattern and elegance of design, are manufactured by Messrs Minton, at Stoke-upon-Trent; and slate-pots have also been more or less employed.

Fig. 263.

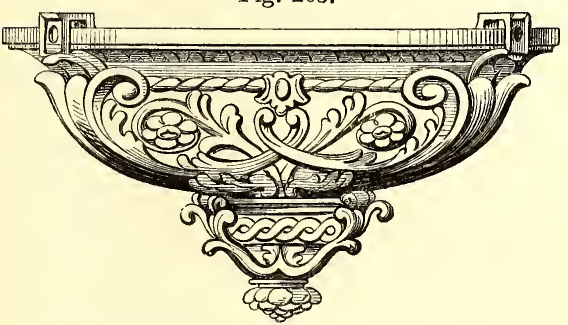

Baskets, chiefly of the rustic description (Fig. 264), are preferred by many, and are capable of being either suspended or set upon the platform of the house. They are exceedingly well adapted to suit genera, as that of Stanhopea, which send their flower-spikes out from toward the region of their roots, and which, if kept in pots without openings in them, would seldom if ever present us with their splendid flowers; whereas, when placed in baskets, the flower-spikes find their way through between the rods, and so do also the Saccalobiums, Vandas, Gongoras, \&c. The best form of the baskets is a square, and one 2 feet on the side need not be more than from 9 to 12 inches in depth. Deep baskets are unnecessary, as there is no space taken up in drainage, and the roots seem to prefer to run close to the surface. The best material we have found for making such baskets are the shoots of elder, larger or smaller, according to the size of the basket. They last much longer than any other material we have used. The most elegant, however, are made from the rough-barked maple ; in both cases the bark is left on. Baskets of wire, painted with anticorrosive paint, and others of copper and brass wire, we liave tried also; the latter of these are expensive, and the former exceedingly liable to decay from oxydation. The great advantage, however, of such baskets is, that they secure the roots from excess of mois-

Fig. 264.

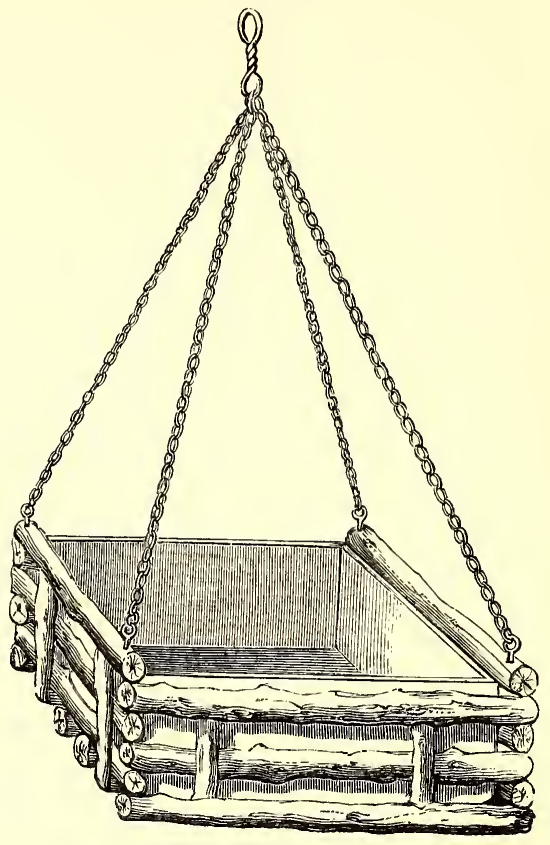

ture, and they seem excellently adapted for the majority of the true epiphytal sorts ; but, like those grown attached to blocks of wood, they require great attention as regards a sufficient amount of moisture. Should this be deficient, they can be taken down and submerged in tepid water. These baskets also admit of placing those species near to the glass which prefer the fullest amount of sun-light. Space is economised by using them, but they should be suspended over the passages so that the drip may not fall into the hearts of others.

Blocks should be larger or smaller according to the plant set upon them; we seldom use them, however, under 3 inches in diameter. The woods we prefer are acacia, cork-oak, and the rough-barked maple. These we find to be durable, and more curious in appearance than blocks of other trees; besides, their bark is not liable to fall off, nor harbour wood-lice. When we employ other than the above, we prefer wood which has been felled the previous year, and has become somewhat seasoned, and these we dis. bark before using. Orchids appear to have no liking to wood of the pine tribe, nor do blocks of that timber last so long in the heat and moisture of an orchid-house as some of the inferior hardwood sorts. Pear and apple wood, when old, and split up into blocks, lasts long, and the roots attach themselves to it freely. Some attach importance to the block being in a green or recently-felled state, and object to those having the old bark attached, as well as to those which have been charred. To the two former points we 
attach little importance, but to charred blocks we object strongly, as they are apt to become too dry, as well as on account of their power of absorbing heat from their black colour. The finer sorts of orchids seldom attach themselves to such blocks, and only the stronger-growing ones do, as they would do to iron, stone, or any other solid material within their reach. The plants should be attached by fine copper or brass wire, and a little sphagnum may be put round the roots at fixing, to retain moisture until the plant is fully established. Blocks should be always suspended perpendicularly, and they should be frequently taken down and examined, particularly during summer, and if too dry, dipped for a few minutes in tepid water.

Potting orchids.-The roots are very liable to attach themselves to the sides of the pots; care should therefore be taken, at turning out of the old pots, that they be not injured. Should they not turn out freely, it will be better to break the pot than injure the roots; and should a few fragments adhere to the roots, they should be allowed to remain. The new pot being thoroughly drained, a little moss should be placed over the drainage, to prevent the finer portions of the soil being washed downwards. The old compost should be entirely removed, and any of the roots that are dead or diseased should be cut away; over the moss place a little of the compost already recommended, the turfier the better; set the plant on this, yet sufficiently high that its crown may be from 1 to 3 inches above the level of the pot, according to its size; work carefully the necessary quantity of soil round the roots. No part except the roots should be covered with soil; and if the plant is of the tall-growing kinds, and likely to fall over, it must be supported by stakes. Water slightly when the potting is finished, and while the operation is going on, keep a sharp look-out for insects. A thorough cleaning of the house should take place at the same time, for their suppression; and if the floors and shelving be watered with boiling water, or caustic limewater, so much the better; for wood-lice and cockroaches, in particular, as soon as the plants begin to be disturbed, will secrete themselves in every available crevice. A very good mode of potting is to invert a pot of rather smaller size within the one in which the plant is to be set; on top of the inner pot set the plant, and if its roots are sufficiently long, allow them to hang down between the one pot and the other; work in the compost carefully in the space between the two, which, if from 2 to 3 inches apart, will be sufficient space for the roots to range in. The roots of most orchids are fond of clinging to the surface of earthenware, and hence some cultivators, instead of filling up the space between the two pots as above, merely throw in a little loose sphagnum to shelter the roots. Those intended to be set in baskets should be similarly treated, covering the bottom with a thin layer of moss, to prevent the finer parts of the soil falling through; a portion of peaty turfy soil, as already referred to, should be laid over the moss, as high as will elevate the plant, when set on it, from 1 to 3 or 4 inches above the sides. The old compost should be removed almost entirely; and if the old baskets, with the plants in them, have been placed in a cistern of water for a day or so previously, the disengagement of the old hard clods from the roots will be more readily effected. It is also an excellent way of dislodging wood-lice and other insects. When the operation is completed, water the new soil thoroughly, support the plant if it require it, and plant pieces of any of the low-growing Lycopodiums on the surface, which will give a healthy green appearance to the plants.

Watering.-It is seldom that orchids, if we except a few truly terrestrial ones, require water at the roots. They, however, during their growing season, require an abundant supply by syringing over their tops; indeed, so often sometimes, during summer, as twice or thrice a-day. The water used should be at least of the same temperature, if not more, than the atmosphere of the house, taking care, however, to avoid throwing it on those that are in bloom. Indeed it is better, where the convenience exists, to place those in flower in a separate apartment, where the flowers may be kept dry and the plants somewhat cool, as both have the effect of greatly prolonging the period of their beauty. During winter, and also during the season of rest in the plants, little water should be given, especially to such as are in pots; but even to this there are exceptions, such as the very beautiful Anœctochilus, which inhabits shaded moist rocks in Borneo, Java, and Ceylon, the Huntleya violacea, H. melagris, \&c., found in similar situations on the river Essequibo. Again, the genera Renanthera, Angræcum, Vanda, Sarcanthus, Phalœnopsis, Aerides, \&c., which have no pseudo bulbs, require more water during the winter than such as are provided with these magazines for storing up aqueous food. They require water most when growing; and here also the quantity greatly depends on the sort, and also upon the stage of its growth; as for example, while the young shoot is beginning to form, and the young roots to appear, little water must be given them, and saturation carefully avoided, particularly during winter, while the shoots are in a young state. It is during spring and summer, when the growth has somewhat advanced, and the sun powerful, that the greatest supplies are required, because evaporation is going on most rapidly, and the plants are in a condition to absorb it in greater quantities.

Shading is important during the intensity of sun-heat; for although some orchids brave the fiercest sun of the tropics, the majority of them are plants growing in moderate shade. (For mode of shading, vide vol. i., p. 555.) It is only, however, during the bright days of summer that shading is required ; for orchids, like most other plants, require all the light our country affords. This points out the absurdity of setting orchidious plants in the most shaded parts of the hothouse; although, under these circumstances, many of the hardier kinds will exist, the finer and more delicate will not, and few even of the former will produce their flowers.

Management of newly-imported orchids.-The plants, being long confined in the dark and close 
air of a packing-case, are ill adapted to being at once placed in fresh air and in the glare of light. Since leaving the tropics they have also been exposed to a much lower temperature than that natural to them; they have been long deprived of their natural supply of humidity; and, indeed, their vital functions may be considered as exceedingly low. To place them at once in the light, heat, and moisture of the orchid-house would be far too rapid a transition. It is better, therefore, to place them on a bed of dry moss in a close pit, the temperature of which is not above $55^{\circ}$ or $60^{\circ}$. Here they should remain partially shaded and slightly moistened until their vital action begins to be excited, and as this takes place they should be removed to another close pit and potted, or attached to blocks according to their nature, gradually stimulated by increased heat, light, and moisture, until they have become sufficiently established. Indeed, it is a good practice to grow all small and weakly plants of this order in such a pit, as in it they will make far greater progress than in a larger house. Orchids are best sent home in a Wardian case, particularly from India, if not forwarded by the overland route. We have often received them by the latter merely packed in light boxes, and packed loosely amongst dry grassy material or inferior cotton. The larger the plants sent home the better they travel, and the more valuable they are.

\section{SELECTION OF ORCHIDS TO BE GROWN ON} BLOCKS.

Barkeria.-The whole genus thrive on blocks, requiring abundance of moisture during their growth, but being kept dry during their period of repose, at which time they shed their foliage. The temperature best suited to the Barkerias, and also, we may state, to all the orchids of Guatemala, is from $60^{\circ}$ to $65^{\circ}$ as a maximum, and from $45^{\circ}$ to $37^{\circ}$ as a minimum. We are informed by Mr Skinner, the well-known importer, that often these plants are exposed to several degrees of frost, and frequently in winter covered with snow. Excessive heat, particularly during their season of rest, or giving no rest at all, is the cause of many of them failing to thrive in this country.

Brasavola.-The whole genus is adapted to blocks, and to the most exposed part of the house, being found in open places exposed to the fullest rays of a tropical sun.

Broughtonia.- When in a growing state, this genus should be kept in a humid and high temperature, but afterwards kept cool and dry.

Burlingtonia.-With the exception of B. candida and rigida, which thrive well in pots, the rest of the genus prefer to be grown on blocks. They require a high and moist atmosphere and partial shade, as they are natives of the dark forests of Brazil.

Camarotis purpurea.-Requires the same culture as Saccolabium, (which see.)

Catasetum.-Although usually cultivated in pots, they grow equally well, if not better, attached to blocks; their natural place of growth being on branches of trees exposed fully to the hot sun of Demerara, they require a temperature equal to from $70^{\circ}$ to $85^{\circ}$ while growing, and a reduction of from $10^{\circ}$ to $20^{\circ}$ while at rest, at which period their leaves are shed. While growing, they require abundance of moisture.

Cattleya, Marginata, Bulbosa, Citrina, Aclandice, and Pumila, prefer block culture, and less heat and moisture than many of the genus. To maintain them in health they also require a pure and free air, and should be kept dry at the roots during autumn and winter.

Cirrhopetalum.-A rare and curious genus, requiring a very moist atmosphere, and a temperature during their season of growth of from $75^{\circ}$ to $85^{\circ}$. During their season of rest the temperature should be reduced to $60^{\circ}$, and little water given.

Loelia. - This is a splendid genus, requiring a medium temperature even during their growing season, and less water than is generally supposed. In their state of rest, the temperature of an ordinary greenhouse is sufficient, and at that period they must be kept dry, but not so much so as to cause their leaves to shrivel. With us most of them flower during autumn and winter, particularly L. superbiens, one of the most magnificent orchids in cultivation.

Oncidium.-This is a very extensive genus, and for the most part fine flowering plants, and, with the exception of 0 . pulchellum, tricolor, uniflorum, uroyphyllum, and triquetrum, are of easy culture. The above exceptions, however, require a warmer and moister treatment, being natives of the West India Islands. They are devoid of pseudo bulbs, and therefore require attention to frequent syringing, as most other orchids so circumstanced do. They require also to be well exposed to light, and kept moderately dry after their season of growth is past.

Phalcenopsis.-A magnificent genus, natives of the warmest climates, therefore requiring more heat than the majority of orchids. They require abundance of moisture during their season of growth, and hence ours aresuspended over the water-tank in which the Victoria Regia is grown.

Renanthera.-Of this genus $R$. coccinea is by far the finest. Being a native of China, it does not require the same temperature as some of the Inảian ones do. It is, however, a capricious plant as regards flowering, and was for years after its introduction considered a difficult plant to manage. It flowered for the first time in Europe in the Royal Gardens at Claremont, into which it was imported from Cochin-China the year before we were appointed to the charge of that establishment, and continued to flower profusely for several years before it did so elsewhere. The plant was merely attached to the stem of a dead tree set at one end of a stove containing a general collection of tropical plants, and no particular care was taken of it; yet it not only flowered profusely annually, but ultimately covered a great portion of the roof of the houseso much so, that we were often obliged to reduce its limits considerably. A portion of the same plant we have in the collection of orchids at Dalkeith, which, although established fourteen 
years, has only once flowered; while a plant we sent to Drumlanrig Castle nearly twenty years ago, and which is grown in a stove along with a mixed collection, flowers most profusely, and covers the whole roof over the back passage to such an extent as to require annual curtailment; yet neither there, nor, as we have already stated, at Claremont, is any other treatment employed than that of the other stove-plants in general. This is a most desirable plant, and has been experimented upon, with a view to insure its yearly production of bloom, by many of our first-rate orchid-growers, whose experience has led to the following conclusions-namely : The plant must be at least 4 or 5 feet in length before it will be in a condition to bloom. Its season of rest should commence about the beginning of November, and be continued till the end of February, during which period it should be placed in a day temperature not exceeding $60^{\circ}$, with a fall during night of $10^{\circ}$. The atmosphere during this time should be kept moderately dry, and the plant deprived of water over its leaves. From the beginning of March to the end of June should constitute its growing season; during March and April it should be kept in a temperature of $70^{\circ}$ by day, and $60^{\circ}$ by night, rising to $85^{\circ}$ by day, and $70^{\circ}$ by night, during May and June, and during the whole of this period supplied abundantly with moisture. July, August, and September will thus become the flowering season, during which the temperature should be reduced to $70^{\circ}$ by day, and $60^{\circ}$ during the night, and now kept comparatively dry, and the plant placed as near to the glass as possible. Should this treatment not induce flowers during the first season, a repetition of the same routine during the second assuredly will. The plant should be secured to the trunk of a tree 4 or 5 inches in diameter, which will retain it in a portable state. Small bundles of moss are fastened to the stems to retain moisture, while the roots are allowed to extend themselves in the air without interruption.

Schomburgkia. - The roots of this fine genus, being impatient of much moisture, should have the blocks upon which they are fixed suspended vertically, and not horizontally, as in general cases. A high humid temperature and atmosphere is required while the plants are growing. When the growing season is past they should be removed to a much cooler and drier house, and water entirely withheld; their period of rest should extend to four or five months.

Sophronitis.-A genus of small-growing plants, growing on the very tops of lofty trees on the Organ Mountains; they therefore do not require a high temperature, but a moderate degree of humidity while growing, and a comparatively dry atmosphere while in a state of rest.

Vanilia.-This is perhaps the most valuable of all tropical orchids, in a commercial point of view, producing as it does the high-priced and rare vanilla of the shops. The vanilla is obtained from the seed-pods of the plant, and was first produced in Europe by Professor Morine, of the University of Liege. It has fruited in various places in England since that time, and may be made to do so freely if attention is paid to fertil- ising the flowers, which, with healthy and freegrowing plants, is all that is necessary. The whole genus Vanilia are true epiphytes, their strong fleshy roots clinging to any solid substance within their reach, the stem of a tree being, however, preferred. The plant roots also freely in half-decayed tan, and grows with great rapidity. Nor is it only the pods of this plant which render it an important inhabitant of our stoves; its flowers are large, beautiful, and agreeably fragrant during the night.

\section{SELECTION OF ORCHIDS WHICH SHOULD BE GROWN IN BASKETS.}

Angrcecum.-Requires a high temperature while in the act of growing, accompanied with a regular but moderate supply of moisture; they should occupy the warmest house or division during that period, and be removed to the intermediate one during their season of rest and drought.

Cirrhcea.-From the manner of their flowering, which is in long drooping racemes, they are better adapted to basket than to pot culture, in which latter they do equally well, only their flowers are shown to much less advantage. Being natives of Brazil, the intermediate temperature is suitable for them.

Dendrobium.-This extensive and splendid genus thrives well when set in baskets, but as many of them attain a considerable height, a block of wood, of from 1 to 2 feet in length, should be planted in the basket firmly, to which the plants will attach themselves as they extend in height. Above the wooden support, which acts as a trunk or stem, the roots will extend themselves in the air, and draw nourishment from it. This genus extends over a considerable extent of latitude ; some, therefore, require accommodation in the warmer compartment, and others in the intermediate one. D. speciosum requires only a temperature of from $45^{\circ}$ to $55^{\circ}$ during winter, and not more than $55^{\circ}$ to $65^{\circ}$ during summer.

Saccolabium. - A splendid genus, requiring abundance of heat and moisture during their growing season; a cooler and drier abode during their season of rest. They should not be disturbed above once in two years, therefore give them plenty of root-room at first starting.

Stanhopea.-The flower-spikes proceeding from the bottom part of the plant often quite horizontally, although sometimes perpendicularly downwards, baskets are found indispensable for their proper cultivation. It is a strong-growing and free-flowering genus, and should have plenty of room at the roots. An intermediate temperature is best for them, keeping them moderately moist while in a growing state. They are also robust in constitution, and will accommodate themselves to a considerable range of temperature, but will require moisture in due proportion to the temperature they are in.

Vanda.-The whole genus requires a high temperature, with corresponding humidity, during their season; ours are kept in the Victoria-house, and seem to thrive well. Their growing season appears to be from May to September. During the rest of the year they should be kept 
rather drier and cooler, and for this reason should be placed in the warmest part of the intermediate house. In the month of April, if they can be accommodated with a temperature of $75^{\circ}$ by day, and kept still moderately dry, their wood will ripen, and flower-buds be induced to form. Afterwards, if again placed in the warmer division with plenty of moisture, they will flower freely.

\section{SELECTION OF ORCHIDS WHICH SHOULD BE} GROWN IN POTS.

Anguloa. - A genus of fine-flowering, freegrowing plants ; they are natives of the temperate parts of South America; therefore during their season of rest they require little more than exclusion from frost. During their growing period they require a day temperature of from $65^{\circ}$ to $70^{\circ}$, accompanied with a corresponding degree of humidity. Their season of rest should extend from the end of September to the middle of March.

Anoctochilus.-A genus of exceedingly beautiful leaved plants, but without any particular beauty in their flowers. They are natives of Borneo, Java, and Ceylon, found on moist shaded rocks; they prosper well in pots well drained and filled with sandy peat, small fragments of rotten twigs, and covered with green moss. (We cover ours with dwarf Lycopods). They should be kept covered with a bell-glass, and never be allowed to flower, particularly while young and weak. As the leaves are their greatest beauty, the flowers may safely be sacrificed to them. A shaded moist part of a warm orchid-house we have found best for these plants.

Arundina. - Require a high temperature, being natives of the warmer parts of India. As they retain their leaves at all times, they require a moderate supply of water, even during the season of rest, which, with our plants, seems to coincide with our natural winter. They seem to prefer a soil of half peat and half semidecayed tree-leaves.

Bletia. - Some of this genus have been amongst our longest cultivated orchids. They are truly terrestrial, being always found growing in soil in their native habitats. They appear to thrive in strong loam in their native country, but the same soil has been found unfitted for them in a cultivated state, light fibrous sandy loam seeming to suit them best; and although some recommend the addition of peat earth, we have not found it beneficial. Deciduous terrestrial orchids, such as these are, are seldom found in anything like perfection in our collections. Their culture, indeed, has been little understood. The season of potting most suitable appears to be February, when the old soil should be completely shaken from the roots. In potting, let the bulbs be just covered, and no more, with the fresh compost, which should be turfy fibrous light loam. The pots should then be set in an intermediate temperature, slightly watered, and placed as near to the glass as possible, until the shoots and leaves appear, after which the quantity of water should be increased; for during the growth of the leaves and the production of the flower stems they can scarcely have too much water, air, and full exposure to light. As the new bulbs attain their full size, the foliage will begin to show symptoms of ripeness by changing to a yellowish hue. After this, water should be gradually withheld, and the pots removed to a cool house or cold pit. Keep them in a state of rest till February, and re-pot them as before.

Brassia.-Although this genus succeeds very well in pots, we grow most of ours in baskets; but these are not suspended. The genus occupies a great range of latitude, and consequently requires some difference in temperature. This must be considered and acted upon.

Burlingtonia candida and regida, as we have already stated, thrive better in pots than treated like the rest of the genus. The latter requires to be placed in a thoroughly drained pot in very porous material; and as it is naturally a plant of shade, it should be placed in the warmest division of the orchid-house in a moderately shaded place, and supplied abundantly with water throughout the spring and summer, which is its growing period. During its season of rest, which coincides with our natural autumn and winter, give it less water and heat.

Calanthe.-The treatment recommended for Bletia is applicable to Calanthe, excepting that the species of that genus which retain their leaves requires more water during their season of rest, as well as a rather higher degree of temperature. They also require a greater degree of heat and moisture while in their growing state.

Catasetum.-We have already stated that this singular genus will thrive when grown on blocks of wood; and we might readily take this for granted, seeing they inhabit the highest branches of trees in the hottest of the South American forests. In culture, however, they are found to thrive equally well when planted in pots in very fibrous and porous material, provided with ample drainage. Being naturally exposed to the sun, they should occupy a place in the orchidhouse where as much light as possible may reach them.

Cattleya.-We have already given the names of those of this genus which prefer growing upon blocks; a much greater number, however, and those of a high degree of merit, in this splendid family, are found to prosper in pots in properly prepared compost. And this compost should consist of the most fibrous material possible, such as the remaining vegetable matter in turfy peat, after the finer or more decomposed parts are separated by beating it well and sifting. To this add sphagnum chopped small, with lumps of sandstone about an inch cube, and a few broken fragments of pots. The temperature throughout the growing season should range from $75^{\circ}$ to $80^{\circ}$, and during sunshine it may rise to $85^{\circ}$, while during night from $65^{\circ}$ to $70^{\circ}$ will be ample. Water freely during the growing time, but be careful that none lodge in the centres of the young shoots. The temperature during the season of rest should be regulated to from $55^{\circ}$ at night to $65^{\circ}$ during the day, at which time watering should be 
almost suspended, and the house kept moderately dry.

Coelogyne. - A very pretty genus. Many of them have long creeping stems which should be trained to a moss-clad block of wood set upright in the pot; if this is not done, they will extend over the edges, and be liable to be broken. During their growing season, which continues over the greater part of summer, give water freely, but chiefly in the morning, that the plants may dry before the full sun shines on them. Shade for an hour or two during very hot weather, and endeavour to have the growth completed before the approach of autumn. During their period of rest, temperature and humidity must be reduced, and watering discontinued upon the approach of cold weather.

Coryanthes. - Drainage, in the case of this genus, is of the greatest importance, as the roots are impatient of moisture; the same compost as recommended for Cattleya should be used. Growth should be encouraged about the beginning of March, and as the summer advances the temperature should be raised to $85^{\circ}$ by day and $70^{\circ}$ by night, as a maximum. While the pseudo bulbs are forming, water should be given freely; and when they have attained their full size, excitement should be checked to bring on a season of rest, during which they must be kept both cool and dry.

Cycnoches.-The growing season of this genus begins in general in March, at which time they should be shifted. The soil to be used, and the treatment generally, should be the same as for Coryanthes. The genera Cycnoches, unlike most other plants of this order, flower as soon as the pseudo bulbs are fully formed; whereas, in the case of most other orchids, flowering does not take place until the pseudo bulbs are not only fully formed, but have rested some time afterwards. As soon as the flowers fade, their period of rest commences, when water should be withheld, and the bulbs be allowed to remain dry till the potting season again returns, which is about March.

Cymbidium.-A genus of very easy culture, requiring a light porous soil, composed of fibrous loam, leaf-mould, and half-rotten twigs. They all require a high temperature, say from $70^{\circ}$ to $80^{\circ}$, and abundance of water at their roots, as well as occasional syringings during their growing season. After their new leaves are made and fully developed, reduce humidity, but do not withdraw it entirely, as the plants require a moderate supply, even during their season of rest, at which time the temperature may be allowed to fall to from $55^{\circ}$ to $60^{\circ}$.

Cypripedium.-All the genus, whether tropical or extra-tropical, are terrestrial, and delight in a soil abounding in vegetable matter, such as leafmould, rotten twigs, turfy peat, with a portion of sand to keep the whole open. They should be potted in March in well-drained pots, rather deep than otherwise. Their growing season is from March to August, during which they should have abundance of water; and the tropical ones will do better in a close pit during summer than elsewhere. From August to March they should be kept rather dry, giving just enough to keep them from flagging. In general they are kept too hot in cultivation, and are more adapted to the greenhouse or close pit than the regular orchid-house.

Cyrtopodium.-These are also true terrestrial plants; they require large pots, well drained, and a soil composed of very turfy light loam, a little leaf-mould, and a few crocks broken small and intermixed. They should be potted just when they show signs of vegetation, and placed in a warm part of the house, supplied at first with a moderate supply of water, which must be increased as growth proceeds. After flowering, and when the stems have completed their growth, water should be at first reduced in quantity, and while they are at rest it should be entirely withheld. They winter best in a temperature of from $55^{\circ}$ to $60^{\circ}$.

Dendrobium.-We have already stated that many of them may be grown in baskets, if sufficient compost be afforded them, and only those suspended that are of dwarf growth; the whole family may be so treated, leaving the baskets to stand on the stages or platforms. Again, on the other hand, if care be taken to give very ample drainage, and a sufficiently turfy porous compost, they may be all grown in pots also. One important consideration in their culture is to bear in mind that Dendrobiums have naturally three seasons annually. In the rainy and warm season they make their growths, in the cool season they rest, and in the hot and dry season they flower. With what has been already said regarding seasons of growth, and those of rest, the above may serve as a key to the cultivation of this noble tribe to cultivators of ordinary understanding.

Epidendrum.-A large family, but much less interesting than many of those we have named. Some of them thrive well in pot culture, and the majority may be grown in blocks or in baskets, and treated like Cattleyas, \&c.

Galeandra.-Chiefly from the warmest parts of South America, consequently requiring a temperature, while in a growing state, of from $75^{\circ}$ to $85^{\circ}$ during the day, and of $70^{\circ}$ during the night. The usual turfy compost used for orchids, and the necessary precautions of drainage, are required. During the season of rest, the day temperature should not exceed from $60^{\circ}$ to $65^{\circ}$, falling during night to $55^{\circ}$.

Govenia.-Are natives of the plains of Mexico, where the heat is excessive, and the rains almost continuous, during their growing season, which extends from March to September. During their season of rest, which occupies the rest of the year, they are exposed to great drought, with not much of diminished temperature.

Grammatophyllum.-A fine but rare genus, whose cultivation is not as yet sufficiently well known to admit of any precise directions being given.

Houlletia.-A temperature of from $70^{\circ}$ to $75^{\circ}$ by day, and $65^{\circ}$ during night, is sufficient for it during its growing season; while during its period of rest, from $50^{\circ}$ to $60^{\circ}$ is abundant. They 
require abundance of humidity while growing, and to be kept very dry at the root, yet in a somewhat moist atmosphere, during the first part of their period of rest, and afterwards much drier, taking care, however, that the pseudo bulbs and foliage do not actually shrivel.

Lissochilus.-A true terrestrial genus; natives of the warmest parts of Africa, growing in open and exposed places. Contrary to the majority of even terrestrial orchids, they require a pretty strong soil, three parts loam, with a little leafmould, sand, and turfy peat. A day temperature of from $70^{\circ}$ to $80^{\circ}$ is required, falling to $65^{\circ}$ during the night. During winter the roots should be kept rather dry, but with a moderate humidity in the atmosphere, with a temperature of from $55^{\circ}$ to $60^{\circ}$ during the night.

Lycaste.-Turfy peaty soil, with thorough drainage; a temperature of from $65^{\circ}$ to $70^{\circ}$ by day during their growing period, and from $55^{\circ}$ to $60^{\circ}$ by day during their period of rest. While growing, they require abundance of moisture, but diminished when that season is past.

Maxillaria.-Soil very rough, fibrous, peaty turf, with a small addition of chopped sphagnum and pieces of charcoal intermixed. Being chiefly natives of Guatemala, they do not require a great heat, but, like all other plants, they do require a season of rest as soon as their flowering and growth have ceased; and if kept dry at this time, the temperature may fall as low as $45^{\circ}$.

Miltonia.-They will thrive well in a similar soil to the last, and the most perfect drainage. They also require to be set in a somewhat shaded part of the house, as too much light causes the leaves to turn yellowish, and imperfect drainage produces a like effect. They are natives of the woods of Brazil, and therefore impatient of strong sunshine. Their growing temperature should be from $75^{\circ}$ to $80^{\circ}$ during the day, and $65^{\circ}$ during the night. While in a state of rest, it should fall to $60^{\circ}$ by day, and $55^{\circ}$ during the night. During their growth they require abundance of moisture, but this must be gradually lessened after they have completed their growth, and no more given afterwards than is sufficient to keep the pseudobulbs and leaves from shrivelling.

Mormodes.-Soil similar to the last, with a temperature, while in a growing state, of from $70^{\circ}$ to $80^{\circ}$ by day, $65^{\circ}$ by night. During their season of rest it should be allowed to decline to $55^{\circ}$ by night and $60^{\circ}$ during the day.

Odontoglossum.- Soil similar to the last, with a day temperature of from $65^{\circ}$ to $70^{\circ}$ during its season of growth; during its period of rest it may safely decline to from $45^{\circ}$ to $50^{\circ}$. The whole of the genus is of easy cultivation, and highly deserving of being admitted into every collection.

Oncidium.-Many of these thrive well, as we have noticed elsewhere, on blocks; the majority, however, prefer to be grown in pots, in an open porous soil, exactly similar to that recommended for the last three genera. Occupying as they do so large a geographical distribution, some difference, as a matter of course, exists as regards their proper temperature and culture. Those VOL. II. acquainted with the genus will have observed a marked distinction in the form and size of their leaves-so much so, indeed, as to constitute two very distinct sections, the first having large, thick, fleshy leaves, altogether devoid of, or with very slightly marked pseudo-bulbs or swellings at their base; 0 . Cavendishianum, Cavendishi, luridum, Lanceanum, \&c., forming types of this class, while 0 . sphacelatum, leucochilum, \&c., form types of the second class, which have large pseudo-bulbs, and in general smaller, thinner, and longer leaves. For the most part, the plants of the first section are indigenous to the tropical parts of the New World, and consequently require more heat than those of the second section, which are chiefly natives of elevated parts of Guatemala. During the growing season of the first section, abundance of water is required both at the roots and also in the atmosphere of the house, until the young leaves are fully matured. Afterwards lessen the quantity of water gradually, and during their season of repose give little or none; at same time reduce the temperature also. As regards the second section, the soil and shifting are identical with the first, but the temperature must be kept much lower; and their proper place will be in the cooler part of the intermediate house, or even the warmest part of the coldest house. As regards humidity, the same rule must be observed as in the case of the first section, the pseudo-bulbs containing within themselves quite sufficient moisture to maintain the plants in health until the growing season returns.

Peristeria.-A stronger and richer soil is required for this family than for most other orchids-fibrous, sandy, loamy soil, with a portion of leaf-mould, and a small portion of sand, intermixed with pieces of charcoal. Give ample pot-room, as the plants are strong and require nutriment. As natives of central America they require a temperament of $75^{\circ}$ to $80^{\circ}$ by day, and from $65^{\circ}$ to $70^{\circ}$ by night, during their growing season; and while at rest, from $55^{\circ}$ to $60^{\circ}$ will be quite sufficient for them, keeping them moderately dry during their season of repose.

Phaius.-A true terrestrial genus. Soil should be of a loamy nature, mixed with the leaves and twigs of trees. Temperature while growing from $75^{\circ}$ to $80^{\circ}$, and from $65^{\circ}$ to $70^{\circ}$ during the night. They also require a mild bottom-heat and a moderate degree of shade. During the growing season water freely, but during their season of rest water sparingly if at all.

Schomburgkia is often successfully grown upon blocks of wood; but becoming in time too large, and requiring more root nourishment, it has been found expedient to pot them after they have become well established. Thorough drainage, and fibrous turfy soil, with which pieces of charcoal and broken crocks are incorporated, is the most proper. Indeed, some cultivators grow them in broken crocks alone; but this certainly does not afford sufficient nourishment to enable them to make large-sized pseudo-bulbs. A temperature during growth of from $80^{\circ}$ to $85^{\circ}$, with $10^{\circ}$ less during their period of rest, is the heat in which they appear to thrive best.

Sobralia.-This genus prefers a strong loamy 
soil, approaching to clay, and is found growing on little hillocks rising out of marshy places. The growing season is from April to September, during which period they require abundance of water, and in a temperature not exceeding $75^{\circ}$ in summer, or falling below $45^{\circ}$ during winter, which is their season of rest, at which time they should be kept comparatively dry.

Stenorhynchus. - A genus of true terrestrial orchids of easy culture. Soil turfy loam, and temperature that of tropical orchids in general.

Trichopilia.-Requires the usual soil of orchids in general, with a temperature during the growing season of from $70^{\circ}$ to $75^{\circ}$, giving abundance of moisture. From $50^{\circ}$ to $60^{\circ}$ is a good medium winter-heat, accompanied with a much drier atmosphere, as well as much less water at their roots.

Zygopetalum.-This very interesting family of terrestrial orchids is easily managed if potted in light fibrous turfy soil, well drained, and kept in a temperature during their growing season of from $70^{\circ}$ to $75^{\circ}$, with a humid atmosphere, and a moderate supply of water only at the roots, reducing the heat during their period of rest to the extent of $10^{\circ}$ from the above.

The natural order of orchidaceous plants is so extensive, that to do anything like justice to a description of them, or their culture in detail, would require a volume. The excellent treatise on their culture by $\mathrm{Mr}$ Lyons of Mullingar, the papers by Mr Gordon in the "Journal of the Horticultural Society of London," and the excellent articles on Exotic Orchidaceæ, by $\mathrm{Mr}$ Appleby, in "The Cottage Gardener," may all be read with great advantage by the inquiring cultivator. The botanical periodicals, and the splendid works on orchids - such as those by $\mathrm{Mr}$ Bateman, Dr Lindley, \&c.--are almost beyond the reach of readers in moderate circumstances.

A selection of fifty sorts taken from the collection at Dalkeith, with their season of flowering. - Aerides odorata - May, June, and July; Aerides crispa-July and August; Brassia macrostachya-spring and autumn; Barkeria spectabilis-June and July; Cattleya Skinneri -February and March; Cattleya Mossiæ (of this we have four varieties)-May to August; Cattleya crispa-July and August; Cœlogyne cristata-April and May ; C. Wallichiana-September and October; Coryanthes macranthaJune; Chysis bractescens-March and July; Cymbidium Mastersii-August and September; Dendrobium Wallichianum-February to May; D. formosum-February, March, and April; D. pulchellum-April and May; D. Dalhousianum-March and April; D. chrysanthumFebruary, March, and April ; D. cucullatumMarch and April; Lycaste Skinneri-November to April ; L. aromatica-February and March; Lælia superbiens-November to January; L. anceps-November and December; L. Perrinii -October and November; L. acuminata-No. vember and December; Mormodes luxatumOctober and November; Miltonia spectabilisAugust and September; M. candida, of which we have two varieties-August and September; Odontoglossum grande-September and October; O. citrosmum-September and October;
Oncidium Lanceanum-June, July, and August: O. guttatum-May and June; $O$. sphacelatum - May and June; O. flexuosum - May and June; O. tricolor-May and June; Phalœnopsis amabilis--nearly the whole year; Peristeria elata - August and September; Renanthera coccinea-June to November; Saccolabium guttatum - May to August; Stanhopea aureaJune to September; S. tigrina-June to August; S. grandiflora-July and August; Sobralia macrantha-July and August; Trichopilia tortilisseveral times during the year; Vanda teres-June to August; Zygopetalum Mackayii (of this we have several varieties)-November, December, January, and February ; Cattleya superba-July and August; Cattleya citrina-July and August.

The orchid-house is a very fitting habitation for the genera Tillandsia, Billbergia, Pitcairnia, Echmea, Eschynanthus, \&c., all of which may be successfully cultivated either in pots or suspended baskets, and treated, as to soil, temperature, and water, much the same as has been detailed above for orchids, as well as the singular genus Nepenthus, and the equally curious Cephalotus, or Pitcher plants.

Ferns.-The more shaded parts are also an excellent position for tropical ferns, and many of them may be advantageously grown intermixed with the orchids, their slender and elegant fronds filling up the blanks between, and forming an excellent contrast with the stiff and formal foliage of the latter. Ferns are chiefly natives of shady woods, growing in the half-decomposed vegetable matter formed by the exfoliation of trees and shrubs, sometimes growing out of the crevices of rocks, often in the soil beneath, and also upon old or decaying trees, but in almost all cases accompanied with moisture, and in general where decaying vegetable matter exists. Although they delight in moisture, that must not be in a state of stagnation; and, therefore, when grown in pots, attention should be paid to ample drainage. Spring is the most proper time for shifting them, and water must be administered abundantly; for if ever ferns are allowed to become too dry, their death is next to certain. They are propagated by seed, by division of the plant, and some of them by forming viviparous plants towards the points and edges of the fronds. The seeds or sporules exist on the back of the fronds, and are arranged in different forms in particular genera, some in form of round brownish dots, some along the whole margin of the leaves, and others in lines running from the midrib to the margin, \&c. In either case the seeds or sporules, when ripe, may be brushed off with a soft brush, and, if allowed to fall upon a smooth surface of damp soil, in a shady warm place, but not covered as in ordinary seed-sowing, on account of their extreme minuteness, they will vegetate freely. We have often, by brushing the seed of even dried specimens, and allowing them to fall upon the surface of a piece of soft sandstone, reared fine crops of young plants. In the Royal Gardens at Kew, Mr Smith, one of our first cryptogamic botanists and cultivators, has a shelf filled with rather strong loam, upon which he scatters the sporules of many rare ferns, and 
in this simple way rears abundance of plants. Water, when applied to keep the soil in a proper degree of moisture, must be applied with the greatest care, and administered with the finest perforated cap on the syringe or watering-pot possible, else the seeds will run a great risk of being washed off. It is well in all cases to cover the surface on which the sporules are sown with hand or bell glasses, and to allow them to remain until the plants have attained a size nearly fit for potting off. After they are transferred to the pots, even these should be again covered for some time, as the young plants are extremely delicate, and require to be grown for a time in a still humid atmosphere.

The vapour arising from carbonate of ammonia has been applied to orchids with marked advantage, causing them to assume a much more bealthy and vigorous appearance. It may be applied by dissolving about two ounces of carbonate for a large house, and sprinkling the hot-water pipes or flues about twice a-week. A strong ammoniacal sinell will be immediately perceptible, similar to that of stable manure in a high state of fermentation.

Insects and diseases.-The most destructive of the former are the cockroach, fig. 265, and the woodlouse, fig. 266 ; the latter is the most to be dreaded on account of its powers of multiplying and the difficulty of combating it. They are sel-

dom seen during the day, and only
The Cockrodich. Male and Fernale.

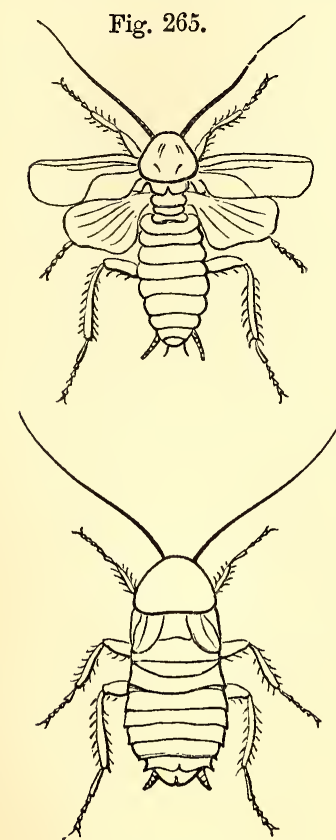
feed during the night, or in the dark, and secrete themselves in the warmer parts of the house, generally in the vicinity of the boiler or under the hot-water pipes. They also find their way within the pots, locating themselves amongst the crocks, and feeding upon the roots unseen. They dislike water, and may be dislodged if the plant is plunged in that element. We find keeping the places near the boiler and under the pipes saturated with water prevents them taking up theirabode there, while it also secures the amount of humidity necessary in the atmosphere of the house. The slug, snail, white scale, brown scale, mealy bug, black and green aphides, thrips and red-spider, all attack them, but none are so much to be dreaded as the two first. Indeed, the aphides, thrip, and red-spider only appear upon them when something is wrong in the art of culture. Excessive heats, droughts, \&c., are the cause, the appearance of the insects the effect. Not so with the two former, and especially as regards the cockroach, for every additional plant added to the collection may carry with it, if not the perfect insect, its eggs ; and this is very much the case when orchids are imported, it not unfrequently happening that whole cases of these plants are entirely devoured by these insects during their passage home. On the arrival of plants from abroad, the utmost vigilance should be exercised in searching for such dangerous enemies. We, in general, after examining the plants of orchids carefully, and after removing all dead or decaying matter attached to them, plunge them two or three times in water heated to $100^{\circ}$, and afterwards wash them thoroughly in tepid water, using a brush for the removal of their eggs, which water at any temperature less than would destroy the plant would not effect. Cases of imported plants should never be unpacked in or near any of the hothouses, but in some place distant from them in winter, and in summer the operation may be carried on even at a distance from the garden. Amongst traps, we have used common hyacinth glasses, half-filled with sugar and water, or any sweet mucilage, a little of it being smeared round the mouth of the glass, set amongst the plants, but placing a narrow slip of wood as a bridge between the plants and the bottle. The cockroaches or beetles, attracted by the sweetness, fall into the water. Poison is also resorted to, and a mixture of lard, houey, and arsenic is formed into paste, and small pieces of it laid about on the shelving

Fig. 266.
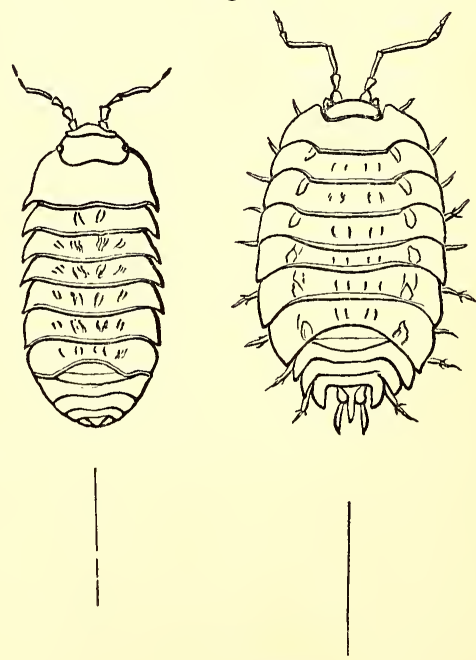

WOOD LICE.

or other parts of the house. Bell-glasses inverted, plunged in sand to the rim, and half 
filled with water, covered with a piece of stout writing-paper having a slit cut out in form of a cross at the centre, and of the length of 3 inches or so each way, will act as an excellent pitfall for beetles, if a little sugar or honey be placed over the slit. In attempting to devour the bait, the paper opens, and they fall through, while it regains its former state unless the house be kept very damp, which destroys its elasticity. Great attention should be paid to cleanliness in every part of the house, which is one of the best means of keeping insects away. The best trap for wood-lice is cutting the stalks of beans into short lengths and placing them on the soil or near the plants; the wood-lice, which feed during the night, will take shelter within the stalk, and may be readily captured in the morning by blowing in at one end, holding the other end over a pot of water. Slices of potatoes, carrots, turnips, \&c., laid about, will also make an excellent bait for them as well as slugs, and if examined early in the morning they may be readily destroyed.

The white and brown scale are only to be got rid of by washing them off with soap and water, using a small hard-brush-an old tooth-brush, for example.

The aphides are destroyed by means of tobacco smoke, but they are seldom very injurious.

The great and decisive combat against insects should take place at the time of the general shifting of the plants; and to render this the more effective, the plants should be all removed to another house for a time, to admit of a thorough cleansing of the walls, woodwork, shelving, floor's, \&c., each plant being carefully examined before it is brought back. The plants which may not have required re-potting, or taking out of the baskets or from the blocks of wood, should be submerged for a minute or so in a tub of tepid water, which will dislodge any insects that may be lurking about their roots. This process is often necessary at various seasons of the year, particularly if insects be suspected to be preying upon the roots. The brown ant is also often vexatious, but being a day-feeder is easier detected, and is destroyed by a mixture of loaf-sugar and arsenic mixed together, and laid on broken crocks placed amongst the pots or on the surface of the blocks or baskets.

\section{$\S$ 3.-BUIB-HOUSE.}

Bulbous - rooted plants of great beauty and interest constitute a very large portion of the rich floras of the Cape of Good Hope, Chili, Brazil, Peru, Mexico; and various parts of South America and Australia, China, the East and West Indies, contribute their share, although to a considerably less extent. The art of man has greatly added to the number of varieties, both in our own country and on the Continent; some of the genera, such as Amaryllis, have been hybridised to such an extent that hybrids far exceed the number of admitted species. Tropical bulbous plants, notwithstanding their gorgeous display while in bloom, have by some strange caprice found comparatively few admirers in this country, and hence their cultivation has with few exceptions been greatly neglected. Their habits being peculiar may to some extent account for this. Being indigenous to countries so wide apart, and so diversified in regard to climate and other natural circum. stances, it might be supposed that considerable difficulty would attend their cultivation in an artificial state. Such is, however, by no means the case ; and those who have paid even a moderate degree of attention to their culture have found this to be so. Three structures are necessary for their successful cultivation; one for their accommodation during their season of repose, another for them while in a growing state, and a third in which a higher temperature can be kept up for growing and flowering those from the warmest parts of the tropics.

As the great majority of these plants, even when in flower, do not exceed 3 feet in height, lofty houses are exceedingly ill-adapted for them; and as they for the most part grow naturally under an almost perpendicular sun on the most exposed plains, they should be grown near the glass, and the structure should be constructed so that the greatest amount of the sun's rays may act upon it. No form, therefore, is so well adapted as that of the span or curvilinear mode of roofing; and no internal arrangement better than flat platforms, on which to place the plants so that their foliage may be fully exposed to light on all sides. The house may be 60 feet in length, divided into three compartments of 20 feet in length each, and 15 feet in width within, the apex of the roof 8 feet, and the sides (if perpendicular) 5 feet high. A platform 3 feet broad should occupy the centre, having a passage of the same breadth passing round it leaving a space on each side between it and the fronts of 3 feet for side-platforms, under which the hot-water pipes should be placed. These platforms, to be economical, durable, and elegant, should be in part covered with Welsh slate, supported on iron brackets ; and if the platforms be furnished with a neat beading of slate along their edges, so much the better. Or a covering of ornamental cast-iron grating supported in the same manner will be found in some cases even more suitable, as it will admit of a circulation of air being kept up around the plants. During the flowering season most bulbs prefer a moderately humid atmosphere, and during the growth of most of them this is essential. The beading, therefore, will admit of water being kept on the surface of the slate platform, and the pots may occasionally stand immersed to the depth of half an inch or so; or those kinds may be elevated whose roots do not require the same degree of moisture to that extent, by placing slips of slate under them. It is better humidity be supplied in this way than by syringing the plants too often over the foliage. The taller-growing kinds, and those having long flower-stems, should occupy the centre platform, and those of more humble growth the two side ones. The sandy or alluvial deposits in which most bulbs grow in the tropics, are heated to a very considerable degree by the vertical sun; and the capacity of 
such soils for absorbing and retaining heat is very great. To imitate nature in this respect, the platforms in the division destined for the truly tropical species, or others while in a state of growing excitement, should be constructed on the tank principle, the heated water being supplied from the pipes, as shown in various figs., vol. i., art. TaNK-HEATING, \&c. : over these tanks a bed of clean river-sand should be placed, and the pots set on or plunged into it.

The internal arrangement of the other two divisions need differ in no other respect from this, excepting dispensing with the tank under the platform. One boiler placed in the centre of this structure will be ample heating power. The warmest division, for very obvious reasons, should be the centre one.

Season of rest.-From bulbous plants associating so ill, both in appearance and culture, with other stove and greenhouse plants, and their giving more evident signs of a season of torpidity, by the partial or total dying away of their leaves, they are too often cast aside immediateiy after flowering, placed in some obscure nook, or perched on a lofty shelf to be out of the way, and in all these cases next to totally neglected. When their season of rest arrives, they should be placed in the third division, kept dry, but by no means, as is too generally the case, kept cold, for all exotic bulbs require nearly the same degree of heat while lying dormant as when they are growing. Some have adopted the practice of shaking the bulbs out of the soil after their leaves begin to wither, and keep them in drawers, or on open shelves, in a dry, warm apartment, or under the shelving of the stove, where they can be kept free from damp, and allow them so to remain, watching carefully the first symptoms of excitement both in the roots and embryo leaves, at which time they select such as are fit, and pot them immediately, supplying them with water moderately at first, but more copiously afterwards, and placing them in their growing station. In this way the late $\mathrm{Mr}$ Sweet, the most successful bulb-grower of his day, treated the splendid collection of the late $\mathrm{Mr}$ Colvill, who had at that time the largest collection in Europe. The late Dean of Manchester, the Hon. and Rev. W. Herbert, followed a somewhat similar routine. This mode of wintering bulbs has the advantage of occupying little useful space-no small consideration to those having limited means; and to those who grow bulbous plants to a limited extent, we would say they cannot follow a better rule. This, however, although applicable to a vast number of bulbs, is not without its exceptions, and the splendid genus Amaryllis offers a case in point. Amaryllis reticulata and striatifolia, or the hybrids raised from them, will flower much better by remaining in pots all the year, as do also A. aulica, calyptrata, *solandræflora; but these, of course, must be kept dry, so as to remain quite dormant, otherwise they will not flower with certainty. Again, A. reginæ, crocata, rutila, acuminata, fulgida, *Johnsonii, psittacina, and the mules between them, are much better turned out.

In 1838 we wrote as follows (vide the GreeNноUSE), and subsequent experience has not al- tered the opinion therein expressed : "The whole art of cultivating bulbs well depends on the attention paid to two particular pointsviz., the season when they are put into, and the length of time they are to remain in, a state of rest, and the perfection to which their foliage is brought during the season of growth. The most rational season for putting all bulbous plants into a state of rest is soon after they have flowered, and while their leaves are beginning to decay. It is extremely injurious to take up any bulb, or even to destroy a single leaf, while it is in a growing state; for it should be remembered that it is the leaves which bring the bulb to maturity, and prepare it for flowering the following year. The length of time that bulbs should be kept out of the ground, orkeptin a state of rest, depends on their habits as to flowering. Some kinds require one month, while others of the same genus may be kept for the space of three months without injury. It may be laid down, however, as a rule, from which there are very few exceptions, that no bulb should be kept out of a state of growth after it has once shown symptoms of vegetation; nor should that vegetation be impeded in the slightest degree from the period of its first commencement till the foliage is perfectly matured, and beginning to decay." No precise period of the year can therefore be set down as that at which bulbs should be potted, because where a large and varied collection is grown, there will be some that will demand this attention every month, nay, almost every week in the year. As the roots become excited before the foliage, it will be unsafe to delay re-potting till the latter give indications of returning growth; and here the turning out of the bulbs, and keeping them in drawers, \&c., has its advantages, because the first effort of the root is so clearly visible. In the other case, frequent examination will have to be made by turning the ball out of the pot, and examining the state of the roots. From the delicate and fleshy nature of the roots, care must not only be taken that potting be not delayed, but also that the operation be performed carefully, that the points of the young roots be not in the least injured.

Pots.-The pots best adapted for bulbs are much deeper than those used for ordinary purposes, nor need they be of nearly so great a diameter. The roots of most bulbs descend perpendicularly, and that to a considerable depth; those of a healthy Amaryllis, for example, with a bulb 3 inches in diameter, should have a depth of pot not less than 10 inches, while the diameter of the pot at top need not be more than half of that; but for rendering them less liable to be upset, as well as for appearance, and giving more room to the roots, the bottom diameter should be 7 inches. These dimensions may serve as a scale for bulbs of greater or lesser size. Those we use are of these dimensions.

Soil.-Thenatural habitat of most exotic bulbs is in sandy or light loamy alluvial deposits ; and although some are found where vegetable matter abounds, still few, if any, are met with in what we call peaty, heathy, or bog soils. The majority of gardening authorities, however, recom- 
mend peat and loam. Although aware that this is the compost recommended and used for the majority of exotic plants, we cannot subscribe to its being employed for this particular tribe. A better will be found in rich alluvial soil, which is often met with in meadows, by river sides, or by procuring light, rich, turfy loam from an old sheep-pasture, or where the soil has not previously been subjected to cultivation, taking the turf off only to the depth of 3 inches. This, broken down by exposure to the air for a twelvemonth or so, and mixed with equal parts of thoroughly decomposed cow or stable-yard manure, with about an eighth part of sharp riversand, the latter more or less according to the lightness or stiffness of the loam, will make a good compost for the majority of bulbs. Some care, however, will be required that that dangerous enemy the wire-worm be not brought in along with the loam. Charring or roasting the loam, when it is first brought to the compostyard, is the only certain remedy; or to those who have an objection to this, spirits of vegetable tar may be advantageously employed.

Some of the more delicate bulbs will succeed well in soft, sandy, rich alluvial deposit, enriched with guano, liquid-manure, or very much reduced cow-dung gathered from the fields, but before it has been too long exposed to the atmosphere.

Potting.-As it has been already observed, the operation of potting depends more on the state of the bulbs themselves than on any particular season of the year. A general potting cannot take place at stated periods. When in fit condition, those in pots should be carefully taken out, the old soil removed entirely, and all the dead fragments of the old roots, if any, cut away, and also any loose skin that will part from the bulb freely should be rubbed off. When the necessary drainage is put in, a handful of charcoal should be laid over it, the pot filled with the prepared compost to within an inch or two of its top, according to the size of the bulb. A little charcoal dust, or fine silvery sand, should be laid on the top, and the bulb set thereon, and the pot filled up to the brim with just sufficient compost to keep it in an upright position. The majority of bulbs on no account should be buried in the soil; they should at all times stand on the surface, the roots only being admitted into it. Little water should be given until the roots have taken hold of the soil, and the young leaves have begun to develop them. selves; afterwards it must be liberally supplied. It is best to place the bulbs at once in the pots they are to remain in during the season, for shifting, however carefully performed, is always attended with danger.

Propagation.-The majority of bulbous plants seed freely, and are increased by that means. They also, for the most part, form offset bulbs around the sides of the principal ones, which, when removed when ripe-that is, when they part freely from the parent-may be potted into store-pots immediately. Seeds should be sown as soon as they are ripe, if this occurs before the end of July; if not, it is better to keep them till the beginning of the following spring, that the young plants may be strong enough to with- stand the following winter. The seed should be sown in rich, light, sandy loam, and not covered too deeply, as the seeds of most of them are either very small, or else thin, chaffy-looking in appearance. Water moderately at first, and as vegetation proceeds increase it by degrees. Place the seed-pots in a mild temperature at first, and when the seeds have vegetated, increase the heat and other stimulants gradually; for it is of vast importance to insure a vigorous growth, with a view of obtaining as large bulbs as possible the first year ; and to aid in this, excitement should be kept up as late in autumn as possible, so as to give them a long season of growth. When vegetation has ceased, treat them exactly as recommended for established bulbs. Notwithstanding the apparent want of substance in the seeds of bulbs, some of them will remain two years in the soil before germinating, so the seedpots should not be hastily thrown out. Seedlings originated the previous season should be potted off in March or April the following year. 'Their treatment afterwards is exactly that for full-grown bulbs. Every means should be employed to enable them to develop their foliage to the fullest extent, for on this depends the size of the bulbs. Some of the free-growing kinds will flower the third year from seed, but by far the greater number not before the fourth, fifth, and sixth years.

Most bulbous plants propagate freely by offset bulbs. Others do not so readily, as the tunicated ones, which have a number of tunics or coats regularly laid over each other, and of which the onion furnishes a good example. Such bulbs, however, if cut transversely a little above the middle, will form young bulbs along the edges of the outer coat. Such an operation is fatal to the bulb; and to prevent its rotting before the bulbs have time to form, the wound should be dusted over with powdered charcoal or caustic lime once a-day to dry up the sap, and, still farther to prevent decay, it should be placed in a rather warm dry place. Collodion, which has recently been brought into notice as a means of stopping the loss of sap in cuttings, by applying it with a very fine camel-hair brush to the part where the transverse cut is made, might be applied with beneficial effects on the bulb, and, by preventing the escape of sap from the surface of the wound, might, at the same time, induce the formation of bulbs round its edges. Other bulbs difficult to increase may be made to do so by searing their crown with a hot iron. Young bulbs are thus formed round the circumference of the seared part, which, when of a proper size, are removed and potted. Bulbs composed of numerous scales are less difficult to increase, as each of these scales, if carefully removed when of a proper age, has a bud at the base, and if planted, will produce young plants.

Future culture.-In private collections the main object is to have fine flowers, and to insure this, along with other judicious means, the young plants which arise around the flowering plant should be destroyed as soon as they appear. By this practice a great accession of strength is given to the main plant. And in conformity with this rule, every flower should be pinched 
off close to its base as soon as it begins to decay, but not sooner, as an immense expenditure of sap would take place by cutting the flower-stalk while in full vigour of growth. All seeds should also be prevented from forming. The greatest encouragement should also be given to the perfect formation of the leaves. When the object is to increase the stock, then an opposite course should be followed; the young offsets should be encouraged, and left on until they can be removed easily, and without injury to the parent bulb. The plant should also be prevented from flowering, by destroying the flower-stalk while in an embryo state. From the commencement of vegetation care should be taken that no undue excitement be given, but a slow and gradual development of the parts be encouraged, increasing the stimulus as the growth proceeds and as the days lengthen. Over-excitement, particularly by heat, draws up the foliage weak and attenuated; the consequence is, that, if flowers are produced at all, they will be inferior both in colour and size. We have already noticed that during the growing season too much light and sunshine cannot be admitted to them, the atmosphere should be kept humid, and a greater amount of water given at the roots than is necessary for other plants not absolutely aquatics.

General remarks on exotic bulb culture. Amaryllis calyptrata, a native of Brazil, requires a rather high temperature during the season, and is improved if placed on bottom heat. It is one of the sorts which retains its leaves throughout the year, and is, with most that do so, so impatient of cold, that when removed to the greenhouse temperature it often perishes outright. A. formosissima, a native of North America, requires a light rich soil, and is adapted to the greenhouse temperature. The same may be said of psittacina, a native of Brazil; Belladonna, a native of the West Indies, and its variety pallida, from Madeira. Vittata, and the hybrids between it and psittacina, are hardy greenhouse plants, seldom prospering in a higher temperature. Stylosa, solandræflora, reticulata, and striatiflora, are tender stove-plants; while equestris, a native of the warmest parts of the West Indies, is impatient of a high temperature, and will not long exist in active excitement. We give these as examples only. Brunsvigia Josephinæ, contrary to most other bulbs, requires to have its bulbs kept under ground, with their necks left only a little above the surface of the soil : a rich light loam, with abundance of water during its season of growth, and perfect dryness while it is at rest, are the conditions necessary for insuring success in blooming this splendid and now rare plant. Coburghia, Ixia, Sparaxis, Tritonia, Babiana, Trichonema, Moræa, H. Emanthus, Strumaria, Cyrtanthus, Nerine, Grissorhiza, Gladiolus, Phycella, Vallota, Gethyllis, Eucomis, Lapeyrousia, Conanthera, Hypoxis, Albuca, Oxalis, Eriospermum, Massonia, Lachenalia, Tropæo. lum, \&c., require greenhouse ternperature only, but to be kept somewhat warmer while their leaves are forming. They are chiefly natives of the Cape of Good Hope, China, Japan, Aus- tralia, Mexico, Brazil, and Peru. The following, being chiefly natires of the East and West Indies, Senegal, and South America, require a correspondingly higher temperature - namely, Crinum Pancratium, Habranthus, Tacca, Gloriosa, Griffinia, \&c. It may be remarked that the beautiful genus Coburghia, unlike most other bulbs, prospers best in a rather strong, rich, loamy soil. Of Gloriosa there are only four species, one only of which (Superba) is in general cultivation, although the whole ought to be found in every plant-stove in the kingdom. Rarely, however, is this plant well managed. For the guidance of amateurs, with whom the very name may have some attraction, we offer the following as a simple mode of culture, adapted to all who have a stove, however small; premising, however, that the roots - we mean roots proper, not the bulb -should be preserved with the greatest care, for if injured, the plants sustain a check from which they are long, perhaps a year or two, in recovering. In autumn, when the stems have died down, remove the pot containing the bulb to the top shelf of the stove or bulb-house, or place it elevated a few inches above the flue or hot-water pipes farthest from the furnace or boiler, where the mould may be kept dry and warm, and for greater safety an empty pot may be inverted over it. If the tubers be small, several may be kept in the same pot during winter. About the middle of March they should be taken out and potted, one or two, according to their size, being set in each pot of 6 or 7 inches in top diameter. The soil should be similar to that recommended for Amaryllis, only less enriched with manure. Set the roots about 2 inches deep, and plunge the pots immediately in a very moderate bottom-heat at first, increasing it progressively in the course of a few days to $90^{\circ}$ or $95^{\circ}$. Water should be sparingly applied at starting, but after the plants become fully excited a more liberal supply should be given. When the shoots have extended 18 inches or 2 feet, shift into pots 12 inches over, preserving the ball perfectly sound during the operation. If intended to train the shoots up a rafter under the glass, place the pot in the most convenient position to effect this, but keeping it still in a smart bottom-heat. Some cultivators train this plant with good effect to a balloonshaped trellis, and in such a way it has a very good effect.

One of the most difficult to manage well of the bulbous tribe is the genus Cyrtanthus. The greenhouse temperature appears to be too low for it, while that of the stove is as much too ligh. It suffers much if kept in a constant state of excitement. The late Dean Herbert remarks of this genus that it is altogether difficult to manage, the bulbs being more disposed to dwindle and rot than to increase in size. A light soil, and keeping the plants near to the glass in an intermediate tenperature, is most favourable for their growth; and although we have not tried the experiment, still it may be that covering the bulbs with soil, as practised with Brunsvigia Josephinæ, may have its advantages. Of the beautiful Griffinia it behoves us to say 
a passing word. This genus is seldom met with in so satisfactory a state as its merits deserve. From being somewhat difficult to flower, it has, like so many others of its compeers, been abandoned. This being a native of the warmer parts of South America and Brazil, it follows that the temperature of the greenhouse is too low for it, while, as in general treated, the perpetual excitement to which it is subjected in the general stove is equally unsuited. Give it the necessary excitement of abundance of water and a high temperature while in the vigour of growth, but gradually withdraw these as its season of rest approaches, and allow it to remain dormant during three months at least in winter. The want of sufficient excitement at the proper time, and the want of rest for a period yearly, is the cause why that splendid plant $G$. hyacinthina is seen so seldom in flower.

The Cape species, which rank in the natural orders Irideæ, Oxalideæ, Asphodeleæ, Amaryllideæ, Hypoxideæ, \&c., and they are very numerous, are of the most easy cultivation. The coldest division of such a structure as we have described is a very fitting habitation for them. If grown upon a more limited scale, a low pit will be found eminently calculated for them ; and without this, with a glass covering and slight degree of artificial heat at particular seasons, especially when in a growing state, and a due observance of a season of rest, no hopes need be entertained of seeing them in their fullest perfection.

In addition to the genera we have mentioned suitable for such structures and similar management, we may add those of Gloxinia, Sinningia, Gesnera, Codonophora, Pentaraphia, \&c. 'These, however, being for the most part from the West Indies, South America, and Brazil, require accommodation in the warmer compartment, and, like the others, require their stated season of rest.

Insects and diseases. - The white or mealy bug is frequently imported attached to bulbs, and also sometimes makes its appearance on such as have been years in cultivation. Syringing with water at $130^{\circ}$ will destroy them, and dipping the bulb in water at the same temperature previous to potting, will act as a preventive by destroying any insects in a young state that may exist upon them. Bulbs are subject to few natural diseases. They are, however, sometimes attacked by gangrene or rottenness, arising from wounds, too much cold, and damp. When such appears, the decaying portions should be carefully pared away with a sharp knife, and the wound dusted over once or twice a-day with powdered charcoal or caustic lime in powder, kept dry till the disease disappears; or, as has been already suggested, the diseased part, when washed clear of putrescent matter, may be painted over with collodion.

\section{§ 4.-ORANGERY.}

The cultivation of the orange tribe has been much less attended to in Britain than that of any other class of exotic plants. Why this is so, it is difficult to say. The plants make slow growth certainly, and nurserymen cannot, in this country at least, afford to sell them cheap. The supply of small plants, until within these few years, was chiefly from Genoa, Naples, Florence, \&c., the chief importers being the Italian warehousemen. Little regard was paid to selection of sorts, and the plants themselves were, in most cases, of the worst possible description. Of late years, plants of a much better description have been imported, chiefly from France and Belgium, and may be purchased of all respectable nurserymen at very moderate prices. The genus Citrus are chiefly natives of Asia; the principal sorts in cultivation are Citrus Medica, Median Lemon; C. Linetta, Lemon Bergamos; C. Limonum, Lime; C. Aurantium, sweet orange ; C. vulgaris, com mon Seville; C. decumana, Shaddock; C. nobilis, Mandarin. The Maltese orange is a much esteemed fruit, and distinguished by its red pulp; and the navel orange of Bahai is both singular and excellent, the skin being very thin, the pulp almost devoid of seed, while the apex, or crown of the fruit, terminates in a sort of crown or navel-whence the name. We believe this to be a rare variety in Britain, and have lately received two trees of it from its native country. There are a great many other varieties, the Paris nurserymen cultivating about forty sorts, about one-half of which appear in our English catalogues.

The largest collection in Britain is that at Hampton Court Palace, where two large apartments on the ground-floor, having only large glass windows in front, are filled with magnifcent specimens. There are also some very large trees in the Royal Gardens at Kew, kept in an equally sombre apartment. These trees are annually removed to the open air during summer, and arranged along the terrace-walks, after the manner followed over most of the Continent, and especially so in the gardens at Versailles and the Tuileries. Although, from these examples, it will be seen that the orange tribe is capable of withstanding a considerable degree of cold and a long period of diminished light, it does not follow that they would not prosper better if afforded a more transparent accommodation. Plants growing under the brightness of an Italian sky, and exposed to the heat and sunshine of an Asiatic climate, would be in a far more natural position if placed in our light and airy conservatories than in the apartments to which we have alluded. We have referred to this, vol. i. p. 399, and given a design of what we consider an eligible orangery, fig. 550 .

The orange has also been successfully cultivated as a fruit-bearing tree, in several parts of Britain ; and where a judicious selection of the best varieties of the sweet-fruited sorts is made, and duly attended to, they may readily be grown to as great perfection as they can arrive at in their native countries. And for this end they should be planted out in prepared borders, and trained to trellises similar to the peach; and perhaps no better arrangement can be offered than that of fig. 227. The soil, in such a case, should be rich, light, and open, and rendered thoroughly dry below. Those who have suc- 
ceeded best in growing this tribe for their fruit maintain a temperature of $50^{\circ}$ as the minimum during winter, and from $80^{\circ}$ to $90^{\circ}$, accompanied with a moist atmosphere, during summer. If the temperature be kept high during the growing season, it must necessarily be kept comparatively high during the season of rest, the growth of the orange tribe never being totally stopped, if they are not starved, or otherwise mismanaged. Water should be administered rather sparingly to the roots of planted-out trees, the necessary syringings over the foliage being nearly sufficient ; nor should they be so abundantly supplied with stimulating manure as when confined within the limits of pots or tubs. In the latter case, a stronger soil, and that frequently supplied with enrichment, becomes necessary on account of the limited range to which the roots are confined. The branches should also be kept moderately thin if trained to trellises, that the surface of the leaves may be fully exposed to the sun. The fruit being in general produced on the points of the small spurs or branchlets, care must be taken that these be not shortened, unless it be with a view to increase their number. The operation of pruning extends over the whole season, cutting out the old and superfluous branches, and laying in a succession of young ones. This tribe often suffers much from being allowed to carry too large a crop of fruit; and as the young green fruit is used for confectionary purposes, a judicious thinning should take place as soon as they arrive at the size of a small walnut.

Propagation.-The orange is propagated freely by seed, but in this way they require from 3 to 4 years' cultivation before they flower. The seed is sown in rich soil, in pots plunged in a mild bottom-heat; and when the plants have attained the height of 4 to 5 inches, they should be potted singly in rich pretty strong loamy soil, in pots of 4 inches in diameter. If intended to produce fruit, the leading shoot should be carefully preserved until it has attained the height of the desired stem, at which time it should be topped, to cause side branches to be produced. Seedling oranges are, however, for the most part, grown for stocks on which to bud or graft approved sorts. Stocks for this purpose are preferred when originated from seed of the Shaddock, and next to them the Citron, on account of their more rapid growth and greater size. Like all other fruit-bearing trees, the chances of improved sorts from seed are very precarious.

Propagation by cuttings.-All the Citrus tribe strike readily by cuttings, and under good management make excellent trees, coming early into bearing. For this purpose cuttings are taken from the strongest young shoots. As soon as the lower part is somewhat mature, they are cut into 9-inch lengths, the bottom leaves being removed as far as the cutting is to be inserted in the soil, but the greatest care must be taken that those above that be left entire and uninjured. The cutting should be made transversely across, with a sharp knife, close under a bud-that is, where a leaf has been growing. Insert them to the depth of 4 or 5 inches in a pot filled with clean silvery VOL. II. sand, giving them a gentle watering to settle the sand firmly around them; they should then be plunged in a close frame, kept well shaded in a mild bottom-heat, and in this state they are to remain until they are rooted. This is indicated, in general, by the cutting beginning to extend in length, at which time they should be carefully removed from the pot, and planted separately in pots of from 4 to 5 inches in diameter, using rich and rather stiff loamy soil; but, in potting, a little lighter and sandier soil should be placed around the roots, in which they will strike freely, and afterwards extend into the stronger soil. After this potting they will be greatly stimulated in growth if replunged again in a mild bottom-heat, and shaded for a few days; afterwards they should be fully exposed to light and a moderate amount of air. Some propagators make use of wood of the previous year's growth for cuttings; and others, in both cases, use cuttings from 18 inches to 2 feet in length-and this more especially with the Citron, which strikes more freely than any other of the tribe. It is of apparently little consequence at what season the cuttings are put in, if the dead of winter be avoided, and while the plants are making young wood.

Propagation by layers.-Seldom practised in Britain. Plants produced in this way do not make such handsome trees as those propagated by seed, cuttings, budding, or grafting.

Propagation by grafting.-The whip method (vide fig. 100) is usually employed, and the stock should be young and healthy-say one or at most two years' growth, from seed, and about the same thickness as the scion; so that, after the union takes place, no blemish may appear on the stem; and the operation should be perforned within 6 inches of the ground. In such a way nice healthy trees will be produced, having clean stems from 1 to 3 feet in height. The plants should be kept in a close frame or pit until the union has fairly taken place; and if the stock is slightly stimulated at the same time, by being plunged in a moderate bottom-heat, the union will be more speedily accomplished. Indeed, any of the ordinary methods of grafting may be employed, the great object to be kept in view being to have the scion and stock of nearly the same size. Some graft the young seedlings sown in spring the following autumn, as, under good cultivation, they will be fit for this in six or seven months. No clay is used, but a little fine moss is placed over the point of union, and many only secure the scion to the stock with two or three rounds of soft woollen yarn. Some cut off the head of the stock and crown-graft, while others attach the scion to the growing shoot, and cut off the head after the union has taken place. Inarching is frequently practised, and by this means large-headed plants can be speedily obtained, as well as various sorts made to grow on the same stem.

Propagation by budding.-This is the usual method employed by the Italians, who insert, in general, a bud on each side of the stem, which is usually 4 feet high, and often from 1 to 2 inches in diameter. The stock is cut horizontally off at that height, and, as may be supposed, 
seldom or never becomes covered with bark, and consequently handsome trees can never be expected. The Maltese cut the head off the stock in a sloping direction, which is a slight improvement on the Italian manner; but even their trees are never so well formed as those of the French, who bud on younger stocks, which, after a year or two, scarcely leave a trace of where the bud has been inserted.

Soil.-A rich, strong, loamy soil is that in which the Citrus tribe naturally delights to grow. These, confined to the narrow limits of a tub, pot, or box, require not only a strong rich soil, inclining to clay, to make up for the limited range of their roots, but also that enrichment should be from time to time added to it. This is applied by top-dressing the surface with cowdung, sheep or deer dung, and more conveniently, and with a much better appearance, by watering once or twice a-week with clear liquidmanure. Those grown in planted-out borders require a lighter soil, as already stated.

General culture.-Although, as already stated, the Citrus tribe will bear a considerable degree of cold, the proper temperature for them, under glass, is from $48^{\circ}$ to $60^{\circ}$, - the former during winter, or while they are in a state of comparative rest; the latter while they are making their wood and perfecting their blossom, and that principally by sun-heat. An excess of heat is most injurious to the orange, when grown as plants of ornament; but when grown for fruit, $10^{\circ}$ or $15^{\circ}$ additional, during the swelling and ripening process, may be accorded them, particularly for Citrons, Lemons, and Limes. During winter, water must be rather sparingly applied, and that in a pure state, while during spring and summer it must be abundantly given, and occasionally in an enriched state; and more will be required for trees growing in pots than such as are in boxes or tubs. A deficiency of water, like excess of heat, is very injurious to them, causing them to shed their leaves, which if they do to any great extent, it will take two or three years' careful culture to recover them. Where slate or wooden boxes with movable sides are used, the state of the soil can readily be examined by removing one or all of the sides, while the ball remains undisturbed. Air should be abundantly admitted on all fine days, particularly during summer; and where the practice is followed of setting them out of doors during June, July, August, and part of September, air should be freely admitted to them for a week or so before removing them, to accustom them to the change, and also after they have been again brought in. The French and Belgians set out their orange trees during summer. The advantages, however, may be questioned; and although it is admissible in Italy, where the temperature is much greater, the practice is extremely erroneous in Britain.

All the Citrus tribe may be grown in tubs, pots, or boxes, and trained as dwarf or half standards; they may be trained also with great propriety to trellises, or, as they often are, to cover the opaque wall of a conservatory where such monstrosities exist. Where the Citrus tribe is to be cultivated for their fruit, the borders of the orangery should be vaulted beneath the soil, and means taken by which heat can be thrown in when necessary, otherwise their fruit can never be expected to attain maturity. Where, however, the common orange is grown for its flowers only, the trees will succeed sufficiently well in a portable state, set on the surface of the floor. The Citron is rather impatient of intense sunshine, they being for the most part natives of woods; they are, therefore, successfully grown when trained to the back walls of vineries. The Seville, bizarade, or bitter orange, is chiefly grown for the production of cut blossoms, and its fruit is imported for making marmalade, bitter tinctures, \&c.

Watering over the foliage is an important element in culture; and this should be attended to at least once a-week, excepting while they are in flower, and during the dark period of winter. In the latter case, once a fortnight will be sufficient. During excessive sunshine in summer, shading should be attended to; and for this purpose thin canvass screens should be mounted on rollers attached to the roof of the house, to be drawn up and down according to circumstances, (vide vol. i. p. 555). Those who have not a regular orangery will find advantage by removing their plants from the greenhouse, and setting them under the shade of the vines in the vinery during summer.

Shifting should be attended to once every two years in the case of large established trees growing in fixed tubs; but where boxes with movable sides are used, they should be examined yearly; and in doing so all the exhausted soil should be removed on one side of the ball, which can readily be done when the side of the box is taken out, the roots examined, and such as are dead or unhealthy cut away; the side of the box is then to be replaced, and the space between it and the ball filled in with rich loamy soil, as already noticed. As each side is opened up, the drainage under that side of the ball should be looked to, and, if defective, taken out and replaced with new material. Such boxes are manufactured by $\mathrm{Mr}$ Edward Beck, of Isleworth, Middlesex, and are of Welsh slate, half an inch thick, or more, according to their size : they are made in five pieces, forming the four sides and bottom, which latter is perforated with many holes an inch in diameter : they are bolted together with screws and nuts; the nuts being on the outside renders their being taken to pieces very convenient : they are made in sizes varying from 12 inches square to 24 inches, and in price from $8 \mathrm{~s}$. to $40 \mathrm{~s}$. each.

Any trees that may have become diseased or lost their branches, should, upon the first appearance of decay, be turned out of the pots or tubs, the soil removed from them, the roots carefully examined, and the remaining branches headed back to healthy buds. They should then be replanted in fresh soil, and plunged in a mild bottom-heat in a deep pit, kept close, shaded, and moderately dry, until they begin to break again, after which more light may be admitted to them, more water given, and a humid atmosphere maintained in the pit around them. A moderate bottom-heat should be continued 
until the new heads are again formed. Such is the treatment, also, for newly-imported plants which may be in a sickly state.

The Continental gardeners attach great importance to pruning, which they perform in rather a wholesale manner, using the hedgeshears, with which they clip the heads as close and symmetrical as if they were more intent on the production of a perfect vegetable sphere than on balancing the top to the capabilities of the roots. Thinning the branches is less regarded than maintaining symmetry of form, although some of the best gardeners give their trees a sort of winter-pruning (talle), and also a summer-dressing (ebouryeonnement). The heads of their trees become, notwithstanding these prunings, in course of a few years both too large and too much crowded with branches to be supported by the roots, and hence every sixth or eighth year a general reduction of the head takes place, which would be avoided were an annual pruning practised. At the general pruning, every shoot on the tree is cut back to within an inch or two of the old wood, leaving them bare and leafless stumps, which require two or three, and often more, years to recover. Where the roots are in a good condition, these trees break into young wood, often with surprising vigour, and afterwards become as large, and are trained as globose as before. The irregular form given the trees in Britain is not only more agreeable to the sight, but is more conducive to their health; they are thinned and trained like any other standard tree, keeping the branches moderately thin, shortening such shoots as greatly outgrow the others. As the blossoms, for the most part, are produced in form of terminating peduncles on the wood of the current year, it follows that in pruning encouragement should be given to the production of young wood in every part of the tree, cutting out all naked wood, and shortening strong shoots where wood is wanted.

Those who have not a regular orangery may place them in the camellia-house, where they will associate well with the tender rhododendrons, Chinese azaleas, and similar plants (vide Camellia-House). Oranges may also be kept in the greenhouse or conservatory, but in either case the temperature is too low for the perfect ripening of their fruit. The following are the most esteemed varieties of the common orange: the China, Blood-red,Sweet-skinned, St Michael's, Mandarin, Fingered, Tiny - fruited, and Pearshaped; and of these the Mandarin and St Michael's are the most proper for cultivating for their fruit.

Insects and diseases.-That general enemy to all plants under glass, the red spider, fig. 22, and the plant aphides, both attack the Citrus tribe. Copious syringing destroys the former, whose appearance is a sufficient indication that the atmosphere has been kept too dry. Tobacco fumigation is fatal to the latter. The orange scale insect, Coccus hesperidum Linn., is a worse enemy to subdue than either of the others. In appearance it resembles an elliptical nut-brown shield, attaching itself to the branches and leaves, particularly when the trees are kept rather warm. The trees should be carefully washed with soap and water, applied by a rather hard brush during autumn, when few young insects are found : the old ones, being displaced, cannot creep up again; but lest they produce their young while on the floor, or the surface of the soil in the pots, it is well to spread a cloth under the trees during the operation of washing, into which the coccus will fall, and hence be removed out of the house.

\section{5.-HEATH-HOUSE.}

No individual genus of plants forms so complete a whole, or is so eminently adapted for occupying an entire house, as the Ericas. The genus is extensive, embracing upwards of 560 species, besides numerous hybrids, originated artificially in various parts of Europe, but principally in Britain. They are of easy culture, of vast variety, and flowering at all seasons of the year. The whole of this vast assemblage are natives of the Table Mountain at the Cape of Good Hope, with the exception of five which are indigenous to Britain, three to Portugal, one to France, one to Northern India, one to Sicily, one to Spain, and four to the rest of Europe. They all occupy elevated ranges, enjoying a pure air, refreshed by copious dews, and exposed for long periods to a dry arid. atmosphere. They are all moderately hardy, with the exceptions we have given, but not so much so as to withstand the severity of our ordinary winters without a glass protection. Enjoying, as we have said, a pure air naturally, they do not prosper in or near towns, and soon perish when placed in the dry close atmosphere of rooms. They are impatient of fire heat, and are found to thrive best in a light airy house, into which artificial heat is rarely admitted.

We have in vol. i., p. 402, spoken of the merits of a complete heath-house, and given examples, ride figs. 552, 554, and 555. We have only further to remark that such a structure should either be span-roofed, as there exemplified, or curvilinear, and that all lean-to houses are objectionable for the purpose. The roof should also be low that the plants may be placed near to the glass; for although many of them may be grown to a large size and considerable height, the majority do not, nor is it for general satisfaction that they be so. From 1 to 3 feet is a very proper height for any heath. Abundant ventilation is of the utmost importance to their welfare; and this should be so arranged that the air may enter close to or near the floor, particularly during winter, and not be allowed to blow directly upon them, unless during summer. The stages or platforms should be of open cast-iron ornamental grating, both for durability and elegance, and also that the air may find its way to their roots, and that superfluous or spilt water may find a ready means of escape. When slate or stone shelving is used (which are the next best materials), the pots should have the bottoms well perforated with holes, and made to stand clear of the table from half an inch to an inch and a half, in proportion to their size. When pots of the ordinary make are used, and set upon a close plat. 
form, the water, in passing through the soil, finds its way through the bottoms of the pots also, and forms a luting between the bottom of the pot and platform, completely preventing the entrance of air to the roots, as well as keeping them in a state next to saturation.

A selection of the very best species, with their varieties, will furnish a large-sized house, and to cultivate any others nowadays but the very best is the height of absurdity. The heath is rather an unsociable plant, and does not succeed well when grown in a mixed collection; yet there are a few genera which may be associated with it with no disadvantage to either-namely, Epacris and Lysinema, the representatives of the heath in Australia; Andersonia and Sprengelia, from the same country; and Astelma, Helichrysum, Aphelexis, and Phœnocoma, its own countrymen. As the heath requires all the light our country affords, training climbers over the roof is injudicious, because they shade at all times alike: they do indeed require a slight degree of shading during the heat of summer, and also after they have been shifted, but this shading should only be permitted during a few hours at mid-day, and its application, unless in the case of newly-shifted plants, must be regarded more as the exclusion of a strong sun on the surface of the pots, which, becoming overheated, parch up the tender rootlets within, than as being of any advantage to the foliage.

Propagation.-The whole family of Erica, or heath, are propagated readily by seed and by cuttings. Seed is often procured from the Cape of Good Hope. Most of them perfect seed in cultivation; but with the exception of a few which are difficult to strike by cuttings, such as elegans, odora - rosea, triumphans, all the rest are propagated by cuttings of the young or nearly-matured shoots, according to the season, kind, and after-mode of treatment. The best time for sowing seed of this tribe is early in spring, so that the young plants may become so completely established before autumn as to enable them to stand the first winter, which, if they are weak, is very trying for them. The seed pots or pans should be prepared by very complete drainage, the broken crocks filling nearly the lower half of the pot; over this a little turfy peat should be placed, and the pot afterwards filled up to within half an inch of the top with finely-sifted sandy peat soil, pressed firmly down and rendered firm and level on the surface, so that the seed may be all covered alike deep. Sow rather thin than otherwise, if the seed is good, and cover to the depth of rather more than the sixteenth of an inch. Give a very gentle watering with the finest rose-pot, and cover the surface of the soil for a few days with a thin sprinkling of moss to prevent evapora. tion and the necessity of watering so often, as the seeds, being small, are apt to be displaced or uncovered. The pots should then be placed in a cool shaded close frame or pit, and kept as near to the glass as possible. The pots must be frequently examined to see they do not suffer from want of water, a deficiency of which at the time of germination is certain destruction to the young crop. Some sorts vegetate quickly, and will be above ground within four weeks; others more slowly, and some will remain even six or seven months before vegetating. Artificial heat should never be applied to them. As the young plants appear, air should be more freely admitted; and when they have attained the height of half an inch, they should be carefully transplanted into pots prepared as the seed-pots were, or they may be set in smaller ones, and arranged around the edges, where they will soon attain sufficient size and strength to admit of being transferred to thumb pots, one plant in each. From the time the seedlings appear above ground, they should be placed as close to the glass as possible. From some cause not easily explained, all young plants make roots faster when in small pots than in large ones, and also when they are placed close to their sides than when set in the centre of the soil within them. After transplanting, they should be kept for a few days rather close, either by covering them with bell or hand glasses, or, when upon a large scale, in a close pit or frame. After they have taken root, air should be gradually admitted to them, until they are so fully established as to support themselves by their roots entirely, at which time they should be placed in a dry airy place, and fully exposed to the sun and air. The usual situation in most nurseries is a broad shelf suspended under the rafters at the top part of the back of an ordinary greenhouse; such, however, has its inconveniences, of which the difficulty of getting up to water and examine them is the greatest. Suspended shelves under the roof of an ordinary pit are better, as tlie sashes can be occasionally removed during fine genial showers; their examination and watering can also be much better accomplished. When the plants have attained the height of 2 or 3 inches, which they will do by the following spring, they should then be shifted into small sixties, one plant in each pot. About this time their tops should be cut or nipped out, to cause side branches to be produced. Their after-treatment is the same as will be noticed below.

Propagation by cuttings.-The best season for this operation is early in spring, as soon as the wood is in a proper state; and when this is not the case naturally, the plants from which they are to be taken should be moderately excited by being placed in as lightly-increased temperature till they have made young wood sulficient. Spring is desirable, because the young plants get sufficiently established before autumn to enable them to stand the winter. In an extensive genus like this, some species differ widely from others in habit, and in the length of time they take to strike; therefore regard should be paid to this, particularly when several sorts are to be put in the same cutting-pot. The late $\mathrm{Mr} M \mathrm{M}^{\prime} \mathrm{Nab}$ has left us most judicious rules as regards this matter. He says (in "Treatise on the Cultivation of Heaths"): "In extensive nursery collections, where great quantities of plants are wanted, one pot may be filled with cuttings of the same species; but in private collections this is not necessary, for a few plants of a sort, in general, is all that is required. When this is the case, 
the kinds selected to be put in the same pot should be as nearly of the same habit as can be judged of at the time. For example, I shall suppose four pots are intcnded to be filled with cuttings-such as the following should be selected for each pot: First pot-Melastoma, Petiveriana, Petiverii, Sebana, penicilliflora. Second pot-pinea, pinifolia, vestita, grandiflora, purpurea. Third pot-ventricosa, prægnans, Linnæana, Linnæoides, colorans. Fourth pot-Aitoniana, jasminiflora, ampullacea, Irbyana, inflata. Unless this is attended to, one sort will be found to strike root in a much shorter time than others in the same pot, which makes it more inconvenient when potting them out. This must, however, always happen to a certain extent, for a little difference in the age or firmness of the cuttings, even when the work is performed by the most expert hand, will often make a difference in the time required to strike root." To this very judicious arrangement we may offer the varieties of the following, which are now pretty numerous of each of the types,--viz., pinea, Ewerana, vestita, exsurgens, tricolor, ventricosa, echiiflora, aristata, Linnæana, \&c.: the varieties of each of these may for the most part be set in the same cutting-pot.

In regard to the state of the wood, that of the present year's growth is to be preferred, some taking it while quite young and soft, others when more firm or nearly matured; while there are cultivators who use it when completely ripened, and all succeed equally well. The former plunge their pots in a mild bottom-heat in a close moist frame or pit, the cuttings rooting rapidly or damping off at once, particularly if the bell-glasses they are covered with be not kept wiped clean of condensed vapour daily. The second generally plunge the pots in coalashes, sand, or similar media, in a rather damp shaded place, covering each pot with a bellglass, and the whole with a close frame and lights. Cuttings thus treated do not root so soon, but they do so with greater certainty. Those who employ wood more fully ripened, treat them much as the last, and wait patiently for the rooting process, should it occupy several months, and even in some cases extend over the winter. Some sorts, it should be observed, strike better by one of these modes than by another; and as some guide to the uninitiated, we may observe that all fast-growing sorts do best by the former, while many of the slow-growing kinds, such as rupestris, Banksiana, \&c., succeed best by the latter process. The length of cuttings depends greatly upon the habit of the species; some of the strong-growing kinds, like versicolor, may be from 1 inch to $1 \frac{1}{2}$ inches in length, while others of the slow-growing kinds may be taken at half that length. In selecting the cuttings, it is of importance that they be taken from healthy plants. At one time a preference was given to the tips of the lateral branches, and some even went so far as to choose them from the side branches nearest the bottom of the plant, and rejected the leading or stronger shoots altogether. This was done from an idea of preventing the plants from growing too robust. The case is now pretty generally re- versed. To have fine healthy plants, we must begin with hcalthy and vigorous cuttings, and depend on topping and after-culture for modelling the plant.

In preparing pots for the reception of cut. tings, no size above that of thirty-twos in England, and thirties in Scotland, which are $4 \frac{3}{4}$ inches, should be employed; and some use pots of that width and only 3 inches deep, thickly perforated with holes in the bottom for insuring drainage. In the former case they should be nearly halffilled with broken crocks, a little turfy peat laid over them, and over that a little finely-sifted sandy peat, and the remainder filled as high as the rim with fine silvery sand for the cuttings to strike root in. When the pots are thus prepared, and the cuttings ready to be set in them, the sand should be well moistened with water, and firmly pressed down.

In preparing the cuttings, the leaves should be cut clean away from the lower half with a very sharp penknife or finely-pointed scissors, and the lower end cut transversely over, making a clean cut. The leaves retained should on no account be shortened, nor should the top of the cutting be interfered with. They should then be set ill the damp sand pretty thickly together, but always in rows across the pot, which will enable the propagator to remove any green $\mathrm{mu}$ cous matter that may afterwards form on the surface of the sand. When they are set, water over-head with a very fine rose-pot, to settle the sand firmly around their stems. When moderately dried in the shade, cover closely with clean bell-glasses, and remove the pots to the place designed for them.

Some cultivators dispense with bell-glasses altogether, but they are chiefly those who employ heat in hastening the rooting process; but at the same time they set the pots on a bed of sand or coal-ashes in a house or pit kept regularly shaded, and exceedingly close and moderately moist. In this way many of the London growers propagate heaths with great rapidity and success. In either way great attention should be paid to watering; indeed, the pots should be kept rather moist than otherwise, for drought and excess of air are equally fatal to them until they have fully established thcmselves. As the roots begin forming, the cutting will begin to elongate at top, which indicates that more air is required; and this demands strict attention, to prevent the young plant from becoming drawn up slender, and to liarden them off so that they may bear transferring into other pots with impunity.

In regard to transplanting, the same rule should be observed as already stated in the case of seedlings, as also their after-culture.

Soil.-The majority of heaths prefer a poor sterile soil upon a dry bottom. There are, however, some exceptions to this rule which will be noticed below. The majority of the plants, also, which we have given at the beginning of this article, agree with them in this respect, and when otherwise, it will be referred to. Peat earth, bog mould, heath mould, \&c.for the soil in question is known by all these names-is to be found chiefly in uncultivated 
elevated situations, in general covered with our native species, E. vulgaris, cinerea, \&c. Along with the decomposed vegetable matter formed by the yearly decay of previous leaves, branches, \&c., and the natural soil of the spot, it should also contain from one-fourth to one-fifth of coarse white sparkling sand, choosing such as lies high and dry, and not that which is exposed to be overflowed with water during the winter. It should be taken off in turfs about from 2 to 3 inches in thickness, carted home, and laid up in a heap to become partially decomposed, and fitted for use by a free exposure to the sun and air. A supply should be procured annually, for to keep it until the whole vegetable matter contained in it becomes entirely broken down, is rendering it exceedingly unfit for the purpose. In from three to four months it is in the best condition for use. Many peaty soils contain oxide of iron and creosote in over-abundance. When such is the case, it should be rejected, as the presence of the lattcr is destructive to vegetation. Some excellent peat soils contain too large a proportion of vegetable matter, and too little sand; the latter can be added to adjust the proportions, yet at the same time peat from deep deposits should be avoided. The fresher the soil is at the time of using, the better; and for shifting established plants it should be in a rough state, chopped up only to the extent that it can be placed between the ball and sides of the pot with freedom. Sifted soil, after the first potting of seedlings, or recently-struck plants from cuttings, should never be employed. Some of the stronger-growing kinds will be improved by the addition of one-third or so of pure sandy loam, while others of the slow-growing sorts will be benefited by the addition of frag ments of broken pots, small pebbles, or, still better, soft sandstone, free of iron, reduced to pieces about the size of small walnuts. The soils natural to the family of Erica on Table Mountain are exceedingly varied, and even very opposite in their natures-a study of which is worth the attention of the cultivator; as, for example, the species vestita, versicolor, discolor, hista, \&c., are found naturally in stiff loam by the margins of woodlands and moist gulleys, growing in company with Pelargoniums, Scirpoidæ, \&c. The former is also found under different circumstances as regards situation, but not of soil, as it flourishes along with triflora, cruenta, filimentosa, versicolor, and cerinthoides, on exposed plains, enduring drought at times for several months; but as regards soil, in deep loam containing iron pyrites. Linnæoides, tubiflora, colorans, \&c., in a black vegetable soil, by the sides of running water, and in springy ground; Sebana, Plukenetii, baccans, sexfaria, \&c., in decomposed schistus, exposed to drought; while persoluta, arborea, urceolaris, are found amongst decomposed schistus, but by the sides of streams and in shady glens; albens, ardens, fasicularis, ampullacea, fastigiata, retorta, \&c., grow on shattered sandstone rocks, with little or no soil about their roots. And in similar situations, but growing more luxuriantly in the moist clefts of rocks, are found gelida, eriocephala, halicacaba, caffra, mammosa, metuliflora, grandiflora, ignessens, cerinthoides, which prefer sandy spots on the lower plains. The rare Massoni, gracilis, Walkeri, calycina, retorta, luxuriate in pure sand, and fully exposed to heat and drought; and viscaria, viridiflora, and blærioides, are found upon decomposed sandstone, shaded by Scirpoidx, the subsoil, into which, however, their roots will not enter, being argillaceous. From this it will sufficiently appear that the Ericeæ enjoy naturally a considerable variety of soils and situations; and although, in cultivation, we cannot conveniently accommodate them to the full extent, yet it should be our aim to approach is as nearly as circumstances will admit of. Practically, we learn from the above few examples that some require a greater degree of humidity at their roots than others, that some require a soil approaching to a sound loam, while others are content to attach themselves to the debris of rocks or fragments of stone, and that some prefer a sheer sand. Peat soil abounding in sand will no doubt enable most of the genus to exist for some years; a stronger and richer soil will evidently tend to their greater luxuriance, as well as to extend their period of existence.

Water is a most important element in their healthy cultivation; that containing mineral matter, particularly lime, is fatal to them. Rain water is the best, and next to that, water that has been well exposed to the sun and air in an open pond. In regard to supply, the roots should never be allowed to become dry, while with the majority an excess is equally injurious; and watering during the summer months overhead in form of dew, applied early in the morning, is exceedingly beneficial, repelling mildew, and imitating the copious dews which fall on them in their natural habitats.

Potting.-In former times, soil sifted as fine as Scotch snuff only was employed, and the shift so closely spun that little more than a quarter of an inch was allowed between the pot and the ball, and even this limited addition was only afforded them once a-year, if as often. A betterpractice is now recognised, and soil chopped up with the spade, containing abundance of vegetable matter, and in pieces from 1 to 2 cubic inches each, and even more, intermixed with the finer particles, is used; and the shift varies, according to the size of the plant, from 1 to 3 inches all round. This, with ample drainage below, and abundance of light and air, with a due supply of water, is all that is required to produce specimen plants in the highest degree of excellence. Early in spring, just before the growing season commences, is the time for a general shifting; and although all may not require it at that time, it is well to turn them out, so that the state of the roots may be ascertained. No plant should be shifted, however, whose roots are not in a healthy state, and have not pretty nigh filled the pots, and these should be placed in the new pots without disturbing the ball. On the other hand, when the soil seems saturated with wet, and the roots black and diseased, the ball should be reduced, the dead parts of the roots cut away, and the plants re-potted in smaller pots in fresh soil; and when, from 
neglect of shifting, the roots have become matted, and the ball hard and almost impervious to water, it should be loosened by gently tapping its sides with the fingers; and in the case of pot-bound ones, the soil may be washed from amongst the roots by water, giving them freedom also at their bottoms, so that the roots may be induced to penetrate the fresh soil. Plants in this state should have a large shift, taking care, however, that for some time afterwards water be so applied that it may find its way into the old ball, and not wholly escape by passing down through the new soil. A general re-potting must not be entirely depended upon, for some may not require it at the spring examination, but may do so a month or two afterwards. Want of shifting is shown by the plants drying up sooner than those around them, by flagging during bright sunshine, by not drying at all, and by the pot feeling heavier than it ought to do. It is absolutely necessary that the soil be quite dry at the time of re-potting, and for this purpose it should be kept under cover of a dry airy shed for some time previous. It is also necessary that the pots be either new or well washed and dried before using. Plants having their roots much disturbed at the time of shifting, should be placed in a close cool pit for a few days after the operation; and even those which have not even been disturbed at all will be benefited by being slightly shaded for a few days after the operation.

We have already adverted to the bad effects of close shifting - that is, transferring the plants from a pot of one size to the one next in rotation of size to it. Another practice prevailed, and to some extent does so still, of potting too highthat is, elevating the collar or neck of the plant above the level of the rim of the pot. Both should be guarded against. Some pot hard, and ram in the new compost as firm as possible between the ball and pot, even using a blunt wedge-shaped piece of wood to render the soil more firm: others, who advocate the theory of porosity, mechanical action, \&c., object to this ; and so would we in the case of a young vine or geranium whose growth is expected to be rapid. The heaths are different; and although the softpotting system will make the plants grow more rapidly, yet in the hard-potted cases, in this tribe, it is found that the plants will be more healthy and vigorous, and attain a greater age.

Winter treatment. - Avoid fire-heat, even should the inside of the glass roof be slightly covered with frost. Most of the family will withstand several degrees of frost; none of them will bear fire-heat with impunity. It is better, therefore, to exclude frost by covering the roof with canvass mounted upon rollers; and even in very severe weather it is better to allow the covering to remain on even for several days together. If frost penetrate, as it sometimes will do, in pits, where the young stock is kept, it is well to allow the covering to remain on until the soil becomes thawed; for heaths, like all other plants, will endure a greater degree of cold if kept dark, than if once frozen, and the light let in immediately upon them. They resist frost better, also, when they are kept rather dry than otherwise. On the subject of low temperature, the late $\mathrm{Mr} \mathrm{M}^{\prime} \mathrm{Nab}$ informs us, in his "Treatise on the Heath," that he has had " all the heaths in the house frozen for days together so hard that the pots could not be removed from their places without breaking them, and fresh air constantly admitted at the time; and I have," he says, "never seen one of them suffer in the smallest degree from it, but on the contrary found them thrive better than under any other treatment. I have," he continues, "several times had the heath-house in winter without fire-heat, when the thermometer out of doors stood at $16^{\circ}$ below freezing. But in these cases the house was always shut close, and I have never seen the heathssuffer from the cold.

"The time," Mr M'Nab concludes, "when these plants suffer most from heat is, when a sharp frost sets in, and no heat is applied until the frost has taken effect in the inside of the house ; then a fire is put on, and the frost is driven out. We know that heaths in the open air will not suffer when the thermometer stands $4^{\circ}$ or $5^{\circ}$ below freezing; and we know, also, that heaths in the house, in winter, will bear the same degree of cold with impunity. Now, we shall suppose the thermometer out of doors to fall to $12^{\circ}$ or $14^{\circ}$ below freezing, and no heat in the heath-house; the thermometer in the inside may then be $4^{\circ}$ or $5^{\circ}$ below freezing. If there be no appearance of a change, then it is necessary to apply heat to the house; but all that is wanted in this case is just enough to prevent the temperature from getting lower than it was when the heat was introduced. Suppose the thermometer to sink to $18^{\circ}$ or $20^{\circ}$ below freezing during the night, the instrument inside should range as near as possible to what it was when the heat was applied. This, however, requires very particular attention. From what I know, heaths will suffer, if, after the thermometer has fallen $4^{\circ}$ or $5^{\circ}$ below freezing inside the house, heat be applied so as to raise the temperature and drive out the frost during the time the thermometer is still sinking out of doors. It would be much better if the house was left without fire-heat, even with the thermometer at $15^{\circ}$ or $16^{\circ}$ below the freezing-point out of doors; such treatment is bad for all plants, but more particularly for heaths." The conclusion drawn by that excellent cultivator was, that were we certain the external temperature would not fall below $10^{\circ}$ or $12^{\circ}$ below freezing, no artificial heat should be applied. Maintaining the temperature of all plant-houses at the lowest point consistent with their perfect safety during winter, is one of the principal conditions in good plant-management. It is their season of rest, and all plants must have such; they have it naturally, and demand it artificially.

The majority of this charming tribe are wintered by the London growers in shallow pits, the pots being plunged in sand or other dry material. By this means ample ventilation is afforded them, upon which so much of their healthy existence depends. Under these circumstances no fire-heat is applied, the frost being excluded by means of covering the glass, which is variously effected, that being the best 
which is most conveniently taken off and put on, and capable of resisting wet and frost. Now, the usual covering employed-namely, Russian mats-are anything but this. They soon become saturated with wet, are not capable of throwing it off, are untidy in appearance, troublesome to put on and remove, become heavy with moisture, break the glass by their weight, and, being of a perishable nature, are very expensive. Vide Covering the Roofs of Glass Houses and Pits for the Exclusion of Cold or the Retention of Heat, vol. i. p. 551. Many of the London growers have excellent pits for this purpose, constructed of 9-inch brick walls, and varying in depth from 12 inches to 2 or 3 feet, according to the size of the plants to be placed in them, but always so that the tops of the plants may be near the glass. They are usually about 6 feet wide. Many, however, use means which are simpler, although less to be recommended, and secure protection by the use of turf pits, garden frames surrounded with dry littering matter, and covered with glass sashes, and, in severe weather, with one or more mats, and sometimes a covering of straw. Although these rude means are suitable to commercial growers, they are not to be recommended for use in private gardens; we notice them, however, to show how easily, heaths, and, indeed, most hard-wooded greenhouse plants, may be protected during winter, and to give confidence to amateurs of limited means. Putting lieaths in order to be wintered in such pits, it is well to examine the state of the roots, by turning them out of the pots to see that the drainage is perfect, and that no worms have taken up their abode in the ball; the branches should be thinned out if requisite, tied up where straggling, and the pots plunged in finely-sifted coal-ashes up to their rims, taking care not to huddle the plants too closely together, but to give such room that no two plants touch one another. The intention of the coal-ashes is to secure the roots from frost, keep them in a uniform state of humidity, so as to lessen the necessity of applying much water during the damp months of winter. The coal-ashes also act as an absorber of humidity, and tend, therefore, to keep the air within the pits free of damp-a thing to be carefully guarded against, particulariy when the pits have been for days shut up, as will be the case during severe frosts. At all other times the more air given the better, even to the extent of taking the lights off altogether on fine days. Ventilation should be secured by openings in the back and front walls, just level with the surface of the pots, so that a circulation of air may pass freely through. All the attention heaths require in such pits during winter is excluding frost by covering, ventilating on all fitting occasions, giving as little water as possible, and taking care in applying it that none be unnecessarily spilt on the plants or surface of the pit. In severe frosts, heaths will submit to being shut up for several days at a time; and should even frost to the extent of several degrees enter, and even should the mould on the surface of the pots become hard, no injury will follow, if the process of thawing be allowed to take place in the dark. It is, therefore, absolutely necessary that the covering, whatever it may be, shall be kept on, and light excluded until the frost has gradually been removed from the pit. In preparing heaths for occupying their winter station in the heath-house, the same care must be taken in examining the roots, state of drainage, \&c., as noticed above. The pots should be cleanly washed, the surface of the soil examined, so that all mucous matter may be removed. The plants should have a general overhaul, their shoots be regulated and tied in, and such as require it be topped and supported with neat stakes. We prefer wire about the thickness of a quill, or that known as No. 4, cut into lengths suitable to the size of the plants, as supports, instead of those made of laths, as they are more durable, cheaper, stronger, and, when painted, more elegant in appearance. In arranging the plants upon the stages or platforms, due care should be taken that they have abundance of room, that each be equally exposed to the light, and that the most delicate be placed in the best situations. At this time the discarding process must take place, and all defective or worn-out specimens be removed. Ample ventilation is now required, and unless damp occur in the house, fires need not be lighted unless in very intense frosts; and when for the removal of damp, they should be lighted early in the morning, and the fullest extent of ventilation given at the same time to allow the damp to escape. And when this can be put at defiance by covering of some sort or other, it is better to adopt this than to risk the danger of one hour's fire-heat. For efficient covering, vide vol. i. figs. $788,789,790$.

Summer treatment.-As the spring advances, more water should be applied; some require it more than others, but these can only be detected by close observation. Air must also be progressively increased as the days lengthen and the warmth of summer approaches, even to the extent of admitting it day and night also. It was long held as a necessary part of good culture to turn the plants out of doors about the month of May, and to allow them to remain so till the beginning or middle of October. The propriety of doing so has been greatly doubted. It is quite evident that many of the very best kinds would suffer by such exposure; and hence all good cultivators retain them in the house during the whole year, giving abundance of air at all times, and keeping them thin on the stages by the removal of the smaller plants to cool pits, and setting only the more hardy and robust in the open air.

The following species, with most of their varieties, should not be set out in the open air during any part of the summer: Massoni, colorans, gemmifera, vestita, Niveniana, tricolor, ferruginea, Irbyana, jasminiflora, ampullacea, ampullaceoides, Bandoniana, Shannoniana, retorta, Russelliana, Aitoniana, aristata, aristella, mutabilis, juliana, elegans, Thunbergia, ardens, physindes, campanulata, propendens, Hartnelli, imperalis, Doniana, Cavendishii, Macnabiana, odorarosea, Murrayana, \&c.

Young plants should certainly be kept under the protection of glazed pits or frames, as then abundance of air can be given them by the 
removal of the sashes in good weather, replacing them during cold winds and soaking rains, neither of which heaths will long bear with impunity. In the south of England the objections to their removal to the open air are not of the same force as in Scotland; but there, again, they are apt to suffer from excessive sun-heat at their rosts - the pots attracting heat on their outer surface, while their inner surface is rapidly absorbing the moisture from the balls, and destroying the tender roots. Plunging the pots to counteract this, is apt to cause stagnation at the roots and an interruption to free drainage. Heaths kept in the house during summer should not be crowded; each plant should stand clear of the next. Abundance of water should be given at their roots, and repeated syringing should be applied to the foliage, and even during very hot dry weather to the extent of twice a-day, early in the morning, and occasionally again towards evening. A lack of humidity will beget mildew, which is the principal disease this tribe is liable to. Some advocate shading during the brightest sunshine, and such we at one time practised ourselves in England, but have never done so in Scotland. When heaths, from want of sufficient accommodation, are turned out for a few months during summer, a well-sheltered but not shaded situation should be selected for them, and the floor upon which they are placed should be dry and impervious to worms: the end of May or beginning of June is a proper time-earlier, of course, in the south. A somewhat elevated platform, either of boarding, or with the sides walled up with brick or stone to the height of a foot or so, having the space within covered with coalashes, upon which to set the pots, will be a good provision against root-damp and worms. To protect them against being blown over by wind, lines of cord or wire could be extended from end to end, and to these the plants should be tied. To prevent too much heat at the roots, and to lessen evaporation, the spaces between the pots should be filled in with sphagnum or other mosses, or the pots themselves may be enveloped in the same material. The middle of September is a proper time to remove the plants again to the house, so that their summer-made wood and buds may be matured before winter. The pots should be carefully washed, and the plants moderately thinned out, removing weak and too crowded shoots; and when carefully arranged by tying in such branches as require it, they are then fit for being placed in their winter abode. The more delicate sorts should be taken in first, the others following according to their various degrees of hardiness.

The treatment we have given above for heaths is applicable to the other genera we have named at the commencement of this article-with this exception, they do not all submit to the same degree of cold; nevertheless, one and all of them will not suffer should the internal temperature fall to $2^{\circ}$ or $3^{\circ}$ of frost. All of them may be propagated by cuttings or seed, as recommended for heaths. Some, however, succeed better by seeds: of these we may name Astelma and Helichrysum, which seed freely in this country; and seeds of them are often imported from the Cape of Good Hope. Epacris, Andersonia, Sprengelia, and Lysinema, strike most readily when the cuttings of the ripened wood are taken off in autumn, planted in sand, and allowed to stand in a cool pit till spring, during which time they form callosities, and, if placed in a gentle bottom-heat in spring, root freely.

In an extensive genus like that of Erica there are many species, of course, unworthy of cultivation, unless in a botanical collection. For private growers and amateurs, in particular, the following selection may be considered very complete, as it embraces eighty species, which, with the many even more beautiful hybrids originated from them and others, but whose names we omit with a few exceptions, will form a selection sufficient for the largest establishment in the kingdom. The cultivator can scarcely err in possessing himself of the different varieties originated by such men as Mr Turnbull of Bothwell Castle, who has, we believe, raised more really excellent varieties than any other man of his day. His crossing is in the right direction; for while most others cross from the rare and choice kinds, creating, no doubt, many novelties, yet these are wanting in constitutional strength, are bad growers, and of short duration. He, on the other hand, breeds with a view to gain strength of constitution as well as beauty of flower, and in this he has admirably succeeded. Many fine varieties have originated with the Messrs Rollinsons of Tooting, Fairbairns of Clapham, and others, amongst nurserymen; and the truly splendid E. regalis, E. laqueata lutea, E. nobilis, E. grandis, \&c., raised by Mr Storey, show what even a keen amateur can do. Then there are the many varieties of E. pinea, vestita, ventricosa, tricolor, \&c., all of which are highly deserving the attention of the heath-cultivator.

SECT. 1. Flowers long and cylindrical. Plukenetii, Petiverii, Ewerana, Rollinsonii, fascicularis, verticillata, Sebana, cruenta, perspicua, splendens, mammosa, carinata, Sparmanni, Archeriana, Hibbertiana, Bedfordiana, Linnæana, onosmæflora, aurea, Bowieana, Massoni, gemmifera, exsurgens, vestita, rosea, Niveniana, transparens.

Sect. 2. Flowers much inflated.-Cerinthoides, princeps, tricolor, acuminata, metulæflora, blanda, Monsoniana, Sprengelii, Templeana, tumida.

SEct. 3. Flowers narrowed upwards, with a spreading border.-Ventricosa, prægnans, Lawsoni, Irbyana, jasminiflora, ampullacea, Bandoniana, Shannoniana, Russelliana, retorta, præstans, hyacinthoides, Aitoniana, muscaria, daphnoides, Humeana, aristata, tricolor, primuloides, mundula, Coventryana, aristella, mirabilis, juliana.

SECT. 4. Flowers enclosed in the inflated calyx.Elegans, baccans, Thunbergia, glauca (supposed to be now lost in Britain), rubella.

Sect. 5. Flowers small, globular. - Ardens, physoides, incarnata, Savileana, aggregata, suaveolens, Persoluta, grandinosa, ramentacea, campanulata, mutabilis, decora.

Secr. 6. Flowers small, not globose--Reflexa, propendens, echiiflora, Bergiana.

SEct. 7. Doubtful to what section they belong.Hartnelli, imperialis, \&c. 
The following heaths flower during the winter: hyemalis, Willmorei, grandinosa, verticillata, mammosa, umbellata, vernix coccinea, Wescotii, arbuscula, Lambertiana, L. rosea, acuminata, Persoluta rubra, P. alba, pyramidalis, transparens, regerminans, purpurea, Linnæana, sanguinea, coccinea, exsurgens and its varieties; vestita, most of the section; gracilis, mundula, muscata, verticillata, arborea, incarnata, pubescens, muscosa, conferta, mutabilis, caffra, echiiflora and varieties; taxifolia, \&c.

Insects and diseases.-Those universal pests the aphides sometimes attack the young shoots of the heath, and should be early assailed by a slight fumigation of tobacco; for if allowed to gain allead, the extent of fumigation sufficient to destroy them would greatly injure the plants. In dry weather, and when the atmosphere of the house is kept too arid, mildew is engendered, and is very fatal, particularly to young plants. The universal remedy, sulphur, must be employed, by throwing it on by means of the sulphurator, or by mixing it with the water with which they are syringed; for syringing, under such circumstances, is highly beneficial in itself. Those who have no sulphurator may dust the flour of sulphur slightly over the plant while yet wet. Removing the plants attacked from the heath-house to the open air, or to a cool pit, will often restore plants affected in the course of a week.

\section{$\S$ 6.-AQUARIUM.}

The cultivation of aquatic plants has hitherto, for the most part, been confined to purely botanical establishments. A more general taste is now prevailing, and we believe there are few who have seen the magnificent Victoria Regia, the Nelumbiums, Euryale ferox, Nymphæas, \&c. in the plenitude of their glory, but will admit that an aquarium furnished with such plants is not the least interesting of all plant-houses. Figs. 566 to 575 , in vol. i., will show their construction. It remains only for us to speak of the culture of the plants. The majority of cultivated aquatics are tropical, and hence, at certain seasons, require a considerable amount of heat, the want of bottom-heat being the chief cause of that want of success which attended our earlier cultivators. This has been pretty well examplified by the Messrs Weeks of Chelsea, who succeeded in flowering the Victoria in a tank in the open air, with the mere protection of a thin awning thrown over it during the night. They maintained, however, a temperature in the tank of from $80^{\circ}$ to $90^{\circ}$ of heat, apparently agreed upon all hands to be the most suitable for it. The heat arising from a large body of water at this temperature would elevate that of the atmospheric air for several inches above its surface to nearly the same height. And this is all that is required, for the leaves, lying flat on the surface of the water, will be just in their element, so far as heat is concerned. The flowers rising only their own height, some 6 or 8 inches above the surface, would be equally comfortable. Now, the reverse of this had hitherto been the prevailing practice, the roots of aquatics being, for the most part, placed in wooden tubs or cisterns - bad conductors of heat, even had it been applied externally to them-while the foliage was exposed to a temperature of from $65^{\circ}$ to $100^{\circ}$, as that of tropical houses often is. Here, it appears, the whole evil rested. Those who succeeded in blooming the Euryale, Nelumbiums, and Nymphras, had the vessels in which they were placed set on the top of flues, or plunged in a strong bed of fermenting material, by which a moderate, but not a uniform, temperature was obtained, and hence their frequent failures. Tc grow tropical aquatics well, a house or pit should be set apart for them, and in which a tank should be introduced sufficient for holding the whole collection. This tank should be heated by pipes of steam or hot water made to circulate through it, and these should be placed at its bottom, for reasons sufficiently apparent to every one who knows anything of the principle of heating.

The following plants, remarkable for their extraordinary forms, are well adapted for entering into the aquarium. They do not, however, require to be grown in water, but thrive best in a humid still atmosphere, and may, therefore, be arranged around the sides of the house. The Nepenthes, being climbers, may be trained over the roof ; while the Cephalotus follicularis, Dionæa Muscipula, the exotic Droseras, \&c., should be placed near to the glass, and in the cooler part of the structure.

Propagation.-The Euryale, Nelumbiums, and Victoria are propagated only by seed, which is produced sufficiently freely. When ripe they should be removed from the capsules, and kept in phials of water till spring, but not corked down, when they should be sown in small pots, set in pans of water sufficiently deep to cover them, one seed in each, and plunged in a strong bottom-heat, yet near to the glass. The Euryale and Nelumbium seed have a cap or valve-covering over the eye of the seed, which should be removed carefully with the point of a penknife, to allow the seed enclosed within the shell-like covering to escape. When the plants have attained leaves an inch or so in diameter, they should be shifted into 6 -inch pots, and replunged again in bottom-heat, still kept in deep earthenware pans filled with water. When the leaves have attained the diameter of from 6 to 9 inches, they are fit for planting out in the tank, the water in which should be brought up to a temperature of from $80^{\circ}$ to $85^{\circ}$. The Victoria, occupying so large a space for the development of its extraordinary foliage, should be planted in a deep tank, in the centre of the one upon which its leaves are to float. Our centre tank is 4 feet square, and the same in depth; 4 -inch hot-water pipes are brought in at its bottom, and made to coil twice round the sides, when the water is allowed to return again to the boiler. This tank is 6 inches below the level of the outer one, and for the first few days it only is covered with water. As soon as the leaves extend to its sides, the outer tank, which is 12 inches deep, is also flooded, and the foliage allowed to extend over it. The same arrangement is suitable to the Euryale and Nelumbiums, only the tank need not be so large. During the growing season, as 
much light as possible is admitted to the plants, the bottom and surface temperature kept both at $80^{\circ}$ to $85^{\circ}$, allowing a rise of several degrees in the latter during sunshine. The night temperature is $5^{\circ}$ less. A constant supply of fresh soft water is admitted by a half-inch pipe, and the overflow escapes at the opposite corner, and is conveyed to a tank in the adjoining house. As the leaves begin to decay, they are cut off, to make way for the young ones, one of which generally presents itself every second or third day. The Victoria Regia is a perennial plant in its own country, and so it has proved itself to be in some collections in Britain, as at Kew, Sion House, \&c. In the former establishment we observed it in flower during its third year's existence. With us, however, it has proved, for three successive years, no better than an annual, dying always towards autumn, but producing abundance of seed : one capsule this year contained 268 seeds. We grow it, therefore, annually, but find great difficulty in getting the young plants along, particularly early in the spring. The cloudy atmosphere and long absence of sunshine may be the cause of this, as well as its not surviving the winter. Almost similar difficulties have occurred at Dalvey, the only other place, we believe, in Scotland where it has flowered.

The Nymphoeas are propagated by seed, and also by division of the roots. Some of them seed so freely, and vegetate in the bottom of the tank, that they require to be often weeded out. As they are much snaller-growing plants than the foregoing, we usually plant them in pans from 12 to 18 inches in diameter, and from 6 to 12 inches deep, and submerge them round the edges of the outer tank. When their season of growth is past, the roots are taken out and placed during winter in smaller ones, the soil of which is kept only moderately damp, and placed in a temperature of from $50^{\circ}$ to $55^{\circ}$. This constitutes their season of rest. They are re-potted again in spring, and treated as above.

Limnocharis and Pontederia are of easy growth, propagating freely by extension, but succeeding better in a much less heat, say from $57^{\circ}$ to $65^{\circ}$.

Papyrus, one of the species of which was a plant of great importance to the ancient Egyptians, furnishing, as it did, a material for writing upon before the invention of paper, is also of easy culture, not requiring much bottom-heat, and succeeding well if planted in a large pot set in a tub of water.

Pistia floats about on the surface of our Victoria tank in great abundance; is difficult to transport, unless sent in a jar of water. It winter's best when its roots are in soil, and plunged in a warm tank; during summer it luxuriates floating on the surface of the water.

Vallisneria is a genus of no particular beauty, but sufficiently interesting in a botanical point of view, being generally selected by the microscopist for the exhibition of the circulation of the sap in plants. The leaves, which resemble the leaves of some long-leaved grasses, evolve a continued stream of oxygen gas, which rises in a current of minute bubbles, and particularly so from parts that may have received slight injury.

The other tropical aquatics we have named are all of the easiest culture, and are readily propagated by subdivision.

Soil.-Contrary to the majority of other plants, aquatics do not require a soil fertile in vegetable matter, at least in a recent state. Their natural soil is the bottom of lakes, rivers, or ditches, composed of alluvial deposit to a considerable depth, although sometimes a gravelly soil. We find a fine rich alluvial soil, formed during ages along the banks of the North Esk, free from stones or the roots of other plants, to answer exceedingly well, and without previous preparation or addition. The soil we refer to is free of mineral ingredients likely to be injurious to the roots. When the plants are finally planted, about an inch of fine silvery sand is laid over the surface of the soil in the case of large plants, while small white pebble-stones are placed over that in which the smaller are set, merely to give the whole a cleaner and neater appearance.

Water.-It is important that the water be pure and not charged with mineral matter. Rain water being the best, we always use it; it being brought from a collecting cistern in a leaden pipe, which is made to pass round the boiler before discharging itself into the tank, to avoid decreasing the temperature of the latter. It is also necessary that it be kept clean and fresh, and free of green slimy or mucous matter, probably the first stages of various species of Confervæ, \&c. This may be partially kept down by skimming the surface of the water daily, and in addition to this a few water-snails. Limnæa stagnalis, if introduced, will breed, and, as they feed upon such matter, render the water clear and wholesome. It is possible, also, that gold and silver fish assist in this nice operation -at all events, we know that they devour the numerous hosts of aphides which prey upon the under surface of the leaves of the Victoria which, until we employed them last year, had nearly devoured the leaves before they were half formed. We believe that there are two species of aphides attacking the Victoria: the one we have referred to, which is an aquatic species, or else capable of adapting itself to a watery element, as it lives on the under side of the foliage; and another, which preys on the upper surface of the leaves, is equally destructive. By their united efforts holes are often eaten quite through the leaves from half an inch to an inch in diameter. Fumigation, both of tobacco and Cayenne pepper, was tried in vain, and no means we used had any effect on the enemy till the introduction of the gold and silver fish. The fish also perform other important offices connected with the growth of the plant. An interesting paper, communicated to the Chemical Society by Mr Warrington, seems to establish this fact. "The fish," he says, " in its respiration, consumes the oxygen held in solution by the water as atmospheric air, furnishes carbonic acid, feeds on the insects, and excretes material well adapted for rich food to the plant, and well fitted for its luxuriant 
growth. The plant by its respiration consumes the carbonic acid produced by the fish, appropriating the carbon to the construction of its tissues and fibres, and liberates the oxygen in its gaseous state to sustain the bealthy functions of animal life, at the same time that it feeds on the rejected matter which has fulfilled its purpose in the nourishment of the fish, and preserves the water constantly in a clean and healthy condition. In reference to the watersnail," he continues, "it finds its proper nutriment in the decomposing vegetable matter and minute confervoid growth, prevents their accumulation by removing them, and by its vital action converts what would otherwise act as a poison into a rich and fruitful nutriment, again to constitute a pabulum for the vegetable growth, while it also acts the important part of a purveyor to its finny neighbours."

\section{LIST OF TROPICAL AQUATICS.}

Ceratopteris thalictroides; Euryale ferox; Limnocharis Humboldtii, Plumierii ; Nelumbium album, Tamara, jamaicense, roseum, luteum, caspicum, speciosum, flavescens; Nymphæa ampla, scutifolia, cærulea, stellata, cyanea, Devoniensis (a hybrid between $\mathrm{N}$. rubra and $\mathrm{N}$. lotus), pubescens, Lotus, biradiata, gigantea, reniformis, macrantha, odorata, pygmæa, rubra, rubra var. rosea; Papyrus antiquorum, odoratus, laxiflorus, elegans; Pistia stratiotes; Pontederia crassipes, dilatata, lanceolata, azurea; Victoria regia; Vallisneria spiralis; Villarsia ovata, indica, lacunosa, chilensis, geminata, sarmentosa, parnassifolia, reniformis; Jussieua, the whole genus; Alisma cordifolia, parnassifolia; Orontium aquaticum; Hypoxis aquatica; Byblis liniflora; Aldrovanda vesiculosa; Oryza sativa (the cultivated rice-plant), latifolia, nepalensis; Saururus cernuus, lucidus, chinensis; Trapa quadrispinosa, bicornis, bispinosa; Elodea guianensis.

\section{$\S$ 7.-THE STOVE OR TROPICAL PLANT HOUSE.}

The plants which come under the denomination stove or tropical plants, are not only vastly numerous, but they differ greatly in character and magnitude. Amongst them are found many of the greatest floral beauty, singularity in foliage or structure, importance in the arts, and many producing fruit of the most delicious flavour. Indeed, so diversified is this vast assemblage of plants, that to attempt to cultivate even a single representative of each family within the limits of one structure is impossible; and hence we have tropical fruit-houses, palm-houses, moist and dry stoves, orchid-houses, aquariums, \&c. The two latter of these we have noticed; of the others we will now briefly speak. In legard to structure, as the majority of tropical fruit-bearing plants and trees attain a considerable altitude, and grow naturally exposed to the full force of a tropical sun, it follows that houses for their cultivation should not only be lofty-some of the bananas and plantains attaining the height of 30 or 40 feet-but that they should also be as transparent as possible. Hence curvilinear or span-roofed houses, constructed of glass to within a foot or two of the ground, are found to be the most eligible. In addition to this, ample means should be secured for not only maintaining a sufficient degree of atmospheric temperature, but for applying bottom-heat also. On abundance of light and heat much of the success depends. Another important part of their culture, we assert, is their being planted in tubs or slate cases instead of being planted out in borders, be they ever so well prepared. We would prefer slate cases, as offering less resistance to the penetration of heat to the roots, as well as being more elegant and durable, and admitting of the renewal of exhausted soil, and examination of the state of the roots. Many of the tropical fruits might readily be ripened if trained to trellises near the glass, after the manner of peaches and vines; but there are others, such as the Musas, which can only be grown when allowed to take their natural habit of growth.

The genus Anona, or custard apple, furnishes nine species of fruits, the most important of which is $A$. Cherimolia. They all require a rich soil, and are readily propagated by cuttings, or by imported seed.

As to Artocarpus incisa, the bread-fruit, $A$. integrifolia, jack-fruit, neither of them, we believe, has fruited in Europe. The former is a plant of difficult culture, and hence rare even in our botanical collections. Notwithstanding, young plants might easily be imported from St Vincent or Jamaica by the West India mailsteamers. A rich soil is indispensable, and either may be increased by cuttings taken from established plants or imported seed. The importation of young plants, however, in Wardian cases would be the most certain.

Achras zapotilla, the Zapotilla plum, $A$. $S a-$ pota, Afgle Marmelos, Bengal quince, are readily propagated by cuttings of the half-ripened wood, and, like most fruits, require a rich soil.

Blighia sapida, (the akee tree), a fruit much esteemed in the West Indies, is readily propagated by seed, layers, or cuttings; but as it is apt to sport into varieties when propagated by the former means, recourse must be had to grafting or budding to continue approved sorts.

Coffea arabica, (the coffee of commerce), is a plant of easy culture, and readily propagated by seed, which ripen freely in our stoves. It is exceedingly liable to the attack of a species of Coccus, seemingly Coccus adonidum ( $\operatorname{Lin}$.) The fragrance of its blossom is delightful, and the abundance of fruit exceedingly great.

Carpodinus dulcis, (sweet Pishamin), a native of Sierra Leone; plant of a climbing shrubby nature, producing fruit resembling the lime, exceedingly grateful and sweet; propagated by seeds or cuttings.

Durio zebethinus.-Said by Rumphuis to be the most excellent fruit of India; propagated by seeds, which may readily be imported from Calcutta.

Eugenia malaccensis, Malay apple; $E$. Jambos, jamrosade, or rose-apple; $E$. macrocarpa, and others of the family, fruit freely, and are of easy 
culture, and readily propagated by cuttings or imported seeds.

Euphoria Litchi, Lee-Chee; E. Longana, Longan, are amongst the best fruits of China. $E$. Nephelium, $E$. verticillata, natives of the East Indies, are also much esteemed; they are all readily propagated by cuttings, layers, or seed, and are of easy culture.

Ficus Brassii, large fig of Sierra Leone, the fruit of which is of excellent quality, and produced in abundance on the old wood, is a plant of easy culture, and not uncommon in collections, but being prevented from attaining a proper size, seldom produces fruit in this country. Like all the genus, readily increased by cuttings.

Garcinia Mangostana, Mangosteen ; G. Gambogia, gamboge; G. cornea, G. Cowa.-The mangosteen is a fruit held in high esteem by all who have resided in India, combining much of the flavour of the strawberry and the grape. The whole genus are readily propagated by cuttings, and also by seeds, which, however, should be sown as soon as possible after they are ripe, as they do not long retain their vegetative properties. Seeds sown in soil in a Wardian case in India, and immediately transmitted to Europe, arrive either when slightly germinated, or, should vegetation have farther advanced, the plants will, in general, be found healthy and in good condition. Young plants transported in close cases seldom suffer much during the voyage.

Grias cauliflora (Anchovy pear) requires a sandy rich soil, and is propagated by cuttings or imported seeds.

Lucuma mammosa, L. Bonplandii, L. ovata, and $L$. salicifolia, are nearly related to Achras, and require the same treatment.

Mangifera indica, mango.-Of this excellent fruit several varieties exist naturally, and two have fruited in this country-namely, the red and yellow Powis mangoes. It is propagated by cuttings, and these should be taken from trees of approved varieties, as, like the apple and pear, many sorts are not worth cultivation.

Musa chinensis, dwarf or Chinese plantain, (Sweet), Cavendishii (Paxt., Mag.ofBot.);M.paradisiaca, common plantain; M. sapientum, banana; $M$. Dacca, and several others, are of easy culture, requiring a rich soil and abundance of root-room. They fruit freely, and are propagated by subdivision, young stems being produced as the older or fruit-bearing ones attain their full growth.

Psidium, (guava), a genus of low-growing trees, $P$. Cattleyanum, only a few of them exceeding 10 or 12 feet in height. The whole are much esteemed for their fruit when in a ripe state, and the delicious guava jelly is also prepared from them. They are of the easiest culture, and fruit freely in any ordinary stove, and may be readily increased by cuttings or by seeds, either imported or ripened in this country.

$P$ assiflora edulus, $P$. maliformis, $P$. quadrangularis, $P$. alata, and $P$. laurifolia, produce fruit of peculiar excellence, and generally known as granadilles. They are easily multiplied by seed, which are produced in considerable abundance.
They are free-growing climbing plants, and therefore should occupy the roof of the tropicalfruit stove. They will set their fruit more readily if assisted artificially by transferring the pollen, upon the point of a camel's-hair pencil, to the pistil, which is large, and situated in the centre of the flower.

Palms require abundance of space both for their roots and also for the full development of their leaves. Their culture is by no means difficult, so long as they enjoy a powerful humid heat and sufficient space-involving, in fact, more the question of expense than that of science. Most of them are found to luxuriate naturally when growing by the margins of springs and streams. Only one of them of which we are aware inhabits the sea-shore, and that is the double-fruiting cocoa-nut, known as the Cocosde-mer, a variety of Cocos nucifer a, or common cocoa-nut. Some are found in the alpine regions of their country; some are gregarious, existing in large groups; while others are scattered about, singly or in clusters, among woods and plains. Some of them, such as Rhapis flabelliformis, increase by division of the roots; others, as Chamcerops humilis, C. serrulata, and some of the Zamias, propagate themselves by suckers, and some by scales, but by far the greater number are only to be increased by seed. The cultivation of palns is mainly confined to strictly botanical establishments; our remarks, therefore, on them are thus necessarily brief.

General collection of stove or tropical plants. The amount of light which plants, not actually inhabiting the shade of impenetrable jungles, enjoy in the tropics, is so much greater than we can accommodate them with in our ordinarily constructed plant-houses, leads us, in the first place, to remark, that the want of complete success in cultivation is more to be attributed to imperfect structures than a deficiency of skill in their cultural details. Houses, therefore, for them, should be of the most light and airy description possible. They should be of glass on all sides, and that extended to within 2 or not more than 3 feet of the ground. An equable distribution of heat and air, and a command of moisture, are equally important. A judicious selection of plants is the next important consideration ; for it will always be found that a limited number of species, if really good, will afford much more satisfaction than a long catalogue of sorts, many of which seldom if ever flower; and others, should they do so, are almost devoid of interest, from the paucity and insignificance of their blooms, and want of elegance of character in the plant. It is not now the number of species, but the excellence of the culture, and the continued display maintained throughout the whole year, that constitutes perfection in plant management; and next to this is the neatness and order exhibited in their arrangement and keeping.

It formerly was the prevailing practice to grow the majority of stove-plants plunged in tan-beds, or other material, in a pretty high state of fermentation. Modern practice has set aside this, unless in some few extraordinary cases, and either places the plants on platforms or stages 
without bottom-heat beyond that of the general temperature of the house, or sometimes upon pavement, under which tanks or gutters of heated water are made to circulate; while others place them on top, or plunge them partially in beds of small stones, heated by means of hotwater pipes. No doubt some tropical plants require the stimulus of a degree of bottom-heat, during their growing season, several degrees above that of the atmosphere their tops are placed in, and all sickly plants do so to bring about a healthy action in them.

It is well, therefore, to have the means of bottom heat at our command, and of these we greatly prefer a bed of small stones or rough gravel, on which to set those plants which do not require such root excitement, or to plunge those less or more that do so. This bed should occupy the centre of the house, and on it the taller and larger plants should be set, the smaller ones being arranged on neat cast-iron ornamental grating tables around the sides and ends next to the glass, as under these the hot-water pipes for heating the atmosphere of the house should also be placed. The heat arising from them through the openings of the grating will keep the roots of the plants somewhat warmer than the general air of the house. The majority of tropical plants, during the commencement of their growth in spring, and also through the whole period of their development during summer, require a slight excitement at their roots, to the extent of placing the roots rather in advance of their branches and leaves. Where a contrary practice is followed, its effects will soon be seen in the sickly and miserable appearance of the plants. The objection to plunging in fermenting material is the want of uniformity in temperature, the exclusion of air to the roots, and the liability of the soil around them to become sodden and waterlogged, to say nothing of the expense and untidiness of appearance. During winter, and under a diminished amount of light and solar influence, there is possibly no necessity for bottom-heat, unless in peculiar instances, such as that of plants of very difficult culture; and even with these extreme caution is necessary, that, while the roots are not allowed to suffer from cold, the plants should not be put into a state of excitement until towards spring, when light and sun-heat become their natural concomitants.

When such plants are planted out in deep borders under the surface line of the heat of the house, and perchance not very effectually drained below, the roots, instead of being in advance of the foliage, are considerably behind them. This must be guarded against; and unless such borders are vaulted underneath, and heated, we would say distinctly that no tropical plant should be planted out. Young tropical plants, whether propagated by seed ol' otherwise, should be forwarded to fit them for being brought into the larger structures, as should also all sickly plants, whether cut down or not; and this is best effected by placing them in low pits, or in a close propagating house, and plunged into a bottom temperature suitable to their various circumstances.
Autumn and winter treatment of tropical plants.-Towards the beginning of September ventilation must be gradually lessened, and the house shut up by three o'clock in the afternoon, so as to enclose a sufficiency of solar heat to maintain the temperatare with the least amount of fire-heat; but fires, from this time forward, supposing them to have been extinguished during the two previous months, should be lighted in the afternoon on all cold or damp foggy days, in particular, if only to the extent of slightly warming the water in the hot-water pipes, increasing it as the days shorten and the nights become colder, to assist in maturing the young wood and buds of the plants, or, in other words, to lengthen out a moderately warm and dry autumn. Growth, however, must not be prolonged, the maturation of the wood and buds being all that is required. Moisture must also be diminished, and the rambling and fast-growing shoots pinched back, unless in cases where the flowers are terminal. At this time, also, a general arrangement of the plants should take place, insects be carefully destroyed, plants requiring it pruned of superfluous branches, and neatly tied up. The whole structure should be carefully cleaned, and all repairs required attended to, the glass washed clean, and, where practicable, the wood-work and walls, where such exist, coloured white. As November approaches, allow the thermometer to fall gradually to $55^{\circ}$ or $60^{\circ}$ as a night heat, and from $60^{\circ}$ to $65^{\circ}$ as a day temperature, unless there be any very rare plants requiring a higher minimum, and these had better be accommodated in a house or pit by themselves during the winter, as any greater heat would be unsuitable to a general collection. Syringing over-head should also be discontinued.

Too much fire-heat, and too little ventilation, are the stumblingblocks with many amateurs and inexperienced gardeners. The lower the external temperature falls, the higher they keep the internal ; and to aid their heating apparatus in effecting this, they all but hermetically seal the ventilation for the time being. In such an atmosphere as is created by this few plants call exist, much less grow ; and hence it is, that with an imperfect house, an apparatus incapable of heating the house to an extreme degree, and the open laps and fractures in the glass roof to admit fresh supplies of air, plants are able to struggle through a winter, even in defiance of the utmost wish of their owner. A lower temperature during the night than during the day, and as free an amount of ventilation as can prudently be given them, with a great diminution of water both at their roots and over their heads, are the principal conditions required for maintaining stove-plants in a healthy condition during the dark months of winter.

Where stove-plants are required to be in flower during this period, they must for the most part be produced in heated pits or small houses, constructed and managed for the express purpose. There are abundance of plants that will flower at the time naturally, or with very little excitement given them. While these are in flower they may be placed in the general 
stove for its adornment; the low uniform temperature, and rather dry atmosphere, will be very favourable for their longer continuance in bloom. When the whole collection is placed in their winter-quarters, care should be taken that they be not set too close together; and here we would recommend a general inspection to take place, and that all sickly or uninteresting plants be at once cast aside. It will not unfrequently happen that several specimens of the same species may be in equally good health and vigour; it is better to throw away one of these than that the others should be spoiled from want of room. In regard to temperature during winter, the occupants of our plant-stoves, in private establishments in particular, are oftener injured by too much heat than the reverse. Both $\mathrm{Mr}$ Beaton and $\mathrm{Mr}$ Spencer, than whom no higher authorities can be given, have shown that in a temperature of from $40^{\circ}$ to $50^{\circ}$ by fire-heat most of the popular stove-plants have not only thriven well, but flowered profusely.

Towards the end of January a general examination of the plants should take place, and all that require potting, either with a view to obtain larger size, or where the soil has become exhausted, should be seen to. This is the advent of their season of growth, and hence the proper time to supply them with fresh food. To delay this is dangerous, as the roots, when once they have begun to grow, will, with all the care that can be taken of them, be liable to be destroyed during the operation. Indeed potting, in all cases where a reduction of the ball has to be made, should be performed before the roots have begun to grow at all. Divide herbaceous stove-plants, top such as require it, train and clean every plant requiring that attention. Lay in a stock of the various soils required, so that it may be dry, warm, and ready for use when wanted. Either use new, or, at all events, clean pots, and let them be always dry before using. Suppress insects by washing, fumigating, or sulphurating. Increase the amount of air as the days lengthen, and progressively raise the temperature also, as well as the humidity of the atmosphere, to meet the demands of the increasing excitement in the plants. For a general collection in a private establishment, the temperature should, when the plants begin to grow, be kept first at from $55^{\circ}$ to $65^{\circ}$ during the day, allowing a few degrees more during sunshine; but the night temperature may be kept $5^{\circ}$ lower than these points. A much higher temperature is necessary in the case of collections containing many of the plants of the torrid zone, and which, to be cultivated in perfection, should be accommodated with a structure for themselves. As the season advances, and the amount of light increases, the stimulants of additional heat, moisture, \&c., must be increased in due proportion. Ixoras, Gardeneas, and similar hardwooded plants, would be better transferred to a close pit, where a little more heat and a much increased amount of humidity could be afforded them, and where also a slight bottom-heat would be of great advantage to them. Plants that are sickly should have provided for them a similar accommodation. The seeds of most tropical plants should be sown towards the end of January, and these should always be placed as close to the glass as possible, yet so as to have the advantage of a moderate bottom-heat, particularly those having hard and thick coverings.

Spring and summer treatment of tropical plants. -If the temperature has been regulated during winter as already directed, the plants will by the latter end of February be in excellent condition, after their long repose, to shoot out into leaf, and flower upon the return of spring. Undue excitement must, however, be guarded against. The sap must be allowed to rise slowly and progressively, and to this end fire-heat must be moderately employed. The influence of sunheat during fine days will bring about a state of matters far nore favourable to the health of the plants than any degree of fire-heat that can be applied, and therefore a rise of a few degrees may be with advantage allowed during bright days. Advantage also should be taken of this for the admission of a greater amount of air than has been given during their season of rest, as this will have the effect of causing the buds to swell boldly, and break into wood or flowers with much greater vigour. A contrary course at this time would be highly improper, and would soon become manifest by the weakly state of the plants, paucity of bloom, and increase of insects. From the middle of February to the middle of April the day temperature should rise gradually, and range, towards the latter period, from $60^{\circ}$ to $70^{\circ}$, the night heat all the while from $5^{\circ}$ to $8^{\circ}$ lower. More air and more water at the roots will be required, increasing both as vegetation proceeds; and towards the end of that period syringing should be occasionally administered, and all the while a moderate humid atmosphere maintained by sprinkling water on the floor of the house. As during winter, so now, the water for all purposes should be used in a tepid state; care should at the same time be taken that too much water be not applied, as the young rootlets are not yet in a state to absorb it, nor do the awakening energies of the plants require it. A general regulation of the plants should take place as early in spring as possible. All will require examination at their roots, many will be in want of training, some of pruning; and in all cases where lean-to houses are in use, every plant will be benefited by being occasionally turned round, that its foliage may be exposed to the light and sunshine.

During this period potting should be attended to ; indeed, every plant should be turned out of its pot, that the state of the roots may be ascer. tained. Some may not require shifting, and in such cases they may be carefully returned again to the pot. The great majority, however, will, and this operation should be proceeded with before the young roots have begun to extend themselves. To facilitate this, a stock of soil should be brought into the potting-house, into which, if contiguous to the stove, the plants should be taken, a few only at a time; but when this is otherwise, the potting had better be carried on within the house, as it would be dangerous to expose the plants at this season to the external atmosphere. Regarding soil, the great 
majority of tropical plants, where we have not stated to the contrary, succeed well in a compost of half peaty soil, and half light rich loam-of course, both containing abundance of vegetable matter; and if the loam is rather strong than otherwise, or the peat deficient in sparkling white sand, then that should be added to the necessary extent. The suil can scarcely be too rough, particularly for large plants; and over the drainage some of the turfiest or most fibrous should be placed, both to prevent the finer particles from being washed down amongst the drainage by the necessary waterings, and also as food on which the roots will feed when they extend to that depth. If the soil is dry and rather light, or abounding much in fibrous matter, it can scarcely be packed too closely around the ball ; if, on the other hand, it be somewhat damp, and of a strong loamy character, then less compression should be used. This is an important matter in pot culture. Regarding the size of pots, this must be regulated by circumstances, as, whether it be desirable to have large specimens, whether the plants are naturally of luxuriant growth, and having large fleshy roots, or whether they be of slender or diminutive habit, with roots of a hair-like nature. All sickly or badly-rooted plants should not only be placed in pots as small, or often smaller than those they have been growing in, but the whole soil, in extreme cases, and the greater part of it in ordinary cases, should be removed, the dead or decaying parts of the roots themselves cut clean away, the plants set in small pots, and plunged in a moderately strong bottom-heat. This treatment should be continued until they are again in a healthy growing state, when they should be again replaced amongst the general collection. Avoid crowding the plants too much. Rapid-growing plants will often require topping to cause them to form a sufficient number of branches, but care should be taken in considering whether the individual produces its flowers at the termination of the shoots, as is the case with most of Melastomaceæ, which should, for the most part, be stopped only once, while in some other orders stopping may be repeated oftener, or, indeed, until the plant has become sufficiently furnished from the surface of the pot upward. Towards the beginning of July many of the hardier stove-plants may be removed to the greenhouse, which will by that time have the majority of its plants set out in cool pits or in the open air. This will give greater room to those which remain, and enable them more fully to develop themselves; and here they may remain till the end of August or beginning of September.

The following stove-plants are highly worthy of cultivation, and indeed no collection can be regarded as approaching perfection if many of them are wanting :-

Achimenes.-A now pretty extensive genus of free-blooming plants, of low habit and of easy culture. They are, however, more properly inhabitants of the bulb house or pit, dying down entirely after flowering, and remaining during their season of rest under ground. They increase rapidly by the roots, which may be kept in a di'y state during winter, and placed in a dry moderately warm chamber, or under a stage in the stove. By February a portion of the roots should be shaken out and potted in light soil, in very shallow well-drained pots or deep pans, and placed in a moderate heat, and at first sparingly supplied with water. A second potting should take place in March, and a third in April, which will produce a long succession of bloom. They are of easy culture, and produce a good effect if planted thickly in the pots. On account of their dwarf habit, few of them exceeding 12 inches in height, they may be grown to great perfection in a low pit, and lemoved to decorate the stove or drawing room when fully in flower.

Achmea.-A genus of plants suited for either pot or basket culture, and hence well adapted to the orchid-house. Their flowers are beautiful and of long continuance. They increase readily by division, and prefer a light turfy soil in welldrained pots.

Aschyanthus.-An interesting genus, requiring similar treatment with the last.

Allamanda.-A splendid genus of climbing and half-climbing plants, producing abundance of large yellow flowers of great beauty. Require a soil of peat and loam in equal parts; propagated freely by cuttings of the half-ripened wood.

Agalmyla.-Requiring similar treatment to Gesneria.

Amherstia nobilis.-A magnificent and hitherto rare tropical tree, requiring a warm stove, a peat and loam soil, abundance of water while in a growing and flowering state, comparatively dry during its season of rest.

Argelonia.-Soil, peat and loam ; propagated readily by cuttings, which should be attended to annually. While young, top the plants till they have made eight or ten shoots, after which allow them to come into flower.

Aphelandra.-A showy genus, several of which flower during the winter ; of remarkable easy culture, striking readily by cuttings ; requiring a soil of half peat and loam.

Batatas Waldeckii.-An" excellent climbingplant, flowering throughout most of the summer. Soil, peat and loam. Propagated by division of the fleshy roots, after the manuer of Ipomea, and also by cuttings of the points of the shoots.

Begonia.-An extensive genus of free-flowering plants, of the easiest culture, requiring a soil of half peat and loam; readily increased by cuttings, and some or others of them in flower throughout the year.

Bignonia.-The store species are chiefly creepers of rapid growth and great beauty ; propagated by cuttings, and requiring a peat and loamy soil.

Billbergia.-A free-flowering genus, requiring the same treatment as Achmea.

Brownea grandiceps.-A plant of great interest propagated by cuttings, and sometimes by layers, requiring a peat and loam rather rich soil.

Caladium.-A genus of the Arum tribe, remarkable for the beauty of their foliage, most of them being variegated. Propagated by division of the plant, and preferring a humid rich soil 
during their growing season, but require to be kept pretty dry during their period of rest.

Campylobotris discolor.-A handsome flowering dwarf plant, propagated by cuttings, and requiring a peat-and-loam soil.

Ceropegia.-A genus of slender creeping plants, more remarkable for the singularity of their flowers than for their beauty. Propagated readily by cuttings, and require a peat-andloam soil.

Charianthus coccinus.-A beautiful slendergrowing, scarlet - flowered, autumnal-flowering plant, related to Melastoma. Propagated readily by cuttings, and thrives in a peat and turfyloamy soil.

Clerodendrum.-A fine genus of free-flowering plants when well grown, some of which are creepers ; the others are of pretty robust habit, therefore requiring plenty of room as well as large pots. They prefer a rich turfy soil, and are readily increased by cuttings.

Combretum.-A splendid genus of climbing plants, requiring a turfy rich soil, abundance of room, and increased by cuttings and layers, with the exception of C. purpureum, which should be grafted on C. Pincianum, or some other free-growing species. As soon as the young wood has ripened and the leaves begin to fall, the lateral shoots should be cut back to within one bud of the base; and if the spurs thus formed along the main stem become too crowded, they should be thinned out to a foot apart. By this means C. grandiflorum has been made to bloom, which is one of the shyest flowerers of the genus.

Cyrtoceras reflexus.-A free-flowering plant, nearly related to Hoya, and requiring nearly the same treatment.

Colvillea racemosa.-A magnificent Madagascar tree, requiring a warm stove, abundance of water while growing and flowering, to be kept comparatively dry during its season of rest. Propagated by cuttings in a brisk heat, and requires a rich turfy peaty soil.

Dipladenia.-A genus separatedfrom Echites, requiring the same treatment, \&c.

Echites.-A genus of climbing plants of great beauty and easy culture, propagated by cuttings, and requiring a rich turfy slightly peaty soil.

Euphorbia.-Propagates readily by cuttings, which should be laid aside after being made for a few hours, to allow a portion of the milky sap to escape, after which collodium may be applied to the wound with advantage. Light well-drained rich soil is most suitable for them.

Eranthemum.-Chiefly half-shrubby plants, free bloomers, and of extremely easy culture; propagated by cuttings, and requiring an ordinary peat-and-loam soil. The young plants require topping in their early stages to induce a sufficient number of branches, after which they should be allowed to extend themselves naturally.

Franciscea.-A genus of low-growing shrubby plants, profuse flowerers, and mostly very fragrant. An intermediate stove temperature seems to suit them best, and a soil three parts of VOL. II. which are peat to one of loam is most congenial to them. They are increased by cuttings, and the plants, under good management, continue for years.

Gardenia.-A very popular tribe of plants, on account of the abundance of their bloom and the exquisite fragrance of several of the species. They are increased by cuttings; prefer a rich peat-and-loam soil ; require a positive season of rest, during which time they should be kept comparatively dry and cool. None of the genus will long withstand perpetual excitement, and this is the principal cause of the want of general success in their cultivation.

Gesneria.-A genus for the most part of tuberous-rooted plants, of easy culture and propagation. During their growing season they require all the stimulants afforded by a well-regulated stove; and as soon as their flowering season is past that of rest begins to take place, and is evinced by the drying up of the foliage and decay of the stems. When this takes place, water must be gradually withheld; and when the stems become withered, the pots should be removed to a dry and moderately warm place, as under the plant-stages, or even into an empty room, and be treated as recommended for bulbs -(vide BulB-House.) After two or three months' rest they may be again set a-growing, taking them in in succession, so as to lengthen out their season of flowering, which can, by a judicious selection of sorts and rotation in exciting them, be made to extend over the whole year. The dried tubers should be shaken out of the soil they had previously been growing in, and planted in fresh compost composed of rich turfy loam and peat, using pots rather deeper than those in ordinary use, to afford room for their large tuberous roots: water sparingly at first, and when vegetation commences, place them in sufficient light, and increase the quantity of water as they progress in growth. They are readily increased by a separation of the tubers. Those species which are not tuberous are propagated sometimes, as in the case of discolor, by seed, and also by cuttings in the ordinary manner.

Gloxinia.-Produces seed in most cases abundantly, which, on account of its very small size, should be sown on a smooth surface of soil, and merely covered with a slight covering of moss laid lightly over it, and kept on till germination has taken place. All the genus, and the now rather numerous garden varieties, propagate readily by the leaves, either by having their foot-stalks inserted in sand, or the entire leaf pegged down on sand, having the ribs on the under side slightly fractured, at which points roots will be formed, and also often along the margin of the leaf. They all prefer a light rich sandy soil, and abundance of water during their growing period, after which, and on the disappearance of the principal leaves, they should be kept comparatively dry, and allowed to take their season of rest. From their low habit they are well calculated for growing in a low pit, being removed from it to the stove when about coming into flower. 
Hibiscus.-Of this extensive genus there are many splendid species; the majority are, however, better adapted to large and lofty stoves than to those of ordinary height. They are all readily propagated by cuttings, and require an ordinary peat-and-loam soil.

Hindsia.-Requires rather an intermediate house as regards temperature. Propagates readily by cuttings, and requires a loam-and-peat soil.

Hoya.-Climbing plants of great beauty, and well adapted to the succulent-house. Propagated readily by cuttings even of the leaves; require a light rich porous soil, as peat and loam, with an admixture of broken bones, brickbats, or small fragments of sandstone. They are all impatient of damp at their roots.

Ipomea.- Very handsome showy climbing plants; the tuberous-rooted sorts increased by division of the tubers, and also by cuttings of the young shoots, by which latter means most of the rest of the genus is increased, as well as by seed. They all require a rich turfy sandy soil. I. Horsfalliæ should be grafted on I. insignis, or any other of the stronger-growing kinds.

Ixora.-One of the finest of all stove families. Seldom met with, however, in a satisfactory state of culture. Perpetual excitement seems to be mainly the cause of this, and hence we find those who give these plants four or five months' rest-that is, from July to February-succeed much better. At the former period they have completed their flowering, and are then removed from the stove to the greenhouse, or to a close pit where frost is excluded. In February they are re-potted, and plunged in a mild moist bottom-heat in a close frame or pit, in which the temperature is maintained at from $60^{\circ}$ to $70^{\circ}$. In this way they make fine strong young wood, show fine heads of flowers, and begin to bloom in April, when, if removed to the plant-stove, they continue in great beauty till the beginning of July. They strike readily by cuttings placed in bottom heat, and thrive best in a rich peaty soil.

Jasminum.-The tender species of this genus prosper best in an intermediate house as to temperature, propagate by cuttings, and prosper in a soil of peat and loam.

Justicia.-Half-shrubby plants of easy culture; free bloomers, continuing most of the season in flower; propagated readily by cuttings, and thrive in a soil of peat and loam.

Lantana.-Readily increased by cuttings of the half-ripened wood, preferring a peat-andloam soil.

Luculia.-Requires also an intermediate temperature, abundance of water while in a growing state, being kept comparatively dry while at rest. They are struck by cuttings, but not very freely, and prefer a soil of peat and loam.

Mandevilla.-A free-growing slender climber of great beauty and profusion of bloom. Propagates freely by cuttings, and thrives in a welldrained peat-and-loam soil. Extremely liable to the attack of thrip, red-spider, and mealy bug. Frequent syringing over the foliage seems to be the only remedy. It suits an intermediate tem. perature better than the excessive warmth of the regular stove, yet it is too tender for an ordi. nary greenhouse. We have found the humid atmosphere of the aquarium a suitable place for it during summer.

Medinella.-Both the foliage and flowers are elegant. The plants strike freely by cuttings, and prosper in a peat-and-loam soil.

Melastoma.-Tall, rather rambling plants, but of great beauty while in flower. Propagated readily by cuttings, and prefer a soil of half peat and loam.

Moussonia elegans.-A pretty Gesneria-like plant, producing its orange and scarlet flowers profusely during winter. Requires similar treatment with Gesneria.

Pavetta borbonica.-An interesting plant of easy culture.

Passiflora.-Climbing plants of great beauty, propagating by cuttings, and a soil of half turfy loam and peat.

Pharbitis.-Elegant climbing plants, nearly related to Ipomea, and requiring the same management.

Philibertia.-A genus of pretty climbing plants of easy culture.

Physianthus.-Climbing plants, some of which, as auricomus, are powerfully perfumed. Propagated by cuttings, and preferring a soil of sandy peat and loam.

Pleroma.-Allied to Melastoma, of easy culture, and profuse bloomers.

Poinciana.-Propagated by cuttings, and requiring a peaty light sandy loam.

Poinsettia.-A genus nearly allied to Euphorbia. While young, the plants should be repeatedly topped to secure a sufficient number of shoots, after which they should be allowed to take their own course, flowering on the tips of the branches, and furnished immediately under the flower with various coloured bracts, which form their greatest attraction. Propagated freely by cuttings, and require a welldrained soil of peat and loam.

Portlandia grandiflora.-An old but elegant plant, producing large pure white flowers like Brugmansia, and exceedingly fragrant. Propagated by cuttings, and thrives in a soil of half peat and loam.

Quisqualis indica.-A charming stove-climber, producing abundance of fragrant bunches of flowers. The young wood should be annually spurred in, as recommended for Combretum. Propagated by cuttings, and requires a turfy peat-and-loam soil.

Rondiletia speciosa.-A shrubby-growing plant, of great beauty while in flower. It requires similar treatment to Ixora.

Siphocampylos.-A genus of free-growing plants, rather of a scandent habit, profuse bloomers, and continuing a considerable time in flower. Readily propagated by cuttings, and requiring a turfy peat and loamy soil.

Stephanotis floribunda.-A charming stoveclimber and profuse bloomer, the flowers of which are very fragrant. This fine plant is very liable to be attacked by the scale and white bug, and therefore requires vigilance in keeping the leaves clean by repeated washing. Propagated 
freely by cuttings, and prefers a rich, light, turfy soil. The humid atmosphere of an aquarium is a very fit place for this plant, if trained near to the glass.

Strelitzia.-An old but interesting tribe, with flowers somewhat resembling the beak and crested head of some species of cockatoo. Propagated by separating the large fleshy roots, having at their tips a portion of the plant; require a rich soil and abundance of root-room.

Talauma.-A fine genus related to Magnolia, producing very fragrant flowers. Propagated by cuttings, and requiring a rich turfy soil.

\section{SELECT LIST OF FINE-FLOWERING STOVE- CLIMBERS.}

1. Allamanda cathartica, Schotti, grandiflora; 2. Bignonia venusta, Chamberlaynii, chirere ; 3. Combretum purpureum, elegans, Pincianum ; 4. Clerodendrum splendens ; 7. Dipladenia crassinoda, splendens, atropurpurea; 8. Echites suberecta, grandiflora, Harrisonii ; 11. Ipomea Learii, insignis, Horsfalliæ, palmata ; 13. Pergularia odoratissima; 12. Passiflora racemosa, alata, quadrangularis, kermesina, amabilis ; 15 . Stephanotis floribunda,Oblisii; 5. Cissus discolor 6. Convolvulus pentanthus; 7. Dioscorea discolor, zebrina; 9. Hoya imperialis, and several others of the genus; 10 . Jasminum sambac; 14. Physianthus auricomus.

\section{LIST OF ORNAMENTAL AND VARIEGATED. LEAVED STOVE-PLANTS.}

Achimenes cupreata, A. picta; Aichmea miniata, discolor; Alloplectus speciosus (capitatus); Anectochilus argentcus (Physurus), A. argenteus pictus, A. Lobbianus, A. Lowii, A. setaceus, A. setaceus cordatus, A. setaceus intermedius, A. xanthophyllus (setaceus pictus); Aspidistra lurida variegata ; Begonia argyrostigma, B. dipetala, B. frigida, B. manicata, B. ramentacea, B. sanguinea, B. stigmosa, B. zebrina; Bertolonia maculata (Erionema cenea), B. marmorea; Bilbergia vittata; Brexia chrysophylla, B. errata; Bucelia violacea ; Caladium pictum, C. bicolor splendens, C. bicolor picturatum, C. hæmatostigma, C. rubricaule; Campylobotris discolor; Coccocypselum discolor; Combretum Afzelii; Cossignia borbonica; Croton longifolium variegatum, C. pictum, C. variegatum; Cypripedium barbatum, C. venustum, C. javanicum ; Didymocarpus crinitus; Dichorizandra vittata variegata; Dieffenbachia maculata; Dionæa muscipula ; Dioscorea variegata (bicolor); Distiganthus basilateralis Dracæna ferrea, D. ferrea versicolor (terminalis), D. maculata, D. nobilis; Eranthemum leuconervum, Erionema ænea, see Bertolonia. Euphorbia pulcherrima, E. alba; Ficus barbatus ; Gesnera zebrina, G. zebrina compacta, G. zebrina splendens; Goodyera discolor, G. repens; Graptophyllum pictum ; Hæmadictyon nutans; Hoya picta, H. variegata; Maranta albo-lineata, M. bicolor, M. eximia, M. glumacea, M. rosea lineata, M. sanguinea, M. vittata, M. zebrina ; Pandanus javanicus variegatus; Pavetta borbonica; Plectranthus concolor picta; Rhopala corcovadensis, R. magnifica; Tillandsia acaulis zebrina, T. acaulis viridissima; Tradescantia zebrina; Vriesia splendens.

Climbing plants are for the most part planted in borders or pits prepared for them in the floor of the structure. Now, we think a worse situation for their roots could hardly be devised; they are, in general, beyond the reach of air or warmth, exposed to damp, and so situated that their roots cannot be examined or fresh supplies of soil added to them. It were better to plant them in slate boxes placed on the surface of the floor, and these even elevated a few inches, to admit of a free passage for the superfluous water to escape, and allow air to reach the roots. Where plant-tables are placed around the sides of the house, these boxes may be set under them, and if in the vicinity of the hot-water pipes, so much the better. The plants, instead of being trained directly upwards under the roof, should be carried along in a horizontal direction; the weaker-growing sorts nearest the bottom, while the stronger-growing ones should be trained nearest the top. This deviation from the usual practice, and using small chains instead of iron rods for conducting the branches along, hung in festoon fashion, would give the roof a more elegant and less formal appearance; besides, the flow of the sap would be moderated, and instead of the flowers and foliage being almost confined to the extremities of the branches, they would be clothed with both from near the bottom of the plant to its farthest extremity. More air and light would also be brought to bear on the foliage, and the whole arrangement could be easily changed to suit the condition of the plants or taste of the cultivator. Too little attention has hitherto been paid to the graceful training of scandent-growing plants not only in our plant-houses, but in the open air also.

\section{§ 8.-SUCCULENT-HOUSE.}

Succulents are of easy culture, requiring, however, abundance of light, a limited supply of water at their roots, and during their growing season a somewhat humid atmosphere; hence they should be grown in pits or low houses, and kept as near to the glass as possible. Naturally, they inhabit the driest situations, where scarcely any other vegetation exists, on naked rocks and sandy hot plains, alternately exposed to the dews at night, and the most powerful rays of the sun during the day. On soil they seem to placc little reliance, and grow in the very poorest, such as sand, gravel, or the debris of decaying rocks, drawing their nourishment through innumerable mouths, which cover their whole surface, and through which aqueous nourishment is conveyed to the juicy beds of cellular tissue which constitute the greater part of their bulk. In imitation of these conditions in culture, they are potted in light sandy soil, with a portion of lime rubbish to render it still more porous; receiving little water at the roots, but abundance once a-day over-head, which, during summer, should be administered in the afternoon, and the house shut closely up: in the winter, this is 
best applied early in the day, and also to a less extent. Many of the Cacteæ, or Opuntiaceæ, may be speedily grown to a large size by being plunged during summer in a moderate bottomheat and kept moist and close; but this must not be attempted during winter, or continued too late in autumn, that the cellular tissue may become properly hardened and matured before the dark days of winter arrive.

Many of the splendid hybrid varieties of Epiphyllum and Cereus are grown successfully in rich well-drained soil, attaining a large size, and producing abundance of gorgeous blooms. Others of the same section, particularly Epiphyllum truncatum, thrive well when grafted upon any of the strong upright-growing species of Cacteæ; indeed, they do better in this way than when upon their own roots. Pereskia acculiata makes an excellent stock for this operation. It is of rapid growth, and, when trained over arches, or longitudinallyalong the roof, and thickly covered with the various varieties of Epiphyllum truncatum, has a fine and novel effect; and these, when wrought upon the upright-growing Cereus speciosissimus, Peruvianus, tetragonus, \&c., may also be made to form in a few years very fine specimens. The operation is exceedingly simple, and in the former case requires only the removal of a thin slice of the stem of Pereskia, to which a small portion of E. truncatum is fitted, and secured by placing a little moss over the bandage of matting, and if placed in a partially shaded place adhesion will take place in a week or two. A slit is usually made in the angular parts of the Cereuses, and the scion is inserted and secured in the same manner. A plant several feet in height may be covered at once by this means, although a portion of the lower part is usually left uncovered, so as to form the specimen in habit of a tree.

With the exception of a few of the Cacteæ, which are natives of the West Indies, and require a pretty high temperature, the rest of the succulent tribes are more injured by heat than the reverse. The great majority being natives of the temperate parts of South America, Peru, the Cape of Good Hope, \&c., prosper better in the temperature of a moderate greenhouse than elsewhere, but always fully exposed to the brightest light. This, however, is not the usual practice, for in nine cases out of ten we find them placed in the most out-of-the-way place, and where scarcely any other plant would live, much less continue to prosper. Few of the tenderest will suffer in a temperature as low as $45^{\circ}$ or $50^{\circ}$. They require seldom to be repotted, unless by inattention their roots have been allowed to suffer from too much wet.

Most of the Opuntiaceæ or Cacteæ propagate readily by cuttings, which should be allowed to remain a day or two after removal from the plant before setting in the soil, that a portion of the sap may escape, and so prevent decay taking place at the part where the incision was made. Some seed freely, and are thus multiplied, as in the case of several Opuntias, Epiphyllums, and Mammillarias, which produce fruit readily. The young shoots of Opuntia and
Epiphyllum, which must be regarded as analogous with leaves, propagate freely, and throw out roots from all parts of their surface whenever they come in contact with the soil. In the case of Mammillaria, Echinocactus, and Melocactus, the case is otherwise; for although described by most botanists as being destitute of a woody axis or central column, this has been discovered by $\mathrm{Mr}$ Beaton to be a "fallacy of the Faculty." In a private communication received some years ago by us from that gentleman, he says: "All the sections of the genus have not only a woody axis, but a medulliferous column inside their woody axis, like other exogenous plants. This axis, however, is not formed during the first few years of their existence, and probably different species require different periods to form it; while, in its turn, it is not at first supplied with its medulla or pith. Both the woody axis and pith make their first appearance at the collar of the plant, at the point where the roots start from the stem. As the axis increases, it throws out fibres into all parts of the succulent portion of the plant, and is the chanuel through which the nourishment is supplied from the roots ; and I am of opinion," he continues, " that the Melocactus, Echinocactus, and Mammillaria, will not form roots from cuttings until they first form their woody axis." This explains clearly enough the difficulty of multiplying the above sections by extension.

The extensivegenus Mesembryanthemum, with the exception of a few in the first section (Acaulia), such as minutum, testiculare, \&c., and a few others which are annuals, propagate readily by cuttings ; the first of these exceptions by division of the plant when sufficiently established, and the second by seed. Almost all other succulent plants, not absolutely annuals, propagate most readily by cuttings; while some which rarely produce branches, as Sempervivum tabulæforme, some of the Echeverias, \&c., propagate by the leaves, these being slightly broken at the edges and laid upon the soil. The latter genus produces abundance of cauline leaves, that is, small scale-like appendages upon the stem. These strike root freely when slightly planted in the soil like ordinary cuttings.

The following genera constitute what are in general called succulent plants :-Dyckia, Echeveria, Echinocactus, Sempervivum, Monanthes, Cactus, Mammillaria, Cereus, Epiphyllum, Opuntia, Rhipsalis, Pereskia, Aizoon, Tetragonia, Mesembryanthemum, Hoya, Stapelia (now divided into nine genera), Crassula, Agave, Furcrœa, Lettæa, Bowiea, Rhipidodendr on, Pachidendron, Aloe, Gasteria, Haworthia, Apicra, Kalanchoe, Bryophyllum, Cotyledon, Portulaca, Talinum, Anacampseros, Euphorbia, Rochea, Kalosanthes, Turgosia, Globulea, \&c. All these require abundance of light to enable them to expand their flowers properly, and some of them, such as the stronger-growing species of Mesembryanthemums and Kalosanthes, make excellent bedding-out plants, particularly in warm and dry situations. The finest flowering plants of this division are to be found in the genera Cereus, Epiphyllum, Hoya, Rochea, Kalosan- 
thes, and Mesembryanthemum; the most curious as to their flowers, although for the most part possessing the disagreeable odour of carrion, are Stapelia and its allied genera; the most singular as to general character are Echinocactus, Mammillaria, Cereus, Cactus, Opuntia, Aloe, and its associates, Apicra, Gasteria, \&c.

\section{§ 9.-THE CONSERVATORY.}

The conservatory and greenhouse, although too often regarded as the same, are by no means synonymous. In vol. i. we have given thirteen illustrations of the former, and also stated so fully our views concerning them that little more requires to be said beyond treating on their culture, arrangement, and management. As the conservatory is, or rather ought to be, the most elegant and perfect of all horticultural structures, it follows that large, elegant, and well-grown plants only ought to occupy it. It ought not to be converted into a mere growing-house, or fitted up with stages covered with a heterogeneous collection of all sorts and sizes, and in all possible states of growth. Every plant admitted should be grown elsewhere, say in the greenhouse or otherwise, and only admitted when coming into flower, excepting in the case of a few rare or very finely grown specimens, which may have outgrown the accommodation they have hitherto occupied; and these should be of evergreen character, or remarkable for their foliage, or some other equally interesting property. Some of our dictionary compilers have fallen into a very general error when they say of the conservatory, "a large greenhouse for exotics, in which the plants are planted in beds and borders, and not in tubs and pots, as in the common greenhouse." This meaning of the word is as contrary to the original as it is to the opinions of most of the best cultural authorities of the present day. The earliest conservatories, either in this country or elsewhere, were for the preservation of plants in a portable state, placed out of doors during summer, and only put under cover during winter. The planting-out system is a modern innovation. The conservatory, more especially when in connection with the mansion, should be, if we may be allowed to borrow a popular phrase, "the show-room" of the garden. It should be kept throughout the whole year as it were in a blaze of flowers; as some begin to fade, others should be brought in to replace them, and neither paucity of bloom nor a sickly or deformed plant should be allowed to appear in it. To this end, however, it is necessary that other houses and pits be at hand to keep up this supply, and without such all attempts to maintain a conservatory as it ought to be will be in vain.

If these are the requirements, therefore, expected in a first-rate conservatory, surely we are justified in saying they never can be realised if the plants are permanently planted out in beds or borders. Every plant has its season of flowering, of growth, and of repose ; it is only during the former of these that it is a fit inmate of the conservatory. These seasons do not take place in all plants at the same time; and as we know quite well that their treatment during each of these periods is widely different, their being associates in the same structure can never. be made reconcilable with good culture. Some of the advocates for planting out have attempted to give a natural, nay, even a picturesque character to their arrangement; but all attempts at producing such effects under glass roofs of the present extent have hitherto proved decided failures, as both the materials we have to deal with - that is, the structure, and the plants as now grown-are so truly artificial in themselves, that it must require the utmost stretch of even the enthusiast's imagination to allow himself to believe that he has produced anything like a natural effect, let him arrange his plants how he will. Let us, therefore, be content to abide by art while contemplating the arrangement of the conservatory, and study how far we can please the eye of taste by the production of a perpetual summer, through the medium of agreeable forms and colours harmoniously combined. The conservatory, more especially when in connection with the mansion, as we have already stated, should be at all seasons, particulary when the flower-garden is denuded of its attractions, kept gay with flowering plants. Now, this cannot be effected if the plants are permanently planted in beds or borders, be they ever so carefully prepared, or the plants ever so judiciously selected. Nor is there any positive reason why they should be so. A few very fine specimens of evergreens, such as camellias, oranges, \&c., may be kept in to give a fulness of effect; but assuredly the great majority should be grown in a supplementary house, and brought in only when about coming into bloom, and again removed when the season of their flowering is past. These remarks, it must be understood, apply to conservatories of the usual dimensions, and placed, as it were, as an appendage to the mansion. When built, however, upon a scale of much greater magnitude, as they assuredly will yet be, and hence coming under the denomination of "jardins d'hiver," or winter-gardens, then their arrangement may be different, and in such many of the plants will undoubtedly be planted out; but, nevertheless, even in them, plants in a portable state will be required to maintain a sufficiently agreeable display of bloom at all seasons. In a conservatory covering an acre ol two of surface, the plants might be arranged somewhat in the natural manner, and this would to some extent be consistent with their natures and habits, particularly in connection with the effects of light upon them. No plant can long exist without light; there are some, bowever, that require less of it than others. One plant is organised to suit the atmosphere of a dense wood, and therefore a diffused light is sufficient to it; others are planted by nature in situations upon which the rays of a shadeless sun are daily shining,- - hence the light necessary to the one would be fatal to the other. The organic difference in such cases as these is believed to consist in the epidermis, a part of the structure of 
plants which regulates their power of perspiration; and hence it is to be inferred that it is not the greatest amount of light that can be obtained, but the greatest quantity that they will bear without injury, that is most favourable to plants.

Plants, therefore, should be regarded as plants of sun and plants of shade; hence the great necessity of arranging them in plant-houses according to the amount of light each specifically requires. This is a matter greatly neglected in arranging plants in conservatories, on greenhouse stages, \&c., as well as in the construction of houses for their accommodation. Heaths, Pelargoniums, and all such plants as inhabit naturally open and sun-exposed situations, should be placed nearest to the glass, and in the most open, airy, and sun-exposed part of the house; while such as become deciduous, or others known to grow naturally in deep woods or shady places, will be better placed farther from the glass, and even under the shade of the former: Others, like the camellia, although growing naturally in exposed enough places, will accommodate themselves in culture to a much less amount of direct sunlight than many others; and hence many growers place them in houses having a northerly exposure. The majority of ferns, and many orchids, are inhabitants of deep shady ravines, under the shelter and shade of rocks, and often growing on the stems and branches of trees in jungles where the rays of sun seldom enter: these should, in culture, be somewhat similarly situated. On the other hand, many plants - the orange, for example-which luxuriate under the brightness of an Italian sky while in a state of growth, will, while in a dormant state during winter, accommodate themselves to a dark orangery or elsewhere, if frost be excluded from them. Plants indigenous to damp and shady places have their leaves very thin, the epidermis pierced by numerous invisible pores called stomata, through which the plant breathes and perspires, and through which it is enabled at all times to procure abundance of liquid food. On the other hand, plants growing on dry and exposed places have their leaves very hard and thick, nature thus adapting them to the circumstances under which they grow. Attention to these points will cousiderably enable the cultivator, even if unacquainted with the circumstances otherwise, to place his plants in those parts of his planthouse most favourable for them. It is, however, only in structures of very large dimensions that these conditions can be rightly fulfilled, as well also as where the planting-out system can be advantageously carried out. The uncovered borders in conservatories, where the plants are planted out, do not at all harmonise with the plants themselves; they should, therefore, be covered in some way or other, and for this nothing is better than the prostrate species of Lycopodiums, which grow rapidly in the shade, and at almost any distance from the glass, and will give the whole a clean and healthy appearance. Plants in tubs and large pots are improved in appearance if covered in this way; and those growing in smaller pots may be covered with various species of lichens, such as Cenomyce rangiferina, the reindeer moss, various species of Ramalina, \&c. These, without exhausting the soil on which they are laid, will last a long time, and remain perfectly fresh if occasionally sprinkled with water.

The larger plants should be grown in highly oruamental vases or cases, in themselves as much the work of art as the house they are to be placed in; and whatever material they may be constructed of, they should be made so as to take to pieces for the examination and adjustment of the roots (vide fig. 260.) These may be grouped or arranged according to fancy upon the floor of the house, which should be paved with encaustic tiles or polished stone; or as exemplified in the large conservatory recently erected by us for the Misses Walker of Drumsheugh, where the centre part of the floor is covered with white sea-gravel over 2 feet of broken stones, to secure ample drainage, and the surrounding passages are of polished Caithness pavement. On the ground the plants are arranged in groups, and elegant vases of various sizes and heights are kept filled with smaller plants in a flowering state, supplied from the other greenhouses. This house is glass on all sides to within 4 inches of the floor-level. The cast-iron columns which support the roof, and the arms proceeding from them, are made conductors for climbing plants, which are trained in graceful festoons, suspended from the upper parts. In a somewhat similar structure, also erected from our designs for A. K. Johnston, Esq., the pavement is of Minton's encaustic tiles, the larger plants are grouped along the centre, with an elevated group of exotic ferns opposite the drawing-room window, which opens into the house. A highly ornamental cast-iron plant-table 2 feet in breadth is arrangea along the sides of this house for the reception of smaller plants in ornamental pots, this table being 2 feet above the floor, which is the height of the stone ashlar parapet in which the bottom glass ventilator's are placed, and which forms the base of the superstructure. In neither of these cases are any of the plants planted out. In another conservatory, at present in course of erection, we have left the centre of the house clear, with the exception of a small fountain in the middle, the plants being arranged on both sides, and the centre portion of the floor paved with polished Arbroath pavement ; thus admitting of a vista-view from the drawing-room window to the farther end of the conservatory, which terminates in a semicircular group of small plants, changed so as to be always in bloom, and rising to the height of 4 feet, over which, and through the glass end, is seen a romantic wooded bank at a considerable distance from the house, but yet, as seen from the drawing-room floor, which is 3 feet above that of the conservatory, appearing to form a continuation of the conservatory itself. Climbing plants are placed in slate tubs, and carried over portions of the roof, but in such a way as to create as little shade, particularly during winter, as possible. The iron columns and diagonal arms which support the roof are made conductor's to such plants, and the $\mathrm{T}$ iron bars, which take a longitudinal 
direction, and to which the astragals are secured, as well as the runners which form the lower part of the top ventilation, are also thinly clothed with climbing plants. During the heats of summer the partial shade afforded by plants thus trained is rather beneficial than otherwise to the plants below, and this shading can be increased or diminished by a judicious arrangement of their branches. Towards autumn these should be pretty well cut in, and, as the sun declines, cut back still farther, so that little more obstruction is caused to the admission of light than that necessarily occasioned by the framework of the structure. Climbing plants are a great ornament to a conservatory, as most of them are profuse flowerers ; but care must at all times be taken that they are not allowed to ramble too much and cause undue shade. This can, however, be always regulated by a judicious use of the pruning-knife, a knowledge of the habit of the plant, and a tasteful eye. Houses arranged as those we have instanced should be of glass, either to within a few inches of the floor, or to not more than 2 feet from it. In such as have opaque side walls, the same effects cannot be produced. Even one opaque side wall, although it may be covered with camellias or other evergreen plants trained against it, will give a one-sided appearance to the structure-a want of balance or uniformity ; and even should it be on the north side, it will exclude light which would be beneficial to the plants growing within. Vide also another of our designs, fig. 511, for J. P. W. Butt, Esq., Grovefield, Gloucestershire.

Undue shade during spring, autumn, and winter, must be guarded against; but, on the other hand, shading by artificial means is of great importance during the powerful sunshine of summer, but this shading must be so regulated that, while the full force of the mid-day sun is modified, light is not intercepted. This is best effected by having the power of covering the roof, during bright sunshine, with thin canvass, which, while it prevents the flowers from fading too rapidly, admits sufficient light in a subdued form, and which is found to be highly advantageous to the plants. By judicious shading, plants may be kept in bloom much longer than they otherwise would, and the enjoyment of the conservatory be greatly increased. We have noticed this, vol. i. p. 555, and given an illustration of our mode, fig. 790. Shading by such artificial means is a very different thing from allowing the roof to become covered by climbing plants; or, still worse, by unnecessary waste of timber in its construction, as is the case with all roofs formed of heavy rafters and framed sashes, or by high architectural ornaments over the sides, with the view, in the estimation of some, of giving effect, or of hiding part of the roof. In these latter cases the shade produced is the same in dull cloudy days as it is in bright sunny ones-in the dark months of winter, when every ray of light is of so much importance, as during the scorching influences of the dog-days' fiercest sunshine. Too much light cannot be admitted into any plant-house ; but by having a properly-appointed shading apparatus we have it in our power to regulate it according to circumstances.

Some persons have an objection to the appearance of large tubs or boxes, be they ever so ornamental in form, when placed on the floor of their conservatories. We have pointed out (vol i. p. 362) how this can be remedied. We have also, at p. 363, noticed the evils of planting out permanently conscrvatory plants. 'To those, however, who differ from us in this opinion, we would say, let the bed or border be elevated at the least 2 feet above the floor level; let it be completely drained below with not less than 2 feet of broken stones, flints, brickbats, \&c. and under that drains should be placed both for the escape of superfluous water, should such happen to occur, and also for the admittance of air to the roots of the plants ; and, as a precautionary measure, such borders should be vaulted beneath, as a substitute for drainage, and hot-water pipes placed either in the vaults, or, on the other hand, amongst the stone of the drainage; so that in the event of the borders becoming cold, or the plants sickly from deficiency of root action, a moderate heat might be readily thrown in by causing a portion of the heated water which warms the atmosphere of the house to flow through them. The depth of conservatory borders should not exceed $2 \frac{1}{9}$ feet, and in their preparation a course of fresh rich turf should be laid immediately over the drainage, to prevent the finer particles of soil above being washed down into it. Upon this a compost consisting of fresh light yellow loam and sandy peat, roughly chopped up, and mixed together in equal parts, should be laid, and in this the plants set, their roots carefully spread out, should they, from long confinement in pots or tubs, have become matted, or contorted into a spiral form. No plant whose roots have been long confined to the narrow limits of a pot should be planted with its ball entire. If the above materials are dry, and have been exposed for a few weeks to the sun and air to sweeten them, they will be in far better condition for the purpose than had they lain for months, and by repeated turnings, chopping, and sifting, been reduced to the state of fine mould, a practice long followed, but now considered obsolete. When the plants are planted out, a little top-dressing of finer compost may be laid over the surface for appearance' sake. During the decomposition of the vegetable matter in such soils as the above, the roots of the plants will be nourished, decomposition and consolidation taking place soon enough, however rough the compost may be at the time of the formation of the beds. The orange tribe, as well as camellias, require rather a stronger compost, and many moderately-rich lightish loams will be sufficient, without the addition of peat, earth, sand, or other admixture. When conservatory plants are kept in a portable state, their arrangement on the floor can be changed to suit their various conditions; those in flower somewhat elevated, or brought more into view, while others that have passed that state may be placed more in the shade. When the house and plants are large, they can be grouped so that a free passage may be had amongst them, which 
could not be if permanently planted out, nor can the plants be individually seen to the same advantage. The surface of the soil, whether the plants are planted out or grown in a portable state, may be covered with good effect with any of the dense-growing species of Lycopodium.

Having thus far spoken of arrangement, it behoves us to refer to another equally important element in the proper management of a conservatory-namely, cleanliness. There is but one step from the drawing-room to the conservatory; it is necessary, therefore, that the one be maintained in as high keeping as the other. It is important, also, that every operation be completed so early in the day that each may be alike ready for the entrance of the owner. Nor is this rule confined to the conservatory adjoining the house; those that are isolated from it require the same attention.

Watering should be performed early in the morning, and during winter it should be administered in a tepid state. All plants derive much benefit from their tops being supplied as well as their roots, but this must always be done with sufficient care that those in full bloom be not injured thereby. The finest cap, therefore, should be used on the syringe or engine, and the water be let fall in the shape of a copious dew rather than of a dashing rain.

The temperature of the conservatory during autumn, spiing, and winter, can seldom be permitted to fall so low as that of the greenhouse, because the plants for the most part, in the former, are supposed to be in a state of pretty active excitement; whereas in the latter, at these seasons, they are approaching to, or in a positive state of rest, or just emerging from that state to one of progressive growth. A moderate degree of heat is also useful in repelling damp, admitting also of a greater supply of fresh air, and enabling the plants to develop their flower's with greater vigour. The temperature, therefore, should seldom fall below $40^{\circ}$, during these seasons, at night, but with sunshine it may be permitted occasonally to rise to $60^{\circ}$. During summer it may range $10^{\circ}$ or $15^{\circ}$ higher, counteracting excessive heat during bright sunshine by partial shading, and keeping the floor moist by frequent waterings.

\section{SELECT LIST OF CONSERVATORY PLANTS.}

Acacia armata, pubescens, juniperina, undulæfolia, affinis, vestita, lunata, dealbata, verticillata, longissima, longifolia, pulchella, grandis (true), lineata; Azalea, any of the Indian varieties; Abutilon insigne, venosum, striatum, trained as climbers; Beaufortia decussata, sparsa, carinata ; Bignonia picta, jasminoides ; Banksia, most of the genus; Billardiera ovalis (climber); Boronia, all the genus; Bossiæa, all the genus; Brugmansia suaveolens, candida, lutea, bicolor, eximia, Knightii; Brachyglottis repanda, for its remarkable foliage; Brachysema platyptera, latifolia (moderate climbers); Bur chellia capensis ; Callistachys lanceolata, ovata, longifolia ; Callistemon salignus, speciosus, lanceolatus, formosus; Calothamnus villosus, macrocarpus, splendens, venustus; Camellia, all the varieties; Cantua bicolor, dependens, trained as creepers; Chorozema ovata, Lawrenciana, varia, spectabilis, Henchmanni; Clianthus puniceus; Citrus, the orange tribe, any of the varieties (vide ORANGERY); Corræa Harrisii, speciosa, pulchella, bicolor; Crotalaria elegans; Crowea saligna, elliptica; Cytisus racemosus, elegans, rhodopnœus; Daphne, any of the tender species; Dryandra, most of the genus; Daviesia latifolia, corymbosa, or indeed any of the genus; Dillwynia, any of the genus; Dacrydium, any of the genus, on account of their graceful habit; Doryanthes excelsa; Enkianthus reticulatus; Epacris, most of the species, or improved hybrids; Eriostemon, any of the genus; Erythrina crista-galli, laurifolia ; Euchilus obcordatus ; Eutaxia, any of the genus; Gastrolobium, any of the genus; Genista canariensis; Glycine secunda; Gompholobium, any of the genus; Goodia latifolia, pubescens; Habrothamnus magnificus; Hovea Celsii, pungens; Humea elegans; Indigofera decora; Jasminum grandiflorum; Kennedya coccinea, monophylla (climbers); Lagerstræmia indica ; Lambertia:formosa ; Lilium Wallichianum, giganteum, speciosum, punctatum; Luculia gratissima, Pinciana ; Magnolia fuscata, pumila, fragrantissima; Mandevilla suaveolans (climber); Mimosa prostrata (slender climber); Mitraria coccinea; Mirbelia, any of the genus; Oxylobium, any of the genus; Passiflora kermesina, Loudoni, Mayani, onychina, and various hybrids (climbers); Phœnocoma prolifera; Phyllocladus, any of the genus, on account of their singular habit; Pimelea, any of the genus; Platylobium, any of the genus ; Polygala, most of the genus ; Prostanthera violacea, rotundifolia ; Pultenæa, all the genus; Rhododendron arborea, Arborea alba, and any of the new tender hybrids, and all the larger-growing Sikkim sorts; Rhodochiton volubile (a slender climber); Rhodoleia Championii ; Rhynchospermum jasminoides (climber) ; Sollya, any of the genus (slender climbers); Statice arborea, macrophylla; Swainsonia galegifolia (trained as a climber); Tacsonia, any of the genus (climbers); Templetonia retusa, Tropœolum, any of the species or varieties (creepers) ; Zichya pannosa, longiracemosa, inophylla, coccinea (climbers.)

\section{$\S$ 10.-THE GREENHOUSE.}

The greenhouse differs from the conservatory, not only in being a smaller structure, but also in having its occupants of much smaller size, and of a far greater variety as to genera and species. Here, however, certain limits should be estar blished, for in a mixed greenhouse-that is, a heterogeneous collection of plants - the best results as to their culture cannot be accomplished, on account of the great dissimilarity which exists in their habits and times of flowering. Where a first-rate establishment is to be maintained, it is necessary to divide greenhouse plants, over and above the divisions we have already described, into two more sections-namely, hard-wooded and soft-wooded. The former is represented by the majority of 
New Holland or Australian plants, and the latter by those of the Cape of Good Hope, the temperate parts of Mexico, Chili, Peru, \&c. For example, the extensive genera of Acacia, Pimelea, Daviesia, Hovea, Dryandria, Banksia, \&c., belong to the former, while Pelargonium, Fuchsia, Lophospermum, Nierembergia, Poly* gala, Salvia, \&c., belong to the latter. The former, for the most part, are capable of withstanding more cold than the latter, and although much longer lived, grow more slowly naturally, and do not admit of the same rapidity of growth which the others do artificially. Indeed, to grow the Pelargonium well, they require even a separate house or pit for themselves. The first principle in obtaining well-grown plants, is to attend strictly to the formation of a selection consisting only of such as have some near affinity to each other, either in being from the same country, or of somewhat similar habits, and submitting to the same routine of treatment. In some respects, however, their management is the same, and where otherwise it will be noticed.

Autumn and winter management of greenhouse plants. - Abundance of air must be admitted on all favourable occasions, more especially after those plants which may have been set out of doors for a season have been brought in again, that the transition to them may be the less experienced. All superfluous shoots should be removed from climbing plants trained over the roof or otherwise, so as to obstruct the rays of light as little as possible, as the plants will now require as much air and light as can be given them, to enable them fully to mature their wood and flower buds. Care must nevertheless be taken that the temperature be not lowered too much, else these objects would be completely defeated. Indeed, all climbers should be at this time cut pretty closely in, as their season of flowering will have ceased, so that the pruning necessary to each, according to its habits, may be safely performed. The plants should be all carefully examined, cleared of insects, and such as appear to be defective in drainage, or in want of potting, attended to. This examination can be more conveniently done in the end of September or beginning of October, at which period those which may have been set out of doors, or set in cold pits during summer, are to be again brought in. It has been customary for ages to remove the hardier kinds for about four months annually - that is, during June, July, August, and September - into the open air, placing them in some sheltered situation, yet where they may be freely exposed to the sun. Of the propriety of this there may be doubts. The practice arose, and is no doubt continued, from necessity. This will be referred to in its proper place. A general regulation of the plants should at the same time take place, both of those which have remained in the house, and those which are about to be brought in; and all necessary pruning and tying up attended to, as it can be better done now than.when the house is crowded with plants. The heating apparatus, whatever it may be, should be examined and put in proper working order, so that no accident may occur during winter; the whole house VOL. II. should be most carefully cleansed, and repairs of glass, painting, \&c., attended to. As the plants are brought in, they should be carefully examined, to see that drainage is all right, and worms extracted where they exist, which is most effectually and conveniently done by applying a little lime-water in a clarified state to the soil. Every pot should be washed clean, the surface of the soil cleaned or top-dressed where necessary, and the plants arranged so that each may enjoy as full a share of light as its nature requires; for there are some which will accommodate themselves to less of that than other's, and these, for the most part, are those having large leaves like the camellia and orange, the others having small leaves like the heath, \&c. Avoid crowding, which, of all things, is the most to be found fault with in greenhouses, and, indeed, in plant-houses in general; and rest assured it is far better, and will, in the end, be found more satisfactory, to throw away a dozen of plants than that a dozen and a half should be spoiled from want of sufficient room. In all collections there are, and must be, plants which have become worn out by reason of old age, bad management, \&c. ; others attain too large a size for the accommodation afforded them, and not a few are found to flower best while young, while it is the natural habit of others to become tall and straggling, naked of branches, and unfit to support themselves without the aid of props. From amongst these a considerable draft should be made, and this is the time to see to it. It is now a clearly recognised principle that every individual plant in such structures should be a model specimen in itself, perfect in its habit, abundantly clothed with leaves and branches, alike perfect on all sides, so far as the nature of the plant will admit, standing, as it were, upon its own legs without the aid of crutches. Collections, unless in the case of strictly botanical gardens, are now less cared for than formerly, and even in these we see an evident desire for fine specimens arising. Selections, and those of the most showy and beautiful kinds, are the order of the day, and the high cultivation of these is the standard by which the merits of the cultivator will be judged. The first step towards arriving at this much-tobe-desired end, is, after forming a judicious selection, to keep up a succession of young plants, not starved in thumb-pots as formerly, but grown from the day the cutting or seedling is potted off for the first time with the view of its becoming a perfect specimen; and this is only to be effected by a speedy growth, the plant supplied with plenty of pot and top room, and otherwise stimulated and encouraged. And that it should not, in consequence of these attentions, become drawn up and deformed, a regular system of training and modelling, as it were, must be carried on, by stopping the leading shoots, and causing others to form at those points most likely to sccure a good foundation upon which to build the future structure of the plant. This will be alluded to more fully afterwards. We refer to it here to show amateurs, in particular, how necessary it is to take courage and fear not, in discarding at this time such 
plants as are likely to become incumbrances of their plant-houses. Every plant, when housed for the season, should stand clear of its neighbour-indeed, not one of the leaves or branches of the one should touch those of the next. As the gloom of November sets in, and onwards till the beginning of February, slight fires should be lighted in the mornings in damp weather, a gentle heat thrown into the house, and ventilation, particularly towards the roof, given at the same time, but not to the extent of exciting the plants, but only to cause circulation in the atmosphere of the house; the impure air will be dislodged, and that great enemy to plants at this season, damp, completely expelled. Moderate supplies of water should be given to the plants, particularly to soft-wooded ones, and the greatest care taken that none be carelessly spilt upon the floor. Hard-wooded plants suffer less from damp, and much less from cold, than such soft-wooded ones as Pelargoniums, \&c.; they also require a very limited supply of water at their roots, as their natural winter is one of drought and diminished temperature. While, therefore, it may be expedient to give a slight degree of fire-heat where soft-wooded plants are grown, to maintain the temperature two or three degrees above the freezing-point, it will not be required for the others till as many degrees of frost actually enter the house ; and if this can be effected by covering, so much the better. During December greater vigilance will be required, as it is seldom till that time that frosts of any intensity of late years have occurred. It should, however, be borne in mind that excitement in the plants at that season should be most carefully guarded against. It is their season of rest, and therefore exclusion of frost is all that is required. A low temperature, with abundance of air and a rather dry atmosphere, are the conditions required to maintain such plants in a healthy state. In regard to ventilation, it should be observed that draughts of cold frosty air, or cold damp foggy air, are equally injurious, and should be avoided by not opening any part of the structure, unless in fine mild clear days. At other times the supply of air should be admitted either through underground air-drains, or by ventilators placed in the side-walls near the level of the floor, so that, on its admission into the house, it shall come in contact with the flue or hot-water pipes, because in either case its temperature will be raised to nearly that of the house before it reaches the plants. It follows, therefore, in cold weather, that a slight degree of heat should be thrown into the pipes or flues some time before ventilation is given. In vol. $i$. many examples are given how this ventilation is to be effected. Both day and night aëration are highly beneficial to plants.

We know such to be the case in nature, and in following her laws in the administration of food to plants we cannot go wrong. Plants, at this season of the year, are more injured by moist stagnant air than by a cold dry atmosphere; and that structure must be pronounced exceedingly imperfect which is not provided with ample means for admitting air continually both day and night, without exposing the plants to cold draughts blowing directly upon them; and that system of culture is assuredly erroneous that would nightly confine a collection of exotic breathing plants within the limited space of a conservatory or greenhouse almost hermetically closed.

In regard to watering, from the beginning of October until the growing season of spring again sets in, great caution is required. It must be progressively reduced in quantity as the plants have perfected their growth, and be entirely withheld from bulbs. Deciduous plants also require less than evergreen ones do.

Regarding temperature, a genial heat should be kept up in autumn, which may in general be effected by shutting up early, and so enclosing a degree of solar heat sufficient for securing the ripening of the wood. Again, in spring, slight fires may be applied to assist in stimulating vegetation in the plants. In both cases, however, it will be most beneficial if applied during the day instead of during the night; for few mistakes are more fatal than keeping up a night temperature by fire-heat, equal to and often greater than that during the day. The very reverse ought to be the case. Amateurs would do well to bear in mind that plants require to go to sleep during the night as well as themselves, and this they cannot do if stimulated by a greater temperature during the night than during the day. There is no country in the world where the night temperature is so high as it is during the day, nor should it be so in our plant or forcing houses. Notwithstanding many of the Pelargoniums and Ericas grow together in the same soil and the same situation at the Cape of Good Hope, their native place of growth, yet, in cultivation, the heaths will withstand a degree of cold, nay, even be improved by it, that would be certain death to the geranium; and the same rule holds good in the case of almost all hard-wooded and soft-wooded plants from whatever country they may come ; and hence the difficulty of cultivating a mixed collection in the same house. To attain anything approaching perfection in the cultivation of greenhouse plants, it is absolutely necessary, more especially during winter, to have all soft-wooded plants in one structure, and hard - wooded ones in another; for the fireheat necessary for the preservation of the former, would be exceeding injurious to the latter. The minimum night temperature which softwooded plants will withstand without injury may be taken at $34^{\circ}$; the maximum during winter at $42^{\circ}$ during the day, as a general rule, allowing $2^{\circ}$ or $3^{\circ}$ of a rise from sun-heat.

The majority of hard - wooded greenhouse plants, on the other hand, will not suffer should the temperature fall to $30^{\circ}$, and many even should it fall to $25^{\circ}-\mathrm{a}$ depression fatal to all plants of a soft-wooded nature, unless such as die down to the ground annually, known as herbaceous plants. Such, therefore, being the case, the importance of having two distinct houses will be sufficiently apparent. No doubt both hard-wooded and soft-wooded plants are too often made to exist in the same house during winter; but it is merely a state of existence, in which either the one or the other, nay, probably both, suffer exceedingly. 
Spring and summer management of greenhouse plants.-As spring advances an increase of temperature should be allowed to take place in a gradual manner; air and water must be increased in a like ratio. A general arrangement will be required towards the end of February or beginning of March. Such plants as require it should be potted, pruned, and neatly trained, and, in the case of lean-to houses, be frequently turned round, so that no part of the plant be too long excluded from the sun and air; due regard should be paid to the suppression of insects, and slight syringings with tepid water should be given to the plants early in the day, to refresh and clear the foliage of dust. Propagation must be thought of, particularly as regards soft-wooded plants; for on kceping up a constant succession of young stock much of the success in greenhouse management depends. The majority of hard-wooded plants, particularly such as are difficult to propagate, or which take a long time to root, never can repay the private grower or amateur. It is better, therefore, to purchase young plants at once from the nurserymen. The majority of greenhouse plants are propagated by seed, as all annuals, many plants otherwise difficult to multiply, like Eucalyptus, Astelma, \&c. ; others, like Kennedya, Chorozema, Eutaxia, Cytisus, Viminaria, Aotus, \&c., seed freely, and are more readily increased in this way than by cuttings: wherever seeds are produced, they should be preferred. Othersareincreased by cuttings, as geraniums, fuchsias, hydrangeas, jasminum, Nerium, \&c.; by cuttings of the roots, as several species of acacia; by budding, inarching, and grafting, like camellias, oranges, \&c.; by cuttings of the leaves, like Hoya, Gloxinia, \&c. ; by layers, like Clematis, Wistaria; by division of the roots, like Calla, Agapanthus, \&c., and by knaurs or dormant buds, which form on the stems of some plants, and the roots of others, like Yucca, \&c. The various processes of propagation have been treated on, vide p. 309-355. We may here, however, remark, that seeds of most greenhouse plants, the production of the preceding summer or autumn, should be sown in February or March, in soil somewhat lighter than that in which the plants are grown, and placed in a temperature a few degrees only warmer than that of the ordinary greenhouse, and in a uniform atmosphere, rather more humid than otherwise. Seed sown at a more advanced period of the season, and particularly during the heat of summer, regetate with greater vigour and more certainty when placed in a house or pit having a northern aspect. Imported seed, particularly those with hard outer coverings, require the stimulus of a slight bottom-heat, and their germination is greatlyinsured by their being steeped for twenty-four hours in tepid water to soften them, so that the embryo bud may protrude more freely. The periods during which seeds remain in the soil before germinating are exceedingly various, some vegetating in a few days, others in a few weeks, and some remaining for one or even two years. Seed-pots, therefore, should not be too hurriedly thrown out. Sow thin, and transplant as soon as the young plants are fit to handle; and the first transplantation may be very judiciously done by pricking them out thinly into store-pots, in which they will soon attain a proper size for planting singly into small 60 -sized pots. Young seedling plants require to be kept in rather a close and moist atmosphere at first, gradually exposing them to the sun and air as they become furnished with roots.

Cuttings of all soft-wooded plants succeed best when planted during February, March, or April, and the majority of them strike roots rapidly if plunged in a mild bottom-heat and kept near to the glass; hence a low pit or garden frame is, in the absence of a regular propagatingpit, the most suitable. Soft-wooded plants, such as pelargoniums, do not require to be covered with bell-glasses. Many hard-wooded ones do; and all which require a long time to strike roots require such covering in a particular degree. From the end of March onward throughout the summer, cuttings either of soft or hard wooded plants strike readier when the structure they are placed in faces the north, because the atmosphere in it can be kept more uniform than if exposed to the full sun; and it lessens the care and trouble of shading, which, however well attended to, is liable to accident; for a few minutes' bright sun striking suddenly upon the unrooted cuttings is in many cases fatal to them. All hard-wooded cuttings should be set in silvery sand, and as soon as the roots have commenced to form, they should be taken up and transferred either to store-pots or to single ones of small dimensions, kept close and humid till fairly established, after which they should be gradually exposed to the sun and air. The majority of soft.wooded plants strike root readily in a light sandy soil, such as sand and leaf-mould, or in light sandy loam. All the other modes of propagation already described should be proceeded with during February, March, April, and May.

Potting.-Early in spring a general examination should take place; those plants intended to be grown as specimens should be shifted into pots two sizes larger than those they have been growing in. Such as are wished to be of moderate size and to bloom profusely, should have a shift of one size only, and all that are defective at the roots should have the soil greatly reduced, often entirely removed, and be set in fresh soil in pots less than those they formerly occupied. The most proper soil for greenhouse plants is sandy peat, and light maiden loam which has not been in previous use. Many plants, such as Ericas, Epacris, \&c., succeed best in the former without any other mixture. Proteas and bulbs do best in the latter, while the great majority prefer a soil composed of these two sorts in equal quantities.

Watering.-As vegetation advances, the quantity of water at the roots should be increased, and such as are not at the time in flower, are much benefited by a gentle syringing over their tops two or three times a-week-regard, however, being paid to the habits of some, which require it in greater quantities than others; and these are those of very rapid growth, and having large fleshy roots, as Richardia Ethiopica, the genus Agapanthus, Crinum, \&c.; and some during the 
period of their making their wood, as rhododendron, camellia, \&c. Soft river or rain water should be used.

Remoring the plants from the greenhouse during summer.-We have already remarked, that want of sufficient room led, in the first instance, to this practice, and habit with many has continued it. There is no doubt many of the hardier kinds of plants may be thus treated with no apparent ill effect, if they are not set out before the beginning of June, and taken in by the end of September, The more tender and choice plants should not be so treated. The smaller ones, that they may enjoy more air, light, and space, should be placed, towards the middle of May, in cold pits, and covered with glass, which, during the heat of summer, may be removed, but kept ready, at the same time, to be replaced during cold nights, and when heavy rains are falling. We have repeatedly stated that, as all plants experience a summer and a winter season naturally, it is only consistent with reason that they should do so in an artificial state. Now, keeping them in a moderately warm greenhouse during winter, and placing them in the open air, in our cold climate, during summer, is assuredly not fulfilling these conditions. The rule should be, keep them cool during winter, and warm during summer, which is, or rather ought to be, their season of growth, flowering, and maturing their wood and buds for another season's growth. This is a matter of much greater importance in Scotland than in the south of England, where many of them would be too hot if kept under glass during the warmest months of summer. Such as are to be placed out of doors should be, so far as is consistent with the welfare of those of more delicate habits, for a week or two before removal exposed to a greater amount of air, by copious ventilation; and on their being taken out, they should be placed for a few days in a rather shaded and sheltered situation, and afterwards placed, not; as is usually the case, under the shade of trees, or behind walls, but fully exposed to the sun, that their growth may be early made and thoroughly ripened before the end of the season. The plants themselves will stand all the sun we have, but the roots are liable to injury if the pots are not protected. Plunging them in the soil prevents this; but, on the other hand, the roots, being deprived of air, and being liable to become saturated with moisture, suffer equally, but from a different cause. They should be, therefore, set upon a dry level floor, rendered impervious to worms, and the pots surrounded with moss, or some other wet-absorbing yet heat-resisting medium; or they may be set within pots of a larger size, and the space between them filled with moss. They should be neatly supported to prevent their being blown over; and this is best effected by fastening long laths to neat uprights of wood, and securing the plants to the former. They should be allowed ample space, and examined night and morning to see that they do not suffer from lack of water. The middle of May in the south, and the beginning of June in the north, is the proper time for setting the plants out; and in the former case they may remain, in most seasons, till the first or second week in October; but, in the latter, the end of September is as late as they can be safely trusted out, unless protected under a portable canvass covering.

During summer those left in the house should be set thinly, abundantly supplied with water, and ventilation given to a greater extent than during any other part of the year. In lean-to houses they should be frequently turned round, that all sides of the plants may enjoy the effects of light and sunshine. Partial shading is only of use to continue the blooming period, the plants otherwise requiring all the sunshine we have; and whatever shading is used, it should be of so thin a material that the rays of light may be intercepted as little as possible.

Training.-During summer the plants should be frequently gone over, and all over-luxuriant and useless shoots removed; others topped, to causea sufficient number of shoots to be produced; such as require it, neatly trained to supports; but plants so treated as to be able to maintain their perpendicular without such assistance always look best. Some, however, of slender growth, do require support, and all scandent or climbing plants do. Training as an art in culture is not, in general, well understood, and hence we have plants trained in the most stiff and formal manner possible, while others are twisted, and constrained to forms of which we have no similar examples in nature. Every plant should be trained or made to assume an appearance as near as possible to that of its natural habit.

\section{SELECT LIST OF GREENHOUSE PLANTS.}

The plants enumerated under the head of Conservatory Plants are all fit inhabitants of the greenhouse also. The following are given in addition to them, and also to those in our former lists. Where the generic name only is given, it is to be understood that the majority of the genus is highly worth cultivating.

Aotus; Aphelexis; Astelma; Arctotis; Adenandra speciosa; Amphicomearguta ; Anagallis; Abelia; Adamia versicolor; Burchellia capensis; Bejaria; Bouvardia; Browallia; Burtonia; Babbingtonia camphorosmæ; Calandrina; Calceolaria; Ceanothus; Chironia; Chorozema; Centradenia; Cosmelia; Coronilla; Cineraria, the garden varieties; Chenostoma; Cuphea; Coleonema rubra ; Dielytra spectabilis;Diplacus; Diploleptis, a slender climber; Dolichos lignosus; Erodium incarnatum; Escallonia macrantha|; Fabiana imbricata; Fuchsia ; Gazania; Gardoquia; Gorteria, Gnidia; Helichrysum, all the tender species; Hardenbergia, agenus of slender-climbing plants; Iberis semperflorens, Gibraltarica ; Loddigesia; Lobelia, most of the tender species; Lantana Sellovii; Lapageria rosea; Lechenaultia, all the genus; Maurandya, slender climbers; Mahernia; Melaleuca; Malva, most of the tender species; Muraltia; Nierembergia; Petunia; Philibertia, slender climbers; Primula sinensis, verticillata; Ruellia ciliata, elegans; Rhyncospermum jasminoides; Russellia juncea; Salvia gesnerifolia, fulgens, splendens, \&c.; Selago Gillii, and most of the genus; Stylidium; Statice foliosa, Holfordii, sinuata; Sutherlandia frutescens; 
Scottia; Sphenotoma gracile; Sprengelia incarnata; Stadmannia australis; Stenocarpus Cunninghamii; Tasmannia; Telopea speciosissima; Torenia; Tetratheca; Triptilion spinosum; Thunbergia, all the hardier species; Thibaudia, all the genus; Tremandra Hugelii, verticillata; Veronica speciosa, Andersonii, formosa; Vaccinium coccineum; Witsenia corymbosa.

\section{SELECT LIST OF EXHIBITION PELARGONIUMS.}

Albira, Amazon, Ariadne, Astrea, Auguste Miellez, Basilisk, Butterfly, Beatrice, Challenger, Colonel Foissy, Commander, Cordelia, Eleanor, Etoile de Jardin, Exhibitor, Extravaganza, Galatea, Gertrude, Général Eugène Cavaignac, Gloire de Belle Vue, Gustave, Gustave Odier, Harriet, Heroine, Incomparable, Jacques Duval, James Odier, Jupiter, Kulla, Lavinia, Leader, Leonora, L'Esperance, Lagoma, Madame de Lamoricière, Magnet, Medora, Mochanna, National, Nonsuch, Novelty, Ocellatum, Optimum, Oscar, Painter improved, Pasha, Portia, Pulcherrima, Purple standard, Queen of May, Rachel, Ringleader, Shylock, Splendidum, Spot, Suzette, Triomphe de la Tour, Vulcan, Zaria.

\section{SELECT LIST OF FANCY PELARGONIUMS.}

Advancer, Argus, Autumna, Barbette, Barrier, Beauty of Blackheath, Bouquet de Venus, Bride, Cassandra, Celestial, Charles Dickens, Conqueror, Conspicuum, Constance, Criterion, Dandy, Duchess of Sutherland, Duchesse d'Orleans, Electra, Eminent, Empress of France, Fanny, Fascination, Figaro, Flora M'Ivor, Flora, Goliath, Illuminator, Isomia, Italian beauty, Kentish beauty, Lady Downes, Lady Hume Campbell (Miller's), Lady Alice Peel (Miller's), Lady Hume Campbell, Madame Sontag, Magnum bonum, Marginata, Mary Howitt, Painted pet, Perpetual, Pet, Princess Alice Maud, Queen of the Fancies, Resplendens, Sambo, Superba, The Ocean Queen, Victoria magna, Viola.

\section{SELECT LIST OF SWEET-SCENTED PELAR- GONIUMS.}

Citrodorum, var. elegantissimum ; do., var. majus; Coquette, Delicatissimum, Exquisitum, Imogène, Innocenza, Lobatum, Odoratissimum, do. variegatum, do. grandiflorum ; Pandora, Pauline, Prince of Orange, var. variegatum, Pandora.

\section{SELECT LIST OF CURIOUS-FLOWERING PELAR - GONIUMS.}

Album plenum, Clown, Coquette, Curiosity, Glaucum grandiflorum, Harlequin, Intermedia, Oddity, Rosea striata, Singularity, Virgineum, Willmore's surprise.

\section{SELECT LIST OF VARIEGATED-LEAVED PELAR- GONIUMS.}

Attraction, Bridal bouquet, Flower of the day, Golden admiration, Golden chain, Golden circle, Lady Plymouth, Mountain of light, Peach blossom.

SELECT LIST OF FUCHSIAS OF RECENT ORIGIN.

Alba floribunda, Annie (Turner's), Beauty (Smith's), Beauty of Devonshire, Collegian
(Banks's), England's glory (Harrison's), Empress (Banks's), Glory (Bahks's), Grandis (Turner's), Gem of the season, Hendersonii, a distinct double variety; Incomparable (Mayle's), King charming (Mayle's), Lady Franklin (Smith's), Lady Emily Cavendish (Banks's), Miranda (Turner's), Model (Turner's), Miniata, Mrs Patterson (Patterson's), Perfection (Banks's), the Queen (Mayle's), Verrio (Turner's), Vesta (Patterson's).

\section{SELECT LIST OF LARGE-FLOWERED CHRYSAN - THEMUMS.}

Atrorubens, Berryer, Campestroni, Conspicua, Cloth of gold, Dorinda, Fleur de Marie, Gluck, King, Lady Hunlake, Le Phar, Madame Andry, Nandee, Nell Gwynne, Norah, Osiris, Peruvienne, Pilot, Pio Nono, Plutus, Prince of Wales, Queen of England, Rabelais, Rebecca, the Warden, Vesta, Vortigern.

\section{SELECT LIST OF LILLIPUTIAN OR POMPON CHR YSANTHEMUMS.}

Adonis, Atropos, Argentine, Autumna, Colibri, Cedo nulli, Cybele, Daphne, Drine drine, Eliza, Etincelle, Fenella, Golden drop, Hendersonii, La Sapajon, Madame Hector Jacquin, Madame Rousselon, Modèle, Ninon, Perfecta, Princess Mathilde, Sacramento, Solfaterre, Surprise, Vitida.

The Sikkim rhododendrons discovered by Dr Joseph Hooker amount to forty-three species. The most striking amongst them are, however, Edgworthii, Griffithii, Dalhousiæ, and Maddeni, remarkable for their large white flowers; Thompsoni and fulgens for their rich blood-coloured flower; argenteum, on account of its fine foliage; Wightii, lanatum, and campylocarpum, for their yellowish blooms; Falconerii and Hodsoni, for their large leaves and dense globular inflorescens; glaucum, nivale, elæagnoides, salignum, pusillum, pumilum, \&c., for their small, neat, and truly Alpine character. These latter are fit tenants for a greenhouse or cold pit; while the former will add greatly to the richness of the greenhouse, conservatory, or camellia-house, especially while in bloom.

\section{$\S$ 11.-FLOWER-FORCING HOUSE OR PITS.}

In every establishment where a profusion of flowering plants is required during winter and early spring, whether for the decoration of the drawing-room, conservatory, or greenhouse, it is quite indispensable that forcing-pits or houses be provided, for without these no great display can be expected. In vol. i. a great variety of examples are given of pits adapted to various purposes; figs. 671, 672, 673, if not perfect specimens, may with slight modifications be adapted to this purpose. Melon pits or houses, which during winter may be unoccupied by their legitimate inhabitants, may be taken advantage of from the beginning of November to the beginning of February or March, for the purpose of forcing flowers; fig. 652 may be offered as an excellent example 
upon a large scale, and those represented by figs. $615,632,648,649$, for demands of less importance. The best example, however, in this work, is fig. 642 , because it is really intended for this purpose, and a more useful structure it would be difficult to devise. With such accommodation, and a judicious selection of plants adapted for the purpose, a fine display of flowers may be maintained from the end of autumn till the beginning of summer. Upon the selection of suitable plants, however, much depends; and here it should be remembered that it is not amongst rare or delicate plants, or those of difficult culture, that we are to look in forming our selection, but to those requiring only a moderate degree of excitement in the development of their flowers, abundant bloomers, brilliant colours, and graceful habits, together with those appreciated for their fragrance and long continuance in bloom. The following selections will afford plants possessed of all these properties, viz. :-

LIST OF FINE - FLOWERING HARDY PLANTS,

(Either to be taken up and potted in autumn, or kept established in pots and plunged in the reserve garden.)

Rhododendrons, preferring the brilliant scarlet and pure white hybrid varieties: Azaleas; Weigela rosea, amabilis; Lily of the valley; Russian, Neapolitan, and double-flowering violets; Mignonette, sown in pots in August, also the giant variety sown in March, and grown during summer into a half-shrubby-like habit; Pinks, perpetual-flowering carnations; Hyacinths; various species of Narcissus, \&c., potted in September or October, and plunged in sand; Daphne Mezereum; Viburnum tinus; Anemone japonica; Kalmia glauca, latifolia, angustifolia; Andromeda polifolia, grandiflora; Hepaticas in variety; Primulas in variety; Iberis Gibraltarica; Dielytra spectabilis; Pompon or Lilliputian, as well as the larger-flowering varieties of chrysanthemums ; perpetual, moss, China, and Tea scented roses; Wistaria sinensis; Tussilago fragrans; Jasminum nudiflorum; Ribes sanguinea, do. fiora alba, do. flora pleno; Cyclamens; Crocus; Tulips; Deutzia gracilis; Forsythia viridissima; Magnolia conspicua; Rhodora canadensis; Ceanothus, various species; Cytisus purpureus, pilosus ; Erica herbacea ; Helleborus, various species; Saxifraga, various species; Scilla, various species ; Clematis azurea grandiflora; Eccremocarpus scaber; Calystegia pubescens; Alyssum saxatile; double-flowering almond; Berberis Darwinii, dulcis, buxifolia, empetrifolia, dealbata; Chinese, French, and Persian lilacs.

\section{LIST OF FINE-FLOWERING, HALF-HARDY, AND GEEENHOUSE PLANTS,}

(Which either flower naturally from the middle of November to April, or do so with very slight excitement in the forcing-pits.)

Abelia floribunda; Abutilon insigne, striatum, pulchellum ; Acacia pendula, armata, vestita, pubescens, undulæfolia, linifolia, affinis, pulchella; Achimenes (most of the genus, by being early potted in autumn, will flower from January to April); Azalea, the Indian species, and their numerous varieties; Bouvardia triphylla, versicolor, splendens, flava ; Camellia, all the varieties; Ceanothus, all the genus; Cestrum aurantiacum; Chorozema, most of the genus; Cineraria, all the varieties; Citrus or orange tribe; Corræa, most of the genus; Cytisus proliferus, rhodopncus, racemosus, filipes; Daphne odora-rubra, indica, papyracea, hybrida, chinensis, japonica; Daviesia ulicina, latifolia, and most of the genus; Dillwynia, most of the genus ; Diplacus puniceus, Californica; Epacris, most of the genus and varieties; Epiphyllum Jenkinsoni, lateritium, truncatum, Russellianum; Eutaxia Baxterii, myrtifolia; Fuchsia, all the species and varieties; Genista virgata; Habrothamnus fascicularis; Indigofera decora; Jasminum azoricum, revolutum, hirsutum, odoratissimum, nudiflorum, grandiflorum, gracile; Lonicera flexuosa; Luculia gratissima, Pinciana; Magnolia pumila, fuscata, anonæfolia, Soulangeana, obovata, tomentosa, conspicua; Muraltia, most of the genus ; Oxalis versicolor, Bowiei, elegans, and various other species of the genus; Pæonia Moutan, papaveracea, rosea, Banksia; Pelargonium, many of the scarlets, and most of the fancy varieties; Phœnocoma prolifera ; Plumbago capensis ; Polygala cordifolia, tetragona, oppositifolia; Pultenæa, most of the genus; Primula sinensis, verticillata; Rhododendron, most of the beautiful hybrids; Salvia gesneriflora; Statice arborea, macrophylla; Tritonia tricolor; Veronica Andersonii.

LIST OF FINE-FLOWERING STOVE-PLANTS,

(Which either flower naturally from the beginning of November to the end of March, or do so with slight excitement in the forcing-pits.)

Amphicoma arguta; Angelonia salicariæfolia, grandiflora, and others; Aphelandra cristata, aurantiaca, fulgens, tetragona, cristata major, like the last, should be propagated by cuttings put in early in summer, and if dwarf bushy plants be desired, they should be topped, the former frequently; Achmea fulgens; Begonia albo-coccinea, sanguinea, incarnata, odorata, coccinea, manicata, fuchsioides, cinnabarina, miniata, Prestoniensis, Zanthina, and several others ; Bignonia Chamberlaynii, æquinoctialis, venusta, speciosa ; Billbergia iridifolia, pyramidalis, cruenta; Browallia grandiflora, Jamesoni, speciosa; Clerodendron scandens, macrophyllum, fragrans; Centradenia rosea, multiflora; Eranthemum strictum, capense, pulchellum; Euphorbia splendens, Bojeri, jacquiniflora, atropurpurea; Gardenia Thunbergii, florida, radicans; Gesnera tubiflora, zebrina; Gloxinia, most of the species and varieties; Heliotropium, all the varieties; Ixora coccinea; Jasminum sambac, do. flore-pleno, gracile, grandiflorum; Lantana aculeata, horrida, mollis, Selloviana; Manettia glabra, coccinea, bicolor, splendens; Mantisia saltatoria; Melastoma corymbosa, and others; Pitcairnia gigantea, micrantha, angustifolia, bracteata ; Plumbago rosea, occidentalis ; Poinciana pulcherrima, regia; Sinningia guttata, velutina; Strelitzia reginæ; 
Thunbergia alata, aurantiaca, albiflora; Tillandsia rubida, rosea, vitellina; Torenia asiatica; Vinca rosea et flora alba.

Great dependence must ever be placed on hyacinths, jonquils, and the plants familiarly known as Dutch bulbs. These having been, it is presumed, potted in September, and buried in dry soil, will be, by the beginning of November, well rooted, and in a fit state for removal into the forcing-pit. We may here remark, that it is of vast importance to procure such roots as early as they are imported, and that they be potted immediately they are received. Allowing them to remain unpotted till spring is exceedingly hurtful to them; and even the old practice of delaying this operation till November is so to a certain extent. The earlier they are potted the better; and, for our earliest show of flowers, we plunge them in dry leaf-mould or sand, in an apartment where the temperature is seldom under $50^{\circ}$ to $55^{\circ}$. For later supplies we bury them in a dry border out of doors, but protected from wet. The intention of potting early is to secure a full development of roots before the plants be put into a growing state, without which the flowers would be of small account, having little to support them beyond the elaborated sap accumulated in the bulb itself during the previous summer; whereas, when well rooted, the roots supply that support so necessary in the production of a strong fine bloom. Want of attention to this simple matter is the chief cause of the frequent failures in their flowering. One advantage of burying them is, that the weight over the bulbs presses them to the soil they are set on,not in - for the bulbs should not be planted deeper than half their diameter-and prevents their being hove up by the slight resistance the embryo roots meet with in endeavouring to descend. From a temperature such as the above they may be at once transferred to the forcingpit; but as they have been hitherto kept in the dark, care must be taken that they be not exposed suddenly to the light; and hence they should be partially shaded for a few days after removal, and only gradually exposed to the light. All plants taken from the reserve garden, particularly in cold weather, should not be at once taken into the warm forcing-pit. The transition is too sudden. It is better to place them for a few days in the intermediate temperature of a protected pit, where they may be secured from frost. The necessary pruning of roses, and such shrubs as require it, should be early attended to; and as scarcely any two species admit of the same mode of pruning, no general rule can be here laid down. Forcing the rose will be detailed in the Roserie. We may state that the common Mezereum and the lovely Wiegelia rosea flower on the shoots of the previous year; therefore any curtailment beyond thinning out illplaced or weak shoots would greatly defeat the object in view. Lily of the valley is prepared for the forcing-pit by being brought into the mushroom-house, or any darkish and mild apartment. There it forms its roots, and in eight or ten days is fit for removal. For the majority of the plants we have enumerated above, with the exception of tropical ones, a moderate temperature only is required; from $50^{\circ}$ to $60^{\circ}$ may be taken as an average. A high temperature and dry atmosphere are equally bad, and few of those named will require bottom-heat beyond that of the temperature of the pit, except in so far as it is necessary for creating action in the roots, which should always have the start of the buds. Where tropical plants are to be excited into bloom at this particular season, bottom.heat and a higher atmospheric temperature are required; and as little dependence can be placed on solar lieat at this time, the bottom and top temperatures may be set down at $70^{\circ}$ to $65^{\circ}$ as an average, allowing, however, the latter to rise several degrees higher whenever there is a little sunshine. As the days turn, and the light and sun-heat are increased, so also increase that of the pit. The great dependence, however, for keeping up a gay appearance in the ordinary greenhouse and conservatory, must be placed on the hardier selection of plants, as those in our previous list of Hardy and halfhardy plants. Many of these, such as rhododendrons, azaleas, and herbaceous plants generally, may be at once taken up from where they have been growing, and potter, well watered, and placed in a cold pit till wanted to be set in the forcing-pit. The great matter to be observed, in all cases, is to bring them into a growing state slowly, for a sudden transition from the temperature they have been growing in, to one much higher, will only defeat the object aimed at; and hence they should be taken up as early in the autumn as possible, and placed under a glass covering, both to prevent their being exposed to an unnecessarily low temperature, and also that they may have time to make fresh roots before they are farther excited; and to aid this, in some cases, it is of advantage to place them in a moderate bottom-heat.

A more tender section of plants, suitable for early spring flowering, consists of pelargoniums, heliotropes, \&c. These should be propagated as early in the spring as possible, frequently shifted, and forwarded in pits or frames until they are fit for their final shifting into pots in which they are to grow during summer. When that season arrives, they should be plunged in the reserve garden, in a situation fully exposed to the sun and air, the greatest attention paid to repeated stopping the shoots during summer, and the removal of every flower, the great object being to cause them to become stout stocky plants, having their wood well ripened before the season of growth closes. Neither of these plants lifts well when grown in the open soil; besides, they are apt to grow too large and straggling, and if allowed to flower in the borders, much of their energies is exhausted, their wood becoming long-jointed, imperfectly matured; and the check occasioned by the loss of their principal roots is such, that they seldom recover even to enable them to drag out a feeble existence throughout the winter.

The finer varieties of Primula sinensis are highly decorative during winter, and not only flower freely, but impart a grateful perfume. The seed should be sown thinly in March, in light sandy soil, and placed in a slight bottom-heat: when the plants have attained two or three 
leaves, they are fit for potting off singly into small 60 -sized pots, in light rich soil. They should be grown in a low pit, with a northern exposure, with free ventilation both day and night. They delight in a rich dry soil, composed chiefly of decomposed cow-dung, light loam, and sharp sand, with a due amount, but not an excess, of moisture during hot dry weather. A moist atmosphere around them is advantageous, and this may be secured by watering the floor upon which they stand once or twice a-day. They do not stand the force of the sun well; shade, if not under the drip of trees, is their favourite situation. They may be set in their flowering pots in June, and kept in an uncovered pit facing the north, in most parts of Scotland; but in most of England they may be turned out into a shady border in the reserve garden, in rather a stiff compost, in which they grow stout, and transplant safely. When potted in their floweringpots, in either case they should be plunged in sand or coal-ashes, allowing them plenty of room, in a free airy exposure, and kept at a very uniform degree of moistness, neither too wet nor too dry. In October they should be placed in a pit under glass, from whence they may be taken into a moderately warm forcing pit or house in succession; but as they will bear neither much heat nor sunshine, these must be guarded against.

The varieties of Cinerarea are great acquisitions during winter and spring. Seed sown about the end of March will produce plants fit for planting out in a north border, by the end of May, in a pretty rich soil of turfy peat and loam. They should be set 12 inches apart, and encouraged in growth throughout the summer, giving the weaker-growing plants a little liquid manure from time to time, and preventing all from flowering by picking out the flower-stalk as soon as it makes its appearance. They will be fine bushy plants by September, at which time they should be taken up with good balls, and potted in pots of a size corresponding with their growth. They should then be treated as recommended above for Primula sinensis, and like it they are impatient of fire-heat. The seed does not always vegetate freely when sown in pots in the usual manner. To overcome this, we have long adopted the following plan: In March, when the earlygrown plants have nearly finished flowering, we select the very finest varieties, and place them under a close garden-frame, covered with glass, and set upon a loose gravel courtyard. The seed naturally sheds itself; and, falling on the surface of the pots, but mostly on the gravel, vegetates freely, and produces thousands of plants. These, when about a quarter of an inch high-that is, as soon as they can be handled-we take up and plant in store-pans, keeping them still in the same frame; and these, by the end of April or beginning of May, are fit for potting off singly into small pots, or of being planted out in a border as noticed above.

The Chinese chrysanthemums are valuable for a late autumnal display, when flowers are most needed, and from their easy production deserve our especial attention. Some propagate them yearly by cuttings taken off in March, or as early as they can be procured; for ourselves, we prefer planting out the old plants in a warm well exposed border early in April. As the plants progress, all the shoots are removed excepting three or four; these are allowed to grow till they begin to show their flower-buds about the beginning of October, when they are laid singly into pots, choosing only about 18 inches of the points of the shoots of the larger-growing sorts, 10 or 12 inches of the dwarf sorts. These soon strike root, and form compact little plants, and are kept in a cool pit, rather close, for a fortnight or so, after which abundance of air is admitted, and frost carefully excluded. They are thus kept till required for flowering, and are taken into a moderately warm pit in succession.

Pinks and carnations, particularly those varieties of the old Tree sort now called perpetual flowering, are valuable in early spring. They should be struck as pipings as early in spring as the "grass"-that is, the young shoots-is procurable. They require to be rooted on bottom-heat, and when hardened off should be planted out in a rich warm border, and kept moderately moist until September, when they must be taken up with good balls of earth, and potted in a compost composed of stiffish loam and very rotten cow-dung, and plunged with the other plants prepared for forcing. Some prefer plants produced by the previous season's pipings or layers, and these no doubt are stronger, and will be in excellent condition for potting in September, if they have been kept in a northern border in rather poor soil, and prevented from flowering.

Some annuals and biennials become very serviceable for this purpose, and none more so than the mignonette, particularly the giant variety, the Double wallflower, Ten-week stocks, \&c. The wallflower we strike by cuttings in April, having prevented the old plants from flowering. When the plants are rooted, they are potted and plunged in a cold north pit, and only encouraged in growth towards the end of summer, when they are placed in their flowering-pots, and afterwards plunged in coal-ashes till wanted. It is unsafe to trust them during winter without protection.

The seed of Ten-week stock is sown in July, and again in August for a succession. When the plants come up, they require abundance of air and little water, and when fit to handle are potted off, sometimes singly, at other times three in a pot, and for this purpose no pot need be more than 6 inches in diameter.

Mignonette is by some sown in pots, and afterwards thinned out to three or four plants in each, the season of sowing being the same as the last. In this way the growers for the London market, \&c., raise it, some of whom have many thousands of pots annually. We adopt a different course, and sow in the open air upon a bed of rather poor soil at the times above specified, and when they have made three or four leaves, choose a dull cloudy day, and take up the plants on the point of a knife, and set them to the number of five or six in 5 -inch pots. These are carried as they are potted to a cool close pit, kept shaded, and slightly watered for a week, after which the 
shading is gradually dispensed with, and less water given, as well as a greater amount of air, until they have begun to grow, after which the glass is removed altogether until the end of October or beginning of November, when it is replaced to guard against frost. The soil we use is comparably poor, and in consequence the plants assume a hardier form of texture, which prevents their suffering from damp during winter. The tops are corltinually pinched off, and no flower is allowed to form until the period arrives when they are required, at which time, and rather before, slight waterings of liquid manure are applied to excite them into bloom. During winter they must be kept pretty dry, and as near to the glass as possible, as well as in a very moderate temperature. Suspended shelves near the roof of a glass house are a very fitting place for them. There are other annuals which may be grown for the same purpose, but their cultivation is the same.

\section{\$ 12.-COLD OR CONSERVATIVE PITS.}

These are of great importance in all plant establishments-and especially so to amateurs who may not have the better convenience of a greenhouse, - their use being to protect halfhardy and many greenhouse plants during winter-that is, those which would not stand the severity of our winters in the open air. They are not heated artificially, the cold being excluded by covering with glass sashes, and, in very severe weather, with the addition of wooden shutters or sheets of tarpaulin. As dryness is so essential in resisting cold, it follows that their floors should be somewhat elevated, the situation well exposed to the sun and air, and that the smallest amount of water should be administered to the plants during winter, and rain or snow prevented from falling upon them. We have given examples in vol.i., figs $671,672,673,674$, and 678 . The management of the plants simply consists in keeping them as dry during winter as is consistent with their safety, admitting air freely on all favourable occasions, removing dampness or decaying leaves where these appear, and, should frost reach them at any time, keeping them dark till it has thawed in the pots. Keeping them close shut up during severe weather for any length of time is to be avoided; and for this reason slight ventilation should be given every day, if only for a few minutes, that motion may be communicated to the air within. Pits constructed as those we have referred to, are capable of preserving a great many plants with very slight attention, if the above remarks are followed up. It is important that in autumn all superfluous or gross shoots should be removed from them, that the plants be set thin, so that the air may circu. late freely amongst them, and that air be freely admitted so long as frost is excluded. Again, in spring, air must be increased gradually, and, as the vital functions of the plants begin to exert themselves, water should be gradually given, and this in small quantities at a time, and applied in a tepid state. During spring and summer their treatment is similar to ordinary green-

VOL. II. house plants; and as the summer advances, the glass protection, instead of being removed, should be continued on them. Plants thus treated enjoy a period of rest somewhat similar to what they do in their native habitats, where, if cold in all cases be not the predominant cause of this cessation in their growth, the absence of moisture produces a nearly equal effect. Plants in cold pits should enjoy abundance of solar heat during summer, which the glass roof will afford them if properly managed. In spring they should be gradually excited, and in autumn the heat maintained, to ripen their wood and perfect their buds. This is placing them nearly in a corresponding temperature to that of their native habitats, and if attended to, most of the plants here enumerated may be cultivated pretty successfully without the aid of fire-heat altogether. But this is not the general rule. No sooner does the cold of winter pass away, and danger from frost is thought to be past, than the glass is wanted for some other purpose, and the plants, at the very time they require the excitement of a genial day-heat, are deprived of it, and exposed to the cold cutting winds of our usual springs. Again, during summer, when they require an amount of solar heat unknown naturally to us, they are left depending on the feeble rays of a sun shining through a dark and cloudy atmosphere, retarding their season of growth till August and September, at which period their full growth ought to have been made, and inducing efforts in them to continue that growth at the very period when it should have been completed, leaving them no time before the return of autumn to elaborate the sap they have accumulated, or to ripen their wood to enable it to withstand the cold of the succeeding winter. Were a contrary course followed, by means of very slight coverings, the majority of our greenhouse plants would be better preserved throughout winter, both in greenhouses and pits, without the aid of fireheat altogether, than they at present are. Vide article HEath-House for the management of plants in such pits.

The following plants form a part of such as may be safely wintered in such structures. The great majority of bulbous, tuberous, and herbaceous exotic plants, which die down to the ground in autumn, and undergo their season of rest during winter, such as most of the genera Oxalis ; Gladiolus ; Alstrœmeria ; Commelina ; Tritonia ; and similar Cape of Good Hope and Peruvian bulbs; Indian Azaleas, the strongergrowing Ericas ; Camellias ; Chinese Chrysanthemums ; Fuchsias; Cinerarias ; Cyclamens ; Petunias; Verbenas; the tender varieties of roses; Sikkim and tender hybrid rhododendrons; European and American ferns; the more tender herbaceous and Alpine plants; the pretty and abundant-flowering genus Cistus; the genus Ceanothus; Escallonia; Buddleya; Clematis ; Coronilla; most of Cytisus; Daphne; Deutzia; Dianthus arborescens ; Enkianthus reticulata; Erythrina; Eccremocarpus scaber ; Edwardsia; Eryobotrya japonica ; Fabiana imbricata; Forsythia viridissima; the genus Garrya; Helianthemum ; Hydrangea; Jasminum nudi- 
flora, heterophyllum, revolutum, affine, Wallichianum; Laurus regalis ; Lemonia laureola ; Magnolia grandiflora, pumilis, conspicua ; Olea fragrans; Pœonia arborea, papaveracea; Rhodolea championii ; Skimmia japonica ; Weigela rosea, amabilis, splendens; Yucca, most of the genus; Chimonanthus; Illicium; Mitraria coccinea; Cantua dependens, bicolor; Aloysia citriodora; Anagallis; Calceolaria ; Cuphea ; Cyananthus lobatus; Campanula pyramidalis, versicolor, Vidalii ; Diplacus; Gazania rigens, pavonia; Humea elegans ; Iberis Gibraltarica, sempervirens, semperflorens; Lobelia, all the herbaceous varieties; Leonotis leonurus; Cineraria maritima; Linum flavum, tauricum; Lilium, most of the tender species and varieties ; Lophospermum ; Maurandya; Nierimbergia ; Nuttalia ; Enothera macrocarpa, taraxifolia; Phlox, the varieties of Drummondii; Plumbago Larpentæ; Rhodochiton volubile; Salvia, most of the greenhouse species; Selago corymbosa; Clianthus puniceus; Sarracenia, all the genus; Sollya; Thea; Tropæolum, \&c.

The conservative pit is also the proper habitation, during winter, for the ever-flowering tree carnations, the more delicate varieties of hybrid Phloxes; Antirrhinums; chrysanthemums; and true Cape geraniums-a section of that extensive order, long neglected, but of late brought again into popular favour. Some of them, as anemonæflorum, shed their leaves annually-others, as echinatum, make short stubby shoots, and become nearly or wholly deciduous during winter, and hence are enabled to withstand several degrees of frost with impunity.

The majority of the following plants are kept in cold pits in the neighbourhood of London : Patersonia, all the genus; Galaxia, all the genus; Cypella; Herbertia; Homeria, all the species; Hovea; Platylobium; Bossiæa; Scottia; Templetonia, all the species of each ; Genista ; Cytisus, all the tender species; Thea, all the genus; Camellia, all the genus and varieties; Polygala and Muraltia, all the tender species; Medicago arborea; Coronilla glauca, C. glauca variegata ; Kennedya, all the genus ; Candollea; Beaufortia, all the genus; Melaleuca, all the genus, with two exceptions, M. leucadendron and cajuputi ; Citrus, all the genus; Cineraria, most of the genus; Agathæa; Gazania, all the species; Arctotis; Othonna, most of the genus; Osteospermum, all the genus; Cypripedium, all the genus, with the exception of venustum, insigne, Irapeanum, and Lowi; Acacia, all the New Holland species; Mesembryanthemum, most of the genus ; Callistemon, all the genus; Sempervivum, most of the genus; Oxalis, most of the genus; Enkianthus, all the genus; Pultenæa; Viminaria; Dillwynia; Eutaxia ; Daviesia ; Callistachys; Chorozema; Podolobium; Oxylobium; Gompholobium, all the species of each; Gnidia; Passerina ; Fuchsia ; Corræa ; Boronia ; Lachenalia; Dianella; Anthericum; Bulbine; Albuca; Eucomis; Tritoma; Veltheimia; Blandfordia; Agapanthus; Alstrœmeria ; Gethyllis ; Doryanthes; Zephyranthes; Habranthus; Vallota; Phycella; Nerine; Anigozanthus, all the species; Statice, most of the genus ; Crassula; Kalosanthes; Rochea, most of the species; Tenoria, all the species ; Phylica; Bırınia; Thomasia; Pittosporum; Diosma; Adenandra; Agathosma; Billardiera; Sprengelia; Andersonia; Dracophyllum; Lysinema; Epacris; Styphelia; Chironia, all the species; Echium, all the Cape species; Blæria; Penæa; Moræa; Marica, all the species; Abelia floribunda, uniflora; Amphicome arguta; Aspidistra elatior, variegata; Benthamia fragifera, $B$. japonica; Berberis, all the tender species; Buddlea Lindleyana; Calampelis scabra; Calandrinia umbellata; Calceolaria, all the species and varieties; Calochortus, all the species; Ceanothus, all the species; Chænostoma polyantha; Cuphea, all the genus; Habrothamnus, all the species; Hardenbergia; Heimia; Hibbertia; Hydrangea, all the species; Illicium; Isopogon, all the species; Jasminum nudiflorum, J. ligustrifolium, J. dianthifolium ; Kadsura japonica; Laurus regalis; Lindleya mespiloides; Lophospermurn, all the genus; Mahonia, all the tender species; Mandevilla suaveolens; Maurandia, all the genus; Plumbago Larpentæ; Prostanthera, all the genus; Salvia Grahami, fulgens, gesneriflora, patens; Sarracenia, all the genus; Sollya, all the genus; Veronica speciosa, salicifolia, formosa, angustifolia, Andersonii; Zichya, all the genus; Zauschneria californica, latifolia; Vittadinia triloba.

\section{$\S$ 13.-CONSERVATIVE WALL AND BOR. DER, PROTECTION OF HALF-HARDY TREES AND PLANTS, \&C.}

The conservative wall and border may be denominated the connecting link between the greenhouse, cold pit, and the open unprotected border. The plants which furnish them are such as will not withstand the cold of our winters without some partial protection, and yet will grow and flower profusely during the sum. mer and autumnal months. The situation should be dry, exposed to the sun, and well sheltered, but not shaded by trees: The soil should be rather poor than otherwise, resting on a dry bottom, and the wall constructed so that a temporary covering may be applied during winter. For construction, vide vol. i. p. 101. The best protection is unquestionably glass, because, unlike all other material employed for a like purpose, it admits light, and to a considerable extent heat also, at least during the day. There are, however, other kinds of protection, which, although incapable of admitting light to the subjects covered, are valuable on account of their non-conducting properties. Of these we may mention the well-known garden bast-mat, canvass, bunting, netting, oiled paper set in frames, branches of trees, fronds of fern, straw ropes, wisps of straw, reed matting, wooden and felt shutters, and the more recently applied Frigi Domo, a canvass made of prepared hair and wool, a perfect non-conductor both of heat and cold. Three important principles ought to be observed in choosing and applying coverings -namely, the exclusion of wet, preventing radiation or escape of heat, and avoiding the exclusion of light. Now the first of these is to be effected by employing a material that is imper- 
vious to wet, or has the property of throwing it off ; the second by placing the protection so far from the plant to be protected as to admit of a body of air being confined between them, and that in a quiescent state; while the third can only be effected by using some transparent or semi-transparent material. For the former of these the bast-mat is ill adapted; its very construction is the antipodes of the principle, and reed mats are little better. Both are expensive in the first instance, and are neither durable nor conveniently put on and taken off. Netting and straw in their various forms, as well as branches of trees, fern fronds, \&c., are only very superficial protections, and greatly tend to shut out the light from the plants; they are useful, however, for protecting deciduous shrubs and trees growing as standards, as netting can be thrown over them, and secured to their stems in form of a balloon; and handfuls of straw, asparagus haulm, fronds of fern, \&c., can readily be tied to the main branches, and so arranged that the leading shoots may be partially covered. Branches of trees, broom, \&c., may be applied in a similar manner, particularly the latter, as it maintains its green colour, and is less offensive to the sight. In this way we protect many tender roses, and various deciduous trees and shrubs, putting it on in November, and allowing it to remain till March, when it is gradually removed as the chances of severe frost disappear. Bunting and canvass, either of which may be dyed a green colour, may be advantageously employed to envelop shrubs and trees, and, like netting, if firmly secured round the stem to prevent the wind from entering, may remain on during the whole winter. Both these last materials, from their flexibility, are well adapted for mounting on rollers or otherwise, and being let up and down in front of plants against a wall. Protection is best secured when it is placed a little distant from the plant, therefore shrubs growing on lawns or in borders should have a framework of wood placed around them, to which the canvass should be secured, one of the sides being so constructed that it may be occasionally opened for the admission of air and light - and this is the more necessary if the canvass be thick and opaque.

Cases of half-inch boarding, covered at top with a movable pavilion roof to throw off the rain and snow, and having the side facing the south partially filled with glass to admit light, and made so as to open for the admission of air, are excellent protectors; but the best of all are glass structures framed in pieces like ordinary handglasses, and of sizes so as to cover the entire plant, so that neither the leaves nor branches come in contact with it. In severe frosts these may be covered with canvass or similar material. Whatever protection be used, it is important that it should prevent the entrance of wet, for the drier the plants are kept, the better they will resist the cold. It is also essential that the roots be prevented from becoming frozen or saturated with moisture, therefore the surface of the ground, as far as they extend, should be covered with moss 3 or 4 inches thick, and secured against displacement; finely-sifted coal- ashes are also both an excellent non-conductor of cold and corrector of damp. That portion of the stem which connects it with the roots, known as the collar of the plant, is the most susceptible of the cold; it is necessary, therefore, that it be protected either by gathering up the coalashes around it, or by enveloping it around with a neat hay-band.

More temporary yet effective protectors may be found, particularly for small shrubs, by covering them with sea-kale pots, lifting the lids off occasionally, inverting large flower-pots over them ; and for plants of larger sizes, empty casks or large boxes are good substitutes.

In the application of covering it should be borne in mind, that anything which obstructs the free aspect of the sky arrests, in proportion, the progress of radiation, and the slightest covering with cloth almost prevents it altogether. Plants, therefore, to derive protection, should have the covering material placed at a moderate distance from them, so as to contain as much confined air as possible. Air in a state of quiescence is one of the worst conductors of heat possible, and no covering will radiate or give out heat till the covering, and the air confined under it, are both above the heat of the atmosphere. Heat, having a natural tendency to escape upwards, must be prevented from escaping in that direction. It will also escape by the sides; and therefore, to confine it entirely, the plant must be covered all round from the external air. It would be difficult to procure, or even apply, a perfectly air-tight covering, but such openings as communicate directly with the external air may be guarded against, so as to prevent the escape of heat. Thus, for example, the orcinary covering of netting, be it ever so fine in the mesh, affords comparatively little protection, because the heat escapes through the meshes; and any number of courses of the same would afford as little, if placed so that the openings be exactly above each other; but by alternately placing over each other the open and closed parts, the escape of heated air, as well as the entrance of cold air, is very much interrupted. The heated air from within would have to deviate as often from its direct line of escape, as would also the cold air from without in endeavouring to enter, as it meets with the obstruction of the closed parts of the netting. Mr Daniell, in Essay on Climate, has clearly explained this important point, and observes: "Almost all the modes in practice of protecting plants are founded on the doctrine of radiation; and hence the fact should be kept constantly in mind, that all bodies placed in a medium colder than themselves, are continually giving out their heat in straight lines, and that these straight lines, when the body is surrounded by air, may always be reflected back on the body from which they emanate, by the slightest covering placed at a short distance from them; while, on the other hand, if this slight covering be applied close to the body, instead of reflecting back the heat, it will carry it off by conduction; that is, the heat will pass off through the covering closely applied, and be radiated from its surface. The radiation is only transferred from the tree to 
the covering, and the cold of the latter will be conducted to the former in every point where it touches. Contact should, therefore, be prevented by hoops, or other means properly applied, and the stratum of air which is enclosed will, by its low conducting power, effectually secure the plants. With their foliage thus protected, and their roots well covered with litter, many evergreens might doubtless be brought to survive the rigour of our winters, which are now confined to the greenhouse and conservatory."

Trees with naked stems and large branches would be benefited if enveloped in hay-bands, or otherwise covered in spring, because it would prevent the action of cold causing the excited sap to descend, by giving a check to the ascending sap, and hence be exceedingly disastrous to the expanding blossom. By this operation, trees suffer more from spring frosts than by their effects directly on the blossom, as generally supposed.

Syringing with cold water in the morning early, if the frost has not penetrated to the juices of the plant, may, by washing off the cold dew, prevent the frost from penetrating; and covering from the sun may save a plant partially hurt by the sudden change of temperature, if a bright sunny day succeed a frosty night; but if the juices have been frozen till the vessels are burst, and a change of colour takes place in the foliage by the suffusion of the sap, no means hitherto known can save the plant from death.

Protection is required for plants on conservative walls, or to such as are grown on the open lawns, particularly those which become excited early in spring, not only from frost, but also from those cold cutting winds so frequently experienced during March and April. Nightcovering counteracts the former, but it requires day-covering to protect against the latter. This protection, however, should be so placed that, while the plant is shielded on the windward side, the other may be exposed to the sun and air.

The winter protection of conservative borders consists in covering their surface with some frost-and-damp-repelling medium; and of these few are better than finely-sifted coal-ashes, moss, vegetable mould, or half-spent tan. Either should be laid on during the first week in November, to the depth of 3 or 4 inches, and allowed to remain till the middle of March, when it should be gathered up and removed.

Both sides of the conservative walls should be furnished with a prepared border, and both surfaces of the wall itself covered with plants, as some prefer the brightest sun, while others delight in a moderate degree of shade. The selection of plants should be made with reference to the situation.

\section{§ 14.-MISCELLANEOUS OBSERVATIONS.}

Our previous remarks on the management of greenhouse and stove plants have been arranged with a view to describe the best mode of treatment required for each natural group; but when taken collectively, they bear upon plants in general, and in such a light will the general cul- tivator view them. Some amateurs and private growers, who abide by the olden practice of attempting to cultivate a small general collection, may expect that we should offer a few hints applicable to their case. We can only remind them of what has been already stated, that high cultivation and a general collection, however small, within the limits of one house, are perfectly incompatible. The season of growth of one favourite genus, or even plant, may be the season of rest in another equally prized; and the temperature to grow the pelargonium, even to mediocrity, will be found too high for heaths, although they are natives of the same country, in consequence of the difference in altitude and other natural circumstances by which the majority of them are affected in their natural habitats. It is in consequence of attempting to grow plants having no natural affinity to each other, as regards their seasons of rest, growth, temperature, and habit, that the well-meant efforts of the amateur are so frequently defeated, and their plants bear no comparison to the plants of those who confine themselves to a selection of such as will admit of nearly the same mode of treatment, or who limit their selection to the extent of their means. Instead, therefore, of forming a distinct section under the head of the Mixed Greenhouse, we will briefly notice a few popular genera which have been only slightly referred to in our foregoing remarks, and which, on account of some peculiarities in their culture, are next to inadmissible into any of the groups treated of.

Pelargonium.-The varieties of this genus originated by the art of man, have years ago amounted to several hundreds, and year after year numbers are discarded to make way for new or more striking novelties. True species are now seldom met with in cultivation, although of late a revival has taken place in a taste for them. By hybridising them in the first instance, we became possessed of numerous varieties; and by rearing by seed from those, in course of time a tribe was produced bearing little resemblance to the originals. This crossing was carried perhaps to nearly its utmost limits, and now even the best of them have almost been supplanted by another race denominated Fancies, which seem to be the favourites at the present time. Pelargoniums are naturally divided into four sections: 1st, Annuals and biennials, few of which are particularly interesting; these are multiplied by seed: herbaceous sorts, not in particular estimation, and increased by division of the plant: tuberous-rooted sorts, some of which are exceedingly rich in colour, and form excellent parents where brilliancy of colouring is in request; these are chiefly propagated by division of the roots, or by cuttings of the roots themselves; but by far the greater part are evergreen shrubs, and are increased by cuttings, \&c.

Propagation by seed.-When the object is to obtain new or improved varieties, the seed process is adopted; and as all, or at least the majority, ripen seed freely, the process, for ordinary purposes, is exceedingly simple. But where improvement is sought for, the points constituting that improvement must be provided for. Those points are, form of the flower, which, ac- 
cording to the rules of modern floriculture, constitutes the first and principal qualification; the second is substance in the petals; the third, size; and the fourth and last, colour. And to these we may add the habit of the plant itself, and the compactness and arrangement of the truss. Our best hybridisers, therefore, only save seed from those already possessing these properties nearest perfection-knowing from experience that the flower to bear the seed should possess the property of form. In order to secure the other points or properties, cut off the anthers of the well-formed variety before impregnation can by possibility have taken place, and protect the female parent by all possible means from being impregnated by other pollen than that of the flower chosen to be the male parent. The seed should be allowed to ripen fully before gathering; and until the ensuing spring it should be carefully kept in a dry and cool room. The beginning of March is a very proper time to sow, using rich light soil, and shallow pans, well drained, placing them in a moderate bottomheat, where vegetation will speedily take place. When the seedlings have attained their two first leaves, and are about an inch in height, remove them from the pit or frame, and place them close to the glass in another pit, or on a shelf in the greenhouse near the glass, watering sparingly, as they are apt to damp off. After a week's exposure in such a situation, they will be fit to pot off singly into small-sized- 60 pots, in light rich loam and leaf-mould, with the addition of about one-eighth of fine river-sand. Replace them in a similar situation, keeping them still close to the glass, and abundantly supplied with air. As the plants advance in size, shift into larger pots, from time to time, as they require it, until the beginning of July, when they may be set out of doors in a warm, airy, but not exposed place, upon a floor of coal-ashes or boarding, where they should remain until the first appearance of autumnal frost, when they should be removed to the geranium-pit or greenhouse. During their first season's growth they should not be topped, as their merits have not as yet been ascertained; and in order to prevent their growing too tall or straggling, they should be kept quite close to the glass; nor should they be stimulated with rich manure. They will flower the following spring, when the selection should take place, and such as are found to possess sufficient merit for retaining should be immediately cut down, the tops used as cuttings, and the plant encouraged in growth to enable it to produce a further supply, if stock be desired. If not, the plant should be treated as established plants noticed below.

Propagation by roots.-The tuberous-rooted, and many of the original true species, as well as some of the new fancy varieties, are found to be best increased by this means. The process is to remove all the soil with care from the roots, and to cut them into short pieces, each portion having a few fibres left attached to it. These are planted in small pots, watered, and placed in a mild bottom-heat, in a close pit or frame. When the young shoots appear, give air progressively. As they will often send up a number of shoots, after these have attained the length of 1 or 2 inches, remove all but such as may be required for forming the future plant, which should not exceed three in number, while some growers retain only one. Whichever of these is adopted, stopping them when about 3 inches in height should take place, with a view to induce them to send out side shoots.

Propagation by cuttings may be carried on from the end of February to the end of August; but the plants are in best condition for affording cuttings at the time they begin to cease flowering, at which time, also, they require to be cut down to insure bushy plants for next season. This usually takes place in June and July. When the number of young plants required is small, then the cuttings may be placed singly in 60 -sized pots, and if these are plunged in a mild bottom-heat in March or April they will root speedily; or if not put in till June, July, or August, they may be set on a floor of sifted coal-ashes under glass in a pit or frame without bottom-heat, and if moderately shaded till the rooting process commences, will strike readily. Where large quantities, however, are required, such as the various sorts of Scarlets, for planting out during summer in the parterres, then March is the proper time, and dung-beds and frames the best means; the plants will be pushed on rapidly, and be in fit condition for planting out by the beginning of June. The pots should be well drained, and not exceeding 5 or 6 inches in width; the soil, light loam mixed with sand. The side shoots which have not flowered make the best cuttings, and should be taken off when about 3 inches in length. The lower leaves should be cut cleanly off with a sharp knife as far as the cutting is to be inserted in the soil ; but those above that should be most scrupulously preserved and left entire. The base of the cutting, being cut transversely over just under a joint, is fit for placing in the soil. When the cuttings are strong and full of sap, they should be laid out on the potting-table in a shady place for a few hours, or if prepared in the afternoon, may remain till morning, to allow a portion of the sap to escape; but such as are weak do not require this precaution. They should then be set in the pots around their edges, keeping them in an upright position ; from four to six or eight, according to the size of the leaves, are sufficient for one pot. Settle the soil about them by a gentle watering, and when dry, either plunge them in bottom-heat or set them on a bed of coal-ashes, according to the season, as noticed above. In either case shading is necessary, but to obviate this as much as possible, we set the frames at either season facing the north, and often behind a north wall, thus preventing the action of the sun upon them till rooted, and yet admitting light, which is of importance to them, which, if covered with ordinary shading, would not be the case. When the cuttings begin to grow, roots will have become formed, and now air must be admitted more freely. If the process is conducted in heat, an atmospheric temperature should be maintained of from $55^{\circ}$ to $60^{\circ}$, with from $5^{\circ}$ to $8^{\circ}$ more of bottom-heat. If bottom-heat is dispensed with, then a close 
atmosphere, with a northern exposure, kept at from $55^{\circ}$ to $60^{\circ}$, which will be the case naturally at that particular season of the year, is to be preferred. Little water should be given them in either case till the callosities are formed at their bases; still the soil should not be allowed to become too dry. We propagate our scarlet geraniums in mild hot-beds facing the north during March and April. Others do so during the summer months, by setting them in prepared soil at the bottom of a wall without pots, covered with hand-glasses; and $\mathrm{Mr}$ Beaton informs us that he prefers striking them on the surface of a vinery border during summer without any covering whatever. When the cuttings have grown to the length of an inch or so, roots will have been emitted, and when that is the case they should be carefully taken out of the cutting-pots or soil, and potted singly into 60 or 48-sized pots according to their size. As this operation goes on, they should be removed; such as were struck in heat, to another frame of equal temperature, and plunged into it, kept close, and partially shaded for a few days, or till they become established. Such as were struck without bottom-heat when potted off, should be set in pits or frames of a corresponding temperature to that they were struck in. When they have filled the pots with roots, they should then be shifted into 32-sized pots, retaining their balls entire, and again into 24's, and so on until they have arrived at the sized pot in which they are to flower, and which may be taken at from 7 to 9 inches in diameter, according to their habit : the Fancy varieties may be flowered in the former of these sizes, while the majority of the tuberous-rooted kinds should be confined to pots of 5 to 6 inches, which are called 24's and 16 's, and none of the Fancies need be flowered in larger than 6 's.

Considerable discrepancy exists between the scale of sizes in England and in Scotland, and even in various parts of the provinces. This, like the differences in weights and measures, appears to be beyond the power of common sense to remedy. We have throughout this

\section{GLASGOW SCALE.}

\begin{tabular}{|c|c|c|}
\hline \multirow{2}{*}{$\begin{array}{c}\text { Pots } \\
\ldots\end{array}$} & \multicolumn{2}{|c|}{2 inches in } \\
\hline & $2 \frac{1}{2}$ & ches by \\
\hline & $2 \frac{3}{4}$ & $\cdots$ \\
\hline . & $3 \frac{1}{4}$ & - \\
\hline . & 4 & $\cdots$ \\
\hline$\cdots$ & 4. & $\cdots$ \\
\hline . & $\sigma_{2}^{\frac{1}{2}}$ & . \\
\hline - & 6 & . \\
\hline$\cdots$ & 7 & $\cdots$ \\
\hline . & 8 & . \\
\hline & 9 & . \\
\hline & 10 & . \\
\hline .. 1 & 11 & $\cdots$ \\
\hline .. 1 & $12 \frac{1}{2}$ & $\cdots$ \\
\hline
\end{tabular}

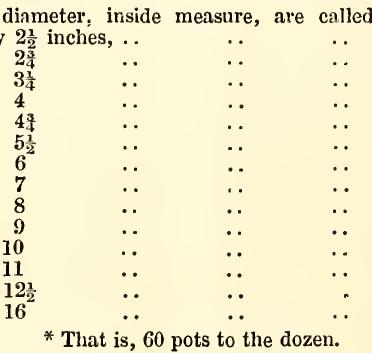

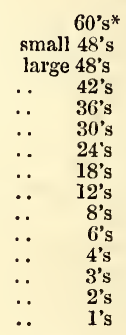

LONDON SCALE.

\begin{tabular}{|c|c|c|c|c|c|c|c|}
\hline Pots & $1 \frac{1}{2}$ inches & by 2 inches, & inside & measure, are & called & thumbs, & or \\
\hline . & $2^{2} \quad \cdots$ & $2 \frac{1}{2}$ & . & . & . & . & \\
\hline$\ldots$ & $\ldots$ & 4 & $\cdots$ & $\cdots$ & $\ldots$ & $\therefore$ & \\
\hline .. & . & 5 & $\ldots$ & $\ldots$ & . & $\ldots$ & \\
\hline . & .. & 6 & . & . & . & . & \\
\hline .. & $\ldots$ & 7 & $\cdots$ & - & .. & $\cdots$ & \\
\hline$\cdots$ & $\cdots$ & 6 & . & $\cdots$ & $\cdots$ & $\cdots$ & \\
\hline$\ldots$ & $\cdots$ & 7 & .. & $\cdots$ & -. & $\cdots$ & \\
\hline .. & . & 8 & .. & $\cdots$ & . & $\cdots$ & \\
\hline 1 & . & 10 & $\cdots$ & $\cdots$ & $\cdots$ & $\cdots$ & \\
\hline$\ldots$ & $\ldots$ & 12 & $\cdots$ & $\cdots$ & $\cdots$ & $\cdots$ & \\
\hline
\end{tabular}

work calculated on the London standard, and as some guide to amateurs and country purchasers, will give the London and Glasgow scales of sizes, which, with some slight allowance, may be taken as representing pretty nearly the sizes used in both countries.

There are also large and small 60's, large and small 48's, large and small 32's; the large in these sizes are of an intermediate size between each.

In Scotland they are sold by the dozen, and in England by the cast; the price is generally the same for all the above sizes, two pots of cast No. 2 costing the same price as eighty pots or a cast of thumbs. And a somewhat similar rule is followed in Scotland, one pot 16 inches by 16 costing the same as sixty 2 inches in diameter.

As the plants increase in size, the leading shoot should have its top pinched off to induce the side shoots to send out branches; and these, in their turn, will require stopping until a sufficient number of branches are formed near the bottom of the plant, to insure its attaining a uniform and bushy habit.

In regard to the propagation of the fancy pelargoniums by cuttings, autumn and spring are the proper seasons, the latter being the best, as plants struck at that time are less likely to become stunted in growth, and cuttings in a very fit state may be then obtained from the young shoots, which will require to be thinned out of the old plants. Therefore, in February or early in March, such plants as it is intended to take cuttings from should be placed in a somewhat moist frame or pit with a temperature from $50^{\circ}$ to $55^{\circ}$, and when the young shoots have made from 2 to 3 inches in length, they should be taken off close to the old stem, and inserted in the cutting-pots. The strongest of 
the plants thus originated, if potted, stopped, and finally re-potted in August, will form nice compact little plants, and will flower throughout the winter if kept in a temperature of from $45^{\circ}$ to $50^{\circ}$. The weakest of such cuttings, if potted, stopped, and prevented from flowering, and firally re-potted towards the end of September, will make fine plants for flowering during the following spring and summer. The section struck by cuttings in autumn, if treated as the above, and finally re-potted in March or April, will flower profusely during the following summer and autumn, thus continuing a succession of bloom throughout the year. The various pottings, it will be understood, are to take place whenever the roots have filled the pots they are in, so that no check may be given to a slow and progressive growth.

Pelargoniums are propagated at times by buds, much in the manner already described for vines. A shoot of moderate growth is selected, from which all the leaves are cut off, but not too close to the stem; the two lowermost buds are cut off, leaving about a quarter of an inch below and above each; the shoot is then split down the centre. When a sufficient number of buds are thus prepared, a well-drained pan or pot being ready filled with soil, and surfaced with sand to the depth of an inch, the buds are inserted in it, so as to leave the point of the bud rather above the surface sand. Place them round the edge of the pot, and give a gentle watering. Set them in a close, warm, shaded frame or pit, keeping them pretty close to the glass, and shade and treat as for ordinary cuttings. The buds will soon begin to spring, and will shortly be followed by shoots and leaves; and when rooted and transferred to small pots, and placed in a mild heat, they will soon make nice little plants.

Soil. - The best compost for pelargoniums is a rich, light, turfy loam, used three or four months after being brought to the compost-yard, and well exposed to the sun and air. It should be chopped up by the spade, retaining its grassy surface and such vegetable fibre as it may naturally contain. If too strong, attenuate it by the addition of pure river-sand; if poor, add a little well-rotted cow-dung. In such a soil they may be grown to great perfection. Cuttings and young seedlings, at their first two shiftings, should have a lighter and more decomposed soil, such as light loam which has been on hand for twelve months, with a little leaf-mould and silvery sand.

General culture.-We have already remarked, that, to grow this charming tribe of plants well, they should be grown in a house or pit by themselves. A pit is the best of all accommodation for growing them in until about to flower, when, both for seeing them to advantage and affording them sufficient air and light, they should be placed in an airy well-exposed greenhouse: that with a span roof is the best, as the plants will enjoy light on all sides, which they cannot do in one of the lean-to form. They should also be placed near the glass, and abundantly supplied with fresh air. When in flower the roof should be shaded during bright sunshine, but at all other times fully exposed to the light. The shading is with a view to preserve the colours, and also to prolong the season of bloom. During summer no artificial heat is required, and if the temperature be kept about $45^{\circ}$ during winter, it will be quite sufficient, raising it slowly as spring advances and the energies of the plants become awakened. Attention must be paid during their growing season that no check be given them; and as the shoots advance after the necessary topping, they should be neatly staked, but the fewer supports the better. The shoots should be trained in an outward direction, so that air and light may reach the centre of the plant; and during their whole growth they must be allowed plenty of roomso, indeed, that no part of one plant touch another. After the early section has flowered, they should be cut down in July to within two or three eyes of the old wood; they should then be placed in a cold pit, and kept rather dry at top by the protection of the glass sashes, yet abundantly supplied with air to prevent too rapid excitement. When the buds have sprung to the length of 2 inches, take the plants out of the pots, and after shaking all the soil from them, shorten the roots a little, and cut out all that are dead or diseased; then re-pot them in two sizes smaller pots, replacing them again in the cold pit, which now must be kept close until the young roots have found their way into the soil, after which more air must be given, and increased so as to accustom the plants to stand the open atmosphere. The second section of flowering plants will be fit for cutting down when their bloom is past; they should be treated in the same manner. The first section now in small pots will be fit for setting in their flowering pots towards the end of October, and those of the second section will require the same attention about the first of January. For List of SElect Sorts, vide p. 729.

The Calceolaria, as a greenhouse plant, is propagated by seed and by cuttings. Seed should be sown as soon as it is ripe, to secure plants to stand over winter and bloom early in spring, particularly in the case of the herbaceous varieties, which should be treated as biennials. To obtain good varieties, artificial impregnation is necessary (vide p. 313), selecting the male parent for imparting colour and the female for shape. Another sowing should take place in March, to secure a late summer display, as well as to provide against accidents befalling the former sowing. Flat pans or boxes, thoroughly drained, and filled with light loamy soil, and rendered quite even and smooth on the surface, being prepared, the seed should be sown thinly, and as it is exceedingly small, it should be covered very slightly. The seed vegetates freely during June, July, and beginning of August, if the boxes or pans are placed behind a north wall, shaded from the sun and covered with handglasses, or a shady pit is equally suitable. The seed sown in spring should be placed in a very moderately warm pit, or the front of a vinery or peach-house, for throughout its whole existence the calceolaria is very impatient of heat. When the plants are up, and just of a size to handle (the smaller the better), they should be carefully taken up and planted, first in store-pots or pans, and when their leaves are as large as a sixpence 
they should be potted singly in large 60-sized pots, and placed on a suspended shelf in a cool pit, and kept moderately damp, yet well supplied with fresh air. Re-pot them as they advance in size until the first flowers show, when, if of sufficient importance, shift again, and encourage growth to the utmost extent. Such as show indifferently plant out in the flower borders, whether they be herbaceous or shrubby.

Both the herbaceous and shrubby sorts are increased by cuttings, which should be attended to in March, the old plants kept over winter. furnishing the supply, and again in August or September, when they have done flowering. The tops of the shoots in all cases are best, and a light sandy loamy soil the most proper for setting them in; and when well watered, and afterwards allowed to dry, the pots should be plunged in a mild bottom-heat, the frame kept moderately close and shaded till the rooting process commences, when more air and less shading should be given them. When rooted, pot them off into 60 -sized pots, and set them on the surface of the bed they have been struck in, partially shaded for a few days to assist the process of root-making; after this is effected they should be placed in a cooler frame or pit, re-potted as they increase in size, and their growth encouraged. Cuttings struck in August or September should be potted off early in March, and those only struck in March as soon as they are sufficiently rooted to bear removal. From this time every encouragement should be given to forward their growth, and no flowers should be allowed to form until they have attained their full size, which they will have accomplished after their third shifting, and when they have been placed in No. 6 sized pots, which is sufficiently large for blooming them in. At each shifting drain thoroughly, for although the calceolarias delight in a humid atmosphere, they are very impatient of damp at their roots. During their whole growth they require abundance of light and air, and therefore should be kept near to the glass. As the plants progress in size, provision should be made for their support, particularly the flower-spikes of the herbaceous kinds, which are naturally long and slender, and produce much larger flowers than the shrubby ones do. The neatest, most durable, and cheapest support, is wire about the thickness of a crow-quill, cut into the required lengths, and painted either green or a soft stonecolour; and in placing them they should be set with their tops inclining outwards, so as to allow room for the free exposure of the flowers. The finer calceolarias should always be grown under glass, their delicate frame, particularly the herbaceous kinds, being ill adapted for resisting winds and heavy rains. Those who have a greenhouse or conservatory to maintain gay with flowers during the summer can scarcely choose a tribe of plants better fitted for the purpose.

Balsam.-This old and justly admired plant, when well grown, is valuable as a decorative plant for the greenhouse or conservatory during summer and autumn. Being annuals they are propagated by seed, choosing that which has been produced from the finest double varieties, and which has been kept for several years, such being found to produce double flowers with the greatest certainty. Sow in pots in March, and again towards the end of April, for succession, using a rich light soil, and the temperature of a moderate hot-bed. Many err in attempting to grow the balsam in too high a temperature, that of its native habitats being only from $50^{\circ}$ to $65^{\circ}$ upon an average, seldom much higher or much lower. When the plants have attained the height of from 2 to 3 inches, pot them in 48-sized pots, one in each, replacing them again in the bed; when the roots have nearly filled the pots, shift again into 24 's, 8 's, and lastly into 4's, keeping them still in the same temperature-say from $45^{\circ}$ to $50^{\circ}$ as a night heat, $65^{\circ}$ to $70^{\circ}$ during the day, with a bottom-heat of from $65^{\circ}$ to $70^{\circ}$. Admit abundance of air so long as the temperature is not lowered, and keep the plants apart from touching each other, and within a few inches of the glass, which will insure nice robust plants, whose diameter through the branches will be nearly equal to that of their height. At each shifting the soil should be richer and stiffer, that of a very rich loam being the best for their flowering in. Water must be applied to the roots freely, even during bright weather, to the extent of twice a-day, and if in an enriched state so much the better. When coming into flower they may be removed to the greenhouse or conservatory, and if elevated on pedestals in the latter, their effect will be enhanced. To prepare them for their removal from the growing-pit to either of these structures, air should be freely admitted to them for a week previously. It is more difficult to procure genuine seed of the balsam than to grow it, as neither its age nor stock can well be depended on; the grower, therefore, should save his own seed. Young seed produces too luxuriant plants, the majority of whose flowers will be single or only semidouble, and their colours inferior, and, strange enough to say, most of them bearing a close resemblance in the latter respect to the parent; whereas when old seed-say from four to eight or more years-is used, the preponderance in the progeny will be double-flowered, and of great variety of colour.

Cockscomb.-Although much less varied in colouring and splendour than the balsam, the cockscomb is, nevertheless, when well grown, a plant of singular beauty, and admirably suited for the conservatory or greenhouse during the summer and autumn months. As to time of sowing and general management, this plant differs little from the balsam, unless in regard to temperature, which can scarcely be maintained too high, if accompanied with air and water in abundance. From $70^{\circ}$ to $85^{\circ}$ may be taken as the average temperature until the flowers are fully formed, after which they will continue to exist in a much lower heat, their vital functions being probably nearly expended. The flowers will remain in tolerable condition should the roots be cut off and the stems stuck into moist sand. Tall and dwarf-grown cockscombs are the result of culture rather than of any seminal difference in the plants. To insure the former, the plants should sustain no check till fully grown; while to effect the latter, when 
the plants have filled with roots the 32-sized pots, they should be retained in them until they begin to assume a stinted appearance, which is insured by giving little water, more air, and keeping them cooler than heretofore. The soil should be rather porous, the pots well drained, and less stimulus given by enriching the soil with solid or liquid manure.

The Globe amaranthe is reared annually by seed, which should be sown early in March. Its cultivation differs from that of the balsam and cockscomb only in respect to temperature, requiring rather more than the former and rather less than the latter. Equally useful for summer and autumn decoration as the last.

The Fuchsia is propagated by seed when new varieties are desired, by cuttings of the young wood for general purposes, and sometimes, but rarely, by the leaves, which may be regarded more in the light of a curious experiment than of practical utility.

To produce improved varieties by seed, it is a necessary condition that artificial impregnation has been made to take place between two existing varieties, possessing some or all of the properties which constitute perfection in the flower and plant. The principal of these properties are, colour, size, form, and habit. Colour should be pure and well defined: for example, in the light-coloured varieties the sepals should be pure white, and the corolla a rich purple; and in the dark varieties the sepals should be of the brightest crimson or scarlet. In both, large size is a qualification. As to form, the sepals should be stout and broad, and well reflexed; the corolla should be large, and extend beyond the sepals,-it should also be circular and cupped. The flower-stalk should be 3 inches or more in length, so that the flower may hang gracefully down. In regard to habit, the foliage should not be too large and coarse, and the flowers should be produced in abundance. In attempting, therefore, to improve a fine-shaped flower, but defective in a good corolla, take the pollen of a variety that has a good corolla, and with it impregnate the stigma of the one with the good tube and sepals, and save the seed produced by the intercourse. The subject of hybridising generally has been already detailed at some length (vide p. 313.) When the seed becomes ripe-and it is important that it be fully so-gather it, and clear it from the pulp by washing; and when thoroughly dried in the sun, keep it in a cool dry place till March, which is the best time for sowing. Prepare shallow pots or pans by draining them well, then fill them to within half an inch of the top, and press the soil pretty firmly down, leaving the surface quite smooth, and on this sow the seed thinly, and cover to the depth of a quarter of an inch. Place them in a pit at a moderate temperature, but near to the glass. When the young plants are from an inch to an inch and a half in height, pot them off into 60-sized pots singly, and place them in a shallow pit, with a usual greenhouse temperature, shifting first into 48-sized pots, and afterwards into 32 -sized, and allow them there to remain until they flower, when a selection of those having good points should be made, and VOL II. the others thrown away. Those retained should be shifted into 24 or 16 sized pots, according to their strength. This constitutes their first season's growth.

Propagation by cuttings is best commenced in February and March, and should the plants from which they are to be taken be not already sufficiently excited, set them in a warm pit for a few days to forward them. The cuttings should be taken when about 2 inches long, cutting them off close to the old wood. Set them in pots filled with light sandy soil to within an inch and a half of their tops, upon which place an inch of fine silvery sand, in which to insert the cuttings. Settle the sand about them by a gentle watering, and when dry plunge them in a mild bottom-heat, as recommended for pelar. goniums, and treat them in the same manner. This mode of propagation may also be carried on throughout the summer, shifting the plants into larger pots as they advance in size.

Soil.-A light rich sandy soil is most suitable for very young plants; but as they attain strength, supply them with stronger soil until they are placed in their flowering-pots, when a compost of strong yellow loam, containing about one-eighth of leaf-mould and one-fourth of very decayed cow-dung, should be used. Young plants will require to have their tops pinched off from time to time, to insure a sufficiency of branches for their proper formation. If they are to be grown as pyramids, which is the form most followed for show plants, one of the leading shoots should be removed as soon as the lower branches have extended to a few inches in length, and the other leader allowed to attain a foot or so more in height, when it should be topped also. This mode is applicable to both old and young plants till the height desired be attained; the side shoots all the while being topped wherever they extend beyond prescribed limits, and also where a thinness of branches is observable. No. 4 sized pots are sufficiently large for any exhibition plant, but for ordinary purposes No. 6 will be large enough.

The fuchsia is often trained as a standard, having a single stem and globular head; they are also sometimes trained as tall pyramids, covering a trellis-work of that form, and clothed with foliage and flowers from bottom to top; and when trained in the manner of creepers over parts of the roof they have a very pretty effect, their long pendulous flowers hanging down in endless profusion. In lean-to houses few plants, if we except Cantua dependens, make a better appearance than they when trained close under the rafters. The fuchsia, being nearly a deciduous plant, is easily protected during winter by being placed, on the approach of frost in autumn, under the greenhouse stage, in a dry shed, or even in a cellar, or anywhere where the frost is excluded. It is important, however, that they be kept dry and brought into gradual excitement, light, and air, in spring, at which time they should be taken out of their pots and the old soil removed, and be re-potted in fresh compost, to carry them through the ensuing season. Smaller plants may be wintered in the cold or conservative pits. 


\section{CHAPTER II.}

\section{THE OPEN FLOWER-GARDEN.}

\section{§ 1.-AMERICAN GARDEN.}

THIs is understood to be a department in large gardens or in extensive grounds, as the American ground is in nurseries, for the culture of plants, comprising many different species, which so far only have an affinity with each other as to require a peculiar soil, without which few of them can be successfully cultivated. There seems, however, to be some incongruity in the term, as a great majority of the plants and trees found in such a department are in reality not of American origin, but from most extra-tropical quarters of the globe; though the types of some of the most important of them are found in America, as Azalea, Rhododendron, Magnolia, \&c., while individuals of even these genera are also natives of China, India, \&c. The term is, however, sanctioned both by long custom and also by cultivators, and is probably, in the main, as correct as rosary, or rosarium, is to a garden of roses, or aquarium to a pond in which aquatic plants are grown.

Situation.-The American garden should occupy a place within the enclosed pleasure-grounds, and, like the rosarium, should form a link in the chain of connection which unites the different parts of the grounds between the mansion and kitchen-garden, or other point of attraction, which the proprietor or his friends may wish to visit. As the proper soil for the plants is of so much importance, it may happen in some cases that such a soil may exist naturally in some other part of the grounds; and if such be within a convenient distance, and otherwise eligibly situated, there is no reason why it should not be adopted, in preference to transporting the soil to another situation; and this would be the more reasonable, if the American ground is to be on a large scale. Although many of such plants require a dry soil, as the azalea-particularly during winter and early spring-still most of them, as the rhododendron, are greatly benefited by partial irrigation during the season of making their young wood: the means of obtaining this irrigation should be thought of in selecting the site; and where this cannot be acquired by natural means, then artificial ones must be had recourse to. Many of this class of plants require full exposure to the sun and air, as the azalea; others, like the rhododendron, thrive well under the partial shade of trees; and the more tender of them require shelter from cutting winds and severe frosts, which shelter may be afforded by surrounding plantations. The more common of them may be planted under the shade of trees in shrubberies, and also for forming evergreen underwood and cover for game, for which they are better adapted than any other evergreen plants, with the exception of the holly, on account of their seldom being injured by frost, even when laurels, \&c., are cut down to the ground. Some of them prosper best in the sun, others in the shade-some in dry ground, others in that which is somewhat moist; but none when constantly kept saturated with water. An American garden upon a large scale would be happily situated in a ravine or rocky glen, in which large patches of peaty soil naturally exist; and if this description of situation had both a south and north exposure, and was sheltered from the east and west winds, it would be all that could be desired. The plants of shade, like the rhododendron, would occupy the northern aspect, which would also have the important advantage of preventing too early growth, and hence escaping the disastrous effects of late spring-frosts, particularly as regards the numerous beautiful hybrids claiming a parentage from R. arborea. So far as our present experience goes, it would also be of all others the best situation for the Sikkim species and varieties, as well as for those of Upper India in general. Northern aspects present another advantage as concerns late spring-frosts, for should the plants be exposed to a more than usually low temperature during night, its effects will be gradually removed before the sun's rays can reach them, thus preventing the consequences so often experienced of warm sunny days succeeding cold frosty nights. Another advantage is that vegetation is not so early excited in northern aspects as in southern ones, and hence plants so situated often escape the effects of late spring-frosts, when the same kinds, planted on a southern and earlier aspect, are considerably injured. All plants disposed to flower early in the spring should, therefore, be placed in a northern aspect, for reasons the intelligent cultivator is well aware of. The southern aspect will accommodate the azalea and such sun-loving plants; the deep deposits, the magnolias and other deep- 
rooting trees; while the barely covered rocks, or rocky debris, would be found fit stations for such as prefer a dry soil, and whose roots love to run near the surface. The Ledums and Andromedas, which naturally grow on tussocks of vegetable matter little elevated above the water's level, could be artifically accommodated with dry seats, while their roots could descend into the semi-fluid bog below. A meandering rill of water should run through the bottom of such a ravine, bordered with rocky or grassy banks, along which also the principal walks should be carried, with branch walks of a less important character winding by easy gradients, or occasionally furnished with stone steps, and leading through the groups of shrubs in such lines as would show here and there the whole, or such parts as possess any particular feature, or even individual groups or single specimens of more than ordinary interest. Such we consider would be the beau ideal of an American garden.

The American garden is not unfrequently placed in sheltered glades in woods where the soil is moist, and the dampest part of the pleasure-grounds is equally suitable; and where no regular garden of this kind is attempted, the majority of the trees and shrubs may be planted so as to form the foreground of large shrubberies, or even plantations, so long as the roots of the trees do not interfere with them. The side of a lake, island, or margin of an artificial piece of water, is also well suited for the purpose; while open and exposed situations, and dry arid soils, are the most unfavourable of all. And where the means are of the most limited description, a border behind a northern wall may be adopted.

The American garden can never be regarded as complete, however rich it may be in the genera, species, and varieties of trees and shrubs, if it be wanting in a selection of the most interesting of the herbaceous plants which form the surface covering where such trees and shrubs naturally exist. The various species of American ferns should be planted either in groups by themselves, or scattered through the masses of shrubs, intermixed with the taller-growing of the rarest and most showy of the herbaceous plants with which that country abounds, the edges of the groups being filled with those of a lower growth; while in sheltered corners the following plants, not often met with in such gardens, should be allowed to ramble into natural patches: Cornus canadensis; Trientalis Americana; Galax aphylla; Lophiola aurea; Hypoxis erecta; Aletris farinosa, aurea; Zigadenus glaberrimus, bracteatus ; Uvularia; Smilacina; Narthecium; Xerophyllum; Trillium; Helonias; Gaultheria; Mitchella; Calopogon ; Arethusa; Pogonia; Calypso; Nuttallia; Sabbatia; Houstonia. The species and varieties of Dodecatheon, and the following: Androsace carinata, linearis ; Anemone Hudsoniana, Nuttalliana; Coptis trifolia; Sanguinaria canadensis; Jeffersonia diphylla; Spigelia Marylandica; Pachysandra procumbens; the American Pyrolas; Dielytra formosa, eximia, canadensis; Cypripedium parviflorum, pubescens, spectabile, album, candidum, arietinum; the American Habenarias; Goodyera pubescens, tessellata; Neottia cernua, æstivalis, \&c. And in damper spots, Hydrocotyle Americana, umbellata ; the American Gentians; Parnassia asarifolia, caroliniana; Drosera Americana, linearis ; Tofieldia pubescens, glutinosa, stenopetala; with beds of the fruit-bearing Oxycoccus macrocarpus, erythrocarpus; the singular genus of Sarracenia, \&c. And"in pools formed where a brook of water flows through the ground, or in a small pond, should be found Menyanthes Americana; Villarsia cordata, lacunosa; Pontederia cordata, angustifolia; Alisma lanceolata, parviflora; Saururus lucidus; Caltha biflora,leptosepala, parnassifolia, flabellifolia; Nymphæa odorata; Nuphar advena, Kalmiana,. sagittæfolia, \&c.; with Yucca gloriosa, acuminata, serrulata, glaucescens, recurva, to give an exotic character to the whole.

Soil.-A soil abounding in, or almost entirely composed of, decayed vegetable matter, with a due portion of sand, is a very fit one for American plants in general. Where the natural soil consists of a peat bog, thorough-drainage is the first operation, that superfluous water may be carried off; and as such peaty soils contain only a small amount of sand, that important ingredient must be added, to the extent of from onefourth to one-half, and thoroughly amalgamated. Where the soil is not surcharged with water, drainage is still necessary, and the beds may be slightly elevated towards the centre. Where beds have to be made artificially, the natural soil should be excavated to the depth of 2 feet, excepting for particular trees, such as the magnolia, \&c., which require a greater depth-the bottom loosened up to admit of filtration; and the imported peaty soil, after being dug up for some months, mixed with the due proportion of sand, well exposed to the weather, and frequently turned over, should be brought and laid in the beds. Under other circumstances, when heath mould can be procured-that is, such as our native heaths thrive in, and are found upon-it forms of itself the best of all soils, and should be taken off to the depth of 3 or 4 inches, laid up in heaps, turning the vegetable productions inward, and allowed so to remain for six months, when it may be chopped down, and brought forward to fill the beds, choosing dry weather for the operation. The best description of peat soil is constituted as follows: Of 400 parts, 156 are fine silicious sand, 2 unaltered vegetable fibre, 110 decomposing vegetable matter, 102 silica (or flint), 16 alumina (or clay), 4 oxide of iron, 4 soluble vegetable and saline matter, 4 muriate of lime, 2 loss. Where neither peat soil nor heath mould is readily obtained, then recourse must be had to substitutes; but even then a portion of these primary soils will be found to be of great advantage. The substitutes are, vegetable mould of decayed tree-leaves; tanners' bark, when the vegetable structure is completely broken down by fermentation and decay; and an equivalent of riversand, or pounded sandstone, to lighten and sharpen the whole. In soils so composed, American plants have been grown to great perfection. Annual top-dressings are, however, necessary, as, on account of the natural decay of such matters, 
the rootsare liable to be left bare. These top-dressings should consist of peat earth or heath soil, and these should be slightly forked in amongst the previous formation, always adding a sufficient quantity of sand. Where the masses are large, these substitutes may be indulged in, but where the beds are small, and the plants rare or choice, then the veritable heath-mould should be accorded them. The roots of most American plants are exceedingly numerous and slender, nor do they range far in search of food, unless the soil be very shallow indeed, and resting on rock or gravel subsoil. To economise the peat earth, therefore, and at the same time give the beds the appearance of fulness without injury to the plants, and even probably with advantage to them, we, in planting, set the plants in their places, and cover the roots thoroughly with good peat soil, and fill in the intermediate spaces between them with pretty large stones, laid in as open a manner as possible, working in the finer portions of the soil into the interstices, and covering the whole with soil to the required height of the border. The plants upon which we have tried the experiment have thriven exceedingly; and when their roots have been examined, they have been found in the greatest abundance close to the stolles, and clinging to them on all sides. American plants which have been planted in pits dug out of the natural soil on a lawn immediately adjoining the borders to which we refer, these pits being filled with their natural peat-earth, have in no case succeeded so well. In some cases, however, American plants are found to thrive well in some sorts of gravel, others in deep sands, and many in soils which have been long covered with timber trees-no doubt, in the latter case, on account of the abundance of vegetable matter existing in the soil from the annual deposit formed by the decay of the foliage and other organised matter; but in clay or chalk soils they cannot be made long to exist. The hardier sorts of rhododendrons and azaleas, however, will flourish to great perfection in a soil composed of thoroughly-rotted tanners' bark and the decayed leaves of trees, if a due proportion of sharp river (but not sea) sand be added. Such a compost, particularly if containing much of the first ingredient, will require two or three years of preparation, by being frequently turned over, and well exposed to the sun and air. To render it more porous and capable of admitting air and the escape of superfluous moisture, a portion of clear river-gravel may be added; but in all cases the leaf-mould should considerably exceed in quantity the tanners' bark. Light sandy loam may be added with advantage, if not exceeding one-eighth in quantity.

Propagation of American plants.-One of the most essential points in growing rhododendrons, hardy azaleas, and, we may say, the majority of American plants, is a certain degree of shade and moisture. In the best peatsoils, without these, they do not succeed ; while with them, in even a very indifferent soil, they are often found to prosper well. There is, however, a great difference between plants merely continuing to exist for a few years, and others in the full luxuriance of healthy growth. The latter can only be expected where soil and circumstances are favourable. In planting the early-flowering rhododendrons, it is well to keep them together in one or more groups, because in such cases protection may be given them in spring ; and the best of all protection is thin canvass, such as is employed for wall-tree screens, thrown over them, and secured round the edges to prevent its being blown off. The lightness of the material is such as to require no support, further than to lie on the tops of the plants in ordinary cases. But in cold localities, subject to late spring-frosts, it would be advisable to erect over the groups or beds of the very early flowering sorts a portable framework of iron or wood, and to cover that with canvass of even a stronger fabric, and to be sufficiently elevated that we could walk comfortably underneath-in fact, to construct a tent for their protection. The canvass should be so arranged as to be readily rolled up and down, so that the plants may be exposed to sufficient light and air on all fine days, and only covered during night and cold windy weather. We have no doubt but the majority of even the Indian sorts of rhododendron will yet becultivated in the American garden in this way; for, unless during very intense frosts in winter, and during early spring, they require no further protection in most parts of Britain, as the mean degree of temperature in their native country is somewhere about $55^{\circ}$. The misfortune is, that with us vegetation is excited often early in March, and nearly paralysed in April or beginning of May, in consequence of late frosts, to which they are not exposed in their native land; our summers are also scarcely sufficiently warm to mature the wood and buds fully, and hence they are less prepared to withstand extreme cold in winter.

Amelanchier.-Increased by layers and seed; free-flowering shrubs of moderate growth, enlivening the grounds during April and May with their profusion of white blossoms. Light sandy soil.

Andromeda.-Generally propagated by layers during the month of September, at which season the following yeur they are fit for removal. They are also increased by seed sown as soon as ripe in pans or flat pots, in sandy peaty soil. Their treatment afterwards is the same as the last. They may also, but less certainly, be struck by cuttings. When seed can be procured, it is the best way to procure a large stock. The treatment of seedlings is the same as azaleas.

Azaleas. - All the hardy American species and varieties, as well as the Ghent sorts. The latter originated with the nurserymen of that city, and are seedlings of the American species, speciosa, nudiflora, calendulacea, and viscosa, and are readily propagated by layers in March, twisted or notched at the part buried in the soil. They are seldom rooted sufficiently to be separated from the parent plant until they have made their second year's growth. They are sometimes propagated by cuttings of the young wood, like the Chinese sorts, but rarely, and grafting the finer varieties on the more common is occasionally practised. The majority of the hardy kinds ripen seed, and are extensively multiplied by this means, as well as 
improved varieties produced by a judicious choice of parents. The seed is sown in March or April in shallow pans, in peat soil rendered smooth and even on the surface, and lightly covered on account of its minute size. The seedpans should be placed in cold frames, partially shaded, and when the young plants are an inch in height they should be removed to a pit with a southern exposure, where they should remain till May the following year, when they should be planted in beds of peat soil 4 inches apart in the open quarters, and the spring following planted in nursery lines, to attain a size fit for sale or planting out. Seedlings from choice sorts, or from flowers carefully crossed, should, when an inch in height, be pricked out into a pit, protected in bad weather with glass, in prepared soil, and the spring following planted in nursery-beds as above.

Bryanthus.-A pretty genus, related to Menziesia, propagated by cuttings and layers.

Calycanthus.-Propagated by layers in September, and allowed one year to root; will accommodate itself to almost any light sandy loamy soil in the absence of peat.

Catalpa syringifolia.-The only American species. A splendid flowering tree, which seldom flowers in Scotland, and, indeed, rarely in the south of England-a consequence, in both cases, of deficiency of heat during our summers to ripen the wood; for on the Continent it flowers abundantly, even in many parts of Germany, where the winter is much more severe than in Britain, but on the other hand the summer is so much more warm. It is propagated by layers and by imported seed.

Chionanthus.-Propagated by layers and im. ported seed.

Cratogus.-A number of this genus are natives of America, and although not directly requiring a peat soil, are admissible into such a garden on account of their abundance of white blossom during May and June, forming a striking contrast with varied shades of azalea and rhododendron, as well as attaining a much greater height. Propagated by budding and grafting, and also by seed, which requires two years to vegetate.

Dirca, of which there is only one species (palustris), a plant difficult to increase otherwise than by seed, which is seldom, if ever, produced in Britain ; dependence, therefore, must be had on an American supply. Layering in autumn is the means usually employed in our best nurseries, and a sandy peat-soil, either naturally damp, or rendered so by artificial means. It takes two years to secure well-rooted plants, and these, when planted out, should occupy a very humid place, the plant growing naturally in moist places, and even deriving its name from Dirke, a fountain.

Empetrum.-The species rubrum, of American origin, is propagated by cuttings under a handlight, put in during summer in sandy peaty soil. Seeds are rarely produced in Britain, and those imported should be sown as soon as received in sandy peat, remaining generally a year before vegetating. It succeeds best in a humid soil, and forms a good edging round taller shrubs.

Epigac.-Propagated chiefly by layers in a sheltered place, kept pretty dry during winter, and rather moist during summer. Being a low creeping plant, it succeeds best under the protection of others, being rather tender.

Ephedra.-Propagated by cuttings, and sometimes by layers and division of the plant. A genus more curious than pretty.

Ericas and Menziesias, although not American plants, are quite admissible into the American garden, on account of their requiring a peaty soil. All the hardy species and varieties are readily propagated by cuttings, planted in sand under bell or hand glasses in a partially shadea situation. The species carnea, herbacea, and such as grow close to the ground, root freely if sandy peat soil be laid over their prostrate branches. When rooted, in either case they should be planted out in nursery-beds in peat soil until sufficiently large for final transplantation. They form excellent groups by themselves, or margins to larger clumps.

Fothergilla.-Readily increased by layers put down in March or in August. They sometimes ripen seed in Britain, and seed can be had from America through such houses as Charlwood \& Cumming, of Tavistock Row, Covent Garden, where, indeed, seeds of most American trees and shrubs can be obtained. They should be sown in spring on a rather shaded border of peat soil, or in pans, and afterwards transplanted into nursery-lines. They rank amongst the few sweetscented shrubs of America, producing their abundant white flowers before the appearance of the leaves.

Galax.-By division of the plant in spring. A pretty evergreen plant of low growth.

Gaultheria.-Propagated by layers and also by seed, the former laid in spring or in autumn, and the seed sown at the former period. The fruit of G. shallon is excellent, and highly deserving the attention of those who are curious in the possession of exotic or little-known fruits. Differing from the rest of the family, this species will grow in almost any soil and situationa moist one, however, is the best. The berries are so much prized by the Americans, that in many parts they bake them into a sort of bread for winter use. They make excellent jellies, and afford a pleasant addition to our desserts.

Gymnocladus.-In consequence of the young shoots being without buds-whence the name Gymnos (naked), and Klados (a branch)-it does not strike root by cuttings of the branches, but does so by those of the roots, cut into pieces about 9 or 10 inches in length, and set in light loam, keeping the top part of the roots uppermost. This is the mode usually followed by the English nurserymen. Seeds are, however, readily obtained from America, and if sown in spring, or as early in the season as they arrive, in general vegetate freely in the temperature of a cool pit. A very elegant tree during summer, and in winter producing a singular effect from its deadlike branches. It should stand singly on the grass, or in any open part of the garden.

Halesia.-Propagated by seed soon in March, and by layers and cuttings of the roots in spring and in autumn. A soil not exactly peaty, if deep and sandy, will suit it exceedingly. 
Hudsonia.-Nearly related to Erica, and requiring the same treatment and situation in the garden.

Kalmia.-This beautiful dwarf flowering tribe is propagated by cuttings of the young wood early in summer, set in silvery sand or very sandy peat, placed in a partially-shaded place, and under the protection of a hand-glass. They also root by layers put down in August or September, but from the very brittle nature of the wood great care is required that the branches be not broken during the operation. The best way, however, is by seed, which often ripens in Britain, and can be readily procured from America. It should be sown in March in sandy peat, and in pans placed in a close frame or pit, until the young plants are up. As soon as they are fit to handle, they should be pricked out into a pit quite close to the glass, and kept close till they have taken with the soil, when air must be gradually admitted to harden them off for standing the sun and air without further protection. The following spring they will be fit for planting out into nursery-beds. The Kalmias are amongst the finest of all American flowering-shrubs, and the most beautiful of the whole is Latifolia, which, however, does not often flower in Scotland.

Laurus.-The hardy deciduous species of this genus are natives of North America and Carolina, and stand the climate of the south of England in most places, not so generally in the north. We have, however, seen some splendid specimens in the American grounds of the south of England. They are propagated by cuttings placed under hand-glasses in the end of summer, also by layers and by cuttings of the roots in spring or autumn. Seed is, however, the best way, and as this is, we suppose, never matured in Britain, dependence must be placed on procuring a supply from America. The seeds, like many other hard-coated ones, require to lie one year in the ground before vegetation takes place. The amateur may, therefore, bury the seed of this and similar plants in a flower-pot mixed with soil in a place where he is sure to find it, and, if possible, in one where vermin will not destroy it.

Ledum.-Is propagated in the same way as Kalmia (which see). They are dwarf-growing shrubs, and with Kalmias, Gaultheria shallon, form fine fringes for groups of azaleas, rhododendrons, and similar taller shrubs.

Lyonia.-A genus nearly related to Andromeda, requiring the same mode of treatment (which see), but rather a moister soil, particularly when planted out for good.

Maclura.-The species aurantiaca is hardy enough for a wall; propagated by cuttings of the roots set in a bottom-heat, and also by layers. Seeds may be abundantly procured from America, where in some parts it is cultivated for hedges, and also for its fruit.

Magnolia.-The most magnificent of all the North American flora. Propagated by imported seed, which is readily procured from America, or from France, where most of the species ripen their seed freely, but seldom in Britain. The seed should be sown in pans placed in a mild bottom-heat in March. They do not vegetate simultaneously, but as they do, they should be carefully taken up and potted singly into 48-sized pots, and placed in a cool pit, and protected during winter, and potted as they require it, until they have attained a size to be planted out, which will be in three or four years, according to the habits of the species. Objections are made by some against plants reared from seed, alleging that they do not flower so soon as those propagated by other means. This is, no doubt, so far true, but, on the other hand, such plants make much better specimens, and continue longer in health and vigour. They are also propagated by layers; indeed, this is the usual way in British nurseries. Layering should be done in September, and in two years they will be rooted sufficiently to be taken off and potted; and then they should bekept in a cool pit till well established. They are found to transplant better from pots than if they were taken from the open ground; but, on the other hand, this dread of injuring the roots is often attended with an equally bad consequence, for if the roots are confined to the narrow limits of a pot of 7 or 9 inches in diameter, sizes seldom exceeded, they become coiled in such a manner that without great care in separating them at planting they will be in the state we have described, p. 370, and illustrated, figs. 130, 131 . Therefore, upon the whole, after they have established themselves in pots for a few months, after being separated from the stool, it is better to plant them out in the nursery, and take the necessary precautions at lifting and packing that will insure success in removal. The stronger growers, like macrophylla and tripetala, are difficult to lay on account of the brittleness of their shoots; these should undoubtedly be originated from seed. Budding, inarching, and grafting, are employed in the case of the weakergrowing species and stronger-growing sorts, such as acuminata, obovata, \&c., are used for stocks; and even in this way a union does not soon take place, often requiring from eighteen months to two years, and after all they do not make such lasting plants as those reared from seed or by layers, although they bloom sooner. The hardy American sorts are glauca and its varieties, longifolia, tripetala, acuminata, auriculata, macrophylla, maxima, cordata. Grandiflora and its varieties, being natives so far south as Carolina, stand only as open standards in the south of England, but to flower them well in most parts of Scotland they require to be planted against a well-exposed wall, with slight protection during winter. A quite dry, deep, sandy, or peaty soil, if we except glauca and its varieties, is the most suitable, the latter requiring peat and a moderate degree of moisture, particularly during its growing season.

Mahonia.-Nearly related to Berberis. The American species are all readily propagated by seed, which in most cases is produced in Britain. The time of sowing is March, in sandy soil, in a cool pit or border. Most of them root freely by cuttings put in in September, and some produce suckers from which good plants may be obtained. Like the Kalmia, \&c., they are of dwarfish 
growth, and form good foregrounds to groups of higher-growing plants. They also form good underwood in plantations, and as such are planted by game preservers. The American species are nervosa, aquifolia, and repens. As the remainder of the genus prefer a peaty soil, they are quite admissible into this description of garden, although not exactly natives of America. They are all propagated by the same means.

Nyssa.-Best propagated by importing seed from America. They are sometimes laid in the British nurseries, but with less success. They are natives of low damp moist situations, therefore a similar site should be chosen for them in cultivation. Their flowers are not conspicuous, but as their foliage assumes a deep scarlet in autumn, they give great enlivenment to the grounds by the brilliancy of their colour.

Prinos.-Propagated by seeds and layers. Does not require an absolute peat soil.

Purshia.-By cuttings of the young wood in sand, under a bell-glass, and also by seed, which often requires two years to vegetate.

Rhus.-The North American species, although deciduous, are nevertheless exceeding ornamental, and attain a considerable size, and are therefore proper for the centre of groups in the American garden. They are propagated by seed, which is usually imported, by layers, and by cuttings of both roots and shoots; will prosper in a soil if light, although not exactly peaty.

Robinia.-The American species are chiefly propagated by seed, either sown in autumn or spring, being preserved during winter in the pods. R. hispida, the well-known Rose-Acacia, being from Carolina, is less hardy than some others. It, however, stands at Dalkeith as an open dwarf standard without any protection. On account of the brittleness of its branches it is very liable to be broken by winds, if not trained to a wall, espalier, or other support. It is also usually grafted on the Pseudo-Acacia at various heights from the ground. In cold situations it deserves a place on a conservative wall. Some of the others are increased by cuttings of the young wood, others by cuttings of the roots, and several by suckers which arise from the roots. The curled-leared varieties of PseudoAcacia, pendula and tortuosa, grafted 4 or 6 feet from the ground, form fine bushy heads, and are largely planted in gardens laid out in the formal style.

Rhododendron.-When propagation is to be carried on upon a small scale, then the seed, which is a better method of proceeding although less expeditious than laying, should be sown in March in flat pans, and placed in a cool rather shaded frame, where they should remain until the plants are fit for pricking out, which they should be as soon as they are fit to handle-say a quarter of an inch in height; the soil used being sandy peat, the pans well drained, and the soil not over fine until within an inch of the surface, where it should be as fine as possible; and when rendered quite smooth, the seed should be sown thinly, and merely covered with a little silvery sand, or finely-sifted sandy peat, to the depth of little more than one-sixteenth of an inch. To prevent the seed being displaced or uncovered during the process of watering, a little soft moss should be laid over the pots, which will counteract excessive evaporation until the plants appear. When upon a large scale, a frame should be placed in some partially shaded place-that is to say, fully shaded from the meridian sun-a course of drainage laid in the bottom; and indeed if the frame is elevated the height of a brick above the surface, so much the better. Over the drainage a foot of sandy peat, broken up by chopping it with the spade but not sifted, should be laid, and firmly trodden down and levelled on the surface. On this $1 \frac{1}{2}$ inches of finely-sifted sandy peat should be laid, and rendered as smooth on the surface as possible, on which the seed should be sown thinly. If various kinds are to be sown, the surface should be divided off in spaces according to the number of sorts and quantity of each, leaving 2 inches between each space to prevent intermixture. When each sort is sown they should be carefully labelled and covered to the depth stated above, and a thin sprinkling of moss laid over all. The whole should then be well watered, the glass lights put on, and the whole kept close until the seeds begin to vegetate, at which time air should be gradually admitted and increased as the plants appear, when the moss should be removed. As soon as the plants are from a quarter to half an inch high, they should be pricked out into other beds similarly prepared, choosing dull cloudy weather for the operation. They should be covered closely and moderately shaded until they have taken root and begun to grow, when air should be more abundantly admitted, removing the glasses entirely during fine genial showers, and hardening the plants by progressive exposure, until they will stand the weather without protection. During winter, however, they should be protected by the glass sashes, and towards the month of May following they will be fit for planting out, with little balls of soil at their roots, into nursery-beds in the open ground, but at the same time in prepared sandy peat soil. The propagation of rhododendrons from seed can hardly be too much insisted upon, seeing that they form by far the most valuable of our evergreens, if we except the holly, even for underwood and cover, while they surpass even that fine plant in consequence of their abundant and gorgeous bloom. It is of little use in these days to breed from the old common sorts, seeing we have so many very superior which seed as freely. Of the old species, the true Catawbiense is the only one we would propagate from. As Scarlets and Whites are so much better for the purpose of display than the ordinary colours, we would increase them largely; and to insure something like certainty in the result, attention should be paid to the plants the seed is taken from. In hybridising, regard should be had to increasing the constitutional hardiness of the stock, as well as obtaining mere improvement in form and colour without that merit; and varieties that will flower late in the spring should be sought rather than such as flower early, as these are so liable to be destroyed by frost.

Approved varieties may be planted out as 
stools in the nursery ground, and the young shoots laid in March or in September. This mode will continue the identical variety without change, but unless there are many stools the stock procured will be limited in number. They are also capable of being increased by cuttings, and for this purpose beds should be prepared as recommended for seed, only double the depth of soil ; and instead of the thin stratum of sand there advised, in the present case it should be from 3 to 5 inches deep, according to the length of cuttings to be used. Young wood should be chosen when it has begun to get firm towards its base; it should be cut across immediately below a joint or leaf, the leaves only removed as far as the cutting is to be inserted, leaving those above perfectly entire. Insert the cuttings pretty thickly, and to the depth of 3 or 4 inches; cover them with a frame and sashes, keeping them pretty close and well shaded, so that the leaves may not fall off or become shrivelled. In a few weeks callosities will form at their bases, and from these the roots will issue, at which time more air must be given, and progressively increased afterwards. Some cultivators set their cuttings in this way, and when they have formed their callosities take them up, having previously prepared a bed upon bottom-heat, and in this insert the cuttings, which, in consequence of the heat below, make roots rapidly. By the former process the operation is less speedily effected, but it is equally certain. Where the number to be struck is small, it is a good method to place the cuttings in pots placed in a cool frame till the callosity is formed, and afterwards to remove them to another frame, where they can be plunged in a mild bottom-heat without disturbing them. Such would be the plan for amateurs to adopt. Grafting, budding, and inarching are adopted in the case of new or rare sorts, the operations being conducted under glass. It is of great importance in such cases that the stock be strong, healthy, and if a vigorous-growing variety so much the better. New seedlings, which might be several years before they flowered naturally, have been made to do so in one year by being grafted on stocks with very long stems. This, which we think an important discovery, was pointed out to us lately by Mr Laing of Dysart House as the means he used in flowering R. Dalhousii, which he has had the good fortune to do before any one else. The stock on which he grafted a shoot of the plant in question is about 7 or 8 feet in length, the pot in which it is growing sunk considerably under the floor of the house and brought up through the stage, and still the top of the plant, with its fine head of bloom, is considerably above the other plants in the house.

Rhodora.-A pretty flowering dwarf shrub, propagated by layers and cuttings, and sometimes by imported seed; forms an excellent foreground to larger masses.

Shepinerdia.-Propagated by imported seed, but generally by suckers which rise from the roots.

Stuartia. -Is, for the most part, increased by layers, and prefers a rather moist peaty soil.
Styrax, of which there are three American species, requires the protection of a wall to bloom it profusely. Propagation is effected by cut tings and layers, and also by imported seed. They also require a pretty deep, dry, sandy, or peaty soil.

Vaccinium.-The great majority of the genus are natives of America, many of them producing pretty flowers, and the greater part fruit, some of which is of considerable merit. They form, in the American garden, a good foreground to shrubs of higher growth. Are all propagated by seed sown in September, and by cuttings during summer, placed in sandy peat, under a handglass. Most of the creeping sorts root freely in the ground, and may be separated, and treated as distinct plants.

Yucca.-The American species, as well as those from other countries, are increased by suckers, which sometimes arise from the roots, and by young shoots which branch from the stems. The best mode, however, is to bare the roots carefully, and remove the knotty protuberances, or underground knars, which are found on the stem underground, and also on the larger roots. These, if removed carefully, and potted, and set in a mild bottom-heat, produce roots, and afterwards leaves and stems, and in time perfect plants. The older the plant, the more numerous these knars are. Seed is rarely produced in Britain, but is occasionally inıported from America and France, in which latter country they seed freely. Although the Yuccas thrive well in a deep peaty soil, they, nevertheless, especially Y. gloriosa, do so in an extraordinary degree when grown in strong deep clay. They produce the most imposing effect when planted singly on the open lawn, or in groups, the taller species occupying the centre.

\section{CLIMBING PLANTS SUITED TO THE AMERICAN GARDEN}

Ampelopsis.-Increased by layers and cuttings in spring.

Aristolochia.--Sipho, tomentosa, and Arkansa, are propagated, like the last, by layers in spring, and both will thrive in a soil not directly peat; and to increase their vigour of growth, which is, in general an object with most climbing plants, enrichment should be accorded them.

Atragene, americana. ? Propagated by layers in Bignonia capreolata. $\}$ spring, laid in pots.

Clematis virginiana, Viorna, cylindrica, Simsii, reticulata, ochroleuca, and crispa, ditto.

Caprifolium dioicum, sempervirens, gratum, flavum, pubescens, ciliosum. - Propagated by cuttings and layers, in March, set in sandy loam.

Lonicera ciliata, oblongifolia.-Propagated by layers and cuttings in March, set in sandy loam.

Menispermum.-By layers and cuttings in sandy loam.

Smilax.-Those of America are propagated by cuttings, layers, and many of them by suckers rising from the roots.

Tecoma radicans.-Propagated by layers and single eyes, like the vine; the former laid in March, in sandy peaty soil, the latter cut into 
lengths of 2 inches each, having an eye or bud. A thin slice of the wood on the under side being removed, the cutting is then set in the soil, having the point only of its bud above the surface. The buds are best planted in single pots, plunged in a mild bottom-heat, when they will root freely.

Vitis, or American vine, of which there are several species, is propagated by layers, cuttings, and buds, similar to the common grapevine. Climbing plants are best introduced into the American garden trained to poles, and sometimes festooned between them-for covering walls where they exist, trained over arches forming arcades by the sides of main walks, or for entirely covering berceaux or covered walks, \&c.

\section{SELECT LIST OF HARDY RHODODENDRONS.}

Rhododendron albo-chloranthum, album elegans, album grandiflorum, alta-clarense, alta-clarense elegans, alta-clarense superbum, amœnum, Alexandrina, Ambrosii, Antagonist, Antonio, aureum, Barber of Seville, Blandyanum, Bouquet de Flore, Broughtonii, campanulatum, captivation, Caucasicum pictum, Cerito, cinnamomeum, Cliveanum, Clowesianum, coccineum, conpactum, constellation, cyanum, compeer, Dauricum, delicatum, delicatissimum, elegans, erectum, Etoile de Flandre, fastuosum, ferrugineum, Gibsonii, giganteum, Gloria Gandavensis, Grisewoodianum, guttatum, hirsutum, Hartley Luttrell, Holfordianum, hyacinthiflorum, illuminator, Ingomar, Ivanhoe, Lowii, Lucifer, macranthum, maculatum, Martianum, Maid of Saragossa, Madame Sontag, metaphor, meteor, metropolitanum, Menziesii, Miss Glyn, Mrs Hemans, multinaculatum, Nell Gwynne, nivale, nivaticum, Nobleanum bicolor, Norma, oculatum, Pamela, pardoloton, Paxtonii, perspicuum, pictum, picturatum, Ponticum, Portia, prœcox, pulchellum, Queen Victoria, Raeanum, Reedianum, reginum, robustum, Rollisonii, Rosalie, roseum argentinum, Russellianum, Russellianum superbum, Sabrina, Sir Isaac Newton, Sir John Franklin, Sir Walter Scott, Sappho, Smithii, Smithii elegans, speculator, Standishii, The Cid, The Fair Carew, The Gem, Titania, Torlonianum, Towardii, varium, varium blandum, varium decorum, varium guttatum, Verschaffeltii, vervæneanum, Vesuvius, Venus, venustum, vivid, Zuleika.

Yellow rhododendrons. - Aureum superbum, decora, luteum superbum, Victoria Regina.

\section{SELECT LIST OF HARDY AZALEAS.}

Adelaide, Admiral de Ruyter, Alba flavescens rosea, amabilis, amœnissima, ardens, atro rubens nana, aurantia major, aurora, Calendulacea, Calendulacea crocea, Calendulacea elegans, Calendulacea grandiflora, Calendulacea incarnata, Calendulacea insignis, Calendulacea mirabilis, Calendulacea pulcherrima, Calendulacea splendens, Calendulacea venusta, capitata incarnata, carnea, cramoisie, coccinea major, coccinea speciosa, cuprea, cuprea splendens, Cymodocée, decus hortorum, Florentina, fulgens, General Chassée, gloria mundi, Glory of Verschaffelt, Grand-Duke of LuxVOL. II. emburg, helvola, imperatrix, Julius Cæsar, macrophylla, Marie Dorothée, Minerva, mixta triumphans, Morterii, multiflora pallida, ne plus ultra, nobilissima, nudiflora bicolor, nudiflora Coburghii, nudiflora double pink, nudiflora grandiflora, nudiflora magnifica, nudiflora variegata, nymphæa, optima, Oscar the First, Pearl of Spring, pontica, pontica alba, pontica amabilis, pontica blanda, pontica double white, pontica eximia, pontica fulgens, pontica globosa, pontica grandiflora, pontica magnifica, pontica mirabilis, pontica princeps, pontica tricolor, præstantissima, Prince Henry, Princess Augusta, Princess Marianne, Princess of Orange, pulchella, punicea, quadricolor, Queen of England, Regina Belgica, Rosalie, rosea flava, Rubens, rubra fulgens, sanguinea, Souvenir de Mortier, speciosa, speciosa aurantia, speciosa major, speciosissima, splendens, Sully, superba, Triomphe de Royghen, triumphans, triumphans gandavensis, triumphans serotina, unique, Van Dyck, venusta, venusta rosea, venustissima, Victoria, viscosa, viscosa double white, viscosa late red, viscosa pubescens, viscosa stricta, vittata.

\section{SELECT LIST OF ANDROMEDAS.}

Floribunda, Rollisonii, phillyrefolia, grandiflora, revoluta, calyculata, mariana, speciosa, polifolia and its varieties, coriacea, acuminata, Catesbæi, racemosa, arborea, dealbata, axillaris, cassinifolia, pulverulenta. Tetragona and hypnoides are rather rare, of very diminutive growth, and fitted for growing on rock-work in a sheltered and rather shaded situation.

\section{SELECT LIST OF MAGNOLIAS.}

Glauca, with its varieties Thomsoniana, Gordoniana, and Burchelliana, requiring a damp soil ; acuminata, maxima, cordata, longifolia, auriculata, pyramidata, conspicua, Hammondii, macrophylla, tripetala, obovata, perfectly hardy, and fitted for single specimens on the lawn. Grandiflora and its varieties are rather tender, and for most of Britain requiring the protection of a wall, which they richly deserve.

\section{SELECT LIST OF KALMIAS.}

Latifolia, angustifolia, of which there are eight varieties in nur'sery collections; glauca, cuneata, rosmarinifolia, and hirsuta.

\section{SELECT LIST OF VACCINIUMS.}

Arctostaphyllos, Madeirense, the first a native of Caucasus, and the second of Madeira, yet sufficiently hardy to stand the open air in most parts of Britain; ovatum and tenellum, along with Madeirense, worth cultivating for their fruit; diffusum, attaining the size of a moderate-sized tree, exceedingly ornamental when planted as a standard on grass; dumosum, venustum, frondosum, stamineum album, ligustrinum, mucronatum, resinosum, corymbosum, amœnum, marianum, grandiflorum, fuscatum, salicinum, prunifolium. This much neglected tribe of plants is exceedingly ornamental when planted either in groups by themselves, or made the foreground of larger masses.

$$
\text { ๖ } \mathrm{C}
$$


These, with the exception of a portion of Conifers, and the genera Juglans, Carya, and a section of Quercus, form the chief American trees and shrubs. The majority of the others have been already referred to. It is, however, only in the milder parts of Britain that the oaks, hickories, and walnuts of America ripen their wood sufficiently-a circumstance the more to be regretted, as their whole character during summer, and the beautiful autumral tints produced by them, render them amongst the most ornamental of decorative trees. The liquidamber, (Platanus occidentalis), and tulip tree, the American rusty-leaved beech (Fagus ferruginea), are also highly ornamental, and form fine specimens when planted on lawns, or as leading features in producing effect in the American garden scenery. These are also less dependent on a peaty soil, and will prosper in most ordinary sandy loams. The hardier species should form the boundary of the American garden, and afford shelter to those of more delicate growth. The majority of American shrubs can only be grown to perfection when grouped by themselves, and hence planting them promiscuously amongst other plants in shrubberies is one of the principal causes of that total want of success and effect so frequently met with in all cases where such an arrangement is followed.

\section{§ 2.-ROSARY.}

Many ages ago Anacreon sung the praises of the rose. He calls it "the most beautiful of flowers," "the delight of the gods," "the favourite of the Muses ;" and since that time it has not inaptly been denominated the Queen of flowers. Two thousand years ago Sappho wrote, " If Jupiter wished to give to the flowers a queen, the rose would be that queen." It is frequently spoken of in Holy Writ; and Homer uses the rose figuratively both in the Iliad and Odyssey. Yet while the rose was the most popular of all flowers both amongst the ancient Greeks and Romans, the time and the means employed to install it an inhabitant of the garden remain perfectly unknown. In regard to its natural geographical distribution, it may be said to be confined to the northern hemisphere, none having yet been found wild very near to or south of the equator. It was long thought that America did not possess a rose naturally,- such, however, is not the fact; but the rast continent of Australia, rich in botanical treasures as it is, has not as yet revealed to us a single species. Siberia, Iceland, Greenland, and Kamtschatka have their roses; and one of those indigenous to Britain (Rosa spinosissima) is the type from which two or three hundred varieties, under the denomination of Scotch roses, have sprung. Warmer climates, however, have afforded us a much finer class, as Chiua, Persia, India, \&c. ; and the art of man has from such materials as the above created those innumerable varieties, which only a reference to the catalogues of such eminent cultivators as Rivers, Paul, Wood, \&c., can give us a correct idea of. The cultural history of even our most common roses is so little generally known that the following brief remarks may be at least interesting :-

The Provence or Cabbage rose (Rosa centifolia), although supposed to be the hundred-leaved rose of Pliny, was only introduced to British gardens in 1596. Mount Caucasus, Languedoc, and Provence, all claim it as their own. Many fine hybrid varieties have been produced both by the French and English growers between this and Rosa gallica, and are known under the general name of Provence roses.

The Unique Provence is supposed to be of English origin, having been observed for the first time in 1777 growing in a cottage garden in Suffolk ; supposed, however, not to be a seedling of the old Provence rose, which, on account of its plenitude of petals, is incapable of producing seed in our climate. The supposition is, that the shoot which produced it had departed from the character of the original plant by one of those unaccountable deviations which sometimes take place in plants-one branch, shoot, or sucker, producing striped or variegated flowers, while the original remains self-coloured. This section contains some of the finest globular roses in cultivation, and most of them are also exceedingly fragrant. To grow them in the highest state of perfection, they require a rich soil, and to be pruned very freely. There is a sub-section which includes the miniature Provence, or Pompon roses, admirably adapted for forming edgings, as elsewhere noticed. They are of dwarf growth, producing flowers in great abundance. Rose de Meaux may be taken as an example of this sub-section.

The moss rose (Rosa centifolia muscosa). - The history of this rose is somewhat obscure; it is most probably an accidental sport from the common Provence rose. The earliest notice we have of the moss rose is, that it was imported from Holland in 1596. Of its origin we have met with no satisfactory account. Mr Rivers throws out the following conjectures: "The origin of the old double moss-rose, like that of the old double yellow rose (Rosa sulphurea), is, therefore, left to conjecture ; for gardeners, in those days, did not publish to the world the result of their operations and discoveries. As regards the moss rose, I regret this, for it would be very interesting to know how and where this general favourite originated. Probably when first noticed gardening was of such small consideration, that the discovery of a rose, however rernarkable, would not be thought worth registering. That it is merely an accidental sport of the common Provence rose, is strengthened by the fact, that plants produced by the seed of the moss rose do not always show moss; perhaps not more than two plants out of three. Those that are not so, are most evidently pure Provence roses, possessing all their characters. To show also the singular propensity of the varieties of Rosa centifolia to vary, I may here mention that the common moss-rose often produces shoots entirely destitute of moss. In the summer of 1836 I also observed a luxuriant branch of the crimson or damask, which is generally more mossy than the old moss-rose, having a remarkable appearance. On examination I 
found it nearly smooth. The next season it had entirely lost its moss, and had produced semidouble flowers, the exact resemblance of the scarlet Provence. The white moss is another instance of this singular quality, for that originated from a sporting branch; the Mossy de Meaux is also a curious deviation." This pretty little moss was found in a garden at Taunton in 1814 , and was in all possibility a sport from the old Rose de Meaux, and not from seed, as it does not produce seed in this country. The white moss, and the crested moss (Rosa crestata), are evidently sports in the same way ; the latter being accidentally found growing out of an old wall at Friburg in Switzerland. The crimson damask moss originated accidentally in a garden at Tinwell in Rutlandshire, and was long cultivated under the name of the Tinwell moss. The miniature moss originated with $\mathrm{Mr}$ Rivers in his endeavour's to raise a superior variety of the single moss-rose. The white Bath moss is the result of a sporting branch of the common moss; and the Moss Unique de Provence is probably a sport from the old Rose unique. Many of the other mosses have been raised from seed in France, particularly in the Luxembourg gardens, and their origin pretty clearly identified. A most extensive family of roses, and from the great number of new hyblids recently added, become a very varied class, many of them partaking largely of the habits of the French and hybrid Chinas, and, therefore, well adapted for growing as pillar roses. Like the Provence, they require high cultivation, their beauty amply repaying the attention accorded them. Some of the section are remarkable for their vigour of growth; these should be pruned very little, removing the weaker shoots, and bending down the strongest, which will induce an abundance of bloom. The moderate and weaker-growing varieties require to be pruned close in. They are very different in constitution: some are of very delicate growth, and will only flourish in a kindly suil; others are very hardy, but all of them delight in a rich soil, and require high cultivation.

The French rose (Rosa gallica).-Indigenous to the hedges of France and Italy. Supposed to be the Rosa Millesiana of Pliny, and amongst the earliest introduced to our gardens. This section contains a large amount of our variegated varieties; indeed, from Rosa gallica versicolor (our Rosa Mundi) all our variegated roses of this section have sprung. This is the most extensive family of roses at present known, and almost unequalled for perfection of form and richness of colour. Mr Wood observes of them : "To the amateur and those who cultivate for exhibition they are altogether indispensable. Being of robust growth and erect habit, they are very suitable for standards in situations where formality of style is preserved. Dwarf plants are well adapted for beds: they require liberal cultivation, and to be pruned rather freely."

Hybrid Provence rose (Rosa centifolia hybrida). -This fine division of roses are hybrids between the French and Provence roses. Nearly all the varieties produce large, well formed, and very fragrant flowers. Some of them are vigorous growers, and are therefore well adapted for growing as pillar or pole roses. They are of easy culture, and require to be moderately pruned.

Hybrid China rose (Rosa indica hybrida).-This section owes its origin to the Bourbon, China, and Tea-scented Noisette, crossed with the French, Provence, and other summer roses, and also to the latter crossed with the former. The varieties first obtained by this crossing arose from accident. Of late years, however, the process has been carried on upon more certain and correct principles. $\mathrm{Mr}$ Kivers, in speaking of these hybrids, remarks : "They give a long continuance of bloom, but they never put forth secondary or autumnal flowers. This is a most peculiarly distinguishing trait, and an interesting fact. Impregnate a Bourbon, China, or Noisette rose, all abundant autumnal bloomers, with the farina of a French or Provence rose, and you entirely take away the tendency of autumnal blooming in their offspring." The plants of this section are of vely vigorous habit, and make magnificent objects when trained as pillar or pole roses. This vigour must be counteracted, when they are grown as standards, by training the shoots downwards in shape of an inverted cone. In pruning, they should be well thinned out, shortening the flowering-shoots, however, but little. Many of the flowers in this section combine all the properties desired in the rose-viz., size, form, fulness, and exquisite colouring.

Hybrid Bourbon (Rosa Bourboniana hybrida). -This very splendid family of roses owes its origin to the Bourbon rose, which is itself a hybrid. Coupe d'Hébé may be given as a specimen of this family, which for the disposition and regularity of its petals is quite unique. They differ from the hybrid Chinas in the greater substance of their flowers and foliage, but, like them, are remarkable for the abundance and beauty of their flowers. They require much the same treatment, but require to be more closely pruned in.

White rose (Rosa alba).-The original species is a native of the central parts of Europe, and was introduced to Britain in 1597-a small but very beautiful section, of which the Celestial rose may be regarded as the type. They are, however, not all white flowered, some being blush, flesh, and pink coloured. Readily distinguished from all other roses by the glaucous appearance of the foliage, which appear's as if covered with a greyish impalpable powder, and the shoots being almost spineless. They are hardy and of easy cultivation, and being for the most part of mo. derate growth, should be pruned pretty closely in.

The Damask rose (Rosa Damascena).-The original single-flowered species is a native of Syria, introduced into Europe in 1573. It is now, as formerly, largely cultivated in the gar: dens of Damascus, where, as is well known, it is cultivated for the purpose of making rose-water, attar of roses, \&c. If Sanuto, a Venetian author, is deserving of credit, no less than five hundred camel-loads of this precious water were employed by Saladin, after the taking of Jerusalem from the Crusaders, to purify the walls of the Mosque of Omer, which had been used as a Christian 
church. Nearly all the perpetual roses now so much sought after owe their origin to the older members of this family, which may readily be distinguished from other roses by their rough spiny shoots and leathery-like leaves. They are all of robust habits, and have large wellformed flowers. Although they are robust growers, they do not require very hard pruning. The name is derived from Damascus, its place of growth, and not from Damask, a red rose, as very generally understood, the majority of the family producing light-coloured flowers, and not red.

The Scotch rose (Rosa spinosissima) derives its origin from the dwarf wild rose indigenous to many parts of Scotland and the north of England. The majority of them originated in the Perth and Glasgow nurseries about the beginning of the present century. They are very undeservedly neglected nowadays; and although the varieties at one time known to exist in cultivation exceeded three hundred sorts, it has been with great difficulty and research that we have been able to bring together above half that number, which are, at the moment these lines are writing, in the full perfection of bloom. Their place of growth in cultivation is on elevated banks forming the margin of the rosary, in groups by themselves amongst other sections, and as standards they have a very pretty effect. They require very little pruning, and will thrive in a poor sandy soil where other roses will not. They are early bloomers, exceedingly fragrant, the flowers globular, small, and double.

Sweet briar (Rosa rubiginosa). - Indigenous to many parts of Britain in its native or single state. Art has added several greatly improved varieties, such as Rose angle sweet briar, with large and very double bright rose-coloured flowers; the Splendid sweet briar, with large crimson flowers, but less fragrant than the common; Celestial sweet briar, flowers very double and fragrant; Carmine sweet briar, with semi-double bright red flowers, \&c.

Austrian briar (Rosa lutea).-A native of the north of Italy. The Persian yellow rose belongs to this section; introduced in 1838; a beautiful yellow double flower with cupped petals. We find the plant a weakly grower even when budded on the Dog rose. None of the section will live in a smoky atmosphere. Mr Rivers asserts that the copper or red Austrian will not put forth a single bloom within ten or twelve miles of London. Rosa Harrisonii, an American double yellow rose, is one of the most beautiful of the yellows, but blooms best in a situation partially shaded from the mid-day sun. Rivers' globe yellow is of Italian origin, delicate in growth, but of great beauty. All this section require a moist and rather poor soil, and moderate pruning. Rosa Harrisonii and the Persian yellow are the two best yellow roses in cultivation.

Double yellow rose (Rosa sulphurea).-This splendid rose has baftled cultural skill to flower it well or with certainty. All admit that it should be planted against a wall in a warm situation, otherwise the flowers will not expand, although they are often enough produced in considerable abun lance, but generally in a malformed state. It is, no doubt, a native of a country possessing a warm summer-some say Syria or the Levant, and that it was introduced to our gardens in 1629 first by Nicholas Lete, though the plants of that introduction did not long survive; afterwards by Jean de Franqueville, who, as well as the former, was a great promoter of horticulture; and being both engaged in mercantile transactions with the Levant, they had the means, and employed them, of introducing many new plants. Rosa sulphurea, in its native state, is probably singleflowered; but of this we have no evidence, as no single-flowered plants have been discovered. If it be truly a double rose as we now have it, it must then relinquish all claim to being a species, for, as Mr Rivers justly observes, "nature has never given us a double-flowering species to raise single-flowering varieties from, but exactly the reverse." So important a matter do we deem the flowering of this certainly the finest of all roses-and we believe this to be a very general feeling - that we will repeat what Mr Rivers says: "The Yellow rose is a native of a warm climate, and therefore requires a warm situation, a fiee and airy exposure, and rich soil. A wall with a south-east or north-west aspect will be eligible." But perhaps this excellent authority-and there is none higher-refers to the climate of Sawbridgeworth, where his extensive rose-garden is situated; for the general climate of Britain, we would say a south, southeast, or south-west aspect would be better. "Give the plants surface-manure every autumn, and water with manure-water in May; prune with the finger and thumb in summer, by shortening the strong shoots so as to make them put out laterals, and there will be no occasion for winter pruning. Thus, by judiciously pinching off with the finger and thumb in June all shoots that are thicker than a straw to about half their length, small lateral twigs will break out, and the plant will be filled with blooming slioots, some of which must be removed in winter if full-sized flowers are desired." This is the mode also recommended by him for pruning the Persian yellow rose. "In unfavourable soils it will often flourish and bloom freely if budded on the Musk rose, the common China rose, or the Blush Boursault; but the following pretty method of culture I beg to suggest, though I must confess I have not yet tried it: Bud or graft it on some short stems of the Rosa Manettii; in the autumn pot some of the strongest plants, and late in spring force them with a gentle heat, giving plenty of air. By this method the dry and warm climate of Florence and Genoa may perhaps be partially imitated; for there it blooms in such profusion that large quantities of its magnificent flowers are daily sold in the markets during the rose season."The Rose Amateur's Guide. There is, however, notwithstanding this very feasible reasoning, a sort of capriciousness about this rose not easily accounted for. We have more than once had splendid specimens in full bloom sent from the Earl of Traquair yearly, produced in his garden in Peeblesshire, nearly 500 feet above the sea 
level; and we know it has flowered in gardens equally elevated. We know of no instance of its having suffered from cold during winter, and are at present growing it in a large pot in rich soil placed in front of a south wall, with a view next spring to remove it to a warm greenhouse, where it may enjoy a temperature somewhat equivalent to that of Genoa, which may probably set the question of temperature at rest. In regard to aspect, upon which much importance is placed by some, we may remark that, to a query put in "The Gardeners' Chronicle," 1841, the replies were as follow: In ten cases success is connected with an east aspect, in eight with a north, in seven with a west, in six with full exposure all round, and in only one case is the south spoken of. And again, as regards soil, the majority of cases of success occur in light land, gravelly, sandy, loamy, and even marly-in some instances in the stiffest land, and in one case even in blue clay, and another in strong, wet, undrained clay. Some, again, grow it upon its own roots, others bud it on the common Dog rose, and some on the Chinese. In regard to latitude, it is reported as flowering beautifully as far north as Ballater in Scotland, and as far south as Suffolk, Essex, Middlesex, and the Isle of Wight. Mr W. Paul in reference to this matter observes, with all the zeal of a rose amateur, "Could any system of cultivation be divulged, which, followed, would insure a successful issue, I would consider pages well occupied in doing so."

Boursault rose (Rosa alpina).-The alpine or mountain rose of the south of France and the Alps is the type from which this very distinct family of roses sprung, the artificial originator being the late M. Boursault of Paris. They are remarkable for the abundance of their bloom, and long, reddish, flexible shoots, and are well adapted for training against walls even with bad exposures, covering arbours, training to poles or pillars, or on festoons formed by connecting the tops of poles with slight iron chains. They are quite hardy, and produce their flowers in immense clusters, suitable for most ordinary soils, and, when pruned, should be well thinned, but slightly shortened at the points.

Banksian rose (Rosa Banksiana).-Natives of China, and therefore, although remarkably fastgrowing plants, too tender to be treated as pole or pillar roses. They require the protection of a wall in most parts of England, and a south, or east, or west one in Scotland. Like most Chinese plants, they flower early, which renders this protection necessary. They require a dry soil, and do not prosper in a damp one; and in regard to pruning, only the luxuriant shoots, or those where they are too crowded, should be removed, while the small and twiggy branches should be retained for flowering. It is very usual with them to send up a number of strong watery shoots; these, unless for filling up blanks on the wall, should be removed early in autumn. They are sufficiently hardy to withstand our ordinary winters without protection; but in cold and exposed places slight protection is necessary in spring, when the buds are forming and expanding. They are well adapted for pot- culture, and may be grown in a small state to great perfection in a low cool pit. Some additions have of late years been made to this section, but their adaptation to our climate has not as yet been fully recognised.

Many-flowered rose (Rosa multiflora).-The original was introduced in 1804 from Japan by the botanist and traveller Thunberg; since then several varieties have originated in Italy, and also in Britain. Rosa Grevillei, or Seven sisters, is an excellent representative of this family, the whole of which are rather tender, and require the protection of a wall of some sort or other during winter. They also require little pruning. In the south, Rosa Grevillei is very successfully trained as a pillar-rose, being one of the strongest growers in the section; but even these require to be planted in a dry soil, and often to be protected during winter. Russelliana is the tenderest of the family, and is seldom seen in perfection in Scotland.

American prairie rose (Rosa rubifolia).-This is comparatively a new family of roses of American origin, where the single-flowered rubifolia is found indigenous to the prairies,--seeds from which produce semi-double and double flowers in the second generation. They are sufficiently hardy to stand our most severe winters, but require a dry warm summer and a moderately dry soil to flower in perfection with us. They are vigorous growers, but require moderate pruning.

Hybrid climbing roses. - The origin of this family is not known; they present traces of various sections. Laure, Davoust, and Russelliana are hybrids of Multiflora. The Garland, or Wood's garland, and Madame d'Arblay, are said to be hybrids of the Musk rose. Sir John Sebright was raised from Italian seed by $\mathrm{Mr}$ Rivers.

Evergreen rose (Rosa sempervirens) is of Italian origin. Most of the early varieties produced from it were raised by $M$. Jacques at the Chateau de Neuilly near Paris. They are amongst the most valuable of all climbing roses, being quite hardy, free growers, and thriving in most soils, producing their blooms in clusters of from ten to fifty flowers each. They require to be well thinned out at pruning, leaving some of the shoots at their whole length, which will flower to their very points. These shoots, partially shortened, will fill up the centre of the space with flowers, which are produced rather later than those on the unshortened shoots. They are often budded on very high stems, and trained in a pendulous manner, forming what are called Weeping roses.

Ayrshire rose (Rosa arvensis).-The origin of the Ayrshire rose is involved in mystery, some believing it to have originated from Rosa arvensis, one of the wild creeping roses of our woods; while others assert that it came from foreign seed. The former opinion is, however, the most feasible. They are all of rapid growth, and admirably suited for planting in situations where few others would live. Some of the finer varieties, such as the Dundee Rambler, Ruga, Thoresbyana, and Queen of the Belgians, are well adapted for pillar roses, or for covering old 
walls, arbours, and some of them, when budded on high stems, even make excellent weepers.

The foregoing roses are denominated Summer roses, from the circumstance of their flowering in June and July. Those which follow are called Autumnal or Perpetual roses, from their flowering from June till November.

Hybrid perpetuals. - This invaluable and popular class has been produced by crossing the hybrid China roses with different varieties of Chinas and Bourbons, the new progeny producing abundance of flowers during the whole of the summer and autumn, and being hence denominated Hybrid perpetuals. While they are perhaps the most useful and interesting of all the classes of roses, they are hardy, and of easy cultivation, calculated for being grown as divarfs or standards, and force with the greatest certainty of producing a fine bloom. In a family so numerous as the Hybrid perpetuals, it is natural to suppose that some are hardier than others, and therefore better suited to colder and more northern localities. Of these, Madame Laffay, Rivers, Mrs Elliot, William Jesse, Dr Marx, Comte d'Eu, Baronne Prevost, Lady Álice Peel, Elizabeth Plantier, \&c., may be planted, with every prospect of success, even in high and comparatively cold situations. Several sorts in this section are well adapted for planting as pole or pillar roses. All of them grow more freely, and flower more abundantly, when budded on stocks of the Dog-rose than when on their own roots. They all require a moderately rich soil and close pruning. Comte d'Eu and $\mathrm{La} \mathrm{Be}$ doyère should, if for standards, be wrought on the Manettii stock, or Boursault rose. De Neuilly, Coquette de Bellevue, General Merlin, Clementine Duval, are rather tender roses, requiring a warm situation, and succeed best when budded on the Manettii or Boursault stocks. Melanie Cornu and Earl Talbot are well adapted for pot-culture and forcing.

Damask perpetuals.-The parentage of this family is difficult to trace. The old white and red monthly roses no doubt have had a considerable share in the production of some of them. They are all remarkably fragrant, and of moderate growth, forming excellent standards from their tendency to form close and compact heads. They are equally adapted for planting in beds as dwarfs, and for pot-culture, and succeed better when budded on low stocks of the Dog rose than on their own roots. They require an exceedingly rich, rather stiff soil, and close pruning. Perpetual roses are difficult to root when layered, which accounts for their being almost universally met with in nurseries budded on stocks of the Dog rose or Manettii stock; but when they can be induced to root, such plants will be found to thrive much better on poor dry soils than when budded. On the subject of soil for this family, Mr Rivers gives the following excellent advice: "Perpetual roses require a superabundant quantity of food ; it is, therefore, perfectly ridiculous to plant them on dry lawns, to suffer the grass to grow close up to their stems, and not to give them a particle of manure for years. Under these circunistances, the best varieties, even the Rose du roi, will scarcely ever give a second series of flowers. To remedy the inimical nature of dry soils to this class of roses, an annual application of manure on the surface of the soil is quite necessary. The ground must not be dug, but lightly pricked over with a fork in November, after which some manure must be laid on about 2 or 3 inches in depth, which ought not to be disturbed, except to clean with the hoe and rake, till the following autumn. This, in some situations, in the spring months, will be unsightly. In such cases, cover with some nice green moss." Our own practice is to apply the manure in a liquid form. Nearly all the Perpetuals force well, and if budded on the Dog rose so much the better, as it is both easily excited and requires little potroom.

Perpetual Scotch.-Hybrids probably between the Scotch roses and the Damask perpetual. The Scotch perpetual and Stanwell perpetual continue long in bloom, the latter commencing to flower in May, and continuing at intervals till the flowers are destroyed by the frost ; it is the only one worth the notice of the private grower.

Buurbon rose, or Isle de Bourbon (Rosa Bourboniana).- - Some of the most beautiful autumnal roses belong to this family, the autumn, indeed, being their peculiar season, few flowering prior to the end of August. The type of the race is a native of the Isle of Bourbon, and conjectured to be a hybrid between the common China and Red four seasons' roses. The first plant was introduced to France in 1822, where it seeded freely; and having attracted the attention of the leading rose-growers at Paris, they set to work and propagated it extensively. It is prone to produce seed, and consequently seminal varieties; and seed being chosen from the best of these, in a few years not less than three or four kindred varieties became registered in the French rose-catalogues. The Bourbons are distinguished for their fine foliage, compact habit of growth, and for the profusion and long continuance of their blooming. They succeed when budded either on the Dog rose or Manettii stock, and also when grown on their own roots. Mr Wood remarks of them, that those of compact habits should not be grown on stocks much exceeding 3 feet in height, as they rarely form fine heads; when grown in beds on their own roots, a moderately rich soil and dry situation should be selected; where these cannot be obtained, budded plants are preferable: the vigorous kinds require moderate, and those of compact habit close, pruning. Augustine Lelieur, Gloire de Rosamene, Dubourg, Splendens, Crimson globe, \&c., form fine pillar-roses in this section from amongst the older yet tried sorts; and Amenaide, Cardinal Fesch, Le Grenadier, Triomphe de la Guillotine, Alfred, Virgil, \&c., from amongst those of more recent origin.

Perpetual moss (Rosa centifolia).-This is a small group, yet the idea of possessing moss roses in October is pleasing; for who does not admire a moss rose in bud? Like the common moss, they require high cultivation, rich soil, hard pruning, and a warm situation. Of them Perpetuelle Mauget is the best. Perpetual white 
moss, like General Drouet, is a bad grower,-at least with us, - and less certain in flowering.

Musk rose (Rosa muscata).-One of the oldest roses in existence, and to be met with in every country where the rose is cultivated. They so nearly resemble the Noisette roses in habit that the same soil and culture may be adopted. They are extremely fragrant, profuse bloomers, hardy, and of free growth, and may be planted as pillar or pole roses, or trained to walls or espaliers. The original Musk rose is a rambling shrub, abounding in Madeira, Persia, and the north of Africa. It seldom flowers till late in summer, at which time the flowers appear in large clusters, and have a peculiar musk-like scent. None of the tribe are very hardy, all of them requiring a sheltered situation, with a humid and still atmosphere. They succeed well in many parts of the west coast of Scotland, and seldom on the eastern.

China rose (Rosa indica), and Crimson China rose (Rosa semperflorens), are both natives of China, the former introduced to Britain in 1789 . These two species are the parents from which a pretty extensive and very interesting family of roses have sprung. The progeny of each remain pretty distinct, those of the latter being amongst our most abundant and brilliantcoloured autumnal sorts. They are also known as Bengal roses, and when compared with others they are of small growth, and bearing a closer resemblance to each other than most other sections. They are very suitable for pot-culture, as they may be with little care had in flower throughout the year. Some of them, such as the common pale China, are pretty hardy-the majority, however, are not, requiring the protection of a wall to secure them from frost during the severity of winter. This is, however, more applicable to the climate of Scotland than to that of the south of England, where they are the ornament of almost every cottage, and stand the winter even when planted in groups in the open rosary with very slight protection. This protection consists in securing the roots from frost, and is best effected by covering the ground with coal-ashes, sawdust, or tanner's bark, to the depth of 2 or 3 inches; and where enrichment is deemed necessary for the soil, to lay on 3 inches of half-decayed stable manure about the beginning of November. The tops may be secured by sticking branches of trees thickly amongst them, by covering them thinly with dry asparagus haulm, fronds of ferns, or wheat straw drawn straight, and made up into handfuls, and tied to the plants; or, better, to a stout stake driven in close to them, and a few inches higher than the plants, so as not to be blown off during winter. The common broom, however, makes the best of all protections, as it retains its green colour pretty well through the winter, and is therefore better suited for roses planted on the lawn, or in a high-kept flower-garden. Whatever protection is used, it is of importance that it be not too early removed in spring, and also that its removal take place by degrees. In all cold localities this section should be accommodated with the protection of a wall, and even then protec- tion during severe frosts will be required. Like many of the more delicate roses, even in otler sections, these might be grown in good sized pots, and plunged in the borders during summer, taken up and placed under the shelter of a cool pit or shed during winter; and many might with great safety be carefully dug up, even when planted out, and laid in by the heels behind a north wall pretty closely together, so that they could be covered over with straw or dry litter during severe frosts. It is by the annual taking up and protecting tender roses in a somewhat similar manner that the French and Germans manage to preserve so many fine roses during their winters, which are so much more severe than ours; their summers also being so much warmer ripens the wood, and enables many even tender roses to withstand a considerable degree of cold during winter. The varieties of the common China succeed well when wrought on stocks of the Dog rose from 2 to 3 feet in height; the varieties of the Crimson China do not, thriving better on their own roots. This section furnishes material for producing groups of distinct colours better than any of the others amongst roses; and when so disposed on the lawn, or in a regular rosarium, produces a very charming effect. The following arrangement has been suggested by $\mathrm{Mr}$ Rivers, and will continue in bloom from June till October : "Clara Sylvain and Madame Bureau - the former, being taller, should be placed in the centre of the bed: for crimson, take Cramoisie superieure ; for scarlet, Fabvier; for red, Prince Charles and Carmine superb ; for deep crimson, Eugene Beauharnais; for blush, Cels multiflore, Napoleon, and Mrs Bosanquet, the latter being planted in the centre of the bed; for a variegated group, take Archduke Charles, Etna, and Virginie; for rose, Augustine Hersent and Madame Breon." These should be pegged down to cover the entire surface; and to prevent the flowers from injury during heavy rains, the whole bed should be coated over with nice green moss.

Tea-scented China rose (Rosa indica odorata). - This section is properly a subdivision of the China group, having for its type Rosa odorata. They are greatly esteemed on account of their fine fragrance and extreme delicacy of colouring. They are, however, of all others, the most tender. The majority of them are unfit for planting in exposed borders in cold situations, yet thriving pretty well when afforded the protection of a wall. To grow them, however, as well as many in the last section, to the highest degree of perfection, they should be planted in a rosary covered with glass. As orchard-houses are now the order of the day, we see no reason why rose amateurs should not have their rose-houses also. This is more to be desired in Scotland than in England, on account of our cold springs, which are so adverse to all tender plants of this tribe. A properly-appointed rose-house would afford a supply of roses throughout the whole year, and in such a structure they would appear in all the splendour they do in the warm climates of France and Italy. Rosa odorata, the type of the section, is a native of China, introduced to 
Britain so recently as 1810 , and with the yellow China, or Tea-scented rose, previously introduced to France, became the parents of all those beautiful varieties we now so much prize, many of which, however, will not flourish in our climate.

It has been said by one of our greatest rosecultivators, that the culture of the Tea-scented rose is quite in its infancy in this country; but this we attribute more to the want of climate than the want of skill in their management; for it must be allowed that climate has much to do in the matter, else why should the roses of the Levant, of Italy, and of France be so superior to ours?

To impart constitutional strength to this class is no doubt a desideratum, but how this is to be effected without deterioration is the question. Budding or grafting them on such stocks as the Blush Boursault, Celine, or Manettii, instead of the Dog rose, as is so frequently done, would, we think, be an improvement. It is well known that these roses, when so wrought, stand the winter better than when on their own roots. These we would decidedly take up in autumn, and protect under shelter till spring. This is, however, at variance with the views of $\mathrm{Mr} \mathrm{Paul}$, who says, "The practice of removing them from the ground for protection during winter, and again transferring them to their places in the rosarium in spring, cannot be too highly deprecated. To remove a plant once, endangers its growth and perfect flowering the first year; and to remove it twice, more than doubles the risk of failure." This is, to some extent, no doubt true, but we would rather run the risk, if such there be, of removal, than the almost moral certainty of having them killed to the ground by frost. The most important rose of this section, raised from seed in Britain, is no doubt Devoniensis, which originated with Mr Foster of Plymouth.

Macartney rose (Rosa bracteata).-The original of this small group is the single Macartney rose, introduced in 1795 from China. The old double variety is not worth growing, and should give place to Maria Leonida, which is a fine border-flower in favourable situations; but, like most of the China roses, tender, and requires protection in cold localities; succeeds in most good garden soils, if moderately light, and when trained upon a wall, and kept rather thin of branches.

Miniature rose (Rosa Lawrenceana).-Considered by botanists to be a distinct species, and of Chinese origin. Mr Rivers, however, says it is nothing but a dwarf variety of the common China rose, like the Rose de Meaux and Pompon, which are dwarf varieties of Rosa centifolia. "Many plants," he remarks, " that have been long under cultivation have a tendency to produce from seed these pigmy likenesses of them. selves." This section prefers a dry light soil and warm situation. They are, in most localities, more fit for growing in pots, kept in a cool pit, than for open-border culture.

Noisette rose (Rosa noisettiana). - The type of this group, the old Blush noisette rose, is of American origin, itself a hybrid, being raised from the seed of the old musk rose, the flowers of which were impregnated by the common
China rose. This is a fine class of roses, mostly producing their flowers in large clusters throughout the summer and autumn. Their flowers are also of good size and fine shape. Some varieties are grown advantageously as standards, and the stronger-growing sorts make excellent weepers. For the most part they are hardy-at least all are which partake of the original-but a large portion of those now in cultivation have deviated much from the characteristics of their first parents, and approach closely to the Tea section, as well as others with which they have become impreg. nated. This is obviously the case wherever the size of the flower has been increased by hybridisation; for here they lose the tendency to produce their flowers in clusters or corymbs, upon which so much of the merit of the veritable Noisette depends. Those hybrids approaching the Teas are more tender, and require the protection of a wall in all cold localities, upon which they will continue to bloom till very late in the season. Most of the free growers are admirably adapted for pole or pillar training in all moderate climates. The class requires a moderately rich soil, and pruning must be modified according to the character of the variety, as some are very luxuriant growers, while others are the reverse, and instead of making long rambling shoots, incline rather to assume a compact habit. During the blooming season it is of importance to the health and vigour of all roses, as well as for appearance' sake, to cut off all decaying flowers as they appear. In the case of the Noisettes this is still more important, as they are so prone to produce seed, the production of one or two clusters of which is more weakening to the plant than the production of its whole display of flowers during the season. All roses, therefore, which have a tendency to produce seed, should have it all removed (if not required for sowing) as soon as the petals droop. The Noisettes are adapted for training and growing in all the various forms-the strong growers for poles and pillars, the more tender for walls, and the dwarf compact sorts for beds on their own roots. Those grown in the latter way may, in situations where they may be endangered by frost during winter, be cut down in November to within 12 inches of the ground, and slightly covered with branches or dry litter, which should be allowed to continue on till the end of March, when it may be gradually removed.

Situation.-The rose can only be cultivated to the highest perfection in an open situation fully exposed to the south, unencumbered by the shade of trees or buildings, as well as far removed from the effects of smoke, and in a position where it may enjoy a free circulation of pure air. Hence the want of success in all attempts to cultivate it in town or city gardens, as well as in close and shaded shrubberies. It also requires climate-and hence the roses of France and the south of England, to say nothing of those of more genial climes, have not their equals elsewhere; and therefore many of the finer sorts do not prosper well in many parts of Scotland. The rose also requires shelter from the wind, and a full exposure to the south, south-east, or south-west. In choosing a site 
for a rosarium, it is important that these points be duly considered. In regard to its geographical position, if we may so speak, in the domain, that must greatly depend on circumstances. It forms with most propriety a link in the chain of connection in the pleasure-grounds, in passing from the mansion to the kitchen-garden or other point of interest; but, like the Arboretum, American garden, \&c., it should form a perfect whole in itself, if the maximum amount of effect be aimed at. Roses interspersed amongst other shrubs produce no striking feature in themselves, neither do they harmonise in character with them; nor, unless in the case of a few of the most common and hardiest, do they succeed, when so grown, to the same degree of satisfaction as when grown in groups by themselves, or planted in a regular rosarium. Where a regular rose-garden cannot be attained, or where the collection is confined to a limited number of the hardier varieties, they may be planted in groups on the lawn, or by the sides of walks through the pleasure-grounds, but unmixed with other shrubs, but never, in places of consequence, so near to the mansion as to be seen from it. The stiff and formal appearance of standard roses, and the artificial character of such as are grown as pyramids or otherwise trained, makes them associate ill with other plants designed to produce a pictorial or natural effect, in connection with architectural buildings, unless they be in the cottage or most simple style. Even on terraced lawns around the mansion, the rose is not a fitting plant. In a terraced flower-garden, at a distance from the house, even should it be of a highly architectural character, standard roses may be with sufficient propriety introduced in lines, either along the top of the slopes or by the margins of the walks.

The above remarks do not, however, apply to villa gardens of small extent, because there a choice of situation is beyond the means of the owner; but even in these the promiscuous style of planting should be avoided. The more tender should occupy a space on a well-exposed wall, the hardier dwarf ones being grown in a bed or beds on the lawn, and the standard or pyramid ones arranged in lines along the sides of the main walks, or grouped in the open spaces.

The best situation for a rosarium is a hollow place, surrounded on all sides but the south by higher grounds, provided it be neither too damp, nor too much shaded, as the plants will there derive shelter from the winds, and be seen to great advantage from the higher grounds above. Next to this is a situation sloping considerably towards the south, because the whole will be seen to greater advantage from the lower part, or front, than were it a dead level.

Arrangement.-For designs, vide vol. i., figs. p. 666 ; and in addition to what we have stated, we may add that the rosarium should be in the geometrical or systematic style, with gravel walks, and box, stone, or slate edgings; the plants being chiefly budded on stems of greater or less height, are so truly artificial in themselves that they harmonise best when surrounded by artificial objects. Mr Paul has sug. gested, and we think with sufficiently good VOL. II. taste, rose edgings, using the Pompon and Fairy roses for the purpose, and keeping them to something under a foot in height. The latter is, however, too delicate for Scotland. Wire basket-work, either left uncovered, or made the conductor of some of the hardy climbing sorts of moderate growth, would have an excellent effect. Grass walks, unless the rosarium be upon a very extensive scale, seldom please, and are difficult to keep in proper order, and, unless in dry weather, are uncomfortable to walk upon. The beds should not be too broad. Those in the celebrated rose-gardens of the Luxembourg at Paris do not exceed 7 feet in breadth; and although the whole is laid out in parallelograms running parallel with each other, and separated by walks from 4 to 5 feet wide, the whole, when seen from a terrace above, produces a brilliant effect. This mode of arrangement admits of each plant being readily examined from the walks, without treading on the borders. Indeed, to see any garden in the formal style, it must be looked down upon, so that the whole may be seen, as it were, isometrically, more especially during the season of flowering. Roses are divided into two very distinct classes-namely, autumnal flowerers, and summer flowerers, from their season of blooming, and in this order we have hitherto treated on them. It therefore would be productive of the best effect, instead of having one large rosarium into which both these classes were introduced, to have two separate rosaries formed, one for each of these classes; or were they grouped according to their habits and time of flowering, a very perfect whole might be produced, even in one rosarium, which could not be the case were they planted promiscuously. This arrangement should be regarded, be the collection ever so small, and in whatever way they may be disposed of through the grounds.

As roses require shelter from winds, this must be provided either by shrubbery all round, or by a wall along the north side, which in itself would afford accommodation for a number of the more tender sorts. Walls, unless under peculiar circumstances, are extremely objectionable in many situations, as they give the appearance of confinement where all should be open and free. The east and west sides, if not sheltered by shrubbery, may be so by a covered walk of roses, communicating with the other principal walks; but on the south nothing should be planted of a height likely to deprive the plants of a single glimpse of sunshine. Wherever shelter is effected by shrubbery, Scotch roses should form the foreground. They flower profusely, if only for a short season; their compact and orderly form, and their not requiring to be pruned or disturbed at the roots, like other roses, for year's together, renders them exceedingly fit for the purpose. They also form the connecting link between roses of high-bred character, and the other plants or trees which form the boundary line.

Where from want of space or other circumstances a regular rose-garden is not indulged in, they may then be grouped through the pleasure-grounds, each group to contain a selection of 
one favourite section. Thus we might have a group, or circular bed, which is the best form, of Damask perpetuals, Hybrid Chinese, Bourbon, Hybrid perpetual, Moss, French, Noisette, Provence, Bourbon perpetual, Hybrid Provence, Damask and Alba mixed, Sweet briar and its hybrids ; and this series might be repeated or transposed, and carried to any extent, according to taste. In regard to the manner of planting these, the tallest plants-and these should not exceed $3 \frac{1}{2}$ feet in height of stem-should occupy the centre, the others gradually decreasing in height towards the edges of the beds. The distance at which the centre or larger plants should be placed from each other should be 3 feet; the smaller growers should have half that distance. If the beds are small, plants of less height ought to be employed, so that the whole, when in flower, may form a pretty regular cone or semi-globular outline. The tallest also should be placed at the back, when the mass is to be viewed from one side; and in the centre when they are to be seen from all sides. Attempts have been made to produce a harmonious arrangement of colours, by introducing into each bed one variety only; this has, however, been seldom happily realised in the case of the rose. For a specimen of this mode of arrangement, see below. When grown as pillars, perhaps the most picturesque way is to group them in clumps of three, five, or seven, according to the height they are intended to be grown to, as well as their number. And as much of their effect depends on height, it is well to avail ourselves of rising ground, or, indeed, to construct artificial knolls on which to plant them. As to the height of pillars, from 10 to 15 feet may be taken as a fair size : below the former they seldom look well; and it is in few situations much north of London that many of the finer sorts can be got to cover a greater height than the latter.

In planting roses in groups on the lawn, much of their effect is lost when they are indiscriminately brought together without regard to their habits and colours. Mr Rivers, in speaking of grouping the Bourbon section of roses, observes: "It is only some of the more vigorous growers that form good standards; they are all beautiful when budded on stems of 1 to 2 feet in height, and form elegant groups; but I will first notice their adaptation for small beds on their own roots. They are too often mixed indiscriminately, without any attention being paid to their widely different habits, or to their grouping as masses of colour. Now this destroys that pleasing unity which every bed of Bourbon roses ought to possess:" thus the Queen of the Bourbons should have a bed to herself, for no other variety is so elegant in its light, rosy, fawn-coloured flowers, and peculiar habit of growth. Souvenir de la Malmaison is another that deserves to be planted in a group by itself, as do also Reine des Vierges, Madame Angelina, Paul Joseph, Proserpine, Bouquet de Flore, and Grand Capitaine. A clump of the following delicate blush-roses, which are all dwarf-growing, and of nearly uniform growth, will have a good effect-Anne Beluze, Comtesse de Resseguier, Madame Nerard, and Reine du Congres; while the following, which are tall and robust growing sorts, with pale flesh-coloured flowers, are suitable for a larger group, and placed at a greater distance from the walks : Imperatrice, Josephine, Madame Lacharme, and Lavinie d'Ost. Of these Mr Rivers observes, that "they put forth in July very robust shoots, 3 or 4 feet in length; if suffered to grow without being shortened, they produce in August and September a large crowning cluster of flowers ; but after the flowers have faded, their shoots have a bare straggling appearance : it is, therefore, an improvement to pinch off the end when the shoot is about $1 \frac{1}{2}$ or 2 feet in length; abundant laterals will then be produced, which will give their flower's in October." A corresponding clump to the last in habit of growth, but with rose-coloured flowers, will be produced by the following-Alfred, Amenaide, Desguches, Gloire de la Guillotière, Madame Aude, Pierre de St Cyr, Triomphe de Plantier, Triomphe de la Guillotière; and a similar bed of crimsoncoloured roses will be found in planting Le Grenadier, Julie de Fontenelle, and Dupetit Thouars; and if the bed be large, the following may be added-Oscar Leclerc, Comte du Rambuteau, Dumont du Courset, Souchet, and Gloire de Paris. A bed of rather dwarf, compact, and free-flowering solts of rosy carmine flowers will be produced with Augustine Leleur, George Cuvier, Lichas, Henri Lecoq, Vicomte de Cussy, and Marquis de Moyra. Gloire de Rosamène and Enfant d'Ajaccio will form a bed of scarlet roses; while another of chelry-coloured blooms will be found in planting Comice de Seine et Marne and Souvenir de Dumont d'Urville, which are both plants of moderate growth. The following, with rose-coloured flowers, moderate growth, well-shaped large blooms, and flowering late in autumn, deserve also to be grouped together-Madame Marget, Emile Courtier, Edouard Defosse, and La Gracieuse.

The roses we have referred to above, being of the Bourbon section, do well in moderately light soils on their own roots, and in this way cover the ground fully better than when wrought on stems of even under 2 feet in height. In strong soils they succeed better when on stocks of the Dog-rose, that soil being naturally favourable to that species of rose. One advantage all roses have when wrought on stems is, that they are elevated above the soil so as to prevent their flowers being splashed with mud during heavy rains, as they will be unless the surface of the bed be covered with moss. They are also brought nearer to the eye of the observer, so far obviating the necessity of stooping to examine them closely. It is only, also, the strongest-growing sorts in the Bourbon section that make fine heads when wrought standard high, the proper height being, for general purposes, from 3 to 4 feet.

Weeping or pendulous roses have their admirers: the open lawn is their proper situation; the pruning necessary is of the simplest kind. If planted in February, which is a good season, they should be immediately pruned, shortening each shoot to six or eight buds. 
This constitutes all the pruning required for several years, beyond the mere removal of dead wood or a few shoots annually when they appear crowded. The selection, however, of strong stocks is important; not less than an inch in diameter at their top, when such can be procured, are the best, and from 4 to 6 feet in height. If the stocks are planted where they are to remain, three shoots, if possible, should be secured, into each of which a bud should be inserted; and when the shoots the following season have attained the length of 6 inches, the top must be pinched off, which will induce the formation of laterals; if all the three buds have succeeded, the shoots proceeding from them should be allowed afterwards to extend without farther shortening. If two only of the buds have taken, then their shoots must be stopped again and again, until a sufficient number be obtained to form a well-furnished head. Roses trained in this way produce abundance of bloom, and are far more graceful and natural than such as are grown as standards in the usual manner. All roses do not alike suit this style of training, and on a proper selection, as well as on the strength of the stock, much of the success depends. The following are well adapted for the purpose-Felicité perpetuelle, Donna Maria, Princess Maria, Myrianthes, Banksiæflora, Princess Louise, Rose Amadis, Boursault inermis, Boursault gracilis, Thoresbyana, Dundee rambler, Ruga, Splendens, Adelaide d'Orleans, Spectabilis, \&c.

In regard to situation, the lawn is unquestionably the most fitting place for weeping roses, as the points of their shoots rest on the grassy surface; and while in bloom, each plant forms a perfect dome of roses. These, if allowed to remain unpruned and unchecked, will continue for several years in all the freshness of unassisted nature.

Hybrid Perpetuals are also well adapted for planting in groups on the lawn; and as they afford us not only our earliest, but also our latest flowers, they should be planted extensively. A rich soil and a warm situation are, however, absolutely necessary to insure this early and late blooming, particularly the latter. This, in favourable situations, is greatly assisted by shortening every shoot that has produced its bloom, as soon as the flower fades, to within three or four buds of its base. Planting this section of roses in a promiscuous manner seldom produces a pleasing effect on the lawn, because they vary so much in their habits of growth; but planting the finer varieties only, and each of these in a bed or group by itself, will be found by far the most satisfactory-preferring, as Mr Rivers has remarked,"to have a small clump of each sort rather than a large one of mixed varieties. The lawn may thus be painted with excellent effect." A bed or group of each of the following is recommended by this enthusiastic cultivatorviz., one of Aubernon, one of Augustine Mouchelet, one of Baronne Prevost, one of Comtesse Duchatel, one of Dr Marx, one of Duc d'Aumale, one of Duchess of Sutherland, one of Lady Alice Peel, one of La Reine, one of Madame Laffay, one of Marquise Boccella, and one of Robin
Hood. Each of these beds containing from ten to twenty plants which will flower from May to November, will afford much more gratification to the owner than if his collection extended to a much greater number of varieties planted in the mixed manner. To the above list we may add that rose of roses, Géant des Batailles, Mrs Elliott, Comte de Montalivet, Mrs Rivers, Sidonie, \&c.

Standard roses are often planted as single specimens on the lawn, sometimes in groups of three, five, or seven, and not unfrequently in lines by the sides of the walks, \&c. Now, all this, so far as the arrangement goes, is very well, while in nine cases out of ten the planting is very ill. The plants, when received from the nursery, are thrust into holes scarcely large enough to contain their roots, in poor soil, saturated with wet, or peradventure in sheer sand, incapable of retaining sufficient moisture for their existence. Instead of this, pits should be dug for each plant not less than 3 feet in diameter, and 2 feet in depth, and filled with rich loamy soil. It is not necessary, however, that this space be entirely left uncovered with grass, as in such a case the lawn, if small, would be too much cut up. The space necessary to be left uncovered may be restricted to 2 feet in diameter, and the remainder turfed over-placing, however, under it 2 inches of sand, to prevent the grass from growing too luxuriantly, or stronger than that of the rest of the lawn.

Each plant should be supported by a neat iron stake, and both that and the stem of the tree, if covered with moss, will have a neater appearance than left bare. The moss also prevents the action of excessive sun-heat on the stem during summer, and, by keeping the bark somewhat moist, favours the free ascent of the sap from the roots to the branches of the tree. Liquid manure should be frequently applied, particularly during the heat of summer and growing season of the plant.

Propagation. - The rose is propagated by seed, by budding, grafting, cuttings, layers, and suckers.

By seed.-The seed-vessels should be left on the trees until they are perfectly ripened, at which time the skin of the hip or vessel becomes almost black. They should then be placed in small pots imbedded in sand, and each sort kept separate, and buried underground till spring. About the beginning of February is a good time to prepare for sowing; at which time the seed-pods should be taken out of the pots and rubbed between the hands until the seed becomes separated from the pulp and skin. They should then be sown in pots or pans, in light loamy soil, and, when watered, covered to the depth of half an inch with the same compost mixed with a little sand. They may then be placed in a cool pit or frame, or plunged at the bottom of a wall, and kept in a uniform state of moisture. The plants will appear during summer, and be fit for transplanting the following spring. Many of the seeds will not vegetate the first year, therefore those which remain dormant should be picked out when the young plants are removed and resown, when they will, for the 
most part, vegetate during the following season. Some of the autumnal varieties will flower the first year, but none of the summer sorts will do so till the second, third, or fourth year. The young plants require shading during hot sunshine, and a regular supply of water, as they are liable to suffer from an excess of the one or a deficiency of the other. As soon as the seedlings have formed their second leaves, they are fit for transplanting; their removal, however, must take place with a due amount of care, lest the seeds just germinating be injured by the operation. Seedlings of the Chinese and Bourbon roses have been known to flower when only five or six weeks old; but this should be prevented rather than encouraged, as it greatly weakens the plants. Upon a large scale, as in nurseries, the seed is sown in the open border, in a sheltered situation, with an eastern exposure; and here they will continue to vegetate throughout the summer and autumn, some appearing so early as April. Amateurs, however, will find rearing them in pots or pans most convenient for their purpose and means. Transplanting any of the seedlings before autumn is to be recommended only as a means of preventing their injuring each other in cases where they have been sown too thick, or where the seed has germinated for the most part during the first season. The middle of October is the proper time for the general transplanting, where they have come up moderately thick; at which time they should be taken up with care, so that the still dormant seeds be not disturbed, as they will in all probability vegetate early in the following spring. The seedlings should have their roots and tops somewhat shortened, and be transplanted into a sheltered border in light rich loamy soil in lines, from 9 to 12 inches apart, according to their size. Many of them will begin to show flower during the following summer and autumn, at which time the selection should be made. Those which come single or deficient in colour should be destroyed, retaining those only whose colour is clear, outline good, and although not a perfect double flower, yet approaching thereunto; for its plenitude of petals will increase by culture and another year or two's growth, for as yet its properties may not be fully developed. Those which appear to possess more than ordinary merit may be hastened in their development by being grafted on a free-growing stock to the extent of a year or two sooner than if left to flower in their natural course. The transplanted seedlings should be protected from frost during the first winter, particularly the delicate kinds. A thin coating of tanner's bark or sifted coal-ashes is an excellent preservation to their roots, and a few branches of trees stuck thickly amongst them a security to their tops. Some sow in autumn in preference to spring: the former no doubt produces stronger plants, but the seeds are liable to be destroyed by mice, \&c., during the long time they remain dormant in the soil, from which danger those sown in autumnare exempt, in consequence of their vegetating with greater rapidity.

The process of rearing roses from seed is comparatively a modern branch of the gardener's art in Britain, being almost confined to France ; and hence the opinion arose - which is, by the way, a pretty extensively received one-that the climate of this country was insufficient to ripen the seed to full maturity. From the statements of Mr Paul and others of our first-rate rosegrowers, this appears not to be the case, at least to the extent supposed. The Tea-scented and Chinese are almost the only sections that require the protection of glass to bring to perfection their seed; and this is very much owing to the greater length of time they require to do so than the majority of others. To the amateur it may be interesting to know that the following selection of first-rate roses, out of a more extended list, ripen their seed perfectly in ordinary situations and seasons in Britain: Athelin, Bouquet de Flore, Chenedole, Charles Duval, Celina, Comtesse Duchatel, Ceres, Duke of Devonshire, Du Luxembourg, Gloire de Rosamène, Great Western, General Allard, Harrisonii, Marechal Soult, Madame Laffay, Malvina, Rosa Mundi, Russelliana, William Jesse, \&c. There appears to be scarcely any limits to the varieties of roses that may be produced from seed, and so far the amateur need not restrict himself to the crossing of individuals of the same group, nor confine himself to the degree of fulness in the flower, as some which are too full, or double, to open their flowers at all times, ripen their seeds, while many semi-double varieties rarely form a seed-pod. "If," says Mr Paul, "the hybrid Bourbons crossed with the Moss produce perfect seeds, we may presume that the intermixing of the pollen grains of other species will be productive of like results. This, it will be seen, demolishes the idea of the necessity of restricting ourselves to the crossing of individuals of the same group. Not only may we choose the parents from different groups, but from different species." We have thus briefly dwelt on the rearing of roses from seed, as it opens a field of considerable interest to the amateur and private grower.

$A$ few remarks on the sorts most likely to produce improved varieties by crossing or hybridising. - In the Provence section, Wellington may be fertilised by the pollen of the Crimson moss, the Triomphe d'Abbeville with the Luxembourg moss : if seeds are procured, some fine Crimson and pure Provence varieties may be expected. In the Moss section, the Luxembourg moss pollen impregnating the single Crimson moss may produce varieties of great interest. In the French or garden roses, Rosa Gallica section, the following process is recommended by Mr Rivers : "To raise self-coloured French roses from seed, they should be planted in a warm dry border, sloping to the south, in an open airy situation. The shade of trees is very pernicious to seed-bearing roses; and in planting roses for the purpose of bearing seed, it must be borne in mind that it will give great facility to their management if the plants are planted in pairs, close to each other : accident will often then do as much as art in fertilising them. Thus the Wood-pigeon rose may be planted with, and fertilised by, the Tuscany, Vesta with Feu brilliant, Jean Barte with Princess Victoria, Superb 
Tuscany with Leopold, and La majestueuse with the latter. To raise variegated roses, the following, which also bear seed freely, should be selected, and planted as before directed-Aglae Adanson with Donna Sol, the Village maid with Duc d'Orleans, Tricolor and Tricolor pompon also with Village maid: the last union will probably produce some pretty striped roses. Berlese may also be planted with Seguier, Duc d'Orleans with André Thouin, and the Leopard rose with Anacreon : these would most likely give pretty spotted roses. Picotee might be fertilised with the Village maid, and if seed could be procured, some fine varieties must be the result; but the central small petals should be removed by a pair of tweezers from the former rose when its flowers are half expanded, as they are too much crowded to allow it to bear seed. The Tuscany rose, which is a most convenient variety for imparting dark colours, as it abounds in pollen more than any cultivated rose, should be planted in every rose seminary." This fine rose is, we regret to see, seldom now found in rose-gardens. The above quotation will, we think, explain clearly enough the process of hybridising roses. In the section hybrid Provence rose, the Globe hip seeds freely, and if impregnated with any favourite variety, is most likely to become the mother of an interesting progeny. In the hybrid China section, Aurora, which seeds freely, if impregnated by Athelin, would produce self-coloured flowers. Aurora impregnated by the Village maid will produce striped ones, and a durable combination would possibly take place between the Duke of Devonshire and the French Tricolor.

In the hybrid Bourbon section many of the varieties bear seed in abundance, and it is to this group we are indebted for the majority of our hybrid Perpetuals.

In the White rose (Rosa alba) section little is to be expected, as, on account of the plenitude of the petals, they do not often bear seed in this country.

The Damask rose section offers few seed-bearing kinds, the Purple Damask being almost the only one. The Scotch rose section affords abundant seed-bearing sorts, and when planted in mixed masses, as they generally are, impregnate one another, the seed producing endless varieties. In the Austrian briar section, Harrisonii, is almost the only one that bears seed from which much need be looked for. The Persian yellow is mostly barren, but, if crossed with Harrisonii, might produce something interesting amongst yellows, of which there has hitherto been a great scarcity. The Ayrshire section seeds freely, and some fine climbing roses have been producer, such as the Queen of the Belgians and Ruga-the latter said to be a hybrid between the Tea-scented China rose and the common Ayrshire. The Evergreen (Rosa sempervirens) section holds out little prospect of producing much in this way, and the same may be said of the Banksian section. Few of the hybrid climbing roses, with the exception of the Ayrshire rose, will bear seed in this country. Perpetual Damask roses are not free seeders in Britain. The hybrid Perpetual section being deficient in yellow, white, and fawn-coloured roses, this desideratum may be partially made up by crossing the Duchess of Sutherland with the Tea-scented roses Victoria and Safrano, and the same with the China rose, Clara Sylvain.

Of all roses, excepting the Scotch, none are multiplied so easily by seed as the China section; but this is chiefly effected in the climate of France, and the results have been the production of those first-rate roses, Clara Sylvain, Eugene Hardi, Mrs Bosanquet, Augustine Hersent, Cramoisie superieure, Madame Bréon, Sully, Tancrede, \&c. The Tea-scented are too delicate to ripen their seed freely in our climate, unless under the protection of glass; and the same may be said of the Banksia and Laurenceana.

The Noisette section, the original of which was itself a hybrid, has increased by crossing, particularly in France, to an extraordinary extent, many of these being hybrids of the Teascented and other roses; so that, in fact, some of those called Noisettes have almost lost the character of the group.

Propagating the rose by budding.-This is the mode in general use in nurseries for the multiplication of the finer varieties, and for those intended for standards. For mode of proceeding, vide p. 343 .

That excellent rose-cultivator, $\mathrm{Mr}$ Rivers, adopts the plan of budding his roses in pots, choosing the Blush Boursault as a stock, on account of its striking readily by cuttings planted in autumn. His practice is: "The strongest shoots are selected early in July for layering; flower-pots of the size 48 are taken, and the aperture in the bottom is enlarged, so as to allow the end of the shoot to be passed through. After doing this the shoot is tongued: the pot is drawn up till the tongue is about its centre ; it is then filled with a mixture of rotten dung and sand in equal parts, and well pressed down. The shoot may be budded at the time of layering, or afterwards, according as the buds are ready. The shoots should be headed down at the time of budding to within two eyes of where the bud is inserted. The buds of all Bourbon, Tea-scented, China, and hybrid autumnal roses will push immediately. These may be removed from the stools in August, potted into large pots, and forced with great success the following spring."

Propagating the rose by grafting. - Vide p. $322-338$. We have there stated the sort of stocks adapted to rose-grafting, and to which we now add the Celine; and may here remark that the rose is grafted in general in the wedge, cleft, and whip manners, and that the best season for performing the operation in the open air is in March, and the best of the three methods is the cleft manner. Grafted plants are held in less estimation than such as are budded or struck by cuttings. The best season for grafting plants under glass, and the excitement of heat, is January, the stocks being placed in bottom heat ten days previously, to set the sap in motion; and to prepare them for this, they should be grown in pots one year before they are required for use, and be of about 
the thickness of a quill : the operation is performed close to the ground. Remove all suckers that may arise from the stock as soon as they appear. When the scions begin to elongatewhich they will rapidly do in heat-being still ill supplied with food by the stock, the same attention to shading will be required until a union takes place, as if they were mere cuttings; wheu, however, the scion and stock become united, then less shading will be required, and afterwards it may be discontinued altogether; and a greater amount of air must be admitted to the plants, to harden them progressively for being removed to a cooler and more airy place. The ligatures which hold the stock and scion together should be removed as soon as a union takes place, and to prevent accident they should be supported with a small stick. When the new plant has begun to grow, and has made four or five leaves, it should be topped, which will cause it to send out side shoots, and form a nice bushy plant.

Propagating the rose by cuttings.-This is an expeditious and very certain mode of propagating the majority of roses; and in the case of China, Tea-scented, and Bourbon roses, it is the only proper way of getting plants on their own roots. Summer and autumn are the best seasons, but in the case of rare or new varieties, if placed in a mild heat in February, the process may go on till autumn, as cuttings may be produced by the old plants. The best time, however, as regards those that are growing in the open air, is immediately after they have flowered; for no shoots are mature till their blooming is past; and shoots of moderate strength, and well ripened at their base, are to be preferred. They should be of about the length of from 3 to 4 inches; that is, with three joints or buds : but even greater lengths may be taken, the tops of which should be shortened, so as to leave from three to four perfect leaves, with a bud at their base; and when taken off, a small portion of the old wood, forming what is called a heel, should be removed with them, that portion being more disposed to form a callosity than the young wood above it. The leaves should be retained at planting, and allowed to drop off by degrees, and removed as they fall, to prevent damping. Sixty-sized pots are the most eligible, and these should be filled with light loam and sand, into which the cuttings should be stuck to the depth of an inch, and arranged around the sides of the pot to the number of five or six in each pot. When inserted, settle the soil well about them by watering; and when dry, set them in a close frame or pit facing the north, keeping them shaded while the sun shines upon them. Here they should remain until they have formed a callosity at their base, which will in general take place in about ten or fifteen days. They should then be removed to a pit or frame, and plunged into a slight bottom-heat, and one having a northern aspect will lessen the trouble of shading, and obviate the chances of its being neglected. The roots will soon form, at which time they may be potted off into 60 sized pots, one plant in each, and transferred to a pit or frame with a southern exposure, and again plunged in a mild bottom-heat. Here they should remain for about a month, when they will be completely established, and fit for being placed in a cool pit or frame to harden off, kept at no great distance from the glass, and abundantly supplied with air, even to the extent of uncovering them entirely for days together. This is an expeditious mode. A more slow, but yet equally certain method, is to place them at once in a cool pit, and to allow them to make their roots during the summer, to preserve them from frost during winter, and to pot them off in February following, and then place them in a temperature of from $55^{\circ}$ to $60^{\circ}$, but without bottom heat : they will be in excellent condition for planting out in May following. Those, however, who have the means of following the former plan, will have their plants fit for planting out in the previous August. The above may be termed the summer process, and is best adapted to the autumnal varieties; and the following the autumn process, which is alike suited to both the summer and autumn kinds. About the end of October or beginning of November, before the foliage begins to fall, cuttings should be taken off, close to the old wood, so as to retain a portion of it, as alrcady adverted to; for although cuttings taken from the upper portion of the shoot will root occasionally, they do not so with the same certainty as when furnished with a heel. These are to be set in pots, as directed above, and set in a cool pit, protected during winter from frost, and in April they will be found rooted, and fit for being placed singly into 60-sized pots; when, if placed in a cool close frame, they will soon make plants fit for planting out in May. The stronger and hardier sorts, like the hybrid Perpetual, Ayrshire, hybrid Chinese, hybrid Bourbon, Boursault, \&c., require less care. In October, cuttings of them 10 to 12 inches long, but cut with a heel at their base, may be inserted in the ground, in any moderately sheltered border, to the depth of 5 or 6 inches, placed in rows a foot apart, and 3 inches in the line. A slight protection during winter, by mulching the ground, and sticking a few branches amongst them, will be of advantage. As soon as they begin to grow in spring, it will be seen what success has attended the process, when, if they appear to be too thick, a portion may be carefully taken up and replanted in nursery lines, and the whole encouraged in growth till the following autumn, when they may be taken up and planted elsewhere. Some nurserymen strike immense numbers of roses by placing the cuttings in autumn, not in soil, but pretty thickly under a glass frame in half-decayed flax refuse. Here they stand over winter, and in spring they are taken up with abundance of roots, and are potted and treated as already noticed. By cuttings dwarf plants only are obtained, yet many sorts, such as the China and Tea-scented, do best on their own' roots; and we believe that many others would do so too, if the soil is suitable for them. Those budded on the Dog-rose stock often fail, because the soil in most gardens is too light for that stock to thrive in.

Propagating the rose by layers.-To obtain shoots for this purpose, as they should be strong and freely grown, the plant should be cut down 
near to the surface of the ground: this will cause it to throw out strong and proper shoots during summer, which will be fit for layering in autumn or winter following, which is the usual, although not the most proper season. The process has been described, vide p. 351. Roses layered during autumn or winter will, for the most part, be rooted by the autumn following, and fit for transplanting into nursery rows. The Moss rose, however, and some others, often require two years to furnish themselves with roots; and to remedy this in some degree, it has been suggested to perform the operation during summer, as soon as the shoots are so far advanced in growth as to bear bending. Dwarf roses only are propagated in this way; and to insure success, the whole plant should be cut over, as above; for although a strong shoot or two may be occasionally got near the base of the plant, should these even be laid, it is a chance if they will strike roots while the top remains in action. The best rose-cultivators lay their roses during June, July, and beginning of August, supplying them abundantly with water, should the weather be dry; by this process the free-rooting sorts are ready for removal in November.

Propagating the rose by suckers.--Some few species, as the Scotch and Dog-roses, propagate themselves naturally in this way; others, like the Moss and Provence, do so rarely. Little dependence is placed by the cultivator on this mode of propagation, although advantage is often taken of the circumstance when it occurs.

Soil.-The rose delights in a pretty stiff, holding, rich loamy soil, thoroughly drained and deeply trenched, and further enriched with the cooler manures, such as cow-dung, as a basis, and a portion of super-phosphate of lime to act as a stimulant. Such soils as will grow good wheat will grow roses well; but to grow them fine, it must be much richer. Stubborn clay-soils are unfit, until they are ameliorated either by burning, or by the addition of foreign matter, such as decayed vegetable substances, sand, \&c. In light sandy soils, although they will grow for a time, their existence is of short duration, and their flowers never develop themselves in full perfection. This is more, however, the case with budded plants, than with those on their own roots, as the stocks employed are chiefly those of the wild Dog-rose found in our strong loamy soils. The process of budding has nevertheless, as regards some sorts, something to do in the matter. The hardier kinds of roses will thrive pretty well in a peaty soil, if $d r y$, but much better if strong loamy soil, approaching to clay or brick earth, be laid on the surface during autumn, and forked in during early spring, after it has become pulverised by the action of frost and air. Marl is a good manure for such soils, and lime stimulates it considerably. Very sandy and gravelly soils are the worst of all, because they do not retain sufficient moisture during summer, and are both poor and hungry. Such soils are scarcely capable of improvement, so as to suit the rose, short of replacing them with that of a rich loamy character. Soil abounding in oxide of iron, although injurious to many other crops, rather, it is supposed, improves the rose than deteriorates it. So much is this said to be the case, that the superiority of the roses grown in the neighbourhood of Provence, for medicinal purposes, is attributed to the abundance of this oxide that exists in the soil. The rose also delights in a new soil, and in time dies out of land where it has been long cultivated, unless the soil be naturally adapted to it, and there they will luxuriate for an unknown length of time, if supplied annually with moderate manuring. The rose transplants pretty freely, even when of a considerable age. They may therefore be taken up when the soil appears to become defective, and a portion of it be removed, and replaced with new soil; and when both are properly mixed together, they may be replanted. There are soils in which the rose luxuriates for years; while in others, prepared artificially for them, they will not prosper, and ultimately cease to exist.

Season for planting.-From the beginning of October to the middle of November is the proper autumn season, and from the beginning of February to the end of March is a good season for spring. Some, however, who wish a late display of bloom, either plant their newlyreceived sorts in April, or take up and replant a portion of those already in their possession. The check thus given by late planting causes a later growth, and consequently a later season of blooming. When the plants have been kept long out of the ground, and their roots exposed to drought, it is well to place their roots for a few hours in water, or to dip them in soft puddle previous to planting; for, however hardy the roots of even the Dog rose may be, they suffer much when long exposed to the air; and those of a more delicate constitution, upon their own roots, do so in a still greater degree. If the soil be light, a few spadefuls of rich loamy soil should be afforded to each plant at planting; and if of a strong or retentive nature, the same quantity of leaf-mould and sand will be of great advantage to them, and encourage the speedy formation of roots, more especially as regards Chinas and the Tea-scented varieties. Standards, when planted in beds or groups, should be allowed 3 feet plant from plant, and at this distance dwarf plants may be set between them. Attention should also be paid that the taller and stronger-growing kinds be placed in the centre, that is, farthest from the sides of the beds. Avoid deep planting, which induces the sending up of numerous suckers; and as the standards are planted, supply each with a neat stake for support. Autumn planting is best for all the hardier sorts, but all tender varieties should only be planted in spring. Roses planted in autumn should have the soil over their roots mulched with littering manure for the exclusion of frost, and also that its fertilising properties may be washed down to the roots by the rains of winter. In light dry soils late autumn planting is quite proper, but in strong soils spring is a preferable season.

Pruning.-A judicious system of pruning constitutes the chief part of the rose-cultivator's art. The varieties are now so numerous, some French catalogues extending to two thousand, 
and the difference in habit and character so great, as well as the purposes for which they are grown, that it renders the task of description, at least so far as to be useful to the amateur and tyro, no easy matter. With a view to place the subject in as clear a light as our space will admit of, we will consider the process under the heads of Autumn and Summer pruning. We have also briefly noticed this in our remarks on each section or group.

Autumn pruning.-The beginning of November is the latest period at which roses of even the hardiest kinds should be operated upon. Indeed, it is only those which can with perfect safety be interfered with at this season, particularly in cold localities. Autumn is the season when the sap is in its least active state, for it should be borne in mind that it is never entirely suspended; it would therefore appear to be the most proper for the purpose. And in addition to this, we may add that, while there is rootaction going on, the sap will be accumulating in the shoots and buds; and the more sap there is in them, and the more thoroughly it becomes concentrated, the more certain are we of a vigorous growth in spring, and an abundant display of bloom. Instead, therefore, of this amount of sap being distributed through the whole extent of young wood, a large proportion of which will be removed by spring pruning, it would be retained in the fewer and shorter shoots left, if autumnal pruning were adopted; because, as Mr Paul has wisely remarked, "by cutting away the superfluous shoots in autumn, the buds on those left behind are placed in contact with a greater supply of food by the lessening the number of channels through which the sap has to pass." Autumnal pruning is also said to increase the vigour of the shoots, and flowers are produced earlier, more regular, and in greater abundance. In pruning summerflowering roses in autumn, such as the Provence, Moss, \&c., they should be cut to three or four eyes, according to their strength - that is, after a total removal of all weakly and malformed as well as superfluous shoots. Some of the strongergrowing Damask sorts-Madame Hardy, for example, which is a strong grower-should be cut to from five to seven eyes. The Damask, White Damask, Gallica, or French, often produce shoots from 4 to 5 feet in length, robust, green, and imperfectly formed; these should be cut clean away, and the more moderate in growth shortened to something less than 18 inches in length, according to their strength. In the hybrid Chinese section, such strong-growing sorts as Brennus, \&c., should be shortened to eight or nine eyes; while such weak growers as the Beauty of Billiard should have half the shoots cut away, and the remainder shortened to two or three eyes. The hybrid Provence section are disposed naturally to form dense and compact heads; these should be thinned out to about one-third of their number of shoots, and the remainder shortened to about six or eight eyes. The Macartney rose itself requires very little pruning; while the Maria Leonidas, so nearly related to it, requires pruning to the extent of reducing the strong shoots to eight or nine eyes, and the weaker to three or four. Bourbons and Noisettes should be pruned moderately at this season, in most situations, to five or six eyes when strong, and to three or four when weak, in ordinary situations, but in cold ones defer the operation till spring. The Chinas and Teas, for the most part, had better be deferred to the spring also, unless in warm localities, and when they are planted against walls, in which cases cut to six or seven buds, according to strength; and in warm situations, such as are grown as standards to two or three eyes. Autumn pruning has the advantages stated above, but it has also its disadvantages, in reference to which Mr Paul observes: "A few mild days in winter often excite the buds of autumn-pruned roses, and they push forth ; severe weather follows, the young shoots are frosted, and the bloom injured. This is more particularly the case with the Chinese, Noisette, Bourbon, Teascented, and the hybrids of these kinds, which we shall term excitable, because they are quickly excited to growth. The Provence, Moss, French, Alba, and others, rarely suffer from this cause, as they are not so readily affected by the weather. Be it remarked, however, that the quickness with which buds are roused into action depends much upon how far the shoots were matured the previous autumn ; the less mature, the more excitable. It will be perceived, then, that there is a difficulty in autumn pruning, when applied to excitable kinds, which can only be remedied by affording them protection from frost, should a mild December or January be succeeded by severe weather." Much of all this depends on climate and situation. For ourselves, we prune such sorts only in spring, but that as early as the beginning of March.

With many of the French roses (Rosa Gallica), if they are planted in pairs, and the one pruned in October to six or eight buds if strong, or to two or three if weak, and the other left unpruned till May, a regular succession of flowers will be the consequence. The same rule may be followed in the case of the hybrid Provence section also. The hybrid China, when grown as a pillar rose, should have three of the strongest shoots only retained, and these allowed to grow without stopping till they have attained their full height. The spurs from these shoots will for years give abundance of flowers, and when they cease to bloom should be cut down and re-grown. The shoots of all this group should not, unless they are very weak, be shortened at pruning to less than eight or ten buds. Hybrid Bourbon roses, for an early bloom, should be pruned in November, cutting out all the small branches and their spurs, and shortening the shoots to five or six buds, leaving them at regular distances apart. In April prune in like manner, which will insure a crop of bloom two weeks later than those pruned in November. In pruning every sort of rose, cut about one-eighth of an inch only above the bud.

The Austrian brier tribe require very little pruning; the strong shoots only should be shortened, while most of the twigs being left will produce abundance of bloom. And the same rule applies to the Double yellow and 
Persian yellow. Rosa multiflora requires little pruning; every encouragement should rather be given to the small shoots, which are those which produce the flowers. For this reason they require protection during winter, that their spraylike shoots may not be injured by frost. The Evergreen rose, or Rosa sempervirens, and its near congener, the Ayrshire rose, should, at their winter-pruning, have their branches left at their full length, for if shortened they will not produce flowers the following season. Training them in a tortuous or zigzag manner keeps them within prescribed bounds; and moderate thinning, by cutting out crowded and weakly shoots, is all they require. The Banksian section, on the other hand, should have all the strong shoots not required for extending their size removed in autumn, but the small sidebranches retained, unless unnecessarily crowded, as it is on those the flowers are produced. Regarding the pruning of the perpetual Damask roses, Mr Rivers directs: "In the November pruning cut off from every shoot of the preceding summer's growth about two-thirds; if they are crowded, remove some of them entirely. If this is attended to, there will be, early in June the following summer, a vast number of luxuriant shoots, each crowned with a cluster of buds. Now, as June roses are always abundant, a little sacrifice should be made to insure a fine auturnnal bloom: therefore leave only half the number of shoots to bring forth their summer flowers; the remainder shorten to half their length. Each shortened branch will put forth its buds, and in August and September the plants will be again covered with flowers." The Bourbon section requires little pruning, and that not before the end of March or beginning of April, when all danger of severe frost is past, when they may be thinned of superfluous shoots, those injured by the previous winter removed, and those which are sound reduced to four or five buds. China roses will require pruning, also, about the same time as the last, and also upon the same principle, with this exception, that their shoots should only be shortened to about half their length, unless such as are injured by the frost. Much the same course must be adopted with the Tea-scented group. Noisette roses, as standards, require little pruning further than to thin out their superfluous branches in March; those grown on their own roots may be cut down in November to within a foot of the ground as a safeguard against frost, as they can then be conveniently covered over. The same treatment may be applied to the Musk rose section also.

Summer-pruning.-Roses, when growing in rich soil, often produce many very strong shoots, which the French not inaptly denominate gourmands; and such they truly are, because they rob the other and more moderate growers of their due share of nutriment. These should be cut away as soon as they make their appearance, and so should all suckers rising from the roots, as also such shoots as rise from the stock upon which the rose may be grafted or budded. When a number of shoots are thrown out equally strong, so as to become crowded, one-third of VOL. II. them should be removed entirely; but if it be desired to increase the size of the plant, then a portion only should be removed, and others shortened by pinching off their tops : do not shorten any of the others, as that would cause them to send out a number of small weakly shoots, upon which few or no roses would be produced the following season. Summer-pruning consists chiefly of thinning out superfluous shoots, such as those gourmands we have referred to, and also many of those which are weak or unhealthy, and this is in general done immediately after the season of flowering is past. But there is more required; for even healthy shoots, where they appear crowded or ill-placed, should be thinned out, so that each shoot left should stand clear of others, and be fully exposed to the sun and air on all sides; and this thinning should take place before the formation of the buds, that the strength of the plant may be thrown into the flowers and shoots intended to be retained. The summer-flowering sorts, when so treated, continue their growth with vigour, the buds at the axils of the foliage remaining dormant; while, in the case of the autumn-flowering kinds, the buds push forth the entire length of the shoots, and hence produce their second crop of bloom. Too much dependence has hitherto been placed on winter-pruning alone, and too little on summer-pruning, or summer-thinning of the shoots. When the latter is disregarded, the plants become in a short time exhausted by the production of an immense amount of matter, grown for no apparent purpose but to be cut out in winter and thrown away; whereas if removed early in summer, before the flower-buds form, or, still better, as suggested by Mr Paul, prevented from appearing at all by a judicious process of disbudding early in spring, that sap which goes to the formation of these useless shoots would be thrown into those necessary to be left, as well as into the flower-buds, insuring a much more abundant and perfect bloom, as well as giving additional strength to the tree to resist the attacks both of disease and insects.

Mr W. Paul was the first, we believe, to suggest disbudding the rose. Experience has since proved the correctness of his theory; so that, if disbudding be carefully attended to in early spring and throughout the summer, winterpruning will be reduced to the simple operation of shortening the shoots according to their strength, and the removal of such as may have sustained injury during winter. In support of his practice, Mr Paul observes: "True, it is necessary some ' shoots' should be removed when too many are present; but why are more than will be required suffered to grow? It is questionable whether the theory of branches and leaves elaborating the crude sap, and thereby fitting for assimilation a greater quantity of food, is in favour of their development. A few vigorous branches, with leaves healthy and well developed, must, I think, better accomplish this end than a great number crowded together, the leaves becoming puny and sickly through the exclusion of air and light. Now, by rubbing out a portion of the buds when swelling, and others at any season when they may sprout forth 
in a position where shoots are not wanted, the remaining buds form stronger shoots, and thus, perhaps, a larger, certainly a healthier, surface of foliage is the result. It is bad policy, then, to suffer more buds to be developed as shoots than are required for forming the tree, or for flowering; for, by cutting away these, when pruning, there must be a waste of the elaborated juices of the tree. But this is not the only evil; beyond this, the tree is sorely maimed in the operation. Now, if the nutritive matter which has been supplied in the development and sustenance of numerous branches was confined to a lesser number, they would have been more powerfully developed, and the loss by removal, and the injury the tree suffers by thinning, would have been avoided. I believe," he adds, " disbudding to be the system best calculated to produce flowers in the finest possible condition, to keep a plant in full health and vigour, and to bring it to the highest pitch of beauty." We know the beneficial effect of disbudding on other trees, whether fruit or flower bearing, and analogy points out to us that the rose does not form an exception. Some regard must, however; be paid to the habit of the tree, whether it be a strong or a weak grower. In the former case, much fewer buds should be displaced than in the latter, because, if the whole strength of a strong-growing plant be thrown into the production of a few shoots, these, in all probability, would become too strong, and be more inclined to run into wood than into the production of bloom, at least until a late period of the season. In the latter case, if too many shoots are allowed to form, the energies of the tree are expended in their production, instead of in the production of bloom; and should they even bloom, the flowers will be small and imperfect, and the tree necessarily exhausted. Disbudding should be attended to throughout the whole growing season; and in doing so, those pointing in an outward direction should be preferred, while such as point inward, or in a direction likely to cross others, should be removed. When the buds have extended to the length of half an inch, they are in a proper state for removal, and should be rubbed off close to the wood from which they issue, thinning the centre of the tree most, but retaining such as point to a part of the head deficient in branches, that the proper balance and form may be maintained. Thinning roses of their superfluous shoots before the blooming season arrives, has the important advantage of bringing the blooms more into sight, and exposing the flowers better to the sun and air.

Forcing.-The rose at all times is impatient of a high artificial temperature, and more so during the sunless months of winter. It is equally impatient of a close and impure atmosphere, and requires all the light that can possibly be afforded it. The structure best adapted to it, under winter cultivation, is a low spanroofed house or pit, capable of being heated to the extent of $70^{\circ}$, with fire and sun heat combined, during the day, and to $35^{\circ}$ during the night, whatever the external temperature may be. The interior arrangement should be such that the tops of the plants may be placed within a foot of the glass when coming into bloom; and for this purpose it is desirable to have the platforms upon which they are placed so arranged that they may be lowered or elevated to suit the height of the plants set upon them. It is also desirable that a passage be left within, of sufficient height to admit of head-room for the cultivator to walk freely under the roof; and this is most economically attained by sinking a passage along the centre of the pit, the platforms for the plants being placed on each side; and that all may alike be placed so as to enjoy the light and sun, such pits should run in a north and south direction. The roof may be a fixture, and ventilation secured by openings along both of the side-walls, yet sufficiently low that the air may enter under the platforms, and not come in immediate contact with the plants; its escape should be through an opening along the ridge. The heating should be by means of 3 -inch hotwater pipes. The floor should be thoroughly drained, and covered with sifted coal-ashes or river-gravel, for the absorption of spilt water and the correction of damp. A proper selection of plants should be in readiness, by being established one year in pots, and kept plunged in the reserve garden, in an open and well-exposed situation, till required. That the roots may be prevented from passing through the drainageholes into the soil, it is expedient to remove them frequently; or, what is a more certain and less troublesome operation, to set them on a concrete floor, or on pavement, or tiles, or large slates, and to fill up the spaces between the pots with coal-ashes or sand. Disbudding and pruning should be attended to while in this their preparatory state; their growth should be encouraged, during the same period, by copious waterings in dry weather, and occasional supplies of liquid manure. Although all roses for forcing are better to be one year established in pots, yet, where this lias been neglected, or an insufficient number of plants potted, most of the hybrid and damask Perpetuals will give a good bloom if only potted in October or November previous to forcing; and these will, after blooming, if their young shoots are cut back to within two or three buds of their base, give a second crop of flowers later in the spring, if allowed a week or two to rest in a lower temperature, and again brought into the forcing-pit. The same also holds good with most of the China, Bourbon, and Tea-scented sorts, the last even often producing a third crop of bloom. The beginning of December is a good time to commence forcing, taking in a portion in succession towards the middle of the month, and so continuing every fortnight till the middle of March. If disbudding has been attended to during summer, little pruning beyond shortening the shoots will be required when they are taken in, and this should be carried to the extent of cutting each shoot back to within two or three eyes or buds of their base, the latter for the stronger growers, and the former for the weaker sorts. In regard to temperature, the reverse of ordinary practice should be followed, and instead of lighting the fire in the afternoon, it should be done about 
seven in the morning, and allowed to become extinguished about the same hour in the evening, unless during intense frosts, when it may be kept slowly going till towards twelve at night, the heat in the pipes being sufficient, even in such a case, with very slight covering over the glass, to repel frost, and give three degrees of heat above the freezing point, at which the plants will sustain less injury than were they subjected to a much higher nighttemperature. The roots of the plants should be examined before placing them in the forcingpit, and if any are found defective, they should be turned out of the pots, their roots pruned, be re-potted, and returned to their place in the reserve garden, and kept till another season. Those that are found in a good state, which will readily be ascertained by turning them carefully out without breaking the ball, will be improved if, instead of at once placing them in the forcing pit, they are plunged in a slight bottom-heat for ten days; but, unless during frosty weather, no glass covering should be placed over them-the intention being to give the roots somewhat the start of the buds-after which they are in a condition to be set in the pit along with those already in it. The first section set in do not so much require this attention, as it is presumed the atmospheric heat is kept little in advance of the open air for the first fortnight by means of ample ventilation; the root action will therefore be excited slowly, and in a natural proportion to the ascent of the sap in the buds. Those taken in during the intensity of winter should, when pruned, be placed in a mild bottom-heat in a frame or pit, and covered with glass sashes giving air freely, to induce a slow and gradual action in both roots and buds; and when this is effected they are in a fit state for placing in the coolest part of the forcing-pit. During the whole process of forcing, air should be freely admitted according to the state of the weather, and moderate night-ventilation should not be disregarded. Little water will be required beyond a moderate supply at the roots until the middle of February, when the sun begins to exert its cheering influence, at which time moderate syringings should be applied, but always with water in a tepid state-taking care, however, that as little as possible falls on the flower-buds; and this syringing should be administered about ten o'clock in the morning. Should there be even the slightest appearance of aphides, recourse must be had to tobacco fumigation; but if this be attended to even before any appearance takes place, it will often be found that no aplides will appear at all.

The principal object in forcing roses, unless it be by those who supply the markets with them in a cut state, is to remove them from the forcing-pit, either to the greenhouse or drawingroom, just as the buds begin to expand. To render their removal without injury a matter of certainty, they should be placed in a box of sufficient depth, and instead of having the top movable, one of the ends should be so, that the plants may be the more easily placed in it and taken out again. If kept in the forcing-pit, their flowers will continue only for a few days, while in either of the other cases they may be kept much longer in perfection, the excitement being so much less. When the flowers have faded, the plants may be kept in a cool greenhouse for a week or ten days, pruned back, as already adverted to, and returned to the forcing-pit to produce another crop of bloom. As to the sort of plants, Mr Rivers observes: "Those that are forced with the greatest facility are worked roses; these seldom or never fail to give an abundant crop of flowers: stems from 6 inches to $1 \frac{1}{2}$ or 2 feet are equally eligible; the latter form elegant plants, and I think generally grow with greater luxuriance than dwarfs. China and Tea-scented roses on their own roots are more delicate, and require more care : still one crop of flowers may always bedepended upon even from them. Instead of forcing them for a second crop, it will be better to place them in the greenhouse; they will again bloom finely in May. I find," he continues, "from experience, that all the autumnal roses may be forced every year without any disadvantage. To insure their well-doing, they must be removed from the forcing-house early in June, the surface of the pots dressed with rotten manure, and plunged in the same, or leaves or any light substance. Towards the end of September they should be carefully shifter, removing nearly all the earth from their roots, into a compost of light loam and rotten dung, equal quantities, watered, and again plunged till required for forcing: this shifting would be better performed in June; but as the weather is then often hot and dry, roses wrought on the Dog rose are apt to suffer." This objection as to time has much less reference to Scotland than to the south of England, and even there we would rather incur the additional labour of placing them behind a north wall till the end of August than forego the advantage of a June shifting. The rose, while in bud and in full bloom, is a great acquisition in the decoration of the conservatory or show greenhouse; but before it has arrived at that state, and after it has passed from it, it is almost of all plants the worst calculated for admission into these structures, not only on account of its liability to being attacked by aphides, which would soon spread over other and more valuable plants, but also from its want of association in character with the plants contained in them. It is therefore far better to confine rose culture to pits or small forcinghouses, until the time shall arrive when we have our rose-houses as well as our orchard-houses, heath-houses, \&c. When such is the case, roses will be had in perfection the whole year through, and at much less trouble and expense than by any means at present employed. The sizes of pots for forced roses must ever depend greatly on the sorts and sizes of the plants; 24 's and 16 's are the sizes in ordinary use. (For dimensions of pots, vide p. 738). The soil most eligible is light loam and very rotten manure, of each equal quantities.

Roses in bloom at Christmas are a desideratum with most people, and one which may readily be attained by employing the hybrid Perpetual, Comte d'Eu, and the Bourbon rose, Gloire de Rosamène, the only two of which we are aware 
suitable for the purpose. Plants of these in pots should be plunged in sawdust or rotten tan, and set in the most exposed part of the reserve garden during summer, where they may be allowed to flower during June and July, but from the beginning of August till the end of September every flower-bud should be carefully pinched off. By the end of October abundant incipient flower-buds will begin to form, after which the plants should be removed to an airy pit or greenhouse, kept near to the glass, but without fire-heat, unless during severe weather in November and December, and then only to the extent of excluding frost. Towards the end of the latter month, and throughout January, abundance of bloom will be produced.

The following selection, being moderate growers and profuse bloomers, may be offered: Ne plus ultra, Madame Nerard, Madame Margat, Queen, Miss Glegg, Acidalie, Crimson globe, Lelieur, Grand capitaine, Proserpine, Victorieuse, Phœnix, Persian yellow, Mogador, Duchess of Sutherland, Earl Talbot, Duc d'Aumale, La Reine, Madame Laffay, Prince Albert, De Neuilly, Abbé Mioland, Clara, Clara Sylvain, Barbot, Bougère, Madame Guérin, Miranda, 'Turgot, Mansais, Triomphe du Luxembourg, Charles Souchet, Reine des Viorges, Souchet, \&c.

Propagating stocks for roses. - The wild Dog rose of our hedges and copses is no doubt too indiscriminately employed, probably on account of its being the frst sort used for the purpose, and also as it is found in great plenty in many places, and requires no previous cultivation. It should be sought for in autumn, and those having clean straight stems taken up and transplanted to the reserve garden, where they will be in condition for budding in August the following season. As we have elsewhere observed, this stock is the best for strong soils, but much less adapted for light ones than others which will be noticed below; and as this is an important matter in successful rose-growing, attention should be paid thereto. It has been recommended to bud such stocks prior to their removal, and the process has hence been denominated "hedge budding." The practice is, however, in our estimation, much less satisfactory than that of transferring them at once to the reserve garden, where they should be planted in lines 3 feet apart. The process of removal enables the cultivator to reduce the strong and rambling roots, which, when planted in a richer and more congenial soil, send out a profusion of fibrous roots the first season, rendering their removal afterwards much more certain, as well as adding to the strength of the plant, in consequence of its greater capability of drawing nourishment from the soil. Stocks, at their first removal, should be cut to the height required, weakly plants to the height of 6 inches or a foot, which will be very answerable for dwarfs, while those for half or full standards should be shortened to from 2 to 4 feet. After planting, the surface of the ground should be mulched over with littery manure, both for enrichment and for resisting drought. Hedgebudding is also performed in July or August, and the plants removed to the garden during Novem- ber following. In light soils, roses wrought on the Dog rose are seldom long lived, and there are some roses which do not even take well upon it.

The Celine rose, a very old hybrid Bourbon, makes excellent stocks, especially for roses of the Bourbon or Noisette sections, when planted in a moist rich soil. In preparing stocks, cuttings of one-year-old wood are taken, and should be cut into lengths of 1 foot each, cutting close under an eye or bud in a transverse direction, but taking the top off immediately above a bud in a sloping manner, removing all the buds excepting the two uppermost, planting them in rows, leaving only one eye or bud of the cutting above the ground by drawing up the soil around them to that height; this will keep the stem moist, and prevent exhaustion of its sap during winter and spring. Budding should take place during July and August following, at which time the soil should be withdrawn from around the stem, which, from being kept covered till then, will be in excellent condition for receiving the buds: these should be inserted at about from 6 to 8 inches from the surface of the ground.

Of the Boursault section, the Crimson or Amadis, the Reversa rosa, and the Blush or Rose de Lisle, are the most proper for stocksthe two former, as they never are attacked by mildew ; and the Crimson, in particular, is preferable even to the others, on account of its vigorous growth and abundance of sap. Like the cuttings of the Celine rose, they should be planted early in November in an open and warm part of the reserve garden, and the cuttings treated in the same manner.

Mr Rivers, a few years ago, introduced the Rosa Manettii from Milan, and has used it most successfully in his establishment as a stock, propagating them in the same manner as noticed above for the Celine and Boursault sorts. "All the roses," he observes, "I have budded on this stock have succeeded admirably-above all, the hybrid Perpetuals, which scarcely seem to know when to leave off growing and blooming in the autumn. Indeed, this stock is remarkable for its late growth, for it may be budded during the whole of September. Another excellent quality is, that it never gives any suckers from its roots; and if planted in a stiff 'loam highly manured, it will, if cut down close to the ground, make shoots in one season 6 or 8 feet in length, and will thus form fine standards. It seems to flourish equally in light and dry, as well as in stiff soils; and it will be of much value to the rose amateur, who, if the soil of his rose-garden is light and dry, is so often troubled with the numerous suckers thrown up by the Dog rose." Objections have been started against this rose as a stock by some; the arguments pro and con we need not further allude to than to quote what Mr Paul, who, with Mr Rivers, its advocate, we consider as our highest authority on the subject, says on it: "The Manettii is desirable for hardy kinds when an extremely vigorous growth is desired. It has been recommended for kinds of delicate growth, which do not thrive well on the Dog rose; but my experience 
does not uphold the recommendation. If a change of stock is necessary for such, it would seem that one of a finer, not coarser, nature than the Dog rose should be employed. That the plants grow more vigorously on the Manettii the first year we do not deny, but their subsequent decline is also more rapid." Upon a careful examination recently of the roses in our own collection, we are inclined to agree with the former authority, finding that many of our most delicate roses, in our dry light soil, succeed better on the Manettii than on the Dog rose stock; nor have we found the period of their existence during the course of our observations shortened, but rather the reverse.

T'ender roses.-Many of the more tender and weakly-growing roses may be successfully cultivated if grown on their own roots, and planted in a well-prepared border sloping towards the south, and sheltered from the east and west winds. The plants - of course, we speak of the dwarf-growing sorts-should be set at the distance of 10 or 12 inches apart, and having the spaces between them filled up with large pieces of scoria, stones, \&c., forming a sort of rock-work, which would afford protection to the roots frorn severe frosts, and also to their tops, the stones rising to about half the height of the plants. As a further protection to the roots, as well as giving the whole a more neat and orderly appearance, the spaces between the stones may be covered with green moss. Beds of delicate roses may in this way be introduced on the lawn both with good effect and success, and the more so if they be raised in the centre, particularly in the case of circular beds. In placing the stones, they should be so arranged that while they afford shelter to the flowers, the rays of the sun should not be intercepted, for the object is to afford shelter, and not shade.

Pegging down roses.--The stronger and hardier old sorts may be so treated, and when well selected and managed have a good effect, especially if the ground under them be covered with moss, flints, or small stones, to retain moisture at the roots in dry soils, and to prevent the flowers being splashed with mud during heavy rains. The practice is, however, not good as regards China, Tea, and Bourbon roses, because the shoots, when once bent down, seldom continue to grow longer, on account of a number of strong shoots which will be induced to spring up below the bent parts; and these will monopolise to themselves the sap which would otherwise go to the maintenance of the shoots bent down, and enable them to perfect their blooms. This so far might be corrected, if all such shoots as are not absolutely required for next year's flowering were cut off upon their first appearance. This would not only allow a greater share of nourishment to flow into the bent-down shoots, but it would also have the effect of showing the flower to much greater advantage.

For invigorating roses, particularly such as are grown in a poor light soil, from the time the rose-buds have begun to form until the flowers of the season have ceased to be produced, copious waterings in an enriched state should be applied to the roots, and frequent syringing over the leaves with clear water should be attended to. As soon as the blooms drop, their remains should be cut off; for allowing the rose to produce seed is more exhausting to it than two crops of flowers.

Labels for roses.-Where a collection of roses is grown, it is most desirable that they be labelled, that the owner may know what sorts he has before making further purchases. When roses, like all other plants, are correctly named, it affords no small gratification, and enables the amateur to become conversant with the nomenclature, as well as indicates that system and order prevail in the garden. Of all labels, suspended ones are the best, particularly for pillar or standard plants, as the nanie is brought within a proper distance to the eye ; and also because they are less liable to decay than such as are stuck in the ground, and are beyond the chance of displacement during the process of digging or hoeing the ground. These may be made of pine lath, cut in lengths of 3 inches, and about an inch in breadth, and one-fourth of an inch in thickness, having a perforation near the middle and towards the upper edge, sufficient to admit a piece of brass or copper wire, by which the label may be secured to the stem of the plant, the pole which supports it, or to the branches themselves. On this label, while the paint (which should be white) is still wet, the name of the variety, and the section to which it belongs, should be legibly written with a dark pencil. Such labels will last for two years, and if repainted and rewritten at the expiration of that period, will last as long. Similar labels, but of smaller size, with merely a number written upon them corresponding with the number opposite the name in the rosebook, form even a more economical and less troublesome way, as is also stamping the numbers on leaden labels, painting them on zinc or other metallic ones, or stamping them on thin slips of lead 3 inches long and half an inch in breadth, twisting the end farthest from the number round the stem or branch. Oak or pine labels 12 inches long, 2 inches broad, and $1 \frac{1}{2}$ inches in thickness, having the part stuck in the ground charred or covered with pitch, extending about an inch above the surface of the ground, and having the name written in full, or the number, as above, is also a very good way of labelling. Such labels will, however, last only for a year or two without renewal. In very large collections, cast-iron labels, with the numbers cast on their face, or the name or number painted on them, are more durable. But the most elegant and most durable are porcelain, or copper enamelled on one side, having the name or number first printed or written on it, and afterwards burnt in. Whatever kind of label is used, it is important that they be yearly examined, to see that none are displaced or obliterated. Many other sorts of labels have been recommended; those that are most durable, for very obvious reasons, are certainly to be preferred.

Growing roses in pots. - This is a modern branch of rose-culture, the absolute utility of which, so far as hardy roses are concerned, does 
not very clearly appear, beyond a compliance with the wish of some horticultural societies, who, to enhance the importance of their exhibitions, have held out prizes for roses grown in this manner. It has, however, no doubt produced two advantages - a trial of skill amongst rose-cultivators, and presenting those of the public who frequent such shows with roses in a greater state of perfection than when the flowers are cut and exhibited alone, but which they would, in all cases, see to much greater advantage by visiting the garden in which they are grown, for roses do not travel well when in a flowering state. In unpropitious soils and situations, growing the more tender roses in pots is justifiable, because during winter they can be removed to a place of protection, and during their growth in spring and season of flowering may be placed in a warm and sheltered part of the garden or rosary. Few, we opine, however, excepting those decidedly under the affliction of the "rose mania," will be disposed to cumber their already crowded greenhouses with roses of almost any description, when they can have nearly as good growing in the open air, and that at a season when roses abound. Of course, forced roses flowering at an early season are not included in these remarks. The day is, however, we hope, not far distant when houses will be erected for the sole purpose of growing the finer and more delicate roses, as in such circumstances only, in unfavourable localities, can they be expected to flower in full perfection. The mere growing them in pots, as at present practised, offers but a poor substitute for the more certain and gratifying mode of producing them under the protection of glass.

The plants for this purpose should be selected early in autumn, and, according to some cultivators, consist of such as are wrought on stems from 6 to 10 inches in height, the stems being straight and the root small, so as to be readily admitted into a proper sized pot, and rising exactly from its centre. Some may be employed of even a greater or less height of stem, according to fancy. Mr Rivers says, "from 4 to 6 inches may be taken generally as the most eligible height." The China and Tea-scented roses are found to succeed best when on their own roots, and some of the climbing kinds have been successfully grown in the same way. Plants which have been established in the open borders for one or even two years, if healthy and handsome, are eligible for taking up in October, and potting, using pots of 16 size for the smallest, and 12's for the stronger growers. The soil should be pretty strong turfy loam, to which an equal quantity of rotten cow-dung should be added, the pots thoroughly drained, and plunged in a well-exposed part of the reserve garden, the more tender sorts having the advantage of the shelter of a south wall. If the plants are strong, and have made long shoots during the previous summer, these should be shortened to about half their length at the time of potting, and again to within five or six buds about the beginning of March : the weaker ones, of course, should be cut somewhat closer in, but seldom to less than two or three buds. During April and May, if the weather is dry, water should be administered to the roots daily, and if in an enriched state so much the better: besides this, syringing over-head in the forenoon should not be neglected. About the middle of May they may be placed where they are to bloom; and if for private enjoyment, would be improved if placed in a cool pit, and covered with sashes, admitting air abundantly. This may even be found necessary with the public exhibitor, to forward his blooms to meet the day of competition. On the other hand, if they advance too rapidly, he may retard their blooming by abundance of air and partial shade, or by removing them to a pit with a northern exposure. At any rate, to have the flowers in their fullest state of perfection, they must be sheltered alike from dashing rains, strong winds, and sometimes from too powerful sunshine. We are certain the rose amateur would secure a finer display of roses were he to cover each plant in April with a cloche, or with a glazed case 18 or 20 inches square, filled in with Hartley's patent glass, keeping them a few inches clear of the ground, as by setting them on bricks, for the admission of air; and if a small opening were permanently left in the top, so much the better: in fact, so many rose-houses in miniature. When grown on their own roots, they will require one year's cultivation-that is, not to be allowed to flower until the second, and the weakest even not until the third. Great care must be taken with Chinas and Tea-scented roses that they be not over potted, and also that the compost employed be lighter than that used for the stronger-growing sorts wrought on stocks of whatever kind. During their whole progress the roots must be prevented from extending beyond the limits of the pots, which may be effected by taking them up and examining them when this is suspected to be the case. Some object to plunging altogether on this account, and also from believing that, if the roots are exposed more directly to the heat of the sun, the plants are improved thereby. There is no doubt much truth in this; but, on the other hand, the roots on the outside of the ball, and which are always the best, are liable to sustain injury when the sides of the pots become heated by the sun: of the two evils it is better to choose the former, which is the least. The roots of roses dislike heat and drought. Roses may be grown to a considerable size and for several years in pots, by attending to a regular annual shifting; and this should take place in October, when the old soil must be entirely discarded, the roots thinned, shortened, and, where dead or diseased, totally removed. They are then to be re-potted in fresh soil, and in pots the same size they formerly grew in, unless in cases where it is desired to have very large plants, or where the plants are luxuriant and healthy, when a larger pot must be given them.

Roses suitable for this mode of culture may be taken from most of the groups or sections the family is now divided into. The following are amongst the best : General Allard, General Kleber, Charles Duval, Coupe d'Hébé, Paul Perras, Richelieu, Sophie de Marsilly, Madame 
Legras, Madame Auảot, Auberon, Baronne Prevost, Persian yellow, Dr Marx, Comtesse Duchatel, William Jesse, Robin Hood, Duchess of Sutherland, Comte de Montalivet, Gloire d'Angers, Géant des Batailles, Comte d'Eu, Duchesse de Galliera, Madame Laffay, Duchesse de Montpensier, Eugène Beauharnais, Alcine, Reine d'Angleterre, Triomphe du Luxembourg, Armosa, Ceres, Comice de Seine, Abricoté, Eliza Sauvage, Adam, Souvenir de la Malmaison, Paul Joseph, George Cuvier, Julie de Fontenelle; Vicomtesse de Cazes, \&c.

\section{SELECT LIST OF ROSES;}

\section{Arranged according to the purposes for which they are best adapted.}

1. Winter roses, flowering from November to December under glass.-Comte d'Eu, Gloire de Rosamène, sect. Hybrid Perpetual ; Fellenberg, Vittelina, sect. Noisette ; Bride of Abydos, Laura, Nisida, Fragrans, Goubault, Mondor, La Sylphide, Caroline, Nina, sect. Tea-scented; Alba, Bardon, Fabvier, Belle de Florence, sect. Crimson China; Amarantine, Comice de Seime et Marne, Armosa, Celimène, Splendens, Queen, Justine, sect. Bourbon.

2. Weepiny roses, (or such as, when wrought on stems from 6 to 9 feet in height, are trained in a pendulous direction to a framework of wood or iron).-Du Luxembourg, sect. Noisette ; Félicité perpetuelle, sect. Evergreen; Adolphe, sect. Hybrid Noisette; Amadis, sect. Boursault; Belle Thurette, sect. Hybrid China; Splendens, Thoresby seedling, sect. Ayrshire ; Laure Davoust, sect. Multiflora; Pierre de St Cyr, sect. Bourbon; Princesse de Nassau, sect. Musk.

3. Pillar or pole roses.-Mrs Elliot, Louis Buonaparte, sect. Hybrid Perpetual ; Brennus, Fulgens, Chénédole, Richelieu, Vulcan, Juillet, Decandolle, Petit Pierre, Vingtneuf, sect. Hybrid China; Madame Hardy, La Ville de Bruxelles, sect. Damask ; Emperor, Princess Royal, Princess Adelaide, sect. Moss; Madame Plantier, Madeline, sect. Hybrid Noisette ; Henri Barbet, Victor Hugo, Great Western, Charles Duval, Paul Perras, sect. Hybrid Bourbon; Bouquet de Flore, Acidalie, Madame Desprez, Pierre de St Cyr, Amenaide, Imperatrice, Josephine, Madame Aude, sect. Bourbon; Félicité Parmentier, sect. Alba.

4. Exhibition roses with large full flowers.Eugène Beauharnais, sect. Crimson China; Comte de Paris, Triomphe du Luxembourg, Bougère, Moiret, Devoniensis, Souvenir d'un Ami, Josephine Malton, Silène, La Renommée, sect. Tea-scented; Madame Bréon, Clara Sylvain, sect. China ; Souvenir de la Malmaison, Le Camée, Acidalia, Madame Nerard, Madame Souchet, Le florifère, Lichas, Dupetit 'Thouars, sect. Bourbon ; Baronne Prevost, Duchess of Sutherland, La Reine, William Jesse, Dr Marx, Madame Laffay, Comte d'Egmont, Robin Hood, Reine de fleurs, sect. Hybrid Perpetual; Madame Hardy, Triomphe de Rouen, Madame Soetmans, sect. Damask; Persian yellow, sect. Austrian; Cristata, Laura, Adrienne de Cardoville, sect. Provence; Coupe d'Hébé, Paul Perras, Victor
Hugo, Charles Duval, Légouvé, Tippoo Saib, Comte Boubert, Sylvain, sect. Hybrid Bourbon; Laneii, Atna, White Bath, Alice Leroi, Louise Colet, Blush, Comtesse de Noé, sect. Moss ; Brennus, Chénédole, Galien, Marjolin, Comtesse Plater, Belle Marie, Lady Stuart, sect. Hybrid China; Adèle Prévost, Berénice, Colonel Coombs, Guillaume Tell, La Ville de Gand, Leo the Tenth, Ne plus ultra, Surpasse tout, Sophie Duval, Randolph, Omphale, La Ville de Londres, Ohl, Belle Rosine, Cambronne, Celestine, Couronne d'Amour, Duc de Trévise, Duc de Valmy, Franklin, Gloire de Trevise, Gloire des Amateurs, Jeanne de Laval, Eclat des Roses, sect. French ; La Vestale, Alette, Dubois Dessauzais, Pauline Garcia, sect. Hybrid French.

5. Roses for grouping to produce contrasted colours.-Armosa, Princesse Clementine, Queen, Mrs Bosanquet, Paul Joseph, sect. Bourbon; Beauty of Billiard, Belle Thurette, sect. Hybrid China; Madame Laffay, sect. Hybrid Perpetual ; Miss Glegg, Le Pactole, sect. Noisette ; Fabvier, Belle de Florence, Belle Emilie, sect. Crimson China; Déesseflore, sect. Damask; Madame Plantier, sect. Hybrid Noisette.

6. Yellow roses.-Clara Wendel, Pactolus, Solfaterre, sect. Noisette ; Harrisonii, Persian yellow, sect. Austrian; Eliza Sauvage, Safrano, Devoniensis, Vicomtesse de Cazes, Princesse Adelaide, Pellonia, Diana Vernon, sect. Teascented; Old double yellow, Rosa sulphurea, in situations where it is likely to flower.

7. Climbing roses.-(1) Amadis, Inermis, Elegans, Drummond's thornless; (2) Red Boursault, sect. Boursault; (3) Ruga, Splendens, Countess of Leven; (4) Queen of the Belgians, Splendens, Ruga, Dundee rambler, sect. Ayrshire ; (5) Félicité perpetuelle, Princess Louise; (6) Banksiæflora, Carnea grandiflora, spectabilis, Leopoldine d'Orleans, Rampant, Princesse Marie, sect. Evergreen; (7) Russelliana ; (8) Graulhie, De la Grifferaie, sect. Multiflora; (9) Madame d'Arblay, Garland, sect. Hybrid; (10) Grandiflora, Du Luxembourg, Belle d'Esquermes, sect. Noisette; (11) Beauty of the Prairies, sect. Rubifolia; (12) Princess de Nassau, Double Red, Eponine, sect. Musk.-Those marked 1, 3, 5, 8, 9,11 , and 12, are of extremely rapid growth, and useful for covering surfaces quickly; 2,4 , $6,7,10$, are adapted for a shady wall or norther'n aspect.

8. Roses for covering naked banks, rocks, \&cc., of trailing and rapid-growing habit, amongst which are some fine roses of the kinds, producing a grand effect in places which are often deformities in grounds until so covered.-Leopoldine d'Orleans, Thoresbyana, or Bennet's rose, Alice Gray, Lovely rambler, Rampante, Countess of Leven, Félicité perpetuelle. These are all of the Ayrshire or Evergreen sections.

9. Roses suitable for planting on warm and well-exposed walls, fronts or gables of houses, and in very cold localities requiring slight protection during winter:-La Biche, Solfaterre, Grevillei, Maria Leonida, the White and Yellow Banksian, Cloth-of-Gold, Aurora, Lamarque, Desprez, Triomphe de Macheteaux, Rosa sulphurea, or Old double yellow.

10. Very sweet-scented roses.-Minerva, Riego, 
Aubernon, Adam, Hamon, Rachel, Belle Fabert, Goubault, Louis Puget, Fulgoric, Belle Allemande, Clementine Seringe.

11. Roses with mottled or striped flowers.Perle des Panachées, Rosa Mundi, Village maid, Nero, Madame Campan, Panache pleine, Duc d'Orleans, Unique panache superbe, Tricolore d'Orleans, Donna Sol, Superb marbled, Aramis, Duc de Bassano, Comte de Murinais, Omphale.

12. Select list of much esteemed varieties. (Those marked P. are strong growers, and adapted for growing as pyramids, poles, or pillars, and include most of the finest roses in cultivation).-Adam Paul, Acidalie P., Aimée Vibert, Aristobule, Antenor, Alexandrine Bachmeteff, Angeline Granger, Auguste Mée, Augustine Mouchelet P., Aramis, Archiduc Charles, Abricoté, Adam, Adelaide d'Orleans ; Beauté Lyonnaise, Blanche Lafitte, Bouquet de Flore P., Blanche de Beaulieu, Blanche de Portemer, Bernard, Banksiæflora, Bernard Palissy, Beauty of Billiar'd, Blairii P., Brennus P., Bizarre marbrée, Boula de Nanteuil, Blanchefleur, Baronne Heecheren, Baronne Prerost, Beranger ; Catherine de Wurtemburg P., Celina, Comte Plater P., Comtesse de Segur, Chénédole P., Comtesse Lacepède, Charles Duval, Coup d'Hébé P., Grivellii, Crimson superb, Caroline de Sansalles, Charles Boissière, Chateaubriand, Chereau, Colonel Lorry, Cymedor, Comte de Nanteuil, Comte Montalivet, Comtesse Duchatel, Comtesse de Bathiany, Ceres, Cezarine Souchet, Charles Suuchet. Comte de Bobrinsky, Comte de Paris, Cloth-ofGold, Charles Lawson; Devoniensis, Dupetit Thouars, d'Assas, Diodore, Dr Arnal, Duchesse de Montpensier, Duchesse d'Orleans, Duchess of Sutherland, De la Grifferaie, Dometille Becar, Duc de Trevise, Devigne P.; Eugenie Dessauzais, Eblouissante de la queue, Eugene Sue, Edouard Defosse P., Enfant d'Ajaccio P., Eliza Sauvage, Eugenie Desgaches; Fairy Queen (Paul's), Ferdinand Deppe, Fortuniana, Frederick II. P., Fulgens, Félicité (Parmentier), Fringed Provence; General Foy, Gloire de France, Gloire de Colmar, General Bertrand, General Changarnier, General Bernard, General Jacqueminot, Gloire de Couline, Garibaldi, Gracilis (Boursault), Géant des Batailles, General Negrier, General Cavaignac, General Bedeau, General Castellane, Graziella, General Drouet, George Cuvier, Georges de France; Henri Lecoq, Herman Kegel, Harrisonii; Je me maintiendrai, Jupiter ponctue, Juno, Jaunatre, Inermis, Isocrate, Jacques Lafitte P., James Veitch, Jeanne d'Arc, Joseph Decaisne, Julia; Laneii P., La Ville de Londres P., Ia Ville de Bruxelles P., Lucrèce, La Ville de Gand, Letitia, L'Attrayante, Las Casas P., Laure Davoust, La Reine P., L'Enfant du Mont Carmel, Lady Stuart, Le Lion des Combats, Louise Odier, Louise Peronne, Leonore d'Este, La Favourite, La Sylphide, Le Pactole, Lamarque; Melanie Willermoz, Madame Oger, Madame d'Arblay, Madame Sylvestre, Madame Bréon, Mrs Bosanquet, Madame Angelina, Madame Souchet, Madame Tripet, Menoux, Miroir de Perfection, Madame Campan, Madame Andry, Madame Ducher, Madame Fremion, Madame Hilaire, Madame Lamoricière, Madame Trudeaux P., Mrs Elliot,
Mrs Rivers, Marianne, Mathieu Mole, Madame Legras, Madame Hardy, Madame Zoutman, Madame Alboni, Madame Audot P. ; Napoleon, Niphetos, Narcisse ; Ohl, Eillet parfait; Princesse Adelaide P., Princesse-Royale (Portemer), Princesse Clementine, Princesse Lamballe, Pierre Jaussens, Perle des panachées, Paul Perras, Paul Ricaut, President Mole P., Persian yellow, Princesse Marie, Pius IX., Paul Dupuy, Prince Leon Kotschoubay, Princesse de Nassau; Reveil, Robin Hood, Rosine Margottin, Reine des Fleur's, Reine Blanche, Rose du Roi, Reine d'Angleterre, Rose amiable; Semiramis, Sylvain, Souvenir de la Reine des Belges, Standard of Marengo, Souvenir de Leveson Gower, Salvator Rosa, Souchet, Souvenir de la Malmaison, Souvenir de l'Exposition, Safrano, Souvenir d'un Ami, Solfaterre; Triomphe de Bolwyller, Triomphe du Luxembourg, Triomphe de Paris, Triomphe de Jaussens ; Unique panache, Unique de Provence; William Jesse, William Griffiths, William the Conqueror; Volta, Vorace, Vicomte de Cussy, Vicomtesse de Cazes.

Diseases.-Mildew is by far the most prevalent, and is often very destructive to young roses in their seedling state. It also attacks plants when fully grown, particularly in dry soils, and during dry seasons, and some sorts are much more liable to it than others. The best remedy is to syringe the foliage towards evening, and immediately afterwards to dust it over with flour of sulphur. Where this may be objected to on account of appearance, sulphur in a dry state may be applied by the sulphurator during the day, and while the leaves are dry. Watering with a solution of nitre is said to destroy it. In open well-exposed situations the disease seldom occurs, whereas in pent-up and shaded places its appearance is certain.

The Red fungus, probably a species of Uredo, often attacks roses during autumn. Rubbing the leaves between the finger and thumb is an effectual although a slow process. Dusting them with sulphur, or finely-powdered lime in a fresh state, is more expeditious and certain.

Green centres appearing in roses may be considered a disease. The consequence is the total destruction of such blooms as they occur in. The cause has been very satisfactorily traced to excessive manuring with rank or green manure, and this points out the proper remedy.

For Insects, see end of FLOWER-GARDEN.

\section{$\S$ 3.-THE LAWN.}

The ground immediately surrounding the mansion is by universal consent denominated the lawn; and with the exceptions where modern good taste has overcome the prejudices of a bygone age, and so far restored architectural gardens and terraces on one or more sides of the house, the lawn is made to finish close up to the walls, an unbroken surface of grassy sward, merely reduced in level so far as to render it comfortable to walk upon, and often with no other accompaniment to the shaven grass but some paltry wire fence to divide it from the park, and probably two or three clumps of the most unmean- 
ing for'ms, planted with shrubs as little in keeping with the house or surrounding objects as the forms of the clumps themselves. Such may be considered as the lowest grade of lawn. The natural disposition of the ground is seldom in strict keeping with the building while unaided by the hand of man, even should that building be of the most unpretending description. Sir Uvedale Price observes: "Where architecture, even of the simplest kind, is employed in the dwellings of man, art must be manifest; and all artificial objects may certainly admit, and in many cases require, the accompaniments of art ; for to go at once from art to unadorned nature is too sudden a transition, and wants that sort of gradation and congruity which, except in particular cases, is so necessary in all that is to please the eye and the mind."

In anticipation, as it were, of the taste which is now every day becoming more clearly developed, Sir Thomas Dick Lauder remarks : "It is pleasing to think, however, that those more immediate accompaniments of a house, the terrace, the balustrade, \&c., are now every day becoming more generally considered as essential, and are attended to and studied by the ablest architects ; so that we may hope soon to see the custom of putting down a house in the middle of a shaven grass lawn, like a teabox on a green carpet, altogether exploded." Whatever objections there may be in the minds of some against terraces, parapets, \&c., and symmetry and regularity in the grounds in immedrate connection with the house, the man of refined taste will admit of these being essential, even when carried to the highest degree of decoration, because he knows that they accord with, and are intended to accompany, that which is regular, symmetrical, decorative, and architectural. Sir Thomas Dick Lauder, in allusion to this, further remarks: "There is nothing of which I am more convinced, than of the propriety and necessity of allowing the art to become more apparent in the gardening which comes into immediate contiguity with the mansion. My rule would be, that the house, and its subsidiary buildings, should be directly associated with designs of a character which may have much of architectural regularity, as well as architectural features, about them. By this means the house itself would not only become a more pleasing object to look at from all points, but the different views enjoyed from it become much more interesting from the enrichment of the foreground by minor architectural objects. Straight-lined terraces, bowling-greens, balustrades, vases, sun-dials, architectural seats, fountains, and statues, mingled with a profusion of shrubs, plants, and creepers, are all appropriate and useful decorations in such a place." A lawn without these, be it ever so extensive, or ever so well kept, will always have a tame, naked, and unfurnished appearance. It is not, however, the extent of space over which these accompaniments are disposed-it is their arrangement, so that they shall have a just connection with each other; for as they, one and all, form accompaniments to the house or mansion, they VOL. II. should never be removed to too great a distance from it. Nowhere in Britain is this more beautifully exemplified than at Osborne, the marine residence of her Majesty, in the Isle of Wight. There, on the principal fiont, is laid out a very artistically-arranged terrace-garden, on two levels, connected with spacious flights of steps, with corresponding balustrading, the terminations at both ends, which are on a level with the upper terrace, projecting as far as the front of the lower one, producing those breaks and irregularities in the lines so necessary in the style of architecture there adopted. Gravel or grass, in close connection with architecture, has always a meagre and insipid effect, as if the most classic building were placed in a ploughed field, or surrounded by a dug border. In the case to which we have pointed, that too general error has not been fallen into. The terraces are in part covered with asphalt, so prepared as very closely to resemble stone pavement, and a large portion of them is paved with Minton's encaustic tiles, rendering them at all times dry and fit to walk upon, and forming the proper connection between them and the building. The flower-beds, which are not crowded, are geometrical figures, bordered round with polished stone margins. The balustrading, para pets, and coping, are all in strict accordance with the style of the mansion, which is Italian. Fountains, statuary, niches, and mural decorations, are all correctly disposea, as is also the Italian colonnade, over which roses and vines are trained. Beyond this is the lawn, softened down with great skill, and imperceptibly uniting with the park beyond, which is pastured with sheep up to the base of the external parapet-wall.

Upon a much smaller scale the same thing could be, and in many cases is, admirably accomplished. The smallest villa can have its pavement terrace, its flower-plots surrounded with stone or slate edging, its tiny fountain, its parapet-wall, ornamented or plain, and its flights of steps to the garden below. Such terraces should have a relative proportion in breadth to the height and magnitude of the building. Grass terraces, in all cases except in flower-gardens, and in those ouly when distant from the mansion, have a tame and meagre appearance. They have no just association with architecture, more especially those nearest to the building. The introduction of stone steps in them points out the incongruity, and shows the total want of connection between them; and grass steps betray a poverty of conception in the artist or taste in the owner. Around the house, therefore, be its style what it may, we would have no lawn on what is called the garden fronts until the terraces are first disposed of. The case is, however, otherwise on the carriage-approach fronts, where a reasonable extent of grass lawn may be with propriety permitted. But here, in the majority of cases, a serious difficulty presents itself; for to have a lawn in front of the house, nicely kept with scythe, roller, and broom, it requires to be enclosed from the park for the exclusion of cattle and sheep. This involves the necessity for gates, which are trouble- 
some affairs when crossing the carriage-approach. For this there are only two alternatives; either the inconvenience of the gates must be submitted to, or the sheep and deer must be permitted to feed up to the front door, which, in places of large extent,' is no doubt the most desirable; the shrubbery and dressed ground being protected with wire-fencing so carried that no obstruction may occur in the approach.

In the disposal of the terrace on the garden fronts of the house, we may remark, that in the case of a small villa residence, one terrace may be, and no doubt is, often sufficient; while, in the case of larger structures, two or more may be required-much, however, depending on the inclination of the ground as it recedes from the house; or the provision made by keeping the principal floor-level sufficiently high to admit of the various descents being carried out, as if in reality the terraces were necessary for the finishing of the grounds around. To excavate the ground to a greater depth than that of the natural level, where the last terrace terminates, would, in most cases, produce distortion, and justly create ridicule. The same care should be taken lest the terminating terrace be kept at too high a level, and, as it were, in the air. In the latter case, under certain circumstances, return terraces may be adopted, descending so that the last may nearly unite with the grounds immediately in front of it. In situations where the ground in front is level, or nearly so, with the principal floor of the house, the stone terrace, with a proportionable balustrade, should run parallel with the building, and on the same level with the garden entrance-door; and that terrace may be carried round the ground intended for the principal flower-garden, and at the same level, substituting gravel, however, for stone pavement, where the side terraces join the main one, but carrying the parapets on both sides of the walk all round. The space enclosed may then be excavated to the extent of 3 feet, and the parterres laid out in geometric figures upon a grass ground, if the extent be large, say exceeding two acres; but if not exceeding half an acre, it had better be laid on a gravel ground, with stone or slate margins to the beds. The same holds good with spaces of less dimensions. When grass is introduced in such gardens, it should be limited to broad verges, separating the walks from the parterres. Flights of stone steps should descend from the centre, and also fiom the two ends of the principal terrace, and unite with that in front, ascending by the same means, thus admitting of a gravelled walk surrounding the ground parallel with the terrace above, and half its breadth distant from the surrounding parapet. In large spaces, similar gravel-walks should intersect the ground at right angles with the surrounding ones, to admit of a sufficiently dry promenade when the ground is to be traversed. Where these walks intersect each other, circular spaces of gravel should be introduced, and on them fountains or sculptural ornaments should be placed. If the ground can be rendered sufficiently dry by drainage, many of the flower-beds may be sunk in the panelled manner, which will afford shelter to the plants from the wind should the situation require it. A parterre garden in this style will be viewed with advantage from the terraces which surround it, and more especially from the principal windows, which are in general on the second floor. The stone terrace here described adjoining the house, and the double line of parapets along the other three sides of the garden, will form the architectural accompaniments; the geometrical parterres, fountains, or sculpture, with the straight broad gravel walks, will form, as it were, its episode.

The proper management of terraces is a difficult matter, and is evidently less understood now than formerly. The architectural student and landscape-gardener would do well to consult "James's Translation of La Blond on French Gardening." The great errors fallen into in the present day are, either carrying them too far, or not carrying them out far enough. One general rule should, however, never be lost sight of-namely, if the planes or level spaces between them are to be laid out in parterres, or otherwise planted, the upper parapet should not be carried so high as to hide the others, or their planes, when viewed from the upper terrace, or from those points from which the whole is to be viewed at once. In the case of grass terraces this is a less difficult matter, as the greater length given to the slope in each will throw out the level parts so far as to be readily seen. Another general rule, we think, may be offered, namely, that the upper or principal terrace should be of stone, with its pavement, close parapet, or open balustrade, and corresponding coping. Those under it may be of grass, on the score of economy, at first erection, but their keeping will in time amount to their original cost in stone. The terminating terrace should finish with a retaining wall or ha-ha of sufficient height to render the rest secure against the intrusion of cattle, and also to form the line of demarcation between the highly-dressed or artificial and the natural grounds. Terraces are as much a work of art as the mansion they are connected with, and to attempt to unite them by means of artificial sloping banks of turf, by whatever gradations, with the natural ground around, is, in fact, deserting art, and vainly endeavouring to embrace nature. The line of separation between the lowest or extreme terrace may, in cases where the grounds beyond harmonise in level with it, be a single ha-ha fence; but when otherwise, that ha-ha should be finished at top with a parapet or balustrading not less than 2 feet in height. The end terminations of all terraces-that is, where they join with the natural banks into which they run, or where they are stopped by abrupt declivities, as well as their lengths, breadths, and angles of inclination-can never be correctly determined by general rules; much, therefore, in these respects, must be left to the discretion of the artist upon the spot.

The proper situation for the principal flower- 
garden, in most cases, is on one or other of the garden fronts of the house. This, in some respects, depends upon which front the principal rooms look to. Where they open towards the north, which is, however, not often the case, the flower-garden may be placed there, because the flowers, when looked upon, will have their faces, as it were, turned in the direction of the house, as all flowers turn towards the sun. When the garden is placed on the south front, the backs of the flowers are only to be seen ; and of course, whether upon the east or west front, their side view is only available, in all these cases producing a far less imposing effect than when viewed from the southern front. These are points which should be determined either before the house is built, or before the site of the garden is fixed upon. At all events, the flower-garden should be descended to from the telrace, and not only seen from it, but from the principal windows also, for the enjoyment it affords, as well as for the union it creates between the house and the grounds.

The next in order to the stone terrace is, where a broad gravelly walk bordered with grass surrounds two or more sides of the house. The gravel, no doubt, affords a dry, and so far a comfortable promenade, but its pared edges are meagre and formal. The inferiority of the gravel walk in such a situation, according to the views of Sir Uvedale Price, proceeds from other circumstances besidesthose of formality and want of effect. "Its boundary," he remarks, " is not only meagre as well as formal, but is incapable of receiving any ornament, or of being varied with any effect. The parapet, on the contrary, admits of a great degree of ornament, and also, what is very material, of a mixture of the light and pliant forms of vegetation with the uniform unbending substance of stone and the enrichment of the sculpture. Should the solid wall be thought too heavy, a balustrade, without destroying the breadth, gives a play of light and shadow of the most striking kind, which occurs in the works of all the painters. On the top of the coping, urns, vases, flower-pots, \&c., of every shape and size, find their place; vines, jasmines, and other beautiful and fragrant climbing plants, might add their loose festoons to those imitated in sculpture, twining round and between the balusters, clustering on the top, and varying the height of the wall in every style and degree that the painter might direct." The effect of the former is only somewhat improved by a broad gravel-walk, with a still broader border of scroll-work, either in box embroidery, or filled with plants flowering at the same time, and harmoniously arranged, with a gravel walk of narrower dimensions between it and the terrace, formed by a green sloping bank and grass margin on top. But carry the principle farther, introduce an open balustrade instead of the margin of grass; surmount it with vases or other sculptured ornaments, arrange the flower-beds in geometrical forms with raised margins of stone, and if upon a sufficiently extensive scale, introduce fountains and statuary and stone descents from one terrace to another until the line of the natural ground be arrived at. If these terraces extend to only two, an upper and a lower one, of proportionable length and breadth according to the magnitude of the mansion, the effect will be more pleasing than were they carried farther out, increasing in number, and decreasing in size and importance. All terraces should extend the whole length of the mansion, and only when they cannot well be united with the side grounds should they extend much farther. In such cases they may be made to return along the other sides of the house, rising or falling in level according to circumstances. Nor should they terminate abruptly, especially when the ground beyond falls considerably; and hence the importance of flights of steps at their ends, leading the visitor from one terrace to another, and obviating the unpleasant necessity of retracing his steps. An abrupt termination-where the ground falls suddenly-may often be remedied by placing an alcove at the end; but even here, steps leading to the next terrace, either above or below, become necessary, so that the continuation may not be interrupted. At the ends, again, where the ground rises, such stairs, instead of being placed on the side of the terrace-walk, may be placed in front of it, indicating to the visitor as he approaches it that there are means for his proceeding farther.

The lawn, according to the present meaning of the word, implies greater or lesser breadths of grass, extending, as they often do in large domains, to ten, twenty, fifty, or more acres of ground, kept shaven and shorn by the scythe or machine, and at an expense which would be far better disbursed in the higher keeping of parterres in immediate proximity with the mansion. In smaller places a similar wasteful expenditure takes place, while the effect produced is little better than if the operation of keeping ${ }^{*}$ down the grass were intrusted to the sheep and the deer. Yet we are told by the advocates of modern landscape-gardening that these closely shaven lawns are natural, and in accordance with picturesque beauty - the object they profess to imitate. By carrying them up to the very threshold of the door of the house, they usurp the province of artistic gardening, and substitute for the highest grade of external decoration those imitations which, in good taste, should only be employed in connecting, as it were imperceptibly, art with nature, at a greater distance from the house. Beyond the line we have drawn the lawn should commence. Its keeping and arrangement, as regards planting, levelling, \&c., should be more attended to near the house than at a greater distance from it, because it should in these respects become lost, as it were, in the park or plantations ; and also because it should form the foreground of the piece, while the park should form the middle, and the distant country the extremity of the picture. There are cases, however, where the whole of these must be confined to the lawn and park; such, for instance, as where disagreeable objects might be in sight, which it would be judicious to exclude, and also in exposed situations, where shelter is an object. The size 
of the lawn should be in proportion to the place, as well as in others for privacy, and the means of agreeably uniting one part of the grounds with another. In small places, the lawn may occupy the whole space with the exception of the boundary screen of plantations or shrubberies, kitchen-garden, and such spaces as may be planted for the purpose of effect; and in such, groups of American plants, ornamental trees and shrubs, roses, \&c., may be employed ; nay, by judicious arrangement, the American garden, Rosary, Florist's flower-garden, Alpine garden, Perennial plant and reserve garden, may be each introduced with sufficient effect, when approached, but not to be seen from the windows of the mansion-the parterre being understood to adjoin the house. In places of greater extent, all these can readily be carried into effect without in any way interfering with the general effect of the whole. With the parterre or terraced garden on one or more of the garden fronts of the house, the lawn in sight of the principal windows should not be broken up with flower-plots, the proper furnishing for it being groups of trees and shrubs, either singly or combined; nor should these be so placed or crowded as to reduce its apparent dimensions, and the depth of views seen through unbroken grass glades between them. The trees and shrubs on the lawn should all be disposed of in the gardenesque manner ; that is, so that each individual plant may assume its natural shape and habit of growth. Where masses are planted to produce immediate effect, care must be taken that they be thinned out in time, so that each tree, when separated from the mass, shall appear a perfect specimen of its kind. While we deprecate the idea of crowding the lawn with common oaks, elms, or beeches, we would equally avoid planting such trees as are too tender, or incapable of arriving at their fullest and most perfect development. A flower-garden in the natural style may be placed near the extremity of the lawn, if masked out by shrubs, \&c., till entered; it will invite to frequent visits, and form the termination of the series of distant gardens we have alluded to, and connect them with the kitchen-garden, from which it should be not far distant; while the kitchen-garden itself should be placed at the very extremity. The bowling-green, on the other hand, should be on the lawn, and near to the house; yet it is desirable that it should be planted out, if such can be done without prejudice to general effect: even if exposed, it in no way breaks into the lawn, on account of its being of the same colour.

The bowling-green should be rendered perfectly dry by drainage, and of course made as level as possible, and kept closely mown, and repeatedly rolled. As to form, that of a parallelogram is the best; that of a circle, although the form longest in use, the most absurd for the purpose. The lawn or flower-garden in the irregular style should have a good exposure to the sun and air; yet, to prevent the effects of high winds, it should be sheltered, but not, as is too often done, by high trees, whose lengthened shadows during winter render it cold and damp ; while deciduous ones, in shedding their leaves in autumn, not only give it a littery appearance, but these, if not gathered up in time, injure the late-flowering plants. An espalier or covered walk, planted with ornamental flowering shrubs, will afford sufficient shelter, if backed at a distance by trees, while in itself it associates with the plants it surrounds, and adds to the gaiety of the scene by the profusion of flowers produced on it. Whether the design of the beds be in the geometric or natural style matters little, so long as distorted lines are avoided, and the compartments are not too large; the groundwork or walks between them are of greater consequence. The prevailing taste is in favour of grass, on account, as is said, of the more cheerful and clothed appearance the garden has during winter. It should, however, be borne in mind that a flower-garden at a distance from the house is rarely visited during that season, and that, in a country like most of Scotland, it is not available at all times, even during summer, on account of the dampness of the grass. If grass, therefore, is employed, dry gravel-walks should be introduced to the extent of admitting of its being visited at all times; and these, in whichever of the styles the garden may be laid out, can be introduced without at all affecting the general expression of the whole. Presuming the terraced garden in connection with the house is planted in the grouping manner-that is, with due consideration to the effects of colours when harmoniously arrangedthe lawn flower-garden, if even for variety, may be planted in the mixed manner-that is, having flowering shrubs, roses, herbaceous and annual plants, intermingled together in agreeable variety. In planting such a garden, the greatest attention should be paid, so that its arrangement may not only form an effective whole, but that it shall also display secondary and even minor effects in individual groups, when seen in parts where the whole cannot be comprehended at once. The want of conformity in this respect has no doubt a good effect when seen from certain leading points of view, but when examined in close detail they lose all their interest except what arises from individual subjects, or from these when grouped in masses, when they produce secondary and minor effects. Under ordinary circumstances, such a garden may, with all propriety, contain a certain number of artistic decorations; but when laid out in the highest style of keeping, these may be carried to a much greater extent, particularly as regards fountains and vases. The mixed flower-garden may be laid out on a perfect flat, but where the inequality of the ground admits of it, terraces are quite apropos, and the more especially where conservatories or plant-houses form an adjunct. These, however, should be elevated not less than 3 feet above the garden below, and a terracewalk should separate the one from the other, the terrace to form the base of the building, and to give greater elevation to it. The flower-garden, whether in the irregular or the geometric style, will be seen from this terrace to greater advantage than if viewed as a whole from any part 
within its own boundary, and on the same level as the beds. When the irregular style is adopted, and the beds are considerably elevated above the grass plats or gravel walks, the general effect is lost, and then every turuing must produce its own points of attraction, the one succeeding the other in endless variety.

In connecting the various parts of the lawn, and uniting, as it were, together the American garden, rosary, \&c., with the mixed or lawn flower-garden, kitchen-gardel, \&c. gravel walks become necessary. The lines of these should be so contrived that they be as little seen from the mansion as possible. For, however necessary they are in themselves, if allowed to cut the grounds into pieces, they destroy much of the effect so greatly to be desired in the production of an entire and unbroken lawn. In proceeding from the house they should be carried along the sides of the lawn in a circuitous manner, bending in one direction or another in graceful curves, but always showing some apparent reason for each turning. Thus curves may be taken inwards towards the lawn, at such points as it may be best seen from; others outwards towards the boundary of the grounds, where the finest distant prospects may be viewed to the greatest advantage. A fine tree or shrub, a projecting piece of rock or raised bank, as well as a pit or other inequality in the ground, all offer sufficient reasons for changing the course of the walk, either to see the former to advantage, or to avoid any of the latter. All unnecessary bends should be avoided, particularly in the principal walks. Where any of these obstacles do not occur naturally, they may be made to do so artificially, but seldom with the same good effect. Secondary walks are necessary, particularly in extensive grounds; and these may, by good management, often be made to connect various points of the main ones, to shorten the distance from one point to another, as well as to lead to objects of less importance. The proper management of the direction of walks is an important part of the landscape-gardener's art. They should not be too numerous, else they lead to confusion. They should be made to avoid all disagreeable objects, as well as the least interesting parts of the grounds, and be conducted so that these may be shown to the best advantage. The principal walks should not be less than 7 feet broad, so that three persons may walk comfortably abreast; the secondary ones not less than 5 ; but mere footpaths may only be 3 feet, sufficient for one person to pass along. Resting-places should be provided at convenient distances apart, and in extensive grounds places of shelter as well as retiring to should be formed. In carrying the gravel walks through the lawn, it is always desirable that they be concealed in such a manner that the party traversing them may see no other walk than that which lies immediately before him; so that, as Downing rightly remarks, a person walking in the grounds, in looking across the lawn, can never discover the extent either of what he has seen, or what he has yet to see. To form a great number of walks of this sort, and lead the spectator over them without show- ing him more than one walk at a time, but taking care at the same time to let him have frequent and extensive views across the lawn, and these views always different, constitutes the grand secret of making a small place look large.

The formation of walks. - The proper construction of walks is seldom sufficiently attended to. The great object to be kept in view is to render them dry, and fit to be used at all seasons, and this cannot be doue if they are not efficiently drained below. To effect this in the most substantial manner, the ground should be excavated, in the case of principal ones, to the depth of 12 or 15 inches, depending on the soil and locality, whether it be wet or dry. Upon rocky or gravelly bottoms dryness is easily effected, and such need not exceed half of that depth. In soils of an opposite character, and where much rain falls, such as on the west coast, not only will the former depth be required, but a course of drainpipes, of 3 inches in diameter should be laid along both their sides, with eyes or small cesspools at every thirty or forty yards, on level surfaces - much closer, however, together on steep inclines-furnished with iron gratings laid 2 inches under the level of the surface of the finished gravel, to receive the surface-water during heavy rains. These drains should discharge themselves in hollow places, or be connected with other and larger drains. The bottom of the walk should be filled with the best material the situation offers, such as stones, scoria, brickbats, flints, coarse gravel, or other porous and hard material. Two inches of stones, broken to about 1 inch in diameter, or gravel of the same dimensions, to be laid over the first, and 3 inches of the best pit-gravel employed as a finishing, and which, when laid down, should be well trodden down and rolled until the surface becomes smooth and uniform. The gravel should reach as high as to be within 1 inch of the grass verges, and the centre should have a rise of 2 inches for a 7 -feet walk, to allow the water to run to both sides; if narrower or broader, a rise in the same proportion. Many substitutes for gravel have been recommended; none, however, harmonises so well as gravel of a yellowish tint with the grassy margins.

Grass walks often become necessary in connecting one side of the lawn with the other; and these, if properly laid down in the first instance, require little after-keeping than repeated mowing. Gravel walks are also in cases required for the same purpose; and if these can be rendered sufficientlydry below, they may, to prevent their appearing to cut the lawn in two, be kept from 6 to 10 inches below the grass-level, having the sides sloping down to within 1 inch of the gravel. All deep-cut edges, particularly when not covered with grass, have a harsh and unpleasant effect. For this reason they should be kept straight by using the edging-shears, instead of being cut with the edging-iron. Where pitgravel is of a bad colour, or apt to become muddy after rains, a thin sprinkling of sea-gravel, where it can be procured, may be laid on the surface once or twice a-year, but only to the extent of merely altering the colour, because, if laid on 
to a greater depth, it would render walking uncomfortable on account of its not binding. In extreme cases, where gravel is scarce, and the annual fall of rain much, asphalt laid on a sound and dry bottom of stones or coarse gravel will make a comfortable walk, and its colour may be modified by a mixture of fine sharp gravel laid on at the time of its formation; and concrete walks have been recommended by Mr Beaton, whose mode of construction is as follows: "A layer of stones, brickbats, shells, or clinkers, 6 inches deep, to form a dry bottom; a layer of chalk or lime, in the proportion of one to ten of the stones or other foundation, and well rolled and watered, to the thickness of 3 inches, with a rise of 2 inches in the centre; over this, half an inch of gravel and lime or fine chalk; water and roll well again; add one-eighth of an inch of the best coloured gravel, finely sifted, and again roll until quite solid. Have the walk 2 inches wider on each side than you desire, as this checks the turf and weeds from encroaching, and prevents the rain water getting to the foundation of the walk." Such walks are valuable on steep declivities, and prevent the possibility of the surface being washed away by heavy rains. Such walks are easily kept, as weeds will not grow upon them, and they also require neither hoeing nor rolling. In regard to keeping, the less the surface is disturbed the better, and therefore handpicking and rolling are better than hoeing and raking. Hand-weeding is no doubt a tedious process, and even where boys are employed it is attended with considerable expense. To obviate this, Mr Fleming of Trentham has invented an ingenious machine, consisting of a boiler, furnace, and chimney attached, and mounted on broad wheels, by which it is easily run along the walks. In every gallon of water put into the boiler, about two pounds of common salt is dissolved; and the mixture, while in a boiling state, is dispersed equally over the surface of the walks by means of a common garden watering-pot, having a rose upon the spout; and all plants, seeds, and insects which are touched with the hot solution are immediately destroyed. The only care necessary to be taken in its application is to prevent the solution falling on the box or grass verges.

With a view to effect a somewhat efficient weed-destroyer on gravel walks, without disturbing the surface, we have in course of construction a roller internally heated to almost a red heat, and that heat maintained by means of a fire burning within it. This is drawn over the gravel as in the ordinary mode of rolling; the weeds are burned over by the surface. Where, from the nature of the material, gravel walks are not kept in a hardened state, various kinds of implements have been employed both in this country and on the Continent; one of the best of these is that invented by Mr Carnegie, and manufactured, as is also Mr Fleming's salting machine, by Messrs Shanks \& Son of Arbroath. Some gravels bind exceedingly well, as that known as Kensington gravel, on account of the ferruginous alumina with which it abounds. River and sea gravel do not bind, in consequence of the absence of alumina; and few pit-gravels bind, for the same reason.
The weeds most troublesome in gravel walks are those of various species of grasses, particularly Poa annua, which seeds very abundantly. Other grasses rise in gravel walks in consequence of their seed being blown by the wind from the adjacent lawns, and also from the verges of the walks, when they are allowed to go so long unmown as to give time to the grasses to ripen their seeds. Care, therefore, should be taken that the verges, to the breadth of 2 feet on each side of all gravel walks, be mown frequently, and also that the creeping rooted grasses be eradicated. In damp shady places, gravel walks are often liable to become covered with various species of mosses, but these can be readily destroyed by watering with lime-water in a clarified state, so as not to discolour the gravel. The surface of gravel walks should, if it can be avoided, never be disturbed, unless it be once in one or two years, when it may become necessary to turn the surface to the depth of 2 or 3 inches, to bring up a fresh body of gravel, and to bury that which has become discoloured by use. It is well also, at such times, to add a portion of fresh gravel, which, if the operation be performed during winter, will give the whole a refreshed and new appearance. Frequent sweeping and rolling tend greatly to the suppression of weeds.

Our finely-kept grass lawns and firmly-compressed gravel walks are two of the most -striking features in our English gardens, and the very first thing that strikes all foreigners, for in no other country in the world do such things exist. Much care is necessary to produce a fine lawn, and on its after-keeping much of its beauty depends. No lawn can be fine if badly constructed in the first instance ; and no keeping can maintain them in proper order if plants are allowed to exist in them other than those species of grasses which are of an agreeable green colour, and of a dense and dwarfish growth. Amongst the greatest pests in a grass lawn is the dandelion, Leontodon taraxacum, the broadleaved rib grass, Plantago latifolia, the cocksfoot grass, Dactylis glomerata; and even the humble and beautiful daisy, Bellis perennis, however beautiful its flowers appear during April and May, when our lawns are bespangled with them in myriads, still the spreading of their dense and fleshy foliage destroys the finer grasses with which they become intermixed, and they propagate themselves rapidly by seed. In the formation of lawns, the ground should be thoroughly cleansed of root weeds before sowing. It should be dug or trenched to an equal depth, to prevent unequal settlements; and to secure this, it should be repeatedly trodden, rolled, and levelled, until at last a firm and uniform surface is attained. The selection of proper grass seeds is the next important matter, and this requires more than ordinary attention-that the kinds of grass be suited both to the purpose and to the soil, and that the mixtures of these grasses be proportioned to suit the end in view.

The following list of grasses and their proportions, adapted to various soils, has been carefully drawn up by the Messrs Lawson of Edinburgh, from whose excellent "Treatise on 
Grasses," \&c.- and we have no higher authority -it is taken :-

MR LAWSON'S LIST OF LAWN GRASSES, ETC.

Kinds and quantities of Grass Seeds required for sowing an imperial acre, for fine Lawns and Bowling-greens, \&c., kept constantly under the scythe. Sown without a crop of barley or other grain, a practice which should not be followed where fine lawns are required.

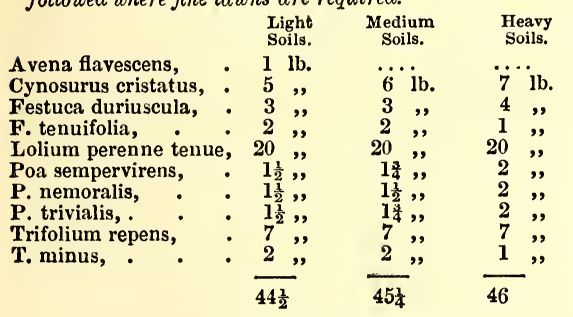

"In cases where primary expense is deemed secondary to ultimate effect, $2 \mathrm{lb}$. of the evergreen wood meadow.grass (Poa nemoralis var. sempervirens) may be added to the quantities given above; and where the ground is shaded by trees, both $P o a$ nemoralis and $P$. nemoralis sempervirens should be substituted for equal quantities of the two Festucas (given above), such quantities being dependent on the extent and depth of the shade. In walks, bowlinggreens, \&c., which are wished to be kept as dry as possible, especially towards the end of the season, Trifolium repens should be sparingly introduced; and when it is intended to mow the grass by machine instead of the common scythes, greater proportions of the hard and fine-leaved Festucas may be sown."

Much of the fine appearance of our lawns depends on regularity in mowing: if they are left too long in the spring before the operation is commenced, or if allowed to grow strong during summer, and more especially if not closely mown at the latest period in autumn, they become coarse, the stronger grasses overgrowing the weaker and best, the smoothness of the surface is destroyed, and ultimately the whole becomes patchy and unsightly. Besides regularity in mowing, sweeping and rolling are important matters in fine lawn management; and hence one of the many advantages of Shanks's mowing machine, which at one operation not only cuts the grass, but gathers it cleanly up, and rolls the ground at the same time. The saving in labour is also material. We calculate, after ten years' use of the largest size of these machines, that we effect a saving of twelve men during the mowing season. To those who have not employed it, we may state that ours has required no repairs during that period, unless when the cutters may accidentally come in contact with a stone, in which case, from their being highly tempered, their cutting edges are liable to become notched. In such cases (which, however, rarely occur) we despatch the cutters to the maker for repair. As it may happen to those at a distance from Arbroath that some time may be lost ere the cutters be returned, we arranged with the Messrs Shanks (and we advise every purchaser to do the same) to supply us with a double set of cutters, so that, should one set meet with an accident, the other is substituted for it. Ours is drawn by a horse guided by a man. There are smaller mowingmachines recommended to be drawn by men; in these we have found no economy whatever. The best scythes are those manufactured by Linley of Sheffield; these we employ in mowing round shrubs, or in small pieces of grass where the machine cannot be conveniently used.

All lawns, unless the subsoil be a porous alluvial gravel. should be thoroughly drained at their formation, and a drain should be carried along the bottom of each terrace slope, so that they may be at all times dry and comfortable to walk upon.

Worms are rather annoying at particular seasons, by casting up the material they have removed during their tunnelling operations. Whether these extraordinary undertakings, by which miles of subterranean tunnels are formed in a single lawn, are merely executed by the worm in search of food, or whether it may not be necessary also for their existence to secure for themselves by this means reservoirs of air, we know not; but of one thing we are certain, that by these very perforations air is admitted to the roots of plants, upon which so much of their health depends. Those who think differently from us will find lime-water sufficiently mortal in its effects; 6 or 8 pounds weight of hot lime being thrown into a hogshead of water, and applied the day after to the grass through the rose of a watering-pot, when it has become sufficiently clarified, and drawn from the vessel by a spigot placed a few inches from its bottom. Those, however, who have sufficient humanity, will sweep the worm-casts up as they appear, or disperse them over the lawn, which will act as a good top-dressing, and encourage the growth of the weaker grasses. A far more useful operation than the destruction of worms, would be the destruction of all coarse grasses, and such weeds as may exist in them - the dandelion and broad-leaved plantain in particular.

Mossy lawns.-Old grass lawns, more especially in humid climates and pent-up situations, are very liable to become overgrown with various species of mosses, rendering them less firm to tread upon, and, by choking out the grasses, destroying that cheerful green appearance which makes our lawns the admiration of all strangers. A writer in the "Quarterly Review" remarks : "Most gardeners strive to eradicate the moss from their grass ; it seems to us that it should rather be encouraged : it renders the lawn much moresoft to the foot, prevents it being dried up in hot weather, and saves much labour in mowing. The most perfect kind of lawns is perhaps that which consists of only one kind of grass; but for the generality a mossy surface would be far better than the mangy bare aspect we so often see." To this we can by no means subscribe, unless it be in shady walks, and places where it would be impossible to get grass to grow. There, indeed, nothing can be better than a mossy covering ; but on the broad expanse of lawn, whether 
in the flower-garden or around the mansion, moss should be extirpated altogether, or so kept under that the healthy growth of the lawn grasses may not be interrupted. So far would we calry our views on this subject that we would advise what we have practised-namely, in the absence of the family, when the lawns assume a mossy appearance, to fold sheep upon them, when of sufficient size to admit of this ; and when either too small, or so intersected with shrubs, trees, or flower-beds as to render such a practice inconvenient or impossible, to have recourse to top-dressing during winter or early in spring, and employing such substances as fertilisers as are congenial to the growth of grass and destructive to the moss. Lime, guano, soot, finely-sifted coal-ashes, \&c., are all of this class of fertilisers ; and where their application may be objected to on account of their colour, we would employ them in a liquid state ; and if applied during rainy weather, neither their colour nor odour would be objected to.

In the formation of lawns where immediate effect is desired, and in all cases of grass terraces, turf should be preferred to sowing down with seeds. Turf of sufficiently fine quality is difficult to procure, unless in the neighbourhood of old and fine sheep-pastures. In such cases the ground to be covered should be previously prepared by thoroughly clearing it of root weeds, by digging or trenching, consolidating and levelling. Turf cut in pieces a yard long, 12 inches broad, and from $1 \frac{1}{2}$ to 2 inches in thickness, and rolled up with the green side innermost, is capable of being transported to any distance. When the ground is ready, these turfs are to be laid down closely together, and when well watered should be beaten to a smooth and compact state by the turf-beater, and afterwards repeatedly rolled, making up from time to time any inequalities that may appear by the unequal sinking of the ground. Upon a large scale, however, and on level surfaces, seeds should be sown as being by far the least expensive. In good soils, and with such grasses as have been recommended, a good firm turf will be obtained in two years. Mistaken notions have long been promulgated, and poor soils recommended for lawns; and even some have gone so far in this direction as to recommend laying turf on a bed of sheer sand. Nothing is more absurd. To have a fine healthy green lawn, the soil must be good either naturally, or made so artificially, and the proper sorts of grasses only sown.

When the grasses become weakly, and the lawn presents an impoverished appearance, which it is apt to do in very dry weather if the soil be thin and poor, a good soaking or two of water, into which a small quantity of guano and muriate of lime is dissolved, will improve the health of the grasses and give them a better colour. A vast deal of labour is thrown away by many in rendering the surface where turf is to be laid as level as a billiard-table, and also on the exact thickness and size the turf is to be cut to. Much of this is labour in vain. Prepare the ground by consolidation, and bring it to nearly the desired level ; lay the turf down, if possible, in damp weather, and beat the whole mass, while wet, until a level surface is attained. If in dry weather, apply water artificially; lay the turfs pretty closely together, filling in any bad joints with fragments, and beat the whole into a pulpy state, which, when dry, will form an entire surface, showing few or no joints.

\section{§ 4.-THE SHRUBBERY.}

The shrubbery, properly speaking, is a department of the grounds in a domain dedicated to the cultivation of shrubs valued for their beauty, variety, or fragrance; and when entering into combination with ornamental trees, particularly those of exotic origin, it produces scenes of the most raried and agreeable character. In its too ordinary acceptation, it means exactly what it represents-a jungle of shrubs and trees, planted with little regard to effect, seldom attended to after planting, the strong being allowed to overgrow the weak, which are often the most valuable; and so long as a moderately healthy surface is maintained, all is allowed to go on in its own way. In course of time, under such circumstances, the plants become naked at bottom; many of the most valuable perish; the stronger sorts, growing beyond the limits originally intended, destroy the character of the scene intended to be produced; and after years of vexatious disappointment, the resolve is at last taken to cut down or grub up the whole, and plant it anew. An error of very frequent occurrence is also fallen into-namely, planting herbaceous plants and florists' flowers around the margins of shrubberies. Than this nothing can be in worse taste. The grass verge should run from the walk as far as the ground is not covered with the shrubs, or those of the most diminutive growth should be planted, so as to unite the taller ones with the grassy margin of the walk. When the walk runs between two distinct shrubberies, or is made to cut through any portion of one, then broad margins of grass should be employed, running back as portions of the shrubbery recede, and narrowing as they advance closer to the walk, to give variety and breadth of effect, and prevent that monotonous appearance that would be produced if the space traversed were of uniform breadth throughout. Where this is not guarded against, and the shrubs are allowed to grow tall, the walk has just the appearance as if it was bounded on both sides by a verdant wall, or placed between two hedges. Few things have a worse effect in landscape-gardening than neglected and overgrown shrubberies, yet there are few places in which this is not most manifestly exhibited. Shrubberies are often planted to afford shelter, to give effect, and also to hide out disagreeable objects, or to mask the limits of the grounds, and to make them appear larger than they really are. Under all these circumstances, it is quite obvious that the closer the masses are maintained-that is, the better they are clothed with branches and foliage close to the ground-the more likely are the desired results to be secured. To produce immediate 
effect, large plants are often employed, and between these smaller ones are thickly set; and so far this is all very well when mere screens are desired; but in course of time, if great attention is not paid to judicious pruning and timely thinning, the object in view is defeated, and the shrubbery ceases to afford that shelter, effect, or screen, for which it was originally planted. The same holds good where the effect intended to be produced is the full development of shrubs of a rare or curious description; the one encroaching on the other prevents either from attaining their full size or natural character. This is also the case where the massed or grouped manner of planting - that is, where one genus is planted in groups following another analogous to it-is followed, and also where attempts have been made at systematic arrangement. In all these cases the shrubbery presents a pleasant and orderly aspect for the first few years, but afterwards it becomes a mass of unintelligible confusion. If there is one department in ornamental gardening more neglected than another, it is assuredly this. As we find shrubberies in general, they present nothing but an almost unbroken surface of foliage, too often as uniform in outline as if clipped with the garden shears ; while below nothing is to be found but dead branches, long naked poles, the remains of most of the choicest kinds that the original planter had thrust in, in a sort of pell-mell fashion, and left to their fate; and others present belts, or screens, of the most heterogeneous materials that can possibly be thought of.

Shrubberies, although almost entirely excluded from the geometrical style of flowergarden, enter largely into what has been appropriately enough denominated the gardenesque style, and by others the picturesque style ; indeed, in both of these they constitute one of the principal features. In the latter style, it matters little what the individuals be, provided they be furnished with foliage, and present sufficient variety of form-the commonest trees and shrubs produce all that is required, and, correctly speaking, only those indigenous to the country should be employed, and these allowed to grow as nature directs them. In the former, the case is different; exotic trees and shrubs enter into its composition, and their arrangement at planting and after-management must be guided by the hand of man. The gardenesque style, if carried to its fullest extent, scarcely admits of other than that each tree or shrub should stand a distinct object in itself-not that these, however, are'to be so scattered as to leave large patches of uncovered ground bare between them. Nor is the grouping principle-that is, planting two or more shrubs of the same species together-entirely excluded from this style. It may be done with great propriety and effect, and the more so if the field of operation be upon an extensive scale. The great object in the arrangement and after-management is, that these groups be allowed to assume their natural character, as each individual would do if planted singly. A thorough acquaintance with the material to be employed is here absolutely necessary in the first arrangement, so that each tree or group may VOL II. occupy the place in which, when fully developed, its natural character will best bear on the general effect. In fact, a shrubbery planted in the gardenesque style should, by the selection and arrangement of the material, show evident marks of the " recognition of art," as much as one in the picturesque style that of "a recognition of nature." The great mistake committed in the planting of all shrubberies is planting too thick, and never thinking afterwards of the necessity of thinning. To produce what is called immediate effect, in inexperienced hands, leads to evils seldom if ever afterwards corrected. Near to the house, and along the principal walks, and in flower-garden borders, let each tree or shrub stand perfectly clear of its neighbour; and if these are judiciously planted, by placing a plant in front so that it shall cover the space between the two immediately behind it, and the border of sufficient breadth, a screen or shelter can be as well attained as if they were planted as thick as an Indian jungle. Here "the recognition of art" should be apparent, and every plant allowed so to grow that its individual form may be fully developed. The sticklers for the picturesque will be alarmed at the idea of any part of the surface being seen between the plants; this may be covered by planting it with various species of cotoneaster, daphne, cytisus, clethra, spiræa, Vinca medea and major, Juniperus prostrata, or similar lowgrowing shrubs; but to the eye of the wellinformed critic in such matters, portions of the neatly-kept naked surface will present no deformity whatever.

Preparing the ground for planting.-In planting shrubberies, too many are content with merely marking out the line of demarcation, and thrusting the plants, as they come from the nursery or elsewhere, into holes scarcely large enough to hold their roots. Such a mode of proceeding can never lead to satisfactory results. The ground should be not only thoroughly trenched from 2 to 3 feet in depth, but, if poor, and more especially if recently covered with trees, \&c., it will require a moderate degree of enrichment, even for the most common shrubs; while for American plants, which seldom thrive if not planted in peat-soil, that ingredient should be procured for them. It is found, however, for the generality of these, that pits dug out to the depth of 2 feet, and say 4 feet in diameter, and filled with peat-soil, will be sufficient to maintain the plants for ten or a dozen years : but where the shrubbery is to be planted entirely with such plants as rhododendrons, azaleas, \&c., it were better to form it entirely of this soil at once. Peat-soil is in many places difficult to procure in such quantities as to form entire borders for such plants, and this difficulty has prevented many from being able to cultivate these to any extent, or with any degree of satisfaction. We may mention, en passant, that decayed tree-leaves, or completely decomposed tanner's bark, mixed with sand, may be used along with peat-soil in the proportion of three to four, and that, in planting, large fragments of sandstone may be advantageously used imbedded in the soil. This we have often done with 
a view to economise the soil, and with the best effects.

Planting shrubberies.-All transplanting, to be successful, must be carefully performed. The operation of taking up and removing has been already referred to (vide p. 356 to 388 ). If immediate effect be aimed at, then large specimens must be employed; and these, if not existing in the grounds, will be both expensive and difficult to procure. It is always a hazardous affair to transplant large trees or shrubs from a distance, and it is almost as great a risk to transplant them from existing shrubberies even in the same garden; while it is still more difficult to select them of proper form, and in fit condition for the purpose. The latter disqualification arises from the neglected state in which our shrubberies are so often found. The plants, being crowded together, become one-sided, if not naked at bottom, and rarely can be taken up with sufficient roots to insure their growing, and more seldom are they to be found of sufficieut symmetry to fit them for the gardenesque style of planting. Under these circumstances, it were better to choose smaller plants; and these, if planted in properly prepared soil, will in a few years conpletely outgrow those that may have been removed of larger sizes. Shrubs which from neglect may have been allowed to become straggling and bare of branches near the ground, may be cut down to within a few inches of their roots, and trausplanted; and if the operation is performed in spring, they will break strong, and become fine bushy plants the season following. Most shiubs may be thus treated, if we except coniferous ones, which seldom break freely after such severe cutting, and few of them will afterwards send up proper leaders. In planting, it is not the present size of the individuals that is to guide us in their arrangement, but the character they will assume in after growth; and hence the importance of the planter's having a thorough knowledge of their habits and characters. In the first stage of proceeding, each tree and shrub should invariably be placed where it is permanently to remain. When this is done, a set of duplicates may be interplanted for immediate effect, or rather for thickening; but this must be done on the understanding that they are to be removed before the branches of any two of the permanent ones touch each other. In planting in the picturesque style, the subjects are placed much closer together, and hence deformities as to form may be much better concealed, the great object being a leafy exterior. In planting, whether in the gardenesque or picturesque styles, care should be taken that the effect intended to be produced be not defeated by the intermixture of too many species, and those of discordant habits. Still, on the other hand, it is exceedingly desirable that our shrubberies should present a more exotic and a more varied character than many of them have heretofore done. This defect does not assuredly arise from a want of sufficient material, for with that our nurseries have been stocked for the last halfcentury or more; nor in all cases has it been from a want of proper discrimination on the part of the planter, but in many cases from a parsimonious disposition on the part of some to purchase good plants; and in the majority from bad management on the part of gardeners, and an unwillingness on the part of their employers to thin out and maintain their shrubberies in a healthy condition; strong and commonplace plants being allowed to overgrow and ultimately to kill their more valuable although less luxuriant neighbours. Portugal and common laurels, hollies, rhododendrons, with an odd mixture of deciduous shrubs having little affinity with these, are the material by which our ordinary shrubberies are furnished, and these are striving with keen rivalry to exterminate each other.

Whatever style of planting may be adopted, it is necessary, for securing ultimate success, that a correct plan of the ground be first made, and upon it should be marked the number and position of such groups or single specimens as are to form the main features of the shrubbery when it has attained its fullest degree of perfection. These positions should be accurately transferred to the ground, and marked by a stake with the name or corresponding number to that on the plan written upon each. When plantiug, these points should be first filled up, and the spaces between planted with such plants as are from time to time to be removed. Unless this or some similar guide be adopted, the whole will go on at random, and the object aimed at by the planter be completely defeated. Still farther to secure this end, the permanent plants should be marked in such a manner as will prevent their being accidentally removed during the future progresses of thinning.

It is the taste of some to plant their shrubberies entirely of evergreens, and no doubt such have at least a more permanent appearance of unison than where deciduous trees and shrubs are intermixed. In such cases, however, unless attention is paid to the introduction of plants of varied colours or habit, the whole will assume a sameness of outline perfectly devoid of character or interest. To break this monotonous appearance, the ground next the margin should be covered with the dwarfest-growing speciessuch as hardy heaths, double-flowering whin, Rhododendron hirsutum and ferrugineum, vacciniums, empetrums, andromedas, helianthemums, mahonias, vincas, cotoneasters, \&c., backed by box, both variegated and green, daphnes, laurustinus, aucubas; and followed by junipers, common laurel, arbutus, rhododendrons, yews ; and, last of all, Portugal laurel, arborvitæ, hollies, both variegated and green, with single specimens interspersed of deodara, araucaria, taxodium, cryptomeria, with some of the most interesting other coniferous trees and evergreen oaks, standing, as it were, in projection. In alranging the other's, the effect will be most striking if they are planted in groups of various sizes and of irregular forms. The mixed or promiscuous manner of planting seldom pleases the eye of taste, and all attempts at systematic arrangement carly with them that methodical stiffness fitted only for strictly botanical gardens. The variegated 
varieties planted in groups by themselves will lighten up and neutralise the monotonous green of the general masses; while the groups of taller trees and spire-like tops of others, with the graceful drooping deodar and rigid-looking araucaria will break the general outline, and produce an agreeably-broken surface.

Renewal of neglected shrubberies.-In the most extreme cases, grubbing up and replanting will be found the most satisfactory, while cutting down to the ground, and allowing them to spring again, may be had recourse to under less desperate conditions. The former becomes most imperative when plants or trees have been introduced which have little or no connection with each other, or with the place and purpose; the latter, when a portion only has to be removed entirely, and the remainder cut down to be again re-grown. In the former case, the ground should be deeply trenched, and a portion of new soil or manure incorporated with it during the operation, as the ground will have become considerably exhausted by the previous crop of trees or shrubs, and ill fitted for the healthy production of a crop so similar. In the latter, such curtailments should take place as to admit of groups of new or more interesting plants being introduced, and a new character given to the whole. Winter and early in spring is the best season for either operation. It is seldom convenient to cut down an existing shrubbery unless during a lengthened absence of the proprietor, on account of the disordered appearance it would give to the place. In such cases, recourse must be had to layering, by which the appearance of keeping need not be much disturbed. This process is also well adapted to shrubberies where, from neglect of timeous heading back, the plants become naked at bottom, while their tops are in a healthy state. It is also valuable when vistas are to be cut out of long-standing shrubberies, and where it would be objectionable to cut down the plants in the line of view to the ground. Layering is performed as in ordinary nursery propagation, when shoots of the current or previous year are to be operated upon, and merely consists of thinning out the superfluous shoots, and layering those most eligible for the purpose, When the operation is to be performed on branches or trees of from $1 \frac{1}{2}$ to 4 inches and upwards in diameter, the process is different, and although not requiring much more skill on the part of the operator, requires a great deal on the part of the director. In such cases, a judicious thinning out of superfluous branches, and often of entire plants, is to be performed. Such branches should be cut out as are not required, and also such as are thin of branchlets at top; such only should be retained as the eye of the judicious operator sees will be required to be laid in certain positions, not only to cover the ground, but also to face up the breach made by the removal of the others, that the vista may appear, when finished, in as natural a state as if it had not been artificially formed. In thinning out, it should be borne in mind that some of the larger branches may be broken during the process, and therefore enough should be left to make up for such con. tingencies. The first thing to be considered is, facing up the sides of the vista by shortening back the leading branches of the shrubs on cach side, yet so as not to let them appear as if they had been interfered with. The branches afterwards, on both sides, are to be foreshortened by layering, so that their green tops may unite with those left on each side, and gradually bring down the green surface, not in a hedge-like form, but in as natural a manner as possible, some branches receding backwards, and falling in height, while others stand in projection, either lowered or at their natural length. Foreshortening-that is, disposing of the long naked stems-is thus performed: A branch is selected out of those left at the first thinning, the top of which is to fill up a certain space; with a sharp bill-hook the side of the branch opposite to that on which it is to be brought down is to be cut away in a slanting direction downwards, and from 1 foot to $2 \frac{1}{2}$ feet (according to its size), or until it will bend down towards the ground without breaking. When this is accomplished-and it should be especially noticed that this cutting away must always be upon one side only, and that opposite to the direction the branch is to fall to- the distance is then to be calculated, which may be done by measurement; at which, and on an opposite side, the branch is to be cut in a similar manner as before, so that it may be bent backwards towards the root, and again cut and bent upwards, so that the top of the branch may come into its proper place for filling up the space allotted to it. When the distance between the two fractures does not exceed 3 feet, a third fracture is unnecessary; and often, where the branch to be laid is short and bushy at top, one fracture will be sufficient. To retain the branches so foreshortened in their proper places, a trench is often cut, into which they are laid, and firmly packed round with soil, although sometimes it is found expedient to fix them down by strong hooked pegs driven in alongside of them. In other cases, this pegging down is found to be sufficient, and the naked stems are laid on the surface of the soil. In most cases, where the branches touch the ground, whether buried in it or not, they strike roots, and are afterwards supported by these roots, should the stem entirely decay. Single specimens of trees and shrubs which may have become one-sided by too close contact with other plants, may by this process be in a great measure restored to symmetry of form and uniformity of outline. All trees and shrubs, with the exception of the conifers, azaleas, and a few others whose wood is too brittle to admit of bending, may be treated in this manner, the proper season for deciduous plants being during winter, while with evergreens the operation may go on at almost any time of the year. Shrubberies which may have overgrown their proper size and height, may be, by this process, reduced to any desired height, and plants of any age may be operated upon. By layering in this manner, and by annual thinning and shortening the tops, shrubs may be kept in health and complete order for many years, while a neglect of thinning, pruning, and layering only hastens the appearance of 
disorder and unhealthy keeping, and ultimately total decay.

Pruning is an important element in the proper management of the shrubbery, and requires quite as much skill and regularity of attention as the pruning of any other trees or plants whatever. The objects of pruning shrubs generally are to modify the form or lessen the bulk of over-luxuriant specimens, the removal of dead, sickly, and misplaced branches, the shortening back even to the extent of cutting over to within a few inches of the roots such as are declining in health and vigour. In the case of many flowering shrubs, other considerations are to be kept in view, and these are founded on their natural habits and mode of flowering. The rose has been already treated on, and what is said of it is applicable to most shrubs which flower in the same way; while the trumpetflower and honeysuckle are spurred in to one or two eyes of the previous year's wood. Rhododendrons require often to have their branches thinned out when too crowded, but their terminal shoots should never be shortened, as it is at the points their flowers are produced. Weigela rosea flowers upon the wood of the previous year, therefore removing much of that lessens the abundance of bloom the following season. The season of pruning the majority of shrubs is during autumn or winter, and that of coniferous shrubs and trees late in spring, after the sap has risen.

Digging shrubberies.-All interference with the roots of trees and shrubs should be carefully avoided; digging, therefore, amongst them, although a rule of long standing, is one more honoured in the breach than "in the observance. It were better practice, indeed, to top-dress the soil over the roots with some rich compost; or, if a vigorous growth is desired, to give them two or three good soakings of liquid manure during their period of growth. All decaying leaves and other vegetable matter which accumulate under them should be annually raked up, and carried to a convenient spot to become decomposed ; and, if afterwards mixed with lime, may be used as a top-dressing with advantage. Shrubberies neglected in this respect become exceedingly unsightly, and even injurious to the health of those who live much amongst them. The vegetable and animal matter undergoing slow decomposition is productive of malaria to a very great extent, and the gases generated are prevented from being diffused in consequence of the want of a sufficient circulation of air. Neglected shrubberies near a house, covering, as they in general do, a mass of decomposing matter, the accumulation of years, are as complete a hotbed of malaria as a common sewer or putrid cesspool.

Selection of material.-Of shrubs and trees, evergreen or deciduous, the proportion which the former bears to the latter in cultivation is something like one to twelve, exclusive of climbers and creepers; but if we include roses, the proportion will be about one to fifteen. These latter should, however, be sparingly introduced even into mixed shrubberies, as they do not associate well with other shrubs; and also, on account of a dissimilarity in their culture, they are found to do best when planted by themselves. To some extent this applies to many American plants also, unless where the grouping system is followed, as there they can form groups or masses by themselves. The season of flowering of trees and shrubs, although of less importance than their forms, is, however, not to be altogether neglected, as are also the colours of their flowers and foliage. The season of flowering extends from the beginning of March to the end of August, and the prevailing colours are white, or various tints of deep red to light pink, yellow and blue being the least abundant. The great distinction, however, to be chiefly regarded, is between those that are deciduous and those that are evergreen. The former produce the greatest amount of bloom during summer, but are deficient of the same amount of interest during winter. Nor are they so well adapted for screening out objects wished to be hid, or for affording shelter during winter, or shade during summer. In planting a shrubbery, therefore, whether in the mixed or grouping manner, the proportion of evergreens should be somewhere about eight to one of deciduous plants. An eye should be kept on those that herald in the spring by their early flowering or foliation, and also on those which possess an unusual share of character, the latter being placed, as it were, in bold relief. Of the former we may mention Daphne mezereum, Ribis sanguinea, Jasminum nudicaule, Rhododendron atrovirens and dauricum, and a host of early spring-flowering hybrids; and of the latter, Araucaria imbricata, Cryptomeria japonica, various of the more rare species of Coniferæ, with trees having singularly-coloured foliage, as well as those of pendent or weeping habits. The latter of these, however, should be planted in the foreground, or more properly as single specimens on the lawn; for, if planted among other shrubs, their stems will become hid, and hence the effect such trees or shrubs are intended to produce is entirely lost. Where shrubberies are to be planted upon an extensive scale, it will be wise to purchase the plants while young in the public nurseries, and transplant them into a home nursery, from which they can be removed as the operation of planting goes on.

Propagation of hardy ornamental trees and shrubs.-Both evergreen and deciduous shrubs are increased by seed, cuttings, eyes, layers, budding, and grafting.

Amorpha.-Deciduous shrubs, requiring common soil, and propagated by layers or cuttings of the ripened wood in autumn.

Ampelopsis.-Deciduous climbers, thriving in almost any soil and situation, propagated by layers or cuttings of the ripened wood in autumn.

Amygdalus.-Deciduous trees and low shrubs. The former propagated by seed, and the varieties by budding on stocks of the common plum; the dwarf varieties by cuttings and layers thriving in any ordinary soil.

Aralia. - Spinosa and japonica, the only hardy species of shrub in this family are de- 
ciduous, thriving in any moderate soil, and propagated by imported seed and by cuttings of the roots.

Arbutus.-Evergreen shrubs or small trees, $A$. unedo and its varieties are propagated by seed sown as soon as ripe, in light sandy soil, under the protection of a cold frame or pit. When the plants are about the height of an inch, they should be pricked off, either into a sheltered border, or where they can be slightly protected during the first winter; afterwards they may be planted out in nursery lines like other ever. greens. The other hardy sorts are increased by being budded or inarched upon stocks of the common arbutus, and all of them are occasionally propagated by cuttings of the half-ripened wood; all thriving in any ordinary garden-soil.

Arctostaphylos. - Low - growing evergreen shrubs, requiring the same treatment as the last.

A ristolochia.-Deciduous climbing plants of very rapid growth, propagated by division of the roots, and also by root cuttings; thriving in any ordinary soil and situation.

Aristotelia.-A hardy evergreen shrub, propagated by layers in autumn, and by cuttings in spring; thriving in any ordinary soil, but requiring a rather sheltered situation.

Astragalus.-Several species of this genus are low under-shrubs, and are propagated by cuttings placed under a hand-glass; thriving in common soil.

Atragene-Hardy, deciduous, rapid-growing climbers, propagated by seed, which sometimes ripens in this country; in general, however, by cuttings and layers, the former taken off during summer, and placed under a handglass, the latter in autumn or during winter; thrive in any ordinary soil and situation.

Atriplex.-Of this the species A. Halimus, a hardy evergreen shrub, is the most ornamental; propagated by cuttings, and requiring no particular soil.

Aucuba.-A hardy evergreen shrub of moderate height, propagated readily by cuttings in spring and autumn in common soil.

Baccharis.-The hardy sorts are propagated by cuttings, and thrive best in a peat and loamy soil.

Berberis. - Propagated by suckers, cuttings, and layers, and also by seed, which, although abundantly produced, is seldom employed in propagation, unless in the case of some of the more recently introduced species. A light sandy calcareous soil is most suitable for them.

Benthamia.-Evergreen shrubs propagated by layers, and seed when procurable; thrives in any ordinary garden-soil, but requires a sheltered situation in most places; ripens its fruit when planted against a wall in the south of England.

Bignonia.-The only hardy species is B. capreolata, propagated readily by cuttings of its roots, or shoots placed under a hand-glass, either in spring or autumn. This beautiful climbing plant requires the protection of a wall in most places.

Broussonetia. - Hardy trees related to the mulberry, propagated by suckers and cuttings of the ripened wood planted in autumn, and also by seed sown when ripe, or kept over till spring; thrives in any ordinary gardensoil.

Buddlea.-The only truly hardy species is $\boldsymbol{B}$. globosa, an evergreen shrub of moderate growth, propagated by cuttings of the ripened wood placed under a hand-glass in September; seeds are sometimes ripened in the south of England, and if sown in spring vegetate freely; thrives in any ordinary garden-soil, but requires a warm sheltered situation against a wall.

Buxus.-For the most part hardy evergreen shrubs of considerable variety as to habit and variegation of the foliage; propagated freely by seeds, which should be sown as soon as ripe, by cuttings of the young wood, and also by layers and division of the plant when used for edgings; thrives in most ordinary soils, best in those of a calcareous nature, and in partially-shaded places. In some soils it almost refuses to grow, and in such cases becomes a source of annoyance to the gardener.

Caprifolium. - Deciduous twiners of rapid growth, requiring no particular soil, but preferring an open situation; propagated by cuttings of the ripened shoots taken off in autumn and planted in a shady border. Also freely by layers in autumn about the period of the fall of the leaf. Cuttings of a foot or more in length are sometimes employed, both ends of which are inserted in the ground, and when rooted they are separated by cutting them over at the middle.

Caragana.-Handsome deciduous shrubs, propagated by seed sown in spring or by cuttings of the roots, and often by layers; the rarer and pendulous sorts by grafting on the common kinds, and all thriving in a sandy loamy soil. This very pretty genus is far too seldom seen in cultivation.

Catalpa. - Deciduous ornamental flowering trees of great beauty ; propagated by seed sown in spring, by cuttings of the roots, by layers, and by cuttings of the ripened shoots in autumn; requires a light dry soil and a warm sheltered situation.

Ceanothus.-Most of the species, with the exception of the truly tropical ones, thrive well when planted against a wall in ordinary warm localities, and in many parts of the south of England they are grown as open shrubs. Propagated by cuttings taken from the side shoots, and planted in sand and covered with a bellglass either in April or August. When established, they are not particular as to soil, but require in most places slight protection during winter.

Celastrus.-The hardy species are $C$. scandens and C. bullatus. The former is propagated by seed, and sometimes, as well as the latter, by layers in autumn: a deep loamy soil is the most proper for both species.

Celtis.-The hardy species are natives of America and Europe, are deciduous shrubs or lowgrowing trees, and are propagated by seed sown as soon as ripe, by layers, and by cuttings of the ripened wood in autumn. Succeeds well in any good garden-soil.

Cephalarthus.-Deciduous shrubs propagated by cuttings set in sandy soil under a hand-glass 
in autumn; also by layers of the ripened wood at the same season. Prefers a light sandy loam abounding in vegetable matter.

Cerasus.-For the most part hardy deciduous trees and shrubs, of which the cherry is one ; also containing evergreens, of which the common and Portugal laurel are examples. The former are propagated by seed, sown when the fruit is ripe, or mixed with dry sand, and frequently turned during winter, and sown in spring following. They are also increased by suckers, cuttings of the roots, and also by layers. The rarer sorts are often budded and grafted on stocks of the more common sorts. The evergreen sorts are increased by layers, and also by cuttings of the ripened wood planted in autumn in a rather moist shaded border. Deep sandy soil is favourable to the whole of the family.

Cercis.-Deciduous low-growing trees, propagated by seed sown in spring, upon a mild bottom-heat. When the plants are $1 \frac{1}{2}$ inches high, they are pricked out in a sheltered place, and hardened off. In the southern counties they succeed when planted on the lawn in warm situations, but in the north require the protection of a wall. Any moderately good gardensoil is quite suitable for thern.

Cistus.-A fine flowering family of shrubby plants, many of them standing our winters when planted in a dry soil in a sheltered situation; the dwarf-growing kinds exceedingly well adapted for rock-work. Propagated by cuttings placed under a hand-glass in May, by seeds when procurable, sown in April, under glass, and also by layers, after the season of their flowering is past.

Ciematis. - Deciduous climbing shrubs of rapid growth, propagated during summer by cuttings of the side shoots when half-ripened, placed under a hand-glass, also by layers in September. A light loamy soil and a dry situation are most suitable to them.

Clethra. - Deciduous low-growing shrubs, propagated by layers in autumn, and by cuttings of the half-ripened wood planted in sand during summer. A soil somewhat moist and inclining to peat the most favourable.

Clianthus.-Requires the protection of a warm wall, and slight protection during winter; propagated by cuttings placed under a glass, and, when rooted, potted in peat and loam soil till of a size for final planting out.

Colletia.-Requires the protection of a wall in cold situations; in moderate ones, C. spinosa and horrida stand in sheltered shrubberies. Propagated by cuttings of the ripened wood set in sand, and covered with a hand-glass. Soil, light sandy loam.

Colutea.-Deciduous shrubs of easy culture, propagated by cuttings set in sandy soil towards the end of summer, and seed sown in spring, which vegetates freely. Soil, any good garden-mould.

Comptonia.-Deciduous low-growing shrubs, propagated by layers in sandy soil.

Cornus. - Deciduous low-growing trees and shrubs, propagated by seeds, layers, and cuttings, preferring a moist situation and common soil.

Cratcous.-Vide page 745.

Cydonia.-Deciduous trees and shrubs, propagated by seed and also by layers in Septem. ber, allowing them to remain twelve months to make roots. A deep loamy soil is the most favourable.

Cytisus.-Deciduous shrubs and low-growing trees, propagated by seed in the case of the commoner kinds; those of greater variety, or for standards, by budding on the laburnum. Any ordinary soil is suitable for them.

Daphne.-Several of them, such as the Spurge laurel, produce seed which vegetates freely, but seldom before the second season, and the plants so produced are employed as stocks, on which the rarer and more delicate sorts are grafted. D. cneorum and similar dwarf sorts are increased by layering, and sometimes wrought on stems 2 or 3 feet in height. Some, as Pontica, are propagated by cuttings set in sand in autumn, which become rooted in spring following. The principal mode, however, is by grafting in March or April, the plants being set in a close frame or pit. A light loanyy soil is favourable for them, and that is improved by the addition of one-third sandy peat.

Deutzia.-Deciduous shrubs, propagated by cuttings placed under a haud-glass, and, when rooted, planted in a sheltered place till fit for final planting. They are all tolerably hardy, and make fine lawn-plants in warm situations, and are worth the protection of a wall in cold localities. Thrive in any good garden. soil.

Elocagnus.-The deciduous species and their varieties are propagated by seeds sown in spring, and also by cuttings towards autumn, planted in a sheltered border. The evergreen sorts by layers in autumn, and cuttings set in sand in summer, and placed under a hand-glass. A soil of half-sandy loam and peat is the most proper for them.

Escallonia. - Evergreen half-hardy shrubs, requiring, in most places, the protection of a wall; propagated by cuttings of the half-ripened wood set in sand under a hand-glass. A light loamy soil with a mixture of peat is the most proper for them.

Euonymus. - The deciduous species are all hardy, the evergreen ones half-hardy. They are propagated by seeds sown either in autumn when ripe, or kept in sand till spring; also by cuttings of the ripened wood planted in a border in autumn. The deciduous sorts require a moist situation, while the evergreen ones, being not so hardy, thrive best in a soil that is dry, and also require slight protection during winter.

Fabiana. - A half-hardy evergreen fine-flowering shrub, requiring the shelter of a wall, and in cold localities slight protection during winter. Propagated by cuttings of the half-ripened young shoots set in sand, and covered with a hand-glass.

Fontanesia - of which there is only one species-an evergreen shrub of moderate growth, and not very hardy. Propagated by cuttings planted under a hand-glass in autumn, by layers at the same season, and also by grafting on the Manna ash, either near the ground or at standard height. Succeeds in any good garden-soil. This beautiful evergreen is seldom met with in gardens.

Forsythia.-Deciduous flowering shrubs, pro- 
pagated readily by cuttings and also by layers, requiring slight protection during winter, thriving in any good garden-soil.

Garrya. - Evergreen shrubs of moderate growth, propagated by cuttings set in sand in August, and covered with a hand-glass; also by layering in autumn. Soil, sandy loam. In very cold situations a little shelter is required, but in ordinary climates they stand our winters without injury.

Genista.-Low shrubs producing abundance of yellow blossom; propagated by seed, which is abundantly produced, and also by cuttings set in sandy soil, and covered with a hand-glass. They are difficult to transplant when large, therefore should be set in their final situation while about two years old. Growing them in pots is practised as a remedy for this, but the consequences are similar to those in the case of coniferous plants so treated, vide p. 369 .

Gleditsclia. - Exceedingly ornamental deciduous trees, propagated by seeds imported from America and the south of France. 'The seeds are very hard, and should, therefore, be soaked in warm water for a few hours previous to sowing, which operation should be performed in March, in common garden-soil. The finer varieties, when seed cannot be obtained, are grafted on the most common, such as $G$. tricanthus, of which there is no difficulty in procuring seed.

Gonolobus. - The hardy species, which are deciduous twiners, are propagated by seed, and prefer a dry sandy soil.

Halesia. - Deciduous shrubs of moderate growth, propagated by seed sown in spring, by layering, and by cuttings of the roots, either in spring or autumn. A deep sandy humid soil is the most favourable for them, and in such they often attain a large size.

Halimoderdron. - Deciduous shrubs, propagated by seed, by layers, and by cuttings of the roots, and sometimes grafted on the laburnum standard high, in which state they form interesting lawn plants. A light sandy rich soil is suitable for them.

Hamamelis. - Deciduous shrubs, producing their yellow flowers during winter after the leaves have fallen; propagated by layers and seeds, which latter require two years to vegetate; they are also increased by cuttings of the roots, and require a sandy moist soil.

Hippophae.-Deciduous shrubs, readily propagated by suckers, layers, seeds, and cuttings of the roots. Excellent for forming shelter from the sea-breezes, as well as for fixing the drifting sands, particularly when intermixed with Carexes and other sea-side grasses.

Hydrangea.-Deciduous fine-flowering shrubs, of moderate growth, sufficiently hardy to stand our ordinary winter's in sheltered gardens, particularly near the sea. Propagated chiefly by cuttings of the half and fully ripened shoots. To produce plants of $H$. hortensis to flower the summer after the cutting has been planted, they should be taken off in August, choosing the terminal shoots, but without shortening them. If set in single pots in sandy soil, they will root before winter sets in, and if kept in a cool pit till spring, and then shifted into a 4 -inch pot, each will produce a head of bloom, the diameter of which will be nearly equal to the height of the plant. In this way many thousands are annually propagated for the London market. These plants, after flowering, and having their leading bud destroyed and again shifted, will send out several shoots, and become good-sized plants by the next season of flowering, producing a bloom in general at the point of each branch. While grown in small pots they require abundance of water, and may be set for days together in pans filled with that element. When grown in the open borders, even in the south of England, they are occasionally cut down to the ground by frost; the roots, however, are seldom injured, especially if a little dry littering matter, or a few inches of sifted coal-ashes, has been laid on the surface of the ground as far as the roots extend. The only culture required is to thin out all the weak shoots as they appear, and to supply them with water during dry weather. We grow some very large specimens in tubs 2 feet in diameter, supplying the plants liberally with liquid manure, and, with the exception of our equally large scarlet geraniums, we know of few plants that can rival either in appearance from June till November. Much has been said about changing the flowers of $\boldsymbol{H}$. hortensis to a blue colour. In some districts we find the majority of the bloorns blue, while in others they are all pale rose. "Notwithstanding all the experiments that have been made," it is rernarked in the Cottage Gardener's Dictionary, "there is still a little doubt as to the cause that produces the change. When iron filings and a solution of alum are used in some soils, the blue colour is produced, while the same means will not produce it in others ; and other soils will almost invariably produce this blue colour without any particular matter being added. The loams at Kenwood, at Hampstead Heath, and Stanmore Heath, and the peats at Wimbledon, as well as some bogs near Edinburgh, are famous for producing this blue in the hydrangea. When trying artificially with iron filings and alum water, we have had different colours on the same plant. This variation is merely temporary -it cannot be propagated like a variety ; a cutting from a blue plant will produce a rose one, unless the peculiar treatment be continued." Our own experiments have alike failed, and even the application of nitrate of silver for two successive seasons had no effect. An amateur friend has, however, recently assured us that using water in which guano has been dissolved invariably, with him, produced the effect desired.

Hypericum.- The hardy section of this family that are of a shrubby character contains both deciduous and evergreen species. Most of them are readily increased by subdivision of the plant, and also by seeds. They luxuriate in almost any soil and situation, and enliven the shrubberies with their fine yellow flowers.

Jasminum.-The majority of the hardy species are deciduous, and are readily increased by cuttings, layers, or suckers; the former set in sandy soil under a hand-glass, and the operation performed when the side-shoots are half-ripened. Layering may be done in autumn, winter, or 
spring. The variegated varieties of officinale are increased by budding or grafting them on stocks of the original. Any common gardensoil is suitable for them. J. nudicaule is valuable for flowering during the whole of the winter, and on this account, as well as its being less hardy than some others, it should be planted against a wall.

Kerria.-A deciduous yellow-flowering shrub. Propagated readily by division of the plant, by suckers and layers, as well as by cuttings of the young wood; planted in sand, and placed under a hand-glass; thrives in any ordinary gardensoil.

Laurus.-Vide page 746.

Lavendula.-Dwarf evergreen shrubs. Propagated by pulling the old plants to pieces, and inserting them in the ground in March or beginning of April; also by cuttings of young shoots an inch and a half long, which, inserted in sand in June, and covered with a hand-glass, will loot before the end of the season, when they should be planted out in lines, and finally planted out the season following. These make not only the nicest plants, but also the most durable. A poor light sandy soil is the most proper for this and all aromatic plants.

Lavatera.- The shrubby section of this genus is propagated by cuttings set in sandy soil, and covered with a hand-glass.

Leiophyllum.-A hardy evergreen shrub, propagated by cuttings, and also by layers; a peaty soil is that in which it prospers best.

Leycesteria.-A half-hardy evergreen shrub; propagates itself abundantly by shedding its seed, by seed sown in March, by cuttings of the young side-shoots planted in May, in sandy soil, under a hand-glass; prefers a light sandy soil, and slight protection during winter in cold localities.

Ligustrum.-Evergreen and deciduous shrubs, propagate freely by cuttings of the young shoots, also by seeds, which may either be sown when ripe, or kept over winter in sand, and sown in spring. The common privet strikes by cuttings of the last or previous season, planted in a shaded border in November: grows in any soil, however poor, and also under the shade of trees better than most plants.

Liquidambar:-Deciduous trees of low growth, chiefly increased by cuttings and layers. When imported seed can be procured, much better plants are produced. The seeds should be kept in their catkins till the time of sowing, remaining often a year in the ground before vegetating. They succeed best in a moist loamy soil.

Lonicera.-Deciduous shrubs, most of them of a twining character and rapid growth, propagated by cuttings of the ripened wood, and also by layering in autumn, the cuttings being placed in a somewhat shaded situation. Layering is preferred for such kinds as have soft pithy wood; they all prefer a good loamy soil.

Menispermum. - Hardy deciduous twining plants, of rapid growth, propagated by cuttings in spring, placed under a hand-glass, by division of the roots, and by seed when it can be procured; elegant twining plants, too seldom met with in our gardens.
Myrica.-Low-growing shrubs, propagated by cuttings, layers, division of the plant, and seeds, which should be sown as soon as ripe; a moist, sandy, or peaty soil is the most proper for them.

Myricaria.-Low evergreen shrubs, propagated by cuttings of the young wood, either in spring or autumn; if covered with a hand-glass so much the better. Soil, sandy loam, leaf-mould, or sandy peat.

- Negundo. - Deciduous trees of moderate growth; propagated by seeds, which should be sown as soon as ripe; also by layers, requiring a moist deep sandy loam.

Nemopanthes. - An exceedingly ornamental deciduous shrub, too seldom met with in our gardens ; propagated by layers in autumn, also by seed, either sown when ripe, or kept in dry sand till spring.

Nyssa.-Deciduous trees, propagated by layering in our nurseries, but much better plants are obtained by importing the seed : a damp deep soil is suitable for them.

Pconia.-The Moutan section, or shrubby deciduous sorts, are of great beauty, and sufficiently hardy to stand our ordinary winters, in most situations, with little or no protection. In colder localities they succeed well against a north wall, a situation they eminently deserve, on account of the great splendour of their flowers. Being natives of China, they become naturally excited very early in spring, and when planted on south-aspected walls this disposition is encouraged; while by planting them against north walls it is retarded to a later period, and hence such escape the late spring-frosts. Propagation is effected by grafting this section on the roots of the herbaceous sorts (vide Rootgrafting, p. 334). They are also struck by cuttings of the young wood in spring, removing with each a small portion of the last year's wood, and placing them under a hand-glass, moderately shaded, till the rooting process commences. They are also increased by layers and suckers; each layer should have a ring of bark removed from around each bud; and if not too deeply covered with soil, each bud will become a distinct plant. They are also sometimes struck from single eyes, after the manner of vines (which see).

Paliurus. - Deciduous shrubs of moderate growth, propagated by suckers, by cuttings, layers, and seeds sown in any common gardensoil.

Paulownia.-A deciduous tree, of moderate growth, very much resembling Catalpa, and scarcely so hardy. It has stood as a lawn plant in the garden at Dalkeith for several years, but has neither bloomed here nor elsewhere in Scotland that we are aware of, and only in a few places in England. Propagated by seed imported from Japan, and probably from single eyes, layers, or cuttings, like Catalpa. A warm and sheltered situation is necessary, and a soil by no means rich.

Pavia.-Deciduous trees and shrubs, propagated by seeds, layers, and the rarer kinds by grafting on the horse-chestnut. In the latter case, the operation should be done early in the spring, before the sap of the stock has begun to 
flow rapidly. They all require a deep sandy loam.

Periploca. - Deciduous twiners of rapid growth, propagated by layers, and also by cuttings set in sandy soil, and protected by a handglass; they succeed well in any good gardensoil.

Pernettya.-Evergreen shrubs of low growth, adapted to the front of shrubberies; propagated by seed, cuttings, and layers; succeeding best in a peaty or light fibrous soil

Philadelphus. - Deciduous free-flowering shrubs, of moderate growth; propagated by suckers, and dividing the plant early in spring, and occasionally by cuttings. When grown as standards trained to a single stem, they become ornaments to the lawn. They succeed in any moderately good soil.

Phillyrea.-Evergreen shrubs of moderate growth, exceedingly handsome, although their flowers are inconspicuous. Too seldom seen in our gardens. Propagated by layering in autumn, by cuttings placed under a hand-glass in spring, and by seed, when it can be procured, which should be buried in sand for a season before sowing.

Phlomis.-The evergreen shrubby section of this genus are propagated by cuttings, planted in common soil, in a shady place. Most of them are free bloomers.

Photinia. - Sufficiently hardy for standing the winter in the south of England: requires, for the most part, the shelter of a wall in Scotland. They are, in general, propagated by being budded on the hawthorn. Thrive in any ordinary soil.

Piptanthus.-A deciduous shrub, or nearly so, requiring the protection of a wall, which it richly deserves on account of the abundance of its yellow flowers early in spring. Propagated by seeds, which ripen in most places, also by cuttings of the ripened side-shoots. It may also be increased by layering in autumn, and by cuttings of the roots at the same season. Thrives in any moderately good soil.

Platanus.-Deciduous trees of great utility in the decoration of grounds, and well suited for city gardens, squares, \&c. Propagated by seeds sown in spring, and also by cuttings put in both in spring and autumn. The nursery practice generally is to propagate them by layers - an expeditious but reprehensible practice. Require a deep rich loamy soil.

Portugal laurel-vide Cerasus.

Potentilla.-The shrubby section are lowgrowing profuse-flowering plants. Propagated by cuttings of the ripened wood in autumn, also by cuttings of the young wood during summer, set in sand, and covered with a hand-glass.

Prinos.-Deciduous low-growing shrubs, with the exception of $\boldsymbol{P}$. lucidus, which is evergreen. Propagated by seeds and layers, requiring a peat-and-loam soil.

Prunus.-Deciduous low-growing trees, producing abundance of white blossom in April, being amongst the earliest flowering plants met with in shrubberies. Propagated by seeds and suckers. The finer sorts are increased by budding or grafting on stocks of the commoner kinds

VOL. II. raised from seeds. They all prefer a deep loamy soil, and if calcareous so much the better.

Pterocarya.-A deciduous tree allied to walnut, upon which it is grafted for multiplying the stock. The young shoots are sometimes laid, but they seldom make so good plants. In cold localities a poor soil is best for it, to check the luxuriance of its growth and insure the ripening of the wood; in warm places a richer and deeper soil may be accorded it.

Punica.-Deciduous trees, requiring in all parts of Britain the shelter of a wall. Propagated by cuttings of the roots and ripened shoots, and also by layering. The double-flowering variety is grafted on the single. A light rich soil is requisite.

Purshia.-An evergreen shrub of moderate growth. Propagated by cuttings of the young wood in May, set in sand, and covered with a bell-glass. Imported seed is sometimes procured, which should be treated as recommended for loses (vide p. 759). It thrives best in a poor sandy soil. An interesting shrub, seldom found in our gardens.

Pyrus.-Deciduous trees producing abundance of snow-white blossom early in the season. The common sorts are propagated by seeds, and form stocks on which the rarer and better sorts are budded or grafted. Layering is also had recourse to both for obtaining stocks and multiplying some of the kinds. They all prosper in a good loamy soil.

Quercus.-Amongst the oak family there are many extremely interesting trees, both evergreen and deciduous. Many of the sorts are propagated by seeds either ripened in Britain or imported from their native country. The rarer, and those of which seeds cannot be procured, are increased by being grafted on those that are so originated. The acorns should be sown as soon as they fall from the tree, or partially dried and kept packed in sand till spring, when they should be sown in good loamy soil in rows, and covered to the depth of half an inch. In importing acorns, they should be partially dried in the sun before packing, and the driest part of the ship should be secured. Sometimes they are packed in dry soil, and should they partially vegetate during the voyage, they should be carefully unpacked and planted immediately on their arrival, shading the embryo shoot for a few days from direct light. The evergreen species and varieties are exceedingly ornamental in the dressed grounds; and the foliage of some of the American species, becoming highly coloured in autumn, produces enlivening tints beyond any of our hardy trees whatever.

Rhus.-Deciduous trees and shrubs. Propagated by seeds, by cuttings of the roots and shoots, and also by layering. They succeed well in any good garden-soil.

Ribis.-Deciduous shrubs of moderate growth, and flowering early in spring. Propagated by seeds, by cuttings of the ripened shoots in spring and autumn, planted in a somewhat shaded border in the open ground. They all succeed in any good garden-soil.

Robinia.-Deciduous fine-flowering trees. The common sorts, such as the locust-tree, are pro- 
pagated by seeds, which should be retained in the pods till March, and then sown in the open ground. This affords stocks on which to graft the finer kinds, which are often wrought standard high, and afterwards planted on the lawn, or in lines by the sides of walks, \&c. They are all sufficiently hardy for our climate, with the exception of $R$. hispida, which requires the shelter of a wall in most places, and its support in all, on account of the extreme brittleness of its branches, which are liable to be broken by the wind. Cuttings of the shoots, and sometimes of the roots, are employed when seed can. not conveniently be proculed.

Rubus.-Deciduous and evergreen rambling rapid-growing shrubs. Propagated in general by suckers, layers, and also by cuttings. The best way, however, is to insert the point of the shoots about an inch into the soil, where they will root in course of a few days. They grow in any ordinary soil, and many of them amongst stones and the debris of rocks, where few other plants could exist.

Ruscus.-Evergreen shrubs, mostly of low growth. Propagated by dividing the roots and by suckers; thriving in most soils, particularly those of a peaty nature.

Salix.-A very extensive genus; some species, however, add greatly to the effect of dressed grounds, particularly in the vicinity of water, an element the whole family delight in. The pendulous sorts, such as S. Babylonica, are indispensable accompaniments to the sides of ponds and streams, and those with white silvery leaves add a feature to the grounds; their graceful branches moving with the wind, and their colour contrasting with the masses of green of which the scene is principally composed. They all strike readily by cuttings of the ripened shoots'; and even truncheons several inches in diameter, if driven into the ground, will strike root and soon become good-sized trees.

Sambucus.-Deciduous trees and shrubs, propagated by cuttings of the ripened wood, either of one or of several years' growth. Thriving in any ordinary soil, and in shady places where few other deciduous trees would live.

Santolina. - Low-growing evergreen shrubs, propagated by cuttings planted either in autumn or spring, in a somewhat shaded situation.

Smilax.-Evergreen climbing moderate-growing plants, propagated by suckers from the roots. Thrive in a soil of peat and loam.

Spartium. - Moderate-sized growing shrubs, producing abundance of yellow or white coloured blossom, enlivening the foreground of the shrubbery. Propagated by seed sown in March, and also by cuttings, which strike freely during summer, planted in sandy soil and covered with a hand-glass. They are difficult to transplant when old, on account of a paucity of fibrous roots; therefore they should be set in their final situation when under two years' growth. They thrive in any poor sandy soil.

Spircea. - The majority are deciduous, moderate-growing shrubs, a few only being evergreen; all are more or less interesting for their flowers, and some particularly so, as S. Lindleyana, S. bella, \&c., the former a fine lawn- shrub. They are all readily increased by cuttings, layers, or suckers, and thrive in any good garden-soil.

Styrax.-Deciduous moderate-growing shrubs, flowering profusely if planted against a wall, or in a dry protected situation. Propagated by imported seeds, cuttings, and layers, and thriving in rich sandy loam or peat.

Symphoricarpus. - Deciduous shrubs, propagated by suckers, and also by cuttings planted in autumn. Succeed in any ordinary gardensoil. The white fruit of S. racemosus imparts an interest to our shrubberies during winter.

Syringa.-Deciduous shrubs, rivalled by few of the inhabitants of our shrubberies in the beauty of their blossoms. Propagated by suckers and layers generally, and by budding and grafting in the case of the finer or rarer varieties. Some of the best are often wrought on stocks of the common sorts at standard height, and as such form great ornaments to our lawns. A loamy soil is the best, although they will accommodate themselves to one of an ordinary description.

Tamarix. - Straggling moderate-growing shrubs, well adapted for forming a barrier against the sea breeze. Propagated by cuttings set in sandy soil in spring under a hand-glass, and when of the height of 1 foot or 18 inches, they should be placed in their permanent place of growth, as they do not transplant readily when too old. A light sandy soil is suitable for them and it is probable that a slight addition of salt would improve their growth.

Tecoma.-Deciduous moderate-growing twining or climbing shrubs, separated from bignonia or trumpet-flower. Well adapted for growing against a wall in cold situations, or over pillars or trellises in more favourable situations. Propagated by cuttings of the roots as well as by cuttings of the shoots; they may also be increased by cuttings of the single eyes, similar to vines. A peat-and-loam soil is the most proper for them.

Ulex. - Low-growing yellow-flowering evergreens. Propagated by seed sown in spring, in any good light garden-soil. The double-blossomed variety, and also the upright or Irish, which latter seldom flowers, and so far as we are aware has not produced seed, are propagated by cuttings planted in sandy soil either in spring or autumn, in a shady place, and covered with a hand-glass. They should be transplanted while young to their permanent position, as they do not-remove with safety when òld.

Viburnum. - Both deciduous and evergreen species exist in this genus. Those which produce seed in Britain should have it sown in spring, after having lain a season in the rot-heap; or upon a small scale, being imbedded in sand in a flower-pot, and buried for the same length of time. They are also increased by layers, and the evergreen kinds, such as the laurustinus, strike readily by cuttings planted in sandy soil in autumn, in a shady border. In two years they will be sufficiently rooted to be taken up and planted in nursery lines. They all succeed in any good garden-soil.

Vinca. - Trailing evergreen shrubs, well 
adapted for covering the surface of shrubberies, rocks, or sterile banks. Propagated freely by layers and cuttings. They will grow in almost any soil, and thrive under the shade of trees where no other plants will grow.

Vitex.-The hardy deciduous species are ornamental, and readily increased by cuttings of the ripened wood planted in autumn, in a shady border, and covered with a hand-glass. Thrive in any ordinary soil.

Vitis. - Deciduous twining, fast-growing shrubs. Propagated by cuttings and eyes of the ripened wood; also by layering, grafting, and inarching. A rich loamy soil is most suitable for them.

Wistaria.-Deciduous rapid-growing climbers, propagated by cuttings made of the strongest roots, also by cuttings of the young wood when half-ripened, planted in sandy soil, and covered with a bell-glass ; most generally, however, by layering the long shoots, tonguing under each bud, and inserting it in the soil to the depth of half an inch; each bud will thus produce a plant, which, when rooted, is to be separated, and either potted or set out in nursery lines. They thrive well in sandy loam and peat.

Zizyphus. - Half-hardy shrubs; propagated by cuttings of the roots, and also cuttings of the ripened shoots planted in sandy soil and placed under a hand-glass. They require a dry deep loamy soil, and slight protection during winter in cold situations.

\section{SELECT LIST OF HARDY CLIMBING OR TWINING SHRUBS.}

(For American Shrubs, vide AMERICAN Garden, p. 749).

Ampelopsis cordata, bipinnata, hederacea; Atragene siberica, austriaca, ochotensis, americana ; Aristolochia Sipho, tomentosa ; Berchemia volubilis ; Bignonia capreolata; Bridgesia spicata; Caprifolium (Honeysuckle), sempervirens, Douglassii, gratum, longiflorum ; Clematis (Virgin's bover), cærulea, campaniflora, crispa, flammula, florida, florida flore-pleno, florida bicolor, tlorida Sieboldii, montana, grandiflora, Viorna, vitalba, viticella; Celastrus (Staff tree) scandens ; Decumaria barbara, prostrata, sarmentosa; Glycine sinensis, frutescens; Hedera (Ivy), many of the curious varieties of $\mathrm{H}$. helix, particularly emarginata, and the yellow fruiting, as also the recognised species canariensis, taurica, and rœgneriana; Jasminum (Jasmine), pubigerum, Reevesii, officinale, with its variegated - leaved and double-flowering varieties, affine, frutescens, heterophyllum, humile; Lonicera flexuosa, japonica, against walls in general ; Lycium (Box-thorn), Chinense, Afrum, Europeum, Barbarum, fuchsioides, lanceolatum, turbinatum ; Menispermum (Moon seed), Canadense, Lyonii, and indeed most of the genus; Passiflora (Passion-flower), cærulea, and in favourable situations incarnata, and the hybrid racemosa cærulea ; Periploca Græca, angustifolia, lævigata ; Rhus (Sumach), radicans, with its varieties, microcarpa, volubilis, vulgaris ; Rubus (the Bramble), bellidiflora, corylifolius, lacinatus flore-pleno, and others; Smilax aspera, auri- culata, sarsaparilla, and several others; Solanum dulcamara variegata, articulatum, crispum ; Tecoma (Trumpet flower), radicans, radicans major, radicans minor, and in warm situations grandiflora may be planted against a wall ; Vitis (the Vine), labrusca, riparia, laciniosa, vinifera, rotundifolia, palmata, cordifolia, dentata, Wallichii.

\section{SELECT LIST OF SHRUBS WHICH THRIVE UNDER THE SHADE OF TREES.}

Evergreens.-Vinca major and minor, with their variegated-leaved and double-flowering varieties; Aucuba japonica ; Androsæmum officinale; Daphne laureola, and its variegated variety, pontica ; Ephedra monostachya, distachya; Buxus sempervirens, and its varieties ; Hypericum calycinum ; Ligustrum vulgare sempervirens ; Viburnum tinus ; the Portugal and common laurel ; Ilex aquifolia (the Holly) and its varieties; Berberis aquifolia, fascicularis, \&c.

Deciduous. -Sambucus niger, fol. variegatus, fol. lacinata, fructu alba, racemosa; Symphoria racemosa, glomerata ; Cornus alba, sanguinea, florida, alternifolia; Mespilus germanica; $\mathrm{Ru}$ bus villosus, odoratus, trivialis; Corylus avellana; Daphne Mezereum, scrotina, alpina; Euonymus verrucosus, angustifolius, latifolius; Lonicera xylosteum, tartarica ; Spiræa sorbifolia ; Hypericum minus, hircinum, prolificum ; Leycesteria formosa.

Creepers or climbers. - Vinca major, minor, and their varieties; Hedera helix, and its varieties; Caprifolium periclymenum ; Clematis flammula, vitalba.

\section{LIST OF SHRUBS WHICH THRIVE IN MARSHY PLACES, AND BY THE EDGES OF RIVERS OR PONDS.}

Evergreens.-Arbutus unedo; Ledum palustris; Pinus palustris ; Atriplex halimus; Andromeda polifolia.

Deciduous.-Taxodium distichum; Alnus pumila; Betula nana, pumila ; Dirca palustris ; Myrica Gale, cerifera, and most of the genus; Salix, most of the genus.

\section{LIST OF SHRUBS, CHIEFLY EVERGREEN, ADAPTED FOR GARDEN HEDGES.}

Buxus sempervirens; Taxus baccatus; Rosmarinus officinalis; Portugal and common laurel; Juniperus communis, and most of the genus; Jaurus nobilis; Ligustrum vulgare, var. sempervirens; Phillyrea, all the genus; Rhamnus alaternus; Thuja, most of the genus ; Ulex Europea and Hibernica; Lavendula spica ; Aucuba japonica; Arbutus unedo; Cratægus pyracantha; Daphne pontica; Ilex aquifolia; Vibernum tinus; Mahonia, several of the genus; Cydonia japonica; Daphne mezereum.

LIST OF SHRUBS OF RAPID GROWTH, FOR PRODUCING IMMEDIATE EFFECT, OR FOR SHELTER AND HIDING OUT DISAGREEABLE OBJECTS.

Evergreen.-Portugal and common laurels; Cupressus sempervirens; Ilex aquifolia; Juniperus virginianus; Rhododendron ; Ligustrum 
vulgare, var. sempervirens; silver and spruce firs, and several other free-growing conifers, whose tops being cut out when they have nearly attained the desired height, they assume a shrub-like character, and often become exceedingly grotesque in habit; Rhamnus alaternus; Spartium junceum; Taxus baccata; Thuja occidentalis. With the exceptions of Rhamnus and Spartium, which should be planted when young, the others may be transplanted when of a large size.

Deciduous.-Sambucus niger and its varieties; Spiræa Lindleyana, sorbifolia ; Viburnum Opulus; Syringa vulgaris; Colutea arborescens ; Cornus alba, florida, sanguinea; Philadelphus coronarius ; Cytisus nigricans, laburnum, alpinus, and their varieties; Symphoria racemosa ; Aralia japonica ; Cratægus, many species ; Kerria japonica ; Salix alba, violacea ; Robinia pseudo-acacia; Tamarix galica; Berberis vulgaris, and several others; Piptanthus nepalensis; Rhus toxicodendron, cotinus, and several other species; Gleditschia triacanthos, sinensis; Gymnocladus canadensis; Pyrus spectabilis, and several other species; Magnolia pyramidata; Lonicera tartarica, alpigena, and several others.

\section{SELECT LIST OF SHRUBS FOR SMALL SHRUBBERIES.}

Abelia floribunda, uniflora; Aralia spinosa; Berberis atro-purpurea, dulcis, repens, Darwinii, elegans, trifoliata, empetrifolia, fascicularis; Buddlæa Lindleyana; Buxus balericus; Calycanthus floridus; Ceanothus azureus, cuneatus, papillosus, dentatus, rigidus; Cerasus ilicifolia; Cotoneaster thymnifolia, denticulata, rotundifolia, mycrophylla; Cytisus nubiginensis, Weldenii, supinus, sessiliflorus, capitatus, nigricans; Coriaria; Daphne cneorum and its variegated variety, alpina, collina, Mezereum, and its varieties, autumnale and alba, pontica, pontica variegata, Tarton-raira, japonica; Deutzia scabra, corymbosa, Mexicana, gracilis ; Escallonia macrantha, rubra, Montevidensis; Euonymus fimbriatus, japonicus, with its gold and silver striped varieties; Eurybia ilicifolia; $\mathrm{Fa}$ biana imbricata; Forsythia viridissima; Fortunea chinensis; Gaultheria procumbens, Shallon ; Garrya elliptica, macrophylla, laurifolia; Kadsura japonica; Laurus glauca, regalis; Limonia laureola; Mitraria coccinea; Myrica Californica; Pernettya mucronata; Spiræa prunifolia, pleno, callosa; Skimnia japonica; Ulex europea flore pleno; Weigela rosea, amabilis ; Helianthemum, all the species and varieties; Cistus, most of the genus in warm situations; Polygonum vaccinifolium; Ononus rotundifolius; Potentilla floribunda, fruticosa.

SELECT LIST OF HARDY AND HALF-HARDY SHRUBS AND LOW-GROWING TREES REMARKABLE FOR THEIR FOLIAGE, HABIT, OR FLOWERS.

Acacia dealbata, Julibrissin, affinis, lophantha; Adenocarpus intermedius; Ailanthus glandulosus ; Abelia floribunda, uniflora; Aralia spinosa, japonica ; Arctostaphylos tomentosus ; Esculus Pavia, rosea, flava, spicata, præcox, spectabilis, pallida; Arbutus unedo, procera,
Croomii, longifolia, Milleri, salicifolia, magnifica, Andrachne; Amygdalus communis, communis-amara, dulcis flo. pleno; Amelanchier florida, ovulis, vulgaris, sanguinea; Anagyris fœtida; Amorpha fruticosa, Lewisii, nana, glabra; Aristotelia Macqui, var. fol. variegata, in warm situations; Asimina triloba; Aucuba japonica, latimaculata; Azara dentata, integrifolia; Benthamia fragifera; Berberis aquifolia, Darwinii, dulcis, sinensis, tenuifolia, fascicularis, Fortunii, Jamesonii, trifoliata, Wallichiana, atro-purpurea, repens, elegans, macrophylla, impetrifolia, aristata, asiatica, angulosa, emarginata, Fischeri, buxifolia, cuneata, nepalensis ; Biota glauca, orientalis; Buxus balerica, arborescens, and its varieties; Broussonetia dissecta; Buddlea globosa, Lindleyana ; Bupleurum fruticosum ; Calycanthus floridus, macrophyllus, lavigatus, glaucus; Calophaca wolgarica; Castanea Californica ; Calycarpa japonica; Ceanothus rigidus, papillosus, azureus, cuneatus, dendatus, integrifolius, divericatus, integerrimus ; Cerasus ilicifolius ; Chionanthus montanus, virginicus ; Chimonanthus fragrans, grandiflorus, luteus (requires a wall) ; Chamœrops excelsa, a palm from the north of China, hardy in most places; Cineraria maritima; Cistus formosus, ladaniferus, and most of the genus; Clianthus puniceus, requires the shelter of a wall in most places ; Cereis japonica ; Coronilla coronata, Emerus, pentaphylla, glauca, and valentina, in warm places ; Cotoneaster buxifolius, thymnifolius, comptus, marginatus, microphyllus, rotundifolia, denticulata, affinis ; Caragana, most of the genus; Cratægus, many of the genus; Clethra alnifolia, pubescens; Comptonia asplenifolia ; Colletia spinosa, ferox ; Colutea cruenta, fruticosa, arborescens; Corchorus japonicus; Cydonia japonica, sinensis; Cytisus, most of the genus ; Daphne, all the genus; Deutziascabra, corymbosa, crenata, gracilis, sanguinea, Mexicana; Dirca palustris; Edgworthia chrysantha; Elæagnus argentea, reflexa, macrophylla; Escallonia, all the genus; Euonymus Javanicus, citrifolius, fimbriatus, japonicus ; Empetrum allum ; Eriobotrya japonica, requires the shelter of a wall ; Ephedra distachya, monostachya ; Eurybia ilicifolia; Fabiana imbricata ; Forsythia viridissima ; Fortunea chinensis; Fontanesia phillyræoides ; Fuchsia, most of the species and varieties, discolor and Riccartonia being the hardiest-the others, although killed to the ground, are seldom killed at the roots ; Garrya elliptica, laurifolia, macrophylla, Macfadyeniana ; Gleditschia horrida, inermis, triacanthos; Gordonia Lasianthus ; Genista tinctoria pleno, and all the genus; Hydrangea arborescens, nivea, quercifolia, and in warm places hortensis; Hibiscus Syriacus, and varieties; Ilex dipyrena, lataspina, latifolia, Torajo, crassifolia, furcata, Sheppardii, with the numerous varieties of aquifolia; Indigofera decora, australis, verricosa; Illicium floridanum, religiosum ; Kadsura japonica ; Kolreuteria paniculata ; Lardizabala biternata ; Laurus benzoin, sassafras, nobilis, regalis ; Leycesteria formosa ; Liquidambar styraciflua, imberbe ; Ligustrum japonicum, ovalifolium, spicatum ; Limonia laureola ; Lindleya mespiloides ; Liriodendron tulipifera; Mahonia aquifolia, 
glumacea, magnifica, repens; Magnolia, all the hardy species of the genus; Morus alba; Maclura aurantiaca, in warm situations; Mitraria coccinea; Myrica Californica latifolia; Nuttalia cerasiformis; Olea europea, and fragrans in warm situations; Ononis natrix, tribracteata; Pavia Californica; Paliurus aculeatus; Pernettya mucronata, speciosa; Pæonia moutan, all its varieties; Philadelphus grandiflorus, Gordonianus, coronarius flo. pleno, fol. variegata; Photinia arbutifolia, serrulata, glabra; Phillyrea latifolia, angustifolia, buxifolia, media, obliqua; Phlomis fruticosa; Planera crinata ; Potentilla floribunda, fruticosa; Prinos glabrus, lavigatus, lanceolatus, prunifolius, fruticosus; Purshia tridentata; Rhamnus Californicus, Alaternus, balericus, latifolius, laurifolius, Rhodothamnus Kamtschaticus ; Rhus Cotinus, elegans, vernix, toxicodendron, copallina; Rubus coronarius flore pleno, bellidiflorus, and others as hardy rambling climbers; Ribes sanguinea flore pleno, flore alba, aurea, speciosa, and others ; Robinia hispida, pseudo-acacia, rosea, viscosa; Solanum crispum ; Spiræa Blumiana, Douglassii, prunifolia pleno, Lindleyana, lancifolia, callosa, sinensis, ariæfolia, Reevesiana, pubescens, bella ; Skimmia japonica; Syringa Emodi, Josikæa, Notyce, persica, siberica, and several interesting varieties of vulgaris; Santolina viridis, chamæcyparissus, squarrosa; Shepherdia argentea, canadensis; Spartium juncum flo. pleno, multiflorum flo. pleno, flo. rosea, scoparium flo. pleno ; Tamarix gallica, germanica; Ulex europea flo. pleno, nanus; Viburnum Opulus flo. pleno, japonicum, Tinus and its varieties, ovalifolium, grandiflorum, macrocephalum, plicatum, suspensum ; Virgillea lutea; Weigela rosea, amabilis, Middendorffiana; Yucca, all the genus.

\section{SELECT LIST OF SHRUBS TO BE PLANTED AS} SINGLE SPECIMENS ON LAWNS.

Aucuba japonica, latimaculata ; Benthamia fragifera; Berberis, most of the genus; Buxus balerica: Ceanothus, most of the genus in warm situations ; Cistus, most of the genus in warm sheltered situations; Colletia ferox, spinosa; Comptonia asplenifolia ; Cotoneaster, most of the genus ; Cytisus, most of the genus; Daphne, most of the genus; Deutzia, all the genus; Dirca palustris; Ephedra altissima, distachya, monostachya ; Escallonia, most of the genus in warm situations; Fabiana imbricata; Fontanesia phillyræoides; Genista, most of the genus; Hydrangea, all the genus in moderately warm places; Laurus nobilis, regalis, sassafras ; Ligustrum japonicum; Mahonia, all the genus ; Magnolia, all the genus in moderately warm places; Pæonia moutan, and its varieties; Paliurus aculeatus; Phillyræa, all the genus; Phlomis fruticosus; Prinos, all the genus; Rhamnus alaternus, balearicus, and its varieties; Santolina chamæcyparissus; Shepherdia argentea; Spartium, all the species and varieties; Spirrea ariæfolia, bella, Lindleyana; Syringa, all the species and varieties; Ulex europeus, flo. pleno sanus; Viburnum tinus, and its varieties; Weigela rosea; Yucca, all the genus.

Half-hardy plants considered in relation to climate and situation.-The vast additions made during late years to our gardens from all parts of the world, have rendered it almost impossible to find protection for them under glass. Nor is this in all cases necessary, as we find many of the trees and plants from India, China, Australia, \&c., sufficiently hardy in many situations to withstand the rigour of our ordinary winters, even when planted in the open air, and many others will do so with very slight protection. The situations in which these succeed best are those within two hundred feet of the sea-level, although some do so at a greater altitude; but in all cases it is a necessary condition that the soil be either dry naturally, or rendered so by artificial means.

Situations best adapted for half-hardy trees and shrubs.-Many are led away with the erroneous notion, that because a plant or tree comes from China, Japan, or Australia, it must needs be placed against a wall, in the most sheltered and sunny spot the garden affords. Than this nothing can be more erroneous, at least as regards most of the plants from the former countries. They flower with us too early in the spring; and when excited by the warmth which these situations possess during March and April, this evil is increased. Many of them, therefore, are found to thrive better when planted in a northern exposure, and many more upon a western one. No doubt a southern exposure is of all the most favourable for ripening the wood and flower-buds, and this is in all cases a most important point to be secured; for unless the sap be maturely elaborated, the trees can neither be expected to survive our winters nor to gratify us with their flowers. For herbaceous plants and bulbs, and all such as die down to the ground annually, no situation can be too warm in this climate ; and hence so many of these natives of the south of Europe, the Cape of Good Hope, and various parts of Australia, continue in our warm borders for years. They have another advantage, also, over trees and shrubs: the vegetable volume is smaller in them, the sap has a much less distance to travel, and it is not improbable that from their structure it is supplied in greater quantity and in less time than in woody plants ; so that the operation of development for the present season, and the elaboration of the sap for the succeeding one, are both effected in less time.

Exotics planted against a warm south wall should be retarded in spring as a means of securing them against the effects of late springfrosts ; and this would be best effected by rearing in front of them, about 2 feet distant, a portable wall of boarding, set perpendicular, and having a covering of the same material as a roof, constructed so that it could be readily opened and shut. The object of this protection is to prevent the vernal sun from shining on the trees, and so as it were placing them nearly in the same condition as if planted against a northern wall. The roof being at all times open, unless during the most intense frosts, would admit sufficient light and air to them while yet in their dormant state. Were the walls upon which such trees are trained heated by hot-water pipes, 
their operation in autumn, for the purpose of maturing the wood, would be, of course, an advantage ; heated during spring would have a contrary effect.

Altitude, as we have already stated, has also much to do in the successful cultivation of exotic trees and plants, and their preservation during winter. Altitude above the sea has been calculated as being equivalent, on the score of climate, to a receding north or south from the equator, 600 feet of altitude being thought to be equal to a degree. The influence of elevation above the sea-level in depressing the temperature has been very little attended to in Britain. Sir Thomas Brisbane and Mr Galbraith (vide "New Edinburgh Journal") have estimated the decrease to be $1^{\circ}$ of temperature for 212 feet of ascent in lat. $55^{\circ}-57^{\circ}$; while $\mathrm{Mr}$ Hewett Watson (vide "Magazine of Natural History") raises it to 239 feet between $53^{\circ}-59^{\circ}$. These estimates do not refer to the winter months; nor is either of them worthy of implicit reliance. According to De Candolle, heat decreases in France at the rate of one degree of latitude for every 540 feet of altitude. In the middle of the temperate zone Humboldt remarked that the mean heat of the year diminished at the rate of $2^{\circ}$ north latitude for every 600 feet of altitude. From the powerful influence on temperature produced by elevation arise those discrepancies which occurin the ripening of fruits and precocity of vegetation between gardens near the sea-level and those at a greater altitude, although at no very great distance from it.

"The mean heat of any situation," Loudon remarks, "does not enable us to judge of what particular species of plants will live there; for the mean temperature found may be deduced from such extremes of heat and cold as would suit but few plants ; or it may be made up from moderate limits, in which many plants will live; as, for example, from the summers and winters of Ireland, or the sea-coast of the middle of Europe. Thus the constitution of a plant, which may be very well suited for the mean temperature of a place, may not be adapted to its extreme differences. Hence many plants, which will live in the open air at Belfast, would perish in the winters at Edinburgh ; and many which would live there, owing to the dryness of the air and the moderate degree of cold from the proximity of the sea, would perish in Yorkshire, where the air is not only more highly charged with moisture, but much colder. Hence the mean annual temperature of any place is of much less consequence, with respect to its influence upon plants and fruit trees, than the mean monthly temperature, and the extremes of each month." The latter form the data, therefore, that cultivators ought to be guided by in determining on their selections of plants and tender fruit trees.

The most serious effects of frost on vegetable bodies arise from the sap becoming frozen in the vessels, which are consequently ruptured by its expansion ; and therefore all soft-wooded plants are much more liable to be affected by intense cold than hard-wooded ones, on account not only of the former being more charged with sap in proportion to their bulk, but also be- cause their bark, or outer covering, is thinner and softer, and their roots nearer the surface. The rugged barks of hard-wooded trees are powerful non-conductors; while soft-wooded plants have smooth and very thin skins, and are less fortified against cold, for want of sufficient non-conducting covering, and hence less capable of resisting cold. The roots of plants, such as trees, \&c., which penetrate deepest into the soil, are, from another cause, better able to resist severe cold, because they penetrate deeper into the soil than frost; and as a slow circulation is carried on in their trunks and branches throughout the winter, the sap they contain is prevented from being frozen, by the heat they obtain from the subsoil. Hence the great advantage of covering the soil above the roots by mulching them over with litter, or some other non-conducting material, to prevent the loss of heat from the soil by radiation. On a similar principle, the practice of covering the stem and main branches, by enveloping them in straw,moss, or other covering, is advantageous, as it assists the bark in resisting frost, or probably it acts rather by preventing the loss of internal heat.

Acclimatation is the doctrine of rendering plants of a warmer country, by cultivation, \&c., capable of thriving in another which is colder. On this subject opinions widely differ. The advocates for it assert that the sensibility of plants may be diminished in habit by a gradation of climate, and by a succession of generationsthat is, by slight protection for a few years after planting, and gradually exposing them to the natural climate of our gardens, and also by rearing plants from seed ripened in the open air of our gardens. Few satisfactory instances have been recorded of such effects being produced. The circumstance, however, that the quality of the products of many plants is greatly changed by raising generation after generation from seed, seems to some extent to favour this idea. That a very considerable difference of constitution exists among individuals of the same species is undeniable, some being far more robust and capable of resisting external influences than others. From these seed should invariably be taken. The majority of the plants and trees, natives of warmer climates, which are found to resist the effects of our severest winters in the open air, never were tender, as may be instanced in the cases of Araucaria imbricata, Aucuba japonica, Kerria japonica, Glycine sinensis, Weigela rosea, \&c. Whereas, on the other hand, no change has been produced on the hardiness of other plants much longer cultivated in our gardens, and annually reproduced from seed ripened in our climate, as may be exemplified in the case of the tomato, kidney-bean, mignonette, and a host of others.

The last winter (1853-4) has been the most trying for many years past. The plants which have survived it in the open air may be regarded as possessing a hardy constitution, compared with others from the same country, and in many cases of the same genus. The following list of survivors, which is partly compiled from the excellent article on "The Effects of the Winter of 1853-4," in the "Journal of the Lon- 
don Horticultural Society," and partly taken from our own observations and correspondence, may be of use to those who, like ourselves, cultivate exotic plants, in general considered tender, in the open air. In all cases where reference is made to Dalkeith, the plants have had no artificial protection beyond being planted against walls of various aspects, or in borders in front of such walls, while many are grown as open standards on the exposed lawns.

LIST OF PLANTS WHICH HAVE STOOD UNINJURED THE SEVERITY OF THE WINTER OF 1853-4-

(When the thermometer indicated, as it did at Chiswick on the night of the 28th December 1853, $8^{\circ}$; and on the night of the $2 d$ January $1854,4^{\circ}$; at Kew, $13^{\circ}$; at Dalkeith, $10^{\circ}$; at Bicton, from $6^{\circ}$ to $10^{\circ}$; at Singleton, close upon the sea, facing the south, $16^{\circ}$; and at other places equally low temperatures.)

Abelia uniflora, rupestris,floribunda, uninjured at Dalkeith; Acacia Julibrissin ; Akebia quinata; Amygdalus angustifolius, incana; Andromeda floribunda, formosa, uninjured at Chiswick ; Anthyllis hestrix, uninjured atDalkeith for years; Araucaria imbricata, injured only in very exposed places, not injured at Dalkeith ; Aralia japonica, as a fine hardy open lawn tree at Dalkeith; Arbutus procera, unedo, suffered only in exposed places, uninjured at Dalkeith ; Azalea ovata, Californica; Bambusa falcata, uninjured at Dalkeith; Benthamia fragifera, uninjured at Dalkeith; Berberis Fortunii, uninjured at Dalkeith and other places; Berberis glumacea, fascicularis, intermedia, Beali, japonica, repens, aquifolia, dulcis, asiatica, aristata, Darwinii, empetrifolia, cuneata, parviflora, dealbata, coriaria, flexuosa, Lycium; Biota orientalis, aurea ; Bignonia grandiflora, at Dalkeith ; Bridgesia spicata, has stood for years at Dalkeith; Bupleurum fruticosum, uninjured at Dalkeith and elsewhere; Buddlea Lindleyana, not injured at Dalkeith; Buxus balearica, uninjured at Dalkeith and elsewhere; Carmichælia australis, uninjured at Dalkeith ; Caprifolium flexuosum, not injured at Dalkeith or Chiswick; Caprosina rhamnoides, uninjured at Dalkeith; Cerasus ilicifolia, reported as injured in many parts of England, not in the least injured at Dalkeith; Ceanothus, all the genus with the exception of rigidus, intergerrimus, and verrucosus, much injured-in many places killedwhile they, along with azureus, remained uninjured at Dalkeith ; Chimonanthus fragrans and varieties not in the least hurt, flowered most profusely during winter at Dalkeith ; Cistus formosus, ladaniferus, and severalothers, escaped at Dalkeith-most of the genus killed elsewhere; Clematis Grahamii, grata, polymorpha, montana, calycina, azurea, tubulosa, grandiflora, uninjured at Dalkeith-the five first not injured at Chiswick; Colletia horrida, although protected at Chiswick, was killed to the ground-escaped in various situations at Dalkeith wholly unprotected, as did also C. spinosa ; C. serrata, escaped at Chiswick ; Colquhounia coccinea escaped injury at Dalkeith without the least protection ; Cotoneaster Roylei escaped at Chiswick; Deut- zia gracilis, scabra, escaped at Chiswick and Dalkeith; D. staminia, escaped at Chiswick ; Diotis ceratoides, escaped at Chiswick ; Duvana dependens, ovata ; Edwardsia grandiflora, uninjured at Dalkeith, only slightly hurt in other places; Elæagnus parvifolius, retusus, apparently quite hardy; Elsholtzia polystachya; Eriobotrya japonica, uninjured at Chiswick and Dalkeith; Escallonia, all killed to the ground at Kew, while macrantha, rubra, montevidensis, were unhurt at Dalkeith, and rubra only escaped injury at Chiswick; Eucalyptus, were all either killed or much injured at Kew, while E. coccifera only escaped at Chiswick, and E. perfoliata at Dalkeith. This plant is 20 feet in height, and has withstood our winters for twelve years without protection. Seven other species were killed with us, but these were young plants only recently planted out. Euonymus japonicus, not injured at Chiswick or Dalkeith; E. angustifolius, Hamiltonianus, survived at Chiswick; Fabiana imbricata, slightly injured at Kew, not in the least so at Dalkeith; Fontanesia phillyrœoides, uninjured at Dalkeith without protection ; Forsythia viridissima, uninjured in most places; Fuchsia, most of the species and varieties stand the winter at Dalkeith. Although cut down to the ground, they start readily in spring, making wood and flowering profusely through the summer and autumn; discolor and Riccartonia are seldom cut down. Garrya elliptica, uninjured at Chiswick, Bicton, Dalkeith, Southampton, and Windermere ; Gaultheria Shallon, procumbens, and nummularia, hardy in most places ; Genista candicans, uninjured at Chiswick, virgata, uninjured at Dalkeith ; Glycine sinensis, and sinensis alba, apparently hardy everywhere; Glyptostrobus pendulus, uninjured at Chiswick and elsewhere ; Gynerium argenteum, not in the least injured at Dalkeith, appears to be hardy in most places; Hydrangea quercifolia, involucrata, uninjured at Chiswick and Dalkeith ; Ilex latifolia, slightly injured in open borders at Chiswick - sickly plant killed at Dalkeith, furcata, perfectly hardy at Bagshot and Alton, cornuta, hardy at Bagshot and Southampton, perado and opaca, hardy. at Chiswick, Taraga, leptacantha, and microcarpa, uninjured at Bagshot, deprina, unhurt at Dalkeith; Jasminum nudiflorum, apparently hardy everywhere, Wallichianum, affine, uninjured at Chiswick and Dalkeith; Laurus regalis, slightly injured at Chiswick, nobilis, perfectly uninjured at Dalkeith in the open quarters, scarcely injured at Kew or Chiswick - much injured in most places, Sassafras, uninjured in most places ; Lagerstrœmia indica, uninjured at Bagshot; Leycesteria formosa, injured neither at Chiswick nor Dalkeith; Ligustrum japonicum and ovalifolium, uninjured at Chiswick, lucidum, very slightly injured in the neighbourhood of London, killed in cold exposed places, quite uninjured at Dalkeith; Lonicera angustifolia, discolor, diversifolia, fragrantissima, escaped injury at Chiswick; Maclura aurantiaca, not the least injured at Dalkeith ; Magnolia grandiflora, not injured at Dalkeith, conspicua, macrophylla, purpurea, auriculata, glauca, obtusifolia, not in- 
jured on the open lawn at Dalkeith; Morina longifolia, not the least injured at Dalkeith; Myrica Californica, not injured at Chiswick or Dalkeith; Myrtus communis, latifolia, uninjured at Dalkeith, although slightly injured about Loudon, and even Southampton ; Nandina domestica, has stood uninjured for twelve years at Dalkeith, although killed on a south wall at Chiswick, and injured at Kew; Neillia thyrsiflora, uninjured at Dalkeith, although killed to the ground at Chiswick; Ornus floribundus, not injured against a south wall at Chiswick, and only slightly at Kew ; Pavia Californica and indica, not injured at Chiswick ; Pawlovnia imperialis, which has stood as a lawn tree for seven years at Dalkeith, so severely injured as to leave little hope of its recovery; Pæonia moutan, escaped at Dalkeith as an open lawn plant, as well as on a northern wall; Phillyreas, not injured at Dalkeith or Chiswick, injured or killed in several other places; Phormium tenex, killed at Dalkeith, the plant being injured by accident the previous season - had stood in an open border for nine years; Photinia glabra, uninjured at Dalkeith or Chiswick; Piptanthus nepalensis, not the least injured at Dalkeith, killed at Chiswick; Plumbago larpenta, quite hardy in the borders at Dalkeith ; Pistacia terebinthus, only slightly injured at Kew; Pittosporum bicolor, slightly injured at Chiswick ; Punica granatum, only slightly injured at Kew, uninjured at Chiswick and Dalkeith; Quercus, - this genus suffered much, the following escaped, Ilex at Kew, Chiswick, and Dalkeith, gramuntia at Chiswick, inver'sa at Bagshot, suber, slightly at Chiswick, not injured at Dalkeith-several of the Kurdistan species were not injured by the winter at Chiswick, but suffered much by the frost of April 24th, dealbata and Cookii, partially, Australis, not the least, glabra, escaped at Bagshot, Bicton, and Exeter, and Mongolica escaped at Shifnal, all the others more or less injured-the Mexican ones all killed; Rhododendron,-the Sikkim sorts are reported to have stood uninjured at Bagshot, with the exception of Edgeworthii, and in several instances they have stood better in peat than in a loamy soil ; R. cinnamomeum, Campbelliæ, Campanulata, stood uninjured at Dalkeith ; Ribes acuminatum and glutinosum, uninjured at Chiswick, and speciosum and glaciale stood without injury at Dalkeith ; Rosmarinus officinalis, although much injured at Kew, did not suffer in the least at Dalkeith; Rosa Banksiæ, lutea and alba, not injured at Dalkeith; the white variety appears to be more hardy than the yellow. Roses in general suffered little at Dalkeith, while in many places the tender varieties were greatly destroyed. Salvia Grahamii, uninjured at Dalkeith for years ; Santolina chamæcyparissis, not injured at Chiswick nor at Dalkeith, although on the open lawn; Skimmia japonica suffered more from the frost of the 25th of April than from the frost during winter; Solanum crispum and jasminoides, escaped unhurt at Dalkeith, both much injured at Chiswick and Kew ; Spiræa Lindleyana, not injured as an open lawn plant at Dalkeith, S. Reevesiana, pubescens, cana, Blumei, prunifolia, uninjured at Chiswick, callosa and prunifolia, uninjured at Dalkeith; Swammerdamia antennaria, slightly injured at Chiswick; Tritoma Burchelli, media, pumila, uvaria, uninjured at Dalkeith; Veronica salicifolia and Lindleyana, uninjured at Dalkeith, killed at Kew, Chiswick, and Bromley; Viburnum tinus, continued to flower throughout the winter as usual at Dalkeith, none of the varieties injured at Chiswick, severely injured or killed to the ground in many parts of England; Weigela rosea, apparently quite hardy everywhere; Yucca gloriosa, flamentosa, and flaccida, uninjured at Dalkeith ; Zauschneria Californica, quite hardy at Dalkeith.

\section{§ 5. THE FLORISTS' FLOWER GARDEN.}

The cultivation of those plants which come under the above denomination has not only been a source of gratification to their admirers for an unknown length of time, but has been, and still is, regarded as a distinct branch of horticultural art. The florist regards his favourite flower, while in its natural state, in much the same light as the pomologist does the fruit of the crab-apple or the uncultivated plum. Each, by an art peculiar to himself, in course of time so completely changes the character of the objects of his care that they no longer resemble the type from which they originated either in form, size, or colouring. Florists' flowers are appreciated whether they are single or double. In the former, although the number of petals is not increased, they are enlarged in size, rendered more circular in form, of greater consistency or substance in petal, and improved in depth and harmony of colouring. In the latter, the petals of the corolla are increased in number, in substance, and in colour. Indeed, so far has the florist carried his art that canons of criticism have been by common consent established by which to estimate the properties of new productions or varieties. Criterions of merit, or the properties of florists' flowers, have from time to time been published, the most complete of which is that by Mr George Glenny, who published in 1847 a pamphlet entitled "The Standard of Perfection for the Properties of Flowers and Plants," which was for a time considered the standard by which their merits were measured. Some difference of opinion has, however, since arisen on the subject. The florists' flower garden occupies comparatively a limited space in gardens generally. Its principal requirements are shelter, exposure to the sun, the means of securing artificial shade at certain seasons, pits or frames for protection during winter, and a glass-house for blooming some of the more delicate productions. Florists' flowers and their culture, when grown to the highest state of perfection, do not harmonise well with the other parts of a general garden; hence the propriety of dedicating a separate department to their accommodation.

The pansy or heart's-ease, one of our most popular florists' flowers, is the progeny of Viola lutea, grandiflora, and amœna, produced by 
fecundating these species together, and the endless varieties continued and increased by crossing one variety with another, or saving seed from approved sorts which of themselves produce considerable variety.

Soil.-For pot-culture the soil should be welldecomposed turfy loam, leaf-mould, and very rotten stable-manure or cow-dung, thoroughly incorporated and turned over several times before using, at which times a careful search should be made for wire-worm, which often abound in turfy loam. The proportions should be half a bushel of the two latter to one and a half of the former, unless the loam be of a light sandy nature, when nearly equal parts of each will be a good compost. When the loam is stiff, a portion of river or washed sand should be added. Although the pansy will grow in most soils, ranging from a stiff clay to sheer sand, still, for the purpose of blooming it in perfection, a prepared bed should be afforded it ; and we have found one constructed as follows to answer the purpose admirably: the sides and ends constructed of 9-inch brickwork, and carried up to the height of 18 inches above the surface of the ground, the width being 4 feet, and the length corresponding to the number of plants to be grown. The object of this is to prevent the intrusion of wire-worm and their natural enemy the mole, and also to elevate the flowers near the eye, so that they may be examined without the necessity of stooping. The bed, thus far prepared, is filled with the compost recommended above for pot-culture, and is removed entirely or partially yearly. During the drought of spring and heat of summer such beds require copious watering, care being, however, taken that, from the time the flowers begin to expand, the water be applied to the surface of the soil only. The best situation for such a bed is one sheltered from the sun during the hottest part of the day; and when that is inconvenient, then recourse must be had to partial shading, by suspending a thin canvass screen along the south side of the bed.

Planting and potting.-About the first week in October a selection should be made of young stout healthy plants for potting. These should be set in 4-inch pots in the compost already recommended, and placed in frames or pits, where they can be protected from heavy rains, but otherwise fully exposed to the weather until frost sets in. During winter abundance of air must be given during fine days, and the plants frequently examined, so that they may not suffer either from damp or excessive drought. About the beginning of February is a proper time for shifting them into the pots in which they are to bloom, which should be from 6 to 8 inches in diameter, according to the strength of the plants and size of the variety. In potting, drainage should be attended to; and over that an inch of rotten cow-dung may be placed to nourish the roots when they descend so deep, which will be about the time the plants are coming into bloom, a period of their existence when they require the greatest amount of stimulant. If the roots and the soil around them are in a good state, the ball should not be reVOI، II. duced farther than taking a little off the surface of the top and softening the sides. The soil used should be tolerably dry, and must not be pressed down too hard. Place the plants in a frame or pit with a full southern and open exposure, setting the pots on a floor of sifted coal-ashes. Place the glass sashes over them, and admit air daily, according to the state of the weather. Water slightly for a fortnight or three weeks, using tepid water, and applying it early in the day. Keep the plants close to the glass, and sufficiently apart from each other, that the air may circulate freely amongst them. If green-fly appears, recourse must be had to fumigation. Turn the plants occasionally round, that each side may alike enjoy the sun. The surface of the soil should be stirred from time to time to prevent the appearance of mucous matter, and a slight top-dressing towards the beginning of March will be of advantage.

The beginning of October is the season for planting in the open beds, because the plants become established before the growing season closes, and are therefore better fitted to withstand the winter. The plants should be healthy and vigorous, but not too large. The stronggrowing kinds, particularly if large blooms are required for exhibition, should be planted 15 inches apart, and 10 inches may be regarded as a proper distance for moderate or weakly growers. If planted on elevated beds, as we have suggested, little other care will be required till spring except the removal of dead leaves, and covering the surface of the soil between the plants with a slight mulching of rotten dung, and in damp localities a thin covering of sifted coal-ashes to counteract damp.

Propagation by seed.-The beginning of May, August, and September, is the best season for sowing the seed saved from approved sorts the preceding autumn, spring, and summer. All seed procured after the middle of September should be kept till the May following. Sow in pans, in a compost similar to that recommended for potting, only in this case it requires to be passed through a half-inch sieve, or otherwise rendered finer than when used for potting. Sow thinly, and place the seed-pans in a gentle heat. When the seedlings appear, great care must be taken in watering, as the young plants are apt to damp off close by the surface. Abundance of air is necessary, and the operation of pricking off into nursery-beds should be attended to as soon as the plants are fit to handle. A partially shaded border is the best place for them, and if covered for ten days after removal with a glass frame, so much sooner will they become established.

Propagation by cuttings.-This operation may be carried on throughout the whole year, with less success, however, during the dead of winter and the excessive heat of summer. In March, April, September, and October, they are found to strike most readily.

In the case of choice or valuable sorts, cuttings should be taken off as fast as they are produced. Those taken off in March, April, and May, should be planted in a northern border, and will produce a supply of bloom until 
November. Those struck during the summer months will flower during autumn, and until arrested by the frost.

"For autumn, winter, and spring striking," Mr Turner, one of our very best florists, recommends, "we use pots, pressing them firmly and closely to the edge, using sandy soil, with plenty of drainage; but for summer propagation we prefer a shady border, which should be well drained, and prepared so that worms cannot disturb the cuttings. They should be protected from heavy rains, and from exposure to the sun; but if they are shaded like most other cuttings, they will most assuredly damp off. All depends on the situation, which should be light, without powerful sun. Another difficulty to contend with at this time (March) arises from harsh drying winds. We use hand-lights and small frames, so that heavy rain and wind can be effectually kept from them. However, not an hour should be lost in keeping them open in favourable weather."

Propagation by layer's.-The best cultivators do not recommend this mode of propagation, unless it be in cases where the plants do not throw up young shoots readily.

General treatment.-The surface of the beds that were planted in autumn should be frequently stirred up, and weeds and decaying leaves removed. Peg down all long rambling branches, and partially cover them with fine soil, allowing the points only of the shoots to appear above the surface. A slight surface-dressing towards spring will be beneficial, and this top-dressing should be somewhat richer than the soil the roots are planted in. Their natural season of commencing growth is in March; and as cold drying winds usually occur at that period, it is advisable to afford the beds some degree of shelter to ward off the cutting winds, and this is best effected by raising a thin canvass screen on their windward side. Where, however, early bloom is required, a top-covering during night of glass or thin semi-transparent canvass is necessary to protect them from frost. Beds for a successional bloom should be prepared about the beginning of March, and planted with the plants kept in store-pots during the winter. During dry weather regularity in watering should be attended to, and this should be administered in the morning until the weather becomes settled and warm, when the afternoon will be a more proper time. Weak liquidmanure may be applied with advantage, particularly to weakly plants. Pansies in pots, and placed during winter in frames fronting the south, should, about the middle of May, have the frames reversed-that is, to face the north. Attention must also be paid that abundance of ventilation be given, even to the extent of drawing the sashes entirely down during the day, and in mild weather leaving them partially open during the night also. When the blooms, however, appear, there is danger in this, as they would be exposed to the wind, which would blow the flowers about, and greatly damage them, particularly if for exhibition. It is better, therefore, to elevate the frames upon bricks, so as to leave a clear space all round of from 4 to
6 inches, through which the air will blow, and come in contact with the pots instead of with the plants. By this mode of ventilation the sashes may be kept constantly on, and so protect the bloom from rain, a very important matter when they begin to fully expand.

The ordinary flower-gardener delights in a full display of his parterre at the same time. The case is different with the florist-he wishes to prolong the period of his bed of flowers, and for this purpose he never allows a flush of blooms to appear at the same time. To effect this he cuts off all small superfluous or inferior blooms, and often destroys the whole of the flowers for a few days to insure a few superior blooms for any particular occasion-a competition, for example.

In July, beds should be prepared for autumn blooming, and planted with plants struck from cuttings during the previous months. These will often produce small flowers at first, but these should be cut off to enable the plants to attain size and strength to produce bloom of larger size and greater merit.

The natural enemies to the pansy are the wire-worm and grub, both of which attack the roots, and the slug and earwig commit great destruction among the leaves and blooms. The wire-worm is easily captured, by placing baits of carrot, potato, \&c., in the bed, in which they will burrow, and thus be readily extracted. The grub is to be sought for about the roots of the plant, and also when the surface of the beds are stirred with a small hand-fork. Finelypowdered hot lime, dusted over the surface of the bed on a quiet day so that it may not fall upon the bloom, will destroy the slug, or cabbage or lettuce leaves laid amongst the plants, will attract it ; and earwigs may be trapped by placing 3-inch pots inverted, having a small portion of dry moss pressed hard into their bottoms, upon the surface of the bed. The leaves and pots should be examined every morning, when the enemy will be found under them taking shelter from the sun.

The hollyhock, at present a very popular flower, is the result of floral improvement upon Althæa rosea, a native of China, and, in one state of perfection or another, an inhabitant of our gardens for about three hundred years, being mentioned by Dr Turner in 1564 as a well-known plant in his time. The hollyhock seeding freely, and being for two hundred and fifty years multiplied by that means, varieties would, as a matter of course, continue to appear. Of their merits we know nothing. Somewhere about twenty years ago, Mr Charles Baron of Walden began to study their improvement, and it is mainly owing to his exertions that we now possess this flower in its present state, his success having given an impetus to others, so that by their united efforts the hollyhock has now arrived at as great a state of perfection as it is possibly capable of attaining. Whether regarded as a mere florist's flower, and grown for the purpose of exhibition, or as an autumnal decorative plant in our flowergardens, the hollyhock is highly deserving of the cultivator's attention, seeing that we have no other plant of equal merit to prolong to 
so late a period the floral interest of our gardens.

The hollyhock is capable of being propagated only by the three following modes:-

Propagation by seed.-Most of the flowers, however double, will be found, on close examination, to contain the necessary parts of fructification; and these often naturally impregnate themselves, and may be made to do so by drawing a camel-hair pencil lightly over the stamens; the pollen which adheres to it can then be readily applied to the styles or female parts of the same flower, or carried to those of another. By impregnating a flower with its own pollen, seedlings of some sorts will come almost identical with the parent; in the majority of cases, differences, less or more marked, will be produced. Other cases, where the form is defective and the colour good, may be improved by crossing with a perfectly formed flower, and vice versâ. In saving seed from very double sorts, they should be planted in a poor soil, the flowers thinned considerably, and the spike allowed to grow to its natural height. Those which are more thin of petals, and intended for seed-bearing, should be planted in a very rich soil, and have their spikes shortened to about half their length. The best time for impregnating the flowers is in the early part of the day, when the flowers are dry; and the process should be continued day after dar, as the flowers expand, until a sufficient number toward the bottom of the spike are impregnated. Such flowers as have taken will in a short time indicate themselves by the swelling of the seedvessel ; and when such is observed, the decaying petals should be carefully removed by the hand. Should dry weather occur during the swelling of the seed.vessel, copious supplies of water should be given at the roots; and as the seed-vessel begins to shrivel and dry up, it is a certain indication that the seed has attained its maturity. When this is ascertained to be the case, they should be removed from the plant when quite dry, and placed in thin canvass bags, keeping each sort separate and correctly labelled, and hung up in a dryairy room or loft; but the seed should not be removed from the calyx. The sorts which flower late, and such as are grown in cold situations, will often not ripen their seed in the open air. In such cases the spikes may be cut off, and placed with one end in vessels of water, and set in a greenhouse or vinery, where they will ripen perfectly.

Autumn, as soon as the seeds are ripened, and also spring, are the periods for sowing. Autumnsowing is preferable, provided the seed is ripened sufficiently early to enable the young plants to attain a size and strength to carry them through the winter. Spring-sowing should not be deferred beyond the beginning of March, to give the plants all the growth possible before their time of flowering in autumn. The seeds, in both cases, should be sown in pots or pans thinly, in light rich soil, and placed in a pit in which a mild bottom-heat can be maintained. When the plants have formed two or three leaves, they should be pricked out singly into 3 -inch pots, and kept close to the glass, and freely exposed to light and air. If strong plants be desired, these should be shifted by the first of March into 4-inch pots, kept under glass, yet abundantly supplied with air, till the beginning of May, when they may be planted out where they are to flower. They should be set in rows, 3 feet apart, and 1 foot plant from plant in the line. The ground should be trenched and moderately manured, and the plants supplied with water during the heat of summer; and if the ground between the rows is mulched with littering manure or tan, a greater degree of moisture will be maintained at their roots, and the ground be more comfortable to walk upon while examining the flowers, as well as during the process of staking and tying them up. The season of their beginning to bloom is one of great interest to the florist. Every flower should be examined, and those which prove of inferior merit should be instantly pulled up and thrown away. Plants from seed are the best; but with a view to secure a stock of any choice variety unchanged, recourse must be had to division of the roots, or increasing them by eyes or cuttings. Some varieties come so true from seed that it is very difficult to trace a difference between them and the parent; but this is not to be depended upon except in a few cases. In cold situations, seed-bearing plants should be planted against a south wall to insure their ripening.

Propagation by division of the root.-Immediately after the plants have done flowering, the roots should be carefully dug up, and divided into two, three, and sometimes four portions, according to their size and number of eyes or buds on the crown. A sharp knife should be used in dividing the crown, but care must be taken that the roots be as little cut as possible, for each portion should have as many roots as can be secured. They should then be potted in pots according to their size, and plunged in a cool pit, and protected with glass till spring. About the end of April or beginning of May, they should be planted out where they are to flower.

Propagation by cuttings and single eyes.-Excellent plants are produced by these means, and the operation may be carried on from March to October-that is, as soon as cuttings can be got off in spring, and as late in autumn as they can be furnished with roots. The cuttings are fit for taking off when they become somewhat hard at their base, and should be set in sandy soil in pots 5 inches in diameter, placing four cuttings in each pot around the edge. Water slightly at planting, and plunge the pots in a mild bottom. heat in a close frame or pit. Some dispense with the bottom heat; the only difference is in the time of rooting. When the plants have made four or five new leaves and corresponding roots, they should be potted off into 4 -inch pots singly, and forwarded in growth by being kept in a cool pit under glass till the end of April in England, or the middle of May in Scotland, when they should be planted out where they are to flower. Planting will depend on circumstances. Nurserymen who plant more for stock than effect, set their plants in parallel 
lines 4 feet apart, and the plants 2 feet asunder in the row. By this means each has space to develop itself fully.

Propagation by single eyes is done with a view to increase stock rapidly, particularly in the case of new or rare varieties. The best time for this operation is September, at which time the stems become somewhat hard. Single eyes are taken from the wood stems, not from the flowering ones, with about an inch above and the same below the eye ; these are set in light sandy soil, in 3-inch pots, and treated as above for ordinary cuttings. In planting the buds, the whole of the portion of wood stem should be buried in the earth, leaving the portion of the foot-stalk only above it. Some, however, instead of placing the eye or bud in a horizontal position, set it vertically.

Soil.-The hollyhock is less fastidious about this than almost any other florist's flower. Its large roots abstract a great amount of nutriment from the soil, and its large bulk of foliage and flowers require it. It is better that the food be placed within reach of the roots, than that they should have to extend far to collect it. It follows, therefore, that the soil should be deep, of a rich light loamy nature, rather than a shallow poor gravel, or, still worse, a cold damp clay. Richness is essential for the perfect development of the plants.

In cold damp soils the roots are liable to perish, and hence many take them up in autumn, and place them in a protected place during winter. This, however, is exceedingly injurious to them. An airy situation, well exposed to the sun, is the best; a shady damp place the worst of all.

Planting.-The season preferred by the English growers for final planting is the latter end of September or beginning of October; and for spring, the month of March, or early in April. In Scotland, autumn-planting should be attended to as early in the season as the plants are fit for removal, that they may become established before winter. Again, in spring, little is gained by planting too early-that is, until our growing season sets in, which is, for the most part, the latter end of April or beginning of May; but much in all these cases depends on the size and state of the plants, and the convenience there may be for keeping them under cover. In planting, the crown of the plant should rather stand above the level of the ground-surface than below it; and in autumn, if the surface is covered with coal-ashes, or rotten tan around the roots, the better will they be protected during winter. With the finer and more delicate sorts some such protection is necessary, for it should be kept in mind that the higher bred or higher the cultivation of a plant is, the more predisposed are such plants to disease, and the more liable are they to sustain injury from atmospheric changes. Mr Paul, in his interesting little work, "An Hour with the Hollyhock," thus speaks of planting: "It is worthy of remark, that the season of flowering may be greatly prolonged by striking and transplanting at different seasons. There is a difference of at least six weeks in the period of flowering between plants removed early in autumn and late in spring; and of this we may avail ourselves to lengthen the succession, or to obtain a full bloom early or late, as particular circumstances may require. Early-rooted cuttings and old plants may be induced to bloom in July, and late-rooted cuttings, and spring-sown seedlings, in November. Hence there will be no difficulty in obtaining a supply of flowers for four successive months."

Situation in the garden.-The hollyhock is not a plant for a small garden, unless it can be trained against a wall or fence. Like all other plants intended to produce striking effects, they should be planted in masses or groups, but these should be upon open lawns or large spaces of grass, and at a considerable distance apart. When planted singly, or scattered through the grounds, their effect is lost; yet, nevertheless, if in groups of ten or a dozen plants, they break the monotonous outline of its planted shrubberies by their spire-like spikes overtopping the other plants. In single line they are not effective, particularly where there is not a sufficient mass of background. In groups by themselves, whether each group be composed of various shades of colour, or confined to one colour only, if such groups be not too large, their effects are the most pleasing.

General remarks. - Three flowering spikes only should be allowed in the strongest plants, two on moderate ones, and one only on such as are weak. The rest may be rubbed off as soon as the selection is made, or they may be taken off for cuttings. As soon as the shoots have attained the height of a foot and a half, they should be staked, to prevent their breaking. In staking, $\mathrm{Mr}$ Paul advises two stakes to be placed to each plant, should it only have one stem, because the stem cannot be drawn close to a single stake without injuring the flowers. "The stakes," he says, "should be placed opposite, and the stem brought between them, so that the three objects form a straight line: pass the bast round the stem of the plant, drawing it to the one stake, and perform the same operation a few inches higher up, tying in the opposite direction, and so on, tying on each stake alternately as the stem rises. When two or three stems rise from one plant, three stakes will be necessary, and they should be placed triangularly, tying as before." Other's run wires or cords along the line of the plants, which are supported every 8 or 10 feet by poles, and to these cords or , wires they attach the plants, without the least injury to the blooms. In the flower-garden, however, the supports should be so placed as to be covered with the foliage, and set on the side of the plant opposite to that from which it is to be seen from the walk or lawn. Where the blooms are set too closely on. the stem, they should be thinned out, so that each flower may have room to expand. Where large flowers are desired for exhibition, a portion of the tops of the flowerspikes is often cut off; but in flower - garden culture this should never be attempted, as it destroys the character of the plant. Shading is also necessary to retard the blooming of individual spikes of flowers to suit the day of exhibition ; it is also employed to preserve colour, as in all other classes of florists' flower's. In flower- 
gardens all these precautions are unnecessary.

Exhibiting.-These flowers were long exhibited as single blooms, just as pansies and dahlias are at the present day. This mode gives no true criterion as to the cultivation or general character of the plant or flower exhibited by the florist. Let us, therefore, have hollyhocks grown in pots; the thing is quite within the range of possibility and practicability. Let us have, instead of single flowers, nay even 2 or 3 feet spikes of flowers, the whole plant set upon the exhibition-table in the pot in which it was grown. We hold that there is as much credit due for growing the plant as for producing the bloom, for the first must be secured ere the other is attained. On the subject of exhibiting Mr Paul remarks: "There are two modes of exhibiting hollyhocks-by single flowers, and by spikes. Some cultivators have advocated the withdrawal of prizes for single flowers, and depending on spikes alone. It is freely admitted that the exhibition of spikes creates a greater display, and affords a truer idea of the nature and properties of the flower. On this ground, then, we admit the desirableness of encouraging this mode of exhibiting, but doubt whether the entire exclusion of single flowers will not prevent many amateurs from entering the list of competitors. To exhibit single flowers does little damage to the garden at home, and they are easily conveyed to the place of exhibition. To exhibit spikes requires too great a sacrifice where a few plants only are grown, and, moreover, they form, however closely stowed away, a somewhat cumbrous package. The most emulous of amateurs would pause ere they cut twelve spikes of their finest kinds from a limited collection; they could not reconcile themselves to behold tarnished in a day, what would give them pleasure for weeks, had the spikes been allowed to remain on the plants. We think, then," Mr Paul very sensibly remarks, and we entirely agree with him, "there should be two classes -one for spikes, and one for single flowers; make the former the more valuable prizes, but do not exclude the other." For village and cottager's' competitions, a thing we have a warm side to, we say, by all means let single flowers be exhibited, but certainly not so at metropolitan and provincial competitions. Either let spikes or the whole plant be shown;-we say the latter.

The ranunculus. - This flower has long been a favourite with florists, who by their art have produced innumerable varieties-so much so, indeed, that above one thousand of these have received distinctive names, and additions are making yearly. The type of this tribe is the Ranunculus asiaticus of botanists, a native of the Levant, and introduced to England prior to 1596 , for at that period we find it was cultivated by Gerard and his contemporaries. The ranunculus is divided into two primary and pretty well-marked sections, the first or common, R. vulgaris, and the second or Turban, R. sanguineus. The Dutch preceded us in its cultivation as a florist flower, and from them we long had large yearly supplies of roots. They also produced the majority of those varieties which are of strong and dark body-colours. The English have, however, surpassed their florist friends in Holland in the production of the finest flowers, although vast quantities of the roots for ordinary purposes are still yearly imported.

Propagation. - This is effected by dividing the roots, which naturally produce offset roots, which attain maturity in one season, and are easily separated at the season of lifting; and by seed, which ripens readily. The former process is so simple as to require no remark; the latter is more important, and deserves some consideration. Flowers possessing the highest properties, as regards size, shape, and colour of marking, should be chosen, as being the most likely to produce first-class seedlings. On this subject the Messrs Tyso \& Son, the well-known cultivators of this flower, observe: "Procure some semi-doubles possessing fine properties, because these produce anthers as well as pericarps, and afford pollen for impregnation, which is very seldom the case in long-established sorts. The more double the flowers are from which the farina is taken, the greater is the probability of procuring double varieties. Apply the farina of any semi-doubles that afford it to the pericarp of the flower selected as a seed-bearer. The most approved method of fertilising is by gathering the semi-doubles, and applying the pollen by lightly pouncing it on the pericarp; but if semi-doubles are scarce, then the application may be made with a large camel's-hair pencil. This operation should be repeated for several days, in fine sunny weather, when the plants are uncovered, shade being inimical to fertilisation. The stems bearing seed-vessels should be supported with sticks and two or three bandages of matting, to prevent the stalks from breaking down, which would deprive the seed of the necessary nourishment. Gather the seed when it turns brown, and keep it loose in a drawer, in a dry place. Ranunculus seed will retain its vitality two, or even three years." The seed should be retained in its natural covering till the season of sowing, when it should be separated.

Sowing the seed.-This operation is best performed in October and in February. Flat pots, pans, or boxes, should be filled with moderately rich loam, with about a sixth part of leaf-mould, carefully examined for the detection of insects, particularly wire-worm. Under the compost sufficient drainage should be placed, and over that the siftings or coarser portions of the soil. The surface being rendered smooth and level, the seed should be sown thinly; for if the young crop come up too thick, the plants are weakened, and many perish. The careful florist will sow his seed so that each may be one-eighth of an inch apart from its neighbour. Cover lightly with finely-sifted soil, and apply a gentle watering with a fine rose watering-pot. Set the seedpans on a dry floor of coal-ashes in an open well-exposed place, and cover them with a frame and glass sashes. Little shading will be required at this period of the year, and a limited supply of water; nevertheless the soil must not be allowed to become at all dry. In four or 
five weeks the young plants will appear, when great vigilance will be required to guard them against the attacks of insects, dry cutting winds, and even severe frosts. In May remove the seed-pans to an open border, where the morning sun only shines upon them, and plunge them in the soil. Here they should remain till the middle of July, when the foliage will begin to ripen and turn yellow: at this time water must be withheld, and if covered with a spare glass frame so much the better. When the foliage has totally disappeared, the roots will be ripe, and in a very proper state for taking out of the soil. This must be done with care, so that none of the very small ones be left behind, as these often turn out the finest varieties. Dry the roots moderately in the shade, and pack them in boxes amongst dry sand.

Planting.--In good seasons, and in dry and favourable situations, the middle of October will be found the best season for planting. The latest period at which this can be safely done is the beginning of February. By following the former course, the roots suffer less from being kept dry; they also begin to vegetate slowly on account of the soil not being as yet cooled down by frost, enabling them to make fresh roots, and so be in a condition to start strongly into growth early in spring. Some object to autumn planting, alleging there is danger to be apprehended from frost. Of this we have no fear; and even were it so, frost could be excluded by a thin covering of litter laid over the beds. The Messrs Tyso appear to be in favour of spring-planting, and remark, "Considerable hazard, however, attends autumn-planting, and it is not recommended, except by way of experiment, to those who possess a large stock, and can afford to risk a portion." Their process of planting in no respect differs from our own; we shall therefore quote from their excellent little work, "The Ranunculus: How to Grow it; or, Practical Instructions in its Cultivation." "In fine weather rake the beds perfectly level, and divide them into six longitudinal rows for mixed roots, allowing 4 inches from the outside row to the edge; or for named sorts, mark the rows transversely, at distances of $\mathbf{5}$ inches asunder, and plant six roots in a transverse row. Draw drills an inch and a half deep, and plant the roots with the claws downwards, with a gentle pressure to secure them in the soil, so as to be an inch and a half from the crowns to the surface. When planting on a small scale, a dibber with a shoulder at the precise depth may be used; but in large quantities it is an inconvenient method; and planting at the bottom of a drill with slight pressure, and without disturbing the subsoil, is attended with similar advantages to the use of the dibber, and in practice will be found to have some points of preference. If the top soil is light after planting, it may be gently beaten with the back of a spade. This operation must be only done in dry weather." Seedlings are to be planted in the same manner as above, and at the same distance; for although the roots are small at planting, they will grow the first year to full size, in some cases making stronger plants than the old-established roots. The Turban ranunculus is hardier than the common, and in all cases they should be planted in October.

Taking up the roots.-The roots are fit for taking up when the foliage has died down; and in dry seasons and soils this should then be done. In wet soils-and such, we may remark, are very unfavourable for this plant-and in late wet seasons, the roots should be taken up before the foliage has completely died down, and just after it has assumed a yellowish colour. It is worthy of remark here that there is danger in allowing the roots to remain too long in the ground, because, particularly in wet seasons, they are apt to begin growing again, so that this must be guarded against. When taken up, the roots should be gradually dried, cleaned of soil, and placed in shallow drawers, or in canvass or paper bags, and kept in a dark dry place, secured from mice, and occasionally examined until the time of planting again arrives.

Soil and situation.-Some florists attach great importance to the soil; and in former times strange composts were formed, and these often of most antagonistic character. Experience teaches us that the Asiatic species and its numerous cultivated varieties will prosper in the highest degree in soil similar to that in which our native species of buttercups are found - namely, a rich hazelly loam. Pastures, therefore, in which these are found to abound, are the places from which to draw a supply for the beds. This, however, requires some slight preparation, and probably enrichment. It should therefore be taken off about 10 inches deep, carted to the compost-yard, laid up in a heap, keeping the green surfaces of the turf inwards; and as the process of forming the heap goes on, lay into it a portion of pretty well decomposed stable or cow dung. Turn the heap over two or three times within a twelvemonth, chopping it down in sections at each turning, that the turfy matter may be well broken down, and the manure thoroughly incorporated with it. The proportion of manure to the soil, in most cases, should be about onethird of the former to two-thirds of the latter. Rank or over-stimulating manure is fatal to the roots, and engenders disease in the tubers. In regard to situation, an open but not an exposed one is the best. Some attach importance to the direction the beds are placed in, some advocating an east and west, while others prefer a south and north direction; for ourselves, we cannot see a shade of difference, all other things being favourable. The Messrs Tyso approve of the former; and their instructions for the formation of the beds, like that of the soil, are so good that we shall transcribe them here. "Remove the earth (the soil of the garden) a foot deep, and from 3 feet to 3 feet 4 inches wide, and fill the bed with the prepared compost to within 2 inches of the surface; leave it thus for a month, and then add the reserved top-soil. These operations are best done in autumn, that time may be allowed for the earth to settle. The surface of the bed should be level, and not more than an inch higher than the paths, in 
order that the roots may be kept regularly cool and moist; and as the ranunculus thrives on a firm bottom, the compost should not be disturbed at the time of planting more than is just needful for that operation. During winter the surface may be pointed up rough to take the benefit of frosts, but in no case should this be done more than 2 inches deep. The beds may be neatly edged with inch boards painted lead-colour (Caithness pavement cut in narrow slips would be more durable, and in the end far more economical), and, in case named sorts are planted, should be numbered with white paint, to correspond with the numbers in the list. As a bed well constructed at the commencement will admit of several successive plantings with an annual addition of fertilising materials, it is worthy of the particular care of the cultivator, though the preparation at first may involve some little trouble and expense."

General remarks. - Mark all seedlings while in bloom that come double and show points of merit, and reserve them for planting, for it is often not until the second or third year's cultivation that perfection is arrived at. The ranunculus may be grown to its fullest perfection in pots, and in this way should they be in some cases exhibited. To this end two or three roots will be sufficient for an 8 -inch pot. Those who are partial to this flower may have a long continuance of bloom by potting in October or November, and plunging the pots in a cool pit till the roots are advanced, when they may be taken into a warmer pit and brought into bloom in March. When the bloom begins to expand in the open-air beds, slight shading will be of advantage during bright sunshiue. To this end gauze netting, elevated somewhat above the flowers, so that air may not be at all obstructed, will be found convenient, but much more so if a framework of iron or wood be constructed over the bed, having a 3-feet passage round it, set in the span-roofed fashion, and sufficiently high at the sides that the cultivator can walk around. The shading should be so constructed that it may be rolled up and let down at pleasure. The sides and ends should be covered with thin canvass, to break the force of the wind; and where flowers are grown for exhibition, a waterproof covering over the roof would be expedient, to avert the consequences of heavy rains. The light-coloured varieties sustain less injury from a powerful sun, as well as from rain, than the dark. After the plants have attained the height of 3 inches, covering over the surface of the bed with decomposed manure about an inch thick will be of advantage to them. As the ranunculus is liable to sustain injury from frosts in spring, those who grow for exhibition, or those who wish for their own gratification to possess a bed of fine flowers, should provide themselves with a framework as mentioned above, so that in spring the beds may be protected by canvass of a thicker description than that employed for the purpose of shade during a more advanced period of the season. Protecting and shading by means of branches, hurdles, mats, \&c., is far behind the age we live in, is not effective, and in the end is far more expensive.

The anemone.-The varieties of cultivated anemones, so far as regards floriculture in its proper sense, are numerous, and, like the ranunculus, it has been brought to great perfection, according to the standard by which florists judge them. They derive their origin from two species, Anemone hortensis and A. coronaria, the former a native of Italy, and introduced from Holland, where it had long before been largely cultivated, in 1596 ; the latter a native of the Levant, also introduced from Holland, where it also had been long cultivated, in 1597. The cultivation of the anemone as a florist's flower is so similar to that of the ranunculus, that to describe it here would be a needless repetition. Nor does it stand in the same estimation amongst florists generally as the ranunculus. Nevertheless it is extremely ornamental as an early blooming border-flower, and a bed of them treated as above for the ranunculus has a very showy appearance. They are sometimes brought forward to exhibitions, and are no doubt worthy of a higher degree of culture than has in general been bestowed upon them.

The dahlia.-The dahlia is a native of Mexico, found in sandy meadows at an altitude of from 4800 to 5600 feet above the sea, which circumstance led, on their first cultivation in England, to the practice of growing them in beds formed of road-scrapings, as was the case in the Ham mersmith nursery in 1815. The first plants introduced to England were brought from Mad rid in 1789 by the Marchioness of Bute. These having been lost, the late Lady Holland reintroduced the plants again in 1804. The present stock, however, originated from plants brought from France in 1814. Those who, like ourselves, recollect the poor single-flowered, bad-coloured dahlias of the latter date, consisting of four or five worthless varieties, little thought to see their offspring ranking as one of the most, if not the most perfect specimen of floricultural skill, the most perfect flower both as regards form and colour in the whole catalogue of florists' flowers.

Propagation.-The dahlia is propagated by seed, cuttings, grafting, and division of the root. The first, with a view to produce new or improved varieties; the second, to obtain stock in large quantities; and the fourth, where there is not the convenience of propagating by cuttings, \&c.

Propagation by seed.-Seed should be saved from the most perfect flowers, and those grown on plants of the best habit, of which compactness of growth forms an important element. Cross impregnation should be attended to when certain forms or colours are desired. New forms and colours can only by this means be obtained. The seed ripens in September and October, but as soon as the petals begin to drop off, and the seed-vessel begins to swell, which is a certain indication that fertilisation or impregnation has taken place, the petals should be removed, and every meaus used to admit sun and air to the swelling seed-vessels, while wet should be carefully excluded, as in damp seasons it is apt to cause the seed-ressel to rot. When 
the seeds are ripe, they should be gathered, cleaned, and, when sufficiently dry, packed in paper bags, and kept in a dry cool place till spring. The middle of February is a good time to sow the seed, which should be sprinkled thinly on the surface of light rich soil, placed in well-drained shallow pots or seed-pans, and these placed in a mild bottom-heat, in a frame or pit kept at from $55^{\circ}$ to $60^{\circ}$ of temperature. Water slightly at sowing, and when the seeds germinate, place them pretty near to the glass, to prevent the plants drawing up weakly. When about 2 or 3 inches high, transplant them into 4 -inch pots, one plant in each, using rich lightish loamy soil. Maintain a steady temperature of $55^{\circ}$ or $60^{\circ}$, still keeping the plants as near to the glass as possible, so that they may become stout and stocky before the beginning of May, at which time they may be planted out in the ground where they are to flower; but till the end of May they should be protected in cold weather and during night with bell or hand glasses, so that they may experience as little check as possible. They will, if staked up as they advance in growth, flower in July or August, when their comparative merits will be developed. As some, if not many seedlings, will not be worth keeping, the plants may be set at the distance of a foot and a half apart, in rich deep soil, and as they flower, the worthless should be pulled up, which will give room to those that are considered worthy of being retained. Such as show bright colours and well-formed petals, if even not very double, may be retained, and have another year's trial. But all such as produce good flowers the first year should be propagated as soon as possible by cuttings, lest the roots perish during winter.

Propagation by cuttings.-To this end the old roots are potted or planted in a bed of soil or decayed leaves in any spare pit, and stimulated into growth in December or January. When the young shoots, which will abundantly spring from the crown of the old roots if kept sufficiently moist, are about 3 inches long, they are in a fit state for taking off for cuttings, and should be taken off close to the old root, and planted in sand singly in 60-sized pots, and placed on a gentle bottom-heat. Some set them in light sandy soil, and lay a little sand only over the surface ; in either way they strike freely. Water moderately and shade from the sun, keeping the pit rather close till the roots begin to form. After this they should be shifted into $3 \frac{1}{2}$-inch pots and kept still close, as well as near to the glass, until the roots are well advanced, after which they should be shifted into $4 \frac{1}{2}$-inch pots, and placed in a cool frame, but protected from frost or wet. On all favourable occasions give air through the day, and stimulate them into growth, to fit them for final planting where they are to flower. Cuttings may also be made of the young side-shoots during the summer until the beginning of August, after which it would be too late to expect them to form tubers sufficiently strong to withstand the winter. In making cuttings of this plant, it must be observed to cut immediately under a joint. Latestruck cuttings, which form nice firm small tubers," if kept in the pots during winter in a state of repose, will often, when started into growth in spring, and forwarded by shifting, make better plants than either the cuttings struck the same spring or the fragments of the old roots. In this state they are very convenient for transport, being less liable to sustain injury during a long journey, and far less expensive in carriage than young plants in pots in a growing state.

Propagation by division of the roots.-Every tuber removed from the mass of roots, having so much of the crown with it as contains one or more eyes or buads, makes a separate plant. This mode, however, is seldom practised, except among amateurs, who have not the convenience for striking cuttings. The roots under these circumstances, when divided, may be potted in pots capable of containing them, and placed under a greenhouse stage, where they will begin to form roots, and be fit for planting out by the time the weather is suitable for the purpose. They have one advantage when thus slightly stimulated,-the root, having been called into action, will immediately push its fibres into the soil. Some amateurs divide their old roots and plant them out about the middle of April, covering them for a time with inverted flower-pots.

Soil and situation.-The soil can scarcely be too deep, rich, and friable. It should be trenched over in autumn to the depth of 3 feet, and heavily manured at the same time. If laid up in ridges, so as to expose as large a surface as possible to the action of the weather during winter, so much the better. When levelled down at the time of planting, a slight enrichment should be added by digging in some well-decomposed cow or stable dung. In regard to situation in the flower-garden, much depends on taste and circumstances. When the object is growing for exhibition of the cut flowers, then any warm sheltered spot, well exposed to the sun, may be chosen; and when such is the object, any place other than the flower-garden should be selected. The manipulations thought necessary by florists, of shading, cutting off all the flowers except two or three, mulching the ground over with rich manure, \&c., are all affair's which would be far better carried on in the reserve garden than in one having any pretension to high keeping and neatness. In the flower-garden much will depend on its size and style. In large ones, clumps or banks of dahlias have an imposing effect, although unfortunately of a very transitory duration. They never produce effect when planted singly, unless when in a shrubbery, and when well backed with masses of foliage. There are some dwarf varieties, such as Zelinda, about 18 inches in height, and flowering profusely above their foliage, which have a pretty good effect; but their flowers for the most part want either that brilliancy of colour or symmetry of form which taller varieties possess. Upon the first appearance of frost the glory of the dahlia is gone, and the blank in the garden, which is in general not a small one, cannot be made up again. The late period also of the season before they come to be effective, particu. larly in many places, strengthens the opinion en- 
tertained by many, that they should be cultivated in a place by themselves, and grown for the purpose of being cut and used in that state for the decoration of the rooms in the mansion. Under these conditions they could be protected by awnings, and kept in bloom to a much later period of the season. The dahlia has never yet had a fair chance under glass ; if it had, it might be continued in bloom a great portion of the year.

Summer culture.-The plants are prepared by being forwarded in heat early in the spring, strengthened in constitution by being kept afterwards in well-aired pits or frames, and when of a good size they are fit for being finally planted out. To state a day or a week when this should take place is impossible, for in some places they may be planted out with safety by the end of April, while in others the middle of June is nearer the mark. That being the case, this matter must be left to the discretion of the cultivator. "The season of planting," says a sagacious writer on gardens, "is as soon as there is no fear of any more frost." Those who grow them to the highest state of perfection, allow them plenty of room; " 5 feet apart every way for the dwarf-growing kinds, and 6 feet for the taller ones, will not be too much." And no doubt all that space is required to admit light and air to all parts of the plants. Regarding staking, it is well to drive in what stakes are required previous to planting, that the roots may sustain no injury by doing so afterwards. As the plants extend in size, the main shoot should be tied to the stakes by soft matting, and the side branches as they extend, but not drawn too close together. The best supports are either iron rods or young larch poles, from 5 to 6 feet in height, according to the size of the variety. Some grow them as an espalier, and we have seen them trained with pretty good effect against a stone wall. Flowers intended for exhibition must be protected from strong sun, rain, and often so shaded as to retard their perfect formation for several days. The shades in ordinary use are oiled canvass caps, tin sconces, wooden boxes, and often inverted flower-pots, \&c. Flowers intended for exhibition should be cut the night before, and placed in a dark cool apartment. Some will keep their flowers in this way for several days, awaiting the day of trial. The dahlia is capable of being transported with safety, if properly packed. The method in common use is thus described in the " Cottage Gardeners' Dictionary :" "Have a box or boxes made with water-tight tin tubes, securely fixed in the bottom, to hold water ; pass the stem of each flower through a plug of wood, with a hole in the centre just wide enough to allow the stem to pass through it, and just thick enough to fit like a cork into the tube. Make the flower quite firm in the wooden plug, and let the lid of the box be so elevated as not to touch the flower."

Autumn and winter culture.-When the frost has slightly blackened the foliage of the plants, their season may be considered finislied: the stems should then be cut over to within a foot or so of the ground, and the roots either immediately taken up, or allowed to remain a week or two longer, laying a few inches of soil over them VOL. II. for the exclusion of frost. The labels by which they have been marked should be firmly tied to the remaining portion of the stem; and if secured with a piece of brass or copper wire, there will be less danger of their being displaced than if they were merely tied with a piece of string. On taking up, the roots should be well dried and divested of soil, a dry day being chosen for the operation. The place where we keep our roots during winter is a dry cellar, perfectly excluded from frost; and they are covered with a little soft hay, to prevent the air drying them up too much. Next to a dry cellar, we would prefer a garret or loft from which frost could be excluded; and those who cannot command better accommodation, may stack them in dry soil, like potatoes. Attempts have long been made to dwarf the dahlia, to fit it for flower borders where tall plants cannot be admitted, by pegging down the branches: no very good effect is produced, and the plants suffer sooner from early frosts. If attempted, the process should be early commenced, on account of the inflexibility of the stem. The flowering season, when they are thus treated, may be more readily prolonged than that of such as are grown to their natural height. The best method would be, upon the appearance of frost, to hoop the beds over with hoops of half-inch iron rods, and to cover them in the evening with canvass, to be removed again in the morning. There is no doubt that much of the beauty of our flower-gardens would be prolonged by hoops adapted to the size and form of our parterres being placed over them in autumn, for the support of canvass coverings, made also to fit the beds. Supposing these hoops painted green and neatly arranged, their effect would be by no means objectionable. The coverings should, however, be cleared away as early every morning as could be safely done for the security of the plants.

Dahlia roots, in diy warm soils, may be left in the ground all winter with perfect safety, particularly if covered with rotten tan, leaf-mould, or, still better, sifted coal-ashes. Care, however, must be taken in spring, when they begin to grow, that all shoots, except one, and that the strongest, should be removed. This may be a suitable practice for cottagers, but to grow this flower in perfection the plants should be annually propagated by cuttings. Liquid manure is of vast importance to this plant, if applied just as the flower begins to form; and the most convenient is guano dissolved in water, pigeon's dung, where it can be got, used in the same way; and half an ounce of sulphate of ammonia to a gallon of water will also prove beneficial, if applied every ten days.

Staking, pruning, and training is of great importance to this flower. Should any of the side shoots grow very strong, and there is danger of their breaking off, either tie them in to the stem or stake, or in many cases it will be better to drive in additional stakes, but these should be so placed that they may be covered by the branches and foliage. Where fine bloom is required, only one flower should be allowed to remain on each shoot. Where, however, a great display is required in the flower- 
garden, the more flowers the better, if the plant is able to support them. This, however, must not be carried too far, else both plant and flower will suffer. A judicious thinning of the buds is highly expedient, removing those first that are towards the centre of the plant, and less likely to be seen; and as soon as the petals fall, remove without delay every vestige of it, unless in cases where seed is desired ; for the effort to produce one seed-vessel is more severe upon the plant than the production of a dozen of blooms.

The carnation and picotee.-The original type of this flower is Dianthus caryophyllis, a native of several parts of England, found on rocks and walls. At what period the carnation became known in Britain as a florists' flower is unknown. It has, however, been cultivated in some state or other as such from the time of our earliest gardening publications, being mentioned in most of them. It is supposed to have reached this country from Italy or Germany; and Gerard, writing in 1597 , informs us that he received it from Poland. The picotee, the near kinsman to the carnation, will be included in the following remarks, as their culture, \&c. is identically the same. Carnations are arranged in three classes -viz., flakes, bizarres, and picotees. Flakes have two colours only, and their stripes large, going quite through the leaves. Bizarres are variegated in irregular spots or stripes, and with not less than three colours. Picotees have a white ground, spotted or pounced with scarlet, red, purple, or other colours. These again are divided into scarlet flake, pink flake, purple flake, yellow flake, \&c.; scarlet bizarre, crimson bizarre, \&c. ; purple picotee, yellow picotee, \&c. Propagation. - The carnation is propagated by seed, with a view to procure new or improved varieties, by pipings or cuttings, and by layers, for the multiplication and continuation of established varieties.

Propagation by seed.-The climate of Britain is unfavourable for ripening the seed of this plant. Our supplies, in general, come from Vienna, and other parts of the south of Germany. Hogg, a writer on this flower, and in his day a most successful cultivator, remarks that it often happens that out of two hundred blooming plants it is rare that more than two pods of perfect seed are obtained, and even from imported seed the chance of getting a good flower is about one to one hundred; and if a florist raises six new carnations in his lifetime, he is to be considered fortunate. This explains pretty clearly why the varieties of carnations are so few compared with other florists' flowers. When seed is procured, it should be sown about the middle of May in pots, in the same soil as is recommended below, covering so slightly as merely to hide the seeds. The pots should be set in an airy part of the garden, partially shaded from the heat of the sun, and kept moderately moist, but not wet. When the young plants have attained six leaves, they should be planted out in a bed, in rich soil, and about 10 inches asunder. During winter they will require the protection of a frame and glass sashes; many of them will bloom the following summer.
Propagation by layering.-The proper season for this operation is the latter end of July and beginning of August, that the young plants may not only become early rooted, but also that they get established in the pots or border into which they are to be transplanted as soon as rooted, before the growing season ceases. This is an important part of their culture; the operation is thus performed: Water the plants at the root before proceeding farther, and have in readiness a number of hooked pegs, about 3 inches in length, made out of worn-out birch brooms, or, where they can be had, pegs made from the fronds of the common brake (Pteris aquilina), also a supply of light sandy rich loamy soil passed through a sieve. With a sharp knife cut close off the bottom leaves of each shoot to be layered, leaving about six nearest the top on, and on no account shorten these, although it is a too common practice. In this way prepare all the shoots intended to be laid on each plant, and then with a thin sharp knife make an incision on the under side immediately below the third joint, and draw the knife upwards through the joint to the extent of an inch if the grass of the shoot is strong, less if it should be weak; bend the layer down, and insert it in the light sandy soil, which should be laid round the plant at the commencement of the operation; in doing so, keep the tongue, or portion partly separated from the shoot, from touching the part from which it has been separated, and set the layer in an upright position; thrust one of the hooked pegs into the ground close to the layer, so that the hooked part may catch the layer as it is pushed down, and thus keep it in its place; cover the layer with the light compost, and so proceed with the others until the whole plant is finished; lay the compost around the plant in a neat manner, covering the parts of the layers, where held down by the pegs, to the depth of an inch and a half. The common practice of watering the plants when the layering is finished is bad; they should have none till after two days, that the wound made by the knife may become partially dried. If there is more grass on the plants than can be conveniently layered, take them off, and make cuttings, or pipings as they are called, of them. -(Vide p. 351, and figs. 127 and 128).

Propagation by pipings is in all respects the same as for pinks (which see, p. 808). The carnation is, however, more difficult to propagate in this way than the pink, and hence it is seldom had recourse to.

Soil.-W onderful composts were recommended by the florists of other days; modern ones, actuated by better sense, employ a simple compost composed of three parts loam, taken from an upland sheep-pasture, cutting it in turfs about 4 inches thick. This they lay up in a heap in the compost-yard for about twelve months, turning it over about once a-month to sweeten and pulverise. At each turning a careful watch should be kept for the detection of wire-worm. To the loam two parts well decomposed cowdung and one part leaf-mould are added, and after three or four turnings it is fit for use.

General culture.-Those who intend to bloom 
their carnations in pots should give them their last shift about the latter end of March. The pots should be 11 -inch ones, and thoroughly drained; it is also of much importance that the compost be dry at the time of potting, and for this end it should be kept for some weeks under cover. It should not be sifted, only broken with the back of the spade. Some people plant them in pairs-that is, two in each pot: this is a matter that ought to depend on the size and strength of the plant. If weak plants, two may be placed in one pot; if strong, and as far as relates to the tree or perpetual bloomers, one is decidedly sufficient. When they are all potted, they should be set upon a prepared bed of coal-ashes, watered with lime-water previous to the pot being set upon it for the destruction of worms. The spot chosen should be somewhat sheltered, yet well exposed to the sun. Attention must be paid to staking the flower-stems as they extend in growth ; the best support is wire of the dimensions known as No. IV. cut into suitable lengths, and painted green or a soft stone-colour. When the flower-buds appear too thick, thin out the smallest with fine-pointed scissors; and as the buds begin to open, place an india-rubber ring or ribbon of soft matting round each, to prevent their opening on one side before the other. At this period of their growth, particularly in cold climates, and such as are very wet, the choicest kinds should be placed under cover, and of course none is so good as a covering of glass. Some occupy the shelves of their greenhouses with them as soon as the other plants are taken out; but this can only be considered applicable to places where greenhouse plants of the most ordinary kinds are cultivated. Shading from intense sun must be attended to while the plants are in bloom; and a similar precaution must be taken, as well as against heavy rains, with those grown in the open air. Carnations grown in beds should be accommodated with the same soil as those in pots, and care taken that they be not destroyed by vermin. When the blooming season is past, the flowerstems should be cut off, and the plants fully exposed to the sun and air. As soon as the layers have taken root, separate them from the old plants, and pot them imimediately. Some use 5-inch pots, placing two plants in each; for ourselves, we prefer to use smaller pots, each to contain one plant. By the latter process the plants can be shifted into larger pots in spring without disturbing the roots; while if two plants are in each pot, the ball has to be broken in two, and some danger to the roots may result. Whichever of these modes is followed, it is necessary that the plants be set upon a dry flooring of ashes, and protected during winter by frames and sashes, admitting abundance of air on all favourable opportunities, even to the extent, during mild weather, of leaving them uncovered during the night. The season in which the carnation naturally flowers is about the end of June and beginning of July. None of the very high-bred sorts submit without deterioration to be forced into bloom much earlier; but the tree varieties do, and these, if intended for forcing, should have a year's previous culti- vation-that is, they should not be allowed to flower at all the year after they have been propagated, but stimulated into vigorous growth, and have the tips of their shoots pinched back to cause them to become bushy and stocky. The autumn of the second year they will show abundance of flower-buds, which should be encouraged, and these will flower during the whole winter profusely. Exhibition plants, as they advance into a flowering state, should be set on stages of a height sufficient to bring the flowers within a proper distance of the eye, and the whole covered with a portable framework, for the support of canvass for protecting them from rains, and at other times of thin gauze netting for shade. Without these precautions the chances are great against securing a perfect bloom. Growers on a small scale shelter and shade their flowers by a variety of means suitable to their circumstances; one of these is a tin cap about 10 inches in diameter, elevated on a stalk a little higher than the bloom, one attached to each plant. All metallic caps have a tendency to forward the opening of the bloom, in consequence of their being conductors of heat. Non-conducting materials, such as wood, felt, \&c., have an opposite effect, and hence the day of blooming may be accelerated or delayed to a certain extent at the will of the florist. Besides the ring of india-rubber above referred to, a little care should be bestowed at the time of the opening of the flower, by gently slitting with a very fine pointed penknife or lancet those sections of the calyx which do not open freely. Something may be done to regulate this opening of the calyx-namely, by turning the plants round, that the side opening first may be set towards the north. The bloom is shown to greater advantage when circular cards of white thin pasteboard, or stiff paper, are placed behind them, on which the petals are spread out. Some object to this as unnatural, but the whole of the manipulations of the florist are so truly artificial that we see little reason for the objection in this case.

The pink.-The origin of the garden pink is involved in great obscurity, although named by Wildenow Dianthus hortensis - a name which cannot well apply to it, as Dianthus hortensis is stated in our leading plant-catalogues as being only introduced to Britain in 1805 from Hungary. However, our recollection of the pink carries us as far back as that period, when it was by no means a rare plant. Some think the pink sprang originally from the carnation ; but others, with more apparent reason, consider it de. rived from some of the smaller-growing species, as armeria, plumarius, deltoides, carthusianorum, \&c. The pink is, as compared even with the carnation, a plant of modern date, being scarcely mentioned by our earliest authors, and until within the last sixty or eighty years it was not regarded otherwise than as a border flower. Pinks are divided into the following sections by growers-viz. Pheasant's eyes, or our most common sorts, thought to have sprung from Dianthus plumarius ; Cob pinks, which are a larger sort, and thought to have sprung from the carnation, and seemingly intermediate between 
pinks and picotees. The early red pinks are smaller than Cobs, but larger than Pheasant's eyes, and are supposed to have sprung from Cobs and Dianthus deltoides or armerius.

Propagation.-By seed, to procure new or improved varieties; by pipings, for general propagation ; and sometimes, but rarely, by layering, and that chiefly in the case of any new or very rare variety, as the process is more certain than by pipings. What has been said of the carnation is quite applicable in this case also. The process of raising the pink by seed is in all respects similar to that of the carnation; but the pink, being a much hardier plant, produces seed very readily in Britain, and hence many new varieties are annually obtained.

Propagation by pipings. - This operation should commence as soon as the grass, as the young shoots are called, is of sufficient length and firmness for the purpose; every day is of importance as regards time, that the young plants may attain size and strength to stand the winter. The usual time directed is immediately previous or while the plants are in bloom. In the neighbourhood of London, about the 20 th of June is a good time, but ten days later is as early in the vicinity of Edinburgh as the grass will be in condition. We do not think it advisable, when many pipings are required, to take them off to such a degree as to leave the plants too bare until the flowering season is past, as the removal of so many leaves at that time must weaken the plants very much. It were better, therefore, to take only a few off each plant to secure an early crop, and make a second batch when the flowers are gone, or, where the stock is large, to have a collection grown in a bed for the express purpose of propagation, without interfering with those in the blooming-bed. We speak here of choice varieties ; for common border-flowers this precaution is much less necessary. Pipings are most successfully rooted on a slight bottom-heat, particularly when they are late in being taken off, and also in all cold localities. In the south this is less important, and many strike them under hand-glasses placed on a warm common border. The earlier in the season they are propagated the better, and as soon as rooted they should be transplanted into a bed, to gain strength, before their final removal to the blooming-bed. By September they should be fit for and set in the bed in which they are to flower; for if this operation be delayed till spring, as recommended by some, the plants will neither be of sufficient strength, nor will they throw bloom so fine as those plants arranged in autumn. It is not desirable, however, to have over-strong plants, and hence over-stimulating must be avoided. Plants of moderate strength are far preferable, as they in general send up only one or two flower-stalks, producing three or four fine fully-developed blooms each. Very weakly plants, on the other hand, send up one small flower-stalk, and the flowers produced are few, small, and deficient in texture. The process of piping is by some done by pulling off the grass and sticking it into the ground without farther ceremony. This is both slovenly and injurious to the parent plant, by lacerating its main shoots to a very unnecessary extent. The proper course is to cut off the best formed grass close to the stem carefully with a sharp knife. The pipings are prepared by cutting off the lower leaves with a sharp knife, not pulling them off, leaving about four leaves at top ; and these are to be left entire, and not cut short, as was long the practice. The base of the piping is to be cut off close under a joint in a transverse form. One variety at a time should be operated upon, and when the number to be struck is small, each sort may be planted in a pot by itself; but when the number required is considerable, it is better to prepare the soil for them, placing it upon a mild bottomheat, such as a nearly exhausted hotbed, setting the cuttings in rows, and covering the whole with a frame and lights. The pipings should be inserted about an inch in light sandy soil, set about 2 inches apart each way, and gently watered when they are put in. After this, shading from the sun is necessary, but to guard against neglect in this matter we place our piping-bed behind a wall, by which shading is scarcely required. Dampness, as well as undue dryness, is equally to be guarded against ; and while the first process of rooting is going on, a close atmosphere is necessary; but as it proceeds, ventilation is day by day increased, until the sashes are to be taken off altogether, which should be the case for a few days before the plants are taken up and transferred to a nurserybed, in which they are to remain till fit for transplanting into the blooming-beds.

Soil.-Although the pink prospers pretty well in most good garden-soils, like all other plants it likes what may be called a new soil, or a portion of such mixed with that of the borders or beds in which it is grown. Of course, when a bed is especially set apart for its blooming, it would be unwise to plant year after year in the same soil. It is better, therefore, to change it in part, if not in whole, annually. The majority of florists recommend a good fresh loamy soil, and of the depth of about 2 feet, manured with cowdung in a very decomposed state. If the loam be stiff, a sufficient quantity of sharp sand and leafmould, in equal proportions, may be added, to reduce it to a proper texture. The whole of this depth need not be made yearly ; if half the quantity of the previous bed be removed, and the deficiency made up of new soil, and both thoroughly incorporated, the compost will be all that is required. The blooming-bed should be elevated from 6 inches to a foot above the alley, and the soil surrounded by thin Caithness pavement set on edge. Indeed, as we have more than once remarked, the beds of all florists' flowers, if we except the dahlia and hollyhock, should be so elevated. It brings the flowers nearer the eye, gives the appearance of greater neatness and order, and in many respects is beneficial to the plants themselves. The bed should be prepared a month previous to planting - say by the middle of August-at which time the bloom will be over, so that planting may be done by the middle of September, or the beginning of October at latest. The blooming plants should 
be set a foot apart each way, a circumstance that will determine the breadth of the bed.

General culture.-During winter, shelter ought to be afforded them, the surface of the soil stirred up, and no weeds allowed to appear. Some mulch their beds over during winter with littering manure, but this has a very untidy appearance. Sifted coal-ashes, to be removed in spring, are at once the neatest and most effectual protector. When the flower-stalks begin to shoot up, neat iron supports, made of No. 4 wire and painted, should be placed to each plant, and the flower-stems secured to them with soft matting or worsted thread. The same precautions should be used to prevent the flowers from bursting as have been recommended for carnations. While in bloom they require protection from strong sunshine, and also from dashing rains. The beds must also be kept in a proper state as regards humidity at the roots, but no water should be spilt on either the foliage or flowers. The florists' flower garden should always be proof against the attacks of hares and rabbits. 'To those, however, whose beds are not so protected, it will be well to use some means or other, for an hour's visit of a hare or rabbit will endanger the whole stock. Traps of sliced potato, \&c., must be set for wireworm, and snails and slugs subdued by some of the means we have repeatedly pointed out in various parts of this work.

The auricula.-The type of this very beautiful tribe is the Primula auricula, a native of the mountainous districts of Switzerland, Austria, Syria, and the Caucasus-not an inhabitant of the rocks, but of the grassy patches at their base, or amongst them. It was cultivated in Britain prior to 1597 . It appears a taste for the cultivation of this plant as a florists' flower began with the English, who long supplied the florists of Holland with plants, and they in after year's repaid the compliment by supplying us. For a long period the auricula was a great favourite amongst florists; it afterwards fell into disrepute, but has again regained a very favourable position. The small space required for its cultivation is convenient for many of limited means, and its capability of withstanding the smoky atmosphere of towns renders it a favourite with those whose garden accommodation only extends to their windowsill or the leads on the top of their house. Florists have divided them into the following sections - viz., Green-edged, Grey-edged, Whiteedged, Selfs or One-coloured, and Alpines, the outer edge of whose petals is shaded by a mixture of two colours, not separated as in the edged varieties; the paste around the tube is also yellow, instead of white, as in the other divisions. The auricula is propagated by seed for the creation of new varieties, and by division of the plant for general purposes.

Propagation by seed. - The parent plants selected for breeding from should be healthy and strong, possessing first-rate properties as to size, form, and colour. Cut out with a pair of sharppointed scissors all the anthers or male parts of the mother-plant just before the pips are quite open, dust the pistil with the pollen of the father-plant, by removing and applying it with a fine camel-hair pencil. Cover the mother-plant, after being thus operated upon, with a bell or hand-glass, to keep the flower impregnated dry, and also to prevent the pollen of any other flower reaching it. Keep this covering on until the flower begins to fade, after which no impregnation can take place, when they may be exposed to the action of the air. If impregnation has taken place, the seed-pods will begin to swell, and by June or July the seed will be ripe and fit for gathering. Place the seed-pods on clean paper in a place exposed to the sun, but secured against wet; and when they begin to burst open, rub out the seed, and sow about the beginning of September. Some recommend keeping the seed in the pods in a dry place till March, which they consider a better time to sow it. It is well to sow the seed in pans or flat pots in light soil, placing them under glass in a cool pit or frame in rather a shady situation. Cover lightly, as the seed is small; a quarter of an inch will be sufficient. When the seedlings have attained the size of four or five leaves, transplant them into a cool shaded border in light soil, setting the plants 6 or 7 inches apart each way. The spring following they will flower, when their merits will be ascertained, and such as are of sufficient merit to be taken into the collection should be potted, and placed along with the potted stock. Some pot their seedlings at once, as soon as they have made four or five leaves, using pots 4 inches in diameter, setting one plant in each. This is no doubt the best plan, when high expectations are entertained of the quality of the plants, and where there is room to spare. Be careful not to throw away the weakly plants; they very often turn out to be the very best varieties.

Propagation by division of the plant.-At the annual re-potting, about the end of June, when the flowering season is past, remove the suckers that have roots to them, where they can be spared without injury to the plant, as each plant is turned out of its pot. Pot them either singly into small-sized pots, or set two, three, or four into larger ones, according to their size and strength, and treat them afterwards just as the established plants. We prefer keeping them in single pots, which renders their shifting into larger pots at a future period more safe.

Soil.-Mr Lightbody, an eminent auricula cultivator at Falkirk, has used for the last twenty years a compost composed of two wheelbarrow loads of cow-dung at least two years old, one barrow-load of leaf-mould, and one of pure light loam from an old pasture. These ingredients are thoroughly mixed, and to them a sufficient quantity of fine silver-sand is added, to keep the mass open and secure drainage. Mr Lightbody seems to attach some importance to having this compost frozen through during the winter, and removes the frozen surface as it occurs to an open shed, where it is kept for use. This is a very safe recommendation for soil.

Mr Cooke, an eminent florist at Coventry, recommends the following compost: About onehalf old hotbed manure, and one-half sound fibrous loam well turned over; and, like $\mathrm{Mr}$ 
Lightbody, he prefers its being frozen through before using. He has also found it preferable to employ charcoal or wood-ashes instead of sand ; they keep the soil equally open, and therefore sweet, and they also help to fertilise it.

General culture.-The auricula cannot be cultivated with the least degree of success without protection; in fact, it ought to be treated as a greenhouse or cool-pit plant. Florists have in use various contrivances, that of Dr Horner of Hull being the best, because, while it secures dryness at the roots, it admits of light all round excepting at the back, which is boarded up, but having a small door in it for getting at the plants, and also to assist ventilation. The frame is elevated between 2 and 3 feet from the ground by strong wooden props or legs; the roof is covered with glass sashes as in an ordinary frame. The front and ends of the frame are also of glass, in sashes made so as to let down, being hinged at their bottoms. There are five rows of shelves for the pots to stand on, and these are set to the same angle of elevation as the glass roof, and placed somewhat apart from each other, so that even when the glass sides and roof are shut up, abundance of air is admitted to the plants between the shelving and the space below, which is open. By letting down the front lights, the Doctor observes, " the plants may be left for days together exposed to all the advantages of light and air without care or notice; and when it is desirable to give them the benefit of a shower, the toplights are removed." Such structures are admirably adapted for all florists' flowers grown in pots, except such as dahlias, hollyhocks, \&c. Those who have not the command of such a frame may, however, grow the auricula to great perfection in covered pits or garden frames, setting them on a dry foundation. The plants should be kept within 9 inches of the glass. During severe frosts the glass roof should be covered with wooden shutters, and in the case of such structures as that described above, the open sides should be boarded up also. The only fault such structures have is, that they do not admit of the proprietor getting inside to examine lis flowers, and attend to the numerous requirements of the plants. During autumn, winter, and spring, advantage should be taken of fine days, when the utmost amount of ventilation should be given, even to the extent of removing the top-lights altogether, shutting up, however, at night, for fear of accident. During winter keep the plants rather dry than otherwise, as they are very tenacious of damp. The plants ought also to be kept clean, dead leaves removed as they appear, the surface of the soil often stirred up to prevent the formation of muscous matter, and the pots frequently washed for the same purpose. In February they should be top-dressed, removing about an inch of the surface, yet not so deep as to interfere with the roots, and laying on new compost as high up as nearly to touch the leaves. Mr Lightbody recommends for this top-dressing a mixture of old sheep-dung with the soil the plants are growing in. This, he asserts, has the effect " of causing the green edges to bloom with a more vivid colour, and increases the size of the pips." The situation of the auricula stages deserves consideration. For ourselves, we would place the plants during summer in a pit, stage, or frame facing the north, and during winter facing the south. More experienced florists, such as $\mathrm{Mr}$ Cooke, prefer a permanent aspect. He says, " East, or east by south, is best for them, placing the frames on bricks in a situation that is dry." Mr Lightbody's stages face the northeast; but immediately after being top-dressed (in February), he removes those plants intended for exhibition to a stage facing due south, and, as they come into bloom, removes them again to the north-east that they may get the morning sun.

About the end of March, when the flowertrusses begin to show themselves, give air freely only on fine days, for at that period the frame must be kept rather warmer, both night and day, than heretofore, to encourage a strong development of bloom. Water must at the same time be given more liberally, and that in a tepid state; none, however, should be allowed to fall on the foliage, and if slightly enriched so much the better. When in bloom, shade from the sun, and guard against any sudden change of temperature, which should be rather diminished when the flowers are fully expanded, with a view to prolong their season of flowering. When the blooming is past, set the plants on a bed of coal-ashes to keep worms out of the pots, and place them in a situation, or shade them so that the sun does not shine upon them after ten o'clock.

Potting. - The period when this operation should commence appears not to be fully determined by florists. Some begin at the end of June, just when the flowering is over, in order, as they say, to have a strong growth to flower freely next season. Others delay till the end of July, so as to have the whole potted by the middle of August, while many do not begin till the month of August. The mode of potting practised by Mr Lightbody is sufficiently feasible. "The plan I follow," he says, " is to shift two years consecutively, leaving a good ball of soil at the roots; and the third year to shake the whole of the compost from the fibres, and pot them afresh. When I require to do this, I re-pot about a month earlier. The method of re-potting is as follows : For a full-sized plant use a pot 6 inches wide and 7 inches deep; and for smaller plants and offsets, pots varying from 3 to 5 inches wide. For draining, I use, first a layer of broken crocks, with a layer of oystershells, convex side up; above this I place some vegetable fibre, to prevent the compost choaking the drainage. I then fill in some compost; when this is done, I examine the plant to be re-potted, and reduce the ball of fibres with a sharp knife. I like to grow my plants with short stems, consequently the lower portion of the stem is well examined annually, and all decaying portions of it cut off, and the wound dressed with wood charcoal to dry it up, and keep the plant healthy. The offsets are then slipped carefully off, and the part of the plant to which they adhered dressed also with charcoal ; the plant is then placed in the centre of 
the pot, which is filled up to within about half an inch of the top, care being taken to keep the foliage clear of the compost: a gentle stroke or two upon the bench will settle the soil, and should it sink much, add the quantity necessary to complete the process."

The auricula, like most other high - bred florists' flowers, is exceedingly liable to be attacked by canker at the roots or on the stems. This is no doubt owing to improper soil; the only remedy is, upon the first appearance of the disease, to take up the plants, and procure a soil more congenial for them.

The polyanthus. - This flower belongs to the same family as the auricula - namely, Primula - and owes its origin to the operations of the florist on the species vulgaris, var. polyanthus. Many botanists believe that the polyanthus, primrose, oxlip, and cowslip are all one species, and the late Dean of Manchester thought he had proved them to be so. This near relationship of the polyanthus with the auricula renders their cultivation nearly the same ; our remarks, therefore, on its culture will be brief.

Soil.- The polyanthus, as well as the florists' varieties of primroses, requires a stronger or more argillaceous soil than the auricula, and also to be kept rather more moist. On examination, the soil in which they are found to luxuriate most will be found to be of this description. They are also much more hardy than the auricula, and seldom perish in the most severe winters. They affect a rather shaded spot, and hence a border with a northern exposure is the most proper for them; while a warm, dry, light, sunny situation is the worst of all. They are propagated by seed and division of the plant.

The hyacinth (Hyacinthus orientalis) is indigenous over most of the Levant, abounding around Bagdad and Aleppo; from thence the Dutch merchants trading to these parts early introduced it to their gardens, and from these it reached the gardens of Britain long prior to 1596, when Gerard, in writing on it, speaks of it as being well known and generally cultivated. We have already treated on this plant as a pot plant; we will consider it in the character of a florists' flower, and its adaptation to out-of-door culture.

Propagation. - Like all other florists' flowers, it is propagated by seed when new or improved varieties are the object in view. By this means the Dutch have wonderfully increased the number of their sorts, while in Britain, although some florists have succeeded in raising many fine flowers, still, from difference of climate, peculiarities in culture, and other causes, the success bears no proportion to that of their neighbours. Mr Maddock, a writer on the culture of this flower, directs that " the seed should be saved from such sorts as have strong and straight stems, and a regular well-formed pyramid of bells (corollas), not perfectly single, but rather semi-double. It should not be gathered until it becomes perfectly black and ripe. Sow in the latter end of October or beginning of March, about half an inch below the surface of the soil, in a deep box filled with good sound garden-mould mixed with sand. It will never require to be watered, or have any other attention paid than to keep free from weeds and frost, till it has remained in the ground two years. It must then, on the approach of winter, have an additional stratum of the compost placed upon it, about half an inch thick; and at the end of the third year, in the month of July, the roots may be taken up, and treated in the same manner as large bulbs or offsets. Some of the roots will flower the fourth year, one-half of them the fifth, but by the sixth year every healthy bulb will exhibit bloom; and then the hopes and expectations of the cultivator will be realised or disappointed. He may think himself fortunate if one-half of the plants that first appeared are in existence at this period; and if he can at last find one flower in five hundred deserving a name or place in a curious collection, he may rest perfectly content, and be assured he has fared as well as could reasonably be expected." This quotation holds out no very cheering prospects to the amateur.

Propagation by offsets. - Offsets are small bulbs that form around the old ones, and should be detached at the period of lifting, dried, and kept for planting in nursery.beds, where they are to be grown until of a proper age and size for blooming. They should be planted in October, setting them about 2 inches deep in light, sandy, rich, well-pulverised soil, not less than 2 feet deep. These will in general bloom the second year, but not in full perfection till the third season; their treatment being the same as for full-grown bulbs.

Soil.-The hyacinth-growers around Haarlem in Holland, where several hundred acres are covered with this plant, enjoy a soil of extraordinary depth, of the nature of a light rich but sandy peat, and in parts merely sea-sand abounding in salts. Their ground is trenched with wooden spades, iron ones not being required, as the soil is completely free of clay, stones, or such impediments as would render the use of the common spade necessary, to the depth of 3 feet, the roots of the plant extending to that depth. Here, at the outset, do Dutch and English culture differ. A writer in the "Florist" remarks, "It is in this that the bulbous and tuberous plants thrive so surprisingly; and," he adds, "I believe it is the soil and mode of cultivation, not the climate, that make the difference between the English and Dutch grown hyacinth. Make the soil, therefore, approximate to that of Haarlem; let half or two-thirds be sea-sand, and one-third peat or leaf-mould, the whole sufficiently watered with ocean salts, chloride of sodium, 'sulphate of soda, and sulphate of magnesia; to which may be added with advantage a little nitrate of soda and muriate of ammonia ; the whole of which salts, sufficient to impregnate compost for a bed to grow one thousand hyacinths would not cost five shillings, and the compost will last for seven or ten years." The bed should be thoroughly drained below to get rid of underdamp; for although this plant will grow and flower in water alone, yet it cannot bear a soil sodden with under-damp; and clays of every 
description are either starvation or rank poison to it, it dwindling year after year, until it totally disappears. The roots also soon exhibit signs of canker and decay when planted in a damp soil.

Planting.-The bed being prepared by being trenched over a month previously, the surface should be forked over and made uniform, rising a little towards the centre. The planting should commence the first week in October; some delay till the middle of November : we prefer the former period in late climates and cold situations; the latter is, however, a better time in such as are differently situated. The danger of too early planting is, that the bulbs would become excited too early, and push through the soil, and be liable to injury during the frosts of winter. On no account, however, should the operation be deferred later than November, as the bulbs would suffer from their natural tendency to vegetate at their proper season. The breadth of the bed should be not less than 4 feet, which will accommodate six rows across. In planting, a little clean silvery sand should be placed under each, and likewise upon the roots, to prevent the compost adhering too closely. Set the bulbs from 3 to 4 inches in the soil, according to their size. The writer in the "Florist" above quoted, says : "As in a show-bed it is very important to have all the colours in bloom at once, and as this is no easy matter, requiring not only a general knowledge of their times of flowering, but a particular knowledge of each variety, it may be as well to know how the Dutch apply this knowledge to compel them to uniformity. This is by planting the latest bloomers deepest; and my impression is, that the difference between the greatest and least depth is as much as a foot; and, as a general rule, I can myself answer for its being very decidedly thus : that the blue are the earliest in flower, and the deepest in colour the first, the red next, the white third, and the yellow last."

Taking up the bulbs.-When the foliage begins to assume a yellowish dried-up appearance, it is pretty evident the growing season has passed. As soon as the flowers have faded, the flowerstalks should be cut off close to the base, but the foliage should be allowed to continue undisturbed until they assume the yellow colour above referred to, at which time the bulbs should be carefully dug up, when the foliage should be cut off close to the crown of the bulb, but the fibres should for the present be left attached to them. Many of the Haarlem growers, however, on digging up their bulbs, lay them on their sides on the surface of the bed, and cover the fibres with soil, leaving them for a fortnight or three weeks with the decaying leaves attached. In this way they dry gradually, and have the protection of the awning, used while in bloom, to keep them dry and shaded from the too drying effects of the sun. At the end of this time they remove the leaves entirely, and rub off the now dry and useless fibres, when they are placed in a dry place, well ventilated, and afterwards cleared of the loose skin and offsets, which must be gently rubbed off, but no force used; they are then wrapped in paper individually, to be ready for sale, and such as are reserved for own planting are buried in dry sand, where they remain till the planting season returns. The Dutch attach, and very properly, great importance to the ripening and drying of their bulbs ; for, unless this is accomplished, they cannot be expected to flower well the following season.

"We often plume ourselves," Mr Beaton remarks in "Cottage Gardener," "on our skilful management of forced bulbs in pots, because we seldom fail of having them very fine the first season." Our failures in succeeding years he attributes to the proper cause, namely, we "overlook or neglect the proper treatment of these bulbs, by cutting off their leaves to make room for other things, or by removing them carelessly from the flower-beds and borders to the reserve ground, just at the very time when the necessary supply of matter for giving a fine bloom the following year is being stored in the bulbs through the agency of the leaves. This explanation reveals the fact-for a fact, and a great fact, it certainly is-that the bloom of this spring was not altogether due to the care we might have taken of the roots or bulbs since we potted them last autumn, but rather to the care that was taken of the leaves last May and June. If it were not so, we could not bloom them so fine in moss and in water-glasses as in a good compost. Therefore it stands to reason, that if we wish for good crops of flowers from spring bulbs next year, we must ripen off their leaves with great care now (May), and this is the proper time to give liquid manure to such bulbs."

General culture.-Bulbs that are allowed to produce seed are greatly weakened thereby; and hence the practice of cutting off, or, !better, breaking off the flower stems as soon as the flower fades, is important, by lessening the draught made upon the plant by its efforts to produce seed. Breaking them off is better than cutting, because the fractured part does not bleed or lose its sap so readily. Bulbs which have been forced do flower again, after a proper period of rest and renewal of their strength. This is not, however, a general practice, but may, under proper conditions, be done with advantage. These conditions are, that the bulbs be allowed to remain in the ground undisturbed for two, and sometimes three years.

Those who intend to grow the hyacinth in perfection should have a portable framed roof placed over their bed, and covered with canvass to exclude frost and rain, and shaded with gauze netting while in flower during the intense heat of the sun. Some florists do not take up their roots annually, and by merely top-dressing their beds secure a brilliant display of bloom. It seems at first sight unnatural that the bulb should be taken out of the ground and kept in a dry state, if even for however short a period; but it should also be considered that high-bred hyacinths are far more delicate than those of their kind growing in their native country, and also, from their disposition to form numerous offsets round their roots, that it is expedient to lift them, were it for no other purpose than their removal, as, if left on, they would, to a very 
considerable extent, rob the flowering bulb of its proper share of nourishment. It also flowers so early as February in its native country ; and having a natural tendency to flower even in Britain before our late spring-frosts are past, if not lifted and retarded in its growth it would flower so early with us, as has been already noticed, that it would be in danger of sustaining injury from that cause. This appears to us to be an important objection to leaving them in the beds for a year or two, as well as to the hopes of their succeeding in a general way when planted out after forcing-that is to say, if left in the ground throughout the year. Our excellent friend Mr Beatson, however, tells that he had hyacinths planted out in a border at Shrublands which had not been disturbed for five years, and, moreover, that they were superior to others that were imported and grown in pots plunged in an adjoining border.

The tulip.-The tulip (Tulipa Gesneriana) is a native of Syria, introduced to Europe in 1559, and to England previous to 1577, as we find it was then cultivated in our gardens. It was early and extensively grown in Holland, and in time the exportation of the roots constituted a very considerable branch of commerce. About 1635 , what is known as the tulip mania was at its height amongst the Dutch, and the malady soon spread over to England, and the prices of single bulbs became enormous. Like most other manias, it had its day; and after a time the taste for this flower greatly declined in Britain, accelerated probably by the introduction of many American plants, an increasing taste for botany, and afterwards by the introduction of plants from the Cape of Good Hope, Australia, \&c. Like most other florists' flowers, little patronage was bestowed upon them by the aristocracy, in few of whose gardens do beds or stands of decided florists' flowers appear. Their cultivation has been carried on chiefly by operative manufacturers and amateurs ; and indeed to this day these are the most zealous and liberal patrons of this class of flowers.

The tulip is propagated by seed when new varieties are sought for, by offsets which are formed round the main bulb, and sometimes by dissecting the bulb-but the latter is a rather precarious method.

Propagation by seed.-The tulip seeds freely, and the selection should be made of such plants as are strong, tall, with well-formed cups, and clear in the bottom, as these are found to produce better breeders than the finest of the variegated or broken sorts. The seed should be fully ripened before gathering, and when cut off remove with the seed-vessel 3 or 4 inches of the flower-stem attached to it. Sow and treat the seedlings as recommended for hyacinths. Seedling plants will flower in general by the fourth or fifth year ; some, however, will stand over till the seventh year. Seedlings are called selfs or breeders until they bloom, and during their period of growth prior to that they should be planted in a dry and rather poor soil, that having the effect of causing them to break sooner-that is, become variegated, and assume new varieties. The process of breaking extends

VOL. II. often over a long period of time, in many cases as much as twenty years, or more, although some will break the first and second year, and some will not break at all. All the art of the florist has not yet accomplished the breaking of these flowers with any degree of certainty, or within any prescribed period of time.

Propagation by offsets.--This is a sufficiently simple operation. The offsets should be planted in a bed of light loamy soil, moderately enriched with decayed cow-dung, and situated in a wellexposed part of the reserve-garden, and treated as to protection, during their growing season, the same as those in the flowering-bed. In this bed they are to be cultivated one season, or more-that is, until the bulbs are of sufficient size to take their place in the bloomingbed.

Soil.-This can hardly be too deep, or too dry at the bottom. 'Three feet should be allowed of prepared soil, and according to the nature of the subsoil, drainage of some sort should be arranged. No better method exists of securing a dry bottom than laying under the compost of which the bed is formed a stratum of 12 or 15 inches of broken stones, flints, or brickbats, with a tile-drain through the centre of it, having a free outlet should water accumulate; over this a course of turf, and on it the prepared soil ; and if the bed is elevated a foot or more above the surface, so mucl the better. The soil or compost found most suitable for them is light rich sandy loam, kept a twelvemonth at least in the compost-yard, and repeatedly turned over and enriched with the drainage of the dunghill or cow-house, and, in the absence of that, with rotten cow-manure added at each time of turning. Excavate the natural soil if a new bed is to be formed; lay in the drainage recommended above, and on that place the compost. If the bed has been in use previously, and thoroughly drained, then a third, fourth, or lialf of the old material may be removed, and replaced with an equal proportion of the new compost.

Situation and preparing the beds.-The situation should be sheltered from high winds, yet open to the sun and air, and by no means shaded. 'The beds should be prepared a month at least before planting is to take place, that they may have time to subside after being so deeply disturbed. The beds should be of a breadth to hold from five to seven rows of plants; broader ones are inconvenient, and narrower ones are deficient in effect when the plants are in bloom. A 5 -feet bed will hold five rows 9 inches apart. The beds should have a walk all round them. The beds should only be prepared in dry weather.

Planting.-The second week in November is the proper season for this operation, and dry weather, if possible, should be chosen. If the bulbs are planted too early, the leaves will be above ground before the cold frosts of spring are past, and if not protected they would suffer thereby. This, however, depends much upon locality, as do all sowing and planting connected with gardening. In most cases we may say from the 20th of October to the 10th of $\mathrm{No}$ - 
vember. The surface of the beds should be rendered smooth by raking, and the distances should be accurately marked out by tracing the lines first longitudinally and then across the bed. The distance between the centre of one root and another is set down by most florists at 7 inches; some, however, allow a little more; but with the tulip, the hyacinth, and similar rooting plants, it should be considered that they send down their roots (the bulbs are not the roots) perpendicularly to a great depth, and not horizontally, as do many other plants ; therefore they may stand much closer together in the bed. Where the squares intersect each other as marked on the bed, there should the bulbs be set, and under and around each bulb a little clean sharp sand should be placed; it tends greatly to prevent the bulb from rotting, and also keeps the soil or compost from adhering to it. When the planting is finished, a top-dressing of strong sound fresh loam, moderately enriched, should be added, so that the bulbs may be covered to a depth proportionate to their size, none deeper than 4 inches, and the weaker at $2 \frac{1}{2}$ or 3 inches at most. In regard to the arrangement of the bulbs in the blooming-bed, some knowledge of the sizes, colours, and habits of the plants is requisite. The largest, tallest, and strongest varieties should occupy the centre row ; the next in order of strength the next; and the weakest and dwarfest of all the outside rows. Some leave the surface of their beds level, so that the rain may be of equal advantage throughout. Others round them off to throw off the heavy rains.

General culture.- It may be necessary, during severe frosts, to mulch the surface of the bed over with some light dry material, to be carefully removed in spring. A more effective covering, however, must be accorded them in spring, as soon as the plants begin to appear above ground, and also while they are in bloom. The protection required should be the same as we have recommended for the hyacinth. The surface of the bed should be kept scrupulously clean and free of weeds, and be frequently stirred on the surface, using a wooden spatula for the purpose. Allow abundance of ventilation to the plants, and water the soil should it seem dry, but let none fall on the blooms, and protect them also carefully from rain and intense sunshine. After the blooming season is past, and the foliage beginning to decay, let the bulbs be lifted on a dry day, and place them under cover to dry in an airy situation. The offsets and loose brown skin should be rubbed off when they are quite dry, taking care, however, not to remove too much of the skin, or to displace offsets very firmly attached to the main bulb. The bulbs should be kept in drawers or in paper bags, in a cool dry room, to await the season of planting.

Several writers on floriculture have gone so far as to include amongst florists' flowers a much greater number than we think come under this direct head. For example, if Lobelias, Rockets, Campanulas, Hydrangeas, Balsams, Fuchsias, Petuneas, Rhododendrons, \&c., are to be so regarded, then Heaths, Epacrises, and almost every other plant found on our exhibition-tables, may be also included.

We deem it of little or no practical utility to give lists of florists' flowers, and therefore beg to refer our readers to the catalogues of growers, who will furnish them gratis upon application. Every year produces a new race of these flowers, and therefore what may be considered a firstrate dahlia, hollyhock, \&c., this year, may be superseded by others in a year or two's time - not, however, that we believe that improvement in all cases keeps pace with this mania for novelty.

\section{$\S$ 6.-THE ALPINE GARDEN AND ROCKERY.}

The cultivation of alpine plants, or, as the term implies, plants natives of alpine or mountainous regions in various parts of the globe but chiefly inhabiting the temperate or frigid zones, has for the most part hitherto been confined to gardens strictly botanical. A taste for their possession is now on the increase, particularly in the private gardens of Scotland. They are exceedingly well adapted for amateurs having limited accommodation; they occupy little room, are not expensive to purchase, are for the most part of easy culture, blooming from early spring to late in autumn, and some even throughout the winter. They are often planted on rockwork or stoneries, but are better grown in pots plunged in sand, and placed within a cool pit, so constructed that a sufficient protection from frost and wet may be afforded them during winter ; for, however strange it may appear, plants from the frozen regions of Spitzbergen, Melville Island, \&c., will not survive our winters without a certain amount of shelter. In their native habitats they are protected from intense frost and damp by thick coverings of snow, during which period they are also confined to total darkness. In imitation, therefore, of these conditions, we cover our alpine pits about the end of November with thin-boarded or felt shutters, which sufficiently exclude the extent of frost that would be hurtful to' them, and keep them comparatively dry, and nearly deprived of light. Towards spring we admit air and light gradually, as it were in imitation of the progressive melting of the snow, until, by the beginning of March, vegetation begins to revive in the plants, when all the light possible is afforded them, covering only during cold or frosty nights; afterwards the covers are removed entirely. During winter, on fine days, the covers are removed, that the plants may be examined, and all dampness removed; for our artificial covering has a much greater tendency to create damp than the constant covering they enjoy in their native places of growth. This may possibly arise from the variableness of our climate com pared with that in which they naturally exist. In March yearly they are re-potted, and such species propagated as are required, by division of the plant, by seed saved during the previous summer, or by cuttings, according to their 
various natures. The soil we use is for the most part sandy peat and loam in equal parts; some in sandy peat alone, and others, as Saxifraga cernua, which is only found amongst the debris of micaceous rocks, in a soil of which mica in a reduced state forms a part; Rubus chamœmorus, and similar true bog-plants, in peat-bog soil; while alpine aquatics, such as Lobelia dortmana, Isoetes lacustris, \&c., are planted in sheer sand, and submerged in tubs of water. The pots in general use in nurseries are small or large sixties. These are no doubt convenient for transport, but to grow the majority of alpines to the fullest extent of perfection, pots 9 inches over should be employed. At Dalkeith, where the largest private collection perhaps in the world is cultivated, we use pots of the latter diameter, but not more than 5 inches deep, perforated at the bottom and round the lower part of the sides with numerous small holes, for admitting air to the roots, and securing ample drainage. From 1 to 2 inches of drainage is placed in the bottom; and when the potting is finished, small white pebbles are set around the plant on the surface of the soil. Upon these many of the creeping kinds recline, and are prevented from damping off, as the air passes under and amongst the stem and foliage. The stones also tend to keep the soil of a uniform moistness, by preventing evaporation; and in many cases the roots of some of the more rare species luxuriate more under these stones than elsewhere in the pot. When their growing season commences, abundance of water is given, often to the extent of twice a-day, for from observation we know that at that stage of the growth of alpine plants they are kept at nearly the point of saturation, in consequence of the melting of the snow above and around them. In all mountainous regions a great amount of atmospheric moisture exists, and hence the practice of watering them over-head with a rose watering-pot is beneficial to them. Many alpine plants are capable of being very successfully cultivated in town, in back-yards and on housetops; and we believe that a very extensive collection could be so grown in the centre of London, if placed in frames covered with glass sashes. The amateur about to form a small collection, say of 100 species, will find the following genera suitable for the purpose :Soldanella, Primula, Aretia, Androsacea, Gentiana, Cyclamen, Illecebrum, Trientalis, the prostrate species of Veronica and dwarf Campanula, Arenaria, Saxifraga, Sedum, Sempervivum, \&c. The arrangements of an alpine pit most suitable for culture are, a span-roofed pit, with a wall along the centre for affording partial shade during the heat of summer to such sorts as are impatient of extreme heat, as well as those, like many ferns, which grow naturally in the mouths of caves, under the shade and shelter of projecting rocks, \&c. ; the front pit for those like the Azalea procumbens, \&c., which grow upon bare exposed places, enjoying the fullest rays of the sun, and which would be ill suited to a northern exposure or cool shaded place. The rockery is also an excellent place for the more hardy, and in its construction regard should be had to securing shade, as well as full exposure to the sun, therefore a bank covered with masses of stones, running in an east and west direction, or one of irregular form, will present these conditions.

\section{§ 7.-THE PERENNIAL-PLANT FLOWER GARDEN.}

Before the grouping and planting-out system came into fashion - that is, the decorating our flower-garden borders with plants such as geraniums, verbenas, \&c., that require to be taken up or propagated annually, and protected under glass during winter-our whole dependence was placed on annuals and perennial plants, intermixed or associated with shrubs, evergreen and deciduous. No doubt that ten out of every twelve sorts of annuals thus grown were useless trash, weedy in appearance, and producing none of those brilliant effects for which our modern flower-gardens are so conspicuous: and the same may be said of the perennial plants existing in those days. The case is now, however, very much altered; a new style of annuals was introduced by the collector Douglas and others, mainly through the influence of the London Horticultural Society, and since through that of some of the London nurserymen. The same means introduced many fine perennial plants before unknown to us. Gardeners of the days to which we refer had little idea of producing pleasing and agreeable effects by means of masses of colour, either harmoniously or contrastedly arranged. Their great aim was to possess a collection of species and genera, without much regard to the beauty of individuals, or the effect they were capable of producing. This mania for collections was never carried to the same extent generally in England as in Scotland, in many gardens of which from five hundred to a thousand species might have been found. Like all reforms speedily brought about, the transition from one extreme to the other had its defects. With the destruction of collections of perennial or herbaceous plants, the name they are better known by, many an exceedingly valuable plant was consigned to the rubbish-heap, which, had it been kept and propagated, would have filled its place in the parterre. Here one of the evils of the modern system stares us in the face. Our parter'es, or, as some will still have it, our flowerplots, are denuded of all their glory on the first visit of frost-say, as in the present year (1854), the 5th October, when heliotropes, calceolarias, and in many places verbenas and geraniums, gave up the ghost ; and if not, they only left the ghost of their former splendour behind them. From the middle of October, therefore, till the beginning of July, unless where the introduction of bulbs is attended to-a practice unfortunately very seldom followed-our flower-gardens are next to a blank. Now, we have often thought-nay, we have proved it-that much of this blank space might be filled up by the proper cultivation of certain perennial plants at present only to be found in very few establishments. We will mention a few of these, and begin with 
Delphinium Barlowii, a deep-blue English hybrid, which flowers twice a-year, first in June, and secondly in October and November; Tritoma uvaria, media, and pumila, flowering till the end of November: Aster strictus, hyssopi folius, rigidus, linarifolius, grandifolius, foliosus, undullatus, dumosus, multiflorus, cordifolius, Novæ Angliæ, sessiliflorus, spurius, cyaneus, lavigatus, serotinus, and blandus. Most of the genus Helleborus flower from January till April. Colchicum autumnale, with its varieties, as vell as several other species of the genus, afford abundant bloom from the beginning of October till December. Achillea ptarmica flo. pleno, micrantha, tomentosa, flower till November; and several of the late-flowering species of Phlox, and hybrids of Phlox omniflora, if grown in a shady cool part of the reserve-garden, may be made to flower from October, until killed down by the November frosts; while Campanula carpatica, if the seed-vessels be picked off as they form, will flower till the middle of November; and Viola palmensis will flower in most places throughout the whole winter, particularly if propagated by seeds or cuttings in June and July. The perpetual or tree carnation comes also within the same category; as do also the autumnal roses, and winter-flowering hardy heaths. The following are a few only among the many of this class that flower early - that is, from February till the end of April or beginning of May: In the genus Corydalis, a number of species which, along with those below, may be grown in pots in the reserve garden, and transferred to the flower-borders in January, will flower during February and on to the end of April or beginning of May. Amongst minute plants the genus Draba affords several species, and Alyssum and Aubrietia afford other examples, while Cheiranthus affords plants of larger growth and agreeable perfume. Anemone offers examples of many species flowering beautifully from March till May, and Hepatica from February till April. Potentilla verna, Phlox verna, divaricata, subulata, nivalis, \&c., bloom from March to May, and P. alba from February onwards. Many of the Oxalis and Viola tribe flower through November, and during mild winters others of them keep up a display till May. Dielytra spectabilis in March and April, and many species of Saxifraga, are amongst our earliest vernal flowers. Muscaria, Hyacinthus, Scilla, Ornithogalum, Gagea, Erythronium, Crocus, Tulipa, Fritillaria, Galanthus, Leucojum, and Narcissus among bulbs, might enliven the dreariness of winter in our flower parterres. The reserve-garden is the place in which they should be grown; those in pots that do not admit of ready transplantation; and such as the Asters, \&c., may be with every safety removed by Mr M'Glashan's smallest flowertransplanting machine, even when coming into flower, giving them a good soaking with water when set in their respective places. Although from June till October we are independent of perennial flowering plants, on account of the great variety we now have of planting-out ones, still there are many of the former that might be introduced with no disadvantage amongst the latter in mixed beds. Those who do not go to the fullest extent in the new method of planting a flower-garden, and prefer a mixed selection, cannot do better than select as many of the best moderate-growing perennials as have decision of character, good habits, brilliant colours, and some duration.

\section{§ 8.-THE PARTERRE GARDEN.}

In vol. i. numerous examples are given of parterre gardens, and some notice has been taken of the arrangemient of the plants in them, so as to produce the best effect in regard to colouring and form. Our remarks here will therefore be more of a collateral nature.

Anruals.- "Annuals," says a very popular writer, "are a somewhat puzzling class : they possess many nice things amongst them, but the question is, how to dispose of them? To reject them from the flower-garden altogether is a most unjustifiable procedure, and what few persons would think of doing; but then they are of such erratic habits-at least many of them-that they ill assort with that dressy character so desirable in the modern flower-garden." We cannot dispense with annuals altogether, but the number worth retaining is exceedingly limited. This we will endeavour to show by-and-by, and in the mean time glance at the disposal and cultivation of them. Annuals of even the very best sorts require cultivation and management, else they become straggling and weedy in appearance, and devoid of that interest they otherwise would have. Sowing too thick and in too rich a soil is a prevailing fault. When sown too thick, they are drawn up weakly, and, never becoming fully developed, produce only a few meagre flowers towards the tops, which, with the plants themselves, are of short duration. When sown in too rich a soil, they overgrow the bounds of orderly decoration, produce a greater proportion of foliage than of flowers, and attain heights their feeble stems are totally unfit to support. To avoid this extreme, some have fallen into another, by sowing on too poor a soil, and unless in some cases, such as that of Calandrina, Talinum, and such semi-succulent plants as require both poor and dry soil, all others require one of sufficient stamina to enable them to develop themselves fully. Some few require a moderately rich soil, such as German and intermediate stocks and aster's. All others require a soil of ordinary richness, and should be sown thin, or, if not, be thinned out after they have attained the height of 2 inches or so, to a distance according to their habit, whether upright, trailing, \&c., so that by the time the plants are coming into bloom the ground may be covered, and not before, so as to enable each plant to develop itself fully according to its habit.

Some, to check the over-luxuriance of annuals, sow the seed in pots 4 or 5 inches in diameter, plunged in the reserve-garden, and brought to the parterre, and plunged in it when wanted. This is no doubt a very commendable mode upon a small scale, and where there is abundance of means; but to attempt to fill 
beds extending over twenty or thirty square vards by such means would be absurd. Sowing in pots in the reserve-garden, however, is absolutely necessary, so as to have a supply of plants just coming into bloom, or nearly so, to fill up such portions of the parterres as have failed from some cause or other, or to replace early bulbs, or auturnn-sown "annuals which have completed their season of flowering. Upon the whole, tempering the soil to suit the annuals, and sowing moderately thin, and thinning afterwards, is the true way to secure a fine bloom through the summer months. To insure an early spring display the seeds should be sown in autumn, and, where needed, protected slightly during the intensity of winter, transplanting them early in spring. Most of the best of the Californian annuals submit to this mode of treatment, and indeed flower fully as well as such as are sown in spring to flower during summer. For a late bloom, many of the same, if sown as late as June or the beginning of July, will flower from the end of August till Novem. ber. To insure a display of hardy annuals throughout the season, sow early in March, middle of April, end of June, or beginning of July, and for an early spring bloom about the beginning of October. Half-hardy annuals, such as stocks, should not be sown in the open ground till the beginning of May, and of those which are rather tender, Petunia, Phlox Drummondi, \&c., should be sown in pots or boxes, under glass, and transplanted about the end of May. All annuals that will submit to it (and most of them will do so) are improved in habit by transplanting, because it checks their over-luxuriance of growth, and causes them to flower in greater profusion. The blooming season of annuals, as well as the duration of their existence, may be considerably prolonged if the flower's are cut off as they begin to decay. Thus the common mignonette may be continued for two or three years, if prevented from forming its seed, and by regularly pinching back a portion of its shoots. Half-hardy annuals should be sown on a gentle hotbed in March and April, afterwards transplanted into another mild hotbed, and finally planted in the parterres about the end of May or beginning of June. Tender or greenhouse annuals require to be constantly grown in heat for the most part; a few of them, however, such as the balsam, may be planted out in warm gardens about the end of June, and will flower in very tolerable perfection.

The after-culture of annuals consists in thinning out the plants, or pruning their branches when they overgrow their prescribed bounds. This is a matter seldom thought of, as is also that of cutting off the decaying flower-stalks, so as to prevent the plants from seeding. We prefer low-growing annuals for our smallest parterres, because they require no supports ; where, however, the parterre or group is pretty large, plants of various heights should be employed, the tallest always towards the middle and the lowest next the margin, attention at the same time being paid to the proper arrangement of the colours. Whether the rows are placed in circles, straight lines, curves, or zigzags, each line should be distinct in itself, and these bands should never be allowed to touch the edging, or the row next to it. Much may be done in securing these distinctive lines, and preventing the plants from running into each other, by sticking in small branches of beech or birch, or any other deciduous tree, along both sides of the lines of plants where these exceed 9 inches or a foot in height, but they must be so regulated that the plants shall completely hide them. Such supports are necessary also when clumps are cut out on grass, to prevent the plants from touching the grass, and to confine them, so that the proper outline, whatever it may be, shall be preserved. Even where annuals are sown in masses, each occupying an entire bed, it is well to introduce such supports, to keep the lower branches from falling down, and so preventing a free circulation of air between the plants throughout. Few annuals require pegging down, as abundance of them are of a trailing habit naturally.

\section{SELECT LIST OF HARDY ANNUALS FOR} PARTERRES.

Ageratum cœlestinum, pale blue ; odoratum, lilac, 18 inches-both may also be continued by cuttings. Alyssum calycinum, white, 12 inches; Anagallis cœrulea, blue ; carnea, fleshcoloured ; indica, purple ; latifolia, blue ; grandiflora, red, 6 inches: may be, like Ageratum, treated as annuals, sown in March, in a warm frame, and also continued by cuttings. Asters, China and German, from 12 to 24 inches, of various colours. Bartonia aurea, 18 inches, yellow. Brachycoma iberidifolia, 12 inches, blue, and also a white variety. Calandrinia ascendens, 6 inches, dark crimson ; glandulosa, 12 inches, red ; grandiflora, 12 inches, rose lilac ; speciosa, 18 inches, maroon. Calendula officinalis flo. pleno, 15 inches, orange ; stellata, 24 inches, yellow. Campanula Loreyi, 9 inches, dark blue ; pentagona, 9 inches, blush purple ; stricta, 9 inches, blue. Centaurea cyanus major 18 inches, blue; depressa, 18 inches, bright blue. Centranthus macrosiphon, 18 inches, scarlet. Chrysanthemum carinatum, 15 inches, white, yellow, and purple. Chœnostoma fastigiatum, 9 inches, pink ; polyanthum, 9 inches, lilac ; viscosum, 9 inches, brown. Clarkia pulchella, 18 inches, reddish purple ; flo. alba, 18 inches, white. Clintonia pulchella, 6 inches, blue, white, and yellow. Collinsia bicolor, 12 inches, purple and white; grandiflora, 9 inches, blue and purple. Collomia coccinea, 9 inches, scarlet; grandiflora, 12 inches, buff. Convolvulus tricolor, 12 inches, blue and white; var. atroviolacea, dark purple. Delphinium (Rocket Larkspur), 18 inches, various; consolida (branching), 24 inches. Dianthus sinensis, 12 inches, various. Erysimum Perowskianum, 24 inches, dark orange. Eschsckoltzia crocea, orange; californica, yellow-both 15 inches. Eucharidium grandiflorum, 12 inches, dark crimson ; concinnum, 18 inches, reddish. Gilia tricolor, 18 inches, lilac and purple. Godetia tenella, 18 inches, purple. Gramnunthes gentianoides, 6 inches, deep orange; var. flora lutea, yellow. Heliophila mabioides, 9 inches, blue. Hemimeris incisifolia, 24 inches, scarlet. 
Hibiscus africanus, 18 inches, pale yellow. Iberis (candy-tuft) coronaria, 12 inches, white ; umbellata, 12 inches, lilac; var. sanguinea, dark red. Isotoma axillaris, 9 inches, blue. Kaulfussia amelloides, 6 inches, blue. Lasthenia californica, 6 inches, yellow. Leptosiphon densiflorus, 12 inches, lilac; androsaceus, 9 inches, rose lilac. Linaria spartea, 12 inches, golden yellow ; tristis, 12 inches, brown. Lobelia crinoides, 6 inches, blue; heterophylla, 15 inches, blue ; ramosa, 12 inches, blue. Lotus Jacobæus, 12 inches, black. Lupinus bicolor, 6 inches, deep blue ; pilosus, 24 inches, dark blue ; nanus, 6 inches, blue and white. Malcomia maritima (Virginian stock), 9 inches, red; flo. albo, white. Mathiola (stocks), 12 to 18 inches, colours various, but can be had distinct. Mesembryanthemum tricolor, 8 inches, red ; pomeridianum, yellow. Nemophila insignis, 9 inches, pale blue; maculata, 9 inches, white and purple. Oxalis rosea, 9 inches, rose-coloured. ()xyura chrysanthemoides, 12 inches, yellow and white. Phlox Drummondi, 24 inches, various colours. Platystemon californicum, 9 inches, pale yellow. Podolepis gracilis, 18 inches, pinkish lilac. Portulaca Thellusonii, 6 inches, orange crimson ; grandiflora, 6 inches, crimson; aurea, 6 inches, bright yellow ; Gillesi, 9 inches, purplish crimson. Reseda odorata (mignonette). Rhodanthe Manglesi, 12 inches, bright rose. Sanvitalia procumbens, 6 inches, yellow. Saponaria calabrica, 9 inches, carmine; ocymoides, 9 inches, red. Schizanthus diffusus, 18 inches, purple and white; venustus, 24 inches, lilac, purple, and yellow; pinnatus, 24 inches, purple and yellow. Śchizopetalon Walkeri, 12 inches, white. Sedum azureum, 6 inches, pale blue. Senecio elegans, 24 inches-the double varieties are of various colours, from dark red to white. Shortea californica, 6 inches, yellow. Viscaria oculata, 12 inches, bright rose.

Biennials are useful in the parterre garden when a judicious selection of sorts is made, and this selection should be so far guided by decision of colour, compact and elegant habits, and plants producing a profusion of bloom, and that lasting for as long a period as possible. Their cultivation should be carried on in the reservegarden, where they are first to be reared from seed; or, in cases of fine varieties, propagated by cuttings, grown moderately thin, and frequently transplanted, so that they may be removed with the greater facility and safety to the parterre when it requires making up. Some of those most difficult to transplant should be grown in pots, which may either be plunged in the parterre, or, better, in most cases carefully turned out, as after their flowering most of them are not worth retaining. Biennials are plants of two years' duration, the seed being sown the first year, and the flowering, seedbearing, and death occurring the next. Many of them, however, may be continued for a longer period if prevented from seeding. Many of these ripen their seed in August or September-those should be sown immediately; and such as do not ripen till a later period may be kept even till April or May following. Double varieties of biennials can only be continued by cuttings. As with annuals, our seed-lists are filled with very trashy things; few out of the many to be had in seed-shops are worth the attention of the tasteful flower-gardener. Th following are the best adapted for the purpose :-

\section{SELECT LIST OF BIENNIALS.}

Ammobium alatum, 24 inches, white and yellow. Catananche cœrulea, 24 inches, blue ; Cheiranthus cheiri (wallflower), 24 inches, in various colours. Dianthus barbatus (sweet-william), 18 inches, in various colours. Hedysarum coronarium, 24 inches, scarlet. Mathiola incana (Brompton stock), 24 inches, in various colours. Enothera taraxifolia, 9 inches, pinkish white; microcarpa, 6 inches, yellow. Seline compacta, 18 inches, pink ; ornata, 24 inches, carmine; biennis, 24 inches, yellow.

Bedding-out plants.-Within the last twenty years a new and better taste has arisen in the planting of parterres. Formerly our subjects, although numerous, were for the most part ill adapted for the purpose of producing those striking cffects we now see in all gardens where taste and discrimination are properly exercised. The introduction of Verbena melindres in 1827; of Calceolaria rugosa, corymbosa, \&c., in 1822 ; of Nemophila insignis in 1833, and various other Californian annuals about the same period, tended greatly to encourage this taste, which, as it extended, called into request plants before that time regarded solely as inmates of our greenhouses and conservatories, as exemplified in the case of the procurable species of Lobelia, Heliotrope, scarlet geranium, \&c., which now form one of the principal features of our best gardens. All of these, however, are too tender to withstand our winters in the open air; and consequently from the beginning of November to the beginning of June our parterres are stripped of their interest, excepting in so far as their forms, when judiciously arranged, display the taste of the landscape-gardener, and the recognition of art in connection with the buildings, \&c. around them. So far the grouping system and the employment of such plants in the production of effect are all that can be desired in gardens where the owners, which is very often the case, do not reside at their country residences before the month of July, when the country season may be said to begin. The parterres during winter, however, may be clothed so as to afford a satisfactory display, although much less brilliant, by removing from the reserve-garden such plants in pots, and many may be removed even without such a precaution-as Laurustinus, which flowers from November till January ; Rhododendron atrovirens in February, March, and April ; R. dauricum at the same time ; the varieties of Andromeda calyculata from February to April; Arbutus unedo and its varieties from October to December ; Gaultheria procumbens, whose beautiful coral-like berries continue during most of the winter, as well as many of the Cotoneasters; Saxifraga oppositifolia in March and April ; Erica arborea and its varieties, E. mediterranea, most of the varieties of E. vulgaris, in February, 
March, and April ; E. herbacea and carnea from January to April ; Primula vulgaris and its varieties from March to May; P. auricula in April and May; P. marginata, March and April, as well as several of the Swiss species; the beautiful species of Soldanella and Cyclamen from Februaly till April ; and of Phloxes, divericata, subulata, setacea, nivalis, crassifolia, verna; many of the genus Narcissus from March to May; Galanthus nivalis, Leucojum vernum, pulchellum, trichophyllum, from January to April ; Tulipa sylvestris, repens, attiaca, biflora, occulus-solis; the varieties of Gesneriana, præcox, and others; many species of Fritillaria, from March to May; Tritoma during the winter ; Erythronium, all the species ; Bulbicodium vernum and Gagea lutea, March and April; many species of Ornithogalum, and also of Scilla, Muscaria, and Hyacinthus, from March to May; several of the genus Berberis, and most of the genus Helleborus, flower during the winter, as do also Eryanthus hyemalis and Sibericus; the whole genus and varieties of Hepatica, most of Anemone, \&c. From such a list there can be no great difficulty in furnishing the parterre garden from the season when the flowering of its summer tenants is past, until the period arrives in spring when they may be again planted with safety. They do not, however, produce such an amount of colour as to warrant their being planted in the grouping manner. They are better adapted to the mixed style: and here probably the taste of many may accord with our own, that this change in arrangement may be equally consistent with good taste ; but, at all events, it produces a pleasing variety, relieving the eye and the mind from dwelling on the monotony and dazzling effects of masses of intense colour which the summer arrangement invariably produces.

Notwithstanding the facility by which a winter and spring display may be effected, it is strange enough that we so seldom see it even attempted, and this more especially where the flower-garden is placed in connection with the mansion, and where the proprietor spends the winter in the country. The plants we have enumerated are neither difficult to procure nor to transplant. All that is required is a good reserve-garden for their propagation and growth, and sufficient industry to remove them at the proper season. Some of them, such as bulbs, may remain in the parterre for two or three years without removal, and as they die down before the time of sowing or planting arrives, if the border's are top-dressed with a little fresh soil, seeds, for example, of annuals, may be sown over them; and when these are removed in autumn, the bulbs will be again starting into growth. Again, bulbs may be planted with very good effect along the margins of the beds in lines within 3 or 4 inches of the edging, which will secure them from being disturbed when the planting season arrives. In small beds intended to be sown with annuals in spring, the whole bed may be planted with bulbs, placing the taller-growing sorts, such as Narcissus, in the centre, dwarf tulips next, and the lower ones, such as Crocus, towards the sides. Here, with the varieties of colours, a considerable effect may be produced. With the variety of shades in crocuses, if attention be paid to their arrangement, a very pretty bed may be produced. All small beds may be planted thickly with bulbs, to be afterwards sown with annuals, or such small-growing plants as the dwarf Lobelias, Anagallis, \&c. As their roots are small, they can be readily introduced amongst the bulbs without injury to them; and as these, when their season is past, are pulled up, the bulbs will remain undisturbed. In the case of larger beds, intended for scarlet geraniums, yellow calceolarias, salvias, \&c., which are grown to some size before turning out, and having considerable balls, these should follow where winter - flowering or evergreen shrubs have been planted, or where such plants as hepaticas and hellebores have been grown during the winter and spring; and even in cases where the summer-flowering plants are pretty large, and not set within a foot or more of the sides, one or two rows of bulbs may be planted, and allowed to remain. In the parterre garden all tall straggling plants, and such as have large foliage, should be rejected ; for unless the beds are very large or broad, no plant exceeding $2 \frac{1}{2}$ feet should be admitted. In the mixed style of planting the case is otherwise; still tall coarsegrowing plants will never produce the same pleasing effect as those of moderate growth and abundance of bloom. One of the greatest errors committed in planting flower-gardens, in whatcver style, is the employment of too many species and varieties, because, where this is carried to the extremc, no harmony can be brought to exist amongst them. Where collections of species, or even of varieties, are cultivated, they should have a place especially assigned to themselves, because they afford a species of gratification peculiar to themselves, and that can never be obtained in the parterre; and if attempted there, it would destroy the whole effect which such scenes are calculated to produce. With seven plants, each of a simple or homogencous colour, all that is capable of being produced in regard to the harmonious arrangement of colour may be effected; and some even produce most satisfactory effects by the employment of only the three primary ones -yellow, blue, and red. We have entered into the details of this subject so fully in the section "Harmony of Colours," vol. i. p. 593, that a reference to that portion of this work renders further allusion to it here superfluous. Many of the plants employed for this purposesome of the strong-growing scarlet geraniums, for example-in rich soils, and even in poor ones in wet seasons, are apt to grow too luxuriantly, producing large foliage, and coming late into bloom. To correct this habit, it is well to retain them in the pots in which they have been brought forward, and to plunge them in the borders 2 inches under the rim of the pots. This also facilitates their being taken up in autumn. Pots about 7 to 10 inches in diameter are well suited to the purpose, and in such pots they may remain two seasons, or may be shifted in spring into a size larger, or have some 
of the soil removed from their roots, and be repotted in the same sized pot. The propagation of all bedding-out plants should be attended to in August and September, that they may become well established before winter. A mild hotbed placed behind a north wall is the most eligible, and in it the pots in which the cuttings are inserted should be plunged. When the cuttings are rooted, they should be planted off into 3-inch pots, shaded for a few days, and afterwards placed in cool pits, or frames covered with glass set in an open sunny situation. On the approach of frost in autumn, the salvias, heliotropes, and such tender sorts, should be removed to their winter-quarters first, followed by geraniums, verbenas, \&c., which are hardier. The best accommodation during winter is shelves near the glass, or in low pits capable of being heated, so that the frost may be excluded and abundance of air adinitted. Verbenas and calecolarias are impatient of fire-heat; they should therefore be kept in pits from which frost may be excluded by covering or otherwise. The same accommodation will be required for such plants as have been plunged during summer: on the appearance of frost they should be taken up, their branches moderately thinned and shortened if required, and be placed in pits or houses for the winter. Abundance of air must be admitted for some weeks after being taken in, and water rather freely given at the roots to prevent the leaves flagging, as some of the roots will liave been destroyed at taking up, having escaped through the holes in the bottoms of the pots. Afterwards and throughout the winter no more water should be given than is sufficient to keep the plants alive, and if possible none be spilt on the foliage, which would encourage dampness. Many make-shifts have to be made to preserve such plants during winter, and those who cannot do so will have much to regret when the planting-out season again arrives. Those who have not the accommodation of pits or houses will have to take up their geraniums, salvias, calecolarias, \&c., and reduce both their roots and branches, and plant them thickly in boxes of sand, and set them in a dry cellar, stable, or other place of shelter, admitting light and air to them on all favourable occasions. Verbenas winter ill in such places, as they require so much light; and heliotropes cannot be saved by such means, on account of want of a sufficient temperature. Where winter accommodation is limited, many defer propagation till spring, keeping only a limited number of plants over winter from which to obtain cuttings, which they strike in bottom heat and grow rapidly, so as to have the plants fit for turning out by the beginning of June. Such plants, liowever, sustain a check at planting-out which those kept over winter do not, as they liave become more woody, better furnished with roots, and hardier in constitution: besides, if shifted into larger pots in March, they have attained a much larger size, and consequently a less number of them covers the same space of ground. The beginning of Jume is as early as bedding-out plants can with safety be planted out, and every means should be employed to get them forward, of a good size, and sufficiently hardened off by ample ventilation and full exposure during fine days, that the transition may be the less felt by them when exposed altogether. A bright sunny day should be avoided, as also when the wind is strong, and a mild cloudy or rainy day chosen for setting them out. Water and shade the most delicate for a few days after planting. Avoid planting too close, particularly geraniums; each plant should have room to develop itself fully. We do not admire pegging down geraniums; and although it is very desirable to have the surface of the beds completely covered, we would rather sow mignonette to effect this end, having the scarlet geraniums growing out of it. Indeed, this makes a very interesting bed, particularly if large. A bed of scarlet geraniums, margined with a ring of the whiteleaved Cineraria maritima, has also a good effect, as has also a bed with variegated-leaved geraniums planted in the same way. Verbenas and similar trailing plants require to be closely trained to the ground, and for this purpose we place small stones on the shoots as they advance, which keeps them in their place, and induces the formation of roots from the joints over which the stones are placed. Verbenas, the dwarf-growing lobelias, cupheas, and similar growing plants, are best when planted in the soil, because they are not required to be lifted in autumn, and also because they do not overgrow their proper limits; but geraniums, calceolarias, heliotropes, and suchlike, we grow in pots, which corrects any grossness of habit a rich soil might induce.

\section{SELECT LIST OF BEDDING-OUT' PLANTS.}

Ageratum Mexicanum, blue; Anagallis Brewerii, carnea, grandiflora, cœrulea compacta, cœrulea grandiflora, rubra grandiflora, Monelli, under 6 inches; Bouvardia aurantiaca (orange), flava (yellow), under 12 inches; Comaclinium aurantiacum, scarlet and orange; Cuphea platycentra, strigulosa var. splendens, under 10 inches; Cyananthus lobatus, light blue, 3 inches high; Calystegia pubescens flo. pleno, peach, may be trained to the ground or used as a climber, rising to 3 or 4 feet; Cheiranthus Marshallii, orange, 12 inches; Cineraria maritima, employed for marginal lines on account of the snow-white colour of the whole plant ; Gazania rigens, pavonia, yellow with dark centres, 9 inches; Hesperis matronalis rubra, et albo pleno, 18 inches; Heliotropium azureum (blue), albicans (white) corymbosa (lavender), gem (blue and purple), Louis Napoleon (deep blue), Perciles (dark dwarf), Voltairianum, (deep blue), Souvenir de Liege, Triumph de Liege (lavender), Voltairianum nanum (dark blue, very dwarf); Iberis Gibraltarica (12 inches), sempervirens (6 inches), saxatilis (6 inches), all white; Lantana florida (rosy pink), purpurea (bright ruby), Sellowii (light pink); Lobelia Ajax (damson), amœna (light blue), azurea (light blue), cœlestis (violet plum), compacta (fine blue), erinoides (light blue), cardinalis (bright scarlet), episcopalis (light blue), favourite (violet), fulgens (intense 
scarlet), ignea (intense scarlet), Roi Leopold (fine blue), marmorata (blue), propinqua (deep crimson), Vesuvius (violet crimson), cornopifolia, erinus compacta, erinus maxima (all blue, and of dwarf habit), lutea (deep yellow, 6 inches); Linum flavum, yellow, 10 inches; Lophospermum erubescens, Hendersonii, scandens, spectabile, climbing plants of great beauty, or may be trained to basket-work round the beds ; Lotus Jacobæa, 12 inches, almost black; Maurandya alba, Barclayana (blue), elegans (fine deep rose), Emeryana (rose), climbing plants, or may be trained like Lophospermum; Mimulus atro-roseus (dark rose), Laingii (salmon rose), lateritius (buff orange), pictus (rich crimson), dwarfish in habit; Nasturtium, double orange, double scarlet, moderate climbers, or may be trained close to the ground; Nierembergia intermedia, calycina, filicaulis, under 15 inches; Enothera macrocarpa, riparia, taraxicifolia; Penstemon azureus (blue), gentianoides of the gardens, and its varieties, coccinea, splendens (scarlet), alba (white), argutus (blue), crassifolia (blue); Rhodochiton volubile, a neat climber, or may be trained to basket-work or hoops over the beds; Salvia azurea (blue), chamædroides (blue), fulgens (scarlet), patens (blue), splendens (scarlet), from 2 to 3 feet; Senecio elegans (red), superba (red), alba (white), 18 inches; Selago corymbosa, blue, 18 inches; Trachelium cœrulea (blue), album (white), 2 feet.

Petunias.-Attraction, deep mottled; Berryer, striped and spotted; Captivation, white; Cassandra, striped; Crimson king, rich deep crimson; Ellen Mavourneen, blush ; Enchantress, large blush; Mrs Low, brilliant crimson; Merides, fine rose; Pourpre royale, crimson purple; White giant, white -2 feet, may be pegged down.

Antirrhinums.-Alba plena, white ; Beauty supreme, purple striped; Brilliant, crimson; Cretia, fine scarlet crimson; Emperor of China, blush, spotted; Hendersonii, white, petals marked with strips of deep carmine; Lord Brougham, crimson splashed; Maid of Pertll, broad stripes; Mountain of snow, fine white; Mr Bowles, crimson stripes; Ochroleuca, pink, buff, and yellow; Queen of the yellows, yellow; Queen Victoria, yellow striped; Tricolor, white, puce, and gold; Van Aerschost, crimson and yellow ; Village maid, white and lilac; Vittatum, rose and crimson-18 inches to 2 feet.

Shrubby calceolarias.-Ajax, brown and yellow, fine habit; Amplexicaulis, fine lemon; Aurantea multiflora, orange; Bertha, yellow and dark spot; Brilliant, rich shiny bronze red; Candidate, fine orange; Crimson king, deep crimson; Gem, crimson; Golden chain, fine golden yellow; Hero, dark crimson ; Kayii, dwarf, early, yellow; Kendle's superb, dwarf crimson; Kentish hero, orange bronze; Multiflora, yellow; Model, brown, shading off to orange yellow; Magnificent, rich crimson, with yellow crown; Phobus, orange, with crimson spotting; Shankleyana, large orange crimson; Sulphurea splendens, fine large yellow; Sultan, fine large crimson ; Tom Thumb, crimson ; Viscossima, deep yellow; Wellington hero, fine golden yellow.

VOL. II.
Verbenas.-Adonis, white and lavender mottled; Albina, fine white; Alboni, blush white with crimson centre; Andrew, violet blue, large truss; Andromaque, deep bright rose; Antigone, best salmon-rose; Avalanche, white; Bride, white; Beauté suprême, white, deep-rose centre; Belle Melanie, white, deep maroon eye; Camille, rose, scarlet eye; Casanova, rich plum crimson ; Celina Mallet, fine white ; Chauvierii, crimson scarlet with white eye; Conquerant, deep orange scarlet; Coquelicot, orange scarlet; Coresus, plum purple ; Defiance (Robertson's), fine orange scarlet; Duchcss of Kent, white, deep crimson centre; Eucelode, rose, dark eye and centre; Favourite, fine purple, white centre; General Rampière, scarlet, and crimson eye ; George Sands, rich scarlet; Gloire de Paris, red crimson; Goethe, deep blue; John Salter, orange scarlet; Juliette, ruby crimson, dark centre; King, pink; La Camarge, good blue, light eye; La Flamboyante, bright deep scarlet, white eye; Louis Napoleon Buonaparte, crimson; Macrantha, fine rose, dark centre; Madame Baenrod, white, with deep crimson eye; Madame Denis, crimson, with large red eye; Madame Legros, pale blue, red centre; Madame Malet, carmine, deep crimson eye; Magnificent, rosy pink, very large; Maria Stuart, good purple blue; Melinda, fine bedding scarlet; Melindres, scarlet; Mirabeau, deep crimson scarlet; M. Jullien, deep scarlet and white eye; Mont Blanc, fine white, large truss; Mrs Mills, deep violet blue ; National, deep crimson ; Olga, white, red eye; Petrus Morel, rosy salmon; Pomone, light blue ; Perfection, white ; Racine, bright rose, dark centre; Richelieu, large rich ruby crimson; Rose unique, cherry colour; Rubens, red carmine ; Standard, bright rose ; Sulphurea, sulphur-coloured; Tweediana, scarlet; Virginius, good violet blue; Zelia, bright rose, crimson eye.

Scarlet, white, and pink flowering geraniums.Andry, fine large scarlet; Beauty of St John's Wood, scarlet, close habit, and free bloomer ; Bishopstowe scarlet, brilliant scarlet; Cerise unique, chcrry red; Cottage maid, dark scarlet; Flamingo, vermilion ; Hark-away, very dwarf, and compact scarlet; Hendersonii, white; Judy, dwarf, rosy salmon; King of Nepaul, bright scarlet; King of scarlets ; Kingsbury pet, bright rosy salmon; Le Titien, fine carmine, very dwarf; Lilliputian, very dwarf scarlet; Nosegay, both the pink and scarlet varieties well worth cultivation, but from their straggling growth are better adapted for training against a wall than for the parterre; Oriflamme, fine scarlet; Pet superb, bright pink; Princess royal (Ingram's), very dwarf scarlet; Punch, large bright scarlet; Skeltonii, pure white; Shrubland superb, scarlet; Tom Thumb, brilliant scarlet; Tom Thumb's bride, bright pink ; Tom Thumb's master, fine; Trenthan scarlet, fine: Triomphe de Meaux, deep scarlet; Triomphe de Paris, intense bright scarlet, dwarf, and profuse bloomer.

Variegated - leaved geraniums. - Attraction, scarlet; Bridal bouquet, fine scarlet; Dandy, quite a miniature, fit for vases; Flower of the day, scarlet, shy grower; Golden admiration, scarlet, foliage finely marked; Golden chain, 
scarlet, and, with the last, good for edgings to other beds; Golden circle, scarlet ; Lady Plymouth, prettily variegated with silver; Lateripes variegata; Mangles', silver-leaved; Mountain of light, fine.

Sweet-scented-leaved geraniums. - Betulæfolium; Citriodorum majus, and its varieties, elegantissimum, roseum, and grandiflorum; Coquette; Delicatissimum ; Delictum ; Emilia ; Exquisita; Fairy; Imogene; Jetty Treffz; Lobatum; Odoratissimum, and its varieties erectum, variegatum, \&c.; grandiflorum; Pandora; Prince of Orange, and its variegated varieties ; Quercifolium ; Rose-scented, with its varieties, majus, odoriferum, and nervifolium.

\section{§ 9. THE RESERVE-GARDEN.}

No flower-garden can be maintained with a succession of bloom throughout the greatest part of the year without having such an adjunct in the same establishment. The situation of such a garden may be in some convenient place, shut out from the principal gardens. It should contain pits and frames for propagating, forwarding, and retarding plants according to circumstances; of these abundant examples are given in vol. i., section PITs, and to these turf pits and other economical contrivances may be added. It is important that such a piece of ground be enclosed, sheltered, and in some parts shaded; if by walls, so much the better. It should also be so arranged that other parts are fully exposed to the sun and air; and it should have prepared beds of various soils, more in the nursery than in the flower-garden style, for the cultivation of plants intended to be removed when coming into flower to the borders of the flower-garden, also beds formed of sand or coal-ashes for plunging pots in. The beds in which perennial plants are to be grown should be composed of turfy or fibrous soil, as plants (not in pots) can be removed from such with much greater safety than were they composed of light finely-pulverised compost. We have found a mixture of various species of the commoner mosses, flax-dressers' refuse, \&c., exceedingly valuable in such cases, the plants attaching themselves by their roots freely to such substances, and lifting with excellent balls. Indeed, as has been exemplified by Mr Ferguson of Stowe, plants, such as verbenas, \&c., struck by cuttings planted in semidecayed moss, about 3 inches in thickness, may be safely removed at an early stage of their growth, by cutting the moss into pieces of 2 or 3 inches square. Successive sowings of the best annuals should be made, that a constant supply may be secured for making up blanks in the parterres; these, if well watered prior to removal, choosing a cloudy or rainy day, remove well. Most of the best Californian annuals are very hardy, and of these several sowings should be made in September and October. Most of them will survive the winter, and will be in excellent condition for transplanting in February for an early bloom, to be followed after their season is past with planting-out things. Roses, particularly of the autumnal-blooming kinds, should be grown in pots, and kept plunged till required; and where the expense of pots is not gone to, and the plants grown in the soil, $\mathrm{Mr}$ M'Glashan's smaller transplanter may be had recourse to. Both roses and all hardy shrubs intended to be kept in a portable state should, if in pots, be taken up during summer, and all roots protruding beyond the pots cut off, while those growing in the free soil should have their roots shortened back by thrusting down a semicircular-shaped sharp spade, of such a radius that two insertions of the instrument may encompass the ball of soil necessary for the support of the plants. All plants grown in such a garden should be allowed plenty of space, so that each may develop itself fully; they should be pruned and trained according to their natural habits, or the purposes for which they are intended. Bulbous-rooted plants remove well; and in the case of scillas, crocuses, and similar small growers, they should be planted in patches so as to be taken up in pieces about 9 inches in diameter. All American plants remove safely; and azaleas, rhododendrons, kalmias, \&c. may be taken up just as they are coming into flower, transferred to the flower-garden, and returned when their bloom begins to fade to make way for other things. In this garden, also, hundreds of scarlet geraniums, heliotropes, verbenas, and similar flower-garden plants, should be brought forward in pots to be ready to fill up beds from whence other plants have been removed. There are some shrubby plants that will not bear removal with safety, unless grown in pots, of which the double-flowering whin, Ulex europea, all the genus Spartium, Genista, Cytisus, Adenocarpus, Ononis, Anthyllis, which are very scantily furnished with fibres at their roots, may be given as examples. Phloxes, œnotheras, campanulas, asters, veronicas, remove freely, but penstemons, alyssums, and similar rooted plants, with much less success. The numerous varieties of wallflower and stocks readily transplant, even when coming into flower. The great majority of showy flowering Alpine plants which do not root deep, such as Sedum, Saxifraga, Draba, \&c., may be lifted in large masses by introducing a broad spade under thern, and placing them on a board corresponding to their size, on which they may be carried to their destination, and the board left under them to facilitate their removal back to the reserve-garden, when it should be withdrawn. The hepaticas; and most of the anemones, remove very freely, while the extensive genus Helianthemum, with its varieties, must be grown in pots ; and those of 6 inches in diameter will sustain a plant 2 feet in diameter. The same may be said of Saponaria ocymoides, many of the Dianthus, most of Gypsophila, Silene, \&c. It is not the number of species and varieties that are adapted to successful transplantation that ought to be so much a consideration with the flower-gardener, as those which are adapted for removal, and are possessed of such properties as are adapted to the parterre-moderate height, fine habit, profusion of bloom, and, above all, that the colours be bright if primary ones, or of suf- 
ficient depth of shade if secondary or neutral ones. Much of the success of these operations will depend on the state of the weather; that which is cloudy and moist being the best, a bright scorching sun or evaporating wind being the worst. The state of the soil is in the hands of the operator; and we need hardly remark that it should be moist from rain, or rendered so artificially, not only where the plants are to be set, but also where they are to be taken from, and as little dependence placed on shading as possible; nevertheless, in some cases this will be found necessary.

\section{$\S 10$. INSECTS INJURIOUS IN THE PLANT-HOUSES AND FLOWER-GAR- DEN.}

In noticing the insects most injurious to plants in plant-houses and flower-gardens, we have deviated from the course followed in the culinary and fruit gardens, where the insects peculiar to each plant and tree are noticed under the head of the plant or tree upon which they are found. In the case of plant-houses and flower-gardens, many species of plants are infested with the same species of insect, and therefore, to prevent repetition, we have placed the insects most injurious to these departments in a section by themselves.

We have noticed those that are most destructive; and although, in some cases, the descriptions are brief, we trust they will be such as will enable the cultivator to determine the genera and species of his insect enemies, while our notices of their habits will enable him to wage a more successful warfare against them. To have even enumerated every species of insect which exists in our gardens, far less to have given a detailed description of them, would have far exceeded our limits, and indeed have been of little practical advantage to the cultivator. We may further remark that whatever directions may be laid down for the suppression of inscets, unless these are followed up with energy and unremitting perseverance until the end is accomplished, all superficial attempts will only be time and labour lost.

The mealy bug (Coccus adonidum Linn.) is a sad pest in tropical-plant houses, some plants being exceedingly liable to be attacked by them, while others are comparatively seldom visited. We have noticed this insect, p. 639; - to what has been there said regarding it, we may add that frequent syringing with pure water applied with force, and keeping up a very high and humid atmosphere, is believed to be the best wholesale mode of destroying them. Such plants as Coffea, Musa, Canna, Hoya, Alpina, Stephanotus, \&c. are most subject to them (ride p. 661). Of the Coccus family, Ruricola, in "Gardeners' Chronicle," speaks in the following terms:"The scale insects vary so considerably in their habits that they may with great propriety be divided into three sections, which will form as many genera. lst, There is the true or tropical coccus, the female of which is covered with a scale inseparable from the animal, - familiar examples are the C. hesperidum and C. vitex of Linnæus. 2d, A large number is distinguished by Bouche, under the name of Aspidiotus, of which C. nerii of that author and C. bromeliæ are examples, - in this group the females are covered with a scale which does not adhere to the animal. 3d, Those species, comparatively few in number, I believe, whose females are not furnished with scales, but are merely powdered over,-they are very different also in not being stationary, but run about when full grown as well as when young, of which C. adonidum is the type."

The oleander scale (Aspidiotus nerii Bouche), fig. 267 , said to have been imported from

Fig. 267.
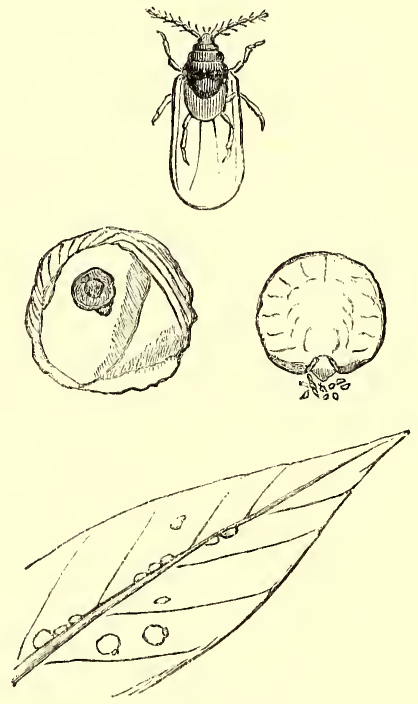

OLEANDER SCALE.

America, is found on most plants of this family. The female is larger than the male, and appears like a round flat shield, inclining to yellow, almost destitute of legs; it feeds on the leaves by sucking the sap out of them with its rostrum. The shield of the male is quite white, the perfect animal brownish yellow, with white wings. Although called the oleander scale, it is found on many other plants, both in the greenhouse and stove. It is most difficult to suppress when once it has been introduced into a collection. One remedy is to cut the plant over by the surface, and re-grow it again in a house or pit where no scales have been admitted. Washing the leaves and branches with soap and water is perhaps the next only remedy.

The orange scale (Coccus hesperidum Linn.)In form similar to the last; in colour differing, inasmuch as this appears as a nut-brown shield; equally abounding with the other, and in similar situations attacking not only the orange tree, but other trees also, the camellia in particular. The same means of suppression must be used as in the former case. 
The pine-apple scale (Coccus bromelice) infests that fruit under bad cultivation.

Aspidiotus echinocacti (Bouche.)-The female very much resembles $A$. nerii, only that the shield is more oblong, and of a darker colour; the male is orange or yellow, the pupa linear, powdered with grey, and doubly furrowed. As the name implies, it is found on the Echinocactus, and also on most other of the Cactus tribe. As it nestles amongst the spines and in the deep angles of the plants, its destruction is difficult. The fumes of a hotbed of stable manure in a high state of fermentation is the only means we have found of destroying them, while in such an atmosphere most of the Cacti luxuriate during their growing season.

The small brown scale (Aspidiotus proteus), fig. 268, had escaped the notice of entomologists

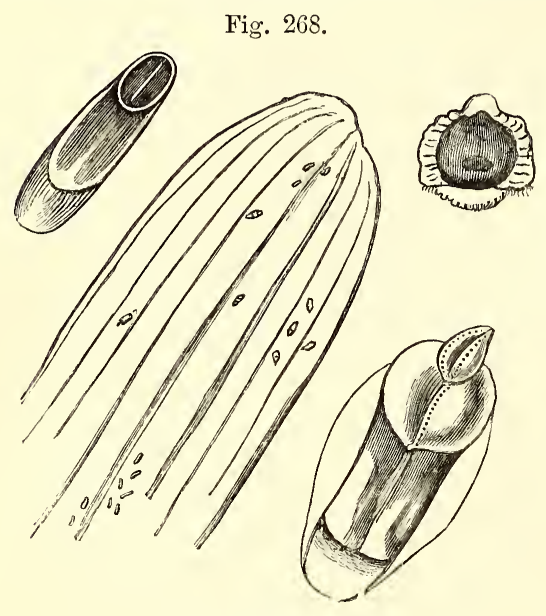

SNALI BROWN SCALE.

until about 1843, when it was detected and described by the writer of the entomological articles in the early numbers of the "Gardeners" Chronicle." It is said to be very abundant in plant-houses, and was first discovered on the leaves of aloes and amaryllises. It belongs to the second section above noticed, and is named proteus, from the variety of forms it assumes. In regard to the destruction of the scale insects in general, the late T. A. Knight tried a dressing of lime and flour of sulphur with beneficial effects.

The brown limpet scale (Coccus patelloformis) is described as being the largest of the family that infests our plant-houses. It is, however, fortunately not very often met with; it is nearly allied to $\mathrm{C}$. hesperidum, but is larger and of greater length. The females are in general found with their heads downwards, and vary very considerably in size and form, some being oval, others more elliptical, while some are slightly curved.

The turtle scale (Coccus testudo), fig. 269, is found chiefly on tropical plants requiring a high temperature. The scale is oval, convex, and of a dark-brown colour. Besides those already de- scribed, there are C. rosæ (rose scale), C. Lauri (sweet-bay scale), and it is possible some others imported along with plants from the tropics.

Fig. 269.

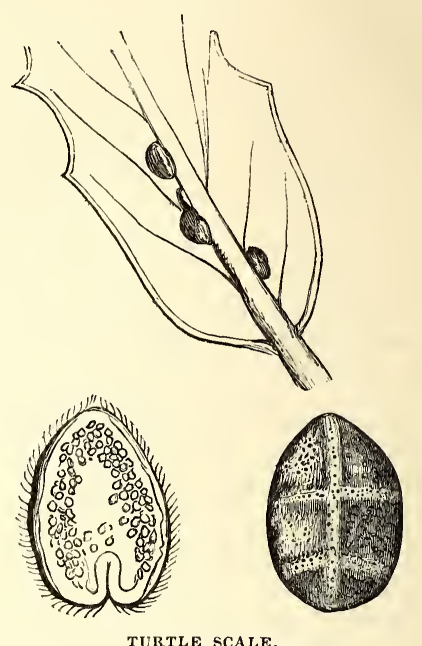

The rocket tinea moth (Tinea hesperidella Hub., T. porrectella Linn.), fig. 270, is often most destructive to the rocket plants, and may

Fig. 270 .

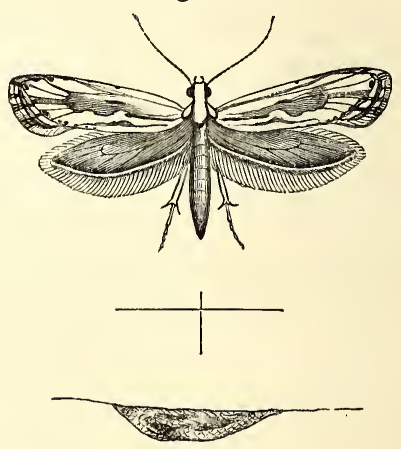

ROCRET TINEA MOTH.

be detected where the leaves are found, as it were, glued together, on opening which a small green caterpillar of different shades of colour, according to its age, will be found feeding on the tender leaves, sometimes alone, and at other times accompanied by five or six in a younger stage. It is conjectured that there are two broods a-year, and in some measure a succession of them, for perfect insects have been found during the entire month of June, as well as of May, and also in August, and occasionally so late as the beginning of October, and that not only on the rocket, but on plants of allied genera. The larva tapers towards both extremities, has six pectoral, eight abdominal, and two anal feet. 
The body is divided into twelve segments, each having about a dozen black spots or warts, each of them producing a short black hair ; a dark line of green runs down the back. The head is somewhat ochreous. They form beautiful cocoons of network, within which the caterpillar changes in a few days to a pale-green chrysalis, slightly tinged with ochre on the head, wing. sheaths, and tail, and with a double brown streak down the back. About the beginning of May the moths appear. The head, feelers, and horns are white, the thorax cream-coloured, the sides brown, upper wings pale clay-brown, with whitish streaks, and lance-shaped; a pale streak runs along the inferior margin, the edges waved and brownish, forming a dark line at the base; a dark streak appears at the centre, as well as a line of black dots along the costal margin; the posterior margin is dark, frcckled with black and light grey, interspersed with specks of ochre; the wings expand to seven lines; the under wings, legs, and abdomen are of a slate colour. The wild rocket, in some seasons, abounds with them; and the fine double flowers of the florist's choicest varieties are often entirely destroyed. The only feasiblc remedy appears to be crushing the young caterpillars between the leaves on their being first observed.

The narcissus fly (Merodon narcissi), fig. 271. -The grubs of this insect are often found in the
Fig. 271.
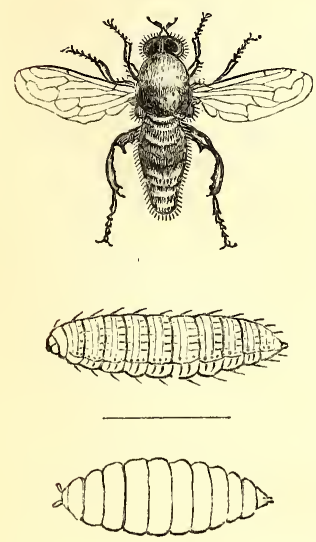

NARCISSUS FLY. very centre of the bulbs of many species of narcissus, which they frequently destroy by eating away their interiors In November, or at the time of planting, the bulbs should be carefully examined, and if one or two pretty large roundish holes be found in their outside, it is pretty certain that the maggot of this insect is within, where it will remain feeding on the heart of the bulb during summer and autumn; the slimy matter discharged from its body during its long imprisonment causing the bulb to rot. About the end of November, the maggot is changed into a pupa, at which period it eats its way out of the bulb near its roots, burying itself in the adjacent earth. They remain in this state till the following spring, when the flies issue from them. Upon what part of the plant the eggs are deposited is not very satisfactorily known. In April, the flies hatch, and have much the appearance of small humble-bces, and are like them in colour, being yellow, orange, and black. The only remedy against their multiplying is to remove every bulb that does not send up leaves at the proper season, and to destroy them, for in such are the maggots to be found. If left in the ground, the increase of the insect would be facilitated.

Tipula oleracea.-The larvæ of this very common insect are very injurious to the roots of the ranunculus. The only remedy appears to be to take up the roots carefully as soon as the leaves begin to flag, and remove the larvæ from them, returning the roots again to their former place.

Psylla buxi Geoffroy, Chermes buxi Linn. -This small homopterous insect is frequently found on the common box (Buxus communis). It attacks the leaves, chiefly of the side branches, and by perforating them, causes them to curl up in a rather remarkable manner, resembling little balls, and more or less hollow. The following curious discription of this insect is given in the "Gardeners' Chronicle," and although somewhat prolix, gives so clear an insight into its economy that we give it entire. "On opening one of these balls about the beginning of June, a quantity of white powdery grains will be found, amongst which is a variable number of minute flattened green insects of an oblong form, with short slender antennæ and six legs; in addition to which there are others of a larger size and somewhat broader form, with flat semi-transparent lobes on the sides of the body; these, as well as the smaller ones, often have attached to the extremity of the body a long white filament, like a short bit of thick coarsc white thread, particles of which, of various shapes, are also to be observed on the leaves of the box, as well as hanging about the ball of leaves. The smaller insects are the larvæ, and the larger ones the active pupæ of the psylla; the flat lobes at the sides of the latter are the cases in which the future wings of the psylla are developed, and the white filaments and grains are nothing less than the excrement of the insects. The instrument by which these insects obtain their nourishment, and at the same time cause the alteration in the growth of the leaves, is a short jointed rostrum attached to the under side of the body, so far back as to be close to the fore-legs; within this rostrum are several delicate threads which the insect has the power of protruding to a great length, and of which the points are thrust into the substance of the leaf, so that it is sometimes difficult for the insect to withdraw them when disturbed. The operation of puncturing the leaf is doubtless attended with the introduction of a certain amount of fluid, which acts as an irritant upon the adjacent vessels, and causes the abnormal growth above described. The precise nature, however, of the action which takes place in this and other analogous cases has yet to be discovered. After remaining a few days in the pupa state, the insect assumes the winged state-the wings which had previously been enclosed in the flattened horizontal lobes being now carried in a slanting direction like the roof of a house; and the insect, instend of being one of the slowest in its movements, has not only now acquired the power of flying, but leaping with great activity to a considerable distance. The perfect insect measures rather 
more than a quarter of an inch in the expansion of its fore-wings. It is of a beautiful green colour, with several reddish-brown spots on the thorax. The fore-wings are of a pale buff colour with green veins, and the hind-wings are quite transparent; the antennæ are yellowish, with tips dusky. The female has a horny ovipositor at the extremity of the body, with which, in all probability, she forms slits in the twigs in order to deposit her eggs, which are not hatched till May in the following year. The place, how ever, in which the eggs are placed has not yet been observed; but the strong horny texture of the instrument for oviposition seems to indicate the habit similar to that of the Cicadæ, or saw-fly." The broad-leaved box, especially when it has attained some size, is more liable to its attacks than the narrow-leaved varieties, and more so when grown as a shrub than when planted as edgings.

The black horned leaf-miner (Phytomyza nigricornis), fig. 11.-This is one of the many of the smaller species of moths, flies, and beetles, which deposit their eggs either on the under side of the leaves, or probably, in some cases, under the skin or cuticle of the leaves of greenhouse and flower-garden plants. Although much less injurious to the general health of plants than many other insects, the whole of these minute miners are vexatious enough from the sickly appearance their presence gives to plants. The Phytomyza nigricornis, in its maggot state, carries on its depredations by burrowing between the upper and lower cuticles of the leaves and devouring the parenchyma, in which it forms long and irregular galleries, and there changes into its pupa state, and afterwards, into the perfect fly. The female is somewhat larger than the male, and both, when fully grown, are of a dull ash or slate colour; the head is ochreous in front, of a dark slate colour behind; the antennæ are small and drooping; they are dark-coloured, compressed, and formed of a small basal joint, a second cup-shaped, and the third orbicular; the eyes are remote in both sexes, small, blackish, and round; the thorax is somewhat globular, quadrate, and very grey, as well as the scutellum; the abdomen is short, narrower than the thorax in the male, slightly tapering towards the point, which is incurved and obtuse; in the female it is broader, ovate and conical at the tip; wings much longer than the body, somewhat dusky, irridescent, ochreous at the base; the costa downy, with a short basal nervure, and two others stout and of a dark colour; there are also three longitudinal ones slightly marked, and uniting at the base, but having no transverse nervures on the disc; legs moderately long and black, tips of thighs and tibia of an ochreous colour; halteres pale yellow and clubbed. They are often found on the foliage of Cinerarias, and similar softwooded plants.

The pansy fly (Agromyza violce), fig. 272.-Of the economy or natural history of this insect little appears to be known beyond its being exceedingly injurious to the flowers of this plant. By May they become abundant in some localities, and continue increasing until the cold of September cuts them off. In August they so abound in some gardens, that three or four may be detected on a single flower, which they perforate in innumerable holes, causing the colours to run, and entirely destroying the beauty and

Fig. 272.

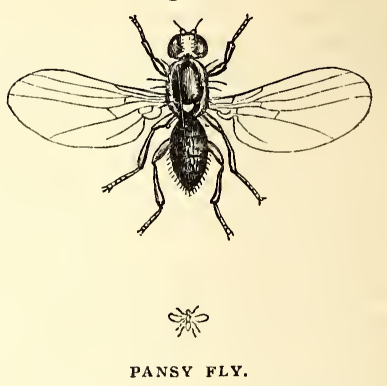

value of prize flowers. The insect is of a shining black colour, and slightly covered with bristles of the same colour, the head and antennæ being bright orange; sides of thorax and scutellum' sulphur colour; wings large, irridescent, and transparent; the sub-costal cell short, divided by a nervure running across it, the three following longitudinal nervures nearly parallel, and extending to the outer margin ; the fourth longitudinal nervure greatly devaricated, and joined to the third by a transverse one, the second and third united near the base by a very short nervure. The whole insect small, about one-eighth of an inch in length; legs pitchy black; anterior tibiæ and tarsi variegated with rusty colour; poisers yellow. In some specimens the thighs are sulphur-coloured on the under side. No satisfactory means have been fallen upon for the destruction of this minute pest; nor is it likely any will be, while its habits are so little known. For prize pansies in frames it is worth trying the fumes of Cayenne pepper, produced by sprinkling it upon hot plates of iron.

Oniscus asellus Linn., O. murarius Fabr., fig. 266.- The genus Oniscus is well known in various parts of the kingdom by the popular names of woodlice, carpenters, slaters, millipedes, sowbugs, pig's-lice, and old sows. There are two distinct species discribed by entomologists, which in gardens are nearly always taken for one-our present subject and the following one.

O. Asellus does not roll itself up into a ball when at rest, which the following one does, a distinction sufficiently obvious to have induced very superficial observers to have drawn a broad distinction between them. $O$. Asellus has its antennæ composed of eight joints, the fourth being nearly as long as the fifth, while the terminating one forms a sharp bristle. The body is ovate, slightly convex, composed of fourteen segments, including the head, which is broad, having two distinct tubercles, one on each side, immediately before the eyes. The tail is furnished with four styles and a central spine. The antennæ are long, angular, and hairy towards the point; the head is of a lead colour ; the back is dark, variegated with yellowish spots; 
seven of the segments have a large dark spot on each; the legs, of which there are seven pairs, are five-jointed, terminated by a claw, and finely ciliated on the inner sides. When young, this species is sometimes red, or of a lead colour ; when fully grown, of a yellowish white, the upper side rough and warty, variegated with brown or lead colour.

Oniscus armadillo Linn., Armadillo vulgaris Isatreille, is at once distinguished from the last by its rolling itself up into a ball when disturbed. The legs are short, somewhat hairy towards the apex, and seven-jointed, the fifth joint twice as long as the fourth. The body is elliptical, very convex, and comprised of fourteen segments ; the head is broad, the tail is not prominent, and the lateral appendages are short and broad. The body on the upper side is of a deep lead colour, and punctured; the sides of the segments are not dilated, but droop below the abdomen; it has seven pair of legs, which are six-jointed. The eggs of the Oniscus family are first deposited in an elliptical bag beneath the abdomen of the female, and when they are hatched, the mouth of the pouch or bag opens, and the young brood sally forth, and as soon as this takes place the mother closes it again. The end of summer is the season when breeding takes place. They shun the light, and during the day hide themselves under boards, pots, or in crevices of walls, or, indeed, in any dry dark place. A toad or two will soon clear a pit or frame of them; and slates or small flower-pots inverted, and set about, will be found excellent traps for them, as they will take shelter under them, and may be afterwards easily caught and destroyed.

The holly-leaf fly (Phytomyza ilicis Curtis), fig. 273 , often attacks the leaves of the holly during the month of May, giving them a blotched appearance, as if the surface were scalded in numerous places. On lifting up the dried cuticle of the leaf where these blotches occur, a small pale greenish larva, sometimes a small ochreous brown oval pupa, will be found. This is the pupa of the Phytomyza ilicis. In its fly state it is of greyish-brown colour, covered with small black hairs interspersed with larger bristles, particularly over the head and thorax. The head is somewhat ovate, transverse, the face concave and yellowish; ocelli three, placed in triangle of the crown; antennæ small; crown inserted in a cavity in frout of the face; the three basal joints are stout, the first small, second bowl-shaped, the third the largest, compressed and sub-orbicular; thorax sub-globose, yellowish on the sides; scutellum trigonate; abdomen elliptical, pale at the base, with six segments in the male, and seven in the female. She is also furnished with a retractile ovipositor. Wings large, broad, and oval, longer than the body, and covered with minute pubescence; nervures brown, subcostal, and very short; legs nearly of equal length, brownish ochre, covered with dark hair; tibiæ short; tarsi as long as the shanks, and five-jointed; thighs stoutish ; claws very small; basal joint elongated. It does not appear, from our present limited know. ledge of this insect, that any remedy can be offered, farther than gathering the affected leaves and burning them. Leaves attacked are

Fig. 273.
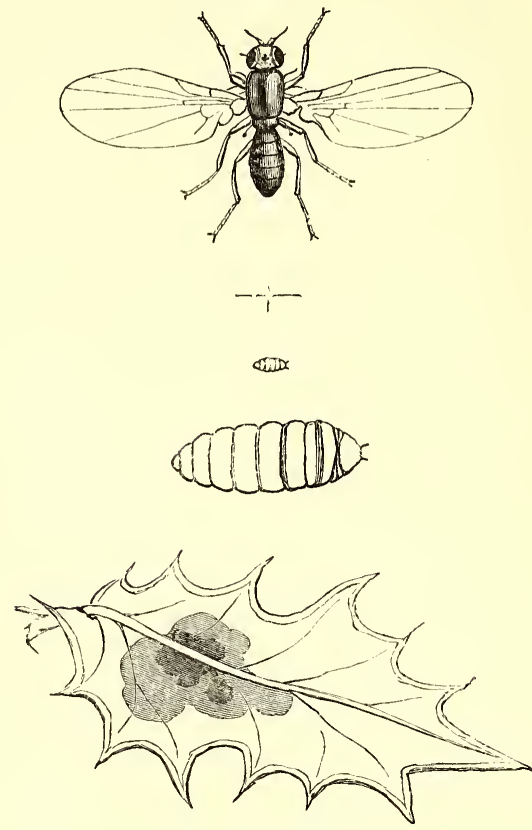

HOLLY-LEAF FLY.

readily aistinguished, from their spotted appearance.

The cockroach (Blatta orientalis). - These are serious enemies when once established in orchid-houses (vide p. 695 , and fig. 265), where they prey upon the fleshy roots, and devour them as fast as they are formed. They also attack the roots of the pine-apple. Their fecundity is as surprising as their voraciousness. They carry on their depredations chiefly during the night, and secrete themselves during the day in holes and places where it is next to impossible to reach them, always choosing both dry and warm quarters. The males alone have wings, and are less in size than the females, which latter; particularly when loaded with a heavy bag of eggs, which they carry about with them for days until they find a place suitable for depositing them in, are ill calculated for flying, and indeed do not travel further than to secure sufficient food. This may be an important reason why they are so difficult to subdue, notwithstanding hundreds may be trapped or killed week after week. It is probable that the majority of these are males, for they are exceedingly nimble, and travel a considerable distance from their nocturnal haunts, as may be seen by entering the places they frequent with a lighted candle, when they scamper off into holes and crevices so quickly that it is difficult to destroy them, although they cover the floor by hundreds. The eggs form an oval mass, enclosed within a 
pitchy black leathery case ; each of these is divided into sixteen cells, and each contains one egg. The young are able to run about as soon as they escape from the egg, and from this time forward they continue feeding and growing, and undergoing the various transformations, but with much less change in their appearance than is the case in most other orders of insects. The cockroach is not easily exterminated, and therefore, in addition to what has been said in sect. ORCHID-House, we may add that phosphoric rat-poison, spread on cucumber rind, and placed near their haunts, has been found by some exceedingly efficacious. They will sometimes eat the poison when laid down in small pieces, but the cucumber is thought to be a great attraction. Some assert that the cucumber rind is of itself poison sufficient for them, but of this we have doubts. A hedgehog kept in the house has been found useful, and wool dipped in spirits of turpentine, and thrust into their holes, will kill and expel them; a tablespoonful of spirits of tar, poured into the crevices they inhabit, is also equally fatal to them.

The rose scale (Aspidiotus rosce Bouche).The female of this species bears a very close resemblance to that of the oleander scale, A. nerii (Bouche). The pupa of the male is linearshaped, doubly furrowed on the back; like that genus, they have two wings, and under them two balancers. The perfect male is pale red, dusted with white, and has white wings; the length of the body is one-third of a line. The female is somewhat larger than the male, and, like it, of a roundish flat shield-like form, almost destitute of limbs ; they feed by sucking the juices of the plant by meaus of their rostrums. They inhabit the stems and older branches of the rose tree, which may be often found entirely covered with them, giving the parts affected a mouldy appearance. They are best subdued by cutting out as much of the parts affected as can be spared, rubbing the remaining scales off with a hard brush, or painting them during winter with spirits of tar.

The rose moth, Tinea (Ornix) rhodophagella (Kollar), appears in the moth state about the end of May. This very small but destructive insect is only about three lines in length; it carries its wings very close to the body, appearing as if wrapped around it. The whole body is of a silvery-grey colour, the upper wings marked with numerous very small black dots; the under wings are narrow, tapering towards the point, and margined with long fringes. The eggs are laid in May on the rose-buds, and the caterpillars are hatched by the end of June, when they immediately form small cases of portions of the foliage, and in them pass the winter, about an inch under the surface of the ground, at the foot of the trees. This indicates the most efficient mode of lessening their numbers, by removing the soil to the depth of 2 inches, and carrying it to a distance, or burning it.

The larva, in form of a small worm, ascends the trees, and commences the work of destruction upon the tender leaves and shoots as soon as they begin to form, devouring one leaf or shoot after anothcr, and often to the extent of not leaving one perfect on the whole plant. This little worm, or larva, is only about three or four lines long, yellow, with a black head and blackspotted collar.

The green rose-chafer, or golden rose-beetle, Cetonia aurata (Scarabcus auratus of some entomologists), fig. 274, is one of our largest and

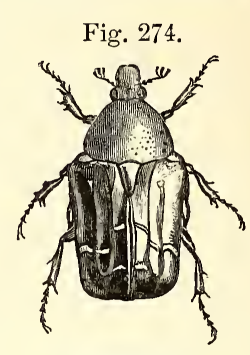
most beautiful beetles, easily recognised by its bright-green colour, sometimes reflecting a rich golden or copper tint. The wings are very long, of a brownish colour, folded under the horny wingcases, which have a few white lines placed transversely, resembling cracks, and scattered over them. On the under side they are of a fine copper tint,

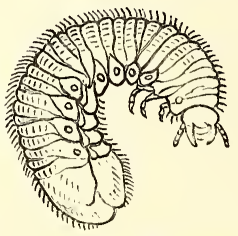

GOLDEN ROSE-BEETLE. looked. The havoc they make in the Parisian rose-gardens is fearful, compared with what we experience in Britain. "Le ver blanc," as they are there called, is the greatest enemy the French rose-growers have to contend with. Their habits in many respects resemble those of the cockchafer: We know of no means more likely to reduce the numbers of the rose-chafer than by capturing them in their beetle state, which, from their large size and conspicuous colours, is no difficult matter.

The rose chermes (Psylla rosa) is destroyed by sjringing the plants with a strong decoction of tobacco, frequently repeated.

The antler rose sawfly, (Cladius difformis Panza), fig. 275.-Several species of Cladius attack the rose in their larva state, devouring not only the foliage, but often the pith also. Their attacks on the foliage are first observed by it appearing perforated with innumerable small holes, which daily increase, as well as portions of the margins being devoured. The cause of this is only discovered by turning up the under side of the leaf, when a small caterpillar will be found either curled up or stretched out, feeding, and holding on by its feet to the under side of the leaf. In size they scarcely exceed half an inch, nearly cylindrical, tapering a little towards the tail; they have twenty feet-twelve abdominal membraceous or false feet, six pectoral, and two anal feet. Their colour is bright green, covered with short erect hairs, with a darkish line down the back, and one much darker on each side; the head is brownish and horny, with two small black dots on the sides. In its fly state, Cladius difformis is black and shining; the antennæ are downy, composed of nine joints; in the male the third, fourth, and fifth joints are hooked at the base, having a long branch at the 
top, while in the sixth it is very short, and altogether wanting in the rest. In the female the

Fig. 275.
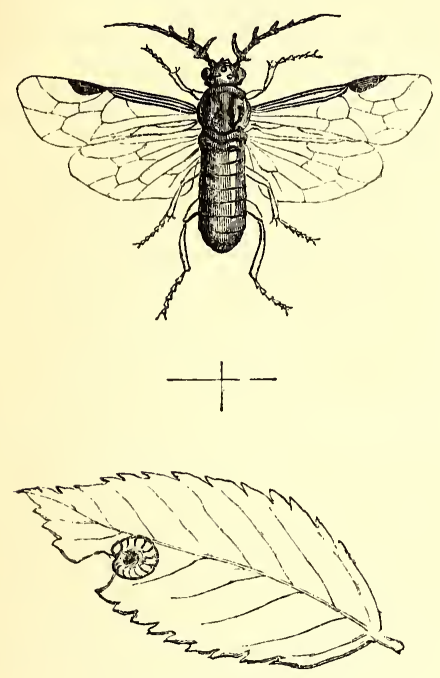

ANTLER-ROSE SAW-FLY.

hol'ns are unbranched and tapering; the wings, of which there are four, are irridescent, and slightly stained with a dark brownish shade; the stigma and nervures brown, the legs ochreous, thighs black, except at the tips. It is diffi-

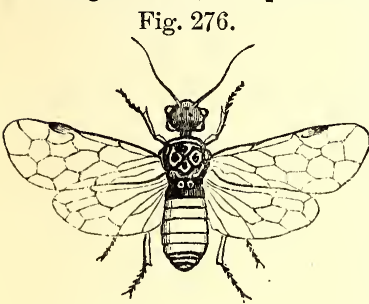

cult to point any remedy farther than what has so often been recommended for caterpillars in general, such as dusting the plants with hellebore in powder, or with snuff, watering with lime or soot-water, shaking the branches suddenly, and catching the enemy on a cloth spread below.

The rose caddice saw-fly $(L y$ -
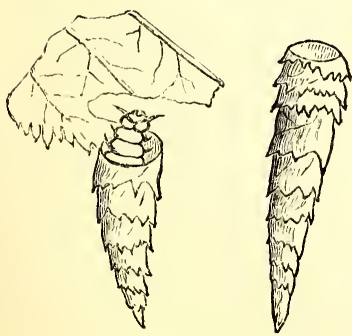

ROSE CADDICE - FLY VOL II. a pair of slender three-jointed feeler-like organs attached to the extremity of the last segment of the body. The fly appears about the end of May or beginning of June, is remarkable for its brilliant golden wings, as it may be seen on the leaves of the rose during hot sunshine, darting off with great velocity on being approached; hence its capture is most difficult. It is by no means a common insect in Britain, but has been found on roses at Hammersmith and elsewhere, com. mitting considerable havoc; and there is little doubt that its existence is more widely diffused, although probably not distinguished from others by cultivators. From the end of June and through July, it has been discovered concealed in curious cases, formed of bits of rose-leaves cut into strips, and carefully rolled up into a long cylinder-like form, one end of which remains attached to the leaf, while in the broken end there is an orifice, by which the caterpillar makes its escape. It destroys the foliage in the construction of its case, cutting off by degrees one edge of the leaf into a strip about one-eighth of an inch in width, while at the same time it sustains itself by feeding on the leaf. This strip is lengthened as the insect proceeds upwards or downwards, one end of the strip being fastened to its body, while it winds it as it is formed round itself, until the case is fully formed, and itself is enshrouded, when it detaches the strip from the leaf, and drops to the ground about July, and remains in the pupa state till the following May. The whole economy of this insect is most curious, and worth the attentive observation of the entomologist.

Megachile centuncularis is one of the carpenter or leaf-cutting bees which attack the leaves of plants, as much, probably, for the purpose of constructing their nests as for actual food. The rose and common laburnum especially suffer from their attacks. The genus is numerous. The present subject may serve as an illustration of the whole. The perfect insect measures about an inch and a half when its wings are fully extended, of a pitchy colour with yellowish legs, the abdomen ringed with white lines, and the whole covered with yellowish woolly hair. In the construction of its nest it cuts out circular holes from the leaves of roses, petals of geraniums, and various other plants. Its e.conomy has been thus described: "The creature -a short, stout, plain bee-mines a tubular channel into some decayed woody substance, boring in the direction of the fibre, making her repository at the bottom sufficiently deep for her purpose. She cuts from the leaf of a rose several large pieces, often a half, conveying them to the bottom of her cave, and rolling them up so as to form a case : in this she deposits an egg. The mouth of the cave is then covered with five or six circular pieces of leaves; fragments of wood like sawdust are then lodged over them, and the remainder of this channel filled up with other patches of the leaf, requiring, perhaps, twenty or more to accomplish it. It has been remarked of this tribe of insects, that they appear to be possessed of habits approaching closely to those of superior intellect. The pupæ are sometimes, but rarely, captured ; the only apparent 
mode of reducing their number is by catching the perfect insect in the act of leaf-cutting; and for this purpose Underwood's wasp-catcher, fig. 253 , will be found most convenient.

Tortrix Bergamanniana, fig. 277.-This differs little in general character from Tortrix nigri-
Fig. 277.
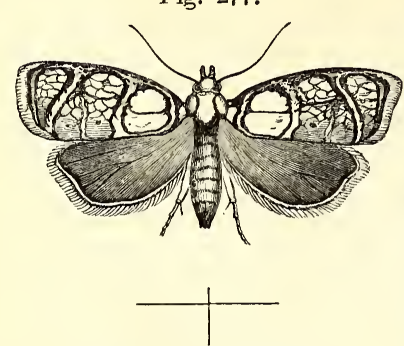

TORTRIX BERGMANNIANA. cana (fig. 233), the plumgrub tortrix, and is even more injurious to the rose than the other is to the plum. When the plants are much infested with the larvæ of this insect, it is better to cut them down at once, and burn the branches. This, and handpicking, seems the only way to get rid of it.

The Garden beetle, Phyllopertha horticolo (Melolantha horticola), fig. 181.-This pretty little beetle is often found during June and July feeding on the petals of roses, particularly white ones. It feeds also on the leaves of the apple and pear, gnawing them full of holes. In its perfect state it is about half an inch long, and nearly half as broad. Its wing-cases are reddish brown, shining, and rather shorter than the body, which, with the head, is dark green; antennæ reddish, with a dark-green club at their ends. The female descends into the ground about the latter end of July, and there deposits her eggs, from which the grubs are shortly produced, and commence feeding upon the roots of plants. The beetle is easily captured in the evening, when she may be found nestling amongst the petals of the white roses. During the day they are very active, and are difficult to catch. Handpicking them in that state, or searching for the grubs under ground, is the only means of destroying them.

The saw-fly of the rose, Tenthredo Athiops, (Klug and Hartig), Selandria Athiops.-During the month of June the leaves of rose-trees are often found assuming a withered brown colour, as if scorched by fire. The cause of this may be traced, by the help of a microscope, to the effects of this insect. The upper cuticle of the leaf will be found nearly eaten away, while the under side remains perfectly entire. A closer examination will show the enemy itself, in colour so nearly resembling the foliage that its detection by the naked eye is almost impossible. In its larva state it is nearly half an inch long, of a cylindrical form, with a dark line down the middle of the back. The head is orange-coloured, with a small black spot on each side. These larvæ change their skins several times before arriving at their full size, and then descend into the earth, where they form elliptical cells, highly polished on the inside, in which they pass the winter, and in which they become transformed, first into a pupa state, and afterwards, towards spring, into perfect insects. I their perfect state they are about one-sixth of an inch long, of a shining black colour, the legs pale buff, the thighs black, except at the tips. They deposit their eggs upon the roses, and the larva is hatched about the end of May. Syringing the roses with water in which hot lime and soot have been steeped, is one of the best remedies for the suppression of all tenderskinned insects on roses and other plants. We place a bag of soot in a tub of water, and add to it a few lumps of unslaked lime, allowing it to stand for a day or two. The water is then drawn off by a tap placed 5 inches above the bottom of the tub, and is applied with the syringe or garden-engine. The soot-bag may remain in the tub for a week or more, and a little lime is added occasionally. The tub is refilled as emptied with soft cold water.

Hylotoma rosa, in general appearance, greatly resembles the gooseberry caterpillar, and is exceedingly injurious to the rose, often stripping the entire foliage, as well as attacking the young wood. Its appearance extends from the end of May till the end of July, during which period brood after brood of larvæ are constantly feeding on the leaves. They deposit their eggs on one side of the shoot, the bark of which soon after dies; the sap is arrested in its upward flow on one side of the shoot, while it goes on to flow in the other, producing a peculiar twist in the branch and deformity in the bark. From ten to twenty eggs are deposited by each female under the bark, and in about twenty days the young larva, which is of a greenish colour, makes its appearance, and soon afterwards reaches the leaves, upon which it feeds. Where the shoots in which the eggs are deposited can be spared, it is well to cut them out, or otherwise to cut out the nest of eggs with a sharp knife. The larva is sufficiently large to be easily detected, and, failing other means, they should be picked off and destroyed.

The rose aphis (Aphis ros $x$ ) is so well known that a very brief notice will be sufficient. In their young state they are usually lightgreen, with brown antennæ and legs, and transparent irridescent wings. As they frequently change their skin, which may be seen hanging about the shoots and leaves, so do they less or more change their colour also. The males are distinguished from the females by a double row of black dots on each of their sides.

Aphis vastator.-This, instead of being an enemy, like the rest of its tribe, to the rose cultivator, is his valuable friend and assistant in the destruction of all the other species of aphides; it should therefore be encouraged rather than destroyed. Its appetite seems extraordinary, as it devotes its whole time and attention to sucking the vitals out of the aphides, and attacks them in all their stages of existence. Besides the Aphis vastator, the following are also valuable in counteracting the ravages of other insects,--viz., the lady-birds, of which we have already spoken, feed upon the aphides both in their larva and perfect state; the aphidivorous syrphidæ in their maggot state live entirely on aphides; the hemerobii, whose larvæ are called aphis-lions, live also upon 
them. Besides these there are a number of parasitic insects which deposit their eggs in the old aphides, and whose young feed upon, and, when fully grown, issue from them, leaving nothing of the body of the aphis but a dry empty skin. The earwig and ant play their part, and a number of soft-billed birds are extremely useful in spring, by devouring the young newlyhatched aphides as they issue from the eggs laid the previous autumn. Amongst birds that are our assistants in this matter, the green and red woodpeckers, the nut-hatch, the tree-creeper, the greenfinch, starling, chaffinch, house-sparrow, ox-eye, tomtit, goldfinch, wagtail, redstart, redbreast, may all be considered as insectivorous; and although some of them do take a little fruit, still this loss is as nothing compared to the immense service they render us in the suppression of our insect enemies.

Destruction of the aphides is effected in various ways ; but whatever mode is adopted, it will be of little use if not done effectually, and followed up as long as a single aphis is seen on the plants. A decoction of quassia, in the proportion of an ounce of the chips to a quart of water, is an excellent remedy, and where the number of plants infested is not great, the branches may be dipped into it; but when the collection is extensive, a more wholesale mode of proceeding must be adopted; and this cannot be better done than by preparing 2 or 3 gallons at a time, and when cold, applying it to the plants by means of the garden-engine or syringe, wetting both upper and under sides of the foliage, so as to reach the aphides upon whatever part of the leaves or shoots they have taken up their abode. This application should be repeated the following day, when few of the insects will be found alive. For greater security, the whole should be syringed with clear water, applied with force, in a day or two afterwards, for the removal of the dead insects and refreshment of the plants. Another remedy we have found of great service in the destruction of aphides on all kinds of plants, is two pounds of bitter aloes dissolved in hot water, and added to 40 gallons of soft river or rain water, and applied in a cool state by the above means. A pint of gas-water added to 10 pints of soft pumpwater is a cheap and useful remedy for destroying aphides on rose-trees. Covering the plants so as to enclose the smoke, and fumigating with tobacco, is a vely effectual remedy; as is also wetting the buds and points of the shoots infested, and immediately dusting Scotch snuff over them. In their very young state, finelypowdered hot lime dusted over them, and repeated two or three times, will destroy those which have just changed their outer skin, or such as have it yet in a soft state.

Lozotcenia rosana lays its eggs in summer and autumn; they hatch along with the opening of the leaves in spring, and often do much injury to the rose. The young caterpillar forms a residence for itself by drawing two or more of the young leaves together, on which it feeds. When this is observed, they should be looked to, and bruised between the finger and thumb.
The common vapourer, or brown tussock moth Bombyx (Orgyia) antiqua, fig. 278.-They feed

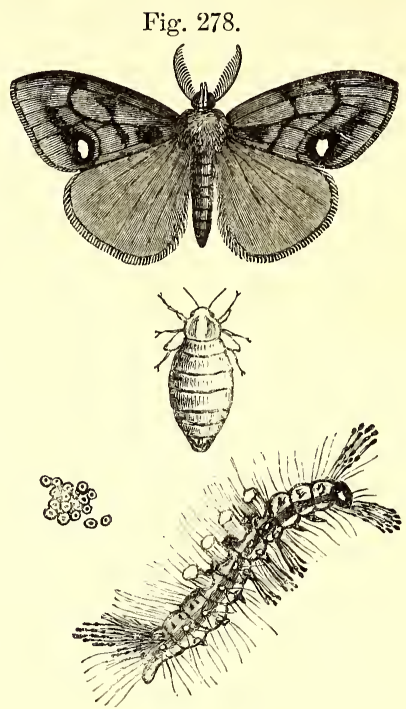

BROWN TUSSOCK MOTH.

on a variety of trees, but principally on the rose and willow. The females are destitute of wings, seldom travelling many inches from the web which they form for a temporary protection, and in which, and on the branch which supports it, they have deposited their eggs; after which they fall to the ground and die. The males being furnished with wings, may be seen from August till October flying about in search of their mates. They are of a rich snuff-colour, the antennæ short, and bipectinated to the tips; the head and thorax are downy, the upper wings forming a triangle, when at rest, of a clouded brownish colour, having two dark irregular lines across them, and a largish uniform white spot near each posterior angle : the under wings, as well as the whole under-side, are of a dark rusty orange colour, the legs ochreous and hairy, the body slender. The females are of a mouse colour, clothed with soft short thick down, oval in shape, and sluggish in habits: head small, with prominent eyes : antenıæ short, and composed of about tiventy joints : thorax small, not winged, but having a pair of small hairy appendages: the abdomen is distended with eggs, which are visible through the skin. The caterpillar is of the sort vulgarly known as hairy-worms. They are of a beautiful lead or light slate colour, richly marked with black velvety spots and red tubercles round the segments of the body. Two long elegant brushes of blackish hairs project like horns on each side of the head, another is elevated at the tail, and three others on each side of the body, giving the animal, when in a state of repose, a very singular and elegant appearance. They have six pectoral horny-like feet, terminated with claws, eight abdominal, and ten anal fleshy 
feet; the males much smaller than the females. The eggs are laid in great numbers, and are fastened by a glutinous substance to the bark of the tree, and even in the web, and are of a light pinky colour.

The orange upper-winged moth (Xanthea croceago) feeds upon almost every species of quercus, and often greatly destroys the foliage of the more ornamental sorts in our arboretums and shrubberies. In its perfect state it measures from an inch to an inch and a half from tip to tip of the wings, which are of an orange or yellowish-red colour, streaked and spotted with brown. The fore wings are marked with six equidistant white spots; the hind wings are of a dirty white, with a dusky spot. The caterpillar is yellowish, inter'spersed with white dots and angulated dark markings along the back, and oblique ones on the sides. The moth appears to survive the winter, being often found during the winter months. Hand-picking the caterpillars, and catching the moths on the wing, seem to be the only means of reducing their numbers.

Bibio marci (Tipula marci Linn.) has been already described, p. 591, fig. 244, and we refer to it here as it is often found to be a formidable enemy in beds of ranunculuses, and also in flower-pots, devouring the roots and often destroying the whole plant. Being gregarious, living in groups of a hundred or more, accounts for the rapid disappearance of the tubers in ranunculus beds, into which they are probably in the first instance introduced along with cow-dung, in which they abound. It should therefore never be used in such beds until thoroughly decomposed.

The March moth, Anisopteryx (Geometra) oescularia.-The caterpillar, which is of a green colour, with pale longitudinal lines along the body, is found in June feeding on the leaves of the horse-chestuut. The moth appears about the middle of March. The males, when their wings are exteuded, measure about an inch and a half. The upper wings are pale glossy ashy brown, having a broad dusky band across them, edged with a white toothed line. The lower wings are paler than the upper ones, with a dark spot and slightly-marked bands. The females are destitute of wings. Of the economy of this insect we know very little, and we believe the only mode of lessening their numbers will be by picking up the caterpillars when they appear.

The large mallow moth Larentia (Geometra) cervinaria, although not a very common insect, is occasionally found on mallows, lavateras, and hollyhocks. The perfect insect measures nearly 2 inches across its expanded upper wings. The upper wings are reddish brown, with bands of dark brown, edged with white. The lower wings are pale brown, with bands somewhat similar to those in the upper. The eggs are laid on various plants of the natural order Malvaceæ, but chiefly on Malva and Lavatera. The caterpillars come forth in June and July, of a dull green colour, with darker lines of the same down the sides, dots of white running across them.

Curculio sulcatus (Otiorhynchus sulcatus).During the winter months the grubs of this beetle do much mischief amongst succulent plants both in greenhouses and out of doors, by devouring the roots, and hence causing the plants to assume a yellow sickly appearance. In its grub state this curculio is about half an inch in length, of a dirty white colour, fleshy, bristly, without legs, but furnished with tubercles at the sides, which aid it in moving. The grubs enter their chrysalis state about the end of May, becoming white, and having the appearance of a beetle stripped of its wings. The perfect insect appears about the end of June, in form of a small beetle scarcely half an inch in length, colour black, slightly glossy, granulated over so as to resemble shagreen. Care should be taken to hunt for and catch this insect in the month of June, for if allowed to deposit their eggs in the ground or the pots, they will breed to a most vexatious extent. In the case of valuable plants when attacked, it will be advisable to shake the soil completely from the roots, and to replant or repot them in new and fresh soil.

The mole-cricket, churr-worm, jarr-worm, eve-churr, or earth-crab (Gryllotalpa europaea Rec., Gryllus gryllotalpa Lin., Gryllotalpa vulgaris Latr.) - for by all these names is this extraordinary insect known in one part of the kingdom or another-from its subterranean habits, commits sad havoc in culinary gardens and even corn-fields, and should it find its way into the florist's beds of choice flowers it causes him great annoyance. It lives chiefly under ground, and is perpetually on the move, bur rowing like the mole, and even, like it, throwing up heaps of earth from its tunnels. The fullgrown insect measures fully 2 inches in length, and 4 lines in breadth. Colour dark brown; head rather smallish, longish, and oval, which it can retract within the prothorax and protrude at pleasure ; thorax very small, like that of a crab ; wing-cases leathery, traversed by large and small veins, and attached to hinder part of the prothorax. The insect, when its wings are extended, assumes a widely different appearance from what might be expected from seeing it when they are folded up. It has six feet, the two fore ones of a peculiar form, short, broad, and very strong, resembling the fore-feet of the mole, and adapted to the same purpose. It is asserted by $M$. Rosel that this insect is capable of pushing forward a weight of 6 pounds with its fore-feet on an even surface, from which some idea may be formed of its power in digging its burrows. The female lays her eggs, to the number of two or three hundred, in the month of June, after having hollowed out a place in the ground about a foot under the surface. The young are hatched in July or August, and commence immediately to feed on the roots of plants. In October and November they bury themselves deeper in the soil for warmth, and come again nearer the surface in March and April. The means recommended for their destruction are various. The most feasible, however, is to dig up their nests in June or July, when hundreds of eggs may be destroyed in an instant.

Cucullia verbasci (Noctua verbasci). - The caterpillars of this moth are found from the end 
of May till August, feeding upon the plants of the genus Verbascum, often completely destroying every vestige of the foliage excepting the mid-ribs. It also attacks the leaves and often the petals of other plants, particularly those of Scrophularia. The caterpillar, when fully grown, is above 2 inches in length, of a dirty white or slaty colour ; each segment is marked with four black dots, sometimes distinct, at other times confluent. There are also smaller black dots along the sides, and a row of yellow ones along the back. The head is yellow, spotted with black. The perfect insect appears in May, about 2 inches from tip to tip of the expanded fore wings, which are of a reddish-brown colour, clouded and lined with black streaks, with a largish white spot on each somewhat resembling the figure 3. The hind or under wings differ little except in size, and being of a lighter colour than the upper ones, sometimes almost white. The eggs are laid upon the Verbascum and allied plants, and if the weather be warm they are hatched in a few days. The caterpillars, when fully grown, descend into the ground near to the roots of the plants upon which they have been bred, and there form cocoons of leaves and earth, so hard cemented together as to resemble hard clods. This, together with the large size of the caterpillar while feeding, affords the means of their destruction, by hand-picking in the one case, and searching for these clods in the other. They remain in the pupa state till the following May, even sometimes for two years.

The spotted buff-moth, Spilosoma lubricipeda (Bombyx lubricipeda), fig. 73, although attacking many of the productions of the kitchen garden, such as turnips, carrots, mint, scarlet-runners, \&c., yet its voracious habits extend to many flowering plants also; indeed, almost no green leaf appears to be exempt from its attacks. For description of it, see page 196.

Melitcea euphrosyne (Papilio and Argynnis euphrosyne).-This is the pearl-bordered fritillary butterfly, and is frequently found destroying garden violets, making its appearance in August and September. 'The wings are about 2 inches across when fully expanded, tawny, redspotted, and lined with black, with a row of silvery spots round the edges. The caterpillar is black and spiny, with two rows of orange spots on its back. It produces two broods yearly, the first in May and the second in September. Netting the perfect insect and fingerpicking the caterpillar from the plants seem the only remedies.

Drosophila graminium Fallen.-The maggot of this insect appears in abundance in spring upon many plants of the order Carophyllaceæ, cutting passages usually terminating in an irregular blotch or blemish, and not unfrequently denuding the plants entirely of their foliage. Amongst flower-garden plants subject to its attacks may be mentioned the common Lobels catchfly, Silene armeria, Visicaria oculata, several species of Lychnis, \&c.

The chrysanthemum leaf-miner (Tryptera $a r$ temisice Fabr., Acidia artemisice Walker, Tephritis artemisice Macquart) is occasionally met with on the leaves of the Indian chrysanthemums, entering the leaves by the upper surface, and penetrating to the parenchyma, which it eats out, particularly towards the centre, and may often be traced scooping out winding and irregular channels between the cuticles of the leaf. The cuticle over the excavated parts rises up like swollen veins above the surface, and in these cavities the eggs are deposited. The lower and older leaves are fortunately only attacked, and hence less injury is caused to the plant than if the younger and upper ones were assailed. We know of no other insect that feeds on this species of plants, therefore think a further description of it here of little importance. The leaves attacked should be cut off as they appear infested, and burnt.

The pine-destroying beetle (Hylurgus piniperda), fig. 279.-The numerous insects which

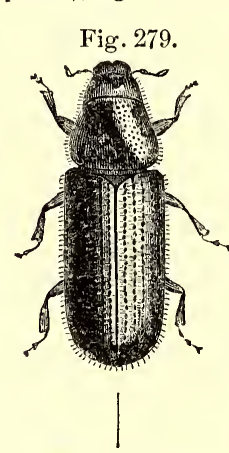

PINE-DESTROYING BEETLE. have been injurious to our pine forests appear, in this country at least, to have been little regarded, until the introduction of so many new and valuable plants of the same natural order from various parts of the globe. The injury inflicted on them by insect enemies has caused investigations to be made regarding their habits and mode of suppression. This subject has been long studied in Germany, as a reference to the entomological authors of that country abundantly testifies. It would far exceed our present limits were we to go into a minute and detailed description of all the insects that infest our woods and forests; we shall therefore confine ourselves to those that are most injurious to trees planted for ornament in the park and pleasure-grounds; and of these the Hylurgus piniperda claims particular attention. This beetle, although an exceedingly small one-not one quarter of an inch in length-has committed great destruction upon our Conifers; and although it is said by some to prefer the Scotch fir (Pinus sylvestris), still it is found, upon closer investigation, to attack the silver fir (Abies picea), the Weymouth pine (Pinus strobus), the spruce fir (Abies alba and excelsa). Kollar describes this beetle as being somewhat shaggy and black ; the wing-cases are pitchy black, irregularly streaked, entire at the tips ; thorax somewhat anteriorly narrowed; antennæ and feet brownish red. The larva is thick, cylindrical, milk-white on the middle of the body; the head dull yellow, also the front of the body and the anal extremity, but somewhat lighter. The pupa resembles that of the common bark-beetle. The mode of attack of the bectle is to drill a hole on the side of a lateral branch, and having reached the pith it directs its course upwards, feeding on the pith at about the rate of one inch in four days, and continuing to ascend until the pith is consumed, when it makes its escape through the terminal bud, although sometimes 
it leaves the shoot before it arrives quite at the extremity. It is supposed by some that it is the males only who perform these borings, while others think that the females are also so employed, and that they deposit their eggs in the perforations. Reasoning from analogy, one would be apt to favour the latter opinion, were it not that no eggs have been detected in them; these are in general found in crevices of the bark, or below the bark of dead or decaying fir trees. When young trees are infested, which will soon be seen by the points of the branches drooping down, turning yellow, and often falling off altogether, such branches should be cut off as soon as the evil is detected. If the insects are found under the bark, the trees had better be cut down and burnt. It is so far fortunate that the abode and propagation of this beetle are in the pith of the young side shoots more generally than in the leading ones. The larvæ feed on the trunks of dead or dying trees, and the beetle only places her brood on healthy trees when compelled by necessity.

The spruce pine weevil, Curculio (Hylobius) abietis Schonh., Curculio pini lin., fig. 232, p. 535. -Although so called, this beetle does not confine its ravages to the spruce alone, but attacks the Scotch fir and larch also. It has been found in some cases exceedingly injurious to rhododendrons, azaleas, and various other trees. This beetle generally attacks newly transplanted trees, or such as are sickly. It operates by stripping off the bark and scooping out the buds of the leading shoots. Kollar conjectured-and subsequent investigation seems to confirm the idea-that the female deposits her eggs deep in the roots of the trees. The passages of the larvæ appear to confirm this opinion, as they generally run upwards. "The favourite resort of the beetles are those spots where the pines were felled, and where the stocks and branches are allowed to remain, although they are found at times, when they have increased very much, in other places, on account of their power of flight. They pass the winter in the felled trees, and are found in spring on the lower branches of the young ones that are covered with grass and moss. The beetles collect in such places in July and August, at the time of laying their eggs, in great number. Of their continuance in the larva state nothing is distinctly known ; they seem, however, to pass the winter unchanged." It appears from observations made at Airdrie and elsewhere, that they attack the young trees in greatest numbers when they have been planted on ground formerly carrying the same kind of crop, and from which the old roots have not been removed. An instance is given in "The Gardeners' Chronicle" of a plantation in Ross-shire, of 10 acres extent, " that was planted with two-years' transplanted larch, spruce, and common fir, upon ground which had been newly cleared of fir timber that had been growing there for upwards of seventy years. The larch plants have been all destroyed by this weevil, which commenced its operations at the root, and stripped every plant of the bark up to the summit; but some larches in the same field, and about 9 years old and 12 feet in height, remain untouched, as well as about 100 acres of larch in the neighbourhood, which were planted about 14 years back, and immediately after an old fir wood had been cut down." We make this quotation to show that the rule is not in all cases the same, but in too many cases it is so to a fearful extent. There can however, be but one opinion on the impropriety of replanting ground with coniferous trees from which a crop of the same has been recently removed, as well as of the impolicy of cutting over the trees and leaving the stumps in the ground, instead of grubbing them up altogether. This beetle measures nearly 6 lines in length, exclusive of its long rostrum, which inclines downwards. The thorax is smaller tlian the abdomen, which is nearly cylindrical ; the other parts of the body are dark brown. The rostrum points upwards, and is very thick ; the antennæ extend nearly to its tip when at rest. The wing-cases have several long and short transverse bands, marked with small yellow hairs. It is more destructive in its perfect state than as a larva, because its transformation never takes place in young living wood. The larva is thick and fleshy, tapering very much towards both ends ; besides the head, the body consists of twelve segments of a milk-white colour ; the head is reddish brown, with very dark jaws and palpi. Instead of three pairs of thoracic feet, there are three pairs of small ones, with from four to six long brown fleshy tubercles, furnished with a few short hairs. The perfect insects do not appear every year at the same time; they are often found in immense numbers in June and July, sometimes in May, and also in August. It has been hinted that two broods may be produced annually, but this is greatly doubted.

The black arch-moth, Psilura monacha (Bombi), Liparis monacha.-This is another of the pine-destroying insects, and although, so far as we are at present aware, it has not visited us as it has for ages the forests on the Continent, of which Kollar and others have drawn up, no doubt, a true but very dolorous account, it does exist amongst us, and may be on the increase; its habits should therefore be known that its progress may be ar'rested. It not only attacks the pines, but the oak, elm, aspen, lime, and willow, and is by no means unknown in our apple and pear orchards. About the end of July and beginning of August the male may be found during the day upon the trunks of the trees it intends to attack. They are smaller than the females, measuring about an inch and a half across the expanded wings, while the females measure nearly an inch more. The colour of the moth is a creamy white, marked with black spots and streaks. The under wings are dusky. The antennæ are black, tinged above with pink. The caterpillars appear in June and July, are of an ashy-brown colour, with tufts of reddish hair on the back, and a black heartshaped spot on the second segment of the body. They are, when fully grown, from one and a half to one and three quarters of an inch in length. The female lays her eggs in the cracks of the bark of old pines, to the number of twenty or thirty, in a bunch resembling a cluster 
of grapes, and moves on and continues to deposit eggs in bunches, until she has been delivered of about one hundred and twenty in all. In March and April they are hatched, after which they sit near the spot where they were hatched in a crowd together for several days, according to the warmth of the weather, as if it were to gain strength to enable them to ascend the trees. At this time their destruction should be attended to, and if omitted an important chance is lost.

The typographer bark-beetle, fig. 280 (Bostrichus typographus Fabr., Tomicus typographiFig. 280. beetles are by far the most formidable of those insects

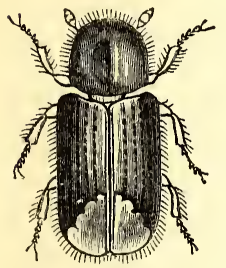
which commit such destruction on the different kinds of pines and firs, and this is the most destructive of them all. The section takes their name from the place of their abode, which is under the bark, where they find their food, which consists of the alburnum and partly of the inner bark itself. B. typographus, for the most part, attacks the silver fir (Abies picea), although it visits other pines also. We are not aware that it has been found injurious to young trees, nor that it abounds to TYPOGRAPHER BARK- a very great extent in Bri-
BEETLE. the comparatively few silver firs we have of from eighty to one hundred years' growth, the age at which they do most mischief. That they do exist we have the evidence of our own eyes, having detected them this season in trees at Braco Castle, Perthshire; they have also been observed in England. This insect, in the beetle state, is nearly two lines and a half in length, and about one and a quarter broad, covered with a hairy coating. While it remains on the bark it is of a rusty yellow colour, becoming of a brownish black after it escapes into the air, jaws sharply toothed, wingcases deeply punctured, broadest behind, deeply and obliquely impressed. The thorax and sternum darker than the wing-cases. The female is distinguished from the male by her thicker abdomen, which is barely covered by the wingcases. The pupæ are white and soft at first, becoming harder and more yellow by degrees. The larva is three lines long, white and wrinkled when it quits the egg, its head changing soon afterwards to pale yellow, the back being reddish striped, jaws sharp, antennæ short, feet six, and of a dirty yellow colour. Two broods appear annually in Germany, while in Britain it is believed there is only one, which makes its appearance in June or July. They pair shortly after quitting the place of their birth, when each pair selects a suitable place on the tree of their choice, and commence boring a hole in the trunk, pointing upwards, to the depth of nearly an inch, or as deep as the sap-wood. Both assist in the operation, and when completed the male dies, and the female proceeds to burrow out a perpendicular canal in the inner bark to the extent of 3 or 4 inches, with shallow branch-excavations on both sides, in each of which she lays an egg, which she carefully covers with a sort of paste made of the finer particles of the bark. In the course of fourteen days, if the weather is good, the larvæ make their appearance, and commence gnawing tortuous passages in a way somewhat similar to what is represented p. 473 , fig. 204, but much more tortuous and curious, and which, on account of their resemblance to letters, have obtained for the beetle the name of typographer. Kollar remarks, that "warm and dry summers, followed by a dry and cold winter, are favourable for the propagation and increase of all insects injurious to forest trees. Hot weather shortens the period of transformation, and thus, by affording time for the maturation of several broods, causes a superabundant number of insects to be found. On the contrary, damp and cool summers and winters, alternating with wet, frost, and cold, are effectual in lessening both the number of the insects and their destructiveness." Trees which begin to appear sickly, at whatever age, should be closely examined, and wherever the insect is suspected to be at work, a small piece of the bark should be removed by a sharp gouge, and when its true position is found, the bark should either be carefully pared away until the enemy is reached and abstracted, or the spot over which they lie should be hit with a wooden mallet several times, which will destroy the insects by bruising them to death. No trees suspected of containing the insects should be allowed to remain after being cut in the neighbourhood of others, and all trees seriously affected should be cut down. The bark in all cases should be removed and burnt.

The pine-tree lappet-moth, Bombyx (Gastropacha) pini Autor, Dendrolimus pini Curtis and Stephens. - The caterpillar of this moth lives on the foliage of Pinus sylvestris and its varieties, and upon none other. When they attack a tree, they entirely consume the leaf and sheath even to the bark; when the shoots are young, they also eat the young tender bark; and when they have stripped one tree of its foliage they retire to another. The perfect insect appears in July and August, the female laying from one hundred to two hundred eggs in a roundish flat heap. In about fourteen days the caterpillars are hatched, ascend the tree, and commence their work of destruction. This is the time to watch for and to destroy them.

Tortrix (Orthotcenia) turionana Curtis and Stephens. - The larva of this very small lepidopterous insect for the most part attacks the Scotch fir, Pinus sylvestris, and the Austrian pine, Pinus nigricans; but it has recently been found upon some of the more rare and valuable, such as Pinus insignis, Llaveana, and ponderosa. The eggs are laid in July, and are hatched in ten or twelve days, when they immediately penetrate into the centre of the bud, which they completely destroy. After descending through the bud to its base, they then attack the adjoining buds in the same manner, not only destroy- 
ing the central or leading shoot, but killing the side buds also. "By the end of October the caterpillar is feasting on the interior of the largest middle bud, beginning below that which was formed for the following year, in which it spends the winter. The larva is of a dark, glossy, purplish brown colour, with the head and a transverse patch on the first segment following the head black; it has three pairs of articulated legs attached in pairs to the three first segments of the body ; the fourth and fifth segments are legless; each of the four following segments has a pair of fleshy prolegs, and there is another pair of the same kind of legs attached to the terminal segment of the body. The caterpillar is full grown about the end of June, when it descends to the lower part of the burrow which it has formed in the bud, and is there transformed into a shining chestnut-brown chrysalis, which, like most of those internal feeding species, has the head furnished with a small point between the eyes and the segments of the abdomen, armed with double rows of very fine points directed backwards, enabling the insect to move itself about in its retreat." - Gardeners' Chronicle.

The perfect insect, which is one of our most beautiful species of Tortricidæ, makes its appearance about the middle of July. Its attacks are confined, so far as we have observed, to the leading bud and the side buds of the trees, and which it often completely destroys, causing, in many cases, secondary leaders to be formed, and thereby greatly damaging the natural character of the tree.

Tortrix (Cocoyx) buoliana Fabr., Tortrix xylostiana Hub., resembles the former in habits, and both may be subdued by following the recommendation given by Kollar-namely, destroying the turpentine tumours, with which the silver fir in particular abounds, about the end of October, while the caterpillars are in them, or cutting off and burning every shoot infected by them. The latter method may be avoided, and the shoot saved, if the resinous tubercle is destroyed before the caterpillar penetrates through it into the alburnum of the tree.

The pine saw-fly (Lophyrus pini Latr., Tenthredo pini Linn., Pteronus pini Klug).-The sexes in this insect differ so much from each other in size, form, and colour, that they might readily be supposed at first sight to belong to two distinct species. The male is always smaller than the female, head dull black, antennæ resemble two bunches of black feather's doubly pectinated, the teeth shorter by degrees as they approach the point, all of which are turned down and bending inwards, so that the antennæ appear hollow. Thorax black, and covered with soft short down, the abdomen shining black, legs dirty yellow, thighs black, wings shining and transparent, upper ones purple and green, sometimes inclining to yellow. The head of the female is brownish black, clothed with very short fine yellowish down. The antennæ consist of nineteen joints, rounded and jointed into each other, somewhat resembling the teeth of a saw ; the three basal joints are yellow, the others blackish. The thorax is divided into four greyish yellowish compartments by impressed lines, and on each of them there is a large black spot. The abdomen, consisting of nine segments, is black from the second to the sixth or seventh segment of the upper side; the under parts are all of a yellow grey,-all the other parts resemble the male. During warm weather, from April to July, the perfect insect or fly appears, the brood of young from May to July. The female lays from eighty to one hundred and twenty eggs, and deposits them where she thinks she can find suitable nourishment for her young. This food is either the foliage of the end of last year's shoot, or that of the shoot of the present season not quite expanded. Having made an incision the whole length of the leaf, she lays her eggs in it to the extent of one or two in each leaf, which she covers over with a resinous matter to prevent their falling out. Thus she proceeds from leaf to leaf until her whole stock of eggs is deposited. In sixteen or twenty-four days the caterpillars appear. When full grown, the caterpillar is rather above an inch in length. The number of legs is twenty-two-six conical, three jointed thoracic feet, which are black, and sixteen shorter abdominal prolegs, yellow or green, cylindrical, obtuse, or broad. The head is of a light rusty brown or dark ochre colour ; the body tapers from the head to the anal extremity; the colour varies according to the age of the insect, the time of changing its skin, or state of the weather. The whole insect is glutinous, and when fully grown devours from six to twelve leaves per day. They seldom attack a tree singly, but always in immense numbers, and are found in greatest numbers on the south side of the tree. They choose for their pupa retreat dry moss or leaves near the roots of the trees, and are found congregated in great num. bers together, and remain in that state from eight to nine months.

Besides the above, there are many other insects injurious to the pine tribe ; indeed, it is probable that most of those described by Kollar and other German entomologists as infesting the pine forests in the north of Europe, may be found in those of our own country also. The investigations of naturalists into our extensive pine woods in Scotland are as yet in their infancy ; a closer examination may trace the failures of our pines and larches more to the ravages of insects than either to soil, situation, or climate. 


\section{GL O S S A R Y}

OF SOME OF THE MOST IMPORTANT TECHNICAL TERMS EMPLOYED

IN THIS WORK.

Adventitious organs, produced in abnormal positions, such as roots from ärial stems or branches.

Albumen.-Matter of a nutritious nature stored up in the ovule, and transmitted into the cavity of the seed either by the umbilical cord, or more probably secreted by the endopleura.

ALBURnUm.-The outer coating of young wood, called often sap-wood.

AxIL.-Literally the arm-pit : in gardening it means the point of the angle between a leaf and a branch, or between a branch and the stem.

Bole.-The trunk of a tree.

Calius, or Callosity.-Granular matter of a soft cartilaginous consistency formed at the base of cuttings, and from which the roots spring.

CAMBIUM.-Mucilaginous matter found between the bark and young wood.

Cellular tissue, or Vascular tissue.-Exists in most plants, constituting the pith and outer bark of trees, the central part of rushes and similar plants. Cultivation has greatly increased it in turnips and other esculent vegetables, forming the terider or succulent part which we eat.

Cloves.-The small side bulbs in shallots, garlic, \&c.

Conm.-A thickened underground stem, as in the Arum, \&c.

Cortex.-The outer bark of a tree.

COTYLEDON LEAF.- The first or temporary leaf of the embryo.

CuTICLE.-That thin transparent membrane which covers the surface of plants, and which is more or less easily separated from the rest of the tissue.

Di\&CIOUS PLANTS.-Having the male flowers on one plant and the females on another.

Embryo state.-- The young plant contained in the seed, and provided with every organ essential for nutrition.

EMBRYo BUDS.-Nodules in the bark of many trees from which branclies spring, sometimes spontaneously, and in general when the top of the tree or main branches are removed.

Epideruis.-The cellular layer covering the external surface of plants.

EPIPHITE. - Attached to another plant, and growing suspended in the air, like many orchids, but not parasitical.

Etiolation.-Blanching, as plants are when growing in the dark.

Fastigiate. - Growing in form of a pyramid.

Fronds.-The leaves of ferns.

Fugacious.-Falling off or fading early; flowers of short duration.

Hilum.-The base of the seed to which the placenta is attached, either directly, or by a cord as in the pea.

VOI. II.
INDIGENOUS.-An aboriginal native in a country.

INTERNODE.-The portion of the stem betweell two joints or leaf-buds.

LIBER.-The fibrous inner bark of trees.

MEDULLARY RAYs.-Cellular prolongations uniting the pith and the bark.

Monacious Plants.-Having male and female flowers on the same plant, but in different flowers.

MoRPHOLOGY.-The study of the forms assumed by different organs, and the laws which regulate such changes.

Mrcelium.-The spawn of fungi.

Parenchrma.-Cellular tissue.

Phanerogamous Plants.-Having conspicuous flowers-not inconspicuous, or invisible to the naked eye, like cryptogamous ones.

Pistil.-The female organ of the flower, while the stamen is the male.

Pollen.-The powdery matter contained in the anther.

Potтle.-A long tapering basket made of deal shavings, holding about a pint and a half.

Pseudo-BULB. - The swelling at the base of the stem of many orchids.

Punnets.-Round shallow baskets made of deal shavings. Of these there are four-namely, mushroom punnets, 7 inches in diameter and 1 inch deep; sea-kale punnets, 8 inches in diameter at top, $7 \frac{1}{2}$ inches at bottom, and 2 inches deep; salading punnets, 5 inches in diameter and 2 inches deep; radish punnets, 8 inches in diameter and 1 inch deep, when intended to hold what is called in the London market six hands: those to contain twelve hands measure 9 inches in diameter and 1 inch deep.

RADICLE.-The young root of the embryo.

RHIzome.-A stem creeping on the ground, and forming buds on its upper and roots from its under side.

Sieve. - A measure of capacity, 15 inches in diameter and 8 inches in depth, containing seven imperial gallons. The half-sieve averages $12 \frac{1}{2}$ inches in diameter and 6 inches in depth, containing three imperial gallons and a half.

SPONGIOLE, OR SPONGIOLET.-The cellular part of a young root, whose chief office is to absorb food from the soil for the use of the plant.

STOMates.-Openings in the epidermis, especially in the foliage. They are the breathing pores, and exist, in the case of some plants, to the number of one hundred thousand to the square inch of surface.

SUTURE.-The groove-like mark in plums and peaches, as if the pulp had been separated and again united.

Viviparous.-Plants producing leaf-buds instead of fruit. These buds serve the same purpose as true seeds. 


\section{N D E X}

\section{SELECT LISTS OF VEGETABLES AND FRUITS.}

Alisander, 129.

ALMOND, 560.

ANGELICA, 239

AnISE, 239.

\section{APPLES.}

Acklam's russet, 414.

Adams' pearmain, 420.

Alban, 432.

Alexander, 425

Alfreston, 423.

American gloria mundi, 425.

American mammoth, 425 .

American Newtown pippin, 419.

American pearmain, 428 .

American summer pearmain, 422

Annat scarlet pippin, 414.

Api noir, 430.

Api petit, 429

Aporta, 425

Aromatic, 417.

Aromatic pippin, 415.

Arundel pearmain, 428

Ashmead's kernel, 415.

Astrachan red, 423.

Astrachan white, 423.

Autumn pearmain, 428

Backhouse's Lord Nelson, 427.

Baddow pippin, 415.

Bain's, 423.

Baldwin, 422.

Baltimore, 425

Baltimore pippin, 423.

Barton's incomparable, 415.

Baxter's pearmain, 428 .

Beachamwell, 415 .

Beachamwell seedling, 415 .

Beaufin, Norfolk, 423 .

Beauty of Kent, 423, 427.

Bedfordshire foundling, 423 .

Bell's scarlet pearmain, 420 .

Belle bonne, 423.

Belle-fleur, white, 422.

Bennet, 432.

Bess $\mathrm{Pool}, 423$.

Best bache, 432 .

Black Kingston, 432.

Black lady, 430 .

Bland's jubilee, 423.

Blenheim orange, 424.

Blenheim pippin, 424

Bonne rouge, 426 .

Borsdorffer, 415 .

Boston russet, 421 .

Bough, 418.

Bovey red streak, 432

Boxbury russet, 4:1.
Brabant bellefleur, 423

Braddick's nonpareil, 419.

Brainton seedling, 432.

Breedon pippin, 415.

Brierley's seedling, 432 .

Bringewood, 432.

Browll cockle pippin, 415.

Buckland, 424 .

Buckland lily, 424.

Burn's seedling, 424 .

Cadbury, 432.

Caldwell, 429

Calville blanche d'Eté, 424.

Calville blanche d'Hiver,

424.

Calville Malingre, 424.

Calville Normande, 424.

Calville rouge d'Eté, 424 .

Calville rouge precoce, 424 .

Cambridge apple, 423.

Cambusnethan pippin, 424.

Carlisle codlin, 424

Catshead, 423.

Cat's head, 424 .

Catshead Beaufin, 423.

Chalmers' Allan Bank

seedling, 424 .

Chandler, 422.

Christie's pippin, 415 .

Cillini, 424

Clara pippin, 415

Claremont pippin, 425.

Claygate pearmain, 420

Clifton nonsuch, 417.

Cluster golden pippin, 417.

Cobbett's fall pippin, 428 .

Cobham, 424

Coccagee, 432

Cockle pippin, 415.

Cockpit, 424.

Codlins, 424.

Coe's golden drop, 417.

Colonel Vaughan's, 415.

Cornish aromatic, 415.

Cornish gilliflower, 416 .

Court of Wick, 415 .

Court pendu plat, 415 .

Cowarne queening, 428.

Cowarne red, 432

Creed's marygold, 416.

Crimson queening, 428 .

Crofton scarlet, 415 .

Crofton white, 415 .

Dainty, 426.

Decroit, 422 .

Devonshire Buckland, 424.

Devonshire Quarrenden, 424.

Devonshire red streak, 432 .

Devonshire wilding, 432.

Dickson's empereur, 425 .

Ditton nonpareil, 419.
Dr Harvey, 429.

Downton nonpareil, 419

Downton pippin, 416, 432

Downton's pippin of Diel, 416.

Downy, 426.

Dredge's fair maid of Wishford, 416 .

Dredge's fame, 416.

Dredge's white lily, 424

Duke of Wellington, 424 .

Dumelow's seedling, 424 .

Dundee, 421.

Dutch mignonne, 425 .

Dymmock red, 432.

Early bough, 418.

Early Crofton, 418

Early French reinette, 416.

Early harvest, 416 .

Early Julien, 416.

Early July pippin, 416.

Early nonpareil, 419.

Early strawberry, 4

Easter pippin, 425.

Ecclenville seedling, 425 .

Edmonton's aromatic pippin, 418.

Eighteen ounces, 424.

Elford pippin, 416 .

Elliot yellow, 432 .

Elton golden pippin, 416.

EIton pippin, 416.

Emperor Alexander, 425.

English nonpareil, 419.

Esopus Spitzenberg, 416

Essex pippin, 416.

Eve apple of Scotland, 424

Fall pippin, 422, 428.

Farleigh pippin, 416 .

Fearn's pippin, 416.

Ferris pippin, 417.

Fill-basket, 425.

Fill-basket, Kentish, 427.

Fine crown pippin, 427.

Florence pippin, 417.

Flower of Kent, 425 .

Flushing Spitzenberg, 416.

Foreman's crew, 417.

Forest styre, 432

Forge, 425, 432.

Formosa pippin, 421 .

Fox whelp, 432

Foxley, 432.

Frankland's golden pippin,

417.

French crab, 425.

French reinette, 421 .

Friar, 432.

Frith pippin, 424

Fry's pippin, 415 .

Garnon's, 415.

Garter, 432.
Gilliflower, 425 .

Gilliflower, Cornish, 417.

Gilliflower, summer, 417.

Glammis Castle, 429 .

Glazen wood gloria mundi, 425.

Gloria mundi, 425, 429.

Glory of England, 425.

Glory of Flanders, 424

Glory of York, 421.

Gogar pippin, 417.

Golden drop, 415.

Golden drop, Coe's, 417.

Golden Harvey, 417, 432

Golden knob, 426.

Golden noble, 426 .

Golden nonpareil, 419.

Golden pearmain, winter, 417.

Golden pippin, 417.

Golden pippin (cider), 432.

Golden pippin, cluster, 417 .

Golden pippin, Frankland's, 417.

Golden pippin, Hughes', 417.

Golden pippin, new, 417.

Golden pippin, scarlet, 417.

Golden pippin, Screveton, 417.

Golden pippin, summer, 417.

Golden reinette, 421 .

Golden russet, 417, 428 .

Golden Worcester, 432.

Gooseberry, 426.

Grange, 432 .

Great pearmain, 428.

Green bellflower, 422 .

Green codlin, 427.

Green gossings, 429 .

Green Newtown pippin,419.

Green tiffin, 426

Green winter pippin, 419.

Greenupp's pippin, 426.

Grey, 421.

Hagloc crab, 432.

Hampshire yellow, 418.

Hampshire yellow golden pippin, 418 .

Hardingham's russet, 421.

Harvey's Wiltslire defiance, 426.

Hawberry pippin, 426 .

Hawthornden, 426.

Hawthornden, new, 426.

Herefordshire pearmain, 420.

Herefordshire queening, 428.

Hick's fancy, 419.

Hoary morning, 426 . 
APPLES-continued.

Hogg's king of the pippins, 418 .

Hogsliead, 432

Holland pippin, 426 .

Hollandburg, 426.

Hollow-cored pippin, 422.

Hormead pearmain, 428

Hormead pippin, 428 .

Horsley pippin, 426.

Hubbard's pearmain, 420 .

Hughes' golden pippin, 417.

Hunt's Newtown pippin, 419.

Iunt's nonpareil, 419.

Hunthouse, 426 .

Hutching's seedling, 429.

Hutton square, 427.

Ingestrie's red, 418, 432.

Ingestrie's yellow, 418, 432.

Irish codlin, 424.

Irish peach, 418 .

Irish pitcher, 424

Irisl russet, 421 .

Iron, 424 .

Ironstone pippin, 425

Isle of Wight pippin, 432

Jansen van Welten, 427.

Joln apple, 428.

Jonathan, 422 .

Jones' Southampton pippin, 418

Josepline, 425 .

Jubilee pippin, 423.

Juneating, white, 418.

Keeping red streak, 428.

Keeping russet, 421 .

Kentish fill-basket, 427 .

Kentish pippin, 423, 427.

Kerry pippin, 418.

Keswick codlin, 424.

King of the pippins, 418.

King of the pippins, II ogg's, 418.

Kingston black, 432.

Kingswick pippin, 415 .

Kirk's golden reinette, 421 .

Kirk's lemon pippin, 427.

Kirk's Lord Nelson, 418.

Kirk's scarlet admirable, 426.

Knight's codlin, 429.

Kniglit's golden pippin, $\mathbf{4 1 6 .}$

Knight's pippin, 416 .

Lacy's non pareil, 419 .

Ladies' sweeting, 423.

Lady, 429.

Lady, black, 430.

Lady de Gray's, 427.

Lady's finger, 427.

Lamb Abbey pearmain, 420.

Lancashire fill-basket, 425 .

Lancaster crab, 427.

Large early yellow bough, 418.

Large fall pippin, 428

Large Newtown pippin, 419.

Large scarlet Siberian crab, 430 .

Large white juneating, 416.

Large yellow bough, 418 .

Late Carse of Gowrie, 429.

Leadington monstrous, 427.

Leathercoat, 429

Le beau rouge, 426 .

Lemon pippin, 427 .

Leyden pippin, 418

London pippin, 427.

Lord Gwydir's Newtown, 423.

Lord Nelson, Kirk's, 418

Loveden's pippin, 419 .

Lucombe's pine-apple, 418.

Lucombe's seedling, 427.

Maclean's favourite, 418 .

Mage's Johnny, 426.

Maiden's blush, 426 .

Mannington's pearmain 420

Manx codlin, 424 .
Margil, 418.

Meggincl favourite, 421

Melrose, 427.

Minchall crab, 427, 432.

Mitchelson's seedling, 427 .

Monkton, 132.

Monstrous bellflower, 422

Monstrous pippin, 425 .

Morris' Court of Wick, 418 .

Morris' russet, 418 .

Motteux seedling, 415 .

Mouse, 422.

Munch's pippin, 418 .

Nelson, 427.

Nelson codlin, 427

Never fail, 418 .

New golden pippin, 417.

New Hawthornden, 426 .

New London pippin, 427

New nonpareil, 419.

New rock pippin, 419.

New York gloria mundi, 425.

Newbold's Admiral Dun: can, 429.

Newbold's Duke of York, 429.

Newtown pippin, 418, 423 .

Newtowı pippin, yellow, 419 .

Nonpareils, 419.

Nonsuch, 427.

Nonsuch, round winter, 427.

Nonsuch park, 419.

Norfoll beaufin, 423

Norfolk pippin, 414, 420

Norfolk stone pippin, $4: 29$

Normanton wonder, 424 .

Northern greening, 427.

Norther'u spy, 422 .

Northwick pippin, 424.

Nutmeg cockle pippin, 415.

Nutmeg pippin, 415.

Ohio favourite, 422 .

Old nonpareil, 419.

Old pearmain, 420,428 .

Oldacre's new, 423

Ord's, 419.

Osterley, 420

Osterley pippin, 420 .

Ox apple, 425.

Oxford peach apple, 420 .

Parmain, 420.

Pawsan, 432.

Peach pond sweet, 422

Pearmains, 420, 428 .

Pearson's plate, 421

Peck's pleasant, 422.

Pennington's seedling, 421 .

Petersburg pippin, 419

Phillip's reinette, 415 .

Pie pippin, 426.

Pile's russet, 429.

Pine-apple pippin, 418

Pine-apple russet, 421

Pitmaston nonpareil, 419.

Pitmaston russet noupareil, 419.

Pomme d'Api, 429.

Pomme grise, 421.

Ponme rose, 429 .

Pope's apple, 428.

Porter, 422.

Powell's russet, 421.

Prince's yellow harvest, 416.

Pumpkin russet, 422.

Putman russet, 422 .

Quince, 427.

Ravelston pippin, 421

Read's baker, 423 .

Red Astrachan, 423.

IRed Cowarne, 432

Red Crofton, 415.

Red Dyınmock, 432.

Red Hawthornden, 426.

Red Ingestrie, 432.

Red Kentish pippin, 427.

Red nust, 432

Red quarrenden, 425.
Red queening, 428 .

Red streak, 432

Red streak, keeping, 428 .

Reinette Blanclie d'Espagne, 428 .

Reinette de Canada, 428.

Reinette d'Espagne, 428 .

Reinette Franche, 421.

Reinette Franche Grauwe, 428 .

Reinette golden, 421 .

Reinette gris, 421 .

Reinette royal, 428 .

Ribston pippin, 421.

Ross nonpareil, 419.

Rosemary russet, 421

Rostoclier, 428.

Round winter nonsuch, 427.

Royal pearmain, 420, 428.

Royal russet, 429 .

Royal Somerset, 427.

Royal wilding, 432 .

Rushock pearmain, 420 .

Russets, 421

Russet, golden, 428 .

Russet, Pile's, 429.

Russet, royal, 420

Russian emperor, 425 .

Rymer, 429.

Sack, 425.

Sack and sugar, 429

St Germain, 428 .

St John's nonpareil, 419.

St Mary's pippin, 416 .

Sam Rawlings, 426.

Sam Young, 421.

Scarlet Court of Wick, 415.

Scarlet golden pippin, 417.

Scarlet non pareil, 419 .

Scarlet pearmain, 420 .

Scarlet queening, 428

Scarlet Siberian crab, 430

Screveton golden pippin, 417.

Shepherd's pippin, 423.

Shippen's russet, 421 .

Siberian bitter sweet, 432

Siberian crab, large scarlet, 430 .

Siberian crab, scarlet, 430.

Siberian crab, yellow, 430 .

Siberian Harvey, 432.

Simpson's pippin, 420 .

Simpson's seedling, 420 .

Sops in wine, 432.

Spring Ribston, 415.

Stagg's nonpareil, 419

Stead's kernel, 432.

Stettin pippin, 425.

Stone pippin, Norfolk, 42.9.

Stonyroyd pippin, $42 \%$.

Straat, 421 .

Striped beaufin, 429 .

Strumer pippin, 429 .

Sudlow's fall, 417.

Sugar-loaf pippin, 429.

Summer gilliflower, 417.

Summer golden pippin, 417.

Summer nonpareil, 419.

Summer pearmain, 428 .

Summer pearmain, Anierican, 422 .

Summer pippin, 426.

Summer queening, 428.

Summer thorle, 421 .

Superintendant, 429 .

Swaar, 423.

Sweet bough, 418.

Sweet liarvest, 418 .

Sweet lading, 432.

Syke House russet, 421 .

Tarvey codlin, 429 .

Thorle, summer, 421.

Tower of Glammis, 429 .

Travers's, 421

Triomphante, 429

True yellow Settin, 429

Wadhurst pippin, 42.9.

Walmer Court, 428 .

Waltham Abbey seedling,

429.

Wanstall, 422.

Warner's king, 429.

Watch, 424 .

Watson's nonsuch, 422 .

Wellington, 424.

White Astrachan, 423

White bellflower, 422 .

White cockle pippin, 415 .

White Detroit, 42\%

White Hawtlıornden, 426.

White Juneating, 418.

White lily, 424

White Melrose, 427.

White paradise pippin

4 97.

White pippin, 427, 429 .

White stone pippin, 429.

Wliorle pippin, 422. 
APRICOTS-continued.

Masculine red, 520.

Masculine white, 520 .

Miss Shipley's, 519.

Moorpark, 520

Musch-musch, 521.

New early Kaisha, 520.

New red-fleshed late Frogmore, 521 .

Oldacre's Moorpark, 521.

Orange, 521.

Peach, 520.

Peach royal, 521

Pêche, 521.

Pêche grosse, 521.

Persique, 519.

Precoce d'Esperen, 521.

Red masculine, 520 .

Roman, 521.

Royal, 521.

Royal George, 521 .

Royal orange, 521 .

Royal Persian, 521 .

Slipley's, 519.

Shipley's large, 519.

Sudlow's Moorpark, 521.

Tardive d'Orleans, 521.

Temple's, 521.

Transparent, 521.

Turkey, 521 .

Walton Moorpark, 521.

White masculine, 520 .

Wurtemberg, 521 .

\section{ARTICHOKES.}

Conical, 133

Dwari globe, 133

French, 133.

Large globe, 133.

Oval, 133.

\section{ASPARAGUS.}

Battersea; 128.

Giant, 128 .

Gravesend, 128.

Grayson's, 128 .

True Reading giant, 128.

BALM, 236.

BASIL, 237.

Bath asparagus, 134.

\section{BEANS.}

Bog, 67.

Broad Windsor, 67.

Child's long pod, 68 .

Common long pod, 67 .

Dutch long pod, 67 .

Dwarf cluster, 67.

Dwarf crimson - seeded, 68.

Dwarf fan, 67 .

Early Aldridge, 67.

Early Bromley, 67 .

Early Lisbon, 67.

Early long pod, 67

Early Malta, 67.

Early Mazagan, 67.

Fan, 67.

Fève de Mazagan, 67.

Fève de Windsor, 67.

Fève de Windsor verie, 67.

Fève naine hâtive, 67.

Fève naine rouge, 68 .

Fève toujours verte, 67 .

Fève très naine rouge, 68 .

Fève verte, 67 .

Fève verte de la Cline, 68 .

Fève violette, 68

Gregory's early hang down, 68.

Green China, 68.

Green cluster, 68 .

Green fan, 68 .

Green Genoa, 67.

Green long pod, 67, 68.
Green nonpareil, 67.

Green Windsor, 67.

Hang down long pod, 67.

Johnston's wonderful, 67.

Kentish Windsor, 67 .

Large long pod, 67.

Lisbon, 67.

Long pod, 67.

Marshall's early dwarf prolific, 67.

Marshall's prolific, 67 .

Moon, 67.

Mumford, 67.

New Windsor, 67

Red Windsor, 68 .

Royal dwarf cluster, 67.

Sandwich, 67.

Sangster's imperial long pod, 68 .

Small Spanish, 67

Stidolph's new early, 67 .

Sword long pod, 67.

Taylor's improved new Windsor, 67.

Taylor's large Windsor, 67 .

Taylor's Windsor, 67.

Thick-seeded Windsor, 68.

Toker, 68.

Turkey long pod, 67 .

Vilmorin's dwarf red seeded, 68.

White blossomed, 67 .

White-blossomed long pod,

Windsor, 67.

Windsor long pod, 67.

Wrench's early moon, 67

Wrench's improved Windsor, 67.

\section{BEET, WHITE OR} SICILIAN.

Brazilian, 140

Common white, 140

Golden veined, 140 .

Green, 140.

Green leaved, 140 .

Large white, 140 .

Red veined, 140 .

Small rooted, 140.

Sea, 140.

Silver veined, 140

White veined, 140.

BLADDER CAMPION, 134.

BLACK BRyONy, 134

Borage, 234.

\section{BORECOLES.}

Anjou, 110.

Asparagus, 110

Buda, 110.

Brown, 109.

Camberwell, 110.

Canada d warf curled, 109.

Cesarean, 110.

Chinese cabbage, 110.

Cockscoinb, 110

Curled brown, 109.

Curled red, 109.

Delaware, 110.

Duke of York's, 110.

Dwarf curled, 109

Dwarf curlies, 109.

Dwarf feathered, 110.

Dwarf German, 109.

Dwarf green, 109.

Dwarf green curled, 109.

Dwarf purple, 110.

Dwarf variegated, 110.

Dwarf winter curled, 109.

Flanders, 110.

French dwarf curled, 109

German cabbaging, 110 .

Great cow cabbage, 110

Green, 109.

Green Scotch, 109

Hamburg, 110.
Imperial hearting or cabbaging, 110.

Jagged, 110.

Jerusalem, 110.

Labrador, 109.

Manchester, 110.

Palm, 110.

Prussian, 110

Purple, 109.

Purple winter, 109.

Ragged Jack, 110.

Red, 109.

Russian, 110.

Scotch, 109.

Tall German, 109

Tall green, 109 .

Tall green curled, 109.

Tall purple, 109.

Tall Scotch, 109.

Tliousand-headed cabbage, 110.

Tree cabbage, 110.

Variegated, 109.

Variegated plumage, 109.

Very dwarf, 109.

Woburn perennial, 110.

Bramble, 594.

\section{BROCCOLIS}

Adam's superb early white, 95

Addison's, 95.

Anierican white, 95.

Chappell's large cream, 95 .

Dancer's pink cape, 95 .

Danish, 95 .

Dilston's bride, 95 .

Early Malta, 95 .

Early purple or sprouting, 94.

Elletson's gigantic late white, 95 .

Gillespie's, 94.

Grange's early cauliflower, 94.

Hammond's white cape, 95 .

llardy green cape, 95 .

Howden's purple, 95 .

Impregnated white, 95

Knight's protecting, 95.

Late dwarf purple Syrian, 95.

Late green, 95 .

Late London white, 94

Miller's dwarf, 95 .

Portsmouth cream coloured, 95.

Purple Cape, 95.

Russian dwarf, 95.

Siberian, 95.

Small green Danish, 95 .

Snow's superb white, 95 .

Snow's winter white, 95

Sumner's late white, 95 .

Stewart's early white, 95

Tamworth white, 95 .

Walcheren, 94.

Willcove late white, 95 .

Winter imperial, 95 .

Brooklime, 172.

Brussels sprouts, 91.

BuRDOCK, 134.

Burnet, 167.

\section{CABBAGES.}

A berdeen red, 91.

Atkin's matchless, 88.

Battersea, 89.

Cattel's dwarf Barnes, 89 .

Cattle, 89.

Chappell's colewort, 89 .

Cornish, 89.

Curled, 89.

Drumhead, 91.

Dutch red, 91.

Dwarf red, 90.

Early blood red, 90 .

Early Dutch twist, 89 .

Early emperor, 89.

Early imperial, 89.

Early nonpareil, 89

Early York, 89 .

Flat pole, 89 .

Fulham, 89.

Imperial, 89.

Kerguelen's Land, 90

King, 89.

Knight's early dwarf, 89 .

Large drumhead, 89

Large red, 91.

London market, 89.

M'Ewan's, 89.

Paignton, 89.

Pentonville, 89.

Pomeranian, 89.

Portugal, 89, 90.

Red, 90.

Red pickling, 90 .

Scotch, 89.

Shilling's queen, 89 .

Shilling's superb dwarf, 89.

Sprotboro, 89.

Strasburg, 89 .

Sutton's dwarf comb, 88 .

Sutton's imperial, 88 .

Tall red, 90.

Tiley's early marrow, 89. 
CAULIFLOWFRS-cont.

Epp's superb, 103

Fine late, 103

Large Asiatic, 103

Large late German, 103.

London particular, 103.

Mercer's new pearly, 103.

New dwarf late Cyprian, 103.

Walcheren, 103

Celeriac, 150 .

CELERY.

Cole's red, 148.

Cole's superb red, 148 .

Cole's superb solid red, 148 .

Cole's superb white, 148

Court hâtif, 148 .

Curled white, 148

Early dwarf solid white, 148.

Fine wlite solid, 148 .

Giant, 148.

Italian, 148.

Large upright, 148

Lion's paw, 148 .

Manchester giant red, 148.

Nain frize, 148

New large purple, 148

New large red, 148

New Russian, 148.

Nutt's champion, 148

Old solid red, 148

Patagonian, 148.

Plein blanc, 148 .

Red solid, 148.

Seymour's white champion, 148.

Shepherd's red, 148.

Striped solid, 148.

Sutton's solid white, 148 .

Turc, 148.

Turnip-rooted, 150

Upright, 148

Upright Italian, 148

Violet de Tours, 148

Wall's white, 148 .

White solid, 148 .

Chamomile, 241.

\section{CHERRIES.}

Amber, 541.

Amber à petit fruit, 513 .

A mber gean, 544

Amber heart, 543

Anglaise, 543.

Anglaise tardive, 541, 512, 543.

Ansell's fine black, $5 \pm 2$.

Archduke, 541.

Armstrong's bigarreau, 541 .

Belle de Choisy, 541.

Belle de Laeken, 544.

Belle d'Orleans, 544.

Benham's fine early duke, 543.

Bigarreau, 541.

Bigarreau, black, 541 .

Bigarreau couleur de chair, 541.

Bigarreau de Hollande, 541.

Bigarreau gros, 541.

Bigarreau, Holland. 541.

Bigarreau, Napoleon, 541

Bigarreau, new large black, 542.

Bigarreau, royal, 541 .

Bigarreau tardive, 541.

Bigarreau tardive de Hildeslieim, 541.

Bigarreau, white, 541

Black bigarreau, 541 .

Black caroon, 542 .

Black caroon gean, 542 .

Black Circassian, 543.

Black corone, $5+3$.
Black Dutch gean, 544

Black eagle, 542 .

Black heart, 542

Black heart, Buttner's, 542

Black heart, Manning's early, 542 .

Black heart, Werder'searly, 542 .

Black morello, 543

Black Russian, 54.

Black Tartarian, $\mathbf{5 4 3}$

Bowyer's early heart, 542.

Buchanan's early duke, 543 .

Buttner's black heart, 542 .

Buttner's October morello, $5 \leftarrow 2$.

Buttner's yellow, 542 .

Castle Menzies gean, 544

Cherry duke, 542,543 .

Coe's late carnation, 544 .

Common red, 542

De Montmorency, 542 .

Donna Maria, 544.

Downer's late, 542 .

Downton, 542 .

Dredge's early white lieart, 543.

Dutch morello, 543

Early black, 542 .

Early duke, 543 .

Early May duke, 543 .

Early Richmond, 542 .

Early white heart, 543 .

Elton, 542 .

Flemish, 542 .

Flesh - coloured bigarreau, 541.

Florence, 542 .

Fraser's black, 543 .

Fraser's black heart, 543 .

Fraser's black Tartarian, 543.

Fraser's Tartarische, 543.

Fraser's white 'Tartarian, 543.

Fraser's white transparent, 543.

Frogmore new morello, 543.

Gean, 543.

Graffion, 541.

Groitte de Portugal, 541.

Harrison's heart, 541 .

11olland bigarreau, 541

Hungarian gean, 544.

Hybride de Laeken, 544 .

Inperial, 541

Italian heart, 541

Jaune de Prusse, 544

Jeffrey's duke, 542 .

Jeffrey's royal, 542 .

Jeffrey's royal caroon, 512 .

Kentish, 542.

Kentish late, 542

Kentish red, 542

Knight's early black, 542 .

Large heart-shaped bigar. reau, 541 .

Large May duke, 543 .

Large morello, 543

Large white bigarreau, 511 .

Late archduke, 541

Late duke, 541,542 .

Late Kentisl, , 542 .

Late morello, 543 .

Manning's early black heart, $5+2$.

May duke, 542 .

Milan, 543.

Millet's late heart duke, 543.

Monstreuse de Bavay, 544.

Morello, 543.

Morello, Frogmore new, 543.

Morello, Rumsey's late, 543.

Morris's duke, 543 .

Morris's early duke, 513.

Muscat de Prague, 542.
New large black bigarreau, 542.

Ox lieart, 541 .

Pie, 542.

Portugal duke, 541, 543

Prussian yellow, 544.

Reine Hortense, 544

Rivers' early amber, 544.

Ronald's heart, 543 .

Ronald's large black lieart, 543

Ronald's morello, 543.

Royal bigarreau, 541 .

Royal duke, 543 .

Royal Kensington duke, 542

Royale, 542 .

Royale liâtive, 543 .

Royale ordinaire, 542 .

Rumsey's late morello, 543 .

Sir Herbert Taylor's morel-

lo, 543 .

Spanish black heart, 542.

Spotted bigarreau, 541

Superb Circassian, 513

Sussex, 542.

Tardive de Mons, 544.

Tartarian, 543 .

Tartarian black, 543 .

Tartarian white, $5 \pm 3$.

Tliompson's duke, 543

Tradescant, $5+1$.

Transparent, 541.

Turkey bigarreau, 5\&1

Turkey lieart, 541 .

Virginian May, $5 \frac{12}{2}$.

Waterloo, 543.

Werder's early black, 512 .

Werder's early black heart, 510

West's white heart, 541 .

White bigarreau, 541 .

Wlite heart, 543 .

White ox lieart, 541 .

White transparent, 543 .

White Tartarian, 543

Yellow Spanish, 541 .

CHERVIL.

Curled, 171.

Frizzled-leaved French, 171

Plain, 171.

\section{CHESTNUTS.}

Devonshire prolific, $5(P 2$.

Downton, 562.

Knight's prolific, 562 .

Marron, 562.

New prolific, 562

Prolific, 562.

Chicory, see Succory.

Chive, 47.

Clary, 235.

CoRIANDER, 172, 240

CORN SALAD, 172.

Costmary, 237.

CotTon thistle, 134

Cranberries, 593.

CR ESSES.

American, 170.

Belleisle, 170.

Broad-leaved, 168

Broad-leaved Normandy, 169.

Garden, 168

Golden, 168 .

Indian, 170 .

Normandy curled, 168

Plain-leaved, 168.

Water, 169.

Winter, 170 .

CUCUMBERS.

Allen's victory of Suffolk,

Brownston's liybrid, 667 .

Conqueror of the west, 667 .

Cuthill's black spine, 667 .

Duncan's victory, 667 .

Early green cluster, 667.

Early long green prickly,

Early short green prickly,

Flannigan's, 667.

Gordon's wlite spine, 667 .

Hamilton's black spine, 667.

Holm Pierpoint wonder, 667.

llunter's prolific, 667 .

Kilway's victory, 667 .

Lord Kenyon's favourite, 667.

Pratt's hybrid, 667 .

Prize-fighter, 667

Ringleader, 667 .

Roman emperor, 667 .

Sion House, 667.

Snow's prize, 667 .

Sussex hero, 667.

Tiley's captivation, 667 .

Tiley's phenomenon, 667

Victory of Bath, 667 .

Victory of England, 667. 
ENDIVE-continued. Chicorée toujours blanche, 163.

Common yellow, 163 .

Curled, 163.

Curled Batavian, 163

Double yellow, 163 .

J)utch green curled, 163.

Fine curled, 163.

Fine curled Batavian, 163.

Green Batavian, 163 .

Green curled, 163

Italian green curled, 163.

Large Batavian, 163.

Large green curled, 163.

Lettuce-leaved Batavian, 163.

Long Italian green curled, 163.

New Batavian, 163

Scarole à feuille de laitue, 163.

Scarole blonde, 163.

Scarole de Hollande, 163.

Scarole grande, 163.

Scarole petite, 163

Scarole ronde, 163

Scarole rouge, 163.

Sinall Batavian, 163.

Sinall French green curled, 163.

Small green curled, 163.

Staghorn, 163

Triple-curled moss, 163

White Batavian, 163.

White curled, 163.

Yellow curled Batavian, 163.

Yellow winter, 163.

\section{FIGS.}

Angelique, 557.

Ashridge forcing, 558 .

Bayswater, 557.

Black Iscliia, 557.

Blue, 558.

Blue Burgundy, 558 .

Blue. Ischia, $557,558$.

Brown Hamburg, 557 .

Brown Iscliia, 557.

Brown Italian, 558.

Brown Naples, 558.

Brown Turkey, 558.

Brunswick, 557 .

Chestnut - coloured Ischia, 557.

Clementine, 557.

Common purple, 558.

Early, 558.

Early forcing, 557.

Ford's seedling, 557 .

Genoa, large white, 557 .

Hanover, 557.

Howick, 558 .

Ischia, black, 557 .

Ischia, brown, 557 .

Ischia, white, 557

Italian, 558 .

Jerusalem, 558 .

Large blue, 558 .

Large white Genoa, 557 .

Lee's perpetual, 558 .

Madonna, 557.

Malta, 557.

Malta white, 557.

Marseilles, 557 .

Murray, 558 .

Nerii, 558 .

Pocock's, 557.

Purple, 558.

Pregussata, 558.

Red, 557

Small blue, 558 .

Sinall brown, 557 .

Turkey, brown, 558 .

Walton, 558 .

White Isclia, 557
White Marseilles, 557 .

Wlite Naples, 558 .

White standard, 557 .

\section{FILBERTS.}

Barcelona, 565

Barr's Spanish, 565 .

Bond, 565.

Cape, 565 .

Cob, 565 .

Cosford, 565.

Downton large, 565 .

Downton large square, 565 .

Downton long, 565 .

Dwarf prolific, 565 .

Frizzled, 565.

Glasgow prolific, 565 .

Great cob, 565 .

Lambert's, 565 .

Lambert's large, 565 .

Large bond, 565 .

Large cob, 565 .

Large round cob, 565 .

Miss Young's, 565 .

Northamptonshire prolific, 565.

Pearson's prolific, 565 .

Prolific, 565.

Red, 565 .

Red hazel, 565

Sir John Aubrey's, 565.

Spanish, 565.

Thin shelled, 565 .

Toker, 565.

Wrotliam park, 565 .

White, 565 .

FUNGI.

Morell, 252.

Mushroom, 243.

Truffle, 253.

GARLIC.

African, 30.

Common, 30 .

Jamaica, 30.

Gakden patience, 138.

\section{GOOSEBERRIES}

Abraham Newland, 578 .

Admiral Rodney, 578 .

Alderman, 579

Alice Hawthorn, 579 .

Amber ball, 578 .

Andrew Faîrservice, 578 .

Antagonist, 579 .

Ardsley beauty, 579 .

Ashton on Mersey, 579 .

Asliton, 578 .

Atila, 579.

Atlas, 578, 579.

A way slie goes, 579 .

Bangor, 579

Banksman, 579

Battle of the Nile, 578 .

Beauty, 579.

Beeswing, 579

Bell's fancy, 578.

Belmont green, 578 .

Birdlime, 578, 579.

Black Prince, 578 .

Bloodhound, 578 .

Blunderbuss, 578 .

Bonny lass, 578 .

Bonny Roger, 578 .

Botherain, 579.

Braid's glory, 578,

Bright Venus, 578.

British crown, 578

British hero, 578.
British lion, 578 .

British queen, 578, 579.

Briton, 579.

Britannia, 578.

Broom girl, 579 .

Bumper, 578, 579 .

Bunker's Hill, 578 .

Cæsar, 578.

Calderbank's, 578 .

Captain, 578.

Captain Cook, 579.

Catherine, 579 .

Champagne, red, 578.

Champagne, white, 578 .

Champagne, yellow, 578 .

Chance, 579 .

Chedle's golden lion, 578 .

Cheshire cheese, 578 .

Cheshire lady, 578 .

Cheshire lass, 578.

Chief, 579.

Chorister, 579.

Columbia, 579 .

Comet, 579.

Commander, 579.

Companion, 579 .

Competitor, 579.

Conductor, 579 ,

Conquering hero, 579.

Conqueror, 578.

Coppice lass, 579

Cossack, 579 .

Cottage girl, 578 .

Crawford's, 578.

Creeping Jane, 579.

Crown Bob, 578, 579.

Crystal, 578.

Dan's mistake, 579.

Dauntìess, 579.

Dean Swift, 579 .

Defiance, 579 .

Delvin's ironmonger, 578.

Derby ram, 579 .

Diogenes, 579 .

Don Joln, 579.

Dreadnought, 578 .

Drill, 579.

Dublin, 579.

Duckwing, 578, 579 .

Eagle, 578, 579 .

Eagle's daughter, 579 .

Early golden lion, 578 .

Early white, 578 .

Echo, 579.

Emperor Napoleon, 578.

English yellow, 578 .

Eliza, 579.

Fair Helen, 579 .

Fair play, 579 .

Fair start, 579

Farmer's glory, 578.

Favourite, 578, 579.

Fletcher's jam, 578 .

Fleur-de-lys, 578, 579 .

Flixtonia, 579.

Flora, 579 .

Foley, 579 .

Forget me not, 579 .

Freedom, 579 .

Gamecock, 579.

Gascoigne white, 578.

General, 579 .

General Moore, 579.

Gillie's late, 579 .

Give it a name, 579 .

Glenton green, 579 .

Globe, 579.

Globe, small red, 578.

Globe, yellow, $5 \div 8$.

Glory, 579.

Glory of Ounsdale, 579.

Glory of Ratcliffe, 578 , 579 .

Gobye, 579 .

Gogar jam, 578

Golden ball, 578 .

Golden chain, 578 .

Golden dross, 578.

Golden lion, 578 .

Golden orange, 578 .

Golden seedling, 578.

Gold finder, 579 .

Goodall's, 578.

Governess, 578

Great Britain, 579.

Great Western, 579

Green, 579.

Green gage, 578.

Green Gascoigne, 579 .

Green globe, 579 .

Green ocean, 579 .

Green prince, 579 .

Green prolific, 578 .

Green providence, 578

Green river, 579.

Green snake, 579 .

Green walnut, 578

Green wonderful, 579 .

Greenock, 579.

Green wood, 578.

Gretna Green, 579 .

Guido, 578, 579.

Gunner, 579.

Harkaway, 579 .

Heart of oak, 578 .

Hebburn, 578.

Hedgehog, 578.
Higllander, 579

Hole in the wall, 578 .

Hollins' green, 579 .

Hopeley's Shannon, 578.

Huntsman, 578.

Independent, 579

Invincible, 579.

Irish plum, 578 .

Ironmonger, 578 . 
GOOSEBERRIES-cont. Napoleon le Grand, 579. Nelson's waves, 578 . Nicholl's, 578 .

No bribery, 578 .

Nonpareil, 578, 579.

Nonsuclı, 578 .

Northern hero, 578

Nutmeg, Scotch, 578 .

Oakmere, 579.

Ocean queen, 579 .

Old Briton, 578 .

Old England, 578, 579.

Oldham, 579 .

Orleans white, 578.

Ostrich, 578, 579.

Overall, 578. 579 .

Peacock, 578, 579

Pecher, 579.

Peover, 579 .

Peover ranger, 579.

Perfection, 579.

Peru, 579.

Philip the First, 579.

Pilot, 578, 579.

Pitmaston, 578.

Platt's white, 578.

Plumper, 579.

Porcupine, 578.

Prince of Orange, 578 .

Prince Regent, 578 .

Princess-royal, 578.

Prize Porterfield, 578.

Providence, 579.

Queen Anne, 578.

Queen Caroline, 578.

Queen Charlotte, 578 .

Queen Dowager, 579 .

Queen Victoria, 579

Queen of Sheba, 578 .

Qucen of trumps, 579 .

Radical, 578 .

Railway, 579.

Random green, 579 .

Raspberry, 578 .

Red champagne, 579.

Red Jack, 579.

Red Robin, 579.

Red Turkey, 578 .

Ricardo, 579 .

Rifleman, 578.

Roaring lion, 578, 579

Rob Roy, 578 .

Rookwood, 578, 579.

Rough green, 579 .

Rough red, 578 .

Royal George, 579 .

Royal oak, 578 .

Royal white, 578 .

Rumbullion, 578 .

Sam Hunter, 579.

Sappho, 578.

Scotch best jam, 578 .

Shakspeare, 578 .

Shaw's, 578 .

Shuttle, 579 .

Sir Francis Burdett, 578 .

Sir Jolın Colgrave, 578 .

Slaughterman, 579 .

Smiling beauty, 578

Smooth, 579 .

Smooth green, 578 .

Snowball, 579 .

Snowdrop, 579.

Speculator, 579 .

Speedwell, 578 .

Sportsman, 578 .

Starling, 578.

Stewart, 579.

Sulpliur, 578 .

Supreme, 578 .

Tally-ho, 579 .

Teaser, 579 .

Telegraph, 579 .

Thrasher, 578.

Thumper, 579.

Thunder, 579 .

Tim Bobbin, 578

Tom:'Joiner, 579

Top-gallant, 579 .
Top-sawyer, 578, 579.

Troubler, 579

Triumphant, 578.

Turkey cock, 579 .

Turnout, 579 .

Twigem, 579.

Two to one, 579 .

Useful, 579.

Victory, 578

Viper, 578 .

Wallace, 579

Walnut, 579.

Walnut red, 578

Warrington, 578, 579 .

Warrior, 578 .

Weathercock, 579.

Wellington's glory, 578 .

White Briton, 578.

White Caldwell, 578 .

White cockade, 579 .

White eagle, 578 .

White hare, 579.

White hart, 579

White lily, 579 .

White lion, 578.

White rasp, 578 .

White rose, 578 .

Whitesmith, 578 .

White wine, 578 .

Wilmot's early red, 578 .

Wilmslow, 579 .

Wistaston, 579 .

Wistaston hero, 579 .

Wonder, 578

Wonderful, 579.

Yaxley hero, 578 .

Yellow, 578 .

Yellow boy, 579.

Yellow companion, 579.

Yellow smith, 578 .

Gourd, 259.

\section{GR A PES.}

Abercairney, 635 .

Admiral, 633 .

Alexandrian Frontignan, 634.

Alicant, 632 .

Amber muscadine, 635

Amiens, 635 .

Black Barbarossa, 636.

Black Constantia, 633 .

Black Damascus, 633.

Black Frontignan, 633.

Black Gibraltar, 633

Black Hamburg, 633

Black Lisbon, 632 .

Black Lombardy, 634 .

Black Palestine, 636 .

Black Portugal, 633

Black Prince, 632.

Black St Peter's, 635 .

Black Spanish, 632.

Black Teneriffe, 633.

Black Valencia, 632.

Blue Frontignan, 633 .

Boston, 632.

Braddick's seedling Hamburg, 634 .

Brown Hamburg, 633 .

Calabrian raisin, 636 .

Cambridge botanic garden, 632.

Cannon Hall Muscat, 635.

Catawba, 636.

Charlsworth Tokay, 636.

Chasselas, 635 .

Chasselas de Fontainebleau, 635 .

Chasselas cioré, 635 .

Chasselas musqué, 632 .

Chasselas precoce, 636 .

Chasselas royal, 636 .

Common muscadine, 635 .

Constantia, 633.

Cumberland Lodge, 633.

Damascus, black, 633 .

D'Arbois, 635.
D'Arboyce, 635

De Clieras, 636.

Dutch Hamburg, 633.

Dutch sweet water, 636 .

Early white Teneriffe, 635 .

Esperion, 633.

Faskbee, 636.

Flame-coloured Tokay, 634 .

Frankendale, 633.

Frankenthaler, 633.

Frontignan, black, 633 .

Frontignan, blue, 633 .

Frontignan, grizzly, 633 .

Frontignan, purple, 633 .

Frontignan, red, 633 .

Frontignan, white, 633 .

Gibraltar, 633.

Golden drop, 633.

Grizeline, 633 .

Grizzly Frontignan, 633.

Gros coulard, 636 .

Hamburg, black, 633 .

Hampton Court, 633.

Hardy blue Windsor, 633 .

Hoolookee, 636 .

Hubshee, 634 .

Isabella, 636 .

Josling's St Alban's, 633.

Judson's Richmond Villa

black Hamburg, 634 .

Kamadanee, 636 .

Keslumushee, 636 .

Kishmish, 636 .

Knevett's black Hamburg, 634.

Kohlauree, 636.

Lashmar's seedling, 634

Lauree, 636.

Le cour, 633 .

Lombardy, 632, 634 .

Lombardy, black, 634 .

Lunel, 634.

Major Moray's, 635.

Malaya, 634.

Millhill Hamburg, 634.

Money's West's St Peter's, 634.

Moschata blanca, 633.

Moscatel commun, 633 .

Muscadine, royal, 635 .

Muscado Bianco, 633 .

Muscat blanc, 633

Muscat blanc de Jura, 633 .

Muscat esclicolota, 634 .

Muscat gris, 633.

Muscat of Alexandria, 634 .

Muscat of Jerusalem, 634.

Muscat rouge, 633 .

Muscateller, 633.

Musk Chasselas, 633.

Nepean's Constantia, 633.

Nice, 635.

Noir d'Espagne, 636.

Oldacre's West's St Peter's, 636.

Parel druyf, 636.

Passe longue musqué, 634.

Passe musqué, 634 .

Pearl, 635.

Pocock's Damascus, 632.

Poonah, 634

Pope's Hamburg, 634.

Purple Constantia, 633.

Purple Frontignan, 633 .

Purple Hamburg, 633.

Queen muscat, 635 .

Raisin de Calabre, 636.

Raisin de Carmes, 634 .

Raisin de Champagne, 635

Raisin de Cuba, 634.

Raisin de Frontignan, 633

Red Constantia, 633 .

Red Frontignan, 633

Red Hamburg, 633.

Red port, 633 .

Reeve's muscadine, 635 .

Round muscat of Alexan-

dria, 634 .

Royal muscadine, 635 .

Saliibee, 635 .

St Alban's, 633.

St Peter's, 636

St Peter's, black, 635 .

Salisbury violet, 633.

Sir Abraham Pytcle's black, 632 .

Sir W. Rowley's black, 633

Stewart's Black Prince, 632.

Still ward's sweetwater, 636 .

Sweetwater, 636 .

Syrian, 635.

Tokay, Charlsworth, 636 .

Tokay, white, 636 .

Tottenham Park muscat,

634.

Trebbiana, 636 .

Turner's black, 633 .

Valentine's, 633 .

Verdal, 636 .

Victoria, 633.

Warner's, 633. 
KIDNEY BEANS-cont.

Early dwarf pink spotted, 75.

Early dwarf purple speckled, 74 .

Early dwarf white, 74

Earliest forcing, 74 .

Early frame, 74.

Early Laon, 74.

Early negro, 75 .

Early purple, 74 .

Early six weeks, 75 .

Early white, 74 .

Early yellow, 75 .

Flageolet, 74 .

Fulmer's dwarf red, 74 .

Fulmer's early, 74 .

Fulmer's early dwarf, 74

Fulmer's speckled dwarf, 74.

Grey, 75 .

Haricot d'Alger, 75 .

Haricot de la Chine, 75 .

Haricot nain noir, 75 .

Haricot sabre à très grande cosse de Soisson, 75 .

Haricot sabre d'Allemagne, 76 .

Haricot solitaire, 75

Large forcing dark red speckled, 74

Large pearl, 75 .

Large running white, 75 .

Large white dwarf Dutch, 74.

Large white sugar, 75 .

Liver-coloured, 75

Long-podded Clina, 75 .

Long-podded negro, 75 .

Long white, 75 .

Magpie, 75 .

Marbled Prague, 75.

Mohawk, 75 .

Nain de Hollande très hâtif, 74

Nain luâtif de Hollande, 74 .

Nain lıatif de Laon, 74 .

Negro, 76 .

Newington wonder, 75 .

Noir de Belgique, 74 .

Robin's egg, 75 .

liound dwarf, 75 .

Runner, 75 .

Sable, 75.

Sabre nain, 74 .

Shilling's new, 76 .

Small pearl, 75 .

Suisse rouge, 74

Tender podded, 75 .

Turkische Blone, 75 .

Victoria speckled, 75 .

Write long pod, 75.

White long pod Dutch, 74 .

Wilmot's forcing cream speckled, 74 .

Wilmot's true forcing, 76

LAVENDER, 261.

\section{LEEKS.}

Dutch flag, 46.

Edinburglı improved, 46. English fiag, 46. Erfurt, 46.

Hollow, 46.

Large flag, 46.

London flag, 46

Musselburgli, 46.

Rouen, 46.

Scotch flag, 46.

\section{LETTUCES.}

A bord rouge, 156 .

Ady's fine large cos, 155.

Aleppo, 156.

Alpliange cos, 156.

Artichoke leaved, 156.
Asiatic, 156.

Batavia blonde, 156 .

Batli cos, 155 .

Bath green cos, 156

Bearfield, 156.

Belle bonne, 156 .

Black-seeded Bath cos, 156.

Black-seeded grotte, 156 .

Black-seeded yellow, 156 .

Blonde à graine noire, $\mathbf{1 5 6}$

Blonde de Berlin, 156 .

Blonde d'été, 156.

Blonde paresseuse, 156.

Bloody, 156.

Bord rouge, 156.

Brighton cos, 156

Brighton green cos, 156 .

Brown Bath cos, 155 .

Brown cos, 155.

Brown Dutch, 156.

Button, 156.

Capuchin, 156.

Chicon, 155

Drumhead, 156

Early cabbage, 156 .

Early dwarf Dutch, 156.

Farly frame, 156.

Early green cos, 156 .

Early green forcing, 156 .

Early yellow, 156.

Egyptian cos, 156.

Egyptian green cos, 156.

Florence cos, 156 .

Golden cos, 156.

Grand admirable, 156.

Green ball, 156.

Green Dutch, 156.

Green Paris cos, 155.

Grosse Allemande, 156.

Grosse brune paresseuse, 156.

Grosse gris, 156

Hardy brown cos, 155.

11ardy green, 156 .

Hardy green Hammersmith, 156.

Hollande, 156.

Ice, 156.

Imperial, 156.

Jaune d'été, 156

Kensington cos, 155 .

Large cabbage, 156

Large drumhead, 156.

Large Mogul, 156.

Large white, 156.

Late cabbage, 156 .

London white cos, 155

Malta, 156.

Metrelle, 156

Mogul, 156.

Neapolitan, 156.

New crystal cos, 156

Nonpareil, 156.

Panachée, $] 56$.

Paris white cos, 155 .

Princess, 156.

Prussian cabbage, 156.

Red-spotted cos, $156^{\circ}$

Romaine à feuilles d'artichaut, 156.

Romaine blonde Maraichère, 155 .

Romaine panachée à grain blanc, 156.

Romaine verte Maraichère 155.

Roman cabbage, 156

Royal à graine blanche, 156 .

Royal à graine noire, 156 .

Sanguine, 156.

Saxony, 156.

Spanish, 156

Sugar, 156.

Superfine new French, $\mathbf{3 5 6}$

Sutton's Berkslire brown cos, 155 .

Sutton's superb green cos, 155.

Sutton's superb white $\cos$, 155.
Swedish, 156.

Tennis-ball, 156

Turque, 156

Turque à graine noire, 156 .

Verde, 156 .

Versailles, 156 .

Versailles blonde, 156.

Victoria cabbage, 156 .

Victoria cos, 156

Waite's white cos, 155 .

White Dutcli, 156

White-seeded Bath cos, 156.

White-seeded brown cos, 155.

White Silesian, 156.

Wond's improved Bath cos, 155.

LIQUORICE, 242.

LOVE A PPLE, 258.

MARYGOLD, 240 .

Marjoram, 238.

\section{MEDLARS}

Broad-leaved Dutcl, 560 .

Common, 560.

Dutch, 560 .

Large-fruited, 560 .

Large German, 560 .

Monstrous, 560 .

Narrow-leaved Dutch, 560

Nottingliam, 560 .

Small-fruited, 560 .

\section{MELONS.}

Beechwood, 671.

Bromham Hall, 671 .

Carolina, 672.

Cassabar, 672.

Cutlill's early cantaleup, 671.

Dampslia, 672 .

Darce, 672.

De la Morée, 672.

Geree, 672.

Green - fleshed beechwood, 671.

Green - fleshed Egyptian, 671.

Green - fleshed Masulipatam, 671 .

Green-fleshed Trentliam hyrid, 671 .

Green Housainee, 672

Green Valencia, 672

Hardy ridge, 672 .

Imperial, 672.

Ispahan, 672.

Keiseng, 672.

Large germek, 672 .

Ostrich egg, 672.

Spanish, 672.

Sweet of Ispahan, 672

Trentham hybrid, 671.

Milk thistle, 134

Mint, 236.

Mulberries, 592

Mushrooms, 243.

Mustard, white, 167BLACK, 168.

\section{NECTARINES.}

Anderdon's, 511

Anderdon's round, 511.

Anderson's, 511.

Aromatic, 512

Barker, 512.

Black, 511.

Bloomfield, 512.

Boston, 512.

Brugnon, 511

Brugnon musqué, 512

Brugnon red at the stone, 512.

Brugnon violette musqué, 512.

Clermont, 511.

Common elruge, 511.

De Tellier's, 510 .

Downton, 511.

Du Tilly's, 510

Duc de Tellier's, 510

Duc de Tello, 510 .

Early black, 511 .

Early black Newington, 511.

Early brugnon, 512

Early Newington, 511.

Early violet, 512 .

Elruge, 510.

Fairchild's, 511.

Fairchild's early, 511.

Fine gold-fleshed, 511

Frencli Newington, 511 .

Golden, 511.

Hampton Court, 512.

Hardwick seedling, 511 .

Hâtive, 512.

Hoy's new seedling, 511 .

Hunt's early tawny, 511.

Hunt's large tawny, 511.

Hunt's small tawny, 511.

Hunt's tawny, 511.

Large scarlet, 512. 
ONIONS-continued.

Lisbon, white, 40.

Madeira, large, 40.

New giant, 40 .

New white.glo be, 40 .

Nocera, 40.

Pale bloodired, 40 .

Pickling, 41.

Portugal, brown, 40 .

Portugal, true, 40.

Portugal, white, 40.

Potato, 41.

Reading, 40.

St Thonias's, 40.

Silver-skinned, 40.

Sinall silver-skinned, 40 .

Spanish, wlite, 40 .

Strasburg, 40.

Tree, 40.

Tripoli flat, 40 .

Tripoli round, 40 .

True Portugal, 40.

Two bladed, 40.

Underground, 41

Welsh, 40.

White globe, new, 40 .

White Portugal, 40

White Spanish, 40 .

\section{PARSLEY.}

Celery, 233.

Englefield extra curled, 233.

Fine curled, 233.

Garnishing, 233 .

Hamburg, 233.

Myatt's, 233.

Naples, 233.

Plain-leaved, 233.

Reid's fine curled, 233.

Triple curled, 233

Very dwarf, 233.

\section{PARSNIPS.}

Batard, 230.

Common long-rooted, 230.

Coquine, 230.

Early short horn, 230.

Guernsey, 230.

Hollow-crowned, 230.

Hollow-headed, 230.

Jersey, 230.

Lisbonnais, 230.

Long Jersey, 230.

Longue, 239.

Rond, 230.

Royal, 230.

Turnip-rooted, 230.

\section{PEACHES.}

Acton Scot, 507.

Admirable, 507 .

Admirable early, 507.

Admirable late, 507.

Alberge jaune, 507, 509.

Alberge yellow, 507 .

A vant, 508.

Avant rouge, 509.

Baltimore beauty, 510 .

Barrington, 507.

Belle bauce, 508 .

Belle bausse, 508 .

Belle bausse or bauce, 507 .

Belle beauté, 508 .

Belle de Vitry, 507 .

Bellegarde, 507

Bergen's yellow, 510

Blood clingstone, 510 .

Boudine, 507.

Bourdine, 507.

Brentford mignonne, 507.

Brevoort, 510.

Buckingham mignomne, 507.

Catherine, 507.

Chancellière, 508 .

Cliancellor, 507.

Chinese, 508.

VOL. II.
Colonel Ansley's, 507.

Columbia, 510.

Cooledge's favourite, 510 .

Crawford's early meloco ton, 510 .

De Troyes, 508.

Double de Troyes, 508

Double Swalsh, 509 .

Downton early, 507 .

Druid Hill, 510.

Du Vin, 509 .

Early admirable, 507.

Early Anne, 508.

Early avant, 509 .

Early Bourdine, 509 .

Early Downton, 507 .

Early French, 508.

Early galande, 507 .

Early May, 508.

Early mignonne, 508.

Early purple, 509 .

Early purple avant, 508 .

Early purple of Kew, 509 .

Early Royal George, 509.

Early sweetwater, 510 .

Early Tillotson, 510 .

Early vineyard, 508 .

Early York, 510 .

Edgar's late melting, 508.

Flat peach of China, 508 .

Foster's, 508 .

Foster's early, 508 .

French Bourdine, 507.

French chancellor, 509.

French Magdalene, 508.

French Royal George, 507

Galande, 507

George the Fourth, 508.

Gold-fleshed, 507.

Golden mignonne, 507.

Griffin's mignonne, 509 .

Grimwood's new Royal George, 508.

Grimwood's Royal Charlotte, 509.

Grimwood's Royal George, 508.

Haine's early red, 510 .

Heatl, 510.

Italian, 508.

Jaune, 507, 509.

Java, 508.

Johnston's early purple, 508.

Johnston's purple avant, 508.

Judd's melting, 507 .

Kensington, 508 .

Knight's early, 507.

La Grange, 510.

La royale, $507,508$.

Large French mignonne, 508.

Large violet, 507.

Large white clingstone,

Late admirable, 507.

Late chancellor, 508 .

Late purple, 507, 508.

Late Warburton, 508 .

Lemon clingstone, 510.

Lockyer's mignonne, 509.

Lord Fauconberg's, 509.

Lord Fauconberg's mignonne, 509.

Lord Montague's noblesse, 509

Lord Nelson's, 509.

Madeleine à petites fleurs, 509 .

Madeleine de Courson, 508.

Madeleine rouge, 508 .

Madeleine rouge à moyennes fleurs, 509 .

Madeleine rouge à petites fleurs, 509.

Malta, 508 .

Mellish's favourite, 509 .

Mignonne, 508 .

Mignonue grosse, 508 .
Mignonne petite,.508.

Mignonnette, 508 .

Millet's mignonne, 509 .

Morris' red rareripe, 510 .

Morris' white rareripe, 510 .

Motteux's, 507.

Neal's early purple, 508 .

Neil's early purple, 508 .

New Royal Charlotte, 509.

New Royal George mignonne, 509 .

Noblesse, 508

Noir de Montreuil, 507.

Noisette, 508.

Old Royal Charlotte, 509

Oldmixon, 510 .

Orange, 510.

Padley's early purple, 508 .

Petite Rosanne, 509

Pool's large yellow, 510.

Pourprée hâtive, 508, 509.

Pourprée hâtive à grandes fleurs, 509.

Pourprée tardive, 507, 508.

President, 510

Purple alberge, 507.

Purple avant, 508 .

Purple hâtive, 508.

Rareripe, late red, 510 .

Red alberge, 507.

Red cheek Melocoton, 510.

Red Magdalene, 509.

Red nutmeg, 508

Red rareripe, 510 .

Ronald's Brentford mignonne, 507.

Ronald's early galande, 508 .

Ronald's seedling galande, 508.

Rosanna, 507, 509.

Rouge paysanne, 508 .

Royal, 507.

Royale, 507.

Royal Charlotte, 509 .

Royal George, 509 .

Royal George mignoune, 509.

Royal Kensington, 508

Royal Sovereign, 508 .

St Laurent jaune, 509

Seott's eariy red, 510 .

Shanghae, 510 .

Small mignonne, 508

Sinooth-leaved Royal

George, 507.

Snow, 510.

Stewart's late galande, 508

Strawberry, 510

Superb, 509

Superb royal, 508 .

Swiss mignonne, 508.

Teton de Venus, 507.

Tippecanoe, 510.

Transparent, 508.

True early purple, 509 .

True red Magdalene, 508.

Vanguard, 509.

Van Zandt's superb, 510 .

Veloutée, 508.

Veloutée de Merlet, 508.

Veritable, 509.

Vineuse, 508.

Vineuse de Fromentin, 508.

Violette hâtive, 507.

Violette hâtive grcsse, 507.

Walter's early, 510.

Warburton admirable, 508

Warburton seedling, 508 .

Washington, 510.

Washington clingstone 510.

Wlite imperial, 510 .

White nutmeg, 508 .

Williams' Catlierine, 507.

Williams' early purple, 509 .

Williams' seedling, 509 .

Yellow alberge, 507 .

Yellow rareripe, 510.

PEARS.

Alèle de St Denis, 459

A court quesse, 455

Althorp crassane, 455 .

Amoselle, 453.

Andrew's, 452.

Ashton town, 452 .

Auch, 455 .

Autumn bergamot, 452

Badham's, 453.

Barland, 463 .

Beadnell's seedling, 452.

Beauchamps, 453.

Beau present, 4.57 .

Beau present d'Artois, 459

Bein Armudi, 454.

Bell, 462.

Belle après Noel, 459 .

Belle et bonne, 45\%.

Belle Hugevin, 459 .

Belle noisette, 461

Bellissime d'été, 457.

Bellissime d'hiver, 461 . 
PEARS-continued.

Beurré de Paques, 453.

Beurré de Payence, 454

Beurré de Ranz, 454.

Beurré des Orphelines, 453.

Beurré de Streckman, 460.

Beurré d'hiver, 455.

Beurré d'hiver de Bruxelles, 453.

Beurré d'hiver nouvelle, 457.

Beurré Diel, 453.

Beurré doré, 453.

Beurré du roi, 453 , 455 .

Beurré Dumortier, 460 .

Beurré Duval, 453.

Beurré, Easter, 453.

Beurré Epine, 454.

Beurré Giffara, 460.

Beurré, golden, 453.

Beurré gris, 453.

Beurré gris d'hiver nou veau, 453,460 .

Beurré, Gurle's, 452.

Beurré Hardenpont, 457.

Benrré in comparable, 453.

Beurré Kenrick, 453.

Beurré Knox, 453

Beurré Langelier, 453, 460.

Beurré lucratif, 460 .

Beurré magnifique, 453, 460.

Beurré,Mollett's Guernsey, 454.

Beurré Monfontaine, 460 .

Beurré Navez, 460 .

Beurré plat, 455 .

Buerré preble, 454

Beurré iRance, 45

Beurré, red, 453.

Beurré Robin, 460 .

Beurré rouge, 453 .

Beurré roupe, 453.

Beurré royal, 453.

Beurré Seutin, 460 .

Beurré Spence, 454

Beurré superfin, 460 .

Beurré suprême, 460 .

Beurré vort, 453

Beurré, winter, 453,460 .

Bezi Clıaumontel très gros, 453.

Bezi de Chaumontel, 455

Bezi d'Esperu, 460.

Bezi d'Heri, 461.

Bezi de la Motte, 454.

Bezi royal, 461 .

Black Auchan, 460.

Bleckers meadow, 454 .

Bloodgood, 452.

Bon Chretien, Flemish, $46^{3} 1$.

Bonchretien fondant, 454.

Bon Chretien nouvelle, 46.

Bon Chretien Turc, 461.

Bon Gustave, 460 .

Bonne de Malines, 458 .

Bonne-ente, 455 .

Bonne Louise d'Araudore, 457.

Bonne rouge, 452 .

Bose sine, 456 .

Bosc's flaskenbirne, 453.

Bosch, 456.

Boston Esparne, 457.

Bouche nouvelle, 456 .

Bourgermester, 459.

Braddick's field standard, 458.

Brilliante, 456 .

Brocas Bergamot, 452

Brougham, 454

Brown beurré, 453

Brown huffcap, 463.

Buffam, 454.

Calebasse, 454.

Calebasse Bosc, 453.

Calebasse d'Hollande, 454.

Calebasse, double extra 454.
Calebasse musque, 454 .

Calebasse vass, 453.

Cambridge sugar, 457.

Canning, 453, 460 .

Capiaumont, 453.

Catillac, 461.

Cellite, 458.

Chambers' large, 462.

Chapman's, 458 .

Chaptal, 454

Charles d'Autriche, 458.

Chaumontel, 454.

Citron des Carmes, 455.

Clion, 459.

Colmar, 455 .

Colmar Deschamps, 453.

Colmar d'hiver, 456 .

Colmar d'or, 458.

Colmar doré, 455 .

Colmar epineux 458 .

Colmar gris, 458 .

Colmar Hardenpont, 458.

Colmar Neil, 455.

Colmar Preul, 458.

Colmar souverain, 458.

Colombia Virgalouse, 455 .

Colombian Virgalieu, 455.

Columbia, 455,460 .

Common bergamot, 452

Compte de Flandre, 461 .

Comte de Lamy, 455 .

Copea, 455.

Courel, 463.

Crassane, 455.

Crassane, Althorpe, 455

Crassane, winter, 455 .

Cresane, 455 .

Cuisse madame, 457.

Cushing, 455 .

Cuvelier, 458.

D'Anans, 458.

D'A remberg parfait, 453.

D'Auch, 455 .

Dean's, 455.

Dearboru's seedling, 455 .

De Bordeaux, 461.

De Bur, 461 .

De cadet, 453 .

Pelice de Jodoigne, 461.

De Louvain, 455 .

De Manne, 455.

De melon, 453.

Deschamps, 453

De Septembre, 455 .

De Spoelberg, 461 .

Dianiant, 452.

Diel's butterbirne, 453.

Dingler, 455 .

Dix, 455 .

Dr Bouvier, Van Mons', 461.

Dorothée royale, 453

Downham seedling, 457 .

Downton, 455 .

Doyenne blanc, 455 .

Doyenne d'automne, 456 .

Doyeune d'Effay, 461.

Doyenne d'hiver, 453.

Doyenne d'hiver nouveau, 453.

Doyeune du printemps, 453.

Doyenne galeux, 456

Dovenne Goubault, 461 .

Doyenne gris, 455.

Doyenne nouveau, 461.

Doyenne rouge, 456 .

Drapiez, 461.

Duc d'Aremberg, 453.

Duchesse d'Angoulêne, 456.

Dumas, 4.59.

Dundas, 456.

Dunmore, 456.

Du Patre, 453.

Easter bergamot, 461 .

Easter beurré, 453.

Eliza d'Heyst, 461.

Elton, 456.

Emerald, 456.
English bergamot, 452.

English jargonelle, 457.

Espargne, 457.

Etrounieau, 458 .

Eyewood, 456 .

Fin or d'hiver, 462.

Flemish beauty, 456 .

Flemish bon chretien, 461

Fondante d'automne, 456.

Fondante de Charmensier, 461

Fondante de Mons, 458.

Fondante de Panisel, 458

Fondante du bois, 456 .

Fondante du bois nouvelle, 456.

Forelle, 456

Forellenbirne, 456.

Forme de Marie Lonise, 458.

Foxley, 463

Franc real, 462

Franc real d'hiver, 462.

Frauenschenkel, 457.

Frederick de Wurtemberg, $456,461$.

French jargonelle, 457.

Fulton, 456 .

Galston's moorfowl egg, 458.

Gambier, 458.

Gansal's bergamot, 452 .

Gansal's late bergamot, 452.

Garde d'Ecosse, 462

Germain baker, 462

Gilogil, 462.

Glout morceau, 456

Gloux morceau, 457.

Gobert, 462.

Golden beurré, 453

Got luc de Cambron, 457.

Gracieuse, 452.

Grande Monarque, 462.

Grand soleil, 461.

Grey, Jean's, 456.

Grey doyenne, 456 .

Groome's Princess-royal, 157.

Groote Mogul, 462.

Gros Dillen, 453.

Grosse cuisse madame, 457.

Gurle's beurré, 452

Hacon's incomparable, 457.

Hampton, rough, 463 .

Hardenpont d'hiver, 457.

Hardenpont du printemps, 454.

Harvard, 457.

Hessel, 457.

Holemore, 463

Huffeap, brown, 463.

Huffcap, red, 463 .

Huffcap, yellow, 463.

Imperatrice de France, 456.

Incomparable, 455 .

Isambert, 453

Isambert le bon, 453 .

Ive's bergamot, 452 .

Jargonelle, English, 457.

Jargonelle, Erench, 457.

Jean de Witte, 457.

Jersey Chaumontel, 455

Tosephine, $456^{\circ}$.

Josephine de Malines, 461.

Kenrick's beurré, 453 .

Kniglit's monarch, 458.

Knox's beurré, 453

La belle de Flandre, 456 .

Jangelier's beurré, 460 .

Lawrence, 457.

Le Curé, 459 .

Lent St Germains, 462

Leon le Clerc, 462.

Leon le Clerc, Van Mons', 459.

Linden d'automne, 457.

Longland, 463.

Lord Cheney's, 453.

L'Orpheline, 453

Louise Bonne d'A vranches, 457.

Louise Bonne of Jersey,

Louvain, 455 .

Madeleine, 455.

Magdalene, 455 .

Manne, 455.

Mansuette, 458 .

Marianne nouvelle, 453

Marie Chretien, 458 .

Marie Delcourt, 458 .

Marie Louise, 458.

Marie Louise, Van Mons', 458.

Marie Louise Nova, 455.

Marie Louise the Second, 455.

Marotte sucrée jaune, 458.

Medaille, 458. 
PEARS-continued.

St Sampson, 457.

Seckel, 459 .

Snow, 455 .

Souveraine, 458 .

Squash, 463.

Squash new meadow, 463.

Staunton, 452.

Striped St Germain, 459.

Stumpfling, 463.

Sucrée dorée, 458.

Suffolk thorn, 461 .

Suprême, 457.

Swan's egg, 459.

Sweet summer, 457.

Teinton, 463.

Terling, 461 .

Teton de Venus, 461

Thomson's, 459

Union, 462 .

Uvedale's St Germain, 462

Uvedale's warden, 462 .

Valencia, 455.

Van Mons' Beurré Colmar, 460.

Van Mons' Beurré de Streckman, 460.

Van Mons'Dr Bouvier, 461

Van Mons' Leon le Cler'c, 459

Van Mons' Marie Louise, 458.

Vermillion d'été, 457.

Vicar of Winkfield, 450

Vicomte de Spoelberg, 461.

Warwick bergamot, 455 .

White autumn beurré, 455 .

White beurré, 455

White Doyenne, 455.

William the Fourth, 457 .

Winter bergamot, 461

Winter beurré, 453,455 460.

Winter crassane, 455

Winter Nelis, 458.

Wolf's, 463 .

Wurtemberg, 458

Yellow liuffeap, 463

York bergamot, 452 .

PEAS.

Adanıson's matcliless marrow, 56 .

American dwarf, 57.

American marrow, 58.

Auvergne, 55

Beck's morning star, 56 .

Bedınan's blue imperial, 58

Bedman's dwarf imperial, 56.

Bedman's imperial, 56 .

Bellamy's early green mar row, 56 .

Bishop's early dwarf, 56 .

Bishov's new long pod, 55 .

Blue imperial, 57.

Blue Prussian, 57.

Blue sabre, 57.

Blue scimitar, 57, 58 .

Blue union, 57.

British Queen, 57.

Burbage's eclipse, 56 .

Clark's Lincoln green, 57.

Cormick's Prince Albert, 58.

Dancer's monastery, 55 .

Danecroft early green, 57 .

Danecroft rival, 57

Dwarf blue prolific, 57

Dwarf blue Prussian, 57.

Dwarf erooked sugar, 57 .

Dwarf green imperial, 57.

Dwarf green marrow, 57 .

Dwarf imperial, 57.

Dwarf sabre, 57 .

Dwarf sugar, 57.

Early Bedalean, 57

Early blue surprise, 57
Early Charlton, 56.

Early Dutch green, 57 .

Early dwarf green, 57.

Early frame, 55

Early green, 57 .

Early hero, 55

Early May, 55 .

Early race-horse, 58

Early railway, 57 .

Early surprise, 57.

Early Warwick, 55, 57.

Early wonder, 57.

En Eventail, 57.

Essex champion, 57.

Extra green marrow, 57.

Fairbeard's champion of England, 56

Fairbeard's early surprise, 56

Fairbeard's surprise, 56 .

Farnes' conservative, 57 .

Fine long - podded dwarf,

Flack's dwarf victory, 56

Flack's new large victory, 56

Green marrow, 57.

Green noupareil, 57.

Green Prussian, $5 \pi$

Green rouncival, 57.

Groom's superb blue, 58 .

Groom's superb dwarf blue, 56.

Grosse Sclıottige Zuckererbse, 57

Hair's defiance Knight's marrow, 57.

Hair's dwarf mammoth, 56 .

Hamilton's November prolific, 56 .

Holloway marrowfat, 57

Hunter's new marrow, 57

Imperial, 57.

Kent's early, 55.

Knight's dwarf marrow, 56 .

Knight's late, 56 .

Knight's tall blue marrow, 56.

Knight's tall green marrow,

Knight's tall marrow, 56 .

Late sugar, 57.

Ledman's dwarf, 57 .

Iynn's prolific, 56

Lynn's wrinkled marrow, 58 .

Mange-tout, 57.

Matchless marrow, 57.

Melford marrow, 57

Ne plus ultra, 57

New dwarf imperial, 57.

New early green, 57 .

New green, 57.

New green nonpareil, 57.

New improved dwarf im perial, 57.

New improved imperial, 57.

New long-podded imperial, 57.

New sabre, 57 .

New tall imperial, 57.

Old dwarf marrow, 57.

Pois sans Parchemin, 57.

Pois san parchemill grandes-cosses, 57.

Prince Albert, 55.

Prince's superfine summer, 57.

Prussian prolific, 57

Queen of England, 57.

Richardson's eclipse, 55

Royal dwarf marrow, 57 .

Royal Prussian blue, 57.

Sabre, 57.

Scimitar, 57.

Shilling's grotto, 58 .

Spanish dwarf, 56

Spanish patriot, 57

Stevenson's railway, 57 .
Stubb's dwarf, 56 .

Sumatra, 57.

Sutton's early champion,

Sutton's early Goliath, 56 . Swerg Zuckerschotte, 57 .

Tall blue imperial, 57.

Tall crooked sugar, 57.

Tall green imperial, 57 .

Tall green marrow, 57.

Tall Prussian, 57.

Tamarind, 57.

Thompson's early dwarf, 56 .

Thurstone's reliance, 57

Torwoodlea, 55 .

Transparent, 57.

True early frame, 56 .

Victoria marrow, 56 .

Waite's king of the marrows, 57.

Warner's early conqueror, 57.

Warner's early emperor, 57.

Warwick, 58.

Weekes', 57.

Wellington, 57 .

Woodford's dwarf, 56 .

Woodford's green marrow, 57.

Pennyroyal, 236.

Peppermint, 236.

PINE-APPLES.

Anson's, 661 .

Ansoll's queen, 660 .

Antigua, black, 660 .

Antigua queen, 661 .

Antigua sugar-loaf, 661 .

Bahaina sugar-loaf, 661 .

Barbadoes queen, 661 .

Birds' eye Bahama, 661

Black Antigua, 660 .

Black Barbadoes, 660 .

Black Caraile, 661.

Black Jamaica, 660

Black prince, 661

Blood, 660

Blood red, 660 .

Bogwarp, 660 .

Brazil, 660.

Broad-leaved queen, 661 .

Brown Antigua, 660

Brown Havannah, 660 .

Brown-leaved sugar-loaf, 661.

Brown - striped sugar-loaf, 661.

Buck's seedling, 660 .

Cape Coast, 660 .

Claret, 660 .

Cochineal, 660 .

Cockscomb, 660

Common queen, 661

Comte de Paris, 661.

Copper, 660 .

Copper-coloured, 660 .

Copper-coloured Antigua, 660.

Demerara, 660.

English globe, 660 .

Enville, 660 .

Globe, 660 .

Green Antigua, 660.

Green Havannah, 660

Green olive, 661 .

Green St Vincent's, 661.

Green-striped sugar-loaf, 661.

Green sugar-loaf, 661 .

Harrison's new, 660 .

Havannah, 660 .

Heaton House, 661 .

Indian Creole, 660 .

Jagged-leaved A ntigua, 660 .

Jamaica, new, 660

Jamaica, old, 660 .

Lemon Antigua, 660 .

Lemon - coloured Barba-

does, 661 .

Lemon queen, 661

Lord Effinghain's, 661

Malacca, 660 .

Mealy - leaved Providence,

661.

ocho, 661 .

Montserrat, 660, 661 .

Moscow queen, 661 .

New black Jamaica, 660 .

New Demerara, 660 .

New Enville, 660 .

New Jamaica, $66^{\circ}$

New Providence, 661

New Ripley, 660 .

old Enville, 660

old Jamaica, 660

Old king, 660 .

Old Ripley, 661.

Orange sugar-loaf, 661

Otaheite, 660 .

Pitcl lake, 661.

Prickly Cayenne, 661 .

Prickly striped sugar-loaf, 661.

Prince Albert, 661

Providence, new, 661. 
PLUMS-continued.

Bradford green gage, 531.

Bruyn gage, 531 .

Buel's favourite, 529 .

Bullace, 534 .

Burgnon gage, 531.

Bury seedling, 529.

Caledonian, 531.

Catalonian, 532.

Chapman's Prince of Wales, 529.

Cheston, 529.

Coe's, 529.

Coe's fine late red, 529 .

Coe's golden drop, 529.

Coe's imperial, 529 .

Columbian, 529 .

Common daurson, 530 .

Conne's green gage, 531 .

Cooper's large, 529.

Cooper's large red, 529.

Cooper's large American, 529.

Corse's admiral, 530.

Corse's field-marshal, 530 .

Corse's nota bene, 530 .

Cruger's scarlet, 530 .

Damas vert, 531 .

Damson, 530.

Dauphine, 531 .

Dean's Jedburgh seedling, 530.

Denniston's superb, 530 .

Denyer's Victoria, 533.

Diamond, 530 .

Diaprée rouge, 530.

Diaprée violette, 529.

Double bearing, 530 .

Downton imperatrice, 530 .

Drap d'or, 530, 531 .

Duane's purple French, 533.

Early black Morocco, 532 .

Early damask, 532.

Early damson, 530 .

Early favourite, 530 .

Early Morocco, 532 .

Early prolific, 530 .

Early yellow, 532 .

Egg, 532.

Emerald drop, 530 .

Florence, 532 .

Flushing gage, 531.

Fair's golden drop, 529.

Fotheringham, 530 .

Gage, green, 530 .

Gage, imperial, 531.

Gage, Lawson's golden 531.

Gage, purple, 531

Gage, red, 531 .

Gage, Woolston black, 531.

Gage, yellow, 531 .

German gage, 529

Gisborne's, 531.

Golden gage, 529 .

Goliah, 531 .

Green gage, 530 .

Grosse Reine, 531.

Grosse Reine Claude, 531.

Grove House purple, 530 .

Guthrie's minette, 532

Guthrie's new apricot, 531.

Guthrie's Tay Bank, 532.

Guthrie's topaz, 532.

Ickworth imperatrice, 532 .

Imperatrice, 529 .

Imperatrice, blue, 529 .

Imperatrice, Ickworth, 532.

Imperatrice violette, 529 .

Imperatrice, white, 532

Imperiale, 532.

Imperial diadem, 530 .

Imperial gage, 531.

Isleworth green gage, 531 .

Jaune hâtive, 532 .

Jefferson, 532 .

Kirk's, 532.

La delicicuse, 529 .
La royale, 533.

Late green gage, 531 .

Lawrence's favourite, 532

I a wrence's gage, 532 .

Lawson's golden gage, 531 .

Little Queen Claude, 530 .

Lucombe's nonsuch, 532 .

Lucombe's seedling, 532 .

Magnum bonum, red, 532.

Magnum bonum, white, 532.

Matchless, 529.

Mimms, 530.

Mirabelle, 533.

Morocco, 532 .

Muscle, 533.

Nectarine, 531.

New golden drop, 529 .

New green gage, 531 .

Orange, 532

Orange gage, 532 .

Orleans, 532

Perdrigon blue, 533.

Pond's seedling, 533.

Prince's imperial gage, 531 .

Purple egg, 532.

Purple gage, 531.

Purple magnum bonum, 532.

Quetsche, 533.

Red apricot, 528 .

Red gage, 531

Red imperial, 532.

Red magnim bonum, 532 .

Reine Claude, 531.

Reine Claude blanche, 531.

Reine Claude de Bavay, 533.

Reine Claude violette, 531 .

Roche corbon, 530 .

Rotherham, 533.

Royal, 533.

Royale hâtive, 533.

St Barnabe, 532 .

St Catherine, 533 .

St Cloud, 531 .

St Martin, 529.

St Martin rouge, 529 .

Schuyler's gage, 531 .

Shailer's white damson, 530.

Sharpe's emperor, 533.

Sheen, 530.

Shropshire damson, 530 .

Standard of England, 533.

Steer's emperor, 531.

Stoneless, 533.

Sucrin vert, 531 .

Superior green gage, 531 .

Veritable imperatrice, 529 .

Vert bonne, 531 .

Victoria, Denyer's, 533.

Violet Queen Claude, 531.

Violette, 529.

Washington, 533

Wentworth, 532.

White damson, 530 .

White gage, 531 .

White gage of Boston, 531 .

White Holland, 532 .

White imperatrice, 532.

White magnum bonum, 532.

White mogul, 532 .

White perdrigon, 533.

White primordian, 532

Wilmot's green gage, 531 .

Wilmot's late Orleans, 531 .

Wine sour, 533.

Winter damson, 530.

Woolston black gage, 531 .

Yellow gage, 531 .

Yellow magnum bonum, 532.

POTATOES.

$\Lambda$ merican, 220.

A merican early, 221.
Ash-leaved early, 220.

Ash-leaved kidney, 219

British queen, 21

Caraccas, 220.

Chapman's early kidney, 220.

Dwarf American early, 220.

Early cliampion, 221 .

Early Cockney, 219.

Early frame, $219,220$.

Farly Manley, 219.

Early seedling, 220.

Early shaw, 221.

Early ten-weeks, 219.

Early Wellington, 220.

Fiftyfold, 221.

Fox's early delight, 220 .

Fox's early globe, 220 .

Fox's John Bull, or early

kidney, 220.

Fox's seedling, 219

Goldfinder, 220

Hopeton early, 220.

Invermay early, 2*20.

Jackson's golden drop, 219

Jackson's improved ash-

leaved kidney, 219.

Kay's early American, 220

Lady's finger, 219.

Lapstone kidney, 221.

Lawhead early red, 220.

Lawhead early white, 220 .

Lawson's conqueror, 220.

London dwarf kidney, 220

London particular, 220

May's parti-coloured seed-

ling, 220.

Musgrove's snow white, 220

New early Windsor seedling, 220 .

Painted lady, 220:

Peffermill prolific, 220

Prince of Wales'early, 220.

Prince Regent, 220.

Ross's kidney, 220.

Rough Robs, 220.

Rufford kidney, 219.

Seek no further, 221.

Shaw's early, 221.

Smith's yellow Peruvian, 221.

Soden's early Oxford, 219.

Tall American early, 221.

Taylor's fortyfold, 221 .

White Sutherland kidney, 221.

Williamson's favourite, 220.

Pumpinin, 25.9.

Purslane, 171.

\section{RADISHES.}

Beck's superb short top, 174.

Chinese rose, 175 .

Crimson turnip-rooted, 174

Early frame scarlet, 174

Grey turnip-rooted, 174

Large Spanish black-rooted,

Large Spanish white, 175.

Long white Naples, 174 .

Pink turnip-rooted, 174

Purple-rooted, 174.

Purple Spanish, 175.

Red Metz, 175 .

Red turnip-rooted, 174.

Rose coloured turnip-rooted, 174.

Round brown, 175.

Salmon-coloured, 174 .

Scarlet, 174.

Scarlet olive-shaped, 174

Scarlet turnip-rooted, 174

Short-topped scarlet, 174 .

Turnip-rooted, 174.

White Russian, 175.

White turnip-rooted, $17 t$

Wood's early frame, 174.

RAPE, 171

\section{RASPBERRIES.}

Barnet, 573

Belle de Fontenay, 573.

Burley, 573.

Cornwall's prolific, 573.

Cornwall's red, 573 .

Cornwall's seedling, 573.

Cornwall's Victoria, 574 .

Cox's honey, 573 .

Double bearing, 573 .

Double-bearing yellow, 574 .

Falstaff, 573.

Franconia, 573.

Howland's red Antwerp, 573.

Knevett's Antwerp, 573.

Large red, 573.

Late cane, 573

Lord Exmouth's, 573.

New red Antwerp, 573.

Perpetual bearing, 573 .

Red Antwer'p, 573.

Red double-bearing, 573 . 
SA VOYS-continued.

Early yellow, 97 .

Feather-stemmed, 97

Globe, 97.

Large green, 97.

Large green curled, 97.

Large green German, 97

Large late green, 97 .

Marcellin, 97.

New early, 97.

Pancalier de Touraine, 97.

Small dwarf green curled, 97.

\section{SCARLET RUNNERS.}

Case-knife runner, 79

Haricot d'Espagne, 79.

Haricot d'Espagne à fleur bicolore, 79 .

Haricot êcarlate, 79 .

Lima pole bean, 79 .

Painted lady, 79 .

Prague bicolor, 80 .

Prague jaspé, 80.

Prague runner, 79.

Red vea, 79 .

Scarlet runner, 79.

Sieva bean, 79 .

Tall scarlet runner, 79 .

White Dutch runner, 79.

York and Lancaster runner, 79

SCORZONERA, 228.

SEA-KALE, 103 .

\section{SHALLOTS.}

Common, 28.

Long keeping, 29.

Russian, 28 .

SkiRRet, 228.

SORREL.

Common, 139.

French, 138.

Mild-leaved, 139

Roman, 138.

Round-leaved, 138

Spearmint, 236

SPINACH

Bordeaux, 137.

Commun, 137.

Fisquermes, 137.
Flanders, 137.

French, 138.

Gaudry, 137

Lettuce leaved, 137.

Mountain, 138 .

New Zealand, 138.

Prickly, 137.

Round, 137.

Wild, 137.

\section{STRA WBERRIES}

Aberdeen, 588

Aberdeen seedling, 588.

Atkinson's scarlet, 588

Barham pine, 589 .

Bath, 590 .

Black pine, 589 .

Blood pine, 589 .

Borsdorf, 589.

Brickley scarlet, 588 .

Caperon hermaphrodite, 590.

Caperon royal, 590 .

Carolina, 589.

Carolina pine, 589

Cisalpine, 591 .

Cockscomb pine, 589

Common Carolina, 589

Comte de Paris, 589.

Conical, 590 .

Coul late scarlet, 589

Cuthill's Black Prince, 588 .

Cuthill's Prince of Wales, 588.

Devonshire scarlet pine, 589 .

Double bearing, 590

Downton, 588

Dwarf, 590 .

Early scarlet, 588 .

Elton, 588.

Elton seedling, 588 .

Formosa, 590

Grandiflora, 589

Grove End scarlet, 587

Hermaphrodite, 590 .

Hovey's seedling, 589 .

Hudson's Bay, 590 .

Ingram's Prince of Wales, 589.

Keen's black pine, 589 .

Keen's new pine, 589 .

Keen's new seedling, 589 .

Keen's seedling, 589.

Kew pine, 589 .

Kittley's Goliah, 589.

Knight's, 588

Knight's seedling, 588.

Large Carolina, 589 .
Large flat hautbois, 590 .

Lowder's, 590

Miss Gunning's pine, 589.

Murphy's child, 589 .

Musk, 590.

Myatt's British queen, 588 .

Myatt's Deptford pine, 589 .

Myatt's Eleanor, 589.

Myatt's Eliza, 588.

Myatt's mammoth, 589 .

Myatt's pine, 588 .

Napoleon, 591.

Nicholson's Ajax, 589.

North's seedling, 589.

old Bath scarlet, 588 .

Old Carolina, 589 .

Old pine, 589.

Old scarlet, 588

Old scarlet pine, 589 .

Original scarlet, 588 .

Pine, 589.

Prince Albert, 589.

Princess Alice Maude, 589.

Princess Royal, 589.

Prolific, 590.

Prolific alpine, 591.

Prolific pine, 588

Pyne Hill, 589.

Red alpine, 591.

Red bush alpine, 591 .

Red wood, 591 .

Regent's, 590 .

Regent's favourite, 589

Rose, 588.

Roseberry, 588.

Ross's Phœnix, 589.

Russian, 591.

Sacombe, 590.

St Andrew's pine, 588 .

Salter's, 590 .

Scarlet, 588 .

Scarlet alpine, 591

Scarlet-fleshed, 589.

Scarlet pine, 589.

Scarlet Virginian, 588

Scotch scarlet, 588 .

Sliew's scarlet, 589

Sir George Mackenzie's late searlet, 588 .

Sir Joseph Banks', 590.

Spring Grove, 590 .

Superior, 589 .

Surinam, 589 .

Trollope's Victoria, 589.

Varnished, 589.

Virginian, 588 .

Weymouth, 590 .

White, 590

White Alpine, 591

White bush Alpine, 591
White wood, 591.

Wilmot's superb, 590 .

Windsor pine, 589 .

\section{SUCCORY.}

Café Chicorée, 166.

Chicorée à Navet, 166 .

Large leaved, 166.

Variegated, 166.

SWeet BAsil, 237.

TANSY, 237.

Tarragon, 166

Thyme, 234

Tомато, 258 .

\section{TURNIPS.}

Autumn stubble, 190.

Early ball, 190.

Early dwarf, 190.

Early six-weeks, 190 .

Finland, 189

French, 190.

Liverpool Preston, 190

Maltese golden, 189 .

Mousetail, 190

Navet, 190.

Red-topped, 190

Robertson's golden stone, 189.

Snowball, 190

Stone globe, 190.

Swedish, 190.

Thorpland globe swede, 190 .

White Dutch, 190.

White garden stone, 190.

White stone, 190 .

Yellow Altringham, 190.

Yellow Dutch, 189.

Yellow globe, 189.

Yellow Liverpool, 190.

Yellow Malta, 189 .

Yellow stone, 190.

VIRgINIAN POKE, 134.

\section{WALNUTS.}

Higliflyer of Thetford, 562 .

Large double - kernelled French, 562.

New dwarf prolific, 562 .

Noyer fertile, 562 .

Tender, 562 .

Thin-shelled, 562.

WorMWOOD, 242.

WOOD SORREL, 172 


\section{N D E X}

\section{SELECT LISTS OF PLANTS.}

\begin{abstract}
Abelia, 728, 730, 734, 792,
796.
Abutilon, 724, 730

Acacia, 724, 730, 734, 792, 795 .
\end{abstract}

Achillea, 816

Achimenes, 716, 719, 730.

Achras, 712.

Aconite, 816 .

Adenandra, 728, 734 .

Adenocarpus, 792

Achmea, 694, 716, 730, 790.

Agle, 712.

Aerides, 694

Aschynanthus, 694, 716.

Esculus, 792.

A galmyla, 716 .

Agapanthus, 727, 734

Agethœa, 734 .

Agave, 720 .

Ageratum, 817, 820 .

Agotliosma, 734 .

Ailanthus, 792 .

Aizoon, 720 .

Akebia, 795.

Akee tree, 712 .

Albuca, 699, 734.

Aldrovanda, 712

Aletris, 743.

Alisma, 712, 743.

Allamanda, 716,719 .

Alloplectus, 719 .

Almond, 730 .

Aluus, 791 .

Aloe, 720 .

Aloysia, 734

Alstrœmeria, 733, 734

Althæa, 796.

Alyssum, 730, 816, 817.

A maryllis, 697, 699 .

Amelanchier, 744, 792.

Amherstia, 716.

Ammobium, 818 .

Amorplia, 784, 792.

Ampelopsis, 748, 784, 791

Amphicome, 728, 730, 734 .

Amygdalus, $784,792,795$.

A nacampseros, 720 .

Anæctochilus, 691, 719.

Anagallis, 728, 734, 817, 820.

Anagyris, 792

Anchovy pear, 713 .

Andersonia, 704, 709, 734 .

Andromeda, 730, 744, 749, $791,795,818$.

Androsace, 743, 815.

Androsæmum, 791 .

Anemone, 730, 743, 803, 816,819 .
Angelonia, 716, 730 . Angræcum, 690 .

Anguloa, 691

Anigozantlius, 734 .

Anona, 712.

Anthericum, 734

Anthyllis, 795 .

Antirrhinum, 821

Aotus, 727, 728 .

Aphelandra, 716, 730 .

Aphelexis, 704, 728.

Apicra, 720 .

Aralia, 784, 792, 795 .

Araucaria, 795 .

Arbutus, 785, 791, 792, 795,818

Arctostaphylos, 785, 792 .

Arctotis, 728, 734

Arenaria, 815 .

Arethusa, 743

Aretia, 815 .

A ristolochia, 748, 785, 791

Aristotelia, 785, 792.

Artocarpus, 712

Arundina, 691 .

Asimina, 792

Aspidistra, 719, 734.

Astelma, 704, 709, 727, 728.

Aster, 816, 817.

A stragalus, 785 .

Atragene, 748, 785, 791.

Atriplex, 785,791

Aubrietia, 816.

Aucuba, 785, 791, 792, 793.

Auricula, 809.

Azalea, 679, 685, 724, 730, $733,744,749,795$.

Azara, 792 .

Babiana, 699 .

Babbingtonia, 728.

Baccharis, 785.

Balsam, 740 .

Bambusa, 795 .

Banana, 713.

Banksia, 724 .

Barkeria, 689, 694 .

Bartonia, 817.

Batatas, 716.

Beaufortia, 724, 734 .

Begonia, 716, 719, 730 .

Bejaria, 728 .

Bengal quince, 712

Benthamia, 734, 785, 792 , $793,795$.

Berberis, 730, 734, 785, 791, 792, 795, 819.

Berchemia, 791.

Bertolonia, 719

Betula, 791 .

Bignonia, 716, 719, 724 ,

$730,748,785,791,795$.

Billardiera, 724, 734 .
Billbergia, 694, 716, 719, 730

Biota, 792, 795.

Blæria, 734

Blætia, 691.

Blandfordia, 734 .

Blighia, 712 .

Boronia, 724, 734.

Bossiæa, 724, 734 .

Bouvardia, 728, 730, 820.

Bowiea, 720 .

Bracliycoma, 817.

Bracliyglottus, 724 .

Bracliysema, 724

Brassavola, 689.

Brassia, 691, 694 .

Bread-fruit, 712.

Brexia, 719 .

Bridgesia, 791, 795.

Brompton Stock, 818.

Broughtonia, 689 .

Broussonetia, 785, 792.

Brownia, 716 .

Browallia, 728, 730.

Brugmansia, 724

Brunia, 734.

Brunsvigia Josephinæ, 699 .

Bryanthus, 745 .

Bryophyllum, 720 .

Brucelia, 719

Buddleya, 733, 734, 785 792, 795.

Bulbicodium, 819.

Bulbine, 734.

Bupleurum, 792, 795 .

Burchellia, 724, 728.

Burlingtonia, 689, 691 .

Burtonia, 728 .

Buxus, 785, 791, 792, 793 795

Byblis, 712 .

Cactus, 720.

Caladenia, 686.

Caladium, 716, 719 .

Calampelis, 734 .

Calandrinia, 728, 734, 816 . 817.

Calan the, 691 .

Calceolaria, 728, 734, 739, 821.

Calendula, 817.

Calla, 727.

Callistacliys, 724, 734 .

Callistemon, 724, 734 .

Calochortus, 734.

Calophaca, 792.

Calopogon, 743

Calothamnus, 724 .

Caltha, 743.

Calycanthus, 745, 792.

Calycarpa, 792

Calypso, 743.
Calystegia, 738, 820 .

Camarotis, 689.

Camellia, 678, 682, 724,

$730,733,734$.

Campanula, 734, 815, 816, 817.

Campylobotris, 717, 719.

Candollea, 734.

Candytuft, 818.

Cantua, 724, 734.

Caprifolium, 748, 785, 791, 795.

Caprosina, 795.

Caragana, 785, 792.

Carmiclılia, 795.

Carnations, 730, 732, 806, 816.

Carpodinus, 712

Castanea, 792

Catalpa, 745, 785 .

Catananche, 818 .

Catasetum, 689, 691 .

Cattleya, 689, 691, 694 .

Ceanotlius, 728, 730, 733,

734, 785, 792, 793, 795 .

Centaurea, 817.

Centrantlius, 817 .

Cereus, 720.

Celastrus, 785, 791 .

Celtis, 785.

Centradenia, 728, 730 .

Cephalanthus, 785

Cephalotus, 694

Cerasus, 786, 792, 795.

Ceratopteris, 712 .

Cercis, 786, 792.

Ceropegia, 717 .

Cestrum, 730 .

Chamærops, 713, 792.

Charianthus, 717 .

Cheiranthus, 816, 818, 820 .

Chenostoma, 728, 734, 817 .

Chimonanthus, 734, 792, 795.

Chinese azaleas, 679, 685 .

Chinese chrysanthemums, $732,733$.

Chinese lilacs, 730 .

Chionanthus, 745, 792 .

Chironia, 728, 734 .

Chorozema, 724, 727, 728, $730,734$.

Chrysanthemums, 729, 730, $732,733,817$.

Chysis, 694.

Cineraria, 728, 730, 732, $733,734,792,820$.

Cirrhœa, 690.

Cirrhopetalum, 689.

Cissus, 719.

Cistus, 733, 786, 792, 793, 795. 
Citrus, 700, 724, 730, 734 .

Clematis, 727, 730, 733, 748, 786, 791, 795.

Clerkia, 817 .

Clerodendrum, 717, 719 730.

Clethra, 786, 792

Cliantlius, $724,734,786$, 792.

Clintonia, 817.

Coburghia, 699 .

Coccocypselum, 719

Cockscomb, 740 .

Cocos, 713.

Codonophora, 700 .

Cœlogyne, 692, 694 .

Coffea, 712.

Colchicum, 816 .

Colconema, 728 .

Colletia, 786, 792, 793, 795 .

Collinsia, 817 .

Collomia, 817.

Colquhounia, 795 .

Colutea, 786, 792

Colvillea, 717

Comaclinium, 820 .

Combretuin, 717, 719

Commelina, 733 .

Comptonia, 786, 792.

Conanthera, 699 .

Convolvulus, 719, 817.

Coptis, 743.

Corcorus, 792

Coriaria, 792.

Cornus, 743, 786, 791, 792 .

Coronilla, 728, 733,734,792.

Corræa, 724, 730, 734 .

Coryantlies, 692, 694 .

Corydalis, 816.

Corylus, 791 .

Cosmelia, 728

Cossignia, 719.

Cotoneaster, 792, 793, 795 818

Cotyledon, 720

Crassula, 720,734

Cratægus, 745,786, 791, 792

Crinum, 699, 727 .

Crocus, 730, 816 .

Crotalaria, 724

Croton, 719

Crowea, 724 .

Cuphea, 728, 734, 820.

Cupressus, 791 .

Cyclamen, 730, 733, 815, 819.

Custard apple, 712

Cyananthus, 734, 820 .

Cyenoches, 692

Cydonia, 786, 791, 792

Cymbidium, 692, 694 .

Cypella, 734 .

Cypripedium, 692, 719, 734 743.

Cyrtanthus, 699 .

Cyrtoceras, 717

Cyrtopodium, 692 .

Cytisus, 724, 727, 730, 733 $734,786,792,793$.

Dacrydium, 724.

Dahlia, 803 .

Daphne, $678,685,724,730$

733, 786, 791, 792, 793

Daviesia, 724, 730, 734 .

Decumaria, 791.

Delphinium, 816, 817.

Dendrobium, 690, 692, 694

Deutzia, $730,733,786,792$, 793,795 .

Dianthus, 733, 817, 818 .

Dichorizandra, 719 .

Didymocarpus, 719 .

Dieffenbachia, 719 .

Dielytra, 728, 730, 748, 816 .

Dillwynia, 724, 730, 734 .

Dionæa, 719 .

Dioscorea, 719

Diosma, 734 .

Diotis, 795 .

Diplacus, 728, 730, 734 .
Dipladenia, 717, 719 .

Diploleptis, 728 .

Dirca, 745, 791, 792, 793.

Disa, 686 .

Distiganthus, 719.

Dodecatheon, 743 .

Dolichos, 728 .

Doryantlies, 724, 734.

Draba, 816.

Dracæna, 719.

Dracophyllum, 734.

Drosera, 743.

Dryandra, 724

Durio, 712.

Duvana, 795 .

Dyckia, 720 .

Echeveria, 720.

Echinocactus, 720 .

Echium, 734.

Echites, 717, 719

Eccremocarpus, 730, 733.

Edwardsia, 733, 795 .

Edgeworthia, 792 .

Elæagnus, 786, 792, 795.

Elodea, 712 .

Elscholtzia, 795

Empetrum, 745, 792.

Enkiantlıus, 685, 724, 733, 734.

Epacris, 704, 709, 724, 730, 734.

Ephedra, 745, 791, 792, 793.

Epidendrum, 692.

Epigæa, 745

Epiphyllum, 720, 730 .

Eranthemum, 717, 719,730.

Erica, 709, 730, 733, 745, 818.

Erionema, 719

Eriospermum, 699

Eriostemon, 724 .

Erodium, 728.

Eryanthus, 819

Eryobotrya, 733, 792, 795.

Erysimun, 817 .

Erythrina, 724, 733.

Erythronium, 816, 819.

Escallonia, 728, 733, 786 , 792, 793, 795 .

Eschscholtzia, 817.

Eucalyptus, 727, 795.

Eucharidium, 817

Euchilus, 724.

Eucomis, 699, 734.

Eugenia, 712.

Euonymus, 786, 791, 792 , 795.

Euphorbia, 717, 719, 720, 730.

Euphoria, 713

Euryale ferox, 710,712 .

Eurybia, 792 .

Eutaxia, 724, 727, 730, 734

Fabiana, 728, 733, 786, 792, 793,795 .

Ferns, 694, 733 .

Ficus, 713, 719 .

Fontanesia, 786, 792, 793, 795.

Forsythia, 730, 733, 786, 792,795 .

Fortunea, 792

Fothergilla, 745 .

Franciscea, 717.

French lilacs, 730

Fritillaria, 816, 819 .

Furcrœa, 720 .

Fuchsia, 727, 728, 729, 730,

$733,734,741,792,795$.

Gagea, 816,819 .

Galanthus, 816, 819 .

Galax, 743,745 .

Galaxia, 73

Galeandra, 692.

Gamboge, 713.

Garcinia, 713

Gardenia, 717, 730.

Gardoquia, 728 .

Garrya, 733, 787, 792, 795

Gasteria, 720 .

Gastrolobium, 724
Gaultlieria, 743, 745, 792, 795,818 .

Gazania, 728, 734, 820 .

Genista, 724, 730, 734, 787, $792,793,795$.

Gentian, 743, 815 .

Geranium, 727, 729, 821 .

Gesnera, 700, 717, 719, 730 .

Gethyllis, 699, 734 .

Gilia, 817.

Gladiolus, 699, 733 .

Gleditschia, 787, 792.

Globe amaranth, 741

Globulea, 720 .

Gloriosa, 699

Gloxinia, 700, 717, 727, 730 .

Glycine, 721, 791, 796 .

Glyptostrobus, 795 .

Gnidia, 728, 734

Godetia, 817 .

Gompholobium, 724, 734.

Gonolobus, 787.

Goodia, 724 .

Goodyera, 719, 743 .

Gordonia, 792 .

Gorteria, 728.

Govenia, 692

Grammatophylluin, 692.

Gramnanthes, 817.

Graptophyllum, 719.

Grias, 713.

Griffinia, 699 .

Grissorhiza, 699 .

Guava, 713 .

Gymnocladus, 745, 792.

Gynerium, 795 .

Habenaria, 686, 743.

Habranthus, 699, 734 .

Habrothamnus, 724, 730 , 734

Hæmadictyon, 719 .

Hæmanthus, 699 .

Halesia, 745, 787.

Halimodendron, 787 .

Hamamelis, 787.

Hardenbergia, 728, 734 .

Haworthia, 720 .

Heart's-ease, 796.

Heatlis, see Erica.

Hedera, 791.

Hedysarum, 818 .

Heinia, 734 .

Helianthemum, 733, 792

Helichrysum, 704, 728.

Heliophila, 817.

Heliotrope, 730, 820 .

Hellebor'us, 730, 816, 819.

Helonias, 743

Hemimeris, 817.

Hepatica, 730, 816, 819.

Herbertia, 734 .

Hesperis, 820.

Hibbertia, 734 .

Hibiscus, 718, 792, 818 .

Hindsia, 718 .

Hippopliae, 787.

Hollyliock, 798 .

Homeria, 734

Houlletia, 692 .

Houstonia, 743 .

Hovea, 724, 734 .

Hoya, $718,719,720,727$.

Hudsonia, 746 .

Humea, 724, 734 .

Huntleya, 688 .

Hyacinths, 730, 811, 816 , 819.

Hydrangea, 727, 733, 734 , $787,792,793,795$.

Hydrocotyle, 743 .

Hypericum, 787,791

Hypoxis, $699,712,743$.

Iberis, 728,$730 ; 734,818$, 820 .

illex, 791, 792, 796 .

Illecebrum, 815

Illicium, $678,734,792$.

Indian azaleas, 679,685 .

Indigofera, $724,730,792$

Ipomea, 718, 719 .

Isoetes, 815 .

Isopogon, 734.

Isotoma, 818 .

Ixia, 699 .

Ixora, 718, 730.

Jack fruit, 712 .

Jamrosade, 712

Jasminum, 718, 719, 724

$727,730,733,734,787$

791,795 .

Jeffersonia, 743.

Juniperus, 791.

Jussieua, 712

Justicia, 718 .

Kadsura, 734.

Kalanchoe, 720.

Kalmia, 730, 746, 749 .

Kalosanthes, 720, 734 . 
Mandevilla, 718, 724, 734. Pancratium, 699.

Manettia, 730.

Mangifera, 713

Mango, 713.

Mangosteen, 713.

Mantisia, 730 .

Maranta, 719.

Marica, 734.

Massonia, 699

Matliola, 818 .

Maurandya, 728, 734, 821.

Maxillaria, 693

Median lemon, 700 .

Medicago, 734 .

Medinella, 718

Melaleuca, 728, 734 .

Melastoma, 718, 730

Menispermum, 748, 789, 791.

Menyanthes, 743

Menziesia, 745 .

Mesembryanthemum, 720 734,818 .

Mespilus, 791.

Mignonette, 730, 732, 818

Miltonia, 693, 694

Mimosa, 724 .

Mimulus, 821.

Mirbelia, 724.

Mitchella, 743.

Mitraria, 724, 734, 792, 793.

Monanthes, 720 .

Moræa, 699, 734

Morina, 796.

Mormodes, 693, 694 .

Morus, 793.

Morissonia, 718.

Muraltia, 728, 730, 734

Musa, 713.

Muscaria, 816, 819.

Myrica, 788, 791, 792, 793, 796.

Myricaria, 788.

Myrtus, 796.

Nandina, 796 .

Narcissus, 730, 816, 819

Narthecium, 743

Nasturtium, 821

Neapolitan violets, 730 .

Negundo, 788 .

Neillia, 796.

Nelumbium, 710, 712

Nemopanthes, 788

Nemophila, 818.

Neottia, 743

Nepenthus, 694 .

Nerine, 699, 734

Nerium oleander, 684, 727 .

Nierembergia, $728,734,821$.

Nuplar, 743.

Nuttalia, 734, 743, 793.

Nymphæa, 710, 711, 712, 743.

Nyssa, 747, 788.

Odontoglossum, 693, 694 .

Enothera, 734, 818, 821 .

Olea, 678, 685, 734, 793 .

Oleander, 684.

Ononus, 792, 793.

Oncidium, 689, 693, 694 .

Opuntia, 720 .

Orange, 700,724

Orchids, 689

Orchis, 686.

Ornithogalum, 819 .

Ornus, 796

Orontium, 712

Oryza, 712.

Osteospermum, 734.

Otlionna, 734.

Oxalis, 699, 730, 733, 734, $816,818$.

Oxycoccus, 743.

Oxylobium, 724, 734

Oxyura, 818 .

Paclidendron, 720.

Pachysandra, 743.

Pxonia, 730, 734, 788, 793, 796.

Paliurus, 788, 793

Palms, 713.
Pandanus, 719.

Pansy, 796.

Papyrus, 711, 712

Parnassia, 743.

Passerina, 734.
Passiflora, 713, 718, 719, 724, 791.

Patersonia, 734.

Pavetta, 718, 719

Pavia, 788, 793, 796

Paulownia, 788, 796.

Pelargoniums, 729, 730, 736.

Penæa, 734.

Penstemon, 821.

Pentaraphia, 700 .

Pereskia, 720.

Pergularia, 719 .

Periploca, 789, 791

Peristeria, 693, 694

Pernettya, 789, 792, 793.

Persian lilacs, 730.

Petunia, 728, 733, 817, 821.

Phaius, 693.

Phalænopsis, 689, 694.

Pharbitis, 718

Pliladelplıus, 789, 792, 793.

Philibertia, 718, 728.

Phillyrea, 789, 791, 793,796.

Phlomis, 789, 793.

Phlox, 734, 816, 817, 818, 819.

Phœnocoma, 704, 724, 730.

Phormium, 796.

Photinea, 789, 793, 796.

Pliycella, 699, 734.

Pliylica, 734

Phyllocladus, 724.

Pliysiantlius, 718, 719.

Picotee, 806.

Pimelia, 724.

Pinks, 730, 732, 807.

Pinus, 791.

Piptanthus, 789, 792, 796.

Pistacia, 796.

Pistia, 711, 712.

Pitcairnia, 694, 730.

Pitcher plant, 694

Pittosporum, 734, 796.

Planera, 793.

Plantain, 713.

Platanus, 789.

Platylobium, 724, 734.

Platystemon, 818.

Plectranthus, 719

Pleroma, 718.

Plumbago, 730, 734, 796.

Podolepis, 818.

Podolobium, 734.

Pogonia, 743.

Poinciana, 718, 730 .

Poinsettia, 718 .

Polyanthus, 811.

Polygala, 724, 730, 734.

Polygonum, 792.

Polupon chrysanthemums, 729,730 .

Pontaderia, 711, 712, 743.

Portlandia, 718 .

Portugal laurel, 789, 791

Portulaca, 720, 818 .

Potentilla, 789, 792, 793, 816.

Primula, 728, 729, 730, 731, 815,819

Prinos, 747, 789, 793.

Prostanthera, 724, 734.

Prunus, 789.

Pterocarya, 789

Psidiuin, 713.

Pultenœa, 724, 730, 734.

Punica, 789, 796.

Purshia, 747, 789, 793.

Pyrola, 743 .

Pyrus, 789, 792.

Quercus, 789, 796 .

Quisqualis, 718.

Ranunculus, 801

Renanthera, 689, 694

Reseda, 818 .
Rhamnus, 791, 792, 793.

Rhapis, 713.

Rhipidodendron, 720 .

Rhipsalis, 720 .

Rhizocarpa, 818 .

Rhodanthe, 818.

Rhodochiton, 724, 734, 821

Rhododendrons, 685, 724 ,

$729,730,733,741,747$, $791,796,818$.

Rhodoleia, 724, 734 .

Rliodora, 730, 748.

Rhodotliamnus, 793.

Rhopala, 719 .

Rhus, 747, 789, 791, 792 , 793.

Rhynchospermum, 724,728.

Ribes, 730, 789, 793, 796.

Richardia, 727 .

Robinia, 747, 789, 792, 793.

Rochea, 720, 734.

Rocket larkspur, 817.

Rondeletia, 718 .

Roses, 733, 750, 771.

Rose apple, 712 .

Rosinarinus, 791

Rubus, 790, 791, 793, 815.

Ruellia, 728.

Ruscus, 790.

Russellia, 728 .

Russian violets, 730 .

Sabbatia, 743 .

Saccolabium, 690, 694

Salix, 790, 791, 792.

Salvia, 7:28, 730, 734, 796, 821.

Sambucus, 790, 791, 792.

Sanguinaria, 743 .

Sanvitalia, 818

Santolina, 790, 793, 796

Saponaria, 818 .

Sarracenia, 734, 743 .

Saururus, 712, 743.

Saxifiaga, 730, 815, 818 .

Schizanthus, 818 .

Schizopetalon, 818 .

Schomburghia, $690,693$.

Scilla, 730, 816, 819 .

Scottia, 729, 734 .

Sedum, 805, 818 .

Selago, 728, 734, 821

Seline, 818 .

Sempervivum,720, 734, 815 .

Senecio, 818, 821 .

Shaddock, 700 .

Sliepherdia, 748, 793 .

Shortea, 818 .

Sierra Leone fig, 713

Sikkim rhododendrons, 729 733.

Sinningia, 700, 730 .

Siphocampylos, 718 .

Skimmia, 734, 792, 793, 796.

Smilacina, 743

Smilax, 748, 790, 791

Sobralia, 693, 694.

Solanum, 791, 792, 796.

Soldanella, 815, 819 .

Sollya, 724, 734 .

Sophronitis, 690

Sparaxis, 699 .

Spartium, 790, 792, 793.

Sphenotoma, 729 .

Spigelia, 743.

Spiræa, 790, 791, 792, 793 796.

Sprengelia, 704, 709, 729, 734

Stadmannia, 729

Stanhopea, 690, 694 .

Stapelia, 720 .

Statice, 724, 728, 730, 734.

Stenorhynchus, 694 .

Stenocarpus, 729.

Stephanotis, 718, 719.

Stocks, 732, 818.

Strelitzia, 719, 731 .

Strumaria, 699 .

Stuartia, 748 .

Stylidium, 728 .

Styphelia, 734.

Styrax, 748, 790.

Sutherlandia, 728.

Swainsonia, 724.

Swammerdamia, 796

Sweet Pishamin, 712

Sweet William, 818.

Symplioria, 791, 79*2

Symphoricarpus, 790 .

Syringa, 790, 792, 793 .

Tacca, 699.

Tacsonia, 724

Talauma, 719.

Talinum, 720, 816.

Tamarix, 790, 792, 793.

Tasmannia, 729 .

Taxodium, 791

Taxus, 791, 792 .

Tecoma, 748, 790, 791. 


\section{GENERAL INDEX.}

A bercrombie on peach-borders, 487on vine-borders, 600 .

A braxas grossulariata, the, 569,580 .

Acarus, the genus of, 77,637 .

A carus telarius, the, 514 .

Acetariaceous plants, see Salad plants.

Achimenes, propagation of, by single leaves, 351 .

Acidia artimisiæ, 833.

Acids, effects of, on the germination of seeds, 313 .

Acton Scott peach, origin of the, 305.

Ageria tipuliformis, the, 570 .

Athusa cynapium, 232.

African garlic, the, 30 .

Agaricus, edible varieties of, $243-$

atrimentarius, 244-campestris, see Mushroom-deliciosus, 244-exquisitus, $i b$.-fusipes, $i b$. - Georgii, $i b$. situs, $i b$. - fusipes, $i b$. - Georgii, $i b$. -orcella, ib. - oreades, $i b$. - personatus, $i b$. - piperatus, $i b .-$ procerus, $i b$. - prunulus, 243-ruber, $i b$. - rubescens, $i b$. - virescens, $i b$. virgineus, $i \vec{b}$.

Agromyza violæ, 826.

Air, absorption of food by plants from the, 21 -importance of, to the roots, 277-necessity of, to the germination of seeds, 310 .

Airthrey, the gardens at, 301 .

Aleyrodes proletella, the, 113.

Alisander, culture of the, 129 .

Alkalies, effects of, on the germination of seeds, 313 .

Alliaceous plants, culture of - The Shallot, 27-Garlic, 29-Rocambole, $30-$ The Onion, ib. - The Leek, 44-The Chive, 47.

Allium, the genus, 26.

Allium ascallonicum, see Shallotcepa, see Onion-porrum, see Leek - sativum, see Garlic-Schœnoprasum, see Chive.

Almond, culture of the, 559-supposed identity of, with the peach, 485 - as a stock for the peacl, 486 .

Almond peach, origin of the, 305 .

Almond stocks, kinds, \&c. of, 328 .

Almonds, management of, in the fruit-room, 676 .

Alpine garden and rockery, the, 814 -its disposal, \&c., $i b$. - plants suitable, 815 .

Alpine strawberries, origin of the, 581 -how propagated, 582 -list of, 590 .

Altica concinna, the, 115 - consobrina, the, $i b$.

Altitude, value of, as regards the selection of fruit-trees, 300-effects of on climate, 794.

Alucita variella or multipunctella, the, 574.

VOL. II.
Alumina, influence, \&c. of, on soils and vegetation, 271 .

America, list of apples for, 430 - the grape vines of, 595

American mode of peach - training, the, 496-of shield - budding, the, 342 .

American blight, the, 433.

American cress, the, 170 .

American garden, the, situation for it, 742-soil, 743-propagation of plants, 744 - list of plants, $i b$. climbing plants, 748 - rhododendrons, \&c. 749

drons, \&c., 749 .
American peaches, list of, 509 - and nectarines, 512 .

American pole saw, the, 408 .

Ammonia, mode of fixing, in liquid manure, 295.

Ammoniacal gases, importance of, to plants, 290

Ammoniacal liquor as a manure, 296.

Amphelexis, culture of, 704 .

Amphidasis pilosaria, the, 440 .

Amygdalus communis and amara, the, 559-Persica, see Peach

Anbury in turnips, the, 198.

Anderson, Isaac, Esq., on liybridisation, 319.

Andersonia, culture of, 704 .

Anemone, cultivation of the, 803 .

Anethum graveolens, 234.

Angelica, culture of, 239.

Animal bodies as a manure, 294

Animal manures, what, 288.

Anise, culture of, 239 .

Anisoplia horticola, the, 437 .

Anisopteryx œscularea, 832.

Anisopteryx pometaria, the, 444.

Annuals, grafting, 335-difficulties of culture, \&c. of, their management, \&c., 816-list of them, 817 .

Annular budding, 344

Ansted, views of, on the crigin of soils, 264.

Anthemis nobilis, 241.

Anthomyia brassicæ, the, 114, $197-$ ceparum or onion fly, $29,42-$ gnava, 197-lactucæ, 157-trimaculata 115, 198.

Anthonomus pomorum, the, 436 pyri, 466.

Antler rose saw-fly, the, 828 .

A pate dispar, the, 442 .

Aphides, peculiar economy, \&c. of the, 193, 469 - attacks of, \&c. on carrots, 181-destruction of, 515 .

Aphidius avenæ, the, 194

Aphidius rapæ, the, 194.

A phis, the, 710-brassicæ, 112, 196 - cerasi, 545-Dianthi, 193-dubia, 196-fabæ, 68-lanigera, 433-lion, 194-pyri-mali, 437, 469-persicæ, 194-pyri-mali, 437, 469 - persicæ, rosæ, 830-rumicis, 515-vastator, $193,221,831$

Apium graveolens, see Celery-petroselinum, see Parsley-petroselinum, var. Tuberosum, 229

A pple, soil suitable to the, 359 -rootpruning the, 400-its natural his. tory, 411-classification of kinds, 412-uses of it, 413-planting it, soil, \&c. suitable, $i b$.-criterion of a good, $i b .-$ comparative sizes and forms, 414-select list of dessert ones, ib-of kitchen, 423 -for ornament or preserving, 429-lists of those suited for peculiar localities, 430 -list of cider, 432 -insects and diseases by which attacked, $i b$. - its European names, 446.

Apple and Pear, pruning and training the, 474-choice of trees, $i b$.- nnanagement of established orehardtrees, $475-$ pruning, \&c. as half standards, $i b$. - as open dwarfs, $i b$. in the conical and pyramidal form, 476-girandole training, $i b$.-Quenouille, $i b$.-Rivers' pyramidal, 477 nouille, $i b$. - Rivers pyramidal, 477 Cappe's method, $i b$.- - espaliers, $i b$. -horizontal espalier's, 479-Rivers' upright mode, $i b$. - pruning and training against walls, 480-Harrison's mode of training, 481-spurpruning, 482-training in pendant pruning, 482 -training in pendant
form, 483 - training to horizontal trellises, $i b$.- summer pruning espaliers, $i b$. - thinning the crops, $i b$.

Apple aphis, the, 437-bark beetle, 442-borer, 444-bug, 433-butter, manufacture of, 413 -cliermes, 442 - saw-fly, 444-stocks, kinds of, \&c., 328-tree mussel-scale, 443scolytus, 436 -weevil, $i b$.

Apples, mode of storing, \&c. in the United States, 674.

Appleby, $\mathbf{M r}$, on propagating the coniferæ by cuttings, 347 -on vine borders, $600-$ on planting vines, 604 -on spur-pruning the vine, 606 . Approach, grafting by, 336 .

Apricot, soil suitable to the, 359root-pruning the, $401-$ natural history of the, $517-$ number of sorts, $i b$.-its propagation, $i b .-$ planting, 518 -pruning and training, $i b .-$ protecting the blossom, 519-thinning the fruit, $i b$.- protecting and gatliering the fruit, $i b$.-summer pruning, $i b$. - root pruning, $i b .-$ select list, $i b$. - selections for particular localities, 521-insects and diseases by which attacked, 522its European names, 523.

A pricot, plum, and cherry, forcing the, 546-liouses, \&c. for them, ib. - Mr Rivers' mode, 547 - kinds $5 \mathrm{Q}$ 
suitable for it, $i b$.- kinds of trees suitable to it, 548-Mr Rivers on it, $i b$ - - time of beginning to force, 549 -temperature, $i b$. - watering, 550 .

A pricot stocks, kinds, \&c. of, 328

Apricots, kinds of, suitable for forcing 547-management of, in the fruitroom, 675 .

Aquarium, management of the, 710 selection of plants for it, and their propagation, $i b$.- -soil, 711 -water, $i b$.-list of plants, 712 .

Aquatic plants, culture, \&c. of, 710 .

Arctia caja, the, 157 .

Arctium lappa, 134

Argillaceous soil, what, 263, 266-

plants indigenous to, 271 .

Argynnis euphrosyne, 833.

Argyromyges scitella, the, 473.

A rmadillo vulgaris, 827.

Armeniaca vulgaris, see A pricot.

Artemisia absinthium, 242 .

Artemisia dracunculus, the, 166 .

Artichoke, Pliny on the, 4-proportion of ground for, 8-natural history of the, 130-its uses, 131mode of propagation, $i b$.-planting, $i b$. -its subsequent cultivation, $i b$.soil and manure for it, 132-gathering and preserving the crop, 133approved sorts, $i b$. - insects and diseases, $i b$.- general remarks, sav ing seed, European names, \&c., $i b$. Artichoke tortoise beetle, the, 133 .

Artificial impregnation, production of improved fruits by, 304 .

Artificial spurs, pruning for, 403-the old system of, 482 .

Arundel, the fig at, 551 .

Ashes as a manure, 298.

Asparaginous plants-Asparagus, 117 -The Alisander, 129-The Cardoon, ib.-The Artichoke, 130The Hop, Milk Thistle, \&c., 134.

Asparagus, culture of, among the Romans, 3-proportion of ground for, 8-natural history of, 117 -its uses, $i b$.-propagation, 118-sowing and planting, $i b .-$ its subsequent cultivation, $121-$ soil and manure, cultivation, $121-$ soil and manure, manent beds, 124 -forcing in permanent pits, 125-amateur forcing, $i b$.- - gatliering, 126-instrument for cutting, 127-list of approved sorts, $i b$. -insects and diseases, 128-general remarks, $i b$.

Asparagus beetle, the, 128

Asparagus copper web, the, 128.

Asparagus cutter, 127.

Aspidiotis concliformis, the, 443 , 516,523 -echinocacti, 824-nerii, 823 - Ostreæfol mis, 472 - proteus, 821-rosæ, 828 .

Astelma, culture of, 704

Astyages hemerobiella, the, 471 .

Athalia centifolia, the, 190 .

Atmosphere, amount of food derived by plants from the, 289 .

Atmospheric action, influence of, in the formation of soils, 264-humidity, importance of, in hothouses, 650 -influence, importance of exposure of the soil to, 282 .

A triplex hortensis, culture of, 138 .

Attar of Roses, 751.

Aucuba, propagation of, by single leaves, 35I.

Auricula, cultivation of the, 809 .

Autumn, transplanting in, 358,360 -as the season for pruning, 391.

Averuncator, the, 409 .

Ayres, Mr W. P., on the culture of the cucumber, 665 .

Azaleas, Clinese or Indian, culture of, 678,683 - select list, 685 .

Bacchus weevil, the, 440 .

Bacon, Lord, singular use of the leek recorded by, 44-on the influence of the stock on the graft, 325 .
Badham, Dr, on edible fungi, 243, 244-on their germination, \&c., 245 .

Baily, Mr, mode of growing cauliflower by, 99 .

Baker, $\mathrm{Mr}$, on the sowing of peas, 50 .

Balaninus nucum, the, 565 .

Baldwin apple, origin of the, 304 .

Balfour, Professor, on the rotation of crops, 13-on the influence of the stock on the graft, 326 - on grafting, 337 -on striking cuttings, $349-0$ n propagation by leaves, 351 - on propagation by single buds, 355on the changes in fruits, 674 .

Balm, culture of, 236 .

Balsam, culture of the, 740 .

Balsamita vulgaris, 237.

Barbarea præcox, 170-vulgaris, $i b$.

Bark as a manure, on, 293.

Barnes, Mr, growth of cauliflower by, 102-on cliarring soils, 282-culture of the pine by, 657 .

Baron, Mr, improvement of the hollyhock by, 798 .

Barral, M., on the fertilising ingredients brought down by rain, 18 , 286.

Barred tree lackey moth, the, 439.

Barron, Mr, invention of wedgegrafting by, 334-grafting conifers by, 335 - on the season for transplanting, 357-on mound planting, 365-large trees removed by him, 367 -his mode of operation, 368 on the evils of growing trees in pots, 369 - on the effects of pot-culture on roots, $i b .-$ his mode of preparing roots in transplanting, 374,379 his large tree-lifting machine, 378 his smaller, 379 .

Basil, history of, 4-culture of, 237 .

Baskets for orchids, 687

Bast mat, the, as a protector, 735 .

Bastard trenching, how performed, 280.

Bath asparagus, the, 134 .

Baumhauer, analysis of a soil by, 272 .

Bean, history of the, 4-proportion of ground for, 8-order of, in the rotation, 16, 17-ingredients removed from the soil by, 17-advantages of transplanting to, 25-its natural history, $62-$ its uses, ib. - mode of propagation, 63 -planting, $i b$.subsequent cultivation, 65-soil and manures, $i b$ - forcing, 67-gathering the crop, $i b$.-lists of approved varieties, and their qualities, $i b$.insects and diseases by which attacked, 68-its foreign names, 70analysis of it, \&c., $i b$.

Bean aphis, the, 68 .

Beaton, Mr, on leaf pruning, 399 on peach training, 495 - on the oleander, 684,685 - on the mammillaria, \&c., 720 - on the hyacinth, 812.

Beattie, Mr, root-pruning as practised by, 399 .

Beck, Mr, his slate tubs, $682,702$.

Bedding-out plants, culture and management of, 818-list of them, 820 .

Beet, history of, 4 -proportion of ground for, 8-place of, in the rotation, 17. See also Red and White Beet.

Belgium, mode of forcing lettuce in, 154 - growing, \&c. of succory in, 165-use, \&c. of liquid manure in, 295-commission for the nomenclature of fruits in, 302-improvement of the pear in, 447

Bell-glasses for cauliflower, 100-use of, for cuttings, 351 .

Belleisle cress, the, 170

Berard on the keeping of fruits, 674 .

Beta cicla, the, 139-Maritima, 226 -vulgaris rubra, see Red Beet.

Bibio marci, the, 591,832 .
Biennials, treatment and list of, 818 .

Bignonia, propagation of the, by eyes, 354

Birds, means of preserving peas from, 60 -means of scaring, 83-the dung of, as a manure, 294-value of, as destroyers of insects, 111, 446.

Bishop, mode of sowing peas recommended by, 52 .

Biston histarius, the, 536 .

Bitter almond, culture of the, 559 .

Black's spade, 279 .

Black arch-moth, the, 834 .

Black Bryony, 134

Black caterpillar, the, 190

Black currant, culture of the, 566-

list of sorts, 569 .

Black fly, the, 68 .

Black gall-midge, the, 472

Black-horned leaf-niner, the, 59,194 , 826.

Black mulberry, the, 592

Black mustard, culture of, 168

Black palmer, the, 190.

Black-spotted turnip-leaf plant-louse, 196.

Black strawberries, list of, 588 .

Black-veined white butterfly, the, 437 .

Black vine-weevil, the, 516, 640 .

Bladder campion, the, 134

Blaeberry, the, 594

Blanching, disadvantages of, for asparagus, $125,126$.

Blanching-pots for sea-kale, 107, 108.

Blatta orientalis, 695,827 .

Bleeding of the vine, the, 631 .

Blind buds, causes of, 340 .

Blistering on the peach, causes, \&c. of, 514 .

Blocks for orchids, 687.

Blood as a manure, 294.

Bloody-veined dock, the, 138 .

Bloomfield lioe, the, 208.

Blossom, protection of the, in the peach and nectarine, 498-in the a pricot, 519 .

Blubber as a manure, 296.

Blue cabbage-fly, the, 115 .

Boletus edulis, 244-scaber, 244, 245.

Bombus terrestris, the, 68

Bombyx antiqua, 831-auriflua, 437 -Cœruleocepliala, 443,515-chrysorrhœa, or phœorrhœa, 438-dispar, 439-ligniperda, 467-lubricipeda, 196, 833-neustria, 439pini, 835 .

Bone-dust as a manure, 293.

Bones, crushed, as a manure, 293.

Bonnet on the absorption of nourisllment by leaves, 290 .

Borage, culture of, 234.

Borders for fruit-trees, the formation of, 359-formation of, in conservatories, 723 .

Borecole, the, its natural history, 108-its uses, $i b$.-propagation, 109 - sowing, $i b$.-subsequent culture, $i b$. - soil and manure, $i b$. - approved sorts and their qualities, $i b$.-European names, 116 .

Bostrichus dispar, the, 442 - typographus, 835 .

Botanical indications, determinations of soils from, 270 .

Botrytis destructor, the, 29.

Bottle gourds, the, 259.

Bottom heat, employment of, for cuttings, 349 .

Boutclier on budding the walnut, 561.

Boussingault on the rotation of crops, 15.

Bowling - green, formation, \&c. of the, 776 .

Box-edging iron, the, 388 .

Braddick, $\mathrm{Mr}$, his mode of grafting the vine, 599 .

Bradley, artificial hybrids first produced by, 314 .

Bramble, the, 594 
Branches of fruit-trees, on bending down the, and its effects, 404.

Brandy, distillation of, from potatoes, 201.

Brassica napus, 171 .

Brassica oleracea, the, 81 .

Brassica oleracea Botrytis asparagoides, see Broccoli.

Brassica oleracea Botrytis cauliflora, see Cauliflower.

Brassica oleracea bullata gemmifera, see Brussels Sprouts.

Brassica oleracea bullata major, see Savoy.

Brassica oleracea capitata, 88 .

Brassica oleracea capitata, var. depressa, 89.

Brassica oleracea var. capitata rubra, see Red Cabbage.

Brassica oleracea, var. oblonga, see Portugal Cabbage.

Brassica oleracea sabellica, see Borecole.

Brassica rapa, see Turnip.

Brassicaceous plants-The Cabbage, 81-The Portugal Cabbage,90-Red Cabbage, $i b .-$ Brussels Sprouts, 91 -Broccoli, 92-Savoys, 96-Cauliflower, 98-Sea-kale, 103-Borecoles, 108-Diseases and Insects by which attacked, 110-saving seed, 115-European names, 116.

Brassy cabbage flea, the, 115-onion $\mathrm{fly}$, the, 42 .

Bread, manufacture of, from carrots, 176-use of potatoes in making, 201.

Brignole plum, manufacture of prunes from the, 525 .

Brindled beauty moth, the, 536 .

Brocl, the Comte de, cultivation of the truffle by, 253.

Broccoli, order of, in the rotation, 17 -natural history of, 92 -its uses, $i b$. - mode of propagation, $i b$.—sowing and planting, $i b$. - subsequent culture, $i b$. - soil and manure, $94-$ gathering, $i b$. - list of approved sorts, $i b .-$ general remarks, 95saving seed, 96, 116 - European names, 116.

Bromelia ananas, see Pine Apple.

Brompton stock, the, 328 .

Brooklime, culture of $17^{\circ}$

Broom, protection of delicate shrubs by, 735 .

Brown, Mr, on the effects of pruning, 405 .

Brown's fumigator, 516 .

Brown limpet-scale, the, 824 .

Brown scale, the, 683, 685 .

Brown-tailed moth, the, 438 .

Brown Tussock moth, the, 831 .

Bruchus flavimanus, the, 69-granarius, the, 59 -pisi, the, $i b$.

Brugmanns on the rotation of crops, 12.

Brussels sprouts, natural history of, 91 -uses, $i b$.- sowing and planting, $i b$. - subsequent culture, $i b$. - gathering, 92 - general remarks, $i b$. saving seed, 116-European names, $i b$.

Brussels stock for apricots, the, 328 .

Bryophyllum, propagation of, by single leaves, 351 .

Budding, propagation by, 338-its nature and rationale, $i b$.- season for its performance, 339 -its advantages, 340-various methods of performing, 341-knives for it, $i b$.various modes of shield budding, $i b$. -American, 342-Saul's, ib.-reversed, $i b$. - Paul's, 343- scallop, 344 -annular or ring, $i b$.-Flute or tube, $i b$.-terminal flute, 345 - with pushing eye, $i b$.- propagation of the vine by, 600 -roses, 760

Budding-knives, kinds of, 341

Buds, propagation by cuttings of, 354 .
Buist, $\mathrm{Mr}$, on the formation of soils, 265.

Bulb-house, management, \&c. of, 696 - plants for it, $i b$. - season of rest, 697 - pots, $i b$. - soil, $i b$. - potting, 698-propagation, $i b$. - general remarks, and lists of sorts, 699 -insects and diseases, 700-soil, 702general culture, $i b$.-pruning, 703insects and diseases, $i b$.

Bulbs, importance of shallow planting to, 28.

Burdock, the, 134

Burnet, culture of, 167 .

Burning soils, benefits of, 282.

Cabbage, early listory of the, 4-proportion of ground for, 8 - order of, in the rotation, 17-advantages of transplanting, 24 - its natural history, 81-its uses, 82-mode of propagation, $i b$. - sowing and planting, 83 - planting-trowels, \&c. for it, 85--its subsequent culture, 87gathering and storing the crop, 88list of approved sorts, 89- saving seed, 115-European names, 116.

Cabbage, red, see Red Cabbage.

Cabbage aphis, the, 112-fly, the, 114 197-moth, the, 1.12, 197-lettuces, distinctive peculiarities of, 155 powder - winged moth, the, 113tribe, the, see Brassicaceous Plants.

Calcareous soil, what, 263, 267-plants indigenous to, 271 .

Calceolaria, culture of the, 739 .

Calderini, Signor, experiments on grafting grapes by, 338 .

Calendula officinalis, 240

Californian seeds, proper time for sowing, 313.

Callow's mode of peach-training, 494 . Camelia, shield-budding the, 341 .

Camellia-box, a, 682 .

Camellia-house, plants admissible to the, 678-the Camellia, $i b$.-its propagation by seed, 679 - by layers, $i b$. -by cuttings, $i b$.- by grafting and inarching, $i b .-$ by budding, $680-$ general culture, $i b$.- potting, $681-$ soil, 682 -planting out in prepared borders, $i b$. - select list, $i b$. - diseases borders, $i b .-$ select list, $i b$.- diseases -diseases and insects, 684 - the Oleander, ib.- the Rhododendron, 685 .

Campoplex paniscus, the, 197.

Canker, the, 190-prevalence, \&c. of, in the apple tree, 432 - of the peacli, on, 512-in the plum, 534-of the cucumber, 668 - of the melon, 672 .

Canker worm, the, 445 .

Cantaleup melon, the, 668 .

Cantharellus cibarius, 245.

Canvass as a protecting medium, 735 protection of the blossom of the peach by, 498 .

Cape bulbs, culture of, 700 -heaths, variety, \&c. of, 703-their culture, ib. et seq.

Cappe's mode of training the apple and pear, description of, 478

Capsicum, culture of the, 260 .

Caraway, culture of, 240 .

Cardoon, place of the, in the rotation, 17-natural history of tlee, 129-its $u$ ses, $i b$.- -sowing and planting, $i b$. -its subsequent cultivation, 130 gathering and preserving, ib.-approved sorts, $i b$. - saving of seed, $\& c . i b$. -its European names, $i b$.

Carduus Marianus, the, 134

Carnation, propagation of the, by layering, 351-cultivation of the, 806 - pipings of, 350 .

Carpenter, Dr, on the germination of seeds, 313.

Carpocapsa pomonana, the, $435-\mathrm{W}$ beriana, the, 516, 522-nigricana, the, 535 .

Carrière, M., on budding witl the pushing eye, 345 .
Carrot, proportion of ground for, 8 order of, in the rotation, 17-its natural history, 176-its uses, $i b$. - propagation, $i b$. - sowing, ib.subsequent culture, 178-soil and manure, 179 -forcing, $i b$ - - rathering and storing, $i b$. - list of approved sorts and their qualities, 180 -insects and diseases by which attacked, 181-saving seed, 182-its European names, $i b$. - its nutritive qualities, $i b$.

Carrot fly, the, 181

Carum carui, 240.

Cassida viridis, the, 133

Castanea vesca, tlie, 562 .

Cataphagus lineatus, tlie, 69,222 .

Cato on the culture of asparagus, 3 , 117.

Cattle, the dung of, as a manure, 291 Cauliflower, place of, in the rotation, 17-distinction between, and broccoli, 95 - its natural lristory, 98 -its use, $i b$,-propagation, $i b .-$ sowing and planting, $i b$.- -subsequent cultivation, 100 -liand-glass protection, $i b$. - wall protection, $i b$. -frame protection, 101-wintering in pots, $i b$. - soil and manure, 102gathering and storing, $i b$.- list of approved sorts, 103-saving seed, 116 -European names, $i b$.

Cawdor castle, gooseberries at, 575 .

Cayenne pepper, culture of, 260

Cecidomyia nigra, the, 472 .

Celeriac, culture of, 150 .

Celery, proportion of ground for, 8place of, in the rotation, 17-natural history of, 141 -its uses, $i b$.propagation, $i b$.- - sowing and planting, $i b .-$ subsequent cultivation, 144-soil and manure, 146-gatliering and preserving, $i b$. - mode of wintering, 147-list of varieties, 148-diseases and insects by which attacked, ib. -its European names, 150 -saving seed, \&c., $i b$.

Celery fly, the, 148-parsley, 233stem fly, the, 149 .

Cerasus Juliana and duracina, the, 537.

Cerapliron niger, the, 195, 196.

Cerostoma Xylostella, the, 197 .

Cetonia aurata, 828 .

Ceutorhynchus contractus, the, 191pleurostigma, the, 196-sulcicollis, the, 114.

Chærophyllum sativum, 171.

Chalk as a manure, 297.

Chambering vine-borders, advantages of, 602 .

Chamomile, culture, uses, \&c. of, 241.

Champignon, the, 244.

Cliandler, Messis, their mode of growing the Camellia, 679 .

Chappel's Colewort, time for sowing, 84.

Charcoal as a manure, 296

Chard, cultivation of artichokes for, 133.

Cliardoon, see Cardoon.

Charring soils, benefits of, 282 .

Chaumontelle pear, origin of the, 304.

Cheimatobia brumata, the, 434

Cliemical analysis, determination of soils by, 271.

Chenopodium bonus Henricus, 137.

Chermes buxi, 825-mali, 442-pyri, 468.

Cherries, kinds of, suitable for forcing, 547.

Clierry, soil suitable to the, 359-difficulty of transplanting, 366-rootpruning the, 401-its natural history, 537-its uses, ib.-its propagation, $i b .-$ soil and planting suited to it, 538-pruning and training it, 539 - select list, 541 -lists for 
particular localities, 544-diseases and insects by which attacked, $i b$. -its European names, 545-forcing the, see Apricot, Plum, and Cherry, forcing the.

Cherry brandy, manufacture of, 537 -saw-fly, the, 545-stocks, kinds, \&c. of, 328 .

Chervil, culture of, 171 .

Chestnut, culture of the, 562 .

Chestnuts, management of, in the fruit-room, 676

Chevandier and Salvetat, experiments on watering by, 287 .

Chick-pea, the, 80.

Chicory, see Succory.

Chile strawberries, origin of the, 582 -list of, 590.

Chilis, culture of, 260

China, growth of the peach in, 485 .

Chinese, use, \&c. of liquid manure by the, 295.

Chinese azaleas, see Azaleas - cabbage, the, 110 .

Chink grafting, 333 .

Chive, the, its natural history, culti vation, \&c, 47.

Chlorine, presence of, in plants, 20 .

Chrysanthemum leaf-miner, the, 833 .

Chrysomela betulæ, the, 197.

Ciboule, the, 41.

Cichorium endivia, see Endive-intybus, see Succory.

Cider, manufacture of, in America, 413.

Cider apples, criterion of, 414-list of, 432-orcliards, slowness of improvement in the, 300 .

Cladius difformis, 828 .

Claremont nectarine, origin of the, 304 - pippin, superiority of the growth of the, 476 .

Clary sage, culture of, 235 .

Clavaria, edible species of, 245 .

Clay, what, 266.

Clay-coloured vine-weevil, the, 640 .

Clayey soil, what, 263, 266-plants indigenous to, 271 .

Claygate pearmain, how originated, 304.

Cleft grafting, 331-the vine, 332

Click-beetles, the, 69 .

Climate, importance of, as to the selection of fruit-trees, 301 -supposed change of, 306 - cliange of, injurious to transplanted trees, 366 - halfhardy slirubs considered with reference to, 793 .

Climbing plants, training of, 719 .

Clingstone peaches, what, 485 .

Clisiocampa Americana, the, 444neustria, the, 439 .

Cloches, employment of, in growing strawberries, 584

Close pruning, purposes, \&c. of, 403.

Cloudberry, the, 594 .

Club in the Brassicæ, the, 111

Cobbett, W., his mode of sowing peas, 50 .

Coccinella, value of the, 68 .

Coccus adonidum, the, $639,661,823$

-bromeliæ, 661,824 -hesperidum, 703, 823-patellæformis, 824-testudo, 824-vitis, 570, 638 .

Cochlearia armoracea, 261.

Cochylis vitisana or reliquana, the, 639.

Cockroach, the, 694,827 .

Cockscomb, culture of the, 740 .

Codlin-moth, the, 435.

Coe's golden drop plum, origin of, 305 .

Coiling, propagation of the vine by, 597.

Cold or Conservative pits, management of, 733-plants suitable to them, $i b$.

Cole, Mr, his mode of growing celery, 150

Coleophora hemerobiella, the, 471 .
Coleworts, culture of, 82,87 .

Collar, importance of the, in plants, 365.

Collards, increasing use of, 82,87 .

Colour, variety and influence of, in soils, 274 -effects of, on the germination of seeds, 311 .

Columella on the culture of the cucumber, 663 .

Common sorrel, culture of the, 139.

Concreting vine-borders, on, 602 .

Conical form, pruning the apple and pear in the, 476 .

Conifers, modes of grafting, 335-propagation of, by cuttings, 347-the pruning of, 406 -transplantation of, 371.

Conservative pits, see Cold Pits.

Conservative wall and border, management of the, 734

Conservatory, management of the, 721 -objections to planting out, $i b$. -distribution of the plants according to light, $i b$. - their arrangement, vases, floors, stages, \&c., 722shading, 723-borders for planting out, $i b$.-cleanliness, watering, and temperature, 724 - select list of plants, $i b$.

Consolidation as a means of improving the soil, 281.

Continent, employment of garlic on the, 30 -extensive employment of haricots on the, 71 .

Cooke, $\mathrm{Mr}$, cultivation of the auricula by, 809 .

Cool tankard, composition of, 234

Copper-coloured weevil, the, 535 .

Coriander, culture of, 172,240 .

Corn salad, culture of, 172 .

Corvisartia helenium, 242.

Corylus avellana, see Filbert.

Cos lettuces, distinctive peculiarities of, 155.

Cosmia pyralina, the, 440 .

Cossus ligniperda, the, 467 .

Costmary, culture of, 237.

Cotton thistle, the, 134 .

Couve Troncliuda, the, 84-culture of

it, 90 .

Cow cabbage, the, 110 .

Coxe on pruning, 394 .

Crab stocks, 328 .

Crace, $\mathrm{Mr}$, on root-pruning, 400

Crambe maritima, see Sea-kale.

Cranberry, culture of the, 593 .

Crane-fly, the, 111

Cree, $\mathrm{Mr}$, lis system of pruning foresttrees, 407

Cresses, culture of, 168

Crioceris asparagi, the, 128

Criptops hortensis, the, 197.

Cropping, simultaneous and successional systems of, 24-injury done to vine borders by, 603 .

Crops, rotation of, 12-various theories on it, ib. et seq.-proper order of it, 16-the successional and simultaneous modes, 24.

Crossing, see Hybridising.

Crown-grafting, 333.

Crowns, propagation of the pine-apple by, 643 .

Cruciferous plants, order of, in the rotation, 16

Cryptogamic plants, way in which nourished, 289 - propagation of, 309.

Cucullia verbasci, the, 833 .

Cucumber, the, its natural history, 663 -its propagation, $i b$. -its cultivation, 664-open-air culture, 666 saving seed, 667 -select list, $i b$.insects and diseases, 668-its European nalues, $i b$.

Cucumis Melo and citrullus, see Melon -sativus, see Cucumber.

Cucurbita melopepo maxima, the 259-ovifera, the, $i b$.-pepo, the, ib.
Culinary apples, criterion of, 414-list of, 423 .

Culinary garden, see Kitchen-garden.

Cultivator, improved, with earthing-up

boards, 210 .

Culzean Castle, list of apples which ripen at, 431 - pears which ripen at, 462-plums grown at, 534 .

Cumberland lodge vine, the, 595.

Cunningham, Mr, results in hybridisation by, 320 .

Curculio abietis or pini, 834-alliaria, 440-Bacchus, $i b$.- betuleti, 640 contractus, 111 -cupreus, 535 -nucum, $565-$-blongus, $436,516,522$ -picipis, 640-pleurostigma, 114, 198 - pomorum, 436-pyri, 466sulcatus, $516,640,832$-tenebricosus, 522, 535-vastator, 443.

Curl on the peach, causes, \&c. of, 514 -in the potato, the, 222.

Currant, advantages of transplanting the, 357-natural history of the, 566-propagation, ib.-soil and situation, 567 -pruning and training, $i b$.-forcing, 568-select list, 569 - diseases and insects, $i b$.569 - diseases and in
European names, 570.

Currant spliynx-moth, the, 570 .

Cuthill, Mr, on the cultivation of Brussels sprouts, 91, 92-of cauliflower, 98-of sea-kale, 106, 107on the culture of asparagus, 119 $122,125-$ on the culture of celery 143,144 - and lettuce, 163-culture of succory by, 165-on the culture of the radish, 173-on forcing potatoes, $216-$ on the culture of red beet, 226, 227-on the cultivation of mushrooms, 248-on the growth of rhubarb, 257-on successional cropping, 284 -his mode of growing the cucumber, 665 - on the culture of the melon, 669 .

Cuttings, propagation by, 346-their form, size, \&c., $i b$. - of hardy fruittrees, 347-of deciduous trees and slurubs, $i b$. - of conifers, $i b$. - of partially-ripened wood, $i b$. - of young wood, $i b$.-mode of striking them, 348-time for it, 349-bottom-heat, $i b$,-pipings, 350 - of the roots, $i b$. - of the flower stems, $i b$. - by leaves, 351-propagation of the potato by, 202-propagating the rose by, 762-propagation of the vine by, 598 .

Cydonia vulgaris, the, 560

Cynara cardunculus, see Cardoonscolymus, see Artichoke.

Dahlia, cultivation of the, 803 .

Dalkeith park, list of apples grown at, 430-pears grown at, 462-age of the peach at, 498-peculiar mode of training the peach at, 506-apricots grown at, 521-plums grown at, 534-cherries grown at, 544 vine-borders at, 601 - the collection of alpine plants at, 815 .

Dalmahoy, peculiar mode of growing carrots at, 178.

Damson plum, propagation of the, 526.

Dandclion, the, 166.

Daniell, Professor, on atmospheric liumidity in hothouses, 651.

Daphnes, culture of, 678 .

Darkness, necessity of, to the germination of seeds, 310 .

Dart-moth, the, 195 .

Darwin, Dr, on pulverisation of the soil, 278.

Daubeny on the rotation of crops, 12 , 13.

Daucus carota, see Carrot.

Davy, Sir H., on the formation of soils, 265 -on clayey soils, 266 -on the heat-absorbing power of different coloured soils, 275 - on pulverisation of the soil, 278-on burning 
soils, 282-on the gaseous products of manures, 289.

Day and night temperature in hothouses, regulation of, 629 .

Death's-head moth, the, 221 .

Decandolle on the rotation of crops, 12-on the distinction between broccoli and caulifiower, 95-researches of, on the nourishment of plants, 290 - on the duration of fruit-trees, 307 -on hybridisation, 315 - on the theory of grafting, 322 -on the roots of plants, 363 .

Deciduous trees, cuttings of, 347 .

Decker, Sir M., introduction of the pine-apple into England by, 641 .

Deep digging, importance of, 23.

Deep planting, evils of, 365 .

Deep sowing, effects of, on germination, 310.

Deer's dung as a manure, 294.

Dendrolimus pini, 835 .

Depressaria applana, the, 182 - pastinacella, the, 231

Dessert apples, criterion of, 413-list of, 414 -pears, list of, 452 .

1 'Hoogvorst, Baron, his mode of growiilg mushrooms, 252

Dibber, sea-kale, 105

Dibbers, various forms of, 85 .

Digging as a means of improving the soil, 279.

Dill, culture of, 234 .

Disbudding, uses, \&c. of, 404-the peach, $500,503$.

Ditula angustiorana, the, 522 .

Dolphin, the, 68 .

Donaldson on the rotation of crops,14on the origin of soils, $264-$ on clayey soils, 266-on sandy soils, $267-$ on loam, $i b$ - on aninial excrements, 290 - on manures, 291-on animal manures, 294-on the application of manures, 298 .

of manures, 298 .

Downing, Mr, views of, on the duration of fruit-trees, 306 - on American whipgrafting,331 - on cleft grafting, 332 - description of the American mode of shield-budding by rican mode of shield-budding by,
342 - on autumn transplanting, 360 -on the effects of pruning on the -on the effects of pruning on the
vigour of the tree, 397 - on pruning transplanted trees, $i b$. - on spring pruning, 394 - accounts of large apple-trees by, 413-account of large pear-trees in America by, 448-acpear-trees in America by, 448-acsap blights by, 465 - on the culture of the peach in America, 486 - on the American mode of peach-training, $496-$ on the soil suitable to the plum, 526 - on American plums, plum, 526 - on American plums, 524 - on the cherry in America, 537
- on the strawberry, $582-$ on its culture, $585-$ on the storing, \&c. of apples, 674 .

Downton imperatrice plum, origin of, 305 -strawberry, origin of the, $i b$.

Draining as a means of improving the soil, 277-mode of its action, $i b$.

Drains, formation of, 277 - their depth, distance, \&c., 278 .

Draw hoe, the, 207-triangular, 208.

Draw hoe and rake combined, 208.

1)rill rake, the, 33 .

Drilling and sowing machine, 211.

Drooping-fan mode of training, the, 390.

Drosophila flava, the, 196-graminium, 833.

Drumlanrig castle, list of apples which ripen at, 431 -pears grown at, 462 -plums grown at, 534-cherries grown at, 544 -vine-borders at, 601.

Drummond, $\mathrm{Mr}$, mode of sowing peas by, 52 .

Du Breuil, Professor, his mode of peach-training, 496 .
Duchess d'Angoulême pear, origin of the, 304 .

Duhamel, researches of, on the nourishment of plants, $290-$ on the bleeding of the vine, 631 .

Duncan, $\mathrm{Mr}$, his mode of earthing-up celery, 145.

Duquesne, the Abbé, improvement of tlie pear by, 447 .

Dwarf fruit-trees, grafting to secure, 325 -standards, pruning of, 394 .

Earthing-up, modes of, as applied to celery, 144 .

Earwig, the, and its destruction, 514. East, introduction of fruits from the, 304.

Easter pippin, peculiarity of the growth of tlie, 476

Edible fungi, the mushroom, 243the morel, 252-the truffle, 253.

Edinburgh, mode of growing celery at, 143.

Egg plant, cultivation, \&c. of the, 259.

Egypt, early use of the onion in, 30 .

Elater, the genus of, 69 -destruction of, 182 .

Elecampane, culture, uses, \&c. of, 242

Elm-destroying scolytus, the, 473.

Elruge nectarine, crigin of the, 304 .
Elton cherry, origin of the, 305 .

Elveston, large trees transplanted at, 367.

Endive, natural history of, 158its uses, 159-propagation, ib.sowing and planting, ib.-subsequewing and planting, 2b.--subsequent cultivation, 160-blanching, ing and preserving, $i b$.-forcing, $i b$. -list of varieties, 163-insects and diseases by which attacked, 164general remarks, $i b$.

Endive blanching-pot, the, 160 .

Enghien, peculiar purple beech at, 316.

England, introduction of the pea into, 48 - of the bean, 62 - of the kidneybean, $71-$ of the carrot, 176-of the turnip, 183- of the potato, 200 - of the Jerusalem artichoke, 223 - of the apple, 411 - of the pear, 447 - of the peach, 485 - of the apricot, 517 - of the plum, 524-of the cherry, 537-of the fig, 551-of the almond, 559-of the currant, 566of the raspberry, 570-of the mulof the raspberry, 570 - of the mul-
berry, 592 - of the vine, $595-$ of the pine-apple, 641 .

En Gobelet management of the apple and pear, the, 475 .

En tonnoir management of the apple and pear, the, 475 .

Epacris, culture of, in the heathhouse, 704.

Episema cæruleocephala, the, 443 , 515.

Eriosoma mali or lanigera, the, 433.

Ermine moth, the, 435 .

Errington, $\mathrm{Mr}$, on stocks for fruit trees, 327-on summer pruning, 391 -on pruning standard plums, 394 - on root-pruning, 399,400 list of pears by, 462 - on pruning the pear, 482 - on pruning the peach, 489 - on summer-pruning the peach, 500 -on forcing it, 504 -on the culture of the cherry, 539 540 - on the culture of the filbert, 564 - on the culture of the currant, 568 -his mode of propagating the vine by mode of propagating the of the vine, 621 .

of the vine, 621 .
Erysiple communis, the, 61 .

Eschalot, origin of the name of, 27see also Shallot.

Esculent-rooted plants-The Carrot, 176-The Turnip, 183-The Potato, 199-The Jerusalem Artichoke, 223 -Red Beet, 226-Salsify, 228-
Skirret, 229-Scorzonera, ib.-The Parsnip, $i b$.

Espalier trees, various modes of training, 390-cherry trees, training of 540

Espaliers, training of the apple and pear to, 478 .

Etna, mount, the chestnut-trees at, 562

Eumerus (Eneus, the, 42.

Euplirates, size of the asparagus of the, 117.

Eupressia caja, the, 157.

Europe, introduction of fruits from the East into, 304 - of the pineapple, 641 .

Evaporation, importance of preventing, in transplanting, 363 .

Evelyn on the pine-apple, 642 .

Evergreens, transplantation of, 363 , 371.

Extension, difference between propagation by, and that by seed, 315 .

Eyes, propagation by, 354-propagation of the vine by, 596

Faba vulgaris, see Bean.

Fairchild's nectarine, origin of, 304

Fan-training, various modes of, 390

the apple and pear as espaliers, 479

-the cherry, 540-the peach, 494.

Fat Hen, culture of, 137 .

Fennel, early listory of, 5.

Fermentation, growing tlie pine-apple by, 654 .

Ferns, culture of, 694

Fertility, effects of grafting in securing, 324.

Ficus carica, the, see Fig.

Fig, soil suitable to the, 359-propagation of, by single leaves, 351root-pruning the, 401 -its natura history and uses, 551-its propagation, $i b$ - - soil, 552-planting, $i b$ pruning and training, 553-protecting during winter, 555-accelerat ing its ripening artificially, $i b$.forcing, 556-select list, 557-diseases and insects, 558-Europcan names, $i b$.

Figure-of- 8 moth, the, 443,515 .

Filbert, natural history of the, 563its propagation, $i b$.- soil and situation, $i b$.- pruning and training, $i b$. - list of sorts, 565 - insects and diseases by which attacked, $i b .-$ its European names, $i b$.

Filberts, management of, in the fruit room, 676 .

Finger-pruning, uses, \&c. of, 404.

Fingers and toes in turnips, the, 198.

Fire-heat, danger of, to heatlis, 707 .

Firs, the pruning of, 406 .

Fish as a manure, 296.

Fistulina hepatica, 245.

Fixing with water, transplanting by 387.

Flat-bodied moths, the, 181, 182, 231

Flat peach of China, the, 486 .

Flea beetle, the, 115 .

Fleming, Mr, his mode of root-pruning the peach, \&c., 401-his systen of spur-pruning the vine, 609 -his mode of vine-forcing, 617-soil used for pines by, 646-liis mode of planting out pines, 657-liybrid melon produced by, 668 .

Florists' flower-garden, situation, \&c. for the, 796-The Pansy, ib.-The Hollylock, 798-The Ranunculus, 801 - The Anemone, 803 - The Dahlia, $i b$. - The Carnation and Picotee, 806-The Pink, 807-Tlı Auricula, 809-The Polyantlus, 811-The Hyacinth, $i b$.-The Tulip, 813.

Flower-forcing houses or pits, management of, 729 -list of plants suitable, 730 - general directions for their management, 731 - annuals and biennials, 732 . 
Flower-garden, the. The American Garden, 742 -The Rosary, 750 bery, 780 - The Florists' flowergarden, 796-The Alpine garden and rockery, 814 - The Perennialplant garden, 815 - The Parterre garden, 816-The Reserve garden, 822 -insects injurious in it, 823 .

Flower-stems, propagation by cuttings of the, 350 .

Flower-transplanter, 86.

Flowers, grafting to continue the purity of, 324 .

Flute or tube budding, 344-terminal, 345 .

Fœnusa pumila, the, 574

Fool's parsley, 232.

Forest trees, Cree's system of pruning, 407.

Forficula anricularis, the, 514

Fork, digging, 89

Forsyth, Mr A., his mode of striking cuttings, 348 .

Forsyth on peach-borders, 487 - on pruning the peacli, 489 -on planting, \&c. the apricot, 518 .

Foster, $\mathrm{Mr}$, asparagus raised by, 123.

Fragaria, the, see Strawberry.

Frames for protecting cauliflower, 101 .

France, the pea originally from, 48culture of asparagus in, 120 -forcing endive in, $16^{\circ} 2$ - uses of the apple in, 413-mode of manufacturing prunes in, 525-culture of the pine-apple in, 645 .

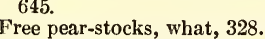

Free stocks for apples, what, 328

Freestone peaches, \&c., what, 485

French, Mr, his mode of growing the vine, 632 .

French bean, see Kidney Beanbudding-knife, the, 341 - mode of peach-training, the, 496-prunes, manufacture of, 525-sorrel, culture of, 138-spinach, culture of, ib.stock for apples, the, 328 - transplanter, a, 86 - turnip, tlie, 184, planter, a, 86 - turnip, the,
190 -whip-grafting, 331 , 333 .

Frogmore, forcing of asparagus at, 125 -culture of the plum at, 526 , 527

Frozen sap blight, the, 465 .

Fruit-gatherers, on, 501-protectors,

Fruit-room, management of the, 673 .

Fruit-trees, selection of kinds, \&c., 299 -the various lists of them, $i b$. recent improvements in kinds, 300 - circumstances to be kept in view in selecting, $i \bar{b}$. - proposed models, \&c. of fruits, 302-on the origin of new of fruits, $302-$ on the origin of new
fruits, 303 -improved varieties accifruits, 303 -improved varieties acci304 -spontaneous change of character in them, 305-on their duration in a healthy state, 306-various modes of propagating, 309-cuttings modes of propagating, 309 -cuttings rect luxuriant growth, 370 -bending down the branches of, 404-laying bare their roots, $i b$.

Fruit-tree borders, formation of, 359 .

Fruits, improved varieties of, 299their nomenclature, $i b .-$ on the origin of new varieties of, 303purity of, grafting to continue, 324 .

Fuchisia, culture of the, 741 .

Fumigators, various, 516

Fungi, various edible, 243-the mushroom and its cultivation, 245 - the morel, 252-the truffle, 253-mode of germination, \&c. of, 245 .

Gamina moth, the, 70 .

Gansel Bergamot, origin of the, 305.

Garden, necessity for constant transplantings in tlie, 357 .

Garden beetle, tlie, 830 -centipede, the, 197-cress, culture of, 168patience, culture of, 138-pebble- moth, the, 262-spades, various kinds of, 279 -swift, the, 222 -tigermotl, the 157 .

Gardeners' Chronicle, the, on rotation, 16-description of the tlirip from, 76 - of the red spider, 77 - of the Aleyrodes from, 113-on forcing mushrooms, 251 - on preparing the soil for next crop, 283 -on budding, 340, 345-on summerpruning, 392-on root-pruning, 400. Gardeners' Magazine, mode of sowing peas recommended by the, 51 .

Gardenesque:style, introduction of the shrubbery into the, 781

Garlic, early history of, 6-natural history of, 29-its uses, $i b$.-its cultivation, 30 -varieties of, $i b$.-its foreign names, $i b$.

Garnishing, plants used for, 232.

Gaseous products of manures, importance of the, 289

Gasparin, M. de, his classification of soils, 263.

Gastropacha neustria, the, 439-pini, 835.

Geans, culture of, 538 .

Geology, relations of, to culture, 301.

Geometra brumata, the, 434-cervinaria, 832-defoliaria, 434-œscularia, 832-pilosaria, 440, 473.

German prunes, manufacture of, 525 .

Germany, forcing of asparagus in, $\mathbf{1 2 4}$ - forcing turnips in, 189-mode of cultivating mushrooms in, 249 uses of the apple in, 413-the cherry in, 537 .

Germination of seeds, the, 309 .

Gesnera, propagation of, by single leaves, 351.

Ghost moth, the, 181

Gibbon on the introduction of fruits, \&c., 304.

Gidney's Prussian loe, the, 208.

Gills, propagation of the pine-apple by, 643 .

Gipsey moth, the, 439 .

Girandole training of the apple and pear, 476.

Glabra, see Liquorice.

Glass screens, protection of the peach by, 499 .

Glasses, forms of, for cauliflower, 100

Glendinning, $\mathrm{Mr}$, on transplanting evergreens, 372 -soil used for pines by, 646 - on watering them, 648 on temperature for thein, 650 .

Glendinning's pick-fork, 208.

Glenny, Mr, on Florists' flowers, 796.

Globe artichoke, Pliny on the, 4-see Artichoke.

Gloxinia, propagation of the, by single leaves, 351 .

Glycyrrhiza, see Liquorice.

Glyphopteryx variella, the, $\mathbf{5 7 4}$.

Goat moth, the, 467.

Gold fislı, use of, in the aquarium, 711.

Golden rose-beetle, the, 828 .

Good King Henry, culture of, 137.

Gooseberry, advantages of transplanting, 357 - natural history of the, 575-soil, \&c. suitable to it, $i b$. 576 -its propagation, 576 -pruning and training it, 577-summer pruning, 578 - select list, $i b$. - insects and diseases by which attacked, 579-forcing it, 581-its European names, $i b$.

Gooseberry-pruner, the, 410-saw-fly, the, 579 .

Gordon of Fountainbridge, seed-list from, 9 .

Gorrie, Mr, new gooseberries origin. ated by, 576 .

Gourd, cultivation and varieties of tlie, 259.

Gowan, $\mathbf{M r}$, on grafting the vine, 599.
Graft, influence of the stock on the, 325 -its influence on the stock,

Grafts, choice of, 325 .

Grafting, propagation by, 322 - its theory, $i b$. -its objects, 324 -to continue purity, $i b .-$ to accelerate flowers or fruit, $i b$. - to induce fertility, $i b$.- to renew the liead, $i b$.several sorts on one tree, 325 -to produce reduction in size, $i b$. choice of grafts, $i b$. - and of stocks, ib. - influence of stock on graft, ib. -and of graft on stock, 326 stocks, 327 - season for it, 329 splice, 330 - whip or tongue, $i b$. splice-grafting the peach, $331-$ saddle, $i b$.- Herefordshire saddle, 332 -cleft, $i b$.-crown, 333-peg, $i b$ shoulder or clink, $i b$. - side, 334root, $i b$. - Herbaceous, $i b$. - rootgrafting the tree peony, $i b$. - expeditious, 335 - conifers, $i b$. - softwooded or succulent plants, 336by approach, $i b$. - clay for it, 337 -and wax, ib.- propagation of the vine by, 599 - of roses, the, 761 .

Grafting clay, preparation of, 337wax, preparation of, $i b$.

Grain beetle, the, 59 .

Grape vine, the, see Vine-thinningscissors, the, 410.

Grapes, sizes of bunches of, 596management of, in the fruit-rooin, 675 .

Grass terraces, situations in which admissible, 773-walks, formation of, 777 .

Grasses, experiments on grafting, 338 -selection of, for lawns, 779 .

Gravel walks, formation, \&c. of 777.

Gravelly soil, what, 263, 268-plants indigenous to, 271.

Grayson, Mr, large asparagus grown by, 117.

Great Britain, origin of the apple in, 411 -introduction of the pear into, 447- of the vine, 595 .

Great yellow underwing moth, the, 196.

Greeks, the turnip among the, 183.

Green's mode of training fruit-trees, 396.

Green, Mr, on layering the vine, 598 .

Green centres, what, in roses, 772gage plum, peculiarities of, as to situation, 527-rose - chafer, 828vegetable manure, on, 292-veined cabbage butterfly, 113

Greenhouse, management of the, 724 -plants for it as soft and hard wooded, $i b$.-their autumn and winter management, 725 - tlieir spring and summer treatment, 727 -propagation, potting, and watering, $i b$.-their removal from it during summer, 728-training of during summer, 728 -training of

Greenhouse plants, propagation of, by cuttings, 348 .

Greens, order of, in the rotation, 17see Borecole.

Grew, discovery of the sexuality of plants by, 314 .

Griffin on vine borders, 600 -on spurpruning the vine, 607 .

Grosse mignonne peach, number of synonyms of, 300 .

Gryllus gryllotalpa, 832 .

Gryllotalpa vulgaris, tlie, 832

Guano as a manure, 294

Guernsey prong, 208.

Guinea pepper, culture of, 260

Gum in the cherry, causes, \&c. of, 544 - on the peach, cure of, 513 .

Gumming of the cucumber, 668-of

the melon, 672 .

Gyde on the rotation of crops, 12.

Gypsum as a manure, 297. 
IIacon's Incomparable pear, origin of, 305 .

Haddington, the Earl of, on pruning conifers, 406 .

Hales on the roots of plants, 362 .

Half standards, management of the apple and pear as, $\mathbf{4 7 5}$.

Halliman's fruit protectors, 501.

Haltica nemorum, the, 191.

Hamburg grapes, treatment of, 619 .

Hamburg parsley, place of, in the rotation, 17-culture of, 229 .

Hamilton, $\mathrm{Mr}$, soil used for pine-apples by, 646 -on watering them, 648 his mode of growing them, 656,659 .

Hampton Court vine, the, 595-the orangery at, 700 .

Hand-glasses for cauliflowers, 100 use of, for cuttings, 351 .

Hard-wooded trees, season for grafting, 330 .

Hardy, Messrs, on the transformation of the shallot into the onion, 29.

Harewood House, mulberry tree at, 592.

Haricot, see French bean.

Haricots, use of, on the Continent, 71 .

Harris, Mr, on mildew of the vine, 637 .

Harrison, $\mathbf{M r}$, on deep planting, 365

- lis mode of training the apple and pear on walls, 480 - on peachborders, 487-on pruning the peach, $489,491-$ on training the apricot, 518 - on the culture of the plum, 526 -on training the fig, 554-on vine-borders, $600-$ on planting vines, 604-his system of spur-pruning the vine, 608 - his system of ing the vine, 608 - his syster
long-rod vine-pruning, 610 .

long-rod vine-pruning, 610 .
Hautbois strawberries, origin of the, 582 -list of, 590 .

Hawkins, Mr, his mode of growing mushrooms, 250

Hawthorn pontia, the, 437.

Hawtlornden apple, origin of the, 304 .

Hay and Sangster, Messrs, propagation of the potato by cuttings by, 202.

Hayward, Mr, on the treatment of fruit-trees, 394-his mode of training them, 3y5-on pruning transplanted trees, 398-liis mode of peach-training, 492-on training the apricot, 518-on propegating the vine, 599 - his mode of pruning, \& c. the vine, 615 .

Hazel-nut, the, 563

Heart and dart moth, the, 195, 221.

Heart's-ease, cultivation of the, 796 .

Heat, influence of colour in soils with regard to, 275-necessity of, to the germination of seeds, 310 .

Heath-house, management of the, 703 -propagation by seed, 704-by cuttings, ib.-soil, 705-water, 706 - potting, $i b$.-winter treatinent, 707-summer treatment, 708-selection of kinds, 709-insects and diseases, 710 .

Heaths, striking of cuttings of, 349 .

Hedwig, researches of, on the nourishment of p'ants, 290 .

Helianthus tuberosus, the, see Jerusalem artichoke.

Helichrysum, culture of, 704 .

IIelvella crispa, 245-lacunosa, ib.

Hemlock, danger of mistaking, for parsley, 232.

Hepialus lumili, the, 181-lupulinus, the, 222 .

Herb patience, culture of, 138.

Herbaceous grafting, 334 .

Herbaceous plants, layering of, 353trausplanting of, 387 .

Herbert, Dean, on hybridisation, 315 , 317 , 318-lis treatment of bulbs, 697.

Herefordshire saddle-grafting, 332.

Herring-bone fan mode of training the, 390 .
Hewitt, Mr, culture of the pine by, 659.

Hitts' mode of lorizontal training, 396.

Hoare, Mr Clement, his mode of vinepruning, $611-$ his rules as to produce of vines, 626 .

Hoes, the Vernon, 38-the Spanish, $i b$.

Hogg, $\mathbf{M r}$, on the origin of the apple, 411 - his classification of apples, 412 - on the growtl of it, 413 -lists of apples for different localities by, 431-lis British Pomology, 299.

Holland, culture of asparagus in, 122 ,

124-mode of forcing lettuce in, $\mathbf{1 5 4}$ -culture of succory in, 165-the tulip mania in, 813 .

Hollyliock, propagation of the, by eyes, 354-cultivation of the, 798 . Holly-leaf fly, the, 827

Holme Lacy, remarkable pear-tree at, 346,448 .

Holmes, Mr, his transplanting machine, 380 .

Honey dew, causes and remedy of, 514

Hoofs of animals as a manure, 293.

Hop, cultivation and uses of the, 134 .

Horizontal espaliers, training the apple and pear as, 479 - mode of training, tlie, 390 -various modifications of, 396-with serew and with double stem, 390-trellises, training the apple and pear to, 483 .

Hormium clary, culture of, 235 .

Horner, Dr, mode of cultivating the auricula by, 810 .

Horns of animals as a manure, 293.

Horse-radish, culture of, $26 \mathrm{l}$

Horticultural Society of London, classification, \&c. of fruits by the, 299.

Hothouses, importance of atmospheric humidity in, 650 .

Hovey, Mr, on grafting the vine, 599.

Humble bee, the, 68

Humidity, importance of, in the atmosphere of hothouses, 650

Humulus lupulus, the, 134

Hungry soils, what, 267.

Hunt's Tawny nctarine, origin of, 305.

Hutchison, Mr, his mode of peachforcing, 505 .

Hyacinth, cultivation of the, 811 .

Hybernia defoliaria, the, 434 .

Hybridising, production of improved fruits by, 304-propagation by, 313 - its advantages, 314 - its laws, 315-Herbert on it, 318-how perfurmed, \&c. by Mr I. Anderson, furmed, \&c. by 19 -roses, on, 760 .

319 -roses, on, 760.
Hydium repandum, 245.

Hydrobolites tulasnei, a substitute for the truffle, 253

Hygrometer, importance of, in the hothouse, 651.

Hylands, cherry orchard at, 538 .

Hylesinus scolytus, the, 473 .

Hylobius abietis, the, 516, 535, 834 .

Hylotoma rosæ, 830.

Hylurgus piniperda, 833 .

Hypogymna dispar, the, 439

Hyssop, culture and uses of, 241

Ichneumon flies, destruction of insects by, 446 .

Impalpable matter, presence of, in soils, 276.

Inarching, propagation by, 336-propagation of the vine by, 599 .

Indian azaleas, sce Azaleas-cress, culture of the, 170 -pole saw, the, 408 .

Inorganic constituents of soils, the, 269-manures, kinds of, \&c., 296.

Inostomma Boscii, the, 472 .

Insect blight, tle, a disease attacking the pear, 465 .
Insects, diminution of, by deep digging, 279 -destruction of, by cliarring tlie soil, 283-uses of transplanting in destroying, 357-enemies to, among birds, 446.

Inula helenium, 242.

Iron, oxides of, influence, \&c. of, on soils and vegetation, 271.

Irregular fan mode of training, the, 390 -mode of peach-training, 495 .

Italy, introduction of the peach into, 485.

Julus, the genus of, 69-pulchellus, the, 114.

Janker tree-lifter, the, 387.

Jerusalem artichoke, natural history of the, 223-its uses, 224 - propagation, $i b$.-planting, $i b$. - soil and manure, 225-gathering and storing, $i b$.- - list of sorts, $i b$. - insects and diseases by whicl attacked, $i b$. - its European names, 226.

Jerusalem artichoke aphis, the, 225 .

John on the mineral ingredients of plants, 21.

Jolinston, Mr A. K., conservatory belonging to, 722 .

Johnston, Professor, on the substances absorbed by plants, \&c., 19-on the formation of soils, 265 - on their examination, 269-on the quantity of niatter removed from the soil by cropping, 273.

Jolinston, G. W., on planting vines, 605.

Johnston, $\mathrm{Mr}$, experiments in growing carrots by, 179 - on syringing the vine, 627 .

Jonghe on the management of the pear, 449 .

Judd, Mr, on the culture of asparagis, 121-on the culture of horse-radish, 262 - on vine-borders, 600 .

Juglans regia, tlie, 561 .

Justice, mode of sowing peas recommended by, 52 .

Keitl, Mr, on the absorption of nourishment by leaves, 290 -on hybridisation, 315-on the theory of grafting, 322, 323-on the spongioles of plants, 362 .

Kendall's mode of peach -training, 494.

Kendrick, views of, on the duration of fruit-trees, 306 .

Kennicott, Dr, anecdote of, 551 .

Kent, mode of growing the filbert in, 564 .

Kerguelen's Land cabbage, the, 90 .

Keswick codlin, peculiarity of the growth of the, 476 .

Kew, orange-trees at, 700 .

Kidney bean, the proportion of ground for, 8 -place of, in the rotation, 17its natural history, 71 -its uses, $i b$. mode of propagation, $i b$.- - sowing 72 - subsequent culture, ib. - soil and manure, $i b$. - forcing, 73approved sorts and their qualities, 74 -insects and diseases by which attacked, 76-vitality of the seeds of, 312 .

Kirschwasser, manufacture of, 537 .

Kitchen-garden, history of the plants cultivated in the, 3-disposition of the crops in it, 7-quantities of seeds, \&c. for, 8-rotation of crops in it, 12 .

Kitchen pears, list of, 461 .

Kniglit, Mr, his mode of growing onions, $35-$ on the seeding of early potatoes, $203-$ on the culture of horse-radish, $261-$ on the origin of new varieties of fruits, 303 - on changes of character in fruit, $305-$ views of, as to the duration of fruittrees, 306-results obtained by liybridisation by, 316-on the theory of grafting, 322, 323-on the effects of grafting, 324-saddle-grafting as invented by, 332-on budding, 340 
-his mode of layering, 352-on propagation by single eyes, $354-$ on pruning standards, 393-on ringing and its effects, 402-on stopping the fig, $403-$ on raising the peach from seed, 486-his mode of pruning the peach, $491-$ on mildew, 513-on peach, $491-$ on mildew, $513-$ on - on training the fig, 554-on the identity of the almond and peach, 560 - on budding the walnut, $561-$ on the culture of the chestnut, 562 -new currants originated by, 566 -on grafting the vine, 599-on the culture of the pine-apple, 653 .

Kohl rabi, uses of, 109, 110 .

Labels, best, for roses, 769 .

Lackey-moth, the, 439.

Lactuca sativa, see Lettuce-verosa, the, 151.

Lactucarium, manufacture of, 151.

Ladybird, value of the, 68 .

Lambeth Palace, fig-trees at, 551.

Lanıpronia capitella, the, 569 -corticella, the, 574

Lancashire, culture, \&c. of the gooseberry in, 575-prize gooseberries, lists of, 578,579

Langenaria, the, 259.

Langley, dates of fruits ripening given by, 306 .

Larentia cervinaria, 832

Large pear astyages, the, 471-pearmidge, the, 470 - white garden-butterfly, the, 113.

Latitude, value of, as regards the selection of fruit trees, 300 .

Lauder, Sir T. D., on the lawn, 773.

Lavender, early use of, 7-culture of, 261.

Lavendula spica, 261.

Lawn, the, what, 772 - its proper character, 773-terraces, \&c. on it, 774-the bowling-green, 776-the walks, \&c., 777-selection of grasses for it, $779-$ mossy ones, $i b$.

Lawson, Mr, selection of lawn grasses by, 779 .

Layers, propagation by, 351 -its objects, \&c., $i b$. - season for performing it, 352-how performed, ib.-pegs for it, 353-herbaceous plants, $i b$. - propagation of the vine by, 597 .

Layering, how performed, \&c., 351the rose, 762 .

Leaf-pruning, how performed, 399

Leaves, absorption of food by, 21nourishment absorbed by the, 289 , 290 -cuttings of, 351-as a manure, 293.

Leek, early history of the, 6-proportion of ground for, 8 - place of, in the rotation, 17-its natural history, 44 -its uses, $i b$. - mode of propagation, 45 - sowing and planting it, $i b$. - its subsequent management, 46 - soil and manures for it, $i b$. forcing, $i b$. - gatliering and preserving the crop, $i b$. - varieties and their qualities, $i b$.- saving seed, \&c., $i b$. -its European names, 47.

Leguminous plants, order of, in the rotation, $16-$ The Pea, 48 - The Bean, 62 - The Kidney or French bean, 71-The Scarlet runner, 78. Lemon, the, 700 .

Lentils, cultivation of, 80 .

Leontodon Taraxacum, the, 166.

Lepidium sativum, 168 .

Lettuce, the, early history of, 5-proportion of ground for, 8-natural history of the, 151 -its uses, $i b .-$ propagation, $i b$. - sowing and planting, $i b$. - its subsequent cultivation, 154-forcing, $i b$.- - gathering, 155list of varieties, $i b$.-diseases and insects by which attacked, 157saving, \&c. of seed, 158.

Lettuce-fly, the, 157.

Liebig on the rotation of crops, 14
Ligature, ringing by, 402 .

Light, influence of, on the mushroum, 250 -importance of, in the Conservatory, 721 .

Lightbody, Mr, cultivation of the auricula by, 809,810 .

Lima pole-bean, the, 79 .

Limburn, Mr, on the germination of seeds, 313.

Lime, the, 700 .

Lime, presence of, in plants, 20-as a manure, 296.

Limy soil, what, 263, 267 - plants indigenous to, 271.

Lindley, Dr, on the rotation of crops, 12 -on the ingredients of plants, 21 -on the mushroom, 250 -on the importance of air to roots, 278-011 watering, 285 - on the germination of seeds, $309-$ on hybridisation, 315 , 316 -on grafting, 322,323 -on the influence of the stock on the graft, influence of the stock on the graft,
$326-$ on budding, $340-$ on shieldbudding, 342 - on propagation by cuttings, 348, 349-on spring transplanting, $361-0$ mound planting, 365 - on autumn pruning, $391-$ on humidity in hothouses, 651 .

Lindley, G., on hybridising, 305 - on the duration of fruit trees, $307-0$ the rationale of budding, 339-on transplanting, 356 - on preserving the spongioles in transplanting, 361 - on the treatment of the apple and pear as open dwarfs, 475 - on pruning the peach, $489-$ list of apricots by, 521 -list of plums by, 534 - on training the cherry, $540-$ list of cherries by, 544-on training the fig, 554 - on the culture of the filbert, 563.

Lindley's Guide to the Orchard, 299.

Lindon's cast-steel spade, 279.

Link on the spongioles of trees, 362 .

Liparis auriflua, the, 437-dispar, 439-monacha, 834.

Liquid manure, importance of watering with, 285-preparation, administration, \&c. of, 295.

Liquiritia officinalis, 242.

Liquorice, culture, uses, \&c. of, 242.

Livingstone, $\mathrm{Mr}$, on the Petrocarpus marsupium, 346.

Loam, various definitions of, 267.

Loamy soil, what, 263, 267-plants indigenous to, 271 .

Lomba, M., his mode of managing the potato, 212

London, culture of asparagus round, 117 - the sewerage-water of, 295 improved fruits grown near, 300 .

London market, introduction of rhubarb into the, 256 .

London market-gardeners, their mode of growing the onion, 37, 38-culture of peas by, 50,52 - of Brussels sprouts, 91, 92-of cauliflower, 98 -of sea-kale, 104, 107- of asparagus, 119, 121, 122, 125-of spinach, 135-of celery, 142,143, 144-of lettuce, 153 - of endive, 161 - of radishes, 173 - forcing of carrots by, 179-growth of turnips by, 186 - their mode of forcing potatoes, 216 - culture of red beet by, 226 , 227 - of mushrooms, 248-of rhubarb, 257-of the tomato, 258their mode of successional cropping 284.

London market-gardens, the spades used in the, 279 .

London peach-pruner, the, 408.

Long-rod vine-pruning, various modes of, 610 .

Loudon, $\mathrm{Mr}$, oll the rotation of crops, 13 -on transplanting vegetables, 25 -on the mechanical determination of soils, $273-$ on hybridisation, 314 - on the theory of grafting, 323on trausplanting, 356 - on autumın transplanting, 360 - on the effects of pruning, 389 - on autumn pruning, 391-on spur-pruning, 403-on stopping or pinching back, 404.

Lophyrus pini, 836 .

Love apple, culture, \&c. of the, 258.

Lozotœen ia rosaria, 831

Lucas, Mr, sowing of seeds in snow by, 313.

Lunar spotted pinion moth, the, 440 .

Luperus rufipes, the, 467.

Lycopersicum esculentum, the, 258 .

Lycoperdon, edible species of, 245 .

Lyda inanita, 829 .

Lymburn, Mr, on blind buds, 340 .

Lysinema, culture of, 704 .

Macaire on the rotation of crops, 12 , 14.

M'Donald, $\mathrm{Mr}$, his mode of propagating the vine, 598 .

M'Glashan, $\mathrm{Mr}$, his large tree-liftin machine, 383-his smaller, 386his flower-transplanter, 86 .

Mackay's large tree-lifting machine, description of, 381.

M'Nab, Mr, views of, on transplanting evergreens, 371-lis apparatus for removing trees, 375-his large transplanter, 377,378 -on the culture of heaths, 704, 707 .

M'Phail on vine-borders, $600-$ on the culture of the cucumber, 666 .

Madden, Dr, on the constituents of soils, $271-$ on manures, 291.

Maddock, Mr, on the hyacinth, 811 .

Magpie moth, the, 569,580 .

Magnesia, presence of, in plants, 20.

Magnesian limestone as a manure, 297.

Magnolias, house for, 678

Mahalab cherry as a stock, the, 329 , 538.

Maidstone, mode of training, \&c. the filbert at, 564 .

Main's long shoot vine-pruning, 610 .

Maize, vitality of the seeds of, 312 .

Mallow moth, the, 832 .

Malot, M., his mode of peach-training, 496.

Maltese orange, the, 700 .

Maltuen, M., on the germination of seeds, 313.

Malus communis, see Apple.

Mamestra brassicæ, the, 112, 197 oleracea, the, 197 .

Mammoth gourd, the, 259.

Manchester, the mode of growing celery at, 143-gooseberry book, the, 575 .

Mandarin orange, the, 700 .

Manganese, oxide of, influence of, on soils and vegetation, 271.

Manures, kinds of, 288-their subdivisions, $i b$. - mode of their application and action, 289-organic ones, 290 -inorganic, 296.

Manuring, uselessness of, at transplanting, 364 .

Maraschino, manufacture of, 537

March moth, the, 832 .

Marie Louise pear, origin of the, 305 .

Marigold, culture of the, 240.

Marjoram, culture of, 238 .

Marnock, Mr, on the effects of transplantation on fruit-trees, $370-$ on transplanting large trees, 382 .

Martin, Mr, his mode of growing sea-kale, 104.

Mascall on the truffle, 254

Mazagan bean, origin of the, 62 .

Mealy bug, the, $639,661,700,823$.

Mearns, Mr, his mode of propagating the vine by coiling, 597-of pendant training, 483-of vine-pruning, 610.

Mechanical determination of soils, on the, 273.

Medicine, employment of garlic in, 29.

Medicinal plntas. Clamomile, 241- 
Hyssop, ib.-Wormwood, 242Rue, $i b$.-Liquorice, $i b$.-Elecam pane, $i b$.

Medlar, culture of the, 560-stocks, 329

Megachile centuncularis, 829 .

Melagonaster broomeianus, the, 253.

Melissa officinalis, 236.

Melitæa euphrosine, 833

Melolantha horticola, the, 437, 830

Melon, the, its natural history, 668 its cultivation, $i b$. - mode of propa gation, 669-open-air culture, 67 - select list, $i b$. - insects and diseases, 672-European names, ib.vitality of the seeds of, 312 .

Melon, management of, in the fruitroom, $€ 75$.

Melting peaches, what, 485 .

Menthas, culture of the, 236 .

Merodon narcissi, the 825 .

Mespilus germanica, the, 560 .

Meudon, the pines raised at, 655 .

Mice, injury done to peas by, 59 .

Microgaster viridis, the, 195, 196.

Middlemass, $\mathrm{Mr}$, on transplanting, 361.

Mild-leaved sorrel, the, 139

Mild lime as a manure, 296

Mildew, preservation of peas from 61 -on the Brassicæ, 110-on the peach, causes, \&c. of, 513-of the vine, 637 - of the cucumber, 668on roses, cause, \&c. of, 772

Mildew fungus, the, 229 .

Milk thistle, cultivation of the, 134

Millepedes, the, 69 .

Miller, P., general introduction of the kidney bean by, 71-on the influence of the stock on the graft, 325-on autumn transplanting, 360 -on pruning and training the plum 597.

Mineral manures, what, 288 .

Mints, culture of the, 236 .

Mitcliell, Rev. Mr, the first propagator of the vine by single eyes, 596 .

Mitchell, $\mathrm{Mr}$, lis mede of vine-forcing, 617.

Mixed greenhouses, disadvantages of 677

Moccas Court, the weeping-oak at, 315,318

Moisture, value of, in the soil, 276necessity of, to the germination seeds, 310

Mole, destruction of insects by the 111.

Mole-cricket, the, 832

Monck, Sir Charles, experiments by, on the fig, 552

Mouocotyledons, grafting of, 338

Montreuil mode of peach-trainiug, the 496.

Moorpark apricot, disease to which subject, 522 .

Moravia, the cherry in, 537

Morchella esculenta, the, 252

Morel, cultivation of the, 252 .

Morello cherry as a stock, the, 328 .

Morello cherry louse, the, 545 .

Morocco, the bean indigenous to, 62 .

Morton on the origin of soils, 264

Morus alba, the, 593-nigra, 592

Moss, destruction of, on lawns, 779

Mossy soil, what, 263, 268-plants indigenous to, 271.

Moths, means of destroying, 445 .

Mottled umber-moth, the, 434.

Mounds, transplanting on, 365

Mountain spinach, culture of, 138 .

Mudding-in, transplanting by, 387 .

Mulberry, natural history of the, 592 -its propagation, $i b$. - planting, soil, and situation, $i b$. - pruning and training, 593 - insects and and training, 5.93 - insects and diseases, $i b$. - its European names,

terbury, 346

VOL. II.
Mulching, advantages of, at transplanting, 364 .

Mule, the, a species:of spade, 279

Mummy wheat, germination of, 310 .

Muscat grapes, treatment of, 619 620.

Mushroom, the, early history of, 5 its natural history, 243-its uses, $i b$. -its cultivation, 245 - production of spawı, 247-preserving natural spawn, $i b .-$ propagating or trans planting spawn, $i b$. - procuring spawn artiticially, $i b$. - formation of the beds, 248-German mode $2+9$-on the absence of light, 250 production during winter, 251insects by which attacked, 252European names, $i b$.

Musk melon, the, 668

Mussel plum-stock, the, 328, 486.

Mussel scale, the, 443 .

Mustard, early history of, 6-culture of, 167.

Myatt, $\mathrm{Mr}$, introduction of rhubarb in to the London market by, 256 .

Naples parsley, 233.

Napoleon pear, origin of the, 305 .

Nareissus fly, the, 825 .

Nasturtium, culture of the, 170

Natural spuls, superiority of, over artificial, 482 .

Navet, the, 184, 190.

Nectarine, root-pruning the, $401-$ botanical identity of, with the peach, 485. See. Peach and Nectarine.

Nectarine stocks, kinds, \&c. of, 328 .

Nectarines, production of, on peach trees, 305-select list of, $510-$ man agement of, in the fruit-room, 675 .

Neill, Dr, on the influence of the stock on the graft, 326-on the Montreuil mode of peach-training, 496 on propagating the vine by layers, 598.

Nematus trimaculatus or Ribesii, the, 579.

Nemoicus oblongus, the, 436 .

Netting, protection by, 735 .

New Tarbet, fruits ripening at, 553 .

New Zealand spinaclı, culture of 138.

Newington's hand-cultivator, 208liand-row cultivator, 209.

Newtoun pippin, origin of the, 304

Nicol on the cultivation of mushrooms, $246,248-$ on the culture of the cucumber, 666 .

Nigger, the, 190 .

Nightsoil as a manure, 295.

Nitrate of potass as a manure, 297 of soda, $i b$.

Nitrates, action of the, as manures, 297 .

Nitre as a manure, 297.

Nitrogen, presence of, in plants, 20.

Noctua brassicæ, the, 112-cæruleocephala, 443, 515 - exclamationis, 196, 221 - gainma, 70 - pyralina 440 -segetum, 195-verbasci, 833.

Normandy, the apples of, 412 .

North American seeds, proper time for sowing, 313 .

Northallerton, great vine at, 595 .

Nurseries, treatment and selection of apples and pears in the, 474 .

Nut weevil, the, 565 .

Oblong weevil, the, 436, 516, 522 .

Ocymum basilicum and mininum, 237.

Old Alesfoord, mulberry-tree at, 592

Oldacre, $\mathrm{Mr}$, his mode of cultivating mushrooms, 249

Oleander, culture of the, 684-propagation of, by cuttings, 349 .

Oleander scale, the, 823 .

Oleraceous plants-Parsley,232-Dill, 234 - Borage, $i b$. - Thyme, $i b$. Sage, 235-Balm, 236-Mint, ib.Costmary, 237-Tansy, ib.-Basil ib.-Savory, 238-Marjoram, ib.Anise, 239-A ngelica, ib.-Cara- way, 240 - Coriander, ib. - Marigold, $i b$.

Omnivorous weevil, the, 575 .

Onion, early history of the, 6-proportion of ground for, 8 - place of. in the rotation, 17-its natural history, 30-its uses, 31 - its mode of propagation, $i b$. - planting and sowing of it, $i b$.-its subsequent cultivation, 37 - forcing, 38 - gathering and preserving the crop, 39-approved varieties and their qualities, $40-$ diseases and insects by which attacked, 42-the saving of seed, 43

-its European names, 44-trans formation of the shallot into the, 29 .

Onion-fly, the, 29, 42 .

Oniscus armadillo, 827-asellus, 826.

Onopordum Acanthium, the, 134.

Open dwarfs, treatment of the apple and pear as, 475 .

Orange, propagation of, by single leaves, 351 .

Orange-box, a, 682 .

Orange-scale insect, the, 703 .

Orange upper-winged moth, the, 832.

Orangery, management of the, $700-$ varieties, $i b$. - propagation in various ways, 701 .

Orchard, the, work called, 299.

Orchard-house, list of apples suitable to the, 431 .

Orchard, selection of apple and pear trees for the, 474 -management of established trees in it, 475 .

Orchid-house, the, 685 - plants suitable for, 686 -propagation, $i b$.- - season and soil for potting, ib. - pots, baskets, and blocks, $i b$._-potting, 688-watering, $i b$ - - shading, ib management of newly - imported plants, $i b$. - selection of those for blocks, 689 - for baskets, 690 - for pots, 691 -culture of ferns in it, 694 -diseases and insects, 695 .

Organic constituents of soils, the, 269 -manures, kinds of, \&c., 290.

Orgyia antiqua, 831 .

Origanum vulgare, \&c., 238.

Ornament, apples for, 429.

Ornamental shrubs and trees, the pruning of, 406.

Ornithogalum Pyrenaicum, the, 134

Ornix rhodophagella, 828 .

Orthotænia turionana, 836 .

Otiorhynchus picipes, the, 68, 69, 443-sulcatus, the, 575, 832-tenebricosus, the, $52 \%$.

Over-cropping, danger of, to the peach, 500

Oxalis acetosella, 172

Oxycoccus palustris and macrocarpus, 593.

Oxyria reniformis, the, 139

Oyster shells as a manure, 293.

Pædisca angustiorana, the, 470, 522

Pale brindled beauty-moth, the, 440 .

Palestine, the shallot brouglit from, 27.

Pansy, cultivation of the, 796 .

Pansy-fly, the, 826.

Papilio cratægi, the, 437-euprosyera, 833.

Paradise stocks for apples, 328-propagation of, by layering, 352 .

Paradoxical pear platygaster, the, 472 .

Parsley, early history of, 6-natural history of, 232-its uses, $i b .-$ propagation, $i b$.-sowing, 233-subsequent cultivation, $i b$. - soil and manure, $i b .-$ gathering and preserving, $i b$.- list of approved varieties, $i b$. - diseases to which subject, 234 -its European names, $i b$.

Parsnip, the, early history of, 6-proportion of ground for, 8-place of, in the rotation, 17-its natural history, 229-its use, $i b$.-its propagation, 230-sowing and planting, $i b$. -subsequent cultivation, $i b$.- soil and manure, $i b$.- gathering and 
storing, $i b$ - - list of sorts, $i b$. -insects and diseases by which attacked, ib. - its European names, 231saving seed, \&c., $i b$.

Parsnip-fly, the, 148-miner, the, 230.

Parterre garden, the, 816-its arrangement, sowing, \&c., ib. et seq. -list, \&c. of annuals for it, 817 -list, \&c. of annuals - bedding-out plants, $i b$.- list of these, 820 .

Pastinacea sativa, see Parsnip.

Paul, Mr, his mode of shield-budding 343 -on the rosary, 757 - on disbudding the rose, 765-on the hollyhock, 800,801 .

Pavies, what, 485.

Paxton, Sir J., his system of spurpruning the vine, 609

Pea, the early history of, 6-proportion of ground for, 8-order of, in the rotation, 16, 17-advantages of transplanting to, 25-natural history of the, 48-its uses, nutritive properties, \&c., 49-mode of propagation, $i b$.- time, \&c. for sowing, ib.-its subsequent cultivation, 53 -soil and manure for it, 54-forcing, 55- approved varieties and their qualities, $i b$.-insects and diseases by which attacked, 58-general remarks, saving of seed, \&c., 61 -its foreign names, $i b$.- chemical analyses of it, $i b$.

Pea-beetle, the, 59.

Peach, splice-grafting the, 331-soil suitable to the, 359 -root-pruning the, 401-and almond, supposed identity of the, 560-and nectarine, natural history, classification, \&c. of the, 485-varieties of them, $i b$.those of Clina, the United States, \&c., ib.-their propagation, 486situation and soil, $i b$.-preparation of the borders, \&c., 487-planting, 488 -pruning and training on the open walls, ib. - season of pruning, 489-modes of it, $i b$.- Errington's, 490-Harrison's, 491-Knight's, $i b$. - Seymour's mode of training, \&c., 492-Hayward's, and the rationale of it, $i b$.-Callow's, 494-Kendall's, $i b$. - the irregular, 495-the American practice, 496-the French, $i b$. -Du Breuil's, ib.-Mr Thompson's, 497-their longevity, ib.modes of protecting the blossom, 498-thinning the fruit, 499 - principles to be observed for successful cultivation, $i b$. - disbudding or summer-pruning, 500-forcing, 501preparation of the trees for it, $i b$. temperature at various stages, 502 -adjustment of the young wood, 503-importance, \&c. of ventilation, 504-other modes of forcing, 505-training under glass, $i b .-$ select list of peaches, 506-and of nectarines, 510-diseases and insects by which attacked, 512-European names, 516 .

Peaches, thinning of, 499-select list of, 506-management of, in the fruit-room, 675 .

Peach aphis, the, 469-borders, preparation of, 487-pruner, the, 407 -saw-fly, the, 515-stocks, kinds of, \&c., 328-trees, occasional production of nectarines on, 305 .

Pear, the, soil suitable to, 359-rootpruning the, 400-its natural history, 447-planting it, soil, \&c. suited to it, 448 - on quince stocks, sllited to it, 448 - on quince stocks, 449-general management of it, $i b$. - protecting its blossom in spring, 452 -criterion of good, $i b$.- - select list of dessert, $i b$.- recently introduced ones, 459-culinary, 461lists of those suited to particular localities, 462-growing it in pots,
463 - pronunciation of French names, 464-its European names, $i b$. -insects and diseases by which attacked, 465. See also Apple and Pear, pruning, \&c. of

Pears, management of, in the fruitroom, 673 .

Pear and apple aphis, the, 469-blossom, protecting the, 452 -chermes, 468-leaf-miner, 473-midge, 470 moth, ib.-plum.stock, 328, 486saw-fly, 468-stocks, how raised, \&c., 328 - trees, remarkable, at Holme Lacy, 346 - large, in the United States, 448 - tree blistermoth, 473-tree oyster-scale, $472-$ moth, 473 -

Pearl-bordered fritillary butterfly, the, 833.

Peat, origin and nature of, 268.

Peat earth, preparation, \&c. of, 705.

Peaty soil, what, 263, 268-plants indigenous to, 271 .

Peg-grafting, 333.

Pegs for layering, 353

Pelargonium, cutting of, 347-culture of the, 736 .

Pelvilain, M., his mode of growing pines, 655,656 .

Pendant form, training the apple and pear in a, 483 .

Penny-royal, culture of, 236.

Penthina ocellana, the, 466.

Peppermint, culture of, 236.

Perdrigon blanc plum, manufacture of prunes from the, 525 .

Perennial-plant flower-garden, the, 815 - plants suited to it, 816

Perennials, grafting, 335 .

Perry, manufacture of, 447-pears, list of, 463.

Persia, the peach indigenous to, 485 .

Persian melons, the, 668

Persica vulgaris, see Peach.

Peruvians, use of guano by the, 294.

Petrocarpus marsupium, singular pro pagation of the, 346 .

Phalaena grossulariata, the, 569,580 -vanaria or halias, the, 580 .

Phaseolus multiflorus, see Scarlet runner-vulgaris, the, 71 .

Phigalia pilosaria, the, 473 .

Phillips, Mr, on the uses of garlic, 29 $\rightarrow$ on the African garlic, 30-on the origin of the leek, 44

Phœnocoma, culture of, 704 .

Phospliate of lime as a manure, 297.

Phosphoric acid, presence of, in plants, 20.

Phyllopertlıa horticola, the, 437, 830 .

Phytolacea decandria, the, 134.

Pliytomyza ilicis, 827-nigricornis, 59,826 .

Pickling, onions for, 41.

Picotee, cultivation of the, 806 .

Picturesque style, introduction of the shrubbery into the, 781 .

Piedmontese truffle, the, 253.

Pieris cratægi, the, 437 .

Pigeon's dung as a manure, 294.

Pimpinella anisum, 239.

Pinching back, pruning by, 403 .

Pine-destroying beetle, the, 833saw-fly, 836-tree lappet-moth, 835 .

Pine-apple, natural history of the

641 -its propagation, 642 -by seed, $i b$.- by gills, crowns, and suckers, 643-soil, 645-potting, 647-ventilation, 649-shading, $i b$.- atmospheric temperature, $i b$. - atmosphere in connection with humidity, 650 bottom-heat, 653 -its age, 654 growing it without fire-heat, $i b$. growing it planted out in beds, 655 -growing it from suckers on the old stem, 659-expeditious culture, ib.- -select list, 660-diseases and insects, 661-European names, 662 . Pine-apples, nianagement of, in the fruit-roonı, 675 .
Pine-apple scale, the, 661,824 .

Pine strawberries, origin of the, 581list of, 588 .

Pink, cultivation of the, 807 .

Piophila apii, the, 149.

Pipings, propagation by, 350 .

Pislura monaclia, 834.

Pisum sativum, see Pea.

Pitmaston orange nectarine, origin of 305.

Plant-houses, arrangement of, 677 canıllia-house, 678-orchid-house, 685 - bulb-house, 696 - orangery, 700-heath-house, 703-aquarium, 710 -stove or tropical plant house, 712-succulent house, 719-conservatory, 721 - greenhouse, $724-$ flower-forcing house or pits, 729cold pits, 733 -conservative walls and borders, 734-miscellaneous observations, 736 .

Plants, importance of their leaves to, 21 -indications of soils from, $270-$ the sexuality of, 314 .

Planting, preparing the ground for, 358-see Transplanting.

Planting out, objections to, in the conservatory, 721 .

Planting dibbers, various forms of, 85 一trowels, various, $i b$.

Platygaster Boscii, the, 472

Pliny on the globe artichoke, \&c., 4 - on the origin of the leek, 44-on the kidney-bean, 71 - account of asparagus by, 117-on the sexuality of plants, 314-on the pear, 447.

Plum, soil suitable to tlie, 359 - standard, pruning of, 394 -root-pruning it, 401-as a stock for the peach, 486-its natural history, 524-its uses, $i b$.-manufacture of prunes, 525-its propagation, $i b$.- soil suited to it, 526-situation and planting, $i b$.-pruning and training, 527summer-pruning,528-winter-pruning, $i b$.- comparative sizes, $i b$.select list, $i b$. - lists suitable for particular situations, \&c., 534diseases and insects by which attacked, ib.-its European names, 536-forcing the, see A pricot, plum, and cherry, forcing the.

Plums, kinds of, suitable for forcing, 547 - management of, in the fruitroom, 676.

Plum saw-fly, the, 536- stocks, kinds of, \&c., 328 - propagation of thein by layering, 352-tortrix, the, 516,522 -weevil, the, 534

Plisia gamma, the, 70 .

Poiteau, M., on the origin of new varieties of fruits, 303 .

Poland, fungi used in, 243.

Polyanthus, cultivation of the, 811 .

Polydesmus complanatus, the, 181 .

Polydrusus oblongus, the, 436 .

Pomological Magazine, the, 299.

Pomona Londonensis, the, 302.

Pontia brassicæ, the, 113-napi, the, $i b$.-rapæ, the, $i b$.

Ponty on transplanting by fixing witl water, 387.

Poplar saw-fly, the, 515.

Poppy, the seeds of tlie, 309 .

Portliesia auriflua, the, 437 - clirysorrhœa, the, 438 .

Portugal, the onions of, 35

Portugal cabbage, the, 84-culture of it, 90-European names of, 116 .

Portulaca sativa, 171

Potato, the, proportion of ground for, 8 - place of, in the rotation, 17 - composition of, as compared with the turnip, 188 - its natural history, 199-its uses, 201-modes of propagation, 202-propagation by cuttings, ib.-and by seed, ib.planting, 203 - preparation of the ground, $204-$ size and choice of
sets, 206-subsequent cultivation, 
207 - implements used in it, $i b .-$ soil and manure, 212-forcing, 215 - gathering and storing the crop, 217-list of varieties and their qualities, 219--diseases and insects by whicl attacked, $22 \mathrm{i}$ - general remarks on it, 222-its European names, 223.

Potato brandy, distillation of, 201disease, supposed preventive of, 212 -its causes, \&c., 222-onion, culture of the, 41 - seed, gathering, \&c. of, 202-starcl, manufacture of, 201 .

Potash, presence of, in plants, 20 -influence of, on soils and vegetation, 272.

Poterium sanquisorba, 167.

Pot-herbs, proportion of ground for, 8. See Oleraceous plants.

Pots, evils of growing trees in, 369culture of the pear in, 463-growing the peach in, 505-forcing vines in, 621-ornamental, for orchids, 686 -the sizes of, 738 .

Poultry, the dung of, as a manure, 294.

Prague runner, the, 79.

Preserving, apples for, 429-plums grown for, 533 .

Preston Hall, growth of figs at, 301 , 553.

Price, Dr, on mildew of the vine, 637.

Pringea antiscorbutica, the, 91.

Prong spade, the, 279.

Propagation, various modes of, 309 by seed, $i b$. -crossing or hybridising, $314-$ by grafting, 322 - by budding, 338-by cuttings, 346-by layers, 351 -by seed, peculiarities of, 315 .

Protection, various modes of, for halfhardy plants, 734 .

Prunes, manufacture of, 525 .

Pruning and training, importance of and their connection, 389 -works on the theory and practice of, $i b$.their objects, $i b$. - practical rules for, 390 -various modes of, $i b$.the season for, $i b$.-winter-pruning, 391-summer-pruning, ib.-pruning standards, 393-pruning dwar standards, 3.94-spring-pruning, $i b$. - Hayward's system, ib. - the spiral, 395-the liorizontal mode, 396-Green's mode, $i b$.-pruning to increase vigour, 396-pruning newly transplanted trees, $i b$.- leaf-pruning, 399-root-pruning, ib.-rootpruning the pear, 400 - the apple $i b$.-the plum, 401-the peach and nectarine, $i b$. - the cherry, $i b$.- the apricot, $i b$. - the fig and vine, $i b$.ringing in various ways, 402-close pruning, 403- spur-pruning, ib.pruning by stopping, $i b$. - finger pruning or disbudding, 40t-bending down branches of standards, $i b$. - laying bare the roots, ib.-properly and improperly pruned trees, 405 -effects of bad pruning on wood $i b$.-pruning conifers, 406 - ornamental trees and shrubs, ib.-Cree's system of pruning, 407-implements employed in pruning, $i b$. - the apple and pear, see Apple and Pear-the apricot, various ways of, 518-the cherry, 539-season, \&c. of, for the peach, 489-modes of it, $i b$. et seq.of the plum, modes of, 527 .

Pruning of roses, the, 763-of shrubberies, 784 .

Pruning-bill, the, 409-chisel, the and its use, 408-knives, various for'ms, \&c. of, 407-saws, various, 408 -scissors, various kinds of, 410 -shears, various, 409.

Prunils Armeniaca, see Apricot avium, 537-cerasus, 537-domes. ticus, see Plum.
Psilus Boscii, the, 472.

Psylla buxi, 825 - mali, 442 - pyri, 468-rosæ, 181, 828.

Pteronus pini, 836 .

Puddling in, transplanting by, 387.

Puff-balls, edible species of, 245 .

Pulverisation as a means of inproving the soil, 278.

Pumpkin, cultivation of the, 259

Punnet-basket, the, 92.

Purple apple-weevil, the, 440-laburnum, peculiarity of the, 315 .

Purslane, culture of, 171

Pushing eye, budding with the, 345 .

Pyralis luscana, the, 466.

Pyramid pear-trees, winter-pruning of, 478.

Pyradmidal form, training of the apple and pear in the, 476 .

Pyramidal training, Rivers' mode of, 477.

Pyru : communis, see Pear-cydonia, 560-malus, see Apple.

Pyrus malus Brentfordensis, the, 302. Quenouille training of the apple and pear, the, 476-Hayward's, 395.

Quetsclie plum, manufacture of prunes from the, 525 .

Quicklime as a manure, 296 .

Quince, culture of the, 560 .

Quince stocks, raising of, \&c., 328planting the pear on the, 448 -effects of, on pears, 307 .

Quincunx transplanting, on, 388 .

Rabbits' dung as a manure, 294.

Radish, the, early history of, $6-$ natural history of, 172 - its uses, $i b$.-propagation, $i b$.-sowing, $i b$. -subsequent cultivation, 174-soil and manure, $i b .-$ gatliering and preserving, $i b$.-list of varieties, $i b$. - general remarks, saving seed, European names, \&c., 175.

Rain, fertilising ingredients brought down by, 286.

Rain-water, mineral ingredients contained in, 18.

Raised hillocks, transplanting on, 365 .

Rake for drawing drills, a, 33 .

Ranunculus, cultivation of the, 801 .

Rape, culture of, 171 .

Rape-cake, destruction of the wireworm by, 182-as a manure, 292.

Raphanus sativus, see Radish.

Raspberry, the, advantages of transplanting, 357-natural history of, 570 - its propagation, 571 - soil and situation, ib.-plantiıg, ib.winter pruning and training, 572summer-pruning, $i b$.-forcing, 573 - select list, $i b$.-insects and diseases by which attacked, 574 .

Raspberry bud-grub, the, 574-leafminer, $i b$.

Ratifia, manufacture of, 537 .

Rats, injury done to peas by, 59

Red beet, its natural history, 226its uses, $i b$.-propagation, $i b$.- - sowing and planting, $i b$.- - subsequent cultivation, 227-soil and nianure, $i b$.- gatlering and storing the crop, $i b$.- list of varieties, 228-its European names, $i b$.- -saving seed, $i b$.

Red-bud caterpillar, the, 466 .

Red cabbage, culture of, 90-European names of, 116 - currant, culture of the. 566 - list of sorts, 569 footed beetle, 467-fungus on roses, 772-pea, 79-plum grub, 535spider, prevention of, on the kidney bean, 73-description, \&c. of it, 77 . 514, 637, 703-truffle, 253.

Reed mats as protectors, 735 .

Reid, Mr, detinition of loam by, 267 -on the origin of canker in fruit trees, 432.

Reserve-garden, the, 822.

Reversed shield-budding, 342

Revue Horticole, the, on the fruitroom, 673 .
Rheum, see Rhubarb.

Rhizobius lielianthemi, the, 225 .

Rhizoctonia mali, the, 560 .

Rhododendrons, \&c., season for grafting, 330-culture of, 678,685 .

Rluubarb, proportion of ground for, 8-natural history of, 256-its uses $i b$.- - propagation, $i b$. - after cultivation, $i b$.- gathering the crop, $i b$.forcing, 257-saving seed, $i b .-$ list of approved sorts, ib.-its European names, 258.

Rhyuchænus nenuphar, the, 534

Rhynchites alliaria, 440-bacchus, $i b$.-betuleti, 640 -cupreus, 535 .

Ribis album, 566-nigrum, ib.-rubrum, $i b$.-uvacrispa and grossularia, see Gooseberry.

Ridging as a means of improving the soil, 279 -its advantages, 282.

Ring-budding, 344 .

Ringing, various modes of, 402 .

Rivers, Mr, on stocks for fruit-trees, 327 -root-pruning as practised by, 399 -on it, 400 - list of apples from, 431,432 - on the prevention of canker in the apple, 432 -on the climate, \&c. for the pear, 448-on its manageinent, 450 -lists of pears by, 462,463 -his pyramidal training for the apple and pear, description of 477 -his upright training of the apple and pear as espaliers, 479 -his mode of pendant training, 483-list of peaches and nectarines by, 510 -of apricots, 521 -on pruning and training the plum, 527list of plums by, 534-on stocks for the cherry, 538-lists of cherries from, $544-$ on the forcing of plums, cherries, \&c., 547, 548-hybrid between the bramble and raspberry by, 571-on the management of pears in the fiuit-room, $673-$ on the moss rose, 750-on the Hybrid China roses, $751-$ on the Austrian briar, 752-on the yellow rose, ib. -on the damask perpetual, 754on the China rose, 755 - on the miniature roses, 756 - on the Bourbons, 758-his mode of budding roses, 761-on the Manettii rose. 768

Roberts, Mr, his mode of growing strawberries, 584-on vine borders, 601 - his celery blancling tiles, 144.

Robertson, $\mathrm{Mr}$, on propagating the vine, 597 .

Rocambole, natural history, uses, culture, \&c. of, 30 .

Rockery, the, 814

Rocket tinea moth, the, 824

Rogers, Mr, on preserving the spongioles in transplanting, $362-$ on the cultivation of the apple, 413on pruning the peach, 489 -on the almond, 559.

Rogers, J., Esq., on atmospheric humidity in hothouses, 651

Rollinson, Messrs, new lieaths raised by, 709 .

Roman sorrel, culture of, 138.

Romans, culture of asparagus, \&c. among the, 3-introduction of the cabbage by, 81-use of asparagus among the, 117-the turnip among the, 183-apples known to the, 411-pears, 447 - cultivation, \&c. of the fig by the, 551 - forcing of the cucumber among the, 663 .

Romana melons, the, 668 .

Rome, the fungus-market at, 243.

Root, propagation by cuttiligs of the, 350 .

Root extractor, the, 166 .

Ruot grafting, 334 -the tree peony, $i b$.

Root-pruning, modern importance of, 302 
Roots, importance of the admission of air to, 277-importance of attention to, in transplanting, 361the absorbent power of the, $362-$ influence of pot-growing on, 369 -example of well formed, $i b$. - and of ill formed, 370 - of fruit-trees, on laying bare the, and its effects, 404.

Rosary, the. The different varieties of roses, 750-situation for it, 756propagation by seed, 759-crossing and hybridising, 760-propagation by budding and grafting, $761-$ by cuttings, 762 - by suckers, 763season for planting, $i b$._pruning, ib.-autumn pruning, 764-summer pruning, 765-forcing, 766-propagating stocks, 768 - tender roses, 769 -pegging down, $i b$.-labels, $i b$. - growing in pots, $i b$.- - select lists, 771 -diseases, 772 .

Rose, Mr, fruiting of the pine by, 642

Rose, culture of the, see Rosaryaphis, the, 830 - caddice saw-fly, 829-cliermes, 828-maggot, 830moth, 828-saw-fly, 830 -scale, 828 - shield-budding the, 341-stocks, kinds, \&c. of, 329 - water, roses from which made, 751 .

Roses, propagation of, by layering, 352 .

Rosemary, culture of, 260 .

Rosmarinus officinalis, 260.

Rotation of crops, see Crops.

Rubus chamæmones, the, 594-fruticosus, 594-idæus, see Raspberryoccidentalis, 571-strigosus, $i b$.

Rue, culture, uses, \&c. of, 242 .

Rumex acetosa, the, 139-nontanus, ib.-patientia, culture of, 138-sanguineus, culture of, $i b$.-scutatus, uses of, $i b$.

Ruricola on the red spider, 77.

Russia, forcing turnips in, 189 -fungi used in, 243.

Rust in carrots, the, 181

Ruta graveolens, 242.

Rye, vitality of the seeds of, 312 .

Saddle-grafting, 331 .

Sage, culture of, 235

St Catlierine plum, manufacture of prunes from the, 525 .

St David, connection of the leek with, 44.

St Julian stock for apricots, the, 328 . St Mark's fly, 591 .

Salad plants. Celery, 141-Celeriac, 150 - The Lettuce, 151 - Endive, 158-Succory, 164-Dandelion, $166^{\circ}$ - Tarragon, ib. - Burnet, 167 White and Black mustard, $i b .-$ Garden-cress, 168 - Water-cress, 169-American, Winter, and Indian cress, 170-Purslane, 171Chervil,ib.-Rape, ib.-Corn-salad, \&c., 172-The Radish, $i b$.

Salad, early history of, 5-remarks on, 141.

Salsify, place of, in the rotation, 17 -culture of, 228 .

Salt as a manure, 297-value of, as a manure to asparagus, 122 .

Saltpetre as a manure, 297.

Sandy soil, what, 263, 266-plants indigenous to, 271.

Sang, Mr, a properly and improperly pruned tree by, 405 .

Sap, influence of autumn-pruning on the, 391 .

Saperda bivittata, the, 444 .

Satureja montana, \&c., 238 .

Saul, Mr, on the best weather for budding, 339-his mode of shieldbudding, description of, 342 -his transplanter, 85-his transplanting machine, description, \&c. of, 374 .

Saunders, Mr, on vine-borders, 601 on vine-planting, 604 -his system of spur-pruning the vine, 607-his mode of vine-training, $614-$ his trough-culture of the vine, 624 .

Saur kraut, manufacture of, 82

Savage's surface-stirrer, 208.

Savory, early use of, 7-culture of, 238.

Savoy, the order of, in the rotation 17-natural history of the, 96-its uses, $i b$. - propagation, $i b$. - sowing and planting, $i b$.- subsequent culture, $i b$. - soil and manure, 97gathering and storing, $i b .-$ list of approved sorts, $i b$. - European names, 116.

Saw-dust as a manure, 293.

Saynor's botanical knife, 408-budding-knife, $i b$.

Scale insects, the, 516, 523, 823.

Scallions, what, 33 .

Scallop-budding, 344.

Scarabæus auratus, 828

Scarecrow, a, 83.

Scarlet nonpareil apple, origin of the, 304

Scarlet runner, the, its natural history, 78 -its uses, $i b .-$ mode of propagation, $i b$. - sowing and planting, $i b$. -its subsequent culture, $i b$. -soil and manure, 79-forcing, $i b$. - gathering, $i b$.- -list of approved sorts, $i b$. -insects and diseases, $80-$ general remarks, $i b$.

Scarlet strawberries, origin of the, 582 -list of, 587

Sciara pyri, the, 470 -major, $i b$.

Scolopendra electrica, the, 181 .

Scolytus destructor, the, 473-hœmorrhous, the, 436 - pruni, the, $473-$ pyri, the, 465 .

Scopula forficularis, the, 262 .

Scorzonera, place of, in the rotation, 17-culture of, 229.

Scotch cranberry, the, 594

Scotland, use of the leek in, 44-the mode of growing celery in, 143-improvement of the turnip in, 183inferior fruits cultivated in orchards of, 300 - list of apples suitable to, 430 -north of, pears for the, 463 growth of the peach in, 487-lists of plums for, $534-$ new gooseberries originated in, 576-first fruiting of the pine-apple in, 642 .

Sea-beet, 226

Sea-kale, proportion of ground for, 8 -natural listory of, 103-its uses, 104-propagation, $i b$. - sowing and planting, $i b$. - subsequent cultivation, 105-soil and manure, 106forcing, $i b$.- pots for blanching, 108 -gathering, $i b$.-European names, 116.

Sea-weed as a manure, 292

Seasoning, plants used for, 232-early history of them, 5 .

Seaton on spur-pruning the vine, 607 . Secondary crops, what, and their place in the rotation, 17.

Seed, advantages of mixing that of different years, 10-propagation by, 309 -peculiarities of propagation by, 315.

Seeds, quantities of, required for the kitchen-garden, 8-abundance of, produced, 309-their germination, ib. - their vitality, 311 - various, time required for germinating, 312 .

Selandria $A$ thiops, 830-atra, 471

Sensitive plant, vitality of the seeds of the, 312 .

Sewerage - water of London, on the, 295 .

Seymour's mode of peach-training, 492

Shaddock, the, 700 .

Shading, importance of, in transplanting, 360, 363-to transplanted evergreens, 373-and in conservatories, 723
Shallot, early history of the, 6 - its natural history, 27-its uses, $i b .-$ propagation of it, $i b$.-planting, $i b$. -its after management, 28 - soil and manures for it, $i b$.- gathering and storing the crop, $i b$. - varieties of it, $i b$. -insects and diseases to which subject, 29-general remarks on it, $i b$.- foreign names, $i b$. - transformation of it into the onion, $i b$.

Shallot vinegar, manufacture of, 27.

Shallow transplanting, advantages of, 365 -planting, importance of, to the peach, 488, 499.

Shanghae peach, the, 486,510 .

Shield-budding, various modes of, 341 -the Rose, $i b$. - the Camelia, $i b$. American mode, 342-Sanl's mode, $i b$.- - reversed, $i b$.-Paul's mode, 343 . Shortland, Mr, on propagating the vine, 597.

Shoulder-grafting, 333 .

Shrubbery, tlie, its objects, situation, \&c., 780-preparing the ground, 781-planting, 782-renewal of neglected, 783-pruning, 784-digging, $i b$. - selection of material, $i b$. - lists, \&c. of plants, $i b$. - half-hardy plants considered in reference to climate and situation, 793-effects of altitude, 794-acclimatation, $i b$.

Shrubs, cuttings of, 347 .

Sicilian beet, culture of the, 139 .

Side-grafting, 334 .

Sieva bean, the, 79 .

Sievright's improved cultivator, 209.

Silene inflata, the, 134

Silica, presence of, in plants, $20-$ properties, \&c. of, 267-influence, \&c. of, on soils and vegetation, 271 .

Silicious soil, what, 263,266 -plants indigenous to, 271.

Silk-worm, food of the, 593 .

Silybum Marianus, the, 134.

Simultaneous cropping, system of, 24 .

Sinapis alba, 167-nigra, 168.

Single eyes, propagation by cuttings of, 354 .

Sion House, mulberry-tree at, 5.92 .

Sitona canina, the, 68,69 -crinita the, 58-lineata, the, 58,69 .

Skirret, place of the, in the rotation, 17 -culture of the, 229 .

Slimy caterpillar, the, 471 .

Sloe, employment of the, as a stock for plums, 525, 534 .

Slug-worm, the, 471.

Slugs, destruction of, 111.

Small bark-beetle, the, 436 - brown scale, the, 824-ermine moth, the, 435-magpie moth, the, 580-peachpruner, the, 408-pear-midge, the 470 .

Smallage, see Celery.

Smeal, Mr, peculiar mode of growing carrots by, 178.

Smyrnium olusatrum, see Alisander.

Snails, destruction of, 111.

Snake millepede, the, 114 .

Snow, experiments on sowing seeds in, 313 .

Soda, presence of, in plants, 20 - influence of, on soils and vegetation, 272.

Soft-wooded plants, grafting of, 336 .

Soil, ingredients removed by various crops from the, 17-best state of the, for sowing, 312 - preparation of, for transplanting, 358 .

Soils, the origin and nomenclature of 263 - subdivisions of them, ib.various opinions on their formation, 264 - Stephens on them, ib.-Sir H. Davy on them, 265-argillaceons or clayey, 266-silicious or sandy, $i b$. - calcareous or limy, 267-loamy, ib.- - gravelly, 268-peaty or mossy, ib. - their organic and inorganic constuents, 260 - their examination, $i b$. - modes of determining 
their nature, 270-by the plants indigenous to them, 271-by chemical analysis, $i b$. - by mechanical examination, 273-their colour, its effects, \&c., 274 - their mineralogical qualities, 275 - moisture in them, 276 presence of stones in them, $i b$ impalpable matter in them, $i b .-$ their surfaces, $i b$.- their improve-
ment by various means, 277-by draining, $i b$.- by pulverisation, 278 - by digging, ridging, and trenching, and tools for it, 279-by bastard trenching, 280 - by consolidation, 281 -alteration of their constituent parts, $i b$. - their exposure to atmospheric influence, 282 - burning them, $i b$.-preparing them for next crop, 283-watering, 284-manuring, 288 et seq.

Solanum melongena, the, 259-tuberosum, sce Potato.

Solly, $\mathbf{M r}$, on ammoniacal liquor, 296 - on the action of nitrates, 297.

Solubility, importance of, in manures, 289.

Soot, advantages of applying, to the onion, 43-as a manure, 298.

Sorrel, early use of, 7-culture of, 138.

South America, the potato indigenous to, 200.

Spades, various kinds of, 279 .

Spain, the onions of, 35 .

Spanish hoe, the, 38-lentil, the, 80 . Spearmint, culture of, 236 .

Special manures, on, 22 .

Species, difficulty in determining, in plants, 314.

Speechley, Mr, on the culture of

beans, 64-his mode of grafting the vine, 599 - on vine-borders, $600-$ on the culture of the pine-apple, 650.

Spencer, Mr, his pot-culture of the vine, 621.

Sphynx tipuliformis, the, 570 .

Spilosoma lubricipeda, 833 .

Spinach, proportion of ground for, 8 - place of, in the rotation, 17natural history of, 135 -its uses, $i b$. - propagation, $i b$.- sowing, $i b$.subsequent cultivation, 136 - soil and manure, $i b$. - gathering, $i b .-$ list of varieties, 137 - its European names, $i b$.

Spinaceous plants. Spinacl, 135 Wild spinach, 137 - French or Mountain spinach, 138-New Zea land spinach, $i b .-$ Garden patience, Sorrel, \&c., ib.-White beet, 139.

Spiral-cylinder training, Hayward's, 395.

Spiral ringing, what, 402.

Spitzenburgh apple, origin of the, 304.

Splice-grafting, 330 - the peach, 331.

Spongioles, on preserving, in trans planting, 361 .

Spores of fungi, germination, \&c. of, 24.5.

Spotted buff-moth, the, 196, 833 garden gnat, the, 157 - pea weevil, the, 58.

Sprengelia, culture of, 704.

Spring-pruning, on, 394 - transplanting, evils of, 361 .

Spruce-pine weevil, the, 834 .

Spur-pruning, what, $403-$ the apple and pear, on, 482-the vine, various modes of, 606 .

Spurs, superiority of natural over artificial, 476.

Stable-yard manure, importance, \&c. of, 290-its application, \&c., 291

Stables, growth of the mushroom in, 252.

Stafford, Mr, his unode of forcing vines in pots, 621

Staking for peas, substitutes for, 53advantages of, for beans, 65 .
Standard fruit trees, pruning of, 393. Standards, management of the apple and pear as, 475 .

Standish on transplanting, 364

Standish and Noble on the pot-culture of evergreens, 371-their transplanting crate, 375 .

Stellate fan mode of training, the, 390 . Stephens, Mr, on the rotation of crops, 14-on special manures, 22-analyses of peas from, 61 - on manuring beans, 65 - on the origin of soils, 264 - on loam, 267-analyses of soil, \&.c. from, 272,273 - on the depth, \&c. of drains, 278 - on fermenting bones, 293 - on the sewerage water bones, $293-$ on

Steuart, Sir H., his work on transplanting, 367 - his mode of operation, 368 .

Stocks, choice of, for grafting, 325their influence on the graft, $i b$. - and of the latter on them, 326 -general remarks on them, 329-various, for peaches, 486-plunis grown for, 534 .

Stone terraces, situations, \&c. adapted for, their construction, \&c., 773.

Stones, value of, in soils, 276.

Stopping, pruning by, 403 .

Storey, Mr, new heaths raised by, 709 .

Stove or tropical plant house, the, management of, 712 - select lists of plants for it, 712,716 - treatment of palms, 713 - general collecment of palms, 713 - general collection, $i b$.-necessity for bottom-heat, 714- -importance of ventilation, $i b$. -re-potting, 715-spring and sunımer treatment, $i b$.

Strachan, Mr, on the radish, 174

Strawberry, the, necessity for transplanting, 357-its natural history, 581 -its propagation by seed, $582-$ and by runners, $i b$. - soil, and situation, 583-Roberts' mode of growing it, 584-general culture, 585gathering the fruit, $i b$.- forcing, 586 - select list, 587-insects and diseases to which subject, 591-its European names, 592 .

Strong pruner, the, 408

Striped pea-weevil, the, 58

Subsoils, influence, \&c. of, 268,275 .

Succade gourd, the, 259 .

Successional cropping, system of, 24

Succory, natural history of, 164-its uses, $i b$.-propagation, $i b$.- -sowing and planting, $i b$.-its subsequen culture, 165 - gathering, $166-\mathrm{va}$ culture, $165-$ gathering, $166-\mathrm{v}$

Succulent - house, management of the, 719-soil, \&c., ib.-temperature, 720-propagation, ib.-list of plants, $i b$.

Succulent plants, season for grafting, 330 - grafting of, 336 .

Suckers, propagation of the pineapple by, 643-propagation of the rose by, 763 .

Suckling gooseberries, what, 575 .

Sugar peas, uses of, 49 .

Sulphate of lime as a manure, 297.

Sulphur, action of, on mildew, 513 .

Sulphurator, the, 433 .

Sulphuric acid, presence of, in plants, 20 -fixing of ammonia by, 295.

Summer-pruning, on, 391-its effects, 392 - objects aimed at in it, 393the apricot, 519 -espalier apples and pears, 483 - the peach, 500 - the plum, 528-the vine, 615 .

Supporting, importance of, to transplanted evergreens, 373

Swaar apple, origin of the, 304 .

Swayne, G., on training the fig, 554 .

Swedish turnips, culture of, 186 .

Sweet, Mr, his mode of treating bulbs, 697 .

Sweet almond, culture of the, 559 basil, culture of, 237 - or Spanish chestnut, culture of the, 562-marjoram, culture of, 238-William, layering the, 353 .

Swine, the dung of, as a manure, 290. Swiss chard, preparation, \&c. of, 140 Switzer on forcing the clierry, \&c. 546.

(the cherry in, 537 .

Syboes, what, 33 .

Sydenham on the uses of garlic, 29.

Synonyms, number of, in fruit-lists, 299.

Syringing, importance of, in transplanting, 363-to transplanted evergreens, 373-and to the vine, 627.

Tamus communis, 134.

Tanacetum vulgare, 237.

Tanners' bark as a manure, 293.

Tansy; culture of, 237.

Tarragon, culture of, 166

Tarring, the fig at, 551 .

Taylor, $\mathrm{Mr}$ A., culture of the pineapple by, 655 .

Teltow turnip, culture of the, 186.

Temperature, influence of drainage in elevating, 277-day and night, regulation of, 629 .

Tenthredo Ethiops, 830-cerasi, 545 -hæmorrhoidalis, 468-morio, 536 -pini, 836 - populi, 515 - testudinea, 444-ventricosa, or grossulariæ, 579.

Tepluritis artimisiæ, 833-onopordinis, 148,230

Terminal flute-budding, 345 .

Terraces, various kinds of, 773 .

Terrestrial orchids, what, 686 .

Tetragonia expansa, culture of, 138 .

Thick sowing, evils of, 51 .

Thinning, cases in which requisite at transplanting, 369.

Thinning apples and pears, on, 483 -the peach and nectarine, 499the apricot, 519.

Thomery mode of vine-training, the, 613.

Thompson, Mr, select list of peas from, 55-list of savoys from, 97on the varieties of borecoles, 109 on the origin of new varieties of fruits, 303-on the horizontal trairing of the apple and pear as espaliers, 479-on summer-pruning espalier apples and pears, 483-classification of peaches and nectarines by, 485-modes of peach-training tried by, 497-classitication of apricots by, 517-classification of cherries by, $537-$ on the quince, 560 .

Thornlill, Mr, tranplanter invented by, 85 .

Thouin, Professor, on stocks for fruit-trees, 327 - on the varieties of budding, 338 .

Three-pronged fork, the, 225.

Thrips, prevention of, in the kidney bean, 73 -description, \&c. of it, $76,514,535,637$

Thyme, early use of, 7-culture of, 234.

Tiger moth, tlee, 157

Tinea capitella, the, 569-Clerkella, 473-corticella, 443, 574-hesperidella or porrectella, 824-padella, 435-rhodophagella,828-sarcitella, 59 .

Tipula maculosa, the, 157-marci, 591, 832 -oleracea, 111,825 - repens, 170

Tobacco plant, the seeds of the, 309 .

Tomato, natural history, uses, culture, \&c. of the, 258.

Tomicus typographicus, 835.

Tongue or whip grafting, 330 .

Topping, advantages of, for beans, 65.

Tortrix angustiorana, the, 470,522 Bergmanniana, 830 - buoliana or xylostiana, 836 - nigricana, 535ocellana, .466-pomonana, 435 - 
turionana, 836 - vitisana, 639 Wæberiana, 516, 522, 535 .

Tota bona, culture of, 137 .

Totworth, the chestnut tree at, 562 .

Towers, Mr, on the influence of the stock on the graft, $326-$ on the season for grafting, 330 .

Townsend, Lord, introduction of the turnip by, 183 .

Tragium anisum, 239.

Tragopogon porrifolius, 228.

Training, see Pruning.

Training the apple and pear, see Apple and Pear.

Transplanted trees, supports for, 366 -the pruning of, 397.

Transplanters, various small, for vegetables, 85 .

Transplanting, the process of, 356theory of it, $i b$.-Barron on it, 357 - constant necessity for it, ib.- - the season for it, 358 - preparing the ground for it, $i b$.- -early autumn, 360 -reasons for very early autumn, $i b$. - and against spring, $361-$ other seasons for it, $i b$.-preservation of the spongioles in it, $i b$. - prevention of excessive evaporation, 363preparing the places for the trees, $i b$-on treading down the soil, manuring, and mulching, 364-on shallow, 365-that on raised hill. ocks, $i b$. - replanting ground formerly occupied, 566 - supporting the trees, $i b$.- change of climate injurious, $i b$. - some trees more difficult than others, $i b .-$ proper size of trees, 367-the preparation of them, $i b$. - on shortening the roots, 368 - on thinning the branclies, 369 -well - formed roots, ib.--malformed, 370 - that of fruit - trees to aid fertility, $i b$. - that of coniferous plants, 371 - that of evergreens, ib. - that of large trees, 374 -various machines, \&c. for it, - Mr Saul's, ib. - Standisl and Noble's crate, $375-M$ 'Nab's apparatus, $i b$.- - his hand - transplanter, 376-his truck, ib.-raising power in it, 377-M'Nab's large transplanter, ib.-Mr Barron's tree-lifting machine, $378-$ his mode of preparing the roots, 379 -his smaller machine, $i b .-$ Holme's machine, 380-Mackay's, 381-M. Vallet's, 383-Mr M'Glashan's large, $i b$.his small, 386-the janker treelifter, 387 - that of herbaceous plants, $i b$.- by mudding in, $i b .-$ by fixing witll water, $i b .-$ placing the tree, $i b$. - that of turf, 388-in the Quincunx manner, $i b$.- Yegetables, mode of performing, seasons of, \&c., 24.

Transplanting forks, 152

Transplanting machines-Saul's, 374 -Standish and Noble's crate, 375 - Mr M'Nab's, ib. - M'Nab's large, $377-\mathrm{Mr}$ Barron's, $378-$ his smaller, 379- Holme's, 380Mackay's '381 - Vallet's, 383 M'Glashan's large, ib.- and small, 386-the janker, 387.

Traquair, peculiarities of climate of, 300 - the green-gage plum at, 527 .

Tree cabbage, the, 110

Tree-lifting machines, see Transplanting machines.

Trees, large, maclines for transplanting, 374 .

Trenching, importance of, 23-as a means of improving the soil, 279low done, its uses, \&c., 280

Trentham, training of the gooseberry at, 577.

Triangular draw-lioe, the, 208.

Trinidad, soil of the pine-apple in, 645.

Triphœna pronuba, the, 196.
Triple-spotted currant moth, the, 569 . Triptera artemiseæ, 833.

Trochilum tipuliformus, the, 570 .

Tropical-plant house, see Stove.

Tropœolum majus, 170

Truffle, cultivation of the, 253.

Tschudy, Baron, grafting conifers by, 335 .

Tube budding, 344-terminal, 345.

Tuber cibarium and albidum, cultiva-

tion of the, 253-magnatum, cultivation of the, $i b$.

Tulip, cultivation of the, 813-mania, the, $i b$.

Tull, Jethro, on the pulverisation of the soil, 279.

Turf, transplantation of, 388-laying down lawns with, 780 .

Turnip, the, proportion of ground for, 8 - place of, in the rotation, 17its natural history, 183 - its uses, ib. - propagation, 184-sowing and planting, $i b$. - subsequent cultivation, 188 - soil and manure, $i b$. its chemical composition, $i b$.-forcing, 189-gathering and storing, $i b$. -list of approved kinds and their qualities, $i b .-$ diseases and insects by which attacked, 190 - saving seed, \&c. , 199-its European names, $i b$.

Turnip aphis, the, 193 - diamondback moth, 197-flea-beetle, 191gall-weevil, 196- hoe, 185- sawfly, 190-weevil, 191 .

Turuip-rooted cabbage, the, 110 celery, culture of, 150 .

Turtle-scale, the, 516, 824

Typographer bark-beetle, the, 835 .

Tyso, Messrs, on the ranunculus, 801 , 802 .

Underground onion, culture of the,

Underwood's pruning-shears, 409 wasp-catcher, 640 .

United States, the, origin of new varieties of fruits in, 304-supposed degeneracy of fruits in, 306-whipgrafting in, 331-and cleft-grafting, 332 - apples of, 412 - large apple trees in, $\{b$. - uses of the apple in, trees in, the pear in, 448-culture of 413 -the pear in, 448 -culture of
the peach in, 485 -peach-training, \&c. in, 496 - culture of plums in, 524 -injury to plums by insects in, 534-culture, \&c. of the cherry in, 537 - culture of the fig in, 551 varieties of raspberries in, 571 want of the gooseberry in, 576strawberry culture in, 585 - the grapes of, 595-mode of storing, \&c. apples in, 674 .

Uovoli of the olive, the, 355 .

Upright espaliers, training the apple and pear as, 479.

Uredo fabæ, the, 69 .

Urine, value of, as a manure, 295.

Vaccinium oxycoccus, the, 593--vites idæa, 594.

Valentines, great vine at, 595 .

Valerianella olitoria, 172

Vallet, M., his tree-lifting machine, 383.

Van Mons, Dr, improved varieties of fruits introduced by, 299-his mode of obtaining new varieties of fruits, 303, 305-improvement of the pear 303,305
by, 447

by, 447.
Vanessa fluctuata, the, 115.

Vanilla plant, the seeds of the, 309 .

Vaulting borders, importance of, 277 -vine-borders, advantages of, 603 .

Vegetables, cultivated, sketch of history of, 3-transplanting of, 24 purposes of it, $i b$.

Vegetable manures, what, 288-marrow, the, 259 .

Veneer grafting, 334.

Vermin, means of preserving peas from, 59 .
Vernon hoe, the, 38.

Veronica beccabunga, 172 .

Versailles, the orangery at, 700 .

Vertical training with screw shoots, 390 -with upright shoots, $i b$.

Vespa vulgaris, 639 .

Vicia faba, see Bean.

Victoria Regia, tank for the, \&c., 710.

Vieuna, mode of growing asparagus at, 120.

Vine, cleft-grafting the, 332-cutting of the, 346-propagation of the, by layering, $352-$ by eyes, 354 -rootpruning the,401-its natural history, 595 -various modes of propagation : by single eyes, 596 ; by coiling, 597 ; by layers, $i b$.; by seed, 598 ; by cuttings, $i b$. ; by grafting or inarcling. 599 ; by budding, 600 - formation of the borders, $i b$. -cropping and renovating the borders, 603-planting, 604-arrangement and distance, 606 -autumn or winter pruning, $i b$ spur-pruning, $i b$.-Saunders' system of pruning, 607-Harrison's, 608Paxton's, 609-Fleming's, ib.-advantages of spur system, $i b .-$ renewal of plants so trained, $i b$. Mearns' long or succession pruning, 610-Main's long shoot, $i b$.-Harrison's long rod, $i b$. - renewal of plants, 611 -open-air pruning, $i b$. - Hoare's method, ib. - Thomery mode, 613 - various modes of training, 614-Saunders' mode, $i b$. -Hayward's, 615-fruit-tree mode, $i b$. - renewal under any mode of training, $i b$.-summer-pruning, $i b$. -forcing, 617-treatment of Hamburgs, 619-and of Muscats, 620 forcing in pots, 621-Mr Errington's practice, ib.-Mr Spencer's, 622Mr Saunders' trough culture, 624retarding ripe crops, 625 -produce, 626 -training, $i b$.- -syringing, 627 deticiency of heat and liglit injurious, 628-day and night temperatures, 629 -excess of heat injurious, 630 - season of rest, $i b$. - bleeding, 631 -colouring the grapes, $i b$. - thinning them, $i b$. - gathering, $i b .-$ select list of varieties, 632 -diseases and insects by which attacked, 636 -European names, 640 .

Vine-borders, formation of, 600 -soil for them, their depth, \&c., ib. et seq. -draining, vaulting, and concreting, 602-cropping and renovating, 603 -scale, the, 638-tortrix, the, 639.

Vineyards, former existence of, in England, 595.

Vitis vinifera, see Vine.

Virgin Mary's milk thistle, the, 134.

Virginian poke, the, 134.

Vitality, reticence of, by seeds, 311

Wales, use of the leek in, 44.

Walker, Misses, conservatory belonging to the, 722 .

Walks, formation of, 777 .

Wall, pruning and training the apple and pear on, 480 -and the peach, 488.

Wall-trees, various modes of training, 390.

Walnut, culture of the, 561 .

Warrington, $\mathbf{M r}$, on the use of fish in the aquarium, 711 .

Wasp, destruction of the, 639 .

Water, action of, on the formation of soils, 264-cleansing of, in the aquarium, 711

Watering, importance of, 284-how to be done, 285-importance of, to transplanted evergreens, 373

Water-cress, early use of the, 7-culture of, 169.

Water-nielon, 668-snail, use of, in the aquarium, 711 .

Washington plum, origin of the, 304 . 


\section{GENERAL INDEX.}

Wavy-fan mode of training, the, 390 . Wax models of fruits, on, 302 .

Weather, influence of, on transplanting, 357.

Wedge-grafting, 334 .

Weeds, destruction of, on walks, 778 . Weeks, Messrs, culture of the Victoris by, 710 .

Weeping ash, peculiarities of the, 318 -oak at Moccas Court, the, 315, 318.

Weevils, destructive powers of the, 58 .

Welsh onion, the, 41 .

West Indies, growth of the pine-apple in the, 645 .

Whip or tongue grafting, 330 .

White beet, natural history of the 139-its uses, ib. - propagation 140 - sowing and planting, ib. - subsequent cultivation, $i b .-$ soil and manure, $i b$.-gathering, $i b$.-list of varieties, $i b .-$ general remarks, $i b$. -currant, culture of the, 566-list of sorts, 569-garden butterfly, the 113-line brown-eyed moth, 197lupin, 80-mulberry, 593-mustard, culture of, 167-rust on the Brassicæ, 110-scale, 683, 685-truffle 253.

Whitley on the origin of soils, 264 .

Wickham, the Hon. W., on pruning, \&c. the fig, 553 .

Wight, Isle of, the fig in, 551

Wild endive, see Succory-pear stocks, what, 328-spinach, culture of, 137 . Williams' Bon chretien pear, origin of, 304.

Williamson, Rev. W., his mode of growing mushrooms, 251 .

Winter of $1853-54$, plants which have withstood the, 795 .

Winter cress, the, 170-lentil, 80melons, 668-moth, 434-pruning, on, 391 - of pyramids, 478--the plum, 528 .

wintering celery, house for, 147 vines, on, 618 .

Wire-worm, the, 69, 128, 182, 222.

Wishaw House, the vine-borders at, 600.

Withers on preparing the ground for planting, 758 .
Wood, Mr, on the French roses, 751. Wood, effects of compressed roots on, 370.

Wood ashes as a manure, 296-leopard moth, 469-lice, 691-sorrel, culture of, 172-strawberries, origin of the, 581-their propagation, 582 -list of, 5.90.

Wool moth, tlie, 59 .

Woolly aphis, the 433 .

Worms, destruction of, on lawns, 779 .

Wormwood, culture, uses, \&c. of 249

Wrinkling on the peach, causes, \&c. of, 514 .

Xanthea croceago, 832 .

Xyloterus dispar, the, 442.

Yellow-footed bean beetle, the, $69-$ tailed moth, 437-turnip leaf-miner, 196

Yellowlee's fork, 89.

Yester, chainbered vine-borders at, 602.

Yponomeuta, the genus of, 441-ma-

livorella, ib.-padelia, 435 .

Zeuzera æsculi, the, 469 .

THE END. 




$$
\text { BOUND BY }
$$


